Prepared for the U.S. Department of Energy

under Contract DE-AC05-76RL01830

\title{
Borehole Completion and Conceptual Hydrogeologic Model for the IFRC Well Field, 300 Area, Hanford Site Integrated Field Research Challenge Project
}
BN Bjornstad
DC Lanigan
JA Horner
PD Thorne
VR Vermeul

April 2009 


\section{DISCLAIMER}

This report was prepared as an account of work sponsored by an agency of the United States Government. Neither the United States Government nor any agency thereof, nor Battelle Memorial Institute, nor any of their employees, makes any warranty, express or implied, or assumes any legal liability or responsibility for the accuracy, completeness, or usefulness of any information, apparatus, product, or process disclosed, or represents that its use would not infringe privately owned rights. Reference herein to any specific commercial product, process, or service by trade name, trademark, manufacturer, or otherwise does not necessarily constitute or imply its endorsement, recommendation, or favoring by the United States Government or any agency thereof, or Battelle Memorial Institute. The views and opinions of authors expressed herein do not necessarily state or reflect those of the United States Government or any agency thereof.

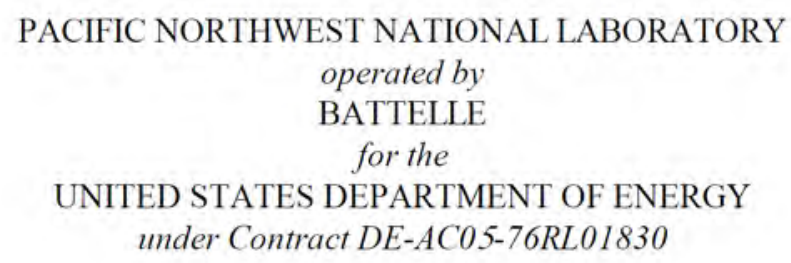

Printed in the United States of America

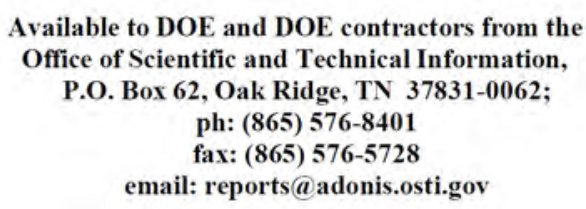

Available to the public from the National Technical Information Service, U.S. Department of Commerce, 5285 Port Royal Rd., Springfield, VA 22161 ph: (800) 553-6847 fax: (703) 605-6900

email: orders@ntis.fedworld.gov

online ordering: http://www.ntis.gov/ordering.htm

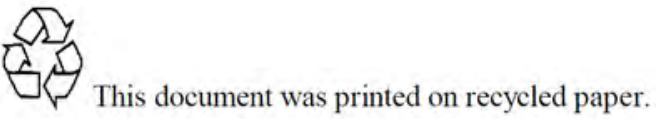

(9/2003) 


\title{
Borehole Completion and Conceptual Hydrogeologic Model for the IFRC Well Field, 300 Area, Hanford Site
}

Integrated Field Research Challenge Project

\author{
BN Bjornstad $\quad$ DC Lanigan \\ JA Horner \\ PD Thorne \\ VR Vermeul
}

April 2009

Prepared for

the U.S. Department of Energy

Office of Biological and Environmental Research

under Contract DE-AC05-76RL01830

Pacific Northwest National Laboratory

Richland, Washington 99352 


\section{Abstract}

A tight cluster of 35 new wells was installed over a former waste site, the South Process Pond (316-1 waste site), in the Hanford Site 300 Area in summer 2008. This report documents the details of the drilling, sampling, and well construction for the new array and presents a summary of the site hydrogeology based on the results of drilling and preliminary geophysical logging. 


\section{Acknowledgments}

Acknowledged is the work of well-site geologists Joe Fritts and Sean Sexton, Gram, Inc. We also thank the Fluor Federal Services drilling and support staff, including Chris Wright, Dorman Blankenship, Paul Lodder, and Les Walker. Rick McCain and Alan Pearson from Stoller Hanford were instrumental in the timely collection of downhole geophysical logs. Finally, we extend our appreciation for the support of others on the Pacific Northwest National Laboratory scientific team, including John Zachara, Mark Freshley, Vince Vermeul, Mark Rockhold, Andy Ward, Jim Fredrickson, Alan Konopka, Jim McKinley, Chris Strickland, and Dean Moore. 


\title{
Acronyms and Abbreviations
}

\author{
bgs below ground surface \\ CERCLA Comprehensive Environmental Response, Compensation, and Liability Act of 1980 \\ DOE U.S. Department of Energy \\ DOE-RL DOE Richland Operations Office \\ ERT electrical-resistivity tomography \\ $\mathrm{ft} \quad$ foot, feet \\ gal gallon(s) \\ gpm gallons per minute \\ ID inside diameter \\ IFRC Integrated Field Research Challenge \\ in. $\quad$ inch(es) \\ $\mathrm{K}_{\mathrm{d}} \quad$ distribution coefficient; measurement of sorbtive capacity of a specific chemical \\ constituent \\ m meter(s) \\ mi mile(s) \\ NMLS neutron-moisture logging system \\ OD $\quad$ outside diameter \\ PNNL Pacific Northwest National Laboratory \\ PVC polyvinyl chloride \\ RCRA $\quad$ Resource Conservation and Recovery Act of 1976 \\ SGLS Spectral-gamma logging system
}




\section{Contents}

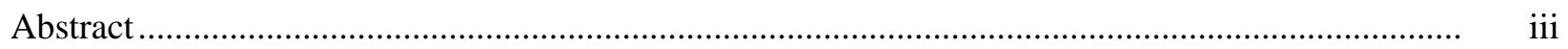

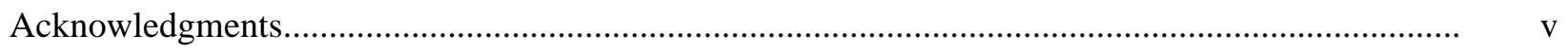

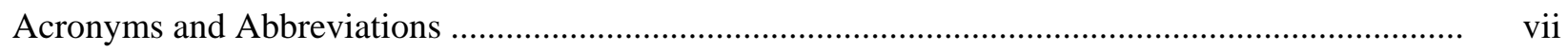

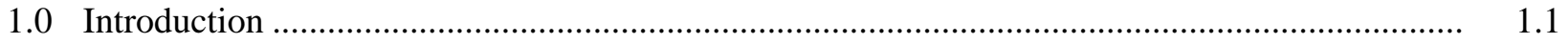

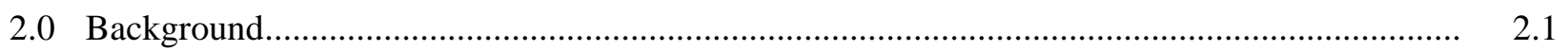

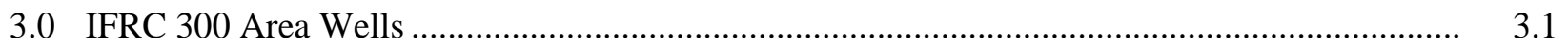

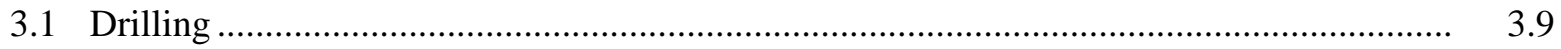

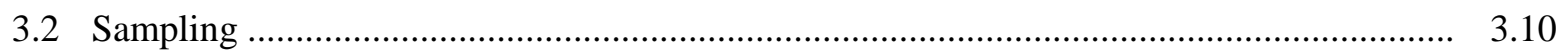

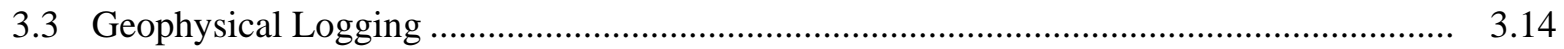

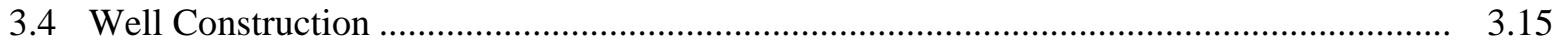

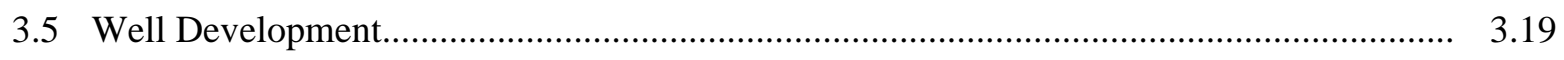

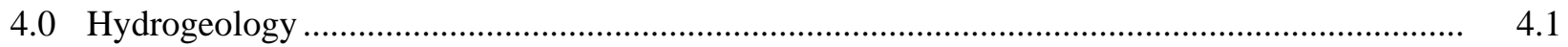

4.1 Columbia River Basalt ................................................................................................. 4.6

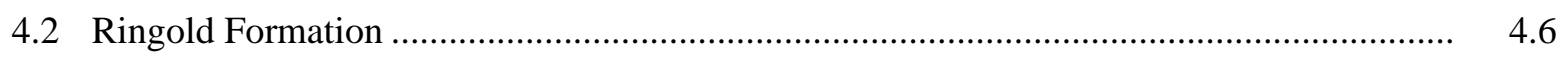

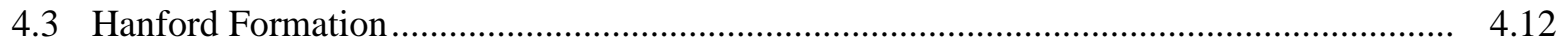

4.3.1 Ringold Formation Rip-Up Clasts..................................................................... 4.19

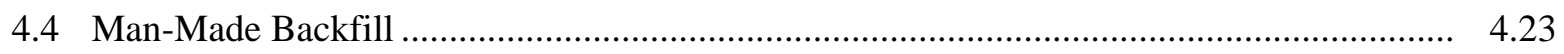

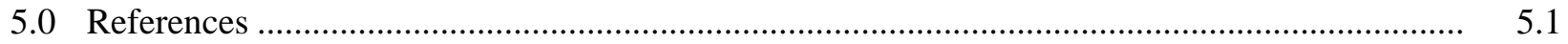

Appendix A - Compilation Borehole Summary Logs ...................................................................... A.1

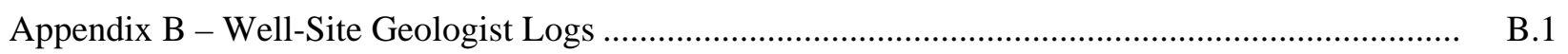

Appendix C - Sample Inventory Sheets ......................................................................... C.1

Appendix D - Field Activity Reports ....................................................................................... D.

Appendix E - Well Development and Testing Data Sheets .......................................................... E.1

Appendix F - Well Summary Sheets ............................................................................................ F.1

Appendix G - Downhole Geophysical Logs …............................................................................ G.1

Appendix H - Survey Reports .................................................................................................. H.1

Appendix I - Chip Tray Photographs .............................................................................................. I.1 


\section{Figures}

2.1 The 300 Area is located in the southeastern portion of the Hanford Site................................. 2.1

2.2 Uranium plume in groundwater beneath the Hanford Site 300 Area in December 2006 .......... 2.2

2.3 IFRC well field location in relation to the former South Process Pond .................................... 2.4

2.4 Vadose-zone backhoe pit SPP\#2 excavated beneath the IFRC site in 2003 ............................. 2.5

3.1 IFRC well field within the 300 Area, looking southeast.....................................................

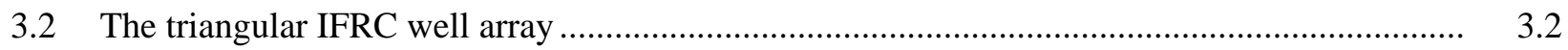

3.3 Drilling, geophysical logging, well construction, and well development were performed

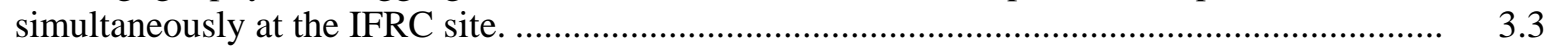

3.4 Sample compilation summary log for well 399-2-9............................................................

3.5 A resonant sonic drill rig was used to drill the 35 new IFRC boreholes.................................... 3.9

3.6 Sediment-filled split-spoon sampler.................................................................................. 3.11

3.7 Capped Lexan-lined cores were collected after the split-spoon sampler was opened................. 3.12

3.8 Bulk core and grab samples were collected from all IFRC wells not sampled via split spoon... 3.12

3.9 Samples were stored temporarily within a locked seatainer on site........................................ 3.13

3.10 A plastic core catcher helped to keep loose Hanford formation sediments from falling out of the split spoon during core sampling. ............................................................................... 3.14

3.11 Downhole geophysical logging was accomplished through the temporary 7-5/8-in. steel casing via Stoller's spectral-gamma logging system.

3.12 ERT electrode and thermistor sensors and cables were affixed at regular intervals down the outside of the 4-in. PVC screen and casing

3.13 Silica sand was added to the well annulus between the 4-in. PVC well casing and 6-7/8-in. temporary steel casing during well construction....

3.14 Comparison between coarser 10-20 mesh silica sand, used around 20-slot well screens, and the finer 40-140 mesh sand placed in the annular space above the screen.

3.15 All but one of the new IFRC wells was completed at ground level to allow for easier and safer movement of vehicles and equipment across the crowded well field.

3.16 Water was pumped out of 4-in. PVC wells into a storage tank during well development.

3.17 The high density of IFRC wells in various stages of completion

4.1 Cross sections through the 300 Area showing major hydrostratigraphic units

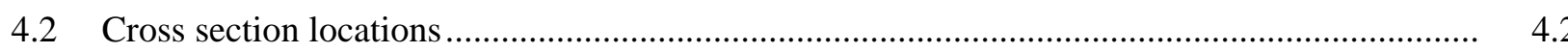

4.3 Hydrogeologic cross section C-C' based on geophysical logs................................................

4.4 Hydrogeologic cross section D-D' based on geophysical logs ............................................... 4.3

4.5 Hydrogeologic cross section E-E’ based on geophysical logs ............................................. 4.4

4.6 Hydrogeologic cross section F-F' based on geophysical logs .............................................. 4.4

4.7 Stratigraphy and lithology represented in deep microbiology characterization well 399-2-25

4.8 Core segment, weathered basalt of the Ice Harbor Member, from the bottom of well 399-2-25 
4.9 The Ringold lower mud unit at 160-ft depth in well 399-2-25 is predominantly a compact, homogeneous, dark greenish-gray, weakly stratified, fine sandy silt.

4.10 Dark fibrous wood fragments in laminated, micaceous, fine sandy silt of the Ringold lower mud unit at 129-ft depth, well 399-2-25

4.11 Ringold Formation Unit E

4.12 Ringold Formation Unit E conglomerate exposed near river level along the White Bluffs within 1 mile of the IFRC .

4.13 Fine-grained strata at the top of the Ringold Formation

4.14 Isopach map showing the thickness of the oxidized zone atop the Ringold fine-grained unit beneath the IFRC site.

4.15 Structure contour map of the top of the reduced zone in the Ringold fine-grained unit

4.16 Model for sediment transport and stratification during an Ice Age flood

4.17 Typical appearance of gravel-dominated facies of the Hanford formation within the 300 Area.

4.18 Network of interconnected braided channels created by the last cataclysmic Ice Age floods....

4.19 Profile of vadose-zone pit excavated in the east side of the South Process Pond in 2003.

4.20 Structure-contour map illustrating the uneven, eroded surface of the Ringold Formation

4.21 Hanford-Ringold formations contact.

4.22 Sharp contact at the top of the Ringold Formation silt-dominated sediment in well 399-2-23 and 399-2-14

4.23 Gravel-dominated facies with openwork fabric within the Hanford formation

4.24 Flood gravels with fine-grained, reworked Ringold Formation matrix in the Hanford formation

4.25 Aerial view, looking northeast, toward the IFRC site

4.26 Rounded rip-up clasts removed from a backhoe excavation in 2003 within SPP\#2 at the south end of the IFRC site.

4.27 Interiors of two fine-grained Ringold Formation rip-up clasts removed by backhoe from beneath the South Process Pond in 2003.

4.28 Olive to brown and yellow fine-grained upper Ringold Formation deposits exposed upriver along the White Bluffs within a mile of the 300 Area

4.29 Loose, basaltic pebbly sand overlies the top of a semiconsolidated Ringold Formation rip-up clast within the Hanford formation at 30- to 33-ft depth, well 399-2-14

4.30 Portion of a 5.5-ft-thick semiconsolidated Ringold Formation rip-up clast, composed of cohesive clayey silt, in well 399-3-26 from 21- to 22-ft depth

4.31 Surface topography in the vicinity of the 300 Area process ponds and trenches. 


\section{Tables}

3.1 Drilling, sampling, geophysical logging, and well construction information for IFRC wells .... 3.5

3.2 Geologic samples by borehole .................................................................................... 3.10

3.3 Well development data for the 35 IFRC wells ...................................................................... 3.20 


\subsection{Introduction}

Pacific Northwest National Laboratory (PNNL) is leading a field study at the Hanford Site in Richland, Washington, to identify new approaches and strategies to help resolve questions about the movement of subsurface contaminants. The field study is part of the U.S. Department of Energy (DOE) Integrated Field-Scale Subsurface Research Challenge (IRFC), a new program that commits multiinvestigator teams to performing large, benchmark-type experiments on formidable field-scale science issues. The field sites will provide capabilities to collect, permit, and ship environmental samples of different types to other program investigators and provide site access to those interested in testing specific concepts or technologies/techniques relevant to the study of subsurface contaminant fate and transport.

The program is managed by the Environmental Remediation Sciences Division, DOE Office of Biological and Environmental Research. Researchers will perform state-of-science field experiments at these sites to resolve the geochemical, hydrophysical, and microbiological factors that control the migration of contaminant uranium through the vadose zone (water-unsaturated sediments below the soil and above groundwater) and groundwater.

The Hanford field study involves the development, characterization, and instrumentation of a vadose zone and saturated zone field site. Researchers are evaluating hypotheses related to a uranium plume that resulted from nuclear fuel fabrication at the Hanford Site from 1943 to 1975.

The research site in the Hanford 300 Area is adjacent to the Columbia River, enabling studies of how the fluctuations in river stage influence contaminant dissipation from the aquifer and discharge to the river. The 300 Area is near the southern boundary of the Hanford Site north of Richland, allowing full access by a diverse and accomplished scientific team involving participants from PNNL, universities, and other laboratories.

In summer 2008, an array of 35 new monitoring wells was installed over the South Process Pond (316-1 waste site), a former waste site in the 300 Area. This report documents the details of the drilling, sampling, and well construction for the new array and presents a summary of the site hydrogeology based on the results of drilling and preliminary geophysical logging.

Section 2 provides historical and other background details about the site of the new well array. Section 3 presents a summary of the methodologies and equipment employed in the steps needed to develop the IFRC 300 Area well field: drilling, geologic sampling, geophysical logging, well construction, and well development. In Section 4, the hydrogeology of the IFRC research site is characterized. Sources cited in the text are provided in Section 5.

Raw data and construction details for each well are provided in a series of appendices:

- Appendix A - Compilation Borehole Summary Logs

- Appendix B - Well-Site Geologist Logs

- Appendix C - Sample Inventory Sheets

- Appendix D - Field Activity Reports

- Appendix E - Well Development and Testing

Data Sheets
- Appendix F - Well Summary Sheets

- Appendix G - Downhole Geophysical Logs

- Appendix H - Survey Reports

- Appendix I - Chip Tray Photographs. 


\subsection{Background}

The 300 Area lies in the southeastern corner of the DOE Hanford Site (Figure 2.1). The site is immediately adjacent to the Columbia River and thus enables studies of how the river stage affects contaminant dissipation from the aquifer and discharge to the river.

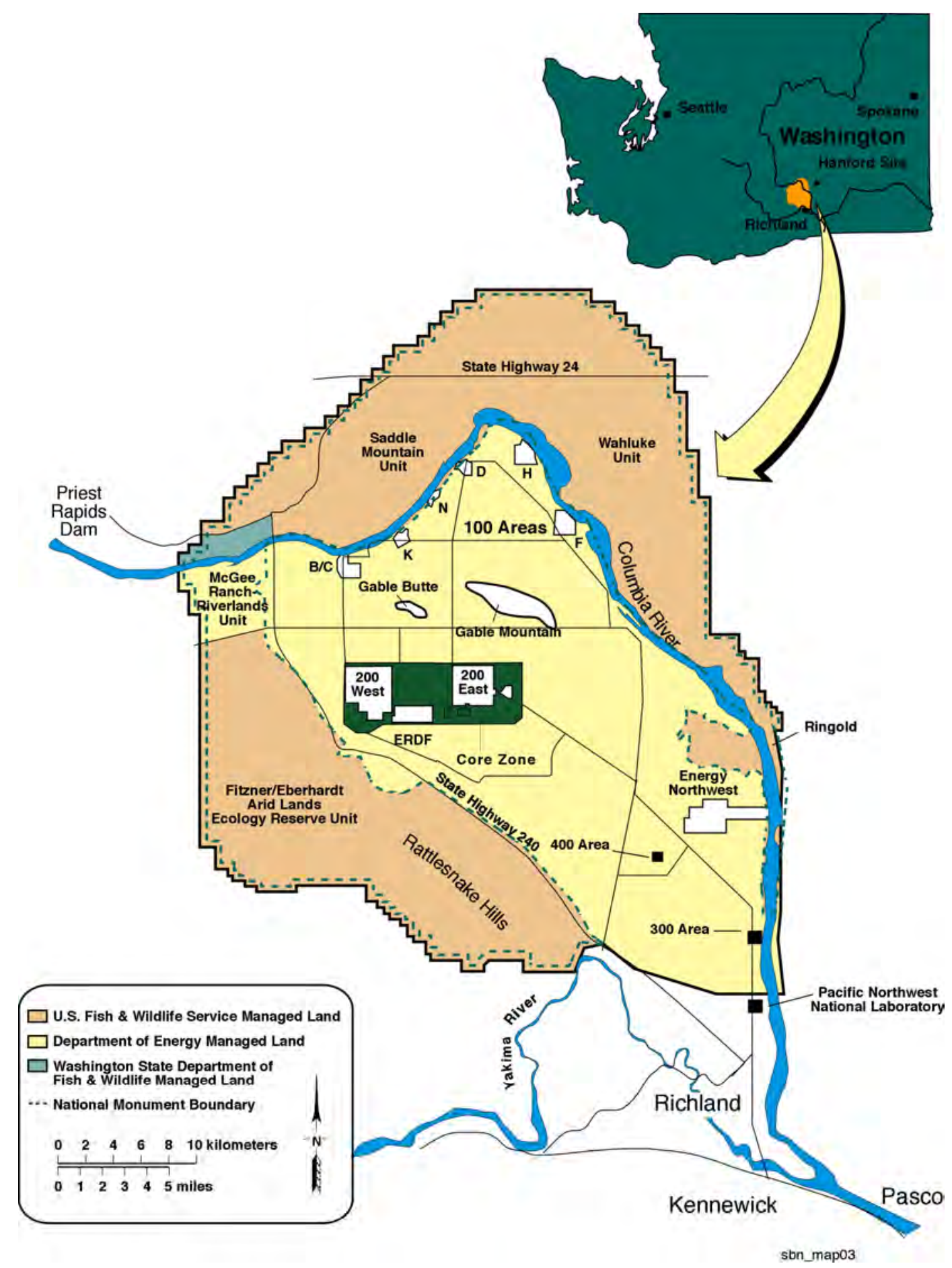

Figure 2.1. The 300 Area is located in the southeastern portion of the Hanford Site. 
A tight cluster of 35 new wells was installed over a former waste site (South Process Pond [316-1]) in the Hanford Site 300 Area in 2008. The wells were drilled to characterize the subsurface and test new approaches and strategies leading to understanding and remediation of a persistent uranium contaminant plume in groundwater beneath the 300 Area (Figure 2.2).

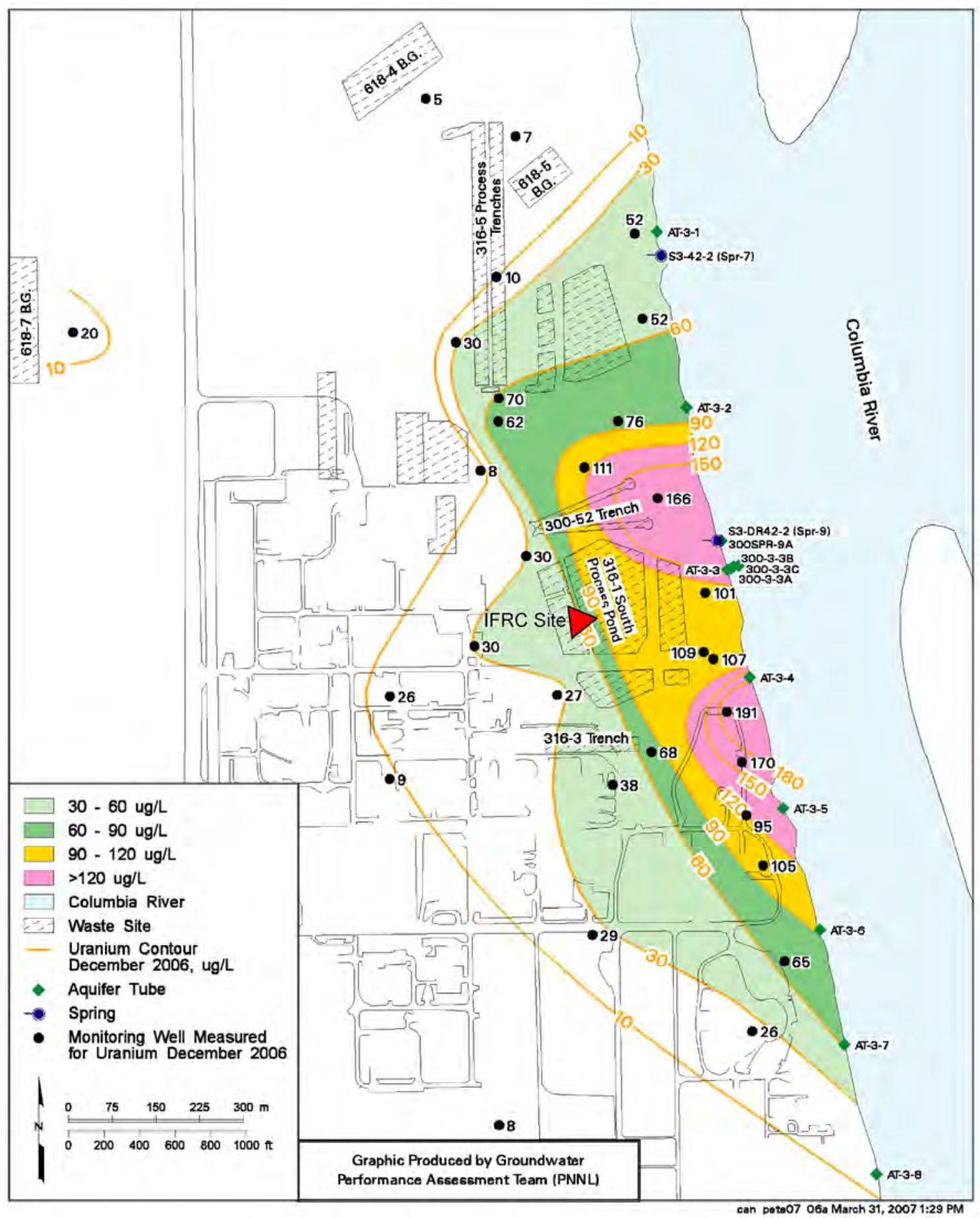

Figure 2.2. Uranium plume in groundwater beneath the Hanford Site 300 Area in December 2006. The IFRC well field is represented by the triangle in the southwestern corner of the South Process Pond, a remediated waste site. 
Large volumes of process waste were disposed of at the 300 Area process ponds, including the since-remediated South Process Pond, over which the IFRC site is located. The process ponds were used for waste disposal between 1943 and 1975. Process waste later was diverted to the 300 Area process trenches from 1975 through 1994. After the ponds were drained, the near-surface contaminated sediments were excavated from the waste-disposal ponds and trenches between 1995 and 2004 as a source-control measure to minimize additional groundwater contamination. ${ }^{1}$ The excavated ponds and trenches subsequently were backfilled and the land surface regraded to a natural state.

Groundwater wells have been installed in the 300 Area since the early 1940s for both subsurface characterization and groundwater monitoring. This monitoring network has been expanded sequentially in response to the growing size and concerns regarding the uranium contaminant plume and attendant investigations under the Resource Conservation and Recovery Act of 1976 (RCRA) and Comprehensive Environmental Response, Compensation, and Liability Act of 1980 (CERCLA) (Lindberg and Bond 1979; Schalla et al. 1988; Swanson et al. 1992). Pump testing and other hydrologic investigations have been ongoing. The results of the monitoring programs and decisions made are summarized in Peterson et al. (2005).

A groundwater uranium plume has existed beneath the 300 Area since the early operations of the process ponds; the highest uranium concentrations were observed from the early 1950s to the late 1980s (Peterson et al. 2008). These concentrations decreased rapidly after disposal activities ceased in the early 1990s, and groundwater uranium concentrations have slowly decreased since then. The plume resulted from liquid process waste infiltrating through the 4- to 10-m-thick vadose zone beneath the disposal facilities. Despite source-term removal and elimination of other leak sources, the general shape of the contaminated groundwater plume has not changed significantly over the last 10 years. The plume occasionally experienced sizable water table fluctuations during pre-dam Columbia River flooding and, to a lesser extent, during present-day dam-controlled river stage fluctuations that appear to be redistributing dissolved uranium into uncontaminated capillary-fringe and deep vadose-zone sediments.

In 2003, after remediation but prior to backfilling and regrading, four deep pits (two each from beneath the North and South process ponds) were excavated with a backhoe to a depth of 10 to $20 \mathrm{ft}$ to the water table (Bjornstad 2004). One of these pits (SPP\#2) was excavated at the southwestern corner of the IFRC well field (Figure 2.3). A profile of the sedimentary materials of the Hanford formation exposed and sampled at that time is shown in Figure 2.4.

\footnotetext{
${ }^{1}$ See http://ifchanford.pnl.gov/history.
} 


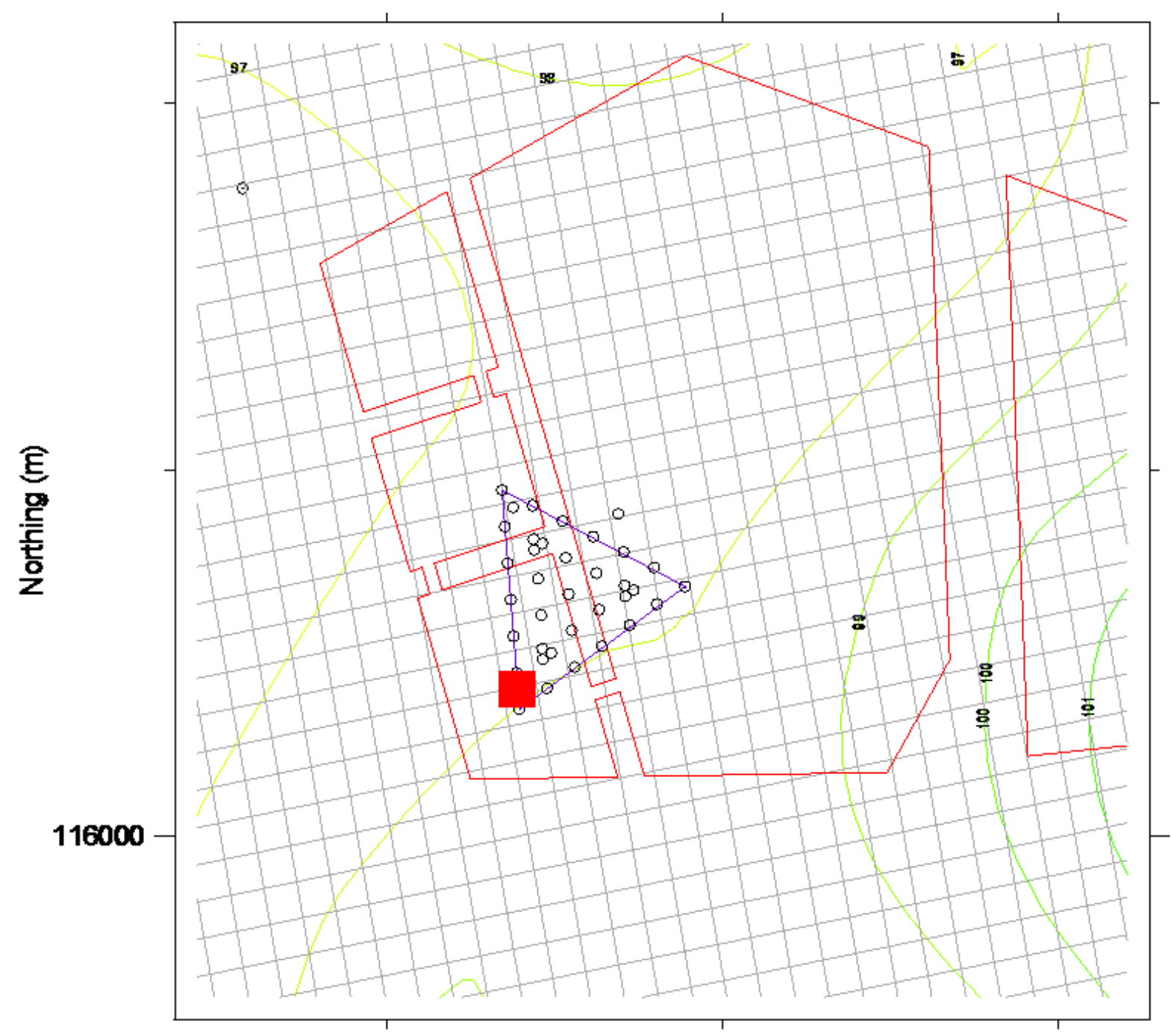

\section{Easting (m)}

Figure 2.3. IFRC well field location in relation to the former South Process Pond (red outline). Grid spacing is $10 \mathrm{~m}$. The red square is the approximate location of backhoe pit SPP\#2 excavated at the base of the remediated South Process Pond in April 2003 (see Figure 2.4). 


\section{South Process Pond - Pit\#2}

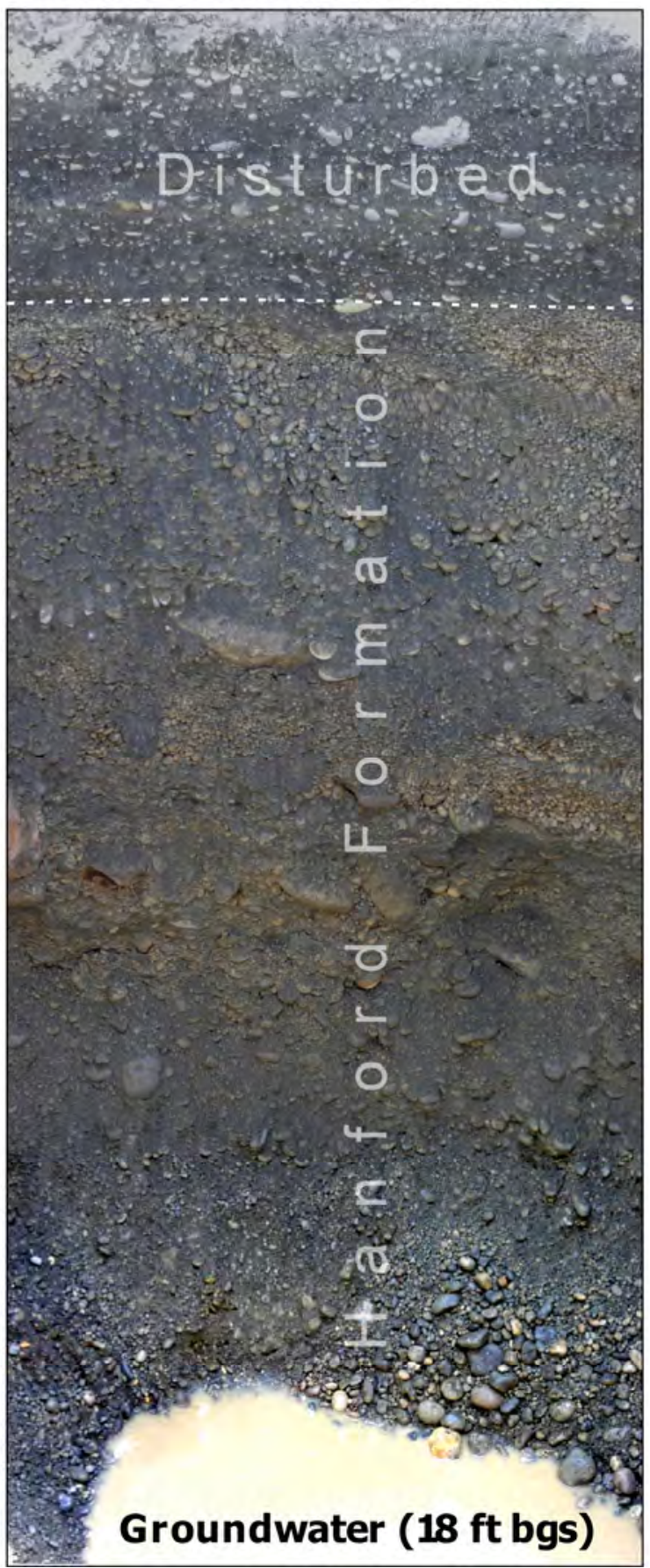

Figure 2.4. Vadose-zone backhoe pit SPP\#2 excavated beneath the IFRC site in 2003. The uppermost $4 \mathrm{ft}$ of the profile lack primary sedimentary structure, apparently destroyed from movement of heavy equipment over the site during remediation. Photo from Bjornstad (2004).
In 1996, a CERCLA interim remedy of monitored natural attenuation was selected for the 300 Area uranium plume based on equilibrium $\mathrm{K}_{\mathrm{d}}$-based reactive transport modeling. The modeling analysis implied that natural processes of groundwater flushing and desorption would lower uranium concentrations below the drinking water standards within 10 years. Subsequent monitoring has demonstrated that groundwater uranium concentrations are not decreasing as projected and persist above the drinking water standards throughout much of the 300 Area. Because of the ineffectiveness of monitored natural attenuation and regulatory mandates, the DOE Richland Operations Office initiated a Phase III Feasibility Study for the 300-FF-5 operable unit in 2005. The feasibility study included a limited field investigation to define the depth-discrete distribution and concentration of uranium in the aquifer and capillary fringe, as well as the nature of the hydrologic boundary between the Hanford and Ringold formations (Williams et al. 2007). Sonic drilling was used to recover continuous and intact large-diameter sediment cores from four limited field investigation boreholes in 2006. One of these wells (399-2-5) comprises the eastern corner of the IFRC well field. Well 399-2-5 was drilled with a cable-tool rig during an investigation of volatile organic compounds in 2007 (Peterson et al. 2008).

Groundwater levels are highly variable at the IFRC site due to seasonal and diurnal fluctuations in the Columbia River, located just east of the site. The normal high water level in late spring and early summer is about $25 \mathrm{ft}$ below ground surface (bgs). The normal low water level is about $35 \mathrm{ft}$ bgs, usually in late fall to early winter. Superimposed on these seasonal variations are daily fluctuations of several feet or more that can occur from fluctuating discharge from Priest Rapids Dam, situated $50 \mathrm{mi}$ upstream. The base of the unconfined aquifer, defined by a fine-grained stratum at the top of the Ringold Formation, varies from 50 to $60 \mathrm{ft}$ beneath the IFRC site. Therefore, depending on the season, the thickness of the unconfined aquifer ranges from 15 to $35 \mathrm{ft}$ beneath the IFRC site. 


\subsection{IFRC 300 Area Wells}

All 35 wells installed over a portion of the former South Process Pond within the 300 Area (Figure 3.1) are contained within a triangle $60 \mathrm{~m}$ on a side (Figure 3.2). The size, shape, and orientation of the well array were designed to take advantage of changes in the groundwater flow fields identified in Zachara et al. (2008). Prior to the start of drilling, a plan for drilling, sampling, and well installation (Bjornstad and Horner 2008) and drilling specifications (Bjornstad and Vermeul 2008) were prepared. Information gathered during drilling, sampling, geophysical logging, and well construction (Figure 3.3) is summarized in Table 3.1.

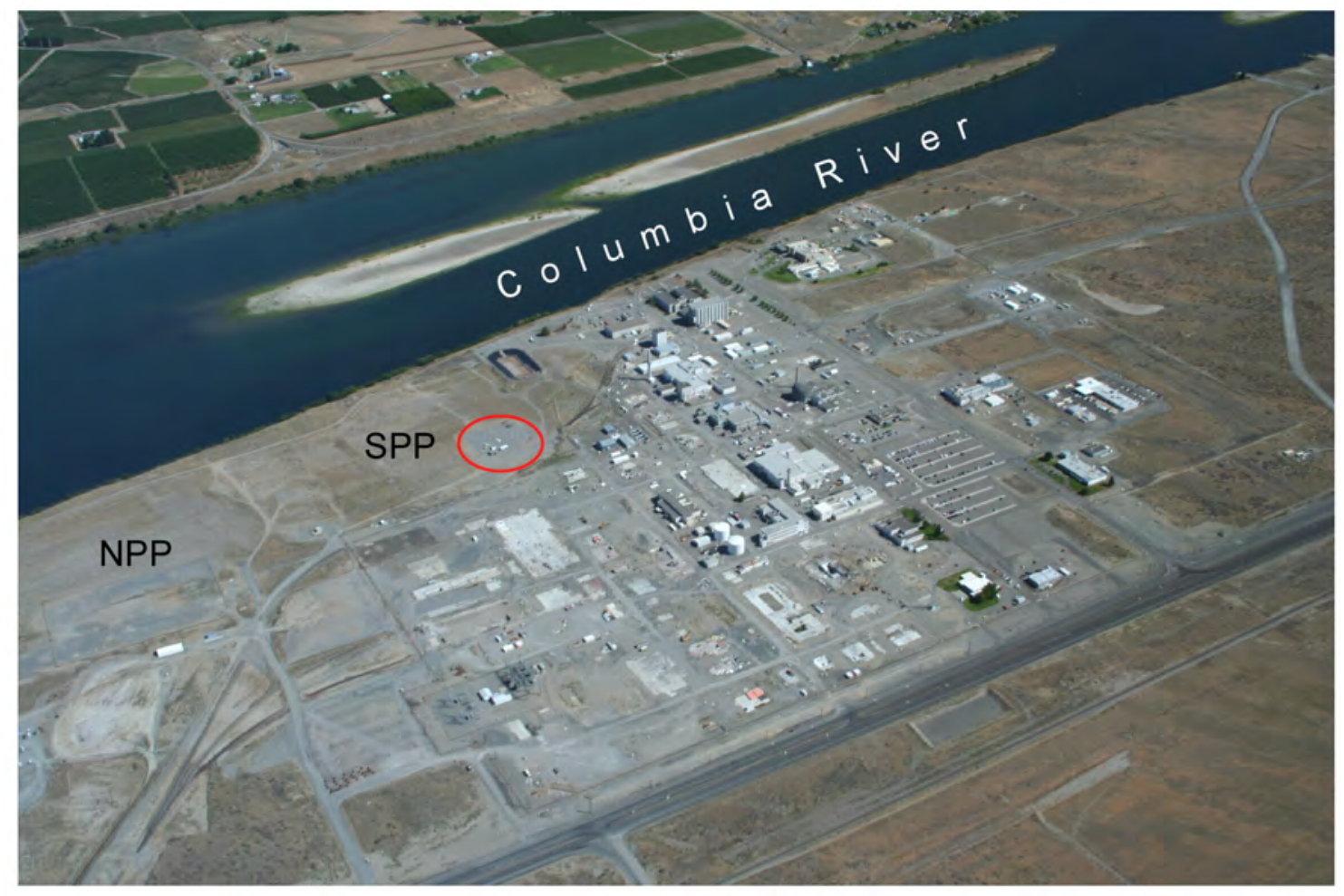

Figure 3.1. IFRC well field (circled) within the 300 Area, looking southeast. Photo: Bob Peterson (PNNL).

Field characterization data are summarized and compiled for each well in Appendix A. An example of a compilation summary log is shown in Figure 3.4. The raw data for each well, used to create the summary compilation logs in Appendix A, are documented in Appendices B through I. 


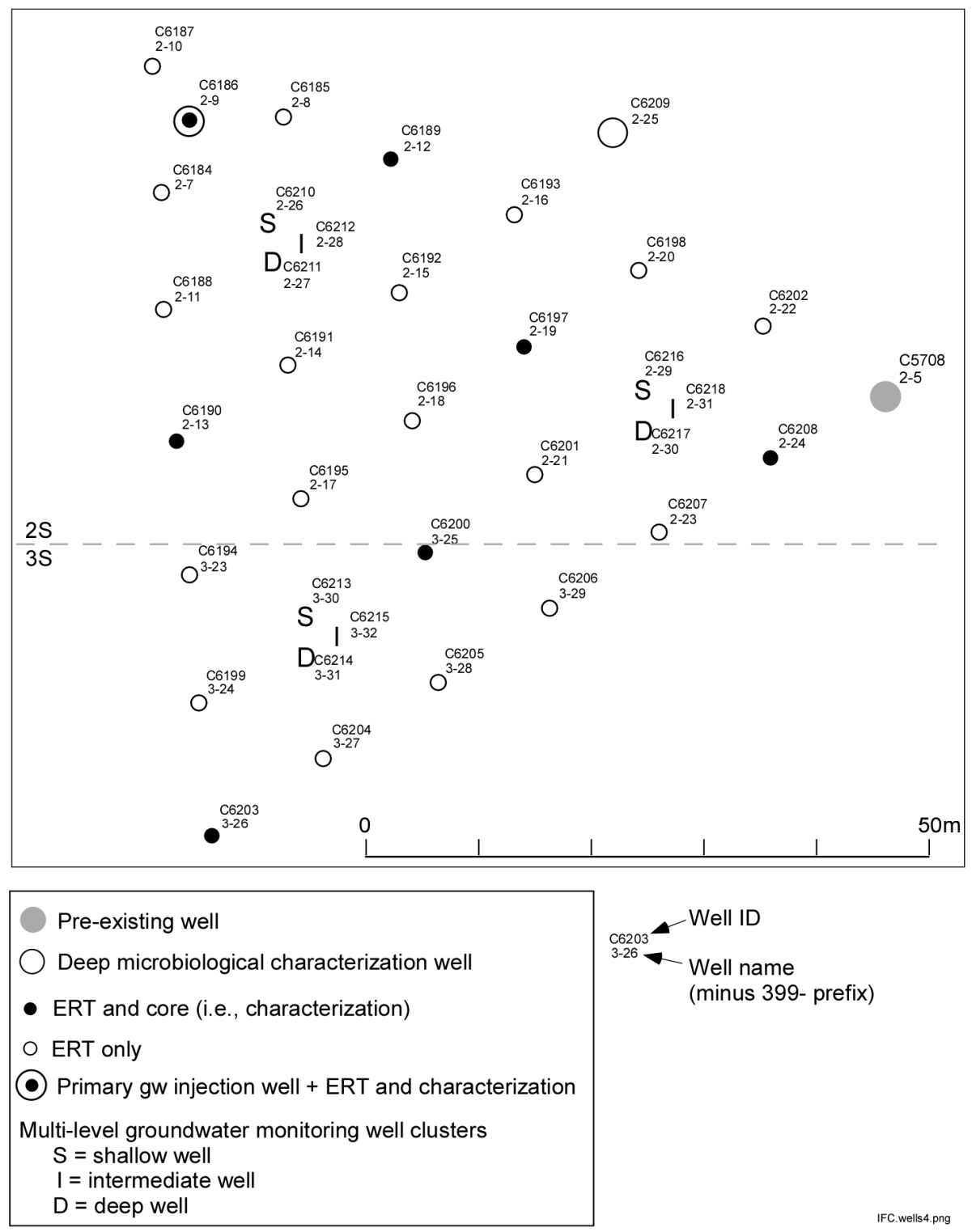

Figure 3.2. The triangular IFRC well array. Wells installed with special electrical-resistivity tomography and thermistor sensors are designated as ERT wells. See Table 3.1 and appendices for details on drilling and well construction. 


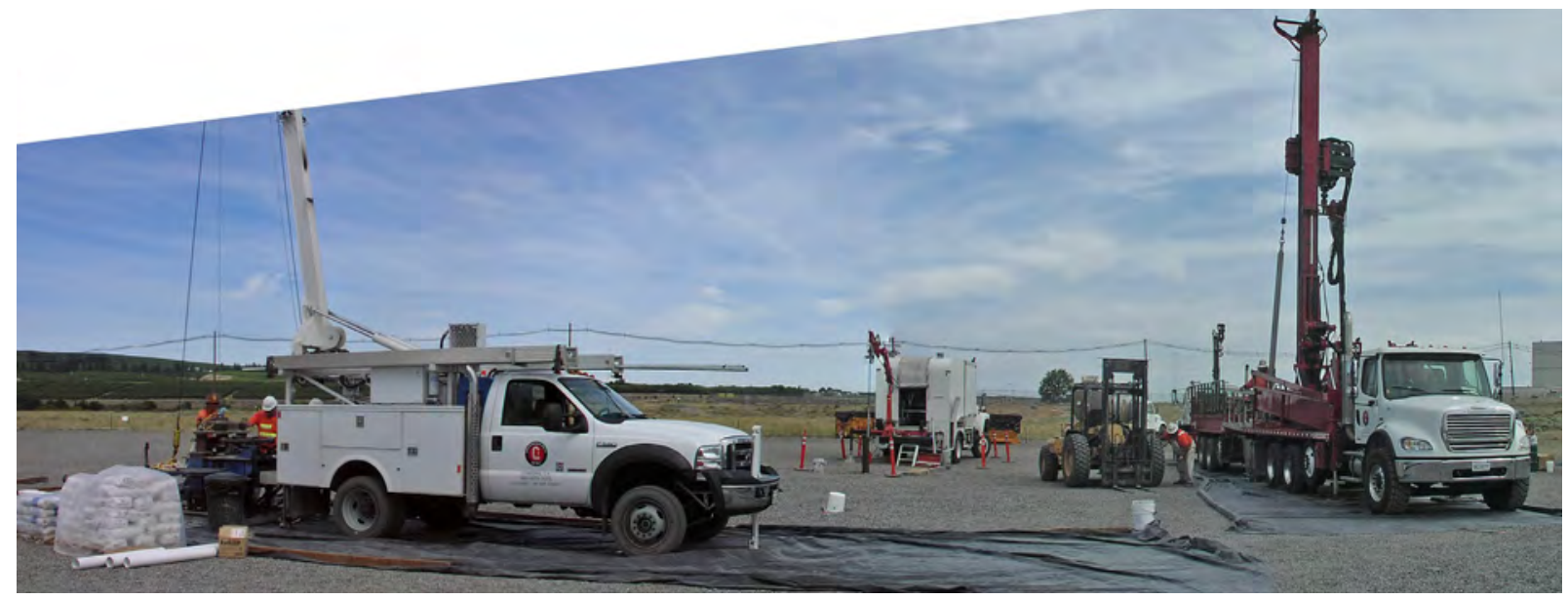

Figure 3.3. Drilling (right), geophysical logging (center), well construction (left), and well development were performed simultaneously at the IFRC site.

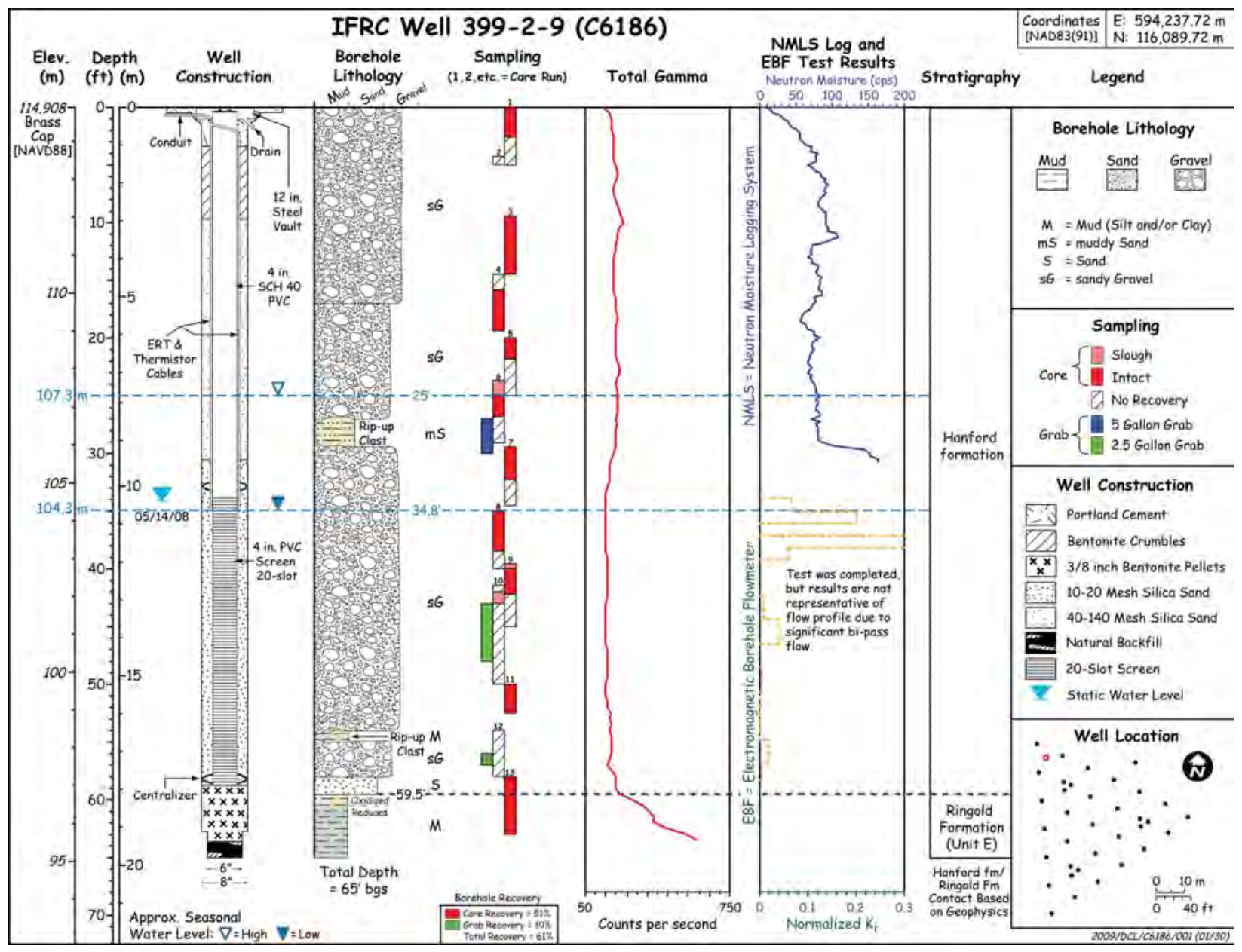

Figure 3.4. Sample compilation summary log for well 399-2-9 (C6186). See Appendix A for summary logs of all 35 new wells. 
Table 3.1. Drilling, sampling, geophysical logging, and well construction information for IFRC wells

\begin{tabular}{|c|c|c|c|c|c|c|c|c|c|c|c|c|c|c|c|c|c|c|}
\hline \multirow[b]{2}{*}{ Well Name } & \multirow[b]{2}{*}{ Well ID } & \multirow[b]{2}{*}{ Function } & \multirow[b]{2}{*}{ Samples } & \multirow[b]{2}{*}{$\begin{array}{c}\text { Drilling } \\
\text { Order }\end{array}$} & \multirow[b]{2}{*}{$\begin{array}{c}\text { Start } \\
\text { Drilling }\end{array}$} & \multirow[b]{2}{*}{$\begin{array}{l}\text { End } \\
\text { Drilling }\end{array}$} & \multirow[b]{2}{*}{$\begin{array}{l}\text { Geophysical } \\
\text { Logging }\end{array}$} & \multirow[b]{2}{*}{$\begin{array}{c}\text { Total } \\
\text { Depth (ft) }\end{array}$} & \multirow[b]{2}{*}{$\begin{array}{l}\text { Length } \\
\text { Screen (ft) }\end{array}$} & \multirow[b]{2}{*}{$\begin{array}{l}\text { Top Screen } \\
\text { Depth (ft) }\end{array}$} & \multirow[b]{2}{*}{$\begin{array}{l}\text { Bottom Screen } \\
\text { Depth (ft) }\end{array}$} & \multirow[b]{2}{*}{$\begin{array}{l}\text { Bottom End } \\
\text { Cap (ft) }\end{array}$} & \multirow[b]{2}{*}{$\begin{array}{l}\text { Instrument } \\
\text { Depth (ft) }\end{array}$} & \multicolumn{3}{|c|}{ \% Recovery } & \multicolumn{2}{|c|}{$\begin{array}{l}\text { Hanford/Ringold Contact } \\
\text { Depth (ft) }\end{array}$} \\
\hline & & & & & & & & & & & & & & Core & Grab & Total & $\begin{array}{l}\text { Geologist } \\
\text { Log }\end{array}$ & $\begin{array}{l}\text { Gamma } \\
\text { Log }\end{array}$ \\
\hline $399-2-7$ & C6184 & $\begin{array}{c}\text { ERT/ } \\
\text { electrodes }\end{array}$ & Buckets & 20 & 27-Jun & 27-Jun & 28-29-Jun & 62.0 & 25 & 31 & 56 & 56.3 & 33.5 & ---- & 75 & 75 & 55.5 & 53.5 \\
\hline $399-2-8$ & C6185 & $\begin{array}{l}\text { ERT/ } \\
\text { electrodes }\end{array}$ & Buckets & 23 & 7-Jul & 8-Jul & 10-Jul & 60.0 & 25 & 31 & 56 & 56.3 & 33.5 & ---- & 80 & 80 & 52.5 & 52.5 \\
\hline $399-2-9$ & C6186 & $\begin{array}{l}\text { ERT/electrodes } \\
\text { characterization } \\
\text { GW injection }\end{array}$ & $\begin{array}{c}\text { Intact } \\
\text { Lexan core }\end{array}$ & 1 & 12-May & 13-May & Complete & 65.0 & 25 & 33.5 & 58.5 & 58.8 & $58.5(?)$ & 52.5 & 9.5 & 62 & 59.5 & 59.5 \\
\hline $399-2-10^{(a)}$ & C6187 & $\begin{array}{l}\text { ERT/ } \\
\text { electrodes }\end{array}$ & Buckets & 31 & 17-Jul & 17-Jul & 18-Jul & 65.0 & 25 & 34.7 & 59.6 & 59.9 & 33.5/58/5 & ---- & 81.5 & 81.5 & 56 & 59 \\
\hline 399-2-11 & C6188 & $\begin{array}{l}\text { ERT/ } \\
\text { electrodes }\end{array}$ & Buckets & 30 & 16-Jul & 17-Jul & 21-Jul & 65.0 & 25 & 31 & 56 & 56.3 & 33.5 & ---- & 78.5 & 78.5 & 55.5 & 55.5 \\
\hline $399-2-12$ & C6189 & $\begin{array}{l}\text { ERT/electrodes } \\
\text { characterization }\end{array}$ & $\begin{array}{c}\text { Intact } \\
\text { Lexan core }\end{array}$ & 11 & 17-Jun & 18-Jun & 23-Jun & 65.0 & 25 & 31 & 56 & 56.3 & 33.5 & 65.9 & 9.4 & 75.3 & 52 & 53 \\
\hline $399-2-13^{(b)}$ & C6190 & $\begin{array}{l}\text { ERT/electrodes } \\
\text { characterization }\end{array}$ & $\begin{array}{c}\text { Intact } \\
\text { Lexan core }\end{array}$ & 4 & 22-May & 22-May & Complete & 62.6 & 25 & 31.2 & 56.2 & 56.5 & 33.7 & 44.5 & 6.5 & 50.8 & 54 & 55.5 \\
\hline $399-2-14^{(c)}$ & C6191 & $\begin{array}{c}\text { ERT/ } \\
\text { electrodes }\end{array}$ & Buckets & 9 & 13-Jun & 16-Jun & 7-Jun & 58.5 & 25 & 30.7 & 55.7 & 56.0 & 33.2 & ---- & 93 & 93 & $52 ?$ & 54.5 \\
\hline 399-2-15 & C6192 & $\begin{array}{l}\text { ERT/ } \\
\text { electrodes }\end{array}$ & Buckets & 17 & 25-Jun & 25-Jun & 28-Jun & 61.0 & 25 & 31 & 56 & 56.3 & 33.5 & ---- & 88.5 & 88.5 & 55 & 55 \\
\hline 399-2-16 & C6193 & $\begin{array}{l}\text { ERT/ } \\
\text { electrodes }\end{array}$ & Buckets & 15 & 24-Jun & 24-Jun & 27-Jun & 62.0 & 25 & 31 & 56 & 56.3 & 33.5 & ---- & 79.8 & 79.8 & 54 & 54 \\
\hline $399-2-17$ & C6195 & $\begin{array}{l}\text { ERT/ } \\
\text { electrodes }\end{array}$ & Buckets & 8 & 12-Jun & 13-Jun & 19-Jun & 63.0 & 25 & 31.2 & 56.2 & 56.5 & 33.7 & ---- & 74.5 & 74.5 & 56.5 & 55.5 \\
\hline $399-2-18^{(\mathrm{d})}$ & C6196 & $\begin{array}{l}\text { ERT/ } \\
\text { electrodes }\end{array}$ & Buckets & 6 & 3-Jun & 11-Jun & 14-Jun & 65.0 & 25 & 33 & 58 & 58.3 & 33.5 & ---- & 95.5 & 95.5 & 57 & 57 \\
\hline $399-2-19^{(e)}$ & C6197 & $\begin{array}{l}\text { ERT/electrodes } \\
\text { characterization }\end{array}$ & $\begin{array}{l}\text { Intact } \\
\text { Lexan core }\end{array}$ & 3 & 20-May & 20-May & Complete & 60.8 & 25 & 31.3 & 56.3 & 56.6 & 33.8 & 66.5 & 0 & 66.5 & 57 & 54 \\
\hline $399-2-20$ & C6198 & $\begin{array}{l}\text { ERT/ } \\
\text { electrodes }\end{array}$ & Buckets & 16 & 25-Jun & 25-Jun & 27-Jun & 62.0 & 25 & 31 & 56 & 56.3 & 33.5 & ---- & 80.6 & 80.6 & 55 & 55.5 \\
\hline $399-2-21$ & C6201 & $\begin{array}{l}\text { ERT/ } \\
\text { electrodes }\end{array}$ & Buckets & 7 & 12-Jun & 12-Jun & 16-Jun & 61.7 & 25 & 31.1 & 56.1 & 56.4 & 33.6 & --- & 79.4 & 79.4 & 55 & 55 \\
\hline $399-2-22$ & C6202 & $\begin{array}{l}\text { ERT/ } \\
\text { electrodes }\end{array}$ & Buckets & 29 & 14-Jul & 16-Jul & 17-Jul & 65.0 & 25.1 & 33.4 & 58.5 & 58.8 & 34.1 & ---- & 72.3 & 72.3 & 56 & 58 \\
\hline $399-2-23$ & C6207 & $\begin{array}{l}\text { ERT/ } \\
\text { electrodes }\end{array}$ & Buckets & 24 & 8-Jul & 8-Jul & 10-Jul & 60.0 & 25 & 31 & 56 & 56.3 & 33.5 & ---- & 78.3 & 78.3 & 55 & 55.5 \\
\hline $399-2-24$ & C6208 & $\begin{array}{l}\text { ERT/electrodes } \\
\text { characterization }\end{array}$ & $\begin{array}{c}\text { Intact } \\
\text { Lexan core }\end{array}$ & 5 & 23-May & 2-Jun & Complete & 65.2 & 25 & 33 & 58 & 58.3 & 33.5 & 35 & 45.5 & 80.5 & 58 & 58 \\
\hline $399-3-23$ & C6194 & $\begin{array}{l}\text { ERT/ } \\
\text { electrodes }\end{array}$ & Buckets & 28 & 10-Jul & 10-Jul & 13-Jul & 65.0 & 25 & 31 & 56 & 56.3 & 33.5 & --- & 63.1 & 63.1 & 52.5 & 53.5 \\
\hline 399-3-24 & C6199 & $\begin{array}{c}\text { ERT/ } \\
\text { electrodes }\end{array}$ & Buckets & 27 & 9-Jul & 10-Jul & 12-Jul & 65.0 & 25 & 31 & 56 & 56.3 & 33.5 & --- & 90 & 90 & 52.5 & 52.5 \\
\hline $399-2-25$ & C6209 & $\begin{array}{l}\text { Deep microbiologgical } \\
\text { characterization GW } \\
\text { monitoring }\end{array}$ & $\begin{array}{l}\text { Intact core } \\
\text { and buckets }\end{array}$ & 34 & 22-Jul & 25-Jul & 27-Jul & 171.0 & 60 & 62 & 122 & 124.9 & N/A & N/A & $\mathrm{N} / \mathrm{A}$ & N/A & 53 & 58 \\
\hline 399-3-25 & C6200 & $\begin{array}{l}\text { ERT/electrodes } \\
\text { characterization }\end{array}$ & $\begin{array}{c}\text { Intact } \\
\text { Lexan core }\end{array}$ & 10 & 16-Jun & 17-Jun & 20-Jun & 65.0 & 25 & 31.6 & 56.6 & 56.9 & 33.1 & 63.8 & 7.7 & 71.5 & 57.5 & 57 \\
\hline $399-2-26$ & C6210 & $\begin{array}{c}\text { Shallow } \\
\text { GW monitoring }\end{array}$ & Buckets & 14 & 23-Jun & 24-Jun & 26-Jun & 62.0 & 5 & 30 & 35 & 59.4 & N/A & ---- & 77.4 & 77.4 & 57 & 58 \\
\hline
\end{tabular}


Table 3.1. (contd)

\begin{tabular}{|c|c|c|c|c|c|c|c|c|c|c|c|c|c|c|c|c|c|c|}
\hline \multirow[b]{2}{*}{ Well Name } & \multirow[b]{2}{*}{ Well ID } & \multirow[b]{2}{*}{ Function } & \multirow[b]{2}{*}{ Samples } & \multirow[b]{2}{*}{$\begin{array}{l}\text { Drilling } \\
\text { Order }\end{array}$} & \multirow[b]{2}{*}{$\begin{array}{c}\text { Start } \\
\text { Drilling }\end{array}$} & \multirow[b]{2}{*}{$\begin{array}{c}\text { End } \\
\text { Drilling }\end{array}$} & \multirow[b]{2}{*}{$\begin{array}{l}\text { Geophysical } \\
\text { Logging }\end{array}$} & \multirow{2}{*}{$\begin{array}{c}\text { Total } \\
\text { Depth } \\
(\mathrm{ft}) \\
\end{array}$} & \multirow[b]{2}{*}{$\begin{array}{l}\text { Length } \\
\text { Screen (ft) }\end{array}$} & \multirow[b]{2}{*}{$\begin{array}{l}\text { Top Screen } \\
\text { Depth (ft) }\end{array}$} & \multirow[b]{2}{*}{$\begin{array}{l}\text { Bottom Screen } \\
\text { Depth (ft) }\end{array}$} & \multirow[b]{2}{*}{$\begin{array}{l}\text { Bottom End } \\
\text { Cap (ft) }\end{array}$} & \multirow[b]{2}{*}{$\begin{array}{l}\text { Instrument } \\
\text { Depth (ft) }\end{array}$} & \multicolumn{3}{|c|}{ \% Recovery } & \multicolumn{2}{|c|}{$\begin{array}{l}\text { Hanford/Ringold Contact } \\
\text { Depth (ft) }\end{array}$} \\
\hline & & & & & & & & & & & & & & Core & Grab & Total & $\begin{array}{c}\text { Geologist } \\
\text { Log }\end{array}$ & $\begin{array}{l}\text { Gamma } \\
\text { Log }\end{array}$ \\
\hline $399-3-26^{(t)}$ & $\mathrm{C} 6203$ & $\begin{array}{l}\text { ERT/electrodes } \\
\text { characterization }\end{array}$ & $\begin{array}{c}\text { Intact } \\
\text { Lexan core }\end{array}$ & 2 & 14-May & 15-May & Complete & 66.0 & 20 & 32 & 52 & 52.3 & 33.5 & 51.5 & 16.5 & 68 & 52 & 52 \\
\hline $399-2-27$ & C6211 & $\begin{array}{c}\text { Deep } \\
\text { GW monitoring } \\
\text { ERT/electrodes }\end{array}$ & Buckets & 32 & 17-Jul & 18-Jul & None & 63.5 & 2 & 54.7 & 56.7 & 59.1 & 58.5 & ---- & 67.7 & 67.7 & 57.5 & ---- \\
\hline $399-2-28$ & C6212 & $\begin{array}{l}\text { Intermediate } \\
\text { GW monitoring }\end{array}$ & Buckets & 21 & 27-Jun & 30-Jun & None & 65.0 & 2 & 42 & 44 & 56.3 & N/A & ---- & 80 & 80 & 56.5 & ---- \\
\hline 399-3-27 & C6204 & $\begin{array}{c}\begin{array}{c}\text { ERT/ } \\
\text { electrodes }\end{array}\end{array}$ & Buckets & 26 & 9-Jul & 9-Jul & 11-Jul & 62.0 & 25 & 31 & 56 & 56.3 & 33.5 & --- & 84.7 & 84.7 & 50.5 & 50.5 \\
\hline 399-2-29 & C6216 & $\begin{array}{l}\text { Shallow } \\
\text { GW monitoring }\end{array}$ & Buckets & 18 & 26-Jun & 26-Jun & None & 61.5 & 5 & 29.7 & 34.7 & 55 & N/A & ---- & 82.9 & 82.9 & 55.5 & ---- \\
\hline 399-3-28 & C6205 & $\begin{array}{l}\text { ERT/ } \\
\text { electrodes }\end{array}$ & Buckets & 25 & 8-Jul & 9-Jul & 10-Jul & 64.5 & 25 & 31 & 56 & 56.4 & 33.5 & ---- & 79.8 & 79.8 & 55.5 & 56.5 \\
\hline 399-2-30 & C6217 & $\begin{array}{c}\text { Deep } \\
\text { GW monitoring } \\
\text { ERT/electrodes }\end{array}$ & Buckets & 35 & 24-Jul & 25-Jul & None & 64.0 & 2 & 54 & 56 & 58.9 & 58.5 & ---- & 95.3 & 95.3 & 55.5 & ---- \\
\hline 399-3-29 & C6206 & $\begin{array}{c}\text { ERT/ } \\
\text { electrodes }\end{array}$ & Buckets & 22 & 30-Jun & 30-Jun & 3-Jul & 64.0 & 25 & 31 & 56 & 56.3 & 33.5 & ---- & 83.6 & 83.6 & 53 & 52.5 \\
\hline 399-2-31 & C6218 & $\begin{array}{l}\text { Intermediate } \\
\text { GW monitoring }\end{array}$ & Buckets & 12 & 19-Jun & 20-Jun & 24-Jun & 63.0 & 2 & 42 & 44 & 56.4 & N/A & ---- & 80.2 & 80.2 & 55.5 & 55.5 \\
\hline 399-3-30 & C6213 & $\begin{array}{l}\text { Shallow } \\
\text { GW monitoring }\end{array}$ & Buckets & 19 & 26-Jun & 27-Jun & None & 61.5 & 5 & 30 & 35 & 55.3 & N/A & ---- & 82.1 & 82.1 & 55.5 & \\
\hline $399-3-31^{(\mathrm{g})}$ & C6214 & $\begin{array}{l}\text { Deep } \\
\text { GW monitoring } \\
\text { ERT/electrodes }\end{array}$ & Buckets & 13 & 20-Jun & 23-Jun & 25-Jun & 63.0 & 2 & 53.2 & 55.2 & 59.6 & 33.6/58.6 & ---- & 76.2 & 76.2 & 55.5 & 55.5 \\
\hline 399-3-32 & C6215 & $\begin{array}{c}\text { Intermediate } \\
\text { GW monitoring }\end{array}$ & Buckets & 33 & 18-Jul & 18-Jul & None & 63.0 & 2 & 42.2 & 44.2 & 56.6 & N/A & --- & 54 & 54 & 56 & ---- \\
\hline $\begin{array}{l}\text { (a) Borehole } \\
\text { (b) Shallow } \\
\text { (c) Redrill o } \\
\text { (d) Bottom t } \\
\text { (e) Tagline I } \\
\text { (f) Unintent } \\
\text { (g) No recor } \\
\text { N/A = not app }\end{array}$ & $\begin{array}{l}\text { e was deepe } \\
\text { refusal; off } \\
\text { out bottom } \\
\text { bentonite se } \\
\text { lost; redrill } \\
\text { tionally dril } \\
\text { very } 45-55 \\
\text { plicable. }\end{array}$ & $\begin{array}{l}\text { hed to } 67.5 \mathrm{ft} \text { (casing } \\
\text { et hole } 1 \mathrm{~m} \text { east. } \\
\text { hole. } \\
\text { l bridged; redrill bo } \\
\text { ole. } \\
\text { ed an extra } 10 \mathrm{ft} \text { dur } \\
\text { t. }\end{array}$ & $\begin{array}{l}\text { tom. } \\
\text { ng final cleanc }\end{array}$ & out. & iysical logg & & & & & & & & & & & & & \\
\hline
\end{tabular}




\subsection{Drilling}

The IFRC well field consists of 36 evenly distributed wells (Figure 3.2). The electrical-resistivity tomography (ERT) wells were spaced $10 \mathrm{~m}$ apart. At three locations, a tighter three-well cluster also was installed to monitor shallow, intermediate, and deep levels within the unconfined aquifer. All of the wells were drilled using the resonant sonic drilling method (Figure 3.5). One of the wells (399-2-5) at the eastern corner of the well field was a pre-existing well, drilled with the cable-tool method in 2007 to a depth of $131 \mathrm{ft}$ (6 ft into the Ringold lower mud unit) as part of a volatile organic compound investigation (Peterson et al. 2008). Only a single deep well (399-2-25), a new microbiological characterization well, was drilled to the top of basalt bedrock at $171 \mathrm{ft}$ depth. The remaining 34 wells were drilled to depths of 50-60 ft into the top of a fine-grained subunit of the Ringold Formation at the base of the unconfined aquifer. Drilling of the first new well (399-2-9) began on May 12, 2008. Drilling ended with well 399-2-25 on July 25, 2008 (Table 3.1). The total drilled footage for the 35 new wells was $2318 \mathrm{ft}$.

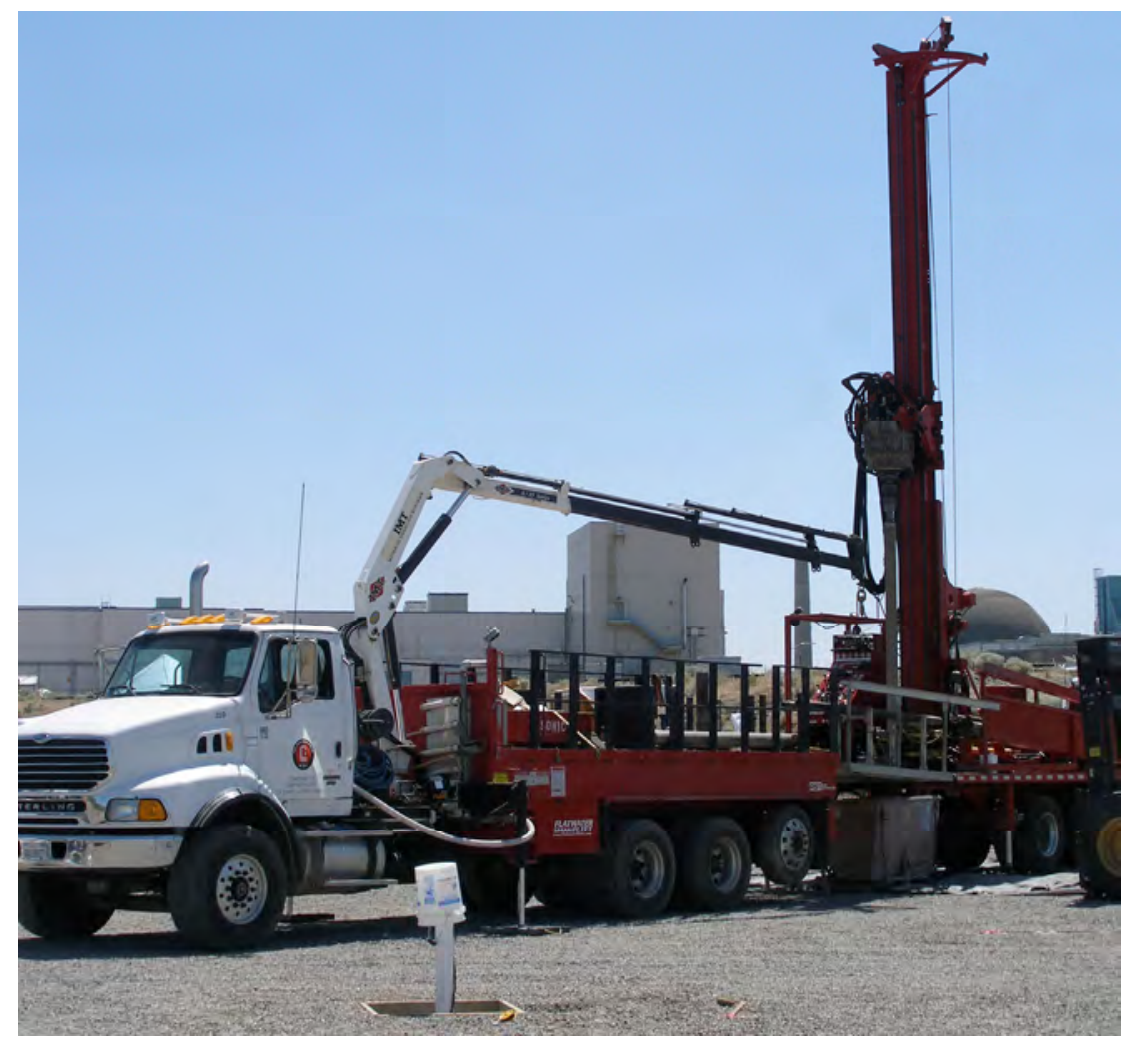

Figure 3.5. A resonant sonic drill rig was used to drill the 35 new IFRC boreholes.

A single string of 7-5/8-in.-0D (6-7/8-in.-ID) carbon steel casing was used to maintain an open borehole in the 34 shallow boreholes. During drilling, boreholes were advanced by vibrating a 6-in. core barrel into the formation to obtain intact core material below the bottom of the casing. Next, the casing was advanced, via sonic vibration, over the cored interval, and the hole was cleaned out to the bottom of the casing before the next core sample was collected. In a single deep well (399-2-25), three strings of telescoping casing were used to preclude aquifer intercommunication between the unconfined and deeper aquifers. Drilling details and other documentation for each of the new wells are provided in Appendices $\mathrm{A}, \mathrm{B}$, and D. 


\subsection{Sampling}

More than 1100 geologic samples were collected for physical, chemical, or microbiological analysis from the 35 new IFRC wells (Table 3.2; Appendix C). Seven borings were preselected as characterization holes in which an attempt was made to collect continuous, intact (Lexan-lined) core via 5-ft-long split spoons (Figure 3.6 and Figure 3.7). Lexan core was collected also at selected intervals within the single deep borehole (399-2-25). Up to five core samples ( $1 \mathrm{ft}$ long by 3-3/4 in. in diameter) were collected with each core run. The ends of the Lexan liners were geologically logged and photographed before the core ends were capped and sealed.

Table 3.2. Geologic samples by borehole

\begin{tabular}{|c|c|c|c|c|c|c|}
\hline \multirow[b]{2}{*}{ Well Name } & \multirow[b]{2}{*}{ Well ID } & \multicolumn{4}{|c|}{ No. of Sample Intervals } & \multirow[b]{2}{*}{$\begin{array}{c}\text { Total } \\
\text { Samples }\end{array}$} \\
\hline & & Bulk Grab $^{(a)}$ & Lexan Core ${ }^{(\mathrm{b})}$ & $\begin{array}{c}\text { Smear-Zone } \\
\text { Grab }^{(\mathrm{c})}\end{array}$ & Microbiology & \\
\hline $399-2-07$ & C6184 & 19 & & 1 & 3 & 23 \\
\hline $399-2-08$ & C6185 & 19 & & 1 & 2 & 22 \\
\hline 399-2-09 & C6186 & 2 & 35 & 2 & & 39 \\
\hline $399-2-10$ & C6187 & 21 & & 1 & 1 & 23 \\
\hline $399-2-11$ & C6188 & 17 & & 1 & 1 & 19 \\
\hline $399-2-12$ & C6189 & 4 & 45 & 1 & 1 & 51 \\
\hline $399-2-13$ & C6190 & 3 & 28 & 1 & & 32 \\
\hline $399-2-14$ & C6191 & 40 & & 1 & 1 & 42 \\
\hline $399-2-15$ & C6192 & 22 & & 1 & 1 & 24 \\
\hline $399-2-16$ & C6193 & 22 & & 1 & 6 & 29 \\
\hline $399-2-17$ & C6195 & 31 & & 1 & 1 & 33 \\
\hline $399-2-18$ & C6196 & 46 & & 1 & & 47 \\
\hline $399-2-19$ & C6197 & & 41 & 1 & & 42 \\
\hline $399-2-20$ & C6198 & 22 & & 1 & 3 & 26 \\
\hline $399-2-21$ & C6201 & 32 & & 1 & 1 & 34 \\
\hline $399-2-22$ & C6202 & 19 & & & 2 & 21 \\
\hline $399-2-23$ & C6207 & 17 & & 1 & 4 & 22 \\
\hline $399-2-24$ & C6208 & 18 & 30 & 2 & 1 & 51 \\
\hline $399-2-25$ & C6209 & 70 & 42 & 1 & 15 & 128 \\
\hline $399-2-26$ & C6210 & 23 & & 1 & 3 & 27 \\
\hline $399-2-27$ & C6211 & 19 & & 1 & 1 & 21 \\
\hline $399-2-28$ & C6212 & 27 & & & 3 & 30 \\
\hline
\end{tabular}

Shallow characterization wells.

Deep characterization well.

(a) Collected in 2-gal plastic buckets.

(b) Collected in Lexan liners, $1 \mathrm{ft}$ long x 3-3/4 in. OD.

(c) Collected in 5-gal plastic buckets. 
Table 3.2. (contd)

\begin{tabular}{|c|c|c|c|c|c|c|}
\hline \multirow[b]{2}{*}{ Well Name } & \multirow[b]{2}{*}{ Well ID } & \multicolumn{4}{|c|}{ No. of Sample Intervals } & \multirow[b]{2}{*}{$\begin{array}{c}\text { Total } \\
\text { Samples }\end{array}$} \\
\hline & & Bulk Grab $^{(a)}$ & Lexan Core ${ }^{(b)}$ & $\begin{array}{c}\text { Smear-Zone } \\
\text { Grab }^{(c)}\end{array}$ & Microbiology & \\
\hline $399-2-29$ & C6216 & 21 & & 1 & 3 & 25 \\
\hline $399-2-30$ & C6217 & 30 & 5 & 1 & 1 & 37 \\
\hline $399-2-31$ & C6218 & 27 & & 1 & 1 & 29 \\
\hline $399-3-23$ & C6194 & 22 & & 1 & 3 & 26 \\
\hline $399-3-24$ & C6199 & 18 & & & 3 & 21 \\
\hline $399-3-25$ & C6200 & 2 & 43 & 2 & 1 & 48 \\
\hline $399-3-26$ & C6203 & 2 & 31 & & & 33 \\
\hline $399-3-27$ & C6204 & 18 & & 1 & 5 & 24 \\
\hline $399-3-28$ & C6205 & 22 & & 1 & 5 & 28 \\
\hline $399-3-29$ & C6206 & 23 & & 1 & 3 & 27 \\
\hline 399-3-30 & C6213 & 23 & & 1 & 3 & 27 \\
\hline 399-3-31 & C6214 & 26 & & 1 & 1 & 28 \\
\hline 399-3-32 & C6215 & 25 & & 1 & 1 & 27 \\
\hline \multicolumn{2}{|c|}{ Total No. of Samples } & 752 & 300 & 34 & 80 & 1166 \\
\hline
\end{tabular}

Shallow characterization wells.

Deep characterization well.

(a) Collected in 2-gal plastic buckets.

(b) Collected in Lexan liners, $1 \mathrm{ft}$ long x 3-3/4 in. OD.

(c) Collected in 5-gal plastic buckets.

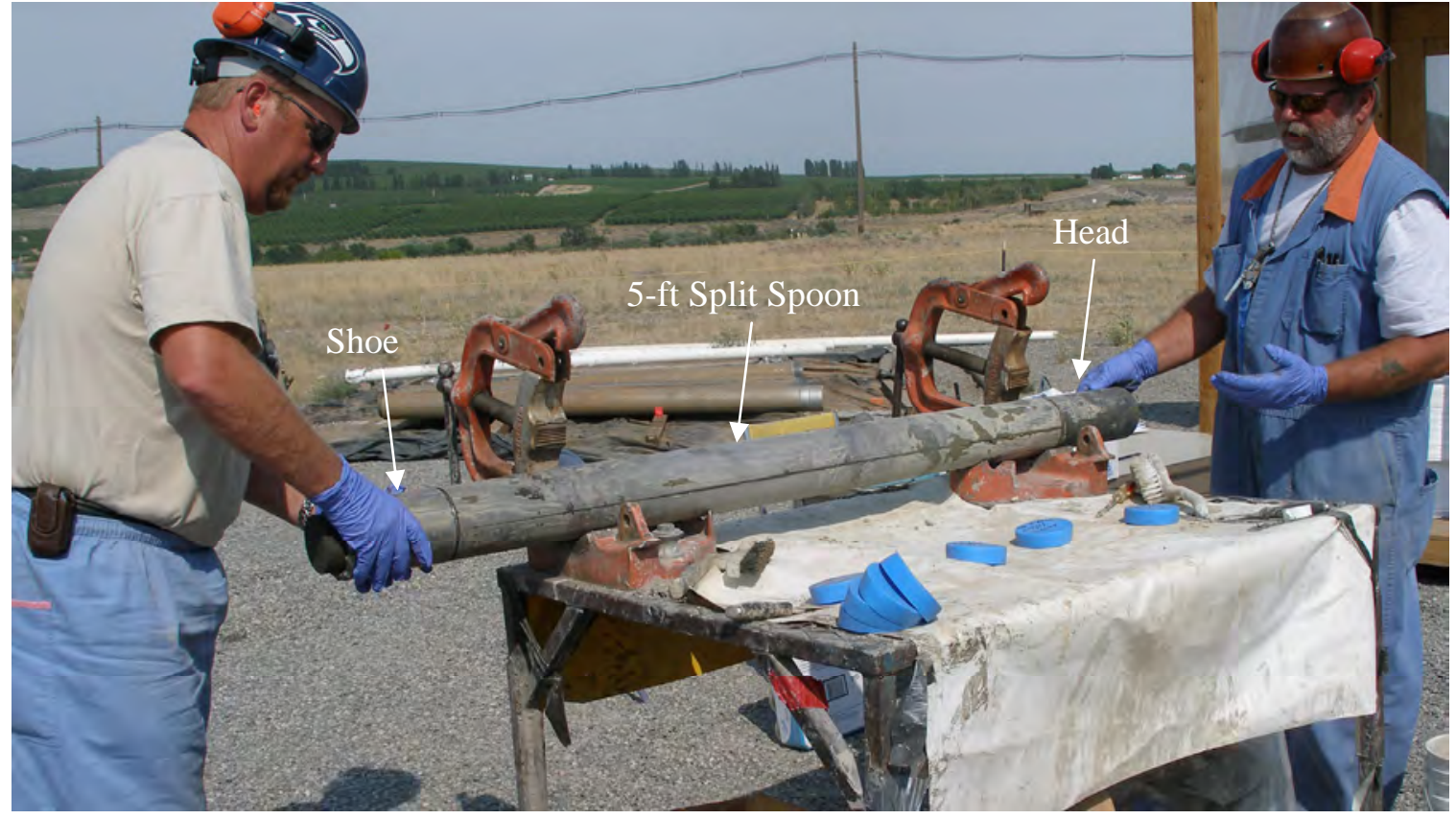

Figure 3.6. Sediment-filled split-spoon sampler. Notice cohesive Ringold Formation core protruding from shoe. Sediment core naturally expanded upon release of highly compressive drilling stresses. 


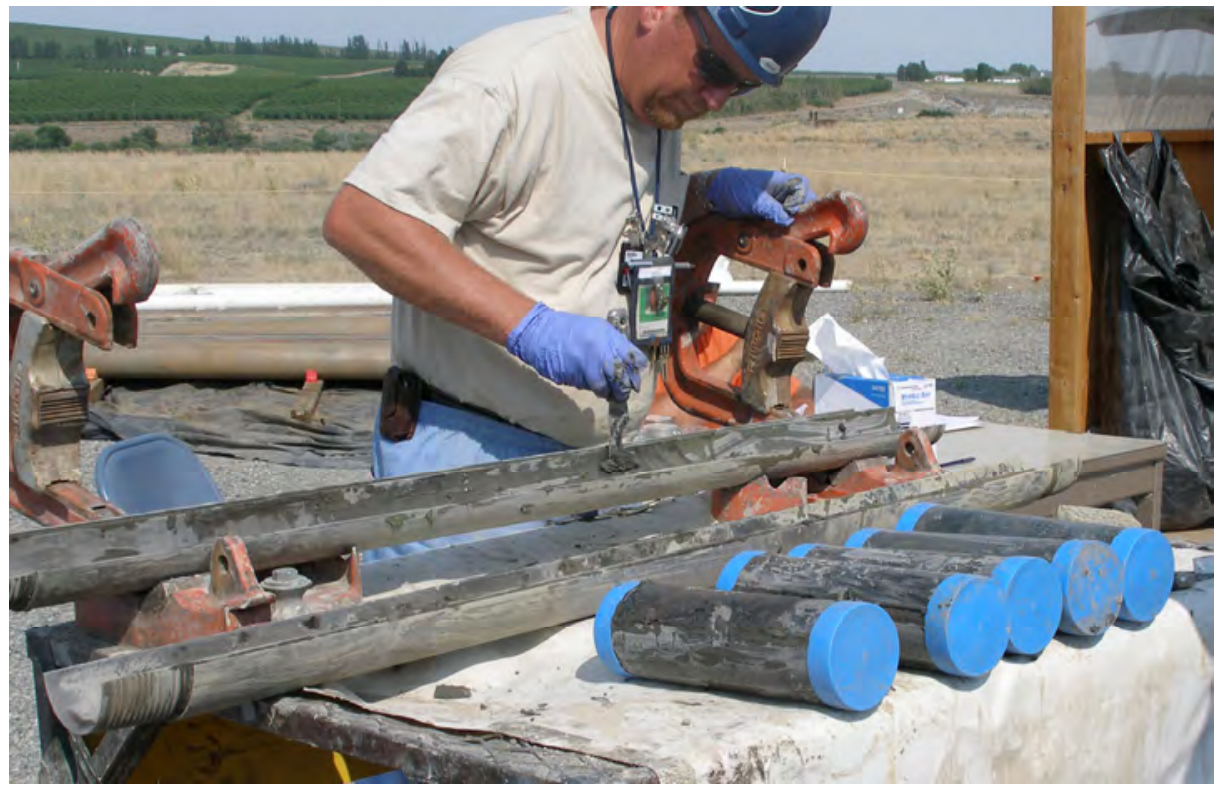

Figure 3.7. Capped Lexan-lined cores were collected after the split-spoon sampler was opened.

In the remaining 27 shallow boreholes, bulk core samples were collected by emptying core runs directly from the core barrel into plastic sleeves approximately $2 \mathrm{ft}$ long (Figure 3.8, left). After the plastic sleeves were sliced open, the sediment inside was geologically logged and photographed before it was transferred into 2-gal buckets (Figure 3.8, right).
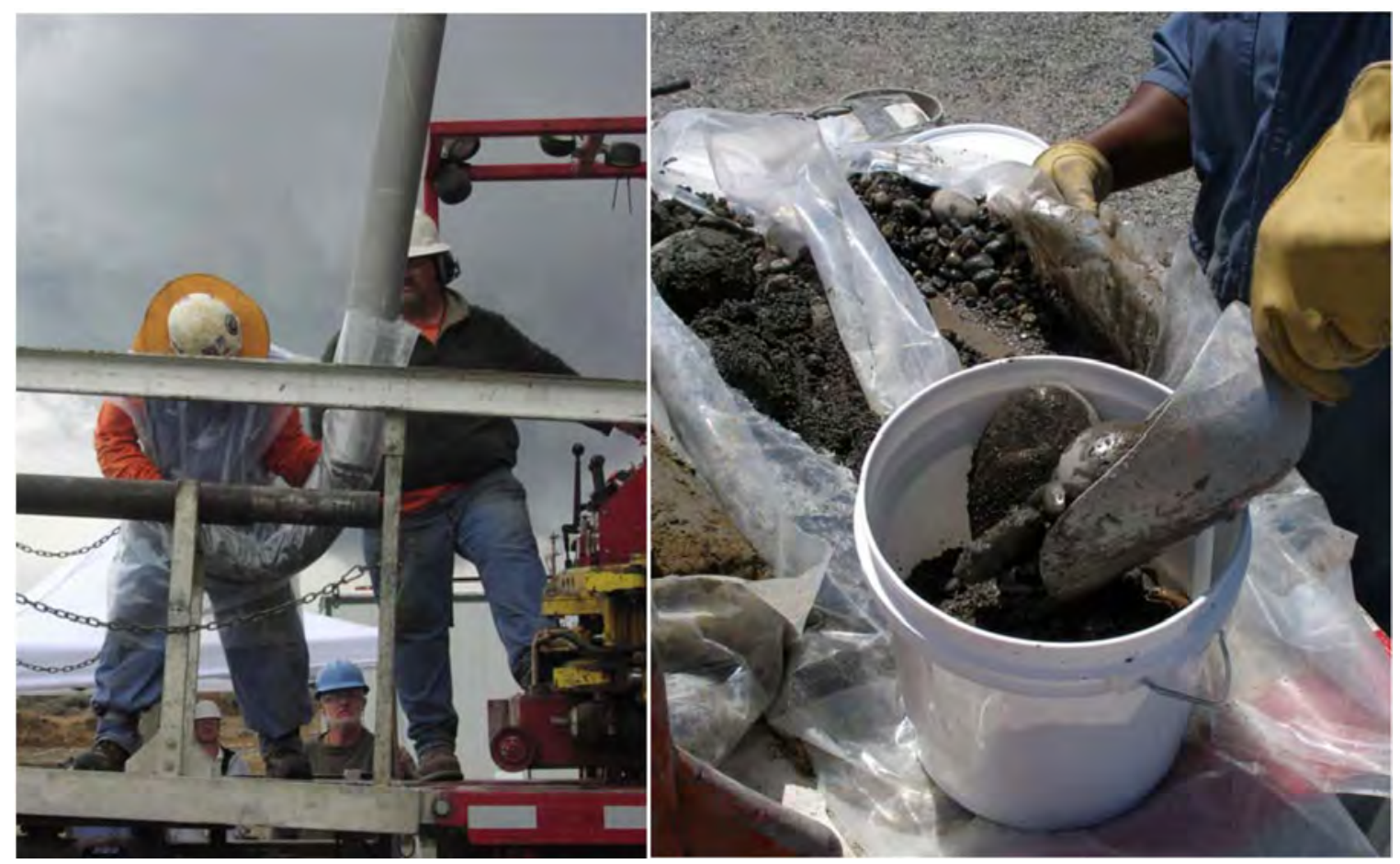

Figure 3.8. Bulk core and grab samples were collected from all IFRC wells not sampled via split spoon. Sediment core (left) was extruded via sonic vibration out of the core barrel into a knotted plastic sleeve. For grab-sample collection (right), core was transferred from the plastic sleeve into labeled 2-gal plastic buckets. Each bucket held 2 to $3 \mathrm{ft}$ of core. 
An additional 34 special bulk grab samples were collected in 5-gal containers from the smear zone (zone of water table fluctuation between 25 to $35 \mathrm{ft}$ depth) for most of the new IFRC wells (Table 3.2). All samples were carefully labeled, inventoried, and transferred to temporary storage onsite (Figure 3.9) or directly to PNNL laboratories.

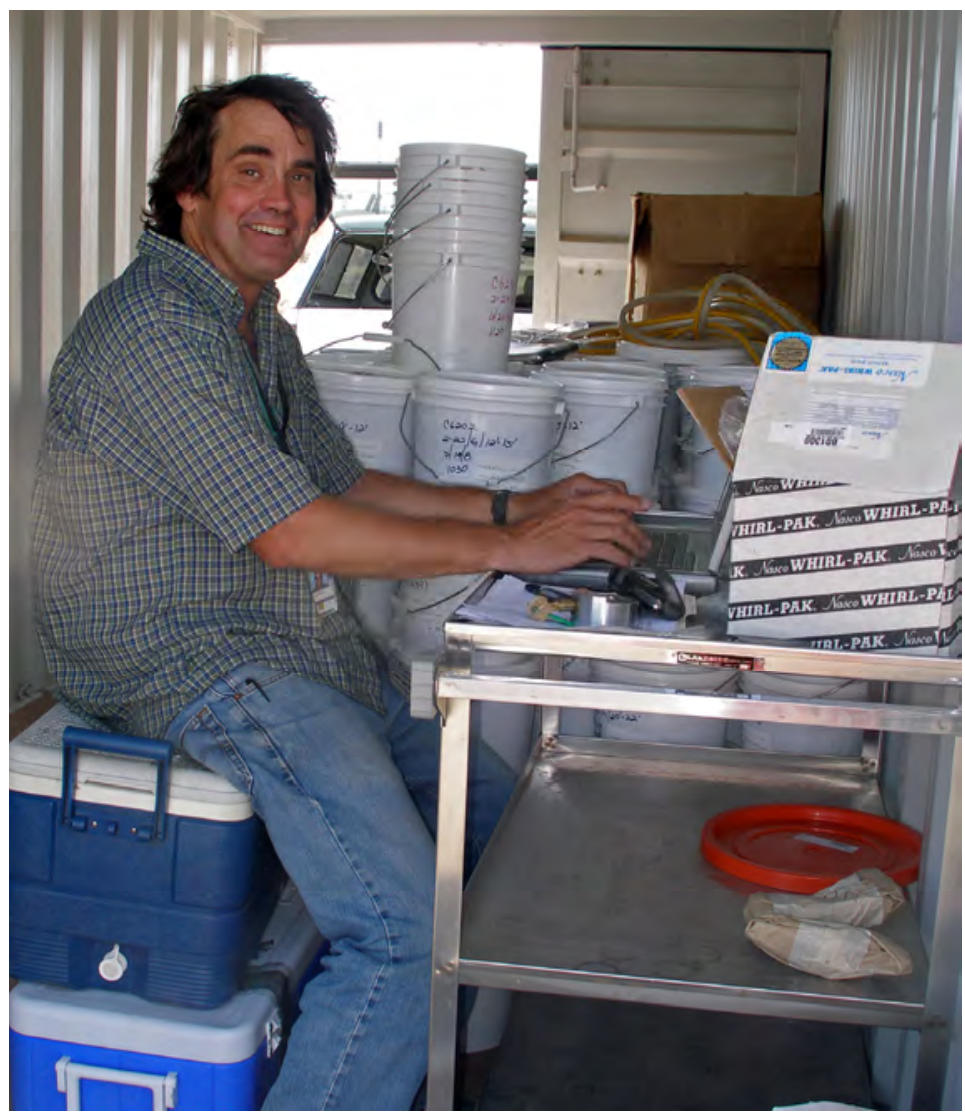

Figure 3.9. Samples were stored temporarily within a locked seatainer on site. All samples were carefully labeled upon collection and later inventoried.

The more than 1100 samples collected included 300 intact Lexan-lined core samples and 752 bulk grab samples (Table 3.2). Sample recovery was fair to excellent. Core recovery in the seven characterization boreholes, which attempted to collect continuous, intact core samples inside the segmented Lexan-lined split spoon, generally ranged from 50\% to 70\%. In one borehole (399-2-24), core recovery was a poor 35\% because the loose Hanford formation material kept falling out of the bottom of the split spoon upon retrieval. Core recovery improved significantly for the Hanford formation after a core catcher (Figure 3.10) was placed at the bottom of the split spoon.

Recovery of bulk grab samples that were emptied directly from the core barrel into plastic sleeves proved more successful (50-95\%) when compared to split-spoon sampling. Core recovery using this method, albeit not preserving the primary fabric and structure, provided good-quality samples believed to be representative of the formation, both texturally and geochemically. Core recovery always improved upon encountering the cohesive and compact fine-grained sediments of the Ringold Formation in each of the holes, irrespective of sampling method. Sample inventory sheets for each of the new IFRC wells are located in Appendix C. 


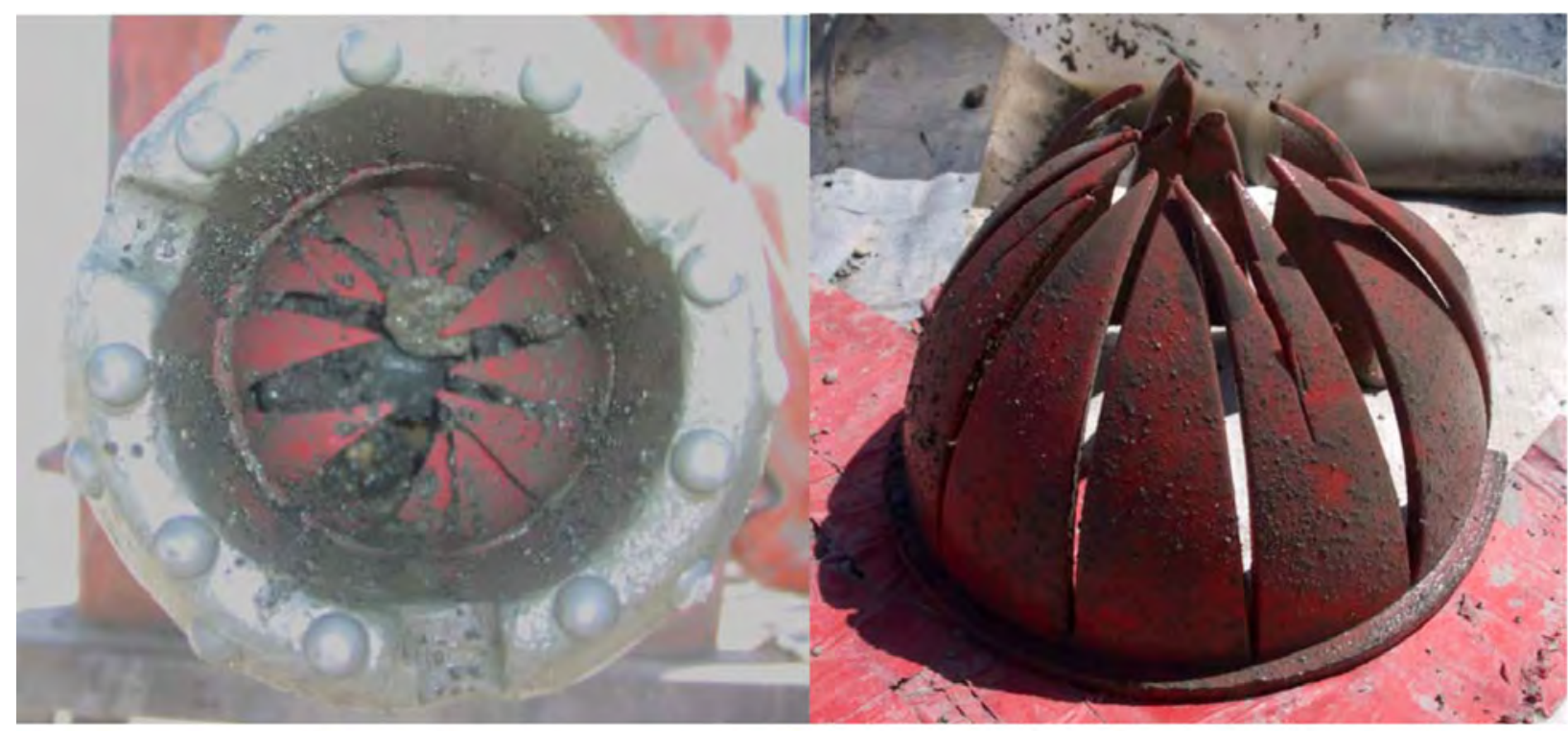

Figure 3.10. A plastic core catcher helped to keep loose Hanford formation sediments from falling out of the split spoon during core sampling.

\subsection{Geophysical Logging}

Downhole geophysical logging was performed on 29 of the 35 new wells (Table 3.1). Immediately after drilling to total depth and prior to well construction, logging occurred with the spectral-gamma logging system (SGLS) and neutron-moisture logging system (NMLS) geophysical logging tools (Figure 3.11). The NMLS was employed for only the vadose-zone portion, while the SGLS probe was used over the entire length of the logged boreholes. The single deep microbiology characterization borehole was logged three times, once for each temporary casing string (0-60, 60-120, and 120-167 ft). Of the nine multilevel groundwater-monitoring wells, only one borehole in each of the three-well clusters was logged with the SGLS and NMLS (Table 3.1). The decision to log only a single well in each cluster was made to save time and expense and reduce redundancy for boreholes that lay in proximity to each other.

Geophysical logs are a useful indicator of vadose-zone moisture as well as lithology and grain-size distribution of the suprabasalt sediments. Downhole geophysical logs for all new IFRC wells are located in Appendix G. 


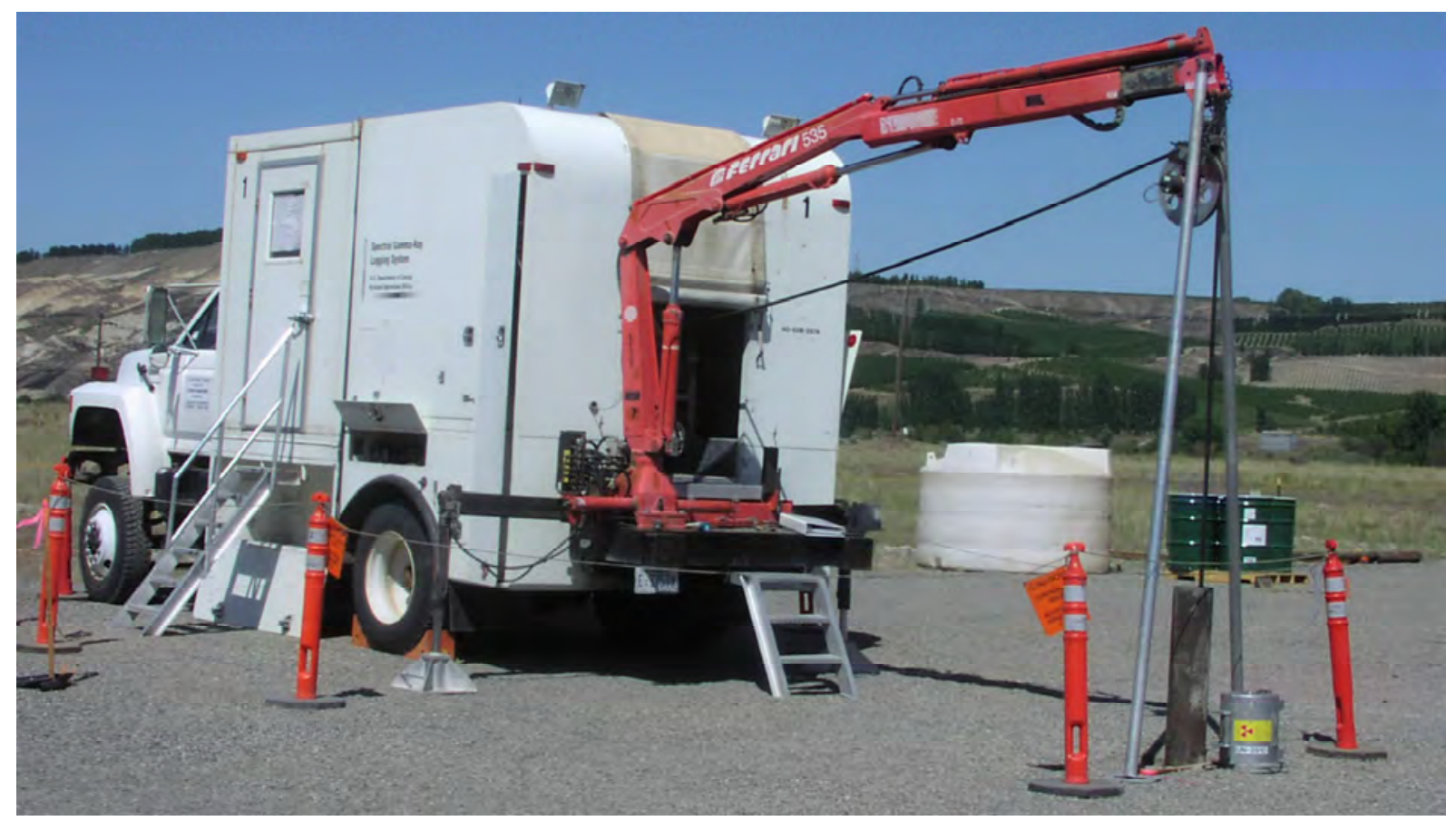

Figure 3.11. Downhole geophysical logging was accomplished through the temporary 7-5/8-in. (OD) steel casing via Stoller's spectral-gamma logging system.

\subsection{Well Construction}

All new wells were completed with 4-in.polyvinyl chloride (PVC) casing and 20-slot screens (see Appendices $\mathrm{A}$ and $\mathrm{F}$ ). The 25 shallow wells not designated as groundwater monitoring wells were constructed with 25-ft well screens over the saturated interval of the Hanford formation. A single exception was well 399-3-26, which was installed with a shorter 20-ft screen. The three multilevel groundwater monitoring clusters each had 2-ft screens installed at the bottom and middle portions of the unconfined aquifer and 5-ft screens installed at the top of the unconfined aquifer. The deep microbiology characterization well (399-2-25) had a single 60-ft screen completed over the semiconfined Ringold Formation (Unit E) aquifer.

Special ERT electrodes in addition to thermistors were installed on the outside of the PVC to the bottom of the screen (Figure 3.12) in 28 of the 35 new wells. Excluded were the 6 shallow and 6 intermediate-depth groundwater monitoring wells (Table 3.1) and the deep microbiology characterization well (399-2-25).

During construction, the annulus of each of the 28 wells was filled with 10-20 mesh silica sand to within $2 \mathrm{ft}$ of the top and bottom of the well screen (Figure 3.13). Above this was placed finer 40-140 mesh sand to within $10 \mathrm{ft}$ of the surface. These specific materials were selected because an annulus filled with permeable sand is needed to maximize the functionality of the ERT and temperature sensors within the well. A coarser sand (10-20 mesh) was used across the screened interval to keep the sand from passing through the screen. Around the casing above the screen, a finer sand (40-140 mesh) that more effectively retains vadose zone moisture was used. See Figure 3.14 for a comparison of these two different sand sizes. Filter-pack sand was not placed in the annulus within $10 \mathrm{ft}$ of the surface. 
However, a waiver was granted by the Washington State Department of Ecology allowing for a less than the 18-ft surface seal stipulated in WAC 173-160, "Minimum Standards for Construction and Maintenance of Wells.”

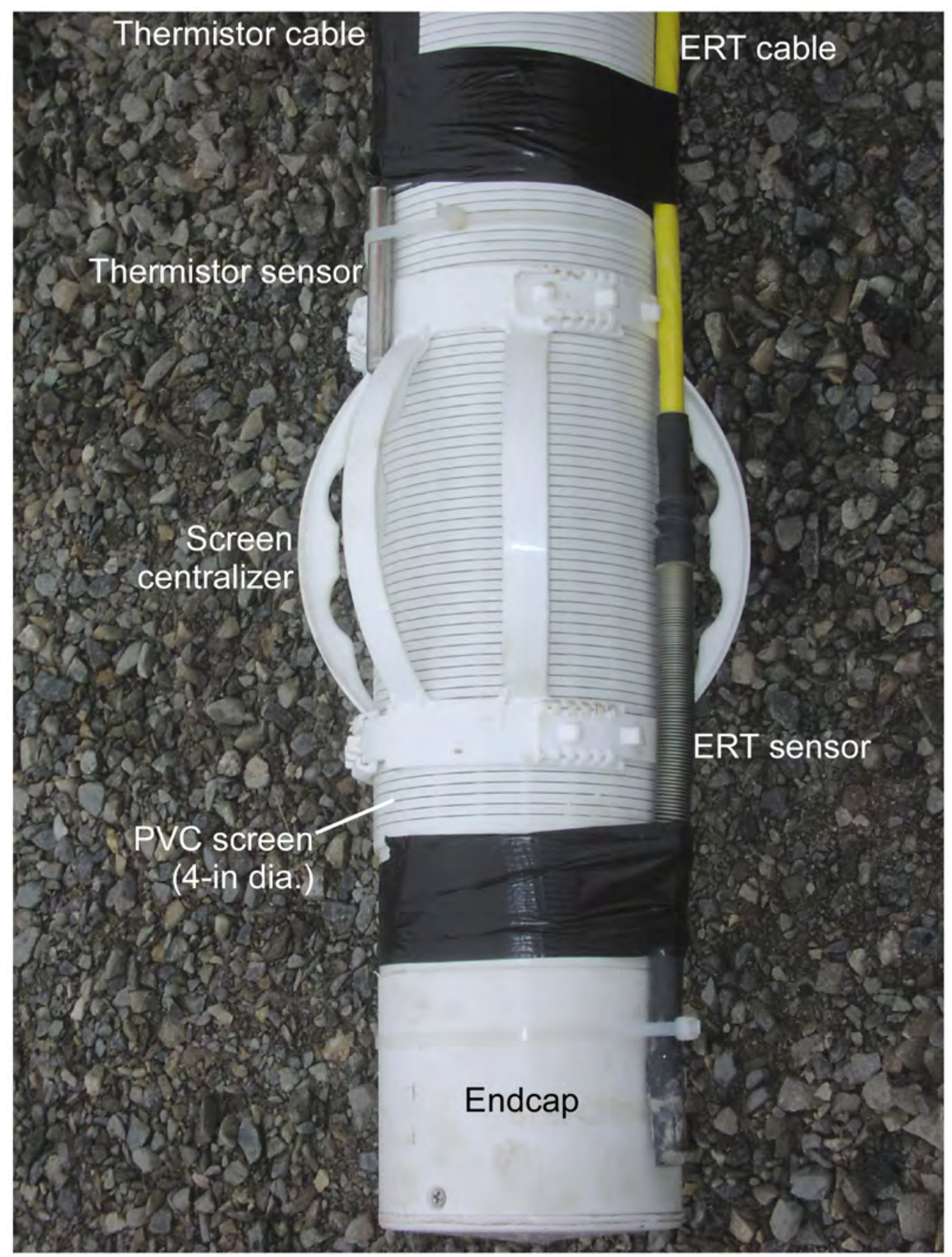

Figure 3.12. ERT electrode and thermistor sensors and cables were affixed at regular intervals down the outside of the 4-in. PVC screen and casing. A centralizer (shown) placed at the top and bottom of the screen kept the screen centered within the well and prevented it from coming into direct contact with the formation. 


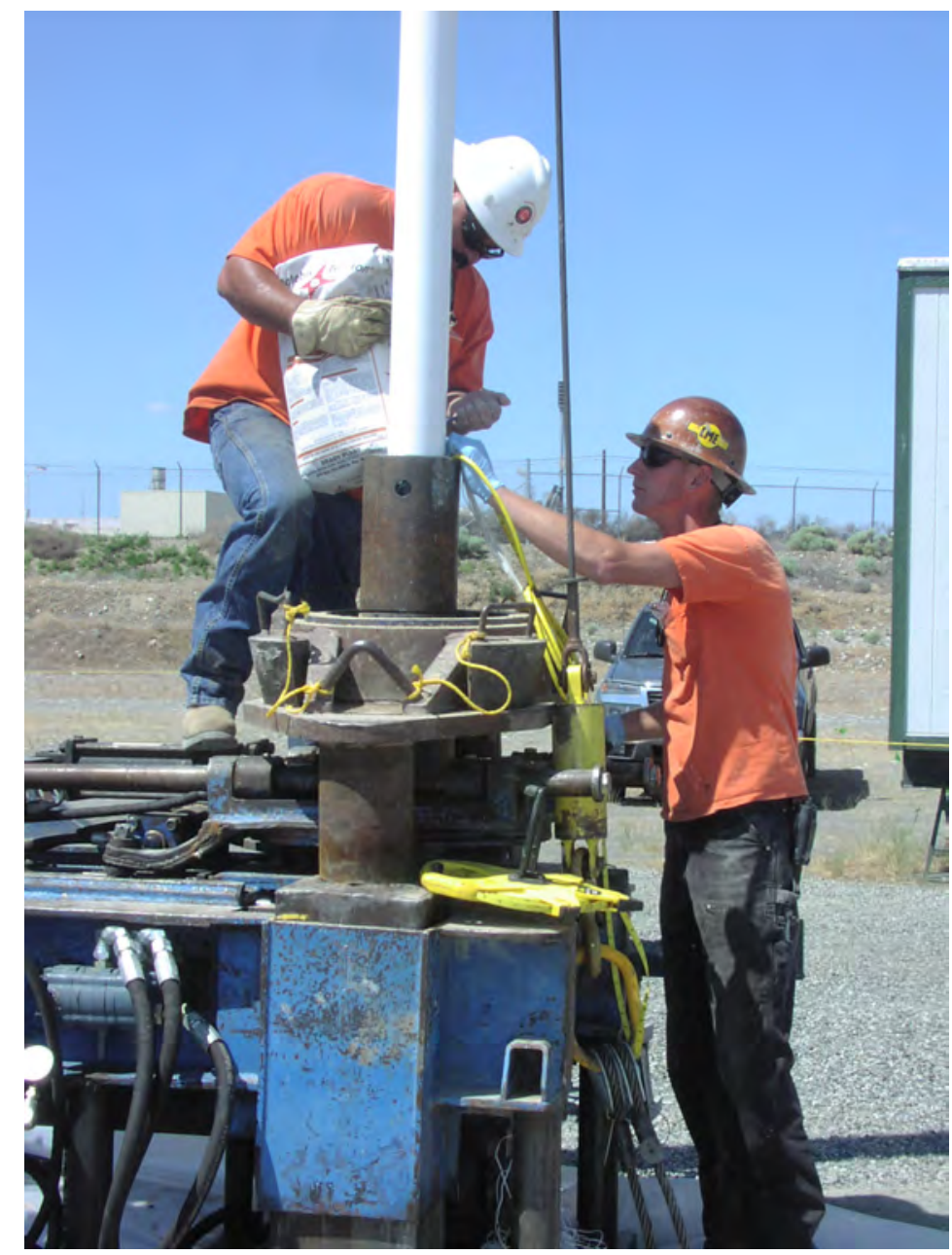

Figure 3.13. Silica sand was added to the well annulus between the 4-in. PVC well casing and 6-7/8-in. (ID) temporary steel casing during well construction. Hydraulic casing jacks, located in center, were used to back pull the temporary casing during well construction.

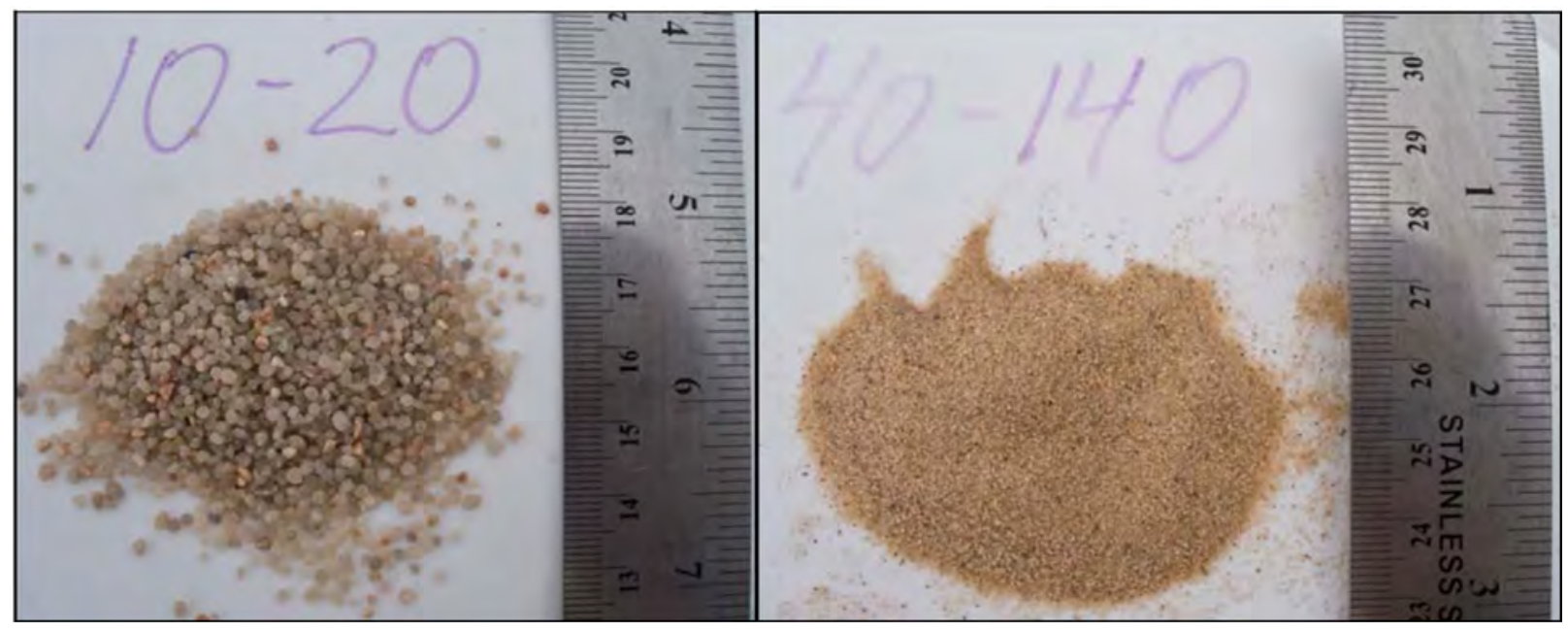

Figure 3.14. Comparison between coarser 10-20 mesh silica sand (left), used around 20-slot well screens, and the finer 40-140 mesh sand placed in the annular space above the screen 
Electrical-resistivity tomography and thermistor sensors were installed also around the outside of the deep groundwater monitoring wells. In these wells, 10-20 mesh silica sand was placed within $2 \mathrm{ft}$ above and below the 2 -ft well screen. Then a 5 -ft bentonite seal was emplaced (via tremie pipe) above the screen before 40-140 mesh silica sand was installed to $10 \mathrm{ft}$ bgs. The other six groundwater monitoring wells were installed as conventional wells with a continuous bentonite seal from near-surface to within $2 \mathrm{ft}$ of the top of the screen. The sand was surged periodically during placement to settle and compress the sand pack, to eliminate the formation of any voids during installation.

Only one new well (the deep microbiology characterization well, 399-2-25) was completed above ground; the remaining 34 wells were installed as surface-mount completions (Figure 3.15). These consisted of a metal well vault encased into a 6-in.-thick cement pad at the surface. Removal of the metal well-vault cover permits access to the PVC well inside near ground level. A diagonal drain hole from the bottom of the well vault through the cement pad was installed to allow any excess condensation, rain, or snowmelt to drain out into the formation instead of collecting in the vault and possibly down the well. A 2-in. PVC conduit passing laterally through a side hole in the well vault to the outside of the surface cement pad was also installed to allow passage of the ERT and thermistor cables out of the well without interfering with closure of the protective vault cover. The compilation borehole summary logs (Appendix A) provide a graphic summary of the well construction details provided in Appendix F.

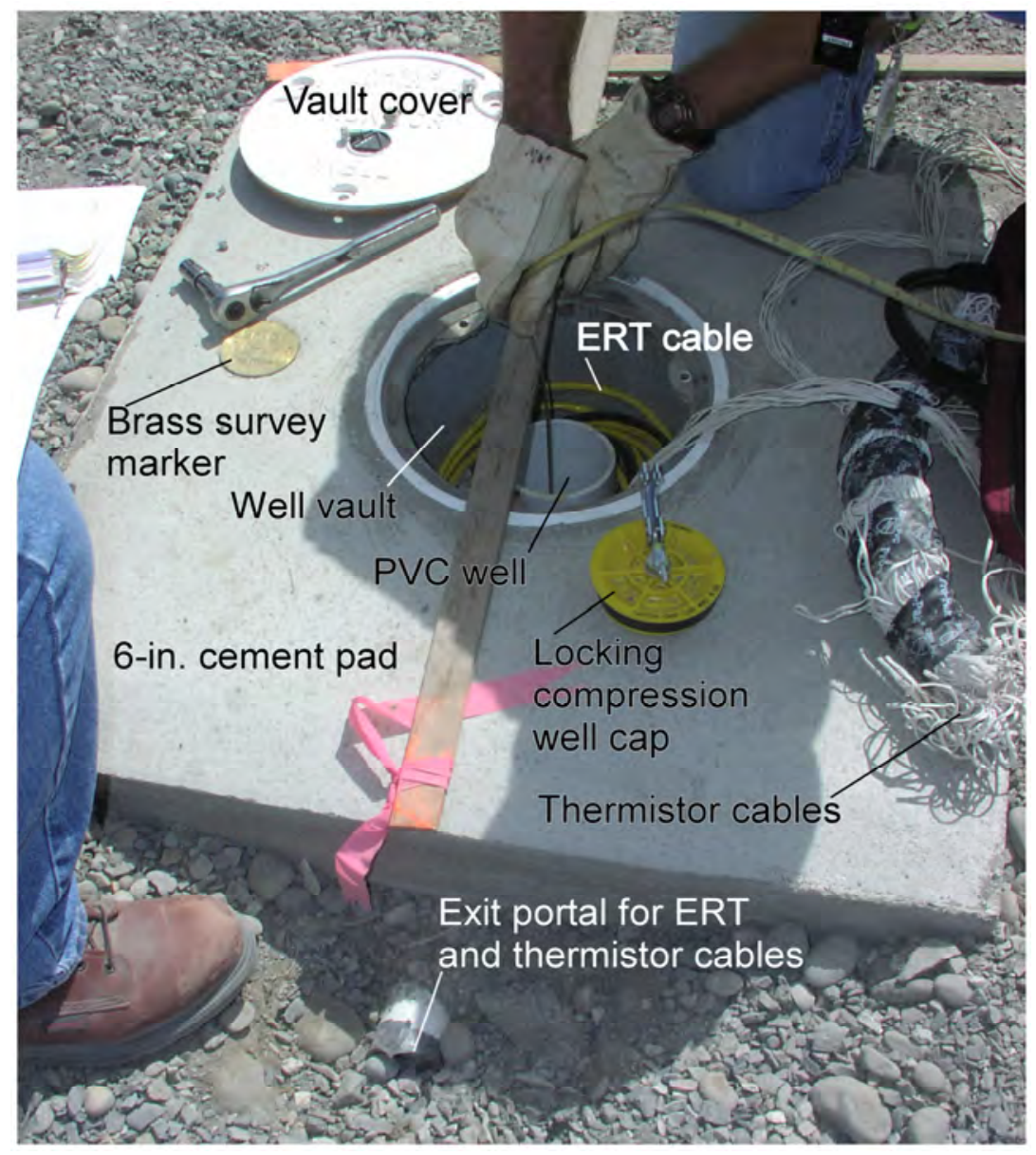

Figure 3.15. All but one of the new IFRC wells was completed at ground level to allow for easier and safer movement of vehicles and equipment across the crowded well field. 


\subsection{Well Development}

After drilling and well installation, it was important to 1) flush out impurities in the well and sand pack, 2) replace the stagnant drilling slurry within the well with fresh formation groundwater, and 3) evaluate the hydraulic properties and integrity of the well. During well development, between 540 and 3700 gal of purge water were pumped from each well at rates from 20 to 90 gpm (Table 3.3). Purge water was pumped into a temporary storage tank (Figure 3.16), and transported to modu-tanks at the Purgewater Storage and Treatment Facility for evaporative storage and treatment.

Drawdown and recovery of water within the wells were monitored during development. In the first few wells, transducers were placed in one or more adjacent observation wells to detect any drawdown during pumping. This practice was discontinued when it became clear that no drawdown was likely because of the extremely high transmissivity of the Hanford formation.

In the 25 ERT wells, pumping was performed twice, at two separate points within the longer 25-ft well screens. With one exception (399-2-9), the upper part of the screened interval was pumped and developed first before the pump was lowered approximately $10 \mathrm{ft}$ to the lower part of the screen. Pumping in the groundwater monitoring wells, on the other hand, was performed at only a single stage because of shorter screen lengths. More details on well development are presented in Appendix E.

Figure 3.17, a panoramic view of the IFRC wells during completion, illustrates the high density of the wells.

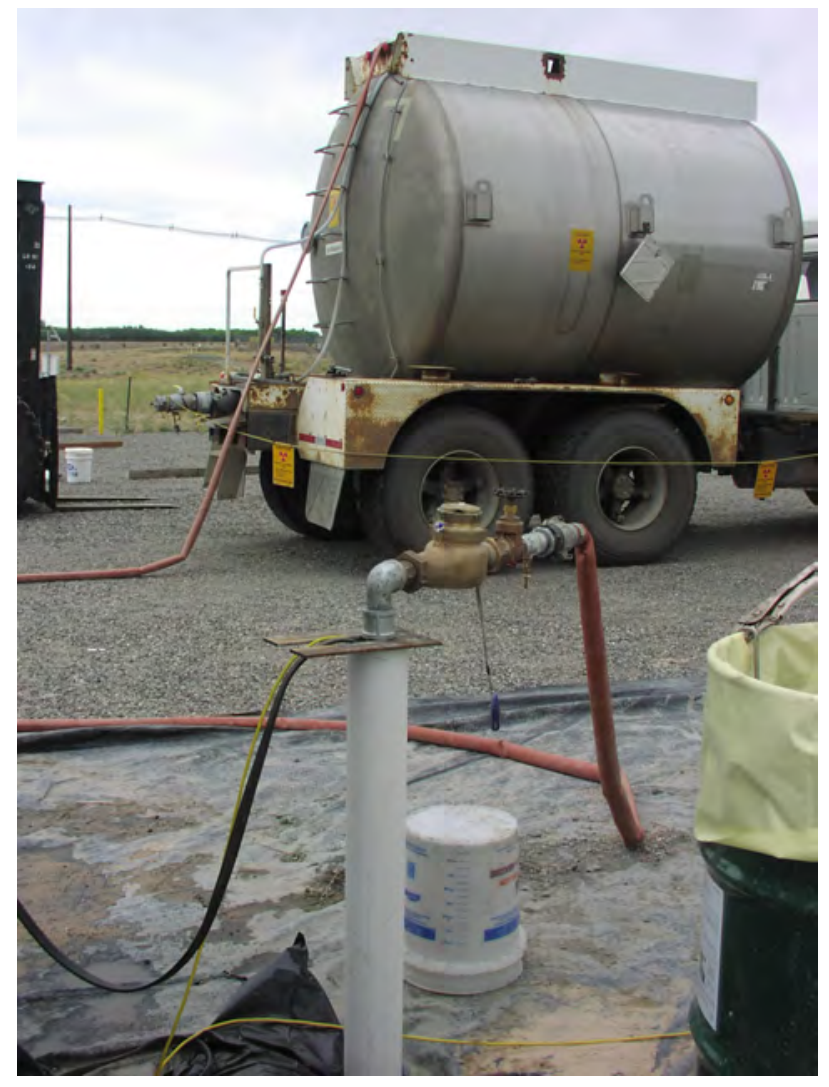

Figure 3.16. Water was pumped out of 4-in. PVC wells into a storage tank during well development. 
Table 3.3. Well development data for the 35 IFRC wells

\begin{tabular}{|c|c|c|c|c|c|c|c|c|c|c|}
\hline \multirow[b]{2}{*}{ Well } & \multirow[b]{2}{*}{$\begin{array}{c}\text { Screen } \\
\text { Depth (ft) }\end{array}$} & \multicolumn{4}{|c|}{ First Pumping } & \multicolumn{4}{|c|}{ Second Pumping } & \multirow[b]{2}{*}{$\begin{array}{c}\text { Total } \\
\text { Volume } \\
\text { Pumped } \\
\text { (gal) }\end{array}$} \\
\hline & & $\begin{array}{l}\text { Pump } \\
\text { Intake } \\
\text { Depth } \\
\text { (ft) }\end{array}$ & $\begin{array}{l}\text { Duration } \\
\text { (min) }\end{array}$ & $\begin{array}{l}\text { Rate } \\
\text { (gpm) }\end{array}$ & $\begin{array}{c}\text { Turbidity } \\
\text { (NTU) }\end{array}$ & $\begin{array}{l}\text { Pump } \\
\text { Intake } \\
\text { Depth } \\
\text { (ft) }\end{array}$ & $\begin{array}{l}\text { Duration } \\
\text { (min) }\end{array}$ & $\begin{array}{l}\text { Rate } \\
\text { (gpm) }\end{array}$ & $\begin{array}{l}\text { Turbidity } \\
\text { (NTU) }\end{array}$ & \\
\hline 399-2-07 & $31-56$ & 40 & 30 & 50 & 1.16 & 50 & 15 & 86 & 2.66 & 2551 \\
\hline 399-2-08 & $31-56$ & 40 & 30 & 50 & 1.63 & 50 & 19 & 86 & 3.72 & 2849 \\
\hline 399-2-09 & $33.5-58.5$ & 50.9 & 38 & $30-60$ & 4.89 & 40.9 & 27 & 60 & 6.79 & 3120 \\
\hline $399-2-10$ & $34.7-59.7$ & 41 & 28 & 45.5 & 2.73 & 51 & 22 & 85.5 & 1.58 & 3281 \\
\hline 399-2-11 & $31-56$ & 41.3 & 33 & 50 & 6.64 & 51.3 & 41 & 87 & 6.29 & 3500 \\
\hline 399-2-12 & $31-56$ & 40 & 30 & $50-55$ & 3.38 & 50 & 17 & 85 & 7.15 & 2834 \\
\hline 399-2-13 & $31.2-56.2$ & 39.6 & 24 & $49-53$ & 2.57 & 49.6 & 29 & $60-80$ & 3.34 & 3115 \\
\hline $399-2-14$ & $30.7-55.7$ & 40 & 31 & $50-55$ & 1.77 & 50 & 19 & $82-85$ & 1.67 & 3146 \\
\hline 399-2-15 & $31-56$ & 40 & 30 & $50-55$ & 3.42 & 50 & 19 & 86 & 3.49 & 3246 \\
\hline 399-2-16 & $31-56$ & 40 & 30 & 50 & 3.97 & 50 & 17 & 86 & 7.57 & 2864 \\
\hline 399-2-17 & $31.2-56.2$ & 40 & 31 & 50-55 & 1.96 & 50 & 19 & 86 & 2.41 & 3146 \\
\hline 399-2-18 & $33-58$ & 40 & 25 & 73 & 5.12 & 50 & 18 & 74 & 2.45 & 3157 \\
\hline 399-2-19 & $31.3-56.3$ & 42 & 31 & $50-64$ & 5.02 & 53.7 & 23 & 50 & 4.14 & 2840 \\
\hline $399-2-20$ & $31-56$ & 40 & 30 & 50 & 3.43 & 50 & 16 & 84 & 5.52 & 2839 \\
\hline 399-2-21 & $31.1-56.1$ & 40 & 20 & 50 & 0.98 & 50 & 13 & 74 & 5.20 & 3025 \\
\hline 399-2-22 & $33.4-58.5$ & 40.2 & 26 & 47 & 1.43 & 50.2 & 13 & 47 & 5.40 & 2209 \\
\hline $399-2-23$ & $31-56$ & 40 & 30 & 50 & 2.41 & 50 & 18 & 86 & 4.49 & 2884 \\
\hline $399-2-24$ & $33-58$ & 40.6 & 25 & 50 & 5.10 & 50.6 & 52 & 75-80 & 3.61 & 2860 \\
\hline 399-3-23 & $31-56$ & 41.5 & 32 & 50 & 1.38 & 51.5 & 16 & 68 & 3.46 & 3744 \\
\hline $399-3-24$ & $31-56$ & 41.3 & 36 & 50 & 3.41 & 51.3 & 10 & 87 & 7.08 & 2500 \\
\hline 399-3-25 & $31.6-56.6$ & 40 & 34 & 50 & 2.85 & 50 & 15 & 85 & 3.95 & 2952 \\
\hline 399-3-26 & $32-52$ & 39.7 & 31 & 55-60 & 4.94 & 48.7 & 30 & $45-50$ & 3.52 & 3280 \\
\hline 399-3-27 & $31.4-56.4$ & 40 & 30 & 50 & 1.13 & 50 & 16 & 84 & 0.97 & 2837 \\
\hline 399-3-28 & 31.6-56.6 & 41 & 32 & 50 & 2.97 & 51 & 21 & 87 & 6.98 & 3500 \\
\hline 399-3-29 & $31-56$ & 40 & 26 & 50 & 1.79 & 50 & 10 & 84 & 2.79 & 2190 \\
\hline \multicolumn{11}{|c|}{ Shallow Groundwater Monitoring Wells } \\
\hline $399-2-26$ & $30-35$ & 41.5 & 41 & 30 & 1.82 & -- & -- & -- & -- & 1200 \\
\hline $399-2-29$ & $30-35$ & 41 & 40 & 50 & 0.71 & -- & -- & -- & -- & 1877 \\
\hline 399-3-30 & $30-35$ & 40 & 31 & 20 & 3.78 & -- & -- & -- & -- & 656 \\
\hline \multicolumn{11}{|c|}{ Intermediate Groundwater Monitoring Wells } \\
\hline $399-2-28$ & $42-44$ & 43 & 35 & 20 & 12.30 & -- & -- & -- & -- & 627 \\
\hline $399-2-31$ & $42-44$ & 40 & 53 & 20 & 5.02 & -- & -- & -- & -- & 768 \\
\hline 399-3-32 & $42.2-44.2$ & 41 & 29 & 18.5 & 7.99 & -- & -- & -- & -- & 537 \\
\hline
\end{tabular}


Table 3.3. (contd)

\begin{tabular}{|c|c|c|c|c|c|c|c|c|c|c|}
\hline \multirow[b]{2}{*}{ Well } & \multirow[b]{2}{*}{$\begin{array}{c}\text { Screen } \\
\text { Depth (ft) }\end{array}$} & \multicolumn{4}{|c|}{ First Pumping } & \multicolumn{4}{|c|}{ Second Pumping } & \multirow[b]{2}{*}{$\begin{array}{c}\text { Total } \\
\text { Volume } \\
\text { Pumped } \\
\text { (gal) }\end{array}$} \\
\hline & & $\begin{array}{c}\text { Pump } \\
\text { Intake } \\
\text { Depth } \\
\text { (ft) }\end{array}$ & $\begin{array}{l}\text { Duration } \\
\text { (min) }\end{array}$ & $\begin{array}{l}\text { Rate } \\
\text { (gpm) }\end{array}$ & $\begin{array}{c}\text { Turbidity } \\
\text { (NTU) }\end{array}$ & $\begin{array}{c}\text { Pump } \\
\text { Intake } \\
\text { Depth } \\
\text { (ft) }\end{array}$ & $\begin{array}{l}\text { Duration } \\
\text { (min) }\end{array}$ & $\begin{array}{l}\text { Rate } \\
\text { (gpm) }\end{array}$ & $\begin{array}{c}\text { Turbidity } \\
\text { (NTU) }\end{array}$ & \\
\hline \multicolumn{11}{|c|}{ Deep Groundwater Monitoring Wells } \\
\hline $399-2-27$ & $54.7-56.7$ & 51 & 31 & $22.5-43$ & 8.36 & -- & -- & -- & -- & 1543 \\
\hline $399-2-30$ & $54.4-56.4$ & 50 & 35 & 26 & 1.44 & -- & -- & -- & -- & 1170 \\
\hline $399-3-31$ & $53.2-55.2$ & 50 & 35 & 55 & 0.42 & -- & -- & -- & -- & 2080 \\
\hline \multicolumn{11}{|c|}{ Deep Microbiology Characterization Well } \\
\hline $399-2-25$ & $31.6-56.6$ & 70 & 57 & 20 & 16.20 & -- & -- & -- & -- & 1078 \\
\hline
\end{tabular}

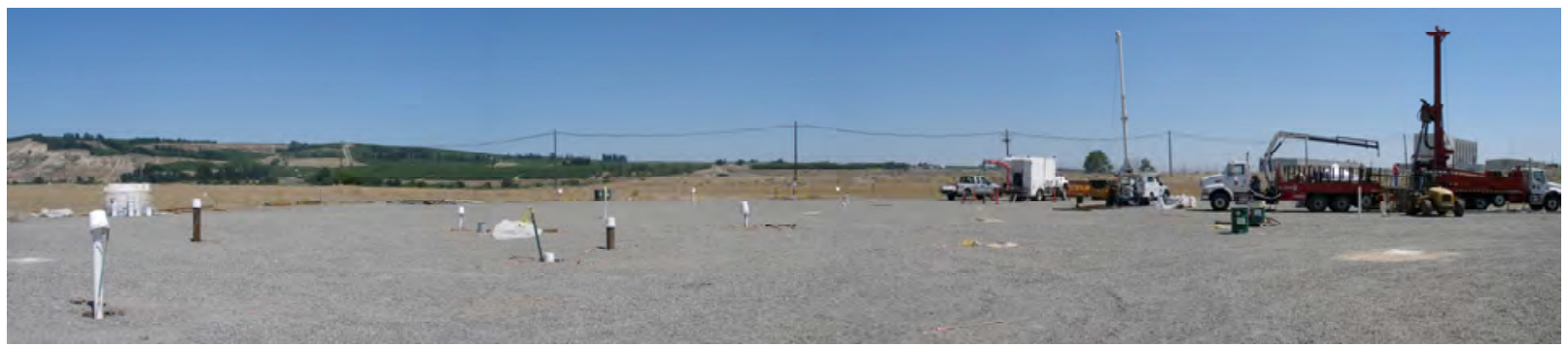

Figure 3.17. The high density of IFRC wells in various stages of completion 


\subsection{Hydrogeology}

Sediments overlying basalt bedrock at the IFRC site belong to three geologic units: 1) backfill materials, 2) the Hanford formation, and 3) the Ringold Formation. The structure and relationships of these stratigraphic units, from oldest to youngest, are shown in Figure 4.1. The water table lies within the Hanford formation everywhere within the IFRC site (Figure 4.1). The locations of cross sections A-A' and B-B' are noted in Figure 4.2. More detailed cross sections (C through F) within the IFRC site are shown in Figure 4.3 through 4.6.
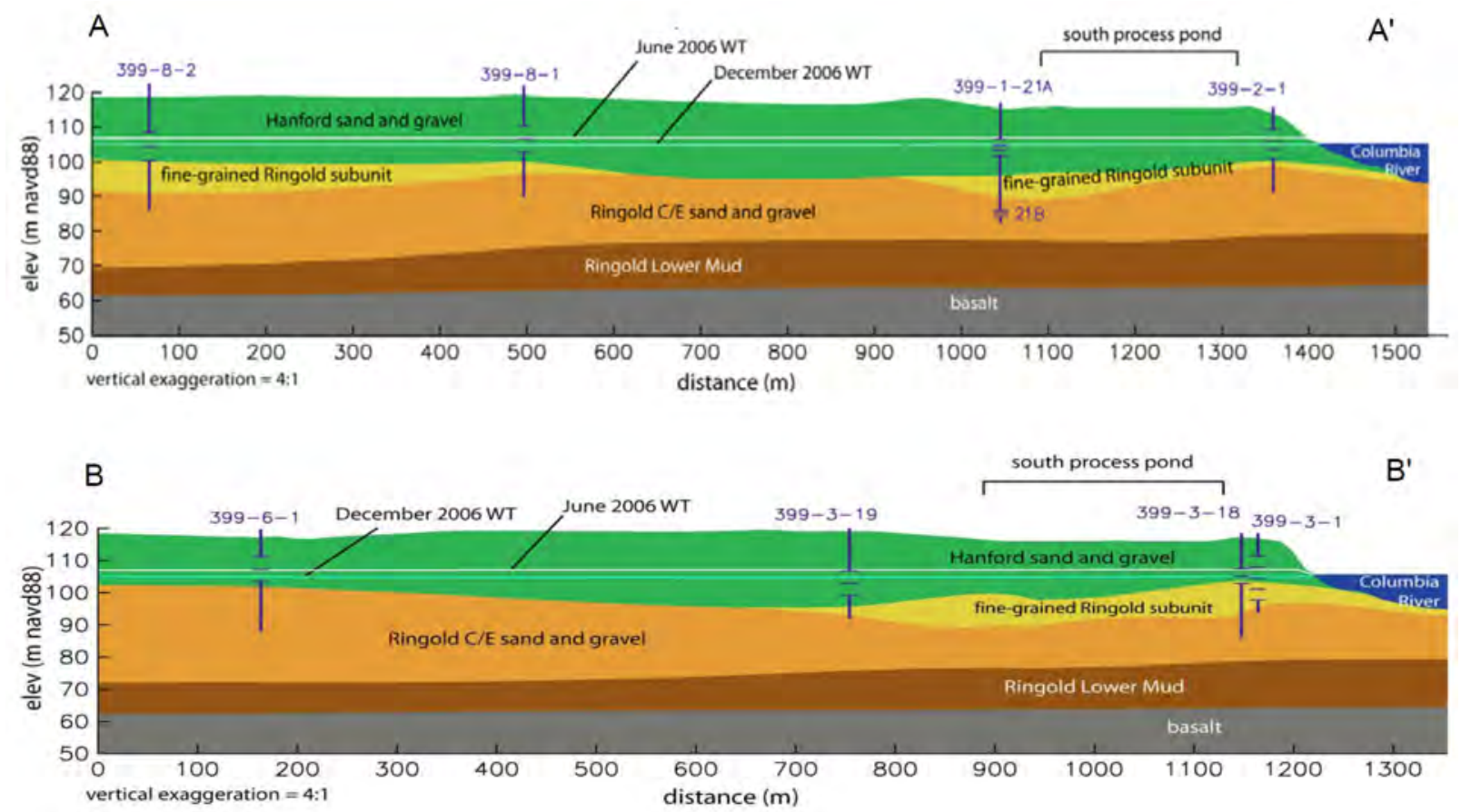

Figure 4.1. Cross sections through the 300 Area showing major hydrostratigraphic units (modified from Williams et al. 2008) 


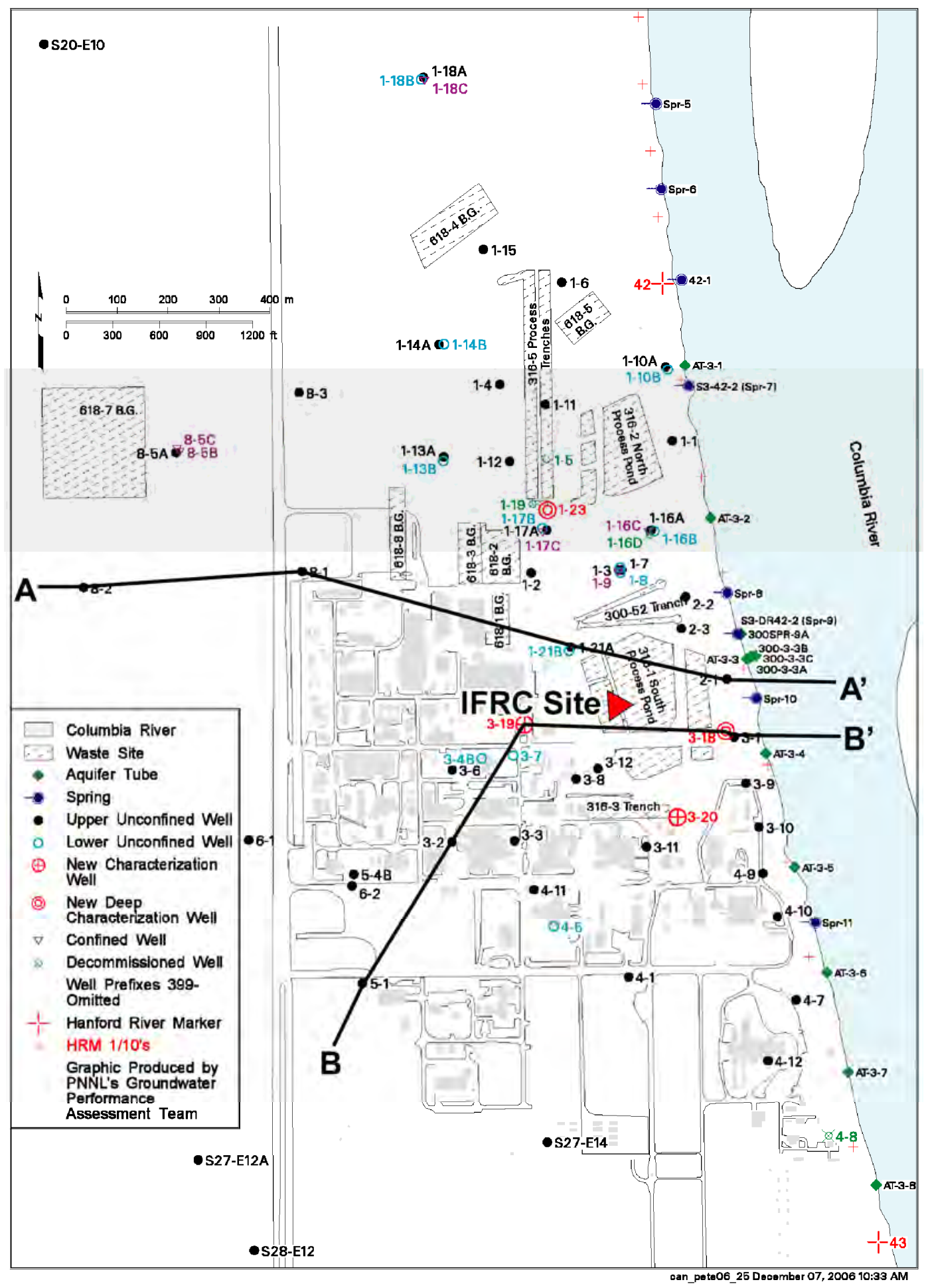

Figure 4.2. Cross section locations 

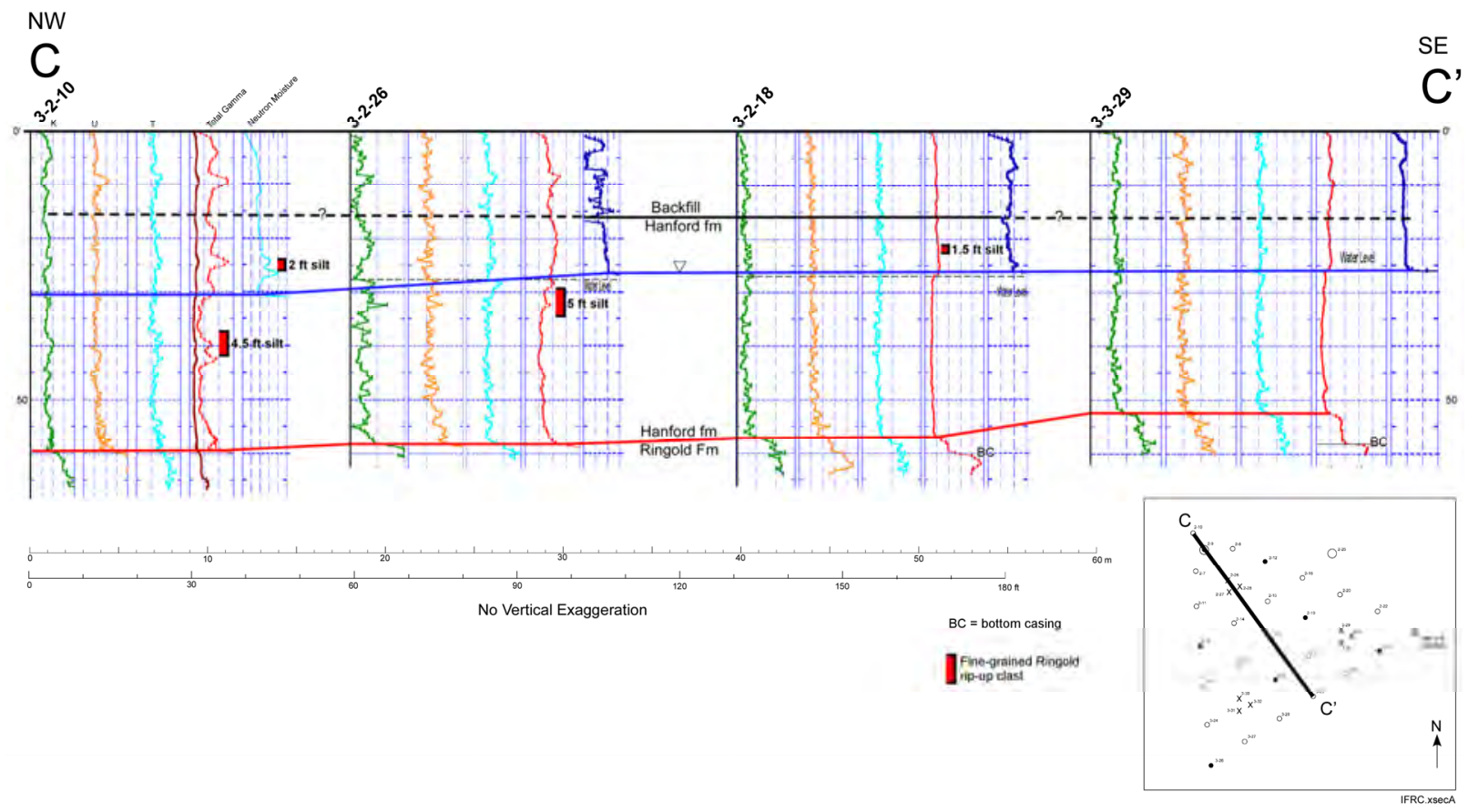

Figure 4.3. Hydrogeologic cross section C-C' based on geophysical logs

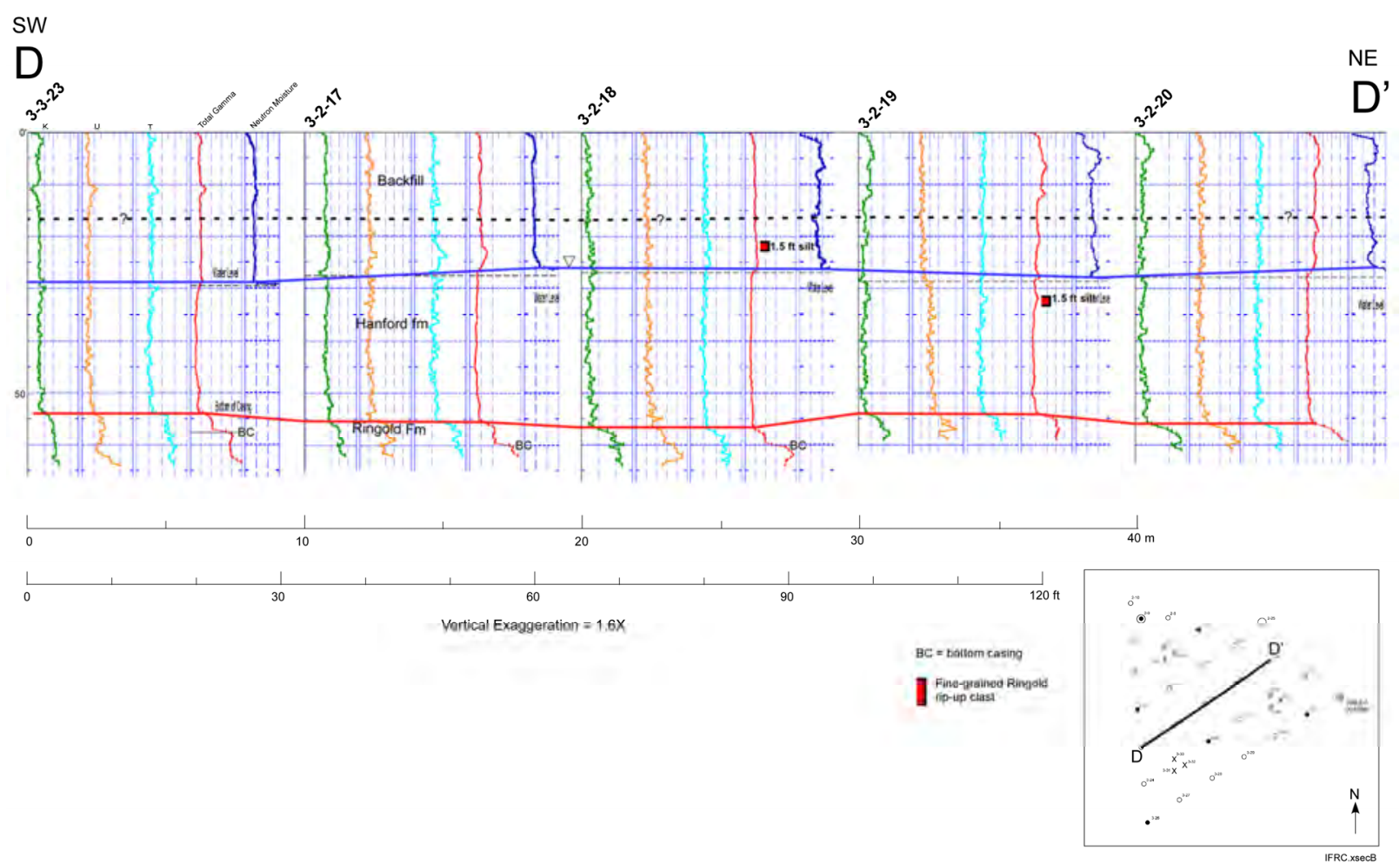

Figure 4.4. Hydrogeologic cross section D-D’ based on geophysical logs 


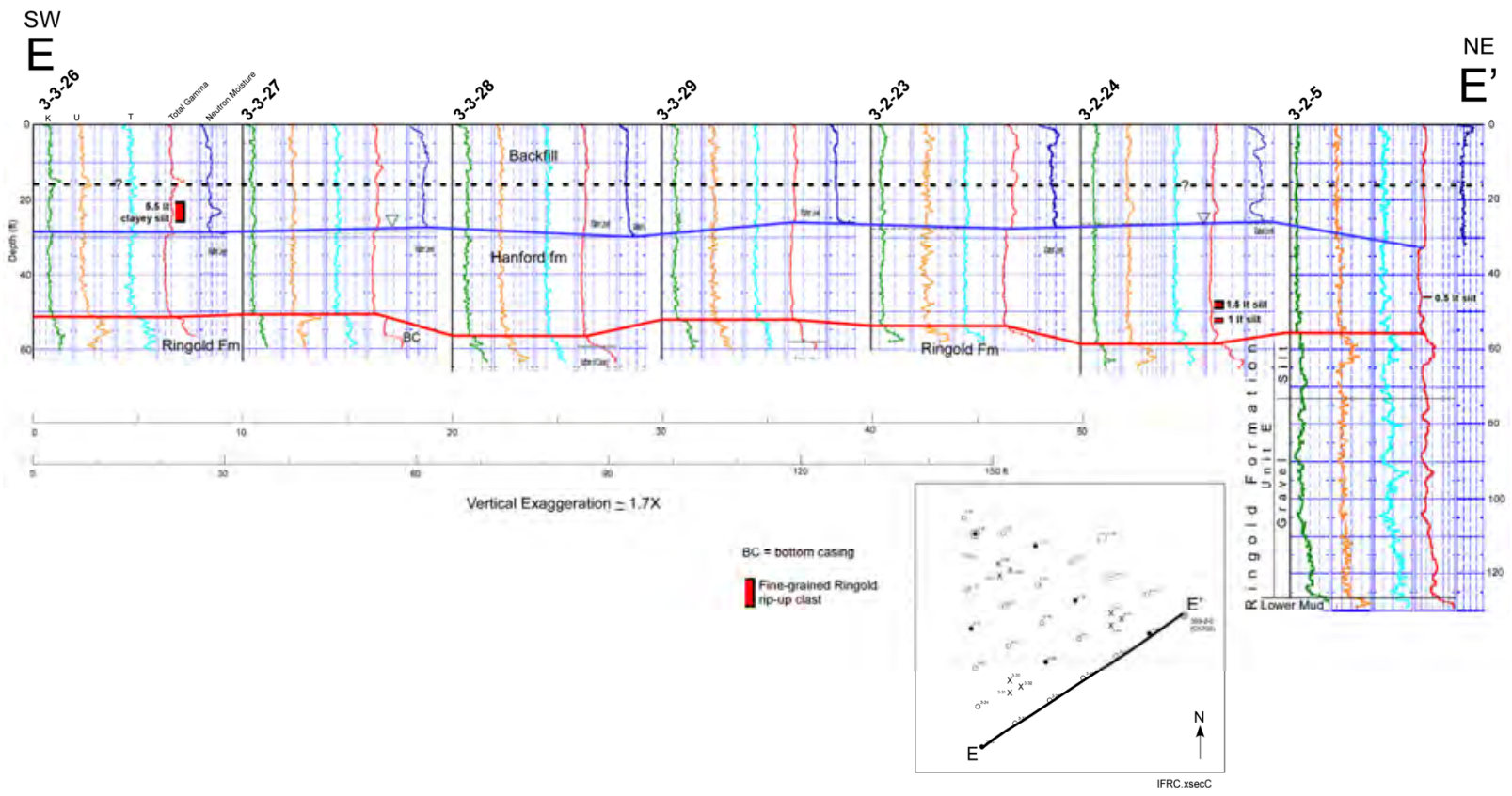

Figure 4.5. Hydrogeologic cross section E-E' based on geophysical logs

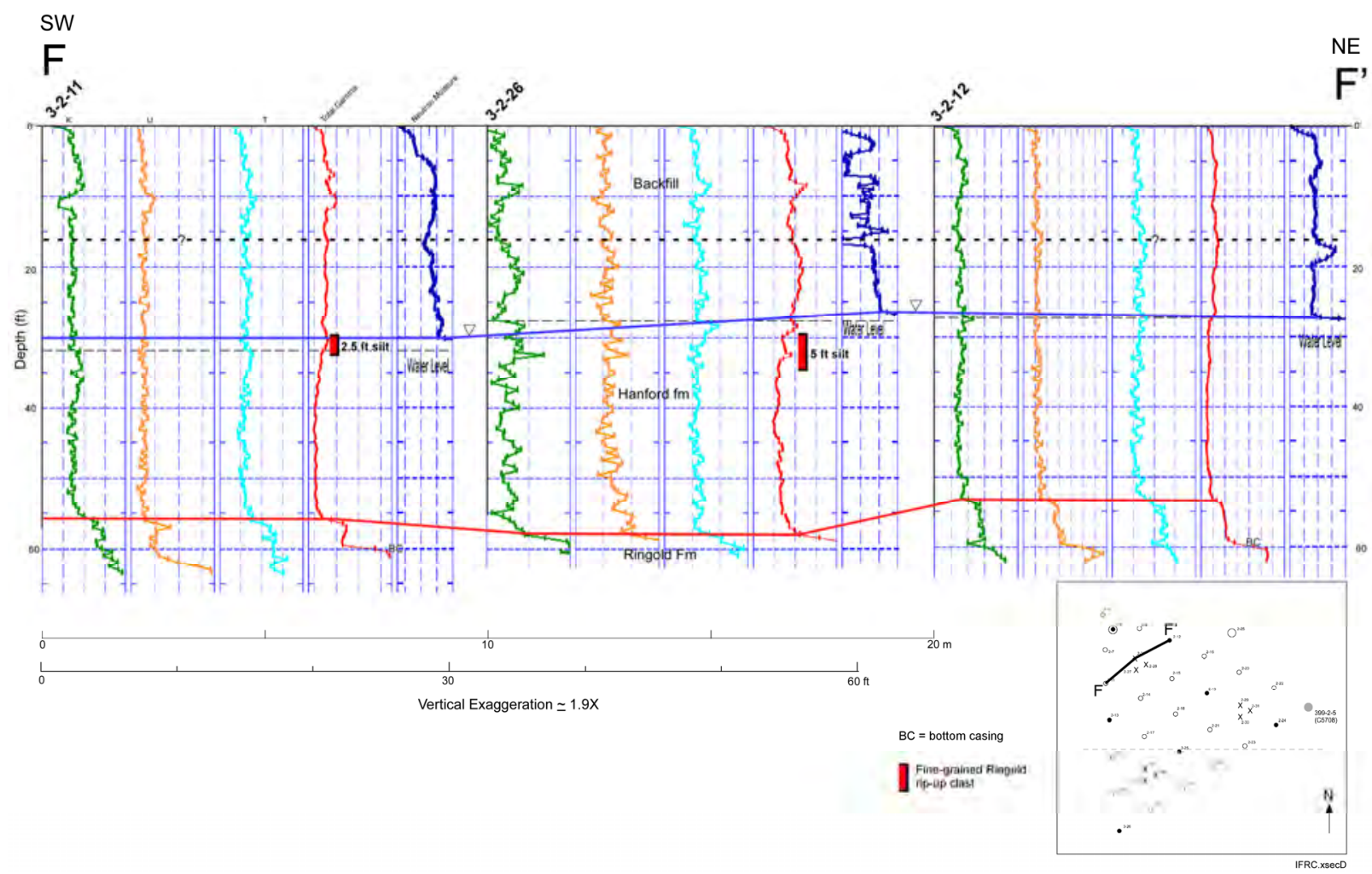

Figure 4.6. Hydrogeologic cross section F-F' based on geophysical logs 
The stratigraphy and lithology of the suprabasalt sediments at the IFRC site are represented in Figure 4.7 for the single deep well (399-2-25) at this location. Close-up photographic images of the different units are presented in Appendix I within small chip tray samples collected for each well.

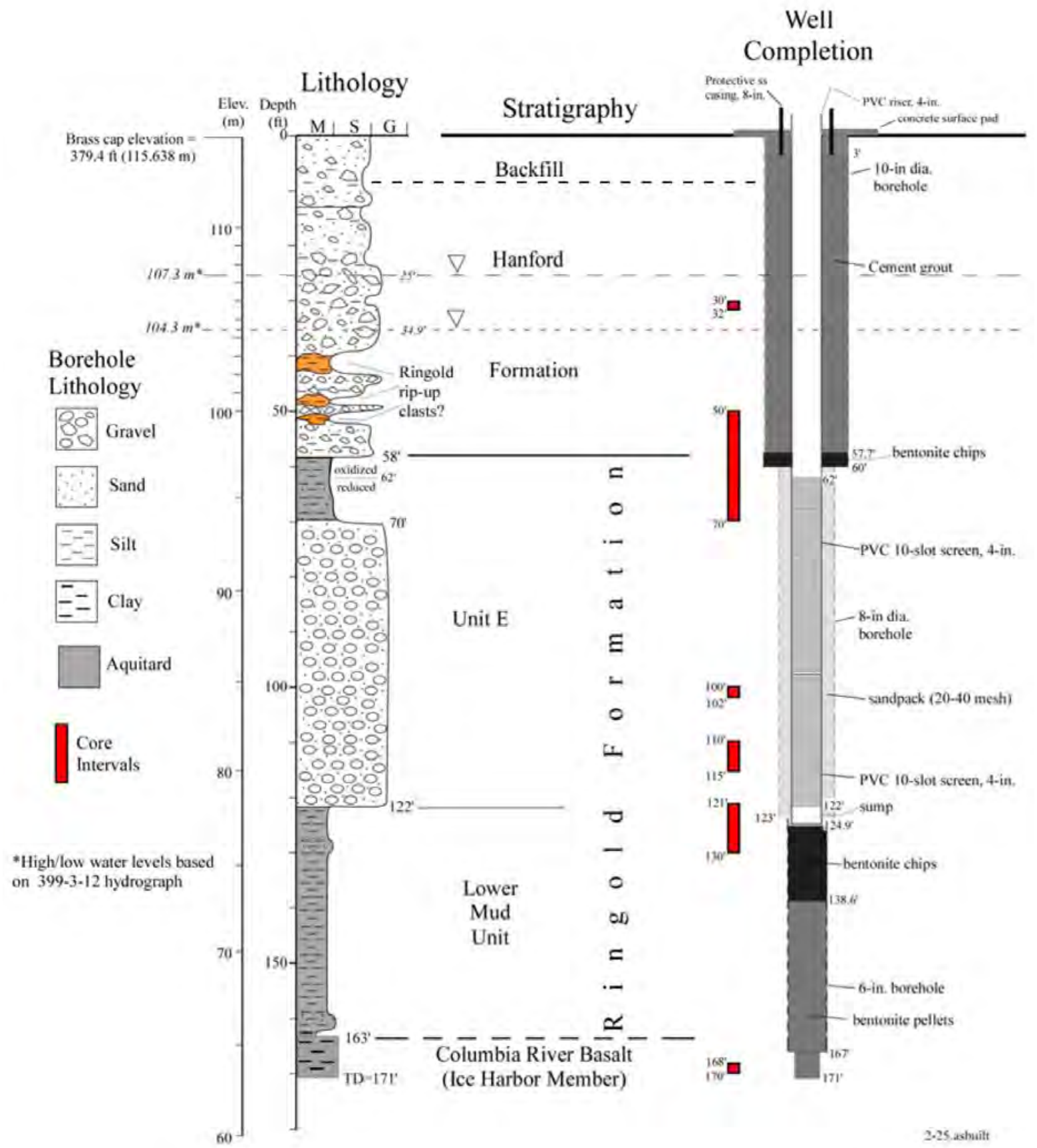

Figure 4.7. Stratigraphy and lithology represented in deep microbiology characterization well 399-2-25 (C6209). See also Compilation Borehole Summary Log in Appendix A. 


\subsection{Columbia River Basalt}

Basalt bedrock lies approximately $160 \mathrm{ft}$ deep beneath the IFRC site. The uppermost basalt flow in this area is the Ice Harbor Member, dated at 8.5 million years before present (DOE 1988). Near the top of the basalt, drilling slowed significantly through a hard, dense clay zone containing vesicular basalt fragments (Figure 4.8) before refusal was ultimately encountered at $170 \mathrm{ft}$ bgs. The clay appears to be an alteration product due to an extended period of weathering of the basalt flow top prior to burial by the Ringold Formation. The color of the highly weathered basalt ranged from black through gray and green with occasional reddish iron-oxide stringers.

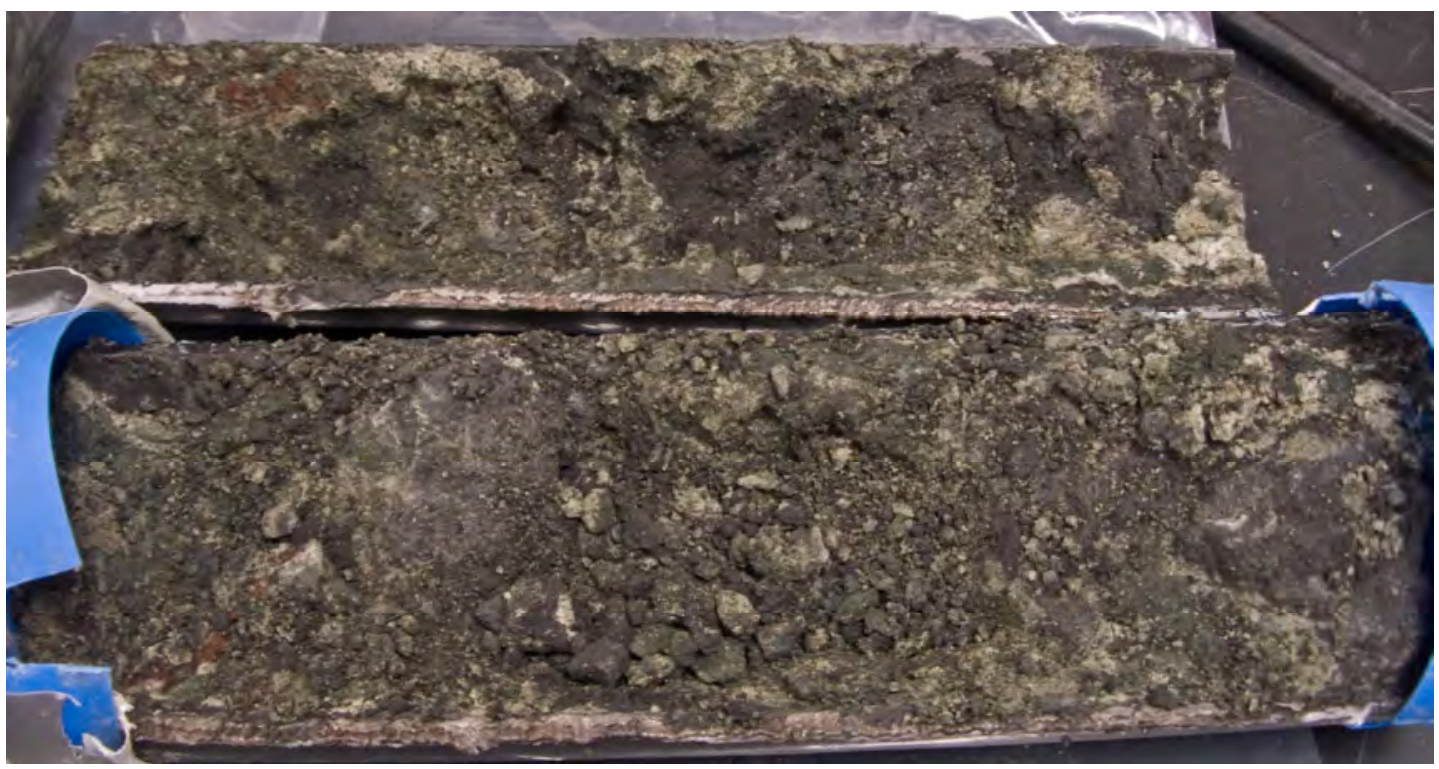

Figure 4.8. Core segment, weathered basalt of the Ice Harbor Member, from the bottom of well 399-2-25. The 1-ft-long Lexan-lined core consists of irregular fragments of black vesicular basalt in a highly weathered and green-gray mottled alteration-clay matrix. Light-colored material effervesces in contact with dilute hydrochloric acid, indicating the presence of calcium carbonate.

\subsection{Ringold Formation}

The Ringold Formation ranges in age from late Miocene to Pliocene (8.5 to 3.4 million years) and consists of mostly fluvial-lacustrine sediments laid down during tectonic downwarping and infilling of the Pasco Basin, one of many synclinal basins of the Yakima Fold Belt (DOE 1988).

Immediately overlying the weathered basalt surface are roughly $40 \mathrm{ft}$ of dark-colored, fine-grained sediments (Figure 4.9) of the Ringold lower mud unit. These sediments are predominantly compact and cohesive, massive to weakly laminated, gray to gleyed-green and blue deposits that vary in grain size among clay, silt, and fine sand. Occasionally, dark fibrous fragments of decomposed wood were found preserved in the Ringold lower mud unit (Figure 4.10). This organic matter, in combination with the fine texture and structure of these deposits, indicates a fluvial-overbank and crevasse-splay origin. 
Overlying the Ringold lower mud unit is coarser, gravel-dominated sediment, about $50 \mathrm{ft}$ thick, belonging to the Ringold Formation (Unit E). This conglomerate unit, approximately 4.8 to 6.7 million years old (Lindsey 1995), is a compacted bimodal mixture of well-rounded and polished clast-supported pebbles and cobbles compacted within a matrix of fine to medium sand (Figure 4.11); variable amounts of silt or clay may occur within the matrix. Within each of the two modes, sediment sorting is moderate to good, with few particles from medium-sand to fine-pebble size. Sand grains within the matrix are predominantly felsic (i.e., composed principally of quartz, feldspar, and mica). Individual pebble and cobble-size clasts, especially those composed of basalt, often display clay skins and weathering (alteration) rinds. Colors within this unit are dark gray to black, indicating this unit is undergoing chemical reduction at the IFRC site. This sedimentary facies represents deposition within a high-energy, shallow, braided-stream (fluvial) environment (Lindsey 1995).

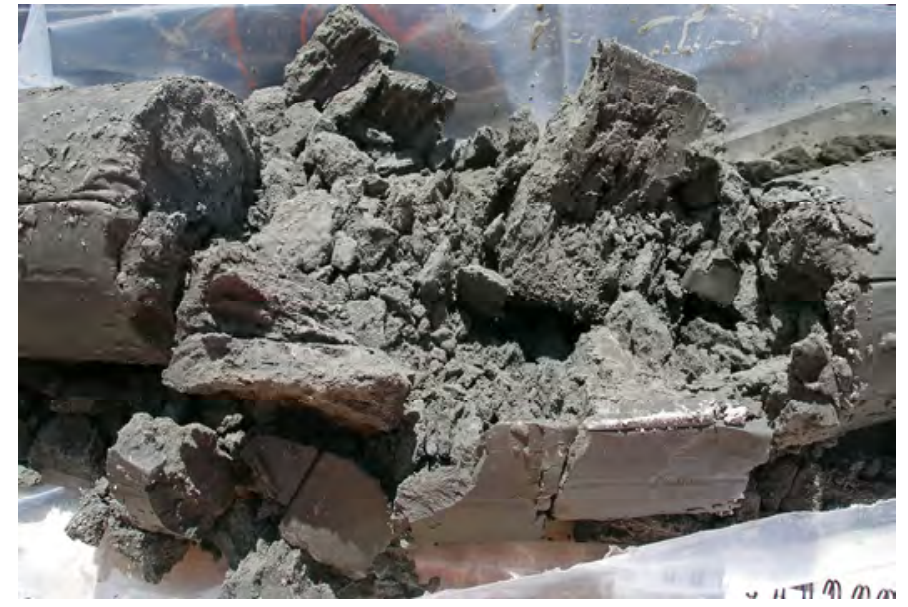

Figure 4.9. The Ringold lower mud unit at $160-\mathrm{ft}$ depth in well $399-2-25$ is predominantly a compact, homogeneous, dark greenish-gray, weakly stratified, fine sandy silt.

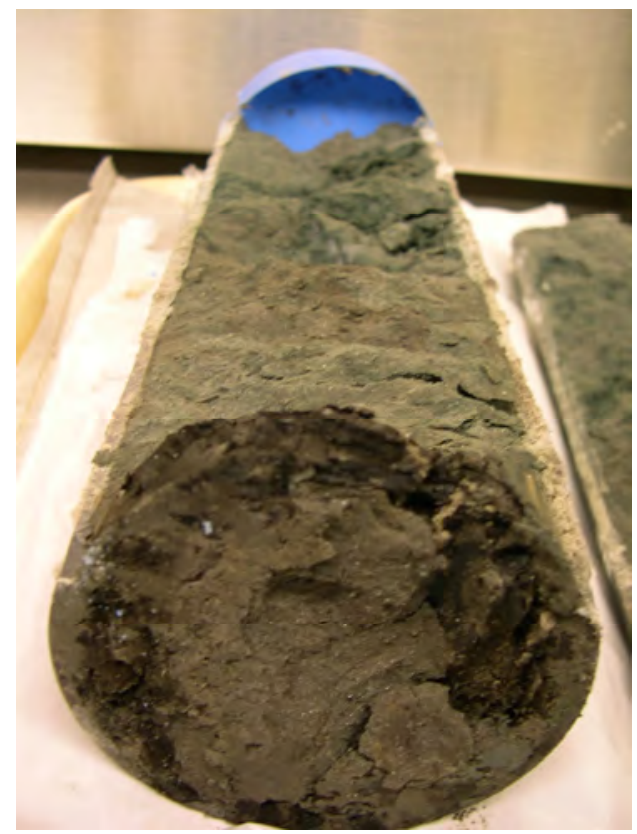

Figure 4.10. Dark fibrous wood fragments in laminated, micaceous, fine sandy silt of the Ringold lower mud unit at 129-ft depth, well 399-2-25 


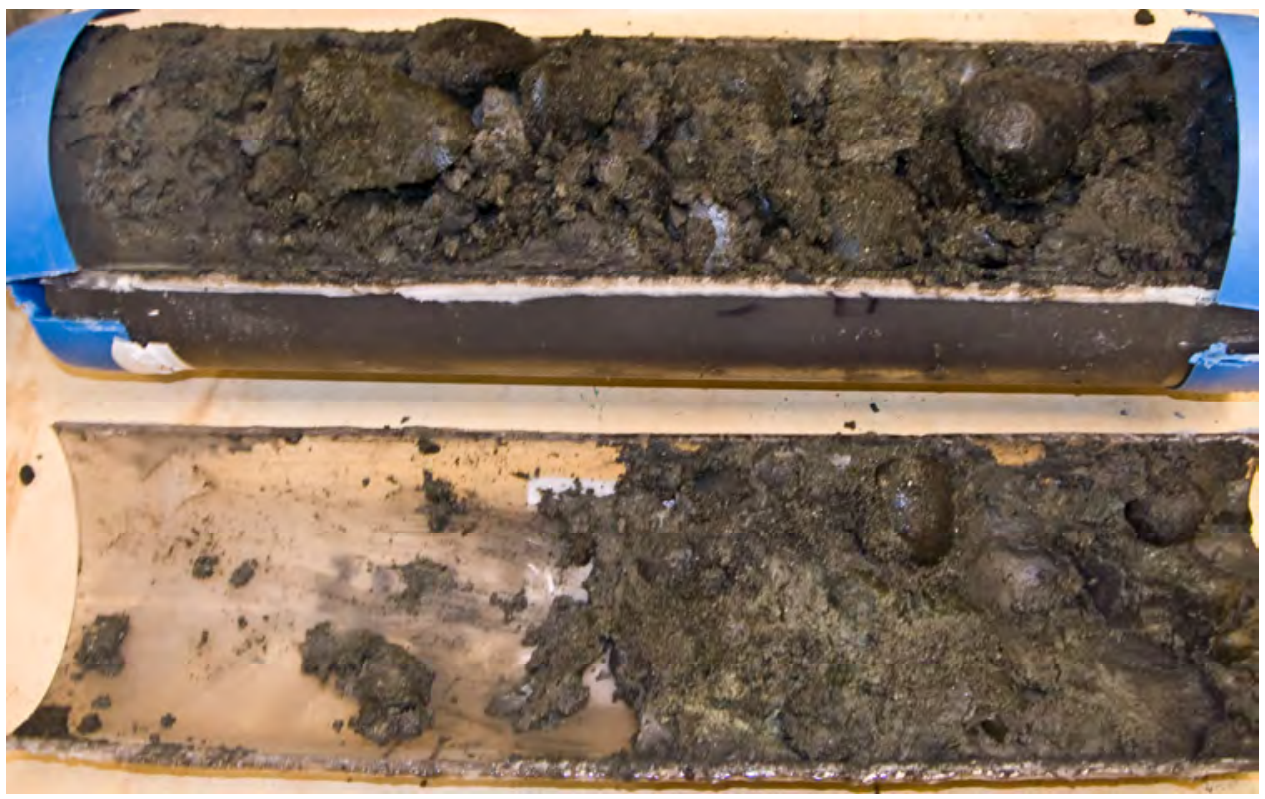

Figure 4.11. Ringold Formation Unit E. Silty sandy gravel of Ringold Unit E from the 110-ft depth in well 399-2-25.

Ringold Unit E gravel-dominated sediments are well exposed at the surface along the base of an erosional escarpment of the Ringold Formation along the White Bluffs (Figure 4.12). These exposures conveniently reveal the two-dimensional stratigraphic and structural characteristics of the sediments that cannot be observed in one-dimensional boreholes.

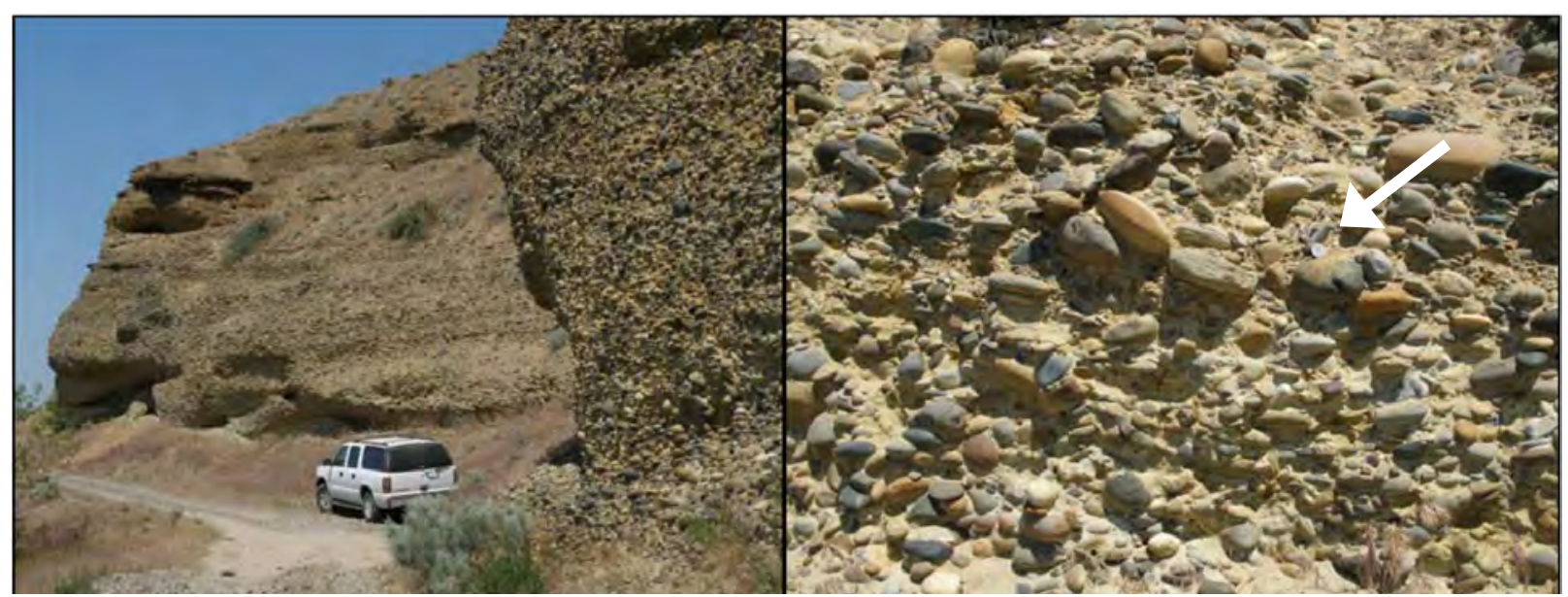

Figure 4.12. Ringold Formation Unit E conglomerate (member of Wooded Island [Lindsey 1995]) exposed near river level along the White Bluffs within 1 mile of the IFRC. Left: In the outcrop, these clast-supported, bimodal deposits may be crudely stratified and weakly imbricated, with occasional lenses of well-sorted fine- to medium-grained sand. Right: Light-colored pebble- to cobble-size clasts are mostly quartzite, granite, and gneiss; dark clasts are dominantly basalt, andesite, and volcanic porphyry (Campbell 1983). Note quarter for scale. Unlike the IFRC site, the Ringold Unit E in these outcrops has undergone chemical oxidation as indicated by a pervasive yellow-to brown iron-oxide stain, which coats all sedimentary particles. The vertical nature of the exposures signifies the semiconsolidated nature of these compacted and cemented sediments. 
Overlying the Ringold Unit E is another fine-grained subunit of the Ringold Formation, up to $12 \mathrm{ft}$ thick within the IFRC. This unit consists of a cohesive and compact, well-sorted fine sand grading upward into silty sand to silt (Figure 4.13). This is also known as the "fine-grained Ringold subunit" identified in previous reports (Williams et al. 2008; Peterson et al. 2008). Away from the IFRC site, the fine-grained Ringold subunit is locally absent or overlain by more of the Ringold Unit E graveldominated sediments, suggesting these fine-grained strata represent a discontinuous lens, stratigraphically equivalent to Ringold Unit E, within the 300 Area.

All but the top of these fine-grained strata show dark, chemically reduced shades of gray, green, and blue. At the top of the unit, however, is a rapid color change to shades of yellow, orange, or brown silt, up to $8 \mathrm{ft}$ thick (Figure 4.14). The color boundary does not appear to occur along a textural or structural boundary because the sediments above and below the boundary appear to be lithologically the same (Figure 4.13, right). For this reason, it appears the color contrast is the result of oxidation along the boundary with the highly permeable and transmissive flood deposits (Hanford formation) that lie directly above.

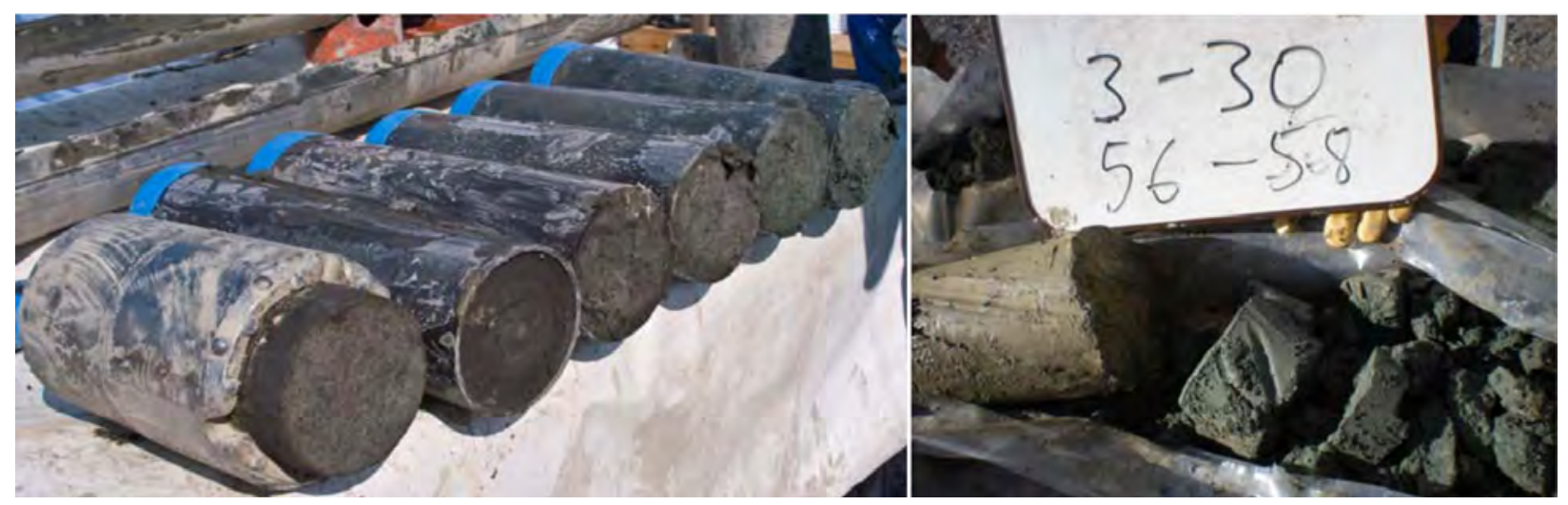

Figure 4.13. Fine-grained strata at the top of the Ringold Formation. Left: core samples collected from 59.5- to 65-ft depth in reduced silts and fine sands of the Ringold Formation in well 399-225. Top of the core is to the upper right. Right: Sharp contact exists between oxidized pale-brown (left) and reduced dark greenish-gray (right) silt layers in well 399-3-30 at the 57-ft depth. Top of the core is to the left.

A map of the top of the reduced zone within the Ringold fine-grained unit is shown in Figure 4.15. The fact that the surface of the oxidized-reduced boundary conforms with the Hanford-Ringold contact suggests a diffusion front of more oxygenated groundwater permeates down into the low-permeability Ringold Formation from the highly transmissive Hanford formation. The oxidized zone at the top of the Ringold Formation (Figure 4.14) is generally about $2-5 \mathrm{ft}$ thick, except to the north where it is up to $8 \mathrm{ft}$ thick. 


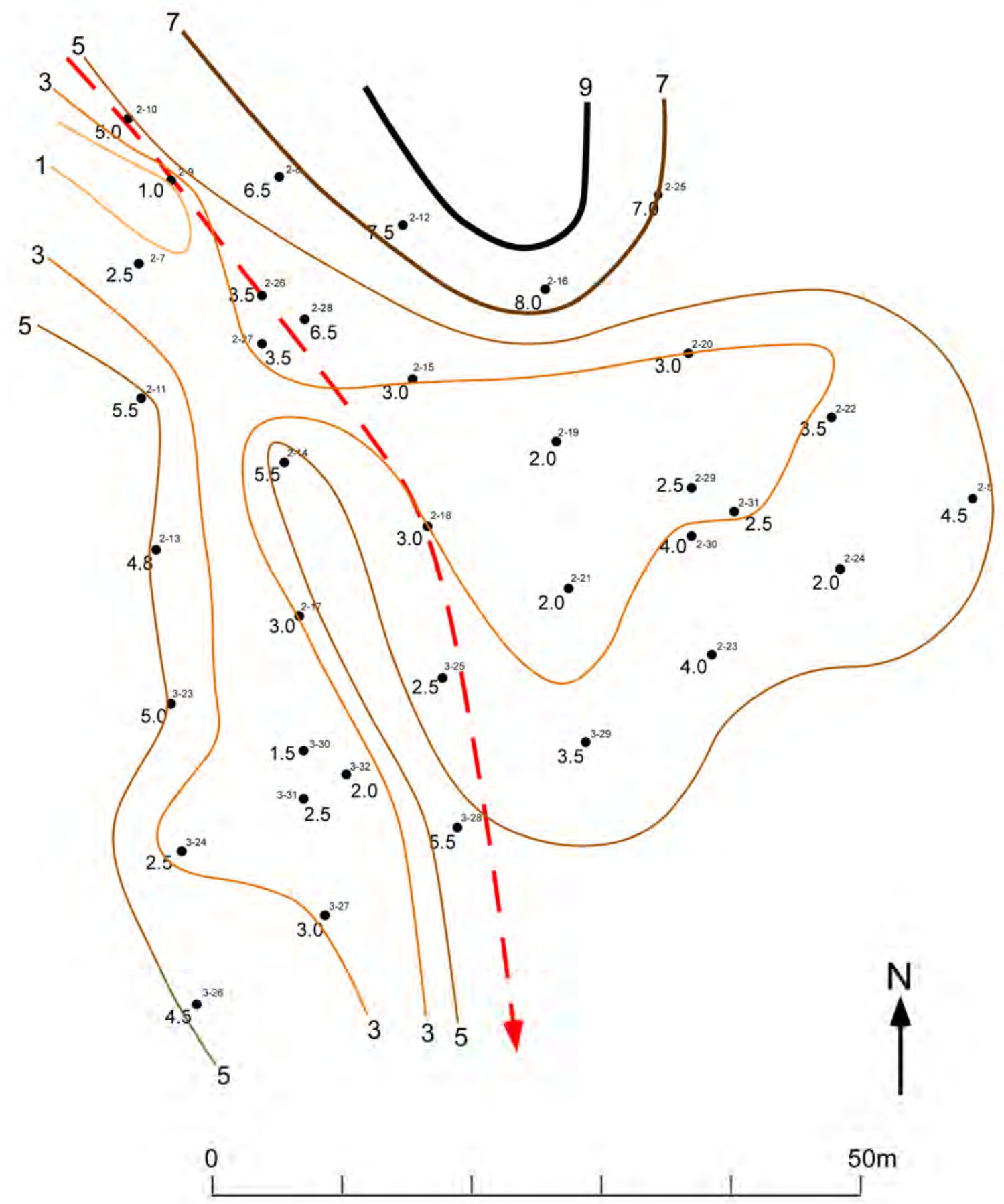

Figure 4.14. Isopach map showing the thickness of the oxidized zone atop the Ringold fine-grained unit beneath the IFRC site. The oxidized zone is thinnest parallel to, but not directly over, the paleochannel eroded into the top of the Ringold Formation (dashed arrow). 


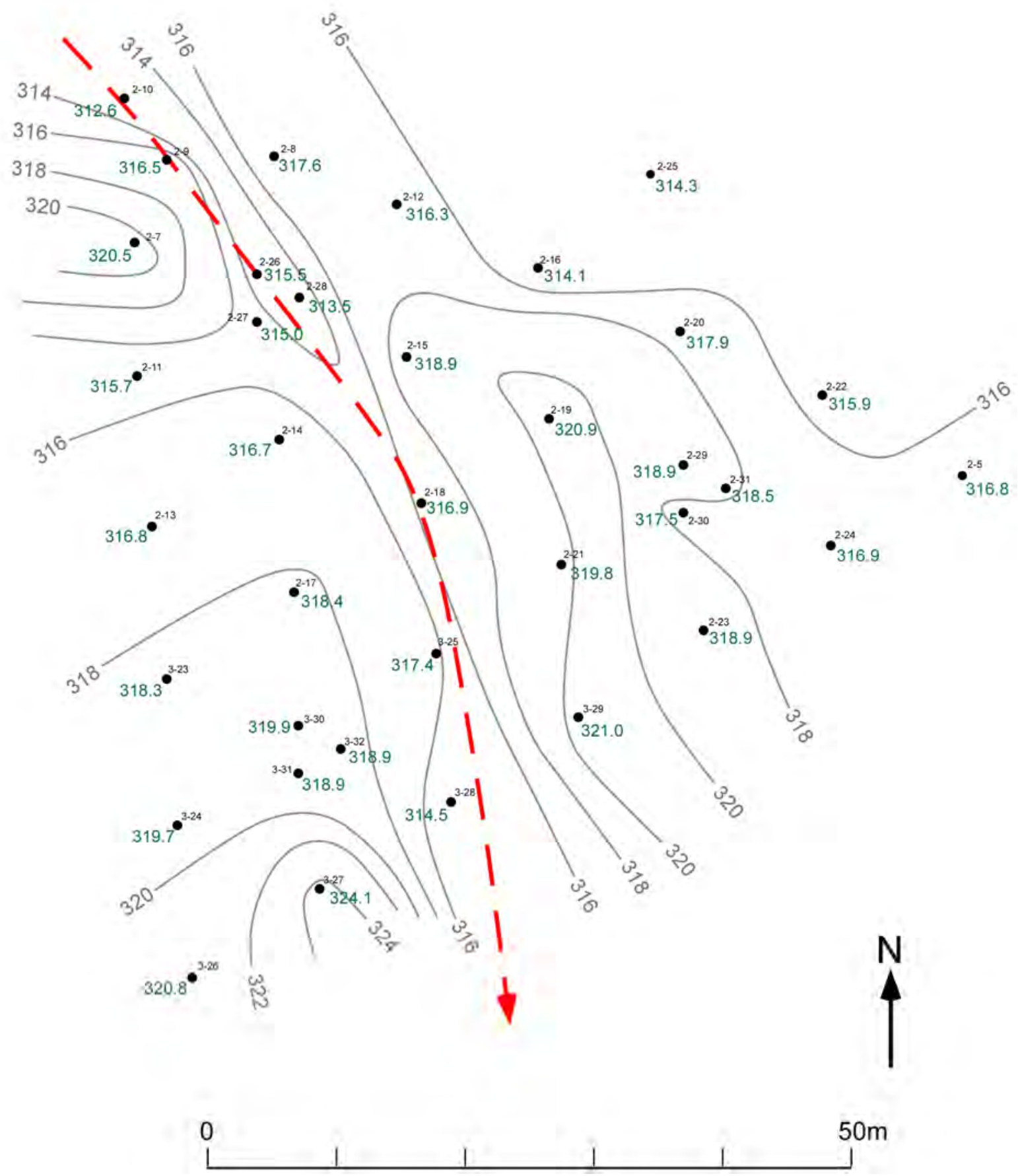

Figure 4.15. Structure contour map of the top of the reduced zone in the Ringold fine-grained unit. The top of the reduced zone generally conforms with a paleochannel (dashed line) eroded into the Ringold Formation. 


\subsection{Hanford Formation}

Overlying the Ringold Formation beneath the IFRC site are up to $50 \mathrm{ft}$ of heterogeneous sand and gravel with variable amounts of silt and clay of the Hanford formation. The Hanford formation encompasses all the sediments deposited during Pleistocene-age cataclysmic floods. Most Ice Age floods originated from periodic outbursts of glacial Lake Missoula, although some floods may have originated from other sources as well (Bjornstad 2006).

Repeated cataclysmic floods stripped away large volumes of Palouse loess, carved deep coulees into the underlying basalt bedrock, and created the Channeled Scabland of eastern Washington. All floodwater converged upon the Pasco Basin before being funneled through a single narrow outlet at Wallula Gap in the southeastern Pasco Basin. Floodwaters ponded temporarily behind this hydraulic constriction and formed Lake Lewis, which locally resulted in thick accumulations of flood deposits upstream within the Pasco Basin. As many as 100 separate flood events may have occurred during the last glaciation, although the exact frequency and number of floods is debatable. However, because no significant discontinuities have been observed within the Hanford formation sequence beneath the IFRC site (see Figure 2.4), it is possible that the entire sequence may have been deposited during the last Ice Age flood about 15,000 calendar years before present.

Each cataclysmic flood transported massive amounts of sediment, all within a period of a week or less. In the turbid column of floodwater was a stratified mixture of sediments being transported by the floods (Figure 4.16). At the base of the flow, along the sediment-water interface, floodwaters carried everything, from large boulders that bounced and rolled along the bottom to finer-grained particles (sand to clay), as tractive bedload. Above the zone of traction, however, only the small particles of sand, silt, and clay were entrained within the highly turbulent floodwaters.

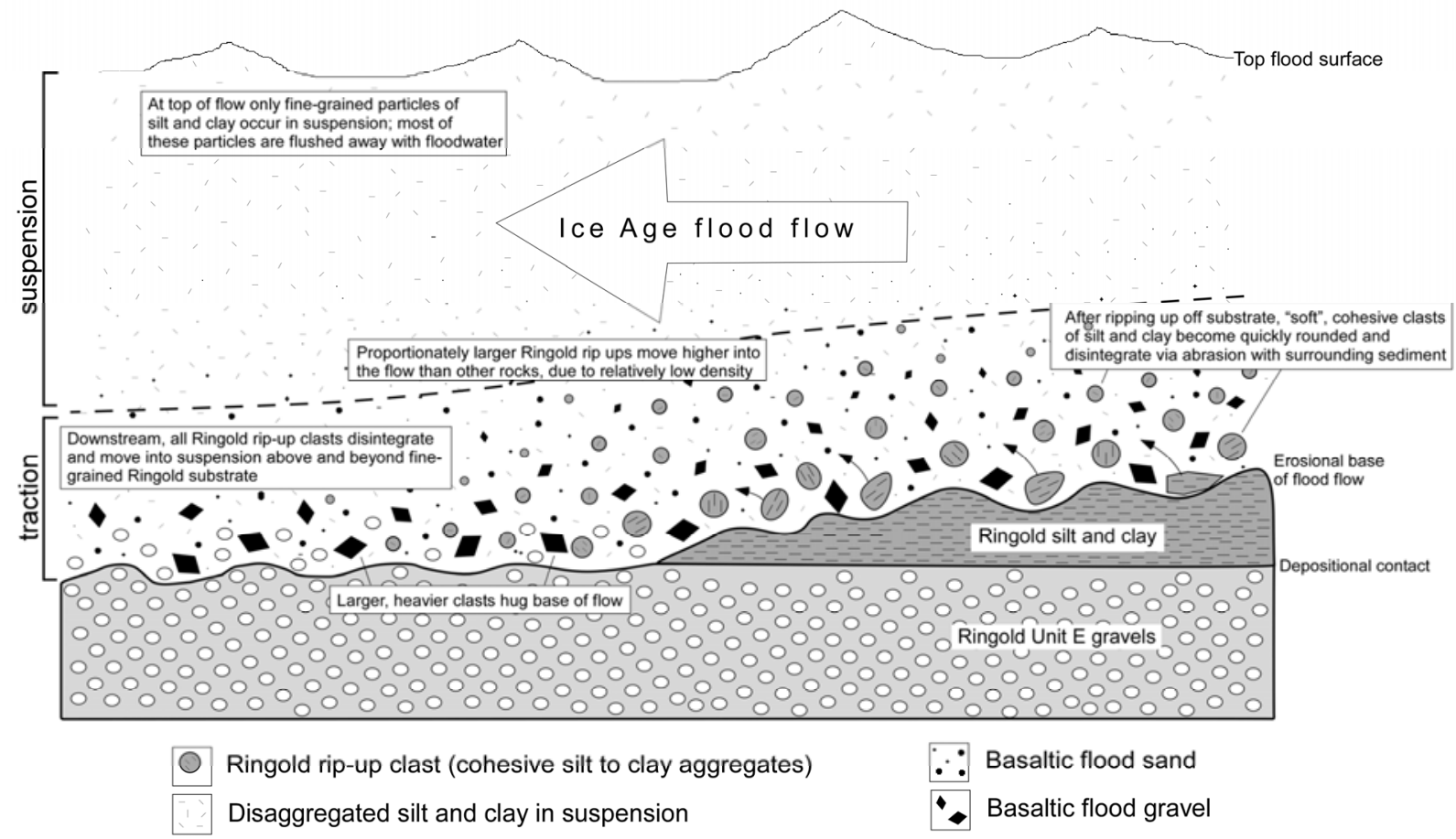

Figure 4.16. Model for sediment transport and stratification during an Ice Age flood 
The Hanford formation in the 300 Area consists predominantly of unconsolidated sediments that cover a wide range in grain size, from boulder-size gravel at one end of the grain-size spectrum to clay at the other end. A poorly sorted mixture, dominated by gravel, with lesser amounts of sand and silt is the dominant lithology for the Hanford formation within the 300 Area (Figure 4.17).

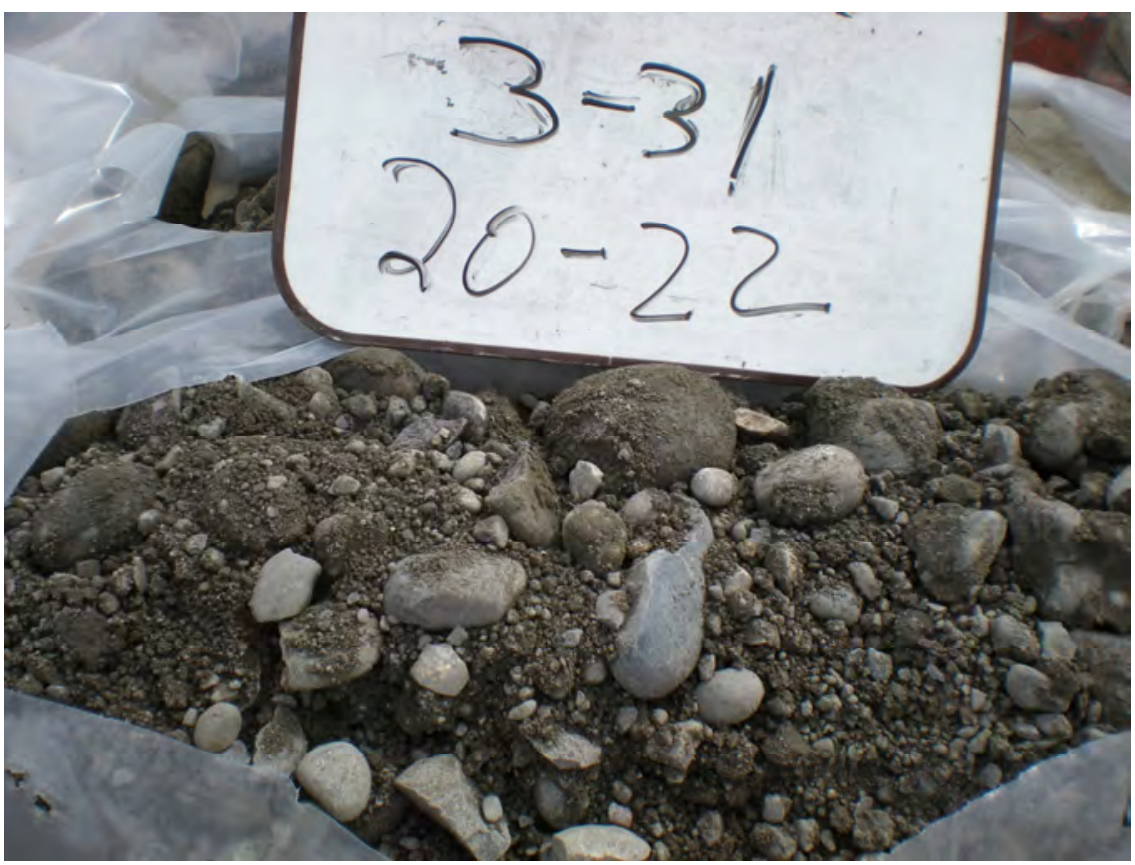

Figure 4.17. Typical appearance of gravel-dominated facies of the Hanford formation within the 300 Area. The sediment is loose, composed of brownish-gray, clast-supported, poorly sorted, silty, sandy, pebble-cobble gravel. Clasts are mostly subangular to subrounded basalt; other clasts include granitics, gneiss, and quartzite as well as other volcanics. Some of the larger clasts were broken apart during sonic drilling, whereas most smaller clasts are unbroken, an indication that the sonic drilling method produces relatively representative samples for characterization. This grab sample was collected from the 20- to 22-ft depth in borehole 399-3-31.

The concentration of basalt rock fragments is much higher in the Hanford formation compared to the underlying Ringold Formation. This is especially true of the sand-sized fraction, which in the Hanford formation consists of up to 70-80\% basalt compared to the sand fraction of the Ringold Formation, which typically consists of only $5-15 \%$ dark mafic grains. The reason for this is the Ice Age floods, which eroded the basalts underlying the Channeled Scabland of the Columbia Plateau. The Ringold Formation, on the other hand, is derived from mostly metamorphic and plutonic rocks eroded from the margins of the Columbia Plateau and transported to the Pasco Basin via the Columbia River.

Three facies exist for the Hanford formation in the Pasco Basin: 1) gravel-dominated, 2) sanddominated, and 3) interbedded sand- and silt-dominated (DOE 2002). The gravel-dominated facies were deposited in the central portion of the basin adjacent to the present Columbia River, at lower elevations and where the energy during flooding was the greatest. The interbedded sand- and silt-dominated facies lie around the margins of the basin and in slackwater areas. The sand-dominated facies, which is the most voluminous facies at the Hanford Site, was deposited in the large area between the other two. The 300 Area, which lies at a low elevation adjacent to the Columbia River, received the full brunt of high-energy 
floodwaters and therefore is composed exclusively of gravel-dominated facies of the Hanford formation. High-energy flood flows are indicated where a train of braided, anastamosing flood channels developed along the center of the basin (Figure 4.18).

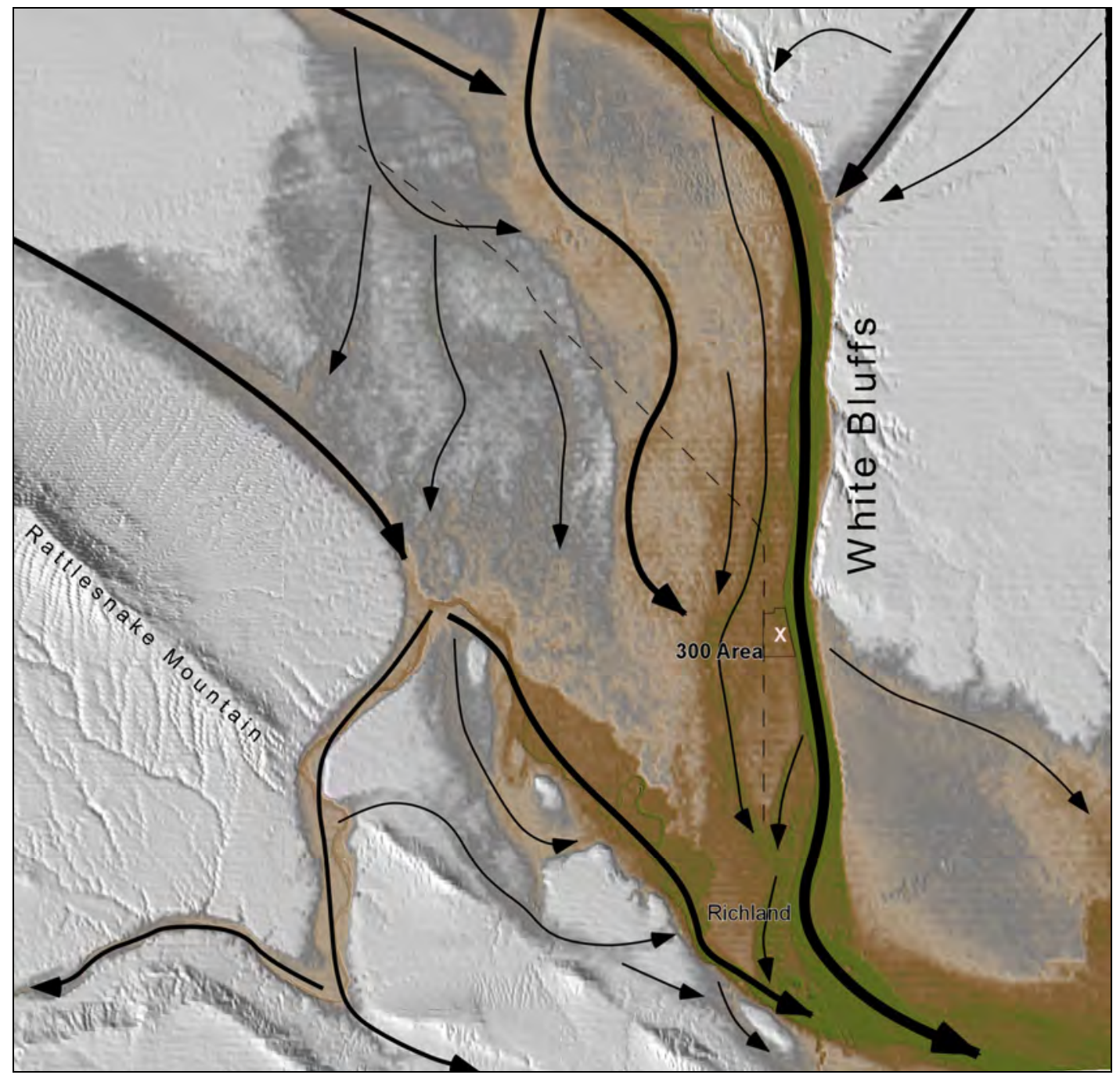

Figure 4.18. Network of interconnected braided channels created by the last cataclysmic Ice Age floods (arrows). During flooding, up to $900 \mathrm{ft}$ of water submerged this entire landscape, except for the highest ridges like Rattlesnake Mountain. Channels were partially to totally backfilled with Hanford formation sediments during flooding. The IFRC site is marked with an X.

Flood deposits of the Hanford formation in the 300 Area are relatively heterogeneous and anisotropic. Some weak bedding and stratification were apparent in excavated pit exposures. However, because of the complex hydrodynamics involved in cataclysmic flooding, individual beds do not appear to extend very far laterally. For example, in Figure 4.19, most beds are discontinuous across the width of the trench. 


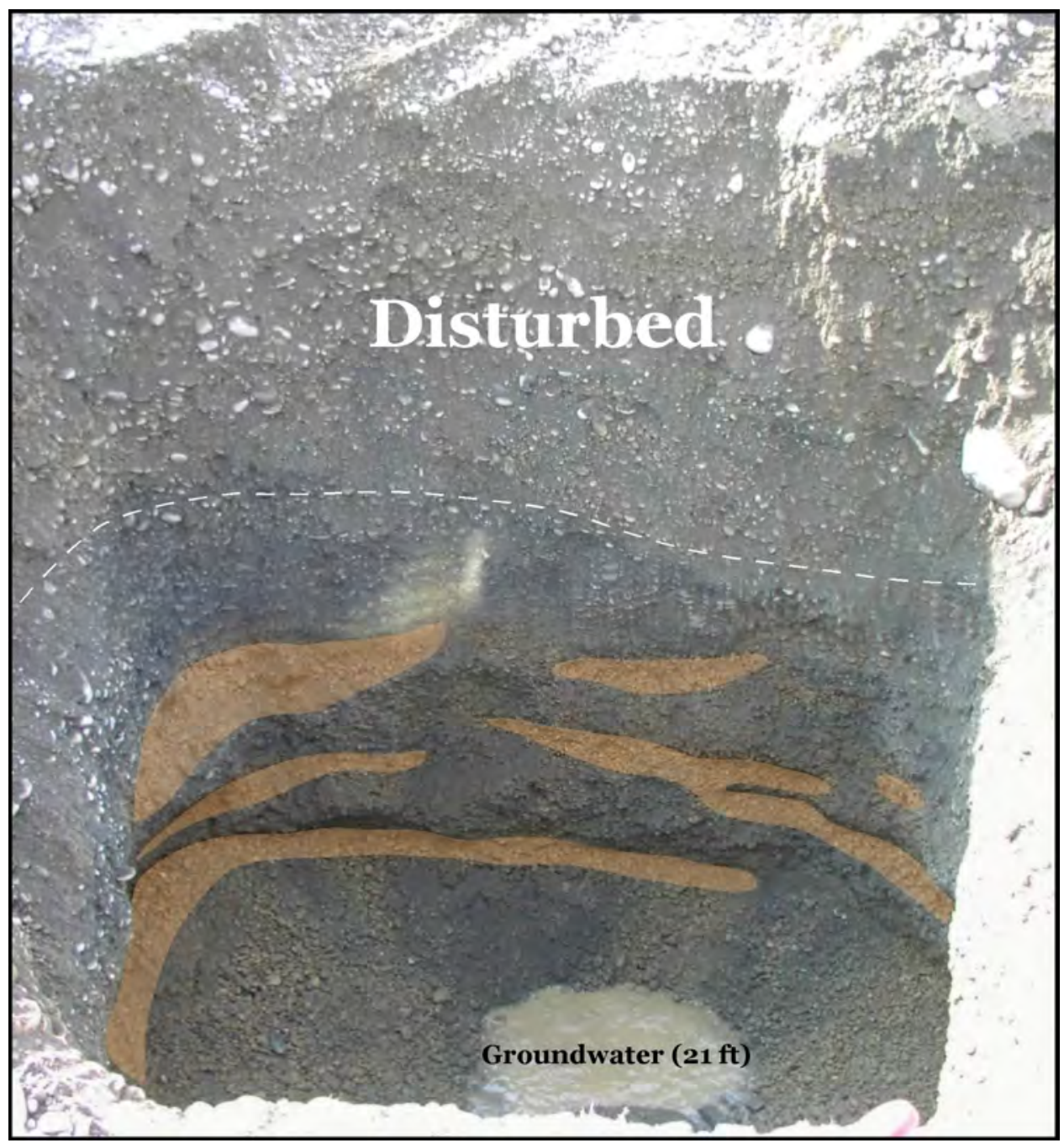

Figure 4.19. Profile of vadose-zone pit excavated in the east side of the South Process Pond (SSP\#1) in 2003. Higher concentrations of oxidized, yellow-brown Ringold silt and clay matrix fill occur within subhorizontal layers (highlighted by artificial coloring). Matrix fill in nonhighlighted areas consists of predominantly higher-permeability basaltic sand with little or no fines.

Discordant clastic dikes (Fecht et al. 1999), which are common to the sand- and interbedded sand- to silt-dominated facies of the Hanford formation, rarely occur in the gravel-dominated facies, including the 300 Area.

A well-defined unconformity exists along the sharp contact between the coarse-grained Hanford and fine-grained Ringold formations. During the main onslaught, floodwaters scoured the Ringold Formation and eroded the surface before backfilling channels with coarse flood deposits during the waning stages of flooding (Figure 4.18). The topography on the top of the Ringold Formation is illustrated in Figure 4.20. The low area that runs diagonally across the IFRC site is interpreted as a channel eroded into the Ringold Formation during Ice Age flooding. The channel is filled with highly permeable Hanford formation sediments and underlain by low-permeability, silty sediments of the Ringold Formation. Therefore, it is a likely place for channelized flow of groundwater to occur. 


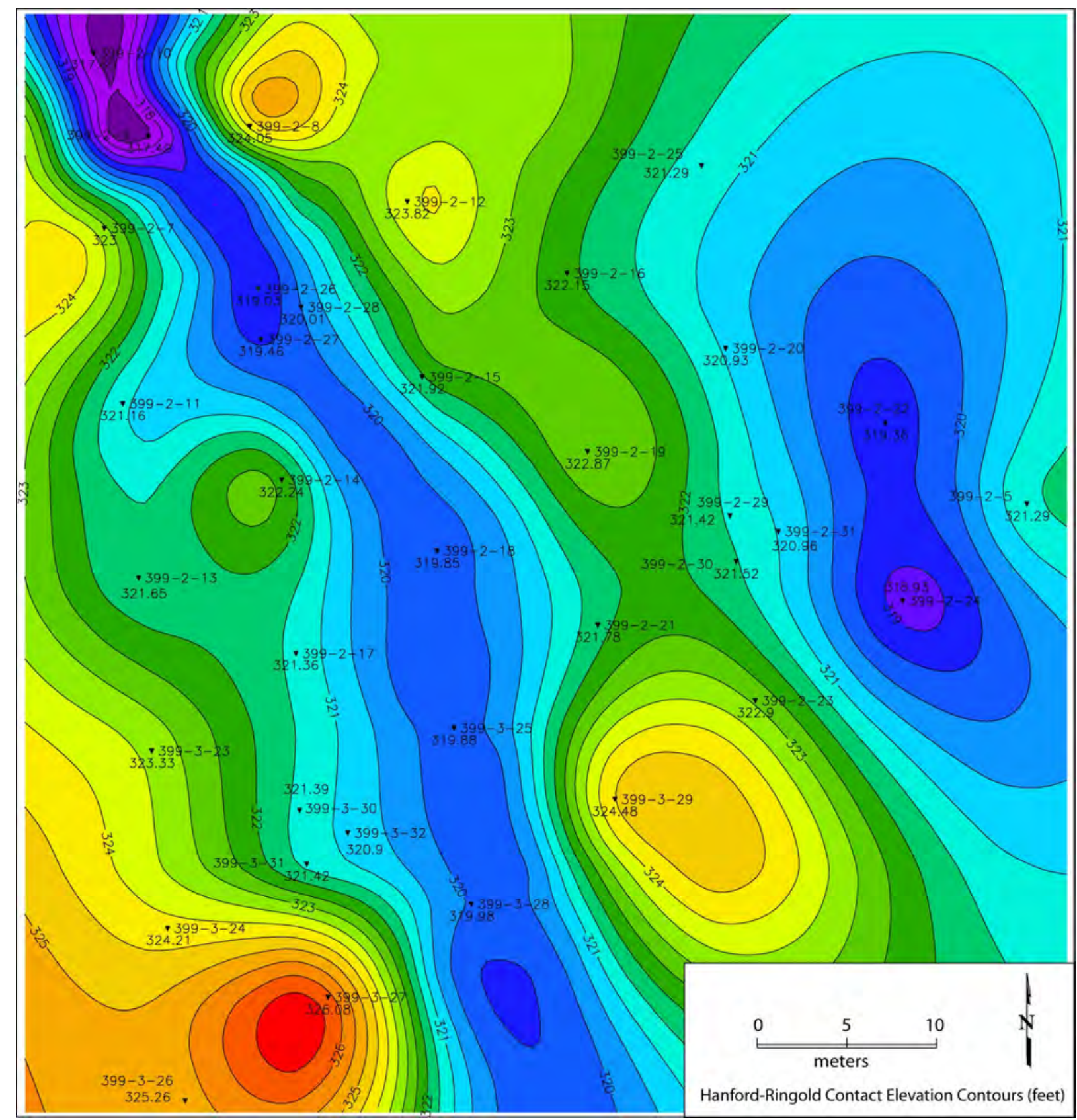

Figure 4.20. Structure-contour map illustrating the uneven, eroded surface of the Ringold Formation. A flood paleochannel appears to run midway, from northwest to southeast through the IFRC site. The channel is flanked with higher-elevation erosional remnants of the Ringold Formation. 
In Figure 4.21 and Figure 4.22 are several images showing the sharp contact between the dark, coarse-grained Hanford formation and oxidized brownish silt at the top of the Ringold Formation.

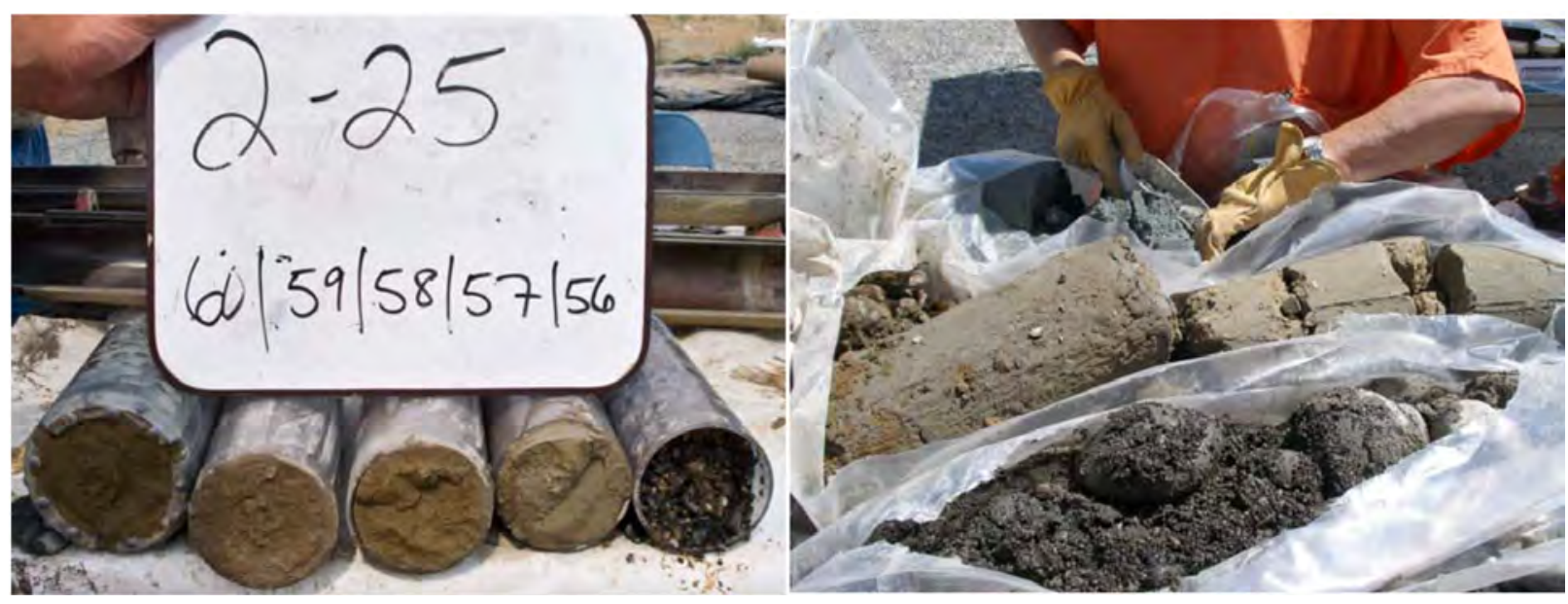

Figure 4.21. Hanford-Ringold formations contact. Left: Contact lies between coarse basaltic sand and gravel in right-hand core and next core, composed of oxidized Ringold Formation silt at the 57-ft depth (well 399-2-25). Right: Loose, basaltic sand and gravel of the Hanford formation (foreground) overlie oxidized (middle) and reduced (background) greenish-gray silt of the Ringold Formation (well 399-3-28).

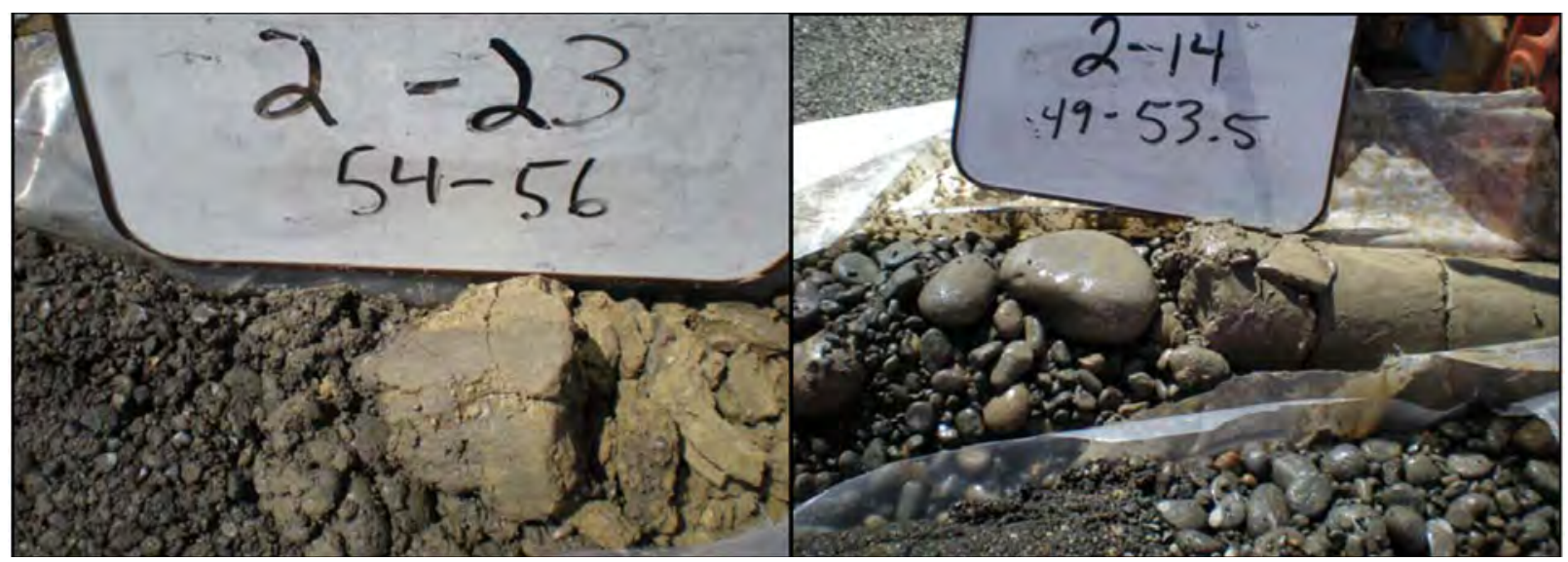

Figure 4.22. Sharp contact at the top of the Ringold Formation silt-dominated sediment in well 399-2-23 (left) and 399-2-14 (right). Notice the loose gravel in right-hand photo has little or no matrix.

Within the Hanford formation, loose, clast-supported, muddy sandy gravel is the predominant sediment type (see Figures 4.17 and 4.23). Lenses of matrix-supported gravelly sand occur sporadically. (The terms mud and muddy are used to describe undifferentiated silt- to clay-size particles.) The maximum size of gravels from the vadose zone observed in excavations within the South Process Pond (Bjornstad 2004) was boulders up to several feet in diameter (Figure 2.4). Roundness on basalt gravel clasts is usually immature (subangular to subrounded) because of relatively recent erosion, transport, and rapid burial of the locally derived basaltic detritus. Gravel clasts of other compositions (quartzite, granite, gneiss, and volcanic porphyries) are commonly more rounded as a result of reworking by the floods of older fluvial deposits (e.g., Ringold and Ellensburg formations). 
The matrix filling between gravel clasts is highly variable. The matrix may be filled or partially filled with sand, silt, or clay, or may be absent altogether, creating an openwork fabric (Figure 4.23).

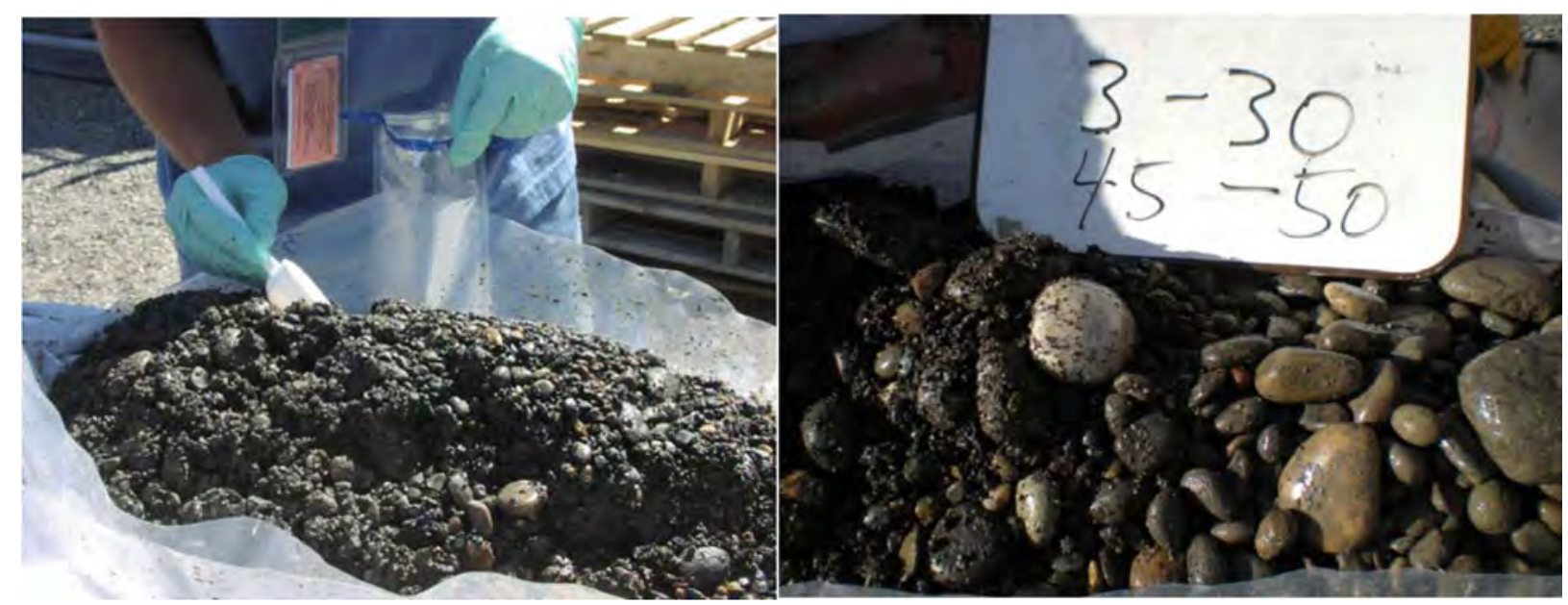

Figure 4.23. Gravel-dominated facies with openwork fabric within the Hanford formation. Left: Grab sample of loose, basaltic, pebble gravel was collected for microbiological characterization. Right: Clean, matrix-free pebble gravel on the right transitions to sandy, matrix-filled gravel to the left.

Occasionally the matrix fill consists of almost pure yellowish silt or clayey silt, apparently derived from reworked Ringold mud (Figure 4.24). Typically, however, silt and clay are not a major component of high-energy, gravel-dominated flood deposits (DOE 2002; Bjornstad 2006). This is because the fine particles of silt and clay tended to remain in suspension during the short-duration (week or less) high-energy Ice Age flood events. Floodwaters never slowed down enough for the fine particles to settle out of suspension before all the water flushed out of the basin. Coarser particles of sand and gravel, on the other hand, could settle out and were deposited during flooding because of their larger size and higher density.

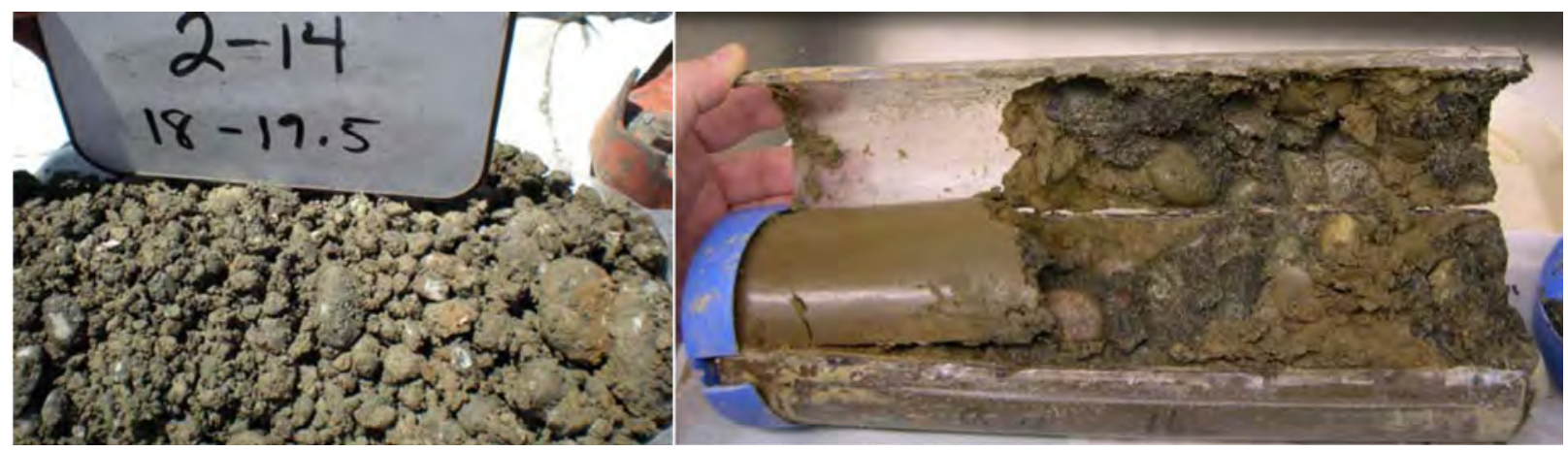

Figure 4.24. Flood gravels with fine-grained, reworked Ringold Formation matrix in the Hanford formation. Left: Matrix of olive to yellow Ringold Formation silt may be derived from erosion beneath the IFRC site or upstream off the White Bluffs. Grab sample from well 399-2-14, 18- to 19.5-ft depth. Right: The matrix surrounding clast-supported gravel in this core consists of a mottled mixture of multi-genetic brown Ringold Formation silt and dark gray basaltic sand (well 399-2-25, 52-ft depth). 
The IFRC site is different from most other locations, however, because it lies close to at least two sources of fine-grained Ringold Formation silt and clay. One source is directly beneath the site, and the other is the White Bluffs erosional escarpment immediately upstream (Figure 4.25). Thus, it appears that significant amounts of fine-grained Ringold Formation silt and clay were incorporated into flood deposits as Ringold rip-up clasts before the clasts had a chance to totally disaggregate and move into suspension with the floodwater (see Figure 4.18). Once in suspension, however, most fine particles of silt and clay were flushed out of the basin along with the ocean-bound floodwater.

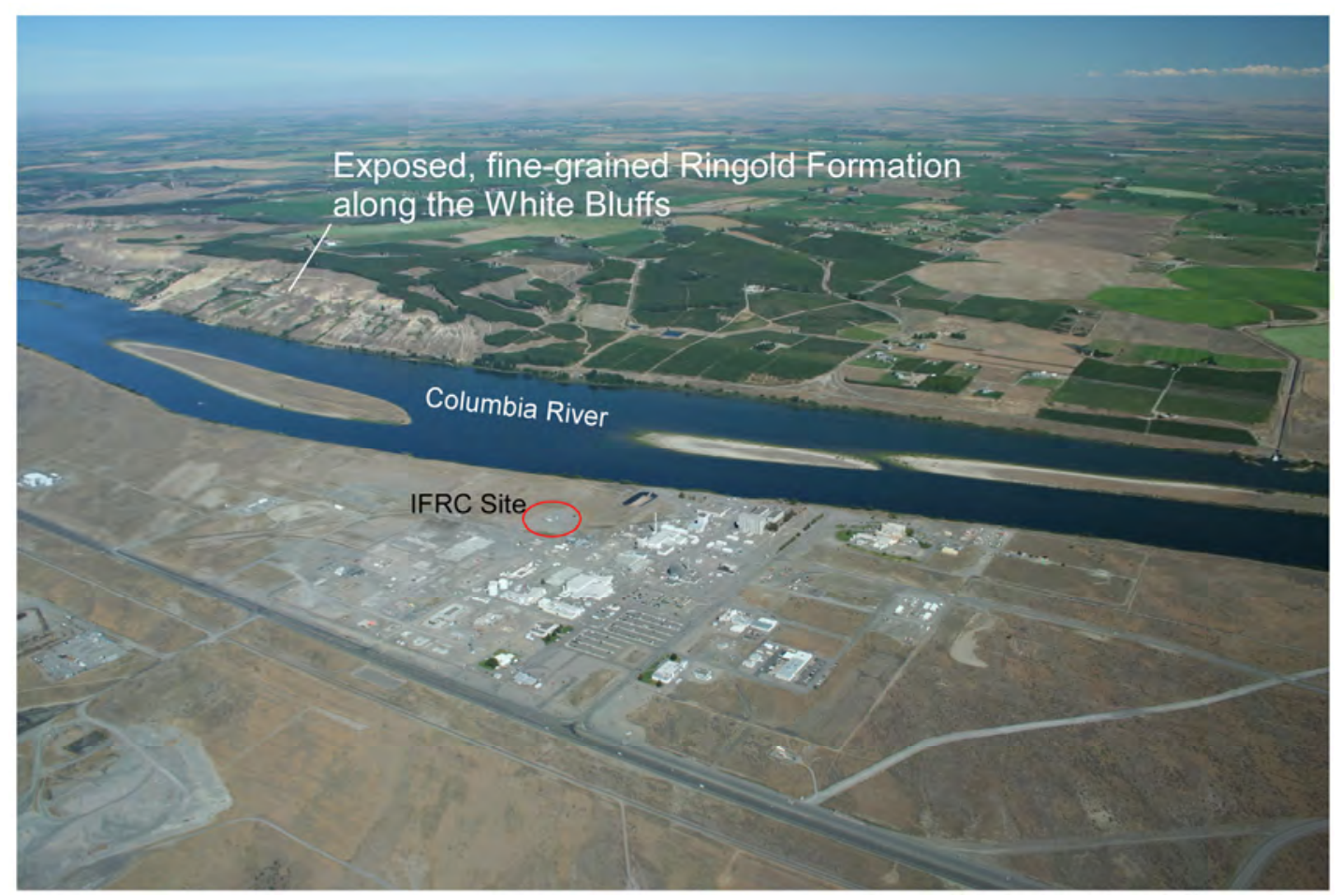

Figure 4.25. Aerial view, looking northeast, toward the IFRC site. The White Bluffs are an erosional escarpment that exposes hundreds of feet of Pliocene-age, fine-grained sediments of the Ringold Formation. Ice Age floods flowed from the upper left and upper right. Photo: Bob Peterson (PNNL).

\subsubsection{Ringold Formation Rip-Up Clasts}

Unique to the flood deposits are rounded rip-up clasts of semiconsolidated, fine-grained Ringold Formation (Figure 4.26 and Figure 4.27). These include clasts of calcium-carbonate-cemented caliche as well as clasts of compacted mud, originally deposited during Ringold time in either floodplain-overbank or lake environments (Figure 4.28). These same types of sediment are exposed in the Ringold Formation within the White Bluffs immediately across the river as well as upstream of the 300 Area. Generally, Ringold rip-up clasts are larger than adjacent clasts (see Figure 4.18), reflecting their short transport distance and lower bulk density in contrast to lithified clasts. The rounded nature of rip-up clasts indicates they were transported as detrital "grains," along with other materials, during flooding. In about half of the new wells (17 of 35) at the IRFC site, fine-grained rip-up clasts were encountered during drilling (see Appendix A). 


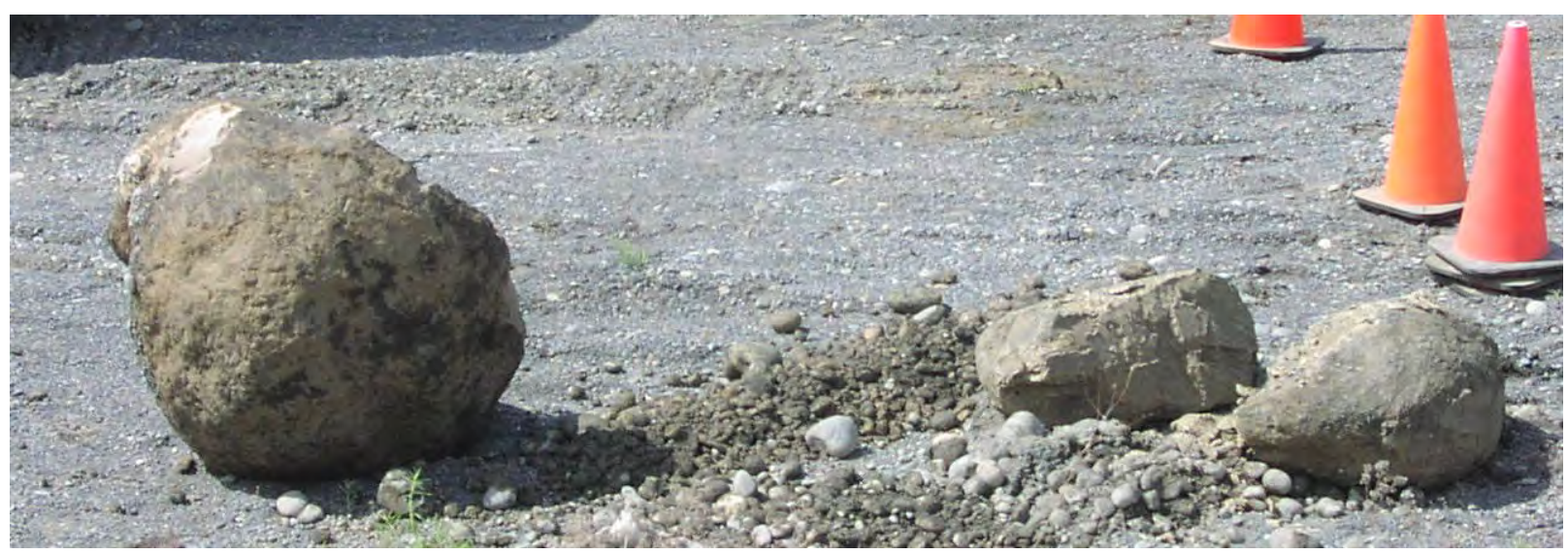

Figure 4.26. Rounded rip-up clasts removed from a backhoe excavation in 2003 within SPP\#2 at the south end of the IFRC site (see Figures 2.3 and 2.4). These boulder-sized clasts, composed of cohesive Ringold Formation silt and clay, were ripped off the White Bluffs escarpment just upstream of, or from beneath, the 300 Area during a cataclysmic Ice Age flood.

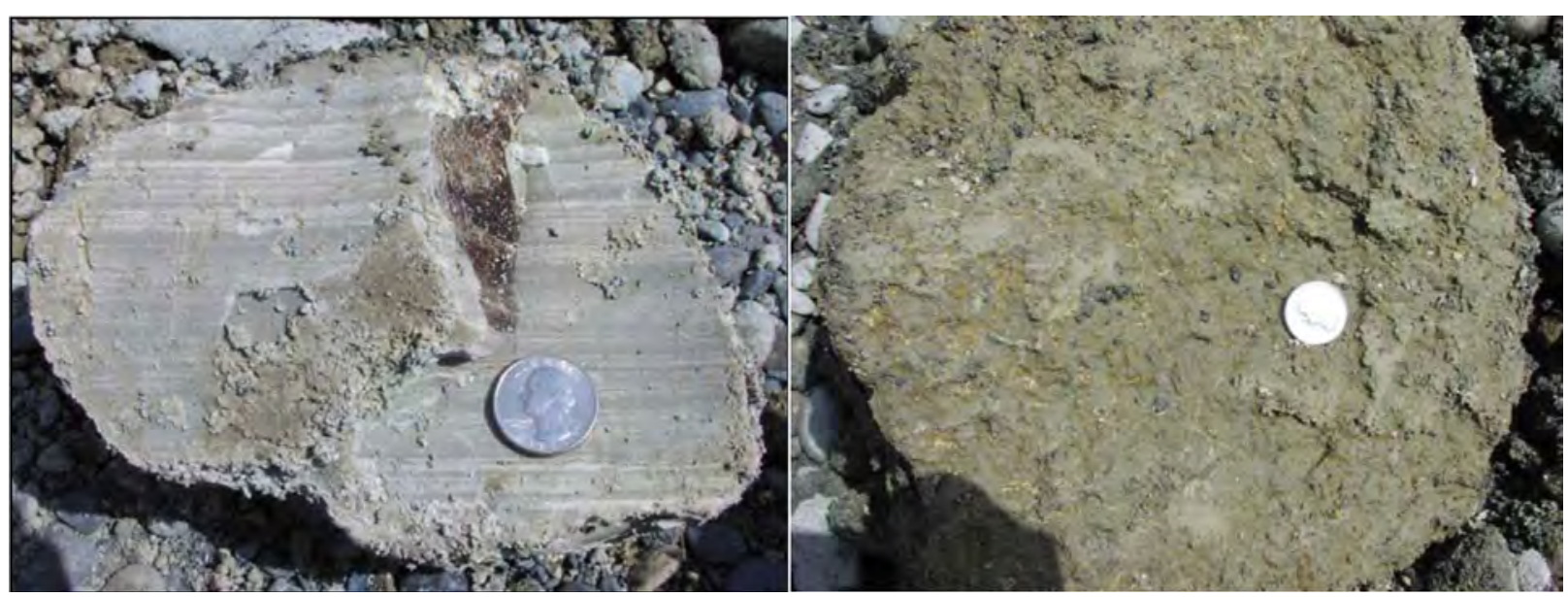

Figure 4.27. Interiors of two fine-grained Ringold Formation rip-up clasts removed by backhoe from beneath the South Process Pond in 2003. On the left is a well-laminated silt, originally deposited in a lake that filled the Pasco Basin between 3 to 5 million years ago. On the right is an olive-yellow silt with relict plant and animal traces from a Ringold Formation paleosol. Both sediment types are exposed in exposures of Ringold Formation in the White Bluffs, upstream of the 300 Area. 


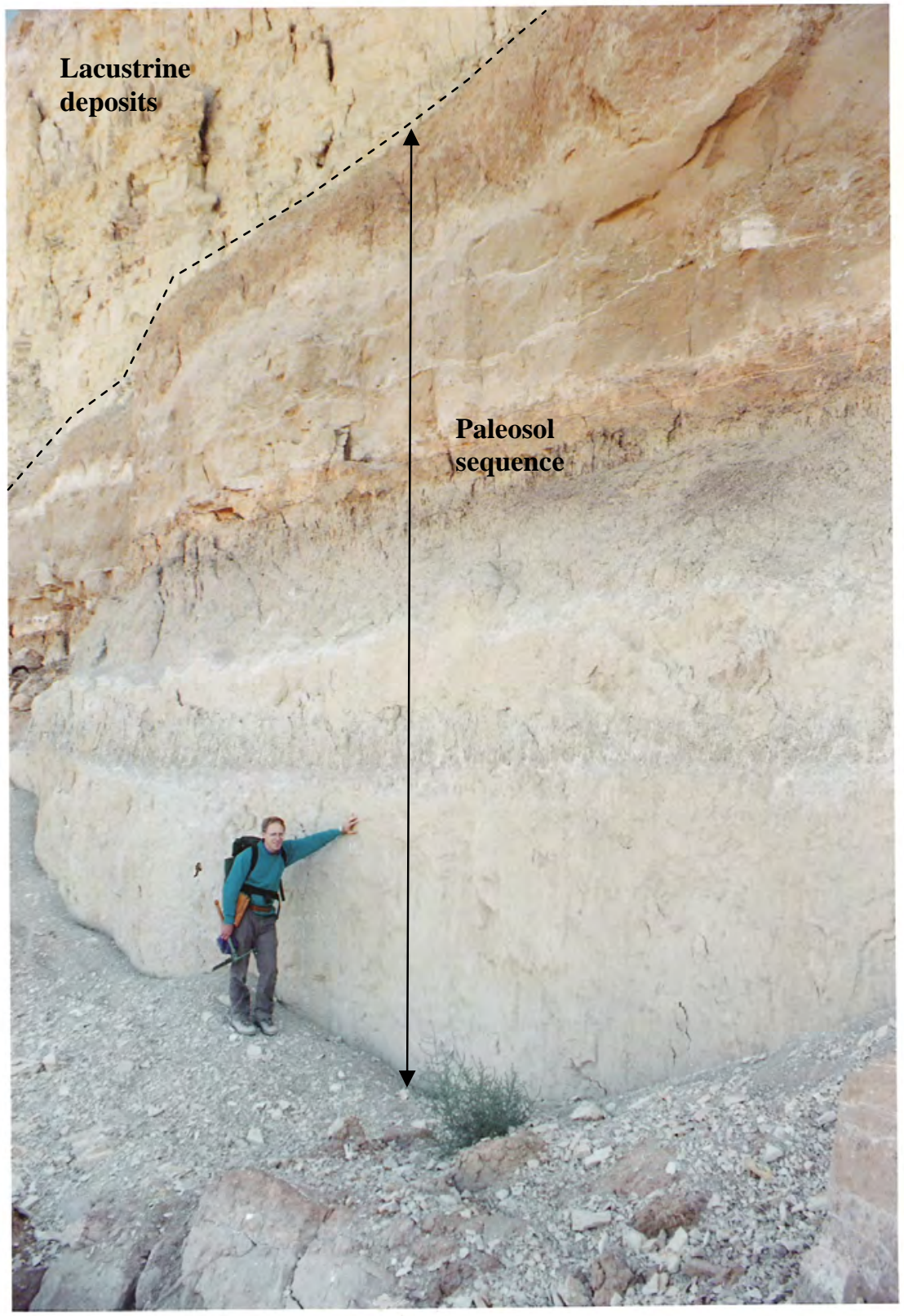

Figure 4.28. Olive to brown and yellow fine-grained upper Ringold Formation deposits (member of Savage Island) exposed upriver along the White Bluffs within a mile of the 300 Area. Here a thick paleosol sequence is overlain by laminated lacustrine (lake) deposits. Deposits like these are a probable source for the many reworked Ringold clasts observed within the Hanford formation in the 300 Area.

Two other examples of rip-up clasts identified in new IFRC wells are shown in Figure 4.29 and Figure 4.30. 


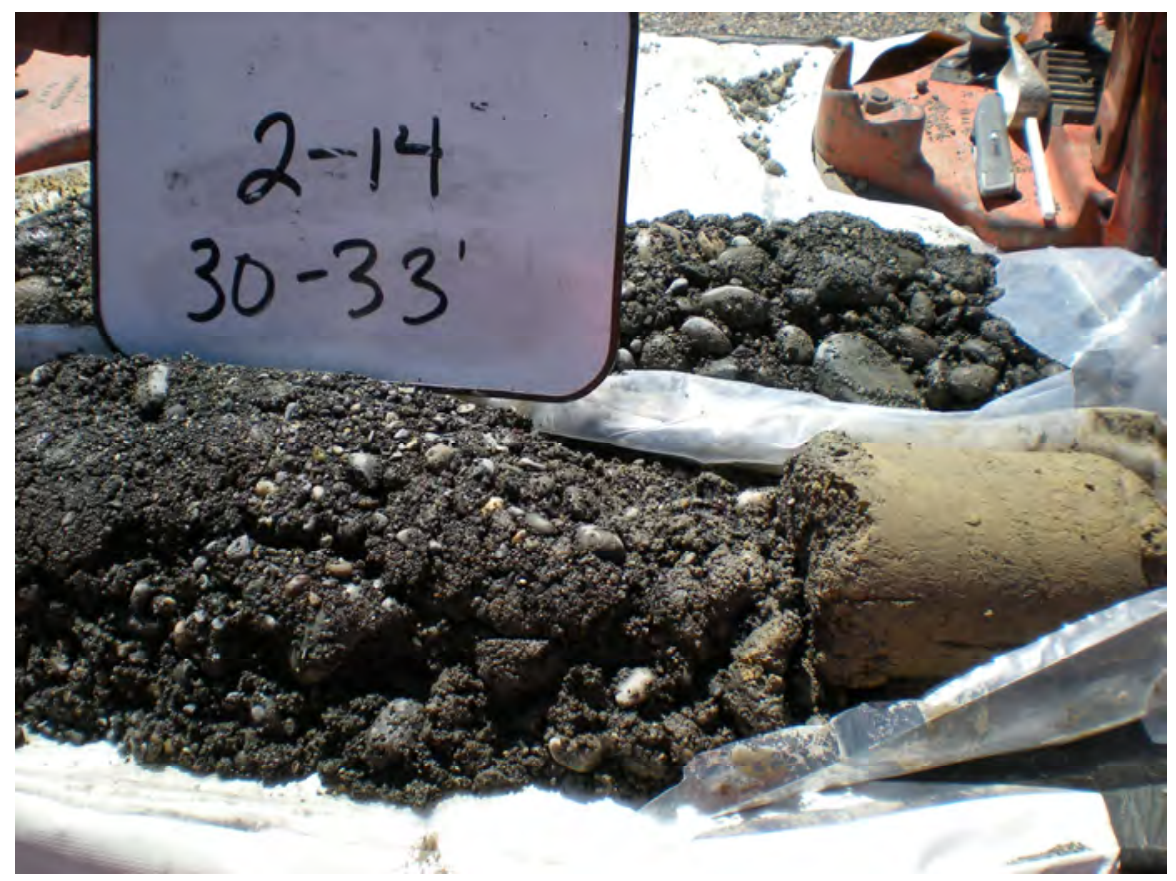

Figure 4.29. Loose, basaltic pebbly sand overlies the top of a semiconsolidated Ringold Formation ripup clast within the Hanford formation at 30- to 33-ft depth, well 399-2-14. This rip-up clast is similar to the oxidized silt of the Ringold Formation that directly underlies the IFRC site.

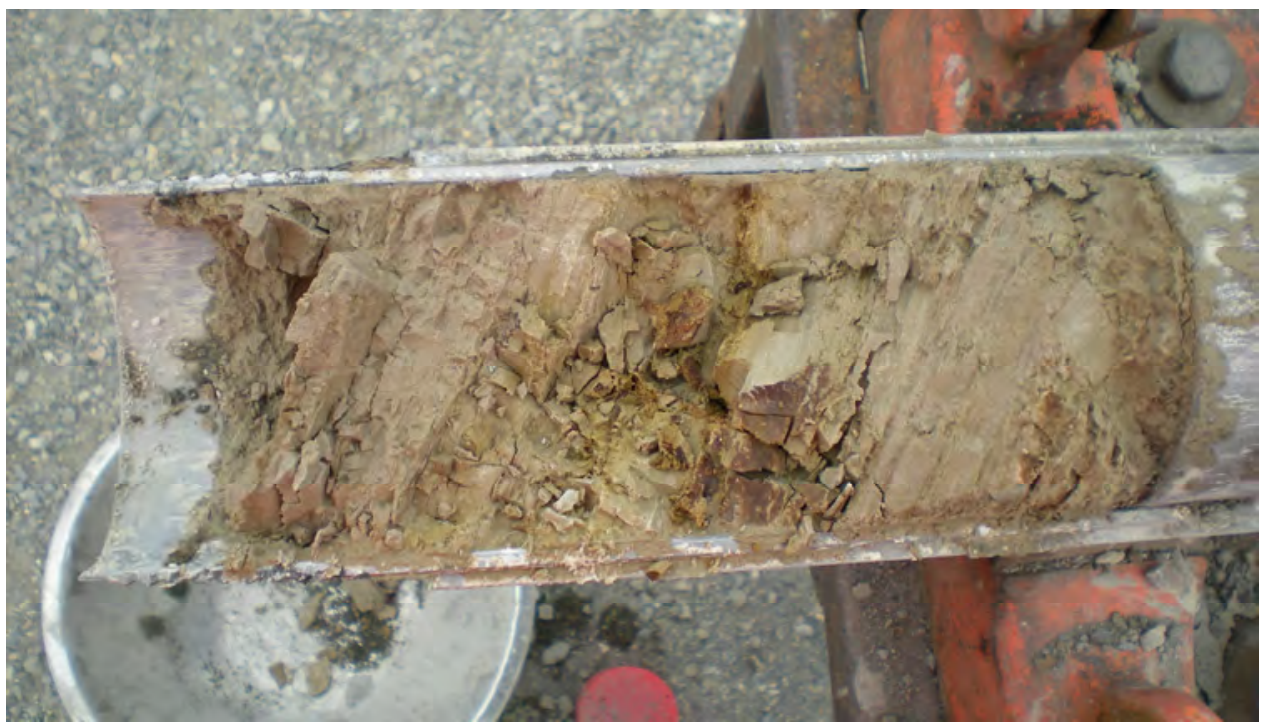

Figure 4.30. Portion of a 5.5-ft-thick semiconsolidated Ringold Formation rip-up clast, composed of cohesive clayey silt, in well 399-3-26 from 21- to 22-ft depth. The inclined lamination in this vertical drill core indicates this sediment was not deposited in situ but rotated in transit and redeposited during an Ice Age flood.

Because some contaminants (including uranium) may have an affinity for mud-sized particles, the character and distribution of concentrated fine-grained material in the subsurface has special relevance. Although clast-supported pebble- to boulder-size gravel is the dominant sediment size in the 300 Area, the matrix between gravel clasts varies significantly between relatively permeable gray to black basaltic 
sand (Figure 4.27) and relatively impermeable brownish mud (see Figure 4.24). The sediment color is an indication of the type of matrix present. The color most often associated with coarse-grained facies of the Hanford formation is dark gray to black, due to a composition of mostly unweathered basaltic rock fragments eroded off the Channeled Scabland during Pleistocene flooding.

However, the character of the flood sediment is different in the 300 Area and unlike most other flood deposits, for it does have more concentrated fines in the form of rip-up clasts as well as beds with finegrained, brown, matrix-filling Ringold Formation materials (Figure 4.24). The brown color, derived from the Ringold Formation, is the result of a much longer period of weathering - over many millions of years - compared to the Hanford formation, which is only about 15,000 years old. As mentioned, the fine-grained Ringold matrix is unusual for the Hanford formation and probably the result of the 300 Area being directly across the river and just downstream of an abundant supply of Ringold detritus during Ice Age flooding (see Figure 4.25). It is possible that some of the brown fine-grained intervals may be associated with concentrations of Ringold rip-up clasts that disintegrated during or soon after deposition by the floods. Rip-up clasts are relatively unconsolidated and, not surprisingly, do not survive flood transport far from their source (see Figure 4.16), which explains why they are not observed far inland of the 300 Area and the Columbia River.

\subsection{Man-Made Backfill}

The contact between backfill and Hanford formation is difficult to discern based on drill cuttings or geophysical logs because the texture of the sediments is essentially the same (compare Figure 4.32 with Figure 4.17). The contact is apparent, however, based on photographs of several backhoe pits excavated in 2003 (see examples in Figures 2.4 and 4.19). Disturbed sediments appear relatively homogeneous with no visible structure; this is in contrast to the in situ Hanford formation, which shows sedimentary fabric in the form of layering, stratification, and clast imbrication.

Based on these photographs, the zone of anthropogenic disturbance extends below the bottom of the process pond excavation. Movement of heavy equipment and other disturbances at the base of the excavated ponds caused disturbance for up to several meters below these excavations. Thus, there may be only a few meters of undisturbed Hanford formation sediments within the vadose zone along the western side of the IFRC site.

The 300 Area process ponds were used for the disposal of uranium-contaminated wastes between 1943 and 1975. Contaminated soils were selectively removed from beneath the ponds from 1994 to 2005. The configuration of the uneven bottom of the process ponds at the end of remediation is shown in Figure 4.31. After remediation, excavations were backfilled with locally derived gravel-dominated sediments of the Hanford formation. Following backfilling, the surface was brought up to a common elevation of about $115 \mathrm{~m}(377 \mathrm{ft})$. 

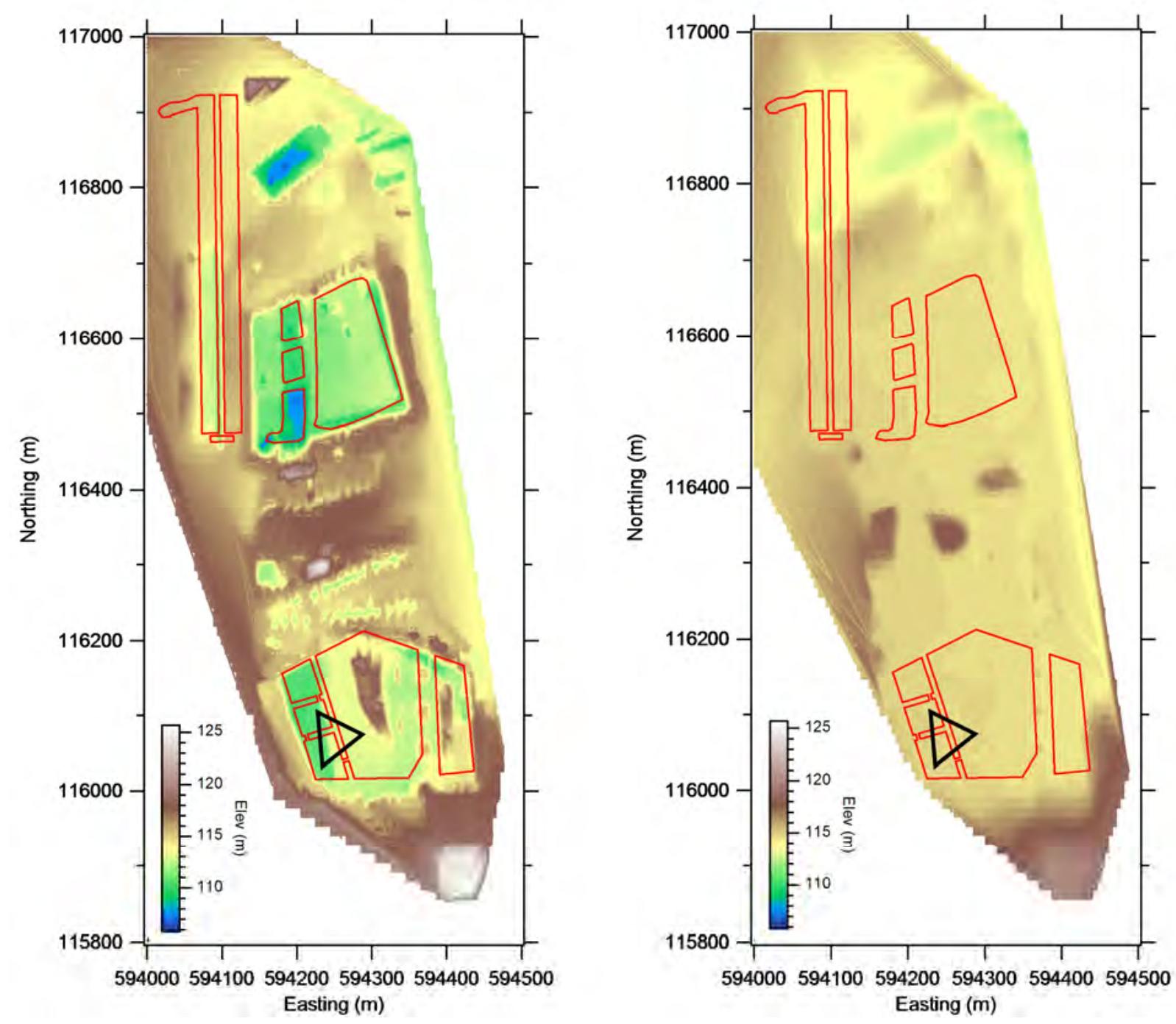

Figure 4.31. Surface topography in the vicinity of the 300 Area process ponds and trenches. Left: topography after remedial excavation and prior to emplacement of backfill. Right: surface as it exists today after remediation and backfilling. Triangle shows the location of the IFRC well field over the southwestern corner of the South Process Pond. Elevation of the land surface over the former South Process Pond today is about an even $115 \mathrm{~m}$ (377 ft).

Backfill materials consist of grayish-brown, poorly sorted, homogeneous mixtures of loose basaltic gravel and sand with lesser amounts of silt (Figure 4.32), derived from excavated and mixed, gravel-dominated facies of the Hanford formation. Figure 4.31 (left) shows the South Process Pond was not excavated to a constant depth. Therefore, the thickness of the backfill varies across the IFRC site. For example, the depth of remedial excavation went to approximately 110-m elevation on the west side in contrast to only about $113 \mathrm{~m}$ on the east side of the IFRC site. This translates to a backfill thickness of about $5 \mathrm{~m}(\sim 16 \mathrm{ft})$ on the western side and approximately $2 \mathrm{~m}(\sim 7 \mathrm{ft})$ along the eastern side of the IFRC site. 


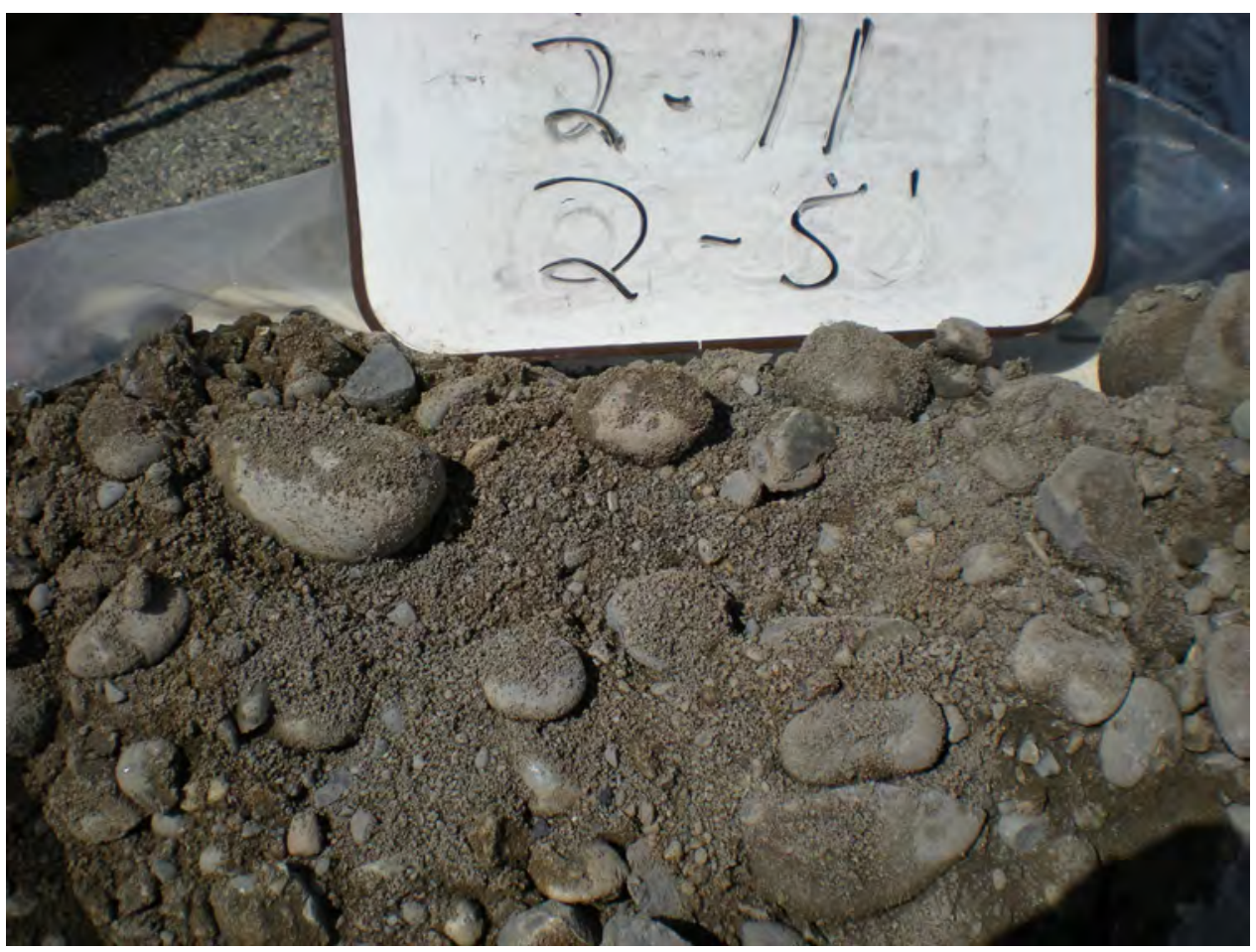

Figure 4.32. Backfill materials from 2- to 5-ft depth in well 399-2-11. This material is very similar to that of the Hanford formation from which it is derived. However, backfill sediments are likely more isotropic than the Hanford formation because the sedimentary fabric (horizontal to subhorizontal layering and stratification) was destroyed during excavation and backfilling. 


\subsection{References}

Bjornstad BN and JA Horner. 2008. Drilling, Sampling Well-Installation Plan for IFC Well Field, 300 Area. PNNL-17512, Pacific Northwest National Laboratory, Richland, WA.

Bjornstad BN and VRVermeul. 2008. Drilling Specifications: Well Installations in the 300 Area to Support PNNL's Integrated Field-Scale Subsurface Research Challenge (IFC) Project. PNNL-17199 Rev. 3, Pacific Northwest National Laboratory, Richland, Washington.

Bjornstad BN. 2004. Sampling and Hydrogeology of the Vadose Zone Beneath the 300 Area Process Ponds. PNNL-14834, Pacific Northwest National Laboratory, Richland, Washington.

Bjornstad BN. 2006. On the Trail of the Ice Age Floods: A Geological Field Guide to the MidColumbia Basin. Keokee Co. Publishing, Inc., Sandpoint, Idaho.

Campbell NP. 1983. Correlation of late Cenozoic gravel deposits along the Yakima River drainage from Ellensburg to Richland, Washington. Northwest Science 57:179-193.

DOE (U.S. Department of Energy). 1988. Consultation Draft: Site Characterization Plan, Reference Repository Location, Hanford Site, Washington. DOE/RW-0164, U.S. Department of Energy, Washington, D.C.

DOE (U.S. Department of Energy). 2002. Standardized Stratigraphic Nomenclature for the PostRingold-Formation Sediments Within the Central Pasco Basin. DOE/RL-2002-39, Rev. 0, U.S. Department of Energy Richland Operations Office, Richland, Washington.

Fecht KR, BN Bjornstad, DG Horton, GV Last, SP Reidel, and KA Lindsey. 1994. Clastic Injection Dikes of the Pasco Basin and Vicinity: Geologic Atlas Series. BHI-01103, Rev. 0, Bechtel Hanford, Inc., Richland, Washington.

Lindberg JW and FW Bond. 1979. Geohydrology and Ground-Water Quality Beneath the 300 Area, Hanford Site, Washington. PNL-2949, Pacific Northwest Laboratory, Richland, Washington.

Lindsey KA. 1995. Miocene- to Pliocene-Aged Suprabasalt Sediments of the Hanford Site, SouthCentral Washington. BHI-00184, Bechtel Hanford, Inc., Richland, Washington.

Peterson RE (ed), EJ Freeman, CJ Murray, PD Thorne, MJ Truex, VR Vermeul, MD Williams, SB Yabusaki, JM Zachara, JL Lindberg, and JP McDonald. 2005. Contaminants of Potential Concern in the 300-FF-5 Operable Unit: Expanded Annual Groundwater Report for Fiscal Year 2004. PNNL15127, Pacific Northwest National Laboratory, Richland, Washington.

Peterson RE, BA Williams, and RM Smith. 2008. Volatile Organic Compound Investigation Results, 300 Area, Hanford Site, Washington. PNNL-17666, Pacific Northwest National Laboratory, Richland, Washington.

Schalla R, RW Wallace, RL Aaberg, SP Airhart, DJ Bates, JVM Carlile, CS Cline, DI Dennison, MD Freshley, PR Heller, EJ Jensen, KB Olsen, RG Parkhurst, JT Rieger, and EJ Westergard. 1988. 
Interim Characterization Report for the 300 Area Process Trenches. PNL-6716, Pacific Northwest Laboratory, Richland, Washington.

Swanson LC, GG Kelty, KA Lindsey, KR Simpson, RK Price, and SD Consort. 1992. Phase 1 Hydrogeologic Summary of the 300-FF-5 Operable Unit, 300 Area. WHC-SD-EN-TI-052, Rev. 0, Westinghouse Hanford Company, Richland, Washington.

WAC 173-160. 2008. “Minimum Standards for Construction and Maintenance of Wells.” Chapter 173160 in Washington Administrative Code. Olympia, Washington.

Williams BA, CF Brown, W Um, MJ Nimmons, RE Peterson, BN Bjornstad, DC Lanigan, RJ Serne, FA Spane, and ML Rockhold. 2007. Limited Field Investigation Report for Uranium Contamination in the 300 Area, 300-FF-5 Operable Unit, Hanford Site, Washington. PNNL-16435, Pacific Northwest National Laboratory, Richland, Washington.

Williams MD, ML Rockhold, PD Thorne, and Y Chen. 2008. Three-Dimensional Groundwater Models of the 300 Area at the Hanford Site, Washington State. PNNL-17708, Pacific Northwest National Laboratory, Richland, Washington.

Zachara JM, MD Freshley, BN Bjornstad, C Zheng, DJ Depaolo, DB Kent, JK Fredrickson, JN Christensen, A Konopka, C Liu, MS Conrad, JP McKinley, PC Lichtner, ML Rockhold, RJ Versteeg, R Haggerty, VR Vermeul, AL Ward, W Nowak, Y Rubin, and Z Zhang. 2008. "Hydrologic and Geochemical Characterization Plan for the Hanford IFRC Well-Field.” PNNL-SA-62816, Pacific Northwest National Laboratory, Richland, Washington. 


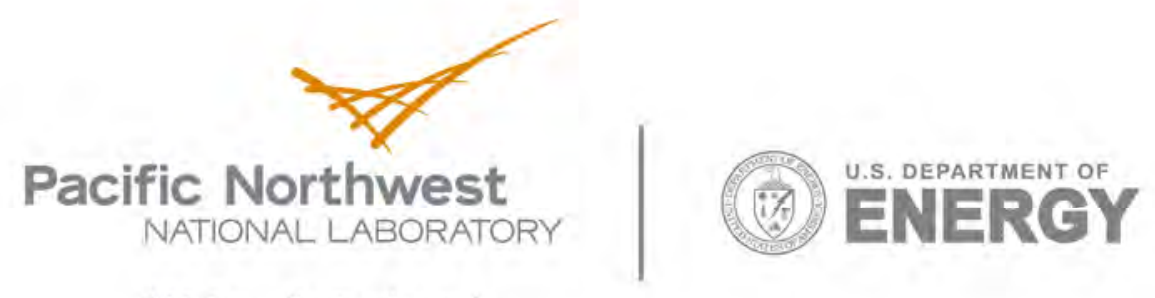

902 Battelle Boulevard

P.O. Box 999

Richland, WA 99352

1-888-375-PNNL (7665)

www.pnl.gov 


\section{Appendix A}

\section{Compilation Borehole Summary Logs}




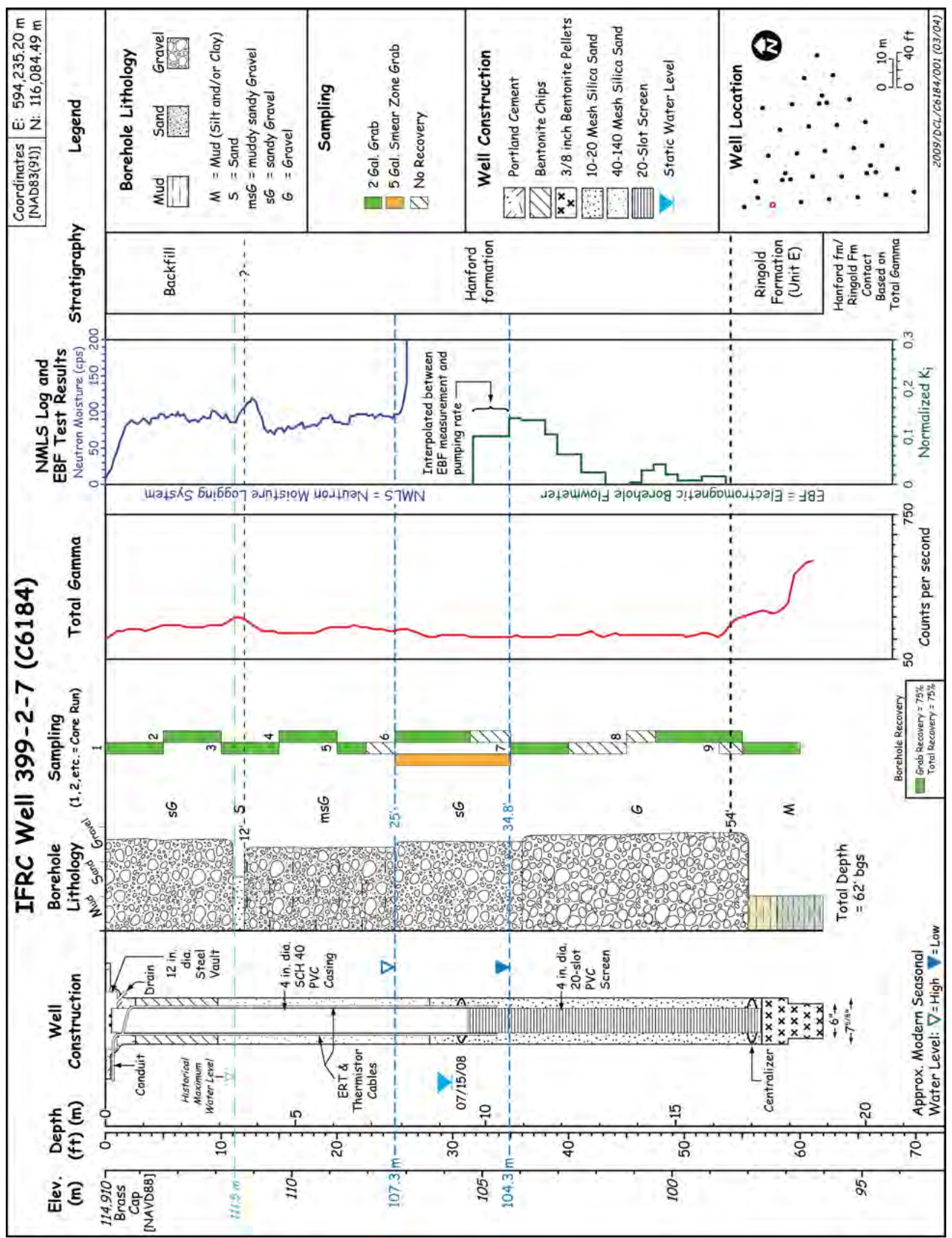




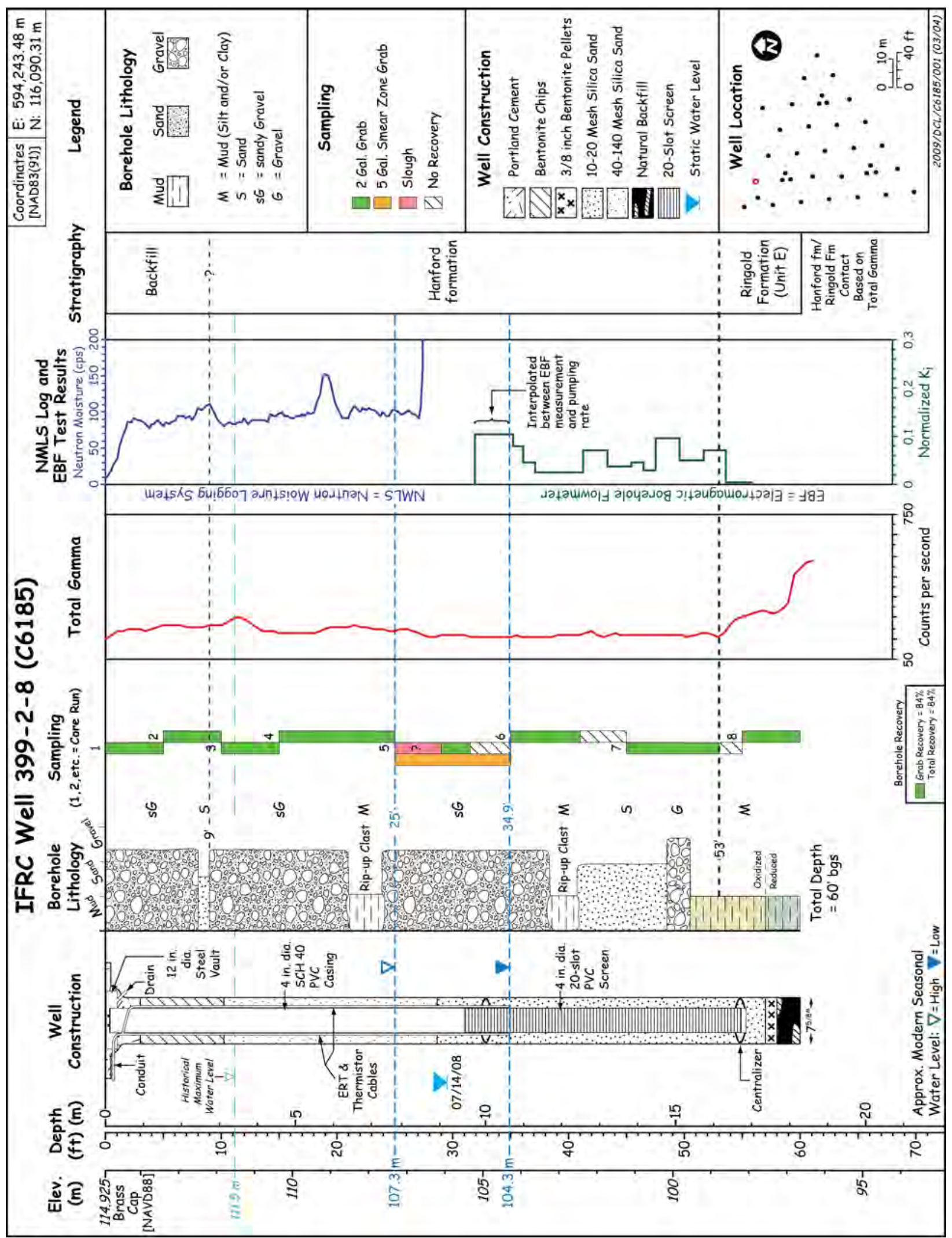




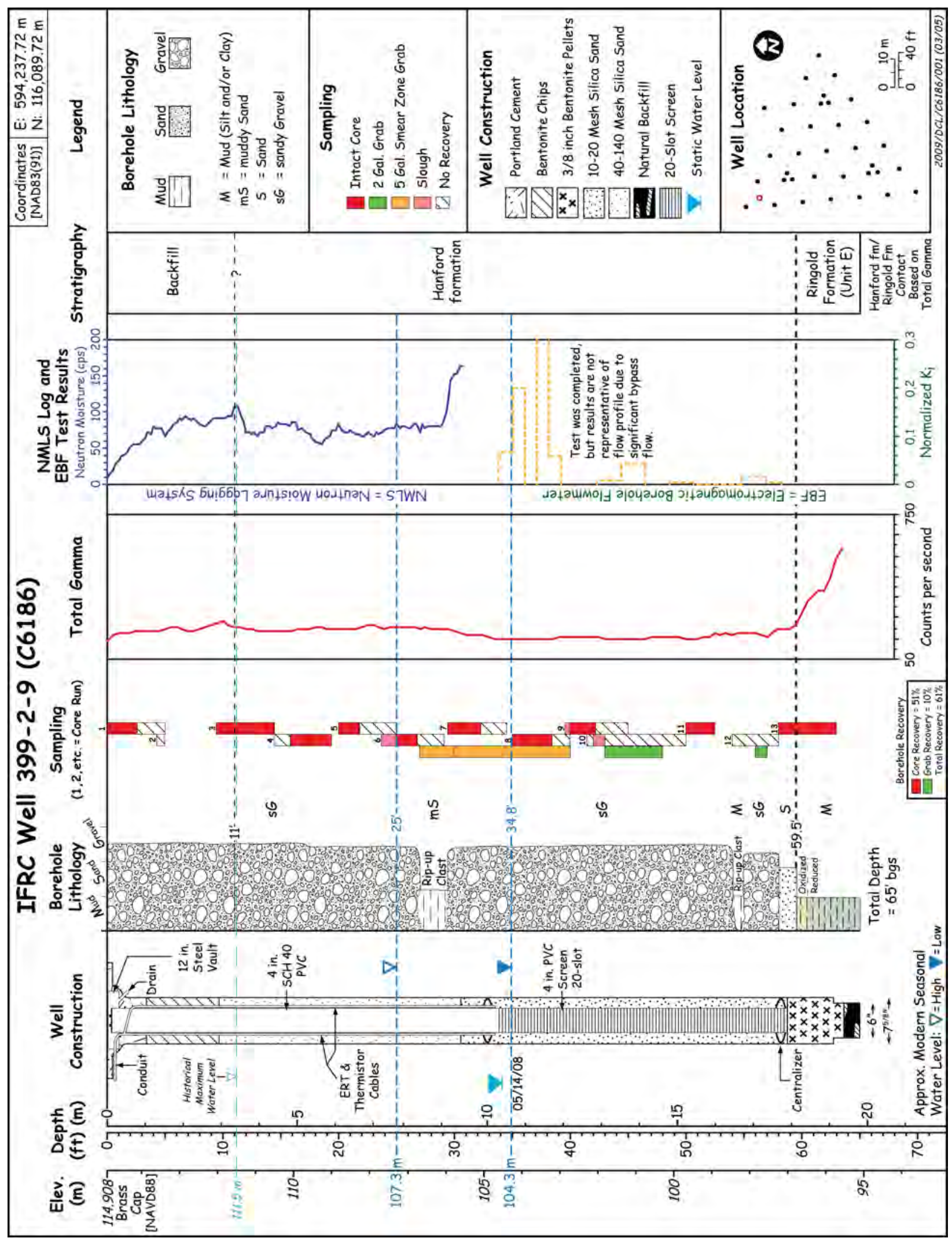




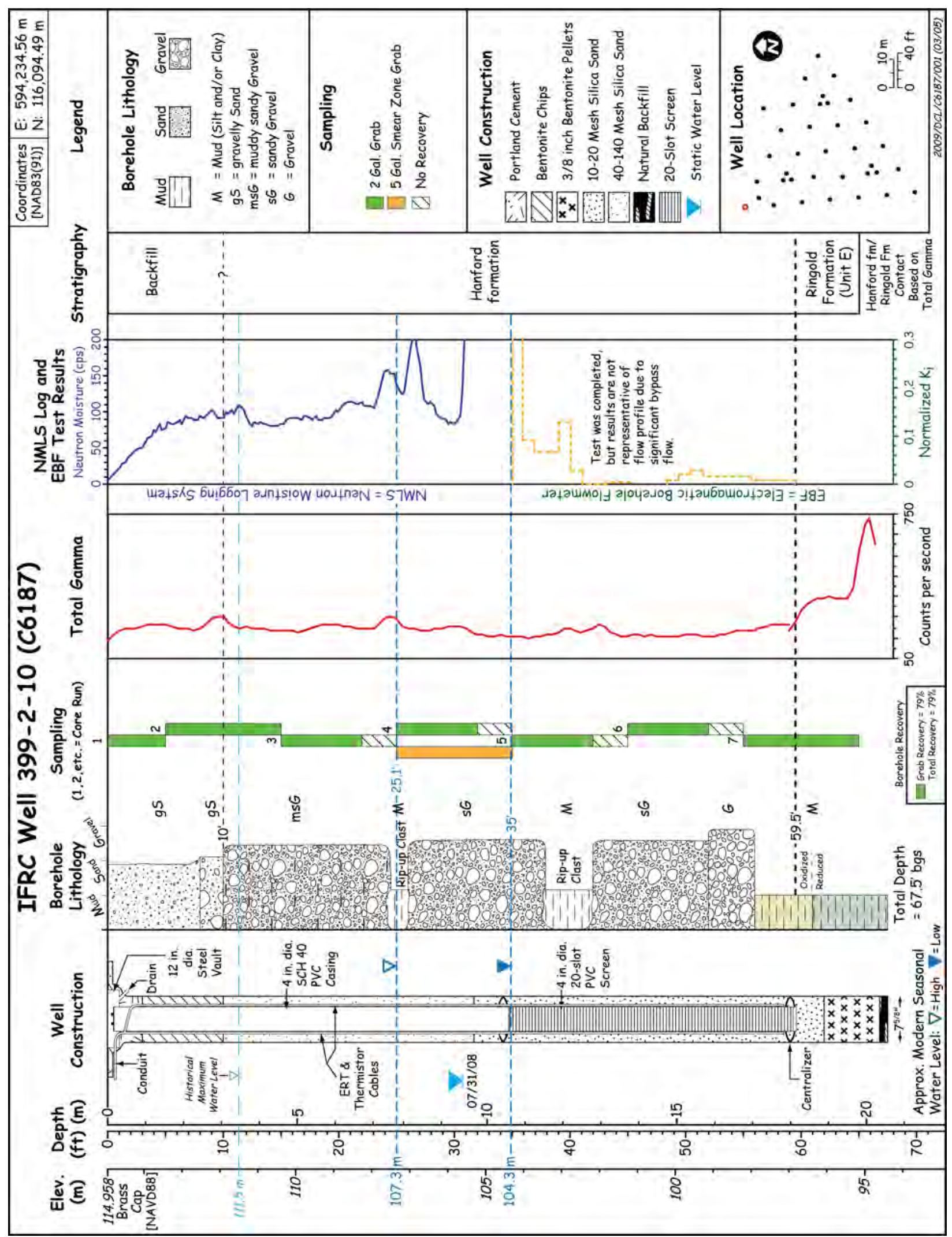




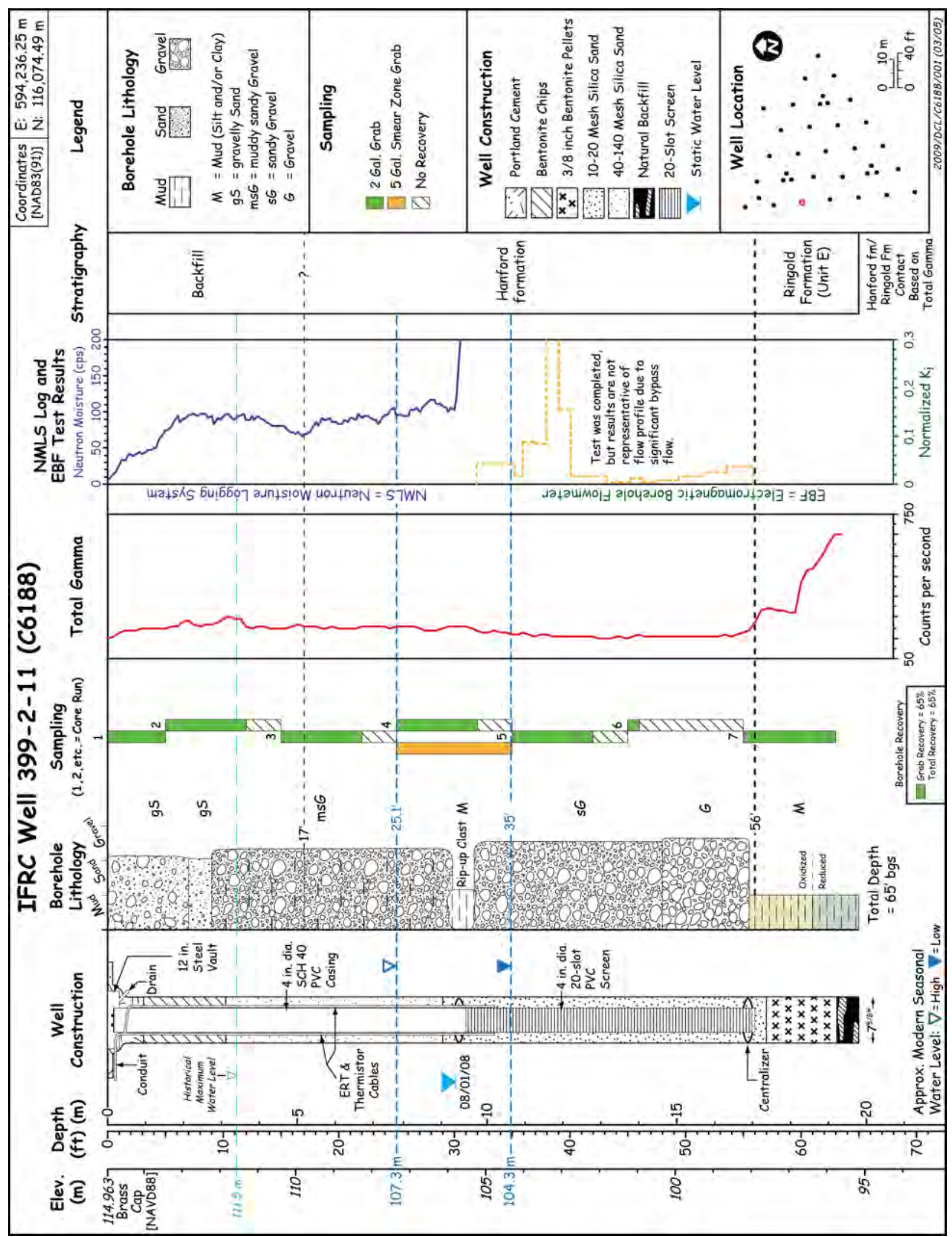




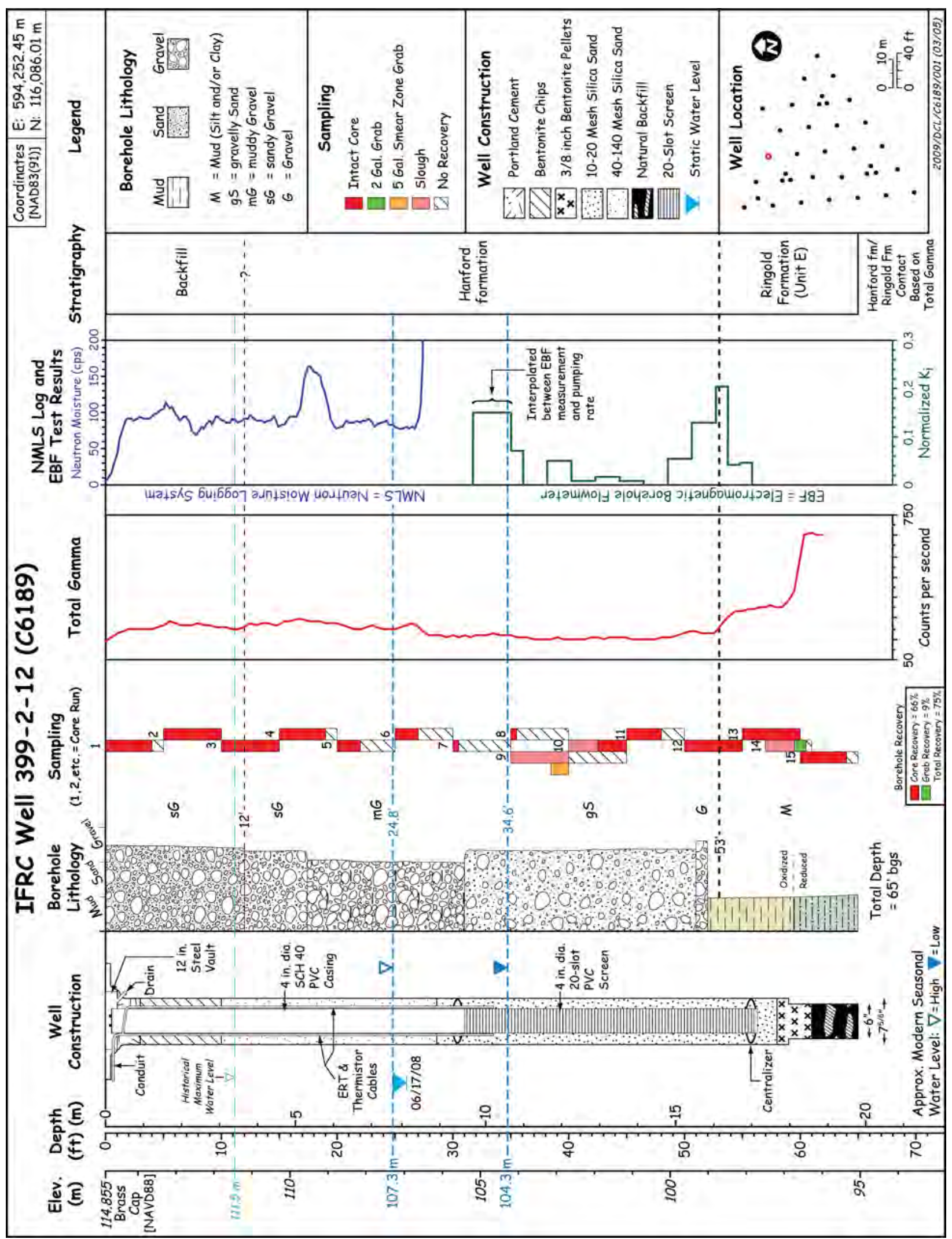




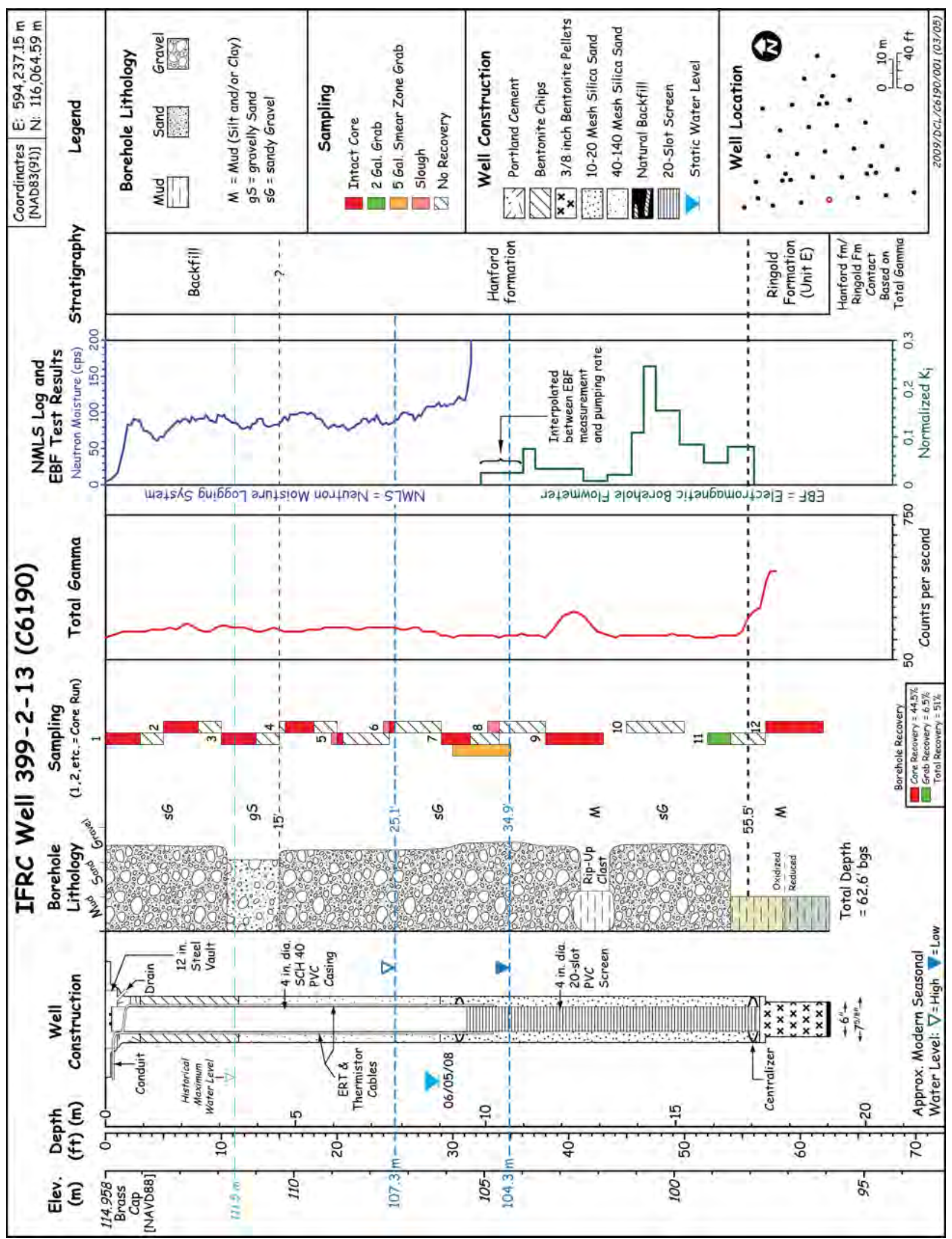




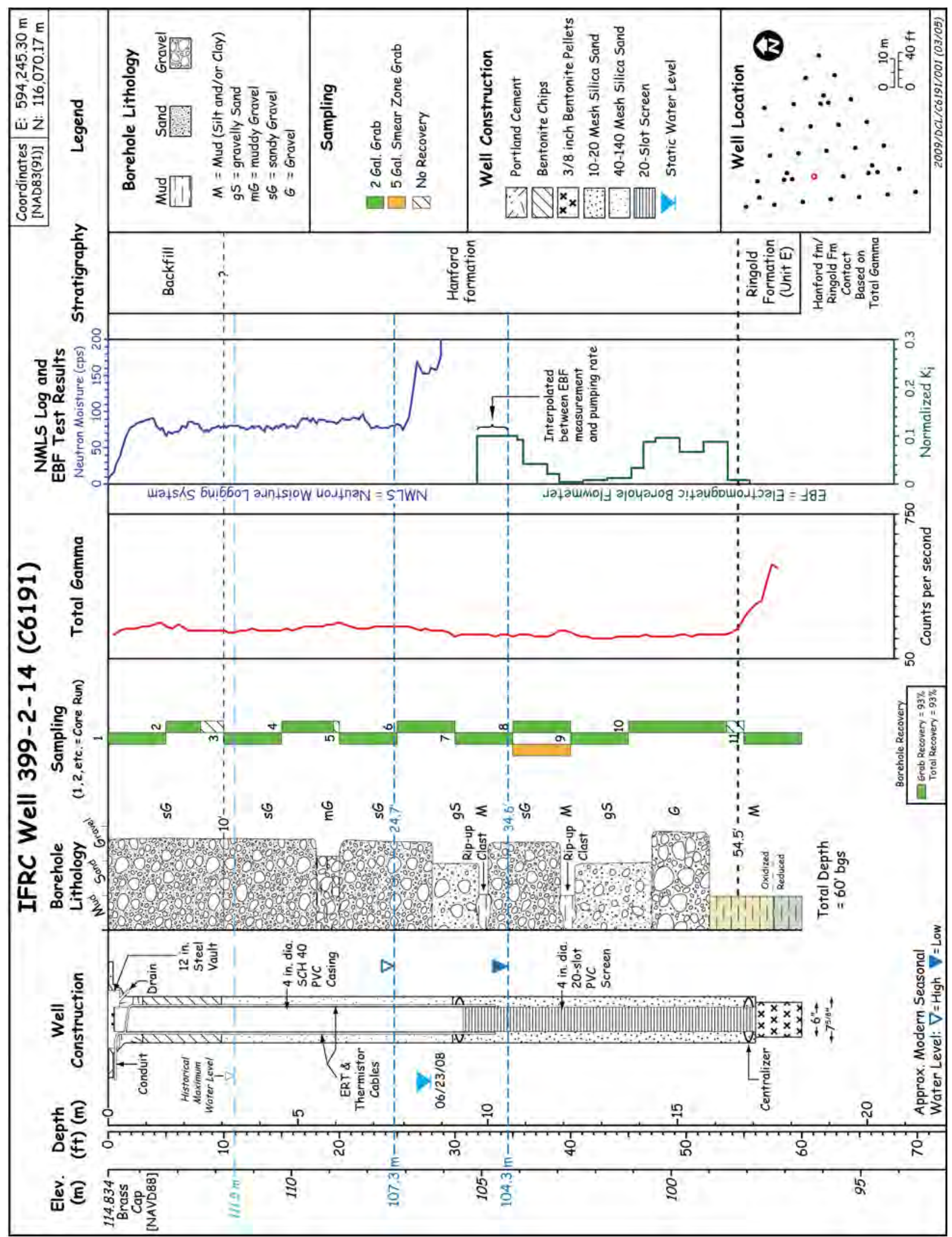




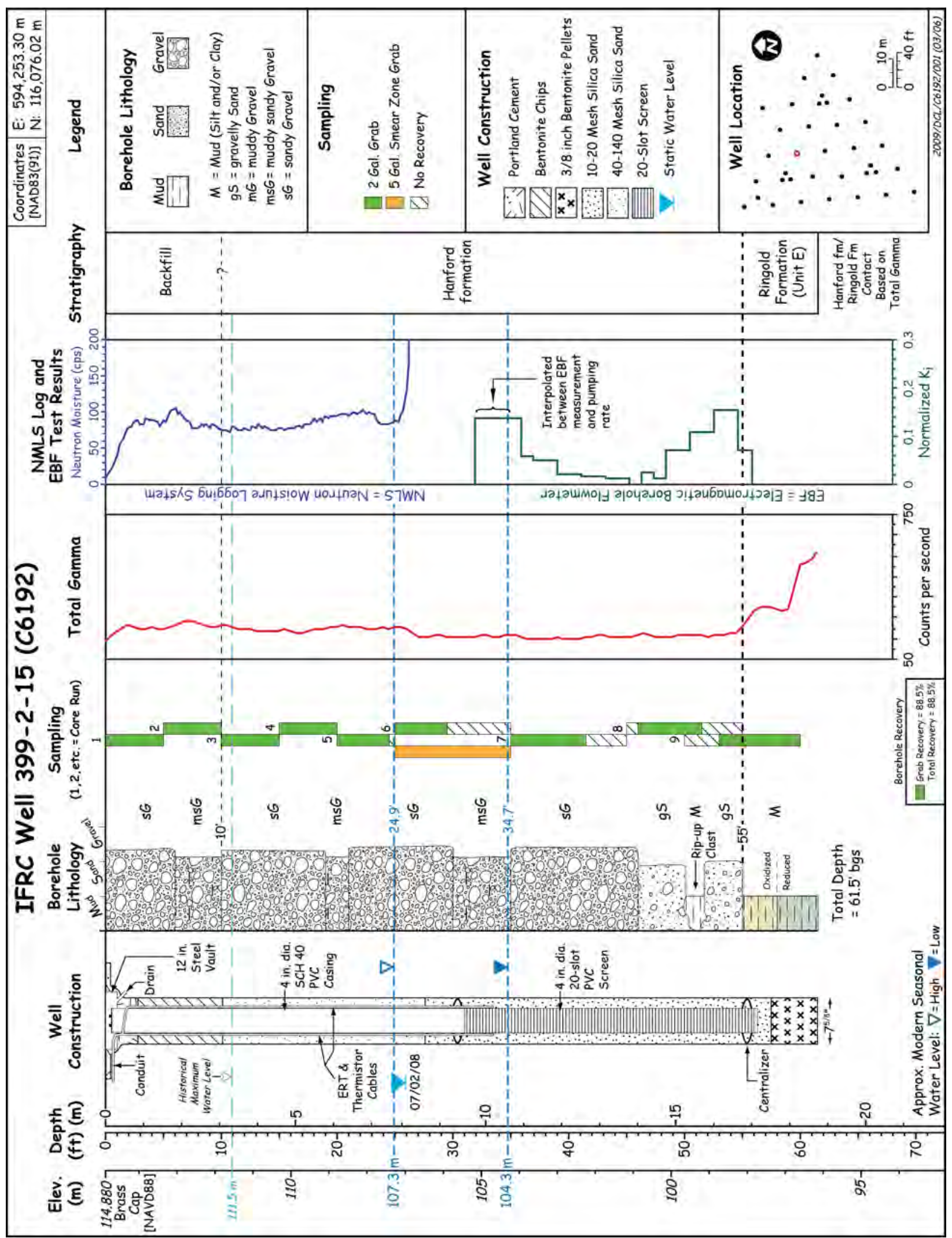




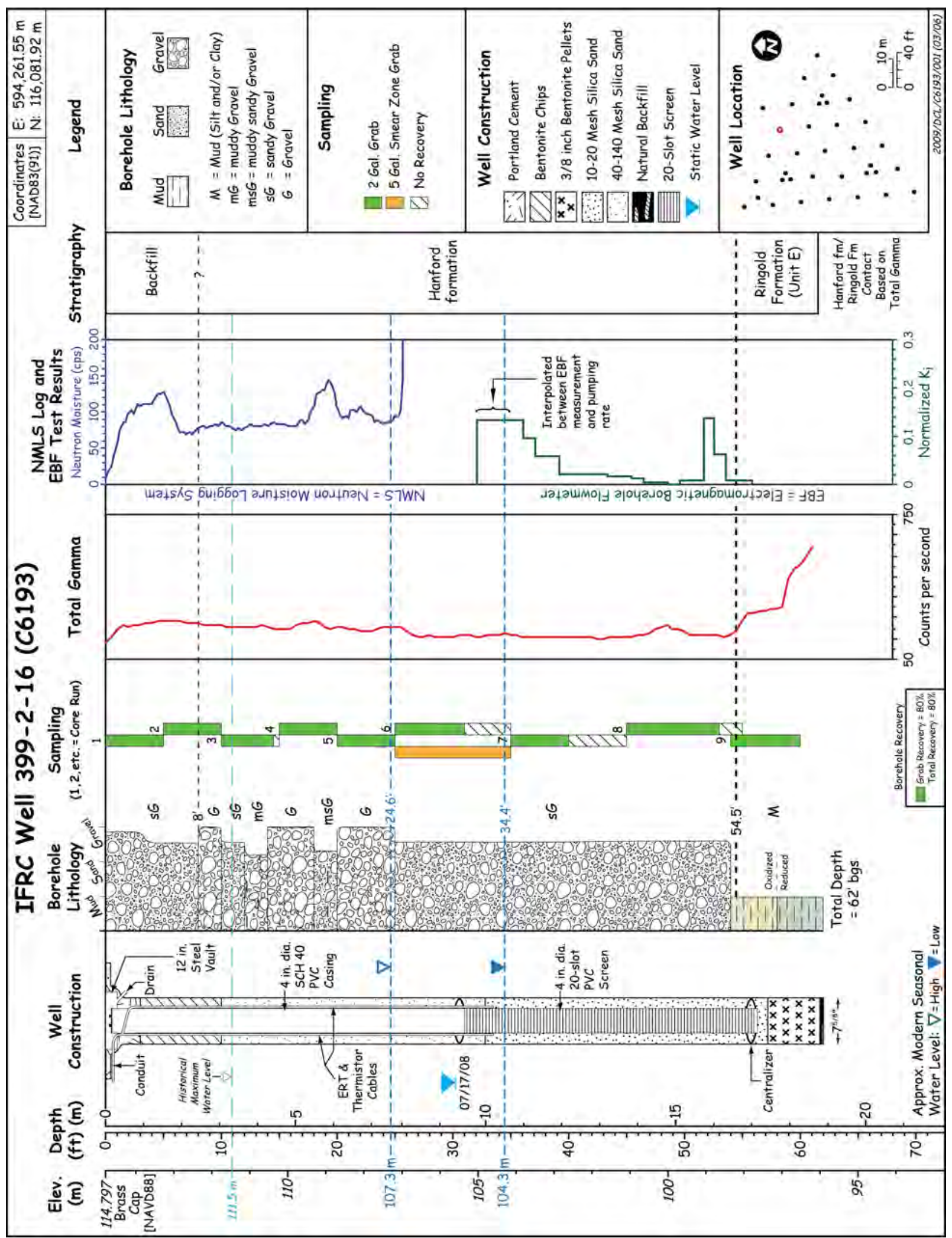




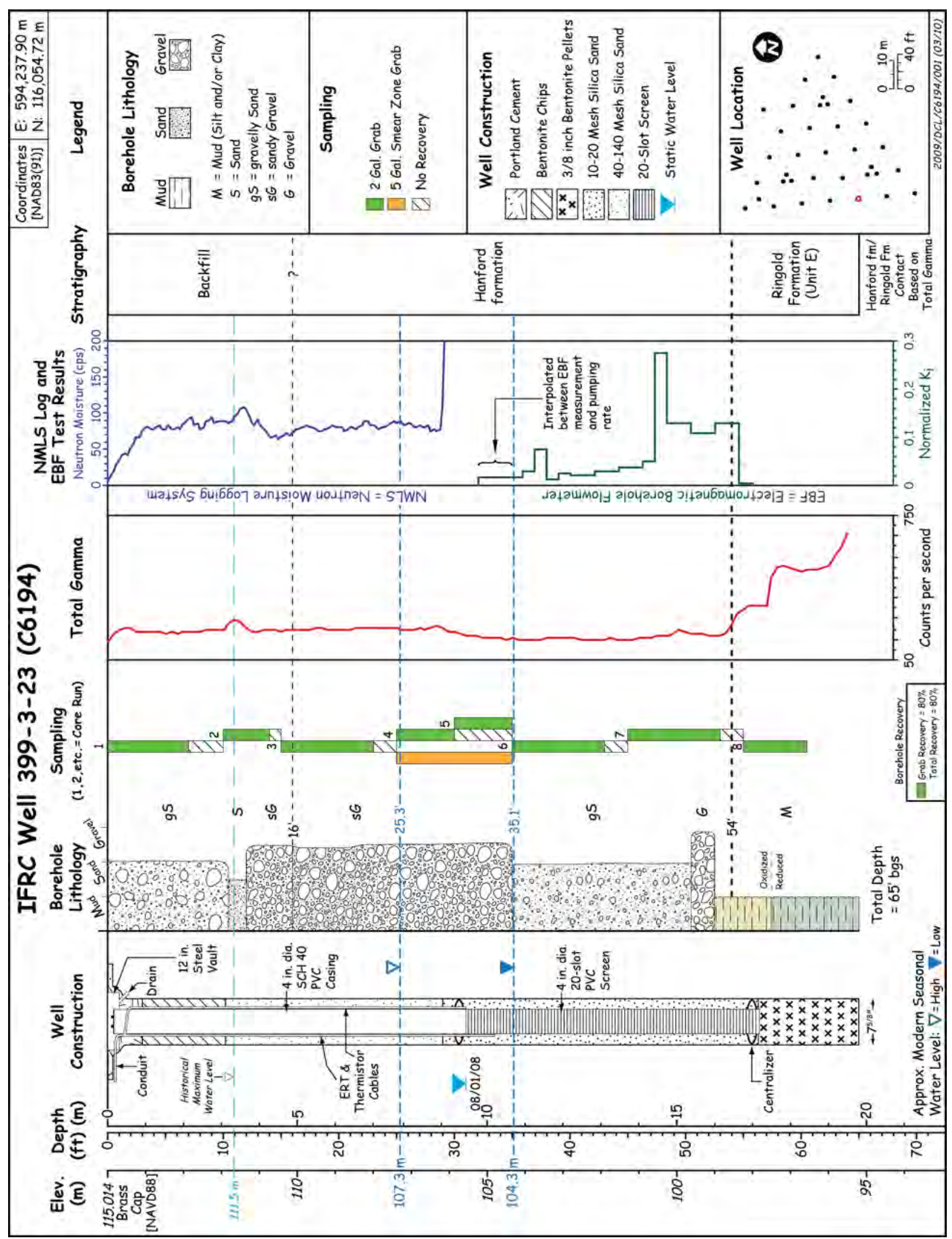




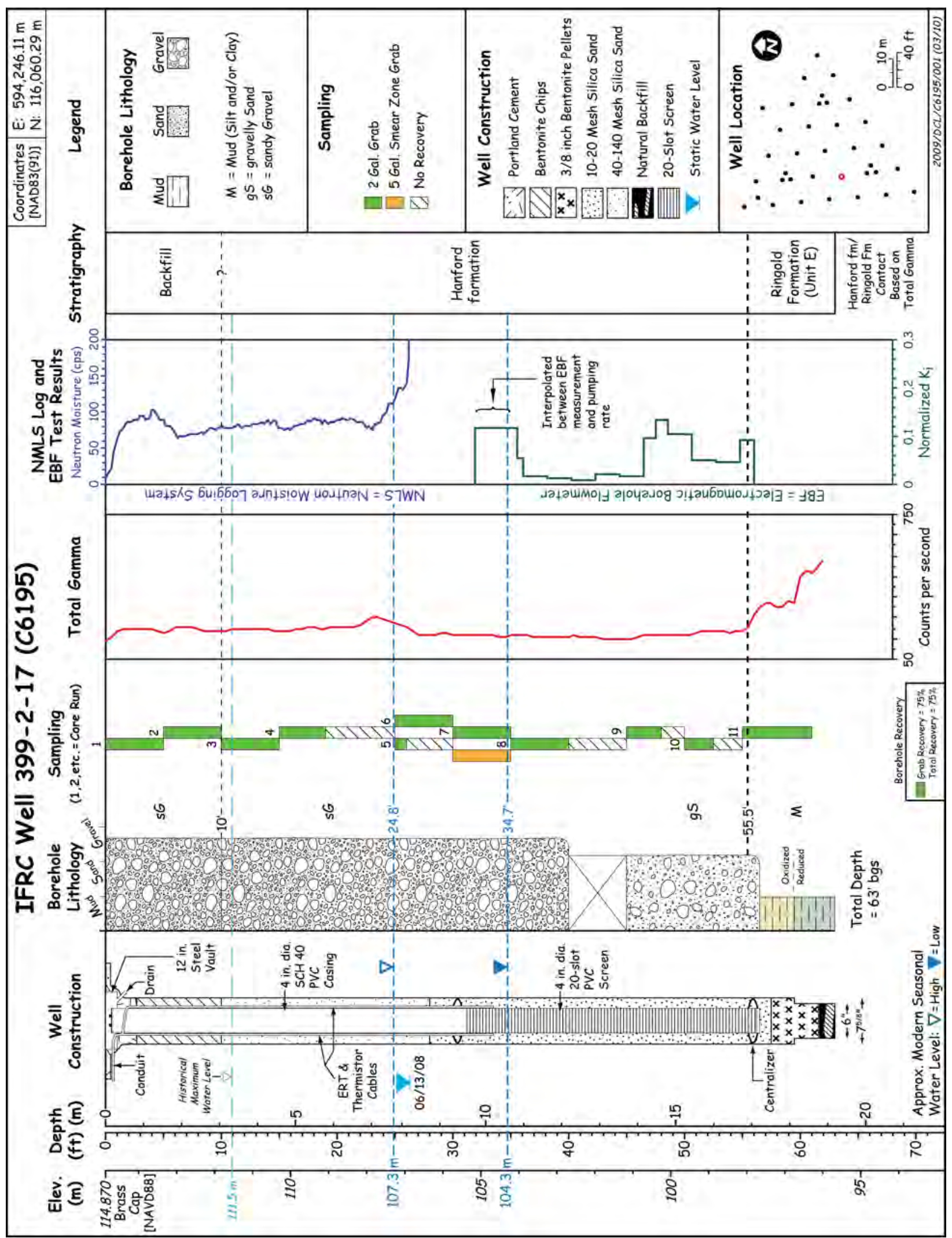




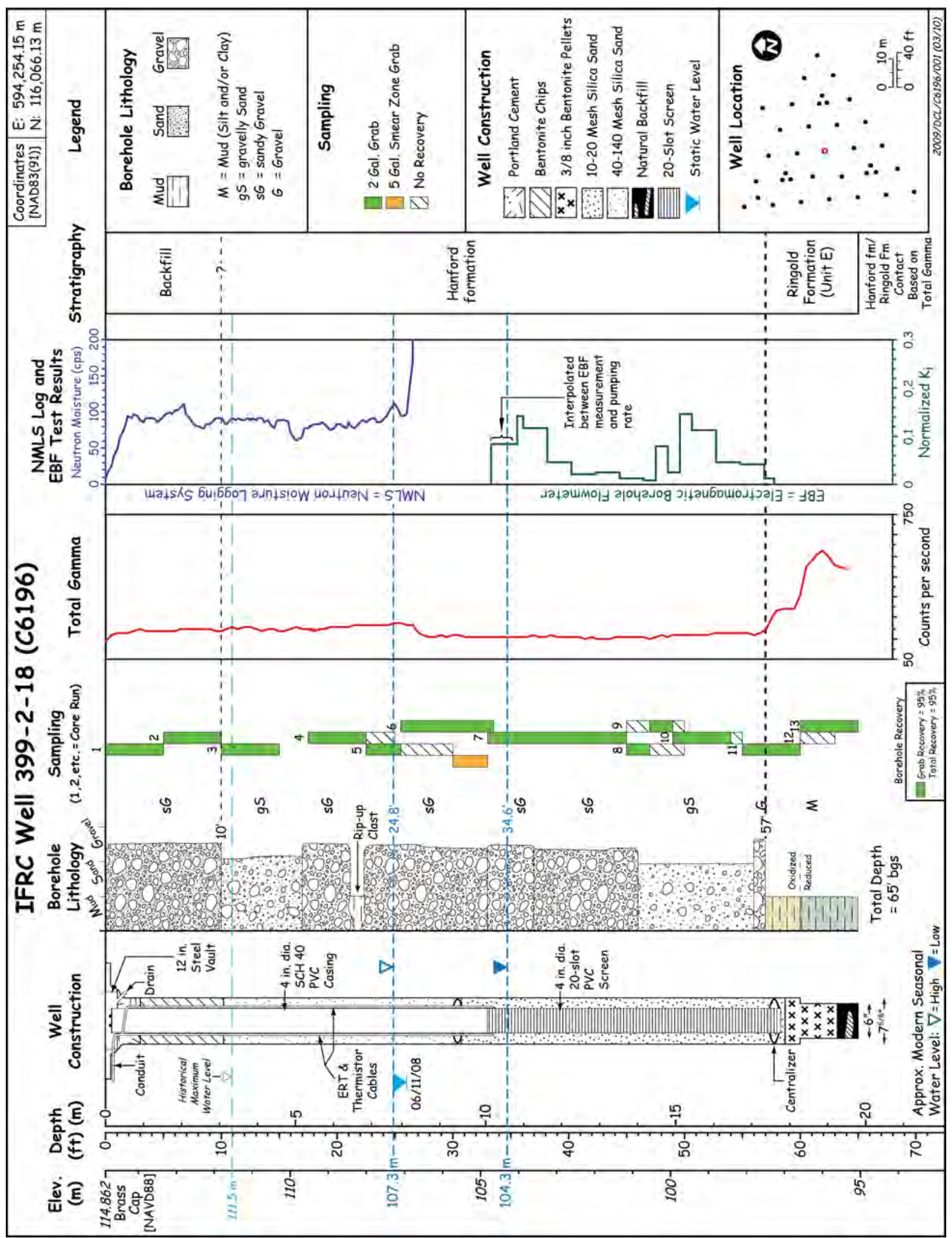




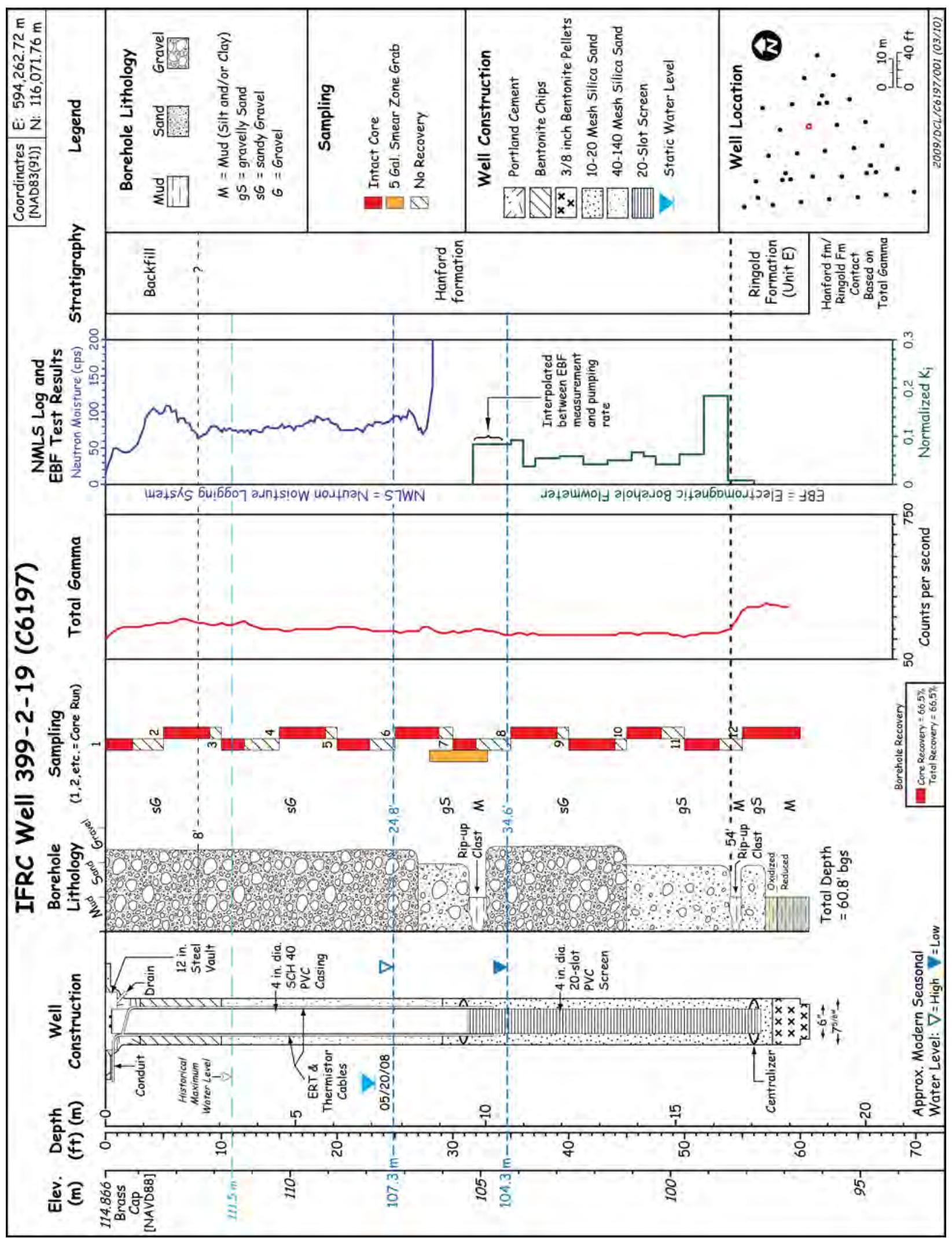




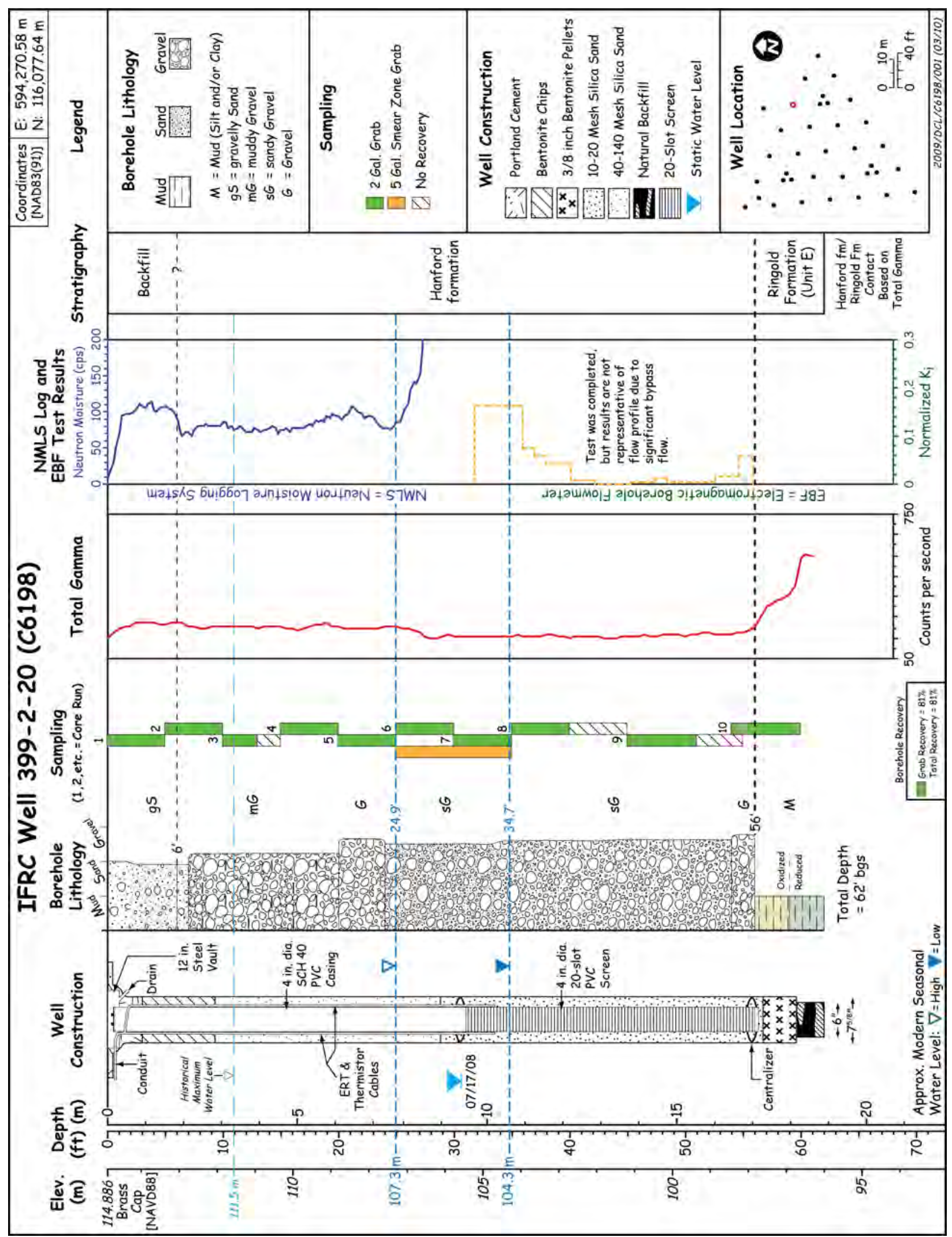




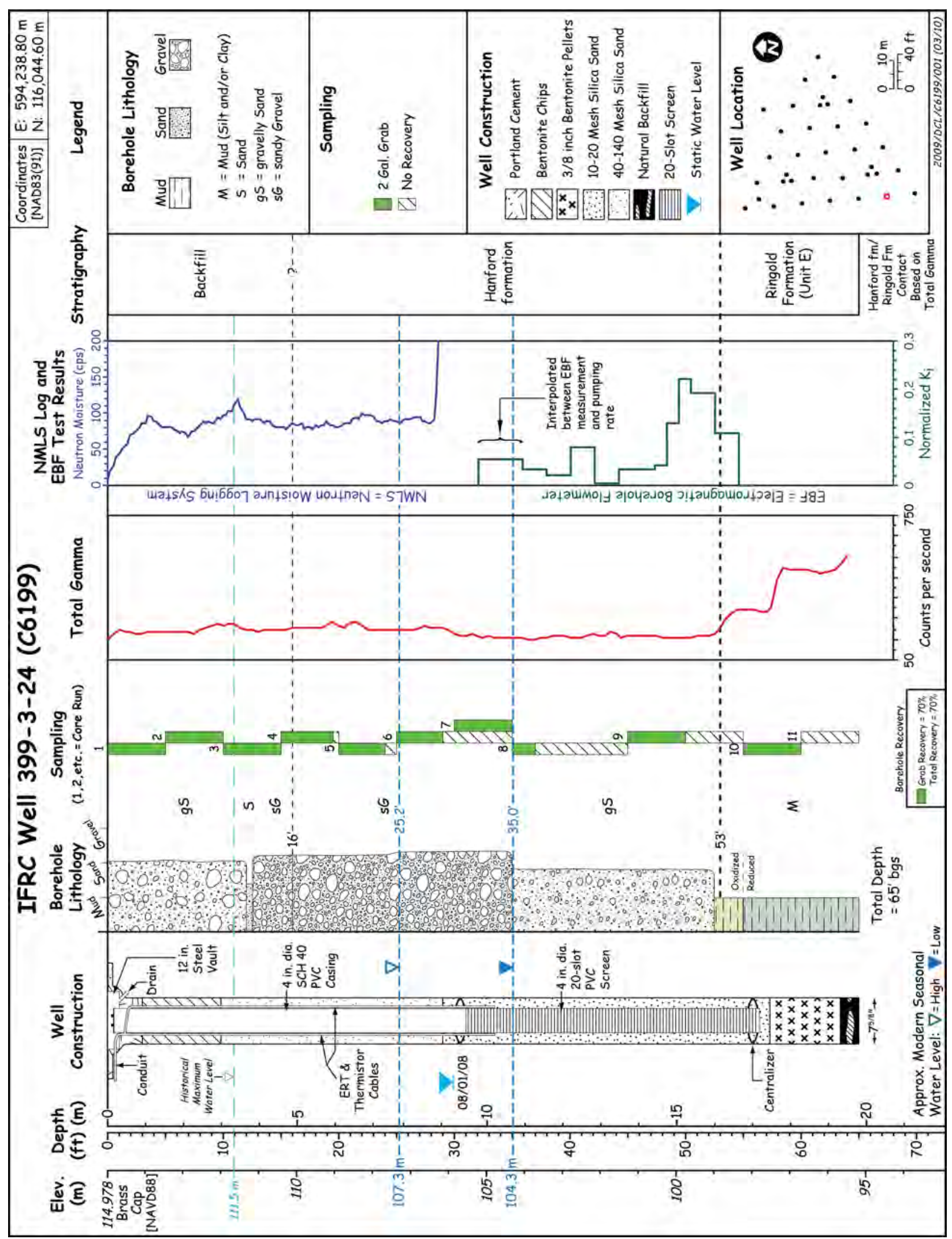




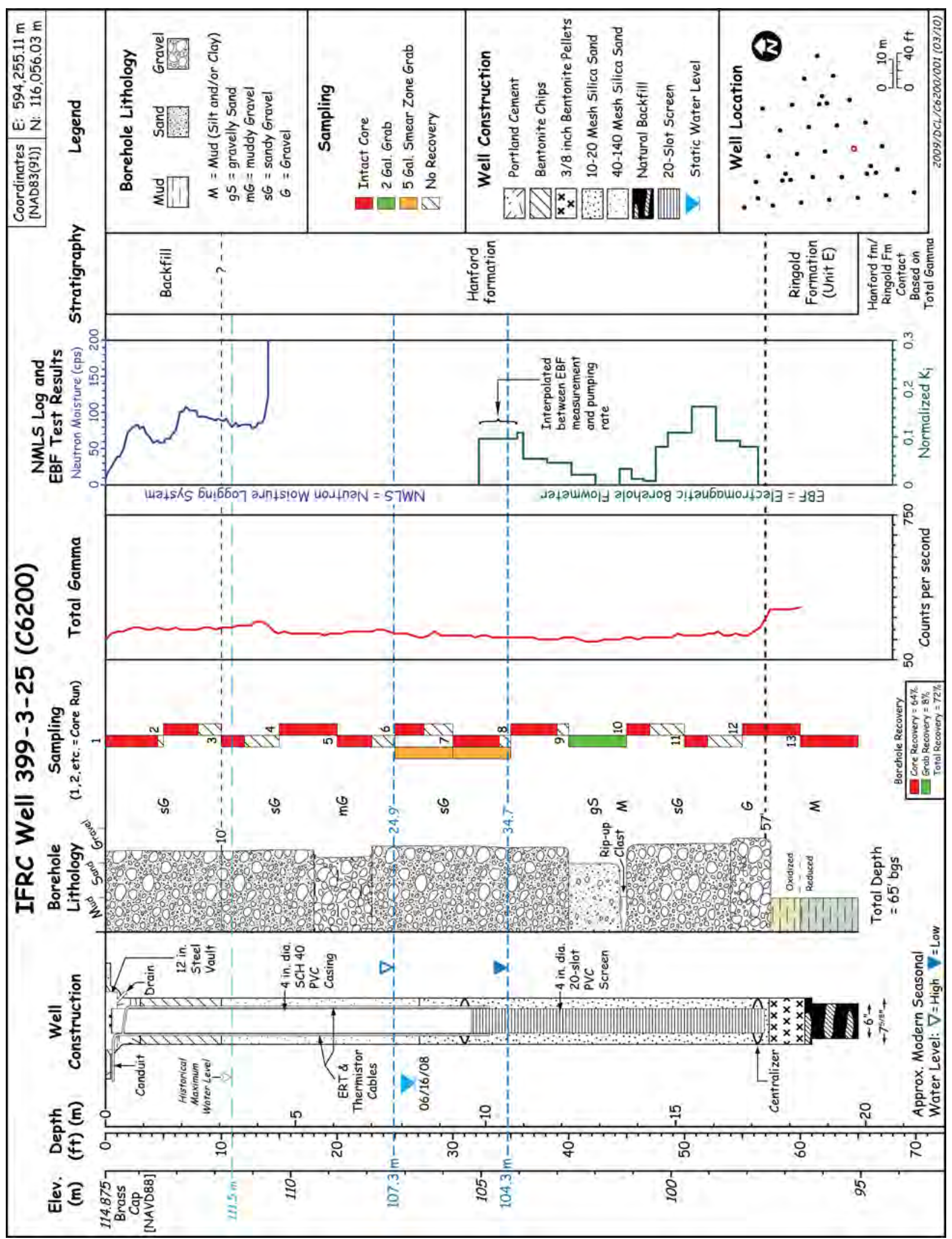




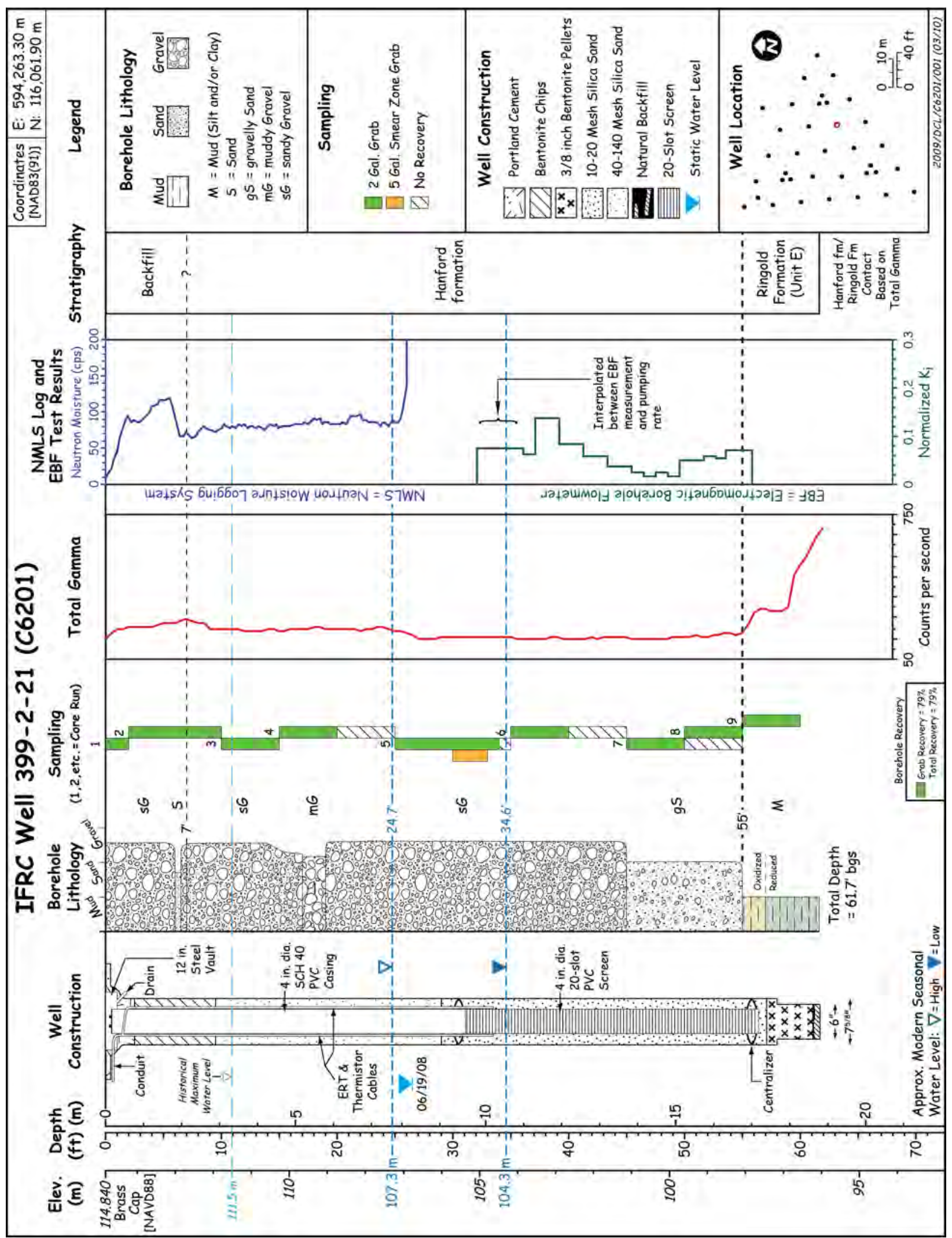




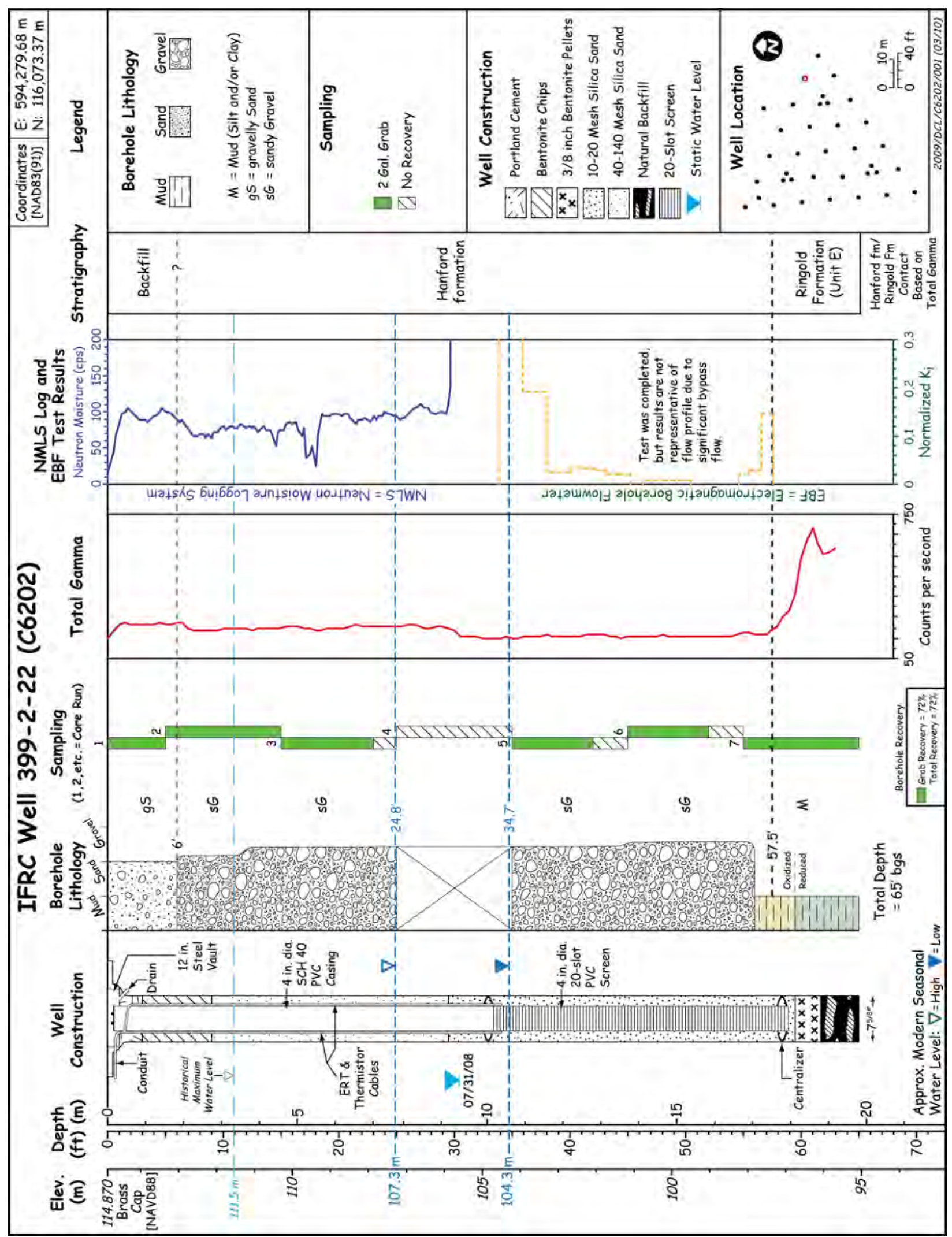




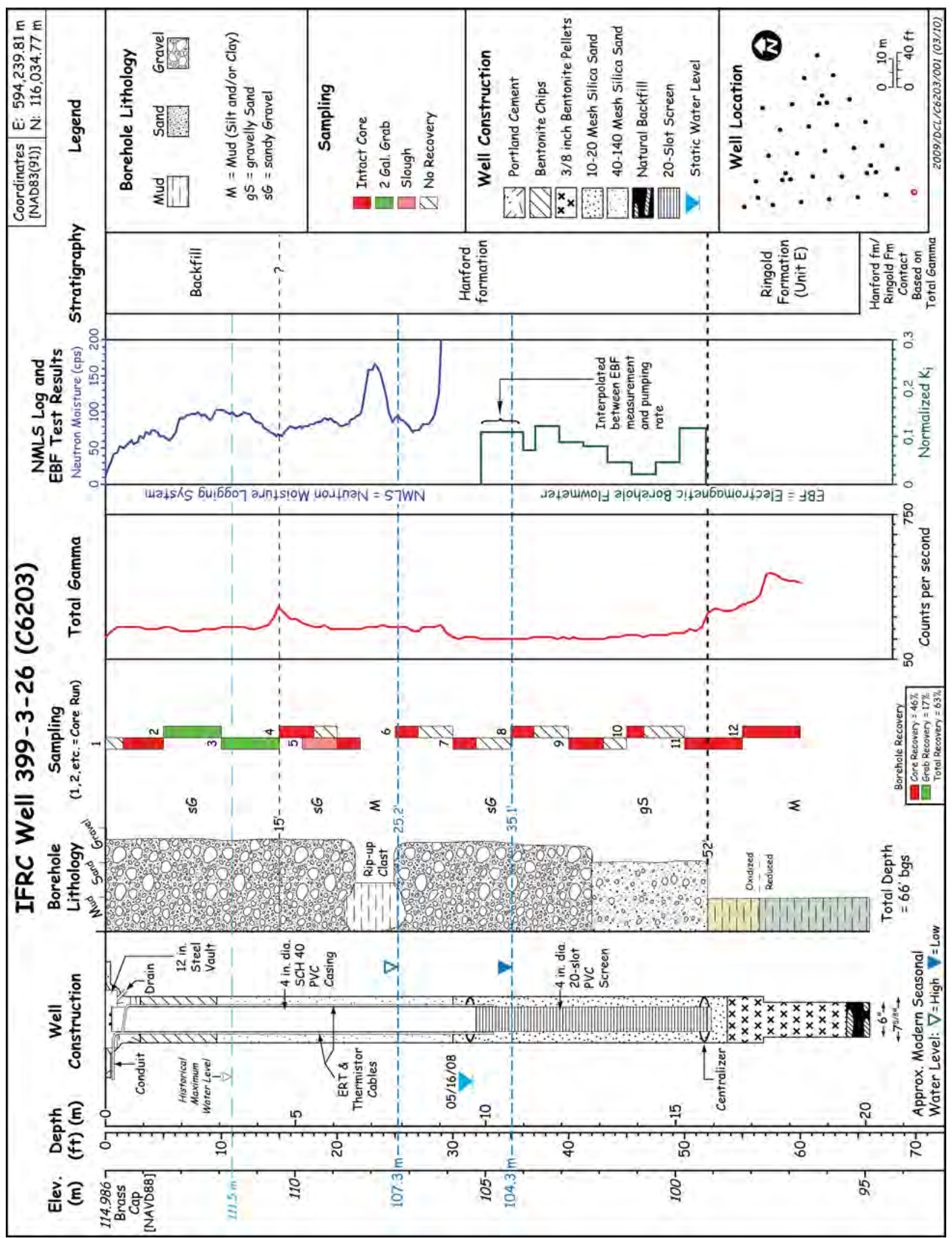




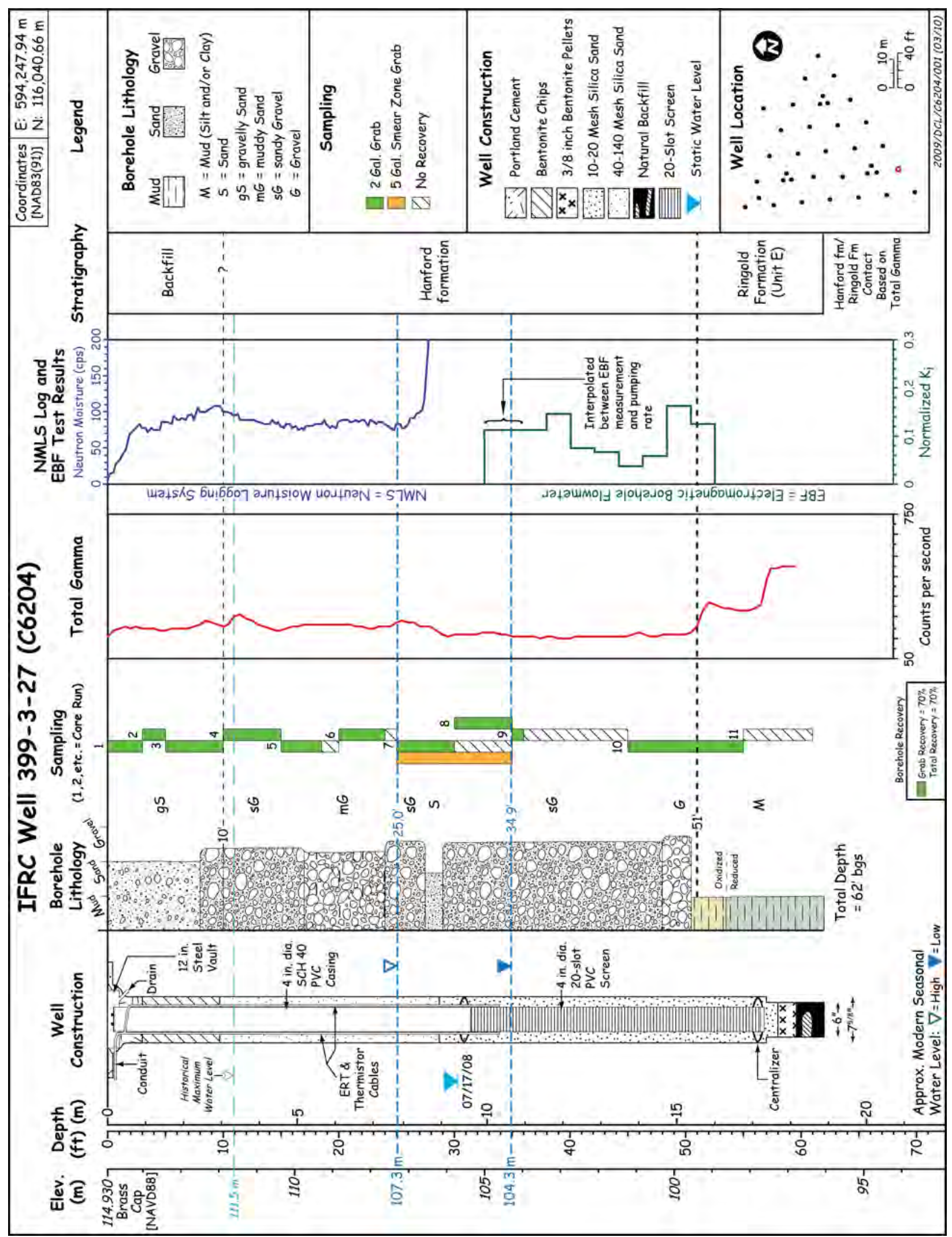




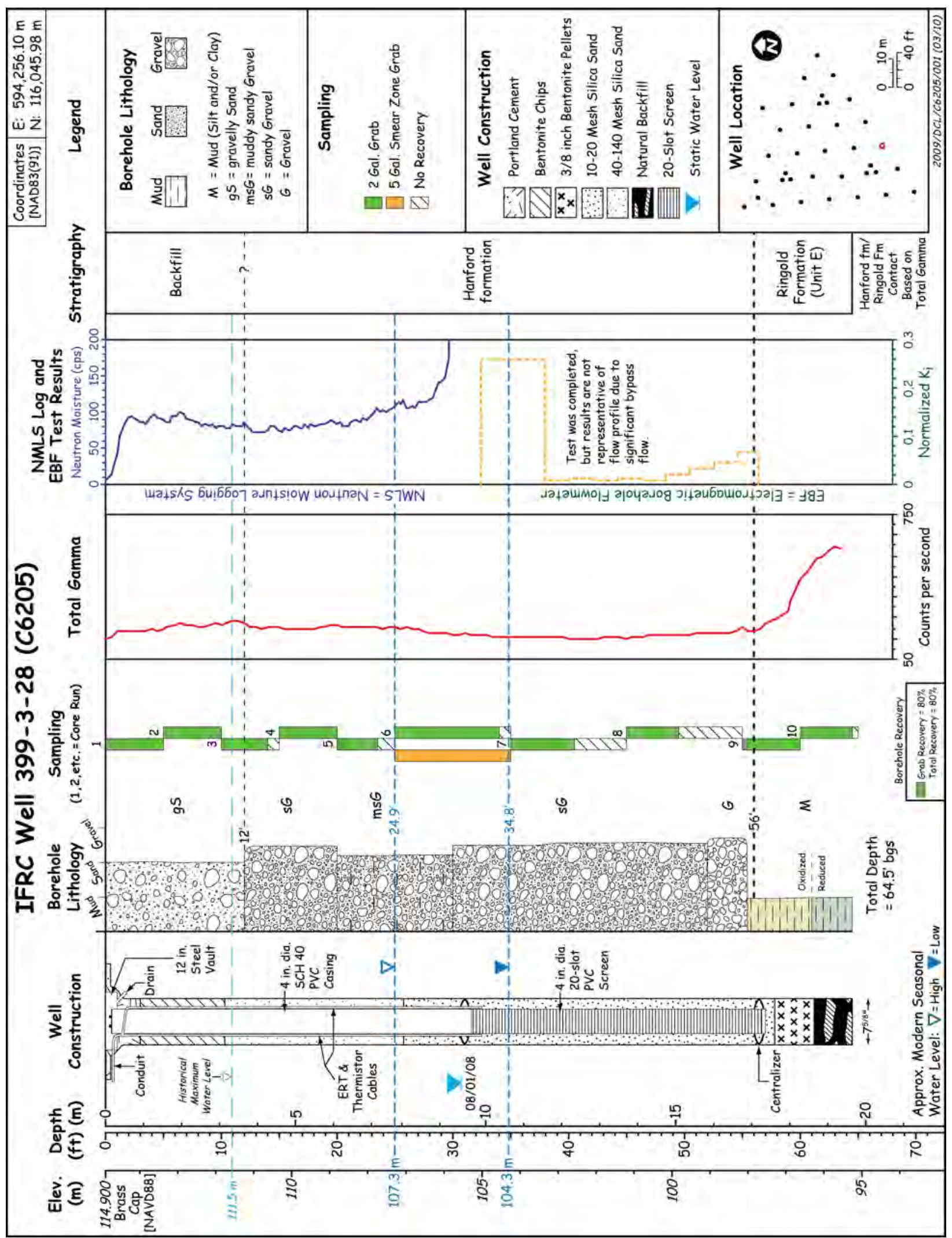




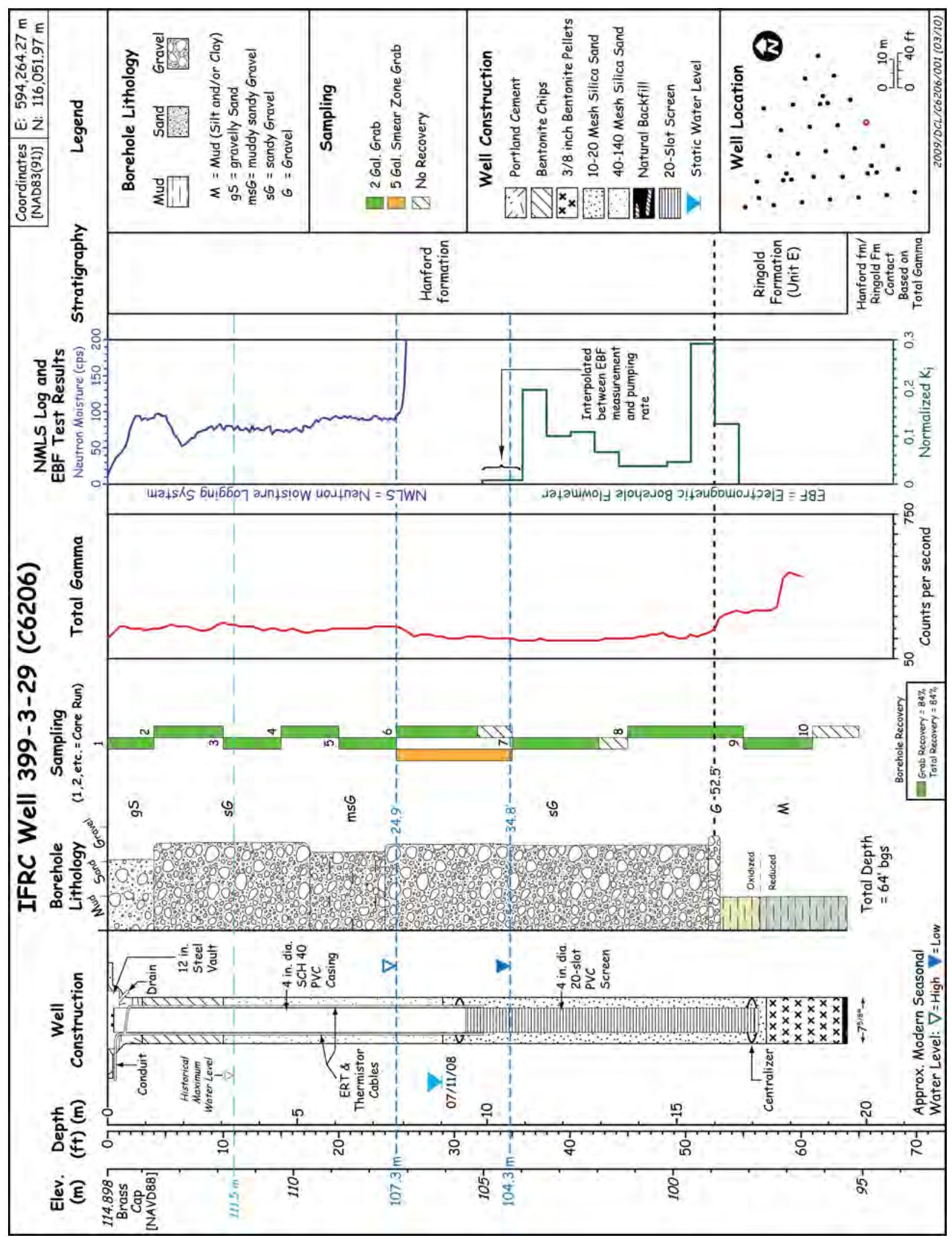




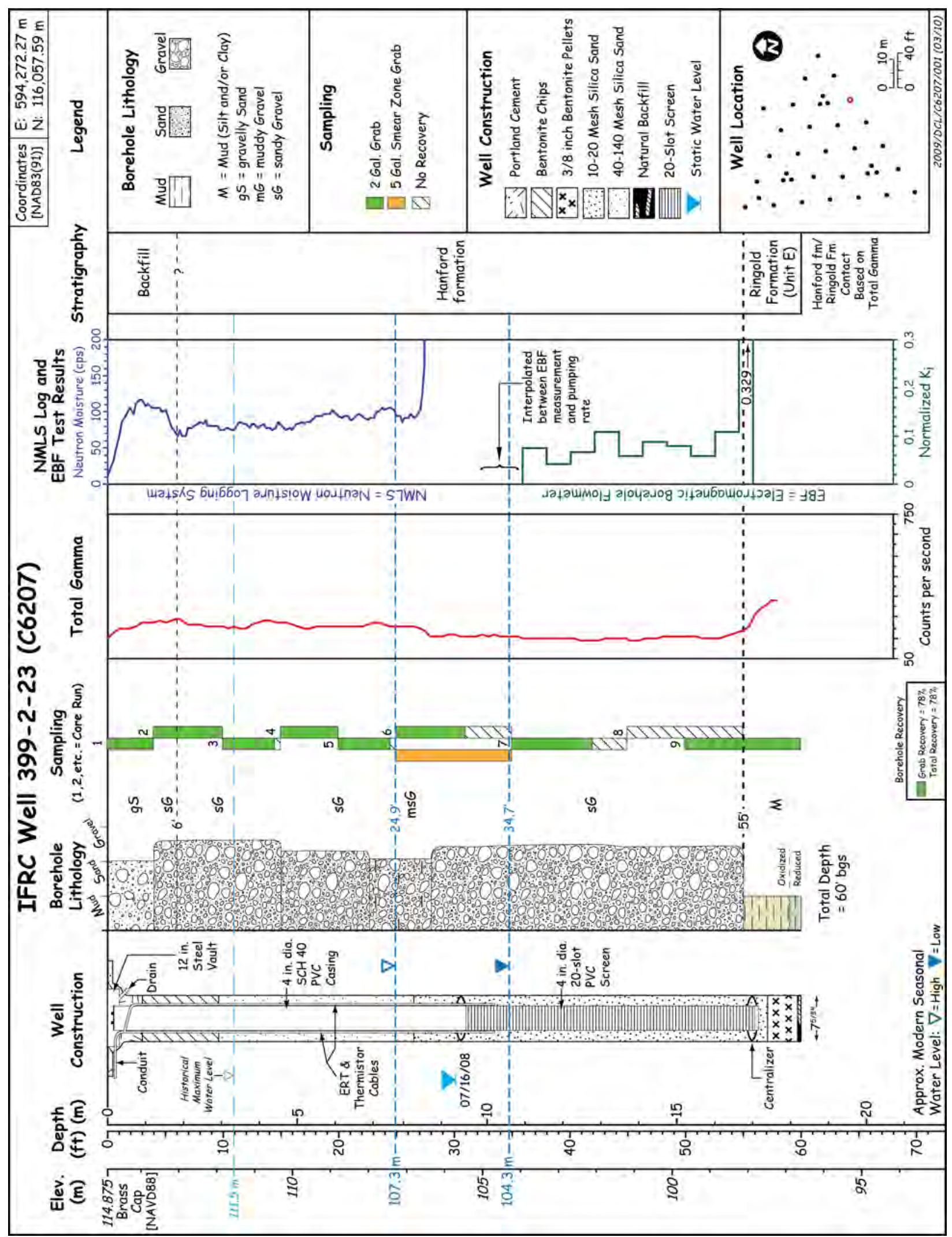




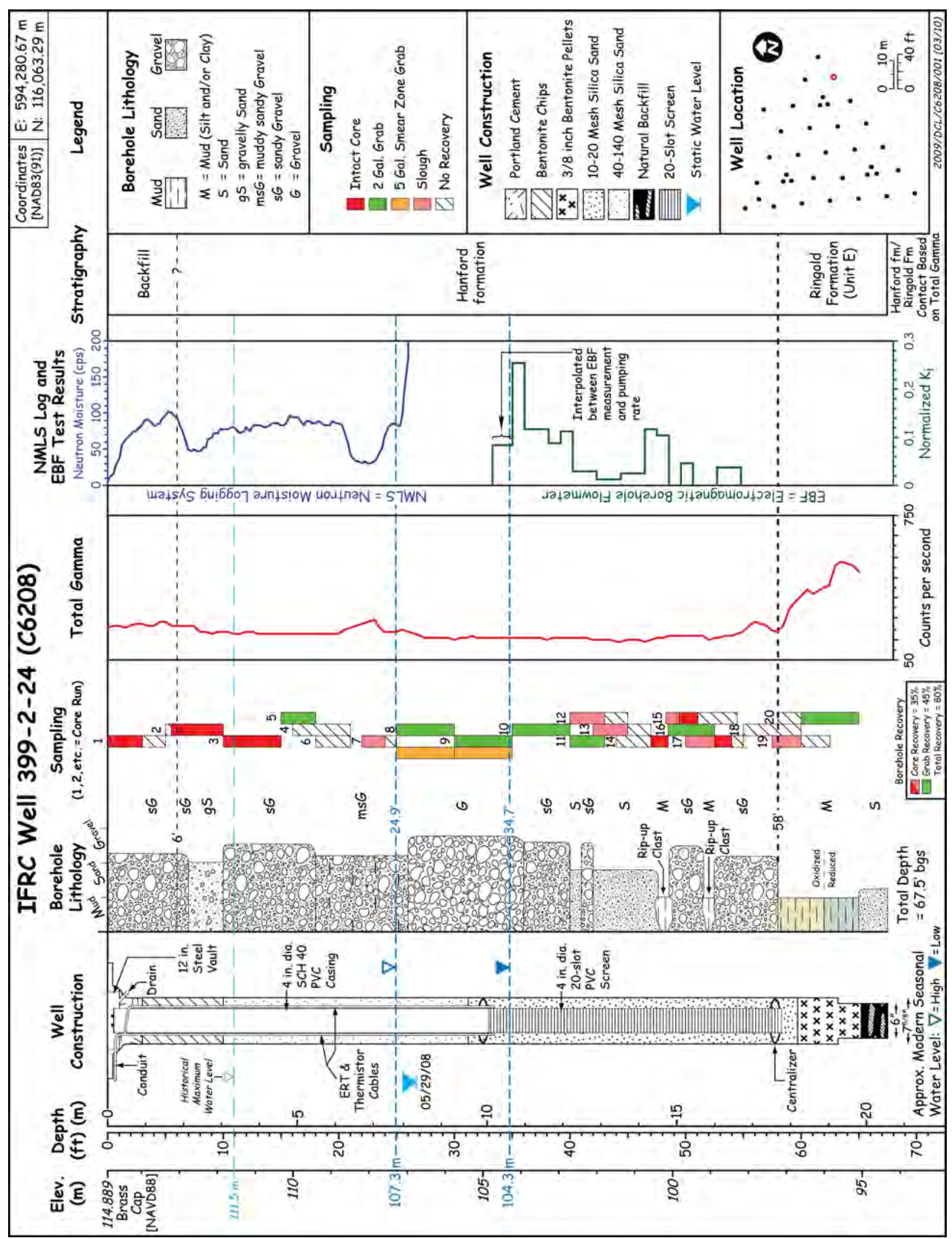




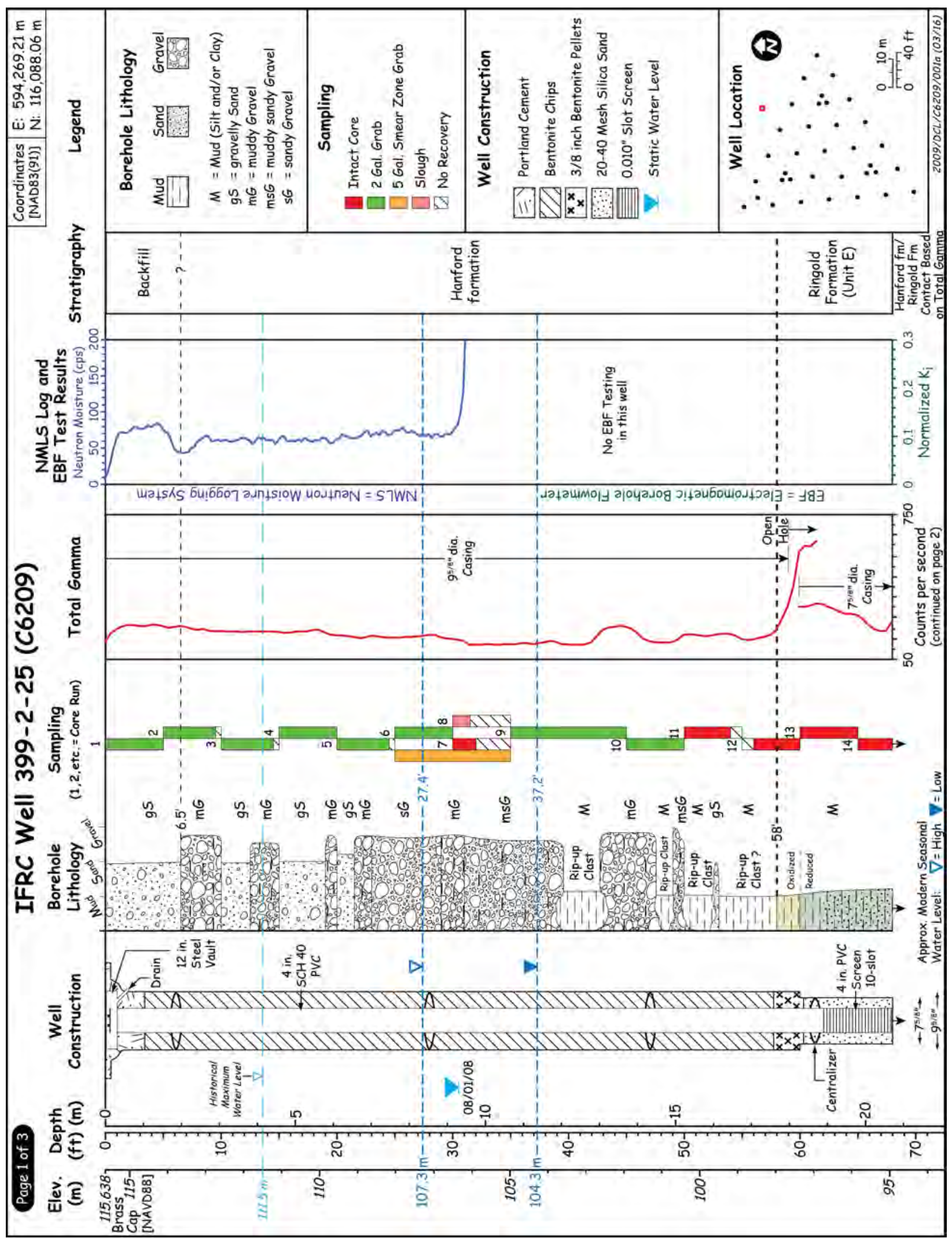




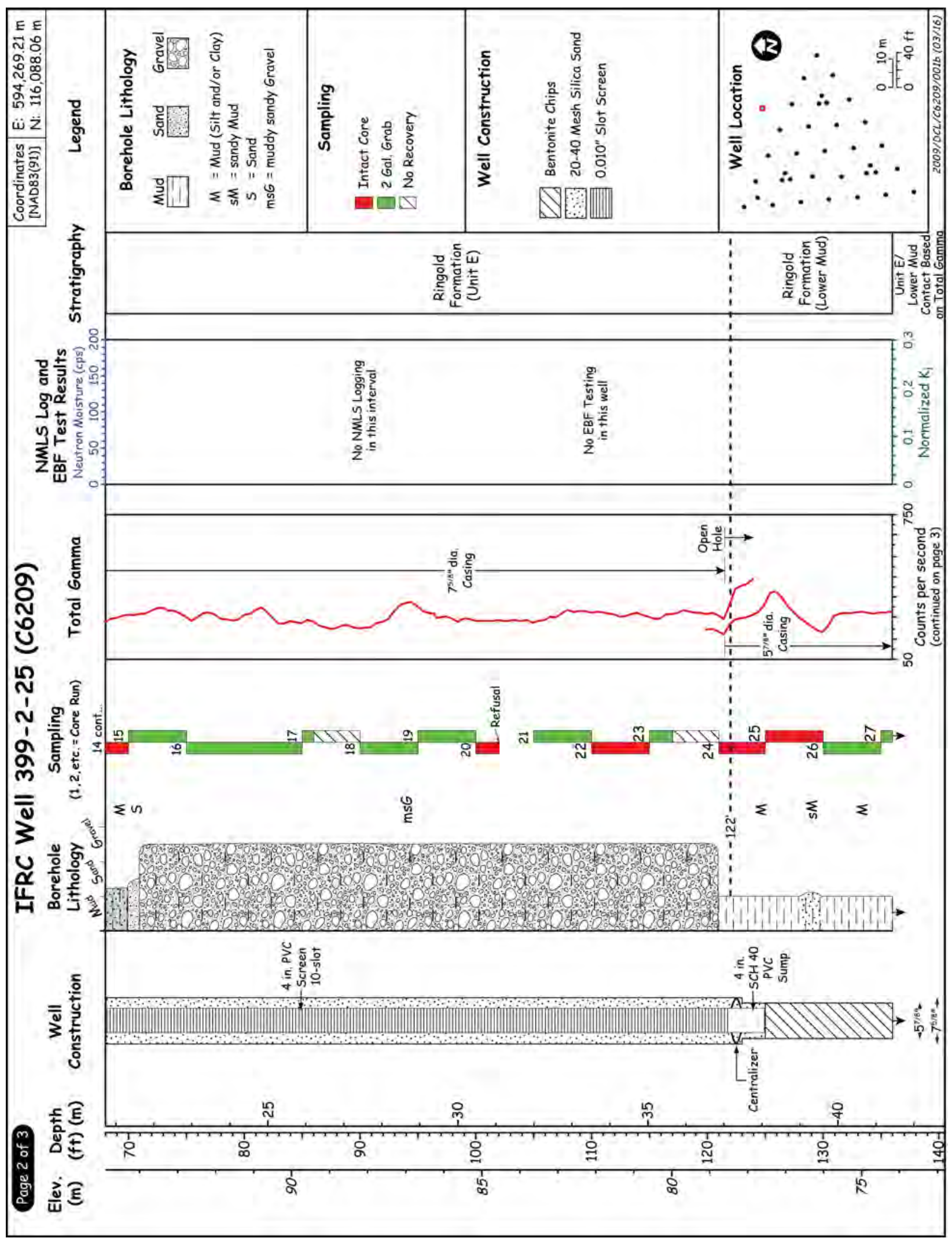




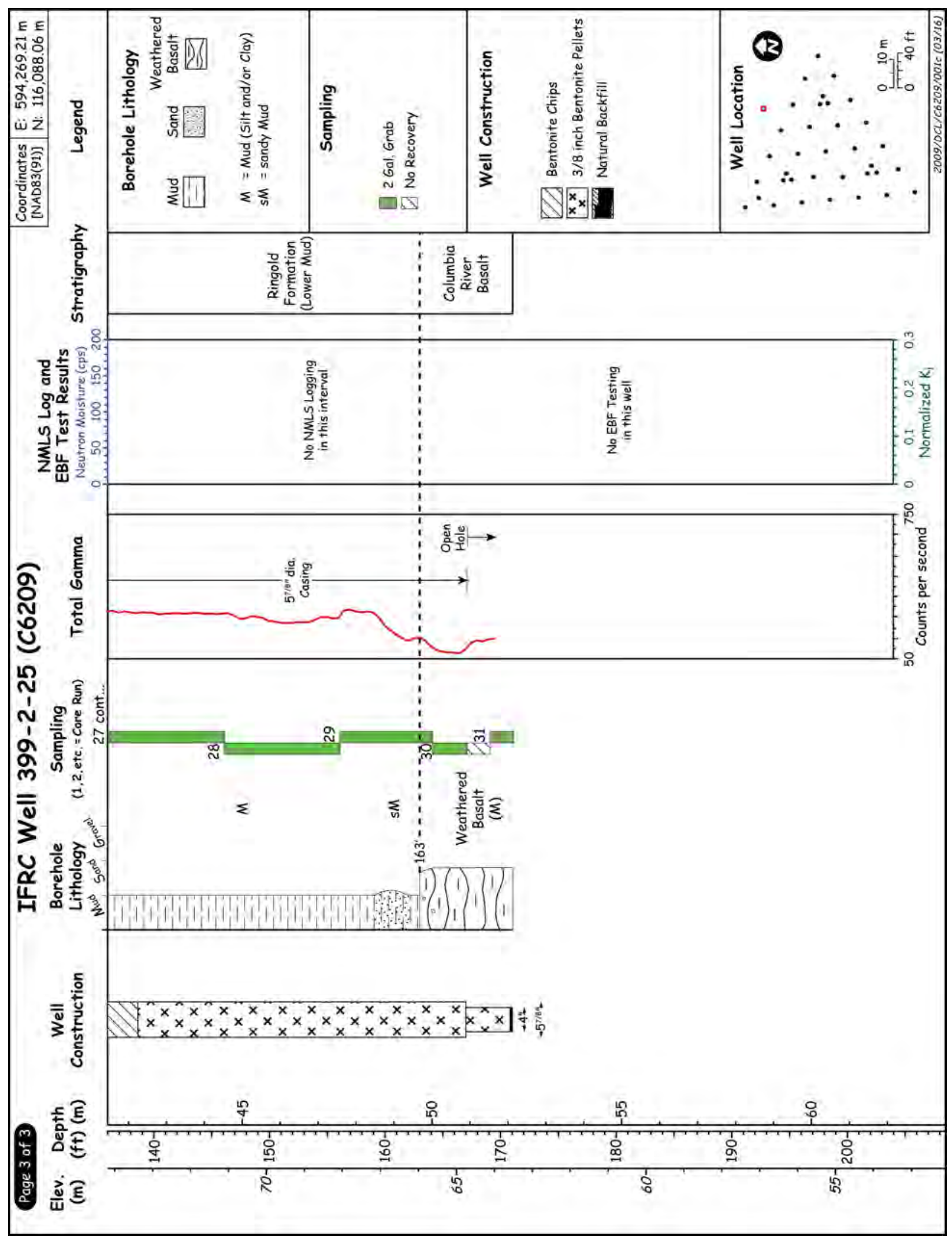




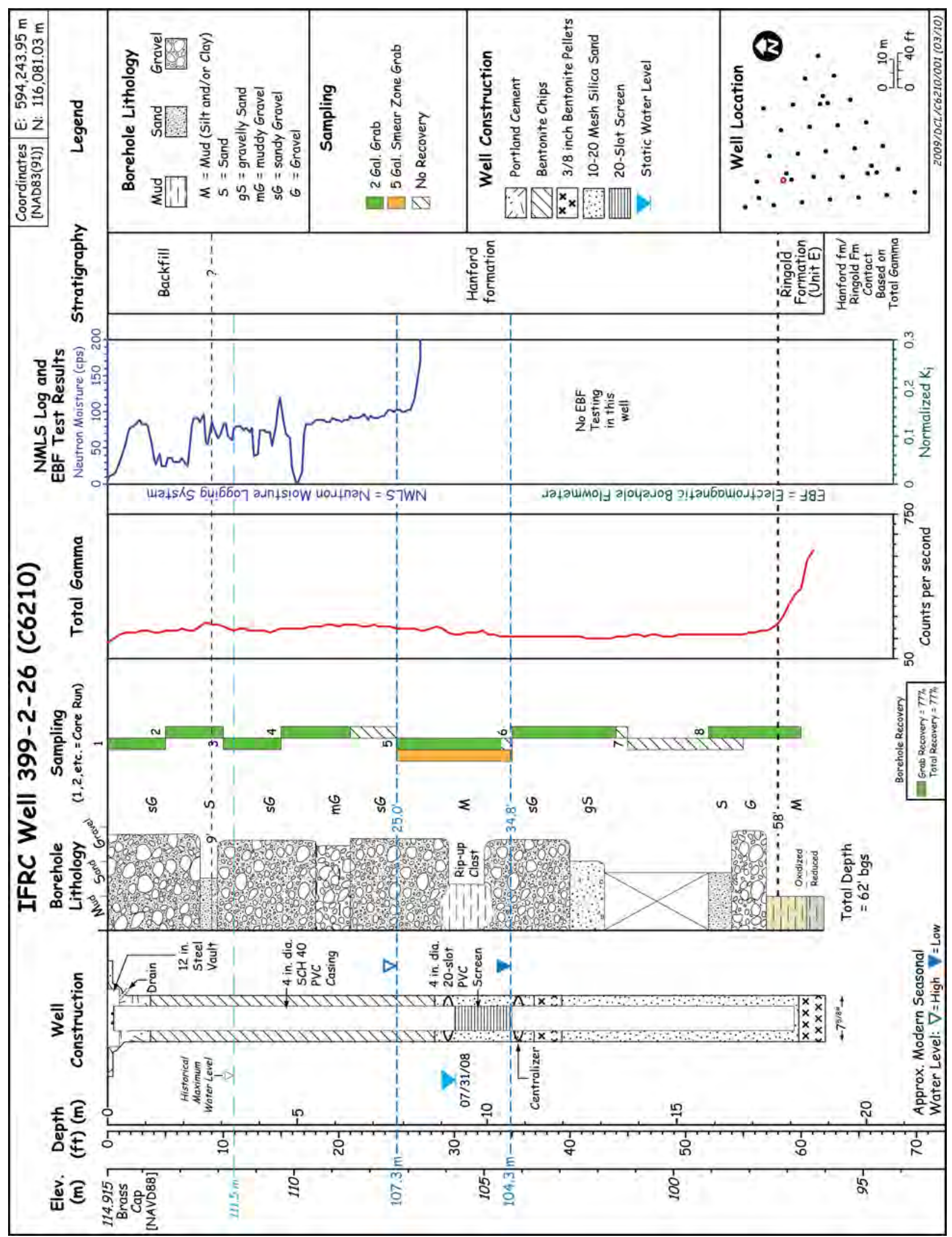




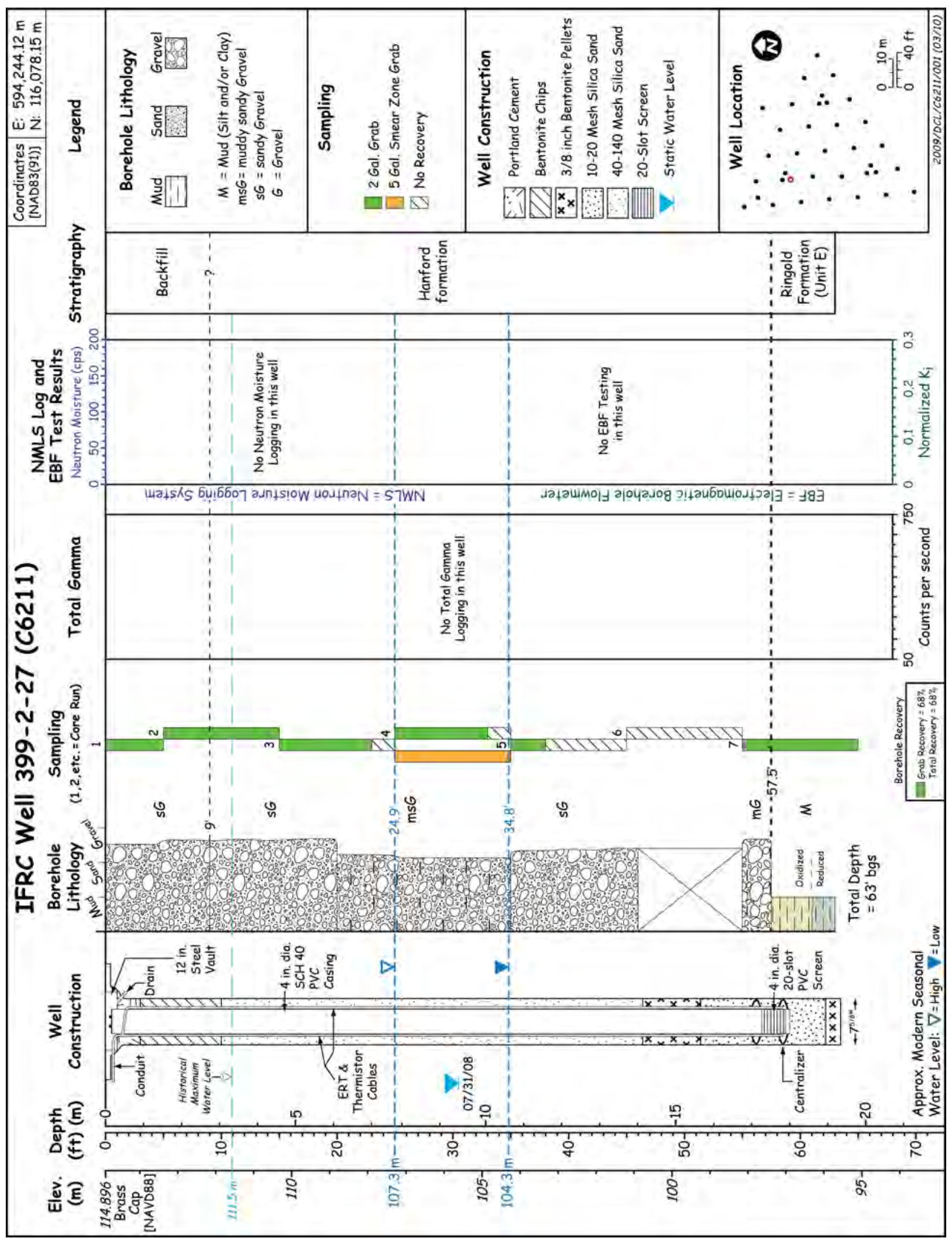




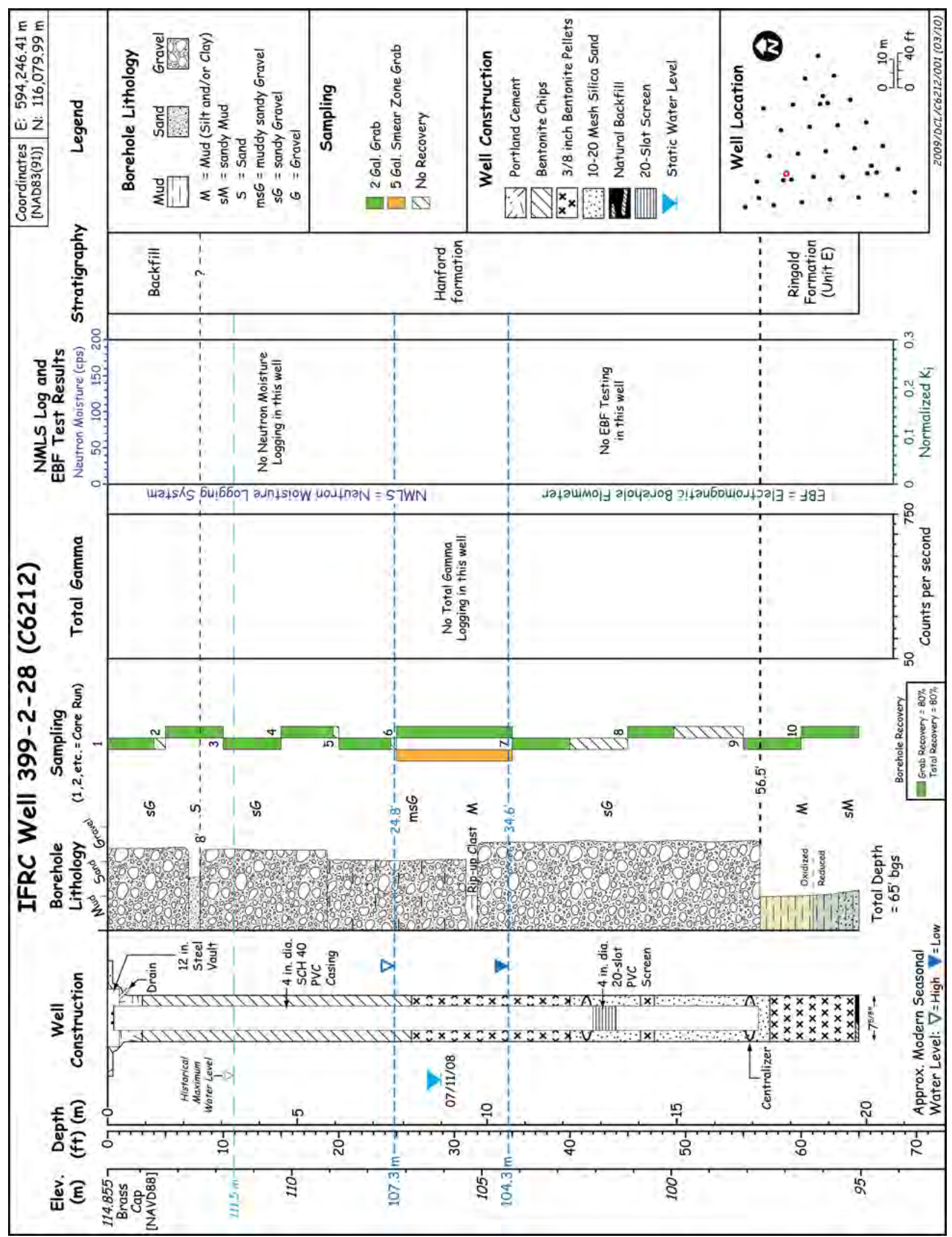




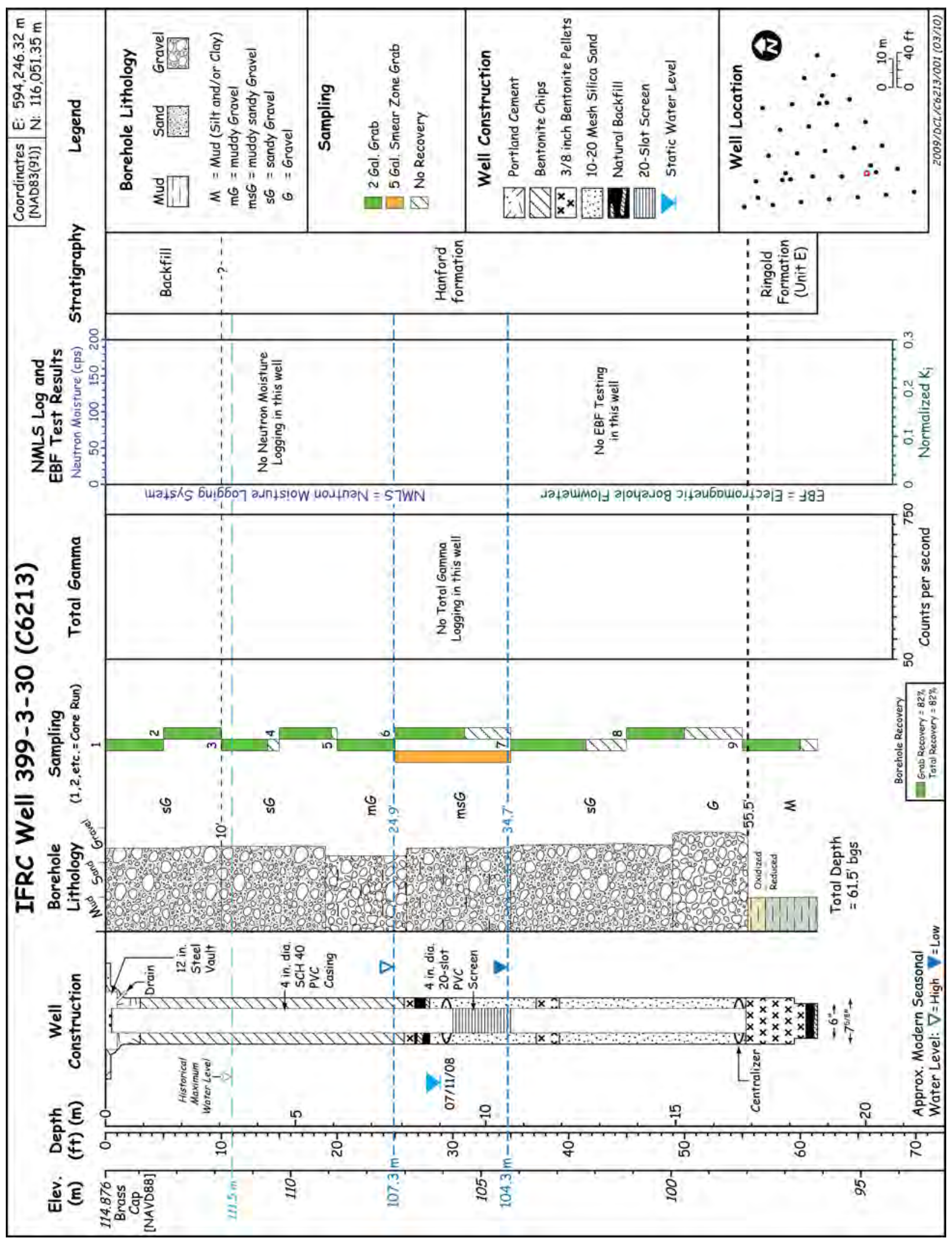




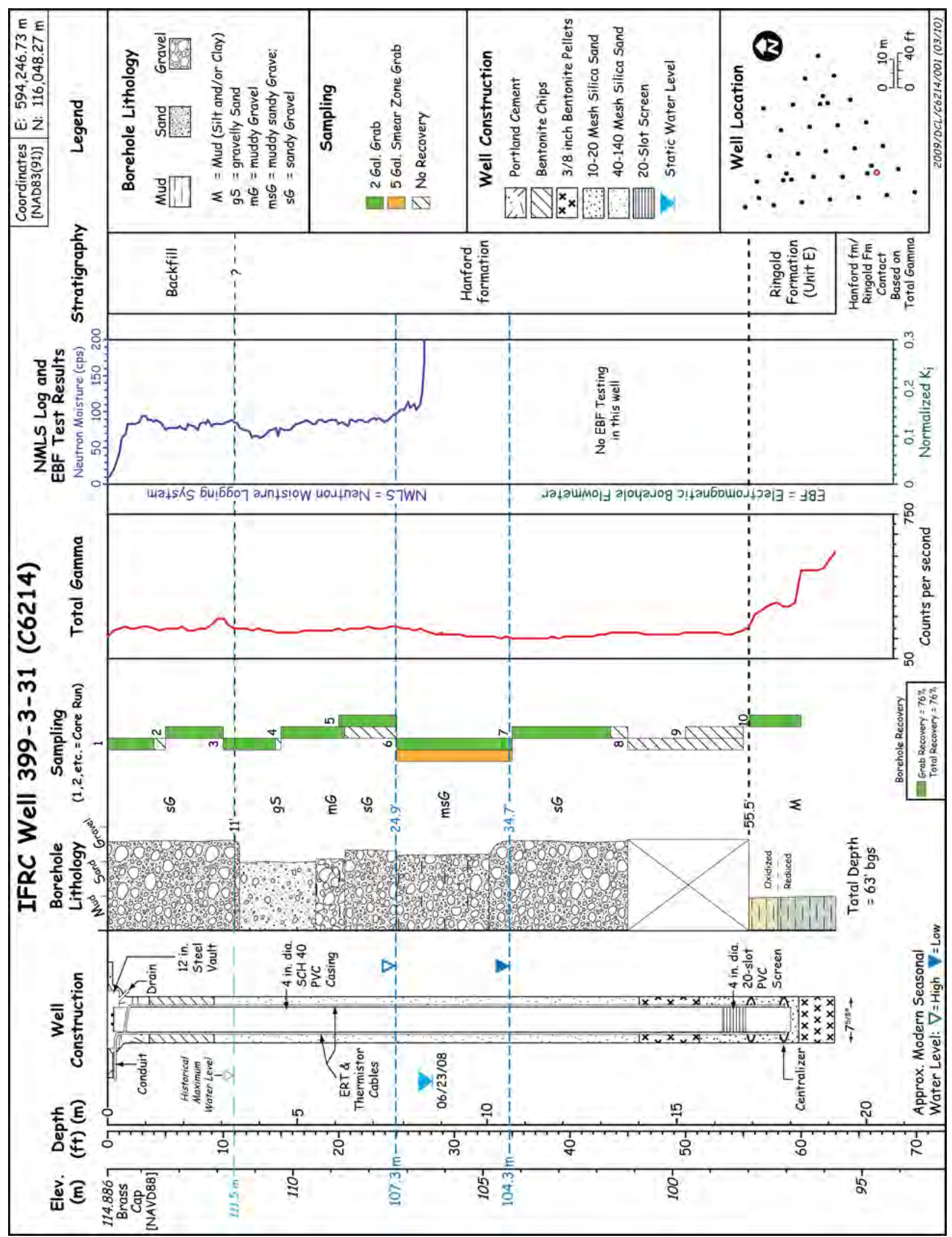




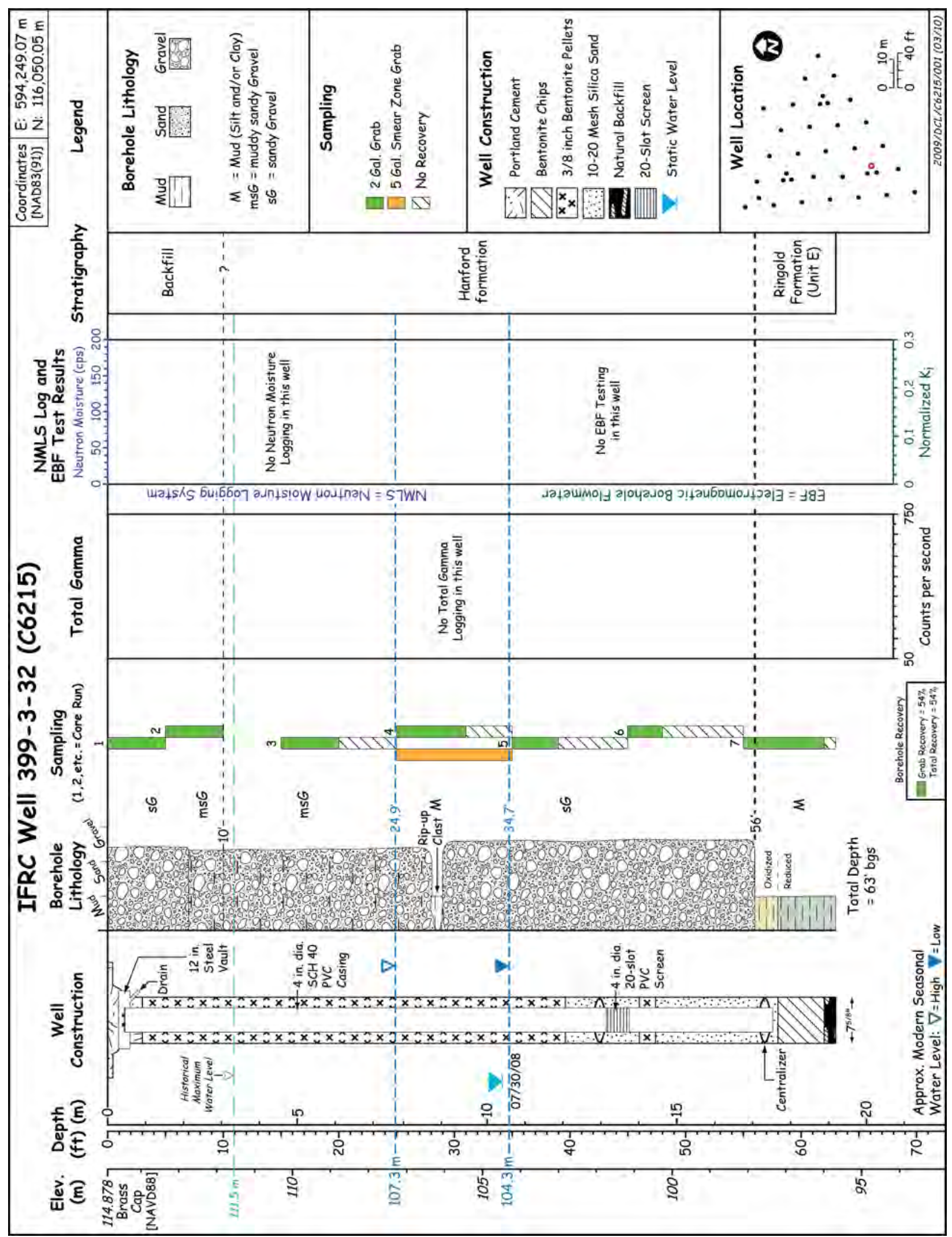




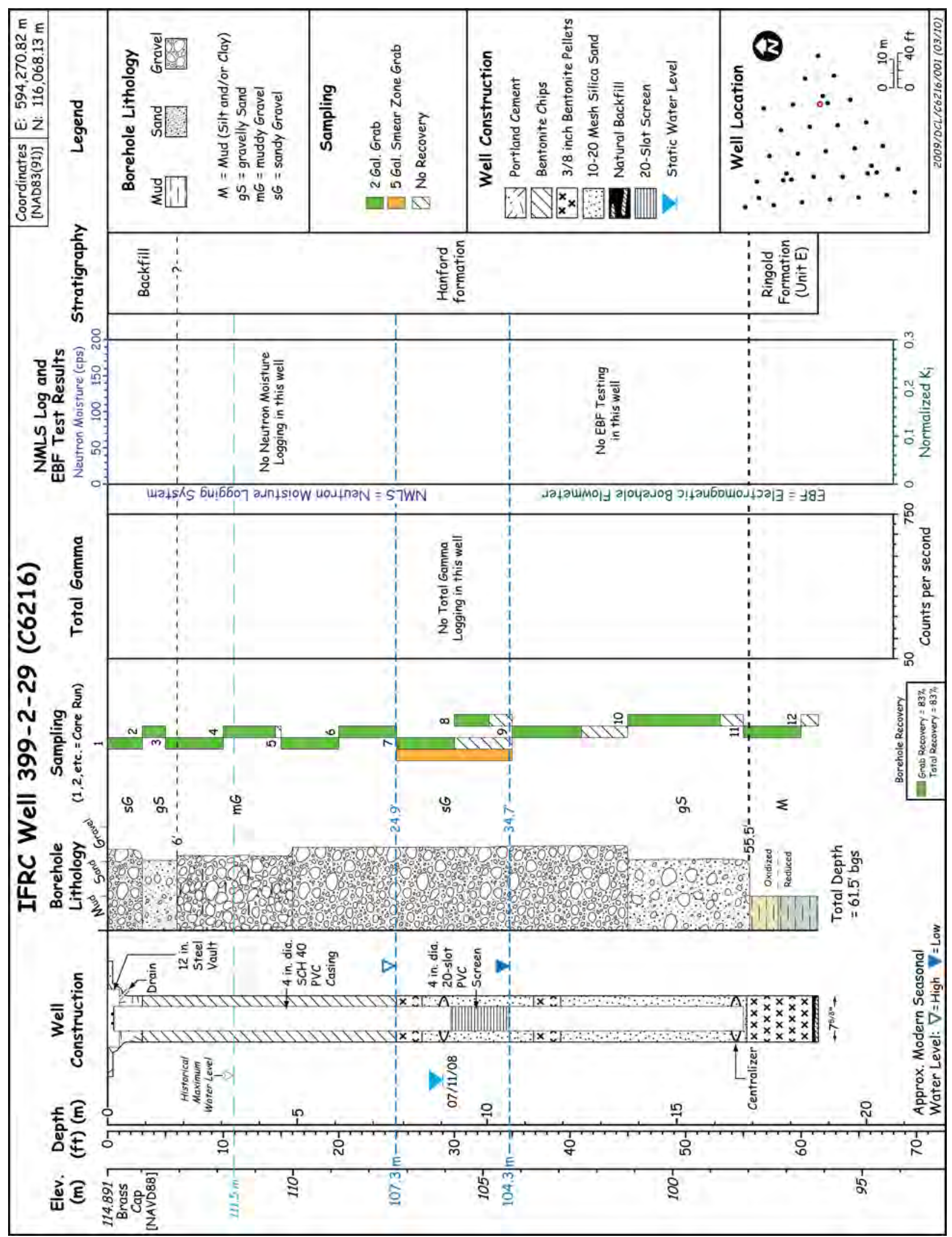




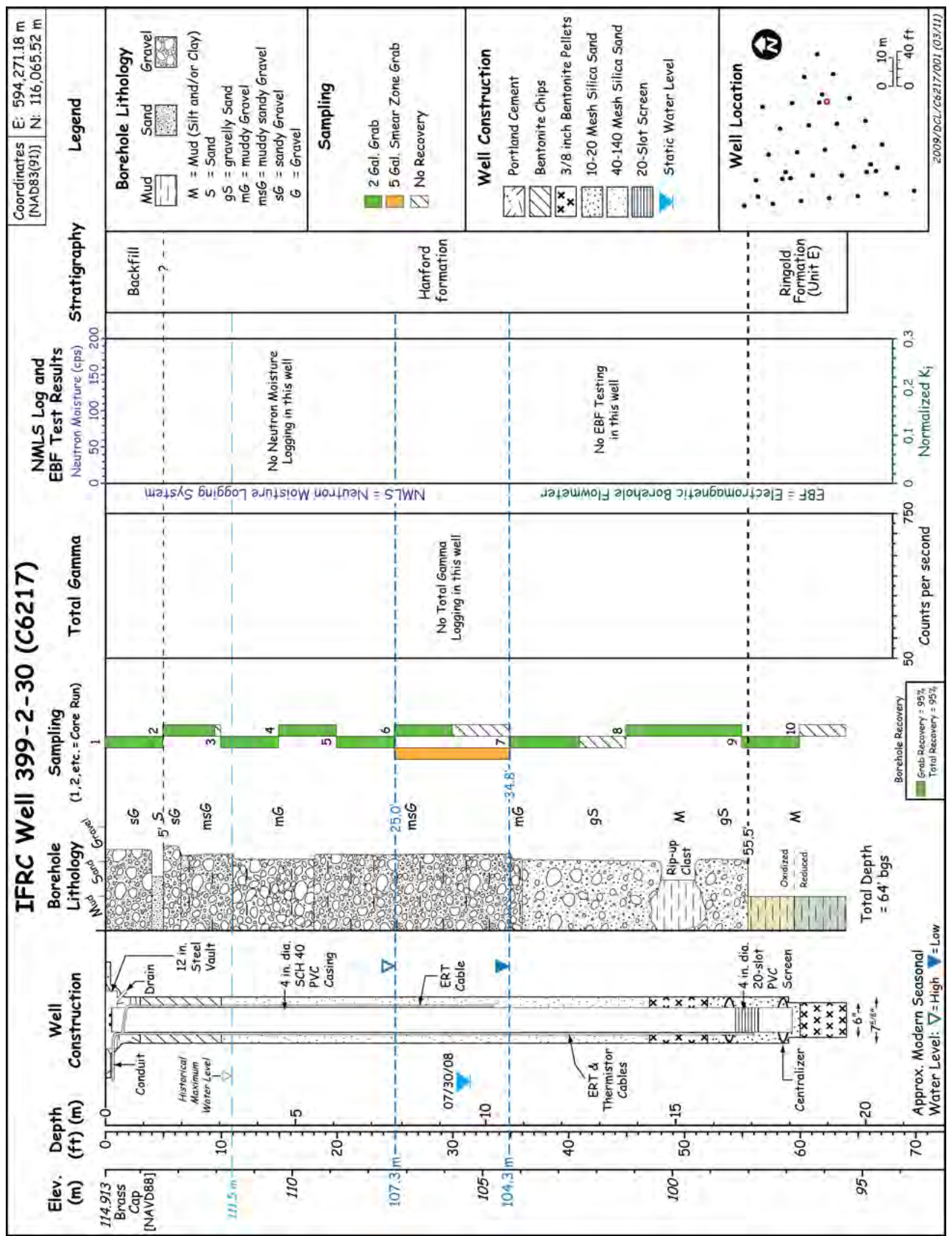




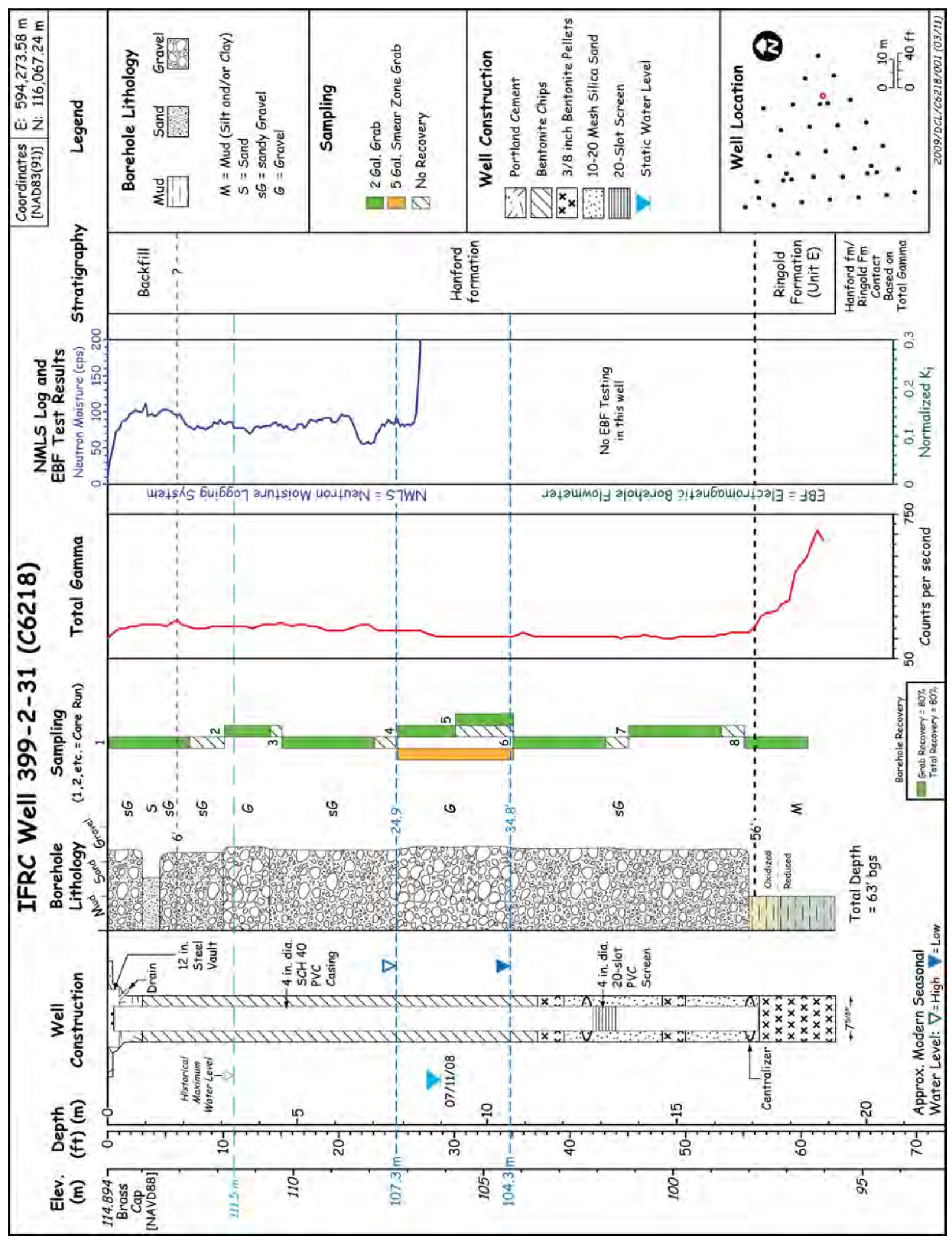


Appendix B

\section{Well-Site Geologist Logs}




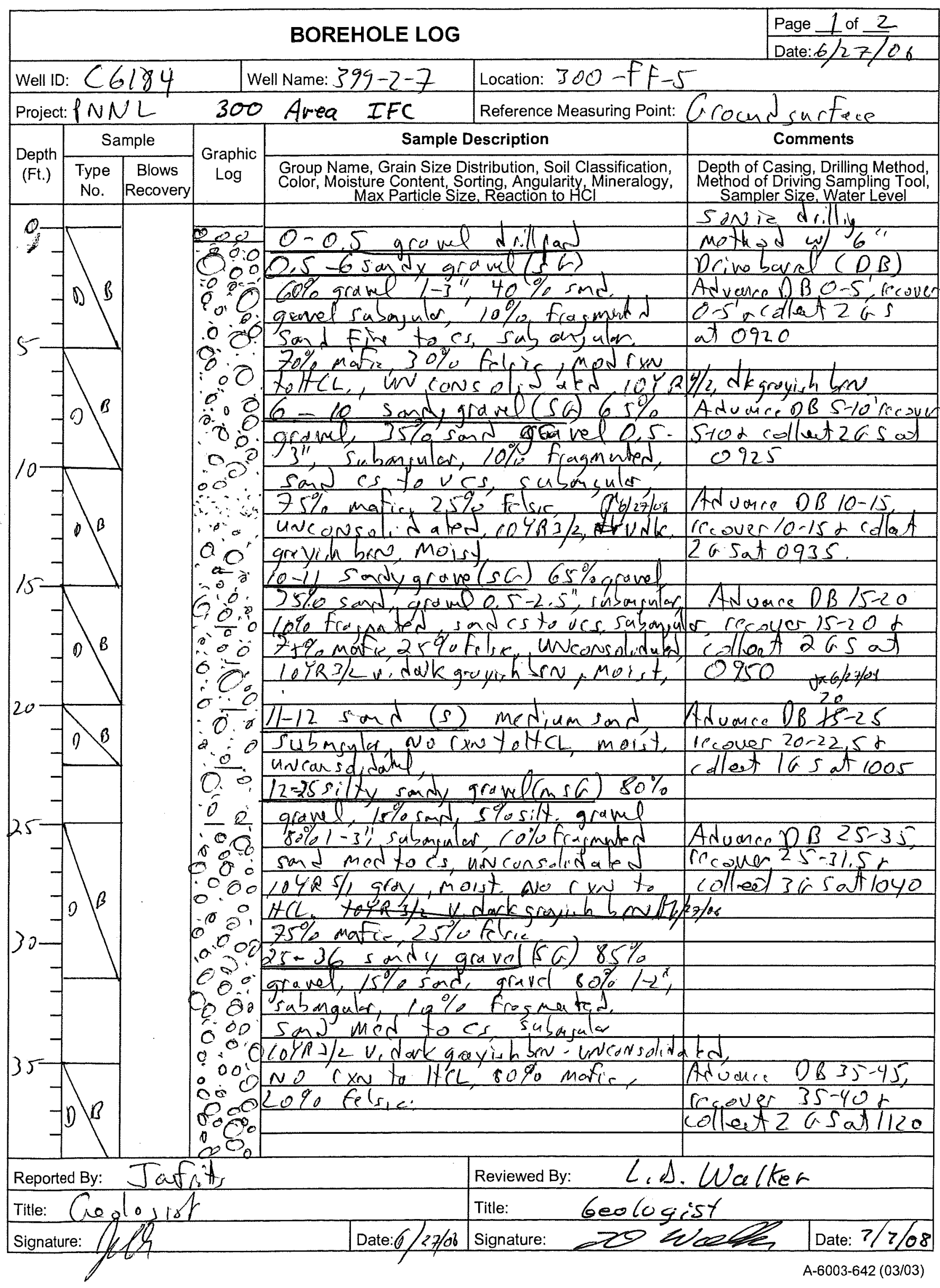




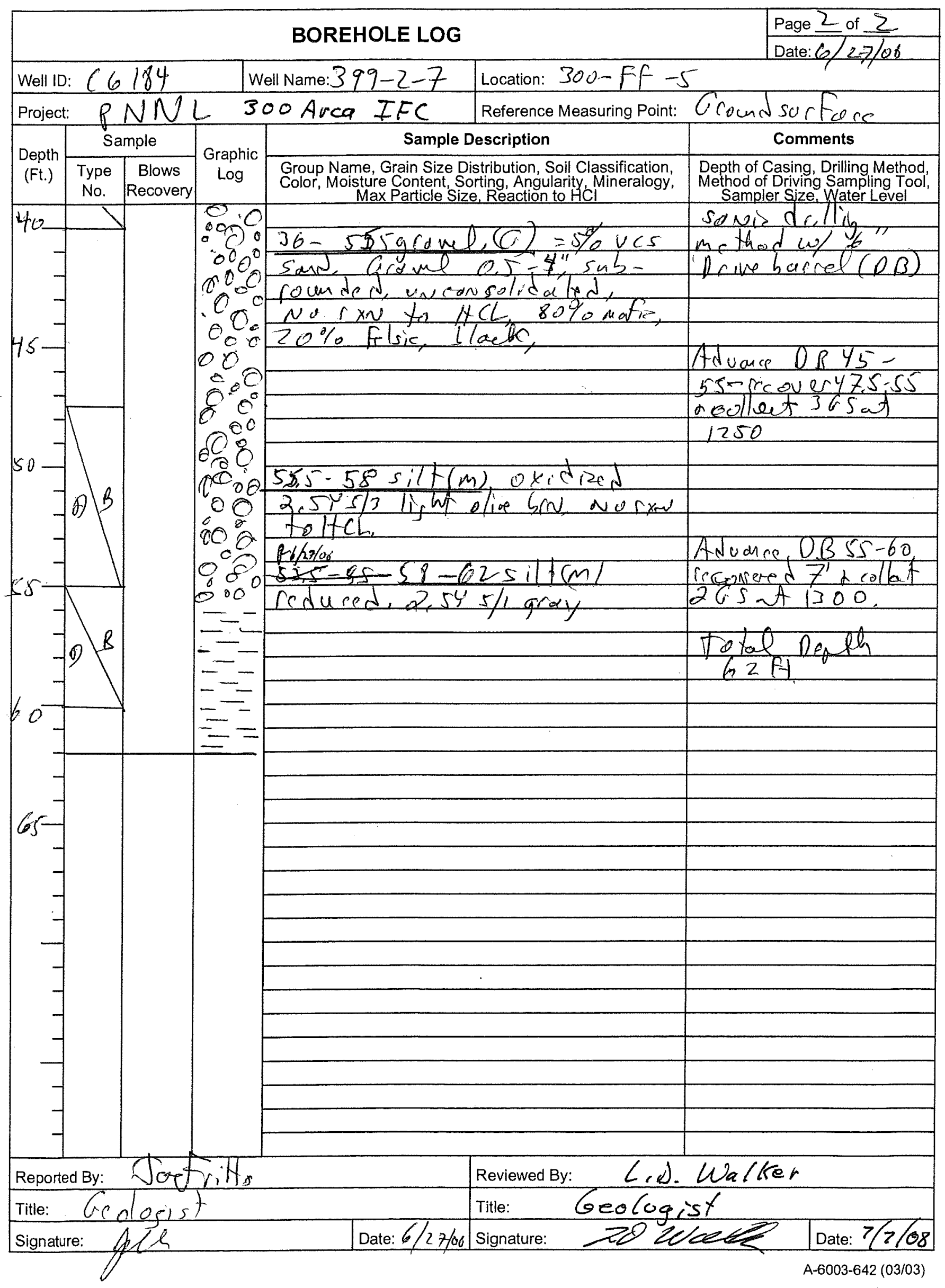

B. 2 


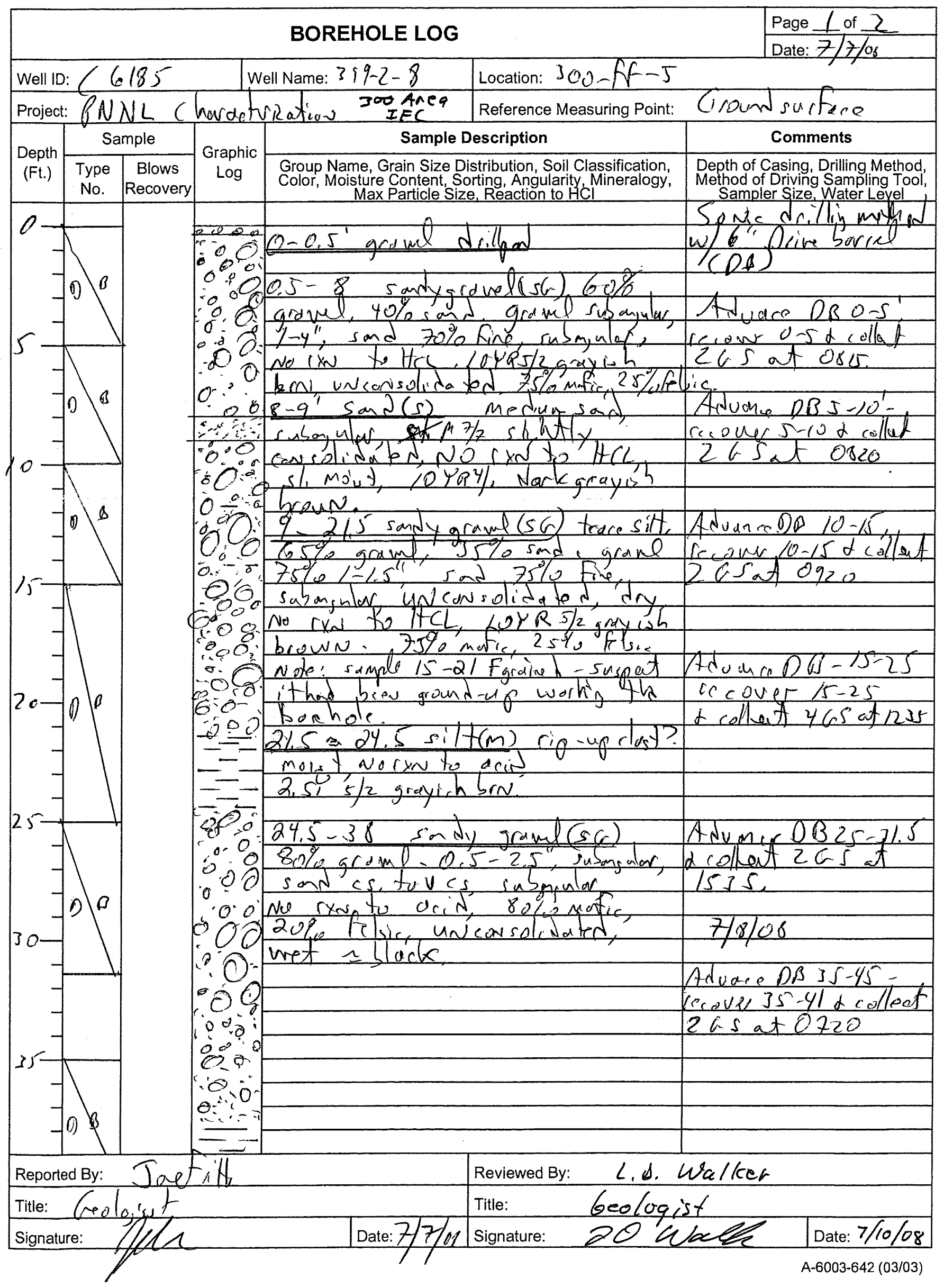

B. 3 


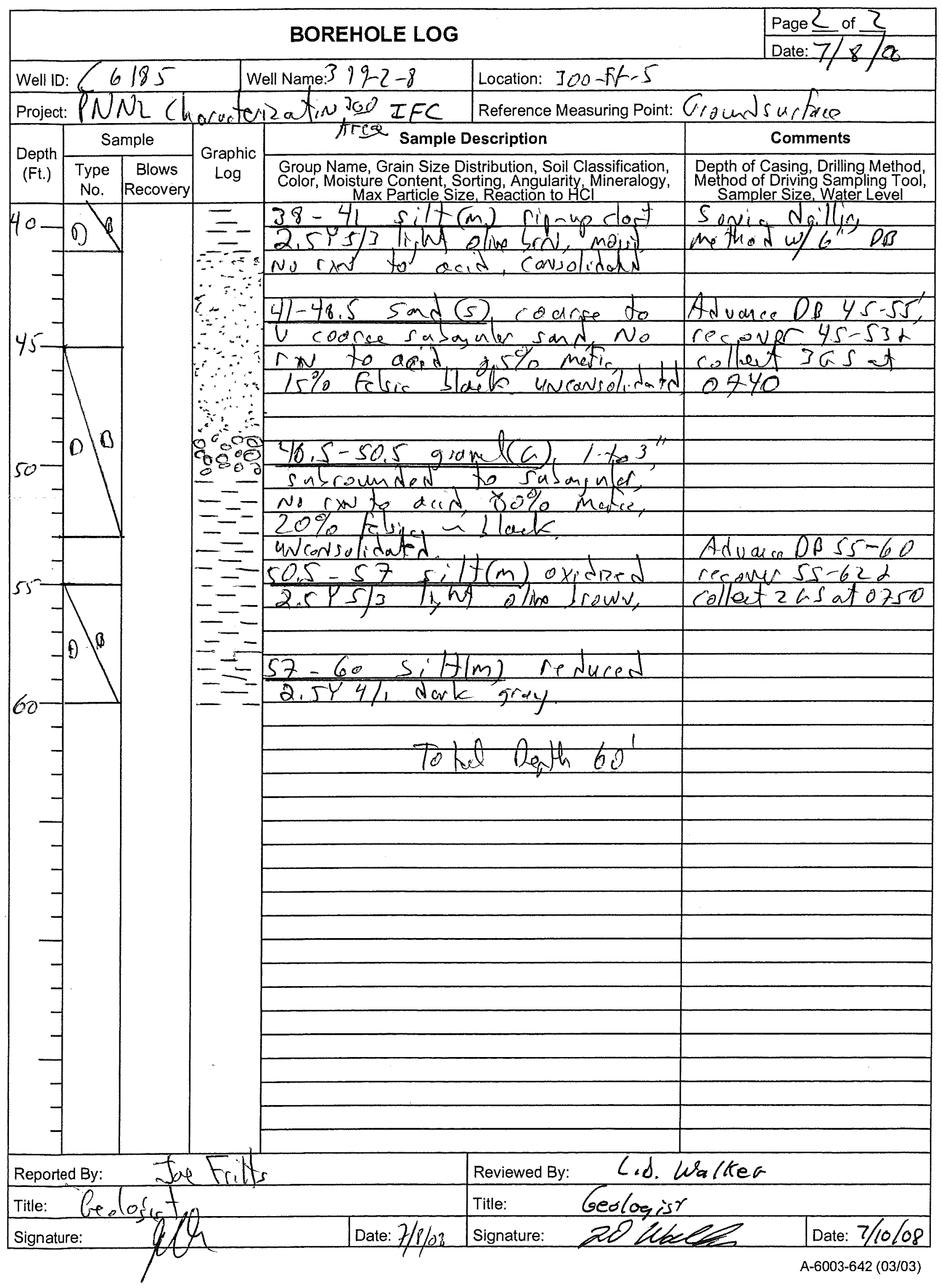




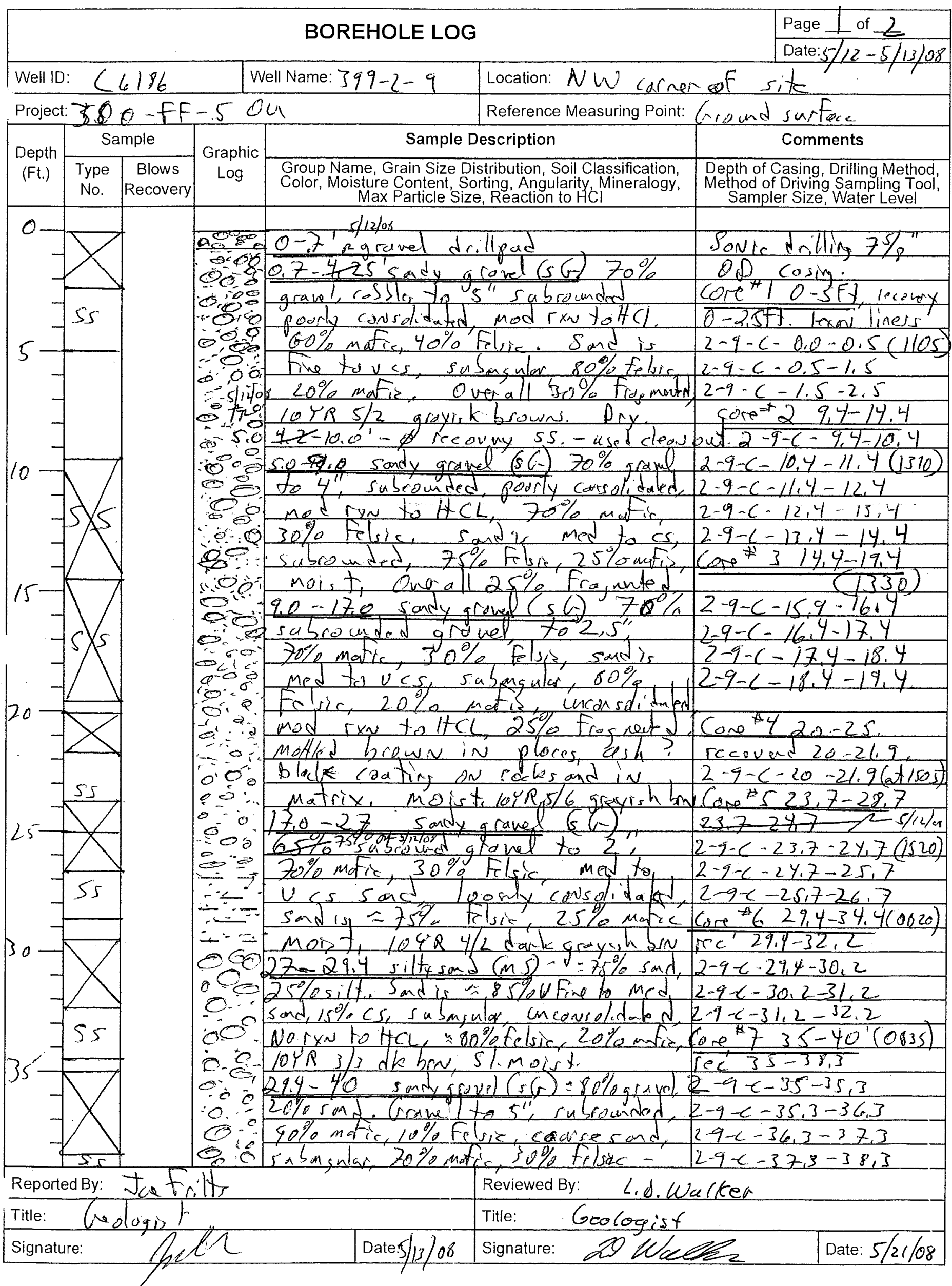




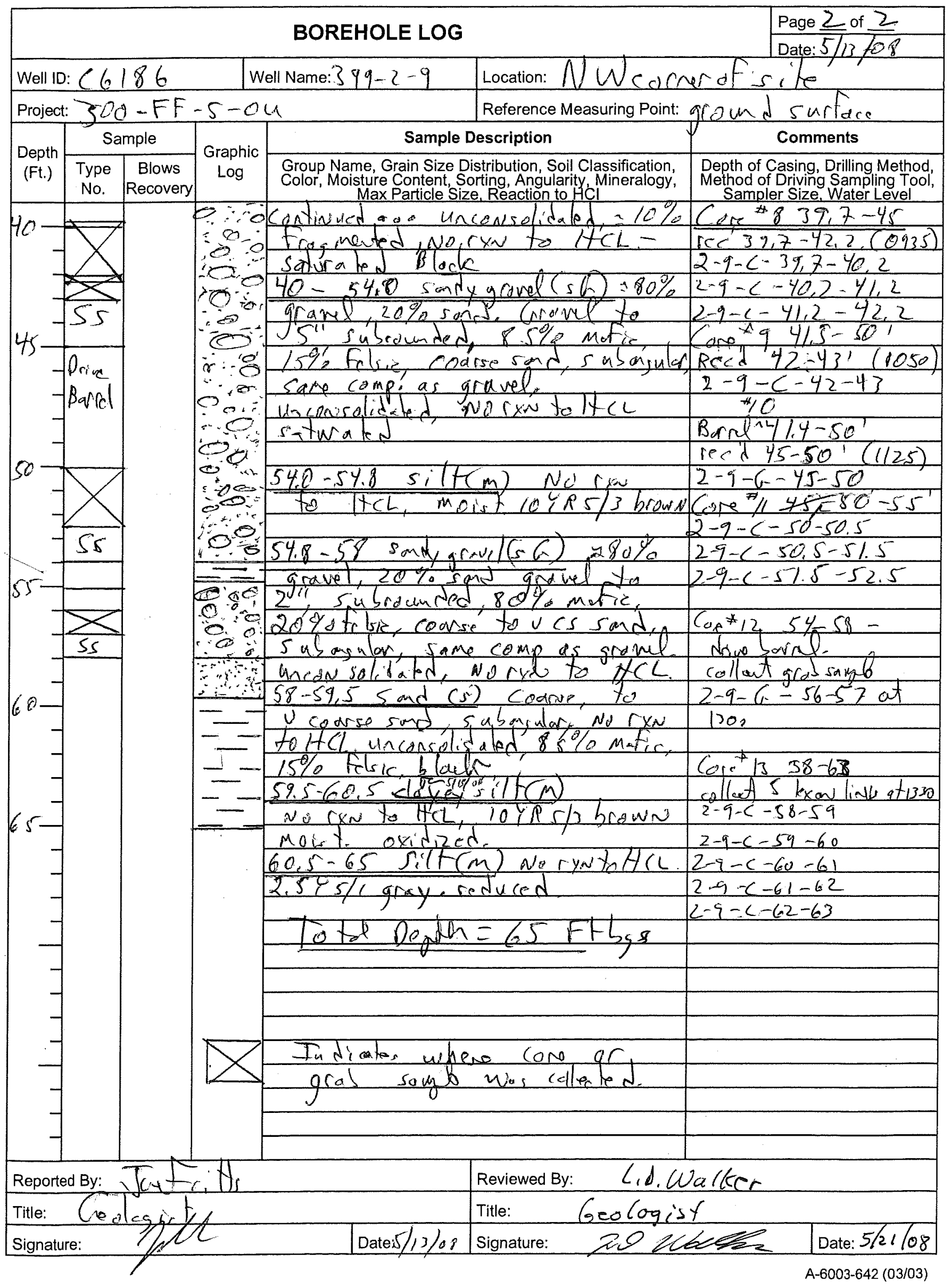

B. 6 


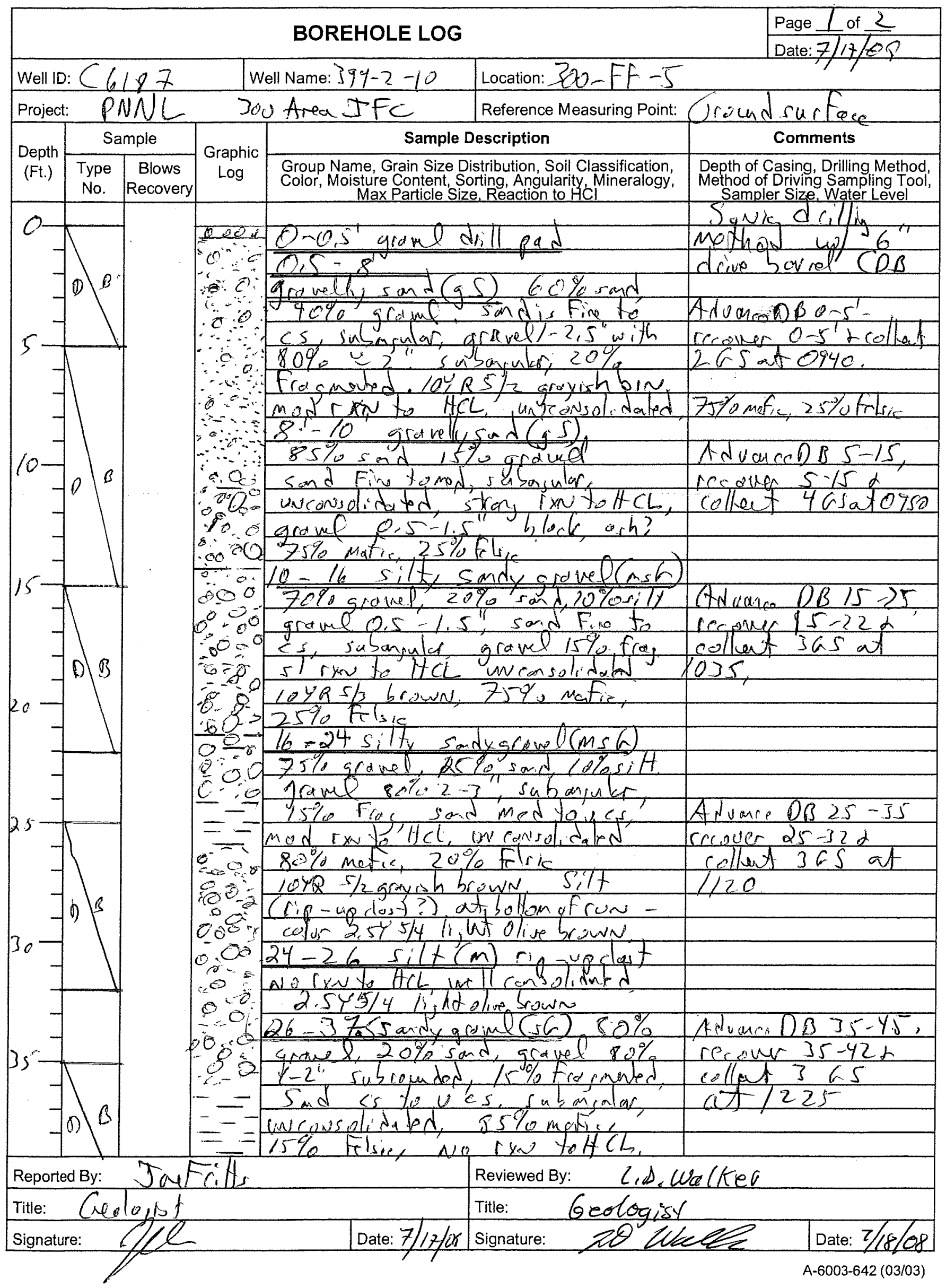

B.7 


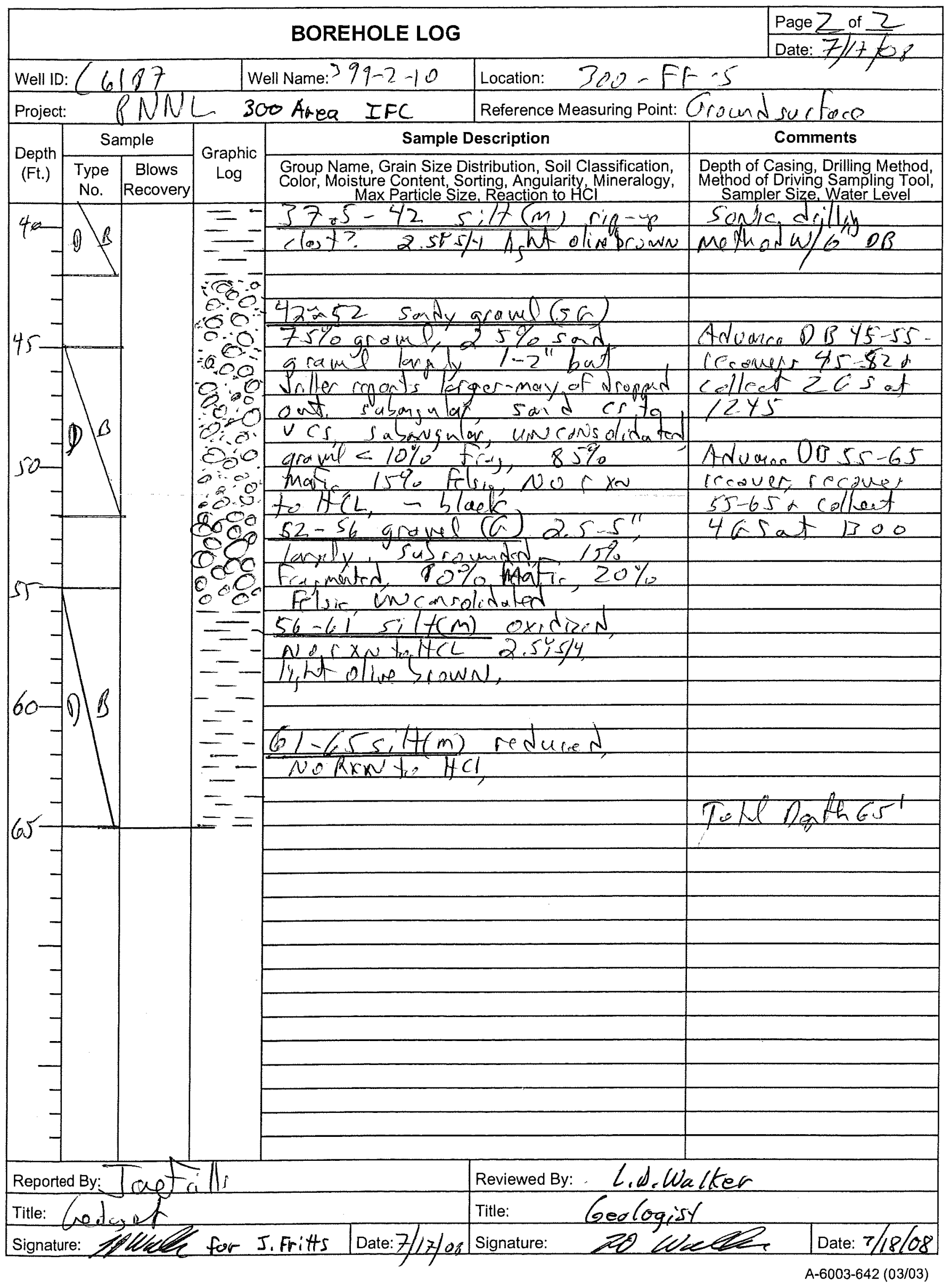


BOREHOLE LOG

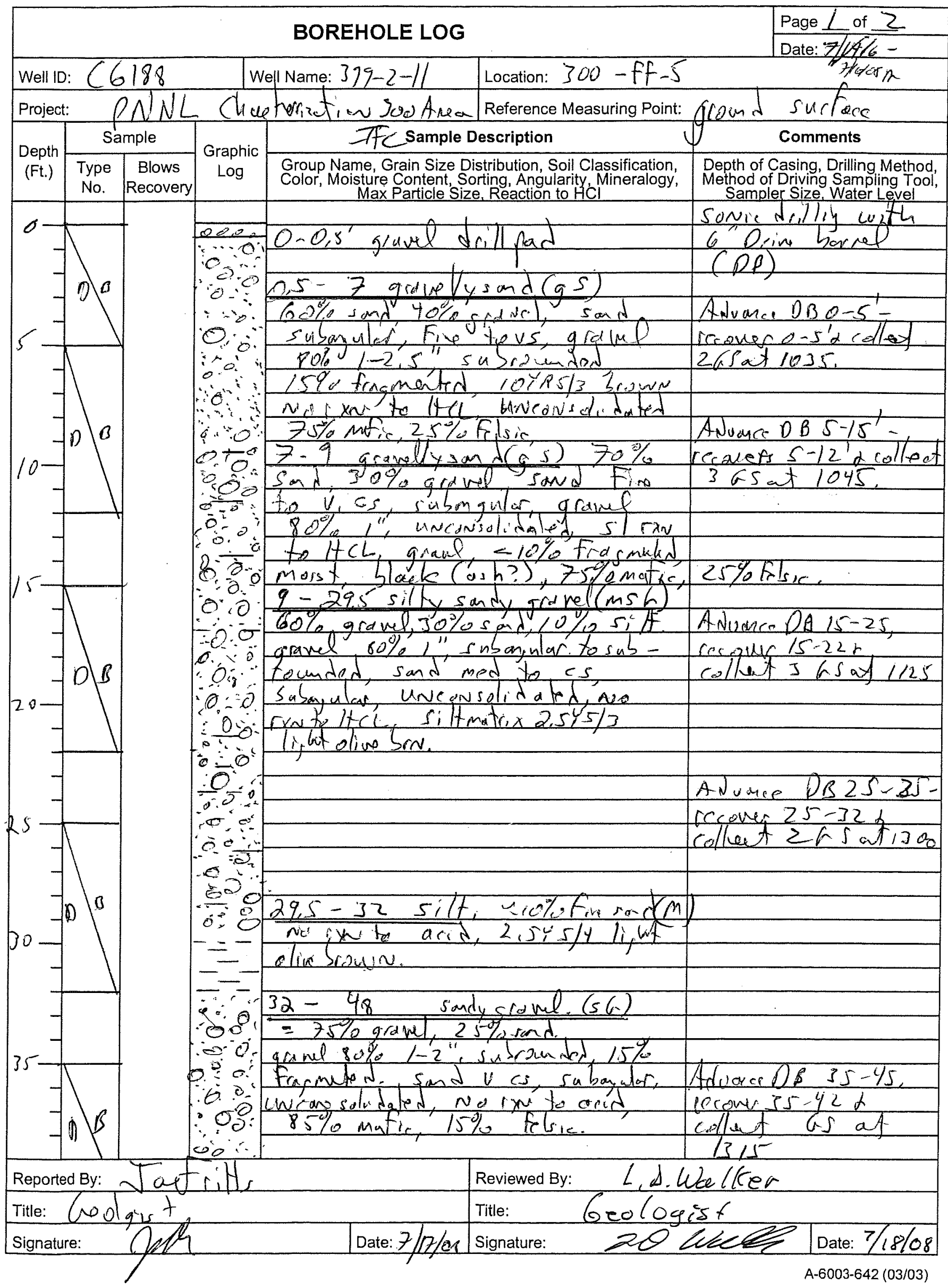

B.9 


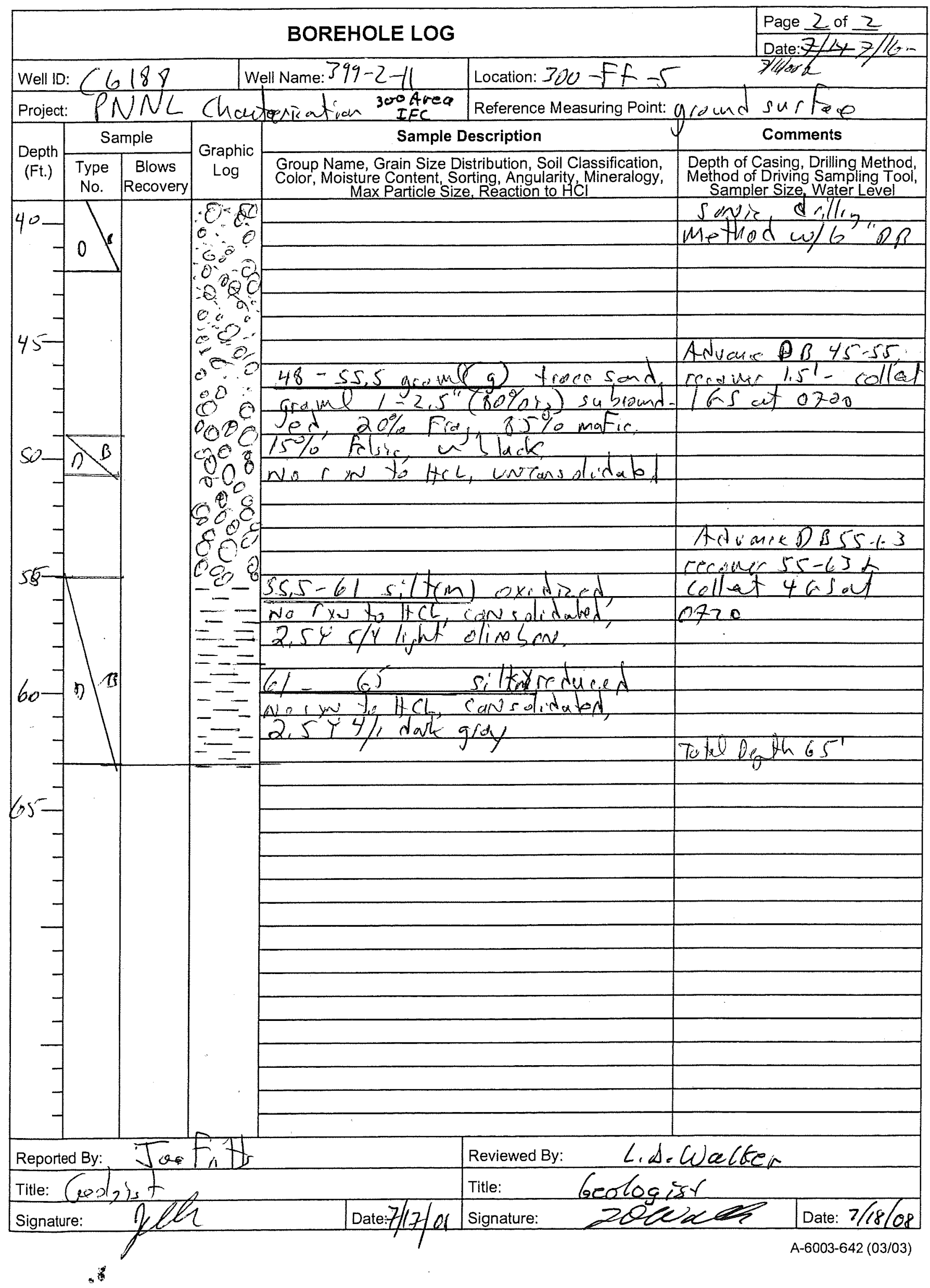




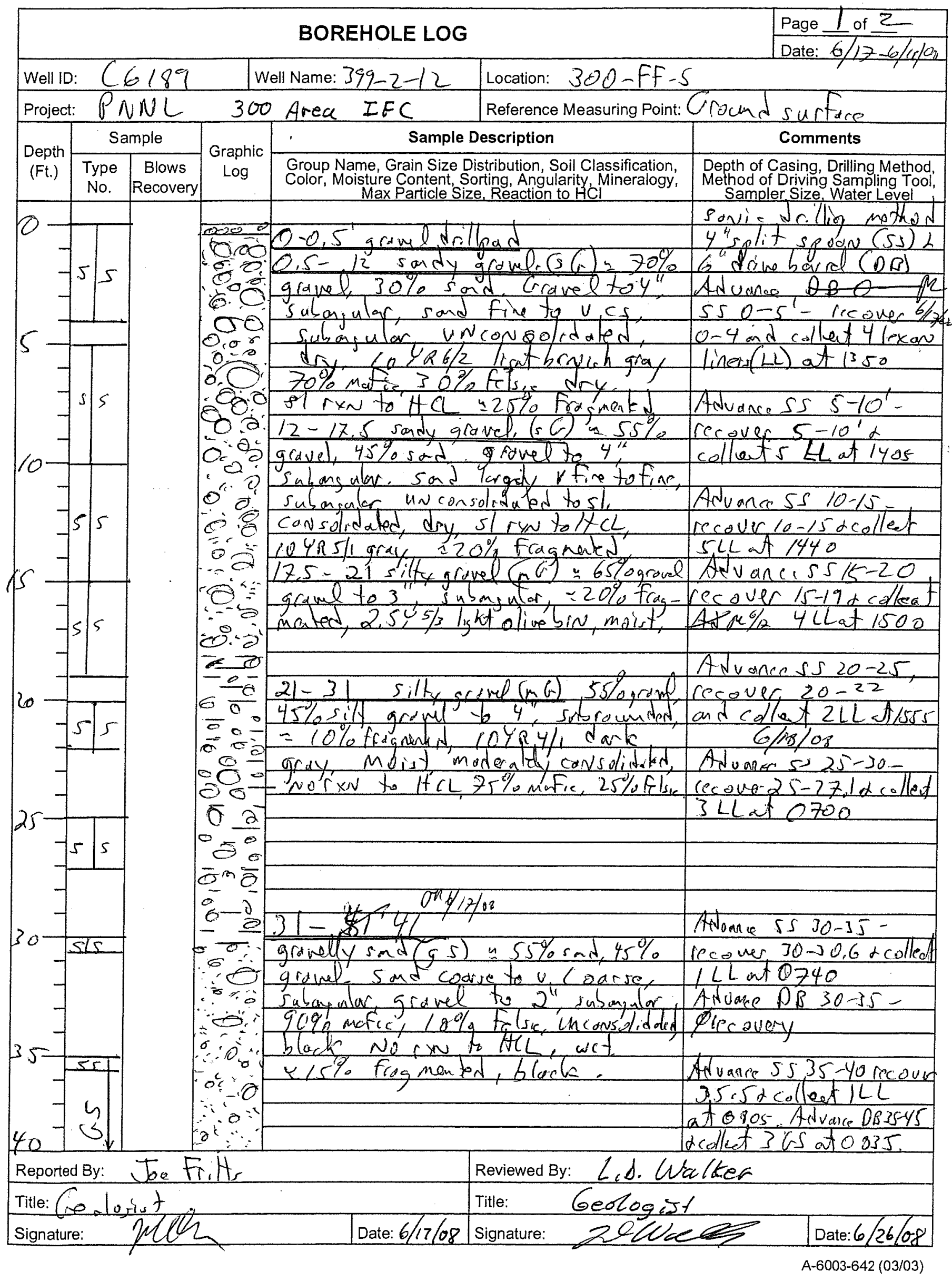




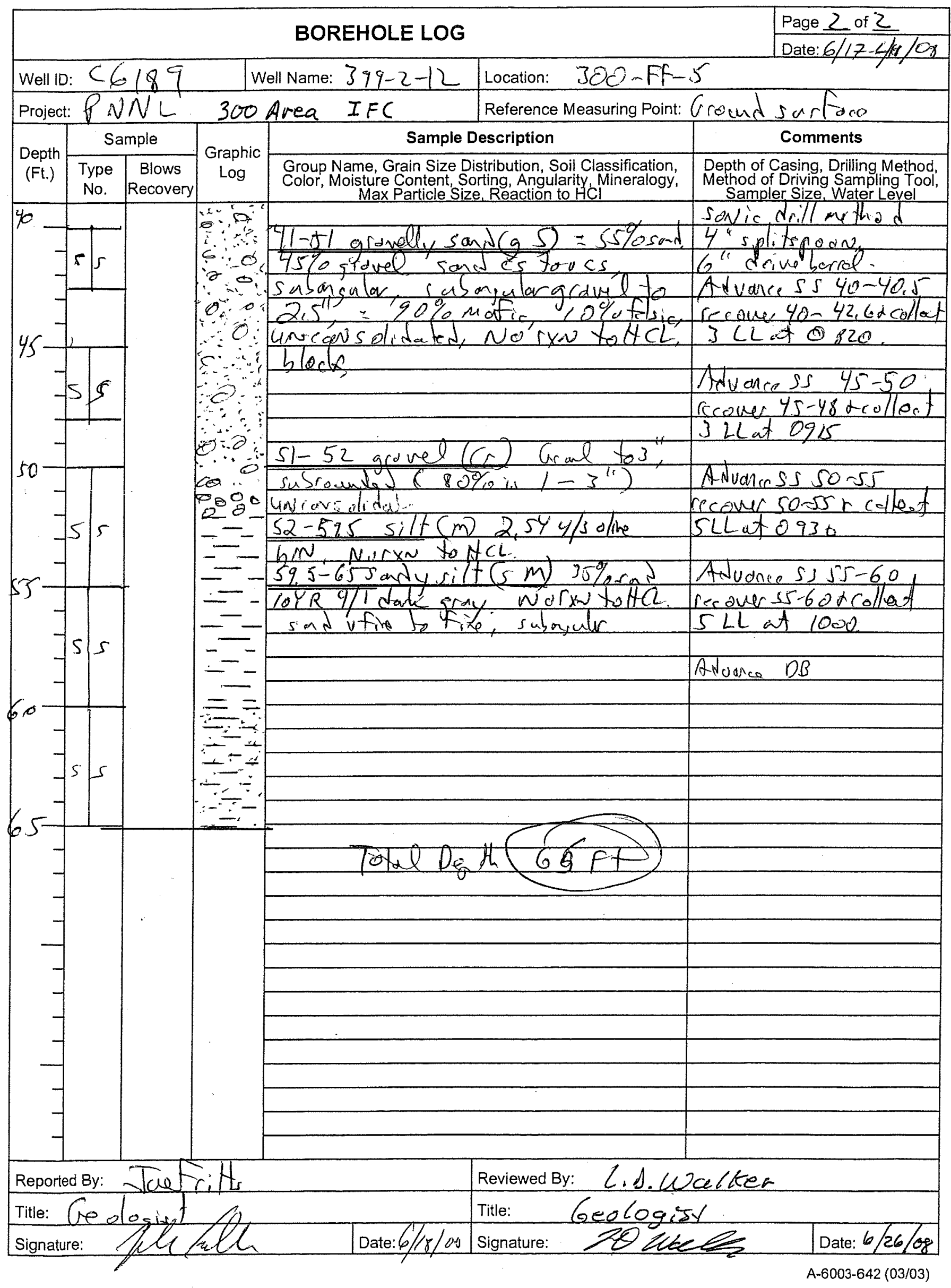




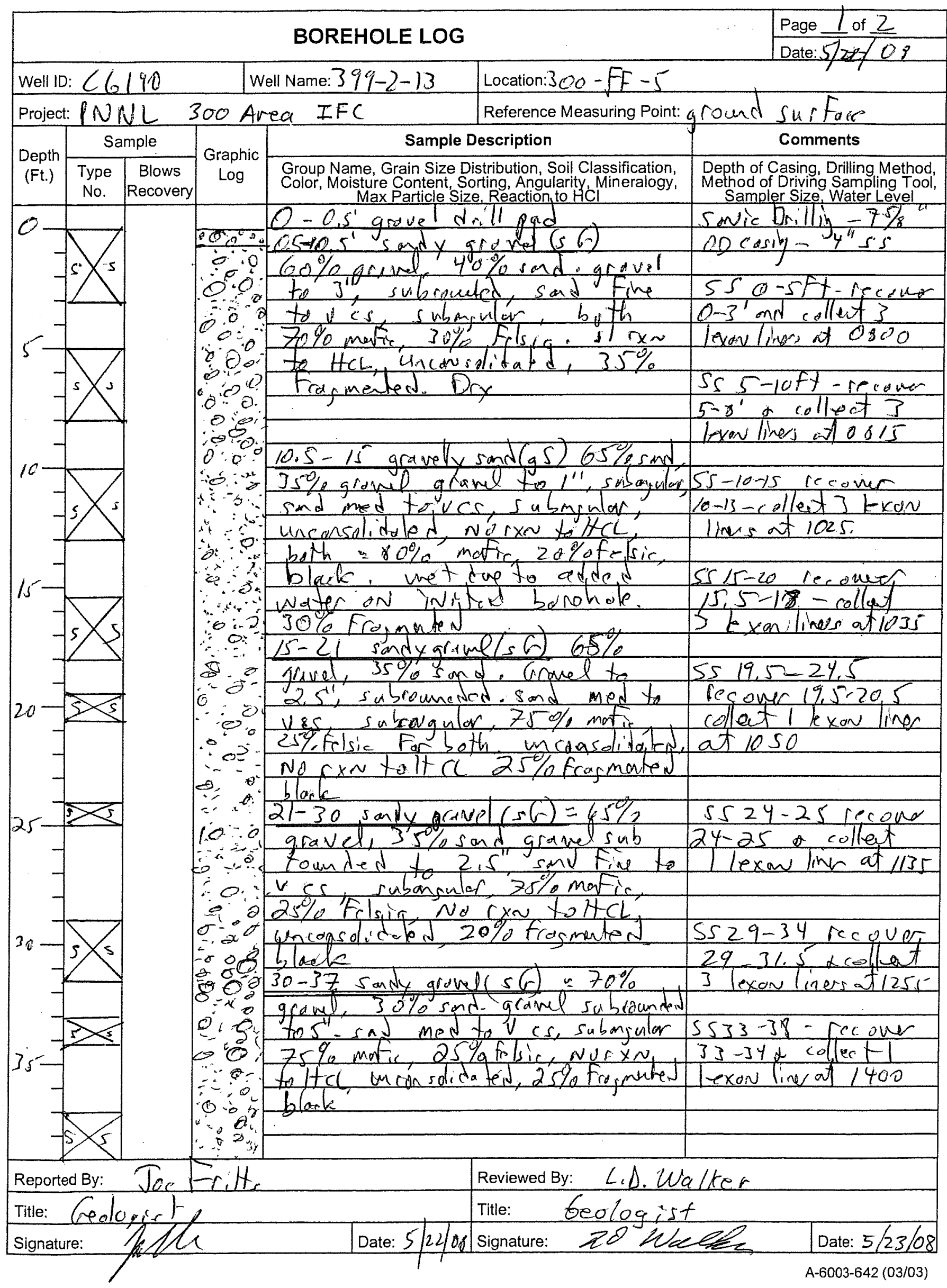




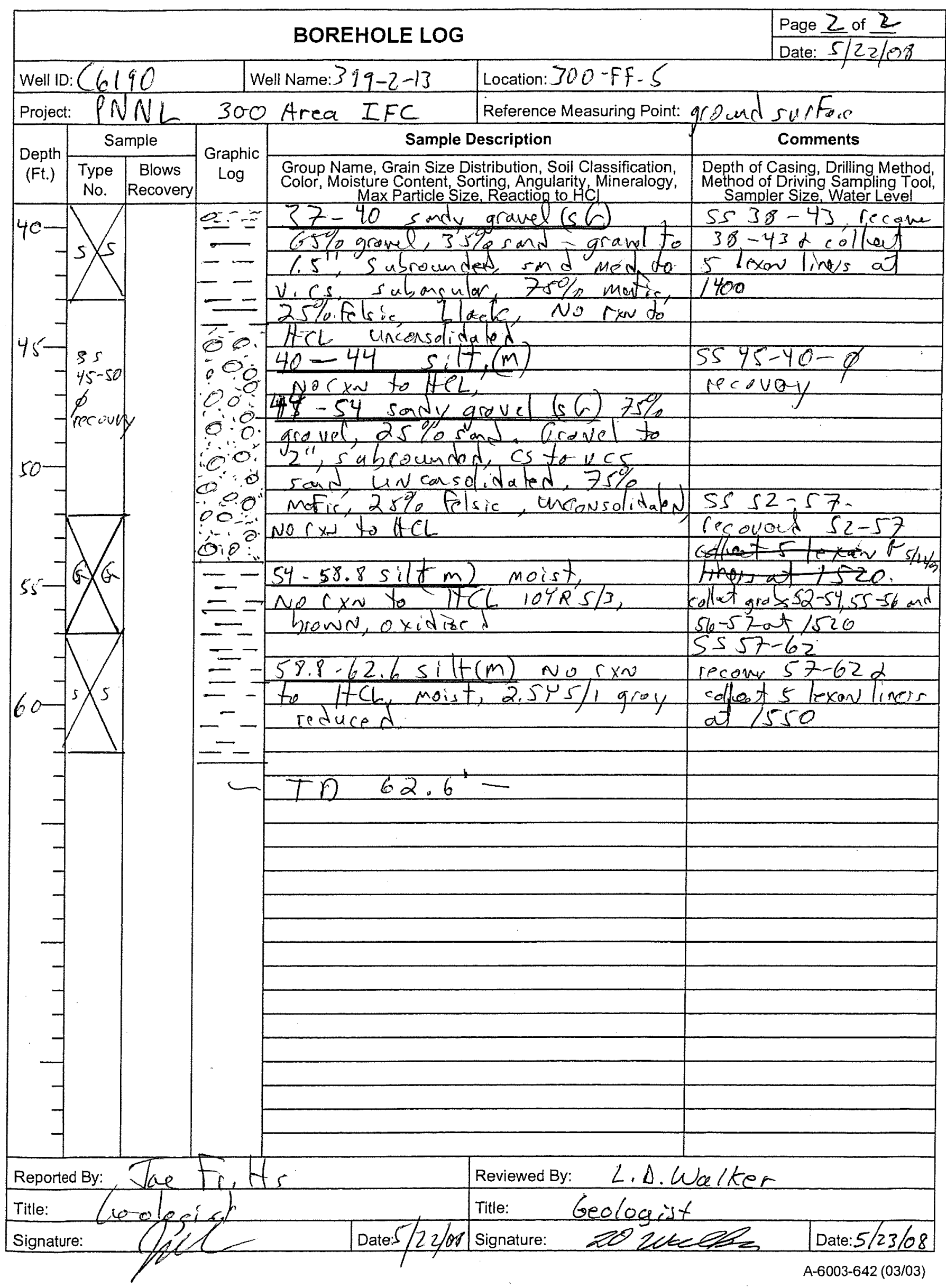




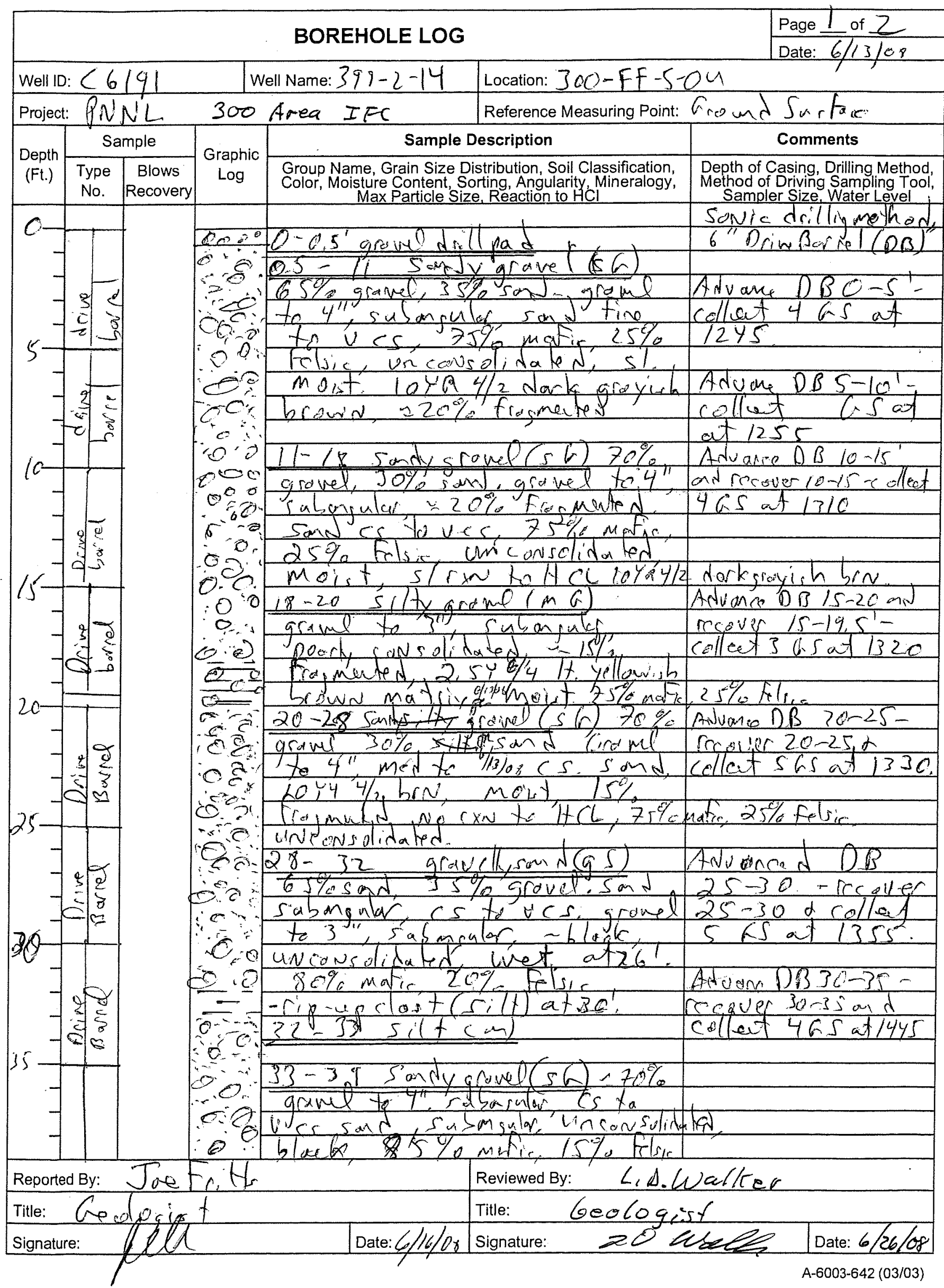




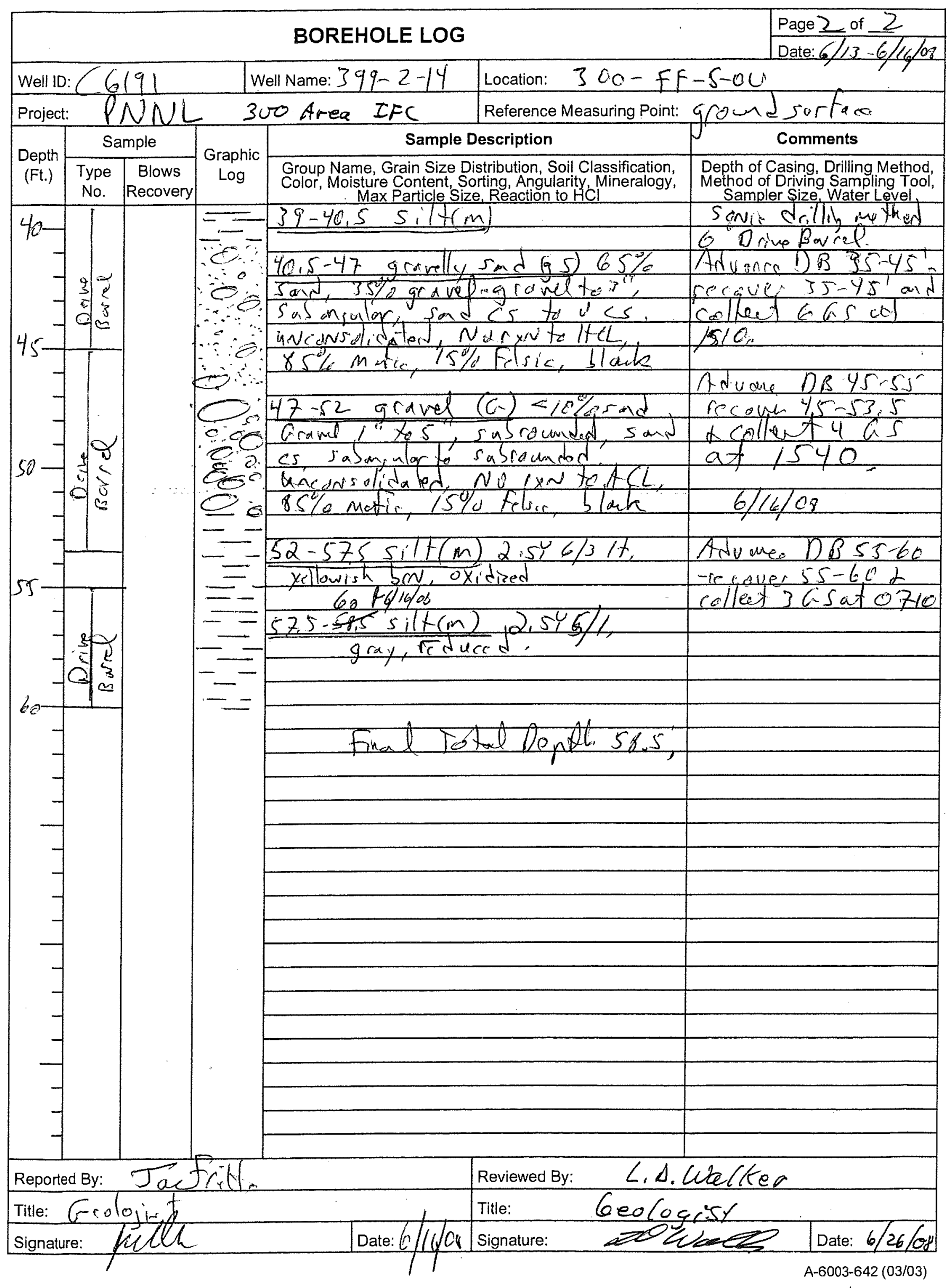




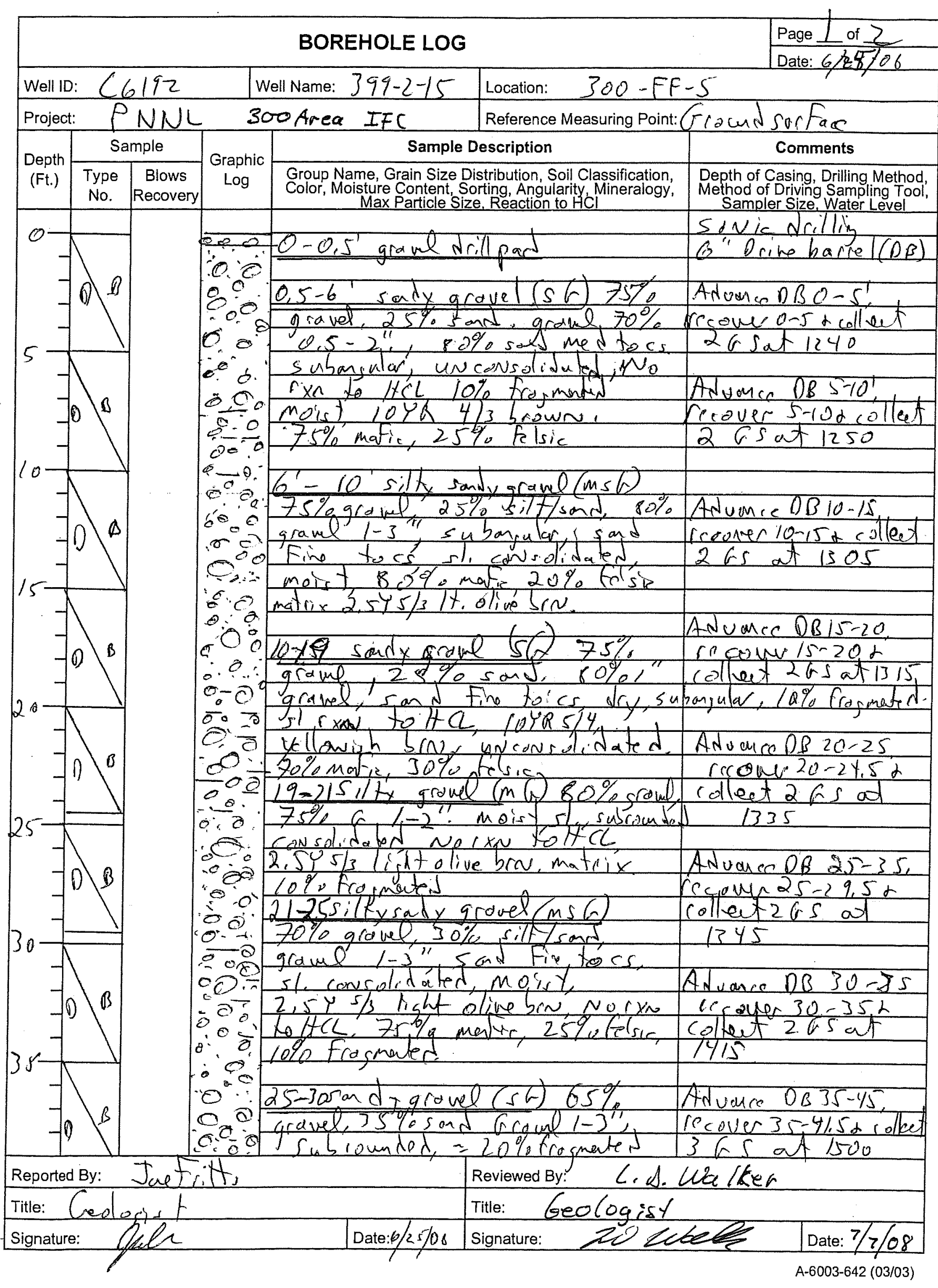




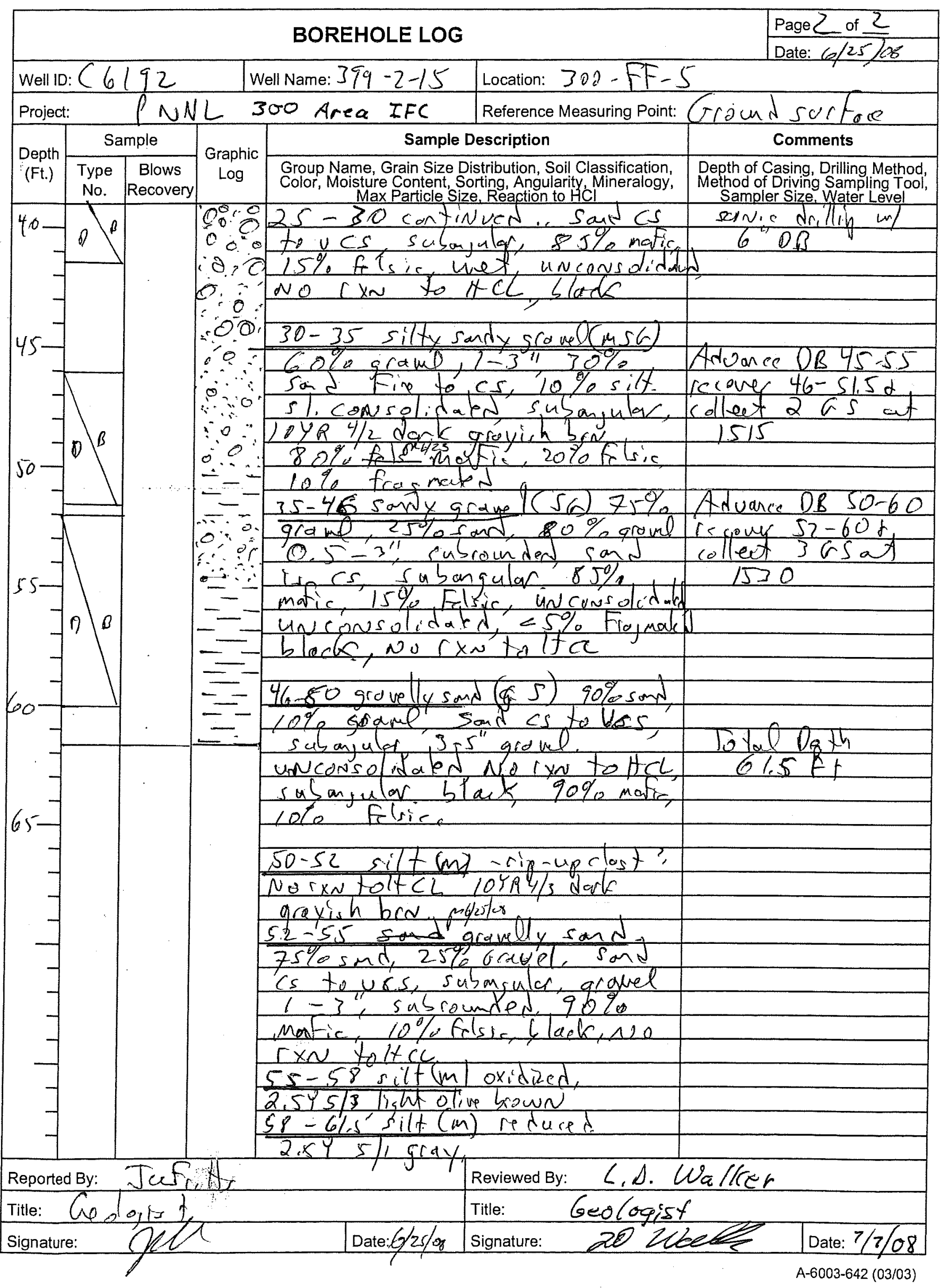




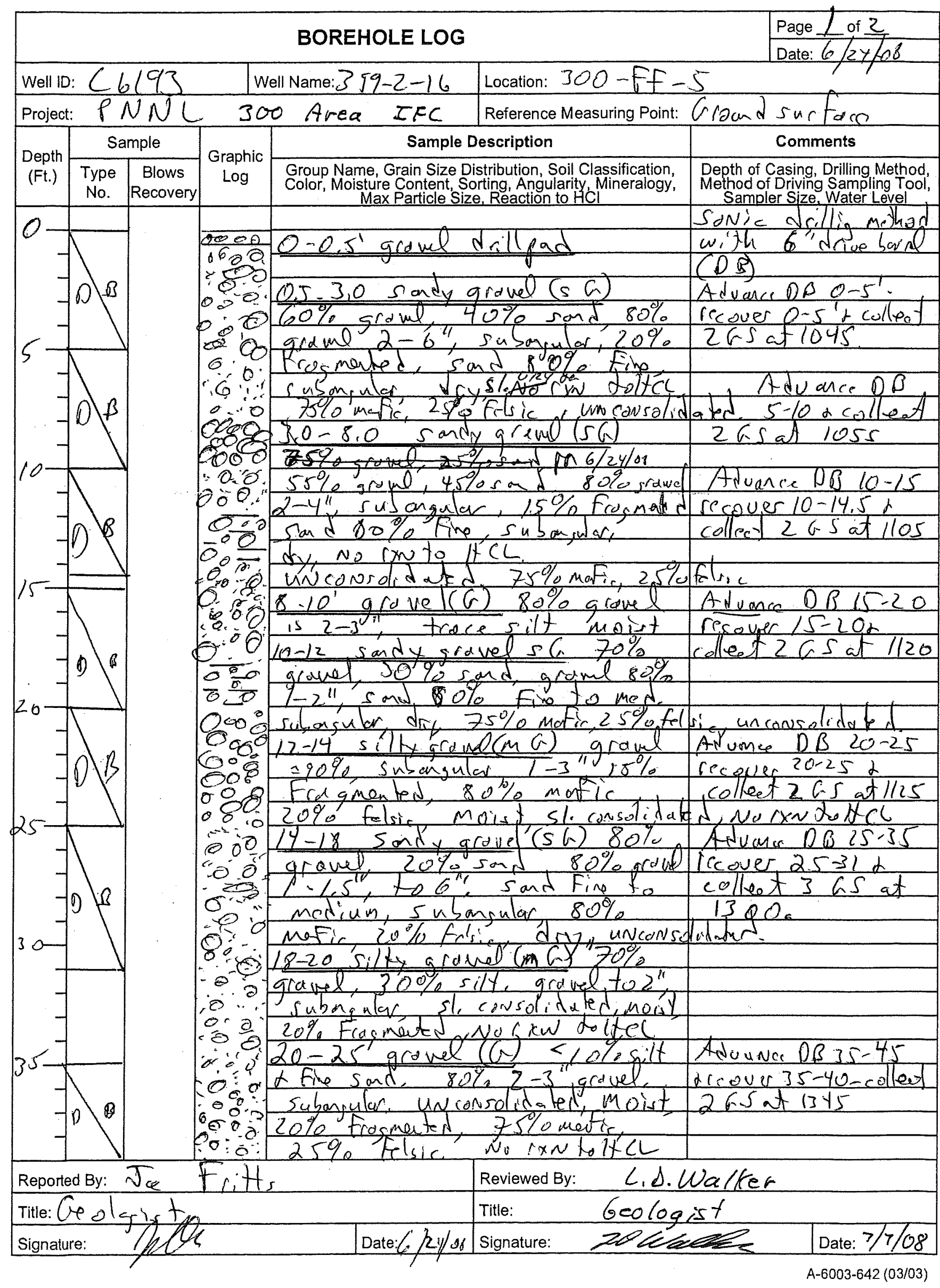




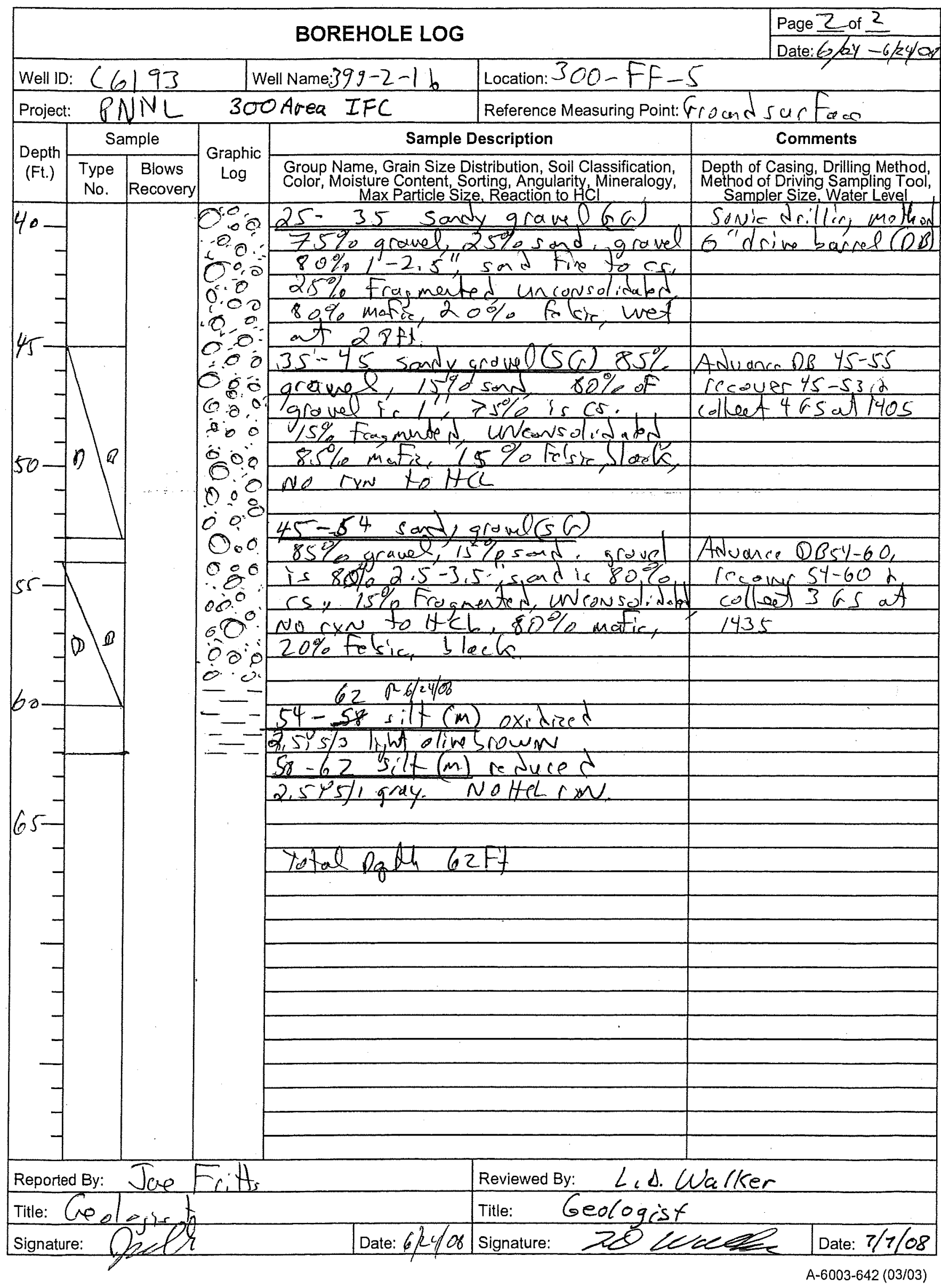




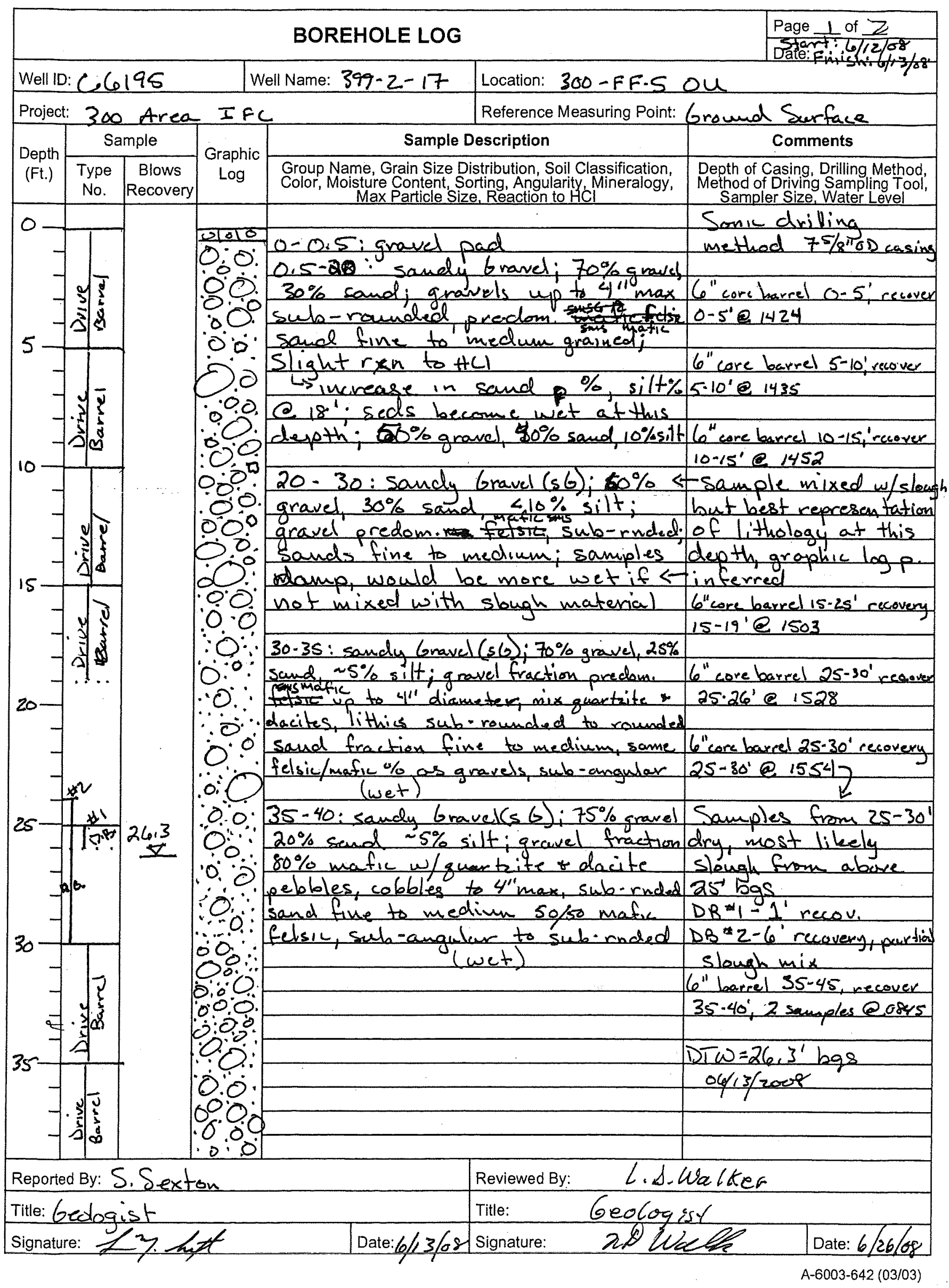




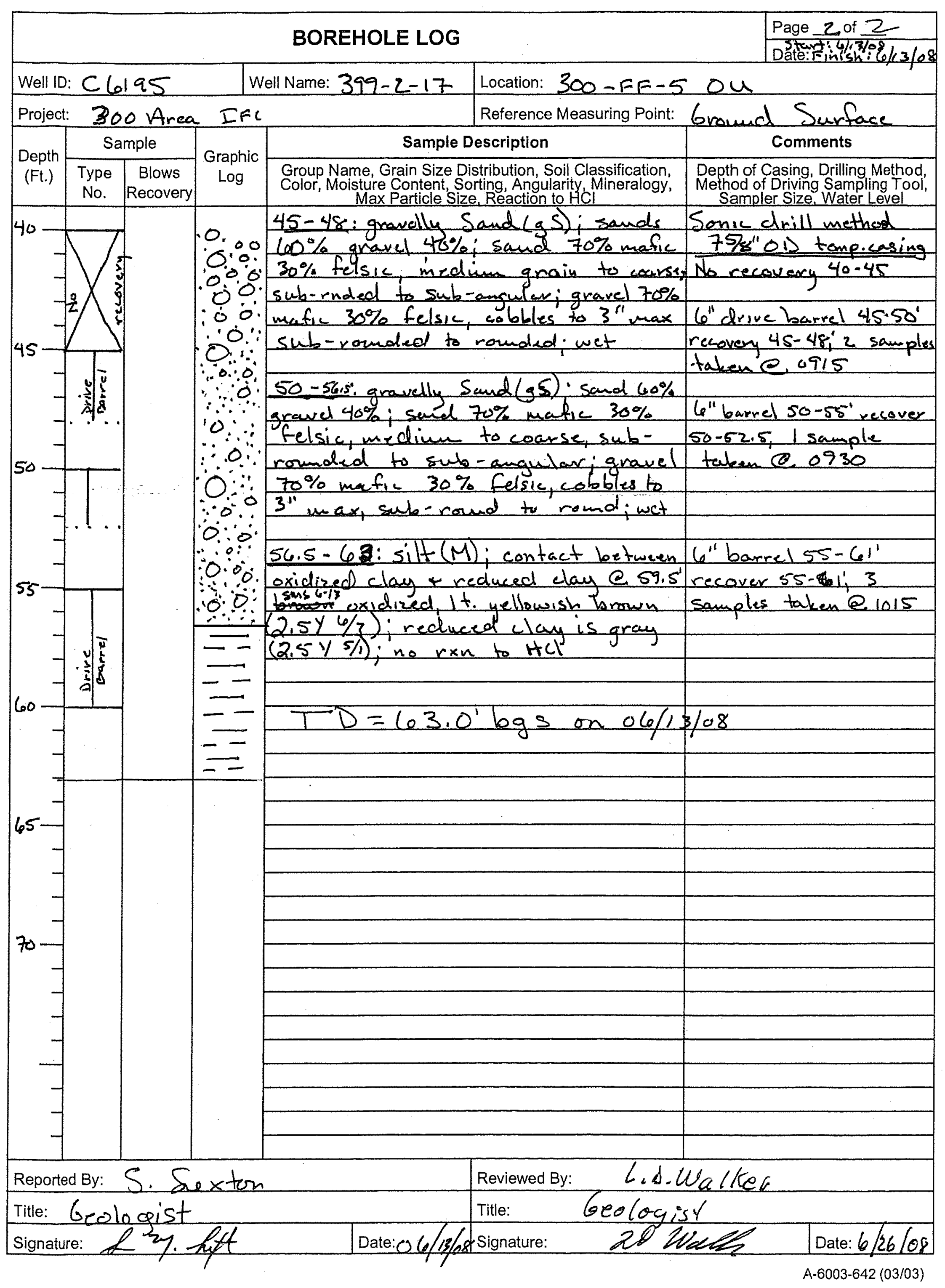




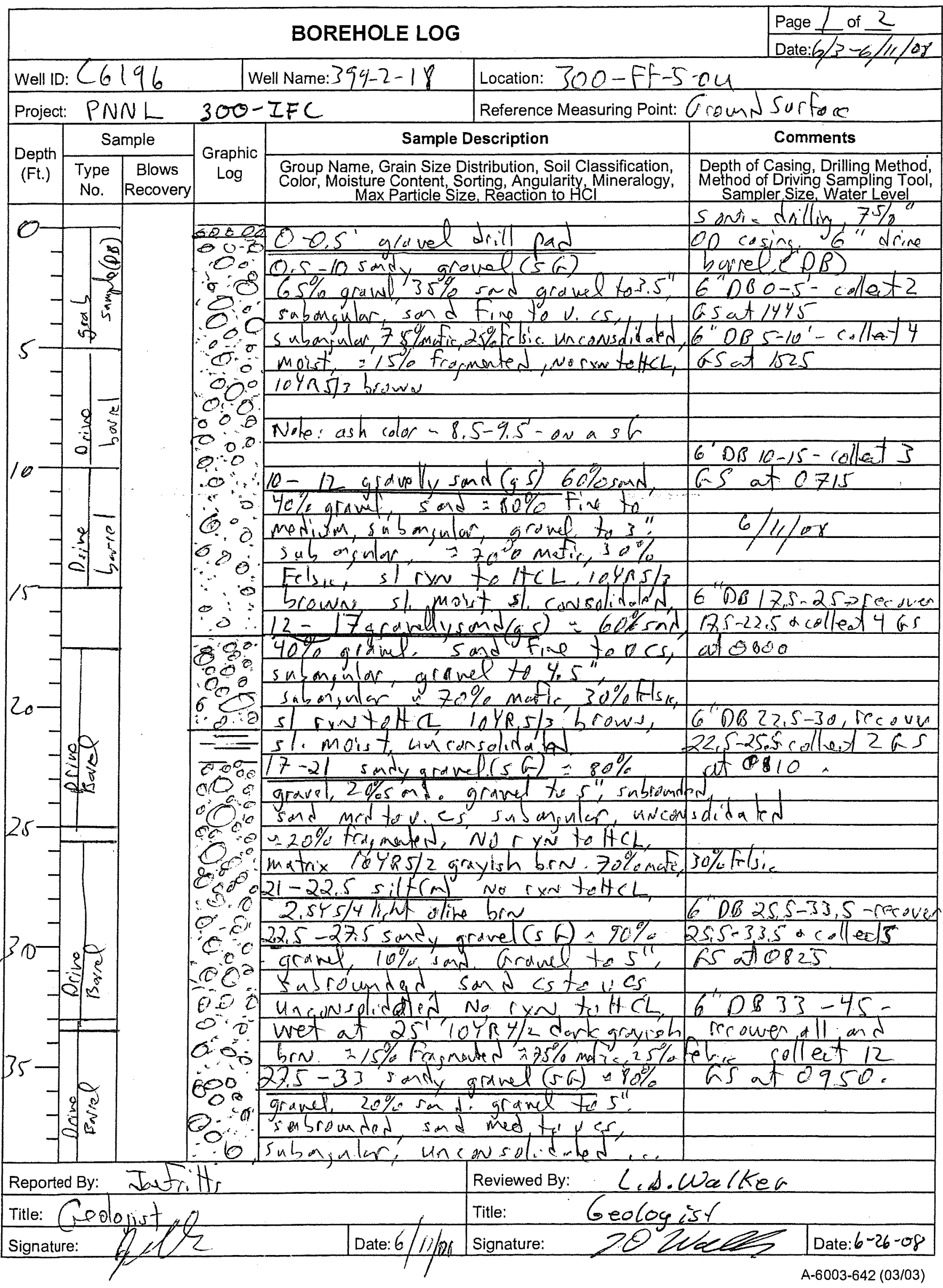




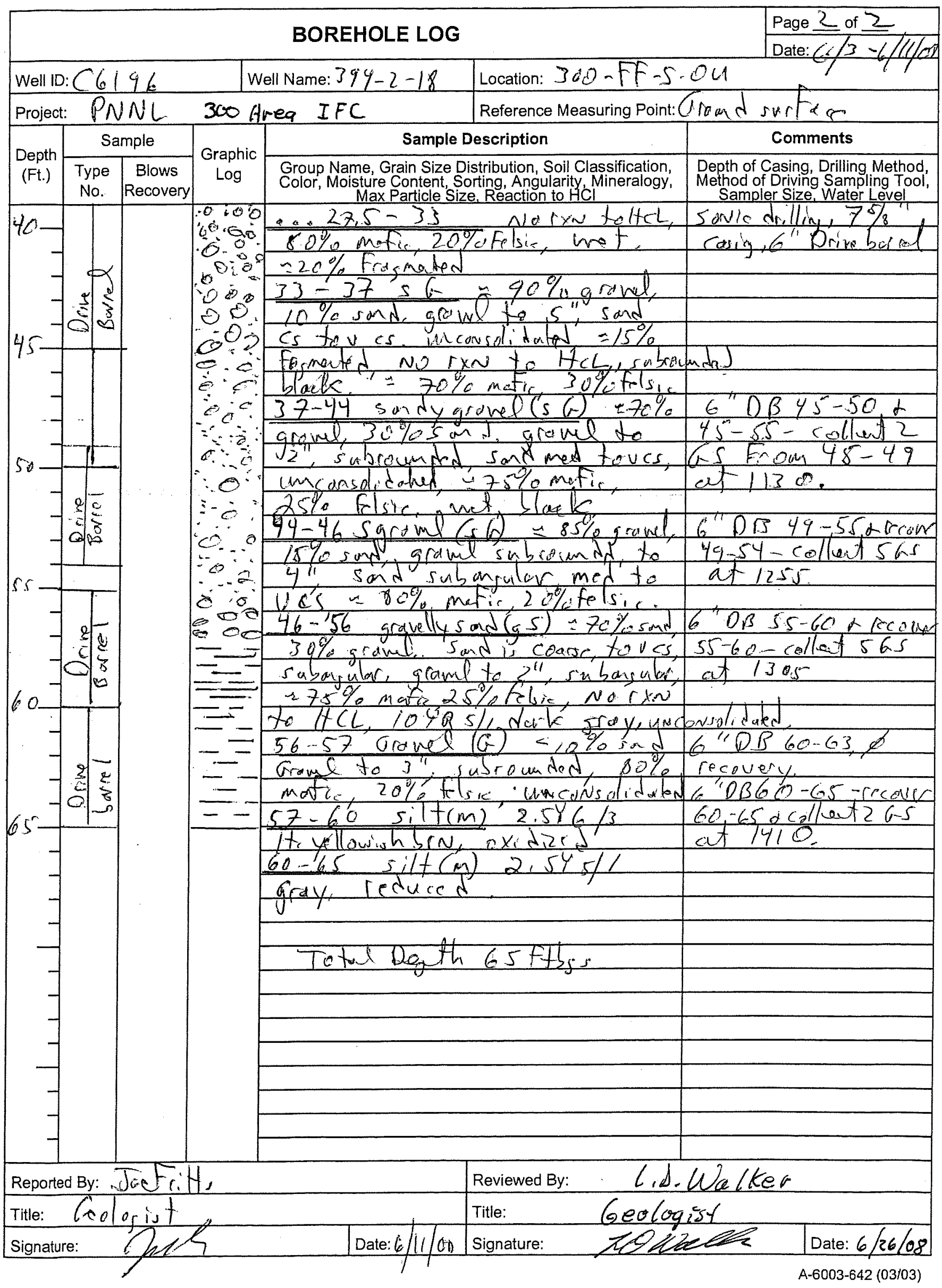




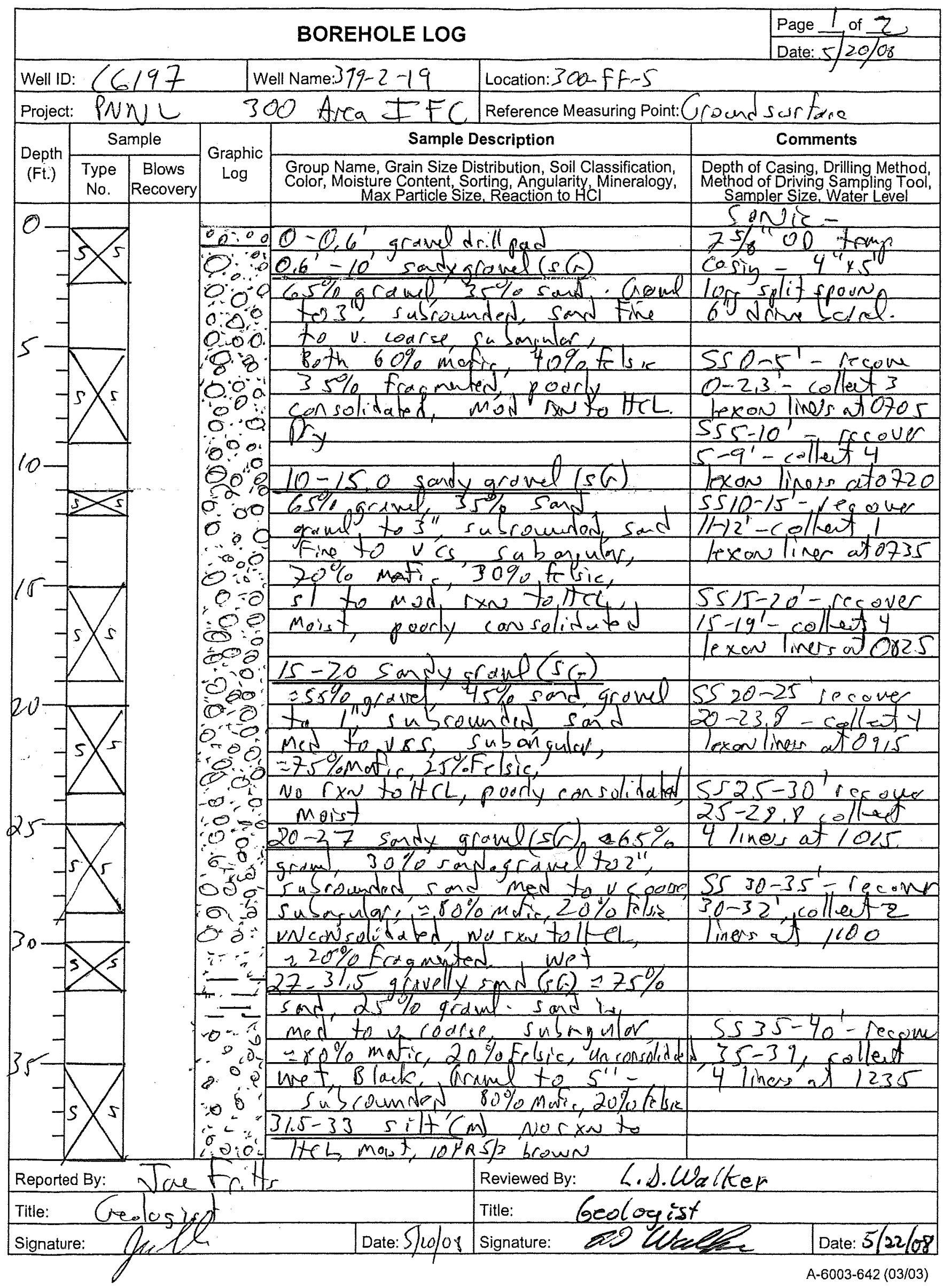




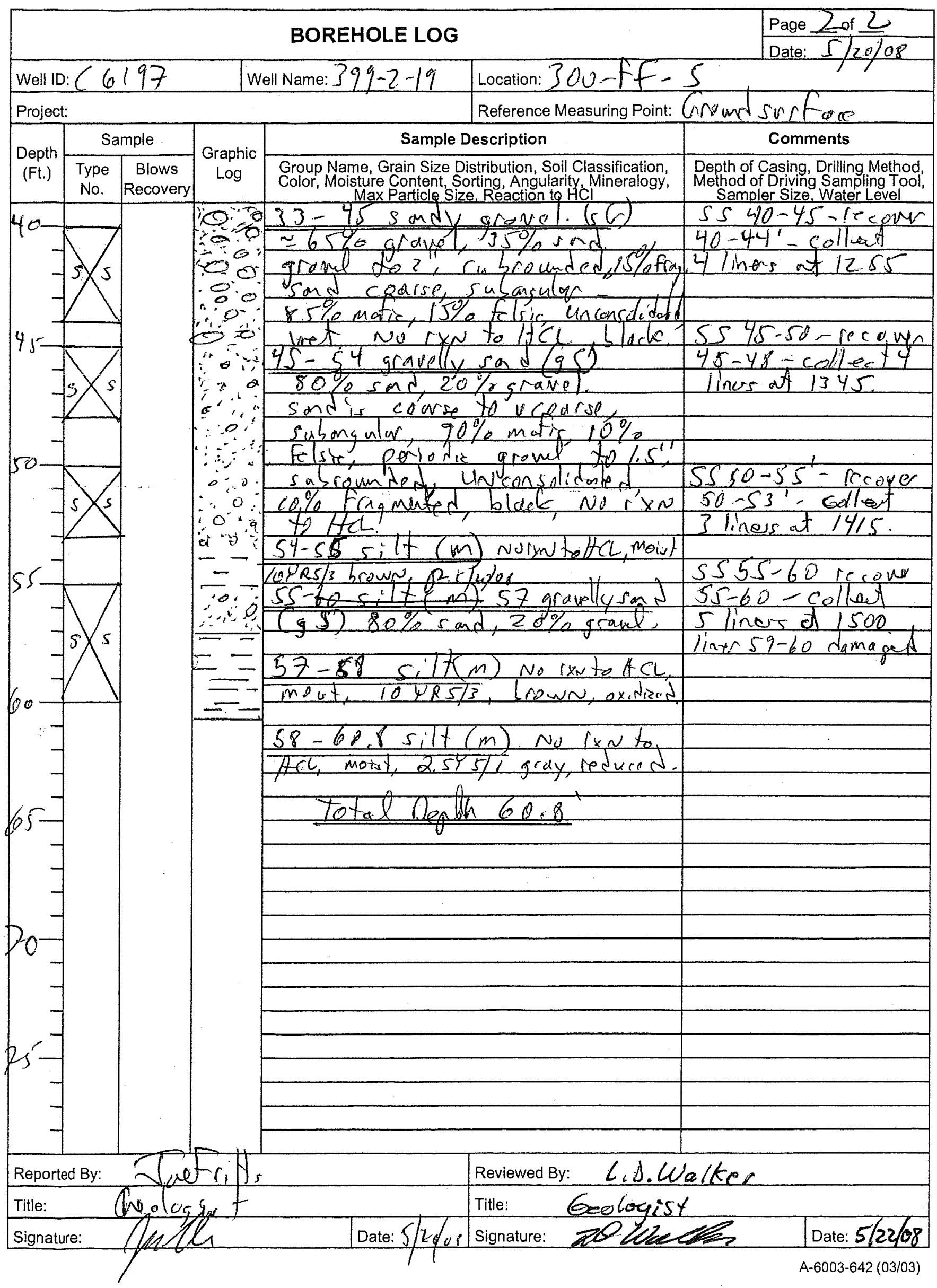




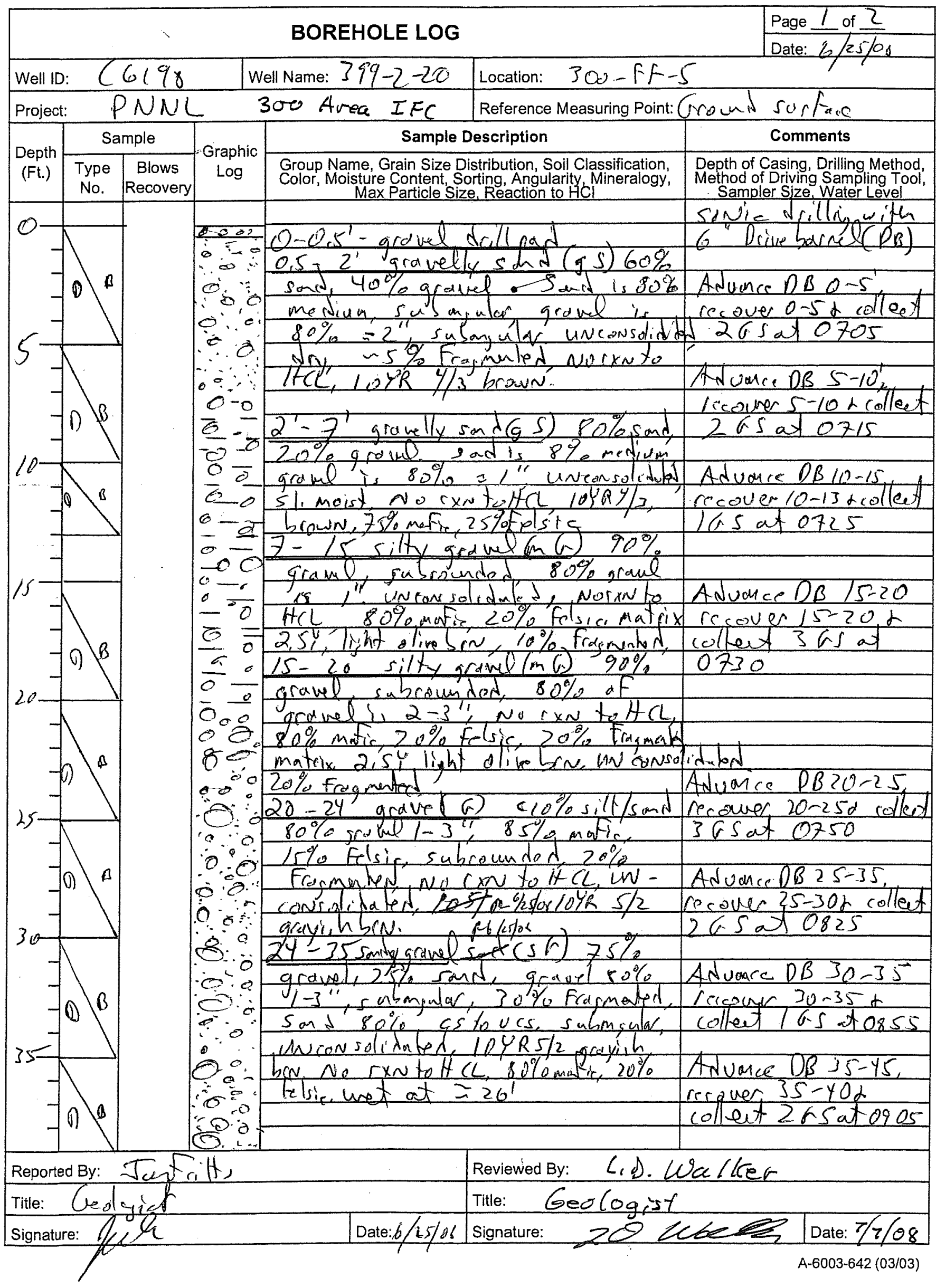

B. 27 


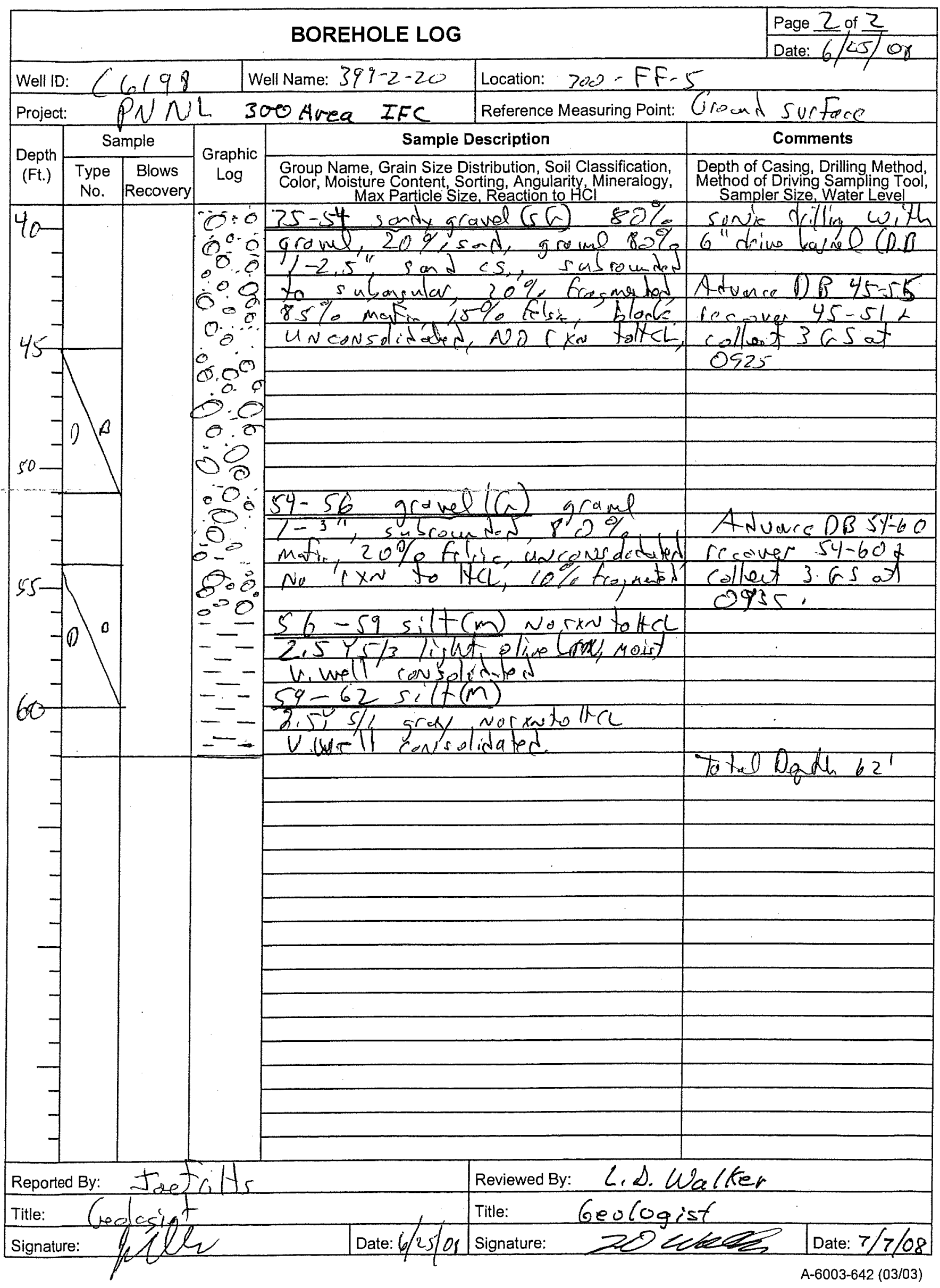




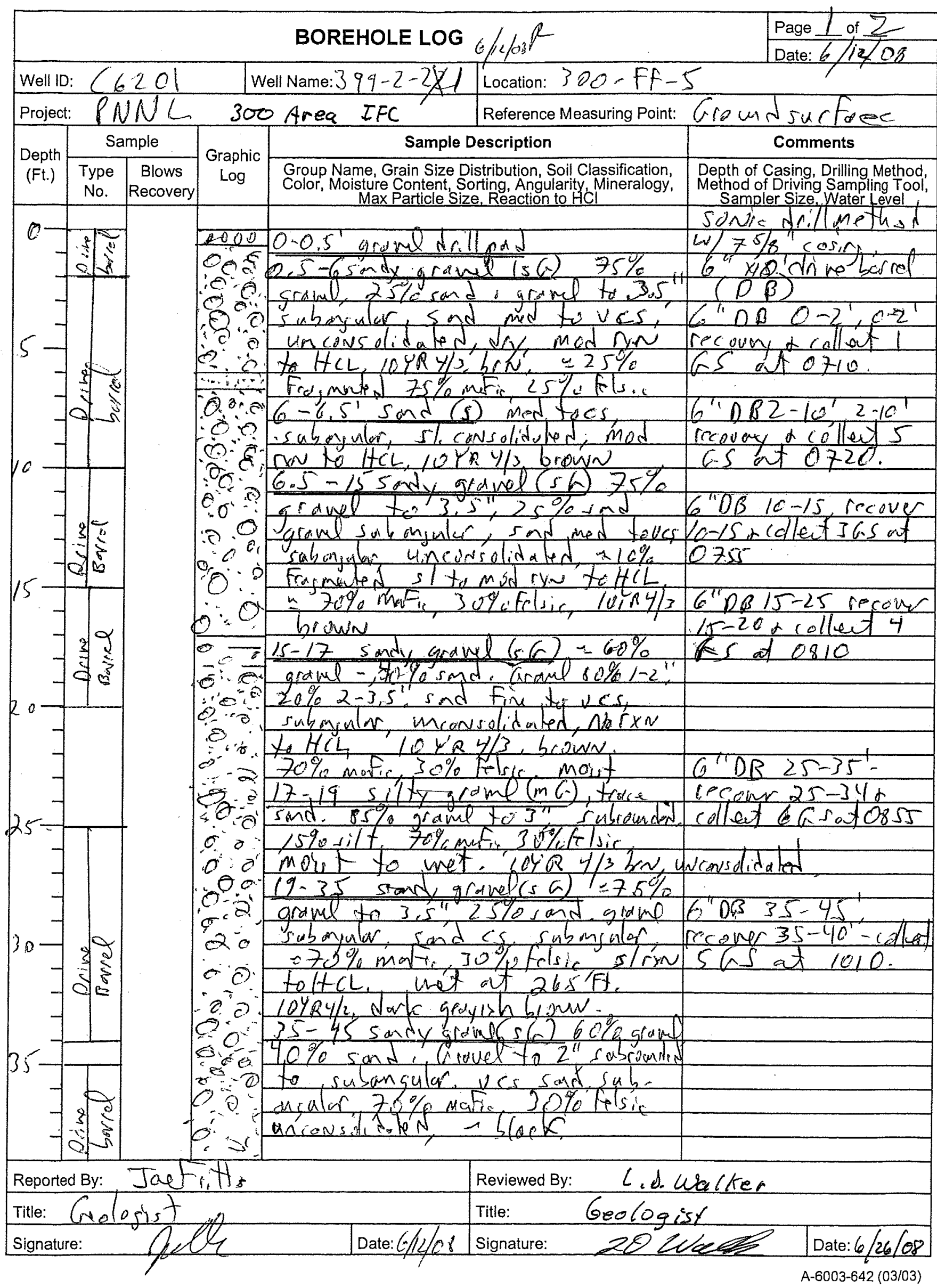




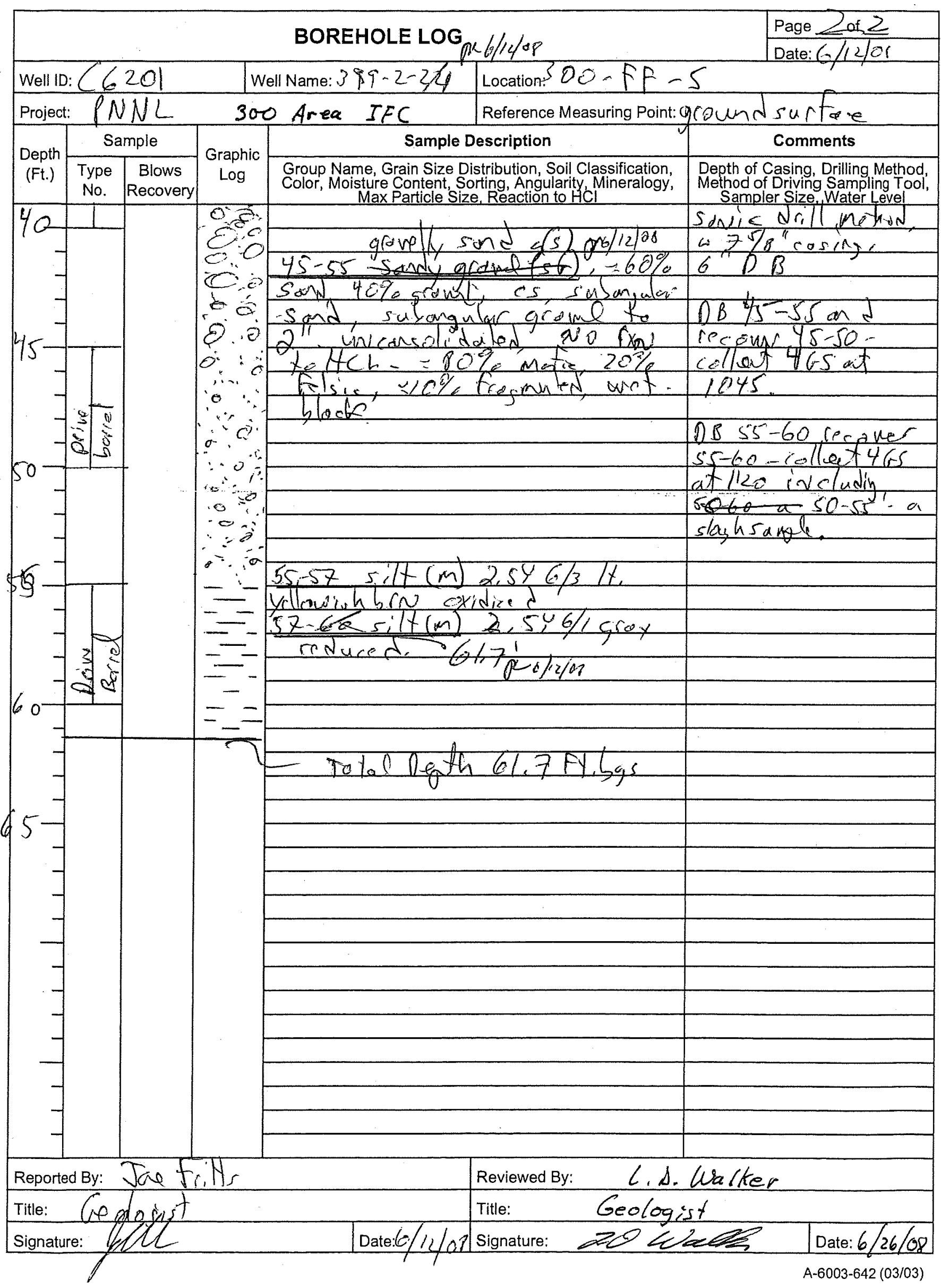




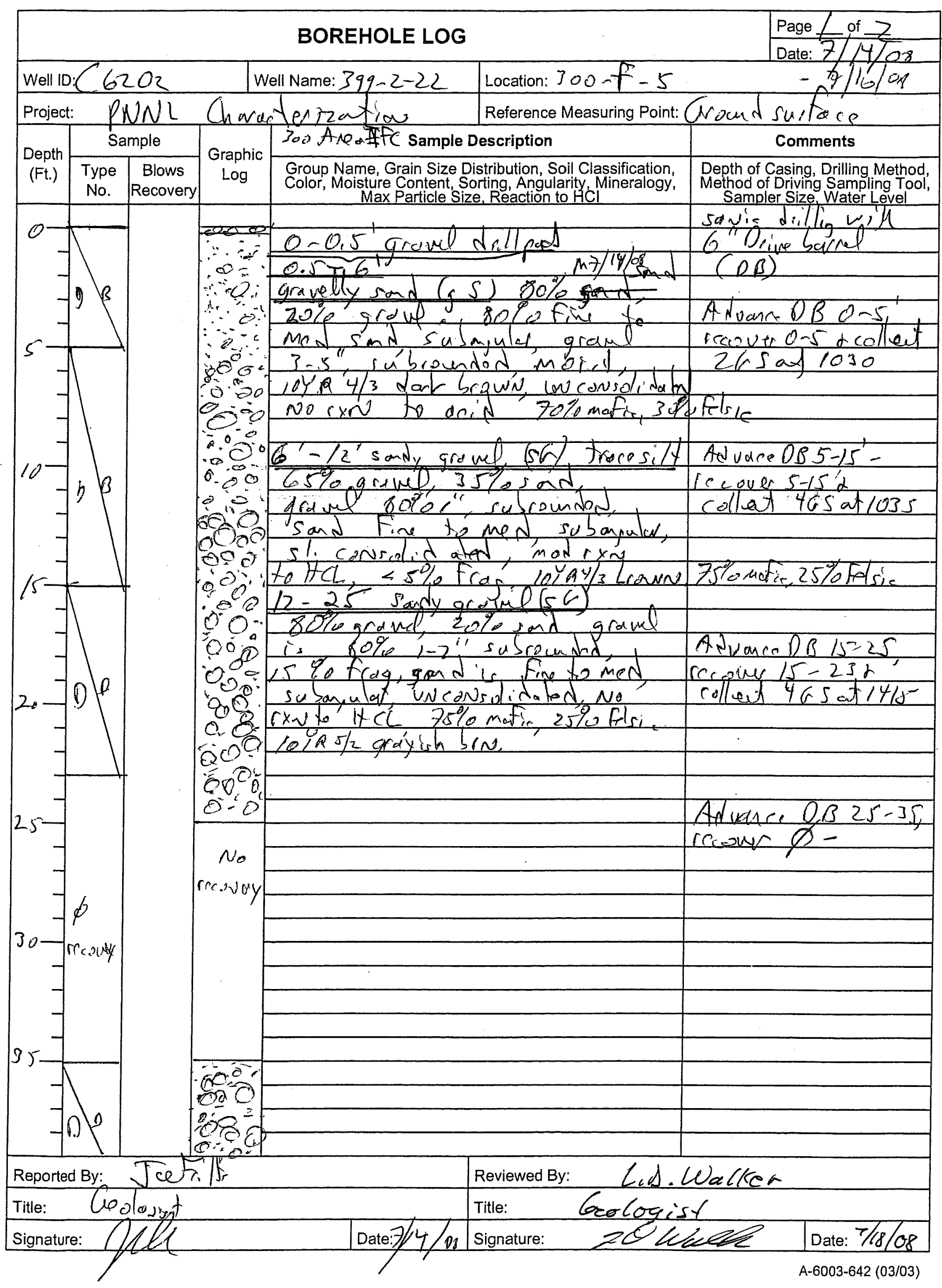




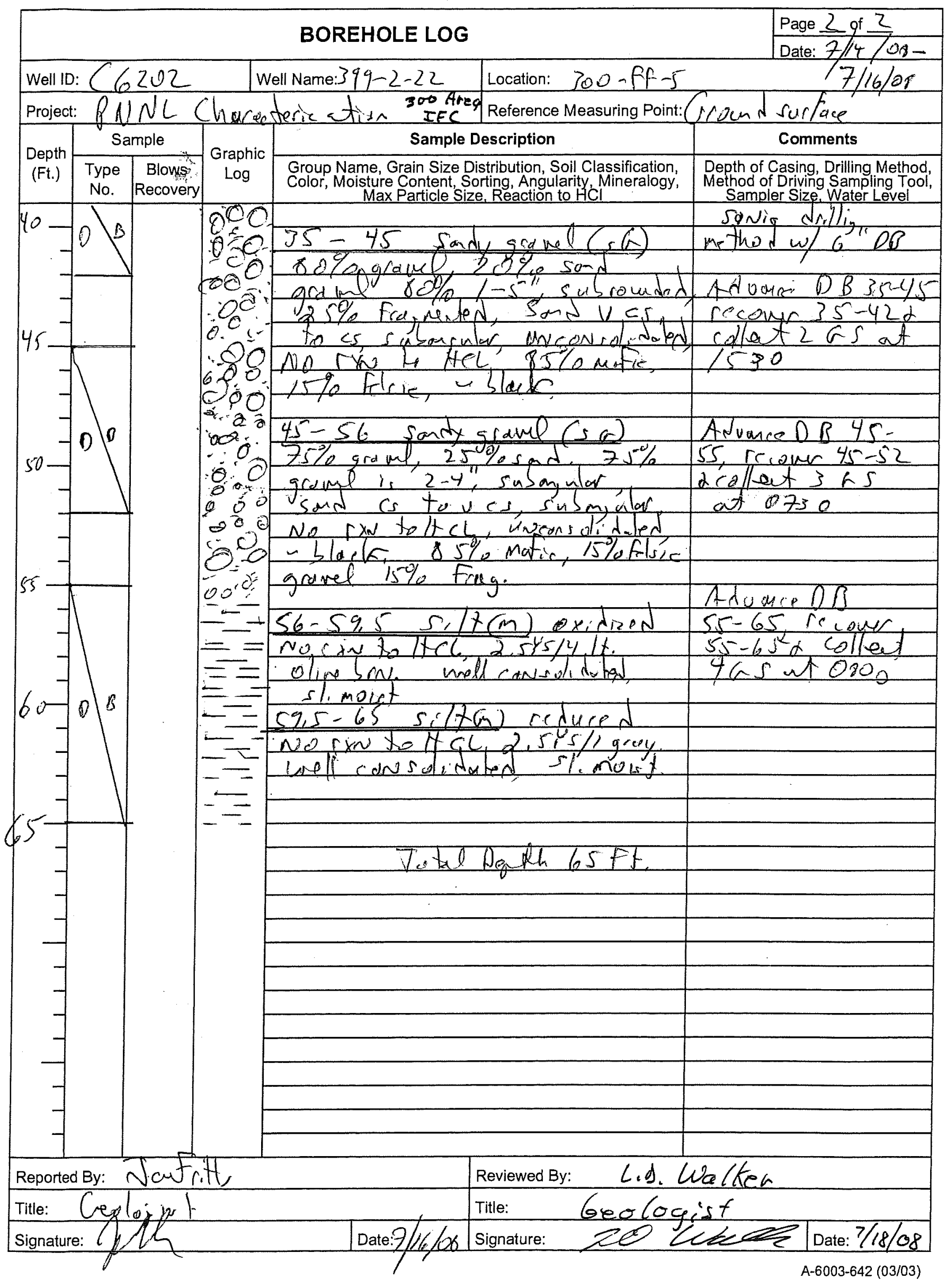




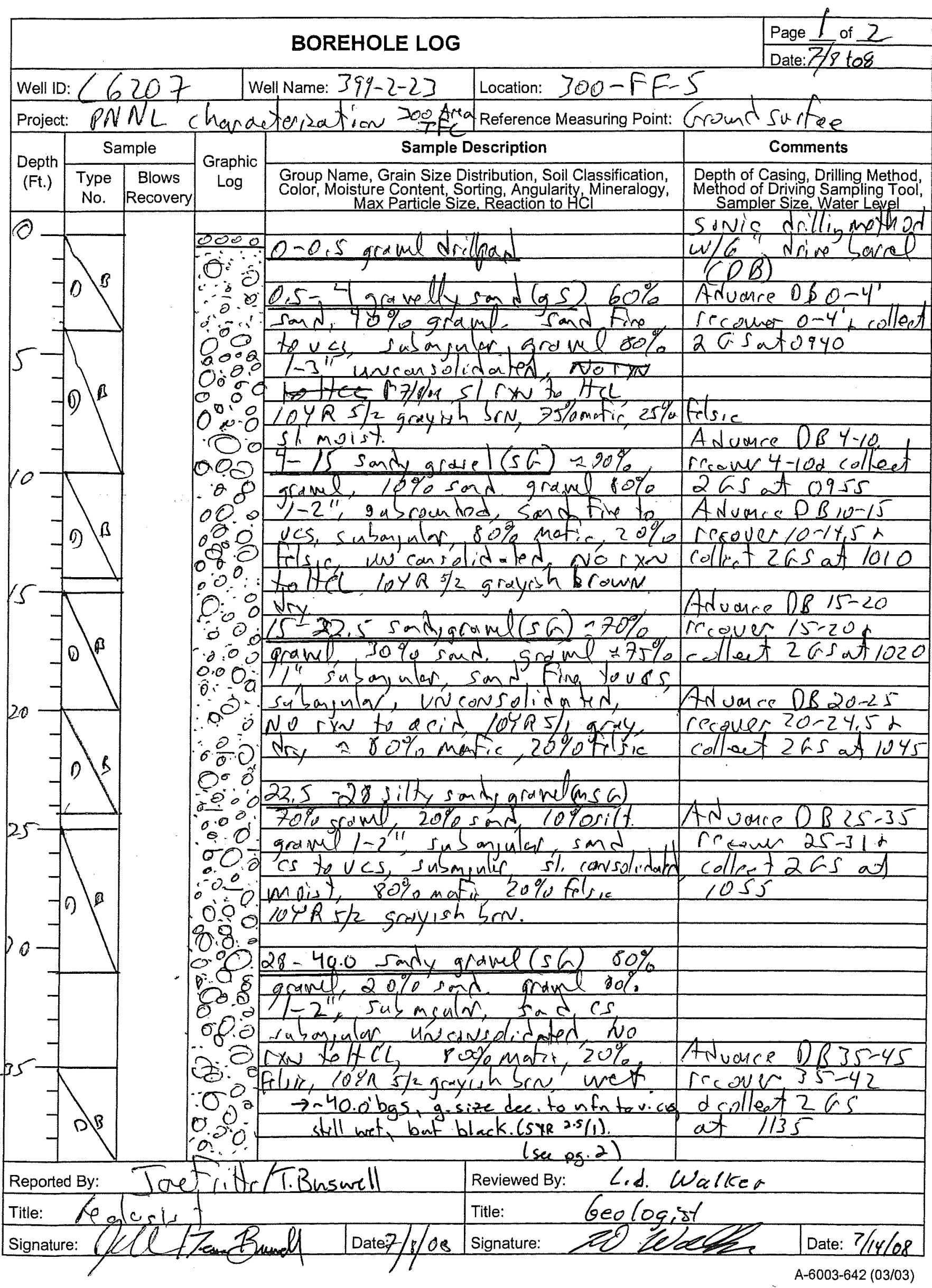

B.33 


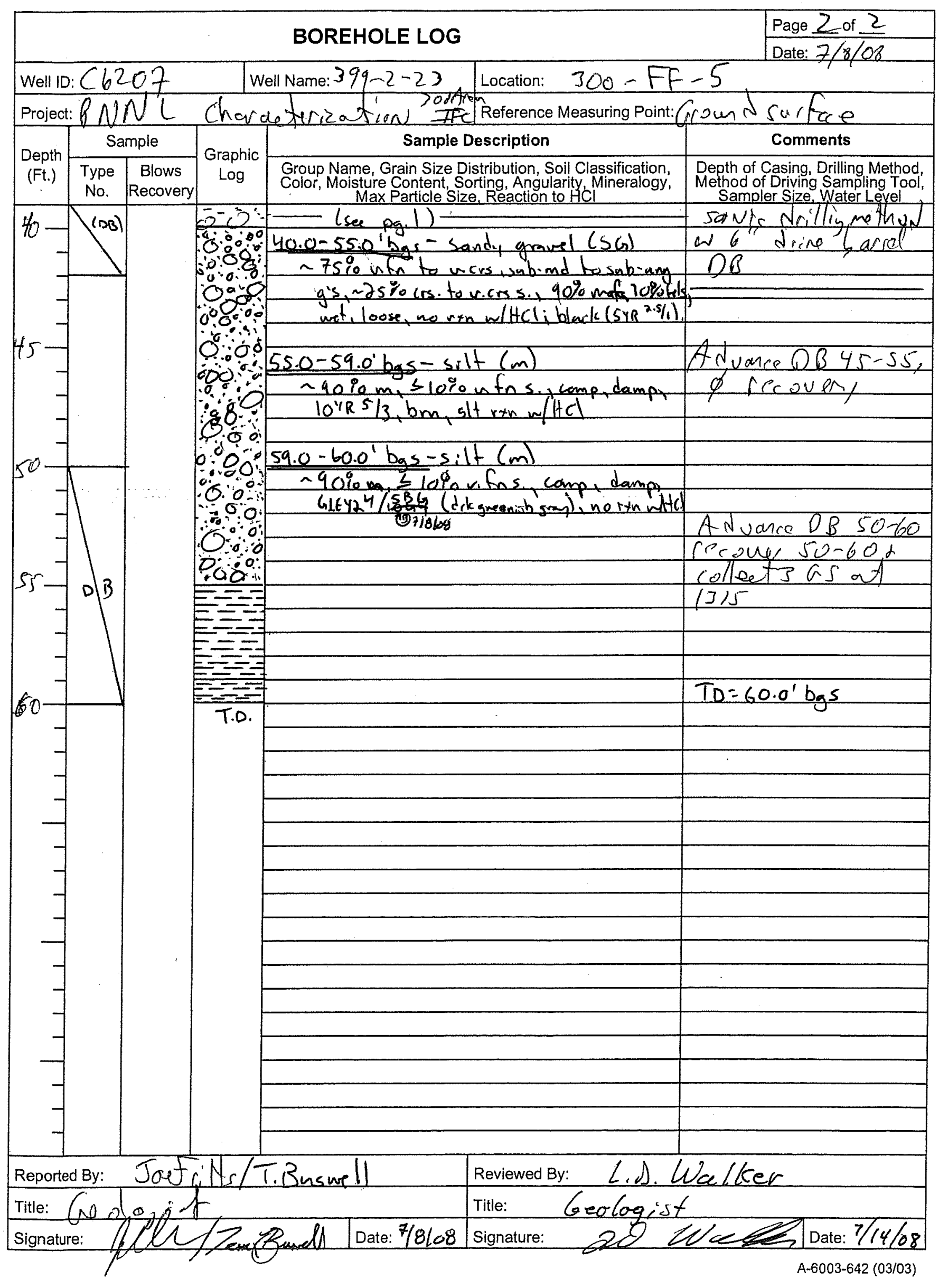

B.34 


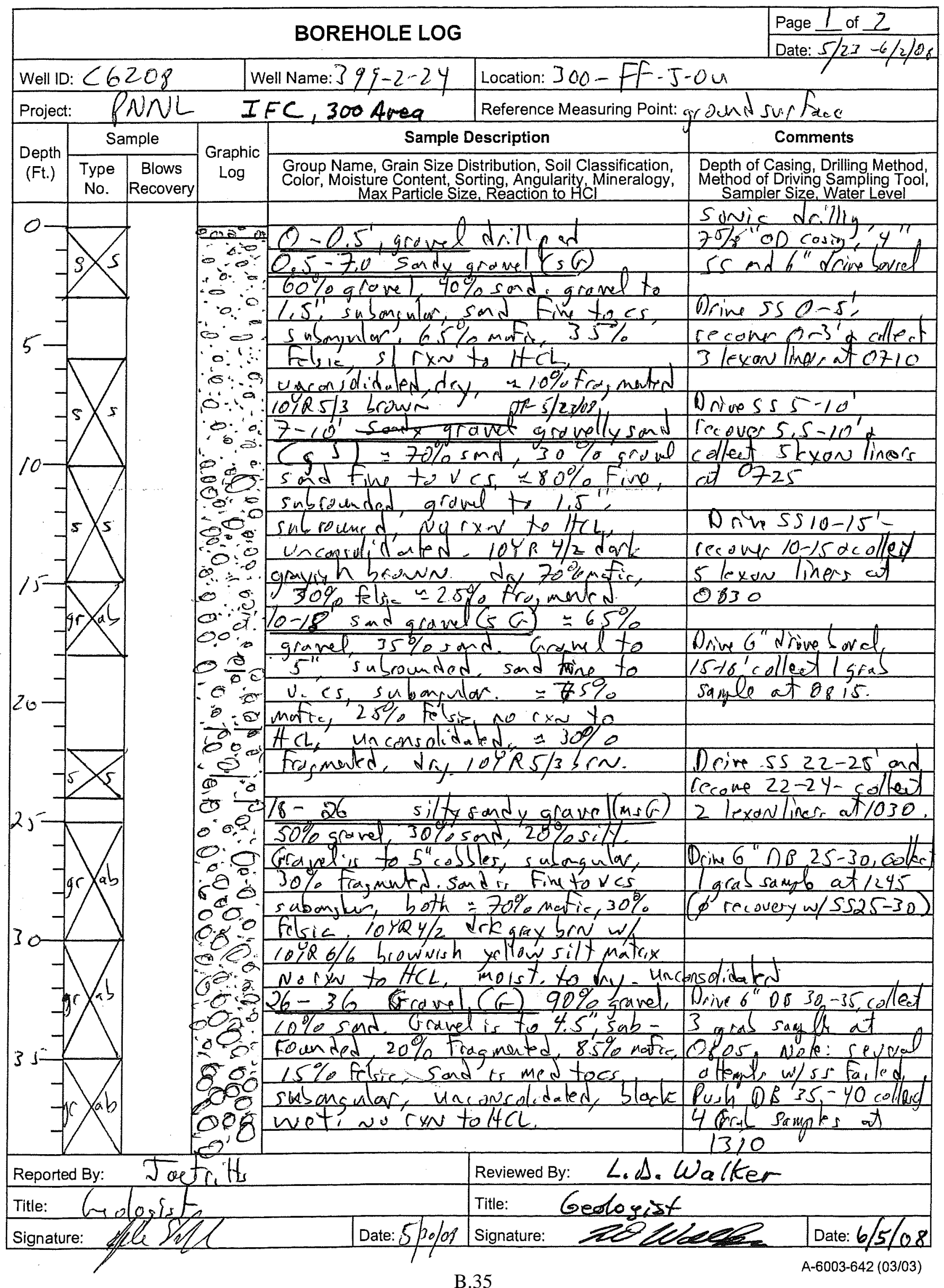




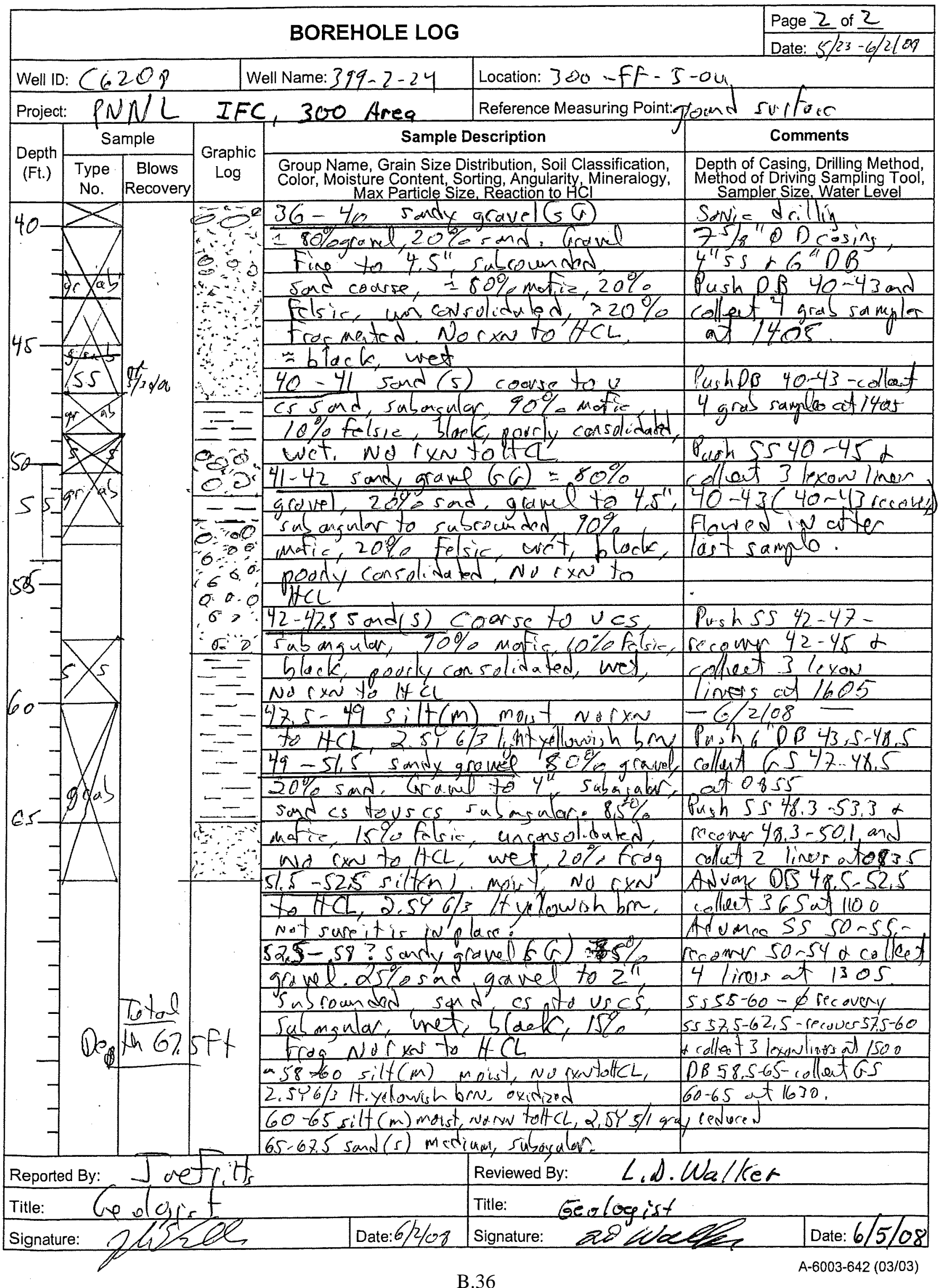




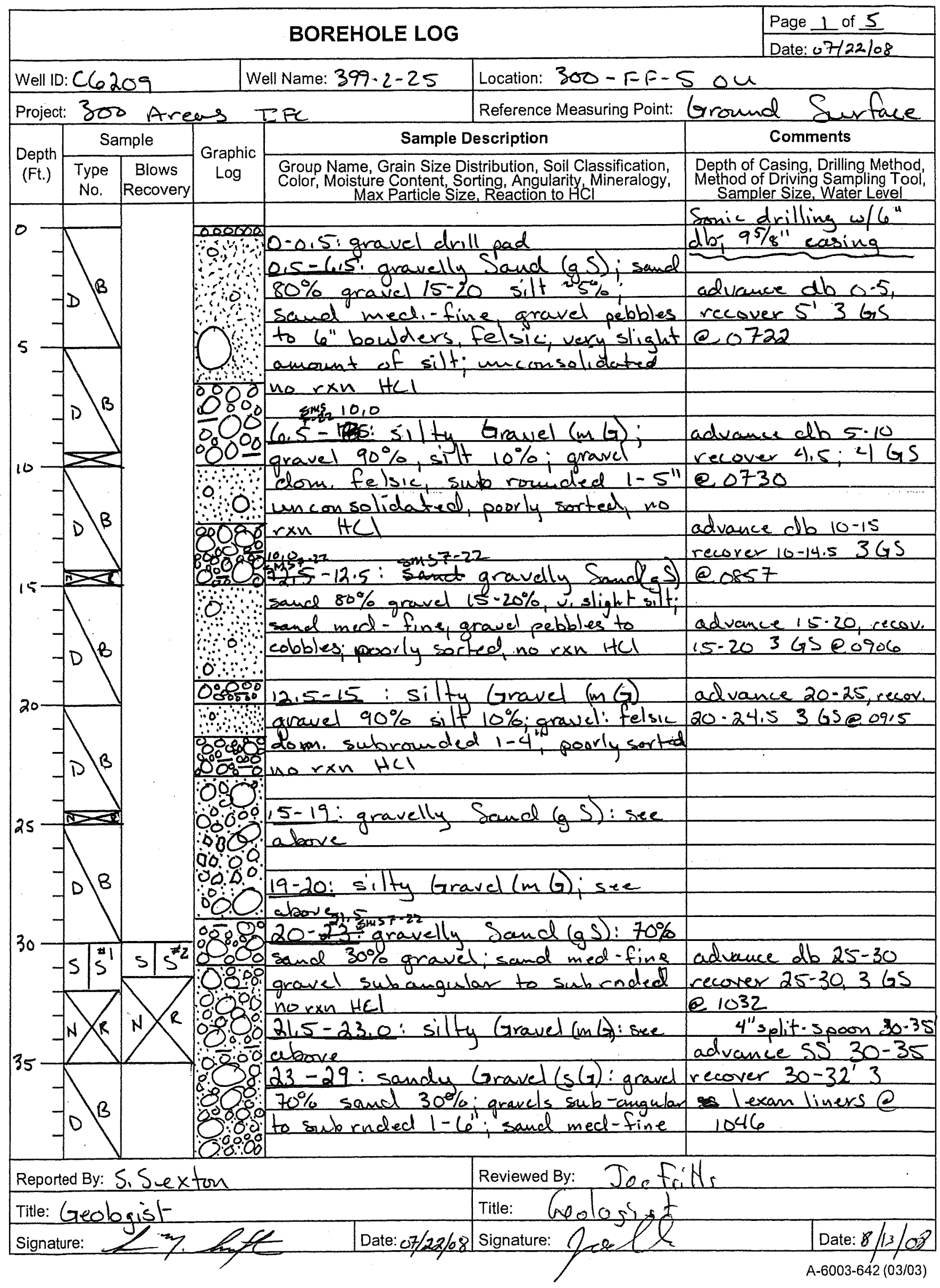




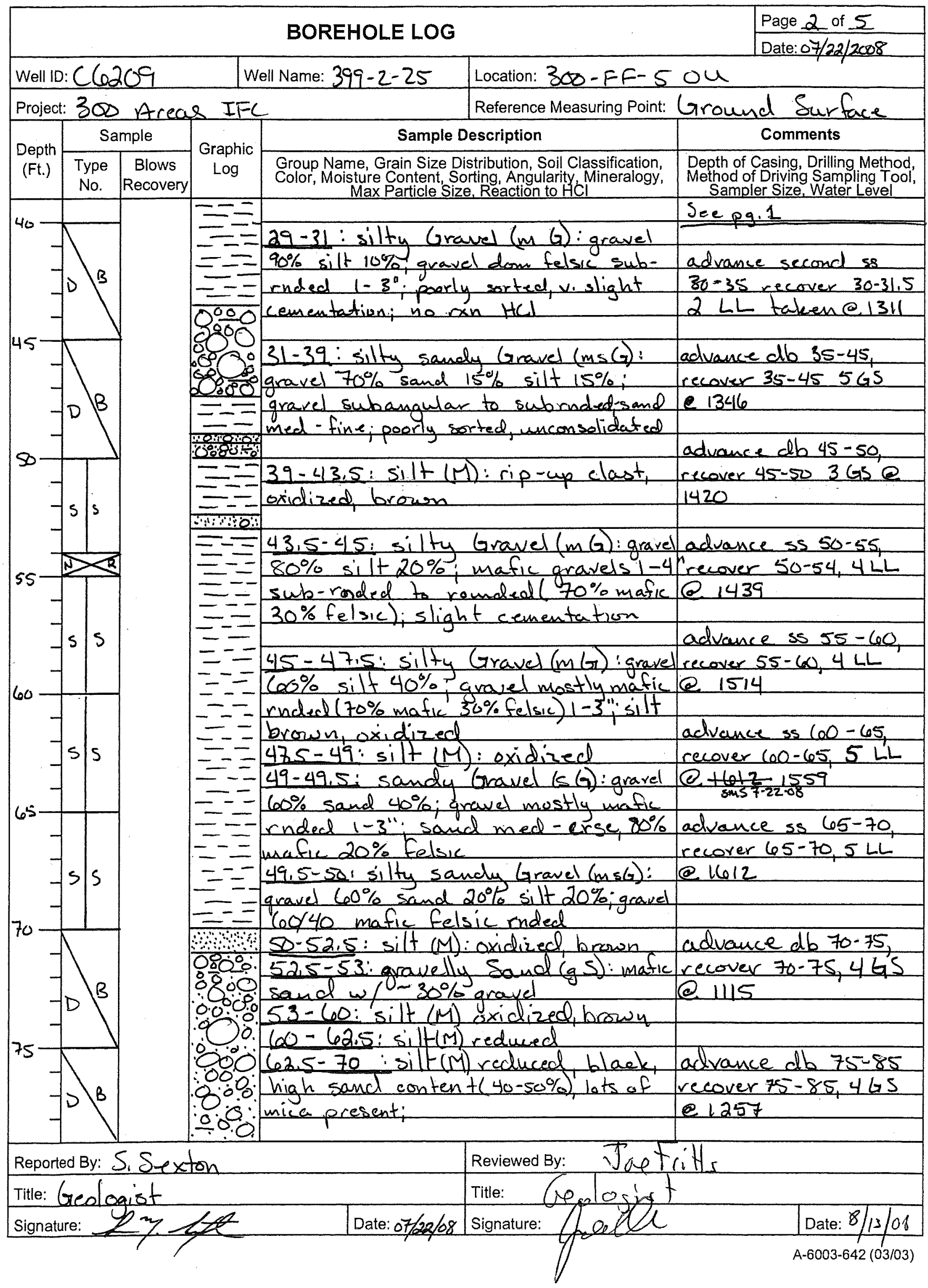




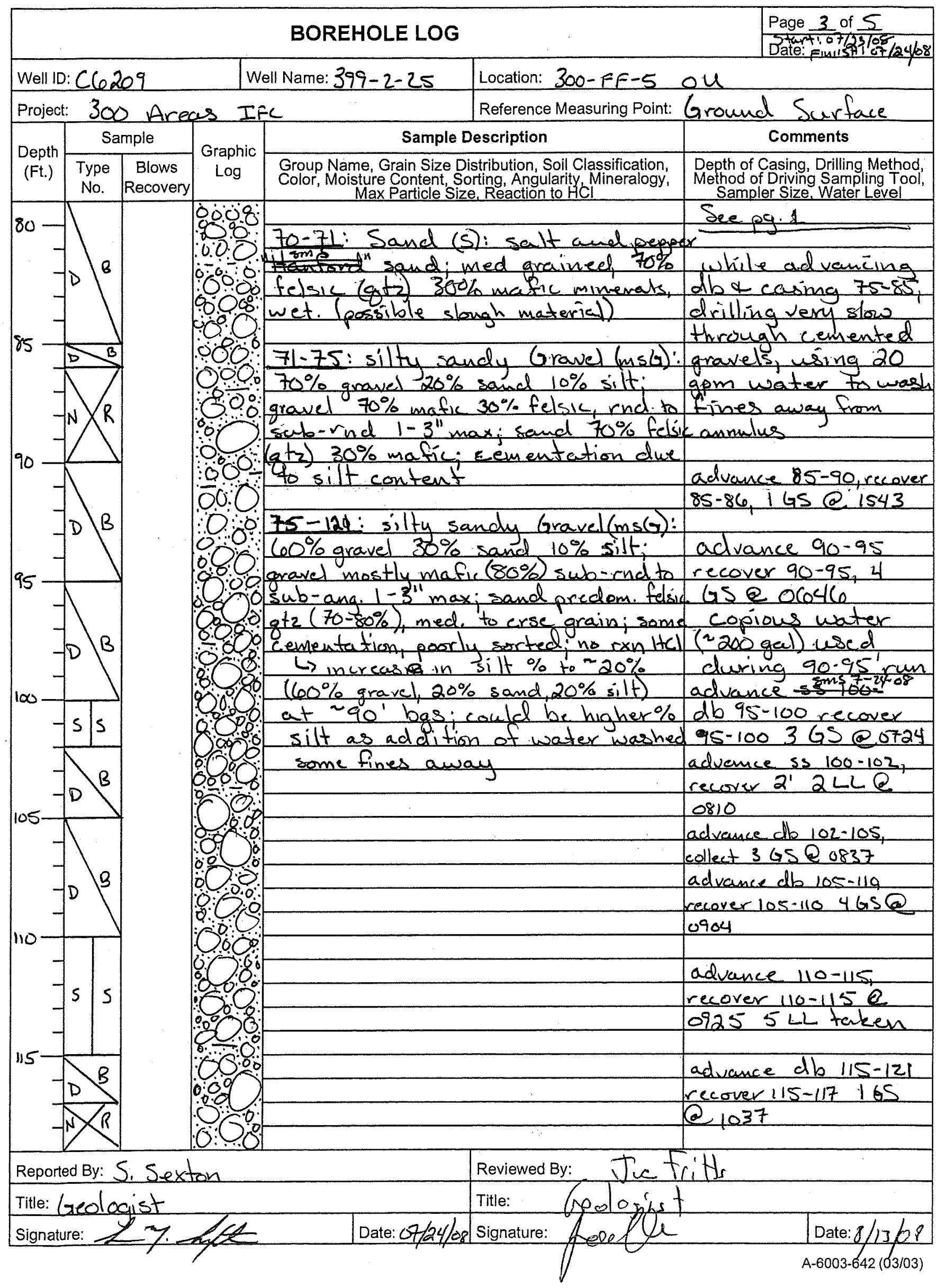




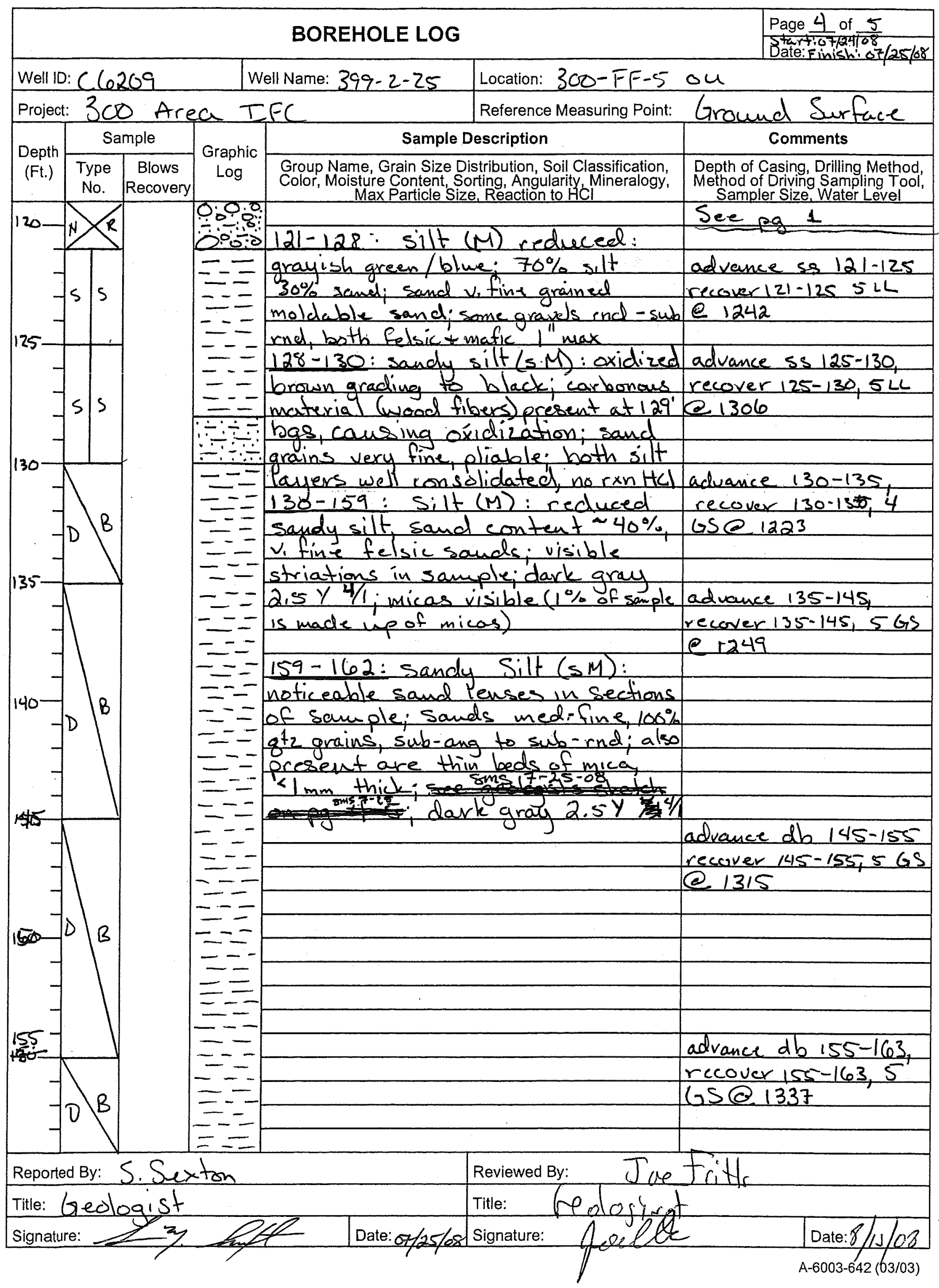




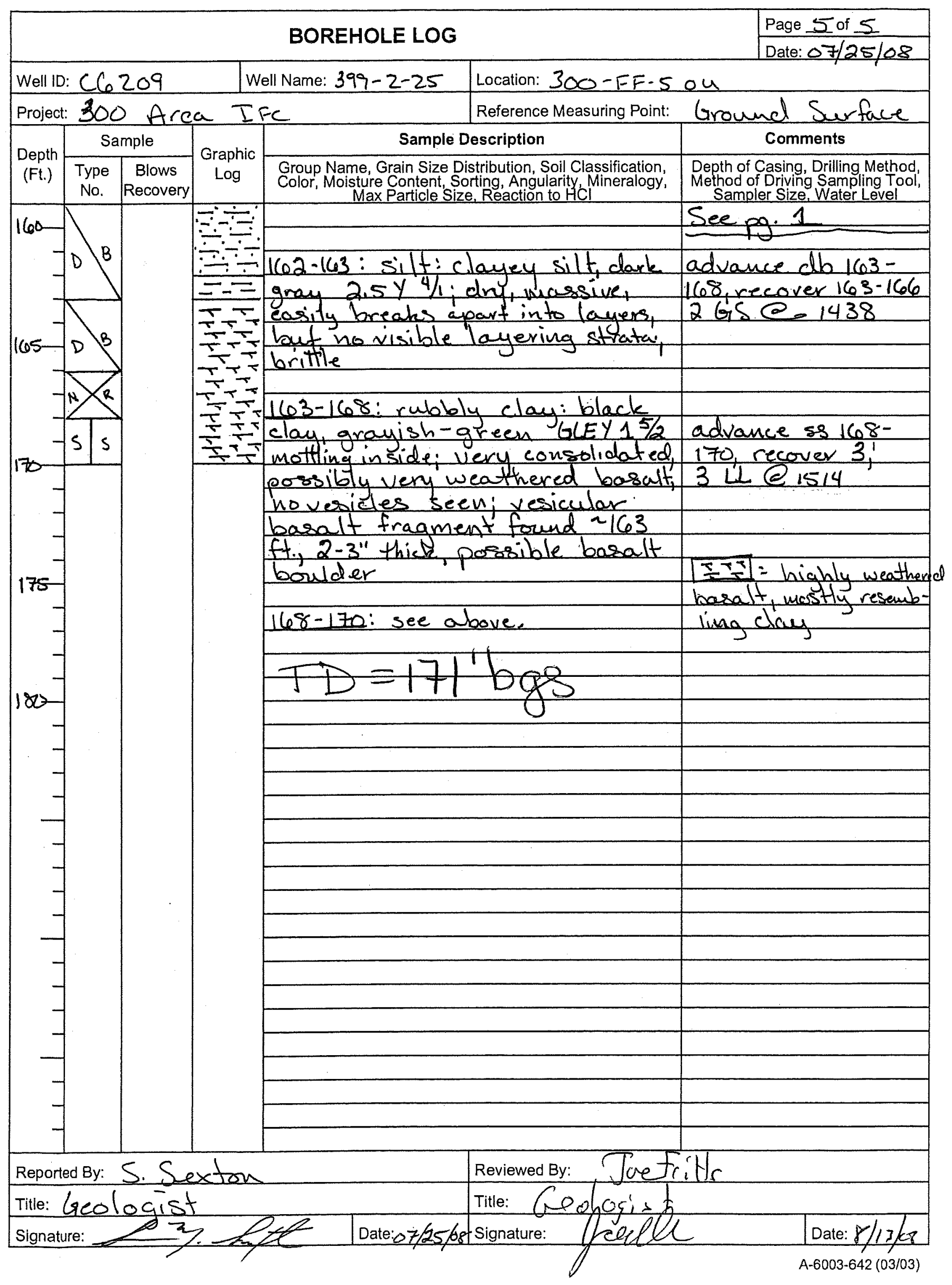


BOREHOLE LOG

$\frac{\text { Page } \frac{1}{\text { of } 2}}{\text { Date: b/23 te/29/08 }}$

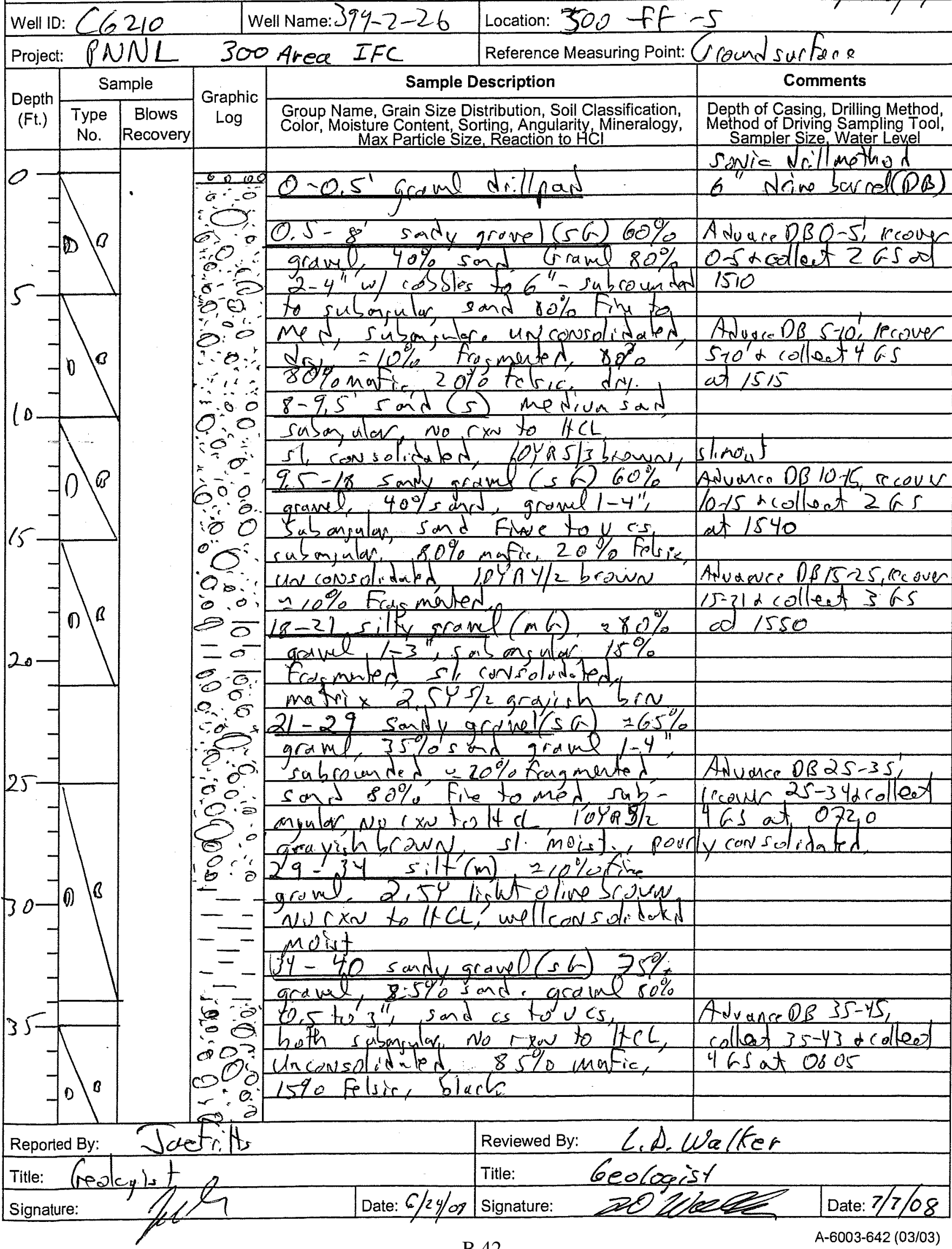




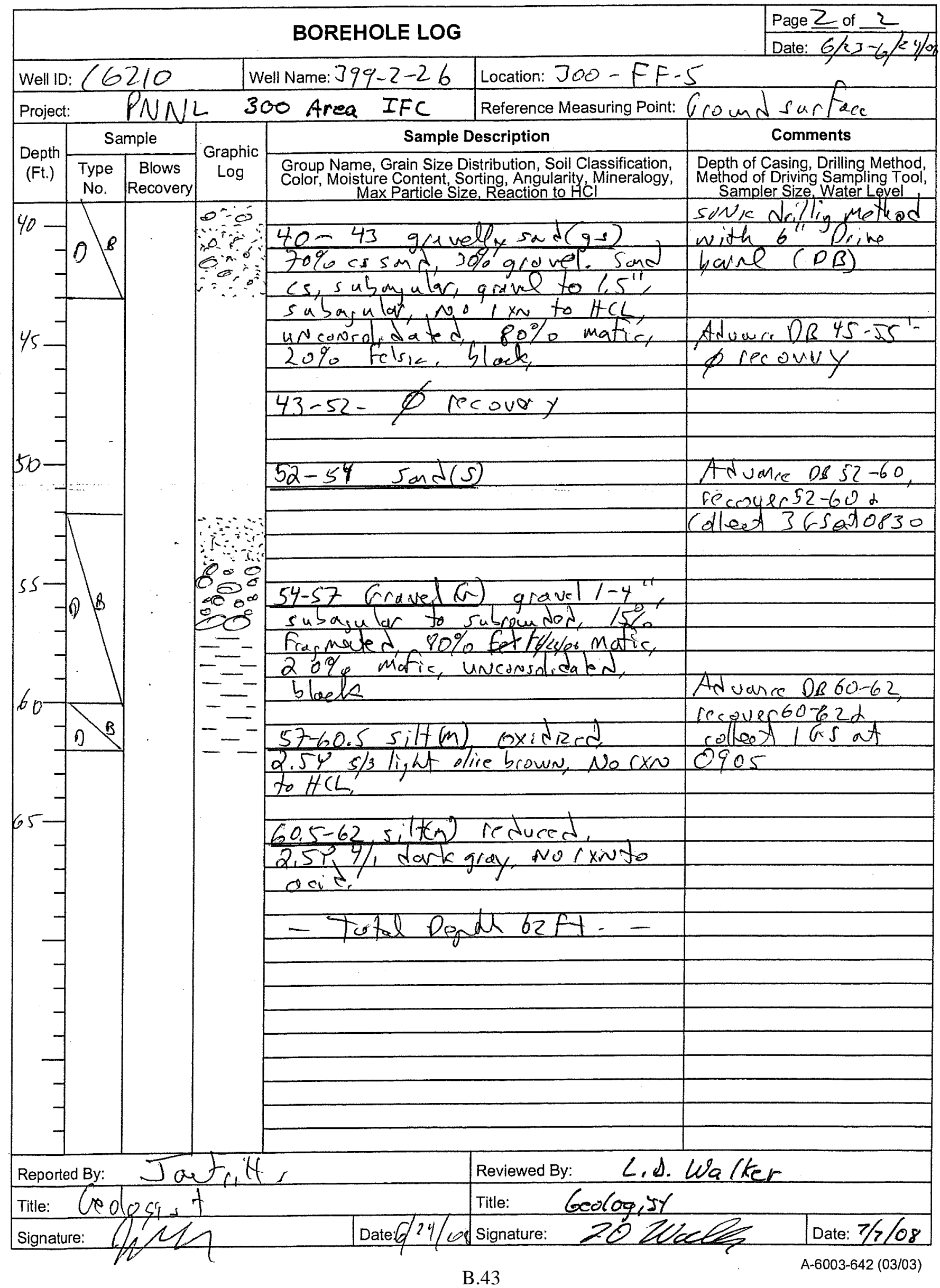




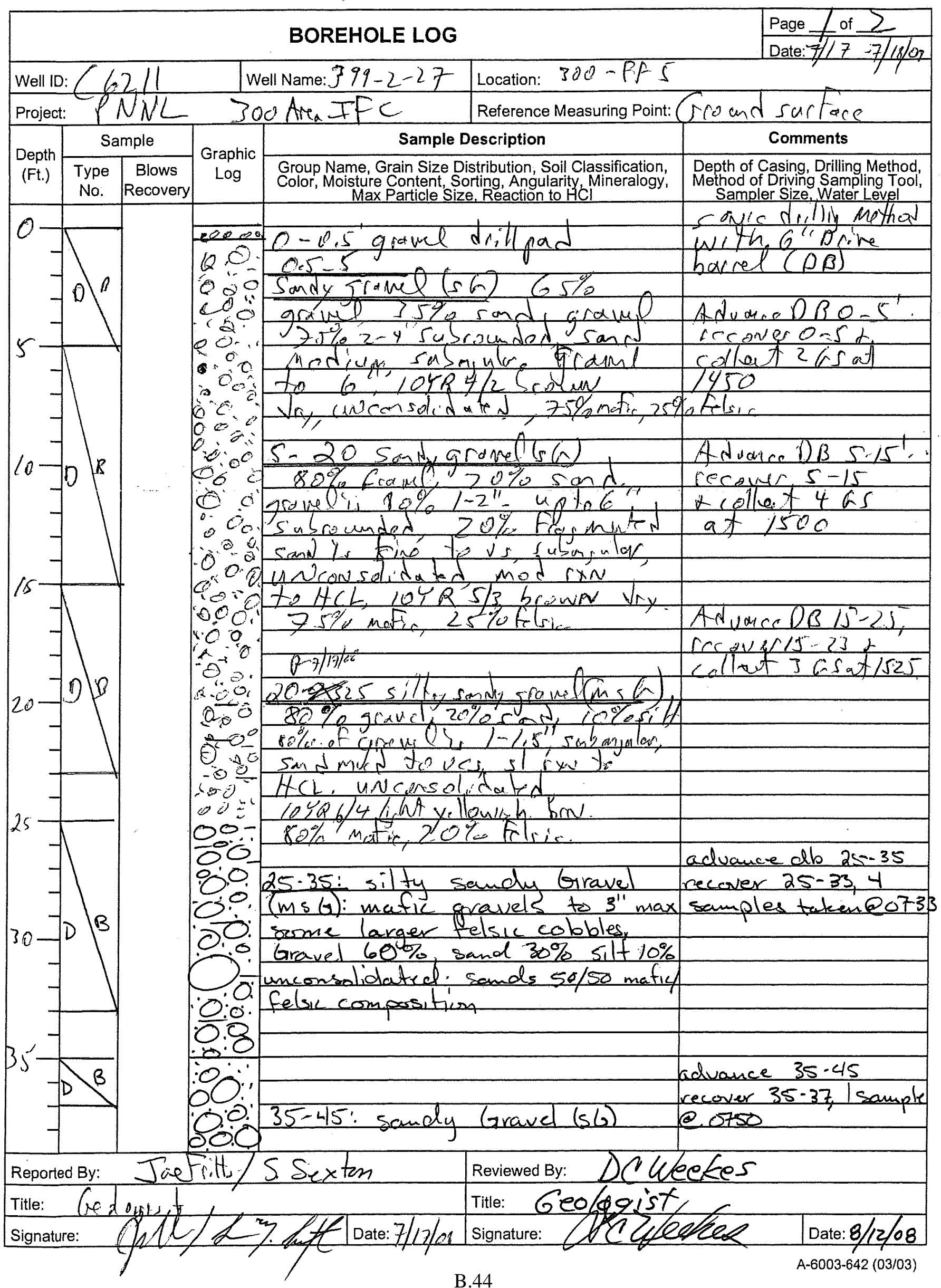




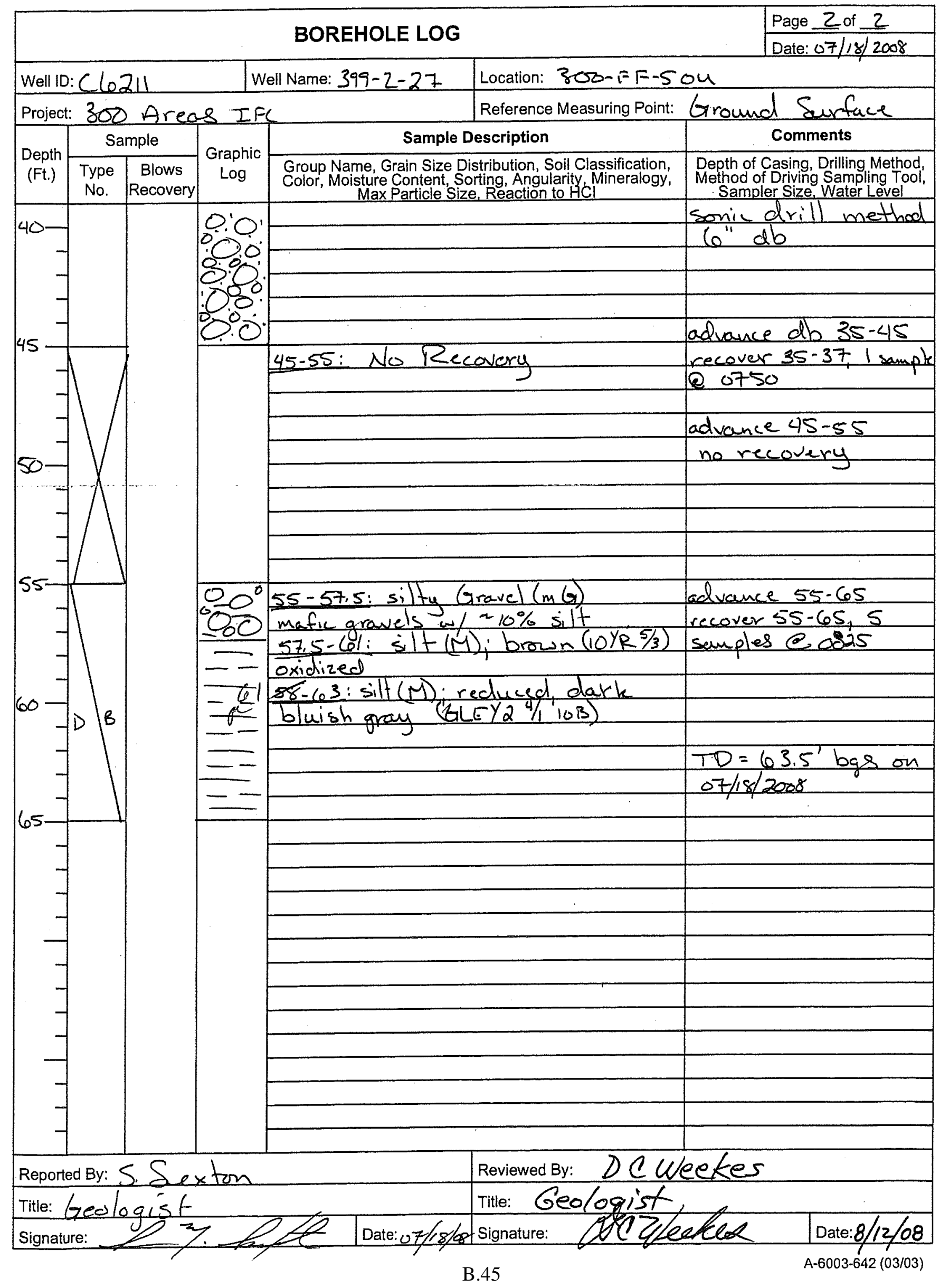




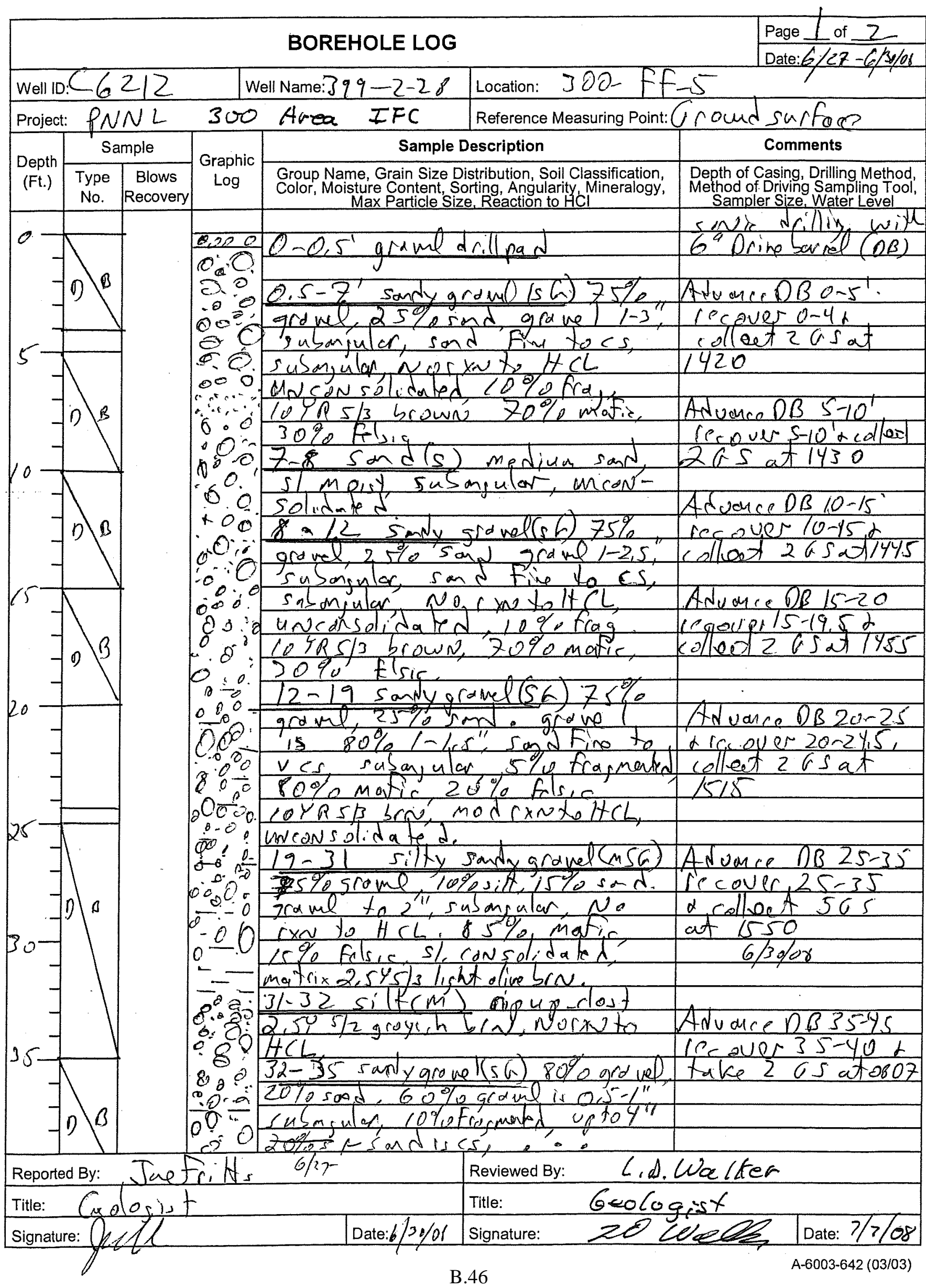




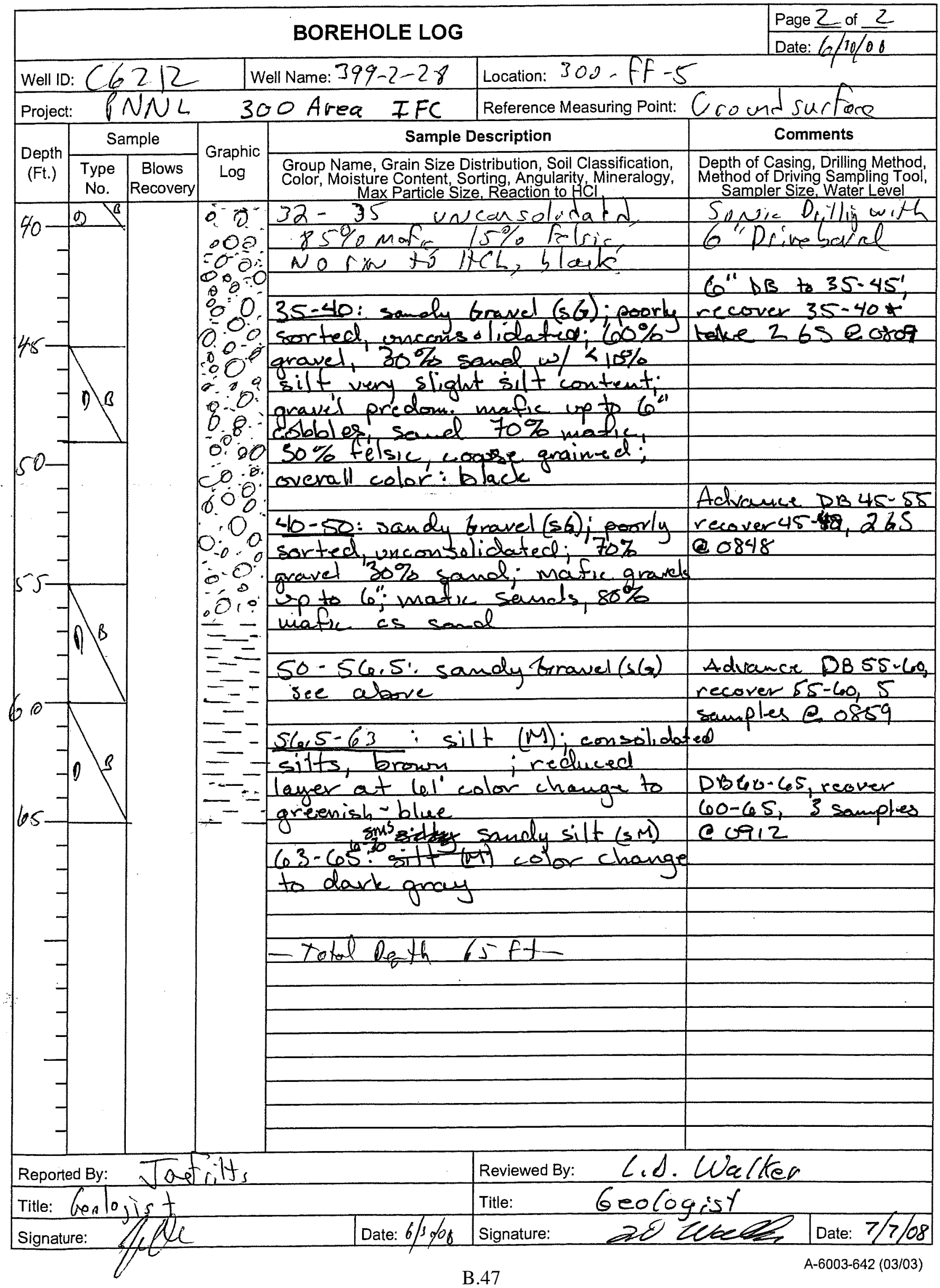




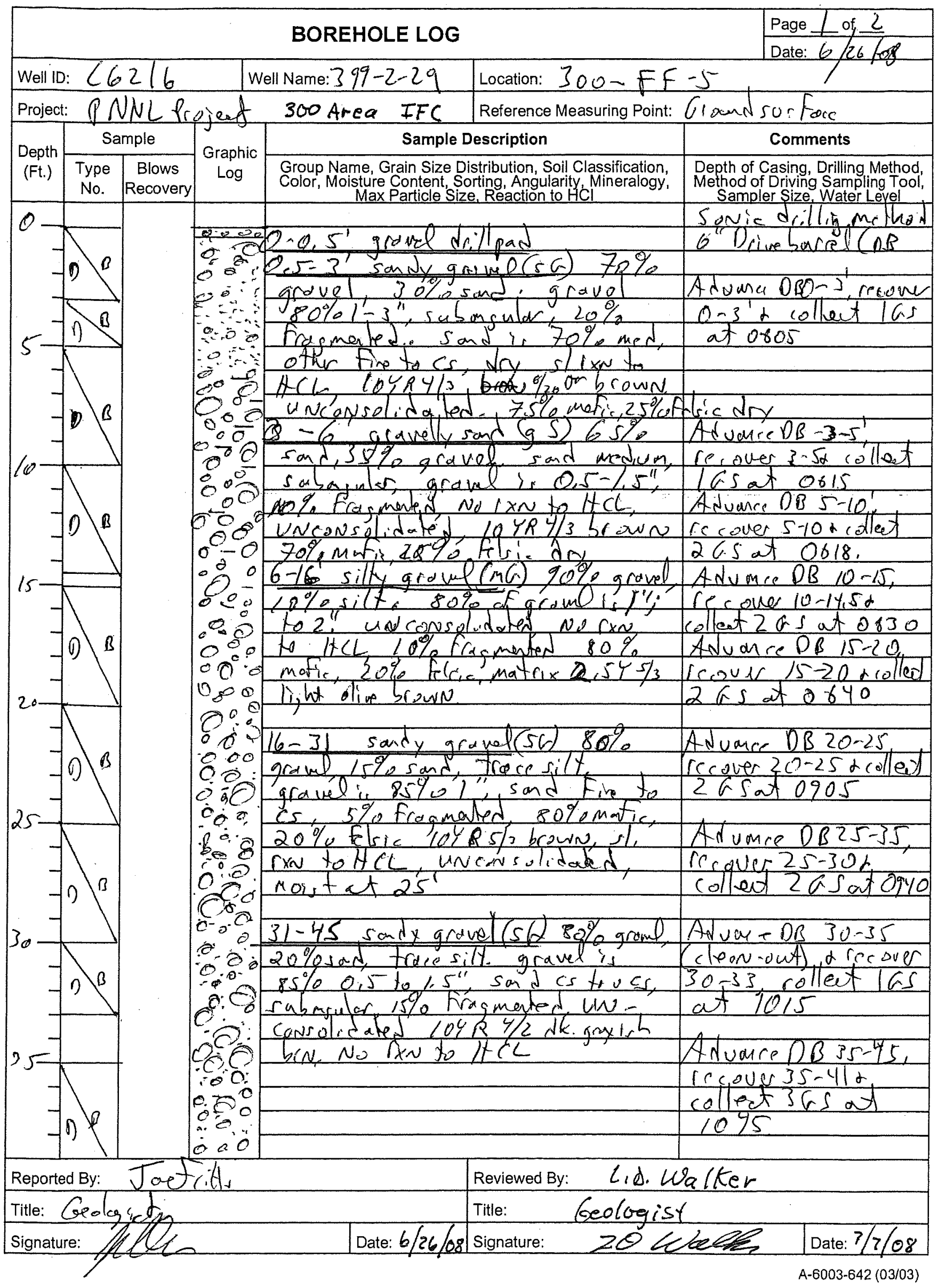




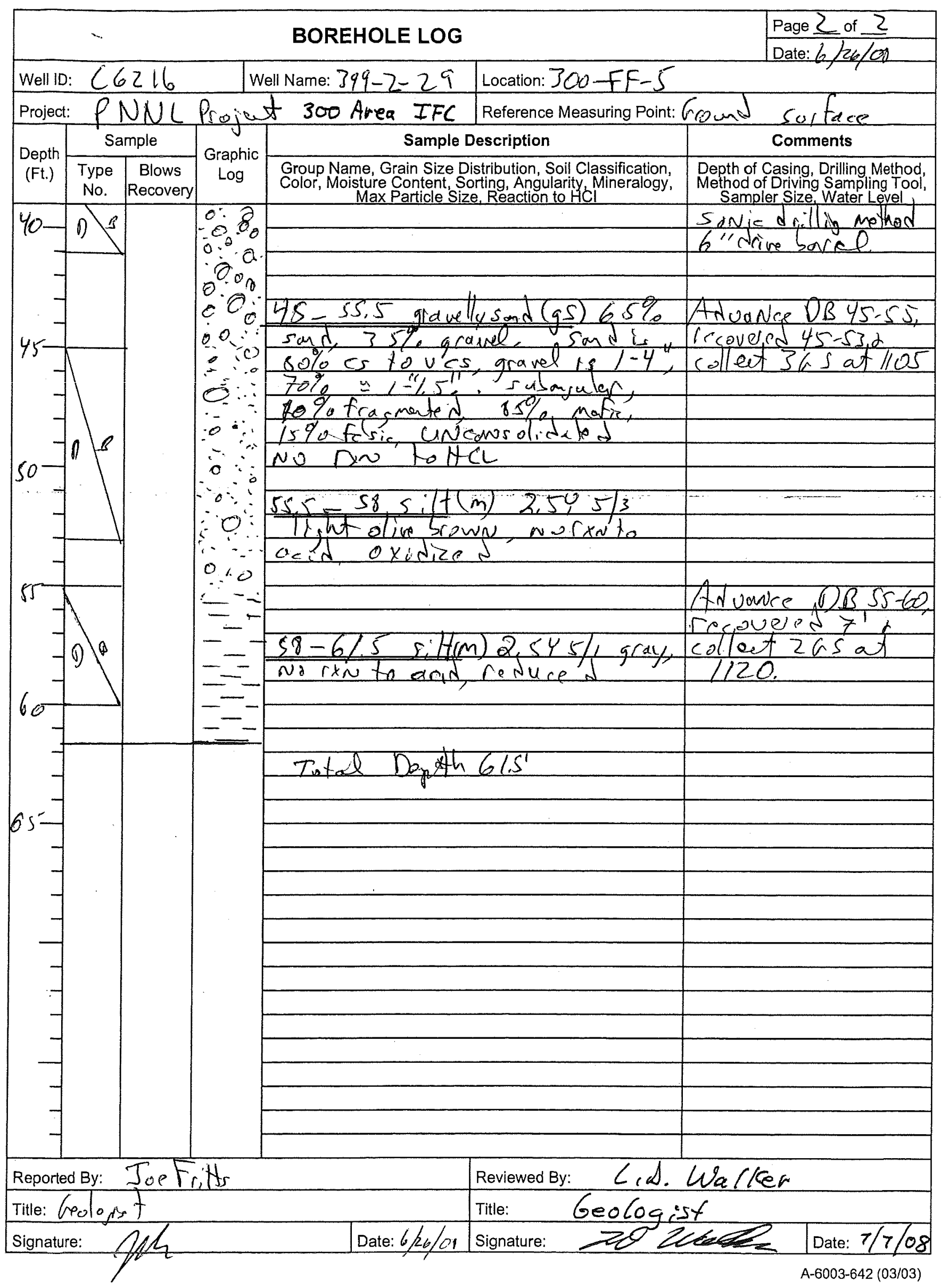




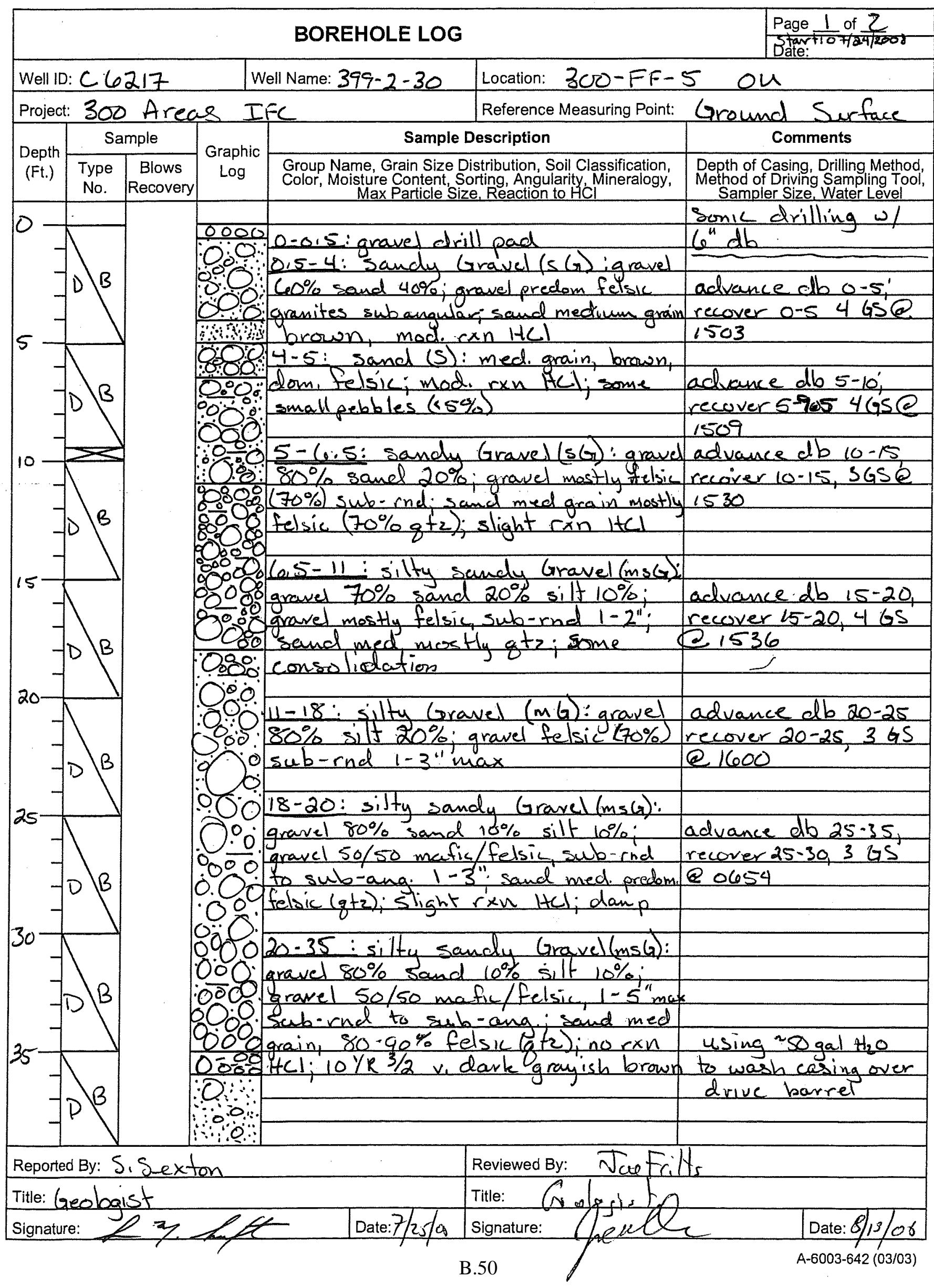




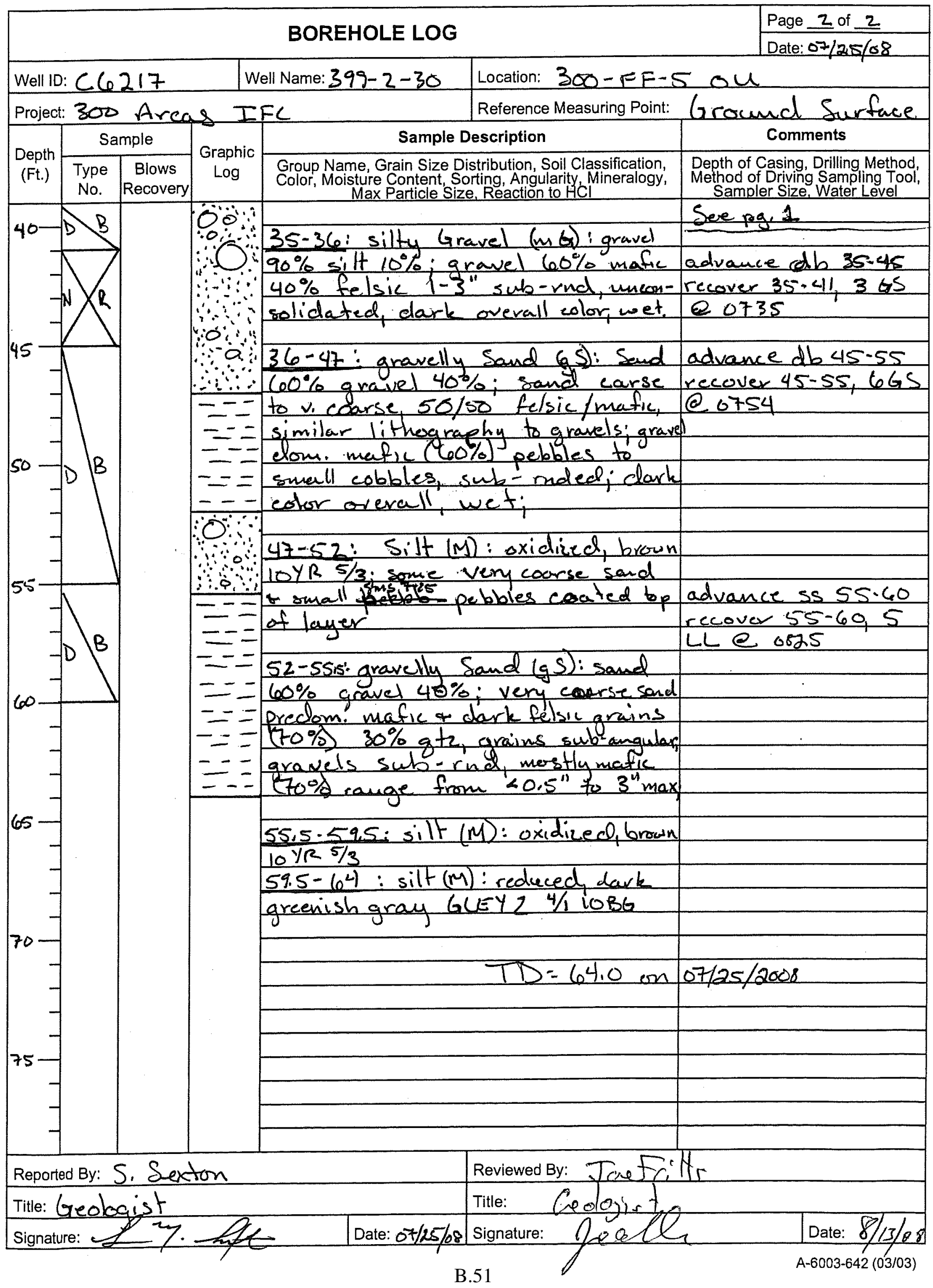




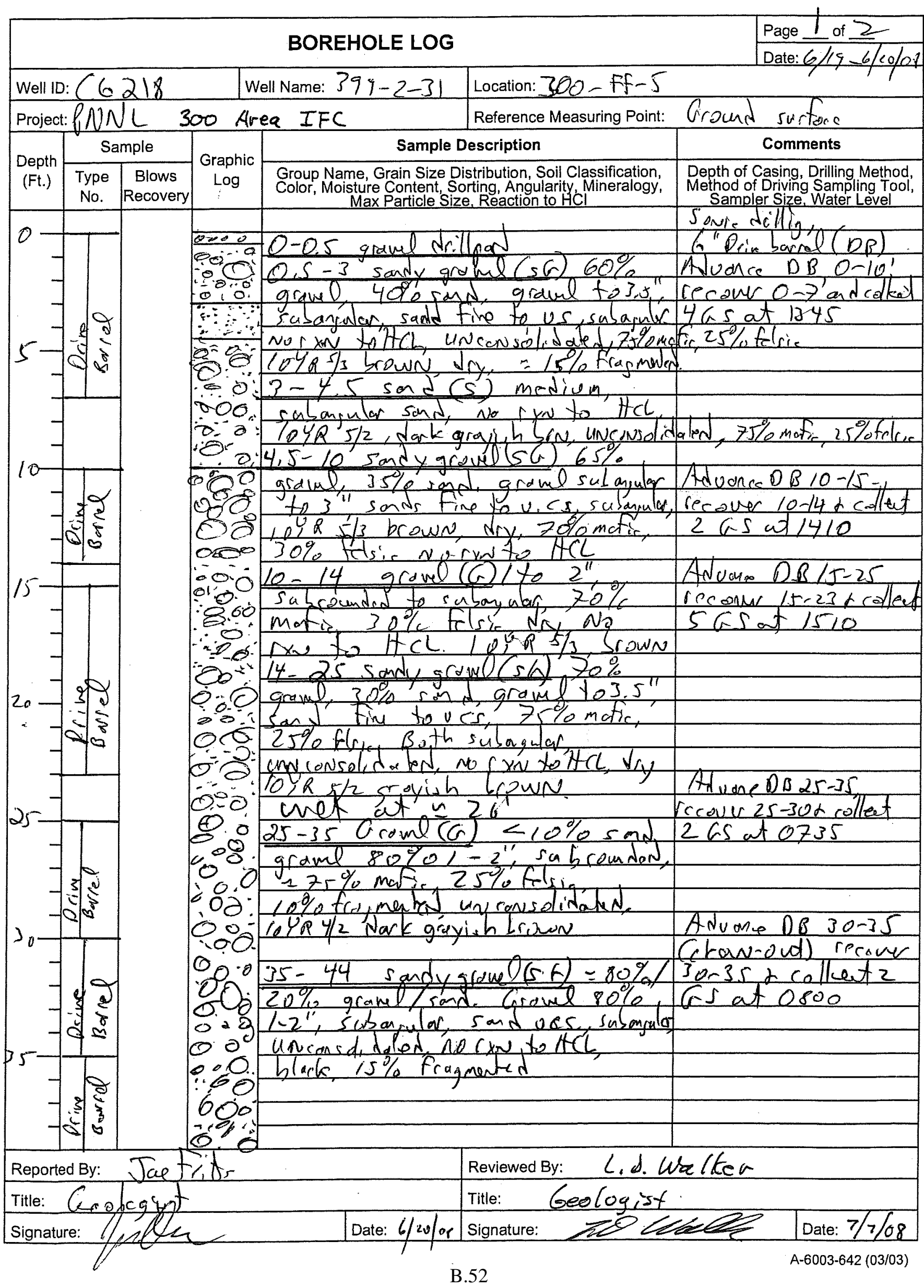




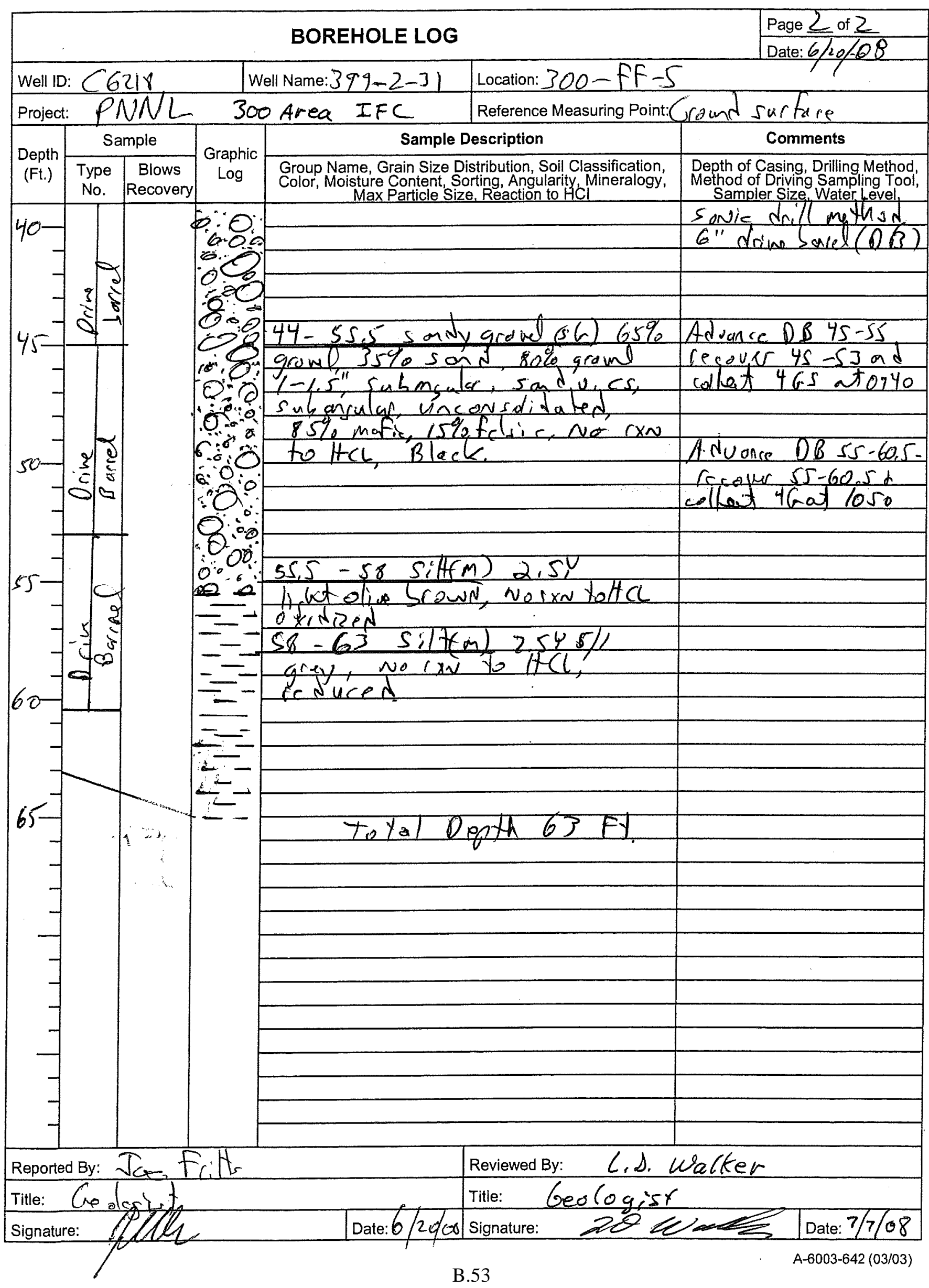




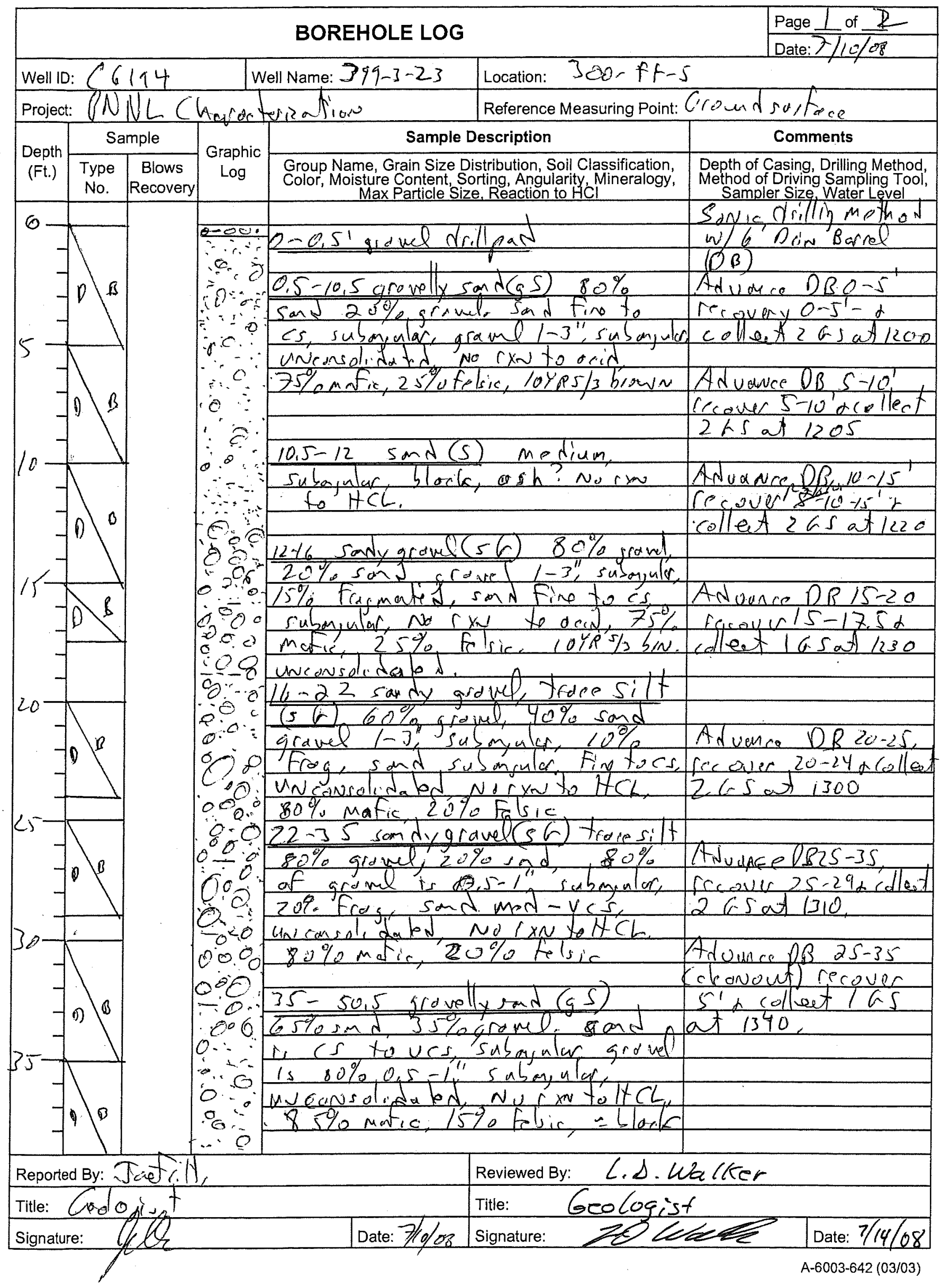


BOREHOLE LOG

\begin{tabular}{l|l|l|l|}
\hline Well ID: $C 6194$ & Well Name: $394-3-23$ & Location: 300 - Ff - 5 \\
\hline
\end{tabular}

Project: PNNChercterization $\quad$ Reference Measuring Point: Cround surfore

\begin{tabular}{|c|c|c|c|c|}
\hline \multirow{2}{*}{ Depth } & Sample & \multirow{2}{*}{ Graphic } & Sample Description & Comments \\
\cline { 2 - 4 } & &
\end{tabular}

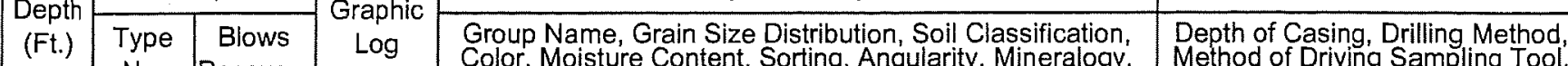
No. Recovery Log Color, Moisture Content, Sorting, Angularity, Mineralogy,

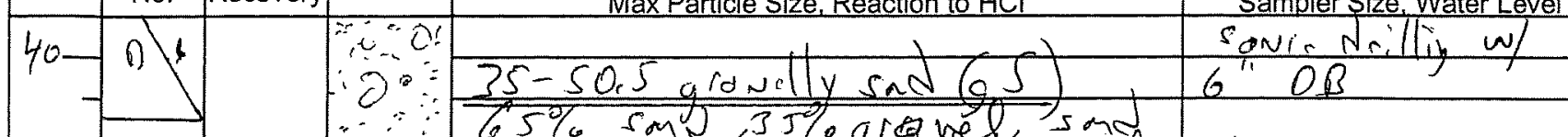

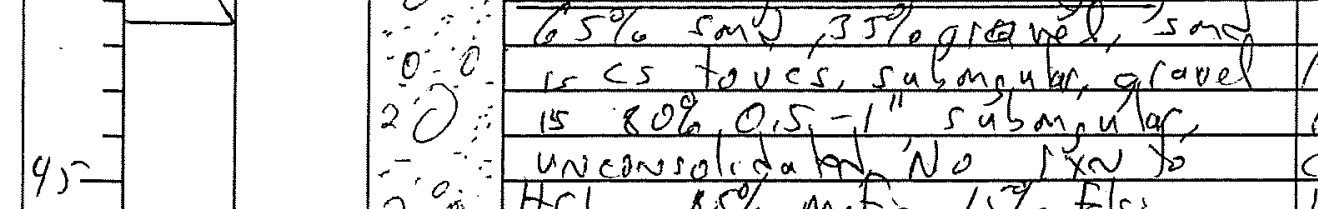

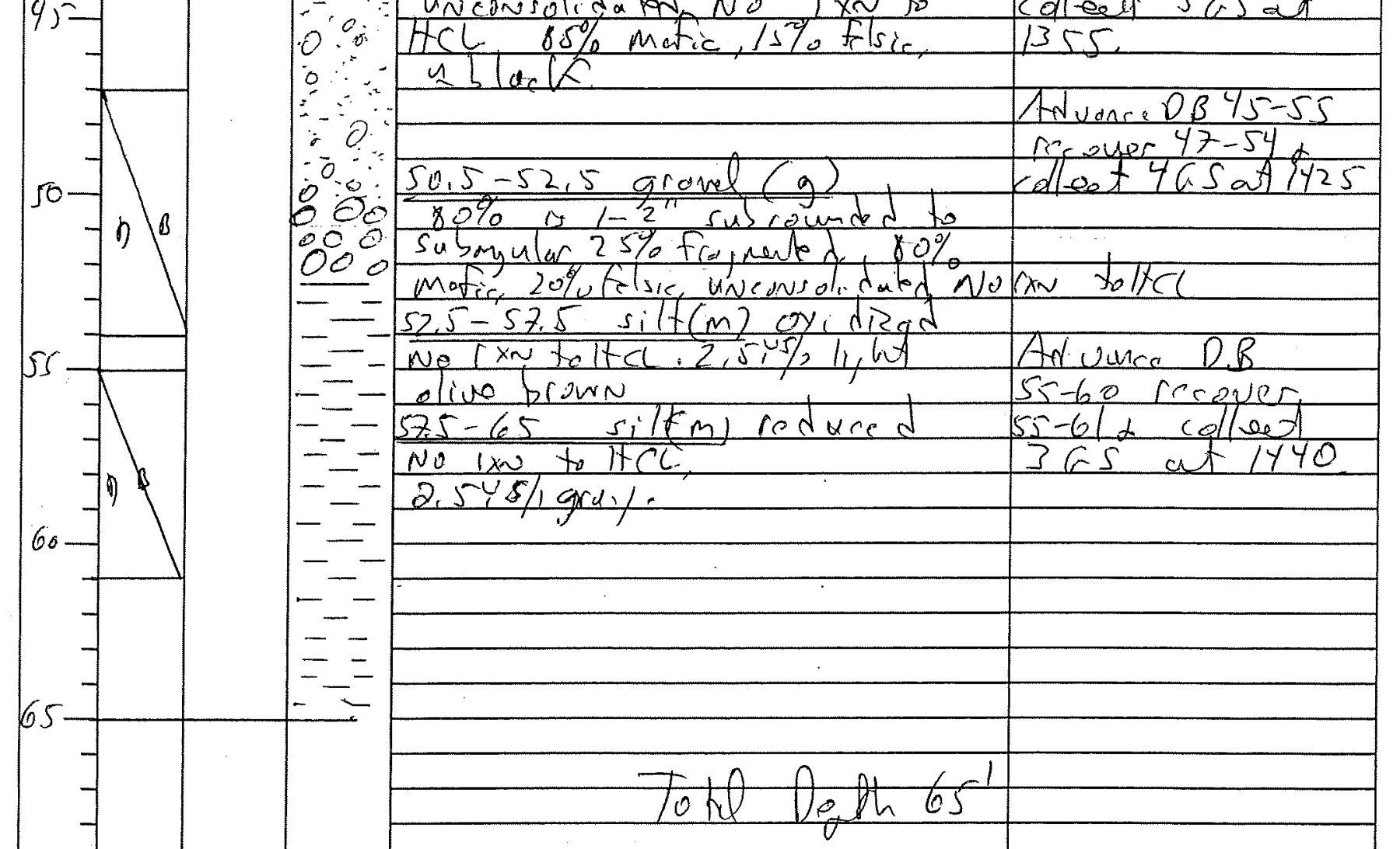

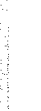

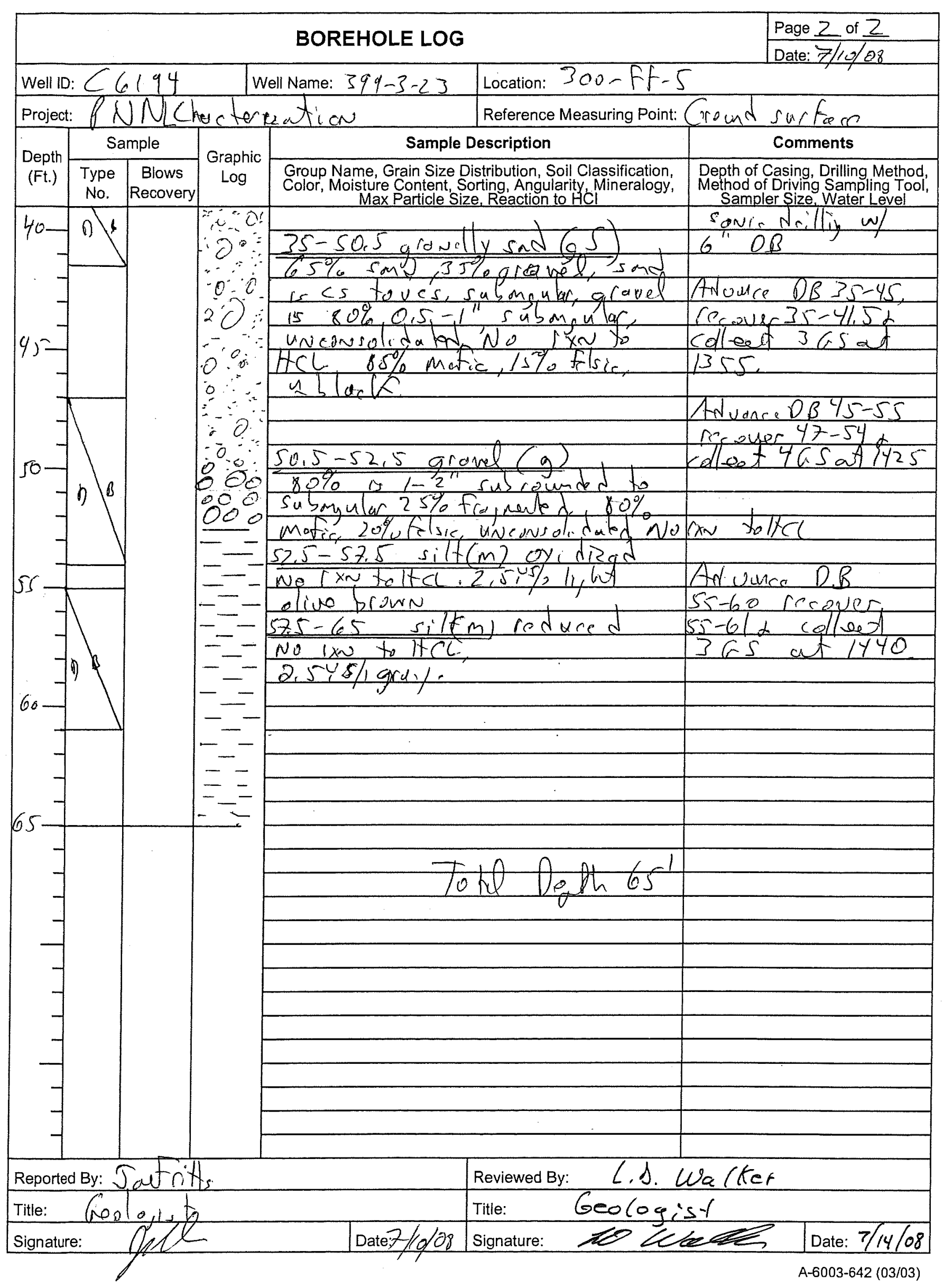




\section{BOREHOLE LOG}

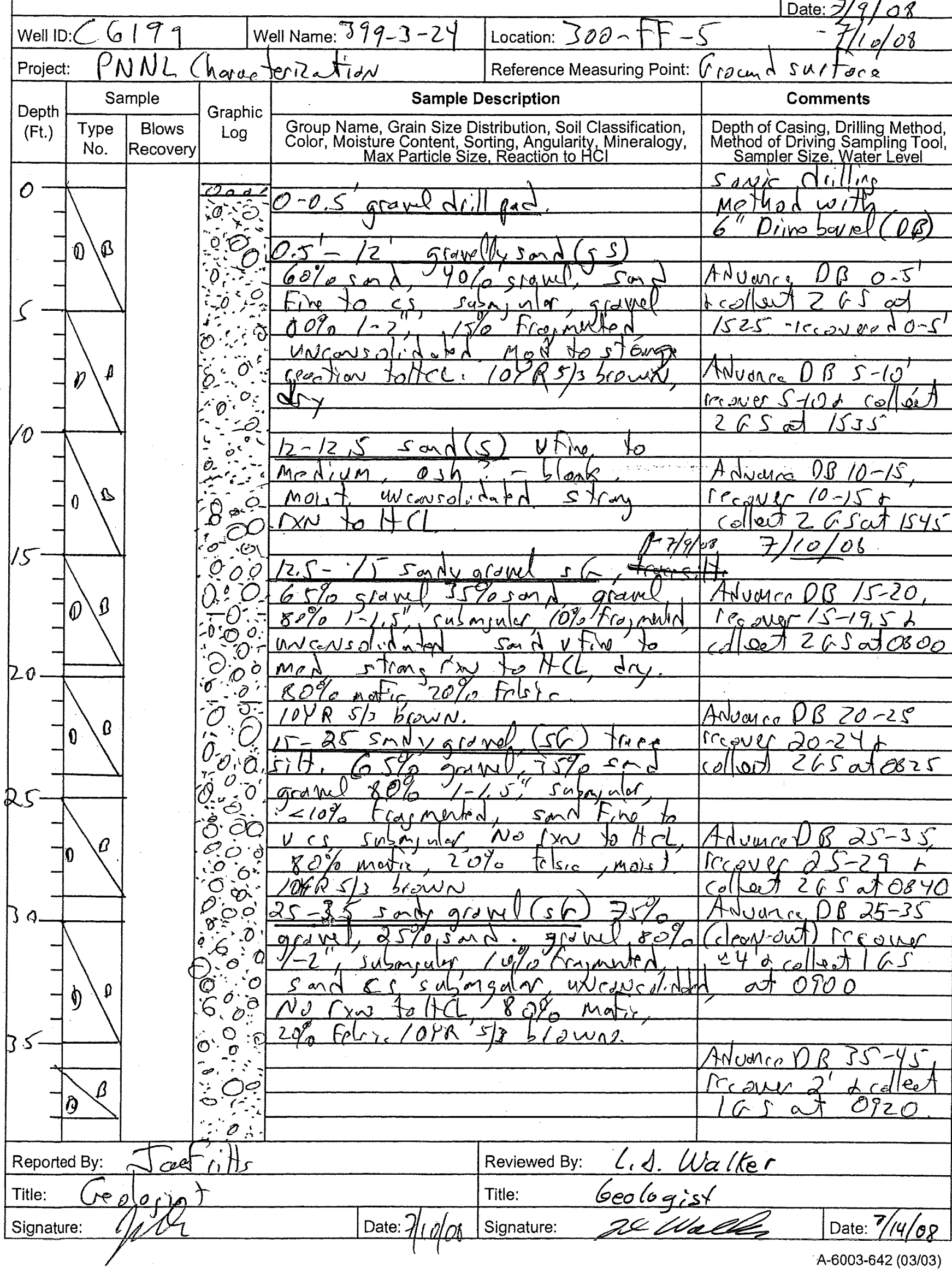




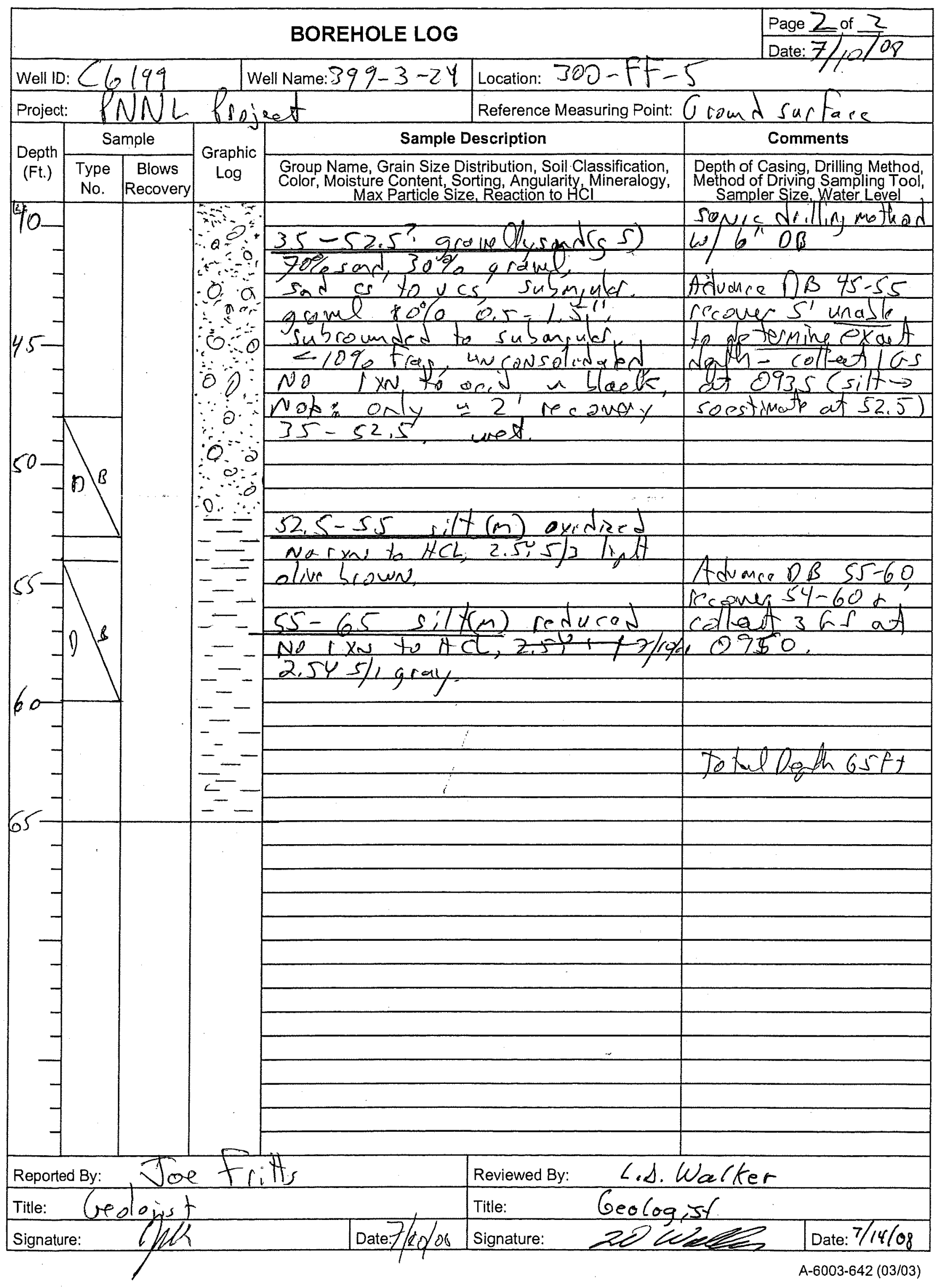




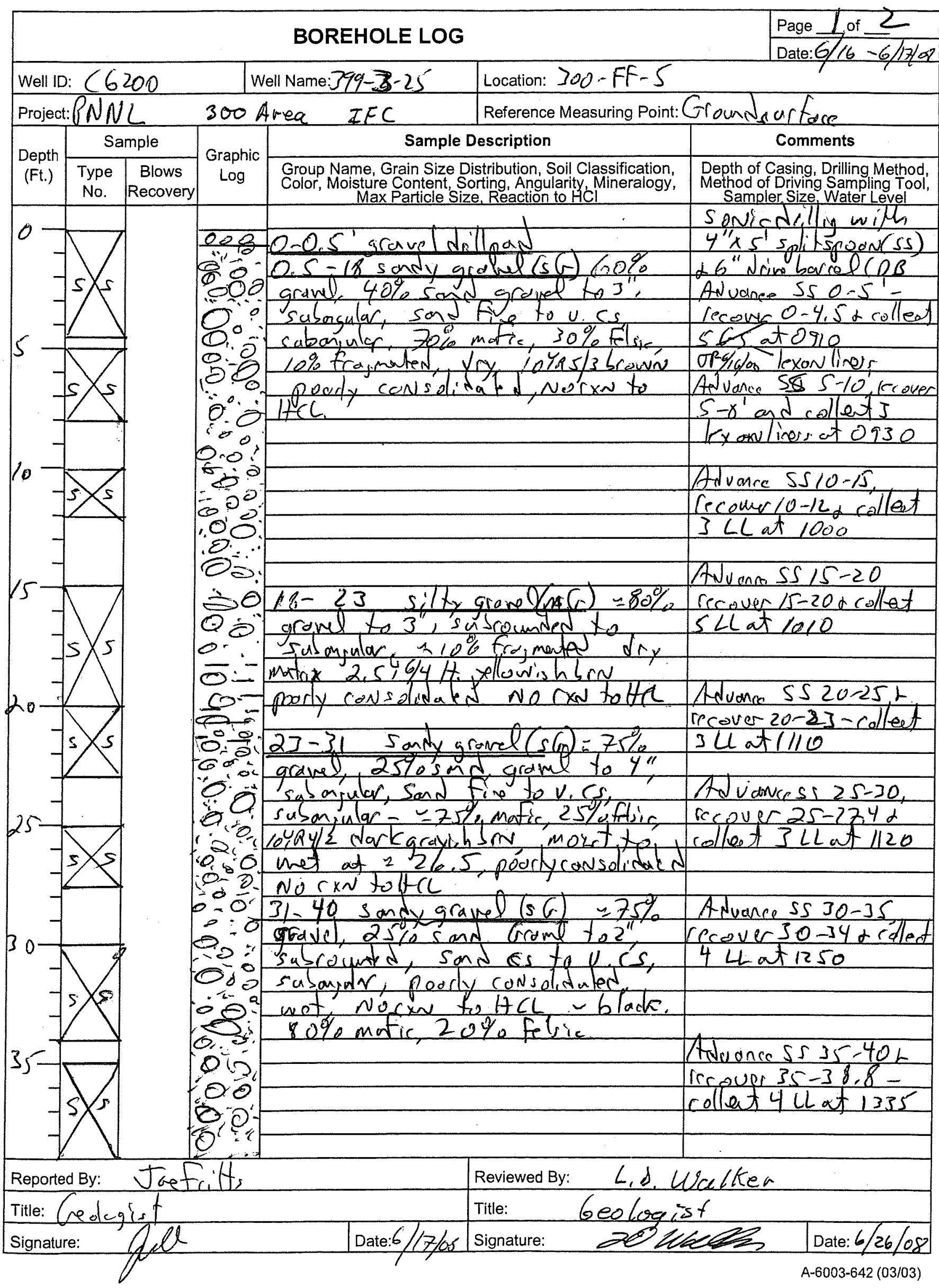




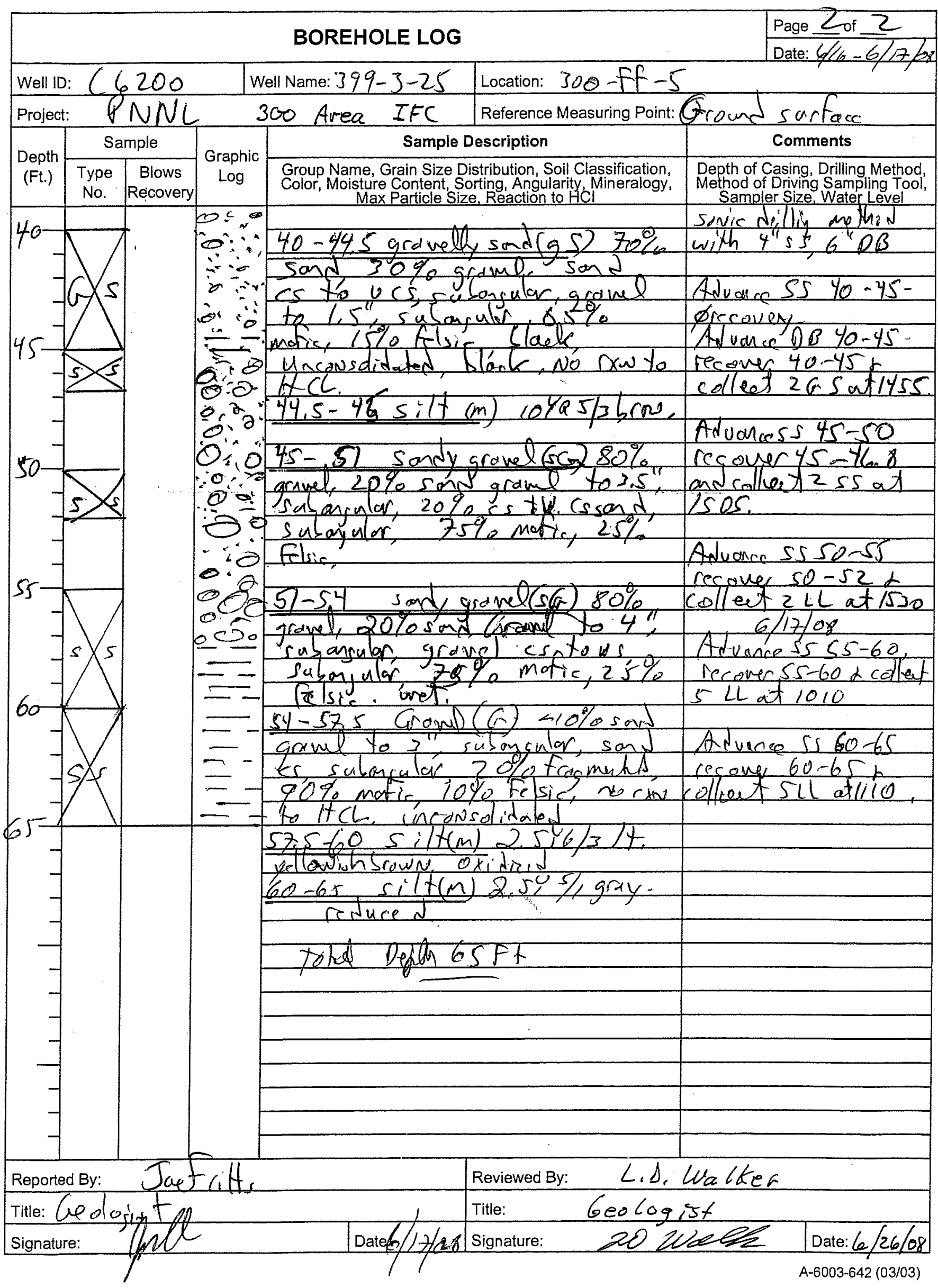




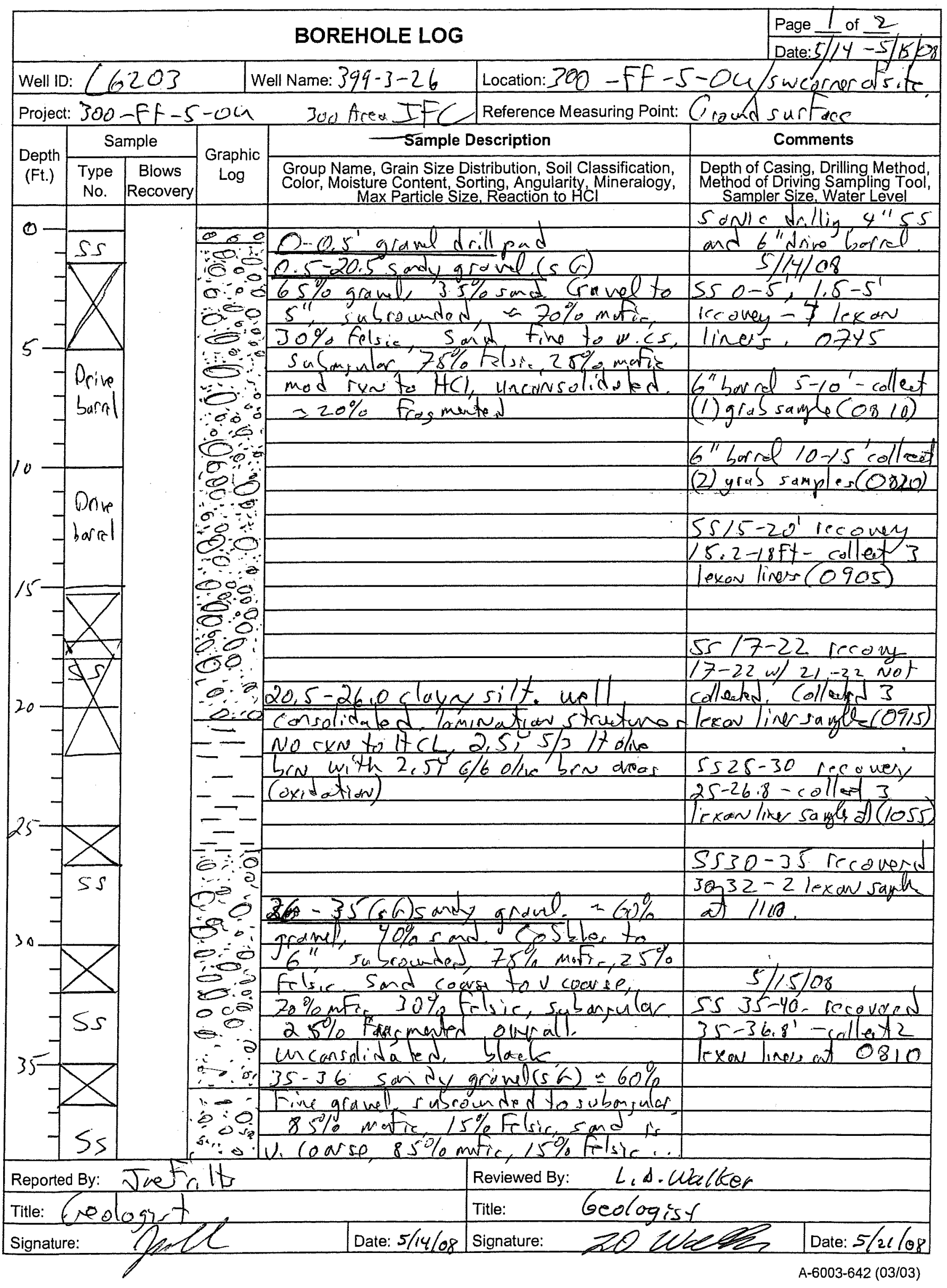




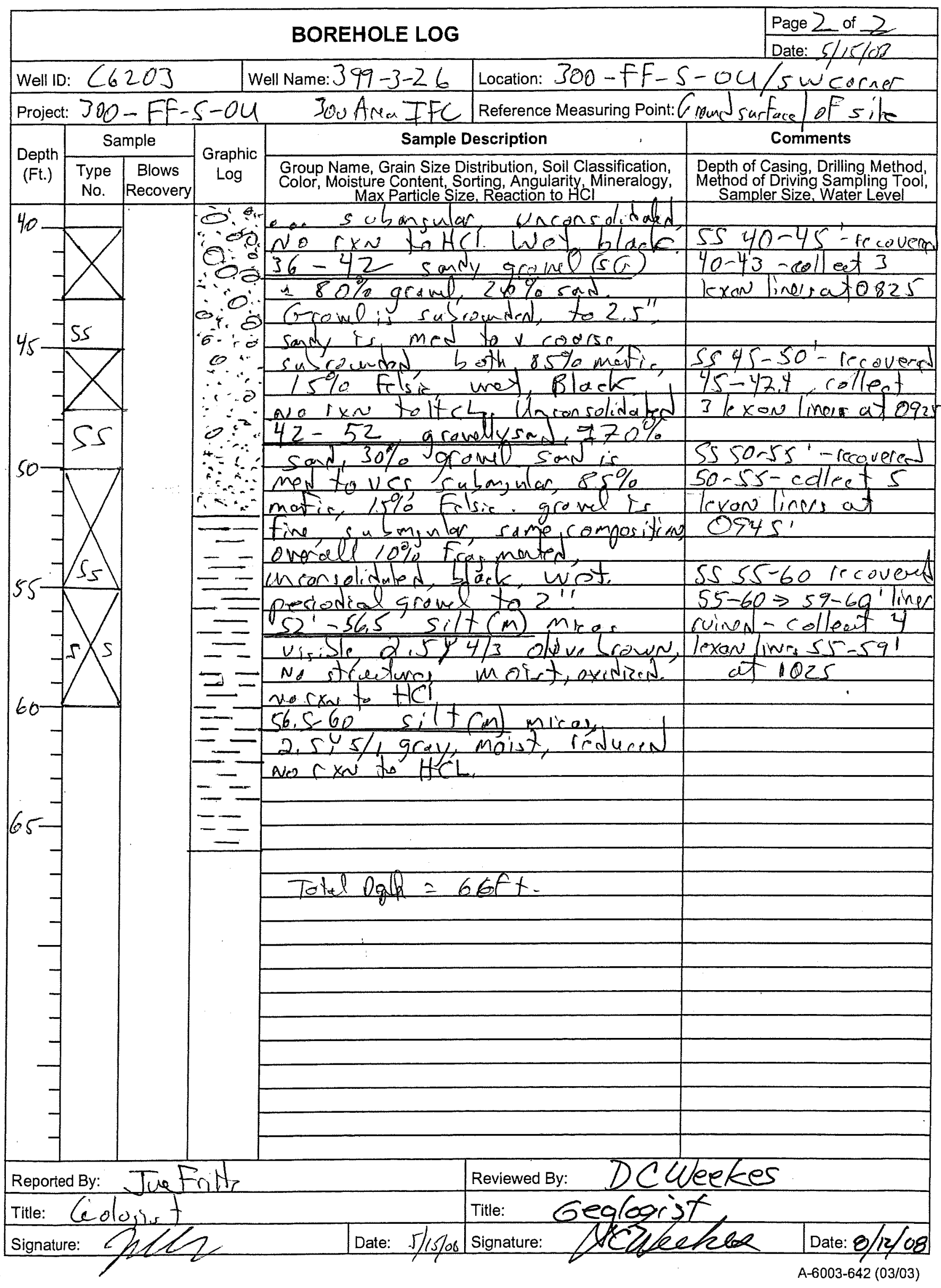




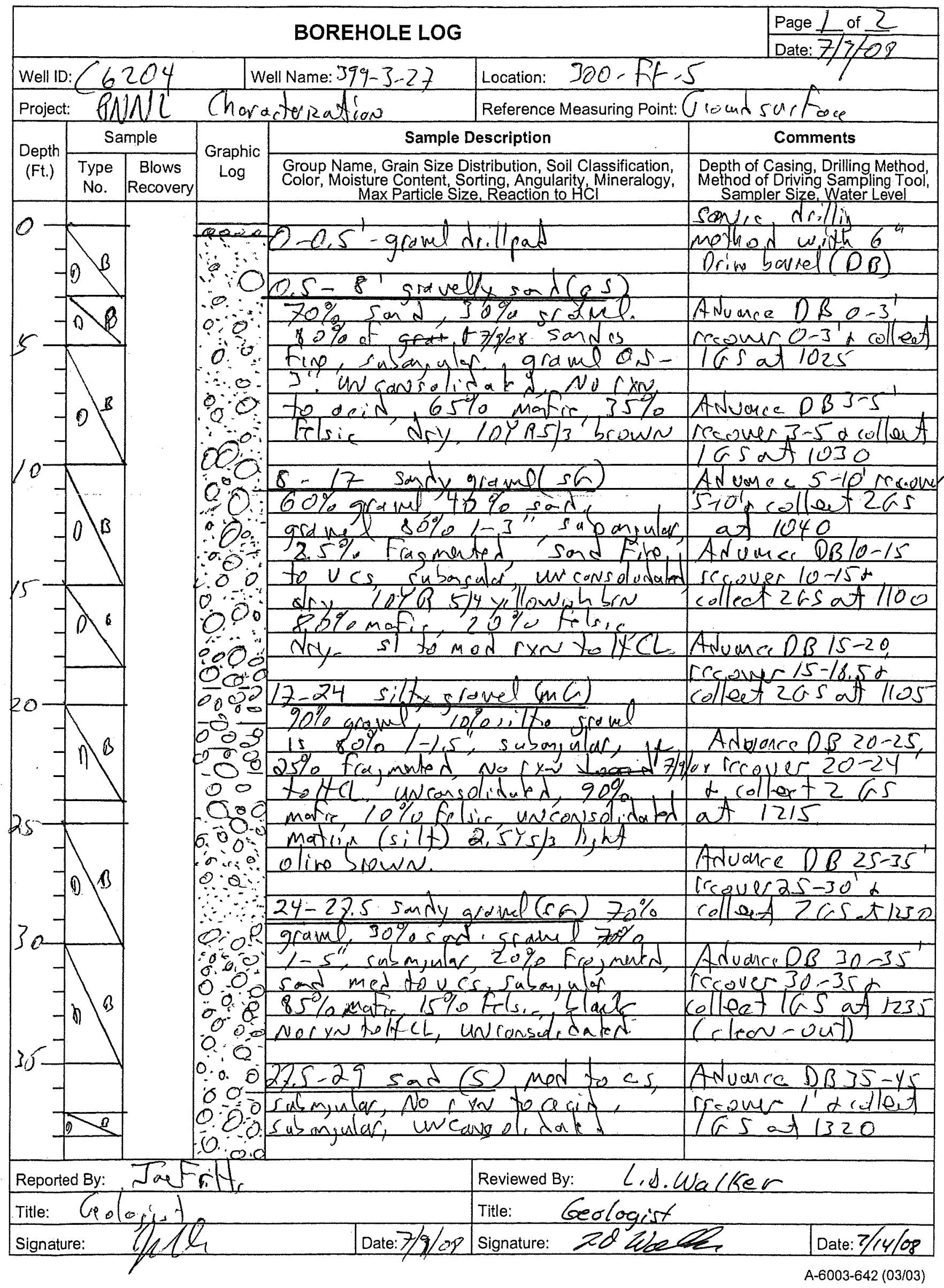




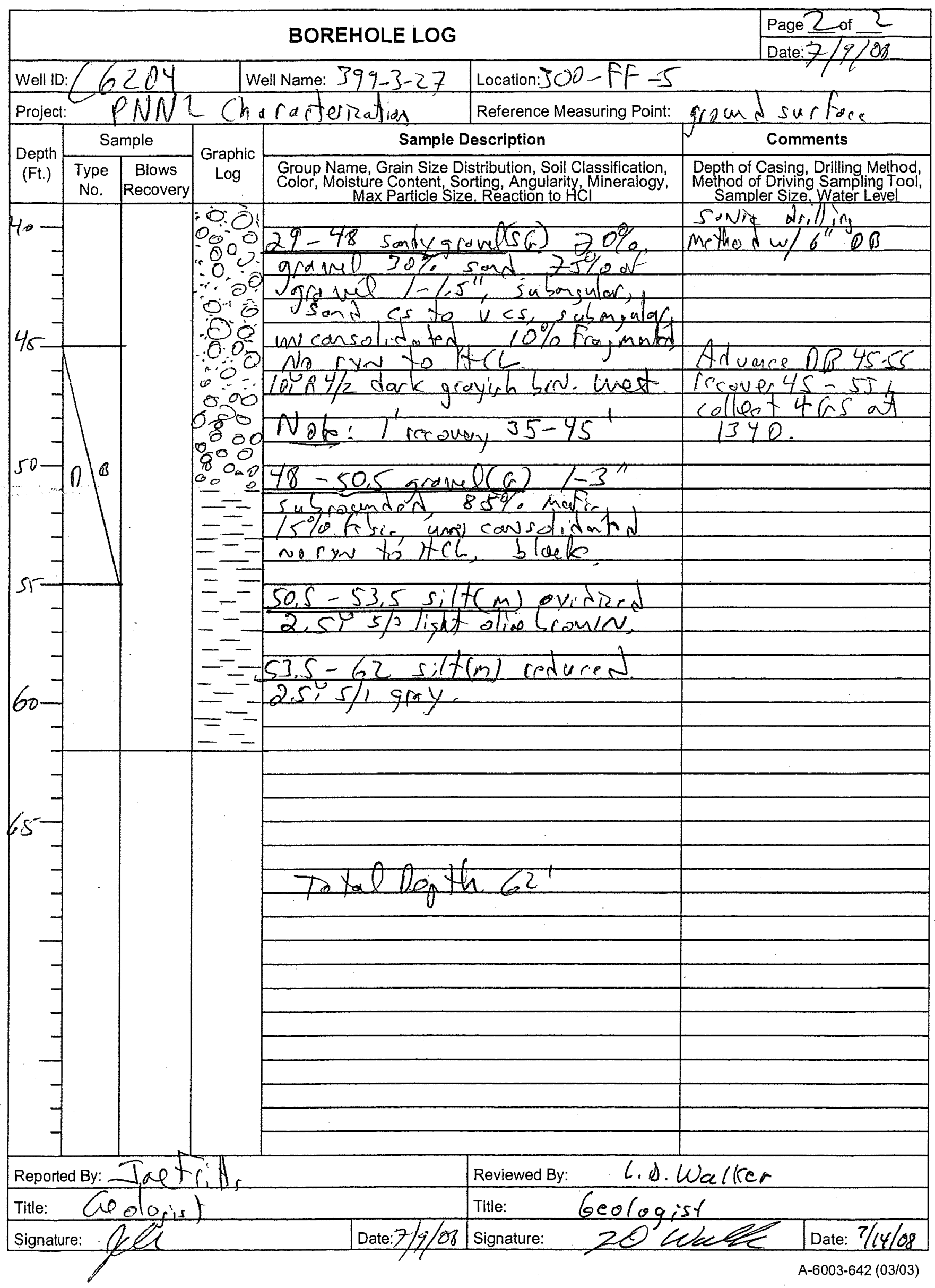




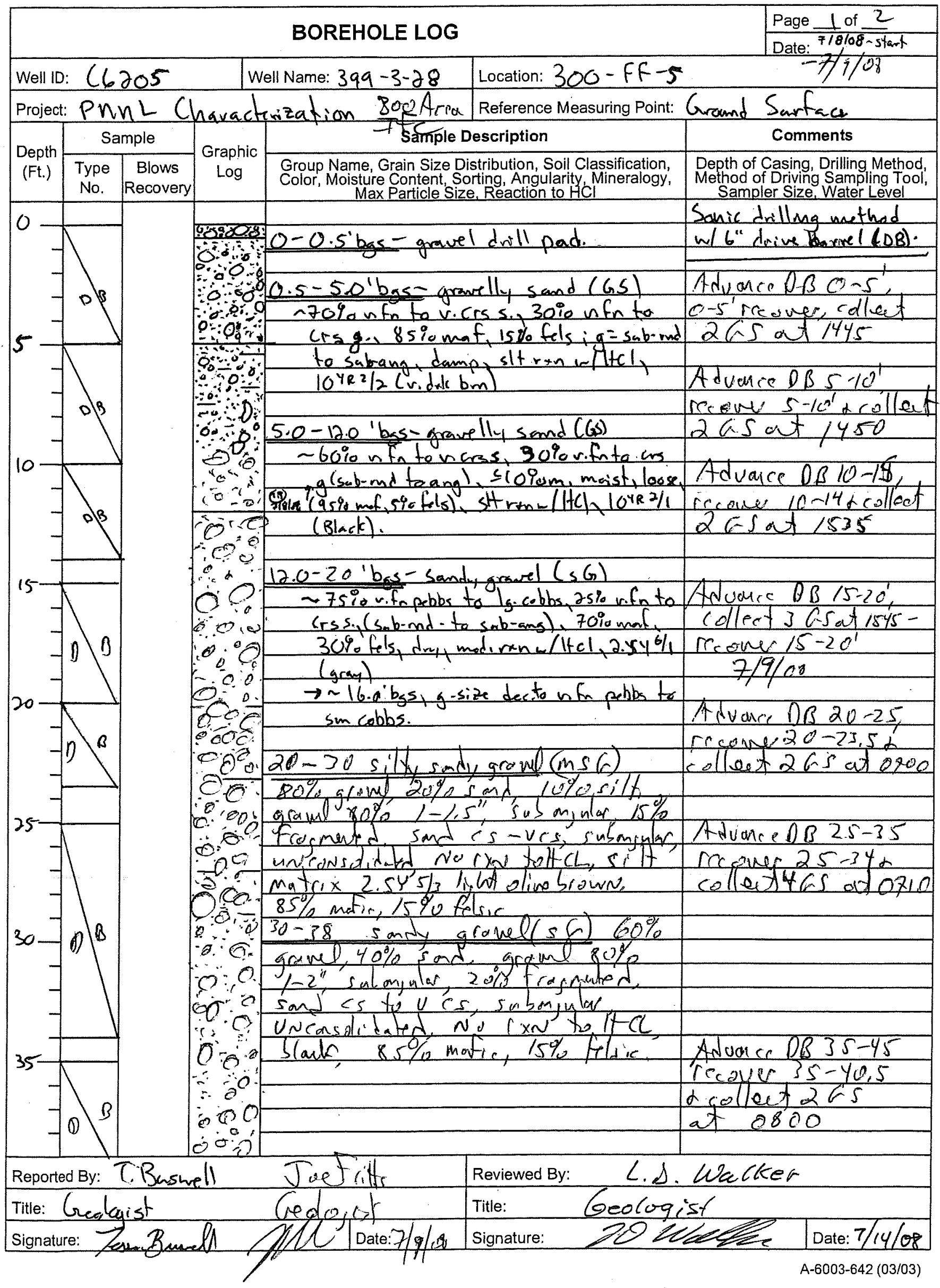

B.64 


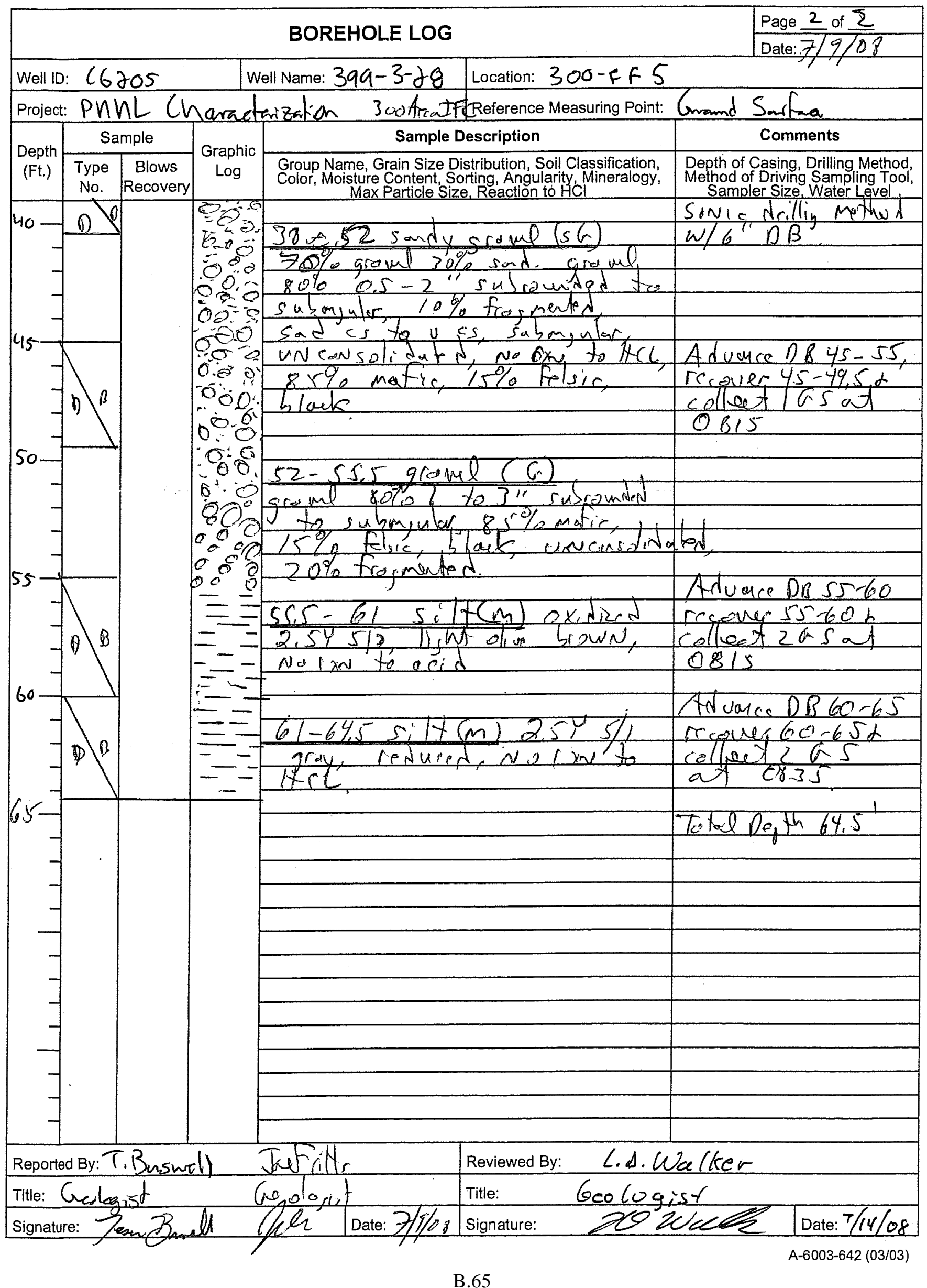




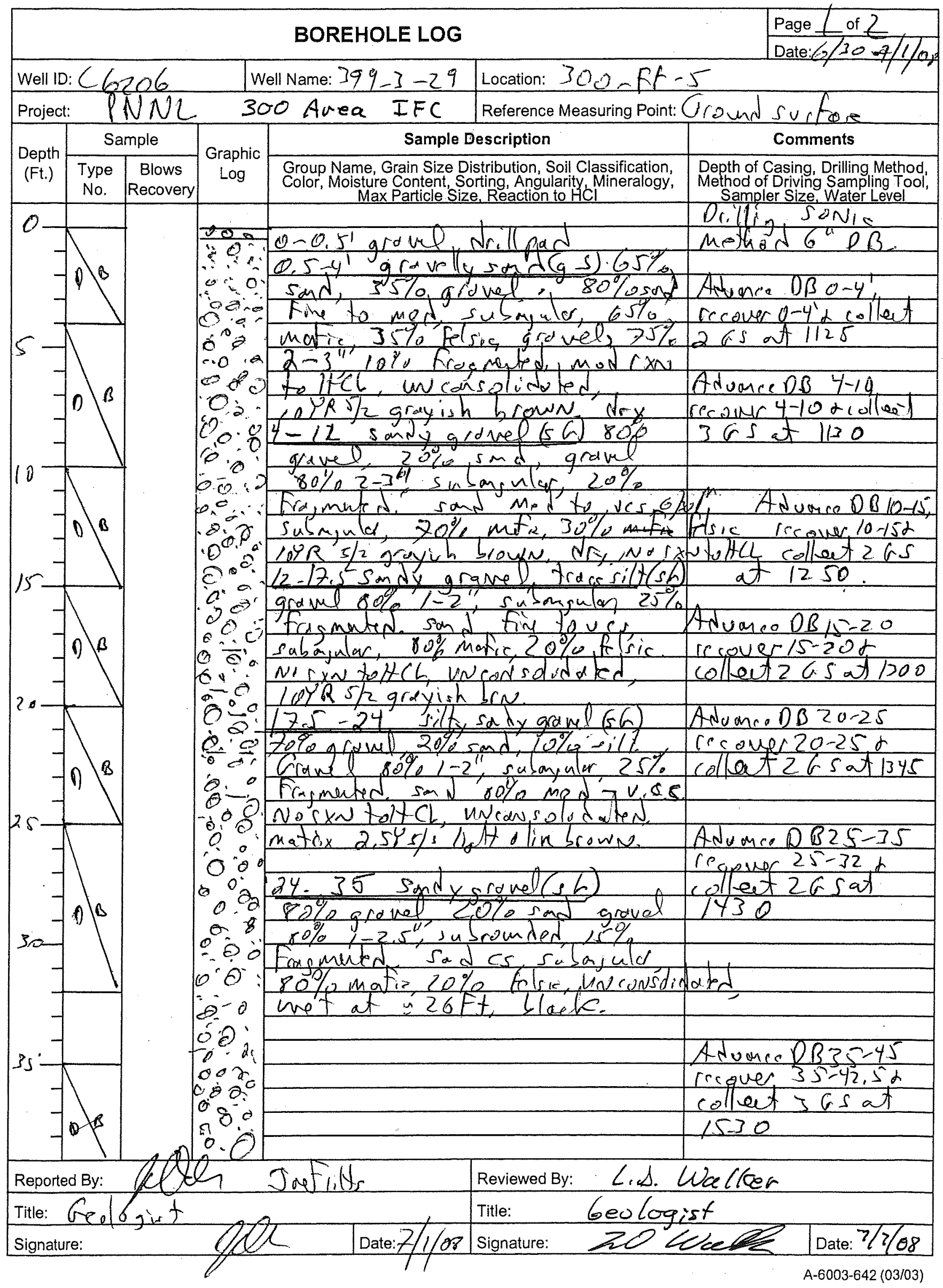




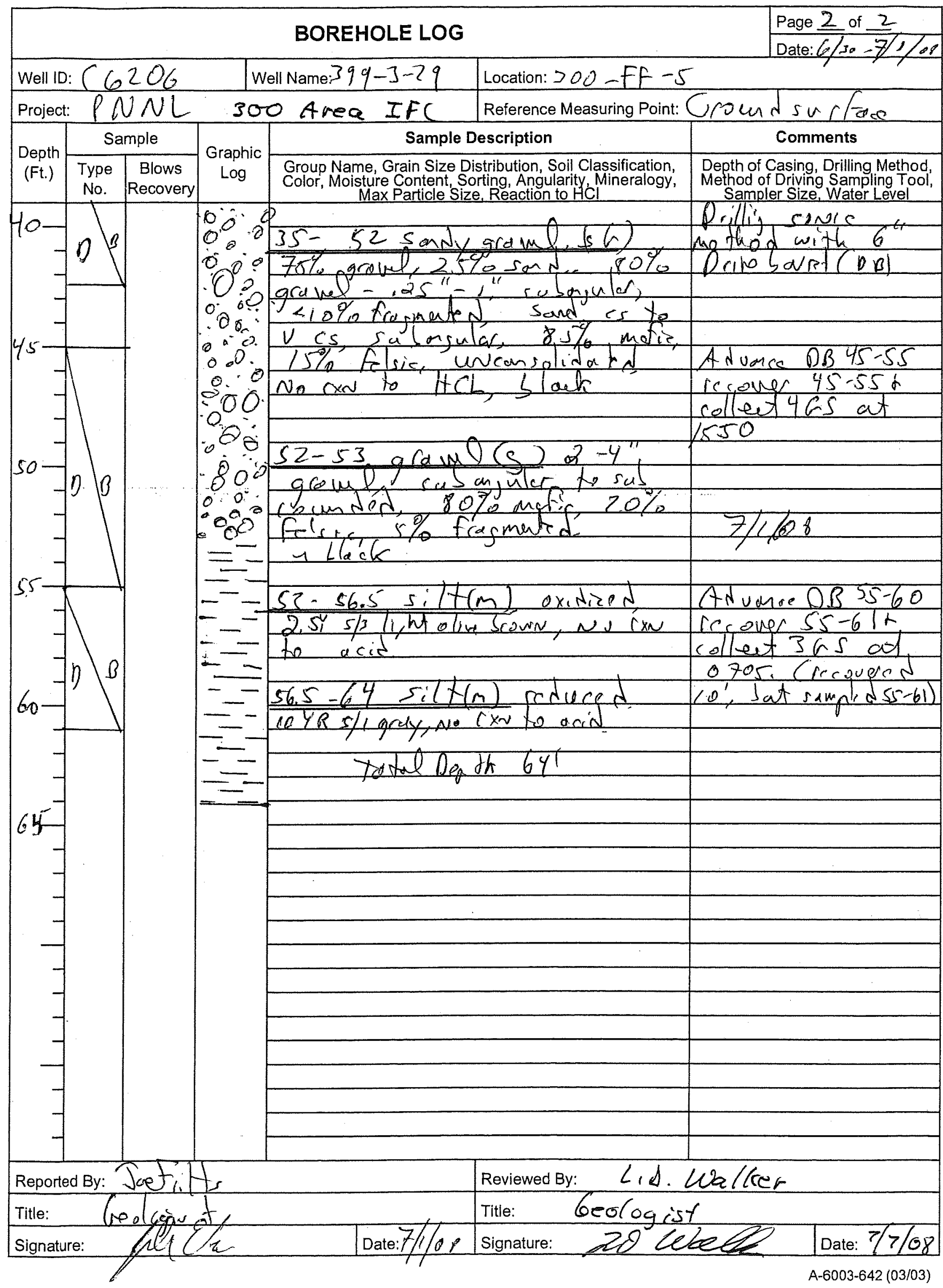




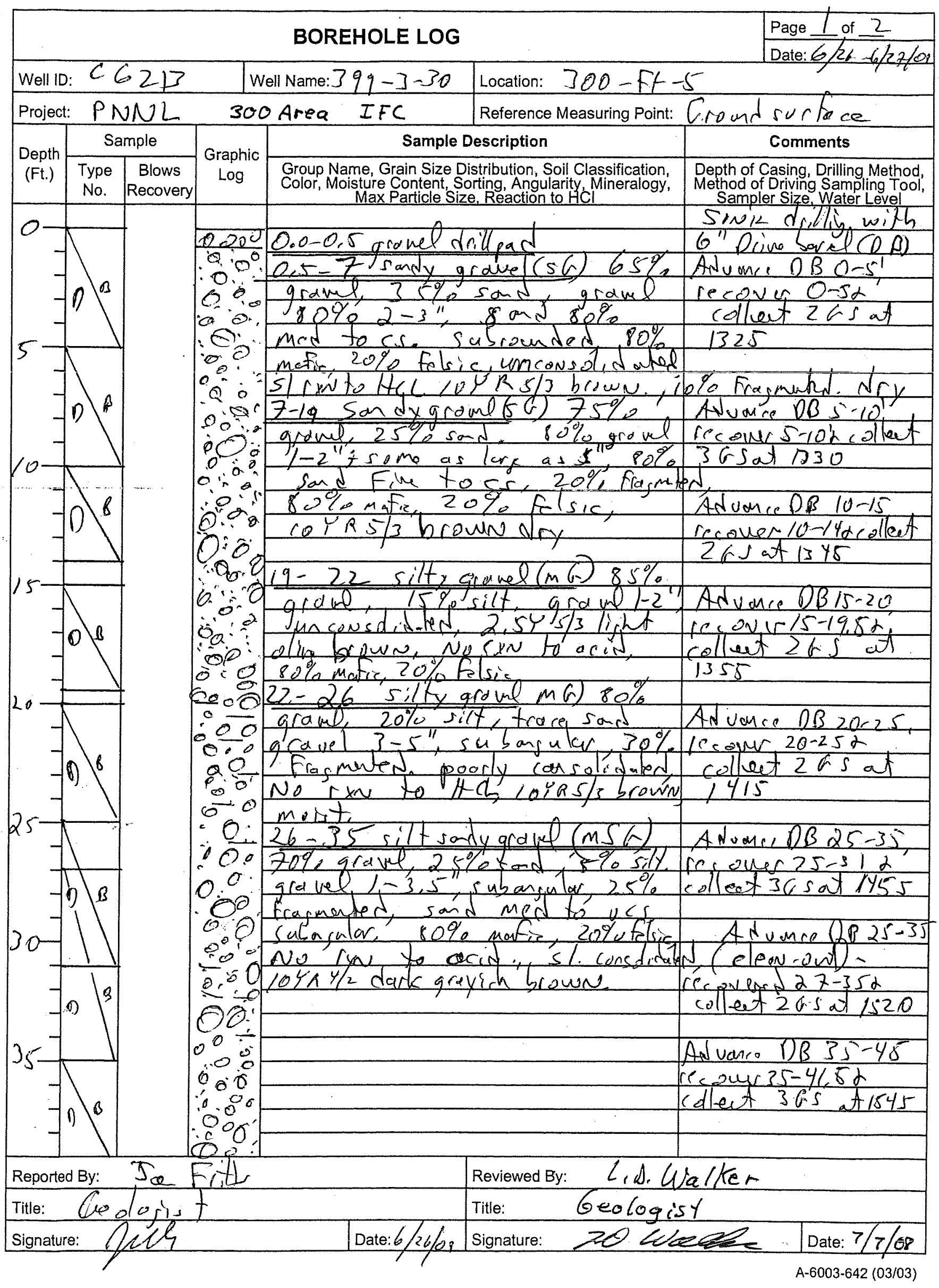

B.68 


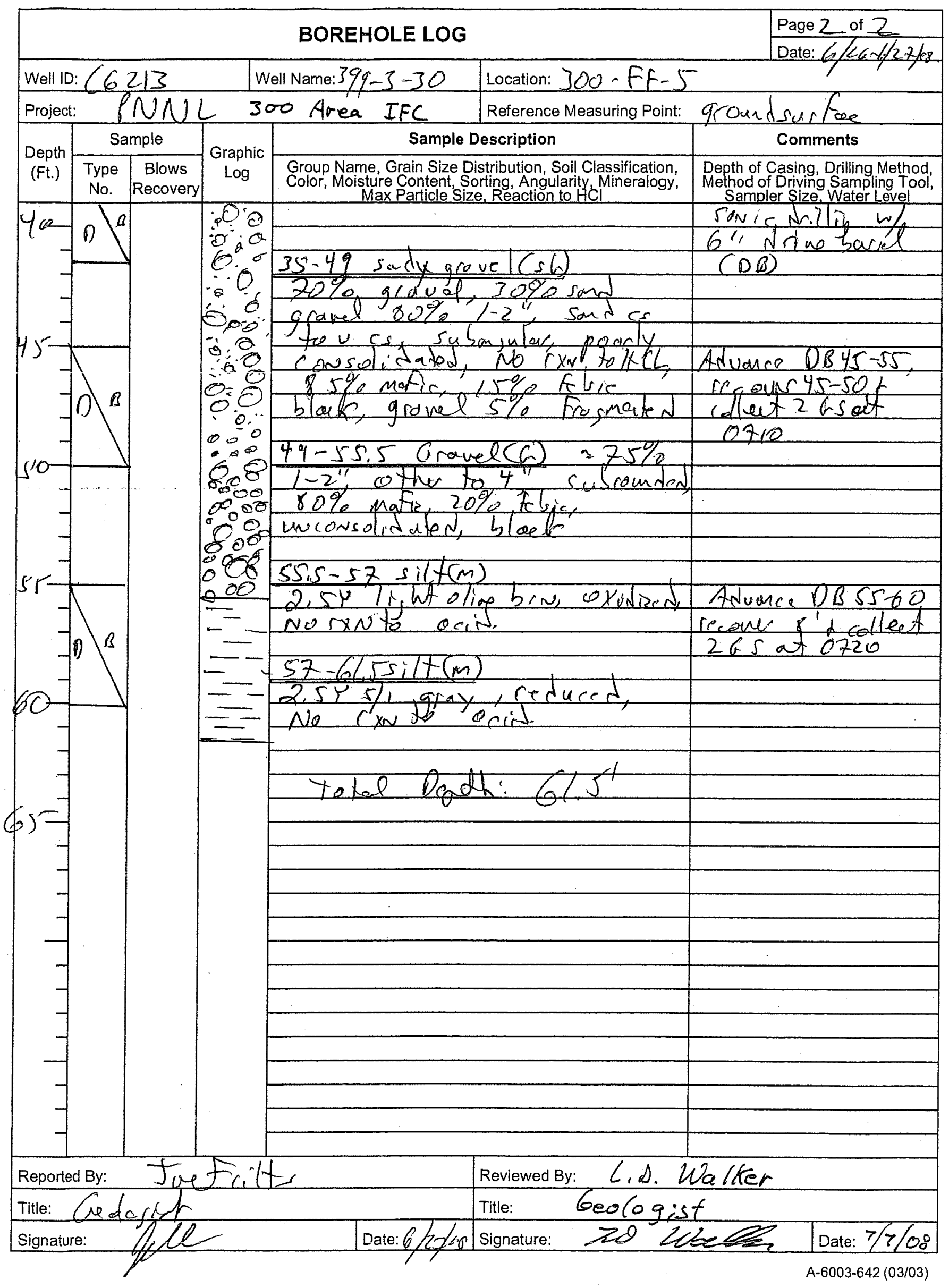

B.69 


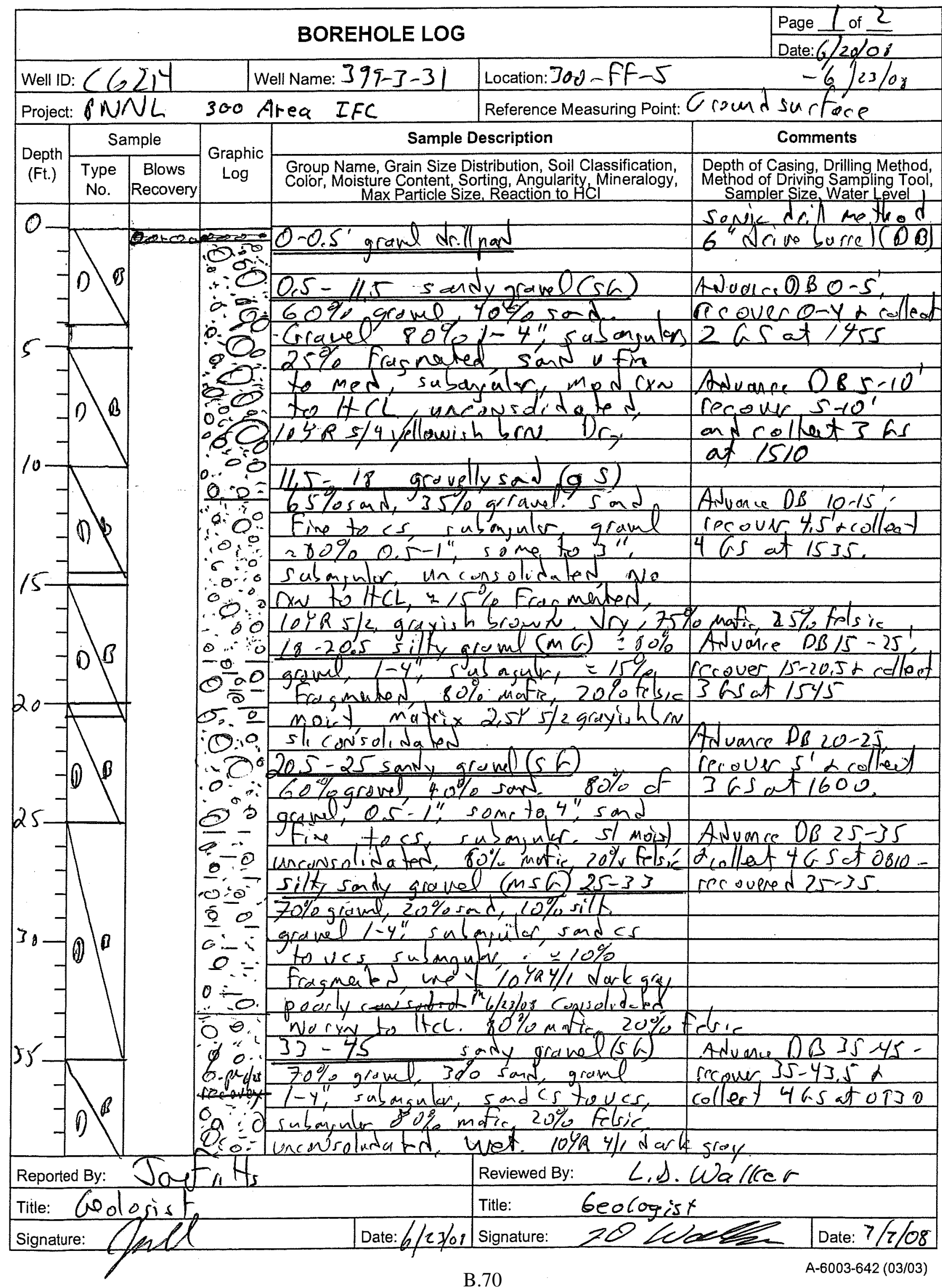




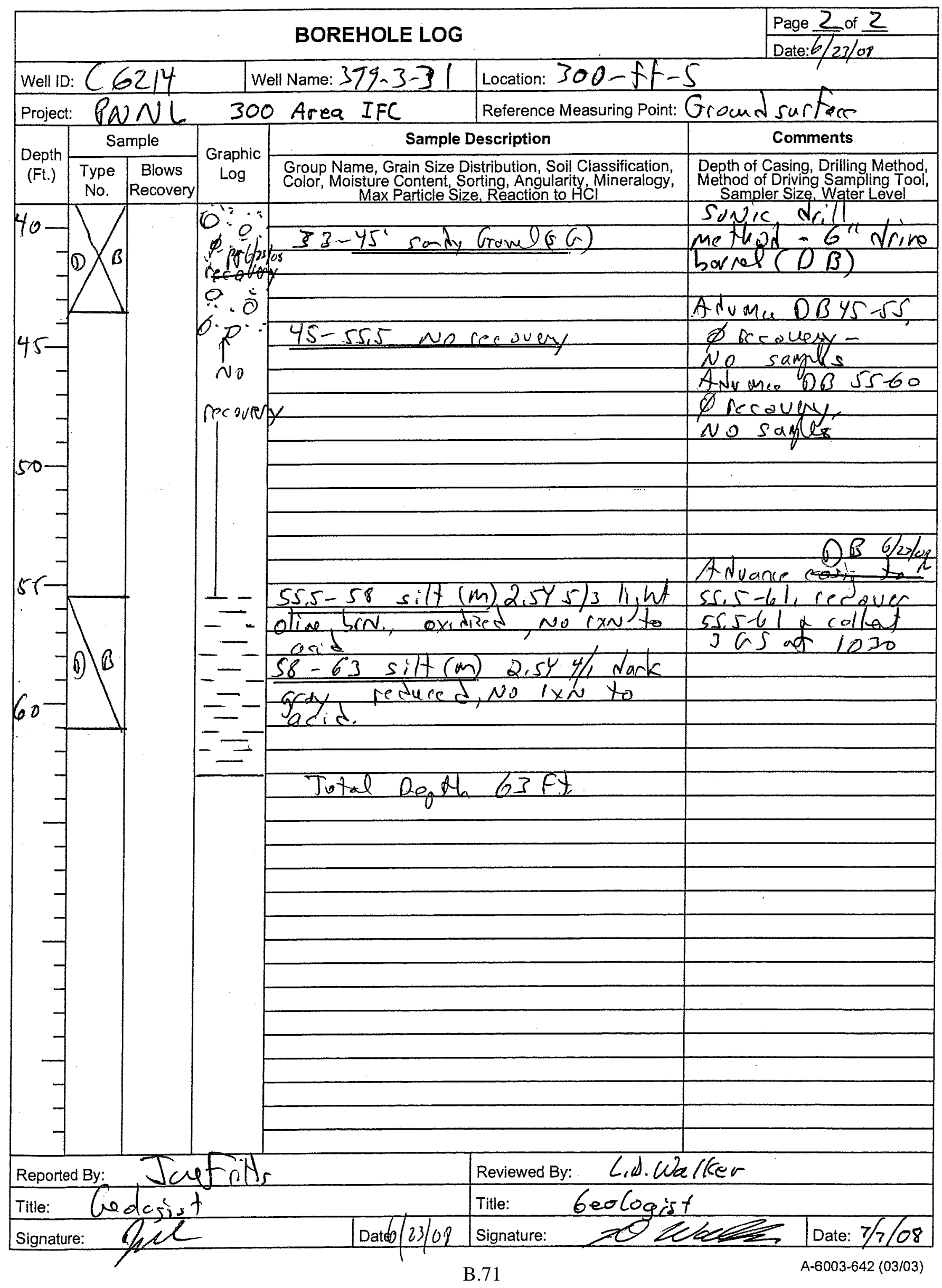




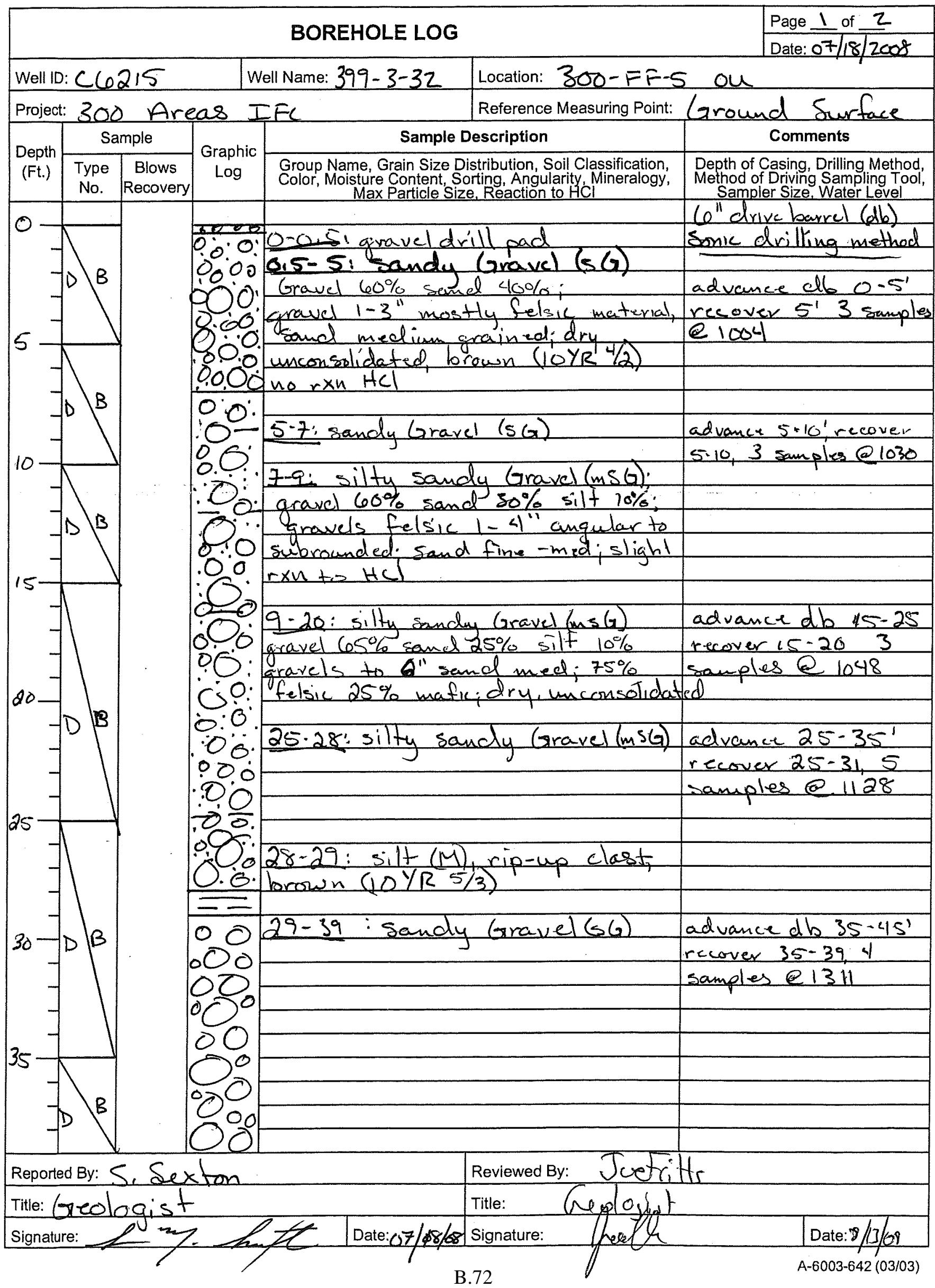




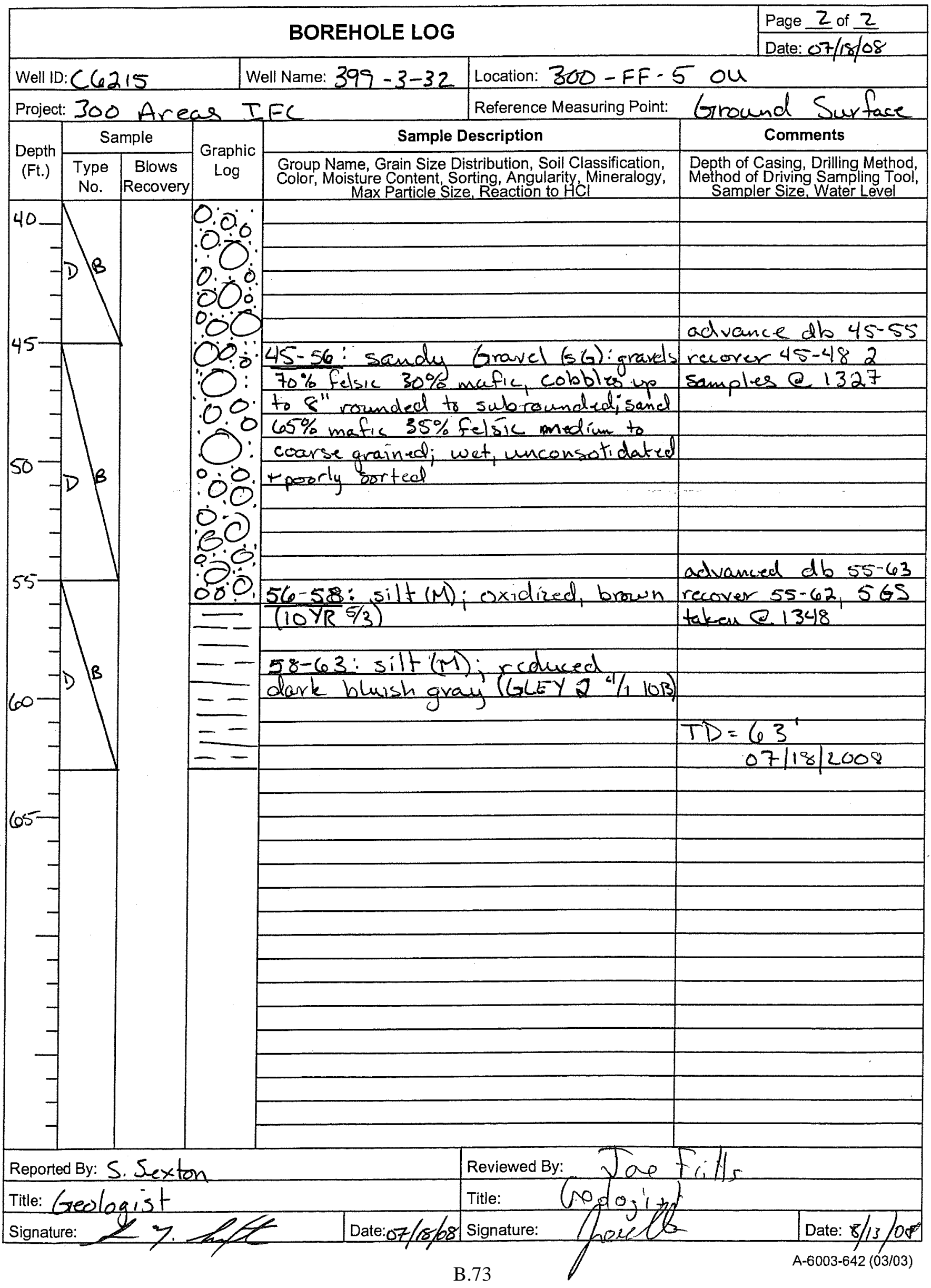


Appendix C

Sample Inventory Sheets 


\section{Sample Summary}

Well ID: $C 6184$

\begin{tabular}{|l|l|}
\hline Depth (ft) & Lithologic Log \\
\hline
\end{tabular}
Well Name: $399-2-7$ Sample Page $1 / 2$
Date $6 / 27$ of $10 / 27 / 00$

( $I|S| G \mid$ \begin{tabular}{|c|c|c|c|}
\hline Graphic & Type & ID & Mass \\
\hline & & & \\
\hline
\end{tabular} Comments

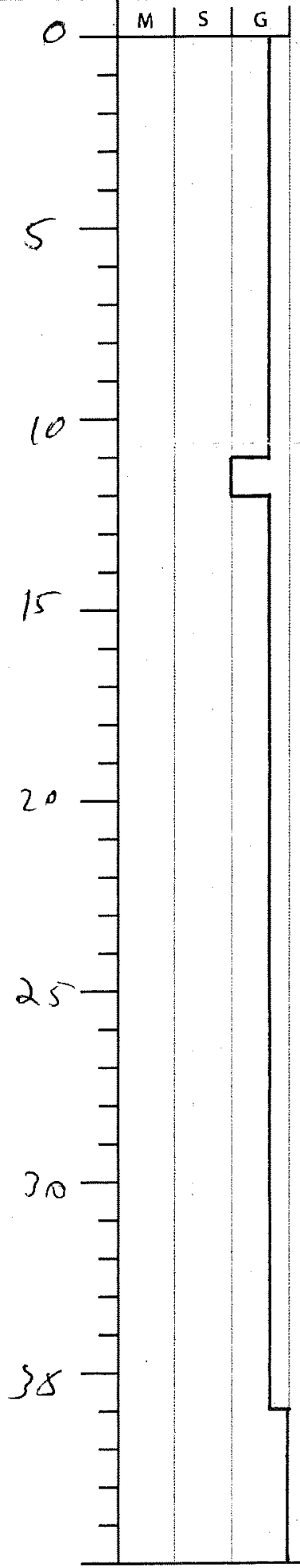

$\theta-2-7-6-0-2.5$

Aduance $D B 0-5$ - recover

c. $2-7-6-2,5-5$ $0-5^{\prime}+$ collet 2 as at o920

$62-7-6-5-7,5$

Aduchace of 5 - $10^{\prime}$ - recover

c. $2-7-6-25-10$

a $2-7-6-10-12,5$

Adume $D B$ 10- $\frac{R-6 / 87 / 01}{25}$

a. $2-7+6+25-15$

coour $10.15+$ collect 2 hs at 0935

G $27-6-15 \cdot 17.5^{\circ} \quad$ Aduace $D \beta 15-20$ a $2-7-6-175=20$ recaver $15-20 \alpha$ collatz 65 at 0950

\begin{tabular}{|l|l|}
\hline$a$ & $2-76-20-225$ \\
\hline
\end{tabular}

Arume ar 20-25, recovier $20-22.5+$ collet 1 as at 100 s

$62-7 \cdot 6-25-27$

Aduance $0 D 25-35$ recoves C. $2-7-6-27-30$ $25-3 / 5$ a collat 3 as at $10 \%$

$62-7-6-30-3 / 5$

c $2-7-6-35-37$ Adumce $D B 35-45-r e c o v e r$ $62-76-37,5-40$ $35-40$ o collat 265 at 1120 


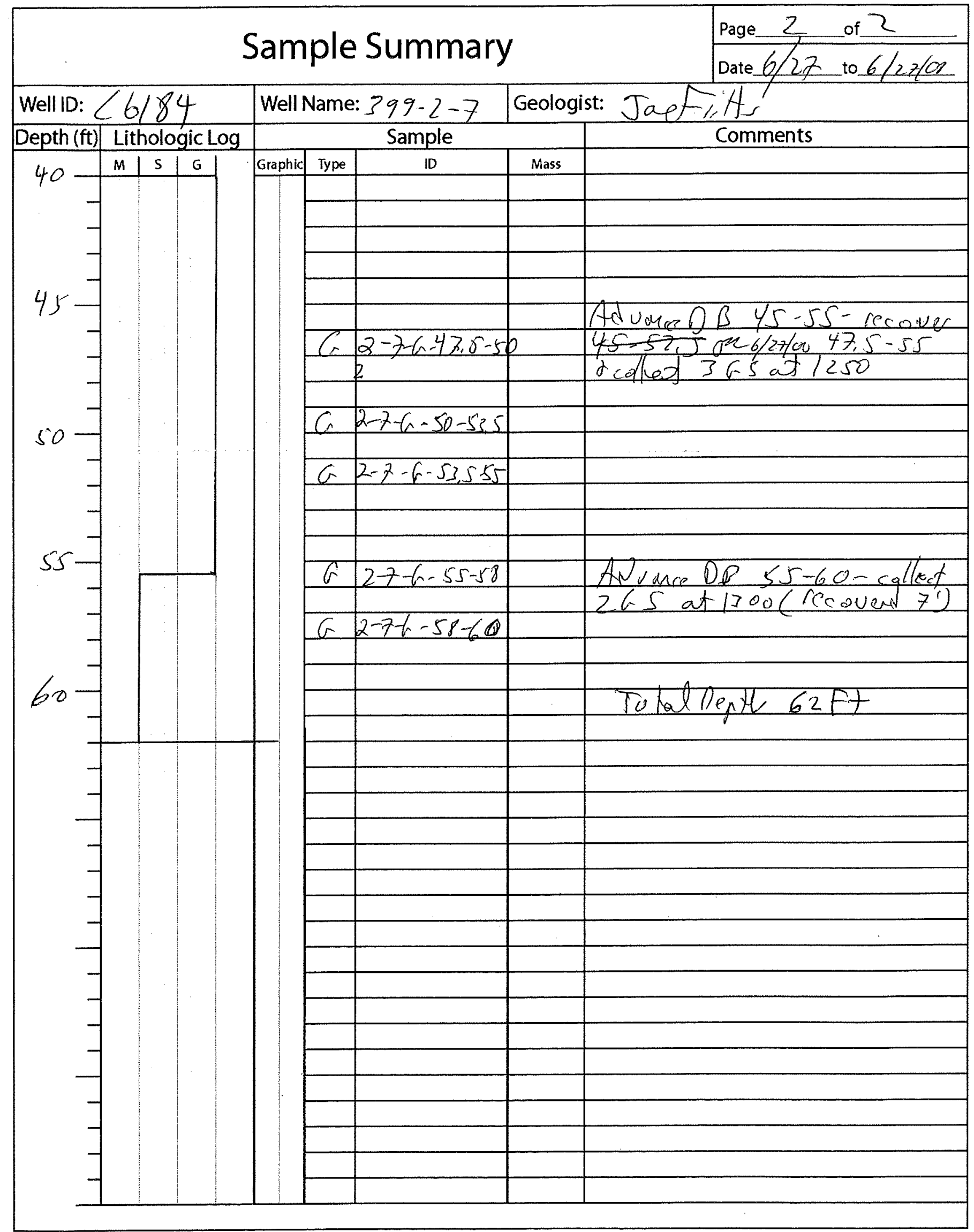




\section{Sample Summary}

Datef/7/0r to $7 / 8 / 08$

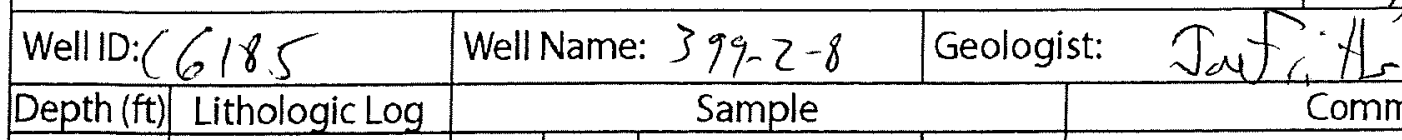
Comments

\begin{tabular}{|c|c|c|c|c|c|}
\hline-7 & \begin{tabular}{l|l|l}
$M$ & 5 & 6 \\
& 1 & 5
\end{tabular} & 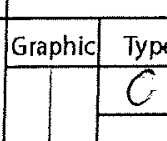 & $\frac{10}{2-8-6-0,-25}$ & Mass & Advonce DB $0-5^{\prime}$ requeve \\
\hline & & & & & \\
\hline & & G & $2-8-6 \cdot 2.5-5$ & & \\
\hline & & G & $2-8-6-5 \cdot 75$ & & 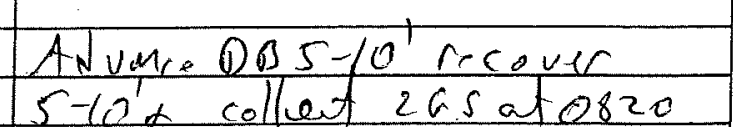 \\
\hline & & & & & \\
\hline & & 6 & $2 \cdot 8 \cdot 6-25-10$ & & Adomace $\rho \beta 10-15, \& c o v$ \\
\hline & & $G=$ & $2-7-6-10-2.5$ & & $10-15$ anc ollet 2 asal \\
\hline & & $G$ & $2-8-6-12.5-15$ & & 0920 \\
\hline & & $\frac{a}{a}$ & $2-8-6-15-125$ & & Adume DB, 15-25, lecaver \\
\hline & & a & $2-8-6-2 z 5-20$ & & $\frac{15.25 \alpha}{1235}$ collect 4 ers at \\
\hline & & & & & \\
\hline & & $G$. & $2-8-6-20-22.5$ & & \\
\hline & & & & & \\
\hline & & e & $32-8-6-225-25$ & & \\
\hline & & a & $2-8-6-25-29$ & & $\begin{array}{l}\text { Advance DB } 25-35 \text { - lecover } \\
25,-31.5 \text { d collect } 2 \mathrm{GS}\end{array}$ \\
\hline & & & & & $\begin{array}{l}\text { at } 1575 \\
\text { Note } 25-29 \text { us neet slough }\end{array}$ \\
\hline & & f & $2-8-6-29-31.5$ & & $7 / 8 / 08$ \\
\hline & & & & & \\
\hline & & & & & \\
\hline & & $G_{-}$ & $2-8-6-75-38$ & & A Adumce D) 35 5 -45 \\
\hline & & & & & 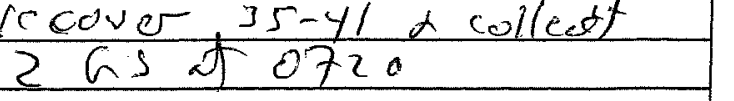 \\
\hline & & a & $|2-8-6-38-4|$ & & \\
\hline
\end{tabular}




\section{Sample Summary}

\begin{tabular}{|l|c|c|}
\hline Well ID: C6185 & Well Name:399-2-8 & Geologist: Jae Fritts \\
\hline Depth (ft) & Lithologic Log & \multicolumn{2}{|c|}{ Sample } & Comments \\
\hline
\end{tabular}

\begin{tabular}{|c|c|c|c|c|c|c|c|}
40 & $M$ & 5 & 6 \\
\hline
\end{tabular}

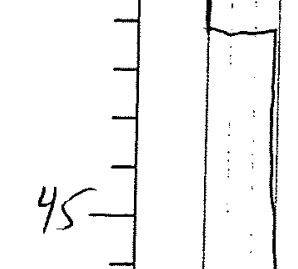

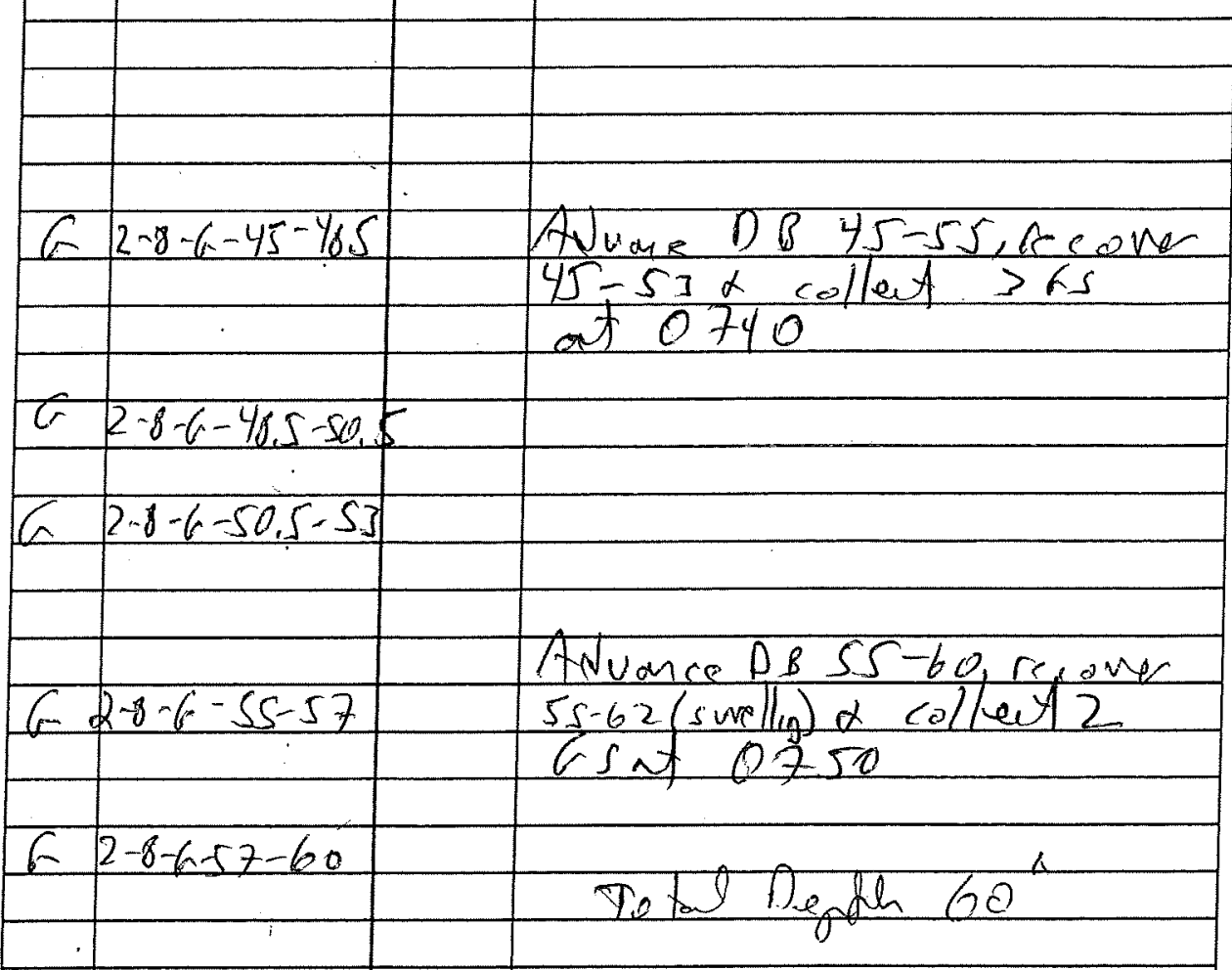

60

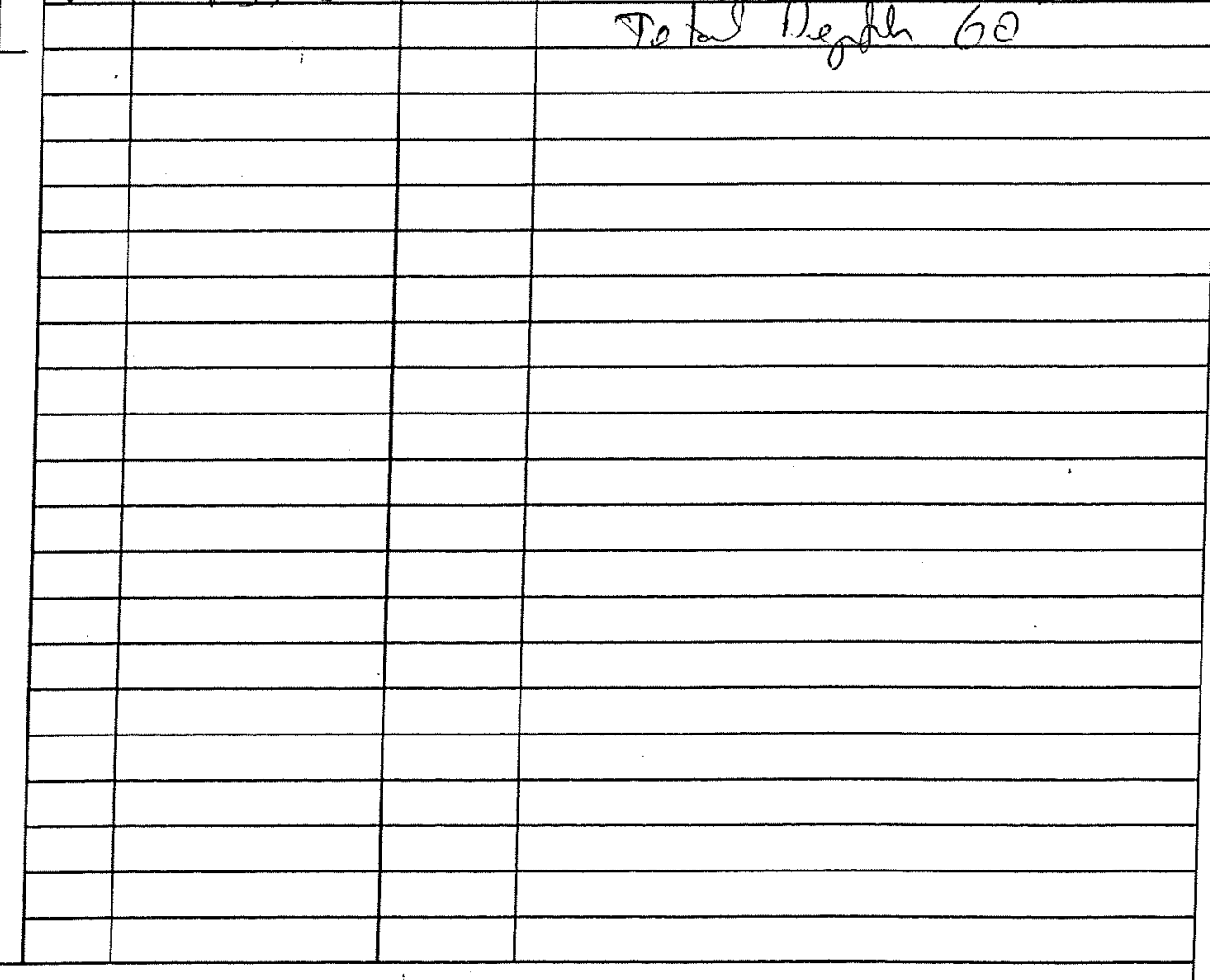




\section{Sample Summary}

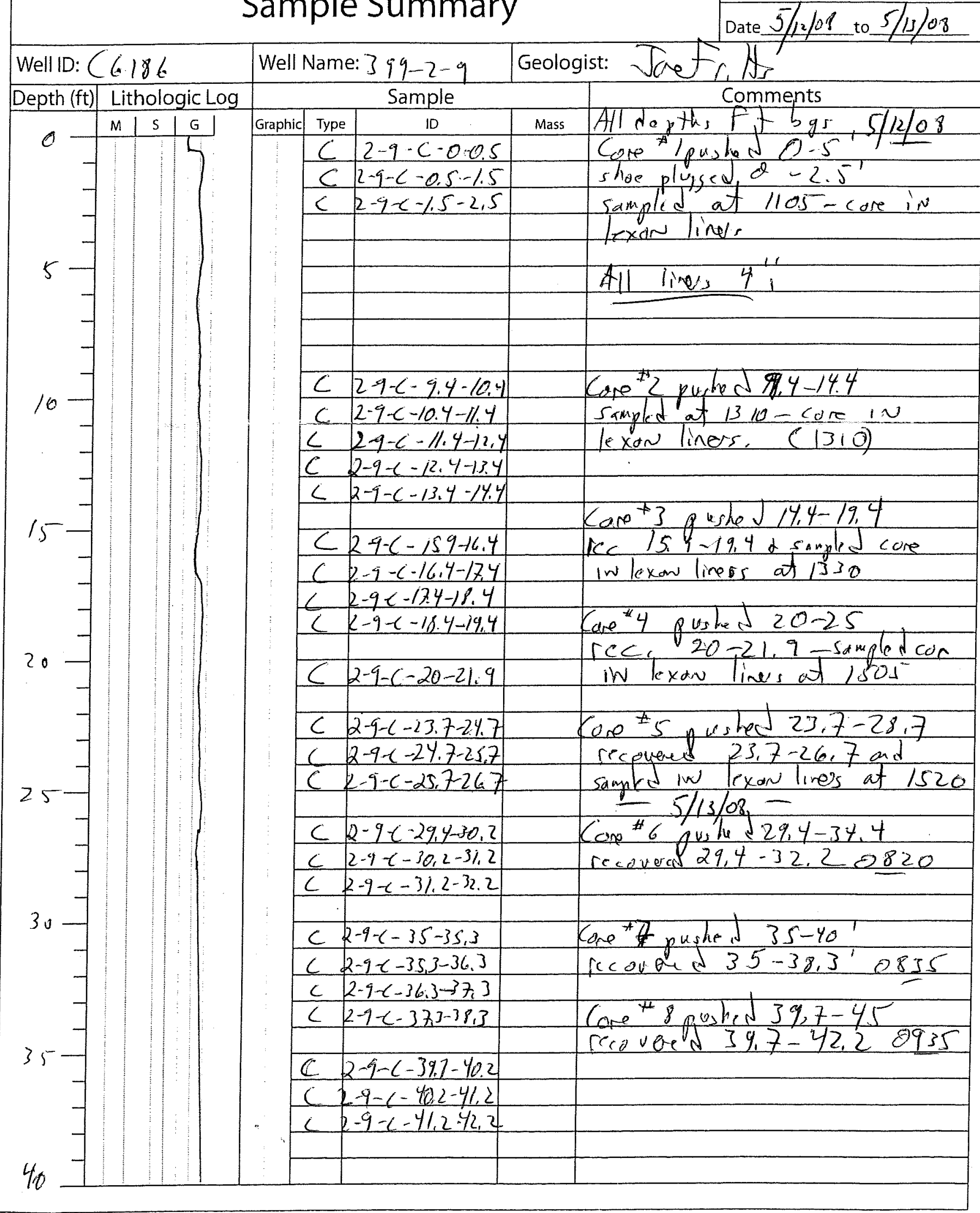




\section{Sample Summary}

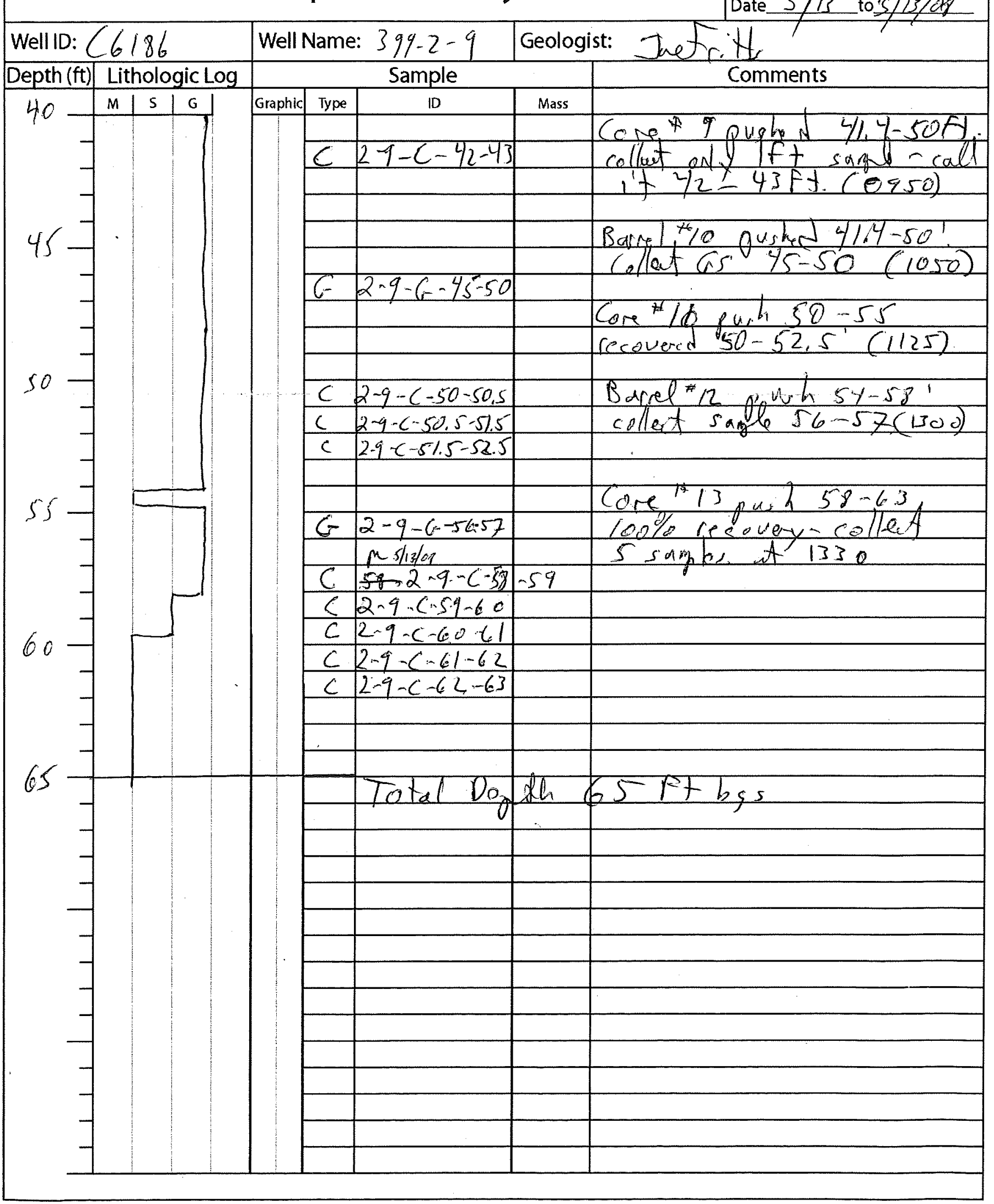




\section{Sample Summary}

Page 1 of 2

Well ID: $C 6187$ Depth (ft) Lithologic Log

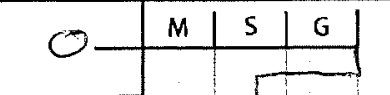

Well Name: $399-2-10$ Sample

\begin{tabular}{|c|c|c|}
\hline Graphic & Type & 10 \\
\hline
\end{tabular}

Geologist: Tagfilts Comments

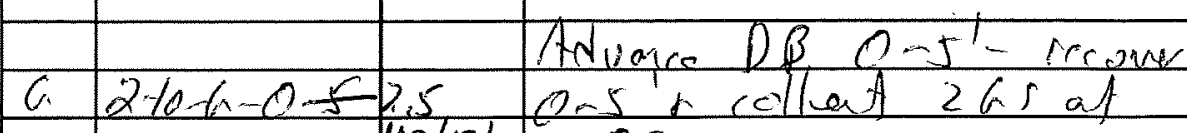

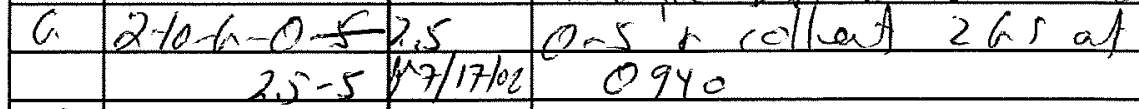

$a 210-6+5-20$

$6+2 \times-6-5-7$

Aduare DB, $5-15-r c c o n e r$ $5 \cdot 15 \alpha$ collert 465

a $2-10-6-7-9$

C $2-10-6-9-12$

G $210-6-12-15$

\begin{tabular}{|c|c|}
\hline & Aduareo DR $15-25-c 0$ counen \\
\hline $62-10-6-15-17$ & $15-22 \times$ collat 365 \\
\hline & $1035-$ \\
\hline
\end{tabular}

\begin{tabular}{lll} 
& & 1035 \\
\hline
\end{tabular}

20

$62-10-t-20-22$

Adumer DB?5-35-1rour

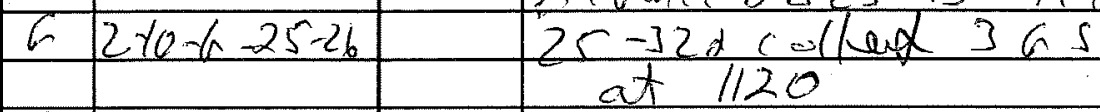

$6 \quad 2-10(-2 t-2)$

$\sigma 2-10-628-32$

$A N 0$ are $D B 35-45$ Treaver

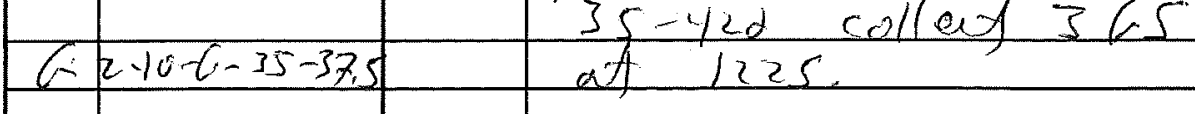

$6-2+10 \cdot 6-52.5+3$ 


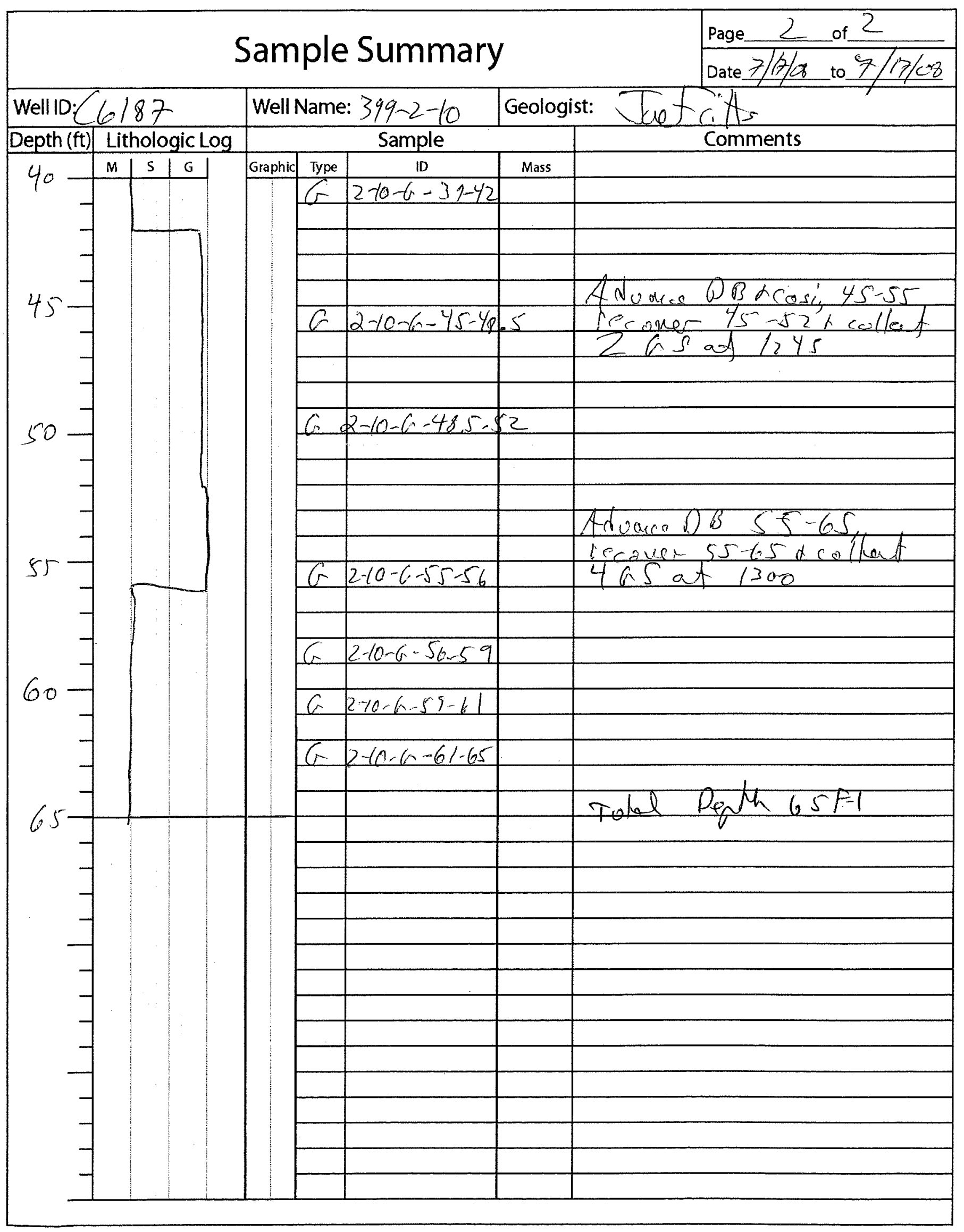




\section{Sample Summary}

Well ID: $C 6 / 88$

\begin{tabular}{|l|l}
\hline Depth (ft) & Lithologic Log \\
\hline
\end{tabular}

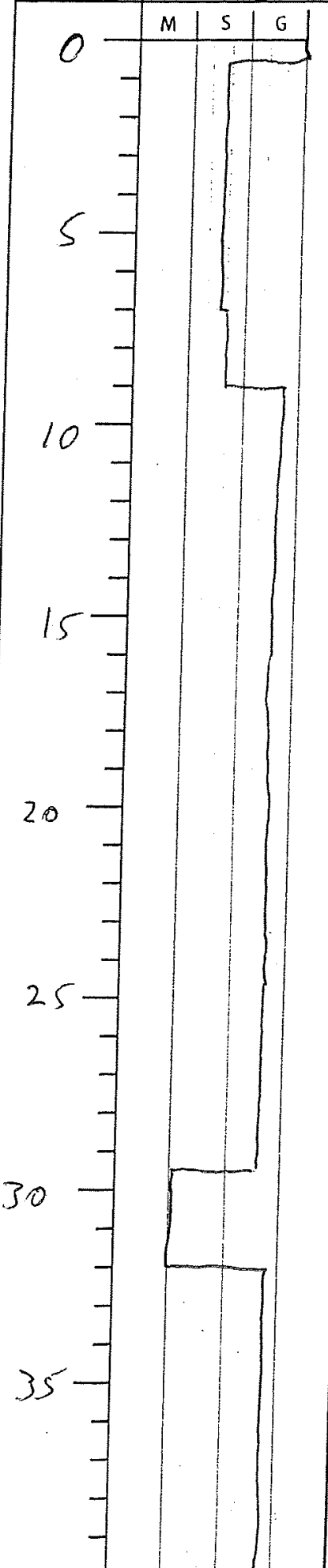

Well Name:399-2 -1 Geologist: Joe tilt

comments

\begin{tabular}{|c|c|c|c|}
\hline \multicolumn{3}{|c|}{ Sample } & Comments \\
\hline Graphic Types & iD & Mass & \\
\hline & $2-16-6-2<$ & & $A$ त $D=B A-5-10$ \\
\hline & & & $0-5+$ colleat $2 \mathrm{asal}$ \\
\hline G & $2-11-6-2,5-5$ & & $1035^{5}$. protol \\
\hline$E$ & $2 y-6-5-7$ & & Aanume D 8 - \\
\hline$G$ & $\mid 2-11-6-7-9$ & & $\frac{5-12}{\text { at } 1045}$ \\
\hline
\end{tabular}




\section{Sample Summary}

\begin{tabular}{|l|c|c|c|}
\hline Well ID: $(6188$ & Well Name:399-2-11 & Geologist: Jo F Frilt, \\
\hline Depth (ft) Lithalogic Log & \multicolumn{2}{|c|}{ Sample } & Comments \\
\hline
\end{tabular}

\begin{tabular}{|c|c|c|c|c|c|c|c|}
\hline $40-$ & m & 6 & Graphic & Type & ID & Mass & \\
\hline 70 & & & & a & $2-11-6-305-42$ & & \\
\hline & & & & & & & \\
\hline & & & & & & & $7 / 17 / 28$ \\
\hline $45-$ & & & & & & & Adumer Dos $45-55$, \\
\hline $75-$ & & & & & & & rerour $1.5+$ cillat 16.5 \\
\hline & & & & & & & at 0700 - degth interval \\
\hline & & $L$ & & & & & hard to idutity \\
\hline & & & & 6 & $2-11-6-45-55$ & & \\
\hline $50-1$ & & & & & & & \\
\hline & & & & & & & Advene $D B 55-63$ cerow \\
\hline & & & & & & & $556^{3}+$ collect $4 a s$ \\
\hline & & & & & & & at 0720 \\
\hline & & & & & & & \\
\hline & & & & 6 & $2-11-6-55-57$ & & \\
\hline & & & & $G$ & $2-11-6-57-59$ & & \\
\hline & & & & & & & \\
\hline $60-1$ & & & & a & $22-11-6-59-6 \mid$ & & \\
\hline & & & & & & & \\
\hline & & & & & $2-11-6-61-63$ & & \\
\hline & & & & & & & $x-101 / 0010$ \\
\hline & & & & & & & Toxel oghn $65 \mathrm{Fy}$ \\
\hline & & & & & & & \\
\hline & & & & & & & \\
\hline & & & & & & & \\
\hline & & & & & & & \\
\hline & & & & & & & \\
\hline & & & & & & & \\
\hline & & & & & & & \\
\hline & & & & & & & \\
\hline & & & & & & & \\
\hline & & & & & & & \\
\hline & & & & & & & \\
\hline & & & & & & & \\
\hline
\end{tabular}




\section{Sample Summary}

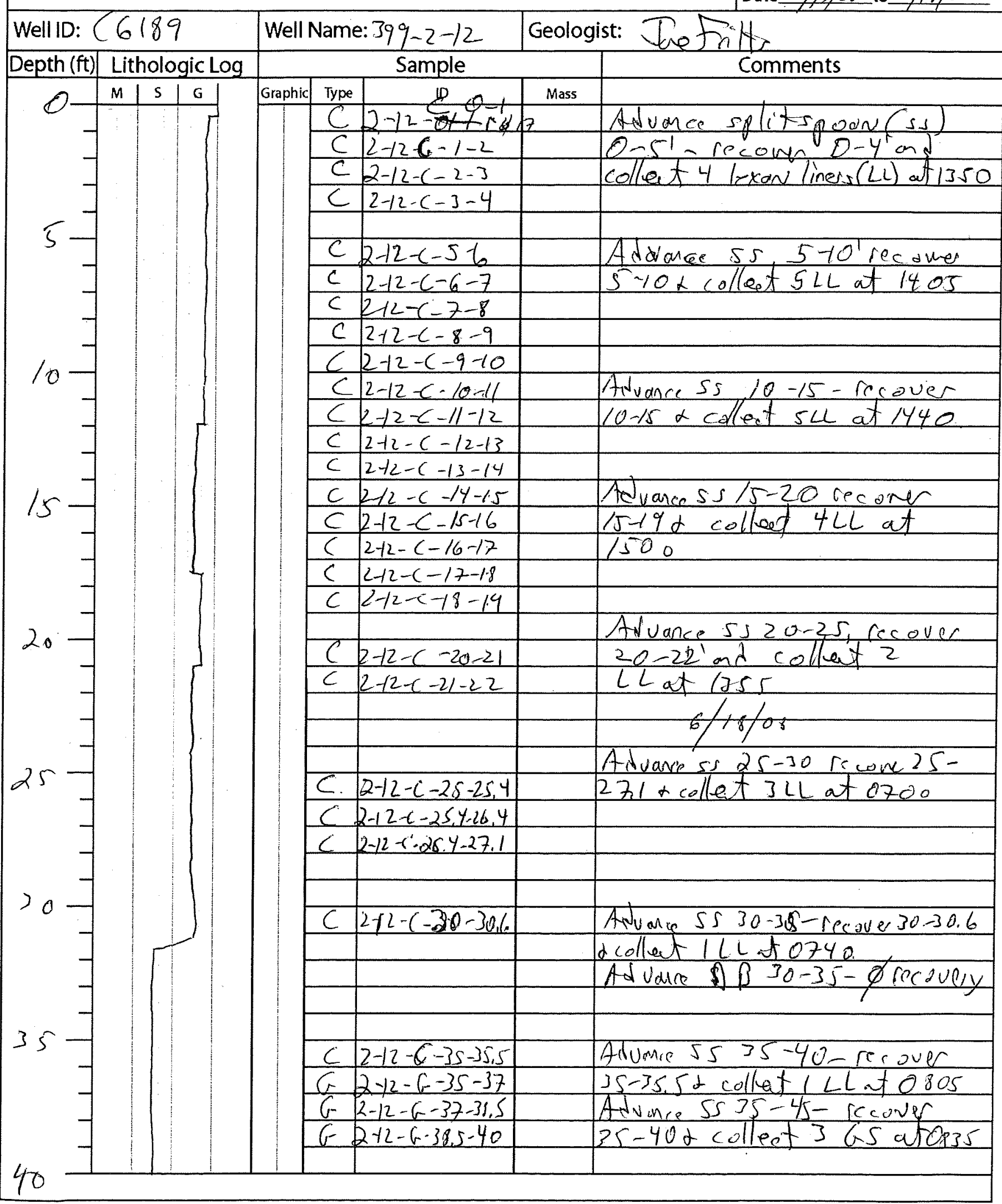




\section{Sample Summary}

\begin{tabular}{|c|c|c|c|c|c|c|c|}
\hline \multirow{2}{*}{\multicolumn{3}{|c|}{$\begin{array}{ll}\text { Well ID: } C 6189 \\
\text { Depth (ft) } & \text { Lithologic Loo } \\
\end{array}$}} & \multicolumn{3}{|c|}{ Well Name:399-2-12 } & \multirow{2}{*}{\multicolumn{2}{|c|}{\begin{tabular}{l|l} 
Geologist: Juefritts \\
\end{tabular}}} \\
\hline & & & \multicolumn{3}{|c|}{$\begin{array}{r}\text { Sample } \\
\end{array}$} & & \\
\hline \multirow{23}{*}{\multicolumn{2}{|c|}{$40 \longrightarrow M$}} & $|G|$ & Graphic & Type & ID & Mass & \\
\hline & & & & C & $2.12 \cdot c-40-40.0$ & & Advarce 55 40-45 drecouer \\
\hline & & & & & $2.12-c-40.6-41.6$ & & $40-42,6$-allowt 3 LLat 0,20 \\
\hline & & & & C & $212-c-41,6.42 .6$ & & \\
\hline & & & & & & & 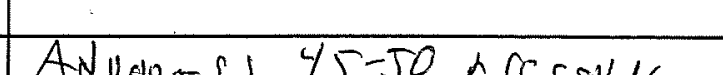 \\
\hline & & & & $C$ & $2+2-c-45-46$ & & $45-47$ - collect 3 LLat 0915 \\
\hline & & & & c & $2-12-c-46-47$ & & \\
\hline & & & & C & $2-12-c-47-48$ & & \\
\hline & & & & & & & \\
\hline & & & & c & $2-12-c-50-5]$ & & Advance $5550-55$ - reconer \\
\hline & & & & & $2 x-c-51-52$ & & $50-55+$ colleet $5 \angle$ lat 0930 \\
\hline & & & & & $2-12-c-52-53$ & & \\
\hline & & & & c & $2-12-53-54$ & & \\
\hline & & & & $c$ & $2-12<-54-55$ & & \\
\hline & & & & $C$ & $2-12-<-\sqrt{5}-56$ & & Anvarcess S5-60-recover \\
\hline & & & & & $2-12-c-56-57$ & & $55-60 \alpha$ collat show al \\
\hline & & & & & $2-17-c-57-58$ & & 1000 \\
\hline & & & & & $2-12-c-58-59$ & & $6 / 18 \%$ \\
\hline & & & & $\leqslant$ & $2-12-6-59-60$ & & ANuance $580 B=57.61$ \\
\hline & & & & C- & $2-12-6-59.5-60$ & 15 & recour 57-60.5 d callentlas \\
\hline & & & & & $2-12-c-60-61$ & & at 1055 \\
\hline & & & & & $2-12-2-61-82$ & & Advace $8560-65-\pi c a n e$ \\
\hline & & & & $\frac{c}{c}$ & $\frac{2-12-c-62-63}{D-12-6-63-64}$ & & 60-65+colleat \& L at $1055-$ \\
\hline \multirow{13}{*}{$65-$} & & & & & & & 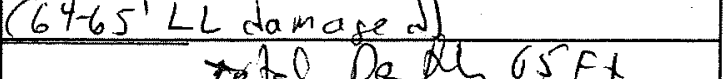 \\
\hline & & & & & & & \\
\hline & & & & & & & \\
\hline & & & & & & & \\
\hline & & & & & & & \\
\hline & & & & & & & \\
\hline & & & & & & & \\
\hline & & & & & & & \\
\hline & & & & & & & \\
\hline & & & & & & & \\
\hline & & & & & & & \\
\hline & & & & & & & \\
\hline & & & & & & & \\
\hline
\end{tabular}




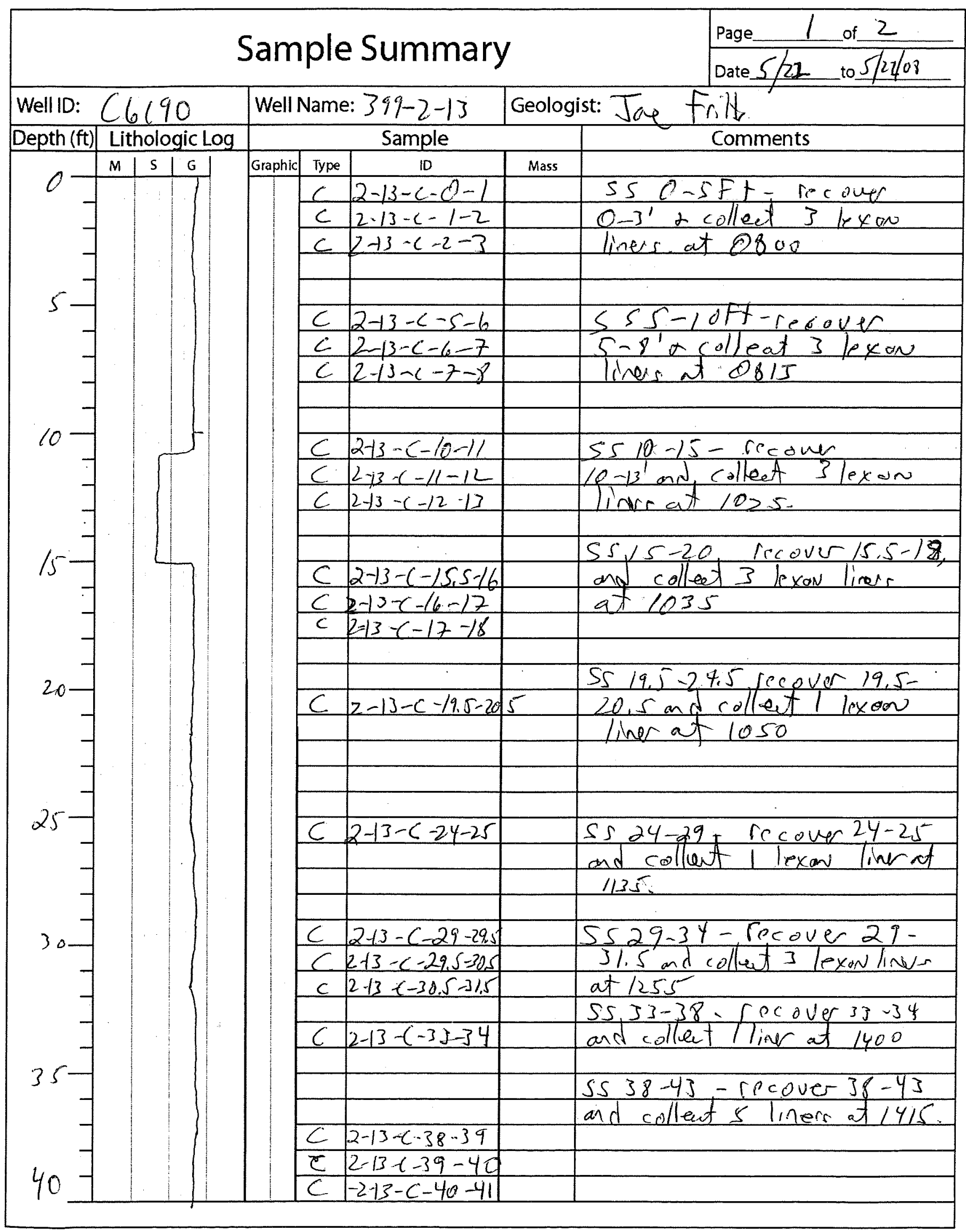

C.13 


\section{Sample Summary}

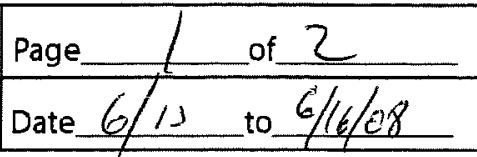

\begin{tabular}{|c|c|c|c|c|c|c|c|c|}
\hline Well ID: & 66 & $(9$ & & Well $\mathrm{s}$ & Name & $399-2-14$ & Geolo & ist: Thetritti \\
\hline Depth (ft) & Lith & olo & gic LC & & & Sample & & Commentspis $6-17$ \\
\hline & M & 5 & $G$ & Graphic & Type & 10 & Mass & $06 / 13$ Sonic 6"core \\
\hline & & & त & & $\zeta$ & $2-14-6-0-1,5$ & & advance drive barrel (db) $0-5^{\prime}$ \\
\hline & & & & & $E$ & $2-14-6-1-5-3$ & & recover $0-5 ; 4$ samples collected \\
\hline & & & & & $\epsilon$ & $2-1<1-6-3-4$ & & 21245 \\
\hline & & & & & $G$ & $2-14-6-4-5$ & & \\
\hline & & & & & 6 & $2-14-6-5-6,5$ & & advance db 5-10' recovery 5-8' \\
\hline & & & & & $\frac{7}{6}$ & $2-14-6-6.5-8$ & & 2 samples taleun e 1255 \\
\hline & & & & & & & & \\
\hline & & & & & & & & \\
\hline & & & & & 6 & $2-14-6-10-11$ & & advance db $10.15^{\prime}$ vecover 10 - \\
\hline & & & & & 6 & $2-14-6-11-12$ & & $15^{\prime} 4$ grab samples e 1310 \\
\hline & & & & & 6 & $2-154-6 \cdot 12-13,5$ & & \\
\hline & & & & & $t$ & $2-14-6-13.5-15$ & & \\
\hline $15-$ & & & & & & & & \\
\hline & & & & & $\frac{G}{1-}$ & $\frac{2-14-6-15-165}{2-14-1-16-18}$ & & advance $2615-20$ recever 15 \\
\hline & & & & & $G$ & $2-14-6-18-19.5$ & & S \\
\hline & & & & & & & & \\
\hline 20 & & & & & & & & \\
\hline & & & & & 6 & $2-14-6-20-21$ & & advance db $20-25^{\prime}$ recover 20 \\
\hline & & & & & 6 & $2-14-6-21-22$ & & $-25^{\prime} 5$ samplese 1330 \\
\hline & & & & & $G$ & $2-14-6-22-23$ & & \\
\hline & & & & & $G$ & $2 \cdot 14-6-23-24$ & & \\
\hline & & & & & 6 & $2-14-6-24-25$ & & \\
\hline & & & & & & & & \\
\hline & & & & & G & $2 \cdot 14-6-25 \cdot 26$ & & advance db $25 \cdot 30^{\prime}$ recover 25 - \\
\hline & & & & & G & $2-14-6-26-27$ & & $30^{\prime} 5$ samplese 1355 \\
\hline & & & & & & $2-14-6-27-28$ & & \\
\hline & & & & & $G$ & $2-14-6-28-29$ & & \\
\hline & & & & & 6 & $2-14-6-29-30$ & & \\
\hline & & & & & 6 & $2-14-6-30-32$ & & advance db $30-35$ recover $30-$ \\
\hline & & & & & b & $2-14-6 x-32-33$ & & $35^{\prime} 4$ grab samples e 1445 \\
\hline & & & & & & $2-14-G-33-34$ & & \\
\hline & & & & & $G$ & $2-14 \cdot G-34-35$ & & advance dlo $35-45^{\prime}$ necover \\
\hline & & & & & $G$ & $2-14-6 \cdot 35-36$ & & $35.45^{\prime} 6$ samples e 1510 \\
\hline & & & & & $G$ & $2 \cdot 14-6-36-37$ & 5 & \\
\hline & & & & & G & $2-14-G-37.5 \cdot 39$ & & \\
\hline & & & & & 6 & $2-14-G-39-40,5$ & & \\
\hline 40 & & & & & & & & \\
\hline
\end{tabular}




\section{Sample Summary}

Page $Z$ of 2
Date_o6/13 to $6 / 16 / 68$

Well ID: $(6191$

Depth (ft) Lithologic Log

\begin{tabular}{l|l|l|l|}
40 & M & 5 & 6 \\
\hline
\end{tabular}

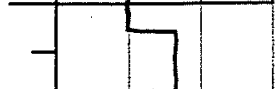

Well Name: 399-2-14 Geologist: TeFrit.

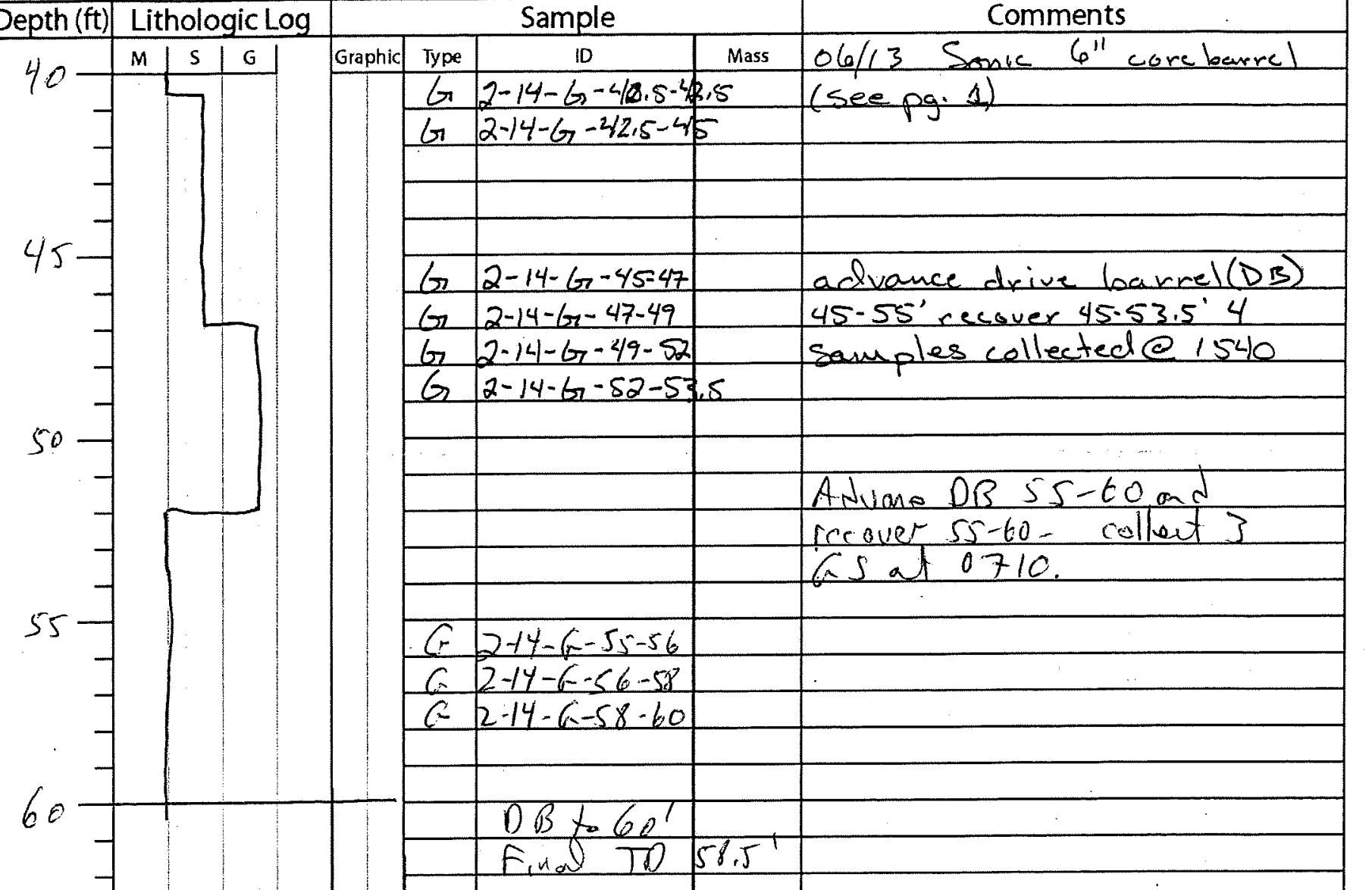

\section{Comments}




\section{Sample Summary}

\begin{tabular}{|c|c|c|c|c|c|}
\hline \multirow{3}{*}{\multicolumn{2}{|c|}{\begin{tabular}{|l|l|} 
Well ID: & C $6 / 42$ \\
Depth (ft) & Lithologic Log \\
\end{tabular}}} & \multirow{2}{*}{\multicolumn{2}{|c|}{ Well Name: $399-2-15$}} & \multicolumn{2}{|c|}{ Geologist: Jae fritts } \\
\hline & & & & & Comments \\
\hline & & \begin{tabular}{|l|l|l} 
Graphic & Typ \\
\end{tabular} & \begin{tabular}{l|l}
$\mathrm{pe}$ & $\mathrm{ID}$ \\
\end{tabular} & Mass & \\
\hline & & 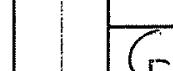 & $2-15-1-0-25$ & & Advance $O B O_{-5}^{-1}$ recover \\
\hline & & I & & & $0-5 \alpha$ collect 2 asat 1240 \\
\hline & & $\Leftrightarrow$ & $22-15 \cdot 6-2,5-5$ & & \\
\hline & & & & & At duance $\cap B S$ Sto' recouer \\
\hline & & $G$ & $2-15+6-5-7,5$ & & $5-10^{\prime}+$ odlet $2 \operatorname{cs}$ at 1250 \\
\hline & & $C=$ & $2-15-6-7.5 \cdot 10$ & & \\
\hline 10 & & & & & \\
\hline & & C & $2-15-6-10-12.5$ & & Adueree DB 10-15, recaver \\
\hline & & $G$ & $2-15-6-12,5-15$ & & 1305 \\
\hline & & & & & \\
\hline & & C & $2-15-6-15-175$ & & 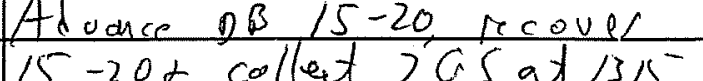 \\
\hline & & & & & 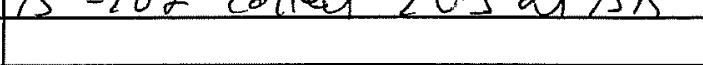 \\
\hline & & f & $2-15+-17 \cdot 5-20$ & & \\
\hline & & & & & \\
\hline & & C & $2-15-6-20-22$ & & Advarce $2 B 2 \partial-25,1000$ we \\
\hline & & & & & $20-24,5-$ colleet $2+5$ at \\
\hline & & $G$ & $2-15-4-92-245$ & & 1335 \\
\hline & & & & & \\
\hline & & G & $52-15-k-25-27$ & & Advance $0 \beta 25,35$, rrcover \\
\hline & & f & $2-15-6-27-29,5$ & & $\frac{25-29.5 \alpha \text { collet } 2 \mathrm{GS}}{\text { at } 1345}$ \\
\hline & & & & & \\
\hline $30-$ & & & & & 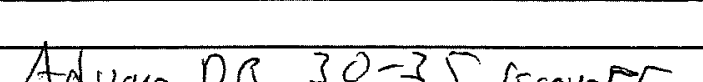 \\
\hline & & $G$ & $2-15-6-30-32,5$ & & 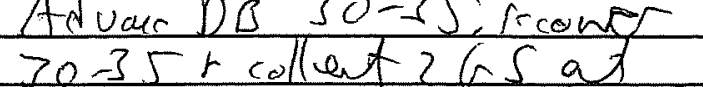 \\
\hline & & $G$ & $2-15-6-32.535$ & & $14 / 5$ \\
\hline & & & & & \\
\hline & & E & $2+5-6-35-36$ & $P$ & Advarce $D B 35-451 e$ cove \\
\hline & & 6 & & 29 & $35-4.5$ drolleet 3 CS at $/ 50$ \\
\hline & & & & & \\
\hline & & & & & \\
\hline
\end{tabular}




\section{Sample Summary}

$\frac{\text { Page } \frac{2}{2} \text { of } \frac{2}{2 / 25} \text { to } 6 / 2 \pi / 08}{\text { Date } 6 / 2}$

Well ID: $(6192$

Well Name: $399-2-15$ Geologist: Joe Frilts

Depth (ft) Lithologic Log

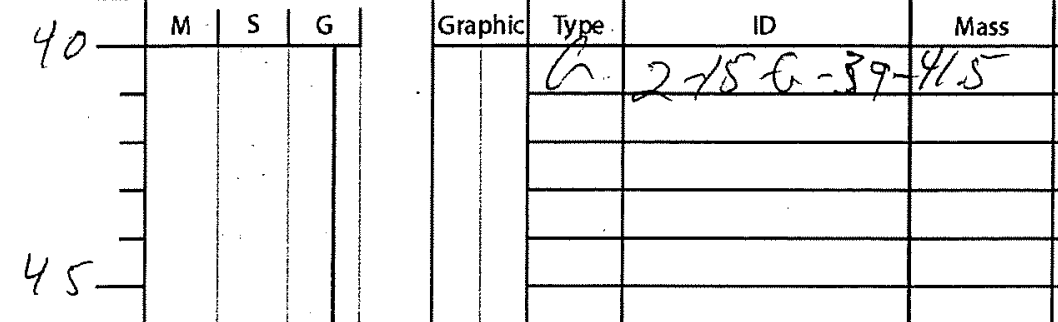

Sample

Comments

\begin{tabular}{|c|c|c|c|}
\hline Graphic & Type & ID & Mass \\
\hline & &
\end{tabular}

Arvance $D B \quad 45-5$, ircam

G $2-15-6-46-50 \quad 46-51.5-\operatorname{colllat} 2 \mathrm{as}$

$-1$

50

G

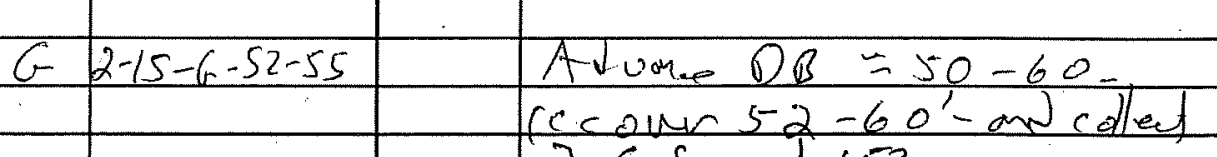

$55-1$

$60-1$.

G $2+15-6-55-50$

7 as at 1530

$6 \quad 2+15-6-58-60$

Te tal Degh $61.5 \mathrm{Fy}$ 


\section{Sample Summary}

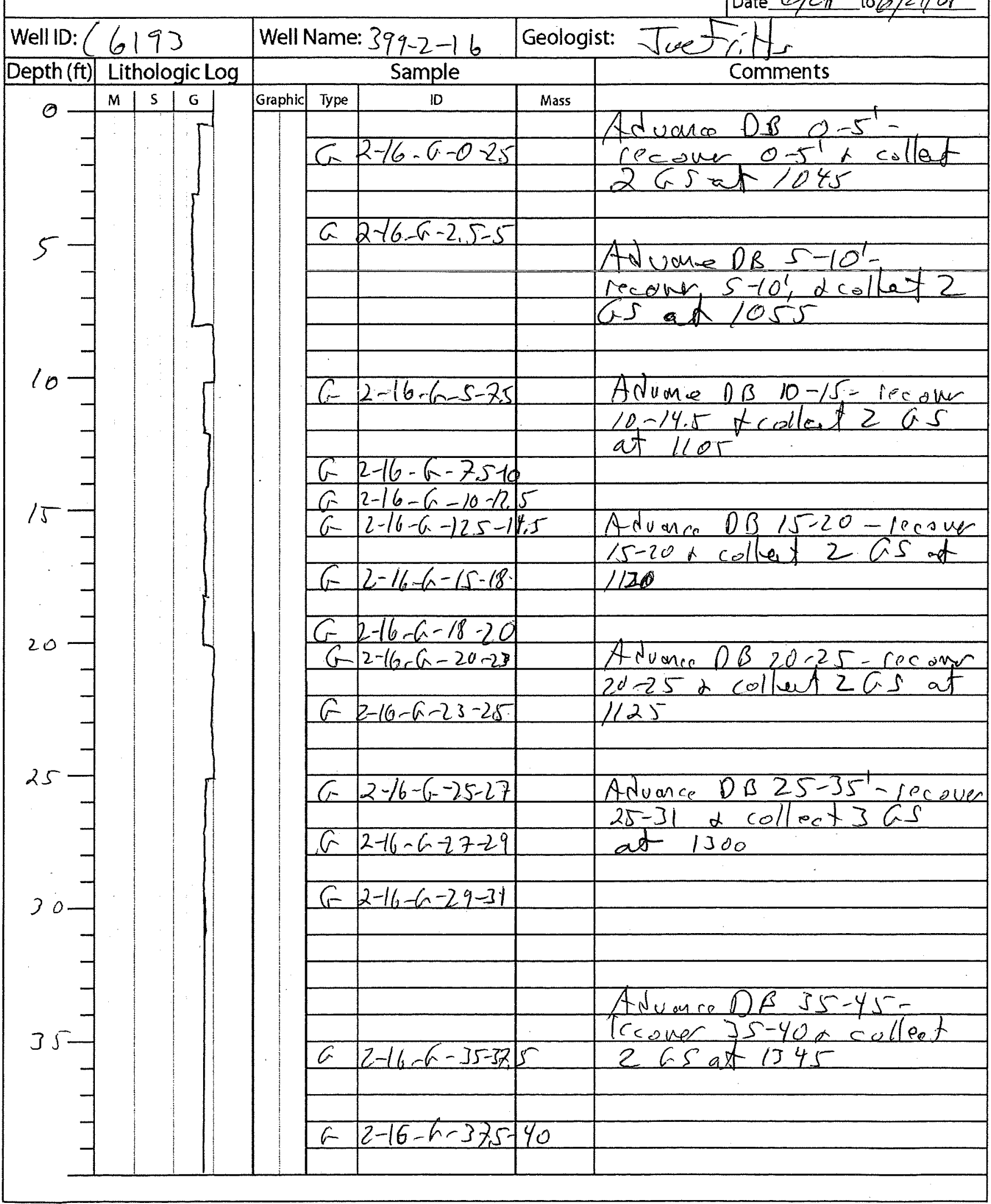




\section{Sample Summary}

Well ID:C6193

\begin{tabular}{ll}
\hline Depth $(\mathrm{ft})$ & Lithologic Log \\
\hline
\end{tabular}

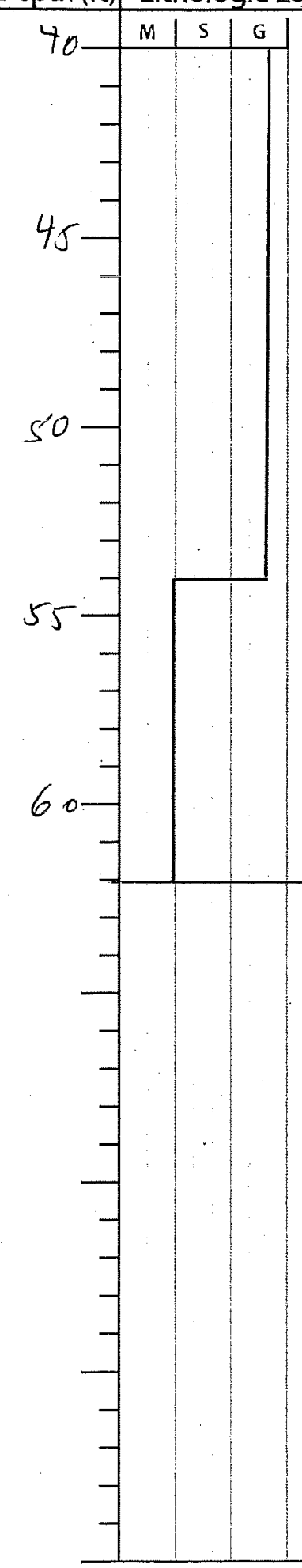

Well Name:399-2-16 Geologist: Jue f citt Sample

\section{Comments}

\begin{tabular}{|c|c|c|c|}
\hline Graphic & Type & ID & Mass \\
\hline
\end{tabular}

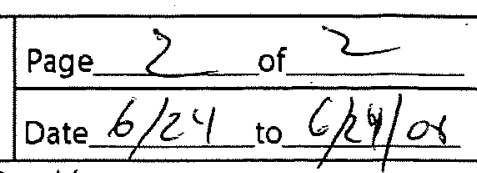

\begin{tabular}{|c|c|c|c|}
\hline पype & ID & Mass & \\
\hline & & & \\
\hline & & & \\
\hline & & & \\
\hline & & & \\
\hline G & $2-16-(-4 c-47$ & & $A D=A B$ \\
\hline & & & Hecouer $45-53$ a collet \\
\hline G & $2-16-6-47-4 \%$ & 5 & 465 at 1405 \\
\hline$G$ & $2-16-6-49.5-5$ & -1 & \\
\hline & & & \\
\hline$G$ & $2-16-c-51-5$ & 3 & \\
\hline & & & Aduance DBS4-60, recoved \\
\hline & & & $54-60 \alpha$ dleet 3 as ad \\
\hline G & $2-16-6-54-56$ & & 1435 \\
\hline & & & \\
\hline$G$ & $2-66-6-56-58$ & & \\
\hline$a^{2}$ & $2-16-a-58-60$ & & Total Dendh 62Ft \\
\hline & & & \\
\hline
\end{tabular}




\section{Sample Summary}

Page 1 of 2

Well ID: C6195

\begin{tabular}{|l|l|}
\hline Depth (ft) & Lithologic Log \\
\hline
\end{tabular}

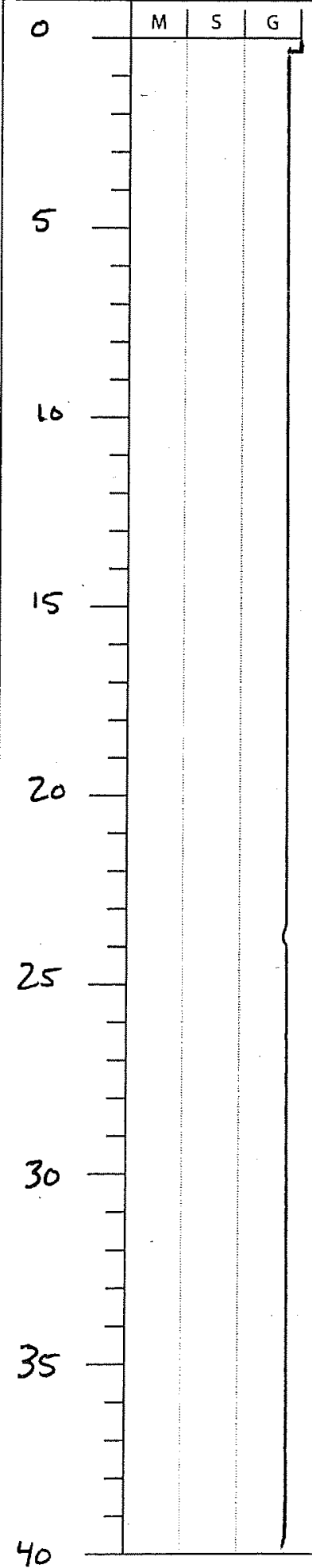

Well Name: $399-2-17$ Geologist: Sean Sexton Sample

\begin{tabular}{c|c|c|c|c} 
Graphic & Type & 10 & Mass & $06 / 12 / 08$
\end{tabular}

drove core barrel $0-s^{\prime}$ recovered $0-5 ; 4$ grate som ples taken e. 1424

core barrel $5-10$, recovered $5-10$, 3 samples e 1435

\begin{tabular}{ll}
6 & $2-17-6-7-8,5$ \\
\hline & $2-17-6-8,5-10$
\end{tabular}

$6 \quad 2-17-2-8,5=10$

b $2-17-6-10-115$

$6 \quad 2-17-6-11.5-13$

b $2-17-6-13-14$

$6 \quad 2-17-6-14-15$

$6 \quad 2-1.7-6-15-16$

$6 \quad 2-17 \cdot 6-16-17.5$

$62-17-6-17.5-19$

\begin{tabular}{l|l|}
\hline 6 & $2-17-6-25-26$
\end{tabular}

$6 \quad 2-17-6-26-27$

$62-17-6-27-28$

$6 \quad 2-17-6-28-29$

$6 \quad 2-17-6-29-30$

core barrel $10-15$, recover 10-15, 4 samples $Q_{0} 1452$

$6 \quad 2-17-6-25-26$ Tsample depths doubled intentionally

\begin{tabular}{|l|l|l}
\hline & & \\
\hline & & $\times$ \\
\hline
\end{tabular}

*ore barrel 25-30' recover 25-26

I sample wllected Q. 1528

core barrel 25-30, recover 25.

30,5 samples e 1554

$6 / 13 / 20086 "$ drive burcel

advance db $30-35$, recover $30-35,3$ samples $e$ o735

$6 \quad 2-17-6-30-32$

$6 \quad 2-17-6-32-33.5$

$6 \quad 2-17-6-33.5-35$

$6 \quad 2-17-6-35-37,5$

l. $2-17-6-37,5-40$
Advance db $35-45$, recover

$35-40,2$ samples e of 45 
Sample Summary

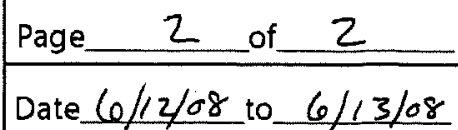

Well ID: C 6195 \begin{tabular}{|l|l|}
\hline Depth (ft) & Lithologic Log \\
\hline 40 &
\end{tabular}

Well Name: 399-2-17 Geologist: Seau Sexton

40

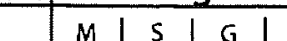

Sample

Comments

\begin{tabular}{ll|l|l|l|l|}
\hline 40 & & & & & \\
\hline
\end{tabular}




\section{Sample Summary}

\begin{tabular}{|c|c|c|c|c|c|c|c|}
\hline \multirow{2}{*}{\multicolumn{3}{|c|}{ 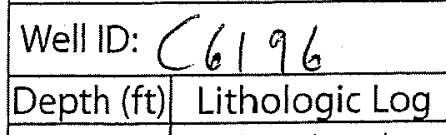 }} & \multicolumn{3}{|c|}{ Well Name: 39 y $-2-18$} & \multicolumn{2}{|c|}{ Geologist: JFritts } \\
\hline & & & \multicolumn{4}{|c|}{ Sample } & \multirow{2}{*}{\begin{tabular}{|c|} 
Comments \\
$G / \mathrm{s} / \mathrm{cg}$ \\
\end{tabular}} \\
\hline \multirow[t]{2}{*}{2} & \multirow{34}{*}{ 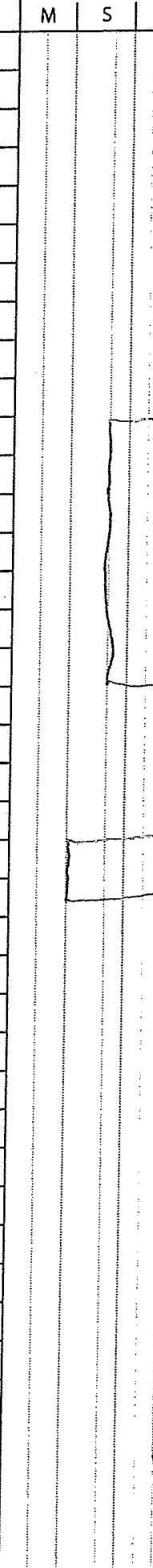 } & \multirow{2}{*}{$\mid \frac{1}{1}$} & \multirow[t]{2}{*}{ Graphic } & \multirow{2}{*}{\multicolumn{2}{|c|}{\begin{tabular}{c|c}
$=$ & 10 \\
$\therefore$ & $2-18-(r-0-2.5$
\end{tabular}}} & \multirow[t]{2}{*}{ Mass } & \\
\hline & & & & G. & & & $6^{\prime \prime} 0 \beta 0-5$ collex (2) \\
\hline & & & & $G$ & $2-18-6-25-5$ & & 05 at 1445 \\
\hline & & & & G & $2+8-6-5+6.5$ & & $G^{\prime \prime} D \beta 5-10^{\prime} \cdot \operatorname{col} l \operatorname{ar}(4)$ \\
\hline & & & & & $2-18-6-6.5-8$ & & fs at 1525 \\
\hline & & & & a & $2-1 x-6-8-9$ & & \\
\hline & & & & $G$ & $2-18-6-7-10$ & & $6 / 4 / 08$ \\
\hline & & & & G & $2-19-6-10-12$ & & 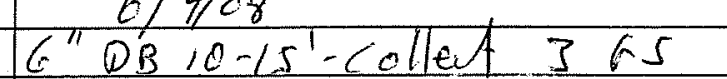 \\
\hline & & & & 6 & $2-18-6-12-14$ & & at 0715 \\
\hline & & & & & $2-18-6-14-15$ & & \\
\hline & & & & & & & $6 / 11 / 48$ \\
\hline $15-1$ & & & & & & & \\
\hline & & & & & & & $6^{10} 017.5-25-i d t e d$ \\
\hline & & & & & $2+18-6-17,5-19$ & & 4 CS at 0800 - reconer \\
\hline & & & & & $2-11-6-19-20$ & & $17.5-22.5$ \\
\hline & & & & & $2-19-a-2 a, 21$ & & \\
\hline & & & & & $2-18-6-21 \cdot 22,5$ & & \\
\hline & & & & & & & $6 " 0022,5-30$ and recour \\
\hline & & & & & $2-19-6-2,2,5-2,5$ & & $22.5-25.5-$ collat 2 as \\
\hline & & & & & $218-6-23.5-2505$ & & at 0810 \\
\hline & & & & & $2-18-6-255-275$ & & $6 " 0 B 325-33,5$ and perom \\
\hline & & & & & $2+18-6-27.5-24$ & & $25,5-33,5-$ alleat 5 G5 ad \\
\hline & & & & & $2+8 \cdot(-29-30,5$ & & 0825 \\
\hline & & & & & $2-10-6-30,5-32$ & & \\
\hline & & & & & $2-18-6-32-3,5$ & & \\
\hline & & & & & & & \\
\hline & & & : & & & & 6" UB 33-45t-t-recone \\
\hline & & & & $G$ & $2-19-6-73-34$ & & 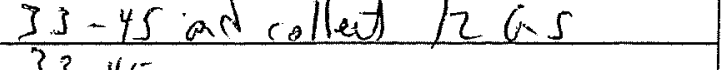 \\
\hline & & & & $Q_{0}$ & $\frac{210-6-3)-51}{2-18-6-34-3-5}$ & & $33-45$ \\
\hline & & & & & $2 \cdot 18 \cdot 6-35-36$ & & \\
\hline & & & & & $2+8-(r-36-37)$ & & \\
\hline & & & & $6: 0$ & $2-18-6-37-38$ & & \\
\hline & & & & 62 & $2+8-6-38-39$ & & \\
\hline & & & & $E$ & $2+1)-(x-39-40$ & & \\
\hline
\end{tabular}




\section{Sample Summary}

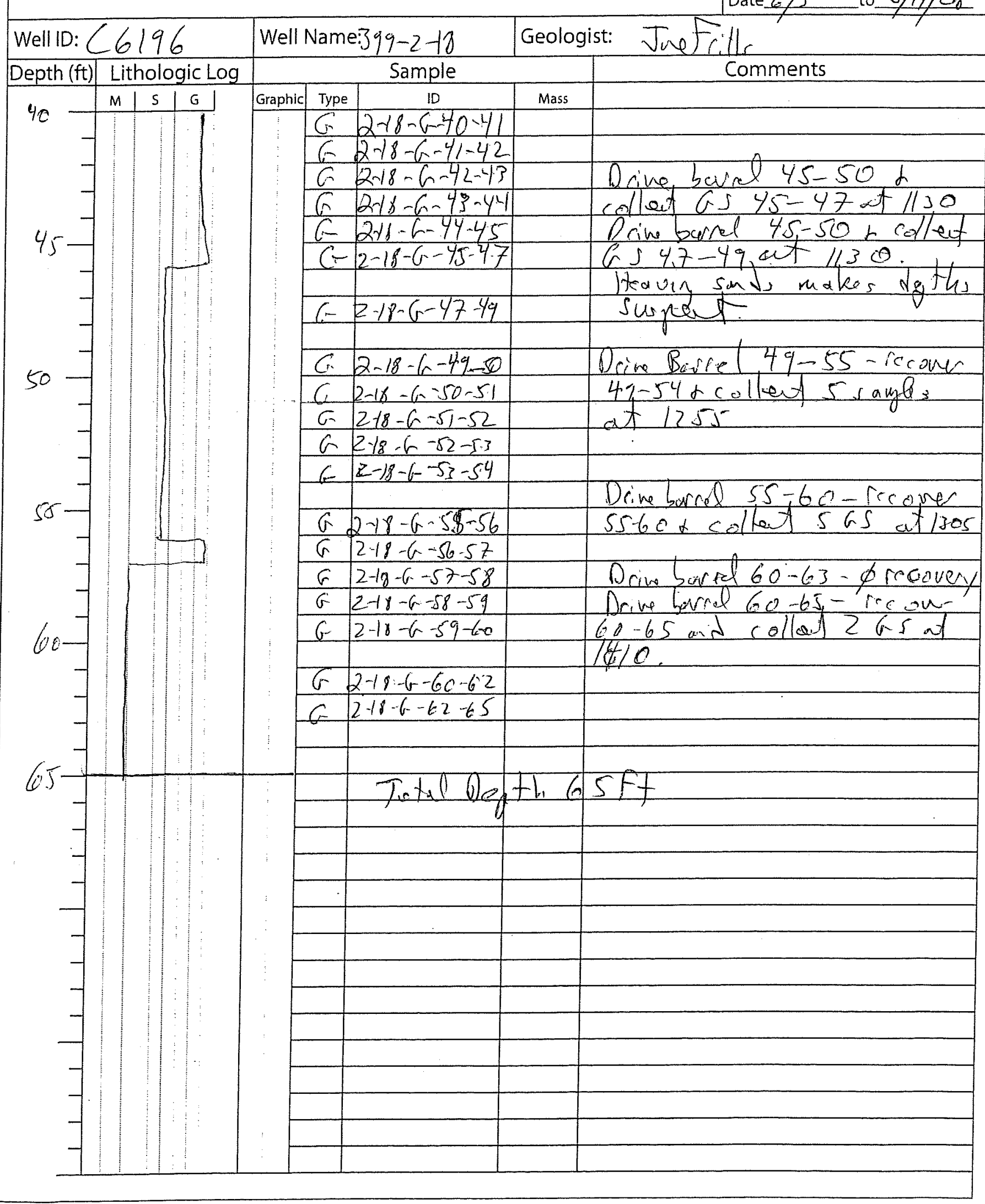




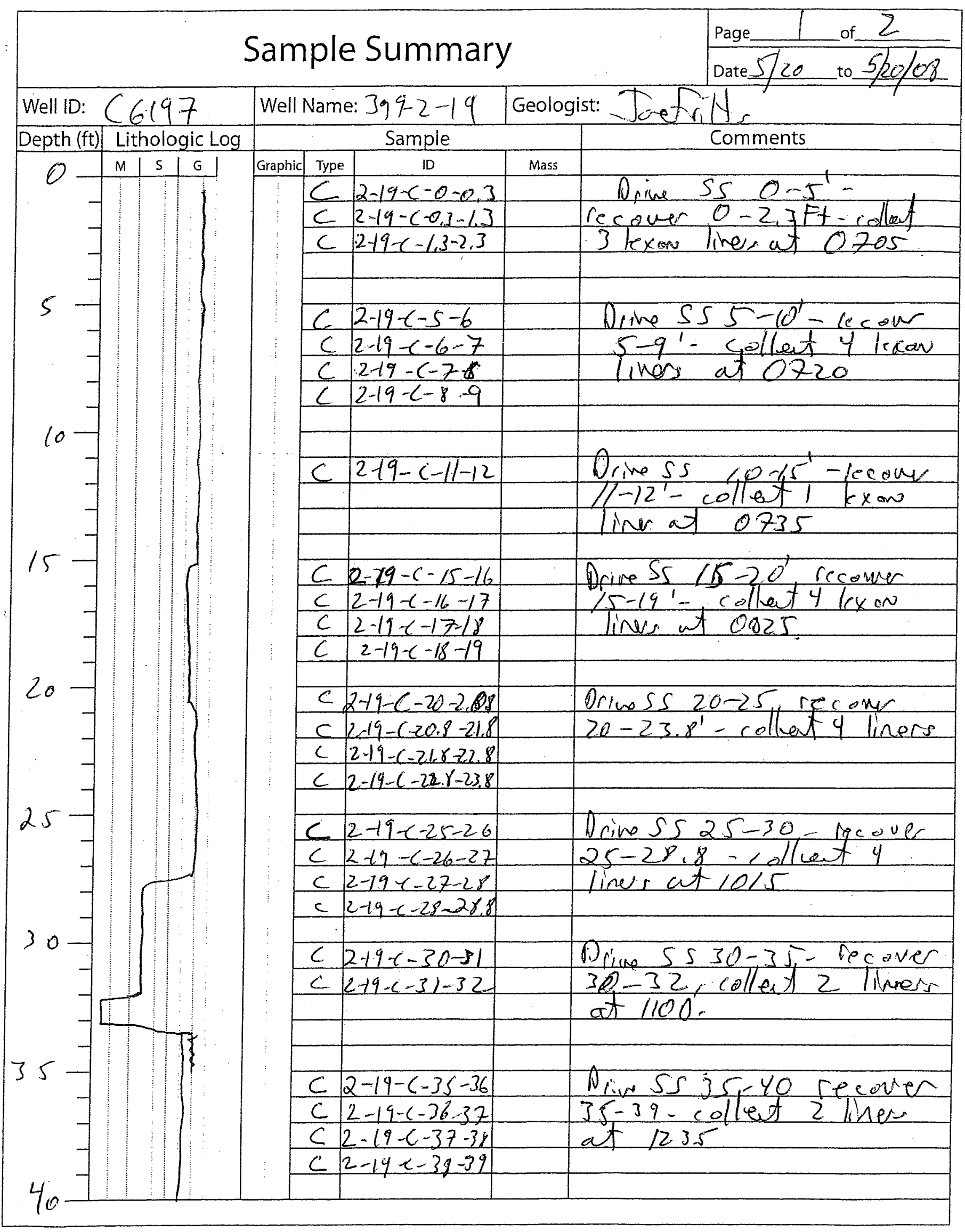




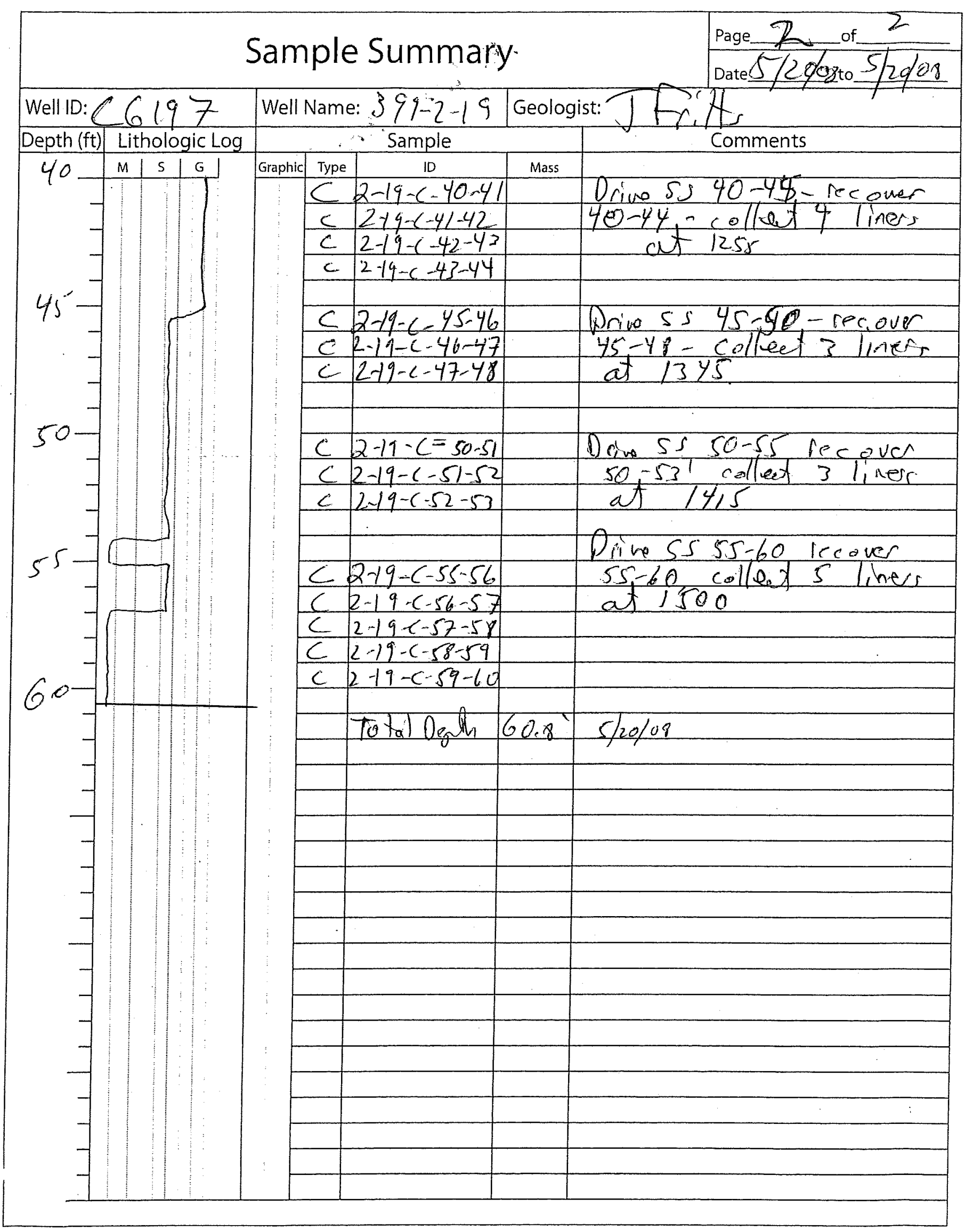

C. 25 


\section{Sample Summary}

\begin{tabular}{|c|c|c|c|c|c|c|c|c|}
\hline Well ID: & -6 & 198 & & Well $\mathrm{N}$ & Name & $399-2-20$ & Geologi & s: Juefrits \\
\hline Depth $(\mathrm{ft})$ & & holos & gic Log & & & Sample & & Comments \\
\hline & M & $s$ & G & Graphic & Type & ID & Mass & \\
\hline & & & & & & $2-20-6-0-2,5$ & & Advance drive barke $0-5^{\prime}$ - \\
\hline & & & & & & & & recaver $0-5+$ collet 2 \\
\hline & & & & & $a$ & $2-20-6-2,5-5$ & & as at 0705 \\
\hline 5 & & & & & & $2-20-6-5-3,5$ & & Aduon $\quad D B$ \& $10^{\prime}$ \\
\hline & & & & & & & & ceser $5-10^{\alpha} \alpha$ collet 2 \\
\hline & & & & & G & $2-20-6-7,5-10$ & & Cs at 0715 \\
\hline & & & & & & & & \\
\hline $10-$ & & & & & a & $2-20-6-10-13$ & & $A d u$ are $D B \cdot 10-15$ recouer \\
\hline & & & & & & & & $10-13+$ collect'16.5 at \\
\hline & & & & & & & & 0725 \\
\hline & & & & & & & & \\
\hline 15 & & & & & & & & \\
\hline & & & & & G & $2-20-6-15-17$ & & Advence $D B / 5=20$ recou \\
\hline & & & & & & & & $15-20+$ collet 365 at 0730 \\
\hline & & & & & $a$ & $2-20-6-17-1.9$ & & \\
\hline & & & & & 6 & $2-20-6-19-20$ & & \\
\hline 20 & & & & & $\frac{v}{6}$ & $2-20-6-20-21$ & & Advan- DQ $20-75$ - lecover \\
\hline & & & & & & & & $20-25 r$ colleet 365 at 0750 \\
\hline & & & & & G & $2-20-a-21-23$ & & \\
\hline & & & & & & 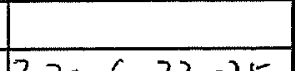 & & \\
\hline 2.5 & & & & & G & $2-20-6-23-25$ & & Aduance $00 \quad 25-35$ - \\
\hline & & & & & ta & $2-20-6-25-225$ & & $25-30+$ colleat 265 at \\
\hline & & & & & & & & 0825 \\
\hline & & & & & G & $2-20-6-27,5-30$ & & \\
\hline 30 & & & & & & & & \\
\hline & & & & & Co & $2-24-4 n-2 n-35$ & & Advance OB 30-35-ircover \\
\hline & & & & & & & & 30.35 reallent as at 0855 \\
\hline & & & & & & & & \\
\hline & & & & & & & & \\
\hline$\sqrt{5}$ & & & & & C & $2-20-6-35-37,5$ & & Adume D) $35-48-$ recover \\
\hline & & & & & & & & $35^{\prime}-400$ acollut 2 as atogos \\
\hline & & & & & E & $2-20-6-37,5-40$ & & \\
\hline & & & & & & & & \\
\hline & & & & & & & & \\
\hline
\end{tabular}




\section{Sample Summary}

\begin{tabular}{|c|c|c|c|c|c|c|c|}
\hline \multicolumn{8}{|r|}{ Date $6 / 25$ to $6 / 28 / 08$} \\
\hline \multicolumn{3}{|c|}{ Well ID: $C 6198$} & \multicolumn{3}{|c|}{ Well Name: $399-2-20$} & \multicolumn{2}{|c|}{ Geologist: Jotrits } \\
\hline \multirow{2}{*}{$\begin{array}{c}\text { Depth }(\mathrm{ft}) \\
40\end{array}$} & \multicolumn{2}{|c|}{ Lithologic Log } & \multicolumn{4}{|c|}{\begin{tabular}{|c|} 
Sample \\
\end{tabular}} & Comments \\
\hline & \begin{tabular}{l|l}
$\mathrm{M}$ & $\mathrm{S}$ \\
\end{tabular} & $\mid \mathrm{G}$ & Graphic & Type & ID & Mass & \\
\hline & & & & & & & \\
\hline & & & & & & & \\
\hline & & & & & & & \\
\hline $45-1$ & & & & & & & AN 0 B 45-5 80 \\
\hline & & & & $a$ & $\alpha-20-(n-75-4 f$ & & $\begin{array}{l}\text { Adume is } 45-35 \text {, reone } \\
45-51+\text { collect? }\end{array}$ \\
\hline & & & & G & $2-20-6-47-49$ & & 0925 \\
\hline & & & & & & & 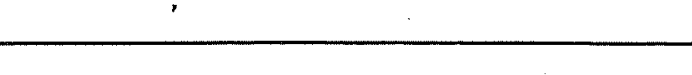 \\
\hline 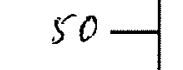 & & $\cdots$ & & $\sigma$ & $2-20-6-47-31$ & & $A d$ vou $D B .54-60^{\prime} \cdot 16004$ \\
\hline & & & & & & & $54-60+$ colleet 365 at \\
\hline & & & & & & & 0935 \\
\hline & & & & $C$ & $3-2$ a d cusil & & \\
\hline $5-1$ & & & & 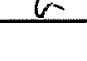 & $2-20-h-34-56$ & & \\
\hline & & & & G & $2-20-6 \cdot 56-59$ & & \\
\hline & & & & & & & \\
\hline & & & & $G$ & $2-20-6-59-60$ & & \\
\hline 60 & & & & & & & \\
\hline & & & & & & & \\
\hline & & & & & & & Tulal Nem 62 \\
\hline & & & & & & & \\
\hline & & & & & & & \\
\hline & & & & & & & \\
\hline & & & & & & & \\
\hline & & & & & & & \\
\hline & & & & & & & \\
\hline & & & & & & & \\
\hline & & & & & & & \\
\hline & & & & & & & \\
\hline & & & & & & & \\
\hline & & & & & & & \\
\hline & & & & & & & \\
\hline C & & & & & & & \\
\hline
\end{tabular}




\section{Sample Summary}

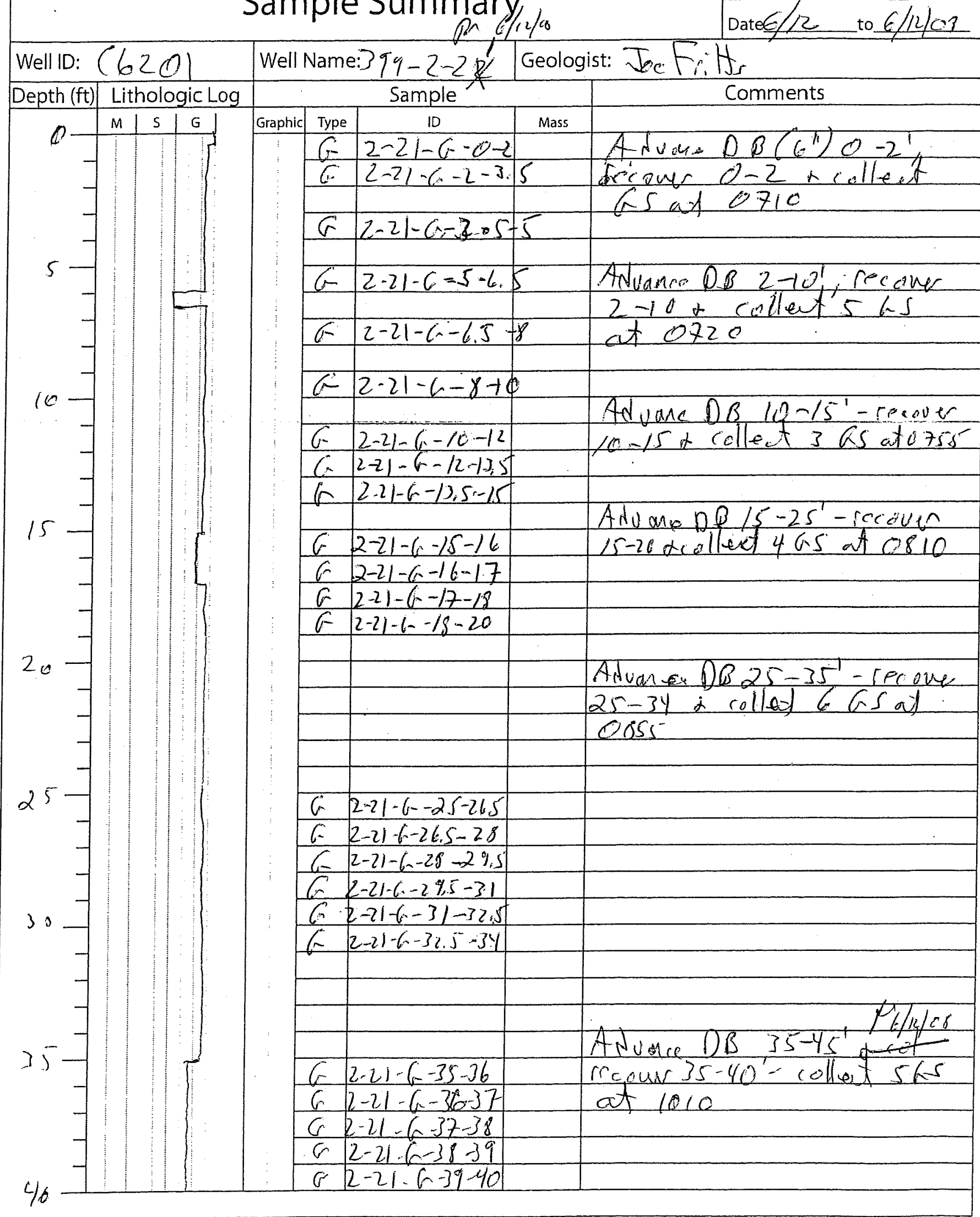




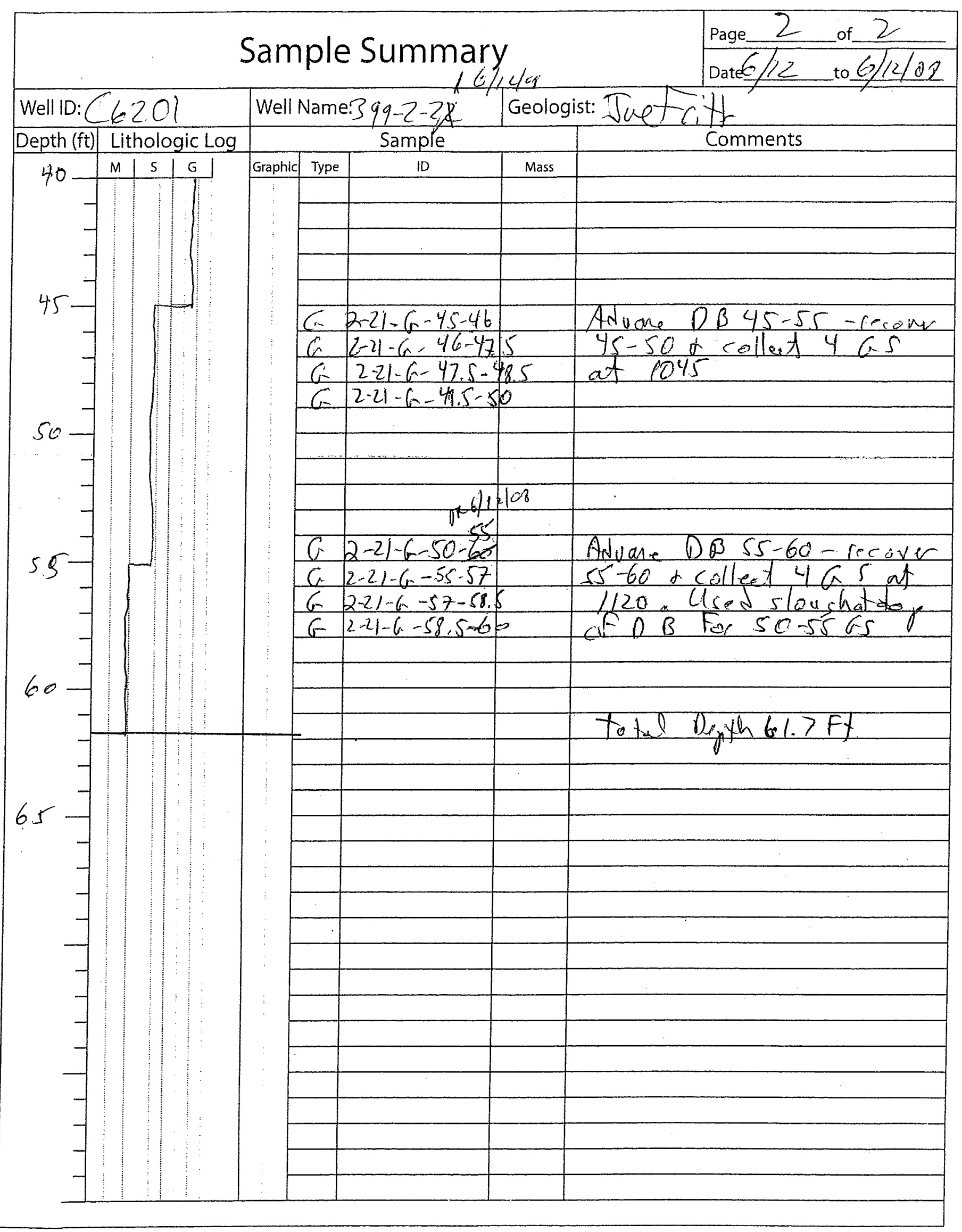




\section{Sample Summary}

\begin{tabular}{|l|c|c|}
\hline Well ID: $(6202$ & Well Name:399-2-22 & Geologist: Toef/its \\
\hline Depth (ft) Lithologic Log & \multicolumn{2}{|c|}{ Sample } \\
\hline
\end{tabular}

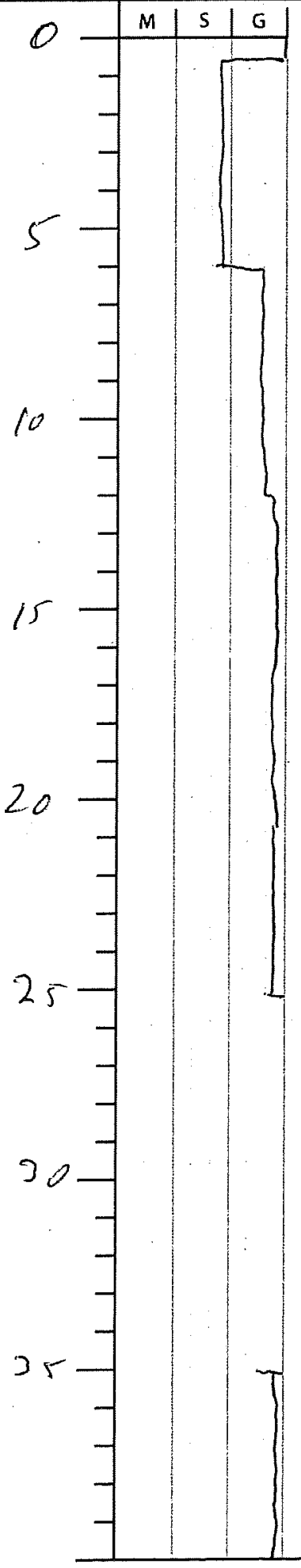

\begin{tabular}{|l|l|l}
\hline Graphic & Type & ID
\end{tabular}

\begin{tabular}{|l|l|}
\hline & Mass \\
\hline
\end{tabular}

Comments

Page $\frac{1}{7 / 14 / 00}$ of 2

\begin{tabular}{|c|c|c|c|}
\hline Type & ID & Miass & \\
\hline & & & Adukce $10 B 0-5^{\prime}=$ \\
\hline C. & $2-22-6-0-2$ & 5 & reoiner $0-5 \alpha$ collent 26 \\
\hline & & & at coes \\
\hline G & $2-22-6-2.5-5$ & & \\
\hline & & & \\
\hline a & $2-22-6-5-7$ & & \\
\hline c & $2-22-\ln -7-9$ & & ANumer DB S - $5-10 \mathrm{co}$ \\
\hline & & & $5-15$ a collect $4 a S$ at \\
\hline & & & 1035 \\
\hline a & $2-22-6-9-12$ & & \\
\hline & & & \\
\hline 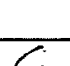 & $102=6-12-15$ & & \\
\hline a & & & \\
\hline E & $372-6-15-17$ & & ANunce DB $15-25$ \\
\hline & & & icrouer $15-23$ a collent) \\
\hline a & $2-22-6-17-19$ & & 4 asat 1415 \\
\hline
\end{tabular}

\begin{tabular}{|l|l|}
\hline$a$ & $2-22-(6-19-2)$ \\
\hline$G-2-22-6-21-23$ & \\
\hline
\end{tabular}

Advenci DB $25-35$,
o rerounery-

G $222+-3.5 \cdot 38,5$

Tduan $0 B 35-45$

cecone $35-42$ a collat

265 at 1530 


\section{Sample Summary}

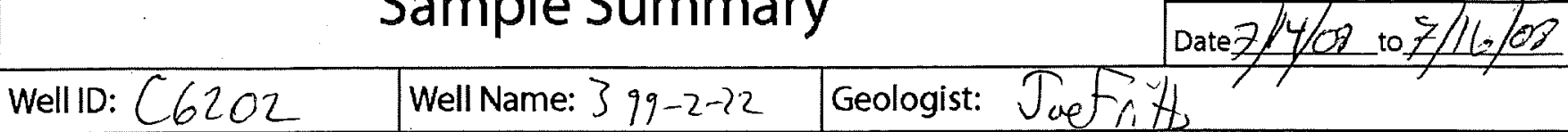

Depth (ft) Lithologic Log
Well Name: 3 99-2-22 Geologist: Joefritt

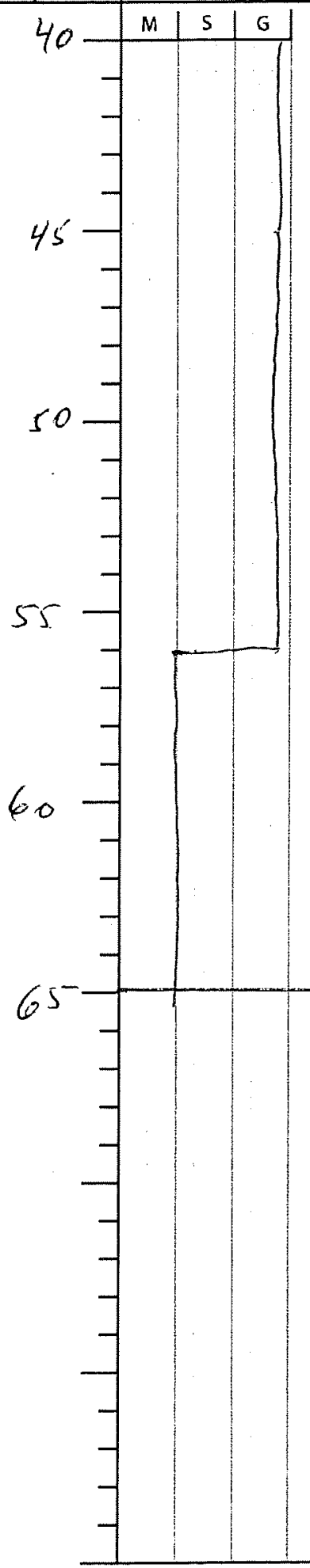
Sample

\begin{tabular}{c|c|c|c|} 
Graphic & Type & 10 & Mass \\
\hline \multirow{4}{*}{$G$} & $2-22-G \cdot 30,5-42$ & \\
\hline
\end{tabular}
Comments

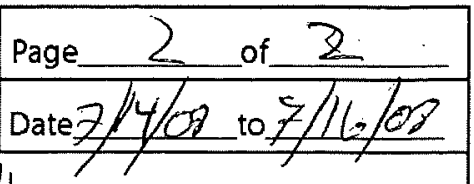




\section{Sample Summary}

Well ID: $C 6207$ Depth (ft) Lithologic Log

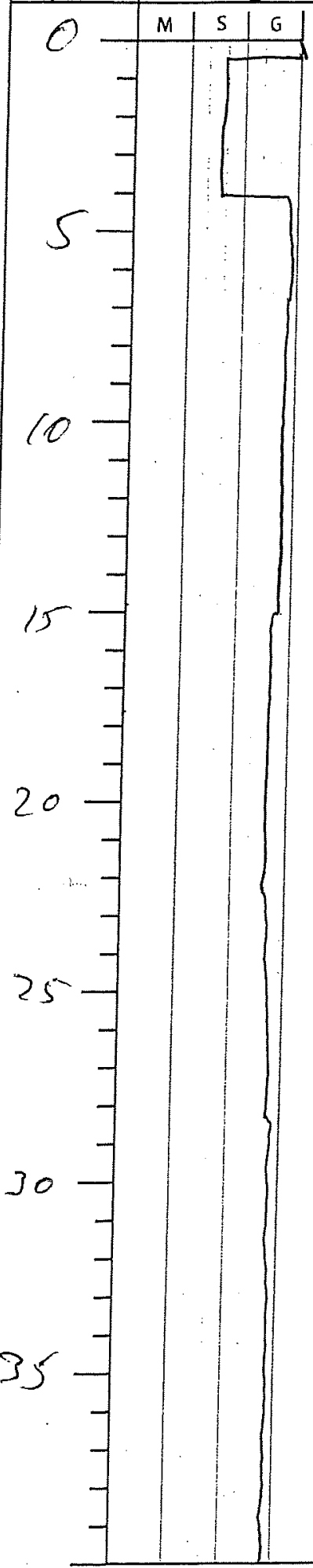

Well Name:399-2-23 Geologist: Juetrily Sample Comments

\begin{tabular}{|c|c|c|c|}
\hline ic Type & ID & Mass & \\
\hline G & $2-23-6-0-2$ & & 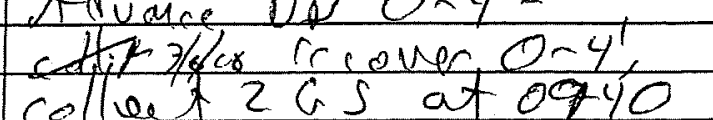 \\
\hline 6 & $2-23-6-2-4$ & & \\
\hline & & & $\frac{1}{10 \mathrm{r} \text { collect }}$ \\
\hline
\end{tabular}

ar $2-23-6=7-10$

a $2-23-6-10-13$ Aduace DB $10-15$, cecover $10-14,5+$ collet 2 as a $2+3-6-13-145$ at 1010

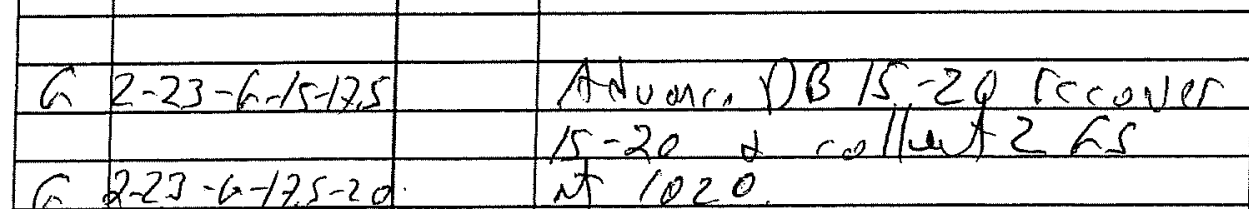

Cn. 2-23-6-20-22.5 Atumace B 20-25, recover \begin{tabular}{|l|l|l|}
\hline & & $20-24,5+$ collut 26 sat \\
\hline$a$ & $2-73-6-22524,5$ & \\
\hline
\end{tabular} C. 2-23-6-25-28 Aduar, DB 25-35, recover $25-31 \alpha$ colles $26 \Omega$ al $0 \quad 2-23-6-28-31$ 


\section{Sample Summary}

Page 2 of 2

Well ID: (6207

\begin{tabular}{|l|l}
\hline Depth (ft) & Lithologic Log \\
\hline
\end{tabular}

Well Name: 399-2-23 $\quad$ Geologist: Tessa Buswrll

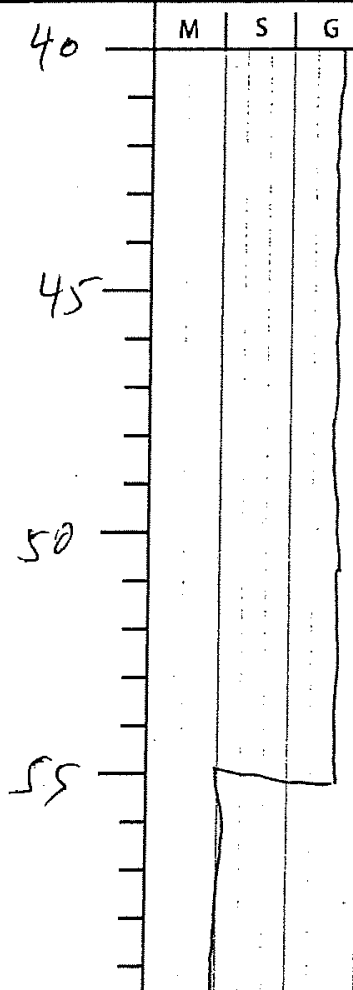
Sample

Comments

\begin{tabular}{|c|c|c|c|}
\hline Graphic & Type & ID & Mass \\
\hline & &
\end{tabular}

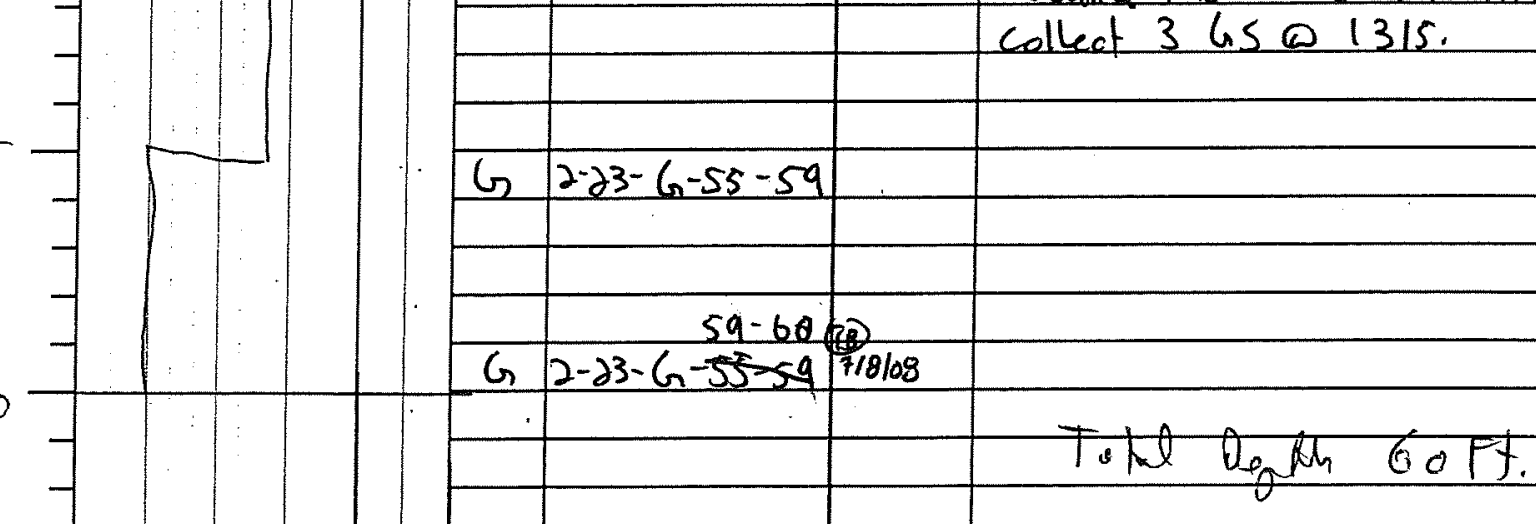

Advance $D B 50-60$, tecover $50-60$. collect 3 LSQ 1315 .

$G \quad 2-23-G-55-59$

Aduana $D B 45-55$, no recovery.

G $2-23-6-50-\$ 5$

$-$

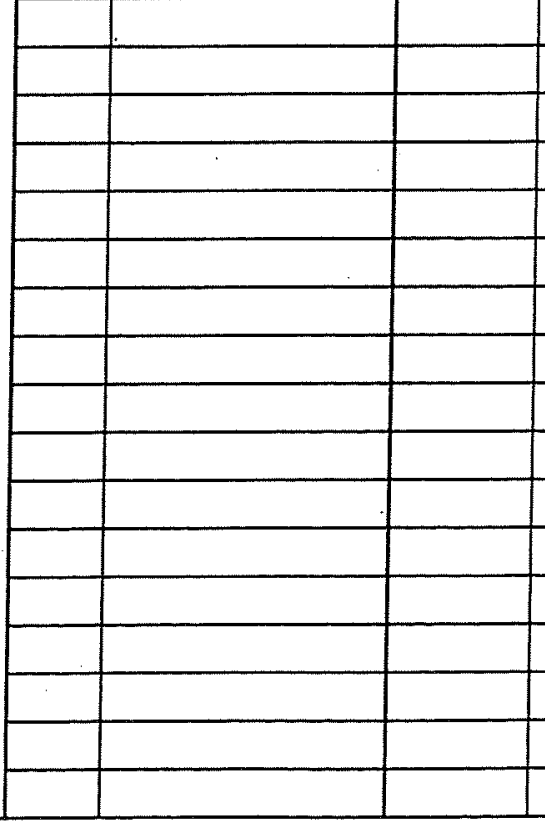




\section{Sample Summary}

Page $\frac{1}{\text { Date } 5 / 23 \text { of } \frac{2}{6 / 2 / 01}}$

Well ID: $C 6208$

Depth (ft) Lithologic Log

Well Name: 399-2-24 Geologist: Joc Frith

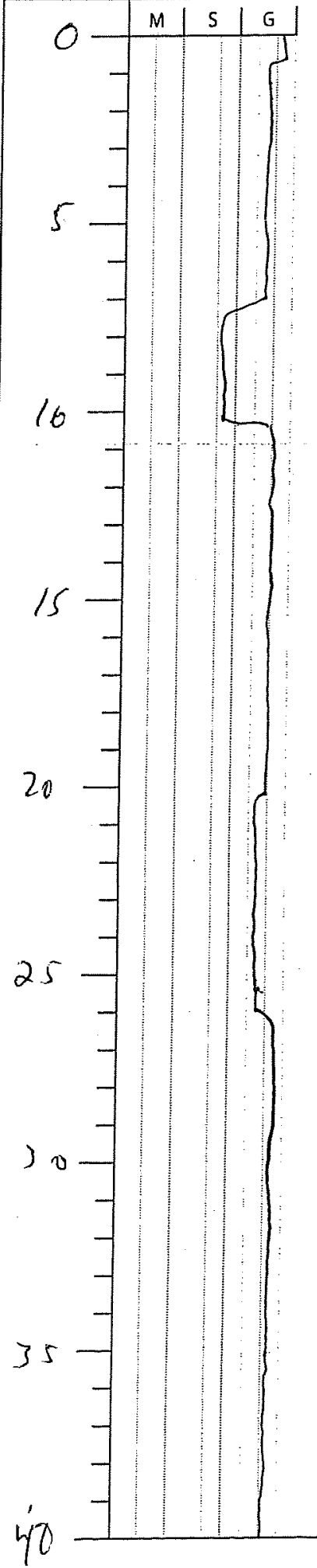

Sample

Comments

\begin{tabular}{c|c|c|c} 
Graphic Type & ID & Mass & $5 / 23 / 08$,
\end{tabular}

$\int 2-2 y-C \cdot 0-1$ Drive $550-S^{\prime}-$ recom

$<2-24-c-1-2 \quad 0-3^{\prime}-$ collect 3 ley

$c 2-2 y-2-3$ lines at 0710 .

$\begin{array}{ll}\text { C } 2-24-c-5,5-6 & \text { Drim } 555-10^{\prime}-\text { recover } \\ \text { C } 2-24<-6-7 & 5,5-10^{\prime} \text { collect } 5 \text { kxon }\end{array}$

c $2-24 x-7-7$ liners at 0725 .

C $2-2 y-c-8-9$

C $2-24-c-9-10 \quad 5 / 28 / 08$

C $2-24 \cdot c-10-11 \quad$ Drwess $10-15^{\prime}-$ recover

c $2-24-c-11-12 \quad 510^{\prime}$ collat 5 lexav lines

c $2-24 \cdot-12-13$ at 0830

c $2-2 y-c-13+14$

c $2-24-c-14-15 \quad$ T5ledor $D \beta \quad 5 / 29 / 08$

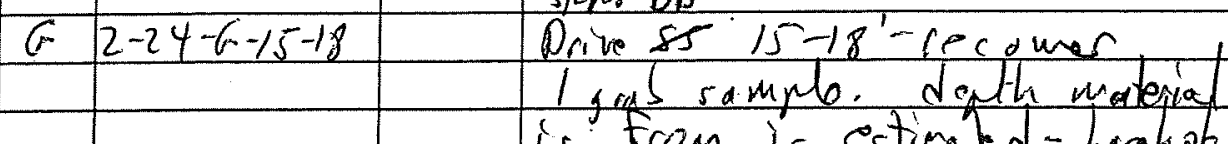

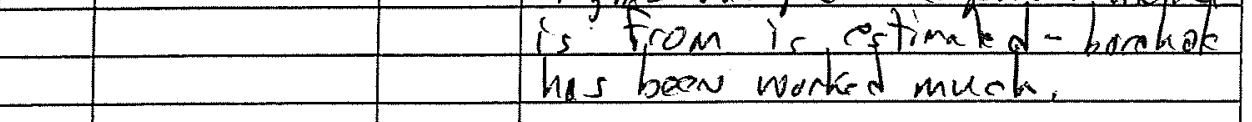

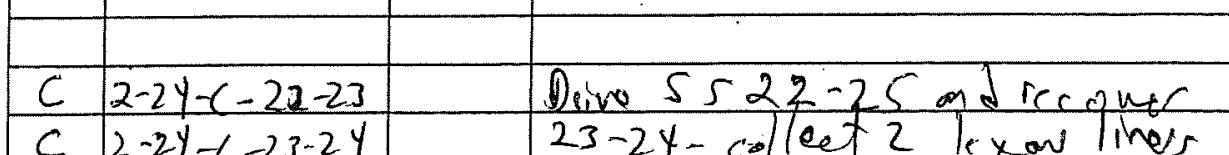

C $2-2 y-<-23-24$ 23-24-colect 2 lexous lines

$G 2-24-6-25-30 \quad$ Orive $5525-30-\phi$ recuvery

- $\quad$ gal gras at 1245

\begin{tabular}{|c|c|c|}
\hline & & $5 / 3408$ \\
\hline$G$ & $2-2+G-30-32$ & Drive drive batrel \\
\hline G & $2-24-6-32-33,5$ & grat samler \\
\hline G & $2-2 y-6-39.5-35$ & $(30-35)$ \\
\hline
\end{tabular}

\begin{tabular}{|c|c|c|}
\hline & & Puish 0dive barre $35-40$, \\
\hline & $2-2 y-6-35-36.5$ & celled 4 grab sambs \\
\hline$G$ & $2-2 y-6-36.5-3 y$ & Pusa o of shollos \\
\hline$a$ & $2-2 y-6-39-39$ & \\
\hline & $2-24-6-39-40$ & \\
\hline & & \\
\hline
\end{tabular}




\section{Sample Summary}

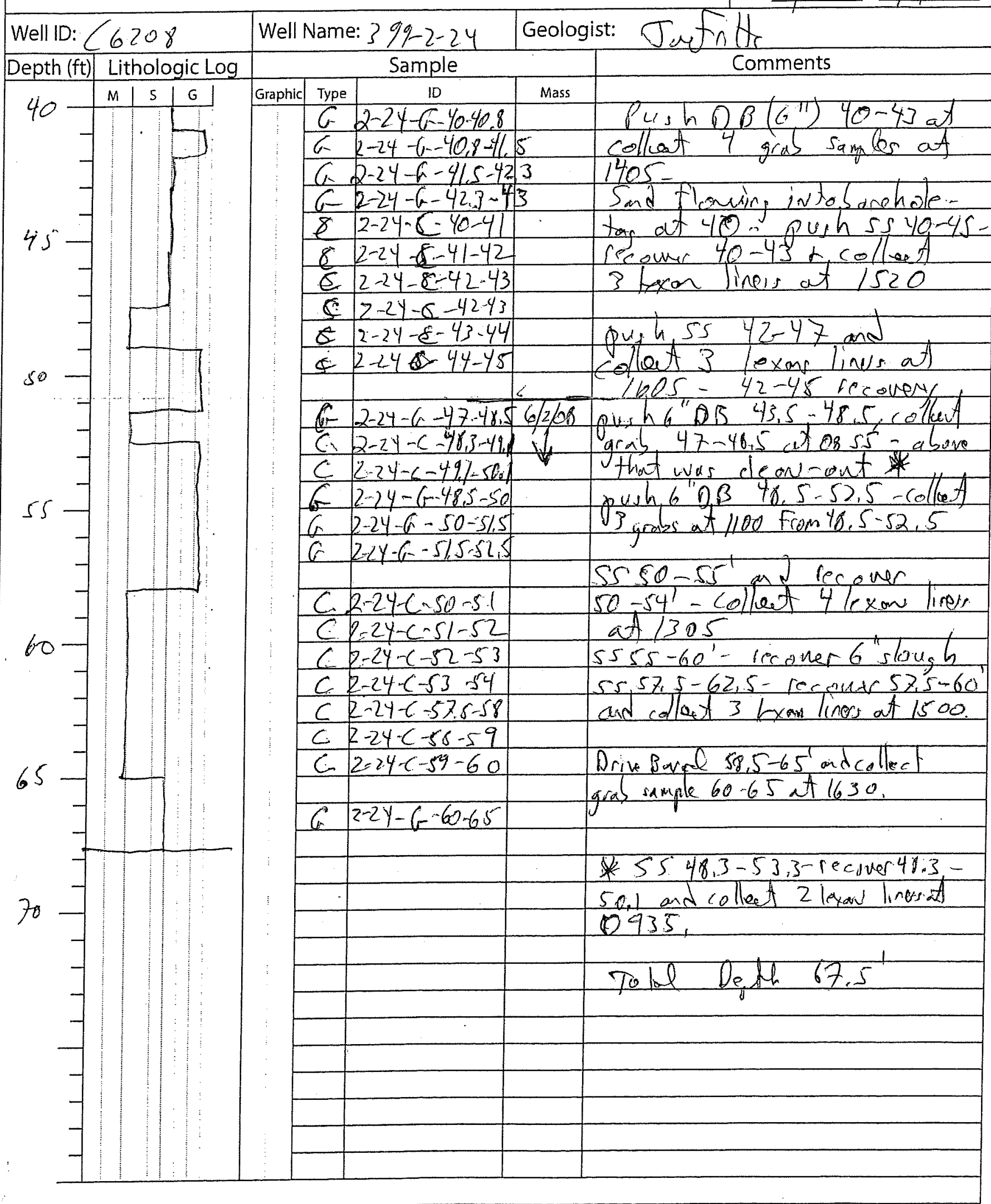




\section{Sample Summary}

Page $\frac{1 \text { of }{ }^{55} z-5}{\text { Date } 7 / 22 \text { to } 07 / 25}$

Well ID: C6209

Depth (ft) Lithologic Log

0

\begin{tabular}{|l|l|l|}
\hline & S & $G$ \\
\hline
\end{tabular}

Well Name: 3eq-2-25 Geologist: S. Sexton Sample Comments

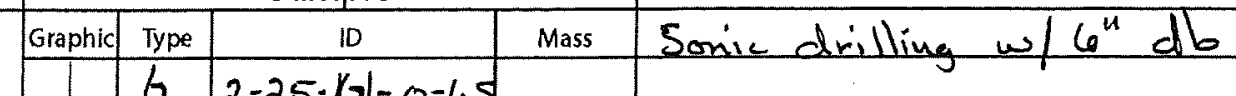

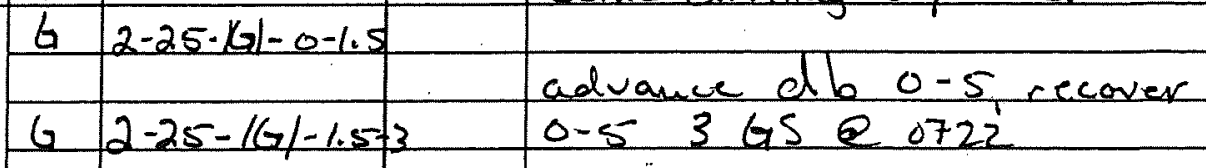

5

$G \quad 2-25-16-3-5$

$G \quad 2-25-|6|-5-6$

$G \quad 2-25-|G|-6-7$

$G \quad 2-25-16 \mid-7-8$

$G|2-25-| 6 \mid-8+9.5$

10

(1)

$G \quad 2 \cdot 25-16-10-12$

$G \quad 2 \cdot 25-16)-12 .-13.5$

$G \quad 2-25-1(5)-13,5-14,5$

15

.

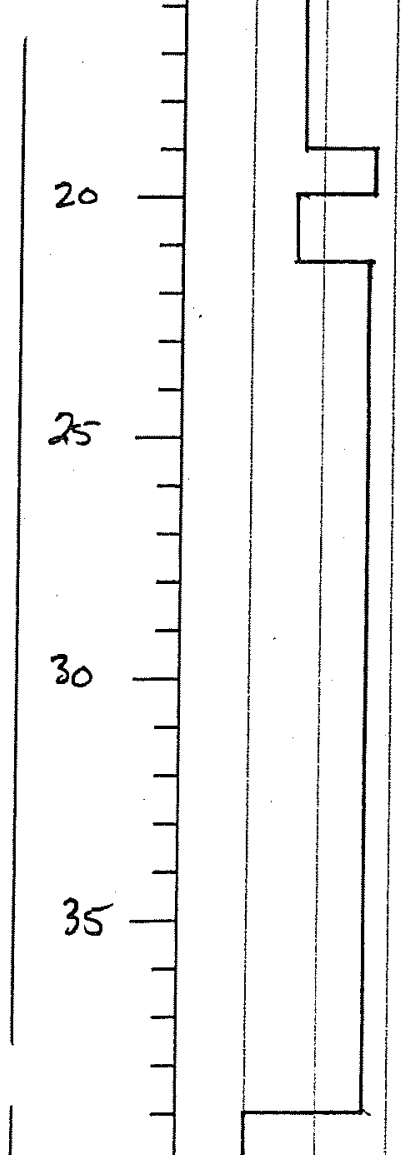

$G \quad 2 \cdot 25 \cdot 16-15-17.5$ advance db $15-20$, recover

$G \quad 2-25-|6|-17.5-69$

G $2-25-|G|-19-20$

$G \quad 2.25 \cdot|G|-20-21.5$ advance db $20-25$ recover

$G \quad 2-25 \cdot 1 G \mid-21.5-23 \quad 20-24.5,3 G 5$ e 0915

$G \quad 2-25-16-23-24,5$

$G 2 \cdot 25-16 \mid-25-26,5$ advance dlo $25-30$, recover

G $2-25-161-26.5-28$

$25-30,3125 @ 1032$

$G \quad 2-25-16-28-30 \quad$ advance $4^{\prime \prime}$ split-sposon $30-35$

ac| $2-25-|c|-30$

צc $2-25-|c|-3 \mid$

(x) $2-25-|c|-32$

\&C $2 \cdot 25-|c|-3 \mid$

$\operatorname{lsc} 2-25-1 d \cdot 31.5$

$G \quad 2-25-|6|-35-36,5$

$G \quad 2-25-16 \mid-36,5-38$

$G \quad 2-25-16 \mid-38-39,5$ 


\section{Sample Summary}

Page $\frac{2 \text { of } \frac{5}{07 / 22 / 08 \text { to } 07 / 25}}{\text { Date } 07}$

Well ID:C6209

\begin{tabular}{|l|l}
\hline Depth $(\mathrm{ft})$ & Lithologic Log \\
\hline
\end{tabular}

\begin{tabular}{|l|l|l|l|}
\hline 40 & $M$ & $S$ & $G$ \\
\hline
\end{tabular}

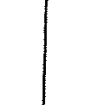

$$
4
$$$$
45
$$$$
4
$$

\begin{tabular}{l|ll} 
Well Name: $399-2-25$ & Geologist: S. Sexton
\end{tabular}

\begin{tabular}{|c|c|c|c|c|}
\hline Graphic & Type & 10 & Mass & Sonic drilling $w / 6^{\prime \prime} d b$ or $4^{\prime \prime} \mathrm{s}$ \\
\hline \multirow{4}{*}{} & $G$ & $2-25-16-39,5-4$ & 3,5 & \\
\cline { 2 - 4 }
\end{tabular}

ls $2-25 \cdot 16 \mid-43.5-45$

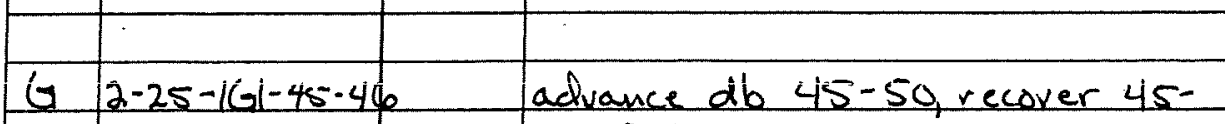

$62-25-19 \mid-46-47.5$

50,3 GS@1420

$6 \quad 2-25-16)-47,5-50$

C 2-25-14-50-50, 5 advance ss 50-55, recover 50

C $2-25-|C| \cdot 50,5-51.5$

C $2.25-|C|-51.5-52.5$

C $2-25-|c|-52.5-33.5$

C $2-25-k \mid-56-57 \quad$ advance ss $55-600$ sms $7-22-056$

C $2-25-|c|-57-58$

c $2-25-14-58-59$

c $2-25-k|-59-60|$

C $2-25-|c|-60-61$

c $|2-25-| c|-6|-62 \mid$

c $2-25-|c|-62-43$

C $2-25-|c|-63-64$

c $2 \cdot 25-|c|-64-65$

C $2-25-(4)-65-66$

c $2-25-|c|-66-67 t$

c $2-25-1 c 1-67-68$

c $2-25-19-68-69$

c $2-25-1 c 1-69-70$

$G|2-25-16-70-7|$

$G|2-25| 6|-7|-72,5$

$G \quad 2-251 G \mid-72.5+74$

$G \quad 3-25|G|-74-75$

$G \quad 2.2516-75-77 \quad$ advance db $75-85$, recover $75-$

$6 \quad 2-25-16]-77-80$
$-54,4$ LL\& 1439 advance is $65-70$, recover $65-$ 70,5 LL 2.1612

advance is $60-65$, recover $60-65,5$ LL Q 1559 $-75,4$ GS Q.1115 $85,4 \operatorname{ls} 21257$ 


\section{Sample Summary}

Page 453 of $\frac{5}{57 / 22 \text { to } 07 / 25}$

\begin{tabular}{|l|l|l|l}
\hline Well ID:C6209 & Well Name: 399-2-25 & Geologist: S. Sexton \\
\hline
\end{tabular}

\begin{tabular}{|l|l|}
\hline Depth (ft) Lithologic Log \\
\hline
\end{tabular} Sample Comments

80

\begin{tabular}{|c|c|c|c|c}
\multicolumn{3}{|c|}{ Gample } & \multicolumn{2}{c}{ Comments } \\
\hline & 10 & Mass & Sonic drilling $w / 6^{n} d$ or $4^{n}$ ss
\end{tabular}
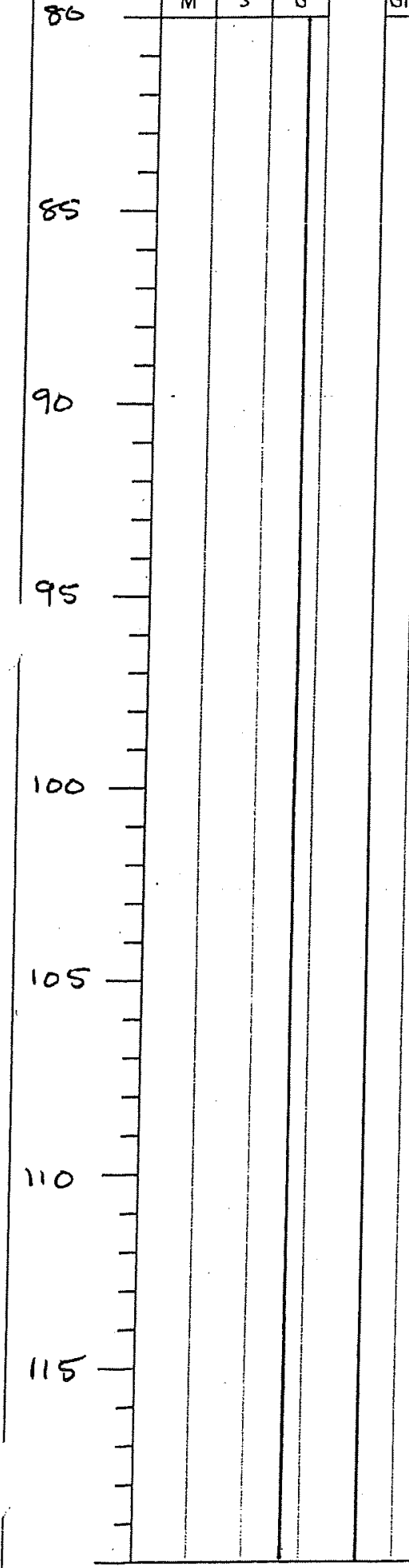

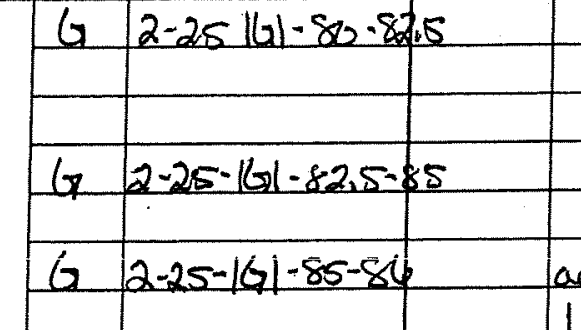

advance $d b$ 85-90, recover $85-86$

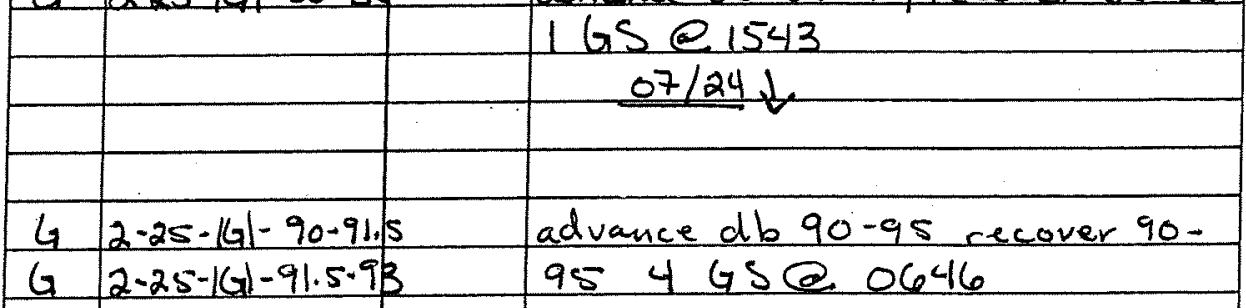

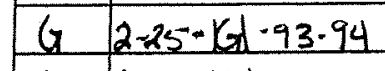

$G \quad 2-25-161-94-95$

$6 \quad 2-25-16 \mid-95-66,5$ advance $d b \quad 95 \cdot 100$, recover $95-$

$G \quad 2-25+16)-96.5-98 \quad 1003$ las 20724

G $2-25 \cdot 161-98-100$

C $2.25-1(2)-100 \cdot 10 \mid$

C $2.25+(-2)-101-102$

advance is $100-102$ recover $100-102$

\begin{tabular}{|l|l|}
\hline & \\
\hline
\end{tabular}

2 LL \& 0810

$G \quad 2-25-16 \mid-105 \cdot 106$ advauce db $105-110$, recover

$G \quad 2.25 \cdot 16) \cdot 106 \cdot 107.5 \quad 105 \cdot 1104 G 5 \propto 0904$

G $2 \cdot 25 \cdot(6) \cdot 107.5 \cdot-109$

$66225 \cdot 161-109-110$

$\operatorname{tac} 2.25 \cdot 16)-110 \cdot 111$

16c $2-25-162-111-112$.

KGe $2-25-161-112-113$

6rc $2-25-16)-113-114$

$6 c 2-25-16)-114-115$

$602.25+61-115-117$

advance ss $110-115$, recover $110-115$ S LL e 0925 


\section{Sample Summary}

\begin{tabular}{|l|} 
Page ${ }^{55} 3-4$ of 5 \\
\hline Date $07 / 22$ to $07 / 25$ \\
\hline
\end{tabular}

Well ID: C6209

Depth (ft) Lithologic Log 120

$$
\begin{aligned}
& 125 \\
& 130 \\
& 138
\end{aligned}
$$$$
\left[\begin{array}{ll}
135 & - \\
140 & - \\
14 & - \\
145 & - \\
150 & - \\
160 & -
\end{array}\right.
$$

\begin{tabular}{|l|l} 
Well Name: 399-2-25 & Geologist: S, Sexton
\end{tabular} Sample Comments

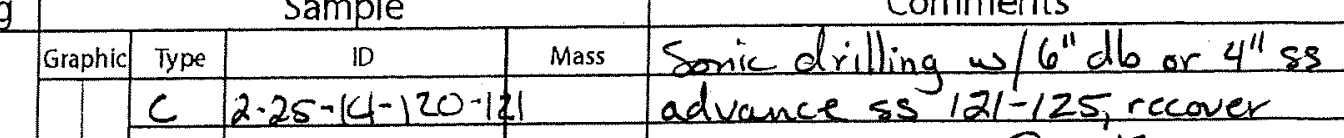

c. $2.25-14-121-12$

c $|2-25-k|-121-122$

c $2-25-141123-124$

c $2-251 c(-124-125$

C $2-25|4|-125-126$ advance ss $125-130$, recover

C $2-25|<|-126-127$

C $2-2514-127-128$

C $2-25(c)-128-129$

c 2 -25k-129-130 $07 / 25: 4^{\prime \prime} d b$ or $4^{\prime \prime}$ ss $\downarrow$

$G \quad 2-25-1(6)-130-13$ advance $d b$ 130-135, recover

$G \quad 2.25-|G|-13 \mid-1325 \quad 130-135,4 G 5$ e 1223

$G \quad 2-25-161-132,5-133.5$

$G \quad 2-25 \cdot 16-133,5-135$

$G \quad 2-25-16)-135-177$ advance db $135-145$, recover

\begin{tabular}{l|l|l|l|}
\hline & & $135-145,5$ GS C 1249
\end{tabular}

$G \quad 2-25-16|-159-14|$

G $2-25-16-141-143$

$G \quad 2-25-16)-143-145$

$G \quad 2-25 \cdot 16 \mid-145-147$ advance db 145-155, recover

\begin{tabular}{l|l|l|l}
\hline & & $145-1555$ GS \& 1315 \\
\hline 0 & $2-25-161-147-149$ &
\end{tabular}

$G \quad 2-25-16 .-149-(5)$

$G \quad 2-25-169-151-153$

G $2-25-1(5)-153-155$

$G \quad 2-25-164-155-156$ advance db $155-163$, recover

$\lg \mid 2-25-161-156-158 \quad 155-163,5$ GS @ 1337

6. $2-25-16) 158+160$ 


\section{Sample Summary}

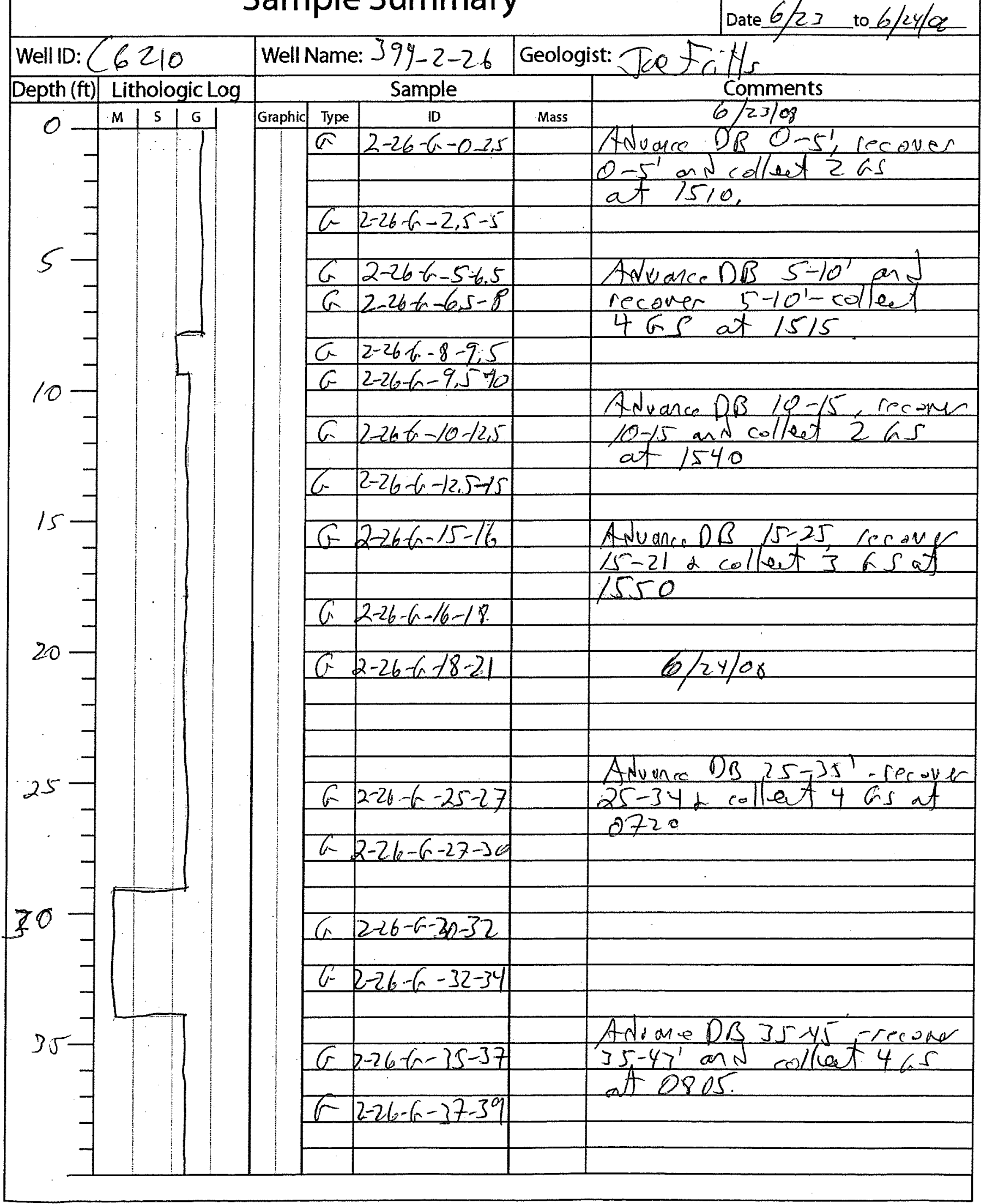




\section{Sample Summary}

Well ID: C6210 $\quad$ Well Name: 399-2-2b Geologist: Taffrid. Depth (ft) Lithologic Log

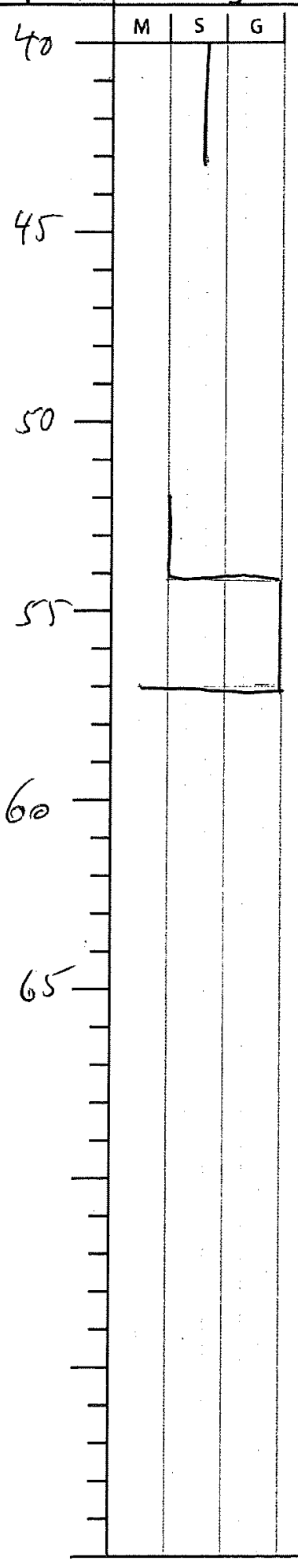

\begin{tabular}{|c|c|c|c|}
\hline \multicolumn{4}{|c|}{ Sample } \\
\hline Graphic & Type & ID & Mass \\
\hline & $C$ & $2-241.39-41$ & \\
\hline & & & \\
\hline & $a$ & $2-26-(r-4)-4]$ & \\
\hline
\end{tabular}

Anvence $O B 45-55-\not$

recover $y$

Aduman D B $52-60^{\prime}-110$ anes

$52-60+$ colleat 3 a 5 ator 30

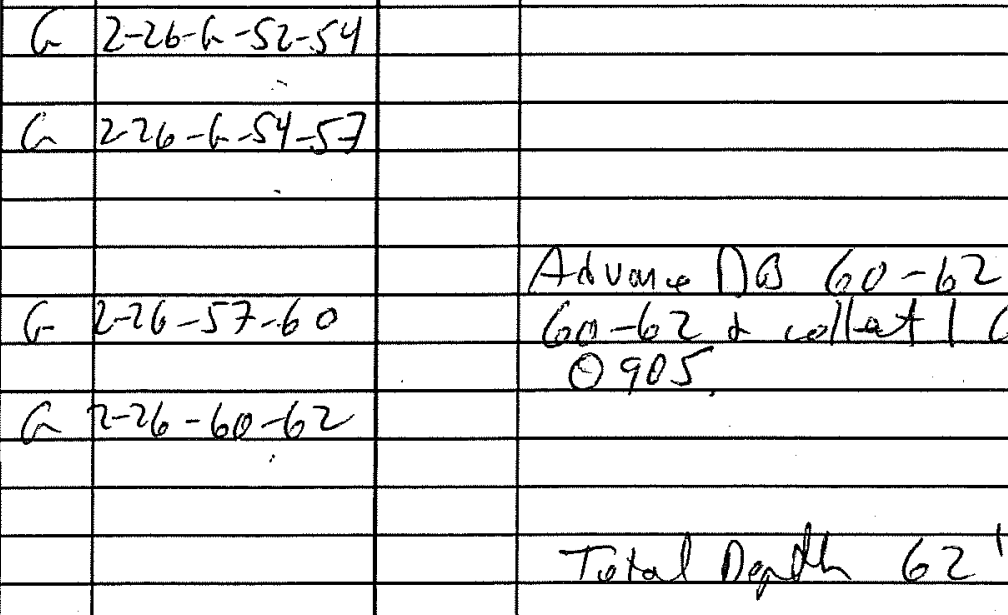




\section{Sample Summary}

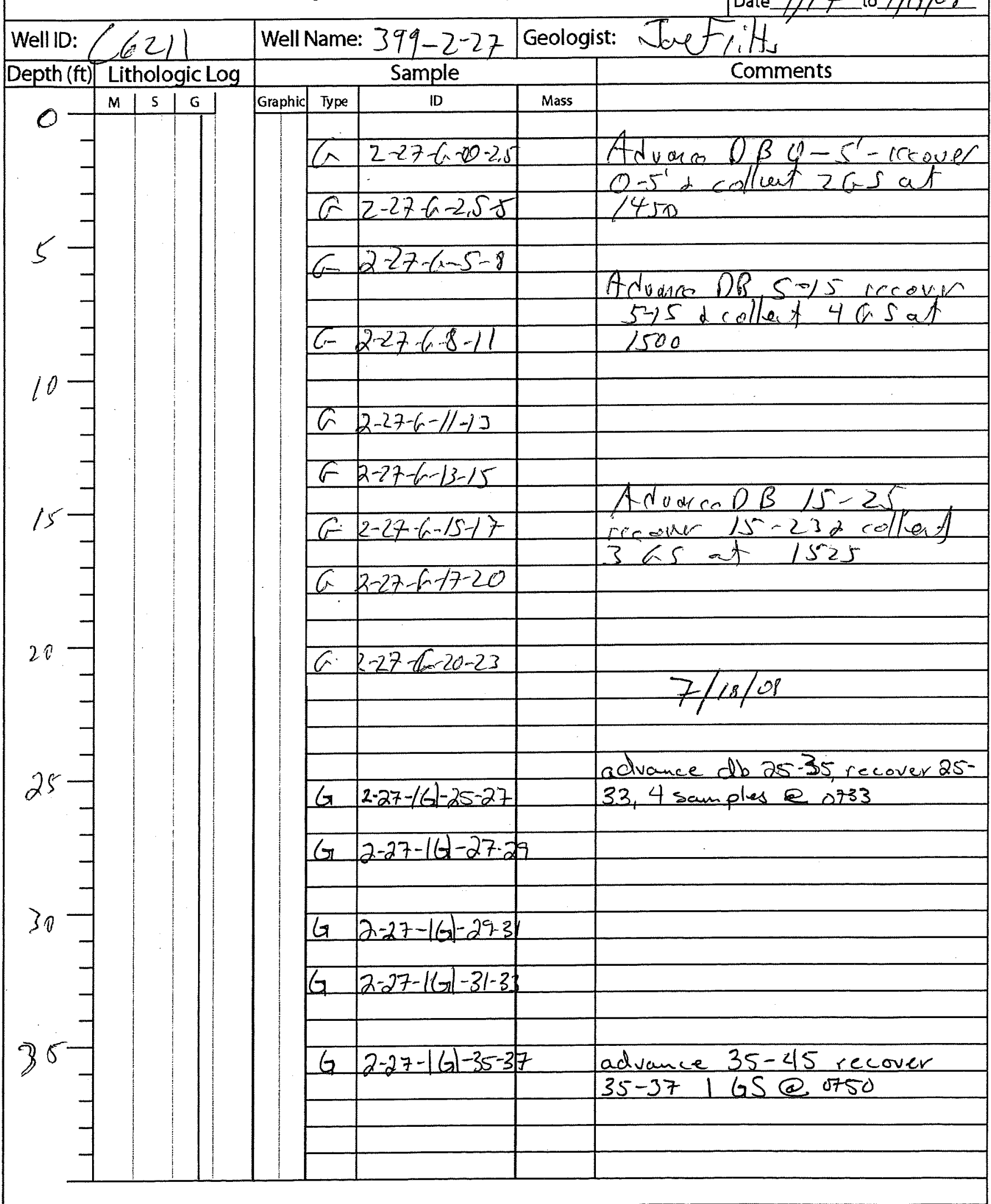




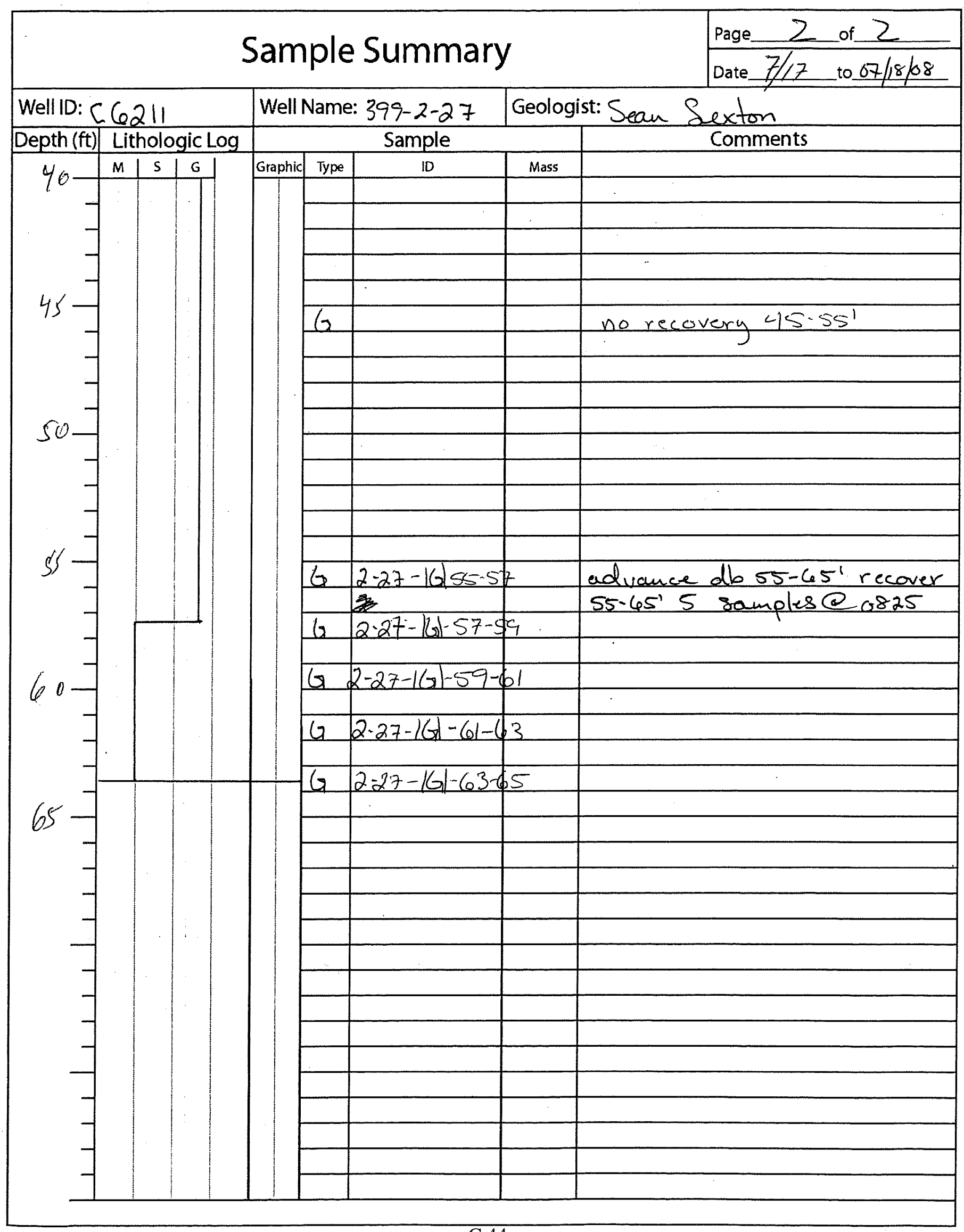




\section{Sample Summary}

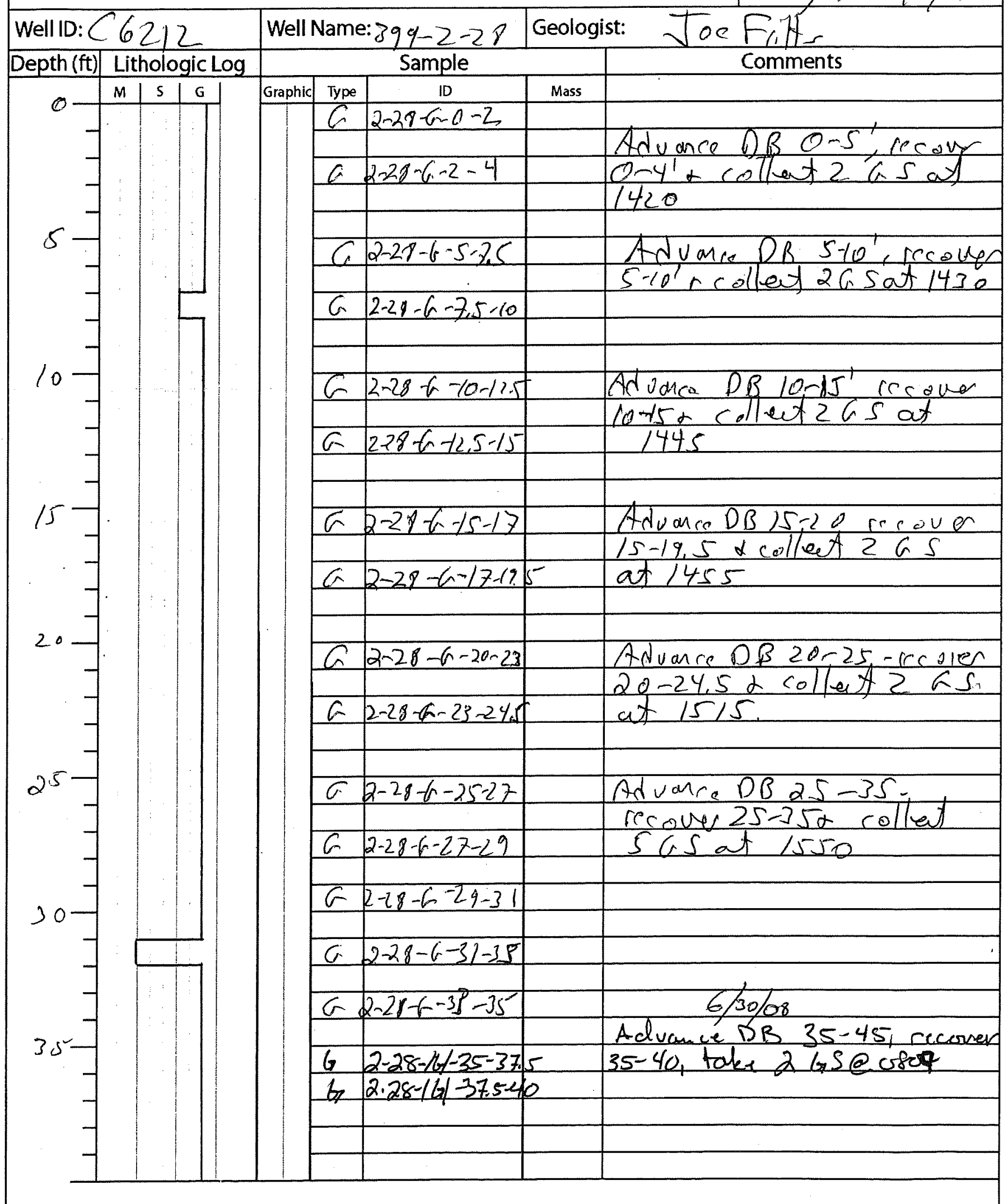




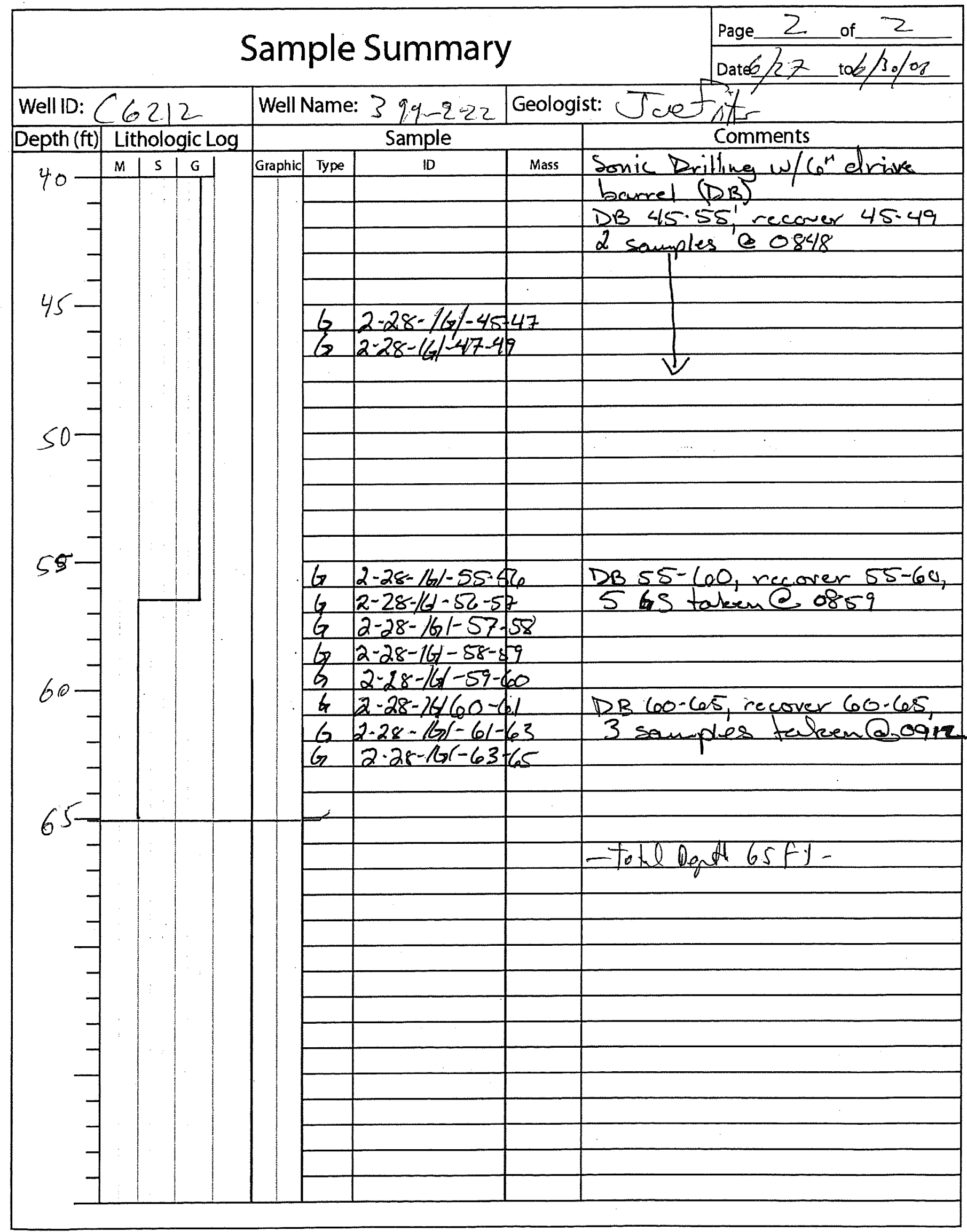




\section{Sample Summary}

\begin{tabular}{|c|c|c|c|c|c|c|c|}
\hline Well ID: & 662 & & Well & Vam & $e: 399-7-29$ & Geolo & ist: Jae Fritt \\
\hline Depth (ft) & Litholc & gic Log & & & Sample & & Comments \\
\hline & \begin{tabular}{l|l}
$M$ & $S$ \\
\end{tabular} & $\mathrm{G}$ & Graphic & Type & ID & Mass & \\
\hline 0 & & & & $C$ & $2-29-a-0-3$ & & Adunce $D B \mathrm{O}^{-3}$, te cover \\
\hline & & & & & & & $0-3^{\prime}+$ collent I' $\mathrm{CS}$ at oros \\
\hline & & & & G & $2-29-6-3-5$ & & Adomo $D B J-5$. recaner \\
\hline & & & & & & & $3-5+$ collat 105 at 0815 \\
\hline $5-$ & & & & C. & $2-29-6-5-7,5$ & & \\
\hline & & & & & & & Aivance $D B S-10^{\prime}$ - recower \\
\hline & & & & a & $2-29-6-75+c$ & & $5-100$ collat 265 al 0818 \\
\hline & & & & & & & \\
\hline & & & & $G$ & $2-29-a-10-12$ & & Advance $D B / 0-15$ recover \\
\hline & & & & & & & $10-14.5+$ colleat 2 as \\
\hline & & & & C. & $2-29-6-12-14.5$ & & at 0830 , \\
\hline & & & & & & & \\
\hline $15-7$ - 15 & & & & C & $2-29-6-15-17=$ & & Aduare DB $15-20$ recoves \\
\hline & & & & & & & $15-20 r$ collect 265 at \\
\hline & & & & G & $2-29-6-175 \cdot 2$ & & 0840 \\
\hline & & & & & & & \\
\hline - & & & & $C$ & $2-2+4-(a-20-1)=5$ & & $A \sqrt{1}$ lum $O B=20-25$ recouer \\
\hline & & & & & & & Anvme $20-25+$ collect 265 at \\
\hline & & & & C & $2-29-6-22,5-28$ & & 0905 \\
\hline & & & & & & & \\
\hline $25-1$ & & & & 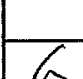 & $2-79-(r-25-2)+2$ & 75 & Aduance DR $25-35$. cecover \\
\hline & & & & $a$ & {$[-(7-2-2)-2$} & tas & $85-30 \mathrm{~d}$ collewt $2 \mathrm{GS}$ at \\
\hline & & & & 6 & $2-29-6-275-36$ & & 0940 \\
\hline & & & & & & & \\
\hline $30-$ & & & & & & & Advance $P B 30^{-1}-5$ (cleonow) \\
\hline & & & & C. & $2-29-6-30-33$ & & afecomer 38$)-33-$ collut \\
\hline & & & & & & & GSat 1015 \\
\hline & & & & & & & \\
\hline & & & & & & & Aduouce OB 35,45 , kcorer \\
\hline & & & & 6 & $2-29-6-35-37$ & & $75-41+$ collat 365 \\
\hline & & & & & & & at 1045 \\
\hline & & & & G & $2-29-6-37-39$ & & \\
\hline & & & & & & & \\
\hline
\end{tabular}




\section{Sample Summary}

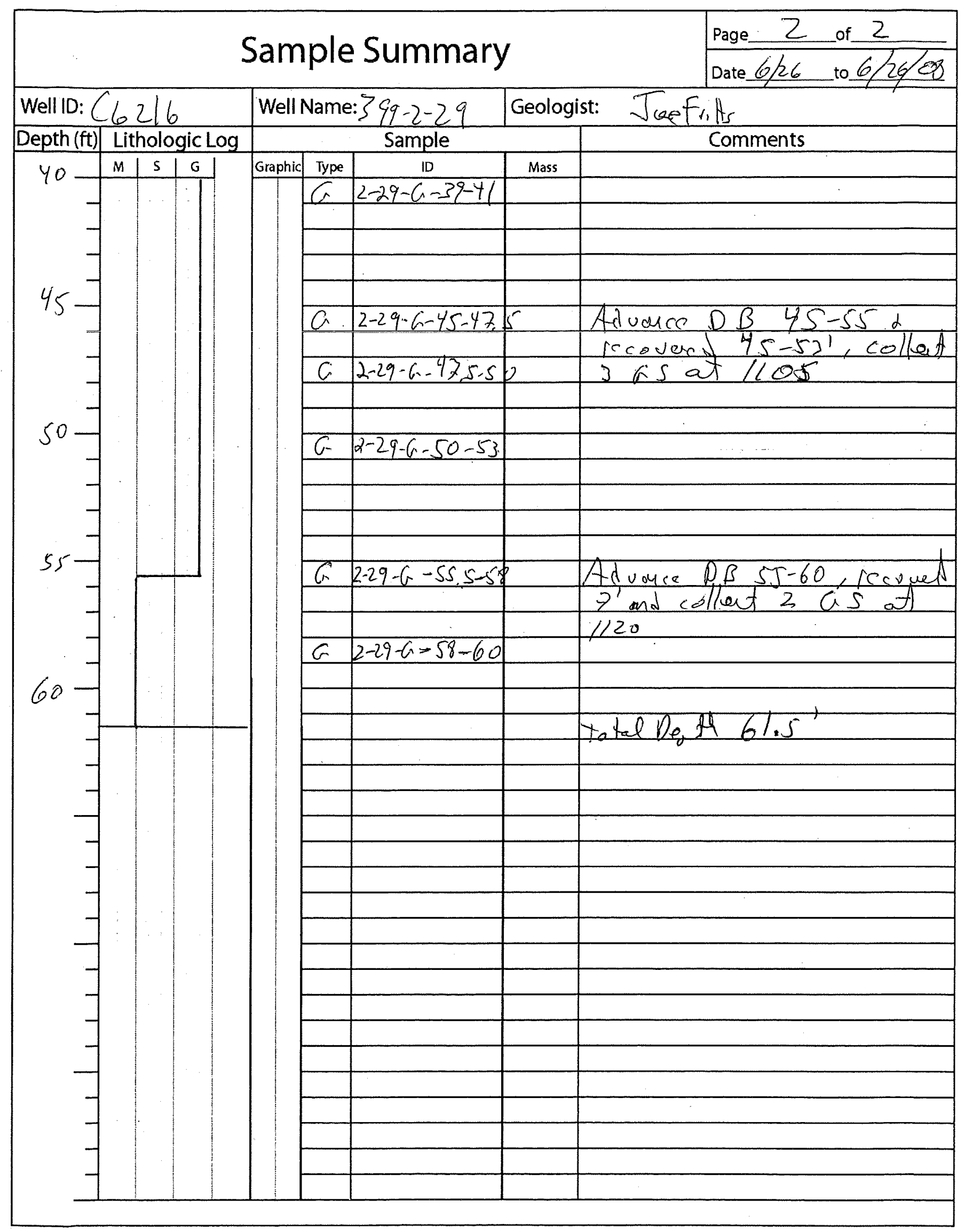

Well ID: $C_{6} 216$

Depth (ft) Lithologic Log

Well Name:399-2-29 Geologist: Juefrits

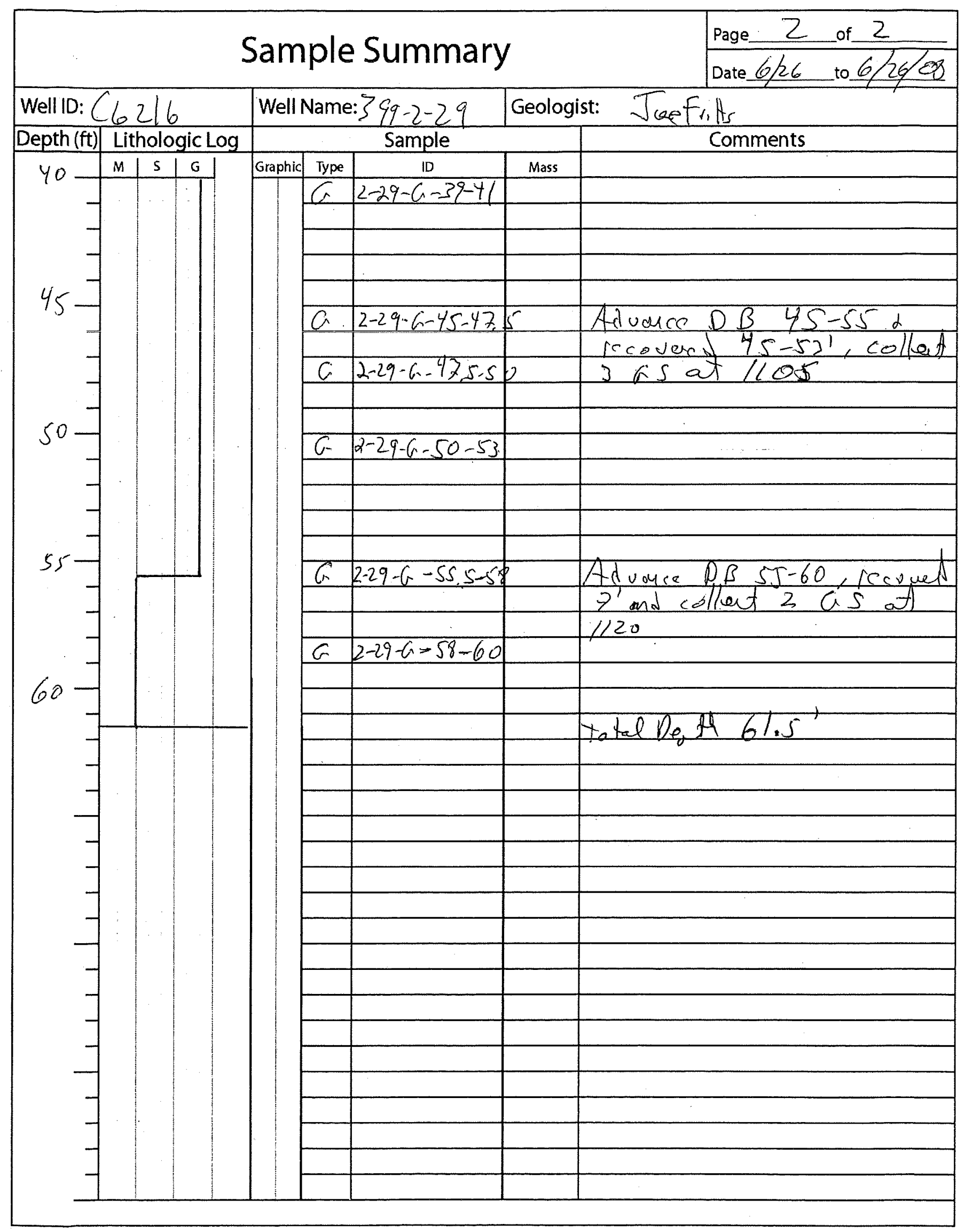




\section{Sample Summary}

Page 1 of $\frac{2}{\text { Date_07/24 to } 7 / 25 / 08}$

Well ID: C 6217

Depth (ft) Lithologic Log

o

0 \begin{tabular}{|l|l|l|l|}
\hline$M$ & $S$ & $G$ \\
\hline
\end{tabular}

5

15

20

25

30

30

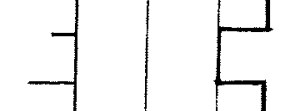

10

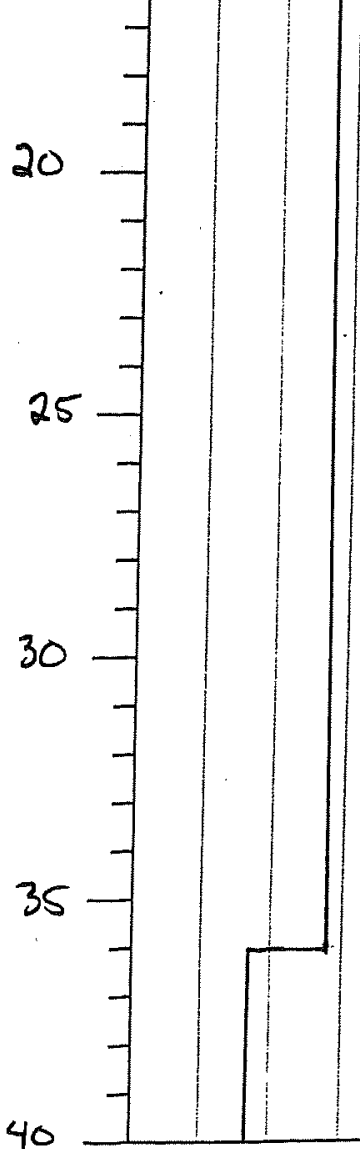

Well Name: 399-2-30 Geologist: S. Sexton Sample Comments

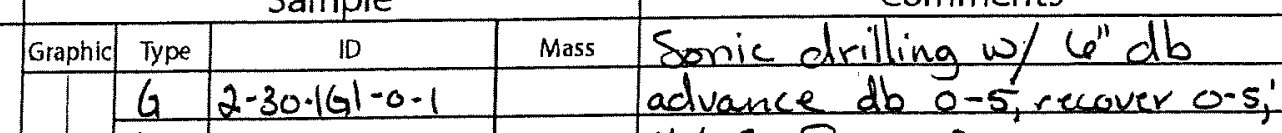

$G \quad 2-30-16 x-1-2$

G $2-30-|| a \mid-2-3$

G $2-30-|G|-3-5$

$G \quad 2-30-|G|-5-6$

G $2-30-|G|-6-7$

advance $5-10$, recover $5 \cdot 9.51$

$G \quad 2-30-|6|-7-8.5$ 4 GS Q 1509 4 GS \& 1503

$G \quad 2-30-|G|-8.5 \cdot 10$

$G \quad 2-30-|6|-10-11$

$G \quad 2-30-|G|-11-13,5$

advance 10-15, recorer 10-15,

3GS@1530

$62 \cdot 30 \cdot 161-13.5-15$

$6 \quad 2-30-15 \mid-15-16.5$

$G|2-30-16|-16.5-18$

$465 \bigcirc 1536$

$G \quad 2-30-19)-18-19$

$G \quad 2-30-1(G)-19-20$

la $2-30-|G|-20-2 \mid$

$G \quad 2-30-|G|-2 \mid-23$

advance 20-25, recover 20-25, 3 GS \& 1600

$G \quad 2-30-19)-23-25 \quad$ of/25 1 sms $7-25-08$

$G \quad 2-30-191-23-25 \quad$ oft25 1 sms $7-25-08$

$G \quad 2-30 \cdot 16 \cdot 25 \cdot 26.5$ advance db $25-35$, recover $25-30,3$ GS $e 0654$

$6 \quad 2-30191 \cdot 26.5 \cdot 28$

$G(2 \cdot 30-79)-28-30$

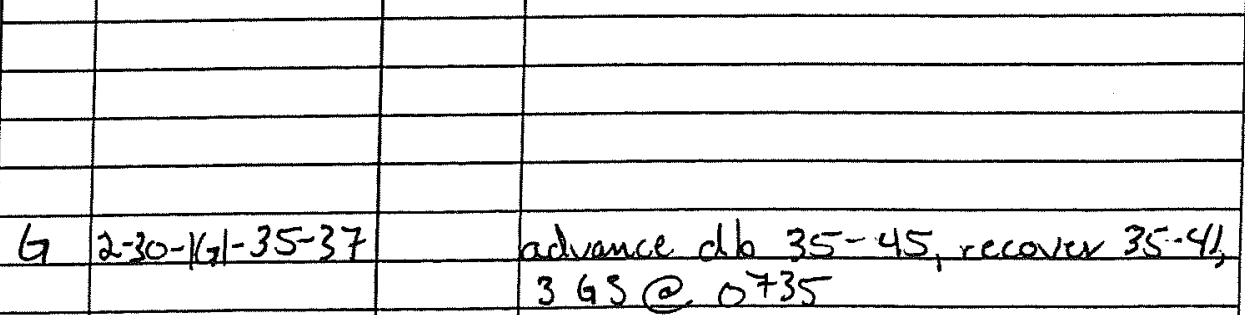

$G \quad 2-30-16)-37-39$ $365 @ 0735$

$G \quad 2.30-1(6)-39 \cdot 4)$ 


\section{Sample Summary}

\begin{tabular}{l} 
Page 2 of 2 \\
Date_07/24 to $07 / 25$ \\
\hline
\end{tabular}

Well ID: C6217

Depth (ft) Lithologic Log

40

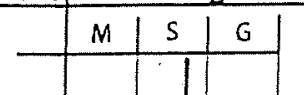

Well Name:399-2-30 Geologist: S. Sexton Sample Comments

\begin{tabular}{|c|c|c|c|c|}
\hline \multicolumn{4}{|c|}{ Damilis } & \multirow{2}{*}{ Somir drillingw/1"dlo or $4^{\prime \prime}$ ss } \\
\hline Graphic & Type & 10 & Mass & \\
\hline & & & & \\
\hline & & & & \\
\hline & & & & \\
\hline & & & & \\
\hline & $G$ & $2-30-16)-45 \cdot 47$ & & advance $45-55$ recover $45-55$ \\
\hline & $G$ & $2 \cdot 30-1(9)-47-48$ & & $6 G 5 Q 0754$ \\
\hline & $G$ & $2 \cdot 30-16)-48-49$ & & \\
\hline & & & & \\
\hline & G & $(2-30 \cdot 16)-49-50$ & & \\
\hline & 6 & $2-30 \cdot 169-50-52$ & & \\
\hline & $G$ & $22-30-10 t-52-55$ & & \\
\hline & & & & \\
\hline & $b c$ & $2-30-|c|-55.56$ & & advance s5 55-60,recover \\
\hline & bes & $2-36-14-56-57$ & & $55-60,54 L @ 0825$ \\
\hline & $6 \mathrm{GCE}$ & $2-30-|c|-57-58$ & & \\
\hline & Ku: & $2-30-144-58-54$ & & \\
\hline & Ge: & $2-30-k 1-59-60$ & & \\
\hline
\end{tabular}




\section{Sample Summary}

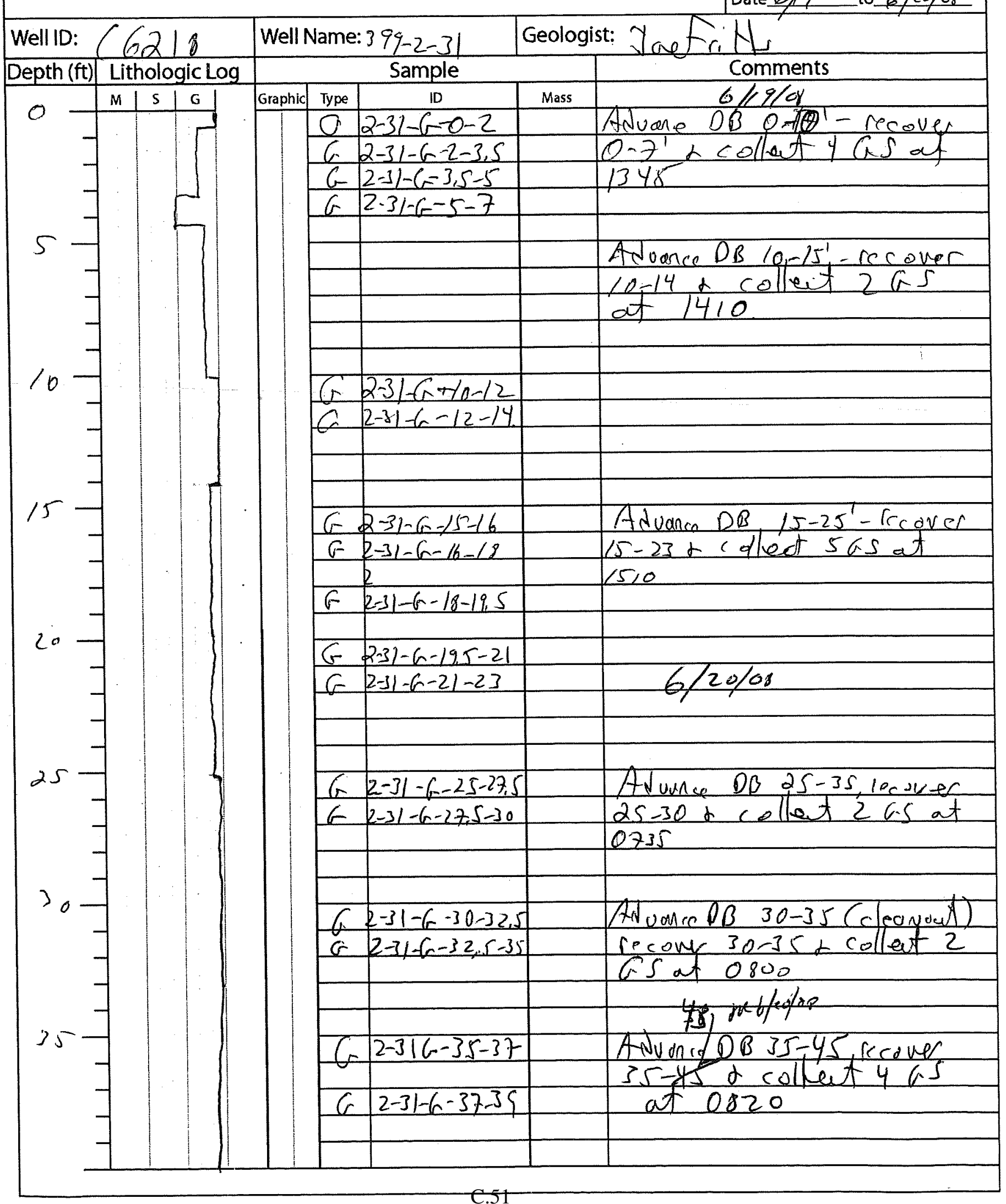




\section{Sample Summary}

Page 2 of 2
Date $6 / 19$ to $6 / 20 / 08$

\begin{tabular}{|l|l|}
\hline Well ID: $(6218$ \\
\hline Depth (ft) & Lithologic Log \\
\hline
\end{tabular}

Well Name:399-2-31 Geologist: Jae Frit)

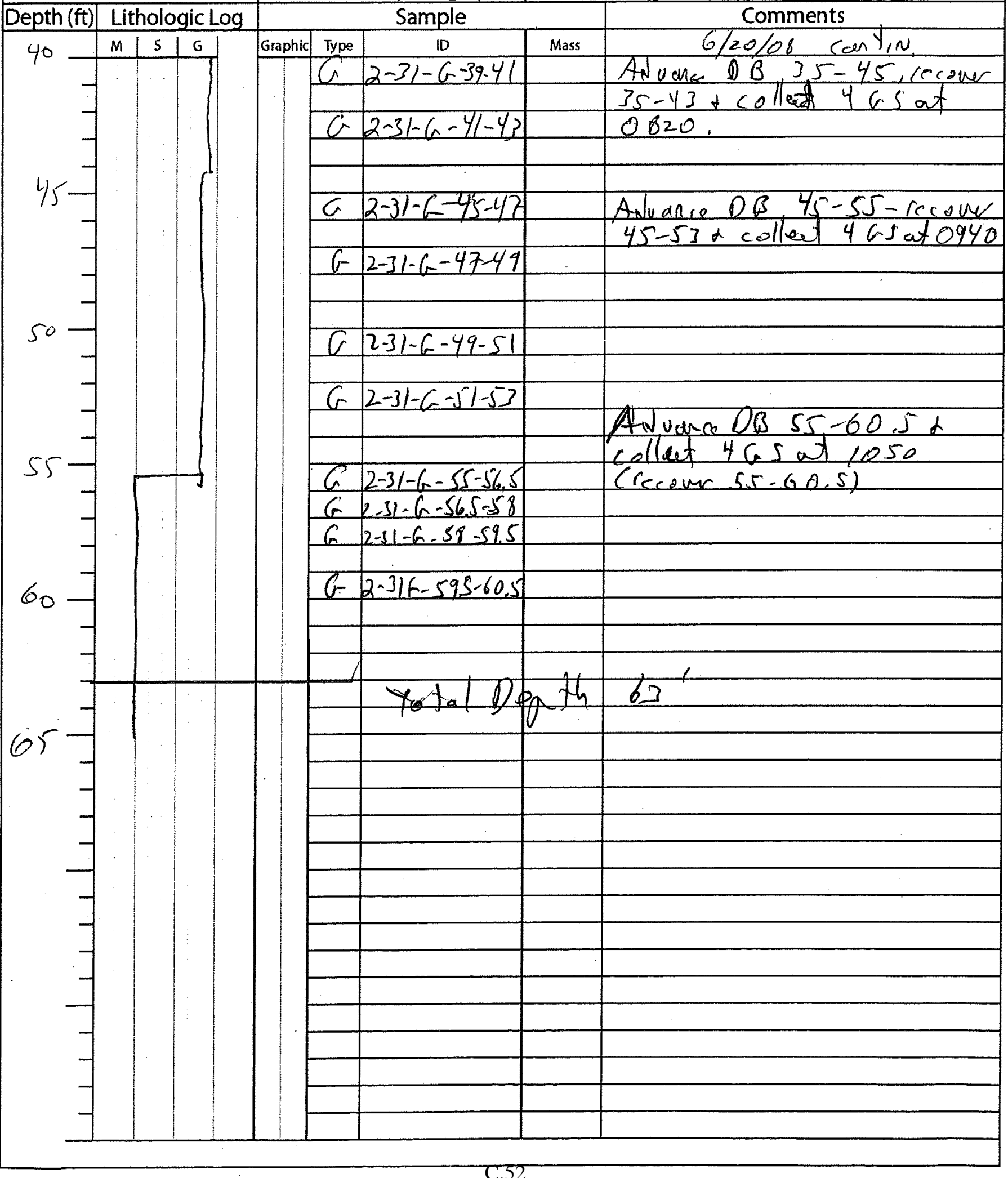




\section{Sample Summary}

Page $\frac{1 \text { of } \frac{2}{7 / 10 / 08 \text { to } 7 / 10 / 08}}{\text { Date }}$

Well ID: 6194

\begin{tabular}{|l|l|}
\hline Depth (ft) & Lithologic Log \\
\hline
\end{tabular}

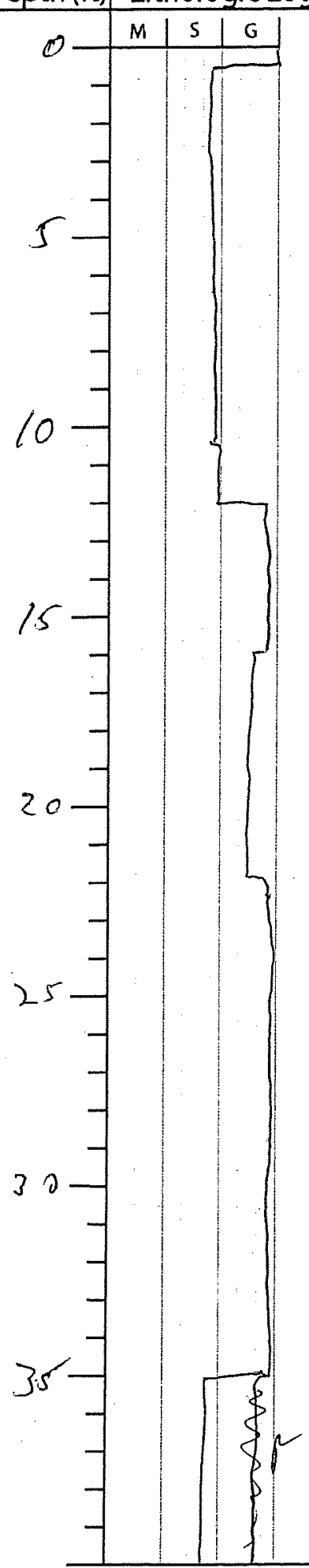

Well Name: 319-J-23 Geologist: Jce Fints Comments

\begin{tabular}{|c|c|c|c|}
\hline \multirow{2}{*}{\begin{tabular}{|l|l} 
Graphic & T \\
\end{tabular}} & & \\
\hline & 10 & Mass & \\
\hline$G$ & $3-23-6-0-8$ & 5 & $A d$ M $O B$ O J \\
\hline & & & $0-5$ a collent 265 al \\
\hline
\end{tabular}

$63.23-6-2.5-5 \quad 1200$

$a 3-23-6-5-7.5$ Adoanco DB $5-10$ recover

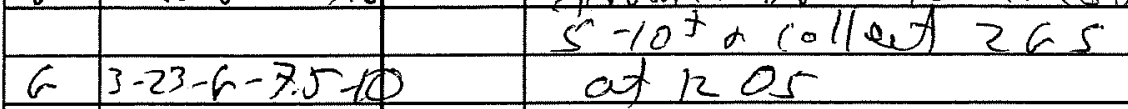

G $33-23-6-10-125^{5} \quad$ Aduence DB 10-15 recouger

$10-15+$ collent 2 as

$6 \mid 23-6-12515$ at 1220 .

$6 \quad 3-23-6-15-17.5 \quad A$ Anumen $O B$ B $15-20$, rec aver

$15-17.5 \alpha$ colbet I as at 1230

\begin{tabular}{l|l|l|l}
\hline$G$ & $3-23-6-20-22$ & Adumed Do 20-25, recone \\
\hline & & $20-24 \alpha$ collat 265 at 1300 \\
\hline
\end{tabular}

$6 \quad 3-23-6-2 L-24$

a $3-23-6-25-27$ Advarn $D \beta 25-35$ rerover

$63-23-6-27-29$ at 1310

Adumre DB $25-35$ Cctedo-

G $3-23-6-30-35$ ont) rrover 5 a collut

las at 1340

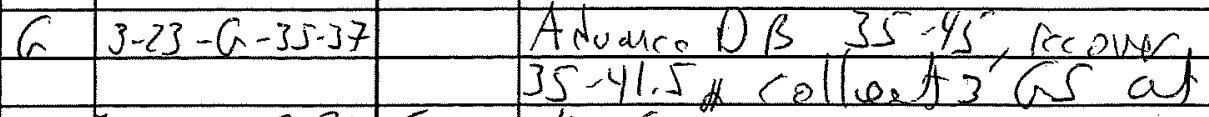

$6 \quad 3-23-6-37-39.5$ 1355 


\section{Sample Summary}

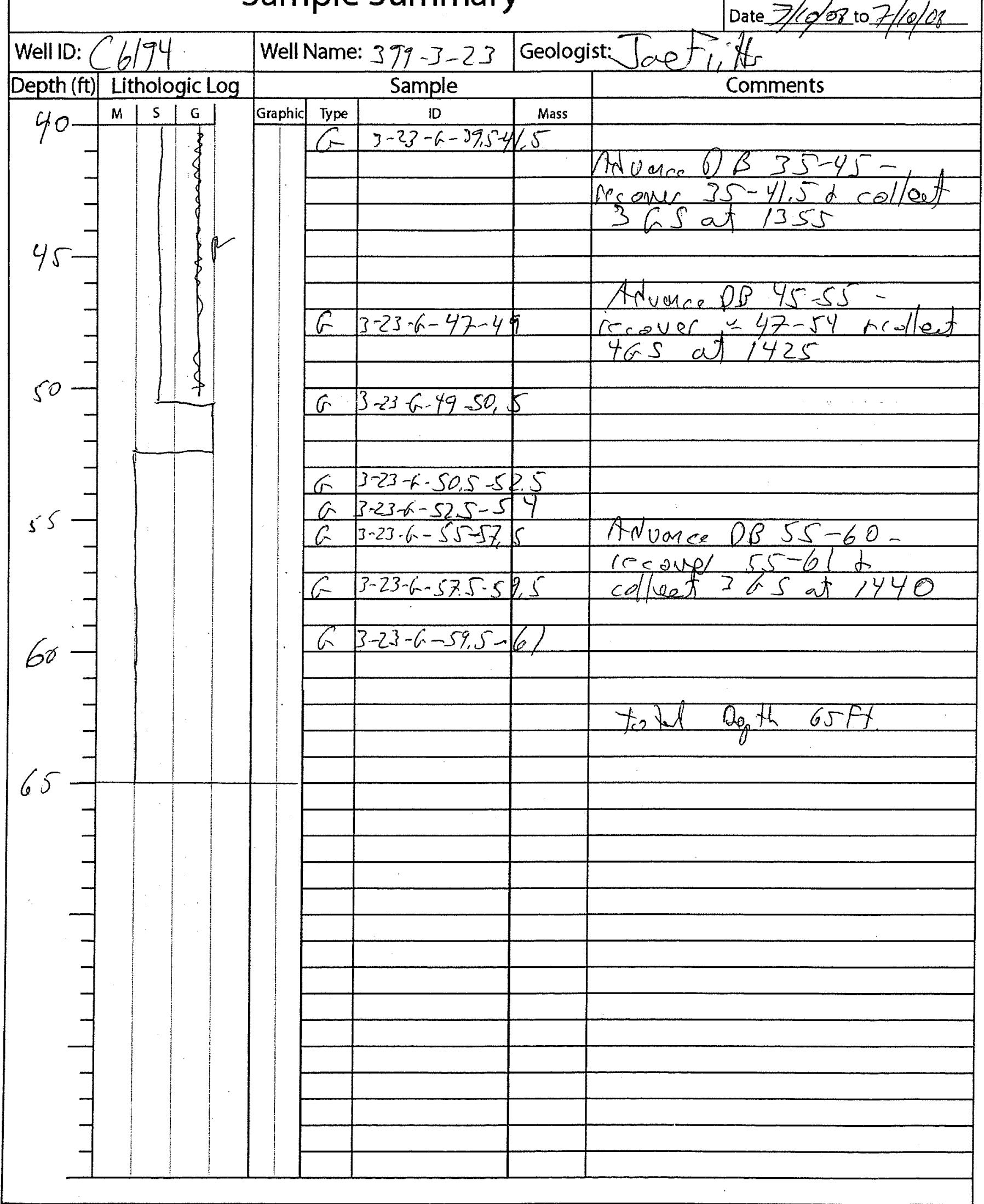




\section{Sample Summary}

Well ID: $C 6199$

\begin{tabular}{lll}
\hline Depth $(\mathrm{ft})$ & Lithologic Log \\
\hline
\end{tabular}

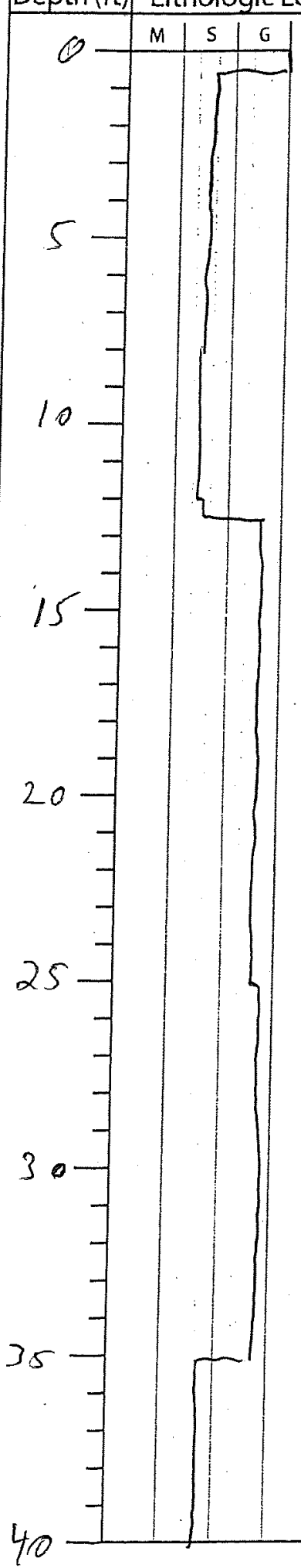

Well Name: 399-3-2.4 Geologist: Jaet ritts Comments

\begin{tabular}{|c|c|c|c|c|}
\hline \multicolumn{4}{|c|}{ Sample } & \multirow[t]{2}{*}{ Comments } \\
\hline Graphic & Type & ID & Mass & \\
\hline & 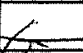 & $A-2+39100$ & & \\
\hline & & & & Aduaice $D B O-5$ \\
\hline & a & $3-2 y-6-0-2.5$ & & recover $0-s^{\prime}$ a rallat 2 \\
\hline & G & $3-24-6-2,5-5$ & & asat 1525 \\
\hline & & & & \\
\hline & a & $3-2^{4}-6-5+25$ & & Anvalce $D B$ S $-10^{\prime}$ recover \\
\hline & & & & $5+10^{\prime} \alpha$ collet 265 \\
\hline & a & $3.2 y-6-7.5-10$ & & ax 1535 \\
\hline
\end{tabular}

G 3-24-6-10.12:S ANuence OB 10-15, recaver

\begin{tabular}{|c|c|c|}
\hline & & $10-15+$ collat $26 \mathrm{~s}$ at $15 \mathrm{~s}$ \\
\hline G & $3+24-6-12.5 \cdot 15$ & \\
\hline & & $Z / 10 / 08$ fitroda \\
\hline & & AN vmce $D B / 50 / 5-201$ \\
\hline G & $3-24-6-15-17$ & Cecouse $15-19.5 \alpha$ colloct 2 \\
\hline & & Es at oros \\
\hline
\end{tabular}

G $3-2 y-6-17-195$

G.3-24-n-20-22 Advone $D B, 20-25$, reover

$20-24+$ rolhat 2 as at 0825

Q $3-2 y-6-22-24$

Advarce $0 B, 25-35$ lecave \begin{tabular}{l|l|l|}
\hline$a 3-2 y-r-25-27$ & $25-29 \alpha$ colleat $2 a 5 a t$ \\
\hline & 0840
\end{tabular} \begin{tabular}{l|l}
\hline & $3-24-6-27-29$ \\
\hline
\end{tabular}

Advare $P B 2.5-35$ (clean-oun) c $3-24-6-30-35$

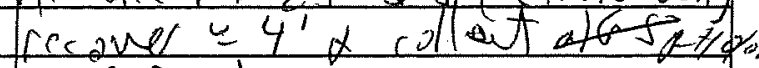
a as at 0900

ANuare DB 35-45-

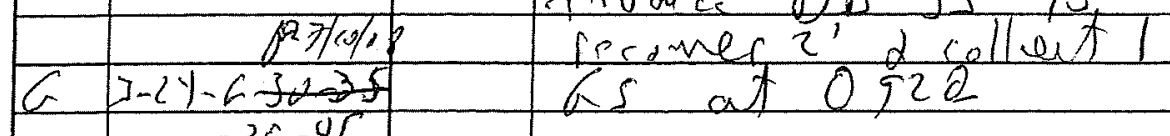
$\frac{-35-45}{5}$ 


\section{Sample Summary}

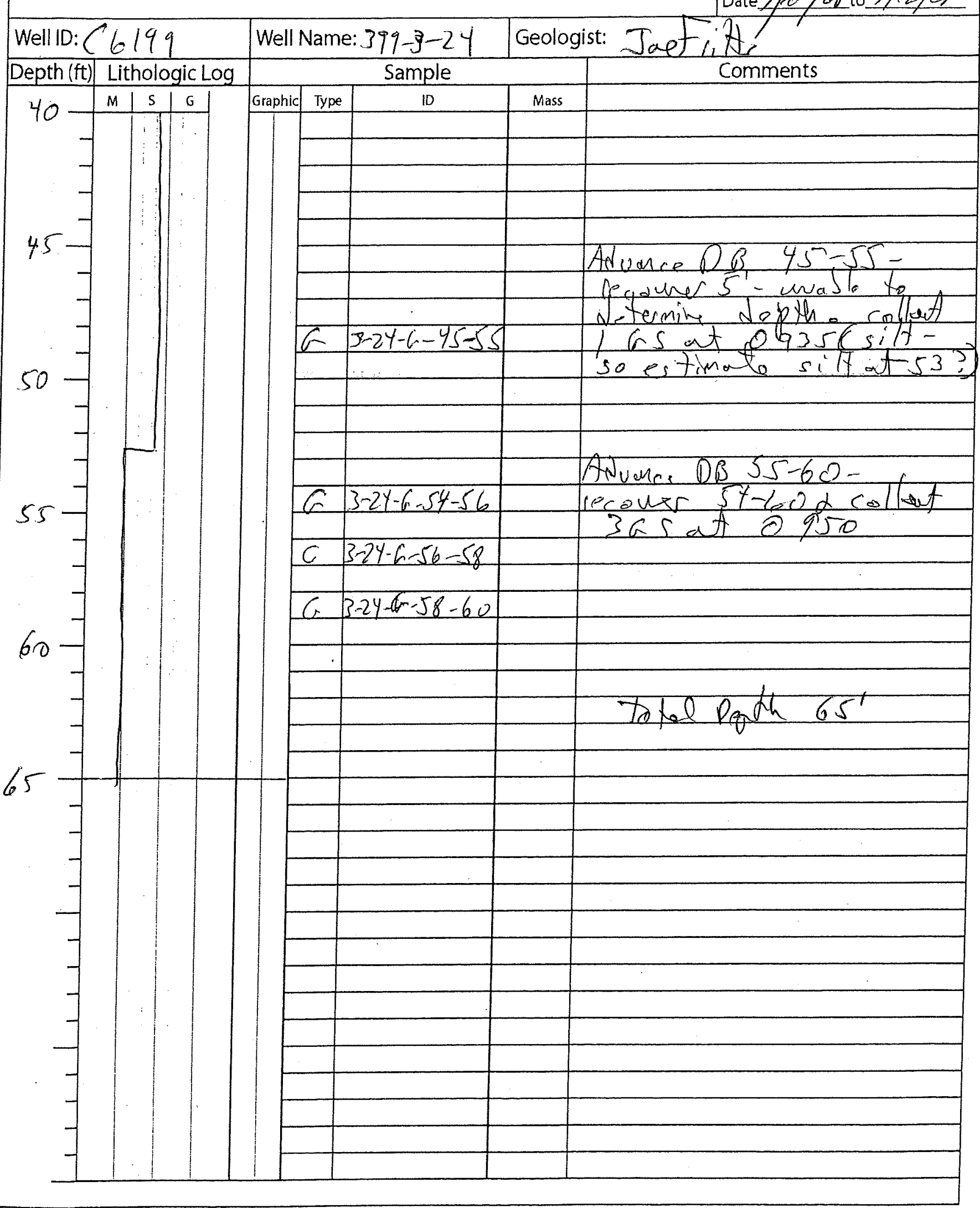




\section{Sample Summary}

Page 1 of 2

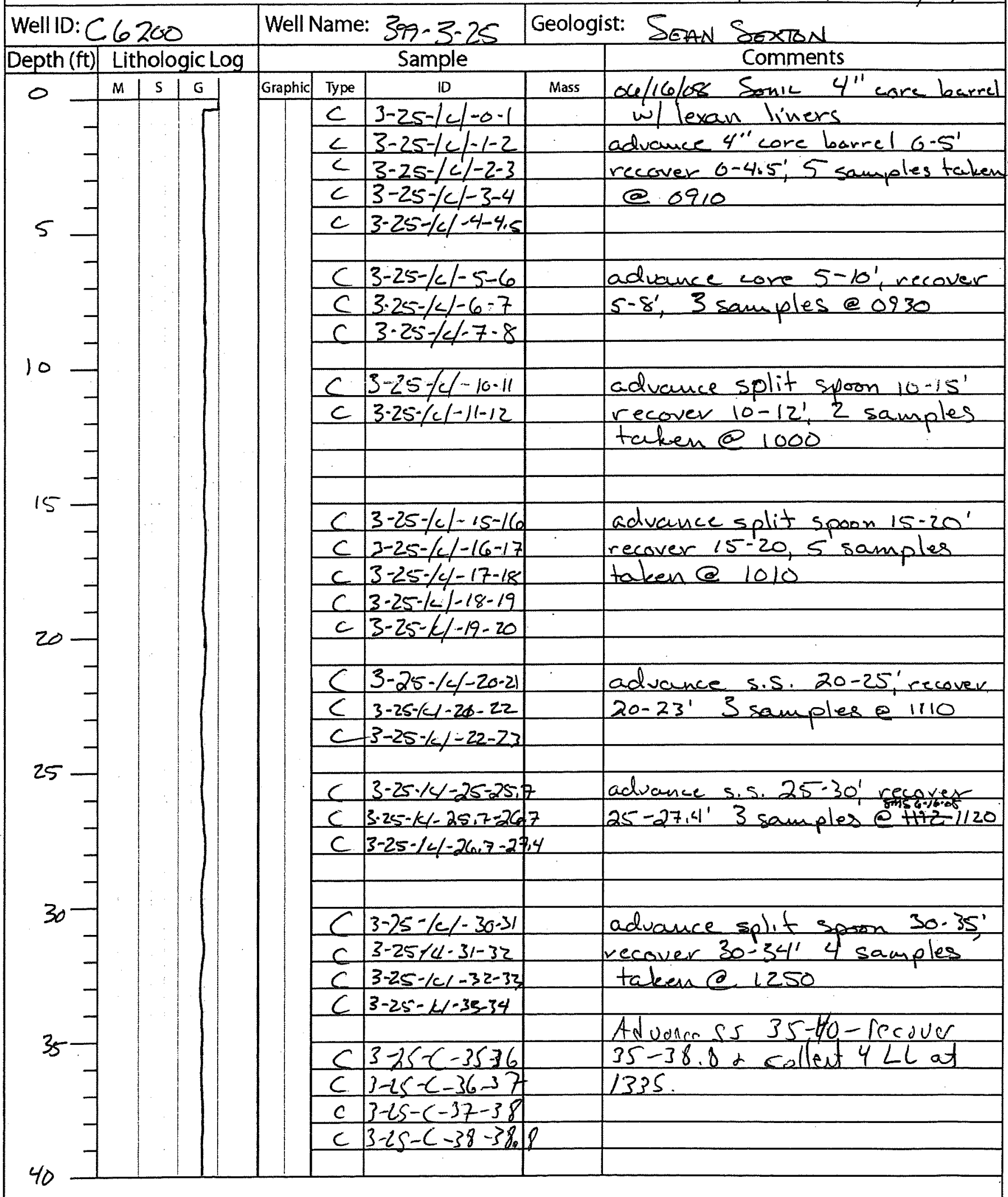




\section{Sample Summary}

\begin{tabular}{|l|l|} 
Page 2 of 2 \\
Date $6 / 46$ to $6 / 17 / 08$ \\
\hline
\end{tabular}

Well ID: $C 6200$

Depth (ft) Lithologic Log

Well Name: 399-3.25 Geologist: S Sexton, J fritt

\begin{tabular}{|c|c|c|c|c|c|c|}
\hline 40 & M & S & G \\
\hline
\end{tabular}

45

$G|3-25-16 ;|-40-42.5$

$6 \quad 3-25-160 \mid-42.5-45$

advance db 40-45; recover 40$45^{\prime}, 2$ grab samples Q 1455

45

$+$

c $3-25-/ c /-45-45,3$ advance ss 45-50, recover

c $3-25-|c|-45,8-46,8 \quad 45-46,8,2$ core samples@ 1505

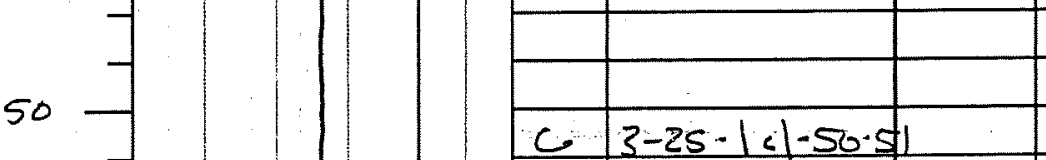

c. 3-25-|c|-51-52 recover 50-52', 2 samples

$55-1$

$+2$ taken \&1530

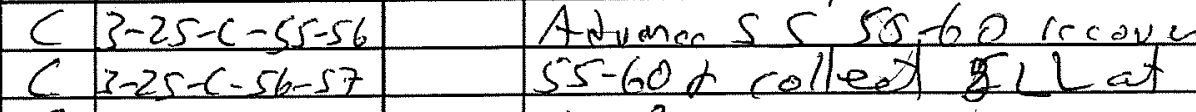

C $3-25-c-57-58 \quad 1010$.

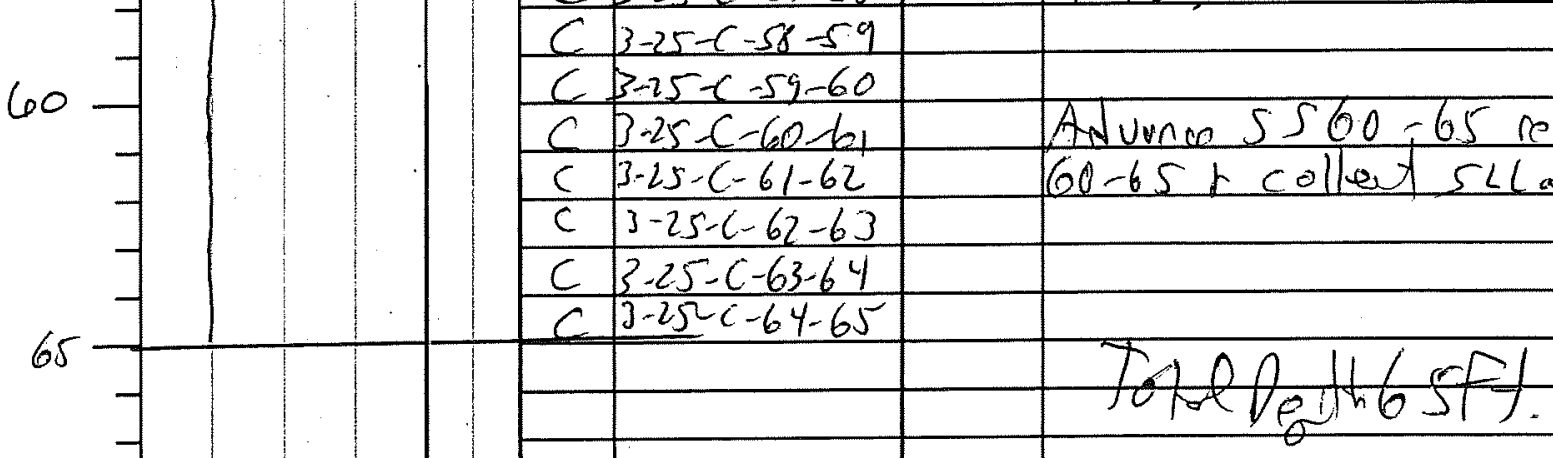




\section{Sample Summary}

Well ID:C6203

Depth (ft) Lithologic Log

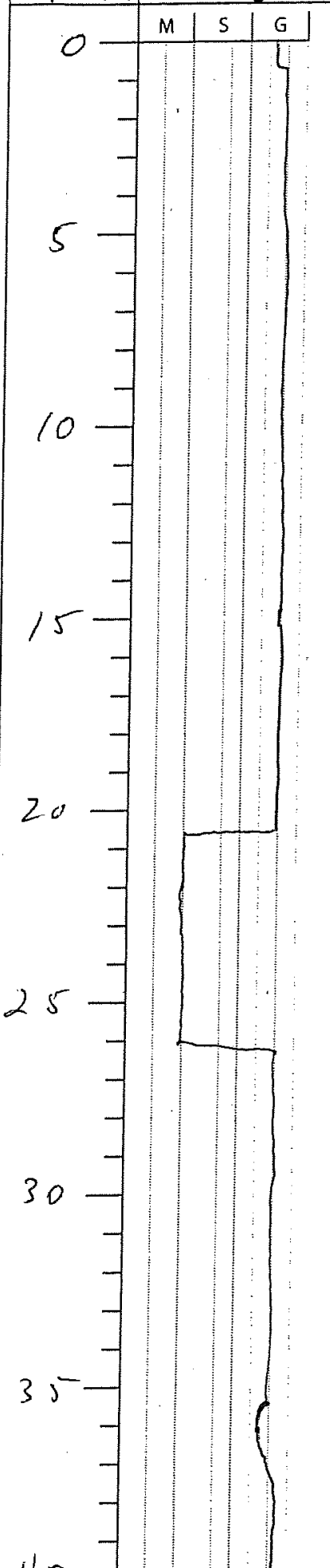

Well Name: 399-3-26 Geologist: Tae frittr Sample Comments

\begin{tabular}{|c|c|c|c|c|}
\hline \multirow{2}{*}{\multicolumn{2}{|c|}{ Graphic Type }} & ID & Mass & $5 / 14 / 08$ \\
\hline & & & & Savic dallin -4 " 55 and 6 " \\
\hline & C & $3-26-C-1,5-2$ & & Nive borrel? \\
\hline & c & $3-26-c-2-3$ & & $\operatorname{ss} 0-5^{\prime}-\operatorname{reconery} / 15-5$ \\
\hline & $C$ & $3-26-c-3-4$ & & 4 sangle lexon lings $(0745)$ \\
\hline & $C$ & $3-26-c-4-5$ & & \\
\hline & $G$ & $3-26-6 \cdot-5-10$ & & 6 hared $5-10$ cafluat 2 galsund \\
\hline & & & & C" Larel $10-15^{\prime}$ collat (2) 2 gall \\
\hline & & & & $.0820)$ \\
\hline
\end{tabular}

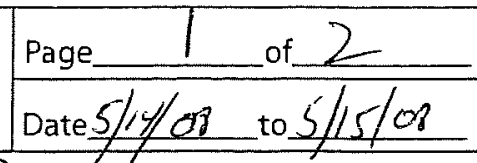

$$
\begin{array}{l|l}
G & 3-26+(r-10-125 \\
G & 3-26-6-12.5-15 \\
\hline
\end{array}
$$

$4^{\prime \prime} 55 / 5-20^{\prime}$ - recound $15.2-18 \mathrm{ft} .(0905)$

$C \mid 3-26-C-15.2-16$

c. $3-26-r-16-17$

c $3-26+-17-18$

C $3-26-c-17+9$

C $3-26 c-19-20$

C $3-26<-20-21$

C $3-26-c-25-2.58$

c $3-26-c-25.8-26.8$ $y^{\prime \prime} 55 / 7-22$ rcound

$17-22-\log t 21-22($ sitt-live, $9000)(0915)$ $17-19$ likely some slaugh.

$4 "$ ss $25-30 \mathrm{Ft}$ - reconend $25-26.8(1055)$ collat 2 lexon lines samper

4 "ss $30-35$ recovered $30-32(1110)$ $c \cdot 3-26-c-30-31$ calciat 2 lexon linersangtes c $3-26(-3)-32$ $5 / 15 / 28$ 4 "Ss $35-40-10$ overe C $3-26-c-35.35 .8 \quad 35-36.8^{\prime}$. ollot 2 $c 3-26-(-35.8-7.8$ lexan lines at $0 \times 10$

40

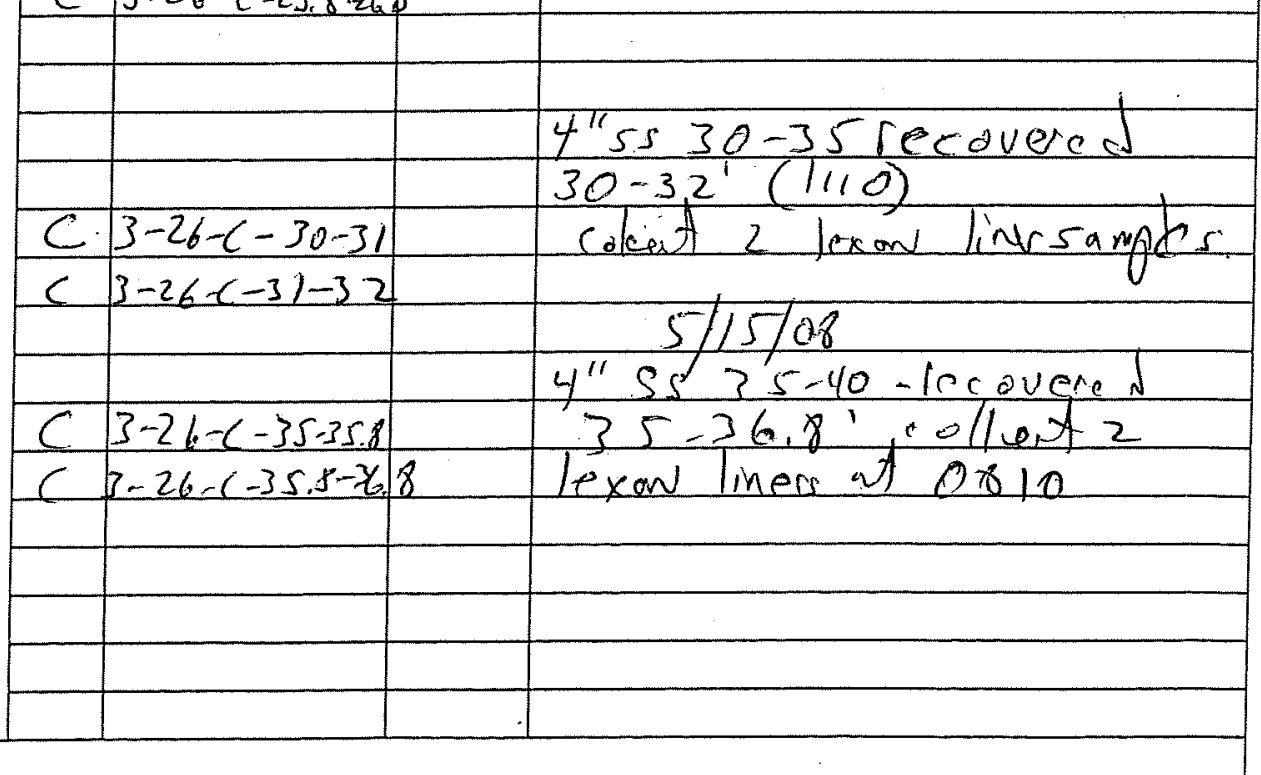




\section{Sample Summary}

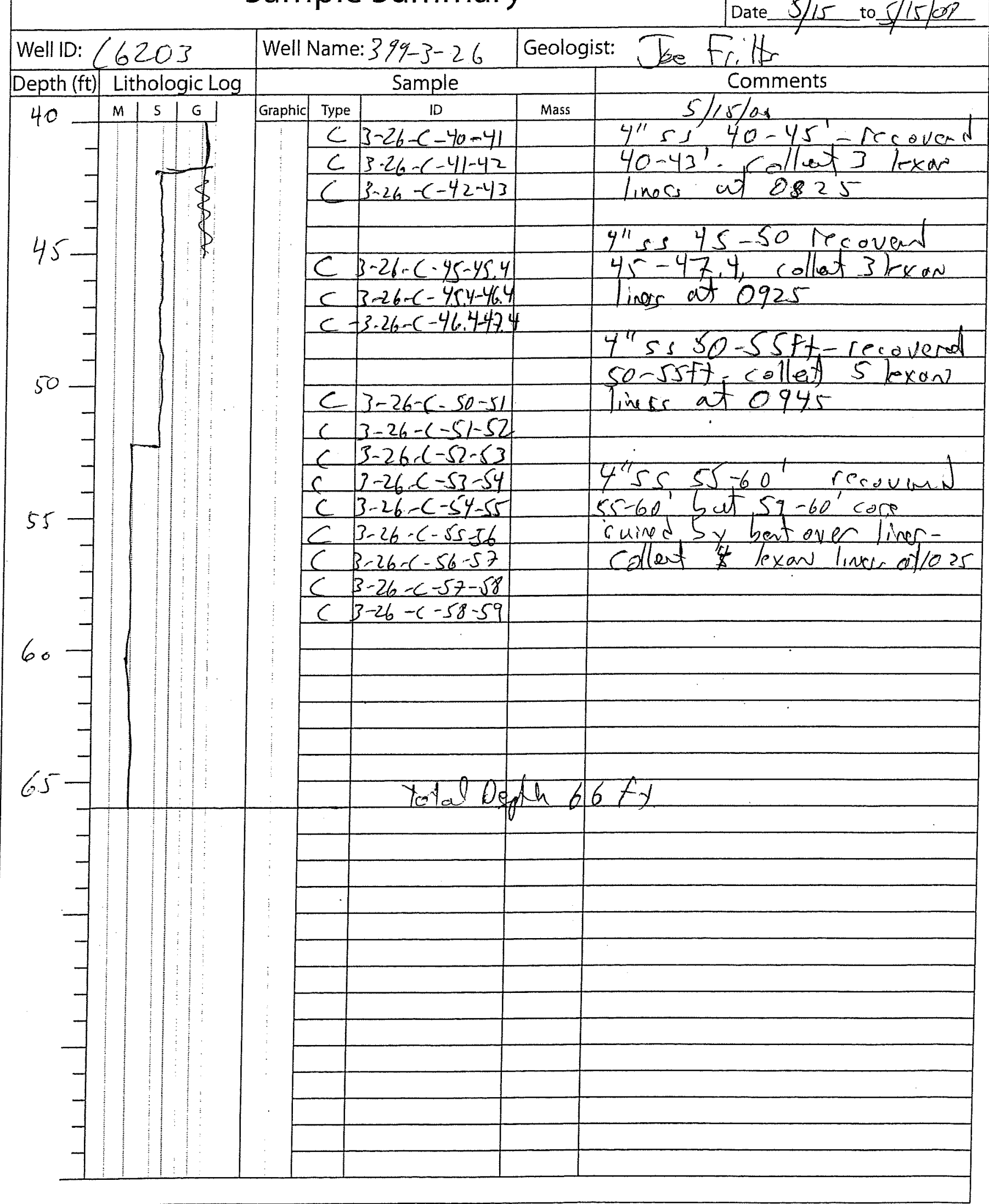


Sample Summary

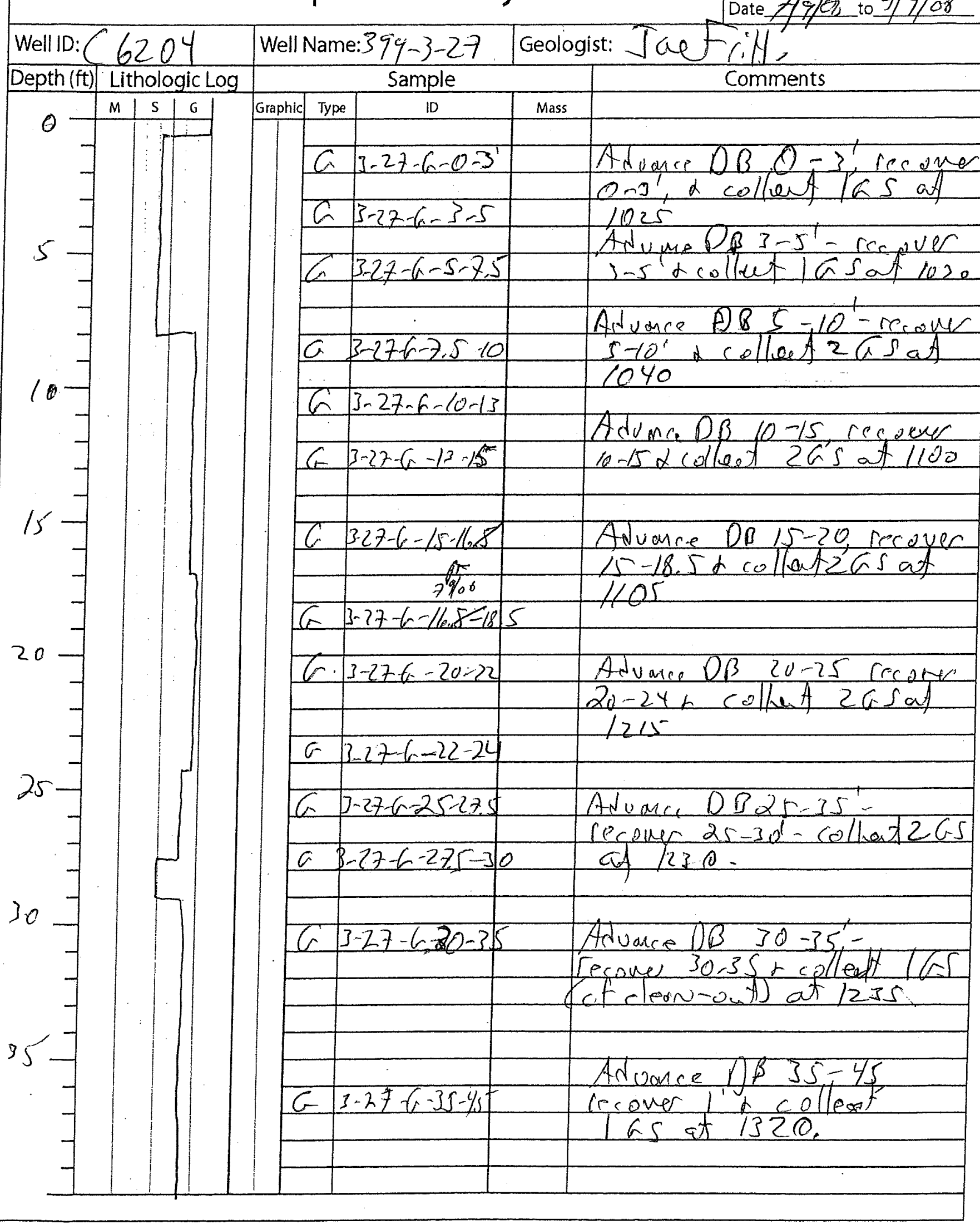




\section{Sample Summary}

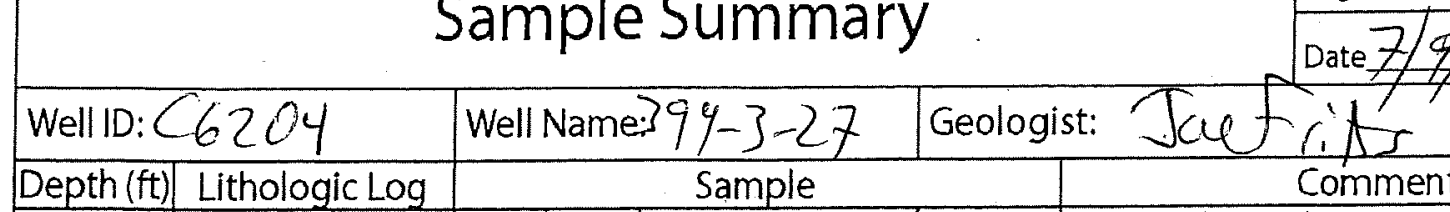
Comments

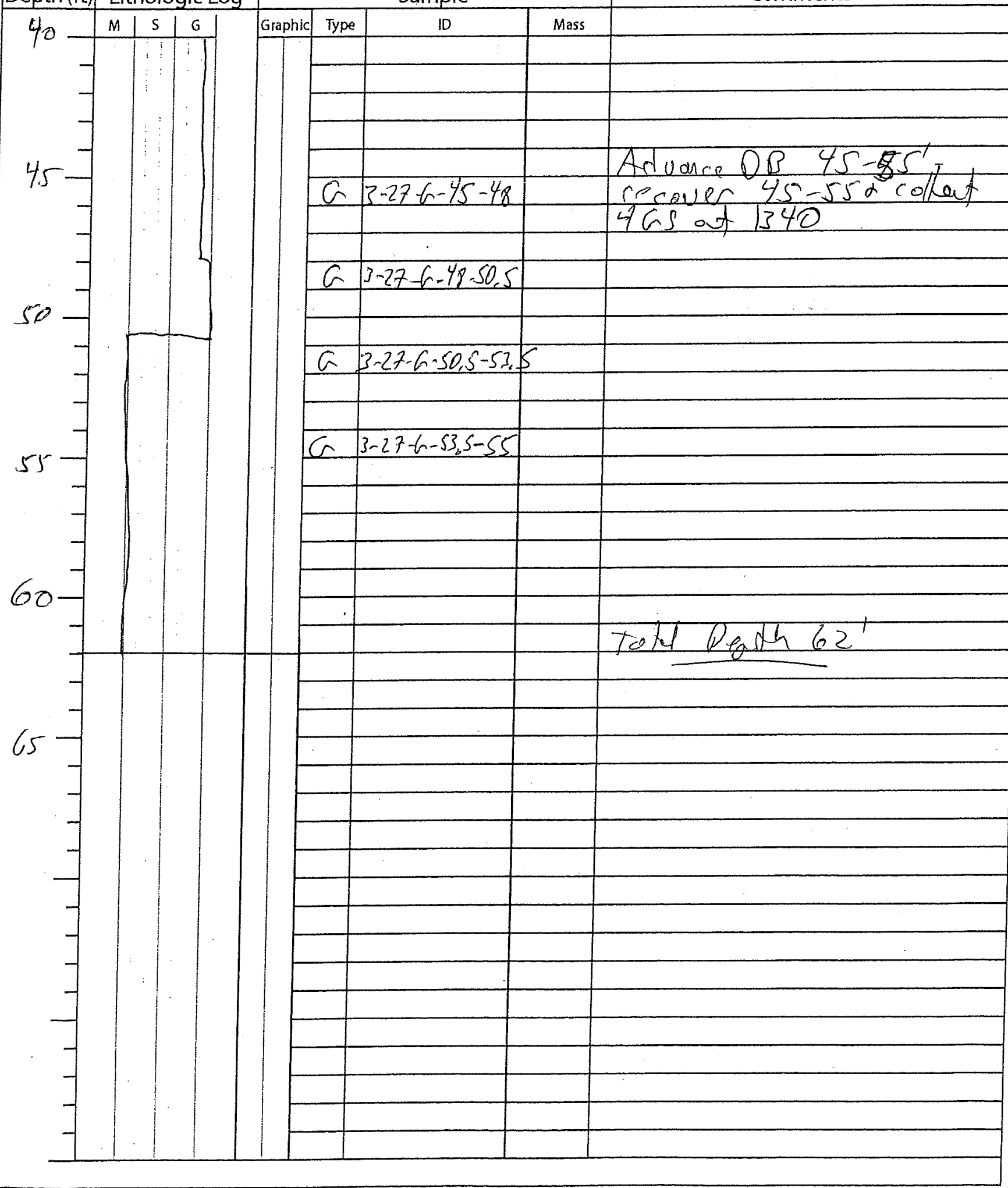




\section{Sample Summary}

\begin{tabular}{|c|c|c|c|c|c|c|c|c|}
\hline \multirow{2}{*}{$\begin{array}{l}\text { Well ID: } \\
\text { Depth (ft) }\end{array}$} & \multirow{2}{*}{\multicolumn{3}{|c|}{$\begin{array}{l}\text { C6205 } \\
\text { Lithologic Log }\end{array}$}} & \multicolumn{3}{|c|}{ Well Name: $399-3-28$} & \multicolumn{2}{|c|}{ Geologist: T. Buswell } \\
\hline & & & & \multicolumn{4}{|c|}{ Sample } & Comments \\
\hline \multirow{4}{*}{0} & $\mathrm{M}$ & & $\mathrm{G}$ & Graphic & Type & ID & Mass & \\
\hline & & & & & & & & Advama DB 0-5'bss, 0-5'reear \\
\hline & & & & & $G$ & $3-28-6-0-2$ & 5 & colleat 24507445 \\
\hline & & & & & $G$ & $3-28-G-2.5-5$ & $z^{2}$ & \\
\hline \multirow[t]{3}{*}{5} & & s & & & $G$ & $3.28-G-5-8.5$ & & Advana $D B 5-10^{\prime} b$ \\
\hline & & & & & & & & recovery, cedlect 24501450. \\
\hline & & & & & $G$ & $3-28-G-8.5-10$ & & \\
\hline \multirow{5}{*}{10} & & & & & & & & \\
\hline & & & & & $G$ & $3-28-6-10-12$ & & Advana $1 D B \quad 10-15^{\prime} b_{g} s, 10-14^{1}$ \\
\hline & & & & & & & & recoveny collect 2 G5@1535. \\
\hline & & & & & $G$ & $3-28-G-12-14$ & & \\
\hline & & & & & $G$ & $3-\partial 8-G-15-16.5$ & & \\
\hline \multirow{3}{*}{15} & & & & & & 300 or 11510. & & Advance DB $15-20^{\circ} b s, 15-20^{\prime}$ hecovern \\
\hline & & & & & $G$ & $3-28-G-16.5-18$ & & colleat 3 GSQ 1545 \\
\hline & & & & & $G$ & $3-28-G-18-20$ & & \\
\hline \multirow{5}{*}{20} & & & & & & & & $7 / 1 / 04$ \\
\hline & & & & & $\mathbb{E}$ & $3 \cdot 28-(-20-2 / 5$ & & \\
\hline & & & & & & & & Advan Da $20-25$, recaver \\
\hline & & & & & & & & $20-23,5 \alpha$ colled 265 \\
\hline & & & & & G & $\beta-28-6-21.5-28$, & $\underline{N}$ & a) 0700 \\
\hline \multirow{4}{*}{25} & & & & & G & $3-28-6-25-28$ & & \\
\hline & & & & & & & & ANure DB 25-35 racoure \\
\hline & & & & & & & & $25-3 y+$ collat 4 as at \\
\hline \multirow{2}{*}{\multicolumn{5}{|c|}{$3-28-6-30-32$}} & G & $3-28-6-28-30$ & & 0710 \\
\hline & & & & & G & $3-28-6-30-32$ & & \\
\hline & & & & & & & & \\
\hline \multirow{6}{*}{$35-$} & & & & & 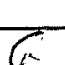 & $3-28+6-32-34$ & & \\
\hline & & & & & & & & $35-45 \cos 20$ \\
\hline & & & & & $G$ & $3-28-6-35-38$ & & $35-40,5+$ collat $2 \mathrm{ks}$ \\
\hline & & & & & & & & at 0800 \\
\hline & & & & & & & & \\
\hline & & & & & G & $3-28-6-3 y-4$ & 9,5 & \\
\hline
\end{tabular}




\section{Sample Summary}

\begin{tabular}{|l|} 
Page 2 of 2 \\
Date $7 / 8108$ to $7 / 9 / 08$ \\
\hline
\end{tabular}

Well ID: (6205

Depth (ft) Lithologic Log

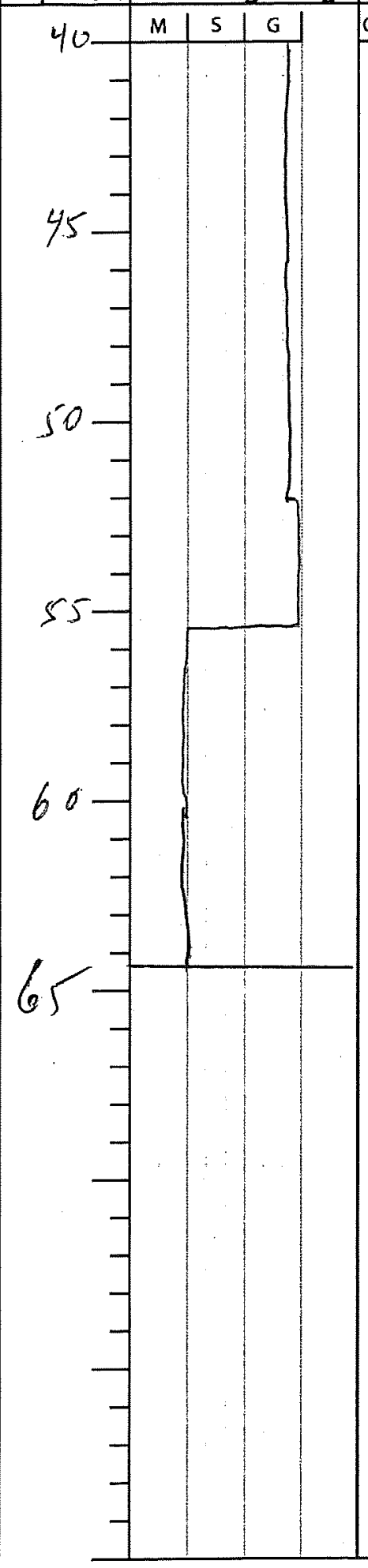

Well Name: 399-2)-28 Geologist: T. Buswell Sample

\begin{tabular}{|c|c|c|c|}
\hline Graphic & Type & ID & Mass \\
\hline & & &
\end{tabular}

Advace 0 $45-56$ recour $45=49,5$ a collect 1 cos at
ANume DQ $55-60$

reone $5560 \alpha$ collent 265

$\left.a^{3} 3-28-6-55-55\right] 5$ at 0815

\section{$63-28-6-555-60$}

Arumen $D B 60-65$, recover $C 3-28-6-60-62,5 \quad 60-65 \alpha$ colleat 2 as

\begin{tabular}{l|l|l}
\hline$G$ & at 0835 \\
\hline & $3-28-6-62.565$ & Total Depth 64.5
\end{tabular}




\section{Sample Summary}

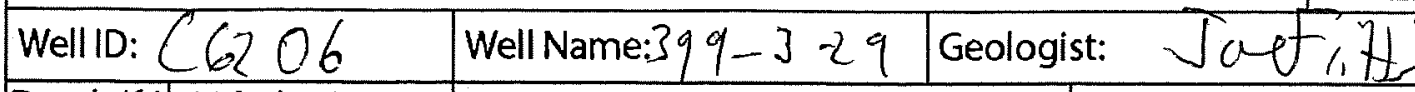

Depth (ft) Lithologic Log

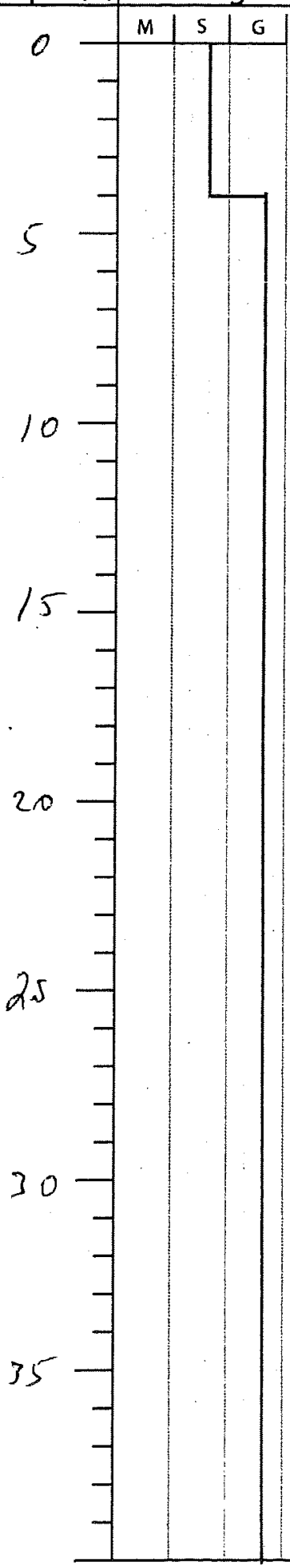
Sample

Comments

\begin{tabular}{|c|c|c|c|}
\hline Graphic & Type & $\mathbb{D}$ & Mass \\
\hline
\end{tabular}

Ca $3-29-6-0-2 \quad$ Arvome Deive barrel( $D \beta)$

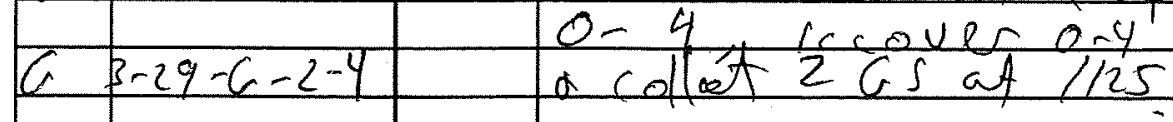

\begin{tabular}{l|l|l}
$G 3-29-6-4-6$ & Avme $D \beta \quad 4-10$, \\
& recover $4-10 \alpha$ collect
\end{tabular}

c 3-29-6-6-8 3 GS at 1100

G $3-29-6-8-10$

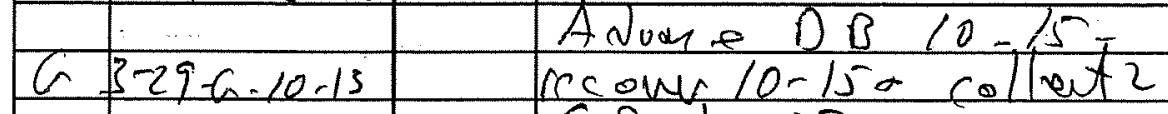

a $3-29-6-13-15$ a sat 1250

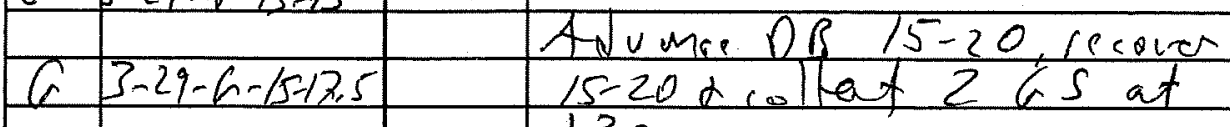

$63-29-6-125-20$ 1300

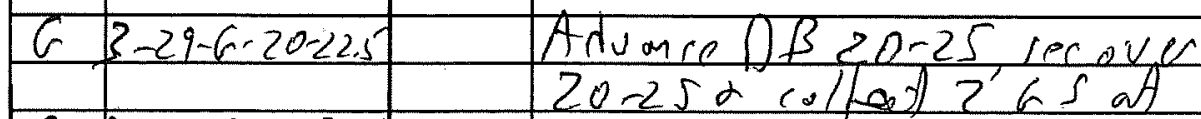

\begin{tabular}{l|l|l|}
\hline$a$ & $3-29-6-22.525$ & 1345 \\
\hline
\end{tabular}

\begin{tabular}{|l|l|l|}
\hline is $3-29-6-2529$ & $25-32 \alpha$ collont $2 C S$ at $1 y_{3} 0$ \\
\hline & & top $2^{\prime}$ slough \\
\hline
\end{tabular}

$6 \mid 3-29-6-29-32$

Adoure $D B 3 S-45-$
recour $35-42,5$ +collat

E $3-29-6-35-37,5$

365 at 1530 


\section{Sample Summary}

$\frac{\text { Page } 2 \text { of } 2}{\text { Date } 6 / 30 \text { to } 7 / i / 02}$

Well ID: $C 6206$ Depth (ft) Lithologic Log \begin{tabular}{l|l|l|l|}
40 & $M$ & $S$ & $G_{f}$ \\
\hline
\end{tabular}
Well Name: $399-3-29$ Geologist: Sample

\begin{tabular}{|c|c|c|c|}
\hline Graphic & Type & ID & Mass \\
\hline & &
\end{tabular}
comments

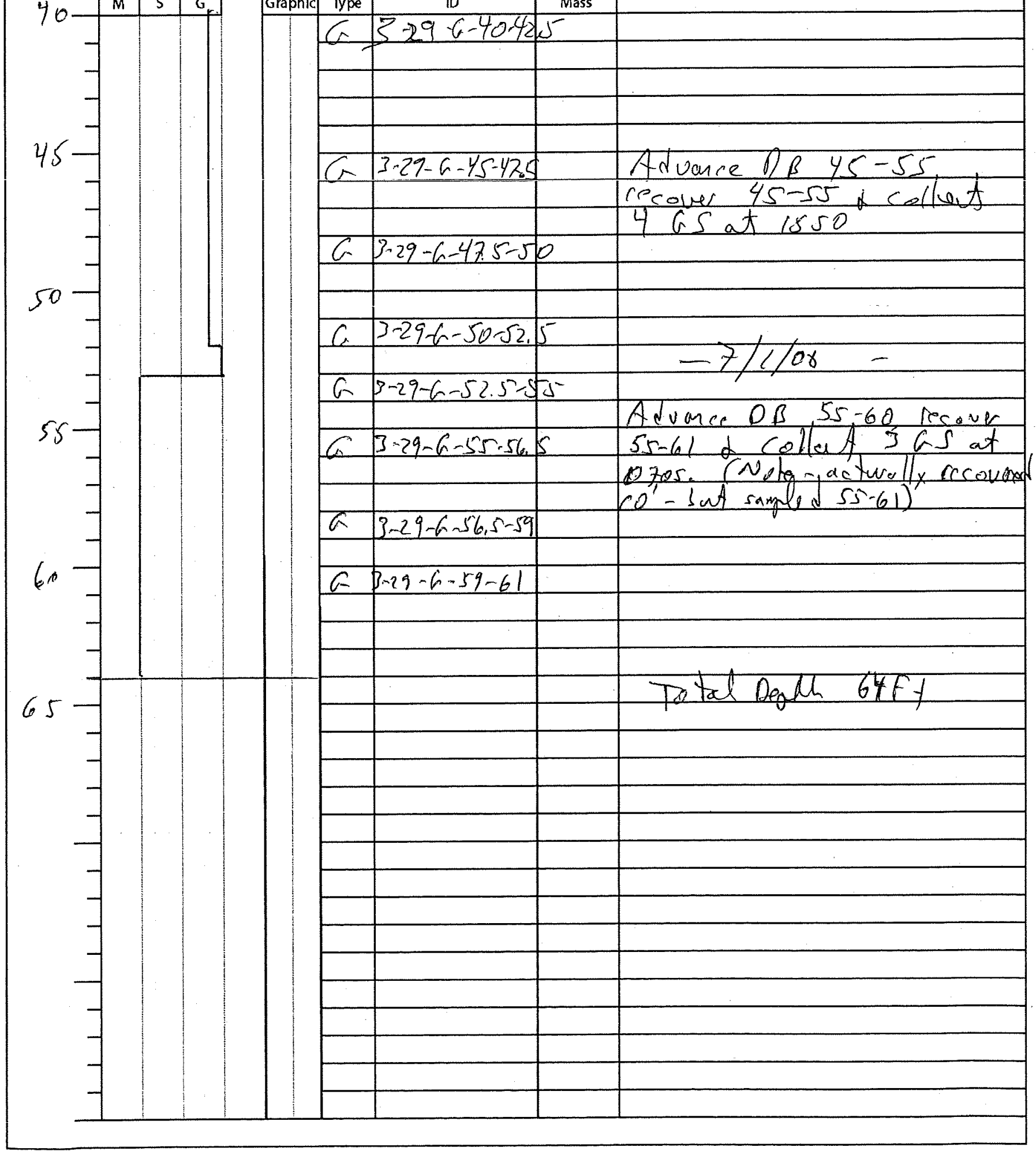




\section{Sample Summary}

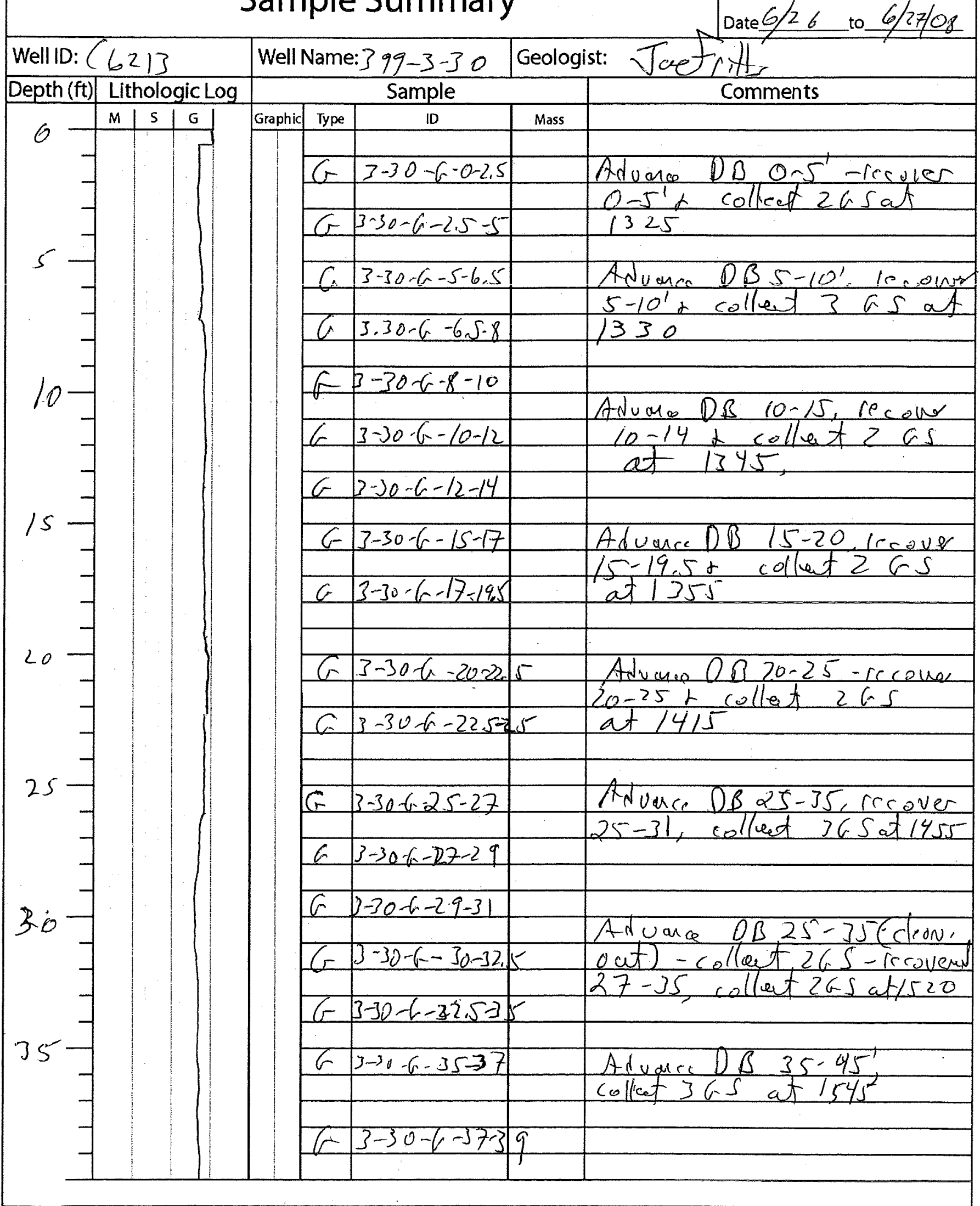




\section{Sample Summary}

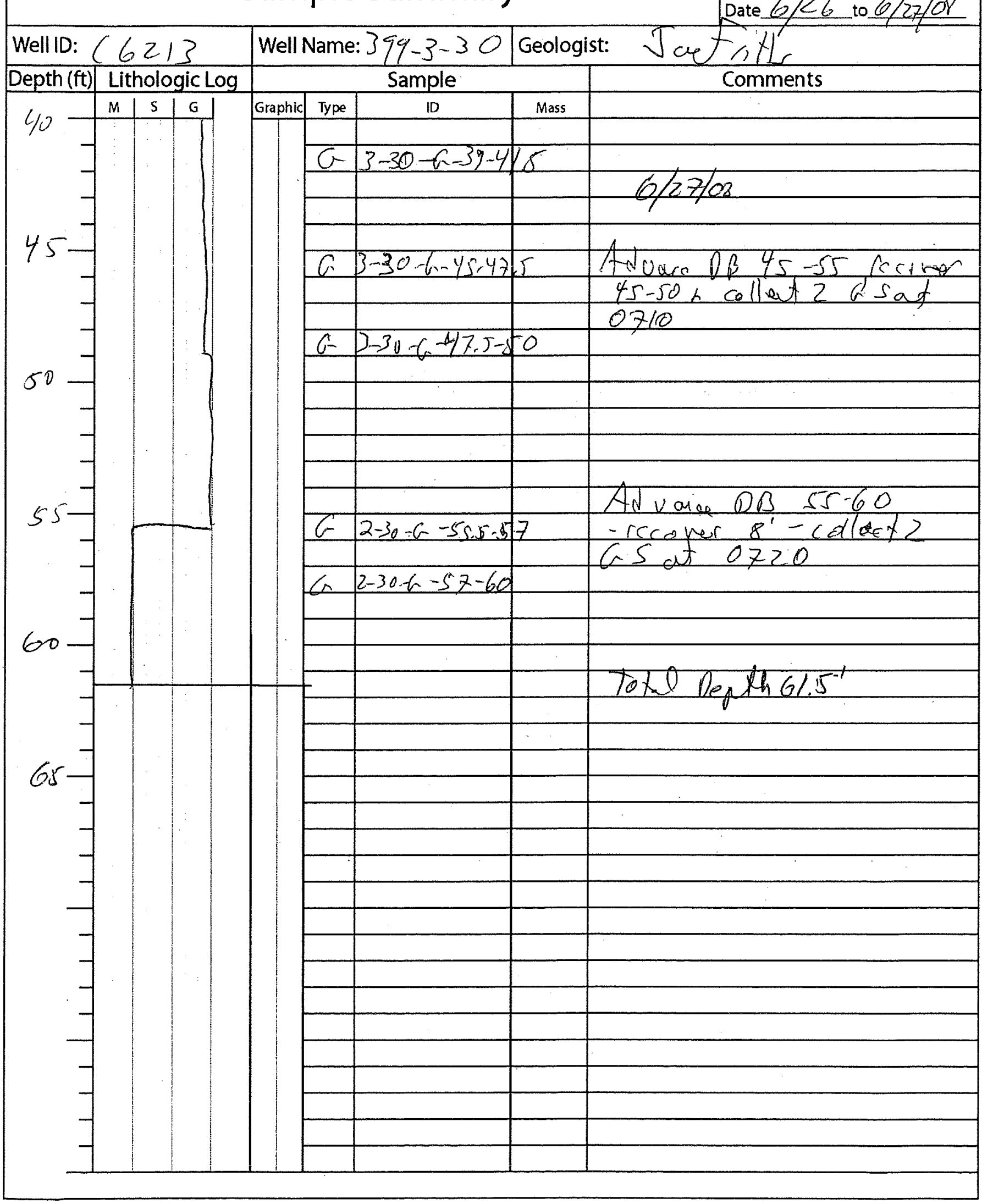




\section{Sample Summary}

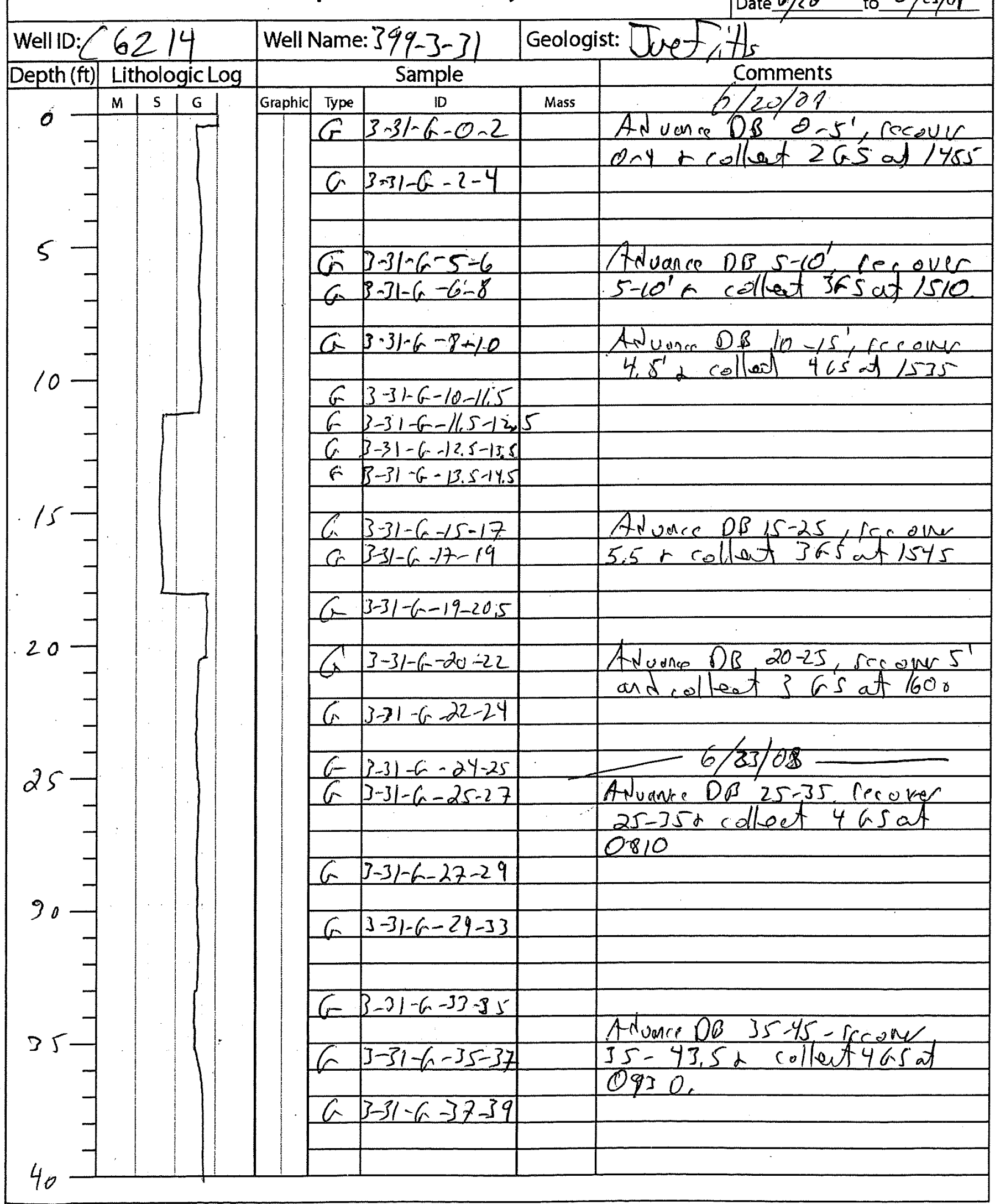




\section{Sample Summary}

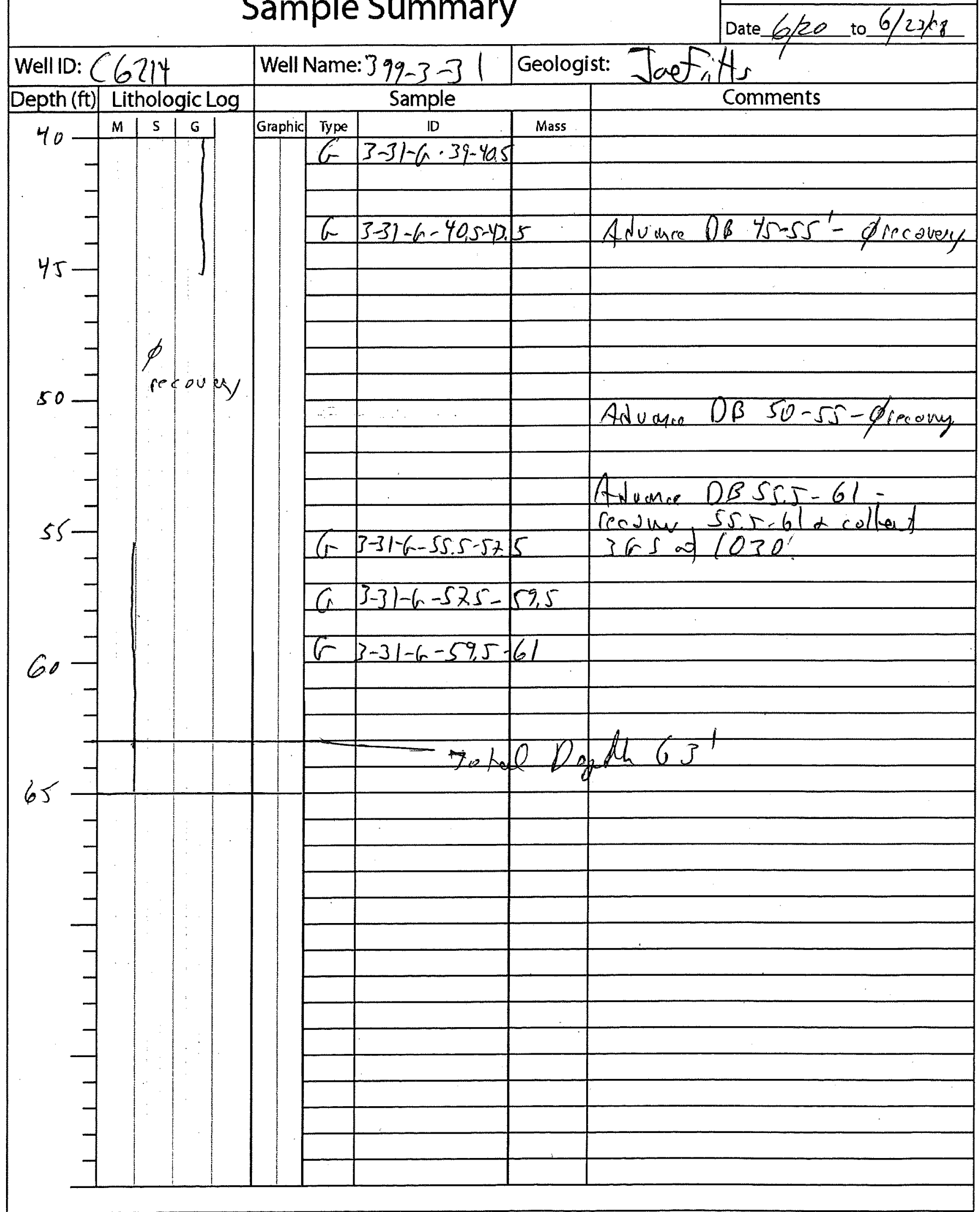




\section{Sample Summary}

Page 1 of 2
Date of/18/08 to of/18/08

Well ID: C 6215

Depth (ft) Lithologic Log

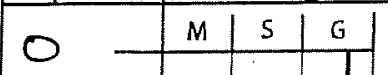

\begin{tabular}{l|l|l|l|}
0 & $M$ & $S$ & $G$ \\
\hline & & &
\end{tabular}

10

5

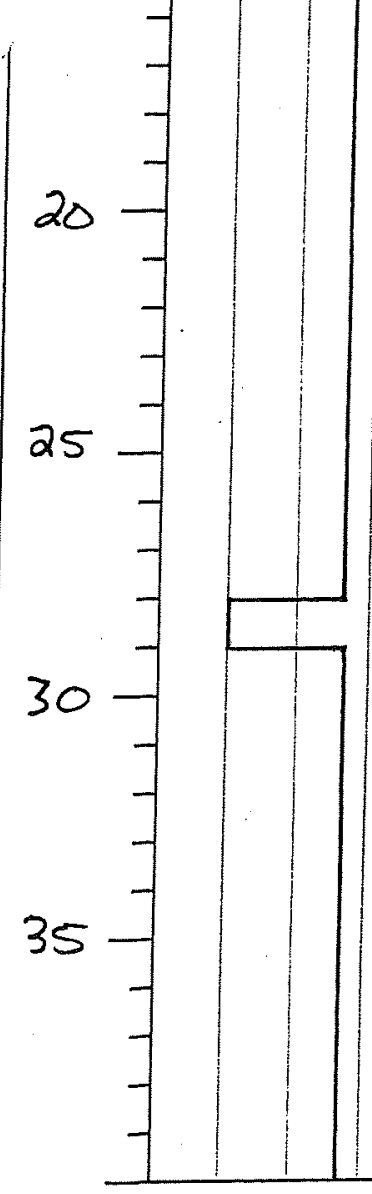

Well Name: 399-3-32 Geologist: S. Sexton Sample Comments

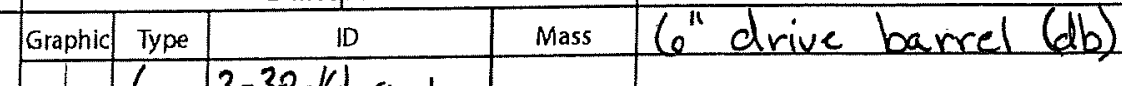
advance $d b \quad 0-5^{\prime}$ recover $0-5$ 3 GS taken @ 1004

$G \quad 3-32-|6|-3-5$

$6 \quad 3-32-|6|-5-7$

adveunce db $5-10$ recover $5-10$

$G \quad 3-32-|6|-7-9$ 3G5@1030 
Sample Summary

Page 2 of 2 Date $07 / 18 / 0807 / 18 / 08$
Well ID: C 6215

\begin{tabular}{l|l}
\hline Depth (ft) & Lithologic Log \\
\hline
\end{tabular}

\begin{tabular}{|l|l|l|l|}
\hline 40 & $M$ & $S$ & $G$ \\
\hline
\end{tabular}

Well Name: 399-3-32 G

\begin{tabular}{|c|c|c|c|c|}
\hline \multicolumn{4}{|c|}{ Sample } & \multirow[t]{2}{*}{ Comments } \\
\hline \multirow{2}{*}{\begin{tabular}{|c|} 
Graphic \\
1
\end{tabular}} & Type & 10 & Mass & \\
\hline & 6 & & & \\
\hline & $t$ & & & \\
\hline & & & & \\
\hline & & & & " \\
\hline & & & & $1115-21$ \\
\hline & $G$ & $\frac{3-32-k a-45-46 a}{3-32 \times 161-46.5-6}$ & & $\begin{array}{l}\text { advance db 45.55 recover } \\
45.48265 \odot 1327\end{array}$ \\
\hline & & & & \\
\hline
\end{tabular}




\section{Appendix D}

Field Activity Reports 


\section{FIELD ACTIVITY REPORT NO. 1 - DRILLING PLAN}

Page 1 of 2

Purpose: PNNL Project JooAraIfC

Well ID: $\quad \mathrm{C} 6184$

Drilling Co.:WOC Drille Bill Zamoz

Casing String No. (1) 2034 Drilling Method

Casing Size $75 \%$ " $6 \%$

Grade

Lbs.Per Ft.

Material Cas on stel

Type:

Welded

Auger

Rotary

Tubex

Cable Tool

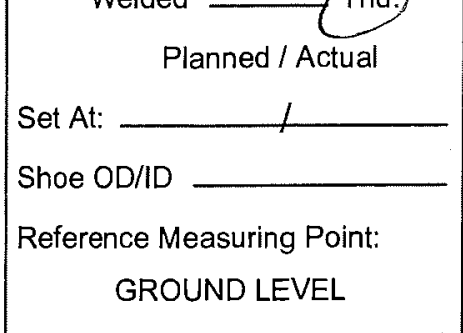

Sonic Supersunic

A.R. w/Sonic की 400

Geoprobe

Other:

Drig. Co.

Casing String No. $\begin{array}{lllll}1 & 2 & 3 & 4\end{array}$

Casing Size

Grade

Lbs.Per Ft.

Material

Type:

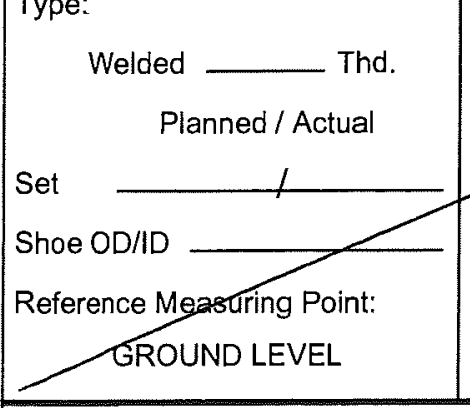

Comments/Remarks:

Rig No.:

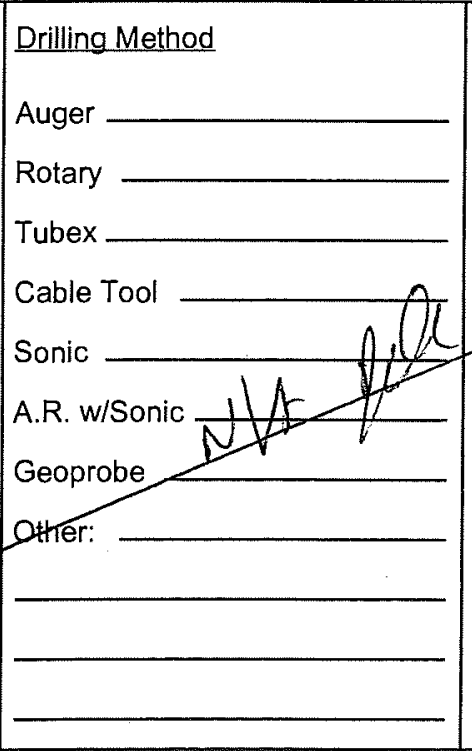

Location: 300-ff- 5

Well Name: $399-2-7$

\begin{tabular}{l|l|l} 
Rig No.: $1 \mathrm{~J} 9$ & Rig Make/Mod.: Suger Sonis
\end{tabular}

Circulation

Air _ Water/Mud

Reverse — Direct

Vol: $\mathrm{cfm}$

gpm

Pressure

Drill Pipe O.D.

Tool Joint Size

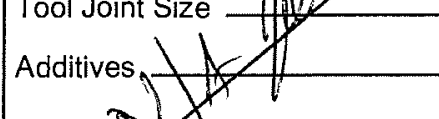

psi

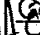

D.H. Hammer

Make

Model

Choke

c. Hammer

Make

Model

Bit Size

Type

Nozzles

$\underline{\text { Rod Size }}$

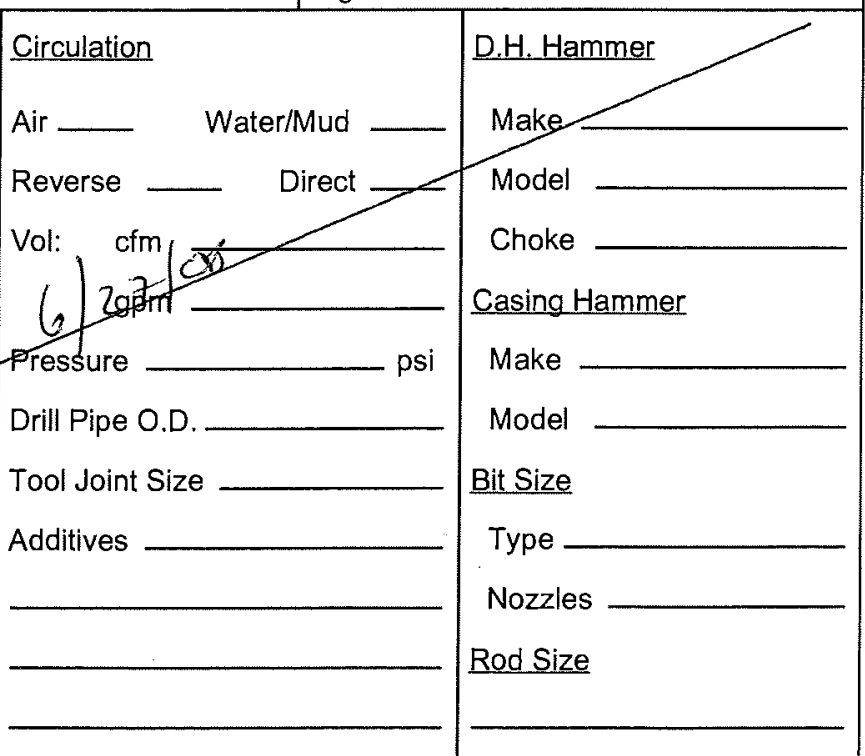

Estimated Depth to Water

Reported By: Jcetrits

NamerTitle: (1)M Ine/Colugh

Signature:

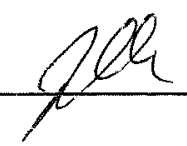

Date: $6 / 27 / 0 \gamma$ 


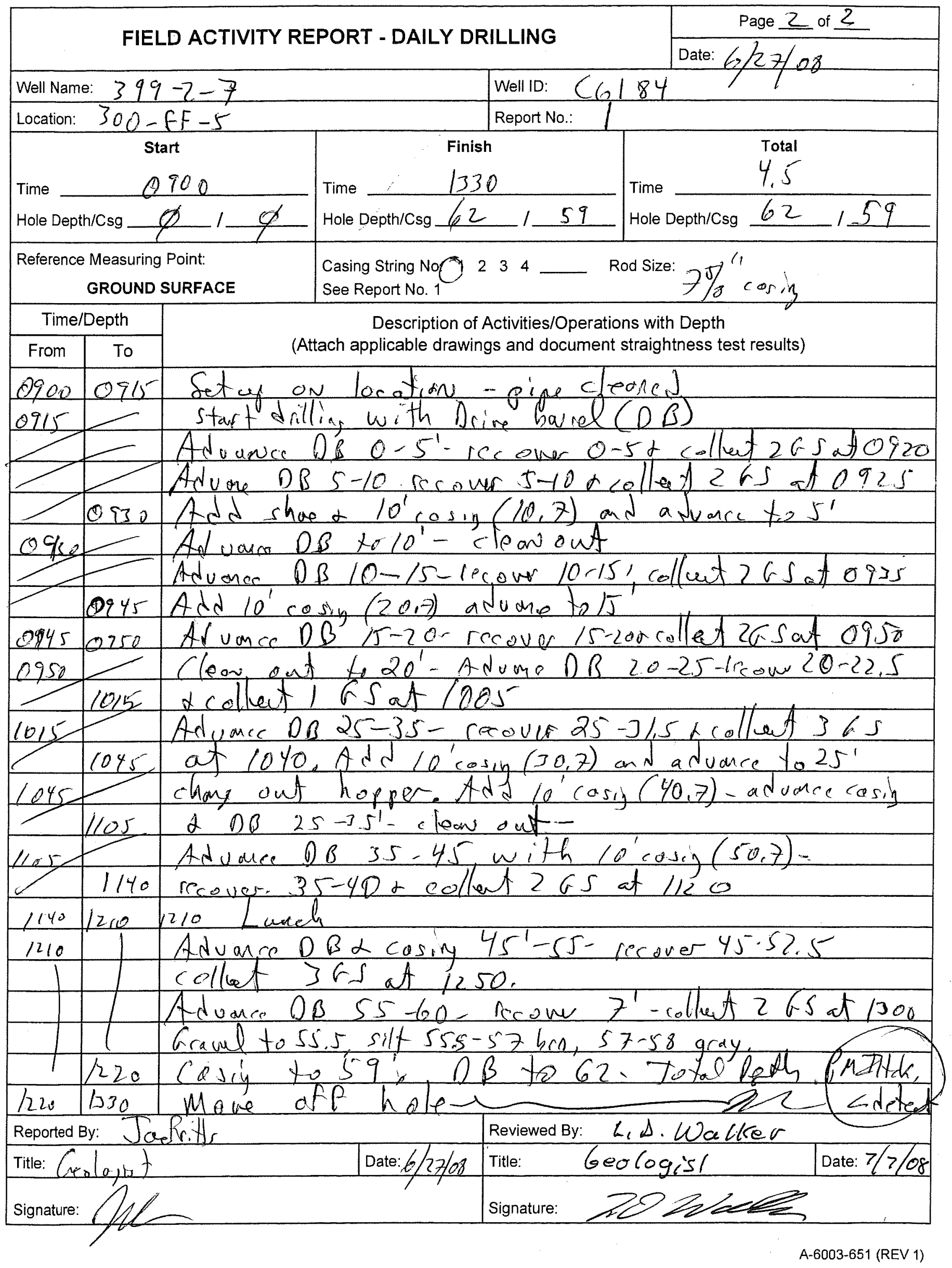

D. 2 


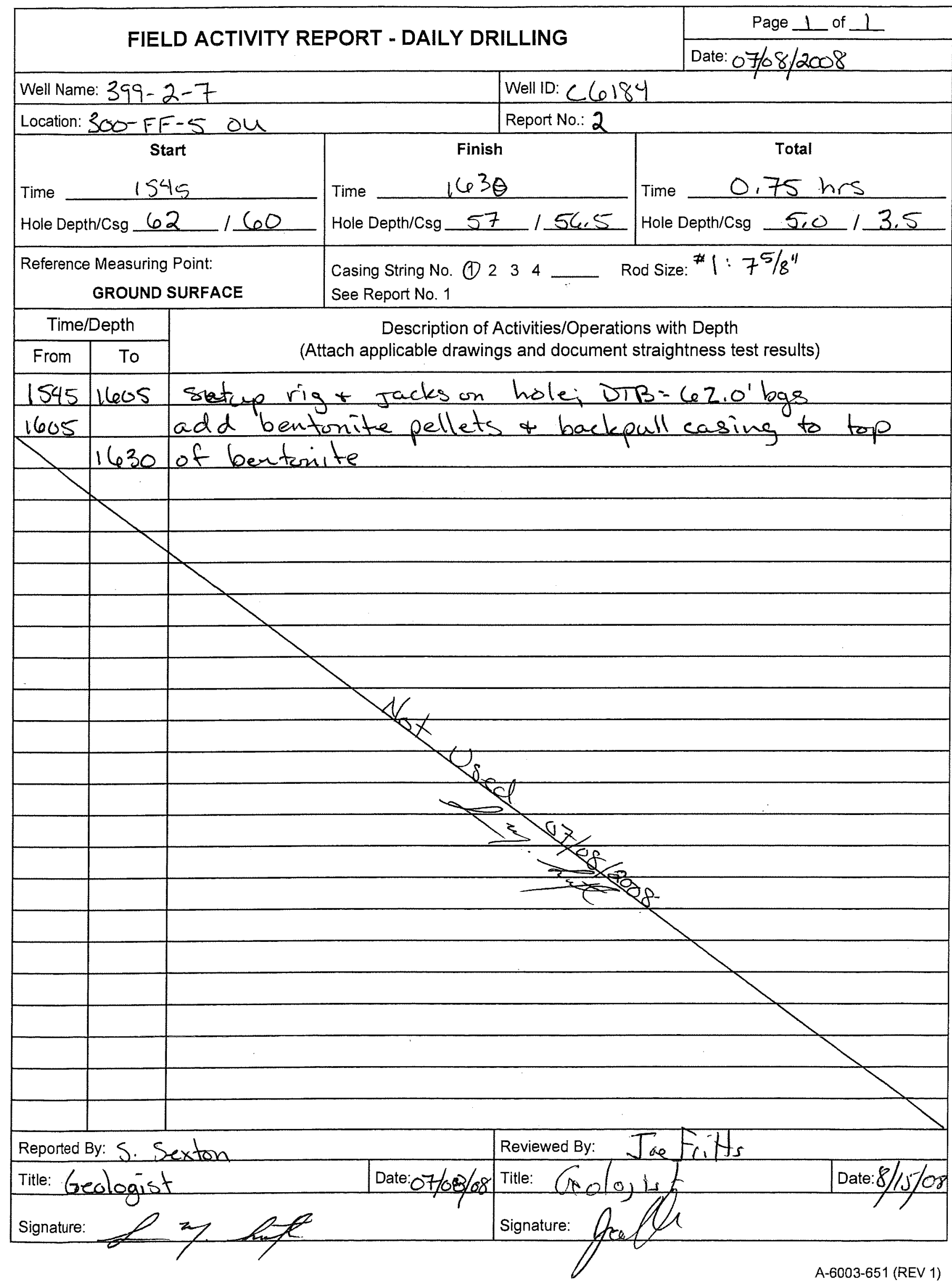




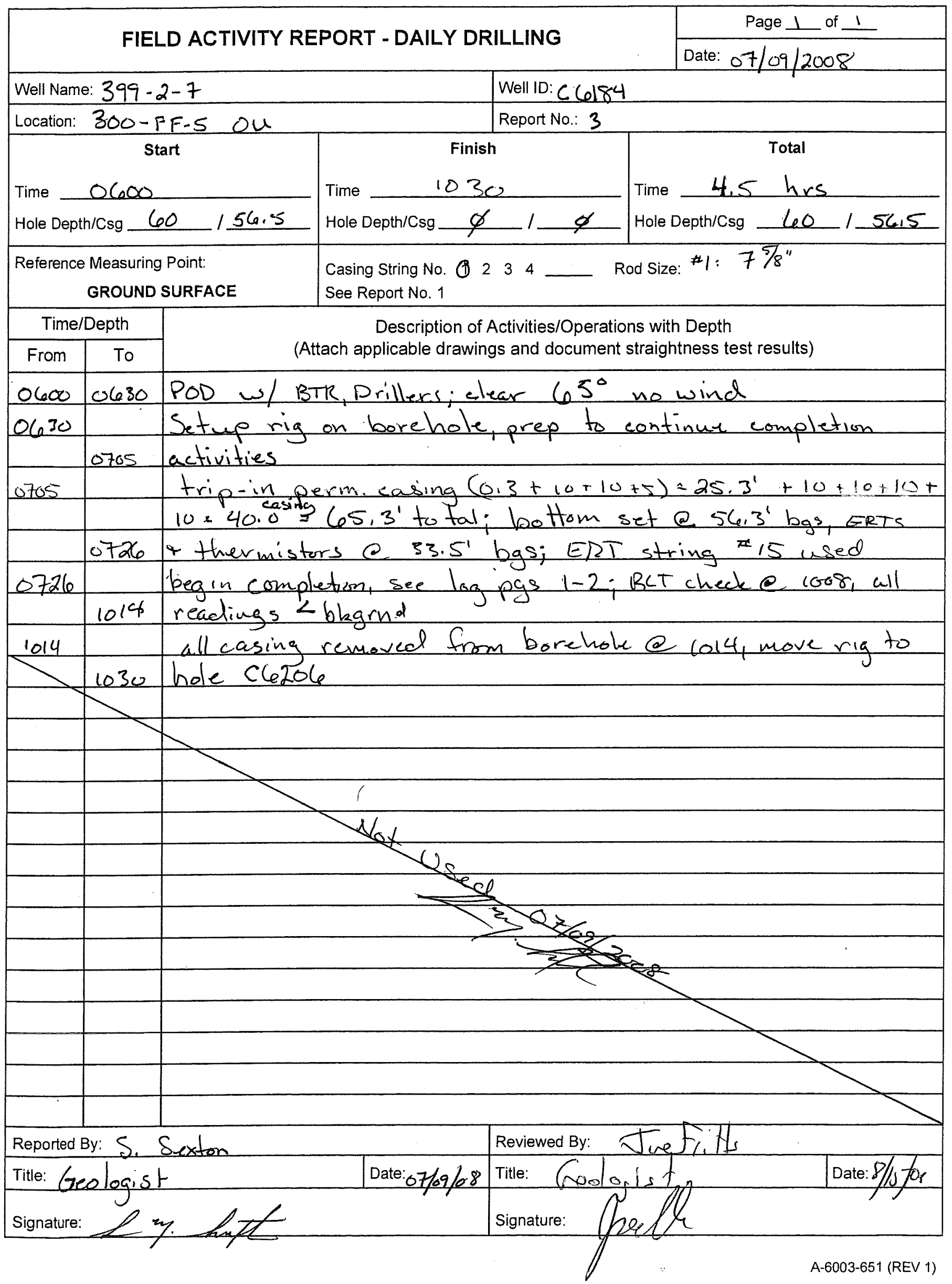

D. 4 


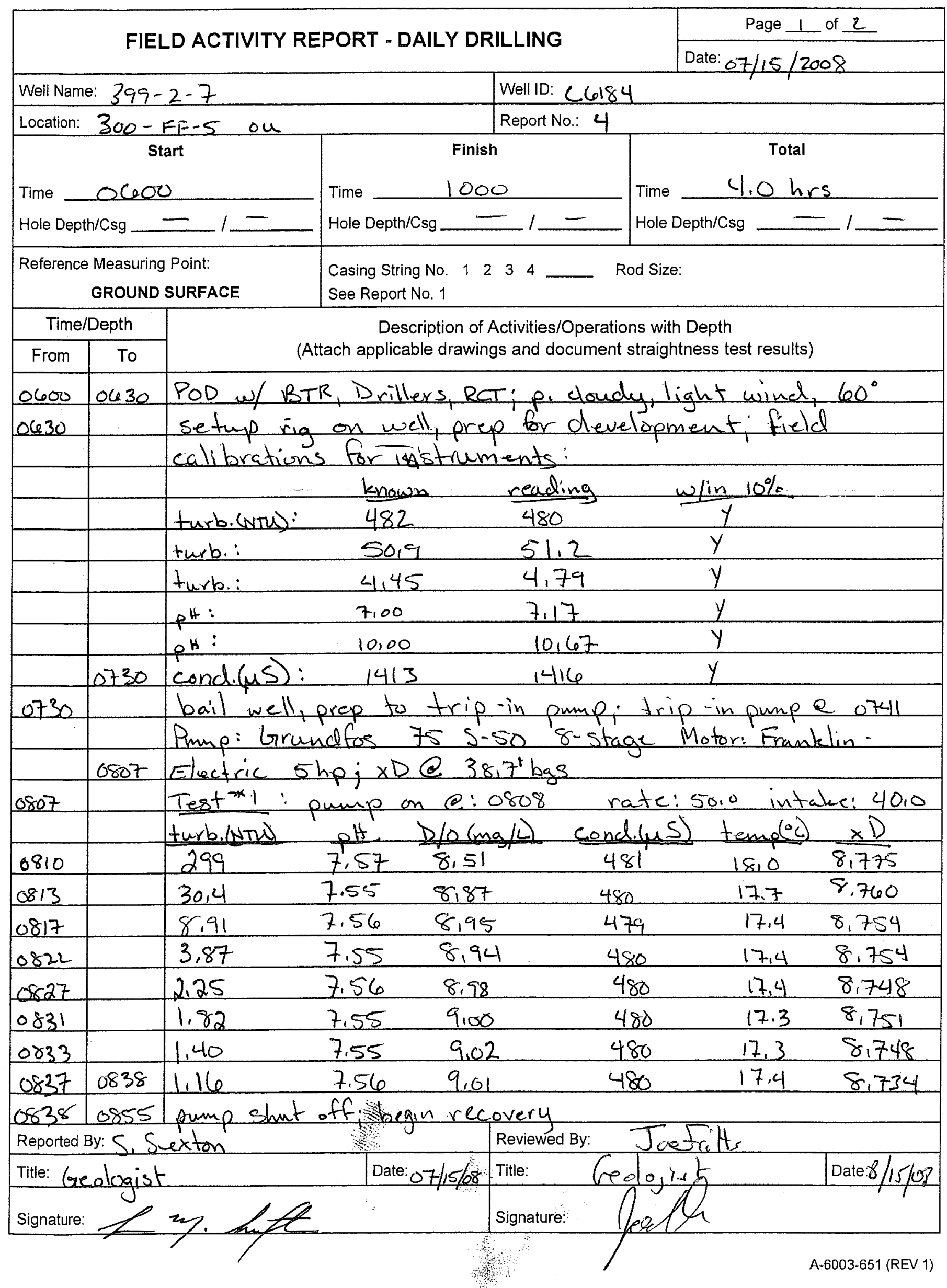

D. 5 


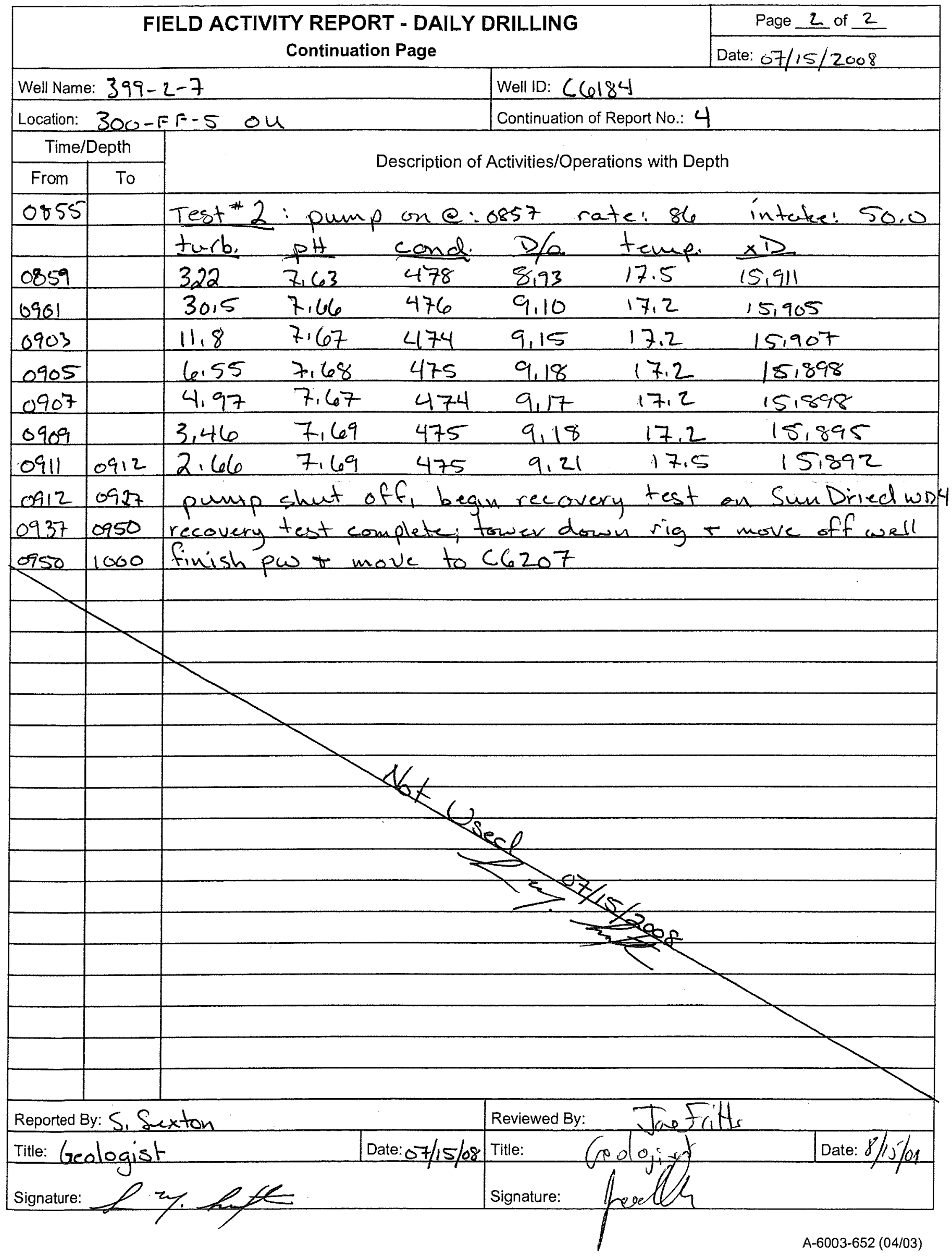




\section{WELL DEVELOPMENT AND TESTING DATA}

\begin{tabular}{|l|l|l|l|}
\hline $\begin{array}{l}\text { Well Name: } \\
399-2-7\end{array}$ & $\begin{array}{l}\text { Well ID: } \\
\text { C6184 }\end{array}$ & $\begin{array}{l}\text { Well Location: } \\
300-5 F-5 \text { ou }\end{array}$ & $\begin{array}{l}\text { Date: } \\
07 / 15 / 2008\end{array}$ \\
\hline
\end{tabular}

Reference Measuring Point (unless otherwise noted): TOP OF OUTER CASING (TOC)

\begin{tabular}{|l|l|l}
\hline Has the well been surveyed? $\bigcirc$ Yes $\otimes$ No & Does the well have a cement pad? $\otimes$ Yes $\bigcirc$ No
\end{tabular}

\section{PART 1}

STATIC WATER LEVEL:

Start of Job 29,85' bas

End of Job $29,7^{\prime}$ bgs

\section{DEPTH TO BOTTOM:}

Start of Job $56.6^{\prime} \mathrm{bgs}$
End of Job $56.60^{\prime} \mathrm{bgs}$
PART 2

\section{WELL DEVELOPMENT DATA}

Pump Model $75 \cdot 5-50$

Intake Depth $40,0 / 50,0$

Starting Turbidity $279 / 322$

\begin{tabular}{|c|c|l|}
\hline \multicolumn{3}{|c|}{ Starting Turbidity $279 / 322$} \\
\hline Pump Start & Stop & Flow Rate \\
\hline 0808 & 0838 & 50 \\
\hline 0857 & 0912 & 86 \\
\hline & & \\
\hline & & \\
\hline & & \\
\hline
\end{tabular}

Total Pumped $1380 / \mathrm{H}^{7} 1$

Final Turbidity $1.16 / 2,66$

XD SN/Range (PSI) $5217 / 20$

PART 3

\begin{tabular}{|l|}
\hline \multicolumn{2}{|c|}{ INSTANTANEOUS SLUG TEST } \\
\hline Static Water Level (TOC) \\
\hline Transducer Depth \\
\hline Baseline Start \\
\hline Injection Start \\
\hline Baseline Start \\
\hline Withdrawal Start \\
\hline Slug Volume \\
\hline XD SN/Range (PSI) \\
\hline Prepared by (Prnt \\
\hline
\end{tabular}

Prepared by (print name):

S. Sexton

Reviewed by (print name):

Tae Fitts

\section{PART 4}

Last Recorded

Measurements

Date: $07 / 15 / 2008$

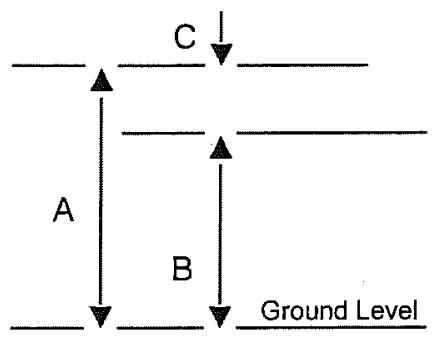

$A=N / A$

$B=N / A$

$c=N / A$
Current

Measurements

Date:
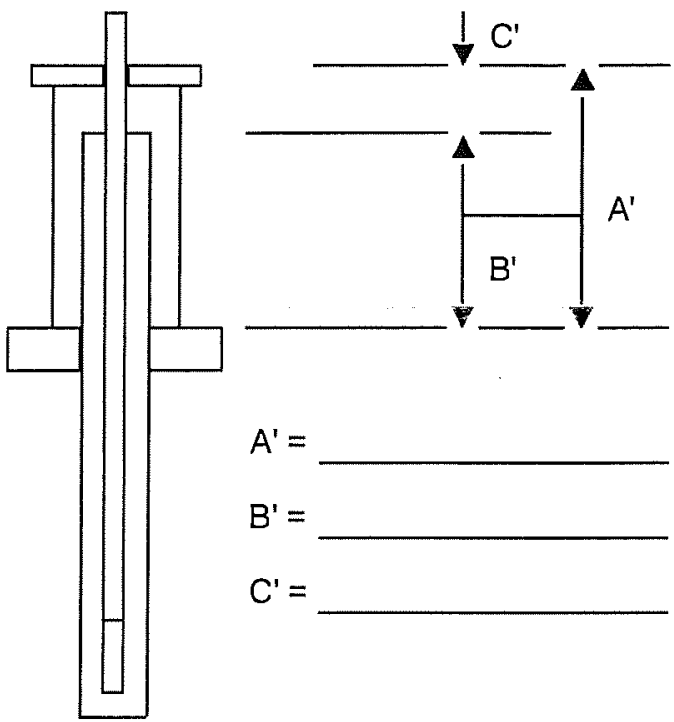

$A^{\prime}=$

$B^{\prime}=$

$C^{\prime}=$

Are there any reference marks on the casing strings? $\bigcirc$ Yes $\otimes$ No

\section{PART 5}

COMMENTS: meter: 45638

Test"1

${ }^{2} D_{i}: 8.813$

xDf:8, 835

$$
\text { meter: } 47018
$$

Tesf 2 : rccorded on SunDried wD3

$$
\begin{aligned}
& \times D_{i}: 16.042 \\
& \times D_{f:} 16.023
\end{aligned}
$$

final meter. 48189

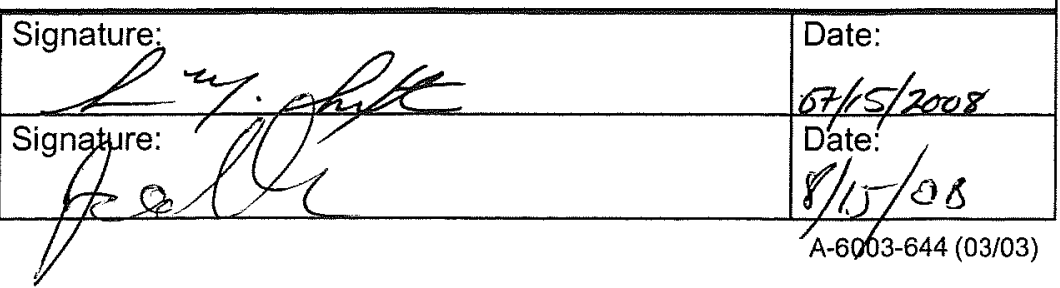

D.7 


\section{WELL DEVELOPMENT AND TESTING DATA}

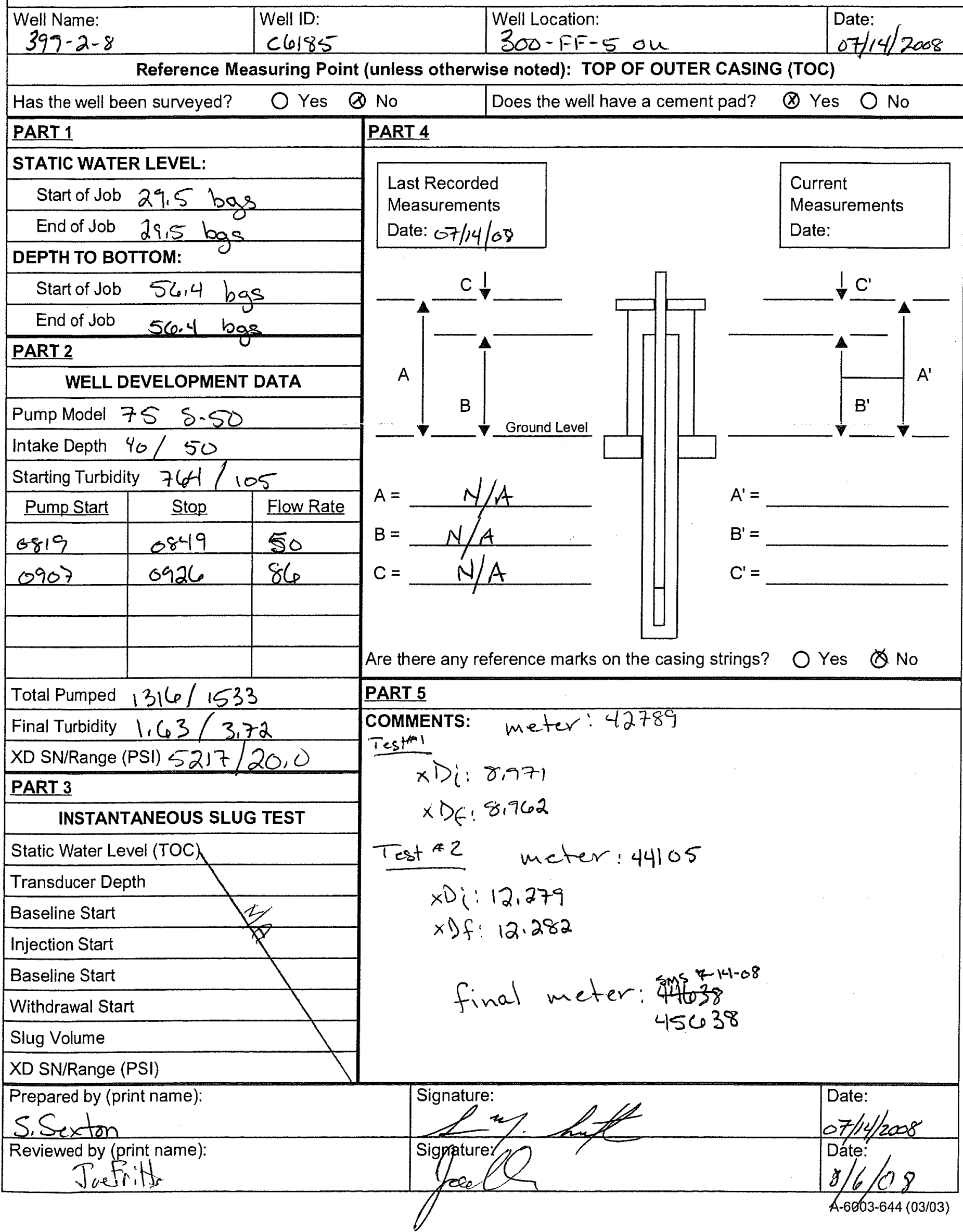

D.8 


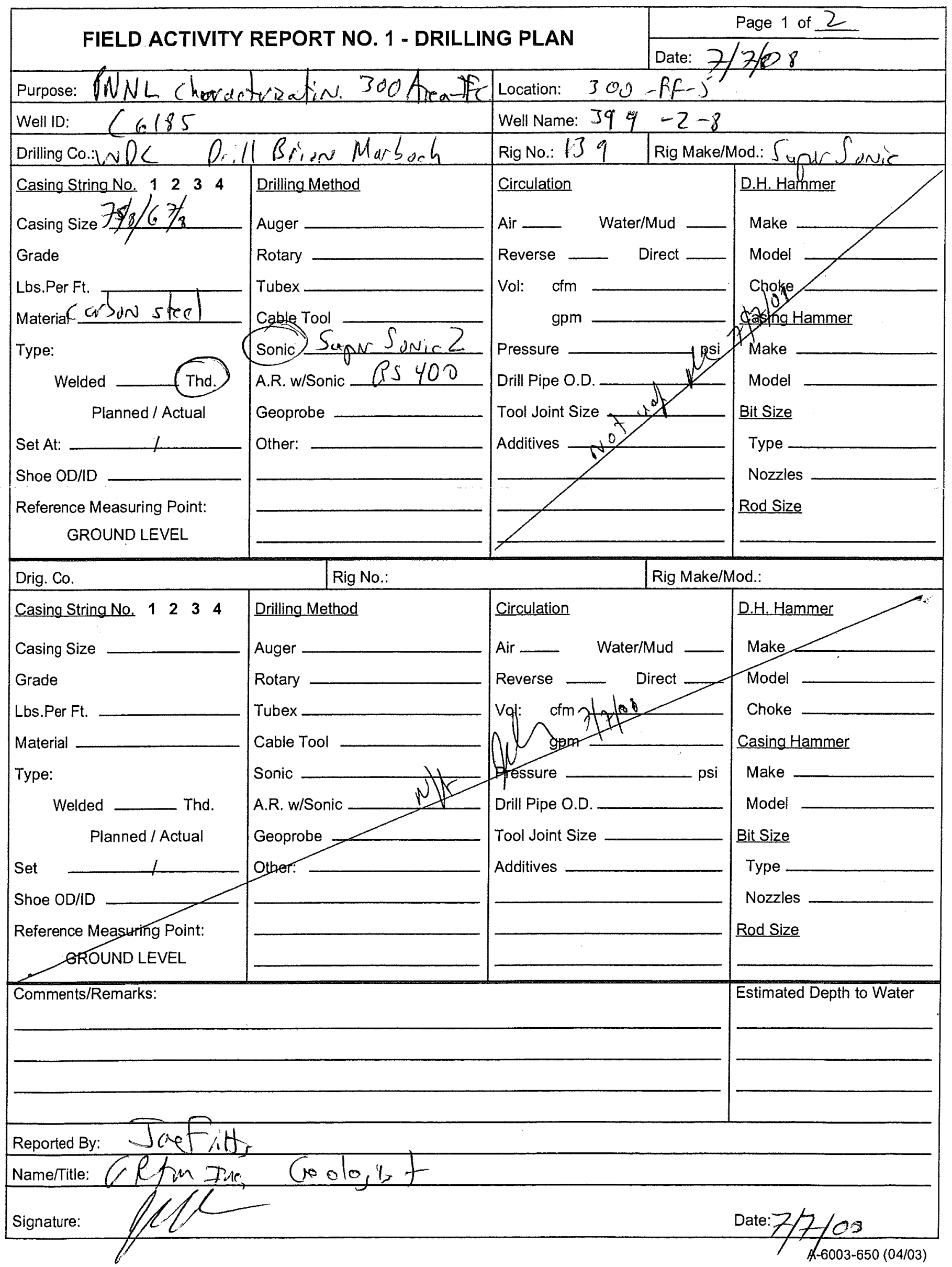




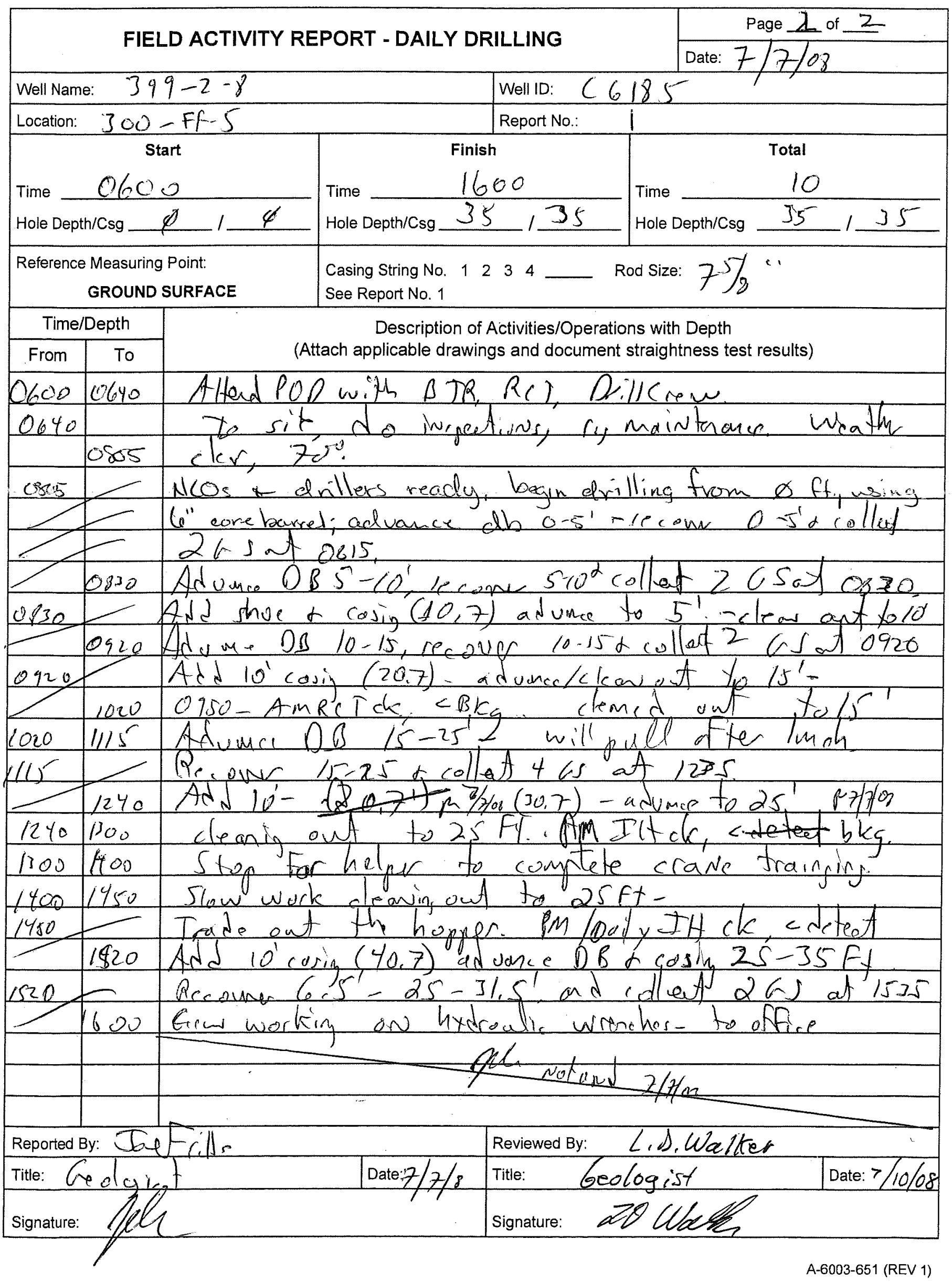

D.10 


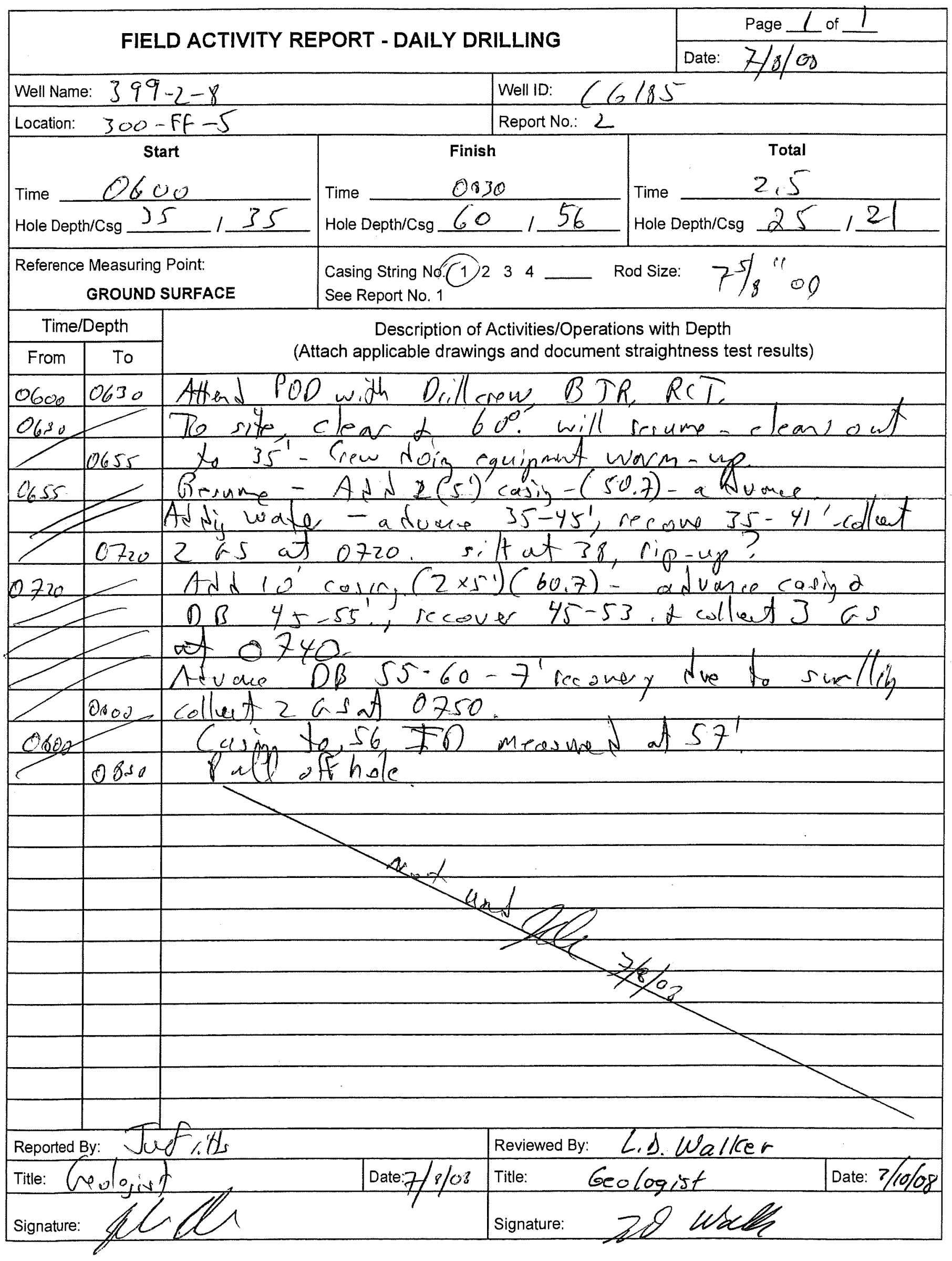

A-6003-651 (REV 1)

D.11 


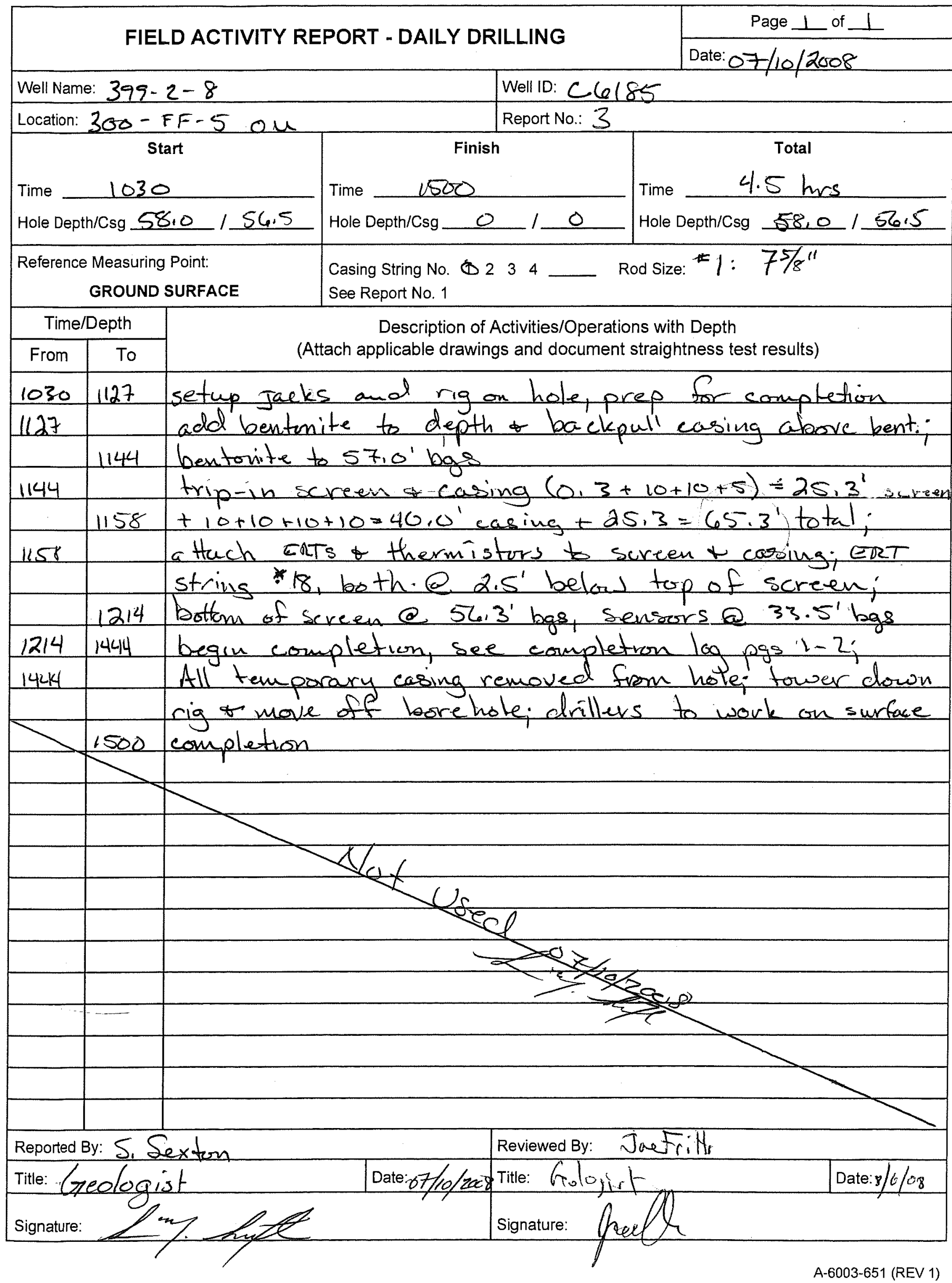

D.12 


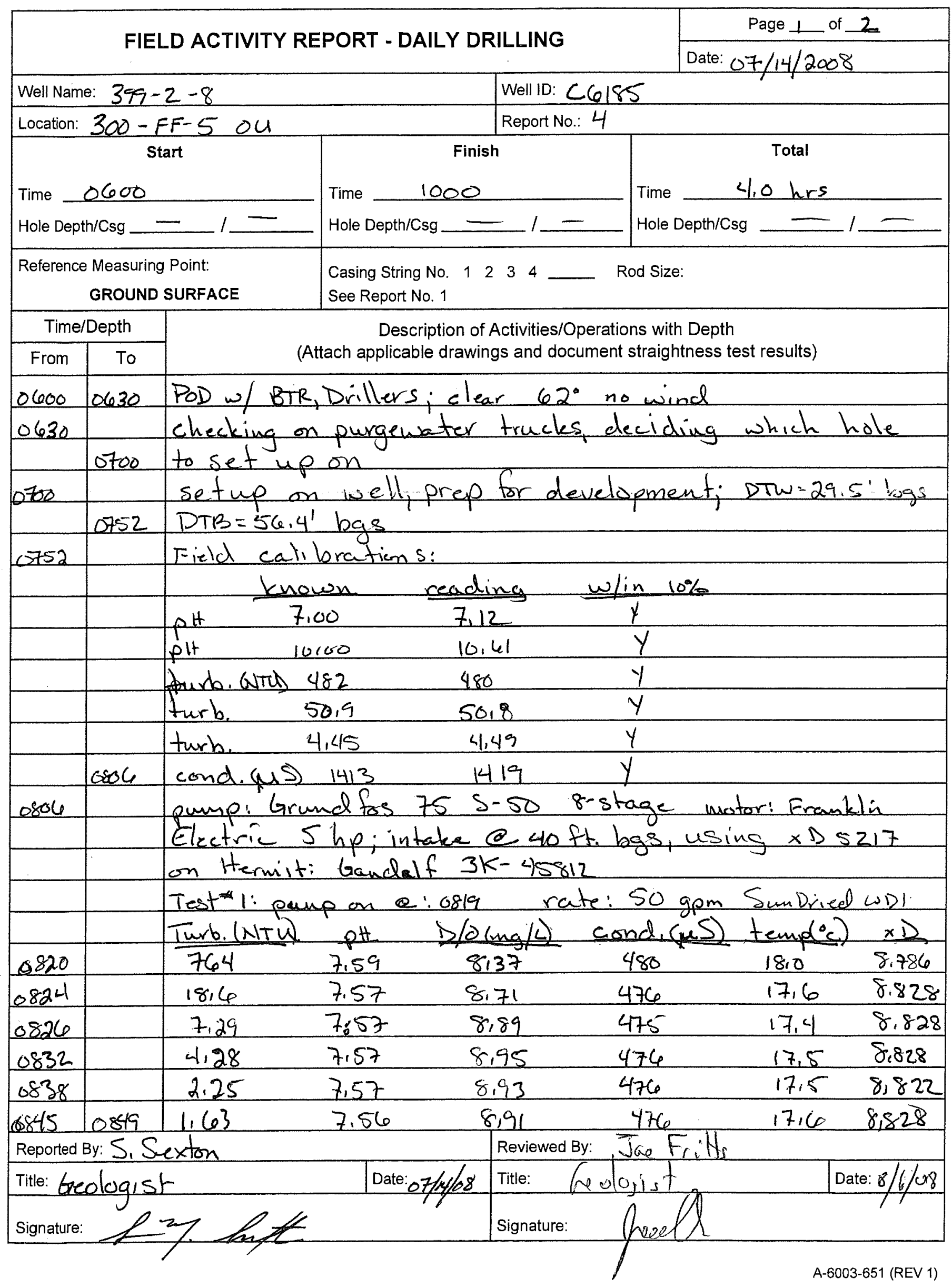




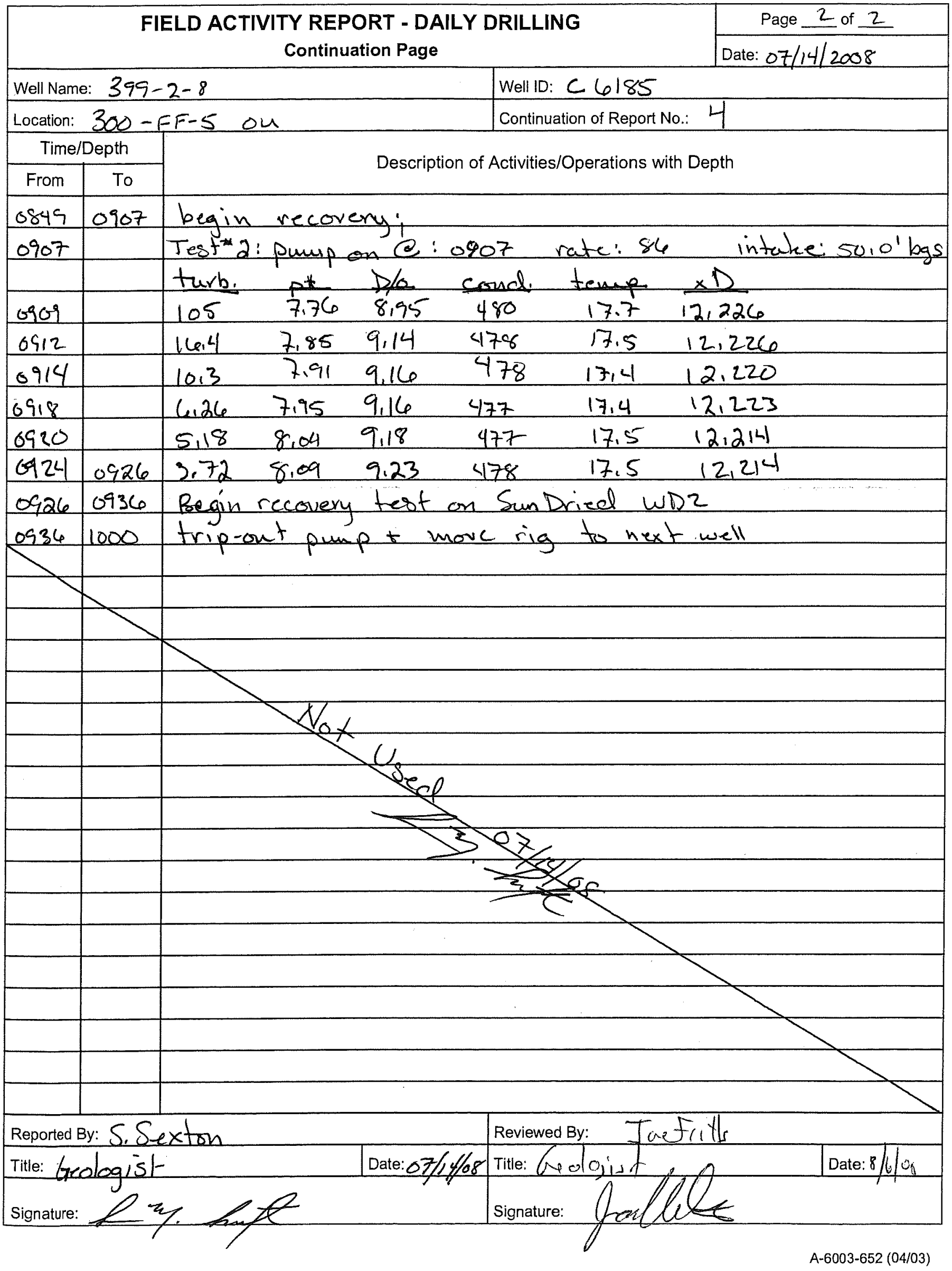

D.14 
FIELD ACTIVITY REPORT NO. 1 - DRILLING PLAN

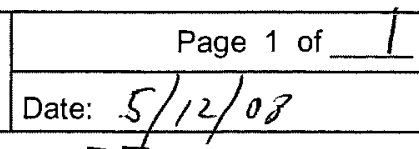

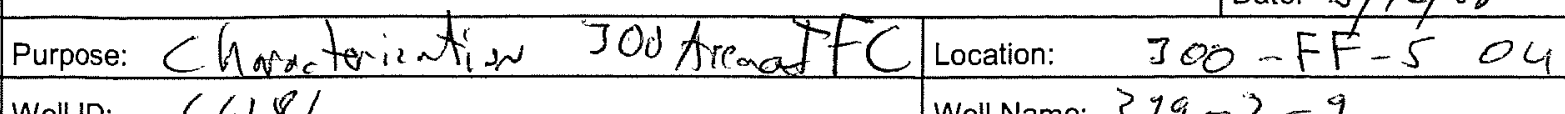

Well ID: $C 6186$ Well Name: $379-2-9$

\begin{tabular}{|l|l|l|l|l}
\hline Drilling co.: Blue Stor $/ W D C M i k e$ & Rig No.: 139 & Rig Make/Mod.: Supu So.vic 2 \\
\hline
\end{tabular}

\begin{tabular}{ll|l|l|l} 
Casing String No. (1) 23 & 4 & Drilling Method WiVen on & Circulation & D.H. Hammer
\end{tabular}

Casing Size $75 / 8^{\prime \prime} 00,67 / 8$ II Auger

Grade

Lbs.Per Ft.

Material Corbow sted

Type:

Welded Thd.

Planned / Actual

Set At: -1628

Shoe OD/ID

Reference Measuring Point: GROUND LEVEL

Drig. Co.

\begin{tabular}{lllll}
\hline Casing String No. & 1 & 2 & 3 & 4
\end{tabular}

Casing Size

Grade

Lbs.Per Ft.

Material

Type:

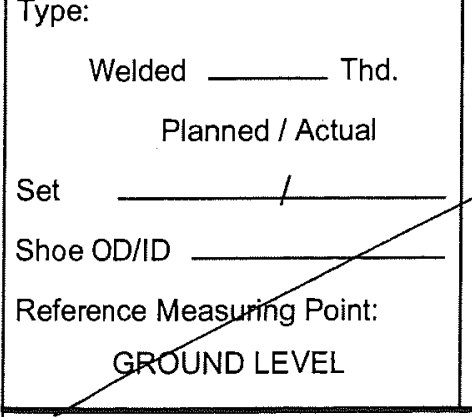

Comments/Remarks:
Rotary

Tubex

Cable Tool

Sonic Superjoxic 2 A.R. w/Sonic RS400* Geoprobe

Other:

\& Built bx Gusfech

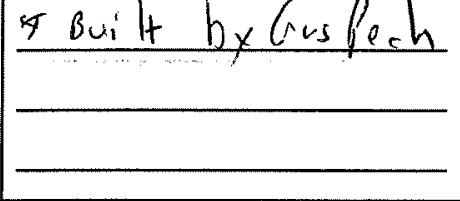

Rig No::

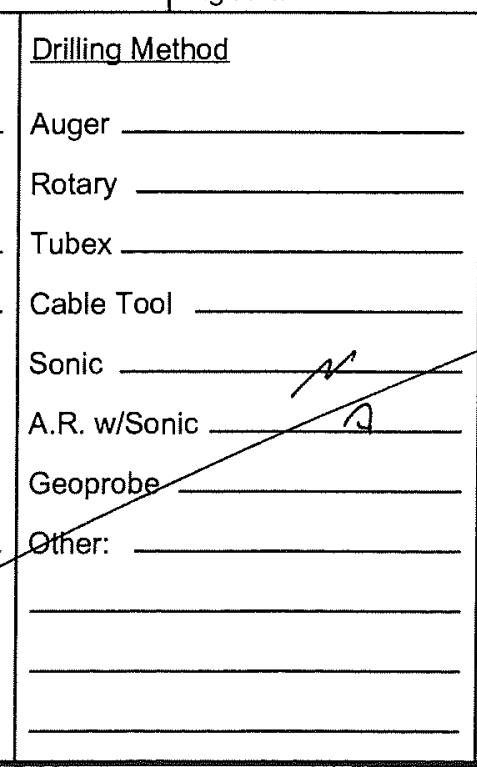

Air _ Water/Mud

Reverse — Direct

Vol: $\quad \mathrm{cfm}$

gpm

Pressure

Drill Pipe O.D.

Tool Joint Size

Additives

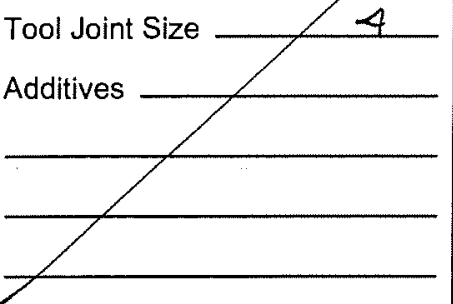

Rig Make/Mod.:

Circulation

Air __ Water/Mud

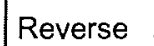

Vol:

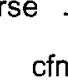

Pressure

Drill Pipe O.D.

Tool Joint Size

Additives
Make

Model

Choke

Casing Hammer

Make

Model

Bit Size

Type

Nozzles

Rod Size

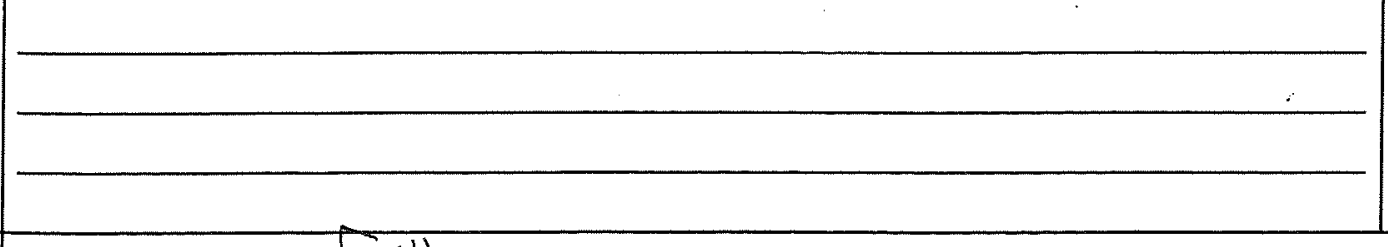

Estimated Depth to Water

$5 / 14 \%$ meas wed $34.10 \mathrm{fth} g \mathrm{~s}$

Reported By: Joe Frill,

Name/Title: Ceolo, ist, GAAMIns

Signature:

Date: $5 / 14 / 08$ 


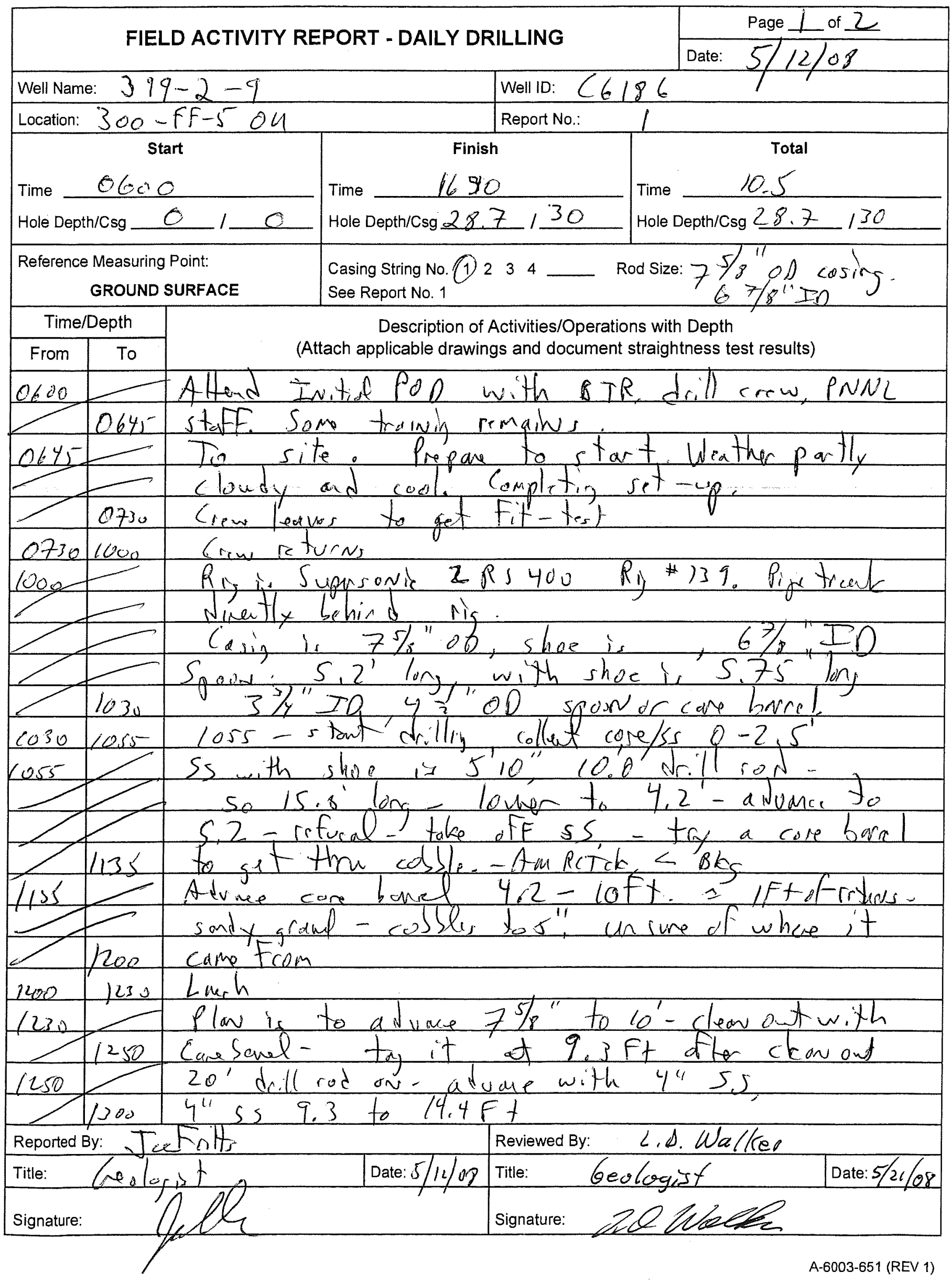

D.16 
FIELD ACTIVITY REPORT - DAILY DRILLING

Continuation Page

\begin{tabular}{l|l}
\hline Well Name: $399-2-9$ & Well ID: C 6186 \\
\hline Location: $300-F F-504$ & Continuation of Report No:
\end{tabular}

Page 2 of 2 Date: $5 / 12 / 08$

\begin{tabular}{|c|c|c|c|}
\hline Time/Depth & Description of Activities/Operations with Depth \\
\hline From & To & & D
\end{tabular}

$1300-559.4-14.4$ cothet 5 sandes in Lexon lives

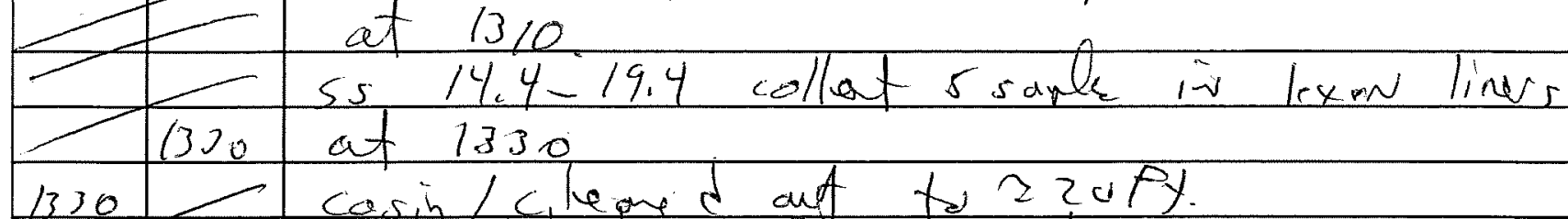

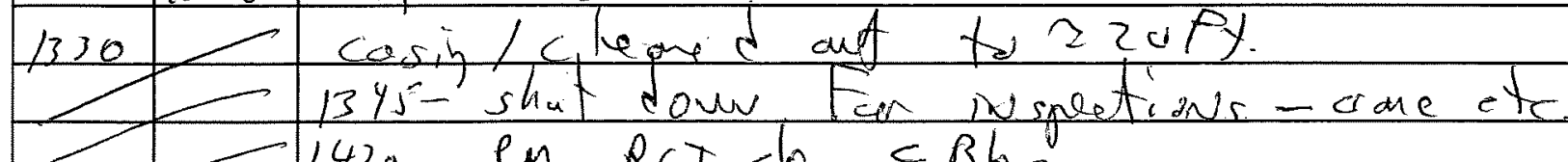

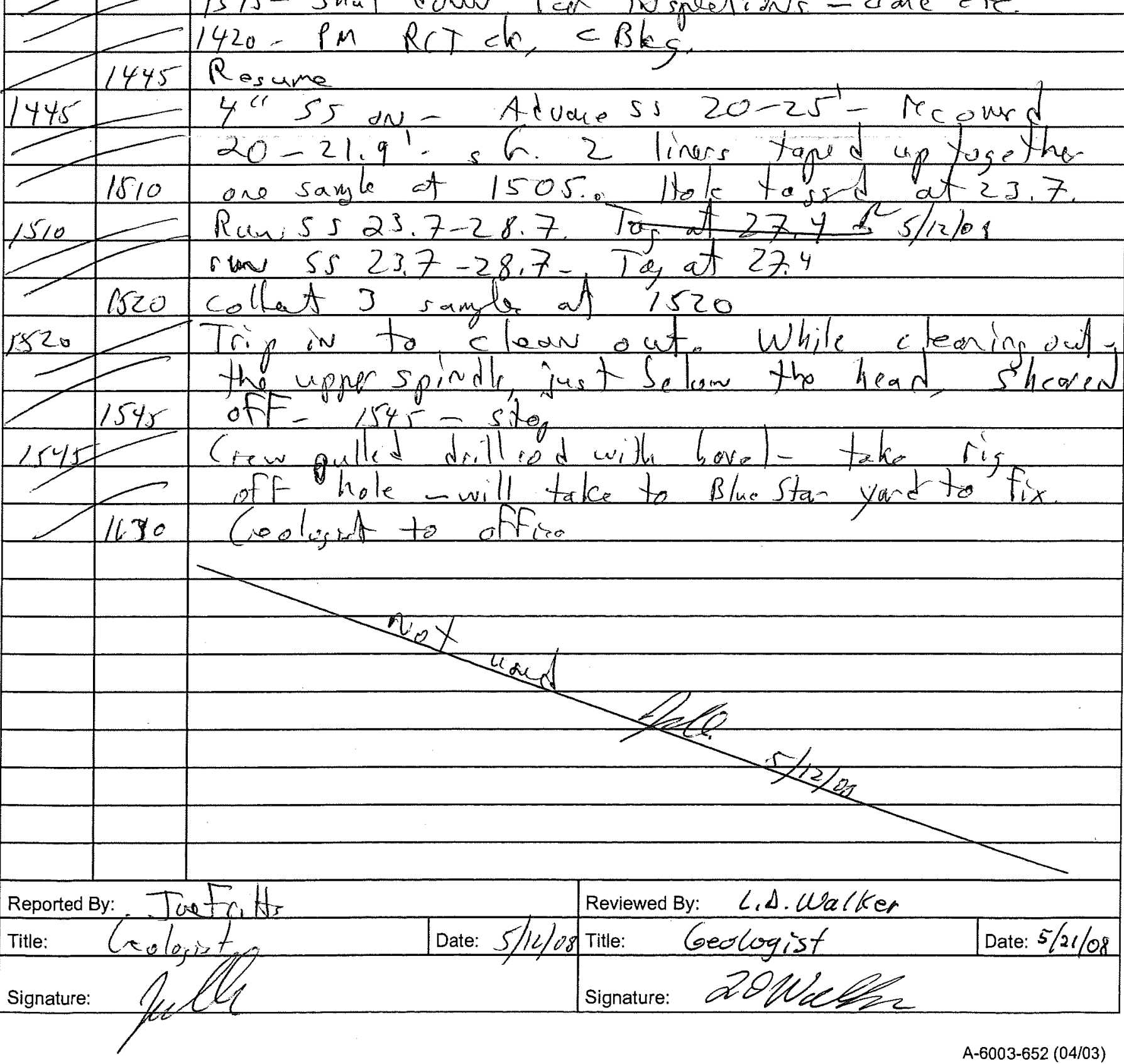




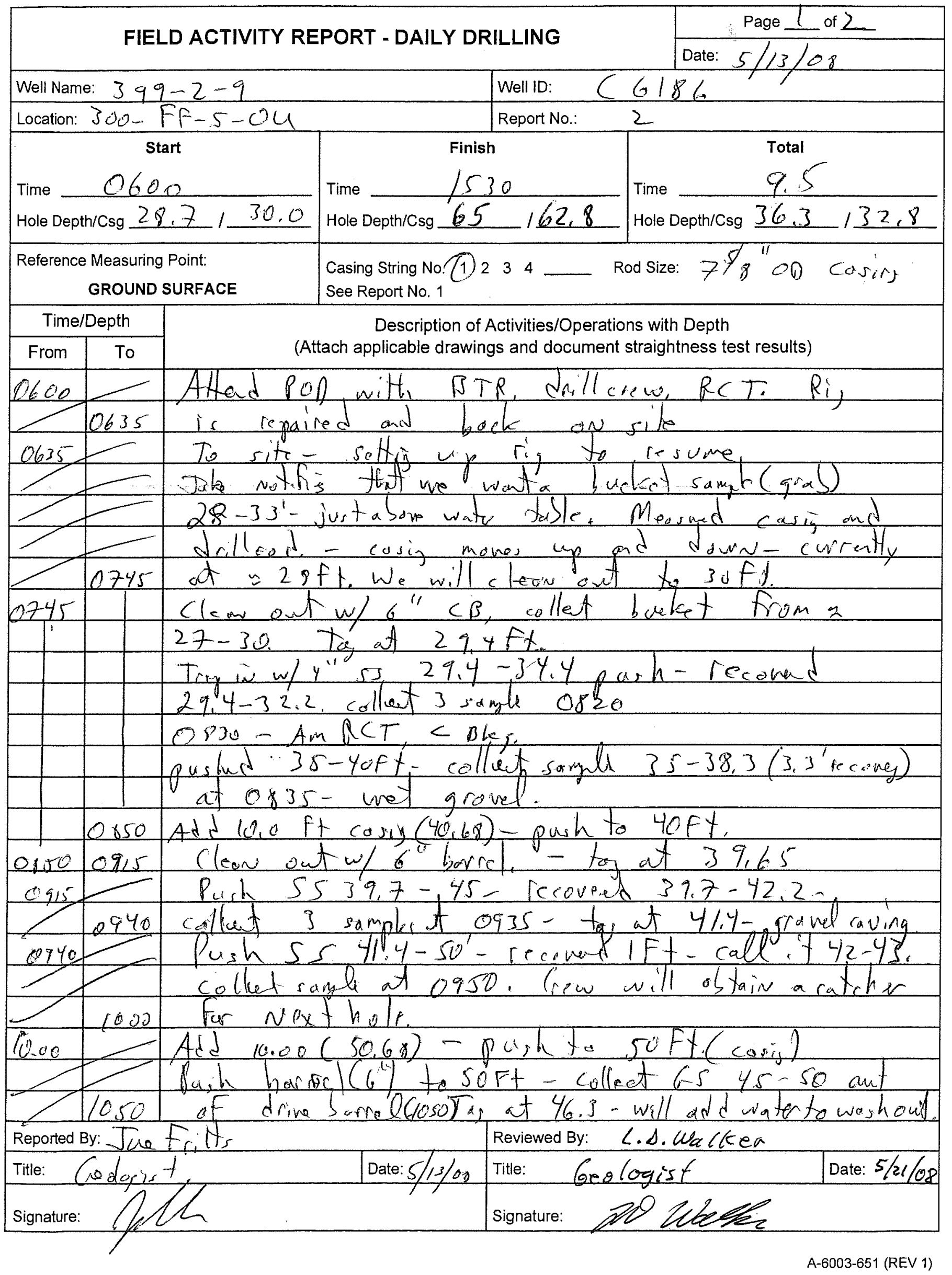

D.18 


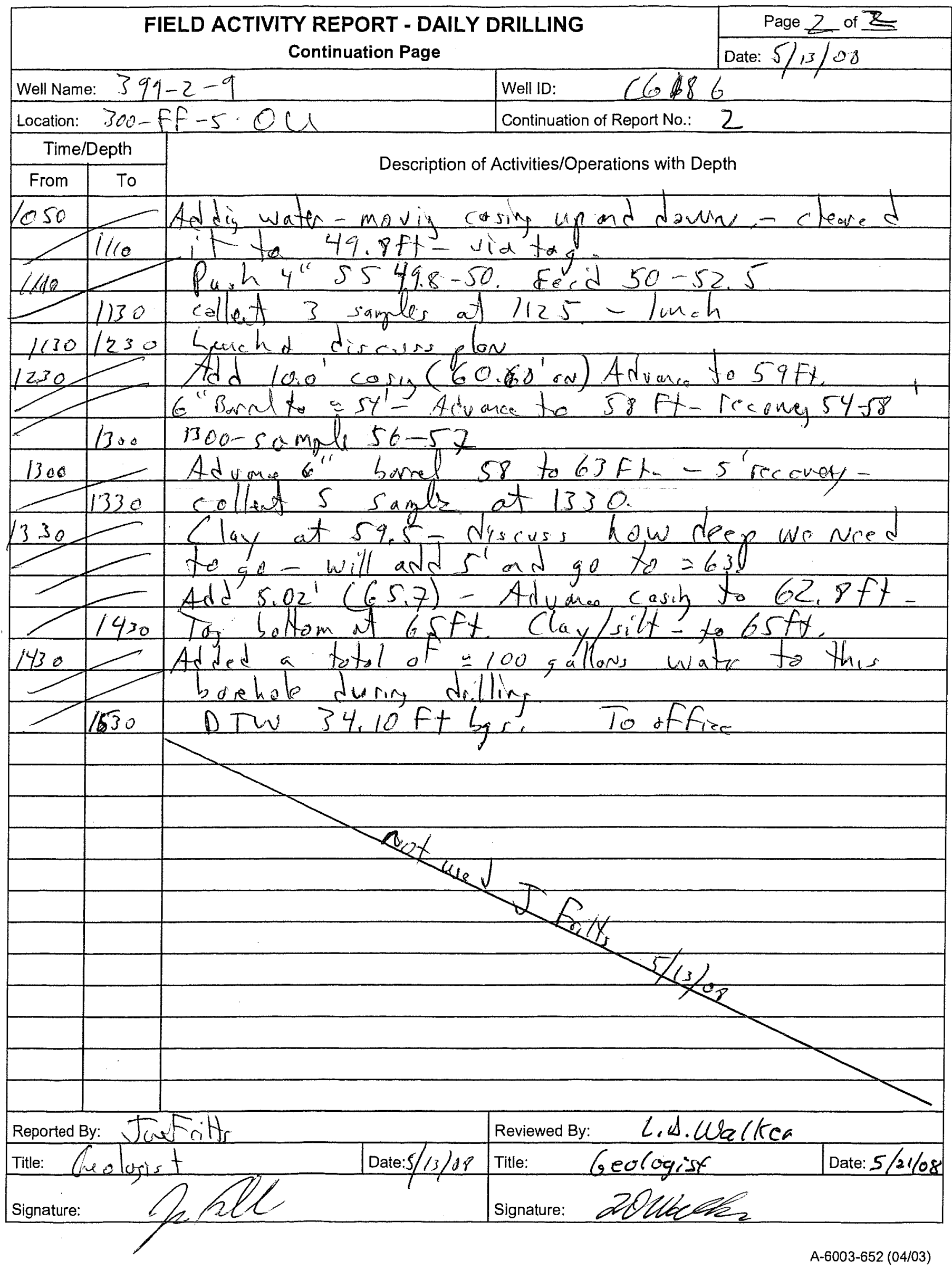

D.19 


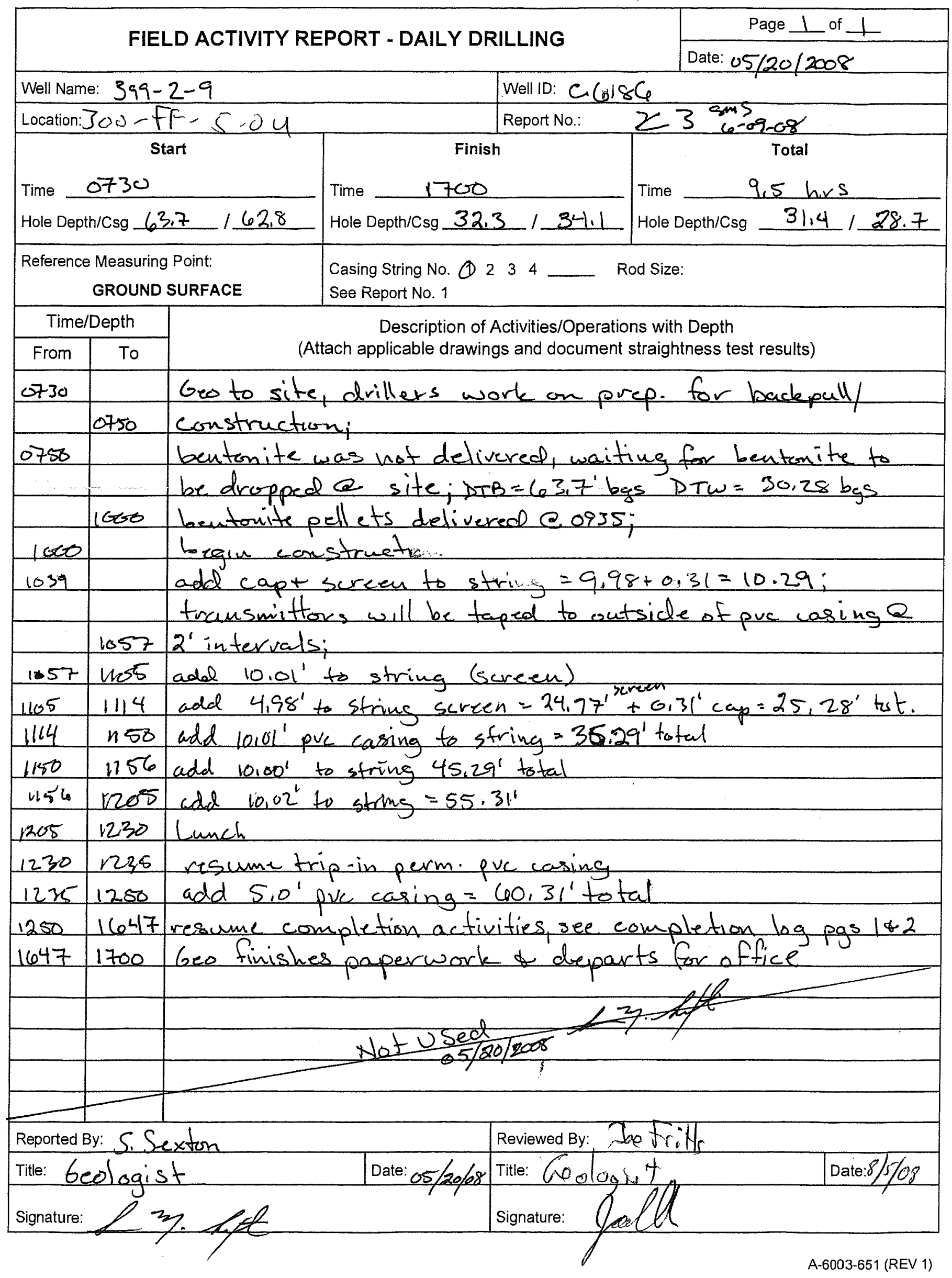




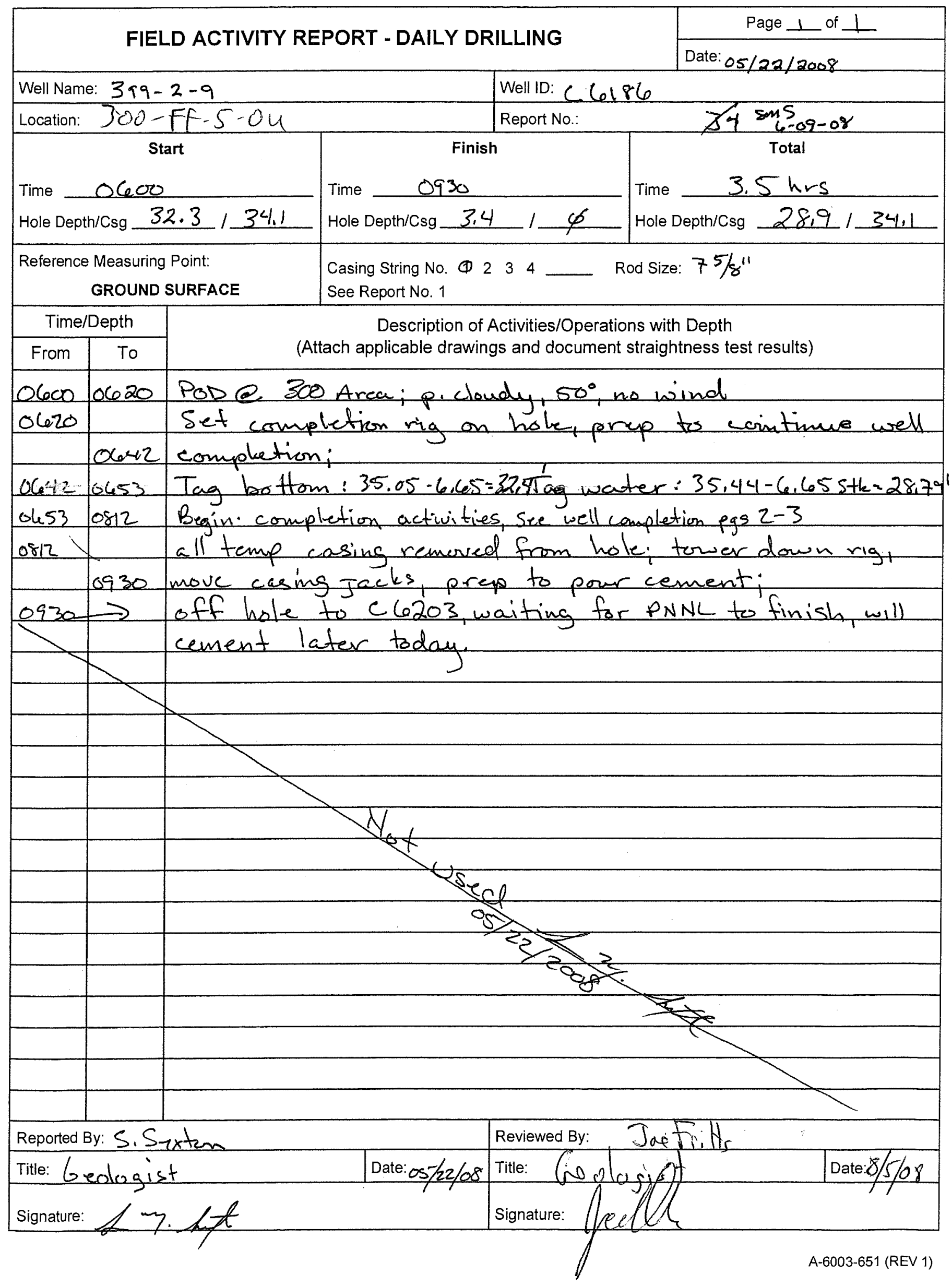




\section{FIELD ACTIVITY REPORT - DAILY DRILLING}

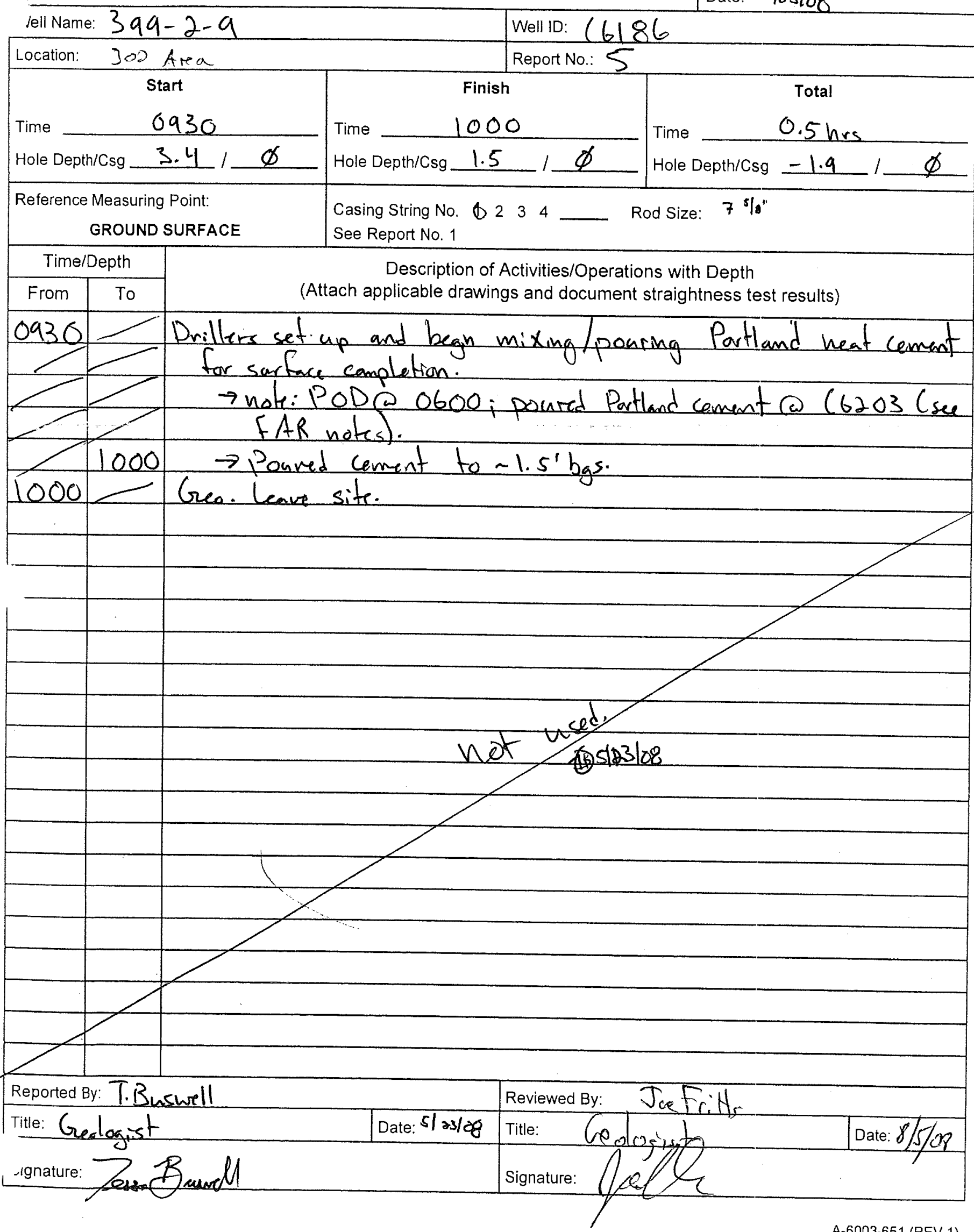




\section{FIELD ACTIVITY REPORT - DAILY DRILLING}

\begin{tabular}{|l} 
Page 1 of 3 \\
\hline Date: $6 / 4 / 08$
\end{tabular}

\begin{tabular}{l}
\hline Well Name: $399-2-9$ \\
\hline Location: $300-$ FF-5 \\
\hline Start \\
Time 0600 \\
Hole Depth/Csg \\
\hline $\begin{array}{c}\text { Reference Measuring Point: } \\
\text { GROUND SURFACE }\end{array}$
\end{tabular}

\begin{tabular}{|c|c|}
\hline & Well ID: $(61$ \\
\hline & Report No.: \\
\hline \multicolumn{2}{|c|}{ Finish } \\
\hline Time & 1630 \\
\hline \multicolumn{2}{|c|}{ Hole Depth/Csg _ _ - 1} \\
\hline $\begin{array}{l}\text { Casir } \\
\text { See }\end{array}$ & 34 \\
\hline
\end{tabular}

6

Time 10,5

Hole Depth/Csg _ 1

\begin{tabular}{|c|c|c|}
\hline \multicolumn{2}{|c|}{ Time/Depth } & $\begin{array}{r}\text { Description of Activities/Operations with Depth } \\
\text { (Attach applicable drawings and document straightness test results) }\end{array}$ \\
\hline From & To & POD 300 area \\
\hline 0630 & PO
\end{tabular}

\begin{tabular}{l|lll}
0630 & 0650 & Move to site-discuss plans w/ driller
\end{tabular}

\begin{tabular}{|l|l|}
\hline 0650 & Driller leaves site to get supplies/Geo. doesn't have notes on previons wells \\
\hline & Geo departs to retrieve notes \\
\hline 0730 &
\end{tabular}

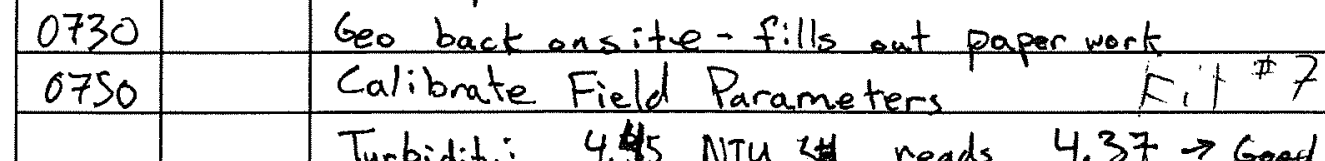

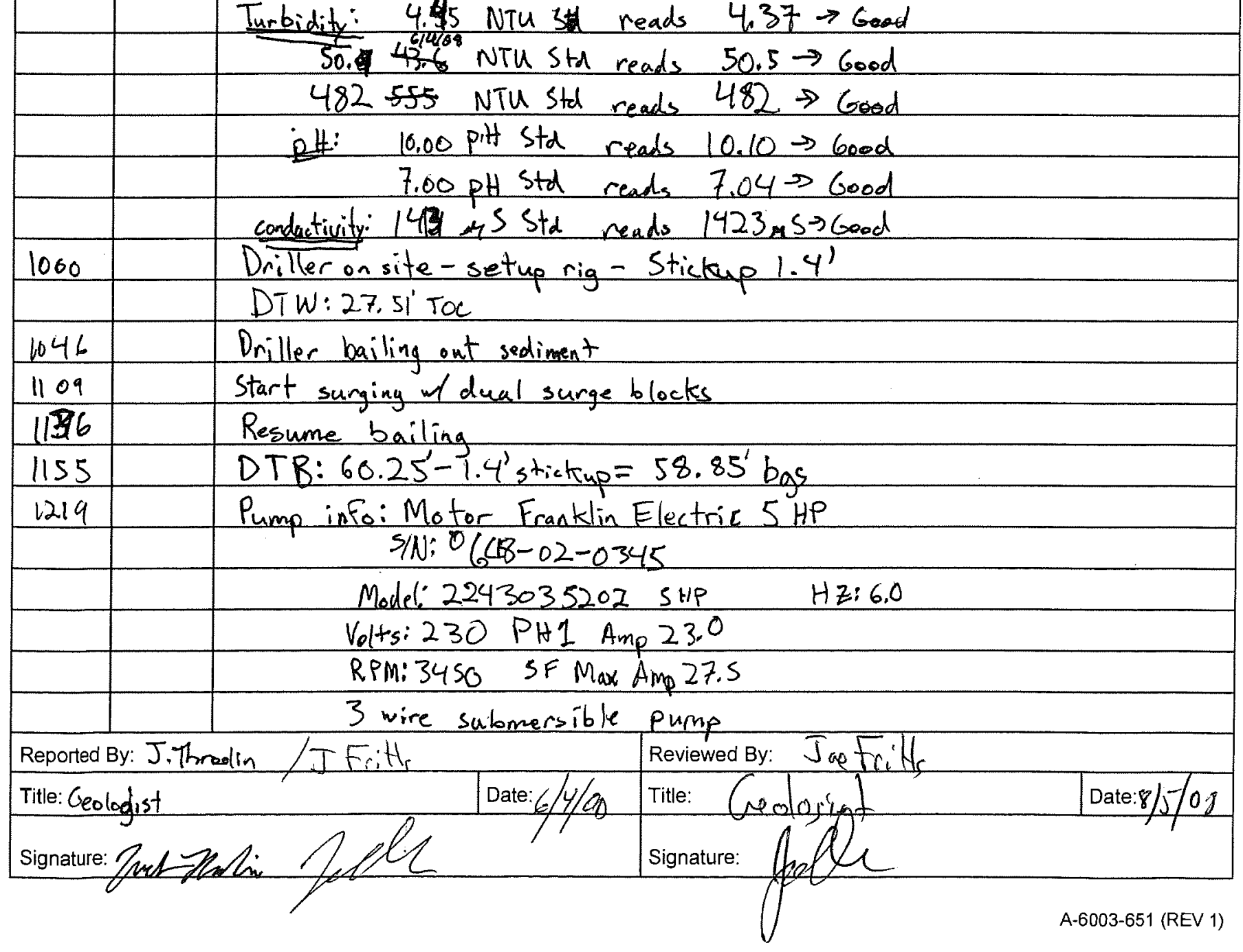




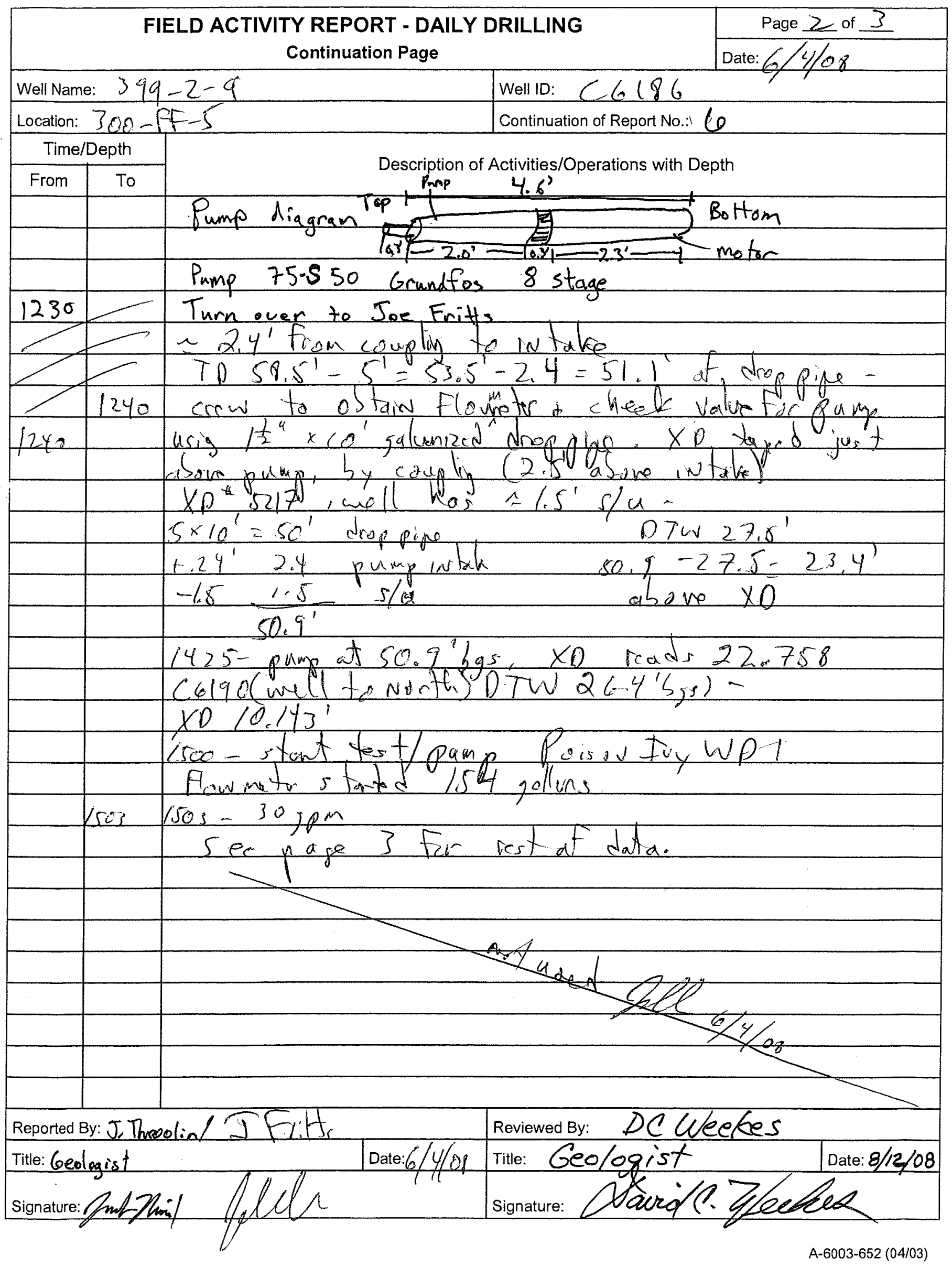

D. 24 


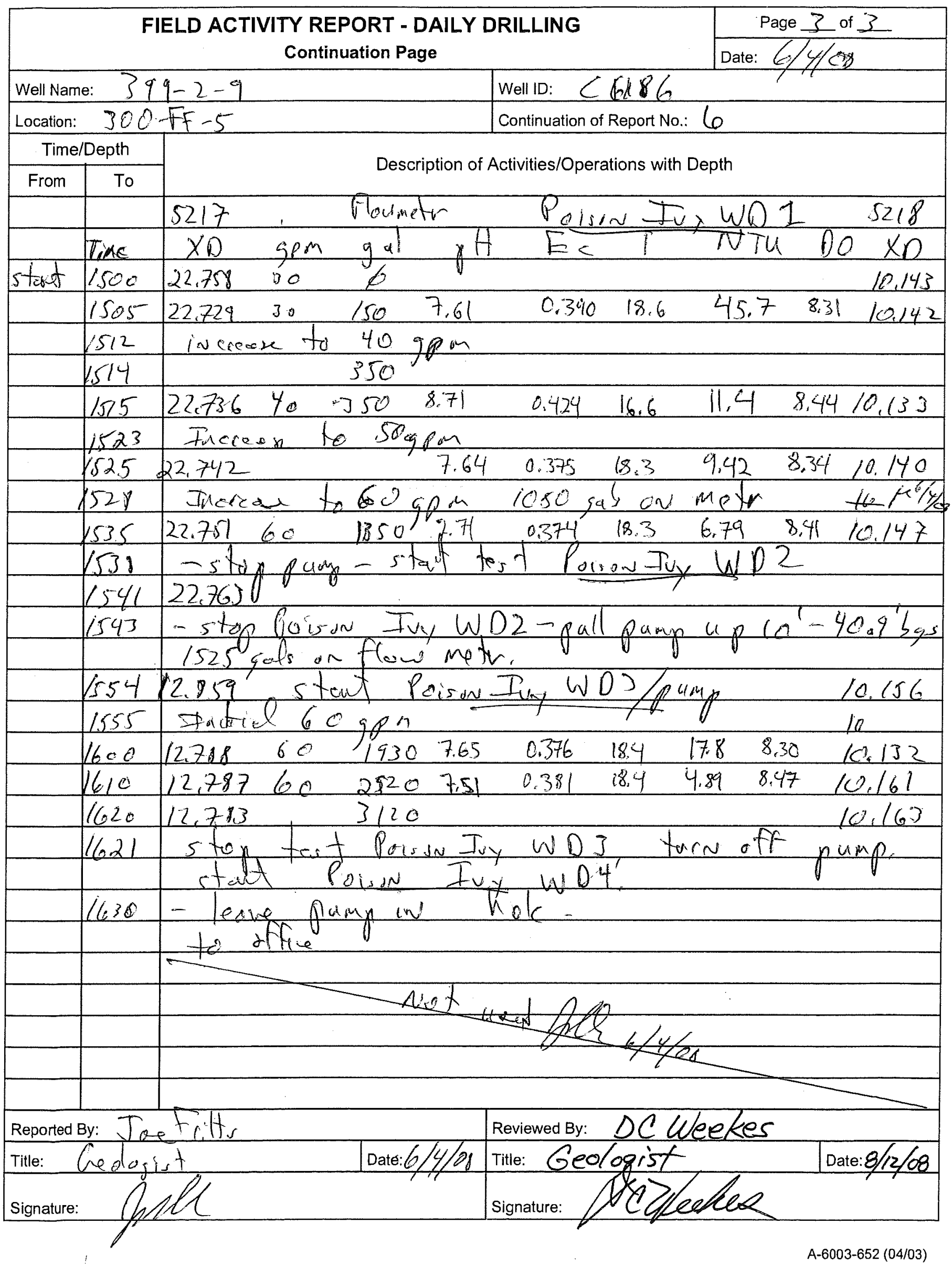

D. 25 


\section{WELL DEVELOPMENT AND TESTING DATA}

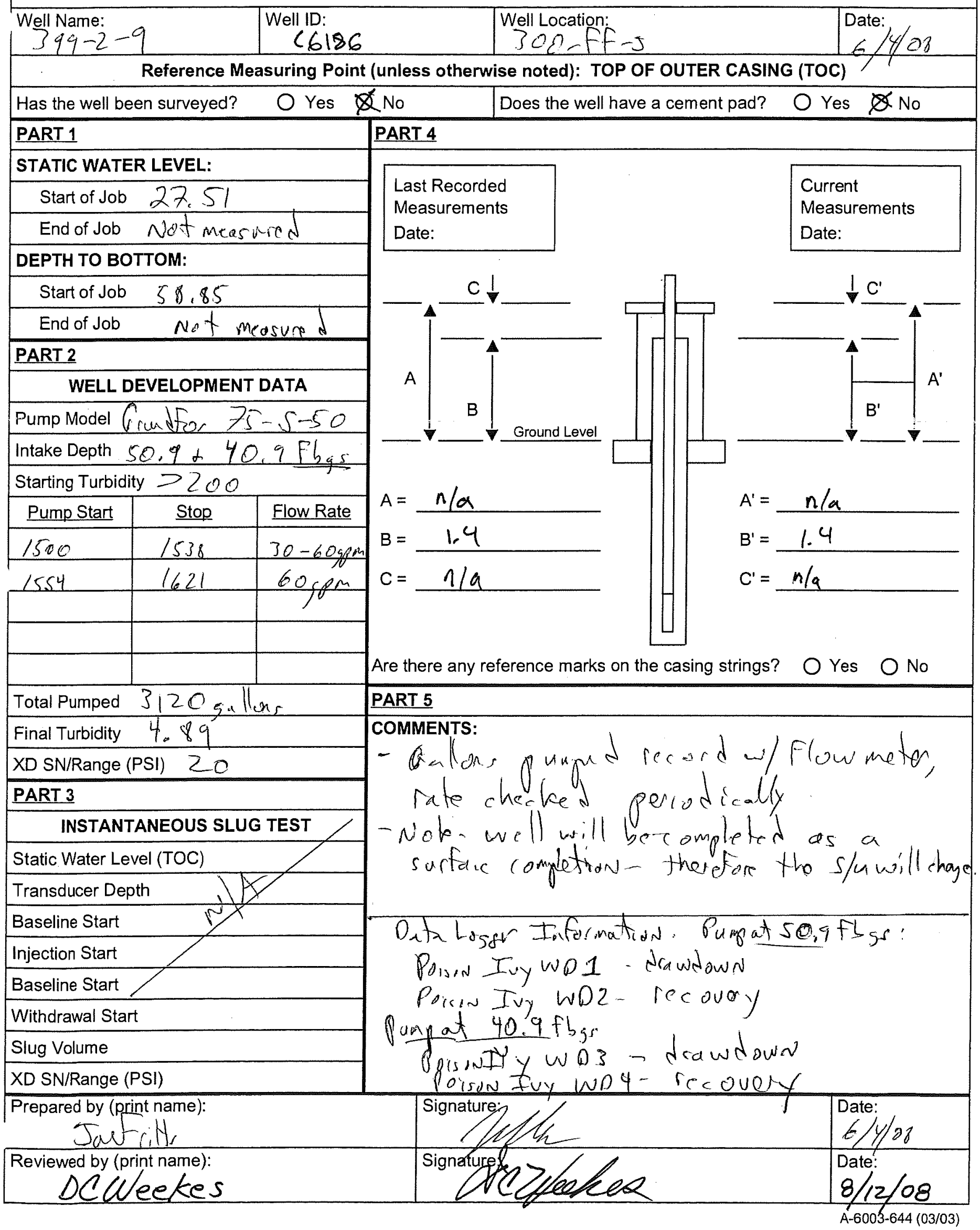


FIELD ACTIVITY REPORT NO. 1 - DRILLING PLAN

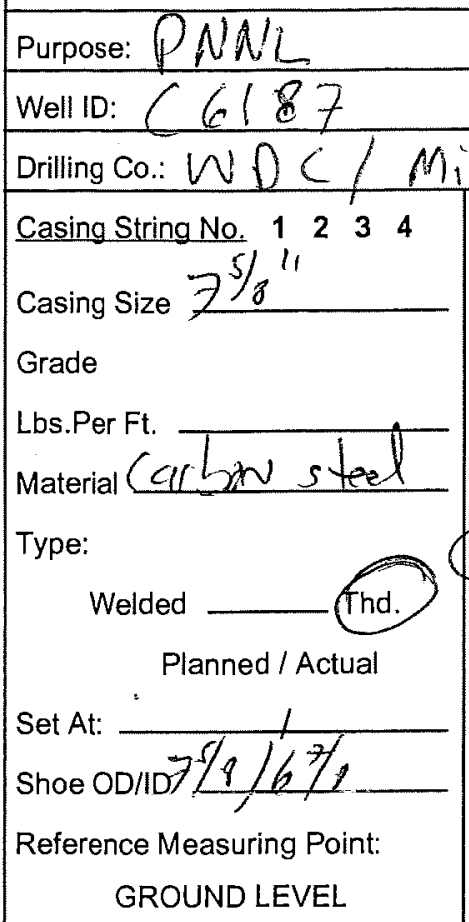

Drig. Co.

Casing String No. $\begin{array}{llll}1 & 2 & 3 & 4\end{array}$

Casing Size

Grade

Lbs.Per Ft.

Material

Type:

Welded Thd.

Planned / Actual

Set

Shoe OD/ID

Reference Measuring Point: GROUND LEYEL
300 Area Ife Location: $300-f 5-5$

Well Name: $399-2-10$

Rig No.: 139 Rig Make/Mod.: Super Sov ic Circulation

Drilling Method

Auger

Rotary

Tubex

Cable Tool Sonic Suner inis A.R. w/Sonic RS 400

Geoprobe

Other:

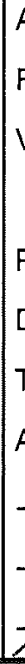

Rig No:

Drilling Method

Auger

Rotary

Tubex

Cable Tool

Sonic

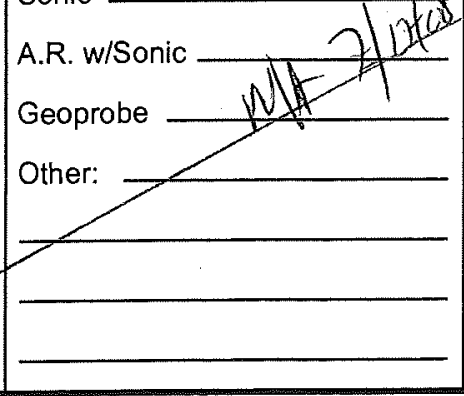

Page 1 of $\&$ $7 / 17 / 08$ 


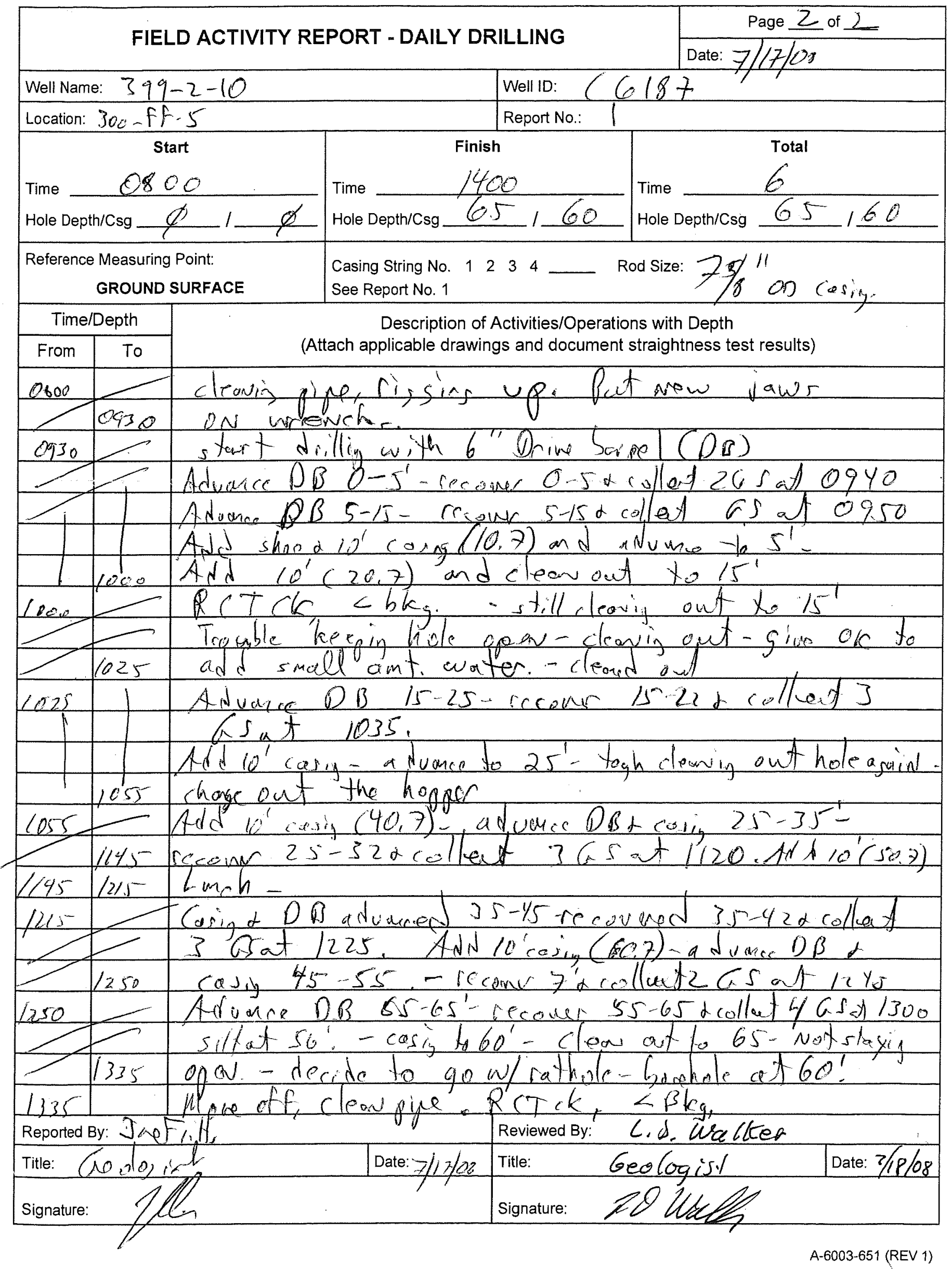

D. 28 


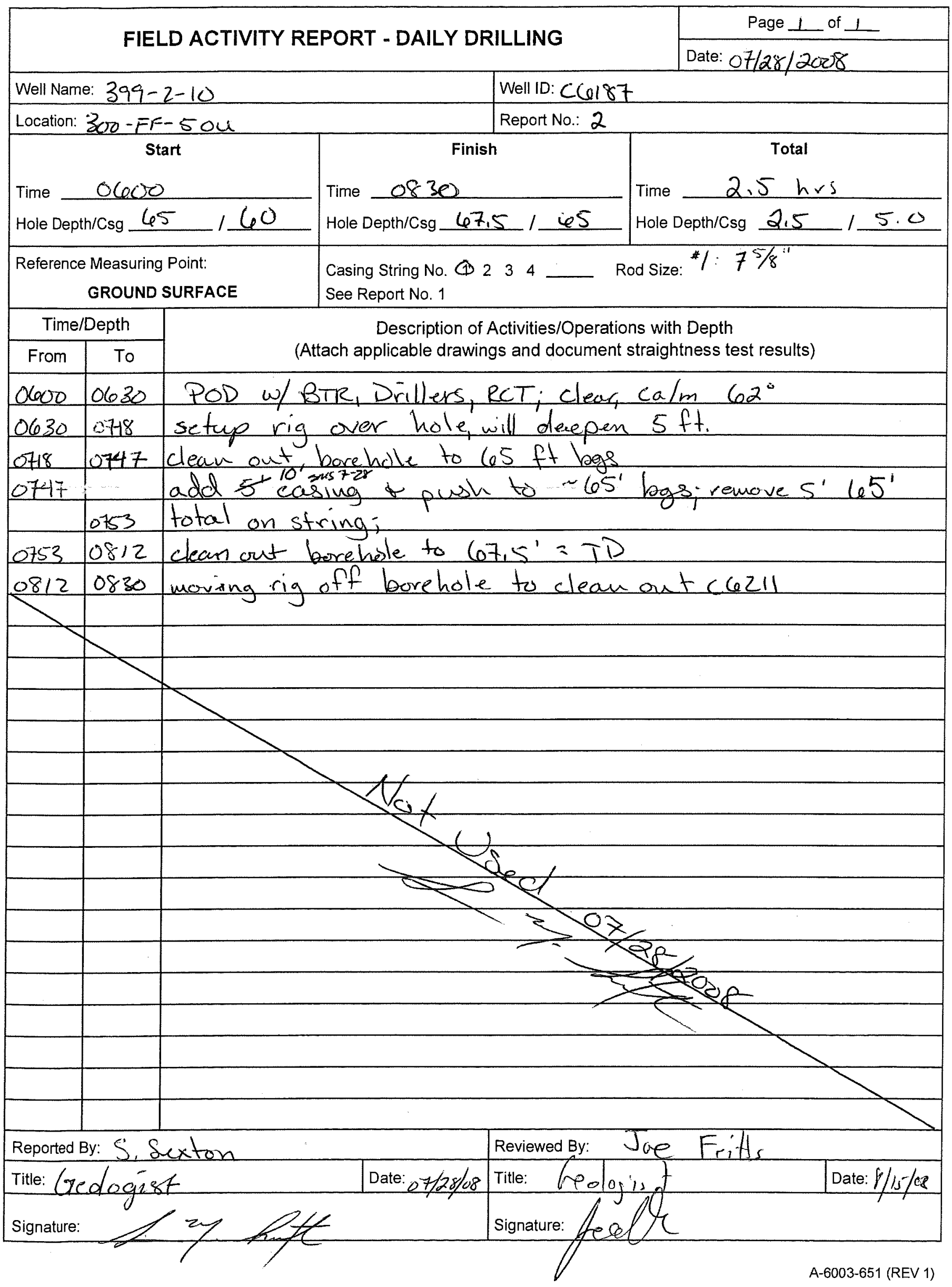

D.29 


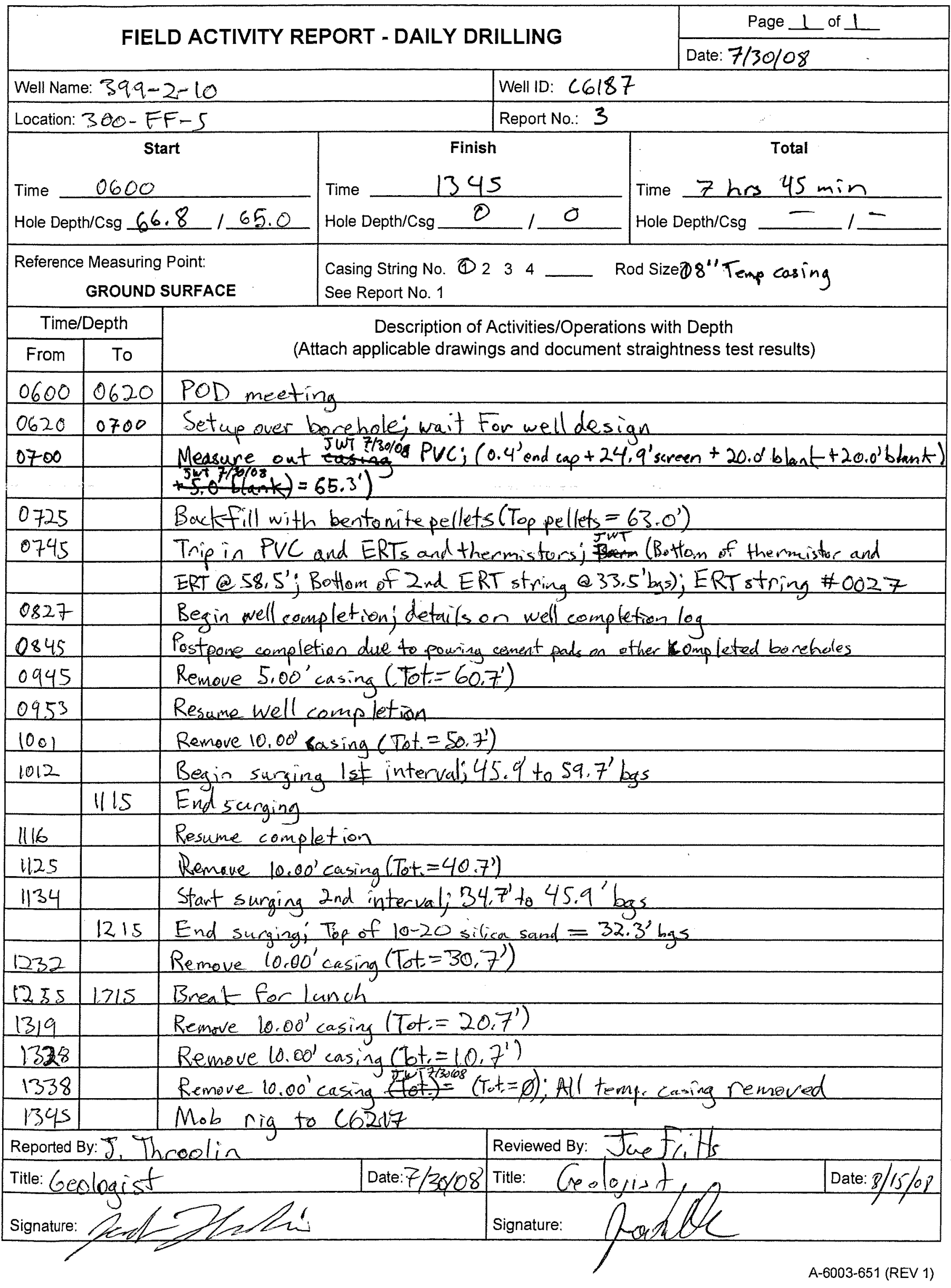

D.30 


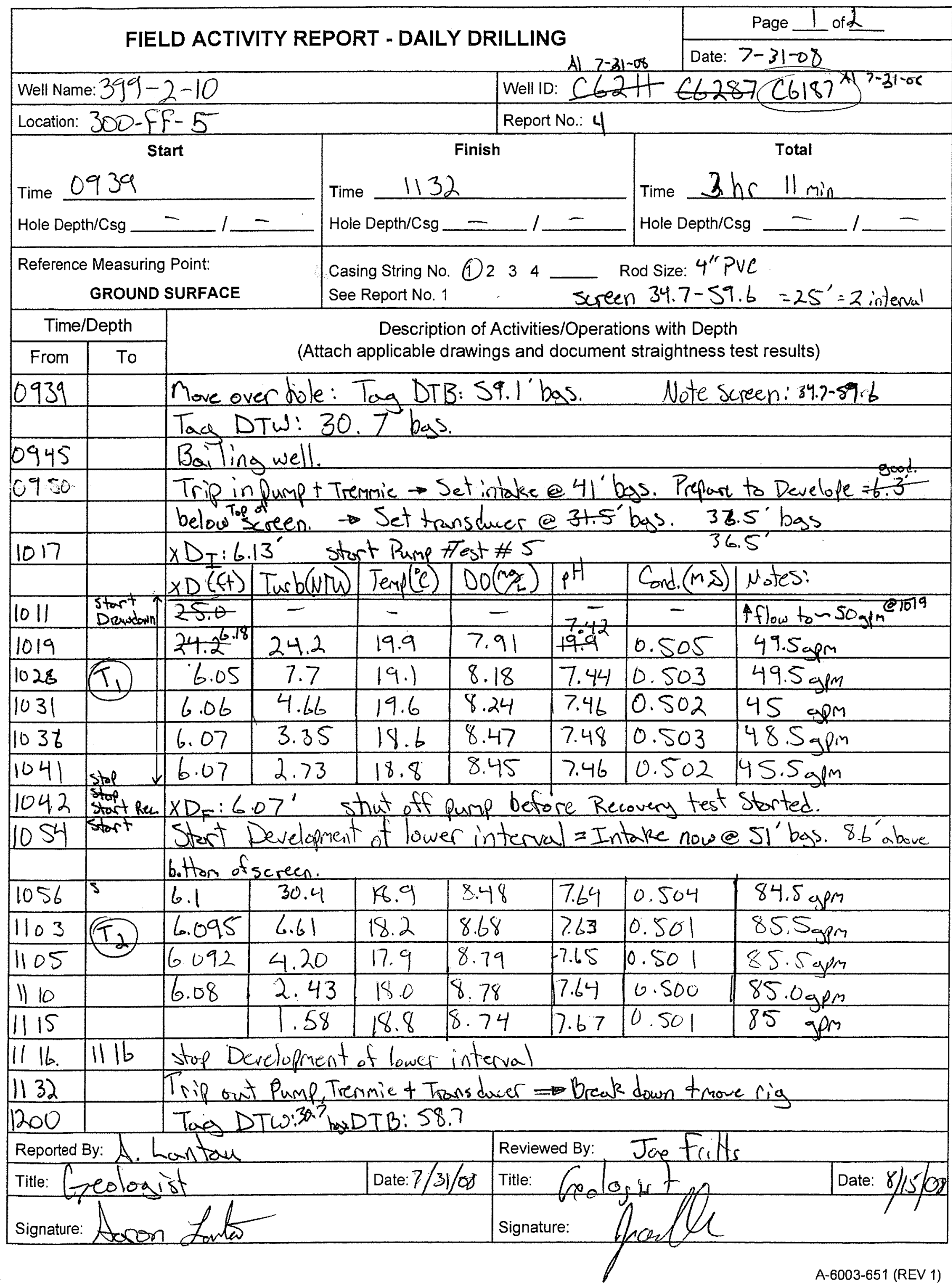




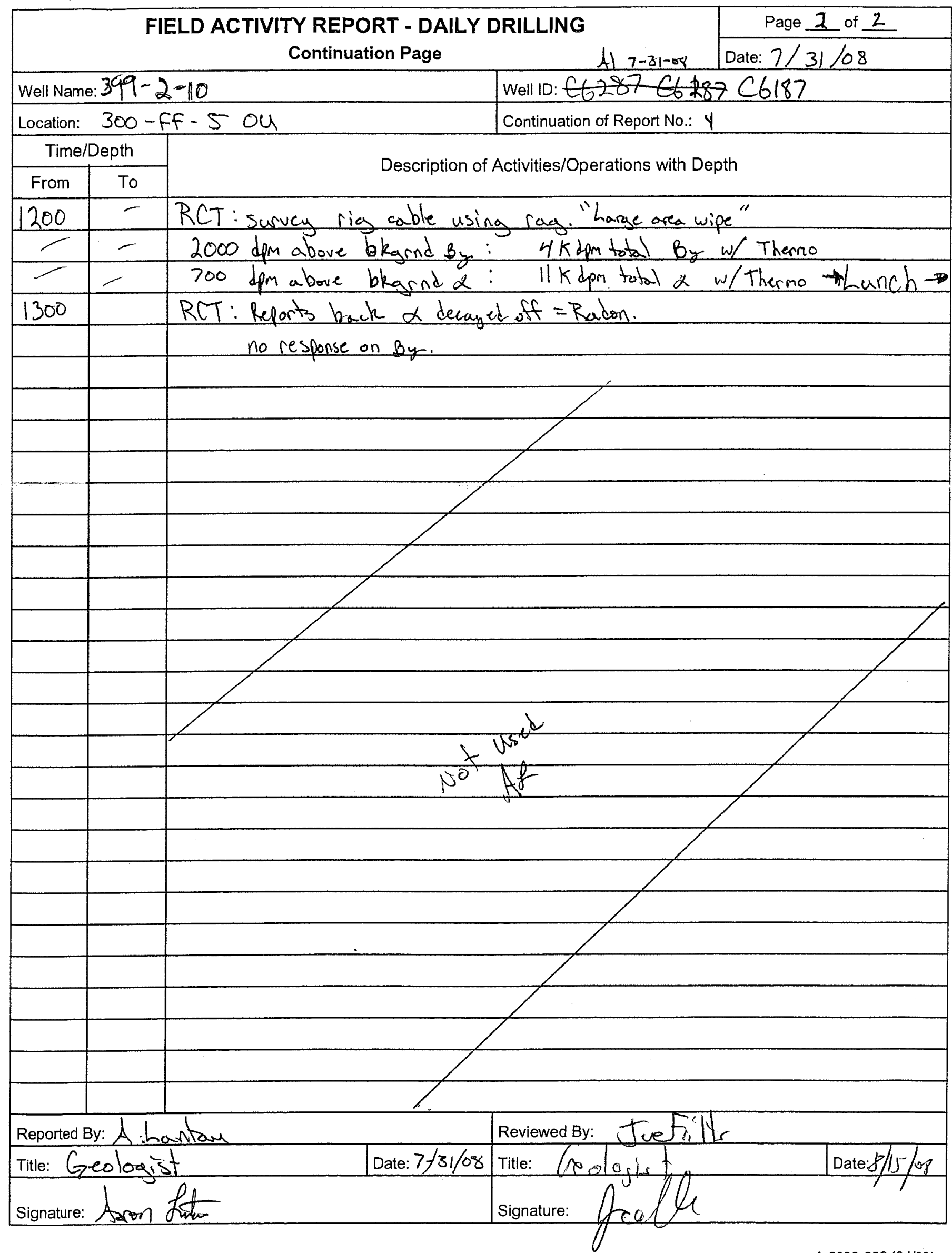




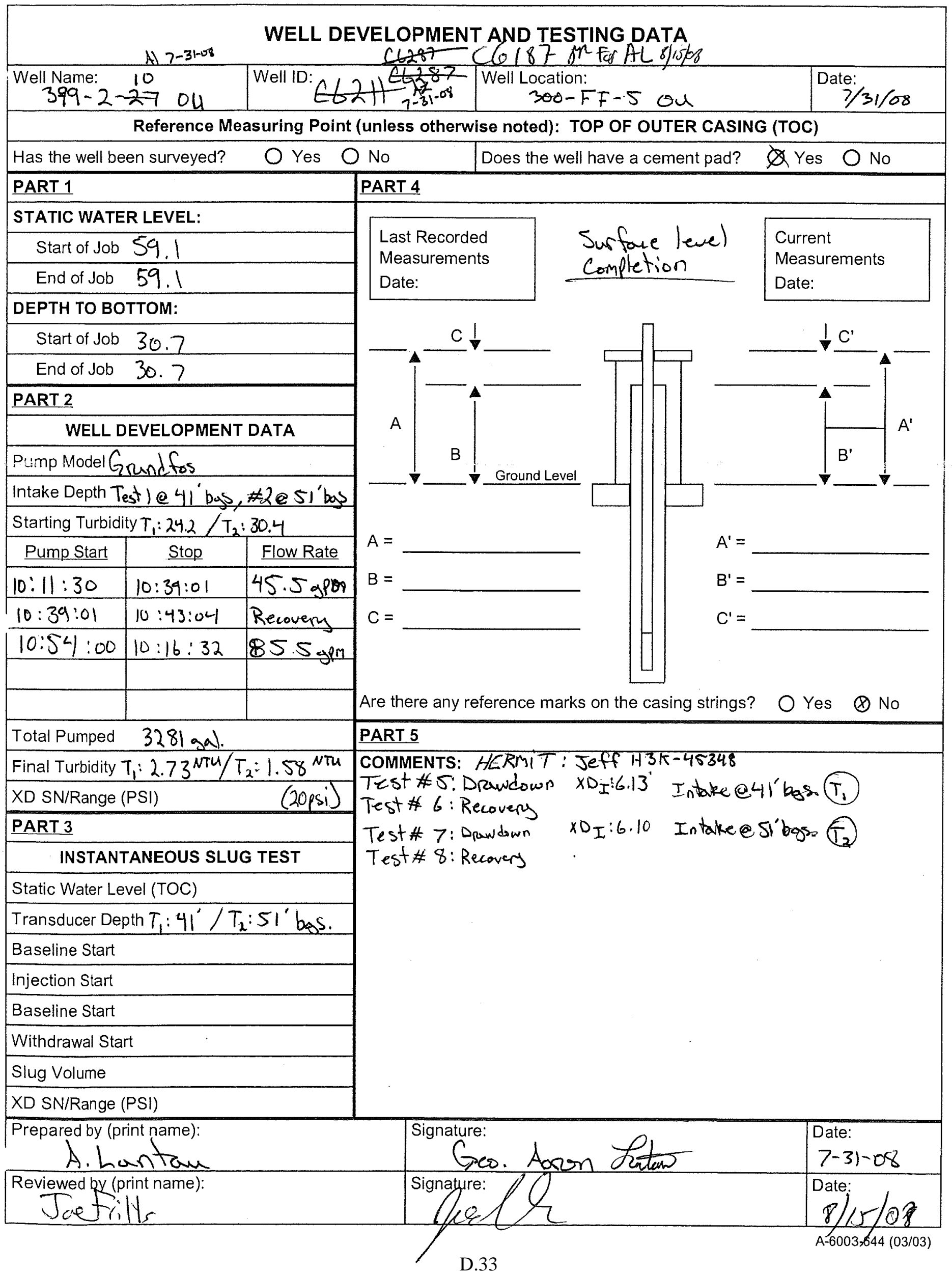


FIELD ACTIVITY REPORT NO. 1 - DRILLING PLAN

\begin{tabular}{l} 
Page 1 of 2 \\
\hline Date: 7 (fifog
\end{tabular}

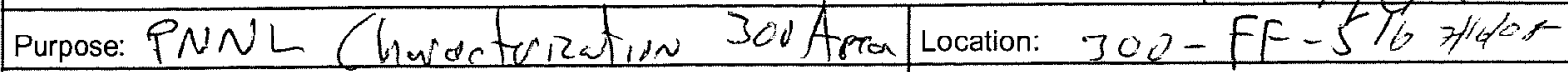

Well ID: $\quad 6189$ FFe Well Name: 394-2-11

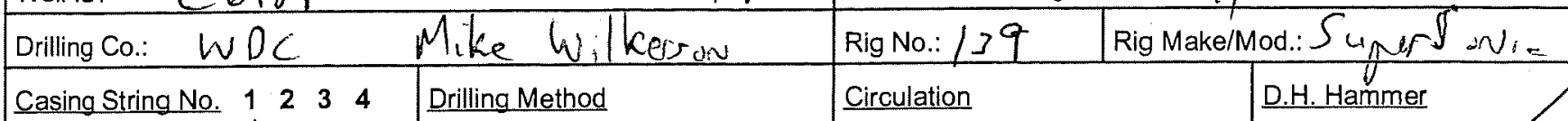

Casing Size $75 / 2$
Grade
Lbs.Per Ft.
Material Car Jnstee
Type:
$\quad$ Welded

Set At: $\frac{\text { Planned / Actual }}{\text { Shoe OD/ID }}$ t5/8/6 /
Reference Measuring Point:
GROUND LEVEL

Drig. Co.

Auger

Rotary

Circulation

Tubex

Cable Tool

Sonic Sunor 5our

Air — Water/Mud

Reverse — Direct

Vol: $\quad \mathrm{cfm}$

A.R. w/Sonic RS 400

Geoprobe

Other:

Drill Pipe O.D.

Tool Joint Size

Additives

D.H. Hammer

Casing String No. 12234

Casing Size

Grade

Lbs.Per Ft.

Material

Type:

Welded Thd.

Planned / Actual

Set

Shoe OD/ID

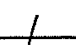

Reference Measuring Point:

GROUND LeVEL

Comments/Remarks:

Rig No.:

Drilling Method

Auger

Rotary

Tubex

Cable Tool

Sonic

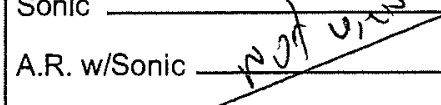

Geoprobe

other:

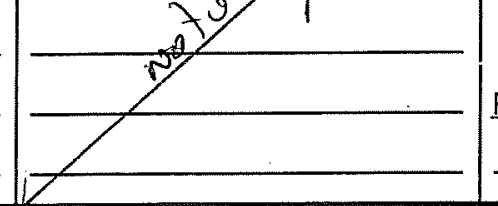

Rig Make/Mod.:

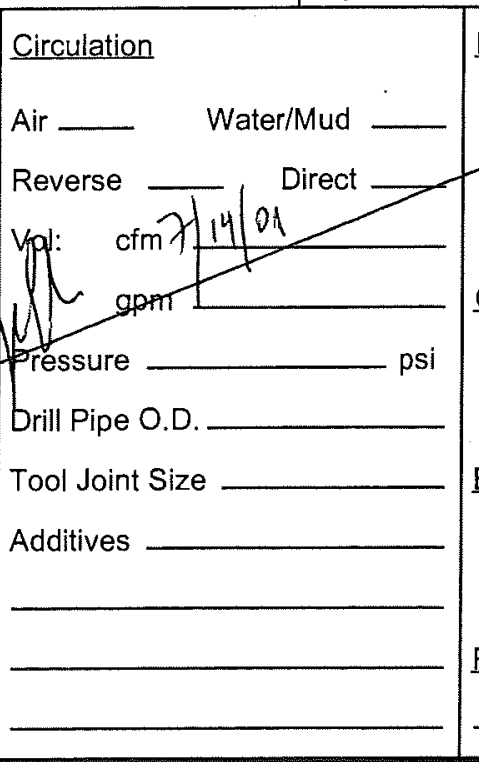

Estimated Depth to Water

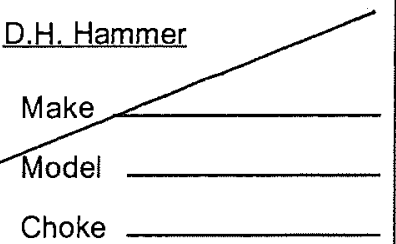

Casing Hammer

Make

Model

Bit Size

Type

Nozzles

Rod Size

Reported By: Jeec ti.t.

NamerTitle: CRAph Ins / Gojogt

Signature:

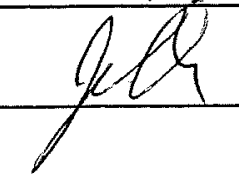

Date

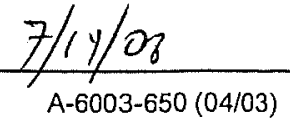




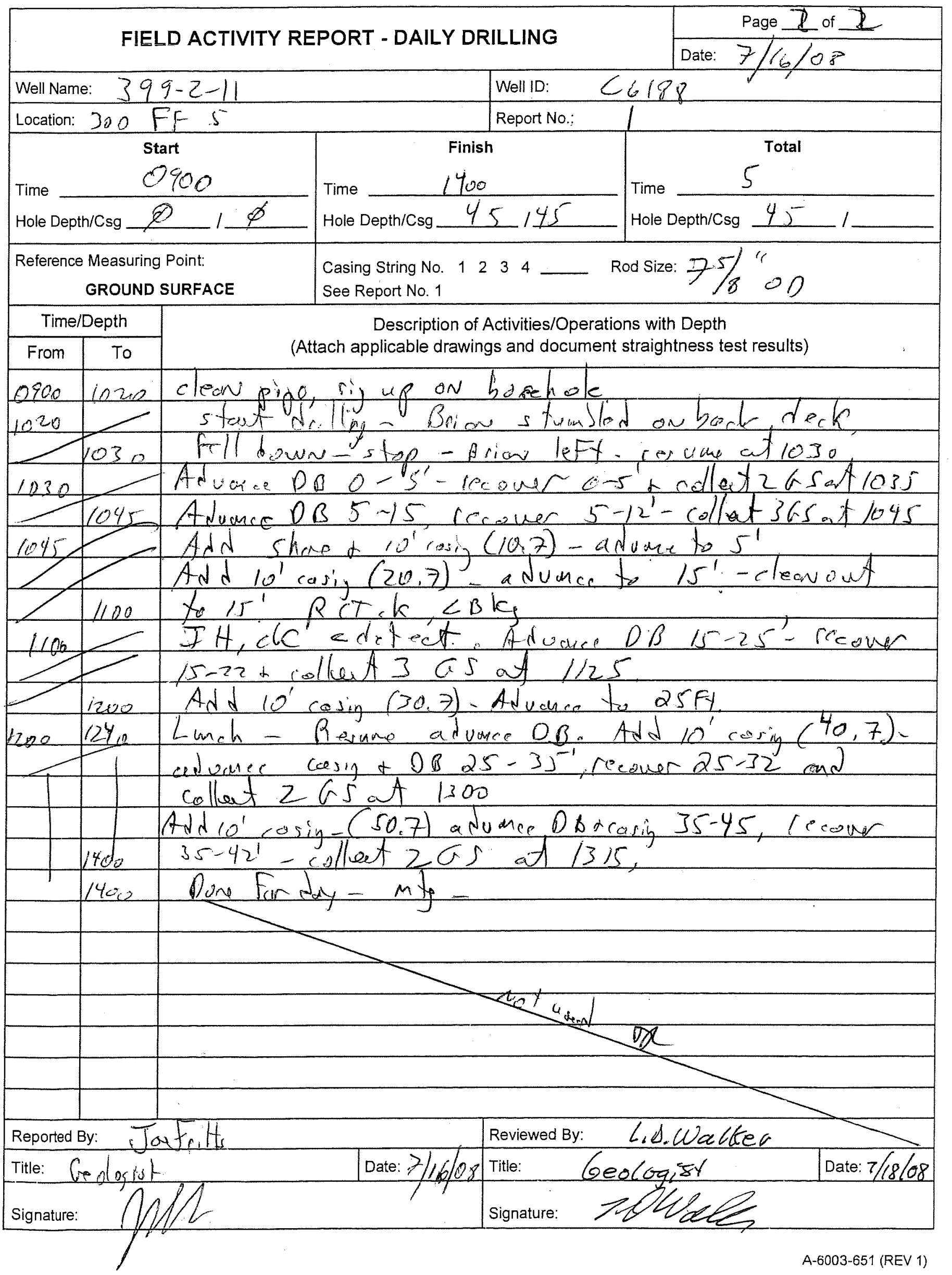

D.35 


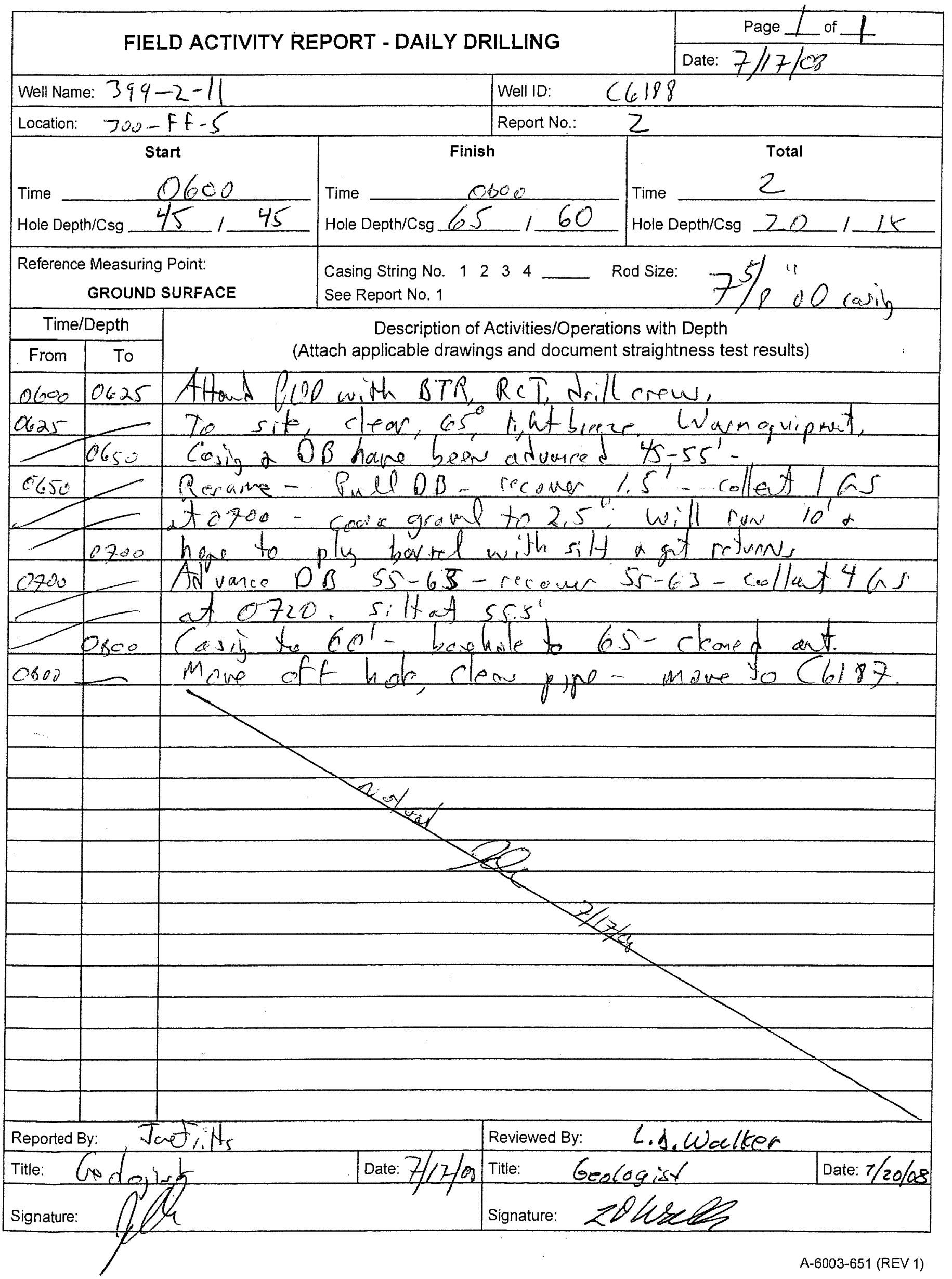

D.36 


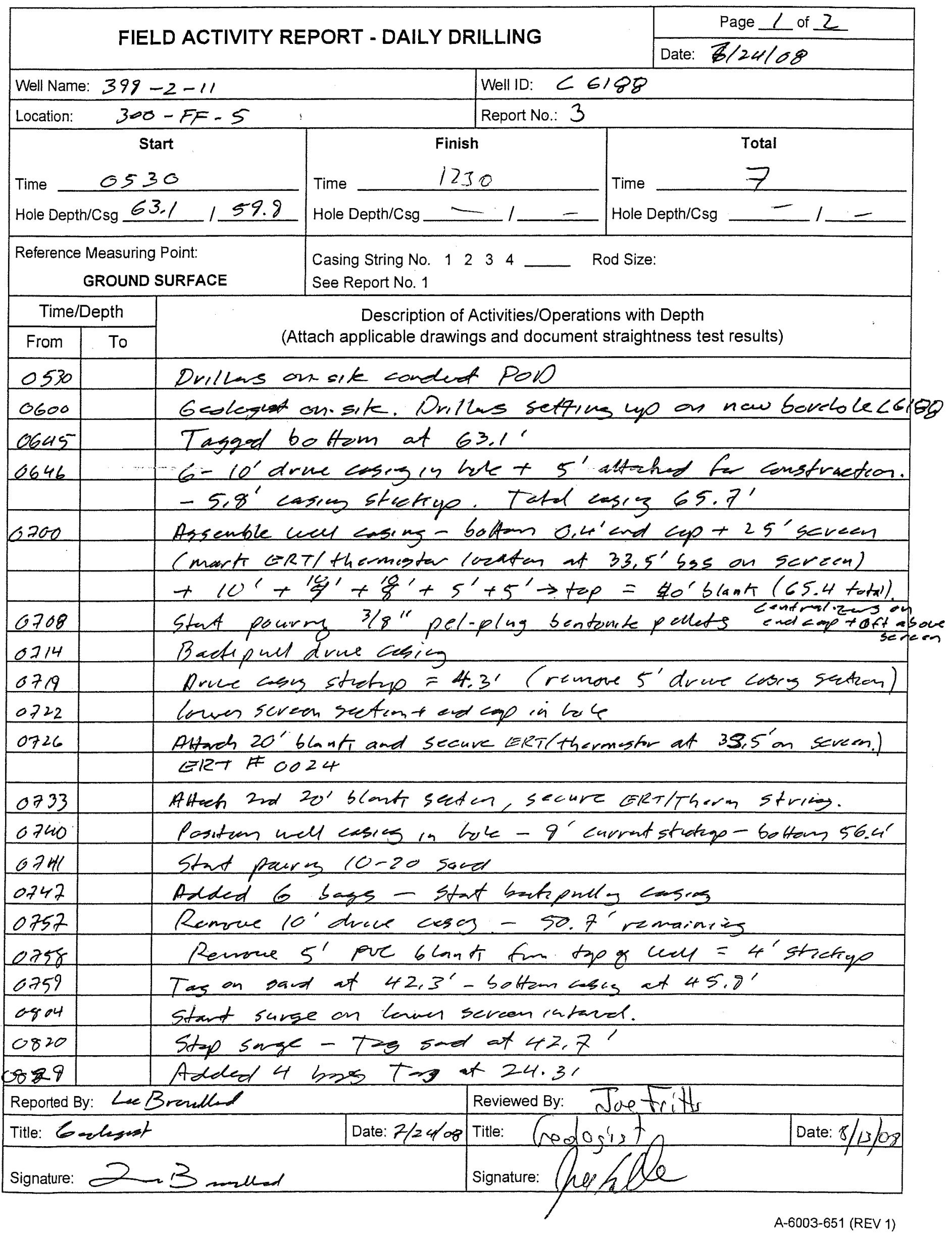

D.37 


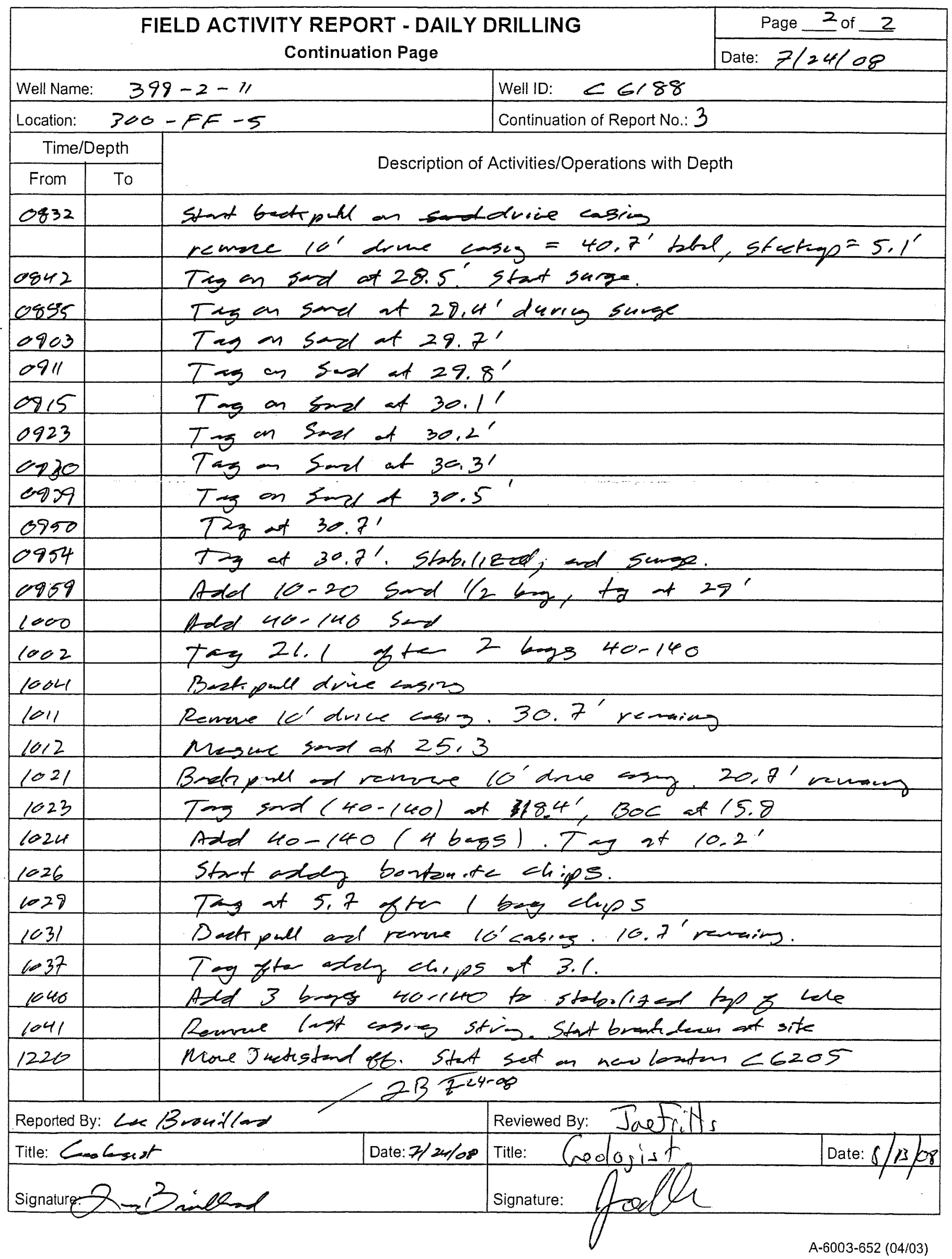

D.38 


\section{FIELD ACTIVITY REPORT - DAILY DRILLING}

\begin{tabular}{|c|} 
Page 1 of 1 \\
Date: $8-1-08$
\end{tabular}

Well Name: $399-2-4$

Location: $300-F F-5$ OM

Start

Time 1230

Hole Depth/Csg $56.5 \%$

Reference Measuring Point:

GROUND SURFACE
Well ID: C6188

Report No.: 4

Finish

Hole Depth/Csg _ _ _ _ _ Hole Depth/Csg _ _ $/ 2$

Casing String No. $\begin{array}{lllll}1 & 2 & 3 & 4\end{array}$

See Report No. 1

Rod Size:

Screen : $31-56$

Description of Activities/Operations with Depth

(Attach applicable drawings and document straightness test results)

Total

Time $3 \mathrm{hr} 20 \mathrm{~min}$
Time 1450

Time/Depth

\begin{tabular}{|c|c|}
\hline From & To \\
\hline 1230 & \\
\hline
\end{tabular}

Mave over hole. Tag: DTB: 56.5 bogs DTw:30.1 bas.

Trip in pump \& Tremie. Set intake e 41.3' bgs.

Tansduver e 37. 3' bys.

$1315 \quad$ Stort Drowdown Test Tanscuuer C $37.3 \mathrm{bgs}$.

$\times D_{I}: 6.69$

\begin{tabular}{|c|c|c|c|c|c|c|c|c|}
\hline & & $X D(f)$ & Turb(NTu) & Temploc & DO(ng) & Cond. $(m)$ & $\rho H$ & Notes: \\
\hline 315 & & 6.65 & 43.3 & 22.0 & 8.31 & 0.505 & 7.68 & 50 \\
\hline 35 & & 6.63 & 11.2 & 20.0 & 8.31 & 0.505 & 7.60 & 50 \\
\hline 30 & & 6.65 & 5.74 & 19.0 & 8.46 & 0.505 & 7.75 & "l \\
\hline 35 & & 6.64 & 2.79 & 18.3 & 8.60 & 0.504 & 7.55 & \\
\hline 40 & & 6.63 & 2.05 & 18.3 & 8.54 & 0.503 & 7.55 & $50 \mathrm{gp}$ \\
\hline 45 & & 6.64 & 1.28 & 18.4 & 8.62 & 0.506 & 7.57 & 11 \\
\hline 350 & & .64 & 0.85 & 18.2 & 8.59 & 0.507 & 7.52 & \\
\hline
\end{tabular}

Start recovery on Test \#2 contractor lifted probe momentas, ly.

1417 lower intake of parp to $51.0^{\prime}$ bgs + Start Development of lower interal.

\begin{tabular}{|l|l|l|l|l|l|l|l|}
\hline 1418 & 6.70 & 123 & 20.0 & 8.42 & 0.502 & 7.62 & $87 \mathrm{gpm}$ \\
\hline 1423 & 6.70 & 27.4 & 18.6 & 8.78 & 0.554 & 7.64 &
\end{tabular}

\begin{tabular}{|l|l|l|l|l|l|l|l|}
\hline 1425 & & 6.710 & 16,5 & 18,4 & 9,00 & 0,506 & 7.67 \\
\hline 1427 & 1430 & 6.698 & 6.29 & 18.2 & 9,00 & 0,504 & 7,67 \\
\hline
\end{tabular}

14301438 pump shut offe 1430 , begin recovery test

\section{Pull pumpt Tremrie. Al zepos \\ 1453 Tas DTB:57.5 bas DTW: 30.530 .1 -Move to next well.} 1450 RCT's on site to check cable on pump rig. Readings: SBkgnd w/Theoro

Reported By: A. Lantau

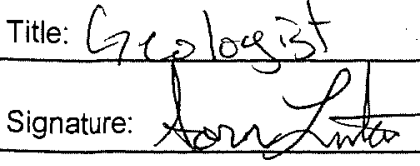

Date: $f / 3 / 0 \mathrm{~s}$

A-6003-651 (REV 1) 


\section{WELL DEVELOPMENT AND TESTING DATA}

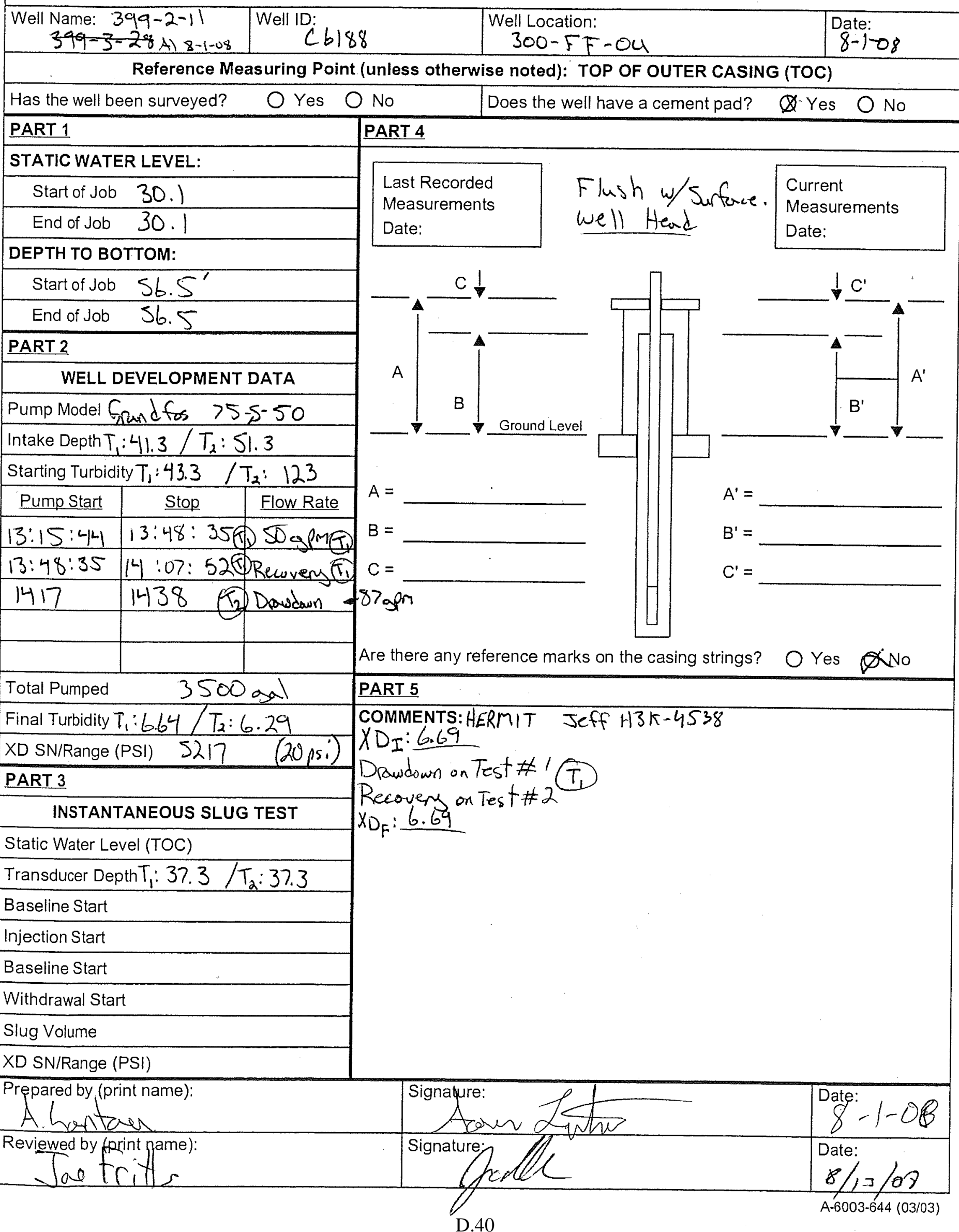


FIELD ACTIVITY REPORT NO. 1 - DRILLING PLAN

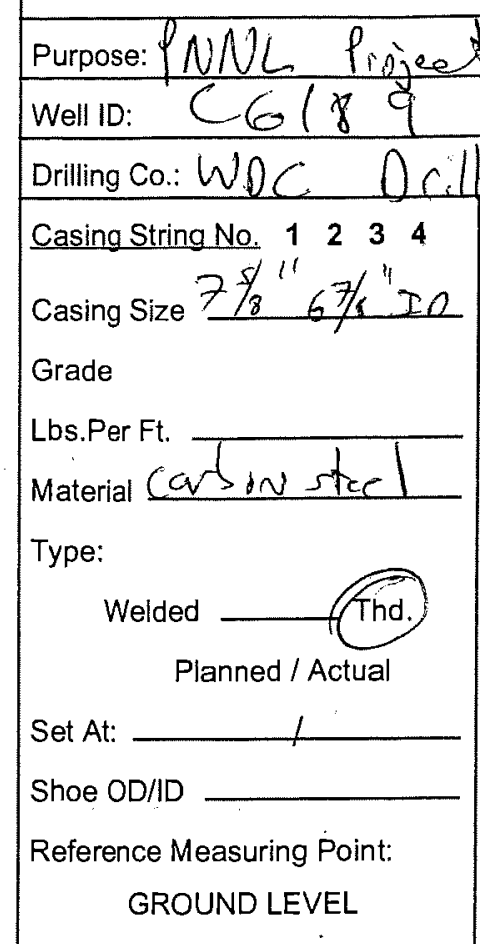

Drig. Co.

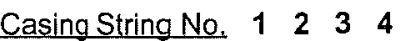

Casing Size

Grade

Lbs.Per Ft.

Material

Type:

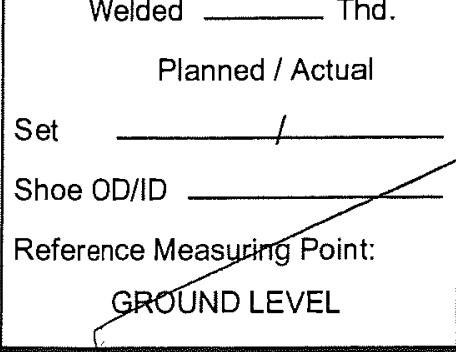

Commenta/Remarks:
Location: $300-F F-5^{\prime}$ Well Name: $399-2-12$ Rig No.: $\int 79$ Rig Make/Mod.: Super. Sonic \begin{tabular}{l|l}
\hline Circulation & D.H. Hammer
\end{tabular} Drilling Method Auger Rotary Air Tubex Cable Tool Sonic A.R. whonic) Surer sovir2 Geoprobe Rs 400 Other:

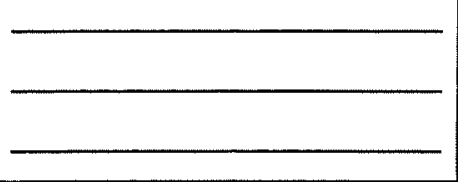

Rig No.
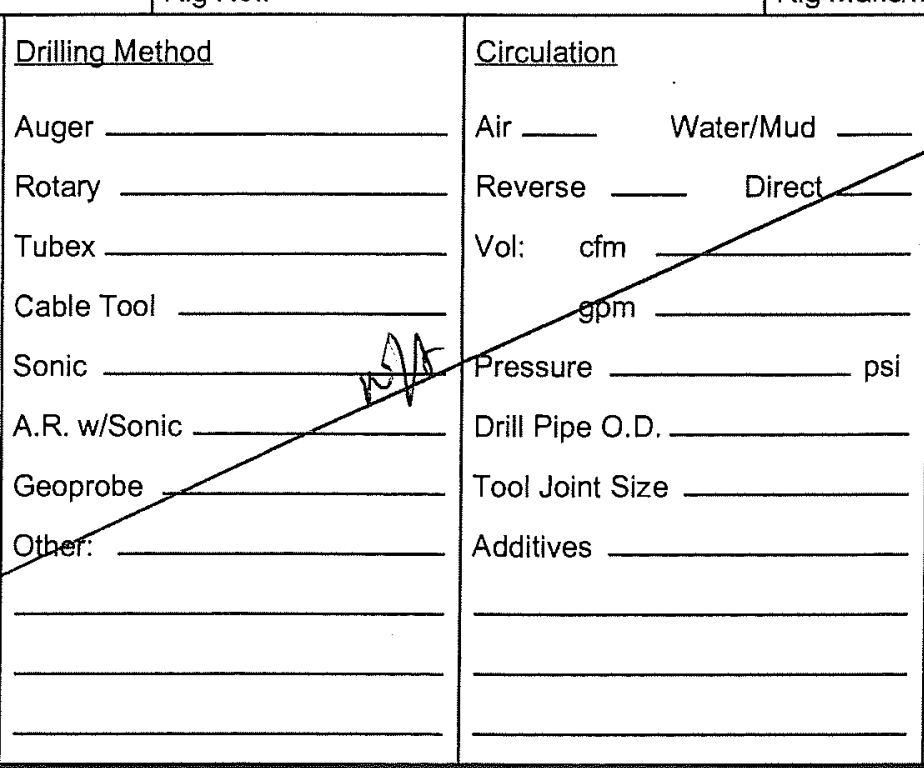

Rig Make/Mod.:

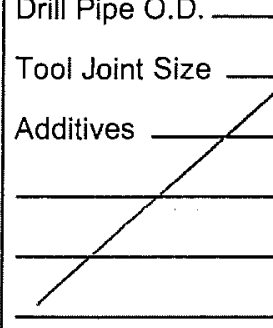

Page 1 of 2

$6 / 17 / 03$ 


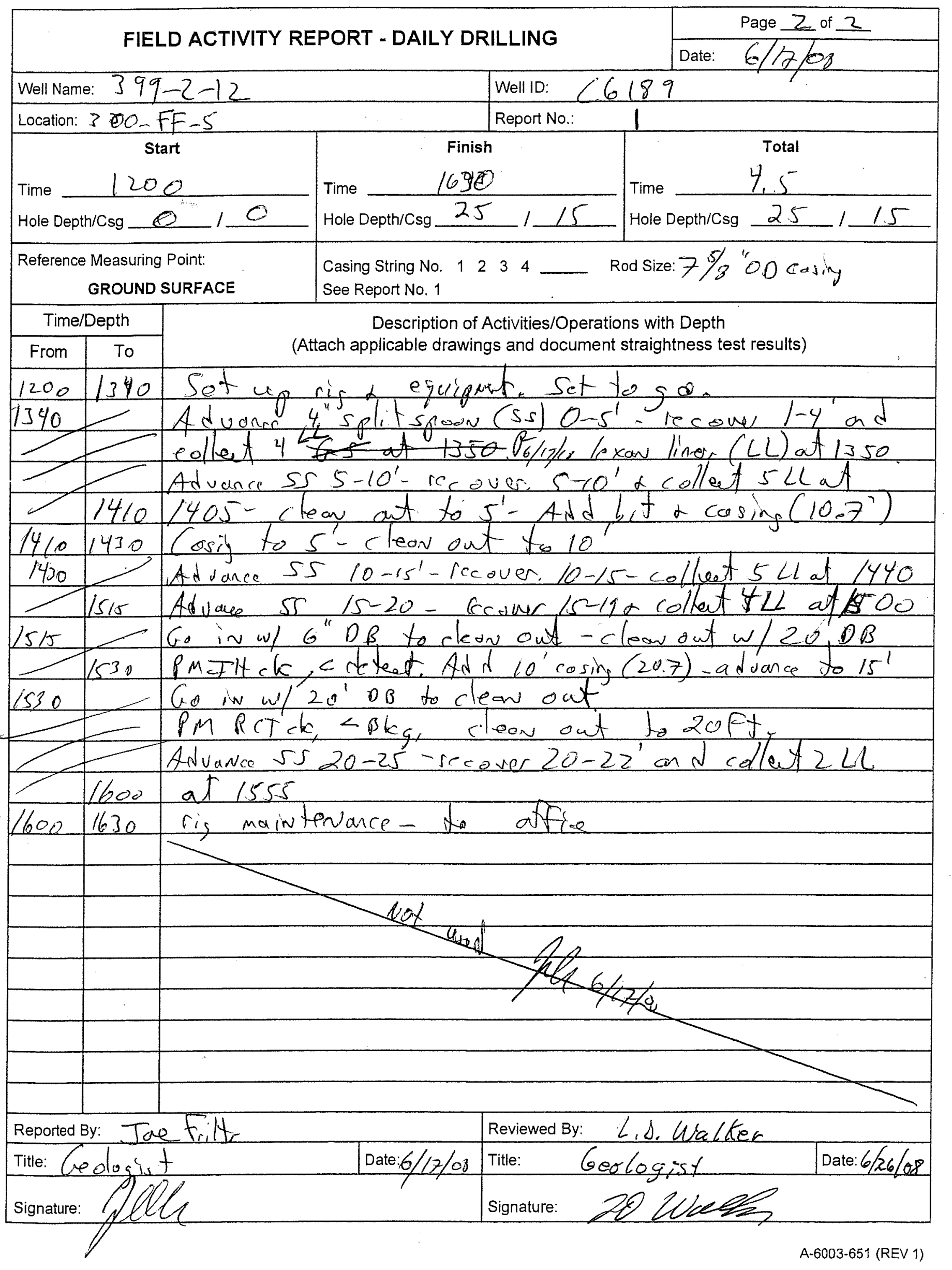

D. 42 


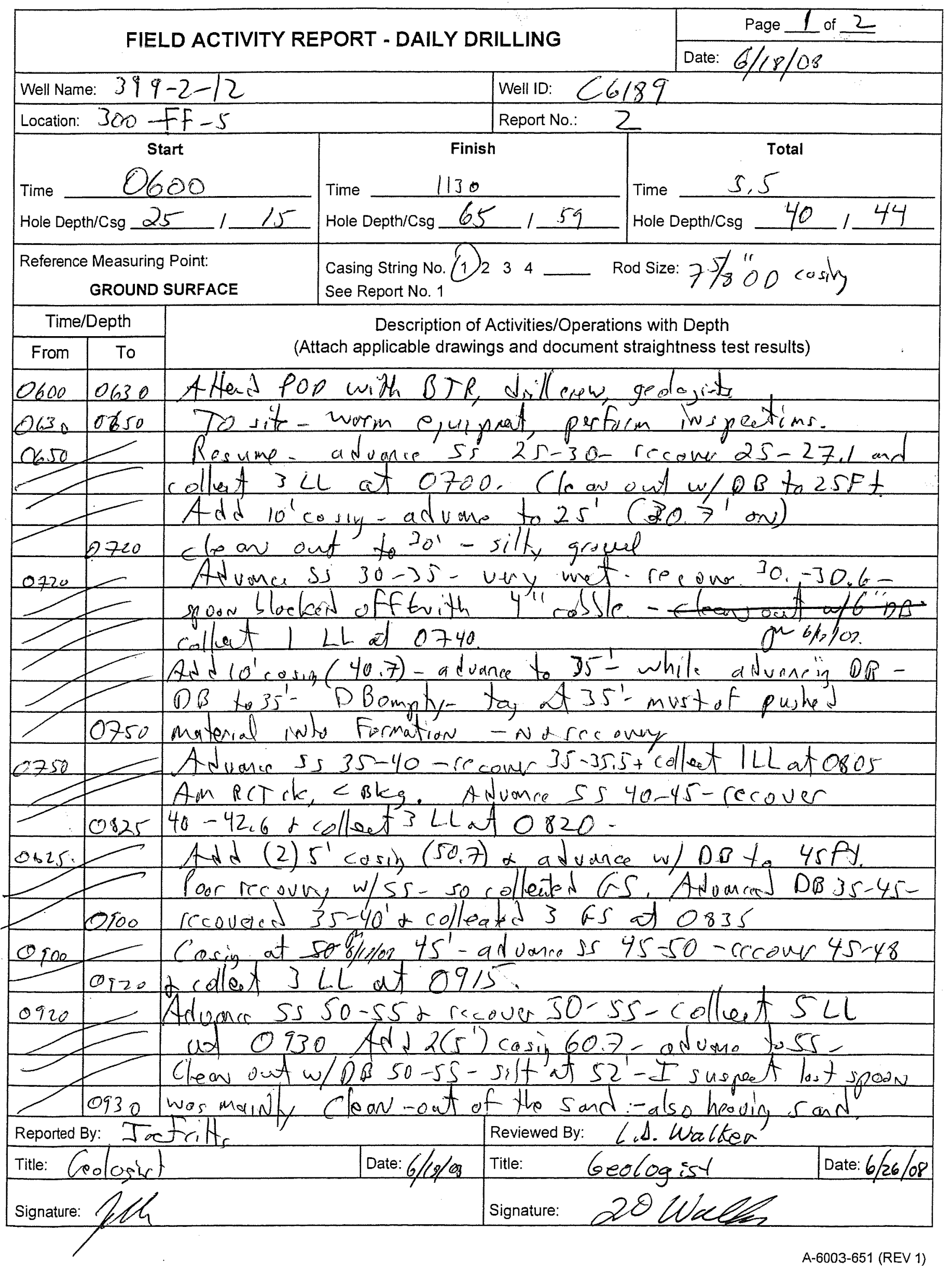

D. 43 


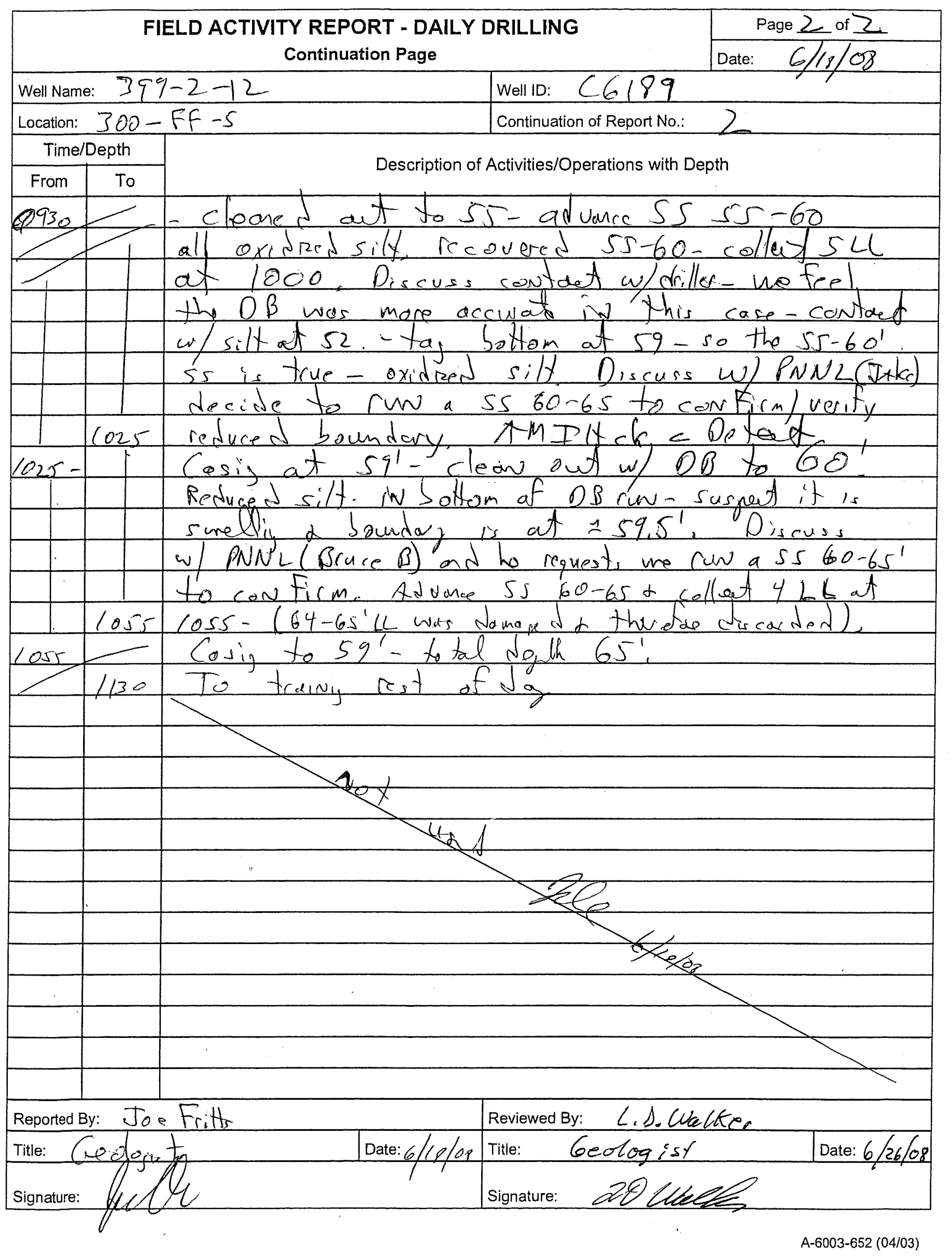

D. 44 


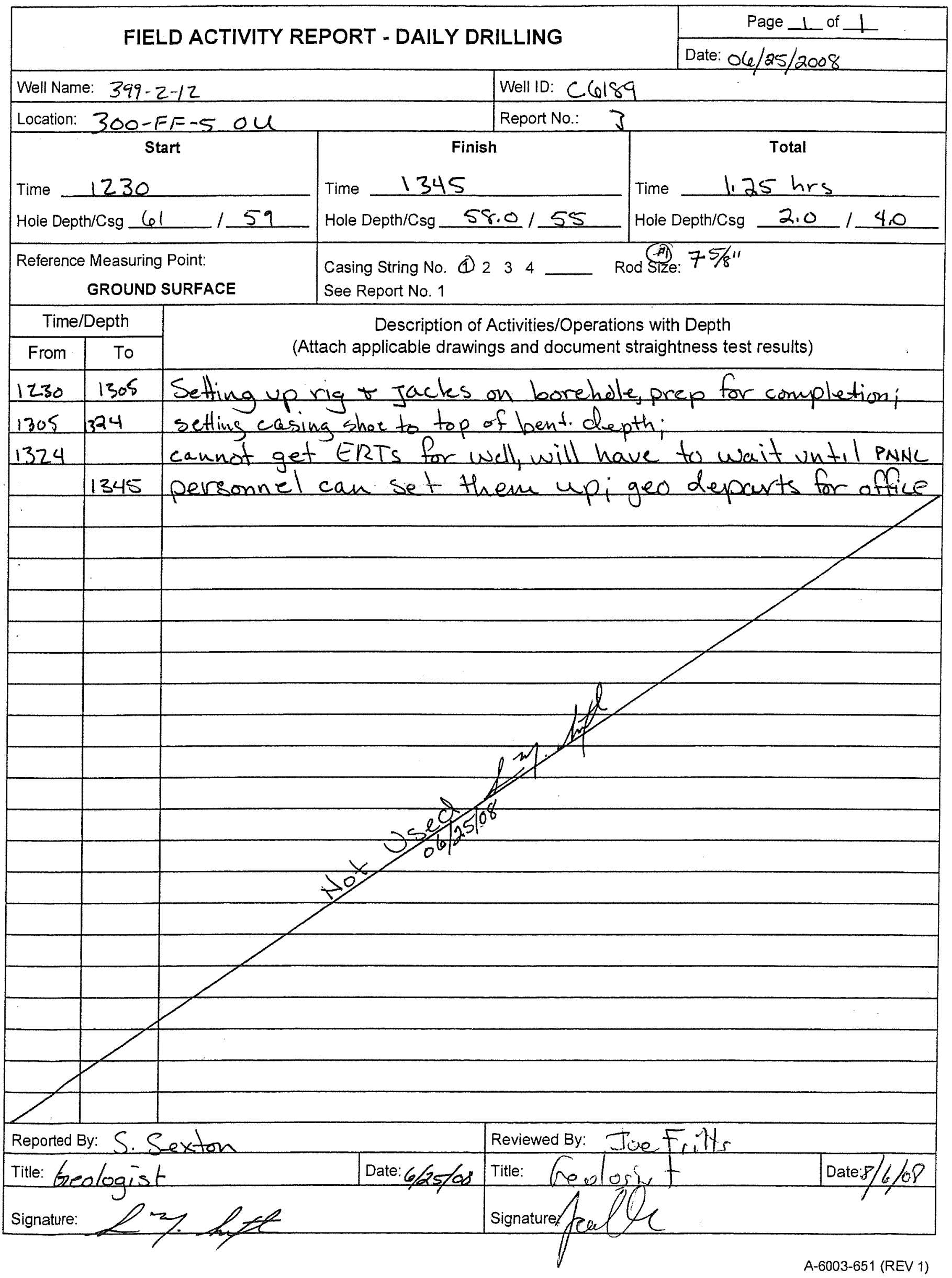




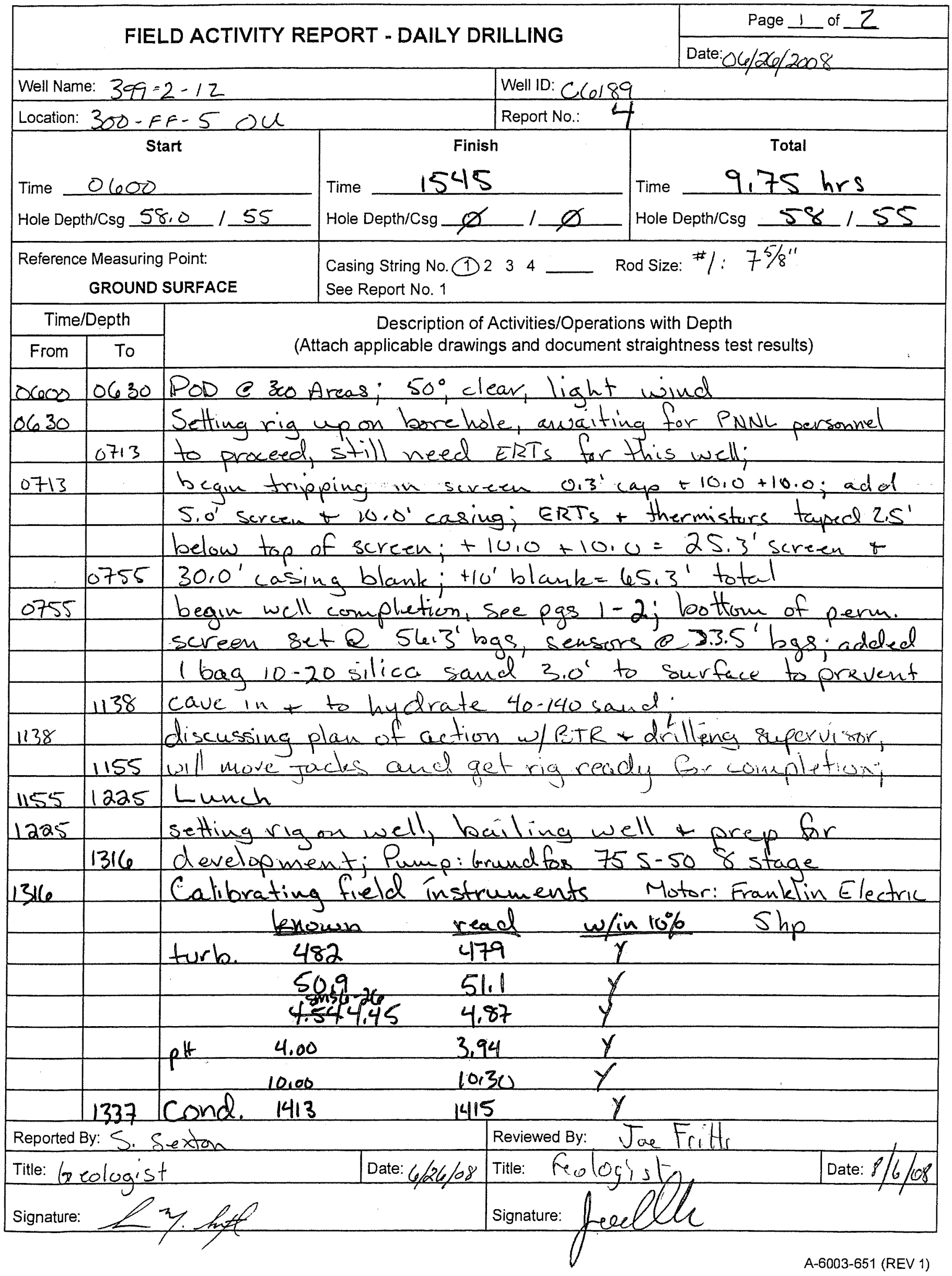

D.46 


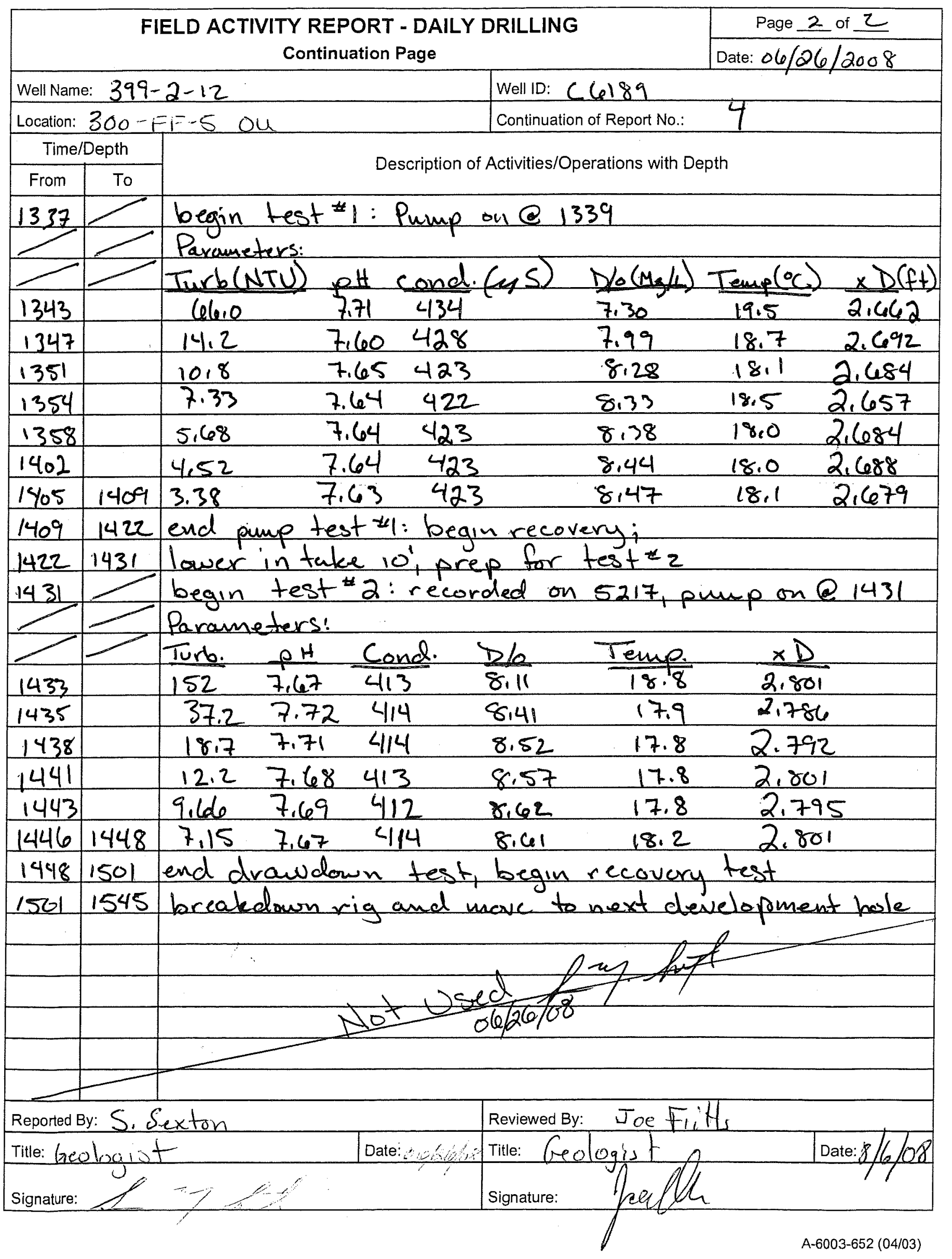

D. 47 


\section{WELL DEVELOPMENT AND TESTING DATA}

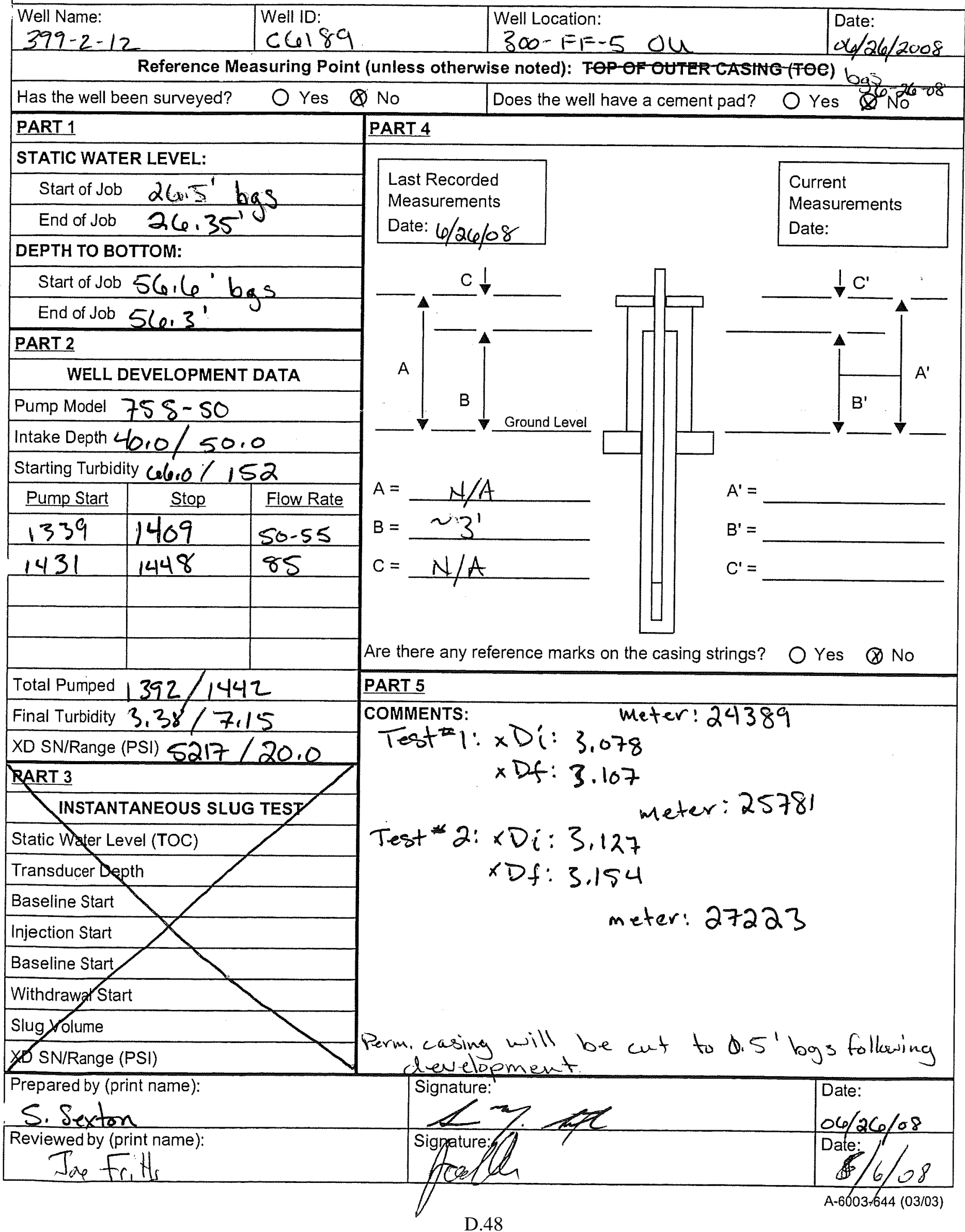




\section{FIELD ACTIVITY REPORT NO. 1 - DRILLING PLAN}

Page 1 of 1

Purpose: PNNLProject SodArea IFC

Location:300 - FF - 5-Ou

Well IDC 6190

Well Name: $3\{9-2-1\}$

Drilling co: WDC Priller Mike Wilkersov

Casing String No. (1) 2

Casing Size $7 \% \%$, 00, $67 \%$, 20

Grade

Lbs.Per Ft.

Material arbon steel

Type:

Welded

(Thd)

Planned / Actual

Set At: -157

Shoe OD/ID

Reference Measuring Point: GROUND LEVEL

\section{Drig. Co.}

Casing String No. $\begin{array}{lllll}1 & 2 & 3 & 4\end{array}$

Casing Size

Grade

Lbs.Per Ft.

Material

Type:

Weided Thd.

Planned / Actual

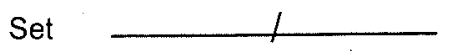

Shoe OD/ID

Reference Measuning Point: GROUND LEVEL

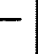

Auger

Rotary

Tubex

Cable Tool

Sonic Suger Sovle 2

A.R. w/Sonic RS 400

Geoprobe

Other:

\&built by Gus Pen

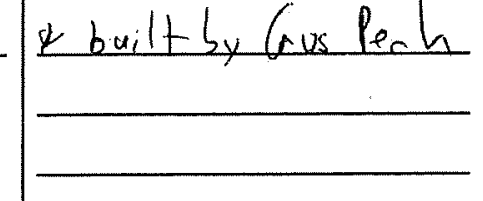

Rig No:

Drilling Method

Auger

Rotary

Tubex

Cable Tool

Sonic

A.R. w/Sonic

Geoprobe

O

Otber:

Comments/Remarks: \begin{tabular}{l|l|l} 
Rig No.: $/ 39$ & Rig Make/Mod:: Sugers. Sowlc 2 \\
\hline
\end{tabular}

Circulation

Air _ Water/Mud _

Reverse — Direct -

Vol: $\quad \mathrm{cfm}$

gpm

Pressure

Drill Pipe O.D

Tool Joint Size

Additives

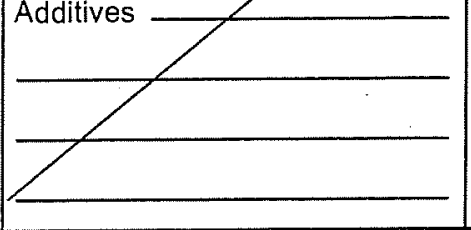

D.H. Hammer

Make

Model

Choke

Casing Hammer

Make

Model

Bit Size

Type

Nozzles

Rod Size

Rig Make/Mod::

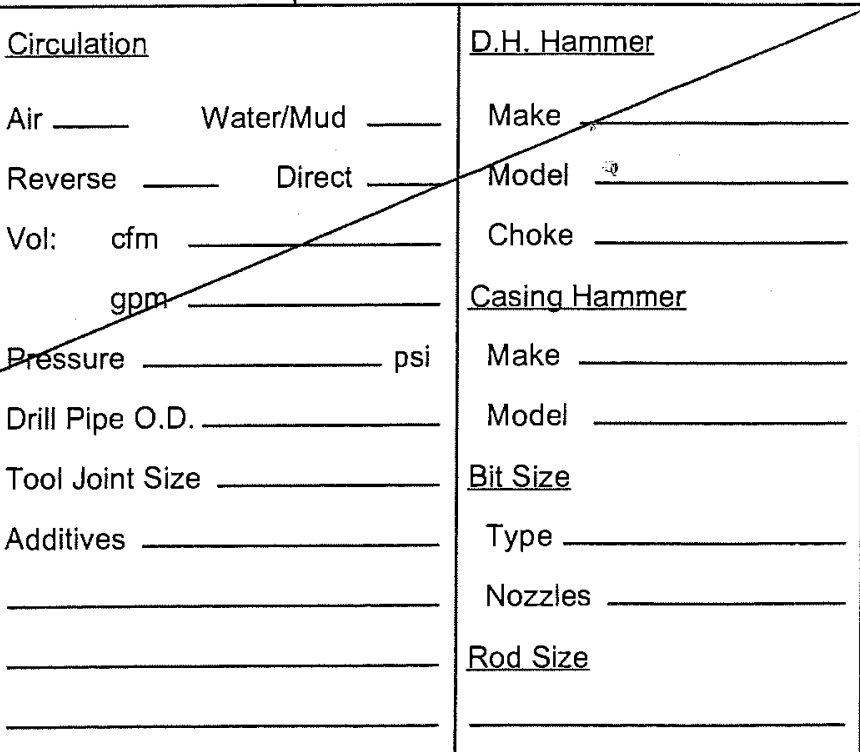

Estimated Depth to Water

Reported By: Jae Frits

Name/Title: CrRtin Ine Geolcgist

Signature:

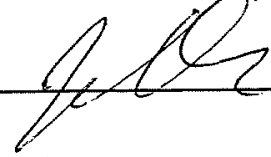

Date: $5 / 22 / 08$

A-6003-650 (04/03) 


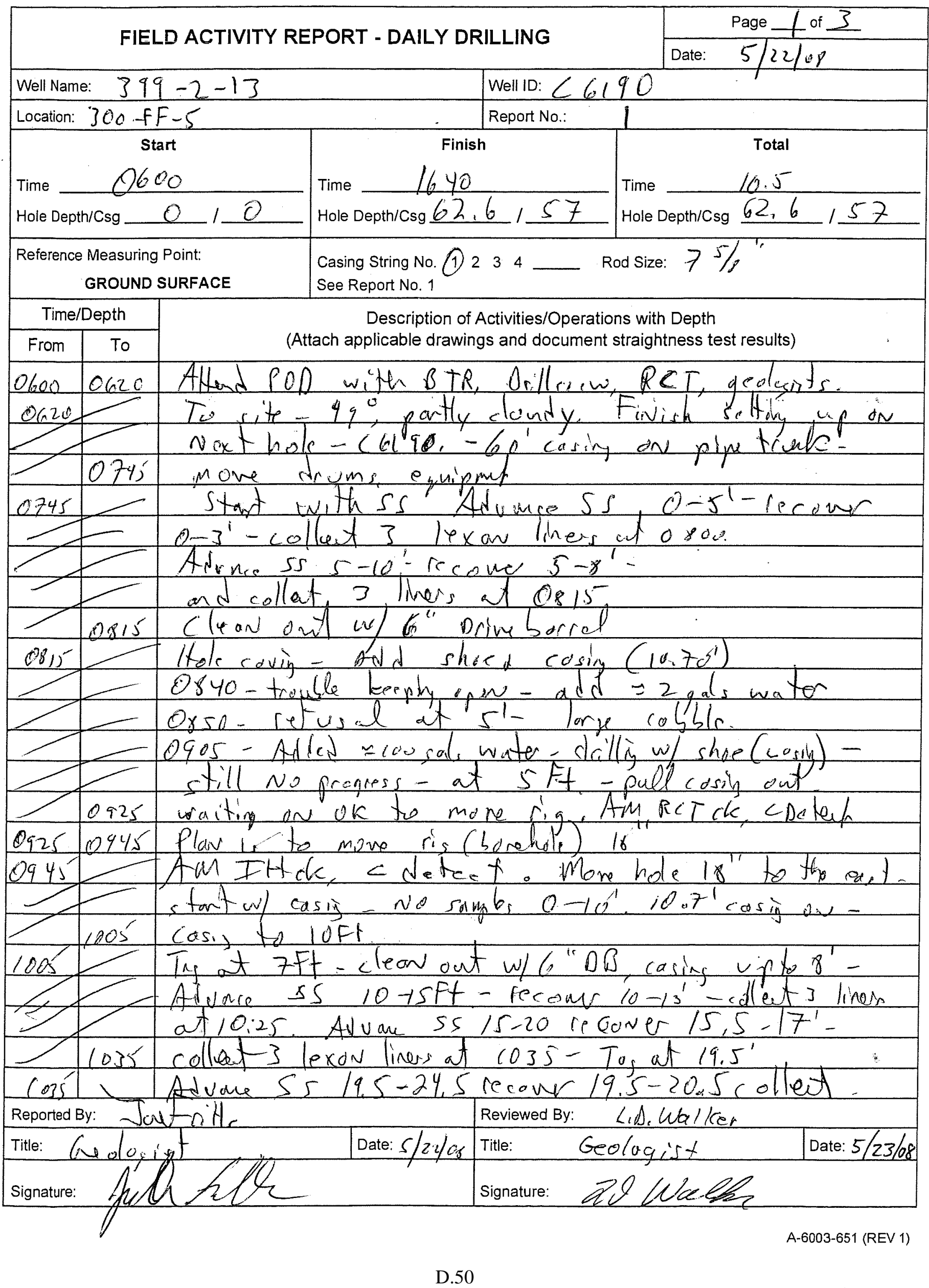




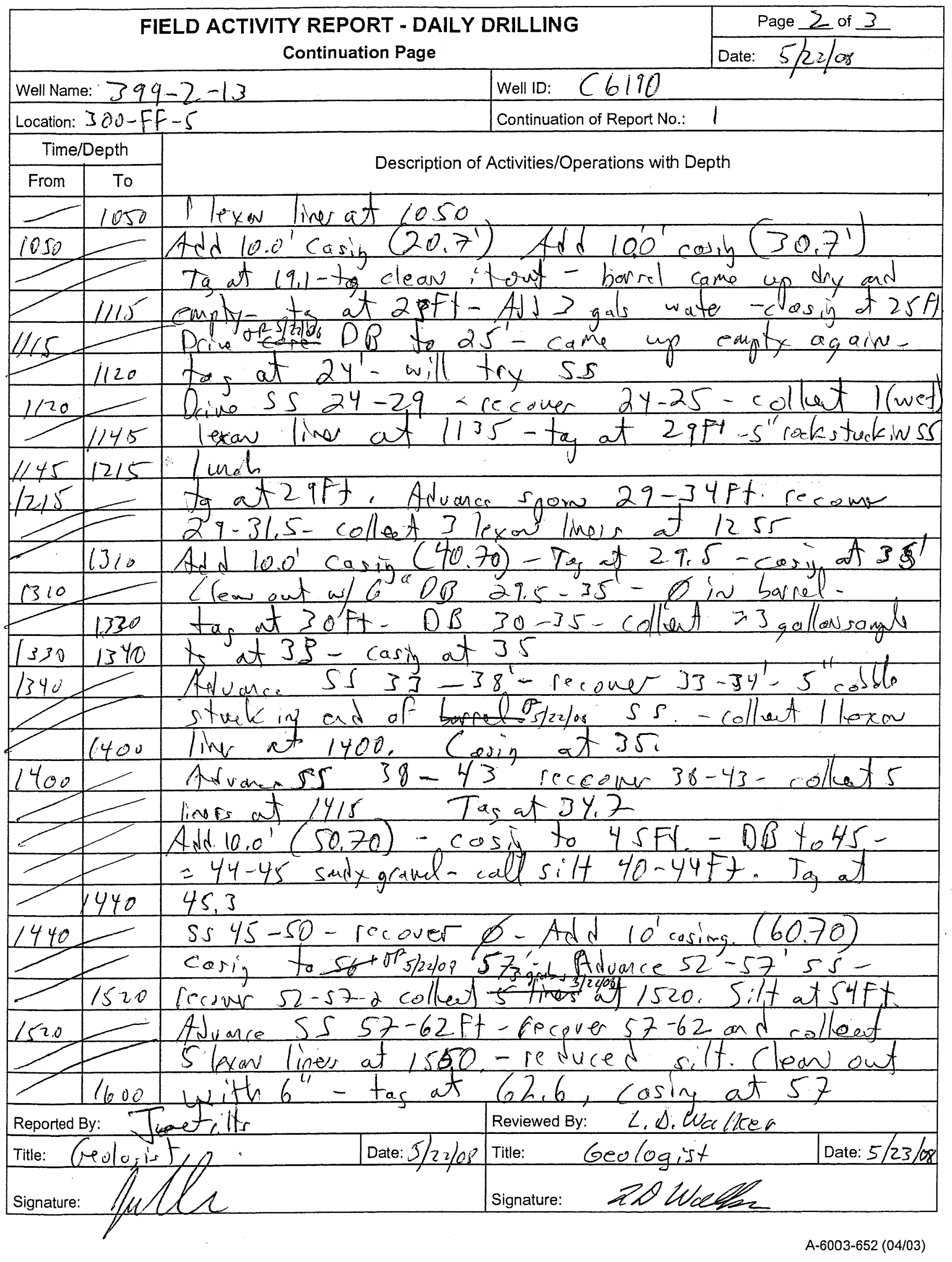

D. 51 


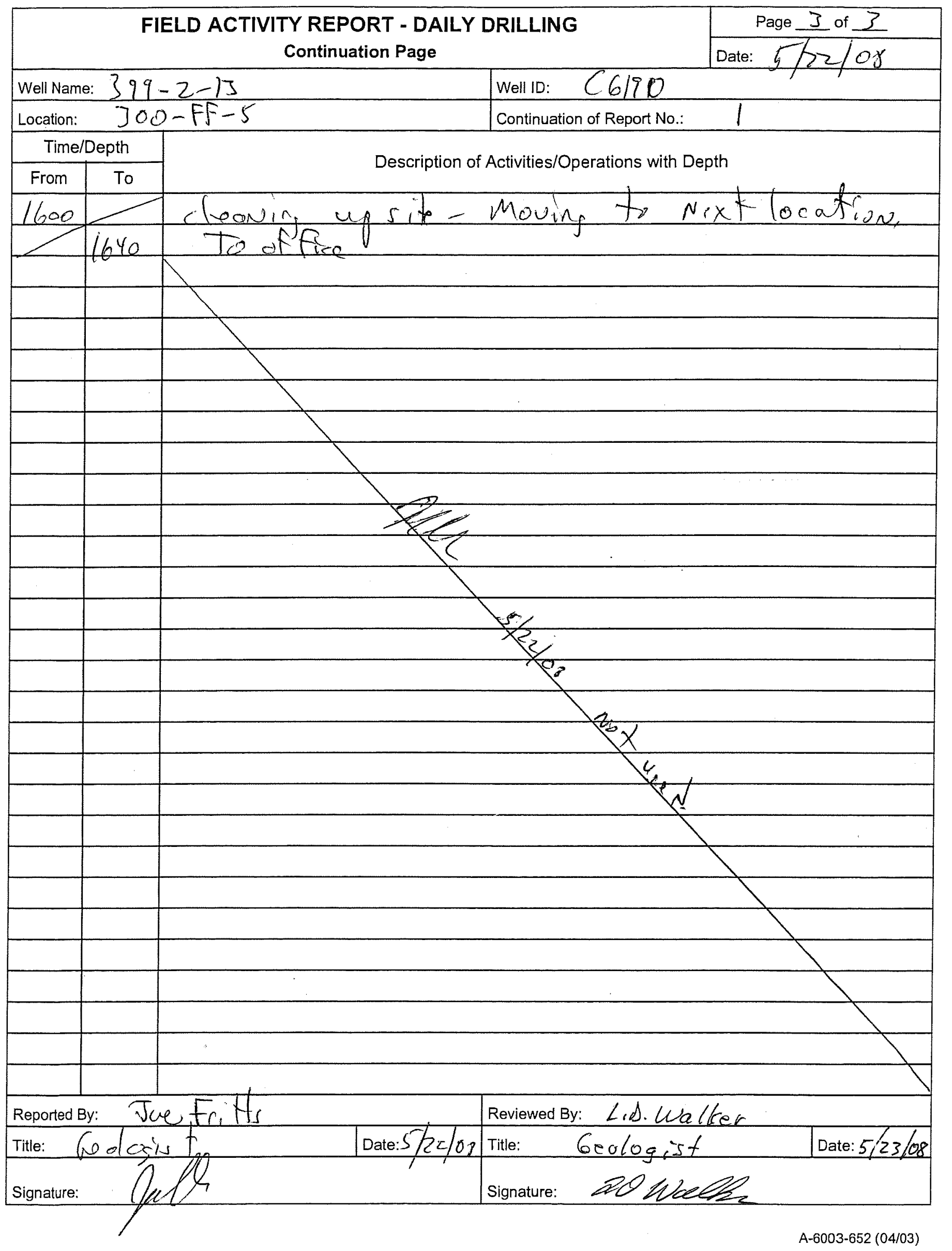

D.52 


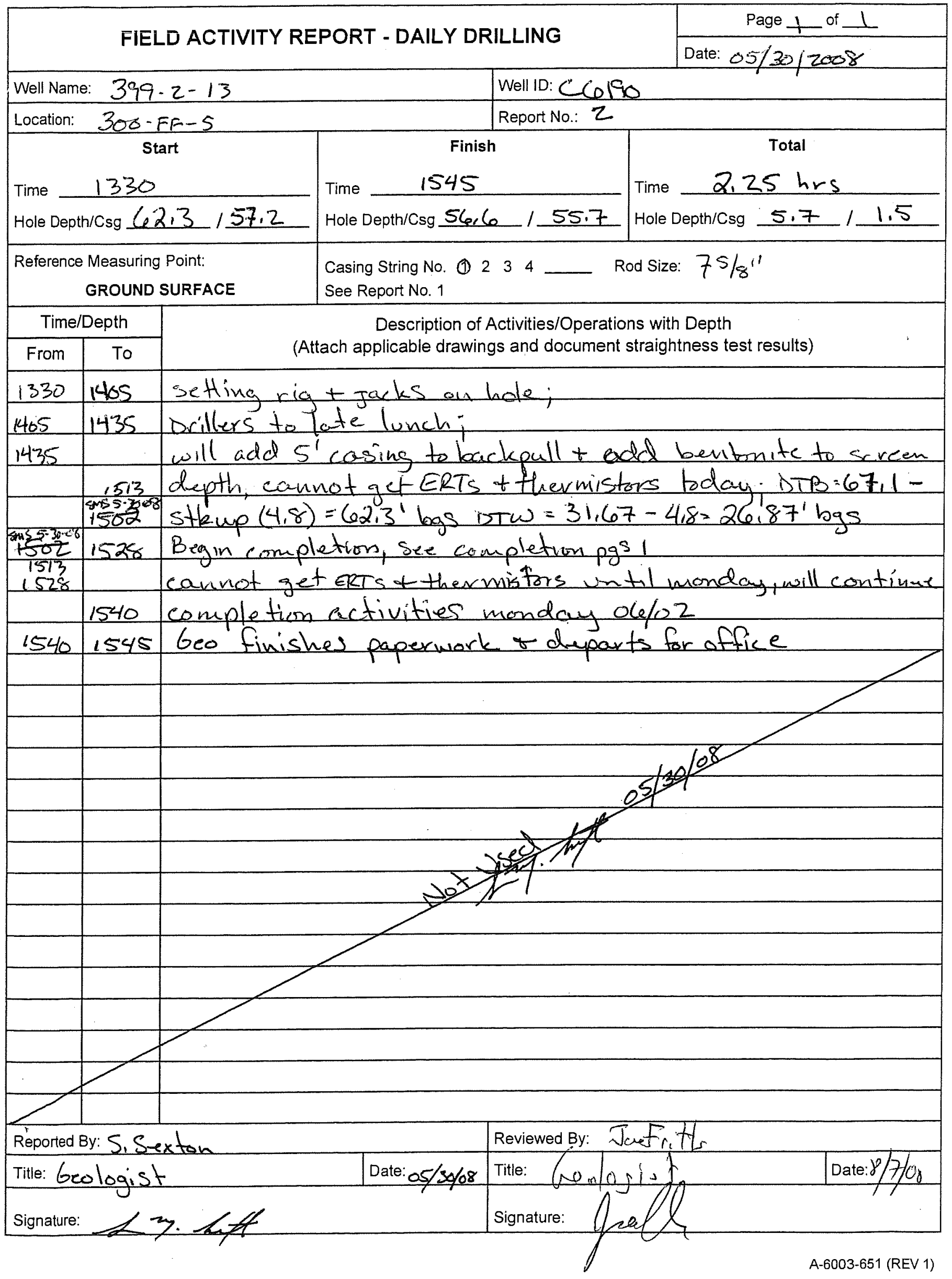

D.53 


\section{FIELD ACTIVITY REPORT - DAILY DRILLING}

Page $\perp$ of 1

Date: $6 / 2 / 08$

Well Name: $399-2-13$

Location: $300-F F-5$

Start

Time 0600

Hole Depth/Csg $56.5 / 55.7$

Reference Measuring Point:

GROUND SURFACE

\section{Time/Depth}

\begin{tabular}{|c|c|}
\hline From & To \\
\hline 0600 & 0630 \\
\hline
\end{tabular}

06000630 PDQ 300 Area

$0630 \quad$ Driller setting up rig on borehole $-D T W=26.4^{\prime}$

$0738 \quad$ Trip screen in hole

0748 Tape ERT and the rmistor 2.5' down from top of screen

$0755 \quad$ Continue tripping permanent well in hole - Botion set $56.5^{\prime}$ bgs

0820 Resume well completion. (Refer to well completion log-pg. 1)

$0900 \quad$ Remove $10.00^{\prime}$ temp casing

09180933 Start surging lot interval from $56.0^{\circ}$ to $45.6^{\prime}$

0933 Resume well completion

MIS $\quad$ Start sunging 2nd interval Frem 45,6'

$1020 \quad$ RCT survags pulled casingi $\leq$ blegd

1025

1057 - Stop Sunging - Resame well completion

12001285 Resume well completion -

1255 Move to $\mathrm{C} 6197$

Total

Time 6 hrs 55 min Hole Depth/Csg

(2) 4 "ID Pue

Description of Activities/Operations with Depth

Attach applicable drawings and document straightness test results)

Reported By: J. Thnoolin $\quad$ Reviewed By: Juetcils

Title: Geologist

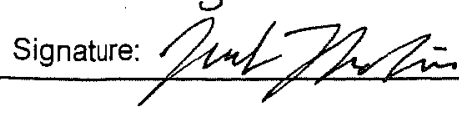
Date $6 / 2108$ Title: (rologis t)

Signature: 


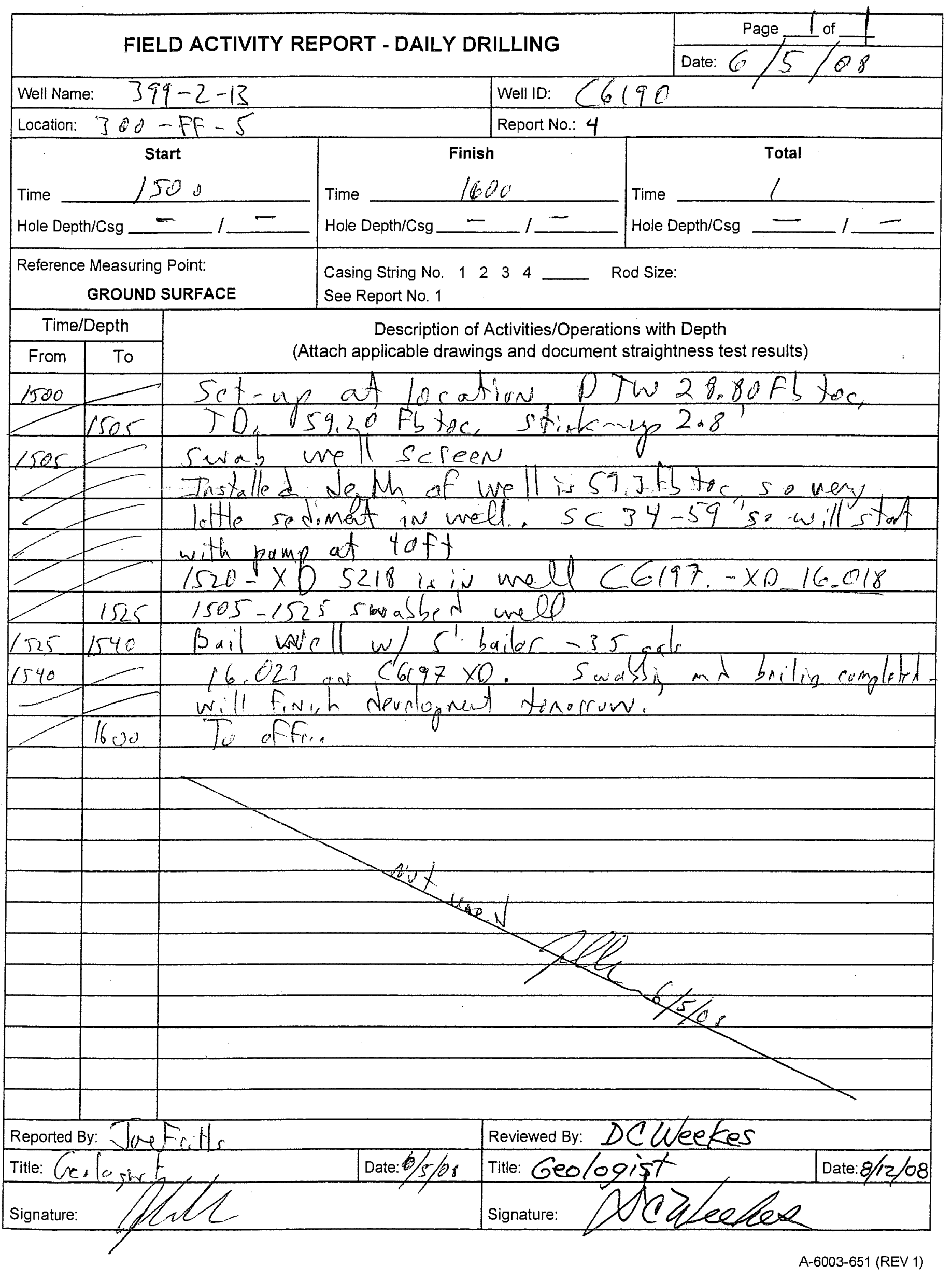

D.55 


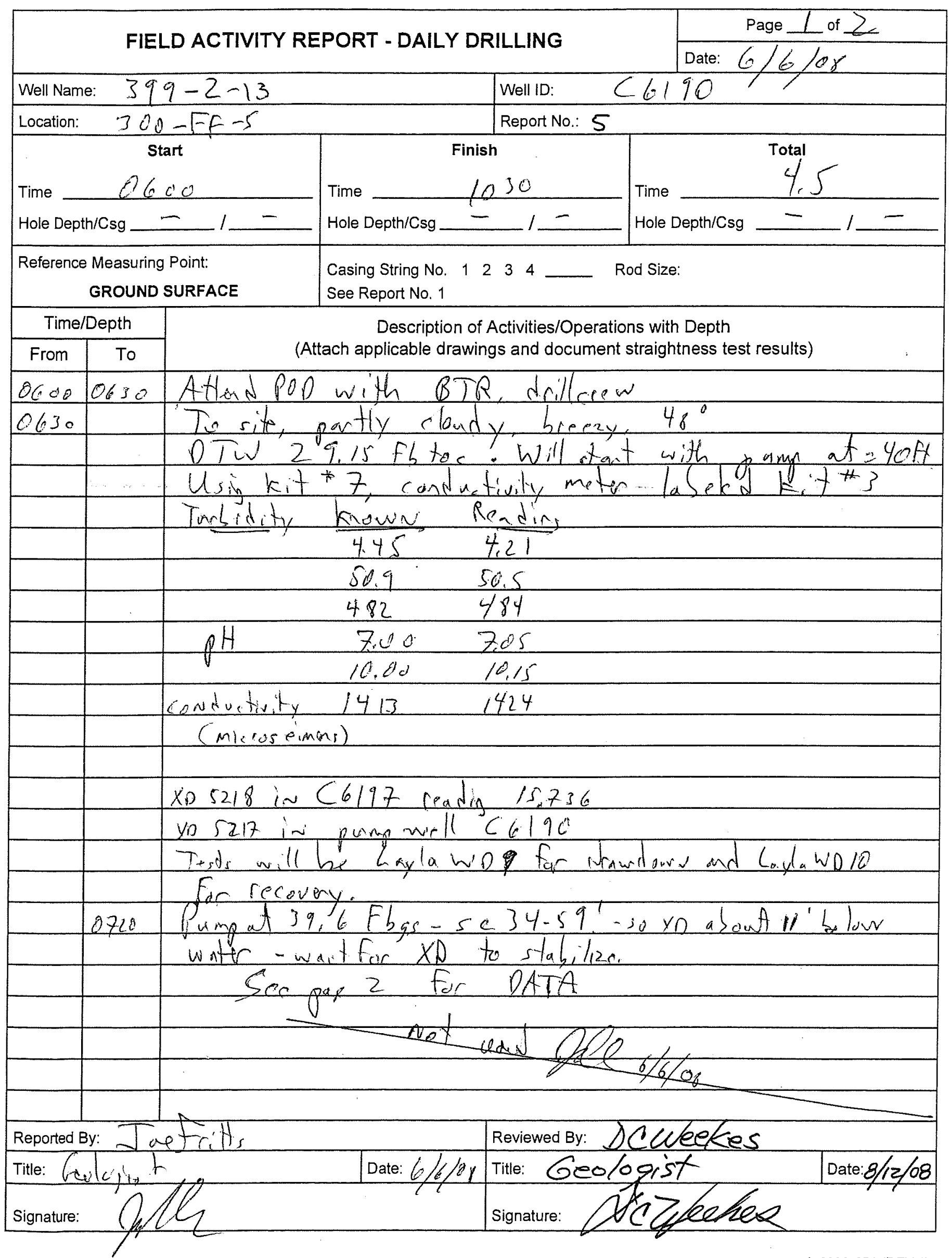




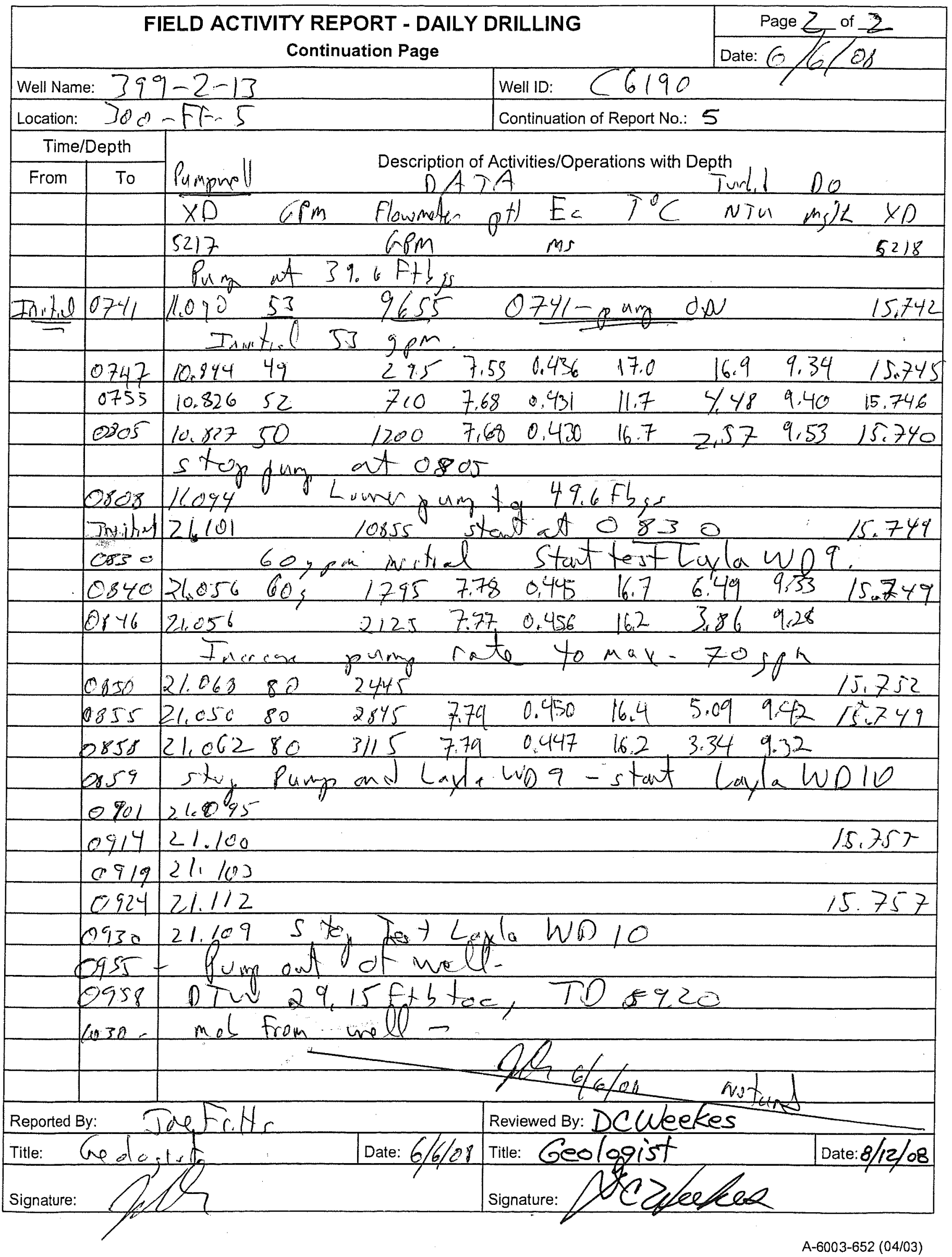

D.57 
FIELD ACTIVITY REPORT NO. 1 - DRILLING PLAN

Page 1 of 3

Date: $6 / 13 / 06$

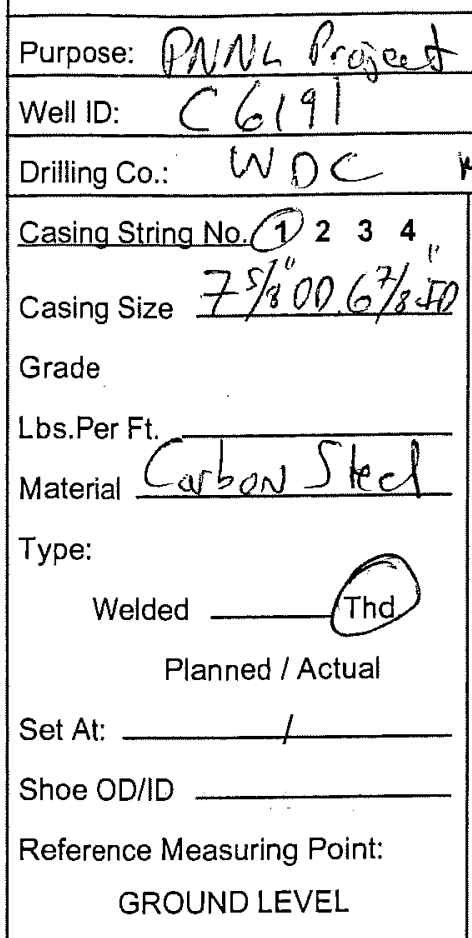

Drig. Co.

Casing String No. $\begin{array}{llll}1 & 2 & 3 & 4\end{array}$

Casing Size

Grade

Lbs.Per Ft.

Material

Type:

Welded Thd.

Planned / Actual

Set 1

Shoe OD/ID

Reference Measuring Point: GROUND LEVEL
300 Arca FFL

Location: $300-F F-5-04$ Well Name: 39 : $2-14$ Rig No.: 139 Rig Make/Mod.: Su jer Sovic

A

Rig No.:

Sonic Sypursonis 2 A.R. w/Sonic 19400

Geoprobe

Other:

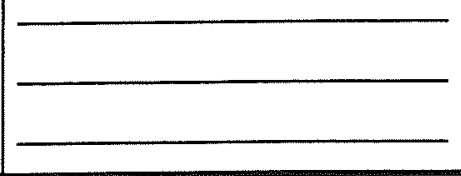

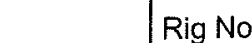

Drilling Method

Auger

Rotary

Tubex

Cable Tool

Sonic

A

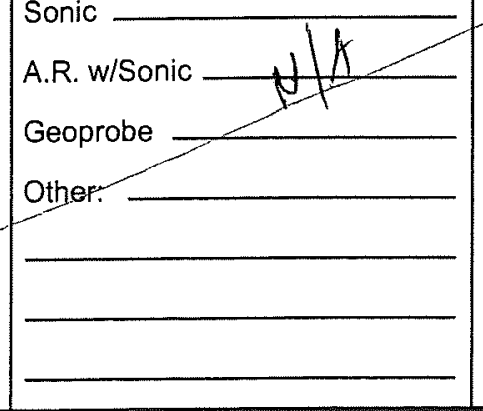

Comments/Remarks: Circulation

D.H. Hammer

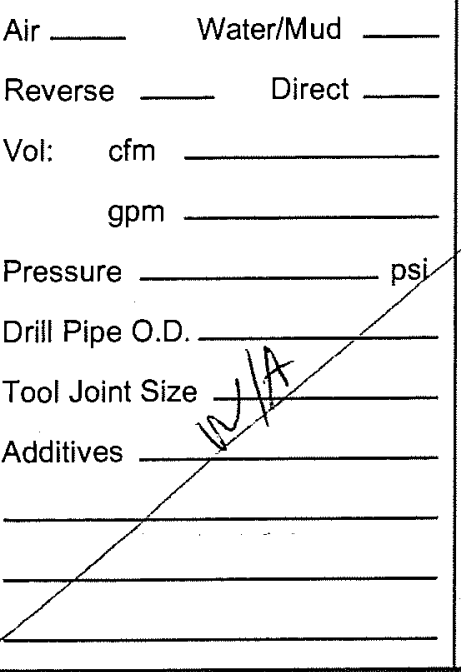

Make

Model

Choke Casing Hammer

Make

Model

Bit Size

Type

Nozzles

Rod Size

Rig Make/Mod.:

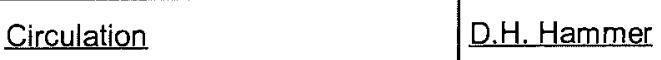

Air __ Water/Mud

Reverse — Direct
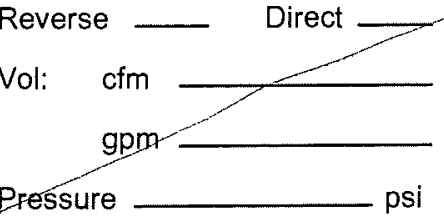

Drill Pipe O.D.

Model

Bit Size

Type

Nozzles

Rod Size

Estimated Depth to Water

\section{Diller. Bill Zamow}

Reported By: Joe frits

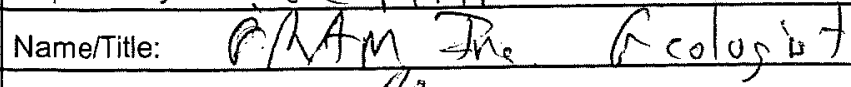

Signature:

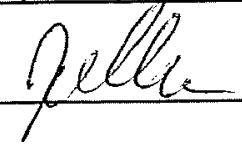

Date: $6 / 3 / 28$ 


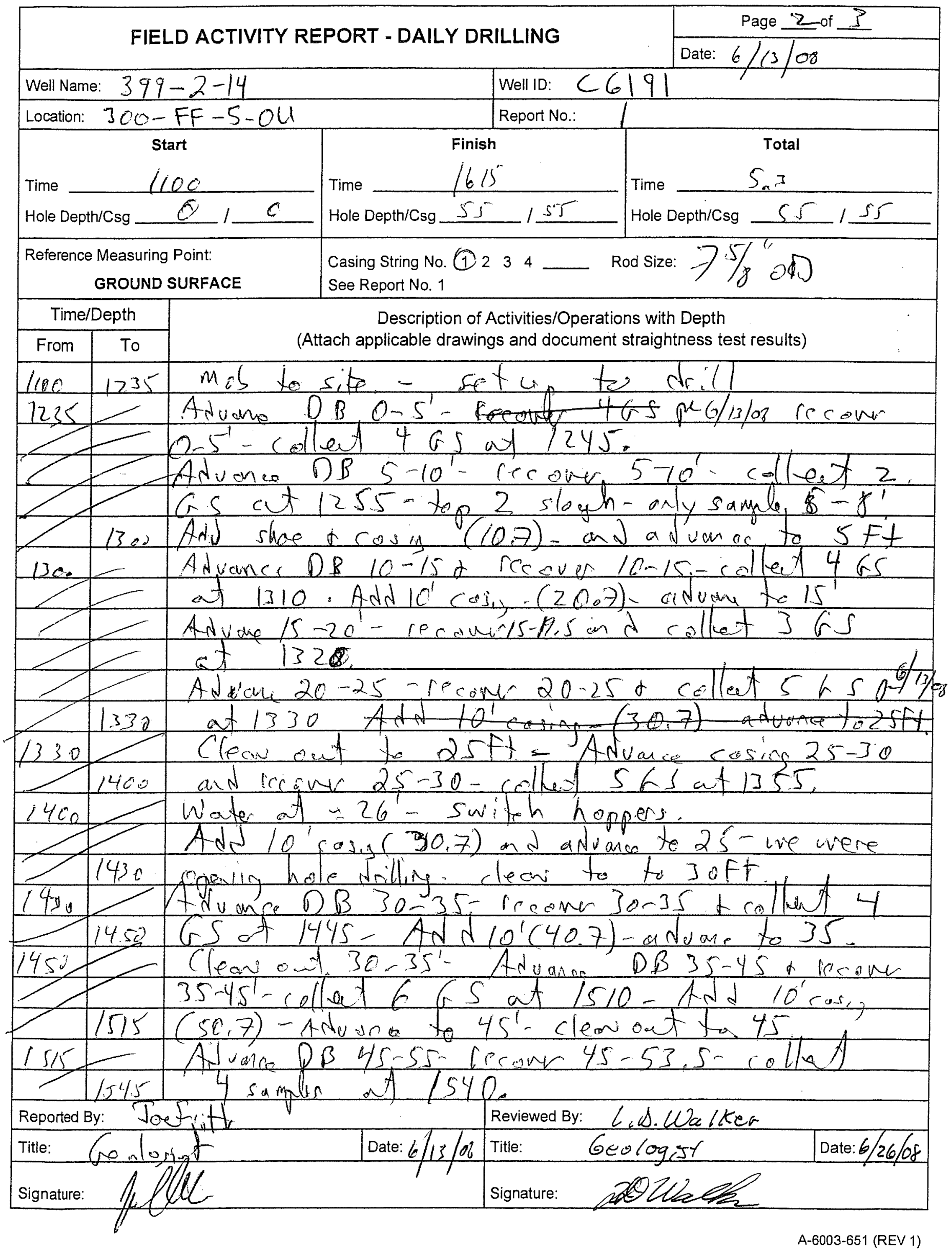

D.59 


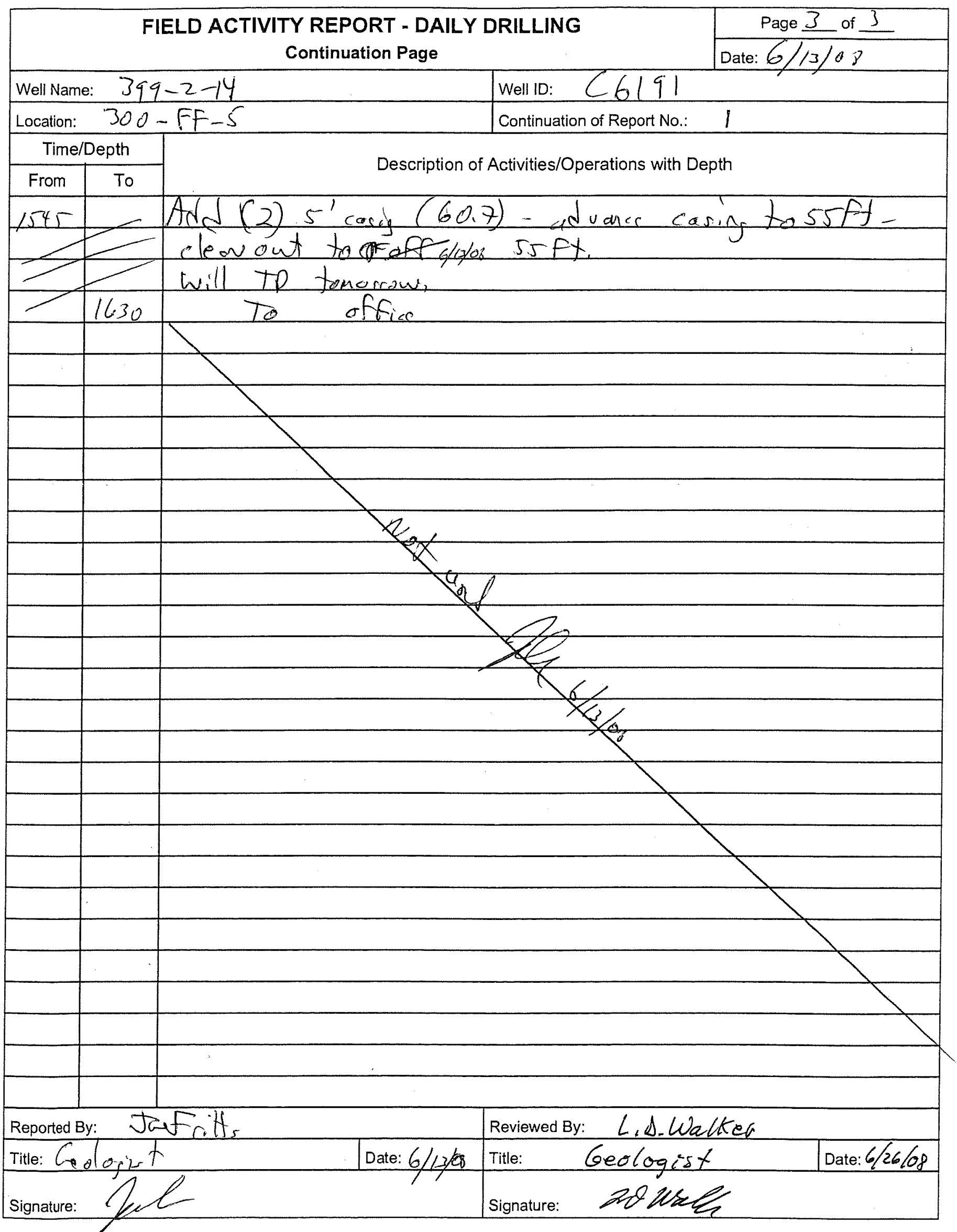




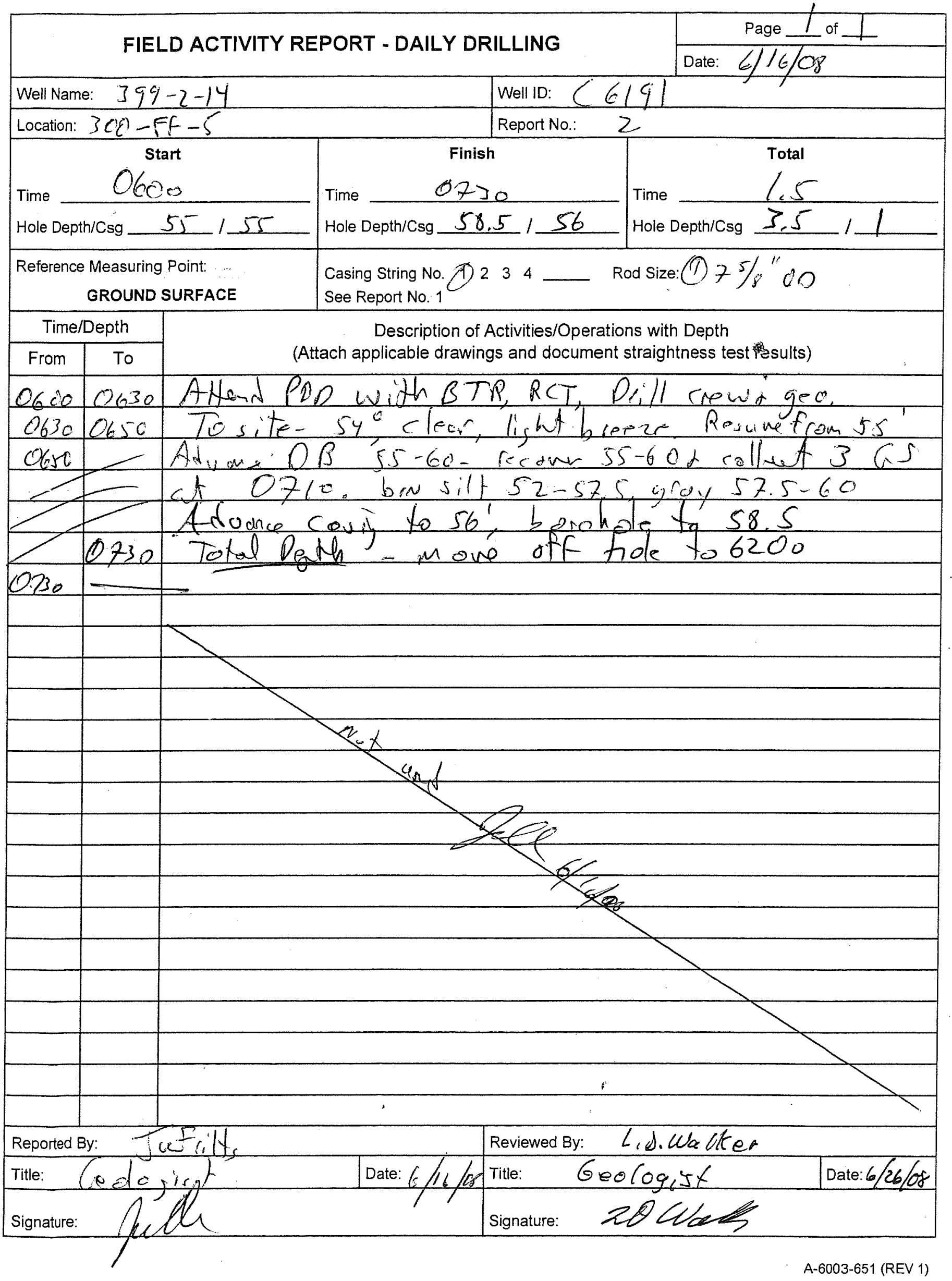

D.61 


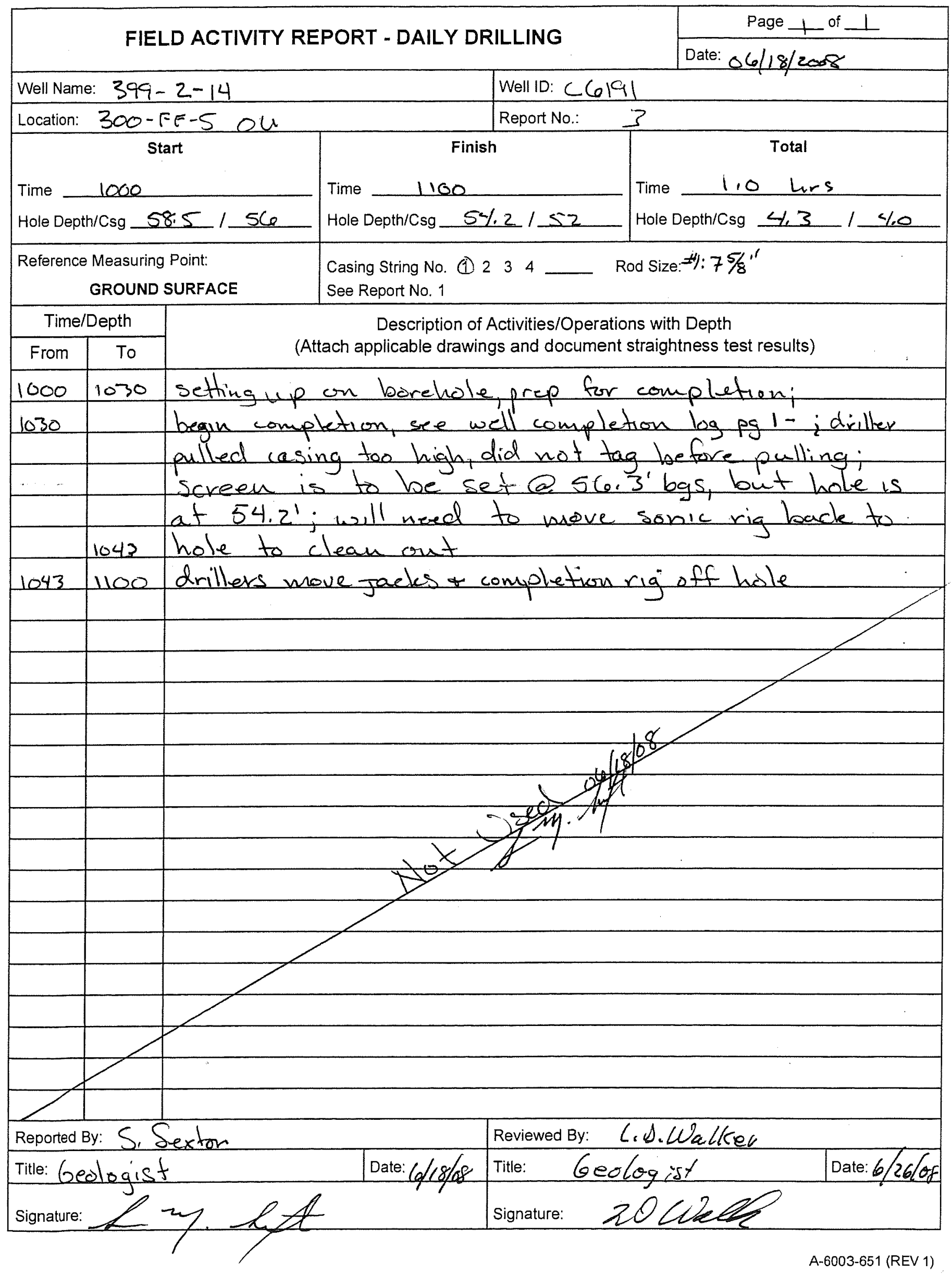




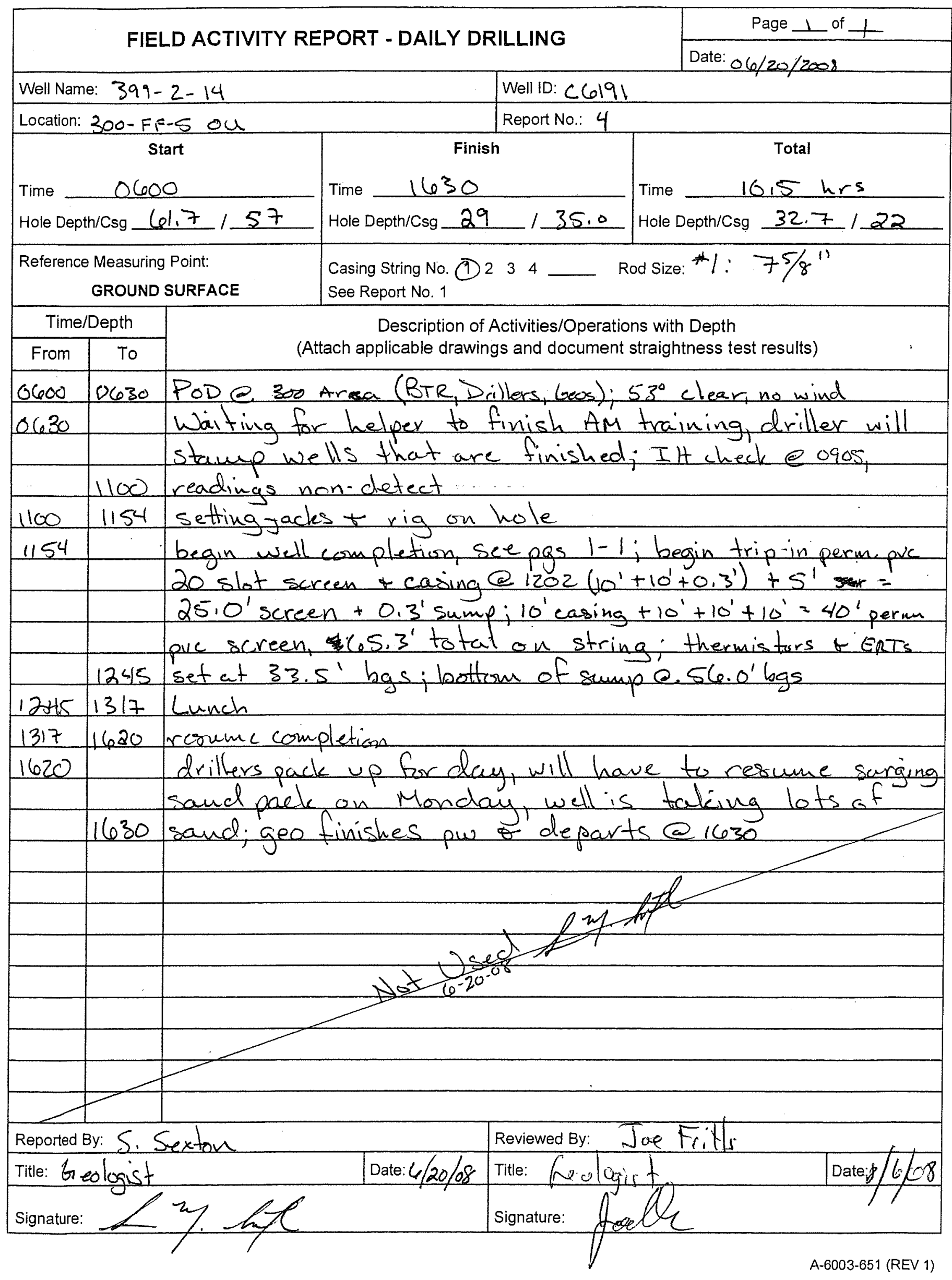




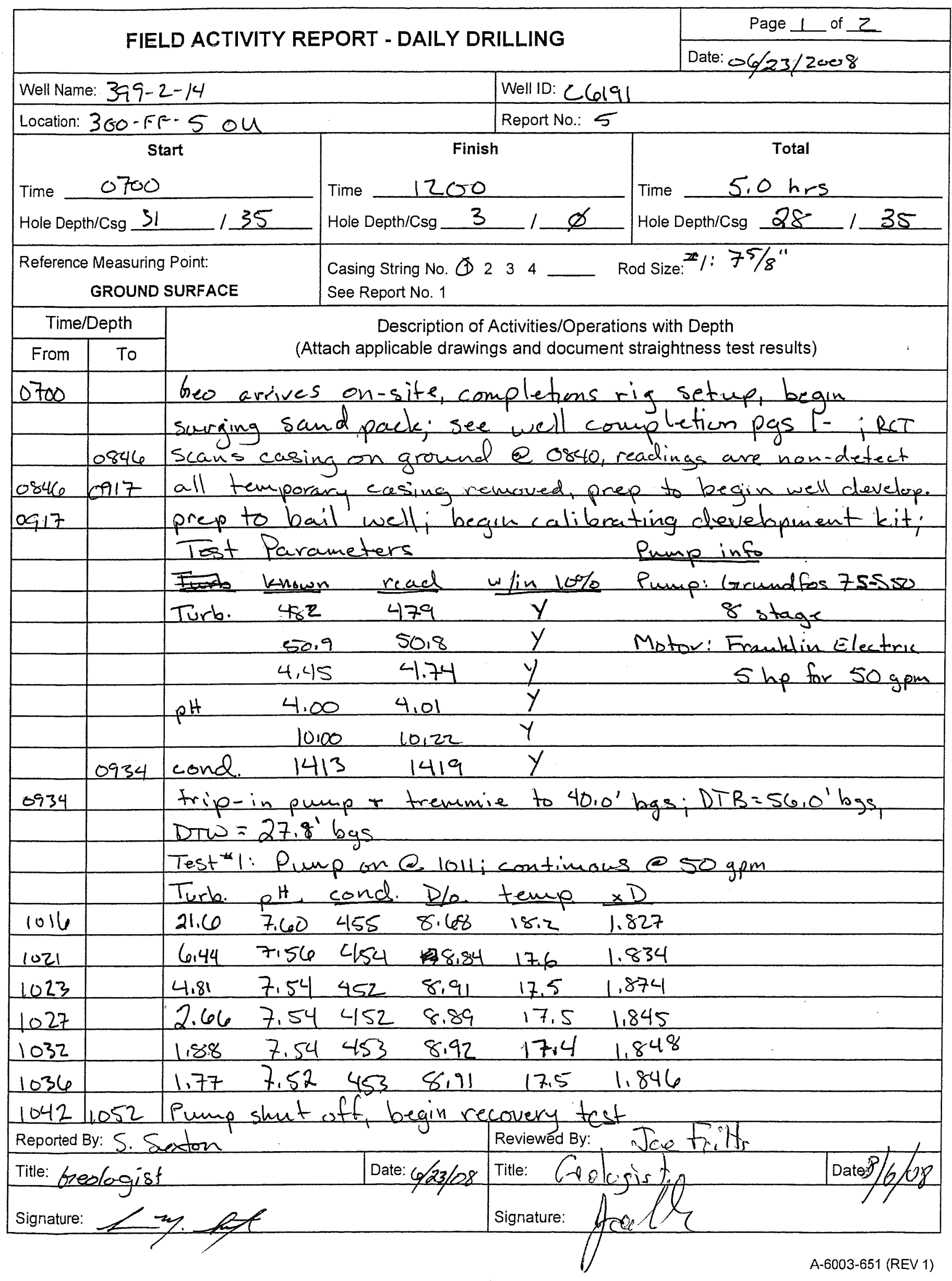




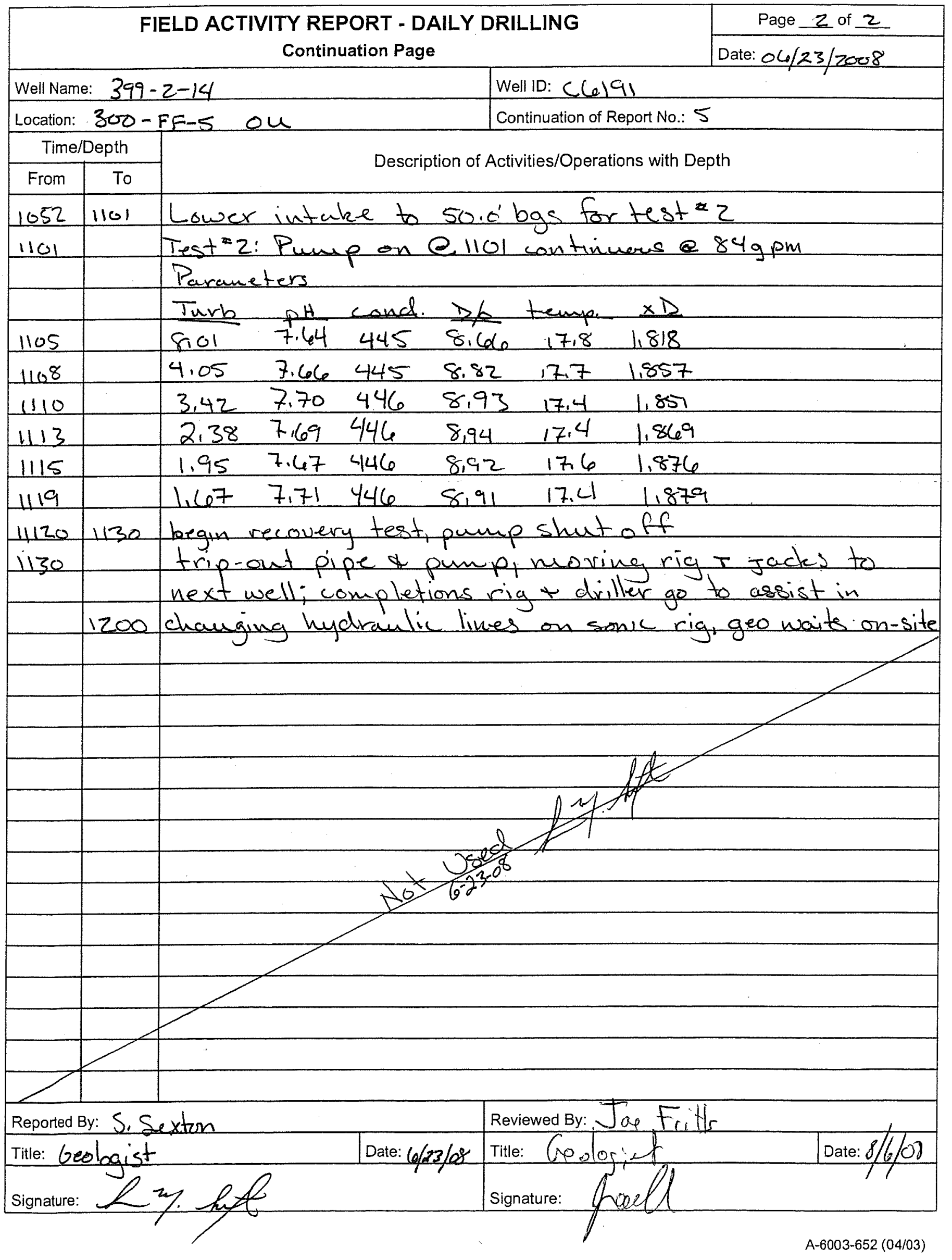

D.65 


\section{WELL DEVELOPMENT AND TESTING DATA}

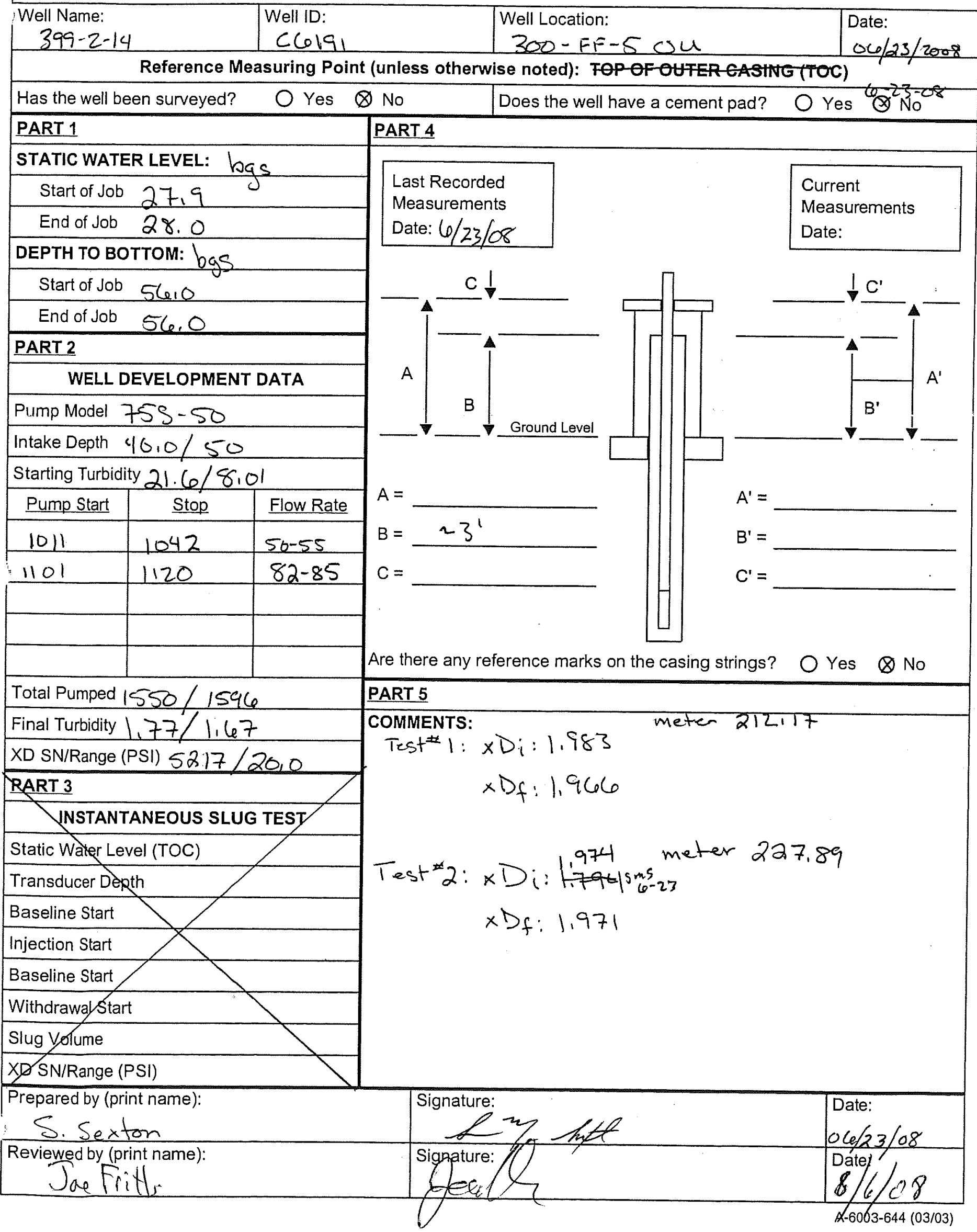

D.66 
FIELD ACTIVITY REPORT NO. 1 - DRILLING PLAN

Purpose: PNNL Project 300 Area IFC
Well ID: $C 6192$

Location: $300-F F-5$

Well Name: $399-2-15$

Drilling co.: W $P C$ Pithe Bill Zamoz

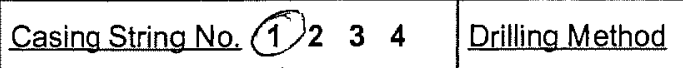

Casing Size $75 / 8 / 6 \%$

Grade

Lbs.Per Ft.

Material Garbous bee

Rig No.:139 Rig Make/Mod.S uper S ovic

Auger
Rotary
Tubex
Cable Tool
Sonis Suger Sonic L R

Circulation

Air _ Water/Mud

Reverse _ Direct

Vol: $\quad \mathrm{cfm}$

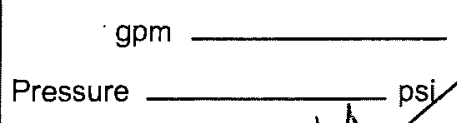

D.H. Hammer

Page 1 of

Date: $6 / 25700$

Type:

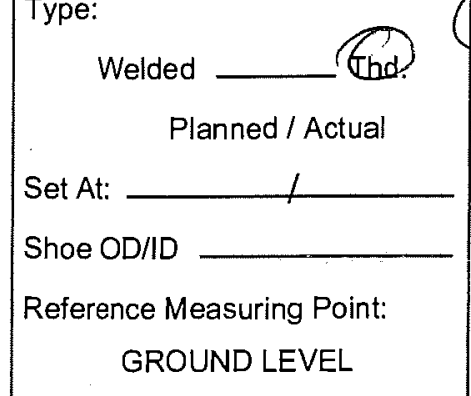

Drig. Co.

Rig No.:

\begin{tabular}{lllllll}
\hline Casing String No. & 1 & 2 & 3 & 4 \\
Casing Size & & & & & \\
Grade & & & & & & \\
Lbs.Per Ft. & & & & & & \\
Material & & & & & & \\
Type: & & & & & & \\
\end{tabular}

Geoprobe

Other:

Pressure

Drill Pipe O.D.

Tool Joint Size

Additives

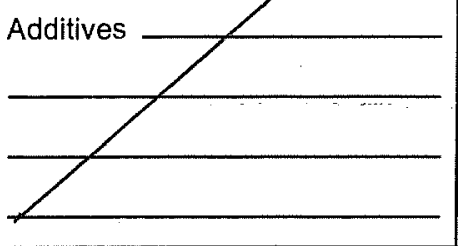

Rig Make/Mod:

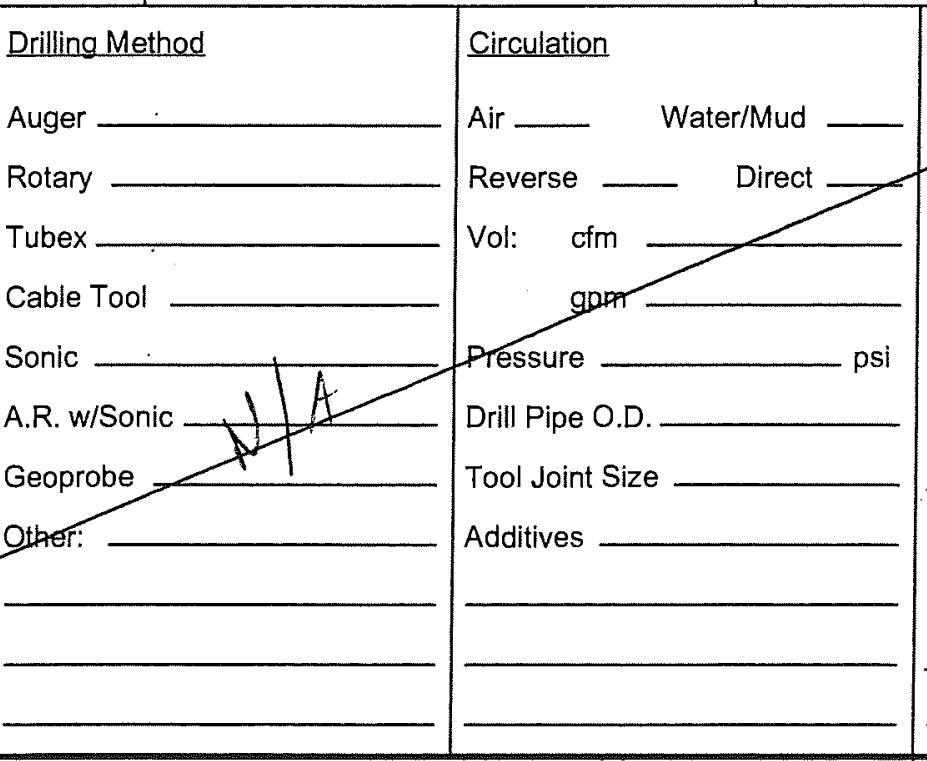

Comments/Remarks:

$$
\begin{aligned}
& \text { Nithe: addad }=200 \text {, allow wate } \\
& \text { duning drilling. }
\end{aligned}
$$

Reported By: Toe Frits

Namertitle: (ARAM, Inc. Geolosist

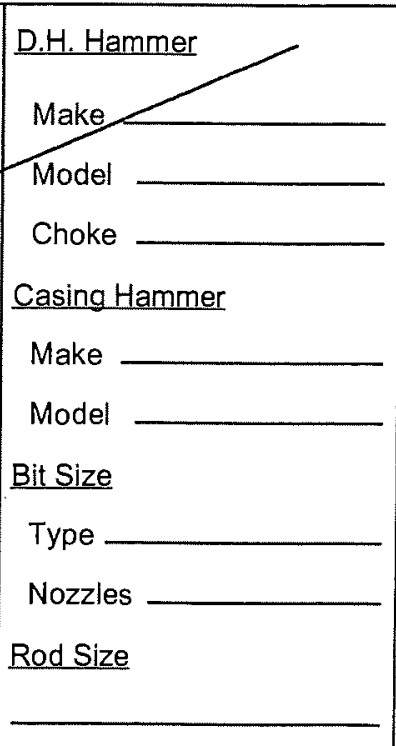

Estimated Depth to Water

Signature:

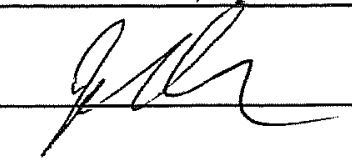

Date: $6 / 24 / 07$ 


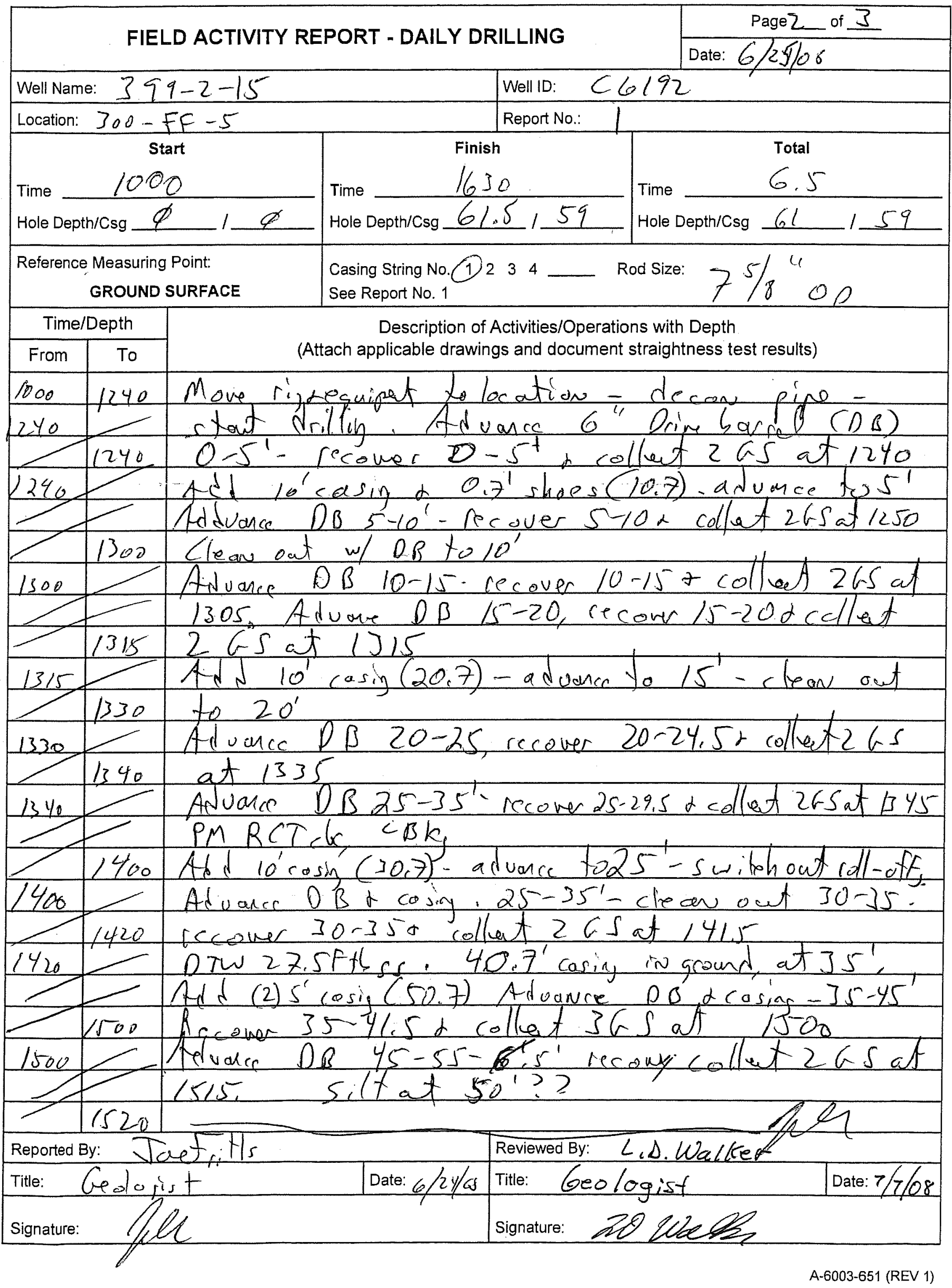

D.68 
FIELD ACTIVITY REPORT - DAILY DRILLING Continuation Page

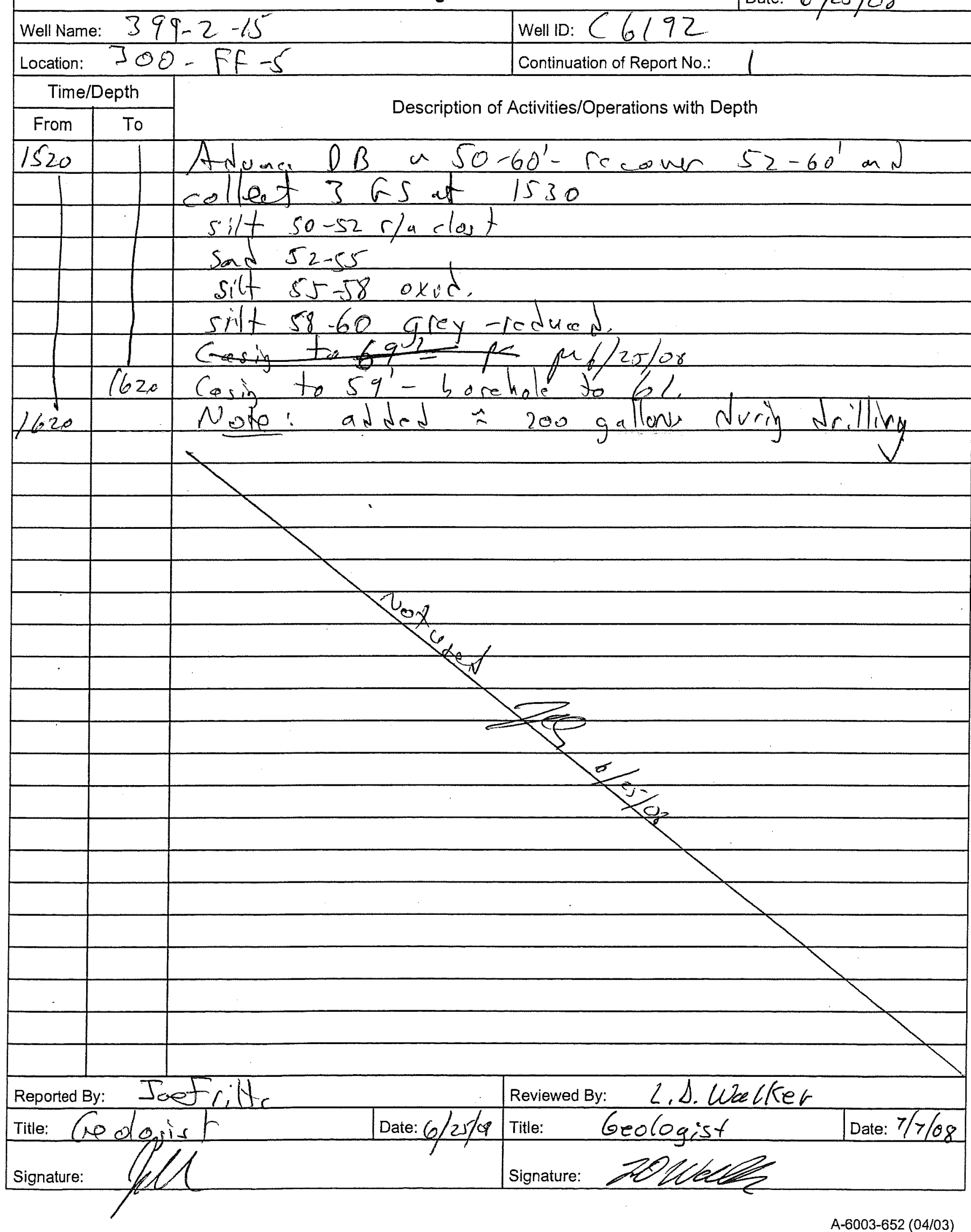




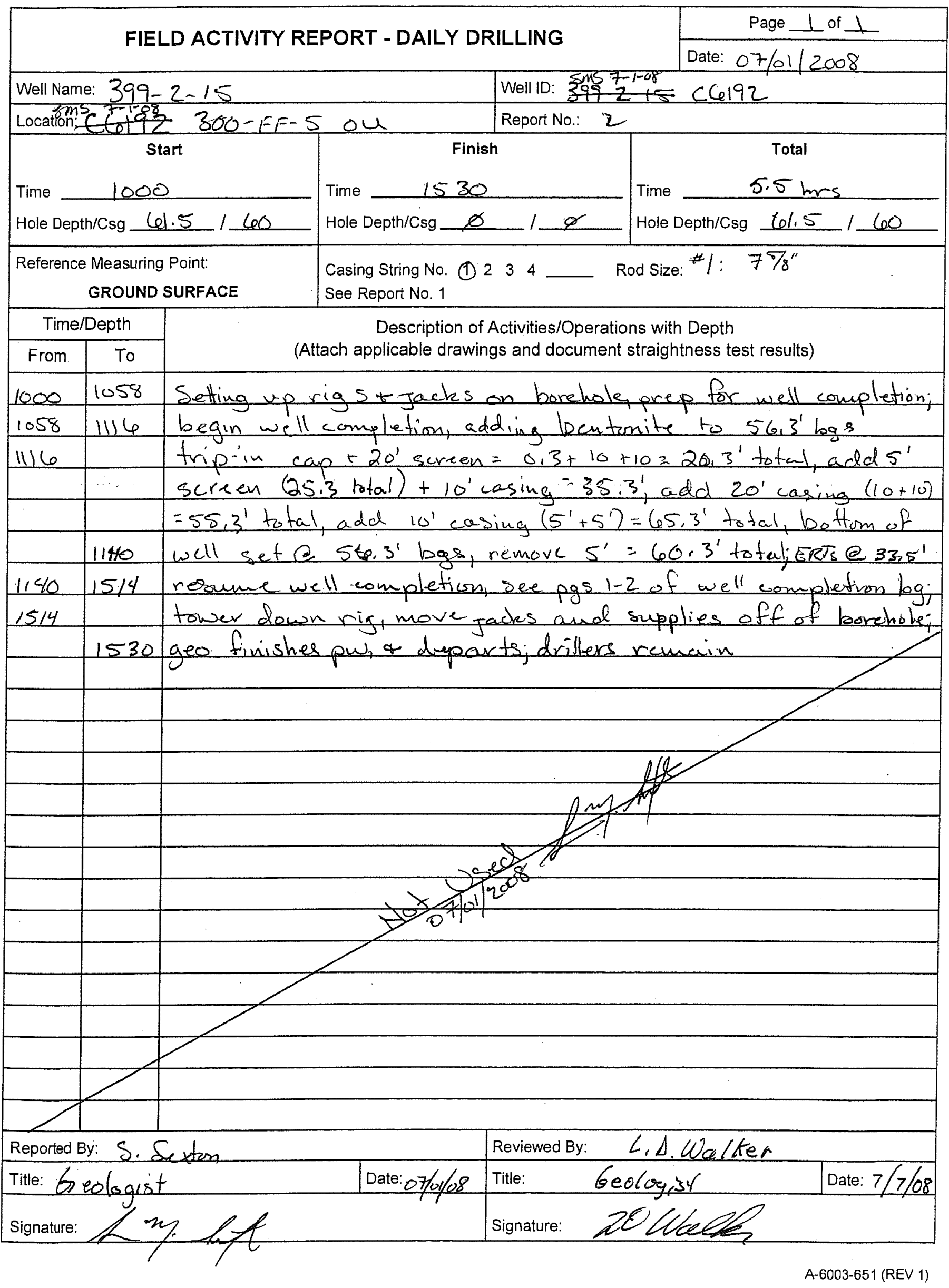

D.70 


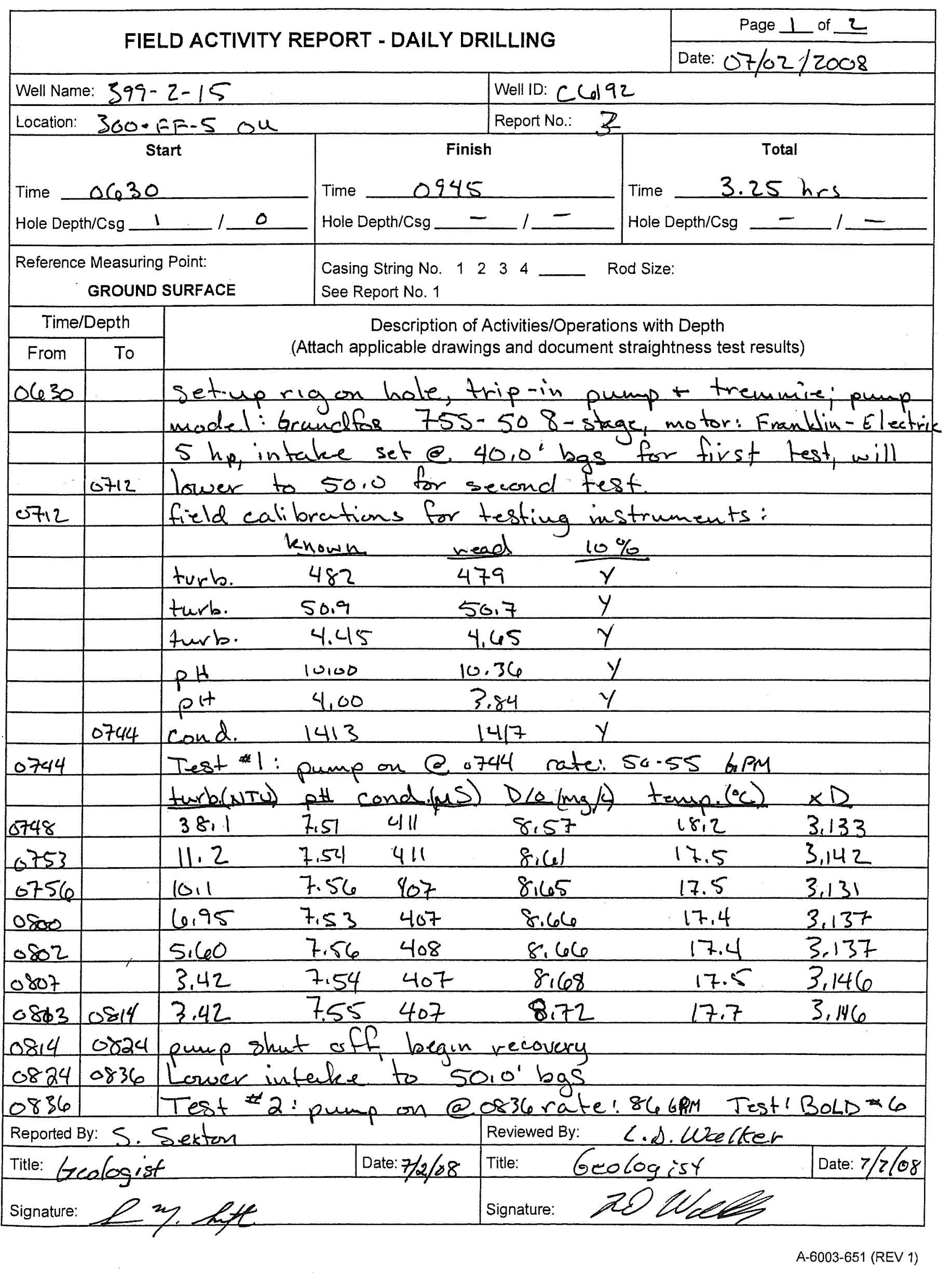

D.71 
FIELD ACTIVITY REPORT - DAILY DRILLING

Continuation Page

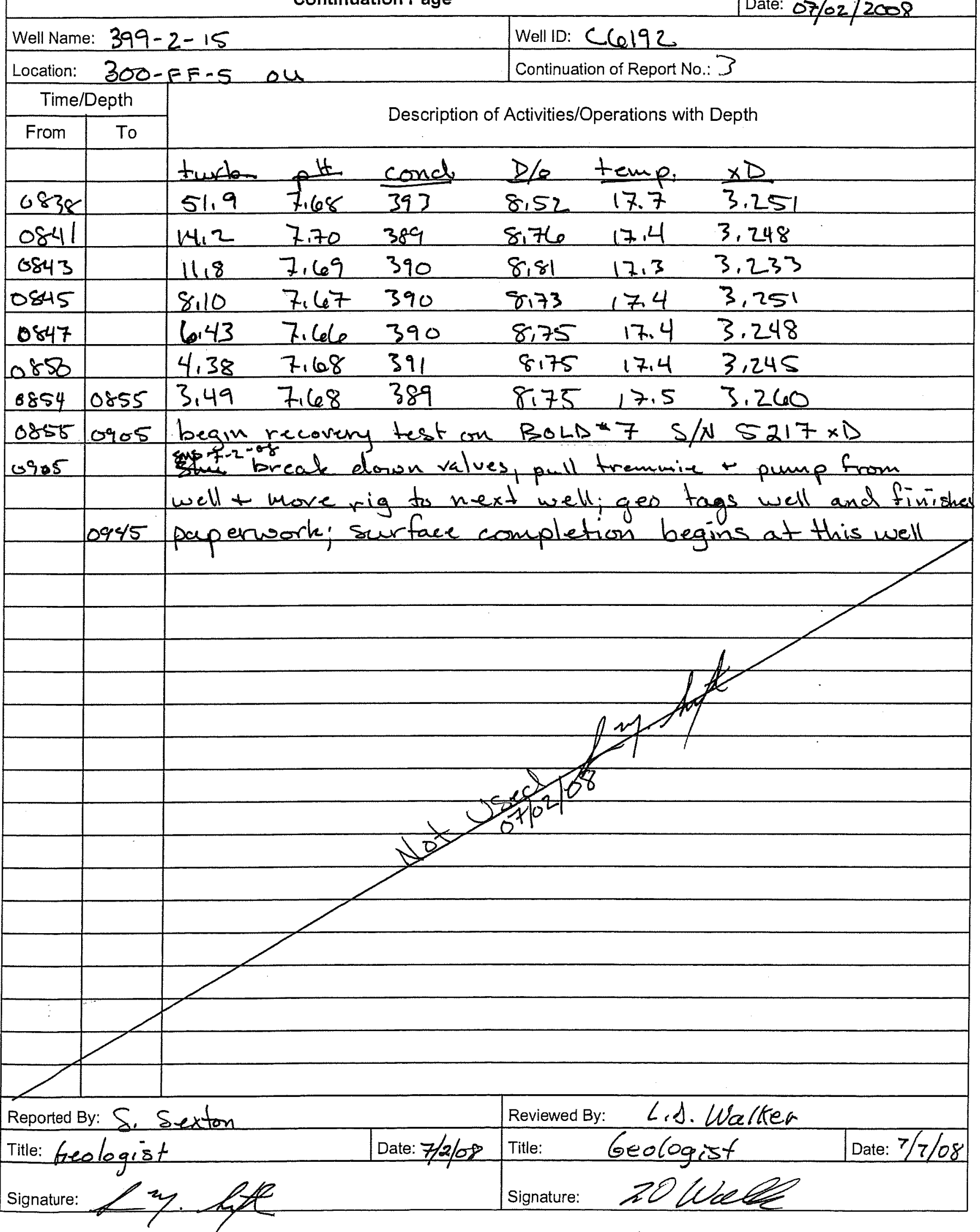

A-6003-652 (04/03) 


\section{WELL DEVELOPMENT AND TESTING DATA}

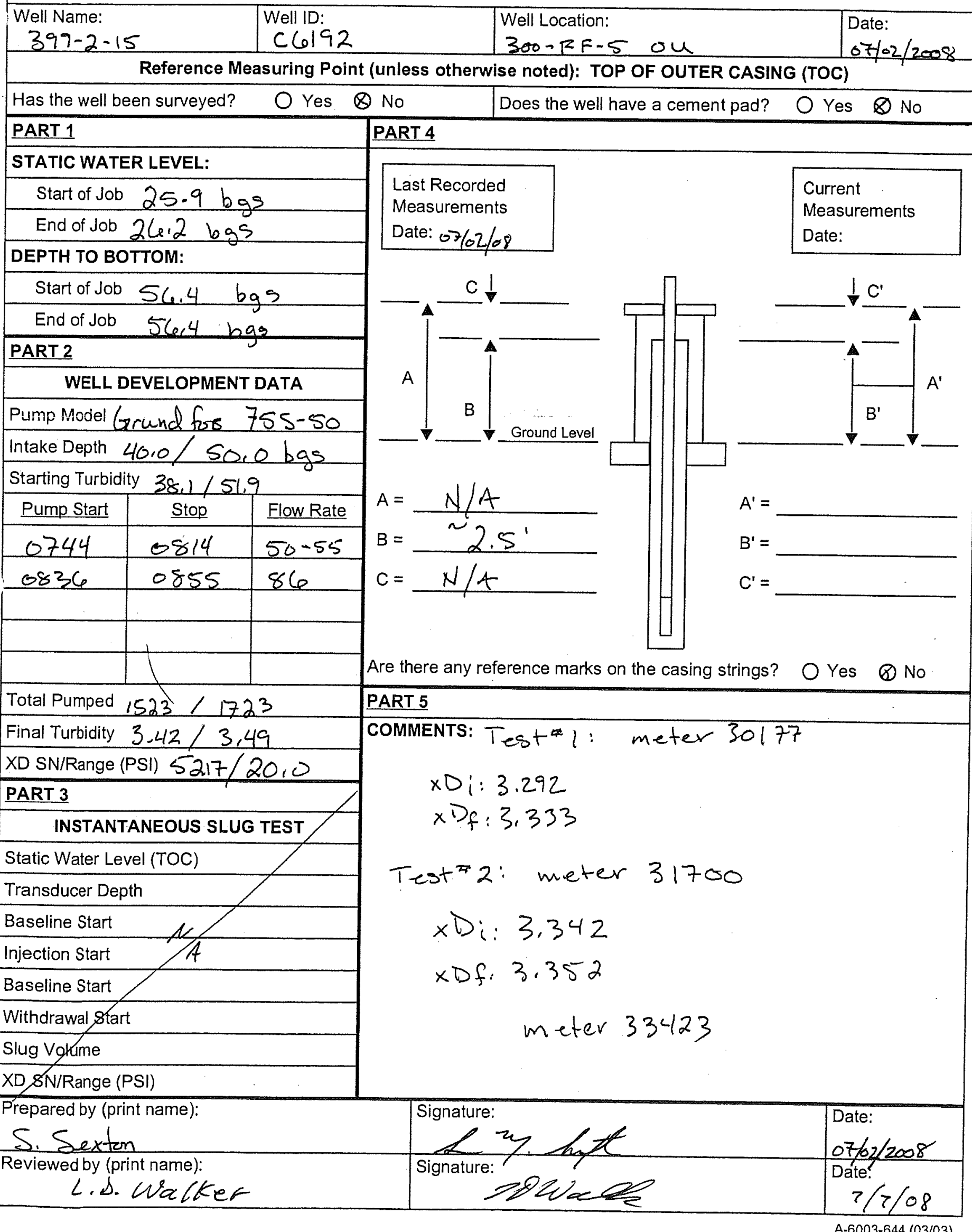




\section{FIELD ACTIVITY REPORT NO. 1 - DRILLING PLAN} Purpose: PNNL Projext 300 Ara FFC Drilling Co.: WDC Ppillis Bill Zamoz Casing String No. $123^{2} 4^{4}$ Drilling Method

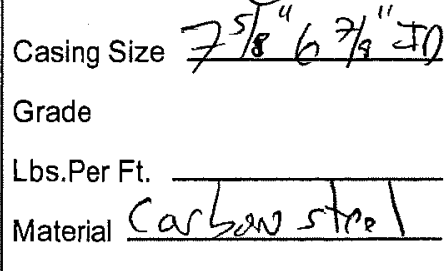
Type: Weided

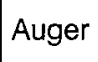
Rotary

Set At:
Shoe OD/ID
Reference Measuring Point:
GROUND LEVEL

Drig. Co. Casing String No. 112034 Casing Size Grade

Lbs.Per Ft.

Material

Type:

Welded Thd.

Planned / Actual

Set

Shoe OD/ID

Reference Measuring Point: GROUND LEVEL Other:
Location: $300-f F-5$ Date: $6 / 24 / 68$ Well Name: $399-2-16$ Rig No.: $139 \quad$ Rig Make/Mod: S Sup en Sojoc Circulation Air — Water/Mud Reverse _ Direct Vol: $\quad \mathrm{cfm}$ Cable Tool Sonic) Surar Sovic 2

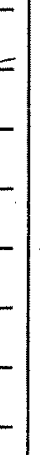
A.R. w/Sonic RS 400 Geoprobe

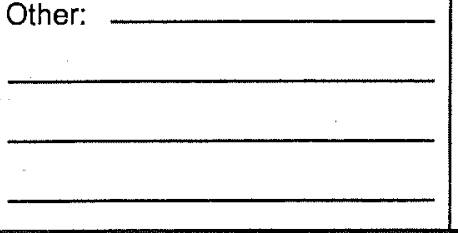

Rig No.:

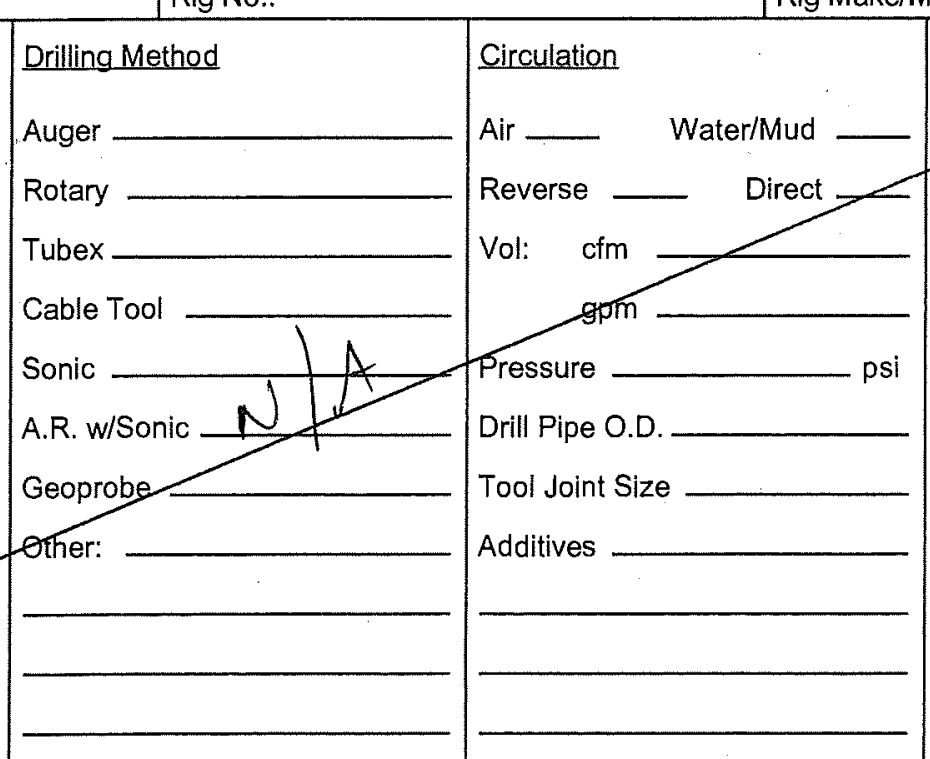

D.H. Hammer Model

Choke

\section{Casing Hammer}

Make

Model

Bit Size

Type

Nozzles

Rod Size

Estimated Depth to Water

Comments/Remarks:

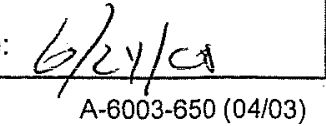




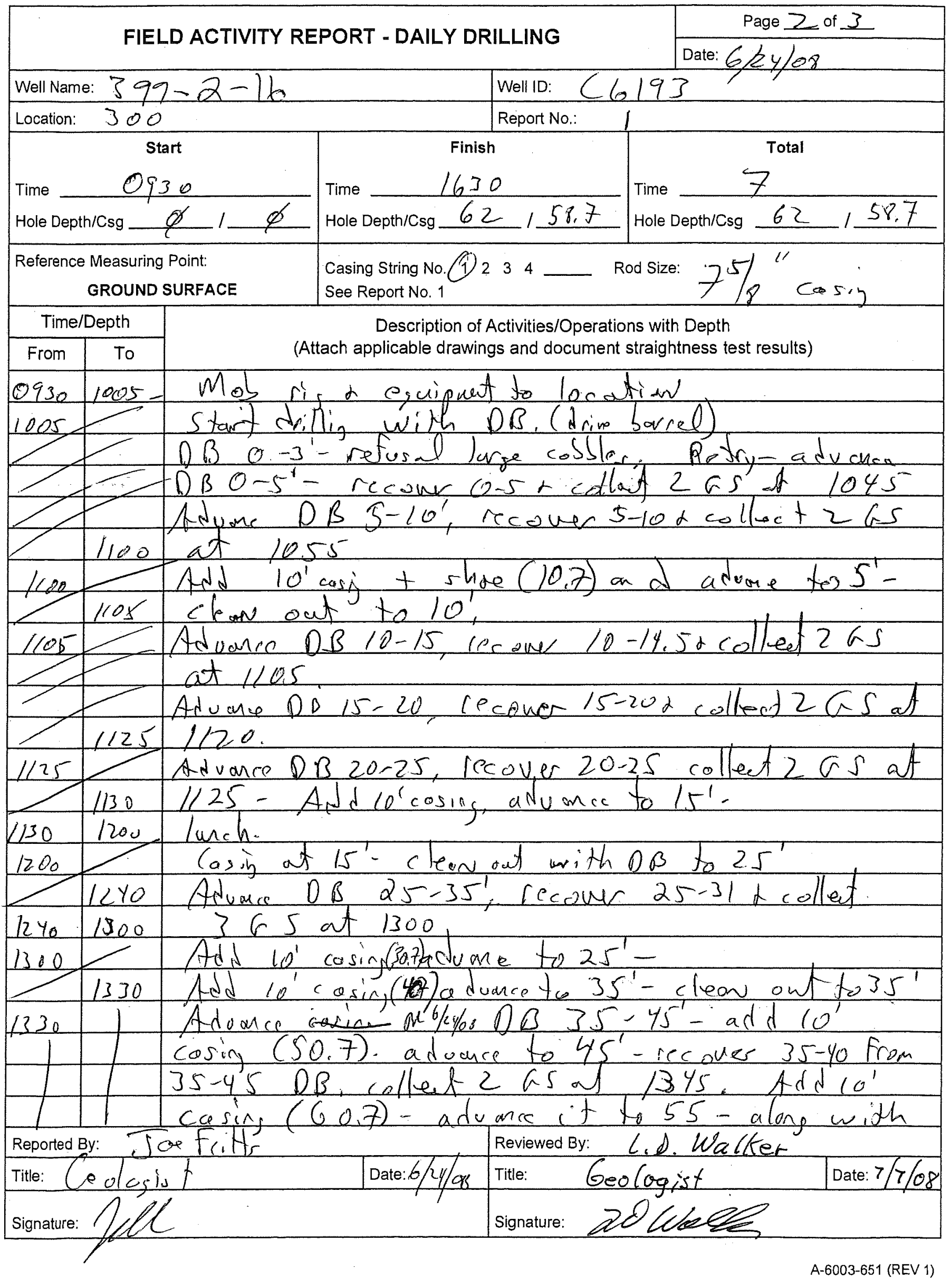

D.75 


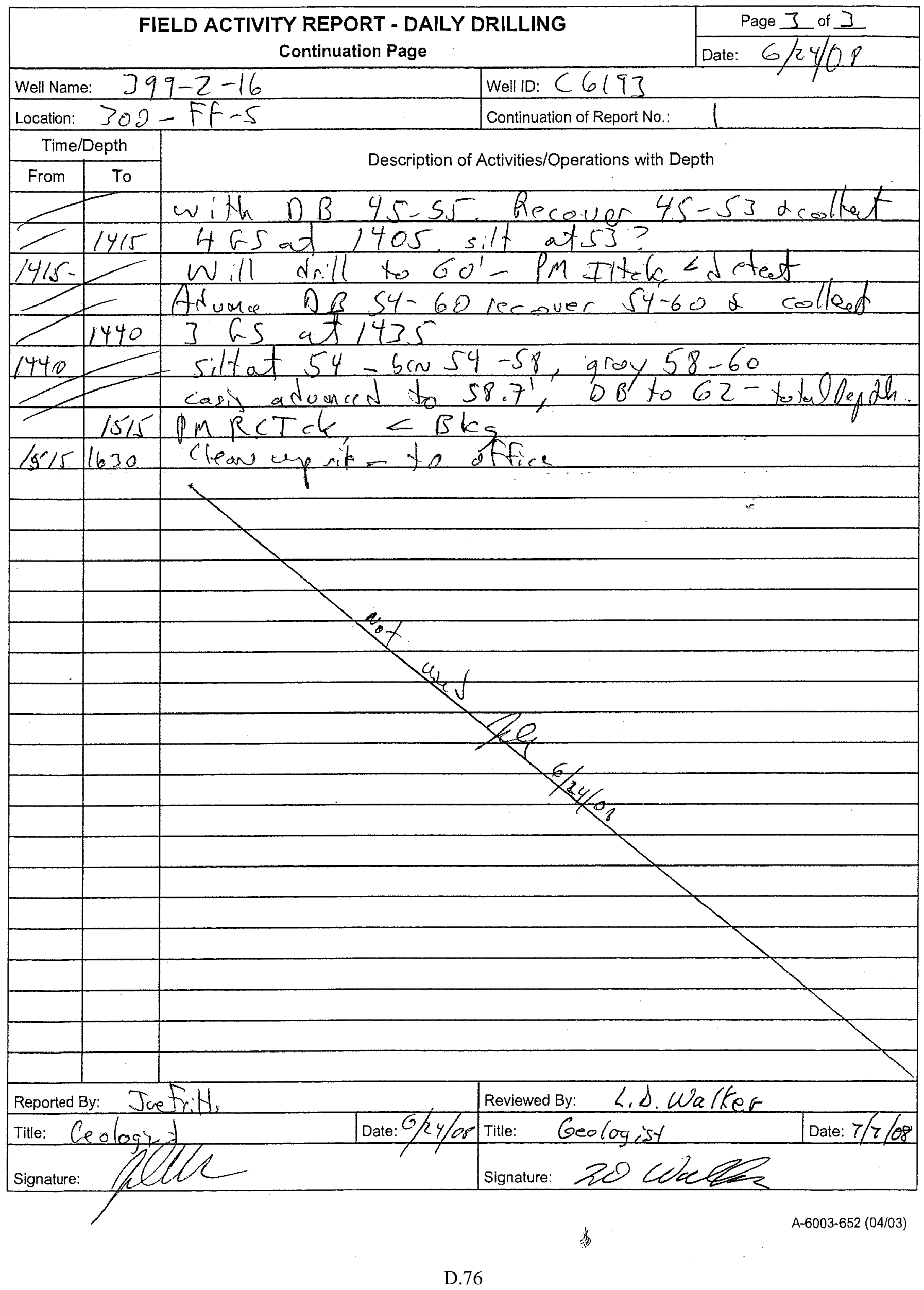




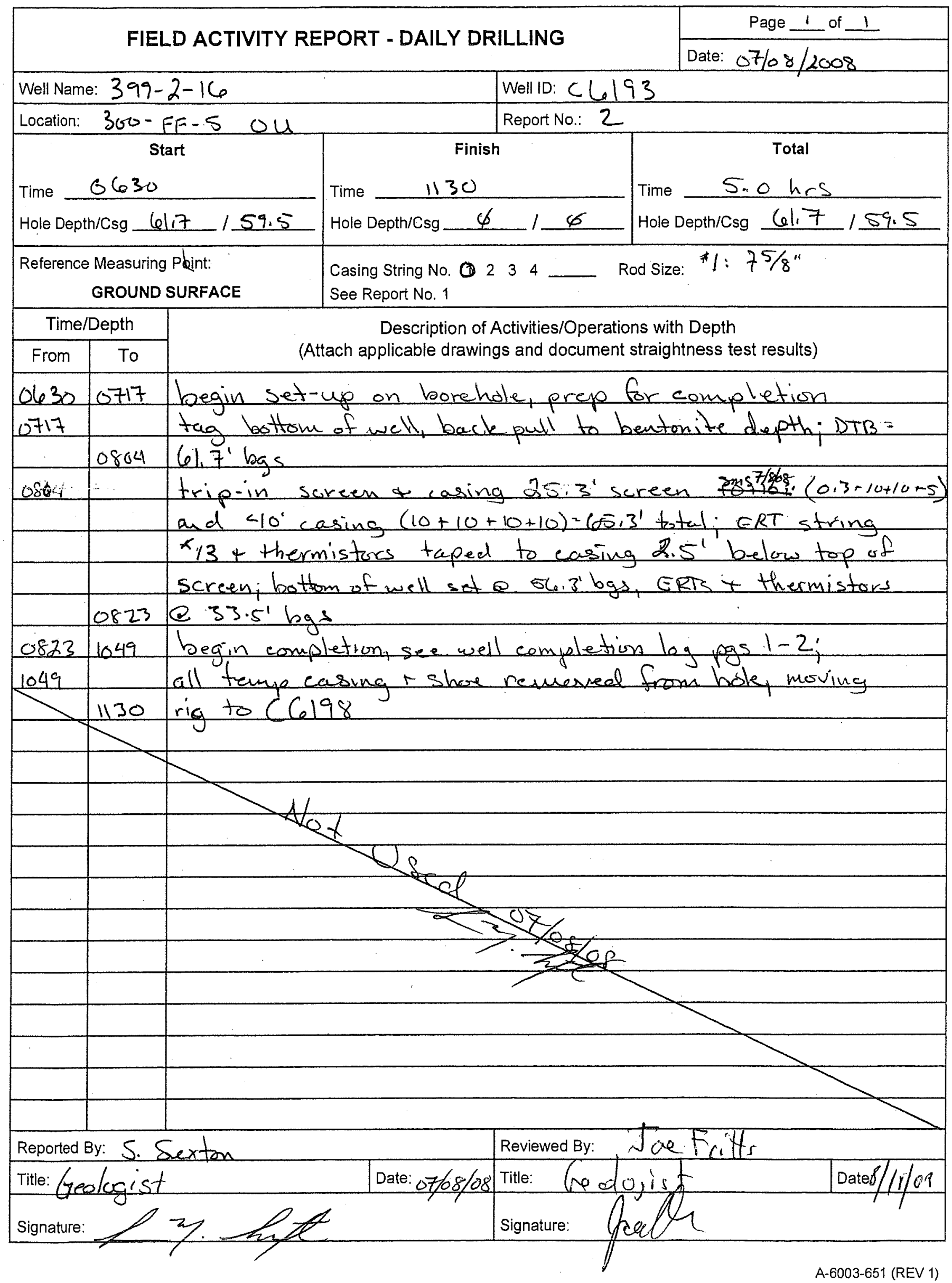




\section{FIELD ACTIVITY REPORT - DAILY DRILLING}

\begin{tabular}{|c|} 
Page $\perp$ of 2 \\
Date: $07 / 17 / 2008$
\end{tabular}

\begin{tabular}{l}
\hline Well Name: $399-2-16$ \\
\hline Location: $300-$ FF -5 ou \\
Start \\
Time $\frac{1000}{\text { Hole Depth/Csg _ _ }}$ \\
$\begin{array}{c}\text { Reference Measuring Point: } \\
\text { GROUND SURFACE }\end{array}$
\end{tabular}

\begin{tabular}{l|l} 
& Well ID: C6193 \\
& Report No: 3 \\
\hline
\end{tabular}

Finish

Total

Time 1230 Time 2.5 hrs Hole Depth/Csg _ _ I _ _ Hole Depth/Csg _ _ I _ Casing String No. $\begin{array}{lllllll}1 & 2 & 3 & 4\end{array}$ Rod Size: See Report No. 1

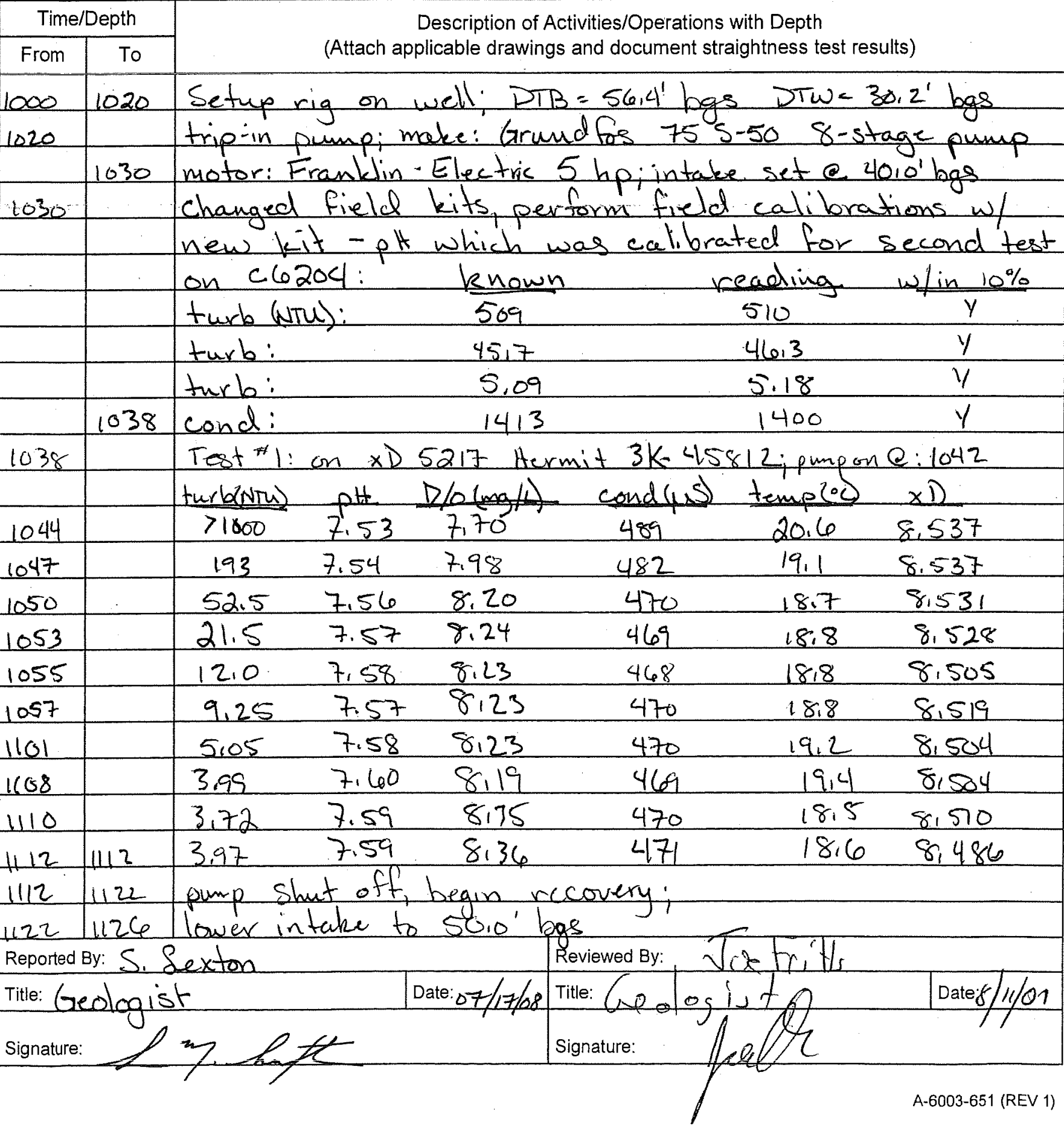


FIELD ACTIVITY REPORT - DAILY DRILLING

Continuation Page

Well Name: $399-2-16$

Location: $300-F F-5$ ou

\section{Time/Depth}

\begin{tabular}{|c|c|}
\hline From & To \\
\hline 1126 & \\
\hline
\end{tabular}

Well ID: C6193

Continuation of Report No.: 3

Description of Activities/Operations with Depth
Page 2 of 2 Date: $07 / 17 / 2008$

\begin{tabular}{|c|c|c|c|c|c|c|c|}
\hline & & \multicolumn{3}{|c|}{ pump on at: 1127} & te: 86 & \multicolumn{2}{|c|}{ intake: 50,0 } \\
\hline & & tusch. & pH & $D / 0$ & cond. & temp. & $\times D$ \\
\hline 1129 & & 224 & 7.63 & 8,30 & 469 & 19,4 & 16,368 \\
\hline 1132 & & 37,9 & 7.60 & 8,57 & 470 & 18,4 & 16,380 \\
\hline 1134 & & 20,0 & 7.58 & 8164 & 470 & 18.2 & $16: 353$ \\
\hline 1136 & & 14,7 & 7.58 & 8,68 & 471 & 1810 & 16,353 \\
\hline 1139 & & 10,5 & 7,56 & 8,72 & 471 & 17,9 & 16.353 \\
\hline 1141 & & 9.32 & 7,57 & 8,77 & 472 & 17.5 & 16,338 \\
\hline 1142 & 1144 & 7.57 & 7.57 & 8.77 & 469 & 18,4 & 16.329 \\
\hline
\end{tabular}

11441154 begin recovery test on Sun Dried wD10

11541230 trip-out punp tower down rig t mave to next well

Reported By: S. Sexton

Title: Geologist

Signature:
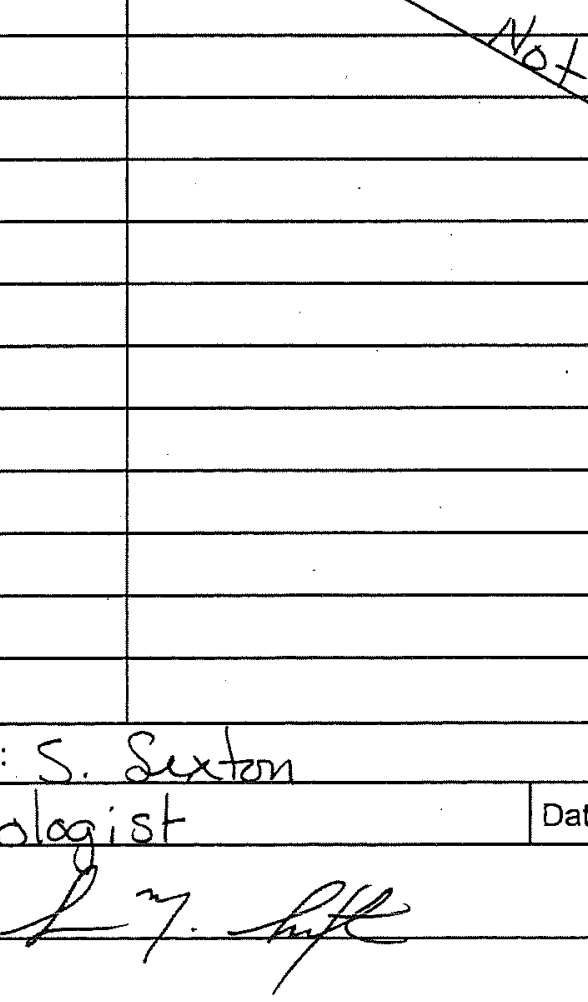

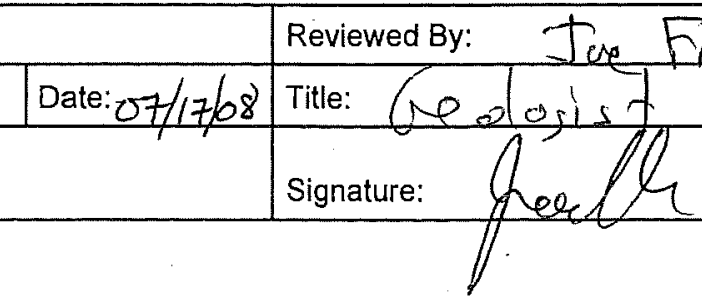

D.79 


\section{WELL DEVELOPMENT AND TESTING DATA}

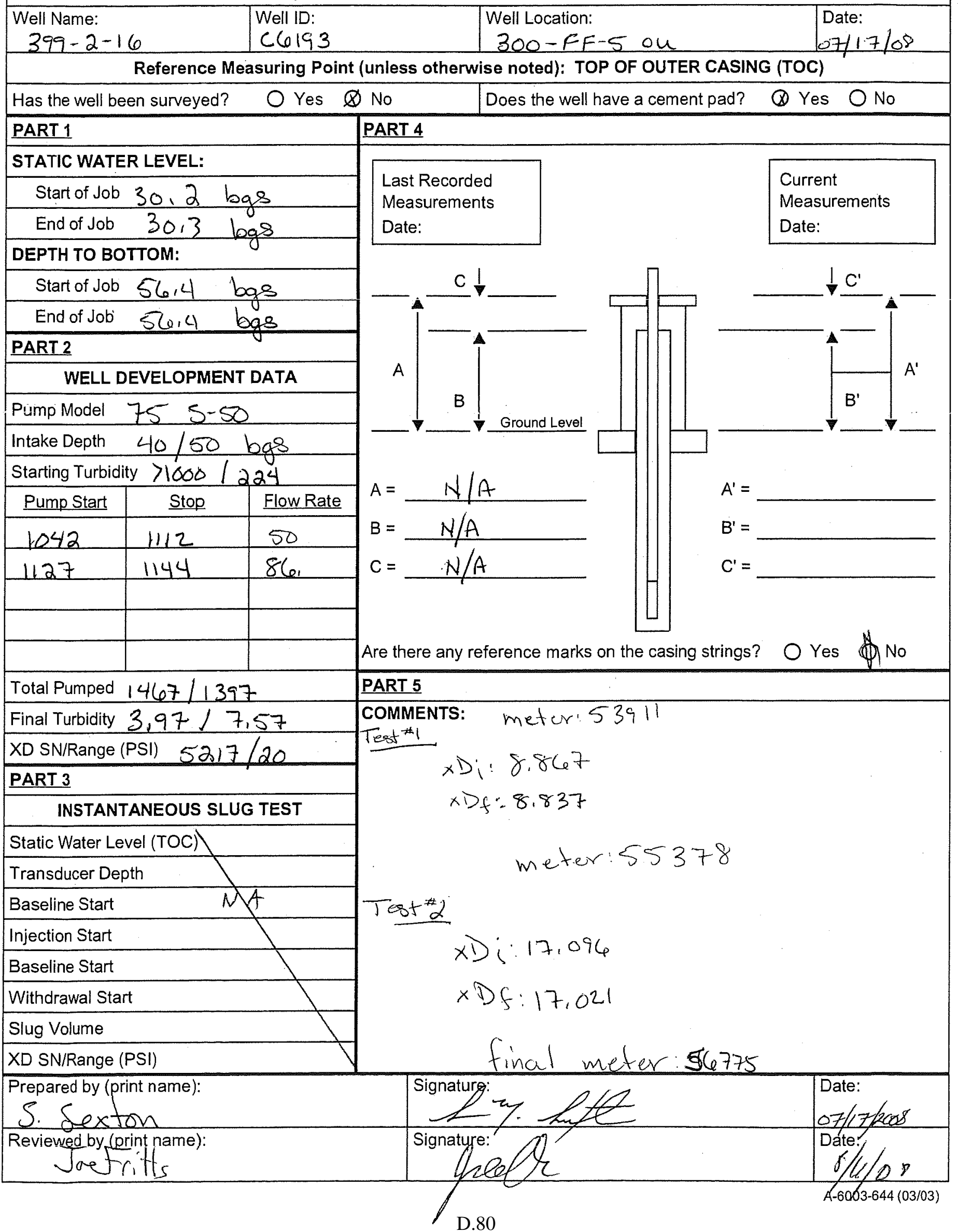


FIELD ACTIVITY REPORT NO. 1 - DRILLING PLAN

Page 1 of 1

Date: $06 / 12 / 2008$

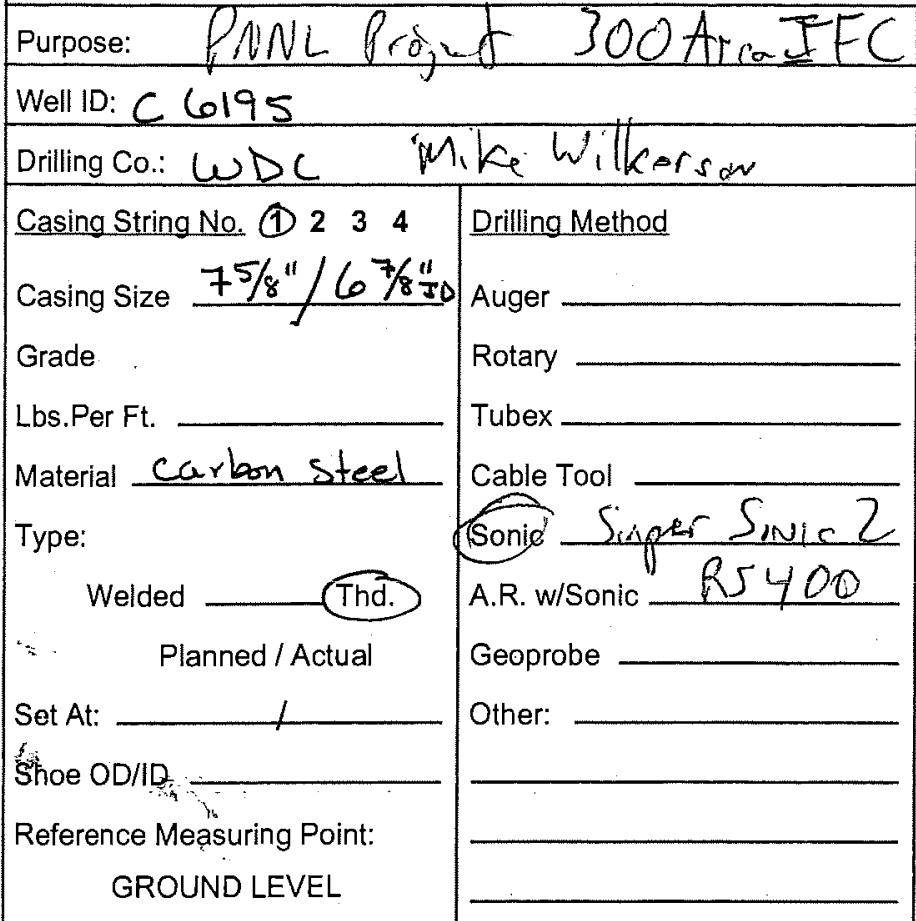

Drig. Co.

\begin{tabular}{lllll}
\hline Casing String No. & 1 & 2 & 3 & 4
\end{tabular}

Casing Size

Grade

Lbs.Per Ft.

Material

Type:

Welded Thd.

Planned / Actua

Se

Shoe OD/ID

Reference Measuring Point: GROUND LEVEL
Rig No.:

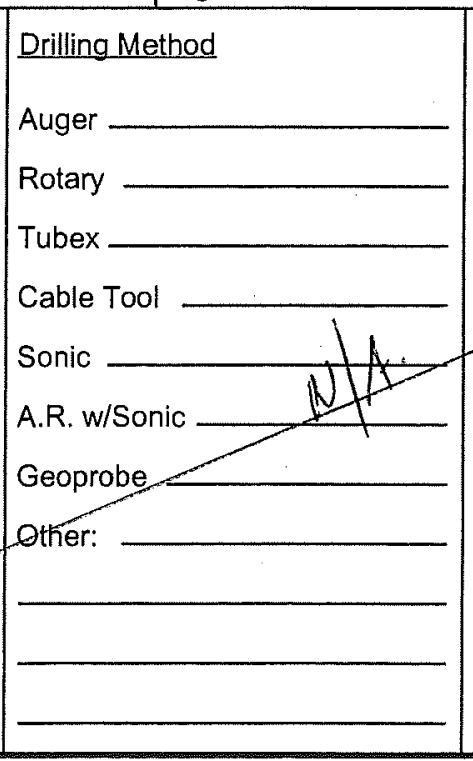

Location: 300 - FF-5 O.U. Well Name: $399-2-17$ Rig No.: $139 \quad$ Rig Make/Mod.: Super Sonic II Circulation Air _ Water/Mud Reverse Direct Vol: $\quad \mathrm{cfm}$

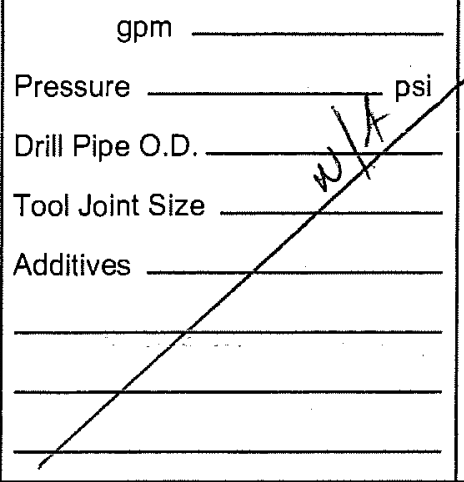
D.H. Hammer

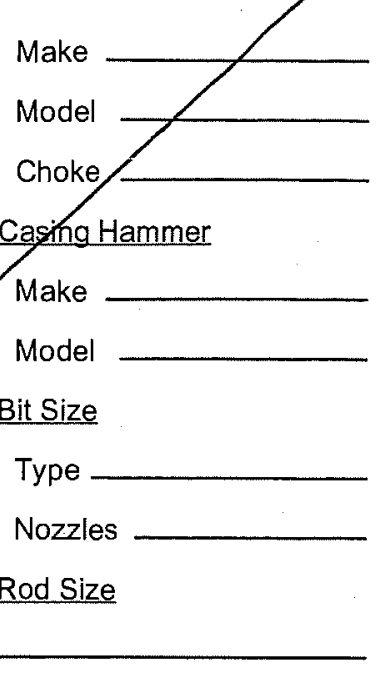

Rig Make/Mod::

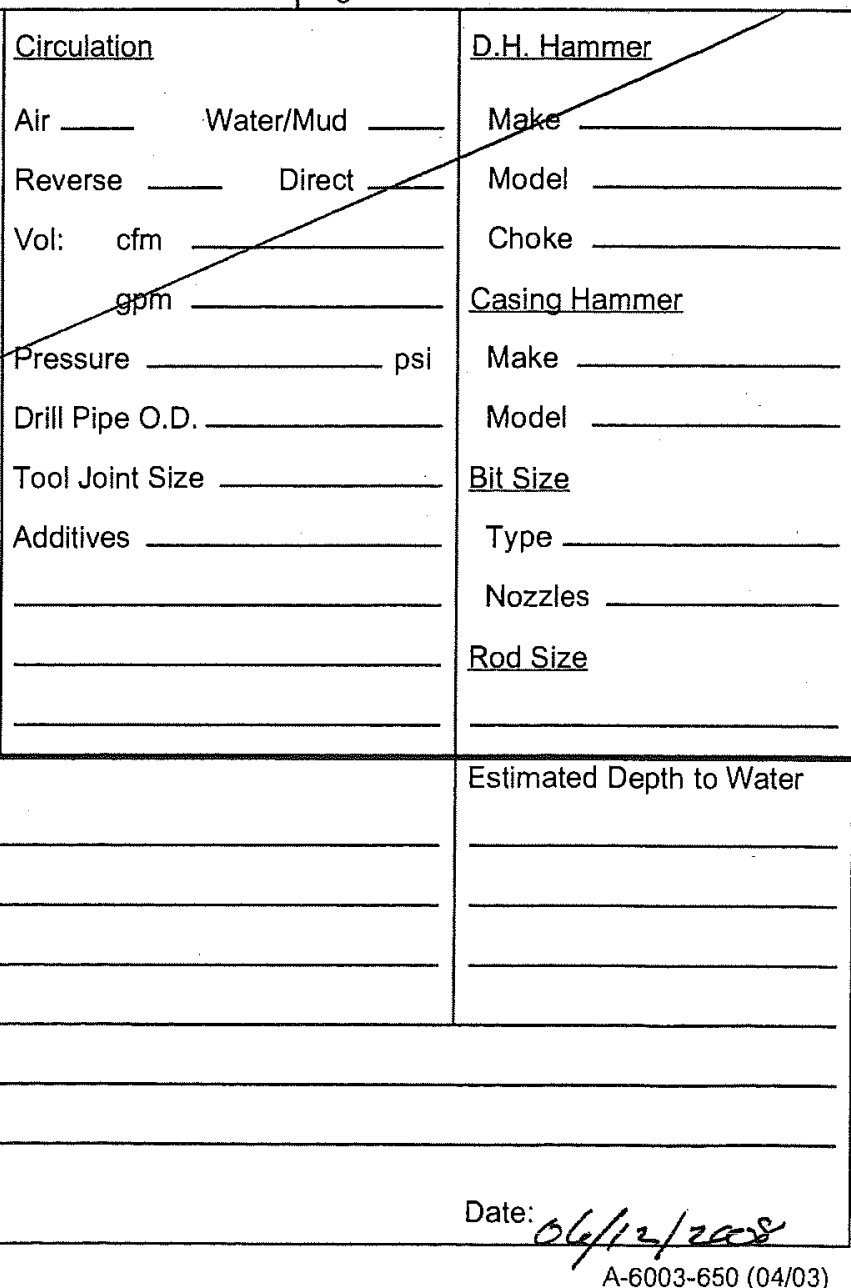




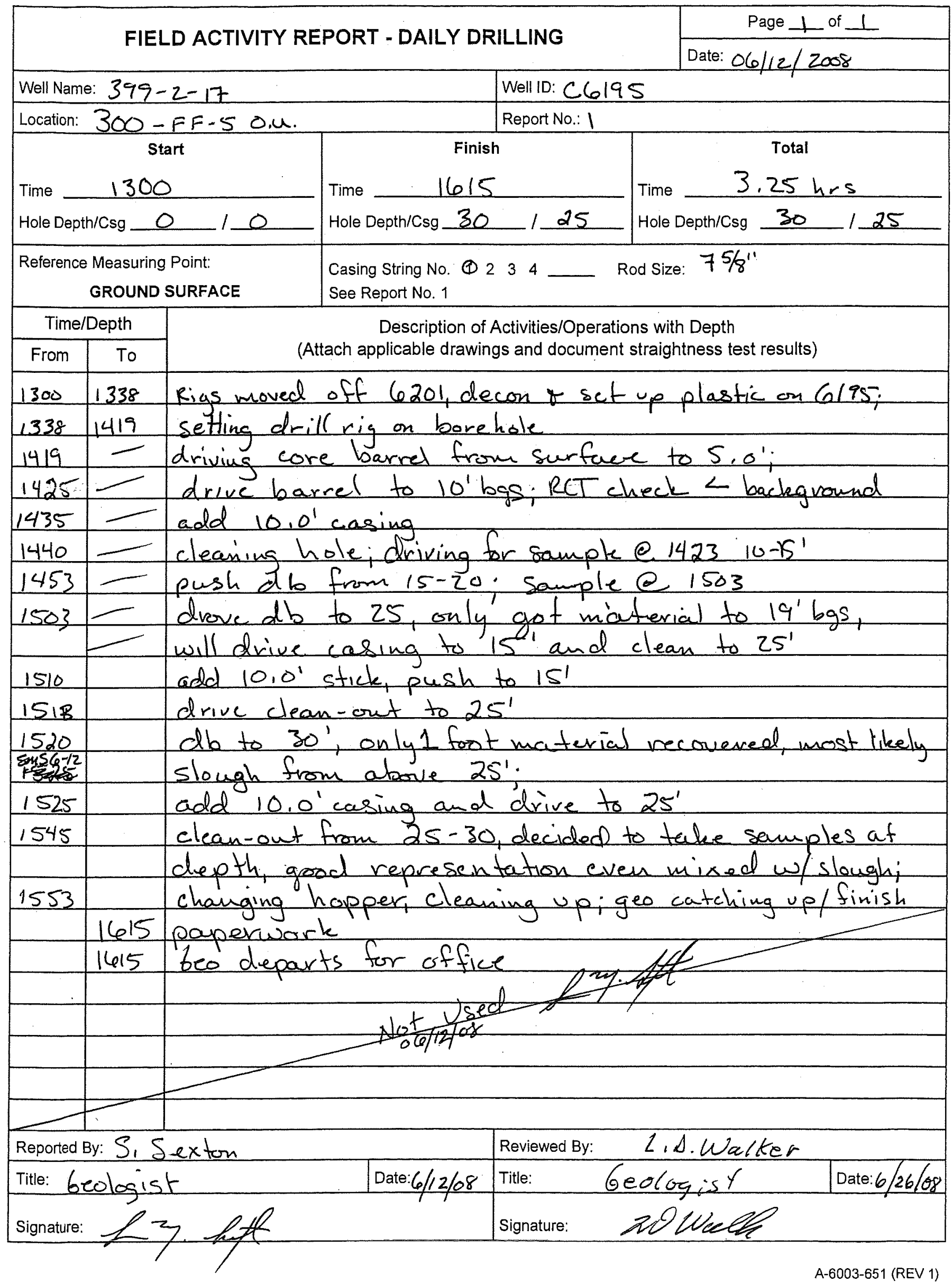




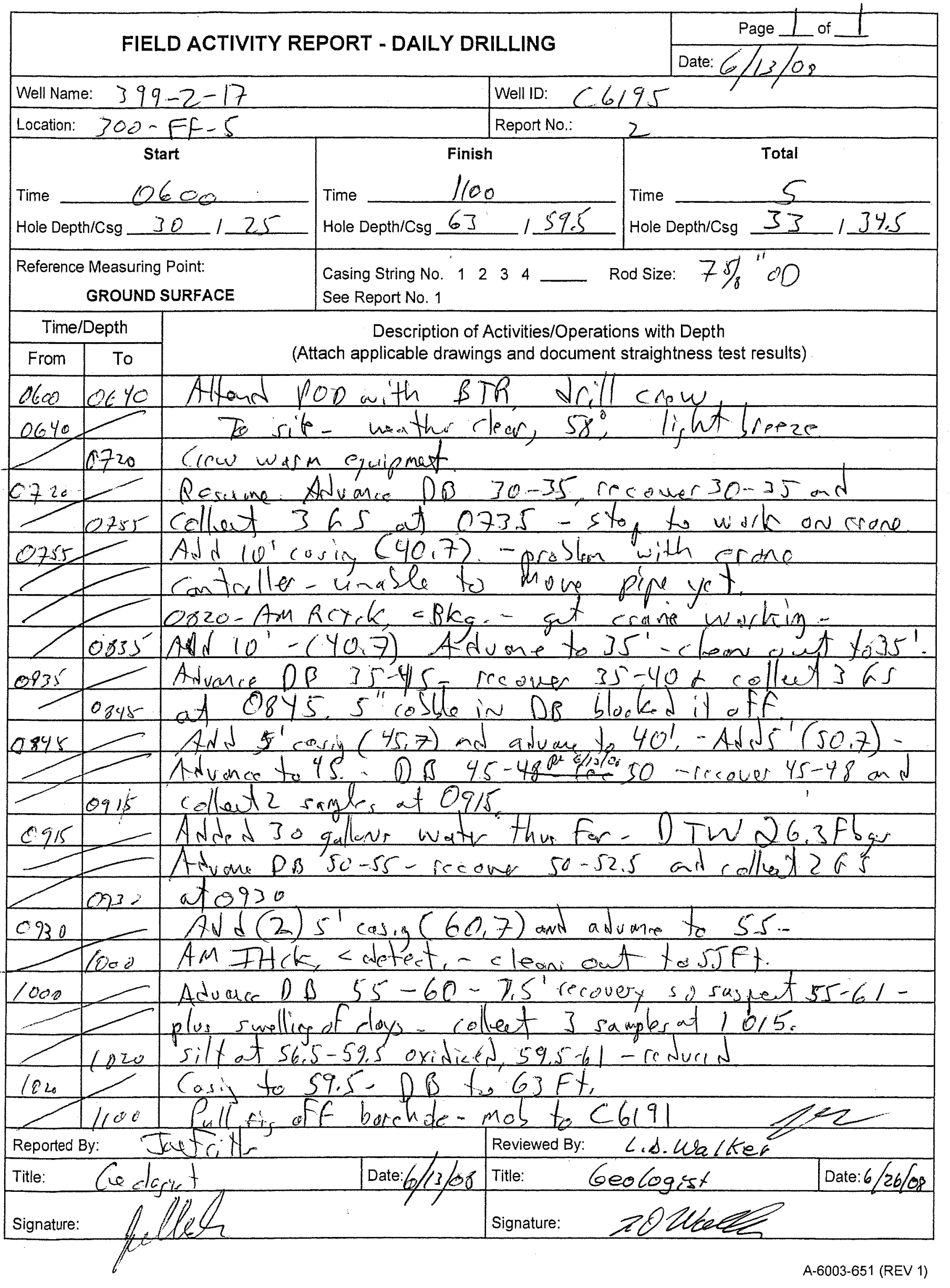

D.83 


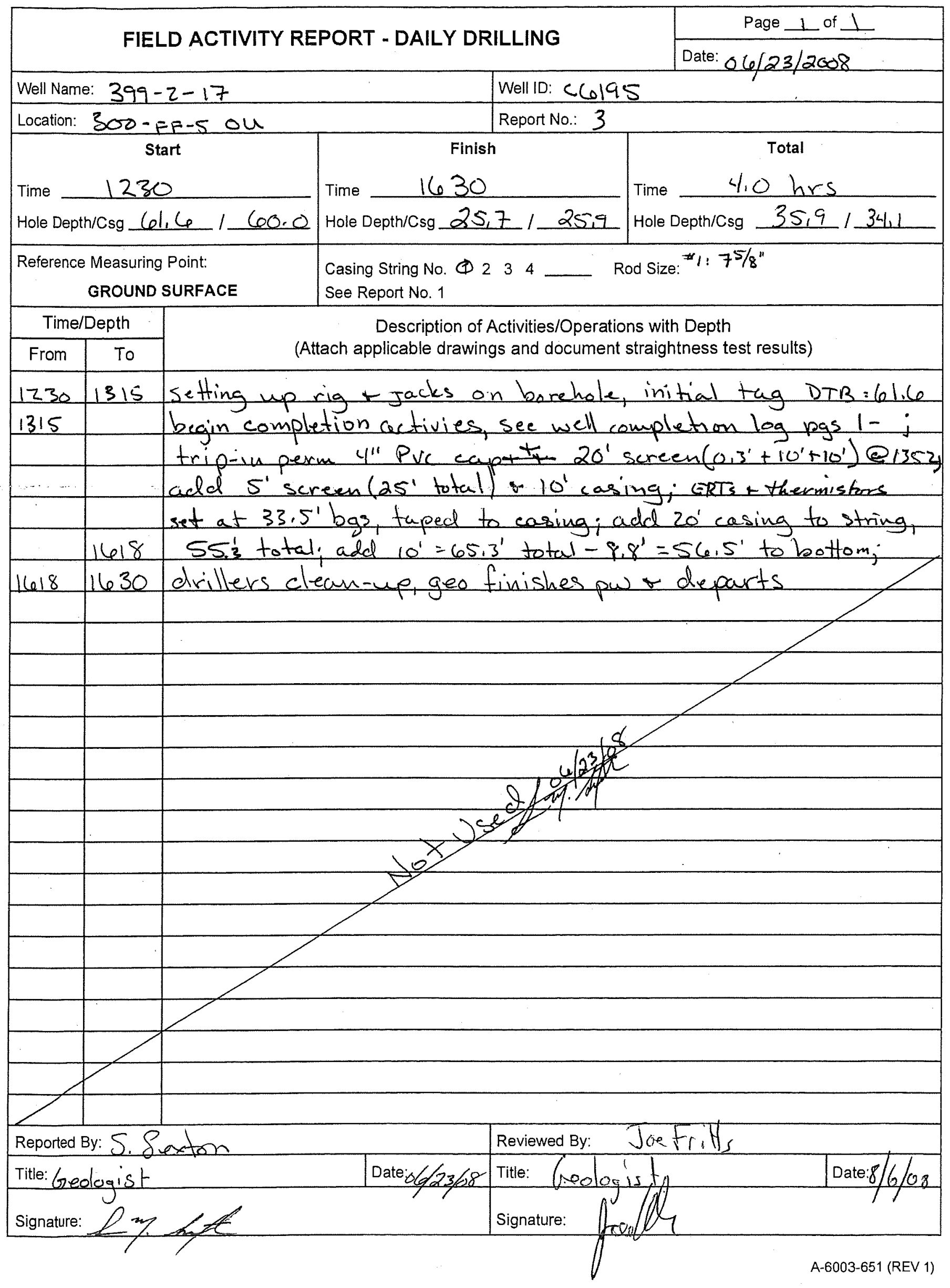




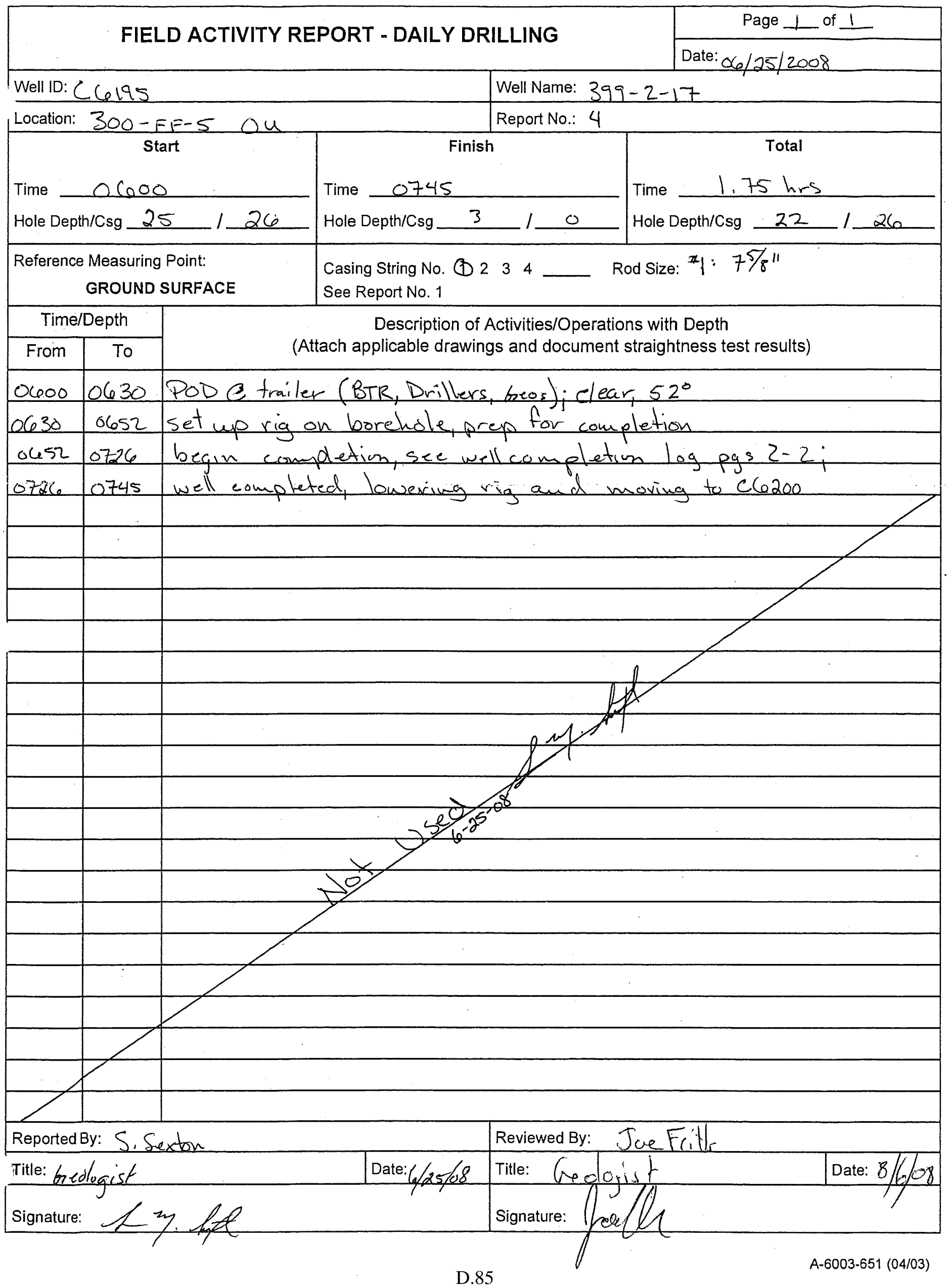




\section{FIELD ACTIVITY REPORT - DAILY DRILLING}

Page 1 of 2

\begin{tabular}{l} 
Well Name: $399-2-17$ \\
\hline Location: $300-F F-5$ OU \\
\hline Start \\
Time 1000 \\
Hole Depth/Csg__ \\
$\begin{array}{c}\text { Reference Measuring Point: } \\
\text { GROUND SURFACE }\end{array}$
\end{tabular}

\begin{tabular}{l|l|l|} 
& Well ID: $C 6195$ \\
\hline & Report No.: 5 \\
\hline
\end{tabular}

\begin{tabular}{|c|c|}
\hline $\begin{array}{l}\text { Finish } \\
1330 \\
\end{array}$ & $\begin{array}{c}\text { Total } \\
\text { Time } 3.5 \text { hrs } \\
\end{array}$ \\
\hline Hole Depth/Csg _ _ & Hole Depth/Csg _ \\
\hline $\begin{array}{l}\text { Casing String No. } \begin{array}{lllll}1 & 2 & 3 & 4 \\
\text { See Report No. } 1\end{array}\end{array}$ & od Size: \\
\hline
\end{tabular}

\begin{tabular}{|c|c|c|}
\hline \multicolumn{2}{|c|}{ Time/Depth } & \multirow{2}{*}{$\begin{array}{l}\text { Description of Activities/Operations with Depth } \\
\text { (Attach applicable drawings and document straightness test results) }\end{array}$} \\
\hline From & To & \\
\hline
\end{tabular}

1000 Set-up rig on well, bail well t setup pump + tremmie 11107 while waiting for purge truck to return; 1107 Purgenater truck on-site, connecting hoses + final prep for testingi instruments calibrated 20730 TestII: Pump: Grund fos $755-50$ Metor: Franklin Electric 5 hp

Pump on at: 1113 rate: $56.55 \times D: 5217$

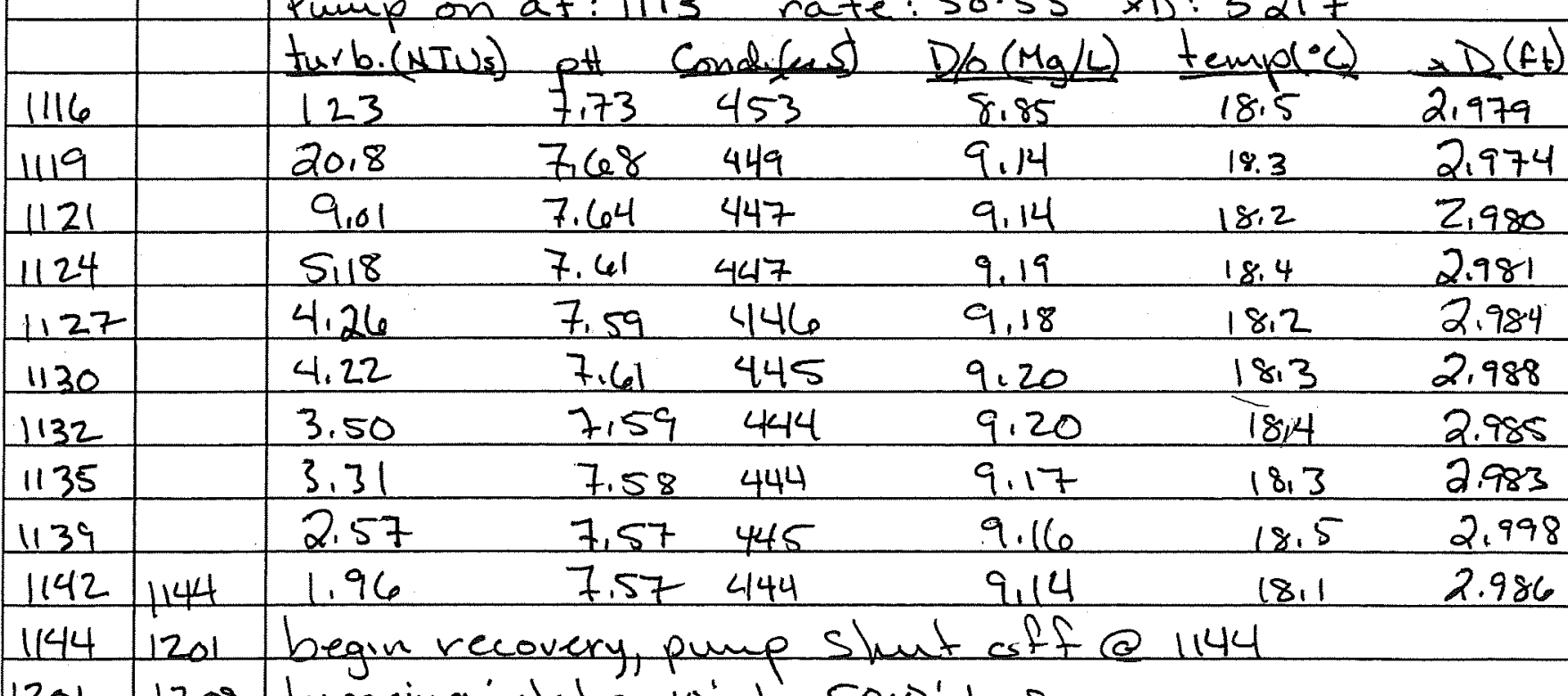

12011209 lowering intalee 10' to $50.0^{\prime} \mathrm{bgs}$

1209 Test 2: Pumpon Q: 1211 rate: XD 5217

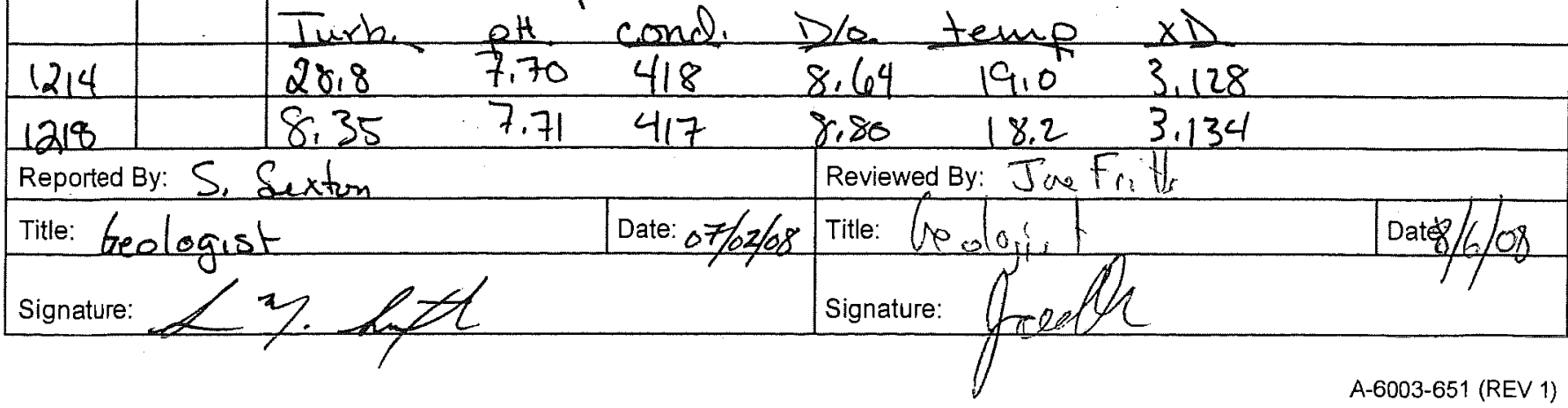




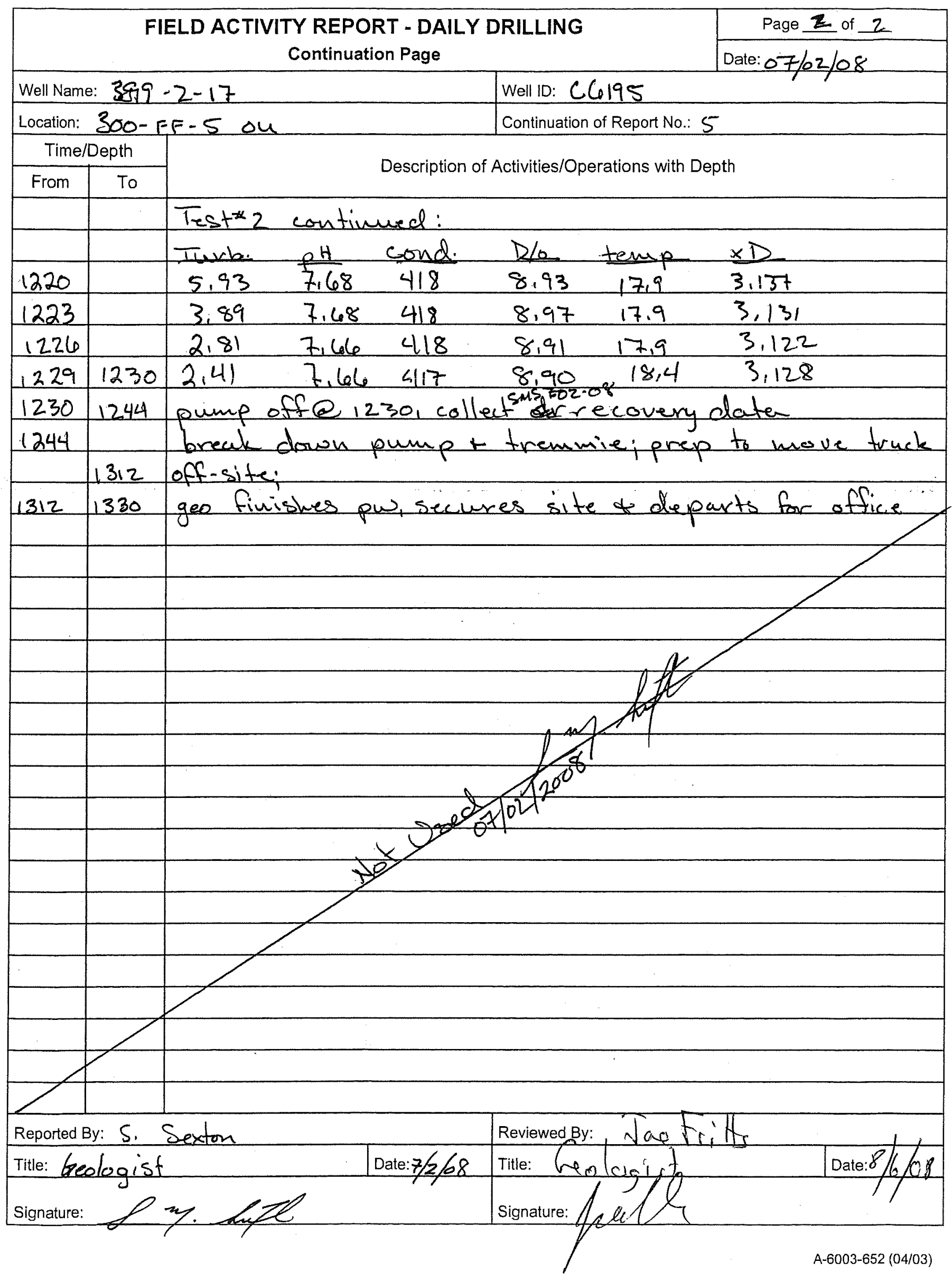




\section{WELL DEVELOPMENT AND TESTING DATA}

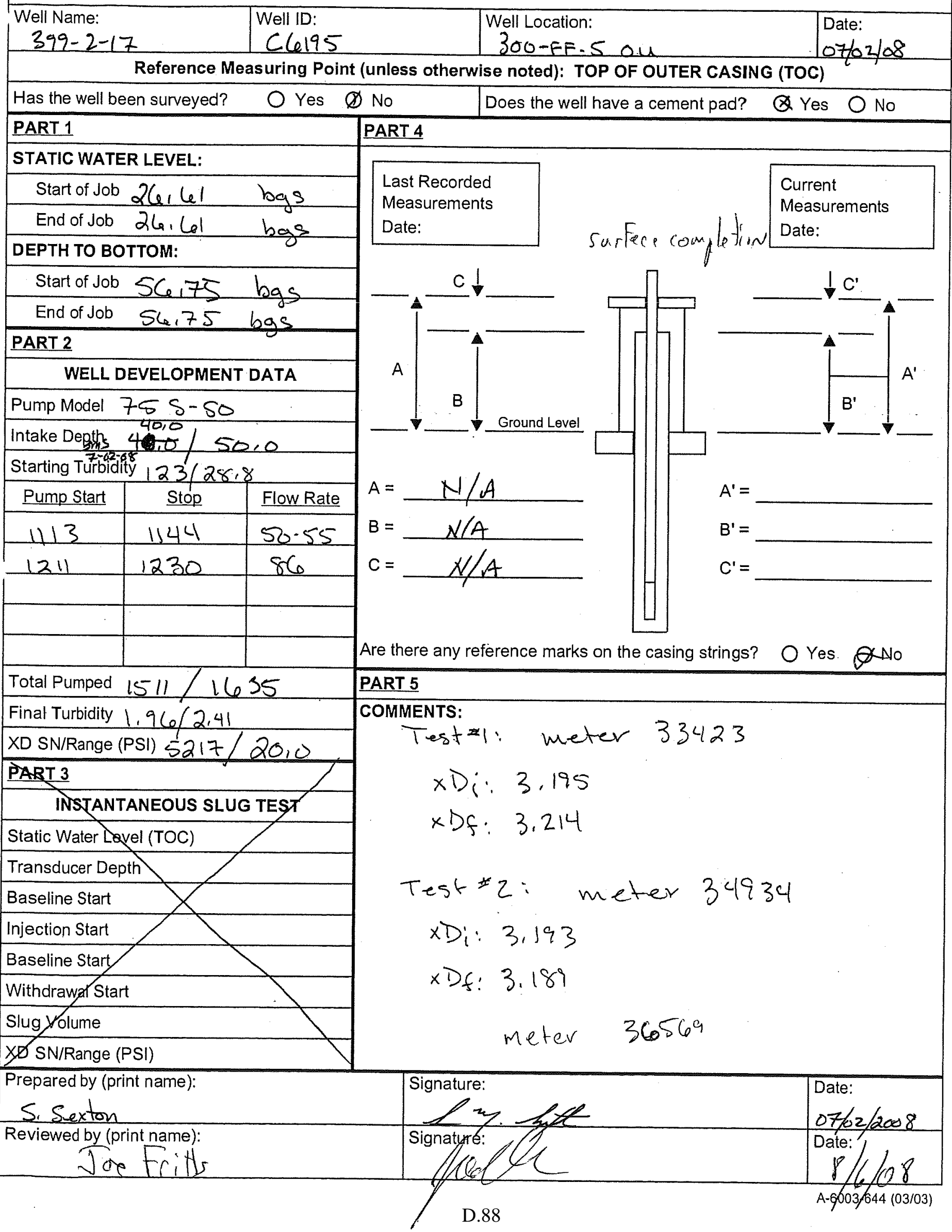




\section{FIELD ACTIVITY REPORT NO. 1 - DRILLING PLAN}

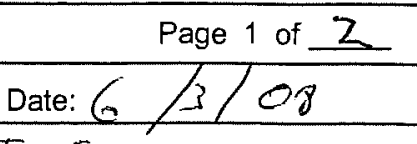

\begin{tabular}{|c|c|c|}
\hline Purpose: PNNL Projed & 300Ateaste & Location: $300-F F-5-0 \mathrm{C}$ \\
\hline Well ID: $\quad C_{6196}$ & & Well Name: $399-2-18$ \\
\hline
\end{tabular}

Drilling Co.: WOC Bdl amowi $_{\text {amollt }}$

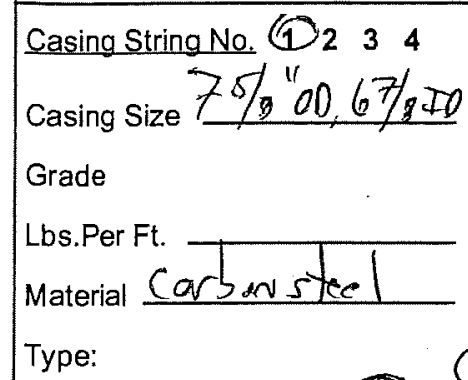

Welded

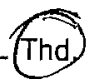

Planned / Actual

Set At:

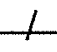

Shoe OD/ID

Reference Measuring Point:

GROUND LEVEL

Drig. Co.

Casing String No. $\begin{array}{llll}1 & 2 & 3 & 4\end{array}$

Casing Size

Grade

Lbs.Per Ft.

Material

Type:

Welded Thd.

Planned / Actual

Set

Shoe OD/ID

Reference Measuring Point: GROUND LEVEL

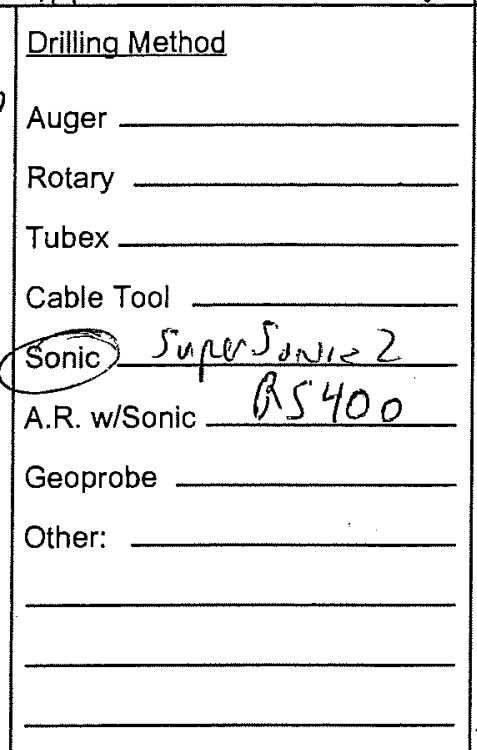

Rig No.:

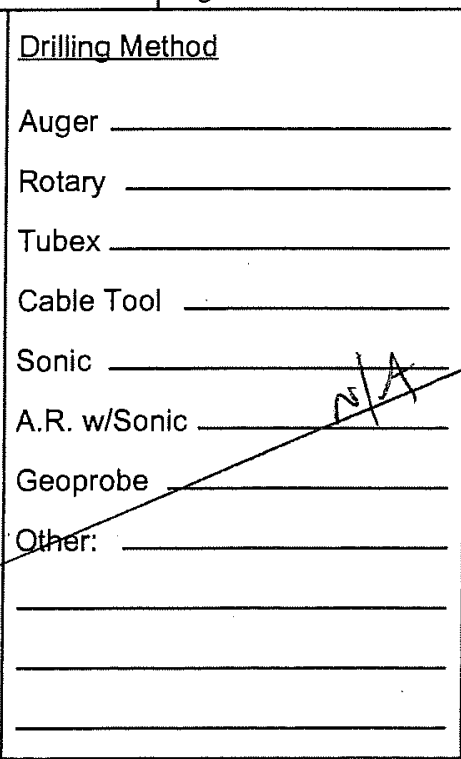
Rig No.: $13 q$ Rig Make/Mod.: Supers In 12 Circulation

A

Wir $-\quad$ Water/Mud -
Reverse $-\quad$ Direct -

Vol: $\quad \mathrm{cfm}$

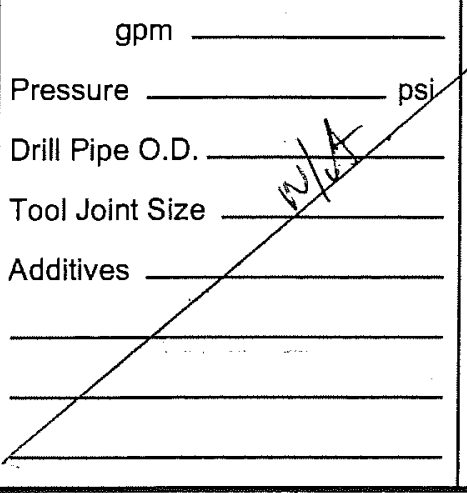

D.H. Hammer

Make

Mode

Choke

Casing Hammer

Make

Model

Bit Size

Type

Nozzles

Rod Size

Rig Make/Mod.:

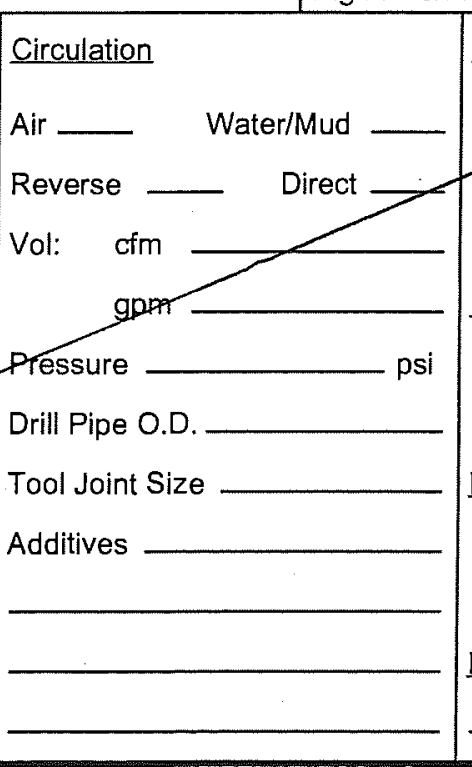

D.H. Hammer

Comments/Remarks:

Reported By: Jyetity

Name/Title: GRAM Ior Credegist

Signature:

Date: $t / 3 / 98$

A-6003-650 (04/03) 


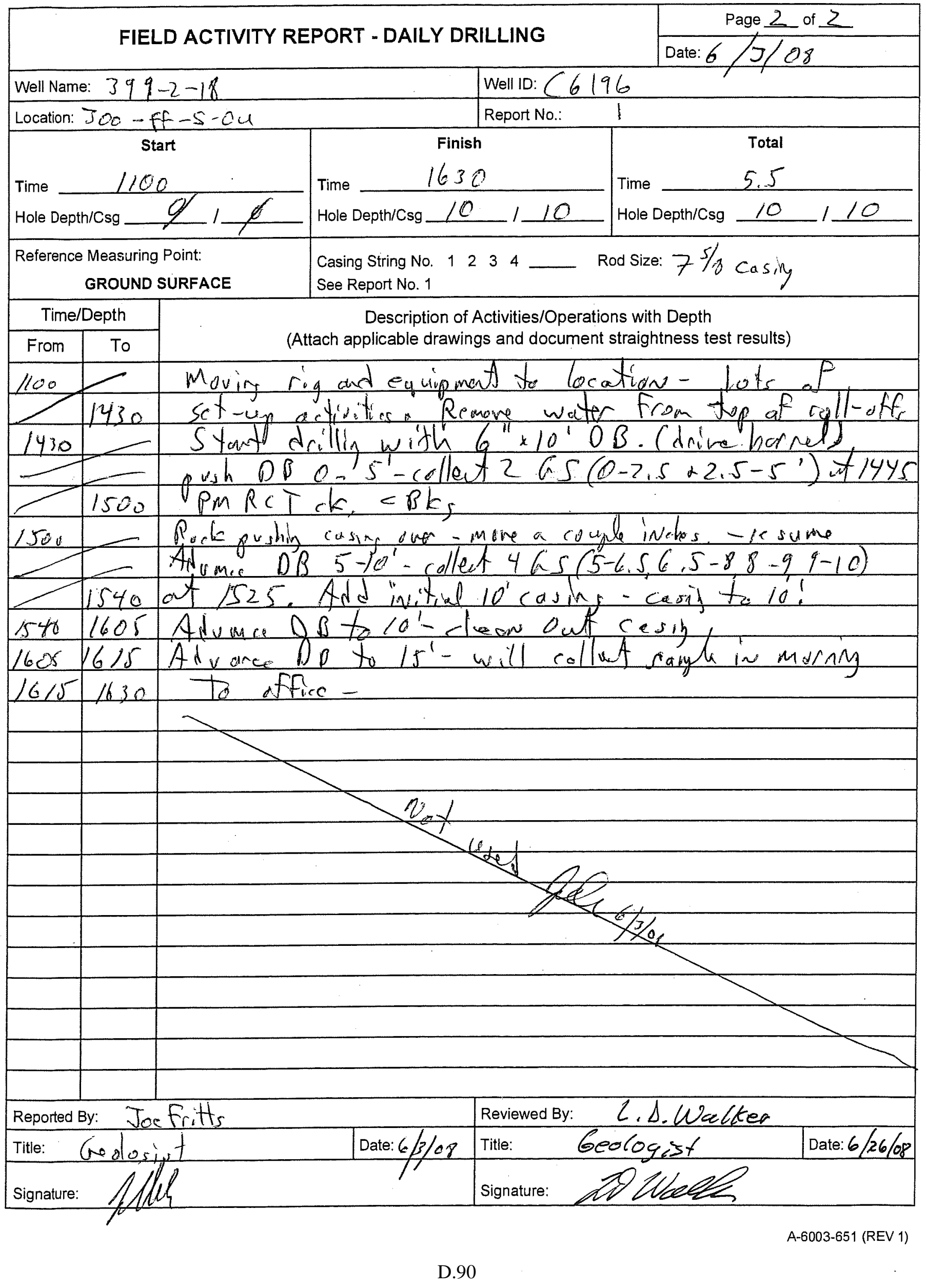




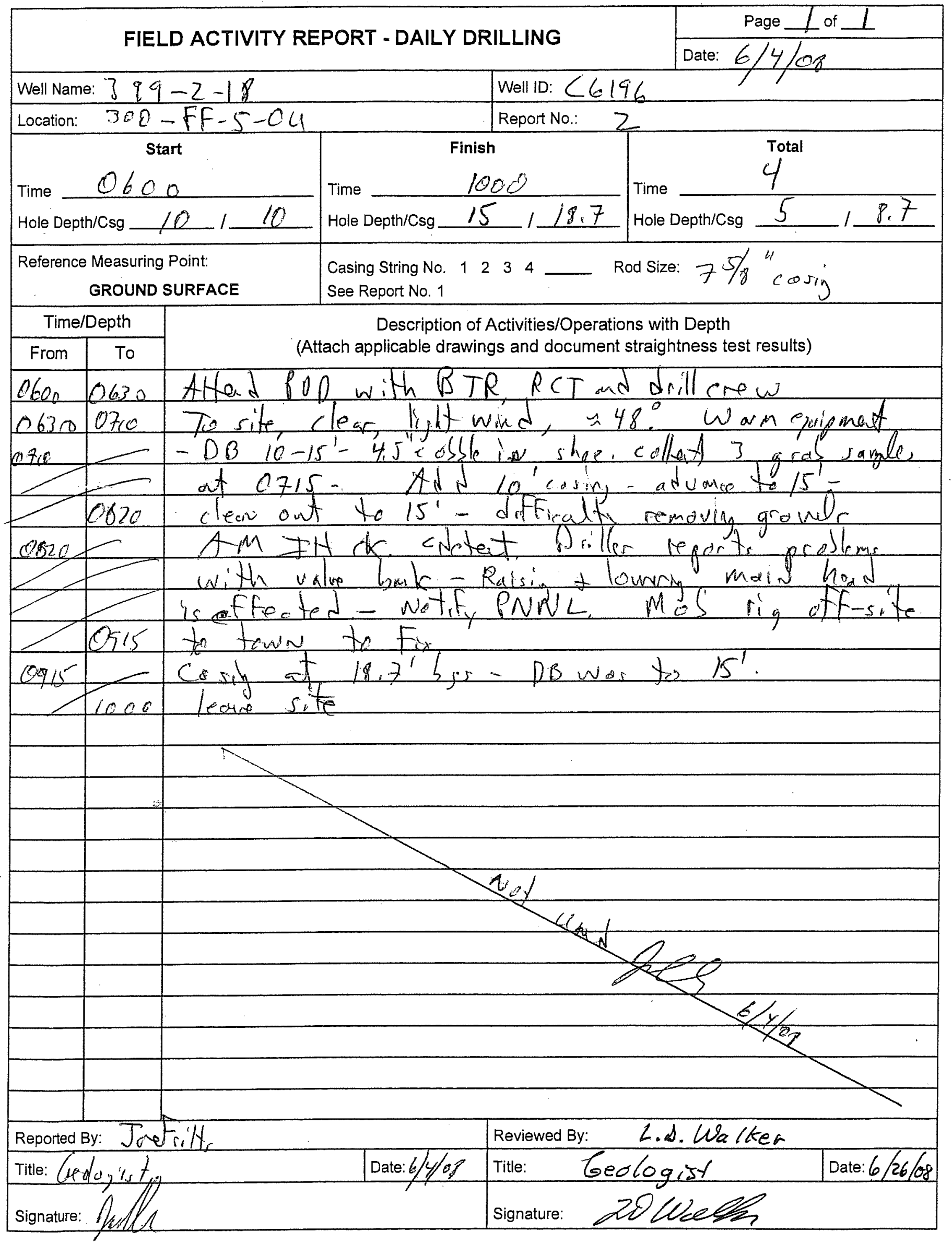

A-6003-651 (REV 1)

D.91 


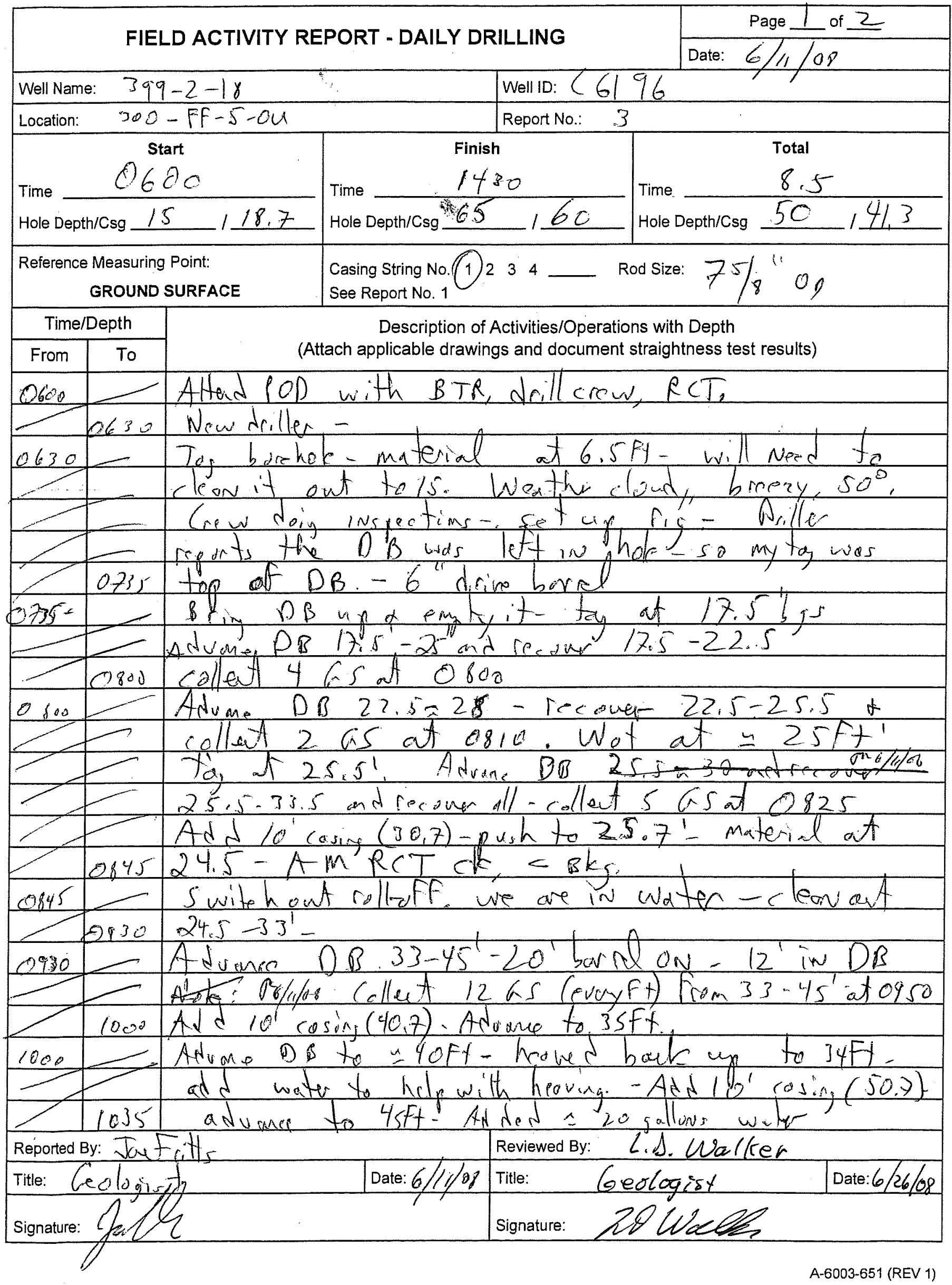

D.92 


\begin{tabular}{l}
\multicolumn{5}{|c|}{ FIELD ACTIVITY REPORT - DAILY DRILLING } \\
Continuation Page
\end{tabular}

D.93 


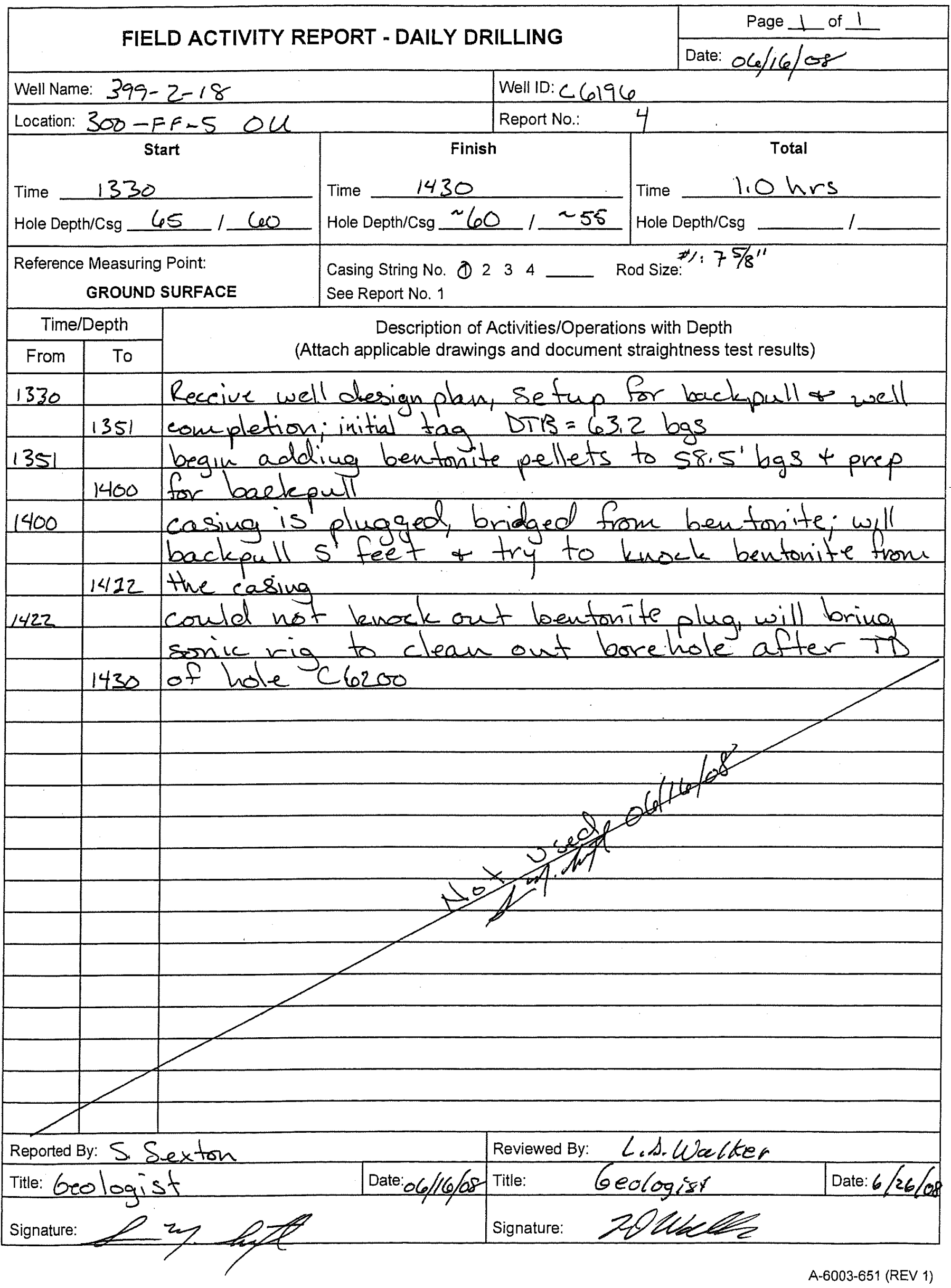

D.94 


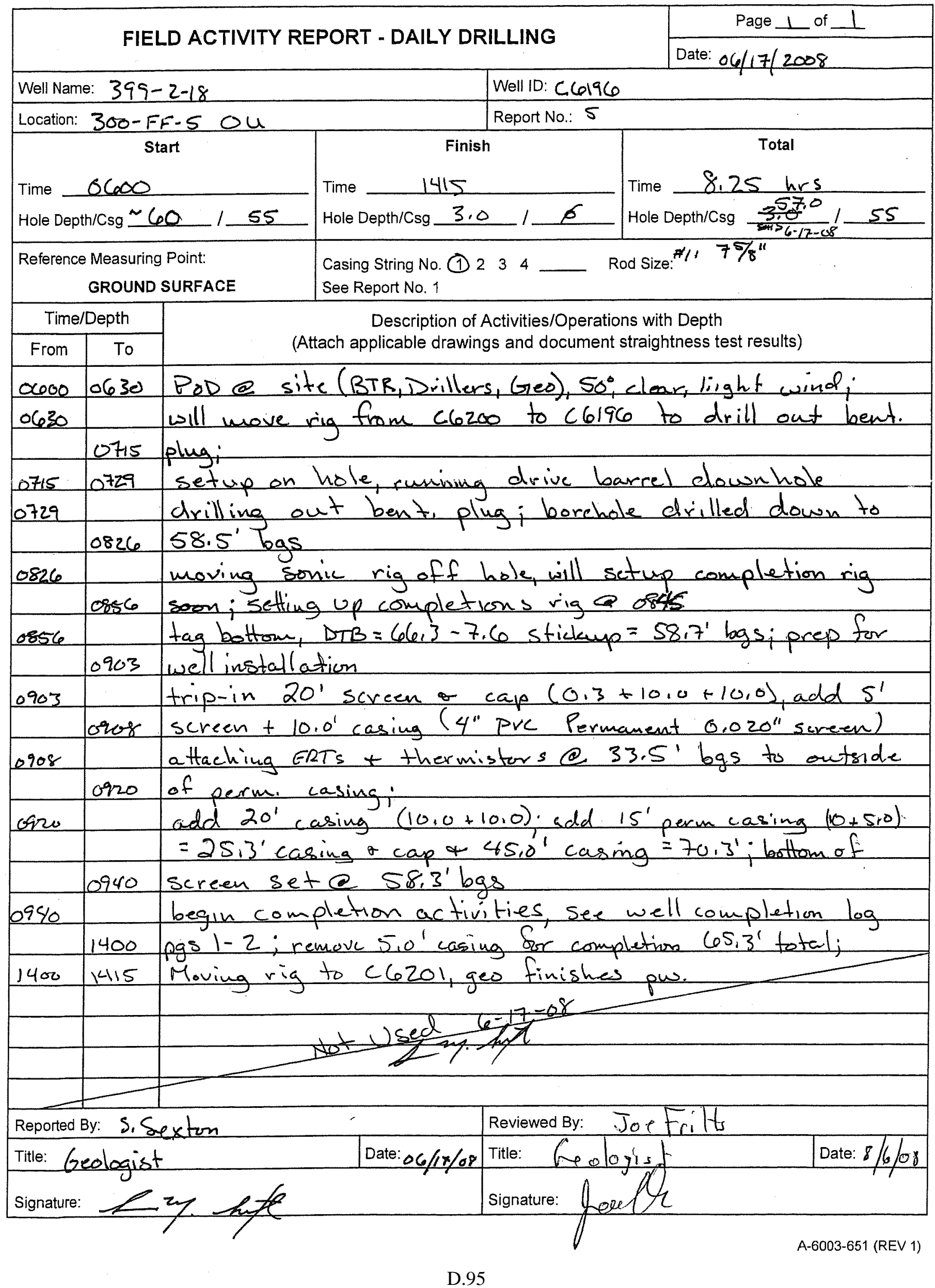




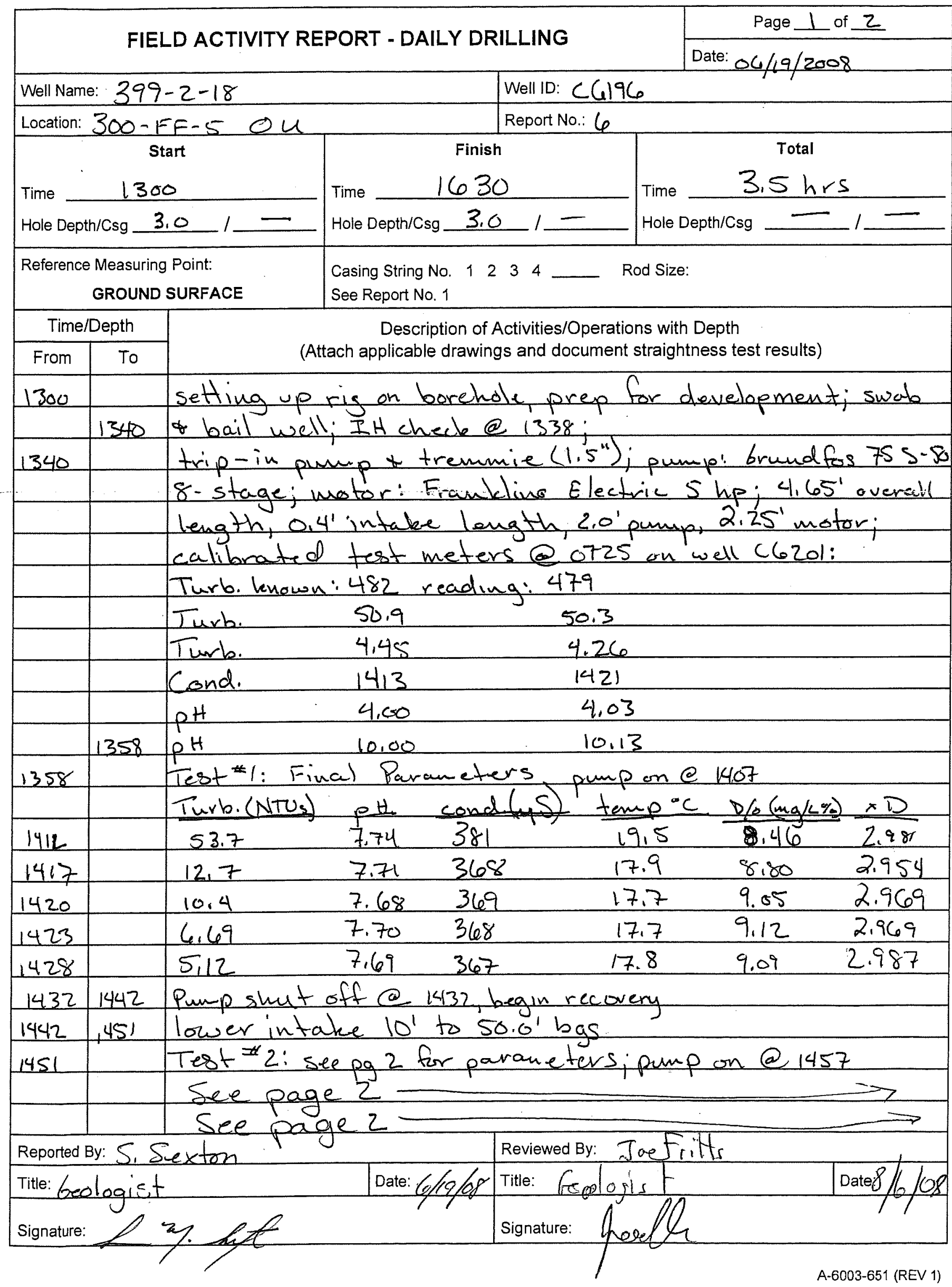


FIELD ACTIVITY REPORT NO. 1 - DRILLING PLAN

\begin{tabular}{|c|c|}
\hline Purpose: PNNL (Proi & est $\$ 0$, Area IfC \\
\hline Well ID: $C 6197$ & \\
\hline Drilling Co.: $W 0 C$ & Mike hillarsos \\
\hline 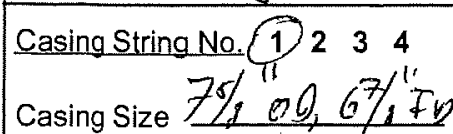 & Drilling Method \\
\hline Grade & Rotary \\
\hline Lbs.Per Ft. & Tubex \\
\hline Material (ors on itee & Cable Tool \\
\hline Type: & Sonid Super Sonie 2 \\
\hline Welded fthd & A.R. w/Sonic \\
\hline Planned / Actual & Geoprobe \\
\hline Set At: $\quad 160,0$ & \\
\hline Shoe OD/ID & buitt to Gupeoh \\
\hline Reference Measuring Point: & \\
\hline GROUND LEVEL & \\
\hline
\end{tabular}

Drig. Co.

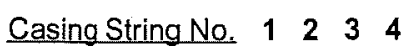

Casing Size

Grade

Lbs.Per Ft.

Material

Type:

Welded Thd.

Planned / Actua

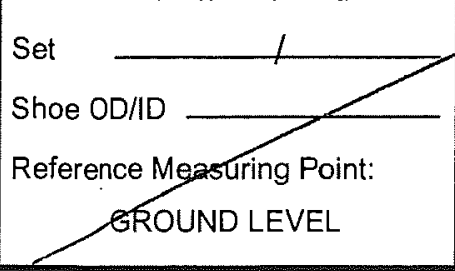

Comments/Remarks:

Rig No::

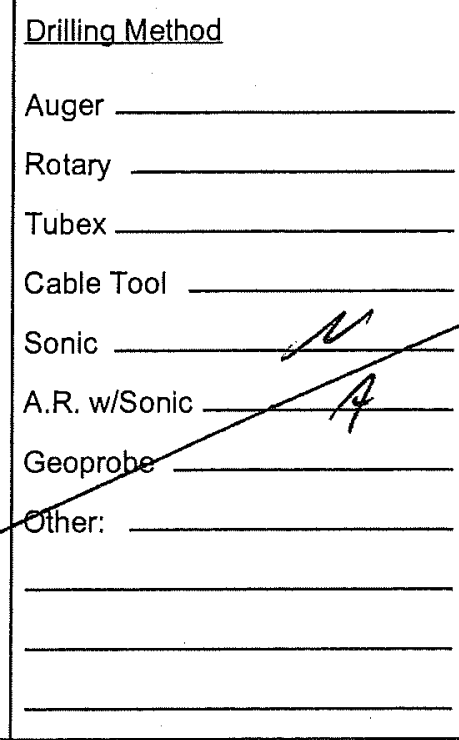

Location: $399-2-19$ Well Name: $399-2-19$

Rig No.: 131 Rig Make/Mod.:Super So vic 2 Circulation

Page 1 of 1 Date: $5 / 20 / 08$

Air __ Water/Mud __ Make

Reverse — Direct

Vol: $\quad \mathrm{cfm}$ gpm

Pressure psi

Drill Pipe O.D.

Tool Joint Size

Additives

Bit Size

Type

Nozzles

Rod Size

Casing Hammer

Make

Model

.

$\longrightarrow$

Rig Make/Mod.:

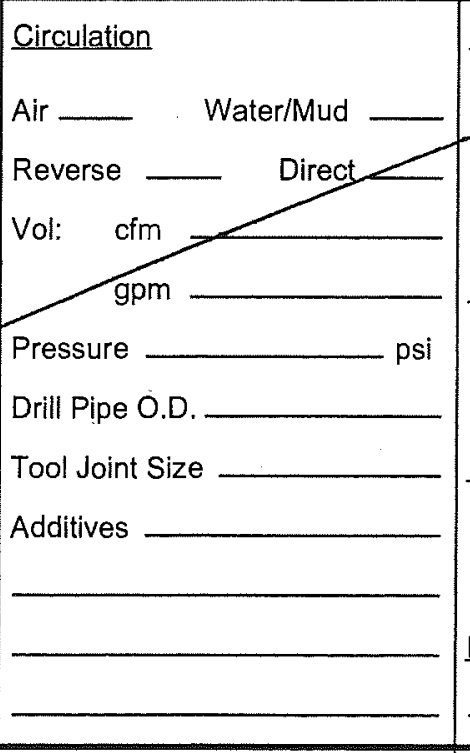

Estimated Depth to Water

(1) Tw 23.3

$\frac{\text { ft } 5,5 \text {. }}{5 / 20 \% 0}$

\section{Reported By: Jatrix}

NamerTitle: GRAm Dac Golegiet

Signature:

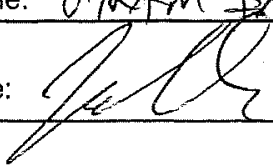

Date: $5 / 20 / 08$ 


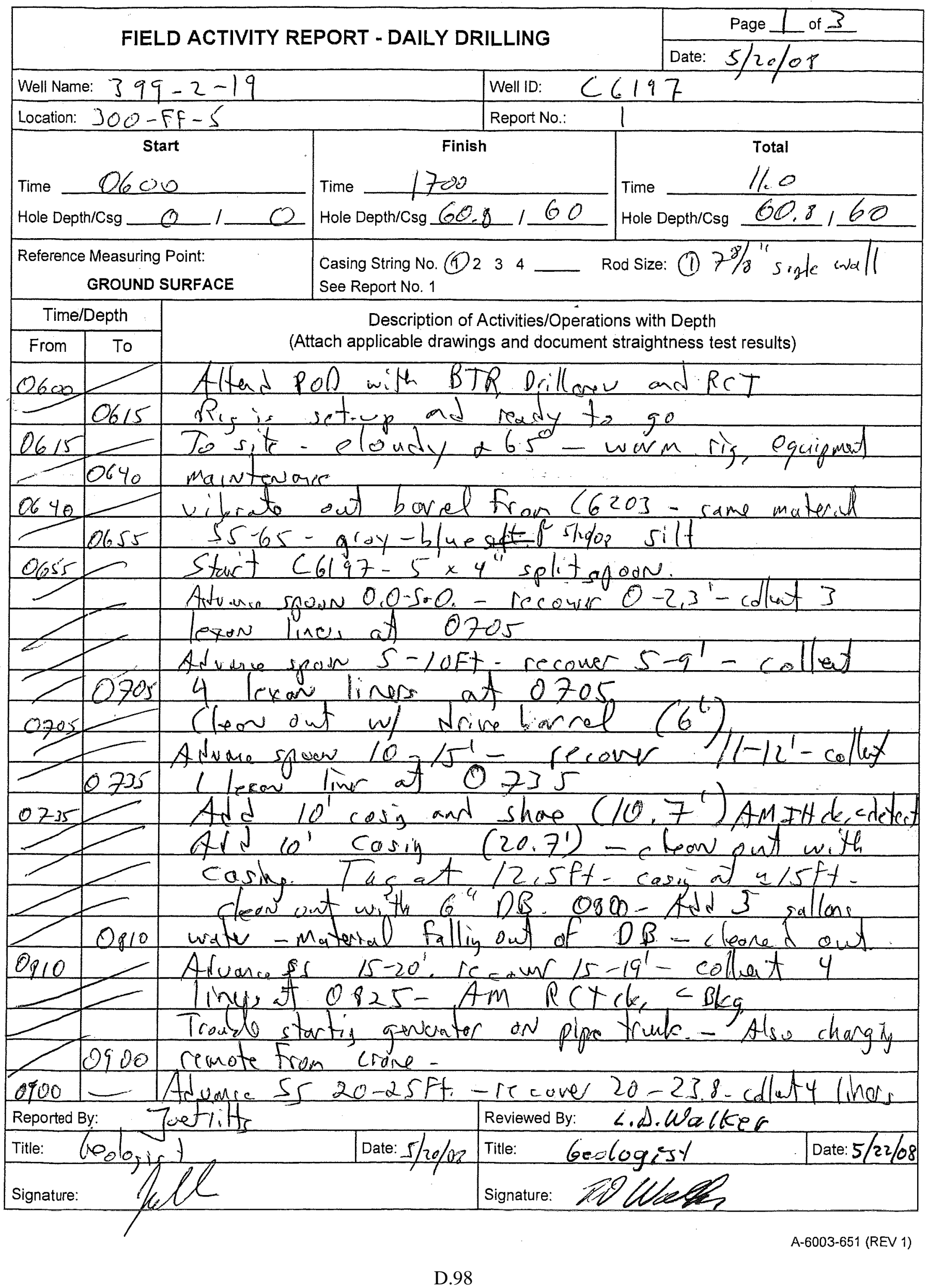




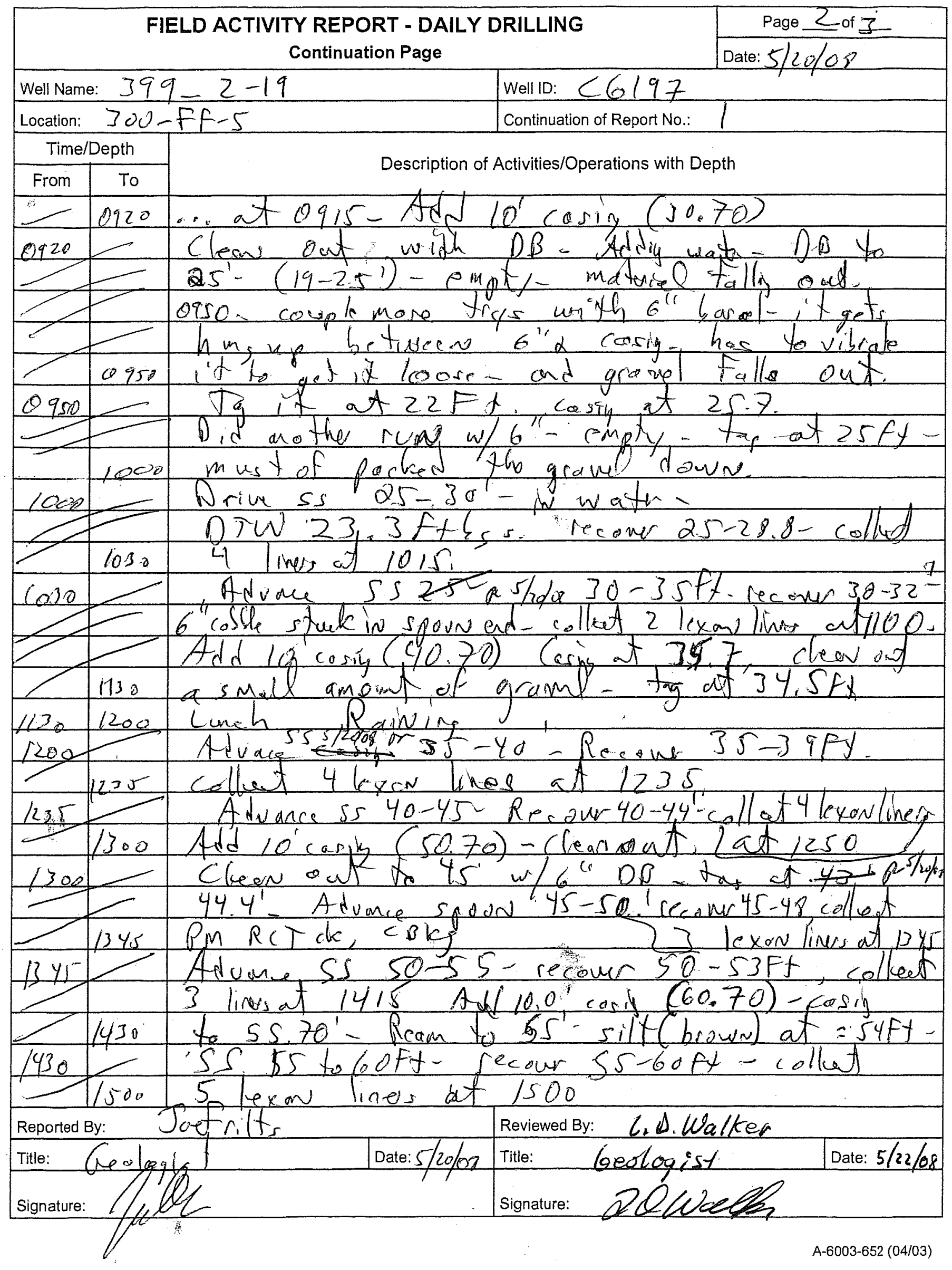

D.99 


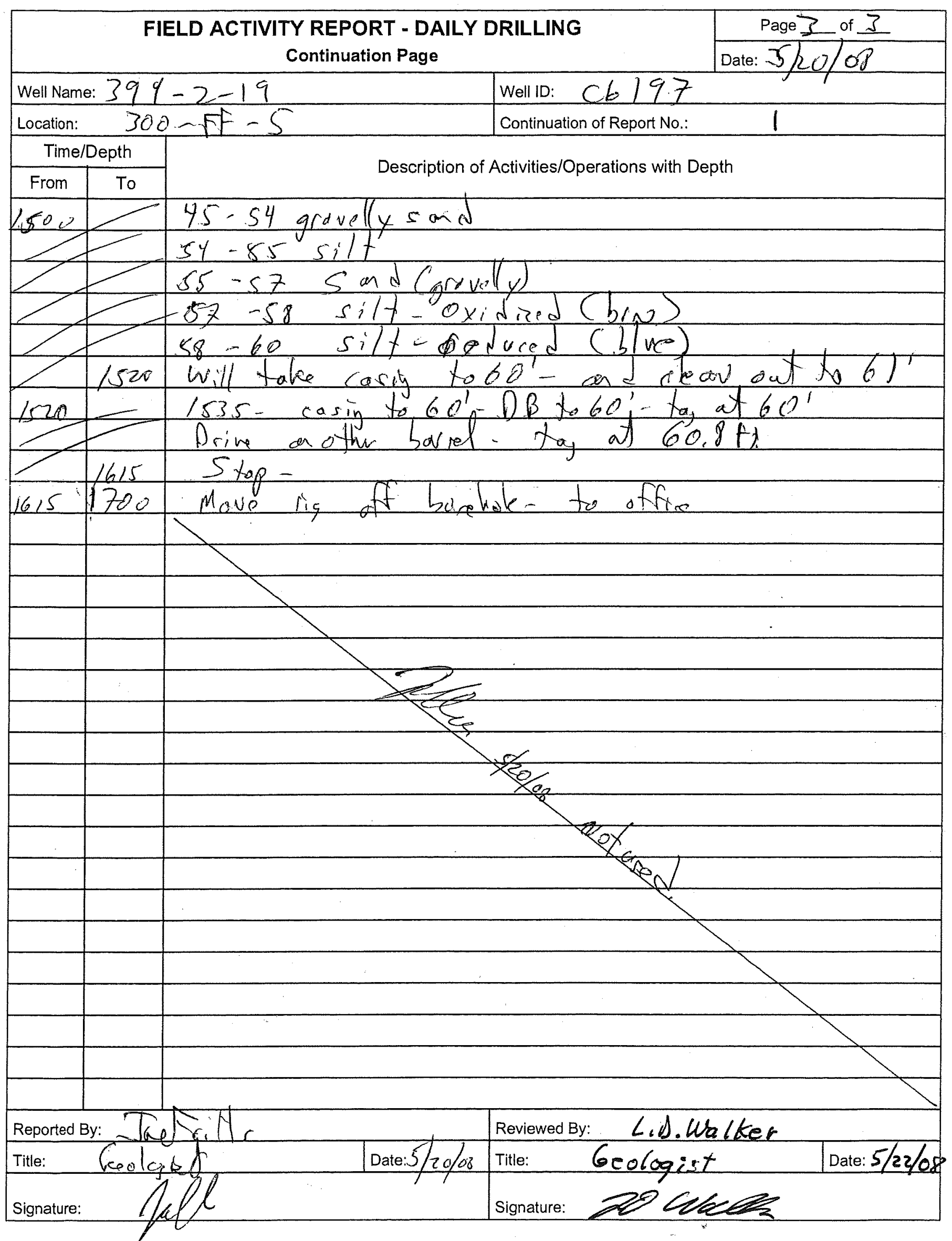


FIELD ACTIVITY REPORT - DAILY DRILLING

Page 1 of 1 Date: $05 / 30 / 2008$

\begin{tabular}{l}
\hline Well Name: $399-2-19$ \\
\hline Location: $300-F F-5$ \\
Start \\
Time 0600 \\
Hole Depth/Csg $100,0 / 60)$ \\
$\begin{array}{c}\text { Reference Measuring Point: } \\
\text { GROUND SURFACE }\end{array}$
\end{tabular}

\begin{tabular}{l|l} 
& Well ID: C 6197 \\
& Report No.: 2 \\
\hline
\end{tabular}

Finish

Total

Time 1330

Hole Depth/Csg $-48 / 54.5$ Hole Depth/Csg $12 / 5.5$

Casing String No. (1) 2344 Rod Size: $75 / 8^{\prime \prime}$

See Report No. 1

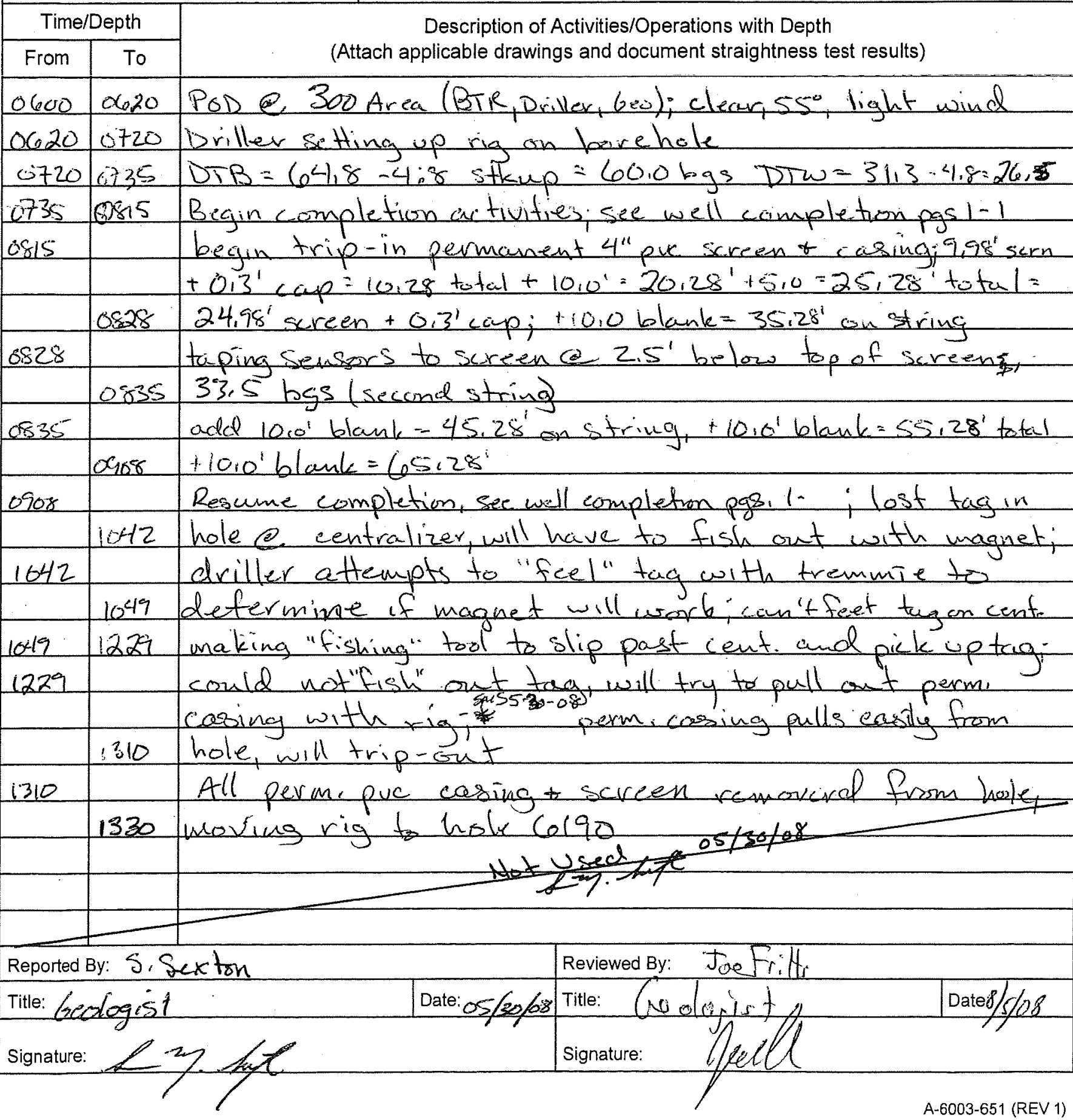




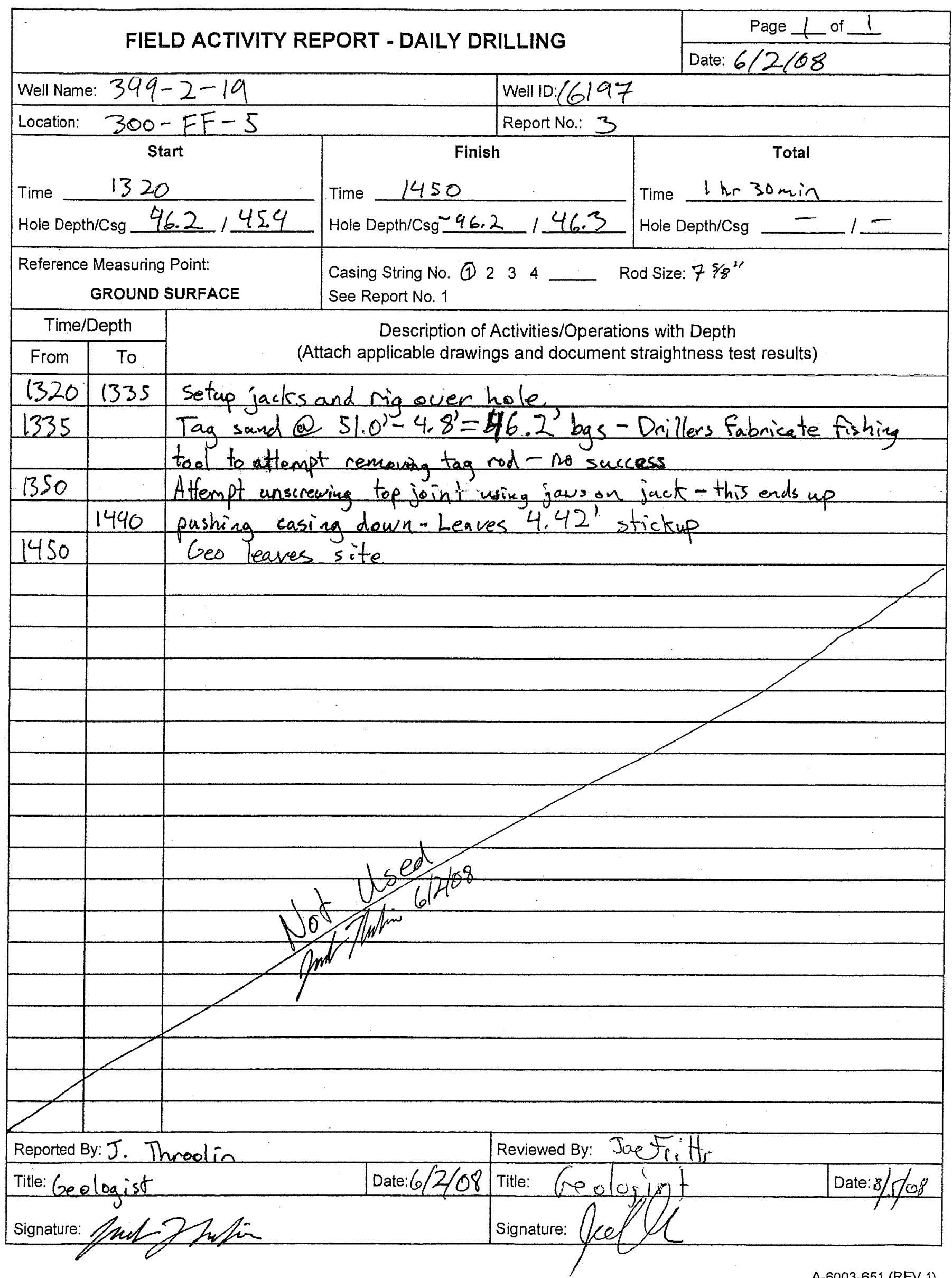




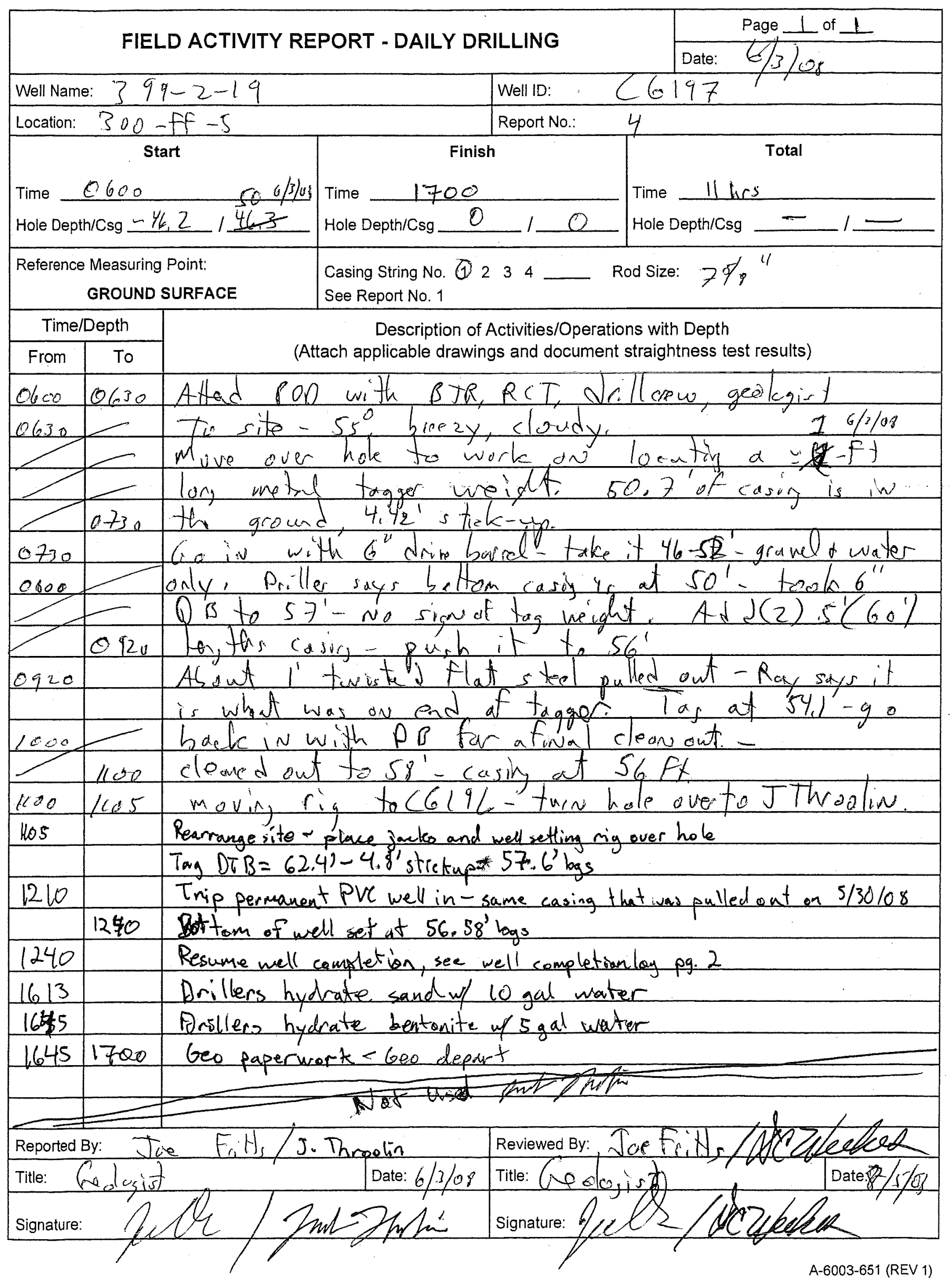

D.103 


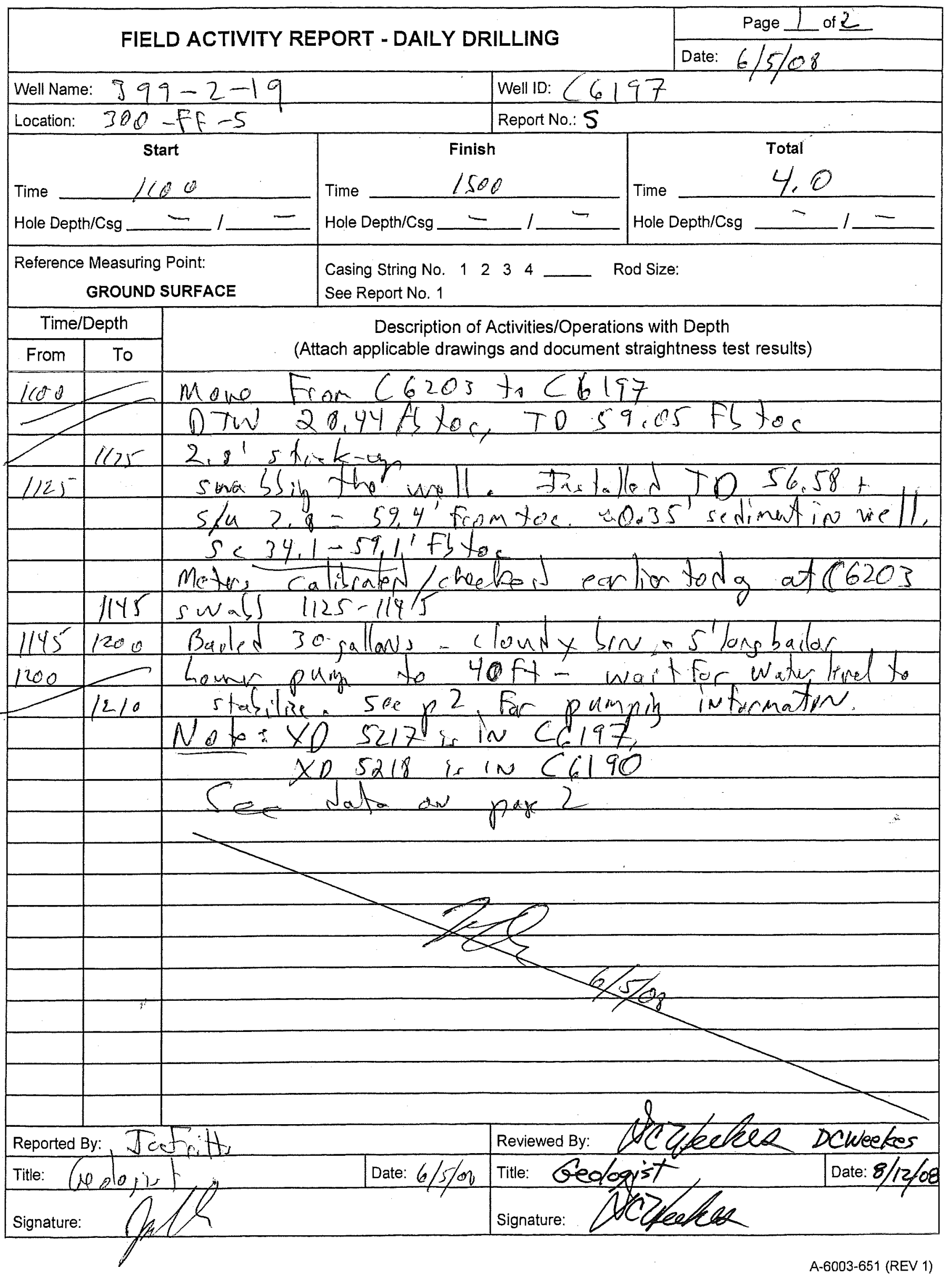

D.104 


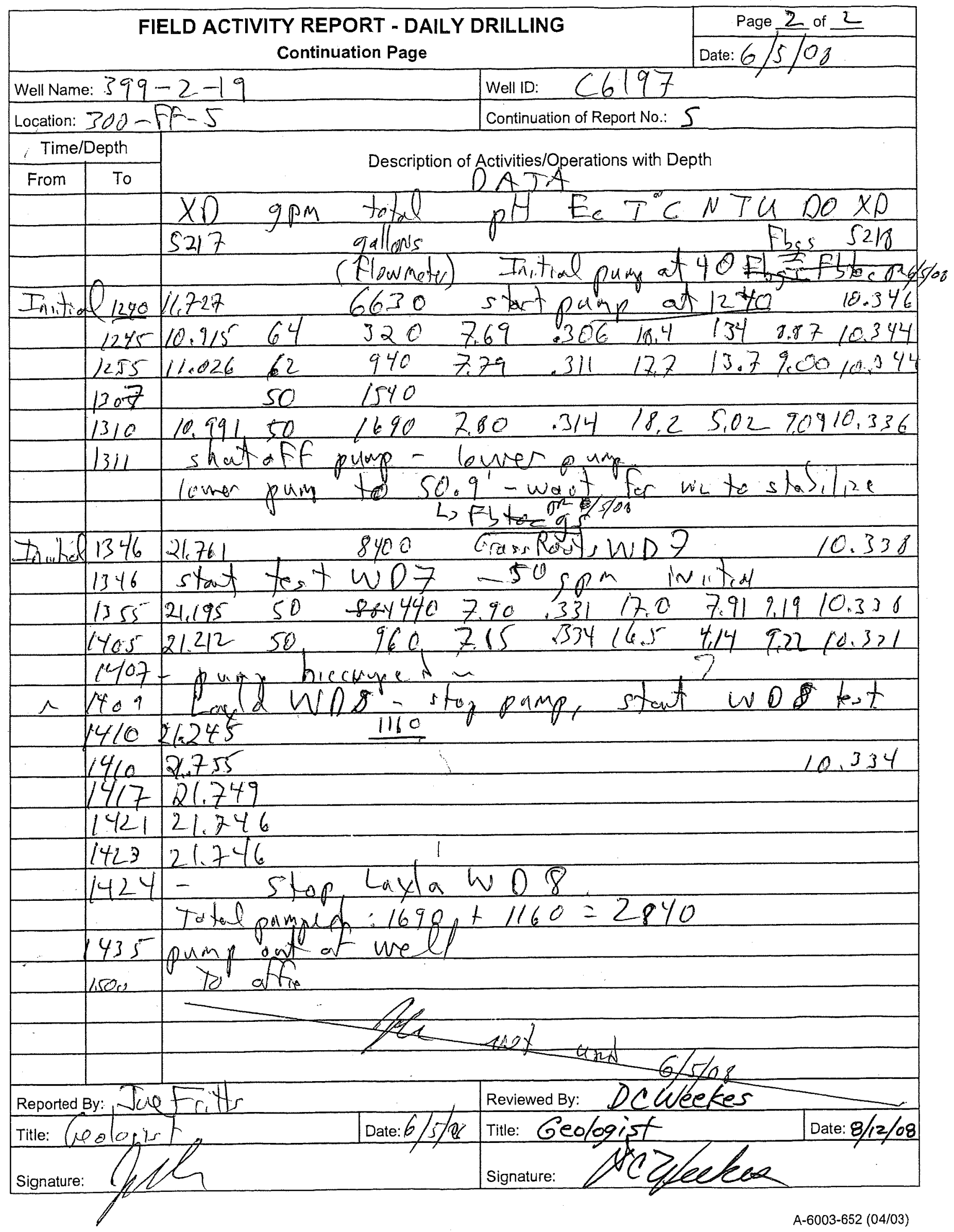

D.105 


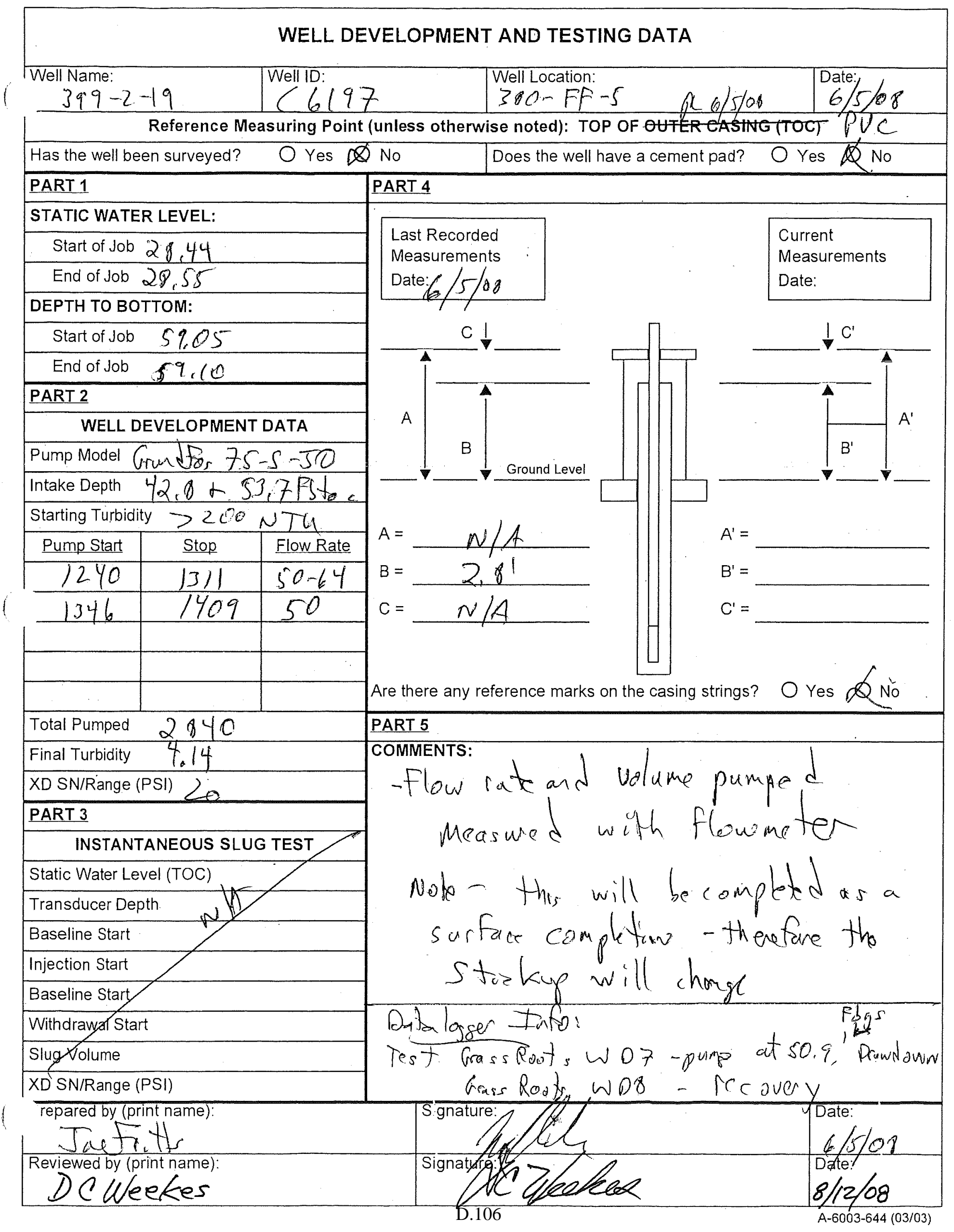




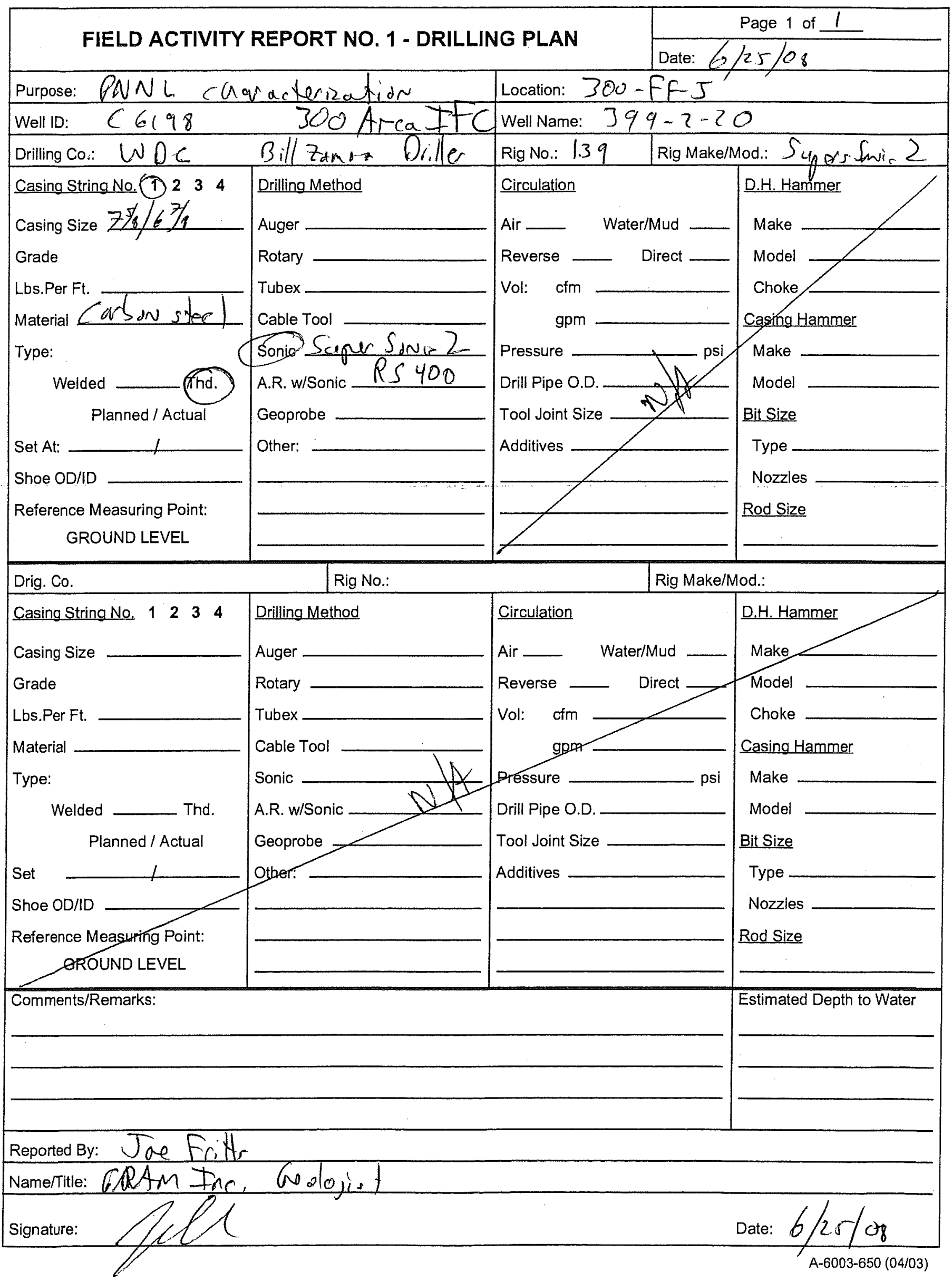




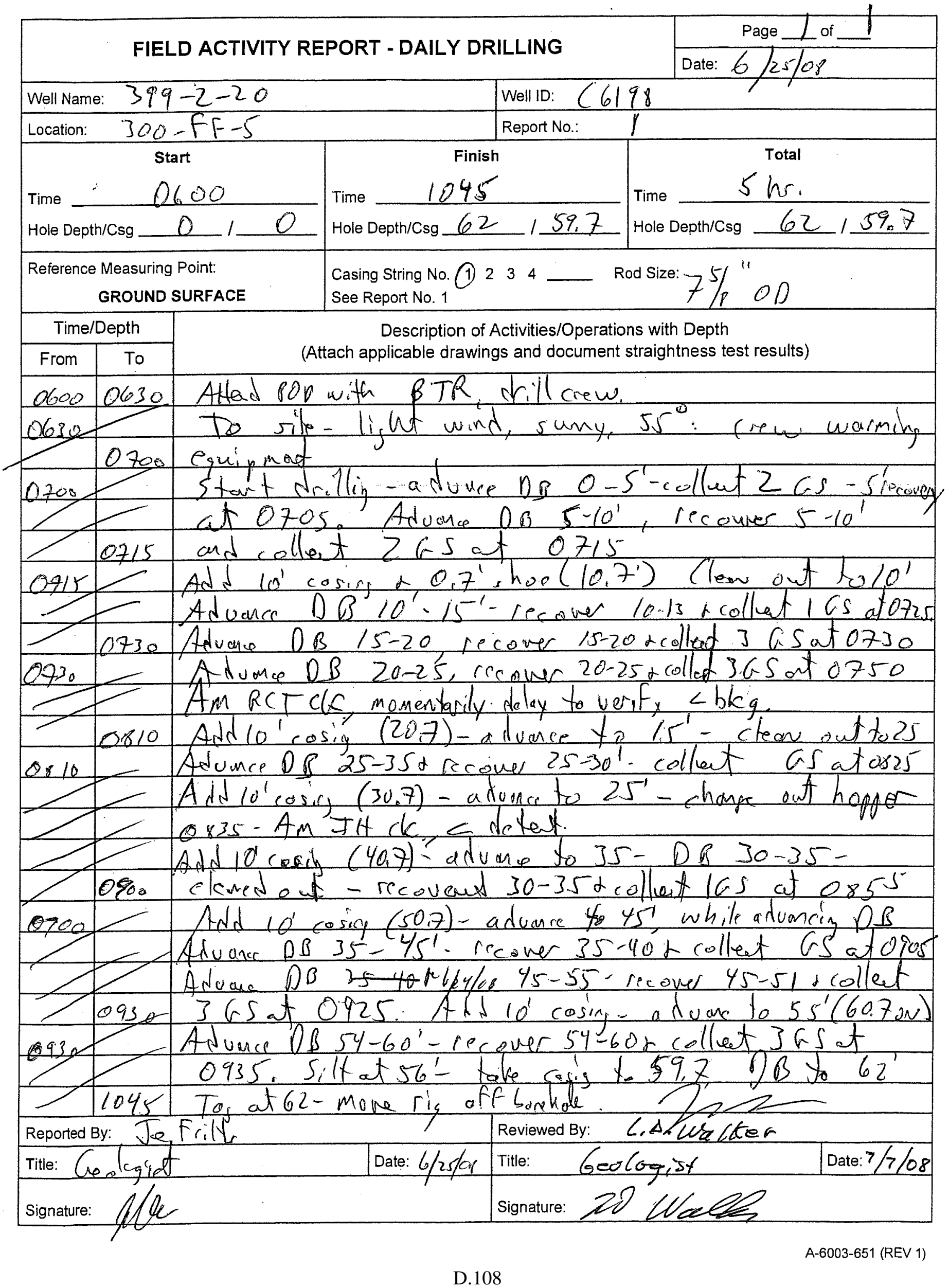




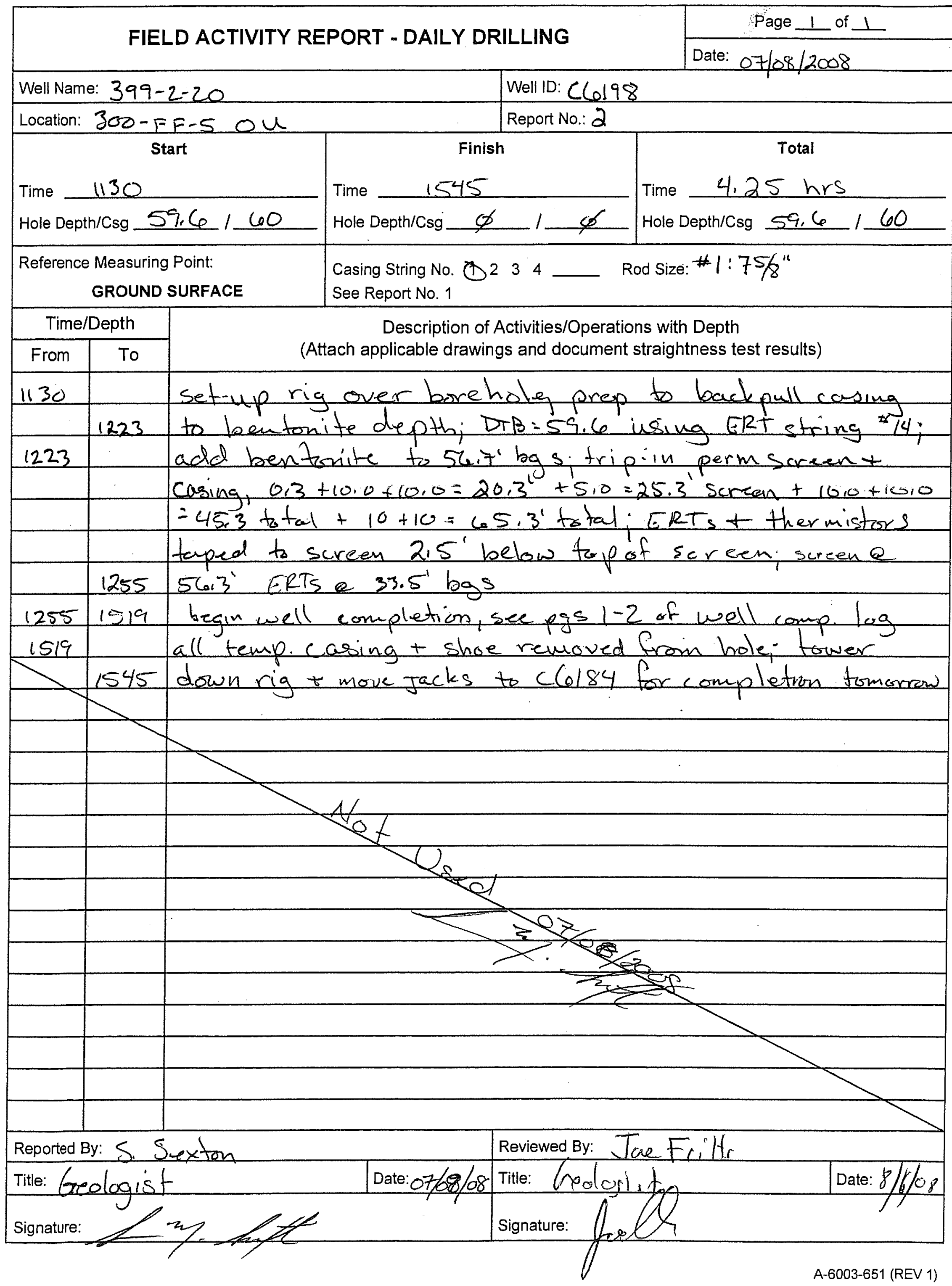

D.109 


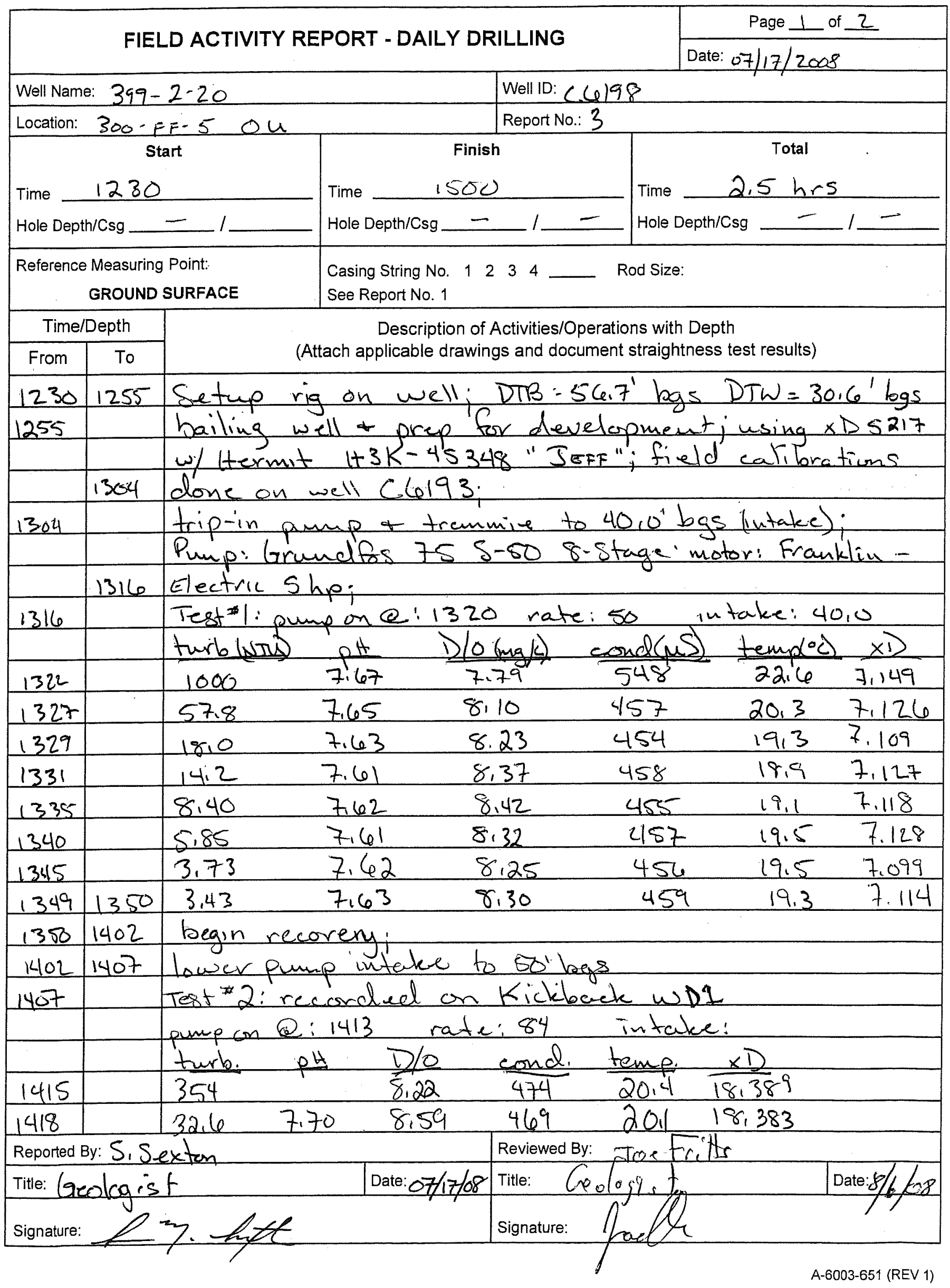




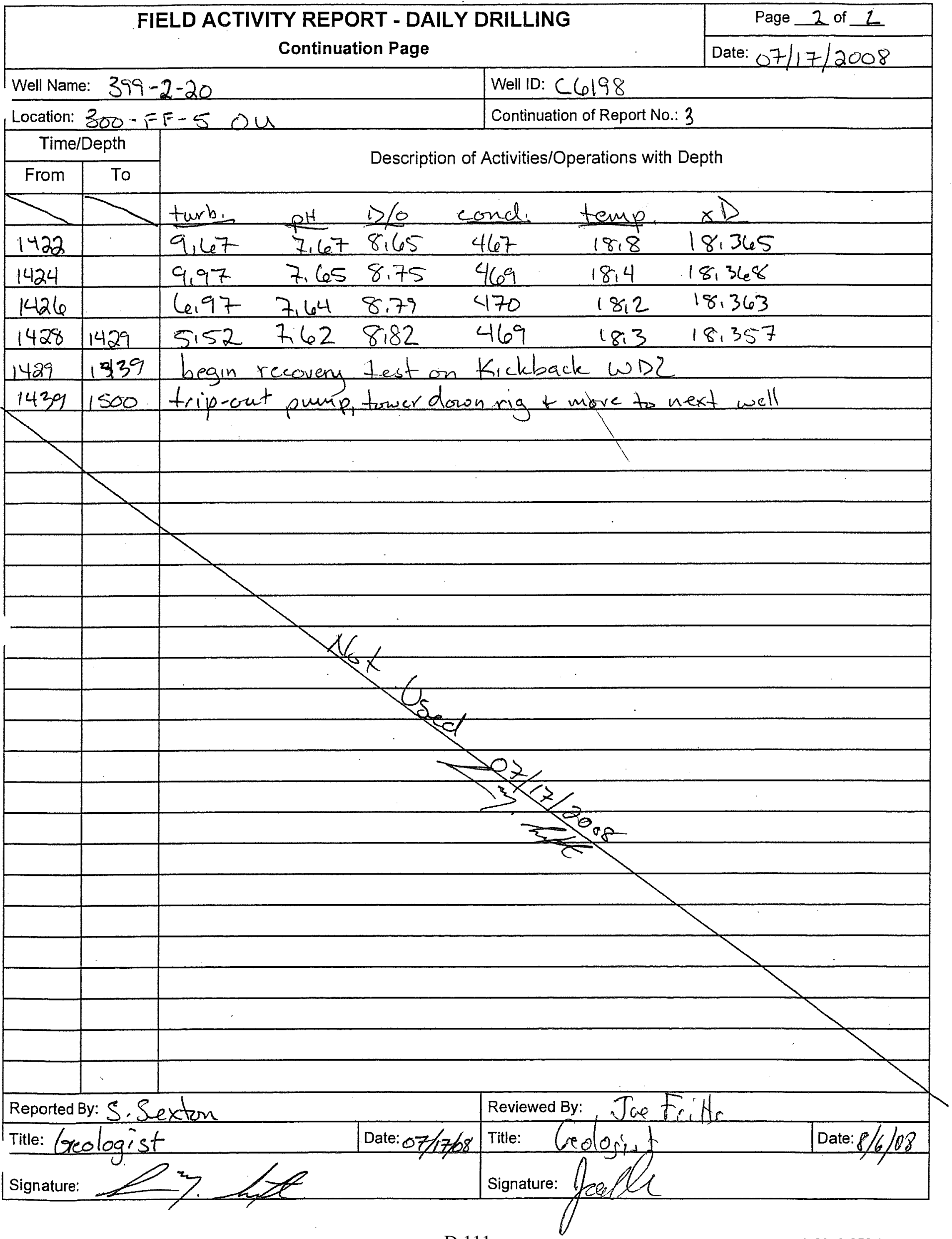

D.111 


\section{WELL DEVELOPMENT AND TESTING DATA}

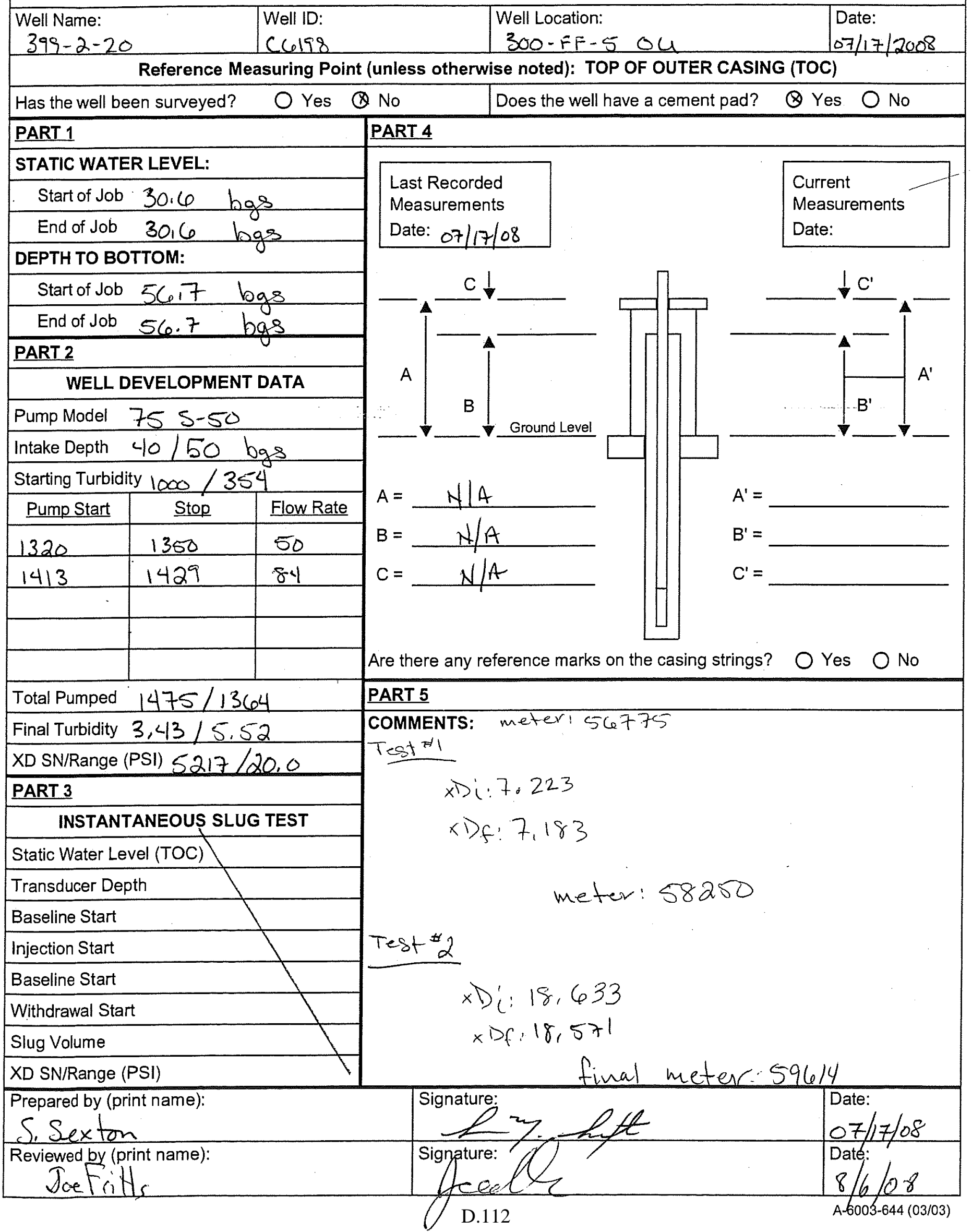


FIELD ACTIVITY REPORT NO. 1 - DRILLING PLAN

\begin{tabular}{|c|c|}
\hline Purpose: PNUL Pigies & Jow Areatife \\
\hline Well ID: $\quad$ C6201 & \\
\hline Drilling Co.: $\cup D C$ & \\
\hline 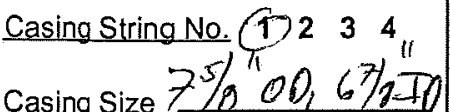 & Drilling Method \\
\hline & Auger \\
\hline Grade & Rotary - \\
\hline Lbs.Per Ft. & Tubex - \\
\hline Material Carsonstes & Cable Tool - \\
\hline Type: & Sonic Supe \\
\hline Welded & A.R. w/Sonic NS400 \\
\hline Planned / Actual & Geoprobe \\
\hline Set At: & Other: \\
\hline Shoe OD/ID & \\
\hline Reference Measuring Point: & \\
\hline GROUND LEVEL & \\
\hline
\end{tabular}

Drig. Co.

Casing String No. $\begin{array}{lllll}1 & 2 & 3 & 4\end{array}$

Casing Size

Grade

Lbs.Per Ft.

Material

Type: Thd.

Planned / Actual

Set

Shoe OD/ID

Reference Measuring Point: GROUND LEVEL
Rig No.:

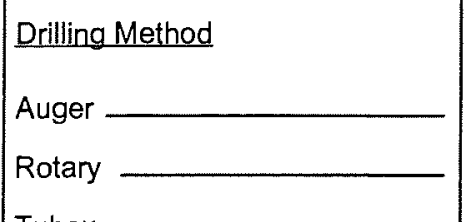

Tubex

Cable Tool

Sonic

A.R. w/Sonic Geoprobe

Other:

Location: I 00 - FF-5-OC Well Name: $399-2-2,216 / 1,2 / z^{3}$ Rig No.:139 Rig Make/Mod.Suger Sinic Circulation

Air — Water/Mud

Reverse — Direct

Vol: $\mathrm{cfm}$

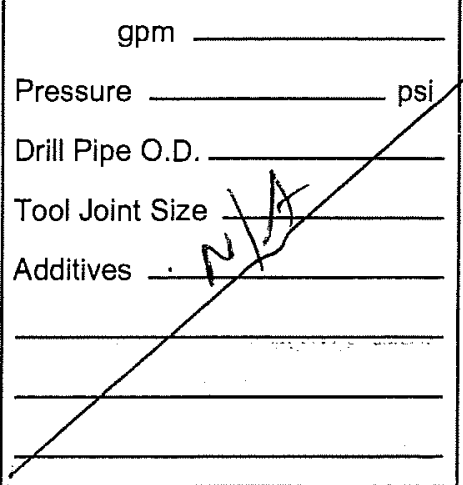

D.H. Hammer

Make

Model

Choke

Caging Hammer

Make

Model

Bit Size

Type

Nozzles

Rod Size
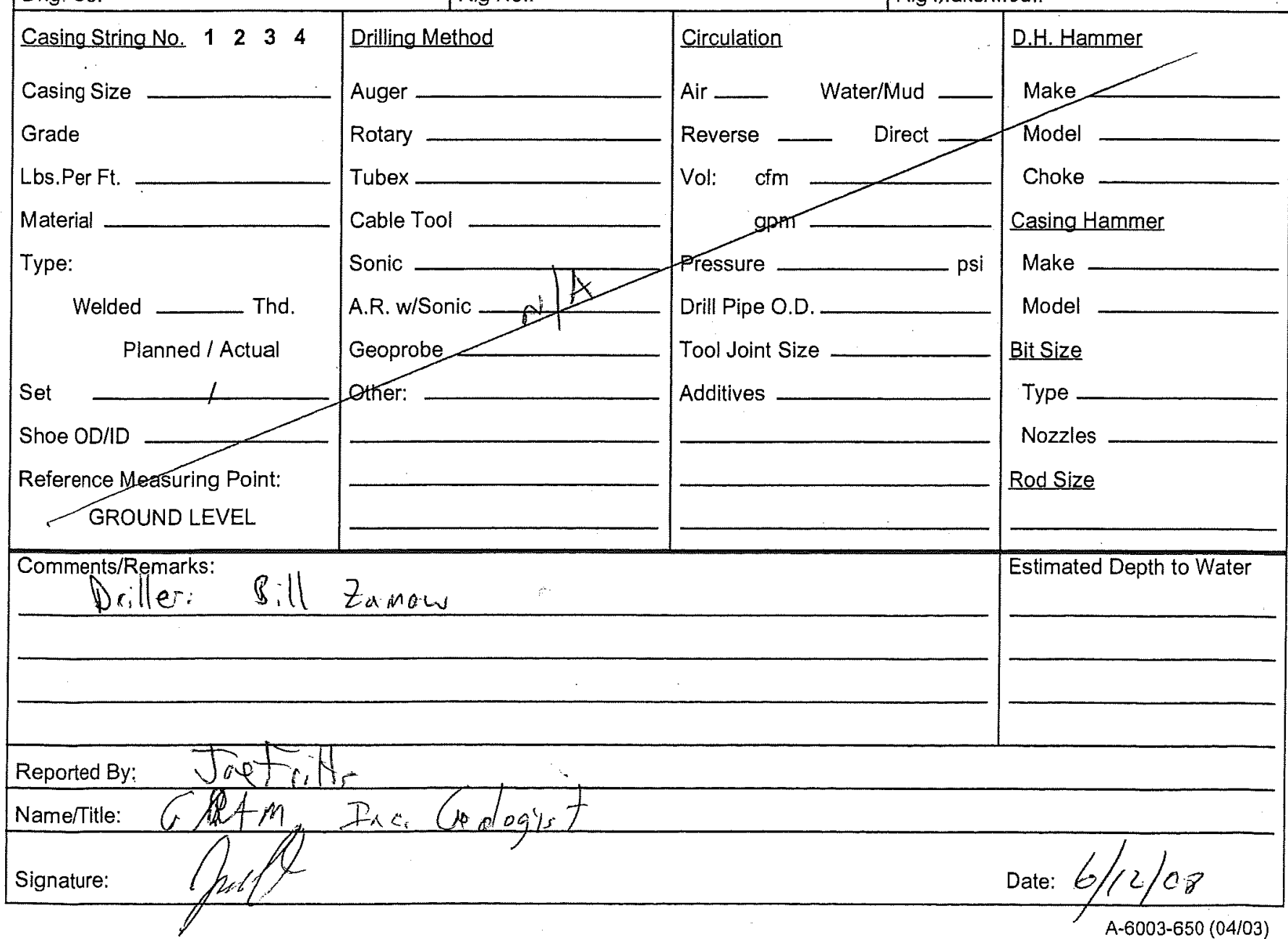


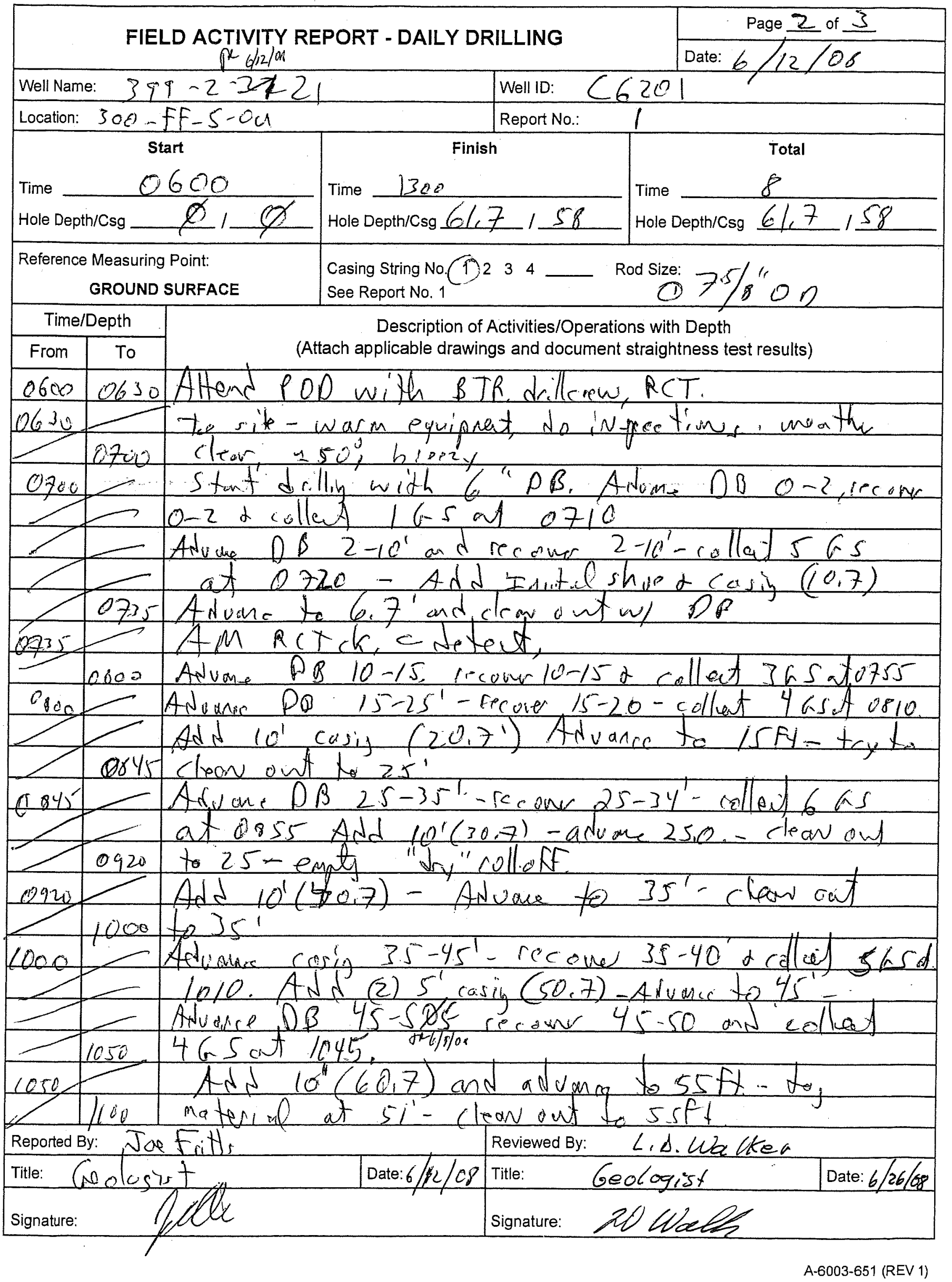

D.114 


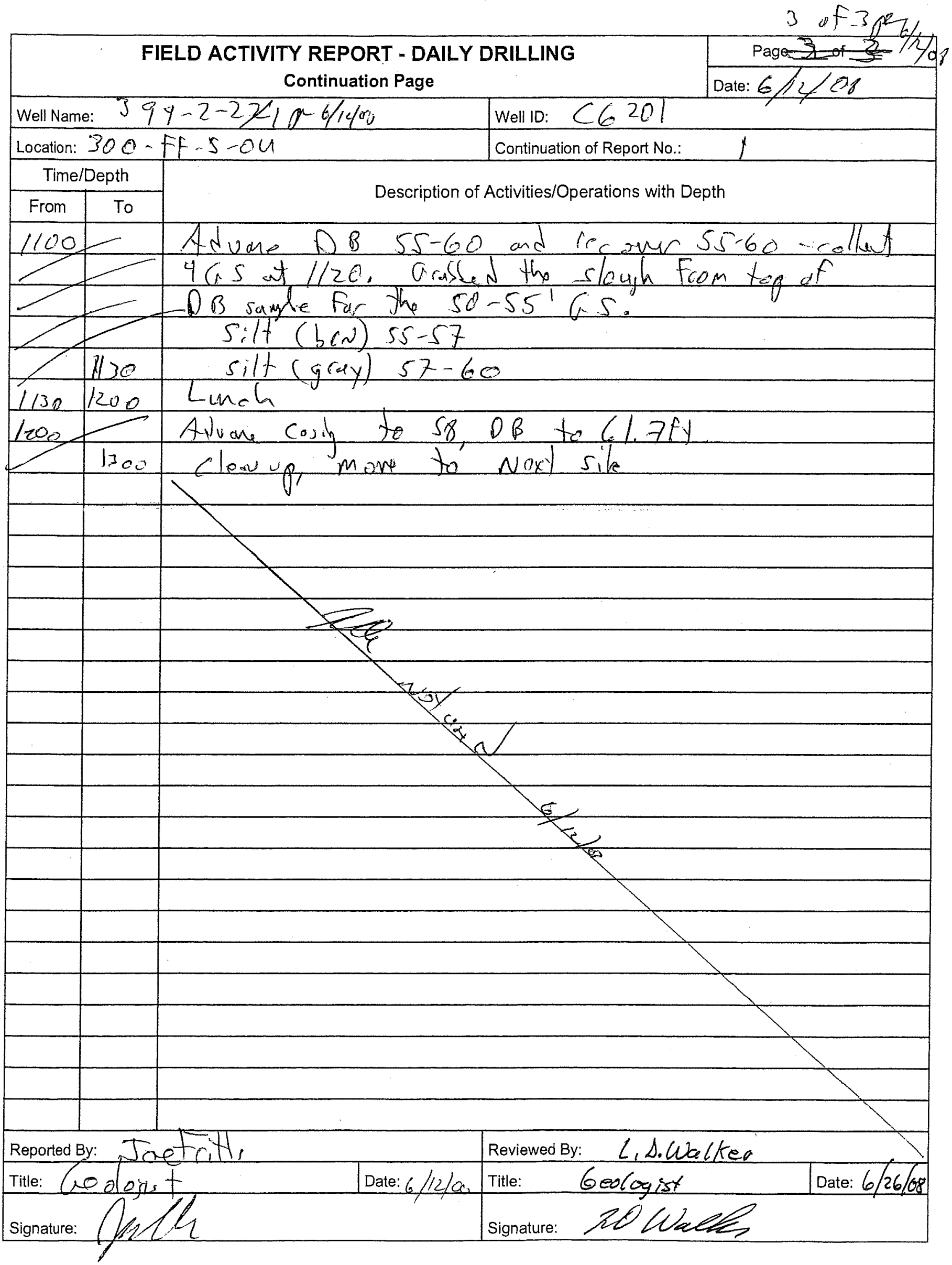

A-6003-652 (04/03)

D.115 


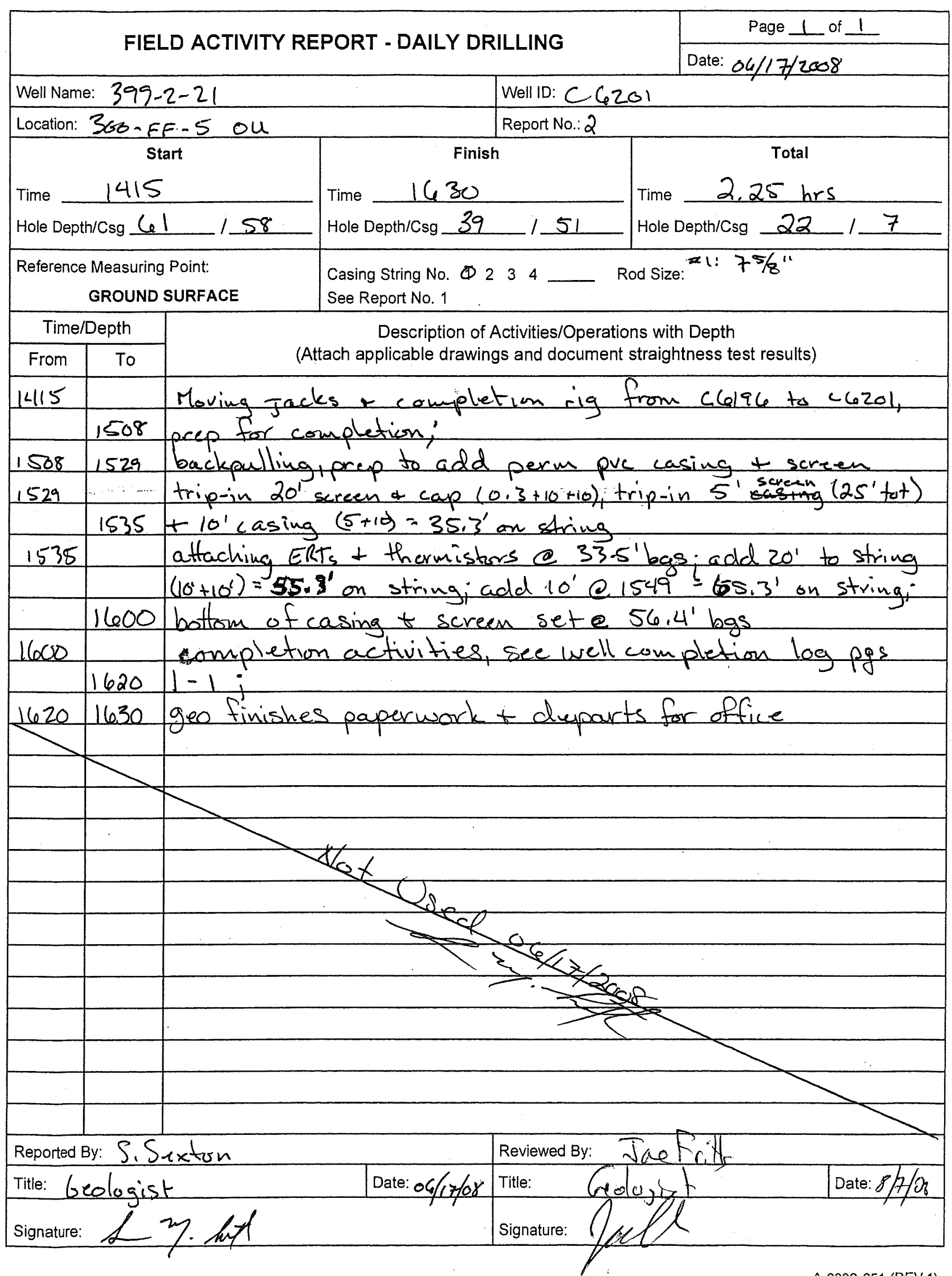




\section{FIELD ACTIVITY REPORT - DAILY DRILLING}

Page $\perp$ of $\perp$ Date: $06 / 18 / 2008$

\begin{tabular}{l} 
Well Name: $399-2-21$ \\
\hline Location: $300-1 F-5$ ou \\
Start \\
Time 0600 \\
Hole Depth/Csg 39 \\
$\begin{array}{c}\text { Reference Measuring Point: } \\
\text { GROUND SURFACE }\end{array}$
\end{tabular}

Well ID: C6201

Report No:: 3

\begin{tabular}{|c|c|}
\hline Finish & Total \\
\hline Time 0945 & 3,75 hrs \\
\hline Hole Depth/Csg 3 & Hole Depth/Csg 36 \\
\hline $\begin{array}{l}\text { Casing String No. (1) } 234 \\
\text { See Report No. } 1\end{array}$ & od Size: $\# /: 75 / 8$ \\
\hline
\end{tabular}

\section{Time/Depth}

\begin{tabular}{|c|c|}
\hline From & To \\
\hline
\end{tabular}

06000630 POD 300 Area; 50 cl light breze

06300658 Setup on hole, prep to continue completion; 0658 completion resumes, see completion pages 1-2; It check $0932 @ 0844$ nom-detect;

0932 completion tinished, drillens mpring jacks and supplies to C6191, geo fimishes paperwork and moves to $C 6189$ to assist

\begin{tabular}{l|l}
\hline & with drilling geo.
\end{tabular}

Description of Activities/Operations with Depth

(Attach applicable drawings and document straightness test results)

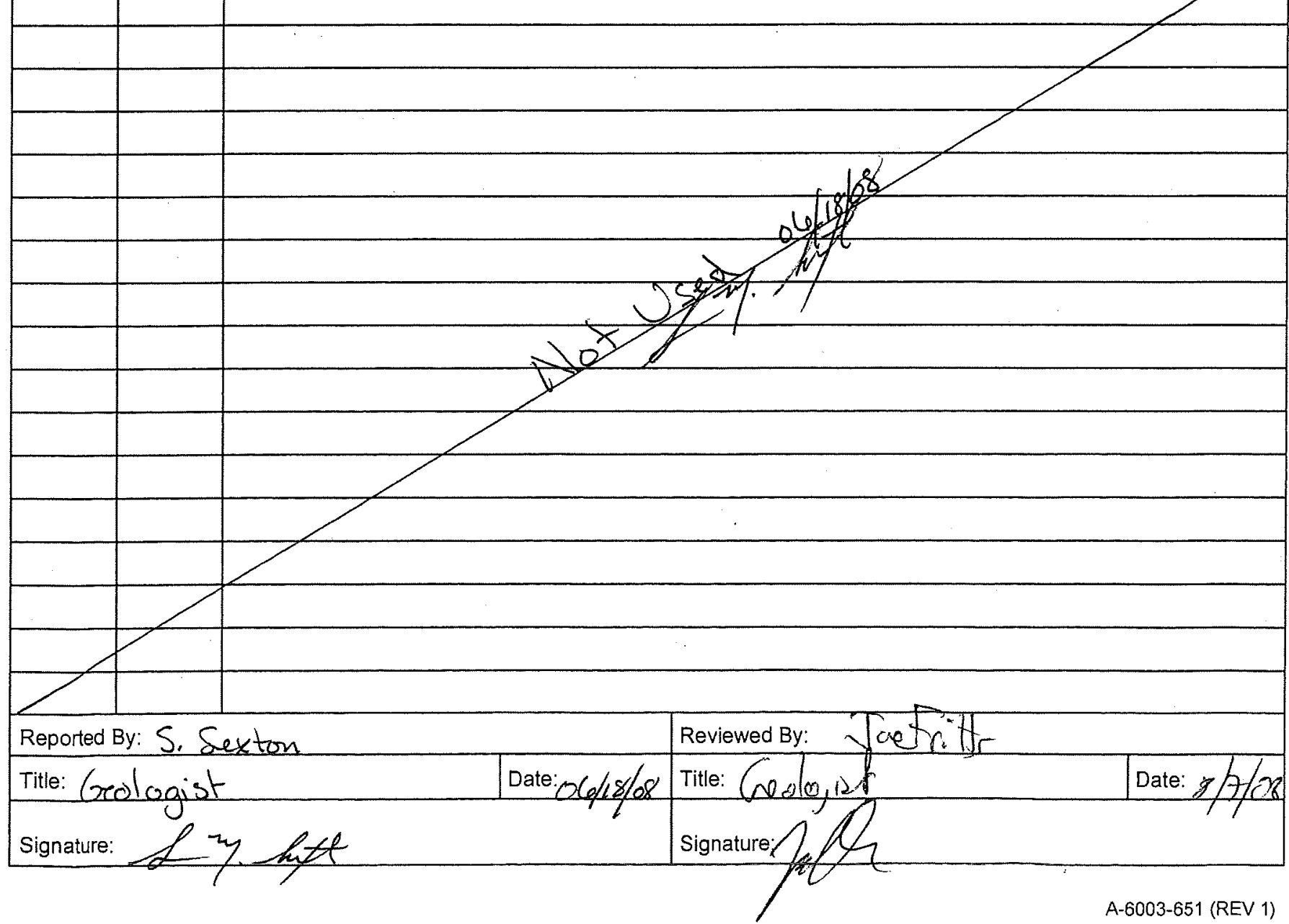




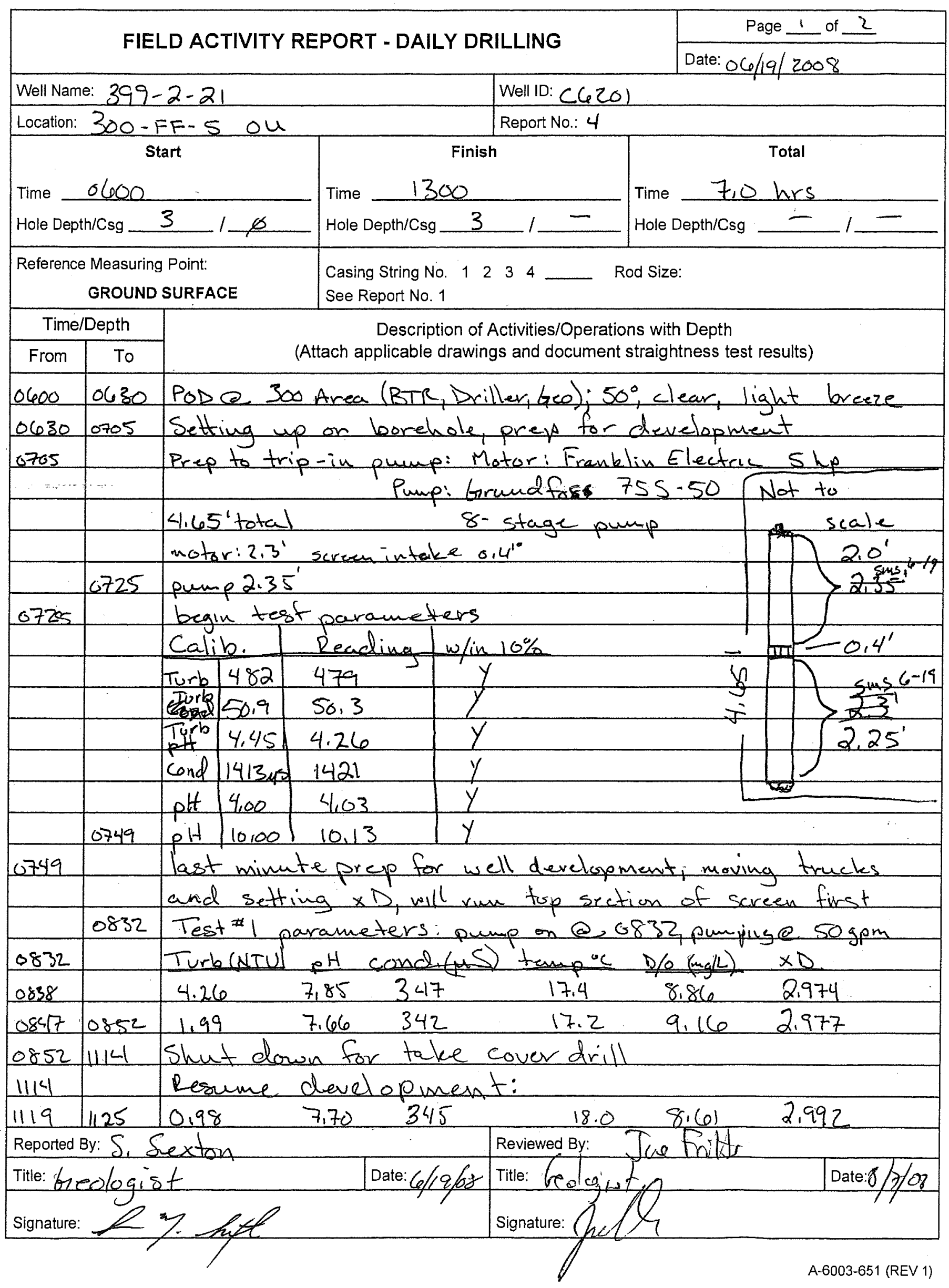

D.118 


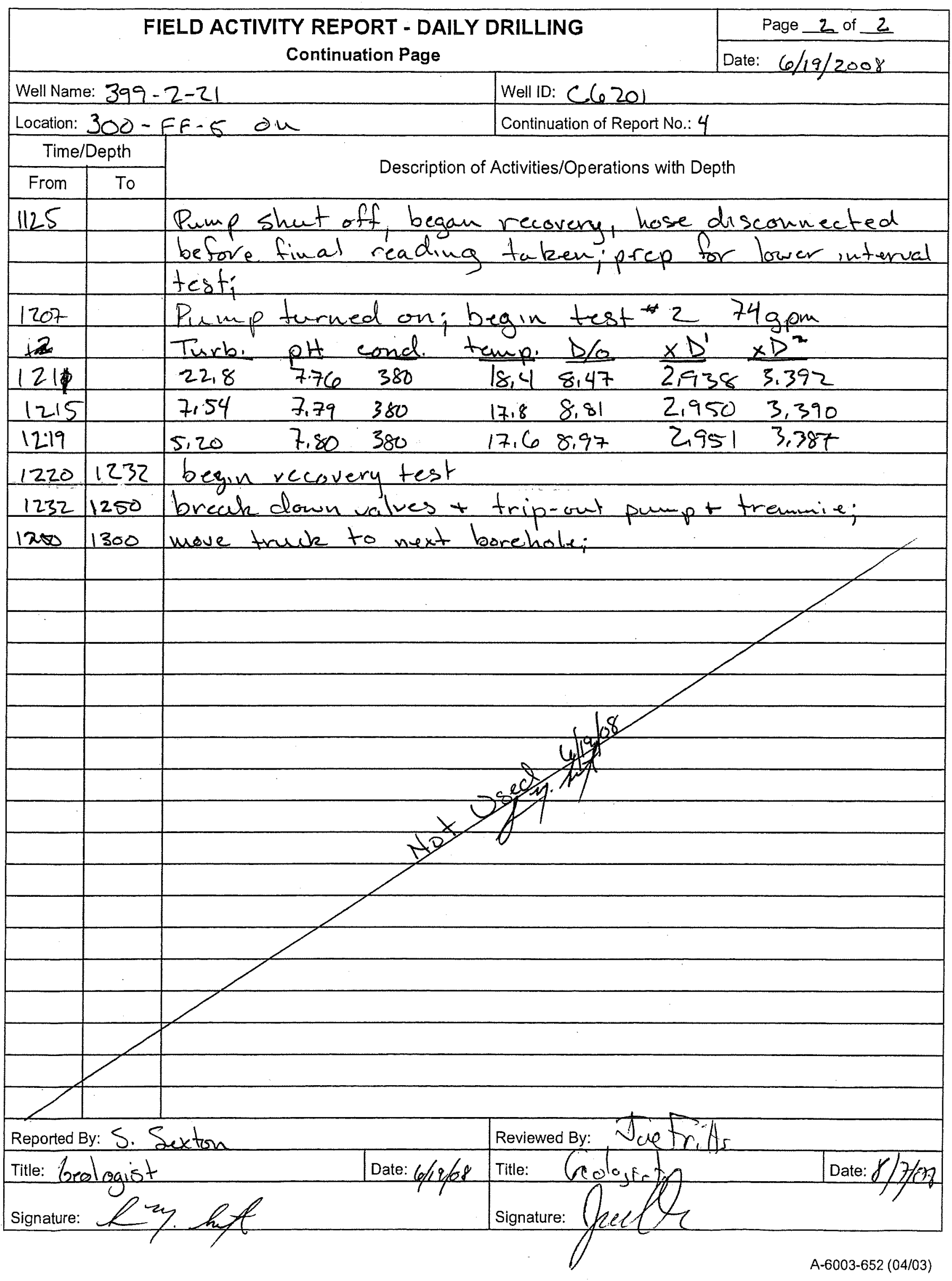

D.119 


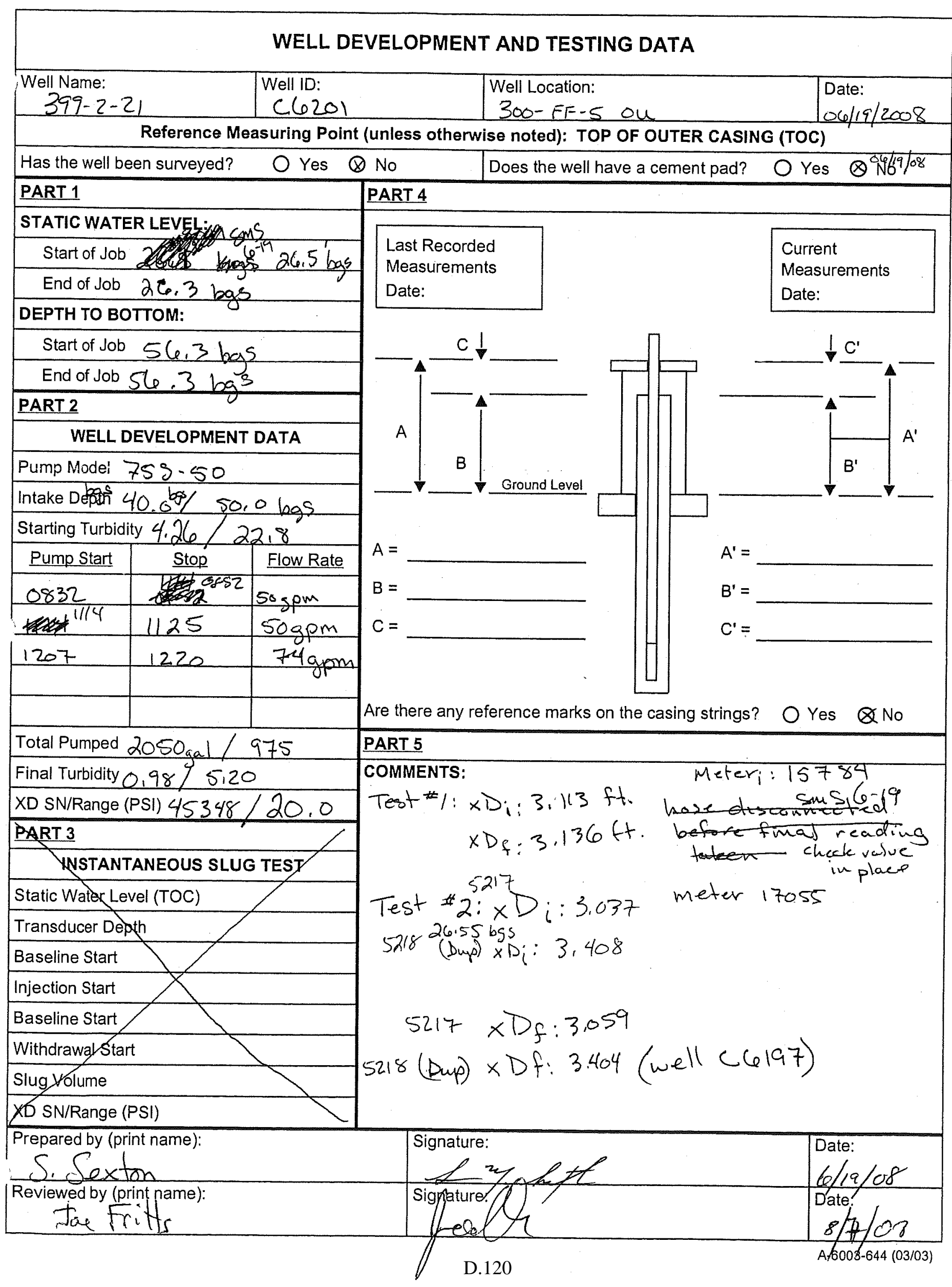


FIELD ACTIVITY REPORT NO. 1 - DRILLING PLAN

Date: $7 / 1+1 / 08$

Purpose: RNNL Cherderication 30oAka Location: 300-FF-5

Well ID: $C 6202$

Ife Well Name: $399-2-22$

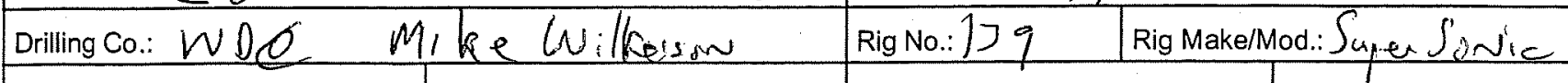

\begin{tabular}{lllll|l} 
Casing String No. & 1 & 2 & 3 & 4 & Drilling Method
\end{tabular}

Casing Size $75 / 6$

Grade

Material Carbun ste

Type:

Welded

Auger

Rotary

Circulation

Tubex

Cable Tool

Sonic $\frac{\text { Sypr Son.e }}{R 5400}$

Set At: $\frac{7 F_{r}}{z^{j} / g / 4 / 4}$

Geoprobe

Reference Measuring Point:

GROUND LEVEL

Drig. Co.

Casing String No. 12234

Casing Size

Grade

Lbs.Per Ft.

Material

Type:

Welded Thd.

Planned / Actual

Set

Shoe OD/ID

Reference Measuring Point:

GROUND LEVEL

Comments/Remarks:

Rig No.:

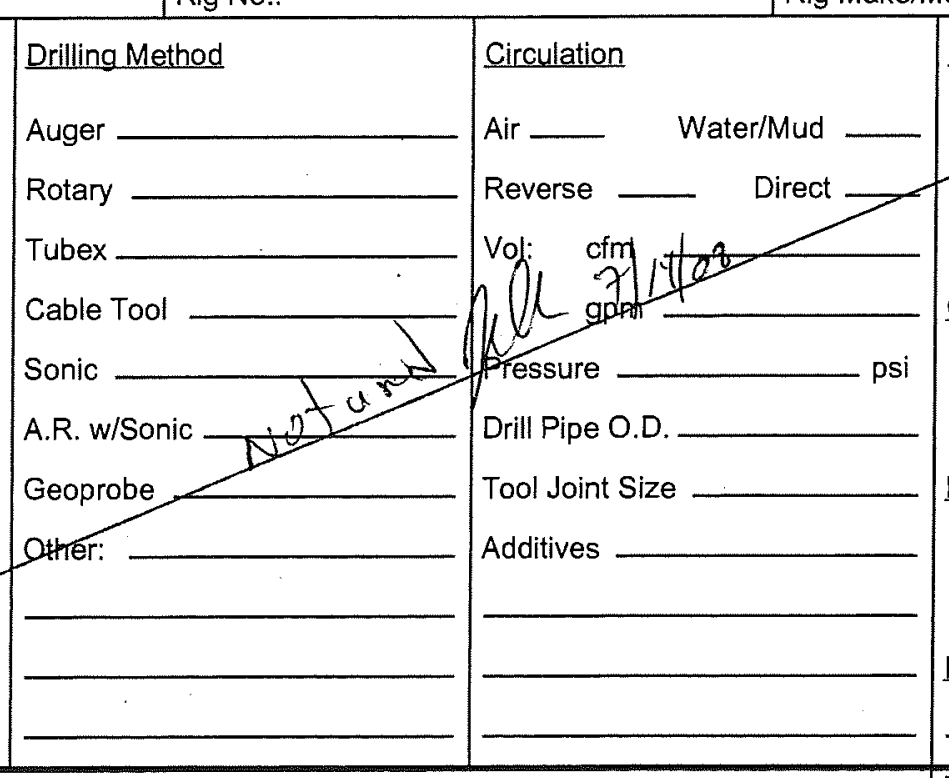

Estimated Depth to Water

Rig Make/Mod.:

D.H. Hammer

Make

Model

Choke

\section{Casing Hammer}

Make

Model

Bit Size

Type

Nozzles

Rod Size

Reported By: Juefrits

Namertitle: G Mtm Dur frodgiet

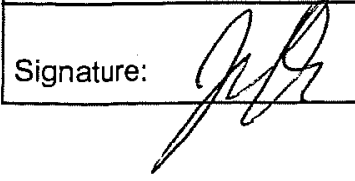

Other:

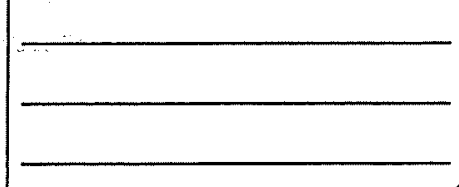

Air

Air

Reverse — Direct

Vol: $\quad \mathrm{cfm}$

Pressure

Drill Pipe O.D.

Tool Joint Size

Additives

D.H. Hammer

Make

Model

Choks

Casing Hammer

Make

Model

Bit Size

Type

Nozzles

Rod Size
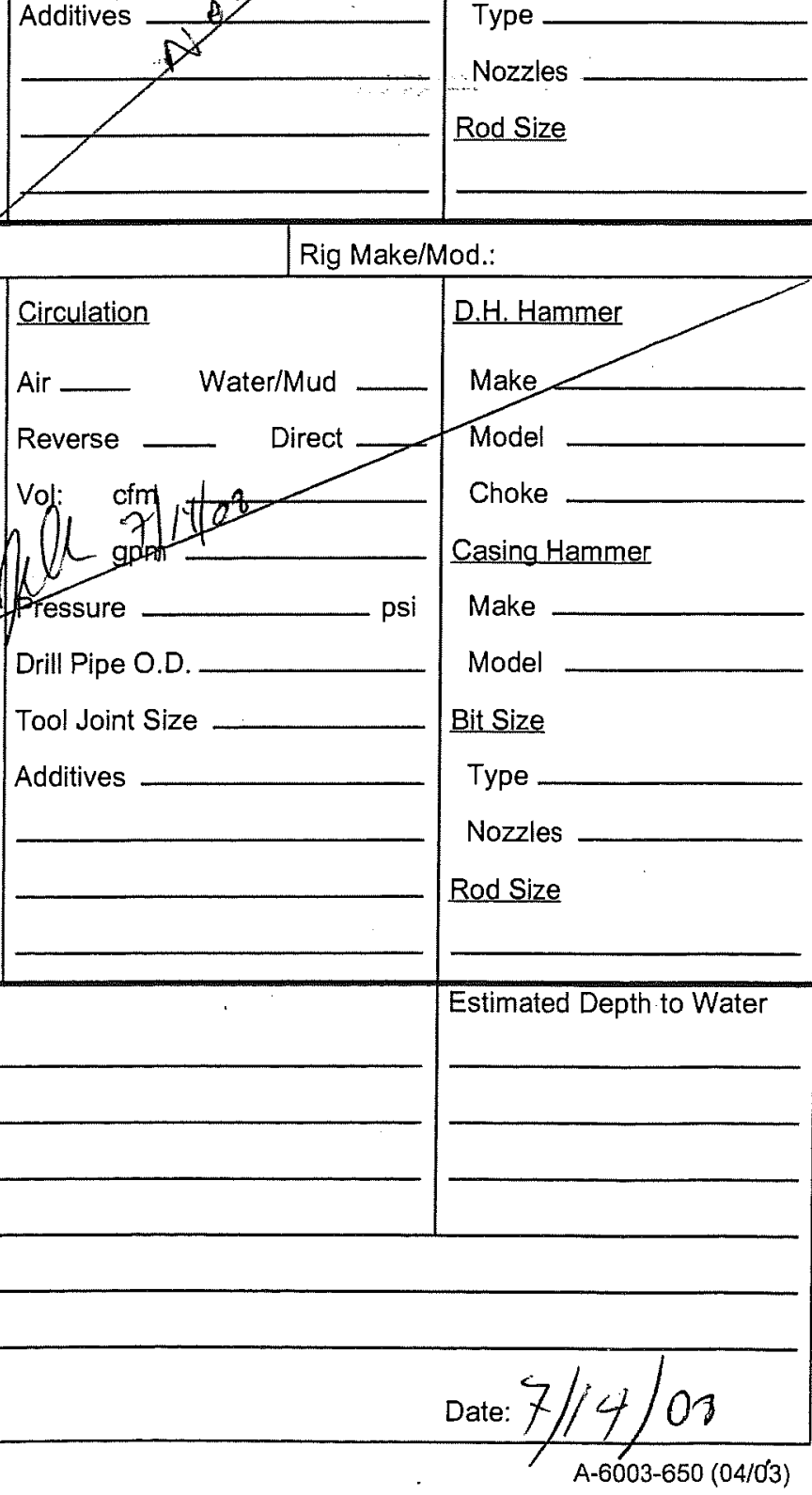


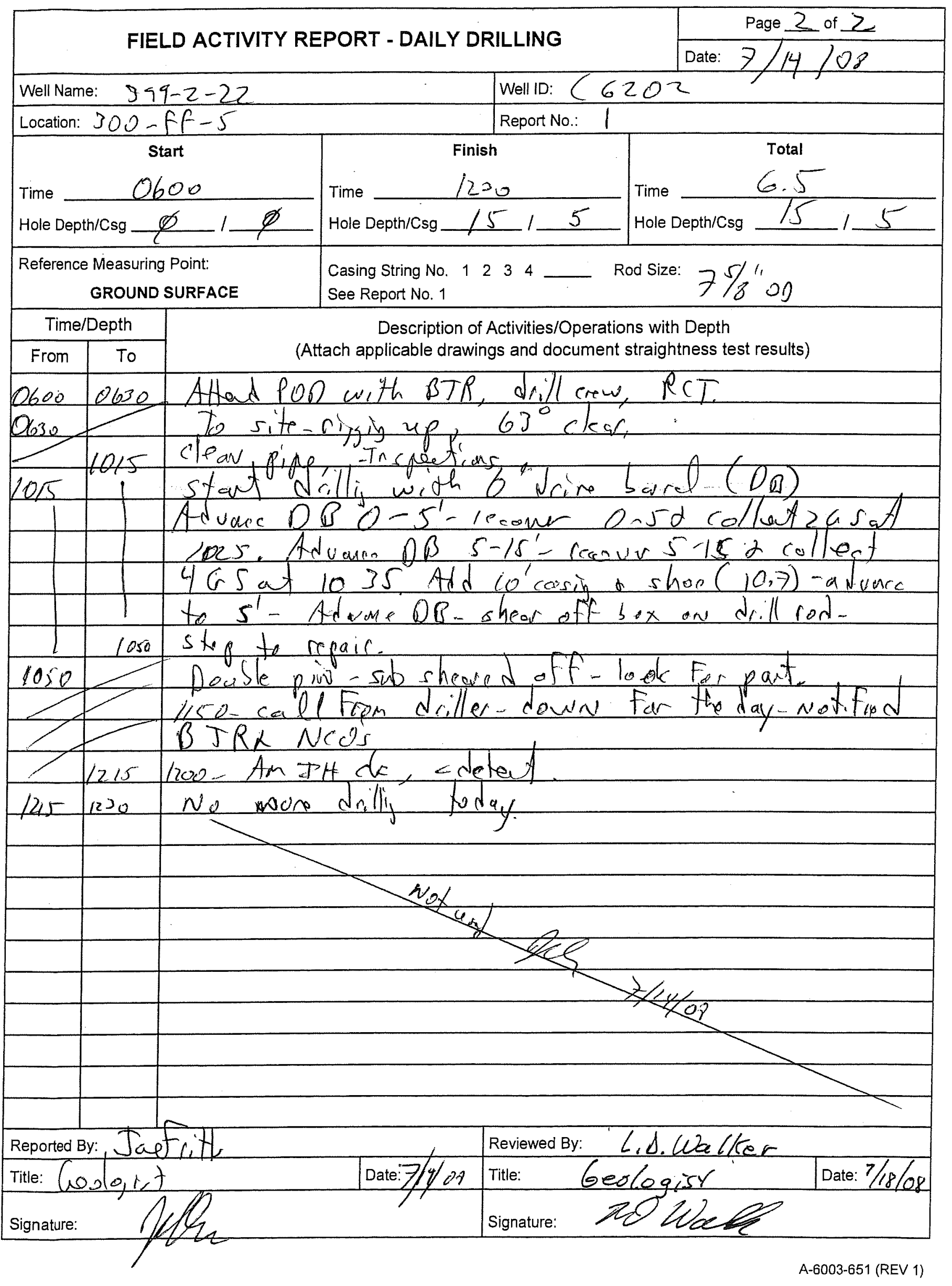

D.122 


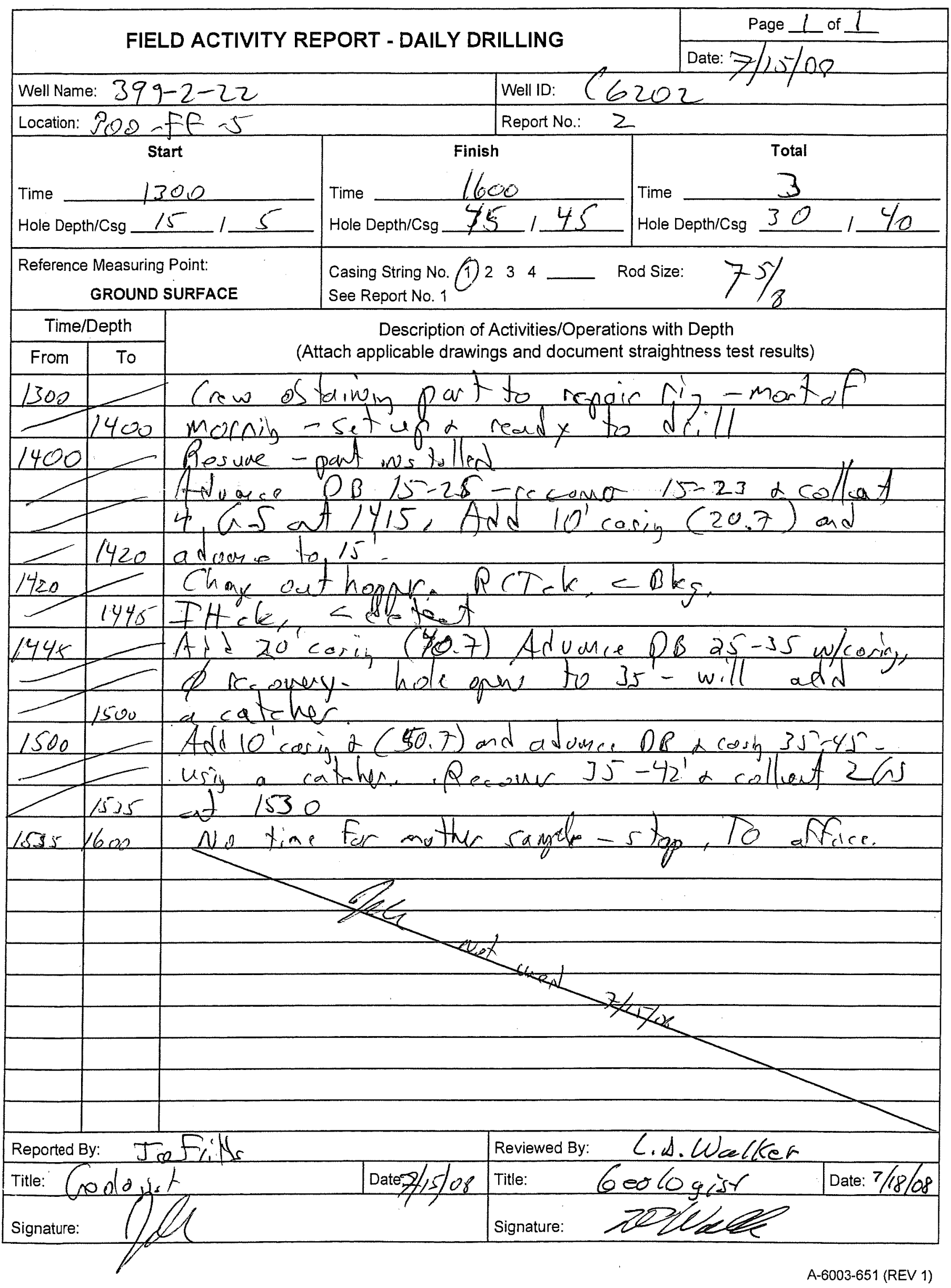

D.123 


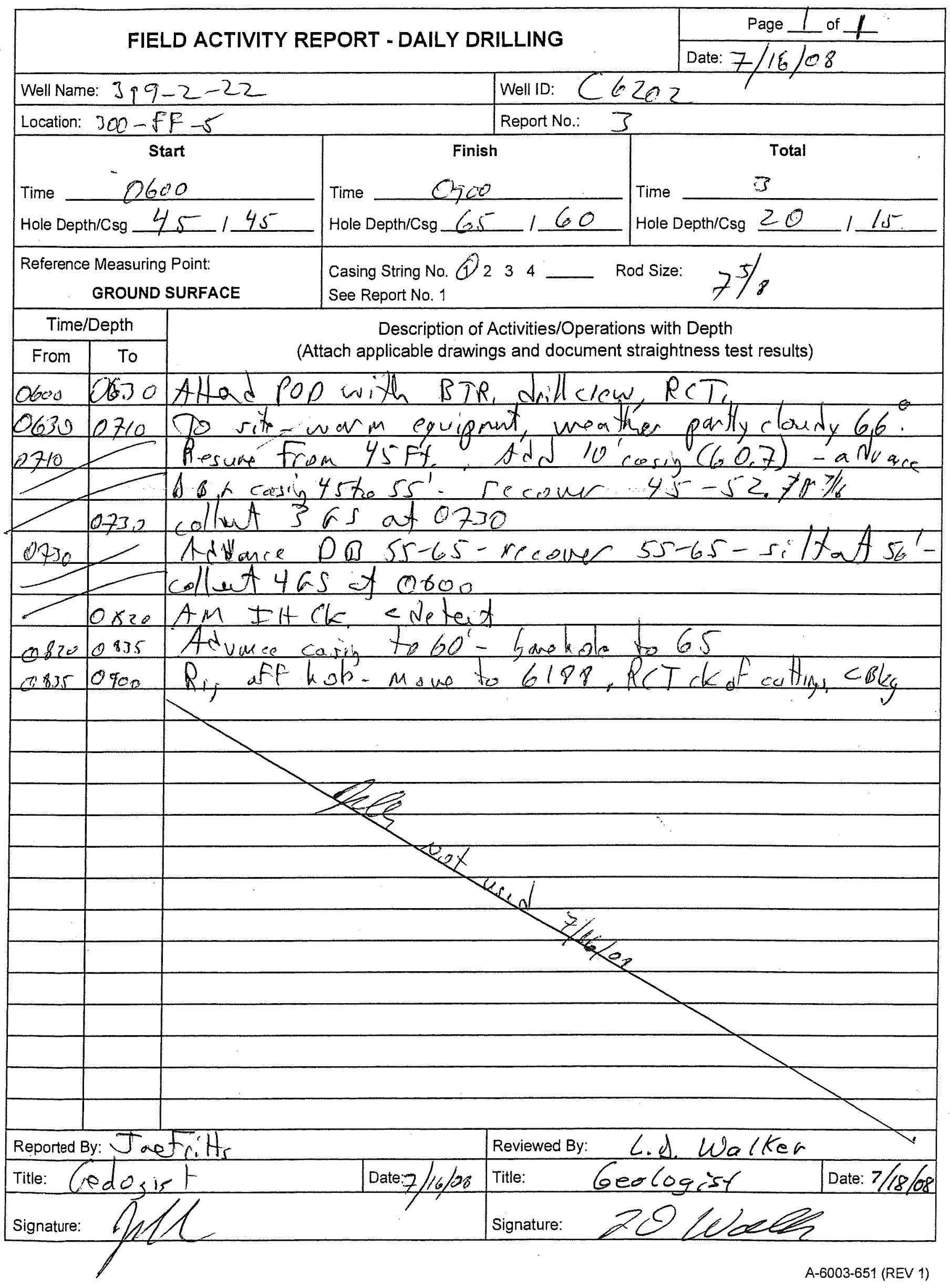

D.124 


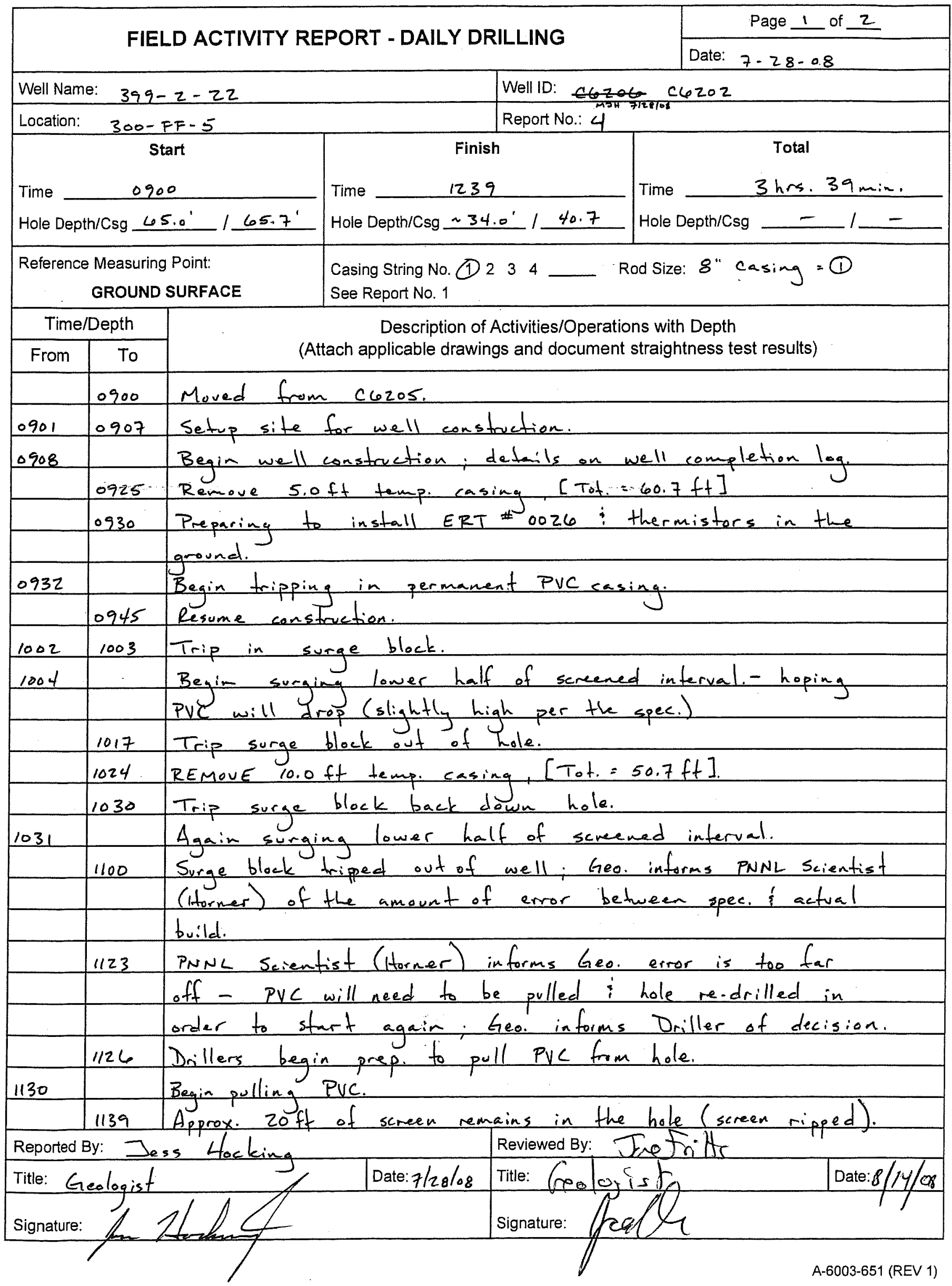




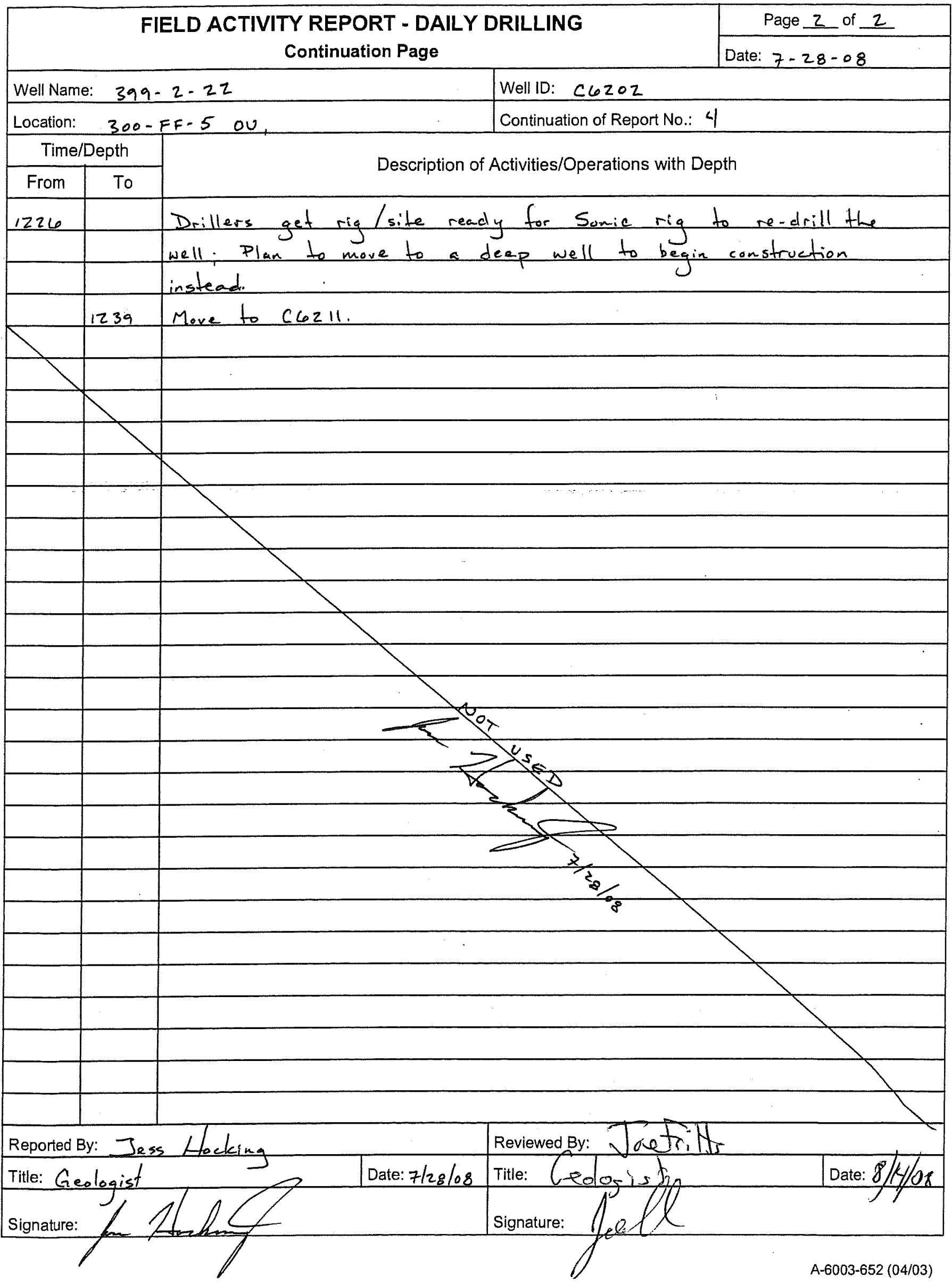

D.126 


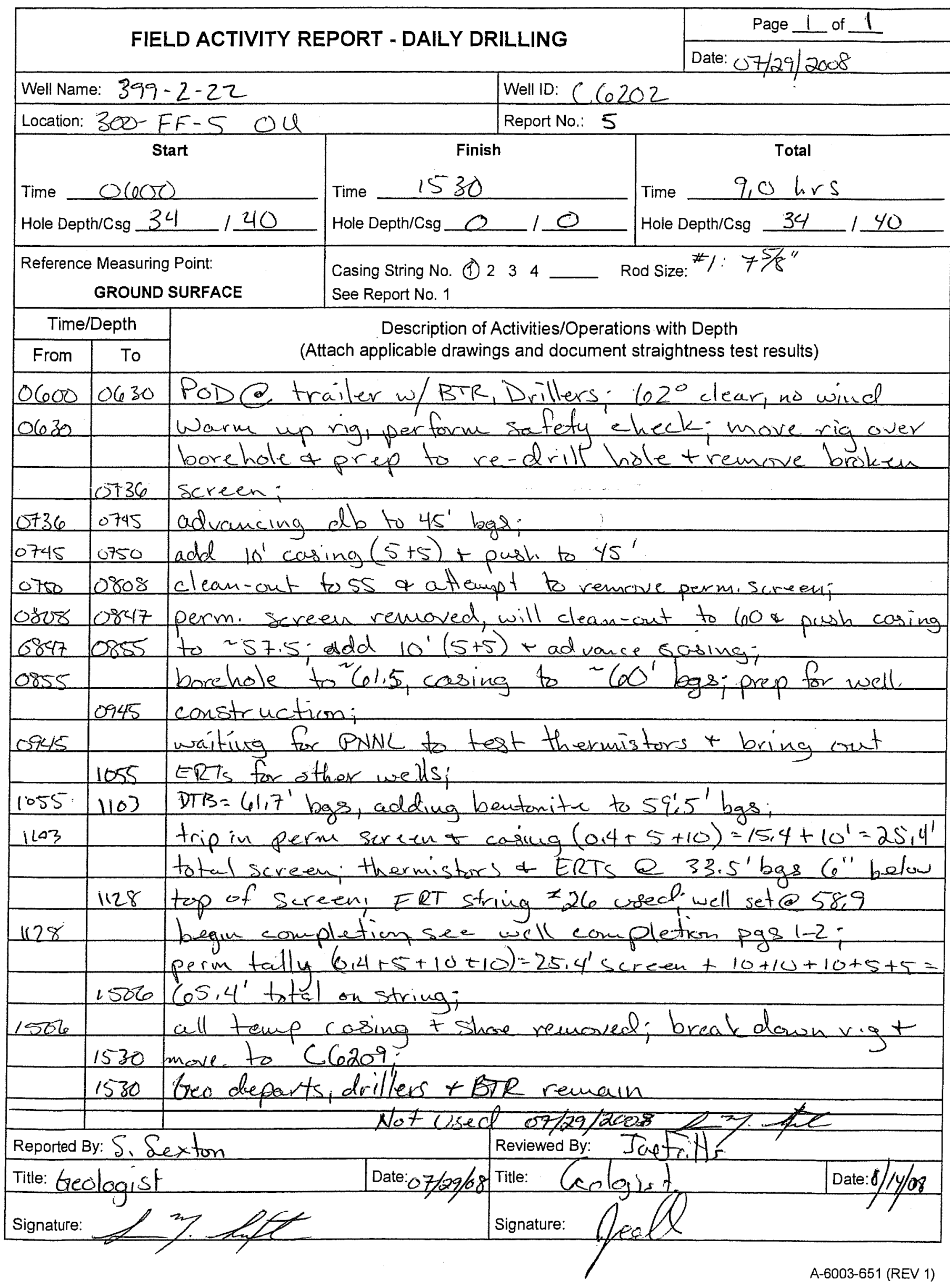




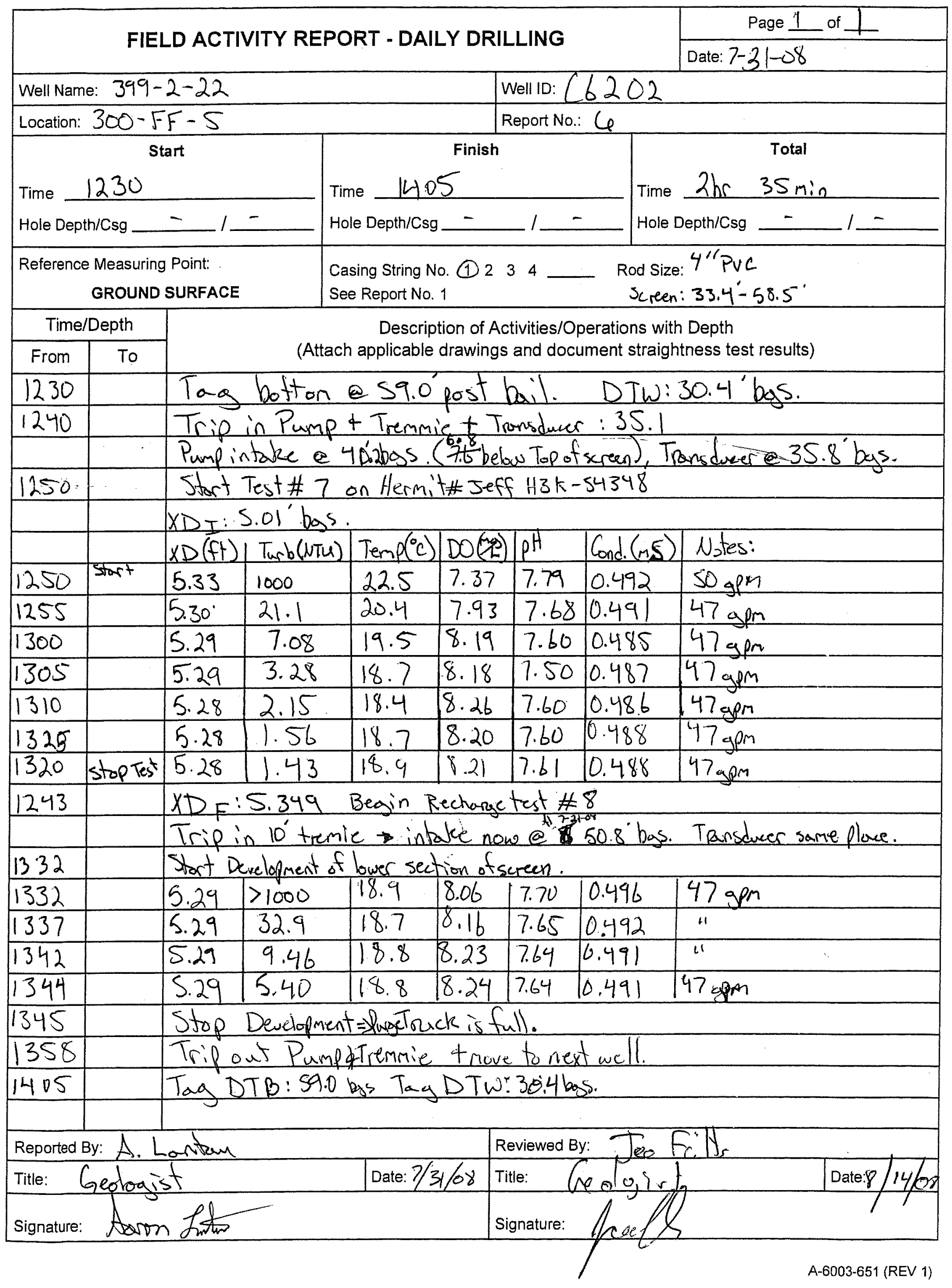

D.128 


\section{WELL DEVELOPMENT AND TESTING DATA}

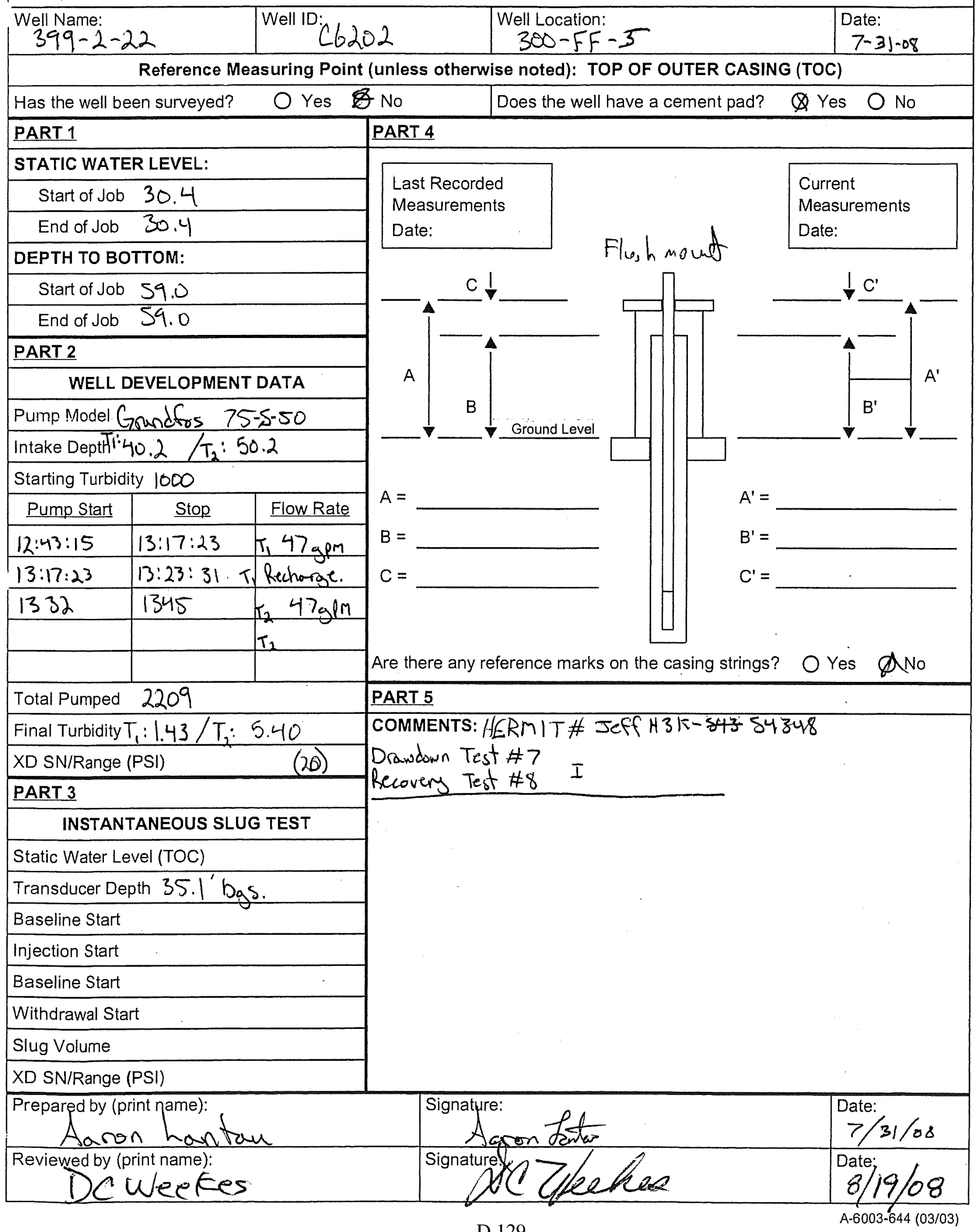




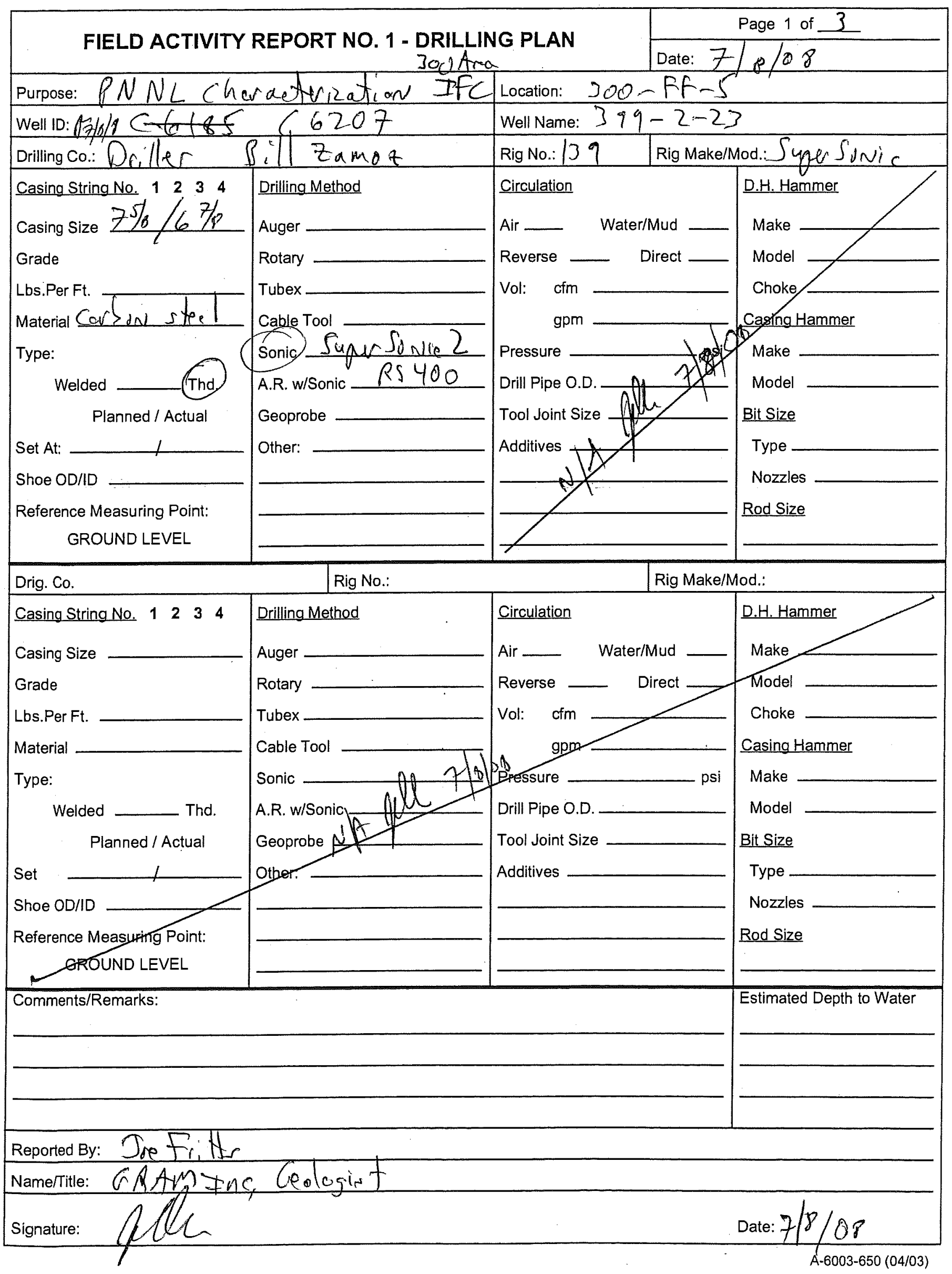




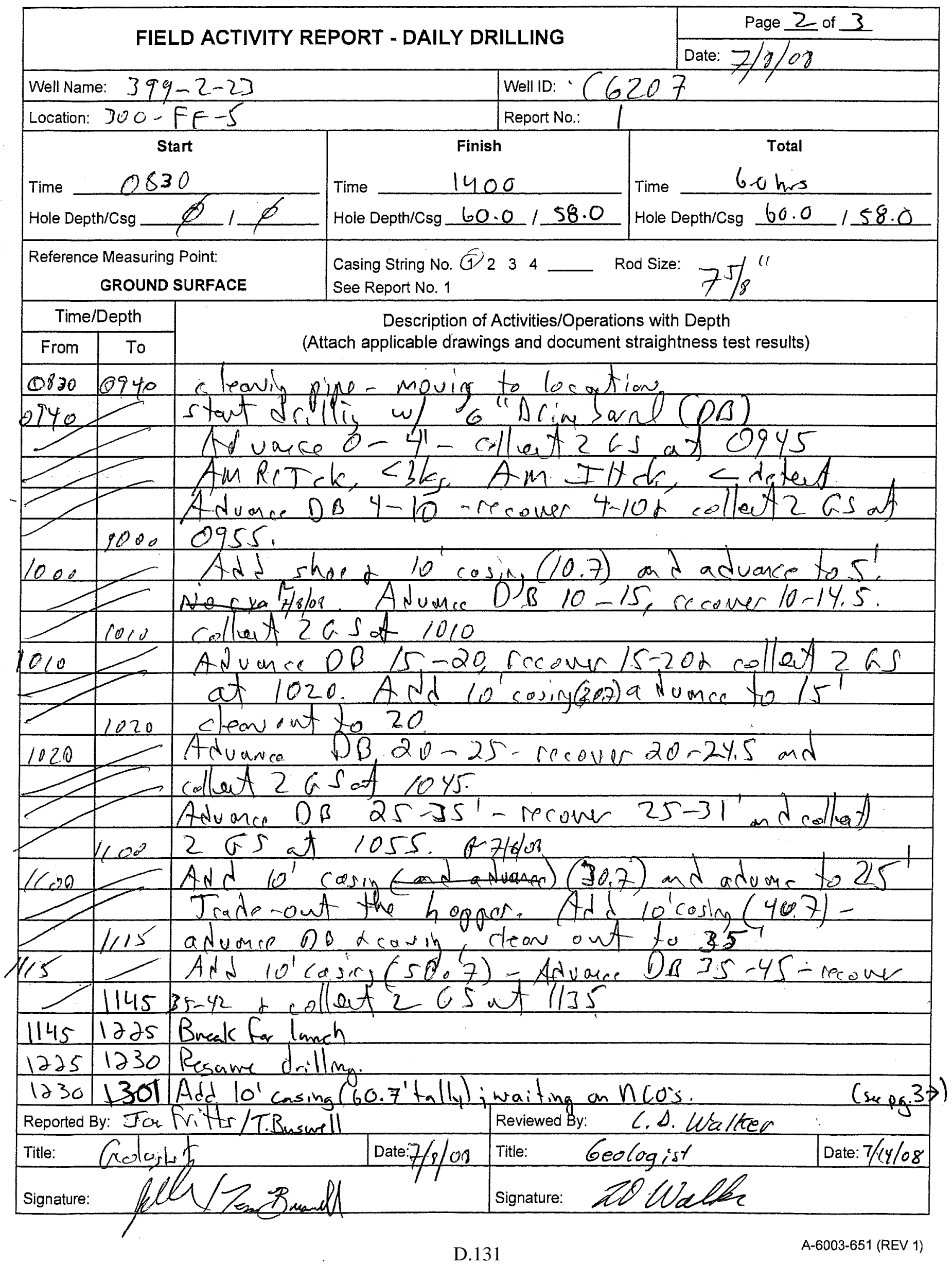




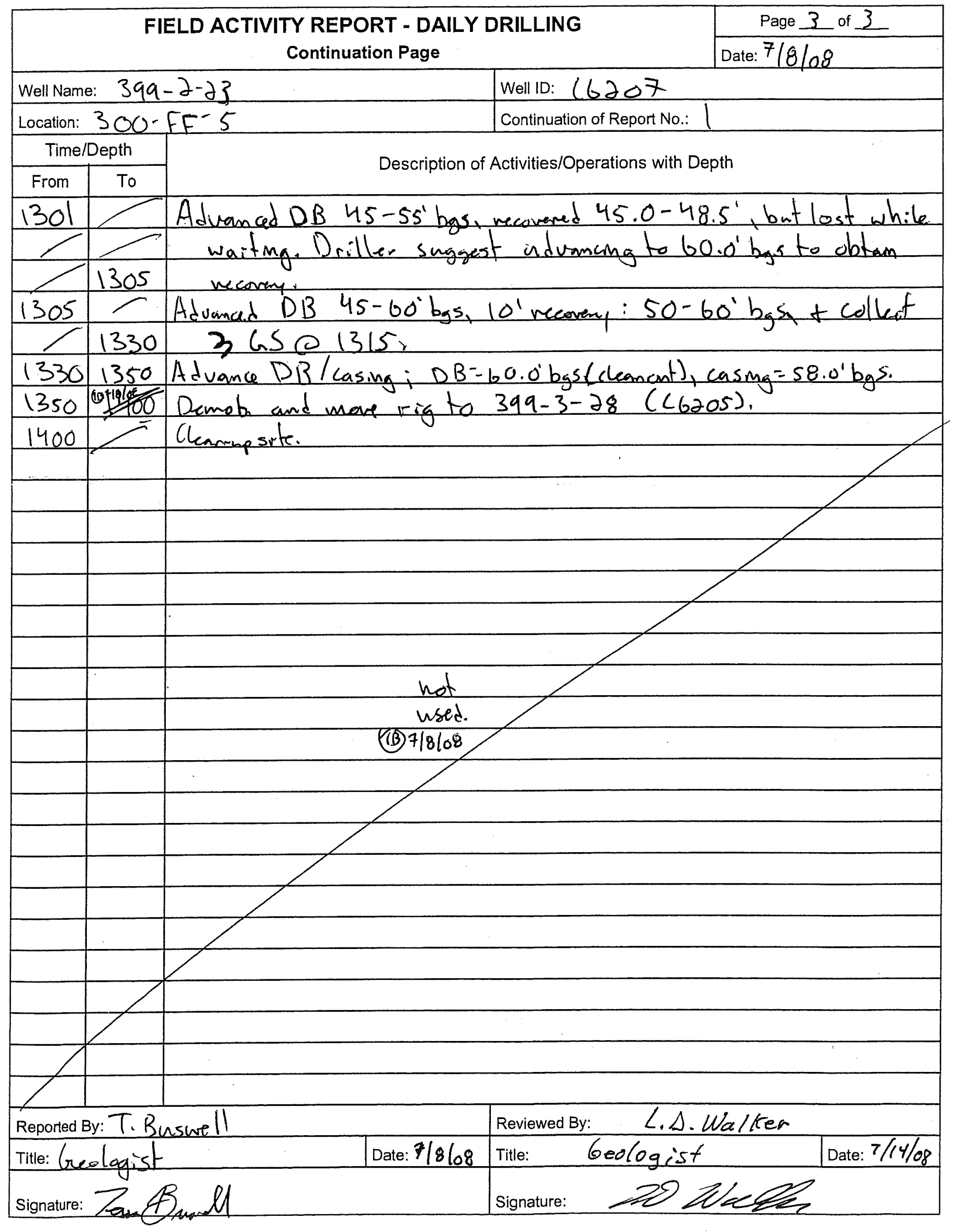

D.132

A-6003-652 (04/03) 


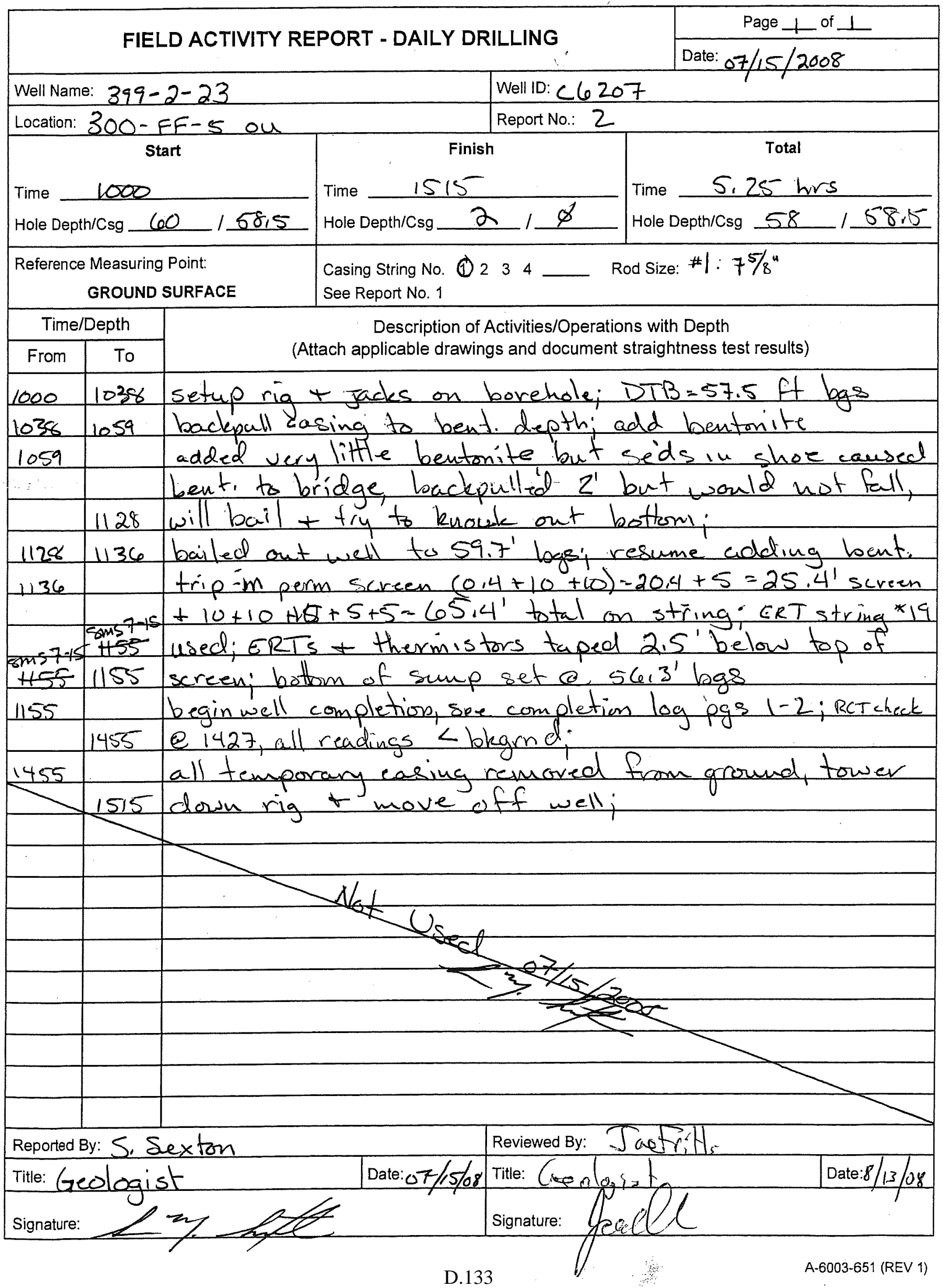




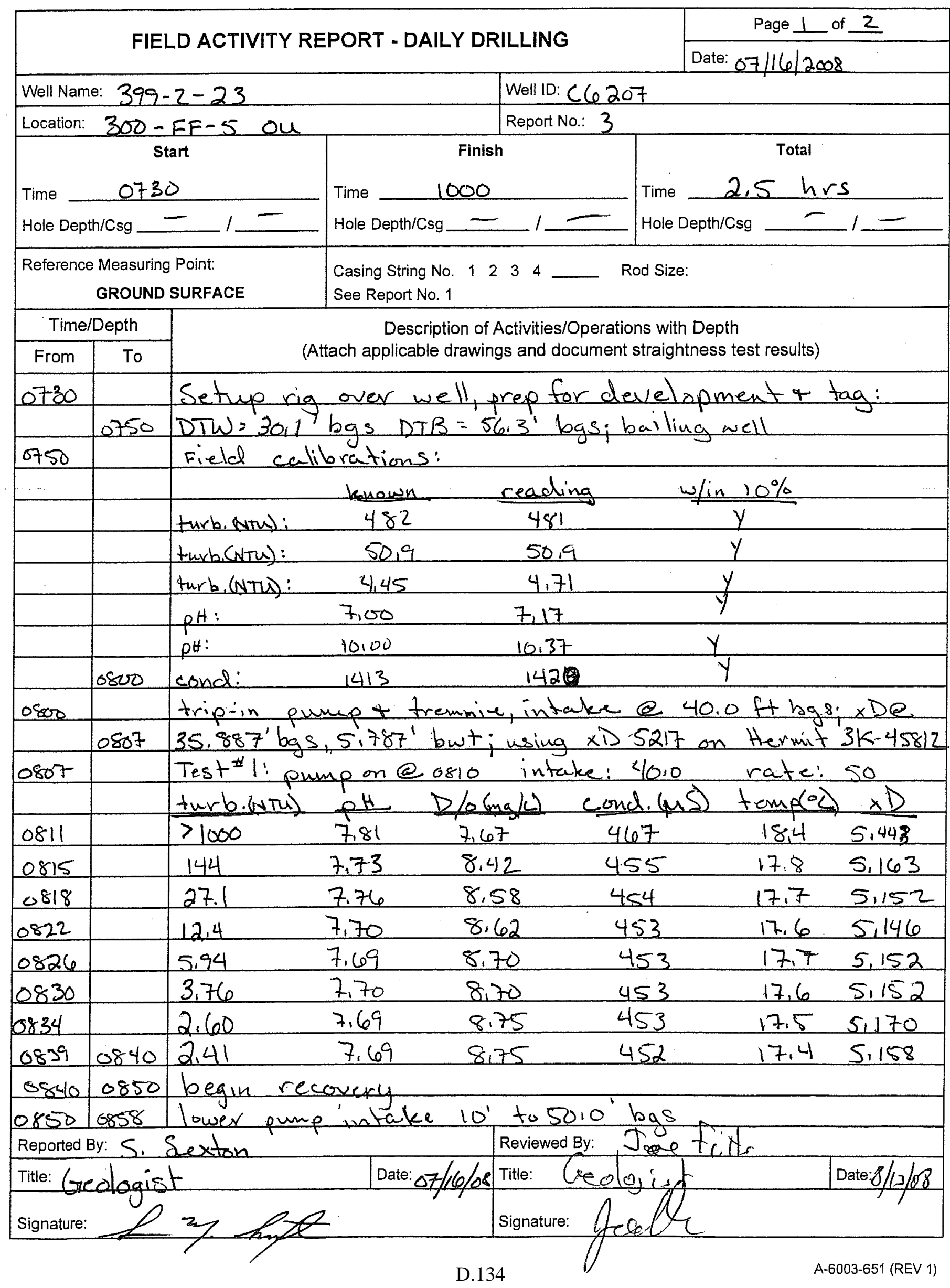




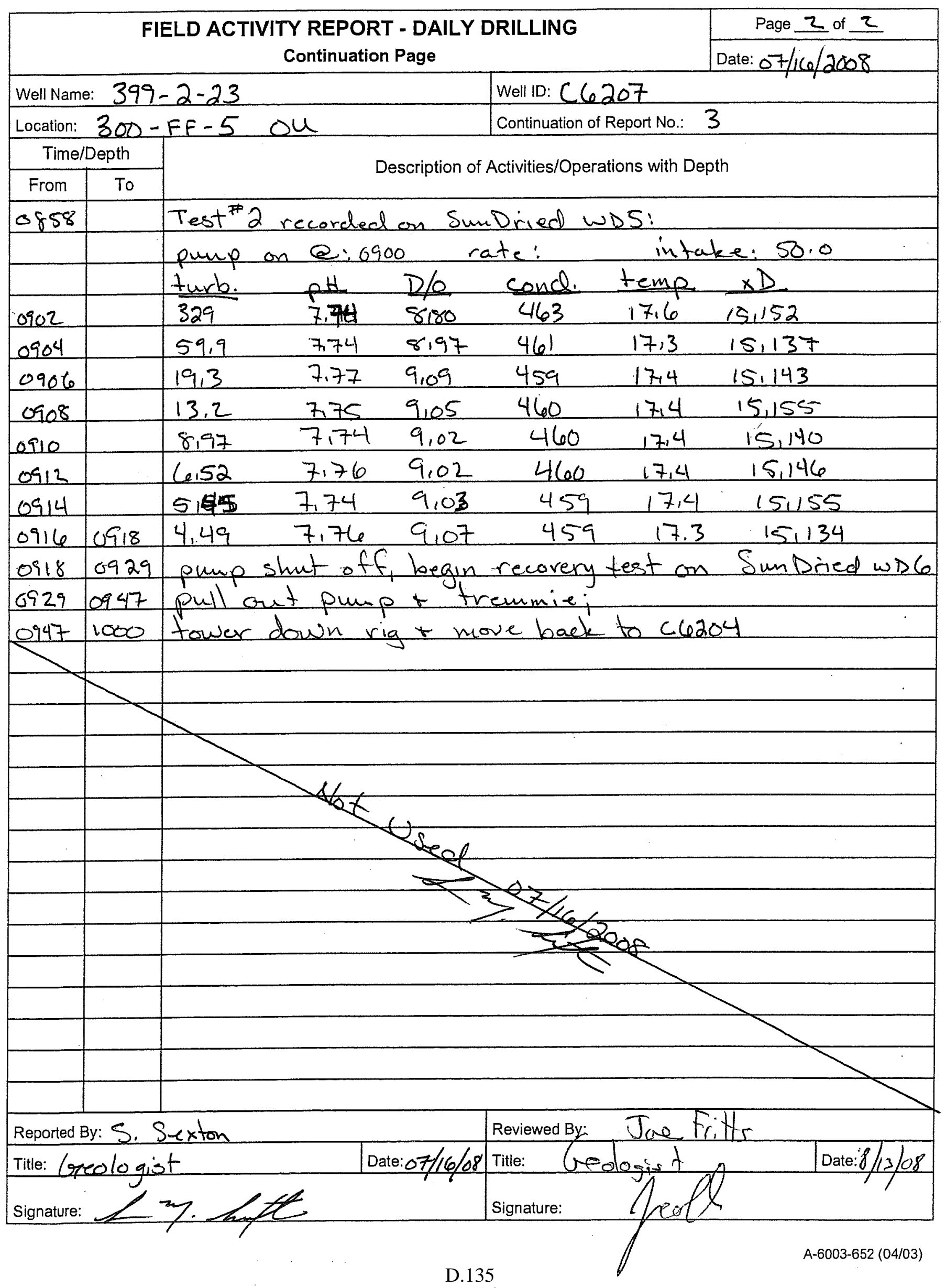




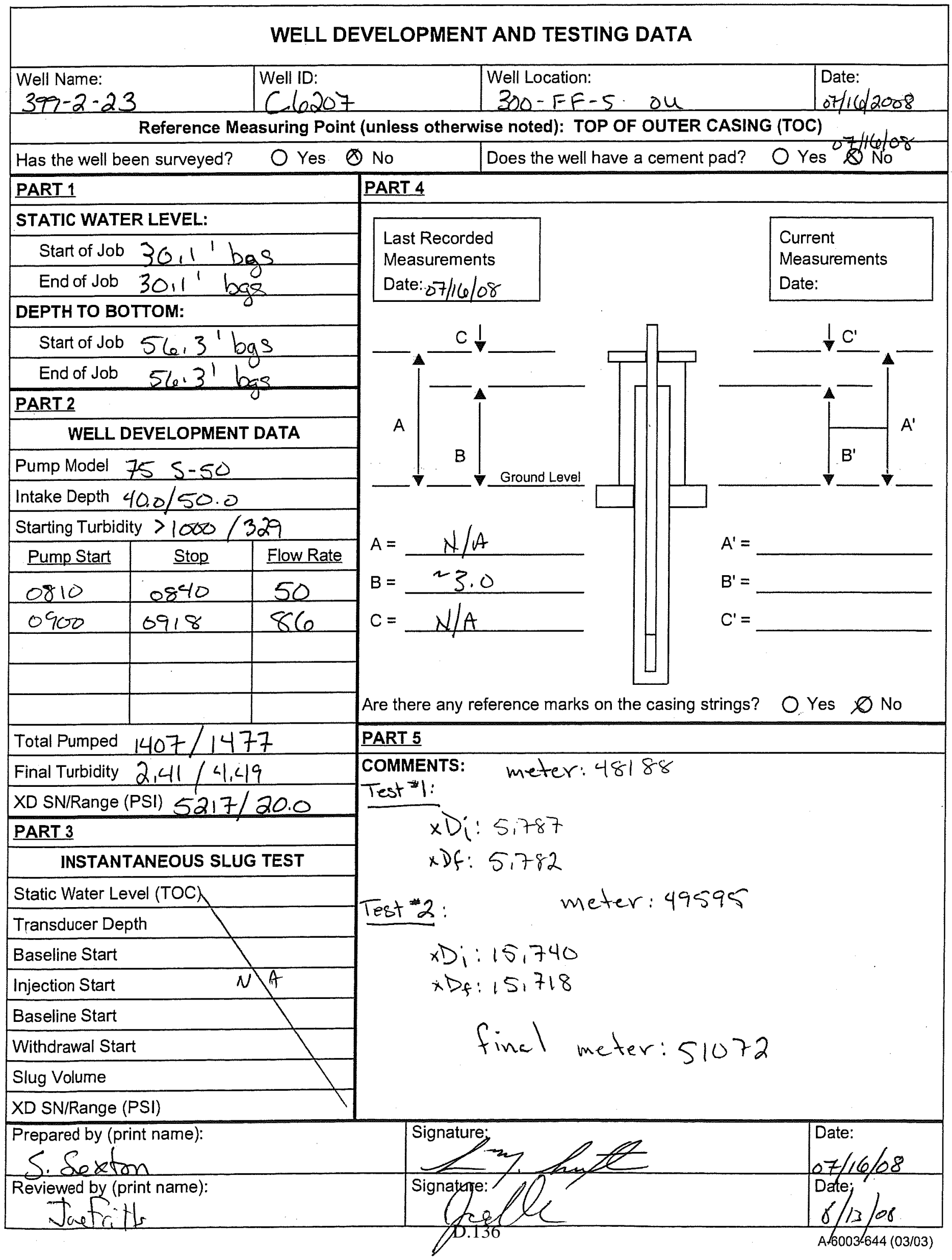




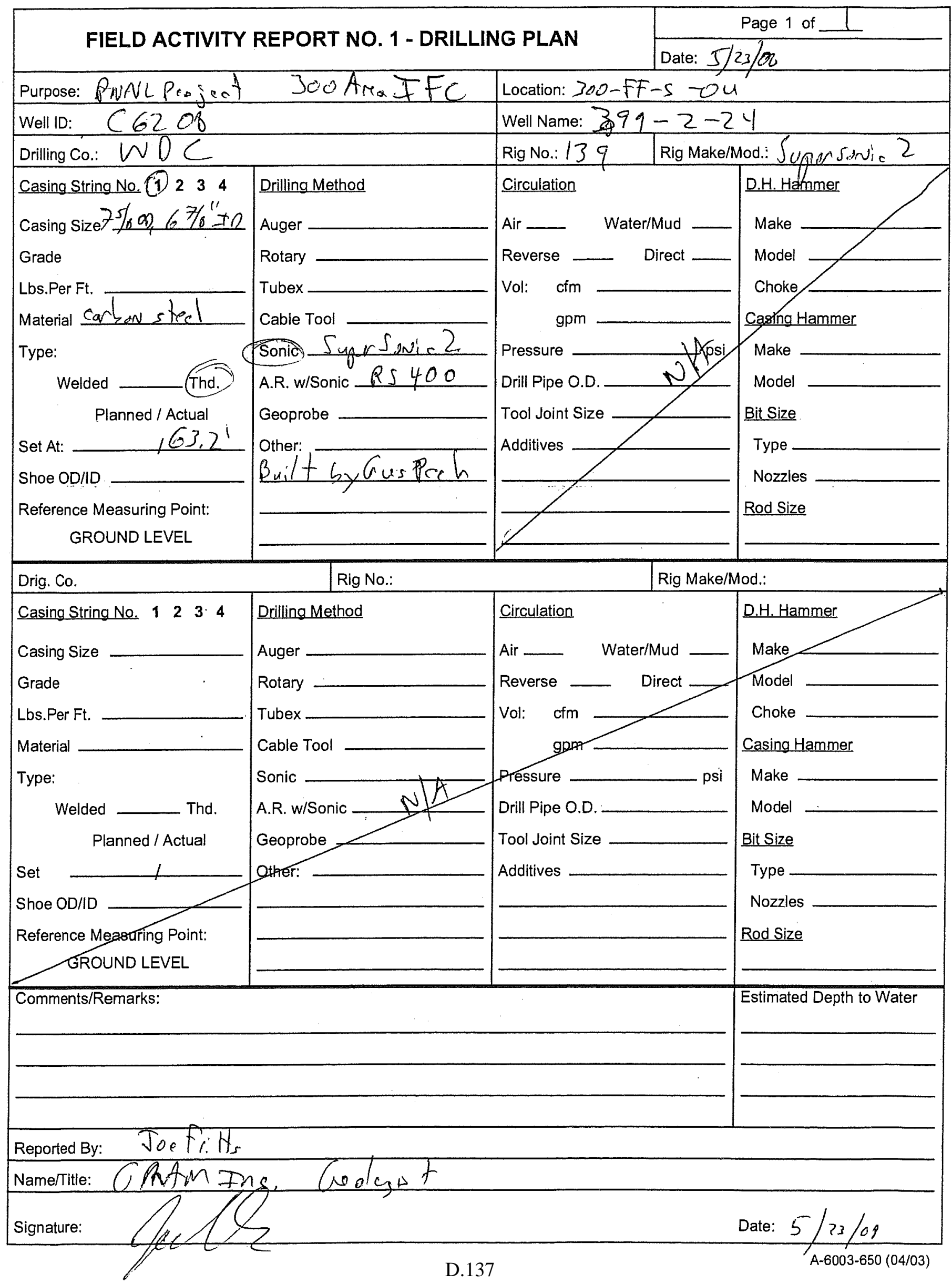




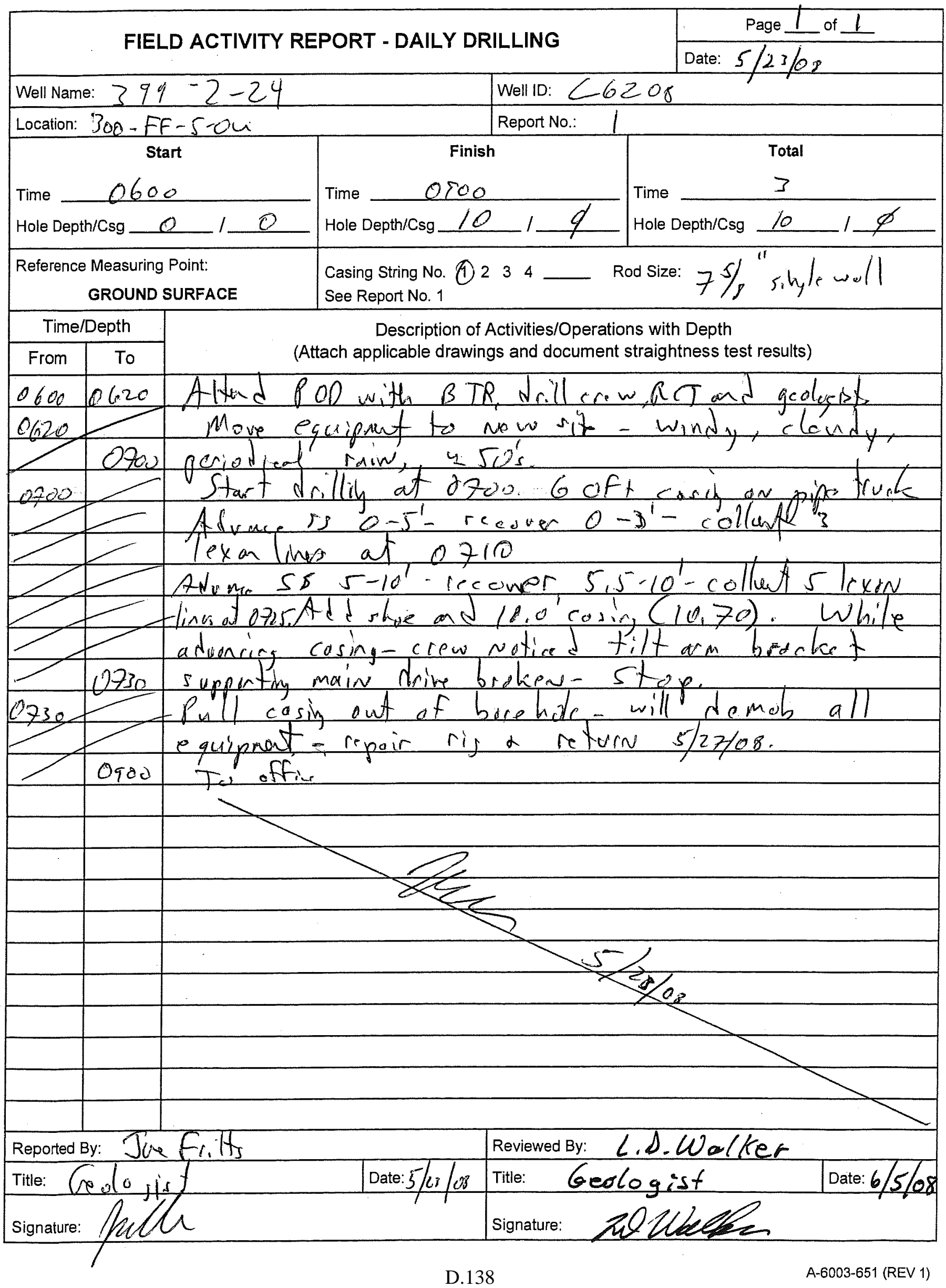




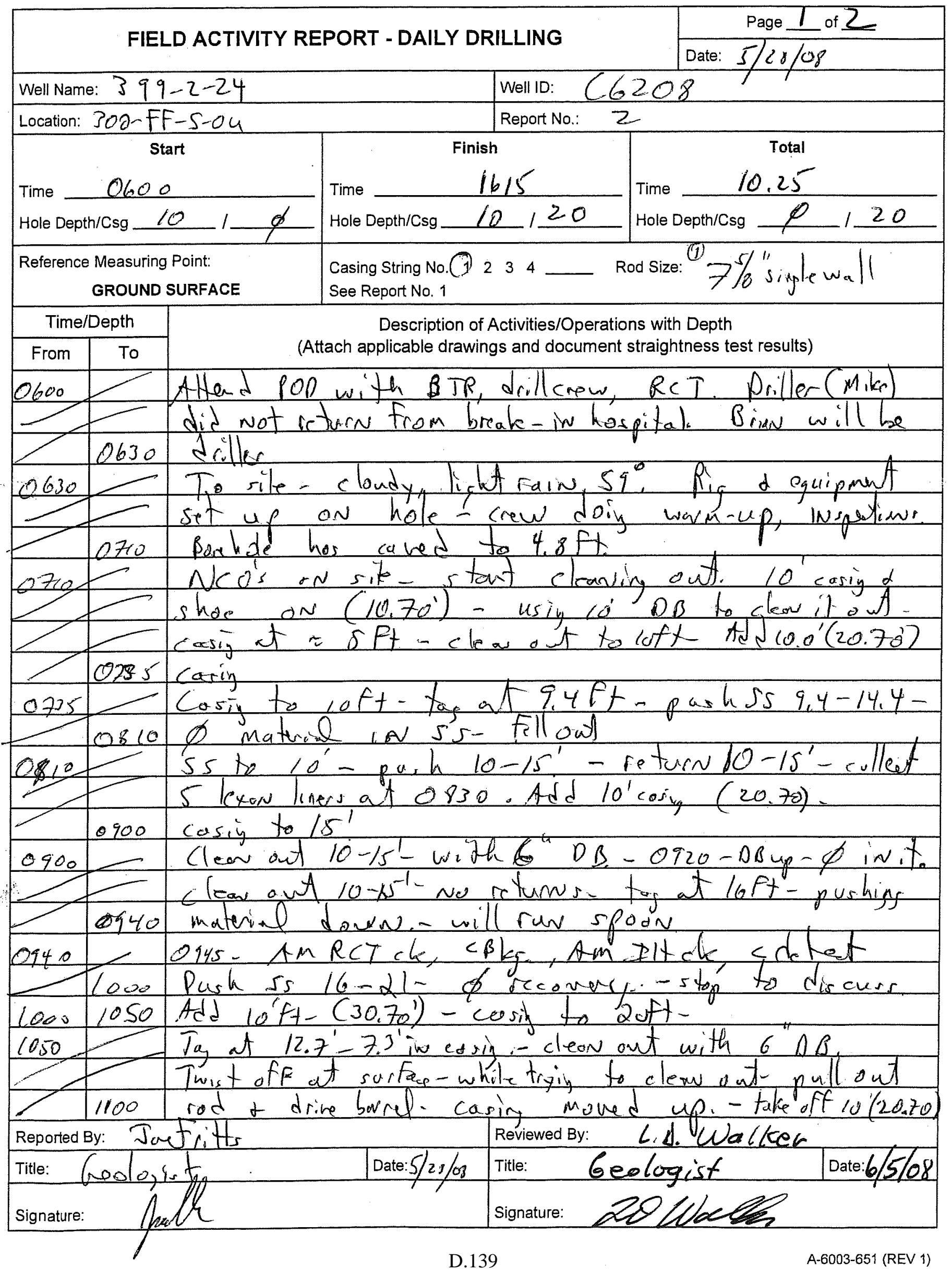




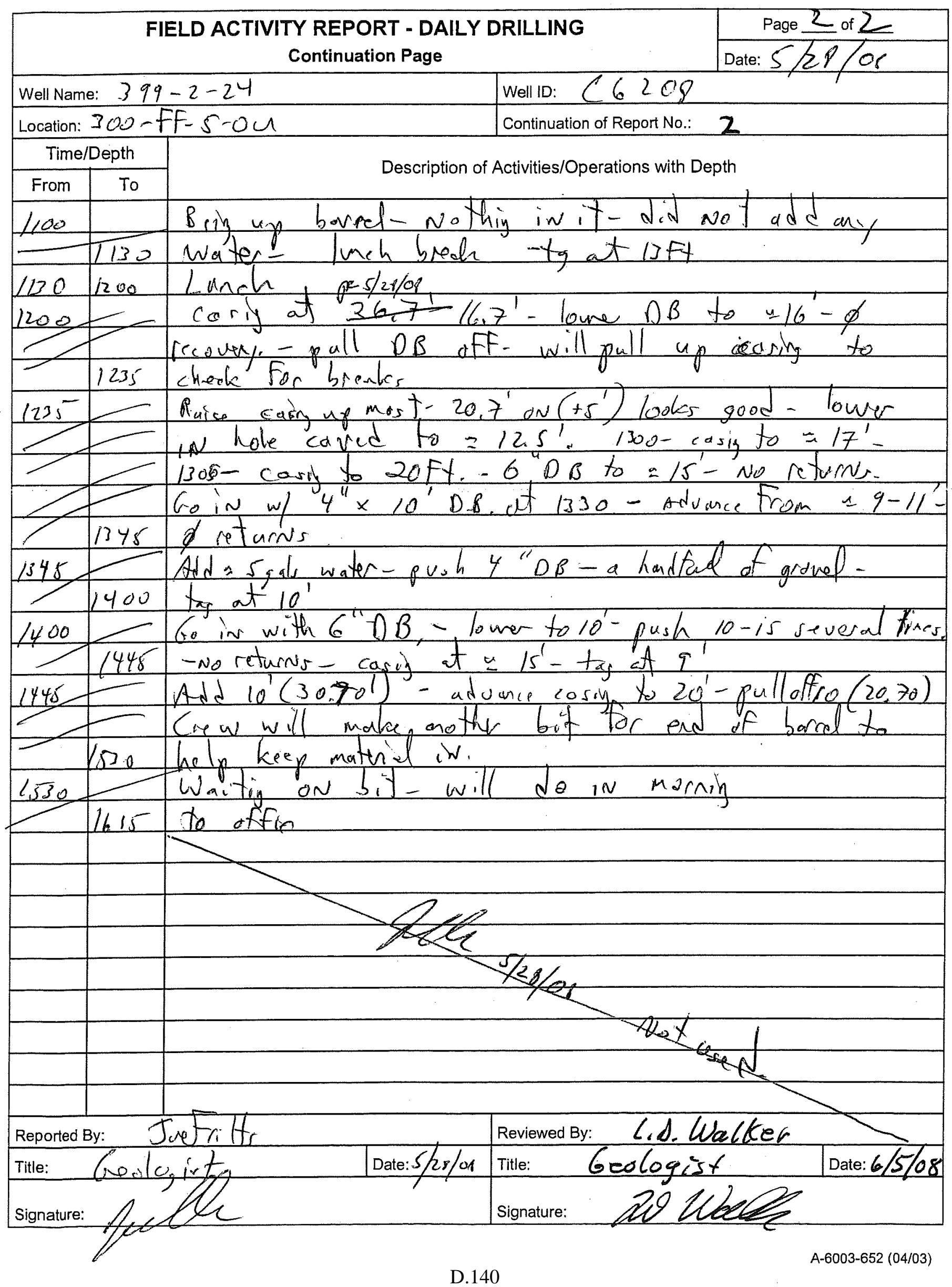




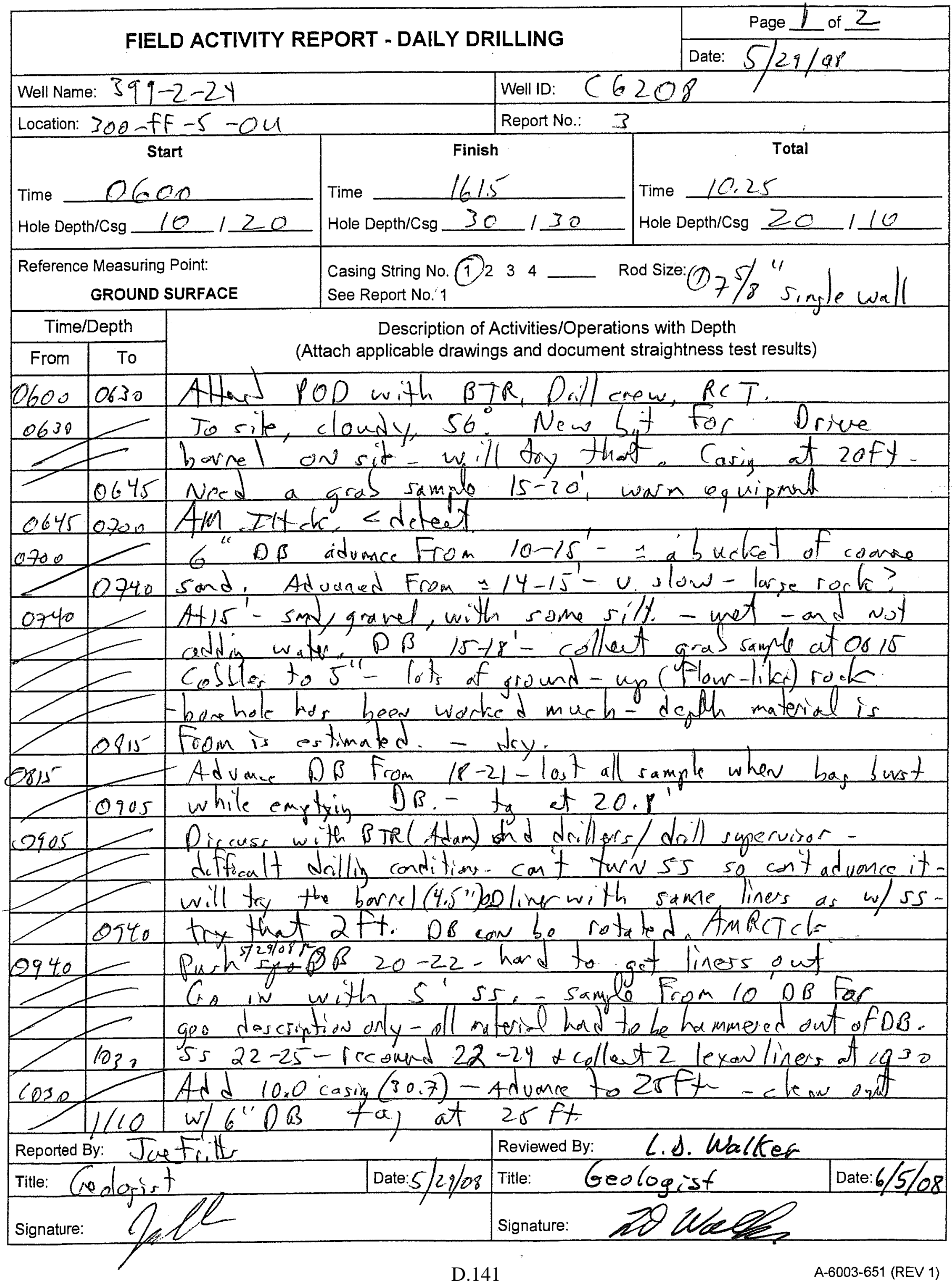




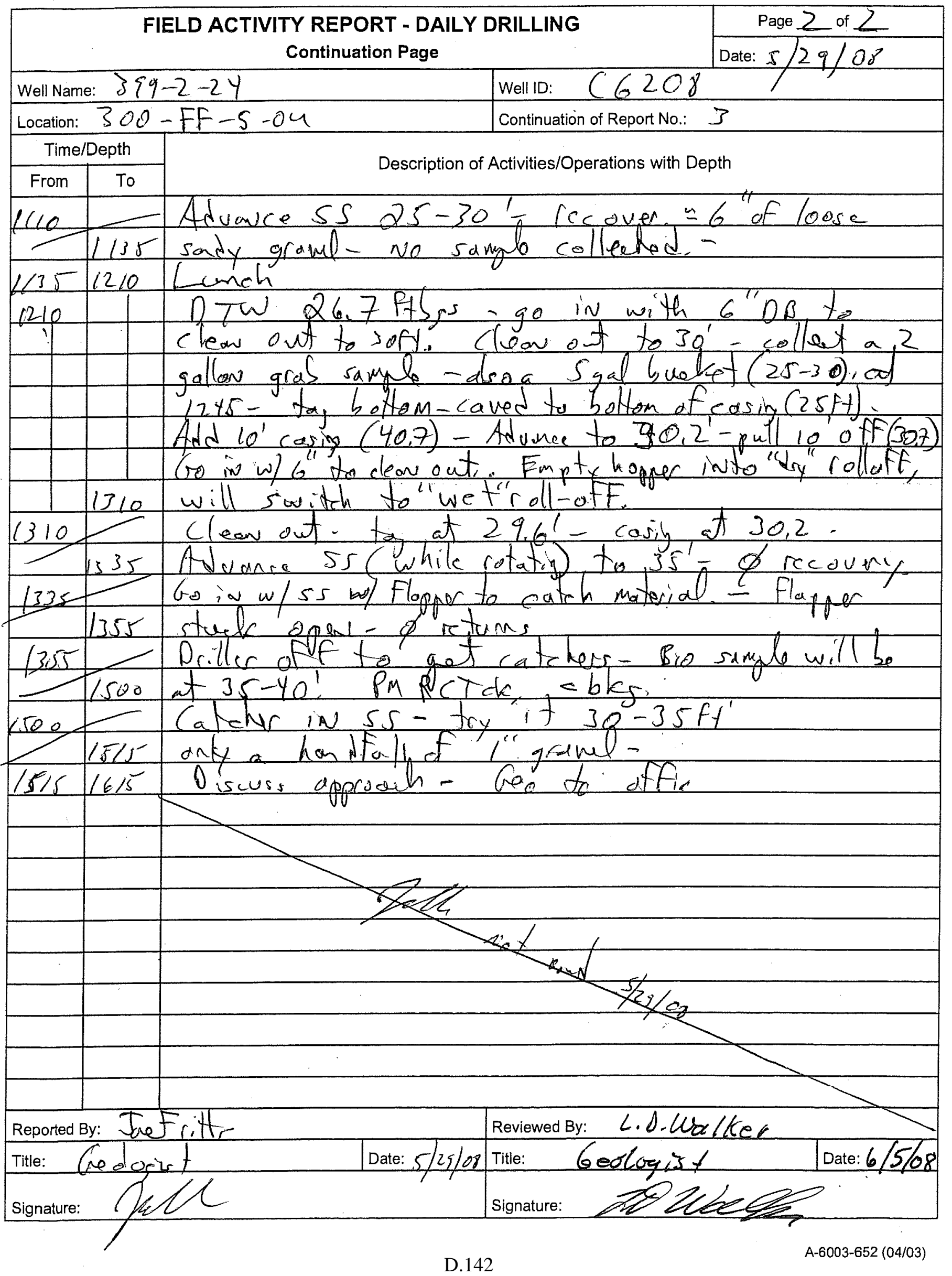




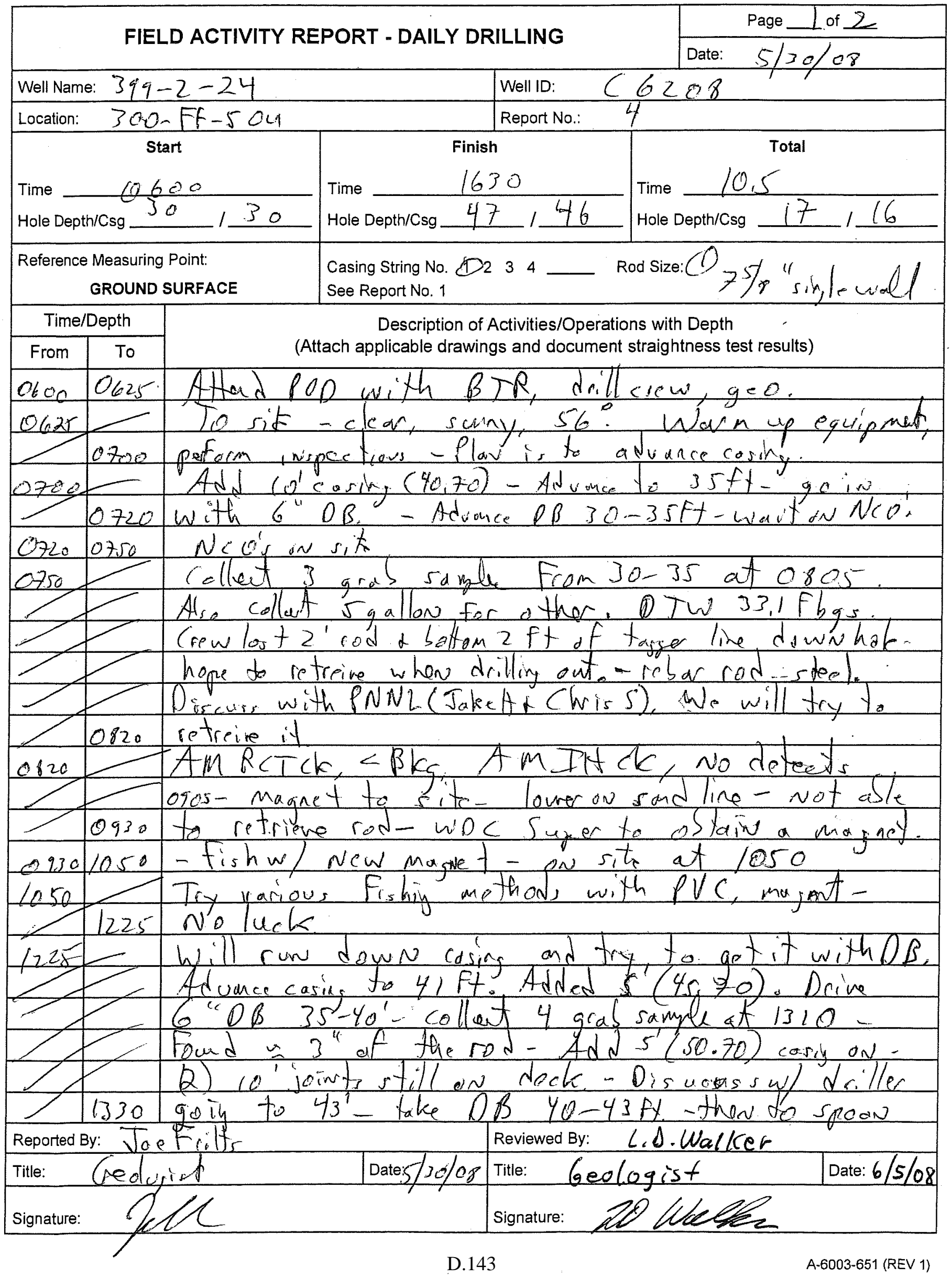




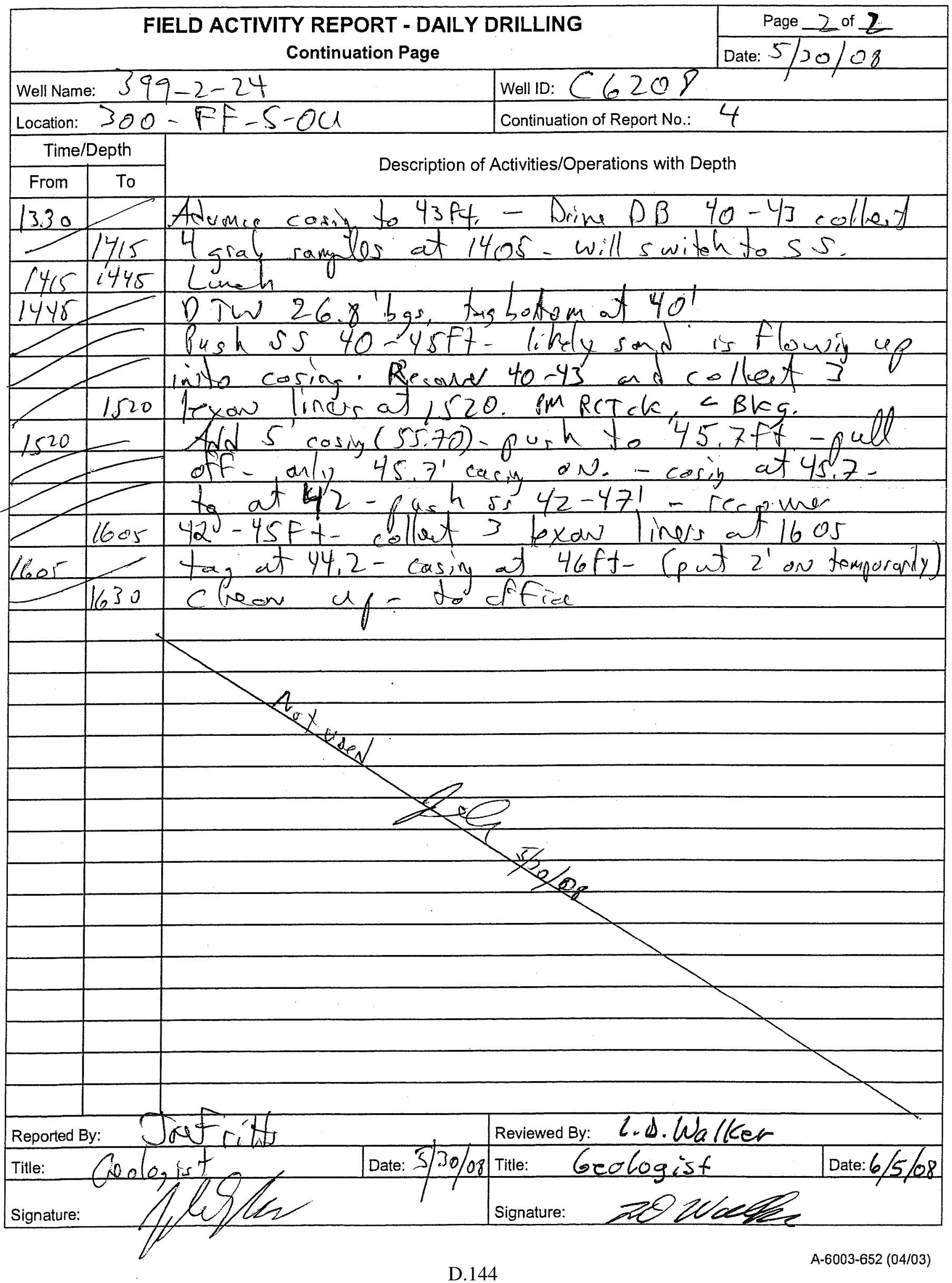




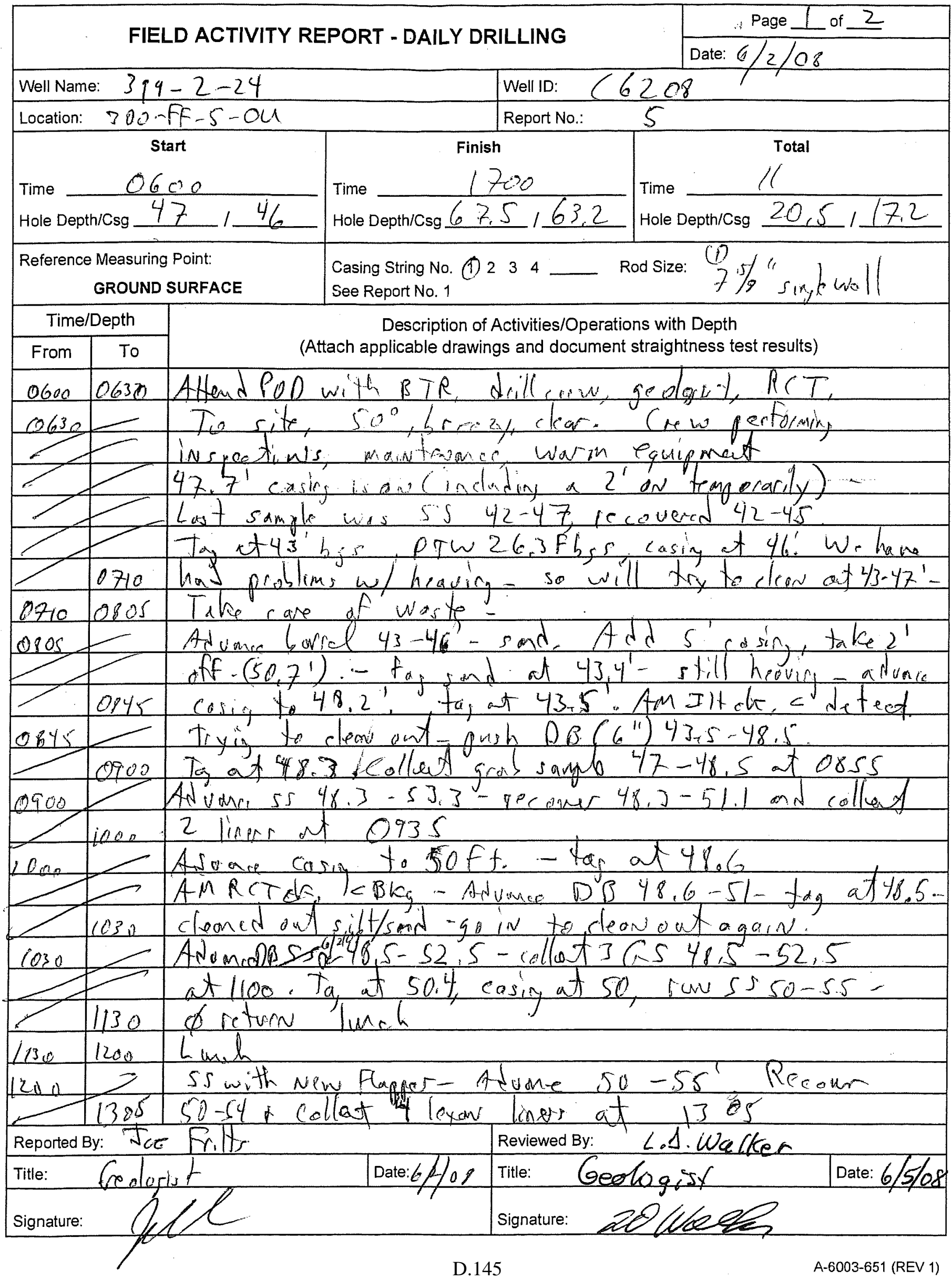




\begin{tabular}{l}
\multicolumn{5}{|c|}{ FIELD ACTIVITY REPORT - DAILY DRILLING } \\
Continuation Page
\end{tabular}


FIELD ACTIVITY REPORT - DAILY DRILLING

\begin{tabular}{|l} 
Page 1 of 1 \\
\hline Date: $6 / 6 / 08$
\end{tabular}

\begin{tabular}{l}
\hline Well Name: $399-2-24$ \\
\hline Location: $300-F F-5$ \\
Start \\
Time $\frac{1015}{\text { Hole Depth/Csg } 65.2^{\prime} / 61.3^{\prime}}$ \\
\hline $\begin{array}{c}\text { Reference Measuring Point: } \\
\text { GROUND SURFACE }\end{array}$
\end{tabular}

Well ID: 6208

\begin{tabular}{|c|c|}
\hline Finish & Total \\
\hline Time $\frac{1730}{-}$ & Time $7 \mathrm{hes} 15 \mathrm{~min}$ \\
\hline Hole Depth/Csg $\quad 0 / 10$ & Hole Depth/Csg _ \\
\hline
\end{tabular}

\begin{tabular}{|c|c|}
\hline \multicolumn{2}{|c|}{ Time/Depth } \\
\hline From & To \\
\hline 1015 & \\
\hline
\end{tabular}

Description of Activities/Operations with Depth

(Attach applicable drawings and document straightness test results)

\begin{tabular}{|l|l|l|}
\hline 1055 & & Mre \\
\hline 1115 & & Bar \\
\hline
\end{tabular}

Mobilize to site from $C 6190-D T B=70.0^{\prime}-4.8^{\prime}=65.2^{\prime} \mathrm{bgs}$

Measure out screen: $9.99^{\prime}+9.99^{\prime}+4.98^{\prime}=24.96^{\prime}+0.3^{\prime}$ cnd cap $=25.26$ Backpull casing - add bentonite to $60 . \mathrm{I}^{\prime} \mathrm{bgs}$ - wait for bentonite to hydrate

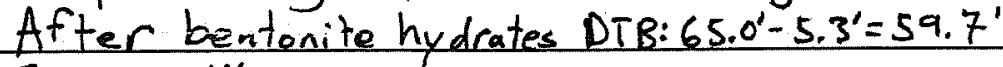

$1224 \quad$ Trip in 4'ID PVC Well screen/casing - screen $25.26^{\prime}+4 \times 10^{\prime}=65.26^{\prime}$

1324 Set pottom of end cap on well e 58,3'

$1325 \quad$ Start adding sand-refer to well completion log pg. 1

\begin{tabular}{|l|l|}
\hline 1720 & Geo Paperiwart \\
\hline 1730 & Geo departs \\
\hline
\end{tabular}

Reported By: JiThroolin

Title: Gedog ist

Signature:

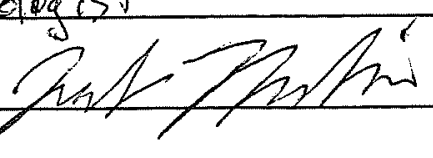

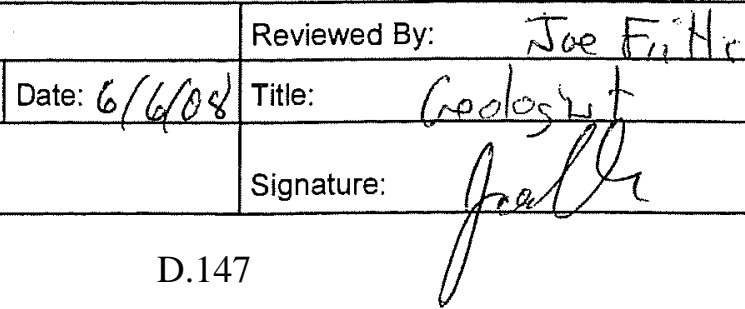

D.147 
FIELD ACTIVITY REPORT - DAILY DRILLING

\begin{tabular}{l} 
Well Name: $399-2-24$ \\
\hline Location: $\overline{3} O \mathrm{C}$ ff -5 \\
Start \\
Hole Depth/Csg \\
$\begin{array}{l}\text { Reference Measuring Point: } \\
\text { GROUND SURFACE }\end{array}$ \\
\hline
\end{tabular}
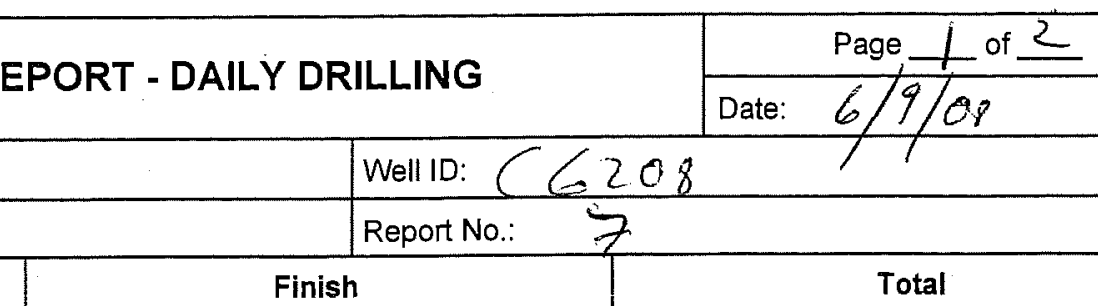

\begin{tabular}{|c|c|}
\hline \multicolumn{2}{|c|}{ Time/Depth } \\
\hline From & To \\
\hline Tho
\end{tabular}

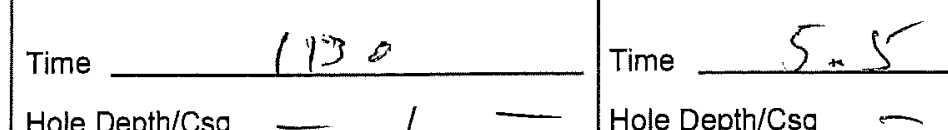

$\begin{array}{llllllll}\text { Hole Depth/Csg ___ } & & & & & \text { Hole Dept } \\ \text { Casing String No. } 1 & 2 & 3 & 4 \\ \text { See Report No. } 1\end{array}$

See Report No. 1

Q600 Q 30 Attad POP with BTR, Prilloww RCT.

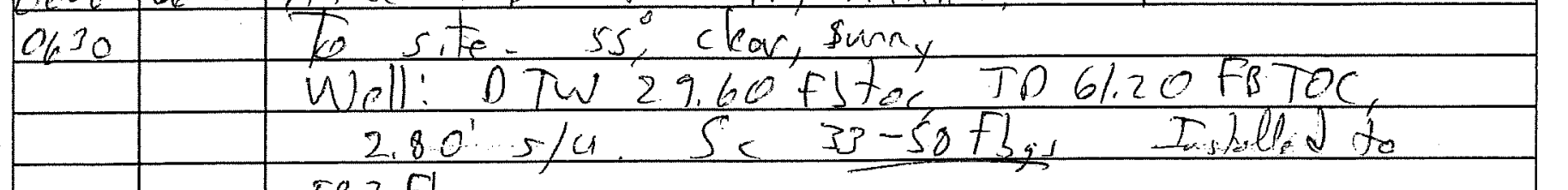

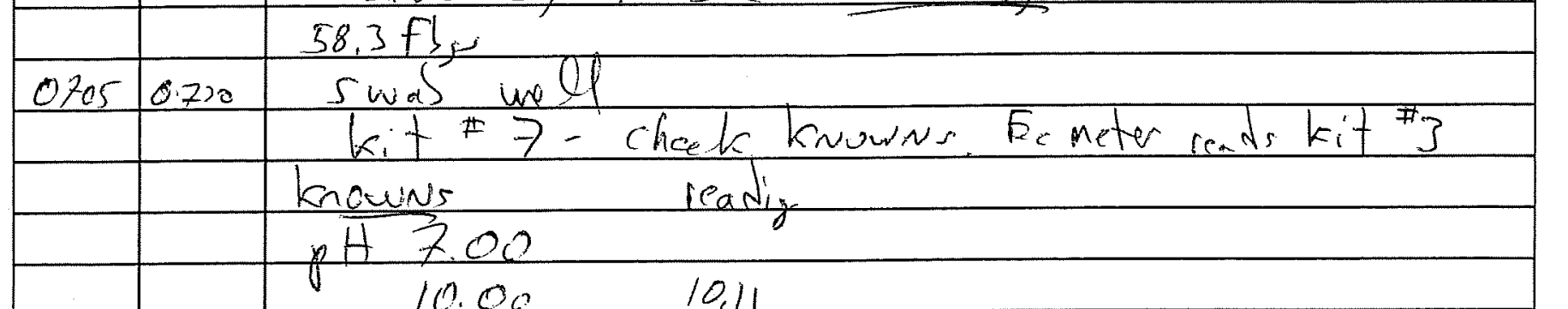

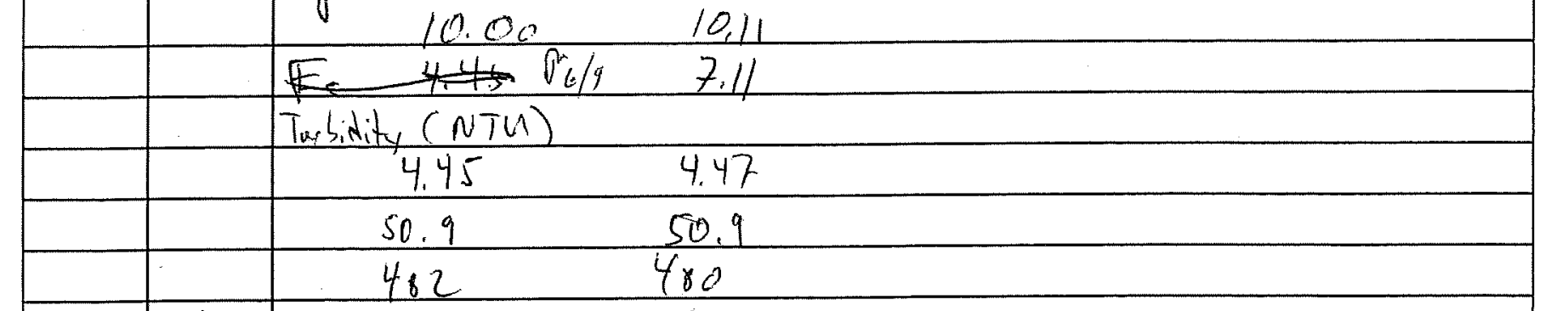

microseinnow/an $E_{E} 1413 \quad 1422$

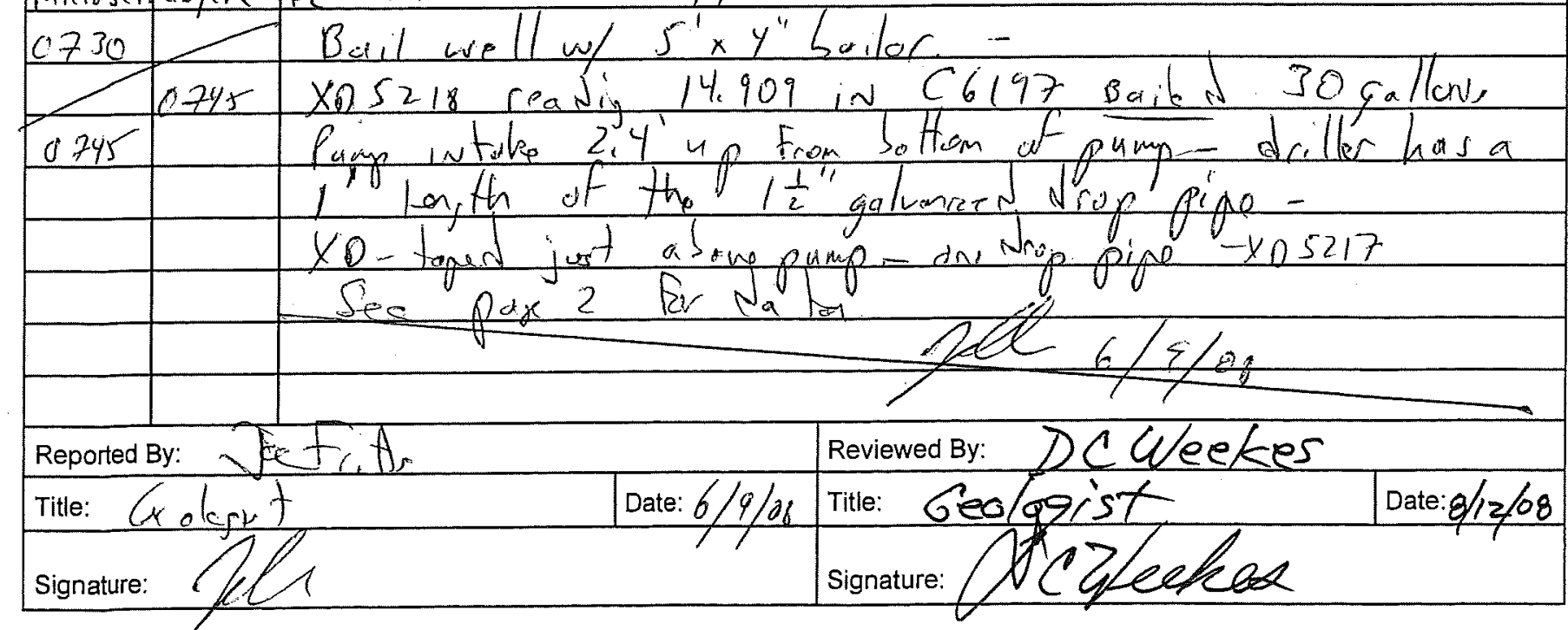




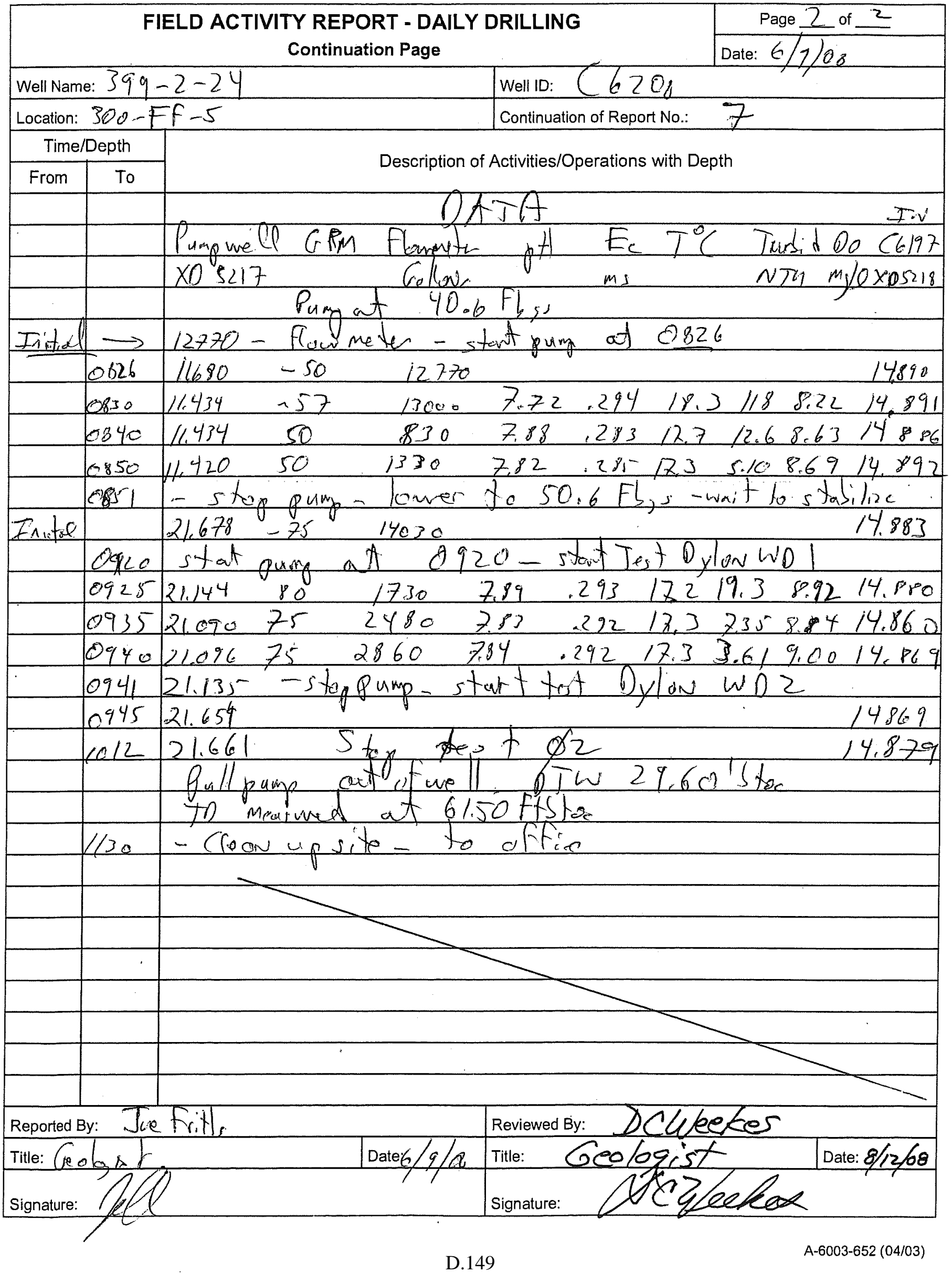




\section{WELL DEVELOPMENT AND TESTING DATA}

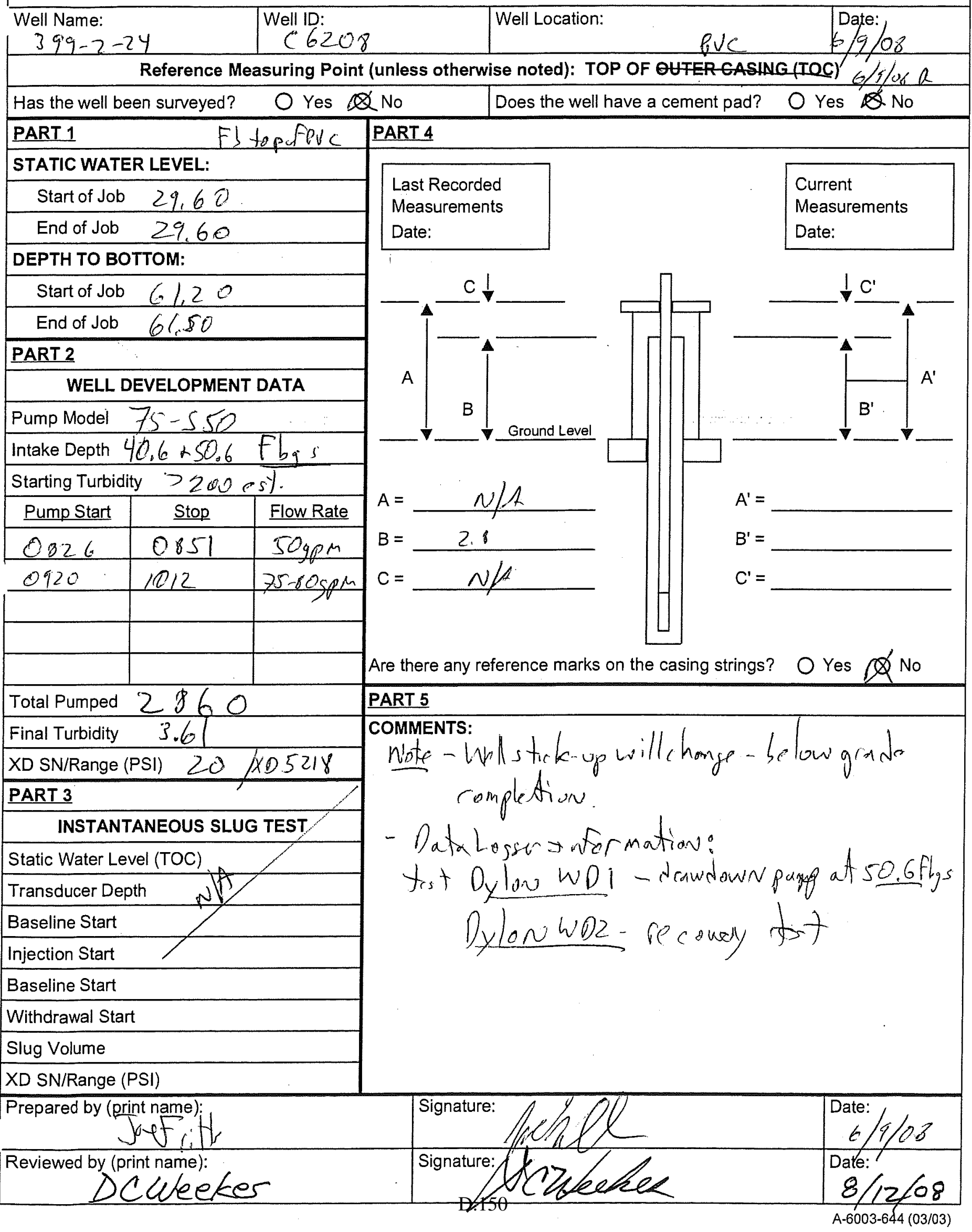




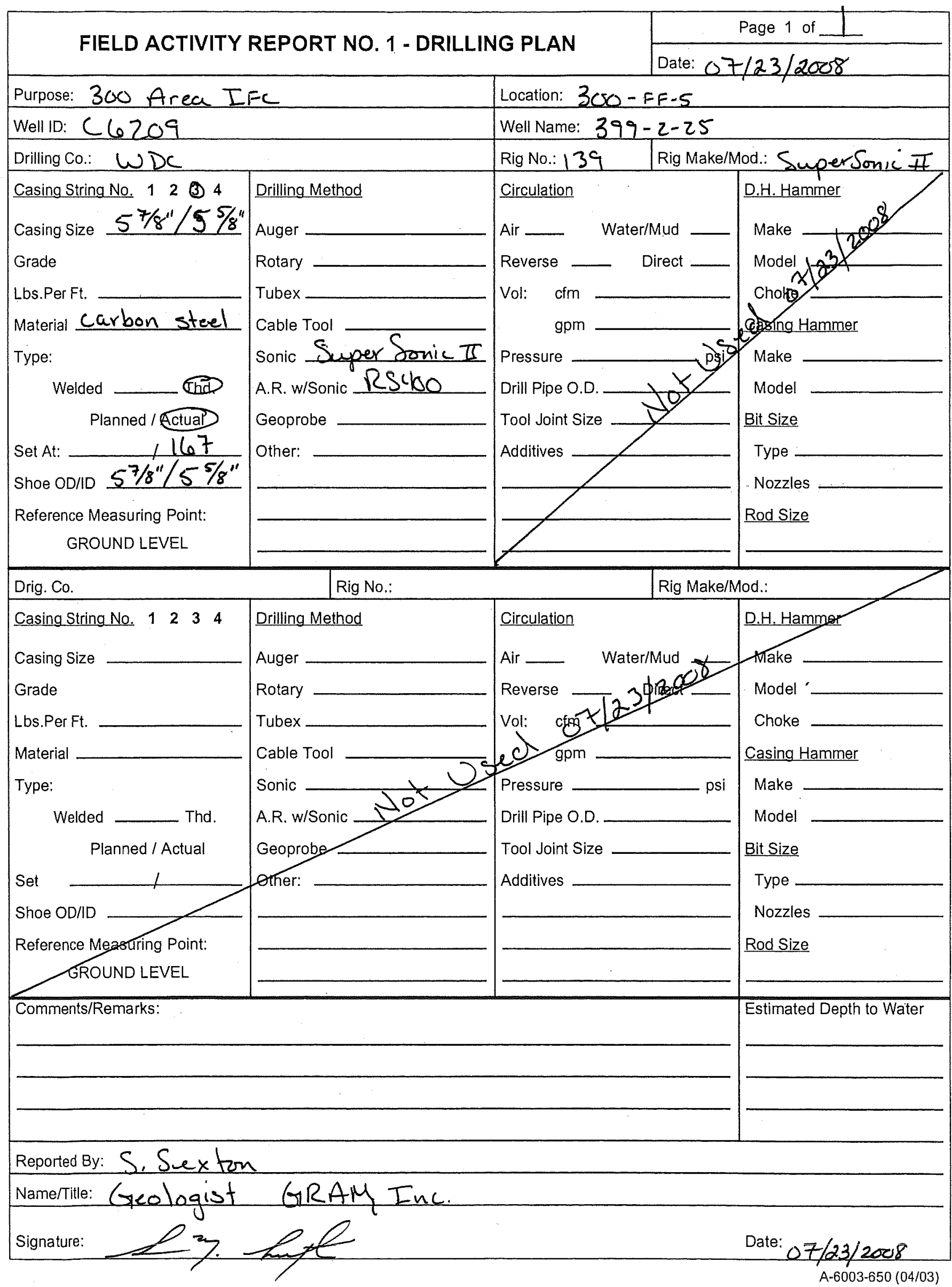




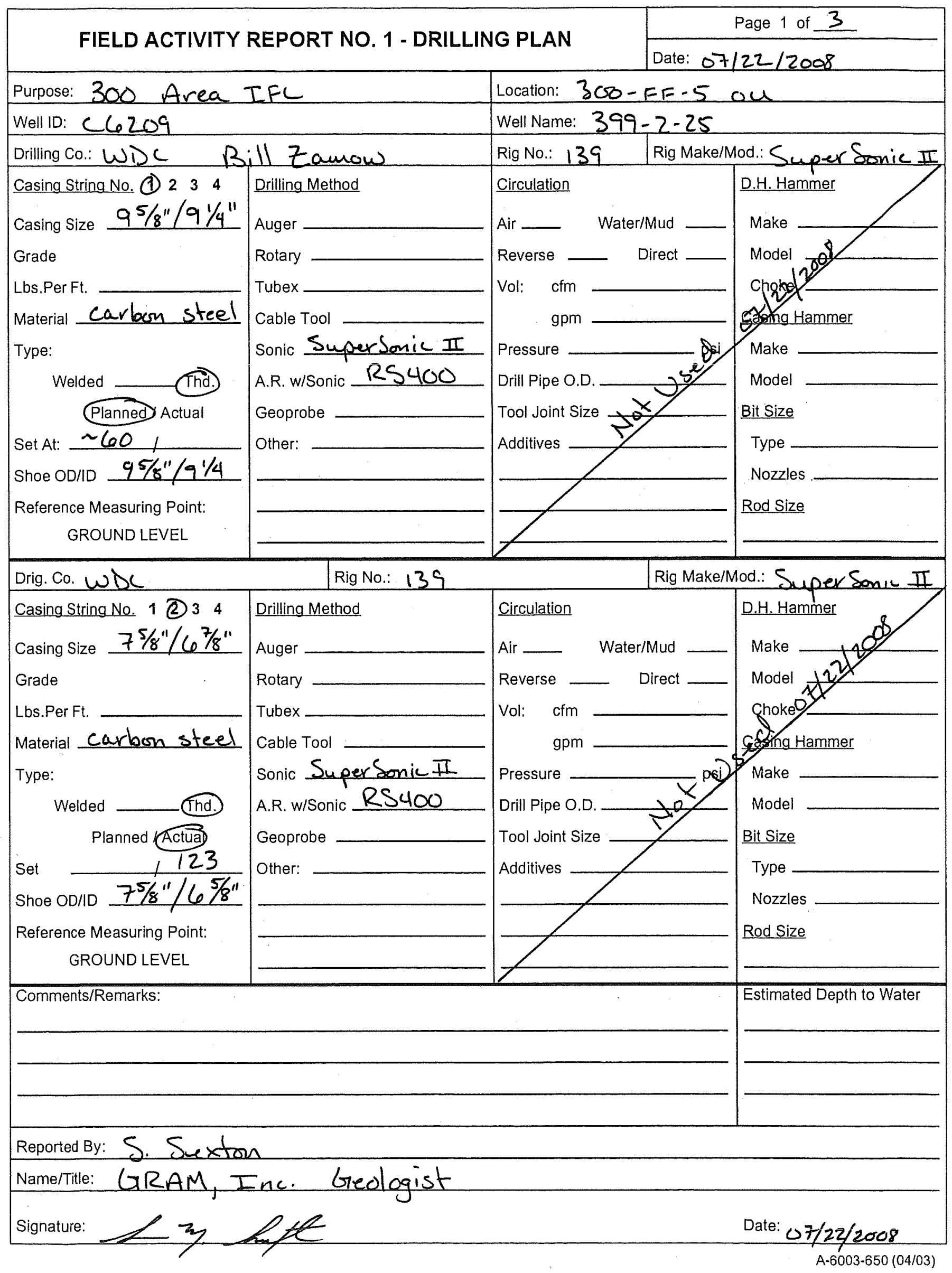


Well Name: $399-2-25$

Location: 300-FF-5 ou

\begin{tabular}{|c|c|c|}
\hline \multicolumn{2}{|c|}{ Time/Depth } & Start: $0600 \quad$ String $1: 95 / 8^{11} \quad$ Fin,sh: 1700 \\
\hline From & To & Total hrs: 11.0 hole \\
\hline 0600 & 0630 & PODe ZOo Aras w/BTR, Drillers, Fit Seffety \\
\hline 0630 & 0718 & warm up rig prep to \\
\hline 0718 & & begin drilling from surface w/ $60^{\prime \prime} d b$; advance db 0 - \\
\hline & & 5'recover 0.5'3GS@0722; advance 5-10 recover \\
\hline & & 5-9.5 \& 0730,4 GS taken \\
\hline
\end{tabular}

0740 . 0747 add 10:0 feet cusing + shoe + advaine to 5 bas 07470838 rig shut down to change out cable on mainline 08380854 advance 20 to clean-out rum to $10^{\prime}$

0854 advance $10-15^{\prime}$ cccover 10-14,5 3GSQ 0857; advance 15-20, recover $15-20,3$ GS@0906; advance db 20-25 recover 20-24,5 3 GS (e) 0915 ;

0922 add $10^{\prime}$ casing, push to $25^{\prime}$ t clean out; RCT check $Q$ 1024 0950, all reading $\angle$ background:

10241037 advance db 25-30, rewver 25-30 3 Gs \& 1032 1037 add split-spoon for sample $30-35^{\prime}$; advance ss $30-35$ \begin{tabular}{|l|l|l}
\hline & 1050 & recover $2, D^{\prime}\left(30-32^{\prime}\right) 3$ samples recovered @ 1046 \\
\hline 1050 & & add $10,0^{\prime}$ casing to string, try to push to 35 but will not \\
& & move, will clean ant w/ db \& try to advance casing again;
\end{tabular} \begin{tabular}{lll}
\hline 142 & 1241 & Lurch \\
1241 & 1252 & cleas out casing to $30^{\prime}$ IH check (2.1243 non-detect;
\end{tabular} 12521312 drive ss 30-35', recover 30-31.5 2.11 taken Q 1311 1312 add $10,0^{\prime}$ casing + advance to $35^{\prime}$ bgsiclean out 30 1337 35'; rum db $35-45$ \& leave in hole, wash casing to 45 13871402 add 10,0 \% push to $45^{\prime}$; recover $35-45^{\prime} 5$ GS Q 1346 1402 clean ant to 45, run db 45-50, add 5.0' to string 1426 and push to $50^{\prime} \mathrm{bgs}$; recover $45-503$ G5 Q , 1420 1447 out to 55 w prep for next ss

Reported By: Si Sexton Title: Geologist

Signature: Ly. hefe
Reviewed By: Joe Fritts Date: $07 / 22 / 08$ Title: Coologint Signature:

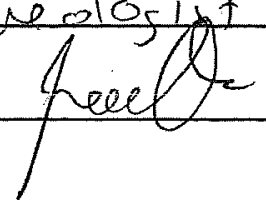

Date: $8 / 18 / 08$ 


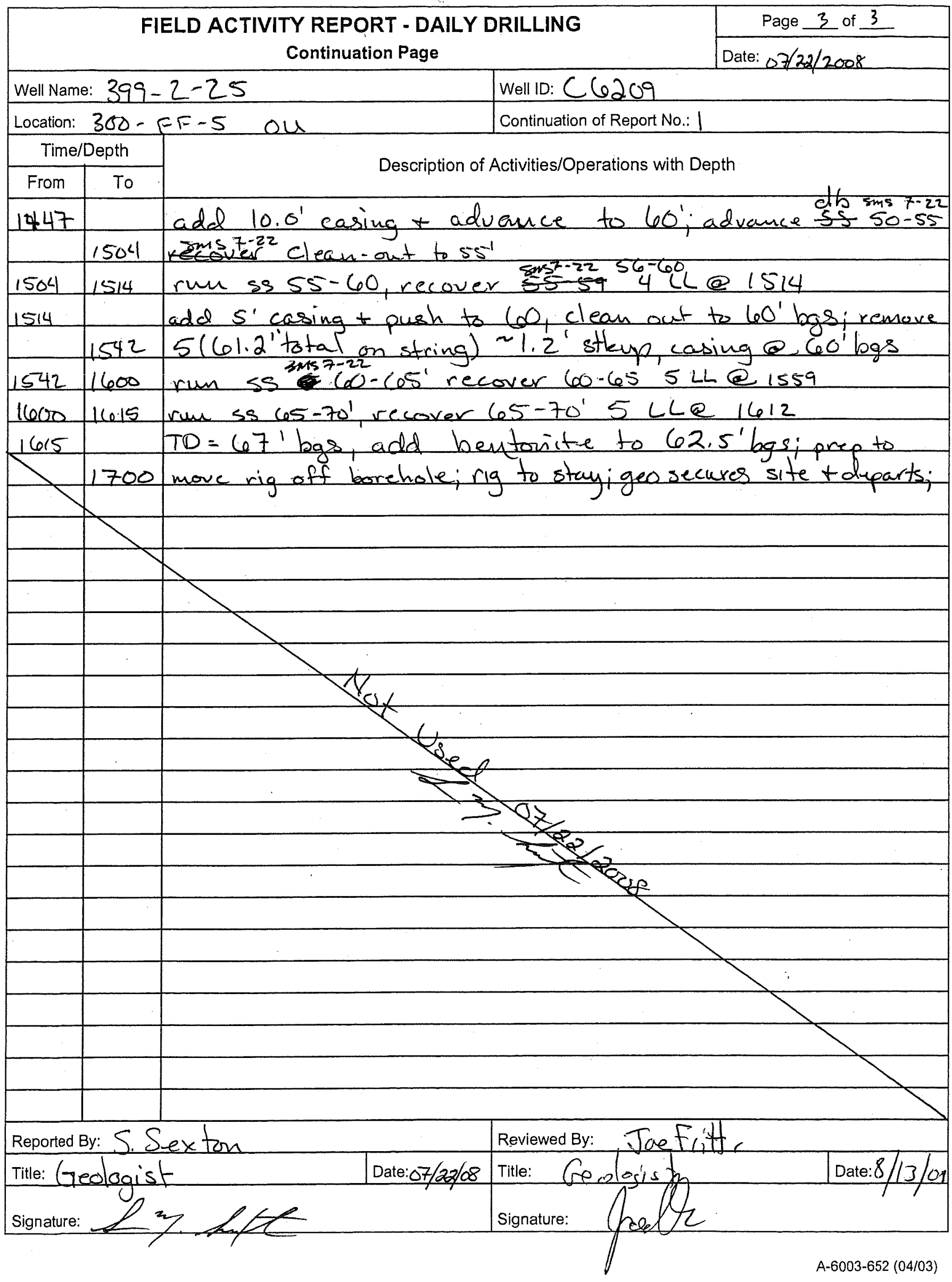




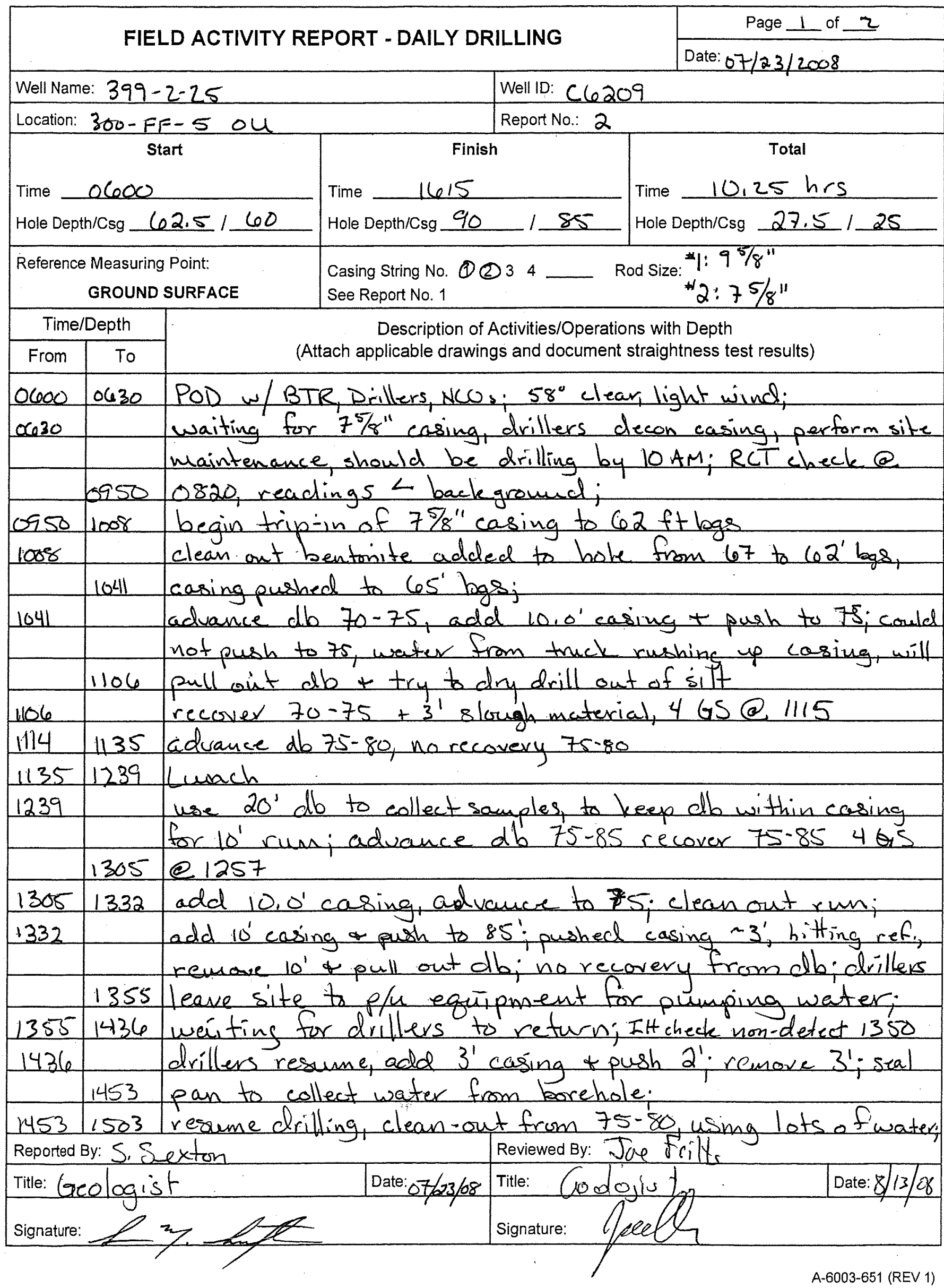




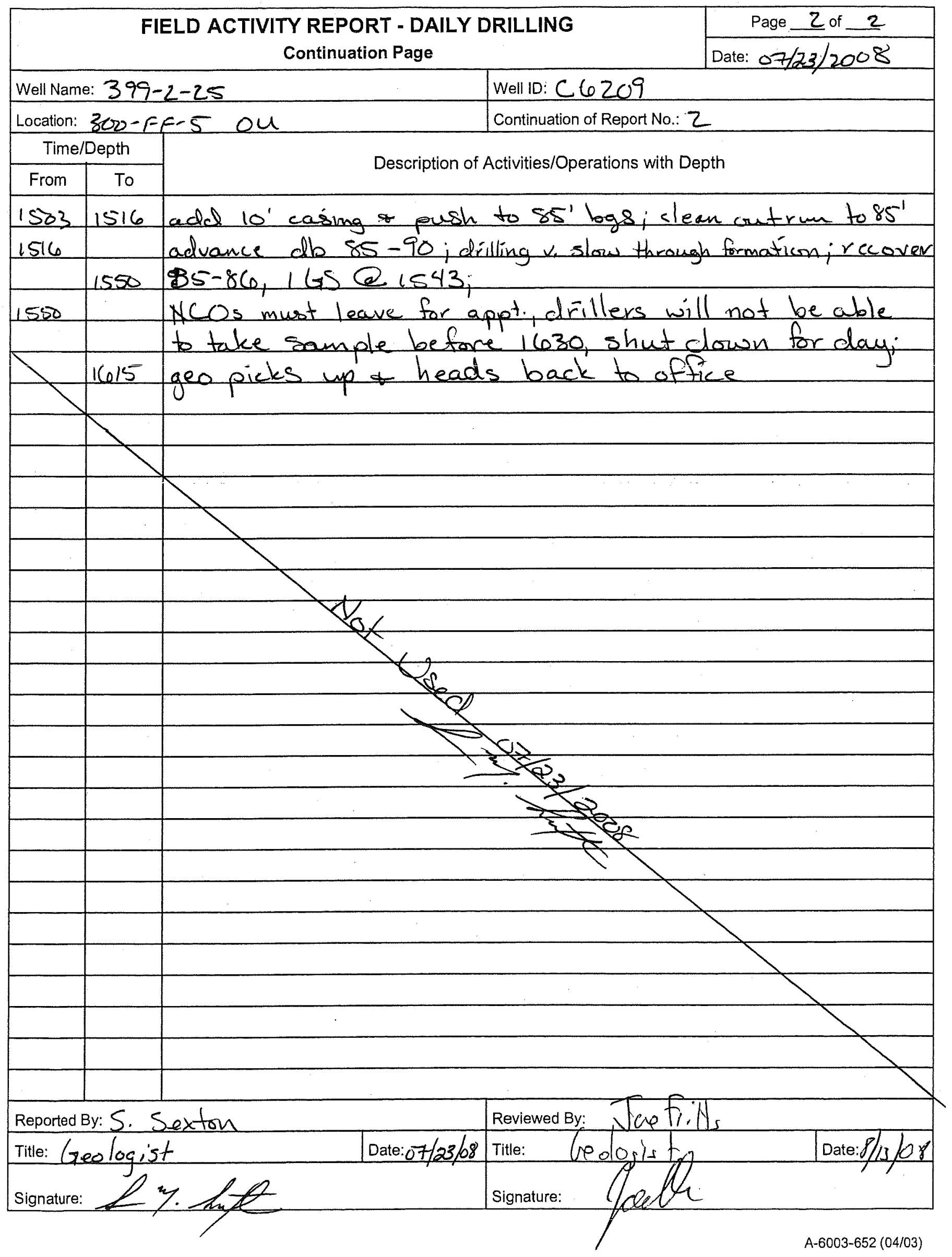




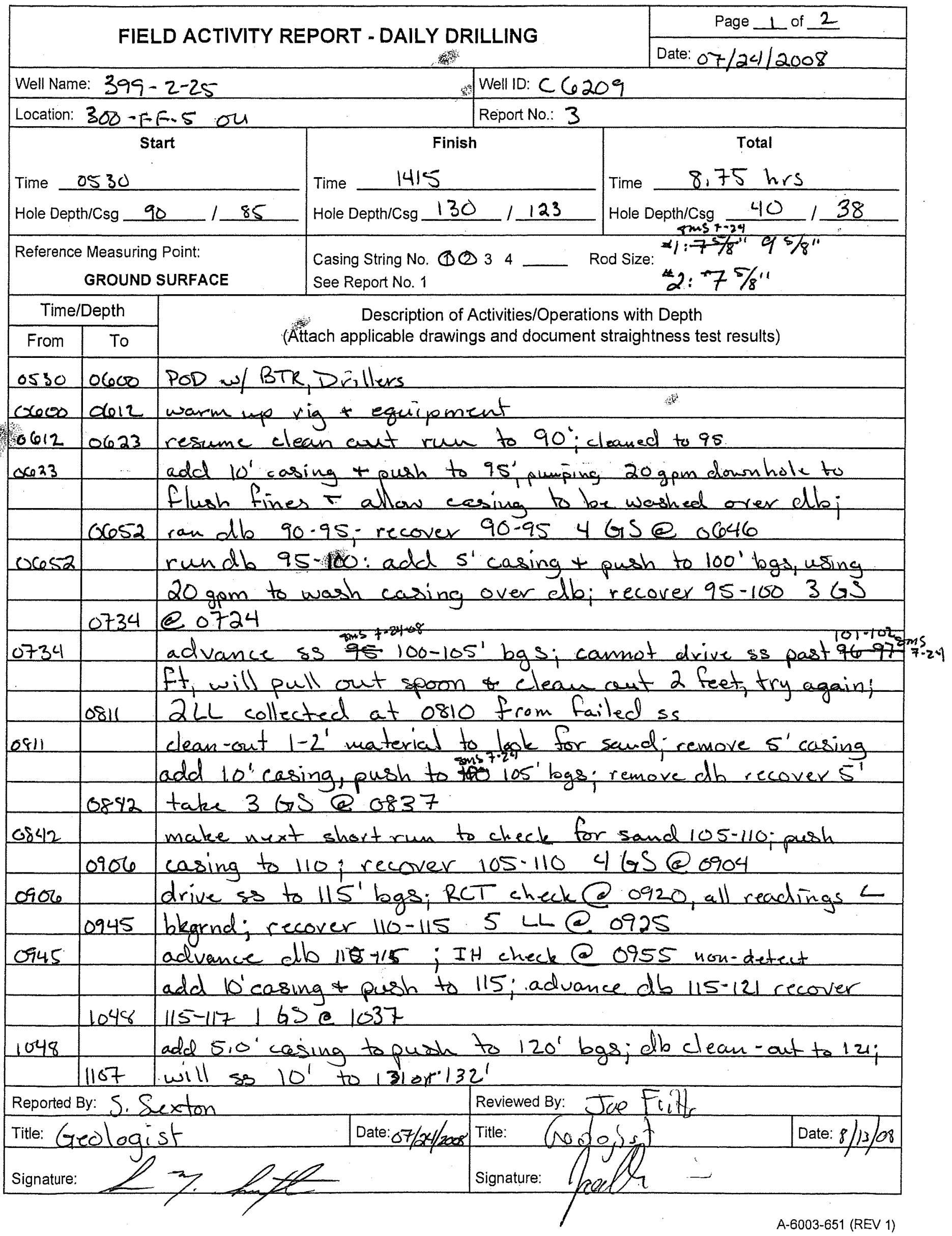

D.157 


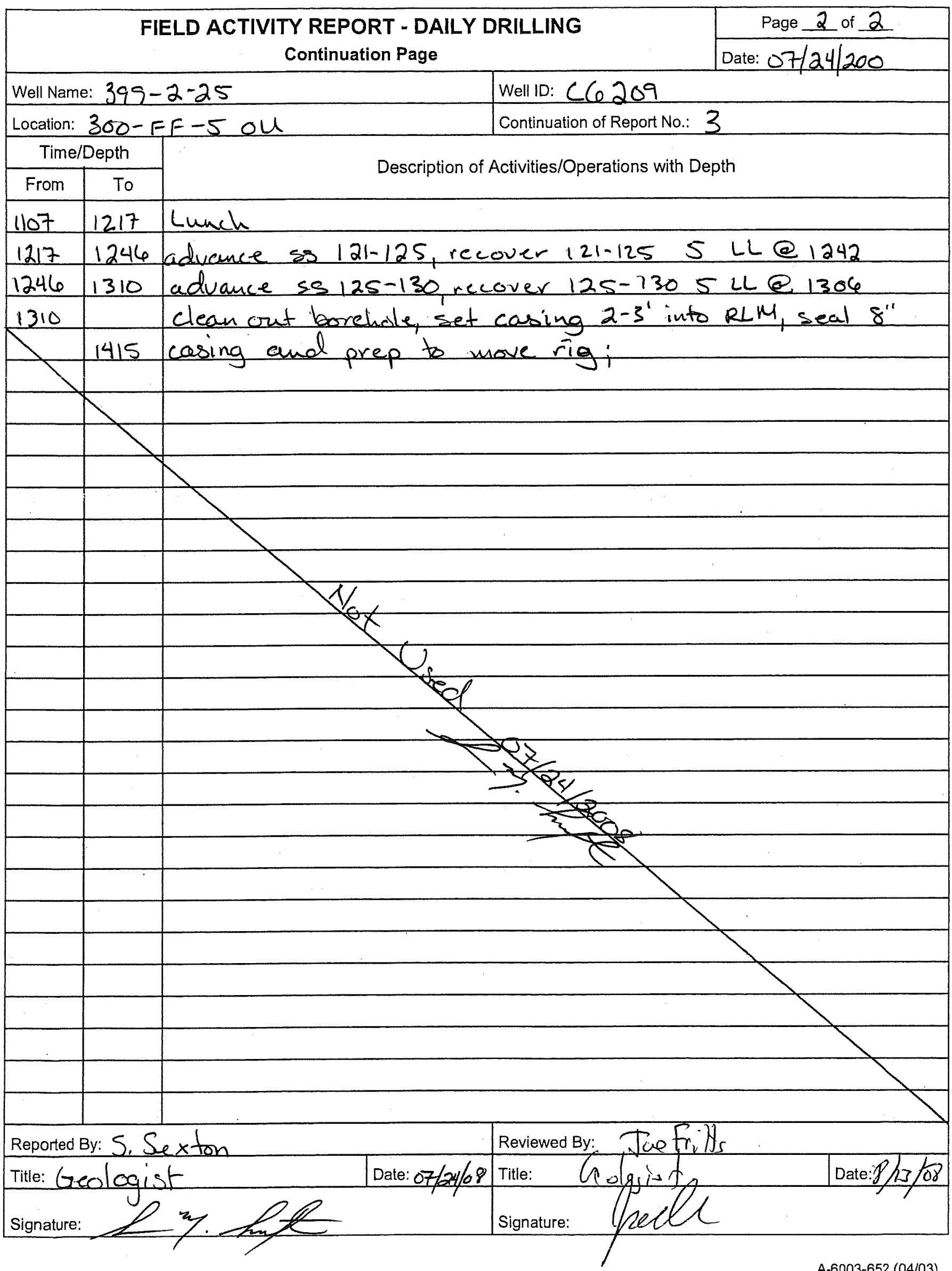




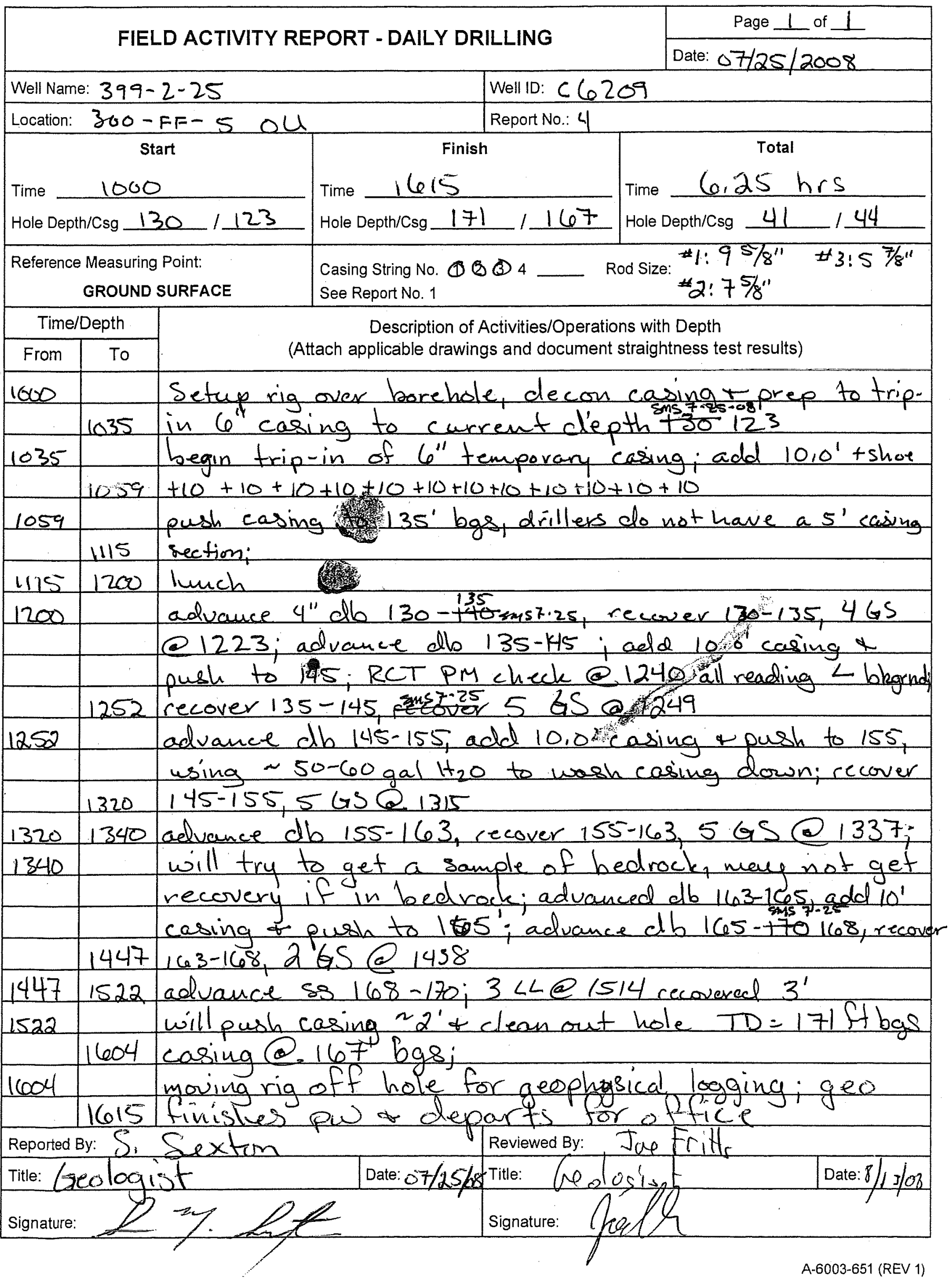

D.159 


\section{FIELD ACTIVITY REPORT - DAILY DRILLING}

\begin{tabular}{|r|} 
Page 1 of 3 \\
\hline Date: $07 / 30 / 2008$
\end{tabular}

\begin{tabular}{l} 
Well Name: $399-2-25$ \\
\hline Location: $300-F F-5$ oU \\
\hline Start \\
Time $\frac{O 600)}{\text { Hole Depth/Csg } 171}$ \\
$\begin{array}{c}\text { Reference Measuring Point: } \\
\text { GROUND SURFACE }\end{array}$
\end{tabular}

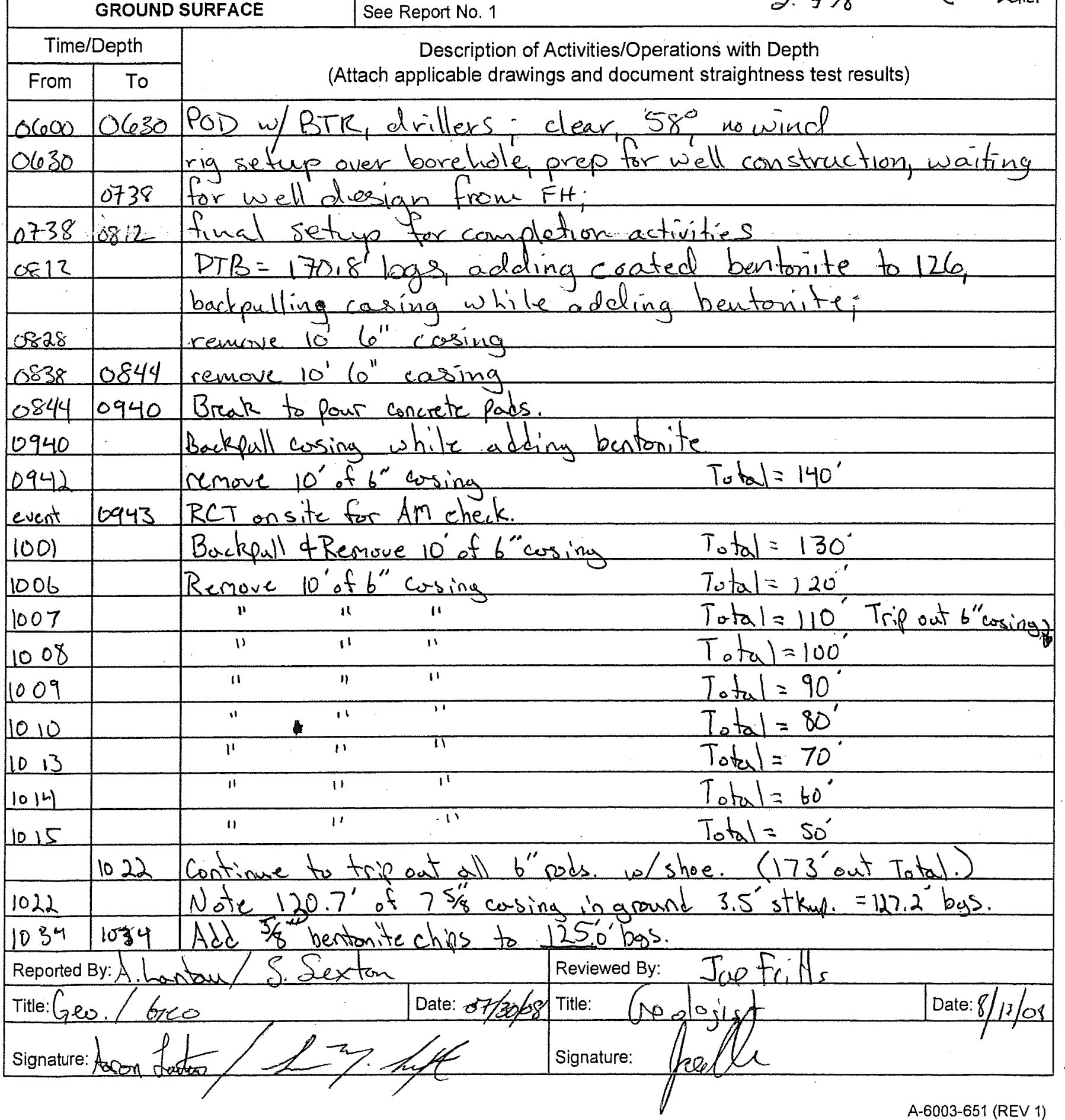




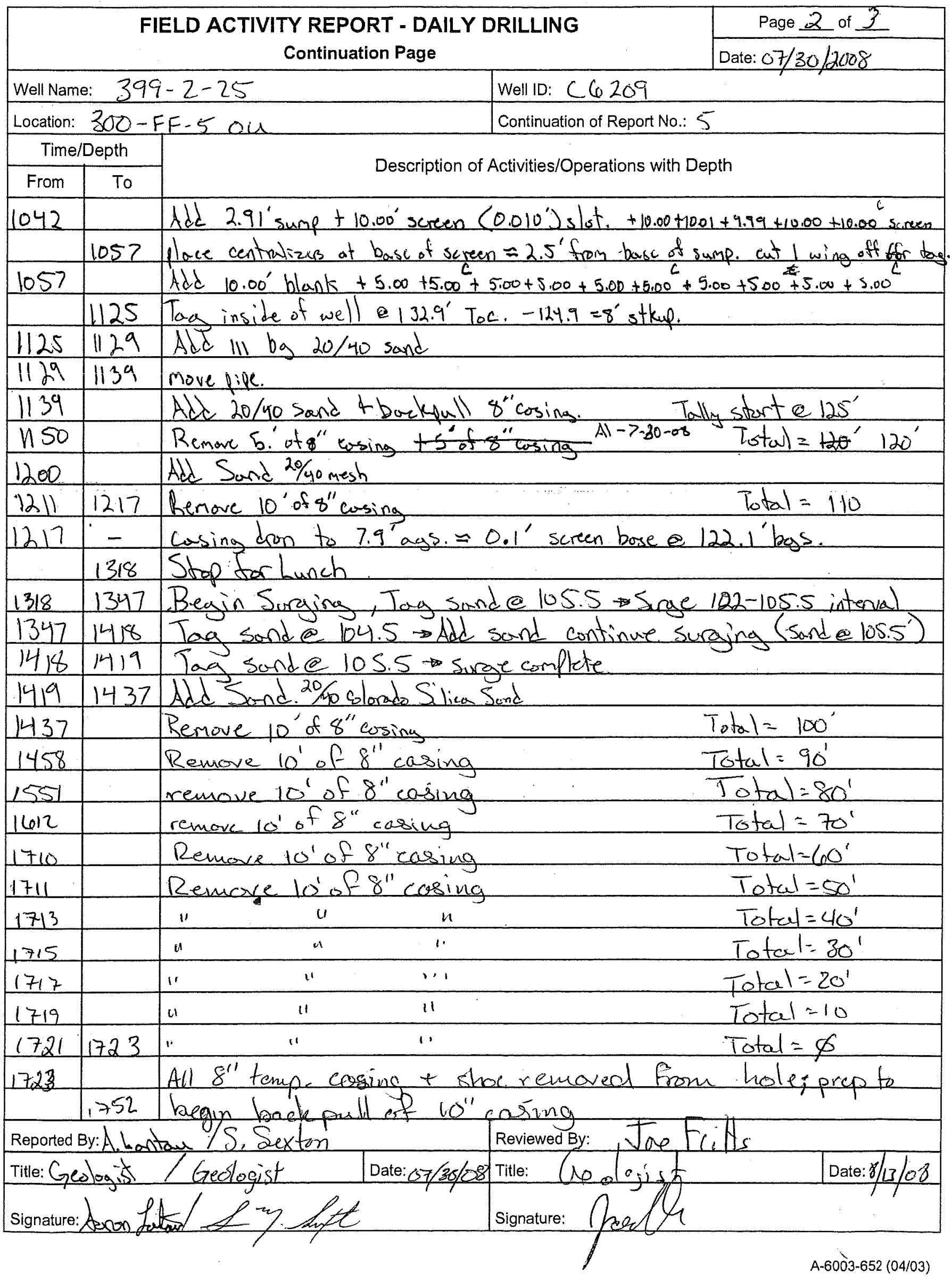

D.161 


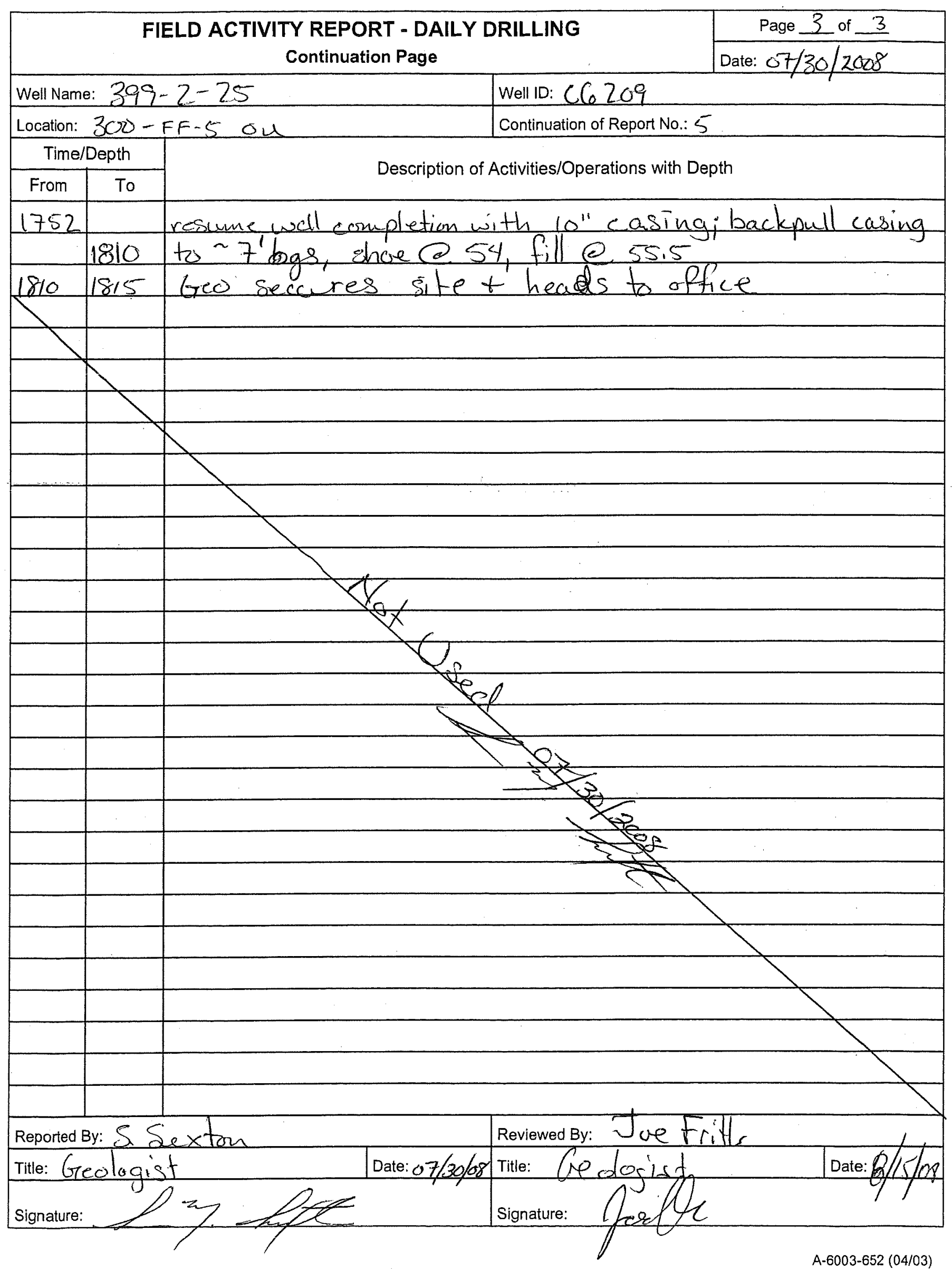

D.162 


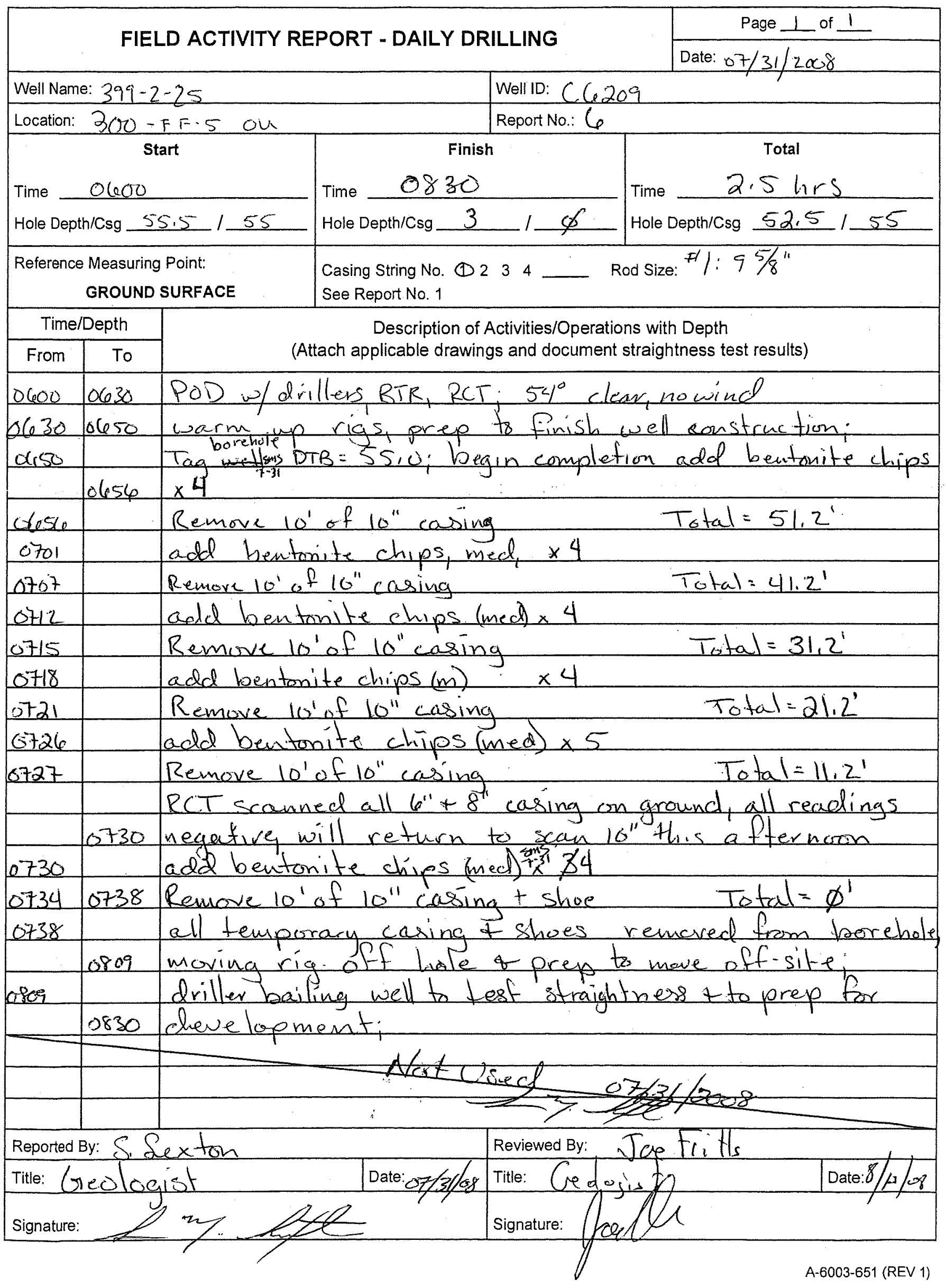

D.163 


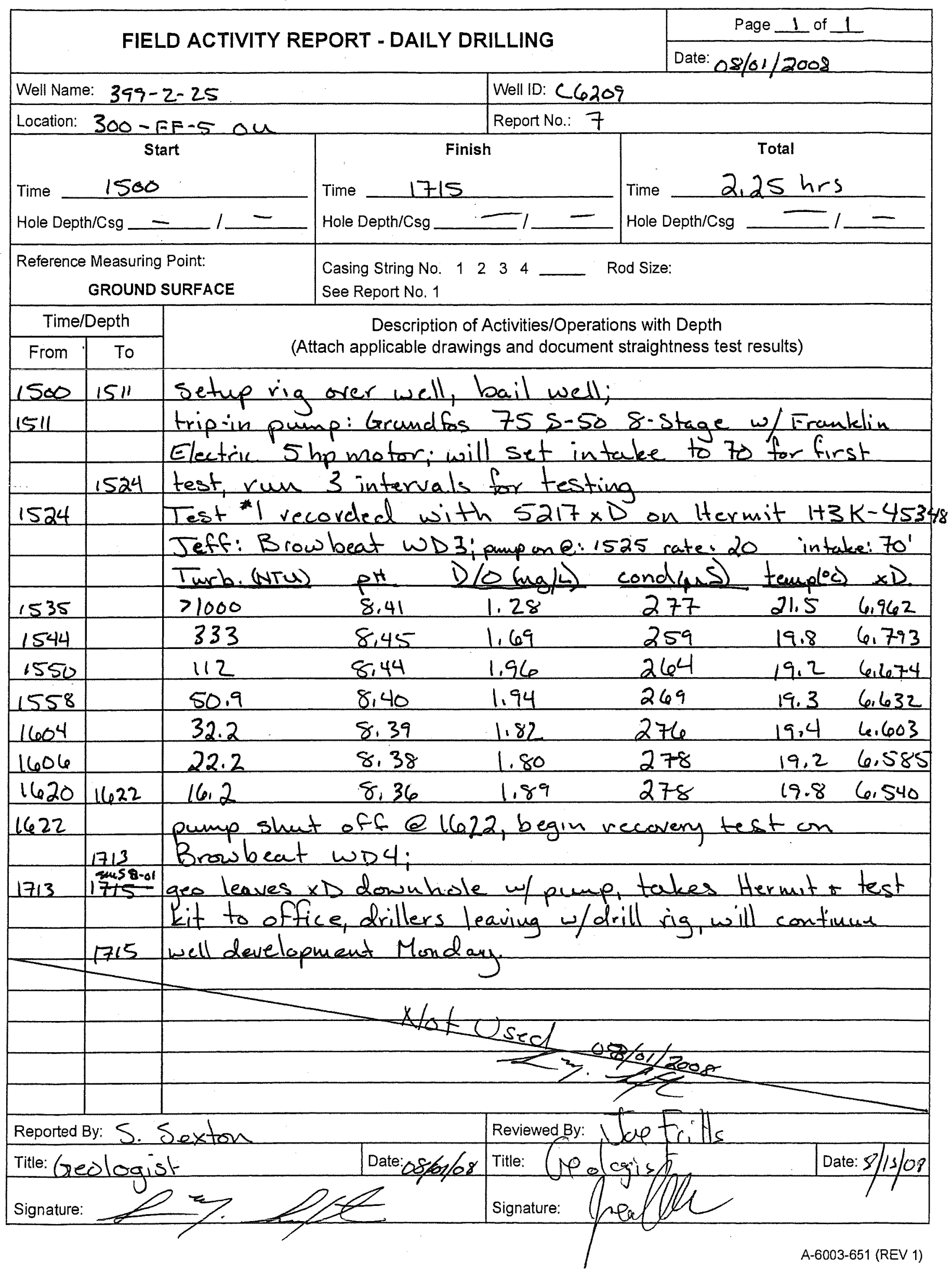




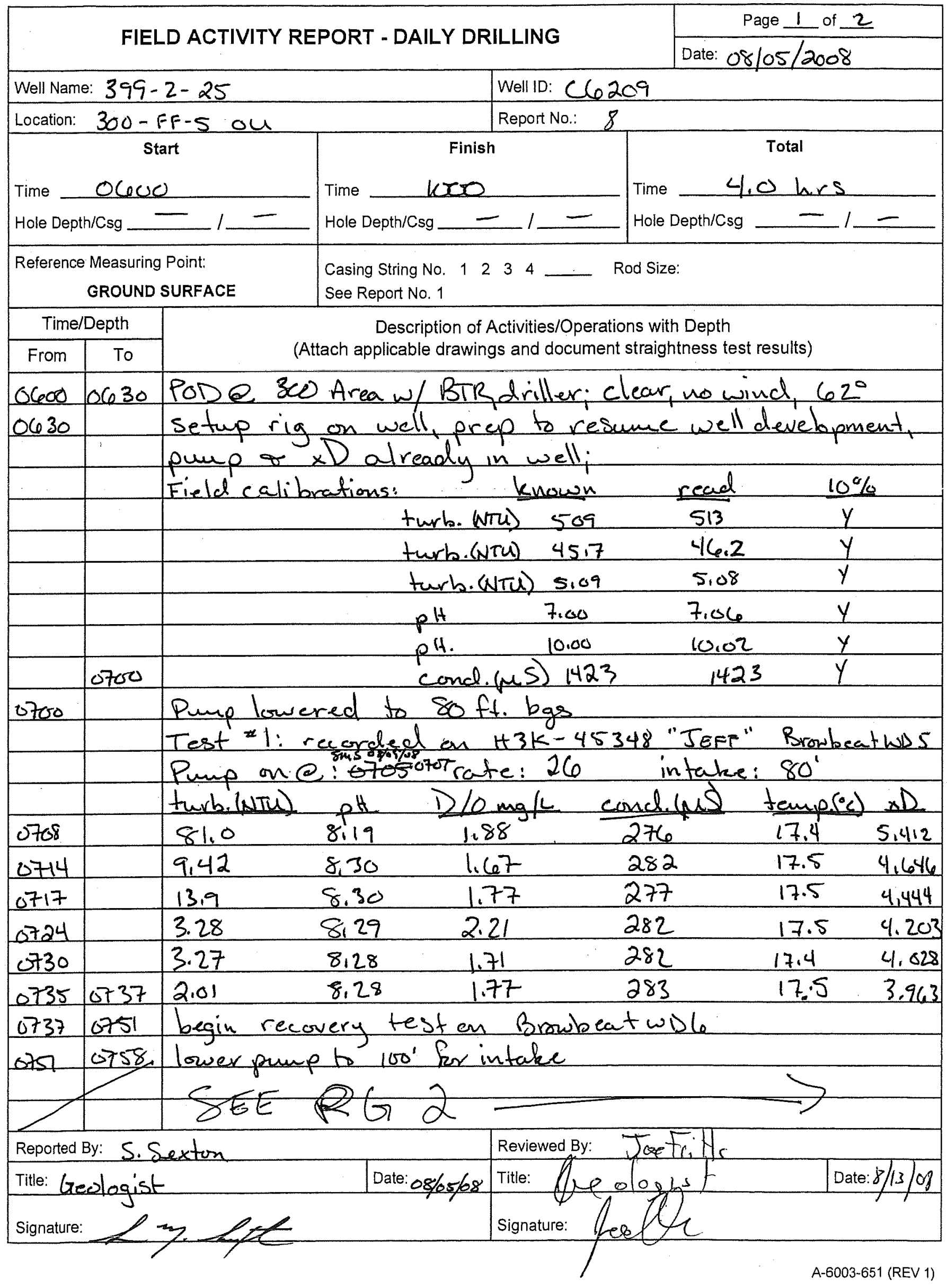




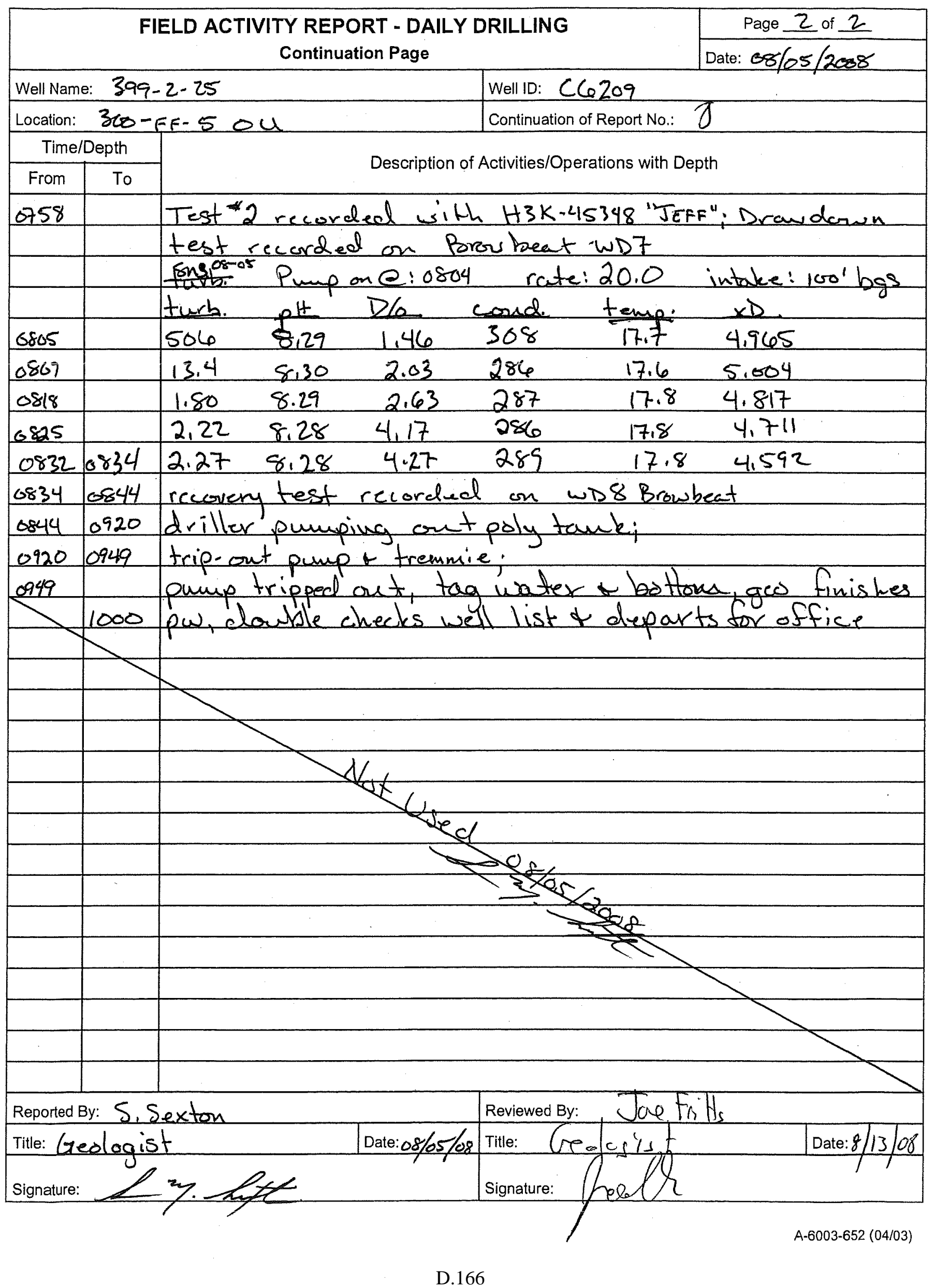


WELL DEVELOPMENT AND TESTING DATA

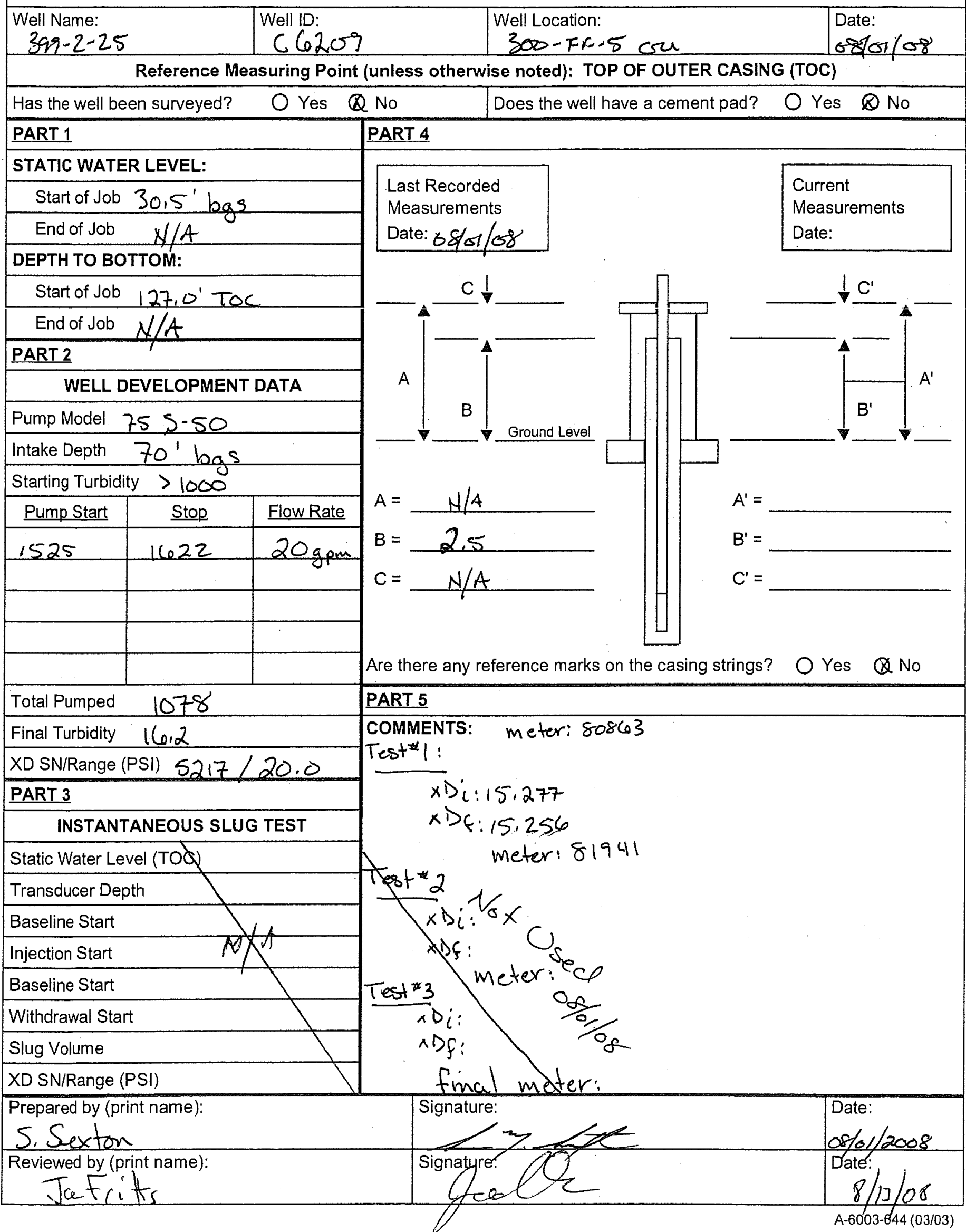




\section{WELL DEVELOPMENT AND TESTING DATA}

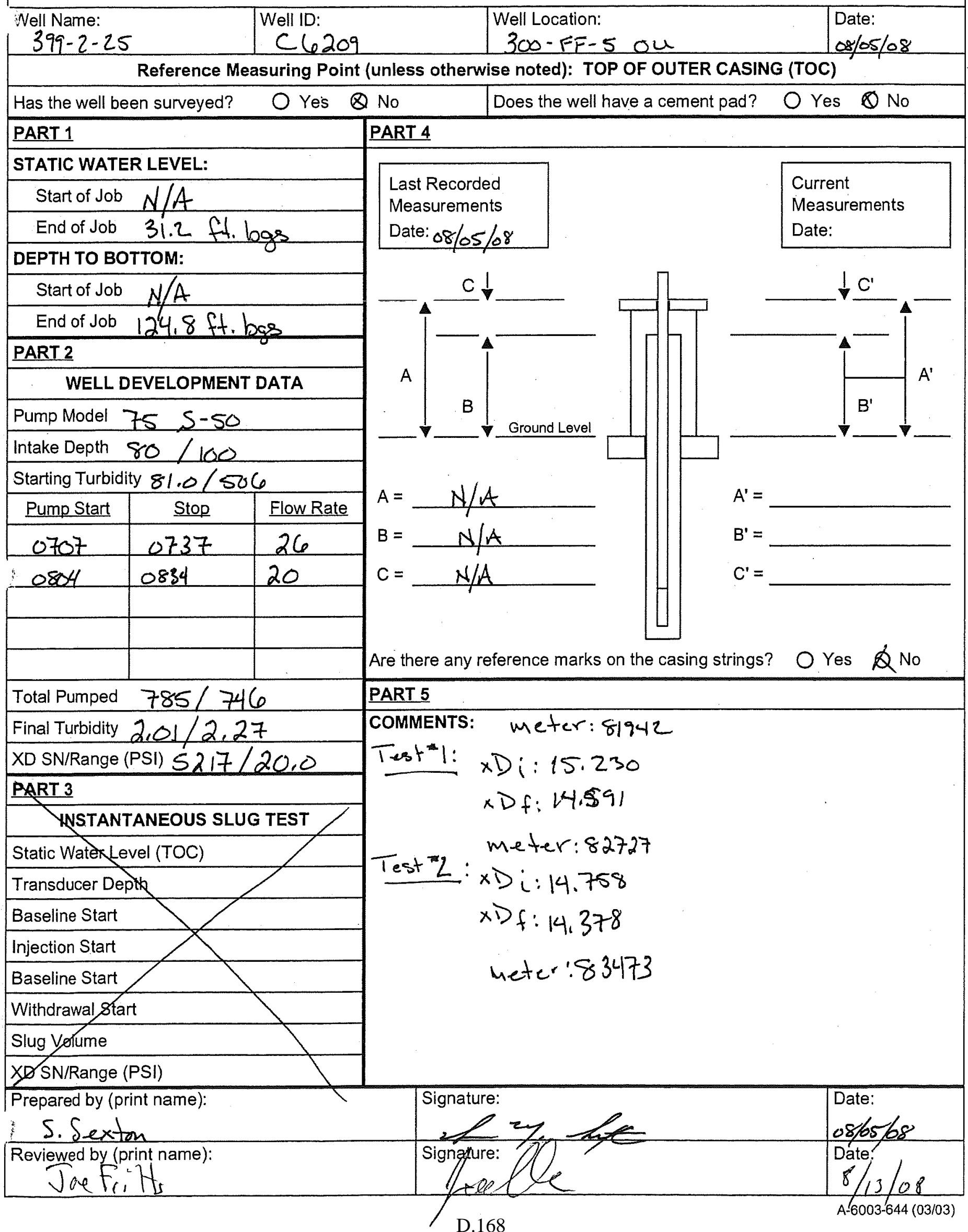


FIELD ACTIVITY REPORT NO. 1 - DRILLING PLAN

Page 1 of 2

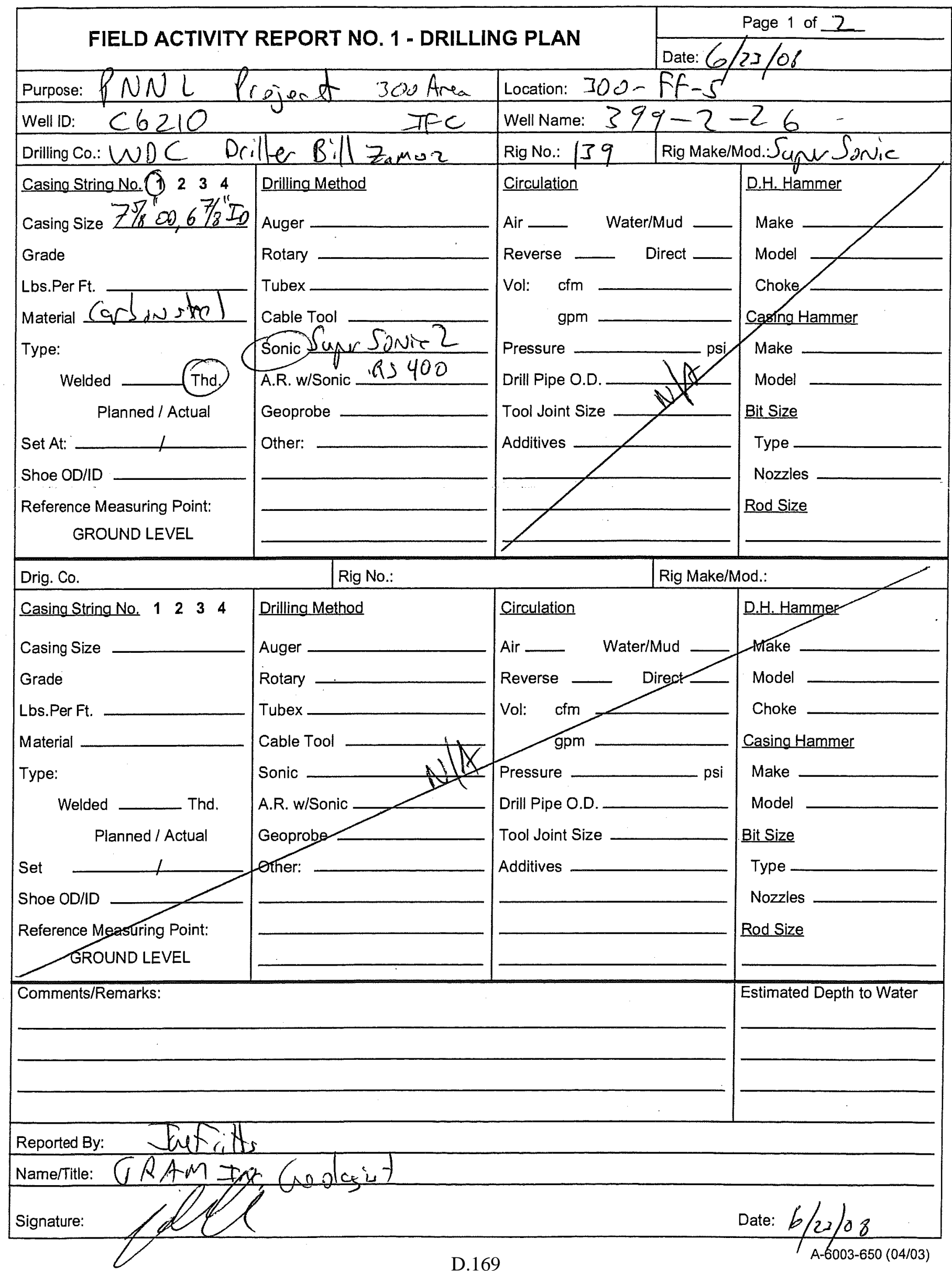




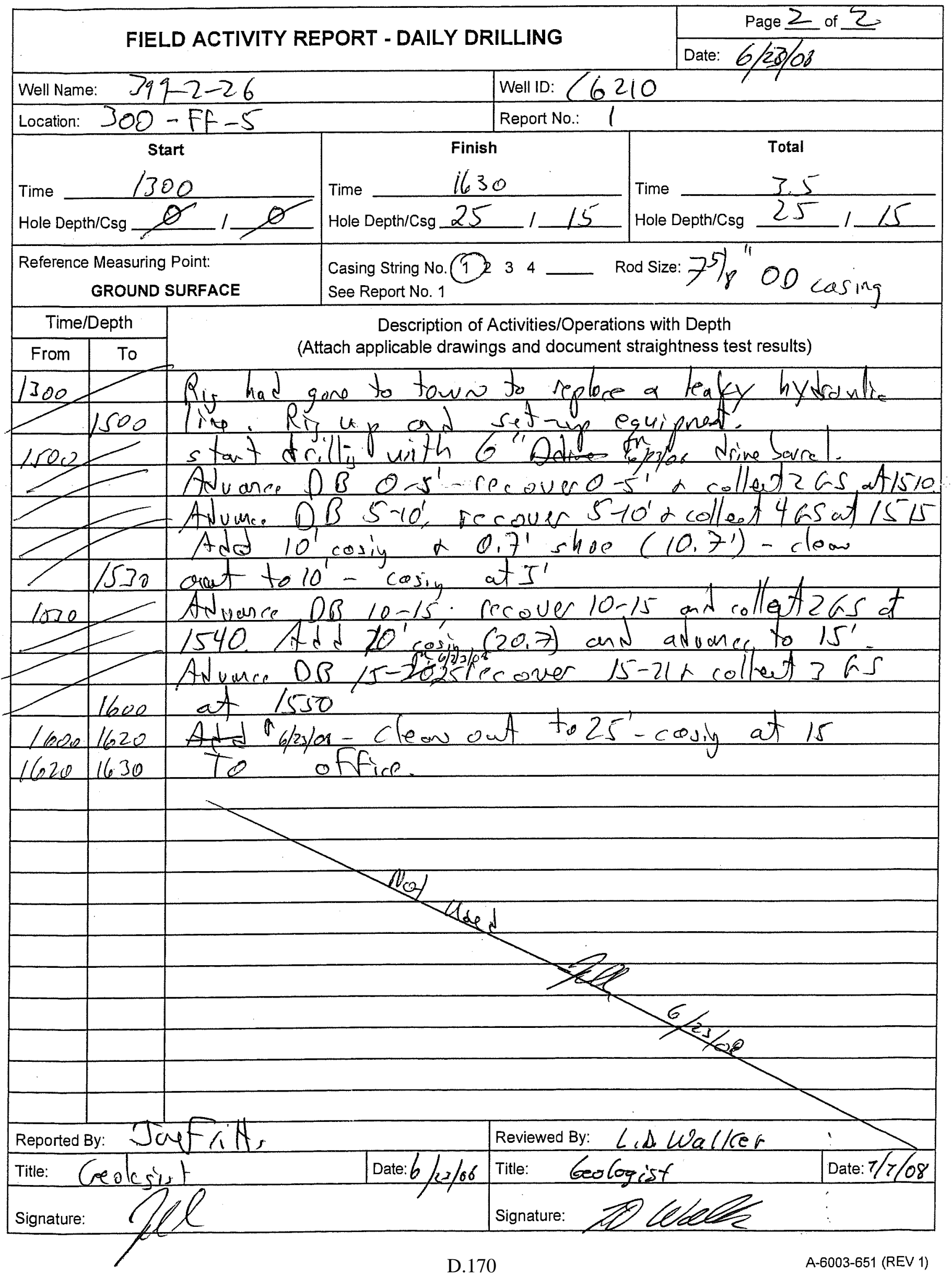




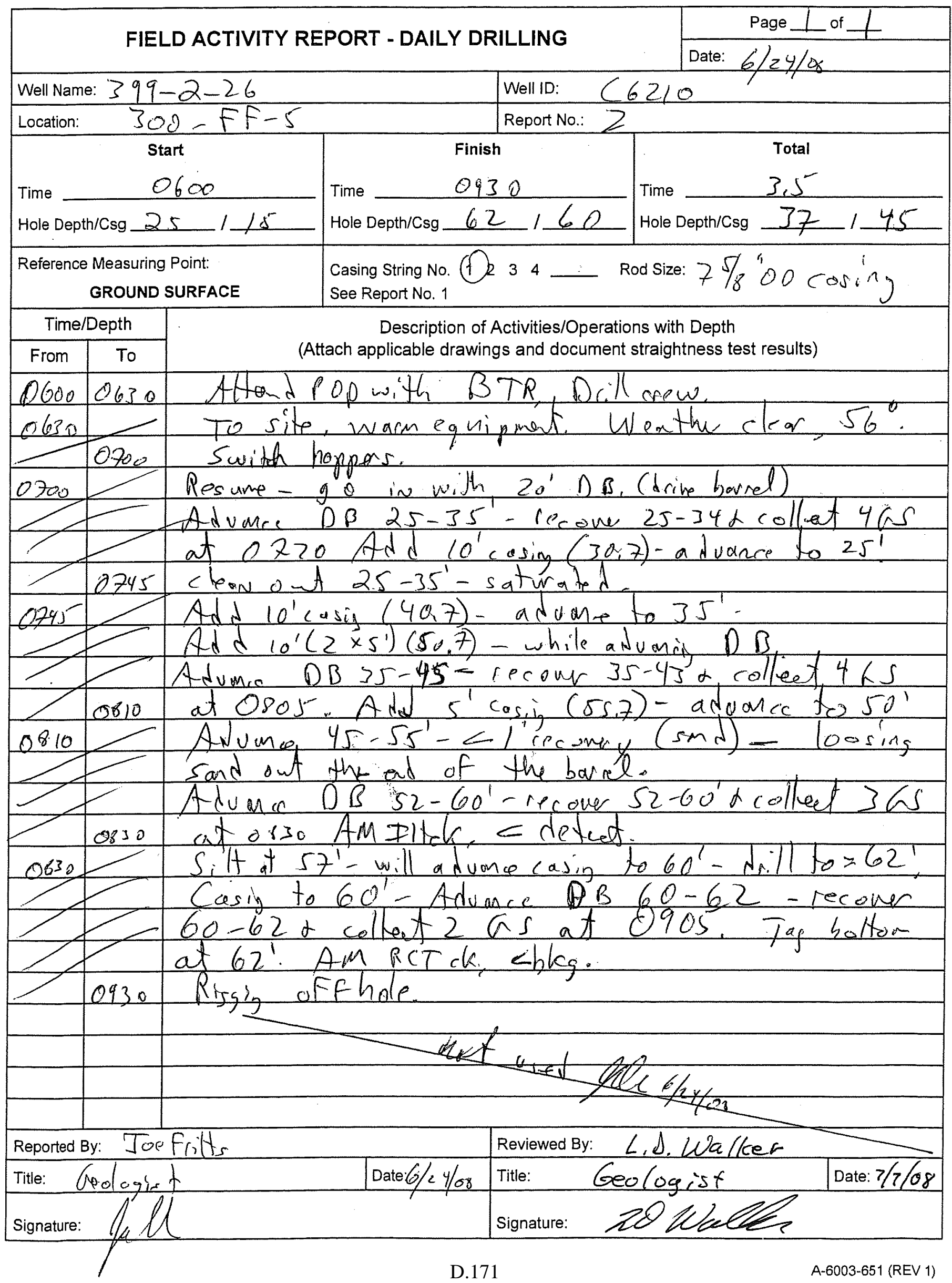




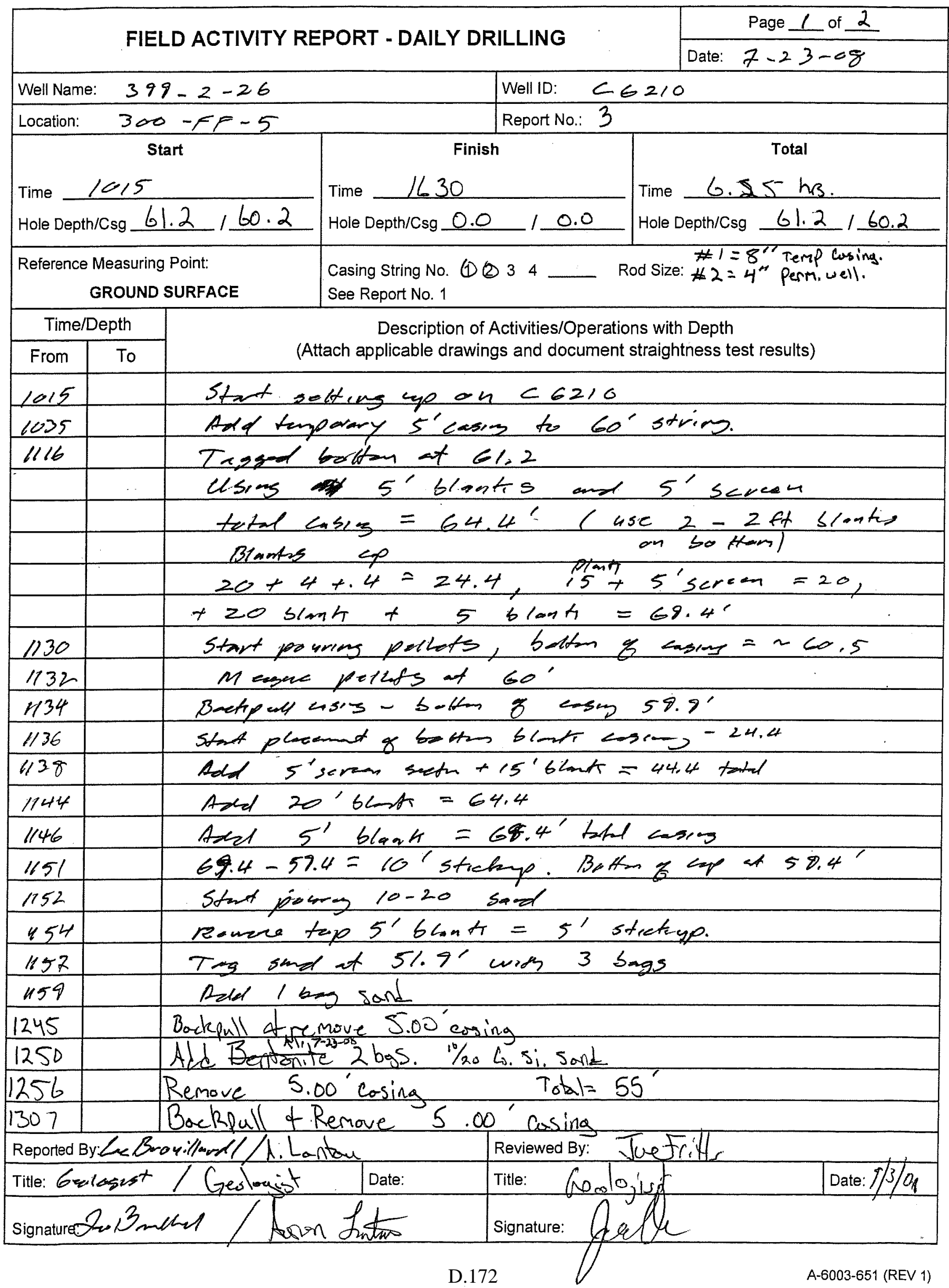




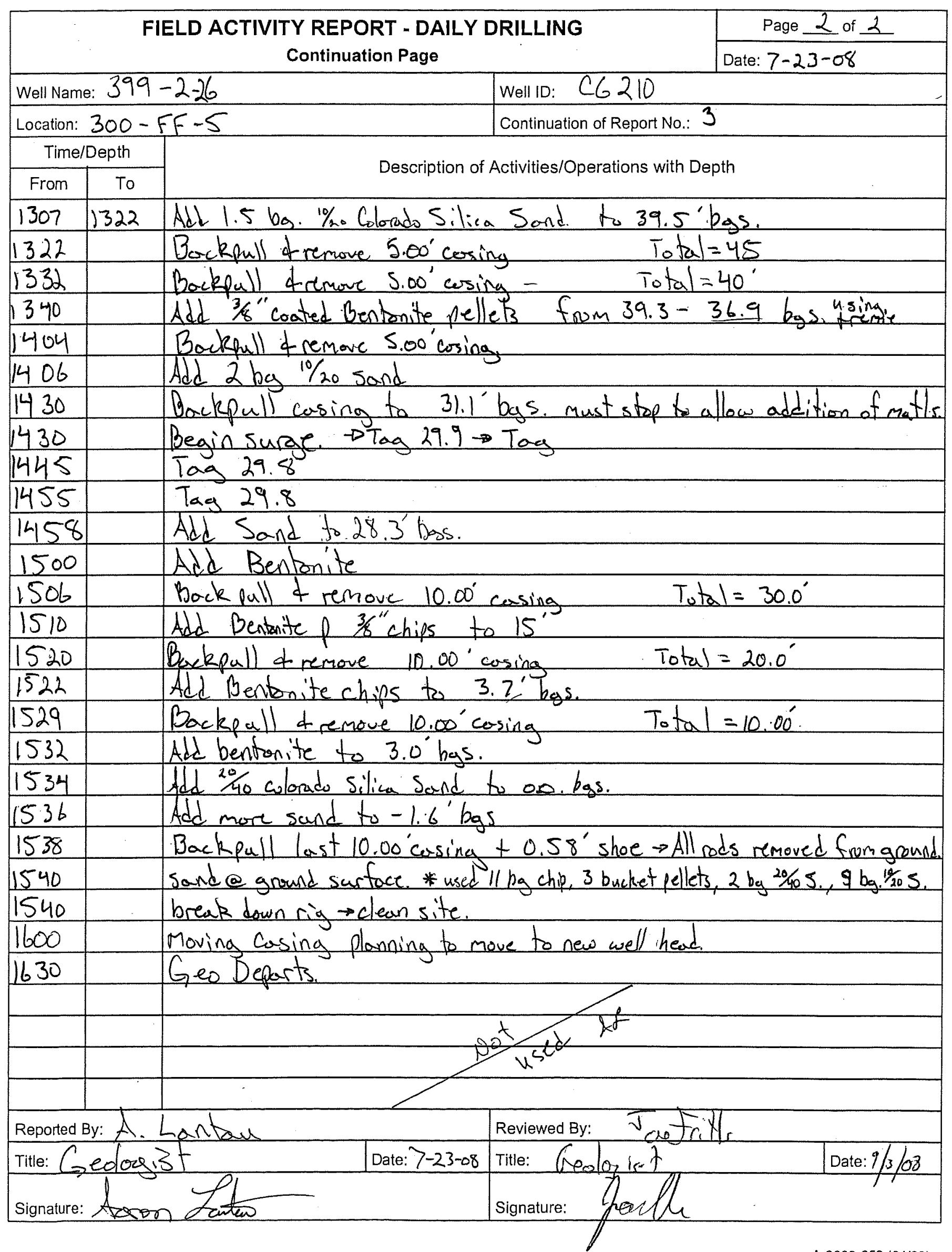




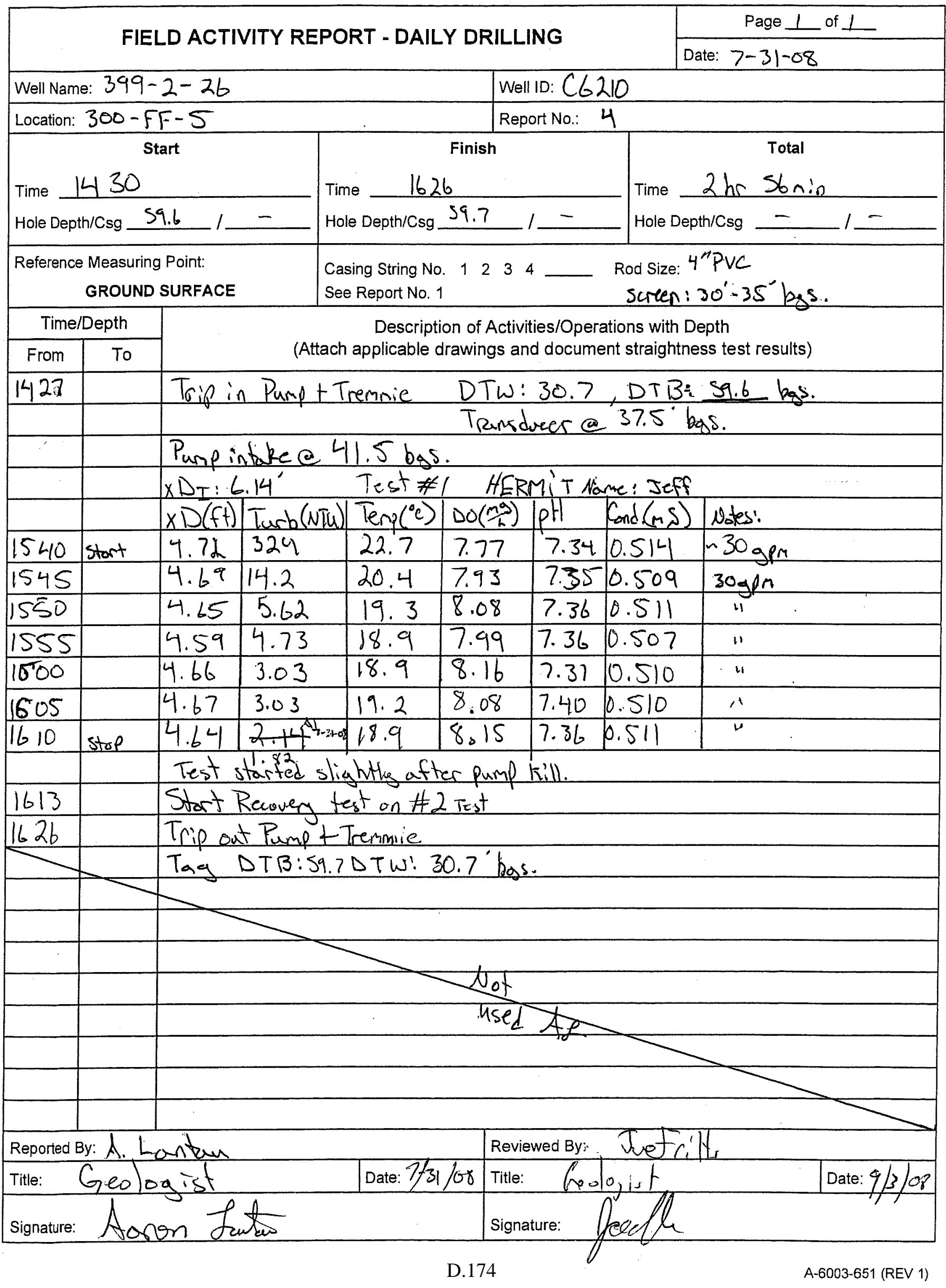




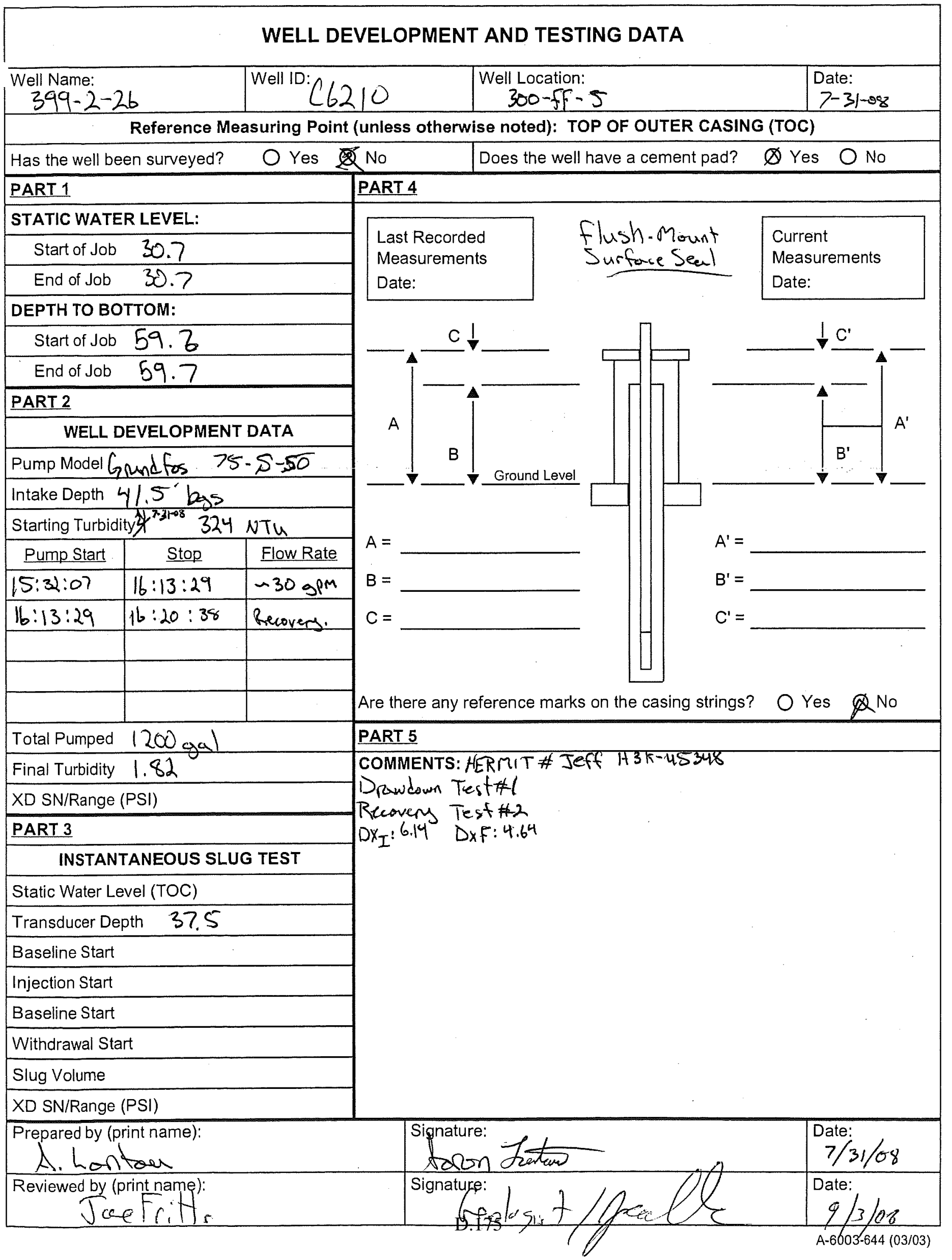


FIELD ACTIVITY REPORT NO. 1 - DRILLING PLAN

$\begin{array}{r}\text { Page } 1 \text { of } 2 \\ \hline \text { Date: } 7 / 17108\end{array}$

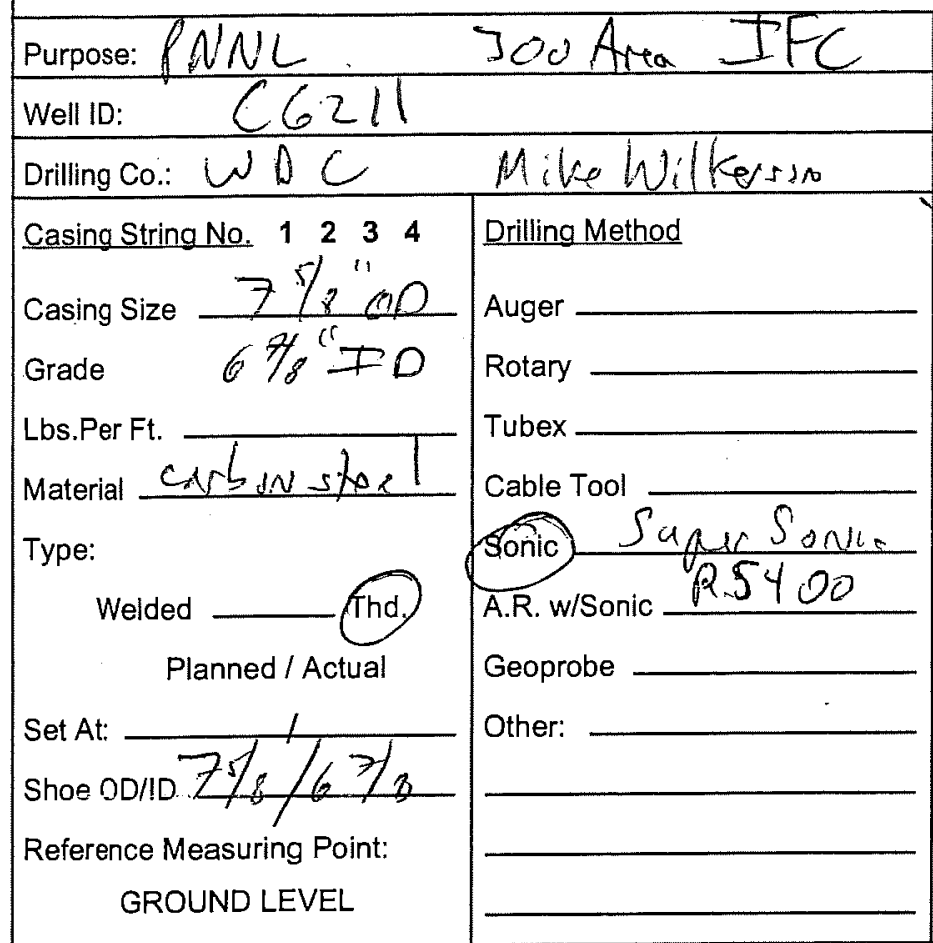

Drig. Co.

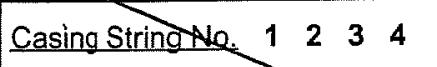

Casing Size

Grade

Lbs.Per Ft.

Material

Type:

Welded Thd.

Planned / Actual

Set

Shoe OD/ID

Reference Measuring Point: GROUND LEVEL
Rig No.:

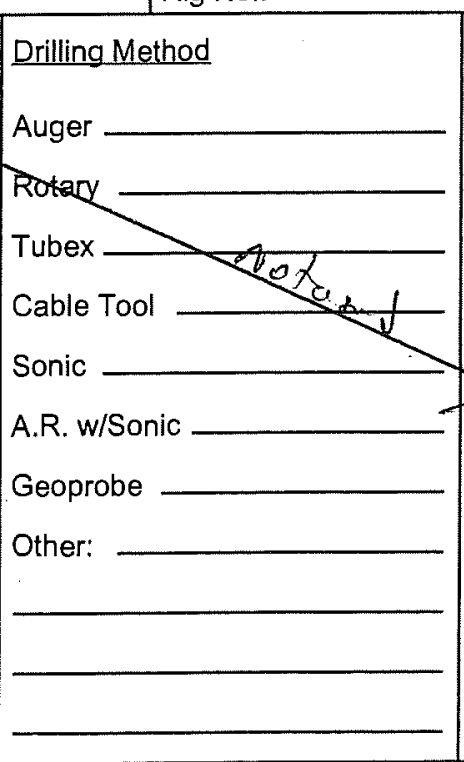

Location:

$300-F F^{\prime}-5$ Well Name: $399-2-12 R \% / 27$ Rig No.: 139 Rig Make/Mod.: Sajer Sinis 2

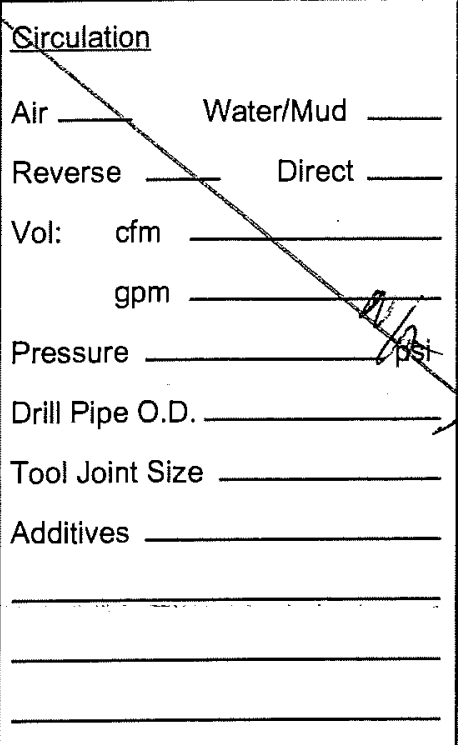
D.H. Hammer Make Model Choke Casing Hammer

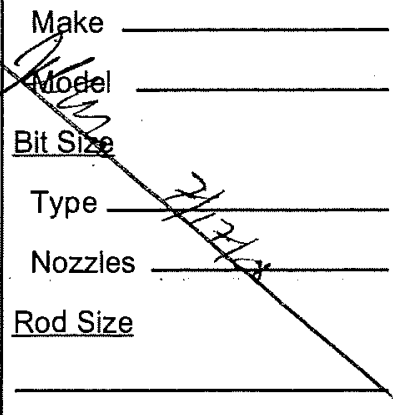
Rig Make/Mod.:

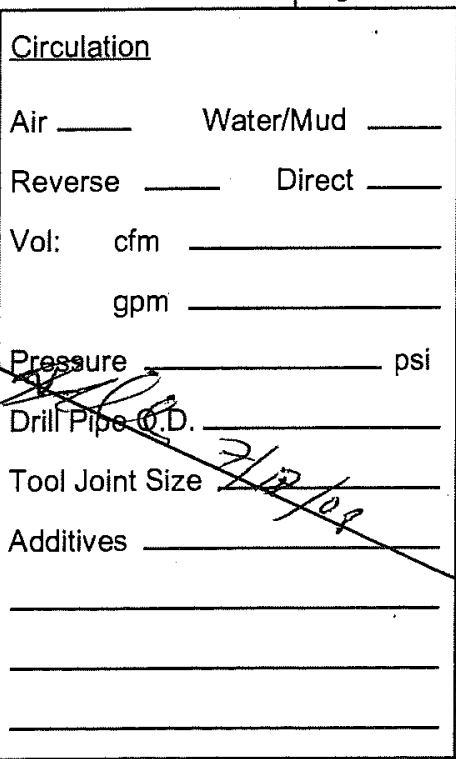

D.H. Hammer

Make

Model

Choke

Casing Hammer

Make

Model

Bit Size

Type

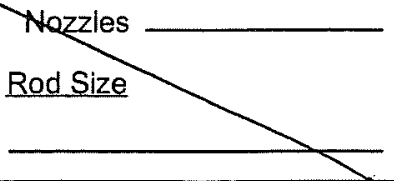

Estimated Depth to Water

Reported By: Tce trits

Namertitle: Cokn the Cologet 


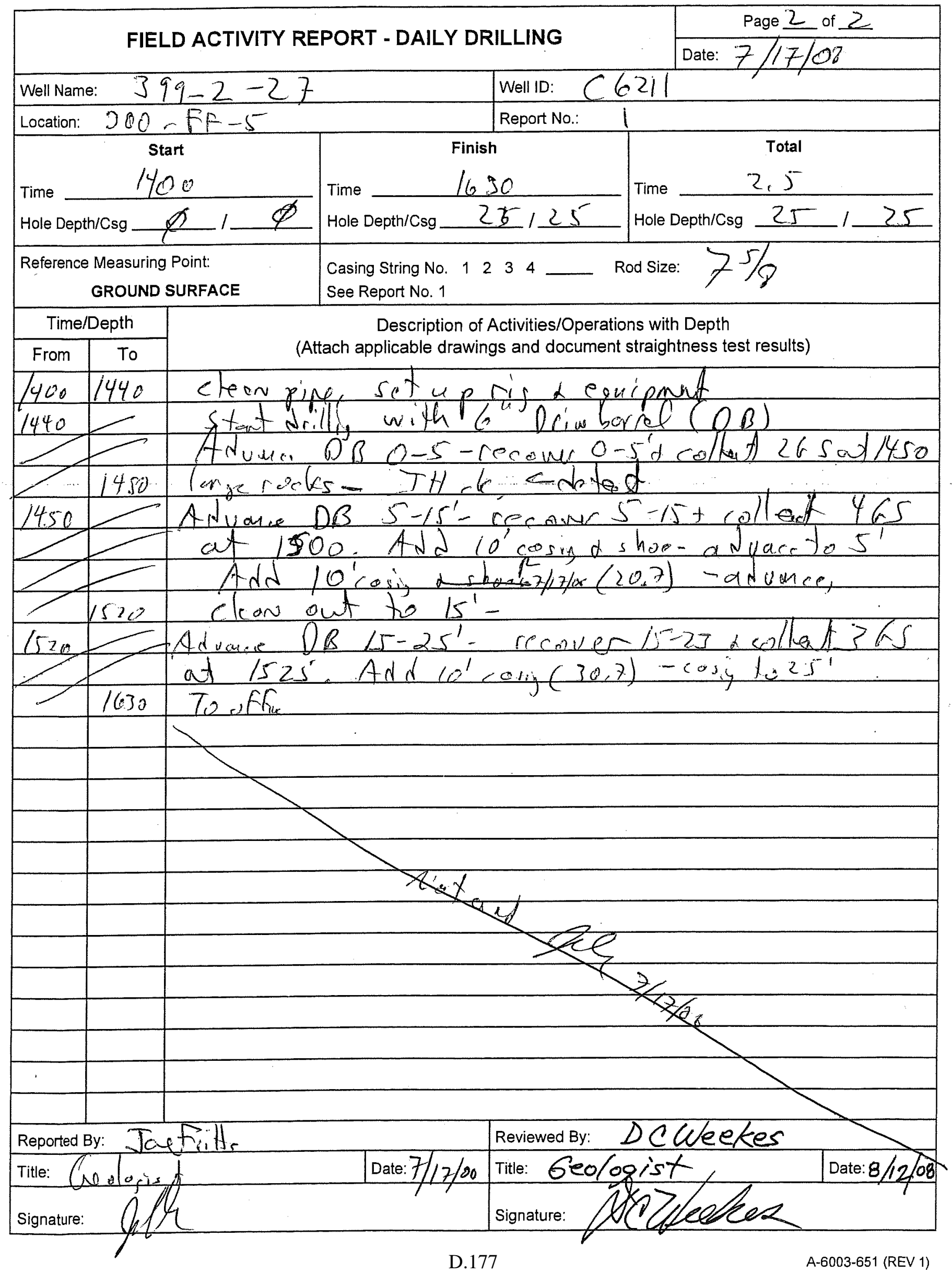




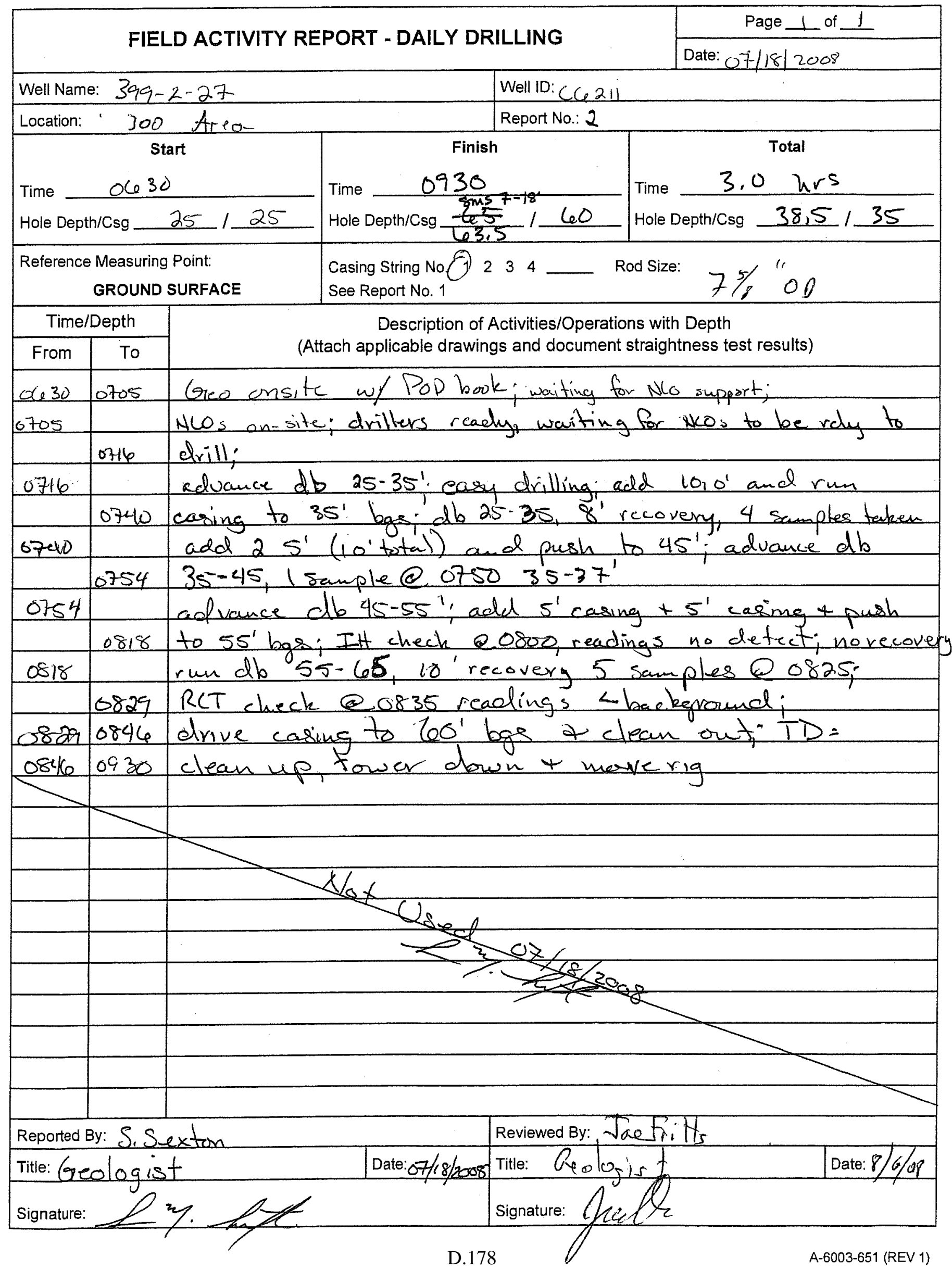




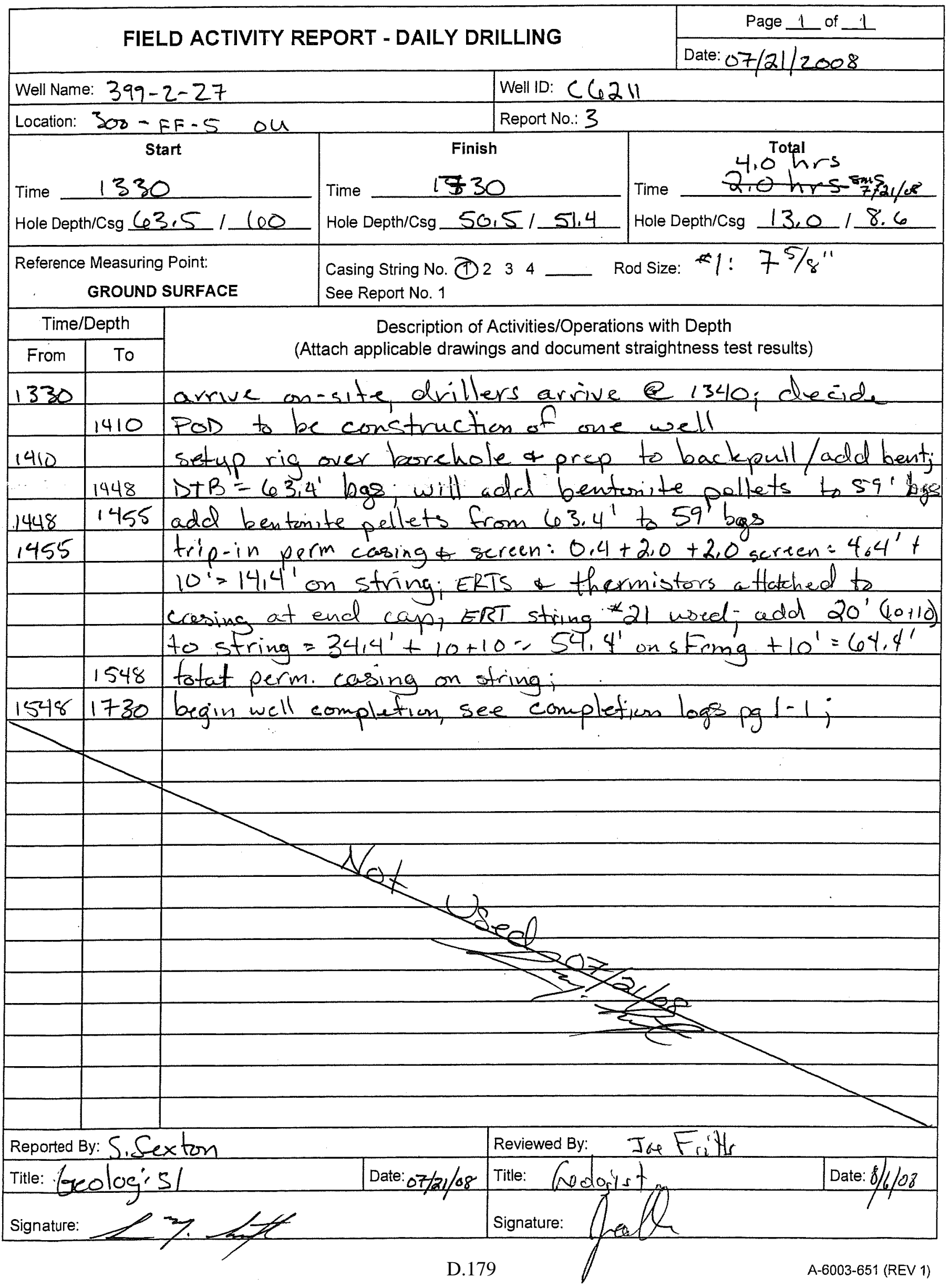




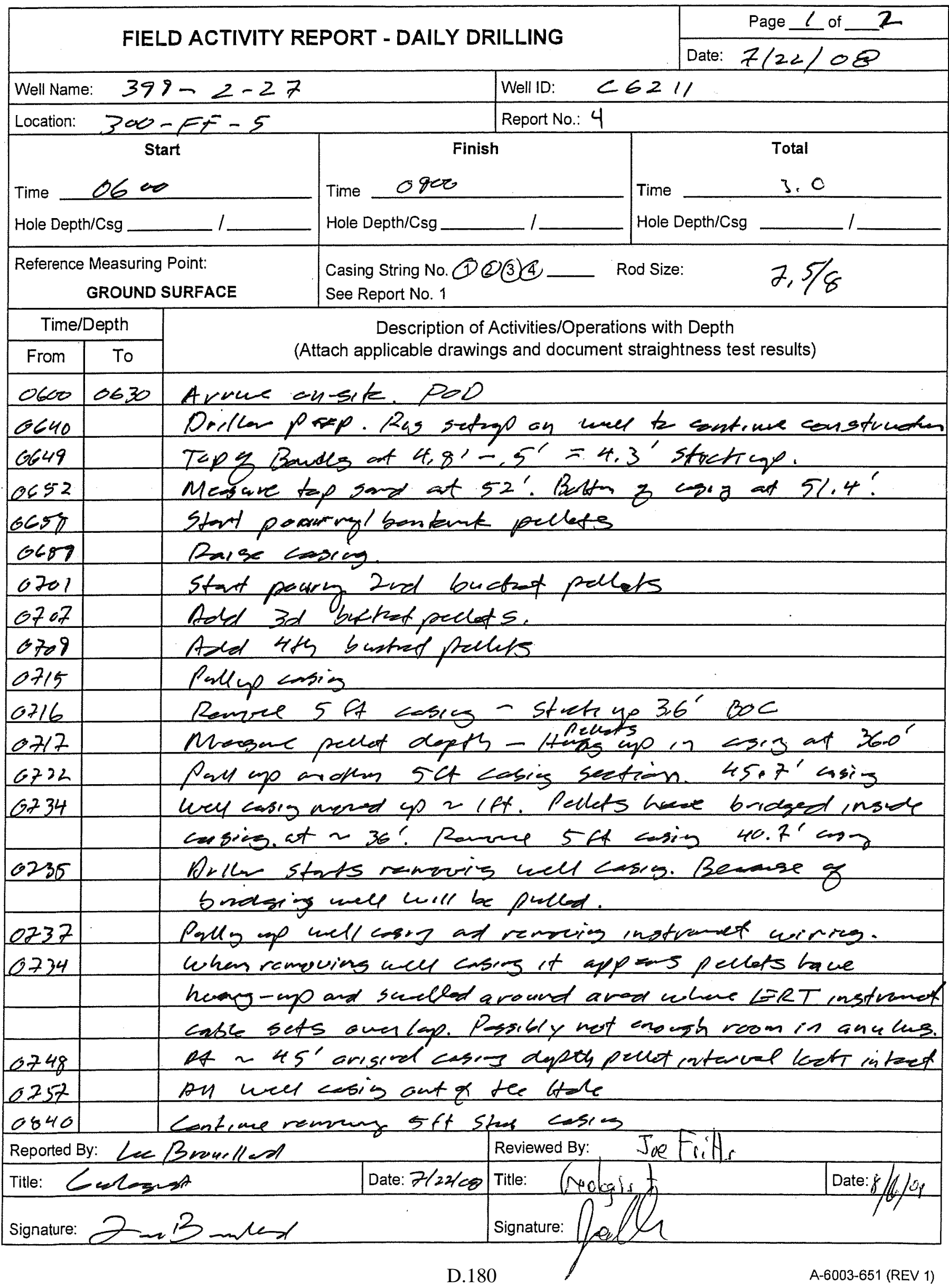




\section{FIELD ACTIVITY REPORT - DAILY DRILLING}

Continuation Page

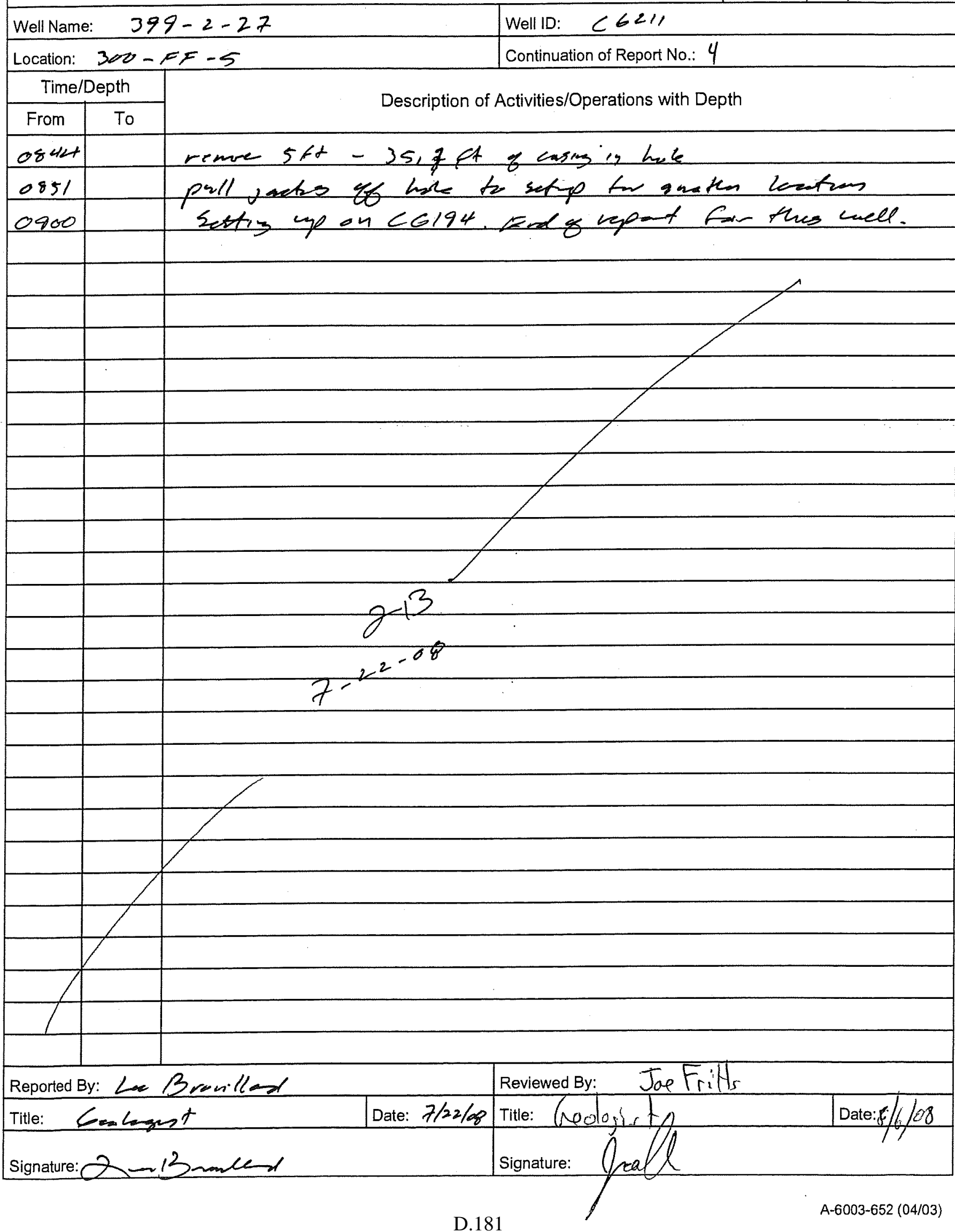




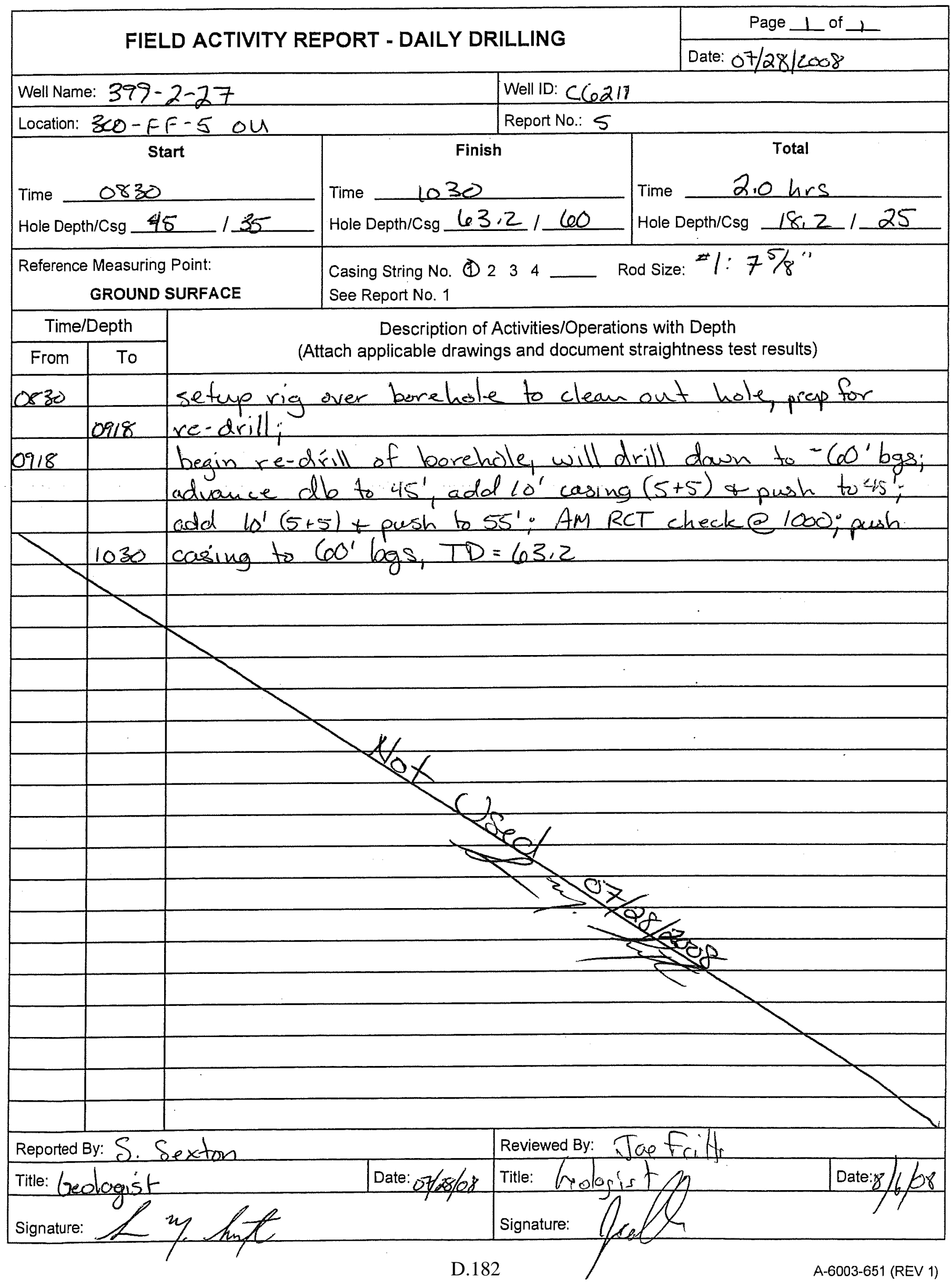




\section{FIELD ACTIVITY REPORT - DAILY DRILLING}

\begin{tabular}{|l} 
Page 1 \\
Date: $7-28-08$
\end{tabular}

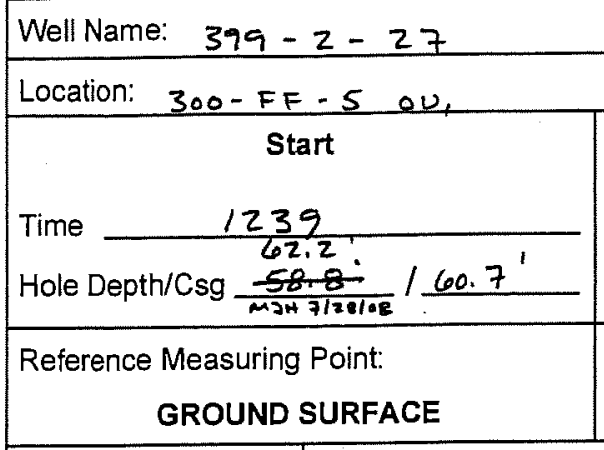

\begin{tabular}{|l|l|} 
& Well ID: C6zII \\
\hline & Report No.: 6 \\
\hline
\end{tabular}

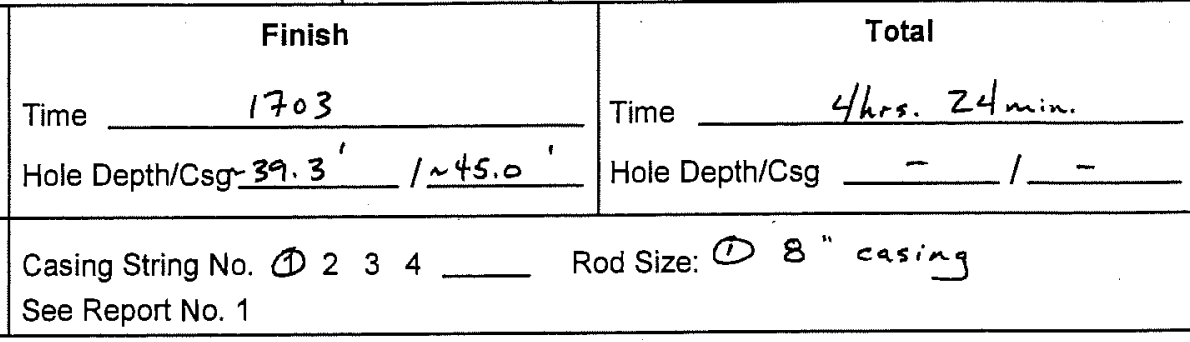

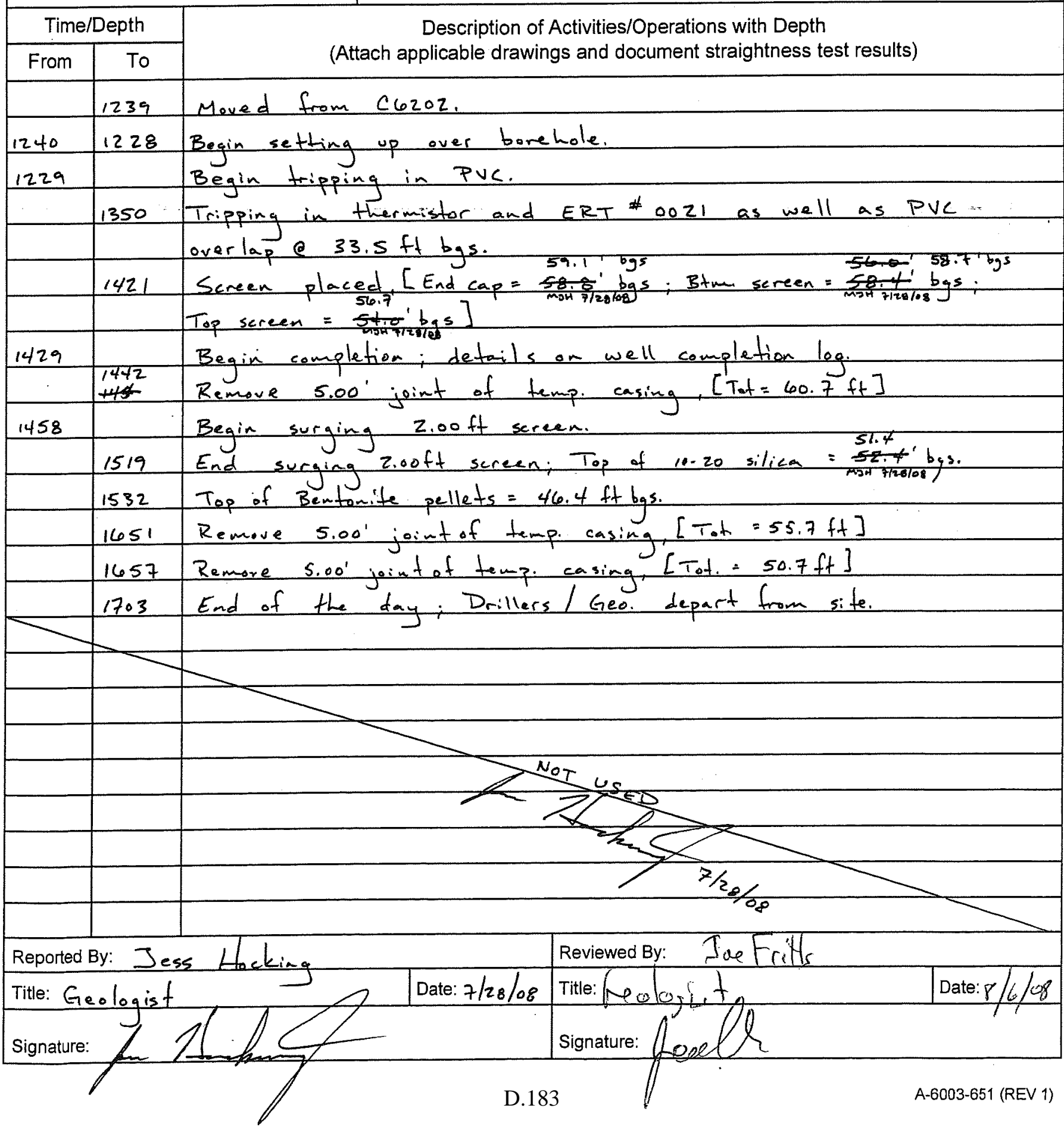


FIELD ACTIVITY REPORT - DAILY DRILLING

Page 1 of 1 Date: $7 / 29 / 08$

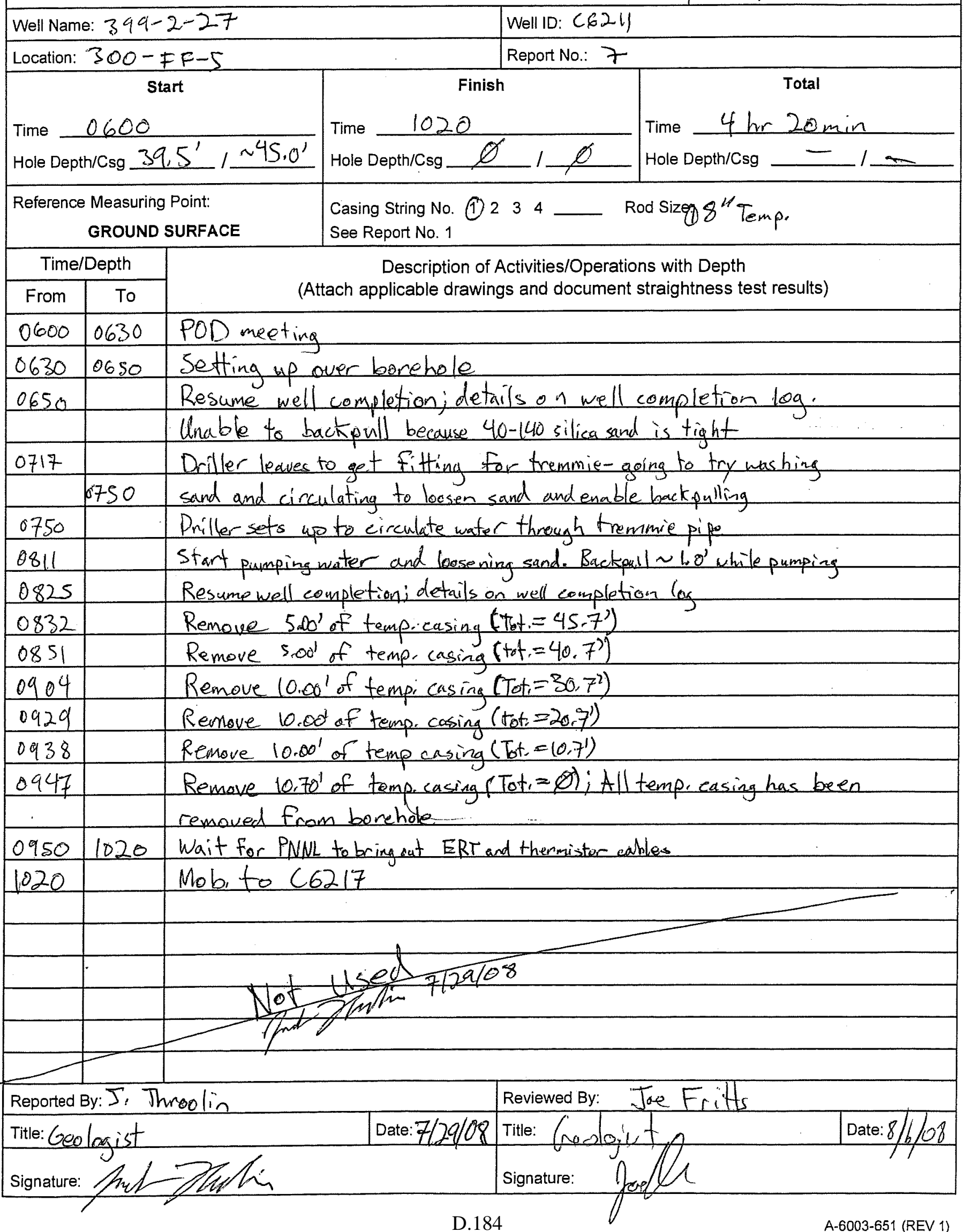




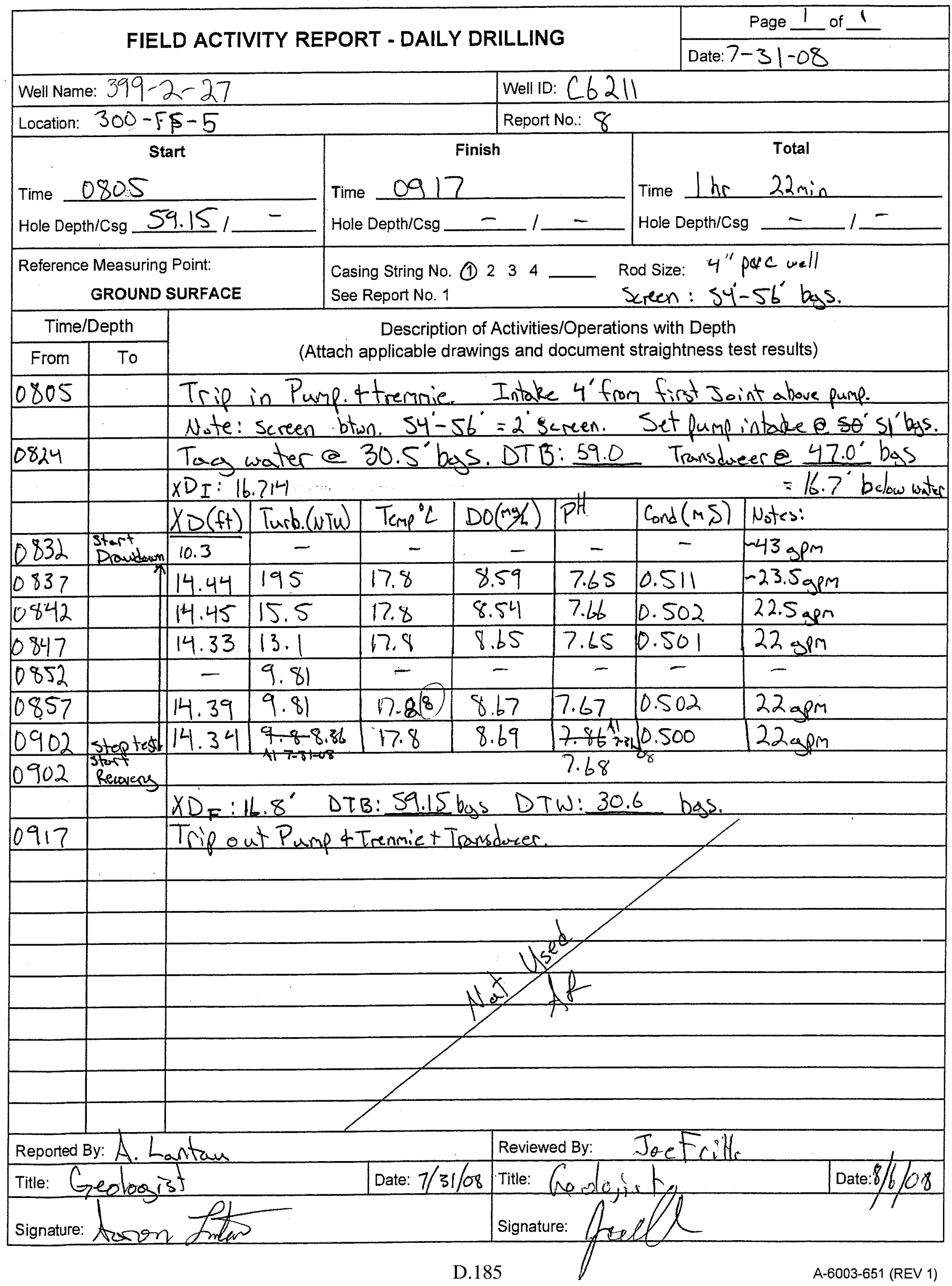




\section{WELL DEVELOPMENT AND TESTING DATA}

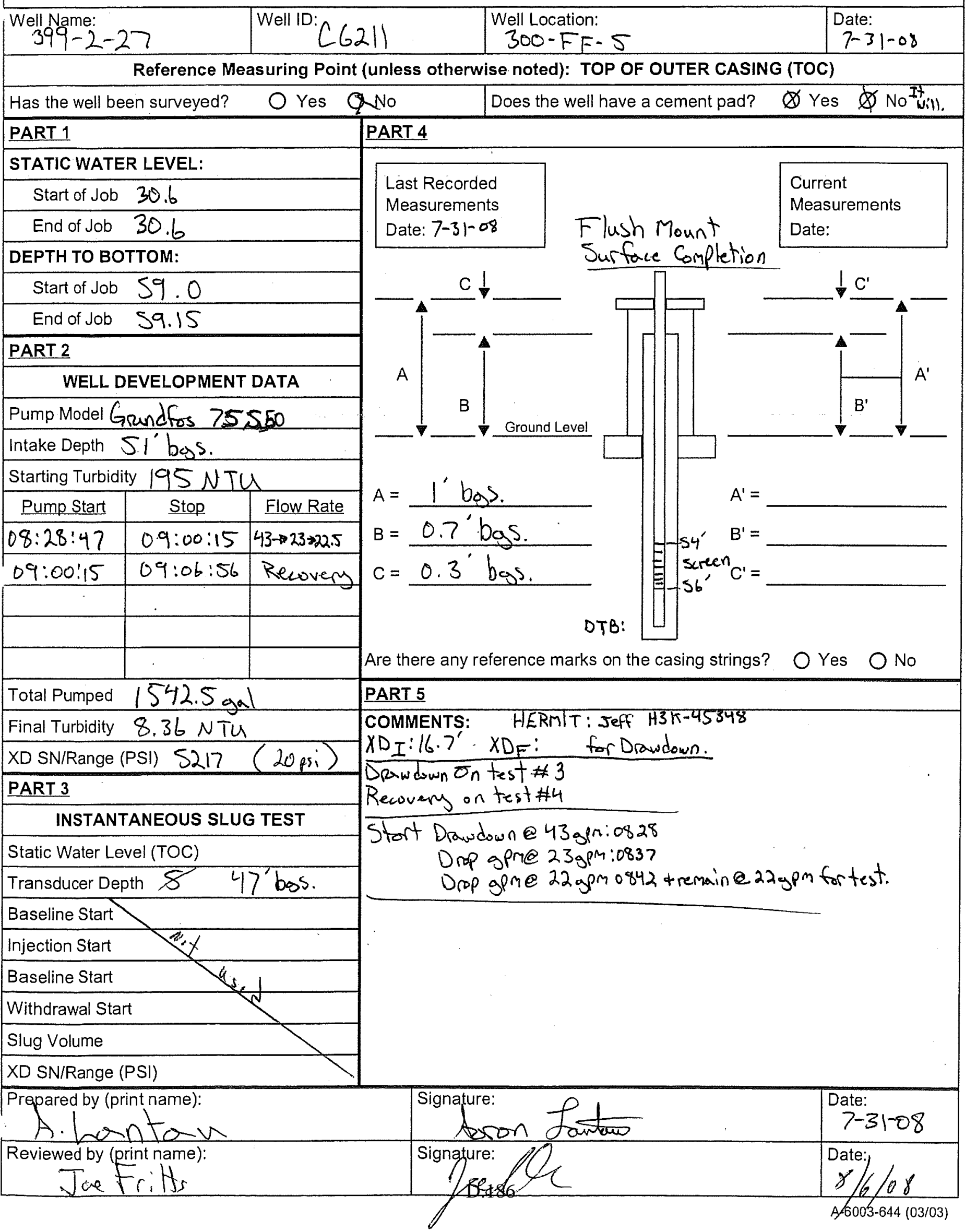




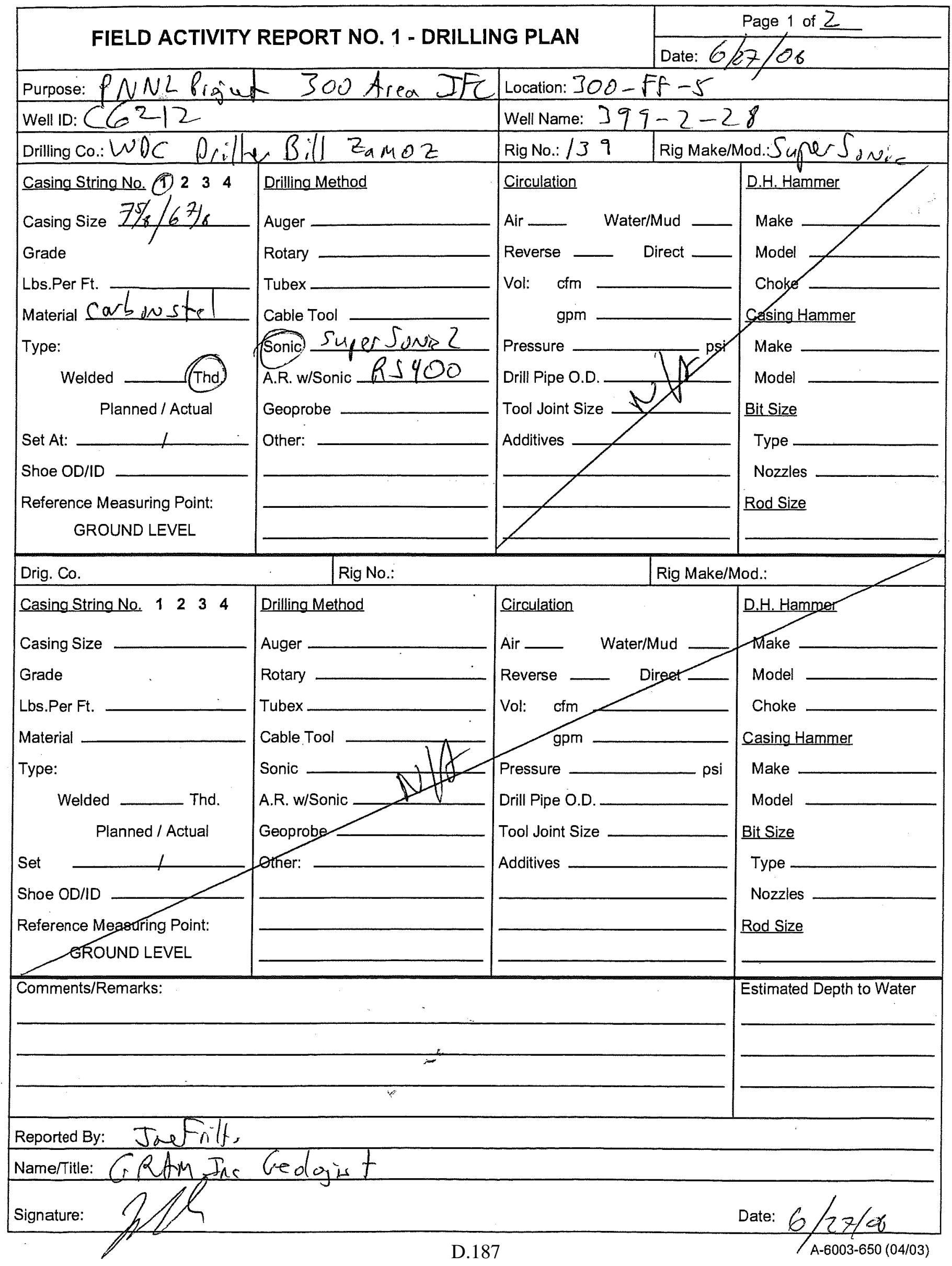




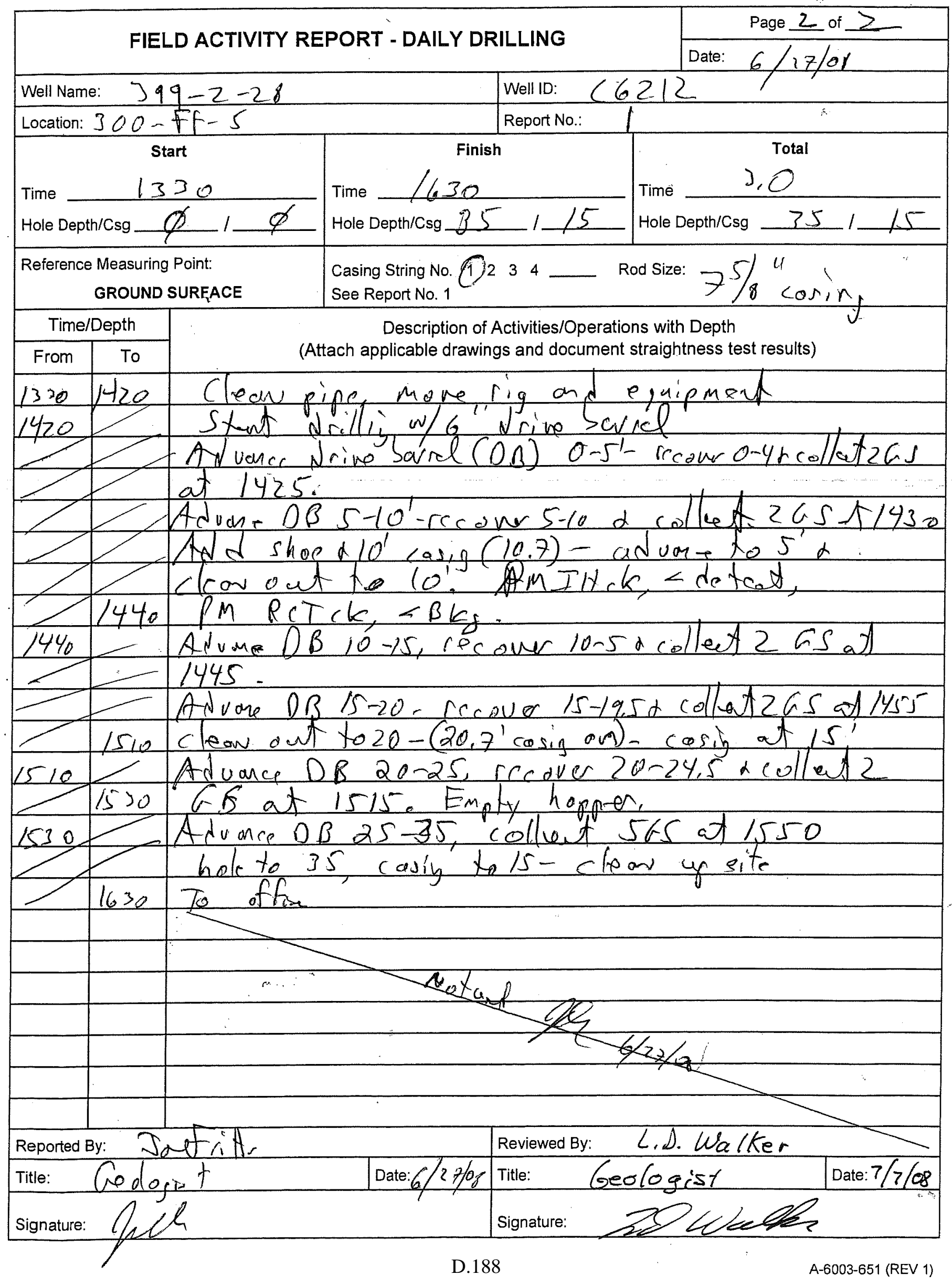




\section{FIELD ACTIVITY REPORT - DAILY DRILLING}

Page 11 of 1
Date: $06 / 30 / 2008$

Well Name: $399-2-28$
Location: $360-F F-5$ oU
Start
Time $\frac{0600}{\text { Hole Depth/Csg } 35}$
$\begin{gathered}\text { Reference Measuring Point: } \\ \text { GROUND SURFACE }\end{gathered}$

Well ID: C 6212 Report No.: 2

Finish

Time 1000 Hole Depth/Csg $65 /\left.63.2\right|_{\text {Hole Depth/Csg } 30 / 48.2} ^{\text {Time }}$ Casing String No. (1)2 $34{ }_{1} \ldots$ Rod Size: $/$ : $75 / 8$ " See Report No. 1

\section{Time/Depth}

\begin{tabular}{l|l} 
From & To \\
\hline
\end{tabular}

\begin{tabular}{|l|l|l|}
\hline 0000 & 0630 &
\end{tabular}

\begin{tabular}{l|l|l|l}
0680 & 0657 \\
\hline
\end{tabular}

0657

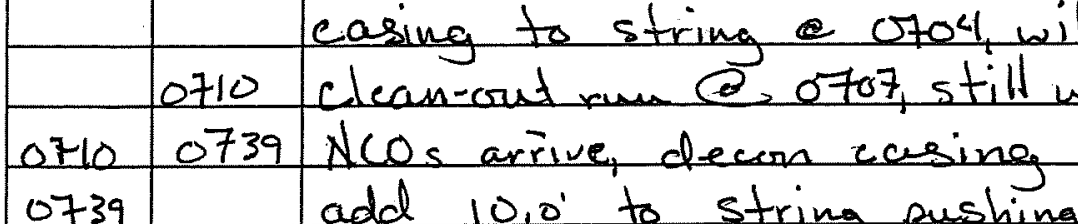

Description of Activities/Operations with Depth

(Attach applicable drawings and document straightness test results)

PODQ 300 Area (BTR, Dïllers, beo); 79; p. Clondy, H. wind AM inspections + rig warm-up;

cunct drive barrel for clean-ent run; add $10.0^{\prime}$ casing to string o 0704 will push to $25^{\prime}+$ cieam-ontif 0710 clean-out rum es 0707, still waiting for NCO support;

0739 add $10,0^{\circ}$ to string, pushing casing to 35'; drillers use "boffle" inside shoc to prevent somple loes; add $5.0^{\circ}$ Q OSO3; drive barrel $35.45^{\prime}$, recover 35.40 2 grab samples taken Q. 0809; cosing C. 45; next db sample $45-55^{\prime} ; A B Q, 55^{\prime}$, add $10^{\prime}$ Laring and push to $55^{\prime}$ b.gs; $D_{B} 45.55$, vecover 45-49, 265 taken Q c848; DB 55-60, recover $55-60,5$ GSQ 6914 0859; DB60-65 recover 60-65, 3 samples 0912 0914 drillers work to TD bowhole, casing set Q C2;

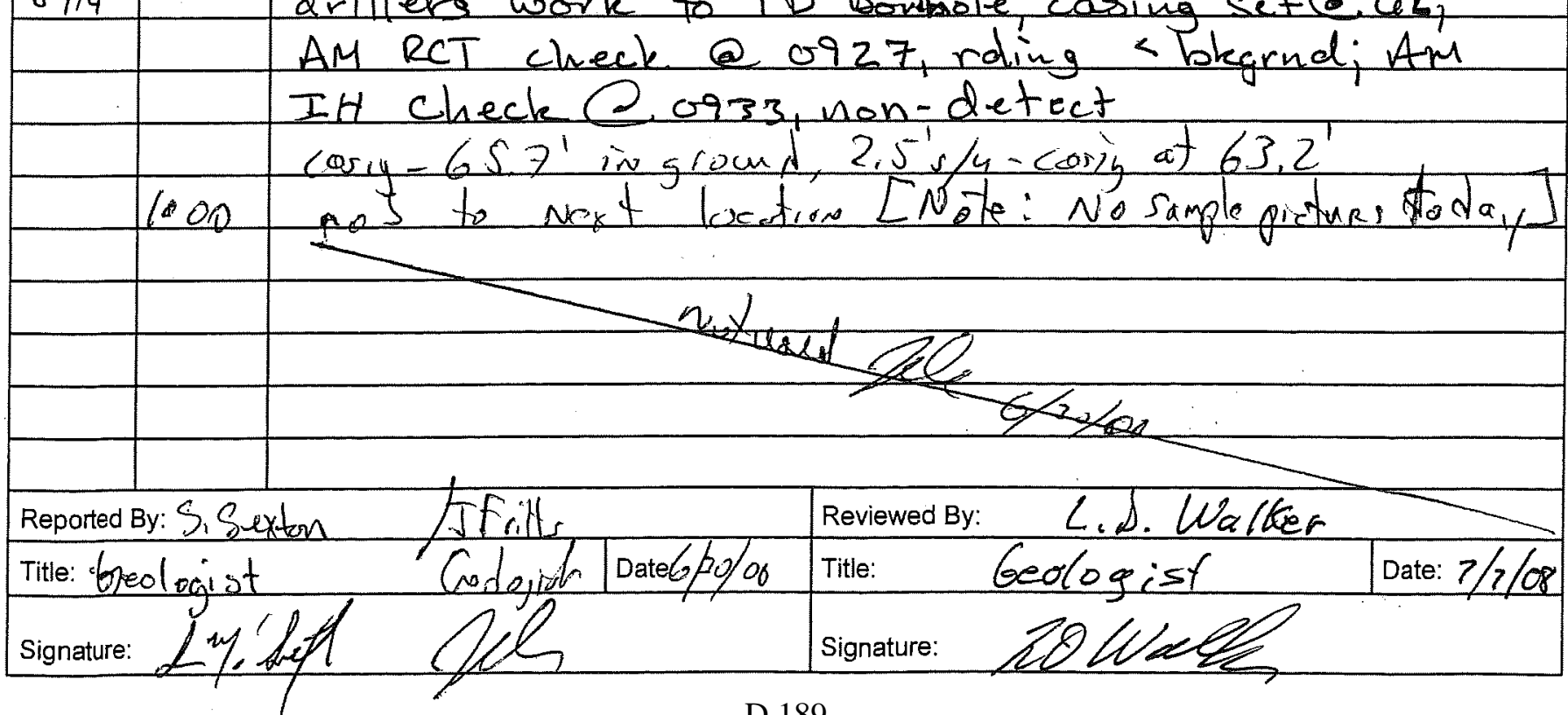
D.189 


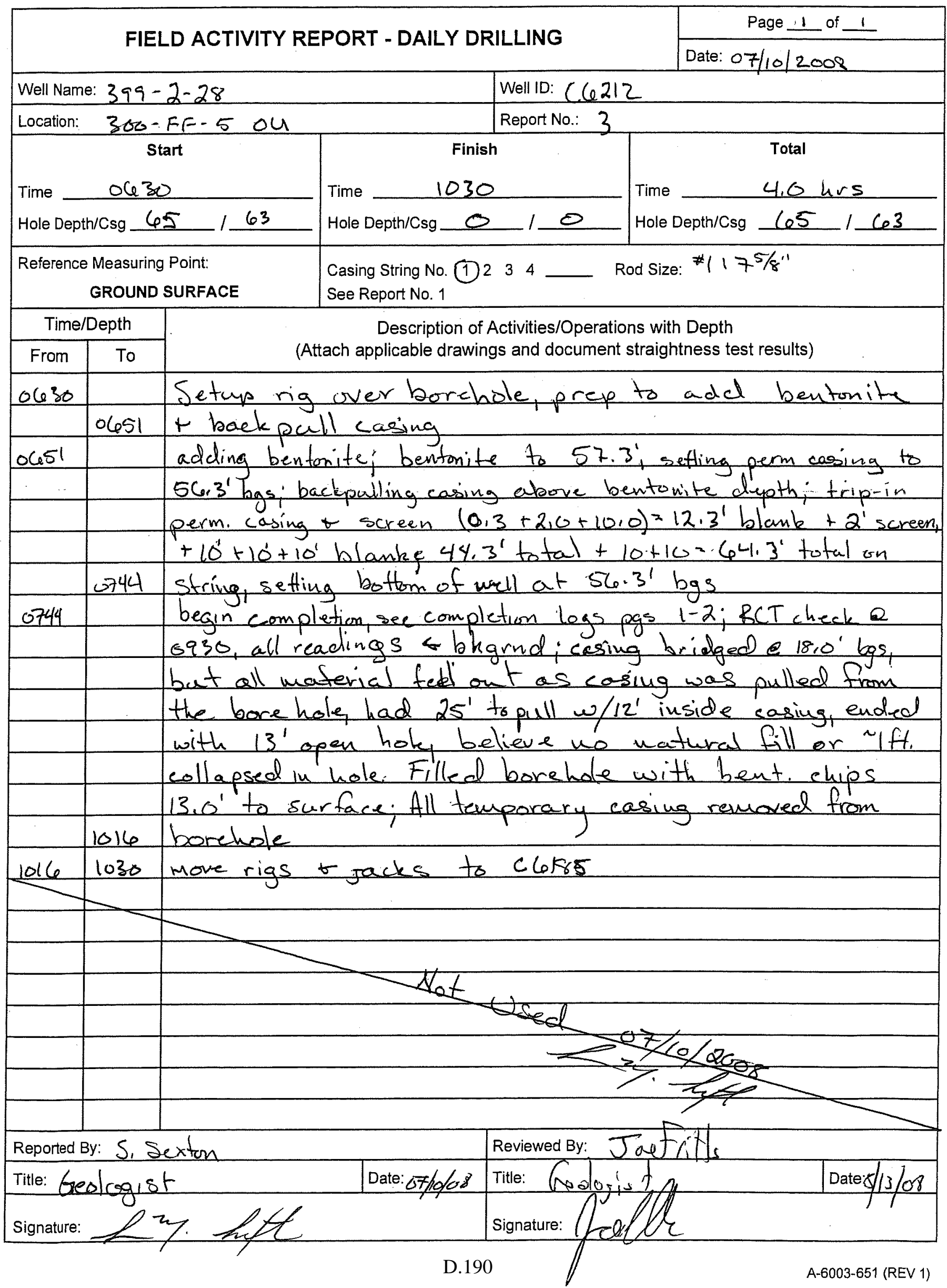


FIELD ACTIVITY REPORT - DAILY DRILLING

\begin{tabular}{l} 
Well Name: $399-2-28$ \\
\hline Location: $300-F F-5$ ou \\
Start \\
Time $\frac{1130}{\text { Hole Depth/Csg - }-1}$ \\
$\begin{array}{c}\text { Reference Measuring Point: } \\
\text { GROUND SURFACE }\end{array}$
\end{tabular}

\author{
Well ID: C 6212 \\ Report No.: 4
}

Finish

Time $\frac{1330}{\text { Hole Depth/Csg - } 1-}$

Time

Total

Casing String No. $123 \quad 34$

Rod Size:

See Report No. 1

Description of Activities/Operations with Depth

(Attach applicable drawings and document straightness test results)

\begin{tabular}{|c|c|c|}
\hline \multirow{3}{*}{$\begin{array}{l}\text { From } \\
1130\end{array}$} & To & (Attach applicable drawings and document straightness test results) \\
\hline & & setup rig on well; tag well: DTB-56.5' logs DTw= \\
\hline & 1145 & bailing well before trip in pump \\
\hline 15 & & trip-in pump: Grundfs 75 S-50 motor: Franklin \\
\hline & 1206 & 5 hp: drandown test recorded on $1+3 K-45348$ Deadlo \\
\hline 206 & & puinp on $Q: 1209$ rate: 20,0 intake. $43.0^{\prime}$ bo \\
\hline & & turh wril D/o(mgh) condus \\
\hline 1212 & & $563 \quad 7.61 \quad 7.09$ \\
\hline 1217 & & 10.260 \\
\hline 1221 & & 10,272 \\
\hline 1225 & & 10.273 \\
\hline 1229 & & 10,276 \\
\hline 1232 & & $7.58 \quad 8.29$ \\
\hline 1235 & 1244 & 7.588 .35 \\
\hline
\end{tabular}

sminti244 1304 pump off Q 1244, begin recovery test on Deadlock wD6

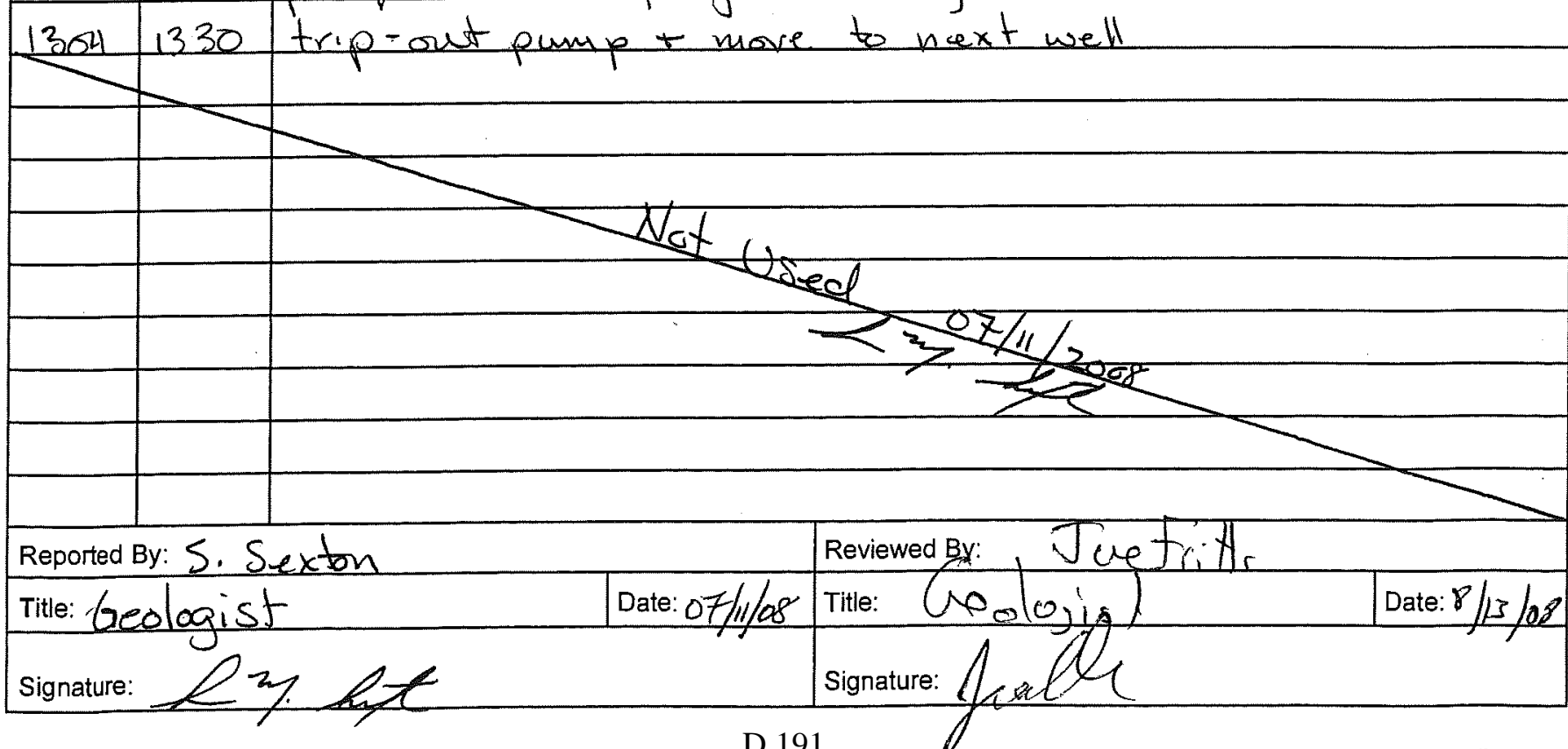

Signature:

D.191 


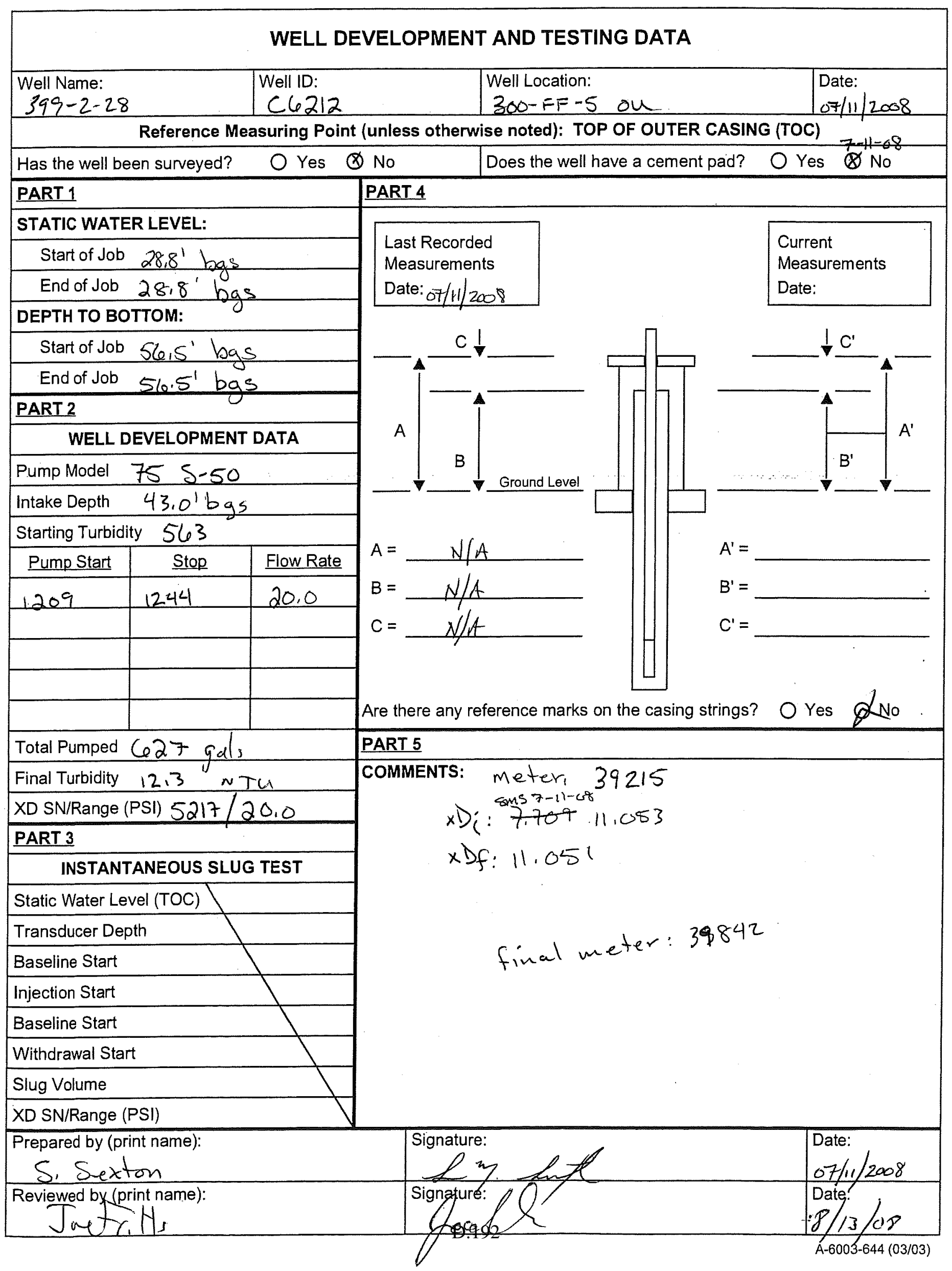


FIELD ACTIVITY REPORT NO. 1 - DRILLING PLAN

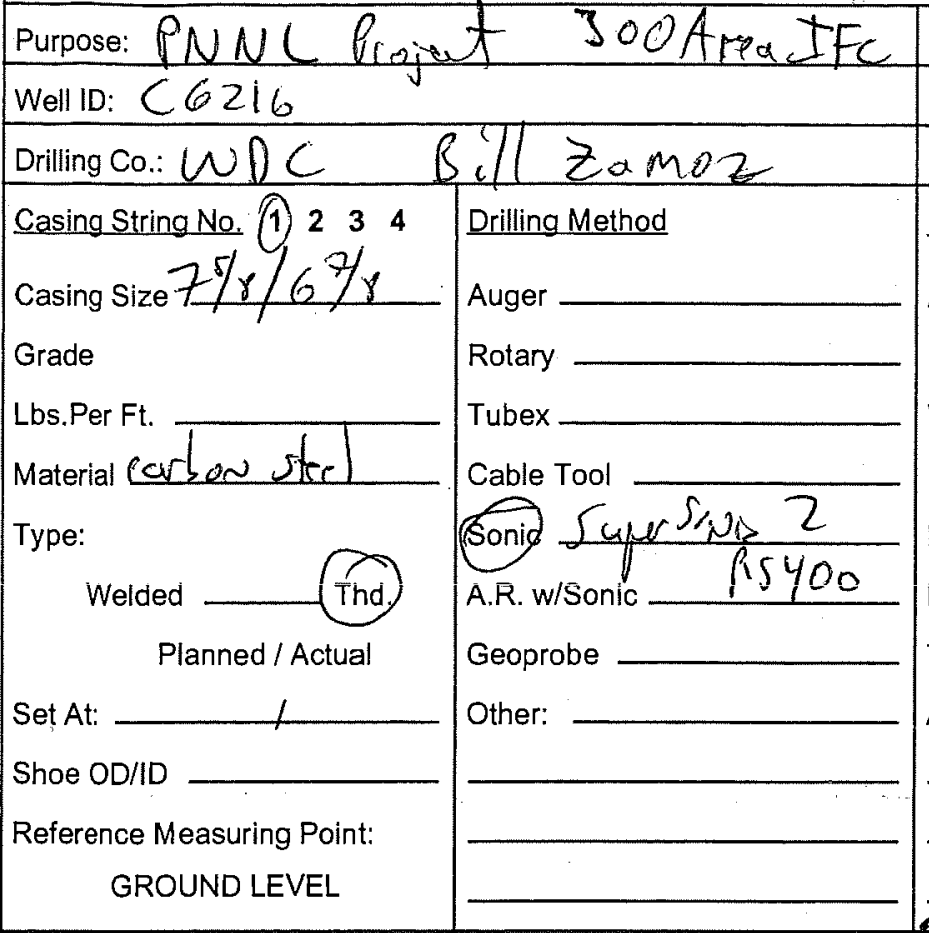

Drig. Co.

Casing String No. $1 \begin{array}{lllll}1 & 2 & 3 & 4\end{array}$

Casing Size

Grade

Lbs.Per Ft.

Material

Type:

Welded Thd.

Planned / Actual

Set

Shoe OD/ID

Reference Measuting Point: GROUND LEVEL
Rig No.:

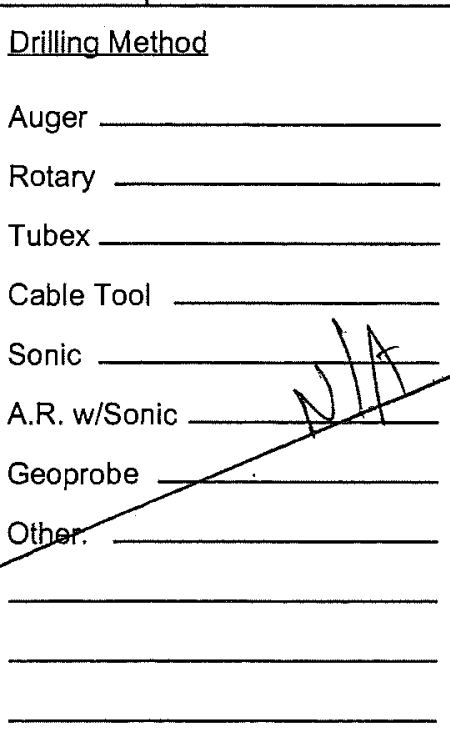

Location: $300-F F-5$ Well Name: $39 q-2-29$ Rig No: $139 \quad$ Rig Make/Mod.S ape SoNic Circulation

Air _ Water/Mud

Reverse —_ Direct

Vol: $\quad \mathrm{cfm}$ gpm

D.H. Hammer

Page 1 of Date: $6 / 26 / 08$

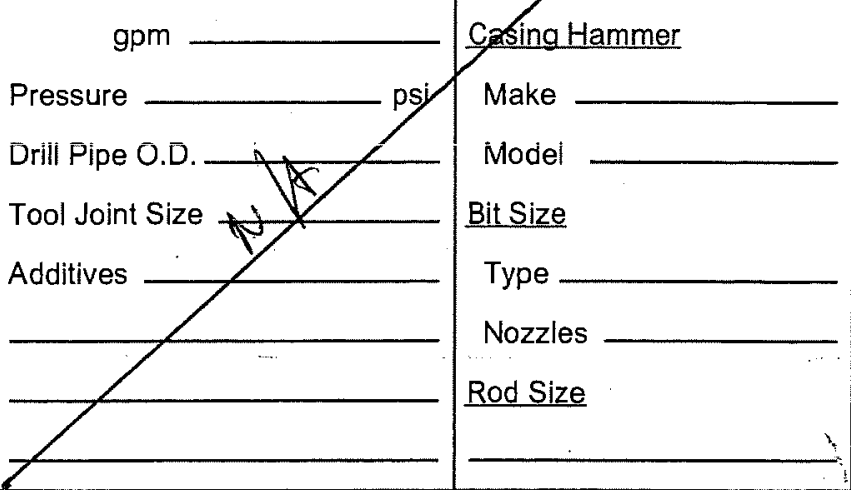

Comments/Remarks:

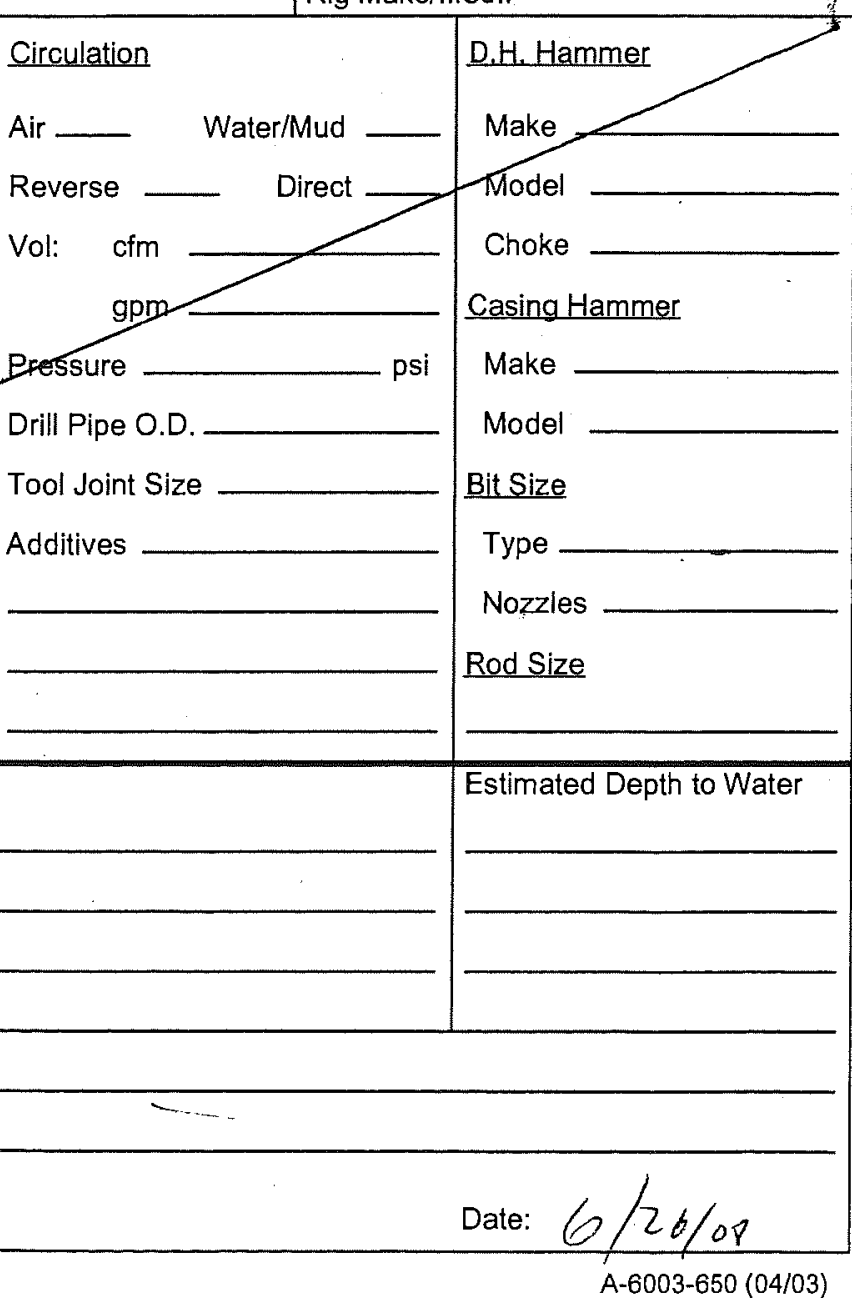




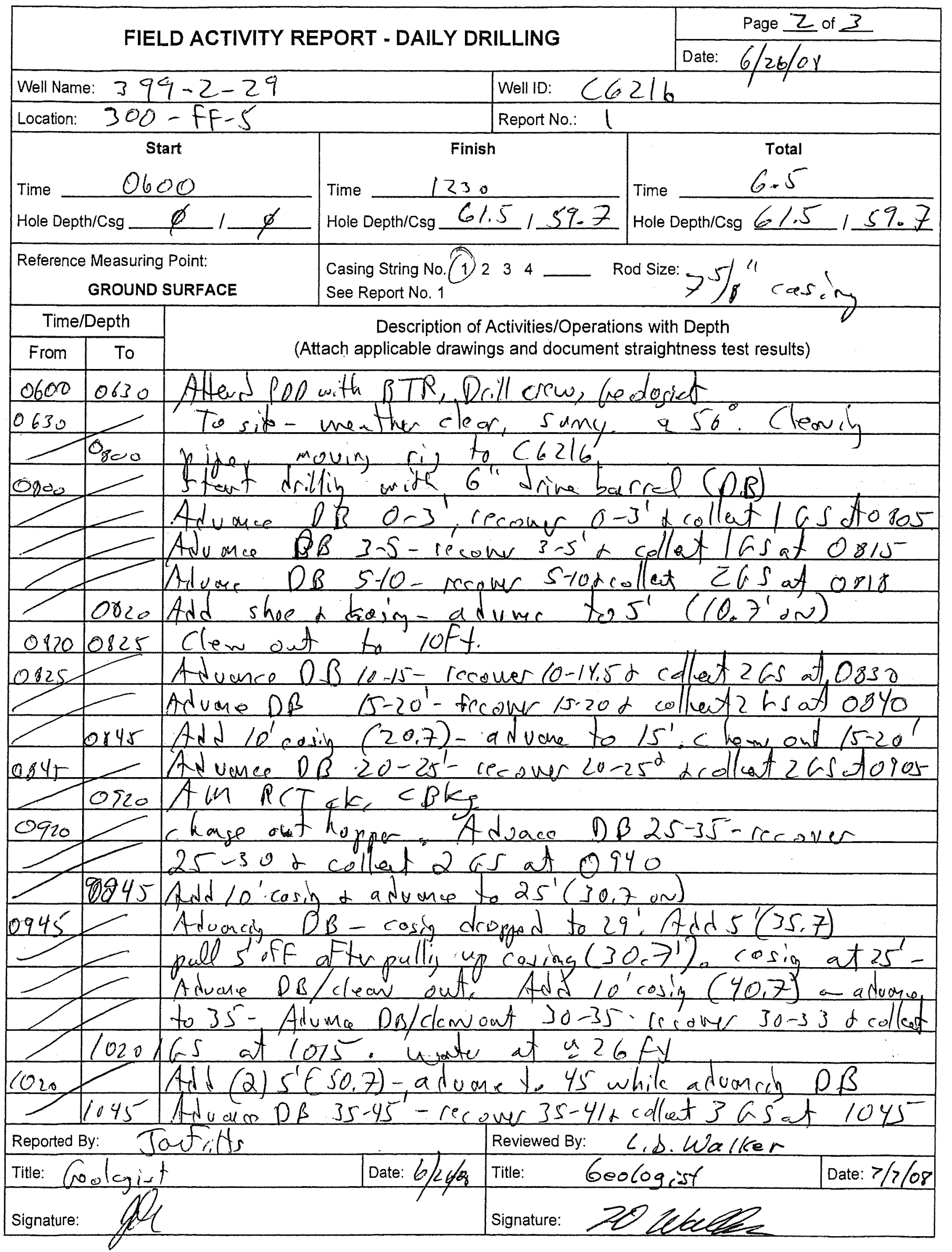

A-6003-651 (REV 1)

D.194 


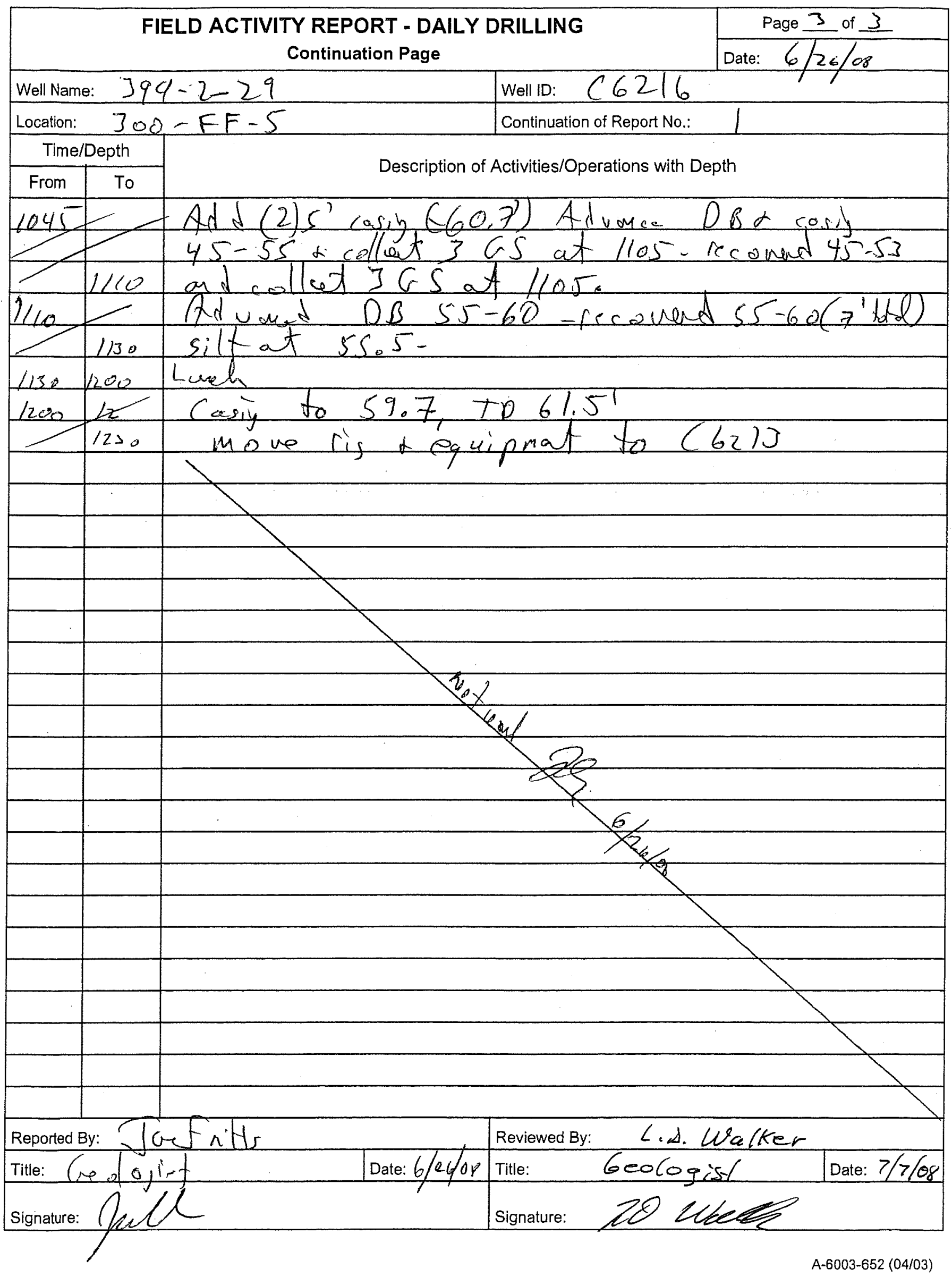

D.195 


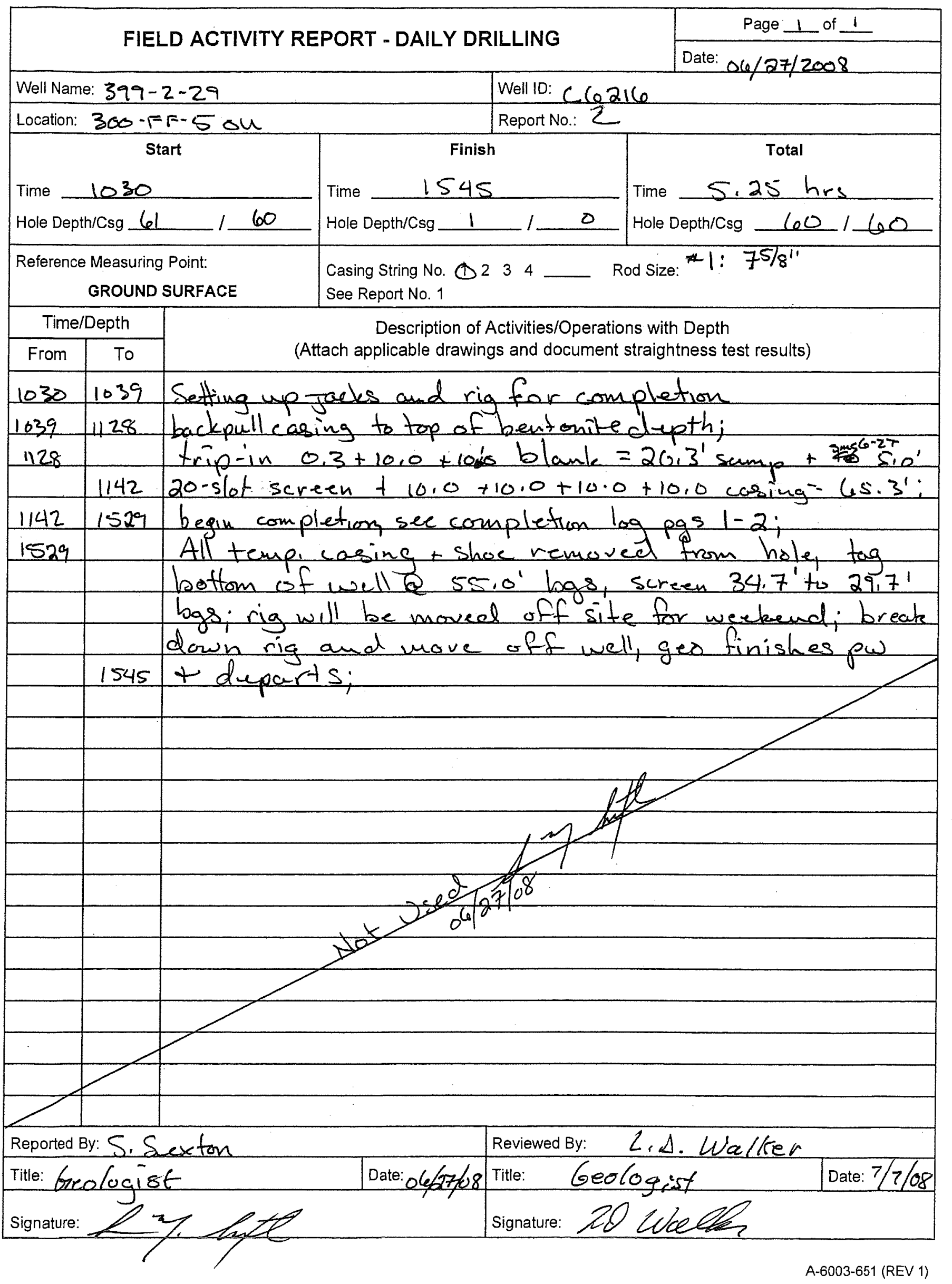

D.196 


\begin{tabular}{l}
\hline Well Name: $399-2-29$ \\
\hline Location: $300-F F-5$ ou \\
\hline Start \\
Time 0945 \\
Hole Depth/Csg _ \\
\hline $\begin{array}{c}\text { Reference Measuring Point: } \\
\text { GROUND SURFACE }\end{array}$
\end{tabular}
Well ID: $(62) 6$ Report No: 3

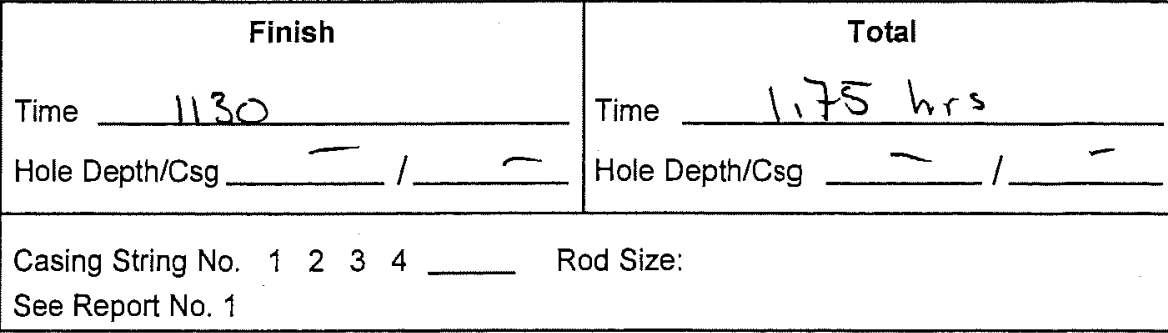

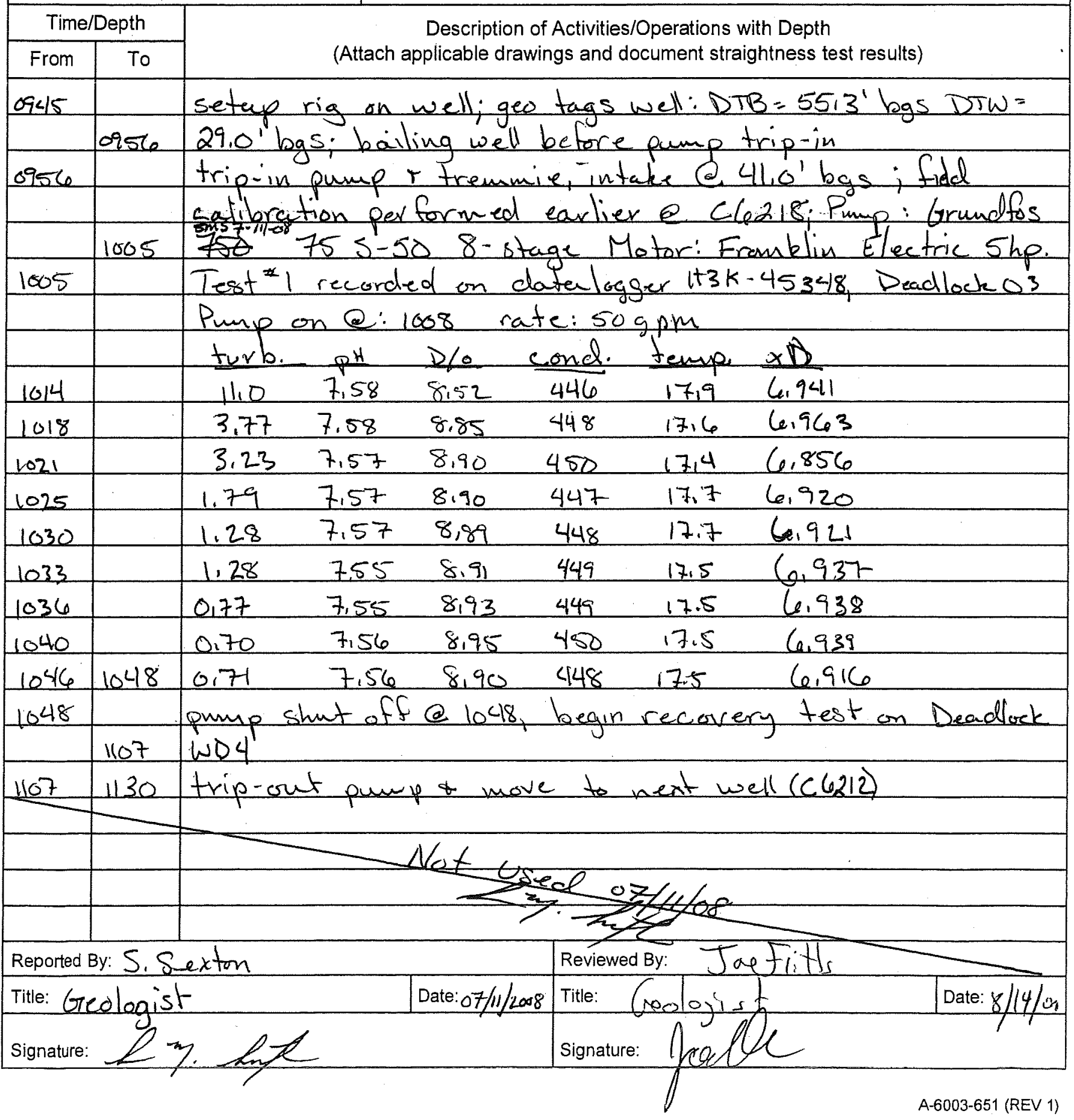




\section{WELL DEVELOPMENT AND TESTING DATA}

\begin{tabular}{|l|l|l|l|}
\hline $\begin{array}{l}\text { Well Name: } \\
399-2-29\end{array}$ & $\begin{array}{l}\text { Well ID: } \\
\text { C6216 }\end{array}$ & $\begin{array}{l}\text { Well Location: } \\
300-F F-5 \text { ou }\end{array}$ & $\begin{array}{l}\text { Date: } \\
07 / 11 / 2008\end{array}$ \\
\hline \multicolumn{3}{r}{} \\
\hline
\end{tabular}

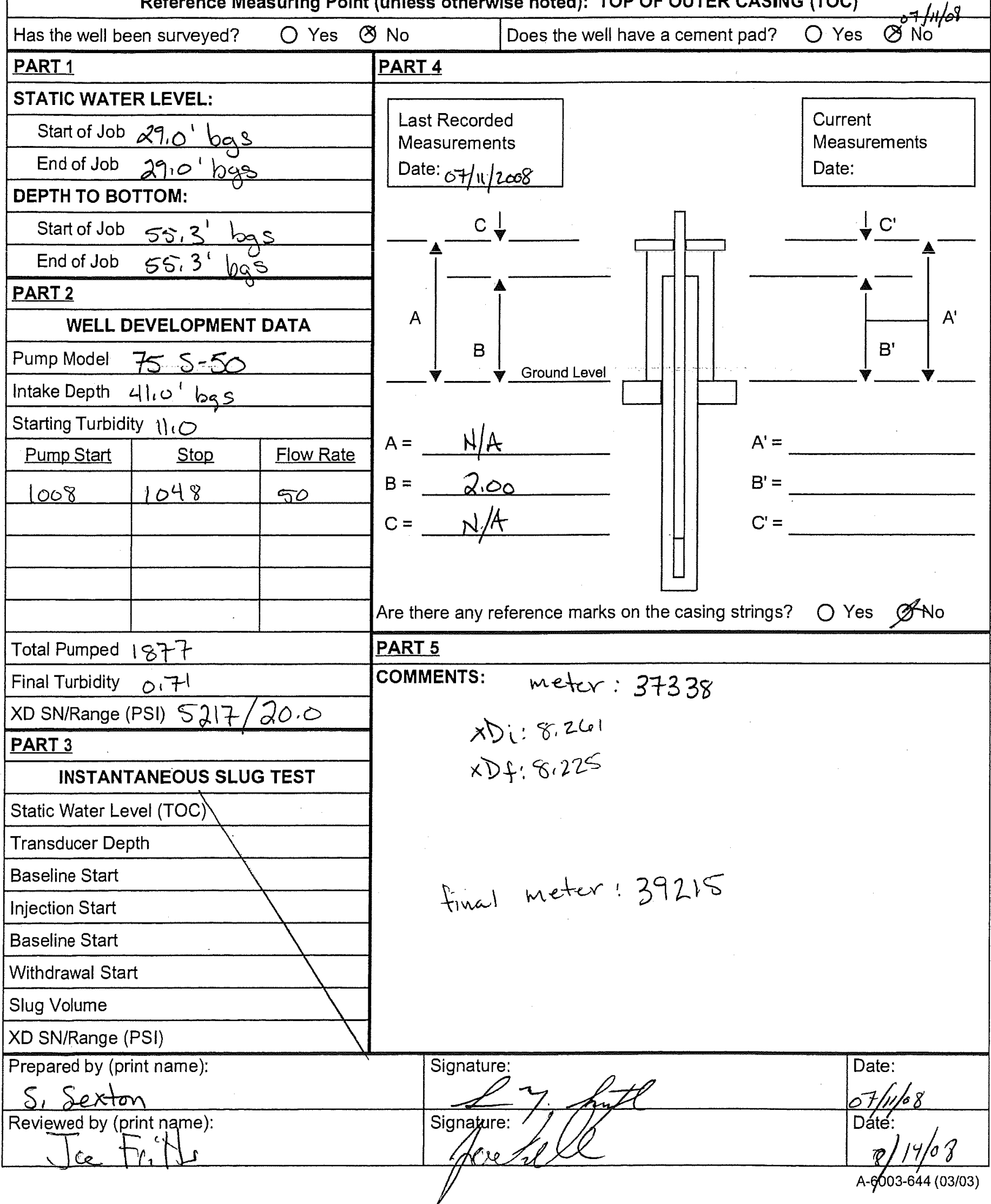

D.198 
FIELD ACTIVITY REPORT NO. 1 - DRILLING PLAN

Page 1 of 2

Date: $07 / 24 / 2008$

Purpose: 300 Areas IFC

Well ID: C6217

Drilling Co.: WDC

Casing String No. (1) 234

Casing Size $75 / 8^{\prime \prime} / 67 / 8^{\prime \prime}$

Grade

Lbs.Per $\mathrm{Ft}$.

Material carbon steel

Type:

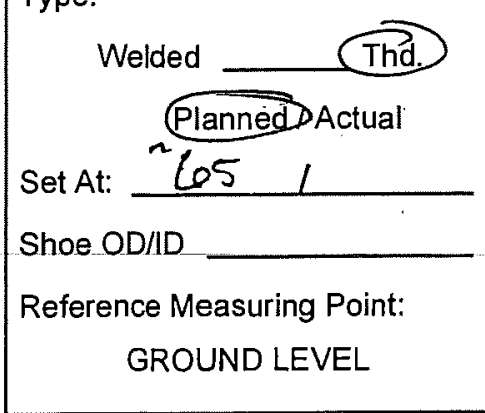

Drig. Co.

Casing String No. $\begin{array}{llll}1 & 2 & 3 & 4\end{array}$

Casing Size

Grade

Lbs.Per Ft.

Material

Type:

Welded Thd.

Planned / Actual

Set At:

Shoe OD/ID

Reference Meastring Point: GROUND LEVEL

Bill Zamow

Drilling Method

Auger

Rotary

Tubex

Cable Tool

Sonic SuperSonic

A.R. w/Sonic RS4050

Geoprobe

Other:

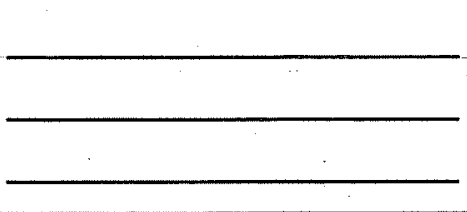

Rig No.:

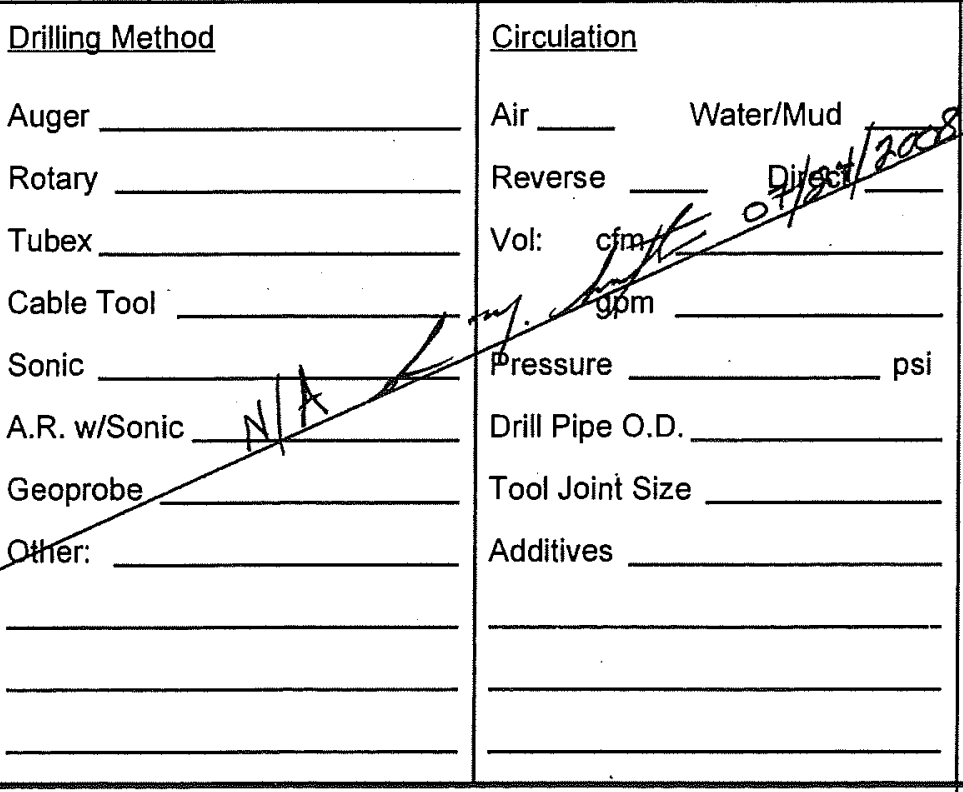

Comments/Remarks:
Location: $300-F F-5$ ou

Well Name: $399-2-30$

\begin{tabular}{l|l|l} 
Rig No.:139 & Rig Make/Mod.: Super Sonic II
\end{tabular}

Circulation

A

Air

Water/Mud

Reverse __ Direct

Vol: $\quad \mathrm{cfm}$

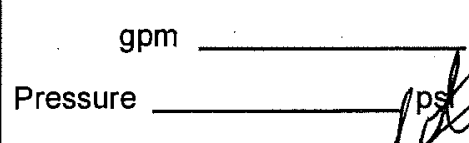

Drill Pipe O.D

Tool Joint Size

Additives

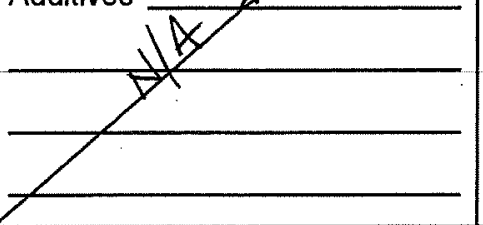

D.H. Hammer

Make

Model

chokels

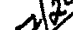

G. 6.jog Hammer

Make

Model

Bit Size

Type

Nozzles

Rod Size

Reported By: S. Sexton

NamerTitle: GRAM, The Geologist 


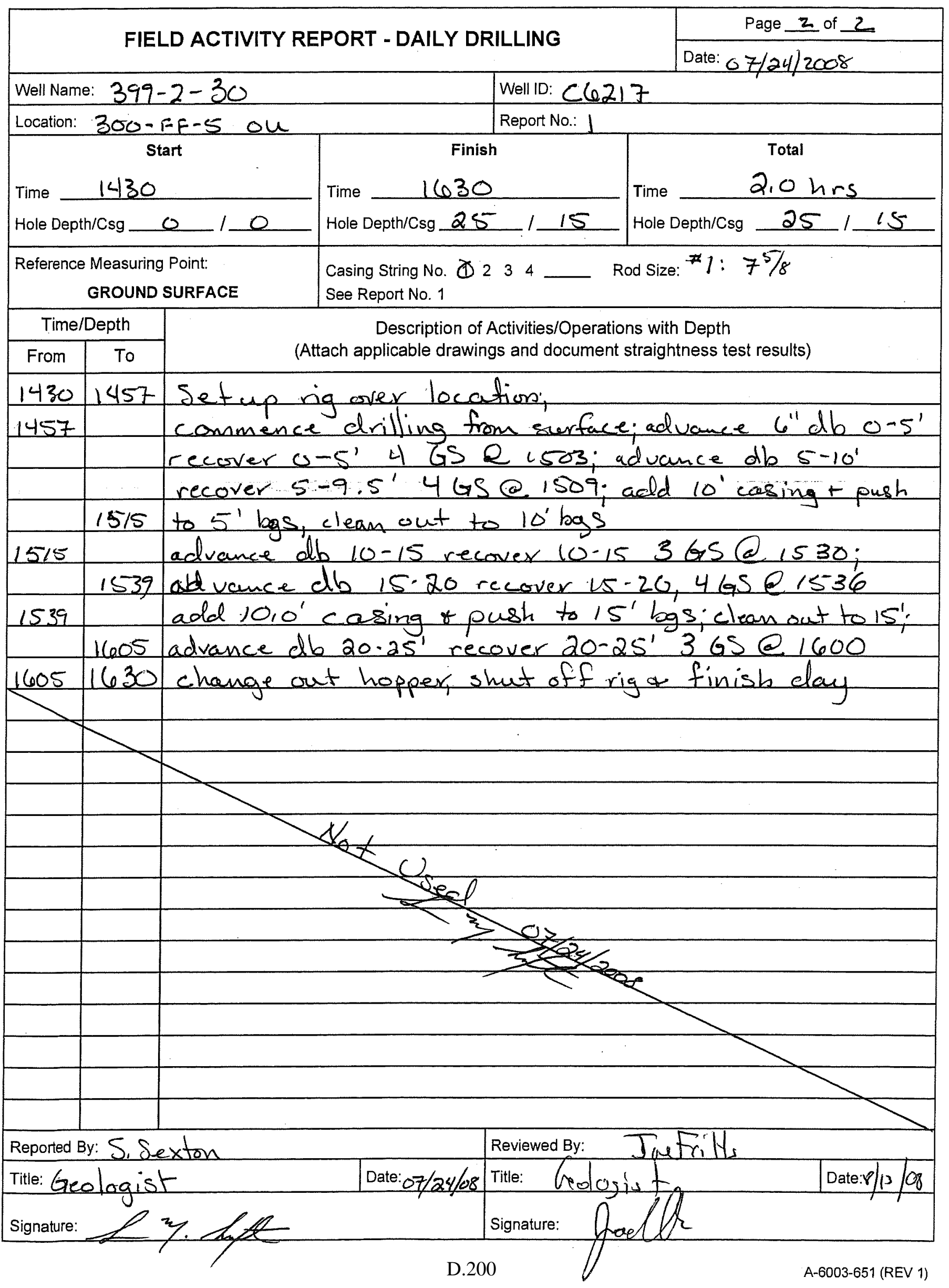




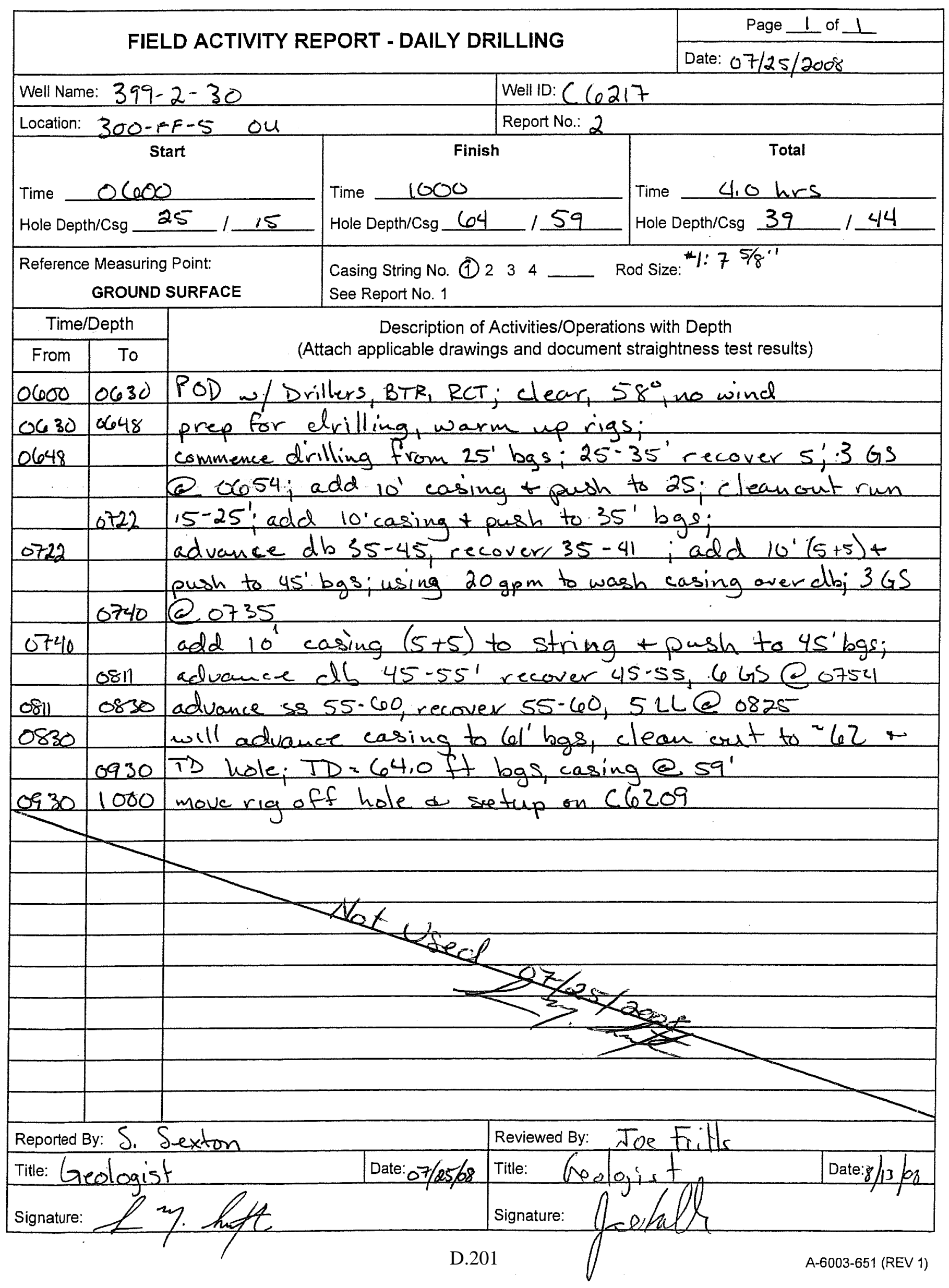




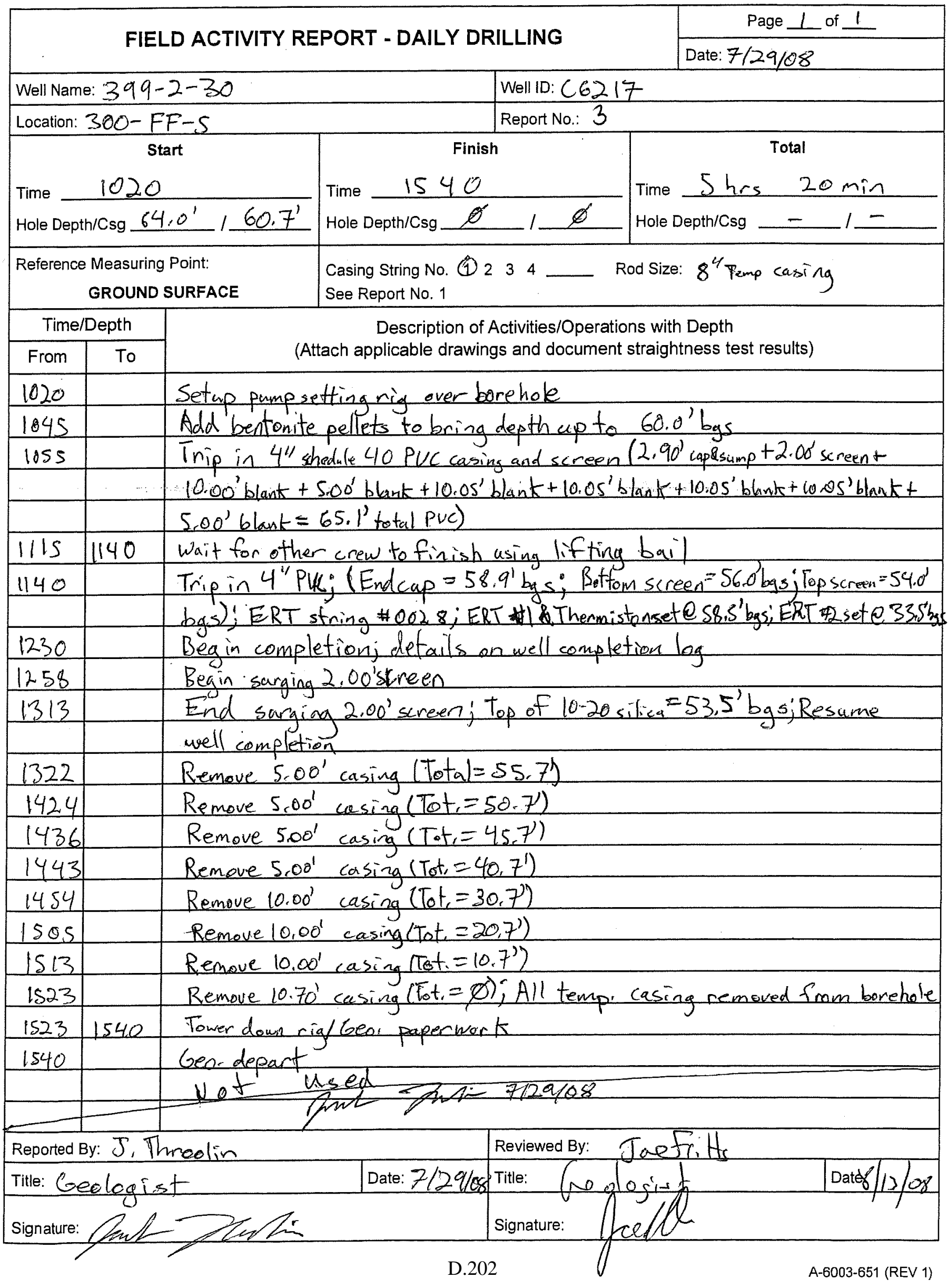




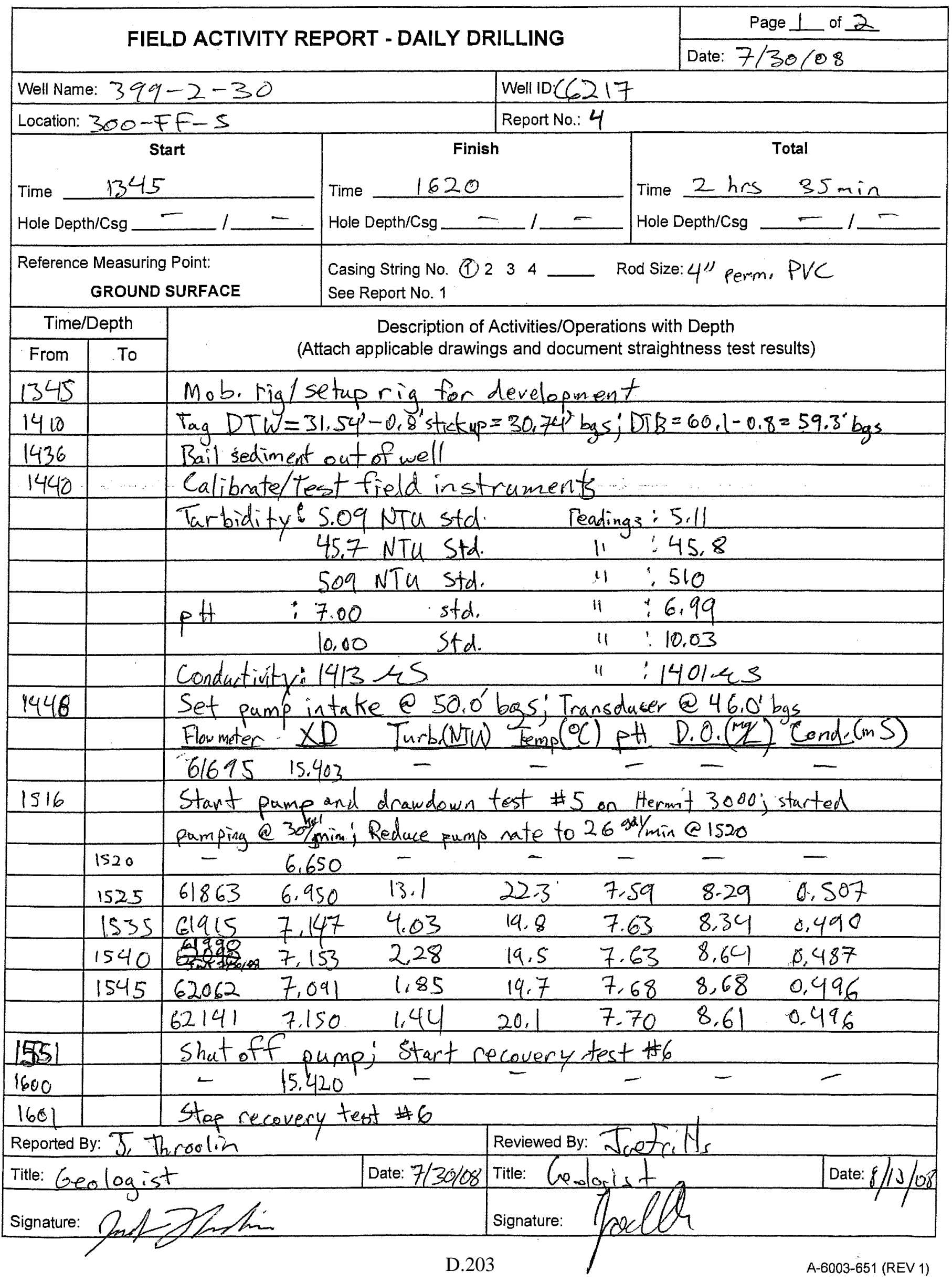




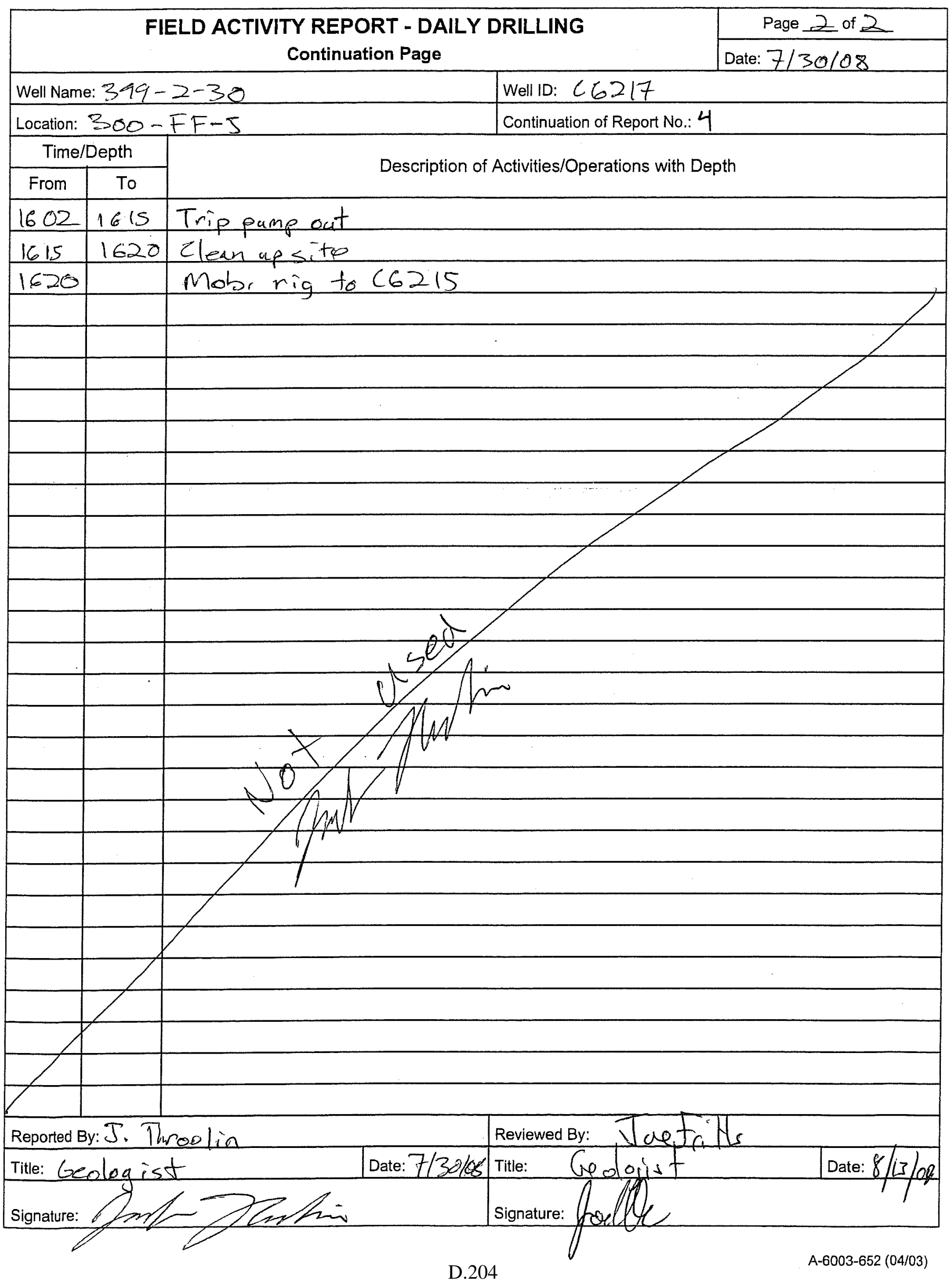




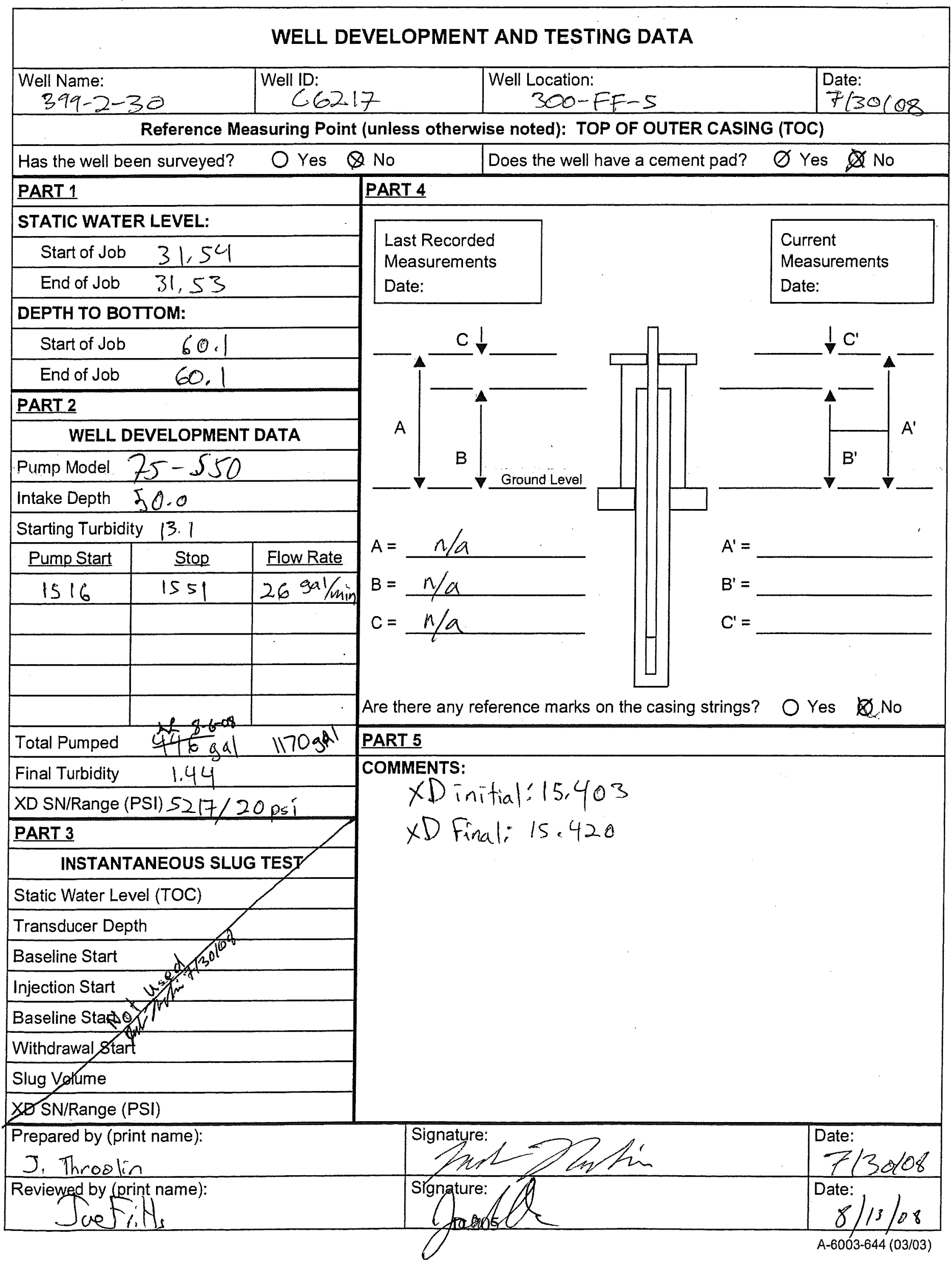




\section{FIELD ACTIVITY REPORT NO. 1 - DRILLING PLAN}

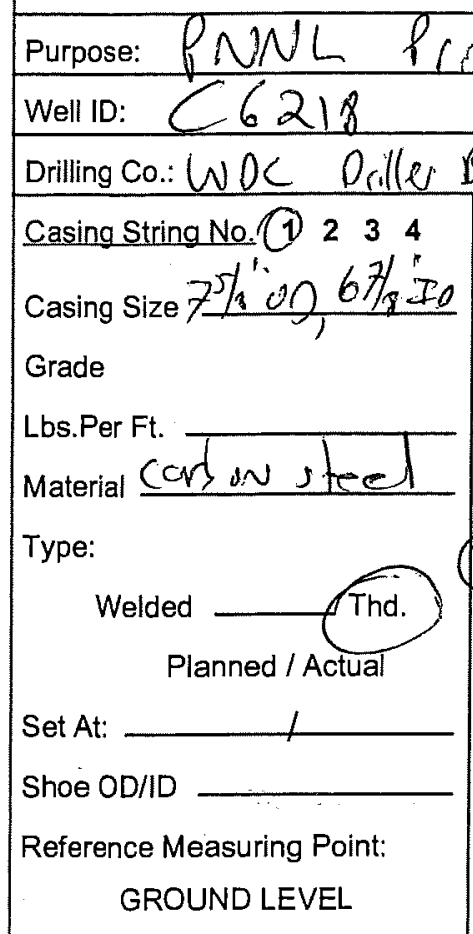

Drig. Co.

Casing String No. $\begin{array}{llll}1 & 2 & 3 & 4\end{array}$

Casing Size

Grade

Lbs.Per Ft.

Material

Type:

Welded Thd.

Planned / Actual

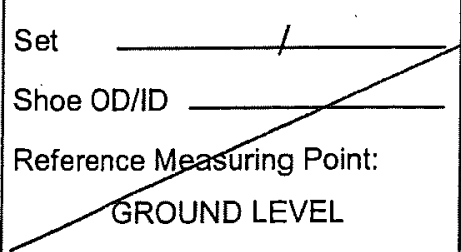

Comments/Remarks:
Location: $300-F F-5$ Well Name: $399-2-31$ Rig No.: 139 Rig Make/Mod.: Syue SUN Circulation Air Rotary Tubex Cable Tool Sonic) Syper Savic 2 A.R. w/Sonic RS 400 Geoprobe Other:

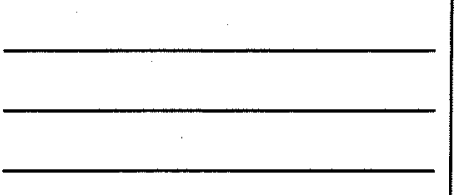

Rig No.:

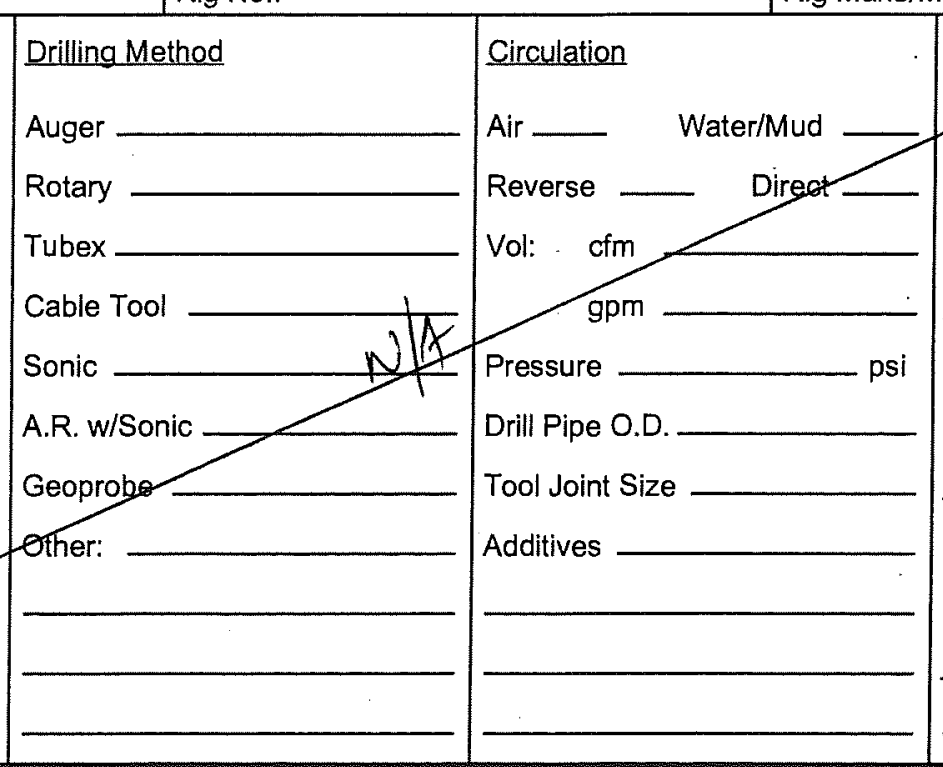

Rig Make/Mod.:

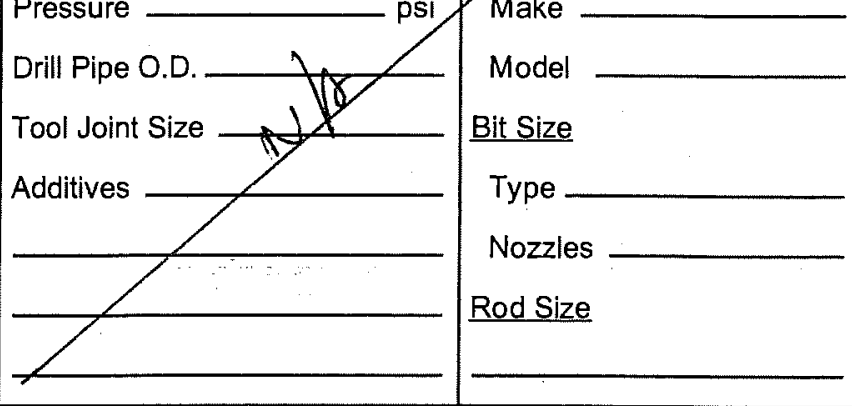

D.H. Hammer

Make

Model

Choke

Casing Hammer

Make

Model

Bit Size

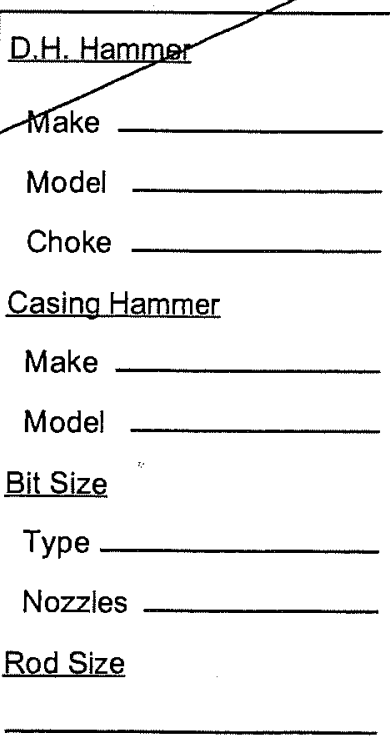

Estimated Depth to Water

Reported By: Jafritts

NamerTitle: GRAM Ine Golugiod

Signature: 


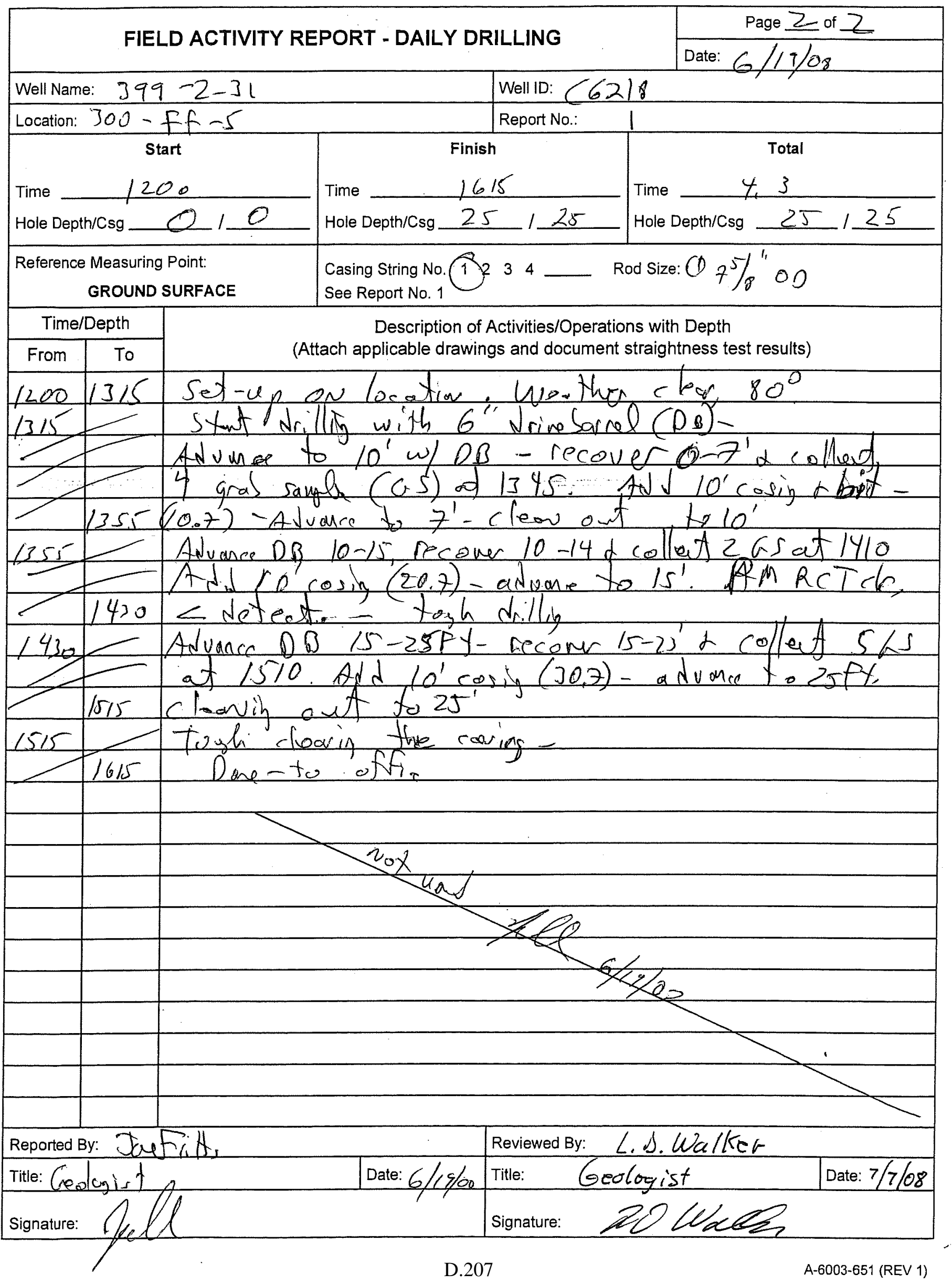




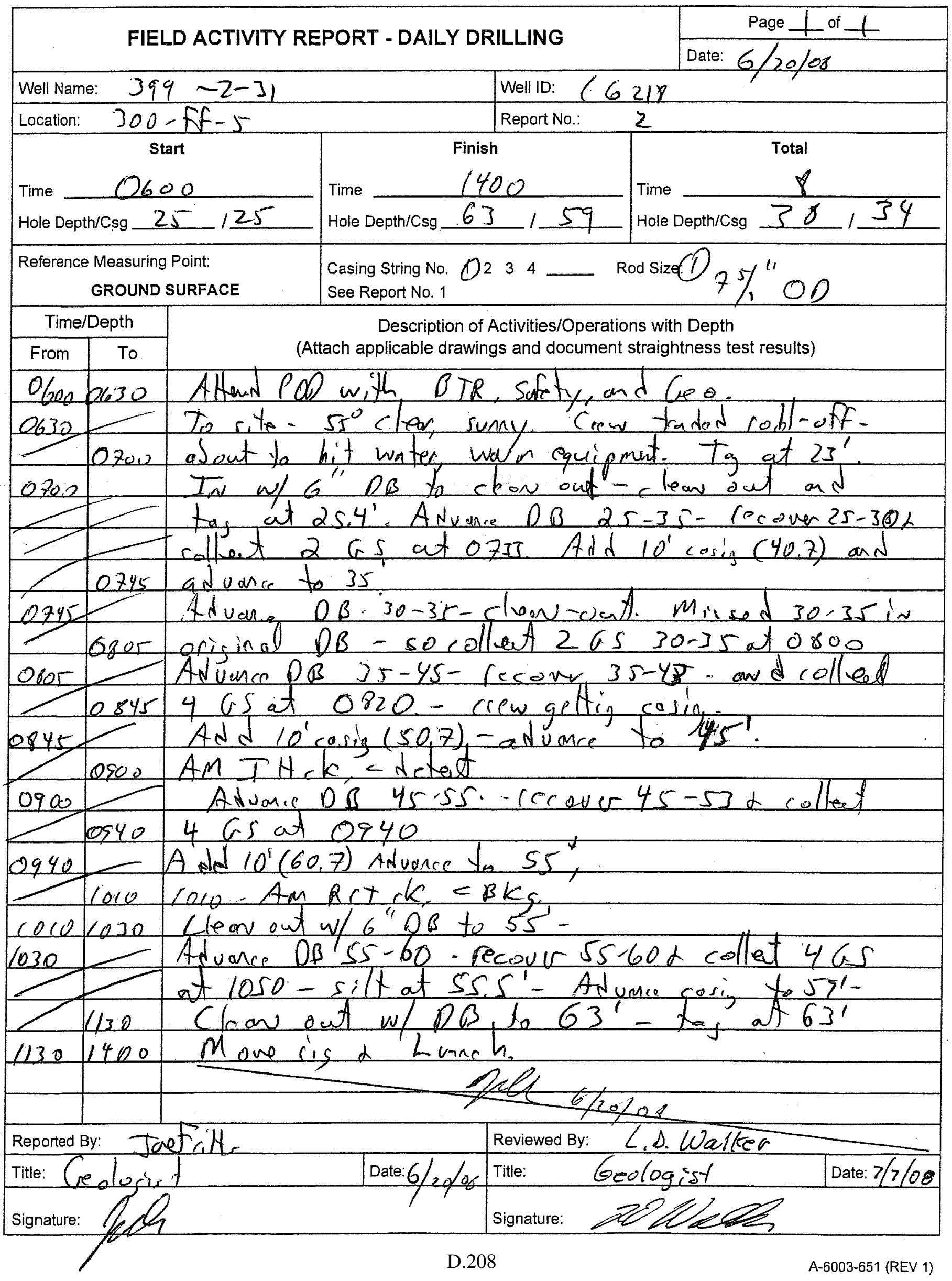




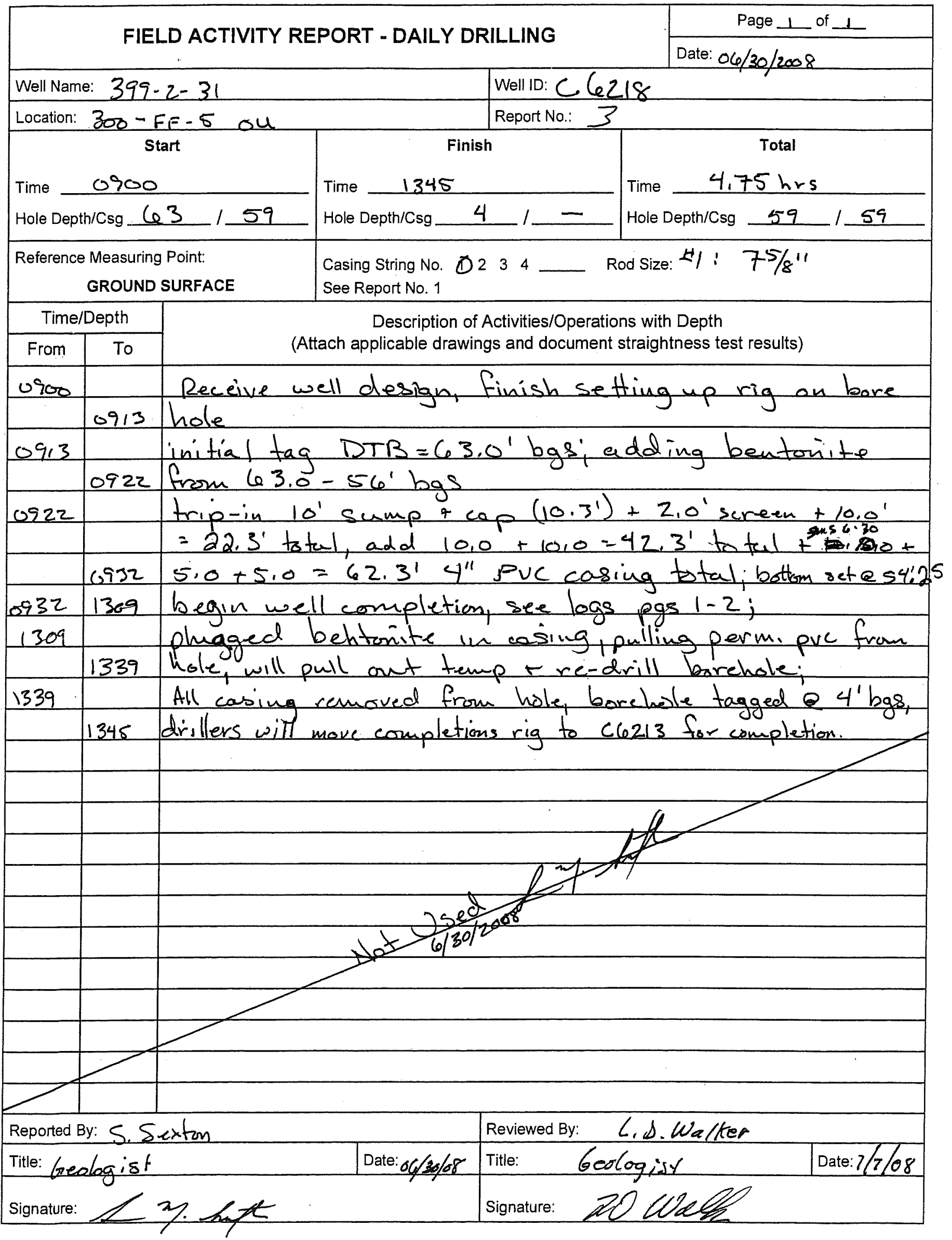




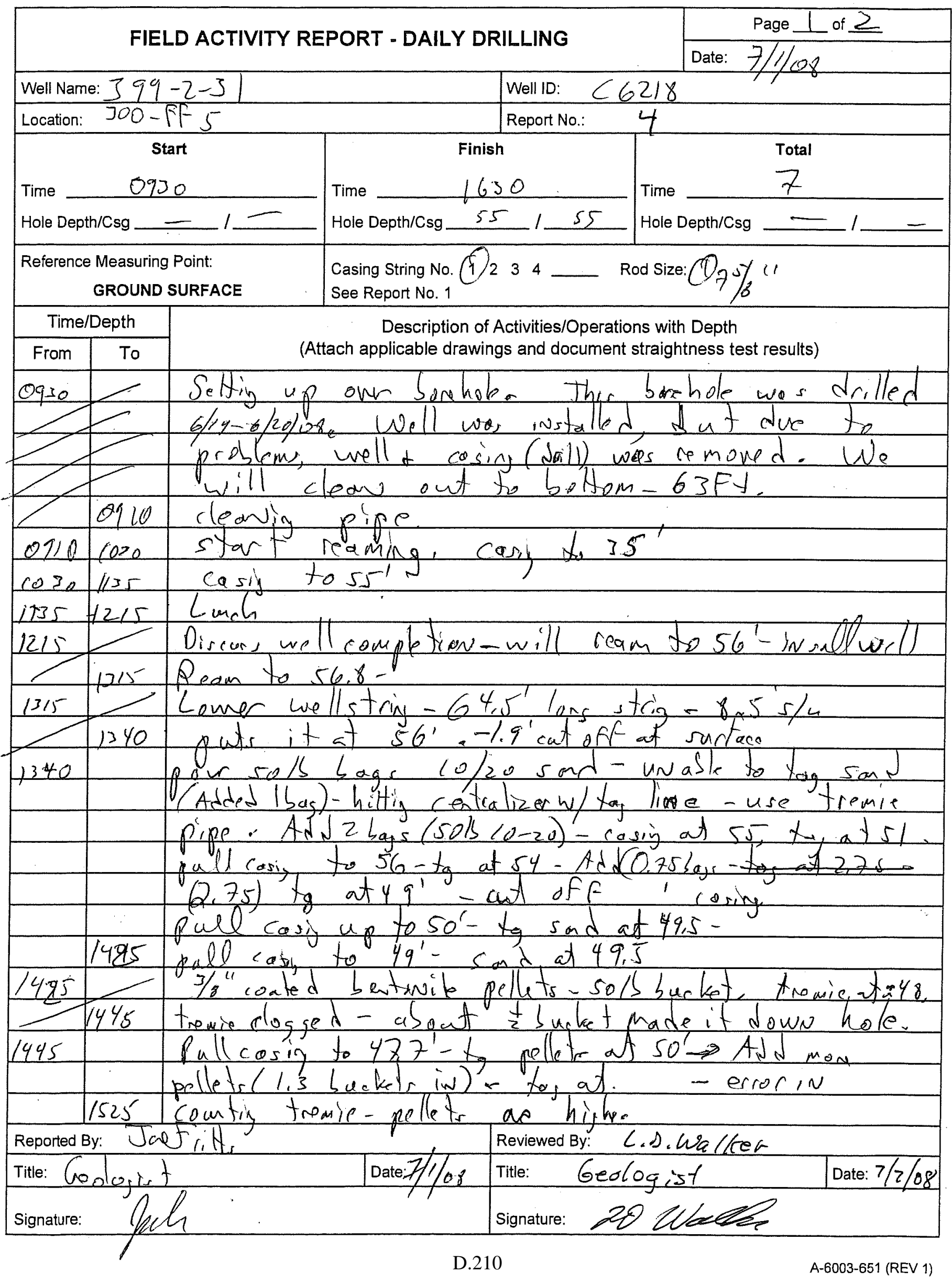


FIELD ACTIVITY REPORT - DAILY DRILLING Continuation Page

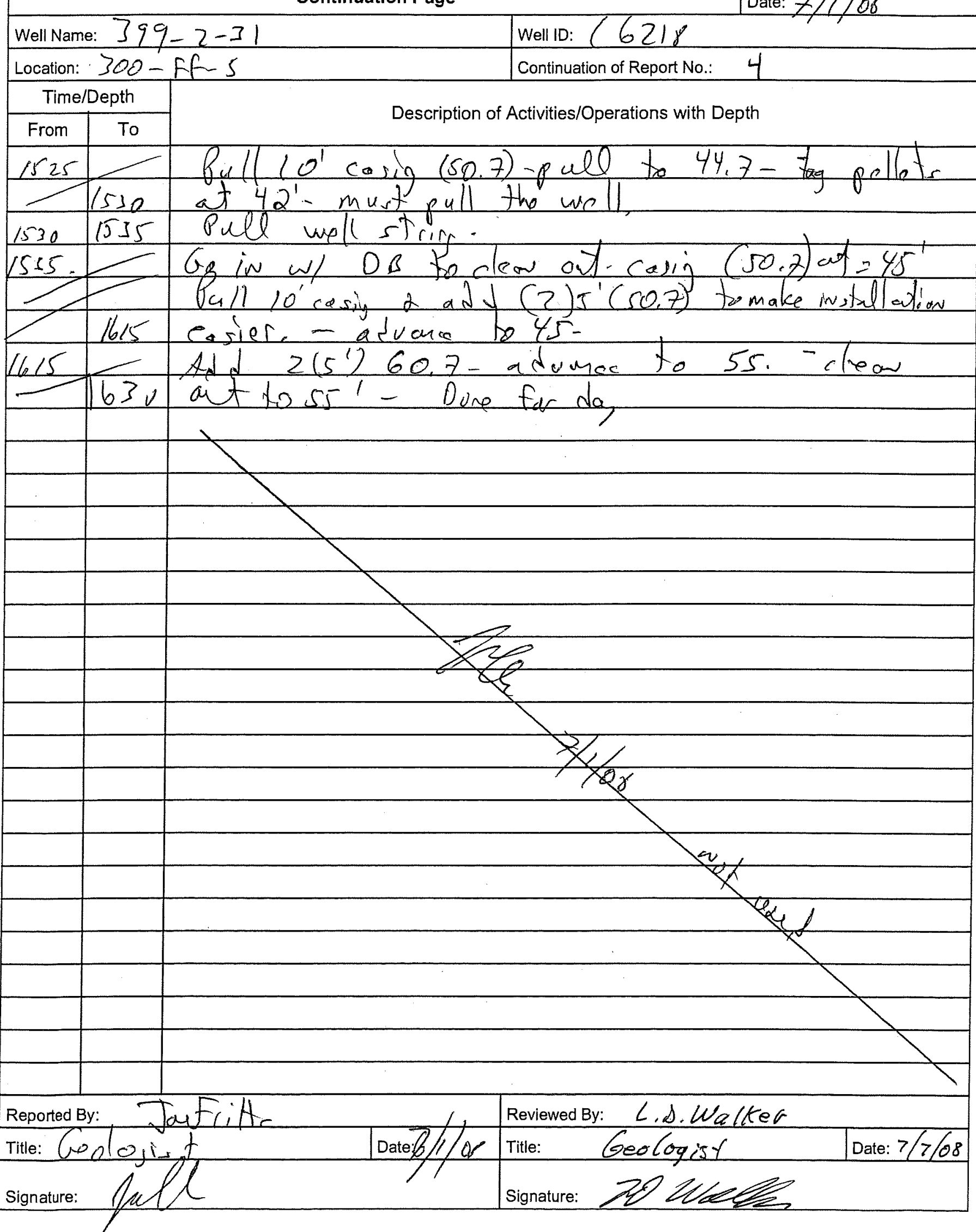




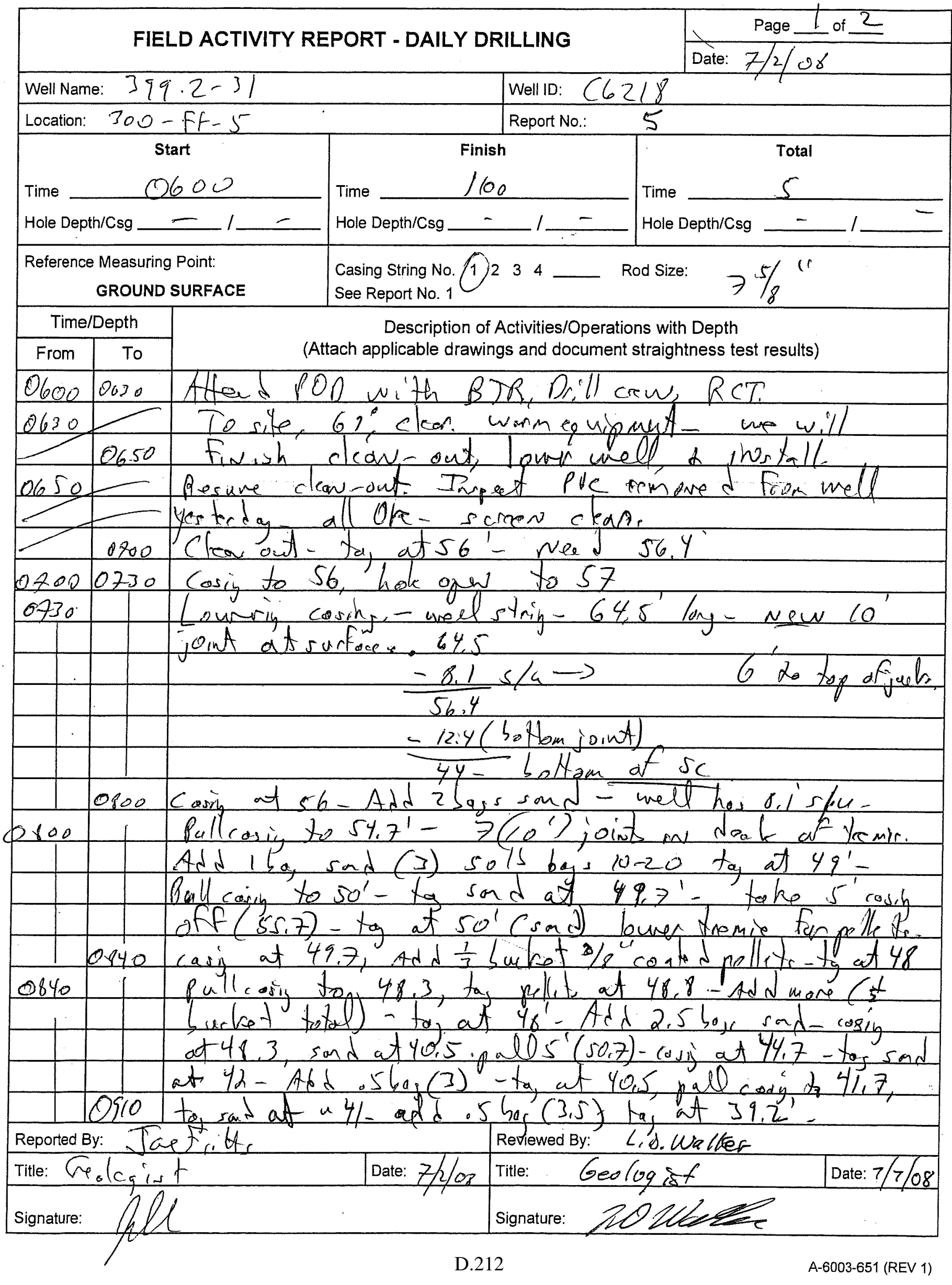




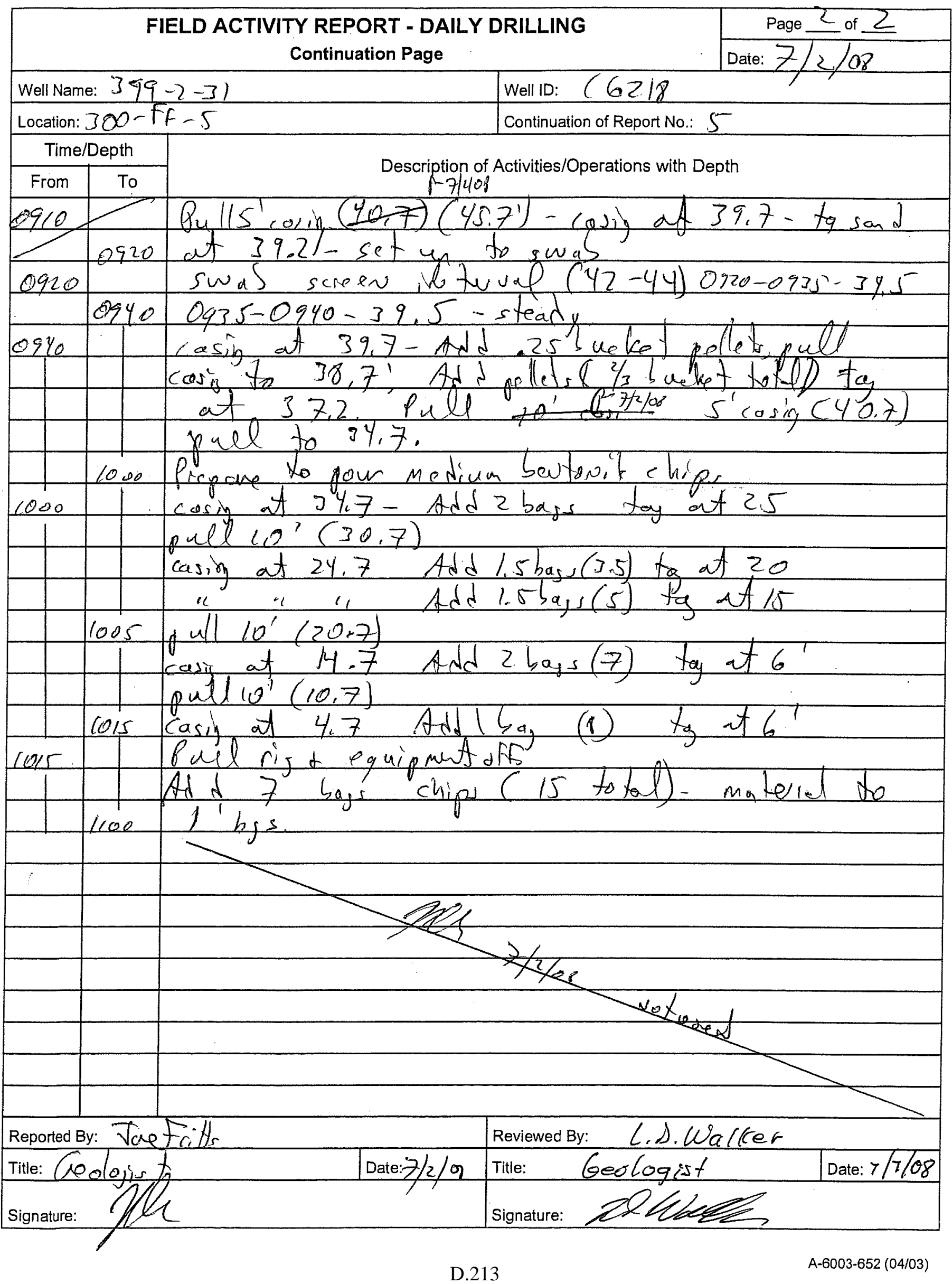




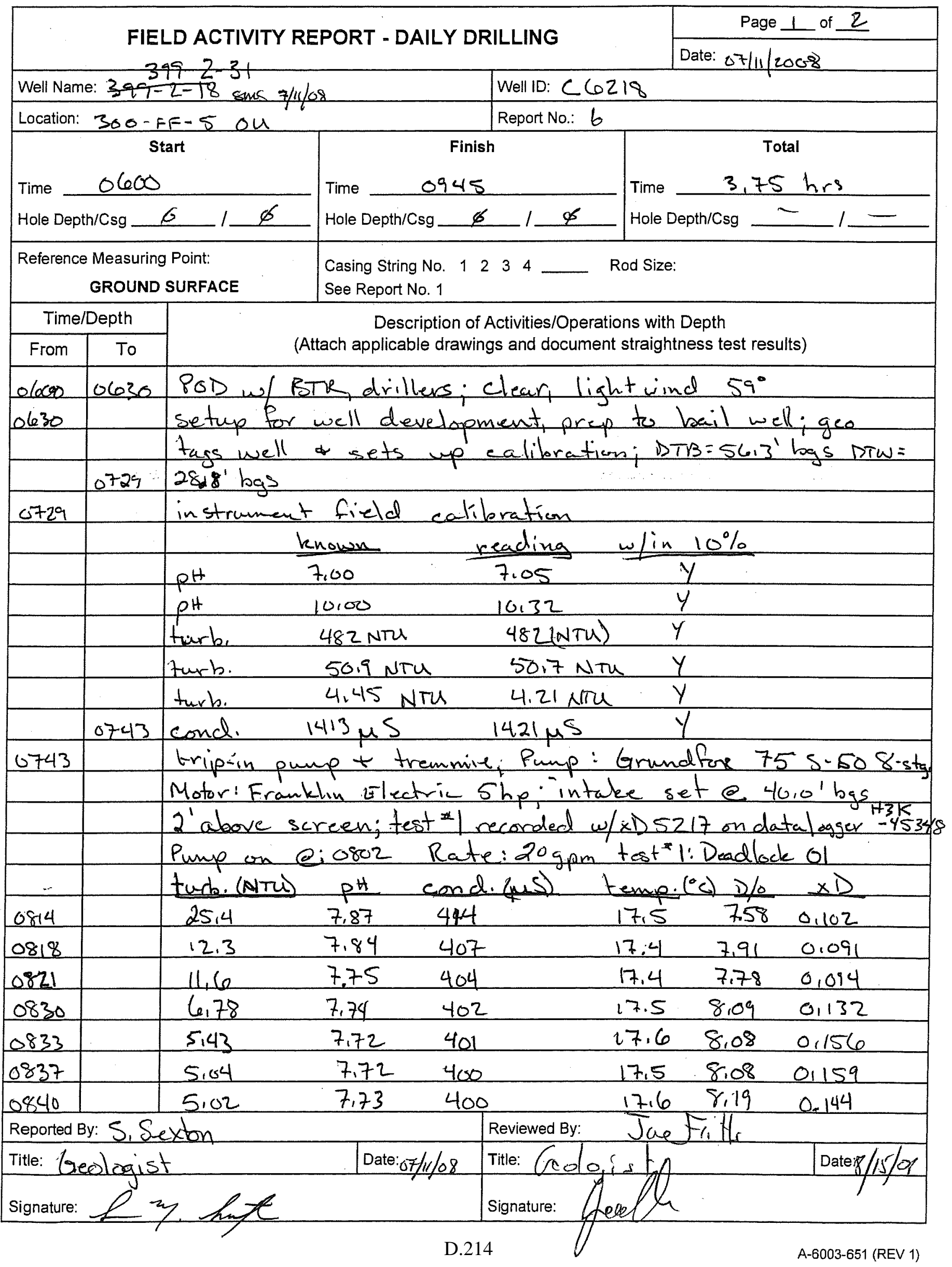




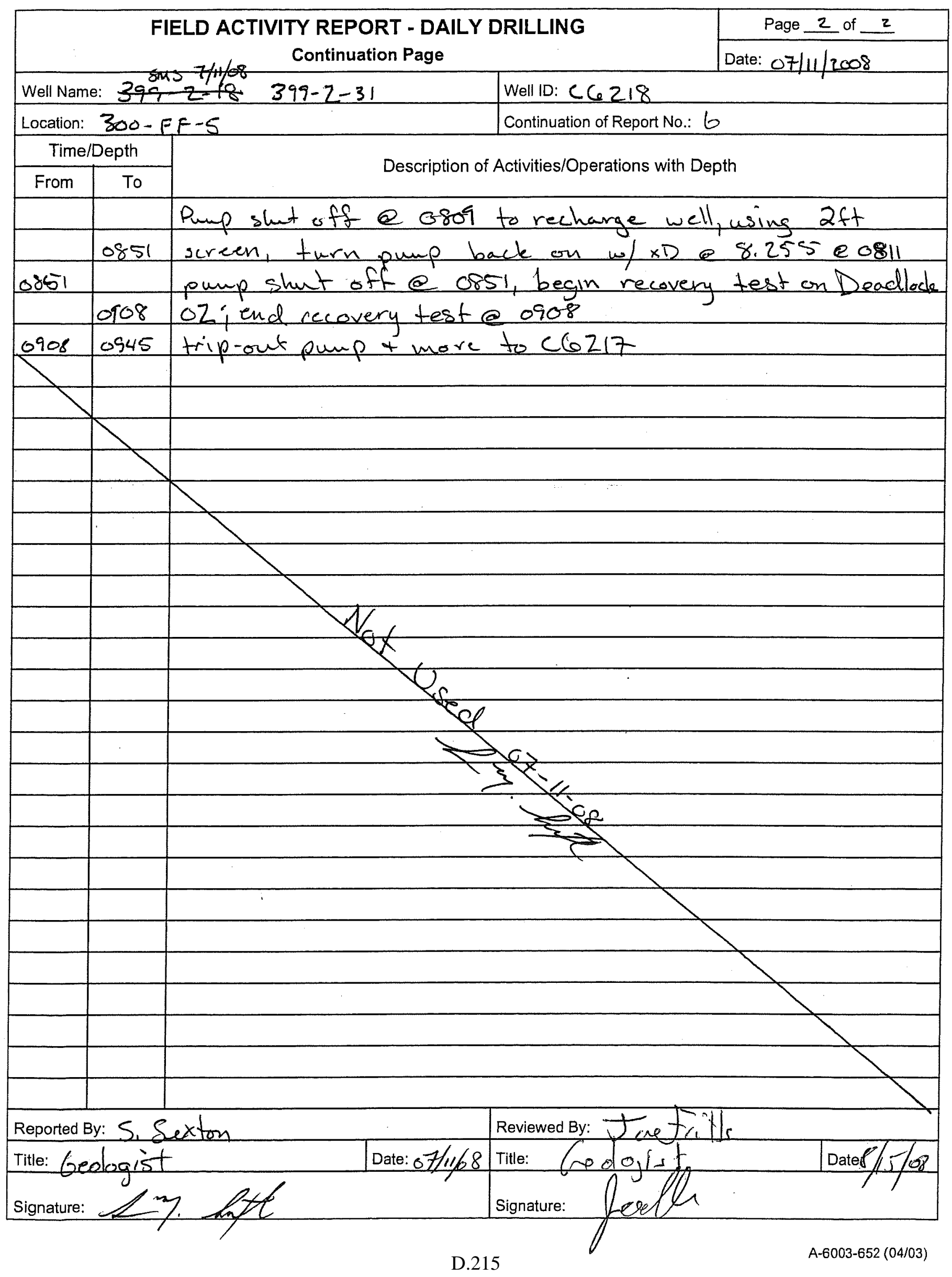




\section{WELL DEVELOPMENT AND TESTING DATA}

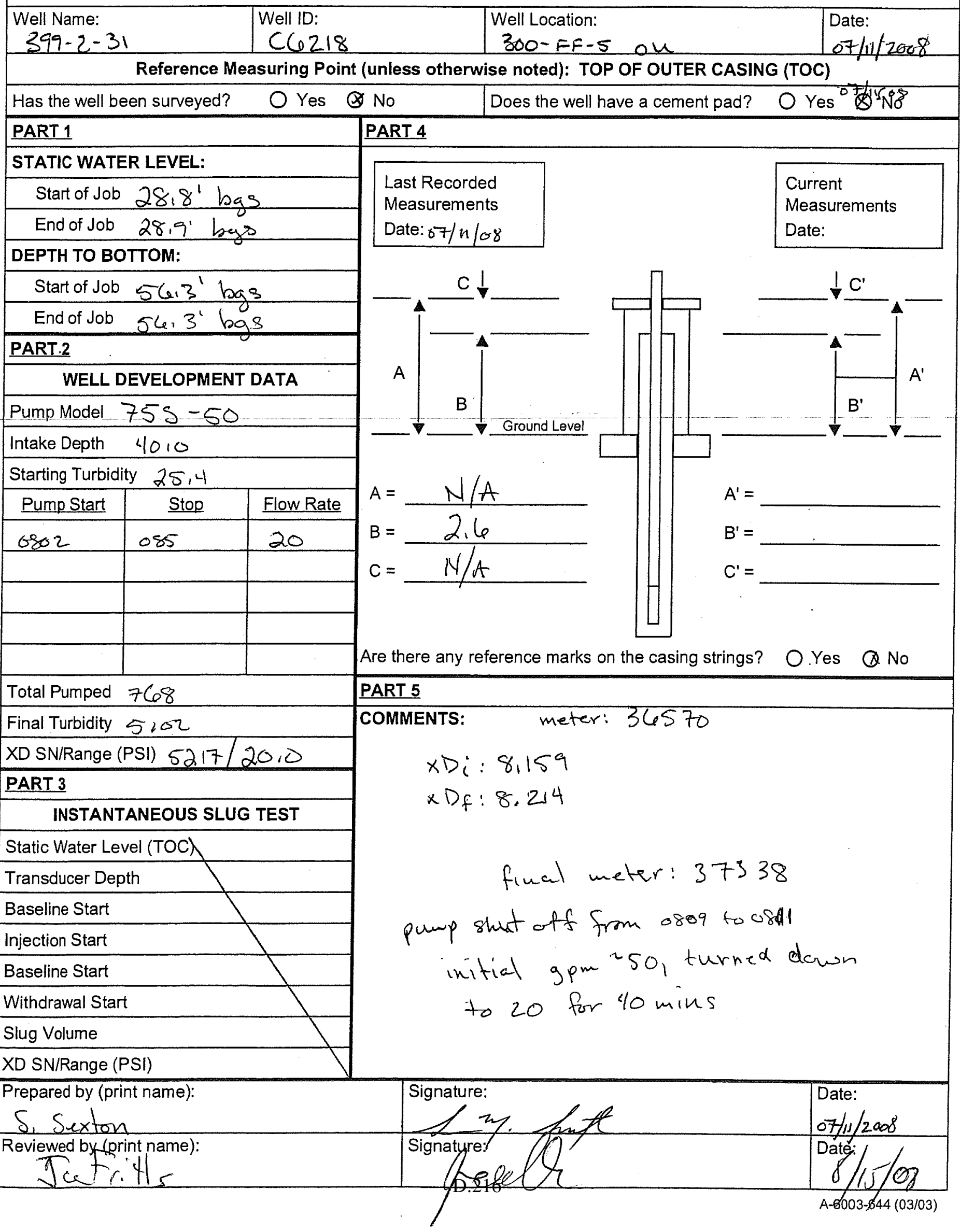


FIELD ACTIVITY REPORT NO. 1 - DRILLING PLAN

Purpose: PNNL Char acterizatiden 300 Are Location: 300 . FF 15

Well ID: $C 6194$ If Well Name: J9T-3-23

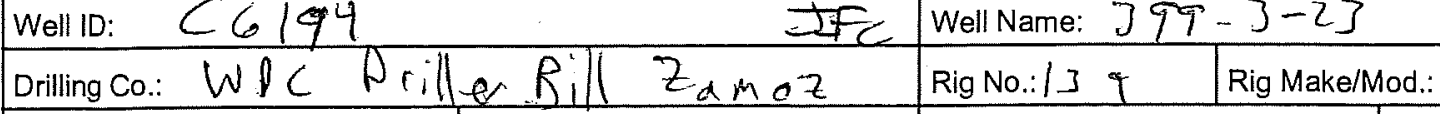

Casing String No. (1) 2334

Casing Size $75 \%$

Grade

Lbs.Per Ft.

Material carbun stel

Type:

Welded

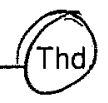

Planned / Actual

Set At: $\frac{1}{75 \% 67 / 8}$

Reference Measuring Point:

GROUND LEVEL

Drig. Co.

Casing String No. $\begin{array}{llll}1 & 2 & 3 & 4\end{array}$

Casing Size

Grade

Lbs.Per Ft.

Material

Type:

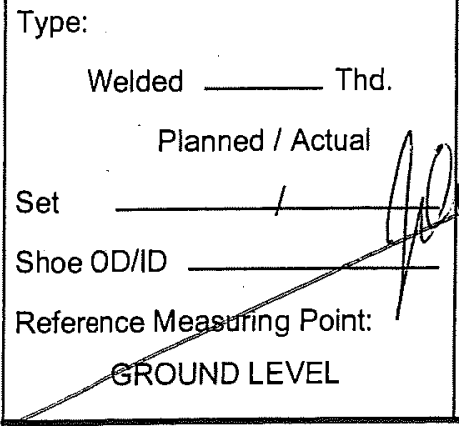

Comments/Remarks:

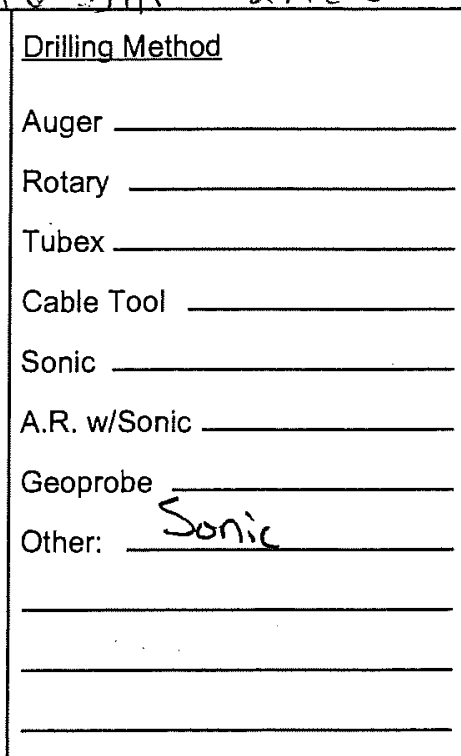

Rig No.:

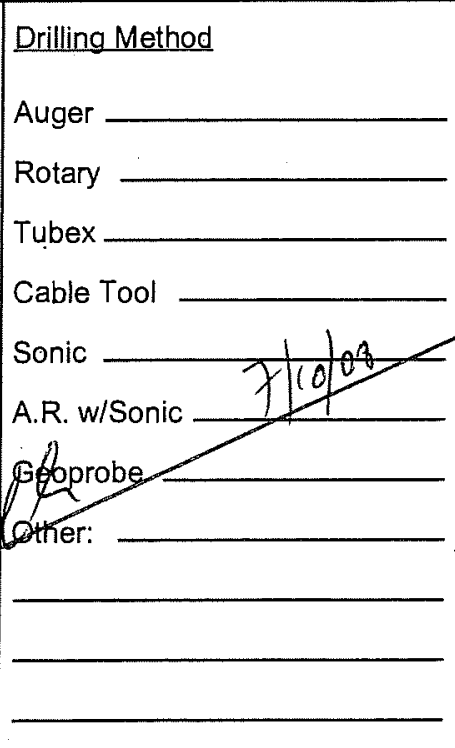

Drilling Method

Auger

Rotary

Tubex

Cable Too

Sonic

A.R. w/Sonic

Circulation

Air _ Water/Mud

Reverse —_ Direct

Vol: cfm

gpm

Pressure

Drill Pipe O.D.

Tool Joint Size
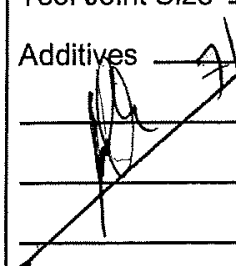

Page 1 of 3

$160 / 08$

5

D.H. Hammer

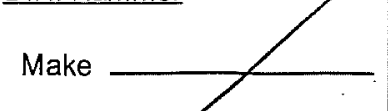

Choke

Casing Hammer

Make

Model

Bit Size

Type

Nozzles

Rod Size

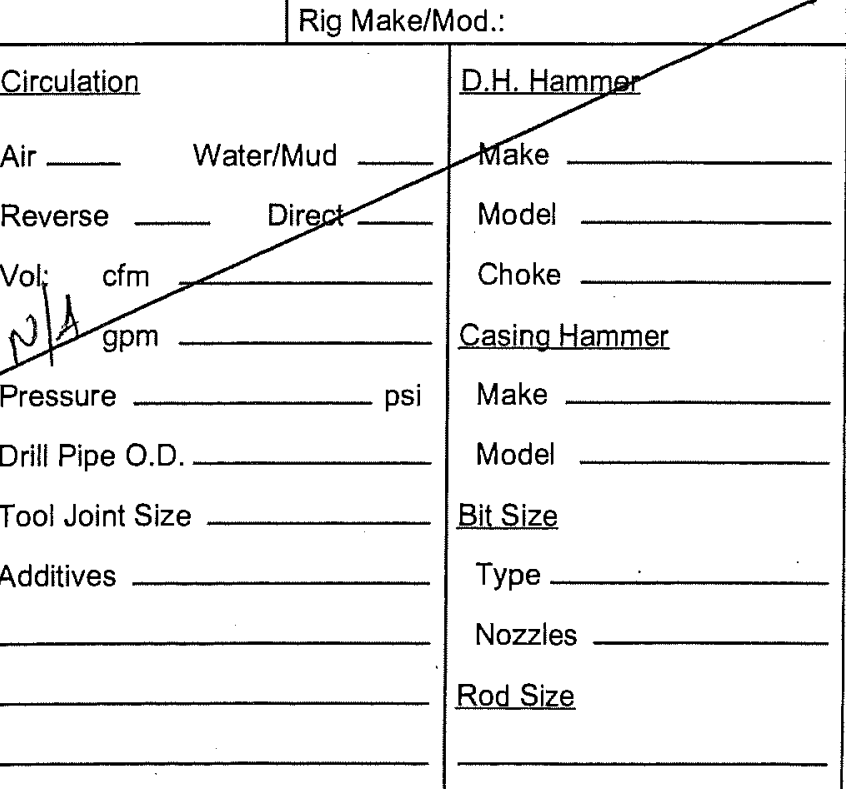

Estimated Depth to Water

Reported By: Juefitt,

Nameritle: GRAMFr, goologirt

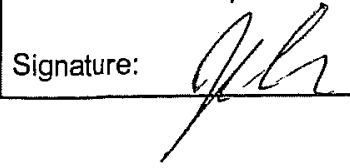

Date: $7 / 10 / 00$ 


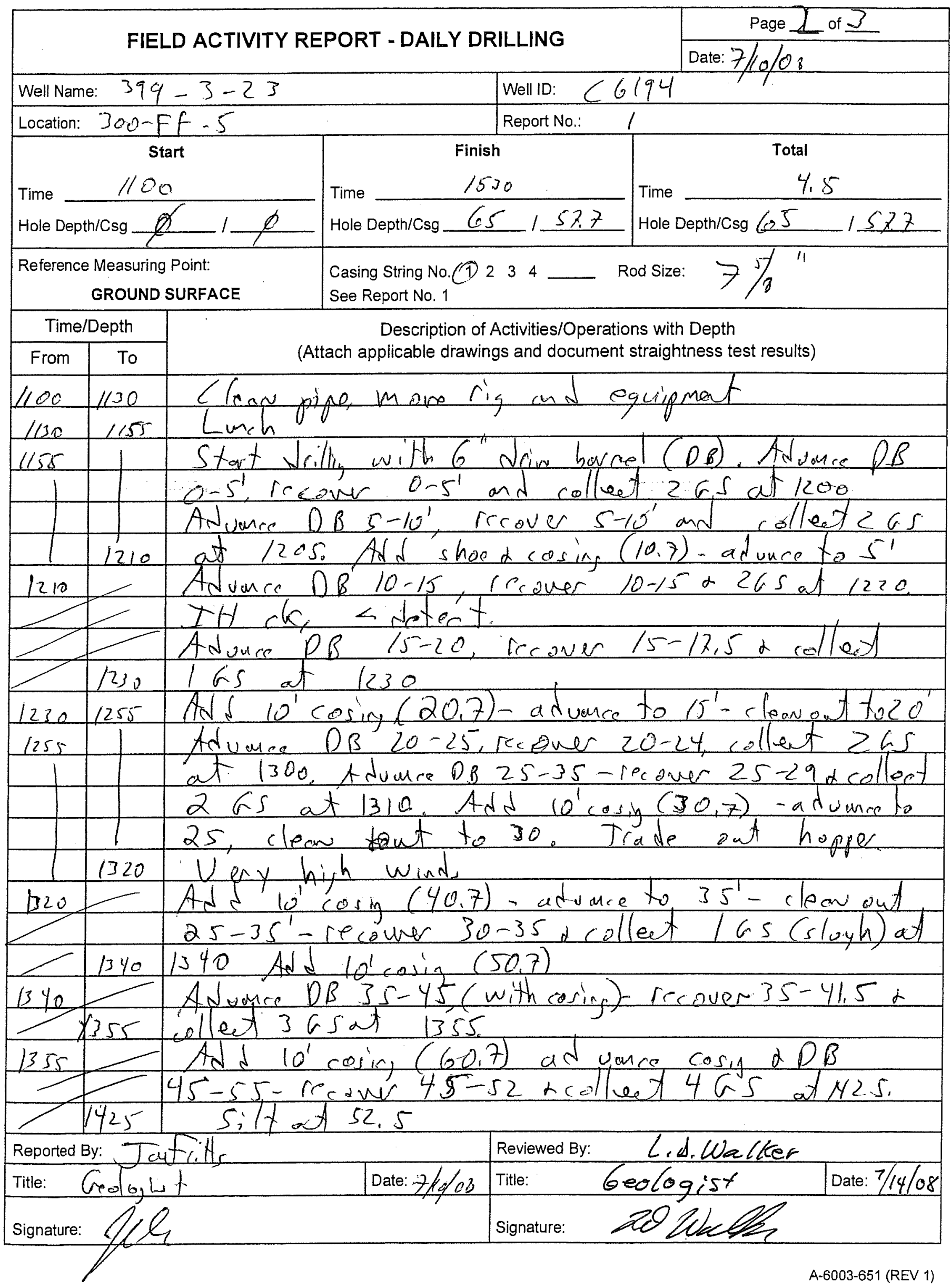

D.218 
FIELD ACTIVITY REPORT - DAILY DRILLING Continuation Page

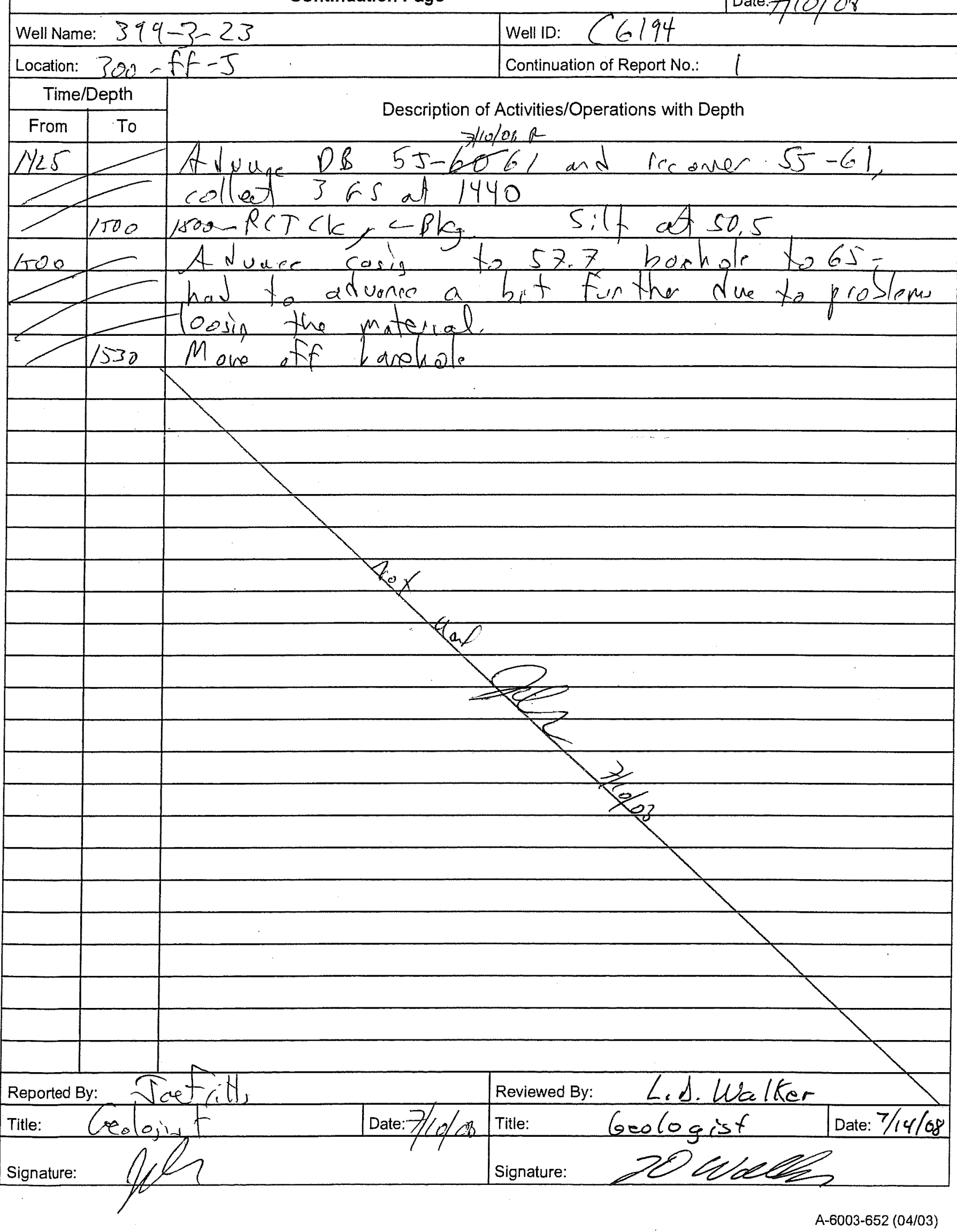

D.219 


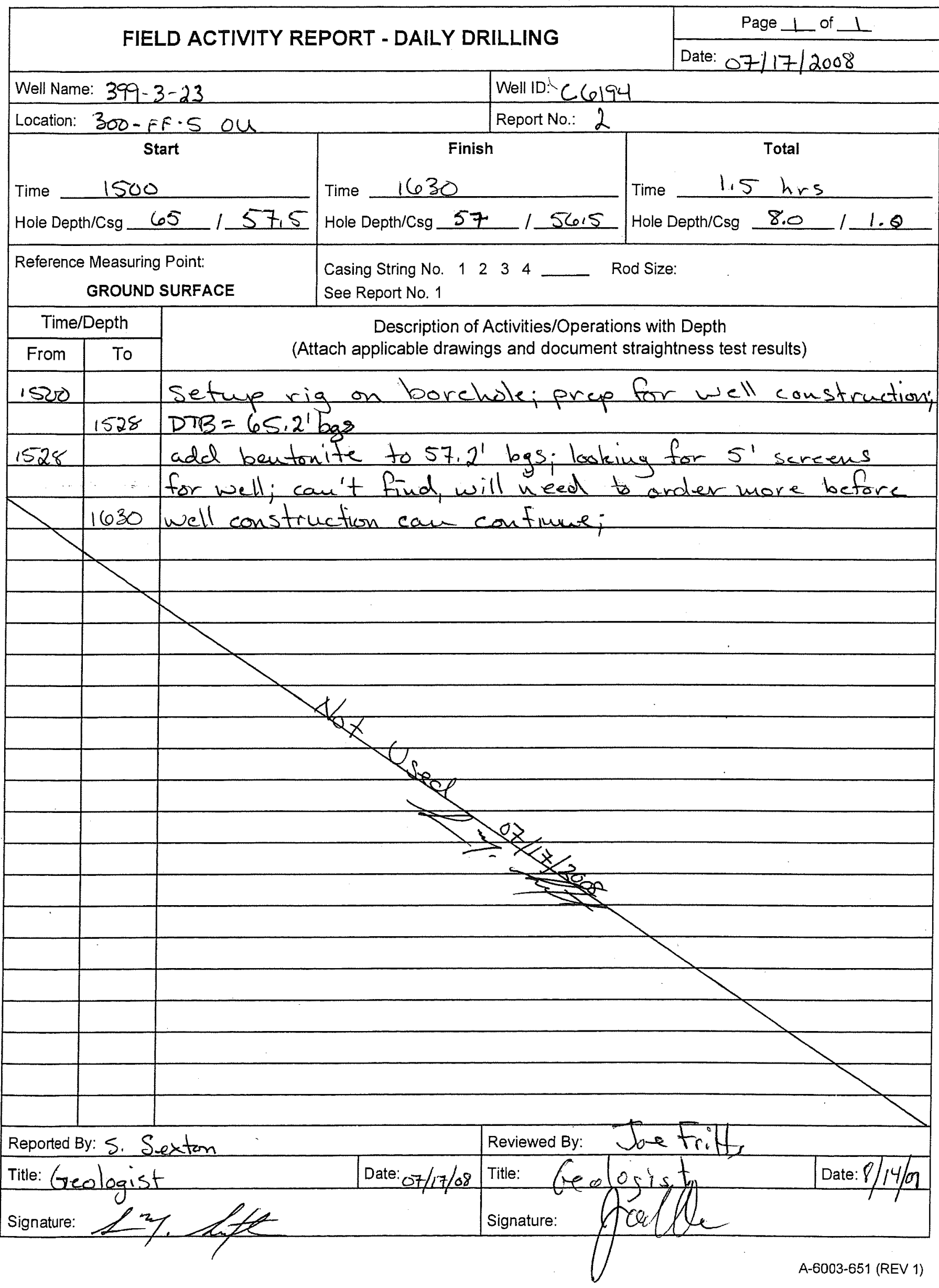

D. 220 


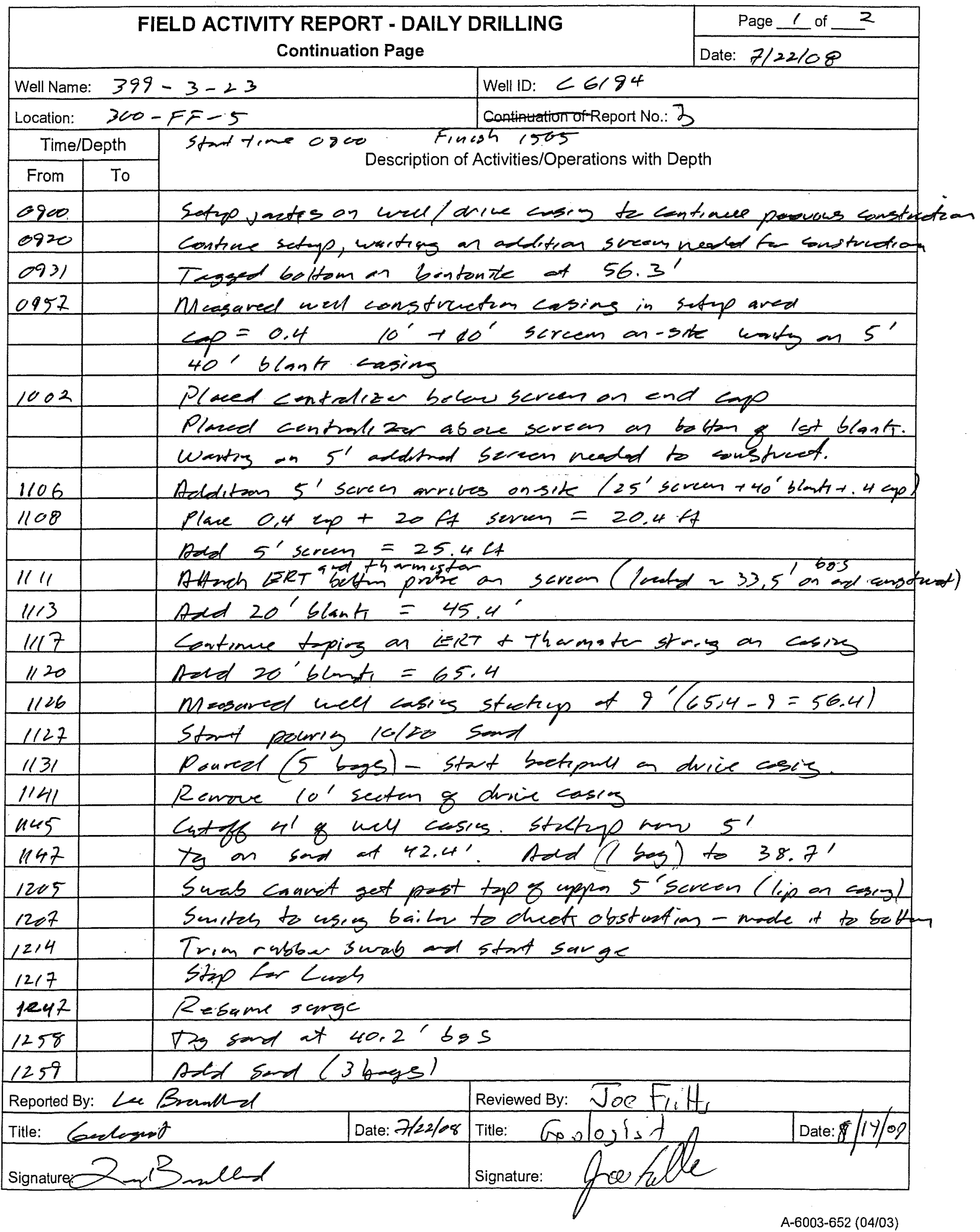

D.221 


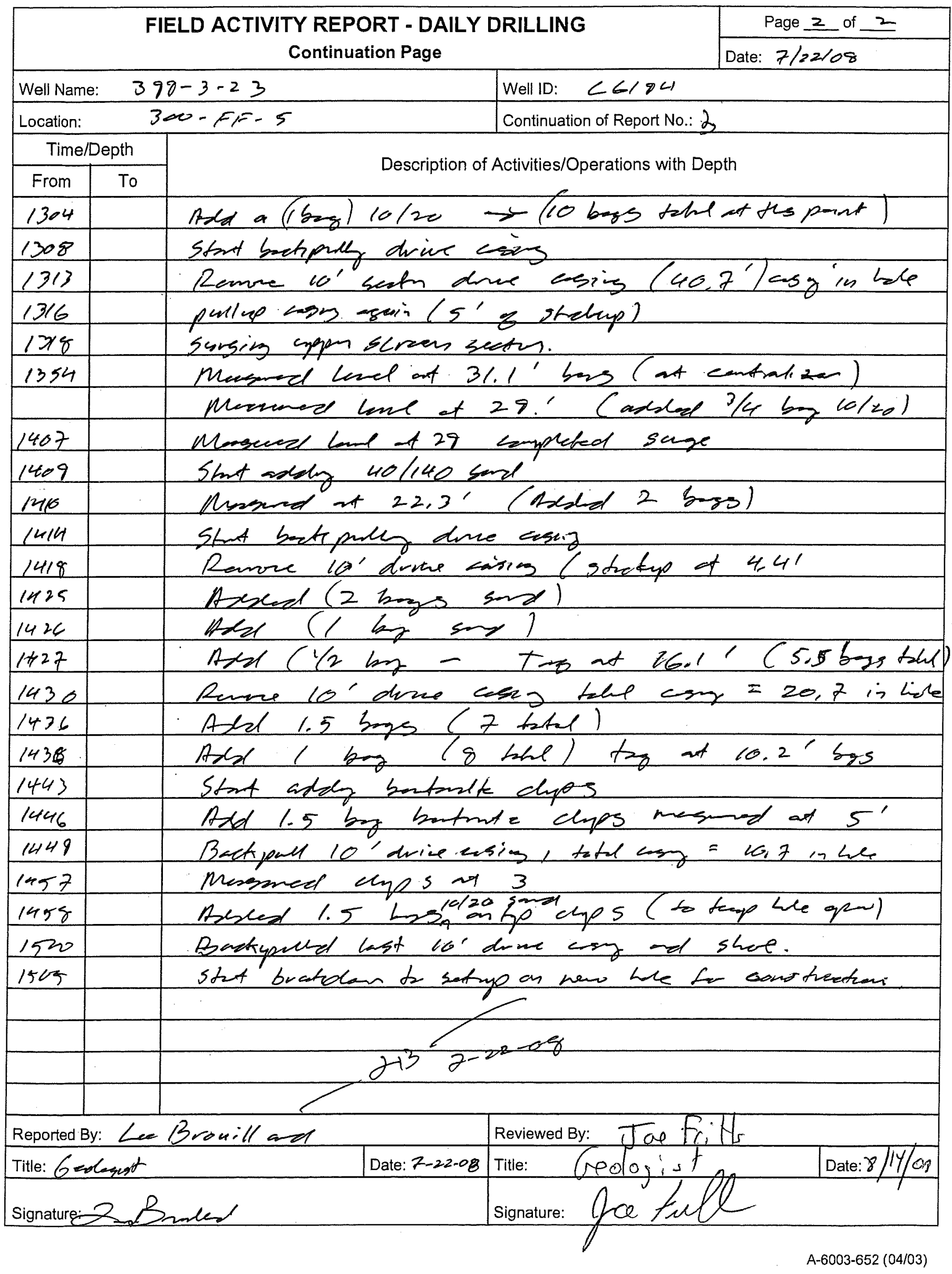

D.222 


\section{FIELD ACTIVITY REPORT - DAILY DRILLING}

\begin{tabular}{|l|} 
Page $\perp$ of 1 \\
\hline Date: $7-31-08$
\end{tabular}

\begin{tabular}{l}
\hline Well Name: $399-3-23$ \\
\hline Location: $300-$ F F - 5 \\
Start \\
Time $\frac{1648}{\text { Hole Depth/Csg } 57.0}$ \\
\hline $\begin{array}{c}\text { Reference Measuring Point: } \\
\text { GROUND SURFACE }\end{array}$ \\
\hline
\end{tabular}

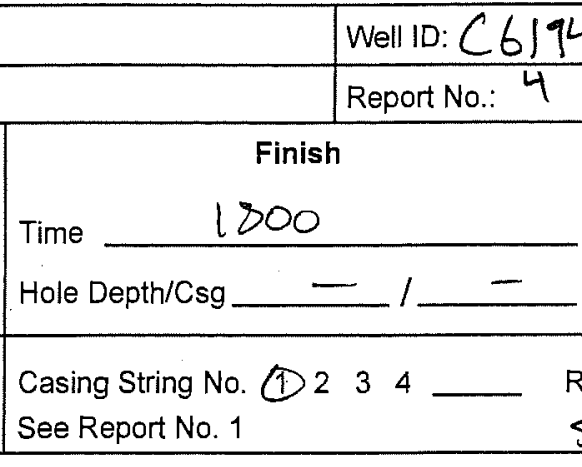

\section{Description of Activities/Operations with Depth}

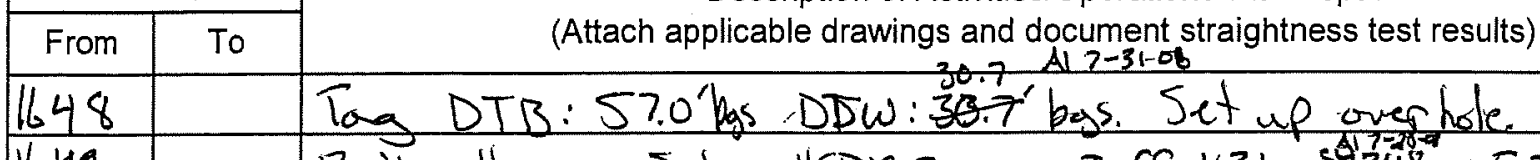

Total

Time $2 \mathrm{hr} 12 \mathrm{nin}$ Hole Depth/Csg _ _ $/$

Rod Size: 4 "PVC 1649 Bail well Set up HERMIT vame: Jeff H3k-\$\$348-45348

$1654 \quad$ Trip in fumpt Tremmic $\rightarrow$ Set pump nake e $41.5^{\prime}$ by $5 .=10.5^{\circ}$ below top of vereen

\begin{tabular}{|c|c|c|c|c|c|c|c|c|}
\hline & Test\# 3 & $X D_{I}: t$ & .76 & & & $=t$ Tian & sodver@ 37 . & 5 bas. 5. \\
\hline 1658 & & $x D(f)$ & Turb(uTu) & $\operatorname{Tem}\left(\Omega^{\circ} \mathrm{C}\right)$ & $D O\left(\frac{n v}{L}\right)$ & $\mathrm{pH}^{\mathrm{H}}$ & Cond. (mS) & Notes: \\
\hline 1700 & & - & - & - & - & - & $\begin{array}{c}7-31-08 \\
-404 \\
\end{array}$ & $\sim 50 \mathrm{gpm}$ \\
\hline 1705 & & 6.38 & 270 & 20.8 & 8.10 & 7.73 & 5.054 & uso gpm \\
\hline 1710 & & 6.39 & 24.8 & 19.3 & 8.46 & 7.56 & 0.501 & 11 \\
\hline 1715 & & 6.38 & 6.0 & 18.7 & 8.50 & 7.51 & 0.498 & " \\
\hline 1720 & & 6.39 & 2.25 & 18.2 & 8.58 & 7.51 & 0.498 & 4 \\
\hline 1725 & & 6.38 & 1.79 & 18.4 & 8.60 & 7.51 & 0.501 & $"$ \\
\hline 1730 & & 6.379 & 1.62 & 18.4 & 8.52 & 7.53 & 0.497 & 11 \\
\hline 1735 & & 6.38 & 1.38 & 18.1 & 8.64 & 7.53 & 0.497 & "Purge Truk foul \\
\hline 1735 & & \multicolumn{7}{|c|}{ Stort Recovery Test on Test \#4 } \\
\hline 1746 & & \multicolumn{7}{|c|}{ Stop finish Test } \\
\hline 1800 & & \multirow{2}{*}{\multicolumn{7}{|c|}{$\begin{array}{l}\text { BTR stop work before Deuctopnent of lower interal begins. } \\
\text { Geo deports for office to conplete poferwork. }\end{array}$}} \\
\hline & & & & & & & & \\
\hline
\end{tabular}

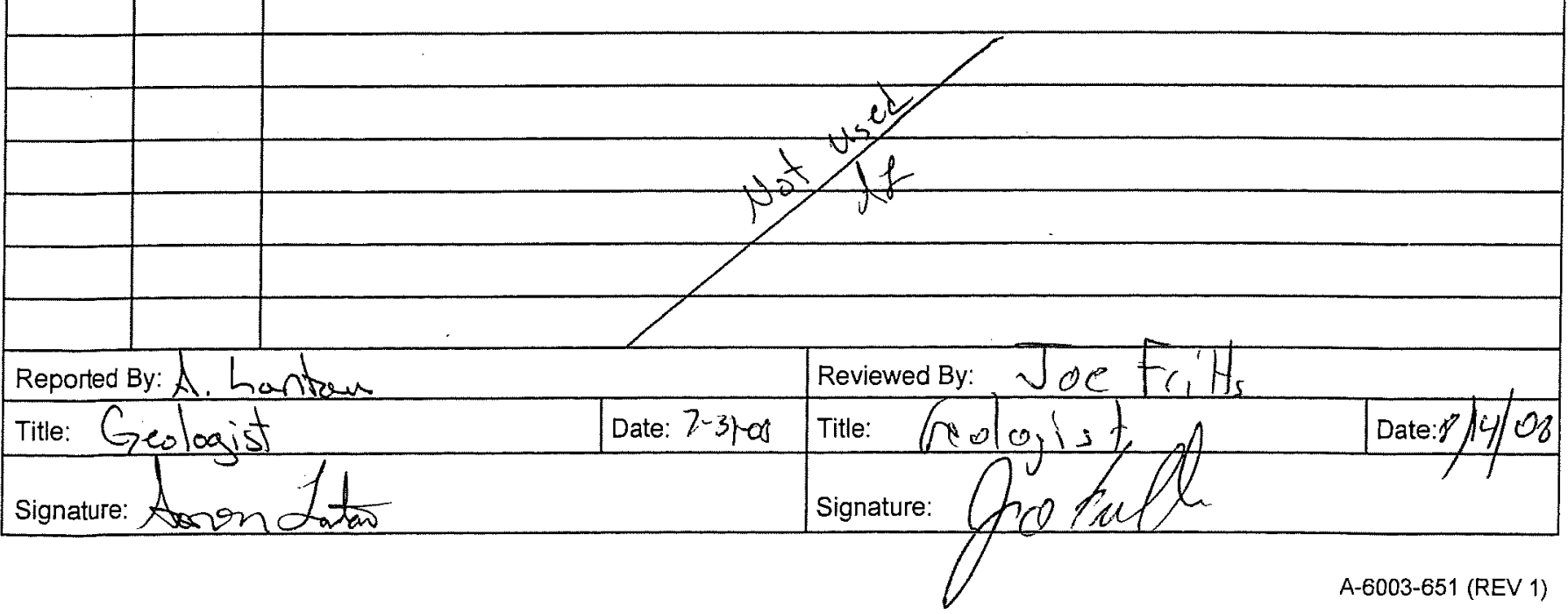




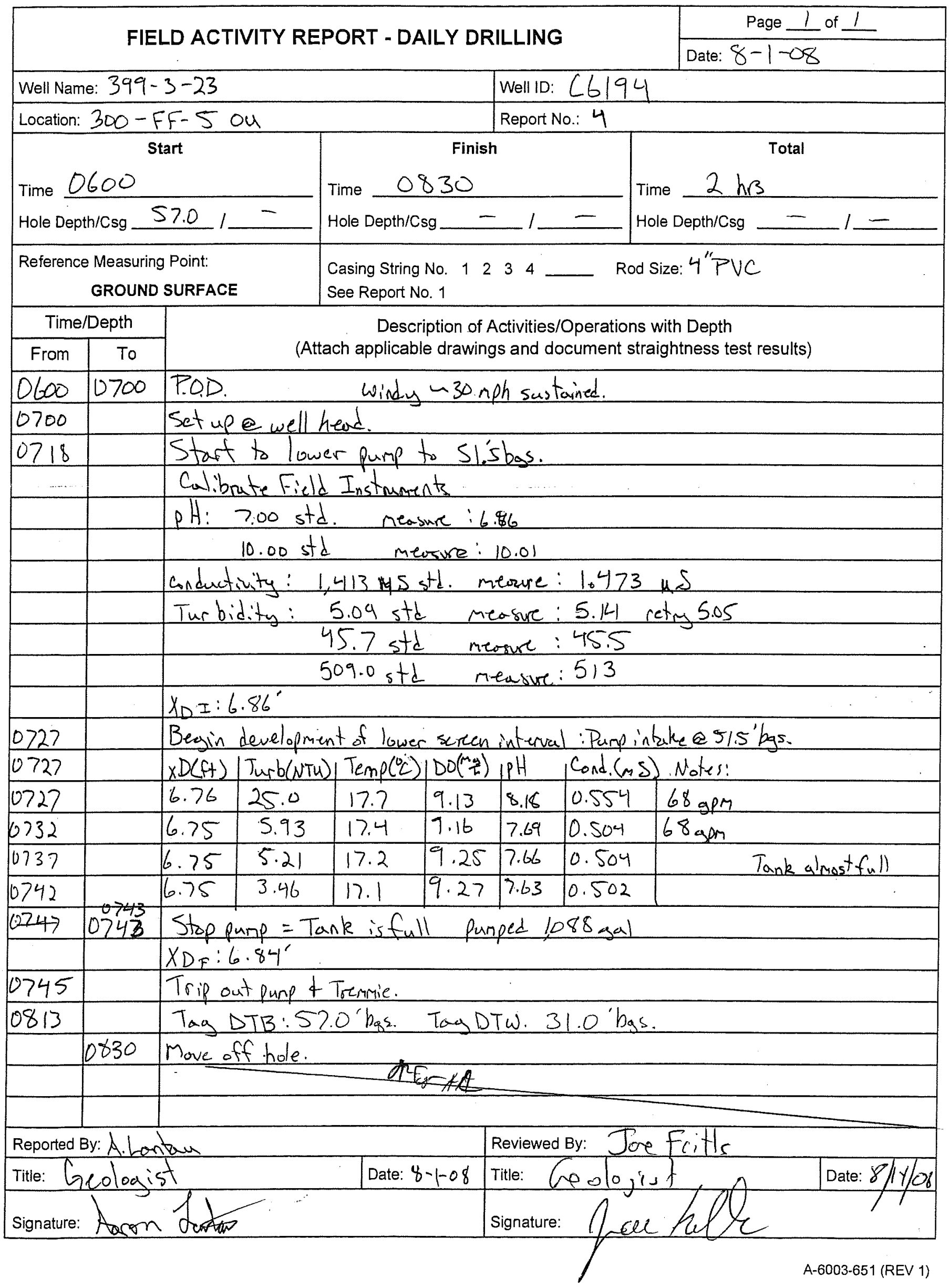

D.224 


\section{WELL DEVELOPMENT AND TESTING DATA}

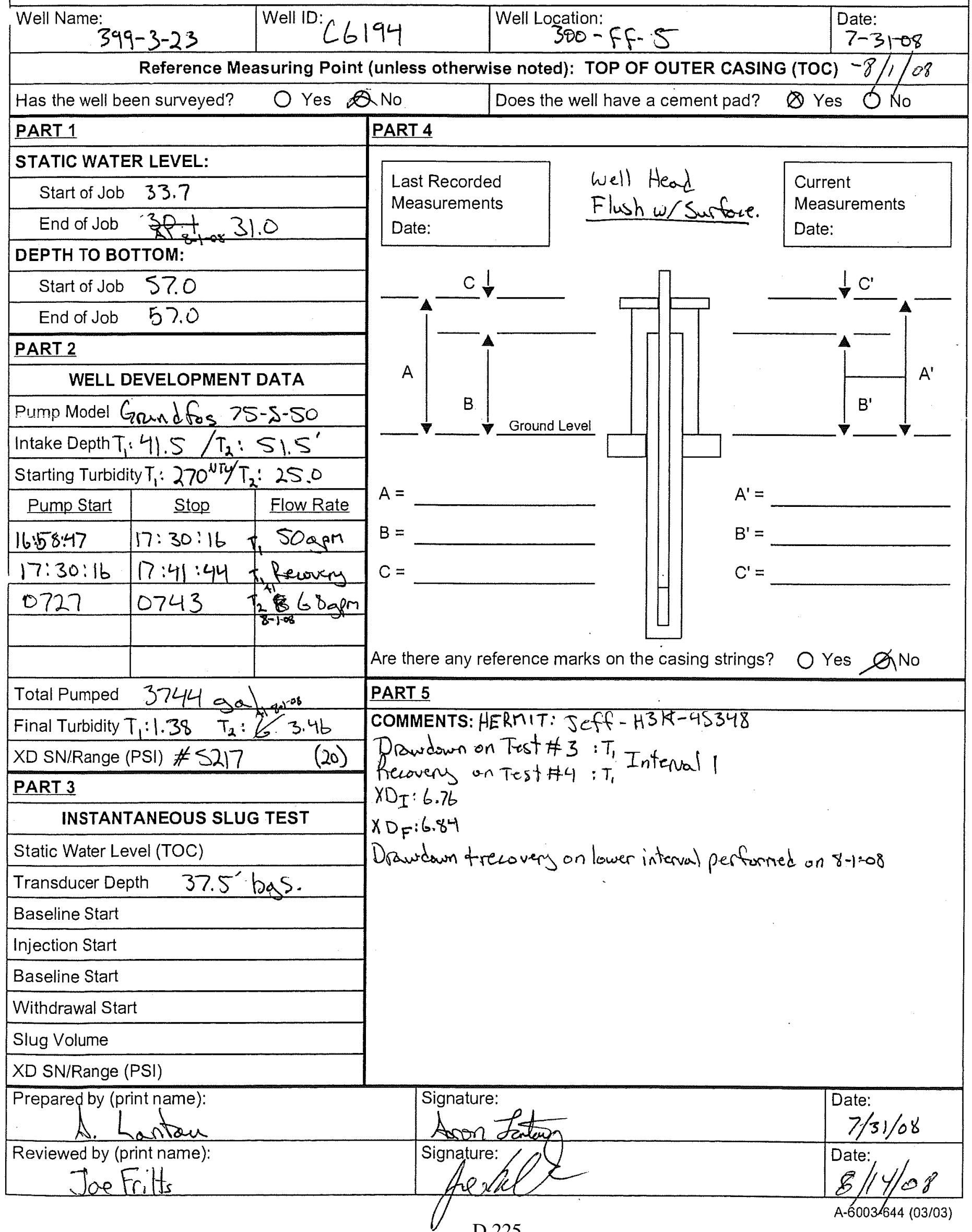


FIELD ACTIVITY REPORT NO. 1 - DRILLING PLAN

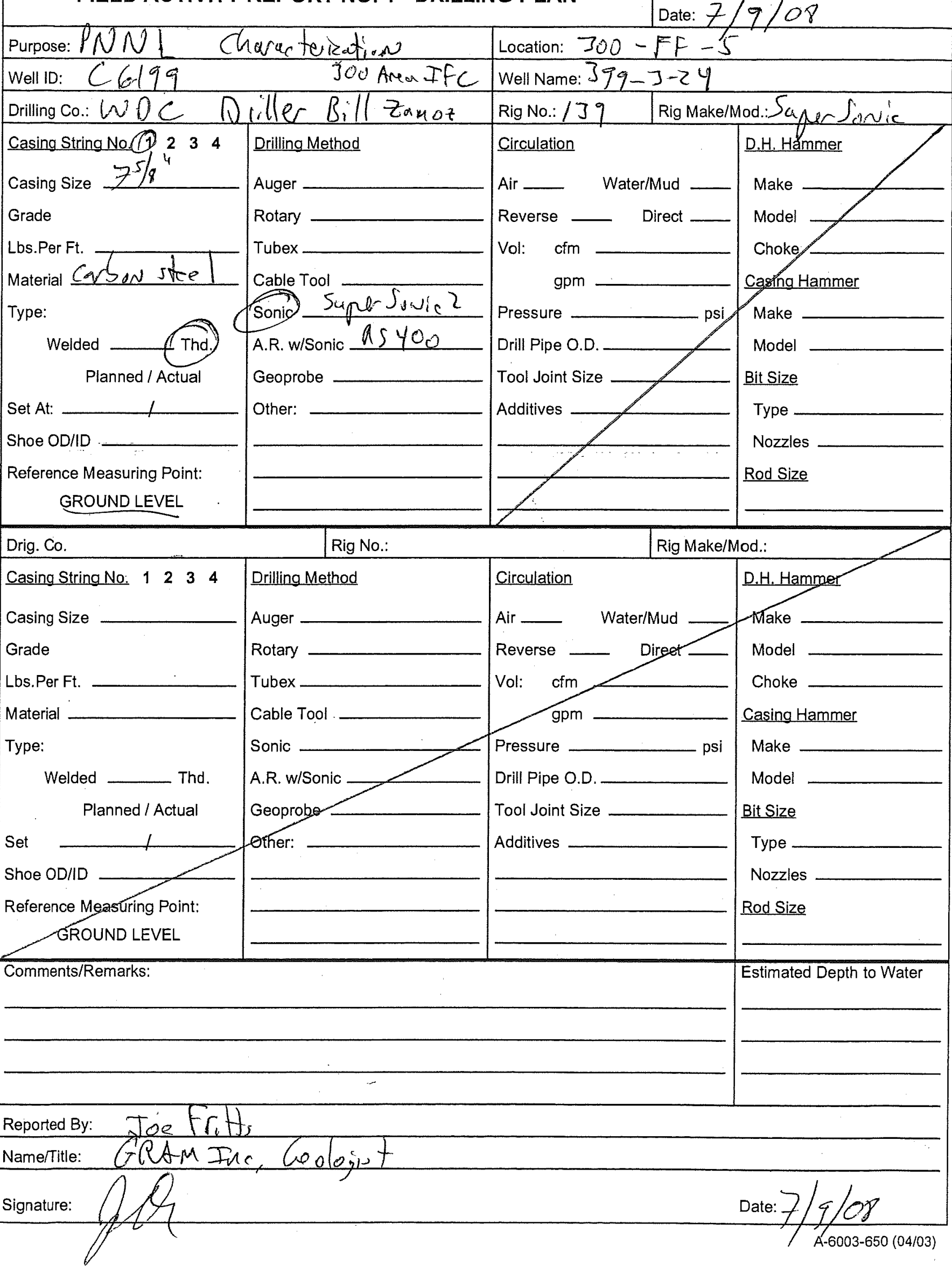




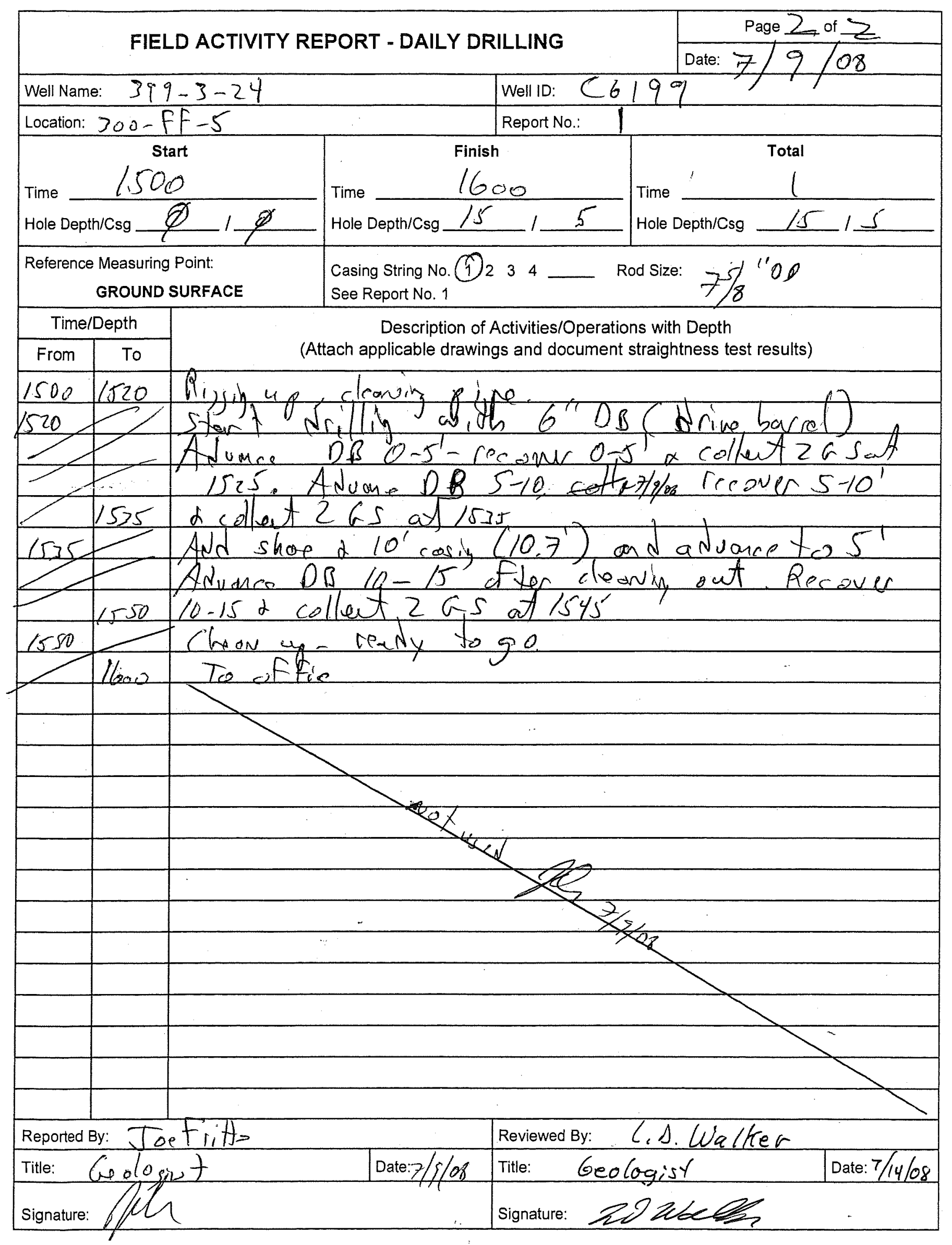

A-6003-651 (REV 1)

D. 227 


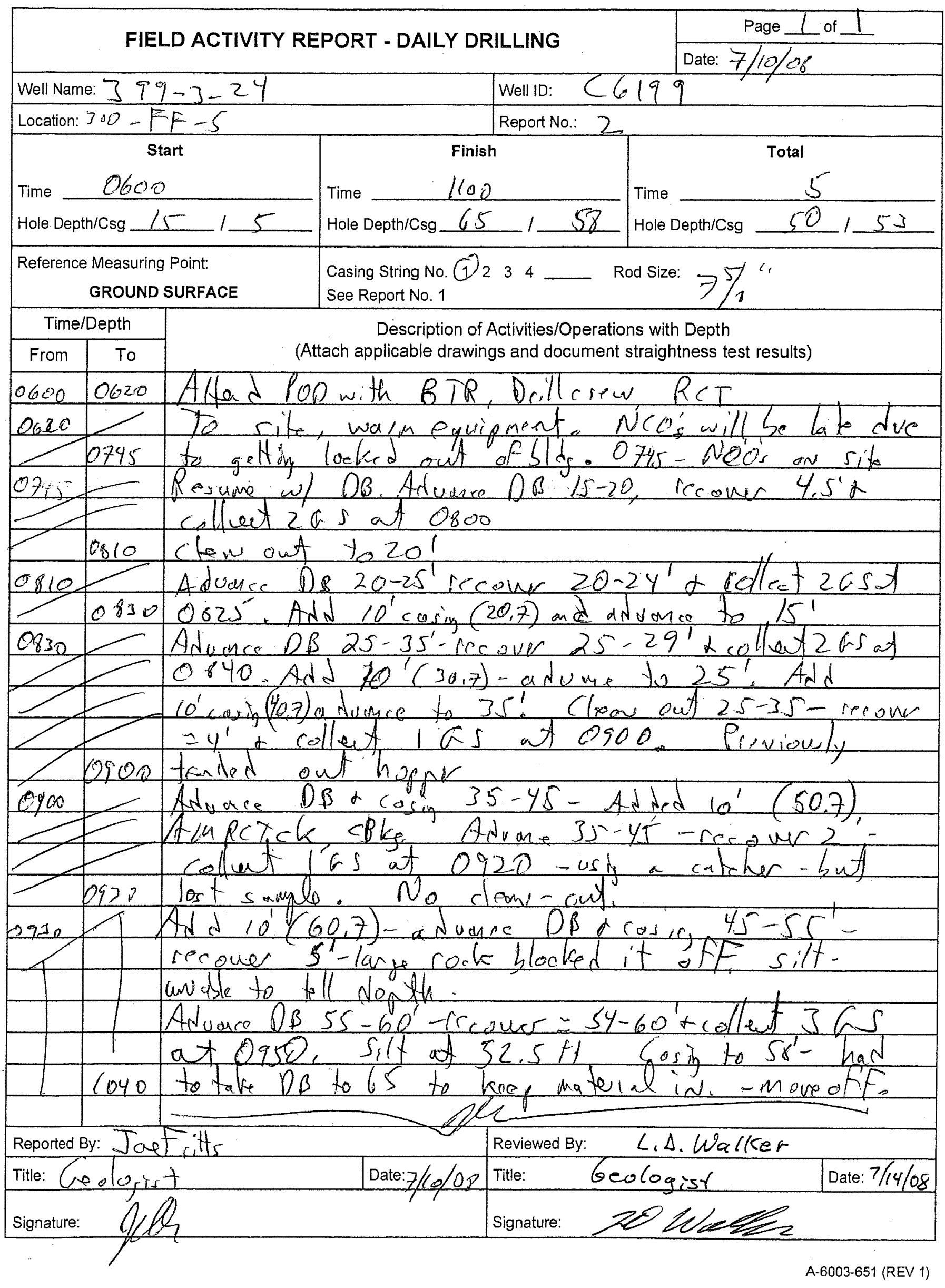

D. 228 


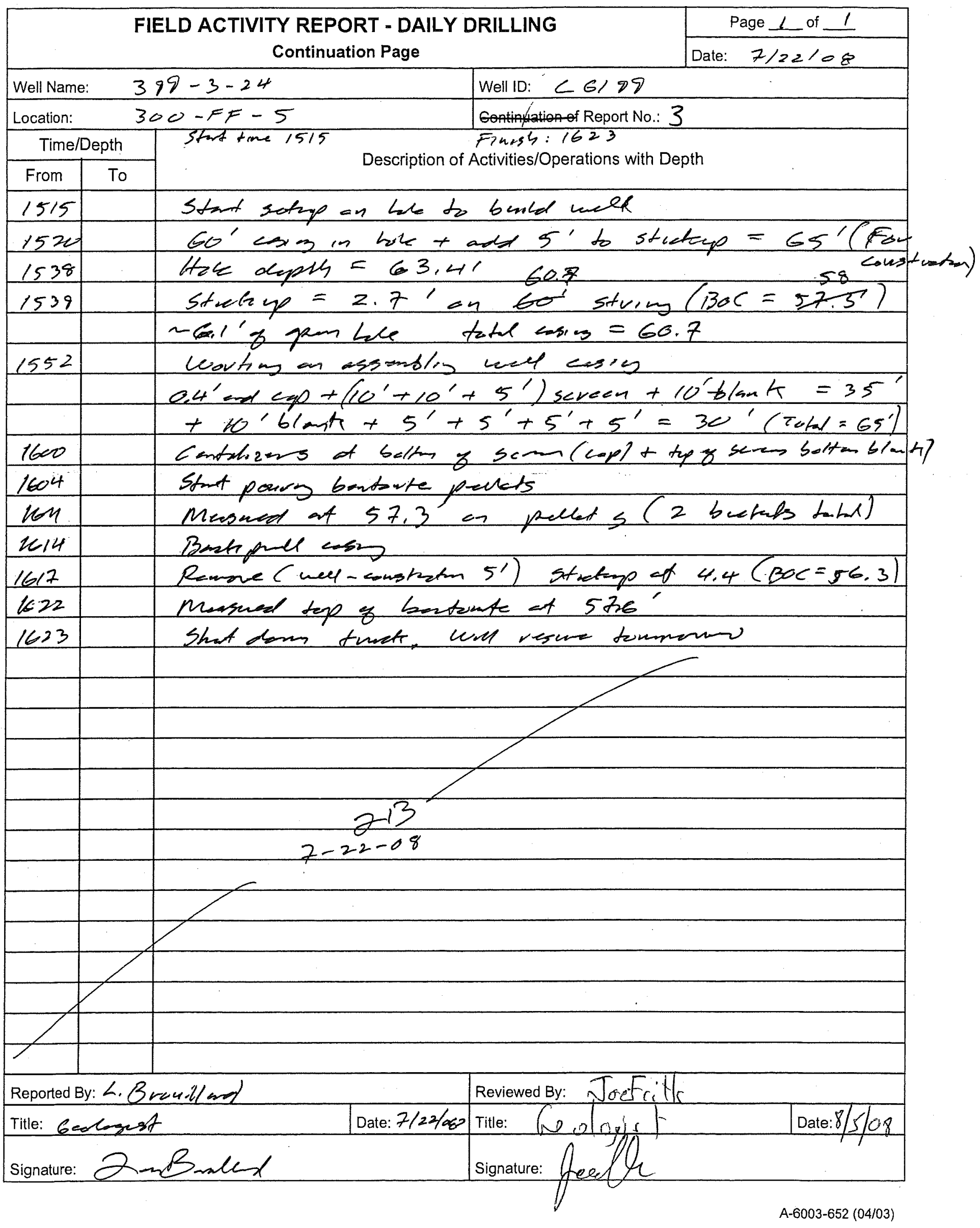

D.229 


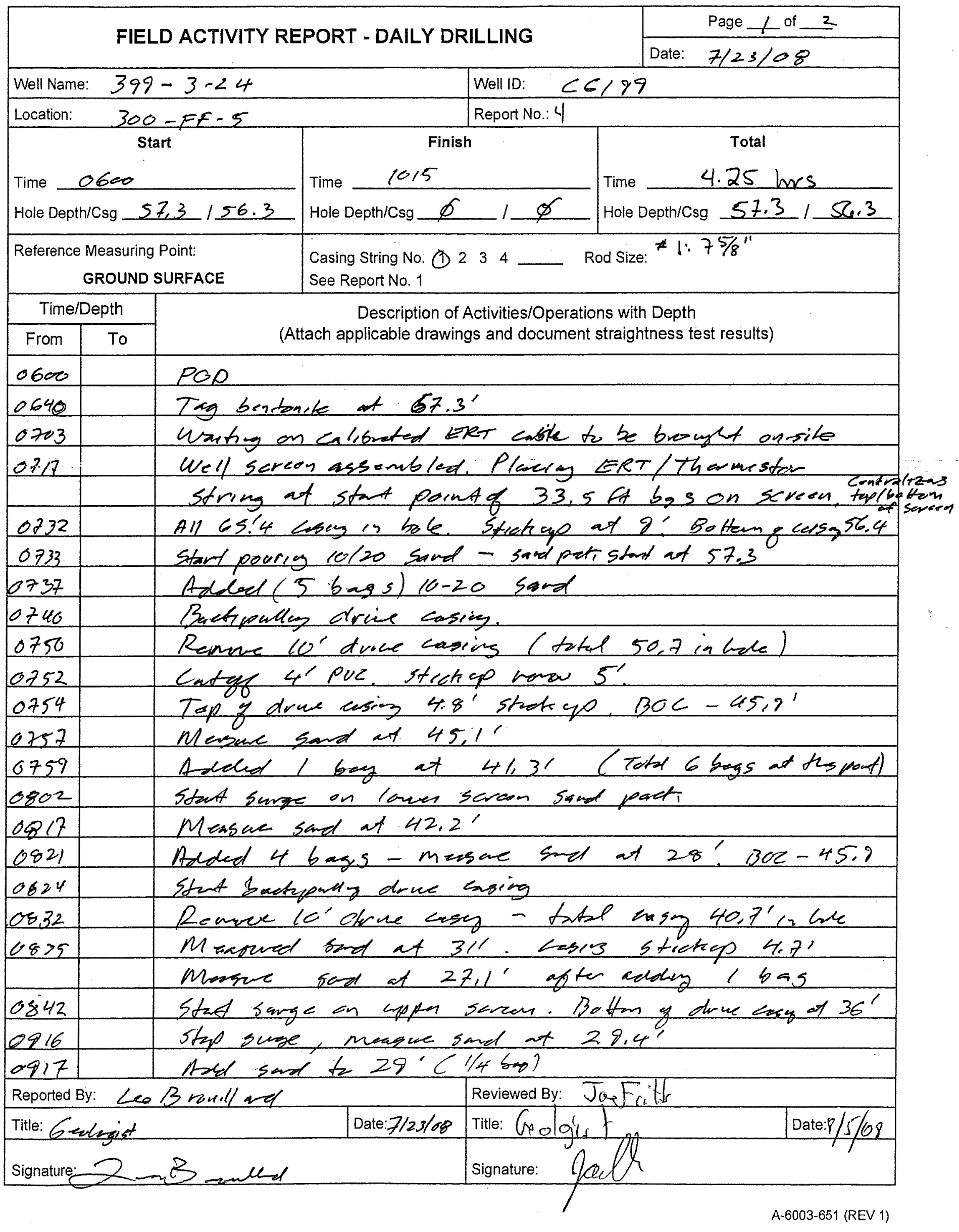

D. 230 


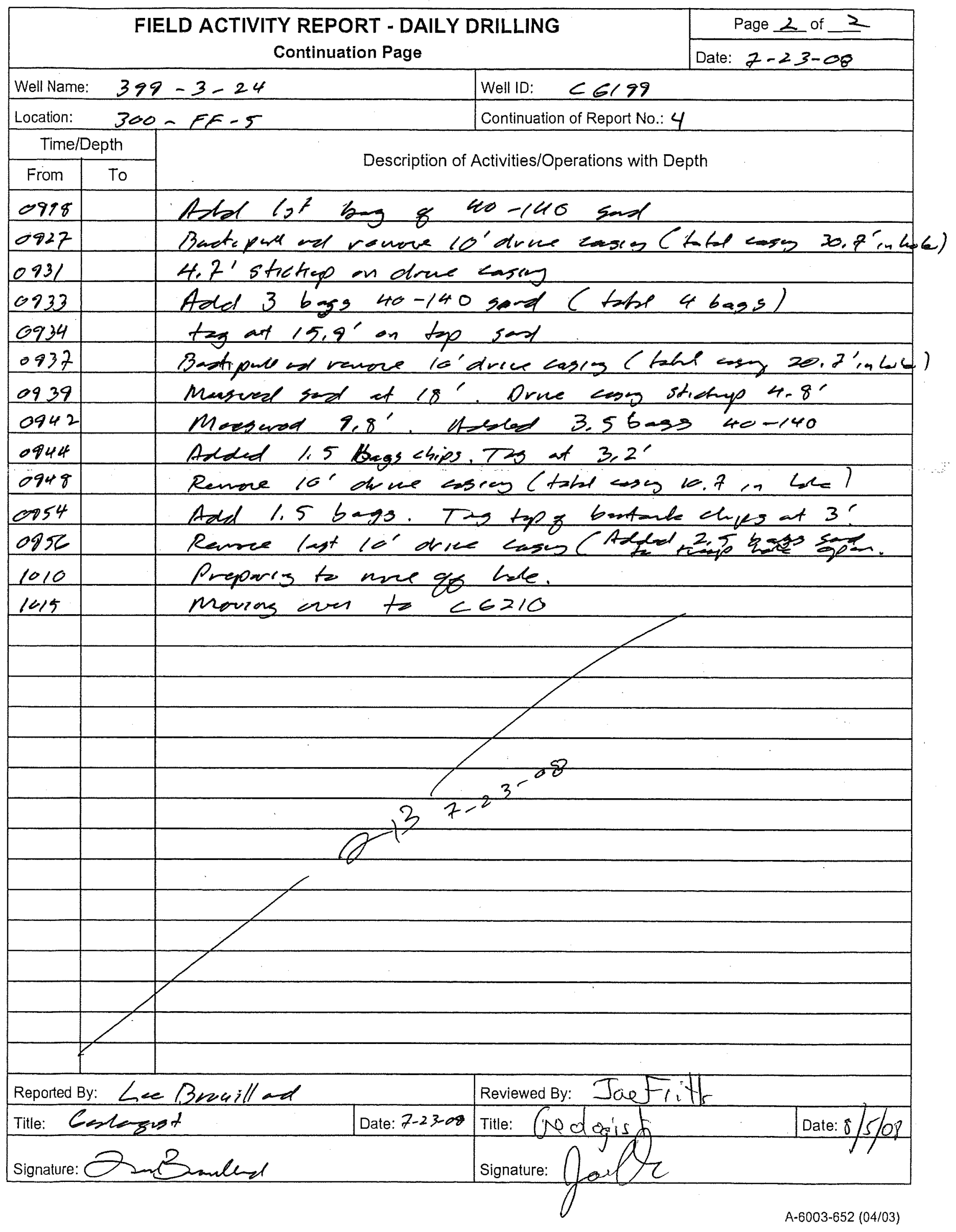

D.231 
FIELD ACTIVITY REPORT - DAILY DRILLING

Page 1 of 1

Date: $81-\infty 8$

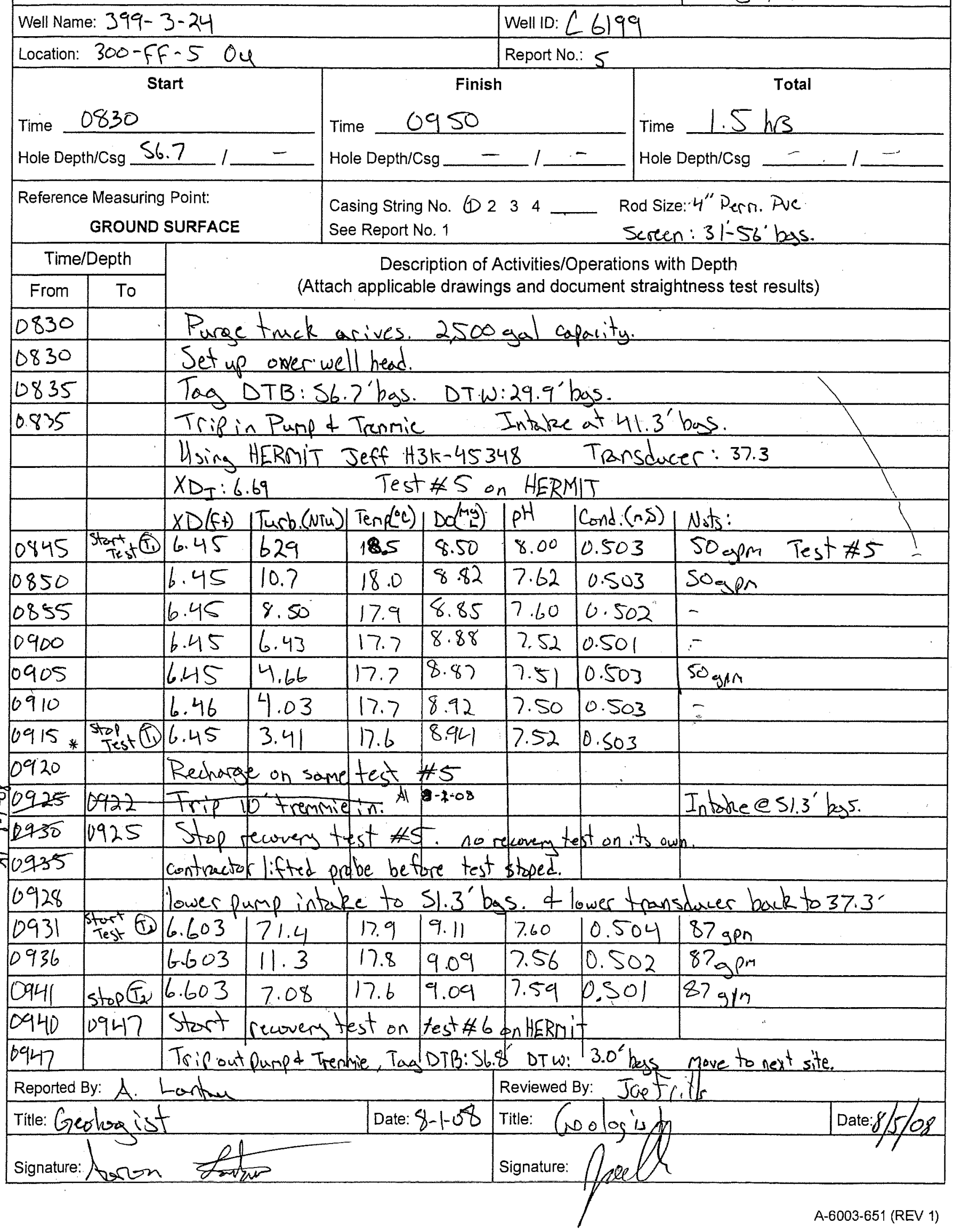




\section{WELL DEVELOPMENT AND TESTING DATA}

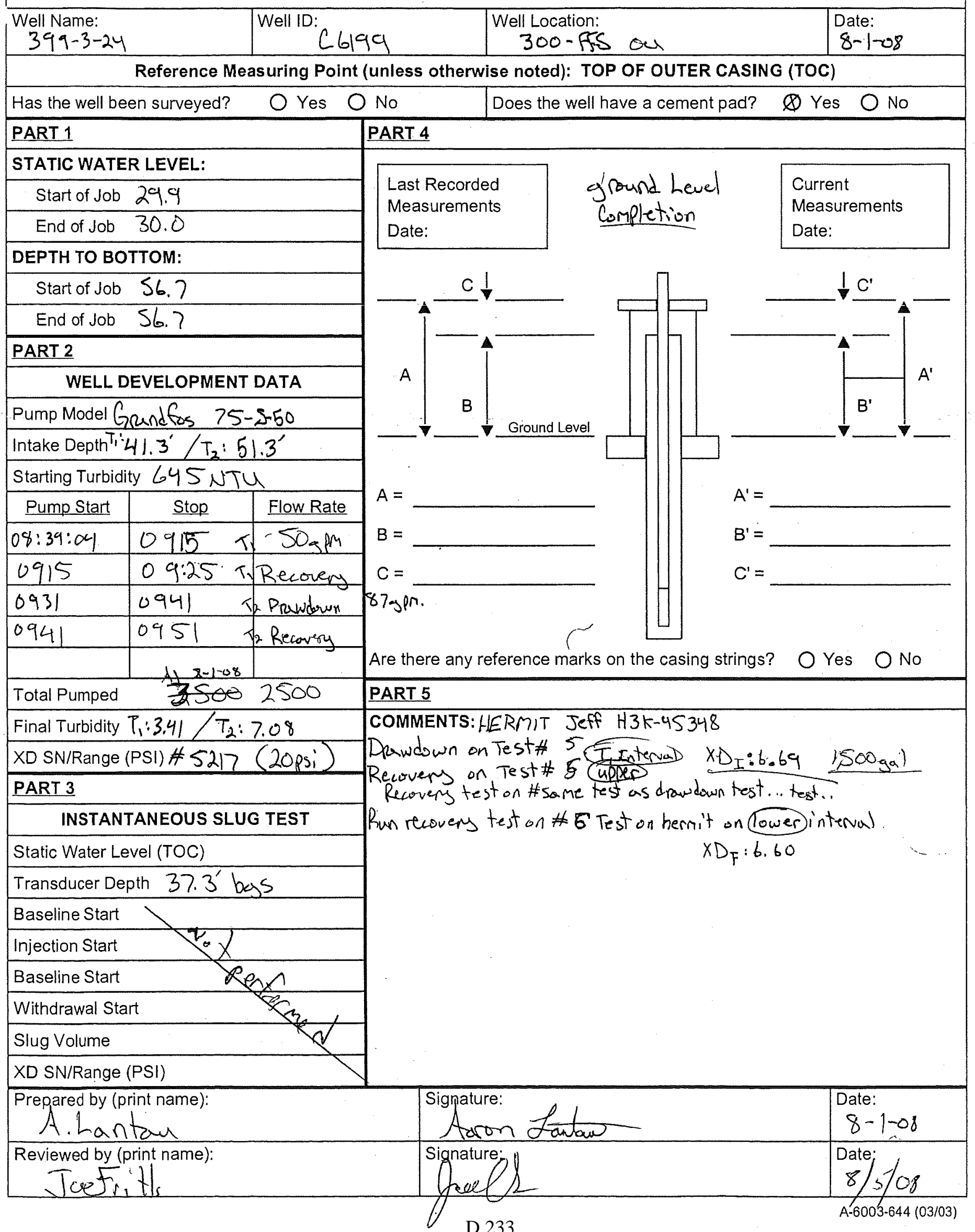


FIELD ACTIVITY REPORT NO. 1 - DRILLING PLAN

Page 1 of 3

Date: $6 / 6 / 63$

Purpose: PNNL Projeet -300 Ara IfC Well ID: $\quad 6200$

Drilling Co.: WDC Driller Billzamaw

Casing String No. (1) $234^{4}$ Drilling Method

Casing Size $75 \% 0^{\circ 00}, 6 \%$ Auger

Grade

Lbs.Per Ft.

Material arbon stoel

Type:

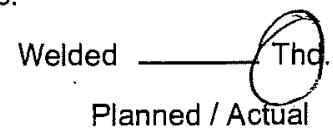

Set At:

Planned / Actual

Shoe OD/ID

Reference Measuring Point: GROUND LEVEL

Drig. Co.

Casing String No. 1 2. 34

Casing Size

Grade

Lbs.Per Ft.

Material

Type:

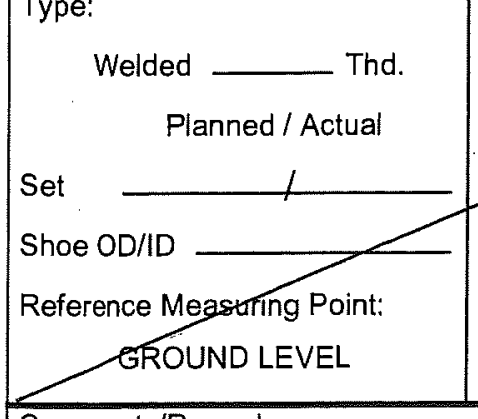

Comments/Remarks:
Rig No.:

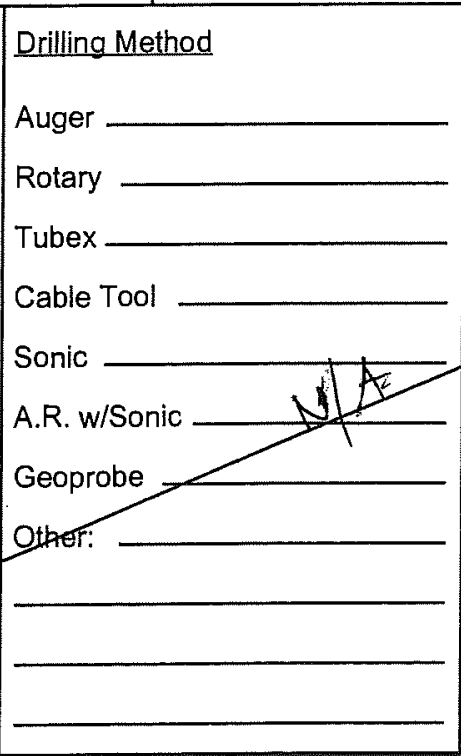

Rotary

Location: 300- FF -5-O4

Well Name: $599-3-25$

Rig No.: 139 Rig Make/Mod.:Super Sinic

\begin{tabular}{l|l} 
Circulation & D.H. Hammer
\end{tabular}

Air __ Water/Mud

Reverse —_ Direct

Vol: $\mathrm{cfm}$

gpm

Cable Tool

Sonic Super SaNic 2

Ge

Geoprobe

Other:

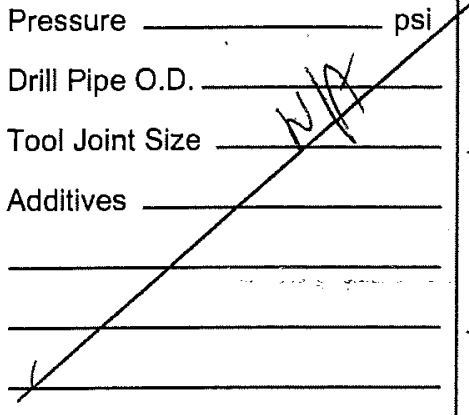

Cogr

Casing Hammer

Make

Model

Bit Size

Type

Nozzles

Rod Size
Rig Make/Mod.

Circulation

Air __ Water/Mud

Reverse _ـ Direct

Vol: $\mathrm{cfm}$

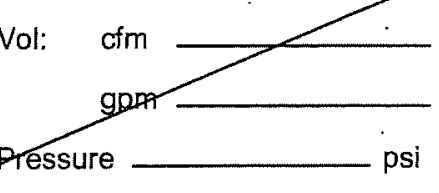

Drill Pipe O.D.

Tool Joint Size

Additives
D.H. Hammer

Make

Model

Choke

Casing Hammer

Make

Model

Bit Size

Type

Nozzles

Rod Size

Estimated Depth to Water

Reported By: ReFrys

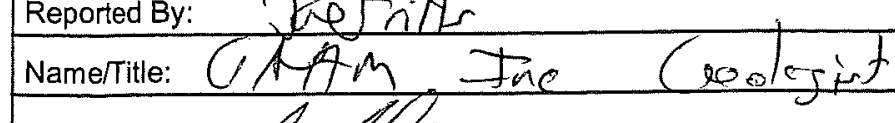

Signature:

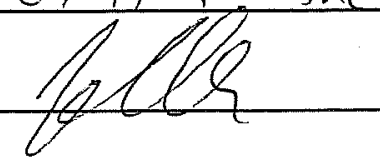

Date: $6 / 13 / 28$ 


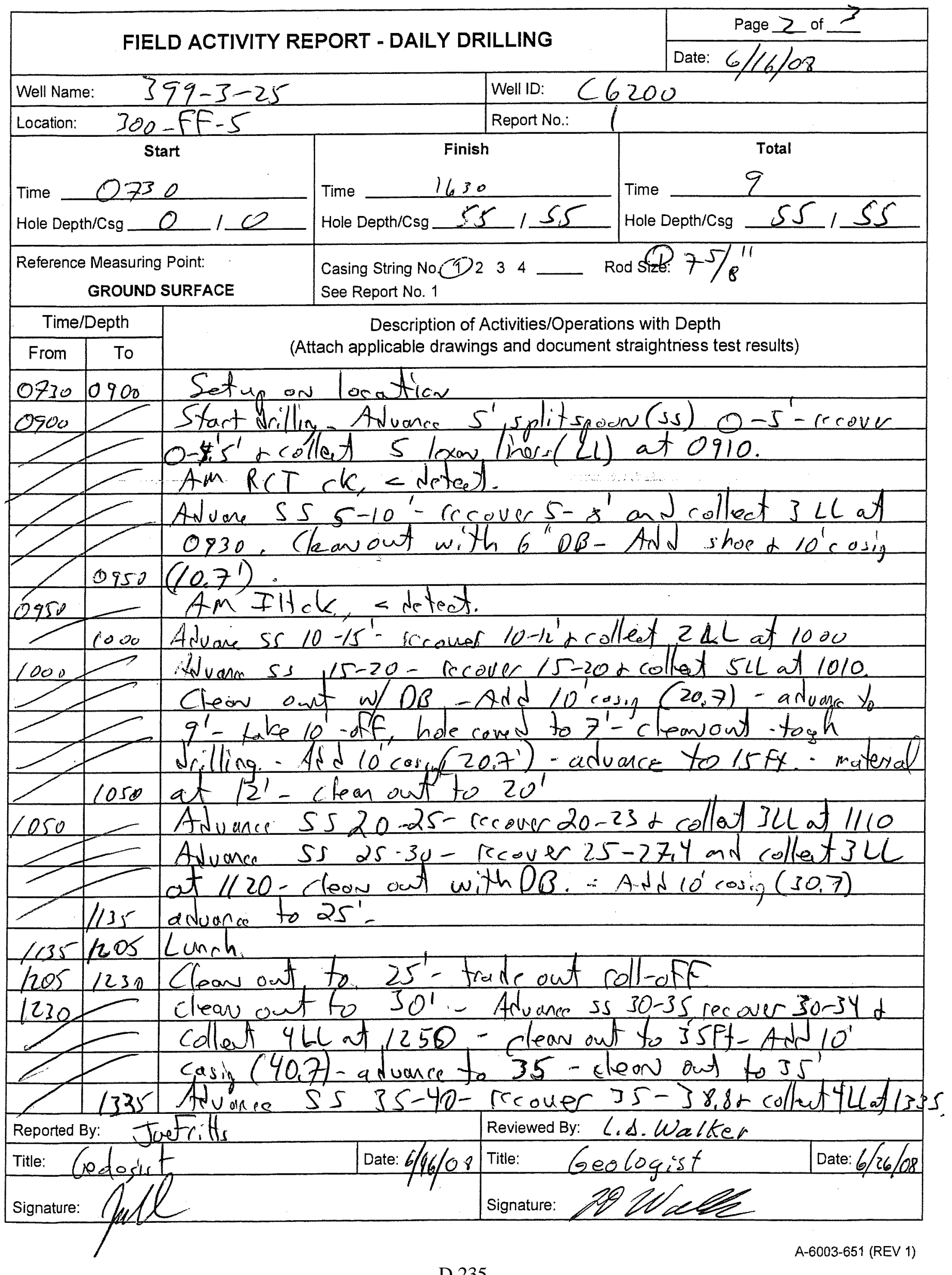




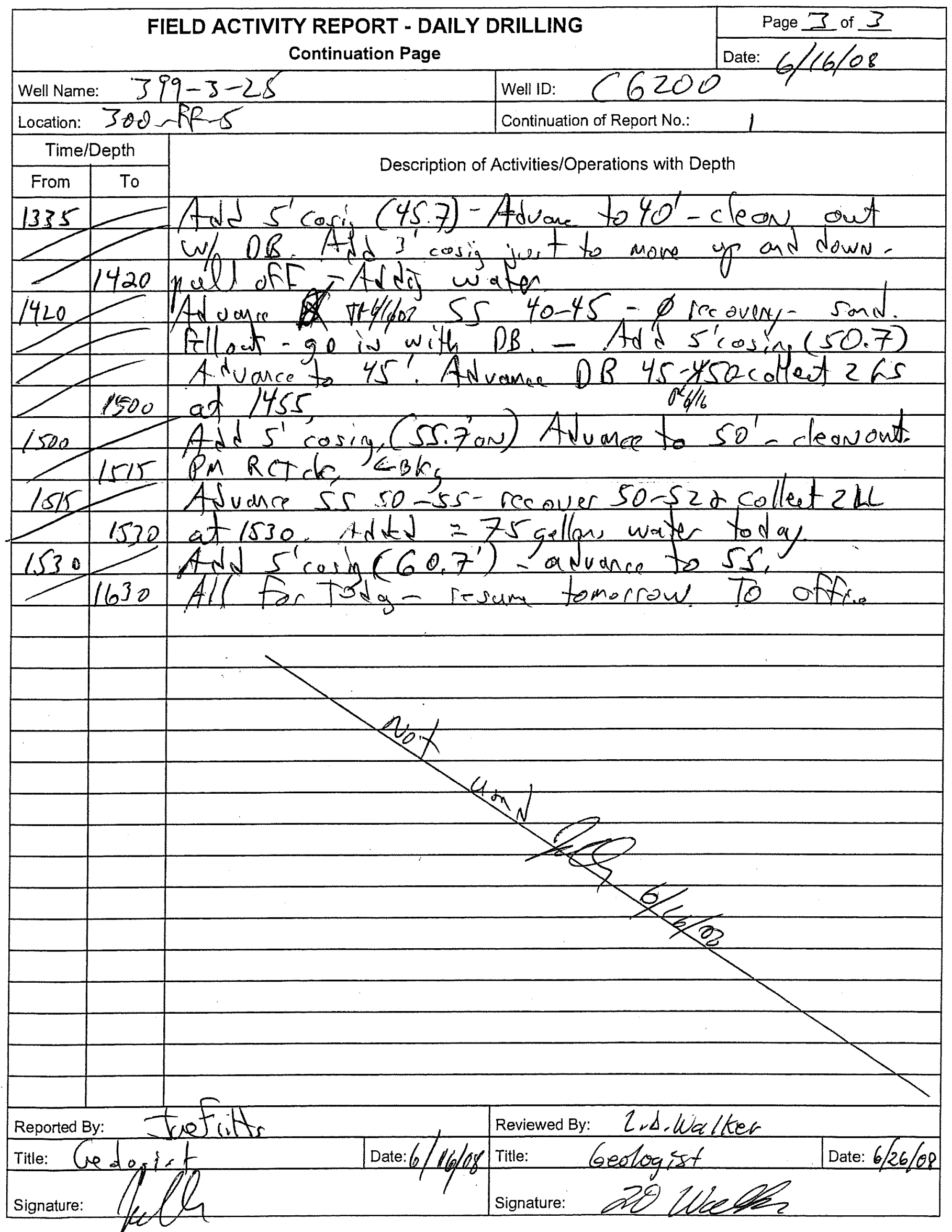




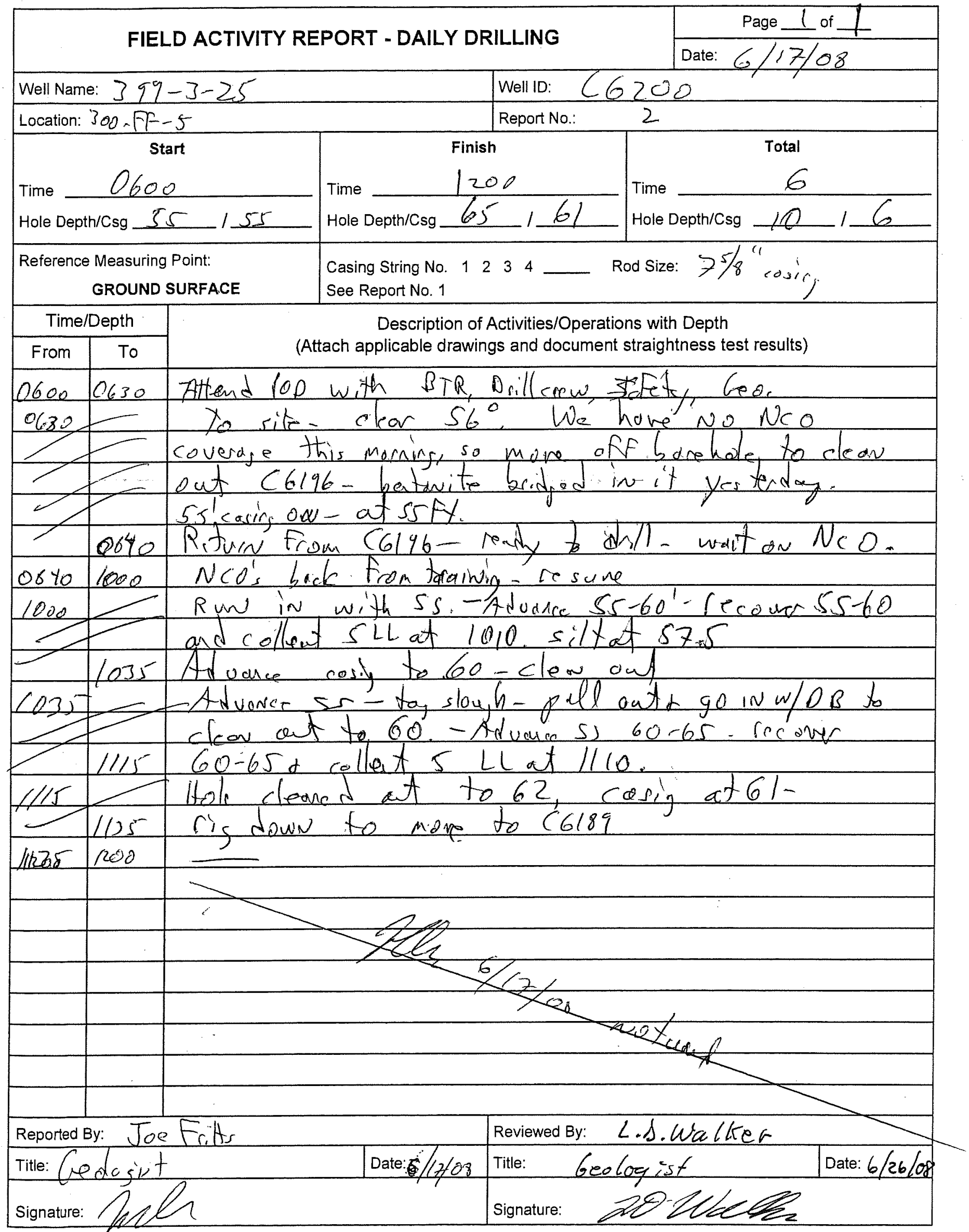




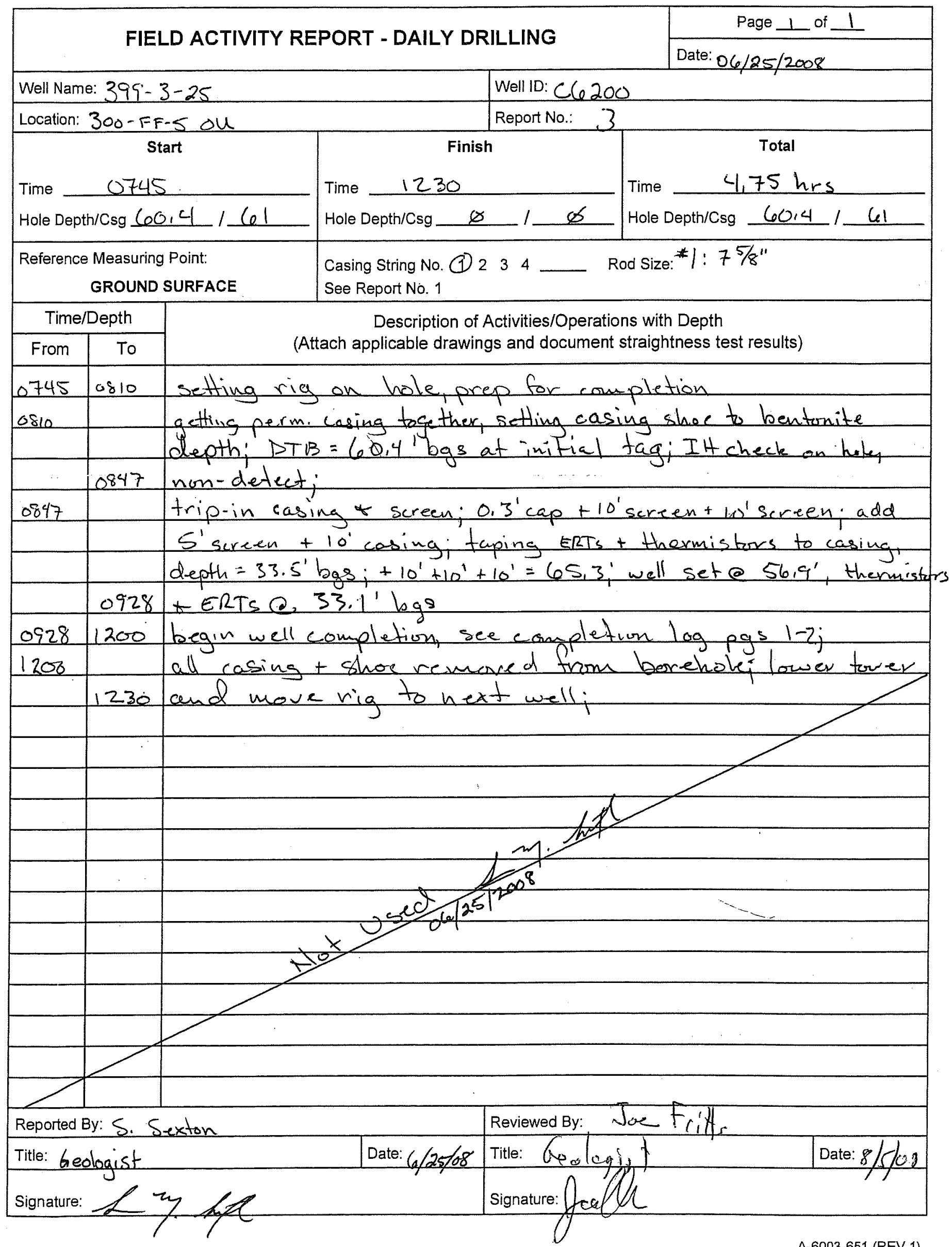

D.238 


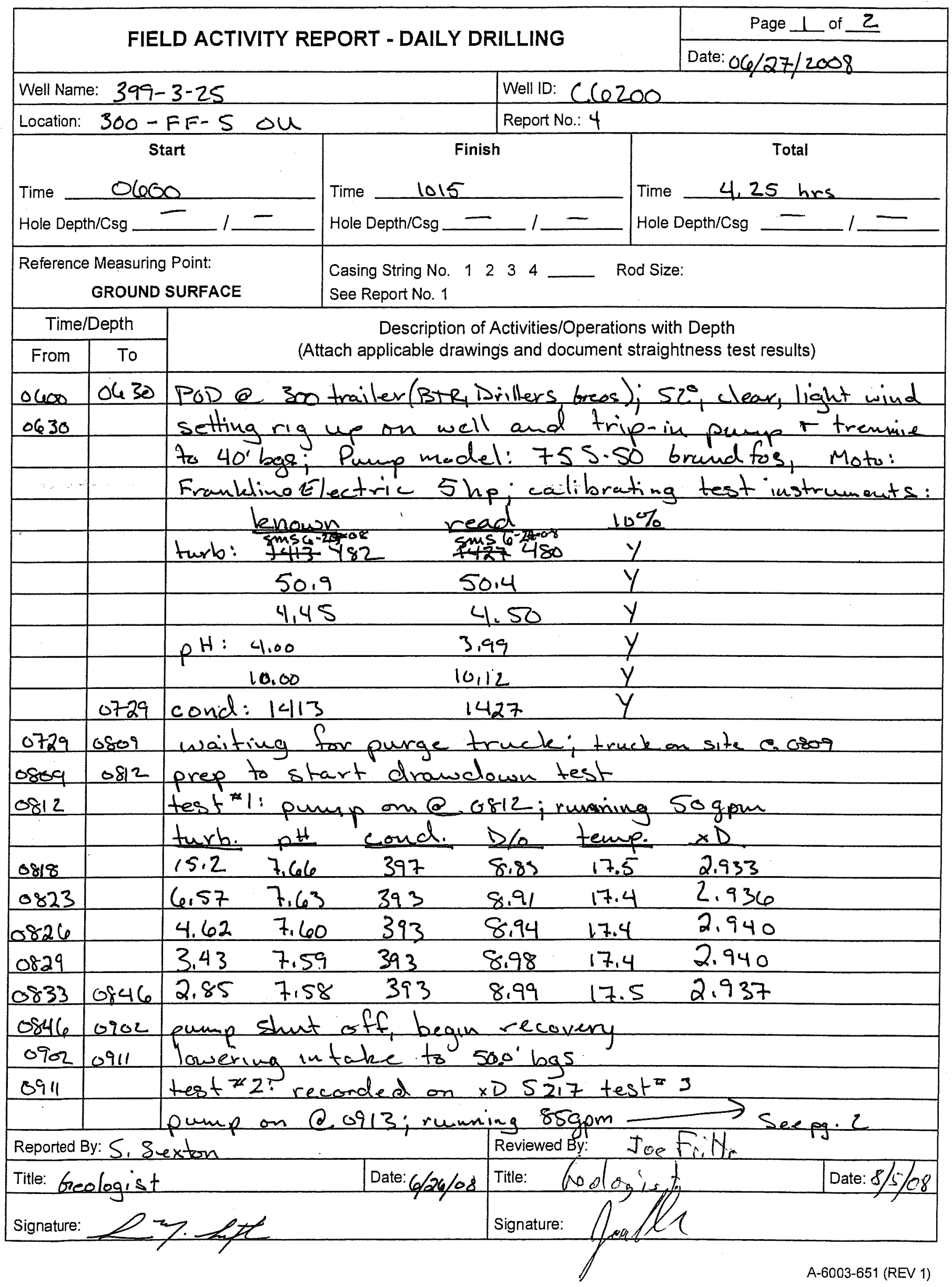

D.239 


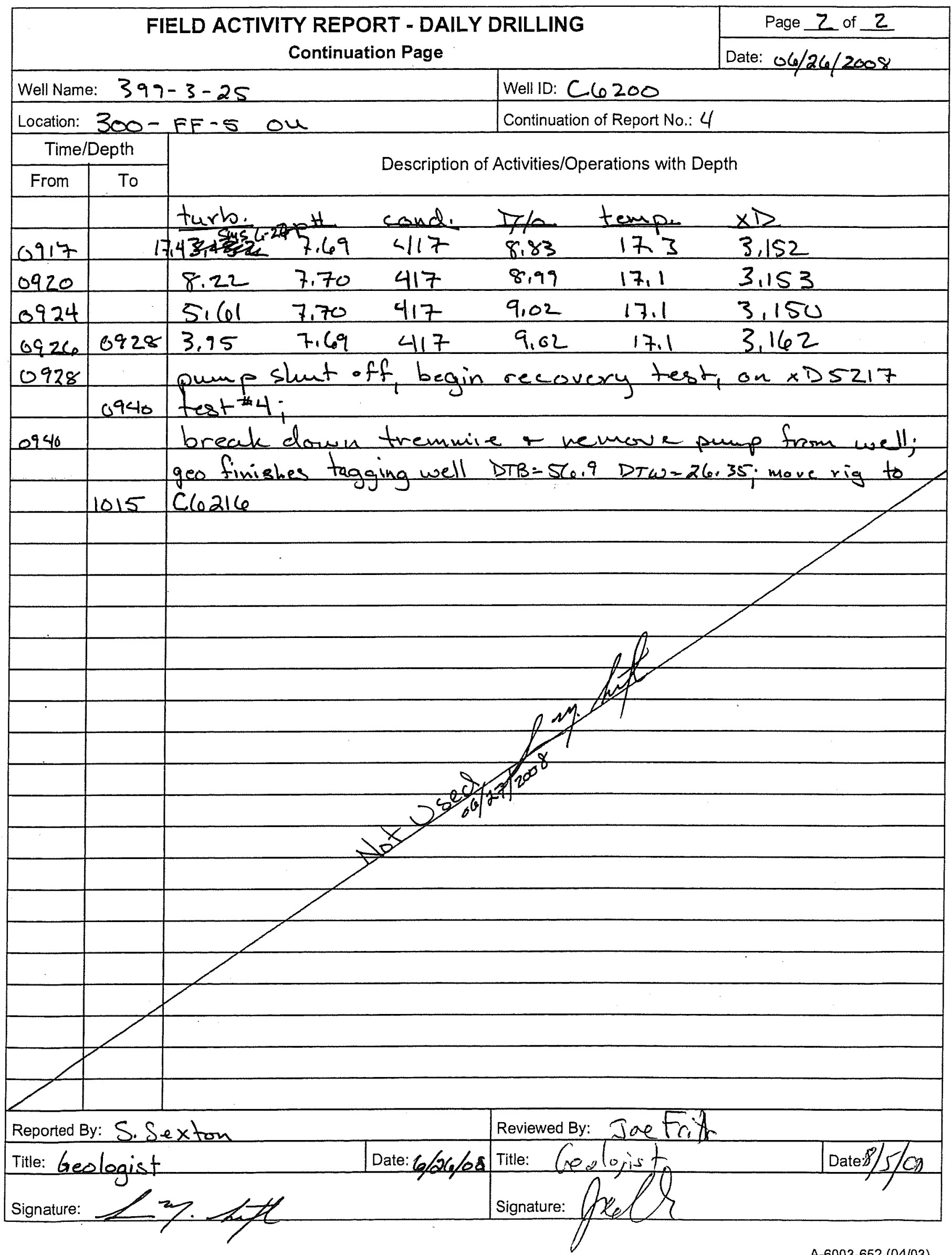




\section{WELL DEVELOPMENT AND TESTING DATA}

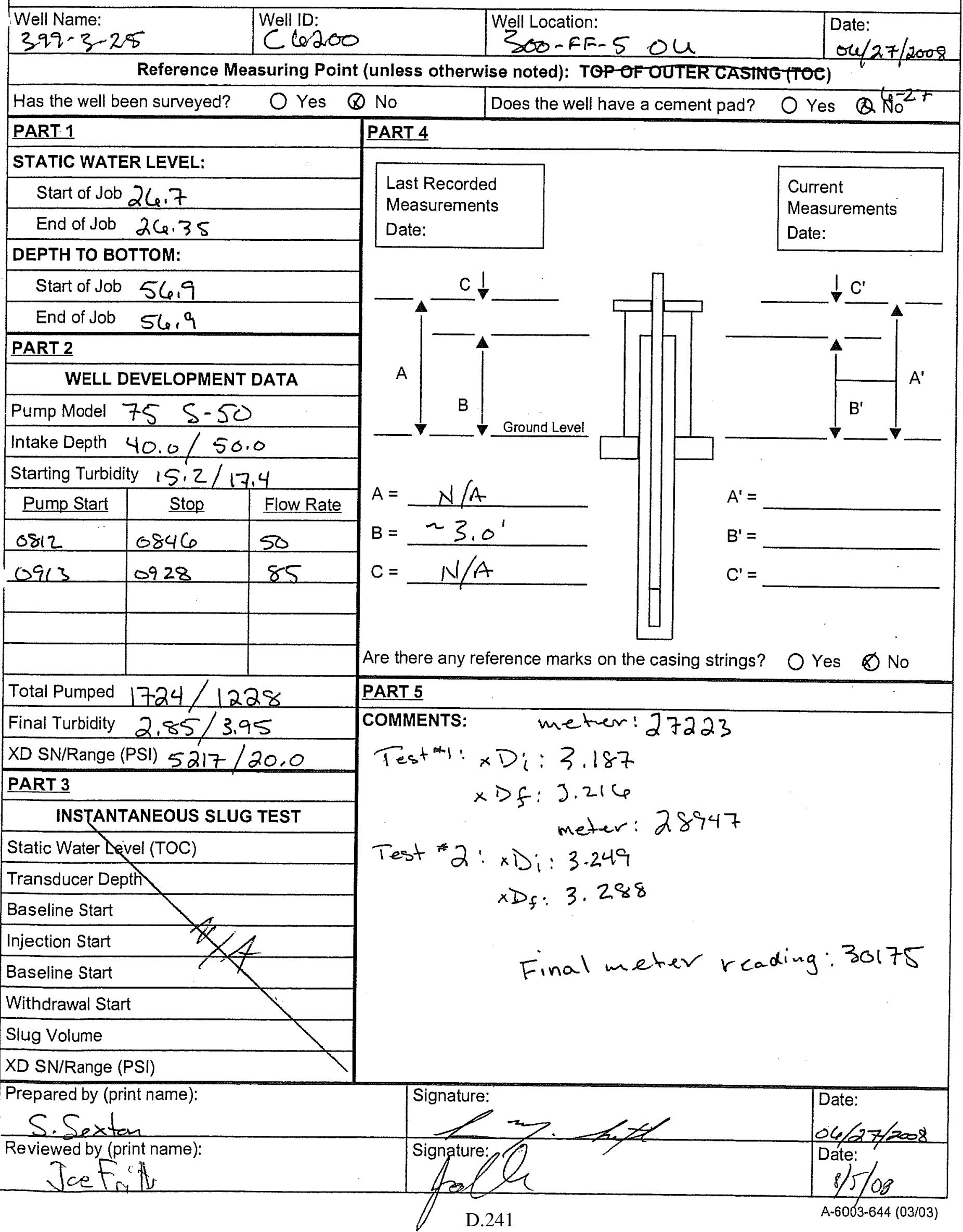




\section{FIELD ACTIVITY REPORT NO. 1 - DRILLING PLAN}

Page 1 of 1

Date: $5 / 14 / 28$

Purpose: Cher actoration Jocinare IfC Well ID: $(6203$

Drilling Co.: $\sim \cap C / \quad B l u+5$ tar

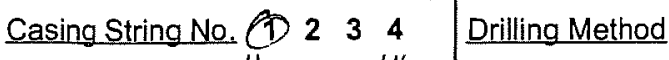

Casing Size $78,00,67 \%^{\prime \prime} \pm 0$

Grade

Lbs.Per Ft.

Material urben stenl

Type:

Welded Thd.

Planned / Actual

Set At: -156.8

Shoe OD/ID

Reference Measuring Point:

GROUND LEVEL

Drig. Co.

Casing String No. $1122 \quad 3 \quad 4$

Casing Size

Grade

Lbs.Per Ft.

Material

Type:

Welded Thd.

Planned / Actual

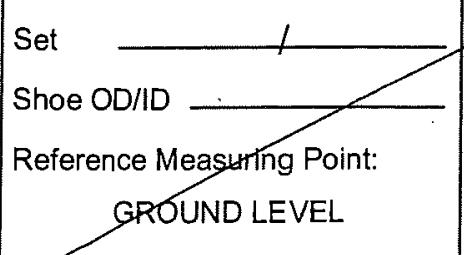

Comments/Remarks:
Auger

Rotary

Tubex

Cable Tool Sonic) Suprsonir 2 A.R. w/Sonic RS 400 Geoprobe Other:
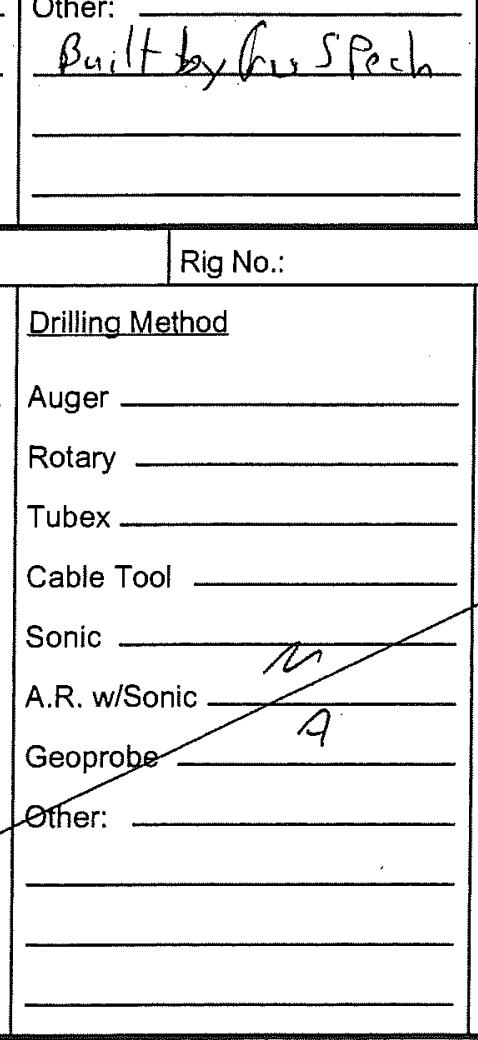

Location: $300-F F-5-O L$ Rig No.: 139 Rig Make/Mod.: Sofer $\mathrm{JN}_{1 \mathrm{C}} 2$ Circulation Air Rever Vo: Vol:
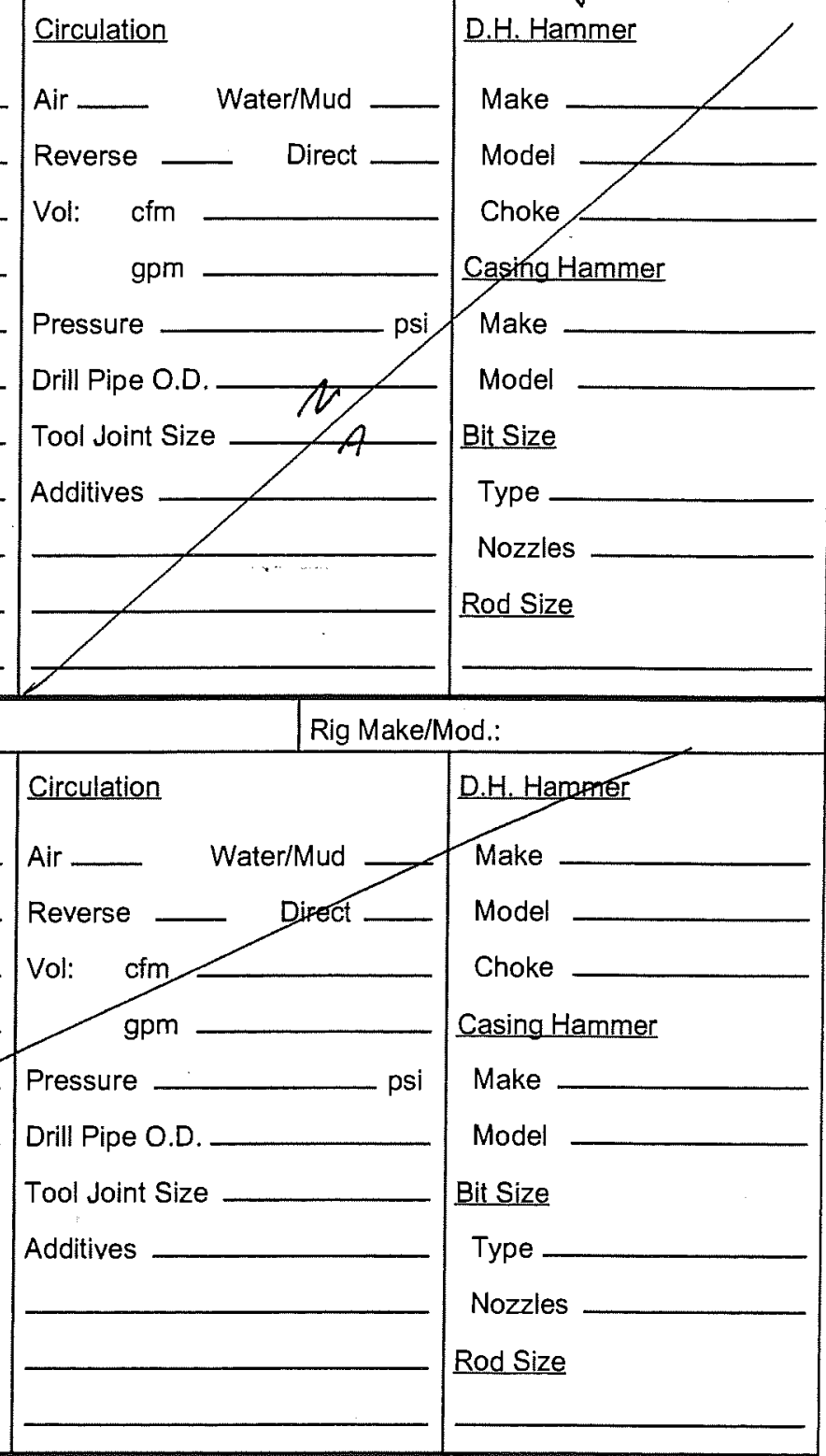

Estimated Depth to Water $\frac{3 / .70 \mathrm{fl}, s \text { on }}{5 / 16 / 0.8}$

Reported By: Jast. Hs

Namertitle: GRAM Ine Golajist

Signature:

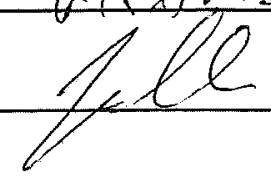

Date: $5 / 14 / 0 \gamma$ 


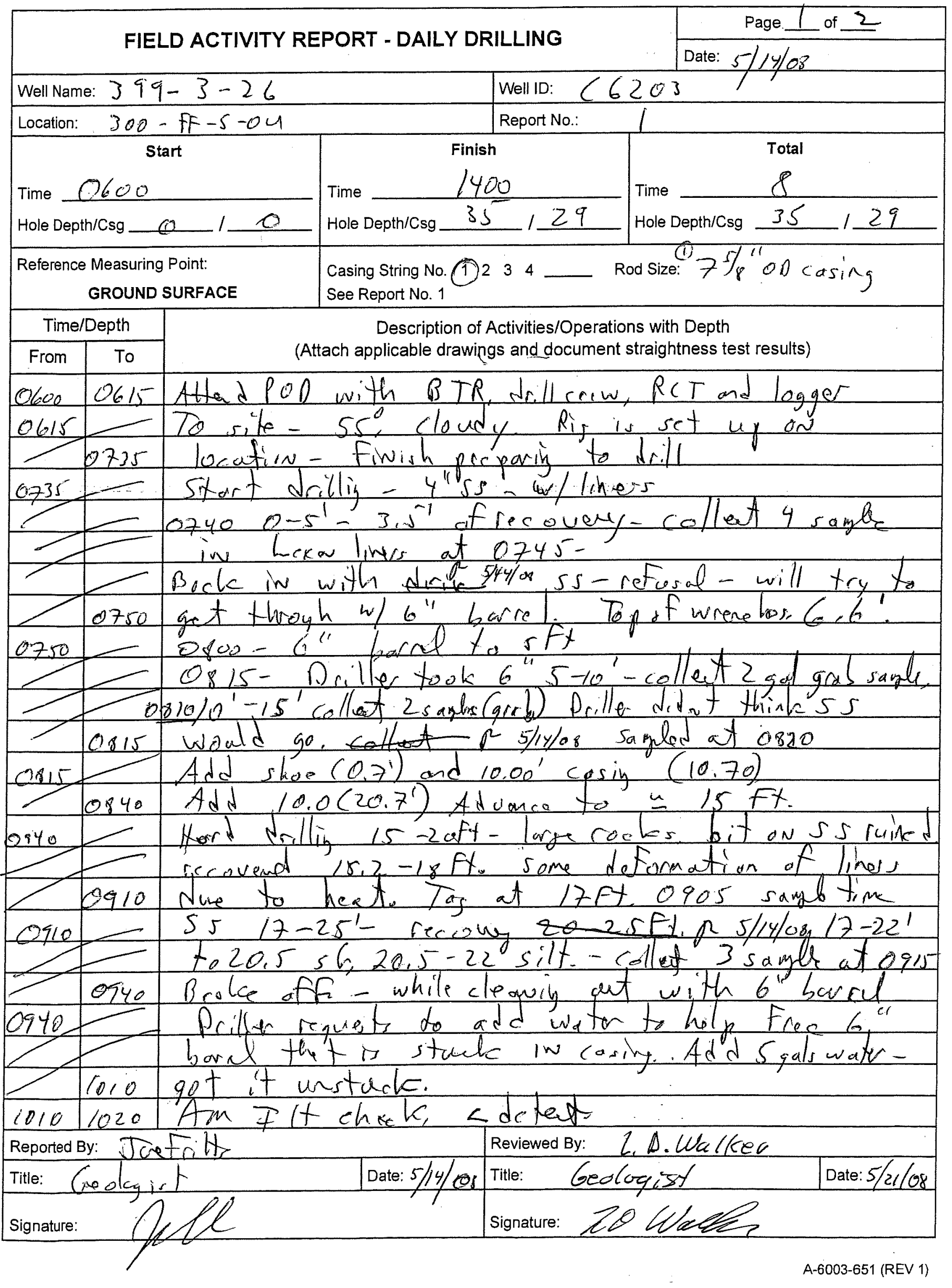

D. 243 


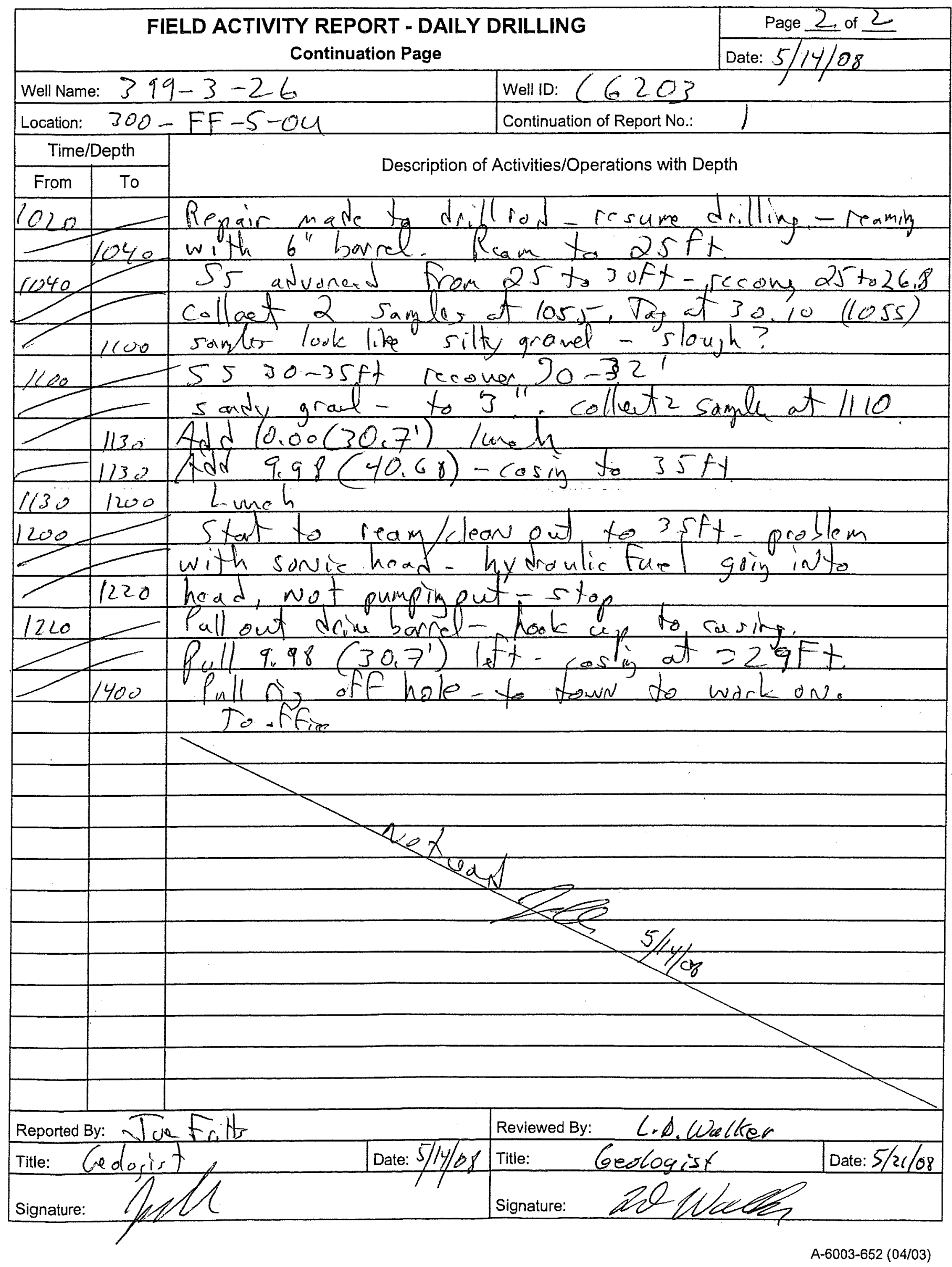

D.244 


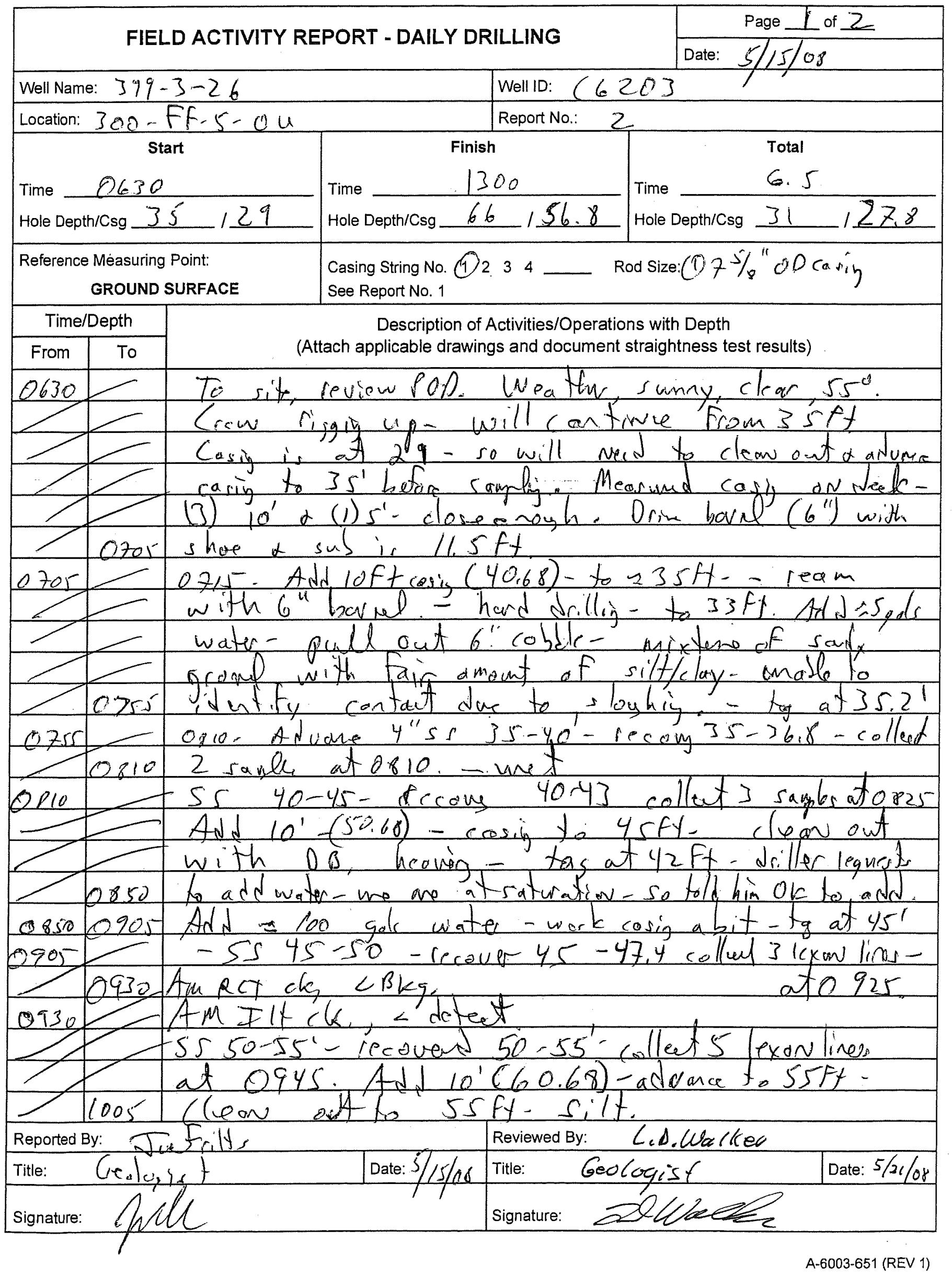

D. 245 


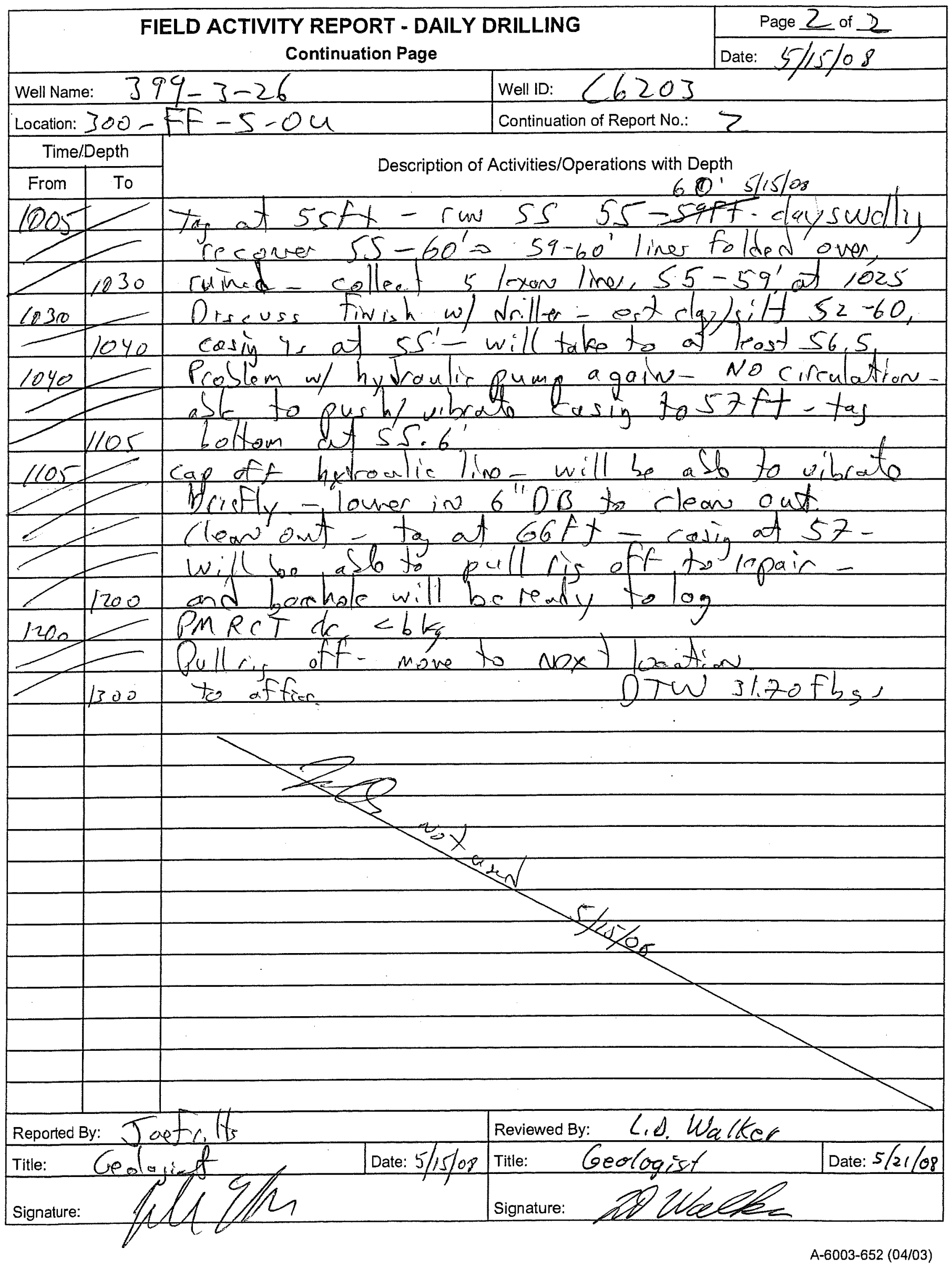

D.246 


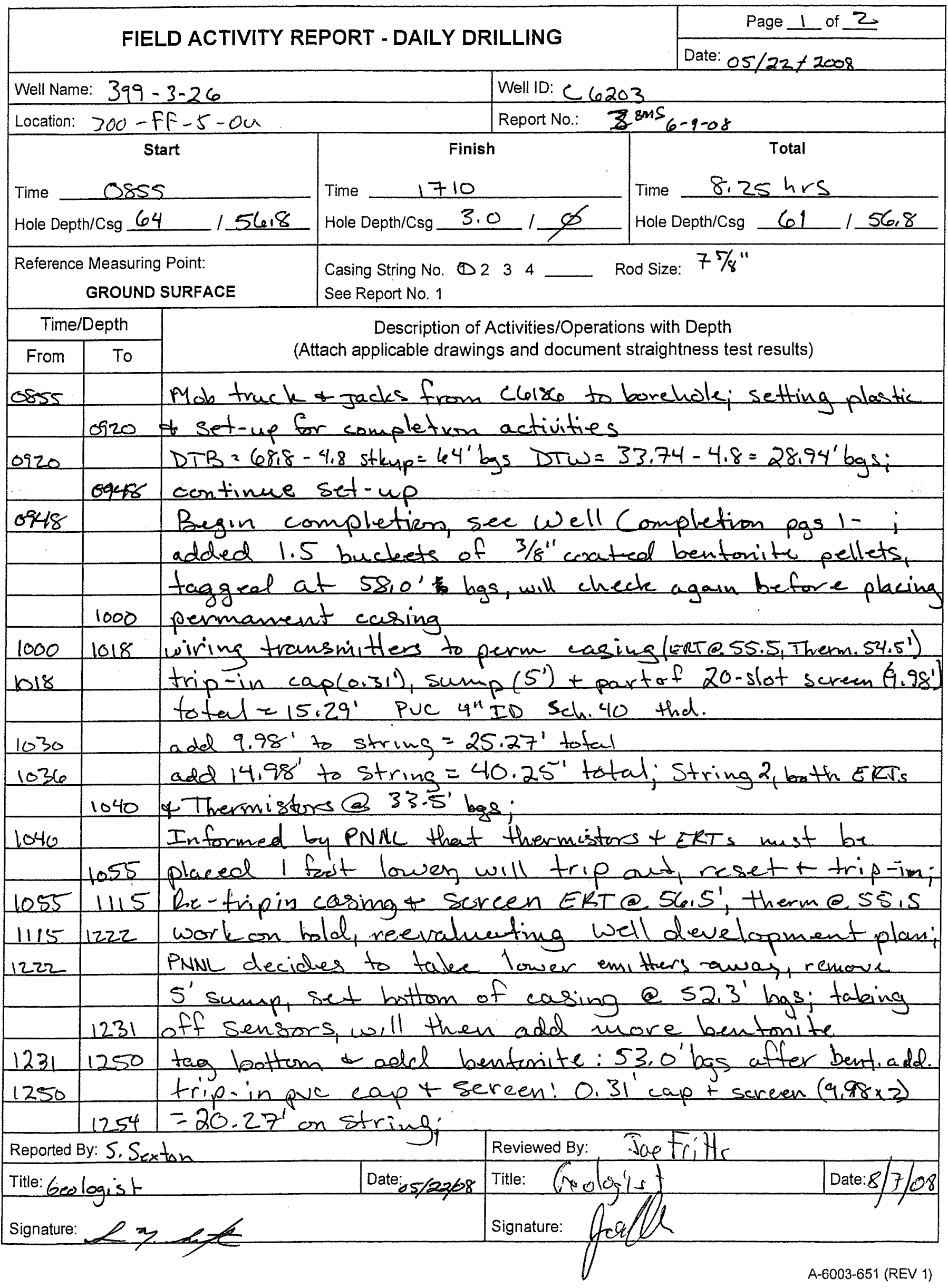

D.247 
FIELD ACTIVITY REPORT - DAILY DRILLING

Continuation Page

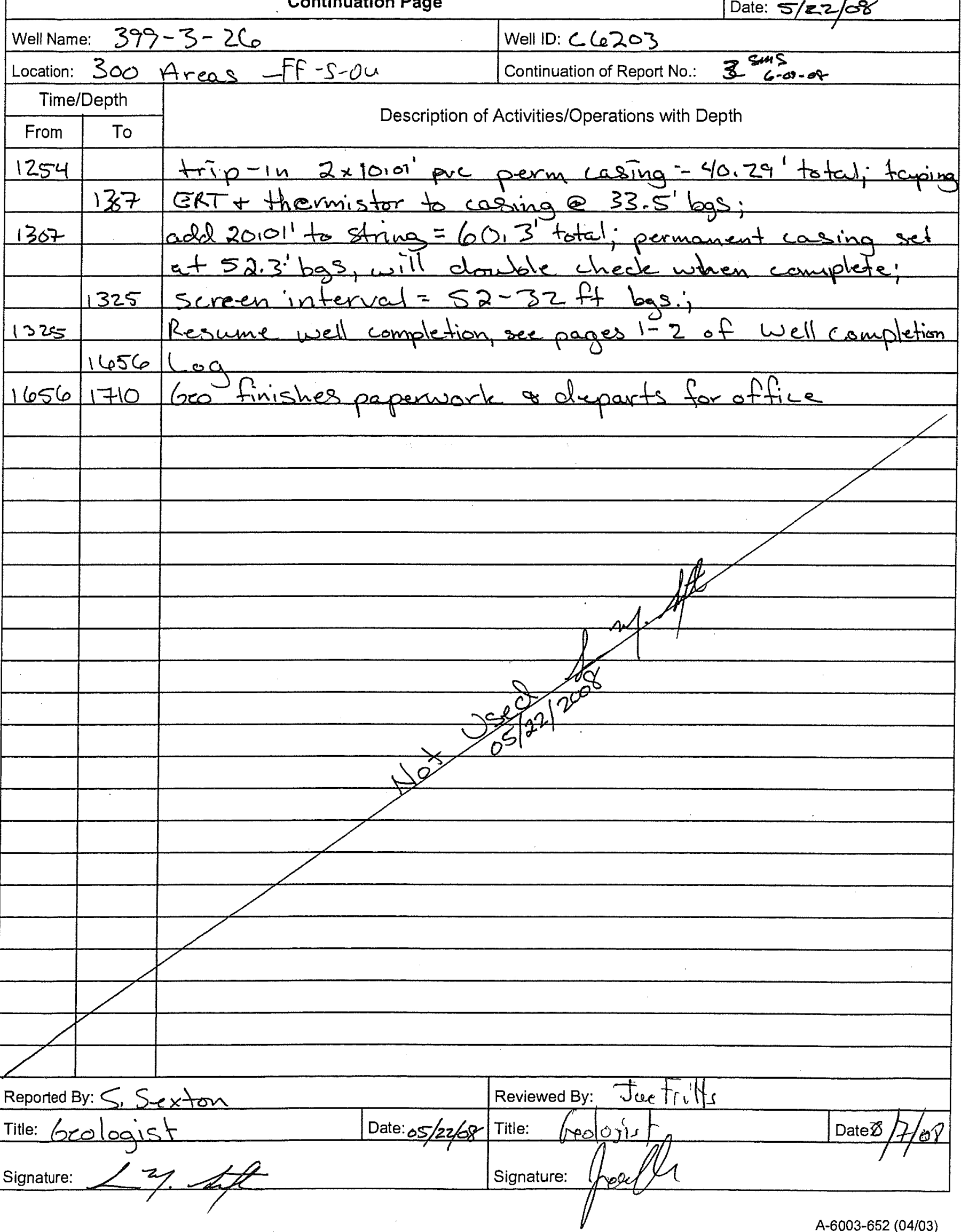




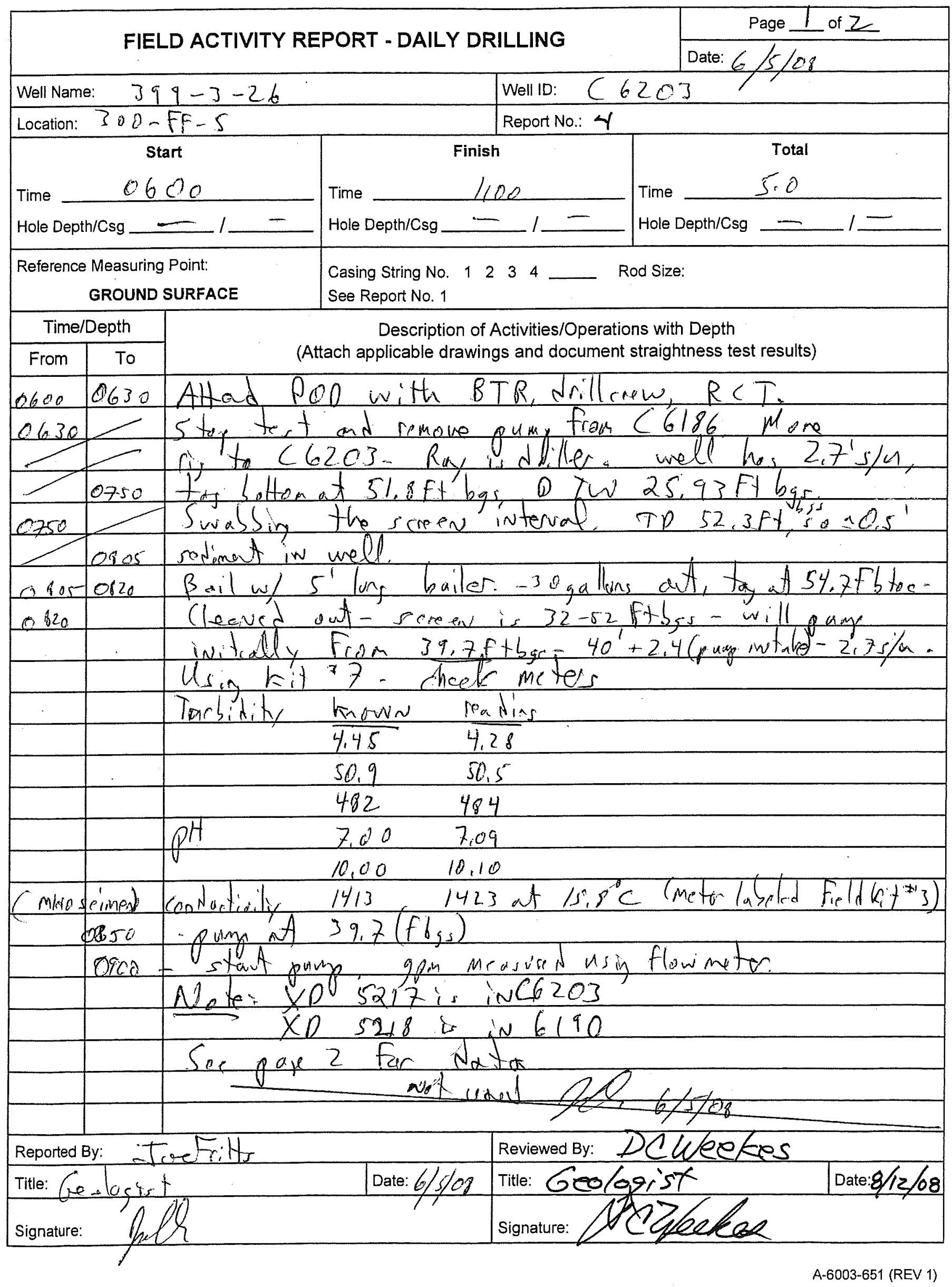

D.249 


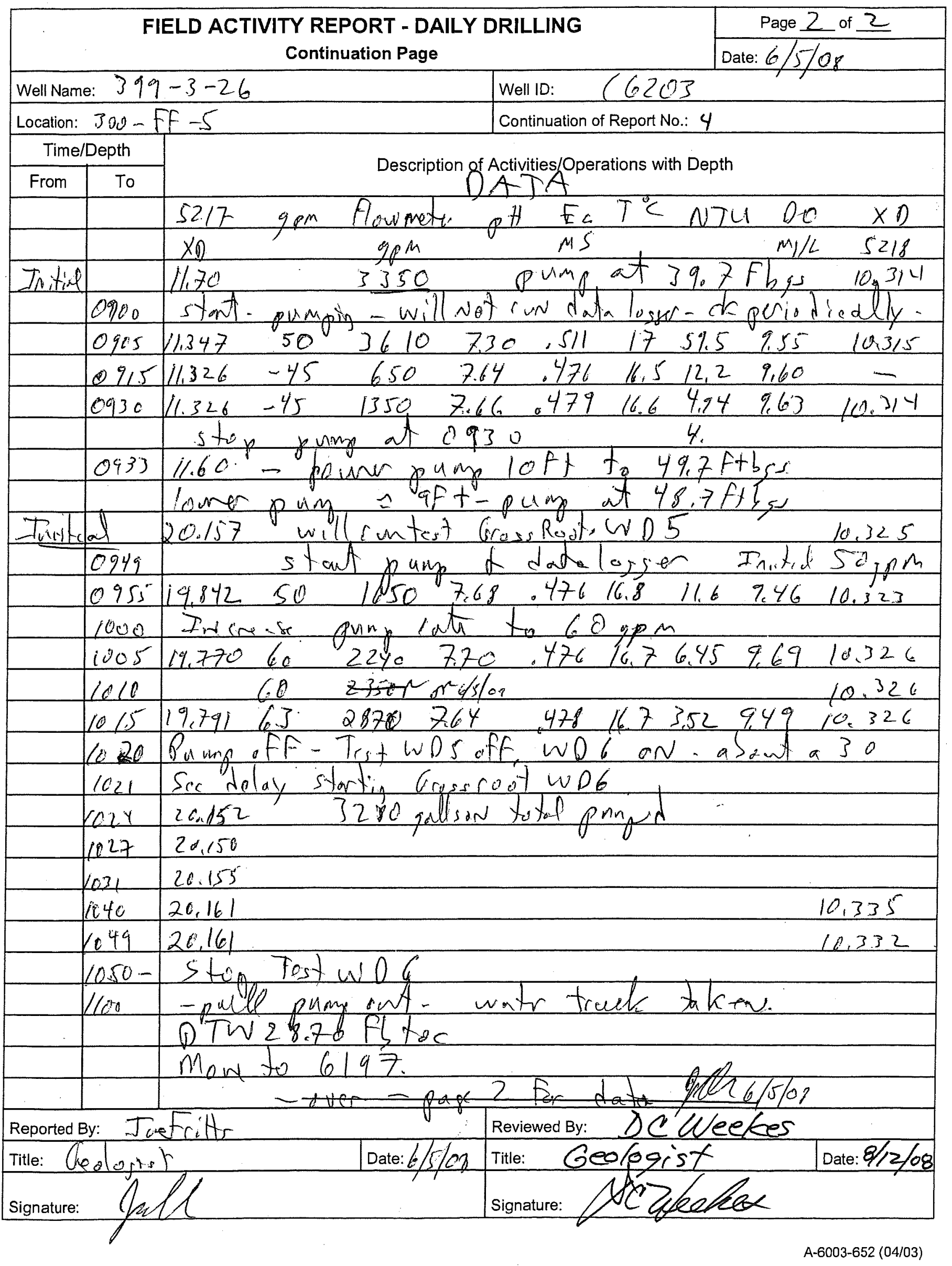

D. 250 


\section{FIELD ACTIVITY REPORT - DAILY DRILLING}

Page 1 of 1

Date:5/23/08

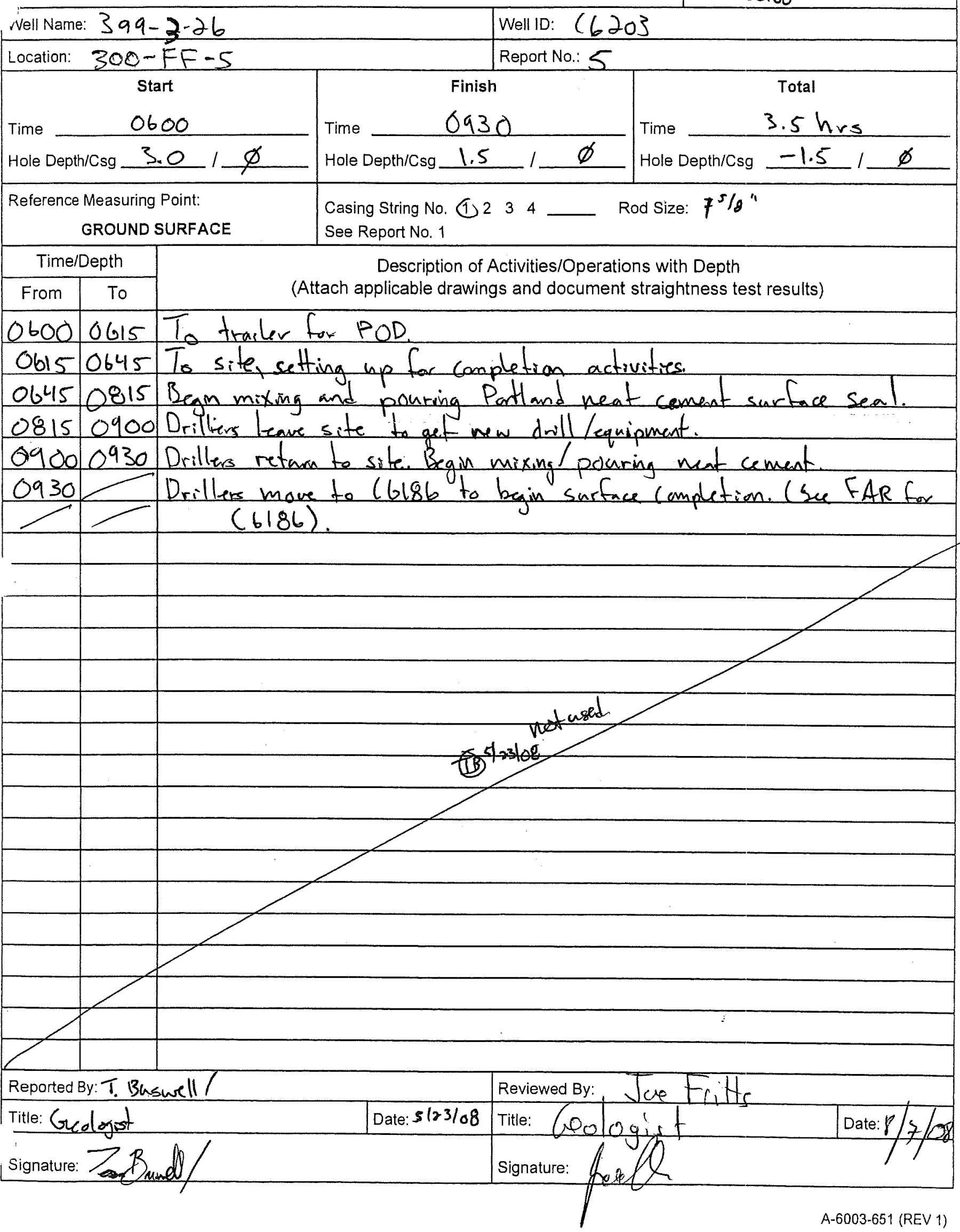

D.251 


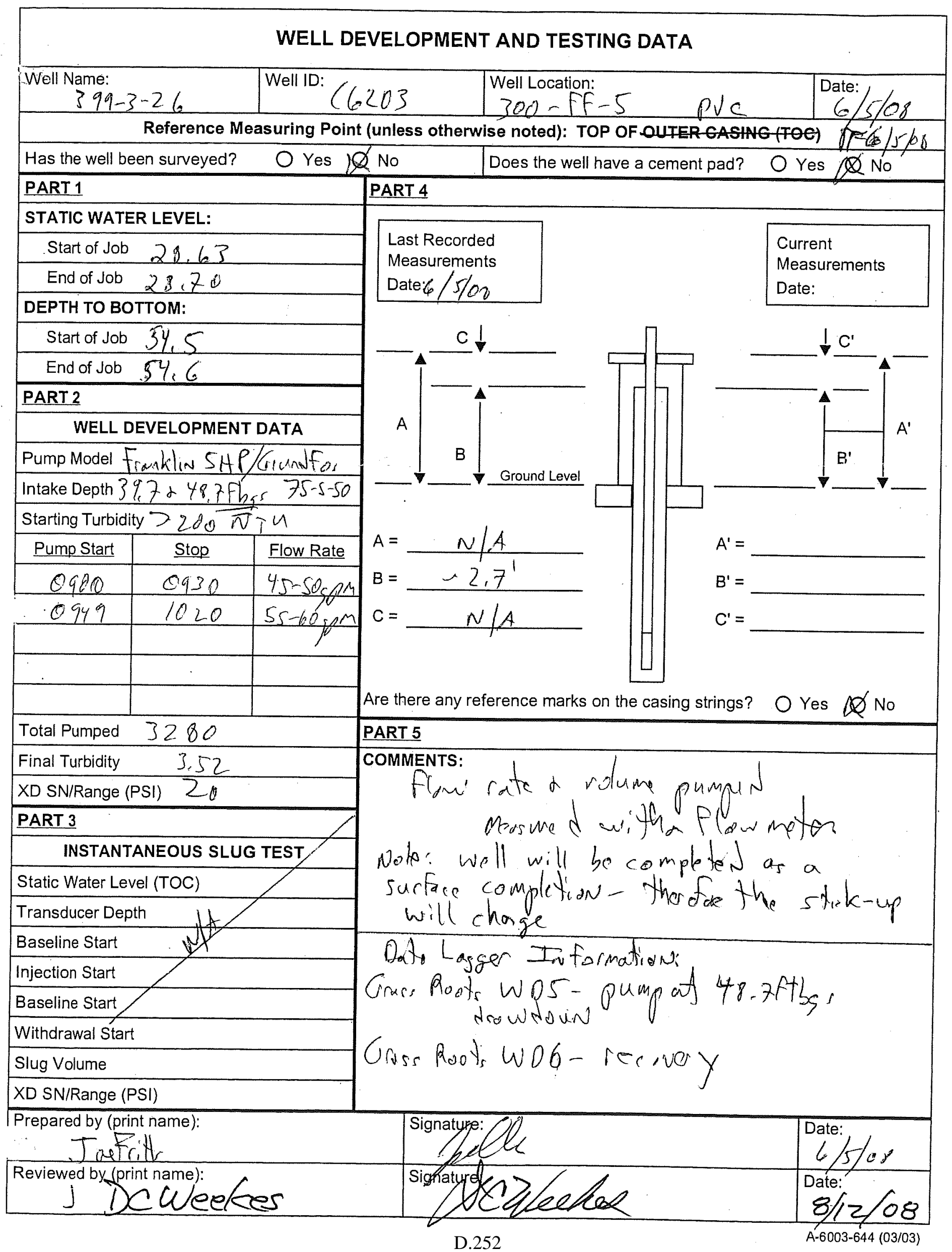


FIELD ACTIVITY REPORT NO. 1 - DRILLING PLAN

Purpose:PNNL Chavactorization 300Area Location: $300-F F-5$

Well ID: C6204 IFC Well Name: $399-3-27$

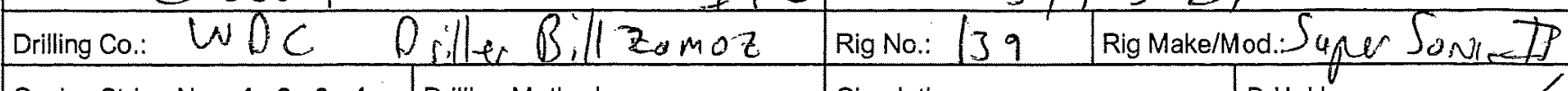

\begin{tabular}{lllllll}
\hline Casing String No. & 1 & 2 & 3 & 4 & Drilling Method
\end{tabular}

Casing Size $75 / 8 / 67 / 8$

Grade

Lbs.Per Ft.

Material Cartin steel

Type:

Welded

(Tho).

Planned / Actual

Set At: 1

Shoe OD/ID

Reference Measuring Point:

GROUNDLEVEL

Drig. Co.

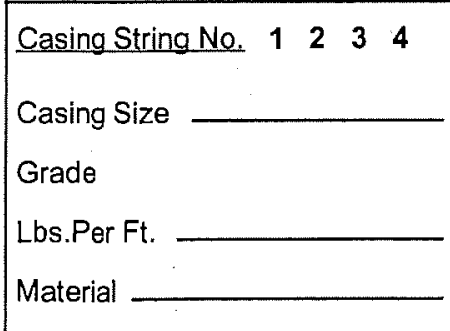

Type:

Welded Thd.

Planned / Actual

Set

Shoe OD/ID

Reference Measurning Point: GROUND LEVEL
Rig No:

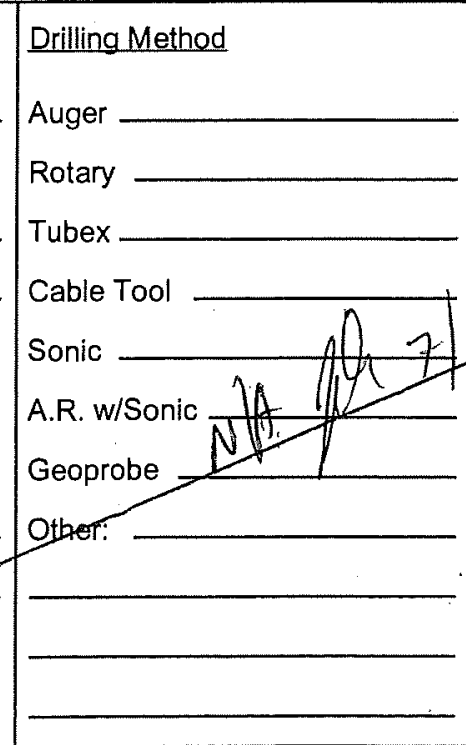

Page 1 of 3

$19 / 08$ 


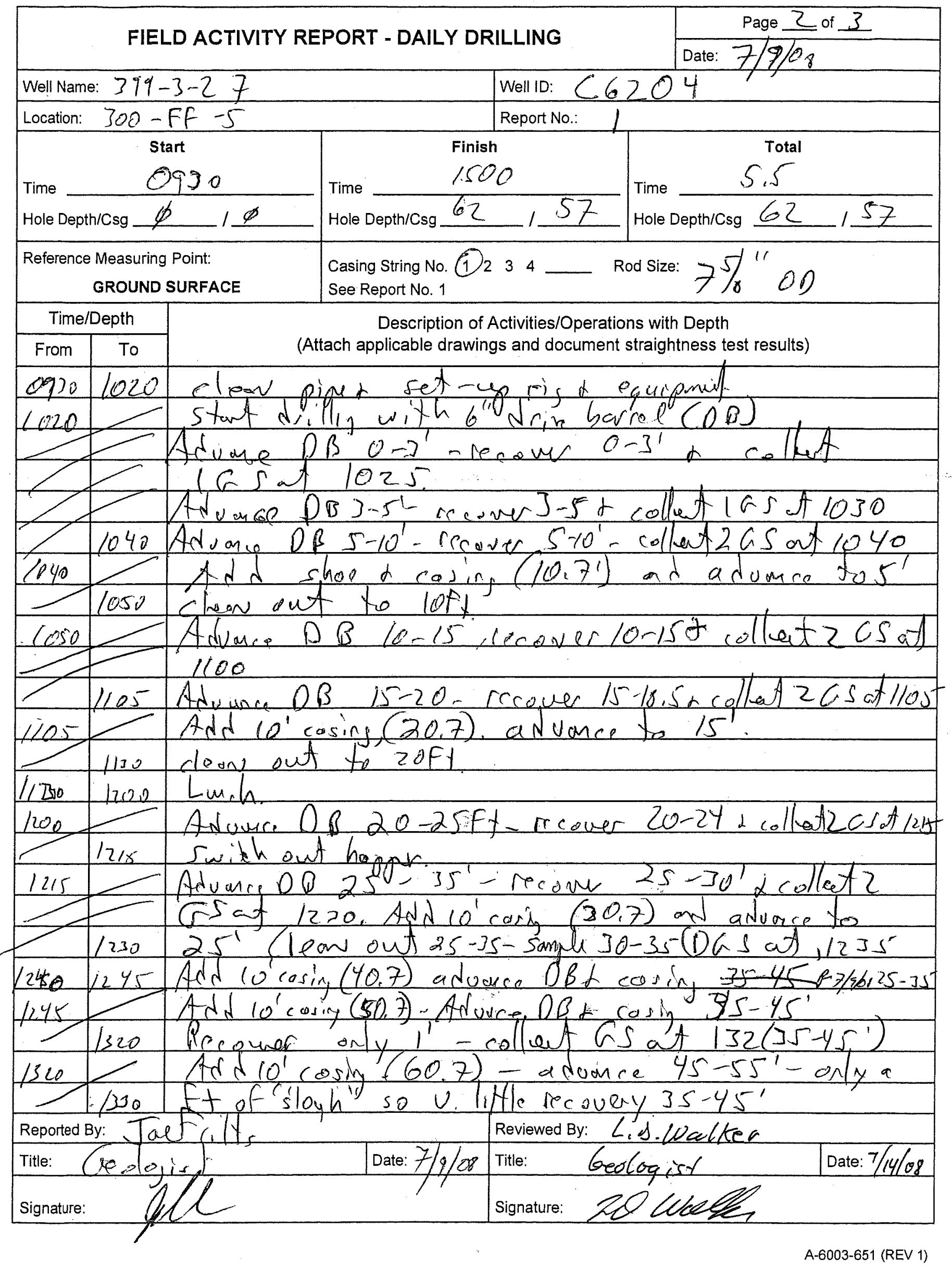

D. 254 


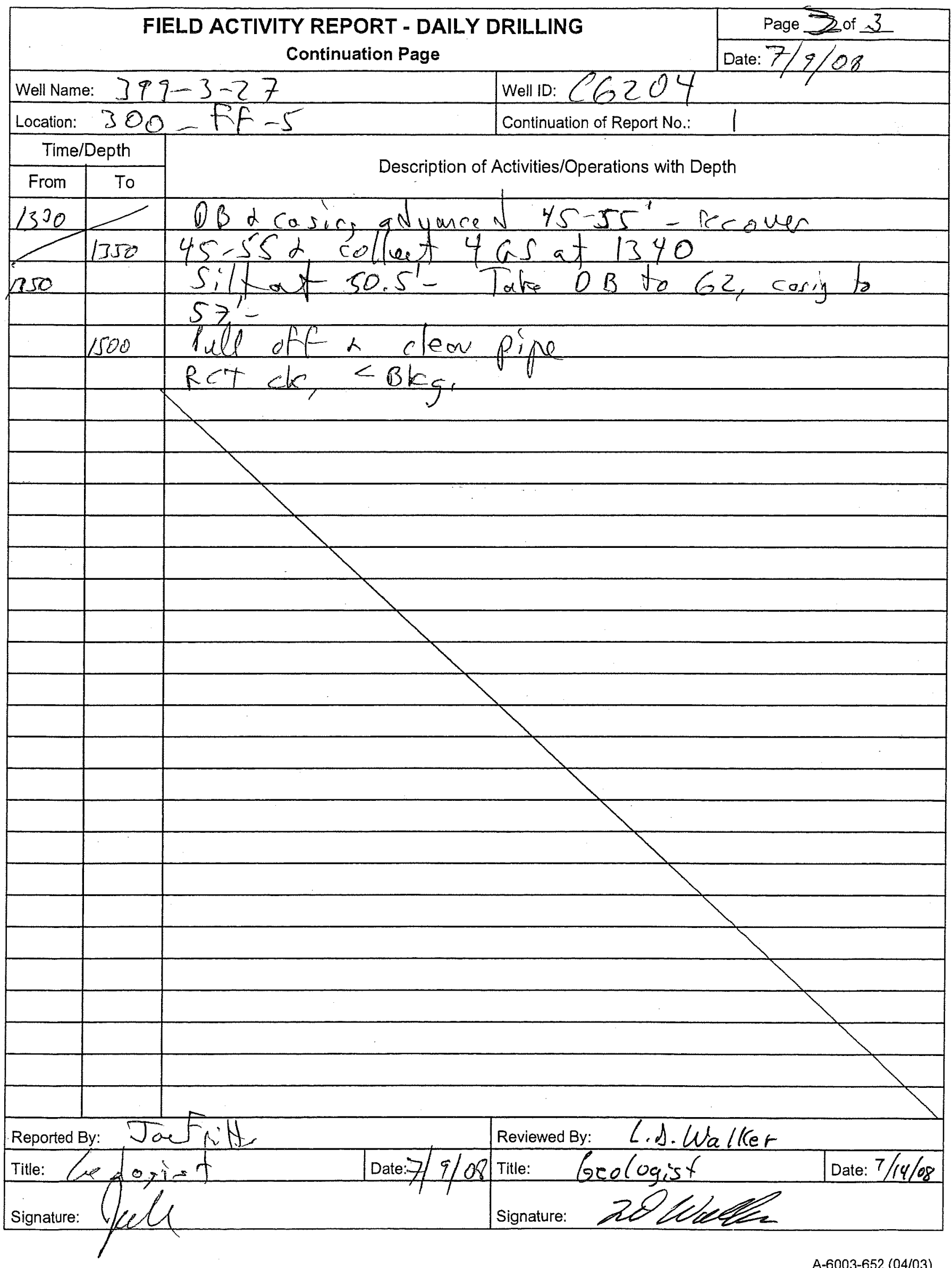

D. 255 


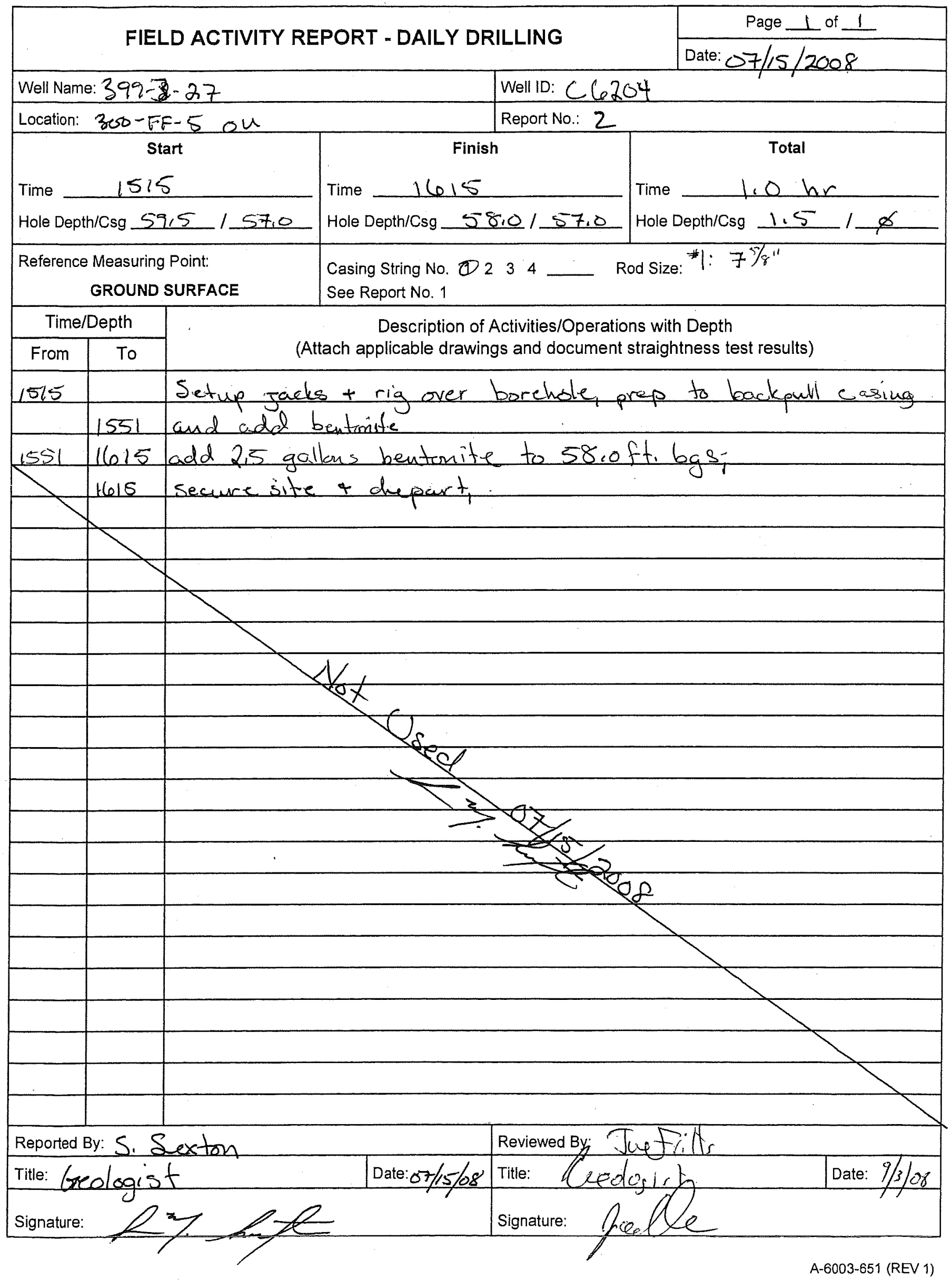

D. 256 


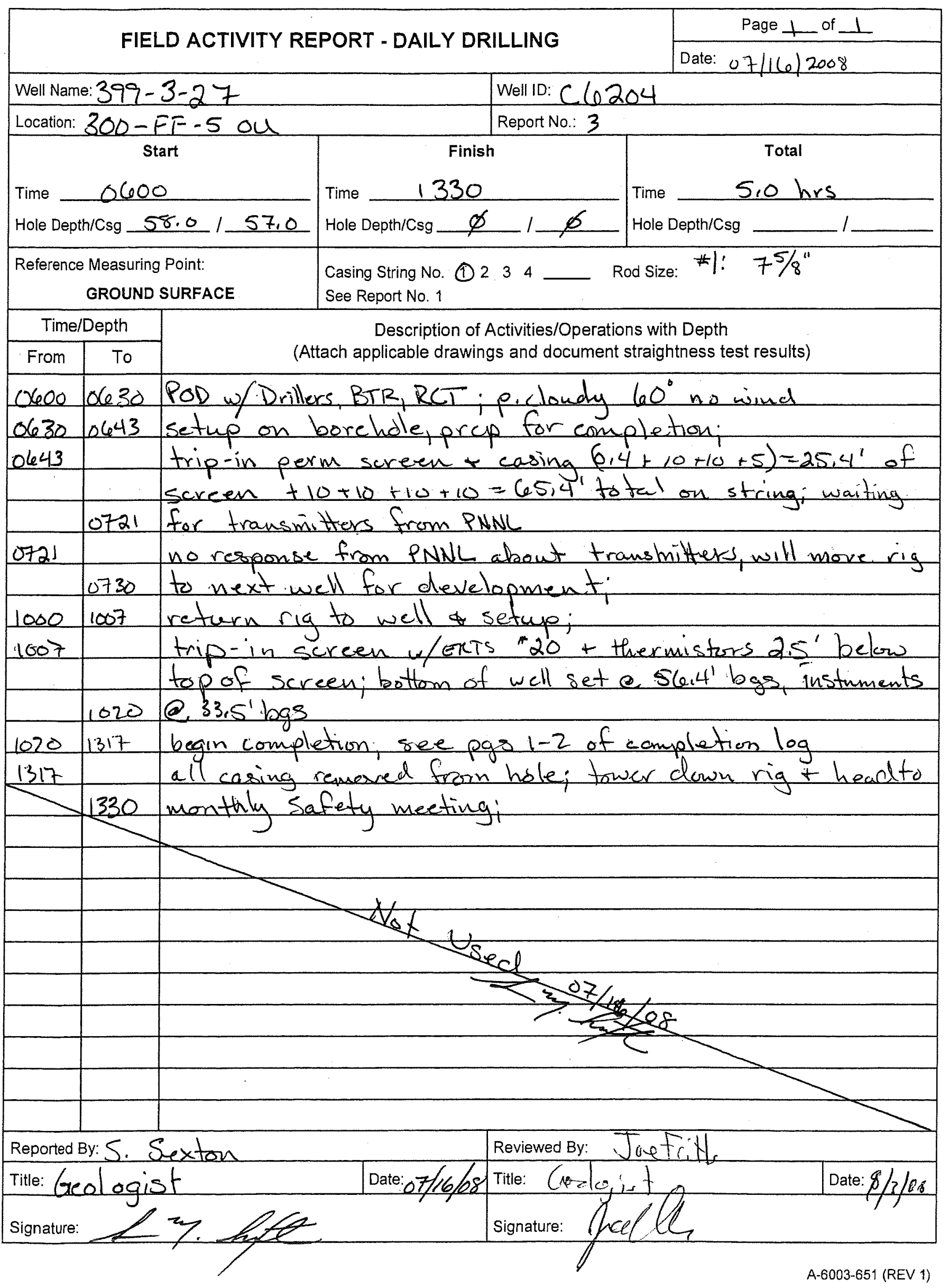

D. 257 


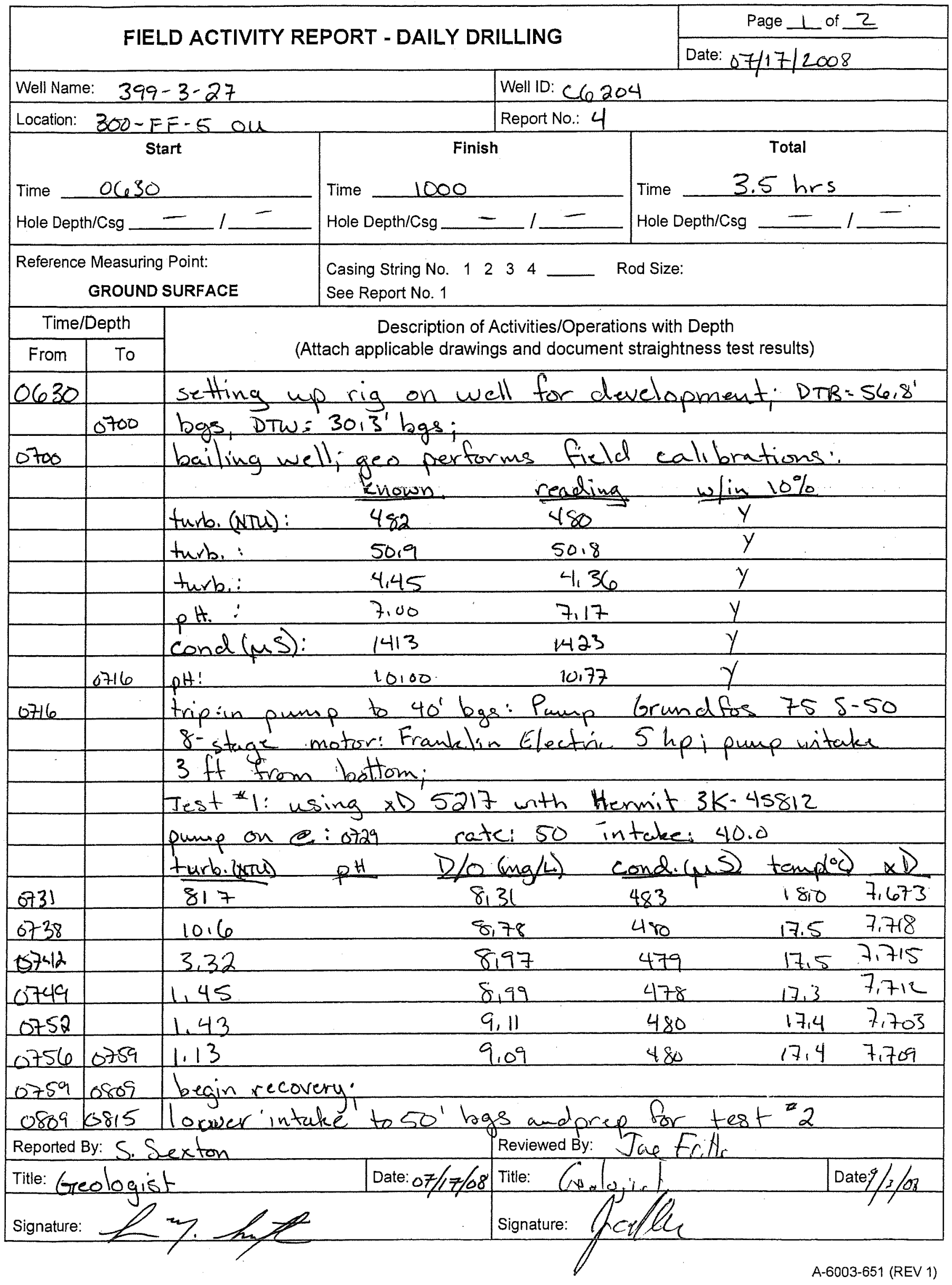

D. 258 
Well Name: $399 \cdot 3-27$
Location: $300-F F-5$ ou

\begin{tabular}{|c|c|}
\hline \multicolumn{2}{|c|}{$300-F F-5$ ou } \\
\hline Time/Depth \\
\hline From & To
\end{tabular}

Well ID: C6204

Continuation of Report No.: 4

Description of Activities/Operations with Depth

08150845 waiting for new pH meter

0845 field calibration for pH: known-7.00 reading - 7.09

0856 known - 10,00 reading - 10,03

0856 Test 2: Draudown recorded an Sun Dried WD7

pump on at:.0858 rate: 84 intake: 50.0

turb. pH D/O cond temp. $x D$

\begin{tabular}{|c|c|c|c|c|c|c|c|}
\hline 0900 & & 36,0 & 7.52 & 8,90 & 485 & 17.8 & 2,235 \\
\hline 0903 & & 4.09 & 7.53 & 9.10 & 481 & 17.4 & 2,224 \\
\hline 0907 & & 2.26 & 7,50 & 9,17 & 480 & 17.5 & 2,221 \\
\hline 6910 & & 1137 & 7,49 & 9.16 & 481 & 17.3 & 2.224 \\
\hline 0912 & 0914 & 0.97 & 7.51 & 9.21 & 481 & 17.2 & 2,212 \\
\hline
\end{tabular}

09140925 pump shut off hegu recovery test on fim Dried wD 8 09251000 tower down rig + move to next well

Reported By: S. Sexton

Title: Geologist

Signature: 2
Page 2 of 2 Date: $07 / 17 / 2008$ 


\section{WELL DEVELOPMENT AND TESTING DATA}

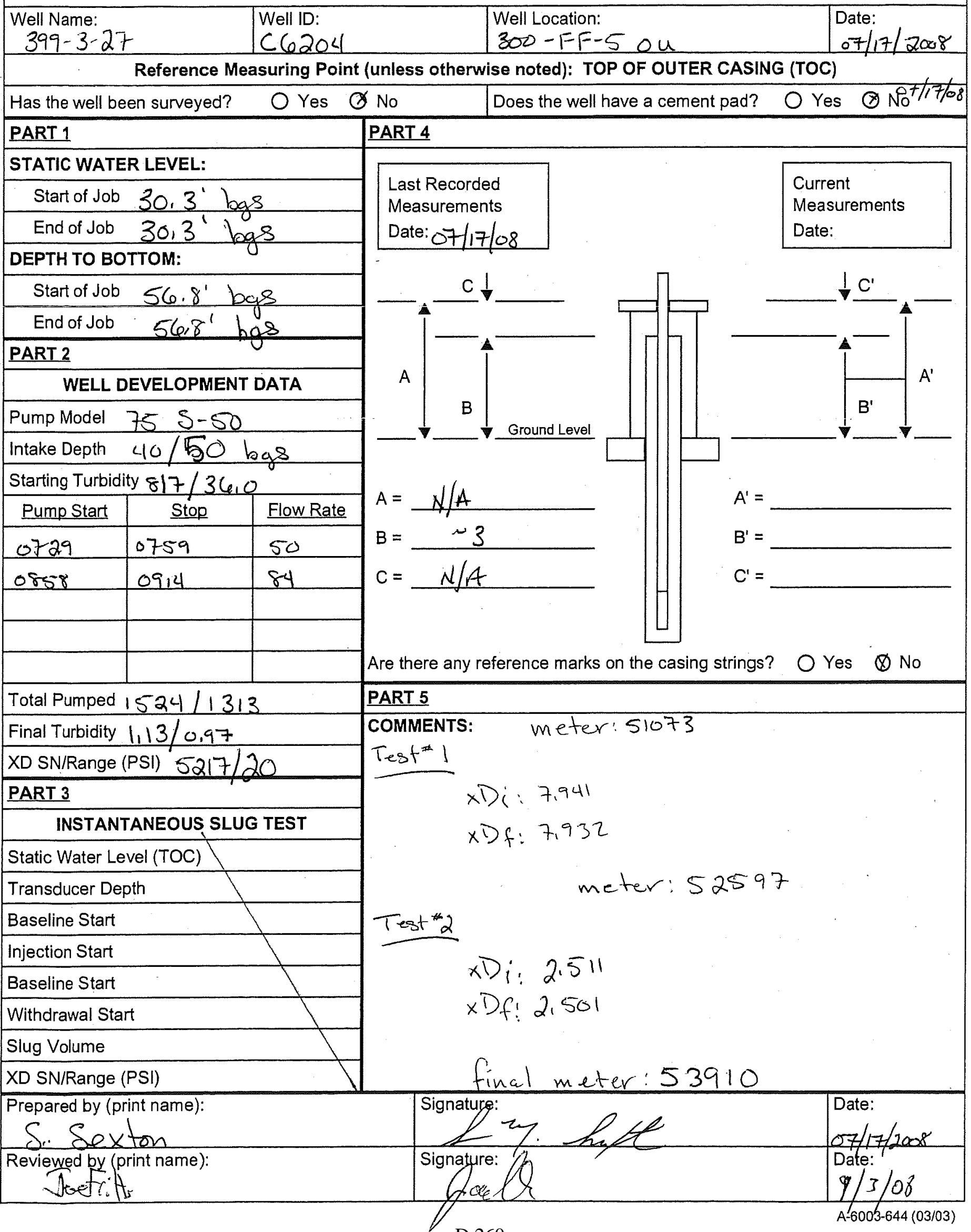


FIELD ACTIVITY REPORT NO. 1 - DRILLING PLAN

\begin{tabular}{|c|} 
Page 1 of 2 \\
\hline Date: $7 / 8 / 08$
\end{tabular}

Purpose: P n L L Chavacterization 300 Area Jfe Location: 300 - FF- 5

Well ID: 26205

Drilling Co.: Driller: Bill Zamoz/WDC

Casing String No. \begin{tabular}{lllll|l}
1 & 2 & 3 & 4 & Drilling Method
\end{tabular}

Casing Size $75 / 8 / 6^{7} / 8$

Grade

Lbs.Per Ft.

Material Carban Stec)

Type:

Weided

(Thd)

Planned / Actual

Set At: ___

Shoe OD/ID

Reference Measuring Point:

GROUND LEVEP

Drig. Co.

Rig No.:

Casing String No, 1 \begin{tabular}{llll}
\hline & 2 & 3 & 4
\end{tabular}

Casing Size

Grade

Lbs.Per Ft.

Material

Type:

Welded

Thd.

Planned / Actual

Set

Shoe OD/ID

Reference Measuring Point:

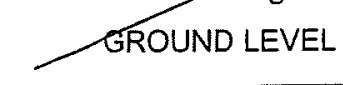

Comments/Remarks:

Rotary

Tubex

Cable Tool

Eonic) Saper Sonie 2

A.R. w/Sonic RS 400

Geoprobe

Other:

Shor $=0.7^{\prime}$
Auger

Well Name: $399-3-28$

Rig No.: 139 Rig Make/Mod.: Smper Senic II

$\begin{array}{ll}\text { Circulation } & \text { D.H. Hammer }\end{array}$

Air _ Water/Mud -

Reverse — Direct

Vol: $\mathrm{cfm}$

gpm

Pressure

psi

Drill Pipe O.D.

Tool Joint Size

Additives

Make

Model

Choke

Casing Hammer

Make

Model

Bit Size

Type

Nozzles

Rod Size

Rig Make/Mod.:

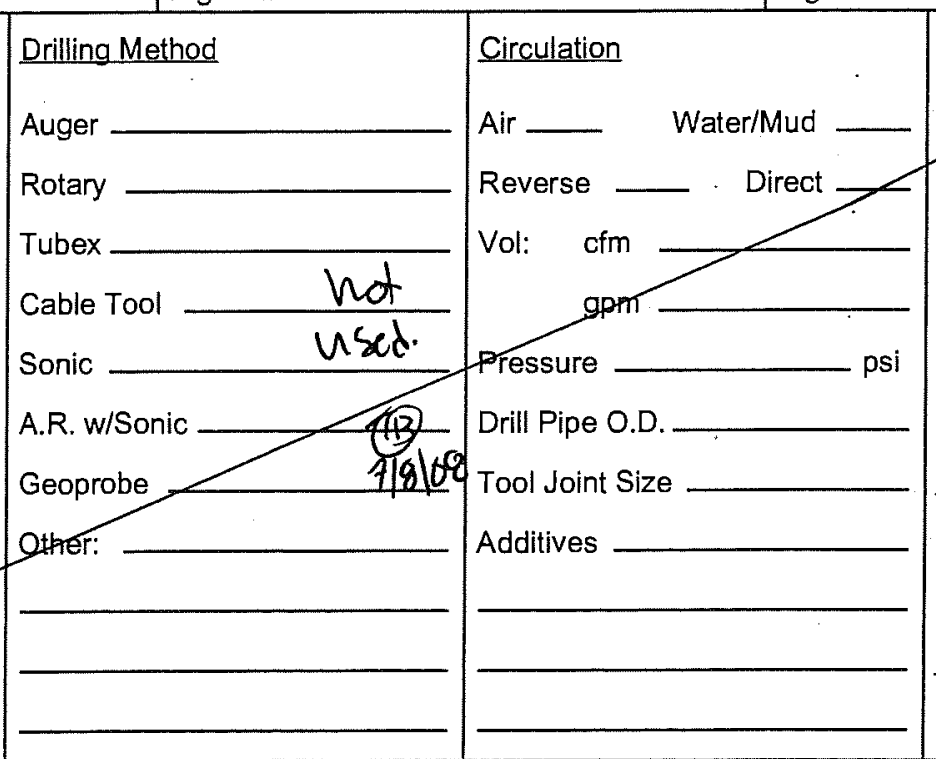

D.H. Hammer

Make

Model

Choke

\section{Casing Hammer}

Make

Model

Bit Size

Type

Nozzles

Rod Size

Estimated Depth to Water

Reported By: T. Buswe ll

Namertitle: GRAm Inc, Gedogist

Signature:

$P$ casar Buell

Date: $7 / 8 / 08$ 


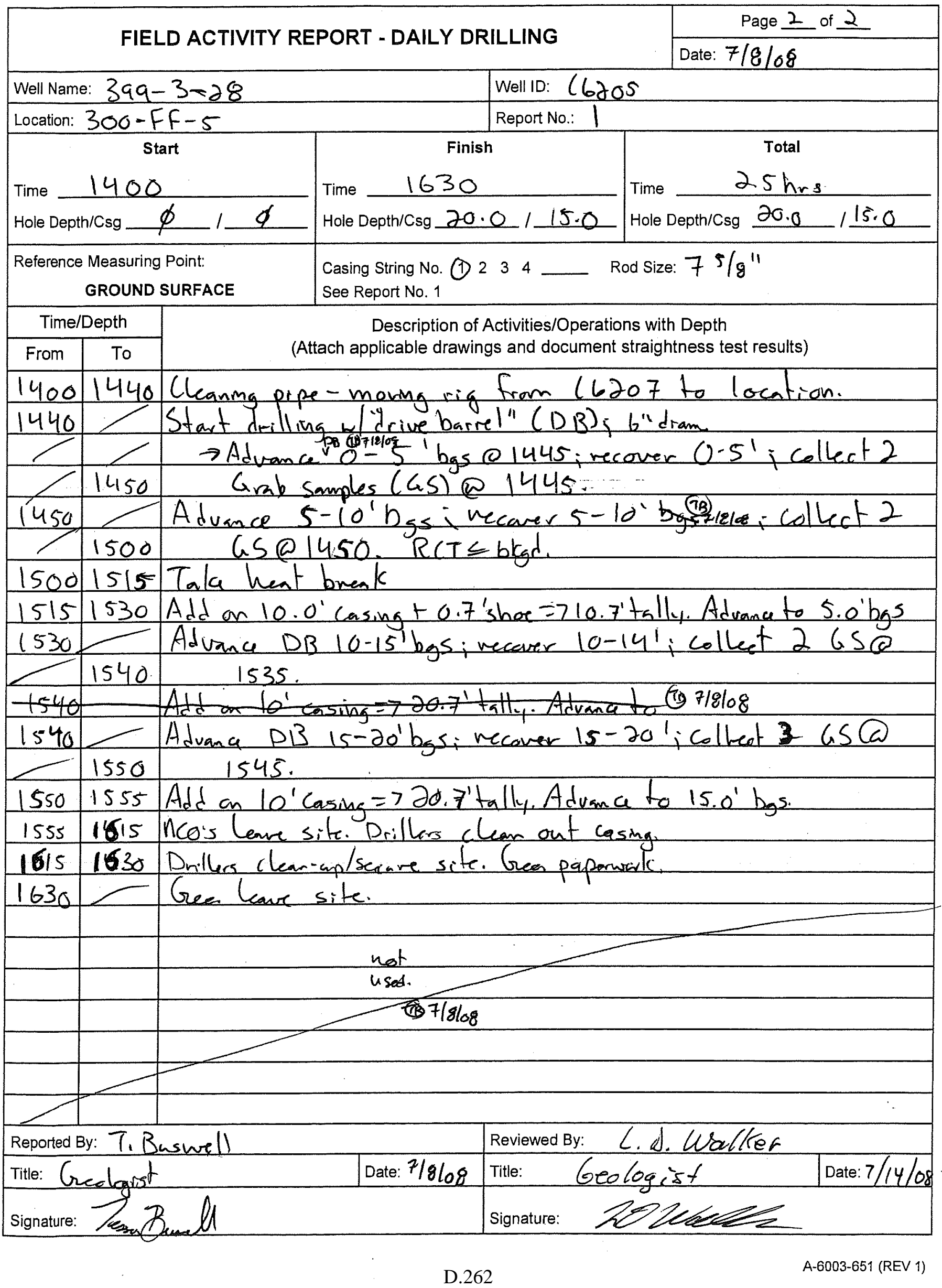




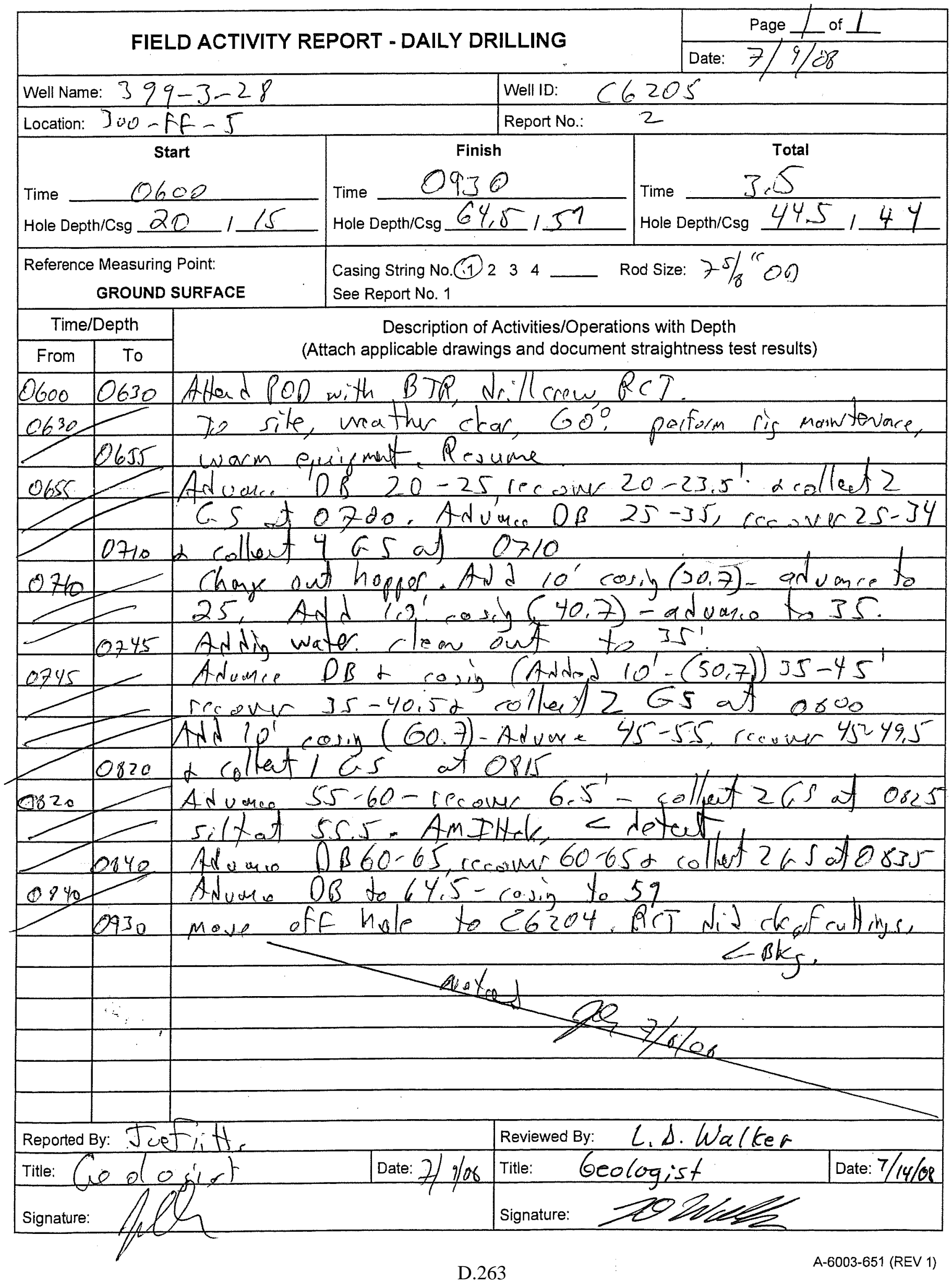


FIELD ACTIVITY REPORT - DAILY DRILLING

Page 1 of 2

Date: $7-24-08$

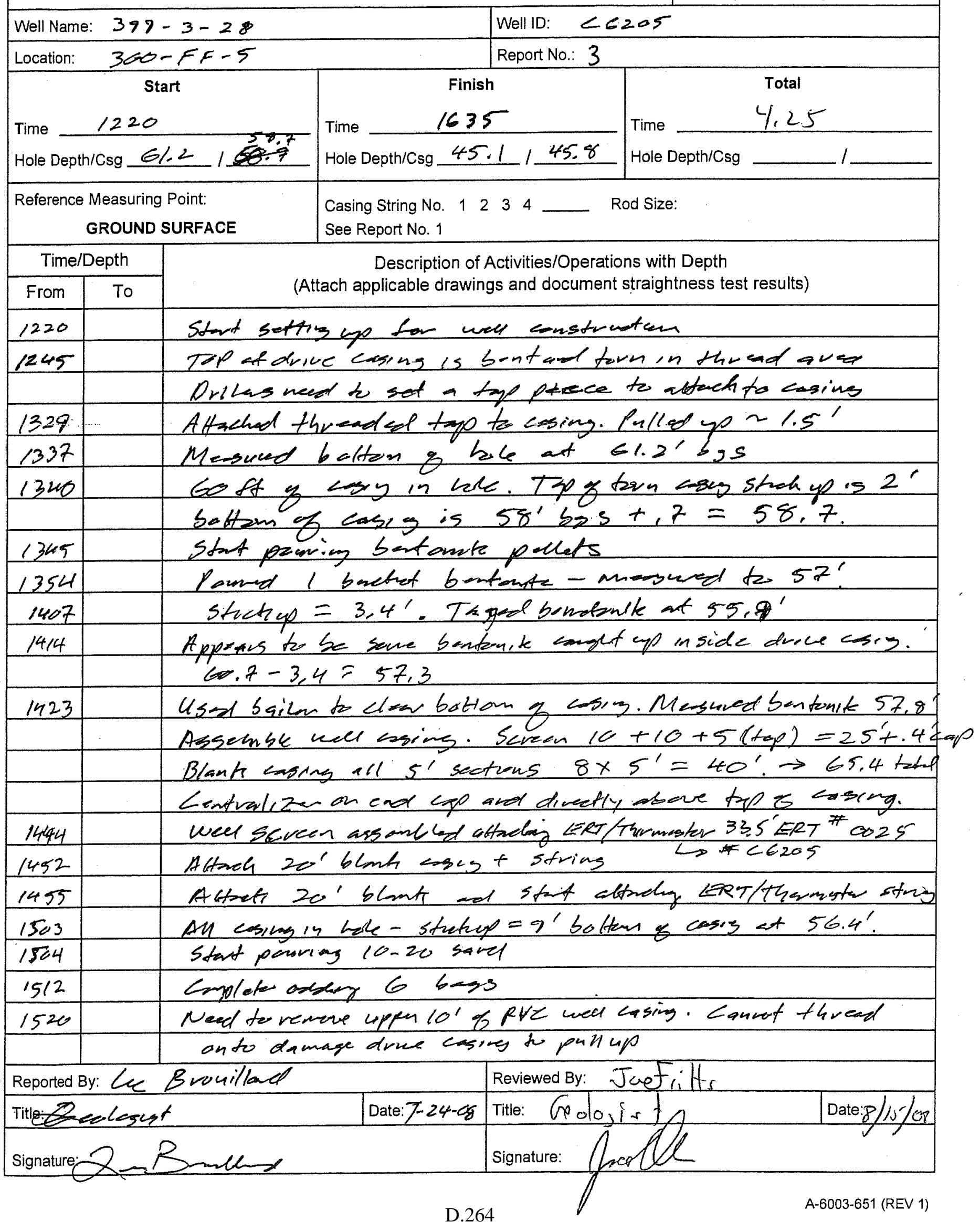


FIELD ACTIVITY REPORT - DAILY DRILLING Continuation Page

\begin{tabular}{|l|l|}
\hline Well Name: $399-3-28$ & Well ID: 06205 \\
\hline Location: $300-F F-5$ & Continuation of Report No.: 3 \\
\hline
\end{tabular}

Page 2 of 2

Date: $7-24-08$

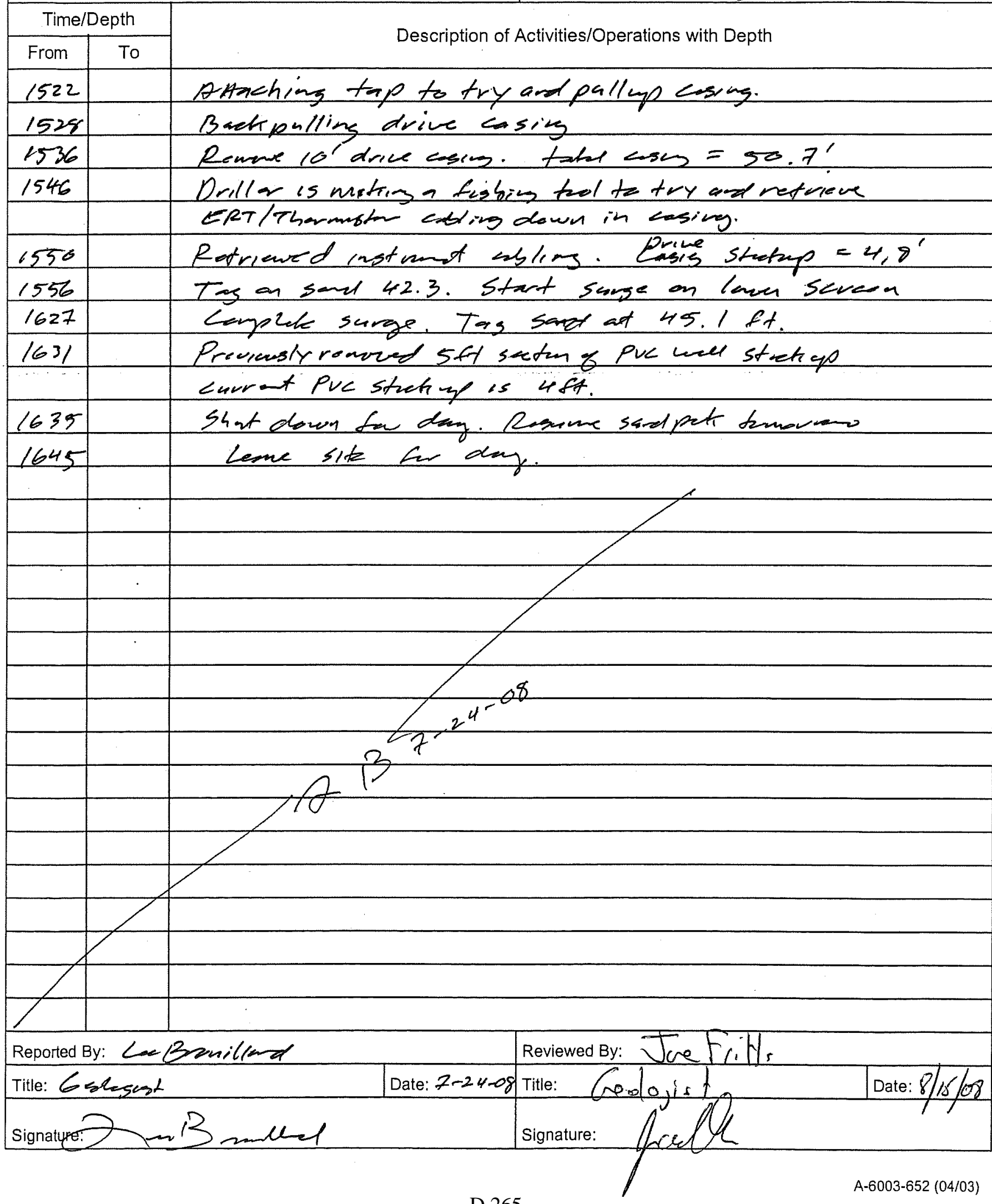




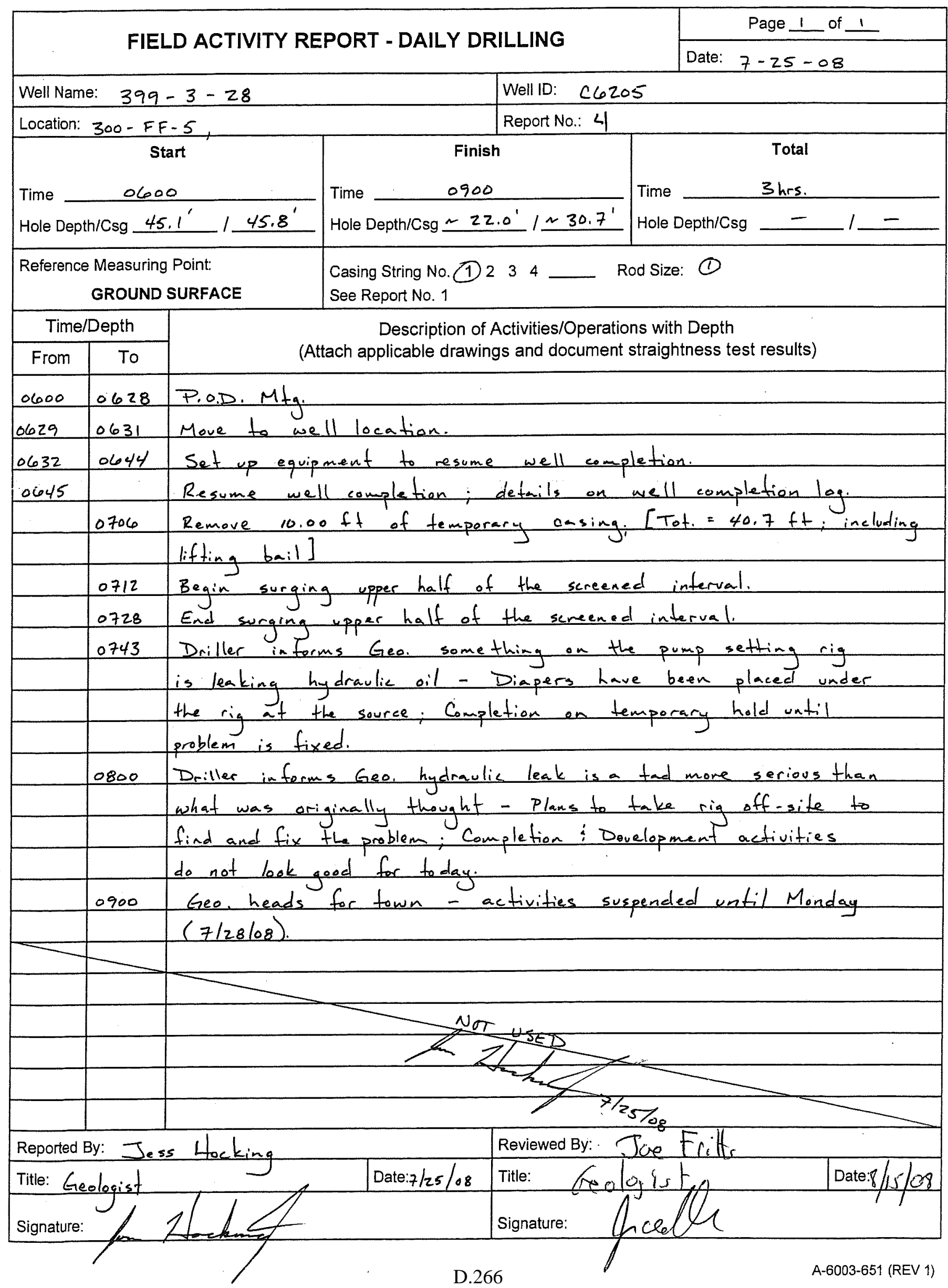


FIELD ACTIVITY REPORT - DAILY DRILLING

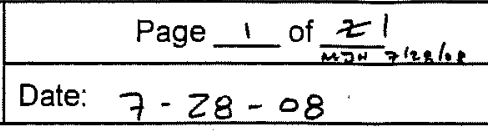
Well ID: C 6205

Report No.: 5

Finish

0754

The/Depth

\section{Time/Depth}

\begin{tabular}{|c|c|c|}
\hline From & To & \\
\hline 0600 & 0625 & P.O.D \\
\hline
\end{tabular}

0626 0628 Move to well site.

\begin{tabular}{|l|l|l}
0628 &. & Drillers set up; make equipment ready to resume \\
\hline 0721 & 0720 & completion \\
\hline & Resume completion; details on well completion log. Remove \\
\hline & $10.00 \mathrm{ft}$ temp. casing $[$ Tot. $=30.7 \mathrm{ft}]$
\end{tabular}

$10.00 \mathrm{ft}$ temp. casing, $\left[T_{0} t .=30.7 \mathrm{ft}\right]$

0731 Remove $10.00 \mathrm{ft}$. temp. casing $\left[T_{0 t}=20.7 \mathrm{ft}\right]$

0741 Remove $10.00 \mathrm{ft}$ temp casing, $[\mathrm{Tot}=10.7 \mathrm{ft}]$

0751 Remove $10.70 \mathrm{ft}$. temp. ansing. $\left[T_{0} \mathrm{t} .=0 \mathrm{ft}\right]$ : All temp.

casing has been removed from the ground.

0754 Driller breaks down pump setting rig - mave to next hole. $(c 6202)$.

Description of Activities/Operations with Depth

(Attach applicable drawings and document straightness test results)

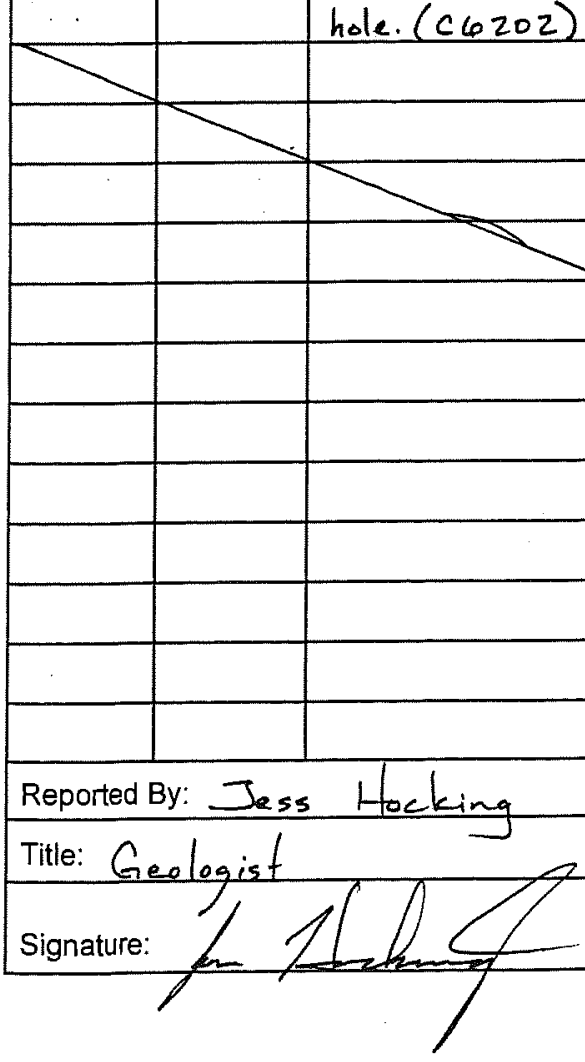

Total

Ihr. 54 min.

Time

1

Casing String No. (1) 234 Rod Size: (1) 8" casing

See Report No. 1

Time $\frac{0600}{\text { Hole Depth/Csg } \sim 22.0^{\prime} / \sim 30.7^{\prime}}$

Reference Measuring Point:

GROUND SURFACE

\begin{tabular}{l} 
Well Name: $399-3-28$ \\
\hline Location: $300-F F-5$ \\
Start \\
Time \\
Hole Depth/Csg $\sim 22.0^{\prime} / \sim 30.7^{\prime}$ \\
\hline $\begin{array}{c}\text { Reference Measuring Point: } \\
\text { GROUND SURFACE }\end{array}$
\end{tabular}




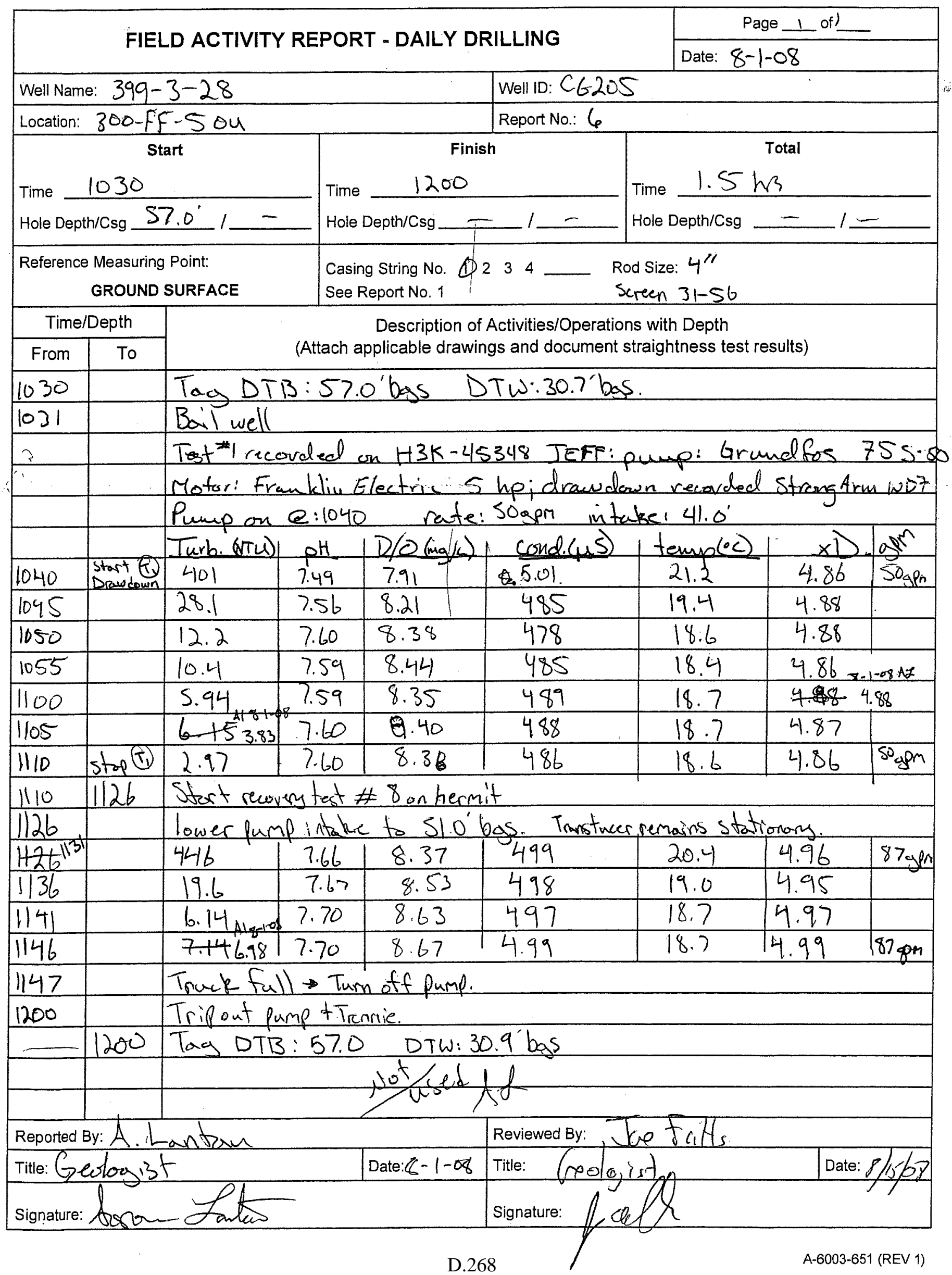


WELL DEVELOPMENT AND TESTING DATA

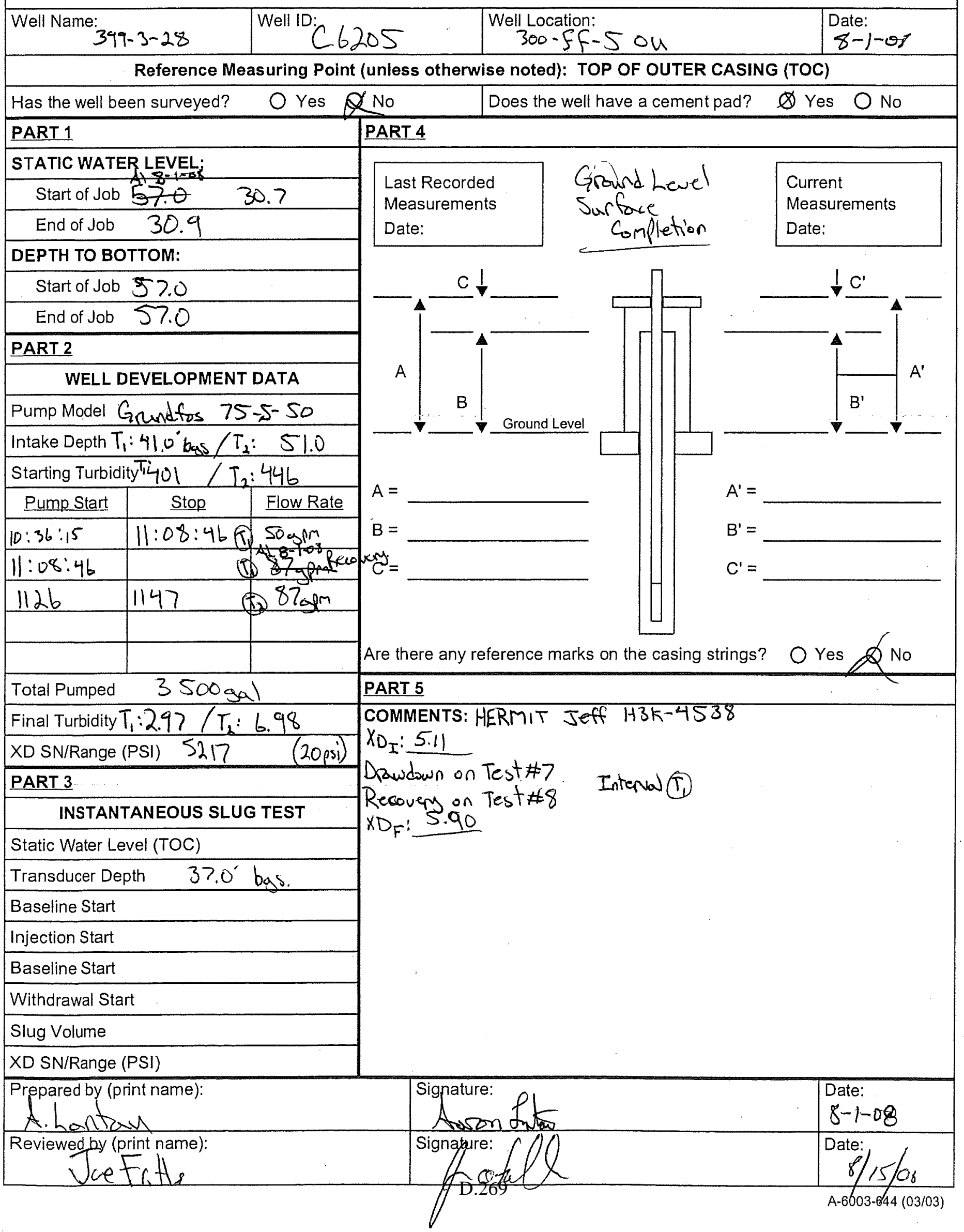


FIELD ACTIVITY REPORT NO. 1 - DRILLING PLAN

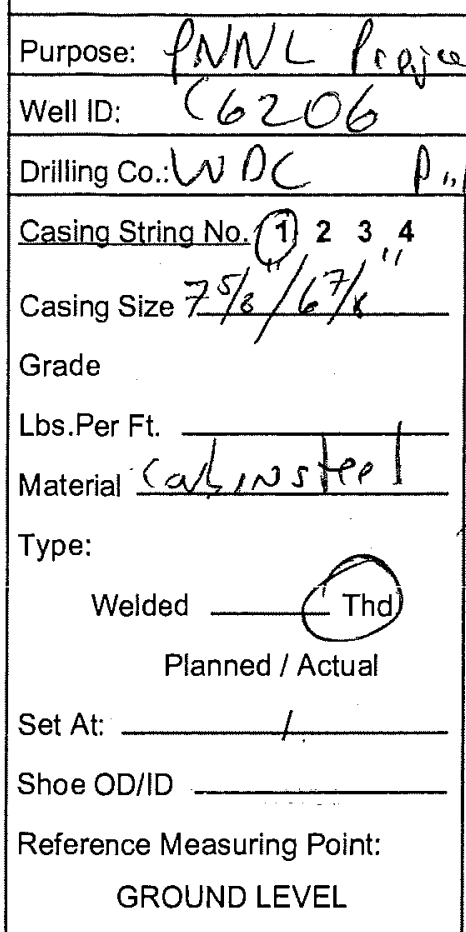

Drig. Co.

Casing String No. 112230

Casing Size

Grade

Lbs.Per Ft.

Material

Type:

Welded Thd.

Planned / Actual

Set

Shoe OD/ID

Reference Measufing Point: GROUND LEVEL
300 Area IFC

Location: $300-F f-5$

Well Name: $799-3-29$

Rig No: $B 9$ Rig Make/Mod.Super Sone

Circulation

Air _ _ Water/Mud

Reverse — Direct

Vol: $\quad \mathrm{cfm}$

Cable Tool $-\mathrm{gpm}$

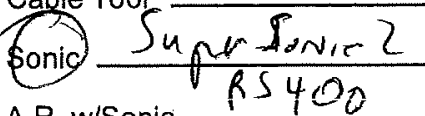

A.R. w/Sonic RS 400

Geoprobe

Other:

Pressure

Drill Pipe O.D.

Tool Joint Size

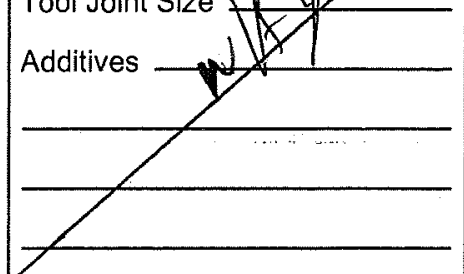

psi

Make

Model

Bit Size

Type

Nozzles

Rod Size

D.H. Hammer

Make

Model

Choke

Casing Hammer

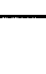

Rig No:

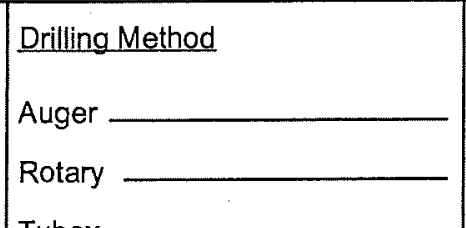

Tubex

Cable Tool

Sonic

A.R. w/Sonic

Geoprobe

Other:

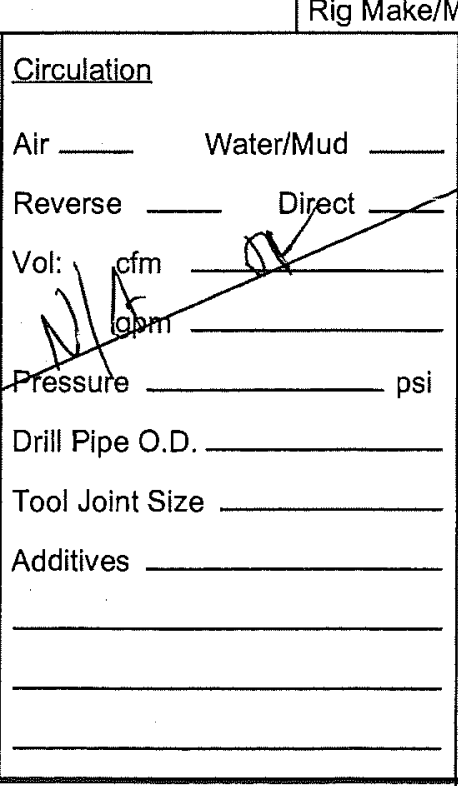

Comments/Remarks:

D.H. Hammer

Bit Size

Type

Nozzles

Rod Size

Estimated Depth to Water

Reported By: Suefits

Name/Title: GRfm Ine/Gedogirt

Signature:

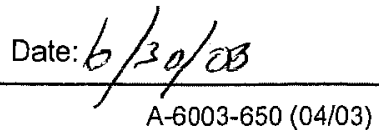




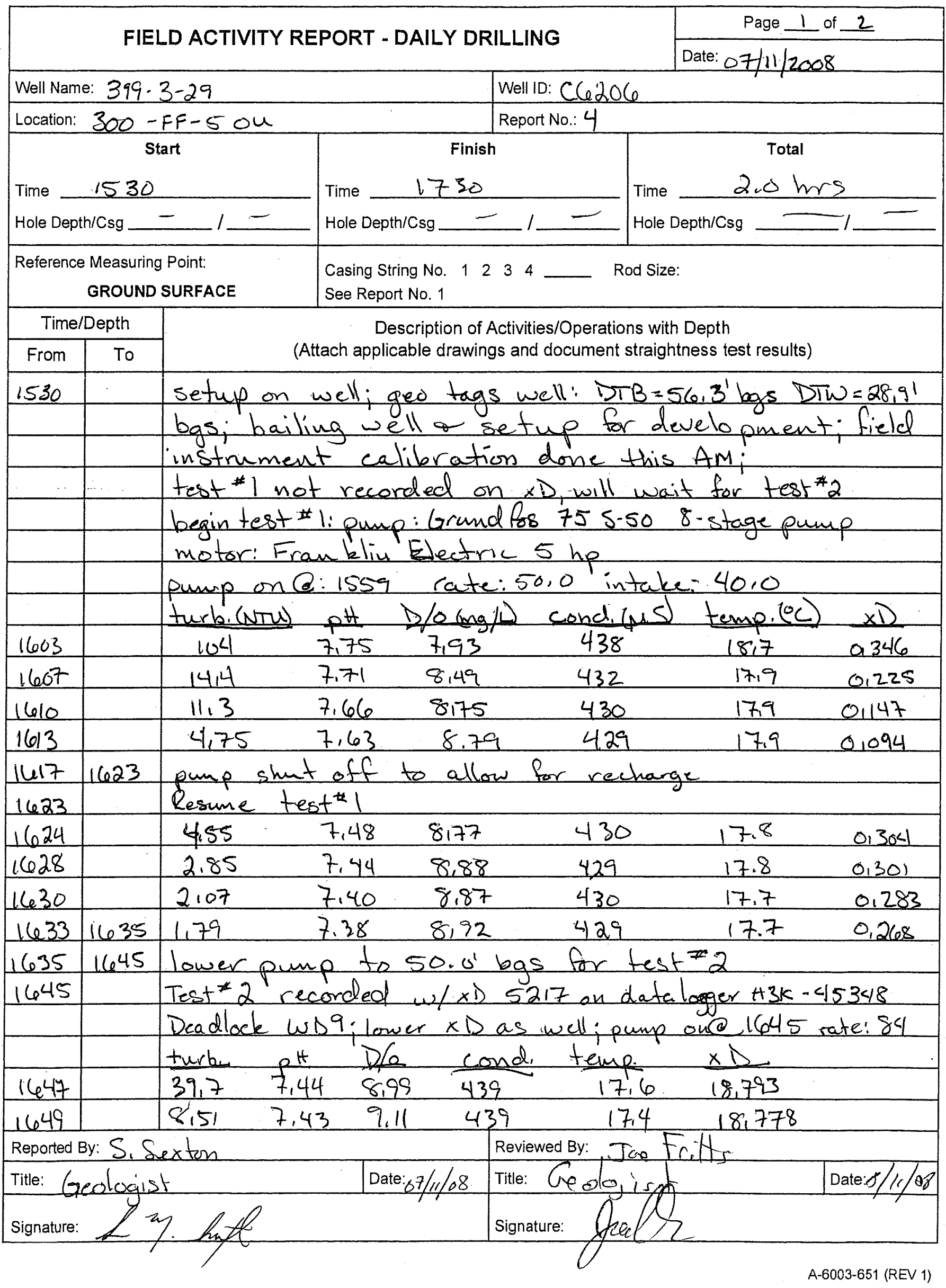

D. 271 


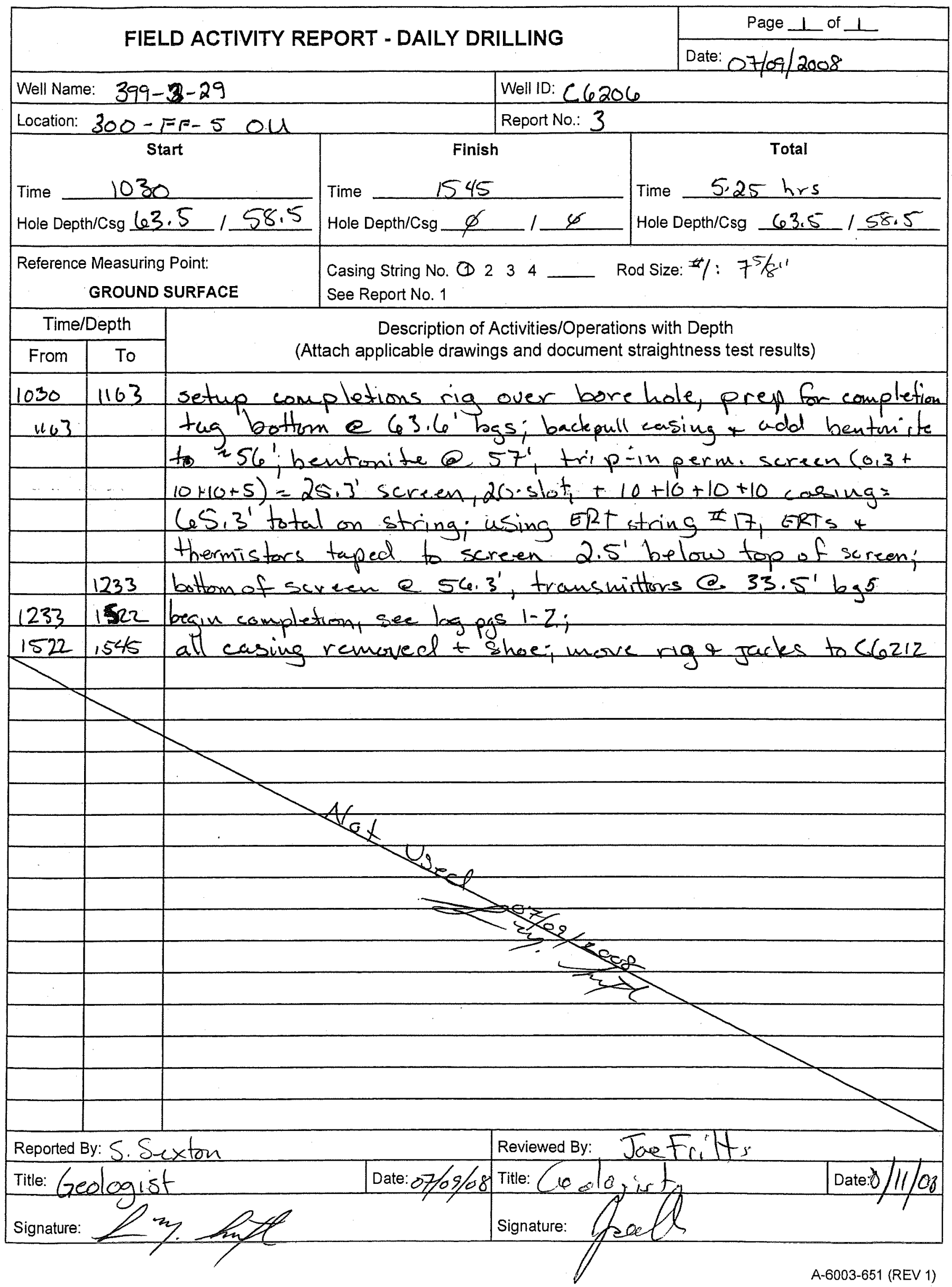

D. 272 


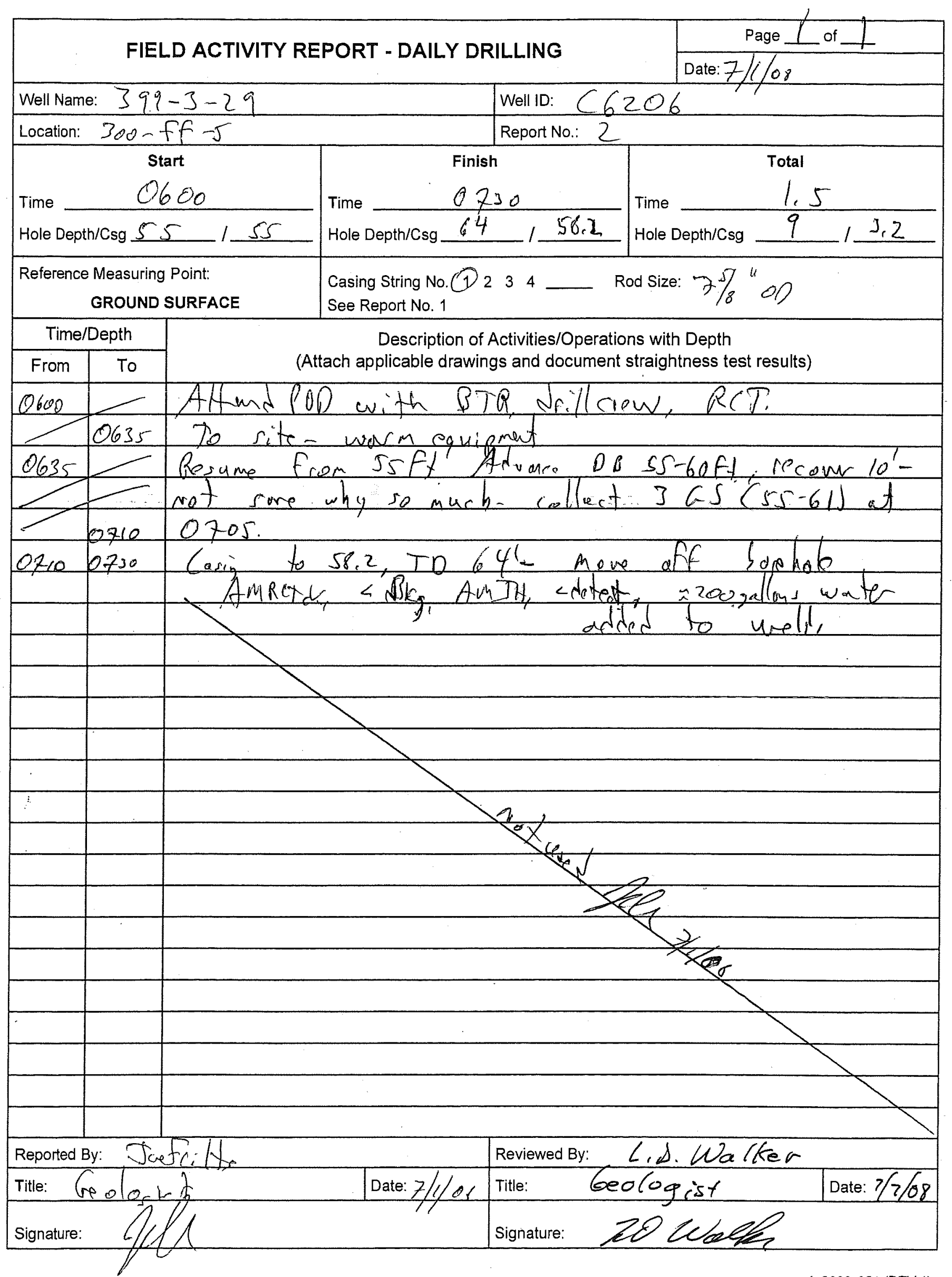

A-6003-651 (REV 1) 


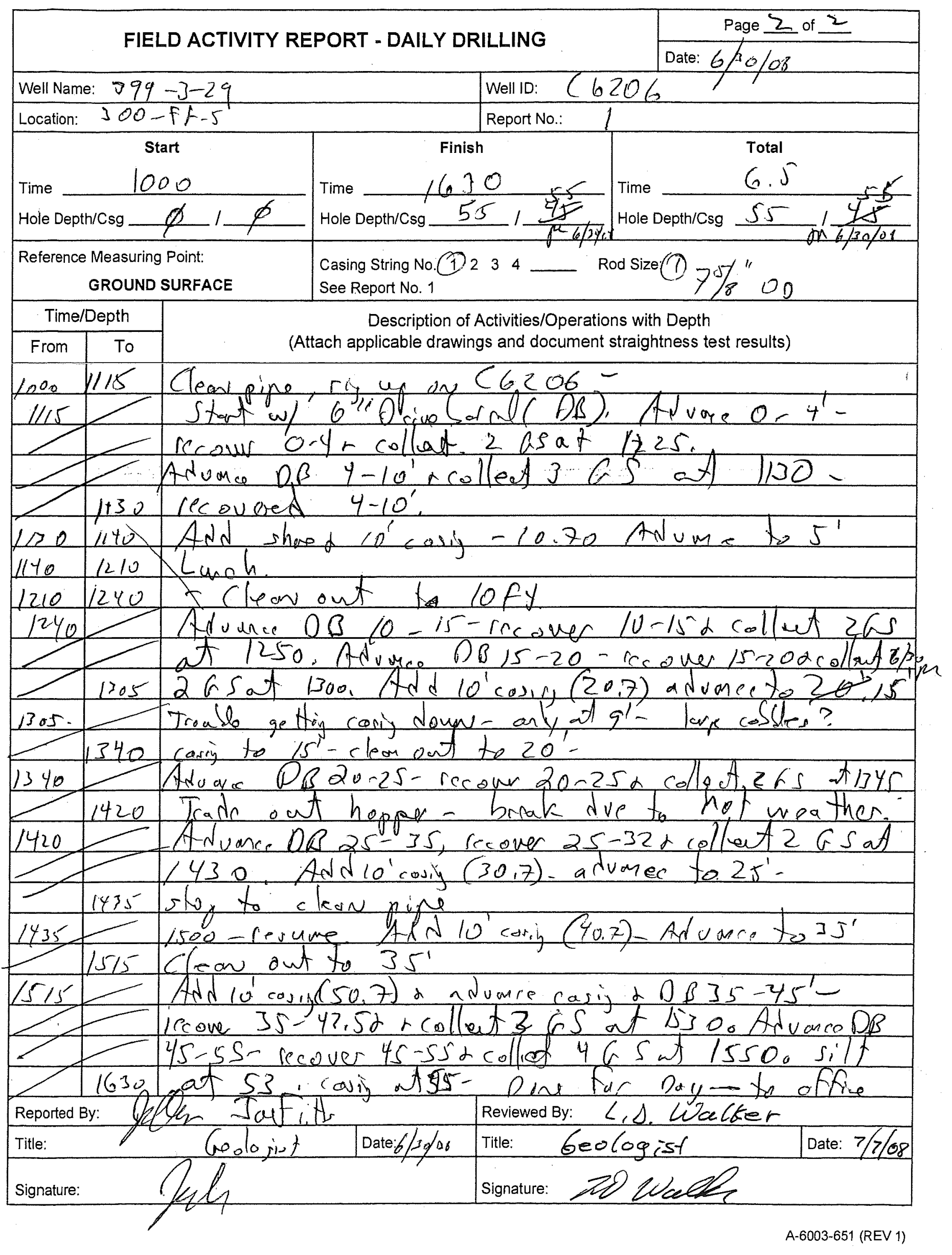

D. 274 


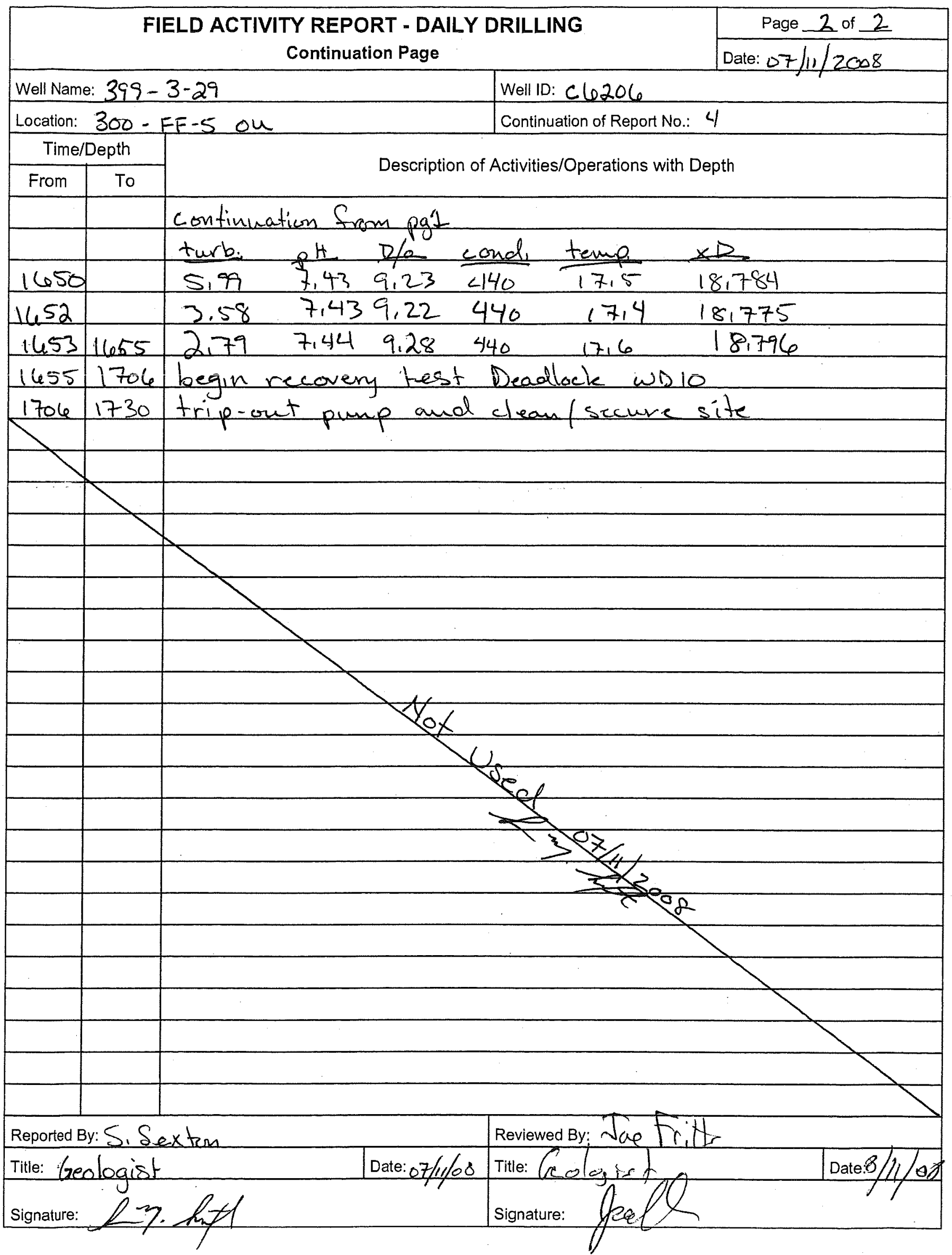




\section{WELL DEVELOPMENT AND TESTING DATA}

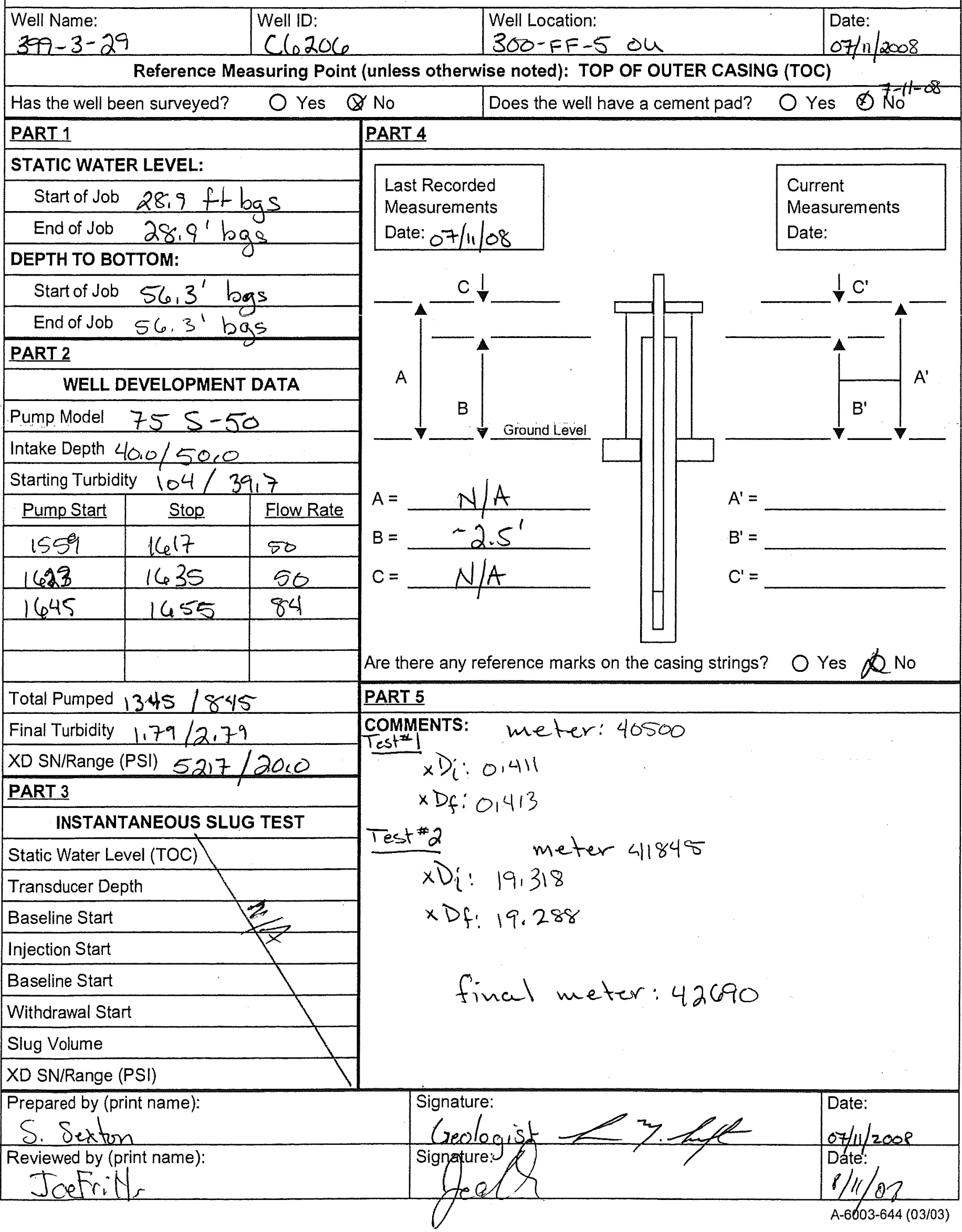

D.276 


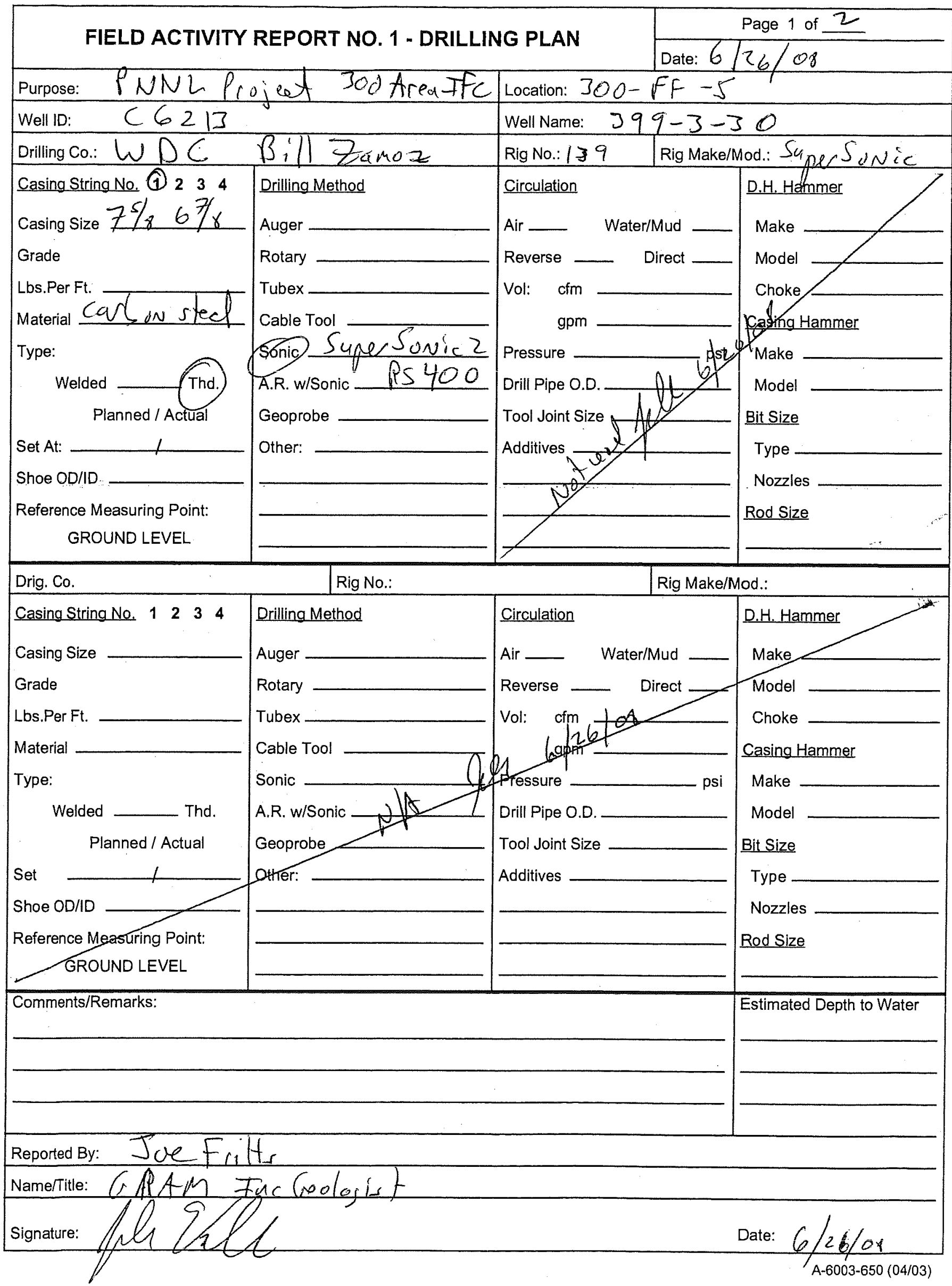




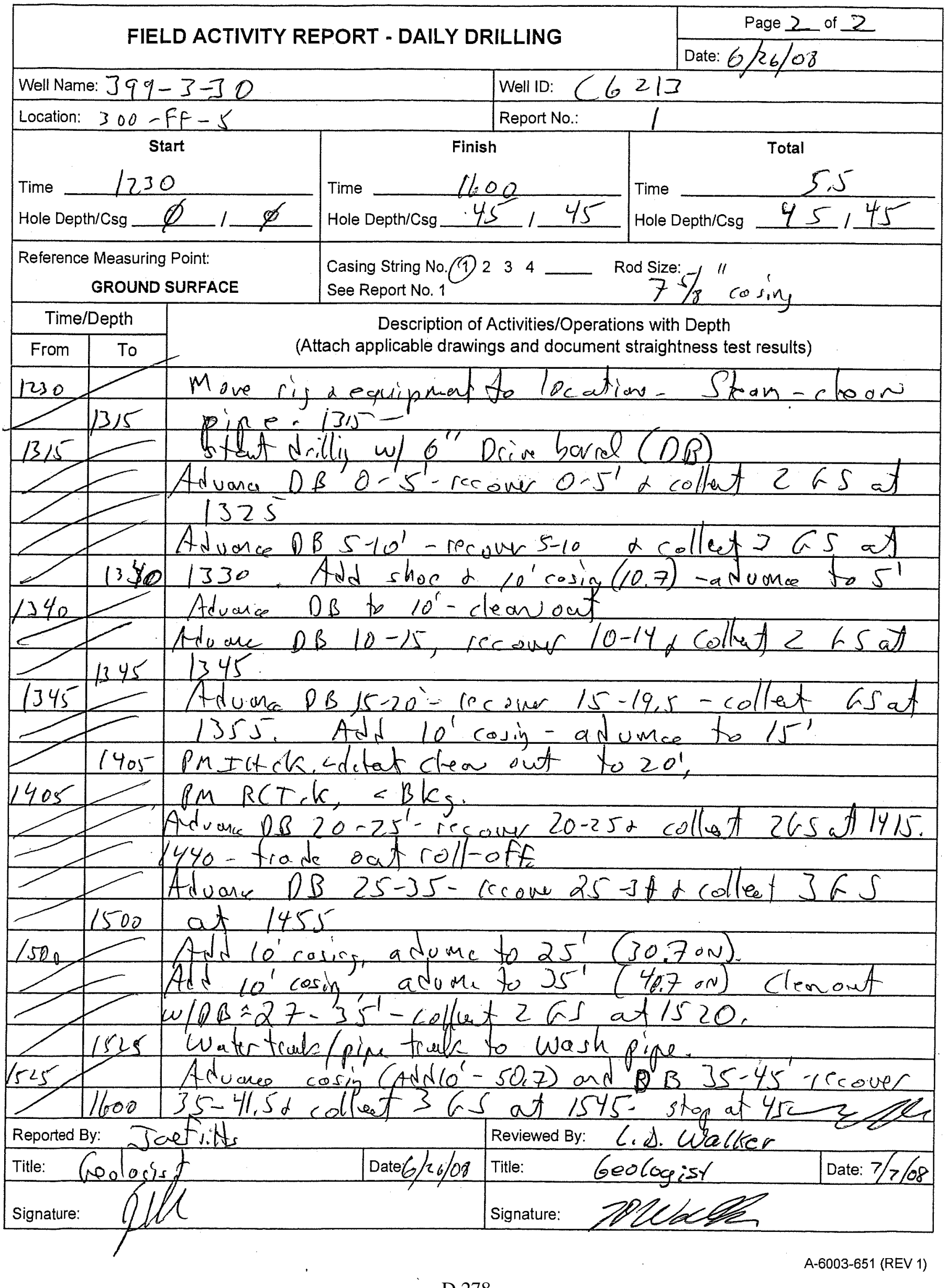




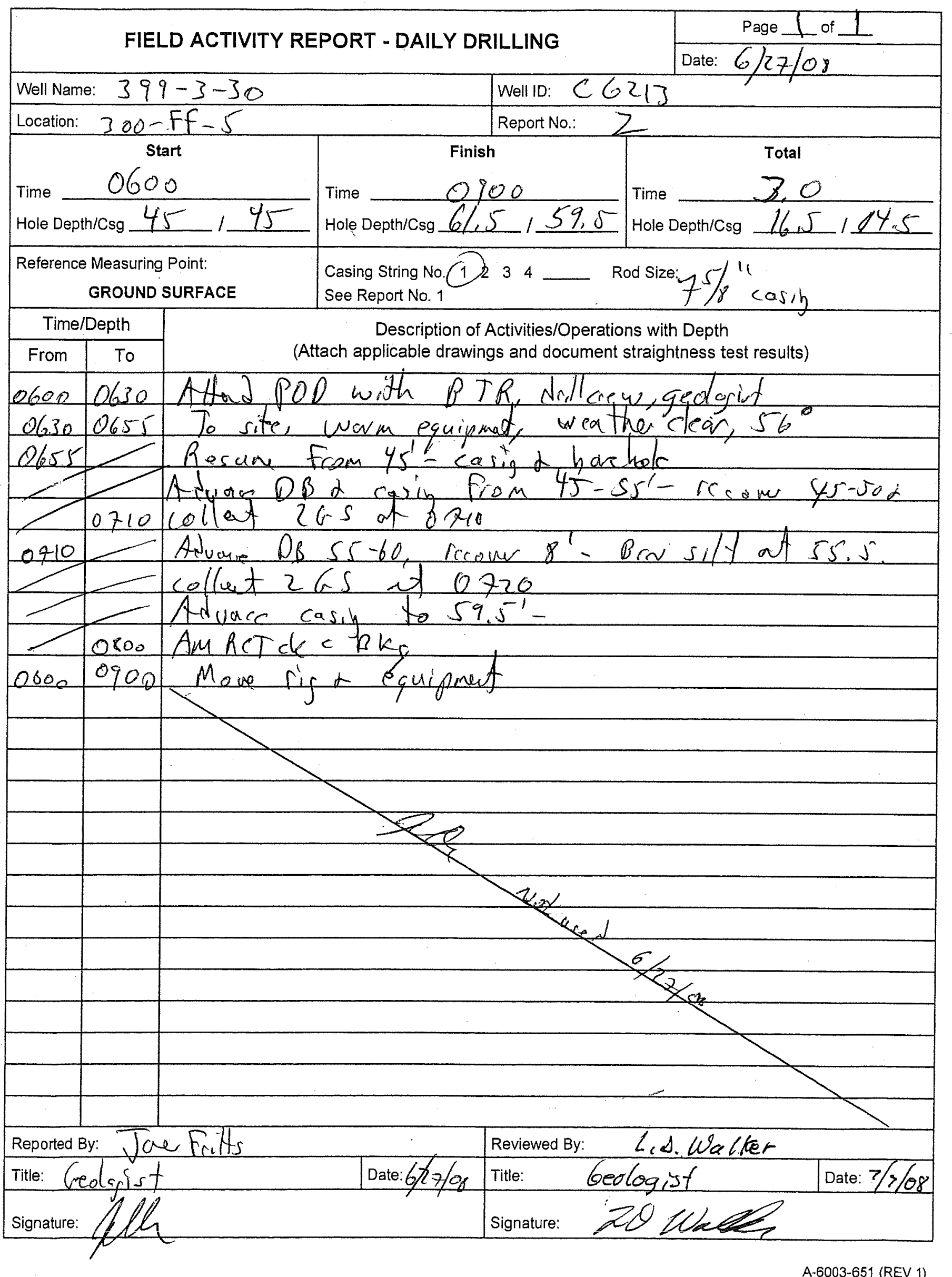

D.279 


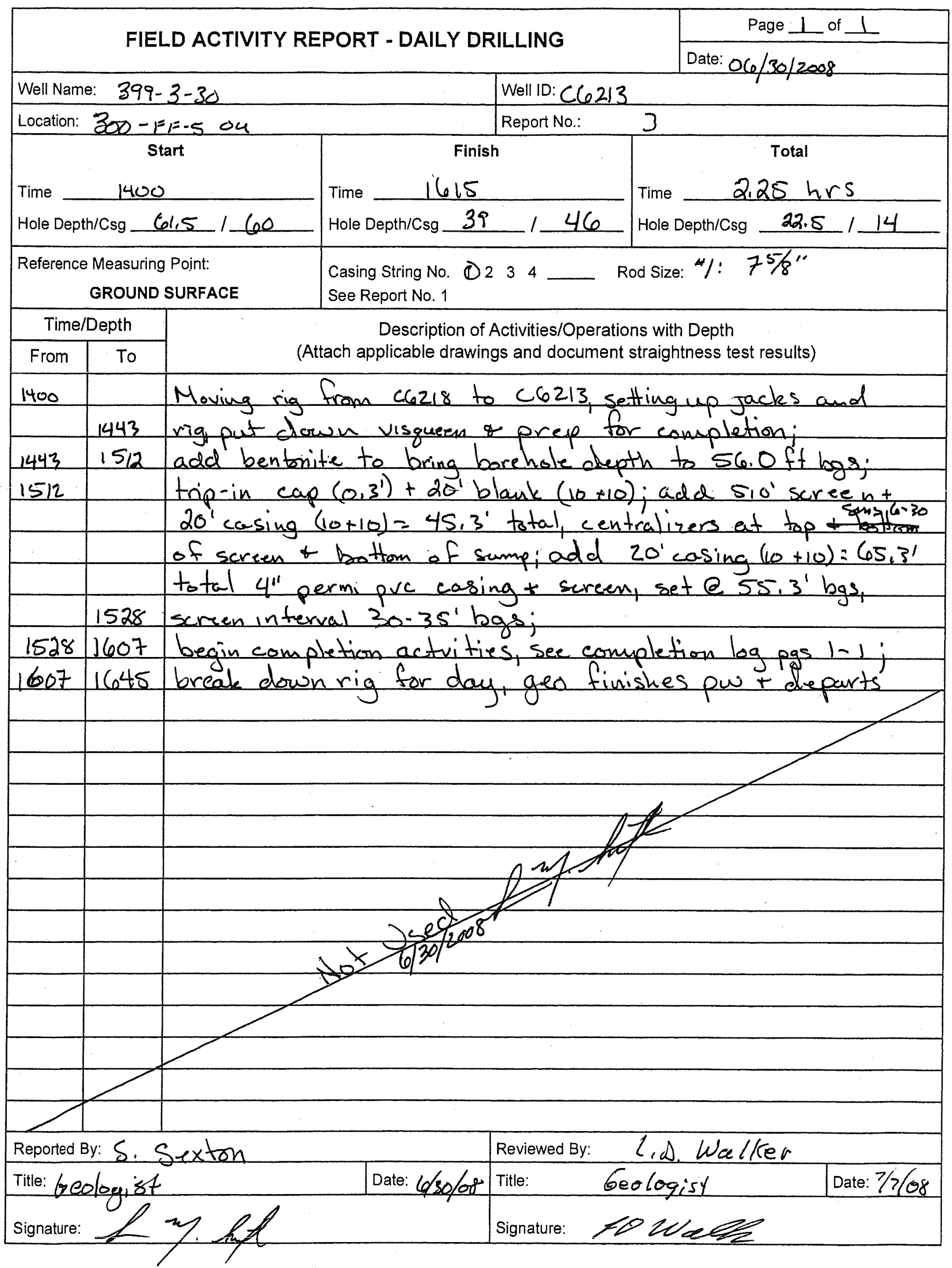




\section{FIELD ACTIVITY REPORT - DAILY DRILLING}

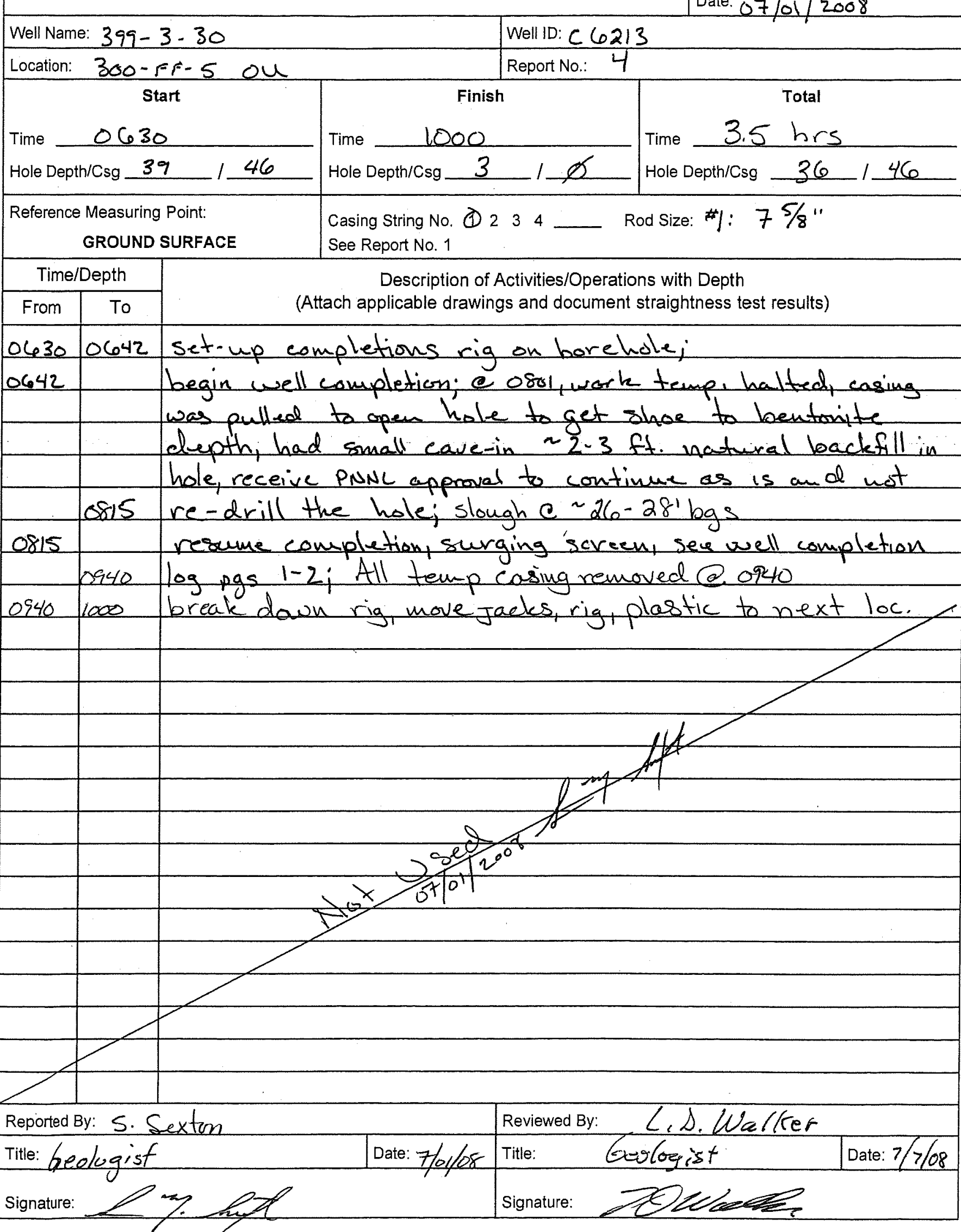




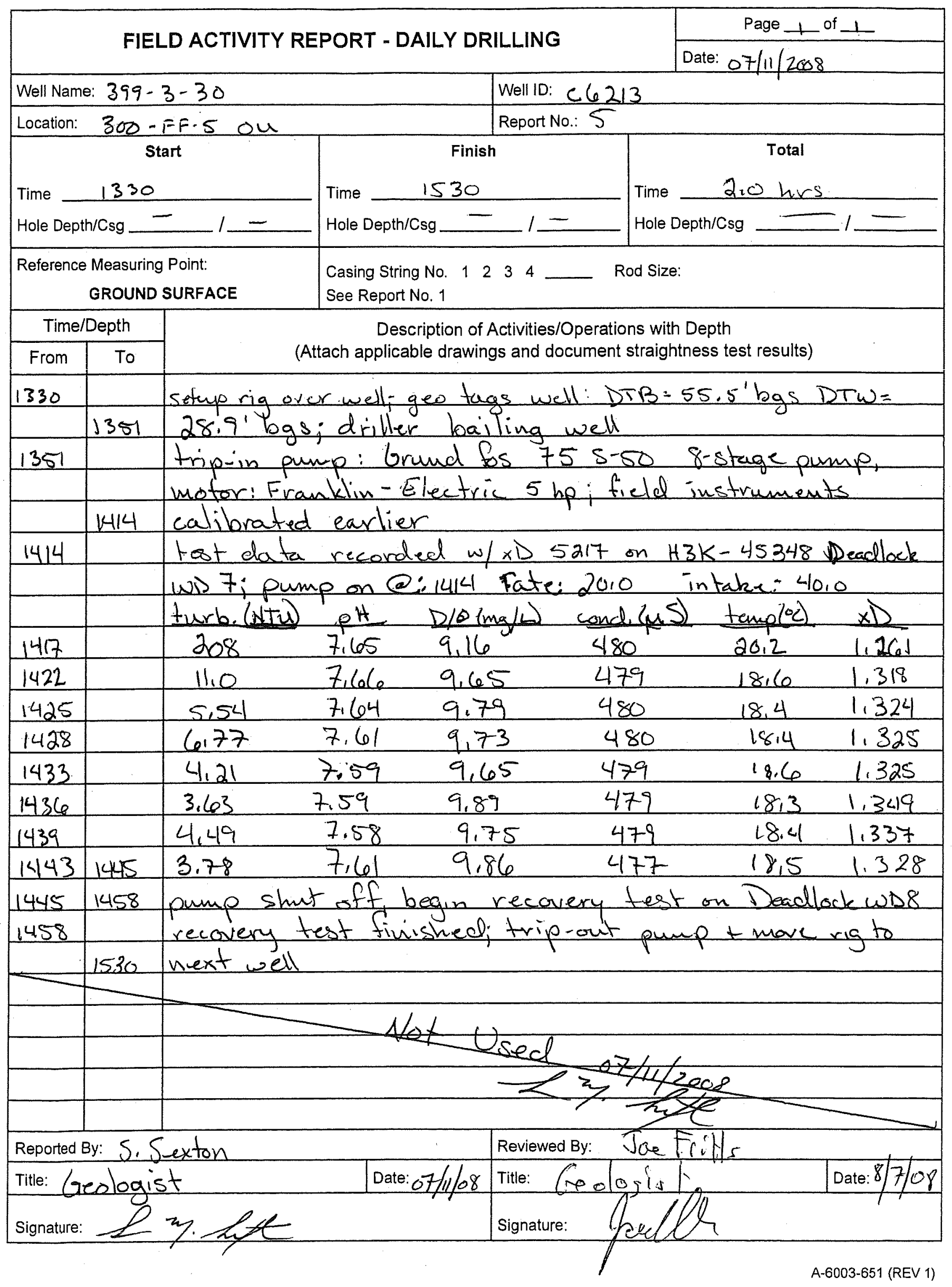




\section{WELL DEVELOPMENT AND TESTING DATA}

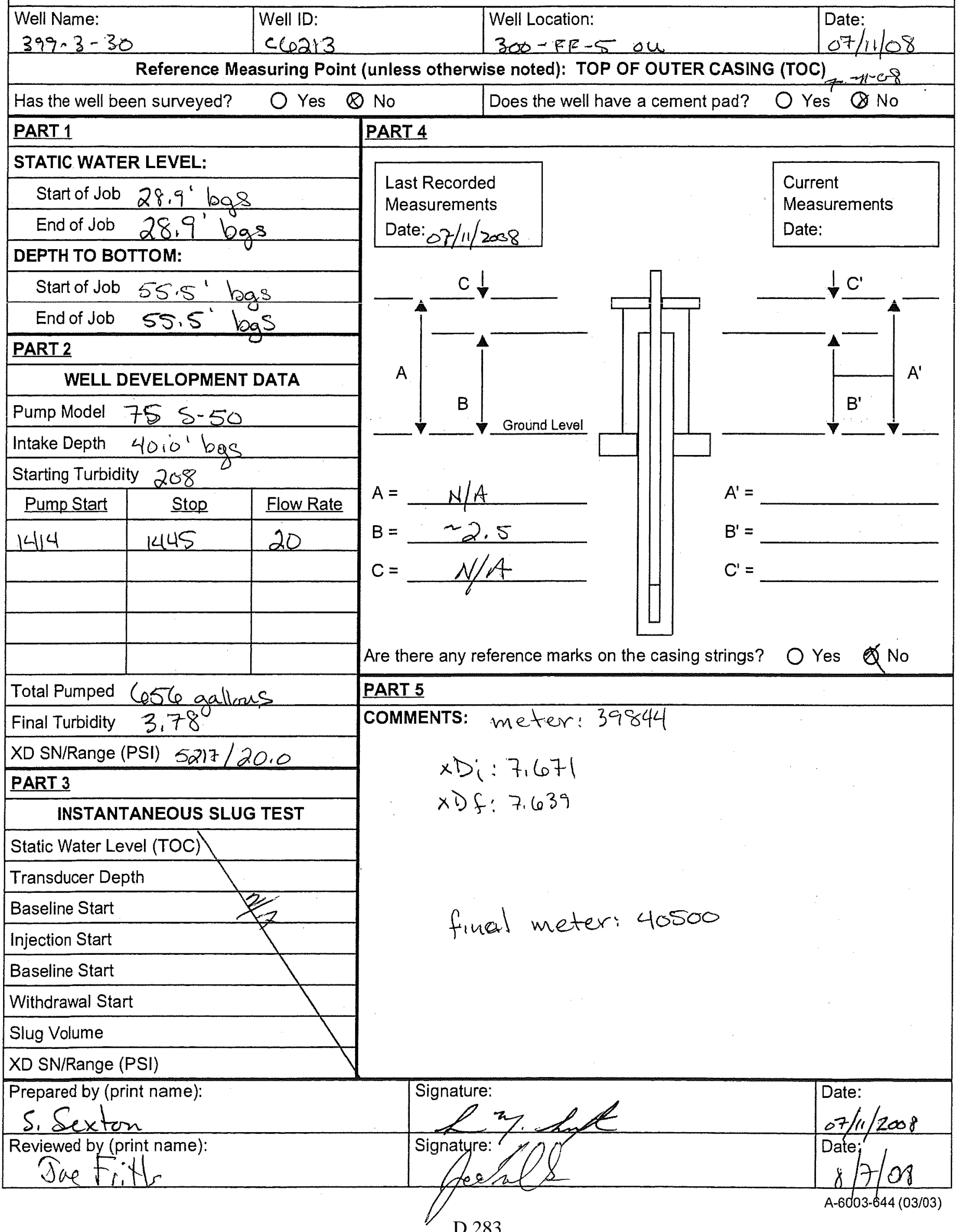


FIELD ACTIVITY REPORT NO. 1 - DRILLING PLAN

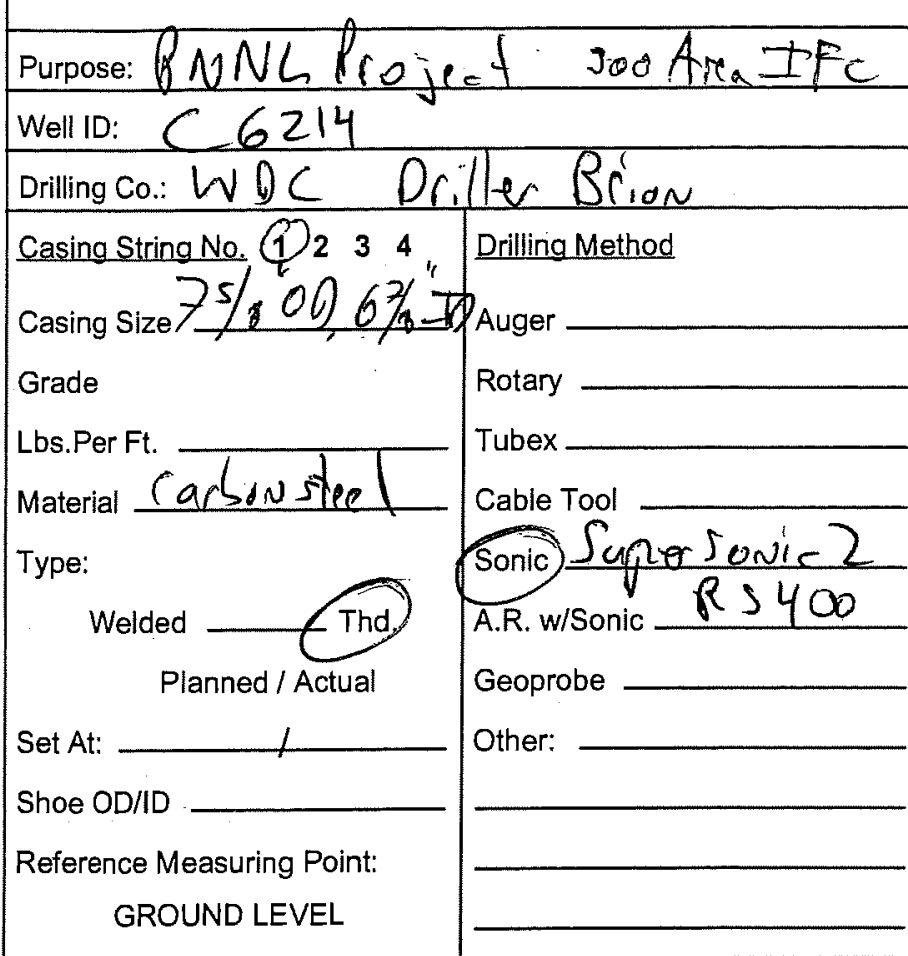

Drig. Co.

Casing String No. $\begin{array}{llll}1 & 2 & 3 & 4\end{array}$

Casing Size

Grade

Lbs.Per Ft.

Material

Type:

Welded Thd.

Planned / Actual

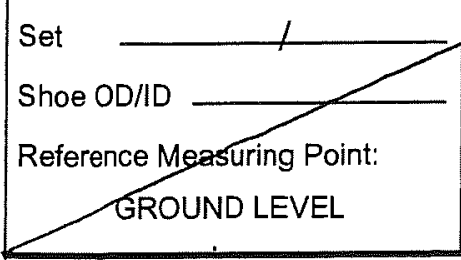

Comments/Remarks:

Rig No.:
Location: $300-F F-S$

Well Name: $399-3-31$

Rig No.: $/ 39$ Rig Make/Mod.: S uper Sonce

Circulation

Air __ Water/Mud

Reverse — Direct

Vol: $\quad \mathrm{cfm}$

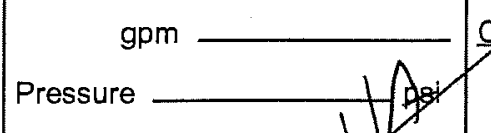

Drill Pipe O.D.

Tool Joint Size

Additives

D.H. Hammer

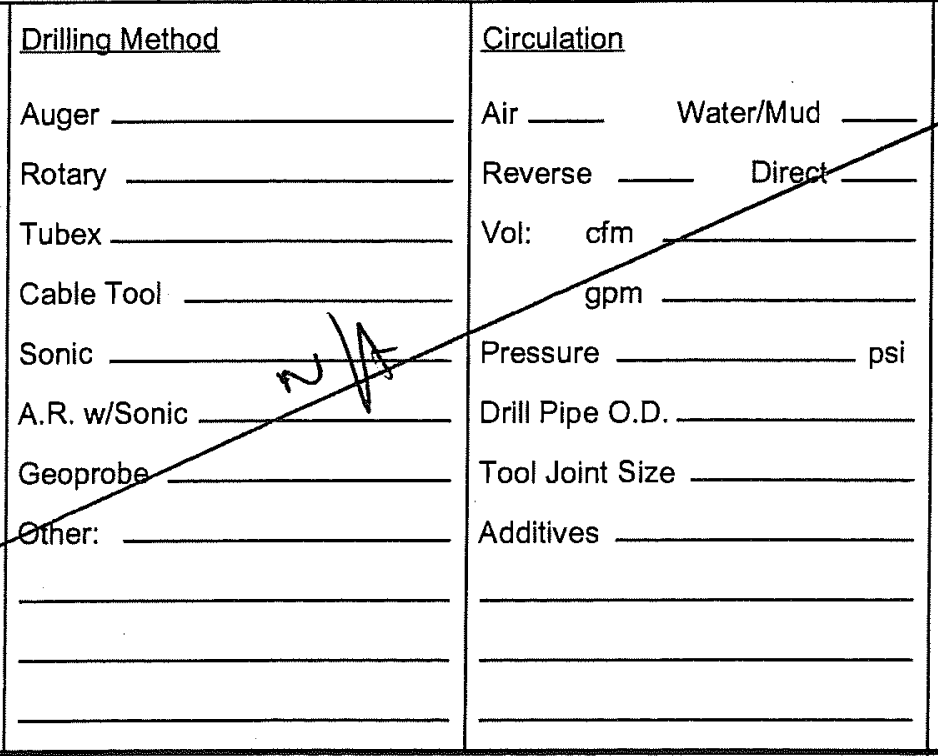

Estimated Depth to Water

Casing Hammer

Make

Model

Bit Size

Type

Nozzles

Rod Size

Make

Bit Size

Type

Nozzles

Rod Size

Model

Choke

\section{Reported By: Juetrits}

NamerTitle: GMA M Fus, (No olosist

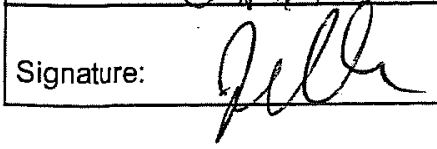




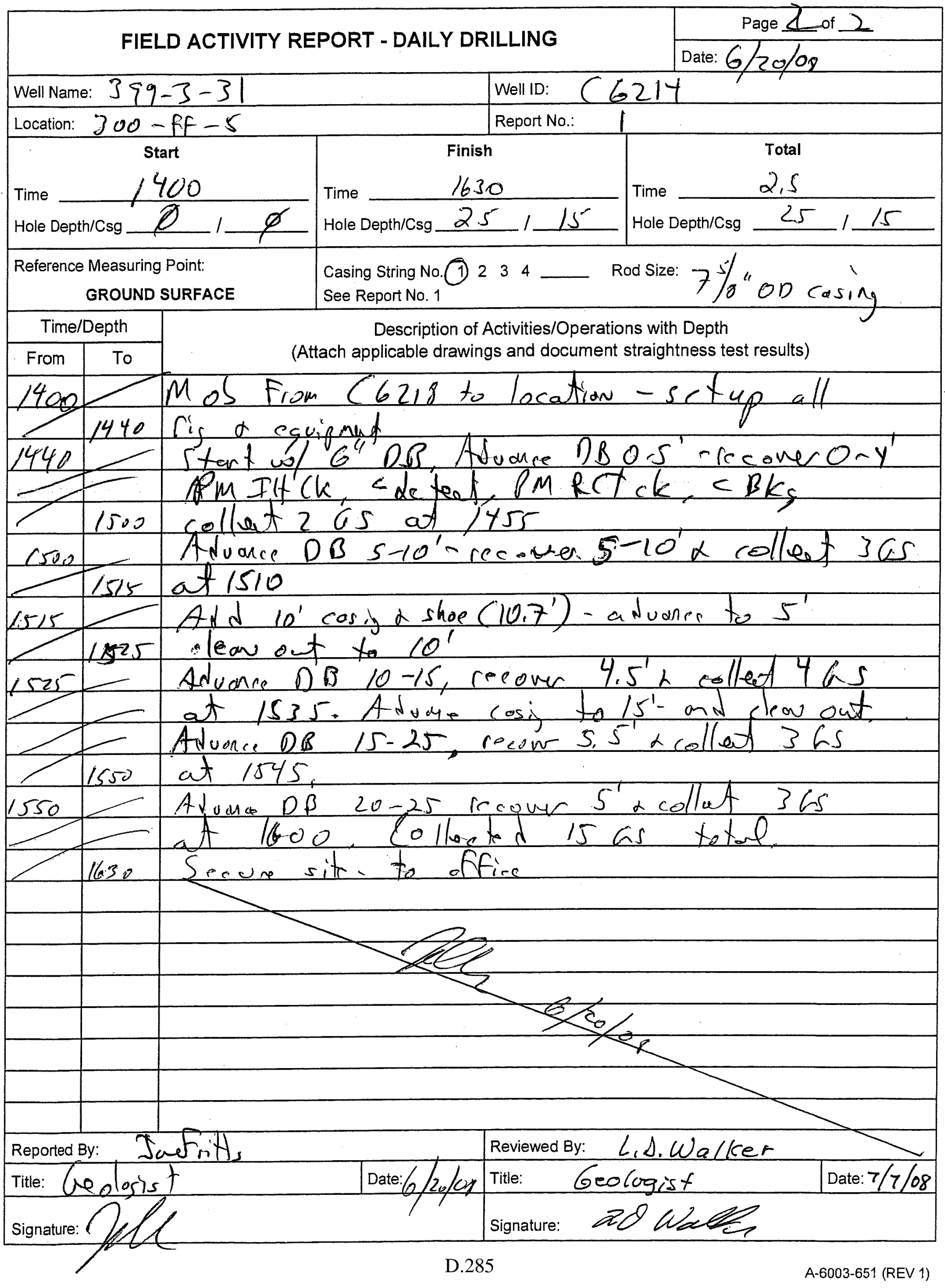




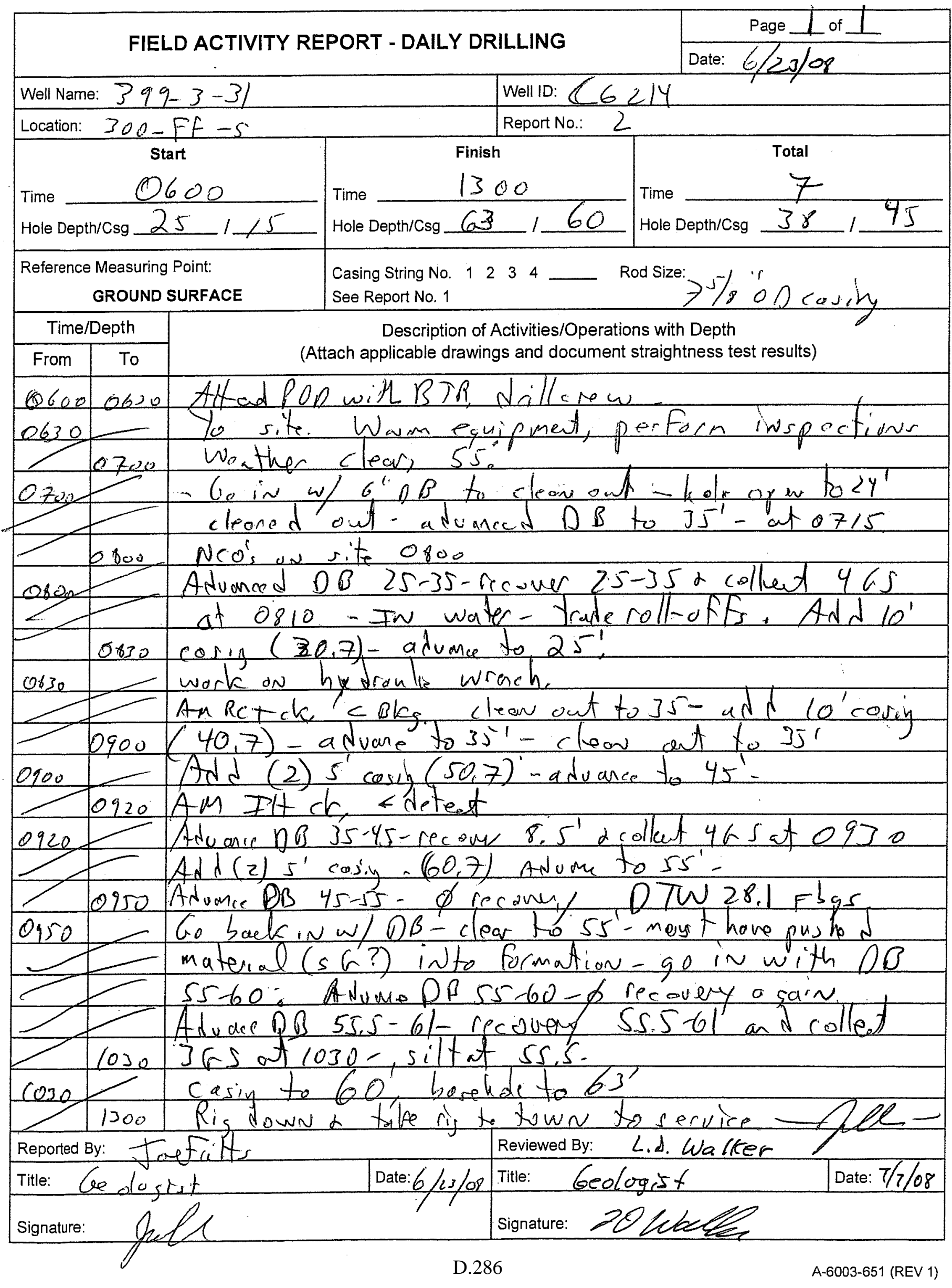




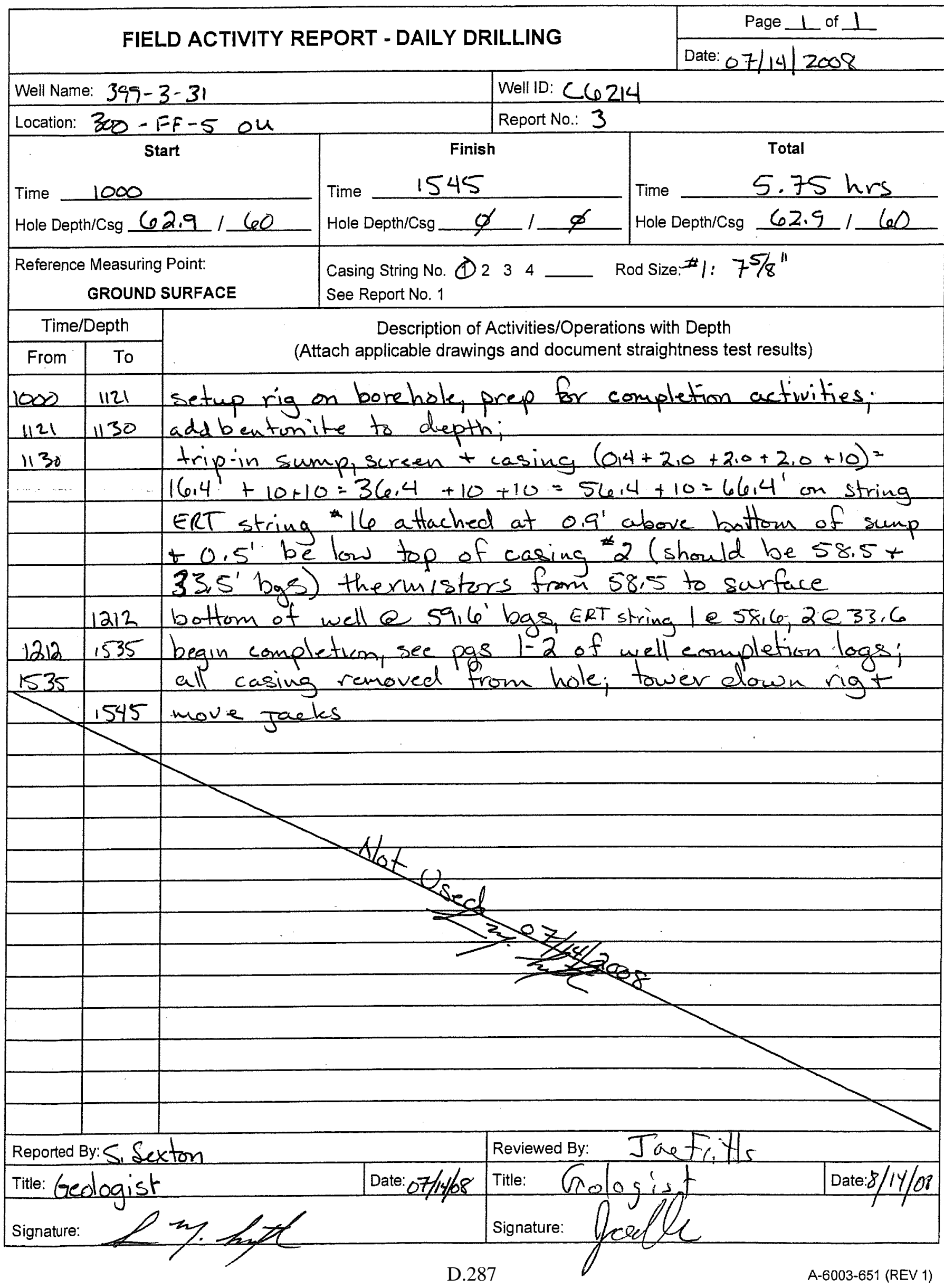




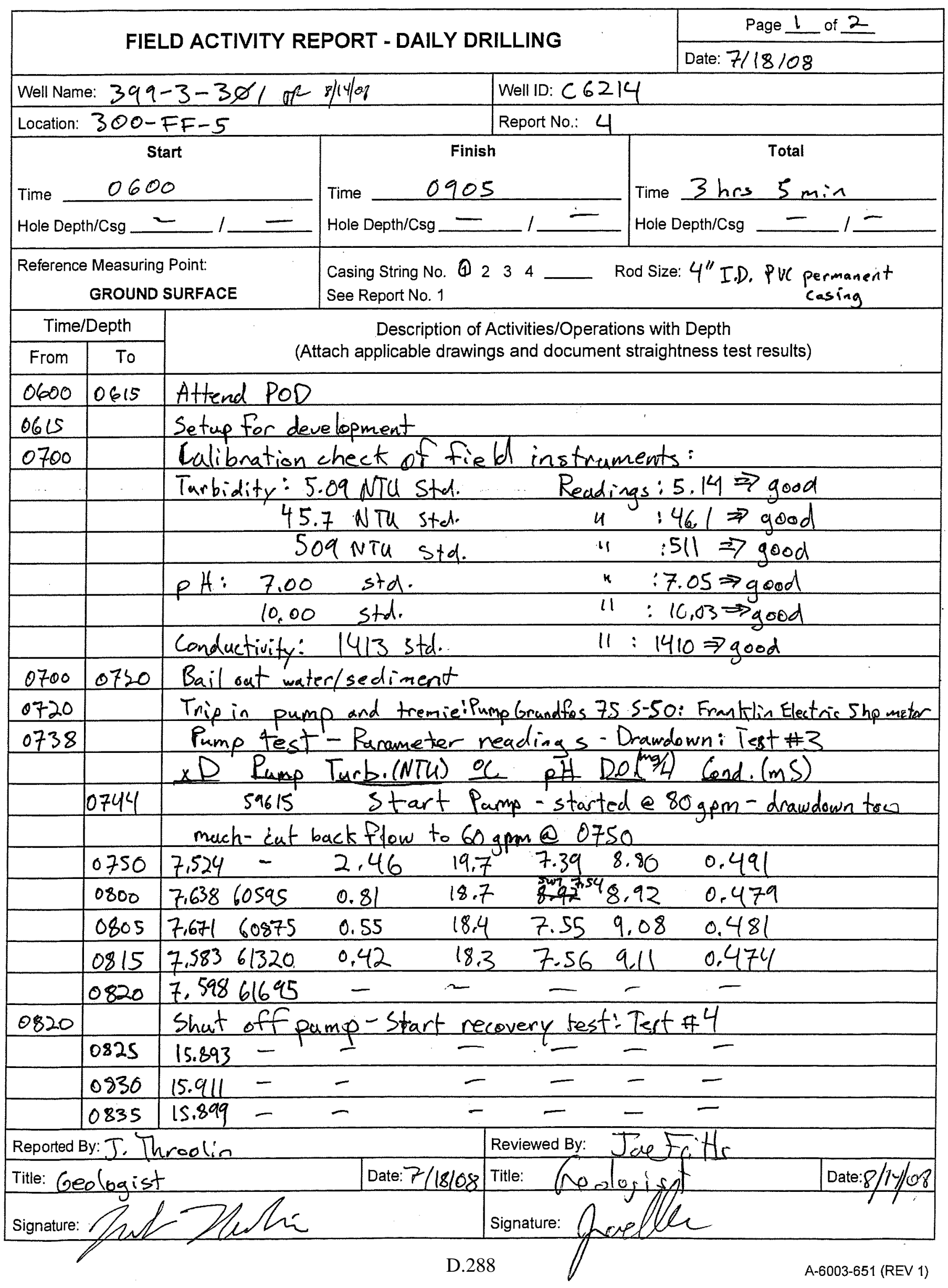


FIELD ACTIVITY REPORT - DAILY DRILLING Continuation Page

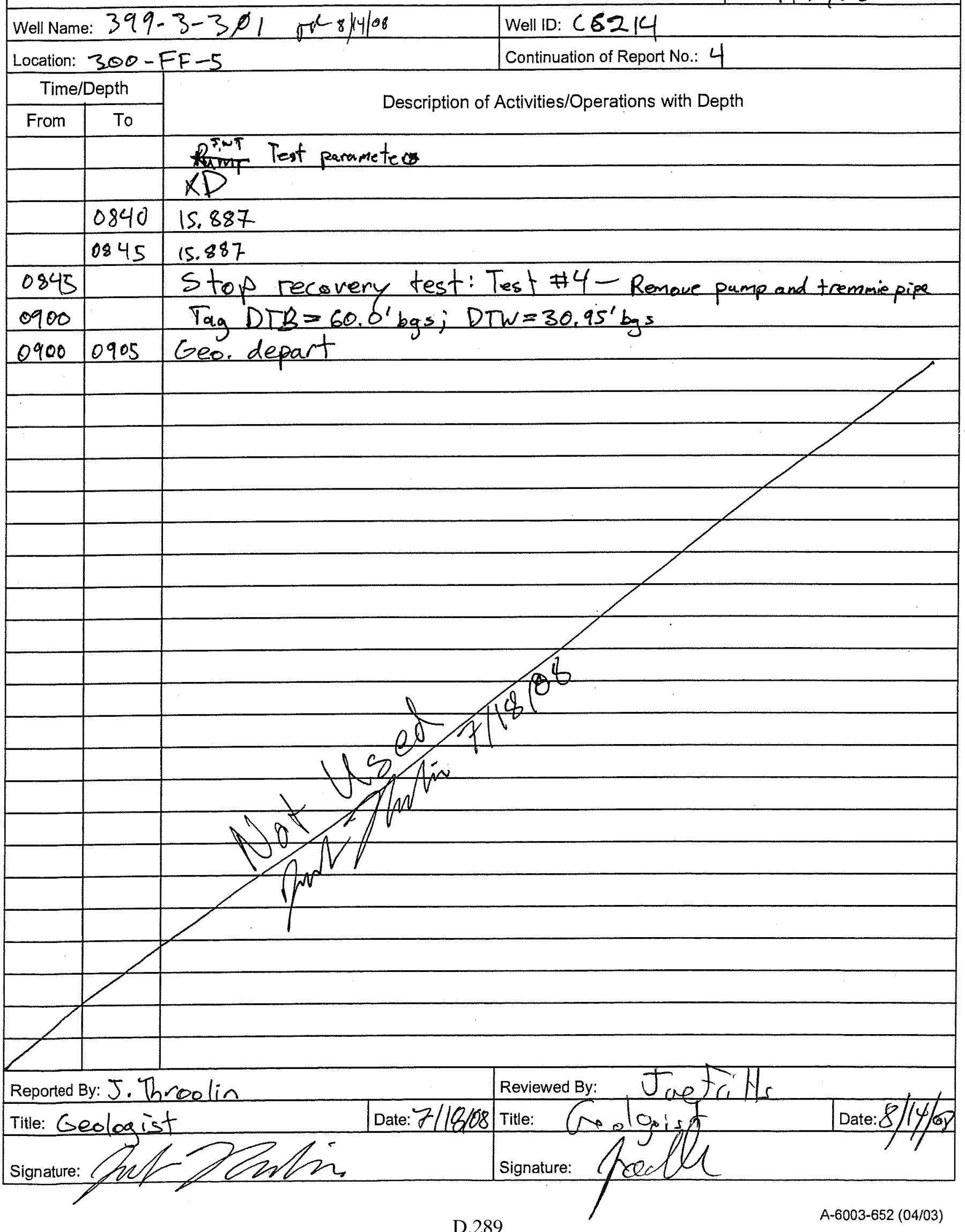




\section{WELL DEVELOPMENT AND TESTING DATA}

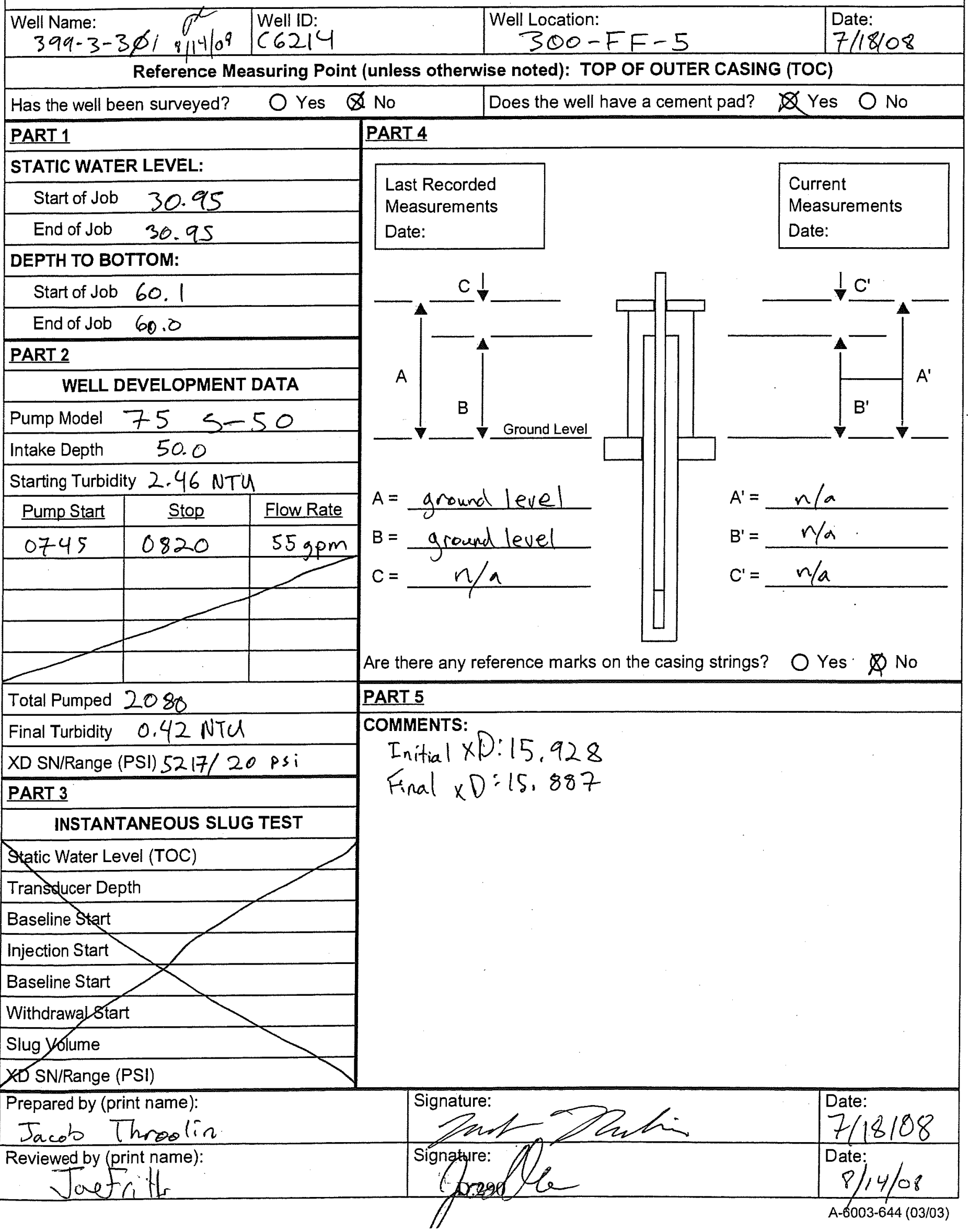




\section{FIELD ACTIVITY REPORT NO. 1 - DRILLING PLAN}

Page 1 of 2

Date: $07 / 8 / 2008$

ᄀurpose: 300 Areas IFC

. vell ID: C6215

Drilling Co: WDC Milee Willeerson

Casing String No. (1) 234 Drilling Method

Casing Size $75 \%$

Grade

Lbs.Per Ft.

Material carbon steel

Type:

Welded

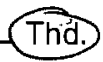

Planned / Actual

Set At: $\frac{1}{7^{5 / 8} / 6^{7 / 8}}$

Reference Measuring Point:

GROUND LEVEL

Drig. Co.

Casing String No. $\begin{array}{llll}1 & 2 & \mathbf{3} & \mathbf{4}\end{array}$

asing Size

Grade

Lbs.Per Ft.

Material

Type:

Welded Thd.

Planned / Actual

Se

Shoe OD/ID

Reference Measuting Point: GROUND LEVEL

Auger

Rotary

Tubex

Cable Tool

Geoprobe

Other:

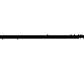

Rig No:

Drilling Method

Auger

Rotary

Tubex

Cable Tool

Sonic

A.R. w/Sonic

Other:

\section{od}

Sonid Super Sonic

A.R. w/Sonic RS400

Geoprobe

Location: $300-F F-5$ du

Well Name: $399-3-32$

Rig No.: 139 Rig Make/Mod.: Scop S J i $_{i}$

Circulation

Air W Water/Mud

Reverse Direct

Vol: $\quad \mathrm{cfm}$

gpm

Pressure

Drill Pipe O.D.

Tool Joint Size

Additives

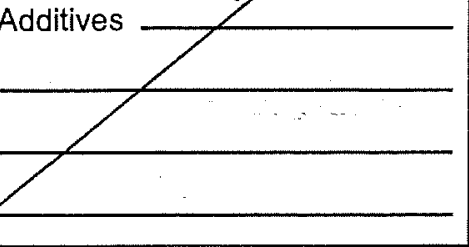

.Rig Make/Mod.:
Nozzles

Rod Size

D.H. Hatmmer

Make

Model

Choke

Casing Hammer

Make

Model

Bit Size

Type

Nozzles

range

Reported By: S. Sextom ame/Title: Gram Ine Geologist 


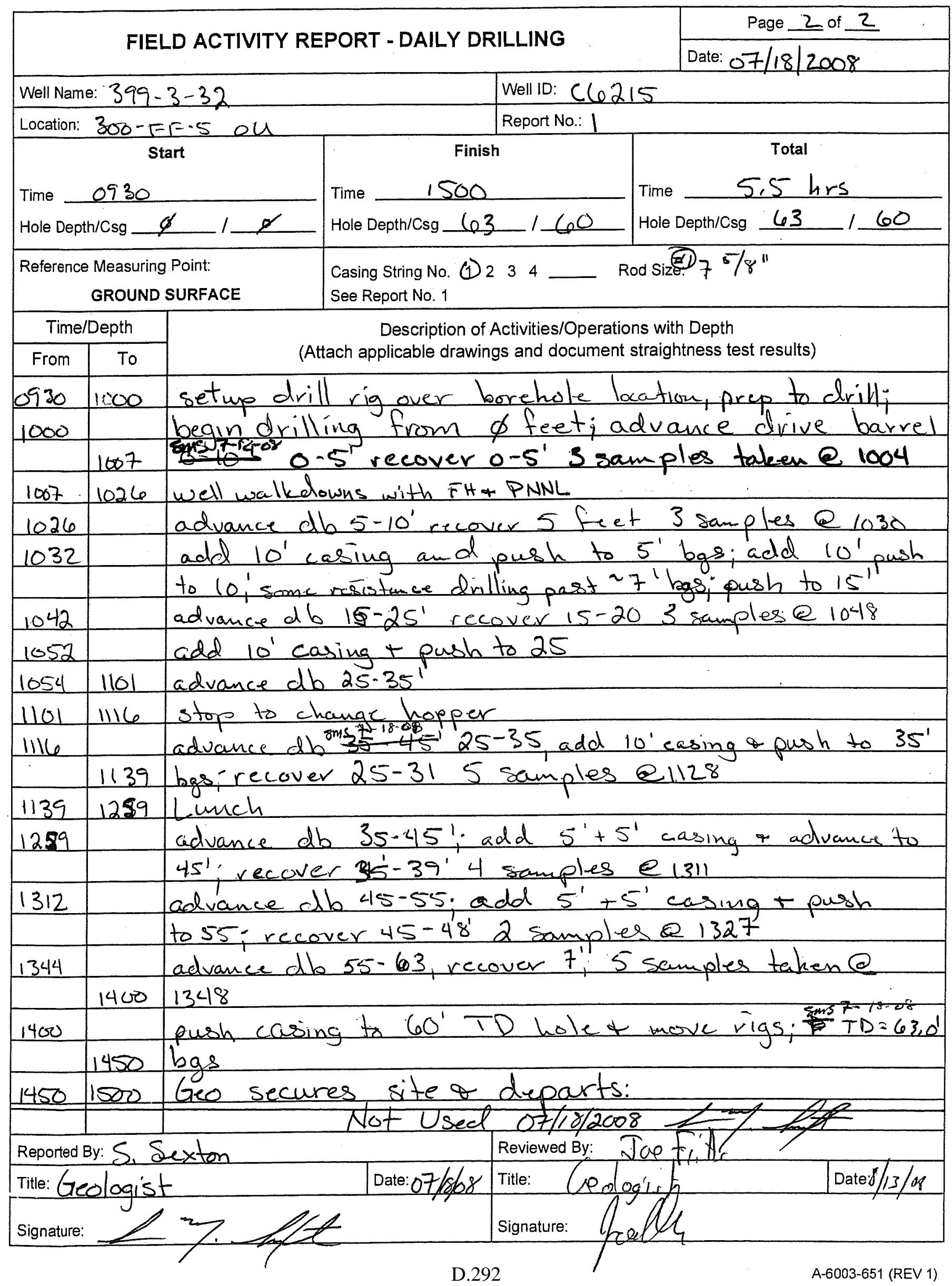




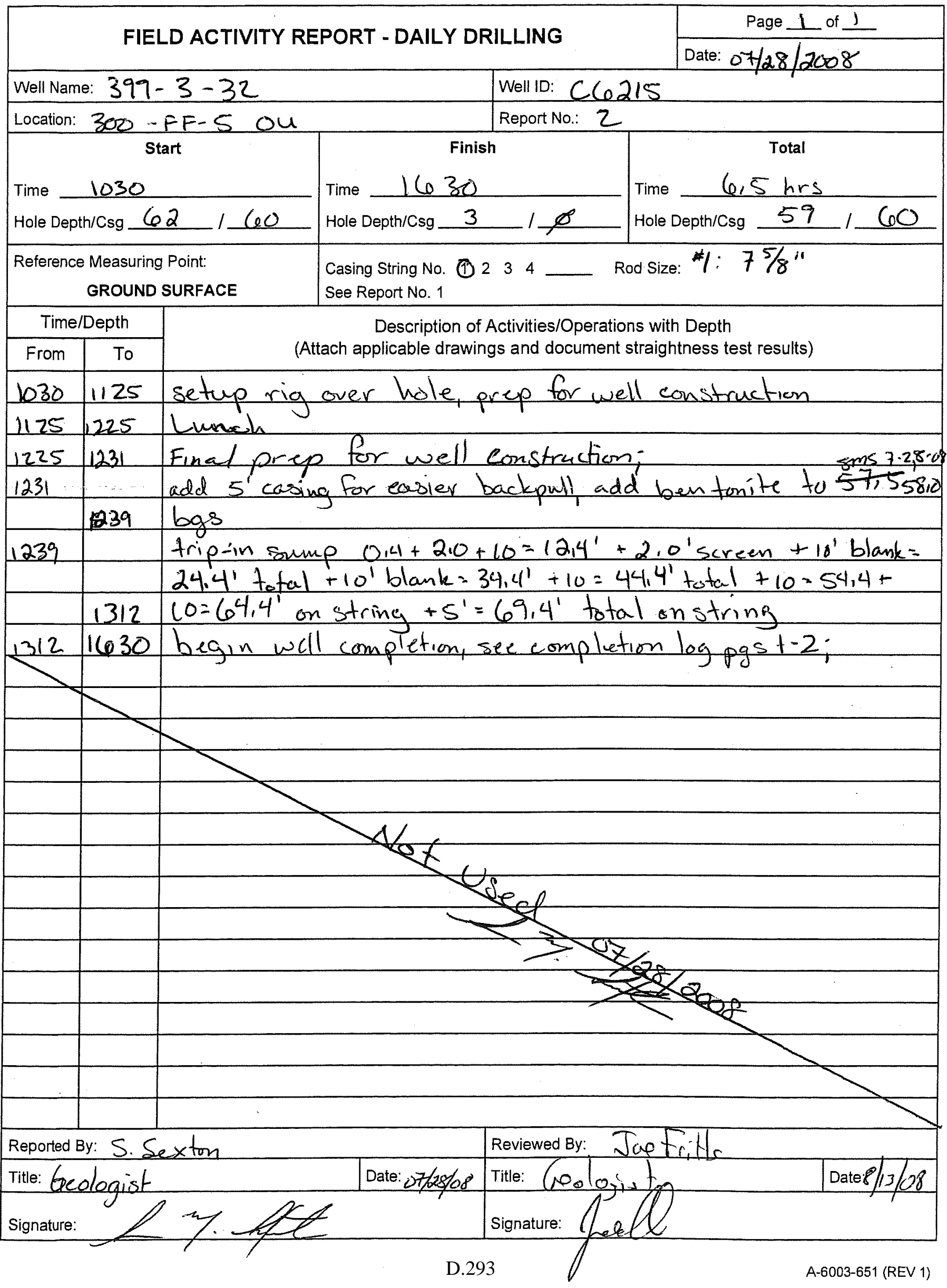




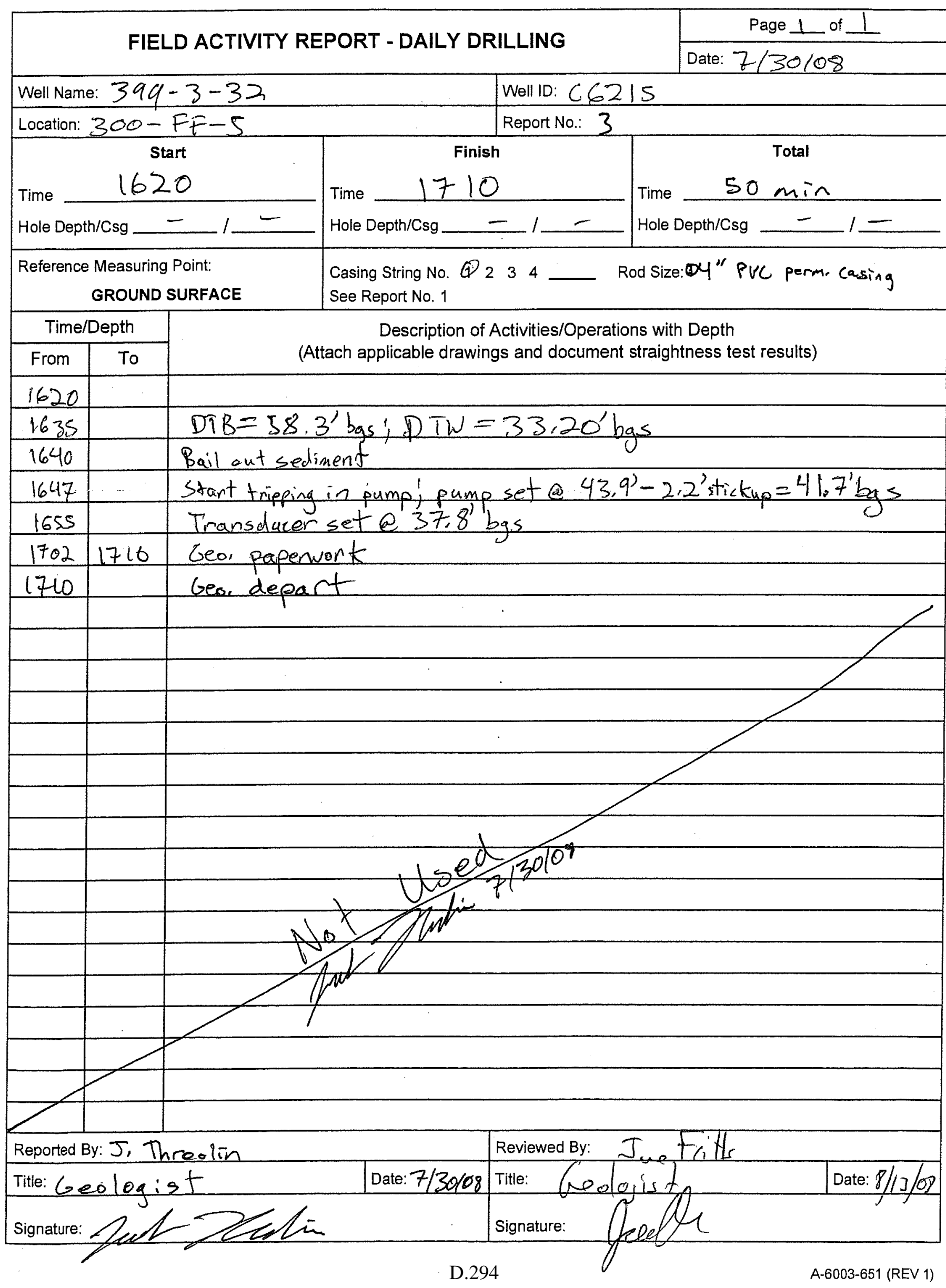




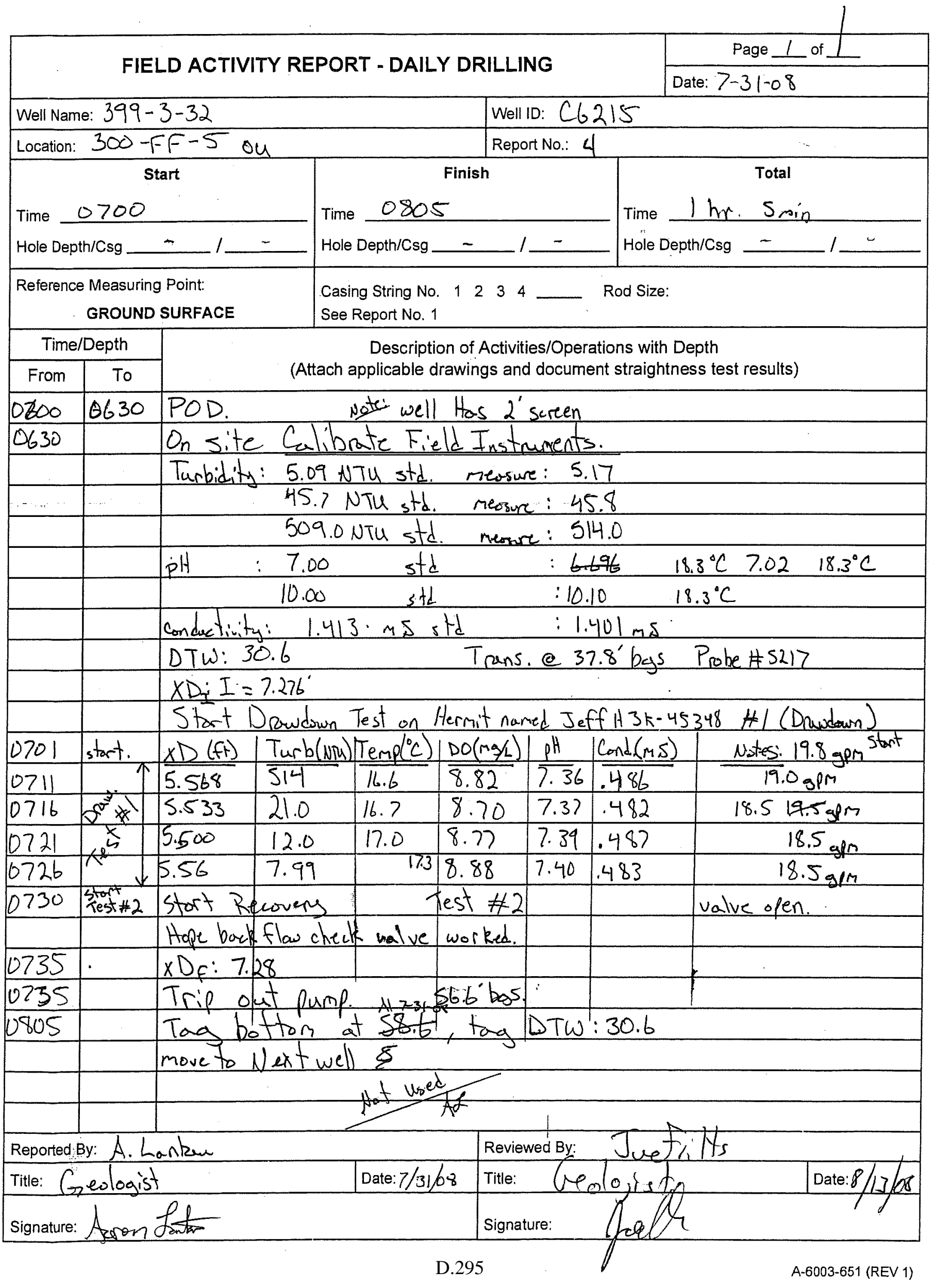




\section{WELL DEVELOPMENT AND TESTING DATA}

\begin{tabular}{|c|c|c|c|}
\hline Well Name: $\quad 399-3-32$ & Well ID: 6215 & $\begin{array}{l}\text { Well Location: } \\
300-F F-5\end{array}$ & 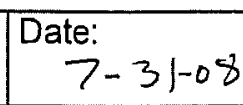 \\
\hline
\end{tabular}

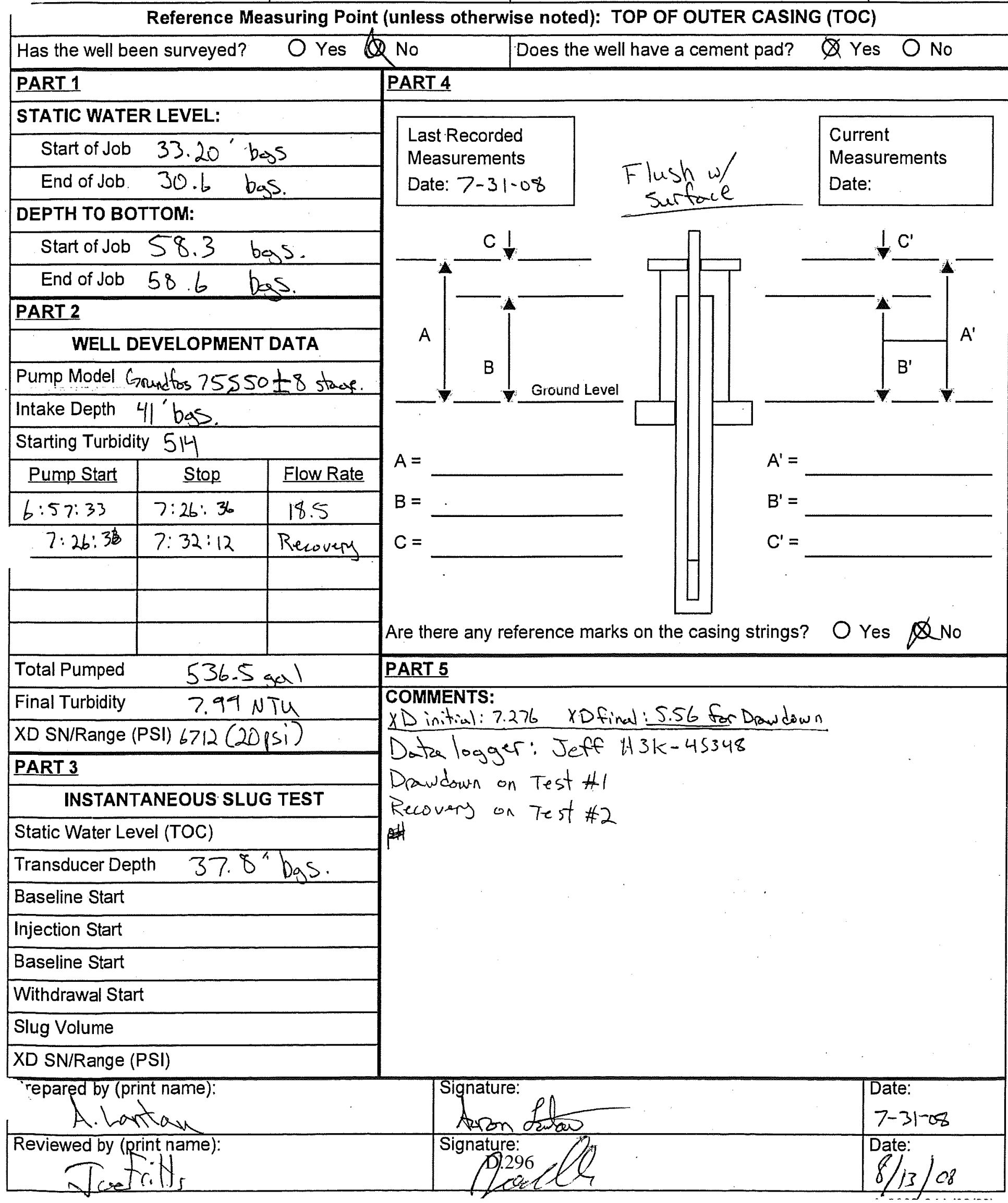




\section{Appendix E}

Well Development and Testing Data Sheets 


\section{WELL DEVELOPMENT AND TESTING DATA}

\begin{tabular}{|l|l|l|l|}
\hline $\begin{array}{l}\text { Well Name: } \\
399-2-7\end{array}$ & $\begin{array}{l}\text { Well ID: } \\
\text { C6184 }\end{array}$ & $\begin{array}{l}\text { Well Location: } \\
300-5 F-5 \text { ou }\end{array}$ & $\begin{array}{l}\text { Date: } \\
07 / 15 / 2008\end{array}$ \\
\hline
\end{tabular}

Reference Measuring Point (unless otherwise noted): TOP OF OUTER CASING (TOC)

\begin{tabular}{|l|l|l}
\hline Has the well been surveyed? $\bigcirc$ Yes $\otimes$ No & Does the well have a cement pad? $\otimes$ Yes $\bigcirc$ No
\end{tabular}

\section{PART 1}

STATIC WATER LEVEL:

Start of Job 29,85' bas

End of Job $29,7^{\prime}$ bgs

\section{DEPTH TO BOTTOM:}

Start of Job $56.6^{\prime} \mathrm{bgs}$
End of Job $56.60^{\prime} \mathrm{bgs}$
PART 2

\section{WELL DEVELOPMENT DATA}

Pump Model $75 \cdot 5-50$

Intake Depth $40,0 / 50,0$

Starting Turbidity $279 / 322$

\begin{tabular}{|c|c|l|}
\hline \multicolumn{3}{|c|}{ Starting Turbidity $279 / 322$} \\
\hline Pump Start & Stop & Flow Rate \\
\hline 0808 & 0838 & 50 \\
\hline 0857 & 0912 & 86 \\
\hline & & \\
\hline & & \\
\hline & & \\
\hline
\end{tabular}

Total Pumped $1380 / \mathrm{H}^{7} 1$

Final Turbidity $1.16 / 2,66$

XD SN/Range (PSI) $5217 / 20$

PART 3

\begin{tabular}{|l|}
\hline \multicolumn{2}{|c|}{ INSTANTANEOUS SLUG TEST } \\
\hline Static Water Level (TOC) \\
\hline Transducer Depth \\
\hline Baseline Start \\
\hline Injection Start \\
\hline Baseline Start \\
\hline Withdrawal Start \\
\hline Slug Volume \\
\hline XD SN/Range (PSI) \\
\hline Prepared by (Prnt \\
\hline
\end{tabular}

Prepared by (print name):

S. Sexton

Reviewed by (print name):

Tae Fitts

\section{PART 4}

Last Recorded

Measurements

Date: $07 / 15 / 2008$

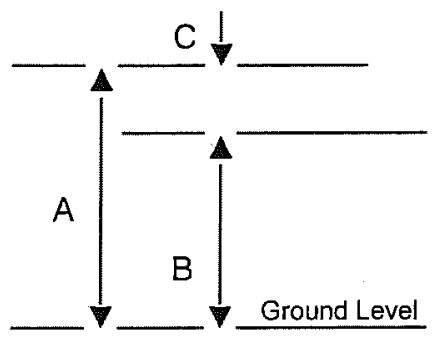

$A=N / A$

$B=N / A$

$c=N / A$
Current

Measurements

Date:
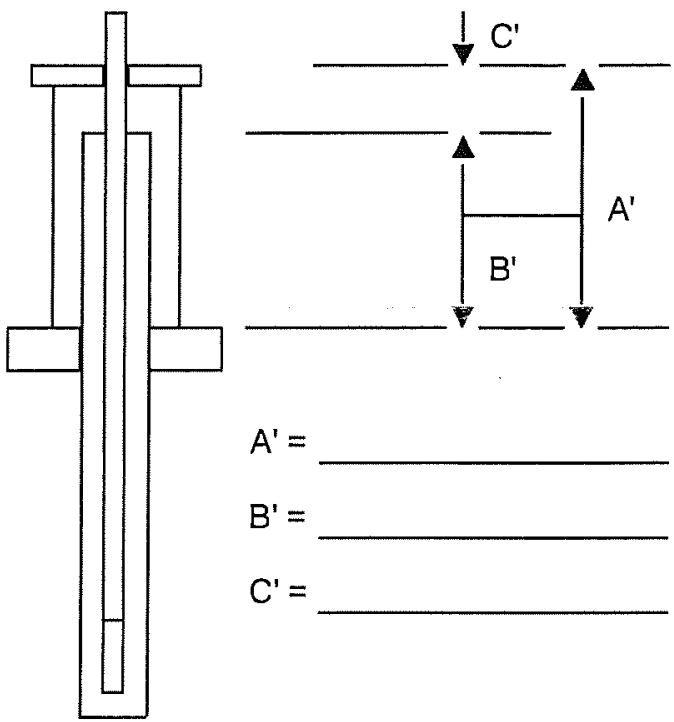

$A^{\prime}=$

$B^{\prime}=$

$C^{\prime}=$

Are there any reference marks on the casing strings? $\bigcirc$ Yes $\otimes$ No

\section{PART 5}

COMMENTS: meter: 45638

Test"1

${ }^{2} D_{i}: 8.813$

xDf:8, 835

$$
\text { meter: } 47018
$$

Tesf 2 : rccorded on SunDried wD3

$$
\begin{aligned}
& \times D_{i}: 16.042 \\
& \times D_{f:} 16.023
\end{aligned}
$$

final meter. 48189

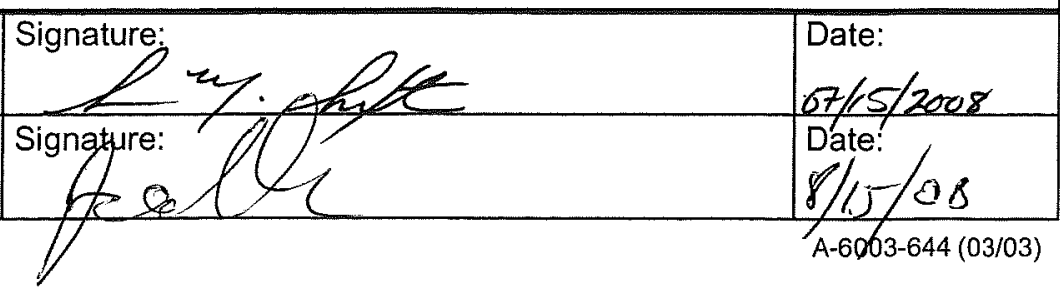

E.1 


\section{WELL DEVELOPMENT AND TESTING DATA}

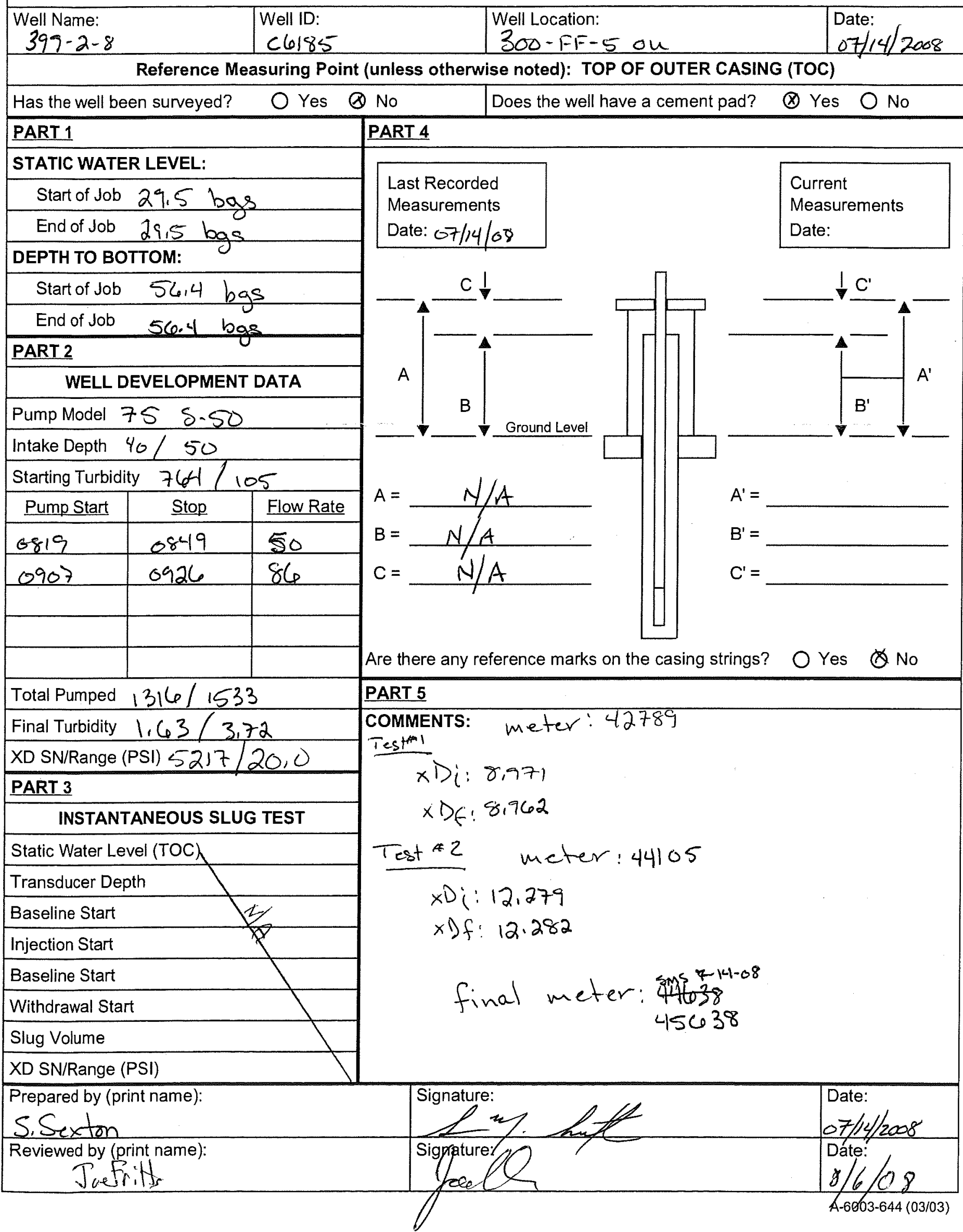

E.2 


\section{WELL DEVELOPMENT AND TESTING DATA}

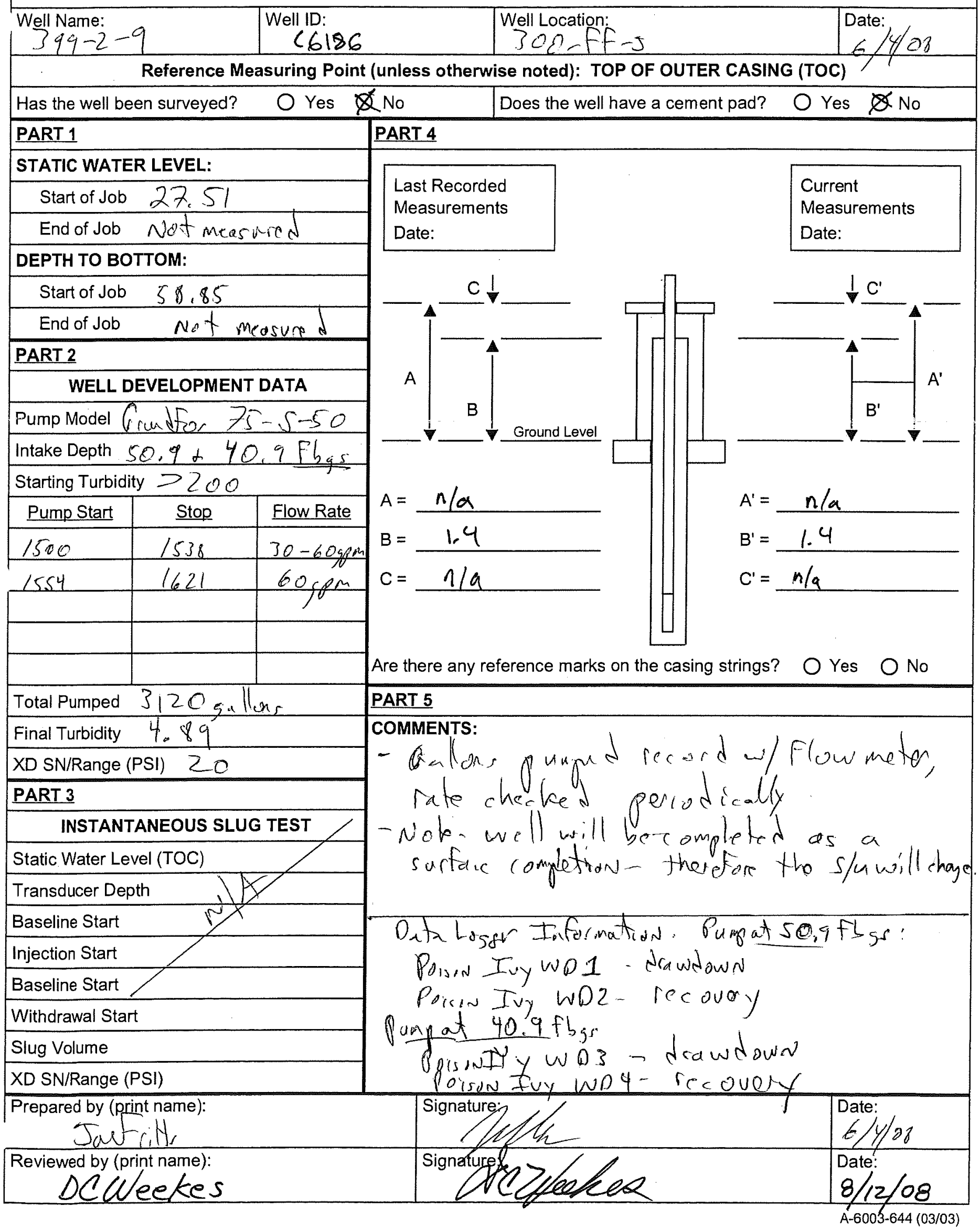




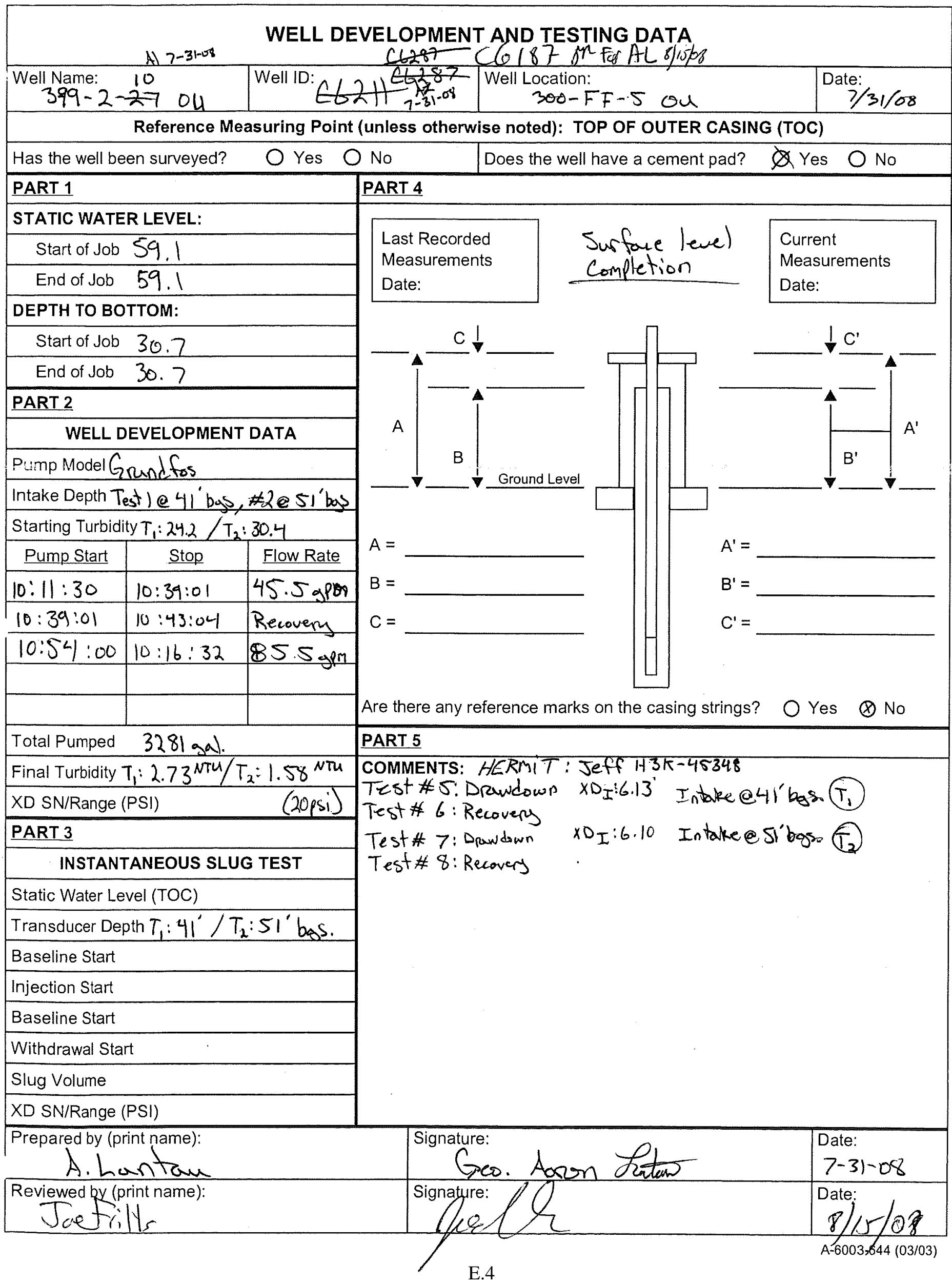




\section{WELL DEVELOPMENT AND TESTING DATA}

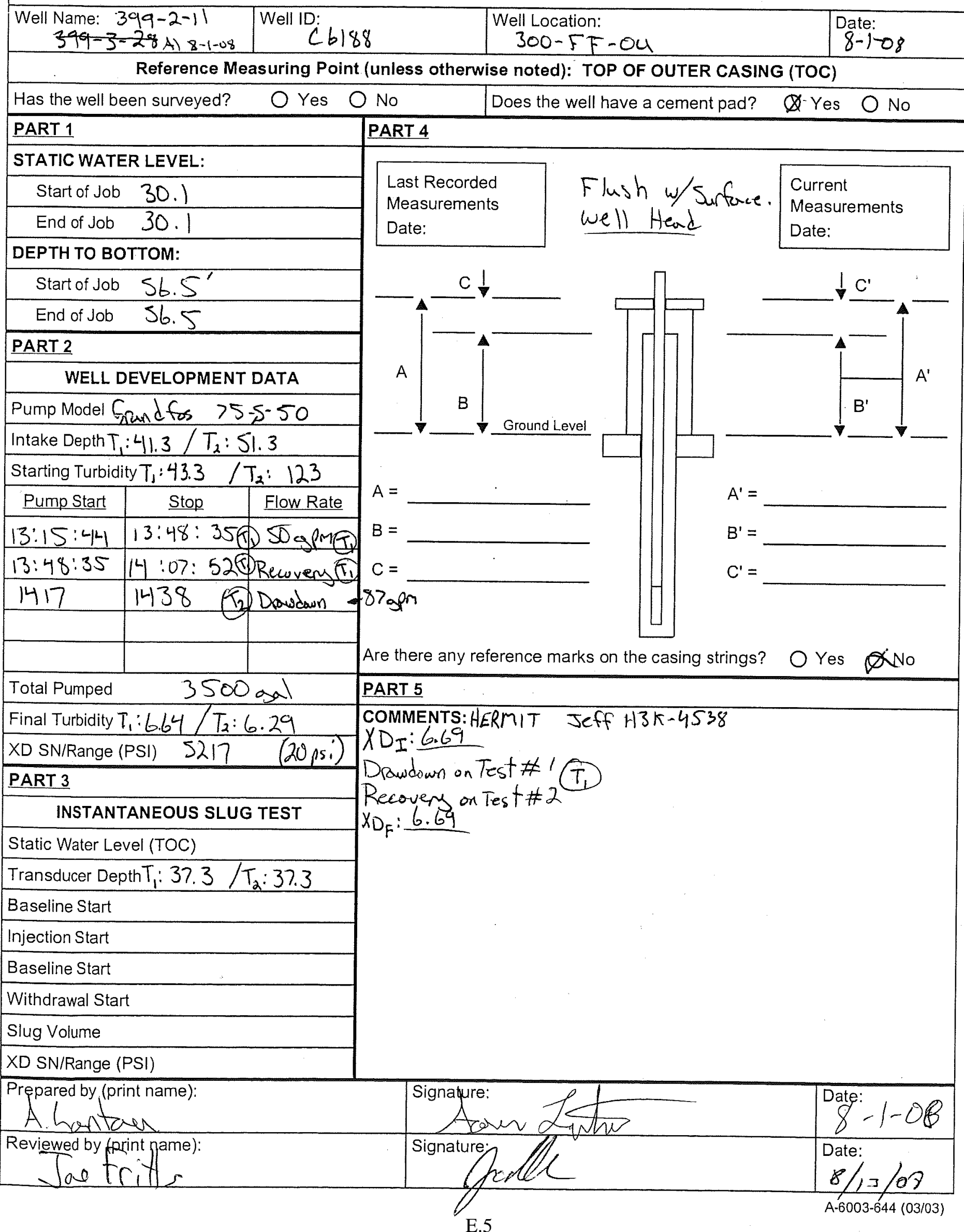




\section{WELL DEVELOPMENT AND TESTING DATA}

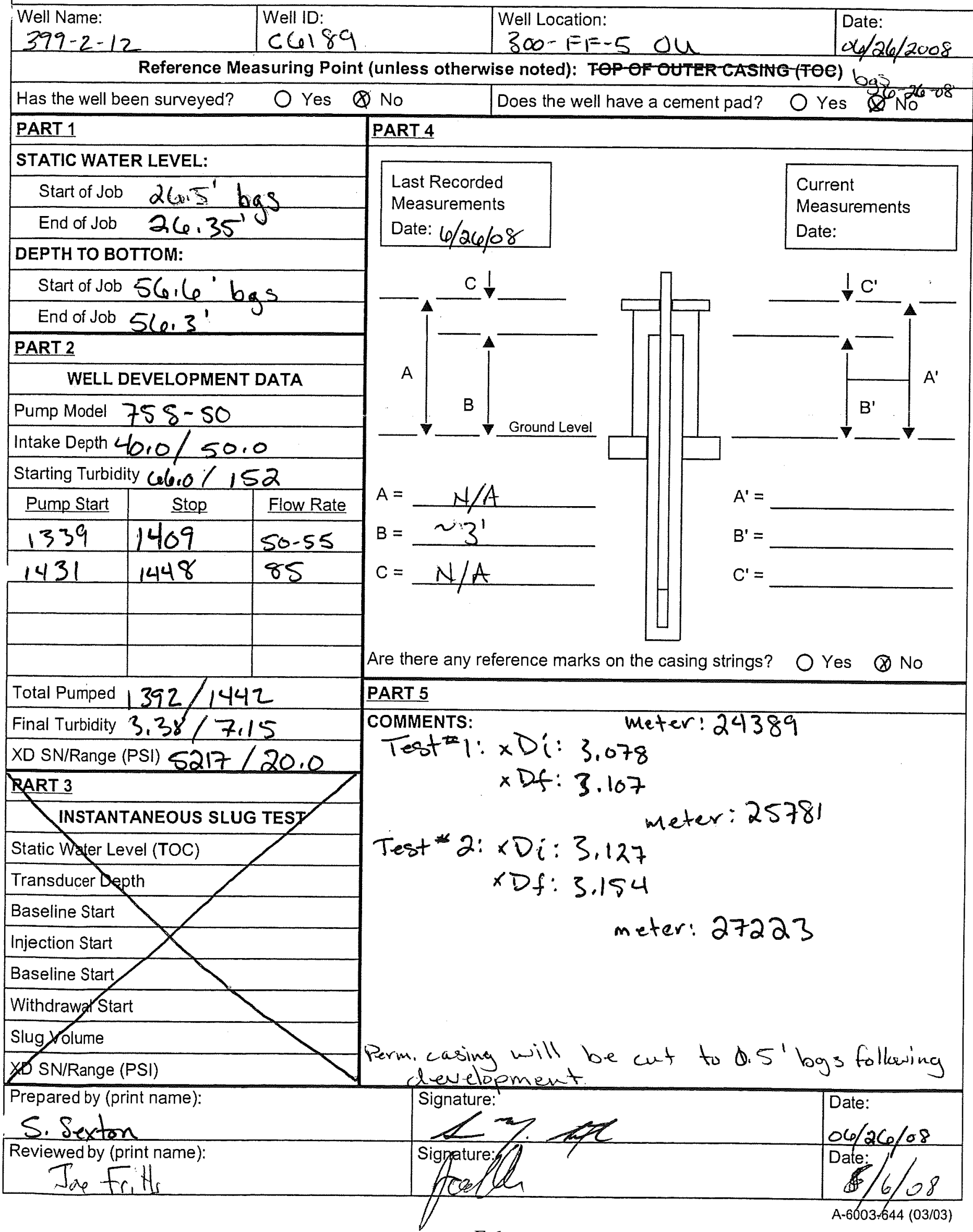

E.6 


\section{WELL DEVELOPMENT AND TESTING DATA}

\begin{tabular}{|c|c|}
\hline Well Name: & Well ID: 6 \\
\hline \multicolumn{2}{|c|}{ Reference Measuring Point } \\
\hline Has the well been surveyed? & OYes $\not$ \\
\hline \multicolumn{2}{|l|}{ PART 1} \\
\hline \multicolumn{2}{|l|}{ STATIC WATER LEVEL: } \\
\hline Start of Job & \\
\hline 29.15 & \\
\hline
\end{tabular}

\begin{tabular}{|l|}
\hline DEPTH TO BOTTOM: \\
\hline Start of Job 59,20 \\
\hline End of Job 59,20 \\
\hline PART 2
\end{tabular}

\begin{tabular}{|c|c|c|}
\hline \multicolumn{3}{|c|}{ WELL DEVELOPMENT DATA } \\
\hline \multicolumn{3}{|c|}{ Pump Model Grendfor 75-s-50 } \\
\hline \multicolumn{3}{|c|}{ Intake Depth $39,6 \alpha 49,6 \mathrm{Fb} 95$} \\
\hline \multicolumn{3}{|c|}{ Starting Turbidity $>200 \mathrm{NTU}$} \\
\hline Pump Start & Stop & Flow Rate \\
\hline 074 & $0 \times 05$ & $49-53 i p m$ \\
\hline 0830 & 0859 & $60-80 \mathrm{gpm}$ \\
\hline & & \\
\hline & & \\
\hline
\end{tabular}

\begin{tabular}{|l}
\hline Total Pumped $3 / 15$ \\
\hline Final Turbidity 3.34 \\
\hline XD SN/Range (PSI) \\
\hline PART 3 \\
\hline
\end{tabular}

\begin{tabular}{|l|}
\hline INSTANTANEOUS SLUG TEST \\
\hline Static Water Level (TOC) \\
\hline Transducer Depth \\
\hline Baseline Start \\
\hline Injection Start \\
\hline Baseline Start \\
\hline Withdrawal Start \\
\hline Slyg Volume \\
\hline XD SN/Range (PSI) \\
\hline
\end{tabular}

Well Location: $\mathrm{F}$. F

Dat
QVC Date: $6 / 5 / 6 / 08$ or $6 / 5 / 08$ Does the well have a cement pad?

$\mathrm{O}$ Yes $\&$ No

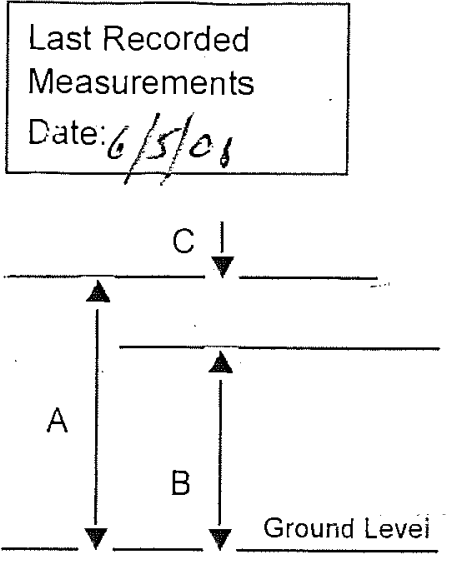

\section{PART 4}

Measurements

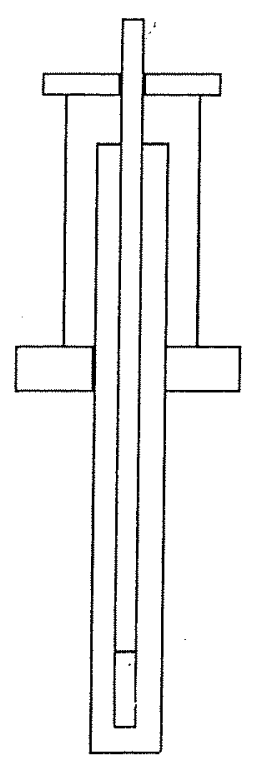

$A=\frac{N / A}{2 / 8}$
$B=\frac{N / A}{A}$

$A^{\prime}=$

$\mathrm{B}^{\prime}=$

$C^{\prime}=$

Current Measurements

Date:

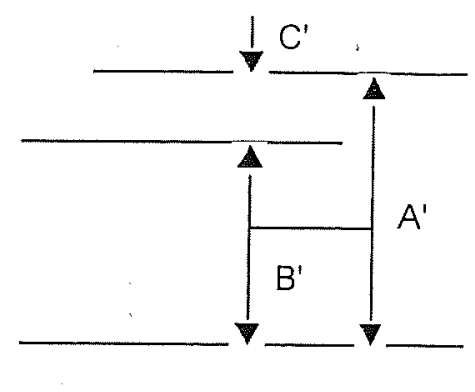

Are there any reference marks on the casing strings? $O$ Yes ${ }_{\text {No }}$

\section{PART 5}

COMMENTS:

- Flow rate and volume pumpe Mraswe $d$ with flowneter

- Note: PVC stick-ap( B obow) will chinge.

Followin below-grade campletion.

Datalogsen Information - pump at $49.6 \mathrm{Fbgs}$

Laylawpq- Drawdown.

Luylaw010 - Recovery

\footnotetext{
repiared by (print name):

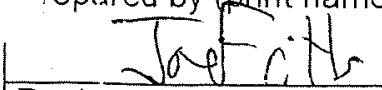

Reviewed by (print name):

DCweekes
}

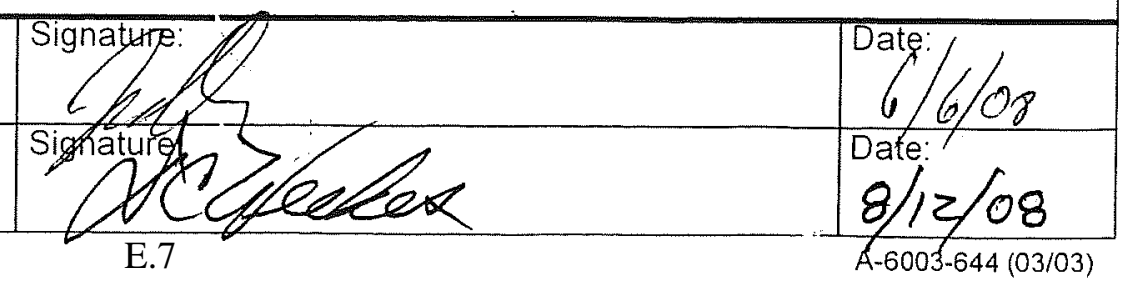




\section{WELL DEVELOPMENT AND TESTING DATA}

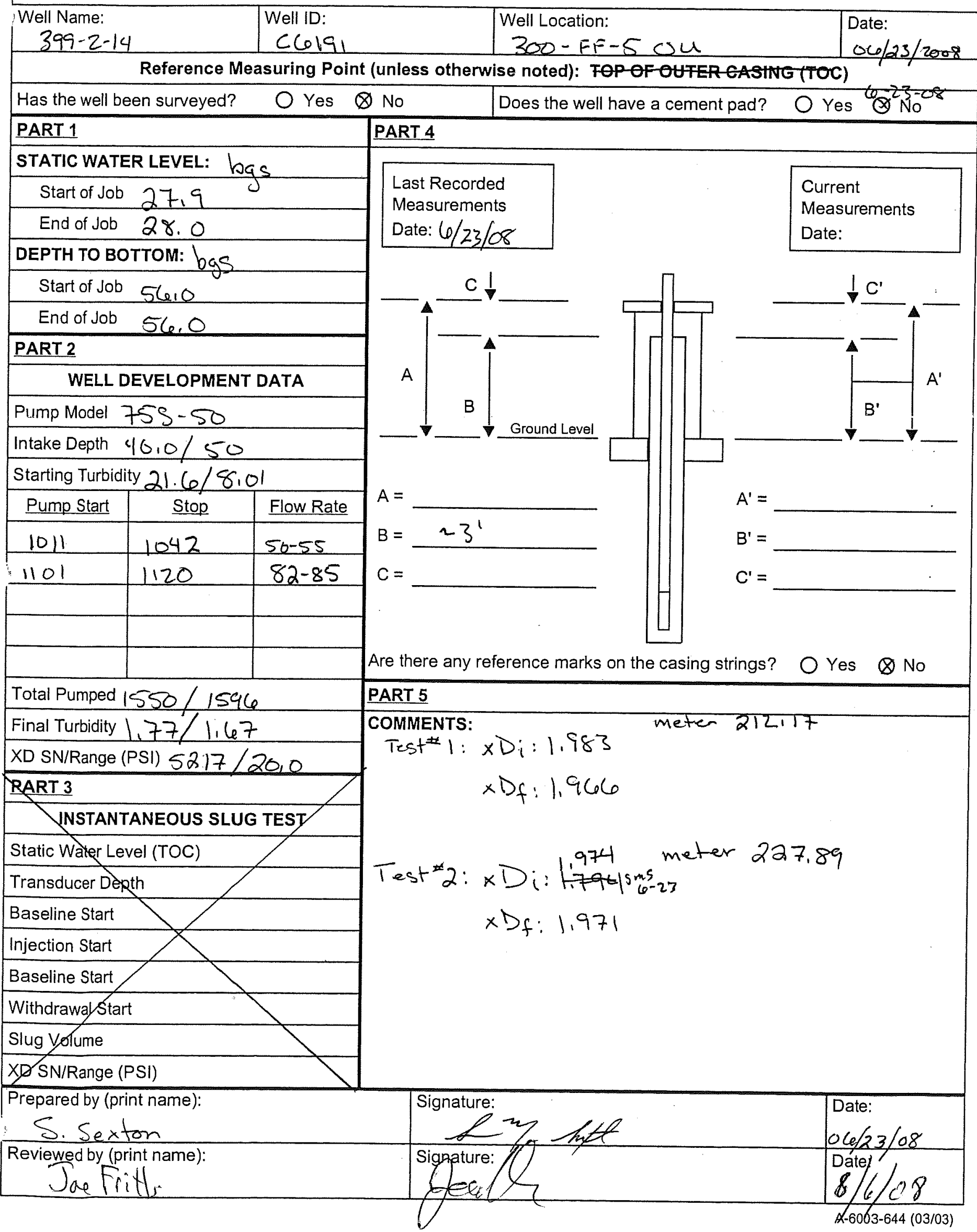

E.8 


\section{WELL DEVELOPMENT AND TESTING DATA}

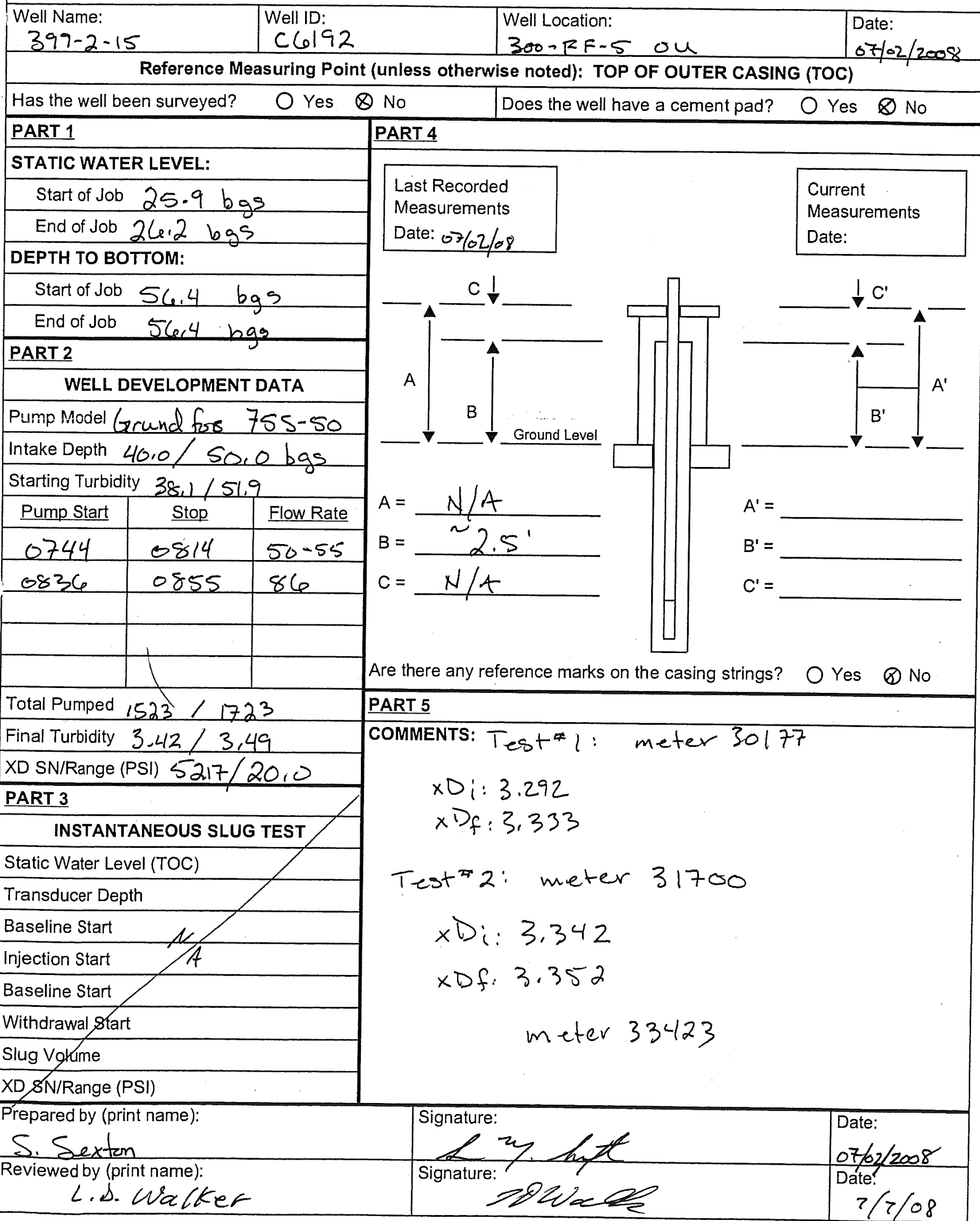




\section{WELL DEVELOPMENT AND TESTING DATA}

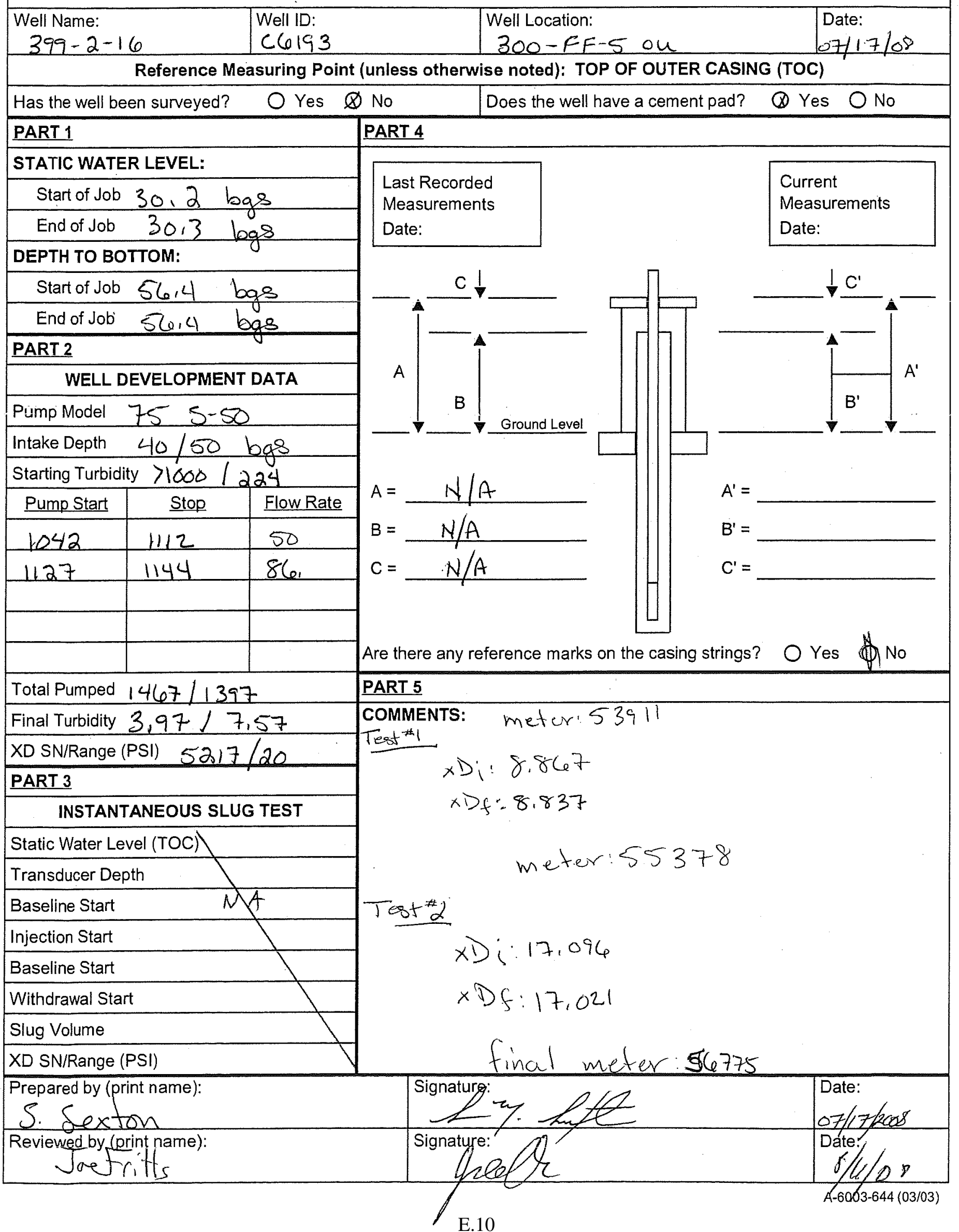




\section{WELL DEVELOPMENT AND TESTING DATA}

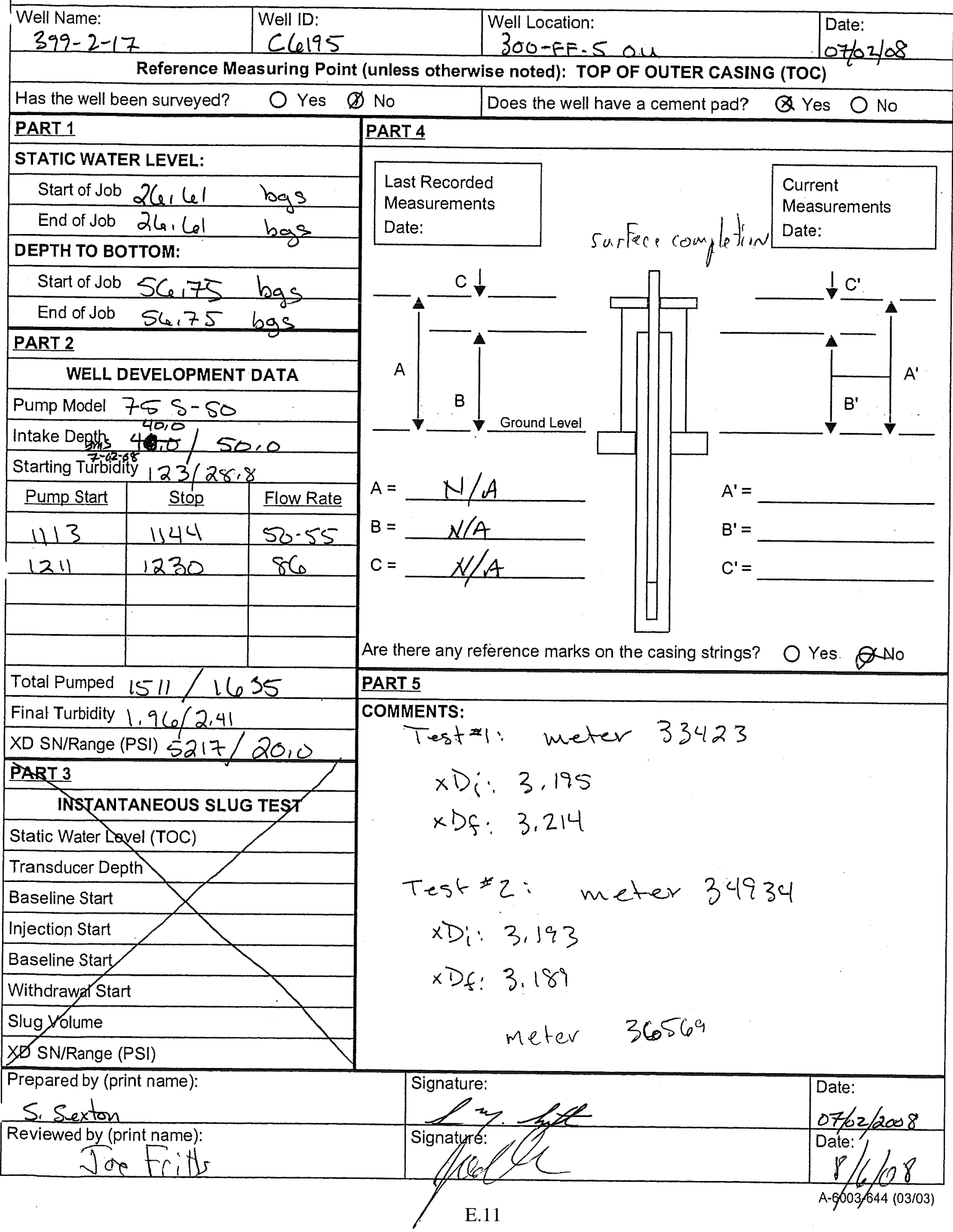




\section{WELL DEVELOPMENT AND TESTING DATA}

\begin{tabular}{|l|l|l|l|}
\hline Well Name: & $\begin{array}{l}\text { Well ID: } \\
\text { C } 699-2-18\end{array}$ & $\begin{array}{l}\text { Well Location: } \\
300-F F-5 \text { ou }\end{array}$ & $\begin{array}{l}\text { Date: } \\
06 / 19 / 20088\end{array}$ \\
\hline
\end{tabular}

\begin{tabular}{|c|c|c|}
\hline \multicolumn{3}{|c|}{ Has the well been surveyed? } \\
\hline \multicolumn{3}{|l|}{ PART 1} \\
\hline \multicolumn{3}{|c|}{ STATIC WATER LEVEL: } \\
\hline \multicolumn{3}{|c|}{ Start of Job 26,15} \\
\hline \multicolumn{3}{|c|}{ End of Job 26.35} \\
\hline \multicolumn{3}{|c|}{ DEPTH TO BOTTOM: } \\
\hline \multicolumn{3}{|c|}{ Start of Job $58, C^{\prime}$ bgs } \\
\hline \multicolumn{3}{|c|}{ End of Job 58,6 bas } \\
\hline \multicolumn{3}{|c|}{ PART 2 } \\
\hline \multicolumn{3}{|c|}{ WELL DEVELOPMENT DATA } \\
\hline \multicolumn{3}{|c|}{ Pump Model $75 s-50$} \\
\hline \multicolumn{3}{|c|}{ Intake Depth $40,6 / 50,0$} \\
\hline \multicolumn{3}{|c|}{ Starting Turbidity $53.7 / 26,5$} \\
\hline Pump Start & Stop & Flow Rate \\
\hline 1407 & 1432 & $73 \mathrm{gpm}$ \\
\hline 1457 & 1515 & $74 \mathrm{gpm}$ \\
\hline & & \\
\hline & & \\
\hline & & \\
\hline
\end{tabular}

\begin{tabular}{|l|}
\hline Total Pumped $1825 / 1332$ \\
\hline Final Turbidity $5.12 / 2.45$ \\
\hline XD SN/Range (PSI) $45348 / 20$-0 \\
\hline RART 3 \\
\hline INSTANTANEOUS SLUG TEST \\
\hline
\end{tabular}

\begin{tabular}{|l|}
\hline Static Wgter Level (TOC) \\
\hline Transducerpepth \\
\hline Baseline Start \\
\hline Injection Start \\
\hline Baseline Start \\
\hline
\end{tabular}

\begin{tabular}{l|}
\hline Last Recorded \\
Measurements \\
Date:
\end{tabular}

$\mathrm{C}=$
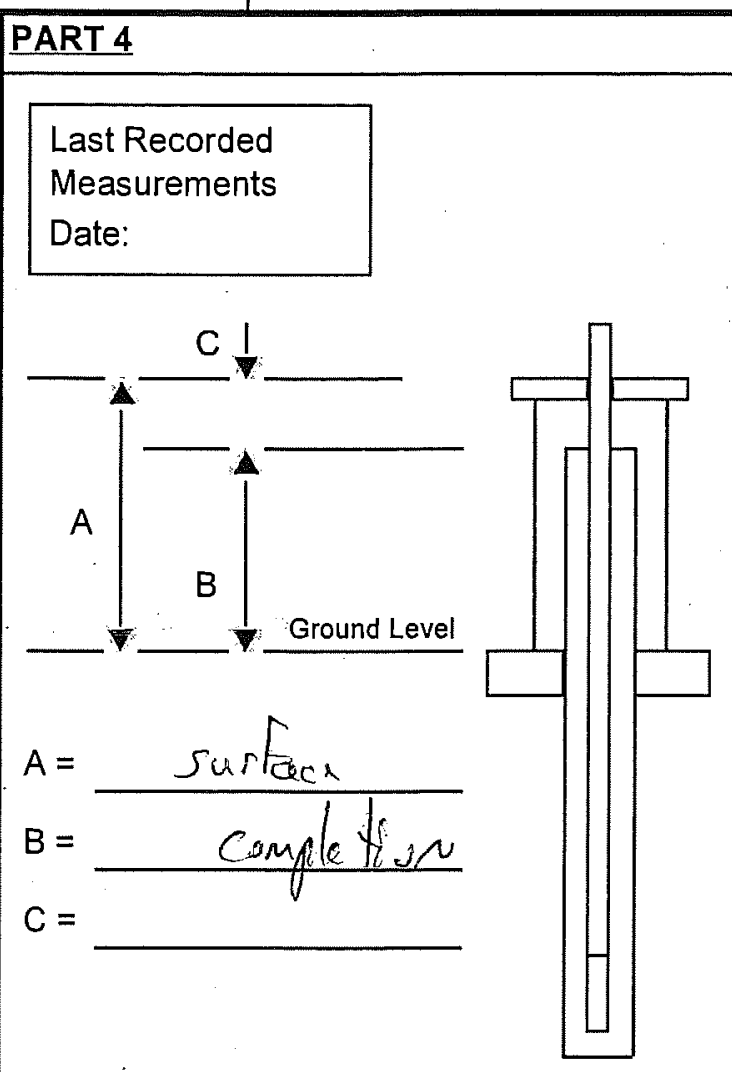

Does the well have a cement pad? $\mathrm{O}$ Yes $6 \mathrm{No}_{\mathrm{S}}$

Are there any reference marks on the casing strings? $\bigcirc$ Yes $\varnothing$ No

\section{PART 5}

COMMENTS: Meter T8188

$$
\begin{aligned}
& \text { Test } 1: \times D_{i}: 3.238 \\
& \times D f: 3.243
\end{aligned}
$$$$
\text { Test } 2: 5217: 3.270
$$$$
5218 \text { (dup) } \times D i: 3.427
$$$$
5217 \times D_{f}: 3.291
$$$$
5218 \text { dup) } \times D f: 3.436 \text { (well C6197) }
$$

final meter reading 21293

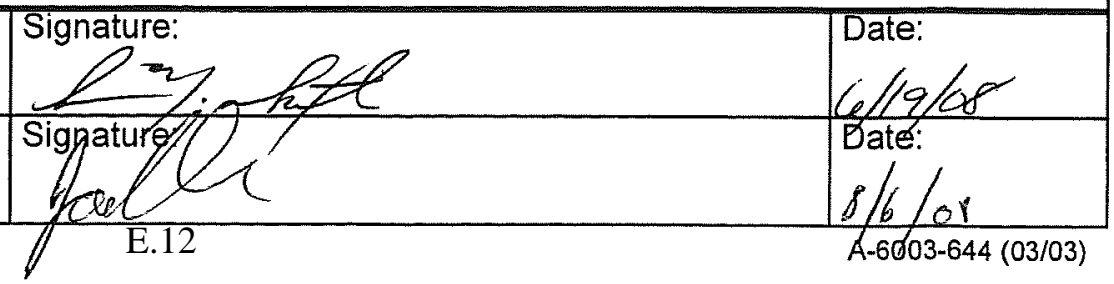




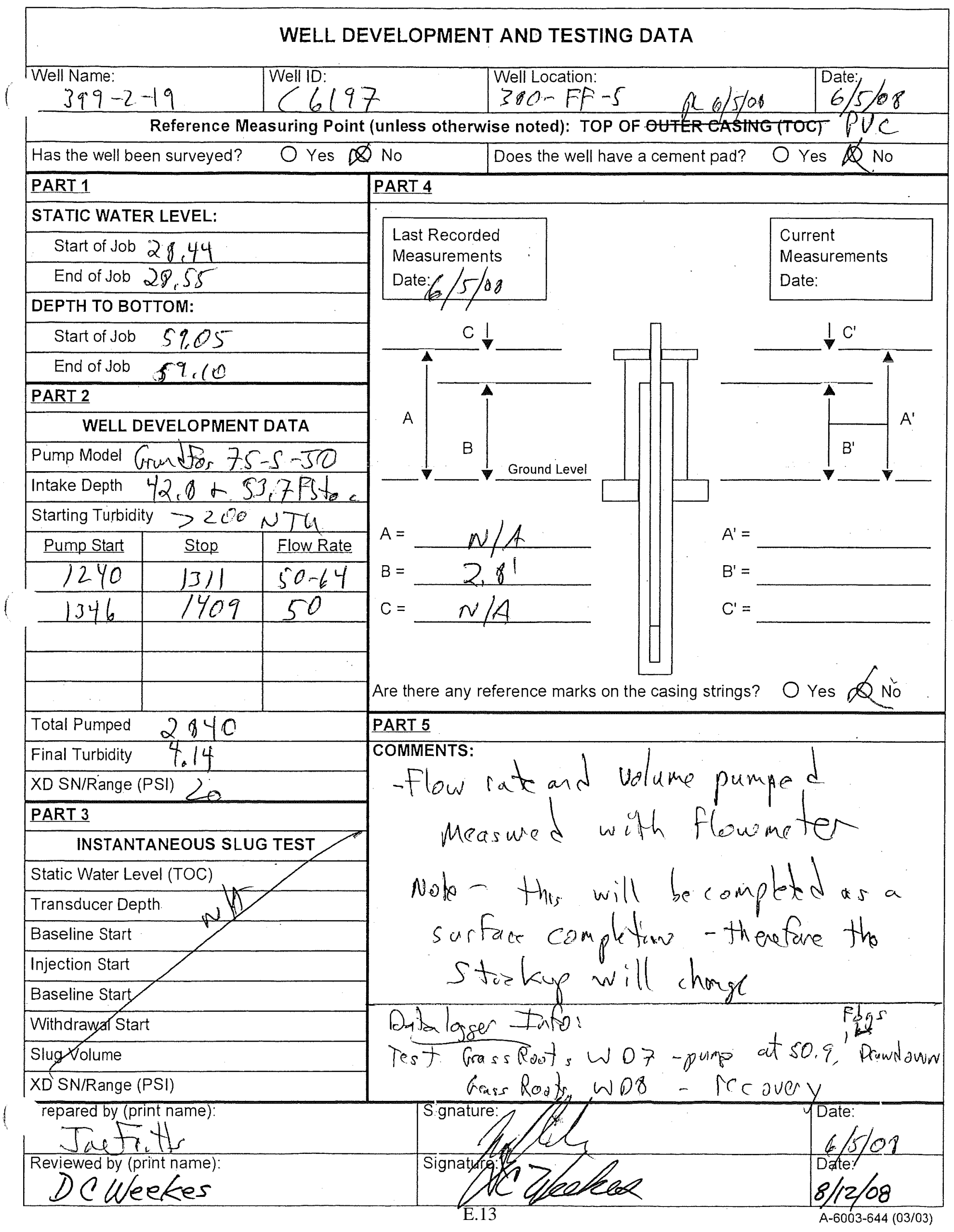




\section{WELL DEVELOPMENT AND TESTING DATA}

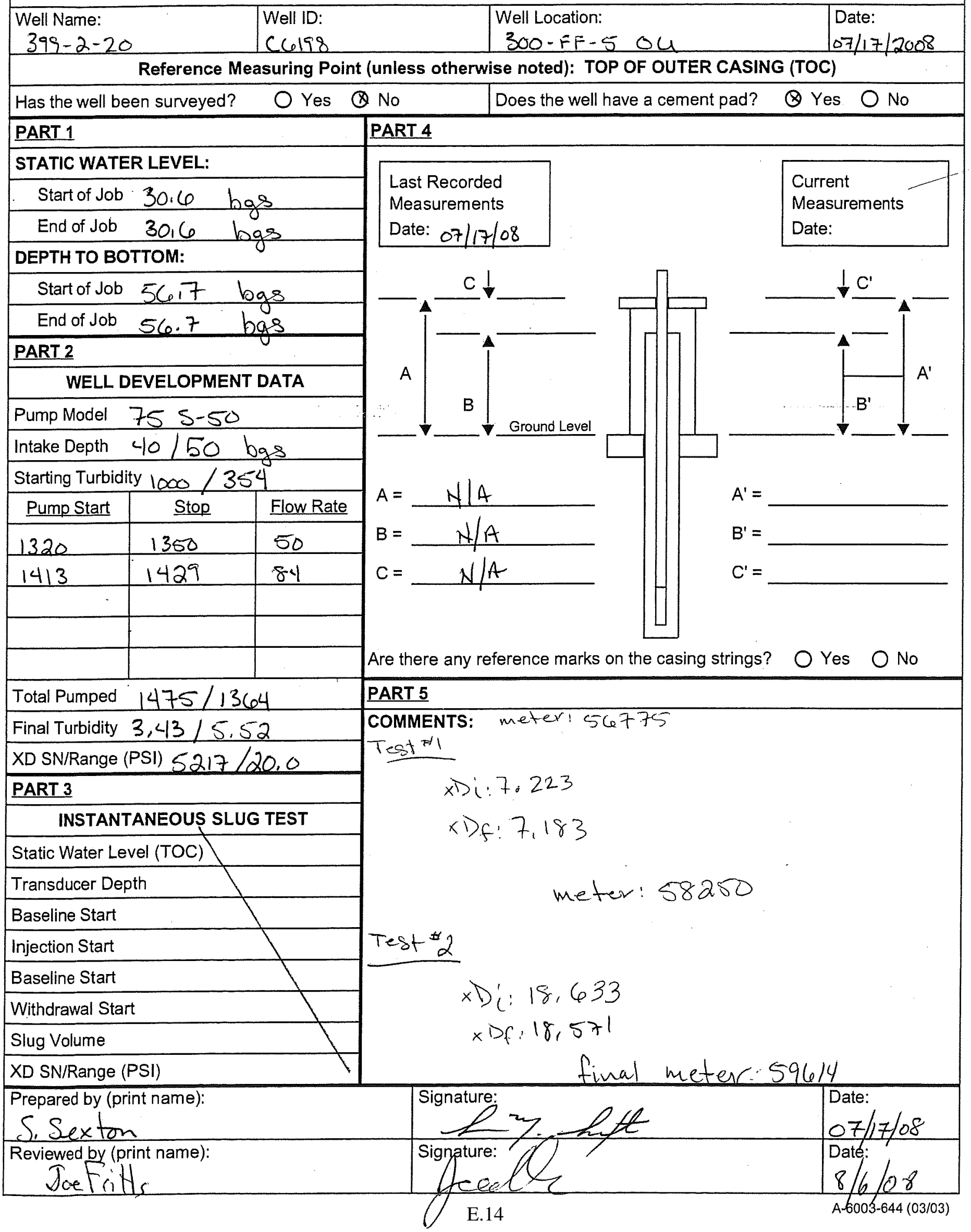




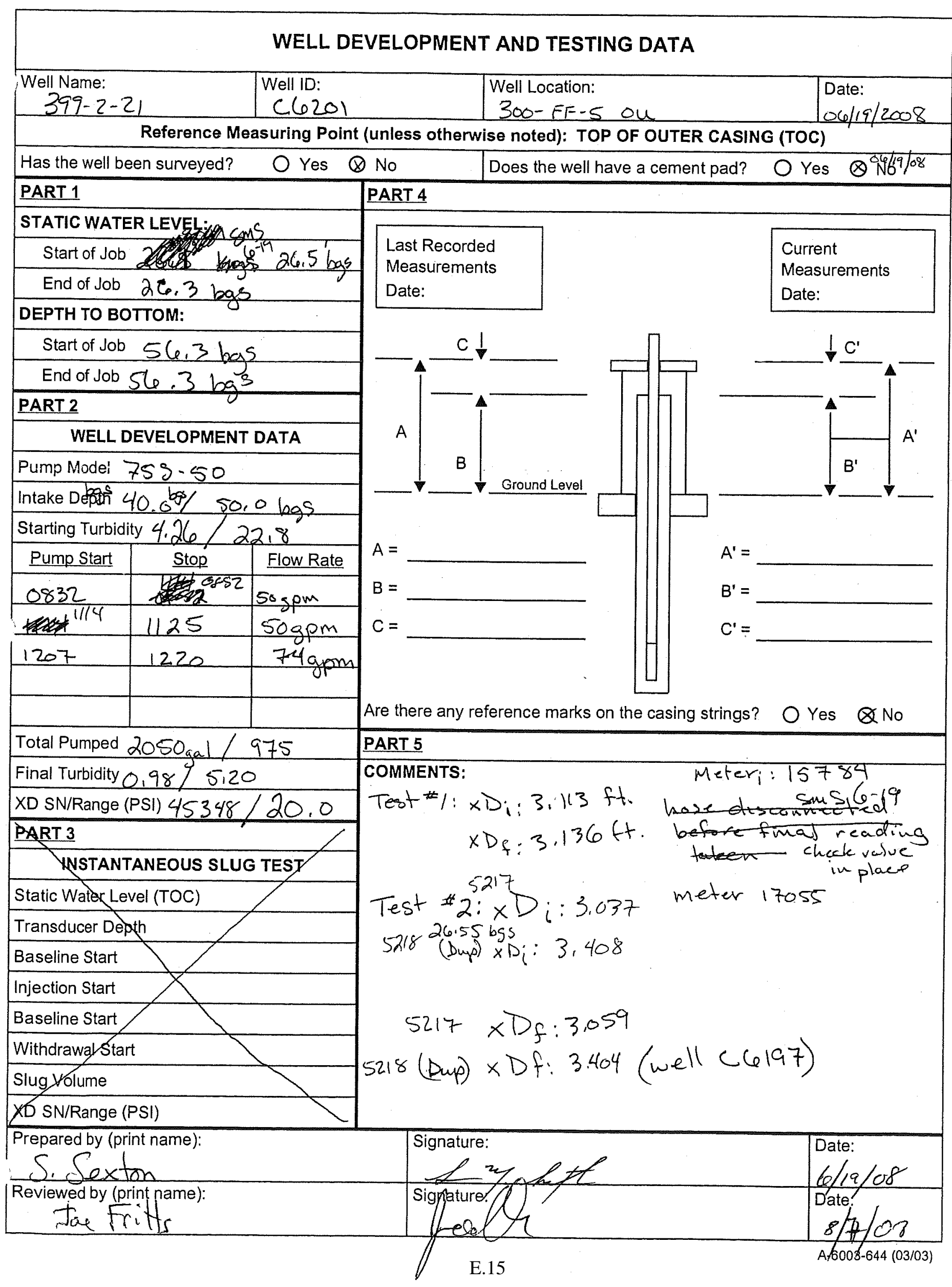




\section{WELL DEVELOPMENT AND TESTING DATA}

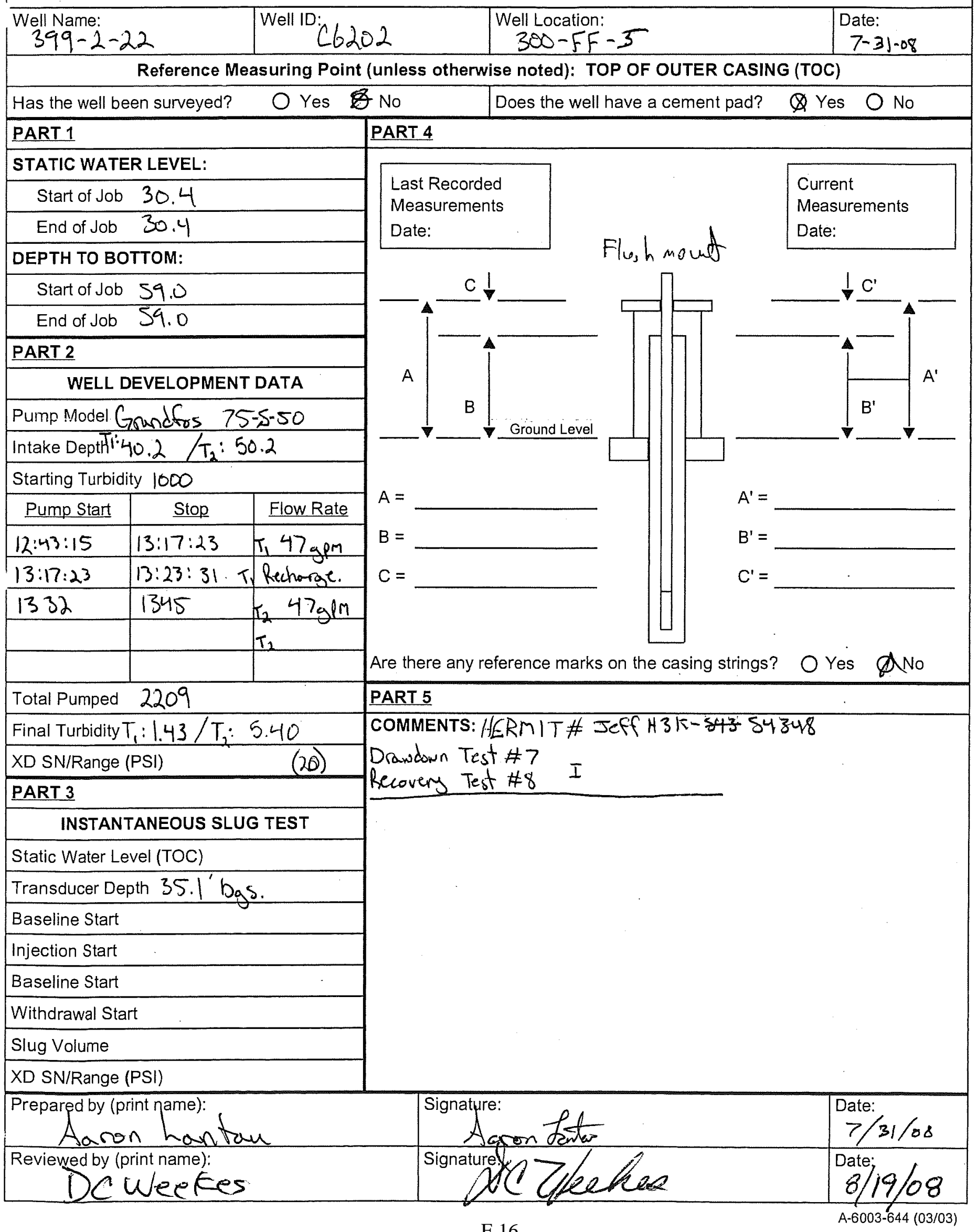




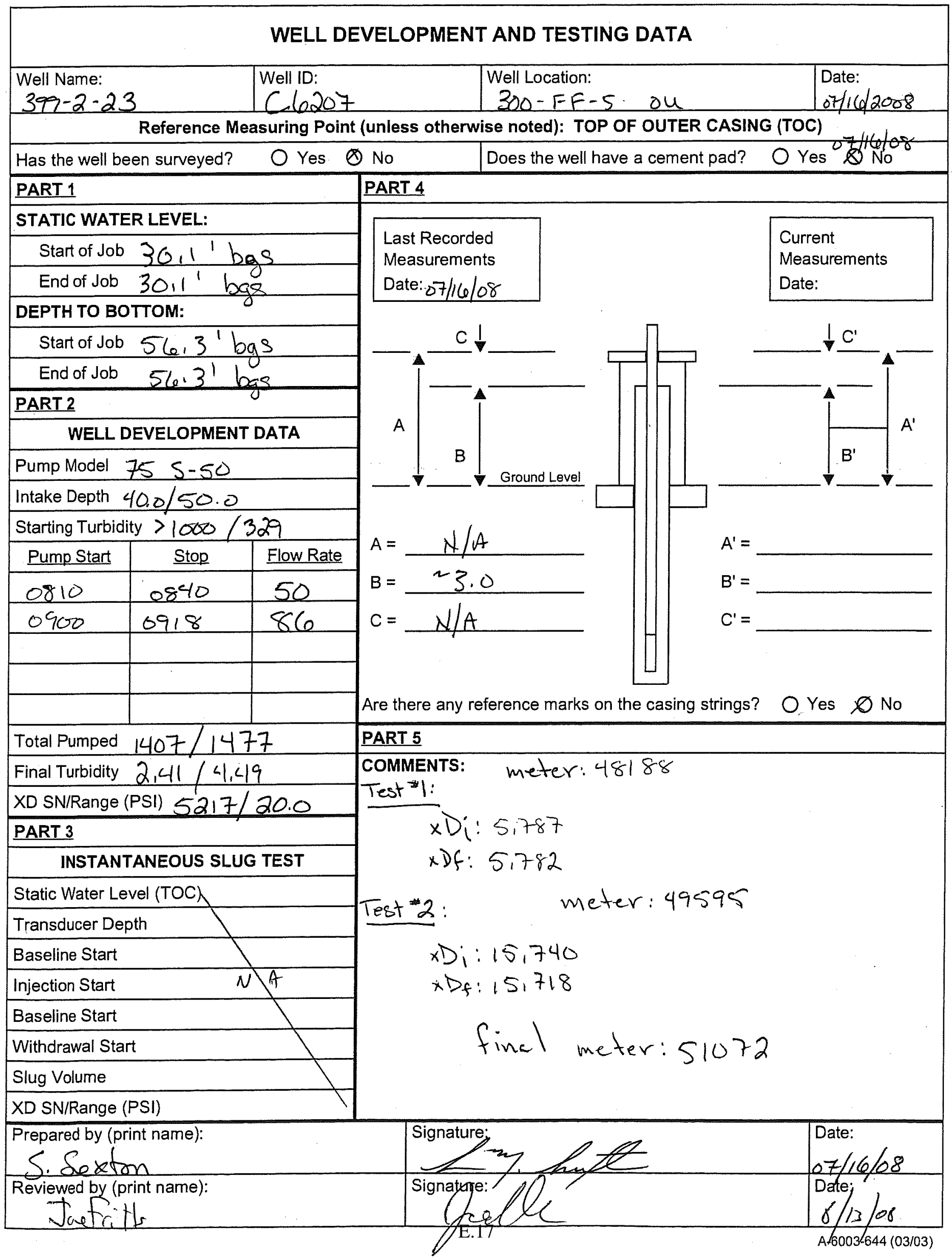




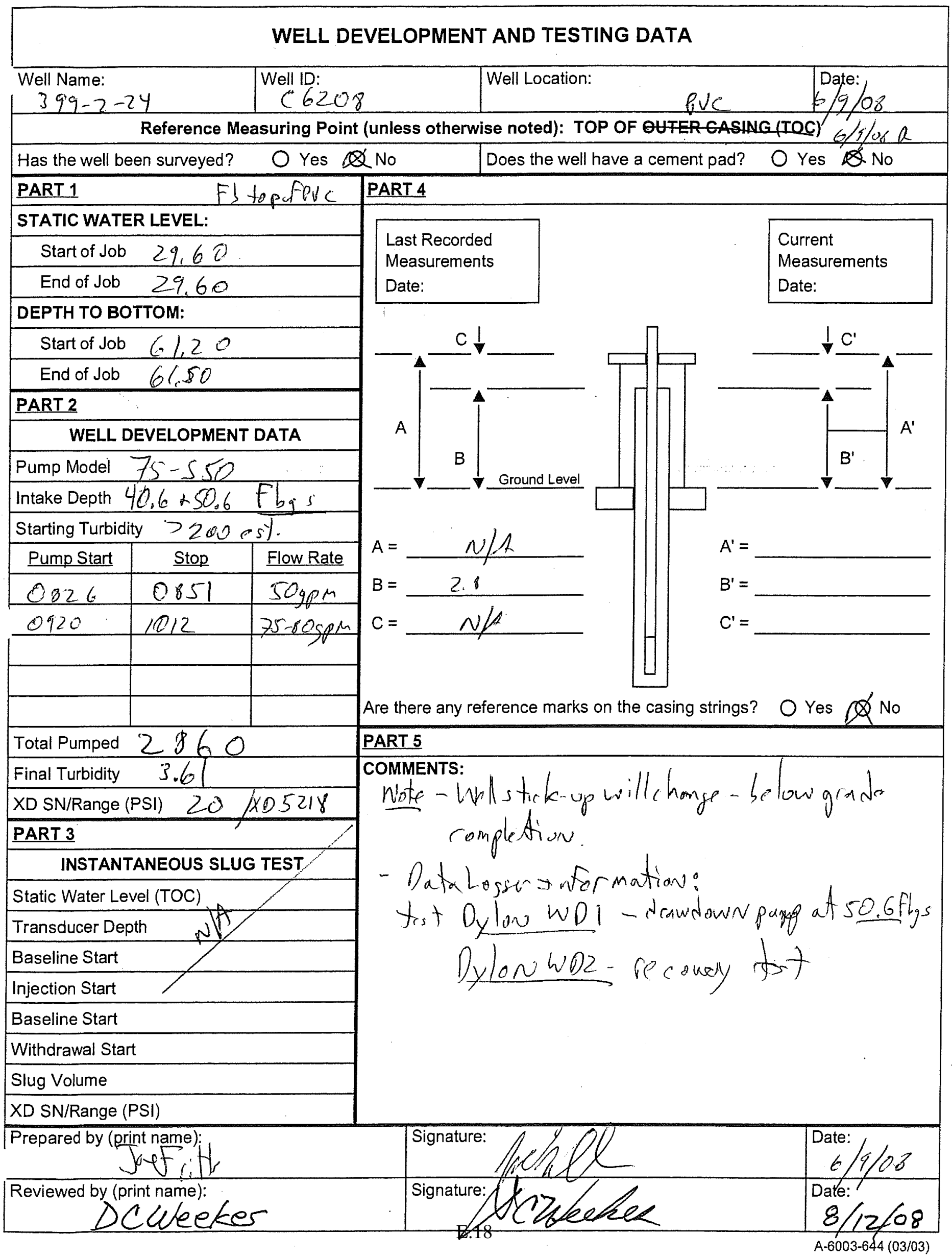


WELL DEVELOPMENT AND TESTING DATA

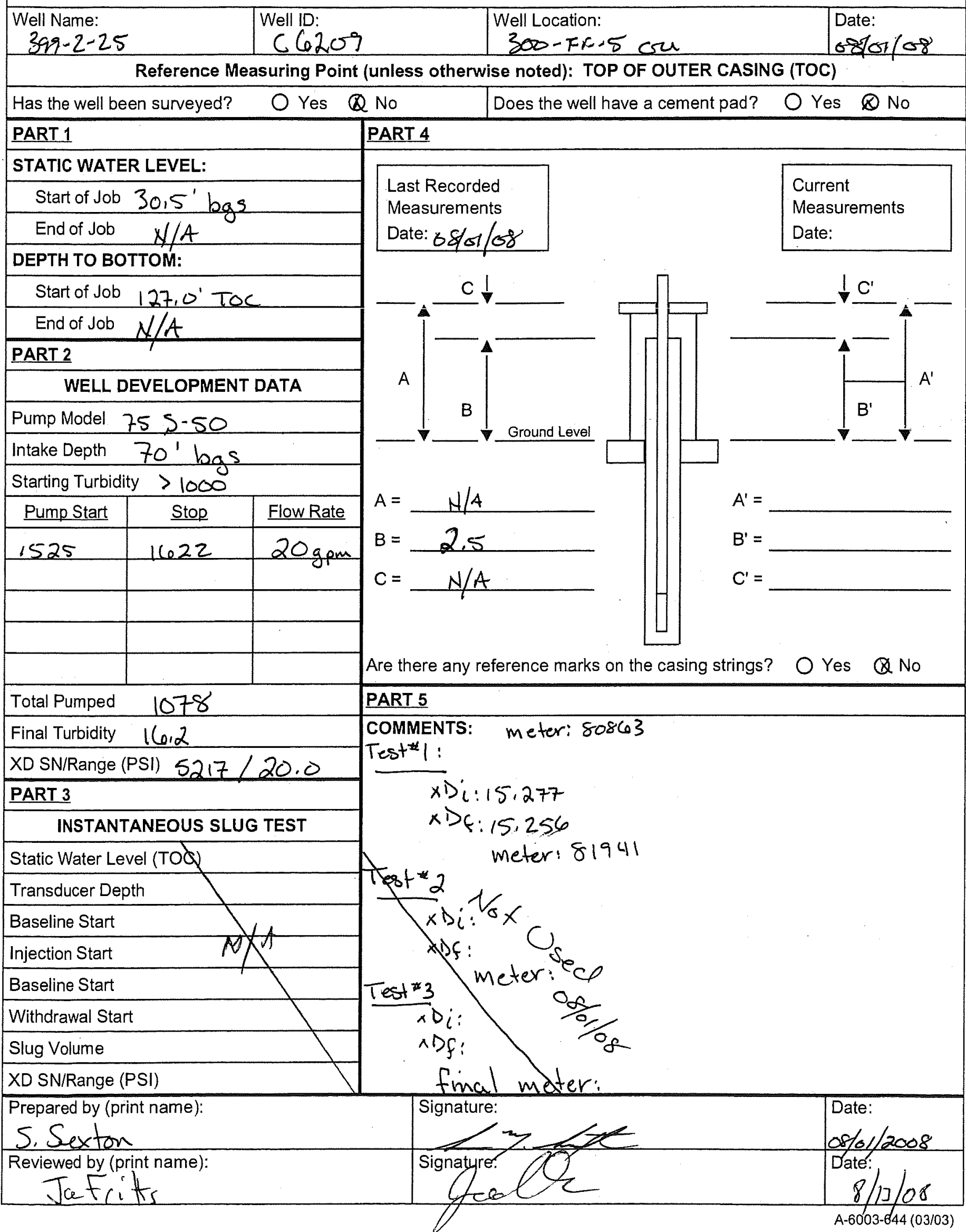

E.19 


\section{WELL DEVELOPMENT AND TESTING DATA}

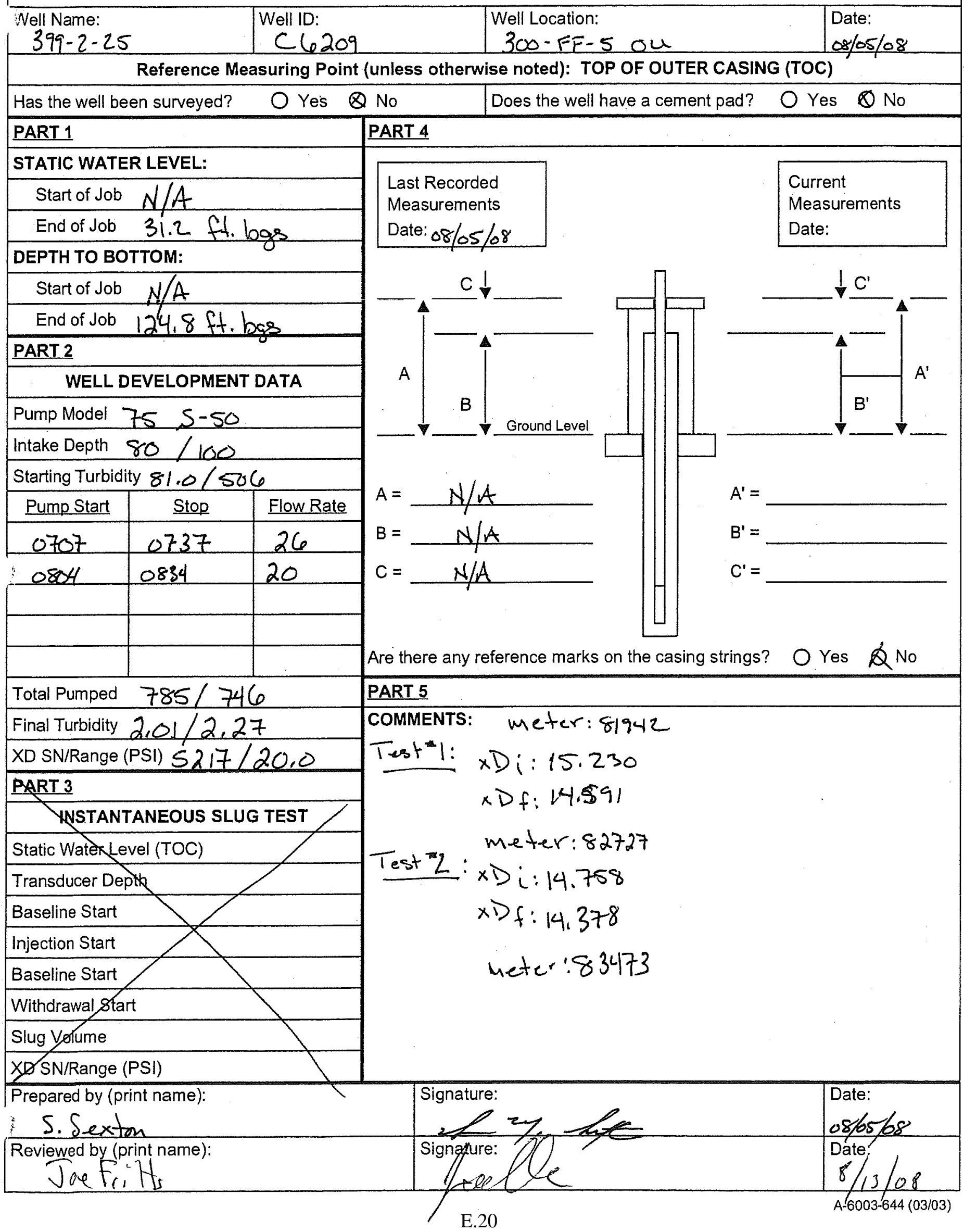




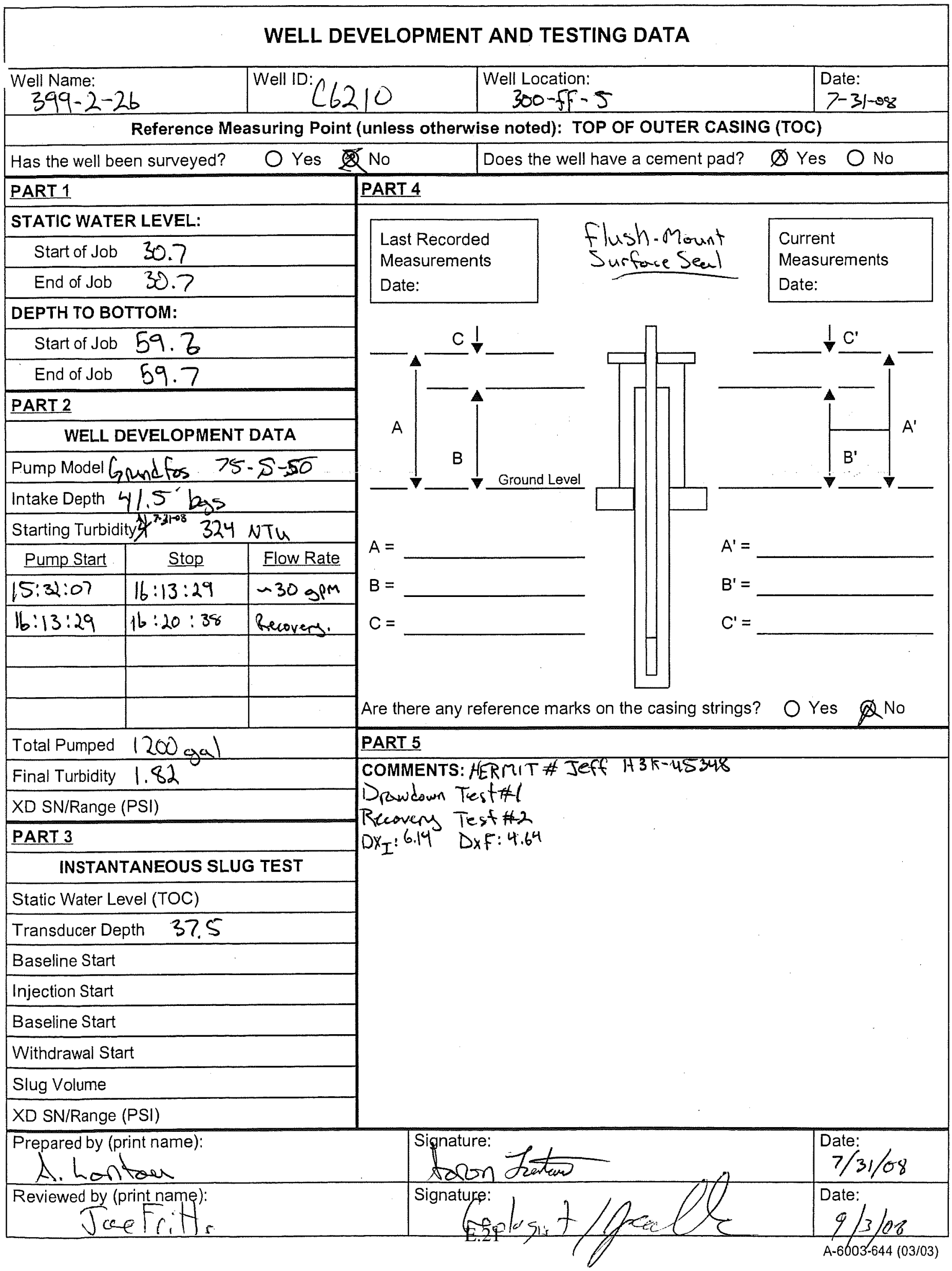




\section{WELL DEVELOPMENT AND TESTING DATA}

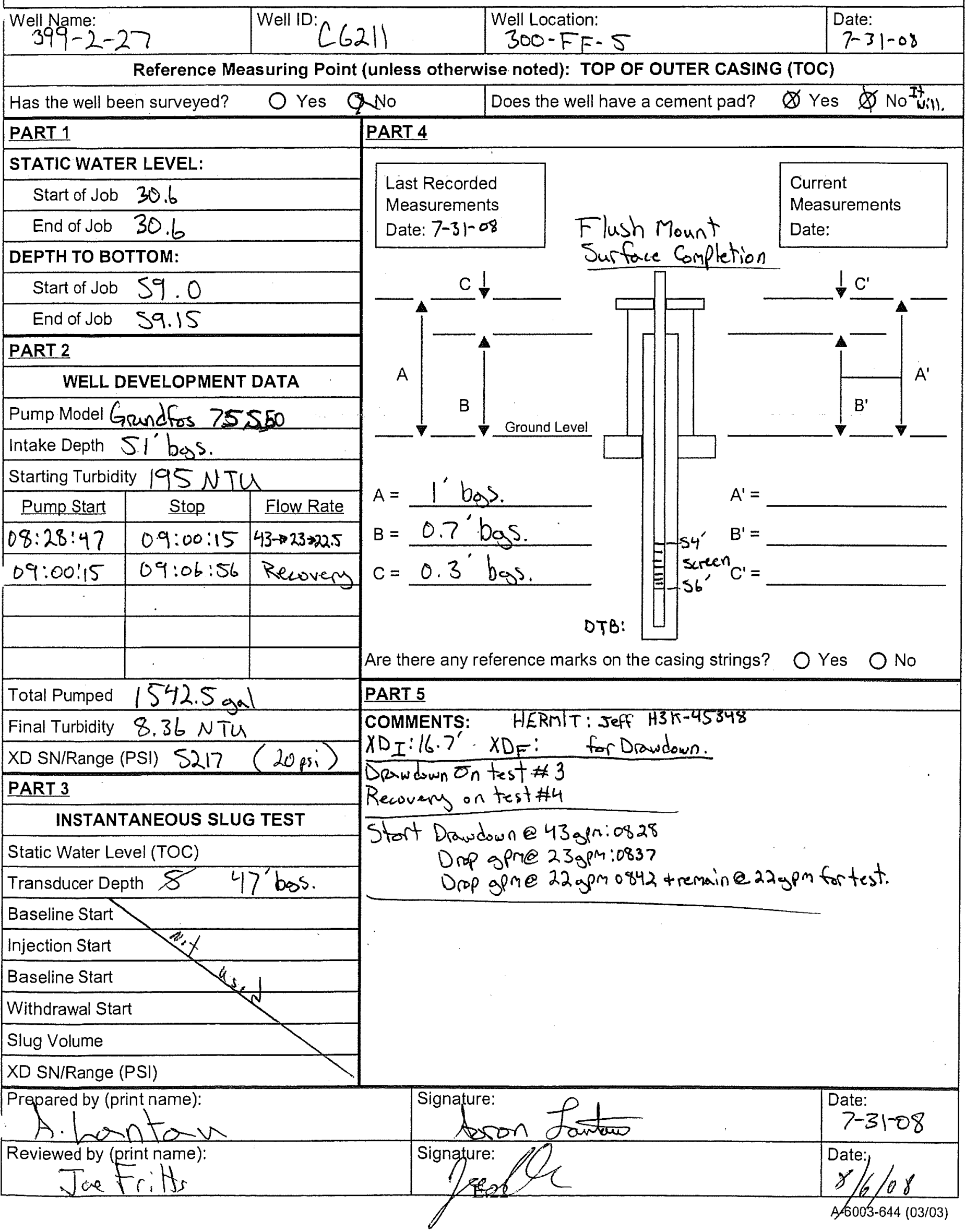




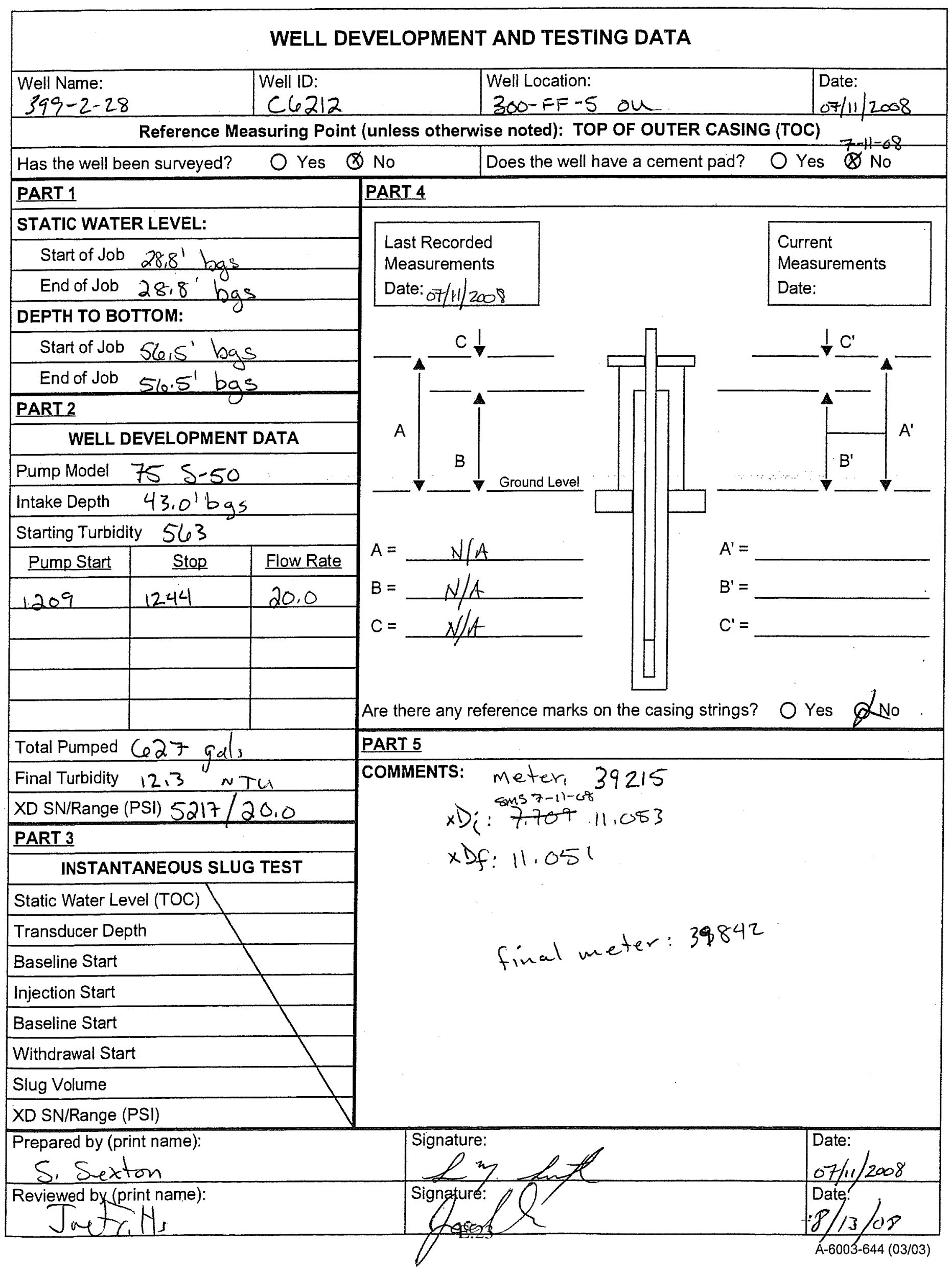




\section{WELL DEVELOPMENT AND TESTING DATA}

\begin{tabular}{|l|l|l|l|}
\hline $\begin{array}{l}\text { Well Name: } \\
399-2-29\end{array}$ & $\begin{array}{l}\text { Well ID: } \\
\text { C6216 }\end{array}$ & $\begin{array}{l}\text { Well Location: } \\
300-F F-5 \text { ou }\end{array}$ & $\begin{array}{l}\text { Date: } \\
07 / 11 / 2008\end{array}$ \\
\hline \multicolumn{3}{r}{} \\
\hline
\end{tabular}

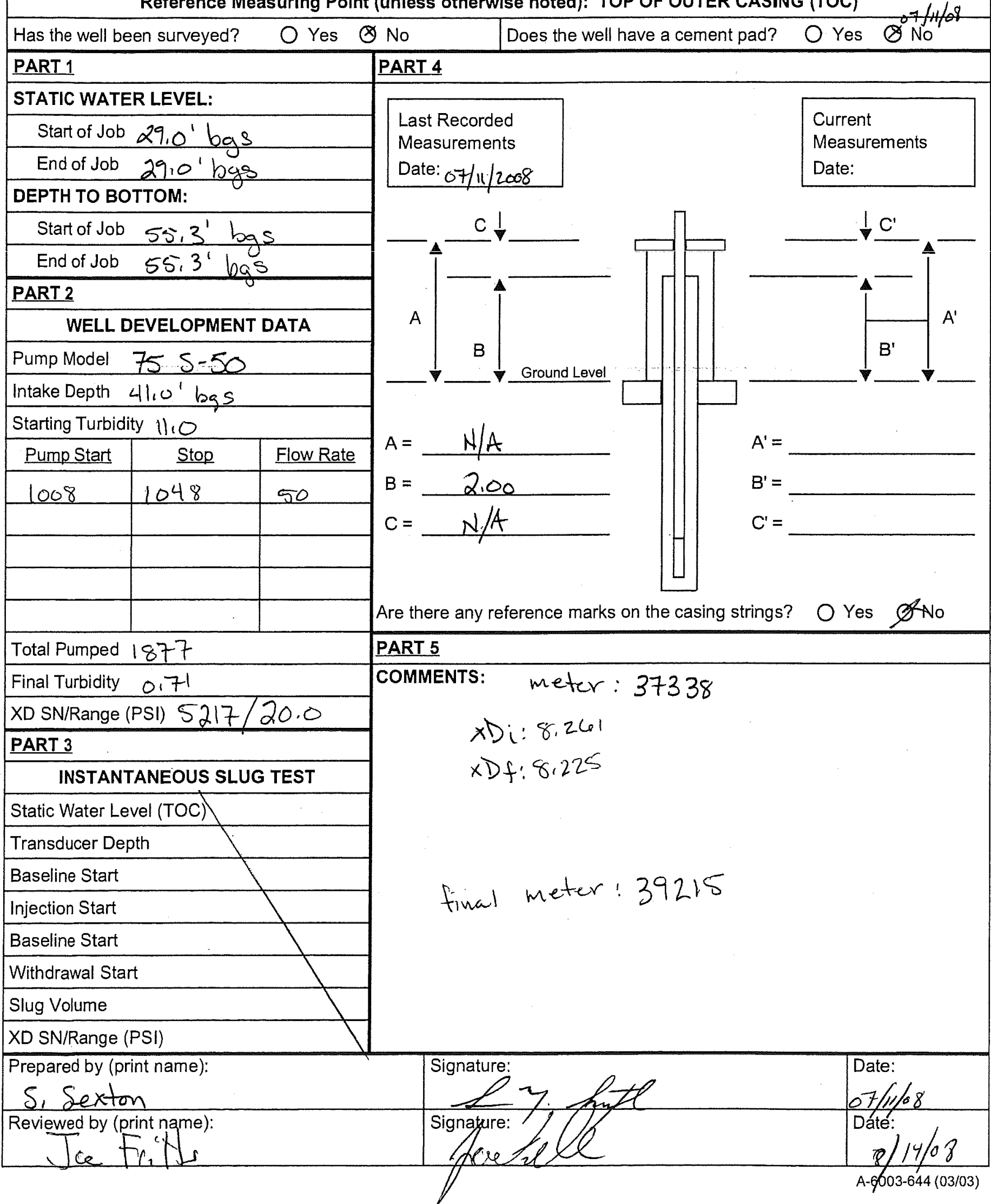




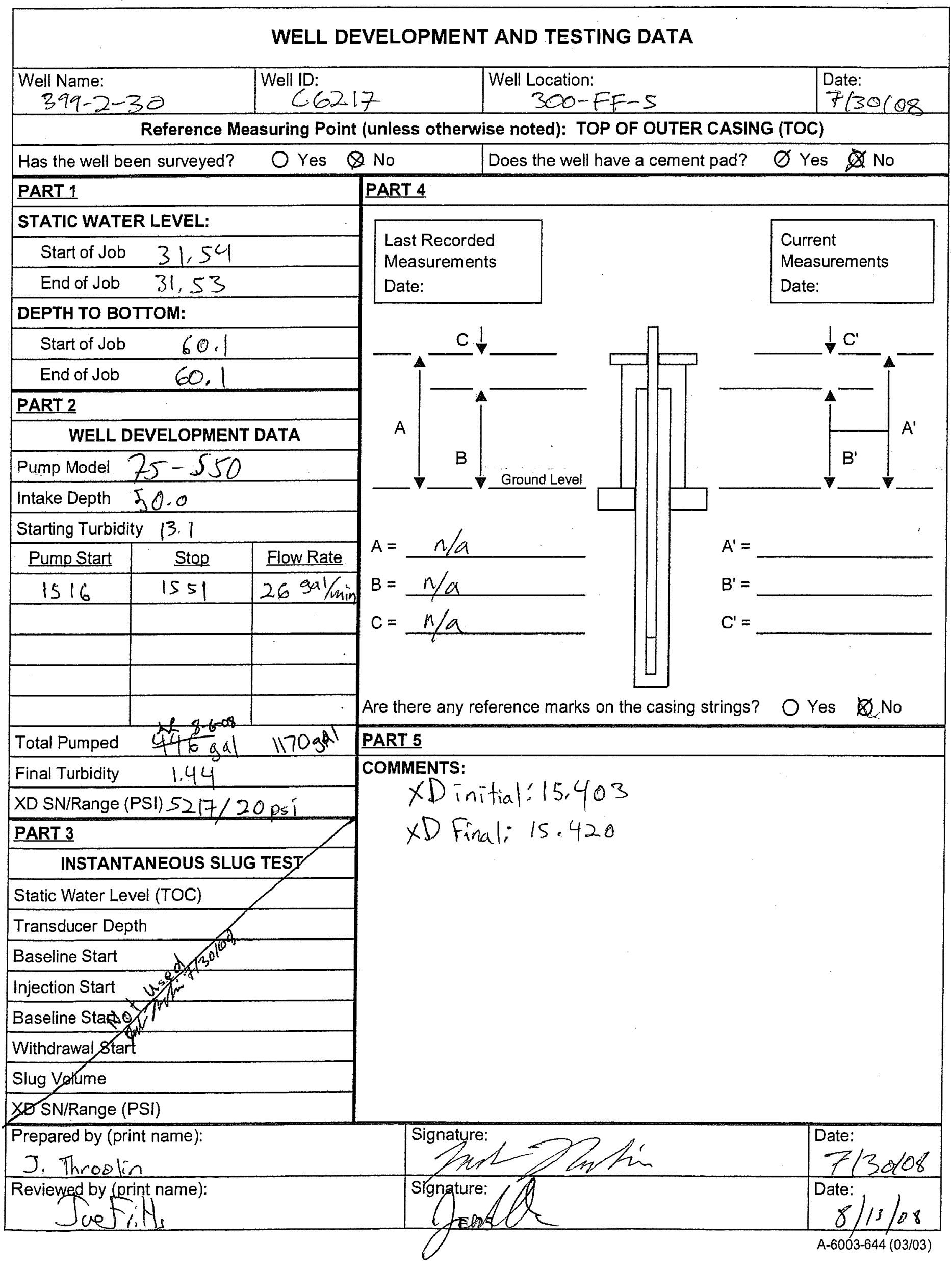




\section{WELL DEVELOPMENT AND TESTING DATA}

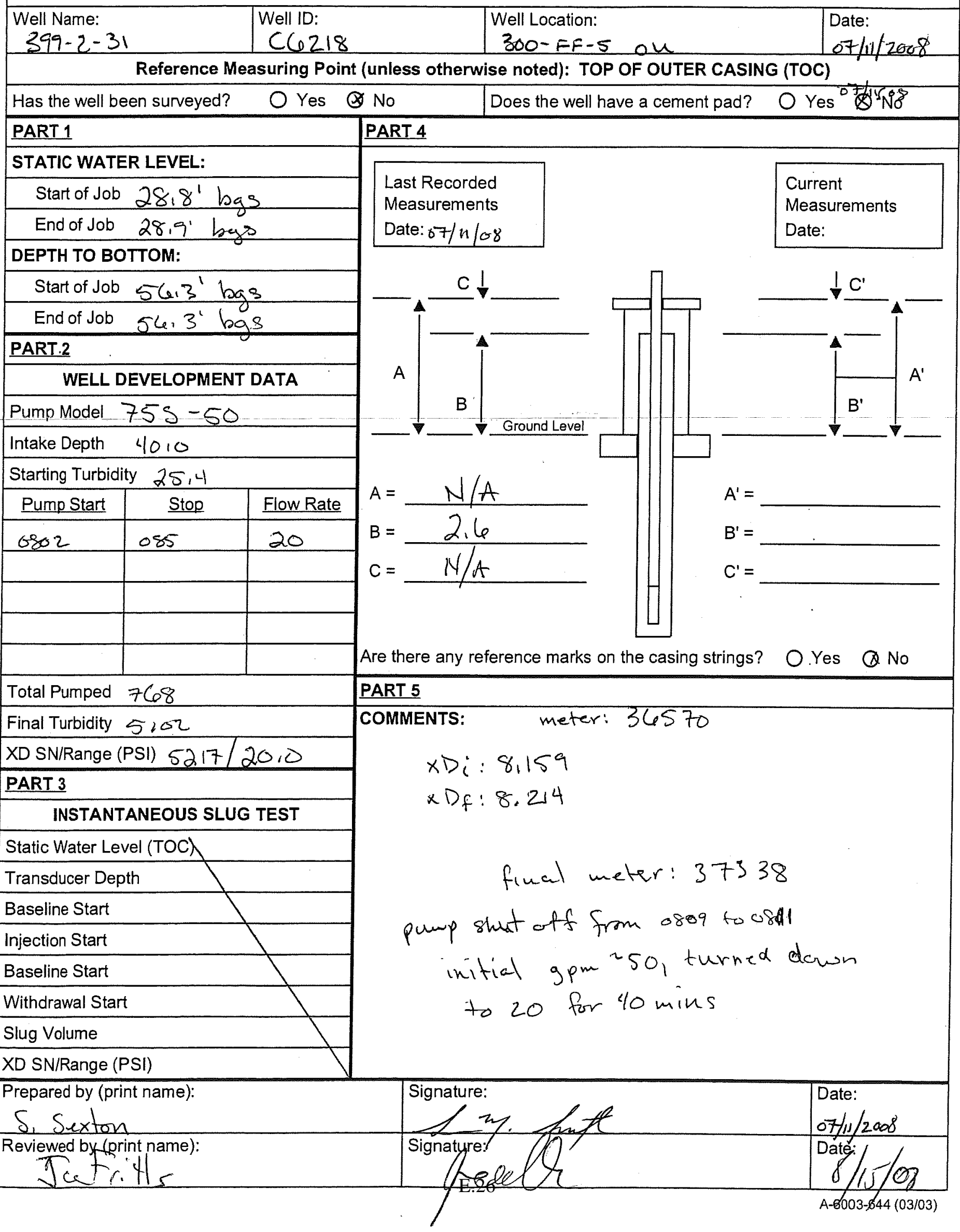




\section{WELL DEVELOPMENT AND TESTING DATA}

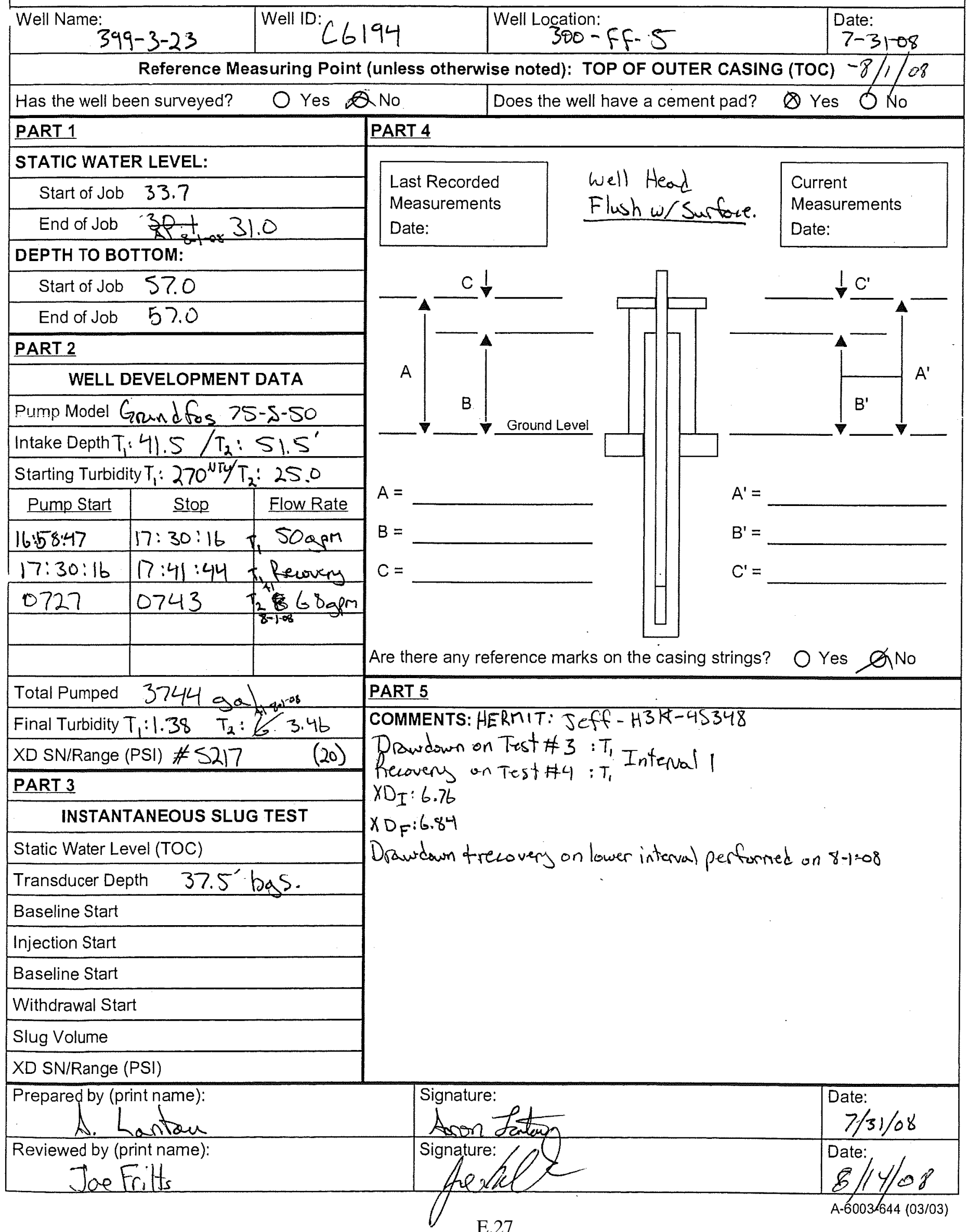




\section{WELL DEVELOPMENT AND TESTING DATA}

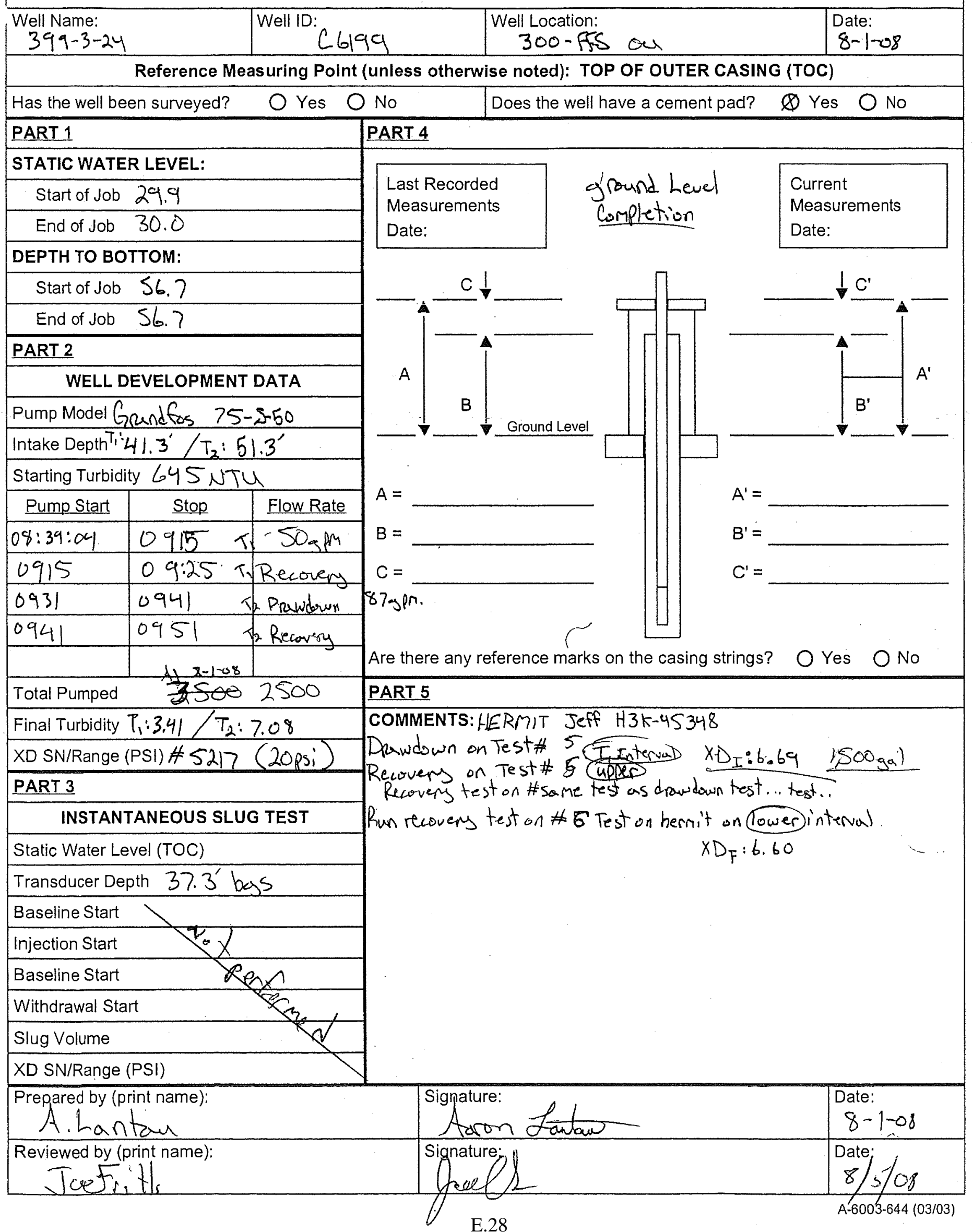




\section{WELL DEVELOPMENT AND TESTING DATA}

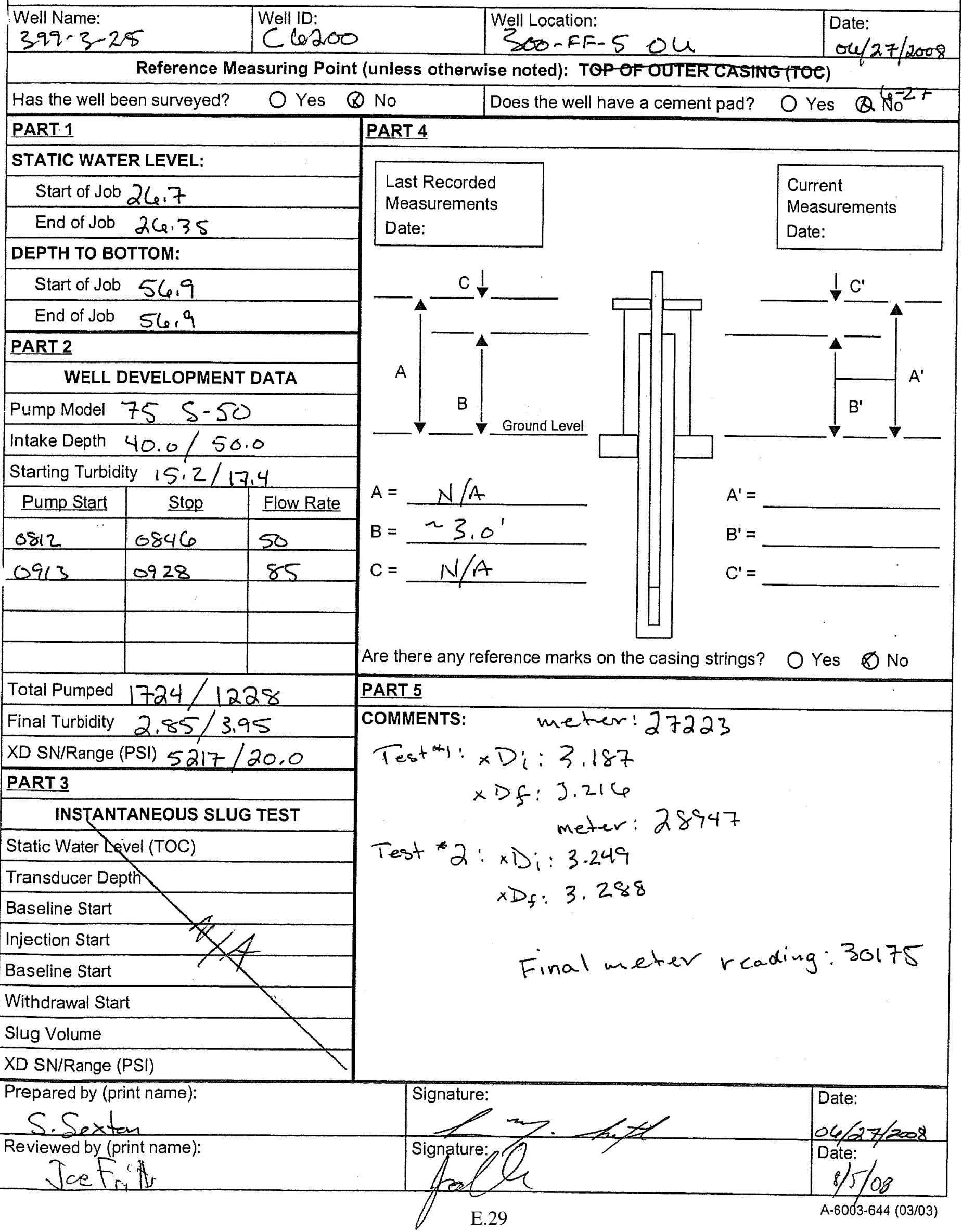




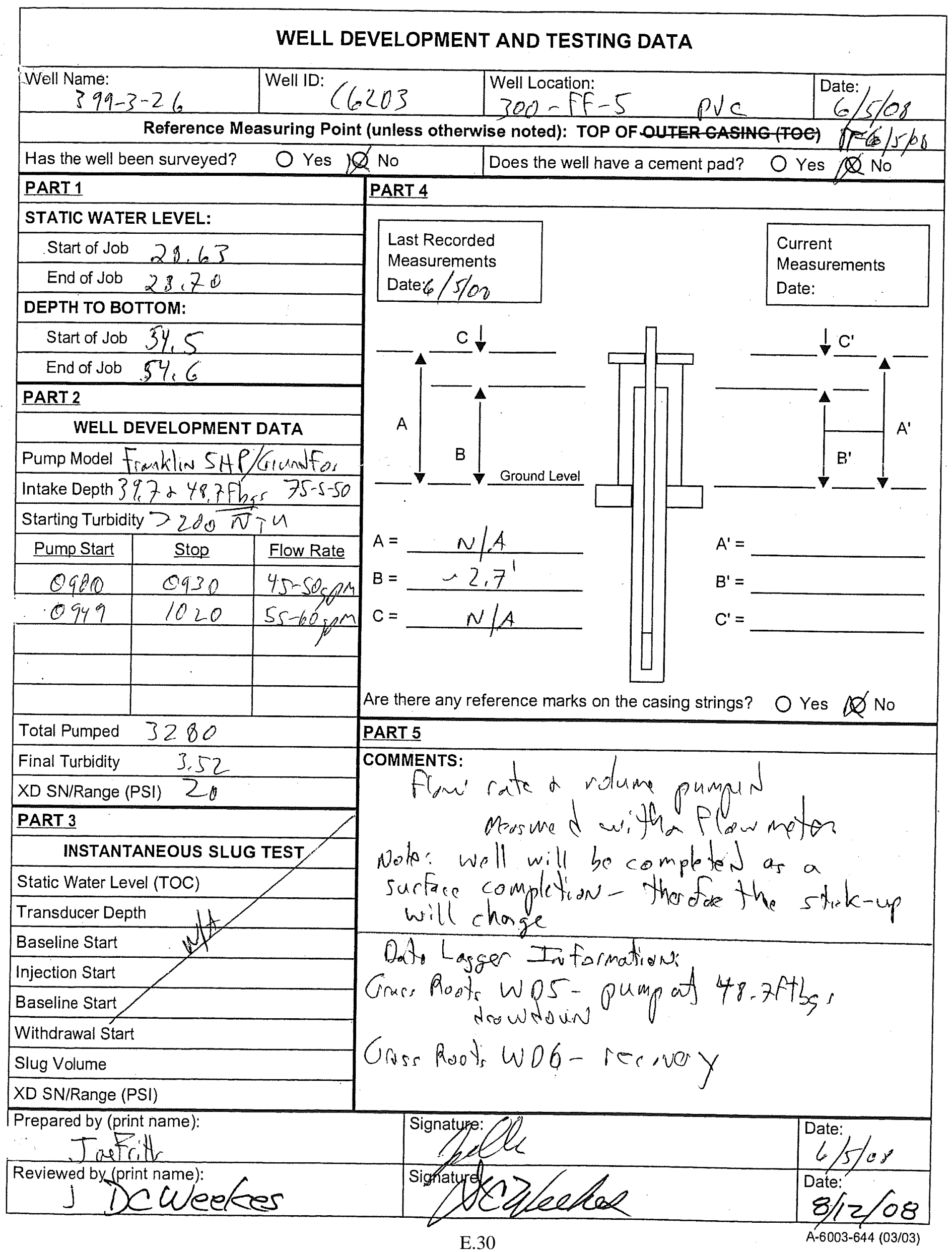




\section{WELL DEVELOPMENT AND TESTING DATA}

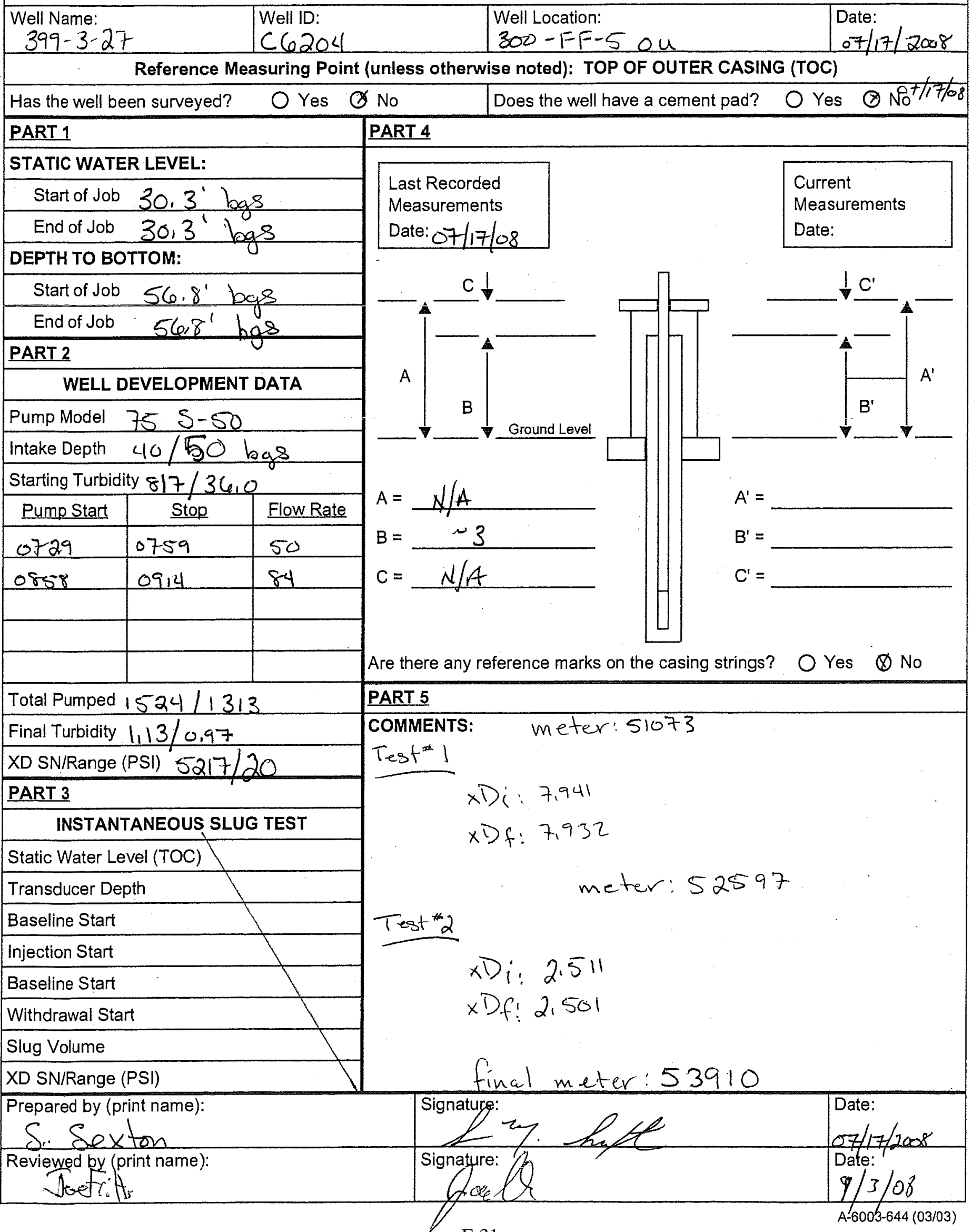




\section{WELL DEVELOPMENT AND TESTING DATA}

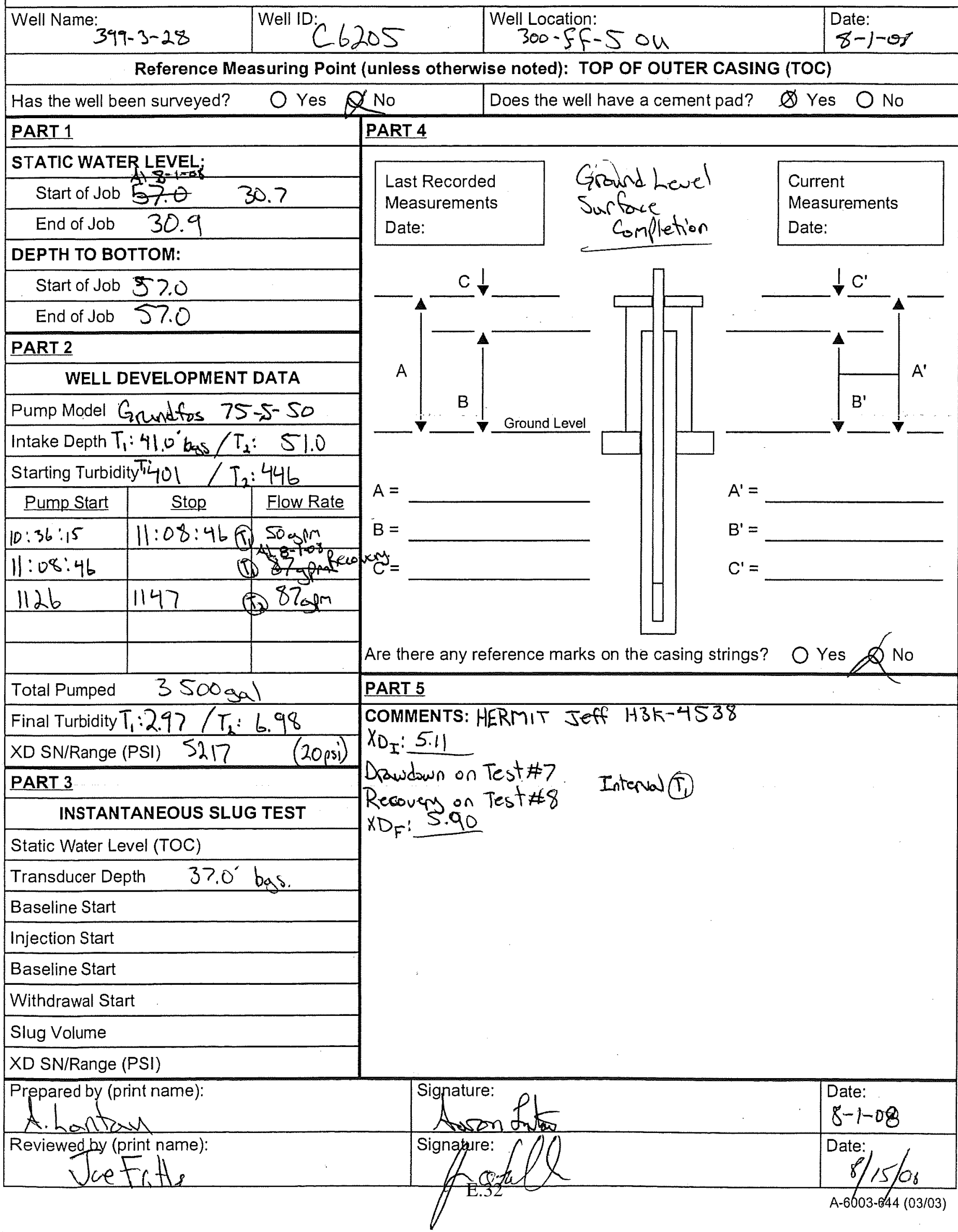




\section{WELL DEVELOPMENT AND TESTING DATA}

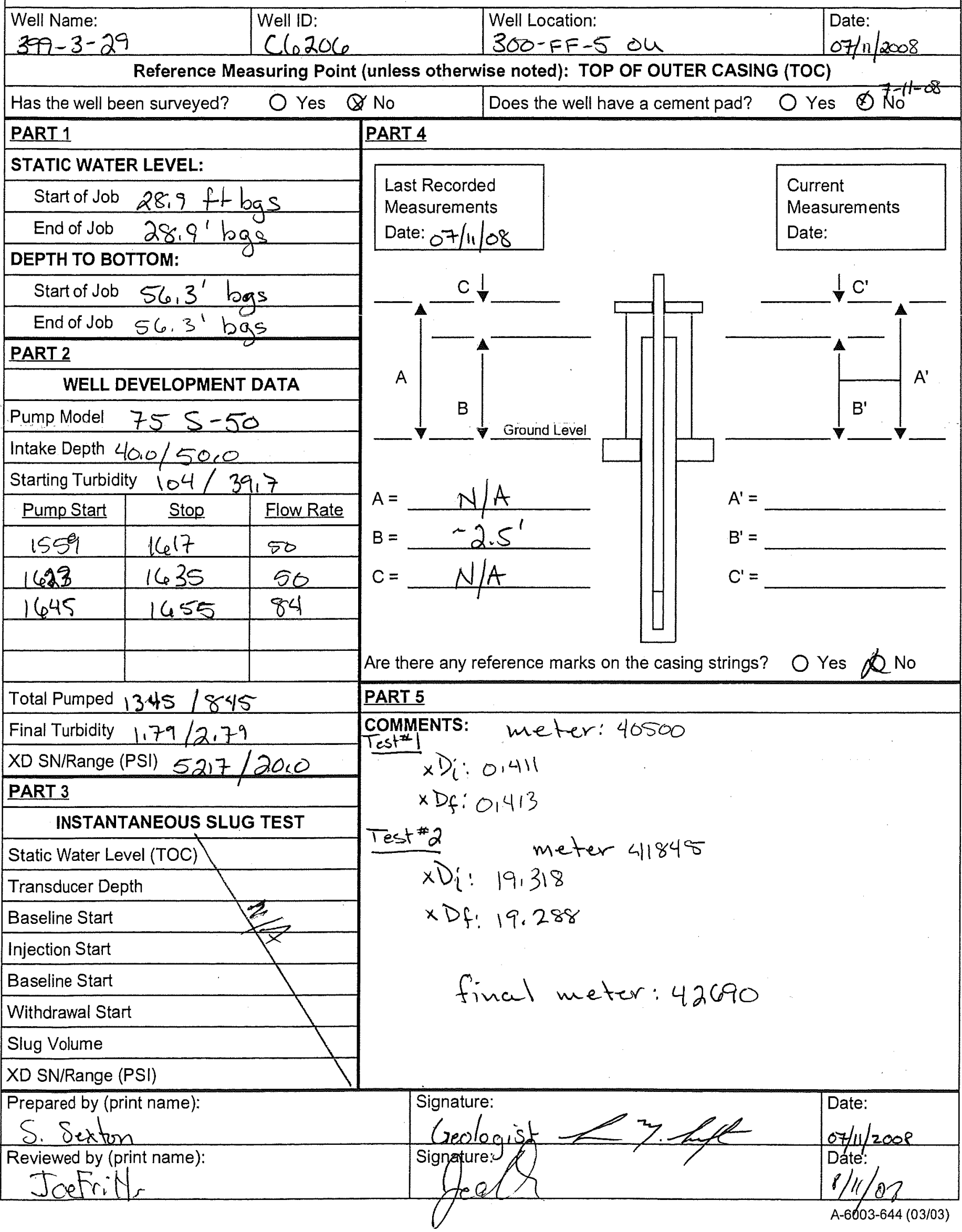




\section{WELL DEVELOPMENT AND TESTING DATA}

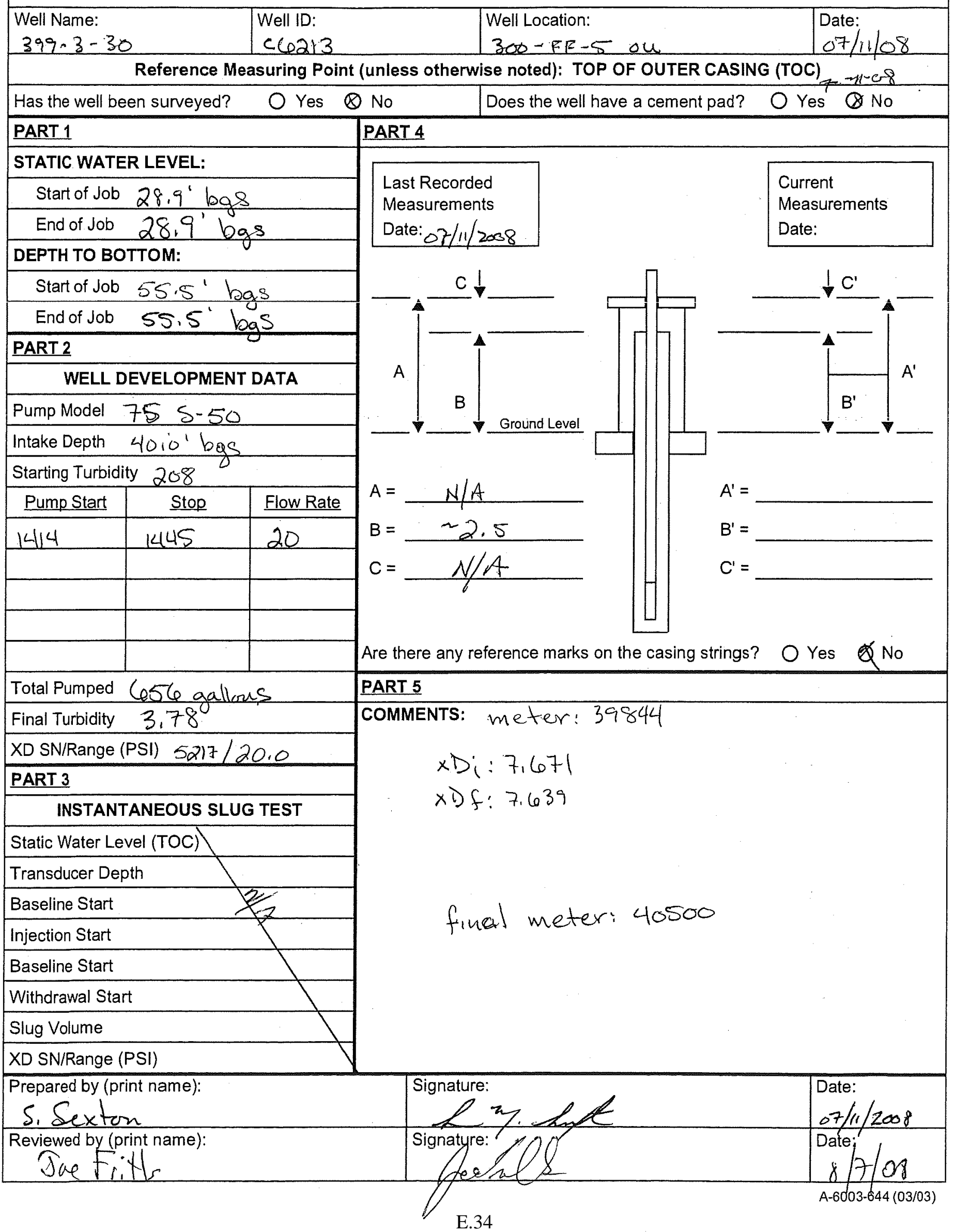




\section{WELL DEVELOPMENT AND TESTING DATA}

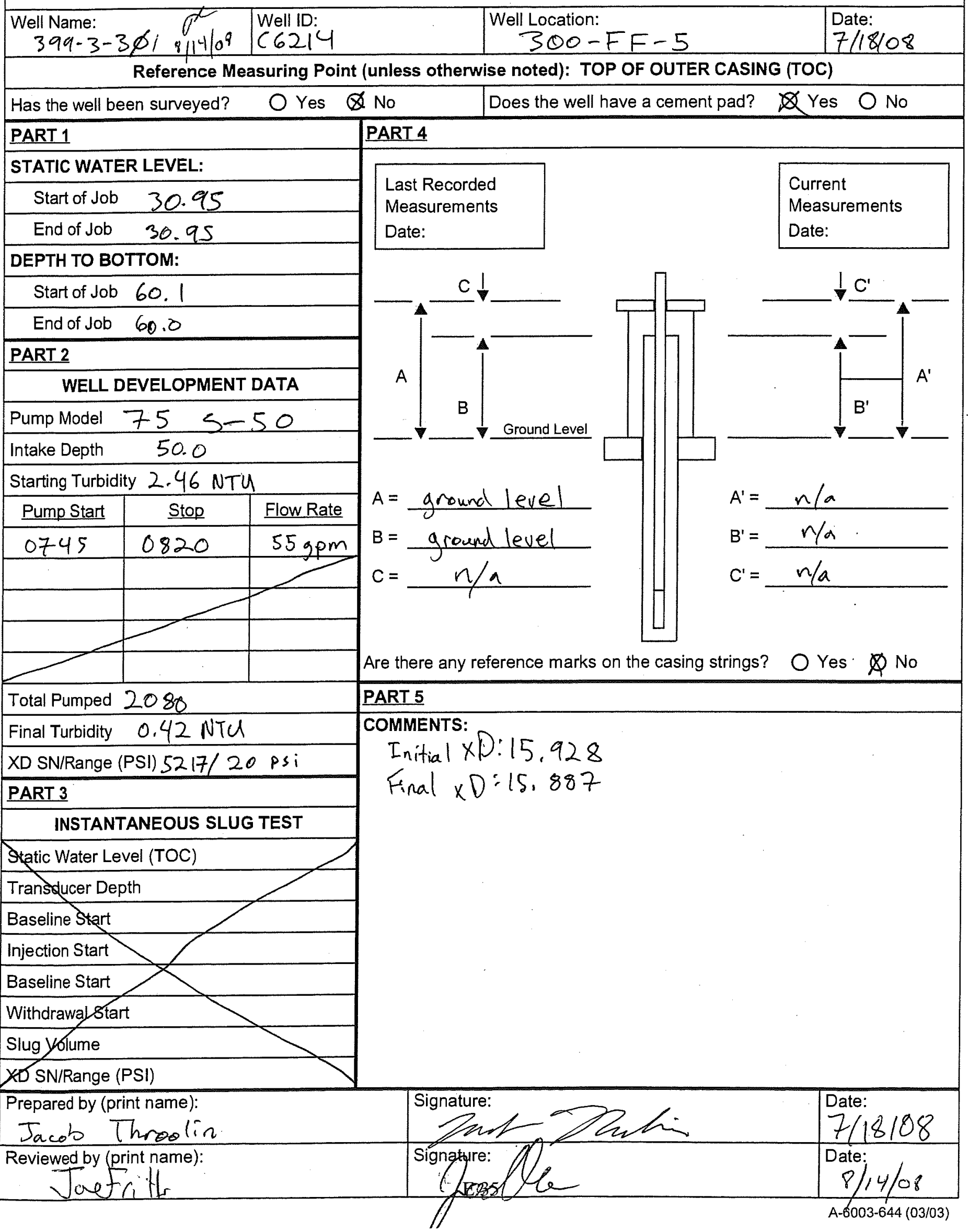




\section{WELL DEVELOPMENT AND TESTING DATA}

\begin{tabular}{|c|c|c|c|}
\hline Well Name: $\quad 399-3-32$ & Well ID: 6215 & $\begin{array}{l}\text { Well Location: } \\
300-F F-5\end{array}$ & 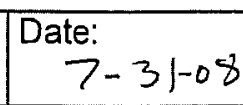 \\
\hline
\end{tabular}

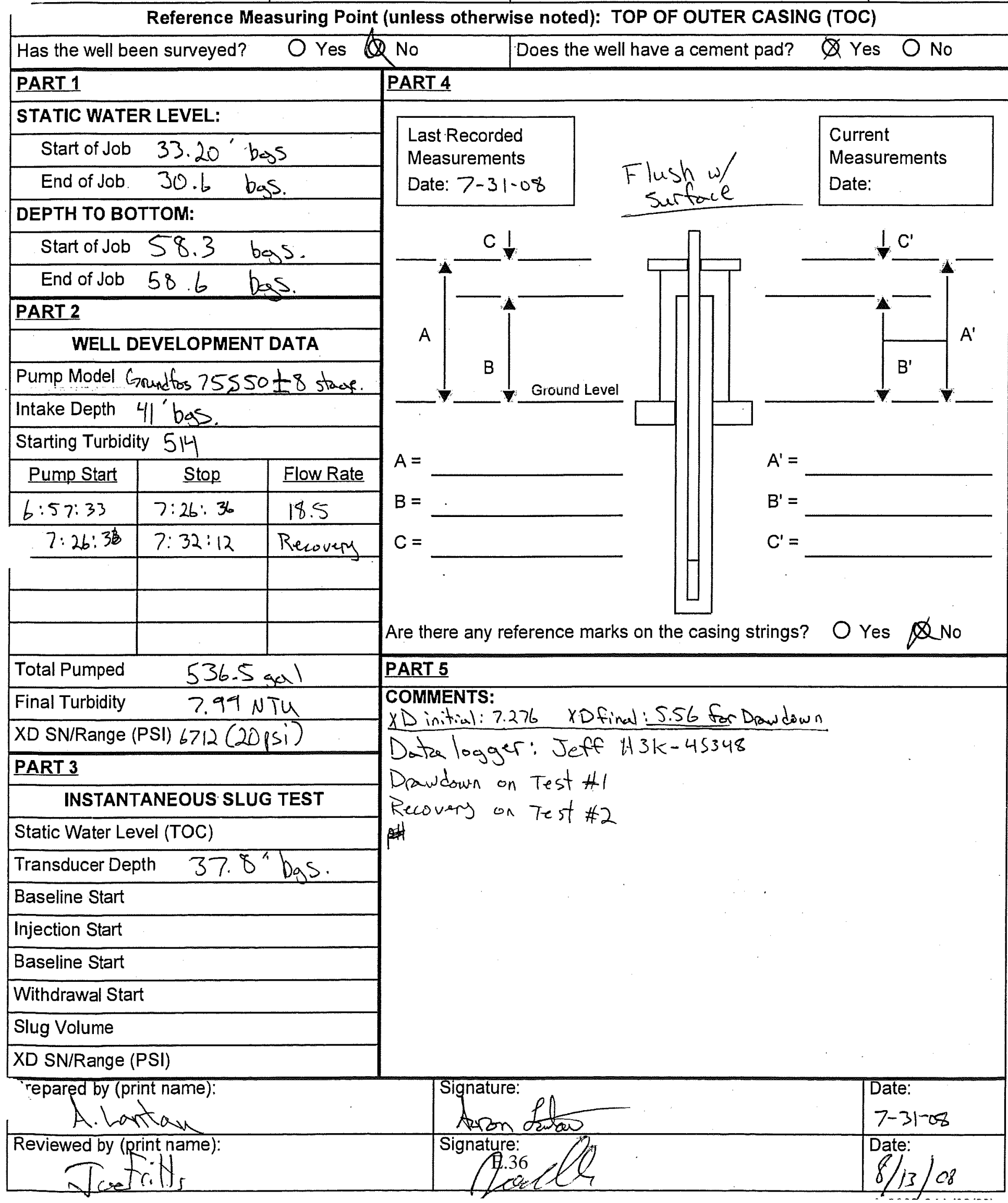


Appendix F

\section{Well Summary Sheets}




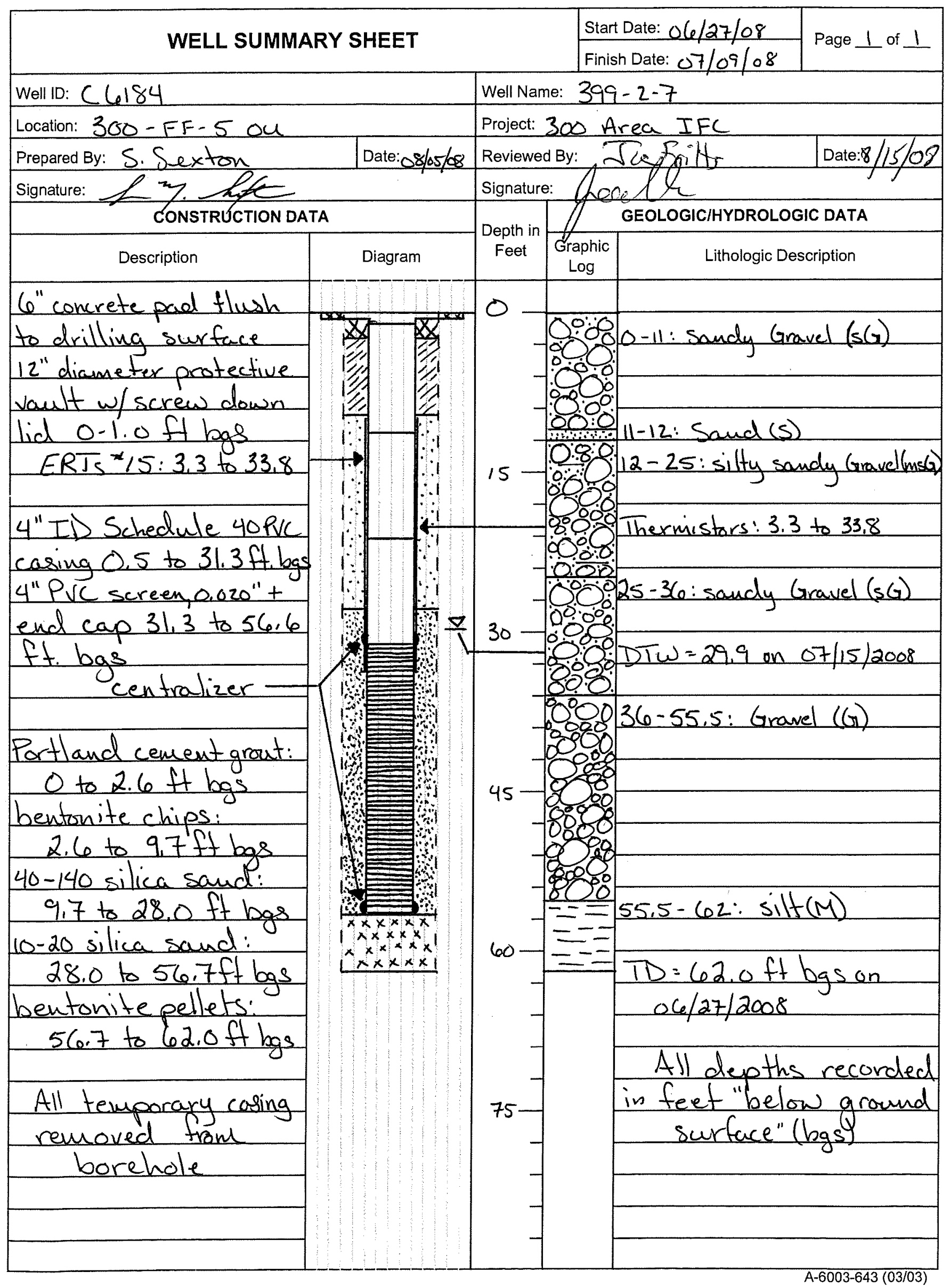

F.1 


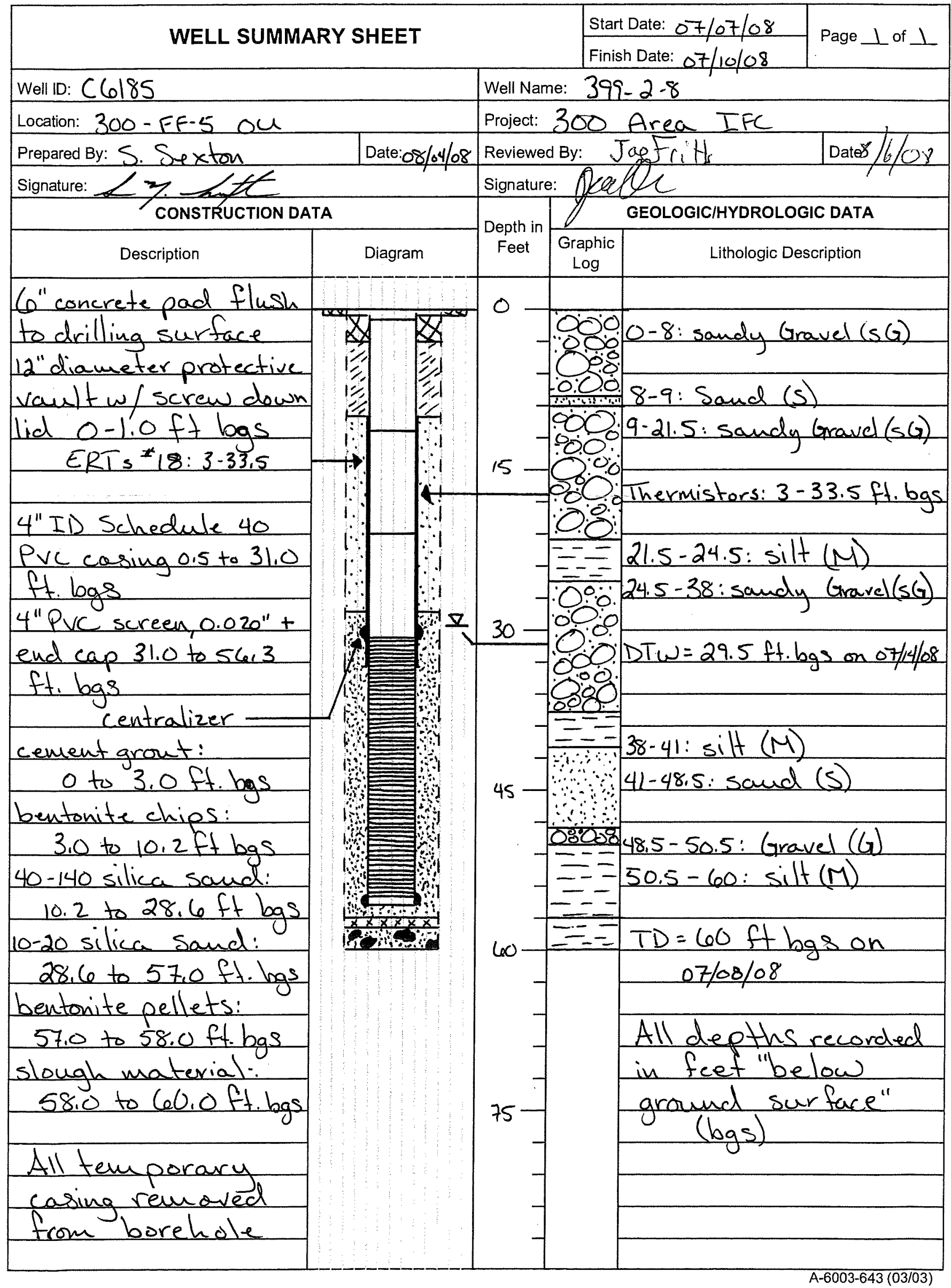




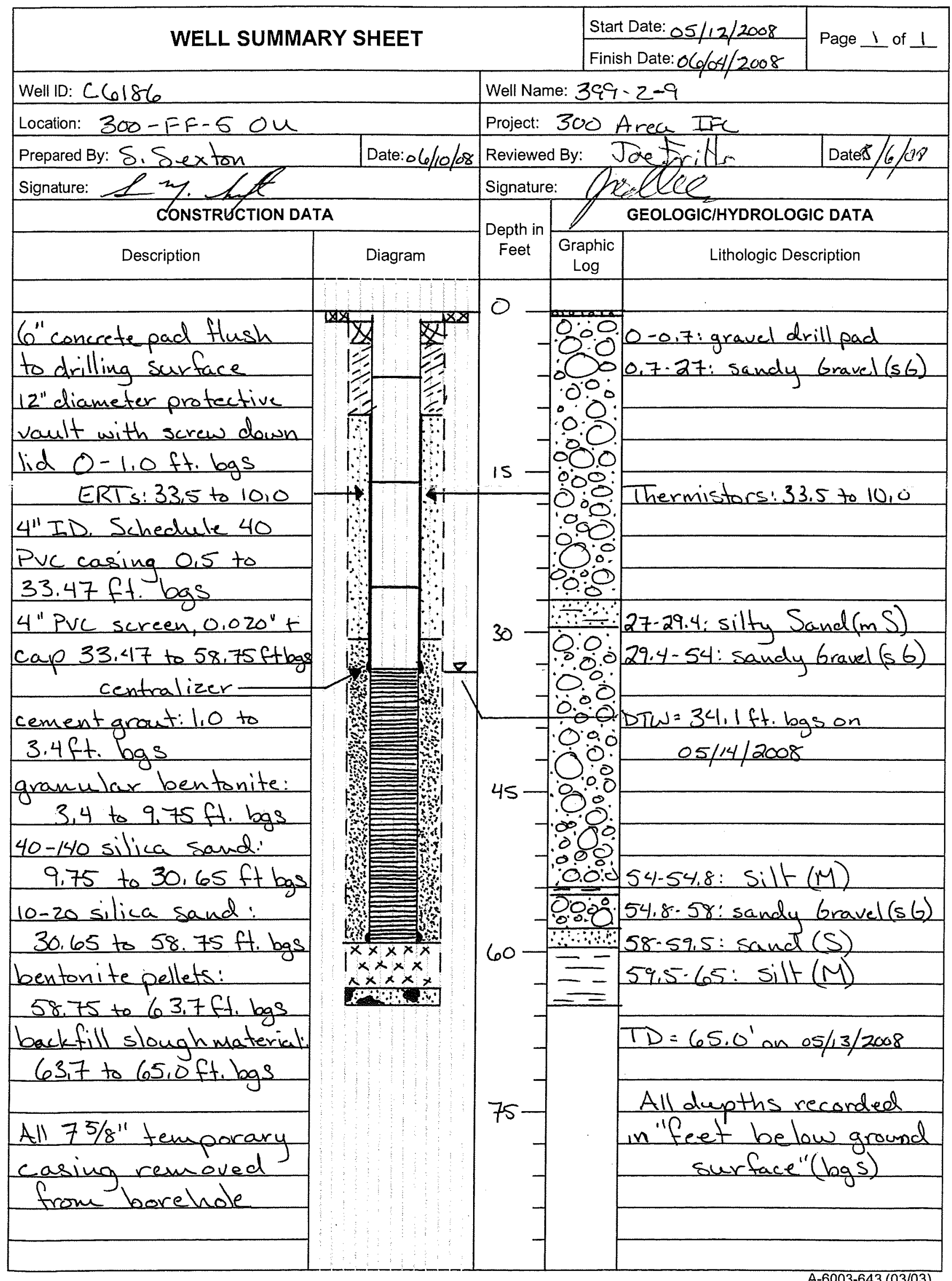




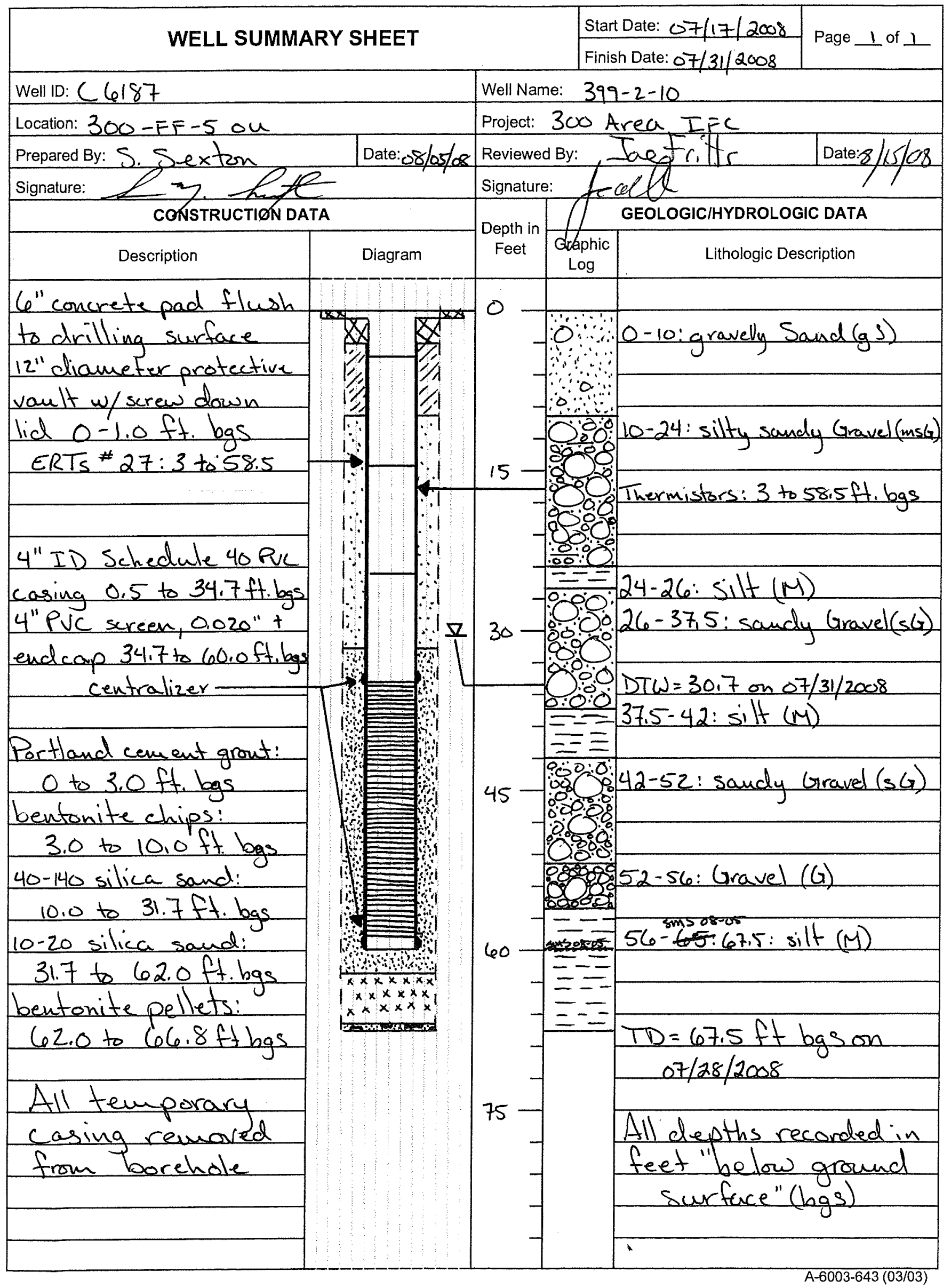

F.4 


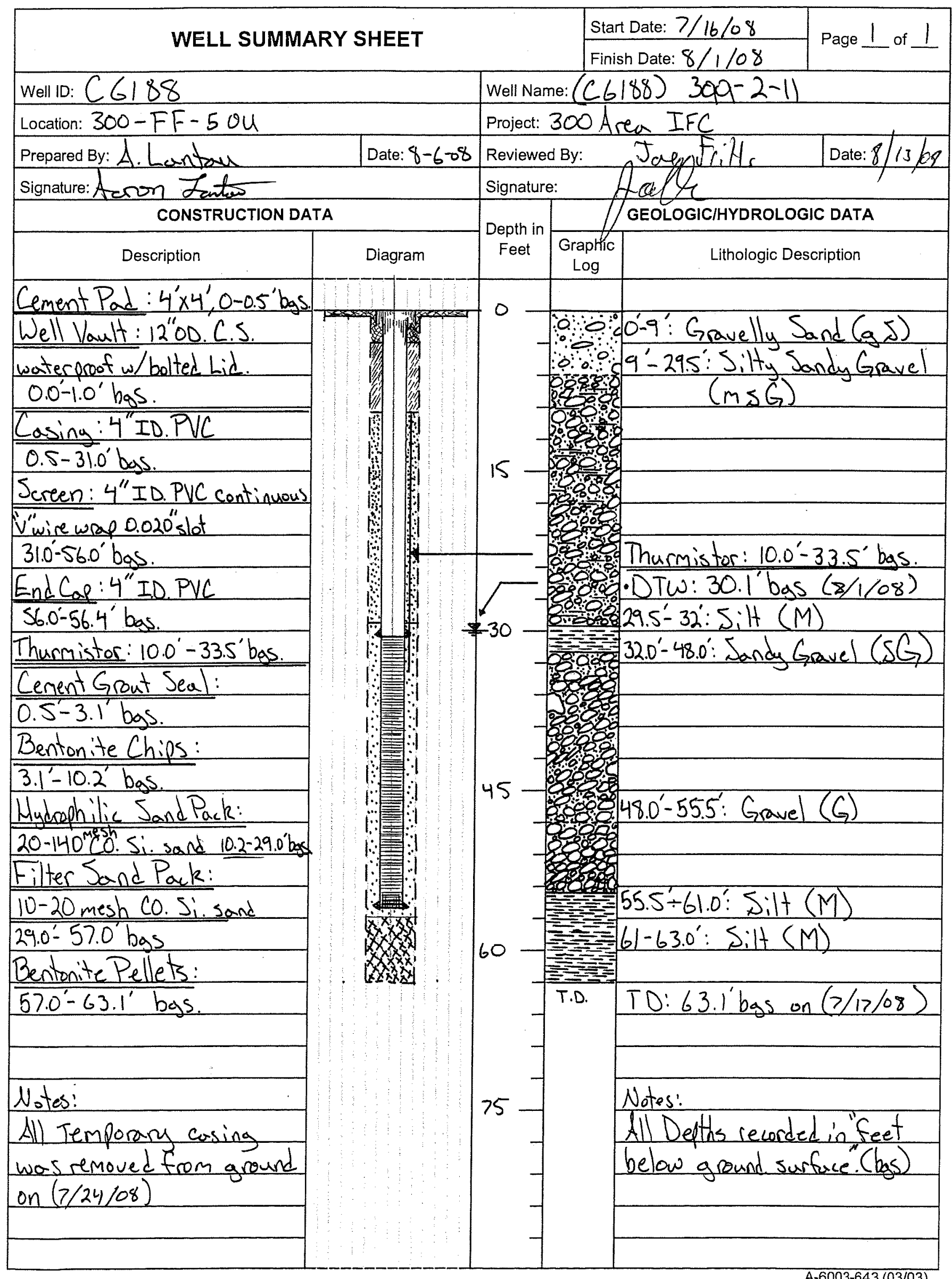




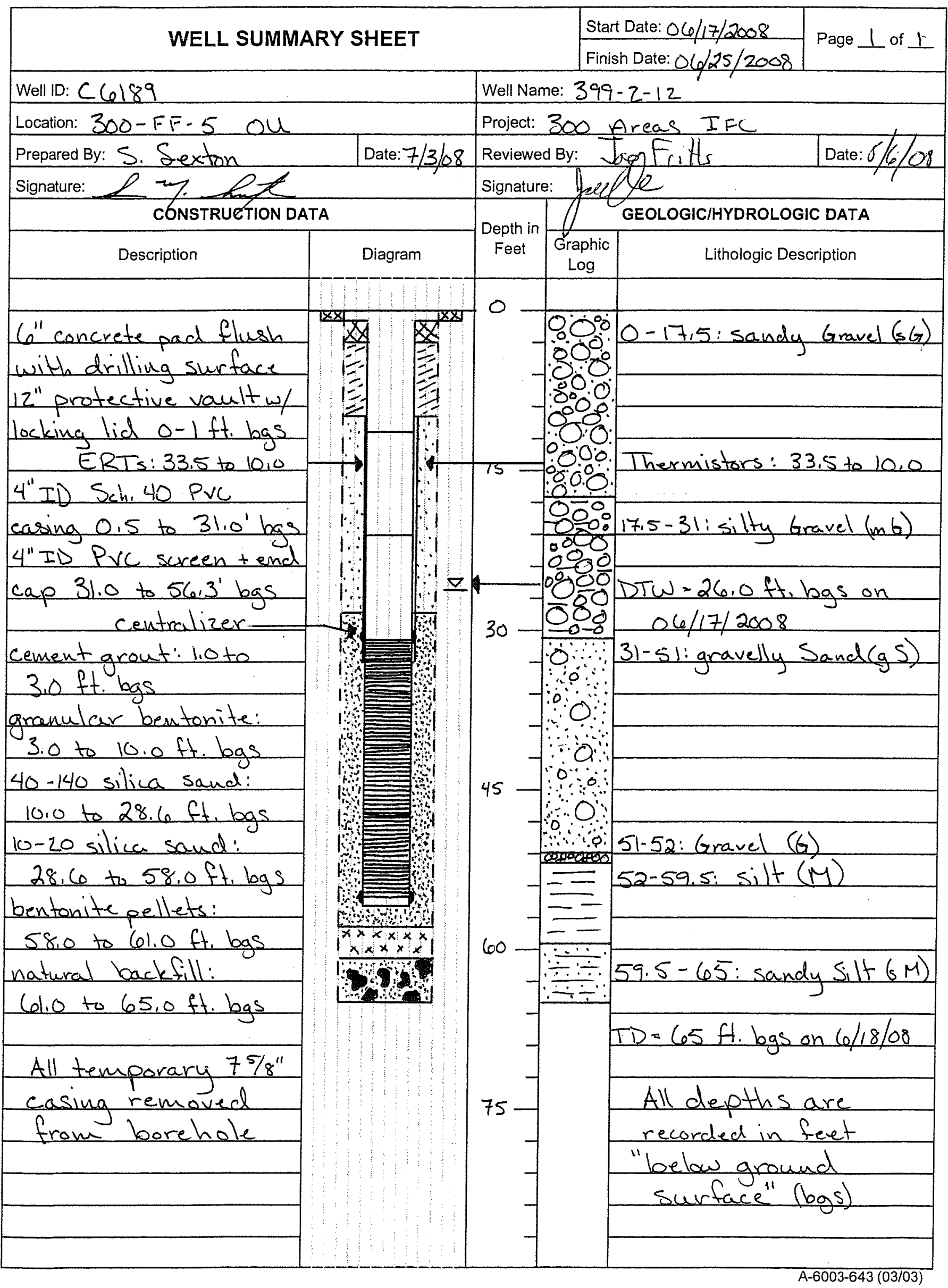

F.6 


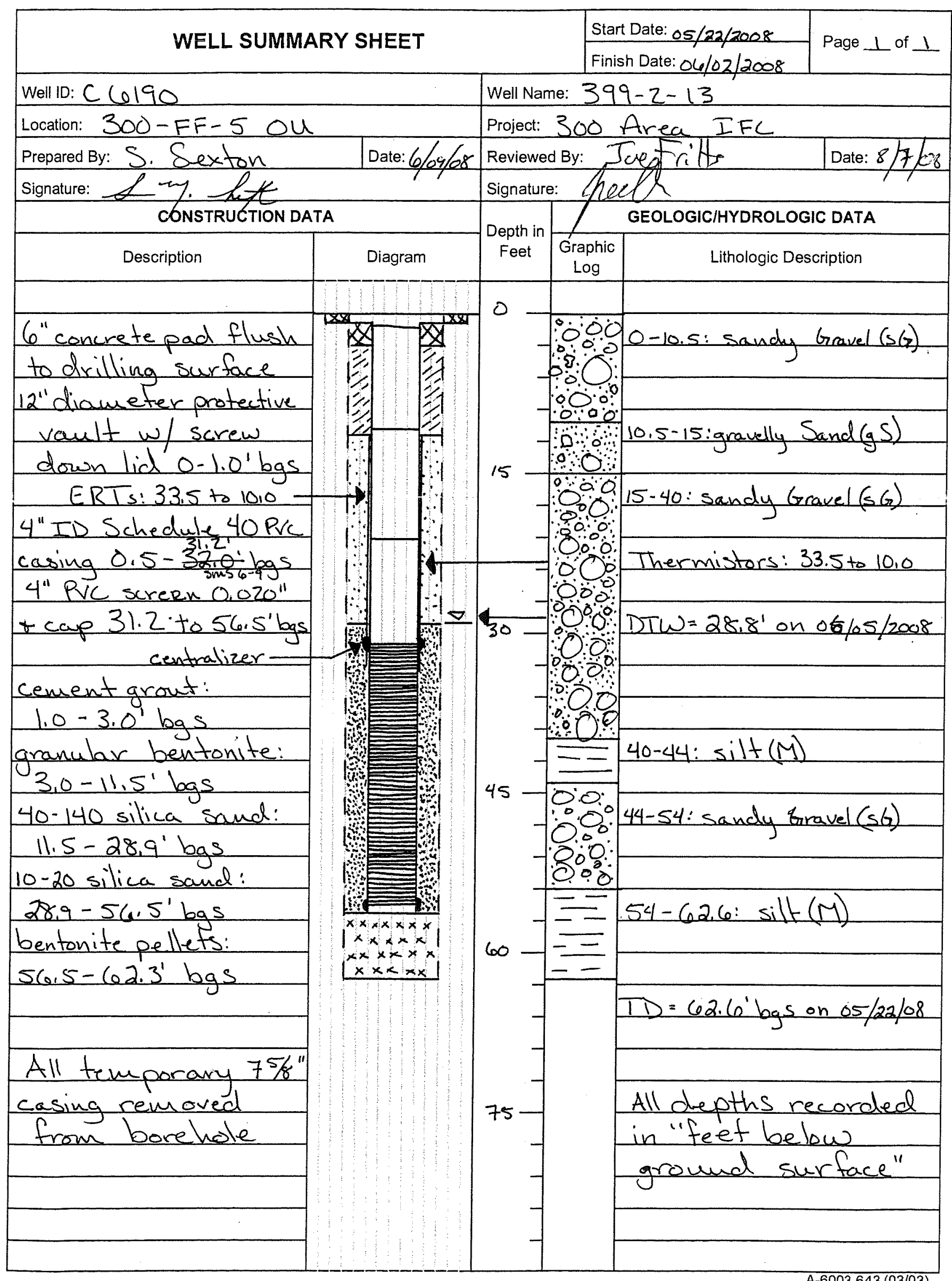




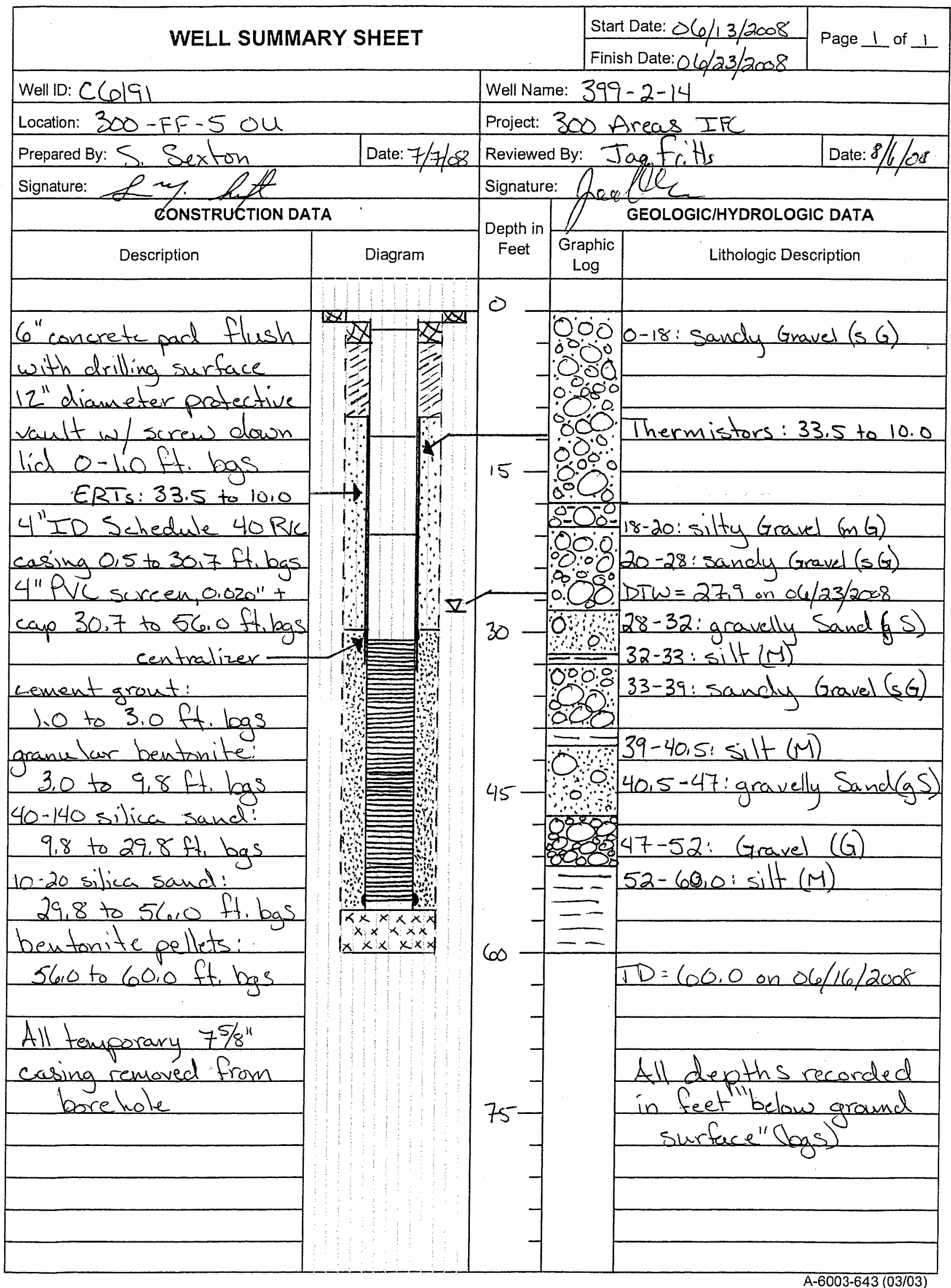




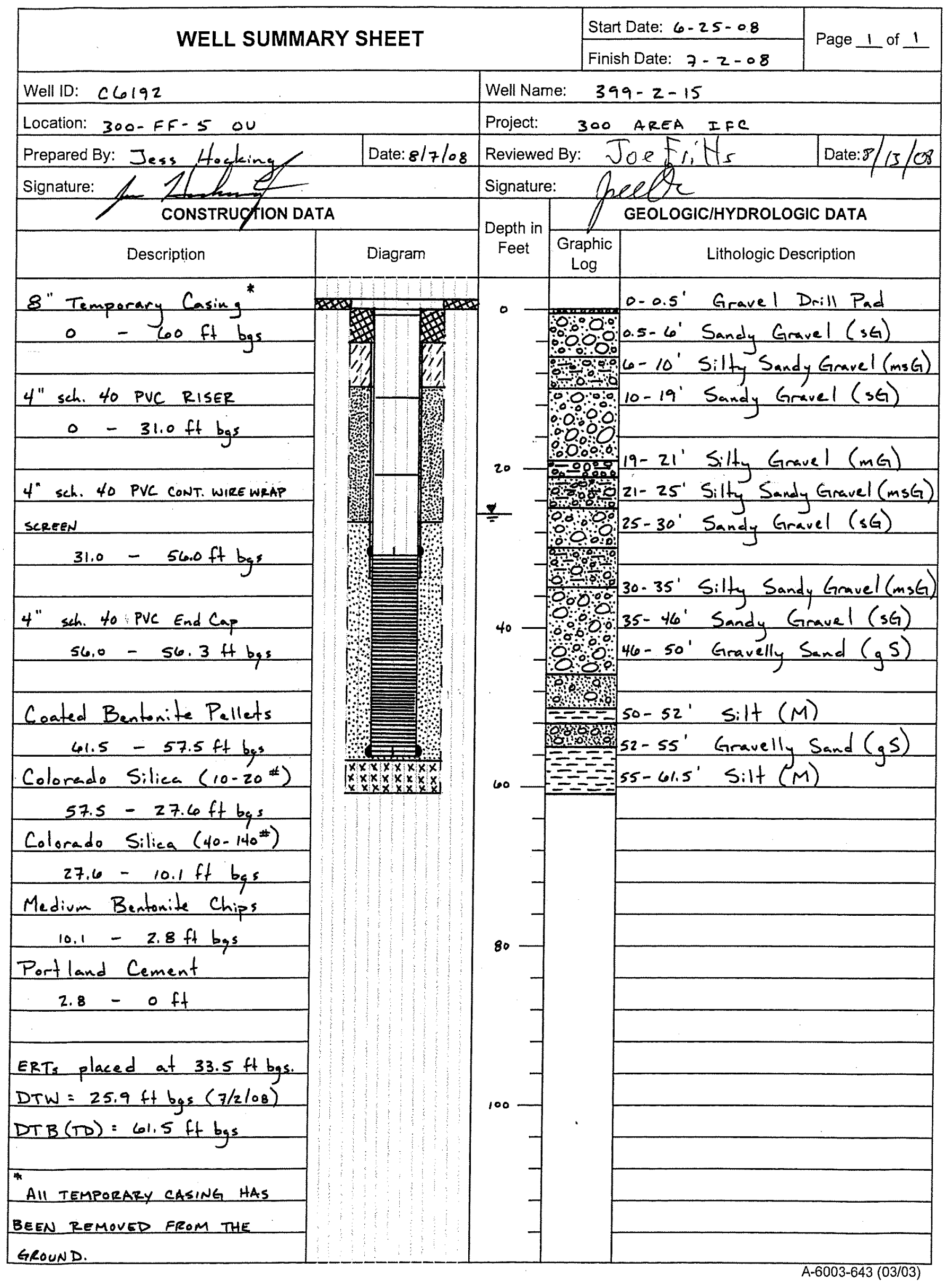




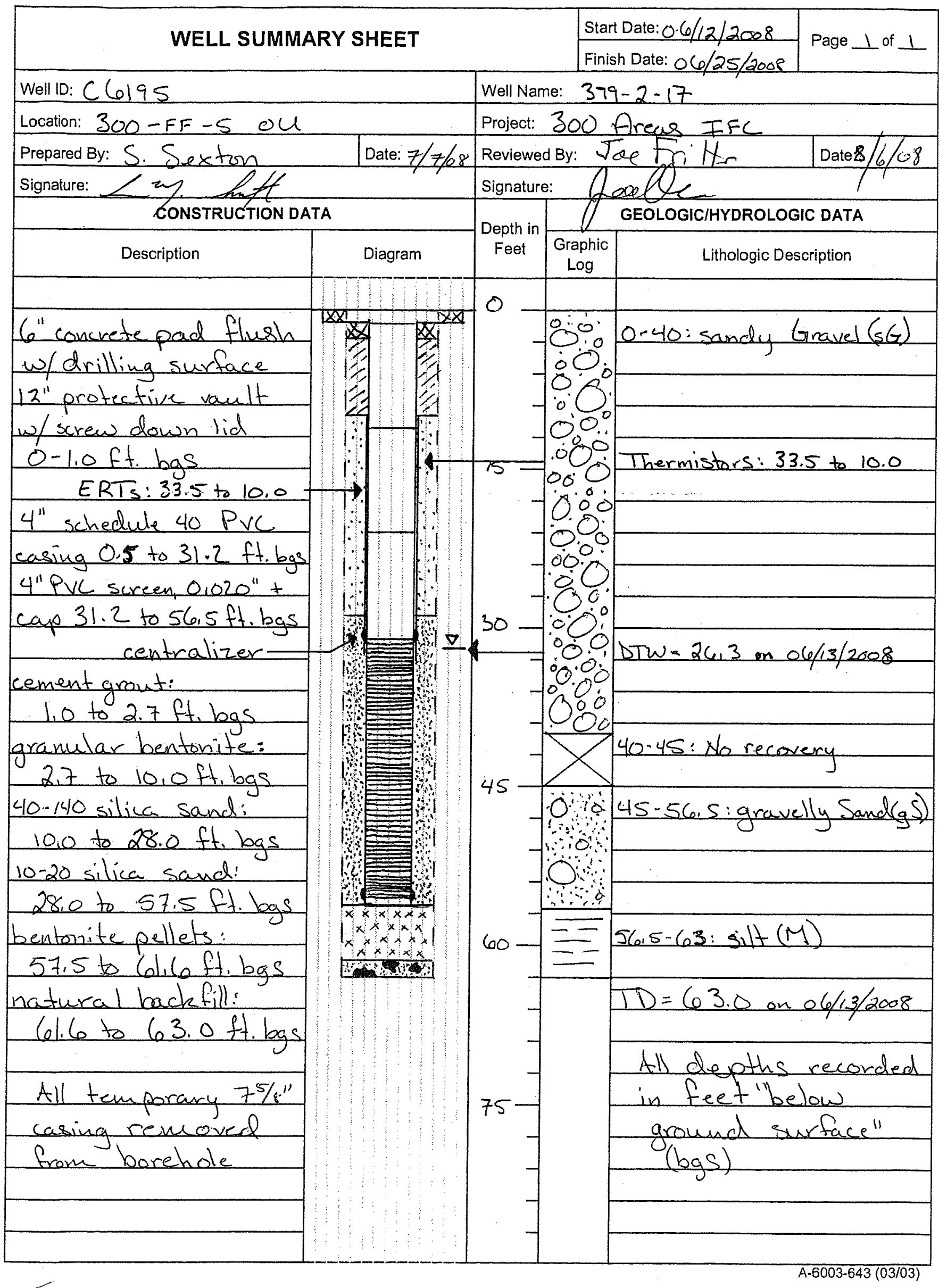




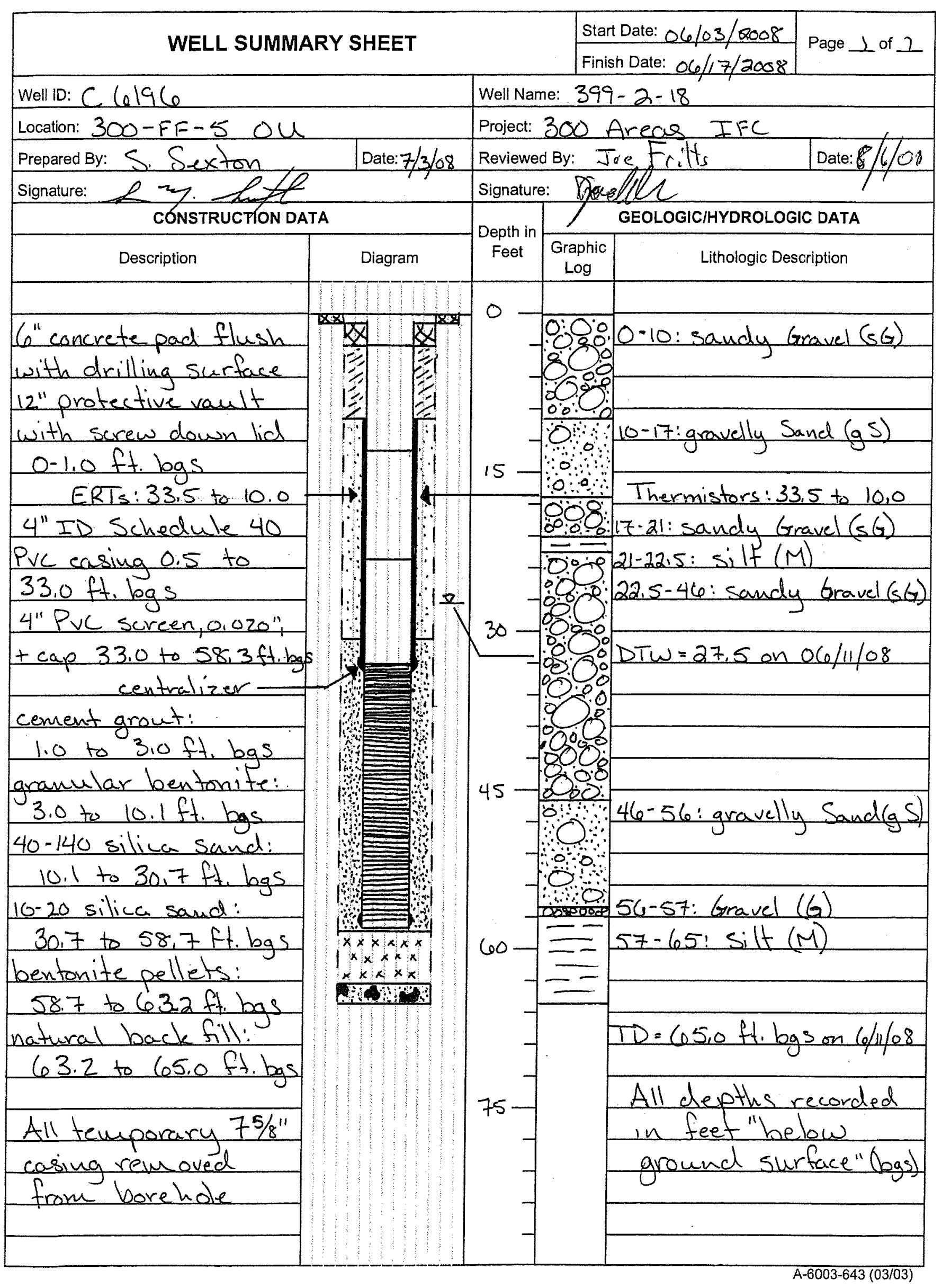

F.12 


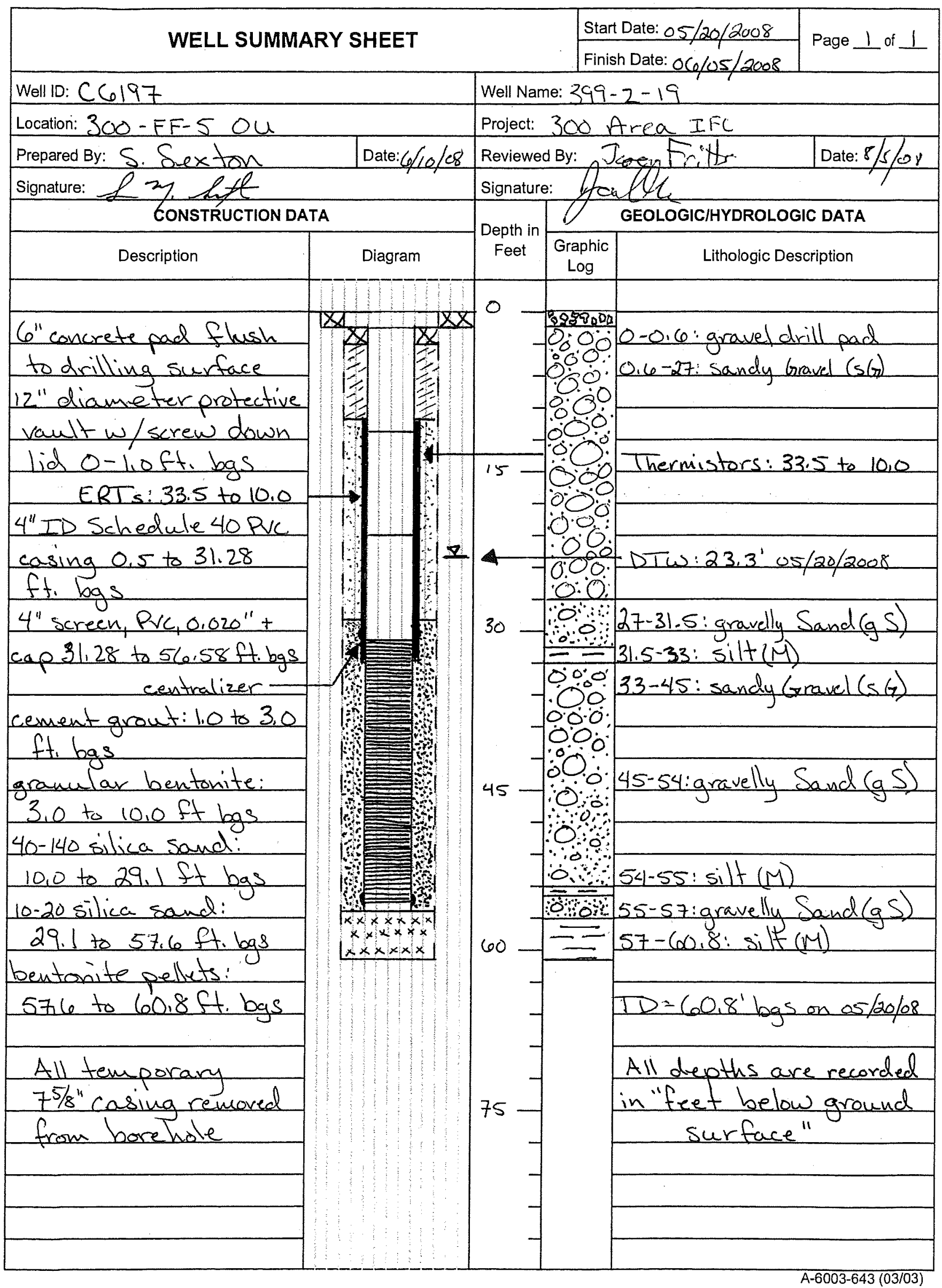




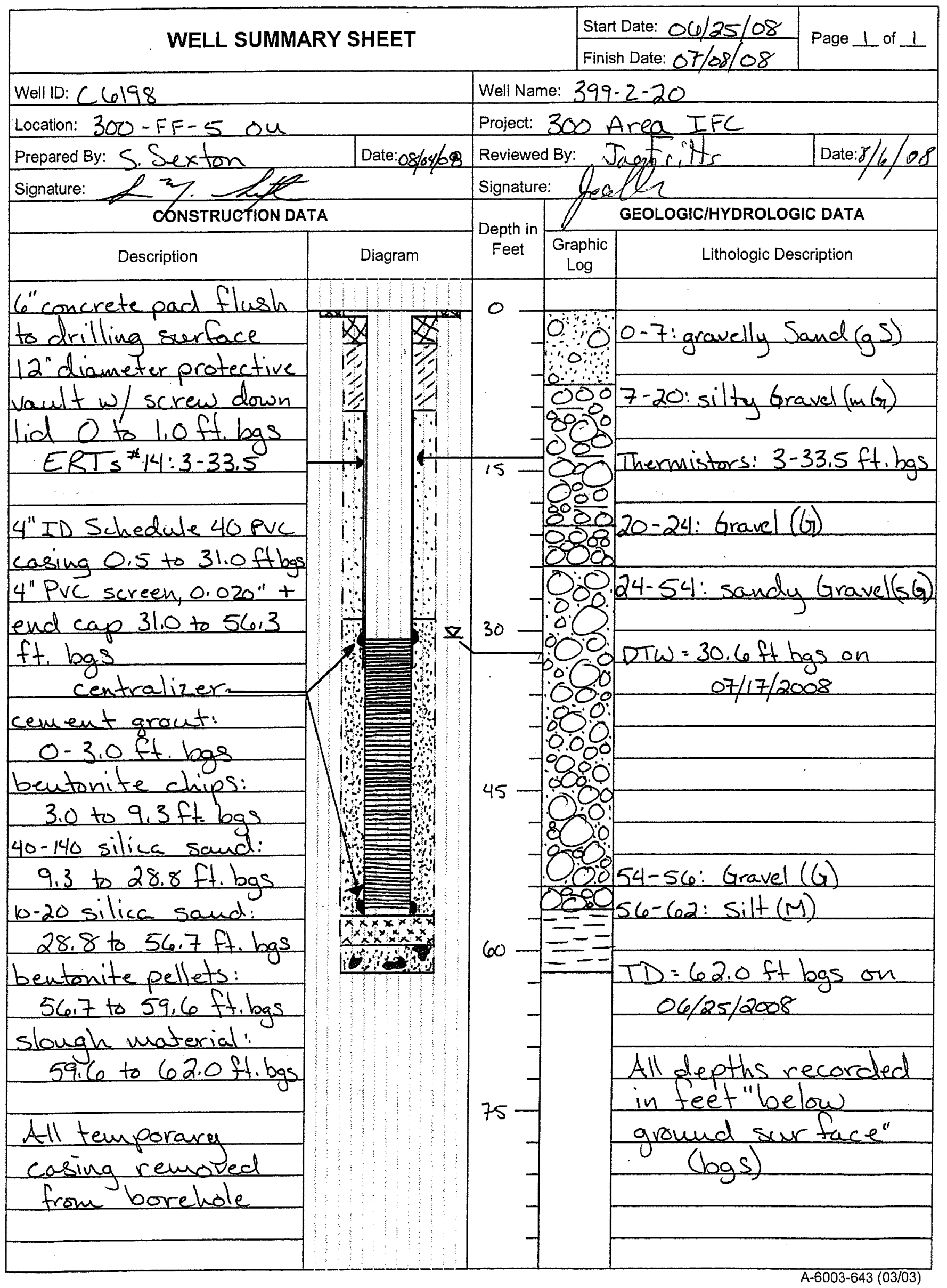

F.14 


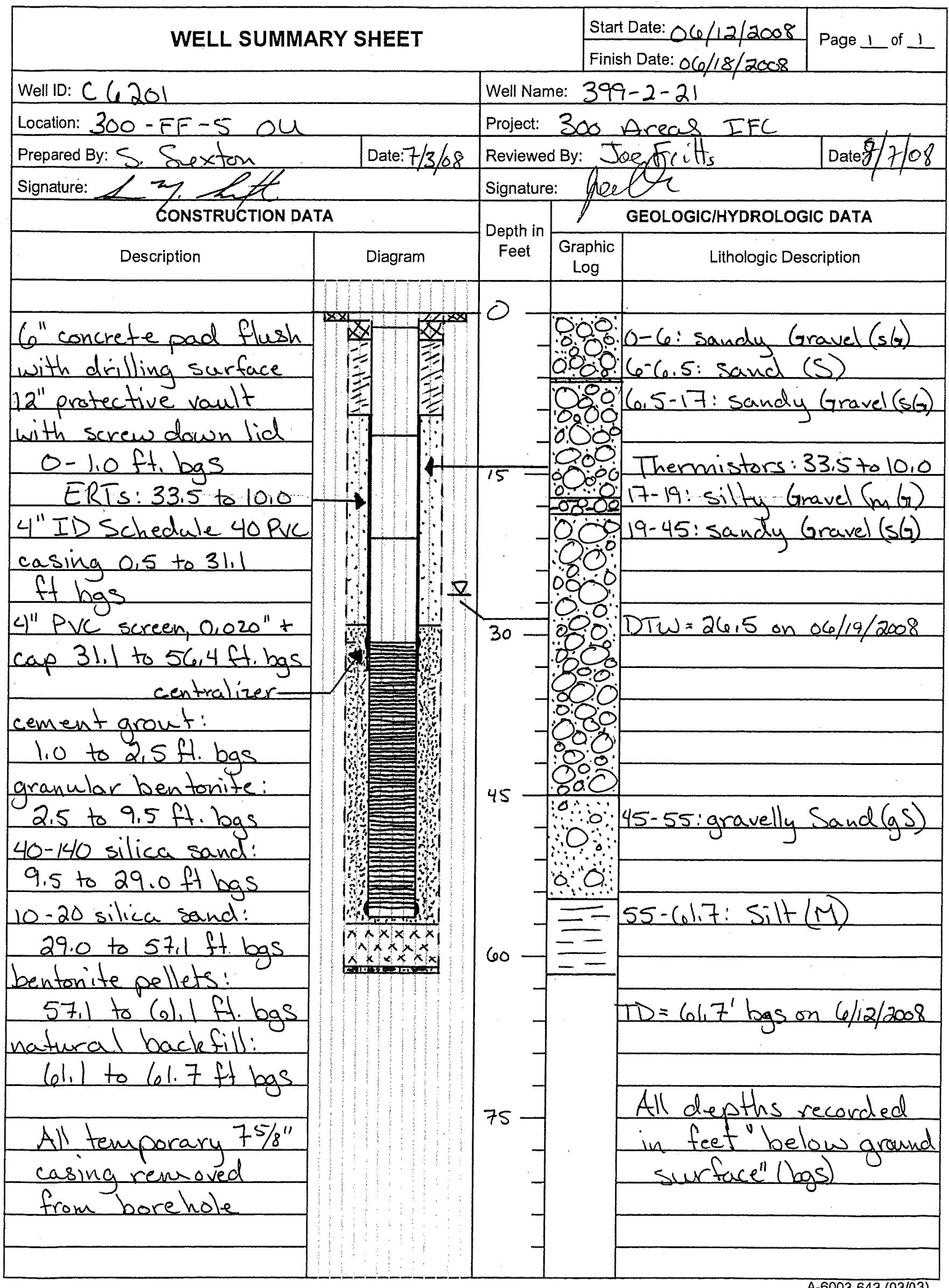




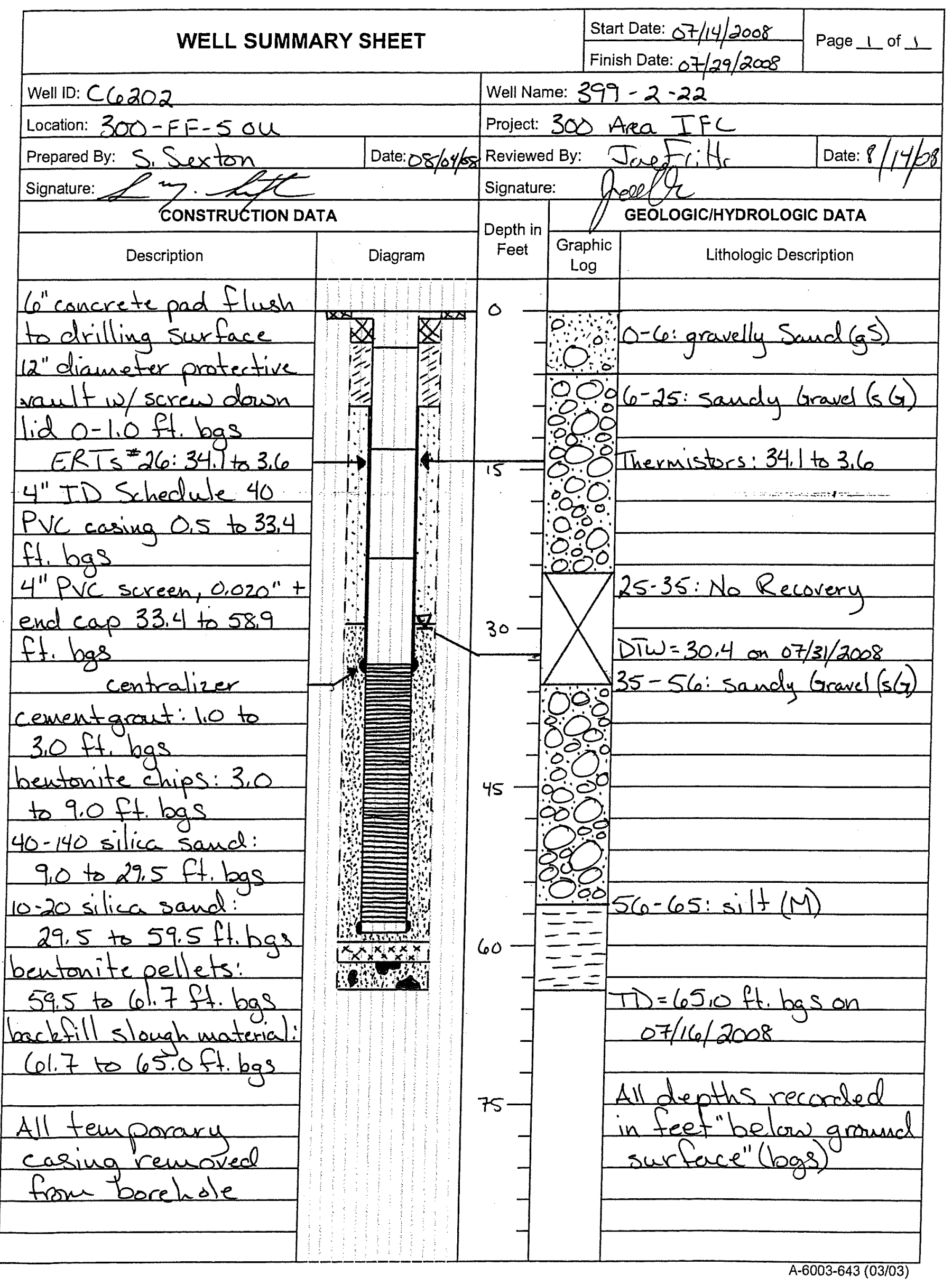

F.16 


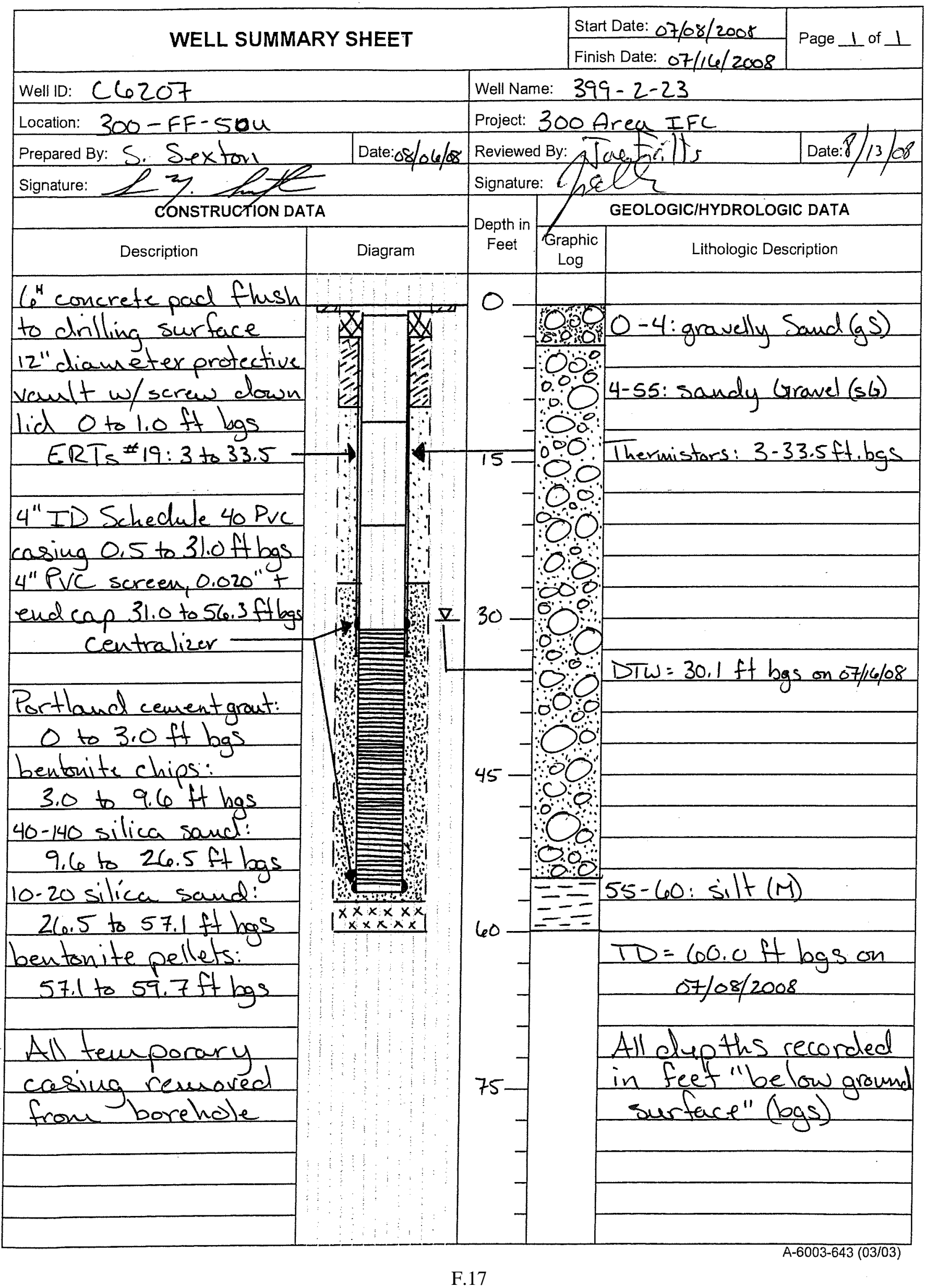




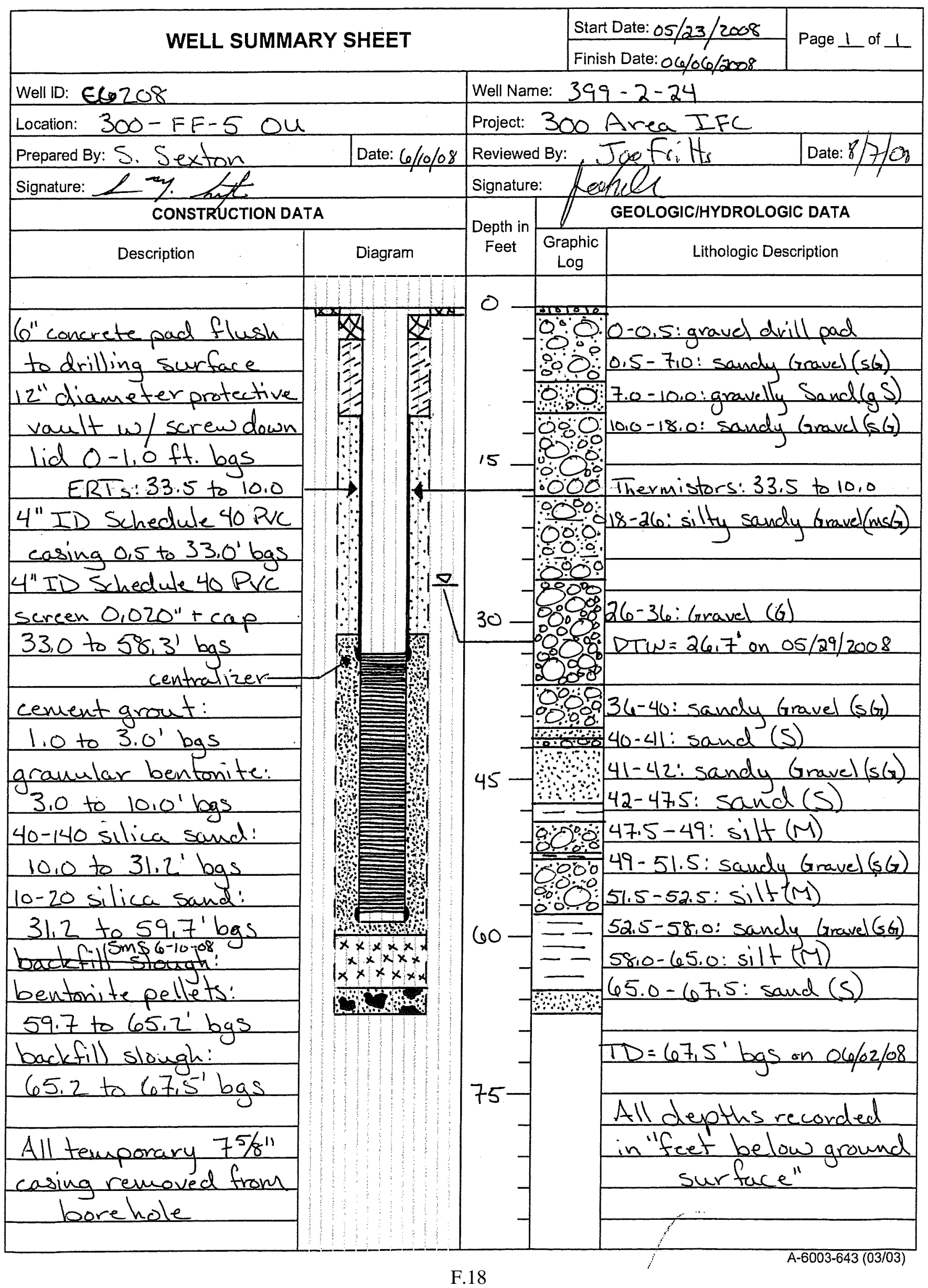




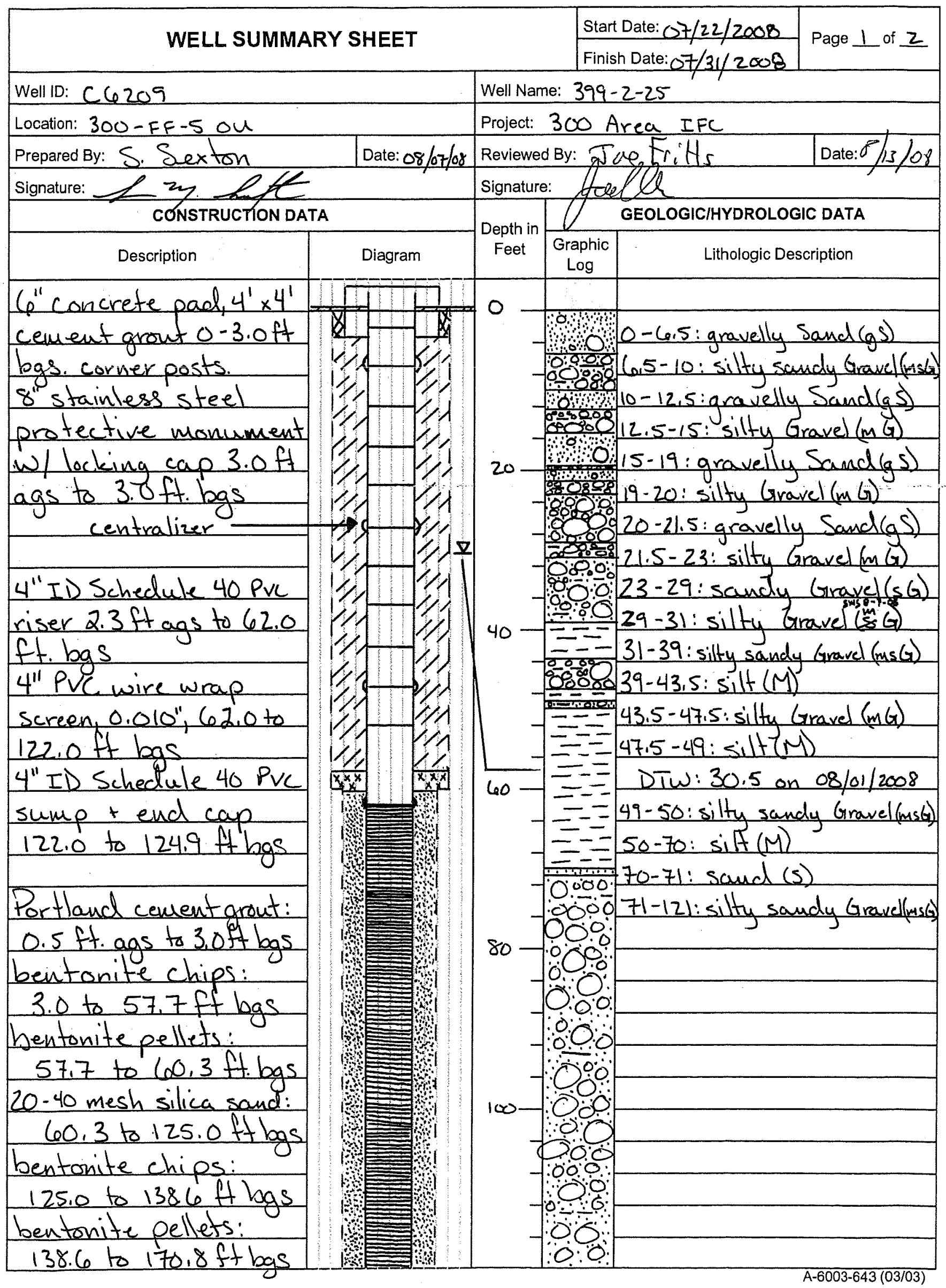

F.19 


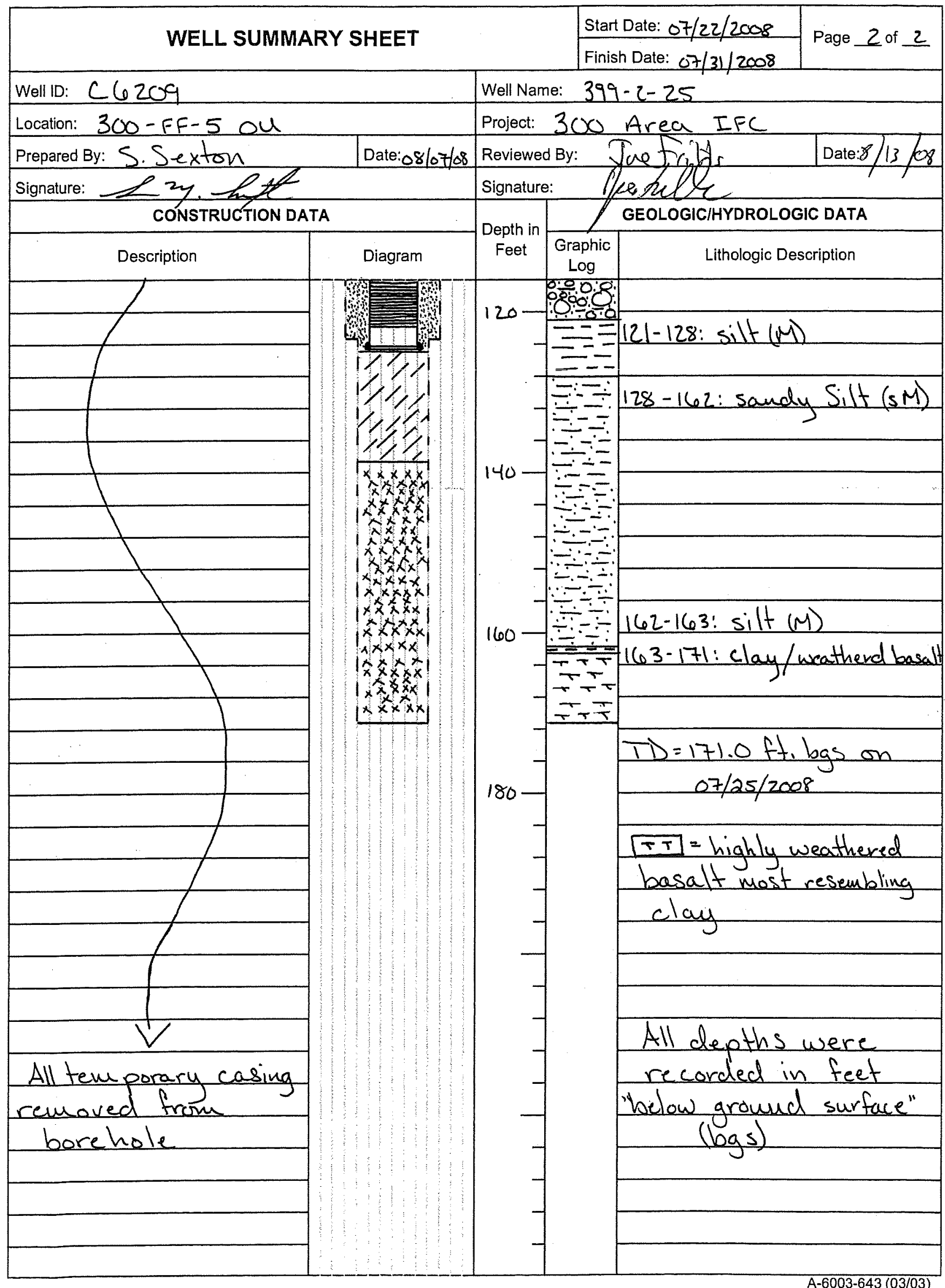




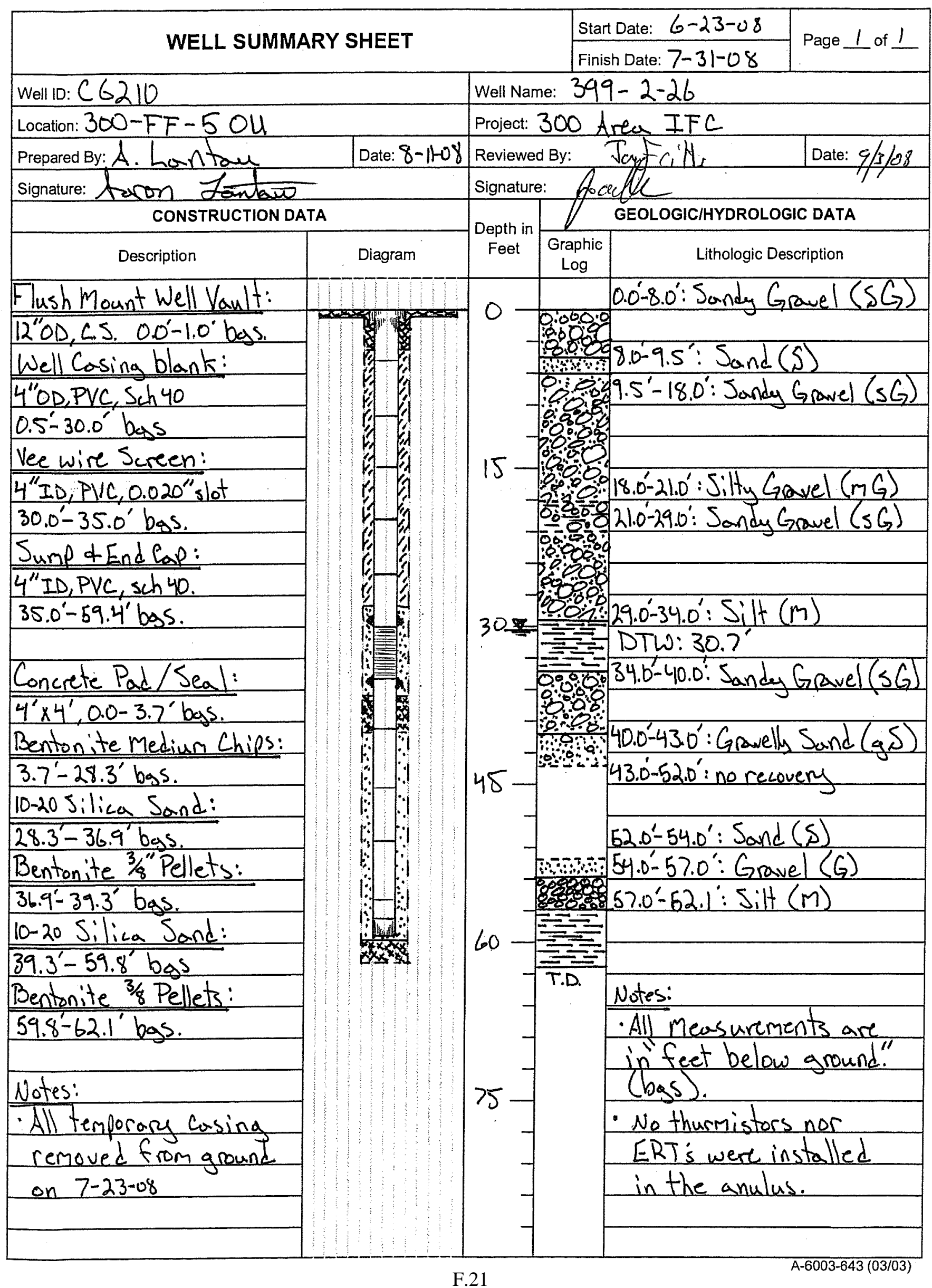




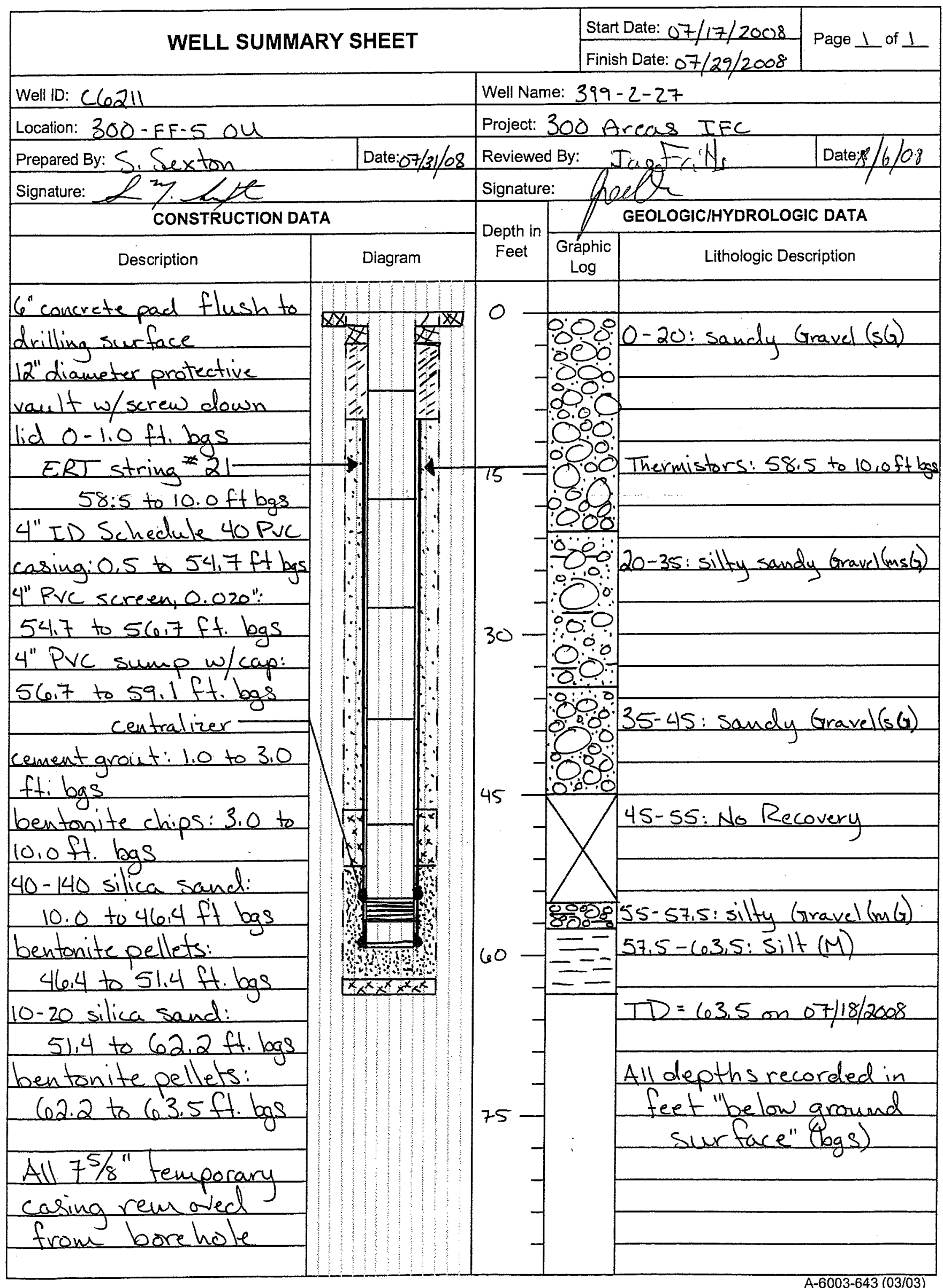




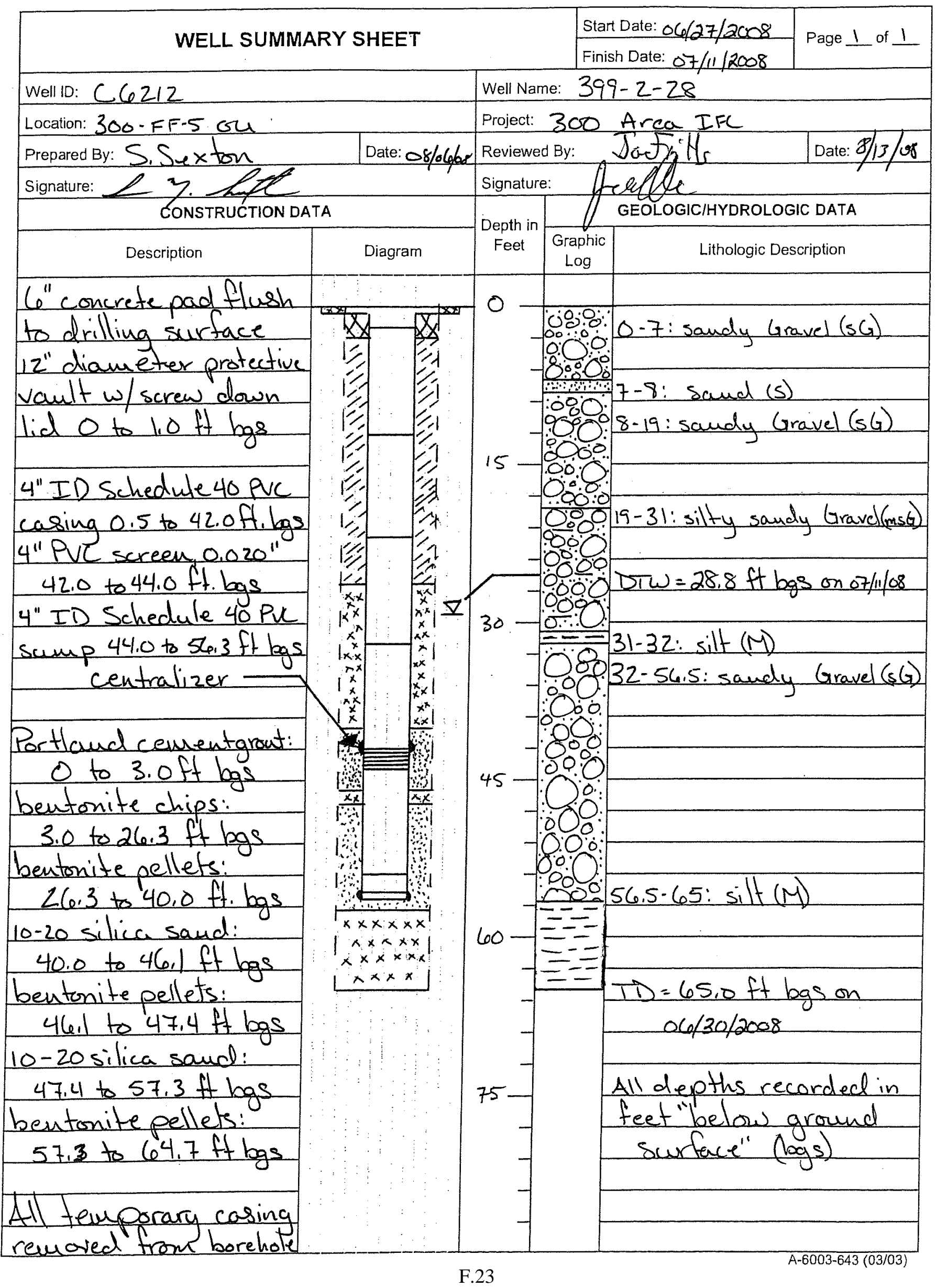




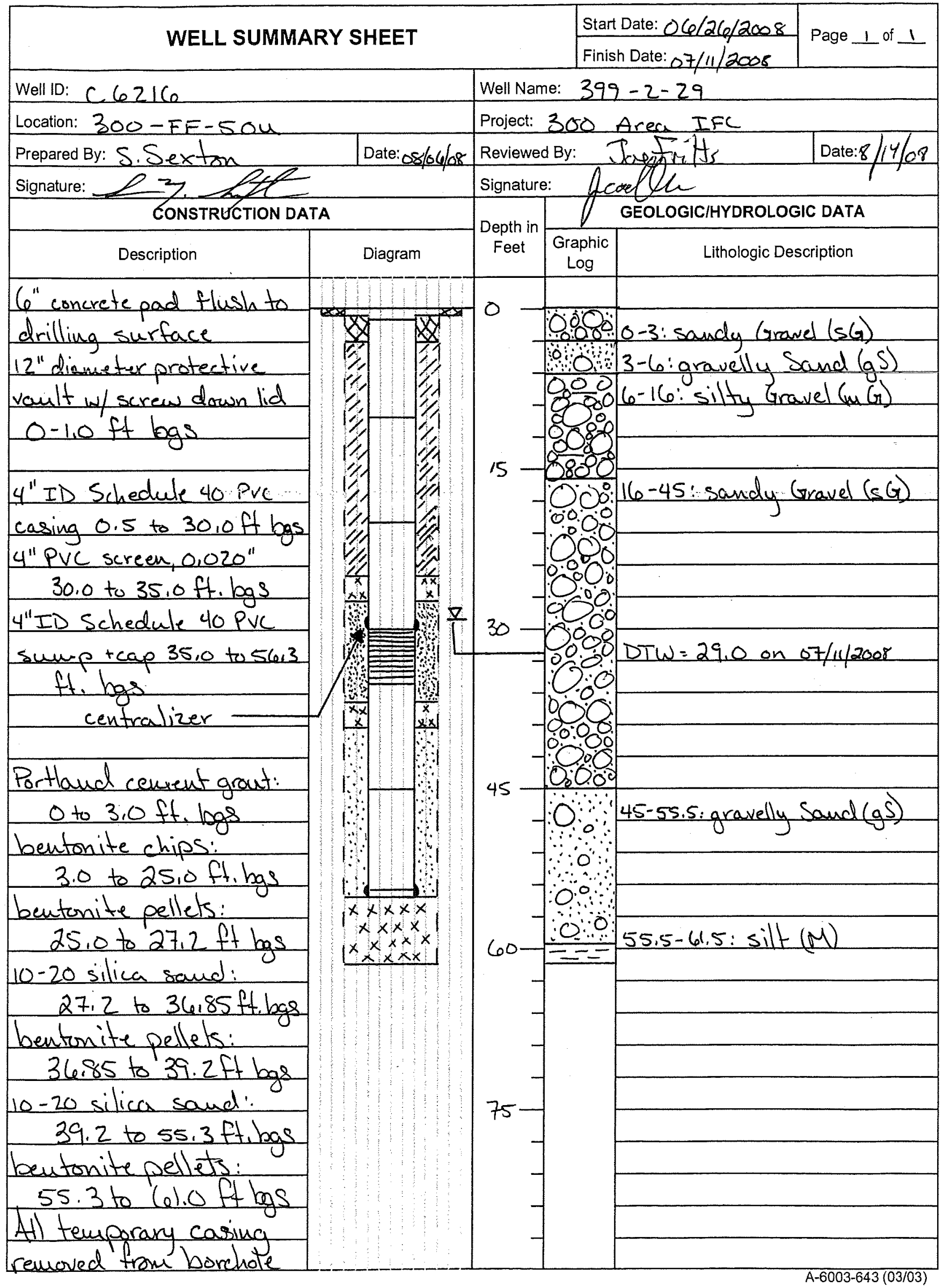




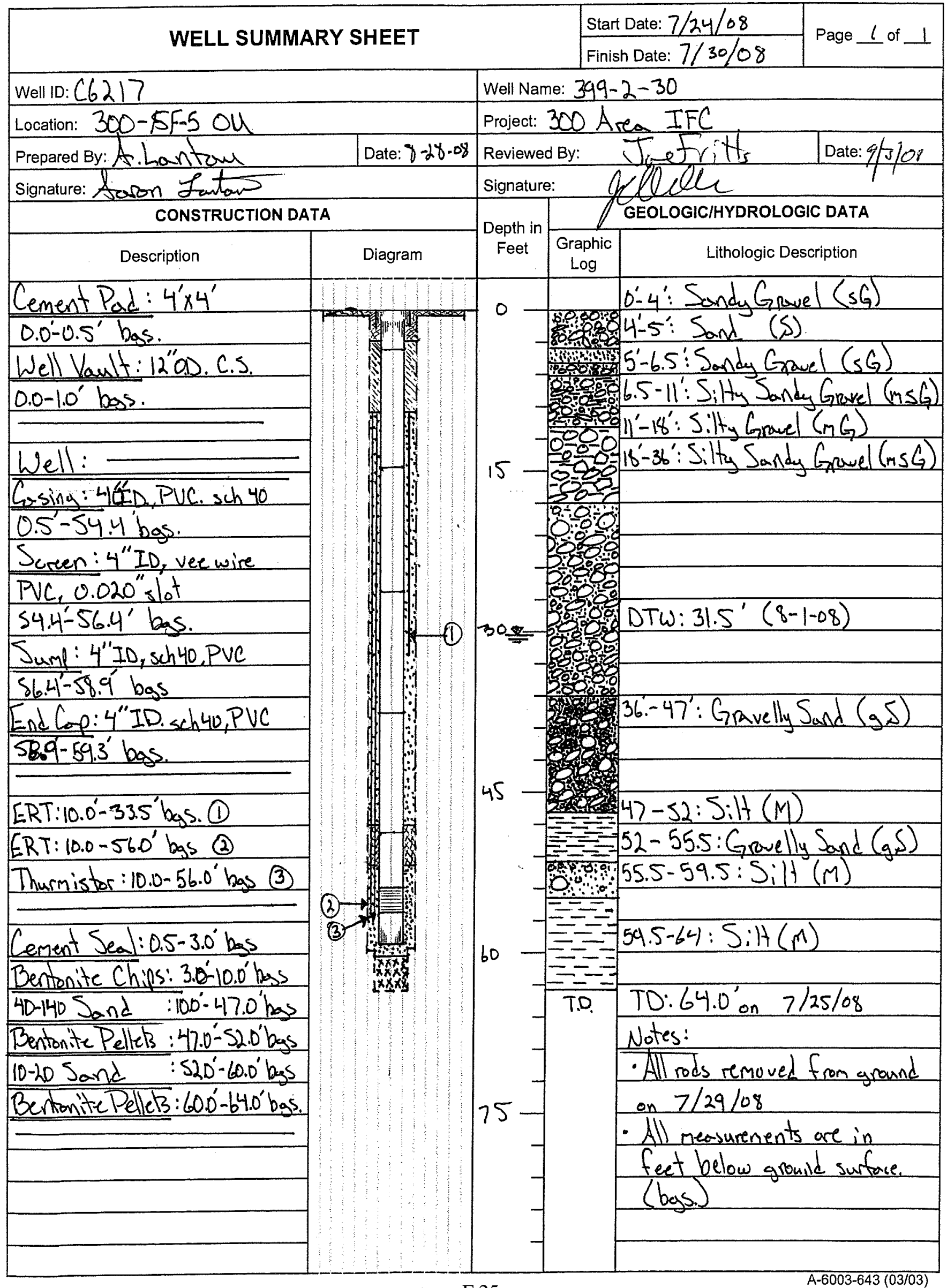




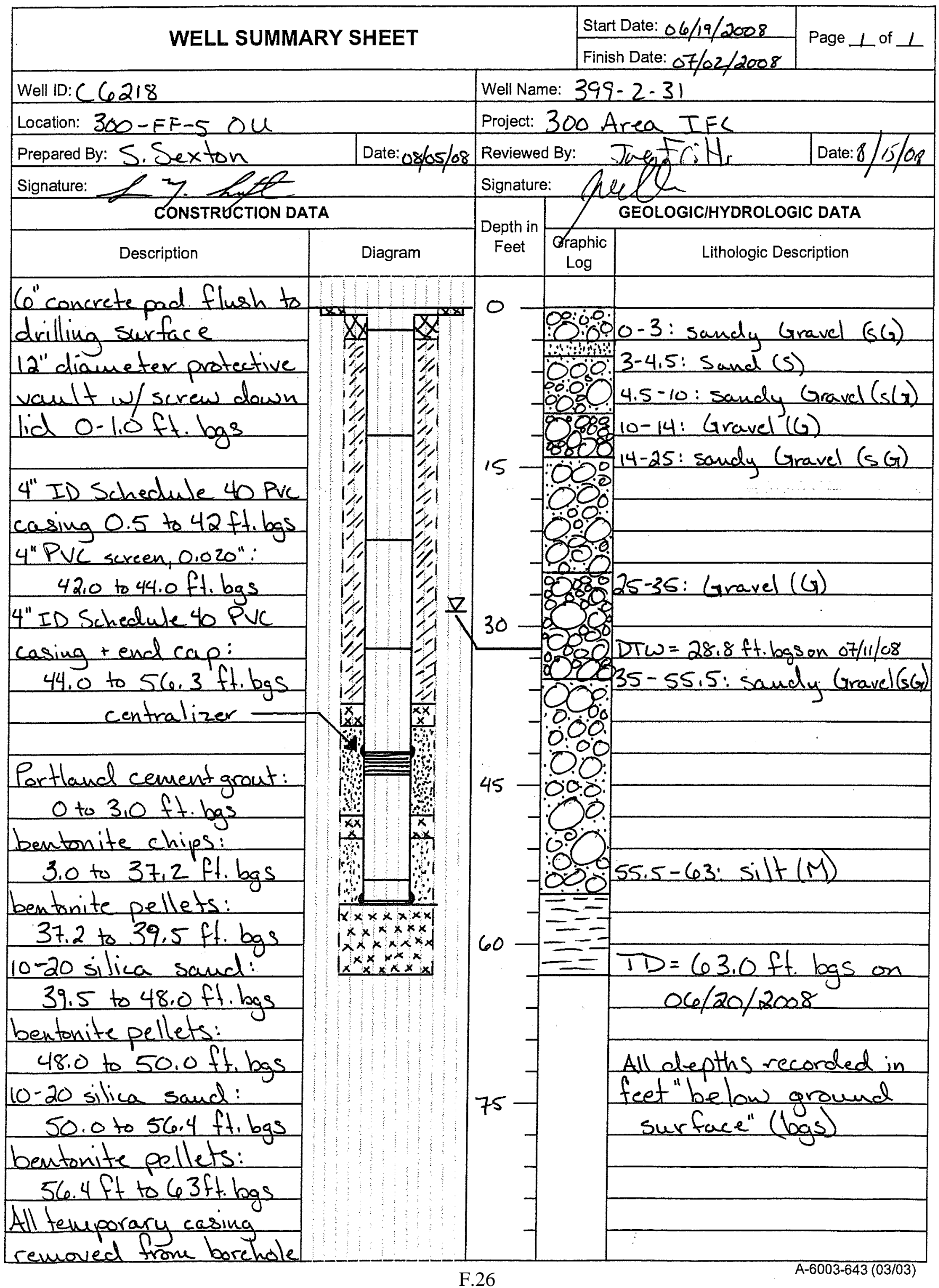




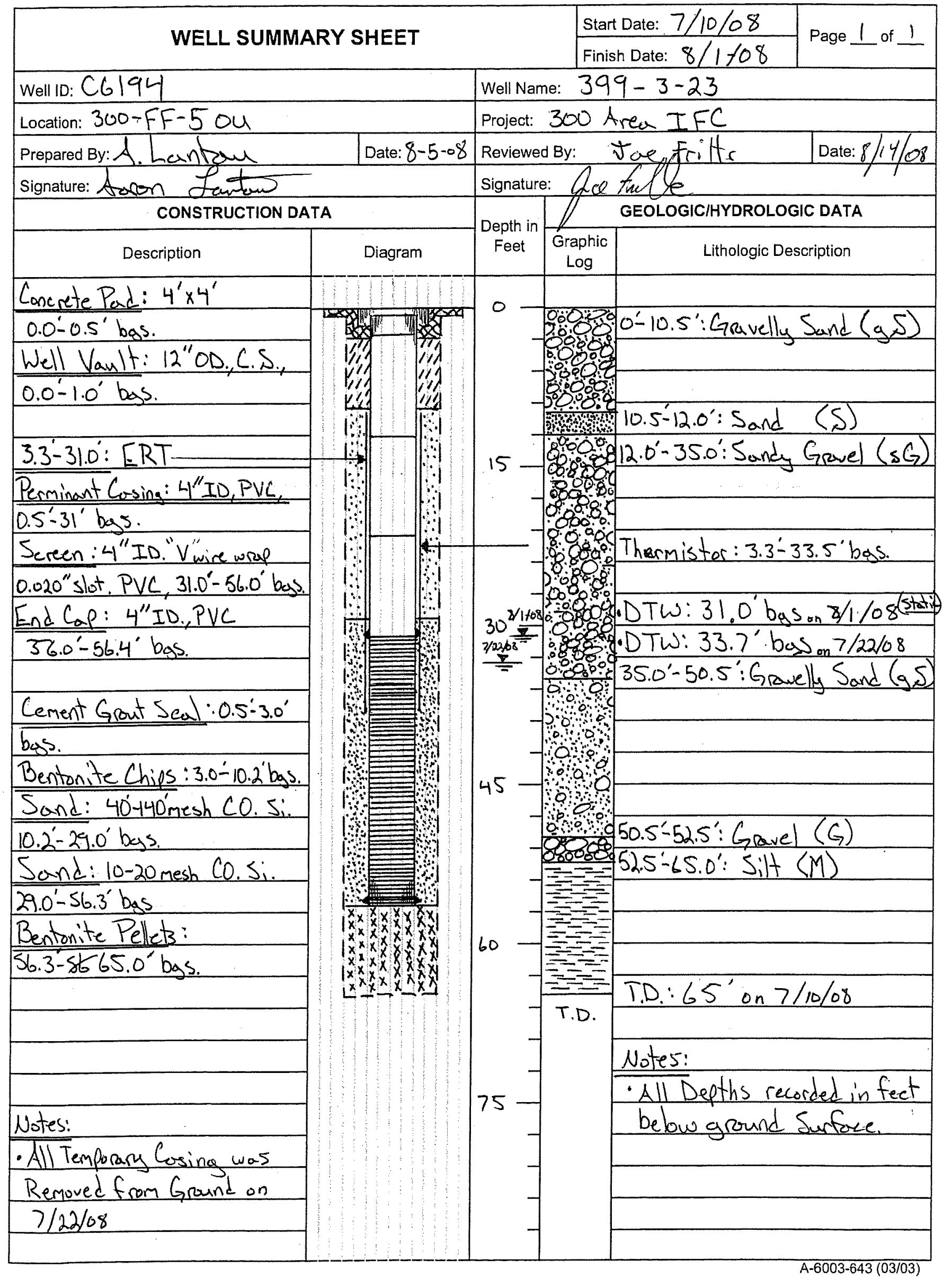




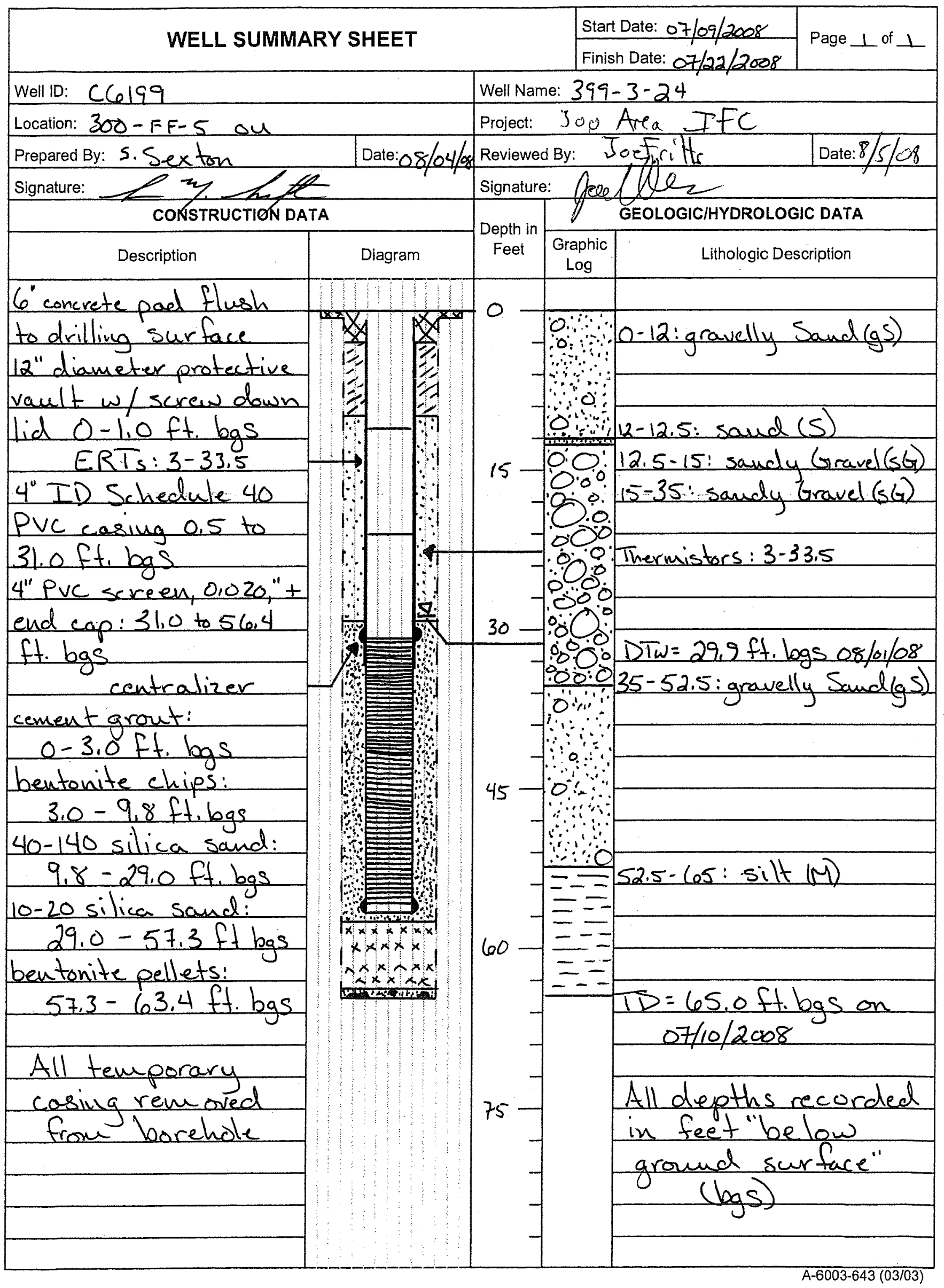

F.28 


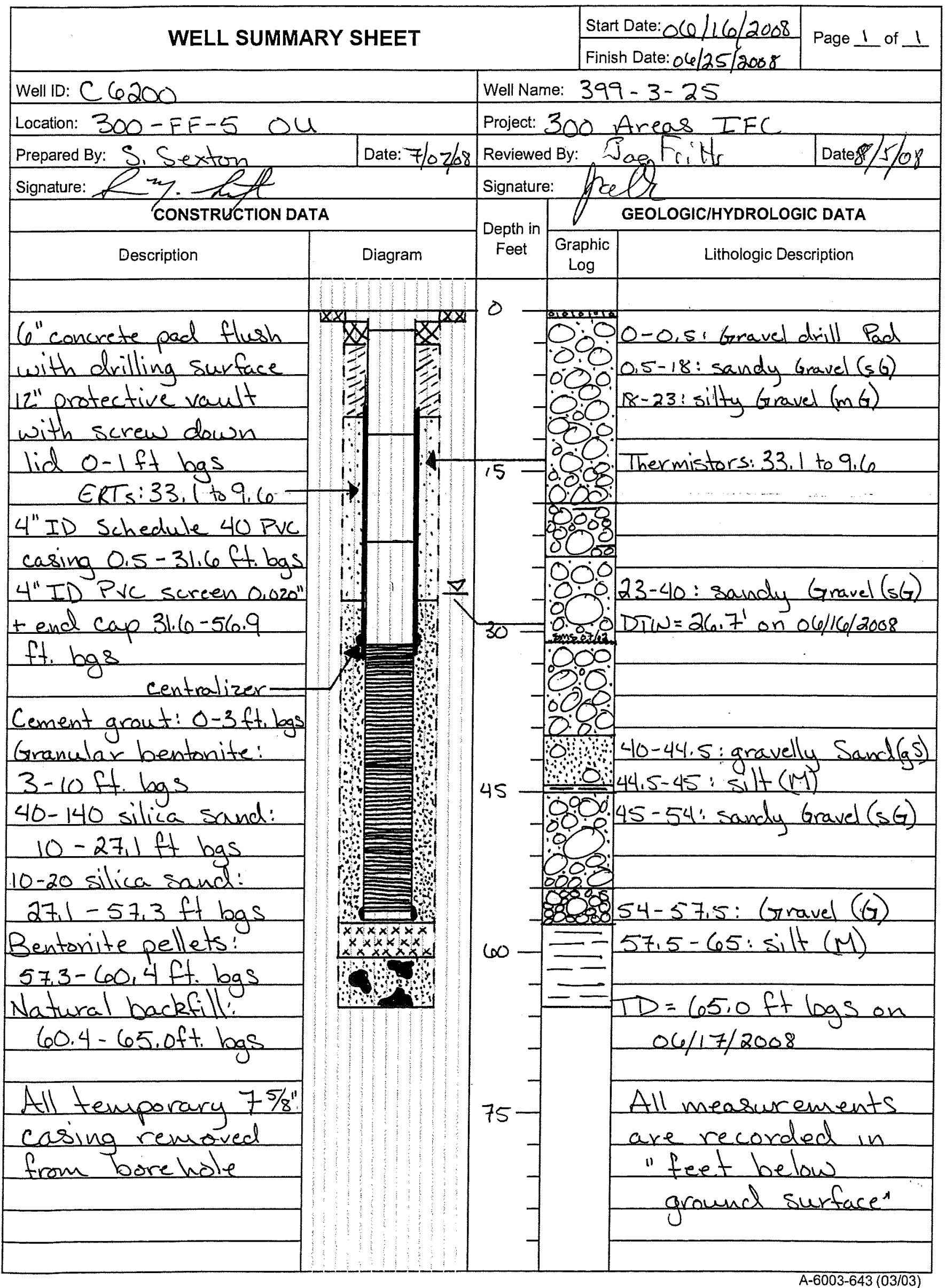




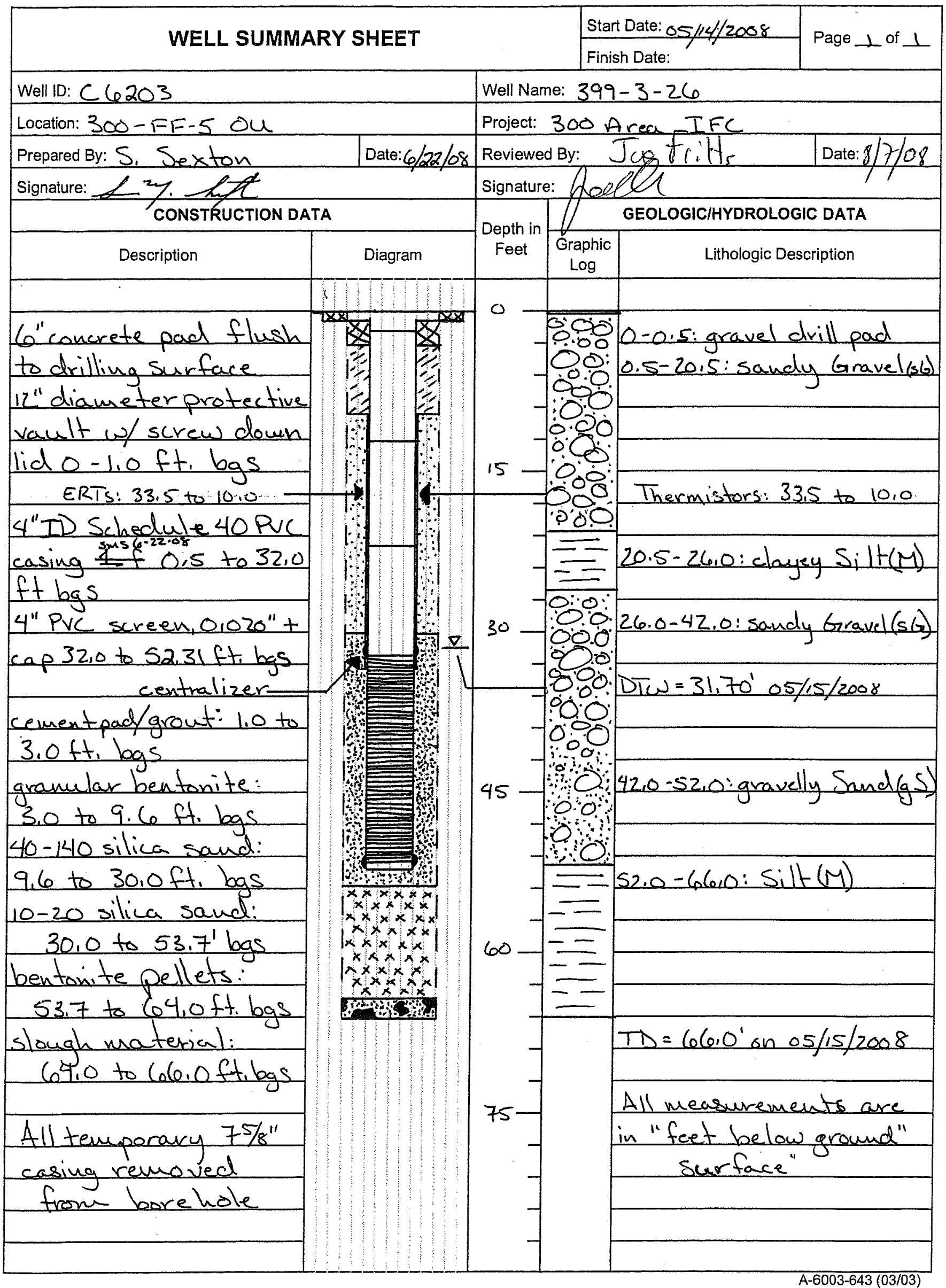




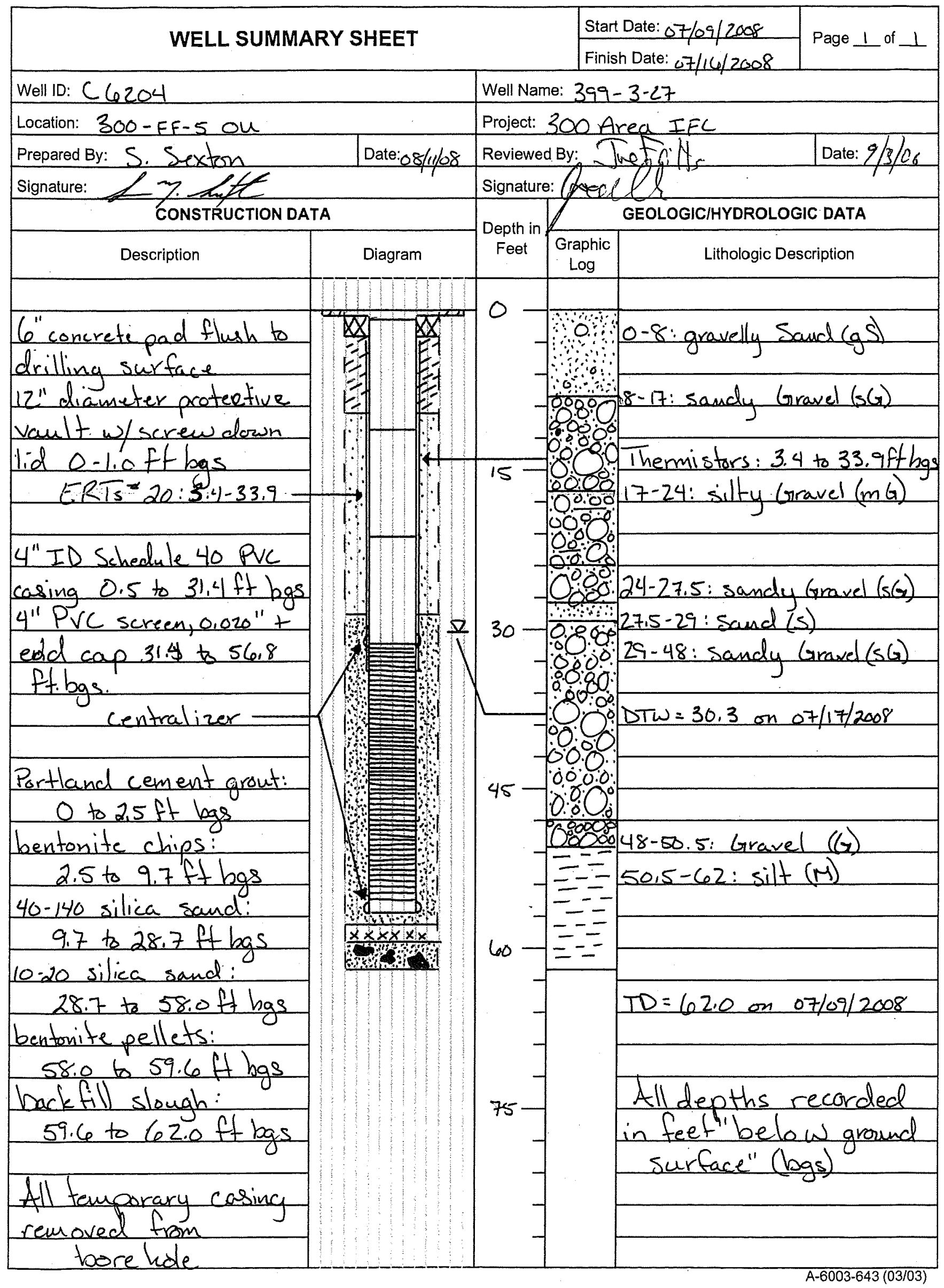




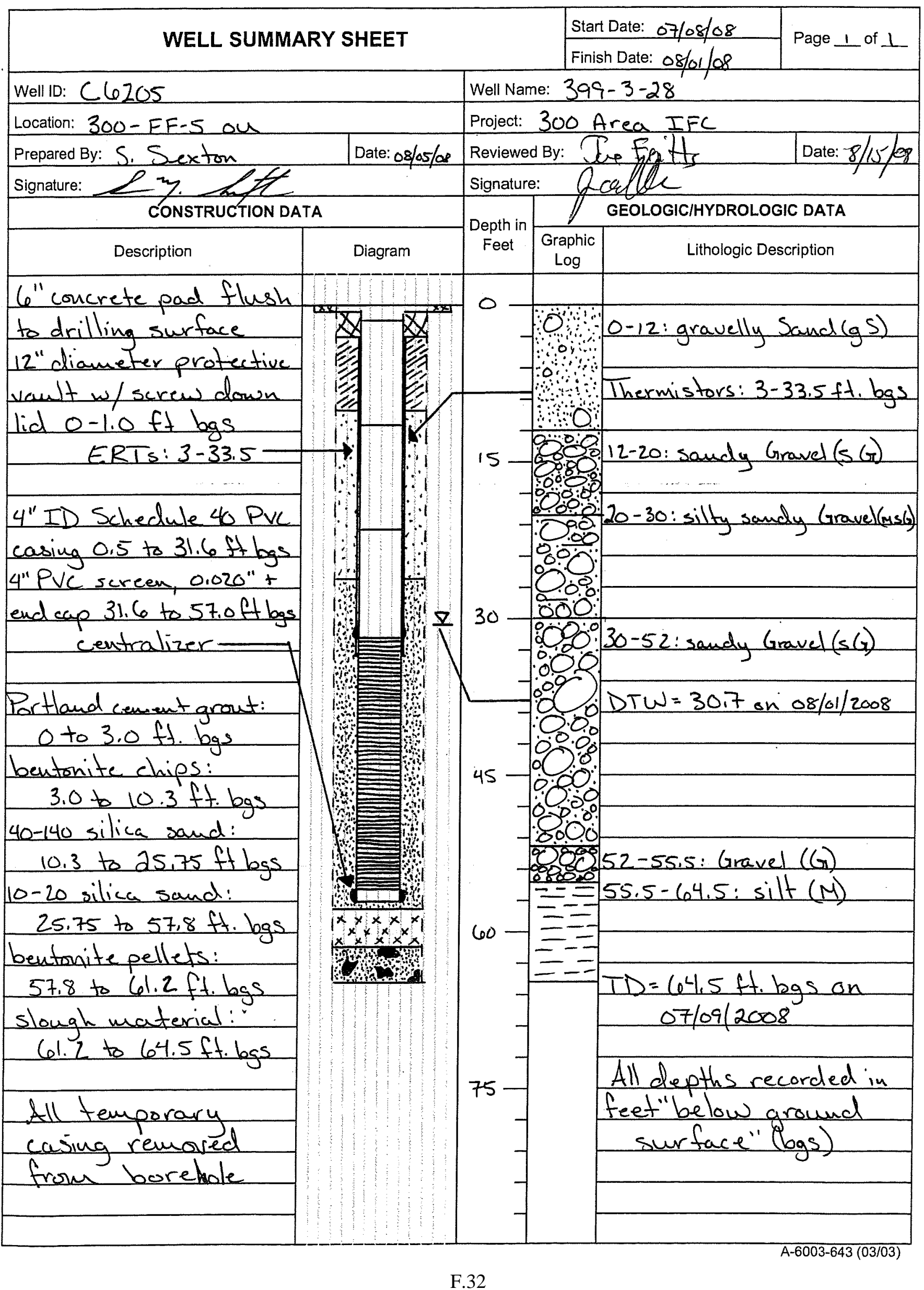




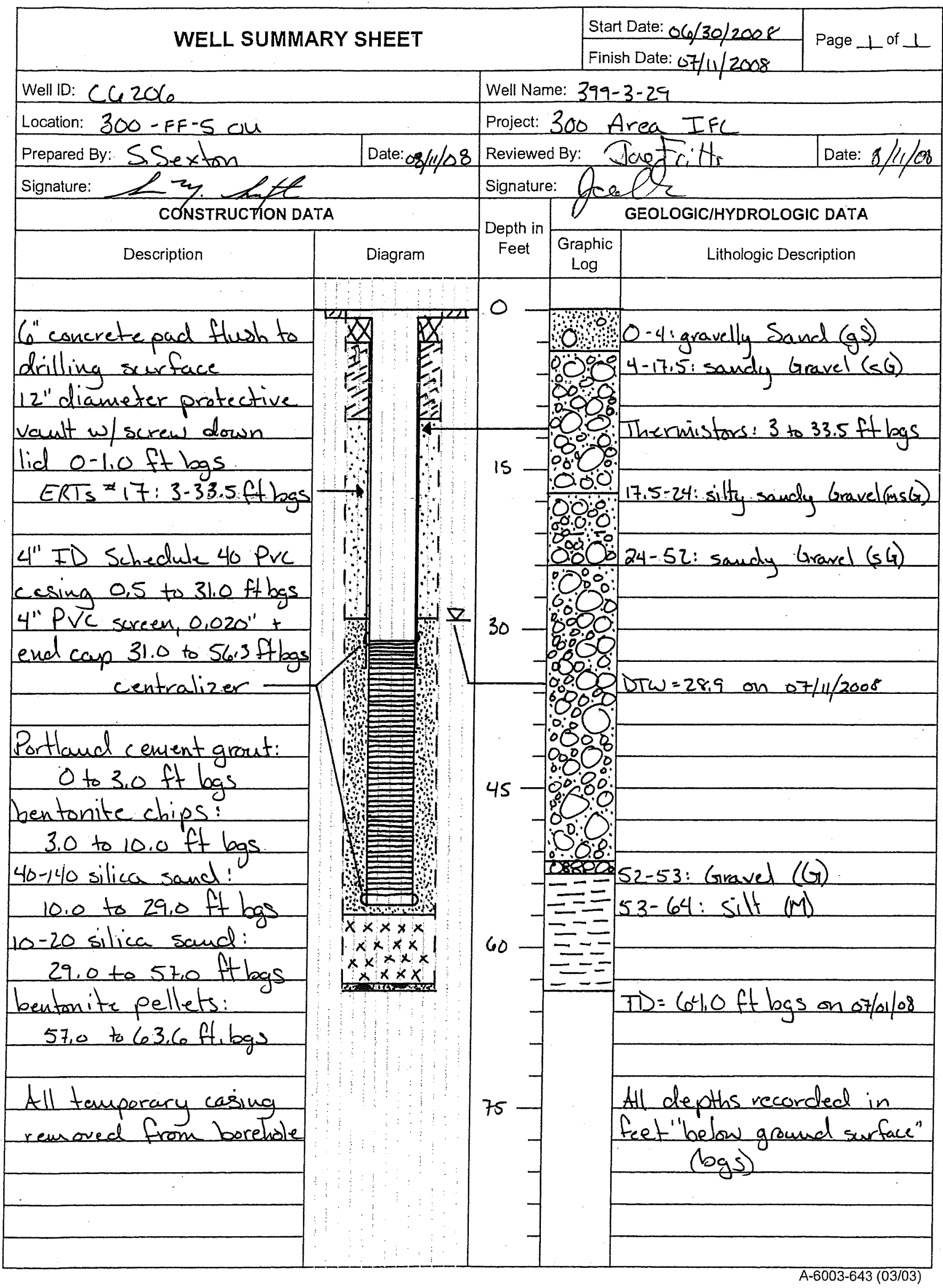




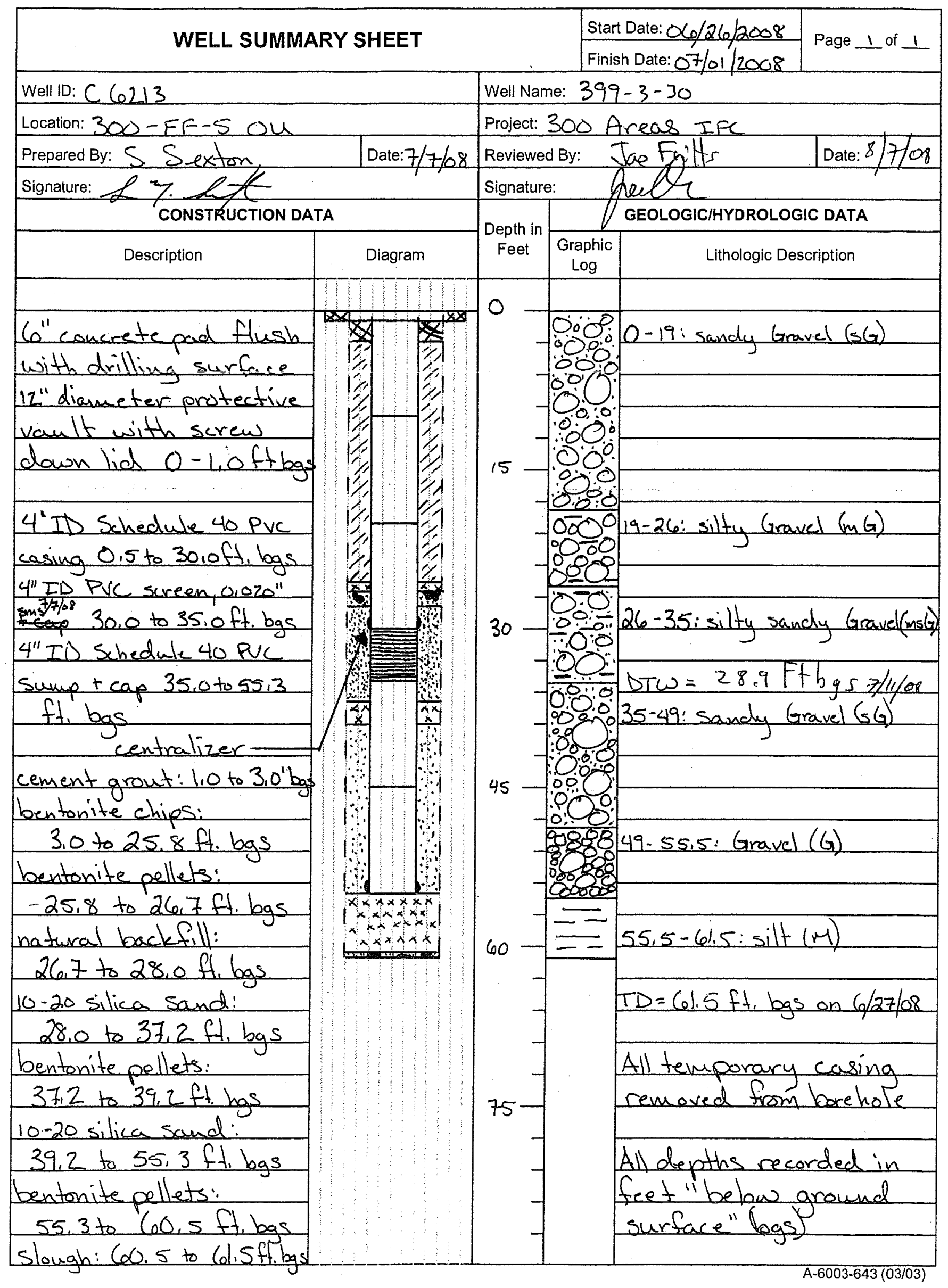




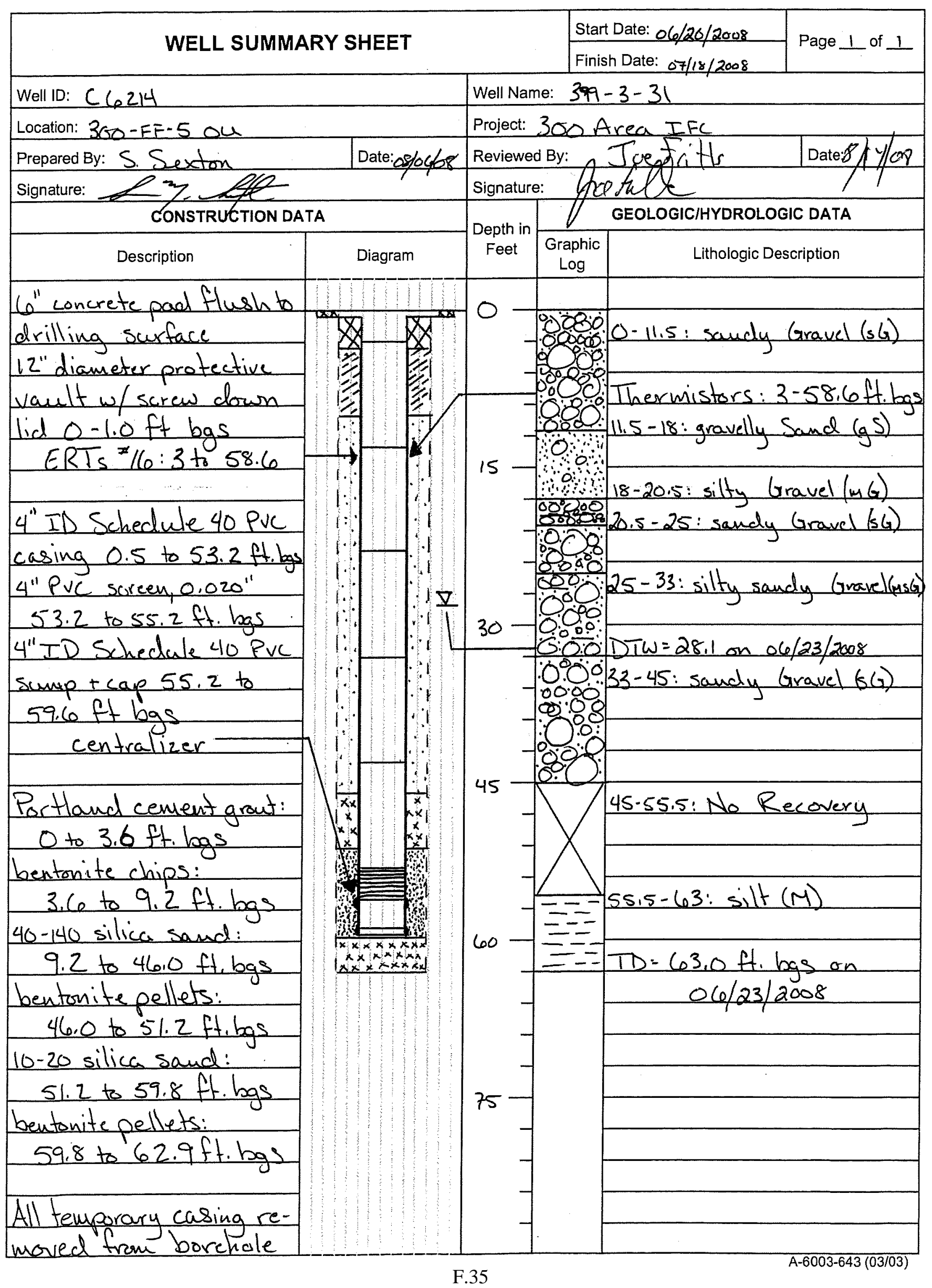




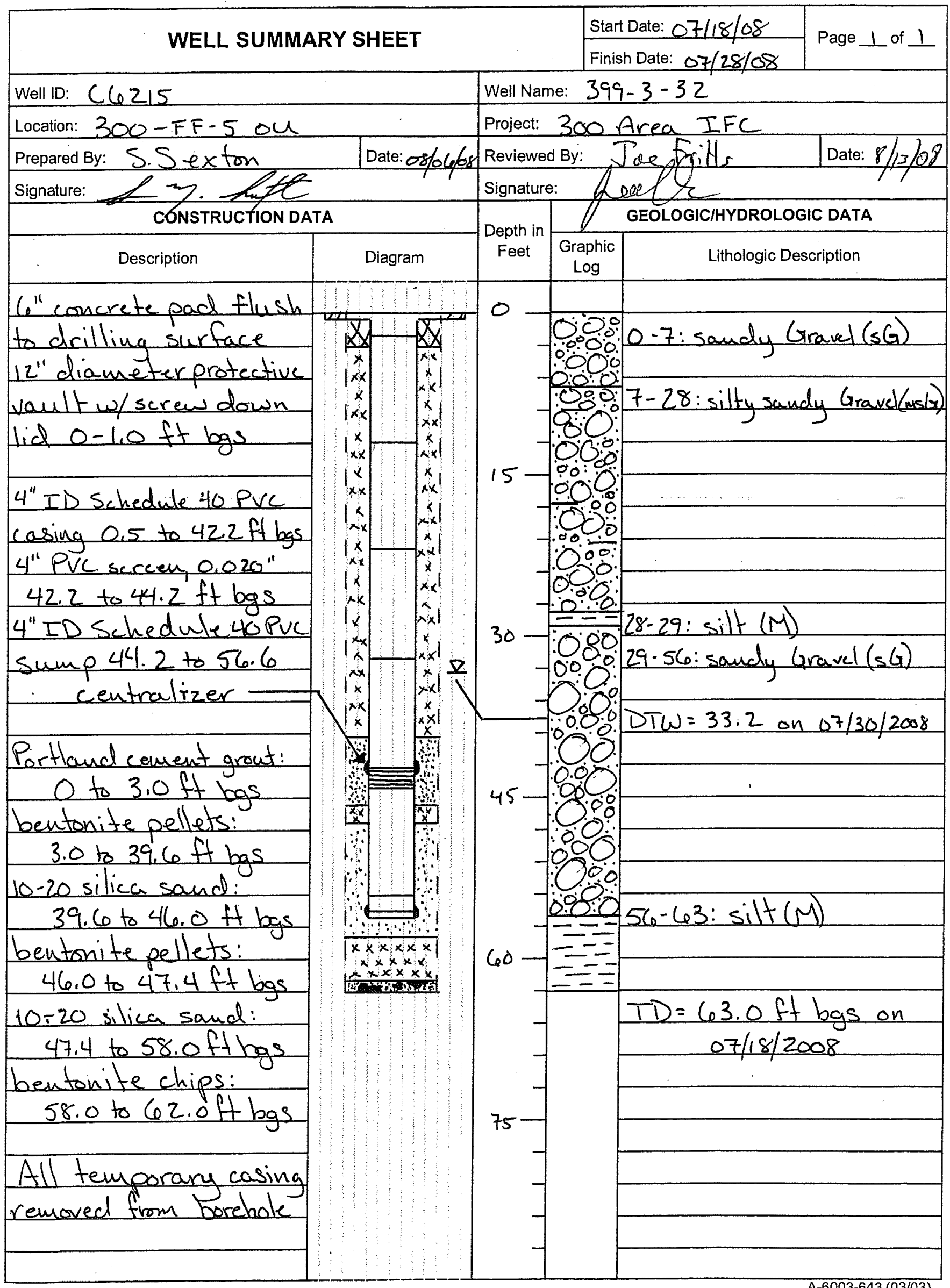




\section{Appendix G}

\section{Downhole Geophysical Logs}




\section{9-2-5 (C5708) \\ Log Data Report}

Borehole Information:

\begin{tabular}{|c|c|c|c|c|c|}
\hline \multicolumn{2}{|c|}{ Borehole: } & \multicolumn{3}{c|}{ Site: } & \multicolumn{2}{l|}{ 300-FF-5 } \\
\hline Coordinates (WA St Plane) & GWL ${ }^{1}$ (ft) : & 33.25 & \multicolumn{2}{c|}{ GWL Date: } & $10 / 01 / 07$ \\
\hline North (m) & East (m) & Drill Date & TOC Elevation & Total Depth (ft) & Type \\
\hline not available & not available & $09 / 28 / 07$ & not available & 129 & Cable \\
\hline
\end{tabular}

\section{Casing Information:}

\begin{tabular}{|c|c|c|c|c|c|c|}
\hline Casing Type & $\begin{array}{c}\text { Stickup } \\
\text { (ft) }\end{array}$ & $\begin{array}{c}\text { Outer } \\
\text { Diameter (in.) }\end{array}$ & $\begin{array}{c}\text { Inside } \\
\text { Diameter (in.) }\end{array}$ & $\begin{array}{c}\text { Thickness } \\
\text { (in.) }\end{array}$ & $\begin{array}{c}\text { Top } \\
\text { (ft) }\end{array}$ & $\begin{array}{c}\text { Bottom } \\
\text { (ft) }\end{array}$ \\
\hline Threaded Carbon Steel & 0.75 & $113 / 4$ & $103 / 4$ & $1 / 2$ & 0.75 & 129 \\
\hline
\end{tabular}

\section{Borehole Notes:}

The logging engineer measured the casing and stickup using a steel tape. Measurements were rounded to the nearest $1 / 16$ in.

\section{Logging Equipment Information:}

\begin{tabular}{|cl|l|l|}
\hline Logging System: & \multirow{2}{*}{ Gamma 4L } & Type: $\begin{array}{l}\text { SGLS (60\%) } \\
\text { SN: 47TP32211A }\end{array}$ \\
\hline Effective Calibration Date: & $07 / 09 / 07$ & Calibration Reference: & HGLP-CC-020 \\
\hline & Logging Procedure: & HGLP-MAN-002, Rev. 0 \\
\hline
\end{tabular}

\begin{tabular}{|cl|l|l|}
\hline Logging System: & \multirow{2}{*}{ Gamma 4H } & Type: $\begin{array}{l}\text { NMLS } \\
\text { SN: H310700352 }\end{array}$ \\
\hline Effective Calibration Date: & $11 / 22 / 06$ & Calibration Reference: & HGLP-CC-020 \\
\hline & & Logging Procedure: & HGLP-MAN-002, Rev. 0 \\
\hline
\end{tabular}

\section{Spectral Gamma Logging System (SGLS) Log Run Information:}

\begin{tabular}{|c|c|c|c|c|c|}
\hline Log Run & 3 & 4 Repeat & & & \\
\hline Date & $10 / 01 / 07$ & $10 / 01 / 07$ & & & \\
\hline Logging Engineer & Spatz & Spatz & & & \\
\hline Start Depth (ft) & 129.0 & 0.0 & & & \\
\hline Finish Depth (ft) & 0.5 & 13.0 & & & \\
\hline Count Time (sec) & 100 & 100 & & & \\
\hline Live/Real & $\mathrm{R}$ & $\mathrm{R}$ & & & \\
\hline Shield (Y/N) & $\mathrm{N}$ & $\mathrm{N}$ & & & \\
\hline MSA Interval (ft) & 0.5 & 0.5 & & & \\
\hline $\mathrm{ft} / \mathrm{min}$ & N/A ${ }^{2}$ & N/A & & & \\
\hline Pre-Verification & DL041CAB & DL041CAB & & & \\
\hline Start File & DL041000 & DL041259 & & & \\
\hline Finish File & DL041257 & DL041285 & & & \\
\hline Post-Verification & DL041CAA & DL041CAA & & & \\
\hline Depth Return Error (in.) & 0 & 0 & & & \\
\hline Comments & $\begin{array}{c}\text { No fine gain } \\
\text { adjustment }\end{array}$ & $\begin{array}{c}\text { No fine gain } \\
\text { adjustment }\end{array}$ & & & \\
\hline
\end{tabular}


Neutron Moisture Logging System (NMLS) Log Run Information:

\begin{tabular}{|c|c|c|l|l|l|}
\hline Log Run & $\mathbf{1}$ & 2 Repeat & & & \\
\hline Date & $09 / 28 / 07$ & $09 / 28 / 07$ & & & \\
\hline Logging Engineer & Spatz & Spatz & & & \\
\hline Start Depth (ft) & 0.0 & 20.0 & & & \\
\hline Finish Depth (ft) & 32.0 & 23.0 & & & \\
\hline Count Time (sec) & 15 & 15 & & & \\
\hline Live/Real & $\mathrm{R}$ & $\mathrm{R}$ & & & \\
\hline Shield (Y/N) & $\mathrm{N}$ & $\mathrm{N}$ & & & \\
\hline MSA Interval (ft) & 0.25 & 0.25 & & & \\
\hline ft/min & N/A & N/A & & & \\
\hline Pre-Verification & DH722CAB & DH722CAB & & & \\
\hline Start File & DH722000 & DH722129 & & & \\
\hline Finish File & DH722128 & DH722141 & & & \\
\hline Post-Verification & DH722CAA & DH722CAA & & & \\
\hline Depth Return Error (in.) & N/A & + 0.5 & & & \\
\hline Comments & $\begin{array}{c}\text { No fine gain } \\
\text { adjustment }\end{array}$ & $\begin{array}{c}\text { No fine gain } \\
\text { adjustment }\end{array}$ & & & \\
\hline
\end{tabular}

\section{Logging Operation Notes:}

Logging was conducted with a centralizer on the sondes. Logging data acquisition is referenced to ground level. Repeat data were acquired in this borehole to evaluate each system's performance.

\section{Analysis Notes:}

\begin{tabular}{|l|l|l|l|l|l|}
\hline Analyst: & Henwood & Date: & $10 / 16 / 07$ & Reference: & GJO-HGLP 1.6.3, Rev. 0 \\
\hline
\end{tabular}

Pre-run and post-run verifications for the SGLS (G4L) were acquired in the Amersham verifier, serial number 115 which is enhanced in the naturally occurring radionuclides K-40, U-238, and Th-232 (KUT). The verification criteria were met.

A casing correction for $1 / 2$-in.-thick casing was applied to the SGLS log data.

SGLS spectra were processed in batch mode using APTEC SUPERVISOR to identify individual energy peaks and determine count rates. Concentrations were calculated with EXCEL worksheet template identified as G4LJuly07.xls using efficiency functions and corrections for casing, water, and dead time as determined from annual calibrations. No correction for dead time was necessary. A correction for water was applied to data acquired below $33 \mathrm{ft}$ in depth.

The NMLS data are presented as counts per second. A calibration for casing inside diameters greater than 8-in. is not available.

\section{$\underline{\text { Results and Interpretations: }}$}

A plot of manmade radionuclides is included for Cs-137 and processed uranium (U-235 and U-238). The plot indicates all detections based on the routine processing software. All of the detections were at or near the respective MDLs. Inspection of each spectrum where a detection was indicated revealed no full energy peaks. Therefore, the detections are considered to be statistical fluctuations and are not considered valid. No other manmade radionuclides were indicated.

There is a strong indication of radon in the groundwater. Comparison of the $1764 \mathrm{keV}$ and $609 \mathrm{keV}$ Bi214 gamma rays show differing concentrations after corrections for water and casing. The casing and water correction factors decrease with increasing energy. Gamma rays originating inside the casing are not attenuated by the steel casing, and the net effect of applying the correction factors is to amplify results from 
HGLP-LDR-096

low-energy gamma rays. The fact that the $609 \mathrm{keV}$ gamma ray results in a higher apparent concentration than the $1764 \mathrm{keV}$ gamma line suggests that radon is present in the groundwater. Typical formation concentrations of naturally occurring U-238 are between approximately 0.5 and $1.5 \mathrm{pCi} / \mathrm{g}$. The concentrations above the groundwater level are consistent with these values for the assays of both the 609 and $1764 \mathrm{keV}$ peaks. Note that enhanced radon is not related to the existence of manmade uranium.

The neutron moisture results are reported in counts per second because no valid calibration is available for borehole inside diameters greater than 8 inches. It is not known to what degree this measurement reflects formation moisture.

The repeat sections generally indicate good agreement of the naturally occurring KUT and moisture.

\section{List of Log Plots:}

Manmade Radionuclides

Natural Gamma Logs

Combination Plot

Total Gamma \& Moisture

Total Gamma \& Dead Time

Repeat Section of Natural Gamma Logs

Moisture Repeat Section

${ }^{1}$ GWL - groundwater level 


\section{9-2-5 (C5708) Manmade Radionuclides}

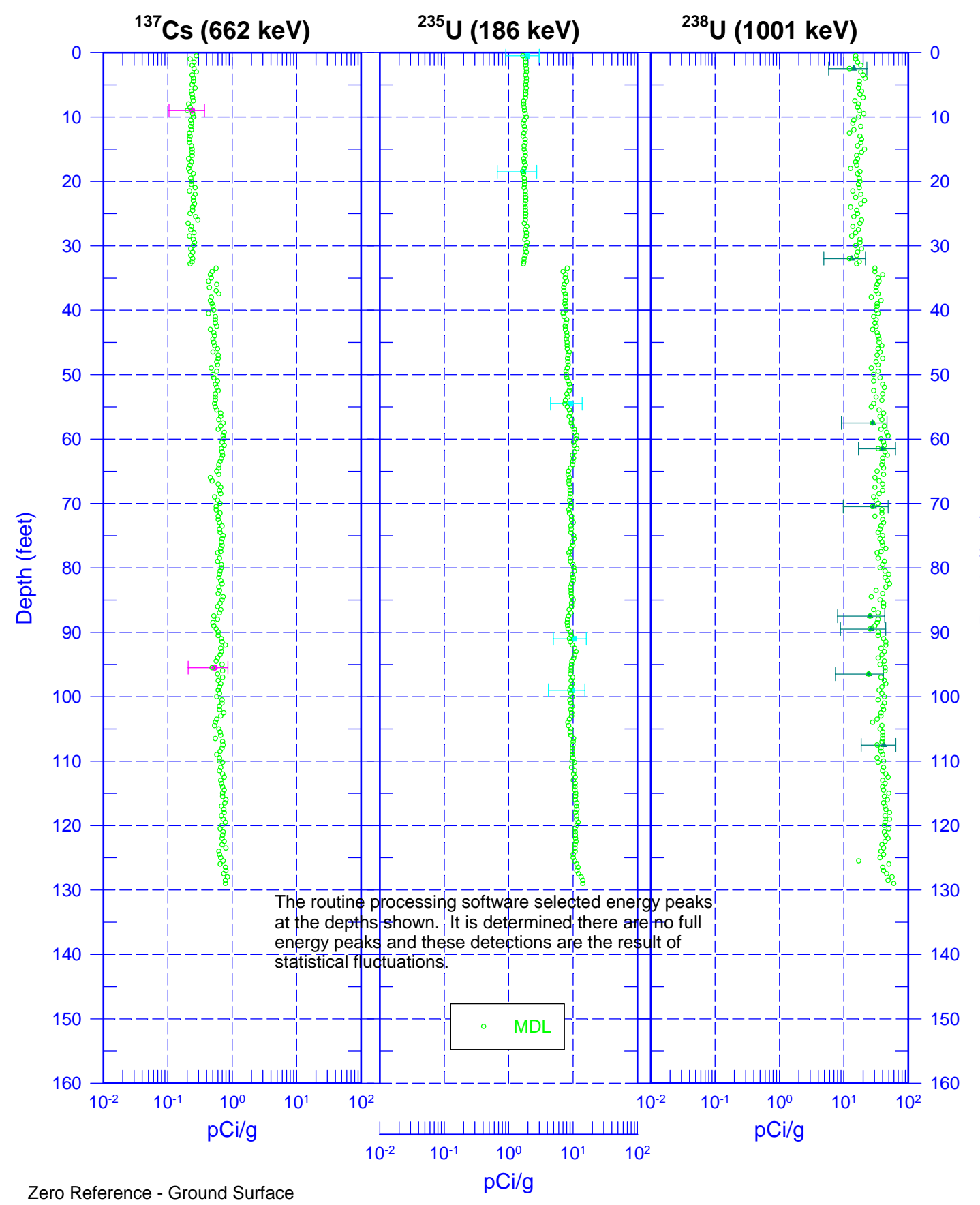




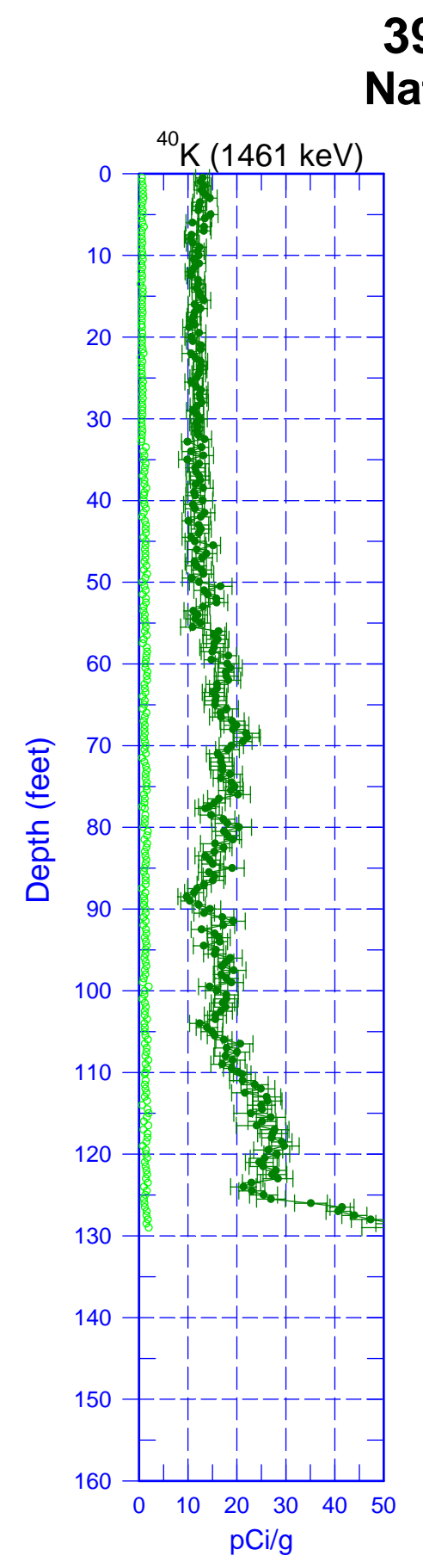

399-2-5 (C5708)

Natural Gamma Logs
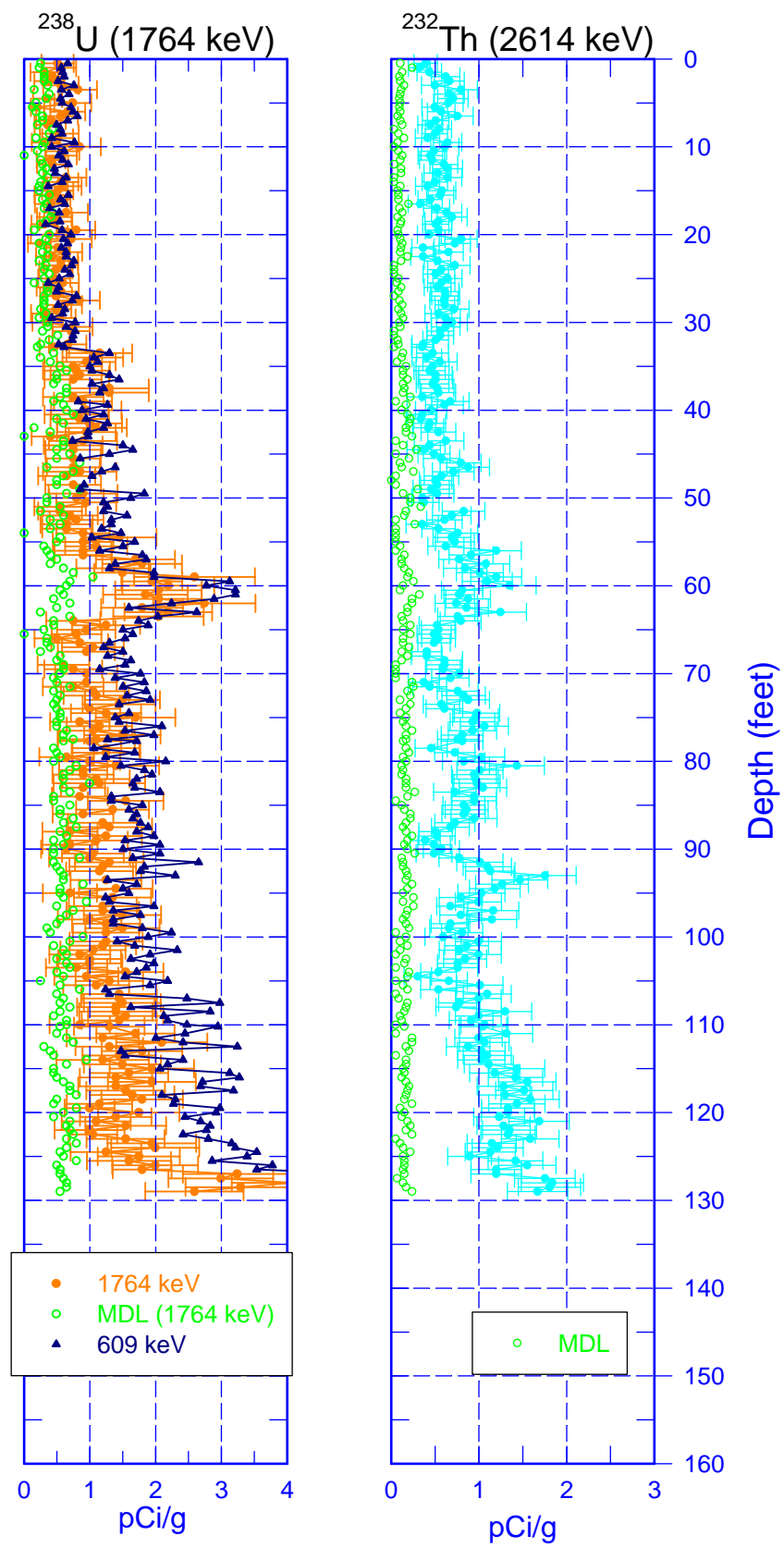

Zero Reference - Ground Surface 
HGLP-LDR-096

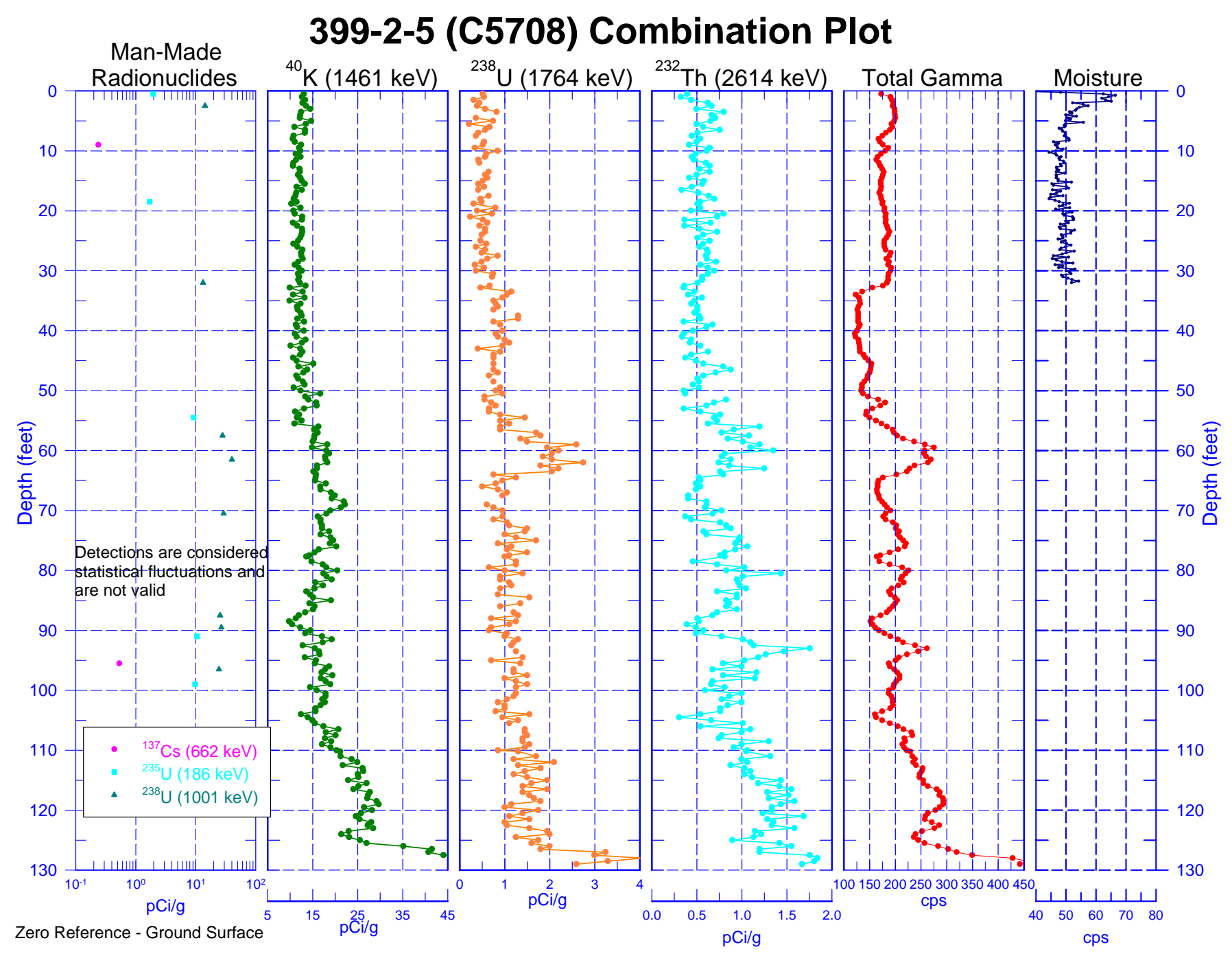

Page 6 


\section{9-2-5 (C5708) Total Gamma \& Moisture}
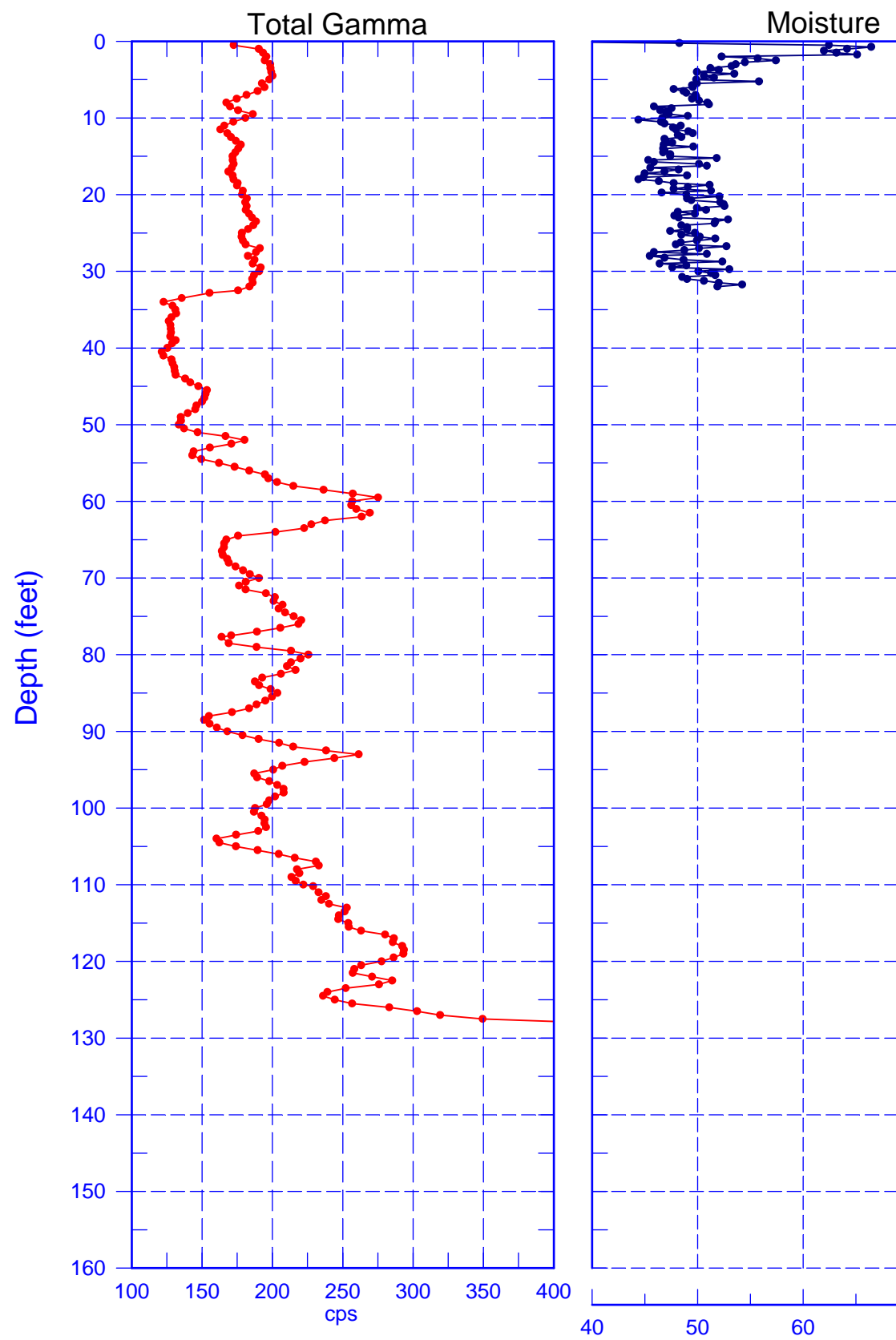

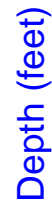

Reference - Ground Surface 


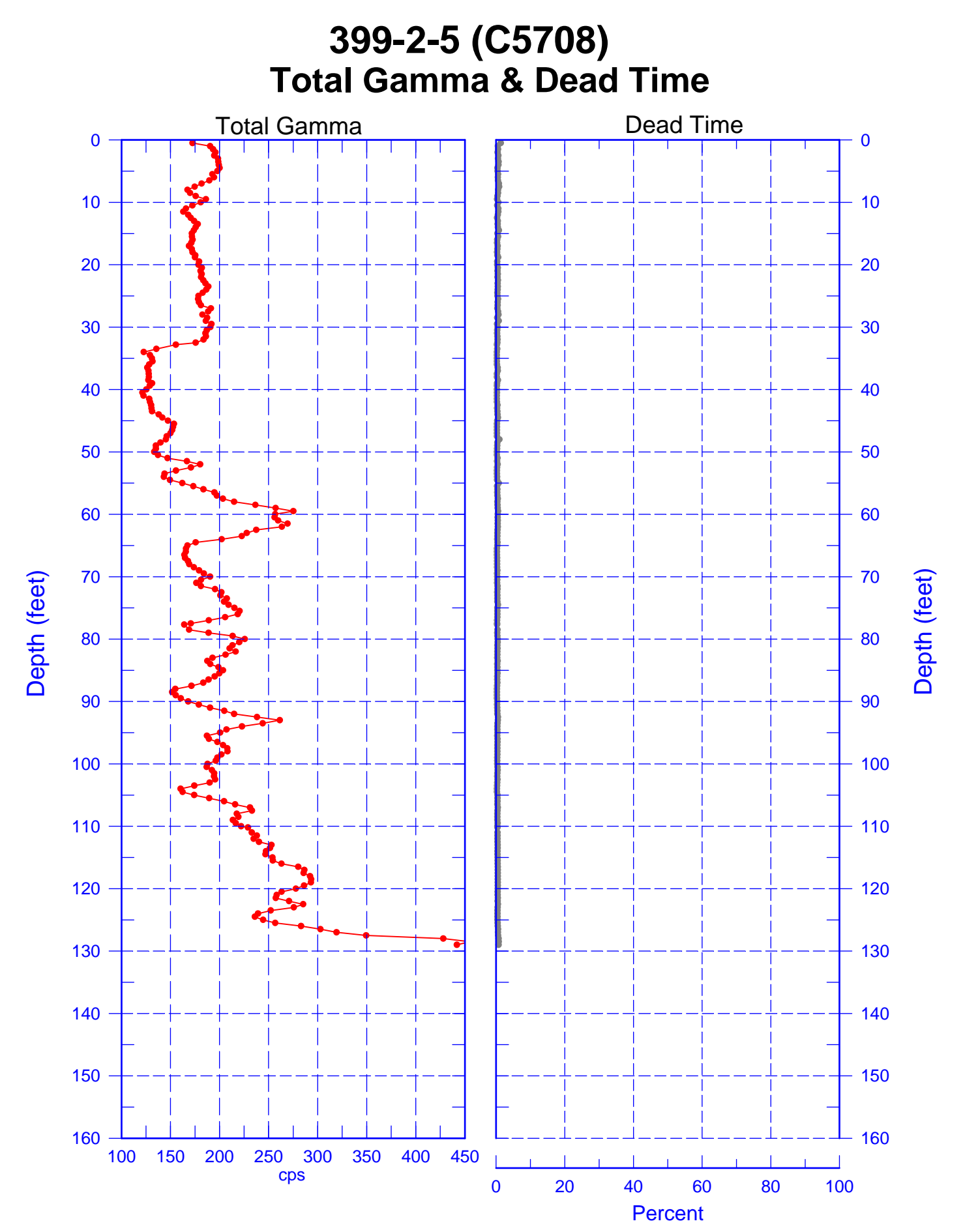

Reference - Ground Surface 


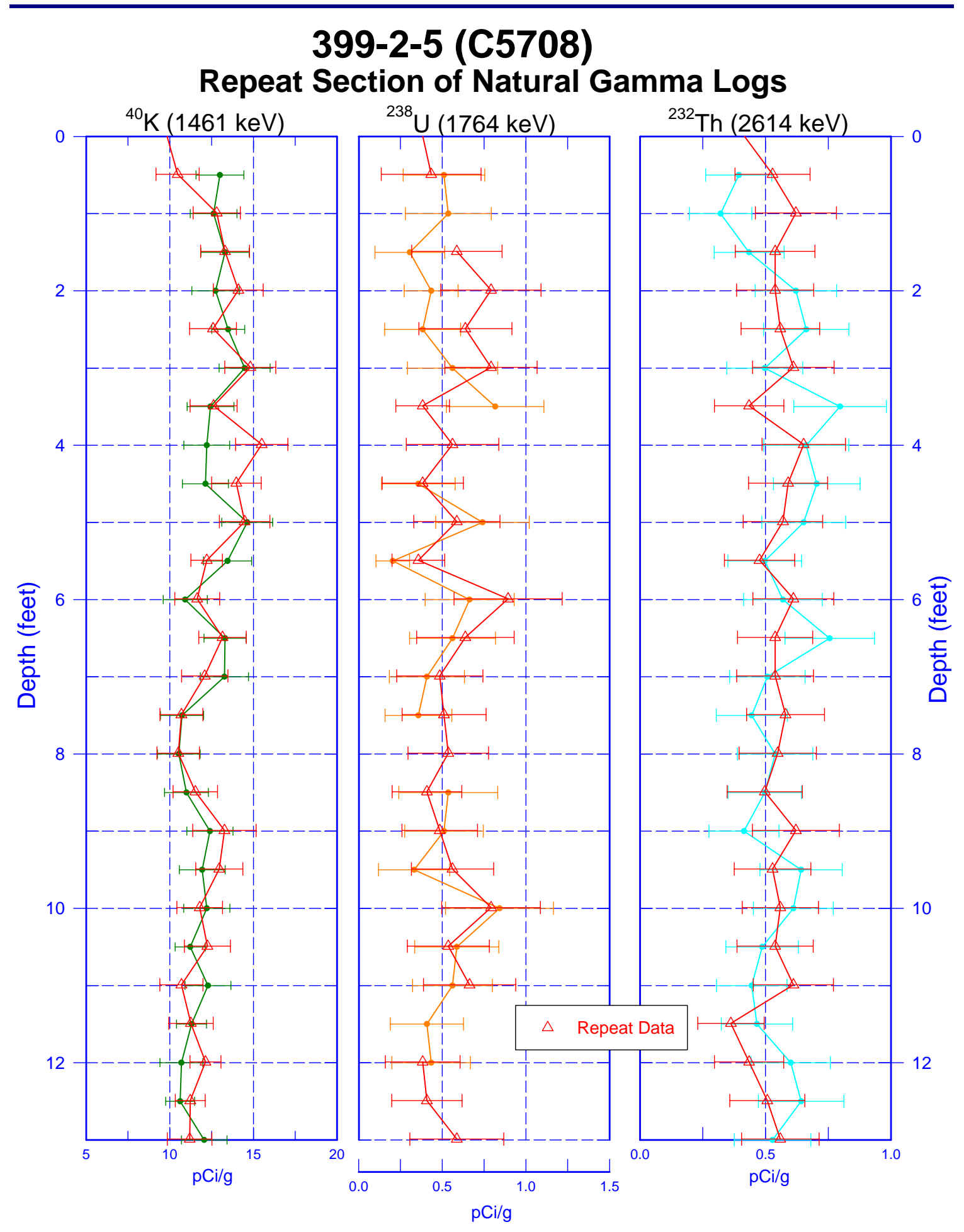

Zero Reference - Ground Surface 


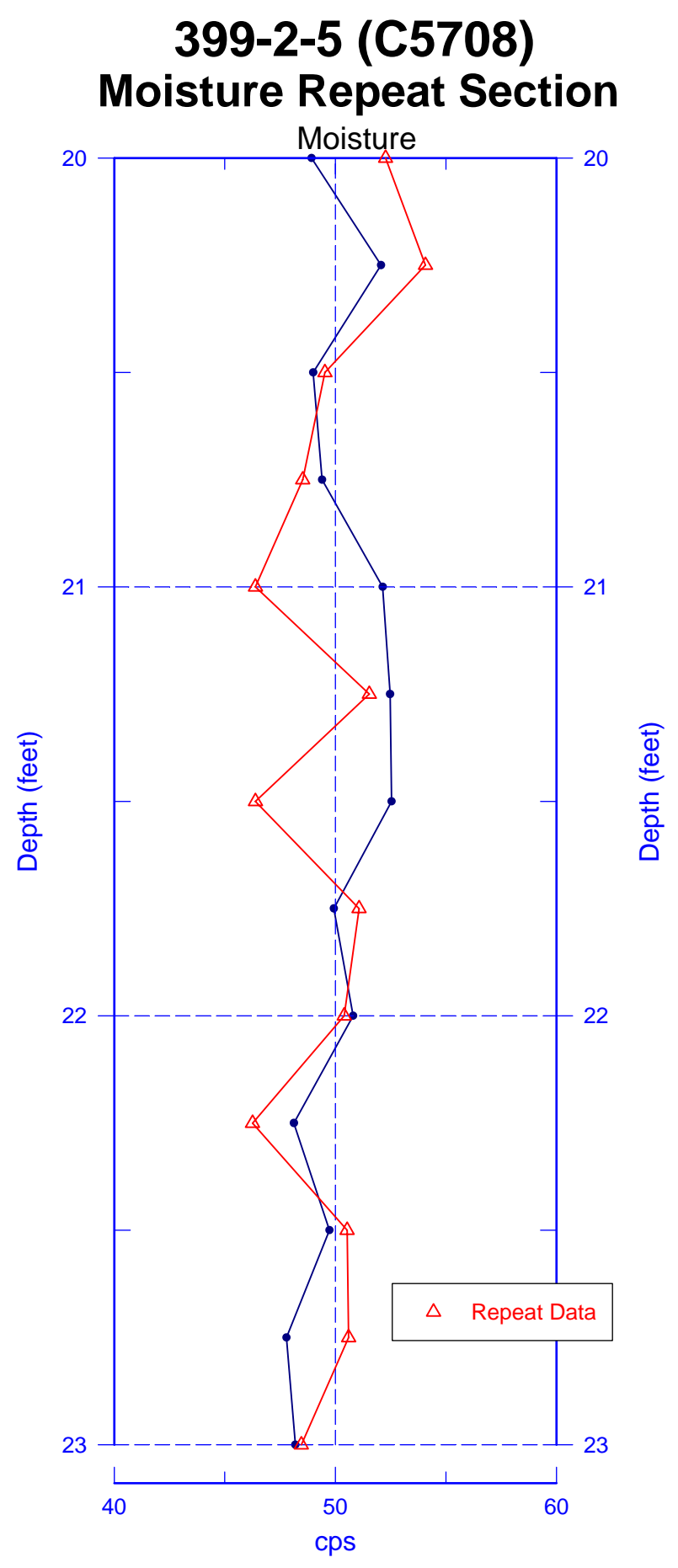

Reference - Ground Surface 


\section{9-2-7 (C6184) \\ Log Data Report}

Borehole Information:

\begin{tabular}{|c|c|c|c|c|c|}
\hline Borehole: & \multicolumn{1}{l|}{ Site: } & \multicolumn{2}{c|}{ G00-FF-5 } \\
\hline \multicolumn{2}{|c|}{ 399-2-7 (C6184) } & GWL $^{\mathbf{1}}$ (ft) : & 27.1 & Date: & 6/28/2008 \\
\hline Nordinates (WA St Plane) & East (m) & Drill Date & TOC $^{2}$ Elevation & Total Depth (ft) & Type \\
\hline 116084.5 & 594235.3 & $06 / 27 / 2008$ & N/A & 62 & Sonic \\
\hline
\end{tabular}

\section{Casing Information:}

\begin{tabular}{|c|c|c|c|c|c|c|}
\hline Casing Type & Stickup (ft) & $\begin{array}{c}\text { Outer } \\
\text { Diameter (in.) }\end{array}$ & $\begin{array}{c}\text { Inside } \\
\text { Diameter (in.) }\end{array}$ & Thickness (in.) & Top (ft) & Bottom (ft) \\
\hline Threaded Steel & 1.1 & $75 / 8$ & $67 / 8$ & $3 / 8$ & -1.1 & 59 \\
\hline
\end{tabular}

\section{Borehole Notes:}

Casing data and total depth was reported by the site geologist. Casing diameter was measured using a steel tape and rounded to the nearest 1/16-inch. The zero reference is the ground surface.

Logging Equipment Information:

\begin{tabular}{|c|c|c|c|c|}
\hline Logging System: & \multicolumn{2}{|l|}{ Gamma $4 \mathrm{~L}$} & $\begin{array}{l}\text { Type: } \\
\text { Serial No.: }\end{array}$ & $\begin{array}{l}\text { 60\% HPGe SGLS } \\
\text { 47TP32211A }\end{array}$ \\
\hline Effective Calibration Date: & $12 / 31 / 2007$ & Calibration Reference: & \multicolumn{2}{|c|}{ HGLP-CC-027 } \\
\hline & & Logging Procedure: & \multicolumn{2}{|c|}{ HGLP-MAN-002, Rev. 0} \\
\hline Logging System: & \multicolumn{2}{|l|}{ Gamma $4 \mathrm{H}$} & $\begin{array}{l}\text { Type: } \\
\text { Serial No.: }\end{array}$ & $\begin{array}{l}\text { NMLS } \\
\text { H310700352 }\end{array}$ \\
\hline Effective Calibration Date: & $11 / 6 / 2008$ & Calibration Reference: & \multicolumn{2}{|c|}{ HGLP-CC-021 } \\
\hline & & Logging Procedure: & \multicolumn{2}{|c|}{ HGLP-MAN-002, Rev. 0} \\
\hline
\end{tabular}

\section{Spectral Gamma Logging System (SGLS) Log Run Information:}

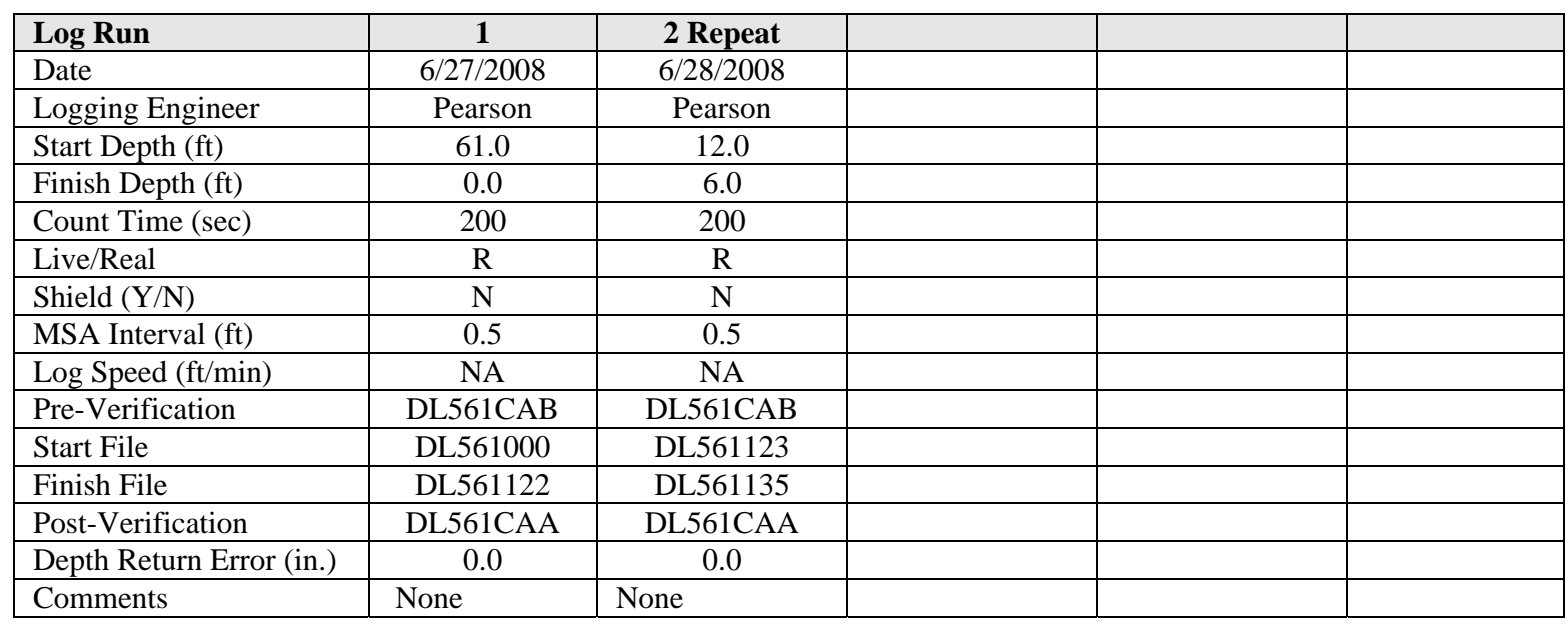


HGLP-LDR-267, Rev. 0

Neutron Moisture Logging System (NMLS) Log Run Information:

\begin{tabular}{|c|c|c|c|c|c|}
\hline Log Run & 1 & 2 Repeat & & & \\
\hline Date & $6 / 27 / 2008$ & $6 / 27 / 2008$ & & & \\
\hline Logging Engineer & Pearson & Pearson & & & \\
\hline Start Depth (ft) & 0.0 & 11.0 & & & \\
\hline Finish Depth (ft) & 26.75 & 14.0 & & & \\
\hline Count Time (sec) & 15 & 15 & & & \\
\hline Live/Real & $\mathrm{R}$ & $\mathrm{R}$ & & & \\
\hline Shield (Y/N) & $\mathrm{N}$ & $\mathrm{N}$ & & & \\
\hline MSA Interval (ft) & 0.25 & 0.25 & & & \\
\hline $\mathrm{ft} / \mathrm{min}$ & NA & NA & & & \\
\hline Pre-Verification & DHG32CAB & DHG32CAB & & & \\
\hline Start File & DHG32000 & DHG32108 & & & \\
\hline Finish File & DHG32107 & DHG32120 & & & \\
\hline Post-Verification & DHG32CAA & DHG32CAA & & & \\
\hline Depth Return Error (in.) & NA & 0.0 & & & \\
\hline Comments & None & None & & & \\
\hline
\end{tabular}

\section{Logging Operation Notes:}

The SGLS data were collected using Gamma 4, HO 68B-3573. Pre and post-survey verification measurements were acquired in the Amersham KUTh-115 field verifier. A centralizer was installed on the sonde. Maximum logging depth was 61.15 feet before the sonde un-weighted.

The NMLS data were collected using Gamma 4, HO 68B-3573. Pre and post-survey verification measurements were acquired in the standard field verifier. A centralizer was installed on the sonde. Maximum logging depth was 26.75 feet before water was encountered.

\section{Analysis Notes:}

\begin{tabular}{|l|l|l|l|l|l|}
\hline Analyst: & SPINNER & Date: & 10/14/2008 & Reference: & GJO-HGLP 1.6.3, Rev. 0 \\
\hline
\end{tabular}

The SGLS AND NMLS pre and post-survey verifications spectra met acceptance criteria.

Analyses of log data suggest slight discrepancies with field reports of depth to water and casing depth. Corrections of log data were applied according to the analysis assumptions in the table below.

\begin{tabular}{|c|c|c|c|l|}
\hline $\begin{array}{c}\text { Top } \\
\text { (ft) }\end{array}$ & $\begin{array}{c}\text { Bottom } \\
\text { (ft) }\end{array}$ & $\begin{array}{c}\text { T } \\
\text { (in) }\end{array}$ & $\begin{array}{c}\text { ID } \\
\text { (in) }\end{array}$ & \multicolumn{1}{|c|}{ Comments } \\
\hline 0 & 27.1 & 0.375 & $67 / 8$ & No water correction \\
\hline 28 & 59 & 0.375 & $67 / 8$ & GWL reported @ 27.1 ft; adjusted to 28 ft. \\
\hline 59.1 & 62 & 0 & NA & Casing depth reported @ 59 ft; adjusted to 59.1 ft. \\
\hline
\end{tabular}

$\mathrm{T}=$ casing thickness used to calculate energy-dependent casing correction factor

ID = casing inside diameter used to calculate energy-dependent water correction factor 


\section{$\underline{\text { Results and Interpretations: }}$}

The Man-Made Radionuclides, Cs-137 (662 keV), U-235 (186 keV) and U-238 (Pa-234m [1001keV]) were identified by the routine processing software at various depth intervals. Scrutiny of the individual spectra indicates that the Cs-137 detections from 10.0 to 11.0 feet are valid. Maximum Cs- 137 concentration is $0.17 \mathrm{pCi} / \mathrm{g}$ at 11.0 feet. Detections obtained outside of this depth range are associated with processing software, are statistical fluctuations, and are not valid. Scrutiny of the individual spectra indicates the U-238 (Pa-234m) detections may be valid at 10.5 and 11.5 feet, however corroborating energy peaks at the $766 \mathrm{keV}$ (Pa-234m) or at $186 \mathrm{keV}(\mathrm{U}-235)$ were not present and the detections were not repeatable. The detections are too near the MDL to determine validity. The remaining U-238 detections outside this range were determined to be statistical fluctuations and are not valid. The detection of U-235 at 49.0 feet is considered a statistical fluctuation and is not valid.

The Natural Gamma Logs (KUT) indicates stratigraphic changes at 12 feet and 54 feet.

The Natural Gamma Logs (KUT) and the Moisture Repeat plots indicate good repeatability.

The Manmade Radionuclides Repeat Log shows a repeatable detection for Cs-137 at 10.0 feet. The U-238 did not repeat.

\section{List of Log Plots:}

Depth Reference is ground surface.

Manmade Radionuclides

Natural Gamma Logs

Combination Plot

Total Gamma \& Dead Time

Total Gamma \& Moisture

Repeat Section of Manmade Radionuclides

Repeat Section of Natural Gamma Logs

Repeat Section of Moisture

${ }^{1} \mathrm{GWL}$ - groundwater level

${ }^{2}$ TOC - top of casing 

Hanford Office Manmade Radionuclides

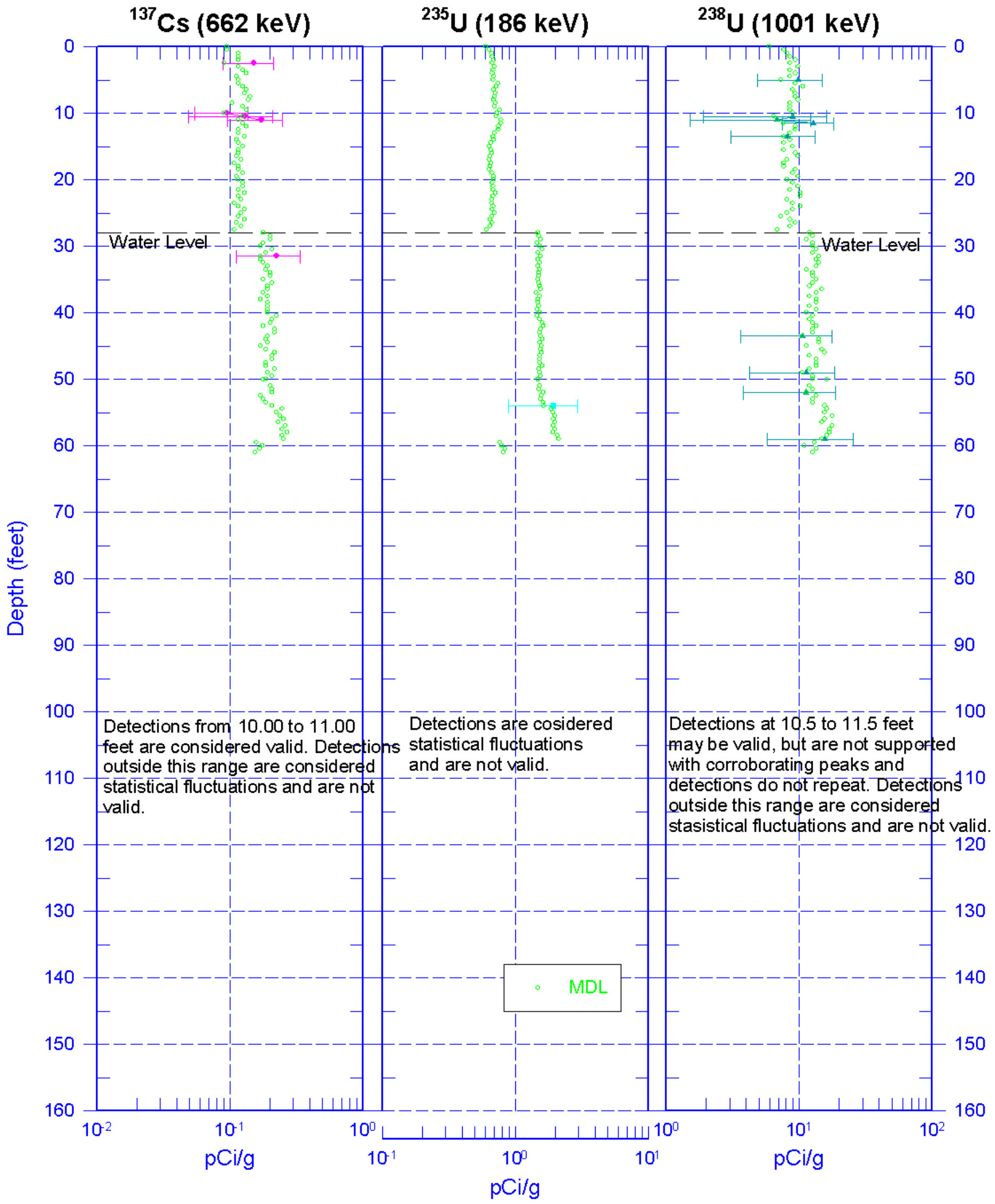

Zero Reference - Ground Surface 


\section{Stoller 399-2-7 (C6184) Hanford Office Natural Gamma Logs}
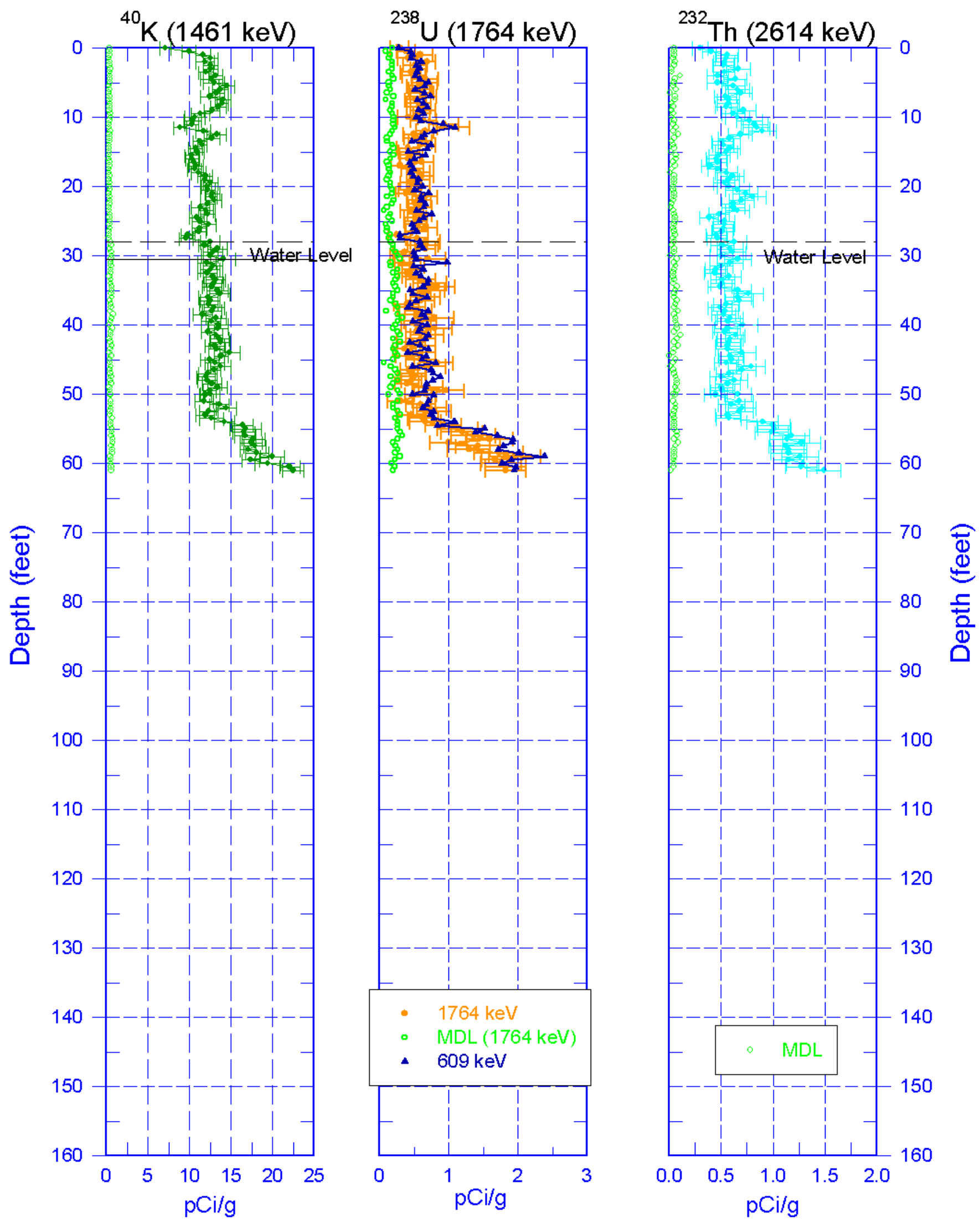

Zero Reference - Ground Surface 


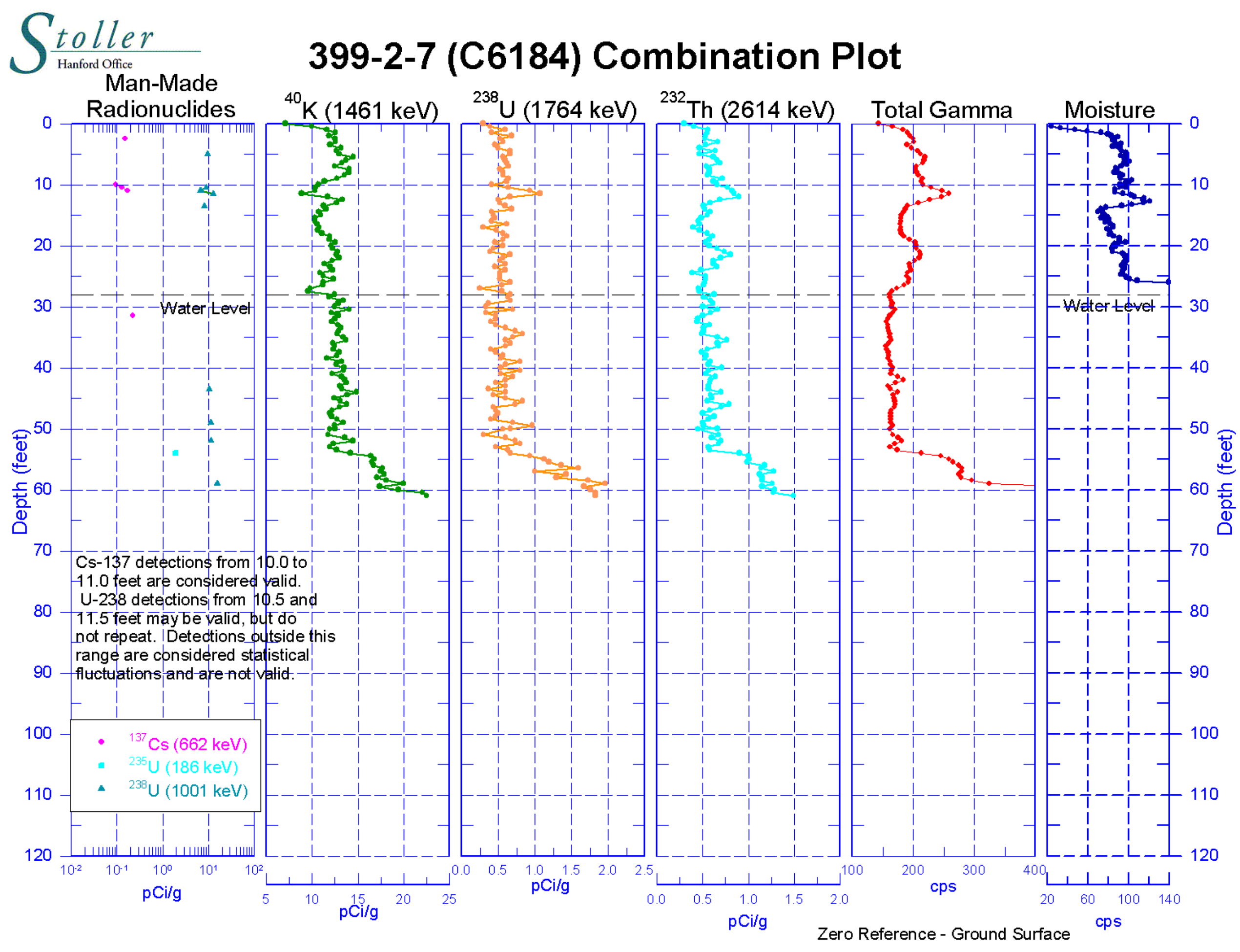



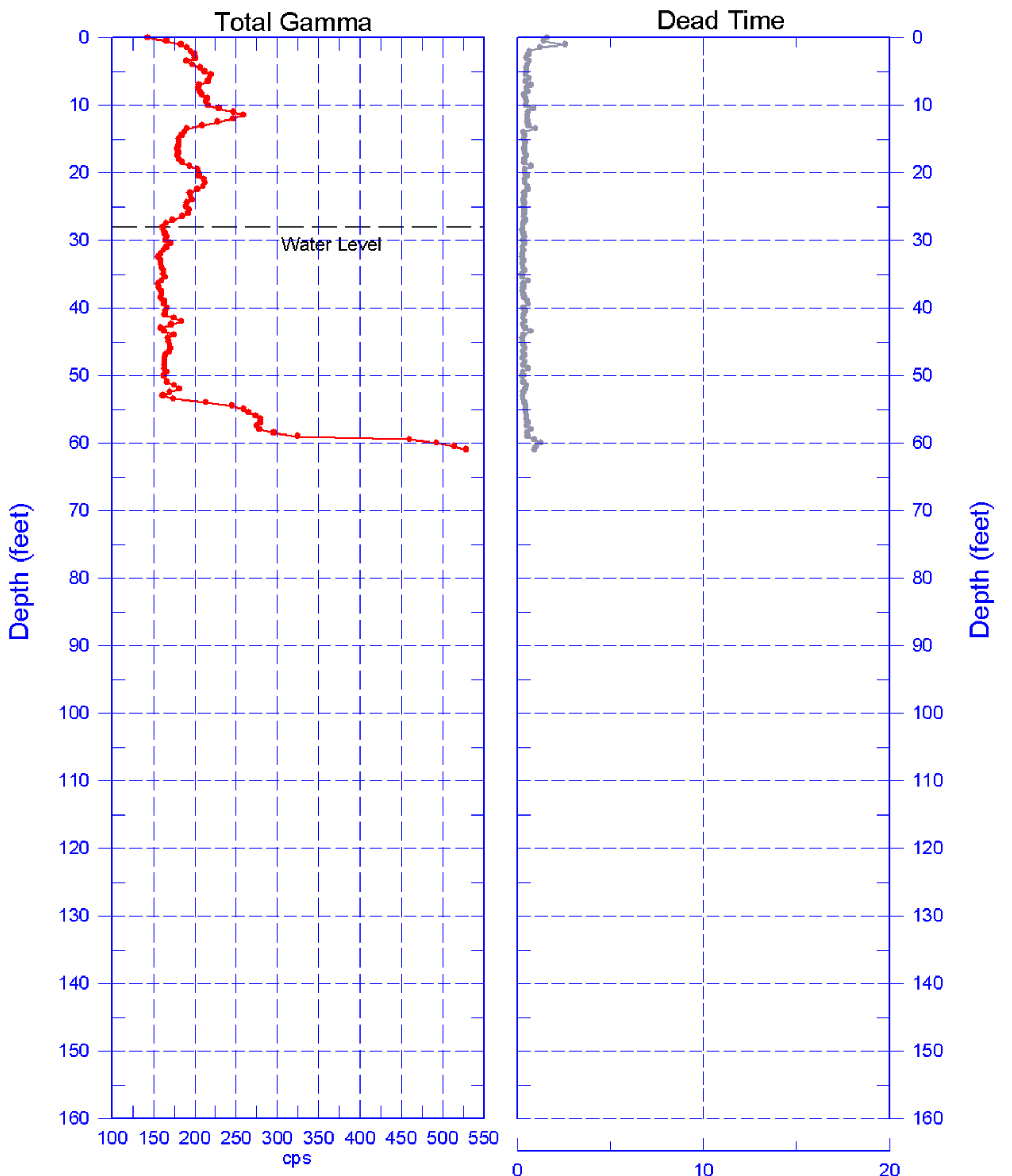

Reference - Ground Surface 


\section{S}

toller $\quad 399-2-7$ (C6184)

Hanford Office

\section{Total Gamma \& Moisture}
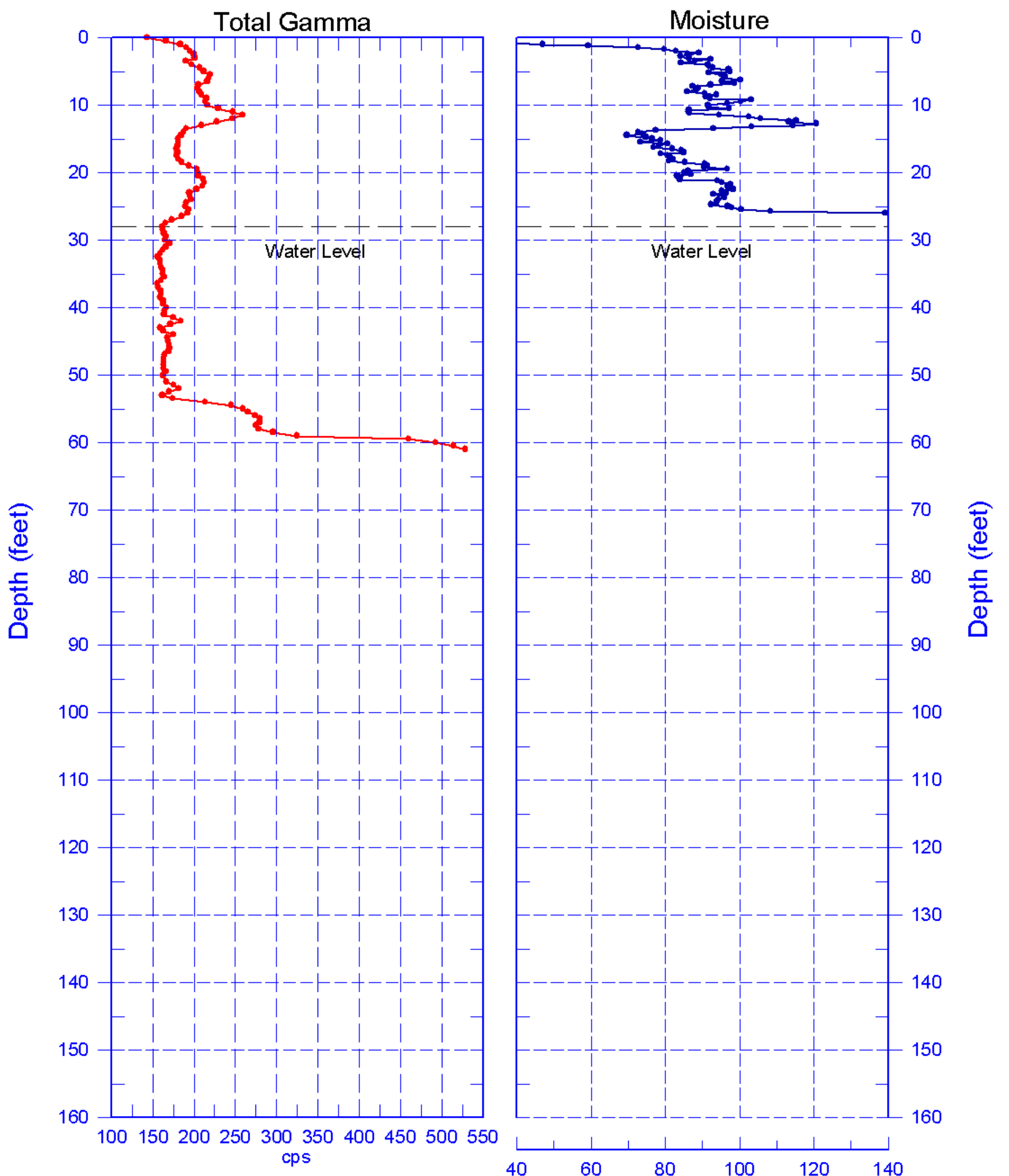

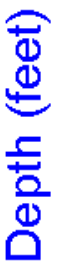

Reference - Ground Surface 
Stoller $\quad 399-2-7$ (C6184)

Repeat Section of Manmade Radionuclides

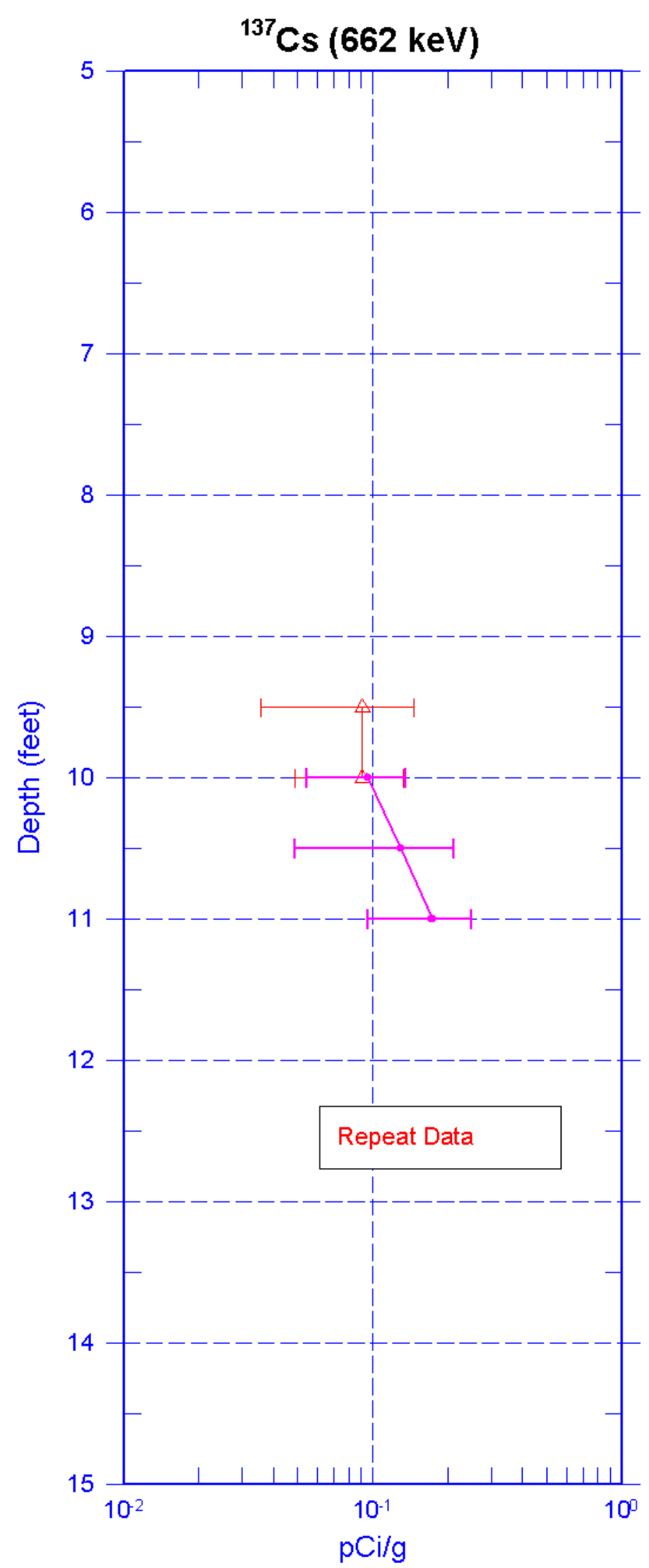

${ }^{238} \mathrm{U}$ (1001 keV)

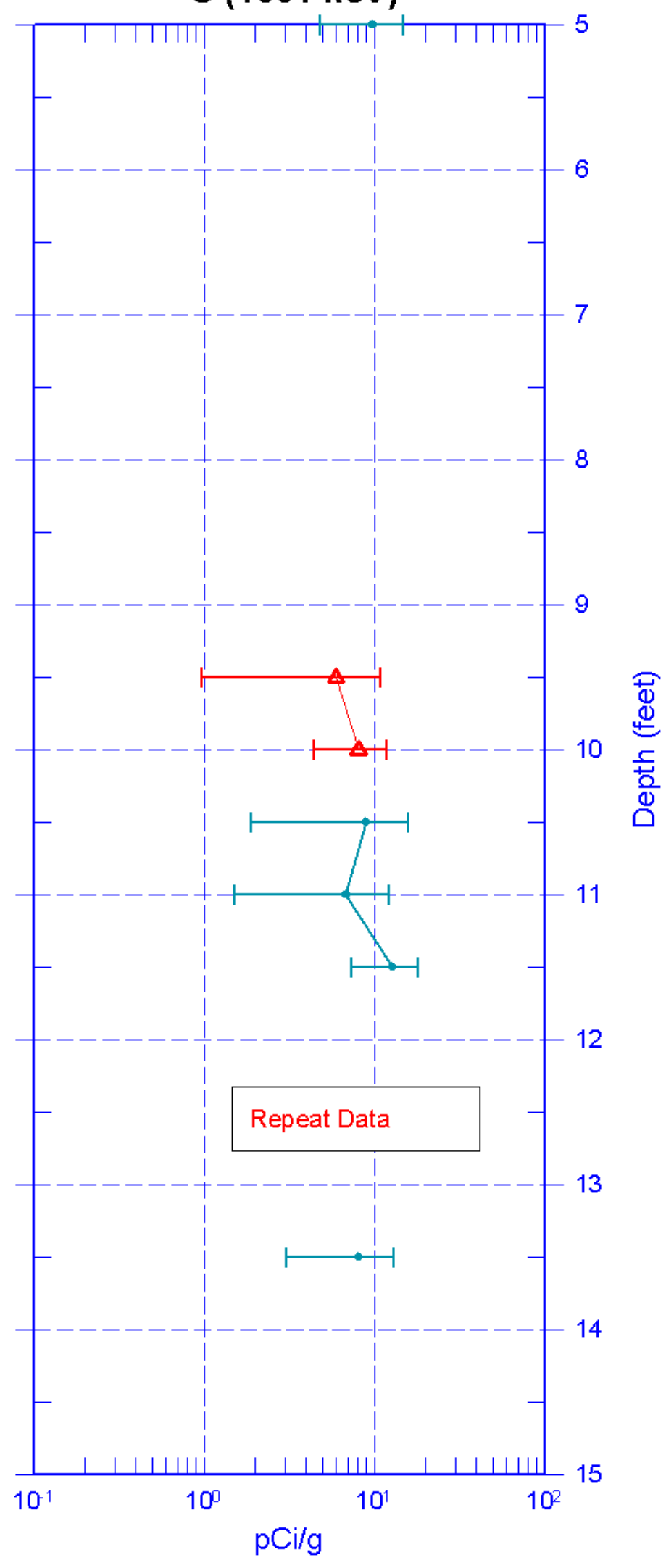

Zero Reference - Ground Surface 


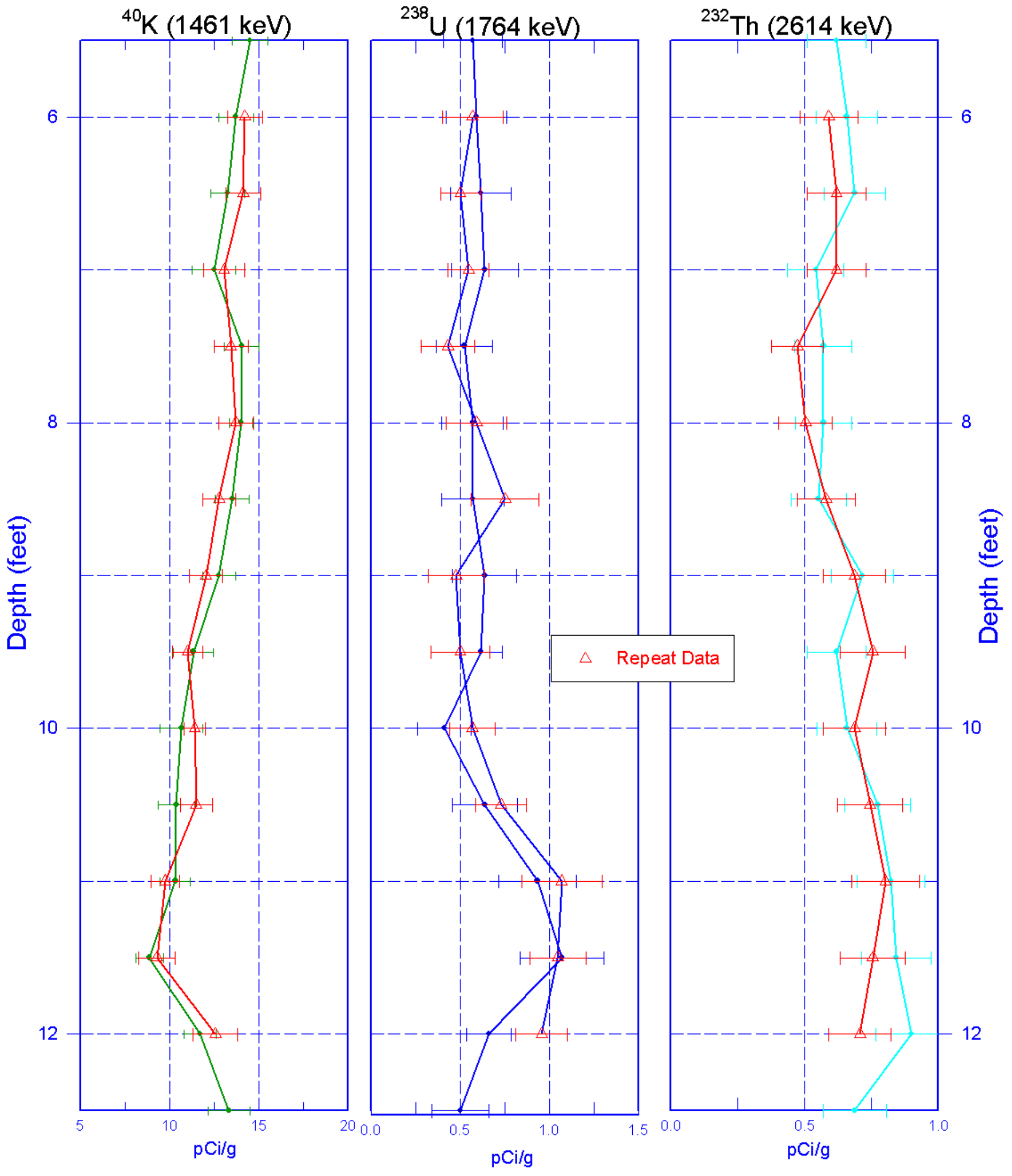




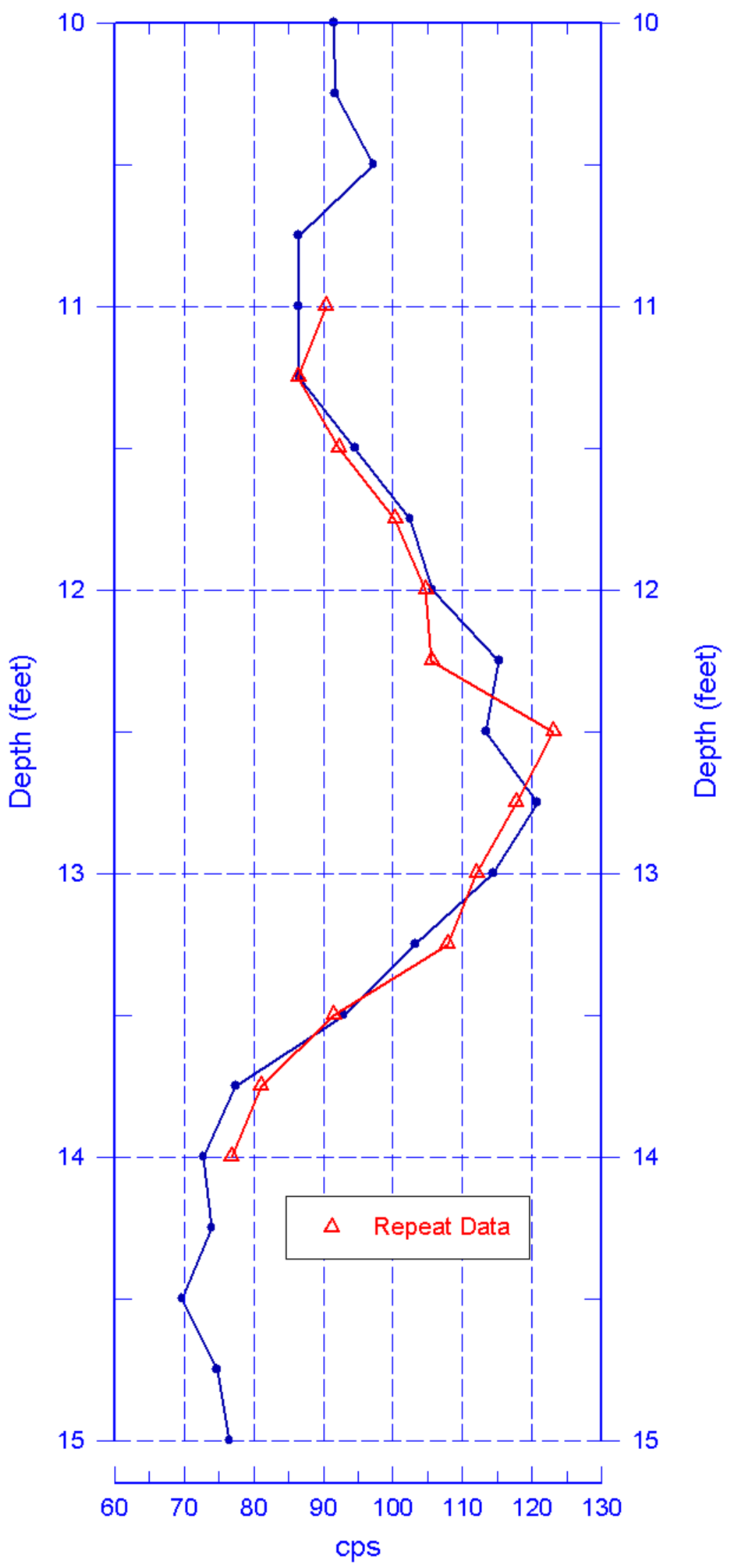


HGLP-LDR-239, Rev. 0

\section{9-2-8 (C6185) \\ Log Data Report}

Borehole Information:

\begin{tabular}{|c|c|c|c|c|c|}
\hline Borehole: & \multicolumn{1}{l|}{ Site: } & \multicolumn{2}{c|}{ GW-FF-5 } \\
\hline \multicolumn{2}{|c|}{ 399-2-8 (C6185) } & GWL $^{\mathbf{1}}$ (ft) : & 27.7 & Date: & $07 / 09 / 08$ \\
\hline Coordinates (WA St Plane) & East (m) & Drill Date & TOC $^{2}$ Elevation & Total Depth (ft) & Type \\
\hline 116090.2 & 594243.4 & $07 / 09 / 08$ & Unknown & 60 & Sonic \\
\hline
\end{tabular}

\section{Casing Information:}

\begin{tabular}{|c|c|c|c|c|c|c|}
\hline Casing Type & Stickup (ft) & $\begin{array}{c}\text { Outer } \\
\text { Diameter (in.) }\end{array}$ & $\begin{array}{c}\text { Inside } \\
\text { Diameter (in.) }\end{array}$ & Thickness (in.) & Top (ft) & Bottom (ft) \\
\hline Welded Steel & 3.7 & $75 / 8$ & $67 / 8$ & $3 / 8$ & 3.7 & 57.0 \\
\hline
\end{tabular}

\section{Borehole Notes:}

Casing data, and total depth were reported by the site geologist. Logging engineer measured depth to water with an e-tape. Casing diameters were measured using a steel tape and rounded to the nearest 1/16-in. The zero reference is the ground surface.

Logging Equipment Information:

\begin{tabular}{|c|c|c|c|c|}
\hline Logging System: & \multicolumn{2}{|c|}{ Gamma $4 \mathrm{~L}$} & $\begin{array}{l}\text { Type: } \\
\text { Serial No.: }\end{array}$ & $\begin{array}{l}\text { 60\% HPGe SGLS } \\
\text { 47TP32211A }\end{array}$ \\
\hline Effective Calibration Date: & $12 / 31 / 07$ & \multirow{2}{*}{$\begin{array}{l}\text { Calibration Reference: } \\
\text { Logging Procedure: }\end{array}$} & \multicolumn{2}{|c|}{ HGLP-CC-027 } \\
\hline & & & \multicolumn{2}{|c|}{ HGLP-MAN-002, Rev. 0} \\
\hline Logging System: & \multicolumn{2}{|c|}{ Gamma $4 \mathrm{H}$} & $\begin{array}{l}\text { Type: } \\
\text { Serial No.: }\end{array}$ & $\begin{array}{l}\text { NMLS } \\
\text { H310700352 }\end{array}$ \\
\hline Effective Calibration Date: & $11 / 06 / 07$ & Calibration Reference: & \multicolumn{2}{|c|}{ HGLP-CC-021 } \\
\hline & & Logging Procedure: & \multicolumn{2}{|c|}{ HGLP-MAN-002, Rev. 0} \\
\hline
\end{tabular}

\section{Spectral Gamma Logging System (SGLS) Log Run Information:}

\begin{tabular}{|l|c|c|}
\hline Log Run & 1 & 2 Repeat \\
\hline Date & $07 / 08 / 08$ & $07 / 08 / 08$ \\
\hline Logging Engineer & McClellan & McClellan \\
\hline Start Depth (ft) & 57.0 & 42.0 \\
\hline Finish Depth (ft) & 0.0 & 36.0 \\
\hline Count Time (sec) & 200 & 200 \\
\hline Live/Real & $\mathrm{R}$ & $\mathrm{R}$ \\
\hline Shield (Y/N) & $\mathrm{N}$ & $\mathrm{N}$ \\
\hline MSA Interval (ft) & 0.5 & 0.5 \\
\hline Log Speed (ft/min) & $\mathrm{N} / \mathrm{A}$ & $\mathrm{N} / \mathrm{A}$ \\
\hline Pre-Verification & DL581CAB & DL581CAB \\
\hline Start File & DL581000 & DL581115 \\
\hline Finish File & DL581114 & DL581127 \\
\hline Post-Verification & DL581CAA & DL581CAA \\
\hline
\end{tabular}




\begin{tabular}{|l|c|c|}
\hline Log Run & $\mathbf{1}$ & 2 Repeat \\
\hline Depth Return Error (in.) & 0 & 0 \\
\hline Comments & $\begin{array}{c}\text { No fine gain } \\
\text { adjustment } \\
\text { made. }\end{array}$ & $\begin{array}{c}\text { No fine gain } \\
\text { adjustment } \\
\text { made. }\end{array}$ \\
\hline
\end{tabular}

Neutron Moisture Logging System (NMLS) Log Run Information:

\begin{tabular}{|l|c|c|}
\hline Log Run & 3 & 4 Repeat \\
\hline Date & $07 / 09 / 08$ & $07 / 09 / 08$ \\
\hline Logging Engineer & Pearson & Pearson \\
\hline Start Depth (ft) & 0.0 & 27.75 \\
\hline Finish Depth (ft) & 27.75 & 24.0 \\
\hline Count Time (sec) & 15 & 15 \\
\hline Live/Real & $\mathrm{R}$ & $\mathrm{R}$ \\
\hline Shield (Y/N) & $\mathrm{N}$ & $\mathrm{N}$ \\
\hline MSA Interval (ft) & 0.25 & 0.25 \\
\hline Log Speed (ft/min) & N/A & N/A \\
\hline Pre-Verification & DHG52CAB & DHG52CAB \\
\hline Start File & DHG52000 & DHG52112 \\
\hline Finish File & DHG52111 & DHG52127 \\
\hline Post-Verification & DHG52CAA & DHG52CAA \\
\hline Depth Return Error (in.) & N/A & 0 \\
\hline Comments & None & None \\
\hline
\end{tabular}

\section{Logging Operation Notes:}

Data were collected using Gamma 4, HO 68B-3573. SGLS pre- and post-survey verification measurements were acquired in the Amersham KUTh-115 field verifier. Maximum logging depth achieved was $57.6 \mathrm{ft}$ before the sonde un-weighted. A centralizer was installed on the sonde. NMLS pre- and post-survey verification measurements were acquired in the standard field verifier.

Analysis Notes:

\begin{tabular}{|l|l|l|l|l|l|}
\hline Analyst: & Henwood & Date: & $08 / 27 / 08$ & Reference: & GJO-HGLP 1.6.3, Rev. 0 \\
\hline
\end{tabular}

SGLS pre- and post-survey verification spectra met the acceptance criteria for the established system. NMLS preand post-survey verification spectra met the acceptance criteria for the established system.

A casing correction for a 3/8-in. thick casing was applied from ground surface to $57 \mathrm{ft}$. A water correction was also applied during analysis from $27.7 \mathrm{ft}$ to total logged depth of borehole.

SGLS spectra were processed in batch mode using APTEC SUPERVISOR to identify individual peaks and count rates. Concentrations were calculated using an EXCEL template identified as G4LDec07.xls using an efficiency function and corrections for casing, dead time and water as determined by annual calibrations. NMLS spectra were processed in batch mode in APTEC SUPERVISOR to identify counts. Count rates were calculated using an EXCEL template identified as G4HNov07.xls. There is no direct calibration data available for a 6 7/8-in. inner diameter borehole casing. Therefore, moisture data are reported in counts per second (cps).

\section{Results and Interpretations:}

U-238 (Pa-234m) was detected at 19 and $19.5 \mathrm{ft}$ at a maximum concentration of $10 \mathrm{pCi} / \mathrm{g}$. Gamma energy spectra at these points show definite photopeaks for the $1001.03 \mathrm{keV}$ gamma ray associated with Pa-234m. A corroborating 
HGLP-LDR-239, Rev. 0

photopeak for Pa-234m at $766.36 \mathrm{keV}$ is not indicated. However, the detection of the $1001 \mathrm{keV}$ gamma ray of $\mathrm{Pa}-234 \mathrm{~m}$ is near its MDL and the gamma ray yield from the $766 \mathrm{keV}$ peak is approximately one-third of the 1001 gamma ray. Therefore, it is expected that the $766 \mathrm{keV}$ gamma ray would not be detected at low concentrations of $\mathrm{Pa}-234 \mathrm{~m}$. Spectra from other detections of Cs-137 and U-238 were individually inspected and it was determined the detections were statistical fluctuations associated with the processing software.

A relatively high concentration of Th-232 is indicated at approximately $39 \mathrm{ft}$. The significance of this "anomaly" is unknown until compared with other boreholes in the vicinity to determine the spatial continuity. The KUT repeat plots indicate good repeatability.

The moisture repeat plot indicates good repeatability.

\section{List of Log Plots:}

Depth Reference is ground surface

Manmade Radionuclides

Natural Gamma Logs

Combination Plot

Total Gamma \& Moisture

Repeat Section of Natural Gamma Logs

Repeat of Moisture

${ }^{1} \mathrm{GWL}$ - groundwater level

${ }^{2}$ TOC - top of casing 
toller 399-2-8 (C6185)

Hanford Office

Man-Made Radionuclides

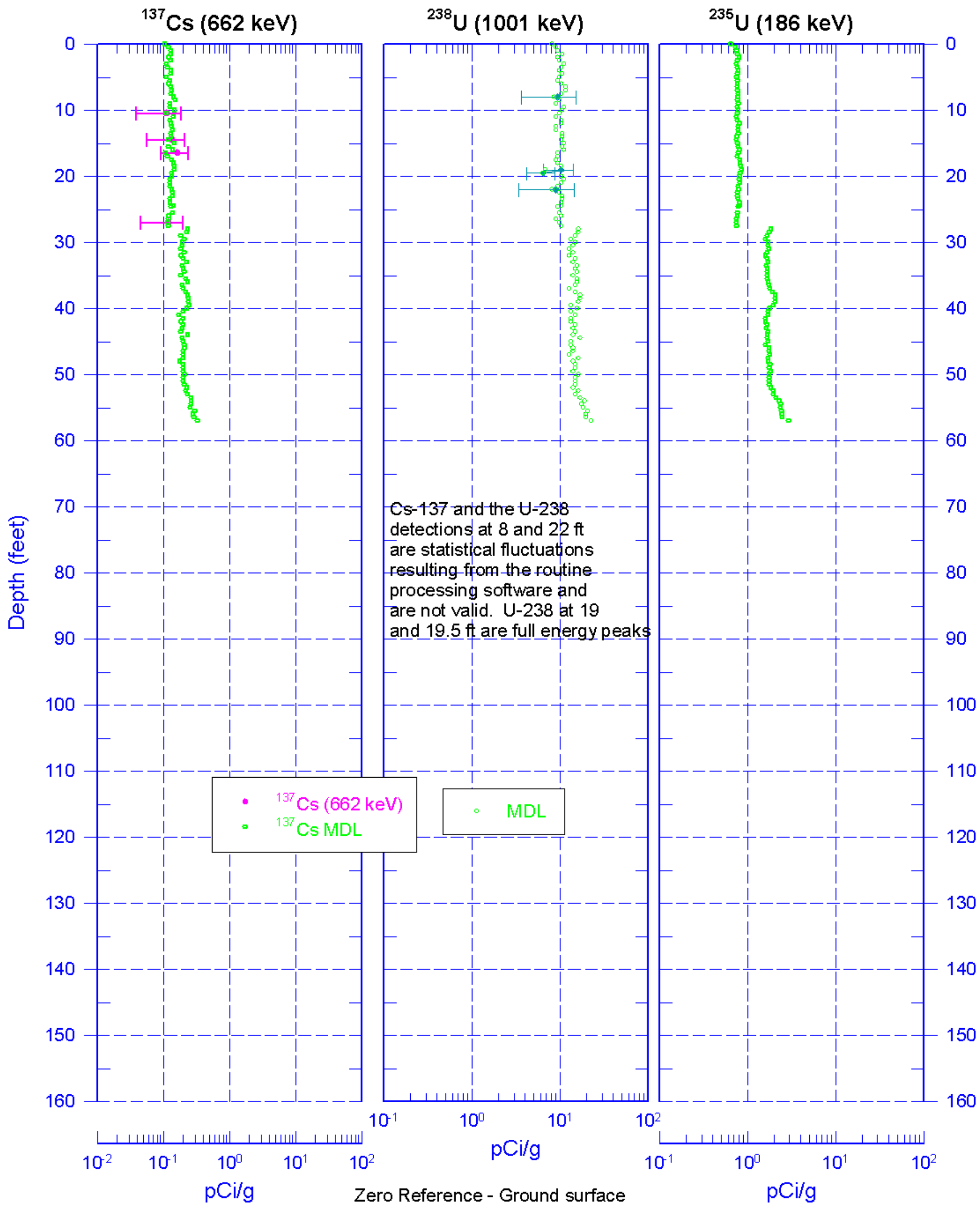


S

toller $\quad 399-2-8$ (C6185)

Hanford Office

\section{Natural Gamma Logs}
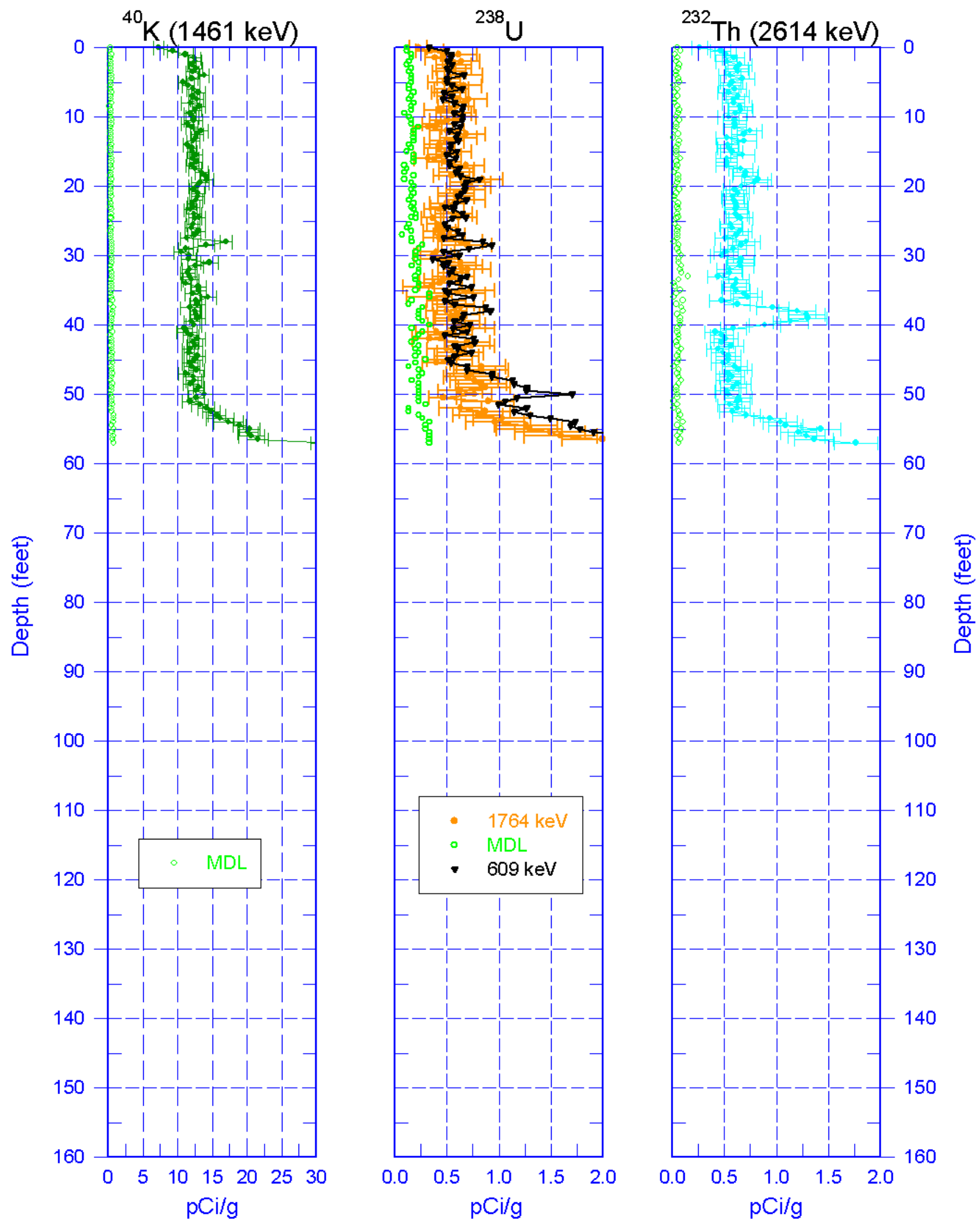

Zero Reference - Ground surface 
Hanford Office

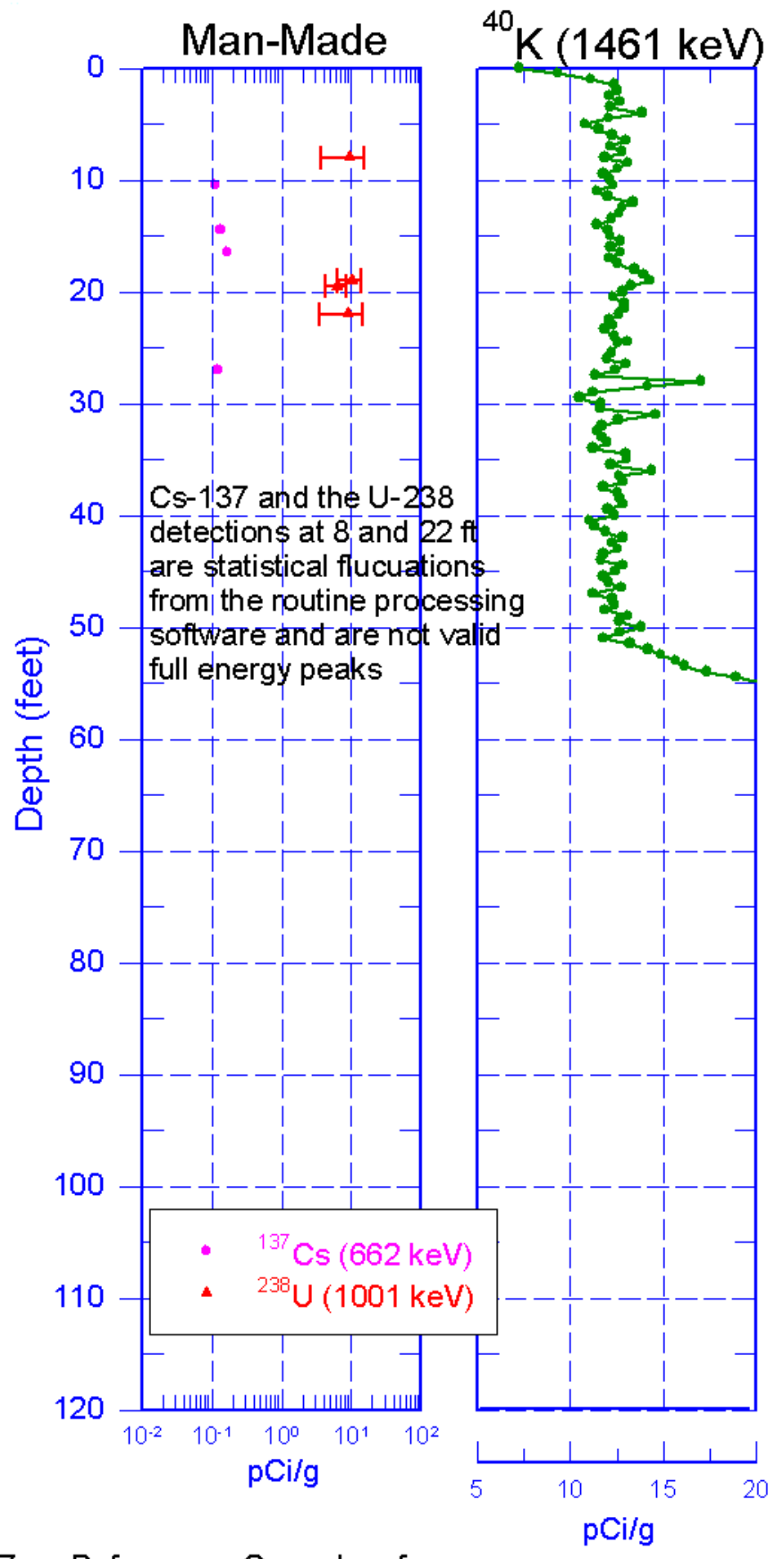

Zero Reference - Ground surface

\section{9-2-8 (C6185) Combination Plot}
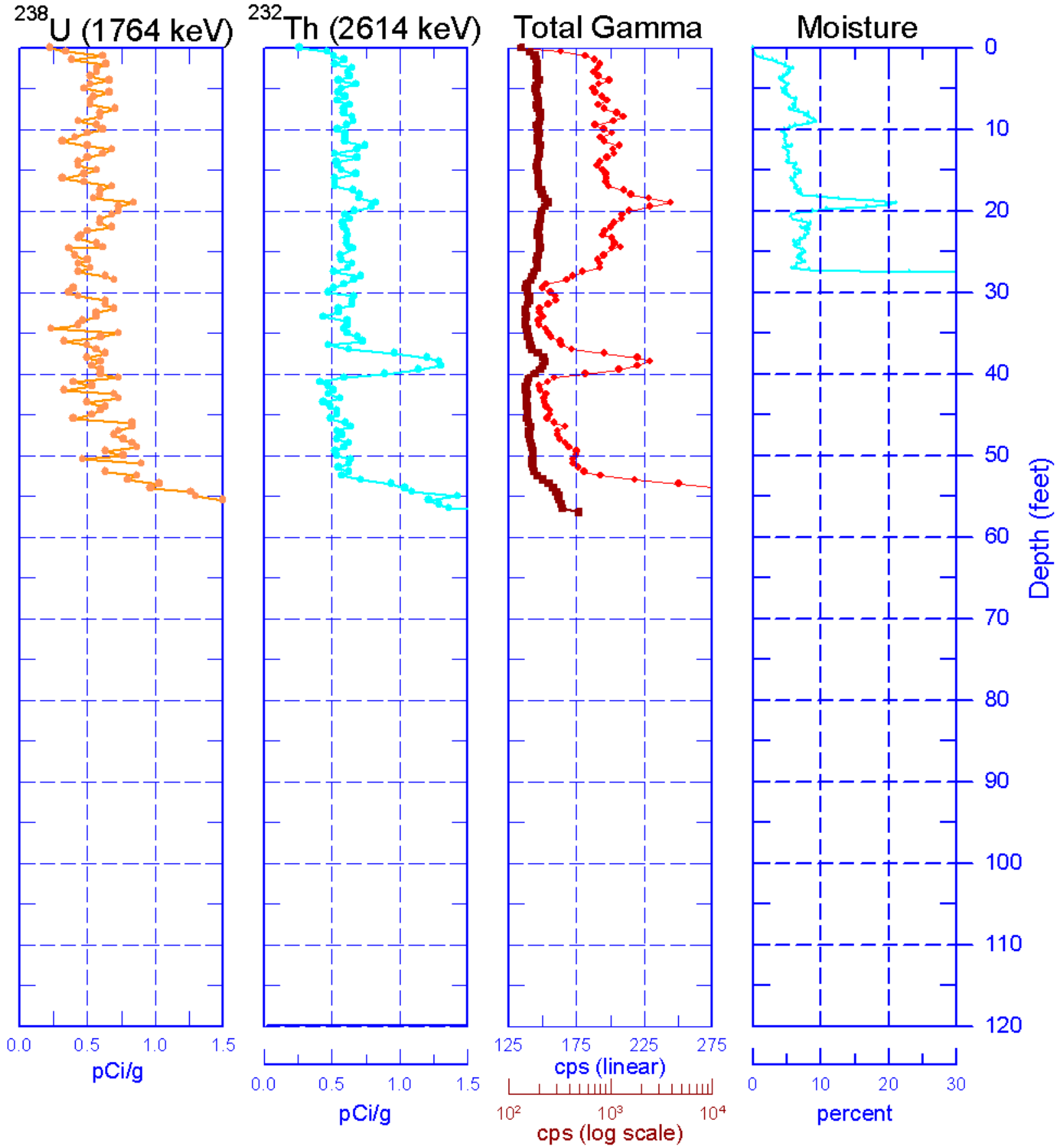

G.27 
toller $\quad 399-2-8(\mathbf{C 6 1 8 5 )}$

Hanford Office

\section{Total Gamma \& Moisture}
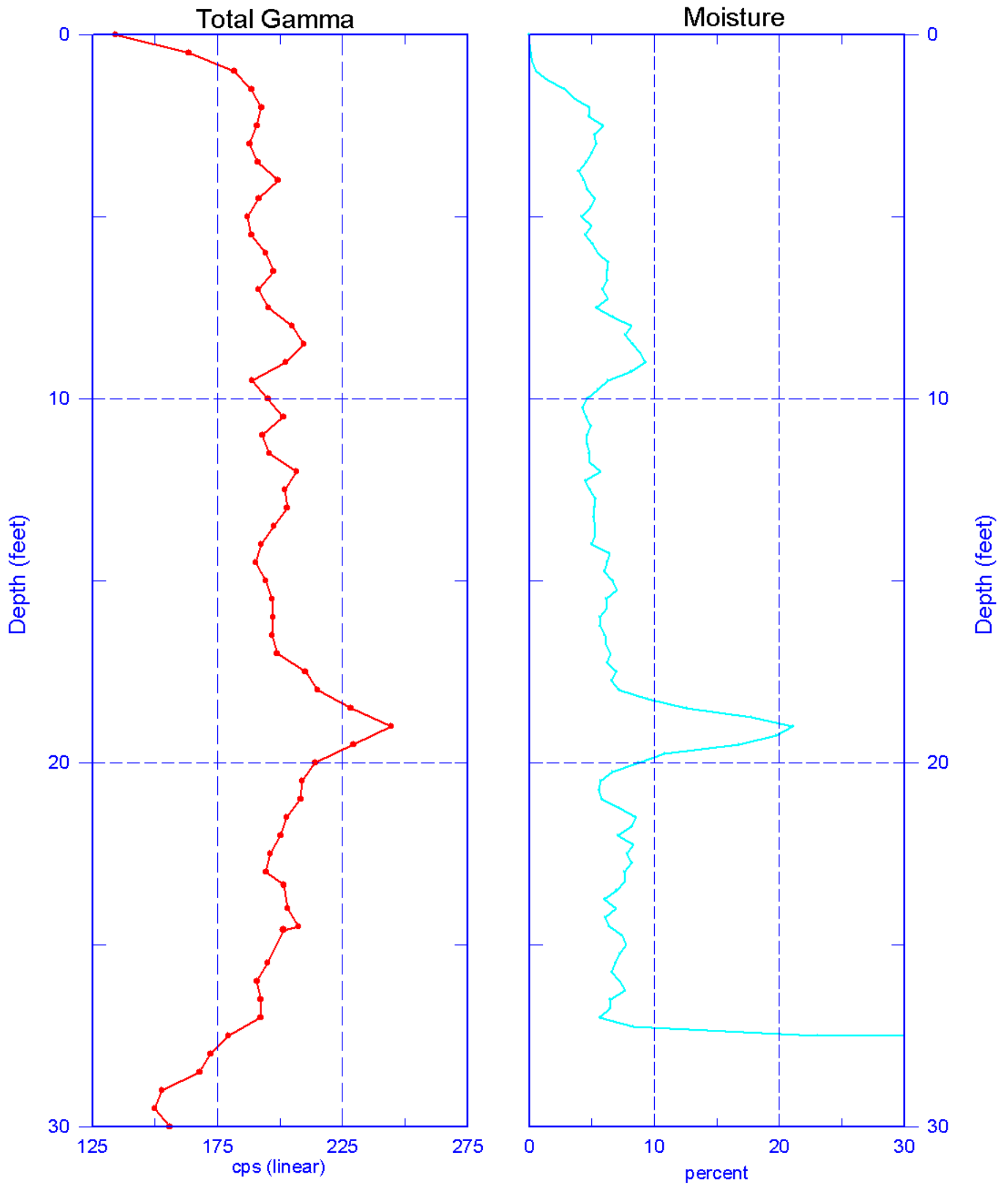

Reference - Ground Surface 


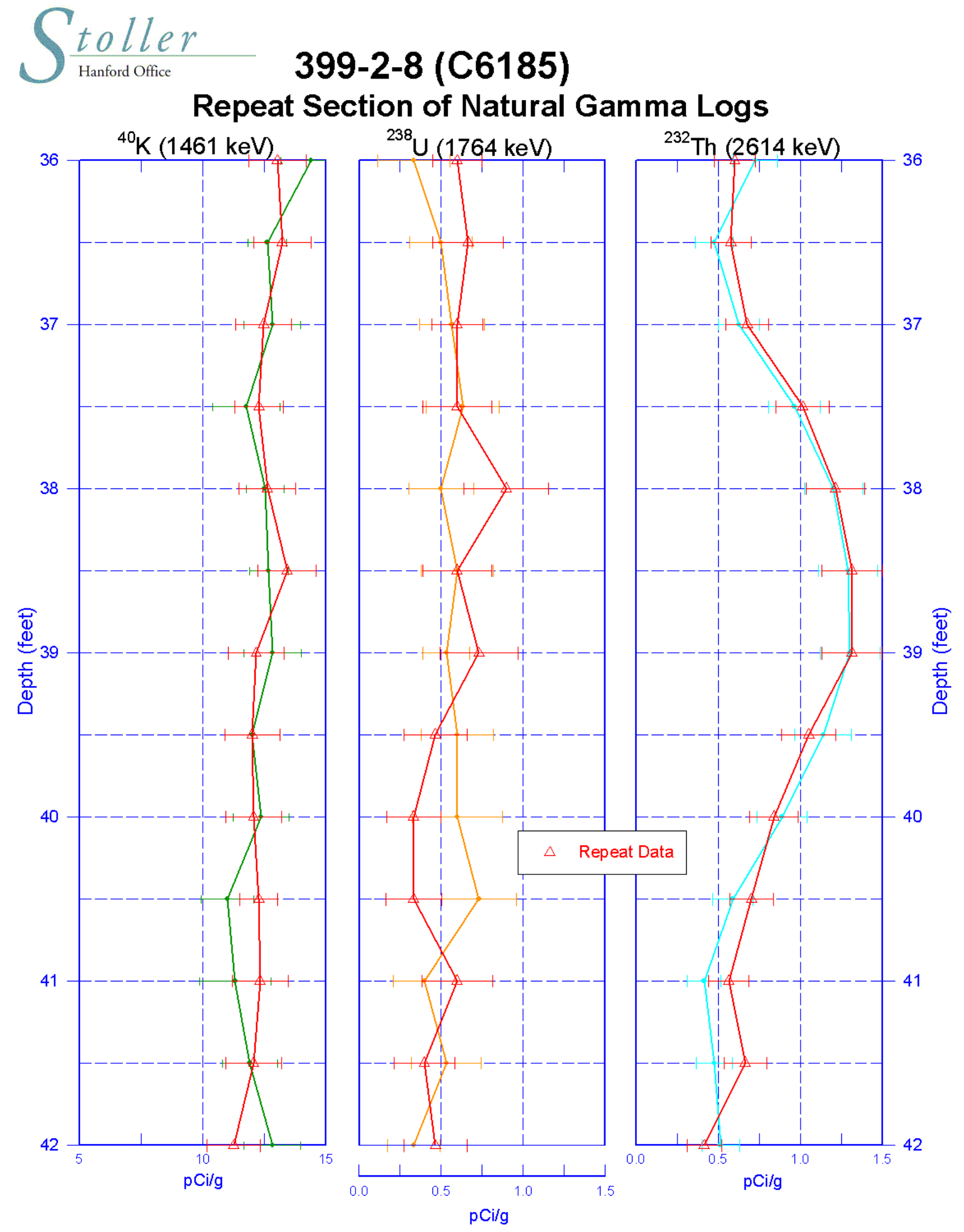

Zero Reference - Ground surface 


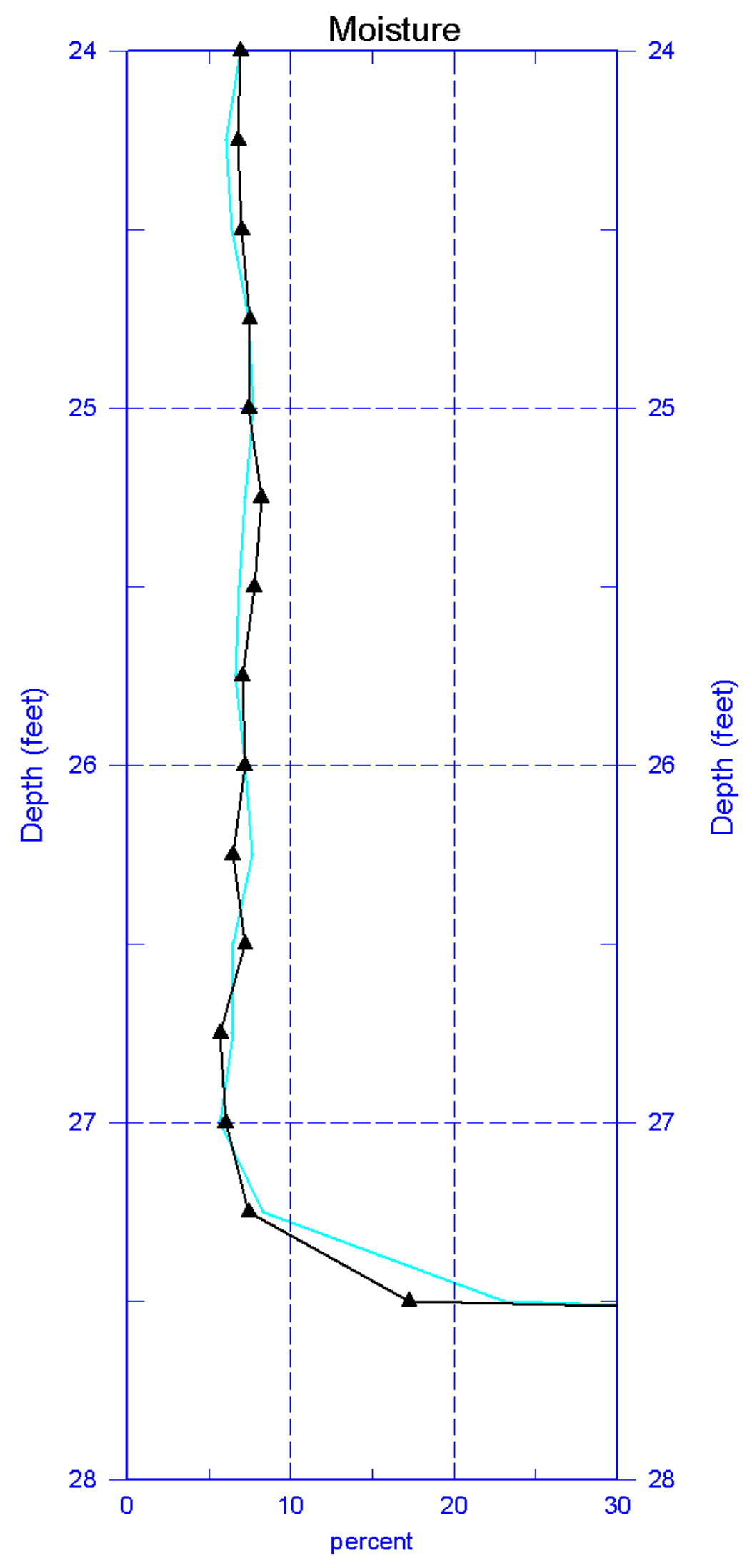


HGLP-LDR-230, Rev. 0

\section{9-2-9 (C6186) \\ Log Data Report}

Borehole Information:

\begin{tabular}{|c|c|c|c|c|c|}
\hline \multicolumn{3}{|c|}{ 399-2-9 (C6186) } & Site: & \multicolumn{2}{|l|}{ 300-FF-5 } \\
\hline \multicolumn{2}{|c|}{ Coordinates (WA St Plane) } & $\mathbf{G W L}^{1}$ (f & 34.1 & GWL Date: & $05 / 14 / 08$ \\
\hline North (m) & East (m) & Drill Date & TOC ${ }^{2}$ Elevation & Total Depth (ft) & Type \\
\hline Unknown & Unknown & $5 / 13 / 08$ & Unknown & 65.0 & Sonic \\
\hline
\end{tabular}

\section{Casing Information:}

\begin{tabular}{|c|c|c|c|c|c|c|}
\hline Casing Type & Stickup (ft) & $\begin{array}{c}\text { Outer } \\
\text { Diameter (in.) }\end{array}$ & $\begin{array}{c}\text { Inside } \\
\text { Diameter (in.) }\end{array}$ & Thickness (in.) & Top (ft) & Bottom (ft) \\
\hline Threaded Steel & 2.85 & $75 / 8$ & $67 / 8$ & $3 / 8$ & -2.85 & 62.8 \\
\hline
\end{tabular}

\section{Borehole Notes:}

Well site geologist reported depth to bottom, depth to water and depth of casing. Logging engineer measured casing using a steel tape and rounding to the nearest $1 / 16$-in. The zero reference is the ground surface for log data.

\section{Logging Equipment Information:}

\begin{tabular}{|l|l|l|l|l|}
\hline \multirow{2}{*}{ Logging System: } & \multirow{2}{*}{ Gamma 1 N } & $\begin{array}{l}\text { Type: } \\
\text { Serial No.: }\end{array}$ & $\begin{array}{l}\text { 60\% HPGe SGLS } \\
\text { 45TP22010A }\end{array}$ \\
\hline Effective Calibration Date: & $03 / 28 / 08$ & $\begin{array}{l}\text { Calibration Reference: } \\
\text { Logging Procedure: }\end{array}$ & HGLP-CC-031 \\
\cline { 4 - 4 } & & HGLP-MAN-002, Rev. 0 \\
\hline
\end{tabular}

\begin{tabular}{|l|l|l|l|l|}
\hline Logging System: & \multirow{2}{*}{ Gamma 1 M } & $\begin{array}{l}\text { Type: } \\
\text { Serial No.: }\end{array}$ & $\begin{array}{l}\text { NMLS } \\
\text { H340207279 }\end{array}$ \\
\hline Effective Calibration Date: & $05 / 06 / 08$ & Calibration Reference: & HGLP-CC-032 \\
\hline \multicolumn{2}{|l|}{} & Logging Procedure: & HGLP-MAN-002, Rev. 0 \\
\hline
\end{tabular}

\section{Spectral Gamma Logging System (SGLS) Log Run Information:}

\begin{tabular}{|l|c|c|c|}
\hline Log Run & $\mathbf{1}$ & $\mathbf{2}$ & 3 Repeat \\
\hline Date & $05 / 14 / 08$ & $05 / 15 / 08$ & $05 / 16 / 08$ \\
\hline Logging Engineer & McClellan & McClellan & McClellan \\
\hline Start Depth (ft) & 0.0 & 50.0 & 63.5 \\
\hline Finish Depth (ft) & 51.0 & 63.5 & 57.0 \\
\hline Count Time (sec) & 200 & 200 & 200 \\
\hline Live/Real & $\mathrm{R}$ & $\mathrm{R}$ & $\mathrm{R}$ \\
\hline Shield (Y/N) & $\mathrm{N}$ & $\mathrm{N}$ & $\mathrm{N}$ \\
\hline MSA Interval (ft) & 0.5 & 0.5 & 0.5 \\
\hline Log Speed (ft/min) & N/A & N/A & N/A \\
\hline Pre-Verification & AN071CAB & AN072CAB & AN073CAB \\
\hline Start File & AN071000 & AN072000 & AN073000 \\
\hline Finish File & AN071102 & AN072027 & AN073013 \\
\hline Post-Verification & AN071CAA & AN072CAA & AN073CAA \\
\hline Depth Return Error (in.) & 0.5 high & 0 & 0 \\
\hline Comments & $\begin{array}{c}\text { Fine gain } \\
\text { adjustment } \\
\text { made before }- \\
086 .\end{array}$ & $\begin{array}{c}\text { Fine gain } \\
\text { adjustment }\end{array}$ & $\begin{array}{c}\text { No fine gain } \\
\text { adjustment } \\
\text { made. Repeat } \\
\text { section. }\end{array}$ \\
\hline
\end{tabular}




\section{Neutron Moisture Logging System (NMLS) Log Run Information:}

\begin{tabular}{|l|c|c|}
\hline Log Run & $\mathbf{4}$ & $\mathbf{5}$ \\
\hline Date & $05 / 16 / 08$ & $05 / 16 / 08$ \\
\hline Logging Engineer & McClellan & McClellan \\
\hline Start Depth (ft) & 0.0 & 30.75 \\
\hline Finish Depth (ft) & 30.75 & 27.0 \\
\hline Count Time (sec) & 15 & 15 \\
\hline Live/Real & $\mathrm{R}$ & $\mathrm{R}$ \\
\hline Shield (Y/N) & $\mathrm{N}$ & $\mathrm{N}$ \\
\hline MSA Interval (ft) & 0.25 & 0.25 \\
\hline Log Speed (ft/min) & N/A & N/A \\
\hline Pre-Verification & AM001CAB & AM001CAB \\
\hline Start File & AM001000 & AM001124 \\
\hline Finish File & AM001123 & AM001139 \\
\hline Post-Verification & AM001CAA & AM001CAA \\
\hline Depth Return Error (in.) & N/A & 0 \\
\hline Comments & None & Repeat section. \\
\hline
\end{tabular}

\section{Logging Operation Notes:}

Data were collected using Gamma 1, HO 68B-3574. SGLS pre- and post-survey verification measurements were acquired in the Amersham KUTh-118 field verifier. NMLS pre- and post-survey verification measurements were acquired in the standard field verifier. A centralizer was installed on the sonde prior to logging. Maximum logging depth achieved was $63.5 \mathrm{ft}$ before sonde un-weighted.

\section{Analysis Notes:}

\begin{tabular}{|l|l|l|l|l|l|}
\hline Analyst: & LEGLER & Date: & $6 / 09 / 08$ & Reference: & GJO-HGLP 1.6.3, Rev. 0 \\
\hline
\end{tabular}

SGLS pre- and post-survey verification spectra met the acceptance criteria for the established system. NMLS preand post-survey verification spectra met the acceptance criteria for the established system, but the verification spectrum file AM001CAB had a measurement above the upper control limit for count rate (cps).

A casing correction for a 3/8-in. thick casing was applied during analysis for depths up to $62.8 \mathrm{ft}$. where casing ends, leaving $0.7 \mathrm{ft}$ of open hole uncorrected. A water correction was also applied during analysis from $33.5 \mathrm{ft}$ to total logged depth of borehole.

SGLS spectra were processed in batch mode in APTEC SUPERVISOR to identify individual peaks and count rates. Concentrations were calculated using an EXCEL template identified as G1NMay08.xls using an efficiency function and corrections for casing, dead time and water as determined by annual calibrations.

NMLS spectra were processed in batch mode in APTEC SUPERVISOR to identify counts. Count rates were calculated using an EXCEL template identified as G1MMay08.xls. NMLS data are presented in counts per second (cps), because no calibration data is available for a 6 7/8-in. inner diameter borehole casing.

\section{Results and Interpretations:}

$\mathrm{U}-235$ was detected at $15 \mathrm{ft}$ and U-238 was detected at $22 \mathrm{ft}$ and $57 \mathrm{ft}$. Further inspection of the spectra at these depths indicate that these detections are statistical fluctuations associated with the processing software and are not considered valid. A plot of the manmade radionuclides for Cs-137, U-235, and U-238 are also included in this log data report.

Repeat sections of SGLS and NMLS logs showed good repeatability. 
HGLP-LDR-230, Rev. 0

The water level was adjusted in the SGLS log to 33.5 from $34.1 \mathrm{ft}$ due to a depth return error of 0.5-in high in the first log run $(0-51 \mathrm{ft})$. The water level in the borehole also appears to have increased to approximately $30 \mathrm{ft}$ based on the observation in NMLS log on 5/16/08.

SGLS data collected on 5/14/08 indicated depth to groundwater at approximately $33.5 \mathrm{ft}$. NMLS data collected on 5/16/08 indicate depth to ground water at approximately $30.75 \mathrm{ft}$. The depth to groundwater appears to fluctuate with changes in river level.

\section{List of Log Plots:}

Depth Reference is ground surface

Manmade Radionuclides

Natural Gamma Logs

Combination Plot

Total Gamma \& Dead Time

Total Gamma \& Moisture

Repeat Section of Natural Gamma Logs

Repeat Section of Moisture

${ }^{1} \mathrm{GWL}$ - groundwater level

${ }^{2}$ TOC - top of casing 

Hanford Office Manmade Radionuclides

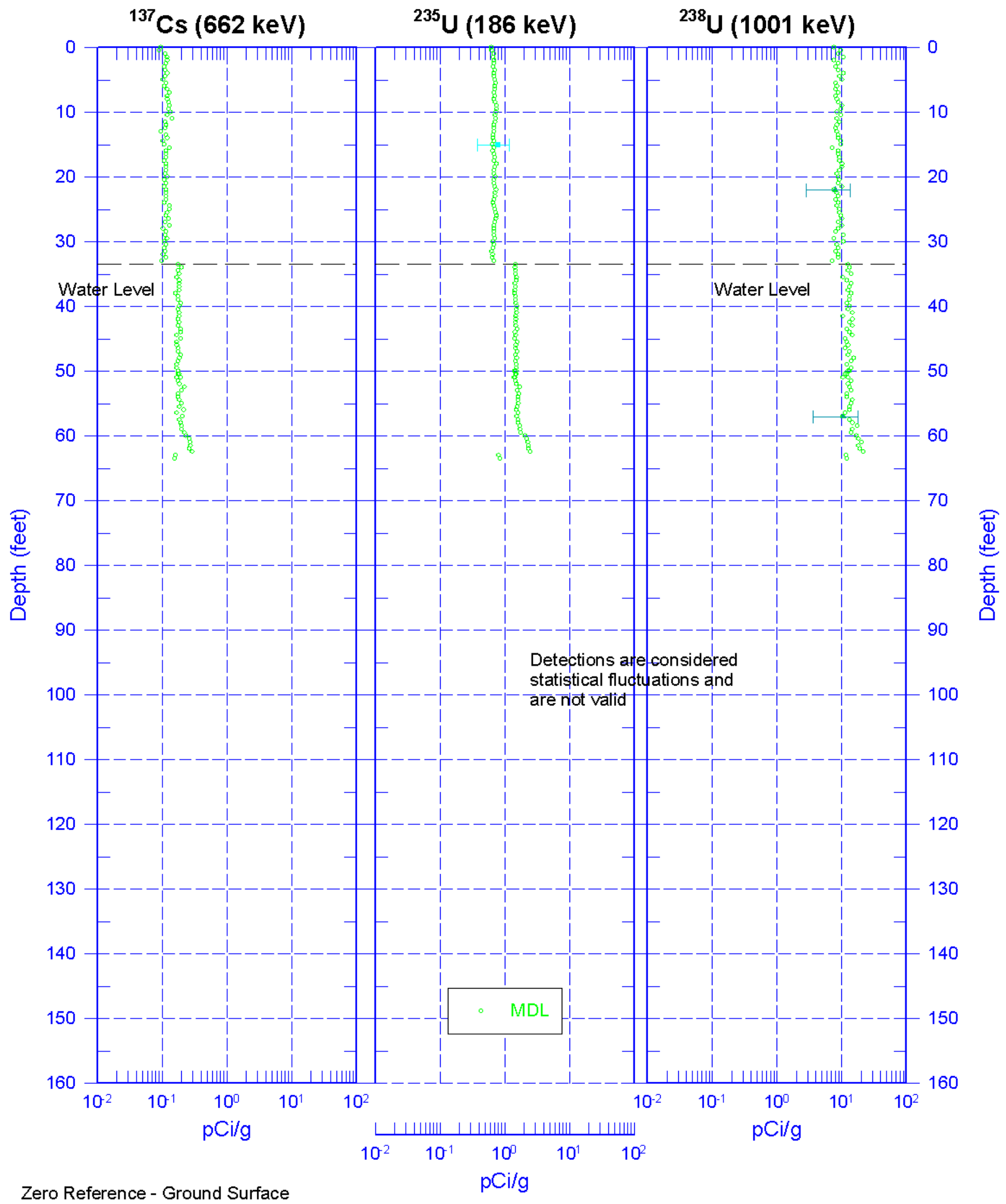

Zero Reference - Ground Surface 
$\cap$

toller 399-2-9 (C6186)

Hanford Office

\section{Natural Gamma Logs}
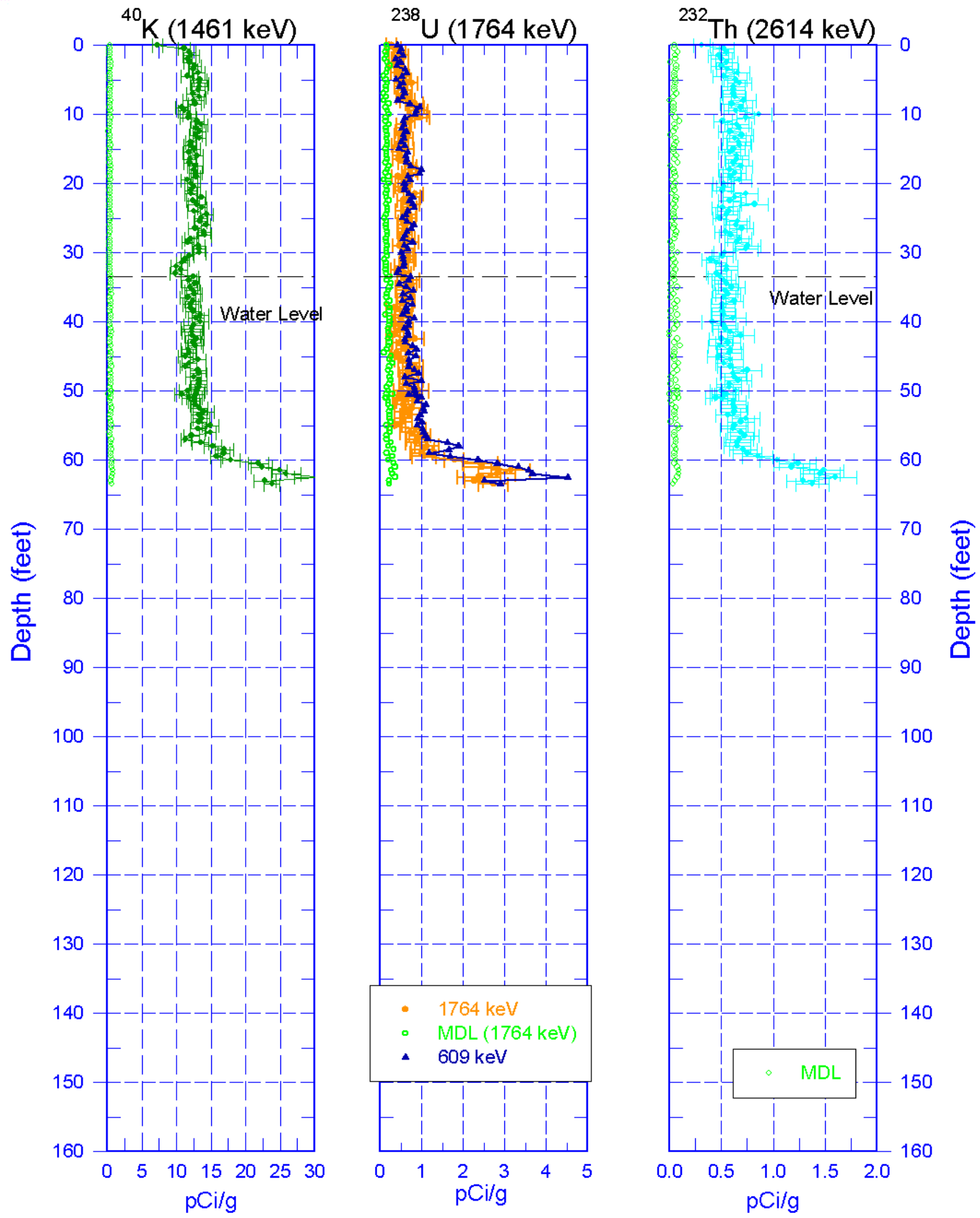

Zero Reference - Ground Surface 


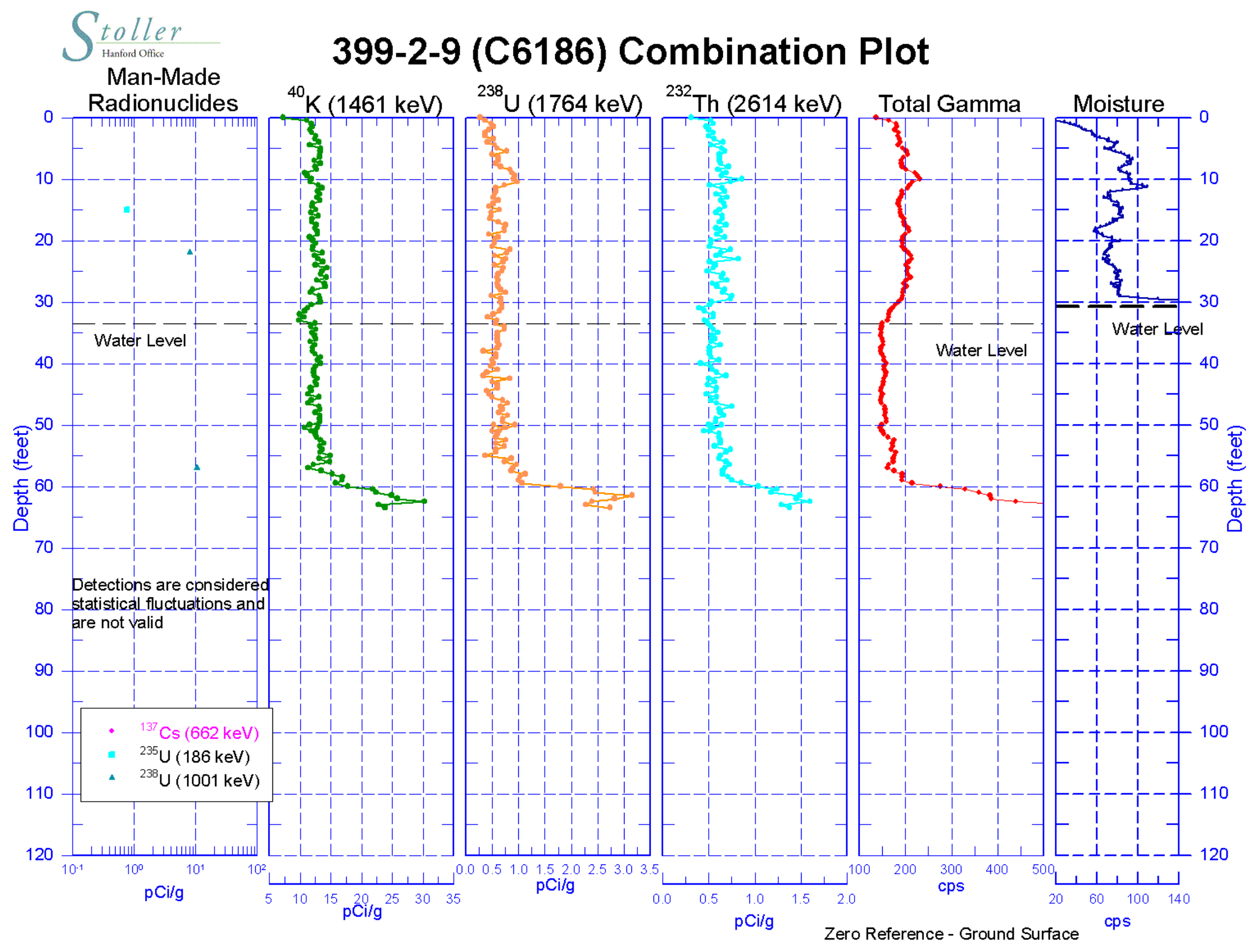



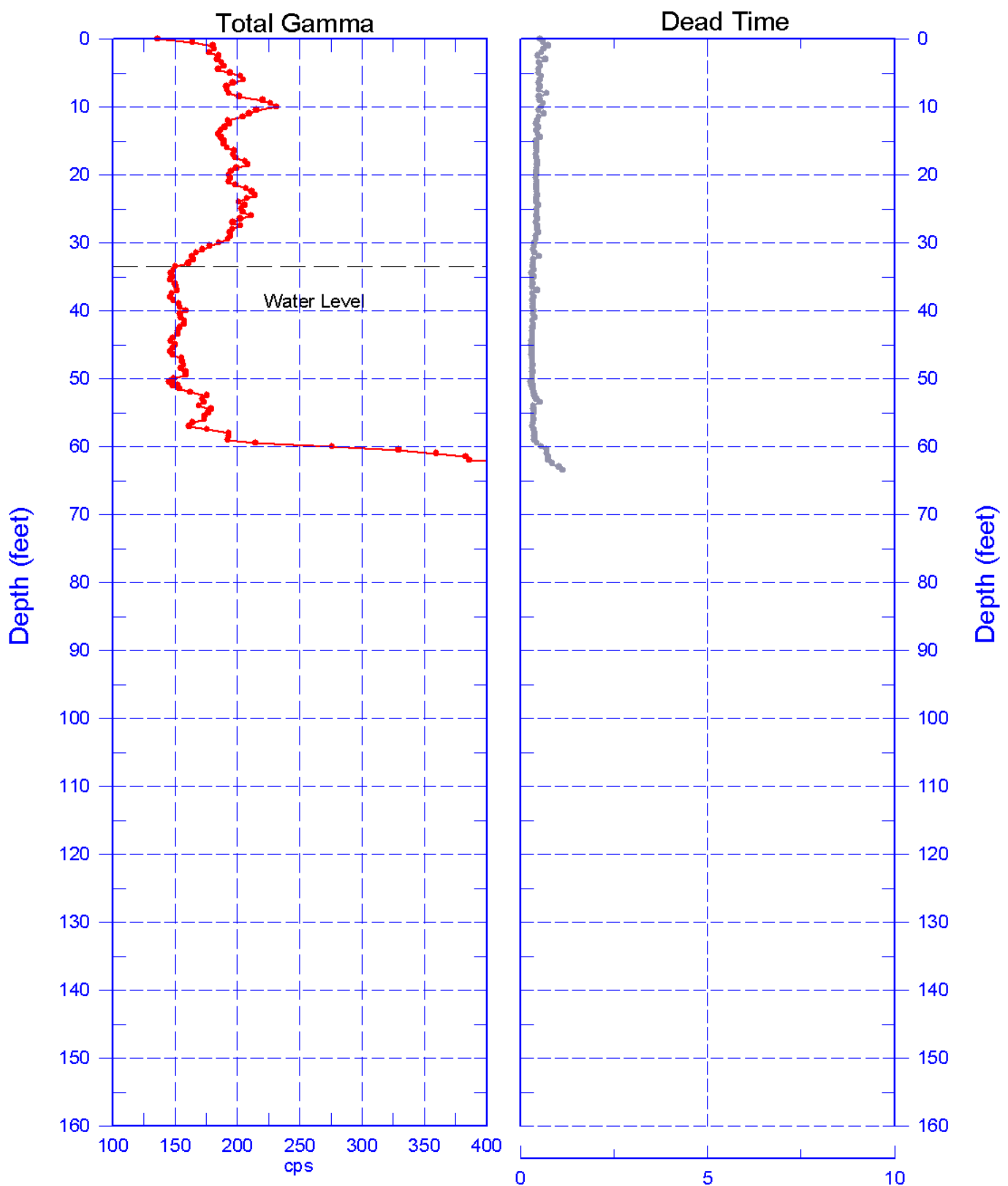


\section{Total Gamma \& Moisture}
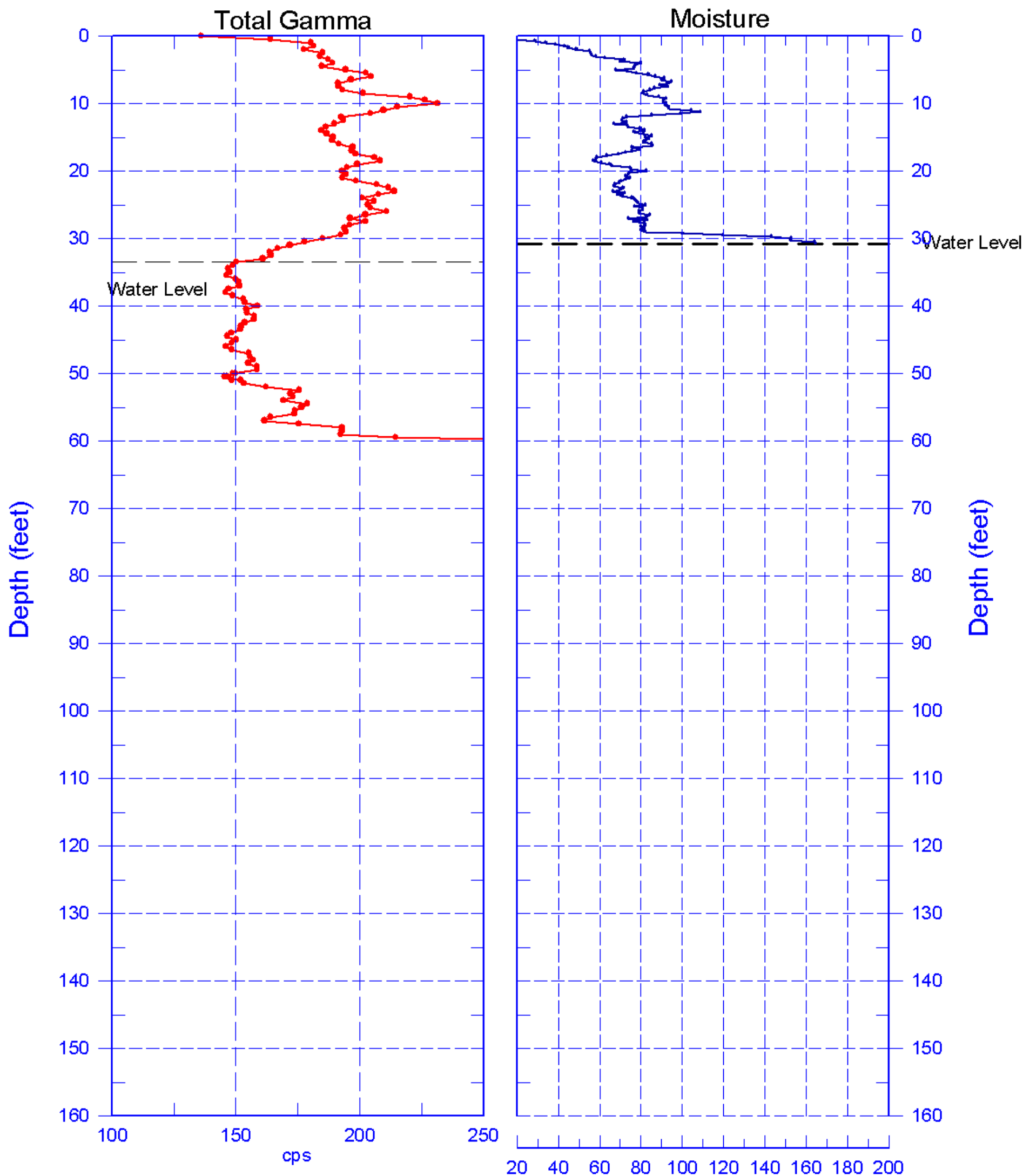

Reference - Ground Surface 
toller 399-2-9 (C6186)

Hanford Office Repeat Section of Natural Gamma Logs

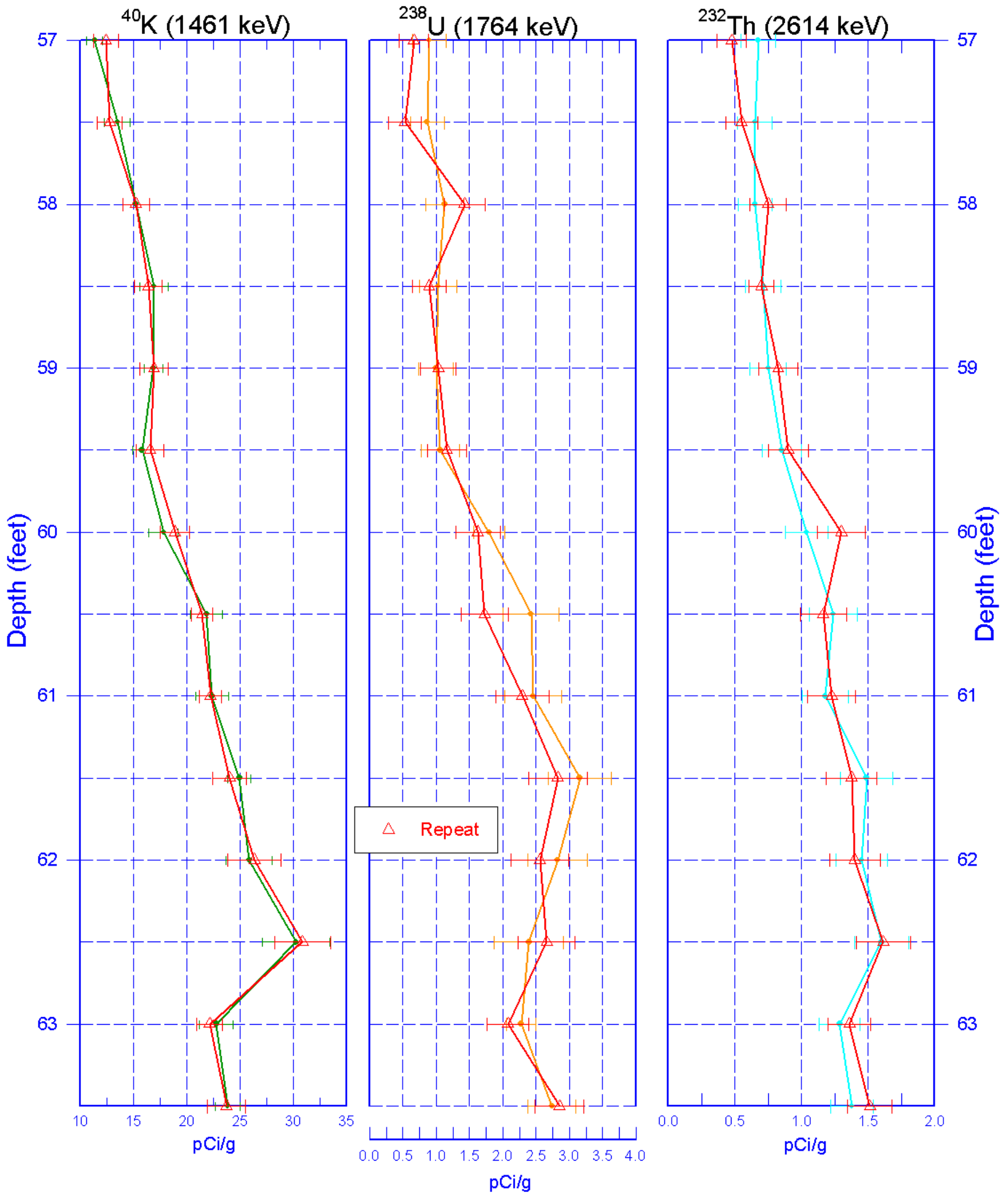




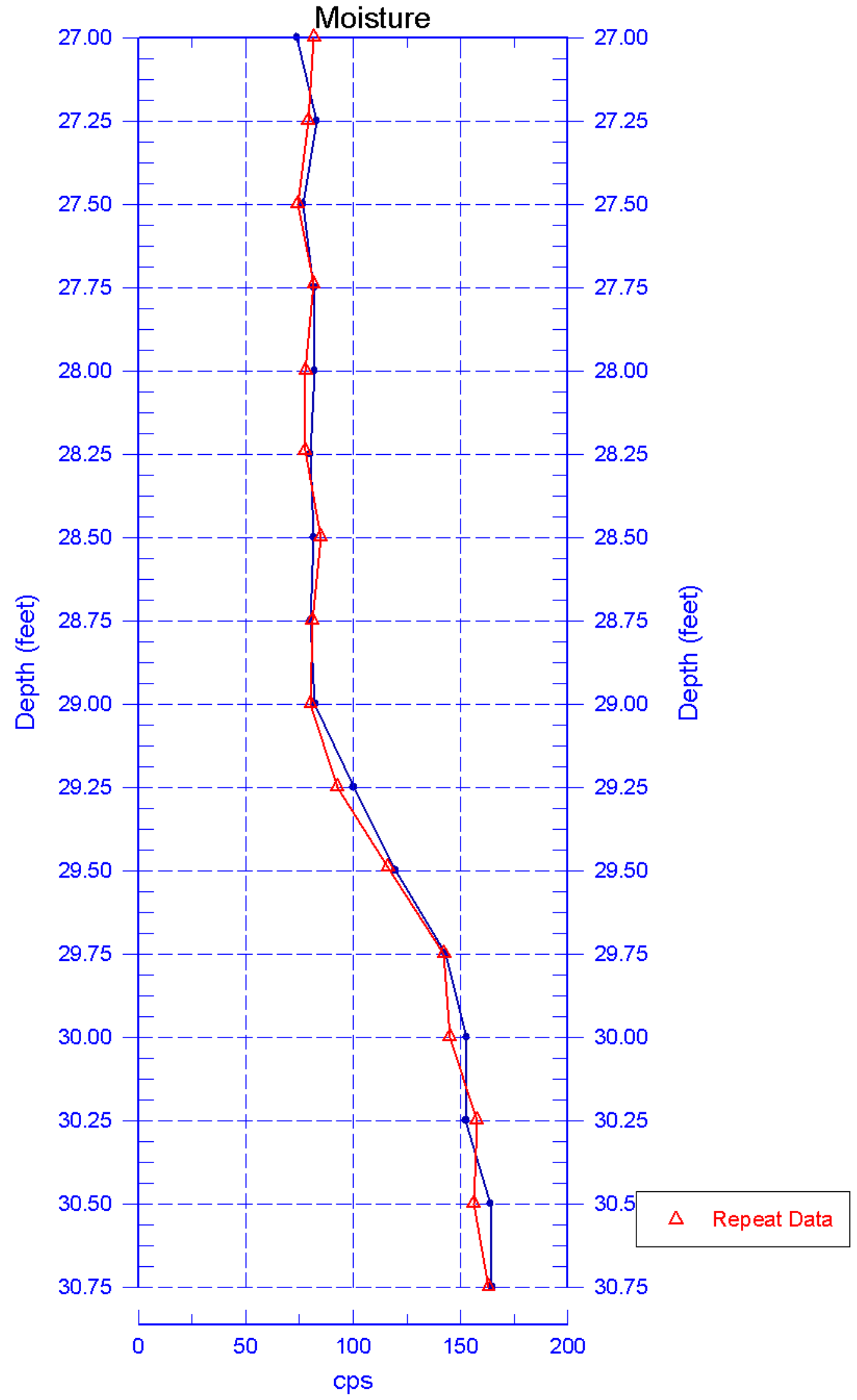


HGLP-LDR-240, Rev. 0

\section{9-2-10 (C6187) Log Data Report}

Borehole Information:

\begin{tabular}{|c|c|c|c|c|c|}
\hline Borehole: & \multicolumn{2}{|c|}{ Site: } & \multicolumn{2}{|c|}{ G00-FF-5 } \\
\hline Coordinates (WA St Plane) & GWL ${ }^{1}$ (ft) $:$ & 31.4 & \multicolumn{2}{c|}{ GWL Date: } & $07 / 18 / 08$ \\
\hline North $(\mathbf{m})$ & East $(\mathbf{m})$ & Drill Date & TOC $^{2}$ Elevation & Total Depth (ft) & Type \\
\hline 116094.5 & 594234.4 & $07 / 17 / 08$ & Unknown & 65 & Sonic \\
\hline
\end{tabular}

\section{Casing Information:}

\begin{tabular}{|c|c|c|c|c|c|c|}
\hline Casing Type & Stickup (ft) & $\begin{array}{c}\text { Outer } \\
\text { Diameter (in.) }\end{array}$ & $\begin{array}{c}\text { Inside } \\
\text { Diameter (in.) }\end{array}$ & Thickness (in.) & Top (ft) & Bottom (ft) \\
\hline Welded Steel & 0.8 & $75 / 8$ & $67 / 8$ & $3 / 8$ & 0.8 & 60.0 \\
\hline
\end{tabular}

\section{Borehole Notes:}

Casing data, and total depth were reported by the s ite Geologist. Logging engineer measured depth to water with an e-tape. Casing diameters were measured using a steel tape and rounded to the nearest 1/16-in. The zero reference is the ground surface.

\section{Logging Equipment Information:}

\begin{tabular}{|l|l|l|l|l|}
\hline Logging System: & Gamma 4 & $\begin{array}{l}\text { Type: } \\
\text { Serial No.: }\end{array}$ & $\begin{array}{l}\text { 60\% HPGe SGLS } \\
\text { 47TP32211A }\end{array}$ \\
\hline Effective Calibration Date: & $12 / 31 / 07$ & Calibration Reference: & HGLP-CC-027 \\
\hline \multicolumn{2}{|l|}{} & Logging Procedure: & HGLP-MAN-002, Rev. 0 \\
\hline
\end{tabular}

\begin{tabular}{|l|l|l|l|l|}
\hline Logging System: & \multirow{2}{*}{ Gamma $4 \mathrm{H}$} & $\begin{array}{l}\text { Type: } \\
\text { Serial No.: }\end{array}$ & $\begin{array}{l}\text { NMLS } \\
\text { H310700352 }\end{array}$ \\
\hline Effective Calibration Date: & $11 / 06 / 07$ & Calibration Reference: & HGLP-CC-021 \\
\hline \multicolumn{2}{|l|}{} & Logging Procedure: & HGLP-MAN-002, Rev. 0 \\
\hline
\end{tabular}

\section{Spectral Gamma Logging System (SGLS) Log Run Information:}

\begin{tabular}{|l|c|c|c|c|l|}
\hline Log Run & $\mathbf{1}$ & 2 Repeat & $\mathbf{5}$ & 6 Repeat & \\
\hline Date & $07 / 08 / 08$ & $07 / 08 / 08$ & $07 / 28 / 08$ & $07 / 28 / 08$ & \\
\hline Logging Engineer & McClellan & McClellan & McClellan & McClellan & \\
\hline Start Depth (ft) & 59.0 & 12.0 & 66.5 & 66.5 & \\
\hline Finish Depth (ft) & 0.0 & 6.0 & 56.0 & 65.0 & \\
\hline Count Time (sec) & 200 & 200 & 200 & 200 & \\
\hline Live/Real & $\mathrm{R}$ & $\mathrm{R}$ & $\mathrm{R}$ & $\mathrm{R}$ & \\
\hline Shield (Y/N) & $\mathrm{N}$ & $\mathrm{N}$ & $\mathrm{N}$ & $\mathrm{N}$ & \\
\hline MSA Interval (ft) & 0.5 & 0.5 & 0.5 & 0.5 & \\
\hline Log Speed (ft/min) & $\mathrm{N} / \mathrm{A}$ & $\mathrm{N} / \mathrm{A}$ & $\mathrm{N} / \mathrm{A}$ & $\mathrm{N} / \mathrm{A}$ & \\
\hline Pre-Verification & DL651CAB & DL651CAB & DL711CAB & DL711CAB & \\
\hline Start File & DL651000 & DL651119 & DL711000 & DL711022 & \\
\hline Finish File & DL651118 & DL651131 & DL711021 & DL711025 & \\
\hline Post-Verification & DL651CAA & DL651CAA & DL711CAA & DL711CAA & \\
\hline Depth Return Error (in.) & +0.25 & 0 & N/A & 0 & \\
\hline Comments & $\begin{array}{c}\text { No fine gain } \\
\text { adjustment }\end{array}$ & $\begin{array}{c}\text { No fine gain } \\
\text { adjustment }\end{array}$ & $\begin{array}{c}\text { No fine gain } \\
\text { adjustment }\end{array}$ & $\begin{array}{c}\text { No fine gain } \\
\text { adjustment }\end{array}$ & \\
\hline
\end{tabular}


HGLP-LDR-240, Rev. 0

\begin{tabular}{|l|c|c|c|c|c|}
\hline Log Run & $\mathbf{1}$ & 2 Repeat & 5 & 6 Repeat & \\
\hline & made. & made. & made. & made. & \\
\hline
\end{tabular}

\section{Neutron Moisture Logging System (NMLS) Log Run Information:}

\begin{tabular}{|l|c|c|}
\hline Log Run & 3 & 4 Repeat \\
\hline Date & $07 / 18 / 08$ & $07 / 18 / 08$ \\
\hline Logging Engineer & Pearson & Pearson \\
\hline Start Depth (ft) & 0.0 & 25.0 \\
\hline Finish Depth (ft) & 31.25 & 28.0 \\
\hline Count Time (sec) & 15 & 15 \\
\hline Live/Real & $\mathrm{R}$ & $\mathrm{R}$ \\
\hline Shield (Y/N) & $\mathrm{N}$ & $\mathrm{N}$ \\
\hline MSA Interval (ft) & 0.25 & 0.25 \\
\hline Log Speed (ft/min) & N/A & N/A \\
\hline Pre-Verification & DHH22CAB & DHH22CAB \\
\hline Start File & DHH22000 & DHH22000 \\
\hline Finish File & DHH22125 & DHH22125 \\
\hline Post-Verification & DHH22CAA & DHH22CAA \\
\hline Depth Return Error (in.) & N/A & 0 \\
\hline Comments & None & None \\
\hline
\end{tabular}

\section{Logging Operation Notes:}

Data were collected using Gamma 4, HO 68B-3573. SGLS pre - and post-survey verification measurements were acquired in the Amersham KUTh-115 field verifier. Maximum logging depth achieved was $66.6 \mathrm{ft}$ before the sonde un-weighted. A centralizer was installed on the sonde. NMLS pre-and post-survey verification measurements were acquired in the standard field verifier.

\section{Analysis Notes:}

\begin{tabular}{|l|l|l|l|l|l|}
\hline Analyst: & Henwood & Date: & 08/28/08 & Reference: & GJO-HGLP 1.6.3, Rev. 0 \\
\hline
\end{tabular}

SGLS pre- and post-survey verification spectra met the acceptance criteria for the established system. NMLS pre and post-survey verification spectra met the acceptance criteria for the established system.

A casing correction for a 3/8-in. thick casing was applied from ground surface to $65 \mathrm{ft}$. A water correction was also applied during analysis from $31.4 \mathrm{ft}$ to total logged depth of borehole.

SGLS spectra were processed in batch mode using APTEC SUPERVISOR to identify individual peaks and count rates. Concentrations were calculated using an EXCEL template identified as G4LDec07.xls using an efficiency function and corrections for casing, dead time and water as determined by annual calibrations. NMLS spectra were processed in batch mode in APTEC SUPERVISOR to identify counts. Count rates were calculated using an EXCEL template identified as G4HNov07.xls. There is no direct calibration data available for a 6 7/8-in. inner diameter borehole casing. Therefore, moisture data are reported in counts per second (cps).

\section{$\underline{\text { Results and Interpretations: }}$}

No manmade radionuclides were detected. Spectra, where detections of Cs -137, U-238, and U-235 using the routine processing software were indicated, were individually inspected. It was determined the detections are statistical fluctuations and are not valid.

The KUT data indicate good repeatability.

The moisture data indicate good repeatability. 
HGLP-LDR-240, Rev. 0

\section{List of Log Plots:}

Depth Reference is ground surface

Manmade Radionuclides

Natural Gamma Logs

Combination Plot

Total Gamma \& Moisture

Repeat Section of Natural Gamma Logs

Repeat of Moisture

${ }^{1} \mathrm{GWL}$ - groundwater level

${ }^{2}$ TOC - top of casing 
Man-Made Radionuclides

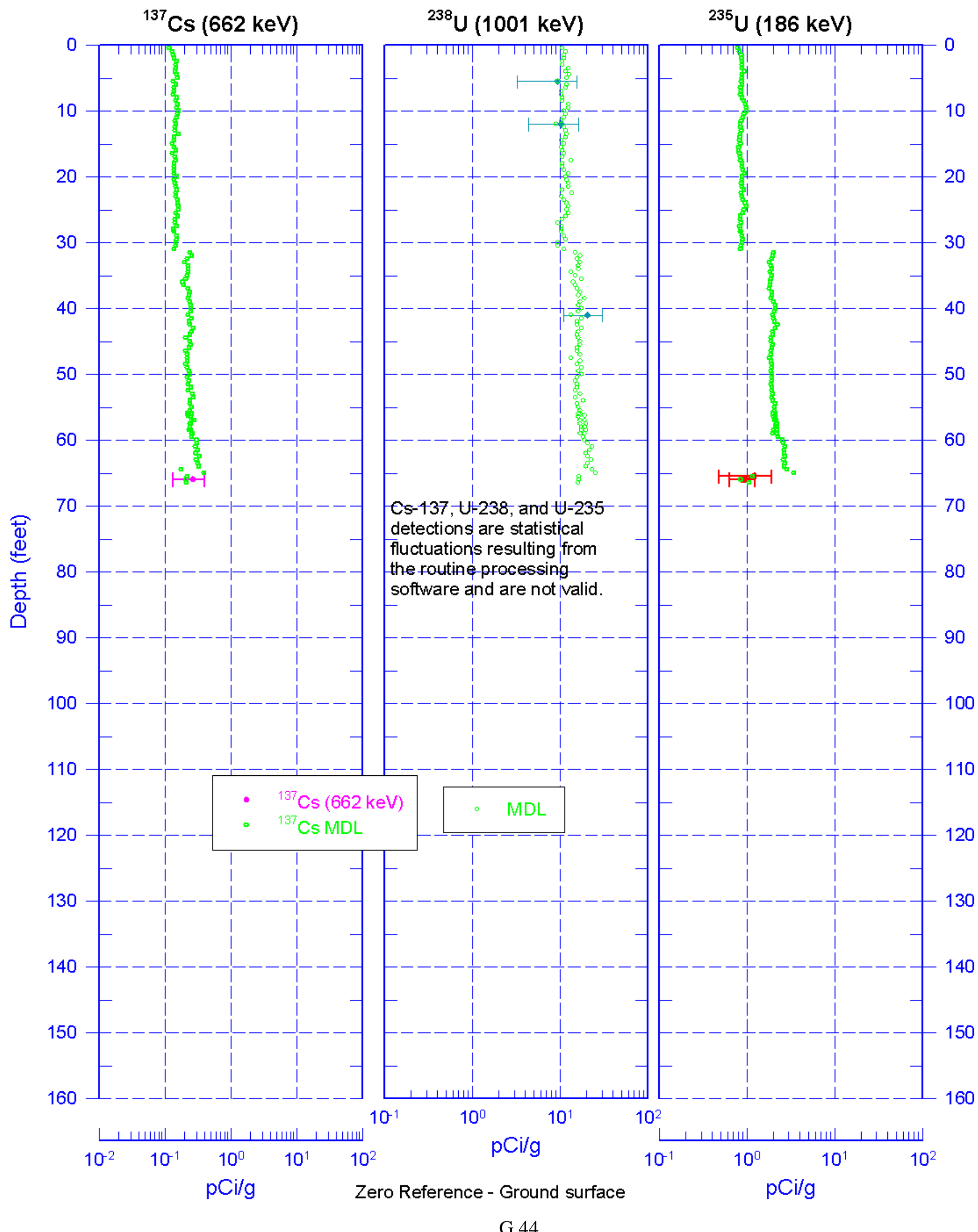




\section{S \\ toller \\ Hanford Office \\ 399-2-10 (C6187) \\ Natural Gamma Logs}
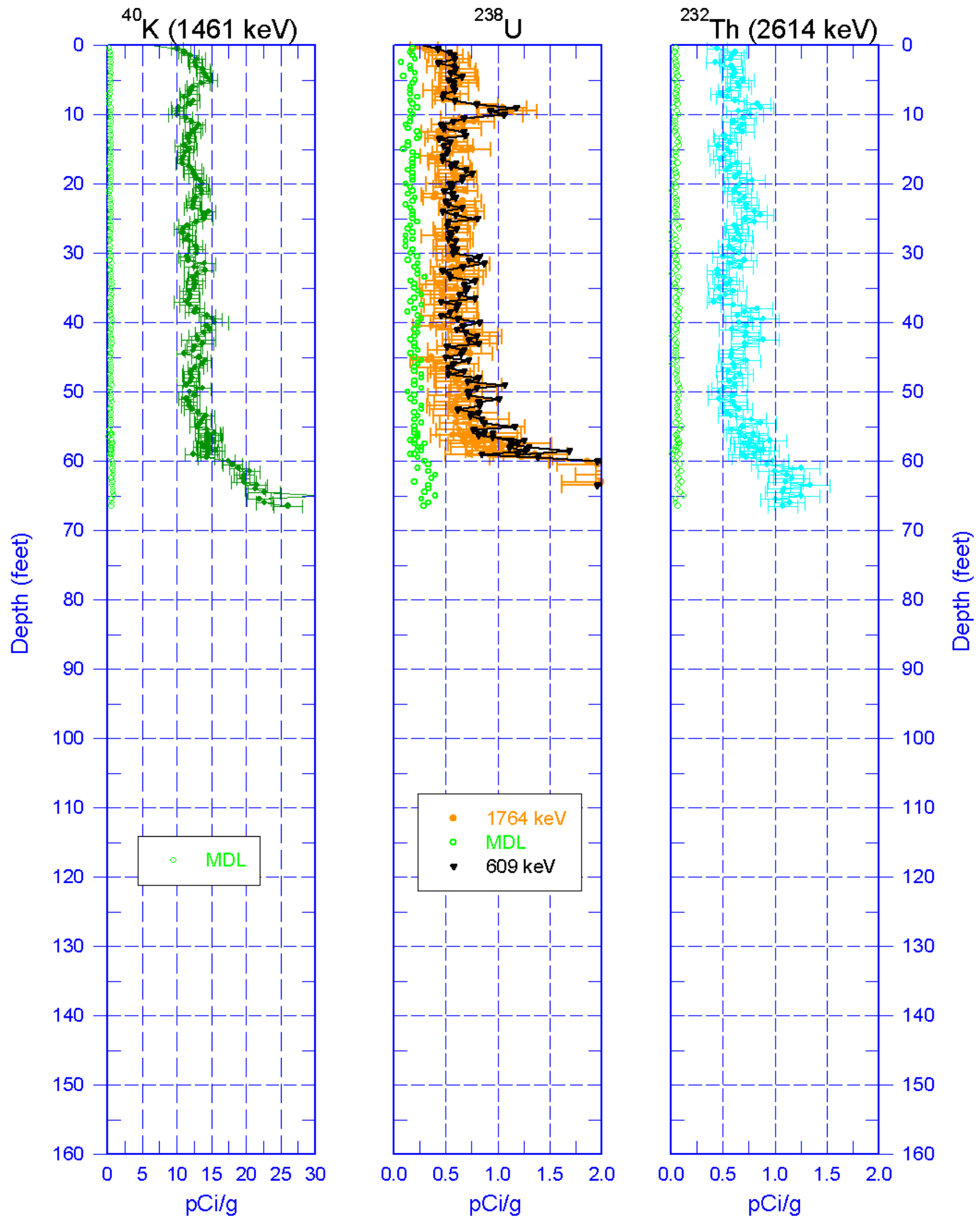

Zero Reference - Ground surface 


\section{9-2-10 (C6187) Combination Plot}
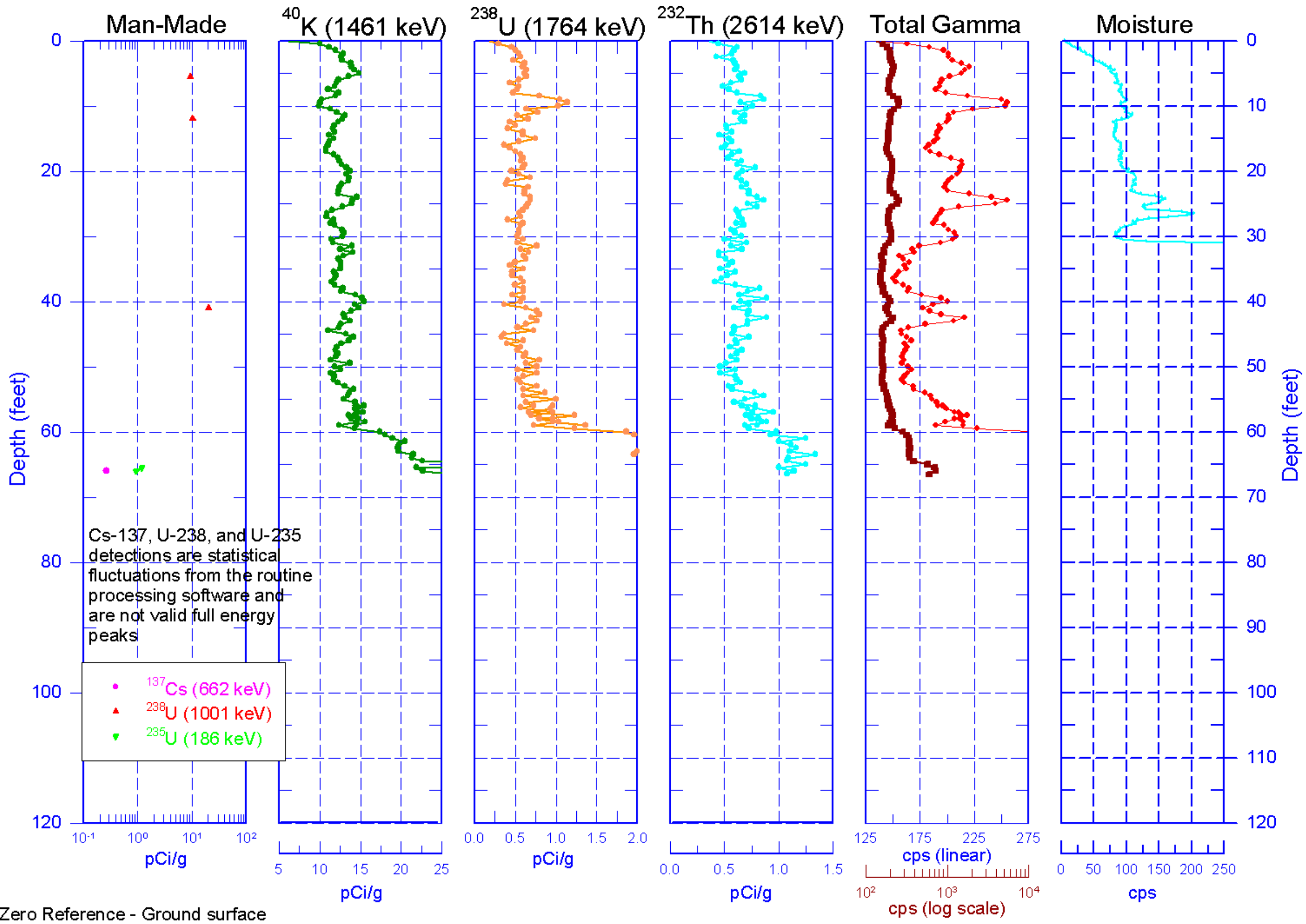
toller

Hanford Office

Total Gamma \& Moisture
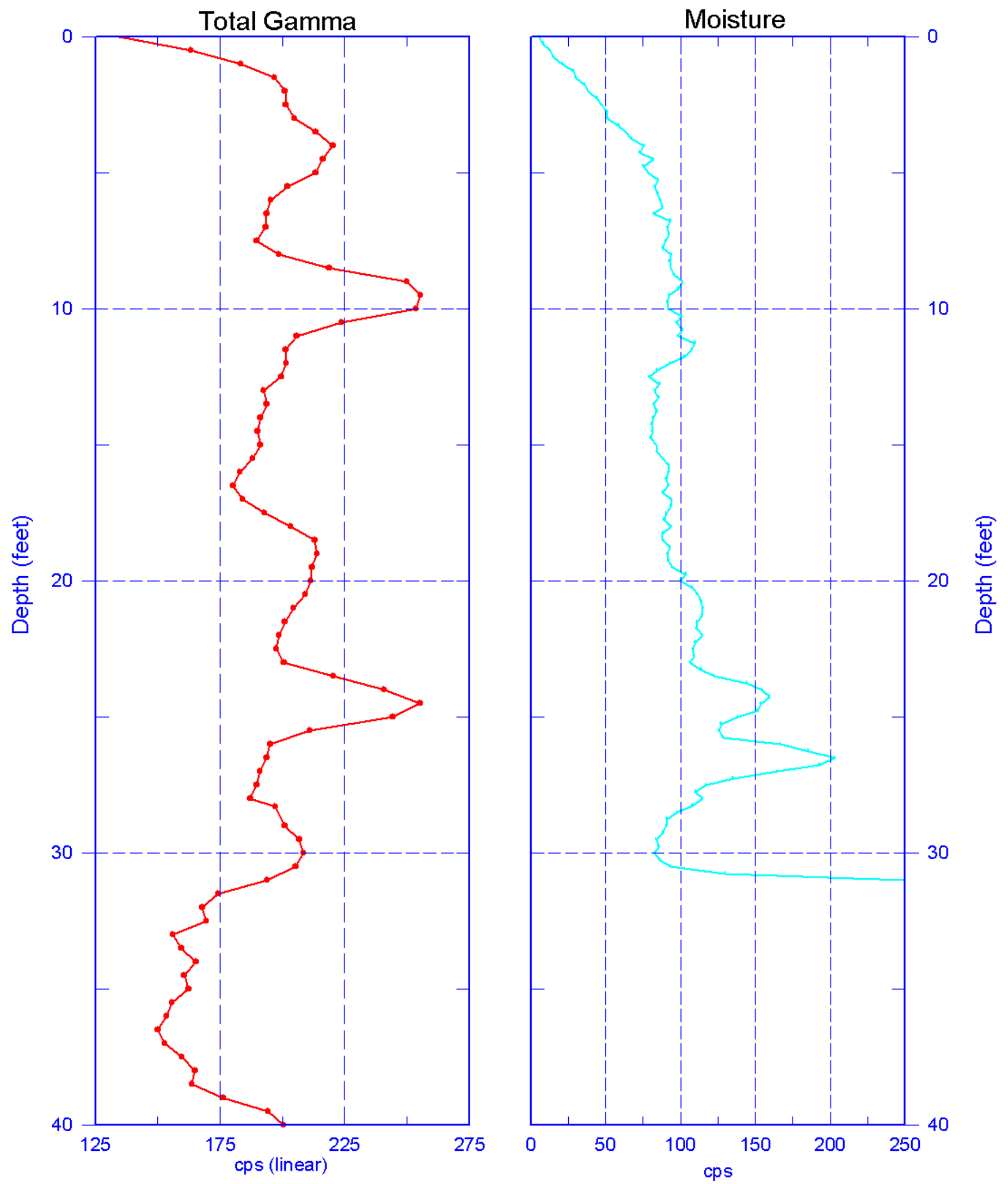
Hanford Office

\section{9-2-10 (C6187)}

Repeat Section of Natural Gamma Logs
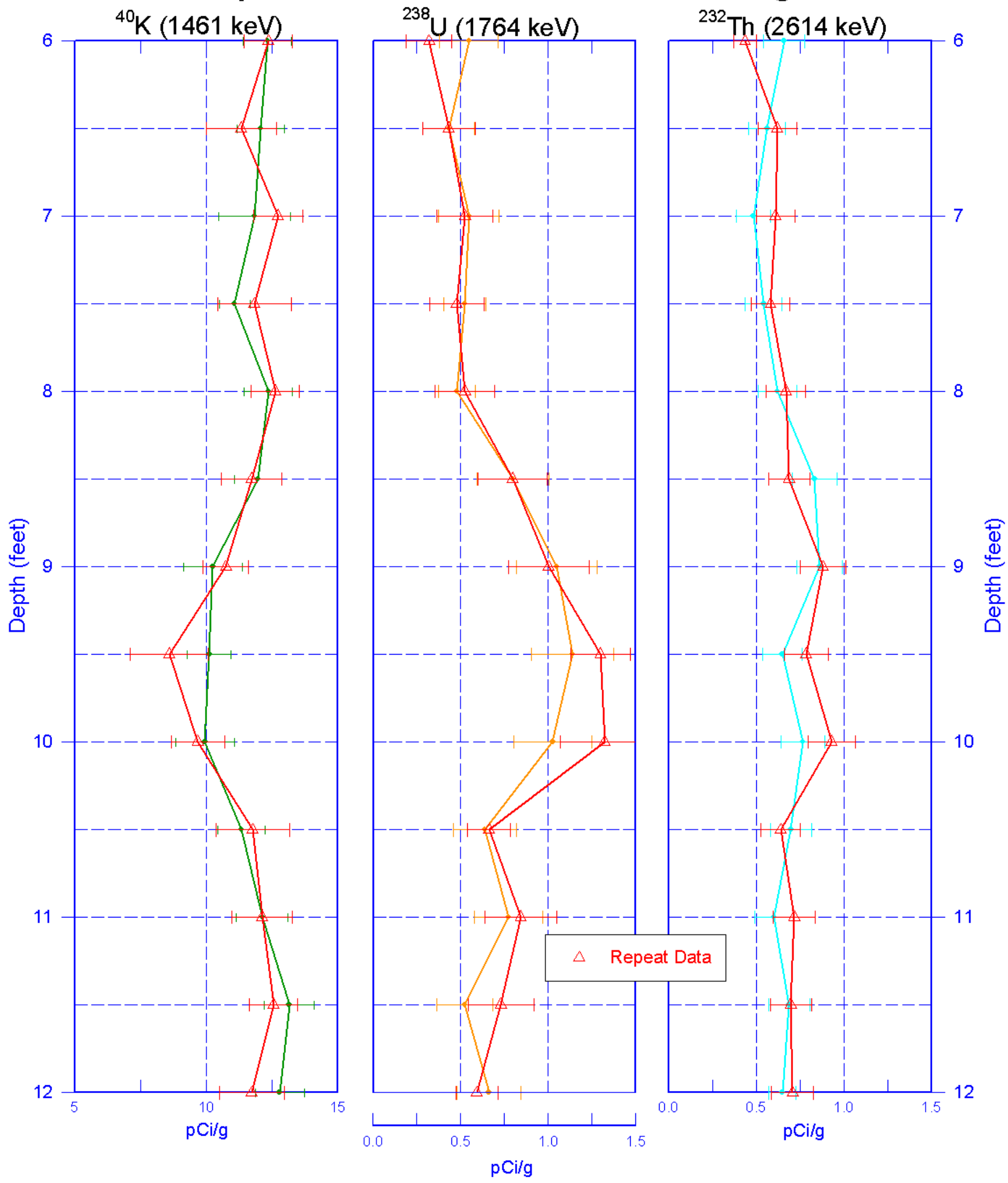

Zero Reference - Ground surface 
toller

Hanford Office
399-2-10 (C6187)

Repeat of Moisture

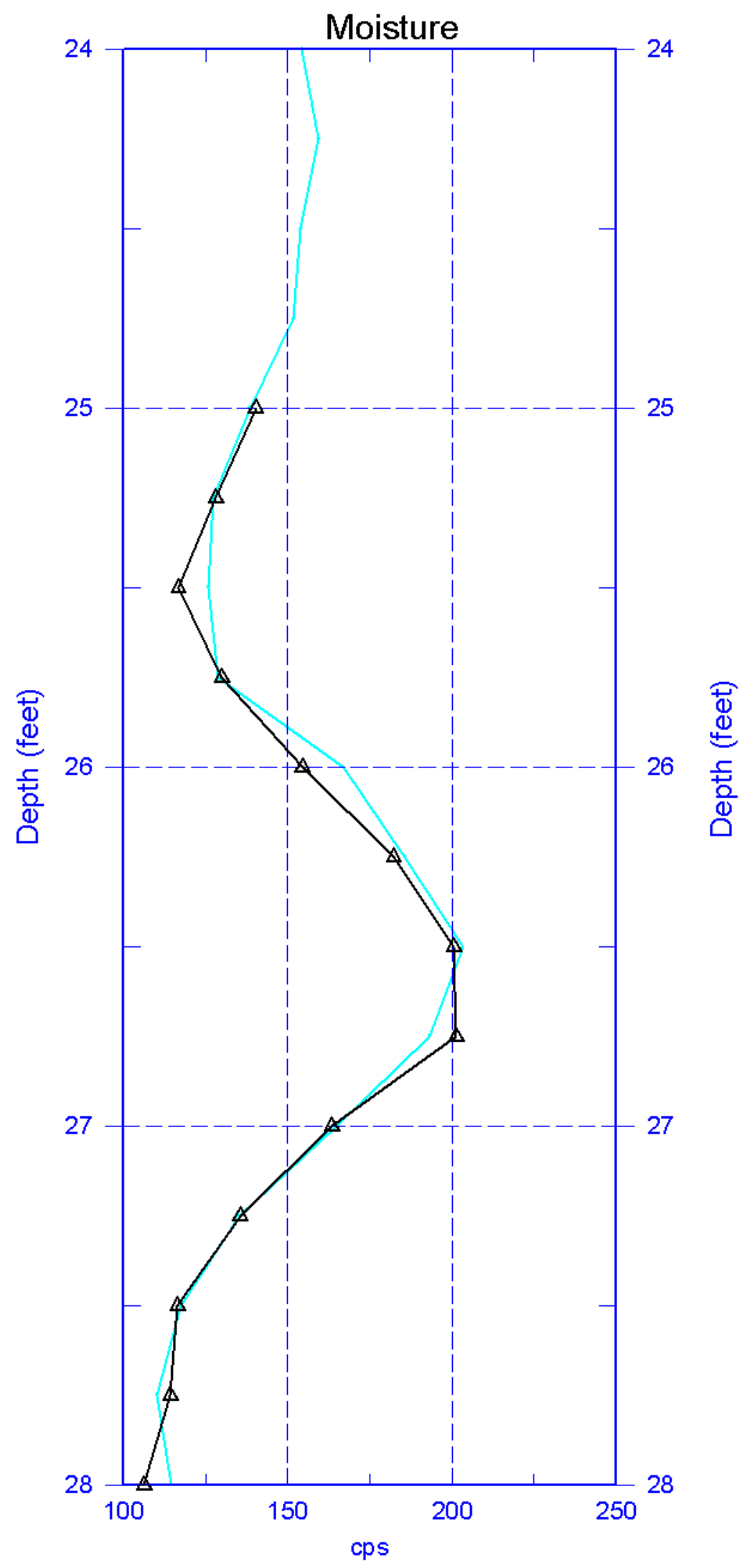




\section{9-2-11 (C6188) Log Data Report}

Borehole Information:

\begin{tabular}{|c|c|c|c|c|c|}
\hline Borehole: & \multicolumn{1}{l|}{ Site: } & \multicolumn{2}{l|}{ 300-FF-5 } \\
\hline \multicolumn{2}{|c|}{ 399-2-11 (C6188) } & GWL $^{\mathbf{1}}$ (ft) : & 30.9 & GWL Date: & $7 / 20 / 2008$ \\
\hline North (m) & East (m) & Drill Date & TOC $^{2}$ Elevation & Total Depth (ft) & Type \\
\hline 116074.5 & 594236.1 & $7 / 16 / 2008$ & unknown & 65 & Sonic \\
\hline
\end{tabular}

\section{Casing Information:}

\begin{tabular}{|c|c|c|c|c|c|c|}
\hline Casing Type & Stickup (ft) & $\begin{array}{c}\text { Outer } \\
\text { Diameter (in.) }\end{array}$ & $\begin{array}{c}\text { Inside } \\
\text { Diameter (in.) }\end{array}$ & Thickness (in.) & Top (ft) & Bottom (ft) \\
\hline Threaded Steel & 0.7 & $75 / 8$ & $67 / 8$ & $3 / 8$ & -0.7 & 60.0 \\
\hline
\end{tabular}

\section{Borehole Notes:}

Casing data and total depth was reported by the site geologist. Casing diameter was measured using a steel tape and rounded to the nearest $1 / 16$ - inch. The zero reference is the ground surface.

Logging Equipment Information:

\begin{tabular}{|c|c|c|c|c|}
\hline Logging System: & \multicolumn{2}{|l|}{ Gamma $4 \mathrm{~L}$} & $\begin{array}{l}\text { Type: } \\
\text { Serial No.: }\end{array}$ & $\begin{array}{l}\text { 60\% HPGe SGLS } \\
\text { 47TP32211A }\end{array}$ \\
\hline Effective Calibration Date: & $12 / 31 / 2007$ & \multirow{2}{*}{$\begin{array}{l}\text { Calibration Reference: } \\
\text { Logging Procedure: }\end{array}$} & \multicolumn{2}{|c|}{ HGLP-CC-027 } \\
\hline & & & \multicolumn{2}{|c|}{ HGLP-MAN-002, Rev. 0} \\
\hline Logging System: & \multicolumn{2}{|l|}{ Gamma $4 \mathrm{H}$} & $\begin{array}{l}\text { Type: } \\
\text { Serial No.: }\end{array}$ & $\begin{array}{l}\text { NMLS } \\
\text { H310700352 }\end{array}$ \\
\hline Effective Calibration Date: & $11 / 6 / 2008$ & \multirow{2}{*}{$\begin{array}{l}\text { Calibration Reference: } \\
\text { Logging Procedure: }\end{array}$} & \multicolumn{2}{|c|}{ HGLP-CC-021 } \\
\hline & & & \multicolumn{2}{|c|}{ HGLP-MAN-002, Rev. 0} \\
\hline
\end{tabular}

\section{Spectral Gamma Logging System (SGLS) Log Run Information:}

\begin{tabular}{|c|c|c|c|c|}
\hline Log Run & 1 & 2 Repeat & 3 & 4 Repeat \\
\hline Date & $7 / 20 / 2008$ & $7 / 20 / 2008$ & $7 / 21 / 2008$ & $7 / 21 / 2008$ \\
\hline Logging Engineer & Pearson & Pearson & Pearson & Pearson \\
\hline Start Depth (ft) & 63.5 & 14.0 & 63.5 & 14.0 \\
\hline Finish Depth (ft) & 0.0 & 8.0 & 0.0 & 8.0 \\
\hline Count Time (sec) & 200 & 200 & 200 & 200 \\
\hline Live/Real & $\mathrm{R}$ & $\mathrm{R}$ & $\mathrm{R}$ & $\mathrm{R}$ \\
\hline Shield (Y/N) & $\mathrm{N}$ & $\mathrm{N}$ & $\mathrm{N}$ & $\mathrm{N}$ \\
\hline MSA Interval (ft) & 0.5 & 0.5 & 0.5 & 0.5 \\
\hline Log Speed (ft/min) & NA & NA & NA & NA \\
\hline Pre-Verification & DL661CAB & DL661CAB & DL671CAB & DL671CAB \\
\hline Start File & DL661000 & DL661128 & DL671000 & DL671128 \\
\hline Finish File & DL661127 & DL661140 & DL671127 & DL671140 \\
\hline Post-Verification & DL661CAA & DL661CAA & DL671CAA & DL671CAA \\
\hline Depth Return Error (in.) & $1 / 4 \mathrm{HIGH}$ & 0.0 & 0.0 & 0.0 \\
\hline Comments & $\begin{array}{l}\text { Adjusted gain } \\
\text { after } \\
\text { DL661041-06 } \\
3 \text { and -125. }\end{array}$ & Repeat Section & None & Repeat Section \\
\hline
\end{tabular}


Neutron Moisture Logging System (NMLS) Log Run Information:

\begin{tabular}{|c|c|c|l|l|l|}
\hline Log Run & $\mathbf{1}$ & 2 Repeat & & & \\
\hline Date & $7 / 20 / 2008$ & $7 / 20 / 2008$ & & & \\
\hline Logging Engineer & PEARSON & PEARSON & & & \\
\hline Start Depth (ft) & 0.0 & 26.0 & & & \\
\hline Finish Depth (ft) & 30.75 & 29.0 & & & \\
\hline Count Time (sec) & 15 & 15 & & & \\
\hline Live/Real & $\mathrm{R}$ & $\mathrm{R}$ & & & \\
\hline Shield (Y/N) & $\mathrm{N}$ & $\mathrm{N}$ & & & \\
\hline MSA Interval (ft) & 0.25 & 0.25 & & & \\
\hline ft/min & NA & NA & & & \\
\hline Pre-Verification & DHH32CAB & DHH32CAB & & & \\
\hline Start File & DHH32000 & DHH32124 & & & \\
\hline Finish File & DHH32123 & DHH32136 & & & \\
\hline Post-Verification & DHH32CAA & DHH32CAA & & & \\
\hline Depth Return Error (in.) & NA & NHA.0 & & & \\
\hline Comments & None & Repeat Section & & & \\
\hline
\end{tabular}

\section{Logging Operation Notes:}

The gain shifted during the first log run resulting in poor peak resolution. The cable head connector was cleaned and a second survey was completed with success. Files DL661000 through DL661140 from the first survey remain unused in the databank. The DL671000 through DL671140 files from the second run were used to complete this survey.

The SGLS data were collected 7/20/2008-using Gamma 4, HO 68B-3573 logging system. Pre and post-survey verification measurements were acquired in the Amersham KUTh -115-field verifier. A centralizer was installed on the sonde. Maximum logging depth was $63.5 \mathrm{ft}$ before the sonde un-weighted.

The Neutron Moisture data were collected 7/20/2008 using the Gamma 4, HO 68B-3573 logging system. NMLS pre- and post-survey verification measurements were acquired in the standard field verifier. A centralizer was installed on the sonde. Maximum logging depth was $30.75 \mathrm{ft}$ before water was encountered.

\section{Analysis Notes:}

\begin{tabular}{|l|l|l|l|l|l|}
\hline Analyst: & L. SPINNER & Date: & 10/1/2008 & Reference: & GJO-HGLP 1.6.3, Rev. 0 \\
\hline
\end{tabular}

The SGLS and NMLS pre- and post-survey verifications spectra met acceptance criteria.

Analyses of log data suggest slight discrepancies with field reports of depth to water and casing depth. Corrections of log data were applied according to the analysis assumptions in the table below.

\begin{tabular}{|c|c|c|c|l|}
\hline $\begin{array}{c}\text { Top } \\
\text { (ft) }\end{array}$ & $\begin{array}{c}\text { Bottom } \\
\text { (ft) }\end{array}$ & $\begin{array}{c}\text { T } \\
\text { (in) }\end{array}$ & $\begin{array}{c}\text { ID } \\
\text { (in) }\end{array}$ & \multicolumn{1}{c|}{ Comments } \\
\hline 0 & 31.75 & 0.375 & $67 / 8$ & No water correction \\
\hline 32 & 59.5 & 0.375 & $67 / 8$ & GWL reported @ 30.9 ft; adjusted to 31.75 ft. \\
\hline 60 & 65 & 0 & NA & Casing depth reported @ 60 ft; adjusted to 59.5 ft \\
\hline
\end{tabular}

SGLS spectra were processed in batch mode using APTEC SUPERVISOR to identify individual peaks and count rates. Concentrations were calculated using an EXCEL template identified as G4LDec07.xls using an efficiency function and corrections for casing, dead time and water as determined by annual calculations. NMLS spectra were processed in batch mode in APTEC SUPERVISOR to identify counts. Count rates were calculated using an 
HGLP-LDR-257, Rev. 0

EXCEL template identified as G4HNov07.xls. NMLS data are presented in counts per second (cps), because calibration data is not available for a 6 7/8 inch inner diameter borehole casing.

\section{Results and Interpretations:}

The Man-Made Radionuclides, Cs-137, U-235 and U-238 (Pa-234m) were identified by the routine processing software at various depth intervals. Scrutiny of the individual spectra indicated that the energy peaks are not valid, and are considered statistical fluctuations.

Co-60 was detected at $0.07 \mathrm{pCi} / \mathrm{g}$ at $13.50 \mathrm{ft}$ and $0.06 \mathrm{pCi} / \mathrm{g}$ at $14.00 \mathrm{ft}$. These detections are valid and the $1333 \mathrm{keV}$ energy peak is corroborated by the Co-60-energy peak at $1173 \mathrm{keV}$.

The peaks at 10.5 and $57 \mathrm{ft}$ in the Natural Gamma Log may indicate stratigraphy changes at these depths.

The Natural Gamma Logs (KUT) and the Moisture Repeat plot indicate good repeatability.

\section{List of Log Plots:}

Depth Reference is top of casing

Manmade Radionuclides (Cs-137, Co-60, U-235 and U-238)

Natural Gamma Logs (KUT)

Combination Plot (MM, KUT, TG, Moisture)

Total Gamma \& Dead Time

Total Gamma \& Moisture

Repeat of Manmade Radionuclides

Repeat Section of Natural Gamma Logs (KUT)

Moisture Repeat Section (26-29 feet)

${ }^{1} \mathrm{GWL}$ - groundwater level

${ }^{2}$ TOC - top of casing 

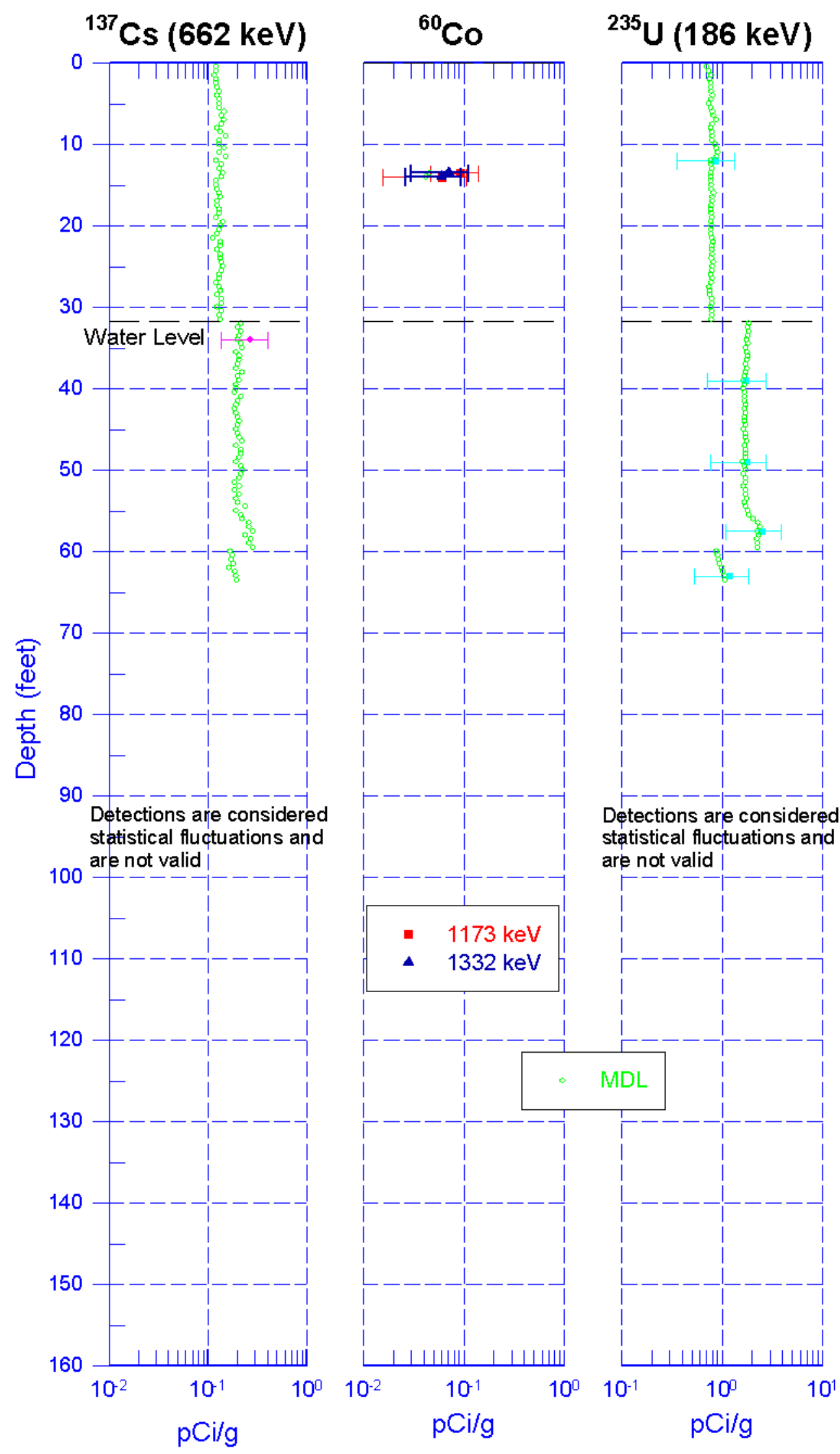

${ }^{238} \mathrm{U}(1001 \mathrm{keV})$

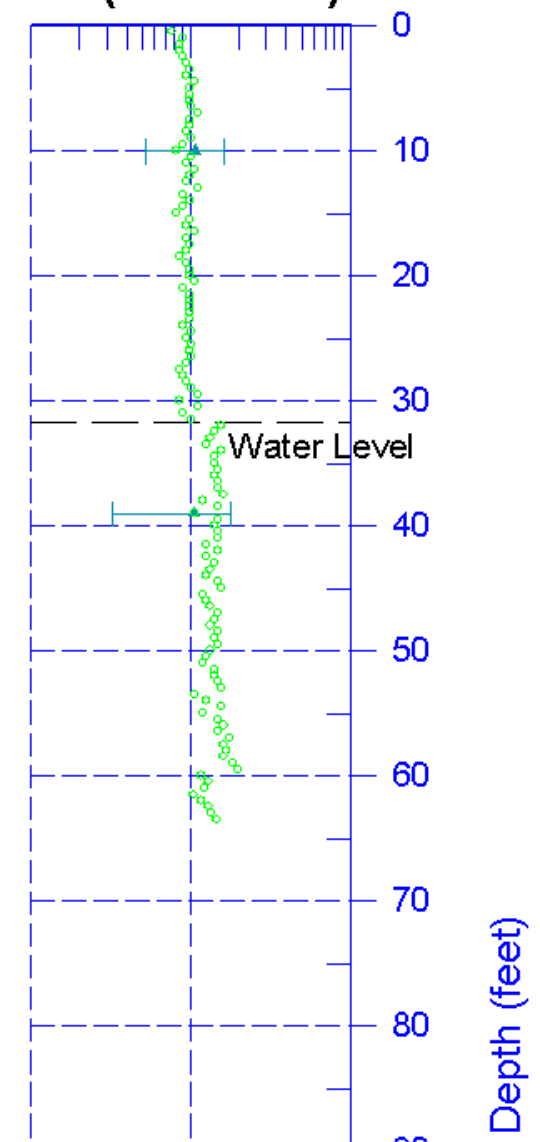

Detections are considered statistical fluctuations and are not valid

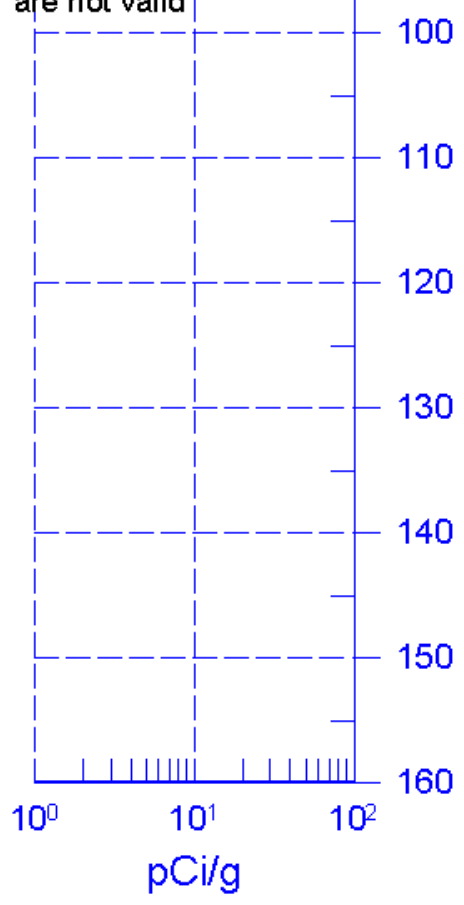




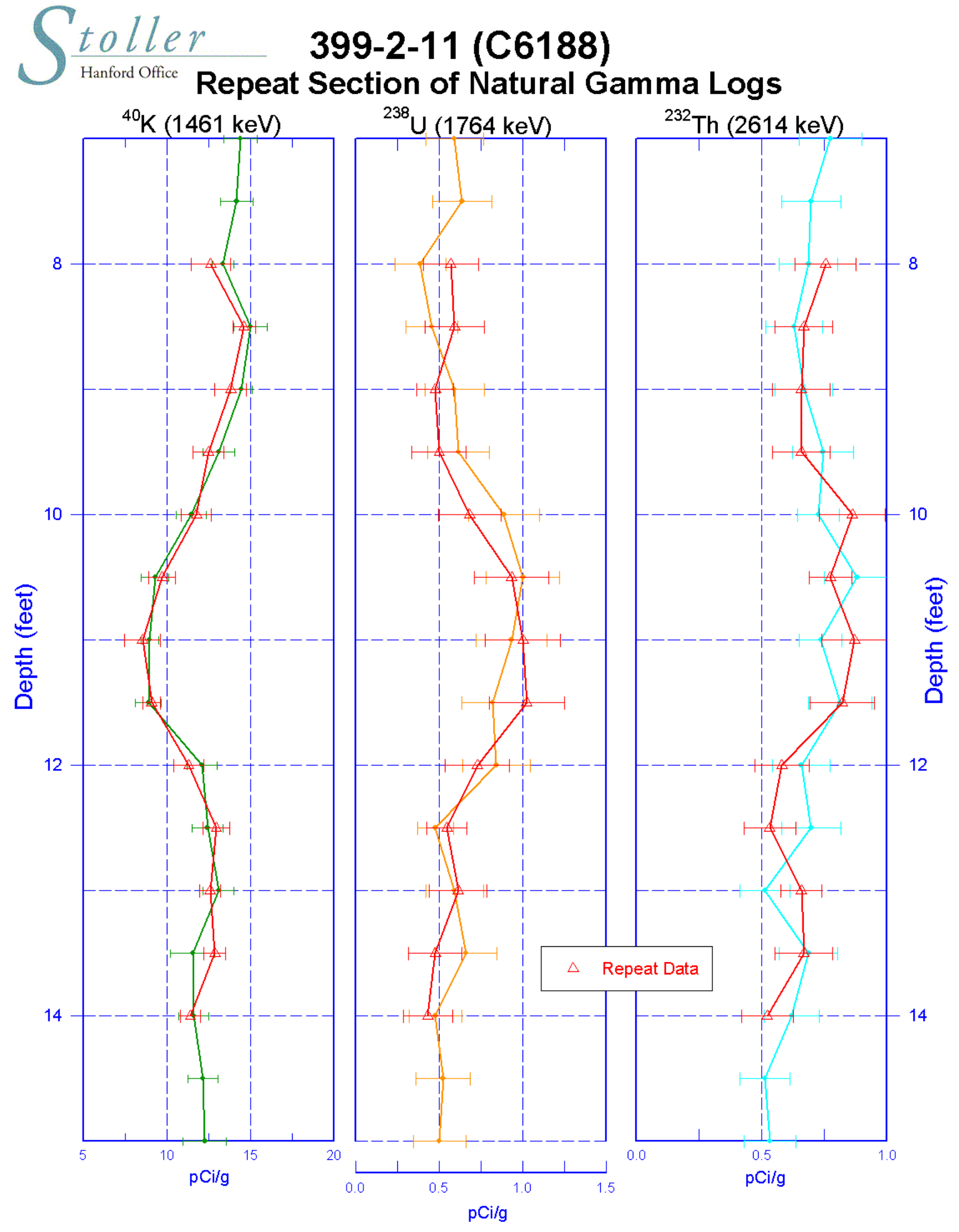




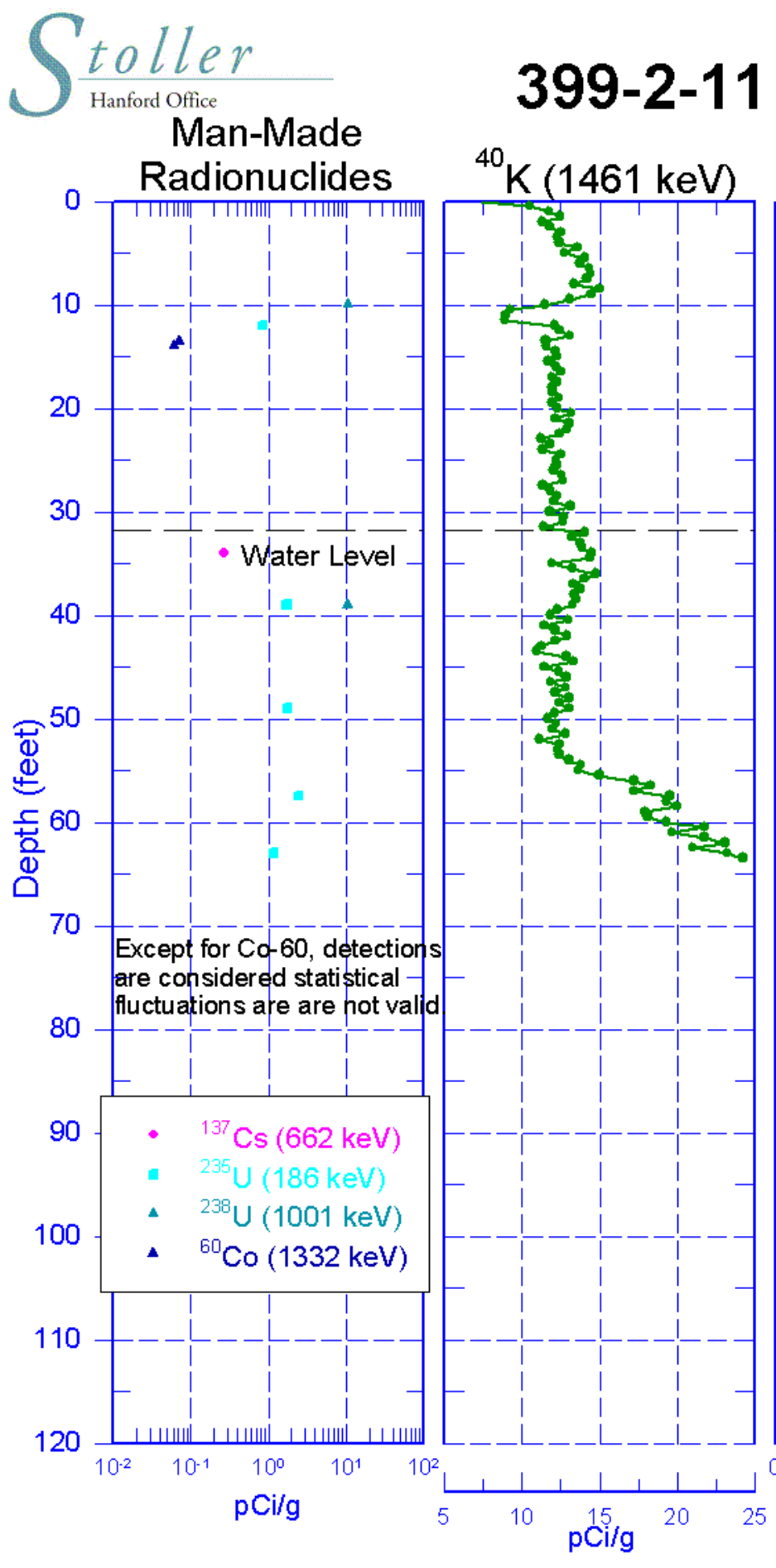

\section{9-2-11 (C6188) Combination Plot}
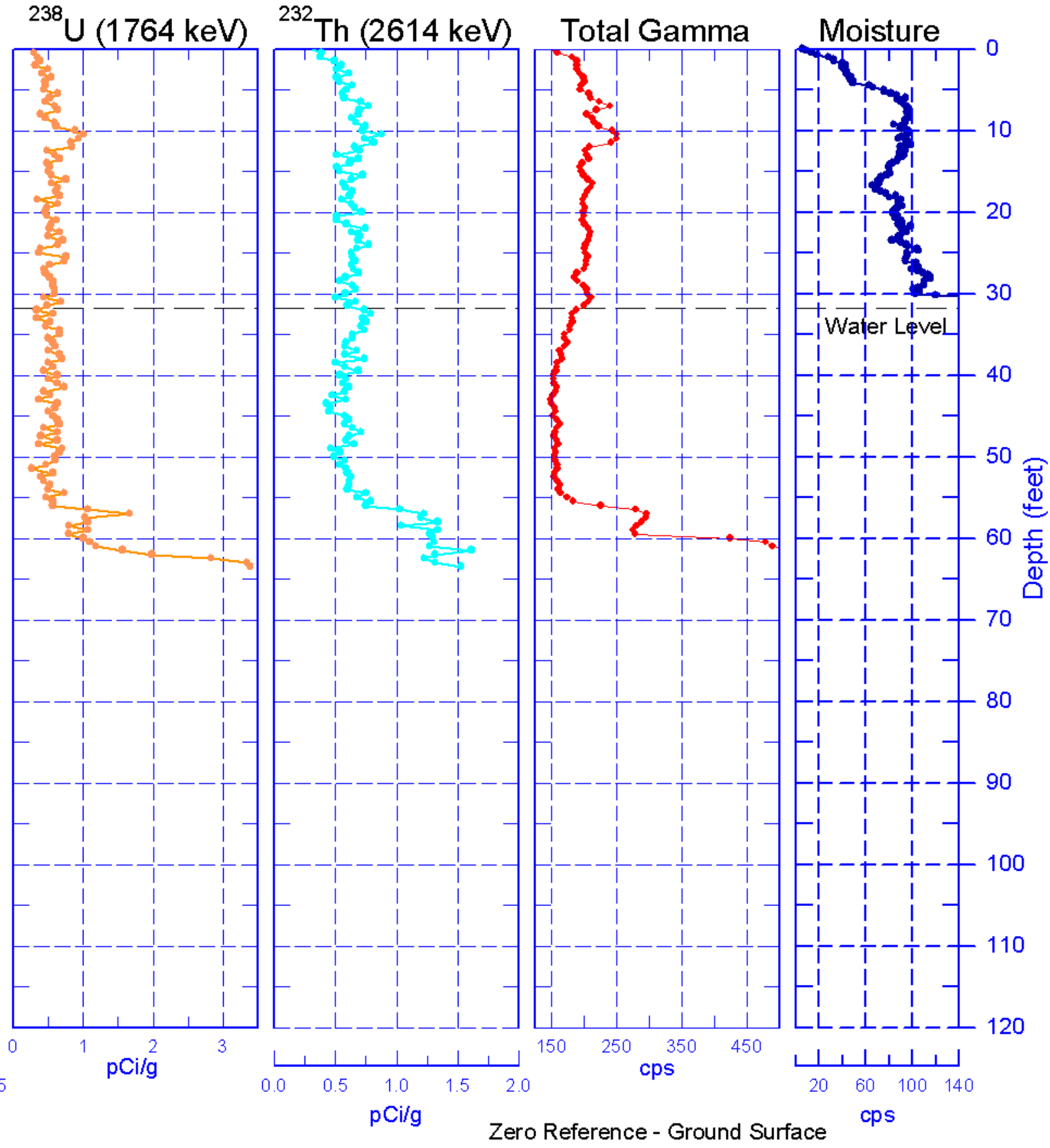

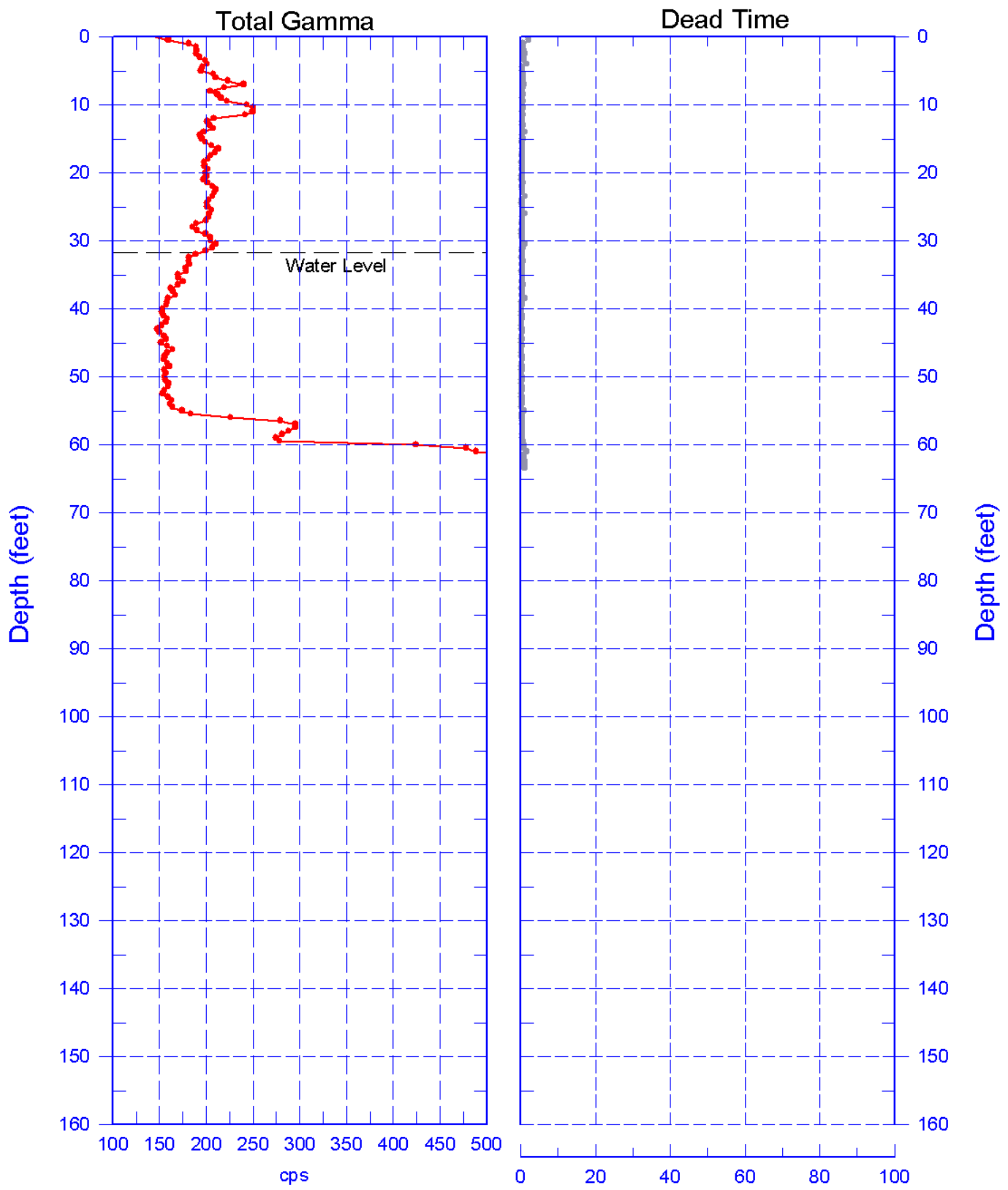

Reference - Ground Surface 


\section{S}

toller $\quad 399-\mathbf{2}-11$ (C6188)

Hanford Office

\section{Total Gamma \& Moisture}
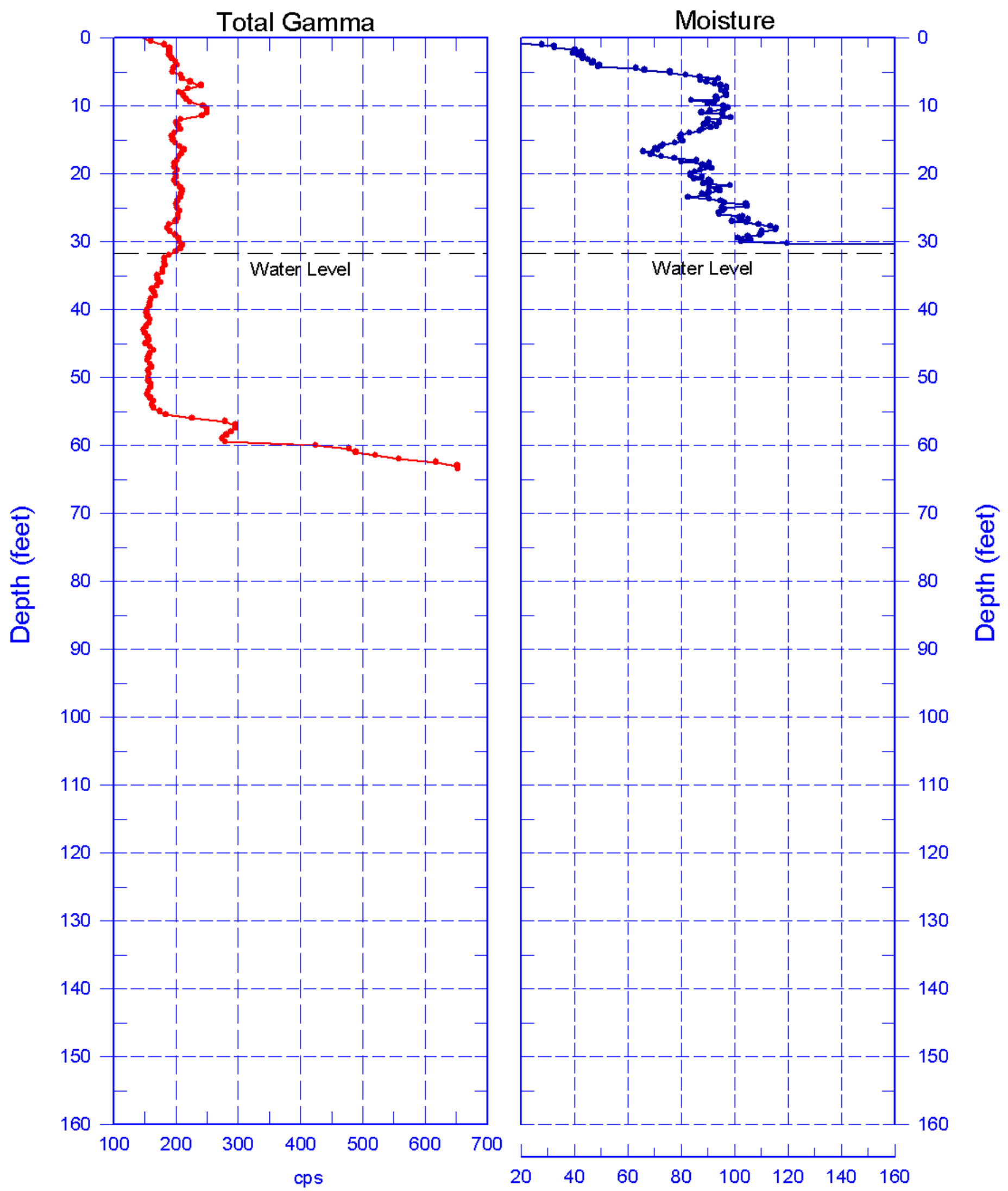

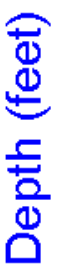

Reference - Ground Surface 


\section{9-2-11 (C6188) Manmade Radionuclides Repeat Section}

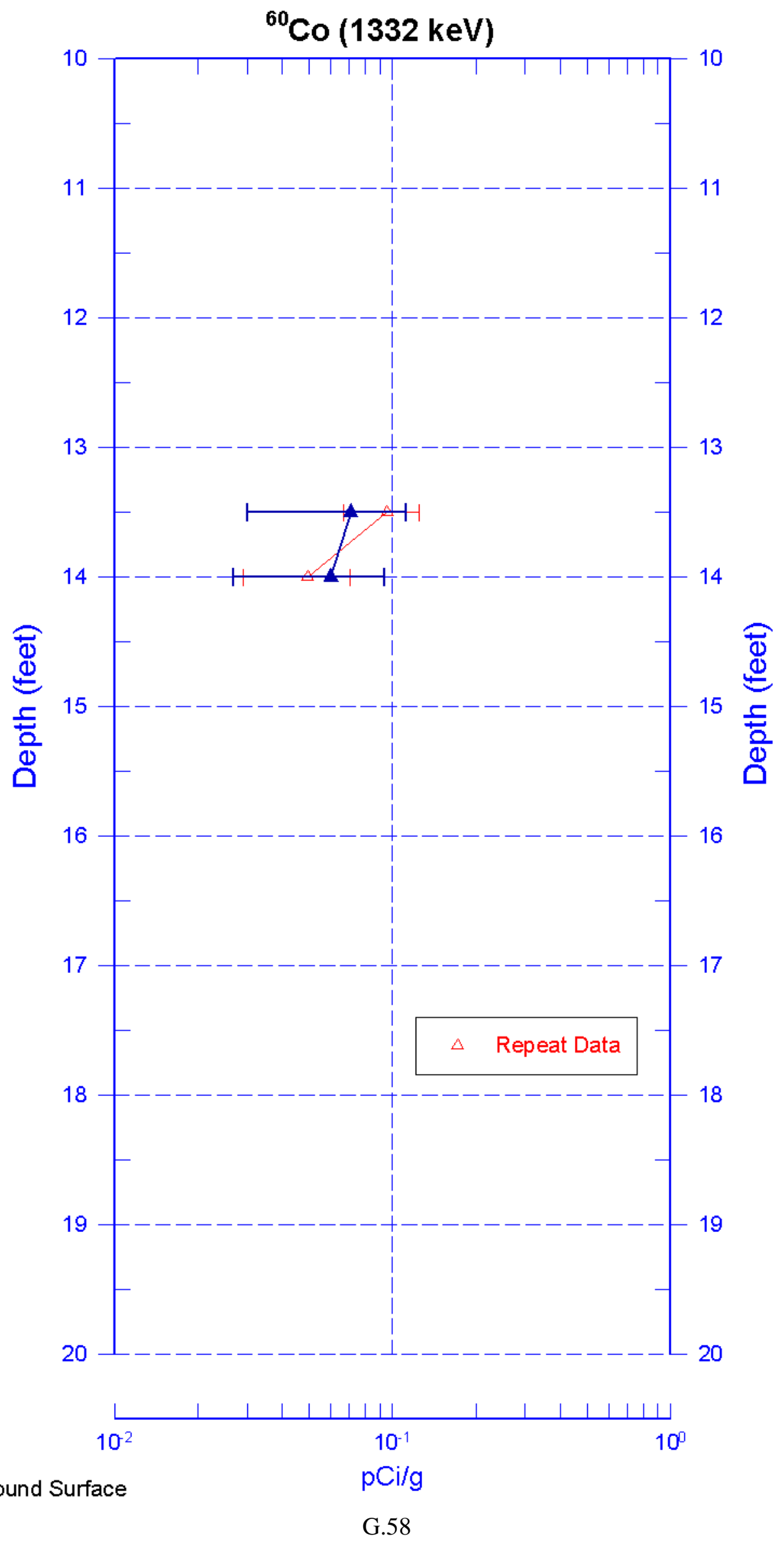




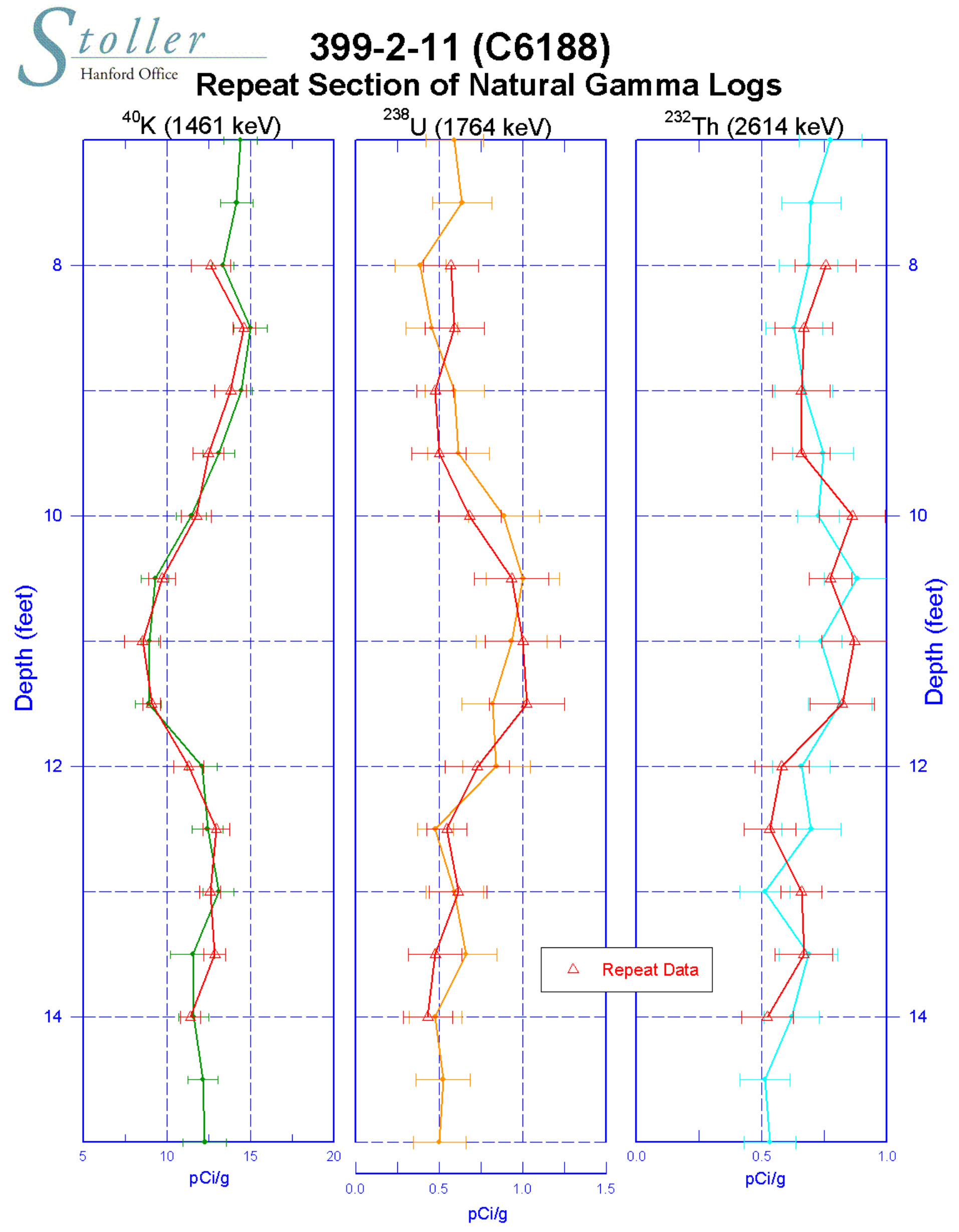




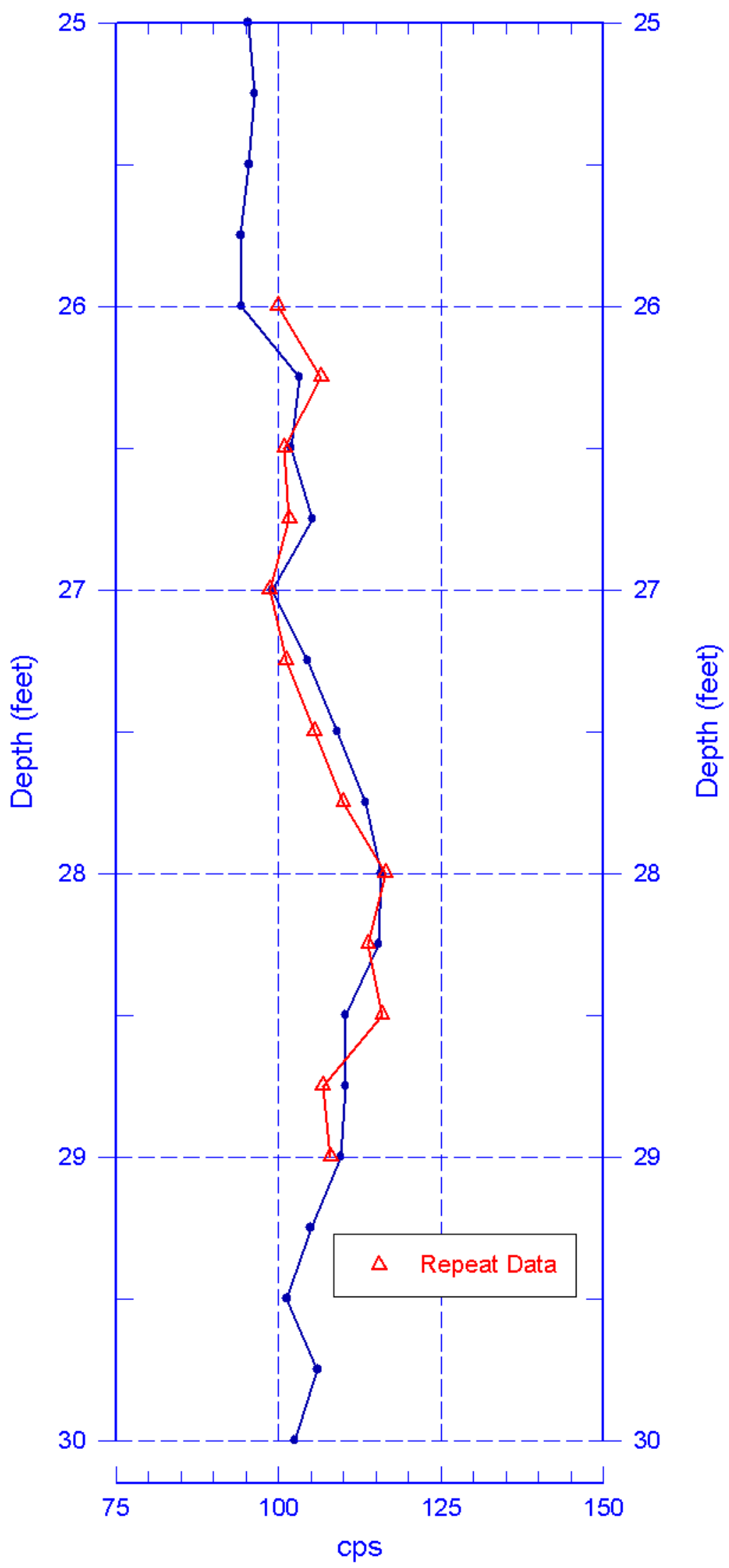




\section{9-2-12 (C6189) Log Data Report}

Borehole Information:

\begin{tabular}{|c|c|c|c|c|c|}
\hline \multicolumn{2}{|c|}{ Borehole: $\quad$ 399-2-12 (C6189) } & \multicolumn{4}{|c|}{ 300-FF-5 } \\
\hline \multicolumn{2}{|c|}{ Coordinates (WA St Plane) } & $G_{W L}^{1}(\mathbf{f}$ & 27.3 & GWL Date: & $06 / 23 / 08$ \\
\hline North (m) & East (m) & Drill Date & TOC $^{2}$ Elevation & Total Depth (ft) & Type \\
\hline 116086.0 & 594252.5 & 06/18/08 & Unknown & 65 & Sonic \\
\hline
\end{tabular}

\section{Casing Information:}

\begin{tabular}{|l|c|c|c|c|c|c|}
\hline Casing Type & Stickup (ft) & $\begin{array}{c}\text { Outer } \\
\text { Diameter (in.) }\end{array}$ & $\begin{array}{c}\text { Inside } \\
\text { Diameter (in.) }\end{array}$ & Thickness (in.) & Top (ft) & Bottom (ft) \\
\hline Welded Steel & 0.8 & $75 / 8$ & $67 / 8$ & $3 / 8$ & -0.8 & 59 \\
\hline
\end{tabular}

\section{Borehole Notes:}

Casing data and total depth are reported by the site Geologist. Logging engineer measured depth to water using an e-tape. Casing diameters were measured using a steel tape and rounded to the nearest 1/16”.

\section{Logging Equipment Information:}

\begin{tabular}{|c|c|c|c|c|}
\hline Logging System: & \multicolumn{2}{|c|}{ Gamma 4 L } & $\begin{array}{l}\text { Type: } \\
\text { Serial No.: }\end{array}$ & $\begin{array}{l}\text { SGLS } \\
\text { 47TP32211A }\end{array}$ \\
\hline Effective Calibration Date: & $12 / 31 / 07$ & \multirow{2}{*}{$\begin{array}{l}\text { Calibration Reference: } \\
\text { Logging Procedure: }\end{array}$} & \multicolumn{2}{|c|}{ HGLP-CC-027 } \\
\hline & & & \multicolumn{2}{|c|}{ HGLP-MAN-002, Rev. 0} \\
\hline Logging System: & \multicolumn{2}{|c|}{ Gamma $4 \mathrm{H}$} & $\begin{array}{l}\text { Type: } \\
\text { Serial No.: }\end{array}$ & $\begin{array}{l}\text { NMLS } \\
\text { H310700352 }\end{array}$ \\
\hline Effective Calibration Date: & $11 / 06 / 07$ & Calibration Reference: & \multicolumn{2}{|c|}{ HGLP-CC-021 } \\
\hline & & Logging Procedure: & \multicolumn{2}{|c|}{ HGLP-MAN-002, Rev. 0} \\
\hline
\end{tabular}

\section{Spectral Gamma Logging System (SGLS) Log Run Information:}

\begin{tabular}{|c|c|c|c|c|c|}
\hline Log Run & 1 & 2 Repeat & & & \\
\hline Date & $06 / 23 / 08$ & $06 / 23 / 08$ & & & \\
\hline Logging Engineer & Pearson & Pearson & & & \\
\hline Start Depth (ft) & 0.0 & 30.0 & & & \\
\hline Finish Depth (ft) & 28.0 & 24.0 & & & \\
\hline Count Time (sec) & 200 & 200 & & & \\
\hline Live/Real & $\mathrm{R}$ & $\mathrm{R}$ & & & \\
\hline Shield (Y/N) & $\mathrm{N}$ & $\mathrm{N}$ & & & \\
\hline MSA Interval (ft) & 0.5 & 0.5 & & & \\
\hline Log Speed (ft/min) & N/A & N/A & & & \\
\hline Pre-Verification & DL491CAB & DL491CAB & & & \\
\hline Start File & DL491000 & DL491125 & & & \\
\hline Finish File & DL491124 & DL491137 & & & \\
\hline Post-Verification & DL491CAA & DL491CAA & & & \\
\hline Depth Return Error (in.) & 0 & NA & & & \\
\hline Comments & $\begin{array}{l}\text { No fine gain } \\
\text { adjustment } \\
\text { made. }\end{array}$ & Repeat Section & & & \\
\hline
\end{tabular}


Neutron Moisture Logging System (NMLS) Log Run Information:

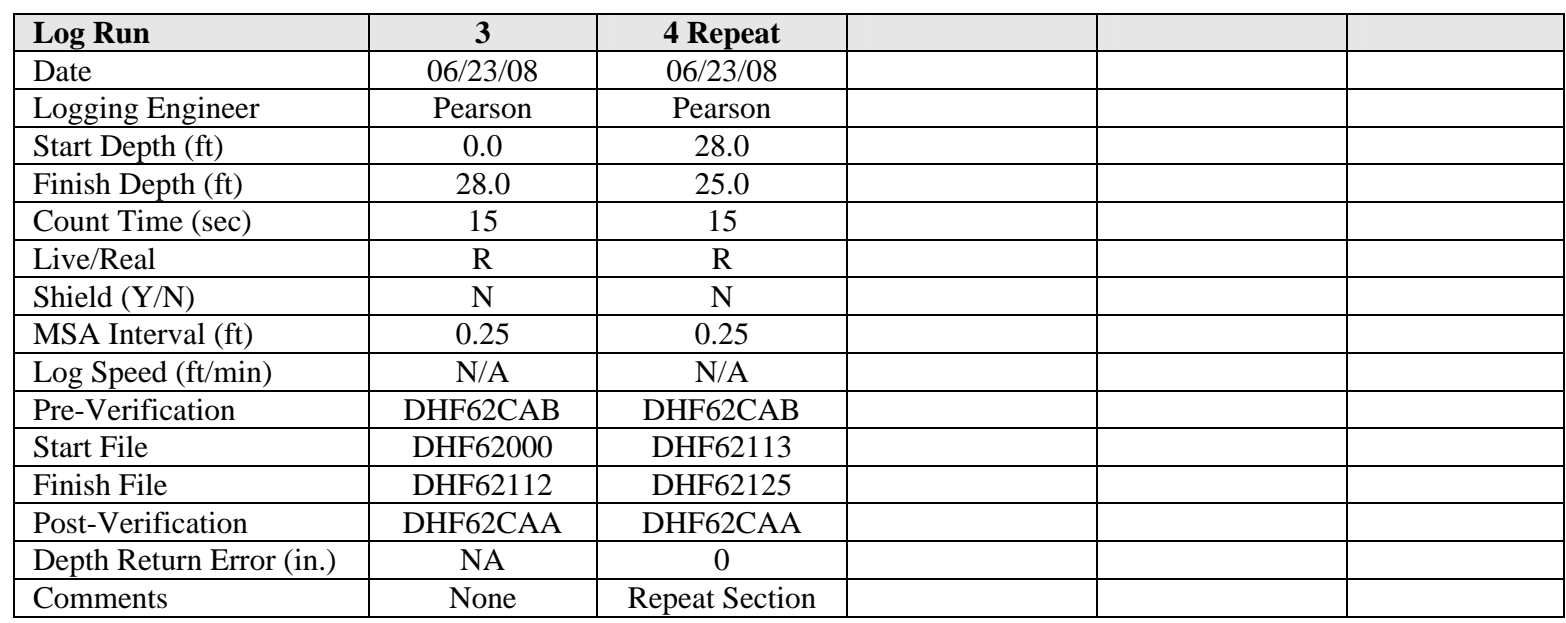

\section{Logging Operation Notes:}

Data were collected using Gamma 4, HO 68B-3573. SGLS pre- and post-survey verification measurements were acquired in the Amersham KUTh-115 field verifier. NMLS pre- and post-survey verification measurements were acquired in the standard field verifier. Maximum logging depth for the SGLS was $62.3 \mathrm{ft}$. before the sonde un-weighted. A centralizer was installed on the sonde.

\section{Analysis Notes:}

\begin{tabular}{|l|l|l|l|l|l|}
\hline Analyst: & LEGLER & Date: & $09 / 15 / 08$ & Reference: & GJO-HGLP 1.6.3, Rev. 0 \\
\hline
\end{tabular}

The SGLS pre- and post-survey verification spectra met the acceptance criteria for the established system. NMLS pre- and post-survey verification spectra met the acceptance criteria for the established system, but the verification spectrum file DHF62CAA had measurements above the upper control limit for counts per second (cps).

A casing correction for a 3/8-in. thick casing was applied during analysis for depths up $59 \mathrm{ft}$ where casing ends, leaving $3 \mathrm{ft}$ of open borehole uncorrected. A water correction was also applied during analysis from $27.3 \mathrm{ft}$ to total logged depth of borehole.

SGLS spectra were processed in batch mode in APTEC SUPERVISOR to identify individual peaks and count rates. Concentrations were calculated using an EXCEL template identified as G4LDec07.xls using an efficiency function and corrections for casing, dead time, and water as determined by annual calibrations.

NMLS spectra were processed in batch mode in APTEC SUPERVISOR to identify counts. Count rates were calculated using an EXCEL template identified as G4HNov07.xls. NMLS data are presented in counts per second (cps), because no calibration data is available for a 6 7/8-in. inner diameter borehole casing.

\section{Results and Interpretations:}

Cs- 137 was detected at $7 \mathrm{ft}, 12.5-15 \mathrm{ft}$, and $54.0 \mathrm{ft}$. Inspection of the individual spectra for these depths, indicate that detections at 7 and $54 \mathrm{ft}$ are statistical fluctuations associated with the processing software and are not considered valid.

Co-60 was detected at $13.5-15.5 \mathrm{ft}$. 
HGLP-LDR-245, Rev. 0

U-235 and U-238 (Pa-234m) were also detected at various isolated depths in this borehole. Inspection of the individual spectra for these depths indicate that these detections are statistical fluctuations associated with the processing software and not considered valid.

A moisture increase is observed at approximately $18 \mathrm{ft}$.

Repeat sections of Natural Gamma and NMLS logs showed good repeatability.

\section{List of Log Plots:}

Depth Reference is ground surface

Manmade Radionuclides

Natural Gamma Logs

Combination Plot

Total Gamma \& Dead Time

Total Gamma \& Moisture

Repeat Section of Natural Gamma Logs

Moisture Repeat Section

${ }^{1} \mathrm{GWL}$ - groundwater level

${ }^{2}$ TOC - top of casing 


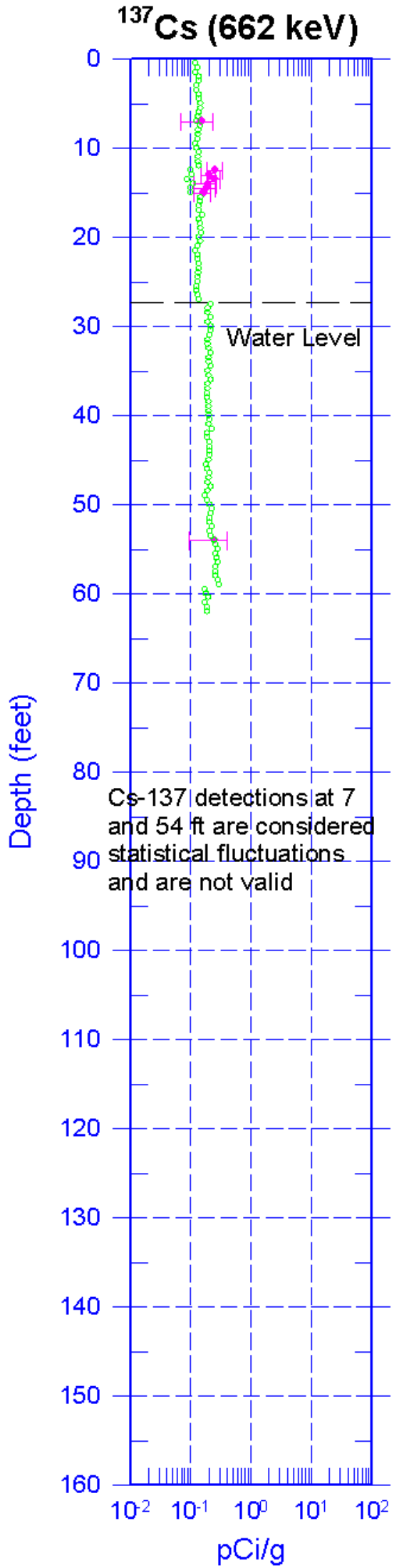

Zero Reference - Ground Surface

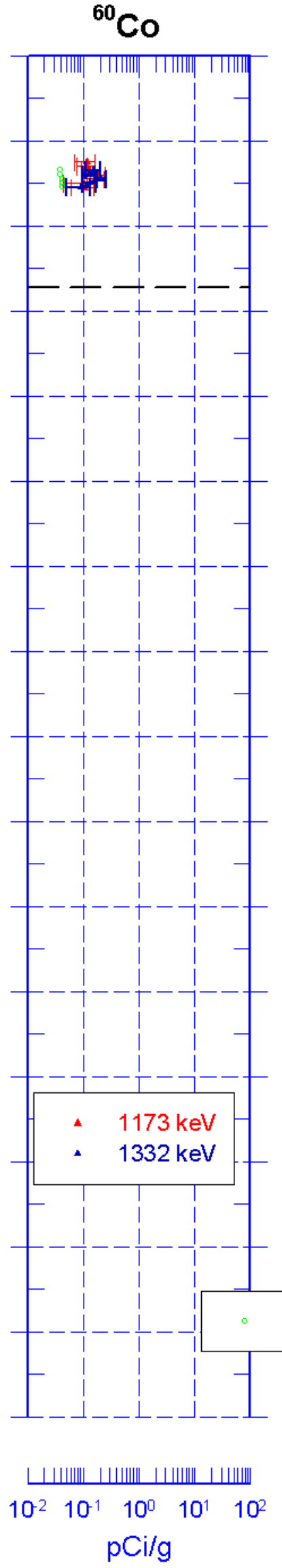

${ }^{235} \mathrm{U}(186 \mathrm{keV}) \quad{ }^{238} \mathrm{U}(1001 \mathrm{keV})$

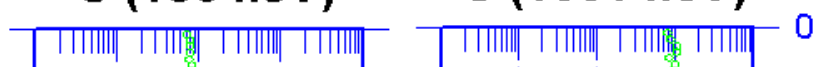

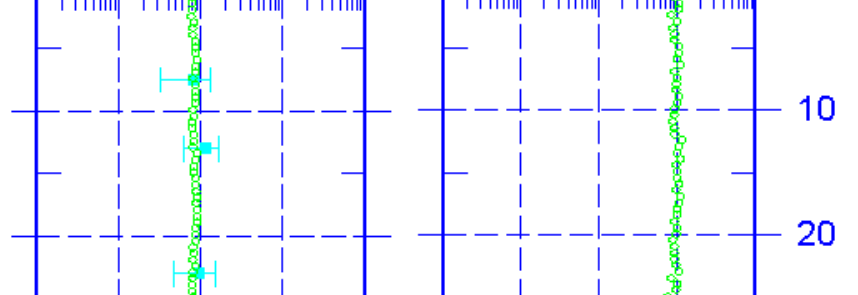

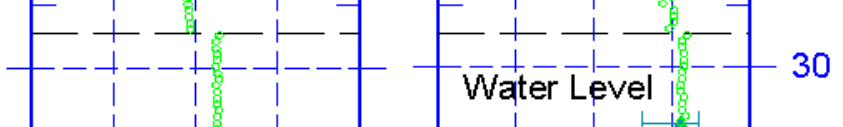
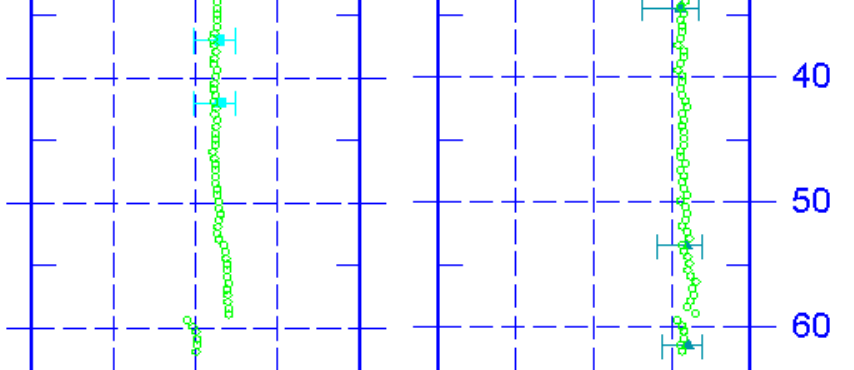

Detections are considered statistical fluctuations and are not valid
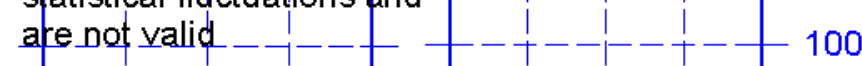

Detections are considered statistical fluctuations and arenot valid- -+-110
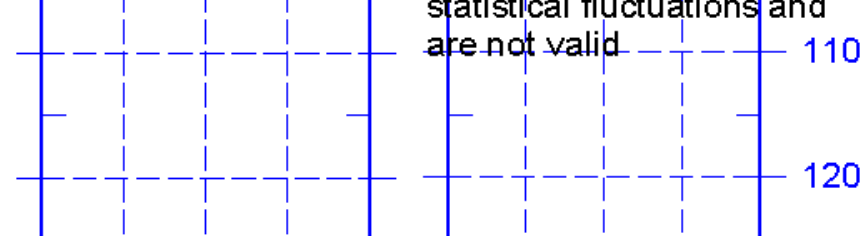
120 130 140

MDL
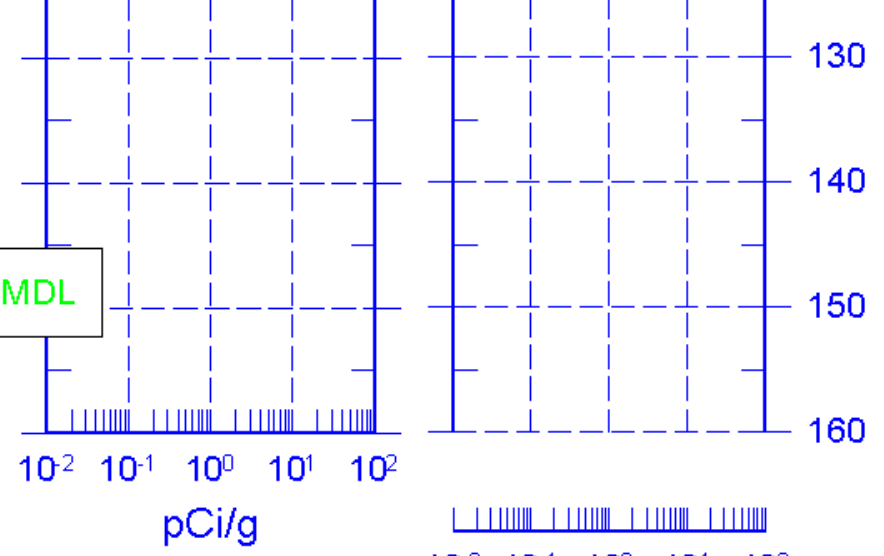

பШ山ШШШШШШШШШШШШ $\begin{array}{lllll}10^{-2} & 10^{-1} & 10^{0} & 10^{1} & 10^{2}\end{array}$ $\mathrm{pCi} / \mathrm{g}$ 
toller 399-2-12 (C6189)

Hanford Office Natural Gamma Logs
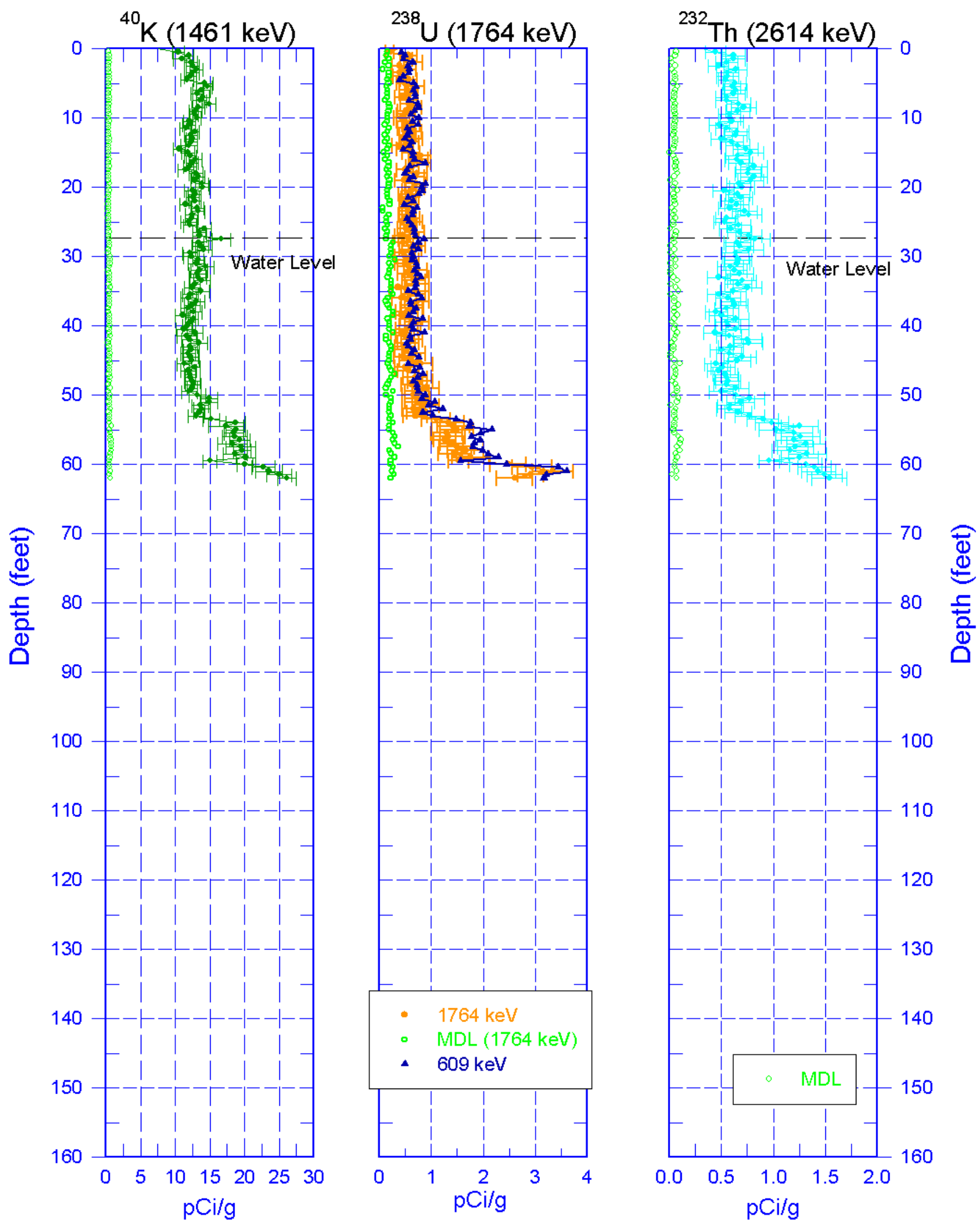


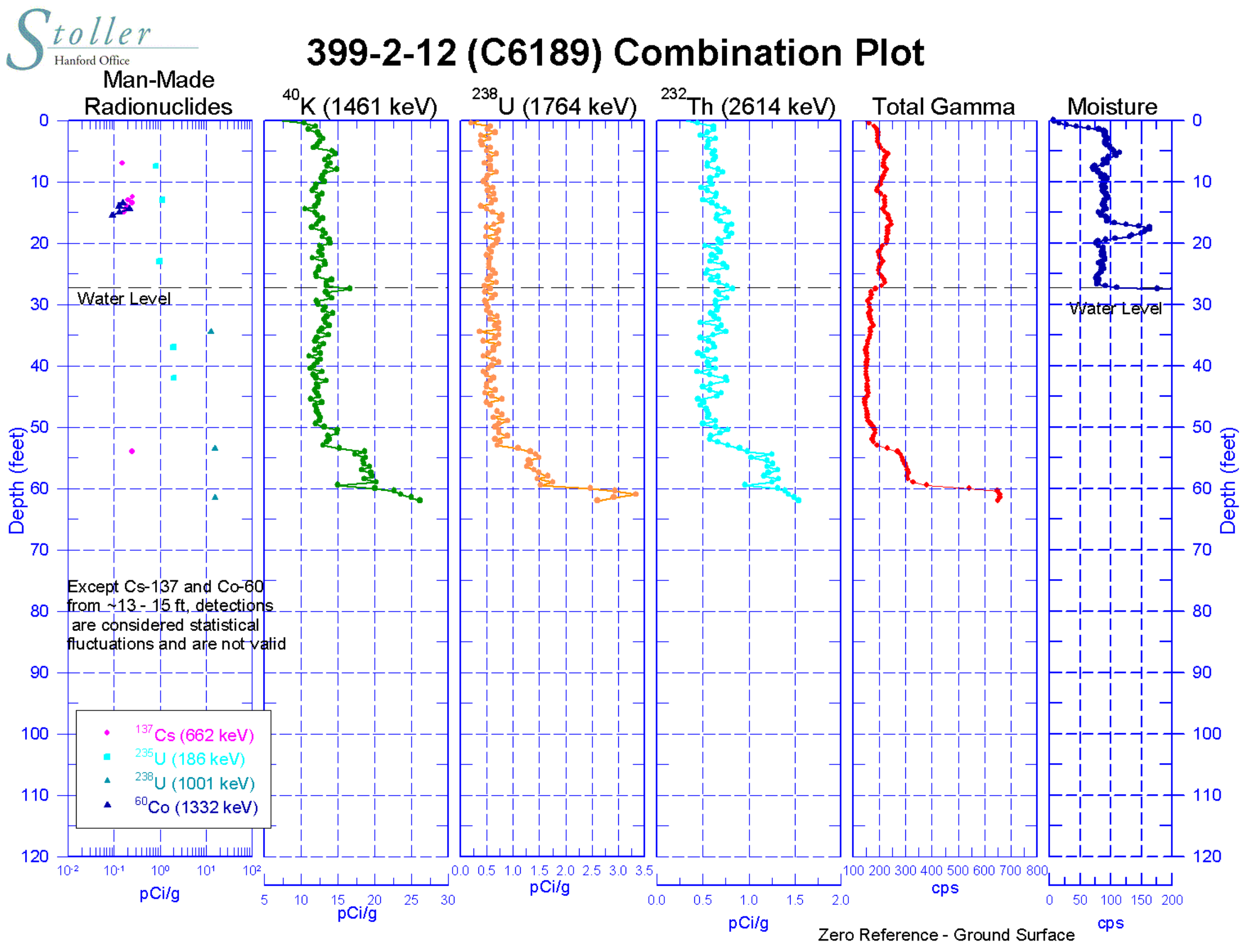



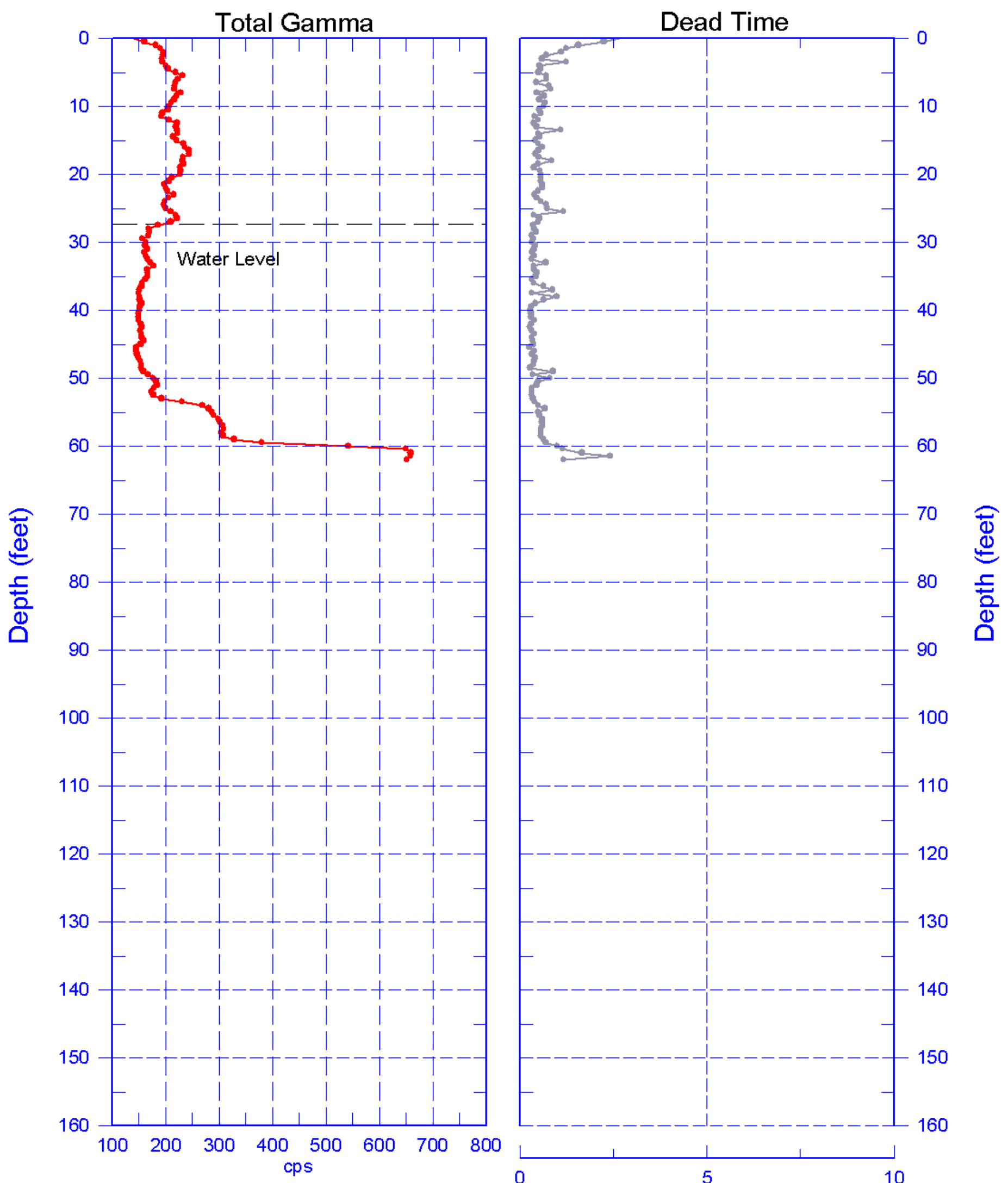

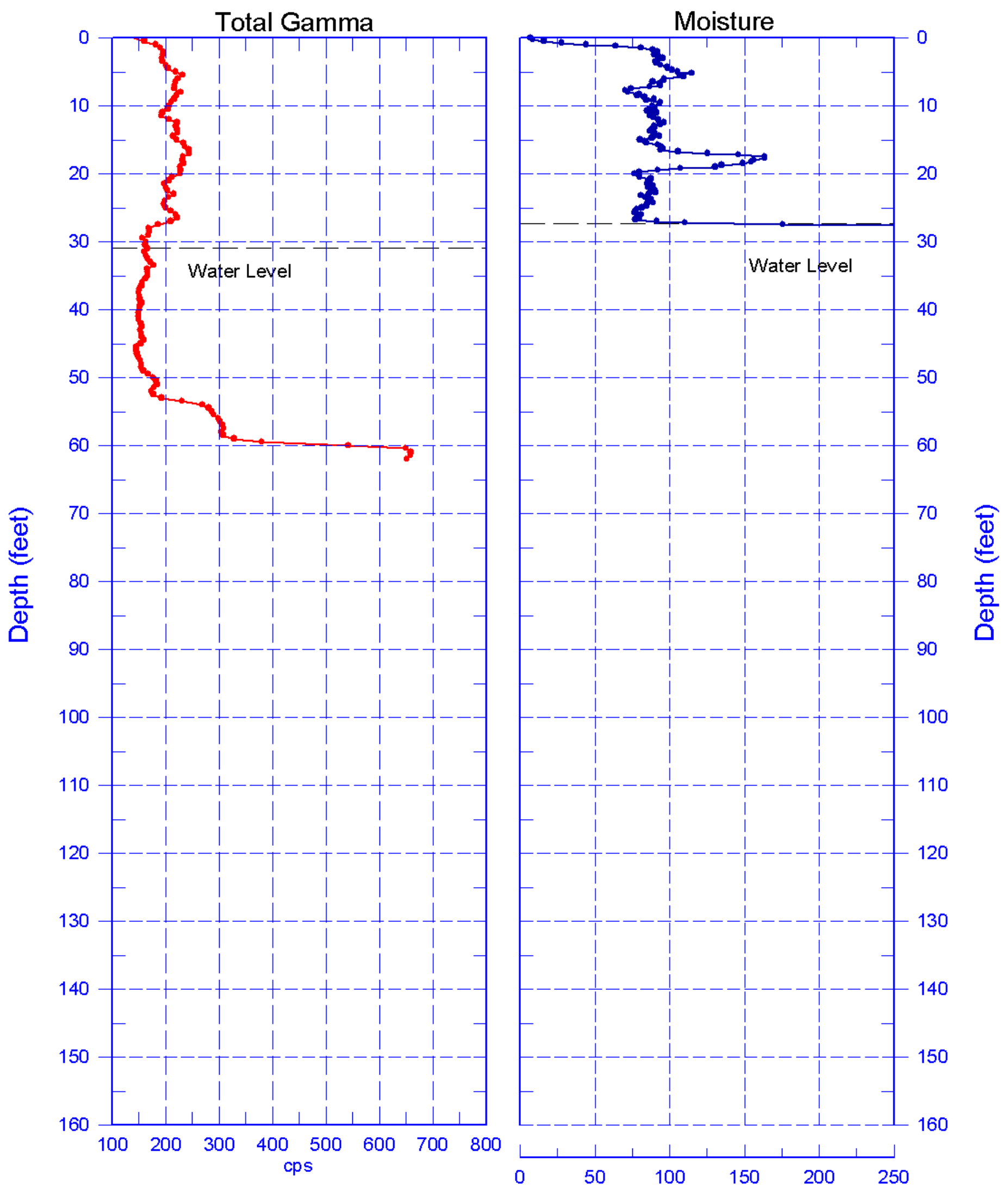


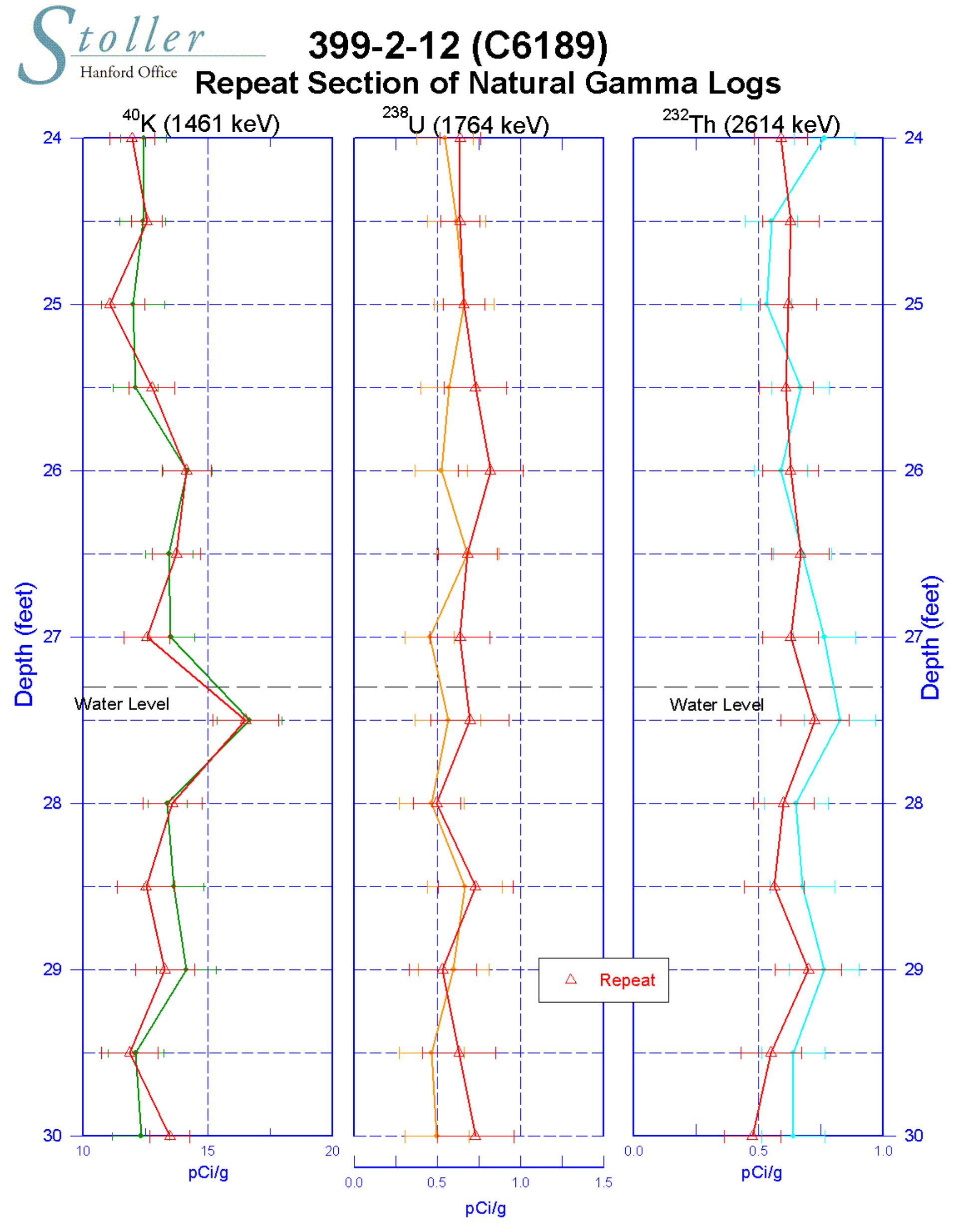

Zero Reference - Ground Surface 
toller $\quad$ 399-2-12 (C6189)

Hanford Office

Moisture Repeat Section

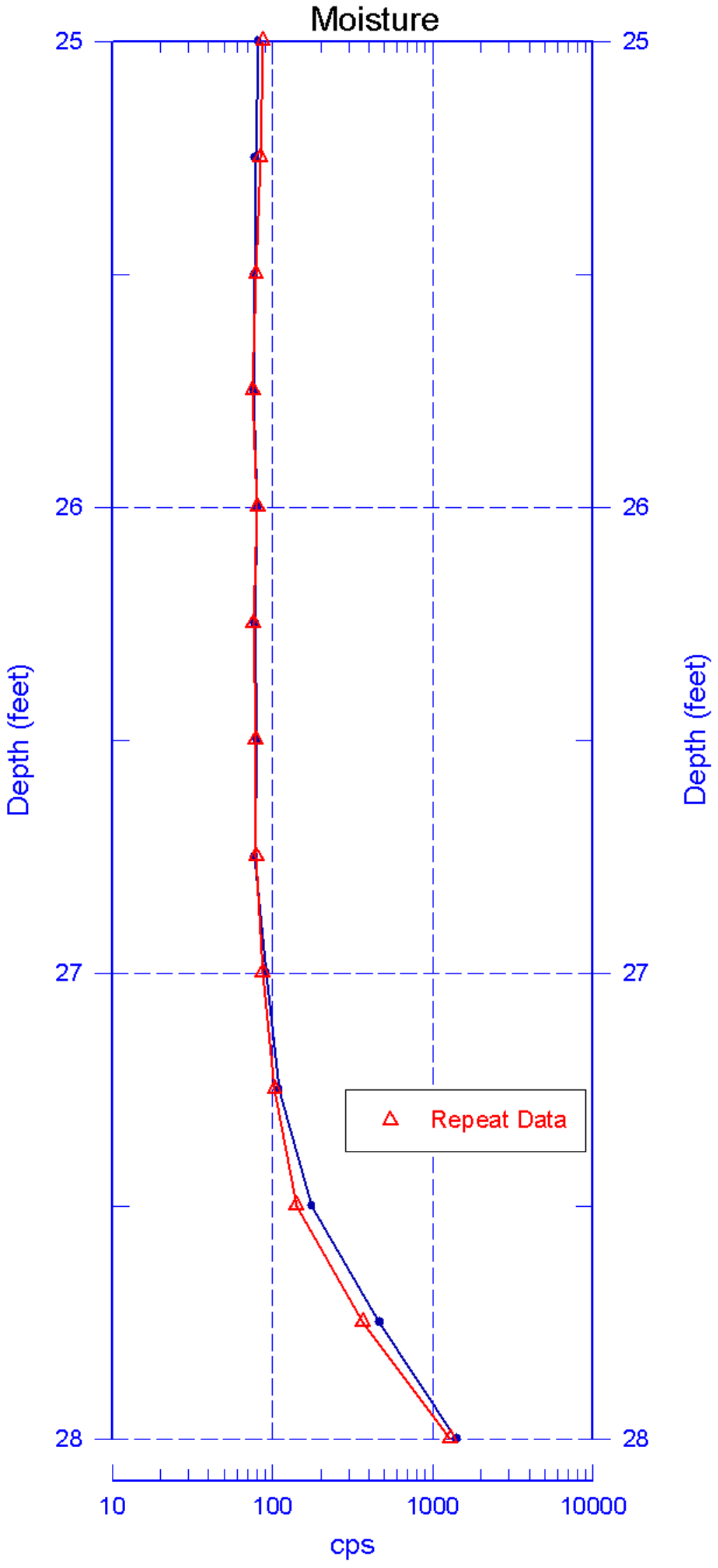

Reference - Ground Surface

G.70 


\section{9-2-13 (C6190) Log Data Report}

Borehole Information:

\begin{tabular}{|c|c|c|c|c|c|}
\hline \multirow{2}{*}{\multicolumn{2}{|c|}{ Borehole: $\quad 399-2-13$ (C6190) }} & \multicolumn{4}{|c|}{$300-F F-5$} \\
\hline & & $\mathbf{G W L}^{1}$ (f & 28.0 & GWL Date: & $05 / 22 / 08$ \\
\hline North (m) & East (m) & Drill Date & TOC $^{2}$ Elevation & Total Depth (ft) & Type \\
\hline Unknown & Unknown & $05 / 22 / 08$ & Unknown & 62.6 & Sonic \\
\hline
\end{tabular}

\section{Casing Information:}

\begin{tabular}{|c|c|c|c|c|c|c|}
\hline Casing Type & Stickup (ft) & $\begin{array}{c}\text { Outer } \\
\text { Diameter (in.) }\end{array}$ & $\begin{array}{c}\text { Inside } \\
\text { Diameter (in.) }\end{array}$ & Thickness (in.) & Top (ft) & Bottom (ft) \\
\hline Threaded Steel & 3.6 & $75 / 8$ & $67 / 8$ & $3 / 8$ & -3.6 & 57.0 \\
\hline
\end{tabular}

\section{Borehole Notes:}

Well site geologist reported depth to bottom, depth to water, and depth of casing. Water level appears to fluctuate with the level of the river. Logging engineer measured casing diameter using a steel tape and rounding to the nearest $1 / 16$-in. The zero reference is the ground surface.

\section{Logging Equipment Information:}

\begin{tabular}{|c|c|c|c|c|}
\hline Logging System: & \multicolumn{2}{|c|}{ Gamma $1 \mathrm{~N}$} & $\begin{array}{l}\text { Type: } \\
\text { Serial No.: }\end{array}$ & $\begin{array}{l}\text { 60\% HPGe SGLS } \\
45 T P 22010 A\end{array}$ \\
\hline Effective Calibration Date: & $03 / 28 / 08$ & Calibration Reference: & \multicolumn{2}{|c|}{ HGLP-CC-031 } \\
\hline & & Logging Procedure: & \multicolumn{2}{|c|}{ HGLP-MAN-002, Rev. 0} \\
\hline Logging System: & \multicolumn{2}{|c|}{ Gamma $1 \mathrm{M}$} & $\begin{array}{l}\text { Type: } \\
\text { Serial No.: }\end{array}$ & $\begin{array}{l}\text { NMLS } \\
\text { H340207279 }\end{array}$ \\
\hline Effective Calibration Date: & $05 / 06 / 08$ & Calibration Reference: & \multicolumn{2}{|c|}{ HGLP-CC-032 } \\
\hline & & Logging Procedure: & \multicolumn{2}{|c|}{ HGLP-MAN-002, Rev. 0} \\
\hline
\end{tabular}

\section{Spectral Gamma Logging System (SGLS) Log Run Information:}

\begin{tabular}{|l|c|c|c|}
\hline Log Run & $\mathbf{1}$ & $\mathbf{2}$ & $\mathbf{3}$ \\
\hline Date & $05 / 27 / 08$ & $05 / 28 / 08$ & $05 / 28 / 08$ \\
\hline Logging Engineer & Spatz & Spatz & Spatz \\
\hline Start Depth (ft) & 0.0 & 54.0 & 38.0 \\
\hline Finish Depth (ft) & 55.0 & 58.0 & 44.0 \\
\hline Count Time (sec) & 200 & 200 & 200 \\
\hline Live/Real & $\mathrm{R}$ & $\mathrm{R}$ & $\mathrm{R}$ \\
\hline Shield (Y/N) & $\mathrm{N}$ & $\mathrm{N}$ & $\mathrm{N}$ \\
\hline MSA Interval (ft) & 0.5 & 0.5 & 0.5 \\
\hline Log Speed (ft/min) & N/A & N/A & N/A \\
\hline Pre-Verification & AN077CAB & AN078CAB & AN078CAB \\
\hline Start File & AN077000 & AN078000 & AN078009 \\
\hline Finish File & AN077110 & AN078008 & AN078021 \\
\hline Post-Verification & AN077CAA & AN078CAA & AN078CAA \\
\hline Depth Return Error (in.) & 0 & N/A & 0 \\
\hline Comments & $\begin{array}{c}\text { Fine gain } \\
\text { adjustment } \\
\text { made after } \\
\text { files 065 and } \\
\text { 090. }\end{array}$ & $\begin{array}{c}\text { No fine gain } \\
\text { adjustment } \\
\text { made. }\end{array}$ & Repeat section \\
& \multicolumn{2}{|l}{} \\
\hline
\end{tabular}




\section{Neutron Moisture Logging System (NMLS) Log Run Information:}

\begin{tabular}{|l|c|c|}
\hline Log Run & $\mathbf{4}$ & $\mathbf{5}$ \\
\hline Date & $05 / 23 / 08$ & $05 / 23 / 08$ \\
\hline Logging Engineer & Pearson & Pearson \\
\hline Start Depth (ft) & 0.0 & 16.0 \\
\hline Finish Depth (ft) & 31.75 & 20.0 \\
\hline Count Time (sec) & 15 & 15 \\
\hline Live/Real & $\mathrm{R}$ & $\mathrm{R}$ \\
\hline Shield (Y/N) & $\mathrm{N}$ & $\mathrm{N}$ \\
\hline MSA Interval (ft) & 0.25 & 0.25 \\
\hline Log Speed (ft/min) & N/A & N/A \\
\hline Pre-Verification & AM005CAB & AM005CAB \\
\hline Start File & AM005000 & AM005128 \\
\hline Finish File & AM005127 & AM005144 \\
\hline Post-Verification & AM005CAA & AM005CAA \\
\hline Depth Return Error (in.) & N/A & 0 \\
\hline Comments & None & Repeat section \\
\hline
\end{tabular}

\section{Logging Operation Notes:}

Data were collected using Gamma 1, HO 68B-3574. SGLS pre- and post-survey verification measurements were acquired in the Amersham KUTh-118 field verifier. Maximum logging depth was $58.0 \mathrm{ft}$ approximately 1-ft below casing. A centralizer was installed on the sonde. NMLS pre- and post-survey verification measurements were acquired in the AmBe standard.

\section{Analysis Notes:}

\begin{tabular}{|l|l|l|l|l|l|}
\hline Analyst: & LEGLER & Date: & $07 / 17 / 08$ & Reference: & GJO-HGLP 1.6.3, Rev. 0 \\
\hline
\end{tabular}

SGLS pre- and post-survey verification spectra met the acceptance criteria for the established system, but the verification spectra files AN078CAB, and AN078CAA had measurements above the upper control limits for the $609 \mathrm{keV}$ energy line and $2614 \mathrm{keV}$ energy line respectively. NMLS pre- and post-verification spectra met the acceptance criteria for the established system.

A casing correction for a 3/8-in. thick casing was applied during analysis for depths up to $57.0 \mathrm{ft}$, where casing ends, leaving $1 \mathrm{ft}$ of open hole uncorrected. A water correction was also applied during analysis from $28.0 \mathrm{ft}$ to total logged depth of borehole.

SGLS spectra were processed in batch mode in APTEC SUPERVISOR to identify individual peaks and count rates. Concentrations were calculated using an EXCEL template identified as G1NMar08.xls using an efficiency function and corrections for casing, dead time and water as determined by annual calibrations. NMLS spectra were processed in batch mode in APTEC SUPERVISOR to identify counts. Count rates were calculated using an EXCEL template identified as G1MMay08.xls. NMLS data are presented in counts per second (cps), because no calibration data is available for a 6 7/8-in. inner diameter borehole casing.

\section{Results and Interpretations:}

Cs-137, U-235, and U-238 were detected by the routine processing software at various depths in this borehole. Inspection of the individual spectra at these depths indicates that these detections are statistical fluctuations and are not valid.

A fine grain sediment layer can be seen at $40.5 \mathrm{ft}$, by the increases in the total gamma, K-40 (1461 keV), natural U-238 (1764 keV), and Th-232 (2614keV). 
HGLP-LDR-232, Rev. 0

The KUT plots indicated good repeatability. Moisture plots indicate some variability.

SGLS log data collected on 5/22/08 indicates depth to groundwater at approximately $28 \mathrm{ft}$. NMLS data collected on 5/23/08 indicate depth to groundwater at approximately $31 \mathrm{ft}$. Depth to water is reported as $33 \mathrm{ft}$ on the Logging Request Tracking Sheet (no date provided). The groundwater level appears to fluctuate with the river level.

\section{List of Log Plots:}

Depth reference is ground surface

Manmade Radionuclides

Natural Gamma Logs

Combination Plot

Total Gamma \& Dead Time

Total Gamma \& Moisture

Repeat Section of Manmade Radionuclides

Moisture Repeat Section

${ }^{1} \mathrm{GWL}$ - groundwater level

${ }^{2}$ TOC - top of casing 

Hanford Ofice Manmade Radionuclídes

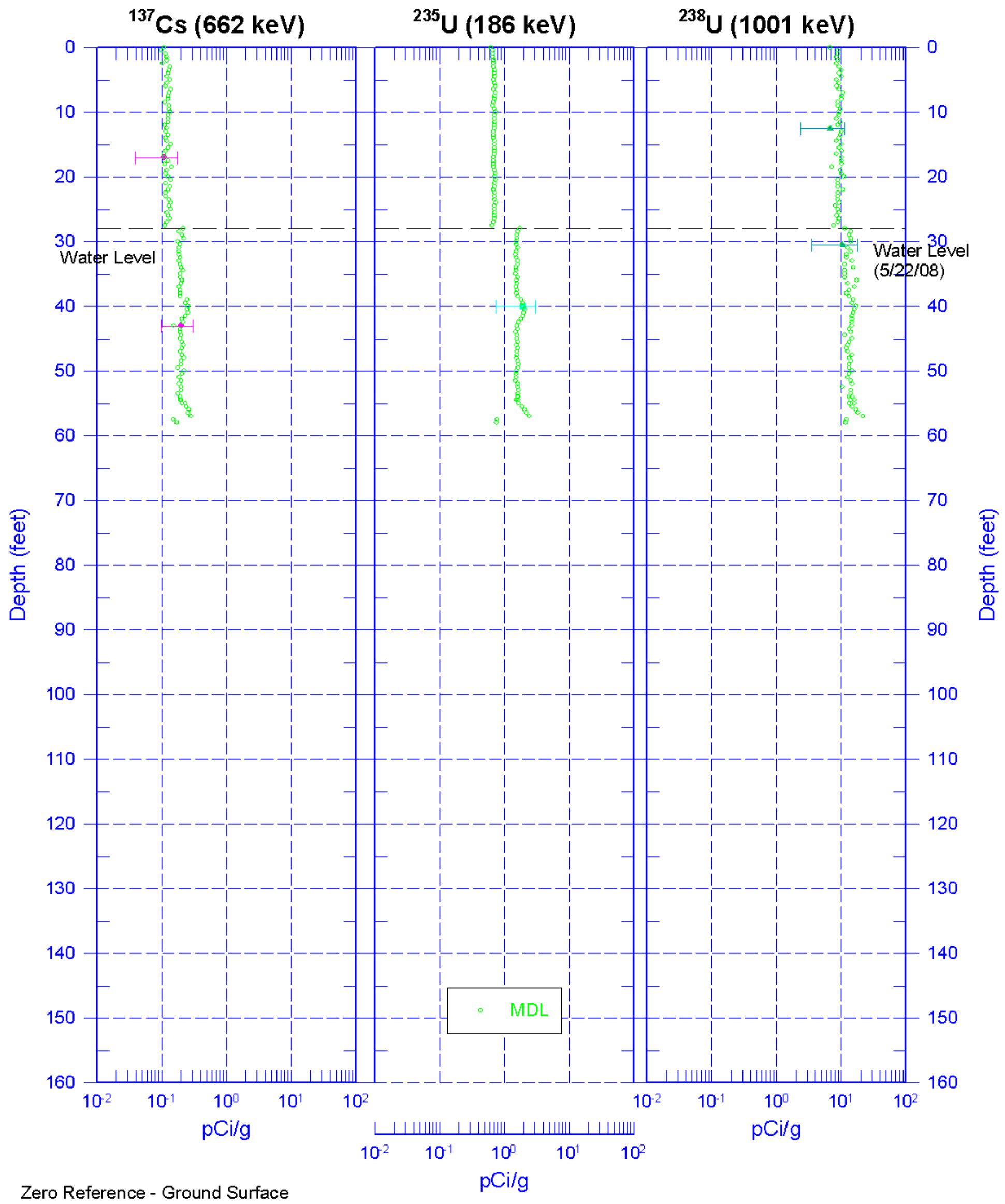


toller $\mathbf{3 9 9 - 2 - 1 3}$ (66190)

Hanford Office Natural Gamma Logs
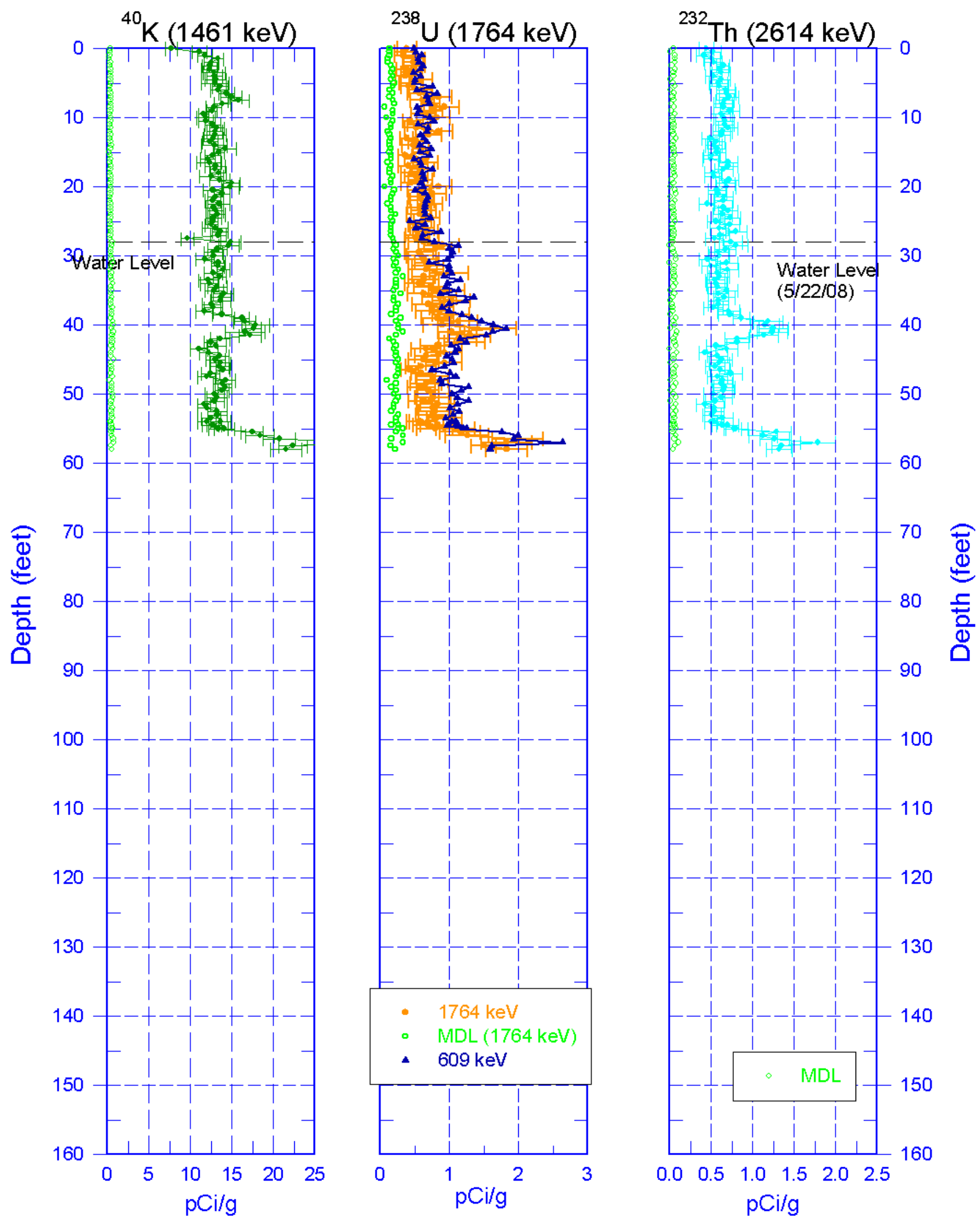

Zero Reference - Ground Surface 


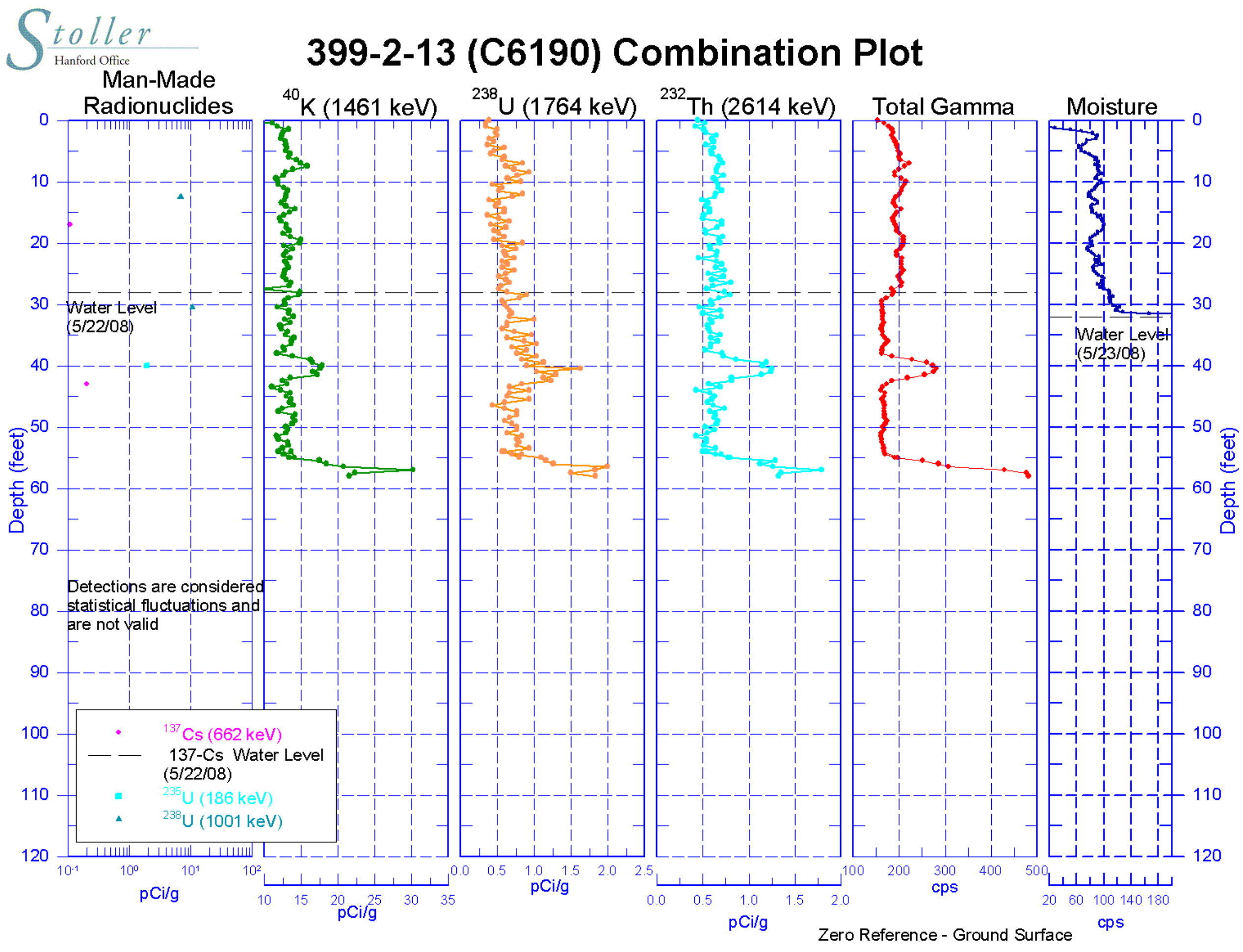



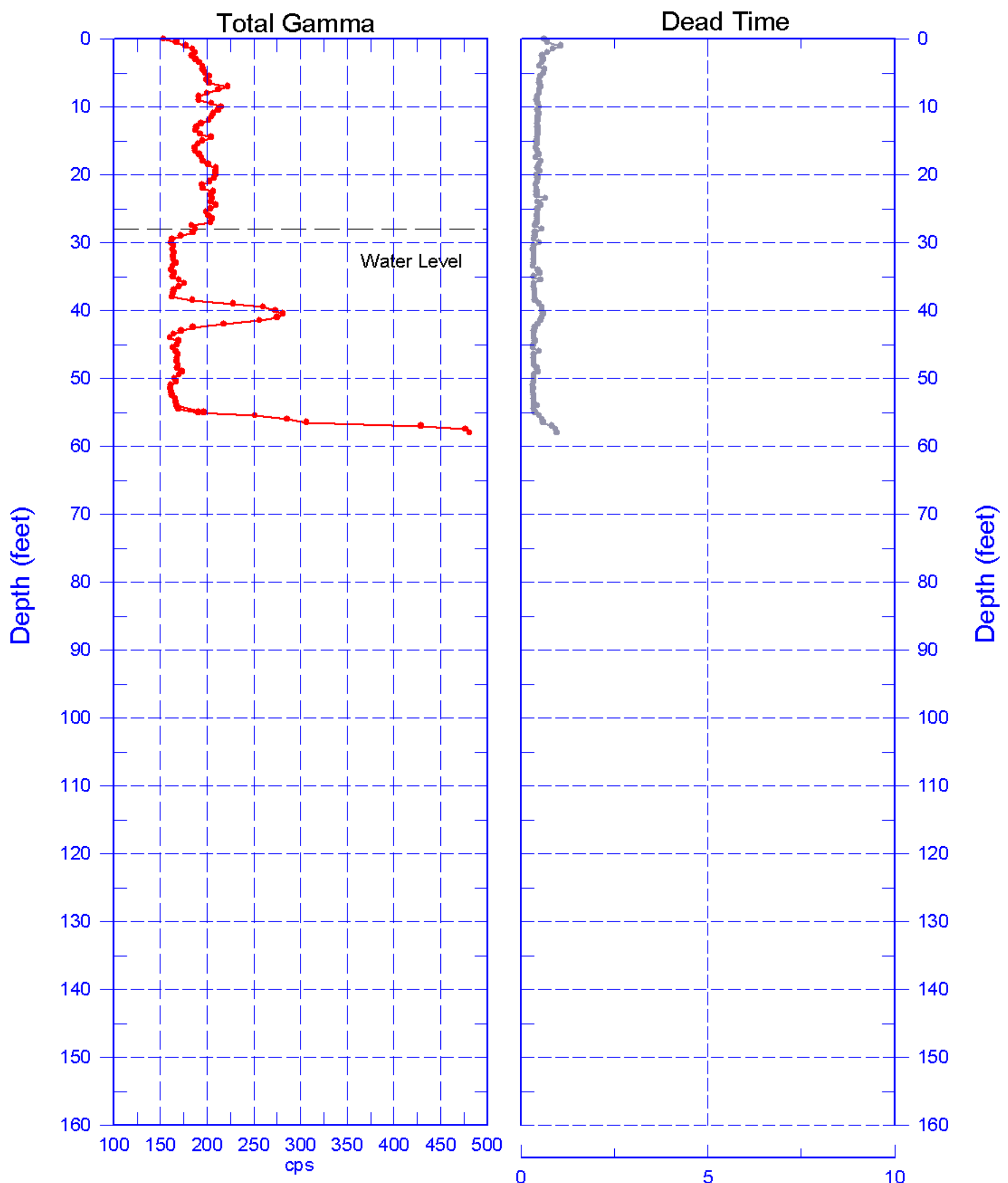


\section{S \\ toller 399-2-13 (C6190) \\ Hanford Office \\ Total Gamma \& Moisture}
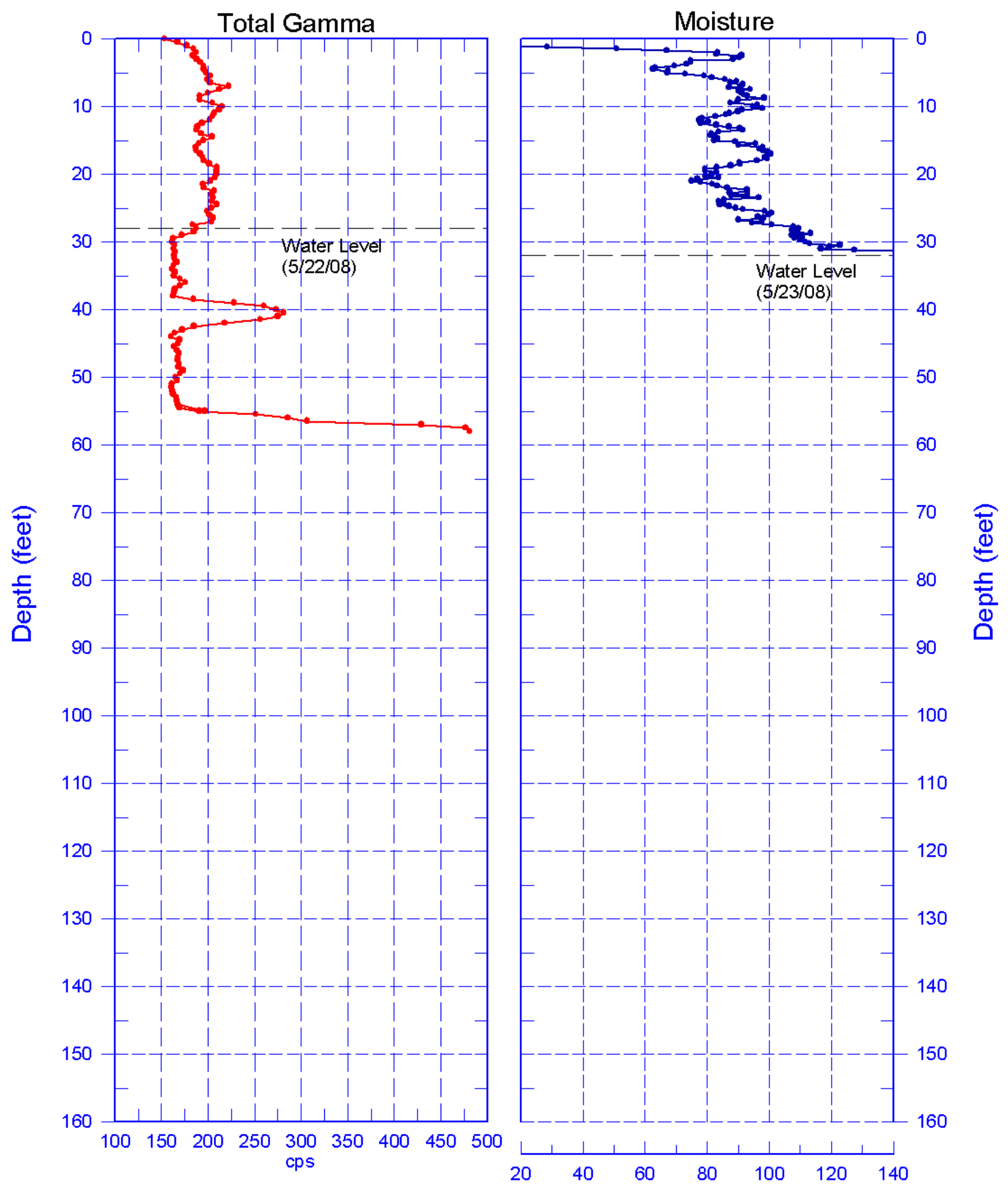

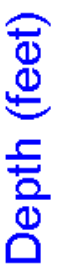

Reference - Ground Surface 


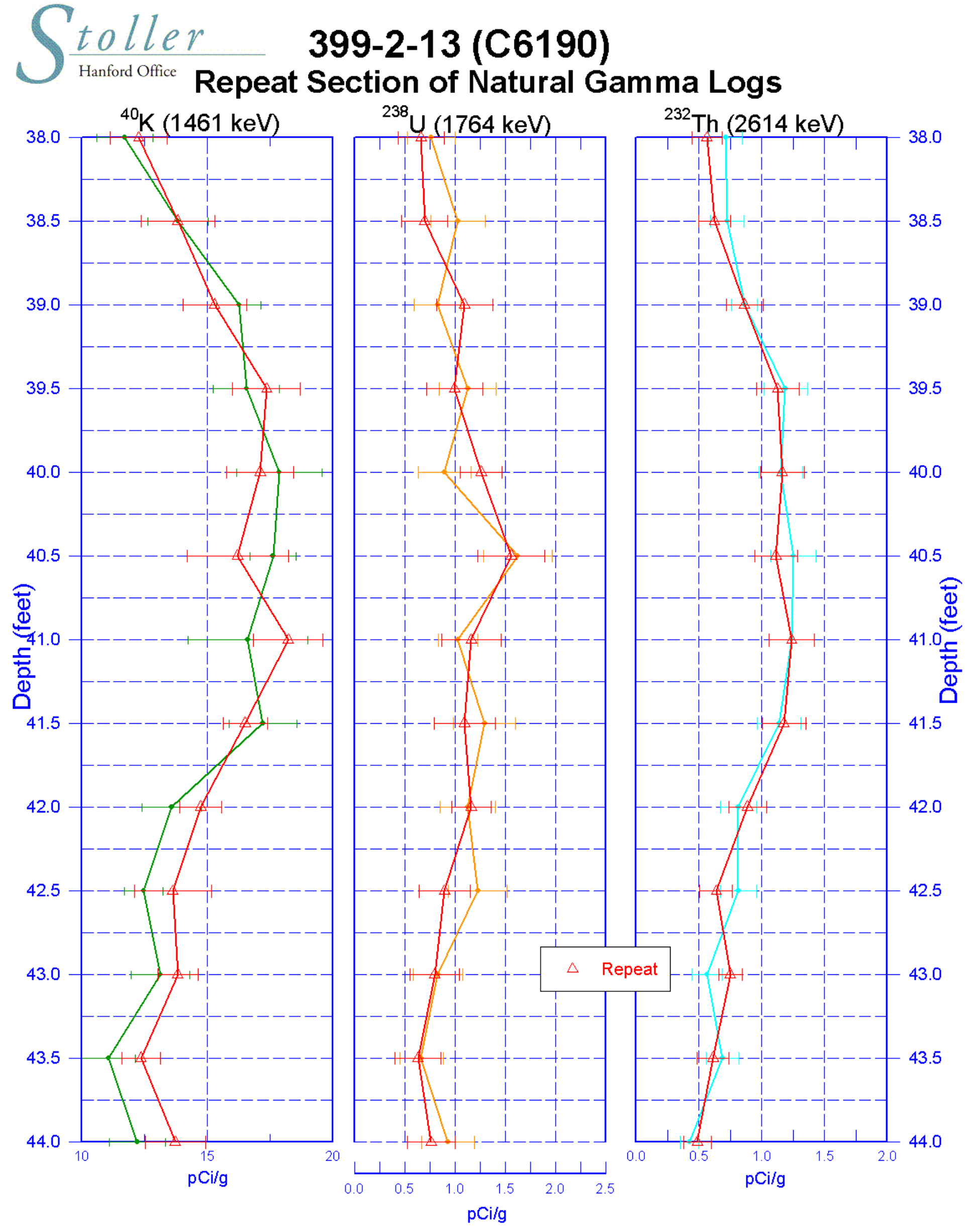

Zero Reference - Ground Surface 


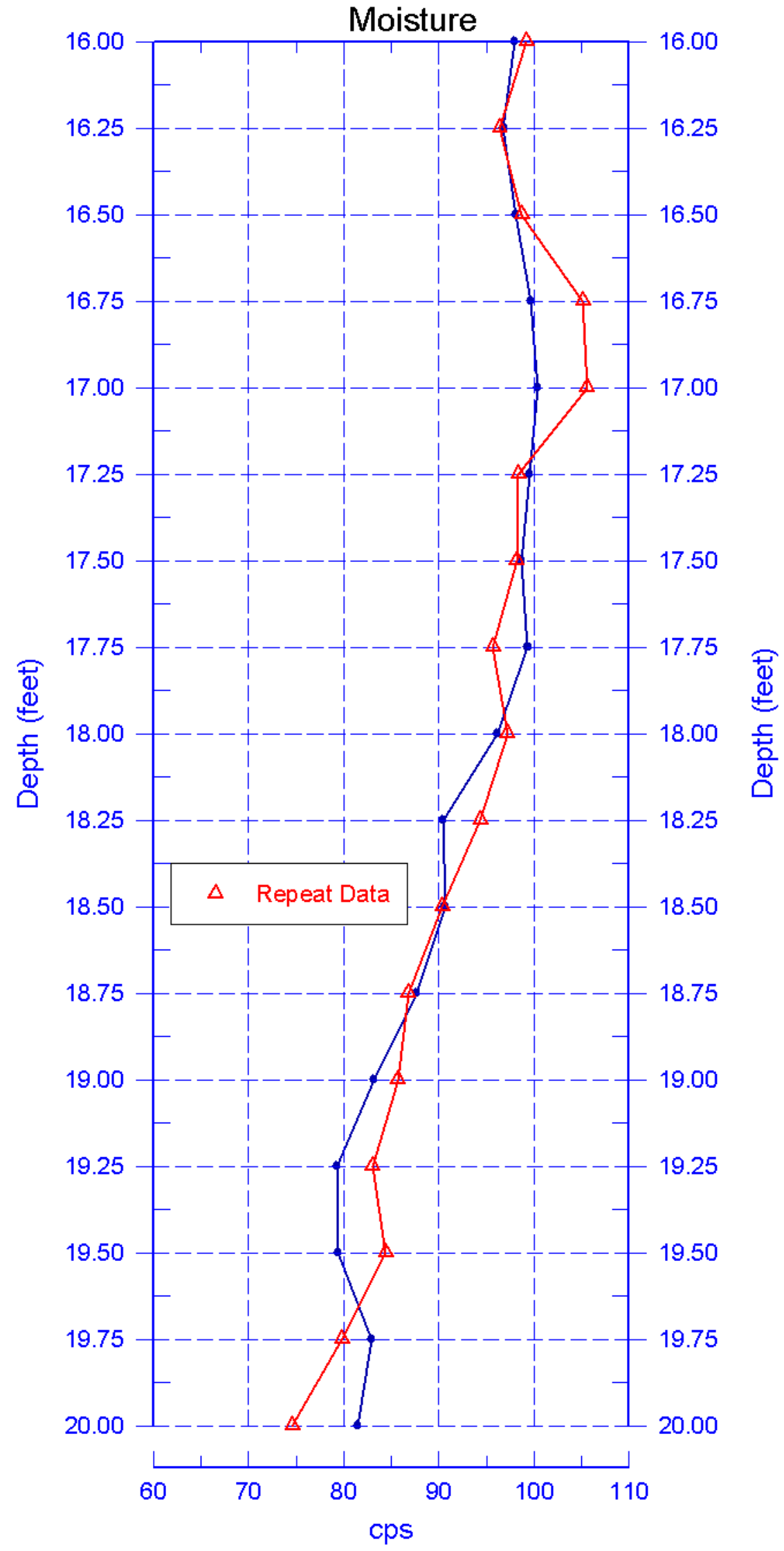


HGLP-LDR-242, Rev. 0

\section{9-2-14 (C6191) \\ Log Data Report}

\section{Borehole Information:}

\begin{tabular}{|c|c|c|c|c|c|}
\hline Borehole: & 399-2-14 (C6191) & & Site: & 300-FF-5 & \\
\hline \multicolumn{2}{|c|}{ Coordinates (WA St Plane) } & GWL $^{1}(\mathbf{f t}):$ & 30.3 & GWL Date: & $06 / 17 / 08$ \\
\hline North (m) & East (m) & Drill Date & TOC $^{2}$ Elevation & Total Depth (ft) & Type \\
\hline 116070.3 & 594245.2 & $06 / 16 / 08$ & Unknown & 58.5 & Sonic \\
\hline
\end{tabular}

\section{Casing Information:}

\begin{tabular}{|c|c|c|c|c|c|c|}
\hline Casing Type & Stickup (ft) & $\begin{array}{c}\text { Outer } \\
\text { Diameter (in.) }\end{array}$ & $\begin{array}{c}\text { Inside } \\
\text { Diameter (in.) }\end{array}$ & Thickness (in.) & Top (ft) & Bottom (ft) \\
\hline Welded Steel & 0.6 & $75 / 8$ & $67 / 8$ & $3 / 8$ & -0.6 & 56.0 \\
\hline
\end{tabular}

\section{Borehole Notes:}

Casing data, and total depth were reported by the site geologist. Logging engineer measured depth to water with an e-tape. Casing diameters were measured using a steel tape and rounded to the nearest 1/16-in. The zero reference is the ground surface.

\section{Logging Equipment Information:}

\begin{tabular}{|l|l|l|l|l|}
\hline Logging System: & \multirow{2}{*}{ Gamma 4 L } & $\begin{array}{l}\text { Type: } \\
\text { Serial No.: }\end{array}$ & $\begin{array}{l}\text { 60\% HPGe SGLS } \\
\text { 47TP32211A }\end{array}$ \\
\hline Effective Calibration Date: & $12 / 31 / 07$ & Calibration Reference: & HGLP-CC-027 \\
\hline \multicolumn{2}{|l|}{} & Logging Procedure: & HGLP-MAN-002, Rev. 0 \\
\hline
\end{tabular}

\begin{tabular}{|l|l|l|l|l|}
\hline Logging System: & \multirow{2}{*}{ Gamma 4 H } & $\begin{array}{l}\text { Type: } \\
\text { Serial No.: }\end{array}$ & $\begin{array}{l}\text { NMLS } \\
\text { H310700352 }\end{array}$ \\
\hline Effective Calibration Date: & $11 / 06 / 07$ & Calibration Reference: & HGLP-CC-021 \\
\hline \multicolumn{2}{|l|}{} & Logging Procedure: & HGLP-MAN-002, Rev. 0 \\
\hline
\end{tabular}

\section{Spectral Gamma Logging System (SGLS) Log Run Information:}

\begin{tabular}{|l|c|c|}
\hline Log Run & 1 & 2 Repeat \\
\hline Date & $06 / 17 / 08$ & $06 / 17 / 08$ \\
\hline Logging Engineer & Pearson & Pearson \\
\hline Start Depth (ft) & 58.0 & 2.0 \\
\hline Finish Depth (ft) & 0.5 & 7.5 \\
\hline Count Time (sec) & 200 & 200 \\
\hline Live/Real & $\mathrm{R}$ & $\mathrm{R}$ \\
\hline Shield (Y/N) & $\mathrm{N}$ & $\mathrm{N}$ \\
\hline MSA Interval (ft) & 0.5 & 0.5 \\
\hline Log Speed (ft/min) & N/A & N/A \\
\hline Pre-Verification & DL461CAB & DL461CAB \\
\hline Start File & DL461000 & DL461116 \\
\hline Finish File & DL461115 & DL461127 \\
\hline Post-Verification & DL461CAA & DL461CAA \\
\hline Depth Return Error (in.) & 0 & 0 \\
\hline Comments & $\begin{array}{c}\text { No fine gain } \\
\text { adjustment } \\
\text { made. }\end{array}$ & Repeat Section \\
& \multicolumn{2}{|c}{} \\
\hline
\end{tabular}


HGLP-LDR-242, Rev. 0

\section{Neutron Moisture Logging System (NMLS) Log Run Information:}

\begin{tabular}{|l|c|c|}
\hline Log Run & $\mathbf{3}$ & 4 Repeat \\
\hline Date & $06 / 17 / 08$ & $06 / 17 / 08$ \\
\hline Logging Engineer & Pearson & Pearson \\
\hline Start Depth (ft) & 0.0 & 0.0 \\
\hline Finish Depth (ft) & 29.25 & 3.0 \\
\hline Count Time (sec) & 15 & 15 \\
\hline Live/Real & $\mathrm{R}$ & $\mathrm{R}$ \\
\hline Shield (Y/N) & $\mathrm{N}$ & $\mathrm{N}$ \\
\hline MSA Interval (ft) & 0.25 & 0.25 \\
\hline Log Speed (ft/min) & N/A & N/A \\
\hline Pre-Verification & DHF32CAB & DHF32CAB \\
\hline Start File & DHF32000 & DHF32118 \\
\hline Finish File & DHF32117 & DHF32130 \\
\hline Post-Verification & DHF32CAA & DHF32CAA \\
\hline Depth Return Error (in.) & N/A & 0 \\
\hline Comments & None. & Repeat Section \\
\hline
\end{tabular}

\section{Logging Operation Notes:}

Data were collected using Gamma 4, HO 68B-3573. SGLS pre - and post-survey verification measurements were acquired in the Amersham KUTh-115 field verifier. Maximum logging depth achieved was $58.0 \mathrm{ft}$ before the sonde un-weighted. A centralizer was installed on the sonde. NMLS pre - and post-survey verification measurements were acquired in the standard field verifier. Maximum logging depth achieved was $29.25 \mathrm{ft}$.

\section{Analysis Notes:}

\begin{tabular}{|l|l|l|l|l|l|}
\hline Analyst: & LEGLER & Date: & 07/29/08 & Reference: & GJO-HGLP 1.6.3, Rev. 0 \\
\hline
\end{tabular}

SGLS pre- and post-survey verification spectra met the acceptance criteria for the established system. NMLS preand post-survey verification spectra met the acceptance criteria for the established system, but spectra file DHF32CAB had measurements above the upper control limit for counts per second (cps).

A casing correction for a 3/8-in. thick casing was applied from ground surface to $56 \mathrm{ft}$ where casing ends, leaving $2 \mathrm{ft}$ of open borehole uncorrected. A water correction was also applied during analysis from $30.3 \mathrm{ft}$ to total logged depth of borehole.

SGLS spectra were processed in batch mode using APTEC SUPERVISOR to identify individual peaks and count rates. Concentrations were calculated using an EXCEL template identified as G4LDec07.xls using an efficiency function and corrections for casing, dead time and water as determined by annual calibrations. NMLS spectra were processed in batch mode in APTEC SUPERVISOR to identify counts. Count rates were calculated using an EXCEL template identified as G4HNov07.xls. NMLS data are presented in counts per second (cps), because no calibration data is available for a 6 7/8-in. inner diameter borehole casing.

\section{Results and Interpretations:}

Cs-137, U-235, and U-238 (Pa-234m) were detected sporadically throughout this borehole. Inspection of the individual spectra at the various depths indicates that these detections are statistical fluctuations associated with the processing software and are not valid.

The KUT repeat plots indicate good repeatability. The moisture repeat plot indicates good repeatability. 
HGLP-LDR-242, Rev. 0

\section{List of Log Plots:}

Depth Reference is ground surface

Manmade Radionuclides

Natural Gamma Logs

Combination Plot

Total Gamma \& Dead Time

Total Gamma \& Moisture

Repeat Section of Natural Gamma

Moisture Repeat Section

${ }^{1} \mathrm{GWL}$ - groundwater level

${ }^{2}$ TOC - top of casing 

Hanford Office Manmade Radionuclides

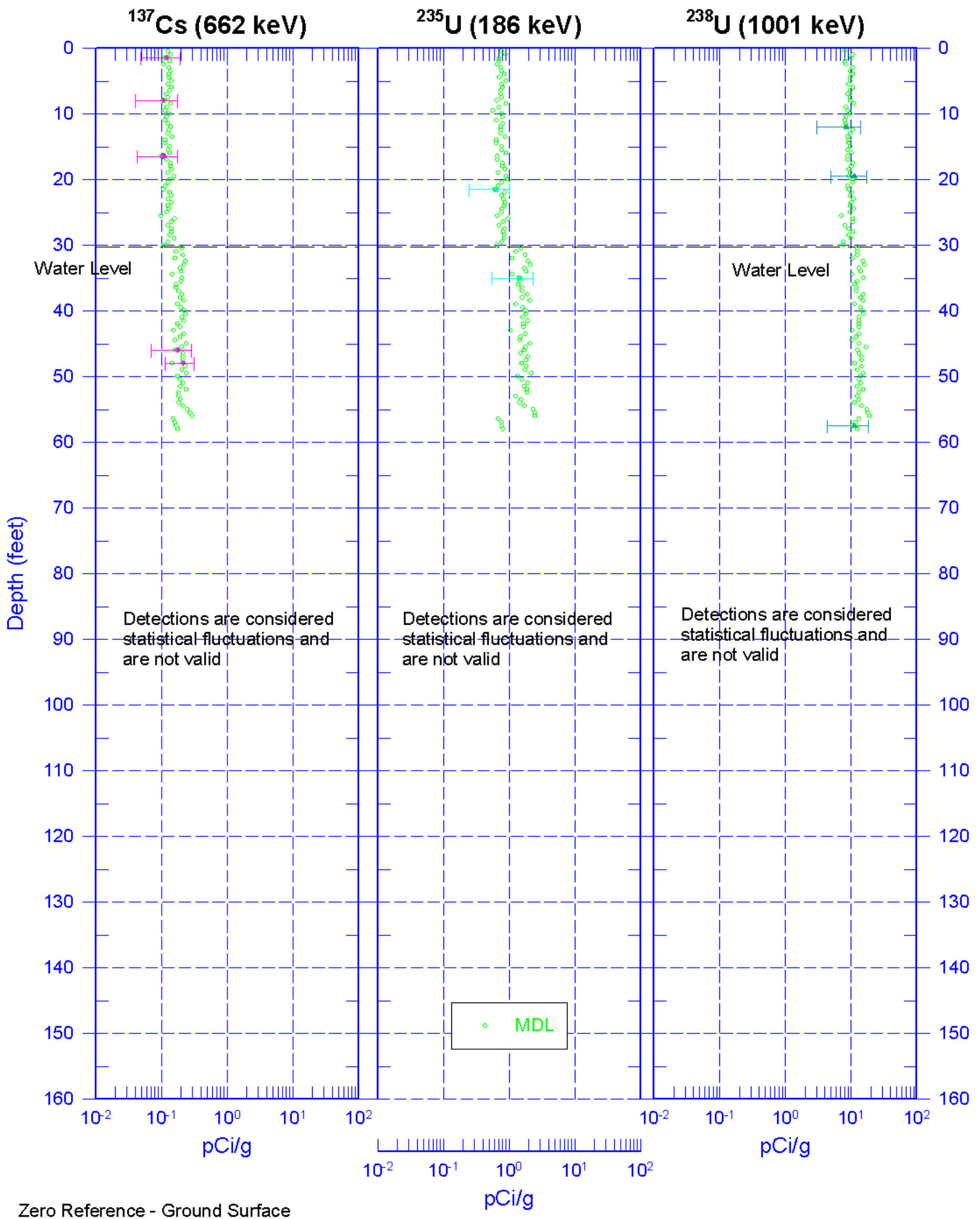


toller 399-2-14(C6191)

Hanford Office Natural Gamma Logs
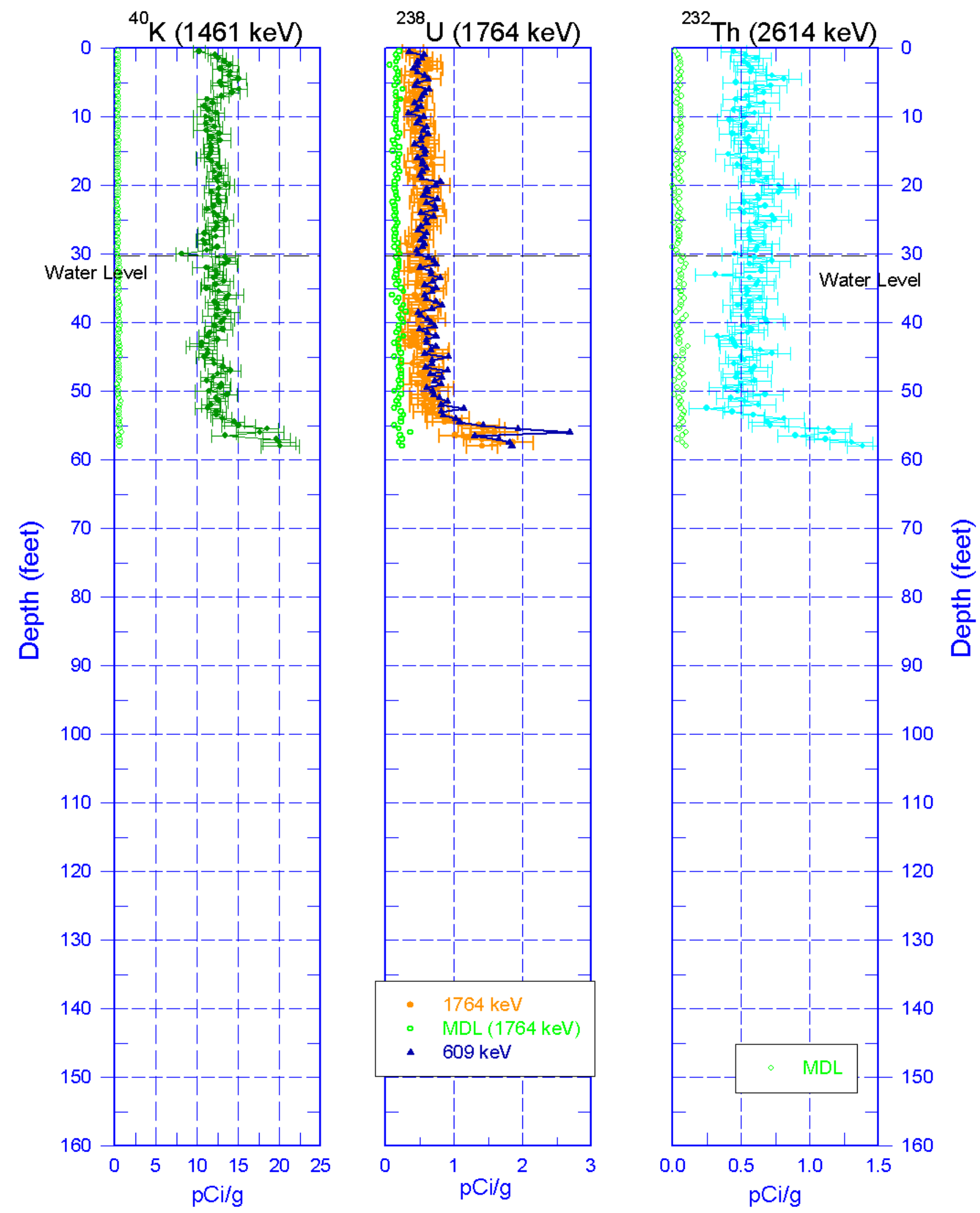

Zero Reference - Ground Surface 


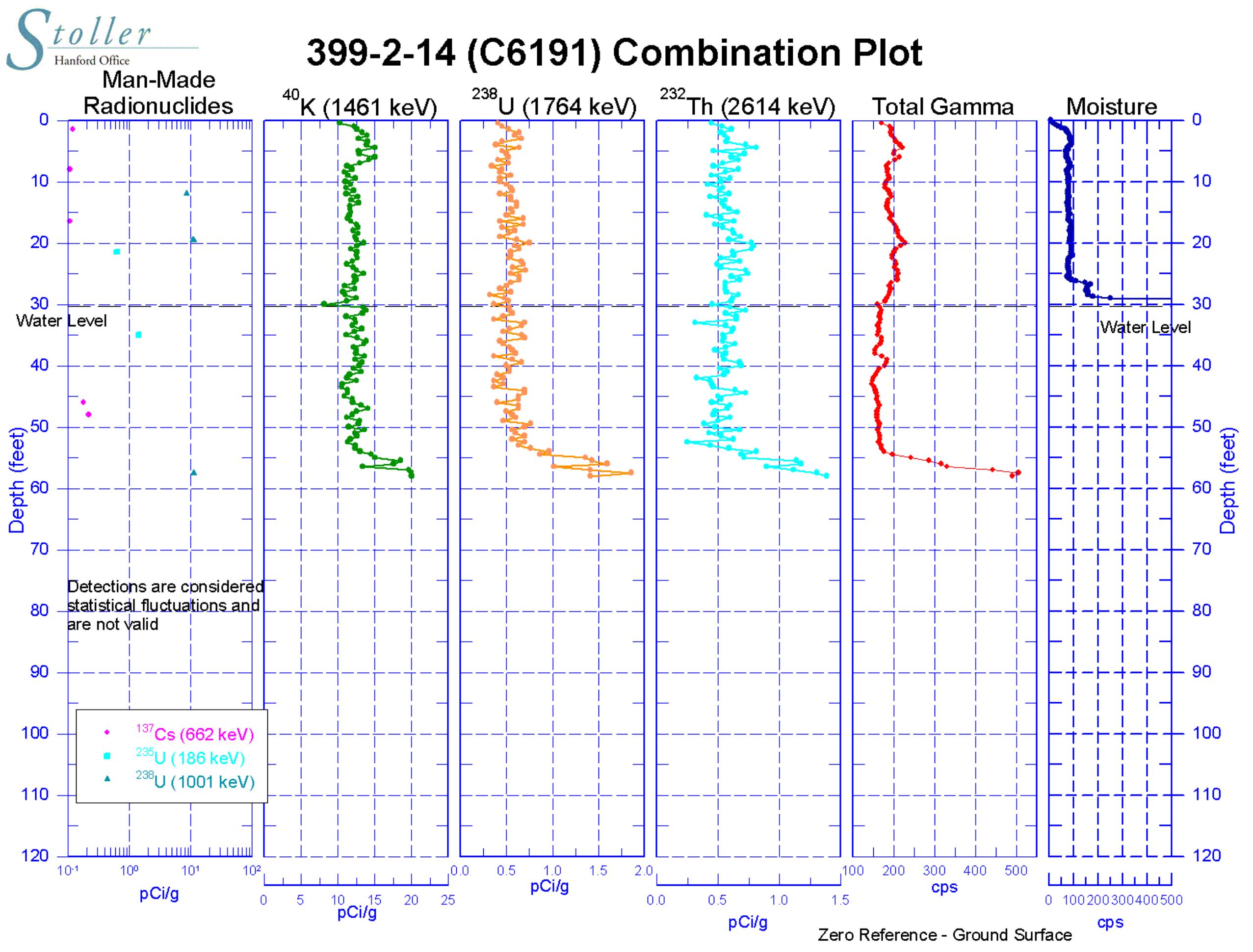



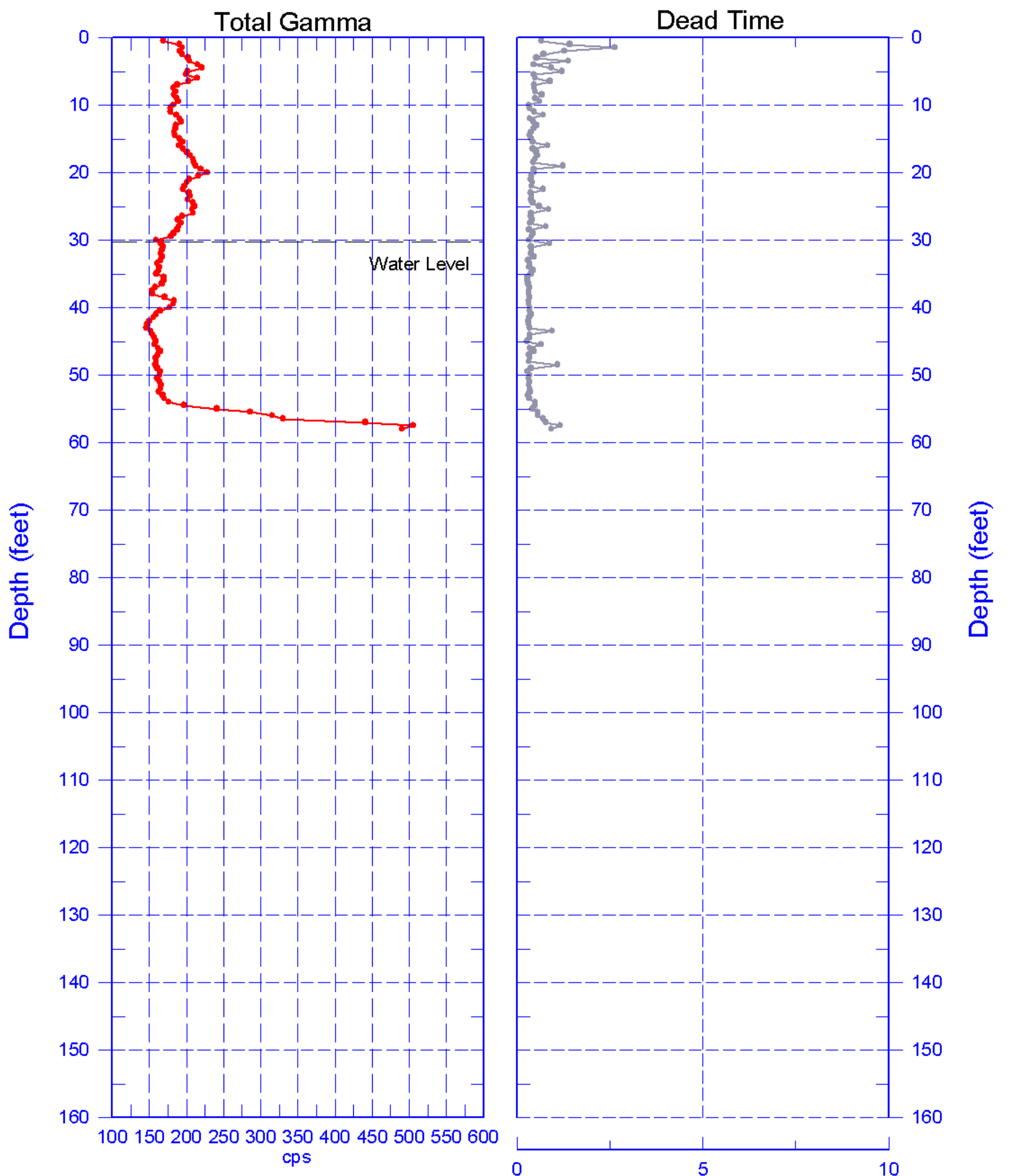

Reference - Ground Surface 


\section{S}

toller $\quad 399-2-14$ (C6191)

Hanford Office

\section{Total Gamma \& Moisture}
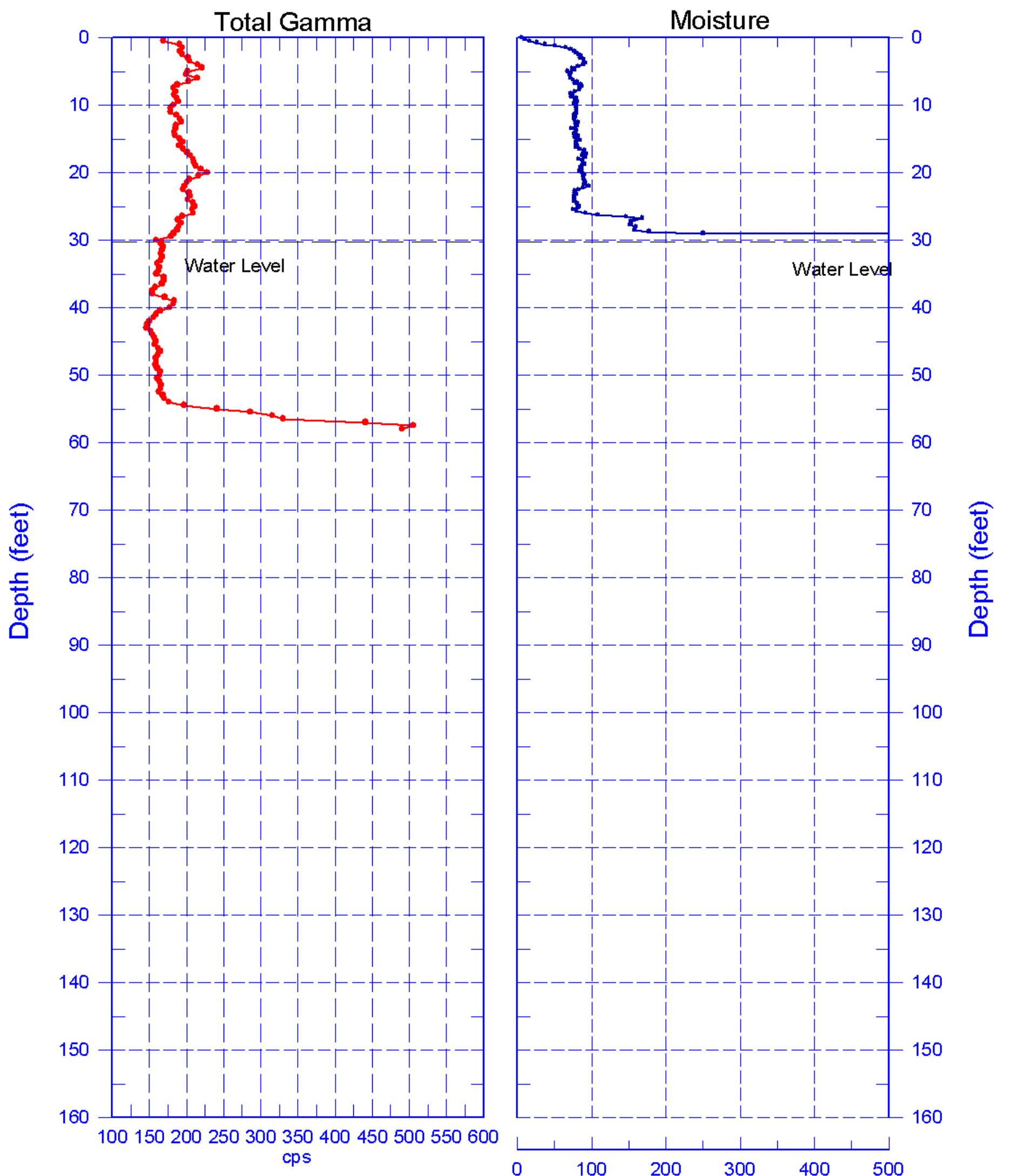

$\stackrel{0}{0}$
$\stackrel{0}{0}$
$\frac{0}{0}$
$\frac{0}{0}$

Reference - Ground Surface 


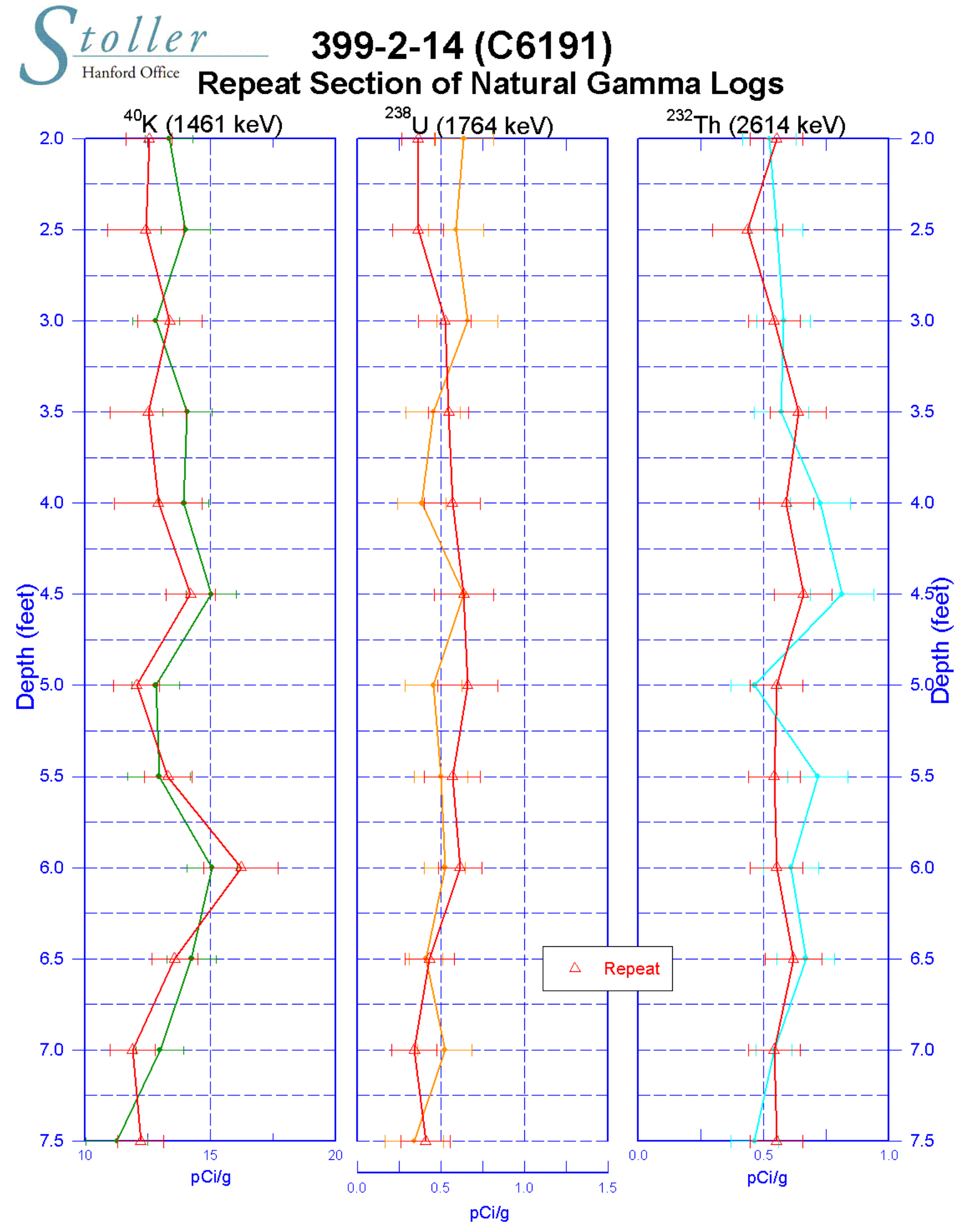

Zero Reference - Ground Surface 


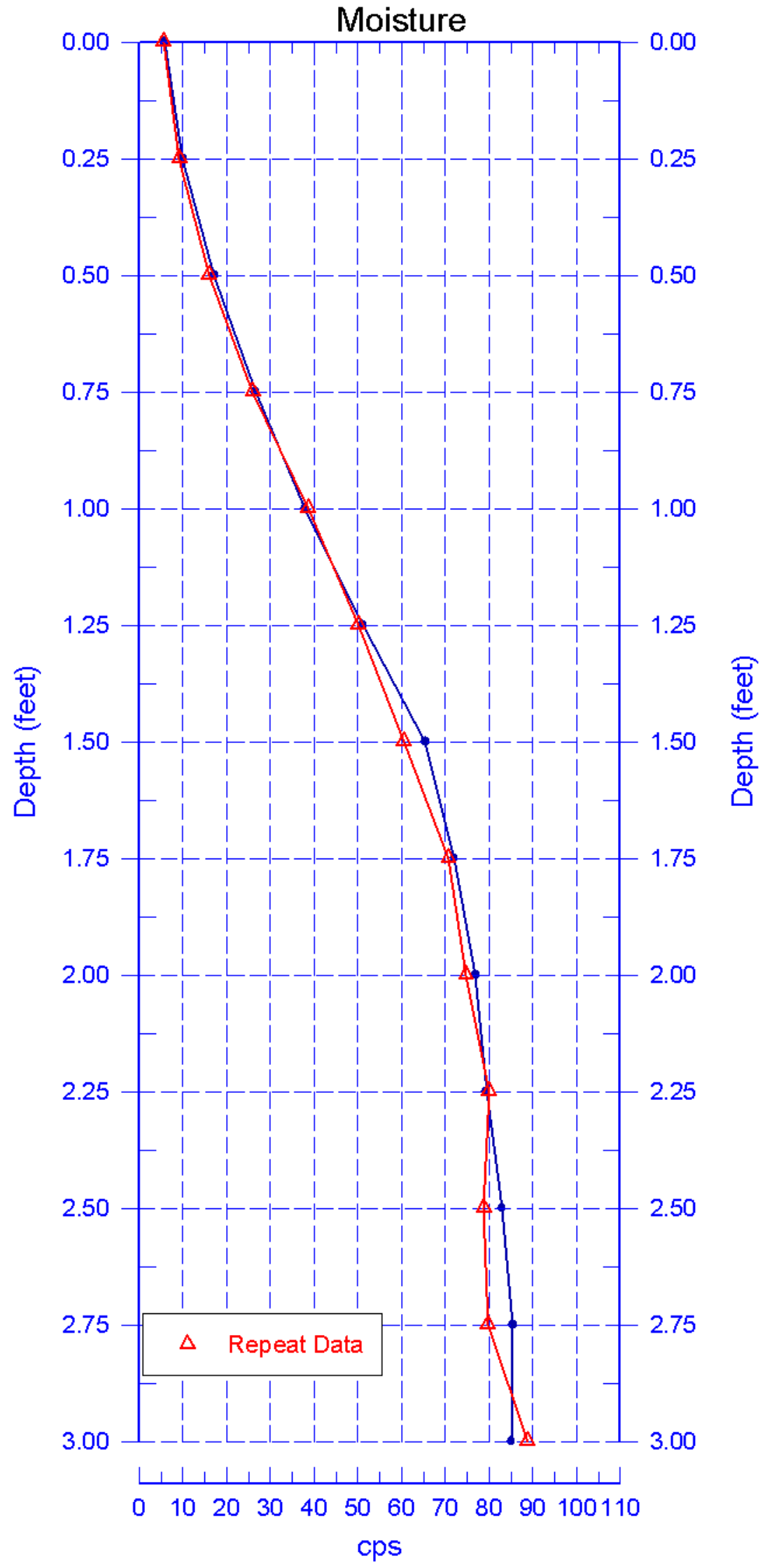




\section{9-2-15 (C6192) Log Data Report}

Borehole Information:

\begin{tabular}{|c|c|c|c|c|c|}
\hline Borehole: & \multicolumn{1}{l|}{ Site: } & \multicolumn{2}{c|}{ GW-FF-5 } \\
\hline \multicolumn{2}{|c|}{ 399-2-15 (C6192) } & GWL $^{\mathbf{1}}$ (ft) : & 26.8 & Date: & $06 / 28 / 08$ \\
\hline Coordinates (WA St Plane) & East (m) & Drill Date & TOC $^{2}$ Elevation & Total Depth (ft) & Type \\
\hline 116076.0 & 594253.4 & $06 / 26 / 08$ & Unknown & 61 & Sonic \\
\hline
\end{tabular}

\section{Casing Information:}

\begin{tabular}{|c|c|c|c|c|c|c|}
\hline Casing Type & Stickup (ft) & $\begin{array}{c}\text { Outer } \\
\text { Diameter (in.) }\end{array}$ & $\begin{array}{c}\text { Inside } \\
\text { Diameter (in.) }\end{array}$ & Thickness (in.) & Top (ft) & Bottom (ft) \\
\hline Threaded Steel & 1.1 & $75 / 8$ & $67 / 8$ & $3 / 8$ & -1.1 & 59 \\
\hline
\end{tabular}

\section{Borehole Notes:}

Casing data and total depth were reported by the site geologist. The logging engineer measured the depth to water with an e-tape. Casing diameters were measured using a steel tape and rounding to the nearest 1/16 inch. The zero reference is the ground surface.

\section{Logging Equipment Information:}

\begin{tabular}{|c|c|c|c|c|}
\hline Logging System: & \multicolumn{2}{|c|}{ Gamma $4 \mathrm{~L}$} & $\begin{array}{l}\text { Type: } \\
\text { Serial No.: }\end{array}$ & $\begin{array}{l}\text { 60\% HPGe SGLS } \\
\text { 47TP32211A }\end{array}$ \\
\hline Effective Calibration Date: & $12 / 31 / 07$ & Calibration Reference: & \multicolumn{2}{|c|}{ HGLP-CC-027 } \\
\hline & & Logging Procedure: & \multicolumn{2}{|c|}{ HGLP-MAN-002, Rev. 0} \\
\hline Logging System: & \multicolumn{2}{|c|}{ Gamma $4 \mathrm{H}$} & $\begin{array}{l}\text { Type: } \\
\text { Serial No.: }\end{array}$ & $\begin{array}{l}\text { NMLS } \\
\text { H310700352 }\end{array}$ \\
\hline Effective Calibration Date: & $11 / 06 / 07$ & Calibration Reference: & \multicolumn{2}{|c|}{ HGLP-CC-021 } \\
\hline & & Logging Procedure: & \multicolumn{2}{|c|}{ HGLP-MAN-002, Rev. 0} \\
\hline
\end{tabular}

\section{Spectral Gamma Logging System (SGLS) Log Run Information:}

\begin{tabular}{|l|c|c|l|l|l|}
\hline Log Run & $\mathbf{1}$ & 2 Repeat & & & \\
\hline Date & $06 / 28 / 08$ & $06 / 28 / 08$ & & & \\
\hline Logging Engineer & Pearson & Pearson & & & \\
\hline Start Depth (ft) & 61.5 & 12.0 & & & \\
\hline Finish Depth (ft) & 0.0 & 6.0 & & & \\
\hline Count Time (sec) & 200 & 200 & & & \\
\hline Live/Real & $\mathrm{R}$ & $\mathrm{R}$ & & & \\
\hline Shield (Y/N) & $\mathrm{N}$ & $\mathrm{N}$ & & & \\
\hline MSA Interval (ft) & 0.5 & 0.5 & & & \\
\hline Log Speed (ft/min) & NA & NA & & & \\
\hline Pre-Verification & DL541CAB & DL541CAB & & & \\
\hline Start File & DL551000 & DL551124 & & & \\
\hline Finish File & DL551123 & DL551136 & & & \\
\hline Post-Verification & DL551CAA & DL511CAA & & & \\
\hline Depth Return Error (in.) & 1 High & 1 High & & & \\
\hline Comments & None & Repeat Section & & & \\
\hline
\end{tabular}


Neutron Moisture Logging System (NMLS) Log Run Information:

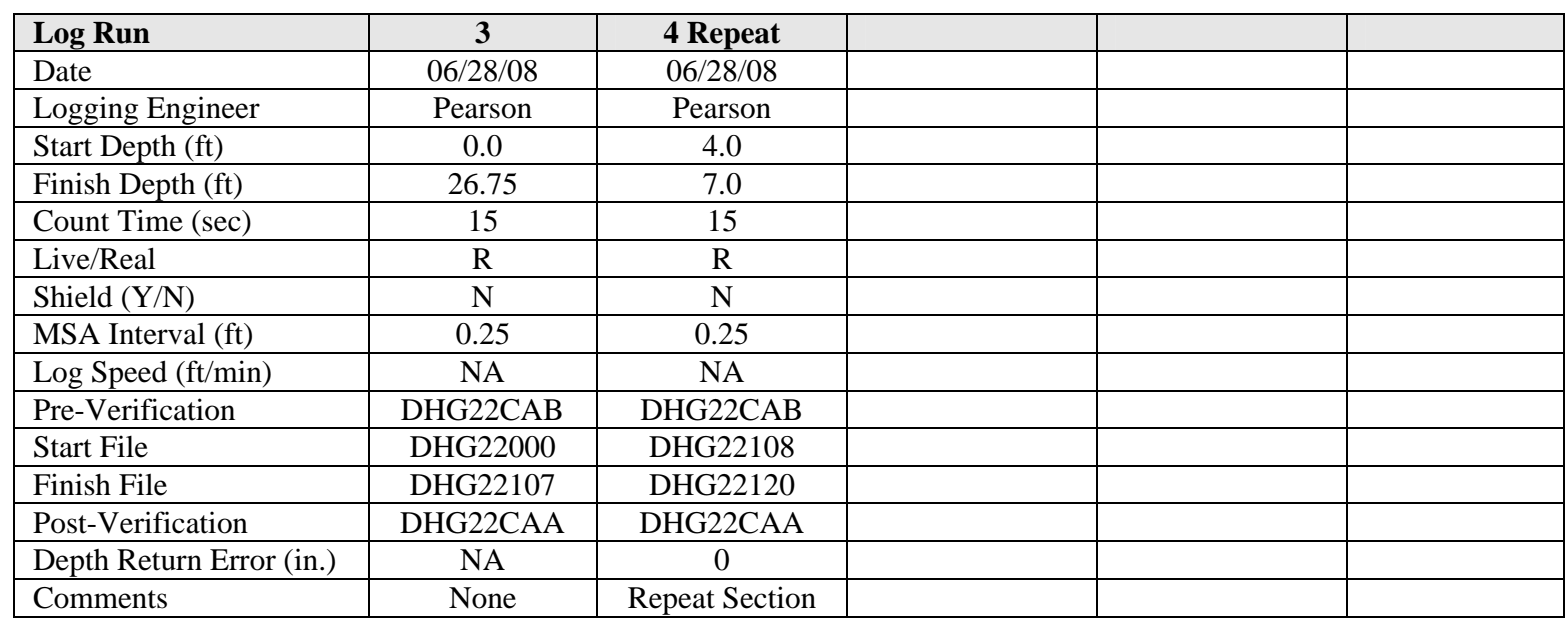

\section{Logging Operation Notes:}

Data were collected using Gamma 4, HO 68B-3573. SGLS pre- and post-survey verification measurements were acquired in the Amersham KUTh-115 field verifier. NMLS pre- and post-survey verification measurements were acquired in the standard field verifier. Maximum logging depth was $61.61 \mathrm{ft}$ before the sonde un-weighted. A centralizer was installed on the sonde.

\section{Analysis Notes:}

\begin{tabular}{|l|l|l|l|l|l|}
\hline Analyst: & LEGLER & Date: & $10 / 01 / 08$ & Reference: & GJO-HGLP 1.6.3, Rev. 0 \\
\hline
\end{tabular}

The pre- and post-survey verification spectra met the acceptance criteria for the established systems. A casing correction for a 3/8-in thick casing was applied during analysis for depths up to $59 \mathrm{ft}$, where casing ends, leaving $2.5 \mathrm{ft}$ of open borehole uncorrected. A water correction was also applied from $26.8 \mathrm{ft}$ to total logged depth of the borehole.

SGLS spectra were processed in batch mode in APTEC SUPERVISOR to identify individual peaks and count rates. Concentrations were calculated using an EXCEL template identified as G4LDec07.xls using an efficiency function and corrections for casing, dead time and water as determined by annual calibrations. NMLS spectra were processed in batch mode in APTEC SUPERVISOR to identify counts. Count rates were calculated using an EXCEL template identified as G4HNov07.xls. NMLS data are presented in counts per second (cps), because no calibration data is available for a 6 7/8-in inner diameter borehole casing.

\section{Results and Interpretations:}

Cs-137, U-235, and U-238 (Pa-234m) were detected in this borehole at isolated depths throughout the borehole. Inspection of the individual spectra at these various depths indicates that these detections are statistical fluctuations associated with the processing software and are not considered valid.

The KUT and Moisture repeat plots show good repeatability. 
HGLP-LDR-253, Rev. 0

\section{List of Log Plots:}

Depth Reference is ground surface

Manmade Radionuclides

Natural Gamma Logs

Combination Plot

Total Gamma \& Dead Time

Total Gamma \& Moisture

Repeat Section of Natural Gamma Logs

Moisture Repeat Section

${ }^{1} \mathrm{GWL}$ - groundwater level

${ }^{2}$ TOC - top of casing 


\section{toller 399-2-15 (C6192) Hanford Office Manmade Radionuclides}

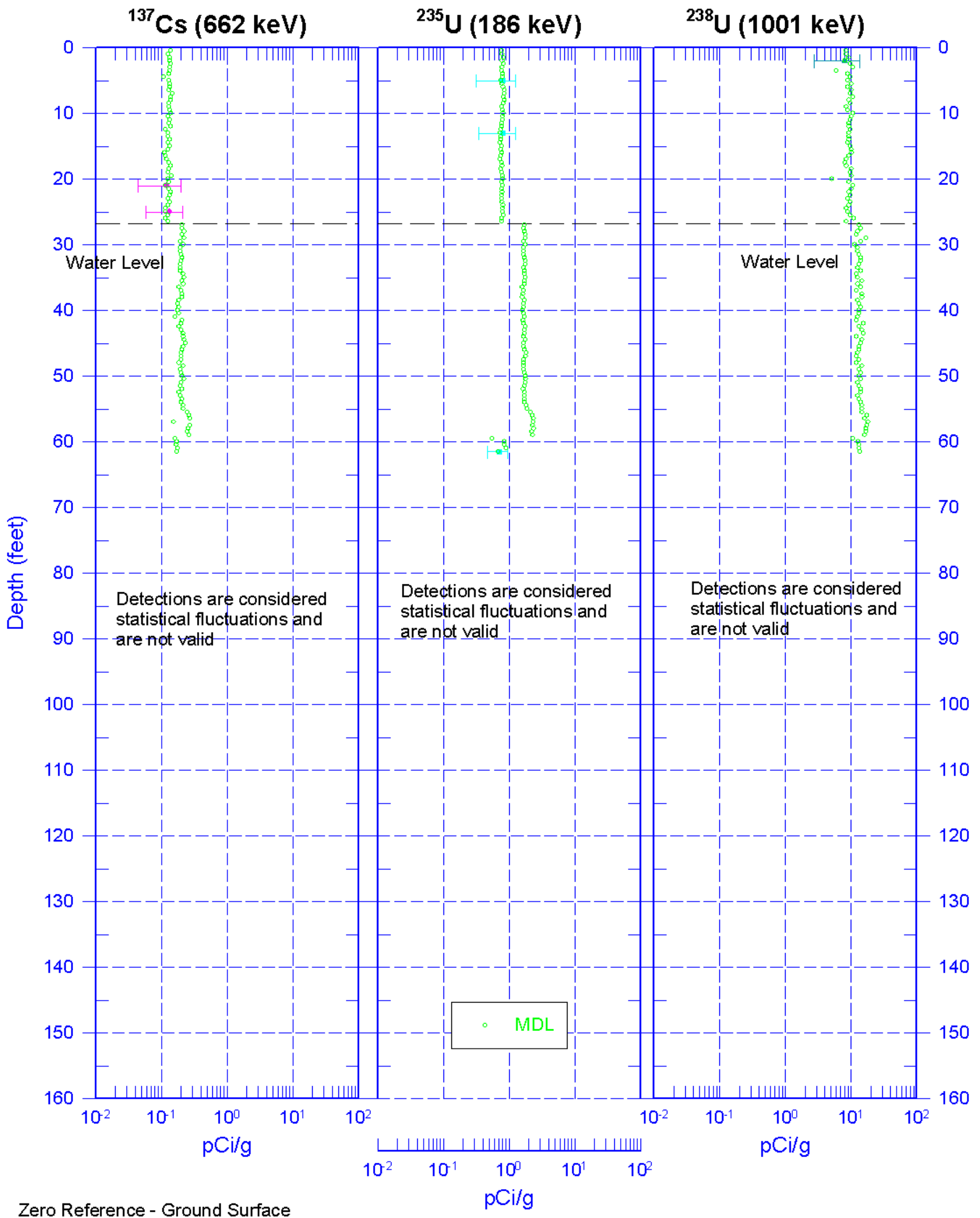



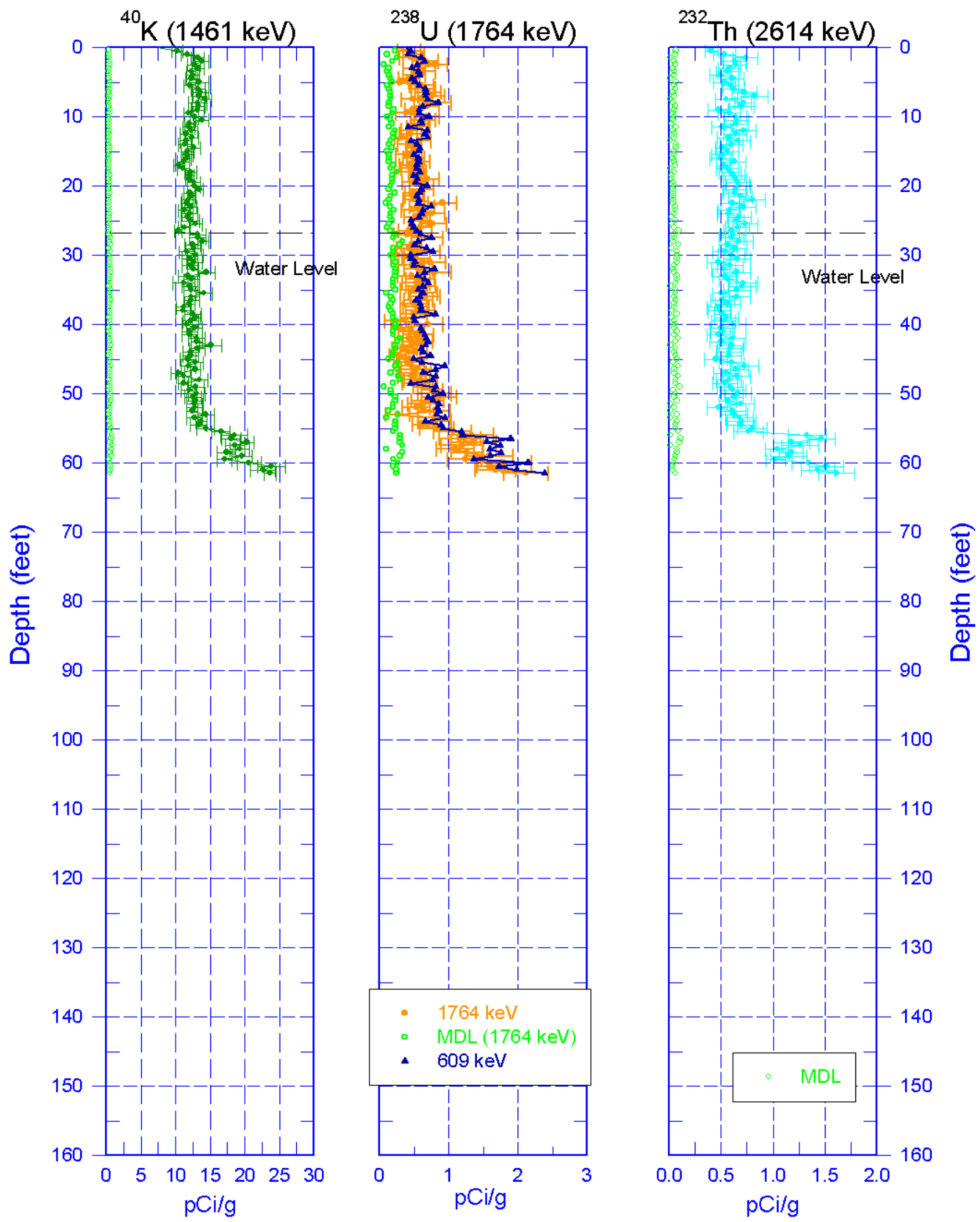


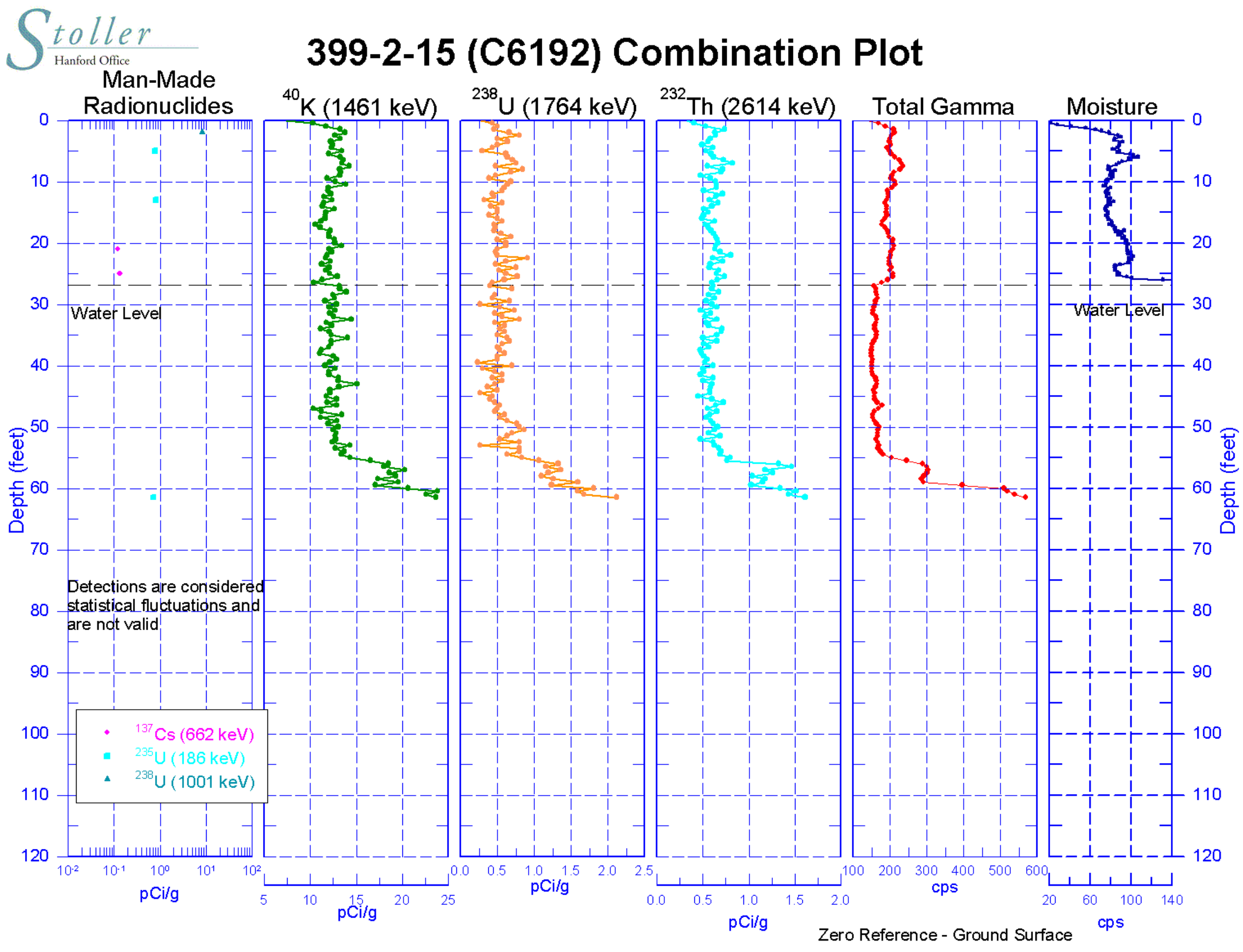



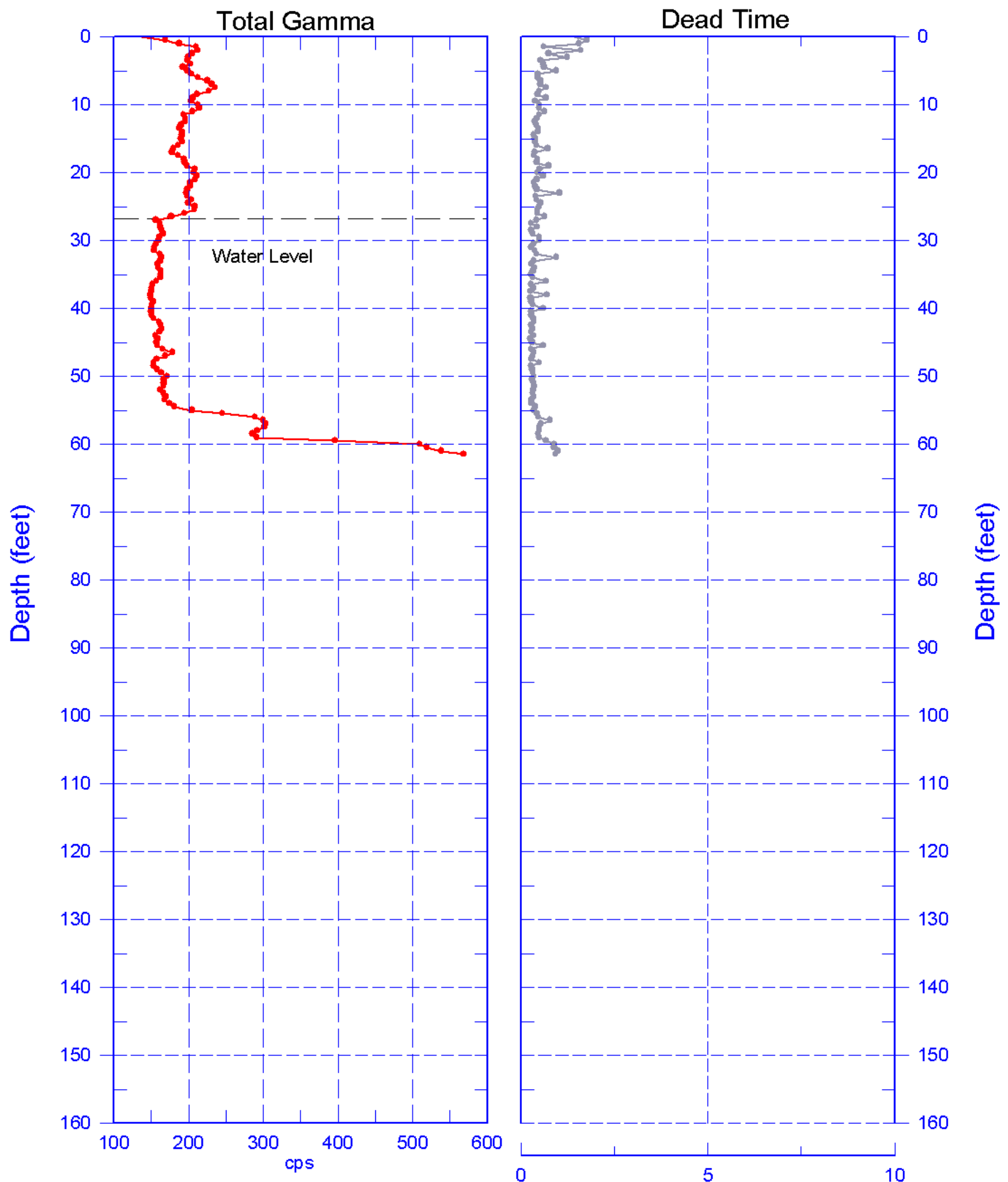

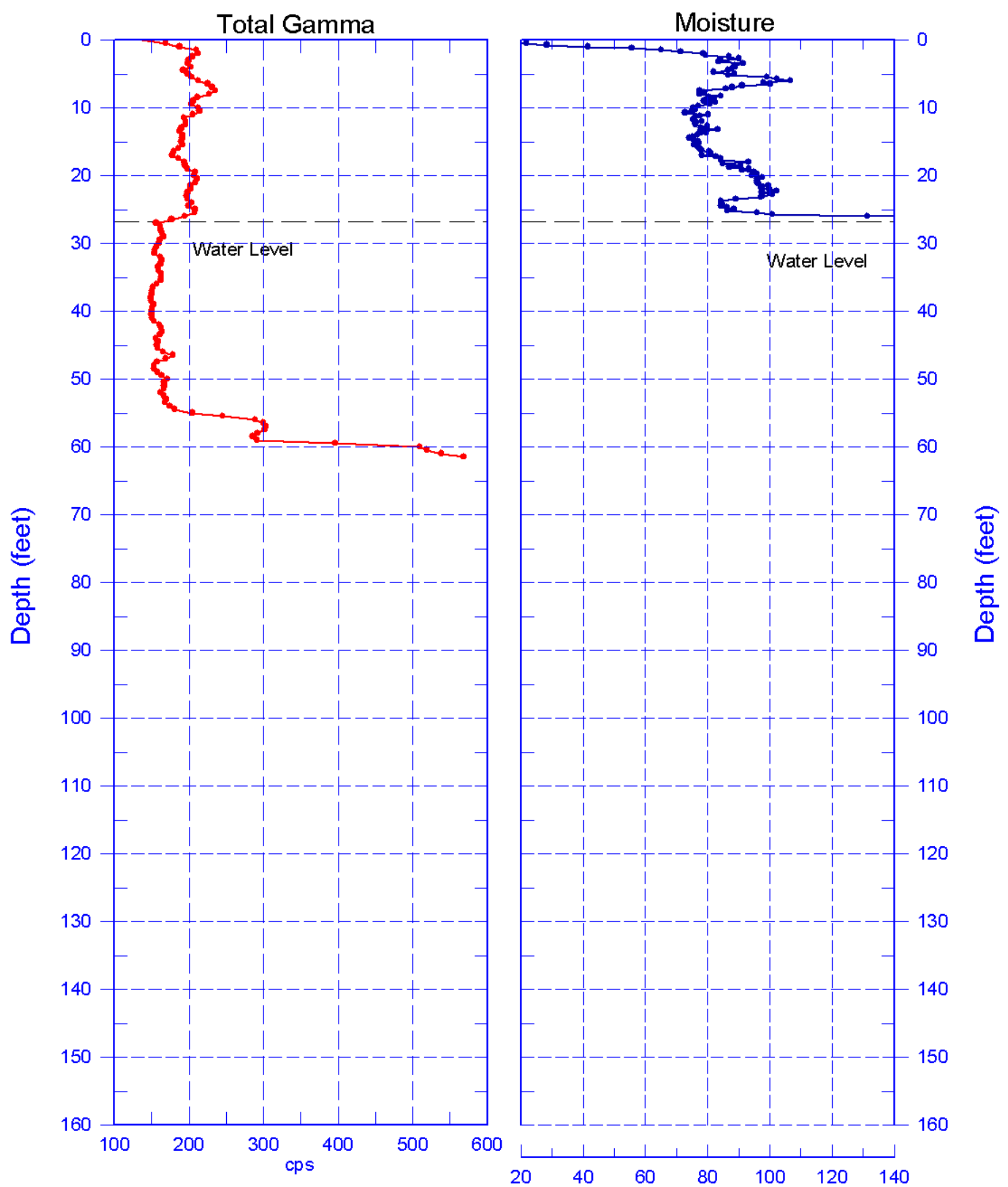


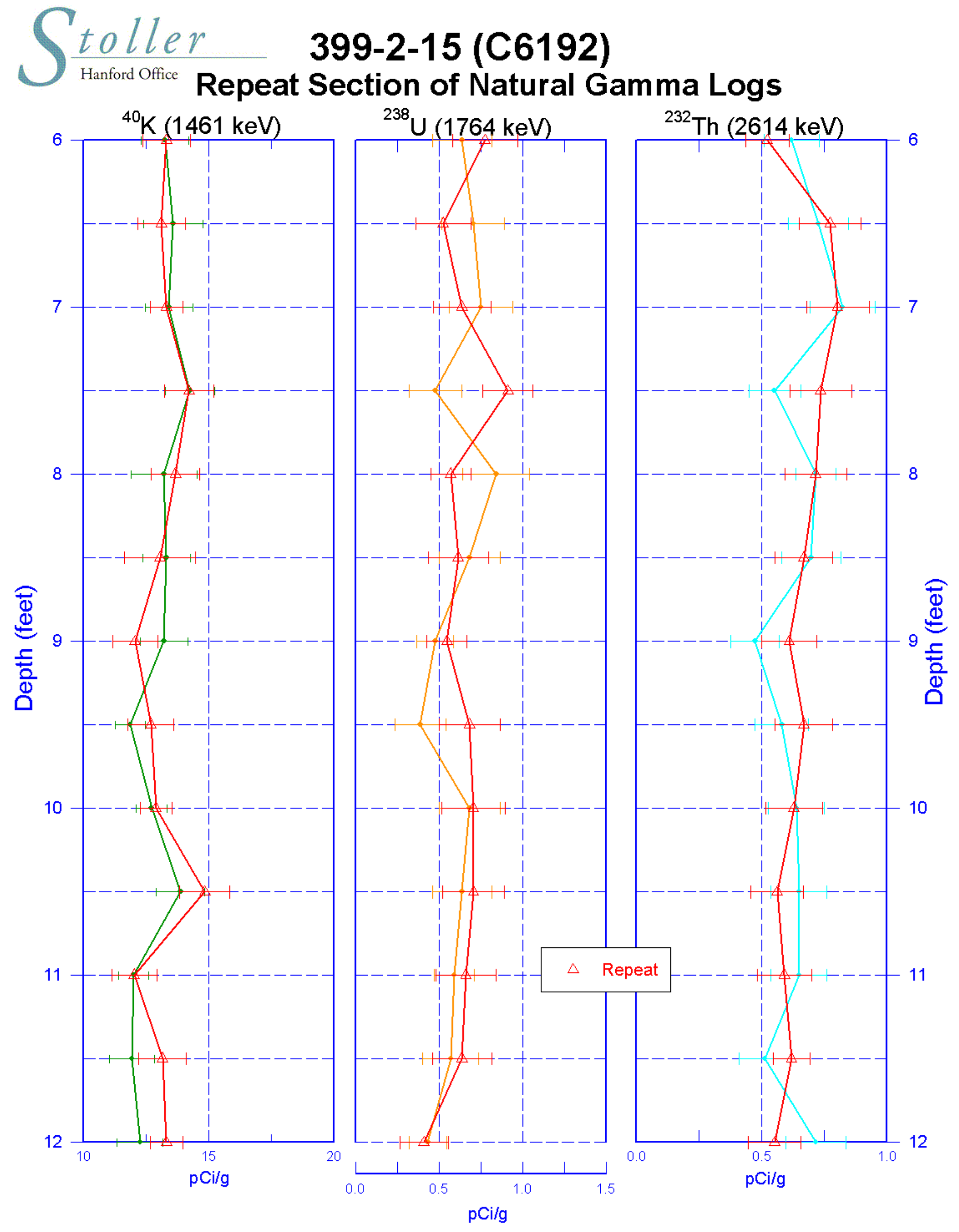

Zero Reference - Ground Surface 


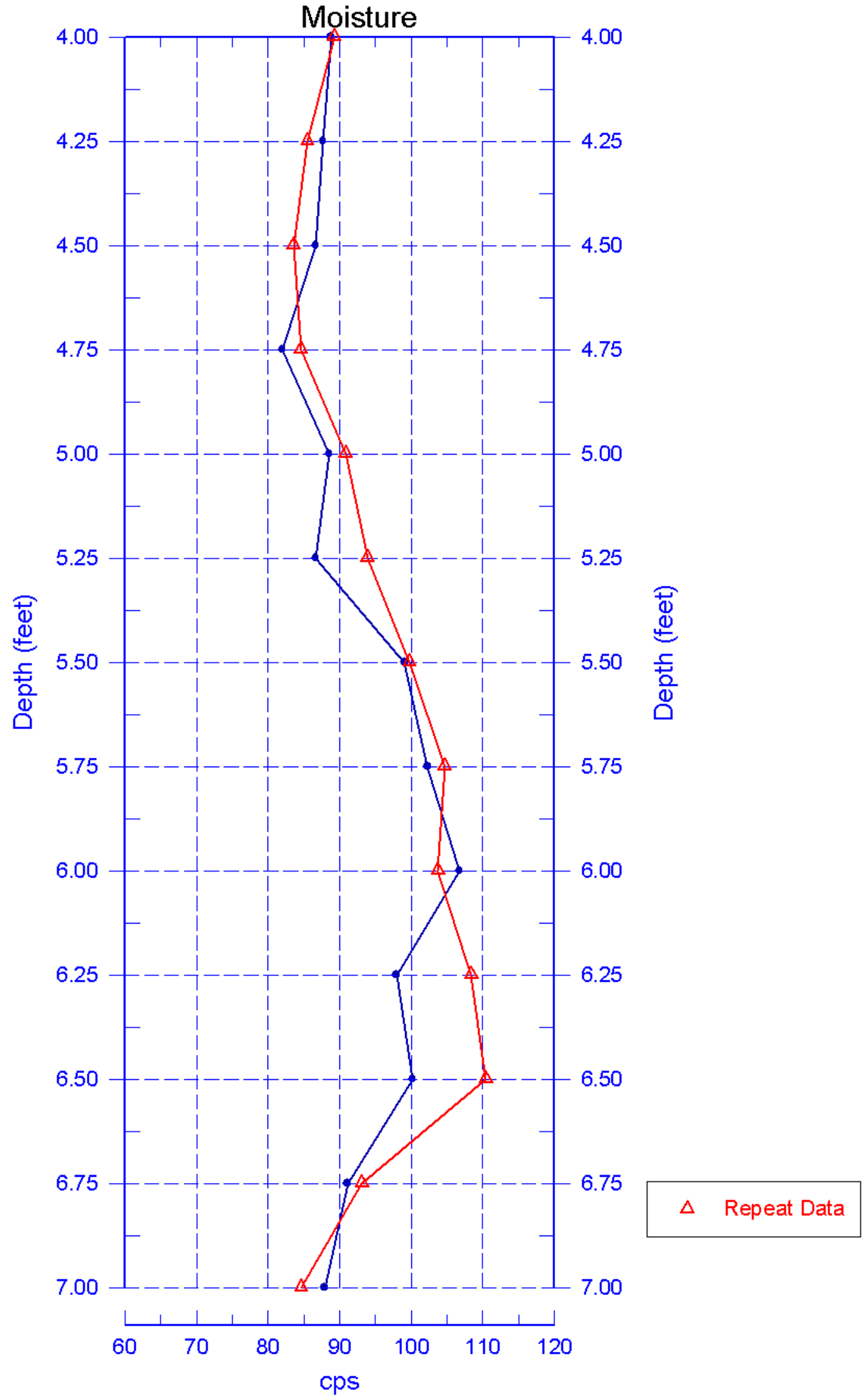




\section{9-2-16(C6193) Log Data Report}

Borehole Information:

\begin{tabular}{|c|c|c|c|c|c|}
\hline \multicolumn{2}{|c|}{ Borehole: $\quad 399-2-16$ (C6193) } & \multicolumn{4}{|c|}{ 300-FF-5 } \\
\hline \multicolumn{2}{|c|}{ Coordinates (WA St Plane) } & $G_{W L}^{1}(\mathbf{f}$ & 26.1 & GWL Date: & $06 / 27 / 08$ \\
\hline North (m) & East (m) & Drill Date & TOC $^{2}$ Elevation & Total Depth (ft) & Type \\
\hline 116081.8 & 594261.6 & $06 / 26 / 08$ & Unknown & 62 & Sonic \\
\hline
\end{tabular}

\section{Casing Information:}

\begin{tabular}{|c|c|c|c|c|c|c|}
\hline Casing Type & Stickup (ft) & $\begin{array}{c}\text { Outer } \\
\text { Diameter (in.) }\end{array}$ & $\begin{array}{c}\text { Inside } \\
\text { Diameter (in.) }\end{array}$ & Thickness (in.) & Top (ft) & Bottom (ft) \\
\hline Threaded Steel & 1.7 & $75 / 8$ & $67 / 8$ & $3 / 8$ & -1.7 & 58.7 \\
\hline
\end{tabular}

\section{Borehole Notes:}

Casing data and total depth were reported by the site geologist. The logging engineer measured the depth to water with an e-tape. Casing diameters were measured using a steel tape and rounded to the nearest 1/16 inch. The zero reference is the ground surface.

\section{Logging Equipment Information:}

\begin{tabular}{|c|c|c|c|c|}
\hline Logging System: & \multicolumn{2}{|c|}{ Gamma $4 \mathrm{~L}$} & $\begin{array}{l}\text { Type: } \\
\text { Serial No.: }\end{array}$ & $\begin{array}{l}\text { 60\% HPGe SGLS } \\
\text { 47TP32211A }\end{array}$ \\
\hline Effective Calibration Date: & $12 / 31 / 07$ & Calibration Reference: & \multicolumn{2}{|c|}{ HGLP-CC-027 } \\
\hline & & Logging Procedure: & \multicolumn{2}{|c|}{ HGLP-MAN-002, Rev. 0} \\
\hline Logging System: & \multicolumn{2}{|c|}{ Gamma $4 \mathrm{H}$} & $\begin{array}{l}\text { Type: } \\
\text { Serial No.: }\end{array}$ & $\begin{array}{l}\text { NMLS } \\
\text { H310700352 }\end{array}$ \\
\hline Effective Calibration Date: & $11 / 06 / 07$ & Calibration Reference: & \multicolumn{2}{|c|}{ HGLP-CC-021 } \\
\hline & & Logging Procedure: & \multicolumn{2}{|c|}{ HGLP-MAN-002, Rev. 0} \\
\hline
\end{tabular}

\section{Spectral Gamma Logging System (SGLS) Log Run Information:}

\begin{tabular}{|l|c|c|l|l|l|}
\hline Log Run & $\mathbf{1}$ & 2 Repeat & & & \\
\hline Date & $06 / 27 / 08$ & $06 / 27 / 08$ & & & \\
\hline Logging Engineer & Pearson & Pearson & & & \\
\hline Start Depth (ft) & 61.0 & 6.0 & & & \\
\hline Finish Depth (ft) & 0.0 & 12.0 & & & \\
\hline Count Time (sec) & 200 & 200 & & & \\
\hline Live/Real & $\mathrm{R}$ & $\mathrm{R}$ & & & \\
\hline Shield (Y/N) & $\mathrm{N}$ & $\mathrm{N}$ & & & \\
\hline MSA Interval (ft) & 0.5 & 0.5 & & & \\
\hline Log Speed (ft/min) & $\mathrm{NA}$ & $\mathrm{NA}$ & & & \\
\hline Pre-Verification & DL531CAB & DL531CAB & & & \\
\hline Start File & DL531000 & DL531123 & & & \\
\hline Finish File & DL531122 & DL531135 & & & \\
\hline Post-Verification & DL531CAA & DL531CAA & & & \\
\hline
\end{tabular}


HGLP-LDR-250, Rev. 0

\begin{tabular}{|l|c|c|l|l|l|}
\hline Log Run & $\mathbf{1}$ & 2 Repeat & & & \\
\hline Depth Return Error (in.) & NA & 1.25 High & & & \\
\hline Comments & $\begin{array}{c}\text { Fine gain } \\
\text { adjustment } \\
\text { made at -087 } \\
\text { and -120 }\end{array}$ & Repeat Section. & & & \\
& & & & \\
\hline
\end{tabular}

Neutron Moisture Logging System (NMLS) Log Run Information:

\begin{tabular}{|l|c|c|l|l|l|}
\hline Log Run & $\mathbf{3}$ & 4 Repeat & & & \\
\hline Date & $06 / 27 / 08$ & $06 / 27 / 08$ & & & \\
\hline Logging Engineer & Pearson & Pearson & & & \\
\hline Start Depth (ft) & 0.0 & 4.0 & & & \\
\hline Finish Depth (ft) & 26.25 & 7.0 & & & \\
\hline Count Time (sec) & 15 & 15 & & & \\
\hline Live/Real & R & R & & & \\
\hline Shield (Y/N) & N & N & & & \\
\hline MSA Interval (ft) & 0.25 & 0.25 & & & \\
\hline Log Speed (ft/min) & NA & NA & & & \\
\hline Pre-Verification & DHG02CAB & DHG02CAB & & & \\
\hline Start File & DHG02000 & DHG02106 & & & \\
\hline Finish File & DHG02105 & DHG02118 & & & \\
\hline Post-Verification & DHG02CAA & DHG02CAA & & & \\
\hline Depth Return Error (in.) & NA & 1/2 high & & & \\
\hline Comments & None & Repeat Section & & & \\
\hline
\end{tabular}

\section{Logging Operation Notes:}

Data were collected using Gamma 4, HO 68B-3573. SGLS pre- and post-survey verification measurements were acquired in the Amersham KUTh-115 field verifier. NMLS pre- and post-survey verification measurements were acquired in the standard field verifier. Maximum logging depth for the SGLS was $61.21 \mathrm{ft}$ before the sonde un-weighted. A centralizer was installed on the sonde.

Analysis Notes:

\begin{tabular}{|c|c|c|c|c|c|}
\hline Analyst: & LEGLER & Date: & $09 / 26 / 08$ & Reference: & GJO-HGLP 1.6.3, Rev. 0 \\
\hline
\end{tabular}

SGLS pre- and post-survey verification spectra met the acceptance criteria for the established system. NMLS preand post-survey verification spectra met the acceptance criteria for the established system, but the verification file DHG02CAA had measurements above the upper control limit for counts per second (cps).

A casing correction for a 3/8-in thick casing was applied to spectral data during analysis for depths up $58.7 \mathrm{ft}$, where casing ends, leaving $2.3 \mathrm{ft}$ of open borehole uncorrected. A water correction was also applied from $26.1 \mathrm{ft}$ to total logged depth of borehole.

SGLS spectra were processed in batch mode in APTEC SUPERVISOR to identify individual peaks and count rates. Concentrations were calculated using an EXCEL template identified as G4LDec07.xls using an efficiency function and corrections for casing, dead time and water as determined by annual calibrations. NMLS spectra were processed in batch mode in APTEC SUPERVISOR to identify counts. Count rates were calculated using an EXCEL template identified as G4HNov07.xls. NMLS data are presented in counts per second (cps), because no calibration data is available for a 6 7/8-in. inner diameter borehole casing. 
HGLP-LDR-250, Rev. 0

\section{Results and Interpretations:}

Cs-137 was detected at 6 , and $49.5 \mathrm{ft}$, but inspection of the individual spectra indicates that these detections are statistical fluctuations associated with the processing software.

U-235 was detected at 15 and $48 \mathrm{ft}$, but inspection of the individual spectra indicates that these detections are statistical fluctuations associated with the processing software.

U-238 (Pa-234m) was detected at $61 \mathrm{ft}$, but inspection of the spectra indicates that this detection is a statistical fluctuation associated with the processing software.

Co-60 was detected at $6 \mathrm{ft}$, and $10-11.4 \mathrm{ft}$, with a maximum concentration of $0.07 \mathrm{pCi} / \mathrm{g}$ at 10 feet.

The KUT, moisture and manmade repeat plots show good repeatability.

\section{List of Log Plots:}

Depth Reference is top of casing

Manmade Radionuclides

Natural Gamma Logs

Combination Plot

Total Gamma \& Dead Time

Total Gamma \& Moisture

Manmade Repeat Section

Repeat Section of Natural Gamma Logs

Moisture Repeat Section

${ }^{1} \mathrm{GWL}$ - groundwater level

${ }^{2}$ TOC - top of casing 

Hanford Office Manmade Radionuclides

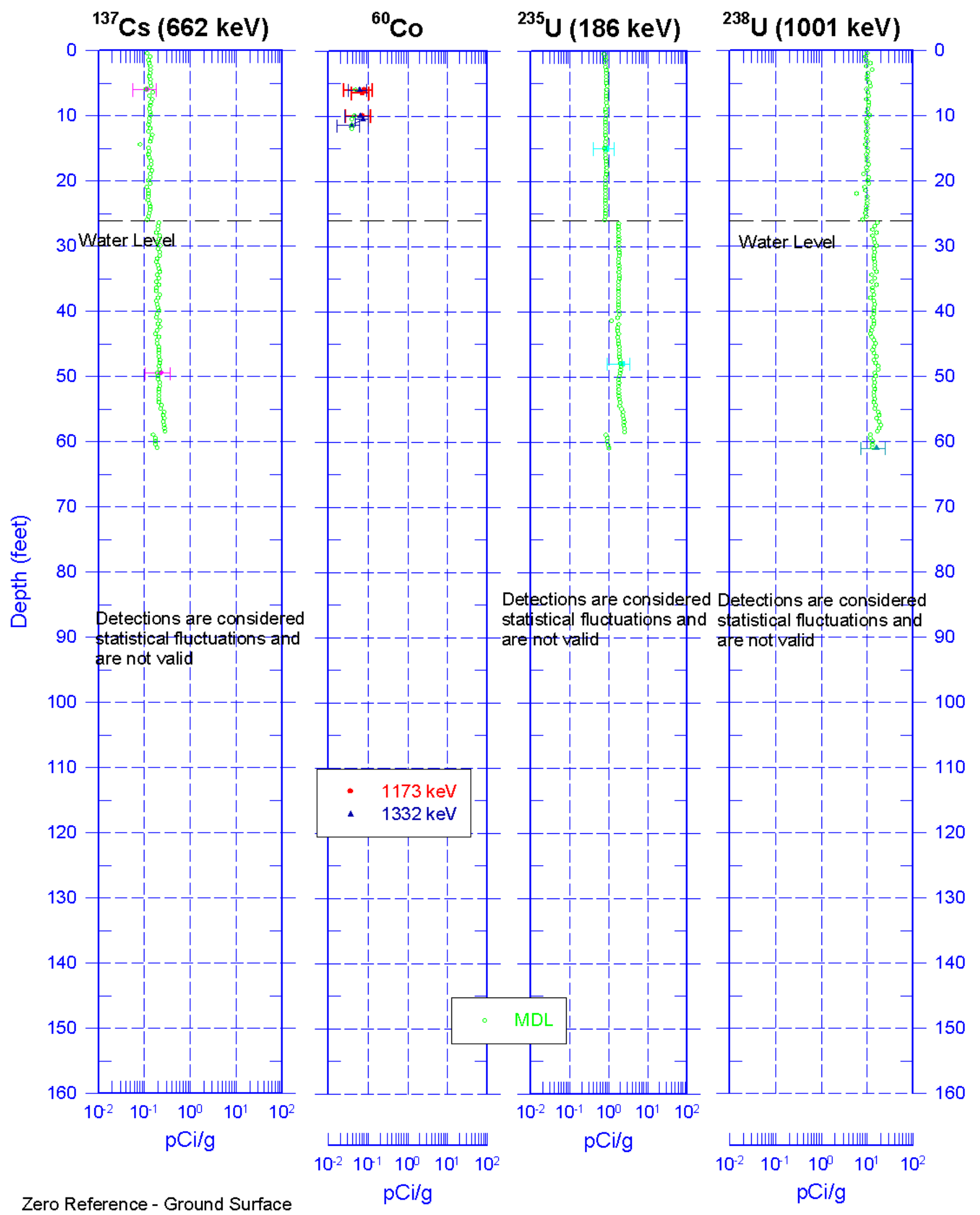


toller 399-2-16 (C6193)

Hanford Office Natural Gamma Logs
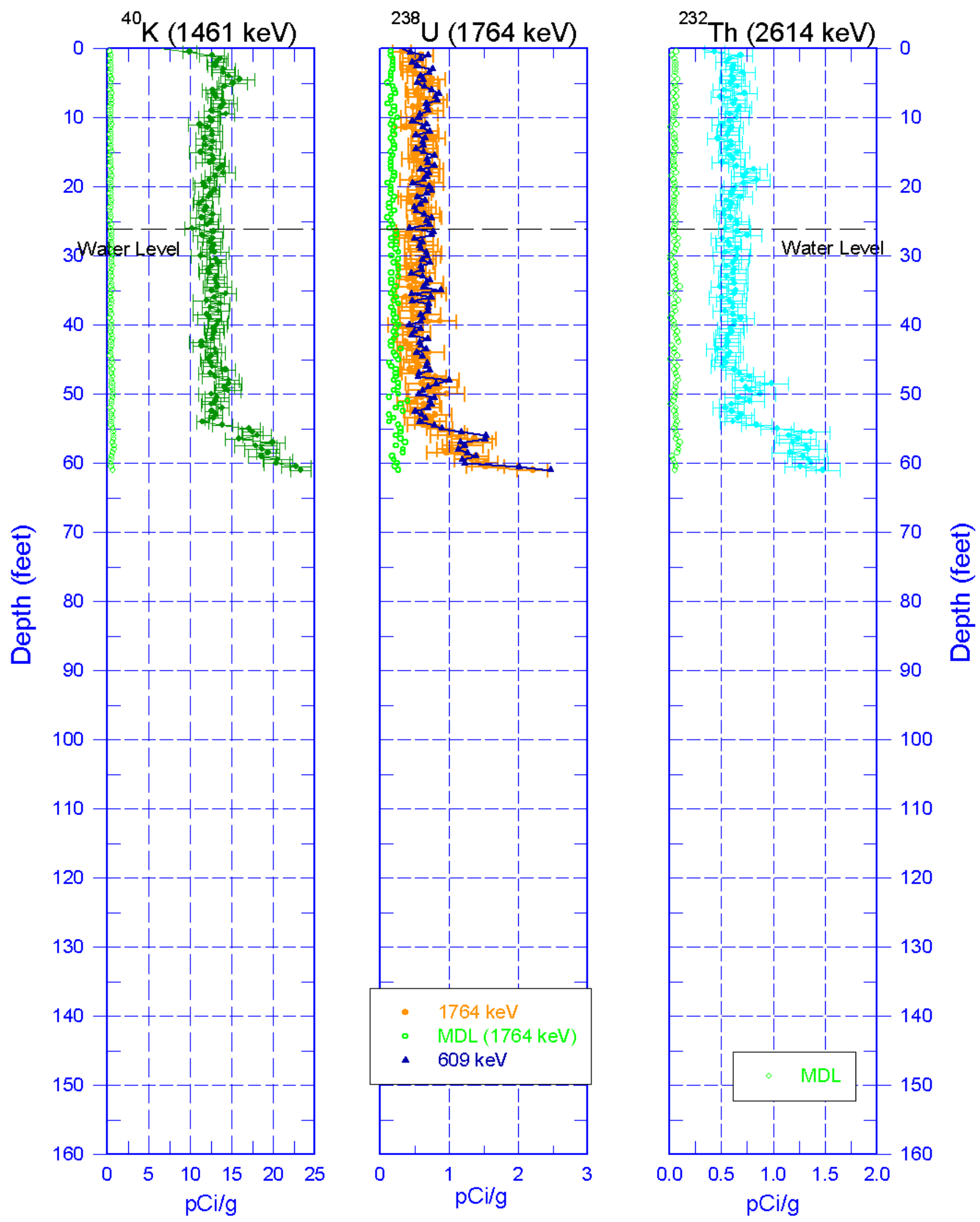

Zero Reference - Ground Surface

G.105 


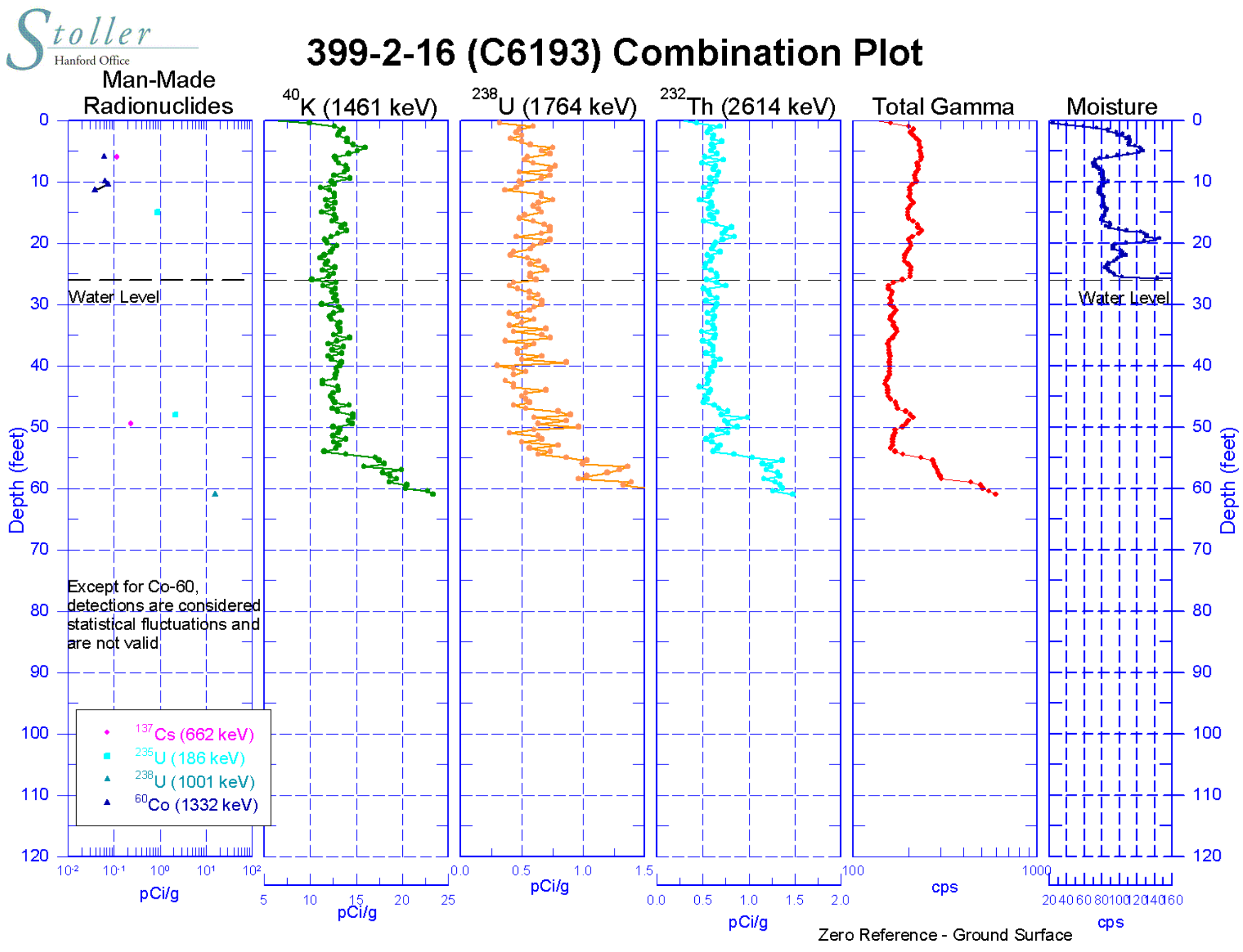


toller 399-2-16 (C6193)

Hanford Office

\section{Total Gamma \& Dead Time}
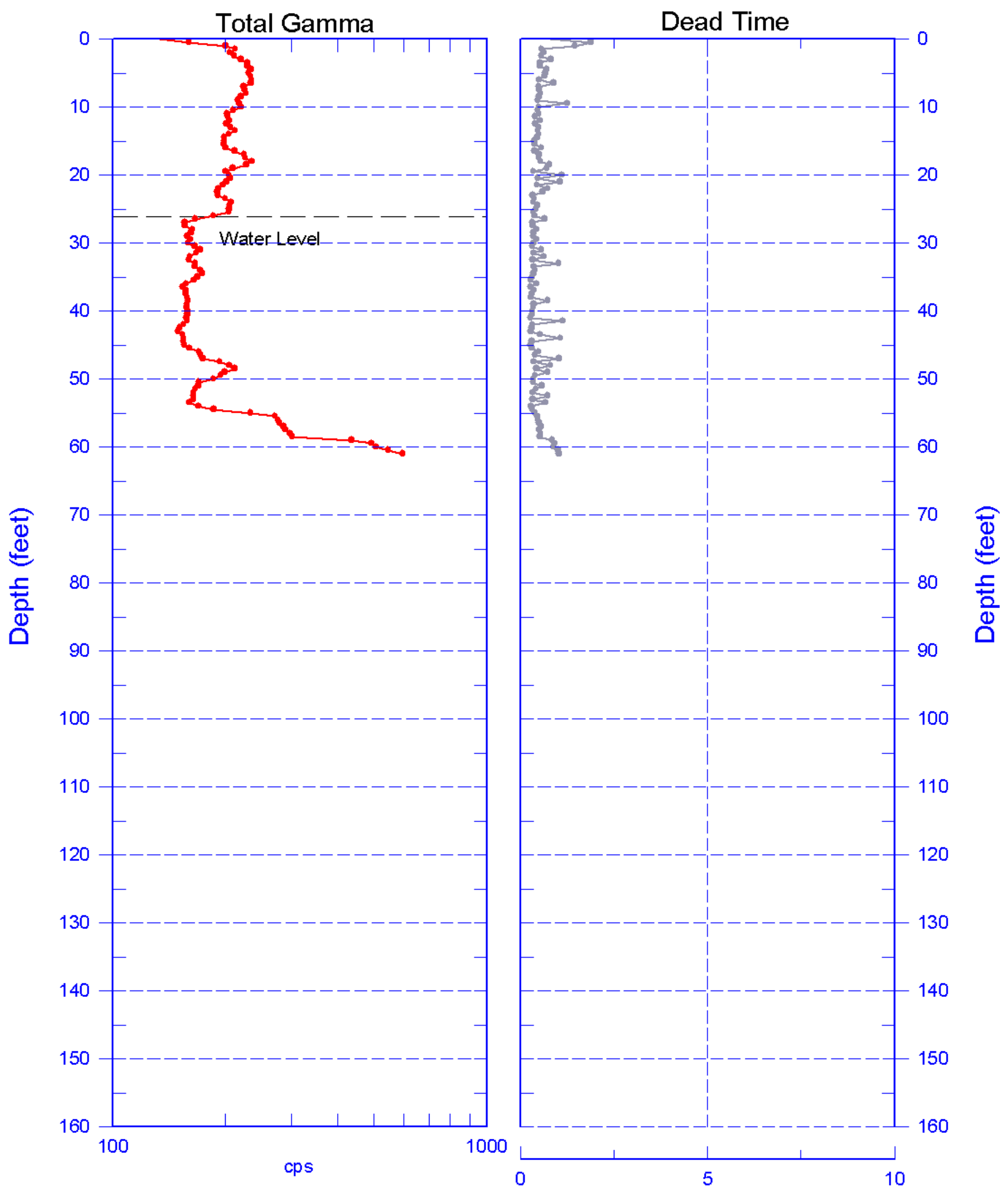

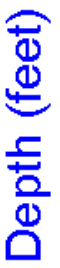

Reference - Ground Surface

G.107 
toller 399-2-16 (C6193)

Hanford Office

\section{Total Gamma \& Moisture}
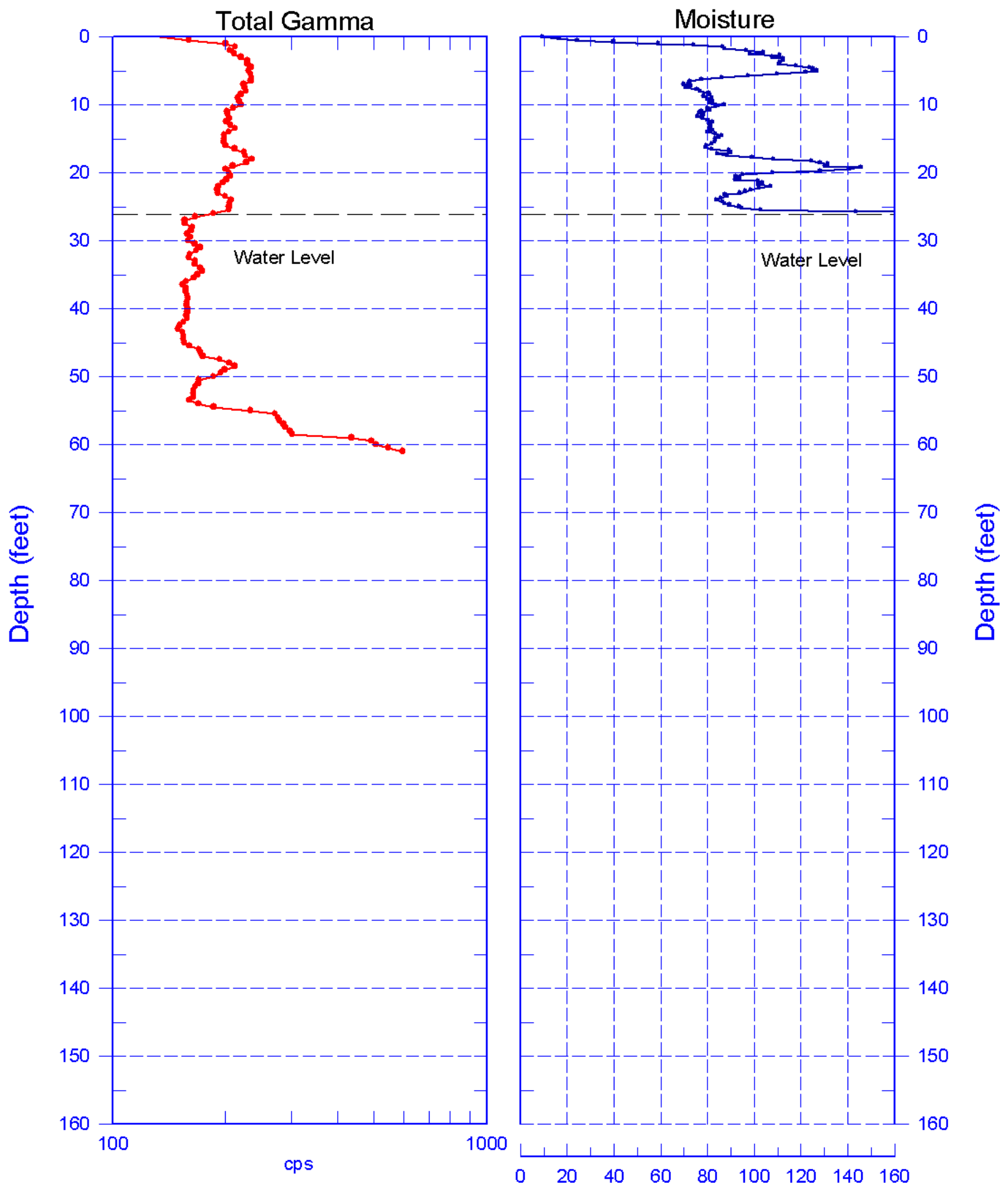

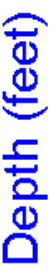

Reference - Ground Surface

G.108 
toller $\quad$ 399-2-16 (C6193)

Hanford Office

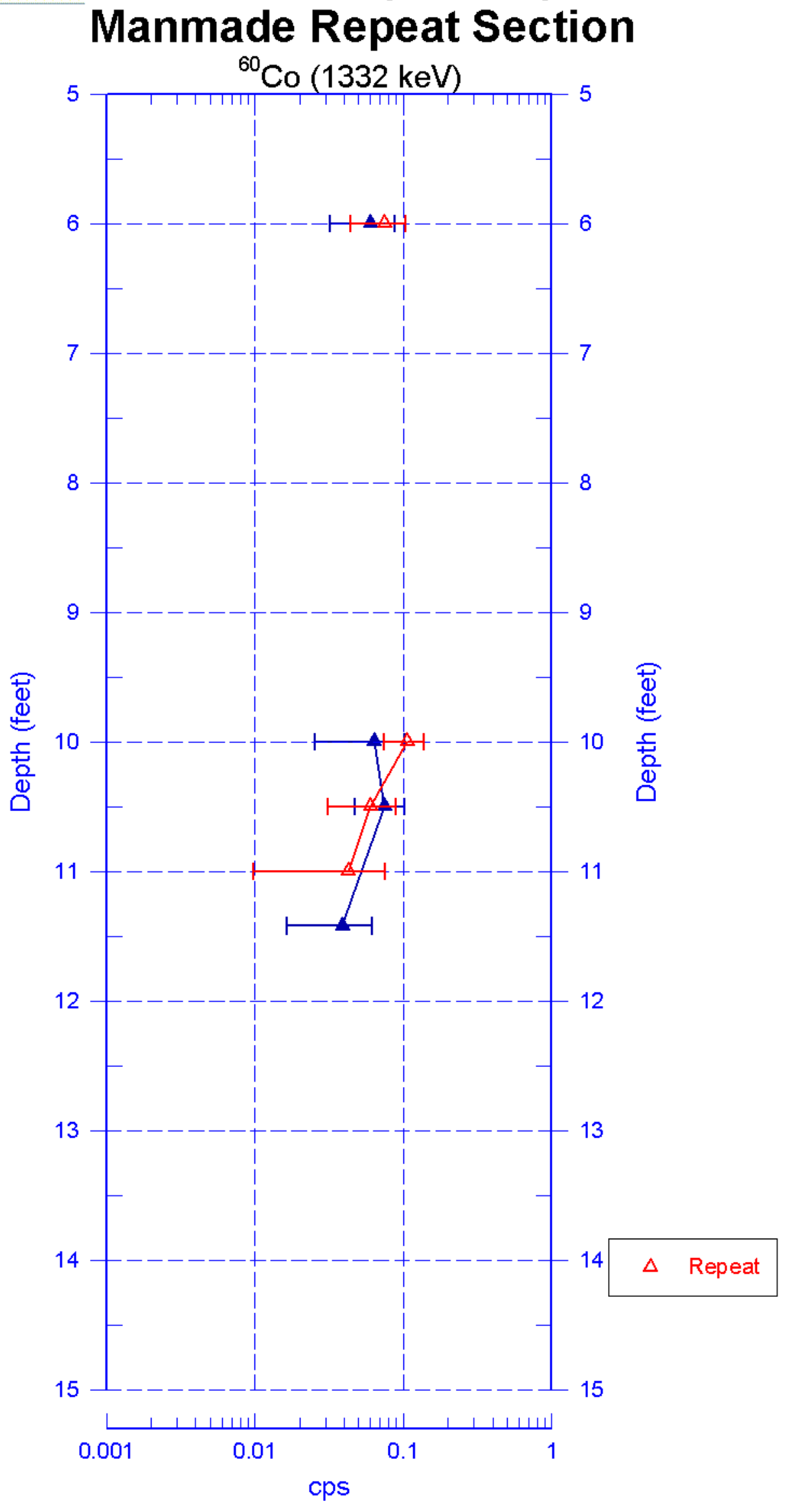

Reference - Ground Surface

G.109 


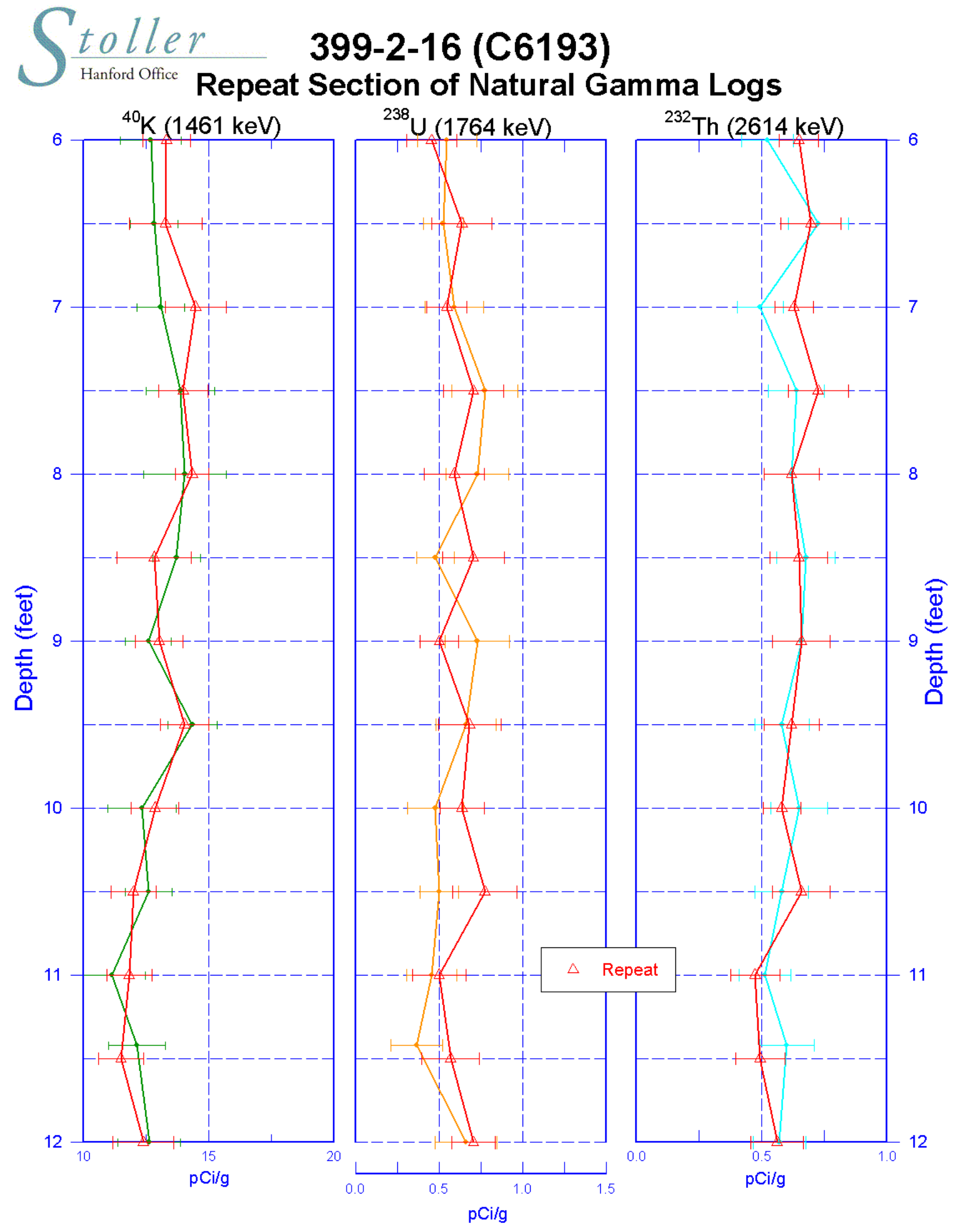

Zero Reference - Ground Surface

G.110 


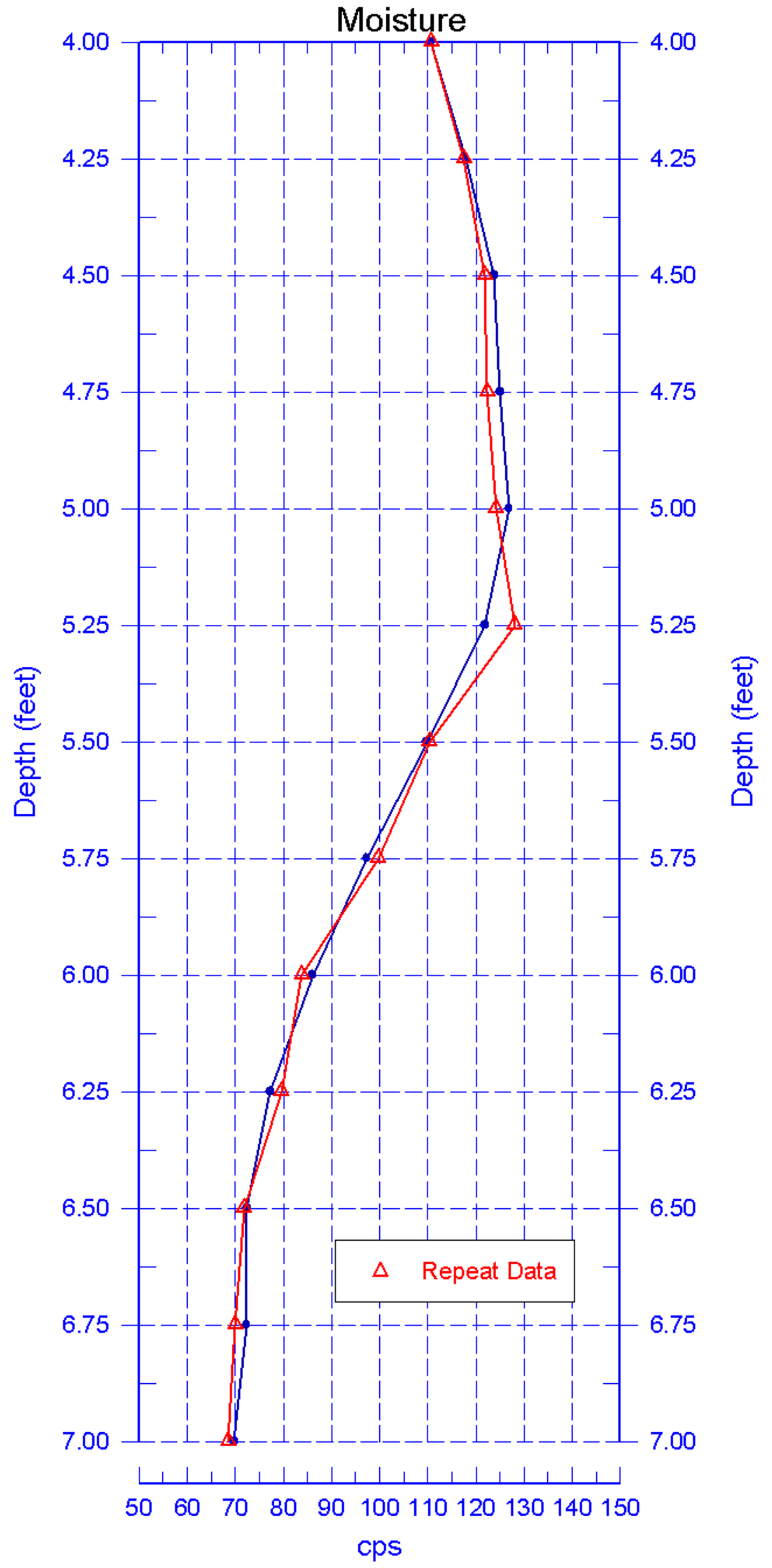


HGLP-LDR-243, Rev. 0

\section{9-2-17 (C6195) Log Data Report}

\section{Borehole Information:}

\begin{tabular}{|c|c|c|c|c|c|}
\hline Borehole: & \multicolumn{2}{l|}{ Site: } & \multicolumn{2}{l|}{ G00-FF-5 } \\
\hline Coordinates (WA St Plane) & GWL $^{1}$ (ft) : & 27.6 & \multicolumn{2}{c|}{ GWL Date: } & $06 / 16 / 08$ \\
\hline North $(\mathbf{m})$ & East $(\mathbf{m})$ & Drill Date & TOC $^{2}$ Elevation & Total Depth (ft) & Type \\
\hline 116060.3 & 594246.1 & $06 / 12 / 08$ & Unknown & 63 & Sonic \\
\hline
\end{tabular}

\section{Casing Information:}

\begin{tabular}{|c|c|c|c|c|c|c|}
\hline Casing Type & Stickup (ft) & $\begin{array}{c}\text { Outer } \\
\text { Diameter (in.) }\end{array}$ & $\begin{array}{c}\text { Inside } \\
\text { Diameter (in.) }\end{array}$ & Thickness (in.) & Top (ft) & Bottom (ft) \\
\hline Welded Steel & 0.7 & $75 / 8$ & $67 / 8$ & $3 / 8$ & -0.7 & 59.5 \\
\hline
\end{tabular}

\section{Borehole Notes:}

Casing data and total depth were reported by site geologist. Logging engineer measured depth to water with an e-tape. Casing diameters were measured using a steel tape and rounded to the nearest 1/16”. The zero reference is the ground surface.

\section{Logging Equipment Information:}

\begin{tabular}{|l|l|l|l|l|}
\hline Logging System: & \multicolumn{2}{|l|}{ Gamma 4 L } & $\begin{array}{l}\text { Type: } \\
\text { Serial No.: }\end{array}$ & $\begin{array}{l}\text { 60\% HPGe SGLS } \\
\text { 47TP32211A }\end{array}$ \\
\hline Effective Calibration Date: & $12 / 31 / 07$ & Calibration Reference: & HGLP-CC-027 \\
\hline \multicolumn{2}{|l|}{} & Logging Procedure: & HGLP-MAN-002, Rev. 0 \\
\hline
\end{tabular}

\begin{tabular}{|l|l|l|l|l|}
\hline Logging System: & \multirow{2}{*}{ Gamma $4 \mathrm{H}$} & $\begin{array}{l}\text { Type: } \\
\text { Serial No.: }\end{array}$ & $\begin{array}{l}\text { NMLS } \\
\text { H310700352 }\end{array}$ \\
\hline Effective Calibration Date: & $11 / 06 / 07$ & Calibration Reference: & HGLP-CC-021 \\
\hline \multicolumn{2}{|l|}{} & Logging Procedure: & HGLP-MAN-002, Rev. 0 \\
\hline
\end{tabular}

\section{Spectral Gamma Logging System (SGLS) Log Run Information:}

\begin{tabular}{|l|c|c|}
\hline Log Run & 1 & 2 Repeat \\
\hline Date & $06 / 19 / 08$ & $06 / 19 / 08$ \\
\hline Logging Engineer & Pearson & Pearson \\
\hline Start Depth (ft) & 62.0 & 28.0 \\
\hline Finish Depth (ft) & 0.0 & 22.0 \\
\hline Count Time (sec) & 200 & 200 \\
\hline Live/Real & $\mathrm{R}$ & $\mathrm{R}$ \\
\hline Shield (Y/N) & $\mathrm{N}$ & $\mathrm{N}$ \\
\hline MSA Interval (ft) & 0.5 & 0.5 \\
\hline Log Speed (ft/min) & N/A & N/A \\
\hline Pre-Verification & DL471CAB & DL471CAB \\
\hline Start File & DL471000 & DL471125 \\
\hline Finish File & DL471124 & DL471137 \\
\hline Post-Verification & DL471CAA & DL471CAA \\
\hline
\end{tabular}


HGLP-LDR-243, Rev. 0

\begin{tabular}{|l|c|c|}
\hline Log Run & 1 & 2 Repeat \\
\hline Depth Return Error (in.) & Low $1 / 4$ & 0 \\
\hline Comments & Fine gain & Repeat Section \\
& adjustment & \\
& made before & \\
& -053 & \\
\hline
\end{tabular}

Neutron Moisture Logging System (NMLS) Log Run Information:

\begin{tabular}{|l|c|c|}
\hline Log Run & $\mathbf{3}$ & 4 Repeat \\
\hline Date & $06 / 18 / 08$ & $06 / 18 / 08$ \\
\hline Logging Engineer & McClellan & McClellan \\
\hline Start Depth (ft) & 0.0 & 26.75 \\
\hline Finish Depth (ft) & 26.75 & 24.0 \\
\hline Count Time (sec) & 15 & 15 \\
\hline Live/Real & $\mathrm{R}$ & $\mathrm{R}$ \\
\hline Shield (Y/N) & $\mathrm{N}$ & $\mathrm{N}$ \\
\hline MSA Interval (ft) & 0.25 & 0.25 \\
\hline Log Speed (ft/min) & N/A & N/A \\
\hline Pre-Verification & DHF42CAB & DHF42CAB \\
\hline Start File & DHF42000 & DHF42108 \\
\hline Finish File & DHF42107 & DHF42119 \\
\hline Post-Verification & DHF42CAA & DHF42CAA \\
\hline Depth Return Error (in.) & N/A & 0.5 high \\
\hline Comments & None. & Repeat Section \\
\hline
\end{tabular}

\section{Logging Operation Notes:}

Data were collected using Gamma 4, HO 68B-3573. SGLS pre - and post-survey verification measurements were acquired in the A mersham KUTh-115 field verifier. Maximum logging depth was $62.2 \mathrm{ft}$ before the sonde un-weighted. A centralizer was installed on the sonde. Due to site-wide “Take Cover” drill, the log includes a 2.5 hour gap between -052 and -053 .

NMLS pre- and post-survey verification measurements were acquired in the standard field verifier. Maximum logging depth achieved was $26.75 \mathrm{ft}$, approximately at groundwater level.

\section{Analysis Notes:}

\begin{tabular}{|l|l|l|l|l|l|}
\hline Analyst: & LEGLER & Date: & 08/04/08 & Reference: & GJO-HGLP 1.6.3, Rev. 0 \\
\hline
\end{tabular}

SGLS pre- and post-survey verification spectra met the acceptance criteria for the established system. NMLS pre and post-survey verification spectra met the acceptance criteria for the established system, but the spectra files DHF42CAB and DHF42CAA had measurements above the upper control limits for counts per second (cps).

A casing correction for a 3/8-in. thick casing was applied from ground surface to $59.5 \mathrm{ft}$ where casing ends, leaving $2.5 \mathrm{ft}$ of open borehole uncorrected. A water correction was also applied during analysis from $27.6 \mathrm{ft}$ to total logged depth of borehole.

SGLS spectra were processed in batch mode using APTEC SUPERVISOR to identify individual peaks and count rates. Concentrations were calculated using an EXCEL template identified as G4LDec07.xls using an efficiency function and corrections for casing, dead time and water as determined by annual calibrations. NMLS spectra were processed in batch mode using APTEC SUPERVISOR to identify counts. Count rates were calculated using an EXCEL template identified as G4HNov07.xls. NMLS data are presented in counts per second (cps), because no calibration data is available for a 6 7/8-in. inner diameter borehole casing. 
HGLP-LDR-243, Rev. 0

\section{Results and Interpretations:}

U-235 and U-238 (Pa-234m) were detected sporadically in this borehole. Inspection of the individual spectra at these depths indicates that these detections are statistical fluctuations associated with the processing software and are not considered valid.

The KUT plots indicate good repeatability. The repeat Moisture plot indicates good repeatability.

\section{List of Log Plots:}

Depth Reference is ground surface

Manmade Radionuclides

Natural Gamma Logs

Combination Plot

Total Gamma \& Dead Time

Total Gamma \& Moisture

Repeat Section of Natural Gamma Logs

Moisture Repeat Section

${ }^{1} \mathrm{GWL}$ - groundwater level

${ }^{2}$ TOC - top of casing 

Hanford Office Manmade Radionuclides

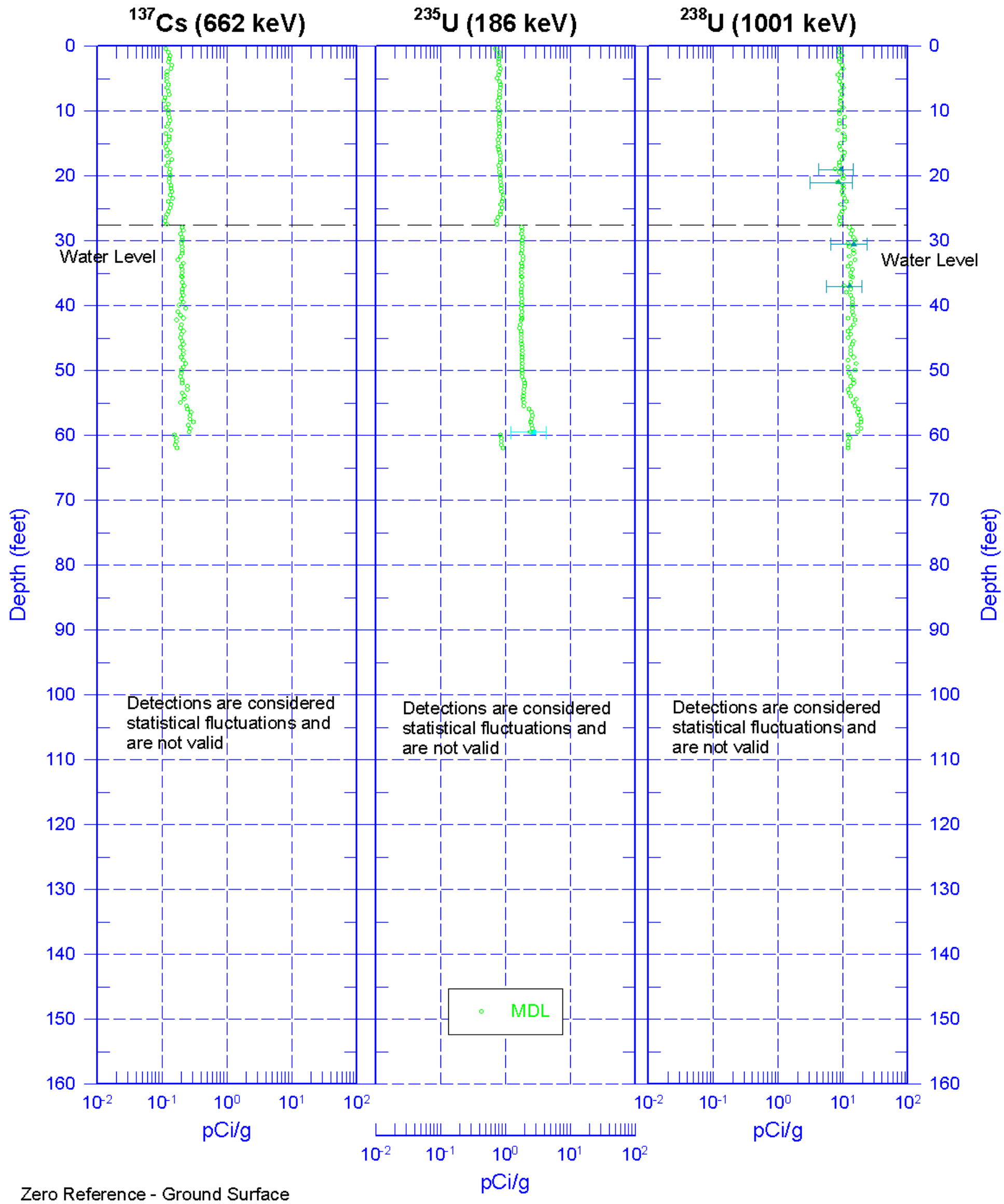


toller 399-2-17 (C6195)

Hanford Office Natural Gamma Logs
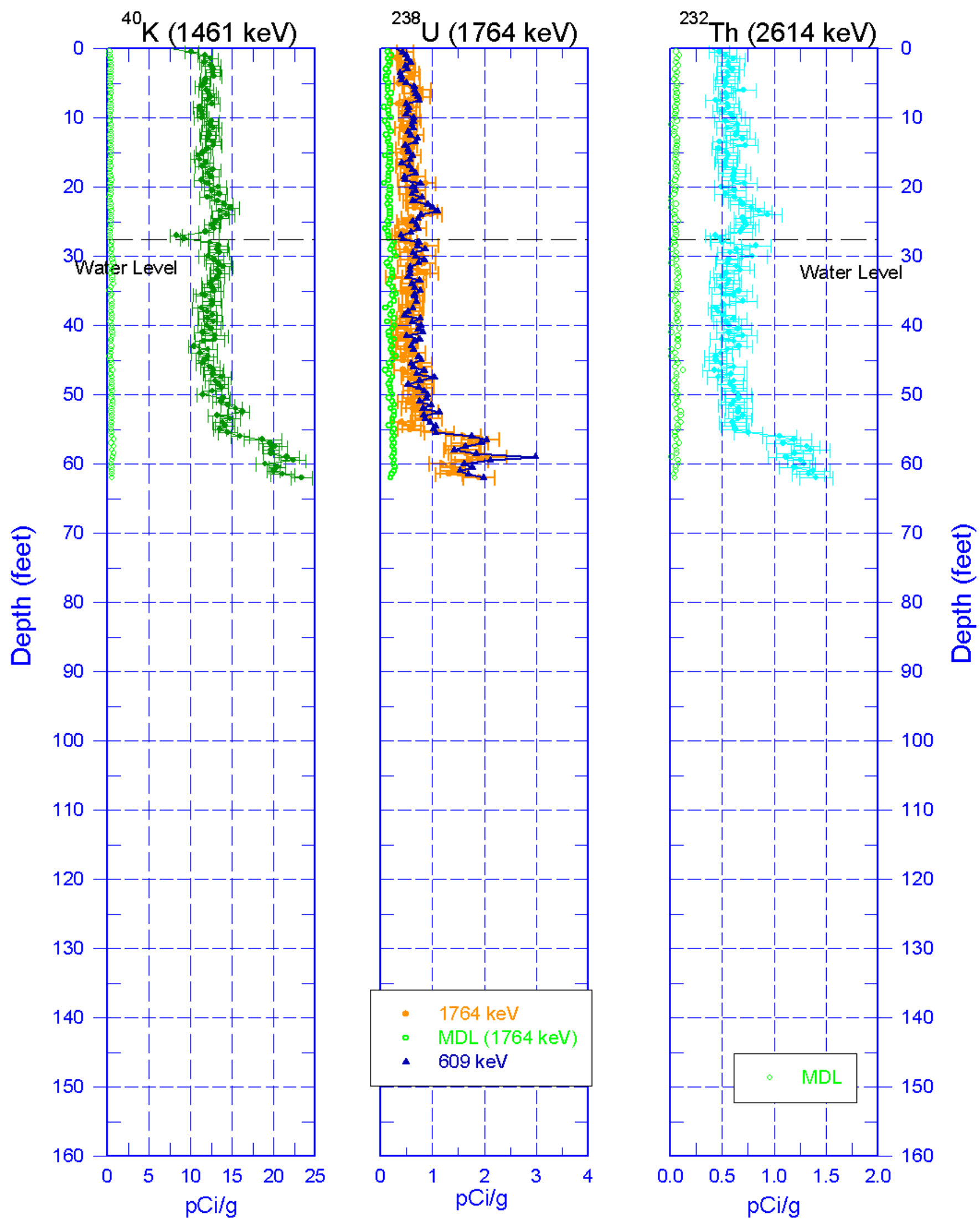

Zero Reference - Ground Surface

G.116 


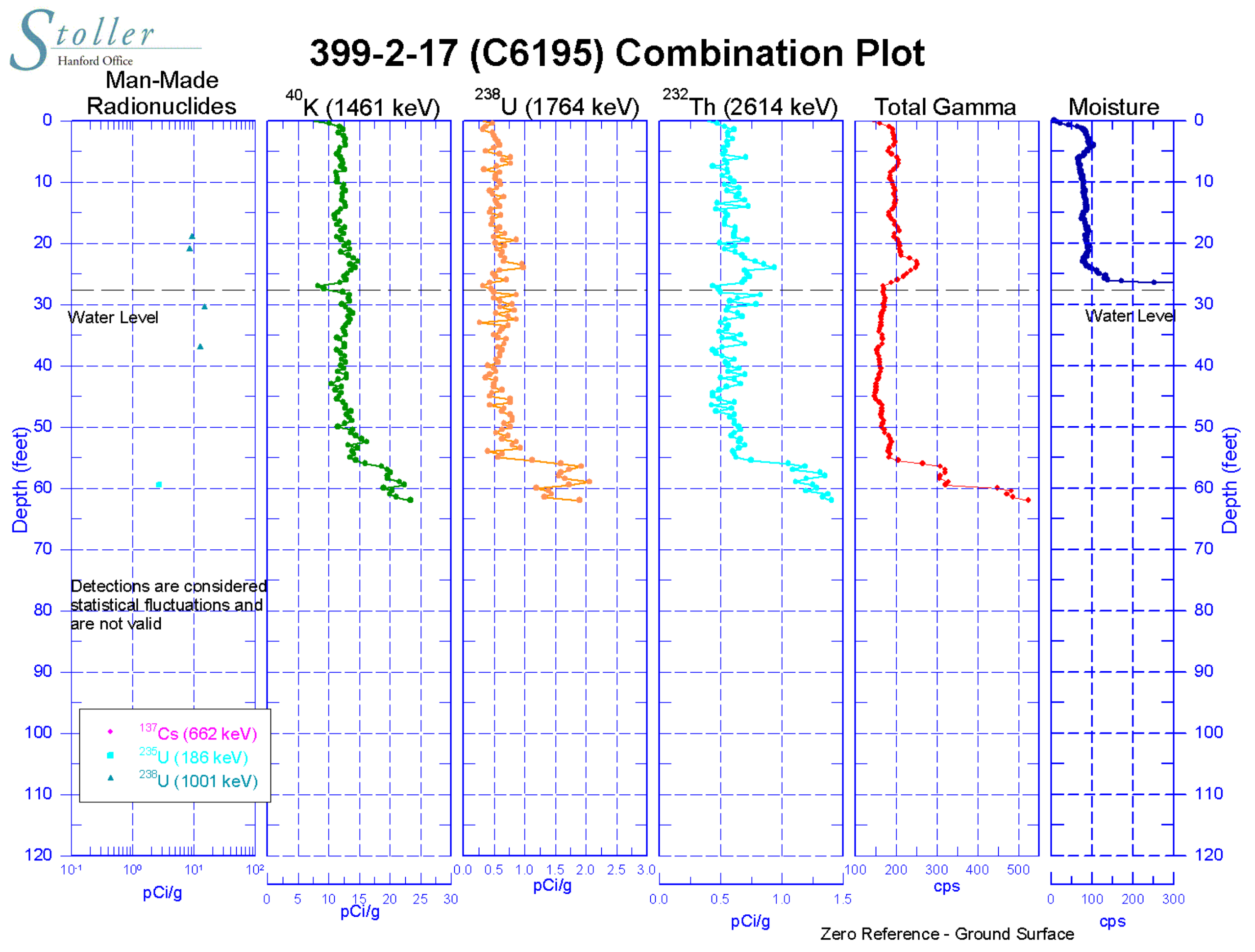



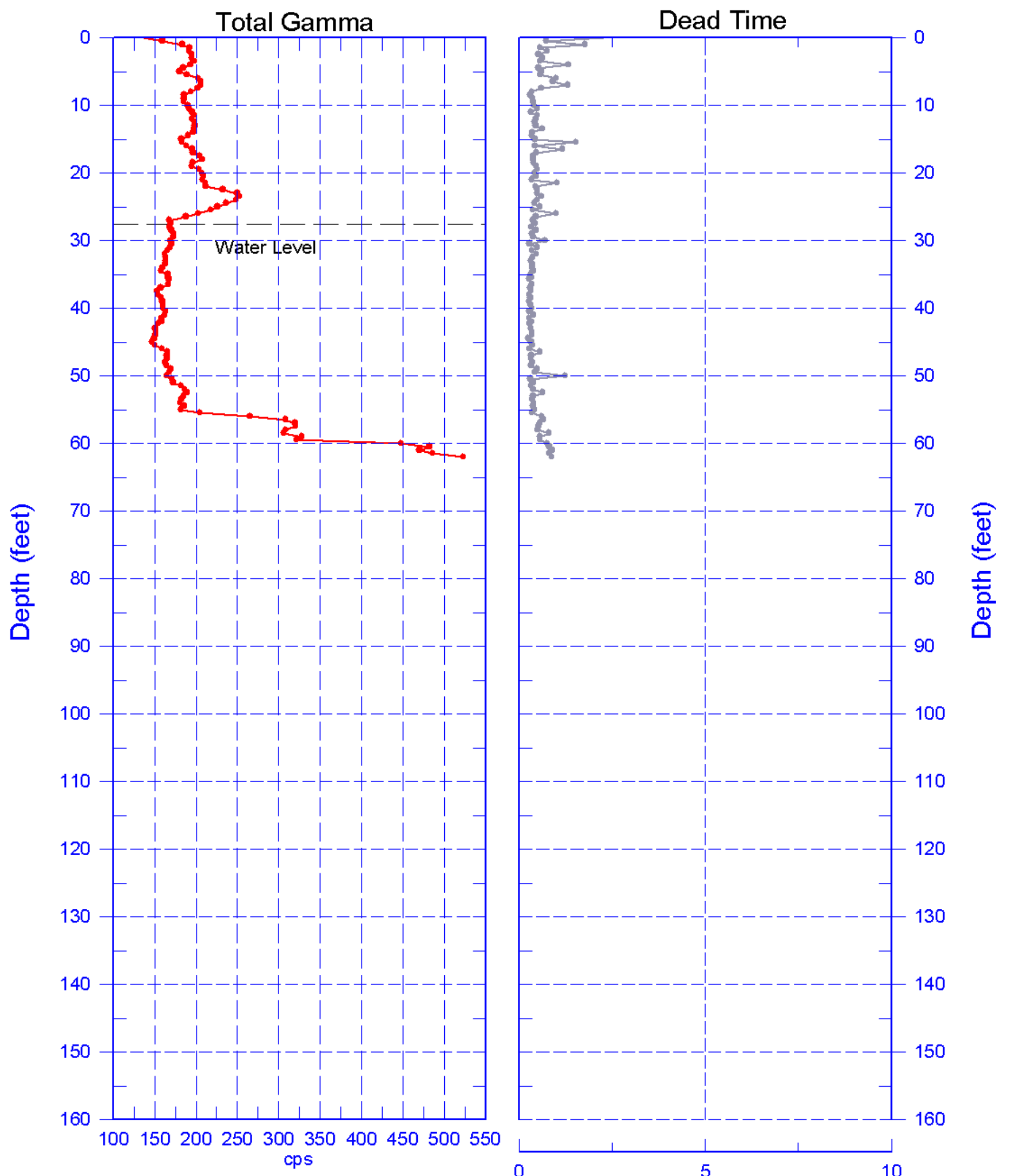


\section{S}

toller 399-2-17 (C6195)

Hanford Office

\section{Total Gamma \& Moisture}
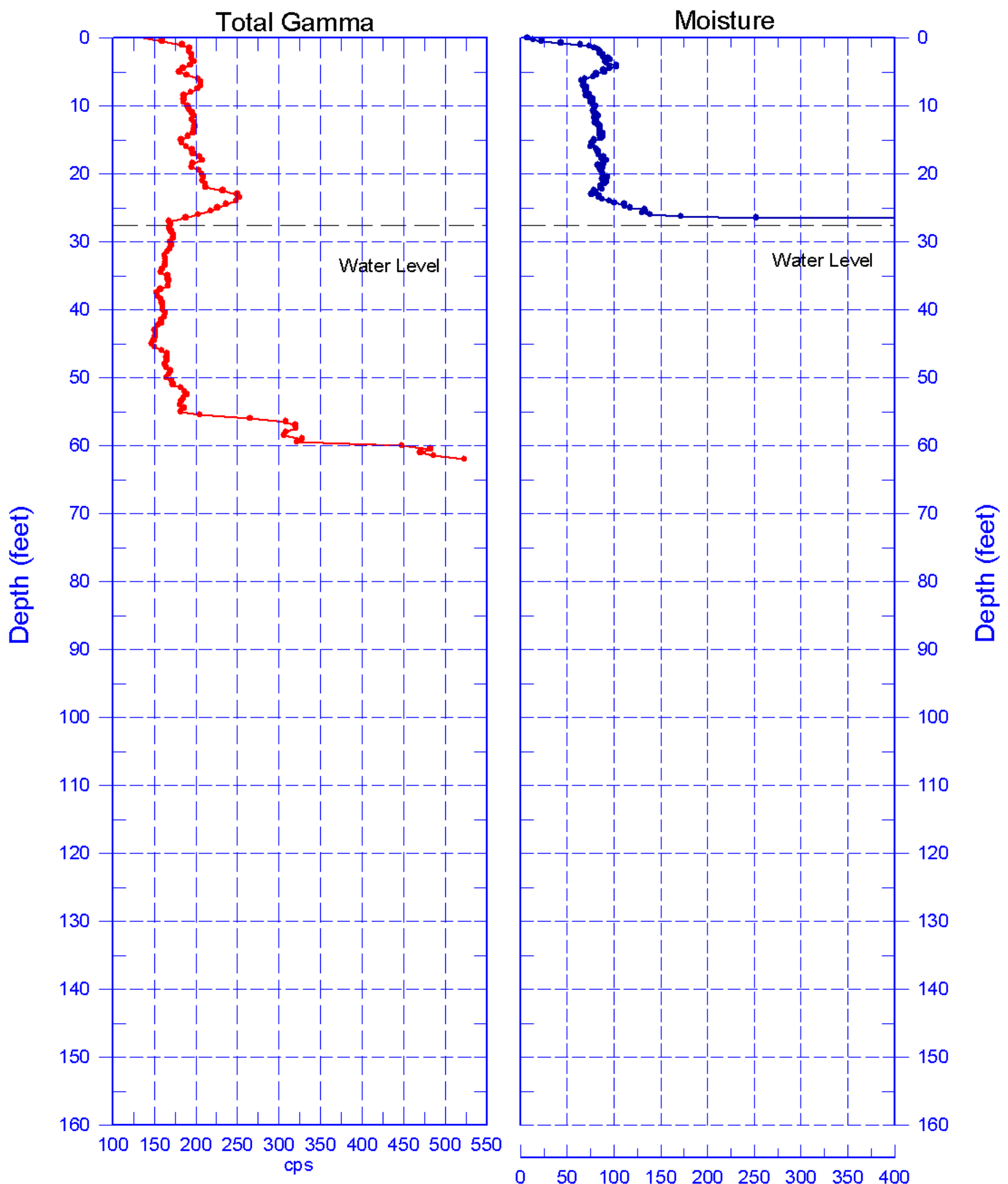

웜
듬
$\frac{1}{0}$

Reference - Ground Surface 


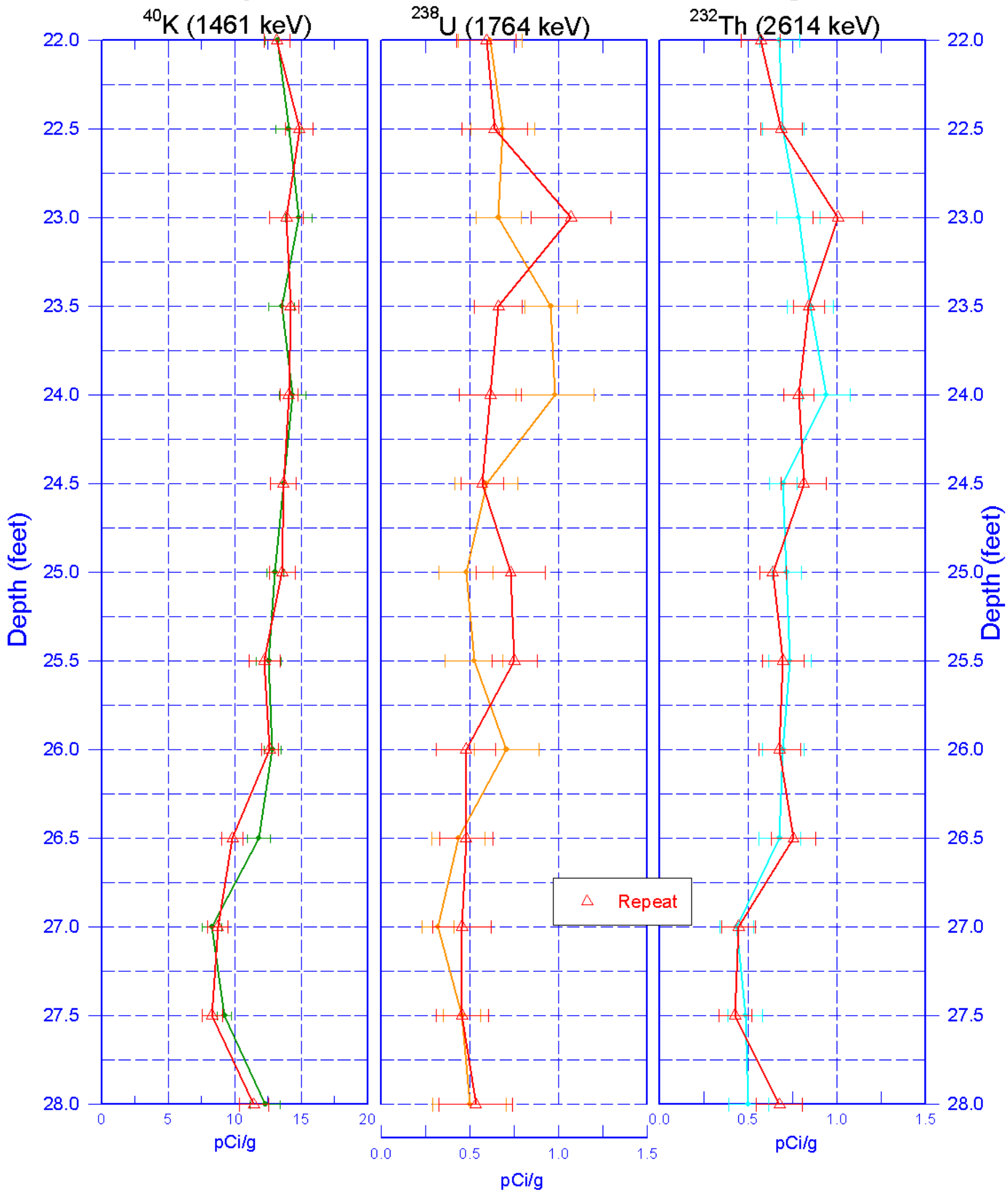




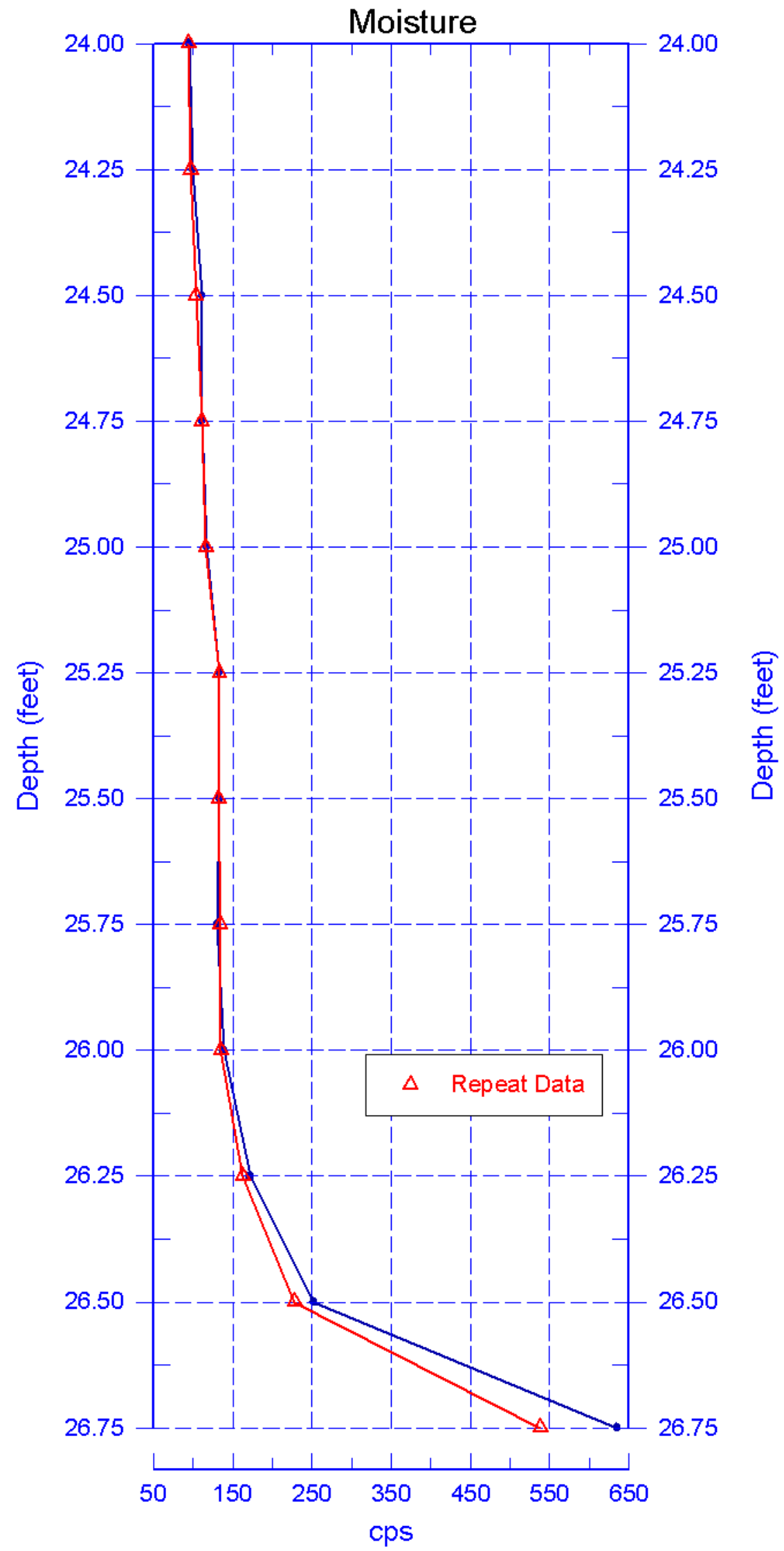




\section{9-2-18 (C6196) Log Data Report}

Borehole Information:

\begin{tabular}{|c|c|c|c|c|c|}
\hline \multicolumn{2}{|c|}{ Borehole: $\quad 399-2-18$ (C6196) } & \multicolumn{4}{|c|}{ 300-FF-5 } \\
\hline \multicolumn{2}{|c|}{ Coordinates (WA St Plane) } & $G_{W L}^{1}(\mathbf{f}$ & 27.0 & GWL Date: & $06 / 15 / 08$ \\
\hline North (m) & East (m) & Drill Date & TOC $^{2}$ Elevation & Total Depth (ft) & Type \\
\hline 116066.0 & 594254.2 & $06 / 11 / 08$ & Unknown & 65.0 & Sonic \\
\hline
\end{tabular}

\section{Casing Information:}

\begin{tabular}{|c|c|c|c|c|c|c|}
\hline Casing Type & Stickup (ft) & $\begin{array}{c}\text { Outer } \\
\text { Diameter (in.) }\end{array}$ & $\begin{array}{c}\text { Inside } \\
\text { Diameter (in.) }\end{array}$ & Thickness (in.) & Top (ft) & Bottom (ft) \\
\hline Welded Steel & 0.5 & $75 / 8$ & $67 / 8$ & $3 / 8$ & -0.5 & 60 \\
\hline
\end{tabular}

\section{Borehole Notes:}

Well site geologist reported casing data and total depth. Logging engineer measured depth to water with an e-tape, and casing diameters using a steel tape and rounding to the nearest 1/16-in.

\section{Logging Equipment Information:}

\begin{tabular}{|l|l|l|l|l|}
\hline \multirow{2}{*}{ Logging System: } & \multirow{2}{*}{ Gamma 4 L } & $\begin{array}{l}\text { Type: } \\
\text { Serial No.: }\end{array}$ & $\begin{array}{l}\text { 60\% HPGe SGLS } \\
\text { 47T32211A }\end{array}$ \\
\hline Effective Calibration Date: & $12 / 31 / 07$ & $\begin{array}{l}\text { Calibration Reference: } \\
\text { Logging Procedure: }\end{array}$ & HGLP-CC-027 \\
\cline { 4 - 4 } & & HGLP-MAN-002, Rev. 0 \\
\hline
\end{tabular}

\begin{tabular}{|l|l|l|l|l|}
\hline Logging System: & \multirow{2}{*}{ Gamma 4 H } & $\begin{array}{l}\text { Type: } \\
\text { Serial No.: }\end{array}$ & $\begin{array}{l}\text { NMLS } \\
\text { H310700352 }\end{array}$ \\
\hline Effective Calibration Date: & $11 / 06 / 07$ & Calibration Reference: & HGLP-CC-021 \\
\hline \multicolumn{2}{|l|}{} & Logging Procedure: & HGLP-MAN-002, Rev. 0 \\
\hline
\end{tabular}

Spectral Gamma Logging System (SGLS) Log Run Information:

\begin{tabular}{|l|c|c|c|c|c|}
\hline Log Run & $\mathbf{1}$ & $\mathbf{2}$ & $\mathbf{3}$ Repeat & & \\
\hline Date & $06 / 12 / 08$ & $06 / 15 / 08$ & $06 / 15 / 08$ & & \\
\hline Logging Engineer & Pearson & Pearson & Pearson & & \\
\hline Start Depth (ft) & 0.0 & 64.0 & 22.0 & & \\
\hline Finish Depth (ft) & 18.0 & 17.0 & 28.0 & & \\
\hline Count Time (sec) & 200 & 200 & 200 & & \\
\hline Live/Real & $\mathrm{R}$ & $\mathrm{R}$ & $\mathrm{R}$ & & \\
\hline Shield (Y/N) & $\mathrm{N}$ & $\mathrm{N}$ & $\mathrm{N}$ & & \\
\hline MSA Interval (ft) & 0.5 & 0.5 & 0.5 & & \\
\hline Log Speed (ft/min) & $\mathrm{N} / \mathrm{A}$ & $\mathrm{N} / \mathrm{A}$ & $\mathrm{N} / \mathrm{A}$ & & \\
\hline Pre-Verification & DL431CAB & DL441CAB & DL441CAB & & \\
\hline Start File & DL431000 & DL441000 & DL441095 & & \\
\hline Finish File & DL431036 & DL441094 & DL441107 & & \\
\hline Post-Verification & DL431CAA & DL441CAA & DL441CAA & & \\
\hline Depth Return Error (in.) & 0 & 0 & 1/4 low & & \\
\hline Comments & $\begin{array}{c}\text { No fine gain } \\
\text { adjustment } \\
\text { made. }\end{array}$ & $\begin{array}{c}\text { No fine gain } \\
\text { adjustments } \\
\text { made. }\end{array}$ & Repeat section. & & \\
\hline
\end{tabular}


Neutron Moisture Logging System (NMLS) Log Run Information:

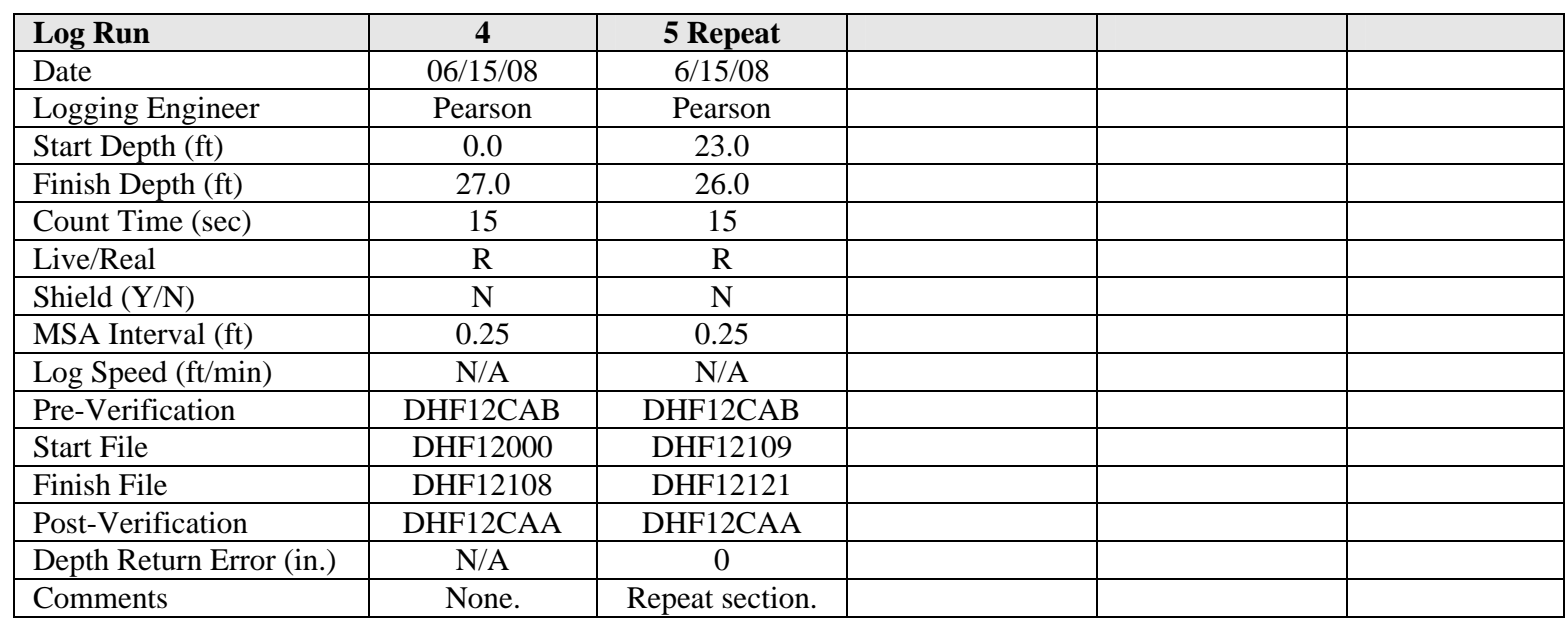

\section{Logging Operation Notes:}

Data were collected using Gamma 4, HO 68B-3573. SGLS pre- and post-survey verification measurements were acquired in the Amersham KUTh-115 field verifier. Maximum SGLS logging depth was $64.4 \mathrm{ft}$ before the sonde un-weighted. A centralizer was installed on the sonde. NMLS pre- and post-survey verification measurements were acquired in the standard field verifier. Maximum NMLS logging depth achieved was $27.0 \mathrm{ft}$.

\section{Analysis Notes:}

\begin{tabular}{|l|l|l|l|l|l|}
\hline Analyst: & LEGLER & Date: & 6/25/08 & Reference: & GJO-HGLP 1.6.3, Rev. 0 \\
\hline
\end{tabular}

The pre- and post-survey verification spectra met the acceptance criteria for the established systems, but verification files DHF12CAB and DHF12CAA had measurements above the upper control limit for counts per second. A correction for a 3/8-in. thick casing was applied to SGLS data from ground surface to $60 \mathrm{ft}$, leaving $4.4 \mathrm{ft}$ of open borehole uncorrected. A water correction was also applied from $27 \mathrm{ft}$ to total logged depth of borehole.

SGLS spectra were processed in batch mode in APTEC SUPERVISOR to identify individual energy peaks and determine count rates. Concentrations were calculated with an EXCEL worksheet template identified as G4LDec07.xls using efficiency functions, and corrections for casing, dead time, and water as determined by annual calibrations.

Moisture data are presented in counts per second because no calibration data exists for a $67 / 8$-in. inner diameter casing.

\section{Results and Interpretations:}

Cs-137, U-235, and U-238 (Pa-234m) were detected at several isolated depths throughout this borehole. Inspection of the individual spectra for these radionuclides at the various depths indicates these detections are statistical fluctuations associated with the processing software and are not considered valid.

The KUT plots indicate good repeatability. The moisture plot indicates some variability. 
HGLP-LDR-228, Rev. 0

\section{List of Log Plots:}

Depth Reference is ground surface

Manmade Radionuclides

Natural Gamma Logs

Combination Plot

Total Gamma \& Dead Time

Total Gamma \& Moisture

Repeat Section of Natural Gamma Logs

Moisture Repeat Section

${ }^{1} \mathrm{GWL}$ - groundwater level

${ }^{2}$ TOC - top of casing 

Hanford Office Manmade Radionuclides

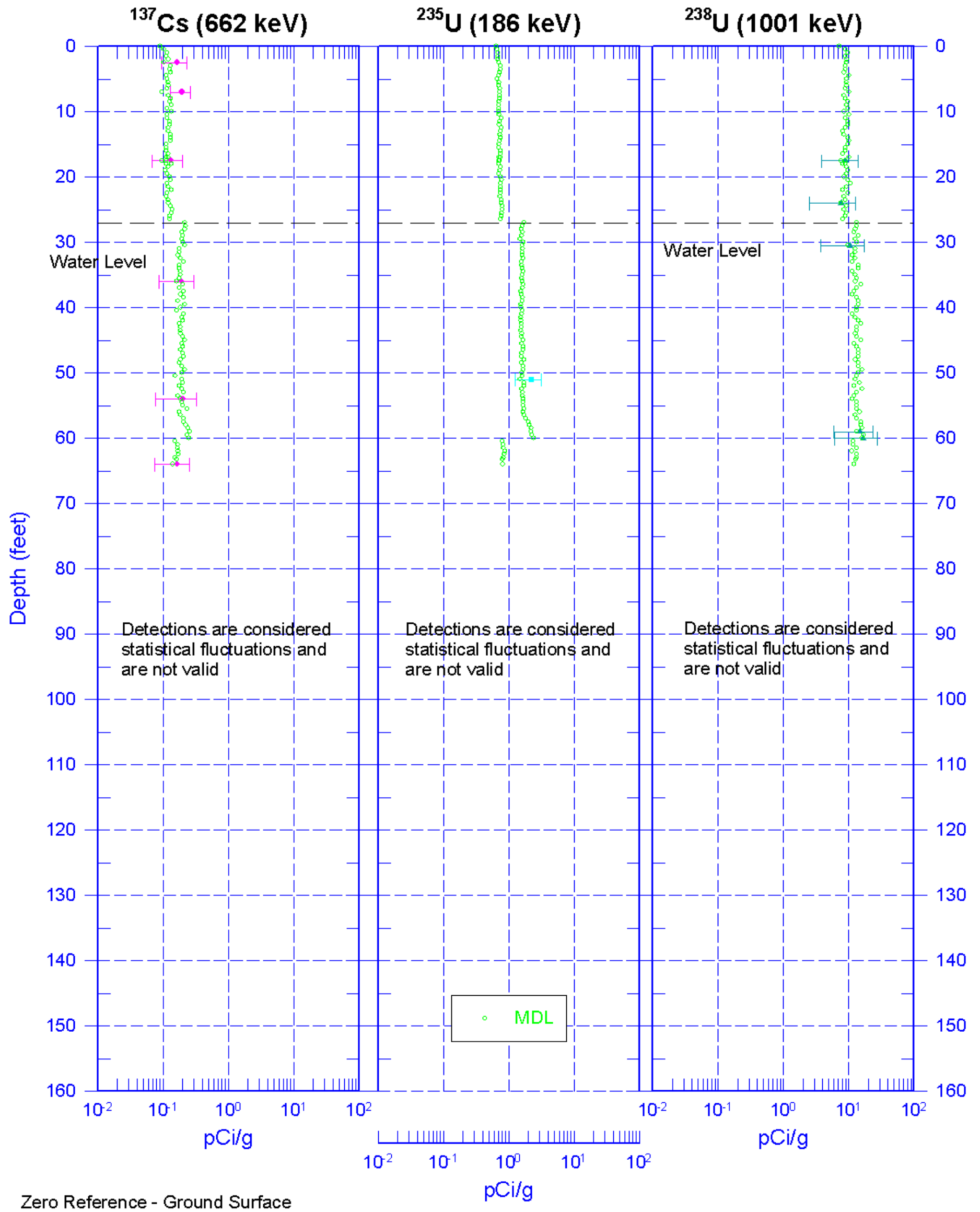

Zero Reference - Ground Surface 
toller 399-2-18 (C6196)

Hanford Office

\section{Natural Gamma Logs}
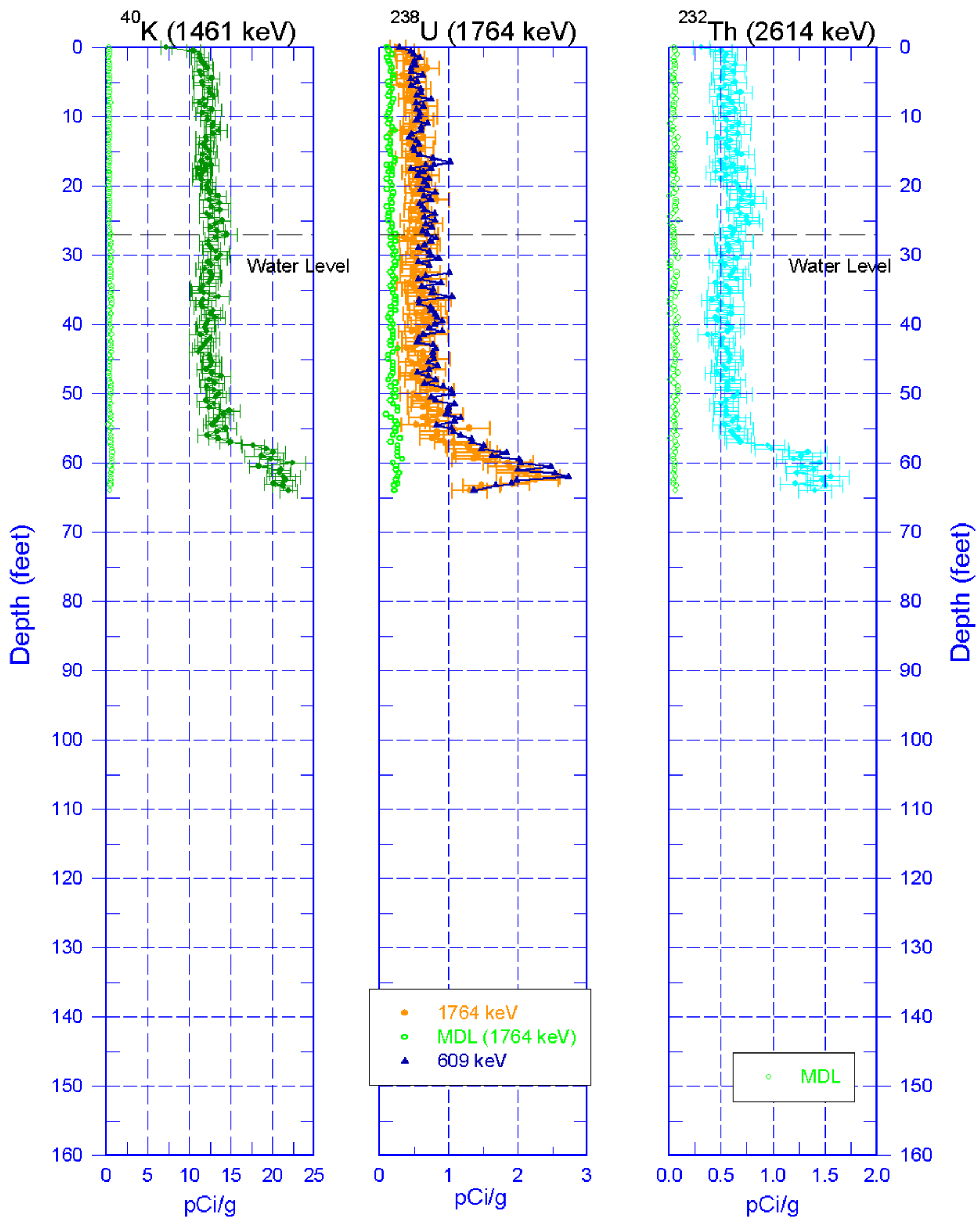

Zero Reference - Ground Surface

G.126 


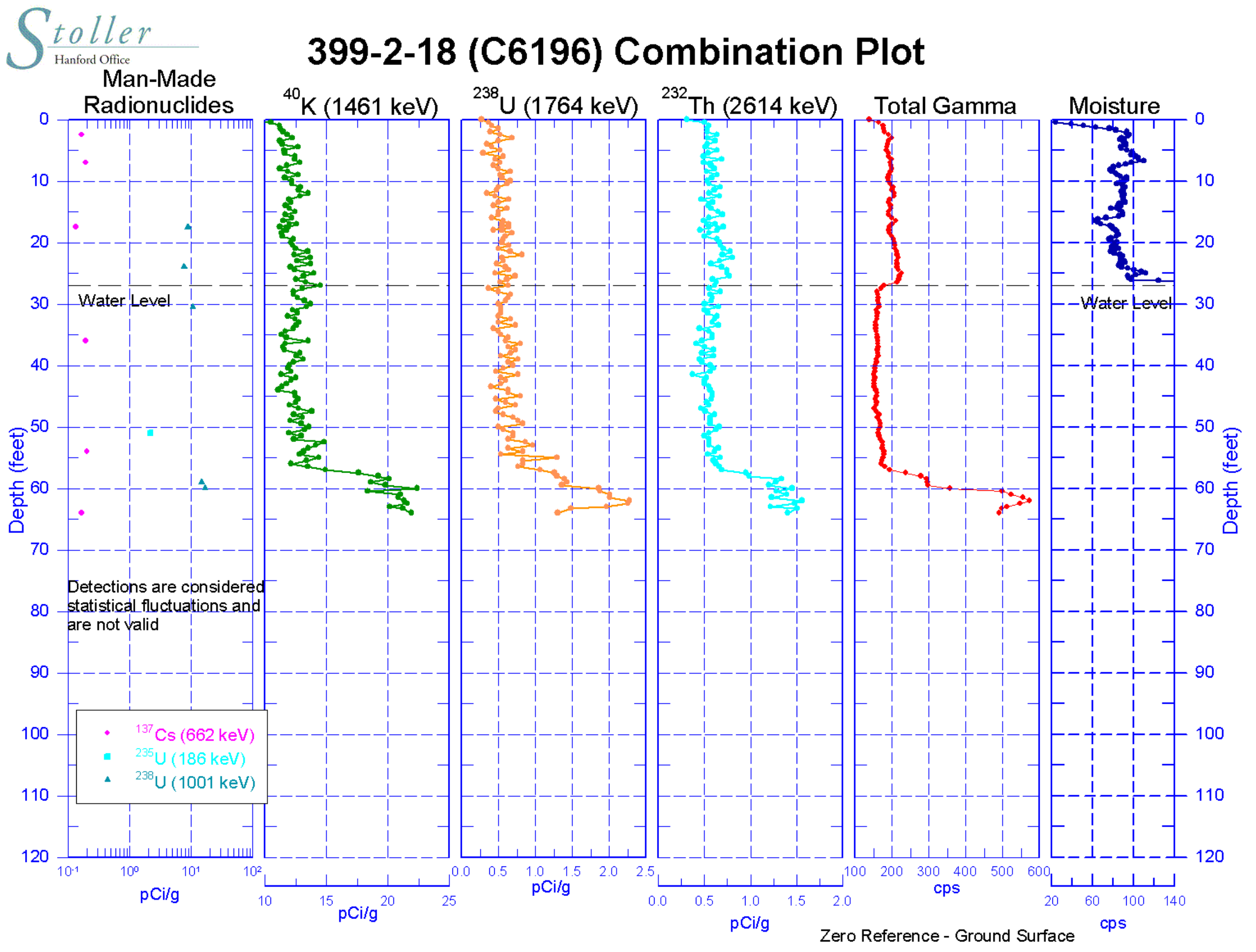


toller 399-2-18 (C6196)

Hanford Office

\section{Total Gamma \& Dead Time}
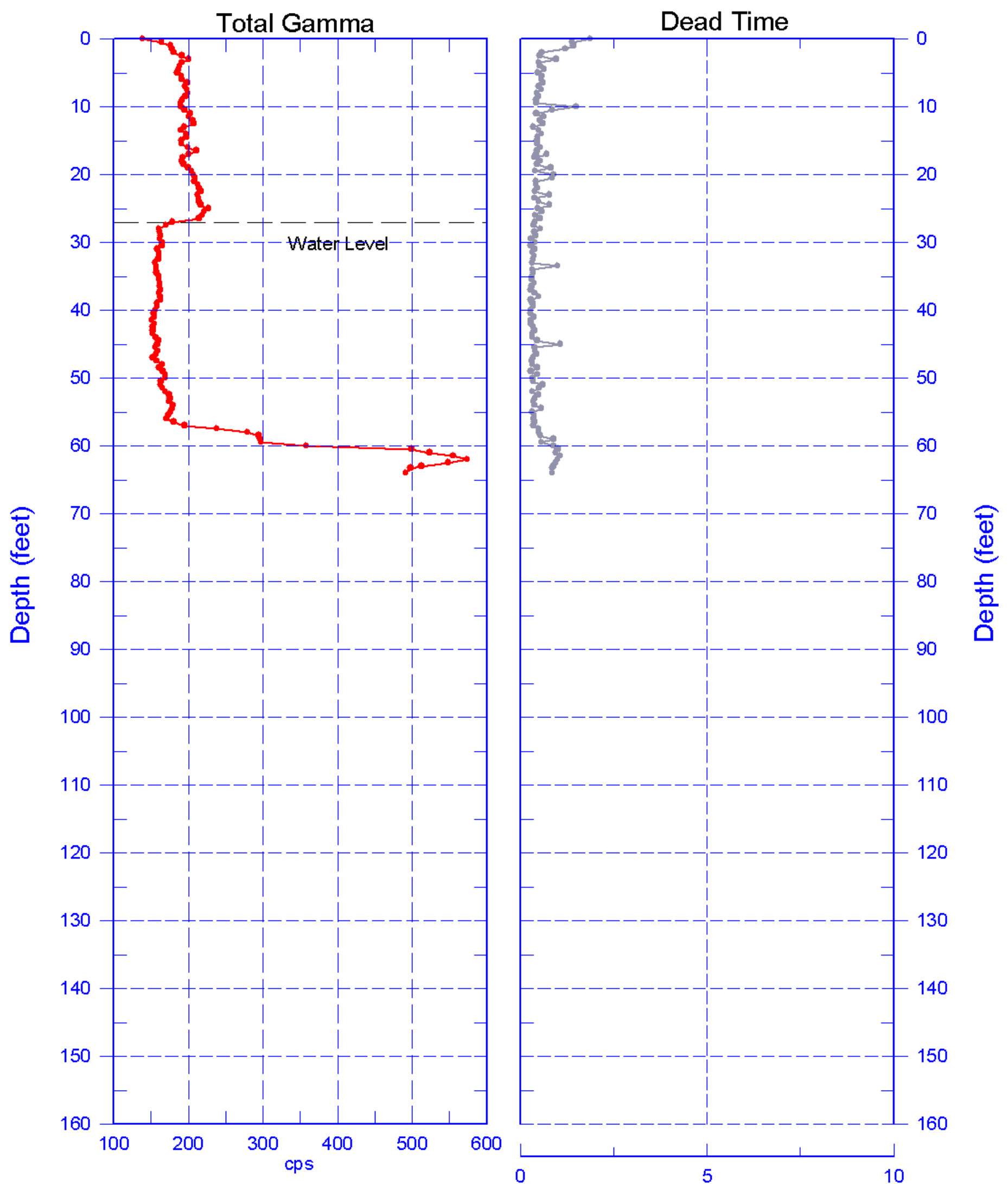

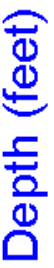

Reference - Ground Surface

G.128 


\section{S}

toller 399-2-18 (C6196)

Hanford Office

\section{Total Gamma \& Moisture}
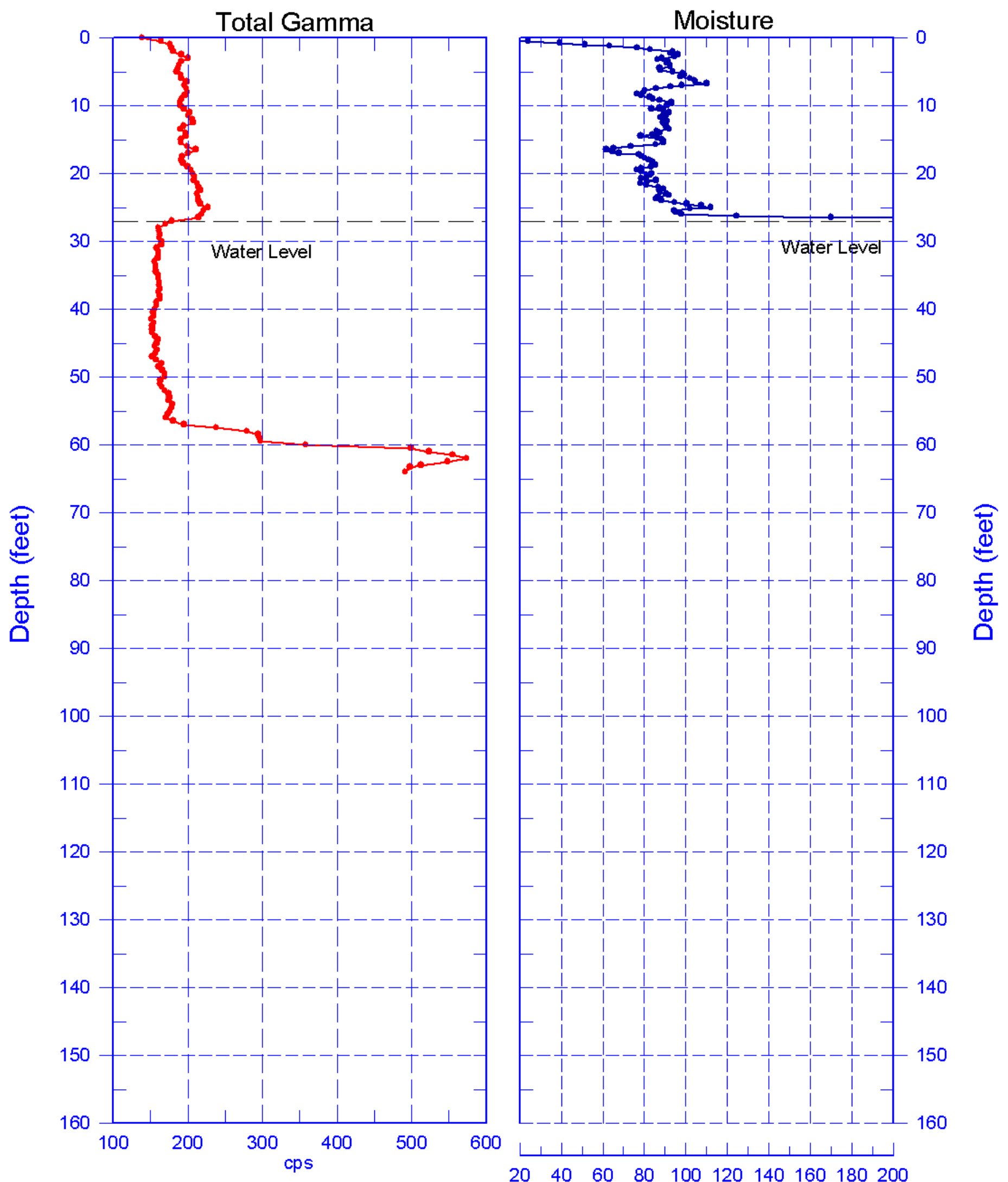

웜
듬
$\frac{1}{0}$

Reference - Ground Surface 


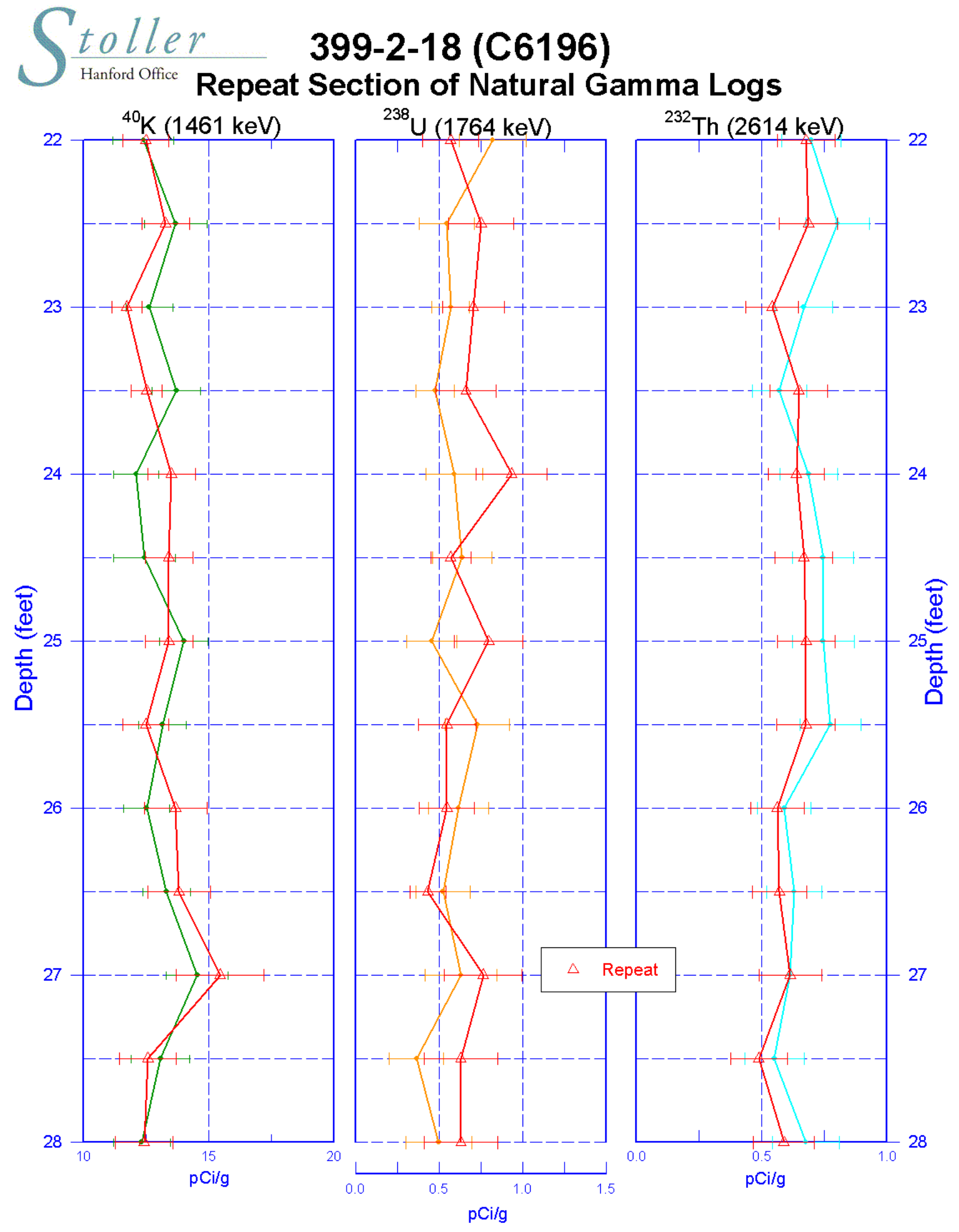

Zero Reference - Ground Surface

G.130 


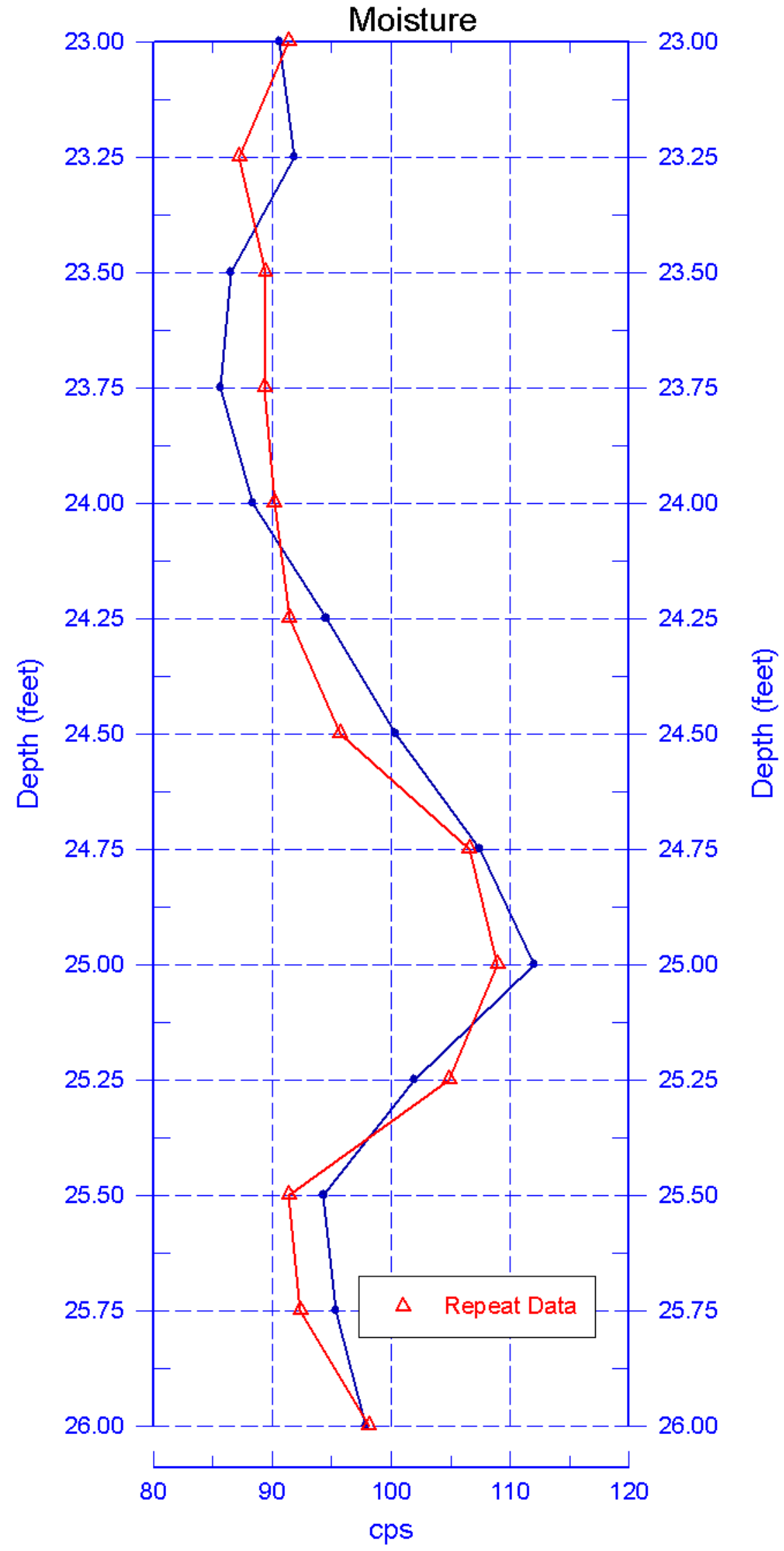




\section{9-2-19 (C6197) Log Data Report}

Borehole Information:

\begin{tabular}{|c|c|c|c|c|c|}
\hline Borehole: & \multicolumn{1}{l|}{ Site: } & \multicolumn{2}{l|}{ G00-FF-5 } \\
\hline \multicolumn{2}{|c|}{ 399-2-19 (C6197) } & GWL $^{\mathbf{1}}$ (ft) : & 23.3 & Date: & $05 / 22 / 08$ \\
\hline North (m) & East (m) & Drill Date & TOC $^{2}$ Elevation & Total Depth (ft) & Type \\
\hline Unknown & Unknown & $05 / 20 / 08$ & Unknown & 60.8 & Sonic \\
\hline
\end{tabular}

\section{Casing Information:}

\begin{tabular}{|c|c|c|c|c|c|c|}
\hline Casing Type & Stickup (ft) & $\begin{array}{c}\text { Outer } \\
\text { Diameter (in.) }\end{array}$ & $\begin{array}{c}\text { Inside } \\
\text { Diameter (in.) }\end{array}$ & Thickness (in.) & Top (ft) & Bottom (ft) \\
\hline Threaded Steel & 0.95 & $75 / 8$ & $67 / 8$ & $3 / 8$ & -0.95 & 60.0 \\
\hline
\end{tabular}

\section{Borehole Notes:}

Well site geologist reported depth to bottom, depth to water and depth of casing. Logging engineer measured casing employing a steel tape and rounding to the nearest 1/16-in. The zero reference is the ground surface.

\section{Logging Equipment Information:}

\begin{tabular}{|l|l|l|l|l|}
\hline \multirow{2}{*}{ Logging System: } & \multirow{2}{*}{ Gamma $1 \mathrm{~N}$} & $\begin{array}{l}\text { Type: } \\
\text { Serial No.: }\end{array}$ & $\begin{array}{l}\text { 60\% HPGe SGLS } \\
\text { 45TP22010A }\end{array}$ \\
\hline Effective Calibration Date: & $03 / 28 / 08$ & $\begin{array}{l}\text { Calibration Reference: } \\
\text { Logging Procedure: }\end{array}$ & HGLP-CC-031 \\
\cline { 4 - 4 } & & HGLP-MAN-002, Rev. 0 \\
\hline
\end{tabular}

\begin{tabular}{|l|l|l|l|l|}
\hline Logging System: & \multirow{2}{*}{ Gamma 1 M } & $\begin{array}{l}\text { Type: } \\
\text { Serial No.: }\end{array}$ & $\begin{array}{l}\text { NMLS } \\
\text { H340207279 }\end{array}$ \\
\hline Effective Calibration Date: & $05 / 06 / 08$ & Calibration Reference: & HGLP-CC-032 \\
\hline \multicolumn{2}{|l|}{} & Logging Procedure: & HGLP-MAN-002, Rev. 0 \\
\hline
\end{tabular}

\section{Spectral Gamma Logging System (SGLS) Log Run Information:}

\begin{tabular}{|l|c|c|}
\hline Log Run & 1 & 2 Repeat \\
\hline Date & $05 / 22 / 08$ & $05 / 22 / 08$ \\
\hline Logging Engineer & McClellan & McClellan \\
\hline Start Depth (ft) & 0.0 & 4.0 \\
\hline Finish Depth (ft) & 59.0 & 10.0 \\
\hline Count Time (sec) & 200 & 200 \\
\hline Live/Real & $\mathrm{R}$ & $\mathrm{R}$ \\
\hline Shield (Y/N) & $\mathrm{N}$ & $\mathrm{N}$ \\
\hline MSA Interval (ft) & 0.5 & 0.5 \\
\hline Log Speed (ft/min) & N/A & N/A \\
\hline Pre-Verification & AN076CAB & AN076CAB \\
\hline Start File & AN076000 & AN076119 \\
\hline Finish File & AN076118 & AN076131 \\
\hline Post-Verification & AN076CAA & AN076CAA \\
\hline Depth Return Error (in.) & N/A & 1.0 high \\
\hline Comments & $\begin{array}{c}\text { Fine gain } \\
\text { adjustment } \\
\text { made before } \\
\text { 089. }\end{array}$ & $\begin{array}{c}\text { No fine gain } \\
\text { adjustment } \\
\text { made. Repeat } \\
\text { Section }\end{array}$ \\
\hline \multicolumn{2}{|c}{} \\
\hline
\end{tabular}




\section{Neutron Moisture Logging System (NMLS) Log Run Information:}

\begin{tabular}{|l|c|c|}
\hline Log Run & 3 & 4 Repeat \\
\hline Date & $05 / 23 / 08$ & $05 / 23 / 08$ \\
\hline Logging Engineer & McClellan & McClellan \\
\hline Start Depth (ft) & 0.0 & 4.0 \\
\hline Finish Depth (ft) & 28.75 & 10.0 \\
\hline Count Time (sec) & 15 & 15 \\
\hline Live/Real & $\mathrm{R}$ & $\mathrm{R}$ \\
\hline Shield (Y/N) & $\mathrm{N}$ & $\mathrm{N}$ \\
\hline MSA Interval (ft) & 0.25 & 0.25 \\
\hline Log Speed (ft/min) & N/A & N/A \\
\hline Pre-Verification & AM004CAB & AM004CAB \\
\hline Start File & AM004000 & AM004116 \\
\hline Finish File & AM004115 & AM004140 \\
\hline Post-Verification & AM004CAA & AM004CAA \\
\hline Depth Return Error (in.) & N/A & $1 / 2$ high \\
\hline Comments & None. & Repeat Section \\
\hline
\end{tabular}

\section{Logging Operation Notes:}

Data were collected using Gamma 1, HO 68B-3574. SGLS pre- and post-survey verification measurements were acquired in the Amersham KUTh-118 field verifier. Maximum logging depth was $59.2 \mathrm{ft}$. before the sonde un-weighted. A centralizer was installed on the sonde. NMLS pre- and post-survey verification measurements were acquired in the AmBe standard.

\section{Analysis Notes:}

\begin{tabular}{|l|l|l|l|l|l|}
\hline Analyst: & LEGLER & Date: & $7 / 22 / 08$ & Reference: & GJO-HGLP 1.6.3, Rev. 0 \\
\hline
\end{tabular}

The pre- and post-survey verification measurements met the acceptance criteria for the established systems. A correction for a 3/8-in. thick casing was applied to spectral log data (SGLS) from ground surface to total logged depth of borehole. A water correction was also applied from $28.75 \mathrm{ft}$ to total logged depth of borehole.

SGLS spectral were processed in batch mode in APTEC SUPERVISOR to identify individual energy peaks and determine count rates. Concentrations were calculated with an EXCEL template identified as G1NMar08.xls using efficiency functions, and corrections for casing, dead time and water as determined by annual calibrations.

Moisture data are presented in counts per second (cps) because no calibration data exists for a 6 7/8-in. inner diameter casing.

\section{Results and Interpretations:}

Cs-137 was detected at 3.5, 6.5, 9.0, 16.5 and $19.0 \mathrm{ft}$. Inspection of the individual spectra at depths 3.5, 9.0, 16.5, and $19.0 \mathrm{ft}$ indicated that these detections are statistical fluctuations associated with the processing software. At $6.5 \mathrm{ft}$, the Cs-137 concentration is $0.2 \mathrm{pCi} / \mathrm{g}$.

Co-60 was detected from 6 to $13 \mathrm{ft}$. The maximum concentration was $0.3 \mathrm{pCi} / \mathrm{g}$ at $6.5 \mathrm{ft}$.

U-235 was detected at 5.0, 14.0, 23.5, 34.0 and $35.5 \mathrm{ft}$. Inspection of the individual spectra at these depths indicates that these detections are statistical fluctuations associated with the processing software.

$\mathrm{U}-238$ (Pa-234m) was detected at depths 6.5, 32.5, 49.5, and $54.0 \mathrm{ft}$. Inspection of the individual spectra at these depths indicates that these detections are statistical fluctuations associated with processing software.

The KUT repeat plot indicates good repeatability. The manmade repeat plot for Cs-137 does not repeat as the detection are statistical fluctuations. The moisture repeat plot indicates good variability. 
HGLP-LDR-233, Rev. 0

Radon is exhibited from 29 - $59 \mathrm{ft}$, as shown by an elevated $609 \mathrm{keV}$ energy peak relative to the $1764 \mathrm{keV}$ energy peak.

\section{List of Log Plots:}

Depth Reference is ground surface

Manmade Radionuclides

Natural Gamma Logs

Combination Plot

Total Gamma \& Dead Time

Total Gamma \& Moisture

Manmade Repeat Section

Repeat Section of Natural Gamma Logs

Moisture Repeat Section

${ }^{1} \mathrm{GWL}$ - groundwater level

${ }^{2}$ TOC - top of casing 
S

toller

399-2-19 (C6197)

Hanford Office

Manmade Radionuclides

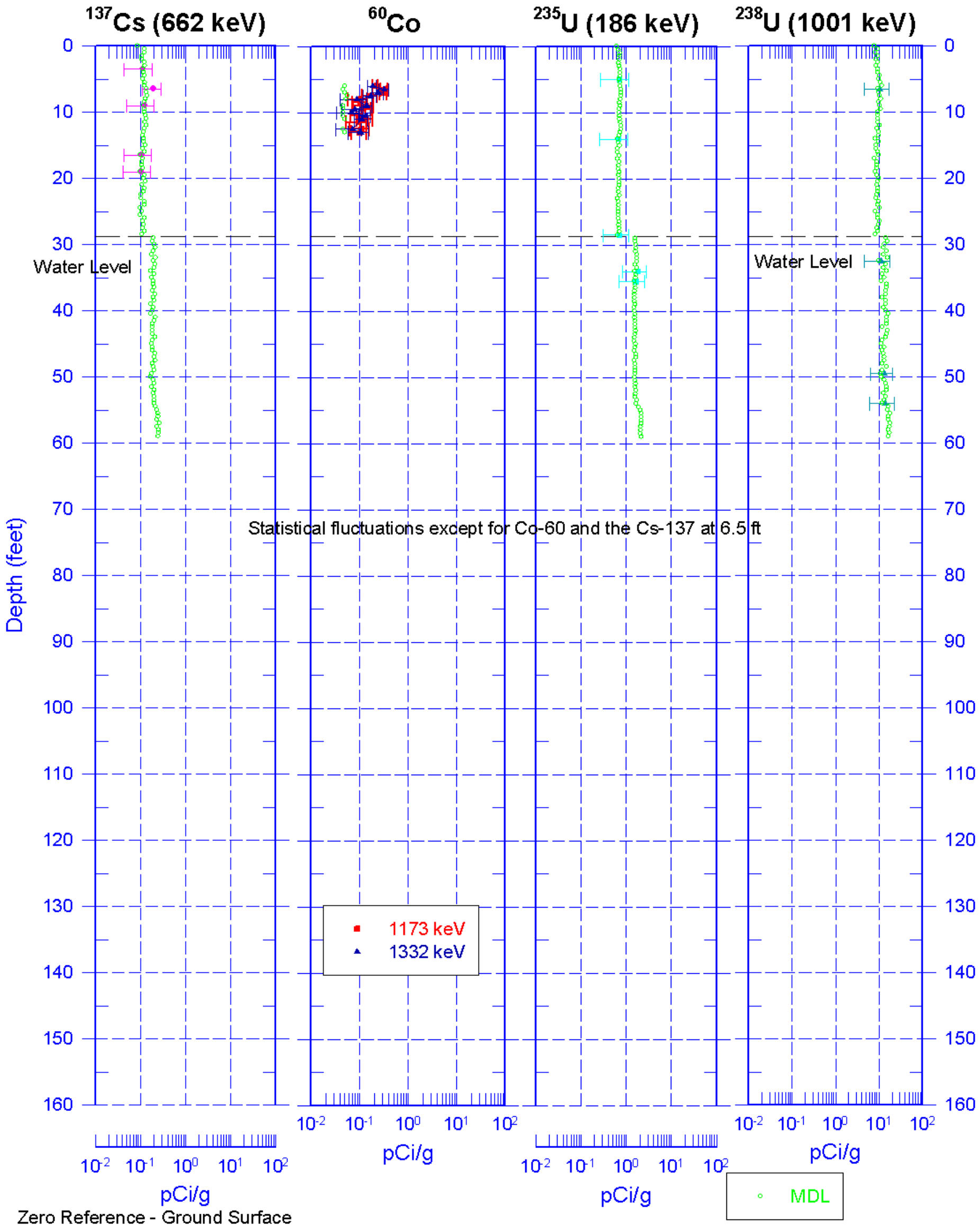

Zero Reference - Ground Surface 
toller $\quad$ 399-2-19 (C6197)

Hanford Office Natural Gamma Logs
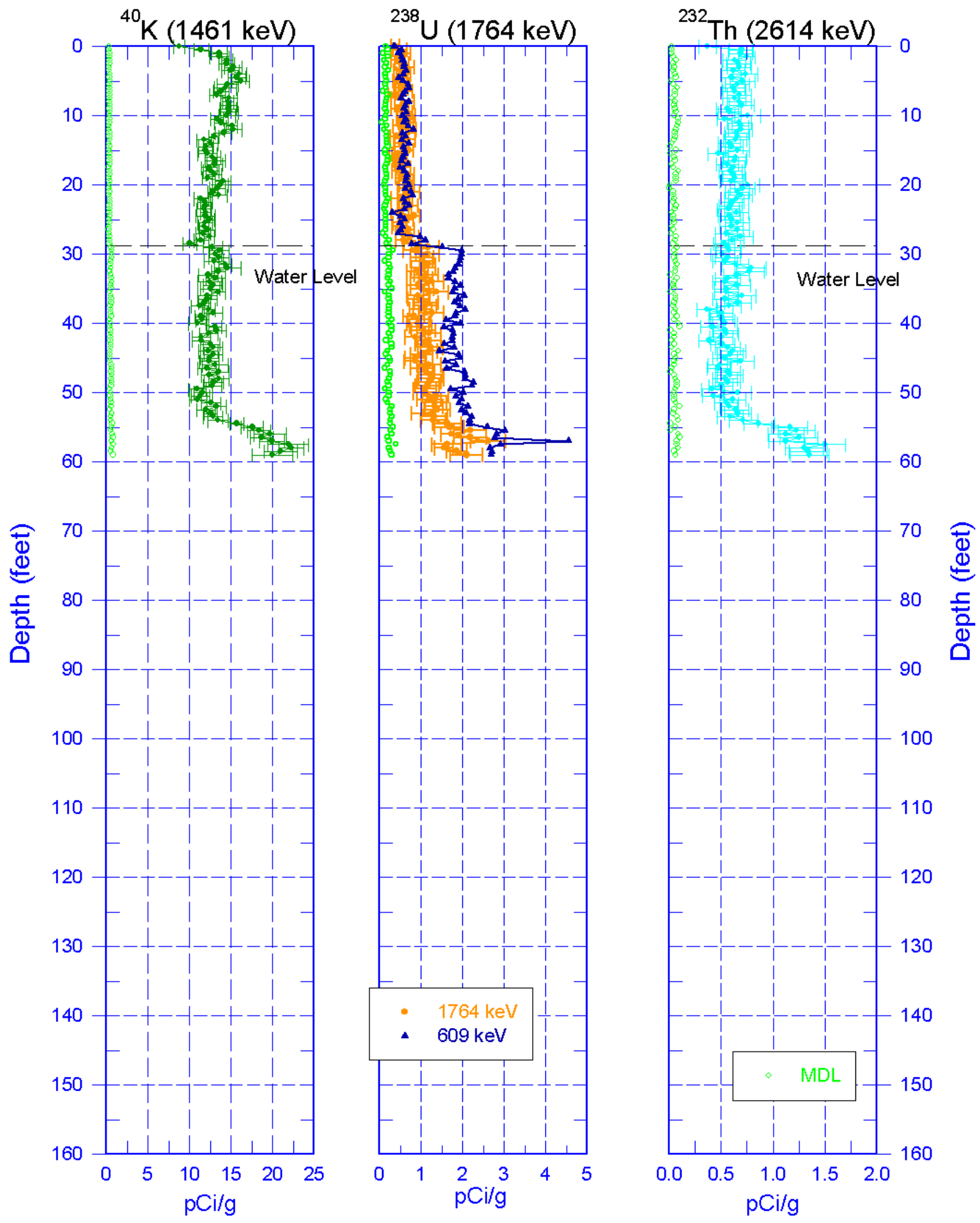

Zero Reference - Ground Surface

G.136 


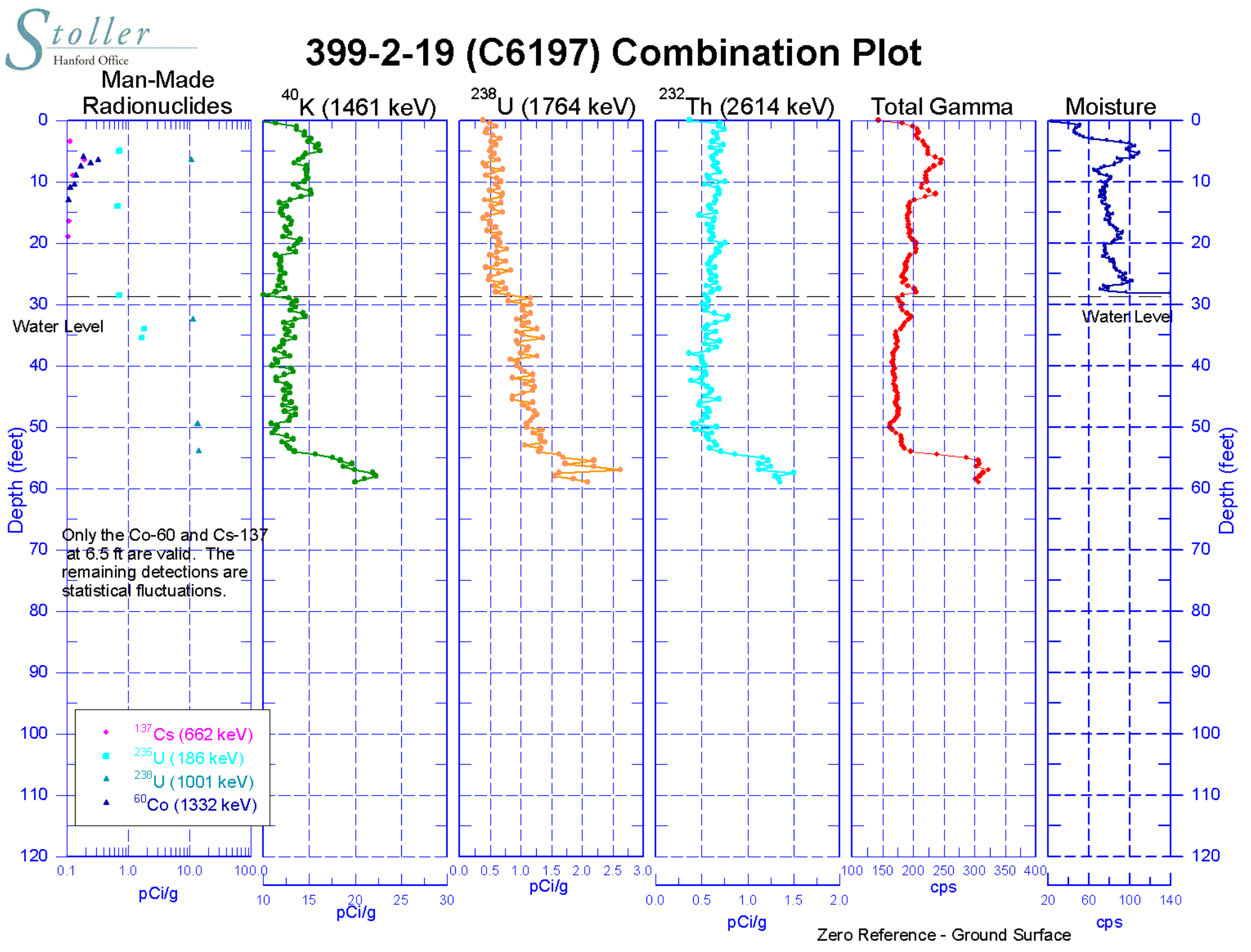



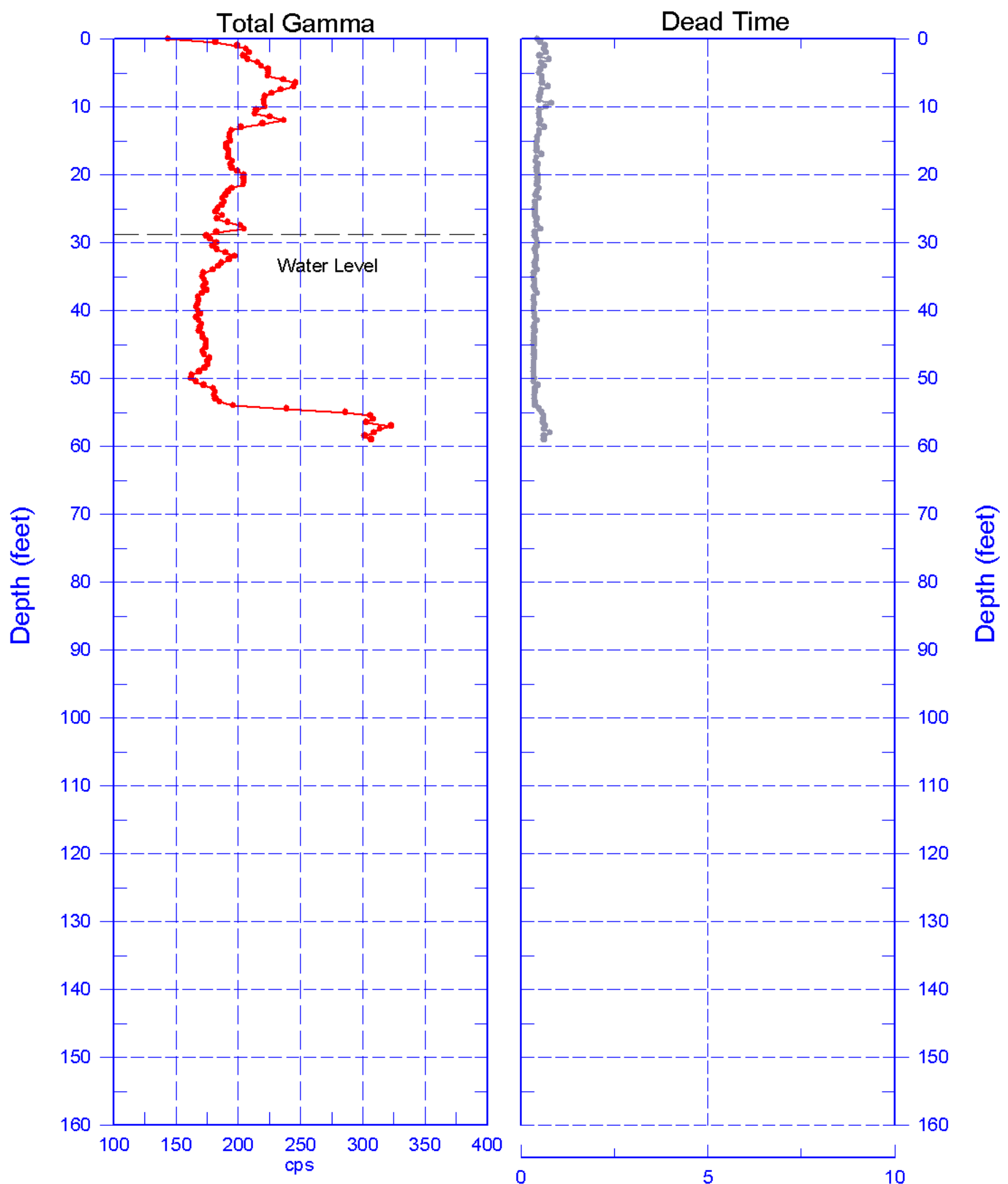

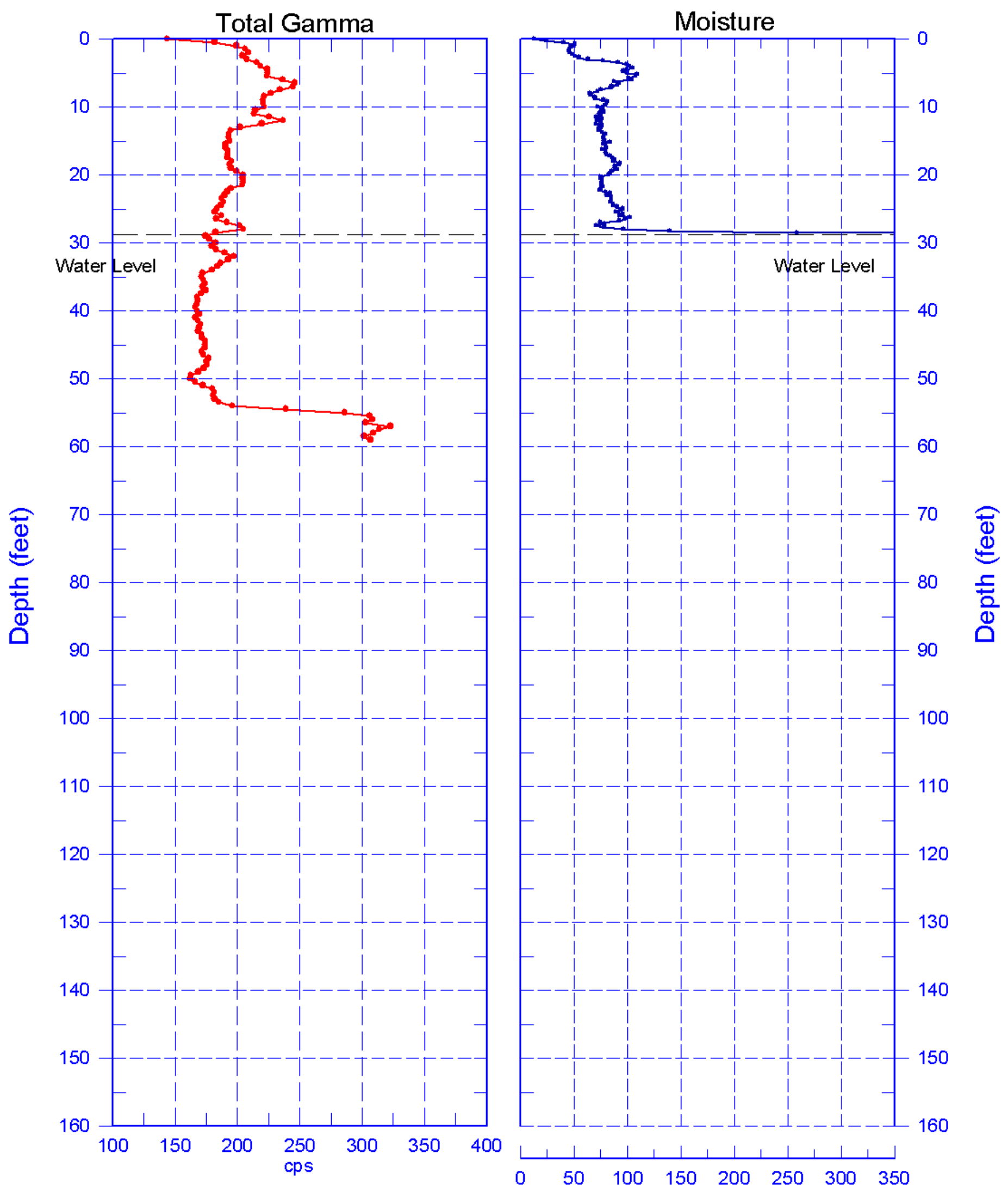
toller 399-2-19 (C6197)

Hanford Office

Manmade Repeat Section

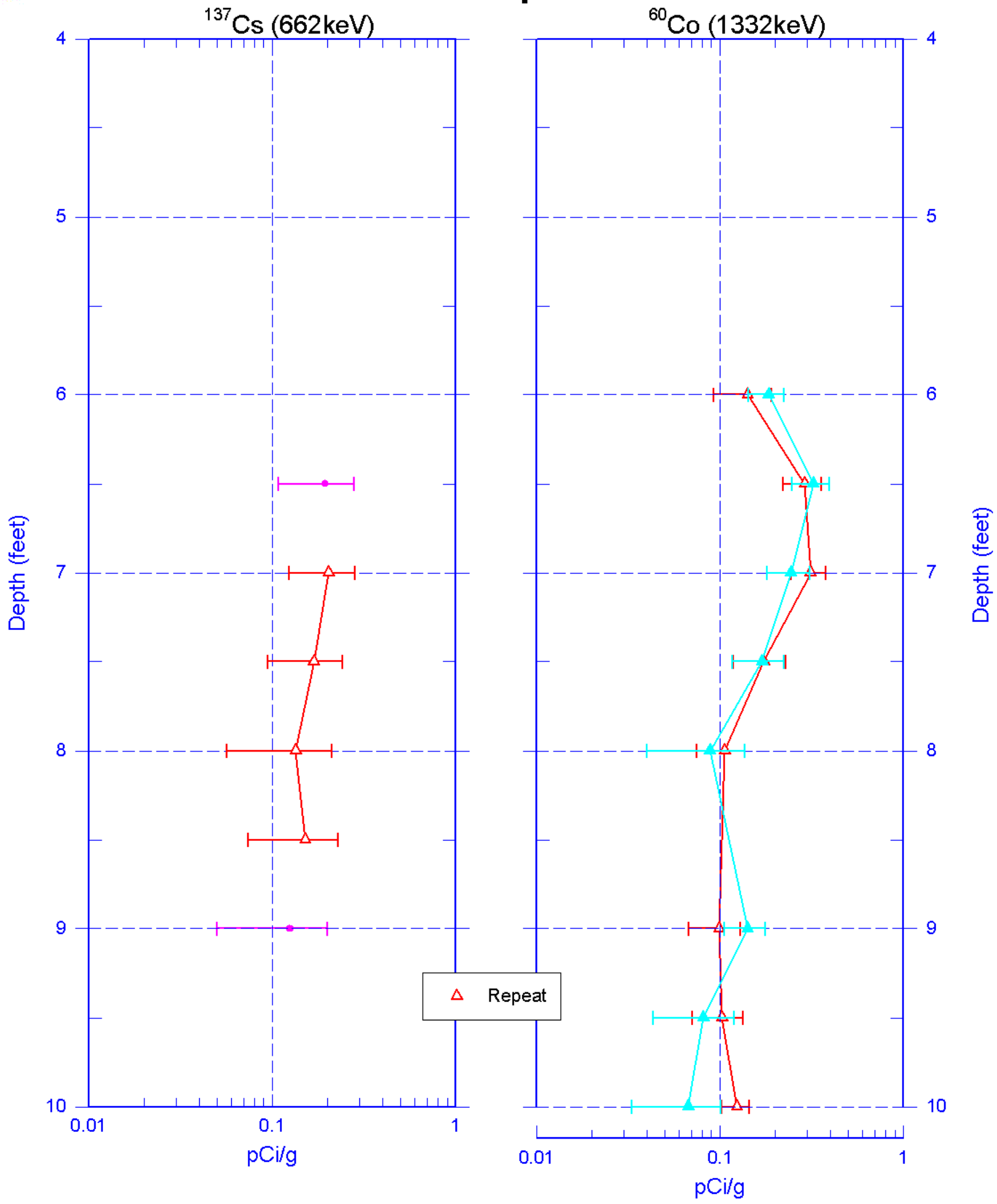

Reference - Ground Surface 


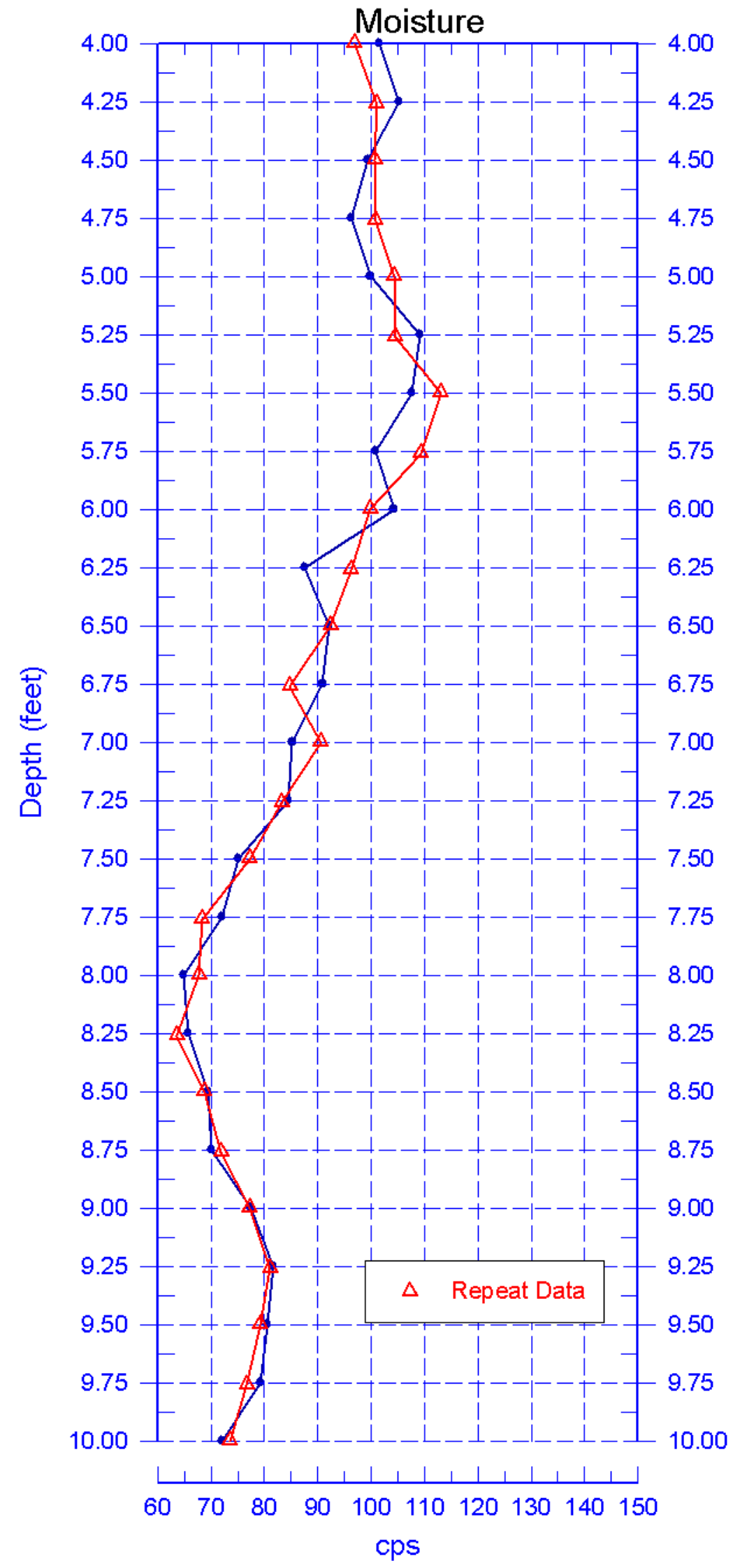




\section{9-2-20 (C6198) Log Data Report}

Borehole Information:

\begin{tabular}{|c|c|c|c|c|c|}
\hline Borehole: & \multicolumn{1}{l|}{ Site: } & \multicolumn{2}{l|}{ GW-FF-5 } \\
\hline \multicolumn{2}{|c|}{ 399-2-20 (C6198) } & GWL $^{\mathbf{1}}$ (ft) : & 27.9 & Date: & $06 / 27 / 08$ \\
\hline Noordinates (WA St Plane) & East (m) & Drill Date & TOC $^{2}$ Elevation & Total Depth (ft) & Type \\
\hline 116077.6 & 594270.6 & $06 / 25 / 08$ & Unknown & 62 & Sonic \\
\hline
\end{tabular}

\section{Casing Information:}

\begin{tabular}{|c|c|c|c|c|c|c|}
\hline Casing Type & Stickup (ft) & $\begin{array}{c}\text { Outer } \\
\text { Diameter (in.) }\end{array}$ & $\begin{array}{c}\text { Inside } \\
\text { Diameter (in.) }\end{array}$ & Thickness (in.) & Top (ft) & Bottom (ft) \\
\hline Threaded Steel & 0.9 & $75 / 8$ & $67 / 8$ & $3 / 8$ & -0.9 & 59.7 \\
\hline
\end{tabular}

\section{Borehole Notes:}

Casing data and total depth were reported by the site geologist. The logging engineer measured depth to water with an e-tape. Casing diameters were measured using a steel tape and rounded to the nearest 1/16 inch. The zero reference is the ground surface.

\section{Logging Equipment Information:}

\begin{tabular}{|c|c|c|c|c|}
\hline Logging System: & \multicolumn{2}{|c|}{ Gamma $4 \mathrm{~L}$} & $\begin{array}{l}\text { Type: } \\
\text { Serial No.: }\end{array}$ & $\begin{array}{l}\text { 60\% HPGe SGLS } \\
\text { 47TP32211A }\end{array}$ \\
\hline Effective Calibration Date: & $12 / 31 / 07$ & Calibration Reference: & \multicolumn{2}{|c|}{ HGLP-CC-027 } \\
\hline & & Logging Procedure: & \multicolumn{2}{|c|}{ HGLP-MAN-002, Rev. 0} \\
\hline Logging System: & \multicolumn{2}{|c|}{ Gamma $4 \mathrm{H}$} & $\begin{array}{l}\text { Type: } \\
\text { Serial No.: }\end{array}$ & $\begin{array}{l}\text { NMLS } \\
\text { H310700352 }\end{array}$ \\
\hline Effective Calibration Date: & $11 / 06 / 07$ & Calibration Reference: & \multicolumn{2}{|c|}{ HGLP-CC-021 } \\
\hline & & Logging Procedure: & \multicolumn{2}{|c|}{ HGLP-MAN-002, Rev. 0} \\
\hline
\end{tabular}

\section{Spectral Gamma Logging System (SGLS) Log Run Information:}

\begin{tabular}{|l|c|c|l|l|l|}
\hline Log Run & $\mathbf{1}$ & 2 Repeat & & & \\
\hline Date & $06 / 27 / 08$ & $06 / 28 / 08$ & & & \\
\hline Logging Engineer & Pearson & Pearson & & & \\
\hline Start Depth (ft) & 61.0 & 10.0 & & & \\
\hline Finish Depth (ft) & 0.0 & 4.0 & & & \\
\hline Count Time (sec) & 200 & 200 & & & \\
\hline Live/Real & $\mathrm{R}$ & $\mathrm{R}$ & & & \\
\hline Shield (Y/N) & $\mathrm{N}$ & $\mathrm{N}$ & & & \\
\hline MSA Interval (ft) & 0.5 & 0.5 & & & \\
\hline Log Speed (ft/min) & $\mathrm{NA}$ & $\mathrm{NA}$ & & & \\
\hline Pre-Verification & DL541CAB & DL541CAB & & & \\
\hline Start File & DL541000 & DL541123 & & & \\
\hline Finish File & DL541122 & DL541135 & & & \\
\hline Post-Verification & DL551CAA & DL551CAA & & & \\
\hline $\begin{array}{l}\text { Depth Return Error } \\
\text { (in.) }\end{array}$ & 0 & $1 / 4$ Low & & & \\
\hline Comments & None & Repeat section. & & & \\
\hline
\end{tabular}


Neutron Moisture Logging System (NMLS) Log Run Information:

\begin{tabular}{|c|c|c|c|c|c|}
\hline Log Run & 3 & 4 Repeat & & & \\
\hline Date & $06 / 27 / 08$ & $06 / 27 / 08$ & & & \\
\hline Logging Engineer & Pope & Pope & & & \\
\hline Start Depth (ft) & 0.0 & 4.0 & & & \\
\hline Finish Depth (ft) & 27.75 & 10.0 & & & \\
\hline Count Time (sec) & 15 & 15 & & & \\
\hline Live/Real & $\mathrm{R}$ & $\mathrm{R}$ & & & \\
\hline Shield (Y/N) & $\mathrm{N}$ & $\mathrm{N}$ & & & \\
\hline MSA Interval (ft) & 0.25 & 0.25 & & & \\
\hline Log Speed (ft/min) & NA & NA & & & \\
\hline Pre-Verification & DHG12CAB & DHG12CAB & & & \\
\hline Start File & DHG12000 & DHG12112 & & & \\
\hline Finish File & DHG12111 & DHG12136 & & & \\
\hline Post-Verification & DHG12CAA & DHG12CAA & & & \\
\hline $\begin{array}{l}\text { Depth Return Error } \\
\text { (in.) }\end{array}$ & NA & 0 & & & \\
\hline Comments & None & Repeat Section & & & \\
\hline
\end{tabular}

\section{Logging Operation Notes:}

Data were collected using Gamma 4, HO 68B-3573. SGLS pre- and post-survey verification measurements were acquired in the Amersham KUTh-115 field verifier. NMLS pre- and post-survey verification measurements were acquired in the standard field verifier. Maximum logging depth for the SGLS was $61.33 \mathrm{ft}$ before the sonde un-weighted. A centralizer was installed on the sonde.

Analysis Notes:

\begin{tabular}{|l|l|l|l|l|l|}
\hline Analyst: & LEGLER & Date: & 09/30/08 & Reference: & GJO-HGLP 1.6.3, Rev. 0 \\
\hline
\end{tabular}

The pre- and post-survey verification spectra met the acceptance criteria for the established systems. A casing correction for a 3/8-in thick casing was applied to spectral data from ground surface to total logged depth of borehole. A water correction was applied from $27.9 \mathrm{ft}$ to total logged depth of borehole.

SGLS spectra were processed in batch mode in APTEC SUPERVISOR to identify individual peaks and count rates. Concentrations were calculated using an EXCEL template identified as G4LDec07.xls using an efficiency function and corrections for casing, dead time and water as determined by annual calibrations. NMLS spectra were processed in batch mode in APTEC SUPERVISOR to identify counts. Count rates were calculated using an EXCEL template identified as G4HNov07.xls. NMLS data are presented in counts per second (cps), because no calibration data is available for a 6 7/8-in inner diameter borehole casing.

\section{Results and Interpretations:}

Co-60 was detected at 5.5 and $6 \mathrm{ft}$, with a maximum concentration of $0.07 \mathrm{pCi} / \mathrm{g}$ at 5.5 feet. Cs-137, U-235, and $\mathrm{U}-238$ (Pa-234m) were also detected at various isolated depths, but inspection of the individual spectra indicates that these detections are statistical fluctuations associated with the processing software.

The manmade, KUT and Moisture repeat plots show good repeatability.

\section{List of Log Plots:}

Depth Reference is ground surface 
Manmade Radionuclides

Natural Gamma Logs

Combination Plot

Total Gamma \& Dead Time

Total Gamma \& Moisture

Manmade Repeat Section

Repeat Section of Natural Gamma Logs

Moisture Repeat Section

${ }^{1} \mathrm{GWL}$ - groundwater level

${ }^{2}$ TOC - top of casing 

Hanford Office Manmade Radionuclides

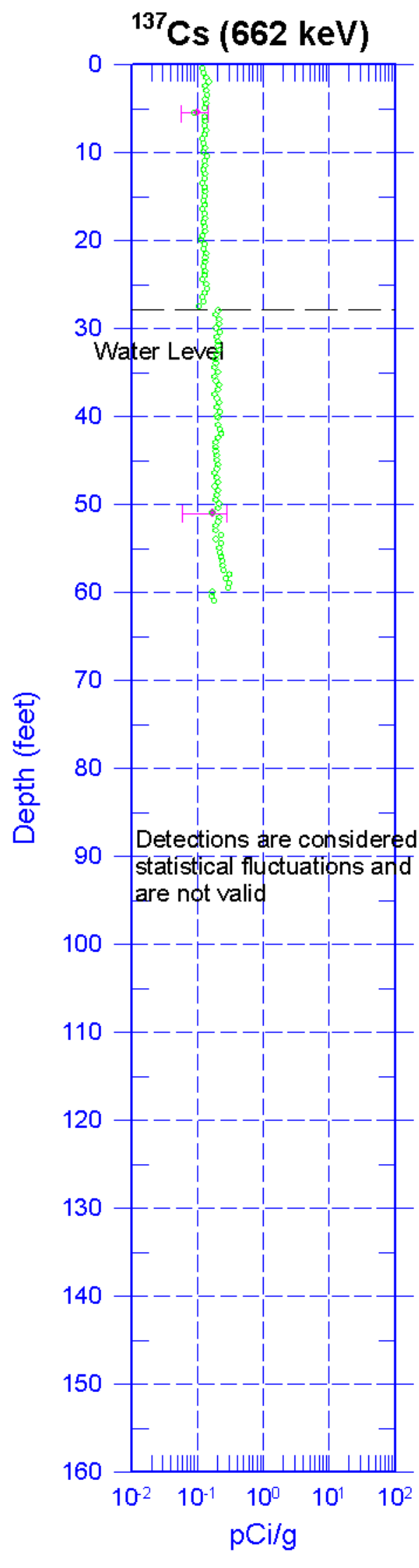

Zero Reference - Ground Surface

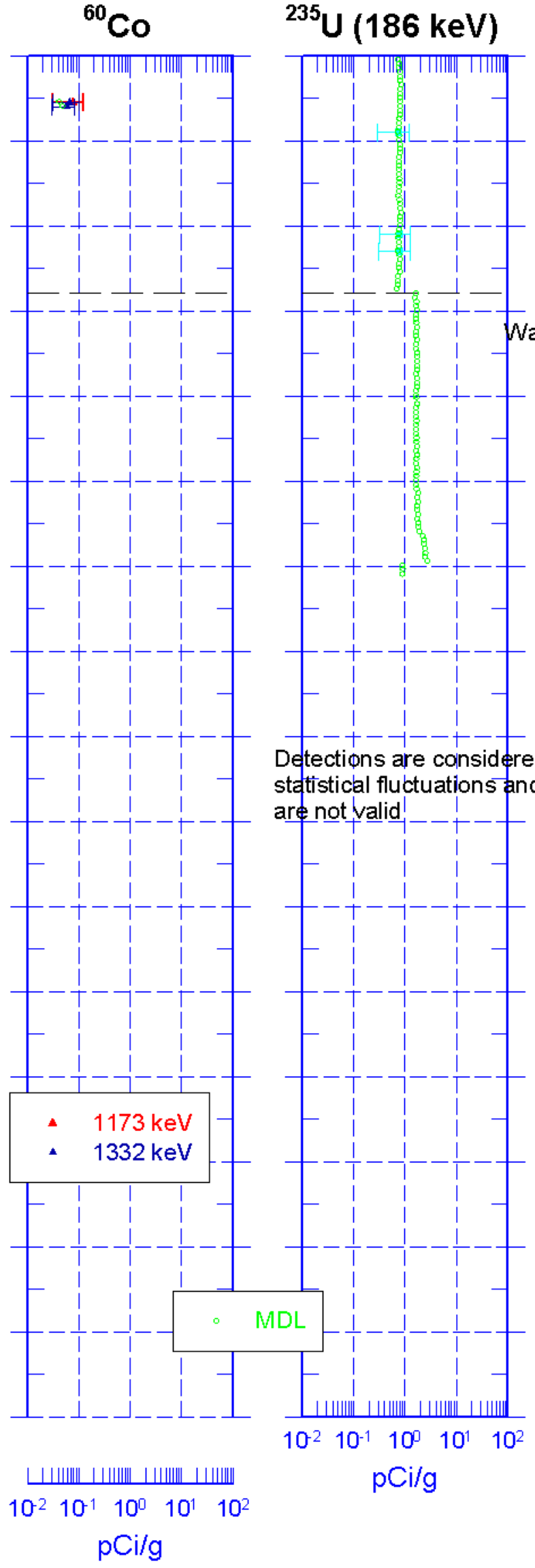

${ }^{238} \mathrm{U}(1001 \mathrm{keV})$

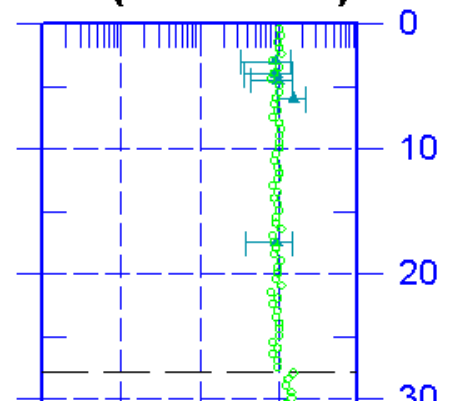

vater Level

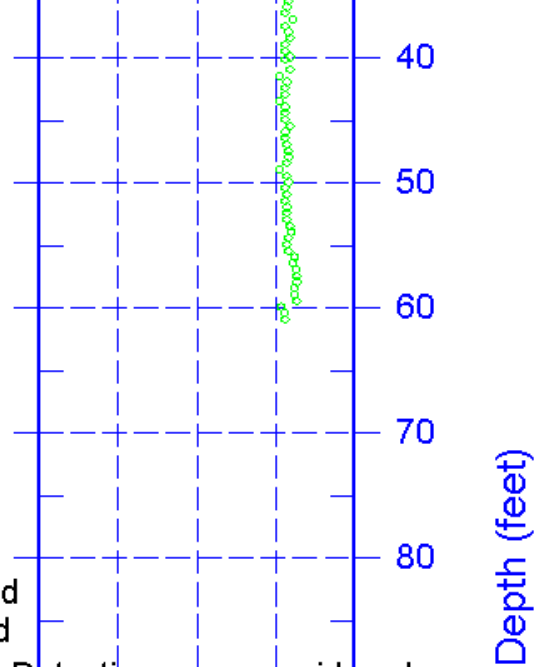

Detections are considerego statistical fluctuations and ane not valid 100

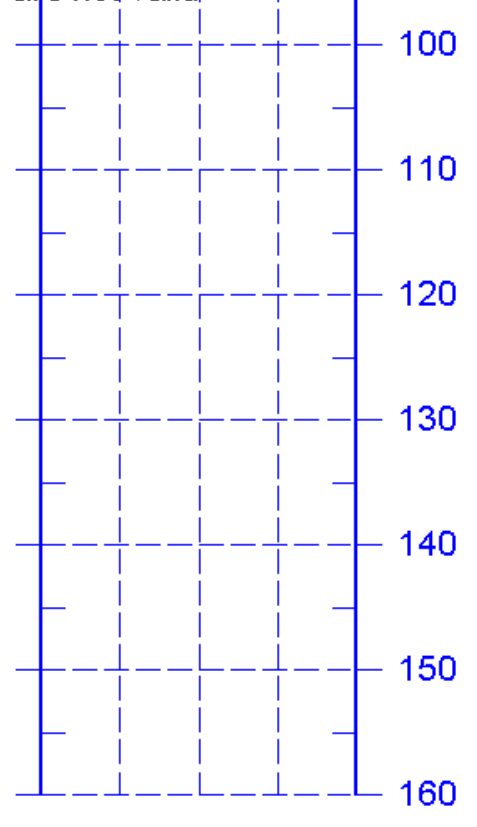

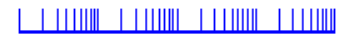
$\begin{array}{lllll}10^{-2} & 10^{-1} & 10^{9} & 10^{1} & 10^{2}\end{array}$ $\mathrm{pCi} / \mathrm{g}$ 


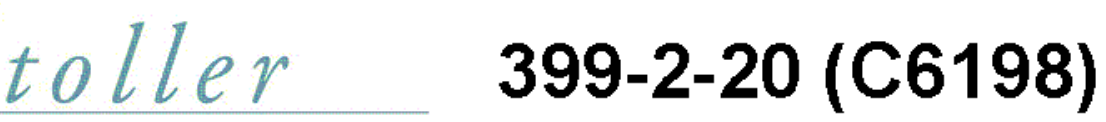

Hanford Office Natural Gamma Logs
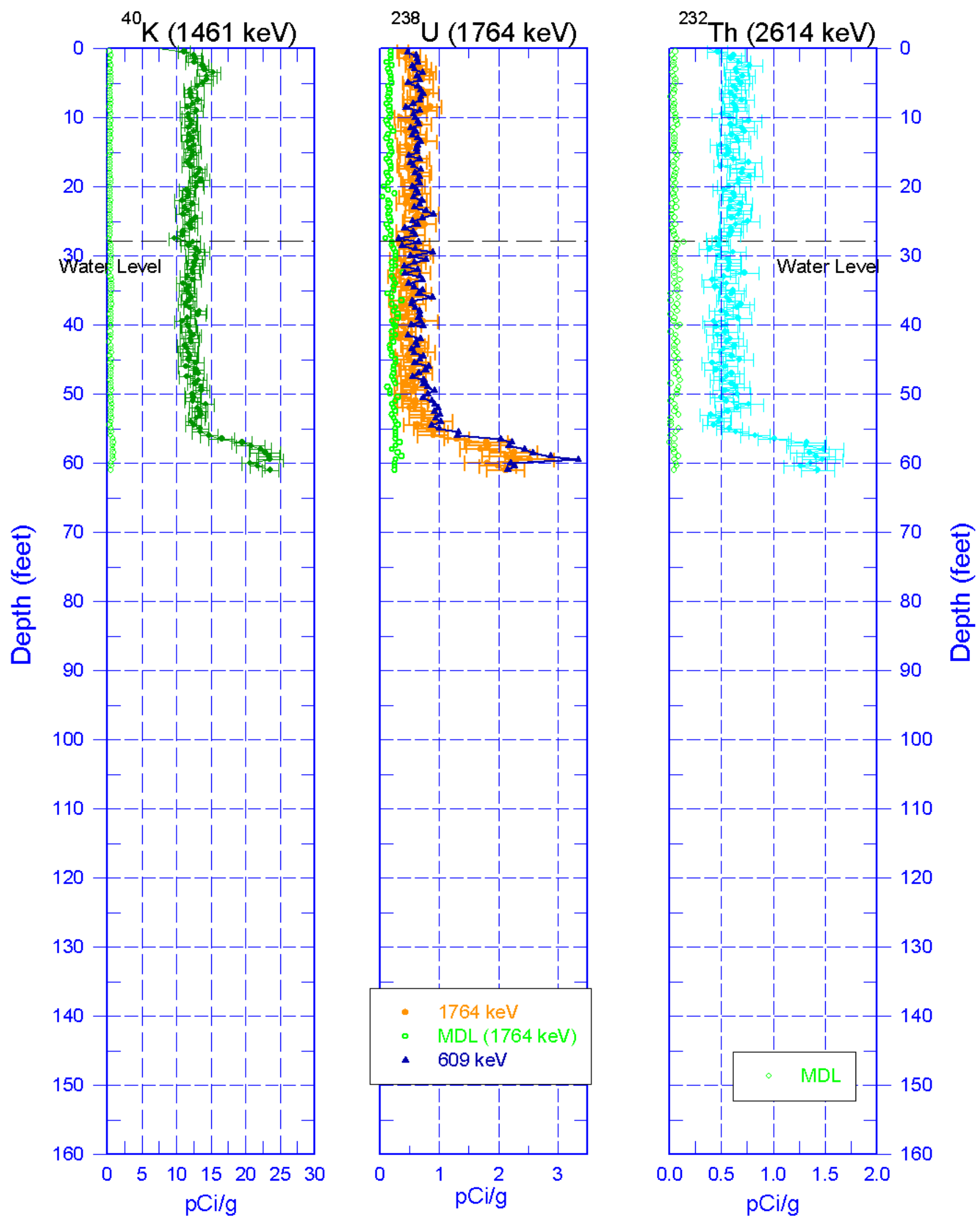

Zero Reference - Ground Surface 


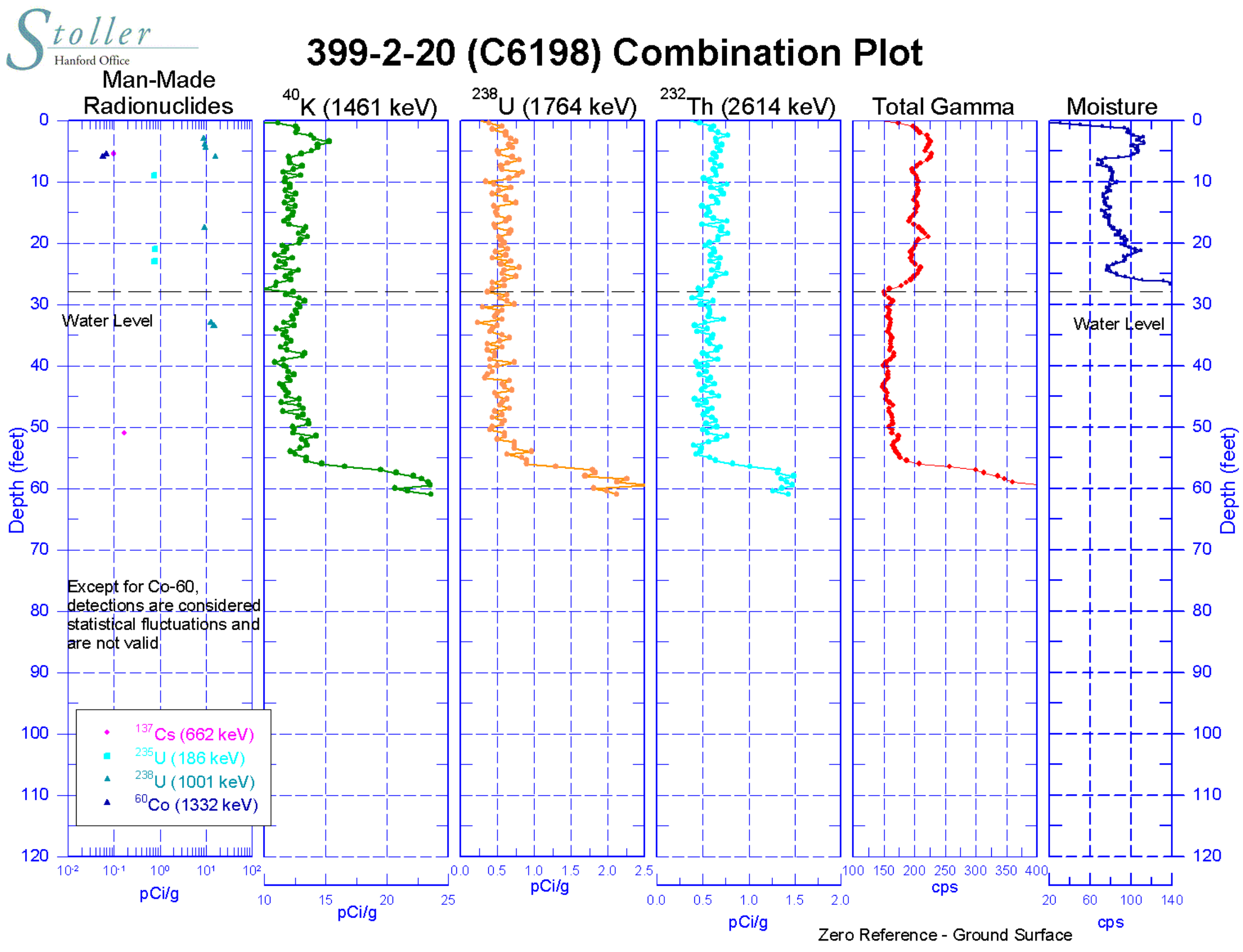


toller 399-2-20 (C6198)

Hanford Office

\section{Total Gamma \& Dead Time}
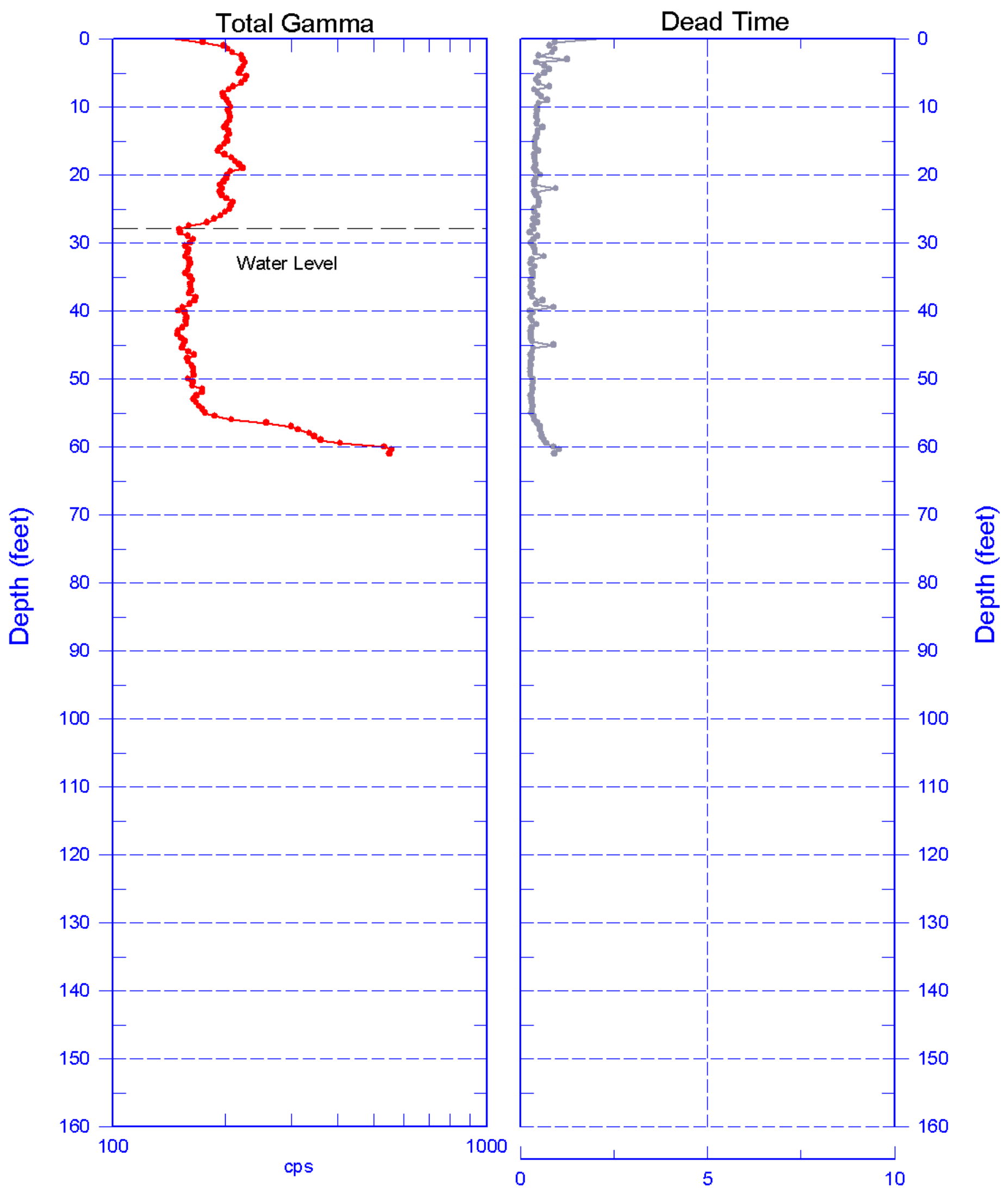

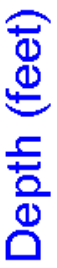

Reference - Ground Surface

G.148 
toller 399-2-20 (C6198)

Hanford Office

\section{Total Gamma \& Moisture}

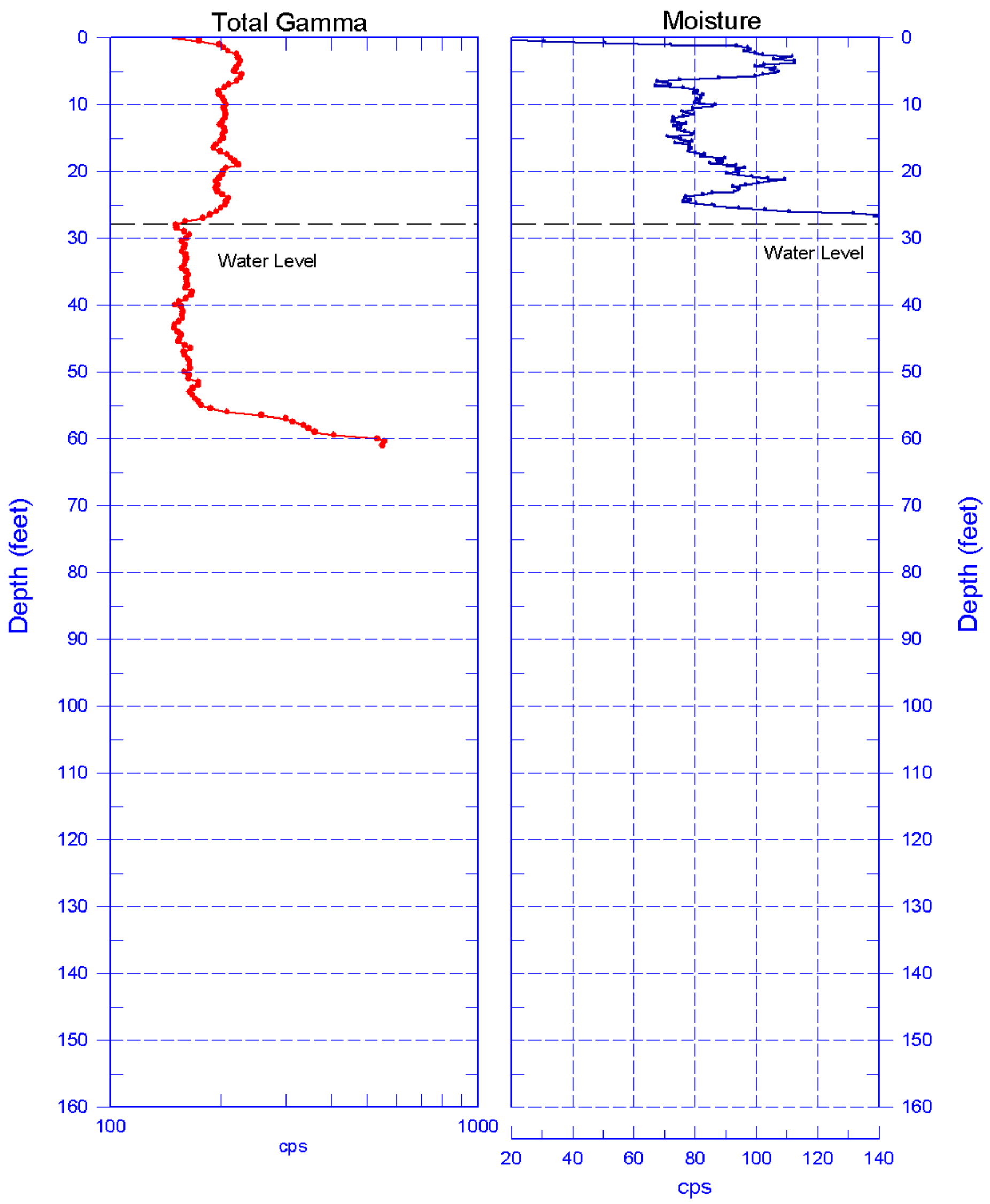

Reference - Ground Surface 
toller 399-2-20 (C6198)

Hanford Office

Manmade Repeat Section

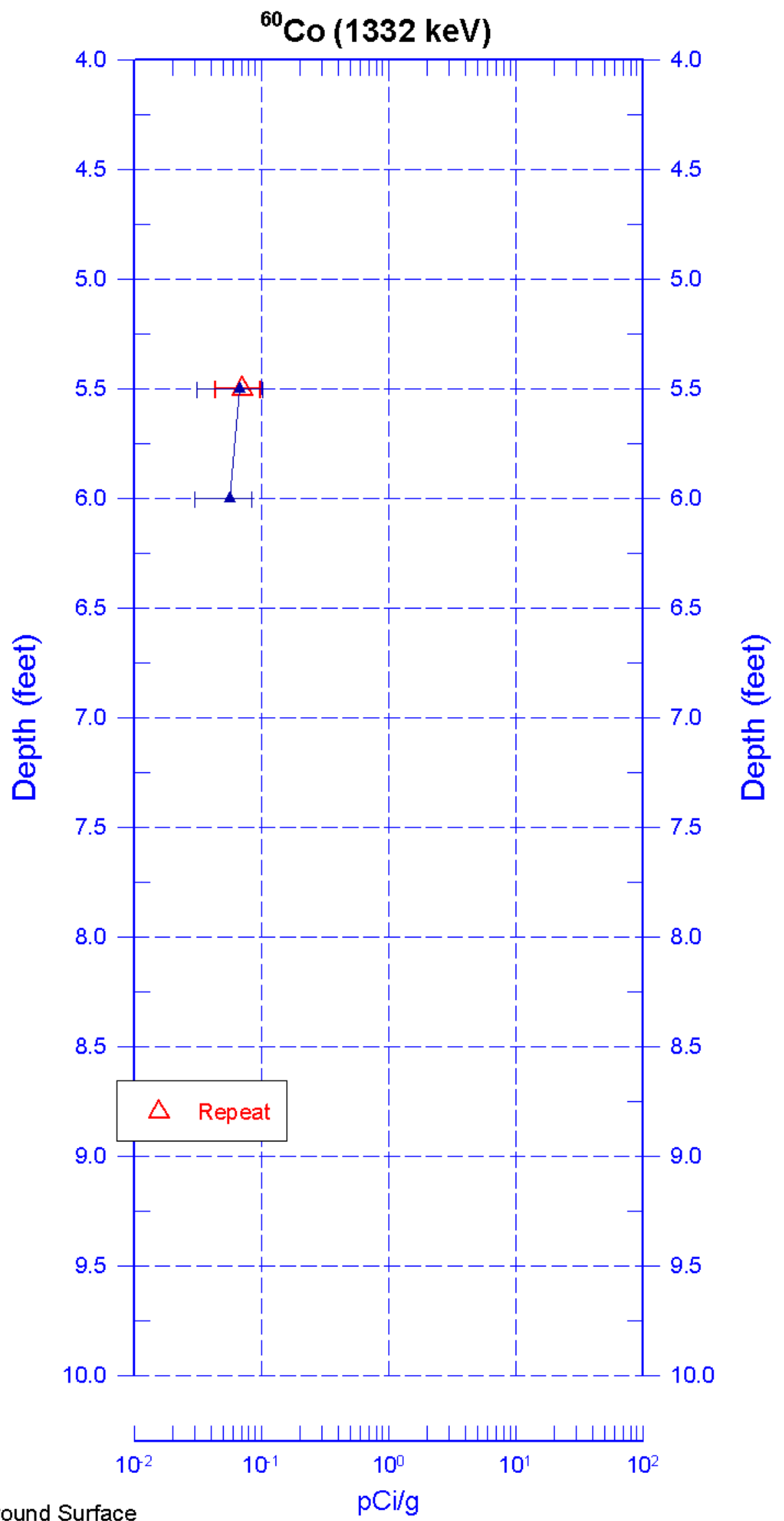

Zero Reference - Ground Surface

G.150 


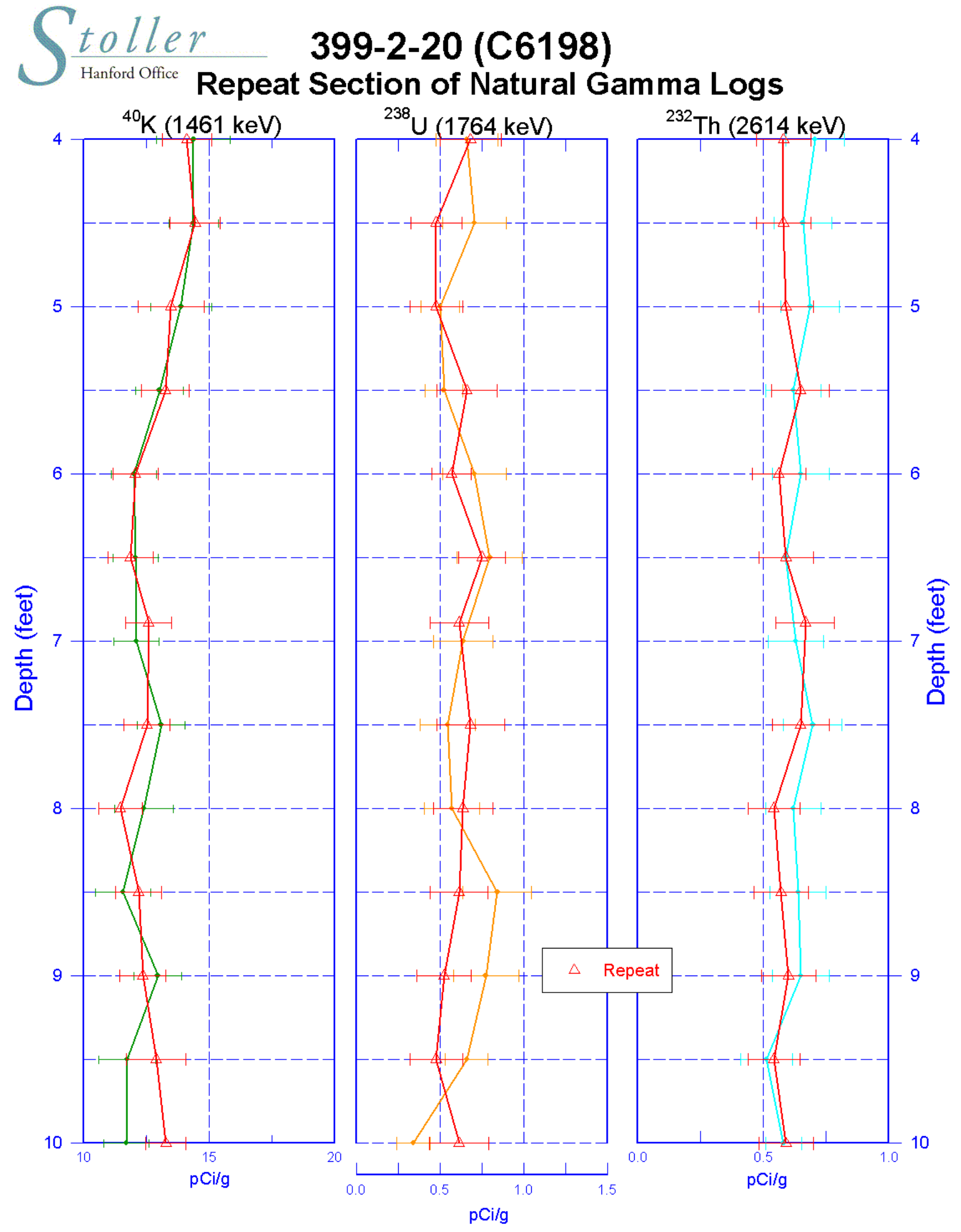

Zero Reference - Ground Surface

G.151 


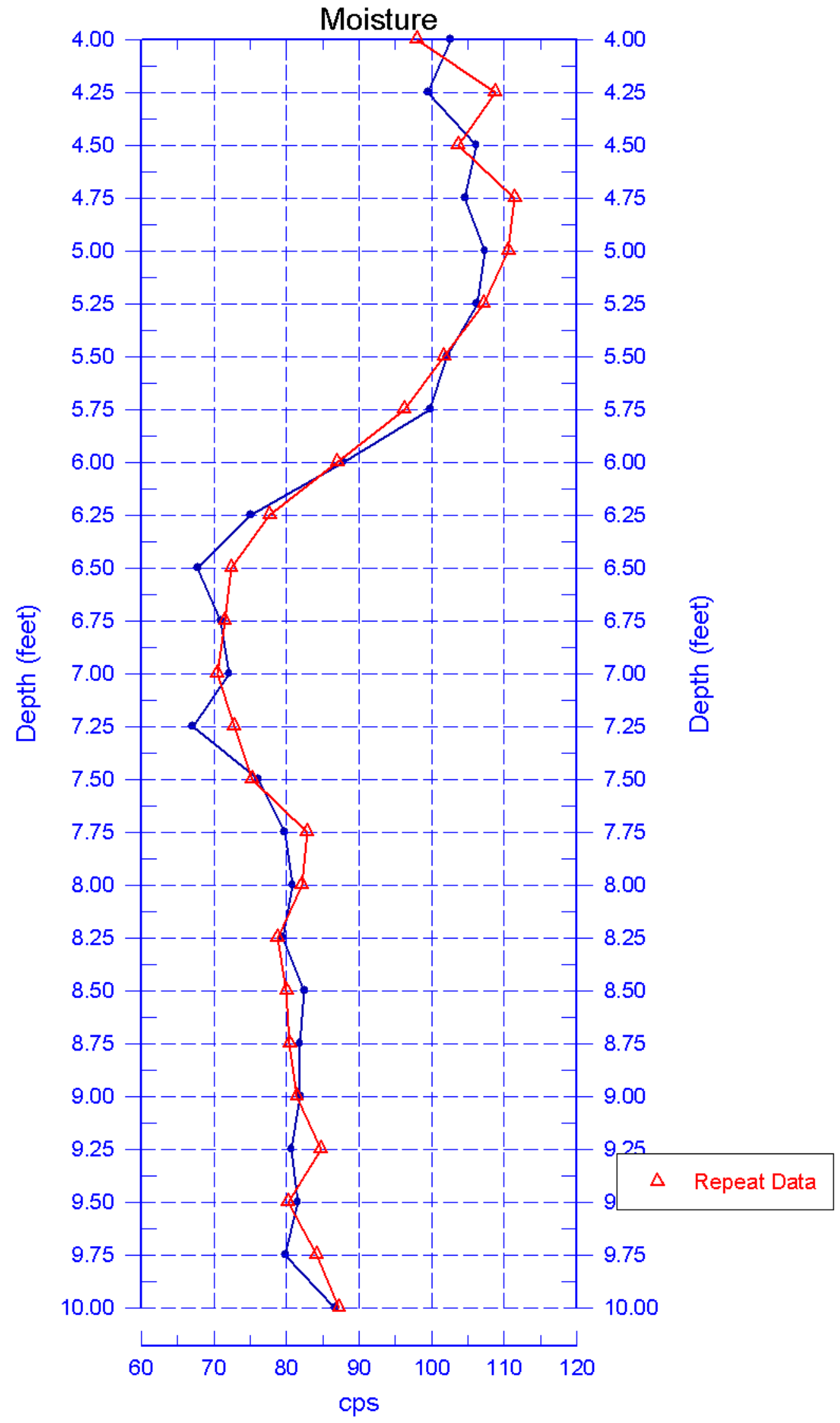


HGLP-LDR-241, Rev. 0

\section{9-2-21 (C6201) Log Data Report}

Borehole Information:

\begin{tabular}{|c|c|c|c|c|c|}
\hline Borehole: & \multicolumn{2}{|c|}{ S99-2-21 (C6201) } & \multicolumn{2}{|c|}{ S00-FF-5 } \\
\hline Coordinates (WA St Plane) & GWL ${ }^{1}$ (ft) : & 26.5 & \multicolumn{2}{c|}{ GWL Date: } & $06 / 15 / 08$ \\
\hline North (m) & East (m) & Drill Date & TOC $^{2}$ Elevation & Total Depth (ft) & Type \\
\hline 116061.9 & 594263.3 & $06 / 12 / 08$ & Unknown & 62 & Sonic \\
\hline
\end{tabular}

\section{Casing Information:}

\begin{tabular}{|l|c|c|c|c|c|c|}
\hline Casing Type & Stickup (ft) & $\begin{array}{c}\text { Outer } \\
\text { Diameter (in.) }\end{array}$ & $\begin{array}{c}\text { Inside } \\
\text { Diameter (in.) }\end{array}$ & Thickness (in.) & Top (ft) & Bottom (ft) \\
\hline Welded Steel & 1.2 & $75 / 8$ & $67 / 8$ & $3 / 8$ & -1.2 & 58.0 \\
\hline
\end{tabular}

\section{Borehole Notes:}

Casing data and total depth was reported by the site geologist. Logging engineer measured depth to water with an e-tape. Casing diameters were measured using a steel tape and rounded to the nearest 1/16”. The zero reference was the ground surface.

\section{Logging Equipment Information:}

\begin{tabular}{|l|l|l|l|l|}
\hline Logging System: & \multicolumn{2}{|l|}{ Gamma 4 L } & $\begin{array}{l}\text { Type: } \\
\text { Serial No.: }\end{array}$ & $\begin{array}{l}\text { 60\% HPGe SGLS } \\
\text { 47TP32211A }\end{array}$ \\
\hline Effective Calibration Date: & $12 / 31 / 07$ & Calibration Reference: & HGLP-CC-027 \\
\hline \multicolumn{2}{|l|}{} & Logging Procedure: & HGLP-MAN-002, Rev. 0 \\
\hline
\end{tabular}

\begin{tabular}{|l|l|l|l|l|}
\hline Logging System: & \multirow{2}{*}{ Gamma 4 H } & $\begin{array}{l}\text { Type: } \\
\text { Serial No.: }\end{array}$ & $\begin{array}{l}\text { NMLS } \\
\text { H310700352 }\end{array}$ \\
\hline Effective Calibration Date: & $11 / 06 / 07$ & Calibration Reference: & HGLP-CC-021 \\
\hline \multicolumn{2}{|l|}{} & Logging Procedure: & HGLP-MAN-002, Rev. 0 \\
\hline
\end{tabular}

\section{Spectral Gamma Logging System (SGLS) Log Run Information:}

\begin{tabular}{|l|c|c|}
\hline Log Run & $\mathbf{1}$ & 2 Repeat \\
\hline Date & $06 / 16 / 08$ & $06 / 16 / 08$ \\
\hline Logging Engineer & Pearson & Pearson \\
\hline Start Depth (ft) & 62.0 & 3.0 \\
\hline Finish Depth (ft) & 0.0 & 9.0 \\
\hline Count Time (sec) & 200 & 200 \\
\hline Live/Real & $\mathrm{R}$ & $\mathrm{R}$ \\
\hline Shield (Y/N) & $\mathrm{N}$ & $\mathrm{N}$ \\
\hline MSA Interval (ft) & 0.5 & 0.5 \\
\hline Log Speed (ft/min) & N/A & N/A \\
\hline Pre-Verification & DL451CAB & DL451CAB \\
\hline Start File & DL451000 & DL451125 \\
\hline Finish File & DL451124 & DL451137 \\
\hline Post-Verification & DL451CAA & DL451CAA \\
\hline Depth Return Error (in.) & 0 & N/A \\
\hline Comments & $\begin{array}{c}\text { No fine gain } \\
\text { adjustments } \\
\text { made. }\end{array}$ & Repeat Section \\
\hline
\end{tabular}


HGLP-LDR-241, Rev. 0

\section{Neutron Moisture Logging System (NMLS) Log Run Information:}

\begin{tabular}{|l|c|c|}
\hline Log Run & $\mathbf{3}$ & 4 Repeat \\
\hline Date & $06 / 15 / 08$ & $06 / 15 / 08$ \\
\hline Logging Engineer & Pearson & Pearson \\
\hline Start Depth (ft) & 0.0 & 3.0 \\
\hline Finish Depth (ft) & 26.5 & 6.0 \\
\hline Count Time (sec) & 15 & 15 \\
\hline Live/Real & $\mathrm{R}$ & $\mathrm{R}$ \\
\hline Shield (Y/N) & $\mathrm{N}$ & $\mathrm{N}$ \\
\hline MSA Interval (ft) & 0.25 & 0.25 \\
\hline Log Speed (ft/min) & N/A & N/A \\
\hline Pre-Verification & DHF22CAB & DHF22CAB \\
\hline Start File & DHF22000 & DHF22107 \\
\hline Finish File & DHF22106 & DHF22119 \\
\hline Post-Verification & DHF22CAA & DHF22CAA \\
\hline Depth Return Error (in.) & N/A & 0 \\
\hline Comments & None & Repeat Section \\
\hline
\end{tabular}

\section{Logging Operation Notes:}

Data were collected using Gamma 4, HO 68B-3573. SLGSL pre- and post-survey verification measurements were acquired in the Amersham KUTh-115 field verifier. Maximum logging depth was $62.2 \mathrm{ft}$ before the sonde un-weighted. A centralizer was installed on the sonde. NMLS pre- and post-survey verification measurements were acquired in the standard field verifier. Maximum logging depth achieved was $26.5 \mathrm{ft}$.

\section{Analysis Notes:}

\begin{tabular}{|l|l|l|l|l|l|}
\hline Analyst: & LEGLER & Date: & $08 / 29 / 08$ & Reference: & GJO-HGLP 1.6.3, Rev. 0 \\
\hline
\end{tabular}

The SGLS pre - and post-survey verification measurements met the acceptance criteria for the established system, but the verification file DL451CAB had measurements above the upper control limit for counts per second (cps) for the $2615 \mathrm{keV}$ energy line. The NMLS pre - and post-verification measurements met the acceptance criteria for the established system, but the verification file DHF22CAB had measurements above the upper control limit for counts per second (cps). A correction for a 3/8-in. thick casing was applied to spectral log data (SGLS) from ground surface to total logged depth of the borehole. A water correction was also applied from $26.5 \mathrm{ft}$ to total logged depth of borehole.

SGLS spectra were processed in batch mode in APTEC SUPERVISOR to identify individual energy peaks and determine count rates. Concentrations were calculated using an EXCEL template identified as G4LDec07.xls using an efficiency function and corrections for casing, dead time and water as determined by annual calibrations. NMLS spectra were processed in batch mode in APTEC SUPERVIOR to compile counts. Count rates were calculated using an EXCEL template identified as G4HNov07.xls.

Moisture data are presented in counts per second (cps) because no calibration data exists for 6 7/8-in. inner diameter casing.

\section{Results and Interpretations:}

Cs-137, U-235, U-238 (Pa-234m), and Co-60 were detected at various depths in this borehole. With the exception of Co-60, inspection of these spectra indicates that these detections are statistical fluctuations associated with the processing software. Co-60 was detected at $7-8 \mathrm{ft}$, with a maximum concentration of $0.9 \mathrm{pCi} / \mathrm{g}$ at $7.5 \mathrm{ft}$.

The KUT plots indicate good repeatability. Moisture plots indicates some variability. 
HGLP-LDR-241, Rev. 0

\section{List of Log Plots:}

Depth Reference is ground surface

Manmade Radionuclides

Natural Gamma Logs

Combination Plot

Total Gamma \& Dead Time

Total Gamma \& Moisture

Manmade Repeat Section

Repeat Section of Natural Gamma Logs

Moisture Repeat Section

${ }^{1} \mathrm{GWL}$ - groundwater level

${ }^{2}$ TOC - top of casing 
toller
Hanford Office Manmade Radionuclides

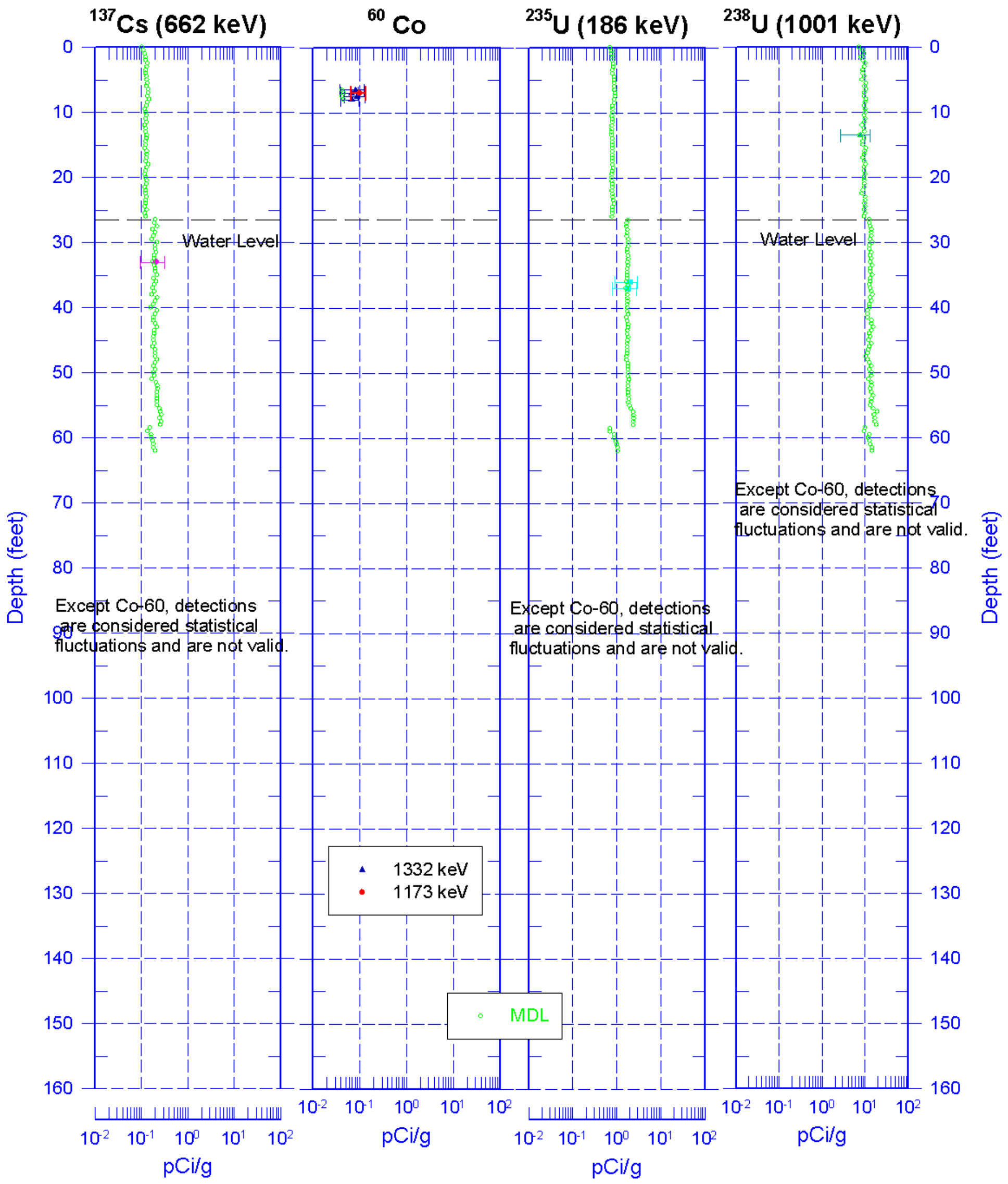

Zero Reference - Ground Surface 
toller 399-2-21 (C6201)

Hanford Office

Natural Gamma Logs
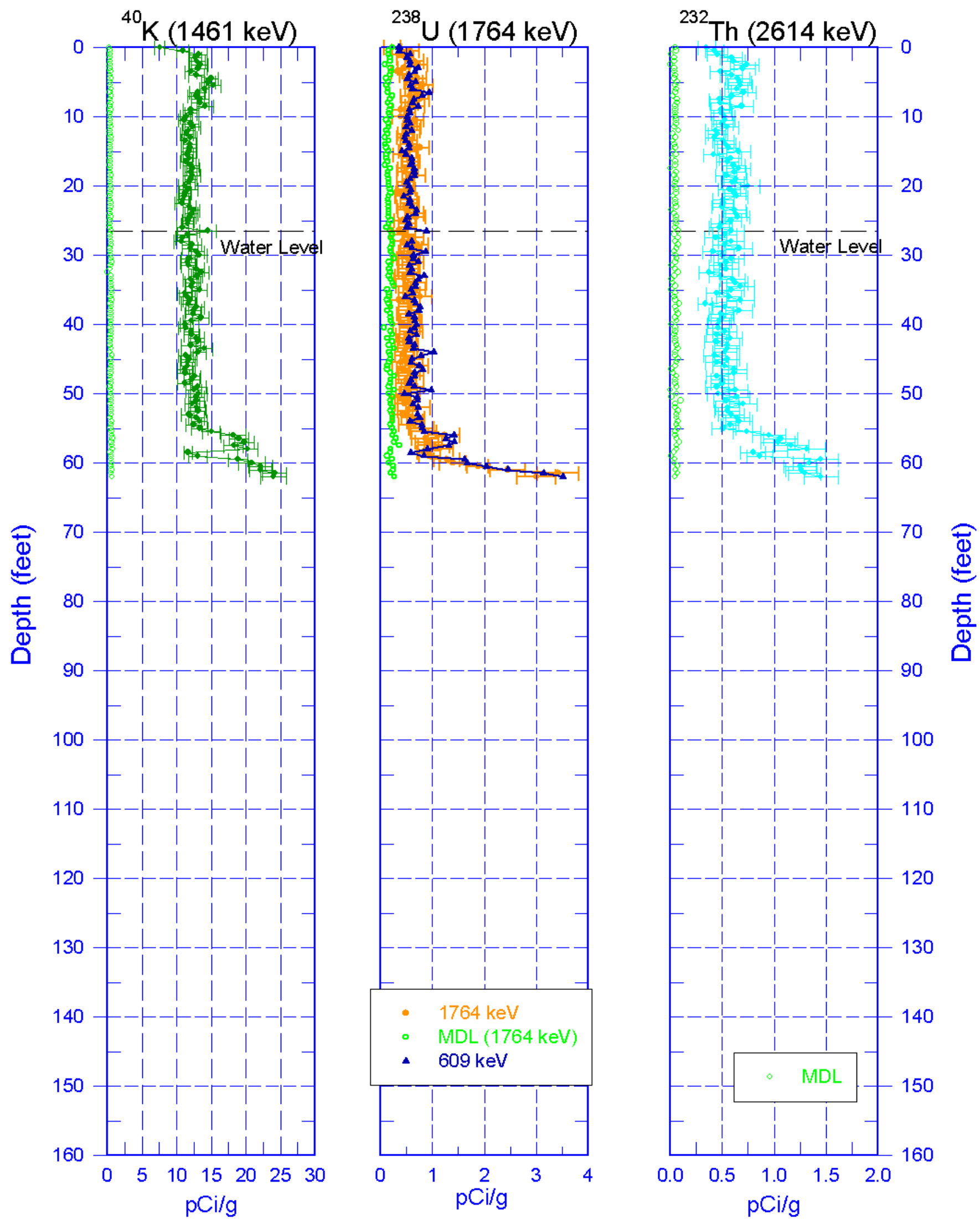

Zero Reference - Ground Surface

G.157 


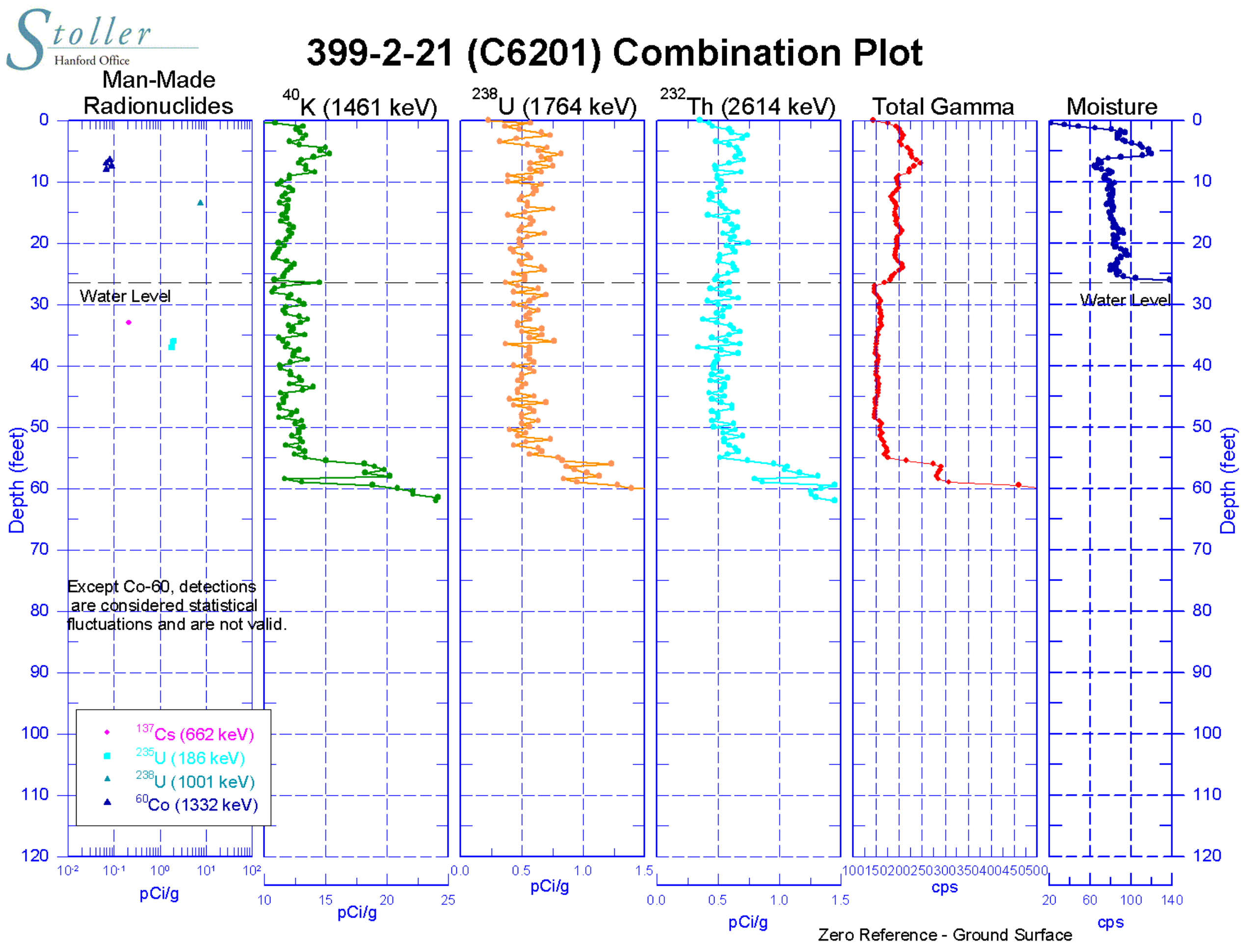


toller 399-2-21 (C6201)

Hanford Office

\section{Total Gamma \& Dead Time}
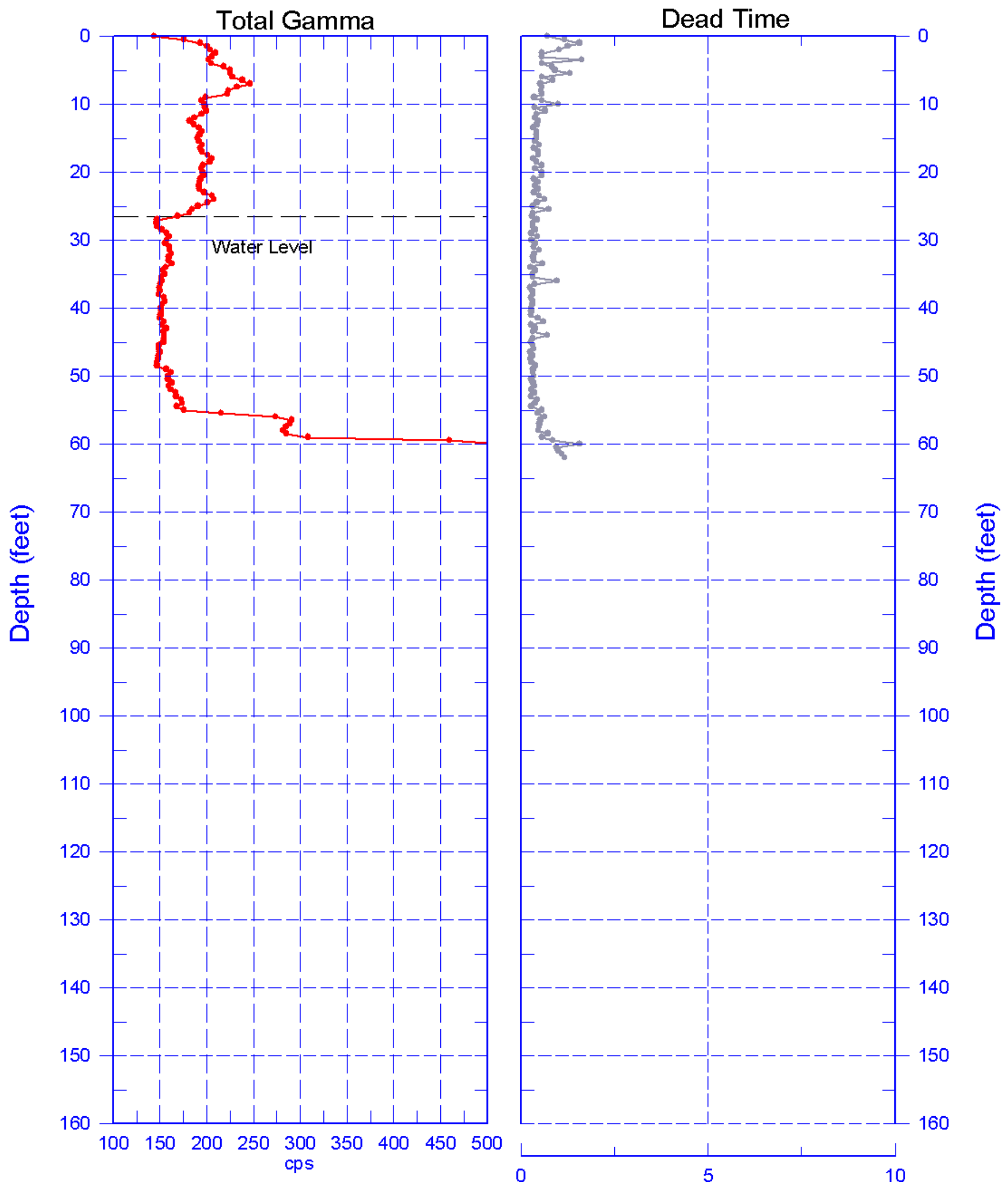

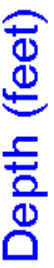

Reference - Ground Surface 


\section{Total Gamma \& Moisture}
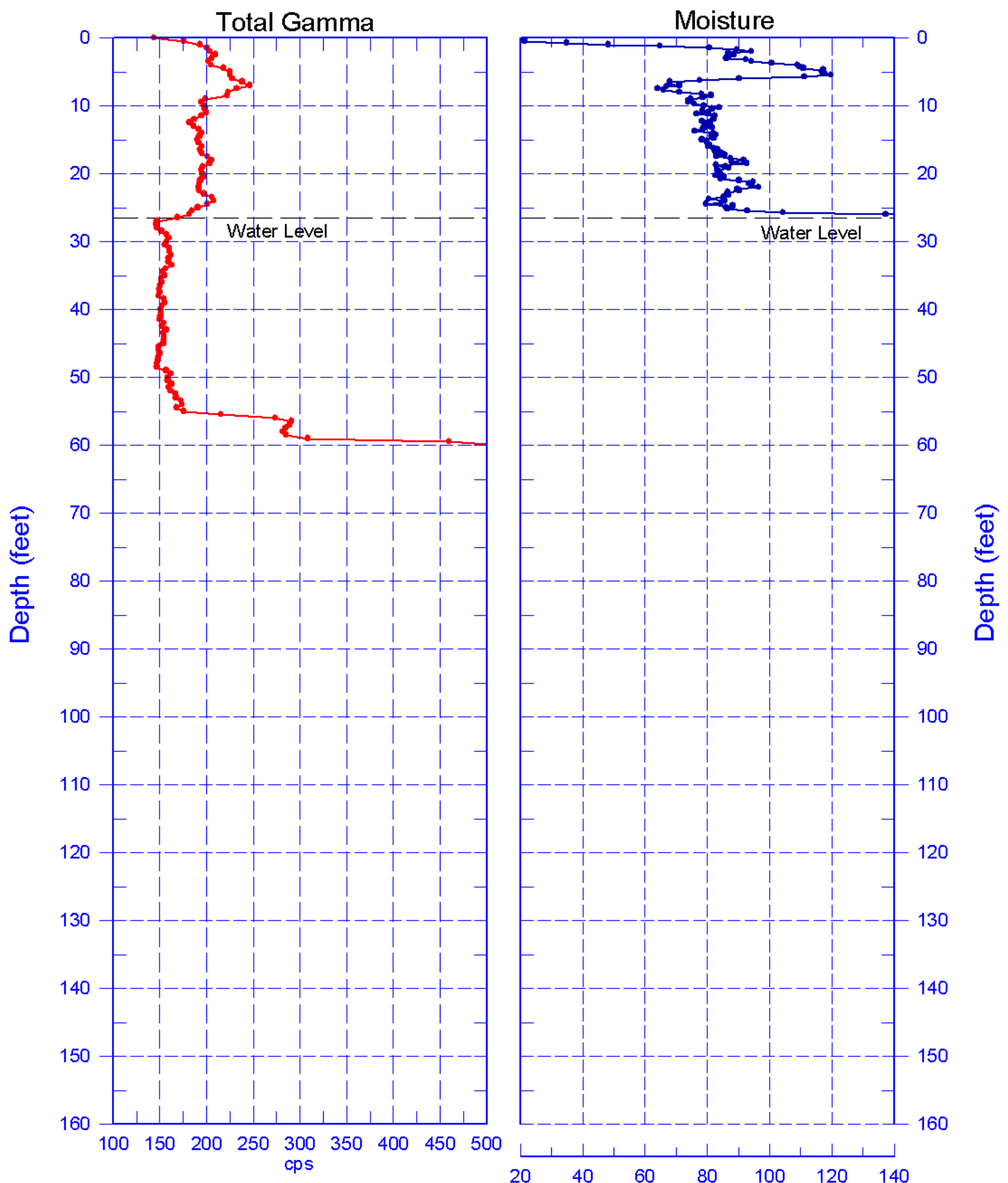
toller 399-2-21 (C6201)

Hanford Office

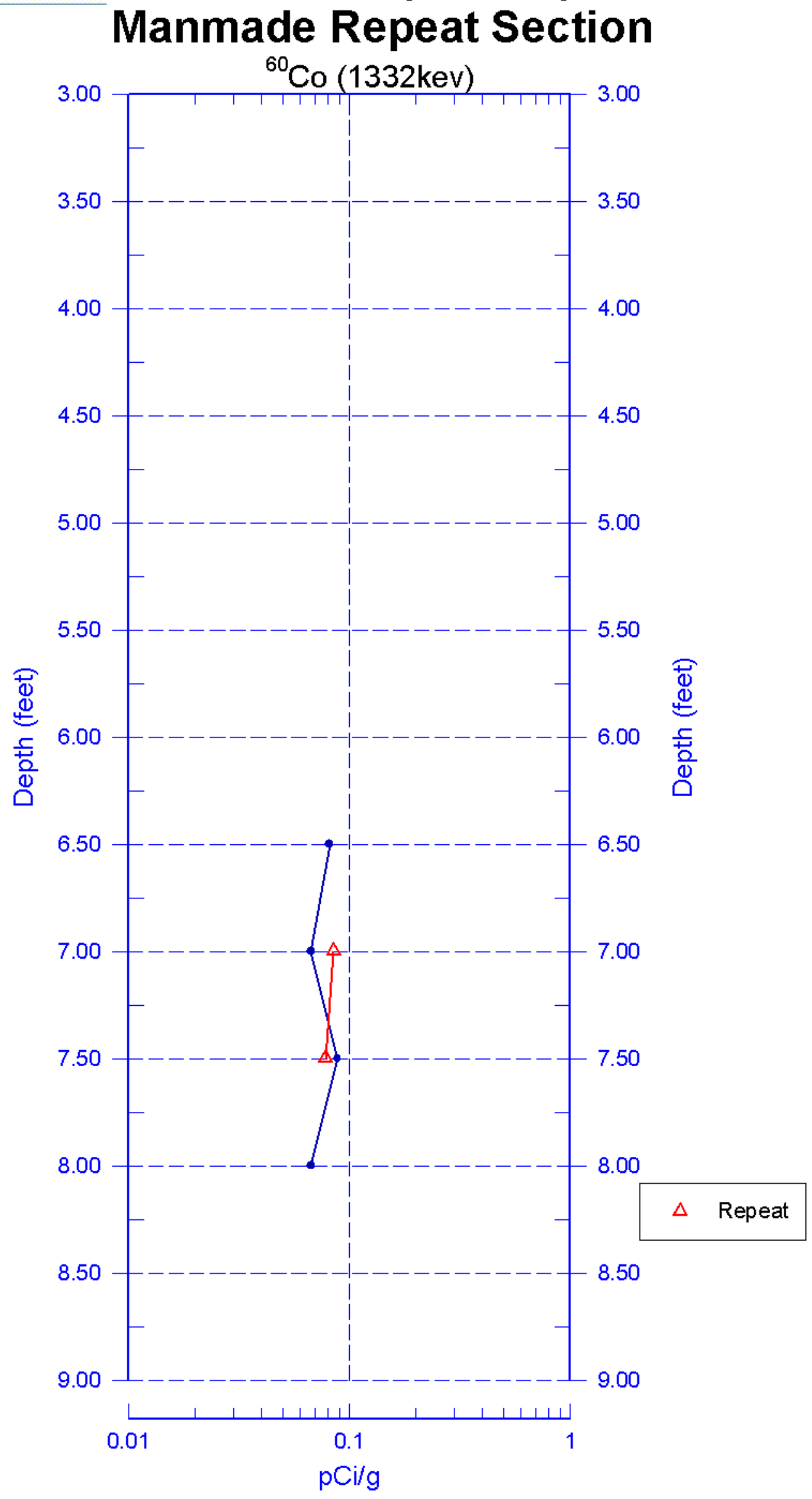

Reference - Ground Surface

G.161 


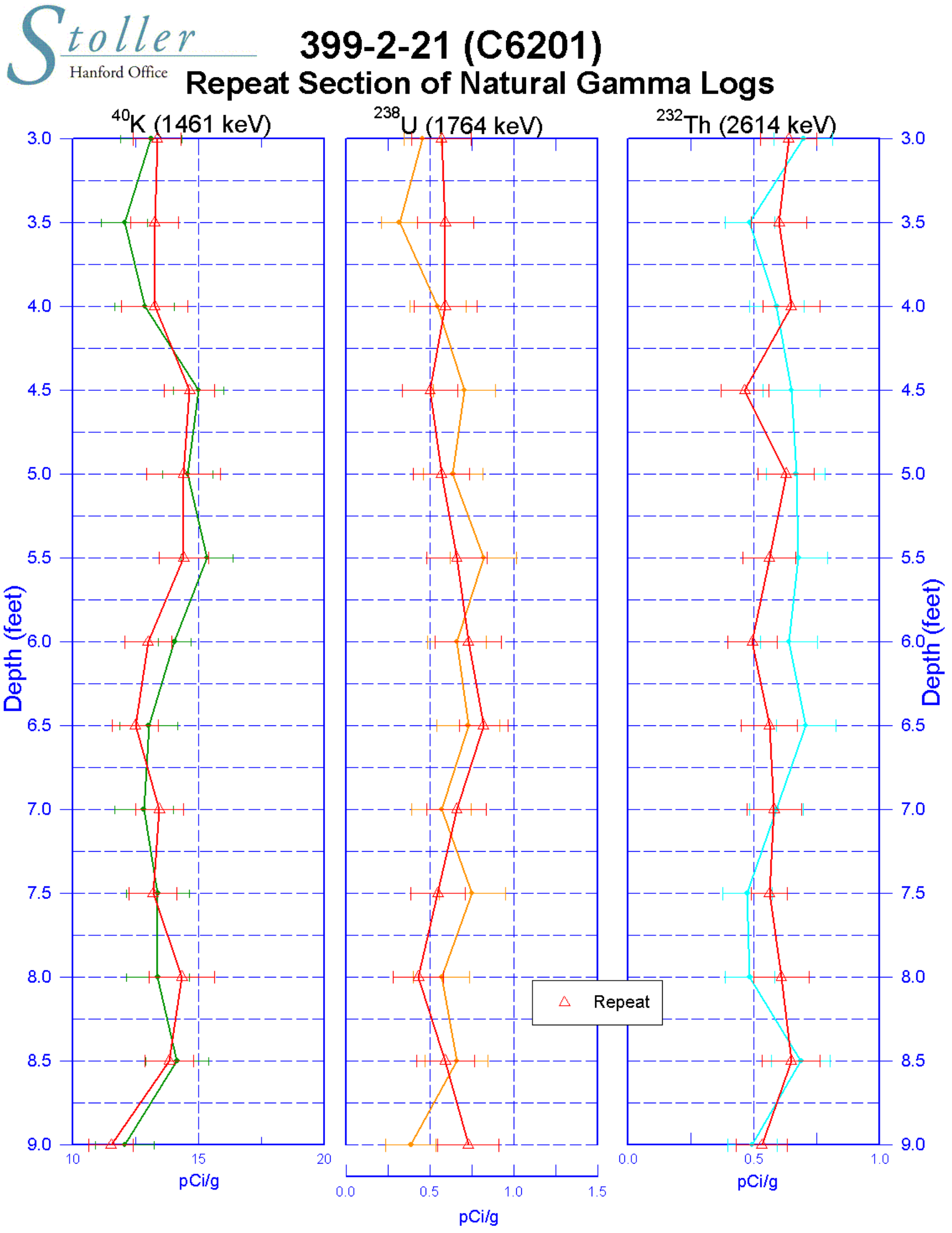

Zero Reference - Ground Surface

G.162 


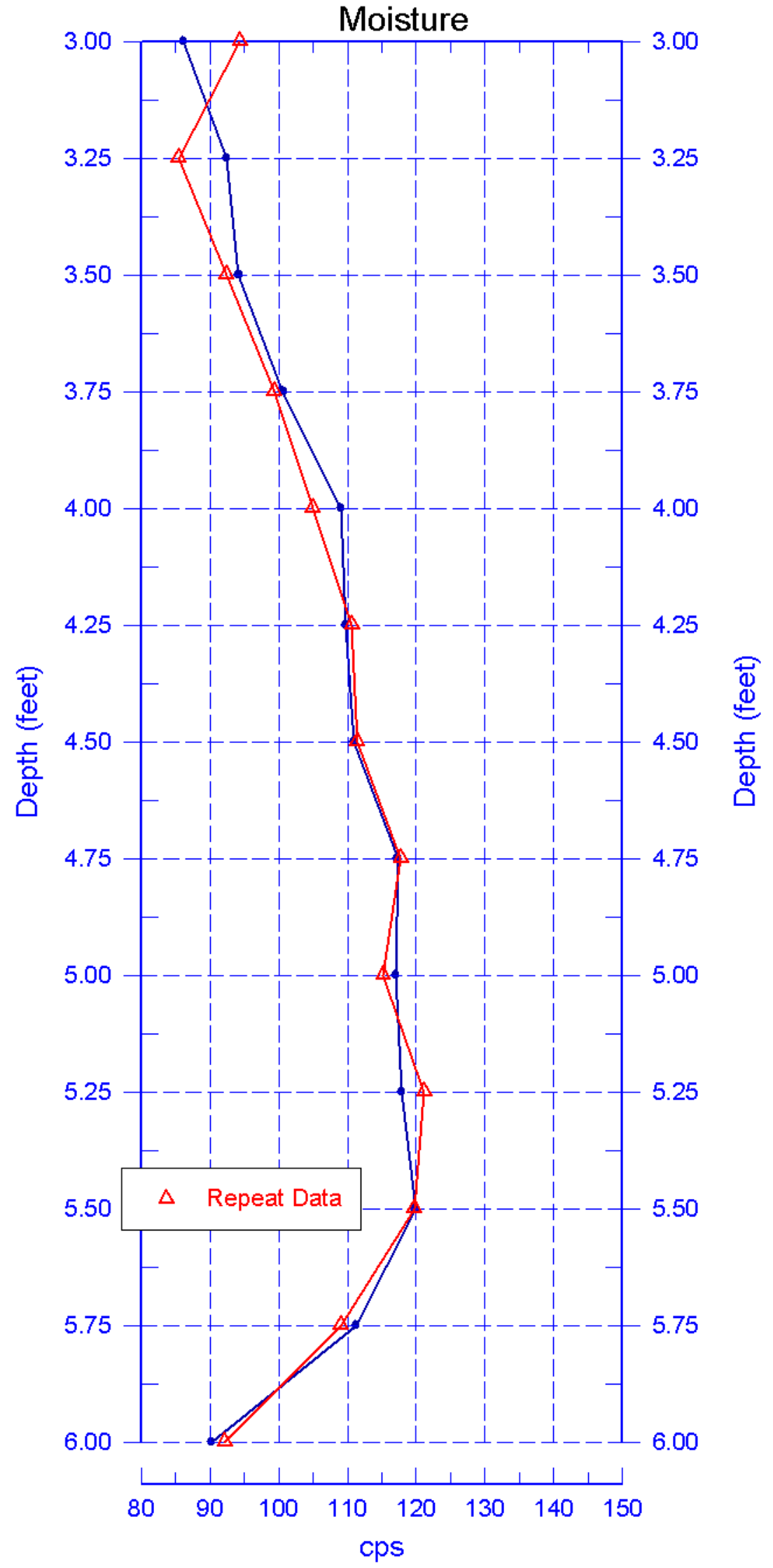




\section{9-2-22 (C6202) Log Data Report}

Borehole Information:

\begin{tabular}{|c|c|c|c|c|c|}
\hline \multicolumn{2}{|c|}{ Borehole: $\quad$ 399-2-22 (C6202) } & \multicolumn{4}{|c|}{ 300-FF-5 } \\
\hline \multicolumn{2}{|c|}{ Coordinates (WA St Plane) } & $G_{W L}^{1}(\mathbf{f}$ & 30.1 & GWL Date: & $07 / 16 / 08$ \\
\hline North (m) & East (m) & Drill Date & TOC $^{2}$ Elevation & Total Depth (ft) & Type \\
\hline 116073.3 & 594279.7 & 07/08/08 & Unknown & 65 & Sonic \\
\hline
\end{tabular}

\section{Casing Information:}

\begin{tabular}{|c|c|c|c|c|c|c|}
\hline Casing Type & Stickup (ft) & $\begin{array}{c}\text { Outer } \\
\text { Diameter (in.) }\end{array}$ & $\begin{array}{c}\text { Inside } \\
\text { Diameter (in.) }\end{array}$ & Thickness (in.) & Top (ft) & Bottom (ft) \\
\hline Threaded Steel & 0.8 & $75 / 8$ & $67 / 8$ & $3 / 8$ & -0.8 & 60.0 \\
\hline
\end{tabular}

\section{Borehole Notes:}

Casing data and total depth were reported by the site geologist. The logging engineer measured depth to water with an e-tape. Casing diameters were measured using a steel tape and rounded to the nearest 1/16 inch. The zero reference is the ground surface.

\section{Logging Equipment Information:}

\begin{tabular}{|c|c|c|c|c|}
\hline Logging System: & \multicolumn{2}{|c|}{ Gamma $4 \mathrm{~L}$} & $\begin{array}{l}\text { Type: } \\
\text { Serial No.: }\end{array}$ & $\begin{array}{l}\text { 60\% HPGe SGLS } \\
\text { 47TP32211A }\end{array}$ \\
\hline Effective Calibration Date: & $12 / 31 / 07$ & Calibration Reference: & \multicolumn{2}{|c|}{ HGLP-CC-027 } \\
\hline & & Logging Procedure: & \multicolumn{2}{|c|}{ HGLP-MAN-002, Rev. 0} \\
\hline Logging System: & \multicolumn{2}{|c|}{ Gamma $4 \mathrm{H}$} & $\begin{array}{l}\text { Type: } \\
\text { Serial No.: }\end{array}$ & $\begin{array}{l}\text { NMLS } \\
\text { H310700352 }\end{array}$ \\
\hline Effective Calibration Date: & $11 / 06 / 07$ & Calibration Reference: & \multicolumn{2}{|c|}{ HGLP-CC-021 } \\
\hline & & Logging Procedure: & \multicolumn{2}{|c|}{ HGLP-MAN-002, Rev. 0} \\
\hline
\end{tabular}

\section{Spectral Gamma Logging System (SGLS) Log Run Information:}

\begin{tabular}{|l|c|c|l|l|l|}
\hline Log Run & $\mathbf{1}$ & 2 Repeat & & & \\
\hline Date & $07 / 17 / 08$ & $07 / 17 / 08$ & & & \\
\hline Logging Engineer & McClellan & McClellan & & & \\
\hline Start Depth (ft) & 63.0 & 8.0 & & & \\
\hline Finish Depth (ft) & 0.0 & 2.0 & & & \\
\hline Count Time (sec) & 200 & 200 & & & \\
\hline Live/Real & $\mathrm{R}$ & $\mathrm{R}$ & & & \\
\hline Shield (Y/N) & $\mathrm{N}$ & $\mathrm{N}$ & & & \\
\hline MSA Interval (ft) & 0.5 & 0.5 & & & \\
\hline Log Speed (ft/min) & NA & NA & & & \\
\hline Pre-Verification & DL641CAB & DL641CAB & & & \\
\hline Start File & DL641000 & DL641127 & & & \\
\hline Finish File & DL641126 & DL641139 & & & \\
\hline Post-Verification & DL641CAA & DL641CAA & & & \\
\hline Depth Return Error (in.) & $1 / 2$ low & NA & & & \\
\hline Comments & $\begin{array}{c}\text { Fine gain } \\
\text { adjusted } \\
\text { before -125 }\end{array}$ & Repeat Section & & & \\
& & & & & \\
\hline
\end{tabular}


Neutron Moisture Logging System (NMLS) Log Run Information:

\begin{tabular}{|l|c|c|l|l|l|}
\hline Log Run & $\mathbf{3}$ & 4 Repeat & & & \\
\hline Date & $07 / 16 / 08$ & $07 / 16 / 08$ & & & \\
\hline Logging Engineer & McClellan & McClellan & & & \\
\hline Start Depth (ft) & 0.0 & 30.25 & & & \\
\hline Finish Depth (ft) & 30.25 & 27.0 & & & \\
\hline Count Time (sec) & 15 & 15 & & & \\
\hline Live/Real & $\mathrm{R}$ & $\mathrm{R}$ & & & \\
\hline Shield (Y/N) & $\mathrm{N}$ & $\mathrm{N}$ & & & \\
\hline MSA Interval (ft) & 0.25 & 0.25 & & & \\
\hline Log Speed (ft/min) & NA & NA & & & \\
\hline Pre-Verification & DHH12CAB & DHH12CAB & & & \\
\hline Start File & DHH12000 & DHH12122 & & & \\
\hline Finish File & DHH12121 & DHH12135 & & & \\
\hline Post-Verification & DHH12CAA & DHH12CAA & & & \\
\hline Depth Return Error (in.) & NA & NHA & & & \\
\hline Comments & None & Repeat Section & & & \\
\hline
\end{tabular}

\section{Logging Operation Notes:}

Data were collected using Gamma 4, HO 68B-3573. SGLS pre- and post-survey verification measurements were acquired in the Amersham KUTh-115 field verifier. NMLS pre- and post-survey verification measurements were acquired in the standard field verifier. Maximum logging depth for the SGLS was 63.4 feet. before the sonde un-weighted. A centralizer was installed on the sonde.

\section{Analysis Notes:}

\begin{tabular}{|l|l|l|l|l|l|}
\hline Analyst: & LEGLER & Date: & 10/06/08 & Reference: & GJO-HGLP 1.6.3, Rev. 0 \\
\hline
\end{tabular}

The SGLS pre- and post-survey verification spectra met the acceptance criteria for the established system, but the verification file DL641CAA had measurements above the upper control limit for the Full Width at Half Maximum (fwhm) for the $2615 \mathrm{keV}$ energy line. The NMLS pre- and post-survey verification spectra met the acceptance criteria for the established system.

A casing correction for a 3/8-in thick casing was applied for depths up to $59.5 \mathrm{ft}$, where casing ends leaving $3.5 \mathrm{ft}$ of open borehole uncorrected. The Borehole Data Sheet lists the casing depth as $60 \mathrm{ft}$, but data from the Total Gamma and KUT plots indicated that the casing ends at $59.5 \mathrm{ft}$.

A water correction was also applied from $30.1 \mathrm{ft}$ to total logged depth of borehole.

SGLS spectra were processed in batch mode in APTEC SUPERVISOR to identify individual peaks and count rates. Concentrations were calculated using an EXCEL template identified as G4LDec07.xls using an efficiency function and corrections for casing, dead time and water as determined by annual calibrations. NMLS spectra were processed in batch mode in APTEC SUPERVISOR to identify counts. Count rates were calculated using an EXCEL template identified as G4HNov07.xls. NMLS data are presented in counts per second (cps), because no calibration data is available for a 6 7/8-in inner diameter borehole casing.

\section{Results and Interpretations:}

Cs-137 was detected at $4 \mathrm{ft}, 6$ to $6.5 \mathrm{ft}$, and 41 feet. Inspection of the individual spectra indicates that detections at 4 and $41 \mathrm{ft}$ are caused by statistical fluctuations associated with processing software. Maximum concentration for detections was $0.13 \mathrm{pCi} / \mathrm{g}$ at 6 feet.

Co-60 was detected at $5.5-7 \mathrm{ft}$, with a maximum concentration of $0.22 \mathrm{pCi} / \mathrm{g}$ at $6.5 \mathrm{ft}$. 
HGLP-LDR-255, Rev. 0

U-235 and U-238 (Pa-234m) were also detected in this borehole at various isolated depths, but further inspection of the individual spectra indicates that these detections are statistical fluctuations associated with the processing software.

The neutron moisture data from 14.5 to $18.25 \mathrm{ft}$ exhibits unusually erratic behavior, dropping to a low of $24 \mathrm{cps}$ at 18 feet. This could indicate low moisture content such as a void next to the casing or an intermittent failure of the detector at these depths.

The manmade, KUT and Moisture repeat plots indicate good repeatability.

\section{List of Log Plots:}

Depth Reference is ground surface

Manmade Radionuclides

Natural Gamma Logs

Combination Plot

Total Gamma \& Dead Time

Total Gamma \& Moisture

Manmade Repeat Section

Repeat Section of Natural Gamma Logs

Moisture Repeat Section

${ }^{1} \mathrm{GWL}$ - groundwater level

${ }^{2}$ TOC - top of casing 


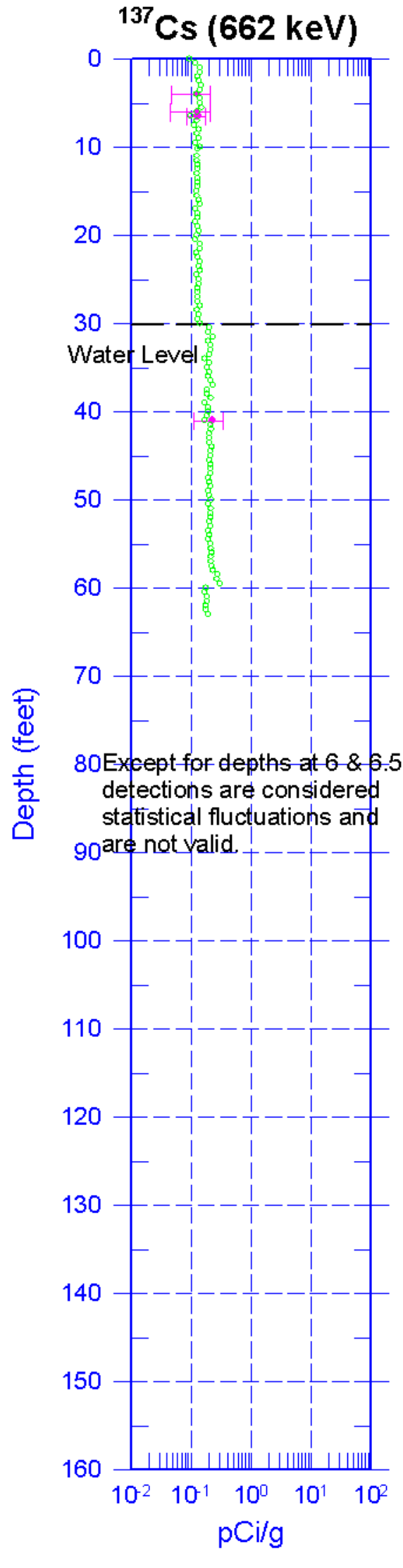

Zero Reference - Ground Surface

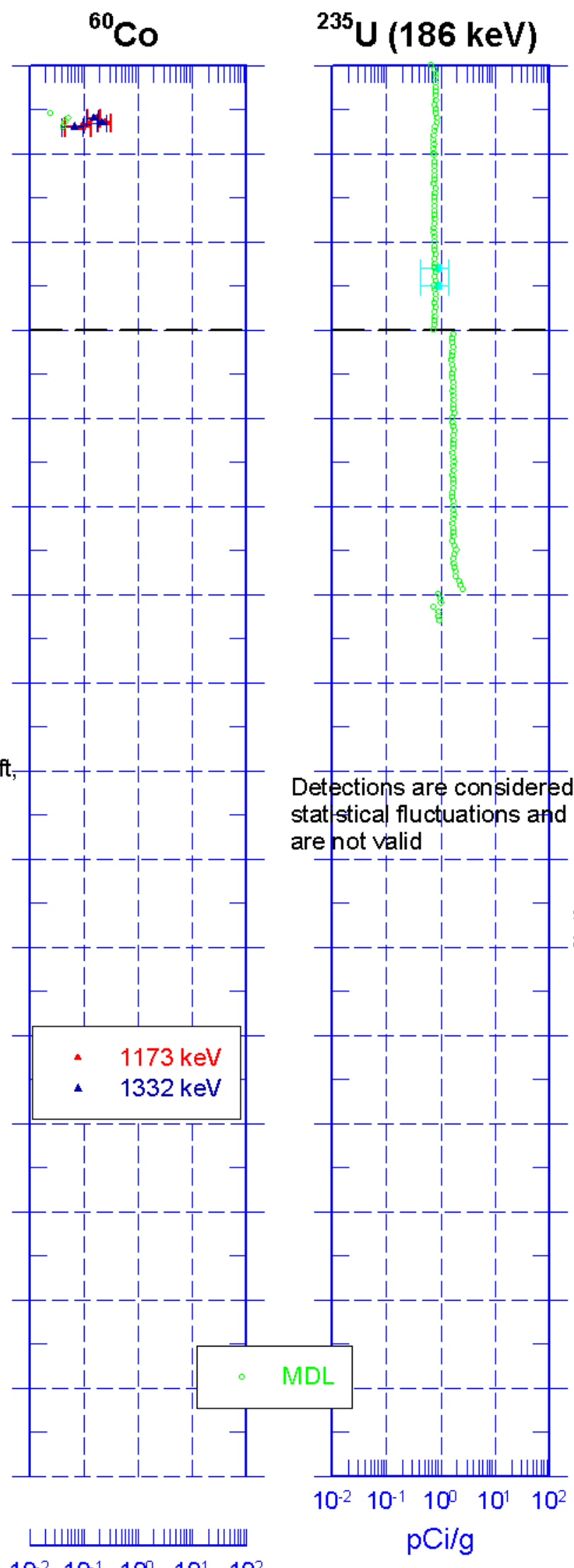

$$
\begin{gathered}
10^{-2} 10^{-1} \quad 10^{0} 10^{1} \quad 10^{2} \\
\mathrm{pCi} / \mathrm{g}
\end{gathered}
$$

${ }^{238} \mathrm{U}(1001 \mathrm{keV})$

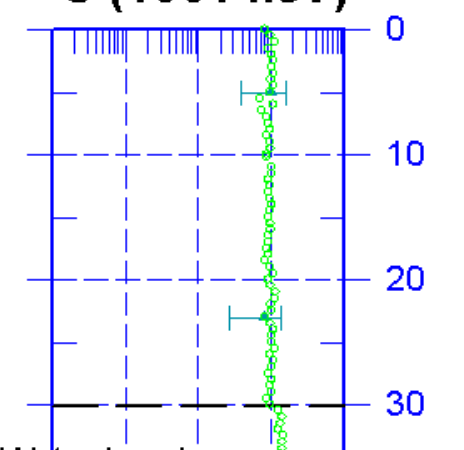

Water Level

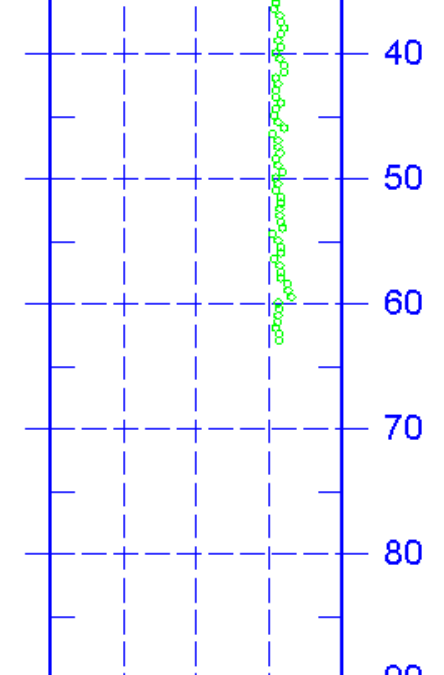

Detections are considered stat stical fluctuations and are not valid -- - - 100

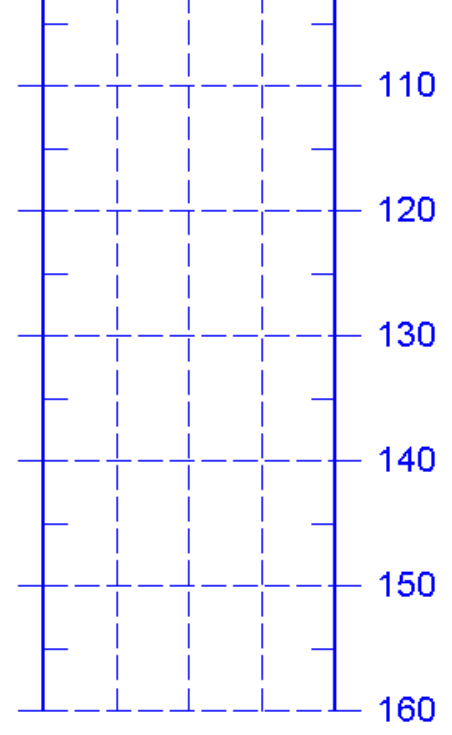

لШ山Ш $\begin{array}{lllll}10^{-2} & 10^{-1} & 10^{0} & 10^{1} & 10^{2}\end{array}$ $\mathrm{pCi} / \mathrm{g}$ 
toller 399-2-22 (66202)

Hanford Office

\section{Natural Gamma Logs}
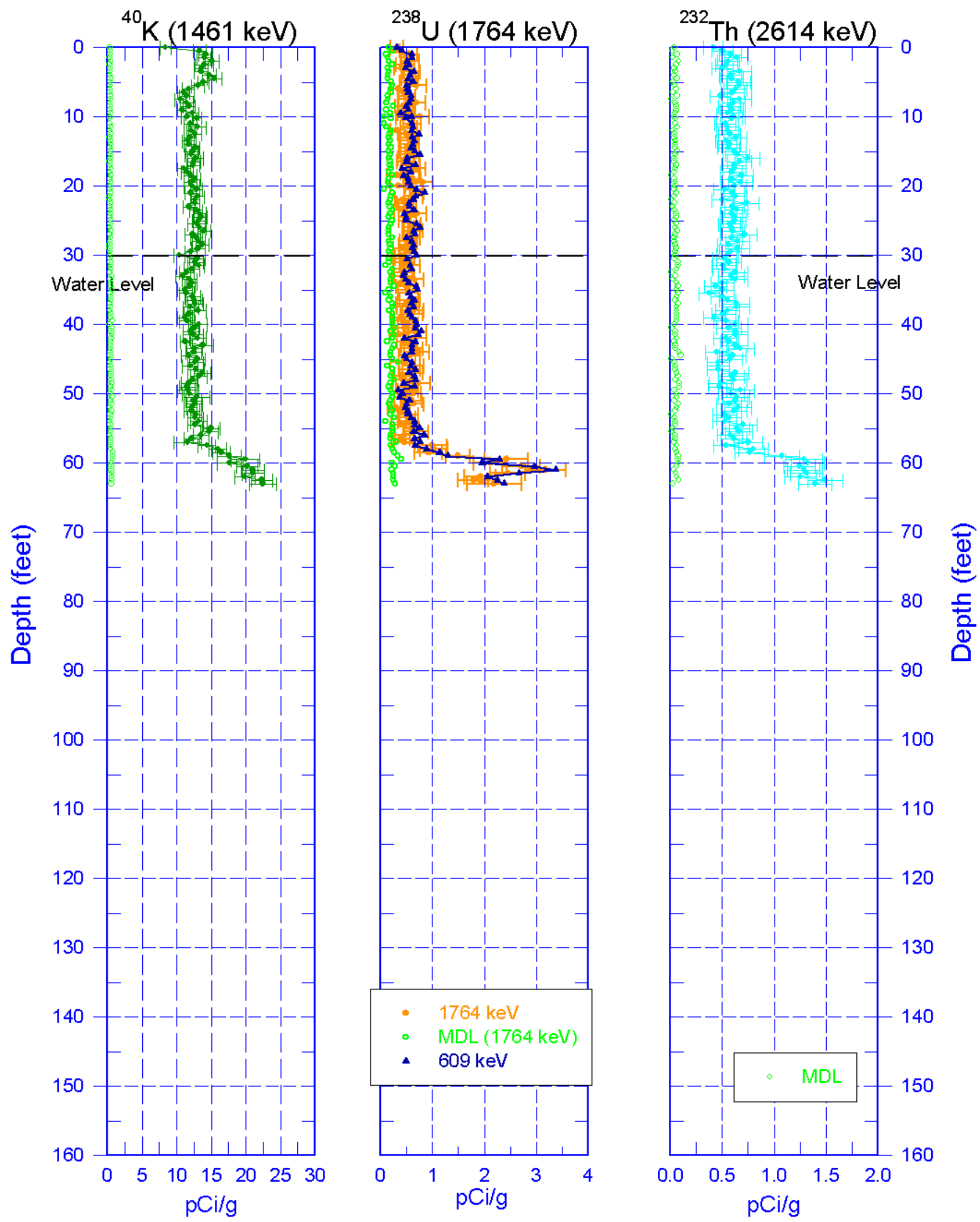

Zero Reference - Ground Surface

G.168 


\section{S}

toller 399-2-22 (C6202)

Hanford Office

\section{Total Gamma \& Moisture}
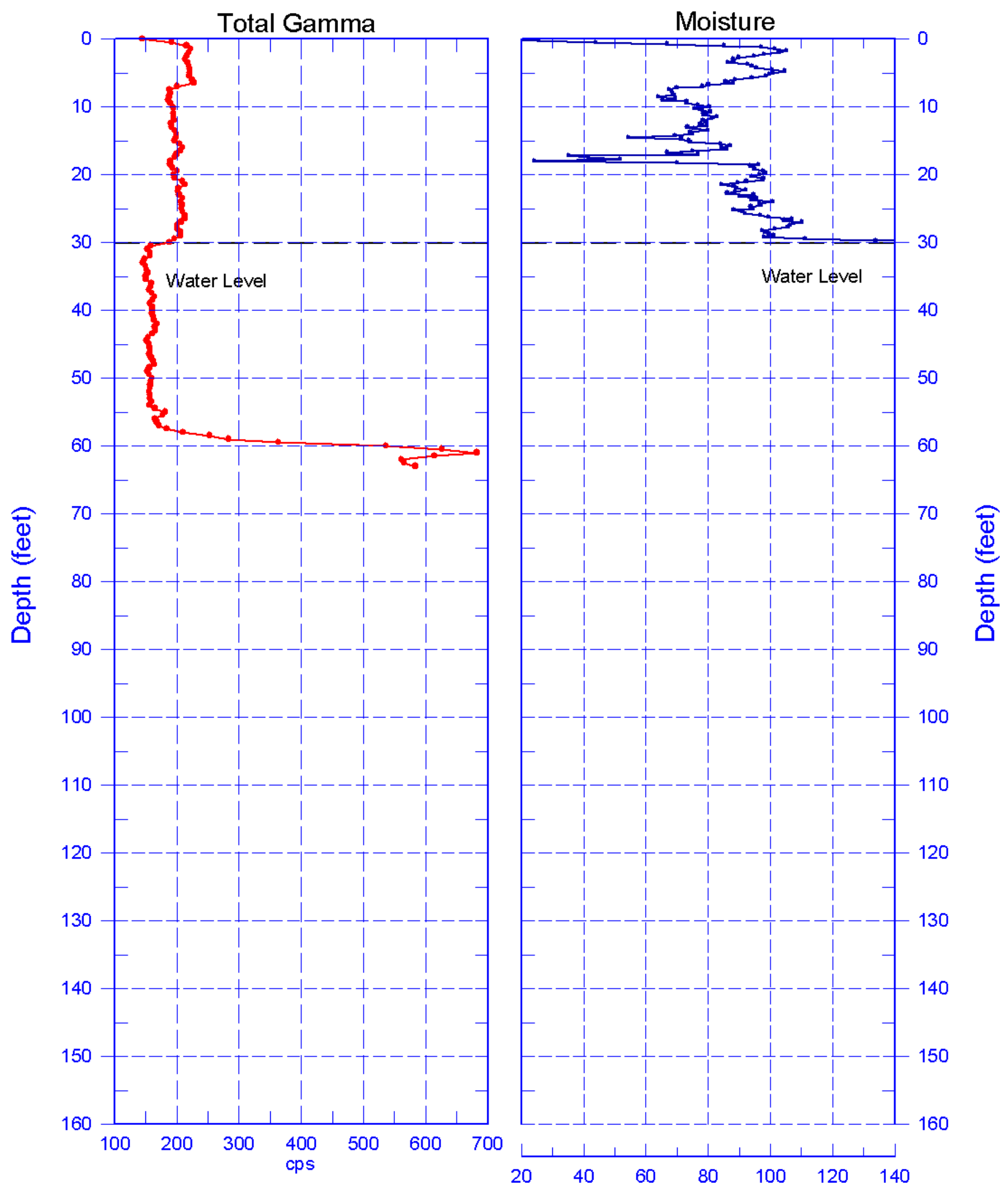

원
듬
$\frac{1}{0}$

Reference - Ground Surface 
toller 399-2-22 (C6202)

Hanford Office

\section{Total Gamma \& Dead Time}
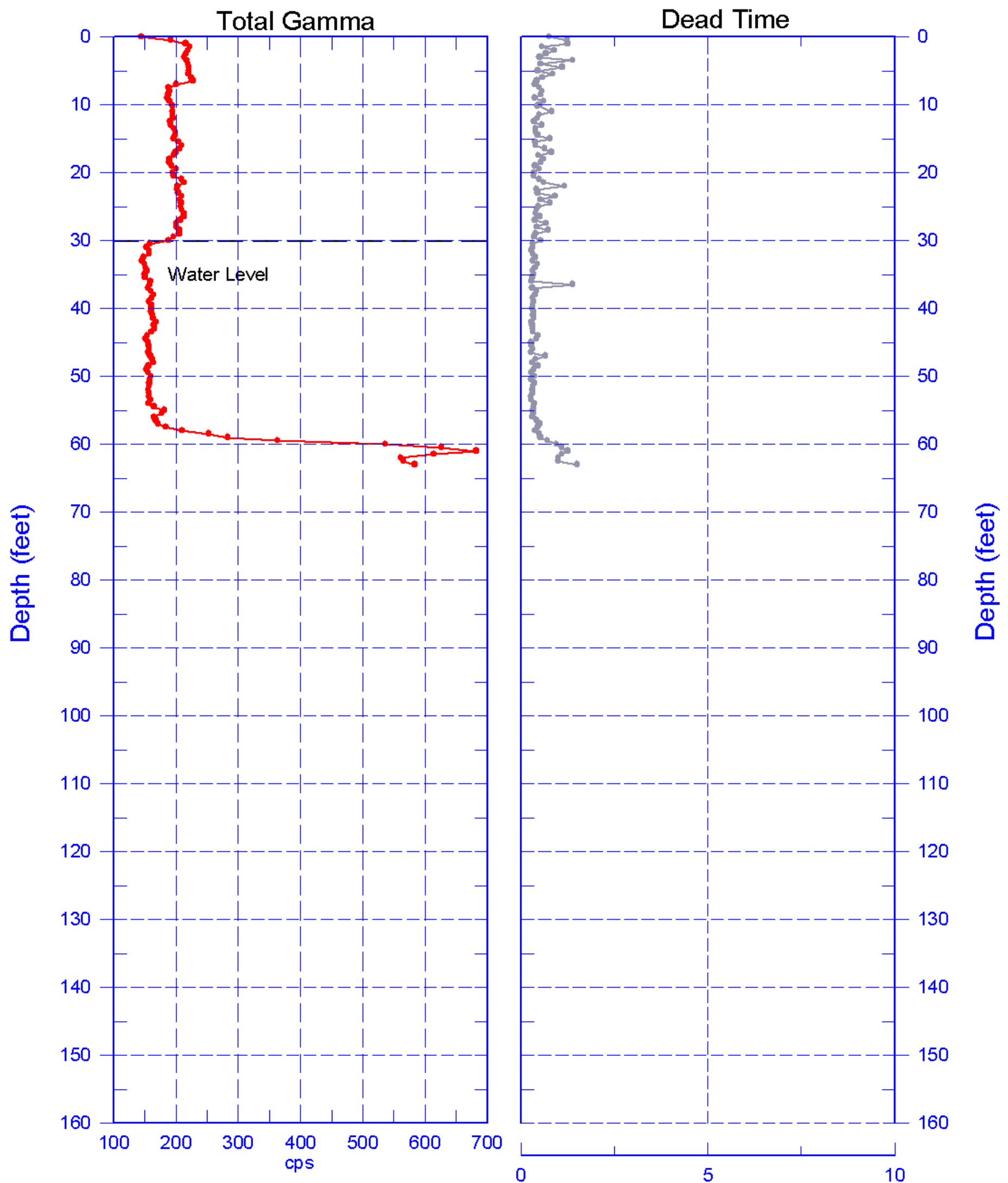

$\frac{\widehat{0}}{0}$
$\frac{0}{0}$
$\frac{0}{0}$
0

Reference - Ground Surface

G.170 
toller 399-2-22 (C6202)

Hanford Office

Manmade Repeat Section

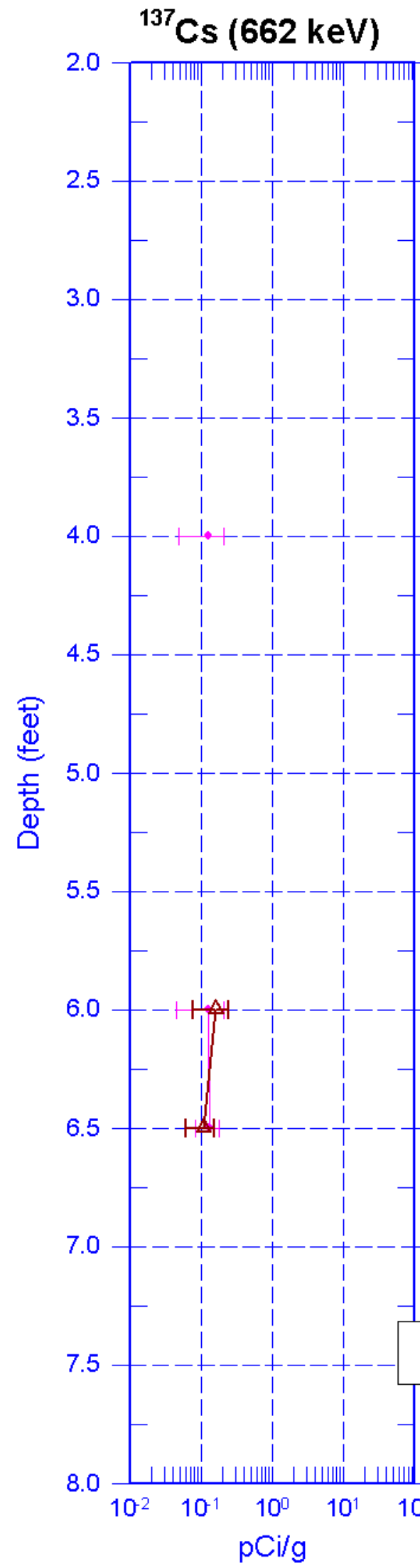

${ }^{60} \mathrm{Co}(1332 \mathrm{keV})$

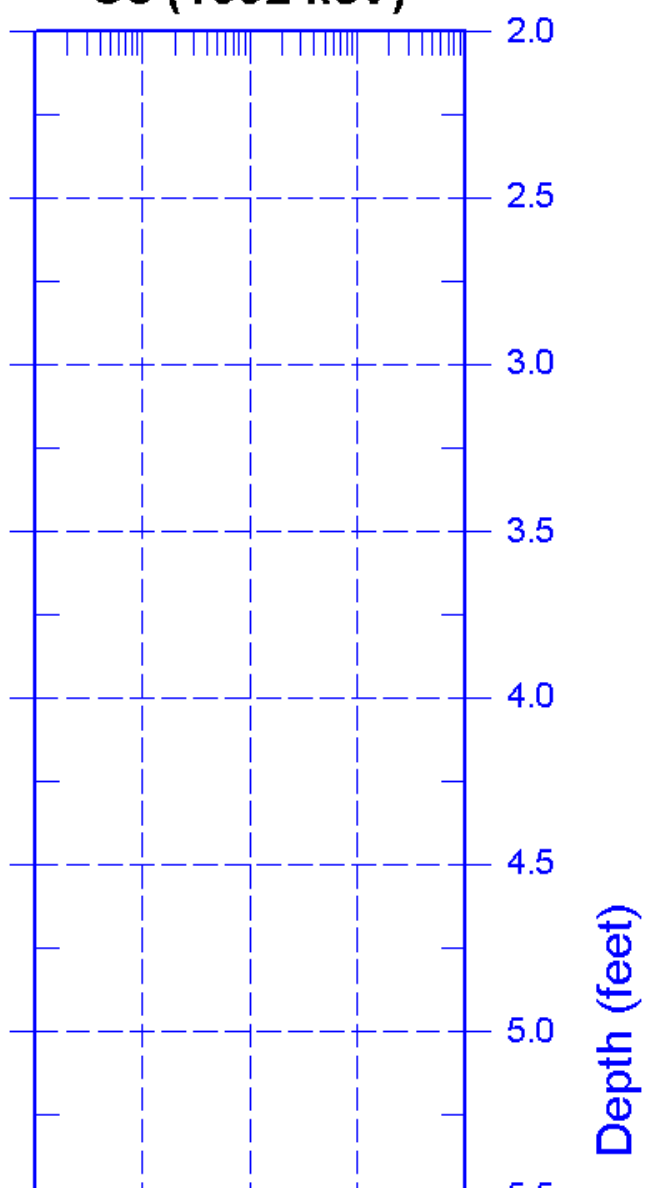

$\triangle$ Repeat

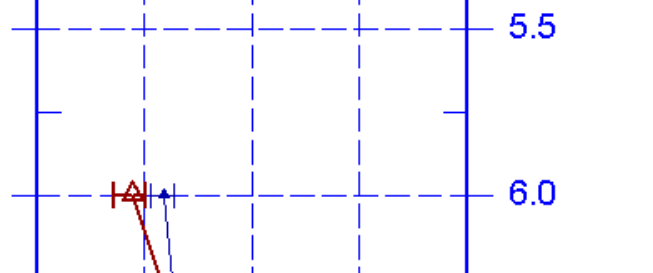

$\mathrm{pCi} / \mathrm{g}$ 


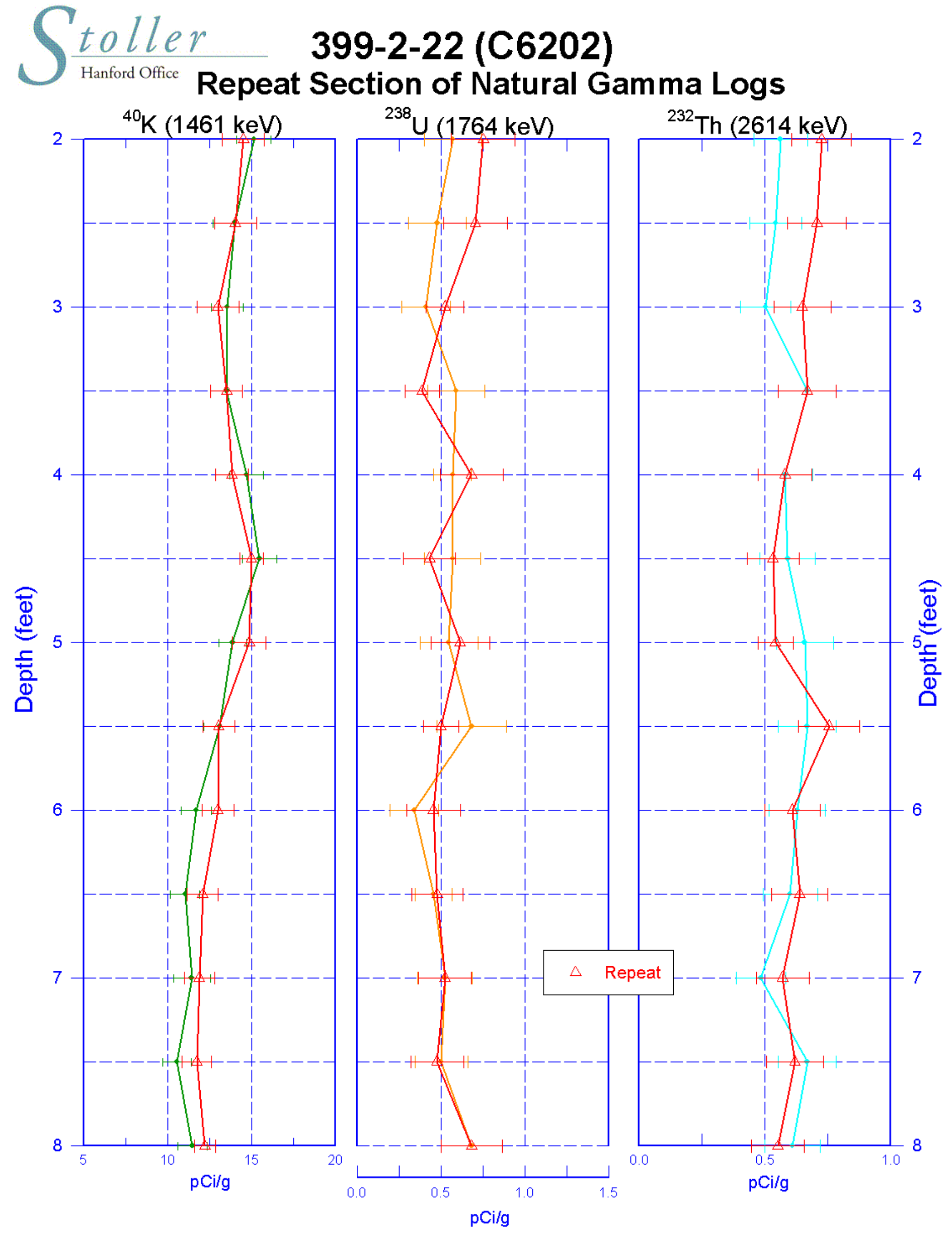

Zero Reference - Ground Surface

G.172 


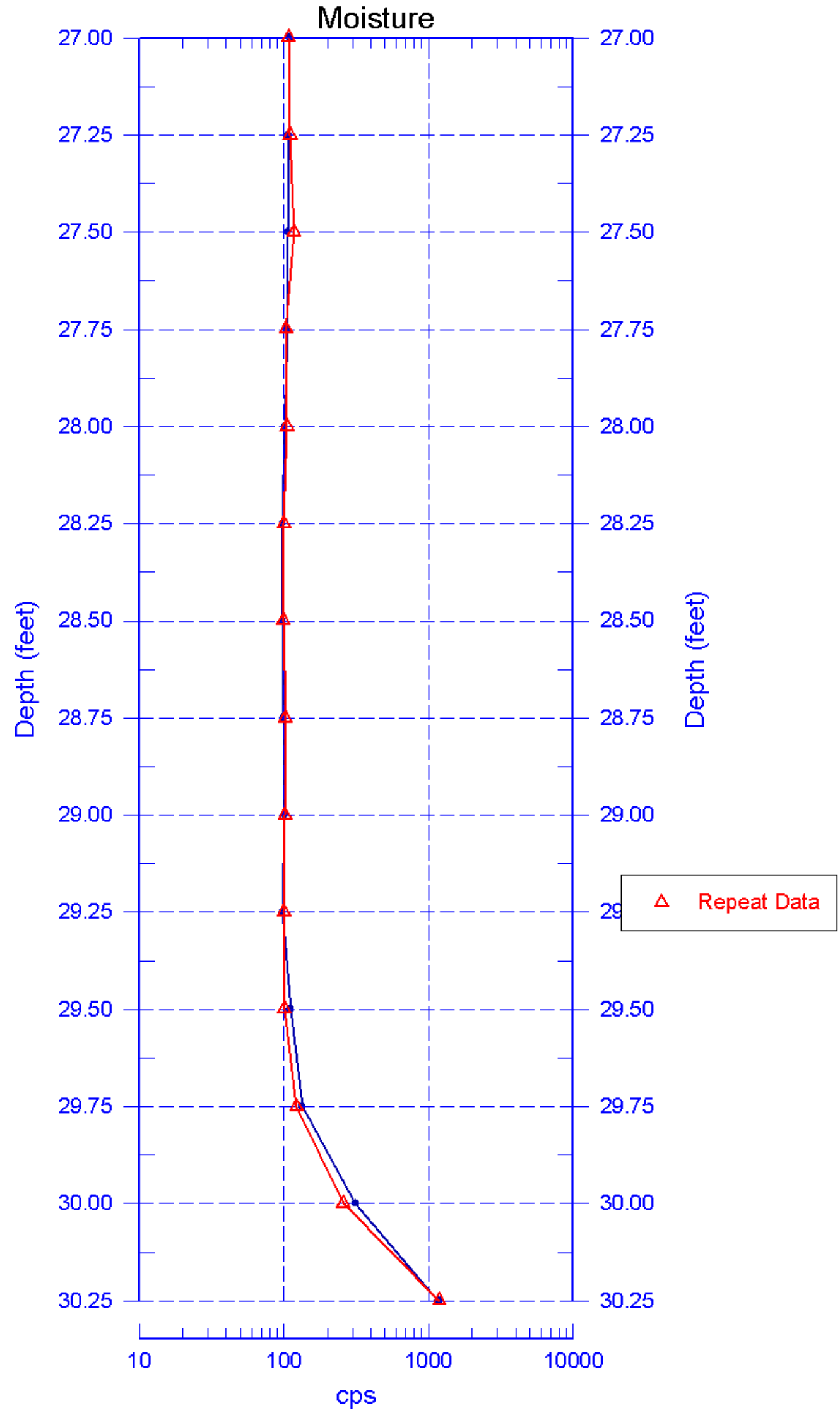

Reference - Ground Surface 


\section{9-2-23 (C6207) Log Data Report}

Borehole Information:

\begin{tabular}{|c|c|c|c|c|c|}
\hline \multirow{2}{*}{\multicolumn{2}{|c|}{ Borehole: $\quad 399-2-23$ (C6207) }} & \multicolumn{4}{|c|}{ 300-FF-5 } \\
\hline & & $\mathbf{G W L}^{1}$ (f & 27.7 & GWL Date: & $07 / 09 / 08$ \\
\hline North (m) & East (m) & Drill Date & TOC ${ }^{2}$ Elevation & Total Depth (ft) & Tyре \\
\hline 116057.6 & 594272.4 & $07 / 08 / 08$ & Unknown & 59 & Sonic \\
\hline
\end{tabular}

\section{Casing Information:}

\begin{tabular}{|c|c|c|c|c|c|c|}
\hline Casing Type & Stickup (ft) & $\begin{array}{c}\text { Outer } \\
\text { Diameter (in.) }\end{array}$ & $\begin{array}{c}\text { Inside } \\
\text { Diameter (in.) }\end{array}$ & Thickness (in.) & Top (ft) & Bottom (ft) \\
\hline Threaded Steel & 2.2 & $75 / 8$ & $67 / 8$ & $3 / 8$ & -2.2 & 57.8 \\
\hline
\end{tabular}

\section{Borehole Notes:}

Casing data and total depth were reported by the site geologist. The logging engineer measured the depth to water with an e-tape. Casing diameters were measured using a steel tape and rounded to the nearest 1/16 inch. The zero reference is the ground surface.

\section{Logging Equipment Information:}

\begin{tabular}{|c|c|c|c|c|}
\hline Logging System: & \multicolumn{2}{|l|}{ Gamma $4 \mathrm{~L}$} & $\begin{array}{l}\text { Type: } \\
\text { Serial No.: }\end{array}$ & $\begin{array}{l}\text { 60\% HPGe SGLS } \\
\text { 47TP32211A }\end{array}$ \\
\hline Effective Calibration Date: & $12 / 31 / 2007$ & Calibration Reference: & \multicolumn{2}{|c|}{ HGLP-CC-027 } \\
\hline & & Logging Procedure: & \multicolumn{2}{|c|}{ HGLP-MAN-002, Rev. 0} \\
\hline Logging System: & \multicolumn{2}{|l|}{ Gamma $4 \mathrm{H}$} & $\begin{array}{l}\text { Type: } \\
\text { Serial No.: }\end{array}$ & $\begin{array}{l}\text { NMLS } \\
\text { H310700352 }\end{array}$ \\
\hline Effective Calibration Date: & $11 / 06 / 2007$ & Calibration Reference: & \multicolumn{2}{|c|}{ HGLP-CC-021 } \\
\hline & & Logging Procedure: & \multicolumn{2}{|c|}{ HGLP-MAN-002, Rev. 0} \\
\hline
\end{tabular}

\section{Spectral Gamma Logging System (SGLS) Log Run Information:}

\begin{tabular}{|l|c|c|l|l|l|}
\hline Log Run & $\mathbf{1}$ & 2 Repeat & & & \\
\hline Date & $07 / 09 / 08$ & $07 / 09 / 08$ & & & \\
\hline Logging Engineer & McClellan & McClellan & & & \\
\hline Start Depth (ft) & 58.0 & 8.0 & & & \\
\hline Finish Depth (ft) & 0.0 & 2.0 & & & \\
\hline Count Time (sec) & 200 & 200 & & & \\
\hline Live/Real & $\mathrm{R}$ & $\mathrm{R}$ & & & \\
\hline Shield (Y/N) & $\mathrm{N}$ & $\mathrm{N}$ & & & \\
\hline MSA Interval (ft) & 0.5 & 0.5 & & & \\
\hline Log Speed (ft/min) & NA & NA & & & \\
\hline Pre-Verification & DL591CAB & DL591CAB & & & \\
\hline Start File & DL591000 & DL951117 & & & \\
\hline Finish File & DL591116 & DL591129 & & & \\
\hline Post-Verification & DL591CAA & DL591CAA & & & \\
\hline Depth Return Error (in.) & 0.0 & NA & & & \\
\hline Comments & None & Repeat Section & & & \\
\hline
\end{tabular}


Neutron Moisture Logging System (NMLS) Log Run Information:

\begin{tabular}{|l|c|c|l|l|l|}
\hline Log Run & $\mathbf{3}$ & 4 Repeat & & & \\
\hline Date & $07 / 10 / 08$ & $07 / 10 / 08$ & & & \\
\hline Logging Engineer & Pearson & Pearson & & & \\
\hline Start Depth (ft) & 0.0 & 1.0 & & & \\
\hline Finish Depth (ft) & 28.0 & 4.0 & & & \\
\hline Count Time (sec) & 15 & 15 & & & \\
\hline Live/Real & $\mathrm{R}$ & $\mathrm{R}$ & & & \\
\hline Shield (Y/N) & $\mathrm{N}$ & $\mathrm{N}$ & & & \\
\hline MSA Interval (ft) & 0.25 & 0.25 & & & \\
\hline Log Speed (ft/min) & NA & NA & & & \\
\hline Pre-Verification & DHG62CAB & DHG62CAB & & & \\
\hline Start File & DHG62000 & DHG62113 & & & \\
\hline Finish File & DHG62112 & DHG62125 & & & \\
\hline Post-Verification & DHG62CAA & DHG62CAA & & & \\
\hline Depth Return Error (in.) & NA & NA.0 & & & \\
\hline Comments & None & Repeat Section & & & \\
\hline
\end{tabular}

\section{Logging Operation Notes:}

Data were collected using Gamma 4, HO 68B-3573. SGLS pre- and post-survey verification measurements were acquired in the Amersham KUTh-115 field verifier. NMLS pre- and post-survey verification measurements were acquired in the standard field verifier. Maximum logging depth for the SGLS was $58.5 \mathrm{ft}$ before the sonde un-weighted. A centralizer was installed on the sonde.

Analysis Notes:

\begin{tabular}{|l|l|l|l|l|l|}
\hline Analyst: & LEGLER & Date: & 10/08/08 & Reference: & GJO-HGLP 1.6.3, Rev. 0 \\
\hline
\end{tabular}

The SGLS pre- and post-survey verification spectra met the acceptance criteria for the established system, but the verification file DL591CAA had measurements above the upper control limit for the Full Width at Half Maximum (fwhm) for the $1461 \mathrm{keV}$ energy line. NMLS pre- and post survey verification spectra met the acceptance criteria for the established system.

A casing correction for a 3/8-in thick casing was applied for depths up to $57.8 \mathrm{ft}$, where casing ends leaving $0.2 \mathrm{ft}$ of open borehole uncorrected. A water correction was also applied from $27.7 \mathrm{ft}$ to total logged depth of the borehole.

SGLS spectra were processed in batch mode in APTEC SUPERVISOR to identify individual peaks and count rates. Concentrations were calculated using an EXCEL template identified as G4LDec07.xls using an efficiency function and corrections for casing, dead time and water as determined by annual calibrations. NMLS spectra were processed in batch mode in APTEC SUPERVISOR to identify counts. Count rates were calculated using an EXCEL template identified as G4HNov07.xls. NMLS data are presented in counts per second (cps), because no calibration data is available for a 6 7/8-in inner diameter borehole casing.

\section{$\underline{\text { Results and Interpretations: }}$}

Cs-137 was detected at $11 \mathrm{ft}, 12.5 \mathrm{ft}, 13-16.5 \mathrm{ft}, 18.5 \mathrm{ft}, 48.5$, and $55 \mathrm{ft}$, with the maximum concentration of $0.22 \mathrm{pCi} / \mathrm{g}$ at $14.5 \mathrm{feet}$. Inspection of the individual spectra indicates that detections at $11 \mathrm{ft}, 48.5 \mathrm{ft}$ and $55 \mathrm{ft}$, are statistical fluctuations associated with the processing software and are not valid.

Co-60 was detected at $6-18.5 \mathrm{ft}$ with a maximum concentration of $0.36 \mathrm{pCi} / \mathrm{g}$ at 13.5 feet.

U-235 and U-238 (Pa-234m) were detected at several isolated depths throughout this borehole, but inspection of the individual spectra indicates that these detections are statistical fluctuations associated with the processing software. 
HGLP-LDR-256, Rev. 0

The KUT, Moisture and manmade repeat plots indicate good repeatability.

Below the $54 \mathrm{ft}$ depth, a probable lithologic change occurs, which is indicated by the increased KUT concentrations and the Total Gamma count rates.

List of Log Plots:

Depth Reference is ground surface

Manmade Radionuclides

Natural Gamma Logs

Combination Plot

Total Gamma \& Dead Time

Total Gamma \& Moisture

Manmade Repeat Section

Repeat Section of Natural Gamma Logs

Moisture Repeat Section

${ }^{1} \mathrm{GWL}$ - groundwater level

${ }^{2}$ TOC - top of casing 

Hanford Office Manmade Radionuclides

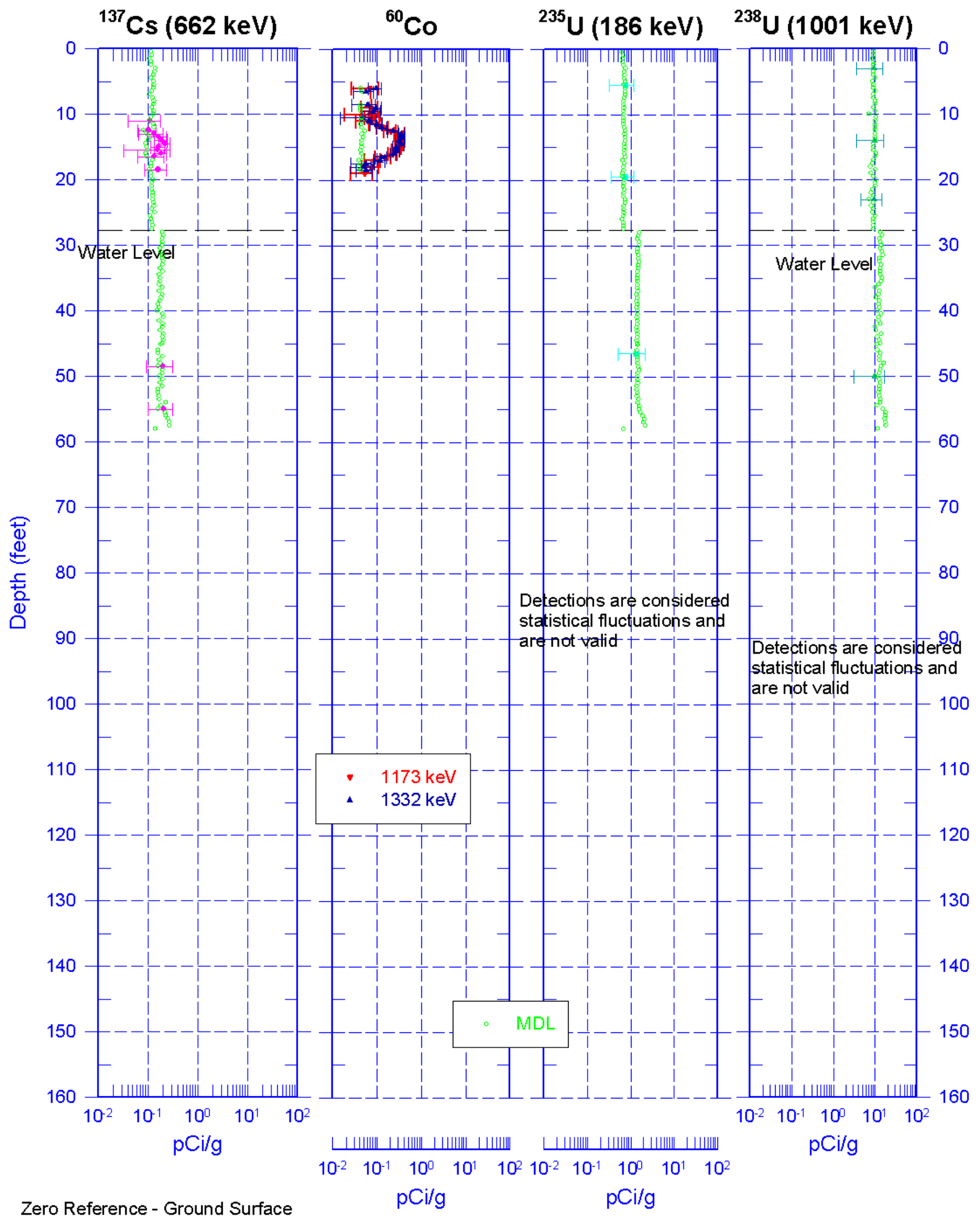


toller $\mathbf{3 9 9 - 2 - 2 3}$ (C6207)

Hanford Office

\section{Natural Gamma Logs}
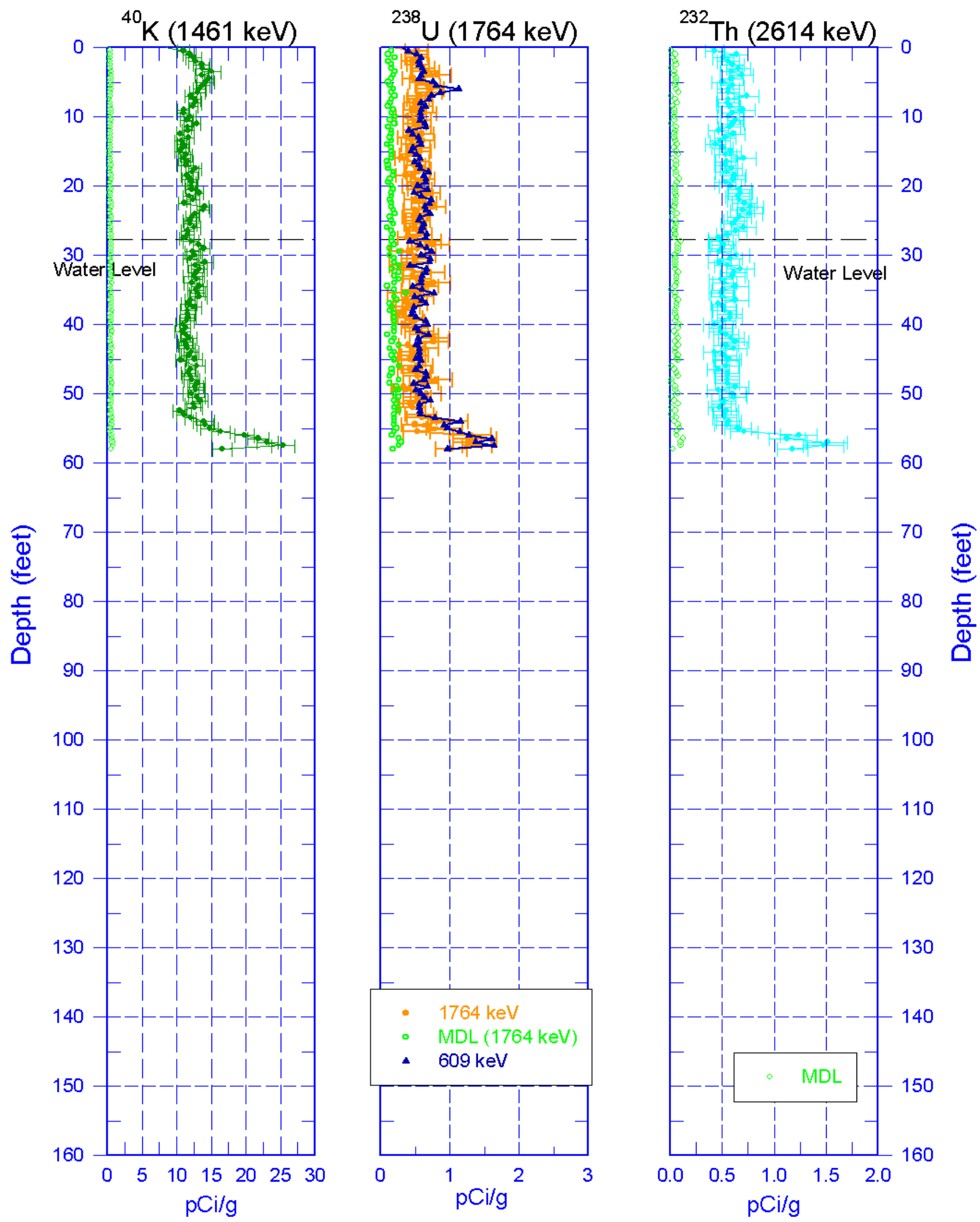

Zero Reference - Ground Surface

G.178 


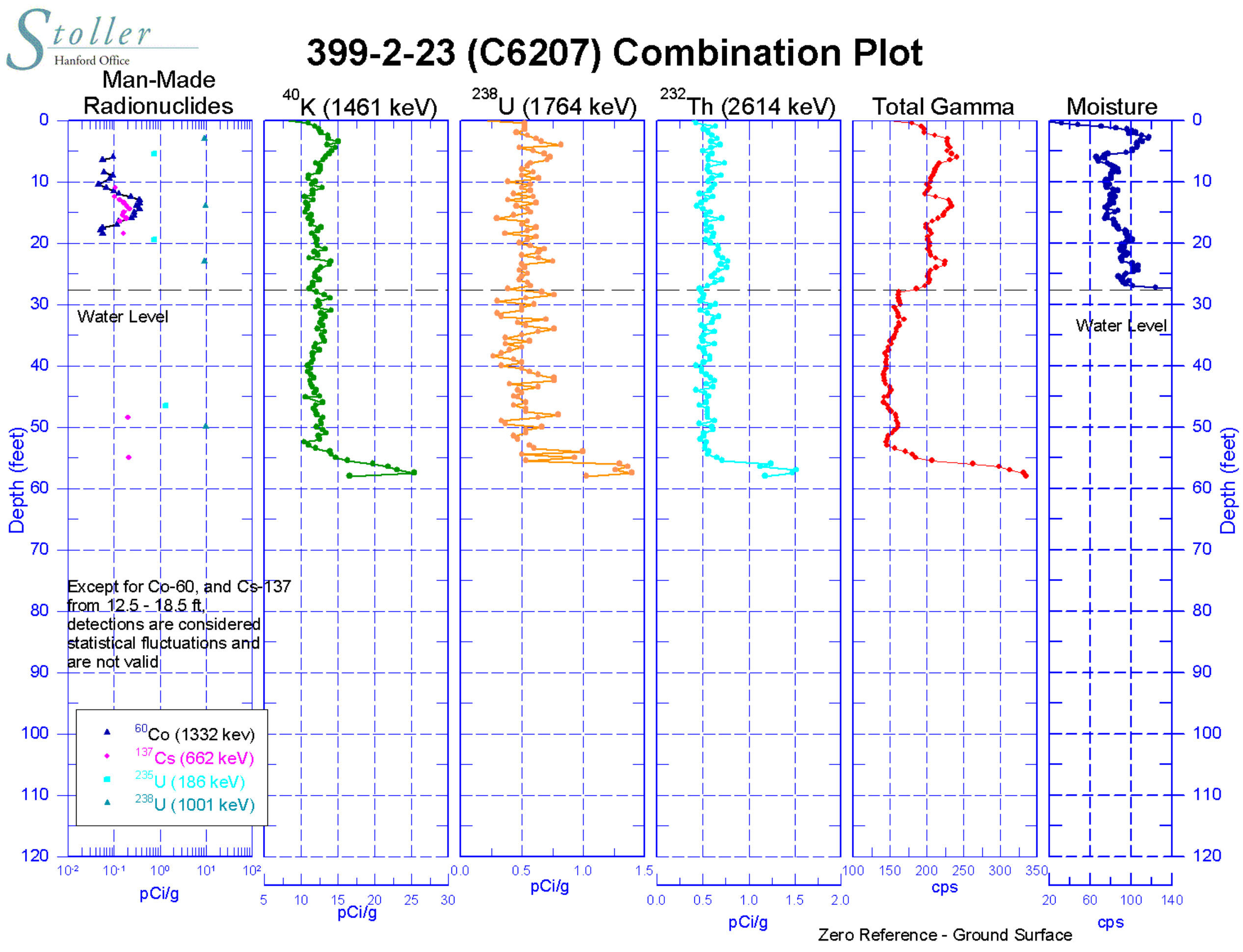


toller 399-2-23 (C6207)

Hanford Office

\section{Total Gamma \& Dead Time}
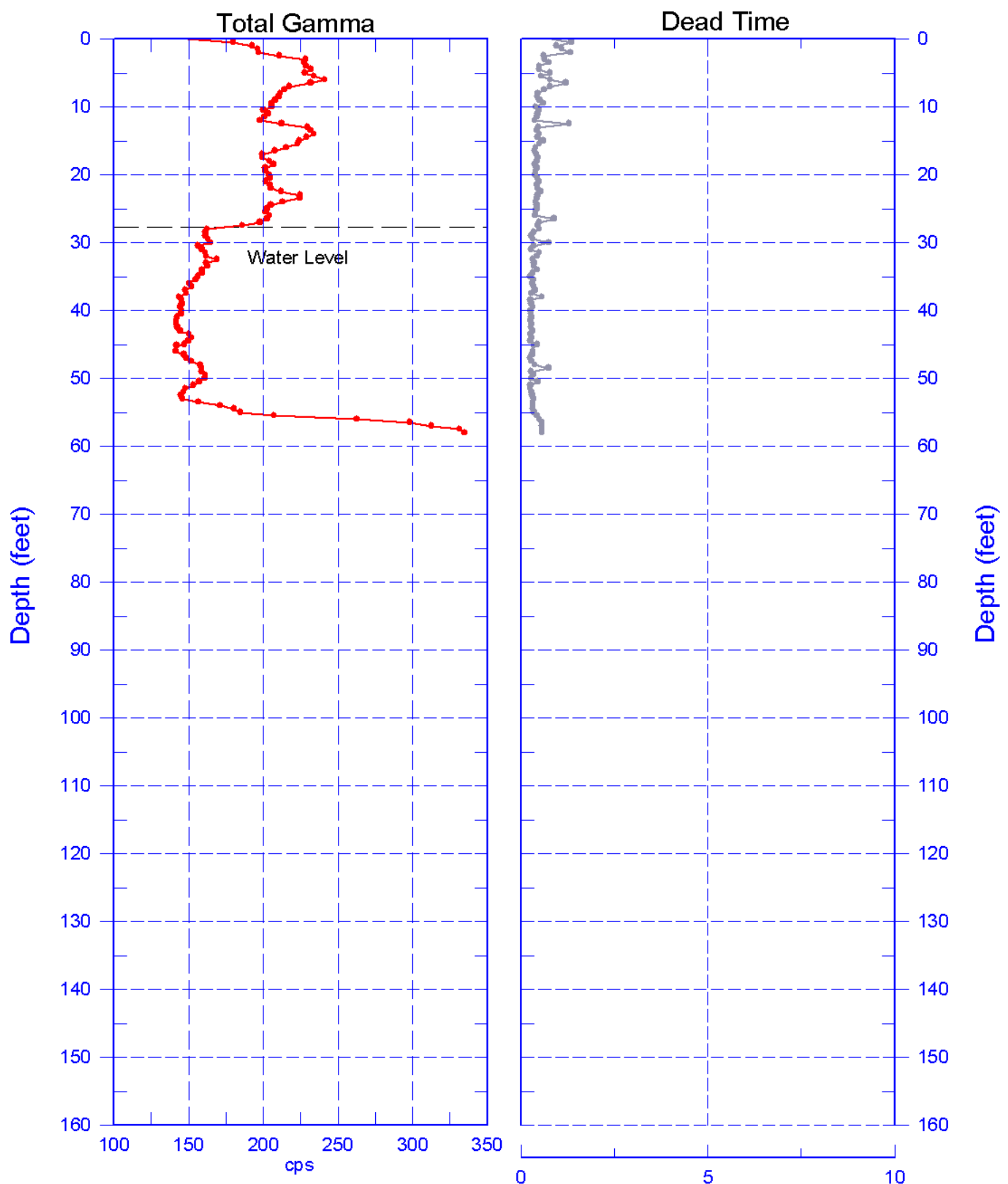

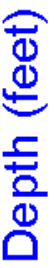

Reference - Ground Surface

G.180 
toller 399-2-23 (C6207)

Hanford Office

\section{Total Gamma \& Moisture}
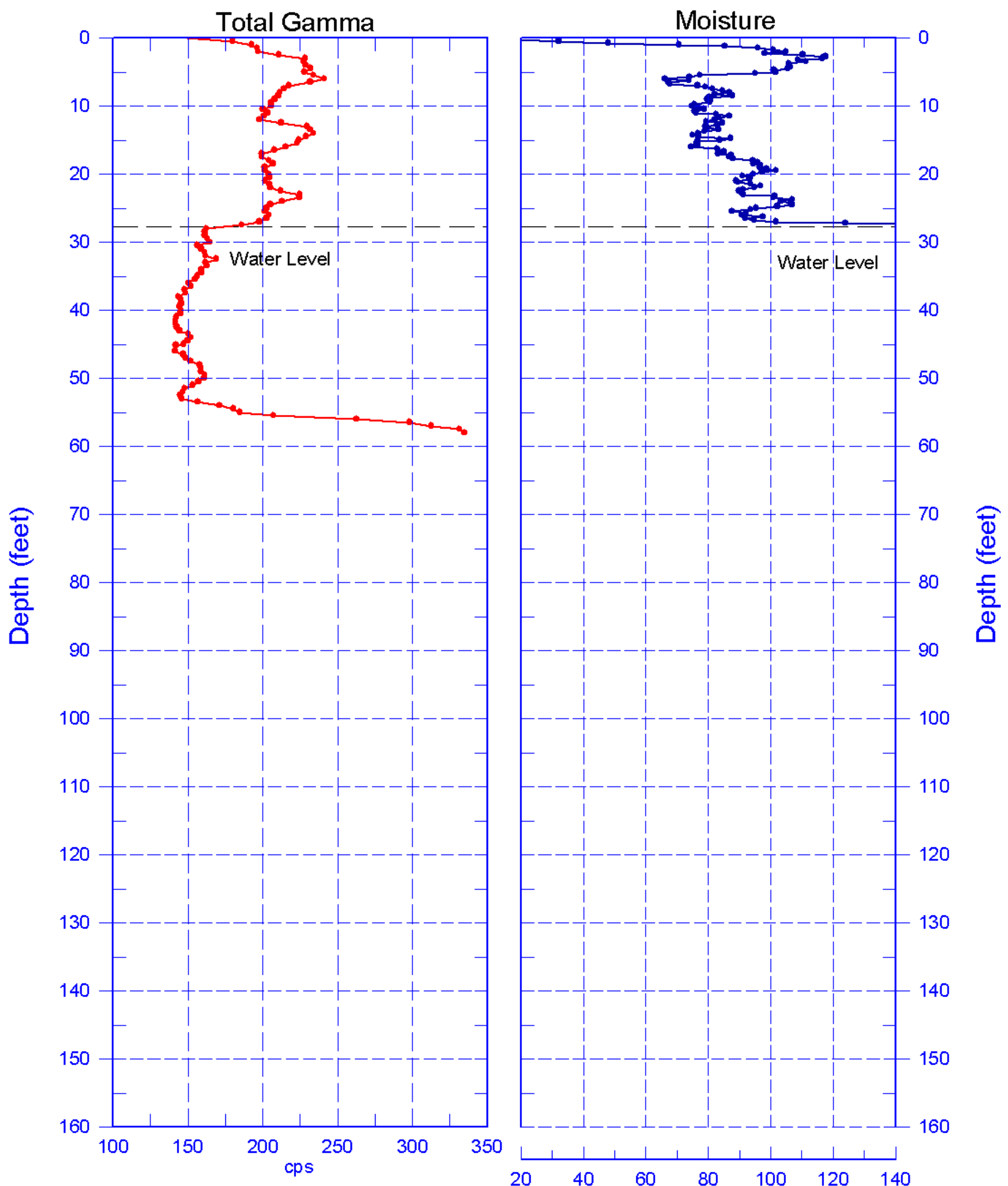

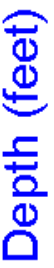

Reference - Ground Surface 
toller 399-2-23 (C6207)

Hanford Office

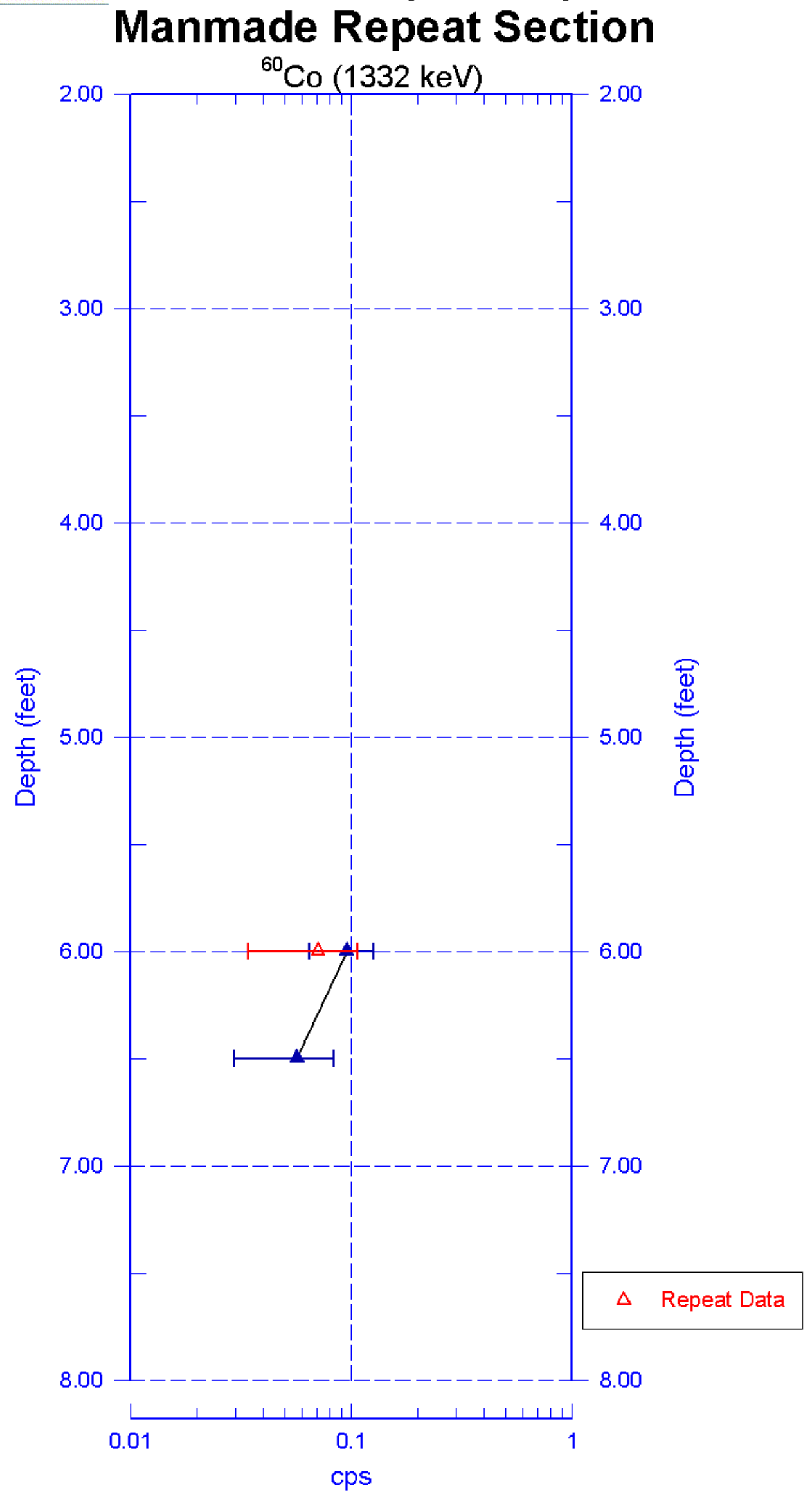

Reference - Ground Surface

G.182 
toller 399-2-23 (C6207)

Hanford Office Repeat Section of Natural Gamma Logs

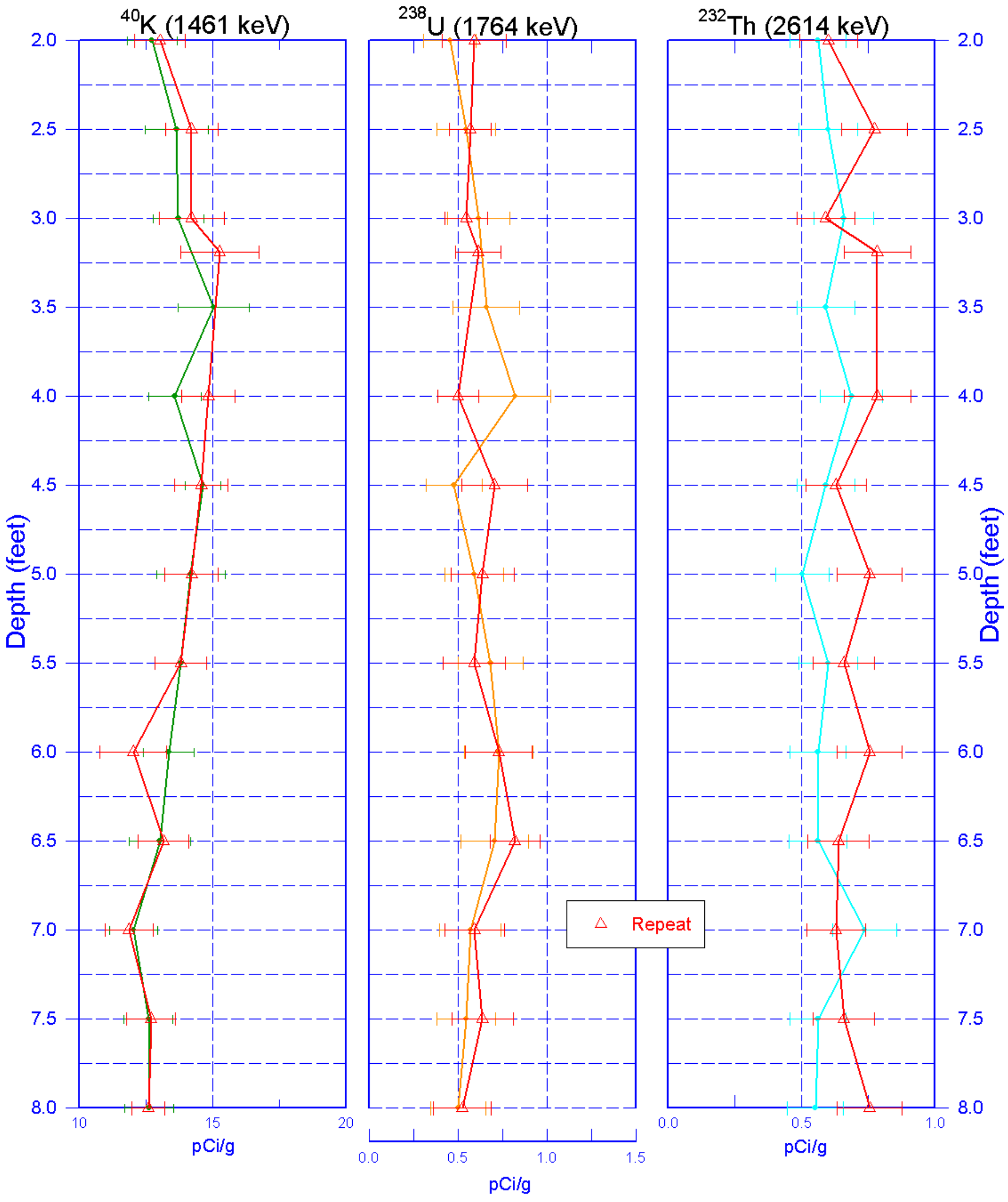

Zero Reference - Ground Surface

G.183 


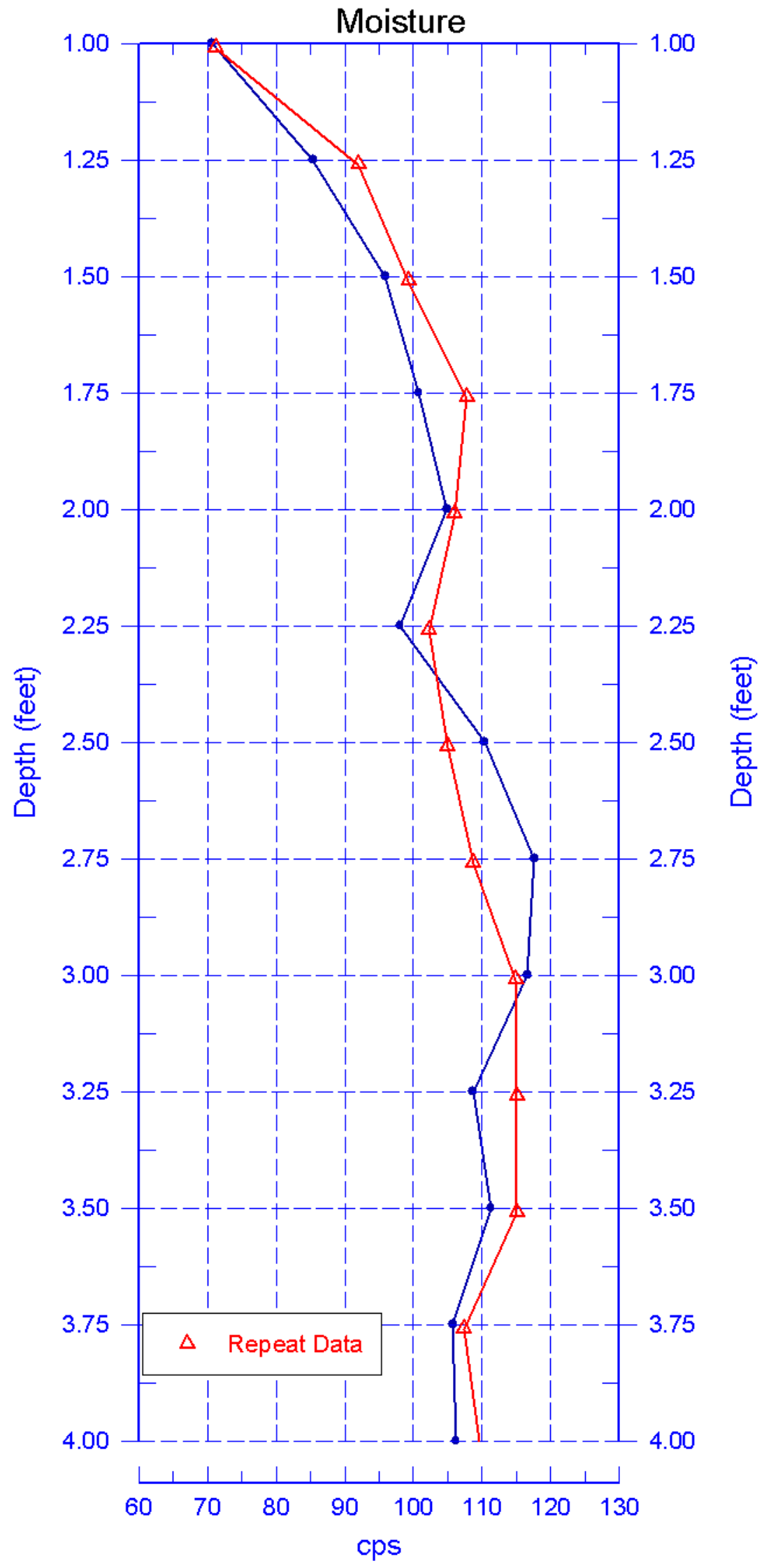




\section{9-2-24 (C6208) Log Data Report}

Borehole Information:

\begin{tabular}{|c|c|c|c|c|c|}
\hline \multirow{2}{*}{\multicolumn{2}{|c|}{$\begin{array}{cc}\text { Borehole: } & \text { 399-2-24 (C6208) } \\
\text { Coordinates (WA St Plane) }\end{array}$}} & \multicolumn{4}{|c|}{ 300-FF-5 } \\
\hline & & $\mathbf{G W L}^{1}$ (f & 26.3 & GWL Date: & $06 / 03 / 08$ \\
\hline North (m) & East (m) & Drill Date & TOC $^{2}$ Elevation & Total Depth (ft) & Type \\
\hline 116063.3659 & 594280.564 & $06 / 02 / 08$ & Unknown & 67.5 & Sonic \\
\hline
\end{tabular}

\section{Casing Information:}

\begin{tabular}{|c|c|c|c|c|c|c|}
\hline Casing Type & Stickup (ft) & $\begin{array}{c}\text { Outer } \\
\text { Diameter (in.) }\end{array}$ & $\begin{array}{c}\text { Inside } \\
\text { Diameter (in.) }\end{array}$ & Thickness (in.) & Top (ft) & Bottom (ft) \\
\hline Threaded Steel & 2.5 & $75 / 8$ & $67 / 8$ & $3 / 8$ & -2.5 & 63.2 \\
\hline
\end{tabular}

\section{Borehole Notes:}

Well site geologist reported depth to bottom, depth to water and depth of casing. Logger measured the casing diameter using a steel tape and rounding to the nearest 1/16-in. The zero reference is the ground surface.

\section{Logging Equipment Information:}

\begin{tabular}{|l|l|l|l|l|}
\hline \multirow{2}{*}{ Logging System: } & \multirow{2}{*}{ Gamma 1 N } & $\begin{array}{l}\text { Type: } \\
\text { Serial No.: }\end{array}$ & $\begin{array}{l}\text { 60\% HPGe SGLS } \\
\text { 45TP22010A }\end{array}$ \\
\hline Effective Calibration Date: & $03 / 28 / 08$ & $\begin{array}{l}\text { Calibration Reference: } \\
\text { Logging Procedure: }\end{array}$ & HGLP-CC-031 \\
\cline { 4 - 4 } & & HGLP-MAN-002, Rev. 0 \\
\hline
\end{tabular}

\begin{tabular}{|l|l|l|l|l|}
\hline Logging System: & \multirow{2}{*}{ Gamma 1 M } & $\begin{array}{l}\text { Type: } \\
\text { Serial No.: }\end{array}$ & $\begin{array}{l}\text { NMLS } \\
\text { H340207279 }\end{array}$ \\
\hline Effective Calibration Date: & $05 / 06 / 08$ & Calibration Reference: & HGLP-CC-032 \\
\hline \multicolumn{2}{|l|}{} & Logging Procedure: & HGLP-MAN-002, Rev. 0 \\
\hline
\end{tabular}

\section{Spectral Gamma Logging System (SGLS) Log Run Information:}

\begin{tabular}{|l|c|c|c|}
\hline Log Run & $\mathbf{1}$ & $\mathbf{2}$ & 3 Repeat \\
\hline Date & $06 / 03 / 08$ & $06 / 04 / 08$ & $06 / 04 / 08$ \\
\hline Logging Engineer & McClellan & McClellan & McClellan \\
\hline Start Depth (ft) & 0.0 & 50.0 & 64.0 \\
\hline Finish Depth (ft) & 51.0 & 65.0 & 57.5 \\
\hline Count Time (sec) & 200 & 200 & 200 \\
\hline Live/Real & $\mathrm{R}$ & $\mathrm{R}$ & $\mathrm{R}$ \\
\hline Shield (Y/N) & $\mathrm{N}$ & $\mathrm{N}$ & $\mathrm{N}$ \\
\hline MSA Interval (ft) & 0.5 & 0.5 & 0.5 \\
\hline Log Speed (ft/min) & N/A & N/A & N/A \\
\hline Pre-Verification & AN079CAB & AN080CAB & AN080CAB \\
\hline Start File & AN079000 & AN080000 & AN080031 \\
\hline Finish File & AN079102 & AN080030 & AN080044 \\
\hline Post-Verification & AN079CAA & AN080CAA & AN080CAA \\
\hline Depth Return Error (in.) & 0 & N/A & 0 \\
\hline Comments & $\begin{array}{c}\text { No fine gain } \\
\text { adjustment } \\
\text { made. }\end{array}$ & $\begin{array}{c}\text { No fine gain } \\
\text { adjustment } \\
\text { made. }\end{array}$ & $\begin{array}{c}\text { No fine gain } \\
\text { adjustment } \\
\text { made. Repeat } \\
\text { section }\end{array}$ \\
\hline
\end{tabular}




\section{Neutron Moisture Logging System (NMLS) Log Run Information:}

\begin{tabular}{|l|c|c|}
\hline Log Run & $\mathbf{4}$ & 5 Repeat \\
\hline Date & $06 / 04 / 08$ & $06 / 04 / 08$ \\
\hline Logging Engineer & McClellan & McClellan \\
\hline Start Depth (ft) & 0.0 & 26.25 \\
\hline Finish Depth (ft) & 26.5 & 20.0 \\
\hline Count Time (sec) & 15 & 15 \\
\hline Live/Real & $\mathrm{R}$ & $\mathrm{R}$ \\
\hline Shield (Y/N) & $\mathrm{N}$ & $\mathrm{N}$ \\
\hline MSA Interval (ft) & 0.25 & 0.25 \\
\hline Log Speed (ft/min) & N/A & N/A \\
\hline Pre-Verification & AM006CAB & AM006CAB \\
\hline Start File & AM006000 & AM006107 \\
\hline Finish File & AM006106 & AM006132 \\
\hline Post-Verification & AM006CAA & AM006CAA \\
\hline Depth Return Error (in.) & N/A & 1.5 high \\
\hline Comments & None & Repeat section. \\
\hline
\end{tabular}

\section{Logging Operation Notes:}

Data were collected using Gamma 1, HO 68B-3574. Spectral log data (SGLS) pre- and post-survey verification measurements were acquired in the Amersham KUTh-118 field verifier. Neutron moisture pre- and post-survey verification measurements were acquired in the AmBe standard. Maximum logging depth was $65.0 \mathrm{ft}$. before the sonde un-weighted. A centralizer was installed on the sondes.

\section{Analysis Notes:}

\begin{tabular}{|l|l|l|l|l|l|}
\hline Analyst: & LEGLER & Date: & 6/24/08 & Reference: & GJO-HGLP 1.6.3, Rev. 0 \\
\hline
\end{tabular}

The pre- and post-survey verification spectra met the acceptance criteria for the established systems. A correction for a 3/8-in. thick casing was applied to SGLS from ground surface to $63.2 \mathrm{ft}$, leaving $1.8 \mathrm{ft}$ of open borehole uncorrected. A water correction was also applied from $26.3 \mathrm{ft}$ to total logged depth.

SGLS spectra were processed in batch mode using APTEC SUPERVISOR to identify individual energy peaks and determine count rates. Concentrations were calculated with an EXCEL worksheet template identified as G1NMar08.xls using efficiency functions and corrections for casing, dead time, and water as determined by annual calibrations.

Moisture data are presented in counts per second because no calibration data exists for a $67 / 8$-in. inner diameter casing.

\section{Results and Interpretations:}

Cs-137, Co-60, U-238 (Pa-234m), and U-235 were detected at several isolated depths in this borehole. Co-60 was detected at $0.12 \mathrm{pCi} / \mathrm{g}$ at $6.5 \mathrm{ft}$. Inspection of the individual spectra for the other isotopes at the various depths indicates these detections are statistical fluctuations and are not valid.

A fine grain sediment layer can be found at around $23 \mathrm{ft}$, as seen by an increase in the total gamma and natural U-238 (1764keV) plots.

The KUT and Moisture plots indicate good repeat ability.

Depth to groundwater is reported to be $33 \mathrm{ft}$ in the Logging Request Tracking Sheet (no date provided). Log data collected on 6/03/08 and 6/04/08 indicate depth to ground water is approximately $26.3 \mathrm{ft}$. The depth to groundwater appears to fluctuate with changes in the river level. 
HGLP-LDR-229, Rev. 0

\section{List of Log Plots:}

Depth Reference is ground surface

Manmade Radionuclides

Natural Gamma Logs

Combination Plot

Total Gamma \& Dead Time

Total Gamma \& Moisture

Repeat Section of Natural Gamma Logs

Moisture Repeat Section

${ }^{1} \mathrm{GWL}$ - groundwater level

${ }^{2}$ TOC - top of casing 


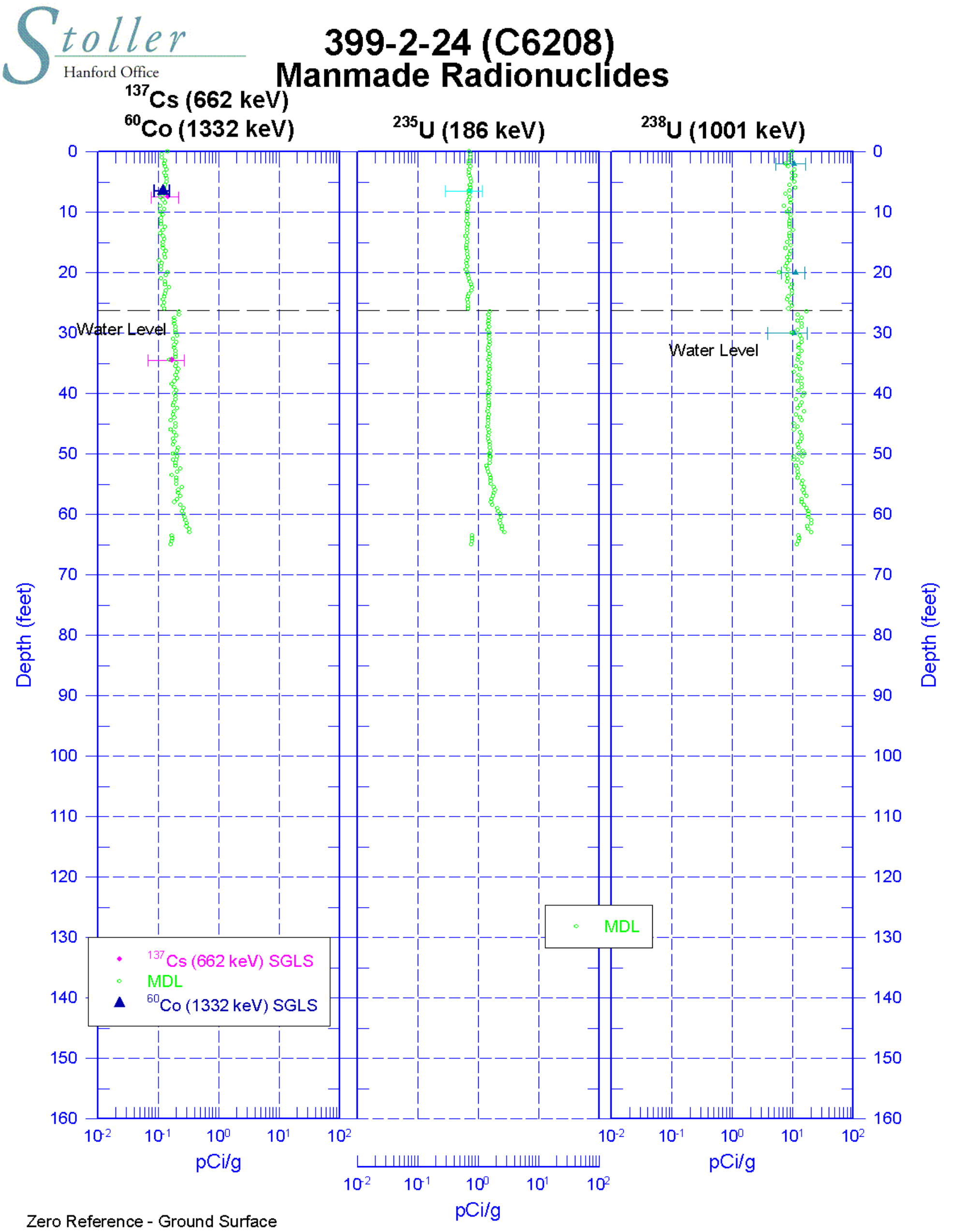

G.188 
toller $\mathbf{3 9 9 - 2 - 2 4}(\mathbf{6 6 2 0 8 )}$

Hanford Office Natural Gamma Logs
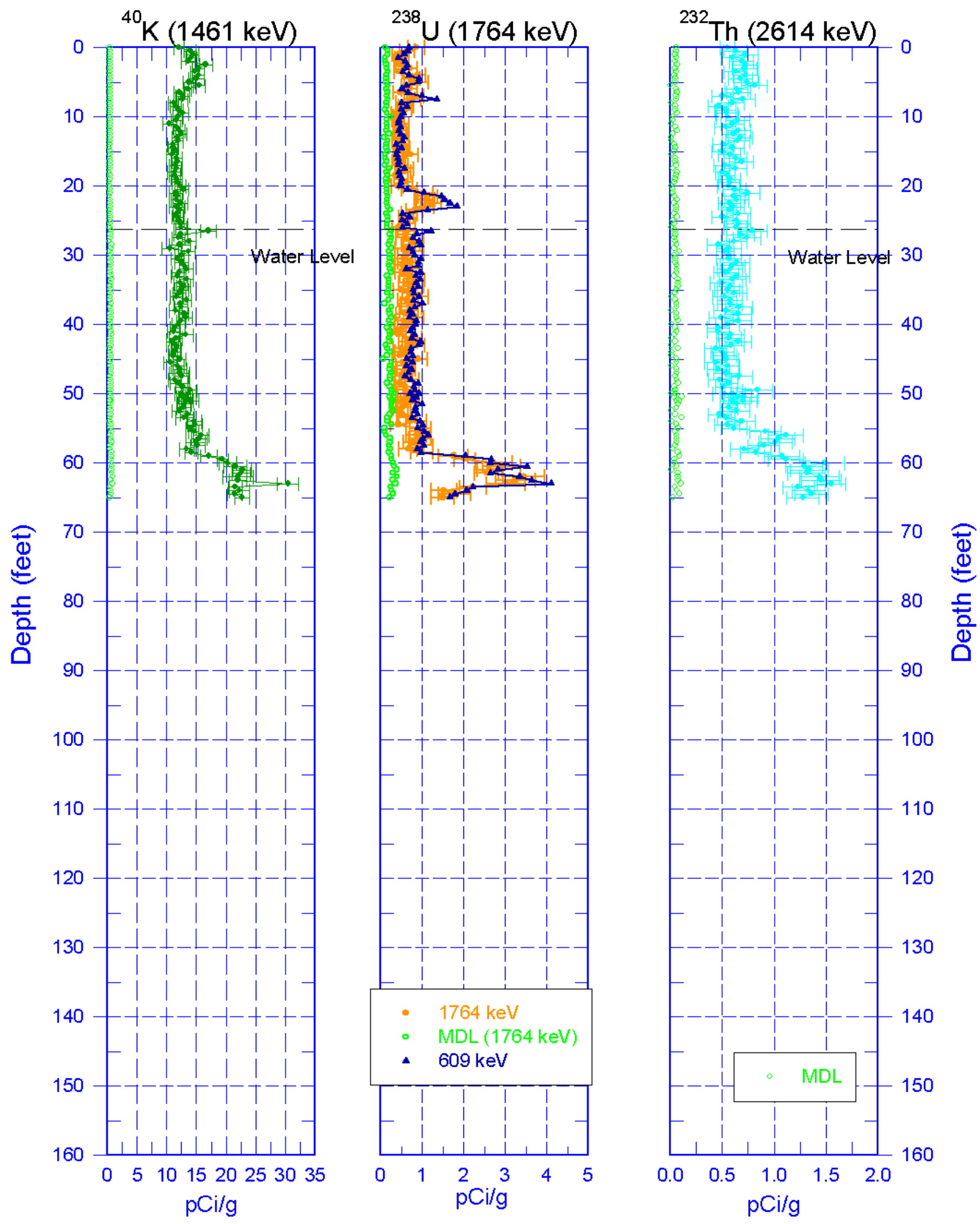

Zero Reference - Ground Surface

G.189 


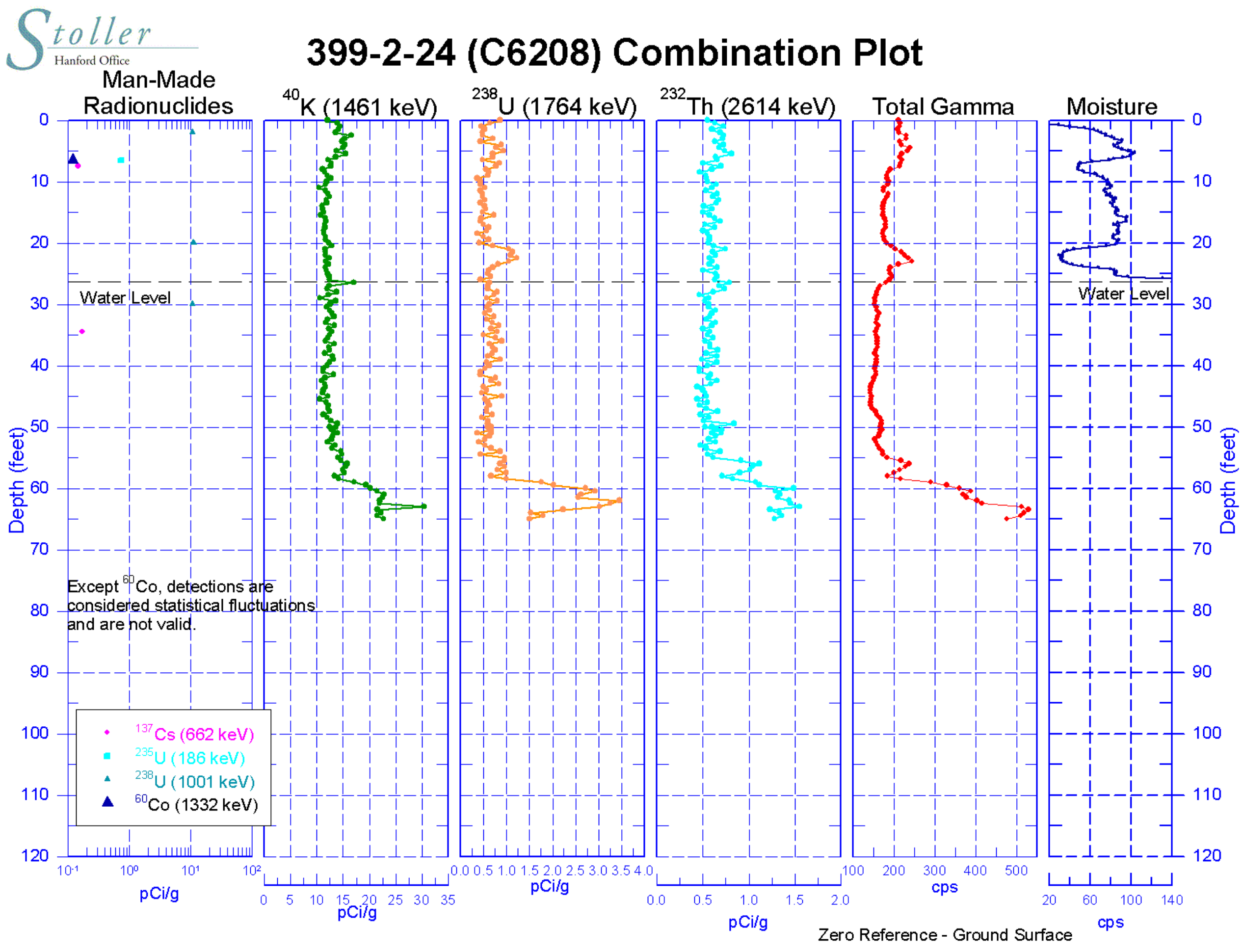


toller 399-2-24 (C6208)

Hanford Office

\section{Total Gamma \& Dead Time}
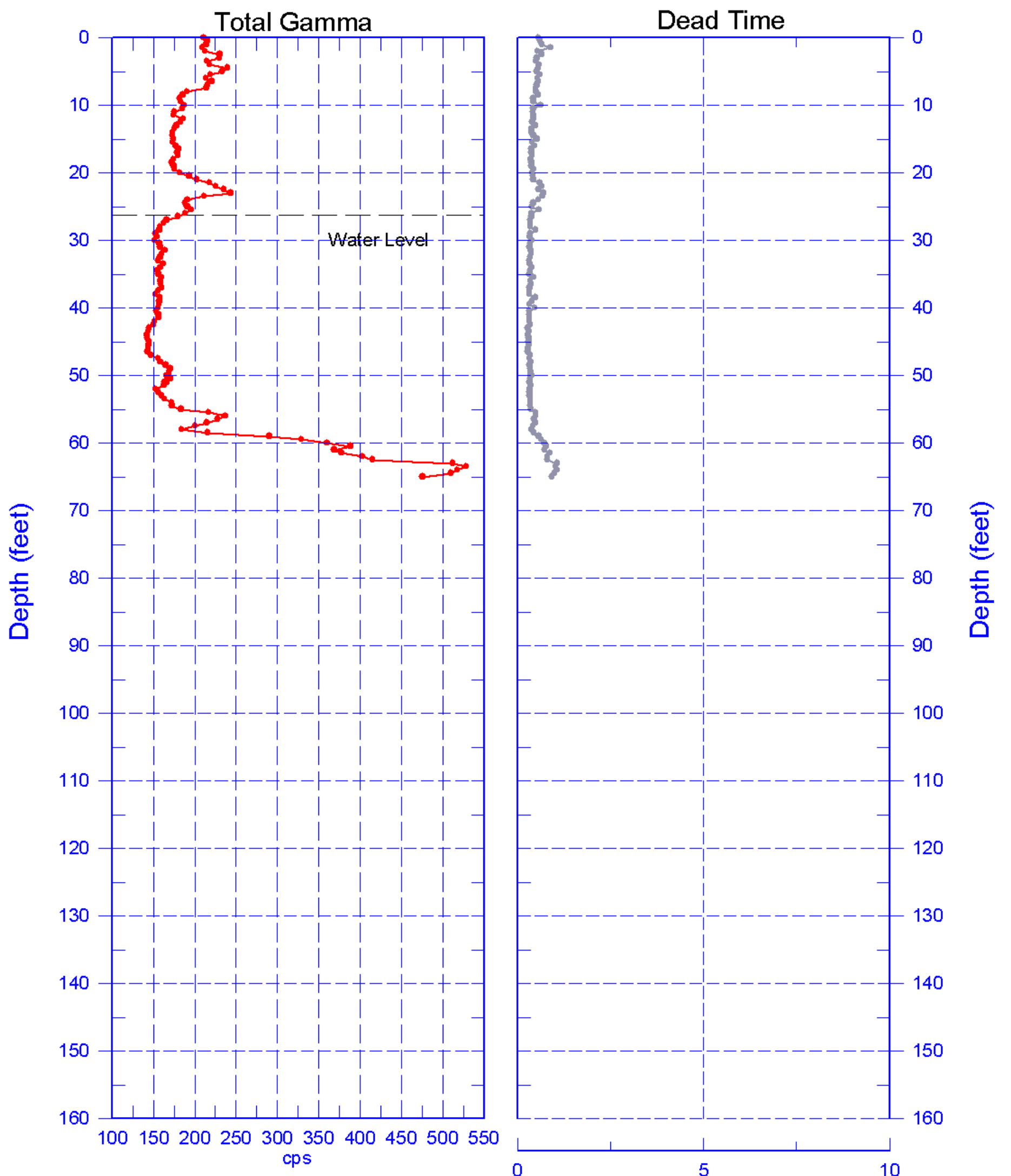

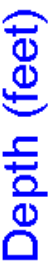

Reference - Ground Surface

G.191 

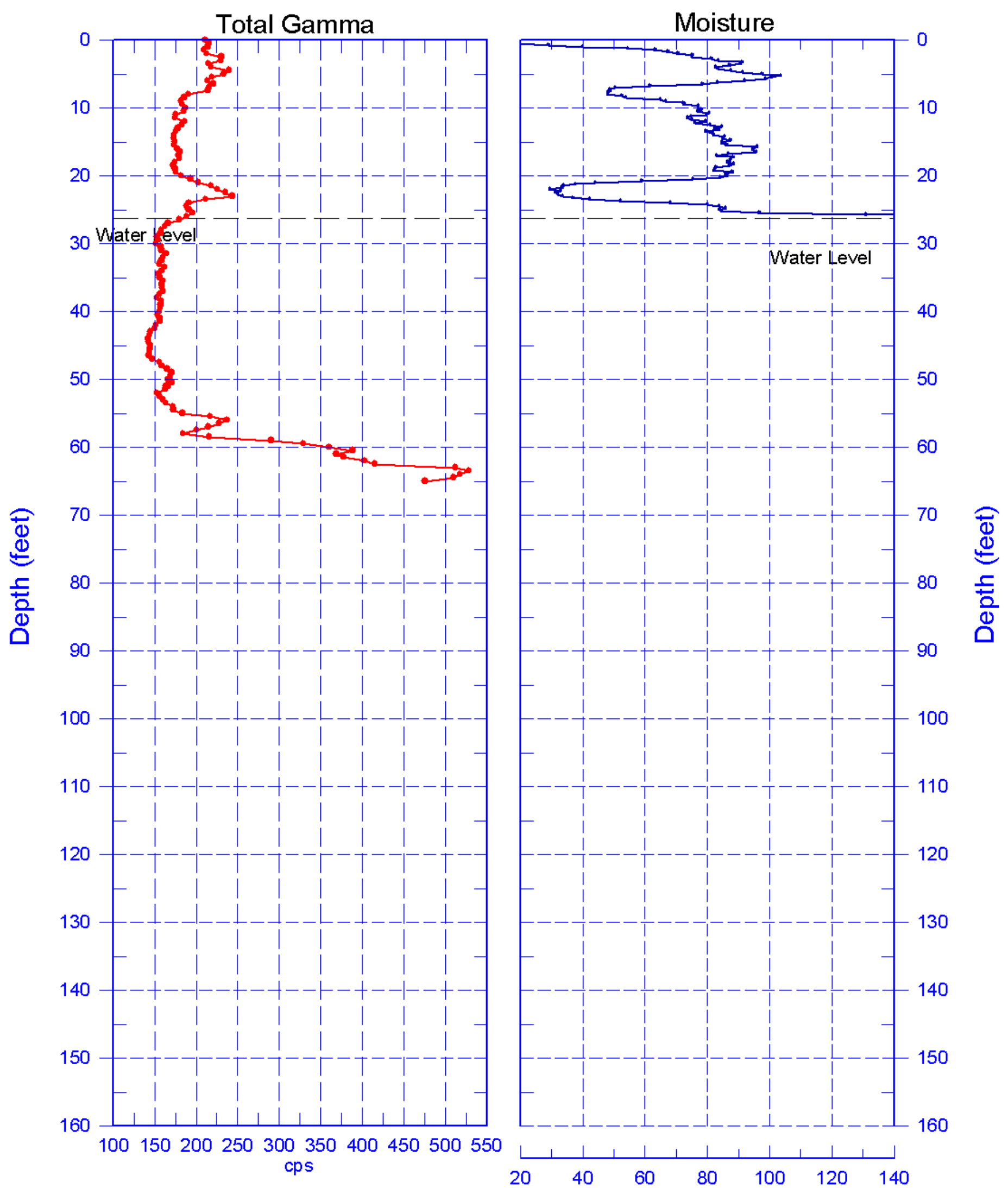


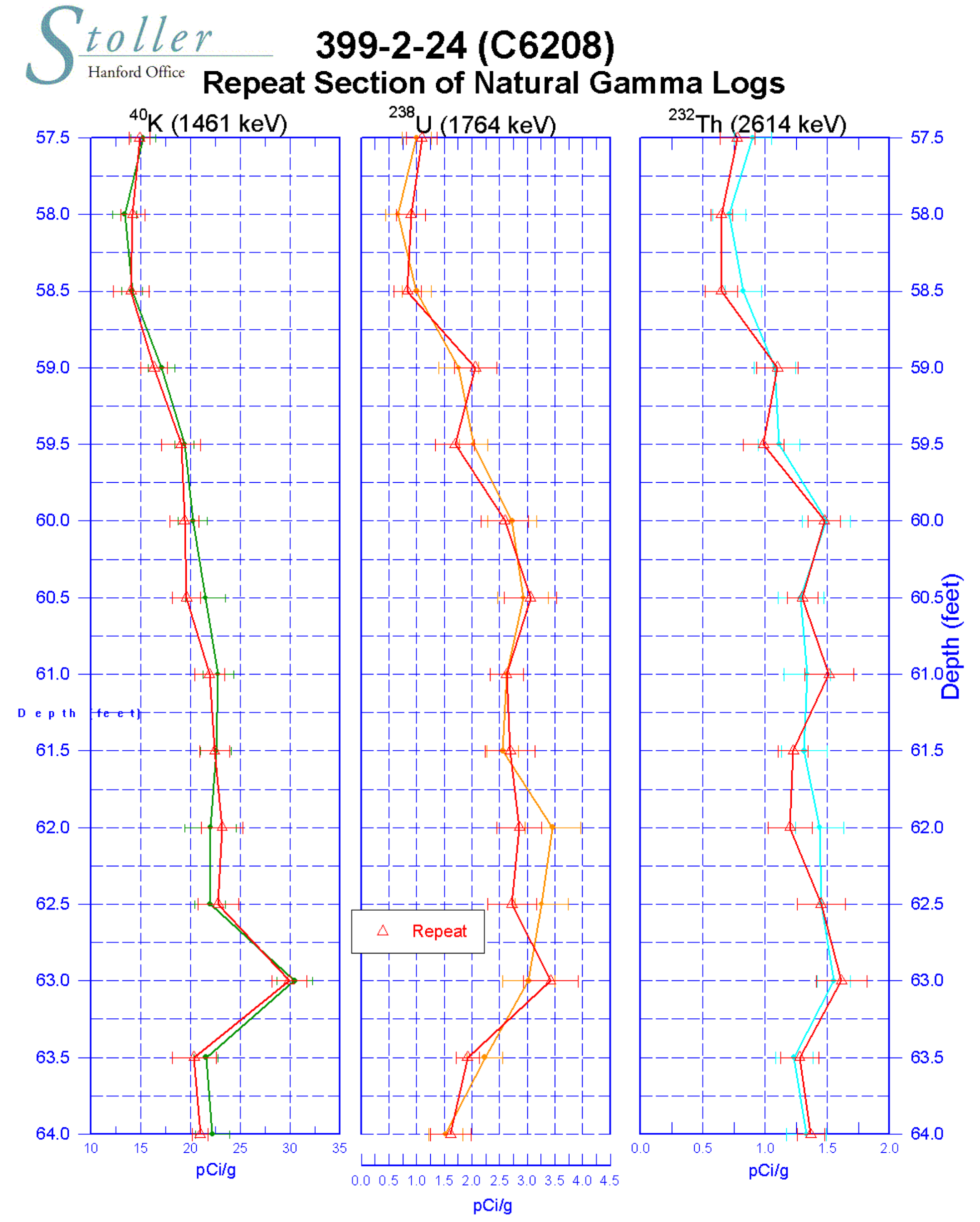




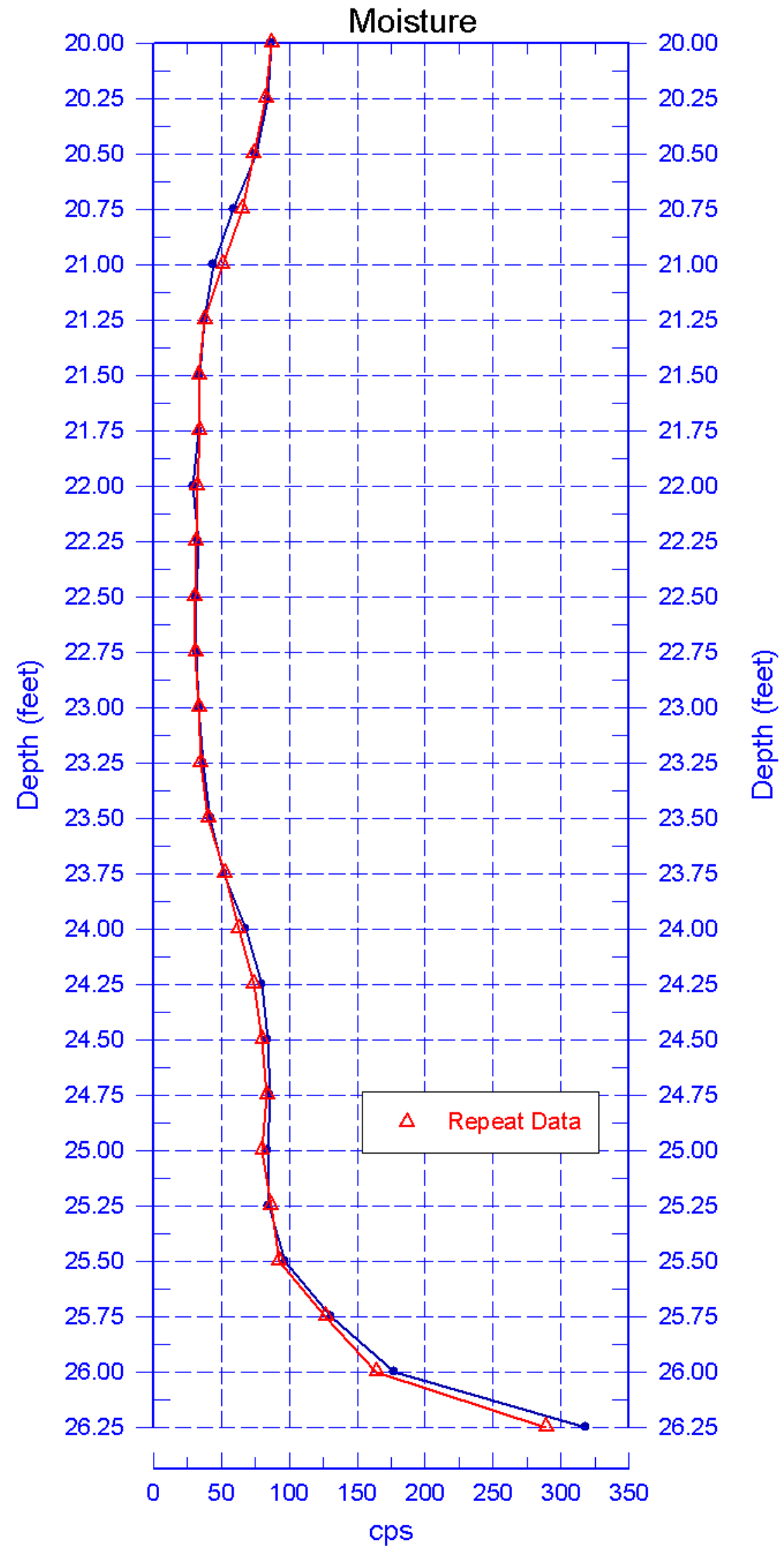




\section{9-2-25 (C6209) Log Data Report}

Borehole Information:

\begin{tabular}{|c|c|c|c|c|c|}
\hline \multirow{2}{*}{\multicolumn{2}{|c|}{ Borehole: $\quad 399-2-25$ (C6209) }} & \multicolumn{4}{|c|}{ 300-FF-5 } \\
\hline & & \multicolumn{2}{|c|}{$\mathbf{G W L}^{1}(\mathbf{f t}): \quad 31.5$} & \multirow{2}{*}{$\begin{array}{c}\text { GWL Date: } \\
\text { Total Depth (ft) }\end{array}$} & $07 / 22 / 08$ \\
\hline North (m) & East (m) & Drill Date & TOC $^{2}$ Elevation & & Type \\
\hline 116008 & 594269 & $07 / 22 / 08$ & Unknown & 170 & Sonic \\
\hline
\end{tabular}

\section{Casing Information:}

\begin{tabular}{|c|c|c|c|c|c|c|}
\hline Casing Type & Stickup (ft) & $\begin{array}{c}\text { Outer } \\
\text { Diameter (in.) }\end{array}$ & $\begin{array}{c}\text { Inside } \\
\text { Diameter (in.) }\end{array}$ & Thickness (in.) & Top (ft) & Bottom (ft) \\
\hline Threaded Steel & 1.3 & $93 / 4$ & $85 / 8$ & $5 / 8$ & -1.3 & 60.0 \\
\hline Threaded Steel & 3.5 & $75 / 8$ & $67 / 8$ & $3 / 8$ & -3.5 & 120.0 \\
\hline Threaded Steel & 3.5 & 6 & $51 / 4$ & $3 / 8$ & -3.5 & 167.0 \\
\hline
\end{tabular}

\section{Borehole Notes:}

Drill site geologist reported casing and total depth information. Casing diameters were measured using a steel tape and rounded to the nearest 1/16-in. An e-tape was employed to measure depth-to-water.

\section{Logging Equipment Information:}

\begin{tabular}{|l|l|l|l|l|}
\hline Logging System: & \multirow{2}{*}{ Gamma 4 L } & $\begin{array}{l}\text { Type: } \\
\text { Serial No.: }\end{array}$ & $\begin{array}{l}\text { 60\% HPGe SGLS } \\
\text { 47TP32211A }\end{array}$ \\
\hline Effective Calibration Date: & $12 / 31 / 2007$ & Calibration Reference: & HGLP-CC-027 \\
\hline \multicolumn{2}{|l|}{} & Logging Procedure: & HGLP-MAN-002, Rev. 0 \\
\hline
\end{tabular}

\begin{tabular}{|l|l|l|l|l|}
\hline Logging System: & \multirow{2}{*}{ Gamma $4 \mathrm{H}$} & $\begin{array}{l}\text { Type: } \\
\text { Serial No.: }\end{array}$ & $\begin{array}{l}\text { NMLS } \\
\text { H310700352 }\end{array}$ \\
\hline Effective Calibration Date: & $11 / 06 / 2007$ & Calibration Reference: & HGLP-CC-021 \\
\hline \multicolumn{2}{|l|}{} & Logging Procedure: & HGLP-MAN-002, Rev. 0 \\
\hline
\end{tabular}

Spectral Gamma Logging System (SGLS) Log Run Information:

\begin{tabular}{|l|c|c|c|c|c|}
\hline Log Run & $\mathbf{1}$ & 2 Repeat & $\mathbf{5}$ & $\mathbf{6 ~ R e p e a t ~}$ & $\mathbf{7}$ \\
\hline Date & $07 / 22 / 08$ & $07 / 23 / 08$ & $07 / 24 / 08$ & $07 / 24 / 08$ & $07 / 27 / 08$ \\
\hline Logging Engineer & Pearson/Pope & Pearson/Pope & McClellan & McClellan & Pearson \\
\hline Start Depth (ft) & 61.5 & 20.0 & 124.0 & 67.0 & 169.5 \\
\hline Finish Depth (ft) & 0 & 14.0 & 60.0 & 61.0 & 120.0 \\
\hline Count Time (sec) & 200 & 200 & 200 & 200 & 200 \\
\hline Live/Real & $\mathrm{R}$ & $\mathrm{R}$ & $\mathrm{R}$ & $\mathrm{R}$ & $\mathrm{R}$ \\
\hline Shield (Y/N) & $\mathrm{N}$ & $\mathrm{N}$ & $\mathrm{N}$ & $\mathrm{N}$ & $\mathrm{N}$ \\
\hline MSA Interval (ft) & 0.5 & 0.5 & 0.5 & 0.5 & 0.5 \\
\hline Log Speed (ft/min) & $\mathrm{NA}$ & $\mathrm{NA}$ & $\mathrm{NA}$ & $\mathrm{NA}$ & NA \\
\hline Pre-Verification & DL681CAB & DL681CAB & DL691CAB & DL691CAB & DL701CAB \\
\hline Start File & DL681000 & DL681124 & DL691000 & DL691129 & DL701000 \\
\hline Finish File & DL681123 & DL681136 & DL691128 & DL691141 & DL71099 \\
\hline Post-Verification & DL681CAA & DL681CAA & DL691CAA & DL691CAA & DL701CAA \\
\hline Depth Return Error (in.) & Low 2.75 & Low 0.25 & NA & Low 2.0 & NA \\
\hline
\end{tabular}


HGLP-LDR-261, Rev. 0

\begin{tabular}{|l|l|l|l|l|l|}
\hline Log Run & \multicolumn{1}{|c|}{$\mathbf{1}$} & 2 Repeat & \multicolumn{1}{c|}{$\mathbf{5}$} & 6 Repeat & $\mathbf{7}$ \\
\hline Comments & None & $\begin{array}{l}\text { Repeat section. } \\
\text { Re-zeroed } \\
\text { detector at start. }\end{array}$ & None & Repeat section & None \\
\hline
\end{tabular}

Spectral Gamma Logging System (SGLS) Log Run Information:

\begin{tabular}{|l|c|l|l|l|l|}
\hline Log Run & 8 Repeat & & & & \\
\hline Date & $07 / 27 / 08$ & & & & \\
\hline Logging Engineer & Pearson & & & & \\
\hline Start Depth (ft) & 130.0 & & & & \\
\hline Finish Depth (ft) & 125.0 & & & & \\
\hline Count Time (sec) & 200 & & & & \\
\hline Live/Real & R & & & & \\
\hline Shield (Y/N) & N & & & & \\
\hline MSA Interval (ft) & 0.5 & & & & \\
\hline Log Speed (ft/min) & NA & & & & \\
\hline Pre-Verification & DL701CAB & & & & \\
\hline Start File & DL701100 & & & & \\
\hline Finish File & DL701110 & & & & \\
\hline Post-Verification & DL701CAA & & & & \\
\hline Depth Return Error (in.) & Low 2.0 & & & & \\
\hline Comments & None & & & & \\
\hline
\end{tabular}

Neutron Moisture Logging System (NMLS) Log Run Information:

\begin{tabular}{|l|c|c|l|l|l|}
\hline Log Run & $\mathbf{3}$ & 4 Repeat & & & \\
\hline Date & $07 / 22 / 08$ & $07 / 22 / 08$ & & & \\
\hline Logging Engineer & Pearson & Pearson & & & \\
\hline Start Depth (ft) & 0 & 4.0 & & & \\
\hline Finish Depth (ft) & 31.5 & 7.0 & & & \\
\hline Count Time (sec) & 15 & 15 & & & \\
\hline Live/Real & $\mathrm{R}$ & $\mathrm{R}$ & & & \\
\hline Shield (Y/N) & $\mathrm{N}$ & $\mathrm{N}$ & & & \\
\hline MSA Interval (ft) & 0.25 & 0.25 & & & \\
\hline Log Speed (ft/min) & NA & NA & & & \\
\hline Pre-Verification & DHH42CAB & DHH42CAB & & & \\
\hline Start File & DHH42000 & DHH42127 & & & \\
\hline Finish File & DHH42126 & DHH42139 & & & \\
\hline Post-Verification & DHH42CAA & DHH42CAA & & & \\
\hline Depth Return Error (in.) & NA & NA & & & \\
\hline Comments & None & Repeat section & & & \\
\hline
\end{tabular}

\section{Logging Operation Notes:}

SGLS and NMLS data were both collected using Gamma 4, HO 68B-3573. Three logging events took place, each after a different casing depth was reached.

SGLS pre- and post-survey verification measurements were acquired in the Amersham KUTh-115 field verifier. Maximum SGLS logging depth achieved was $169.5 \mathrm{ft}$ or approximately $2.5 \mathrm{ft}$ below casing. A centralizer was installed on the sonde. 
HGLP-LDR-261, Rev. 0

Analysis Notes:

\begin{tabular}{|l|l|l|l|l|l|}
\hline Analyst: & R. SPATZ & Date: & $10 / 08 / 08$ & Reference: & GJO-HGLP 1.6.3, Rev. 0 \\
\hline
\end{tabular}

Pre- and post-survey verification spectra for the SGLS and NMLS met the acceptance criteria for the established systems.

SGLS spectra were processed in batch mode using APTEC SUPERVISOR to identify individual peaks and count rates. Concentrations were calculated using an EXCEL template identified as G4LDec07.xls using an efficiency function and corrections for casing, dead time, and water as determined by annual calibrations.

Analyses of log data suggest slight discrepancies with field reports of depth-to-water and casing depth. Corrections of log data were applied according to the analysis assumptions in the tables below.

Casing One

\begin{tabular}{|c|c|c|c|l|}
\hline Top (ft) & Bottom (ft) & $\begin{array}{c}\text { T } \\
\text { (in) }\end{array}$ & $\begin{array}{c}\text { ID } \\
\text { (in) }\end{array}$ & Comments \\
\hline 0 & 31.0 & 0.625 & N/A & No water correction \\
\hline 31.5 & 59.0 & 0.625 & 8.625 & $\begin{array}{l}\text { GWL reported @ 31.5 ft; no adjustment made } \\
\text { Casing bottom reported @ 60.0 ft; adjusted to 59.0 ft }\end{array}$ \\
\hline 59.5 & 62.5 & 0 & 0 & No adjustment made; open hole interval \\
\hline
\end{tabular}

$\mathrm{T}=$ casing thickness used to calculate energy-dependent casing correction factor

ID = casing inside diameter used to calculate energy-dependent water correction factor

Casing Two

\begin{tabular}{|c|c|c|c|l|}
\hline Top (ft) & Bottom (ft) & $\begin{array}{c}\text { T } \\
\text { (in) }\end{array}$ & $\begin{array}{l}\text { ID } \\
\text { (in) }\end{array}$ & Comments \\
\hline 60.0 & 121.5 & 0.375 & 6.875 & Casing bottom reported @ 120.0 ft; adjusted to 121.5 ft \\
\hline 122.0 & 124.0 & 0 & 0 & No adjustment made; open hole interval \\
\hline
\end{tabular}

Casing Three

\begin{tabular}{|c|c|c|c|l|}
\hline Top (ft) & Bottom (ft) & $\begin{array}{c}\text { T } \\
\text { (in) }\end{array}$ & $\begin{array}{l}\text { ID } \\
\text { (in) }\end{array}$ & Comments \\
\hline 120.0 & 167.0 & 0.375 & 5.25 & Casing bottom reported @ 167.0 ft; no adjustment \\
\hline 167.5 & 169.5 & 0 & 6 & Water correction; from 0 to 6-in.; open hole interval \\
\hline
\end{tabular}

Open hole and overlap data were removed from log plots to take away interpretation ambiguities and make the log plots more consistent. Log plot adjustments were applied according to the table below.

\begin{tabular}{|c|c|c|c|l|}
\hline Casing & $\begin{array}{c}\text { Top } \\
\text { (ft) }\end{array}$ & $\begin{array}{c}\text { Bottom } \\
\text { (ft) }\end{array}$ & $\begin{array}{c}\text { Log } \\
\text { Run }\end{array}$ & Comments \\
\hline 1 & 60.0 & 62.5 & 1 & \multirow{2}{*}{ Data removed from log plots } \\
\hline 2 & 122.0 & 124.0 & 5 & \\
\hline 3 & 120.0 & 121.5 & 7 & \\
\hline
\end{tabular}

\section{Results and Interpretations:}

U-238 (Pa-234m) was detected from 4.0 to 5.5 feet. The maximum concentration detected was $16.9 \mathrm{pCi} / \mathrm{g}$ at both the 4.0 and $4.5 \mathrm{ft}$ depths.

Cs-137, U-235, and U-238 (Pa-234m) were detected sporadically in this borehole. Inspection of the individual spectra at these depths indicates that these detections with the exception of Pa-234m between 4.0 and $5.5 \mathrm{ft}$ are statistical fluctuations are not considered valid. No Co-60 was detected. 
HGLP-LDR-261, Rev. 0

Between the depths of 55.0 and $67.0 \mathrm{ft}$, and between 122.0 and $130.0 \mathrm{ft}$, and below the $160.0 \mathrm{ft}$ depth, are probable lithologic changes, which are indicated by the changes in KUT concentrations and Total Gamma count rates.

Increased concentrations of natural uranium occur between 124.0 and $128.0 \mathrm{ft}$, which are shown on the Natural Gamma Log Plots.

The KUT and Moisture plots both indicate good repeatability.

\section{List of Log Plots:}

Depth Reference is ground surface

Manmade Radionuclides

Natural Gamma Logs

Combination Plot (2 pages)

Total Gamma \& Dead Time

Total Gamma \& Moisture

Repeat Section of Natural Gamma Logs (3 pages)

Moisture Repeat Section

${ }^{1} \mathrm{GWL}$ - groundwater level

${ }^{2}$ TOC - top of casing 

Hanford Office Manmade Radionuclides

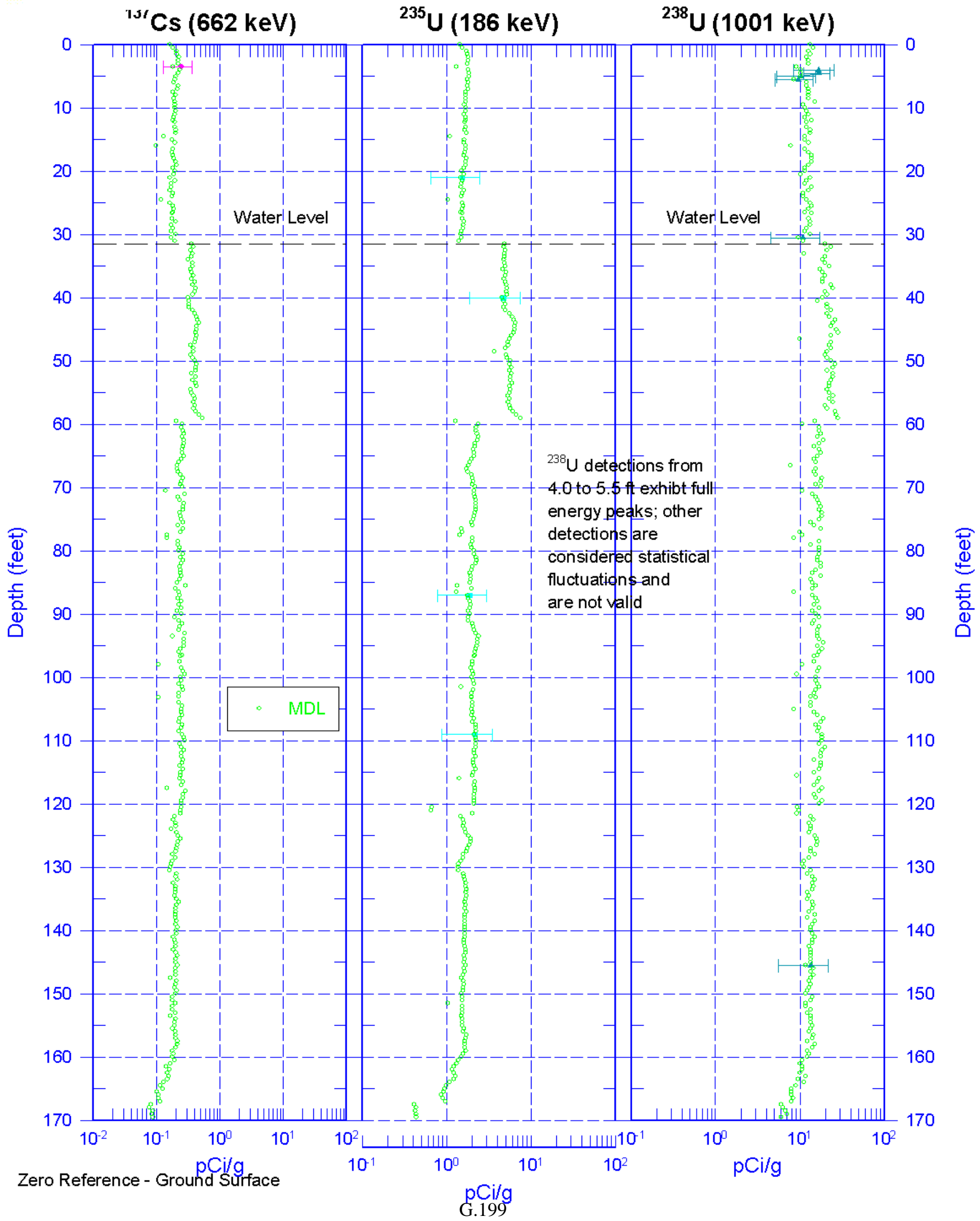


7

toller $\mathbf{3 9 9 - 2 - 2 5}$ (C6209)

Huaford Oficic Natural Gamma Logs

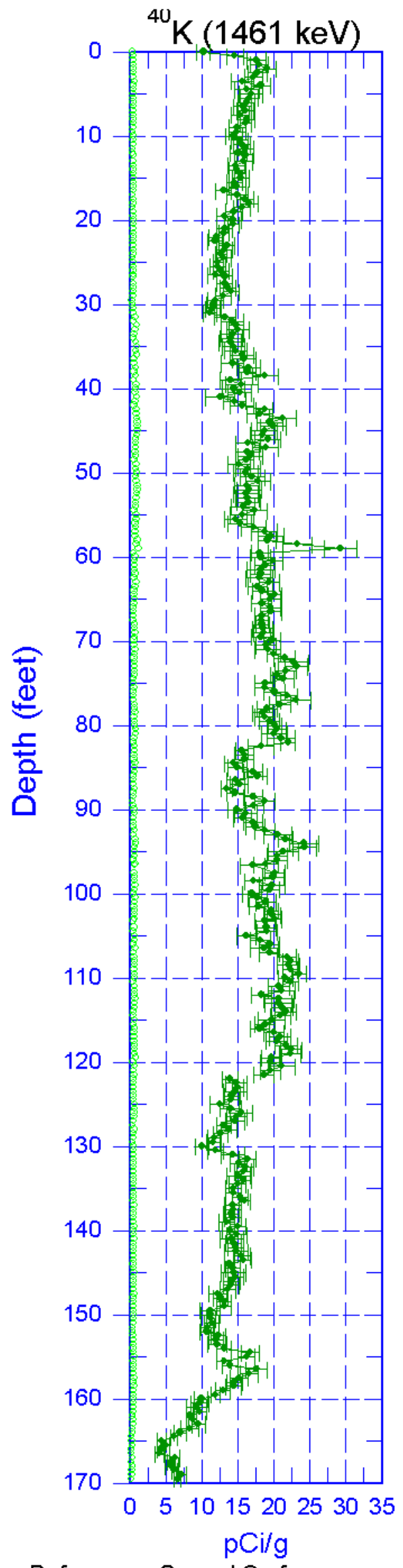

Zero Reference - Ground Surface

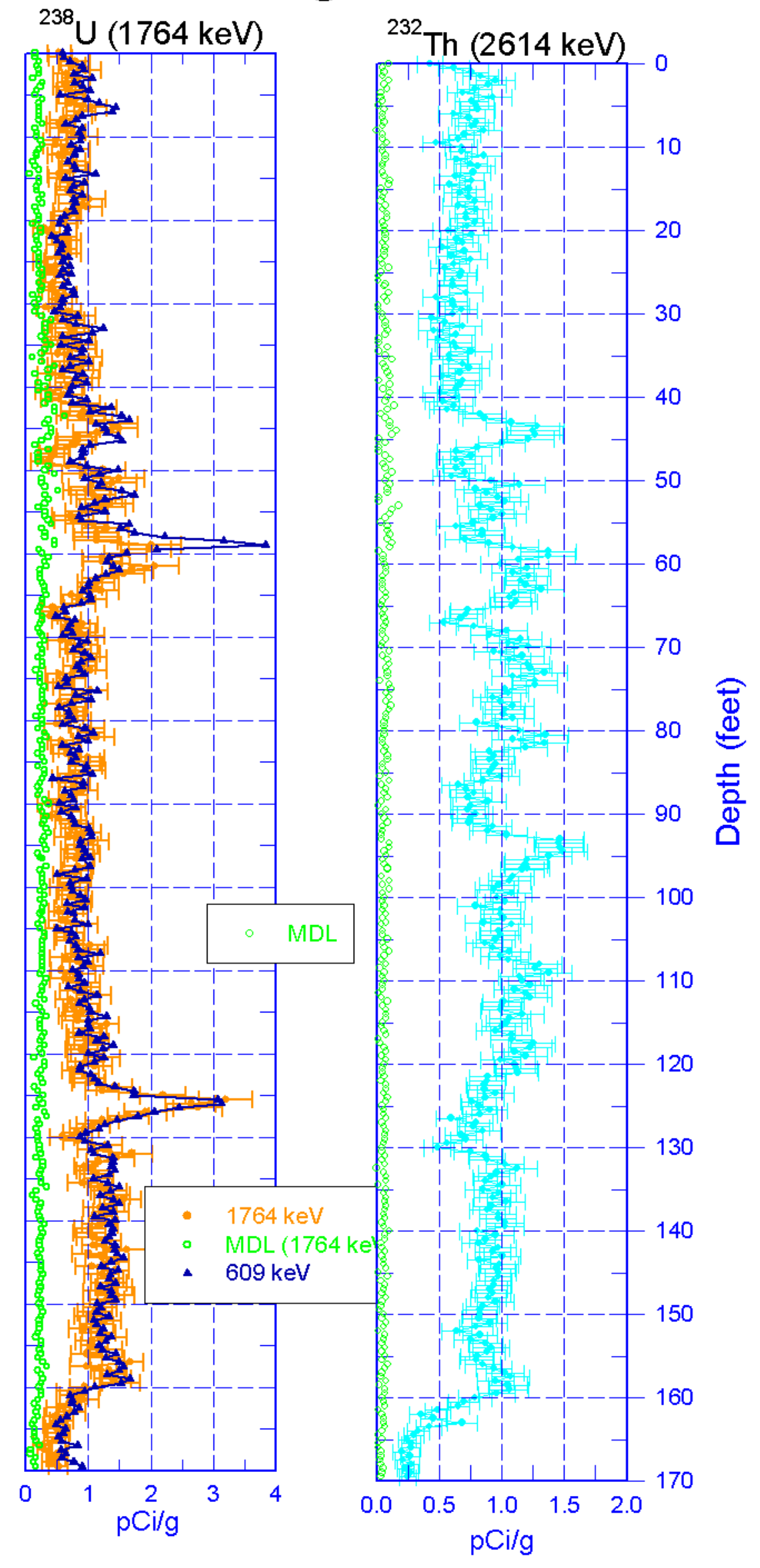

G.200 


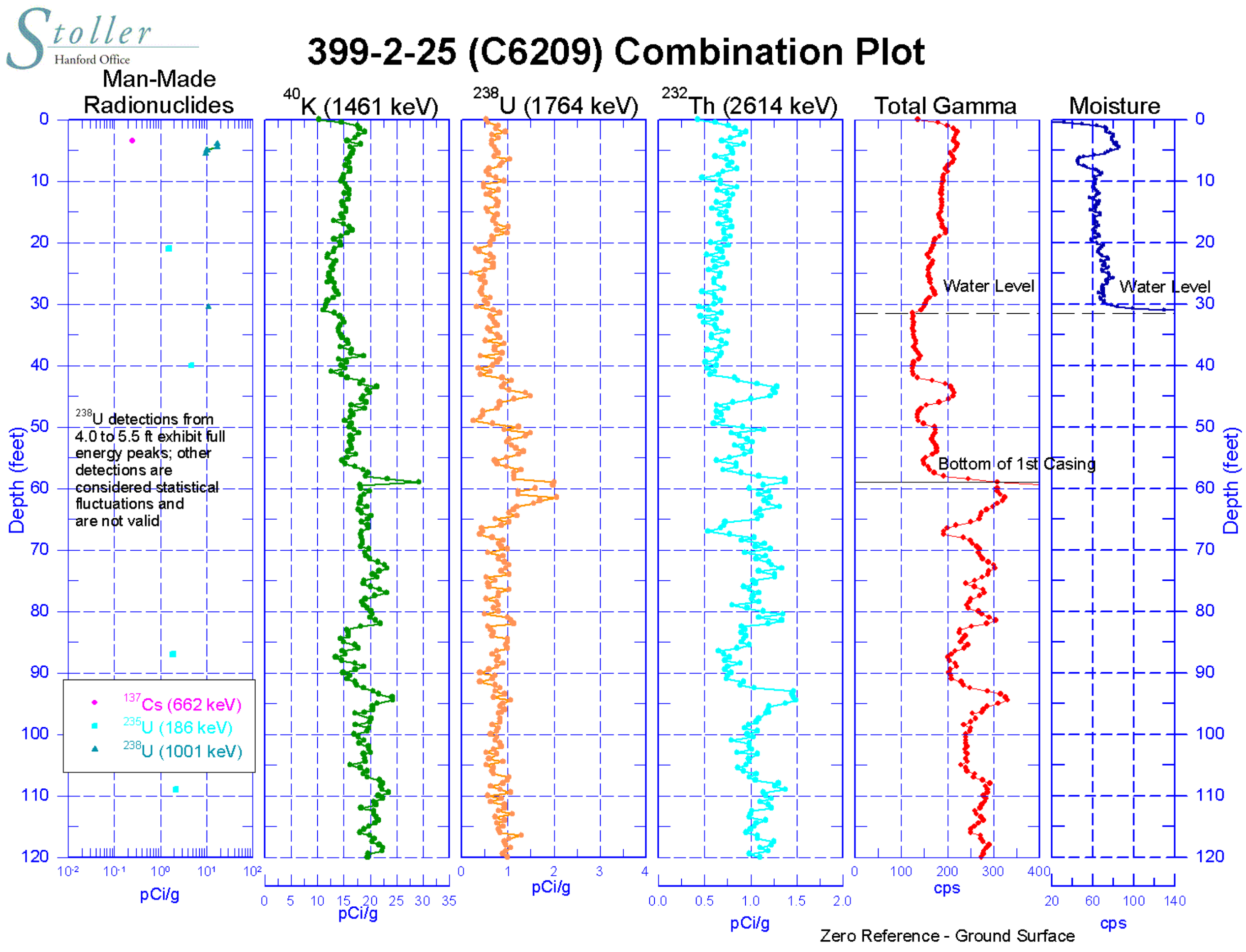




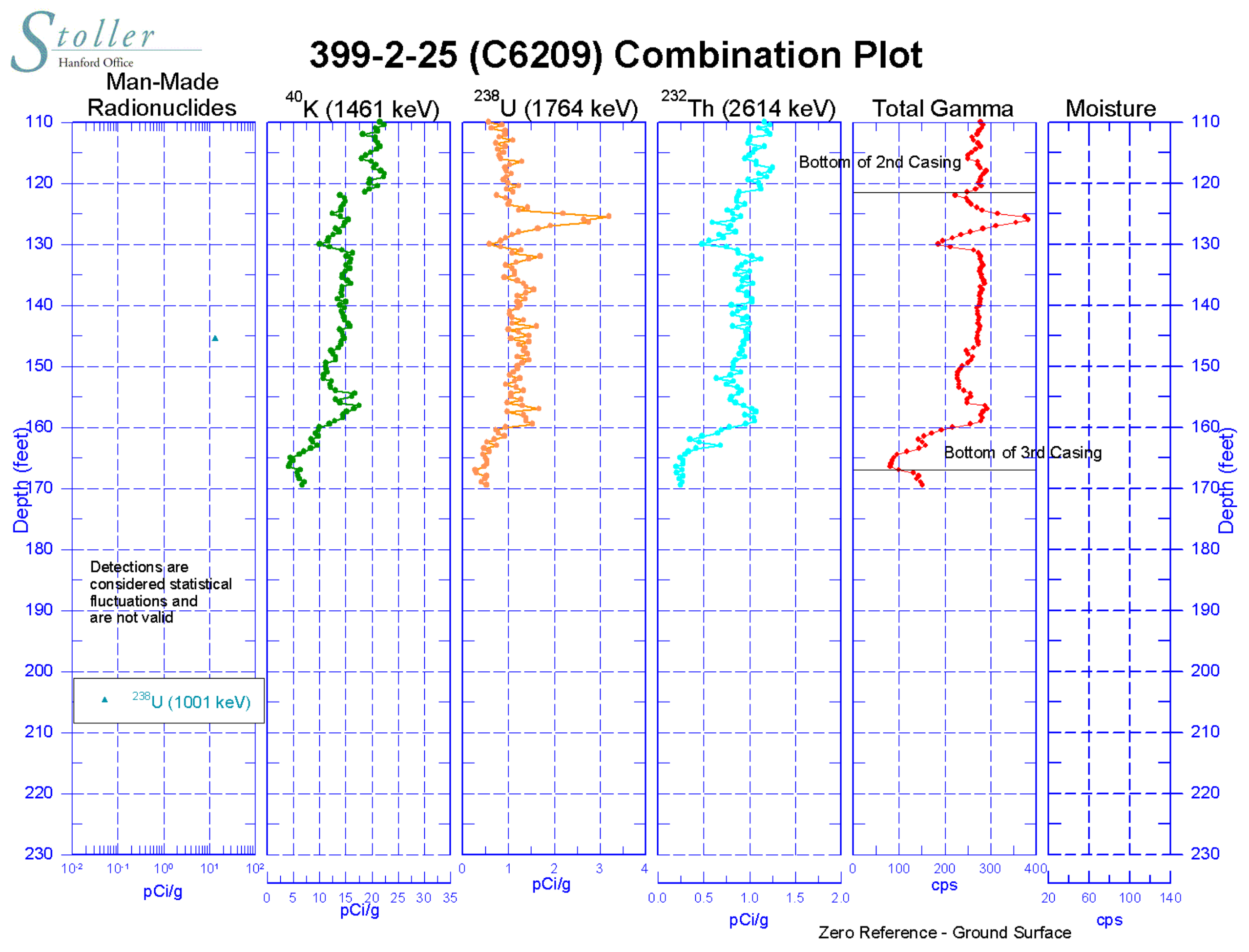




\section{toller 399-2-25 (C6209) \\ Hanford Office \\ Total Gamma \& Dead Time}
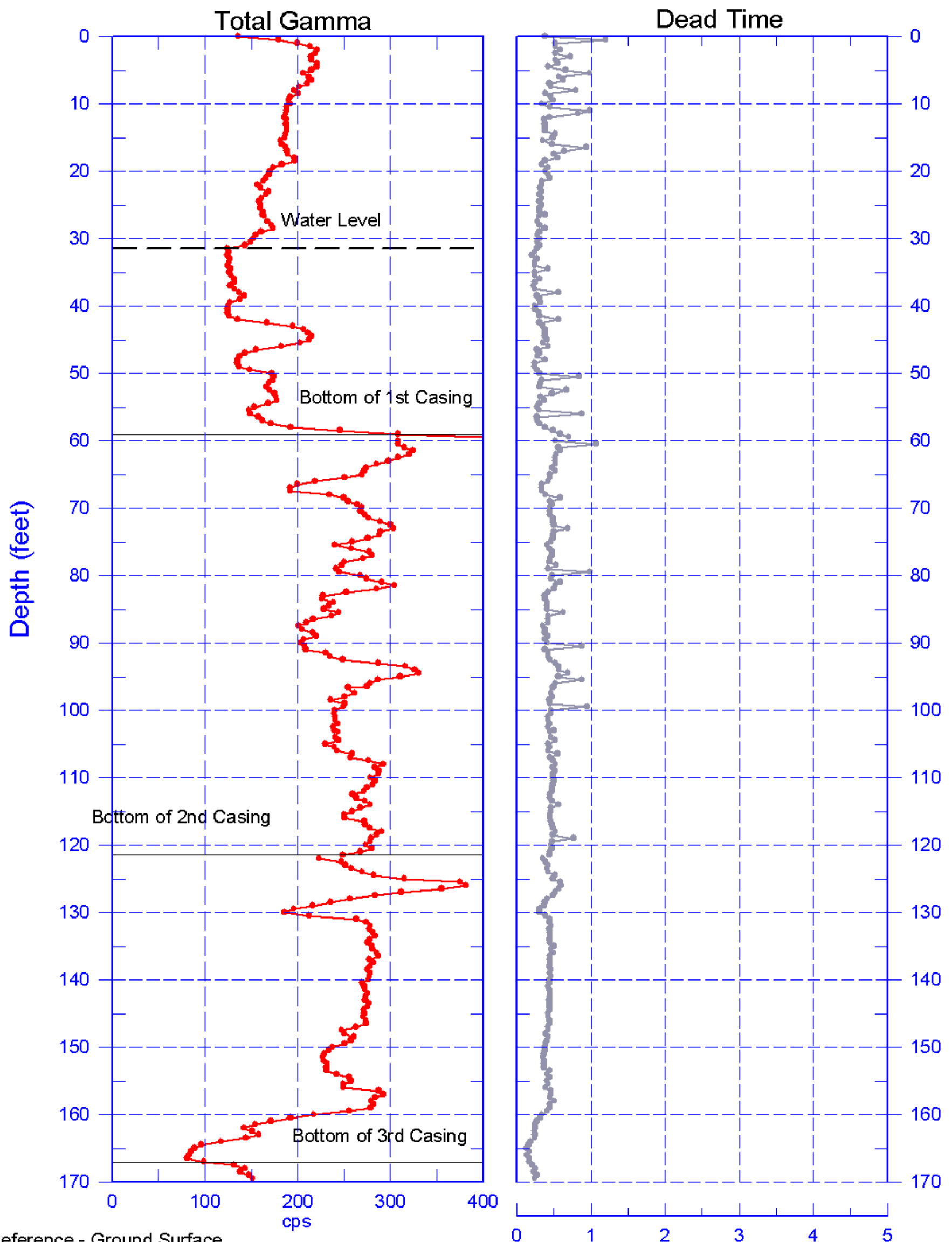

Reference - Ground Surface 
toller $\quad$ 399-2-25 (C6209)

Hanford Office

\section{Total Gamma \& Moisture}
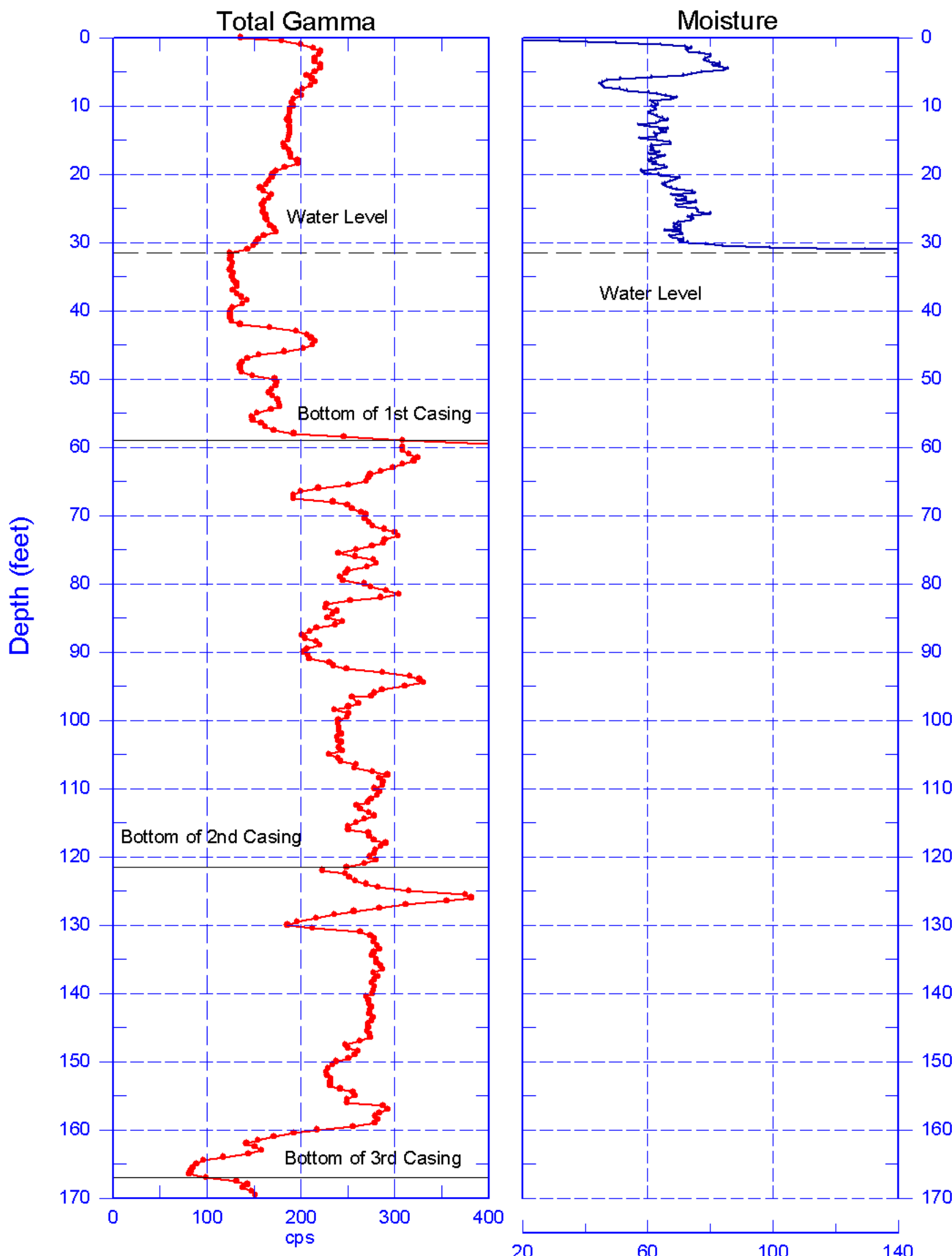

$-30$

Reference - Ground Surface 


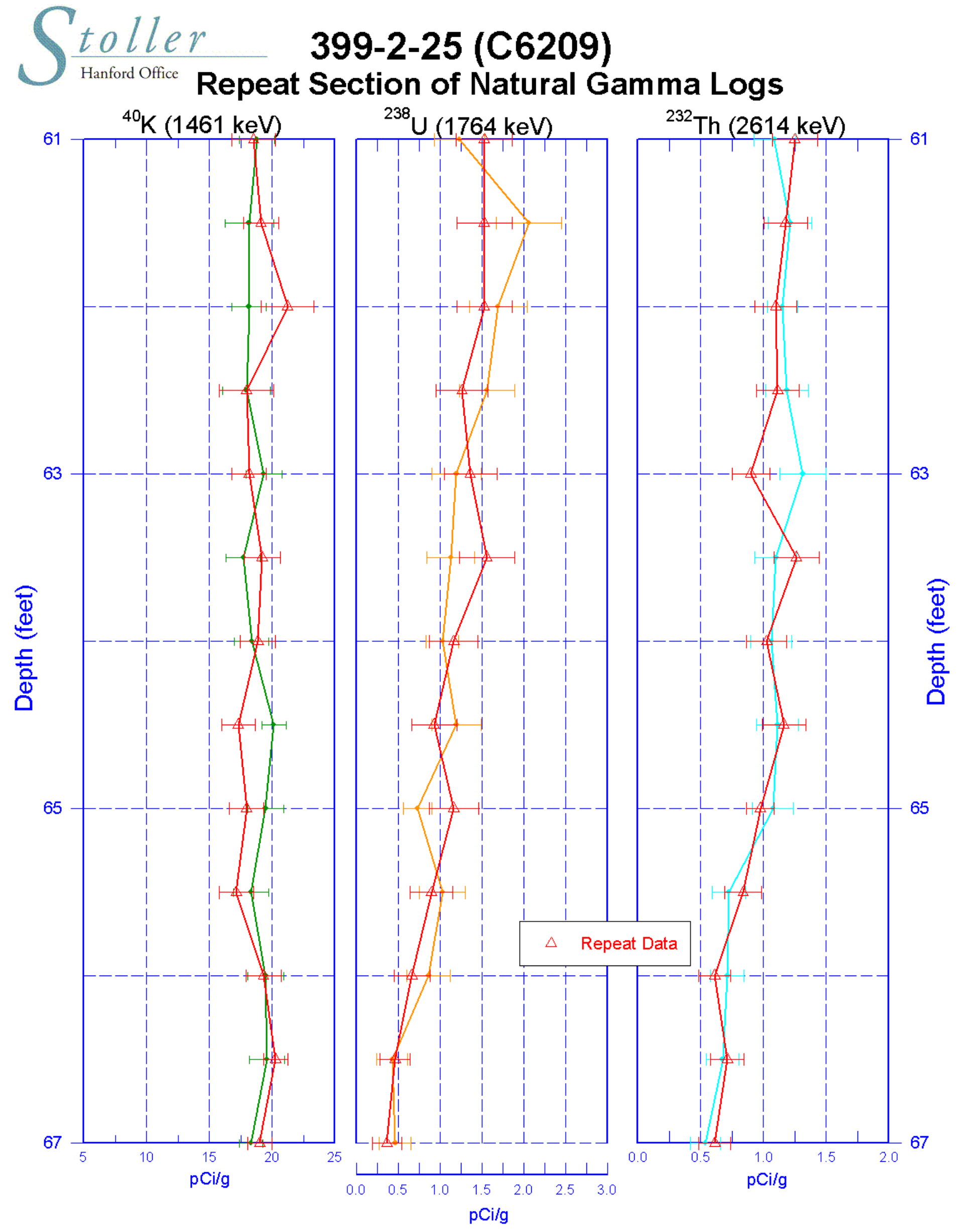




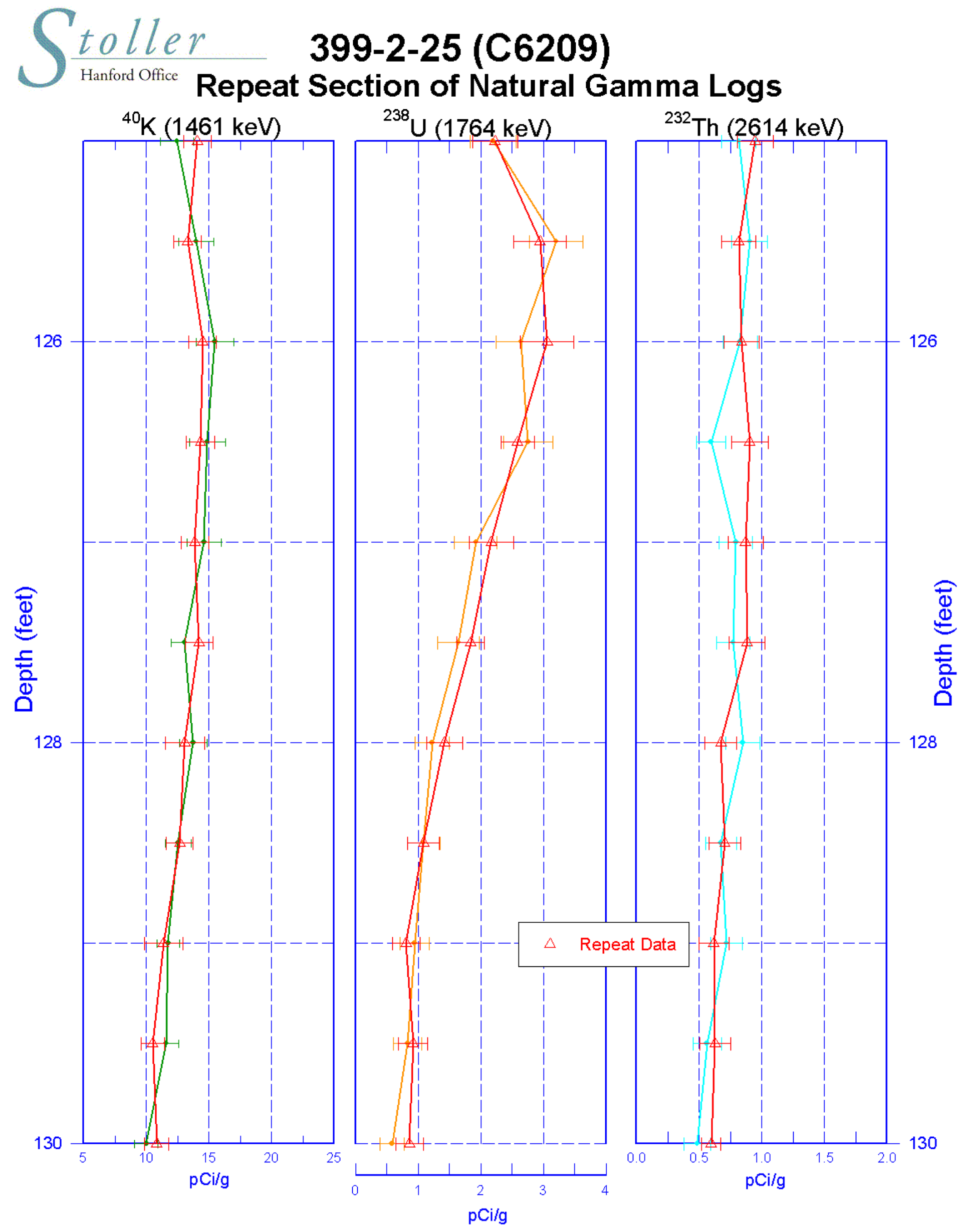


toller 399-2-25 (C6209)

Hanford Office

Moisture Repeat Section

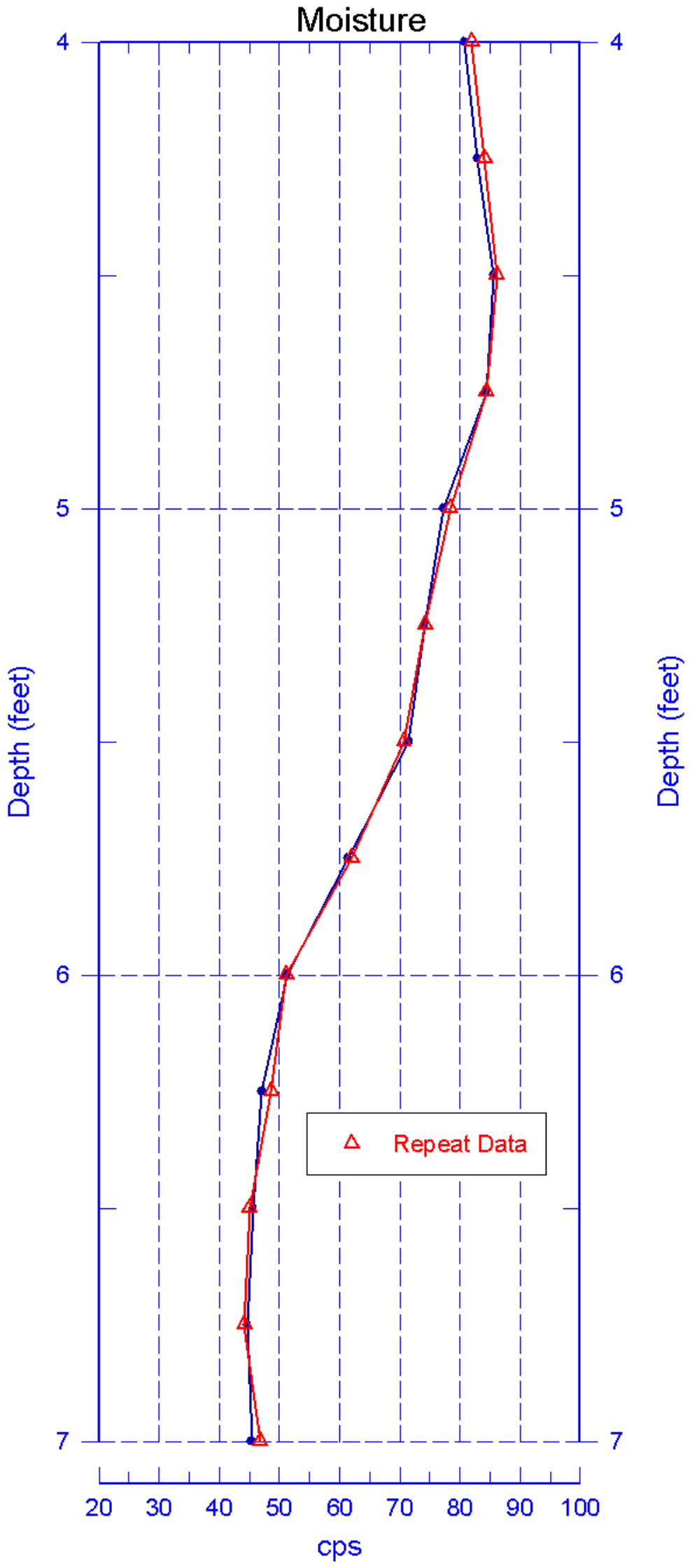

Reference - Ground Surface 


\section{9-2-26 (C6210) Log Data Report}

Borehole Information:

\begin{tabular}{|c|c|c|c|c|c|}
\hline \multicolumn{3}{|c|}{ 399-2-26 (C6210) } & Site: & 300-FF-5 & \\
\hline \multicolumn{2}{|c|}{ Coordinates } & GWL $^{1}$ (f & 27.6 & GWL Date: & $6 / 26 / 2008$ \\
\hline North (m) & East (m) & Drill Date & TOC $^{2}$ Elevation & Total Depth (ft) & Type \\
\hline 116081.1 & 594243.7 & $6 / 24 / 2008$ & unknown & 62 & Sonic \\
\hline
\end{tabular}

\section{Casing Information:}

\begin{tabular}{|c|c|c|c|c|c|c|}
\hline Casing Type & Stickup (ft) & $\begin{array}{c}\text { Outer } \\
\text { Diameter (in.) }\end{array}$ & $\begin{array}{c}\text { Inside } \\
\text { Diameter (in.) }\end{array}$ & Thickness (in.) & Top (ft) & Bottom (ft) \\
\hline Welded Steel & 0.4 & $75 / 8$ & $67 / 8$ & $3 / 8$ & -0.4 & 60.0 \\
\hline
\end{tabular}

\section{Borehole Notes:}

Casing data and total depth was reported by the site geologist. Casing diameter was measured using a steel tape and rounded to the nearest 1/16-inch. The zero reference is the ground surface.

\section{Logging Equipment Information:}

\begin{tabular}{|l|l|l|l|l|}
\hline \multirow{2}{*}{ Logging System: } & \multirow{2}{*}{ Gamma 4 L } & $\begin{array}{l}\text { Type: } \\
\text { Serial No.: }\end{array}$ & $\begin{array}{l}\text { 60\%HPGe SGLS } \\
\text { 47TP32211A }\end{array}$ \\
\hline Effective Calibration Date: & $12 / 31 / 2007$ & $\begin{array}{l}\text { Calibration Reference: } \\
\text { Logging Procedure: }\end{array}$ & HGLP-CC-027 \\
\cline { 4 - 5 } & & HGLP-MAN-002, Rev. 0 \\
\hline
\end{tabular}

\section{Spectral Gamma Logging System (SGLS) Log Run Information:}

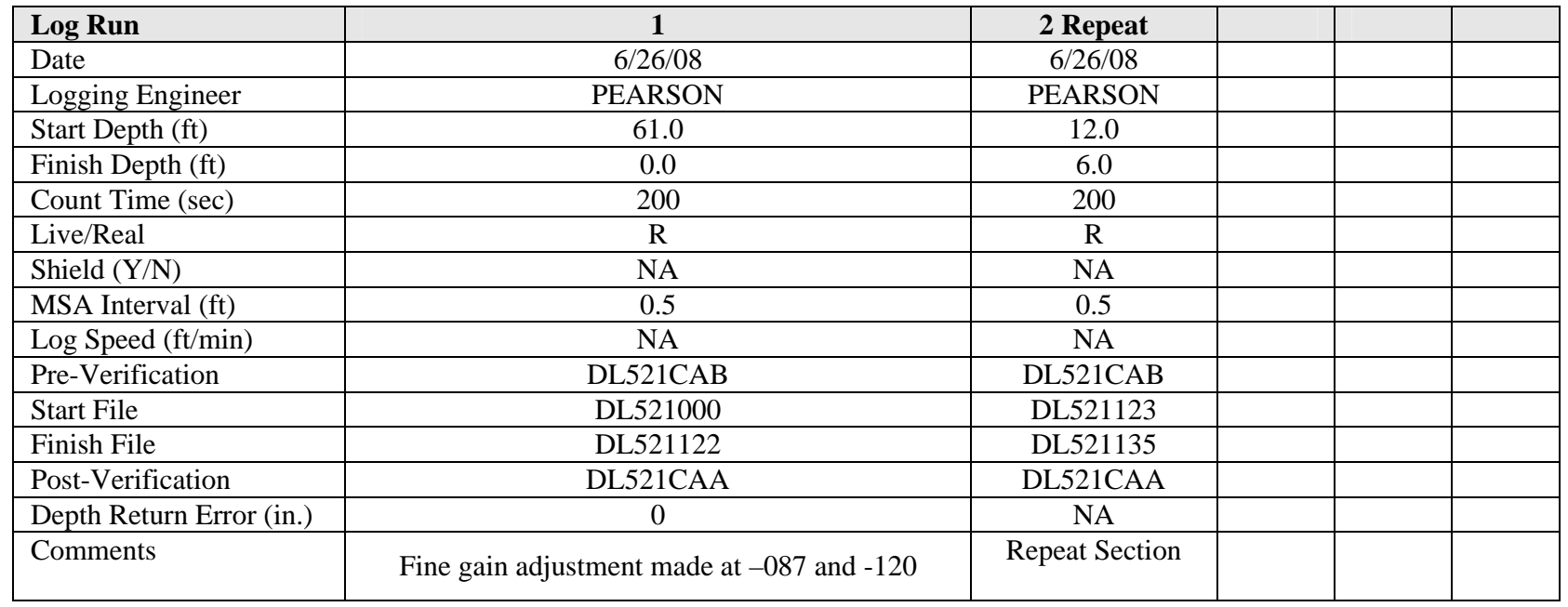

\section{Neutron Moisture Logging System (NMLS) Log Run Information:}

\begin{tabular}{|c|c|c|l|l|l|}
\hline Log Run & 1 & 2 Repeat & & & \\
\hline Date & $6 / 26 / 08$ & $6 / 26 / 08$ & & & \\
\hline Logging Engineer & PEARSON & PEARSON & & & \\
\hline Start Depth (ft) & 0.0 & 27.5 & & & \\
\hline Finish Depth (ft) & 27.5 & 24.5 & & & \\
\hline
\end{tabular}


HGLP-LDR-247, Rev. 0

\begin{tabular}{|c|c|c|l|l|l|}
\hline Log Run & $\mathbf{1}$ & 2 Repeat & & & \\
\hline Count Time (sec) & 15 & 15 & & & \\
\hline Live/Real & R & R & & & \\
\hline Shield (Y/N) & NA & NA & & & \\
\hline MSA Interval (ft) & 0.25 & 0.25 & & & \\
\hline ft/min & NA & NA & & & \\
\hline Pre-Verification & DHF92CAB & DHF92CAB & & & \\
\hline Start File & DHF92000 & DHF92117 & & & \\
\hline Finish File & DHF92110 & DHF92123 & & & \\
\hline Post-Verification & DHF92CAA & DHF92CAA & & & \\
\hline Depth Return Error (in.) & NA & No & & & \\
\hline Comments & None & Repeat section. & & & \\
\hline
\end{tabular}

\section{Logging Operation Notes:}

The SGLS data were acquired 6/26/2008 with the Gamma 4L, HO 68B-3573 logging system. SGLS pre- and post-survey verification measurements were acquired in the Amersham KUTh-115 field verifier. A centralizer was installed on the sonde. Maximum logging depth was 61.23 feet before the sonde was un-weighted.

The Neutron Moisture data were acquired 6/26/2008 with Gamma 4H, HO 68B-3573 logging system. NMLS preand post-survey verification measurements were acquired in the standard field verifier. A centralizer was installed on the sonde. Maximum logging depth was 27.5 feet before water was encountered.

\section{Analysis Notes:}

\begin{tabular}{|l|l|l|l|l|l|}
\hline Analyst: & SPINNER & Date: & 9/16/08 & Reference: & GJO-HGLP 1.6.3, Rev. 0 \\
\hline
\end{tabular}

The SGLS and NMLS pre- and post-survey verifications spectra met acceptance criteria.

A casing correction was applied for a 3/8 inch welded steel casing from 0 to 60 feet, leaving the remaining two feet of the borehole uncorrected. A water correction was applied from 27.6 feet to end of logging depth.

SGLS spectra were processed in batch mode using APTEC SUPERVISOR to identify individual peaks and count rates. Concentrations were calculated using an EXCEL template identified as G4LDec07.xls using an efficiency function and corrections for casing, dead time and water as determined by annual calculations. NMLS spectra were processed in batch mode in APTEC SUPERVISOR to identify counts. Count rates were calculated using an EXCEL template identified as G4HNov07.xls. NMLS data are presented in counts per second (cps), because no calibration data is available for a 6 7/8 inch inner diameter borehole casing.

\section{Results and Interpretations:}

The Man-Made Radionuclides, U-235 and U-238 were detected in the logging survey. These isolated peaks were individually inspected and determined to be statistical fluctuations and are not valid.

The behavior of the neutron moisture log at 16.25 feet and 16.5 feet is suspect. The count rate dropped to 2.47 cps at 16.25 feet and 3.27 cps at 16.5 feet. This is consistent with log response at zero moisture content. It is also possible that the detector experienced a brief failure at these intervals.

The Natural Gamma (KUT) plot and the Moisture Repeat plot indicate good repeatability. 
HGLP-LDR-247, Rev. 0

\section{List of Log Plots:}

Depth Reference is ground surface.

Manmade Radionuclides

Natural Gamma Logs

Combination Plot (0-120 feet), with Moisture

Total Gamma \& Dead Time

Total Gamma \& Moisture

Repeat Section of Natural Gamma Logs (6-12 ft)

Moisture Repeat Section (24.5-27.5 ft.)

${ }^{1} \mathrm{GWL}$ - groundwater level

${ }^{2}$ TOC - top of casing 
S

toller $\quad \mathbf{3 9 9 - 2 - 2 6}(\mathbf{C 6 2 1 0})$ Hanford Office

Manmade Radionuclides

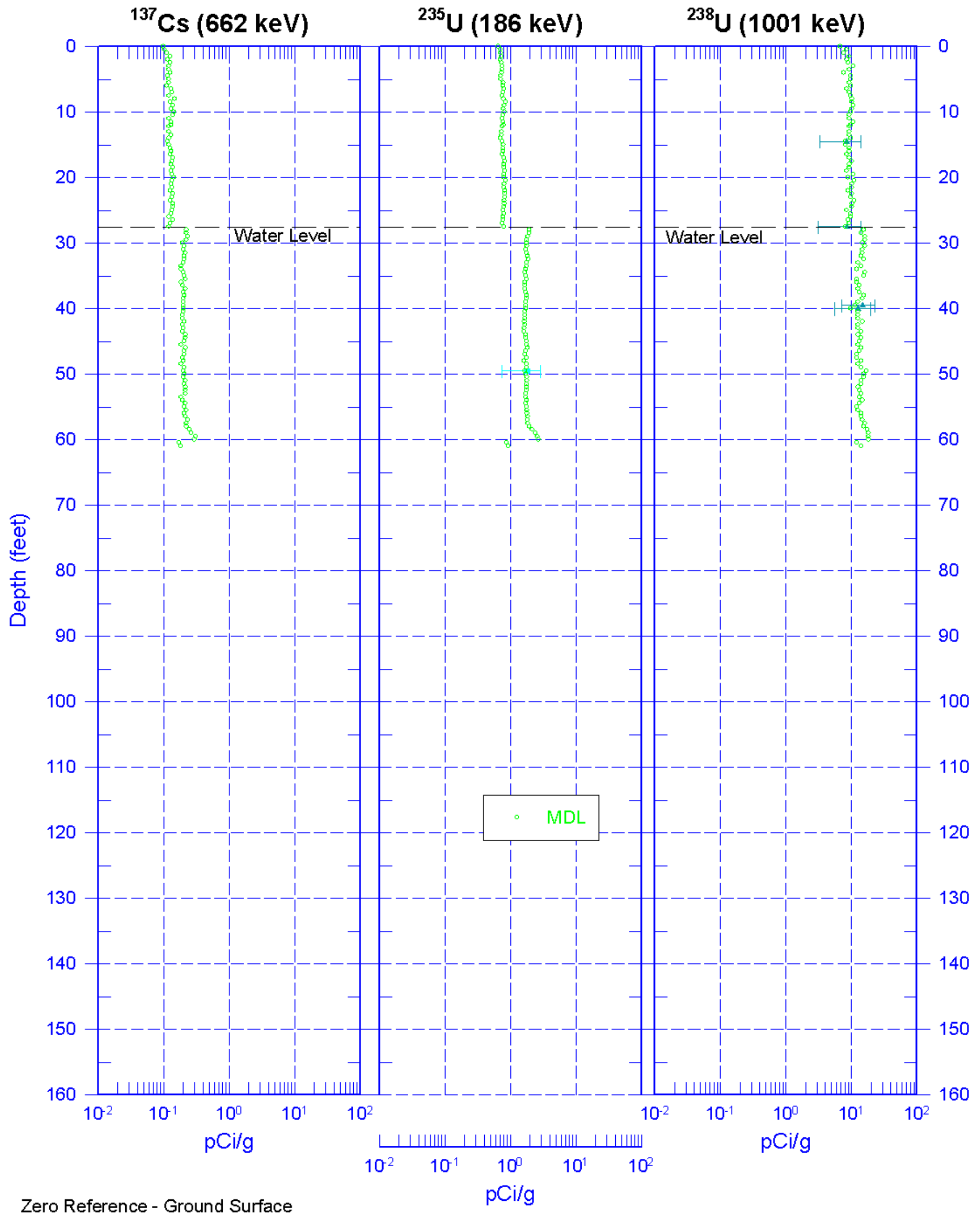


toller 399-2-26 (C6210)

Hanford Office

Natural Gamma Logs
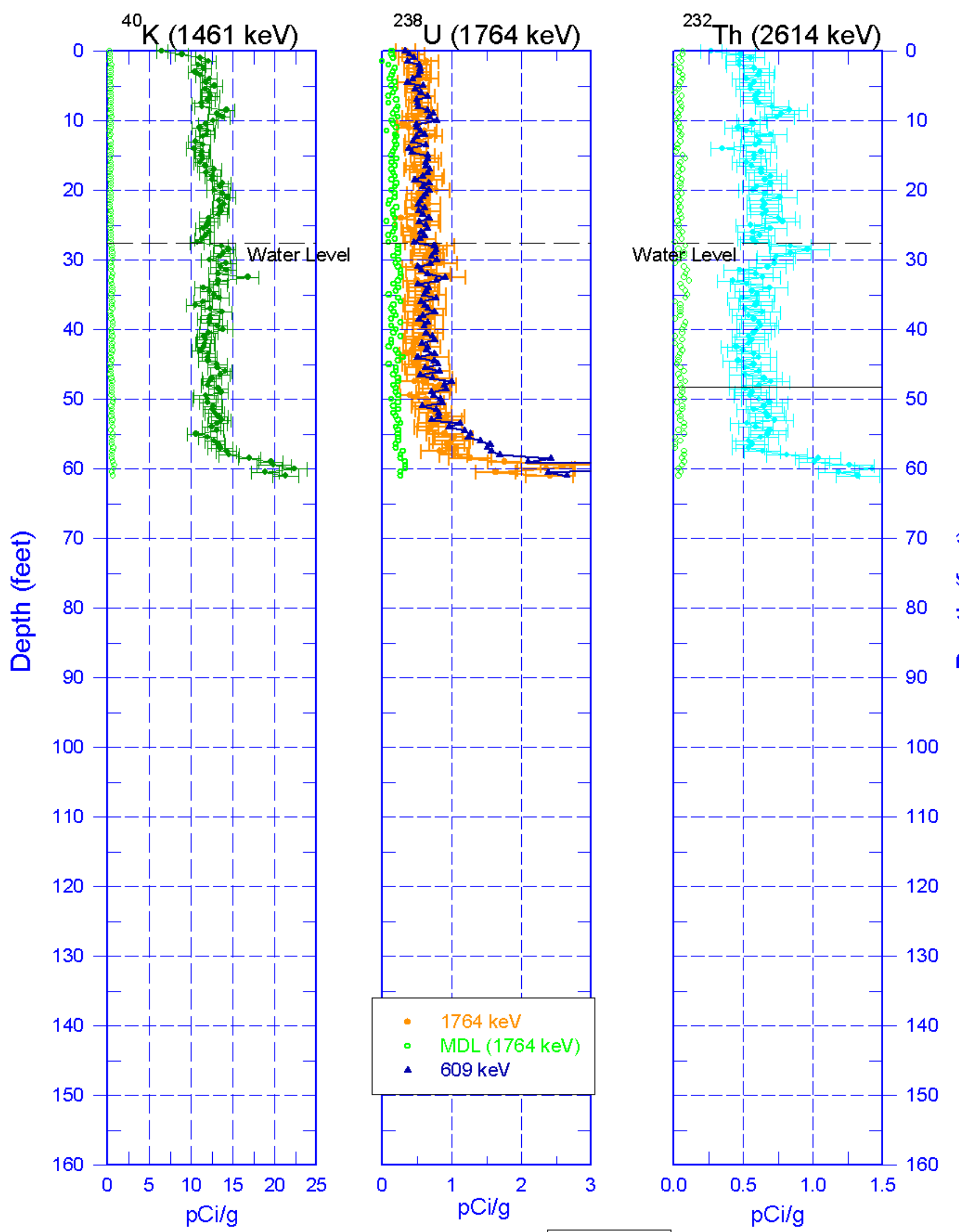

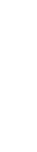

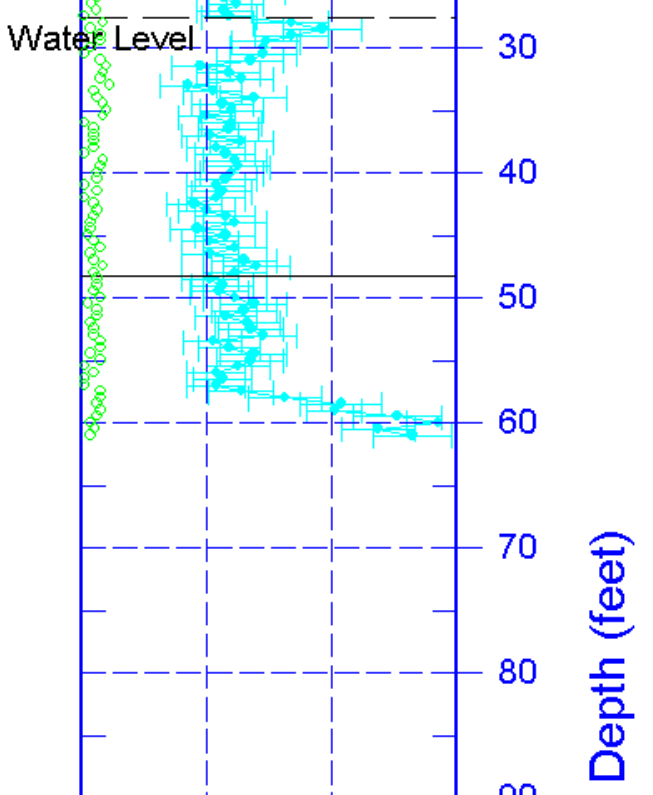

Zero Reference - Ground Surface 


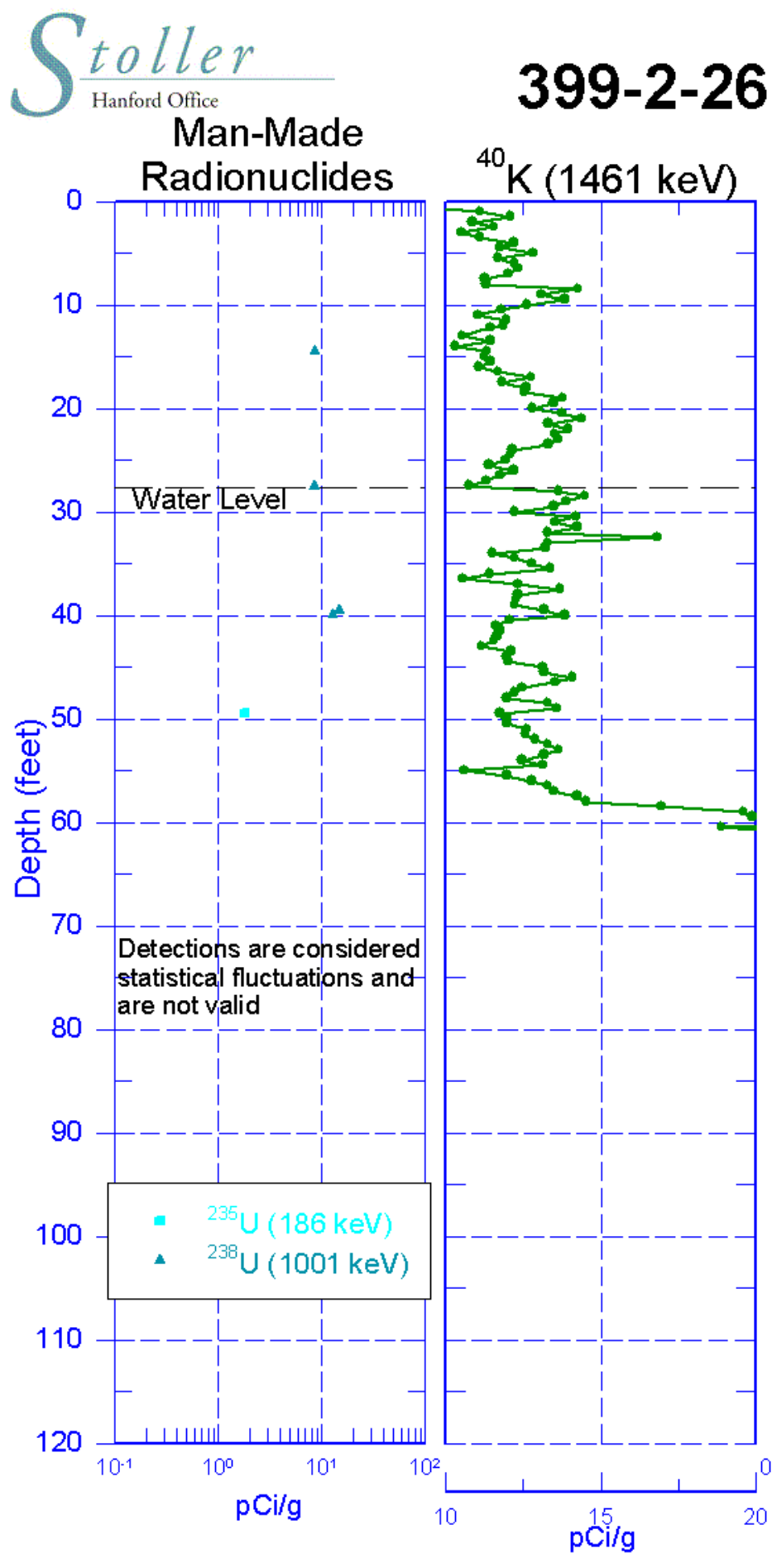

(C6210) Combination Plot
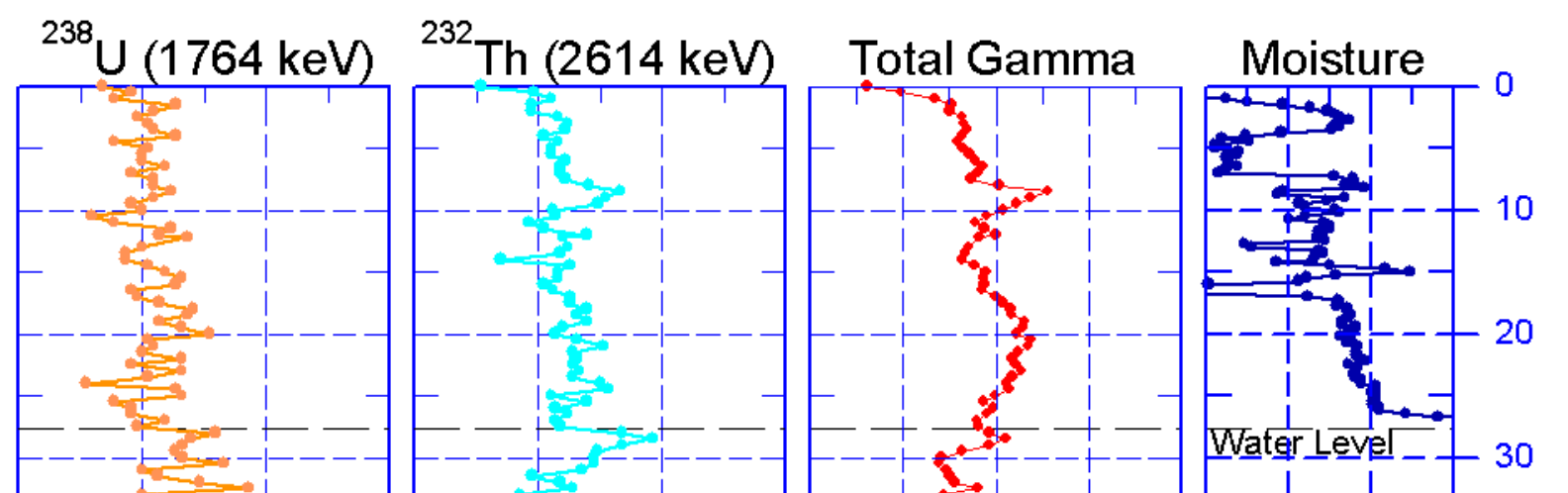
toller 399-2-26 (C6210)

Hanford Office

\section{Total Gamma \& Dead Time}
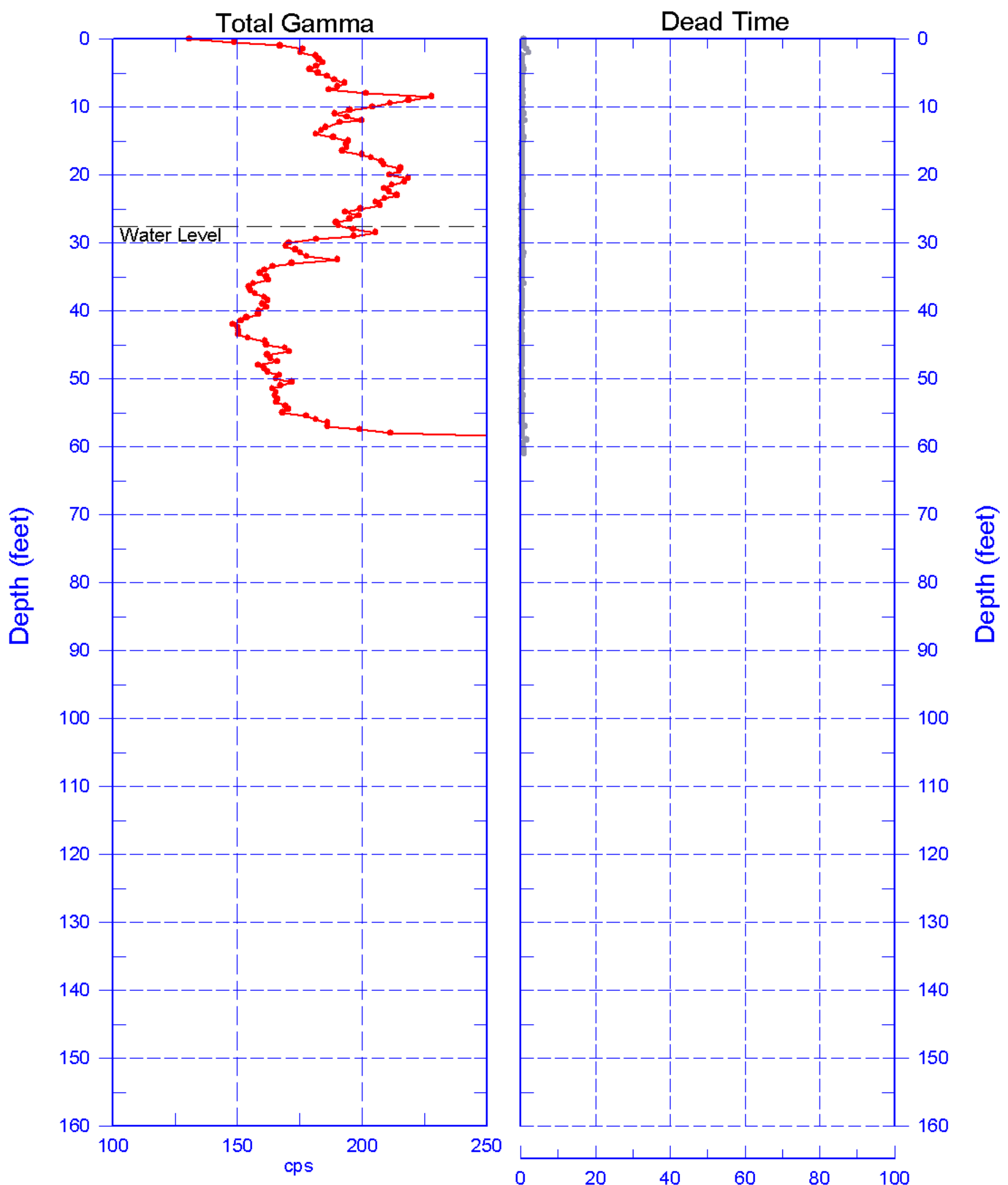

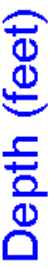

Reference - Ground Surface 
toller 399-2-26 (C6210)

Hanford Office

\section{Total Gamma \& Moisture}
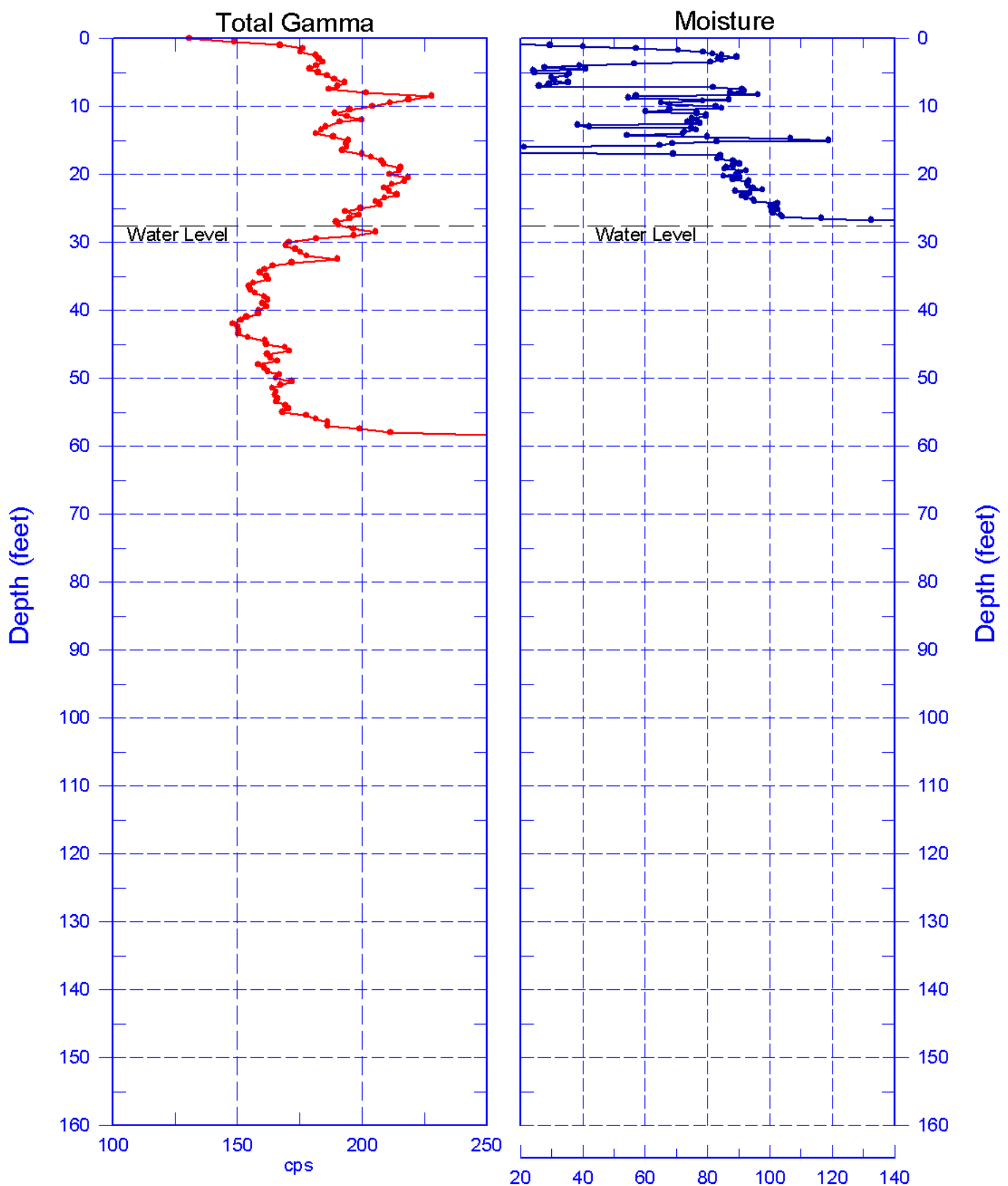

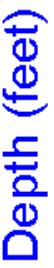

Reference - Ground Surface 


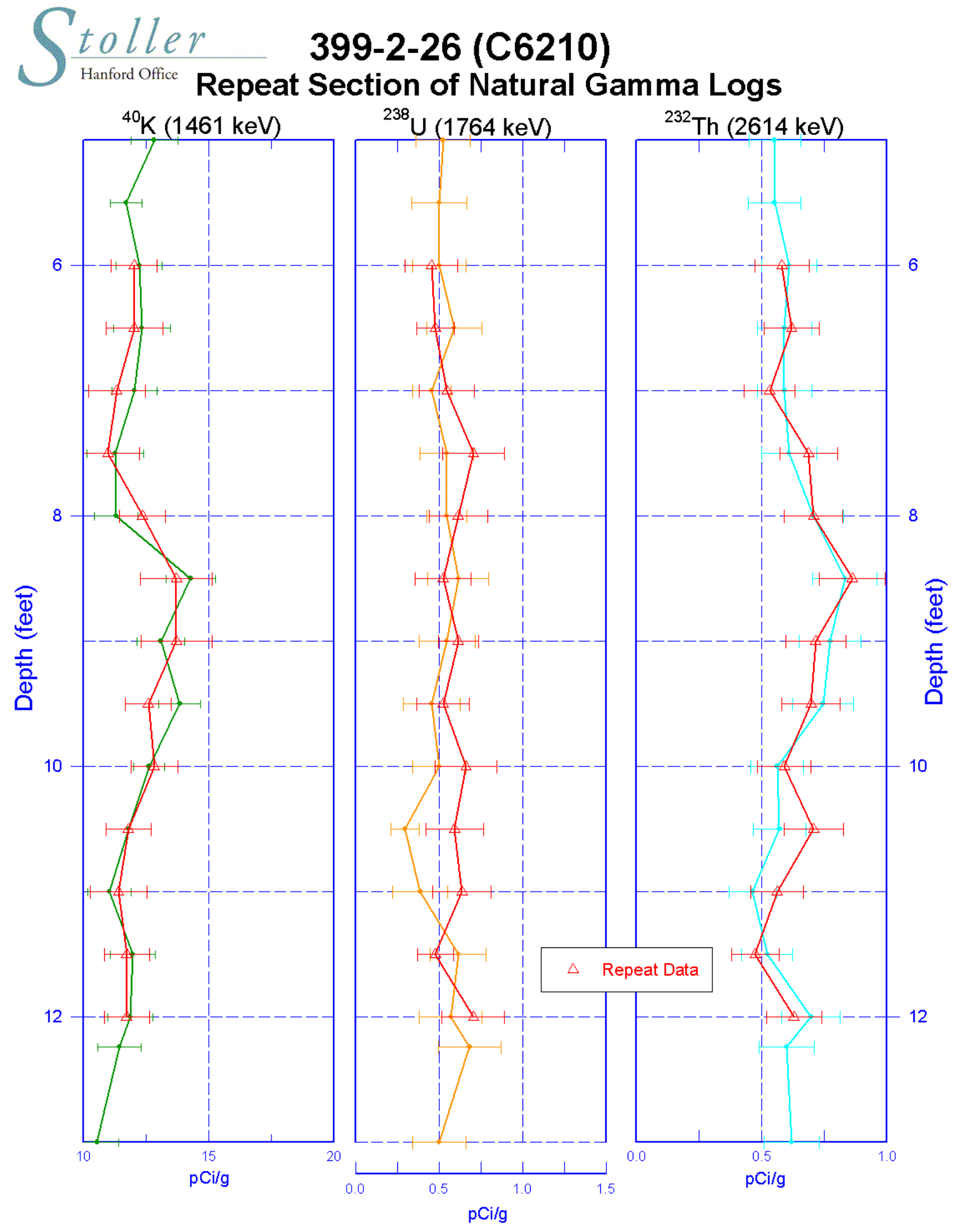

Zero Reference - Ground Surface

G.216 


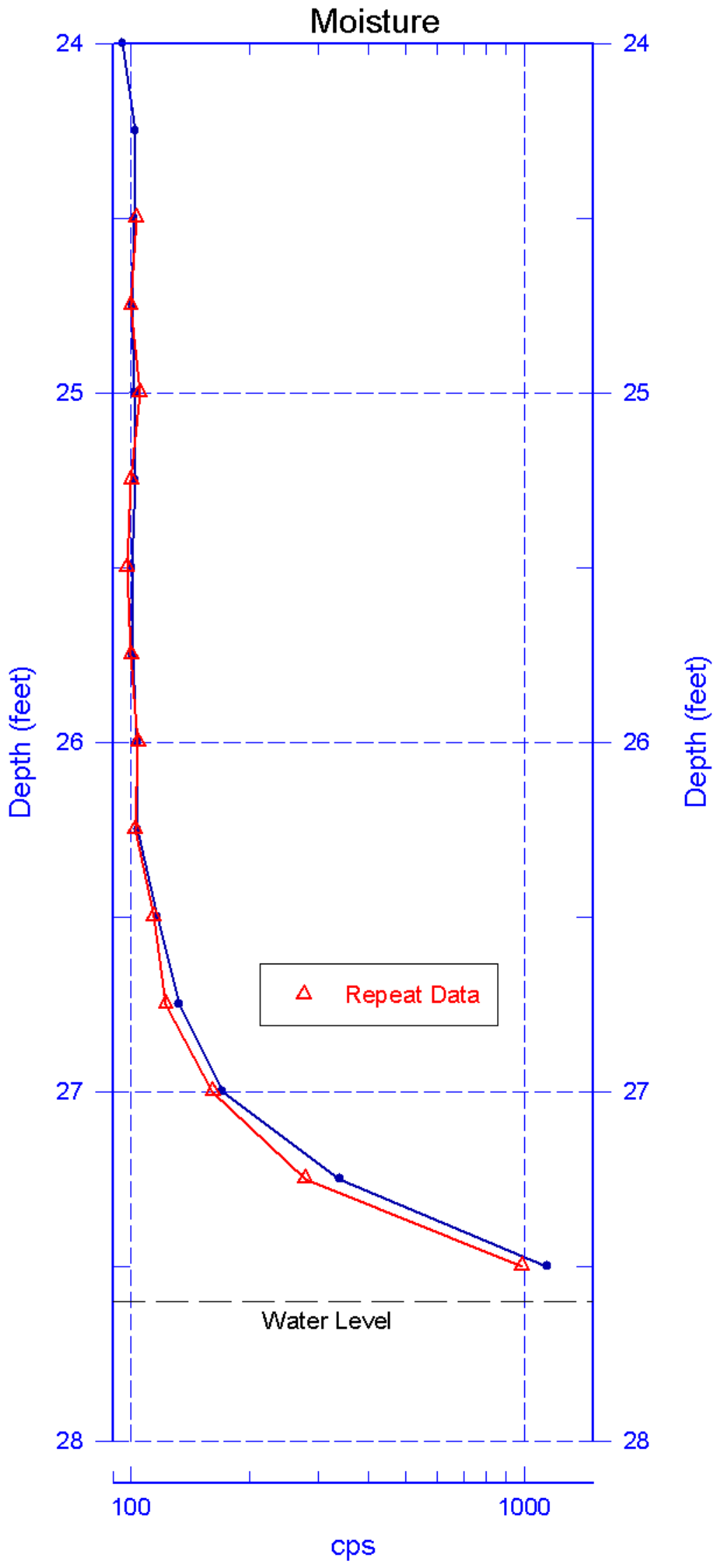




\section{9-2-31 (C6218) Log Data Report}

Borehole Information:

\begin{tabular}{|c|c|c|c|c|c|}
\hline \multicolumn{2}{|c|}{ Borehole: $\quad 399-2-31$ (C6218) } & \multicolumn{4}{|c|}{ 300-FF-5 } \\
\hline \multicolumn{2}{|c|}{ Coordinates (WA St Plane) } & $G_{W L}^{1}(\mathbf{f}$ & 27.3 & GWL Date: & $06 / 24 / 08$ \\
\hline North (m) & East (m) & Drill Date & TOC $^{2}$ Elevation & Total Depth (ft) & Type \\
\hline 116067.2 & 594273.6 & 06/18/08 & Unknown & 63 & Sonic \\
\hline
\end{tabular}

\section{Casing Information:}

\begin{tabular}{|l|c|c|c|c|c|c|}
\hline Casing Type & Stickup (ft) & $\begin{array}{c}\text { Outer } \\
\text { Diameter (in.) }\end{array}$ & $\begin{array}{c}\text { Inside } \\
\text { Diameter (in.) }\end{array}$ & Thickness (in.) & Top (ft) & Bottom (ft) \\
\hline Welded Steel & 1.2 & $75 / 8$ & $67 / 8$ & $3 / 8$ & -1.2 & 59 \\
\hline
\end{tabular}

\section{Borehole Notes:}

Casing data and total depth are reported by the site geologist. The logging engineer measured depth to water with an e-tape. Casing diameters were measured using a steel tape and rounded to the nearest 1/16 inch. Zero reference is the ground surface.

\section{Logging Equipment Information:}

\begin{tabular}{|c|c|c|c|c|}
\hline Logging System: & \multicolumn{2}{|c|}{ Gamma $4 \mathrm{~L}$} & $\begin{array}{l}\text { Type: } \\
\text { Serial No.: }\end{array}$ & $\begin{array}{l}\text { 60\% HPGe SGLS } \\
\text { 47TP32211A }\end{array}$ \\
\hline Effective Calibration Date: & $12 / 31 / 07$ & Calibration Reference: & \multicolumn{2}{|c|}{ HGLP-CC-027 } \\
\hline & & Logging Procedure: & \multicolumn{2}{|c|}{ HGLP-MAN-002, Rev. 0} \\
\hline Logging System: & \multicolumn{2}{|c|}{ Gamma $4 \mathrm{H}$} & $\begin{array}{l}\text { Type: } \\
\text { Serial No.: }\end{array}$ & $\begin{array}{l}\text { NMLS } \\
\text { H310700352 }\end{array}$ \\
\hline Effective Calibration Date: & $11 / 06 / 07$ & Calibration Reference: & \multicolumn{2}{|c|}{ HGLP-CC-021 } \\
\hline & & Logging Procedure: & \multicolumn{2}{|c|}{ HGLP-MAN-002, Rev. 0} \\
\hline
\end{tabular}

\section{Spectral Gamma Logging System (SGLS) Log Run Information:}

\begin{tabular}{|l|c|c|l|l|l|}
\hline Log Run & $\mathbf{1}$ & 2 Repeat & & & \\
\hline Date & $06 / 24 / 08$ & $06 / 24 / 08$ & & & \\
\hline Logging Engineer & McClellan & McClellan & & & \\
\hline Start Depth (ft) & 62.0 & 8.0 & & & \\
\hline Finish Depth (ft) & 0.0 & 2.0 & & & \\
\hline Count Time (sec) & 200 & 200 & & & \\
\hline Live/Real & $\mathrm{R}$ & $\mathrm{R}$ & & & \\
\hline Shield (Y/N) & $\mathrm{N}$ & $\mathrm{N}$ & & & \\
\hline MSA Interval (ft) & 0.5 & 0.5 & & & \\
\hline Log Speed (ft/min) & $\mathrm{N} / \mathrm{A}$ & $\mathrm{N} / \mathrm{A}$ & & & \\
\hline Pre-Verification & DL501CAB & DL501CAB & & & \\
\hline Start File & DL501000 & DL501125 & & & \\
\hline Finish File & DL501124 & DL501137 & & & \\
\hline Post-Verification & DL501CAA & DL501CAA & & & \\
\hline Depth Return Error (in.) & 0.25 low & N/A & & & \\
\hline Comments & $\begin{array}{c}\text { No fine gain } \\
\text { adjustments } \\
\text { made. }\end{array}$ & Repeat Section & & & \\
\hline
\end{tabular}


Neutron Moisture Logging System (NMLS) Log Run Information:

\begin{tabular}{|l|c|c|l|l|l|}
\hline Log Run & $\mathbf{3}$ & 4 Repeat & & & \\
\hline Date & $06 / 24 / 08$ & $06 / 24 / 08$ & & & \\
\hline Logging Engineer & McClellan & McClellan & & & \\
\hline Start Depth (ft) & 0.0 & 27.25 & & & \\
\hline Finish Depth (ft) & 27.25 & 24.0 & & & \\
\hline Count Time (sec) & 15 & 15 & & & \\
\hline Live/Real & $\mathrm{R}$ & $\mathrm{R}$ & & & \\
\hline Shield (Y/N) & $\mathrm{N}$ & $\mathrm{N}$ & & & \\
\hline MSA Interval (ft) & 0.25 & 0.25 & & & \\
\hline Log Speed (ft/min) & N/A & N/A & & & \\
\hline Pre-Verification & DHF72CAB & DHF72CAB & & & \\
\hline Start File & DHF72000 & DHF72110 & & & \\
\hline Finish File & DHF72109 & DHF72123 & & & \\
\hline Post-Verification & DHF72CAA & DHF72CAA & & & \\
\hline Depth Return Error (in.) & N/A & 1.0 high & & & \\
\hline Comments & None & Repeat Section & & & \\
\hline
\end{tabular}

\section{Logging Operation Notes:}

Data were collected using Gamma 4, HO 68B-3573. SGLS pre- and post-survey verification measurements were acquired in the Amersham KUTh-115 field verifier. NMLS pre- and post-survey verification measurements were acquired in the standard field verifier. Maximum logging depth for the SGLS was $62 \mathrm{ft}$ before the sonde un-weighted. A centralizer was installed on the sonde.

\section{Analysis Notes:}

\begin{tabular}{|l|l|l|l|l|l|}
\hline Analyst: & LEGLER & Date: & $05 / 22 / 08$ & Reference: & GJO-HGLP 1.6.3, Rev. 0 \\
\hline
\end{tabular}

Pre- and post-survey verification spectra met the acceptance criteria for the established systems.

A casing correction for a 3/8-in. thick casing was applied to spectral data during analysis for depths up to $59 \mathrm{ft}$, where casing ends, leaving $3 \mathrm{ft}$ of open borehole uncorrected. A water correction was also applied during analysis from $27.3 \mathrm{ft}$ to total logged depth of borehole.

SGLS spectra were processed in batch mode in APTEC SUPERVISOR to identify individual peaks and count rates. Concentrations were calculated using an EXCEL template identified as G4LDec07.xls using an efficiency function and corrections for casing, dead time and water as determined by annual calibrations. NMLS spectra were processed in batch mode in APTEC SUPERVISOR to identify counts. Count rates were calculated using an EXCEL template identified as G4HNov07.xls. NMLS data are presented in counts per second (cps), because no calibration data is available for a 6 7/8-in. inner diameter borehole casing.

\section{Results and Interpretations:}

Cs-137 was detected at $6 \mathrm{ft}, 13.5-16.5 \mathrm{ft}, 18.0,20.0,41.0$ and $51.5 \mathrm{ft}$. Inspection of the individual spectra indicate that detections at 51.5 and $41.0 \mathrm{ft}$ are statistical fluctuations associated with the processing software and are not considered valid.

Co-60 was detected at $5.5-6.5 \mathrm{ft}$, and 13.5 - $19.0 \mathrm{ft}$.

$\mathrm{U}-235$ was detected at $6.0 \mathrm{ft}$.

U-238 (Pa-234m) was detected at 6.0-6.5 ft and $33.5 \mathrm{ft}$. Inspection of the individual spectra indicates that the detection at $33.5 \mathrm{ft}$ is a statistical fluctuation associated with the processing software and is not considered valid.

The KUT and moisture plots show good repeatability. 
HGLP-LDR-251, Rev. 0

\section{List of Log Plots:}

Depth Reference is top ground surface

Manmade Radionuclides

Natural Gamma Logs

Combination Plot

Total Gamma \& Dead Time

Total Gamma \& Moisture

Manmade Radionuclides Repeat Section

Repeat Section of Natural Gamma Logs

Moisture Repeat Section

${ }^{1} \mathrm{GWL}$ - groundwater level

${ }^{2}$ TOC - top of casing 

Hanford Ofice Manmade Radionuclides

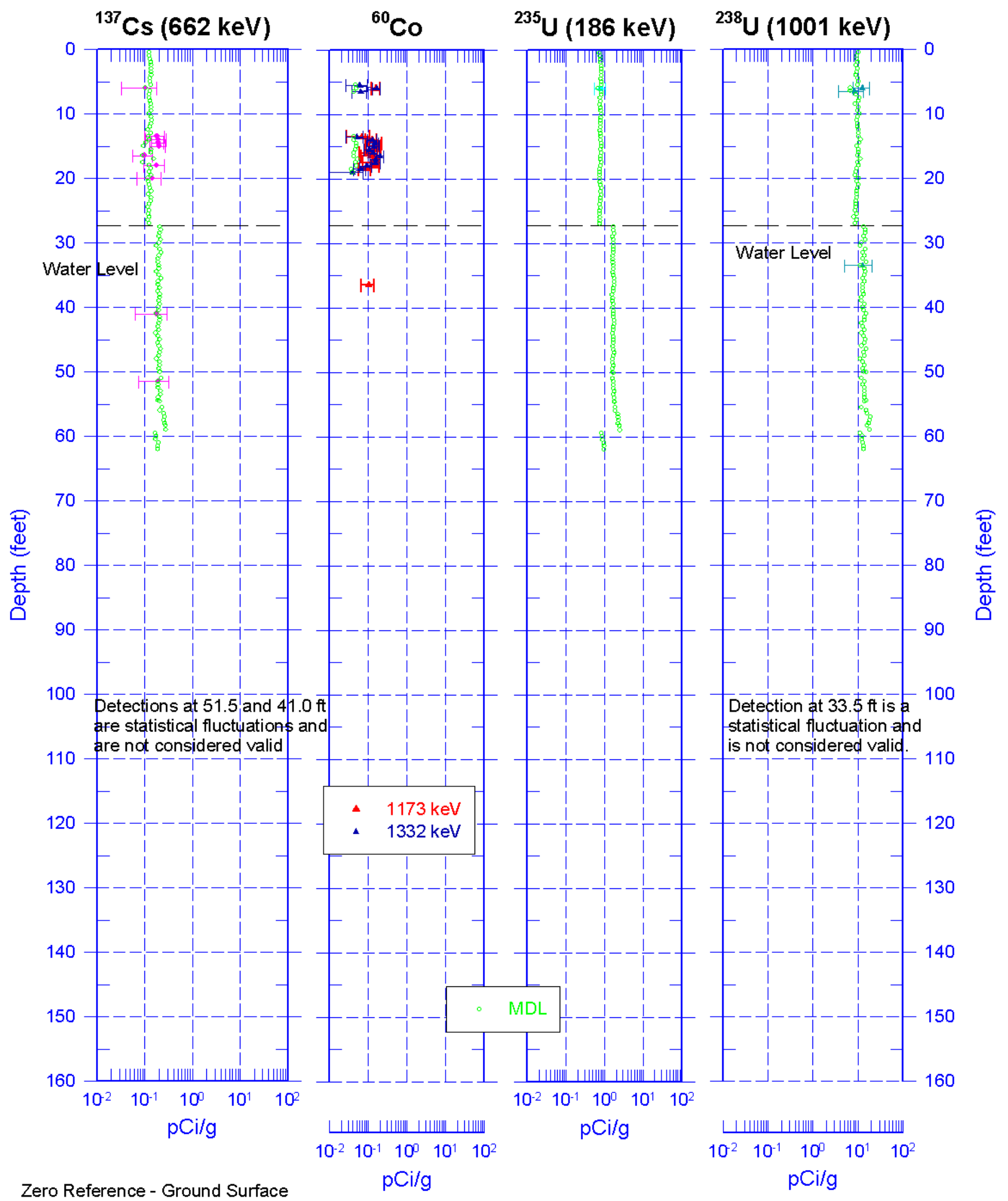


toller $\mathbf{3 9 9 - 2 - 3 1}(\mathbf{6 6 2 1 8})$

Hanford Office Natural Gamma Logs
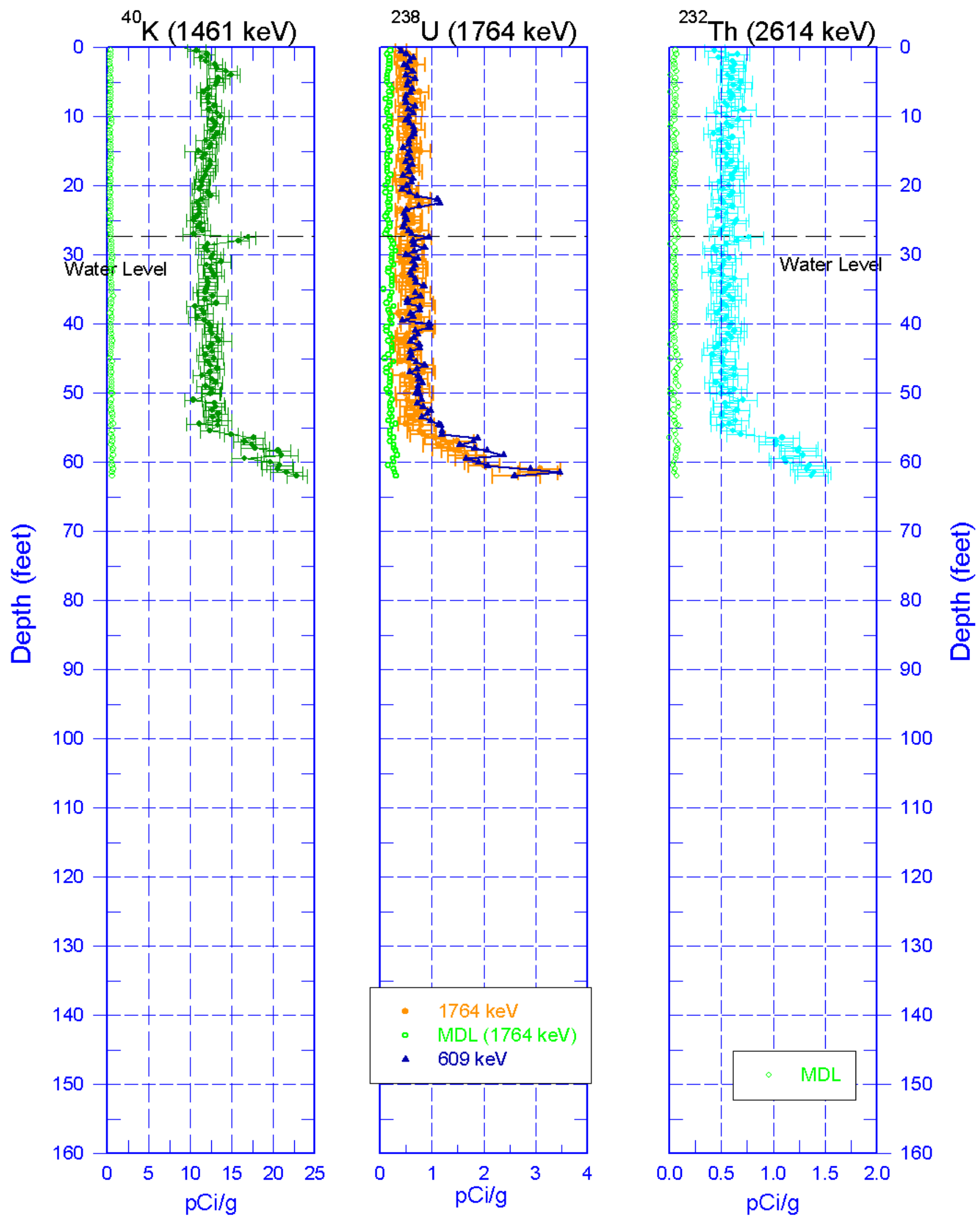

Zero Reference - Ground Surface 


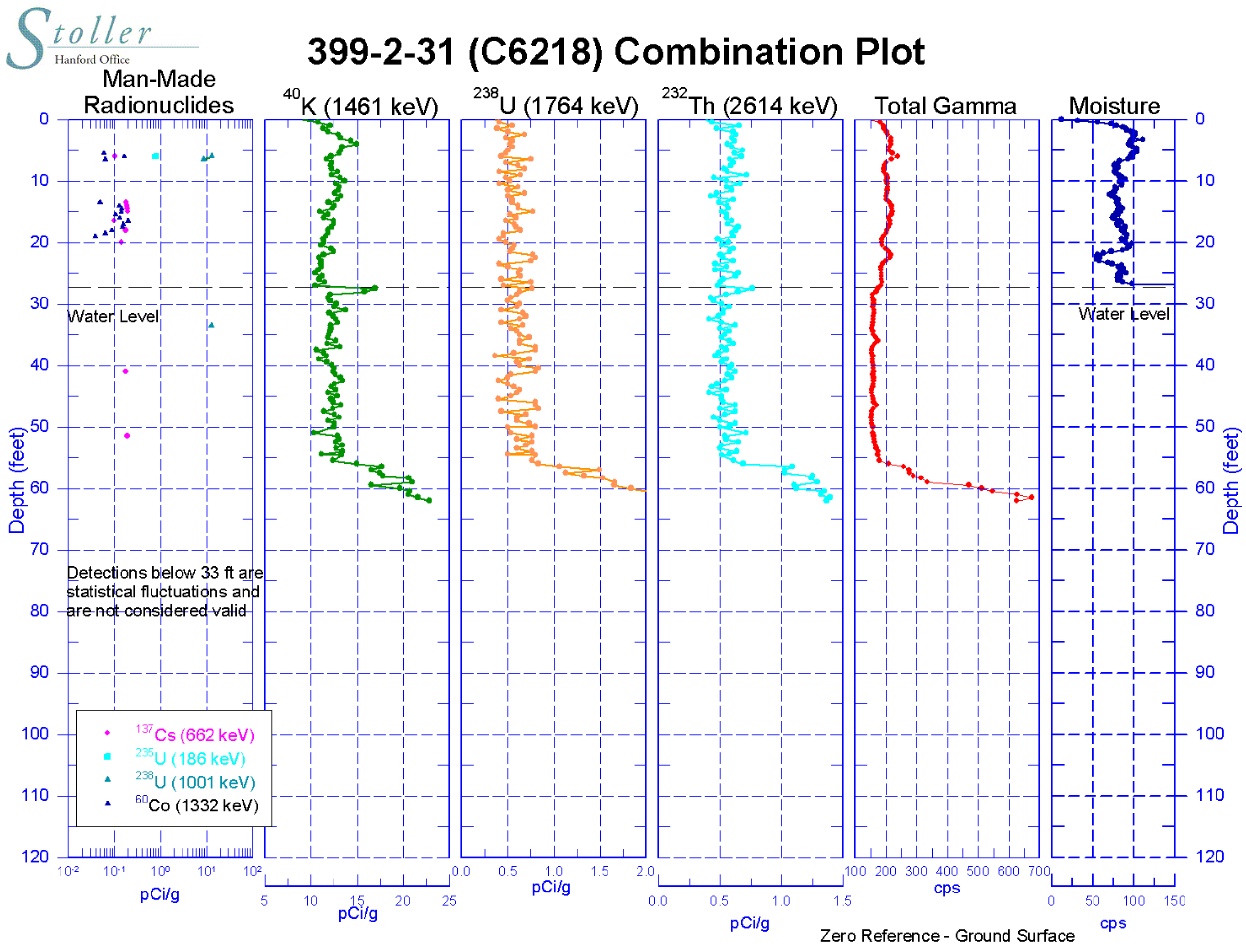


toller 399-2-31 (C6218)

Hanford Office

\section{Total Gamma \& Dead Time}
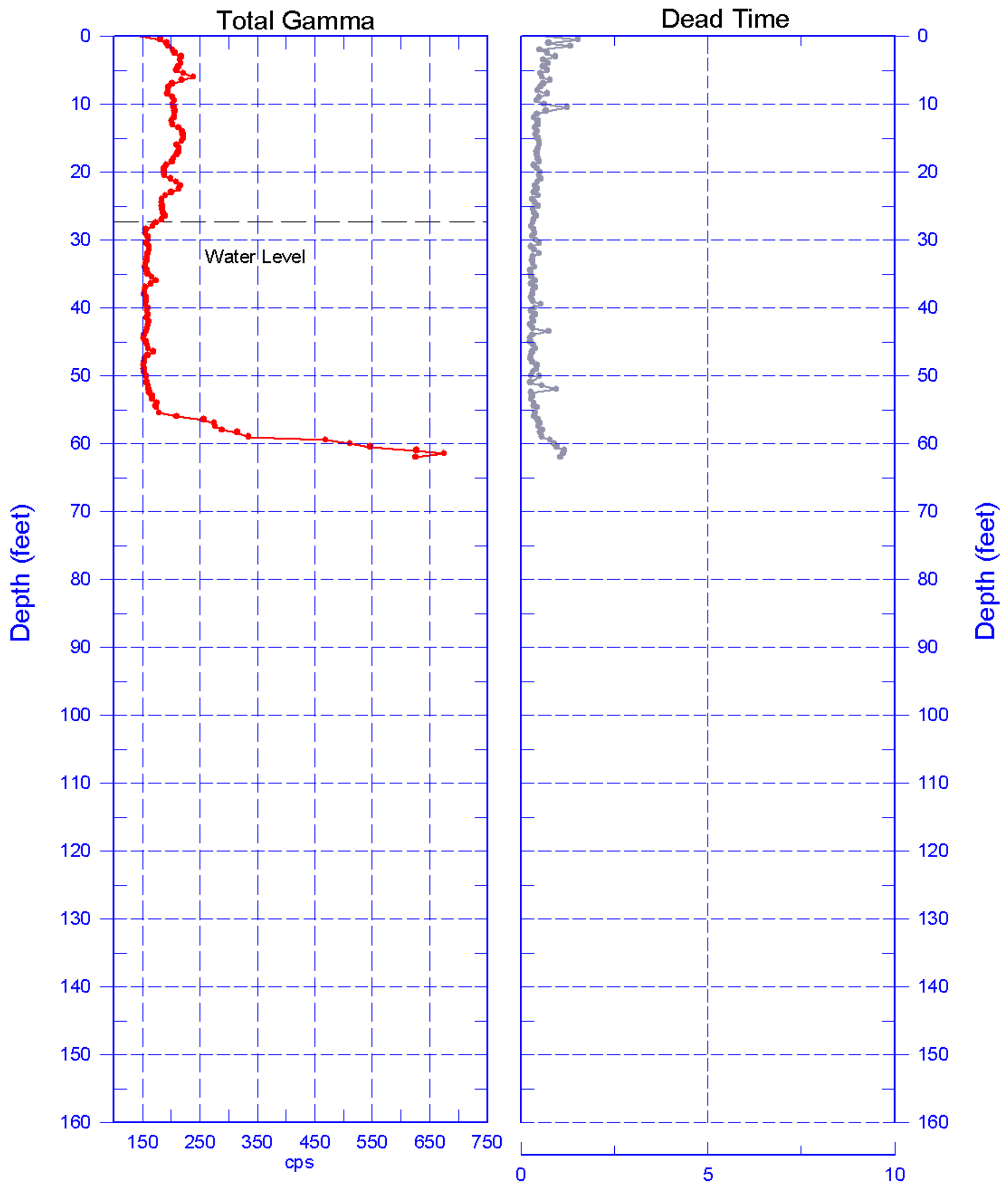

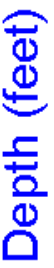

Reference - Ground Surface 


\section{S}

toller $\quad 399-\mathbf{2}-\mathbf{3 1}$ (C6218)

Hanford Office

\section{Total Gamma \& Moisture}
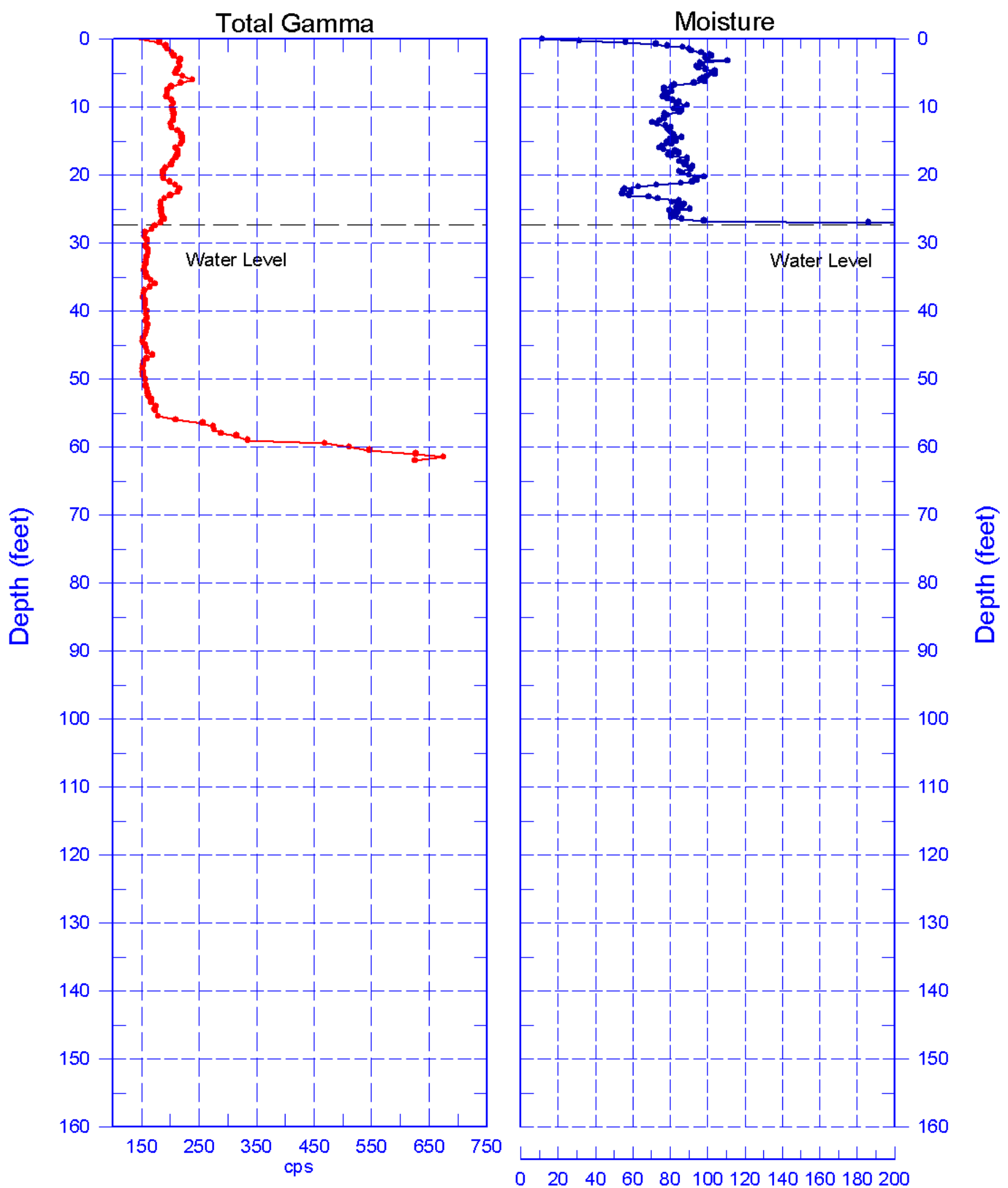


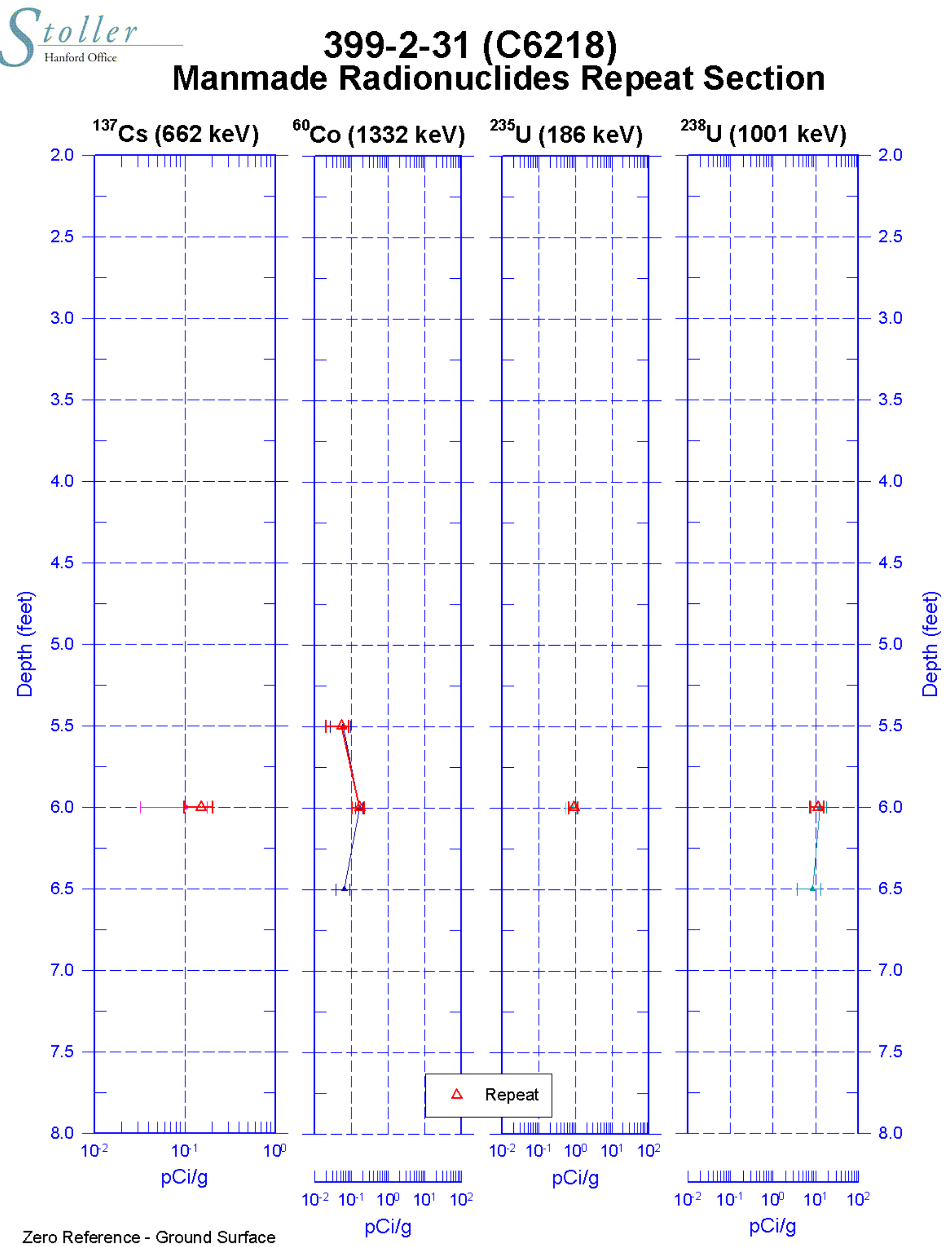




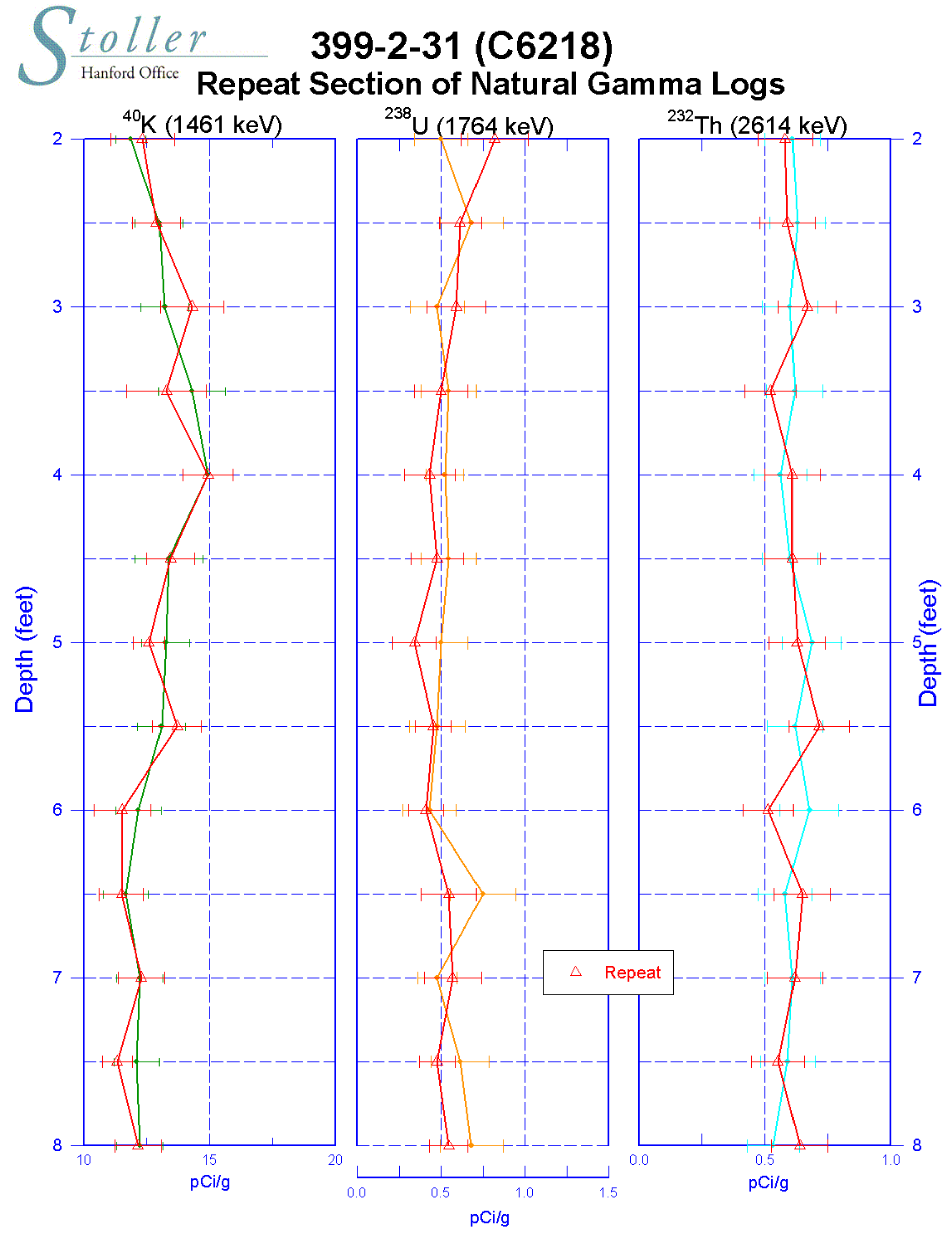

Zero Reference - Ground Surface 


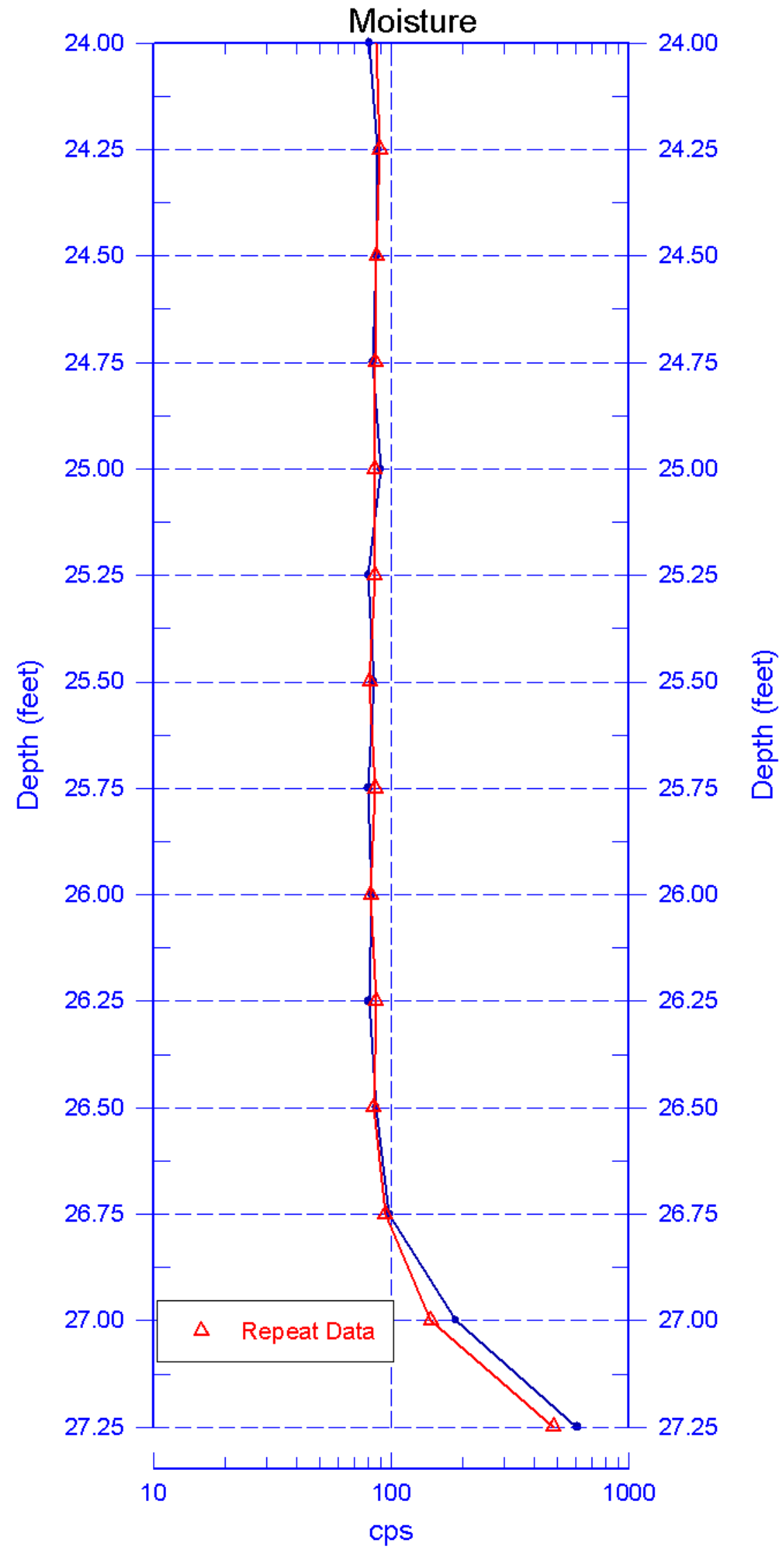




\section{9-3-23 (C6194) Log Data Report}

Borehole Information:

\begin{tabular}{|c|c|c|c|c|c|}
\hline \multirow{2}{*}{\multicolumn{2}{|c|}{ Borehole: $\quad 399-3-23$}} & & Site: & \multicolumn{2}{|l|}{ 300-FF-5 } \\
\hline & Coordinates (WA St Plane) & GWL $^{1}(\mathbf{f}$ & 29.2 & GWL Date: & 07/12/08 \\
\hline North (m) & East (m) & Drill Date & TOC $^{2}$ Elevation & Total Depth (ft) & Type \\
\hline 116054.6 & 594237.9 & $07 / 10 / 08$ & Unknown & 65.0 & Sonic \\
\hline
\end{tabular}

\section{Casing Information:}

\begin{tabular}{|c|c|c|c|c|c|c|}
\hline Casing Type & Stickup (ft) & $\begin{array}{c}\text { Outer } \\
\text { Diameter (in.) }\end{array}$ & $\begin{array}{c}\text { Inside } \\
\text { Diameter (in.) }\end{array}$ & Thickness (in.) & Top (ft) & Bottom (ft) \\
\hline Threaded Steel & 3.1 & $75 / 8$ & $67 / 8$ & $3 / 8$ & -3.1 & 57.7 \\
\hline
\end{tabular}

\section{Borehole Notes:}

Drill site geologist reported casing information and total depth. An e-tape was employed to measure depth-to-water.

\section{Logging Equipment Information:}

\begin{tabular}{|c|c|c|c|c|}
\hline Logging System: & \multicolumn{2}{|l|}{ Gamma $4 \mathrm{~L}$} & $\begin{array}{l}\text { Type: } \\
\text { Serial No.: }\end{array}$ & $\begin{array}{l}\text { 60\% HPGe SGLS } \\
\text { 47TP32211A }\end{array}$ \\
\hline Effective Calibration Date: & $12 / 31 / 2007$ & \multirow{2}{*}{$\begin{array}{l}\text { Calibration Reference: } \\
\text { Logging Procedure: }\end{array}$} & \multicolumn{2}{|c|}{ HGLP-CC-027 } \\
\hline & & & \multicolumn{2}{|c|}{ HGLP-MAN-002, Rev. 0} \\
\hline Logging System: & \multicolumn{2}{|l|}{ Gamma $4 \mathrm{H}$} & $\begin{array}{l}\text { Type: } \\
\text { Serial No.: }\end{array}$ & $\begin{array}{l}\text { NMLS } \\
\text { H310700352 }\end{array}$ \\
\hline Effective Calibration Date: & $11 / 06 / 2007$ & \multirow{2}{*}{$\begin{array}{l}\text { Calibration Reference: } \\
\text { Logging Procedure: }\end{array}$} & \multicolumn{2}{|c|}{ HGLP-CC-021 } \\
\hline & & & \multicolumn{2}{|c|}{ HGLP-MAN-002, Rev. 0} \\
\hline
\end{tabular}

\section{Spectral Gamma Logging System (SGLS) Log Run Information:}

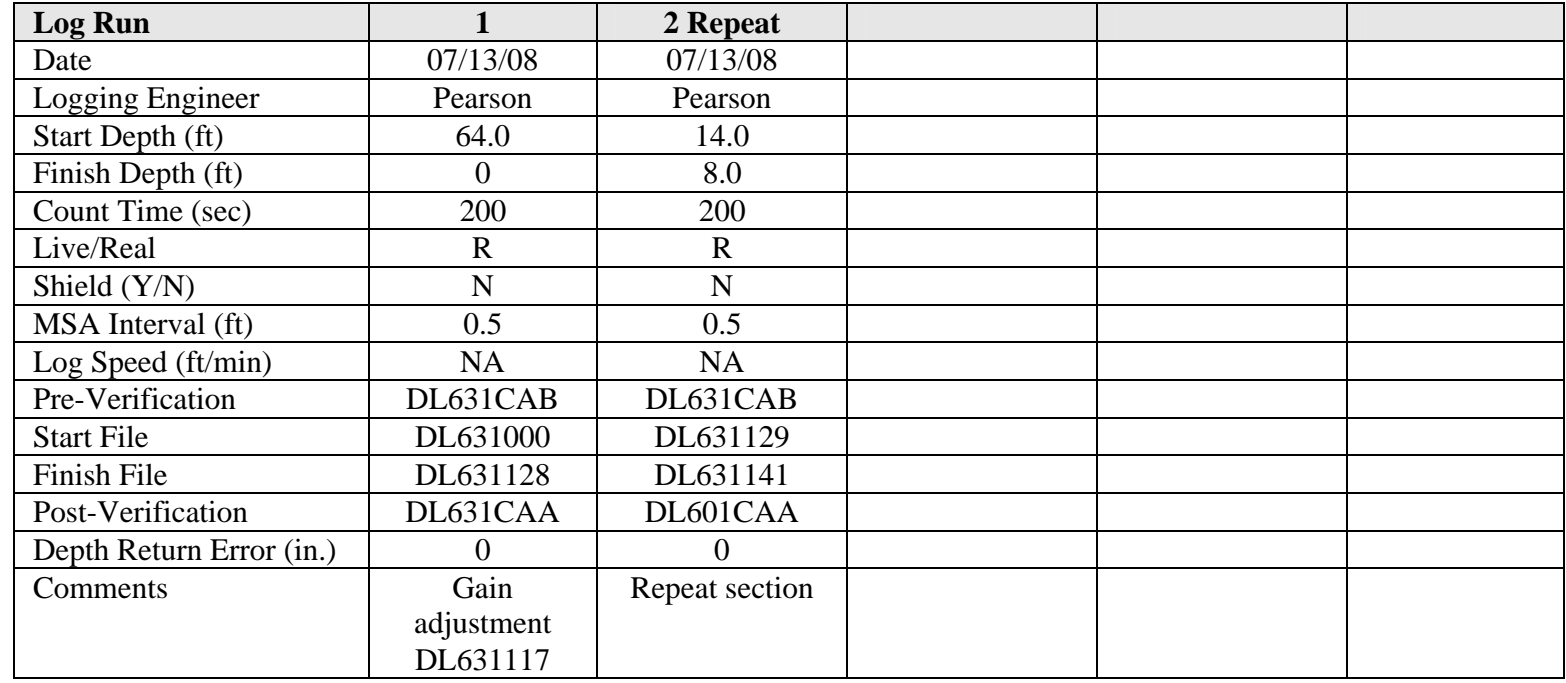


Neutron Moisture Logging System (NMLS) Log Run Information:

\begin{tabular}{|l|c|c|l|l|l|}
\hline Log Run & $\mathbf{3}$ & 4 Repeat & & & \\
\hline Date & $07 / 13 / 08$ & $07 / 13 / 08$ & & & \\
\hline Logging Engineer & Pearson & Pearson & & & \\
\hline Start Depth (ft) & 0 & 11.0 & & & \\
\hline Finish Depth (ft) & 29.5 & 14.0 & & & \\
\hline Count Time (sec) & 15 & 15 & & & \\
\hline Live/Real & $\mathrm{R}$ & $\mathrm{R}$ & & & \\
\hline Shield (Y/N) & $\mathrm{N}$ & $\mathrm{N}$ & & & \\
\hline MSA Interval (ft) & 0.25 & 0.25 & & & \\
\hline Log Speed (ft/min) & NA & NA & & & \\
\hline Pre-Verification & DHH02CAB & DHH02CAB & & & \\
\hline Start File & DHH02000 & DHH02119 & & & \\
\hline Finish File & DHH02118 & DHH02131 & & & \\
\hline Post-Verification & DHH02CAA & DHH02CAA & & & \\
\hline Depth Return Error (in.) & NA & NA & & & \\
\hline Comments & None & Repeat section & & & \\
\hline
\end{tabular}

\section{Logging Operation Notes:}

SGLS and NMLS data were both collected using Gamma 4, HO 68B-3573.

SGLS pre- and post-survey verification measurements were acquired in the Amersham KUTh-115 field verifier. Maximum SGLS logging depth achieved was $64.4 \mathrm{ft}$ or approximately $6.7 \mathrm{ft}$ below casing before the sonde un-weighted. A centralizer was installed on the sonde.

NMLS pre- and post-survey verification measurements were acquired in the standard field verifier. Maximum logging depth achieved was $29.5 \mathrm{ft}$, at approximately groundwater level. A centralizer was installed on the sonde.

Analysis Notes:

\begin{tabular}{|l|l|l|l|l|l|}
\hline Analyst: & R. SPATZ & Date: & 10/08/08 & Reference: & GJO-HGLP 1.6.3, Rev. 0 \\
\hline
\end{tabular}

Pre- and post-survey verification spectra for both SGLS and NMLS met the acceptance criteria for the established systems.

SGLS spectra were processed in batch mode using APTEC SUPERVISOR to identify individual peaks and count rates. Concentrations were calculated using an EXCEL template identified as G4LDec07.xls using an efficiency function and corrections for casing, dead time, and water as determined by annual calibrations.

NMLS spectra were processed in batch mode using APTEC SUPERVISOR to identify counts. Count rates were calculated using an EXCEL template identified as G4HNov07.xls. NMLS data are presented in counts per second (cps), because no calibration data is available for a 6 7/8-in. inside diameter borehole casing.

Analyses of log data suggest slight discrepancies with field reports of depth-to-water and casing depth. Corrections of log data were applied according to the analysis assumptions in the table below. 
HGLP-LDR-260, Rev. 0

\begin{tabular}{|c|c|c|c|l|}
\hline Top (ft) & Bottom (ft) & $\begin{array}{c}\text { T } \\
\text { (in) }\end{array}$ & $\begin{array}{c}\text { ID } \\
\text { (in) }\end{array}$ & \multicolumn{1}{|c|}{ Comments } \\
\hline 0 & 29.4 & 0.375 & N/A & No water correction \\
\hline 29.5 & 57.0 & 0.375 & 6.875 & $\begin{array}{l}\text { GWL reported @ 29.2 ft; adjusted to 29.5 ft } \\
\text { Casing bottom reported @57.7 ft; adjusted to 57.0 ft }\end{array}$ \\
\hline 57.1 & TD & 0 & 0 & No adjustment made \\
\hline
\end{tabular}

$\mathrm{T}=$ casing thickness used to calculate energy-dependent casing correction factor

ID = casing inside diameter used to calculate energy-dependent water correction factor

\section{Results and Interpretations:}

U-235 and U-238 (Pa-234m) were detected sporadically in this borehole. Inspection of the individual spectra at these depths indicates that these detections are statistical fluctuations associated with the processing software and are not considered valid. No Cs-137 or Co-60 was detected.

Between 10.0 and $12.5 \mathrm{ft}$, and below the $54.0 \mathrm{ft}$ depth, probable lithologic changes occur, which is shown by the fluctuating KUT concentrations and increased Total Gamma count rates. The KUT and Moisture plots both indicate good repeatability.

\section{List of Log Plots:}

Depth Reference is ground surface

Manmade Radionuclides

Natural Gamma Logs

Combination Plot

Total Gamma \& Dead Time

Total Gamma \& Moisture

Repeat Section of Natural Gamma Logs

Moisture Repeat Section

${ }^{1} \mathrm{GWL}$ - groundwater level

${ }^{2}$ TOC - top of casing 

Hanford Office Manmade Radionuclides

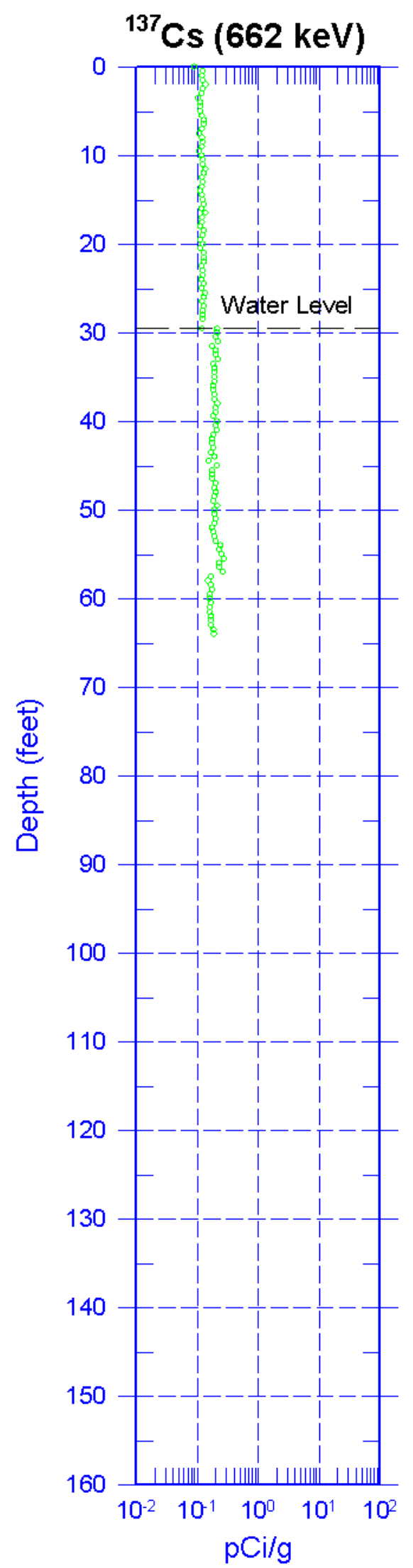

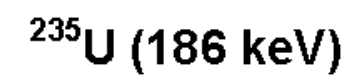
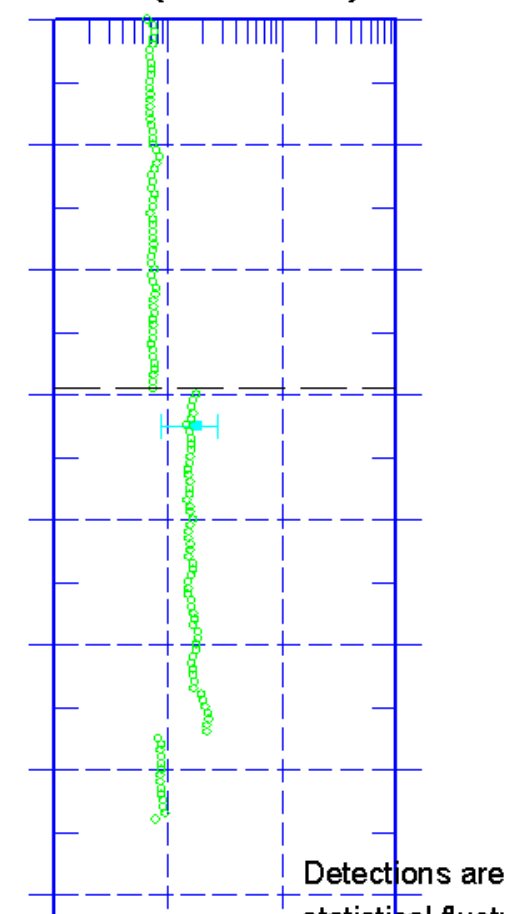

statistical fluctuati

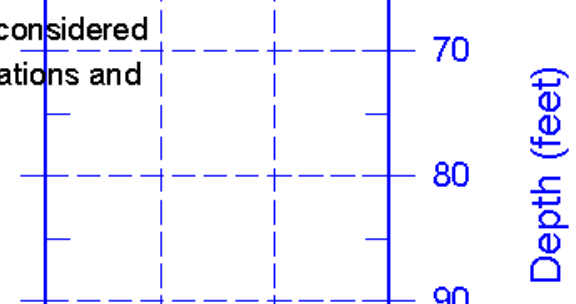

are no valid

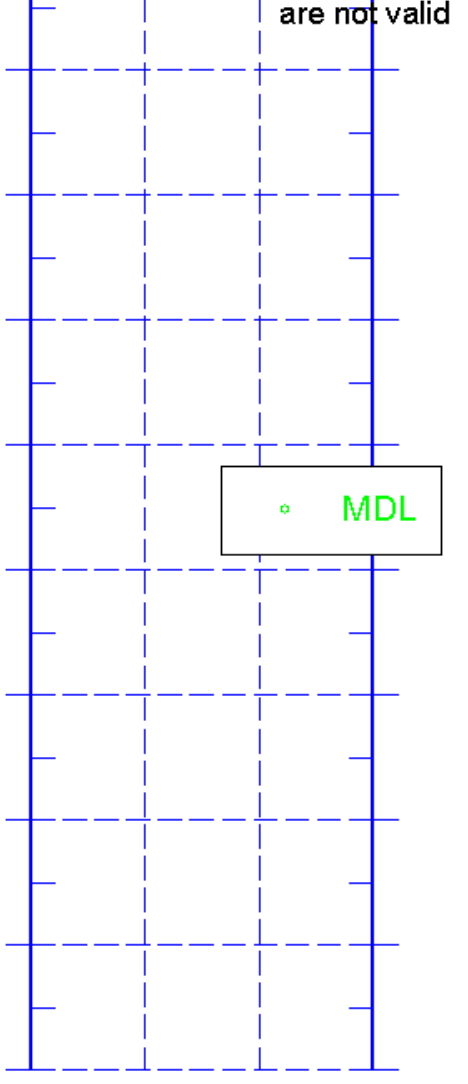

$$
\begin{aligned}
& \text { பـШШШ_ШШШ_ШШШ } \\
& \begin{array}{llll}
10^{-1} & 10^{0} & 10^{1} & 10^{2}
\end{array} \\
& \text { pCi/g }
\end{aligned}
$$


toller

399-3-23 (C6194)

Hanford Office

Natural Gamma Logs
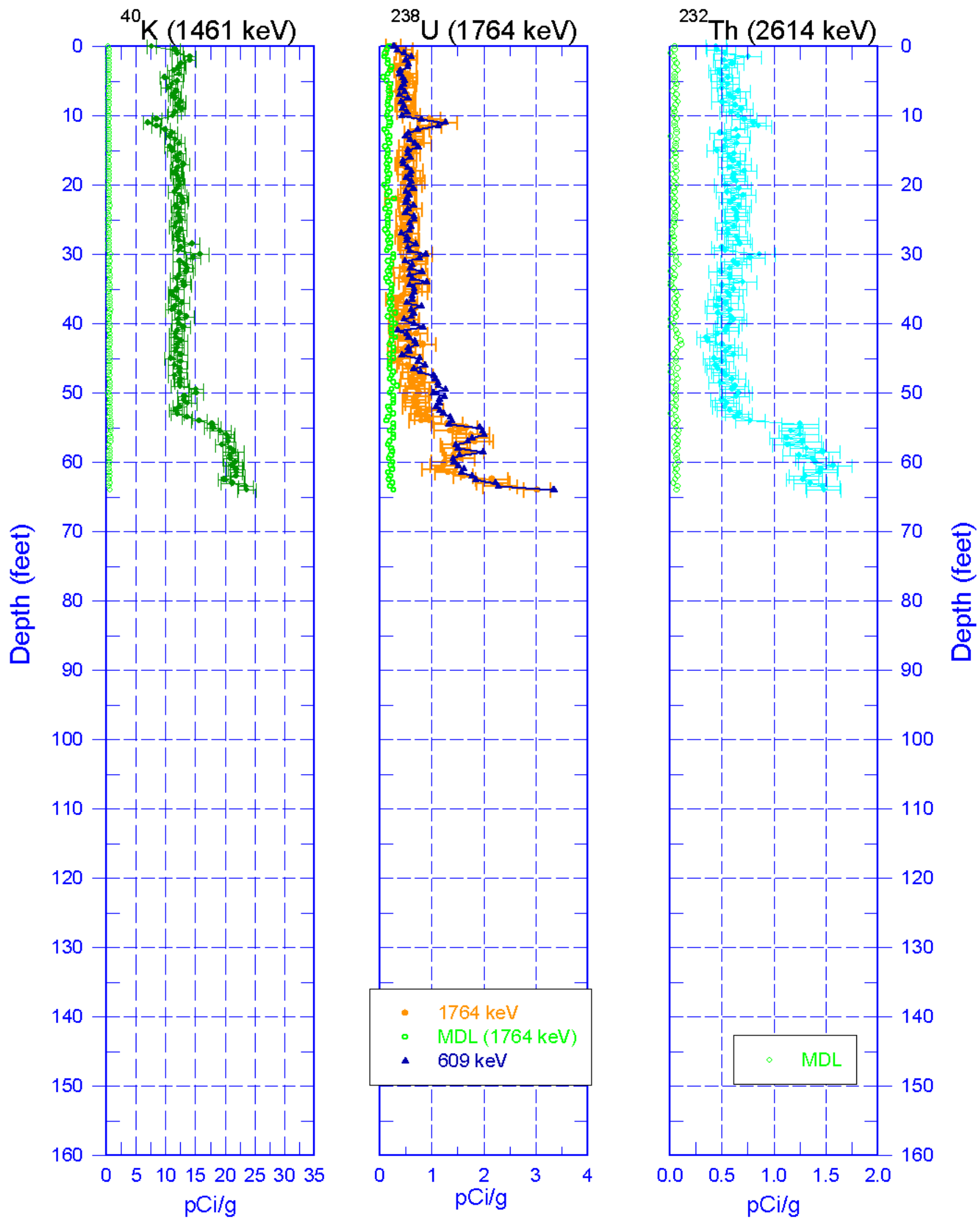

Zero Reference - Ground Surface 


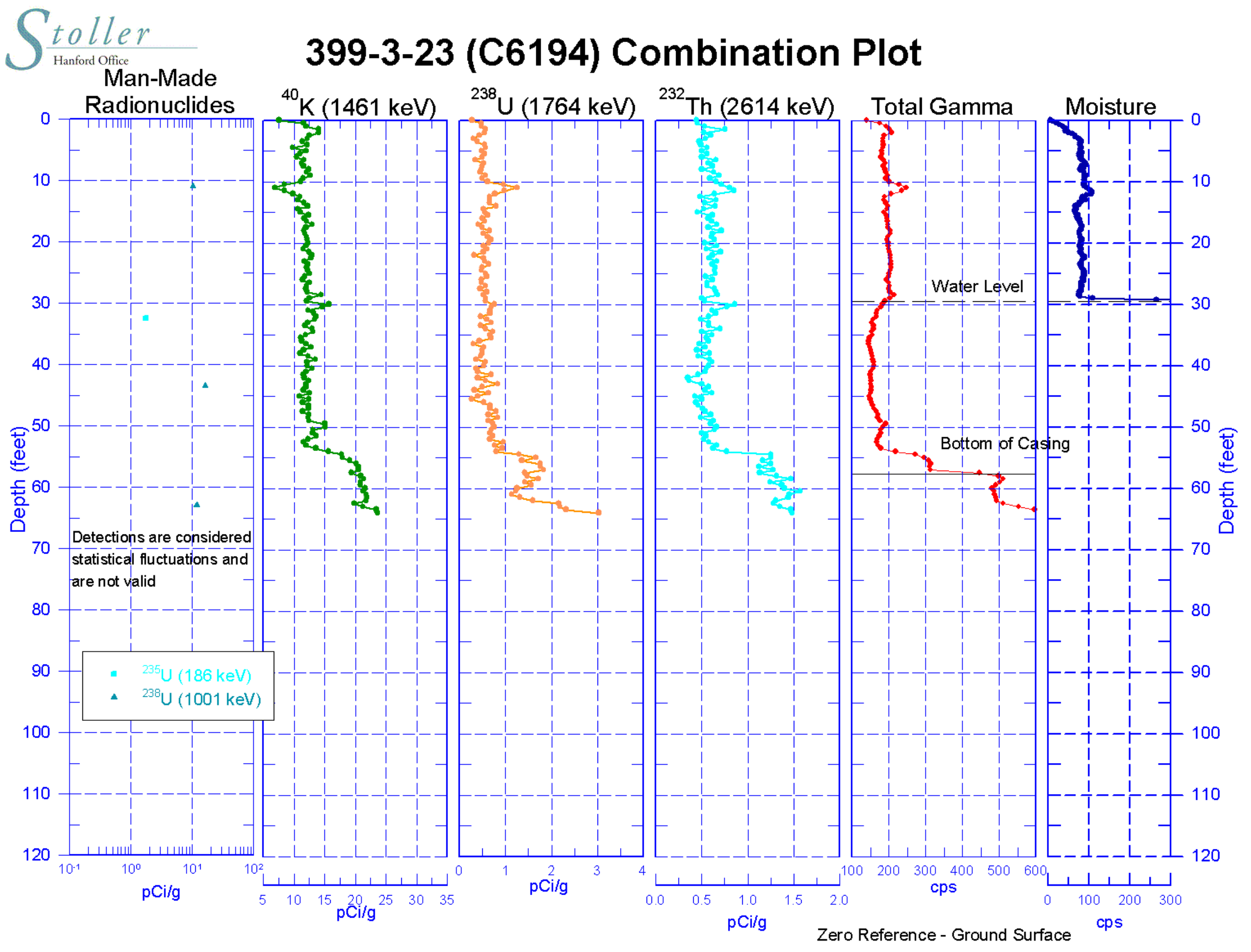



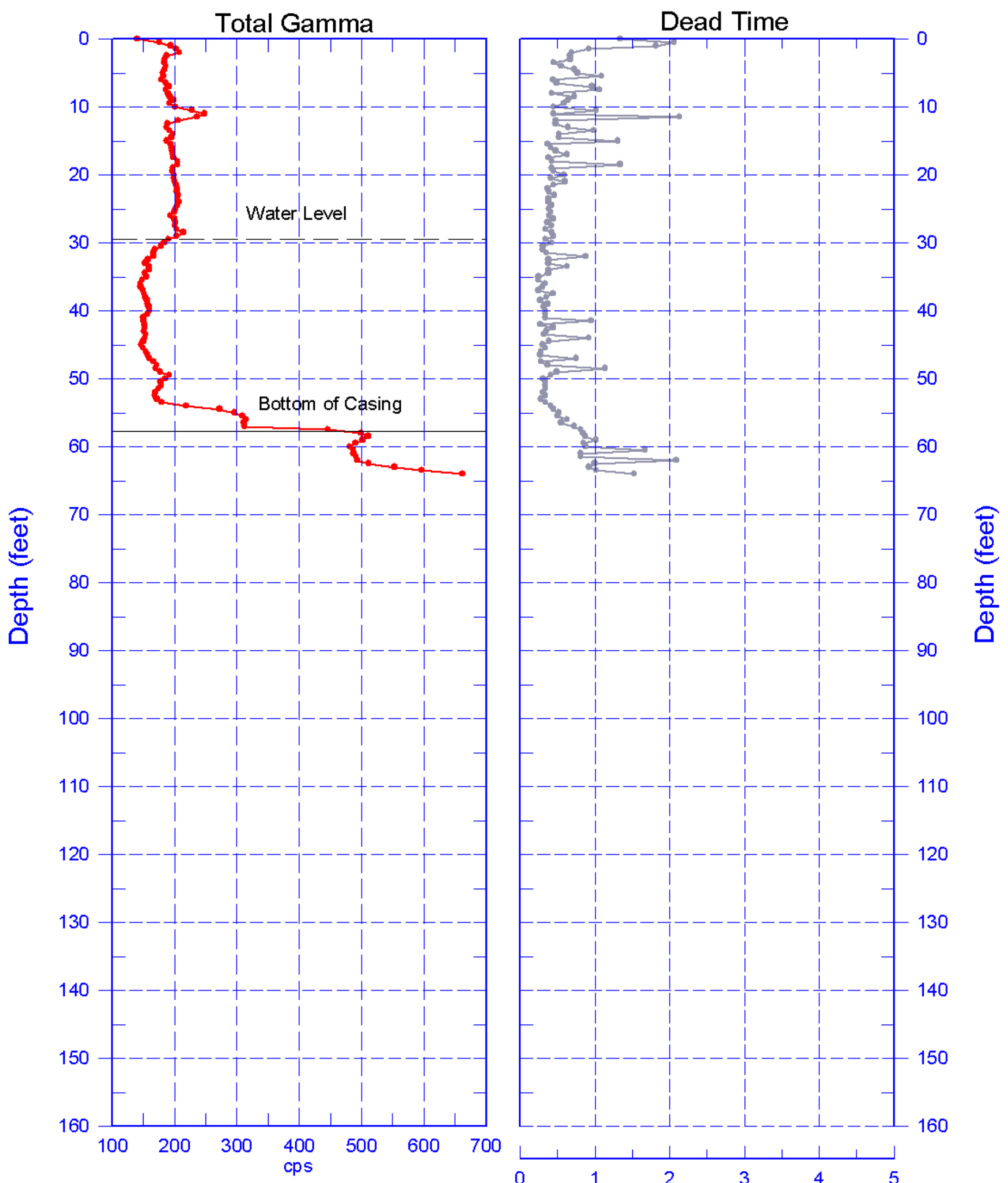

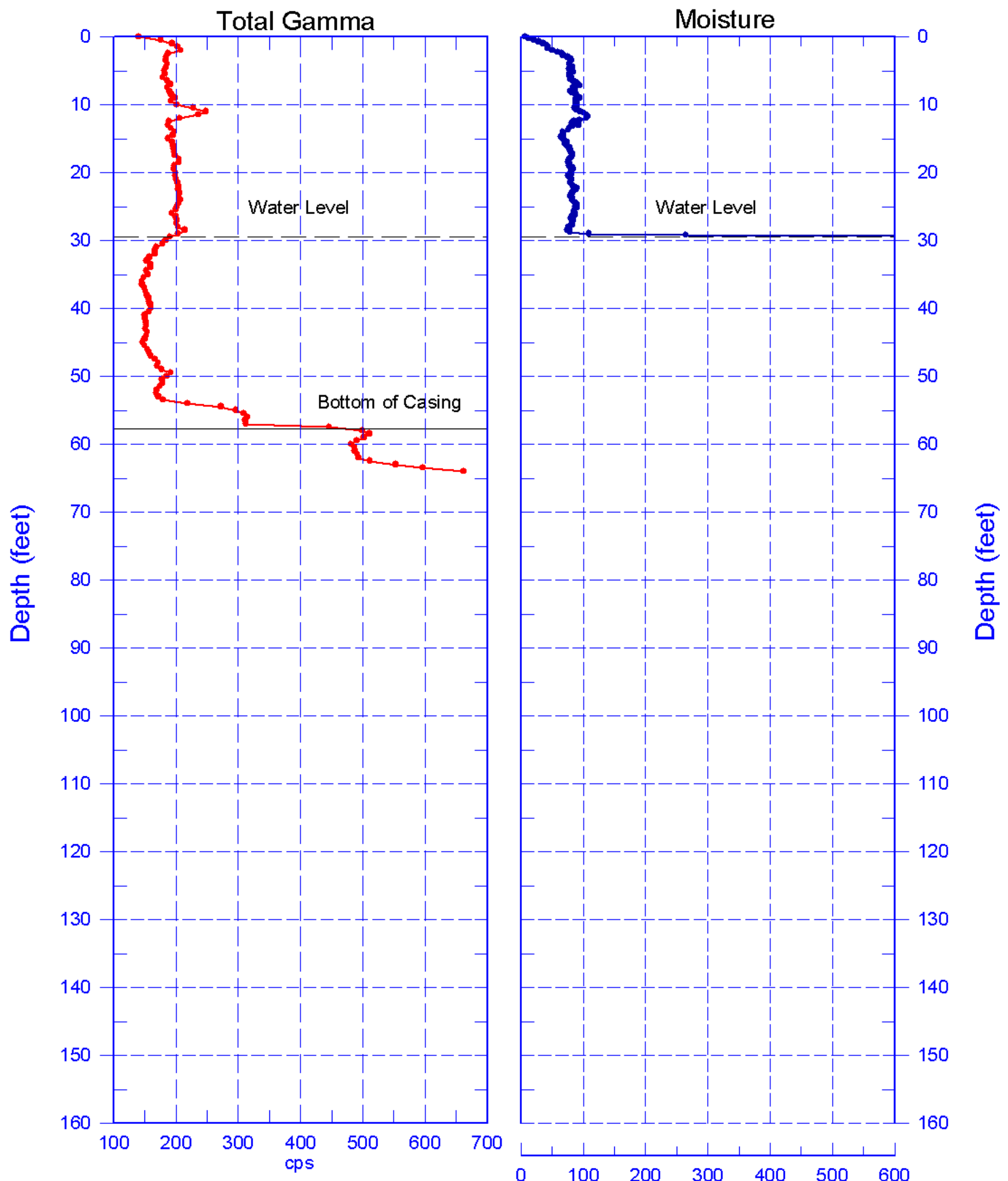


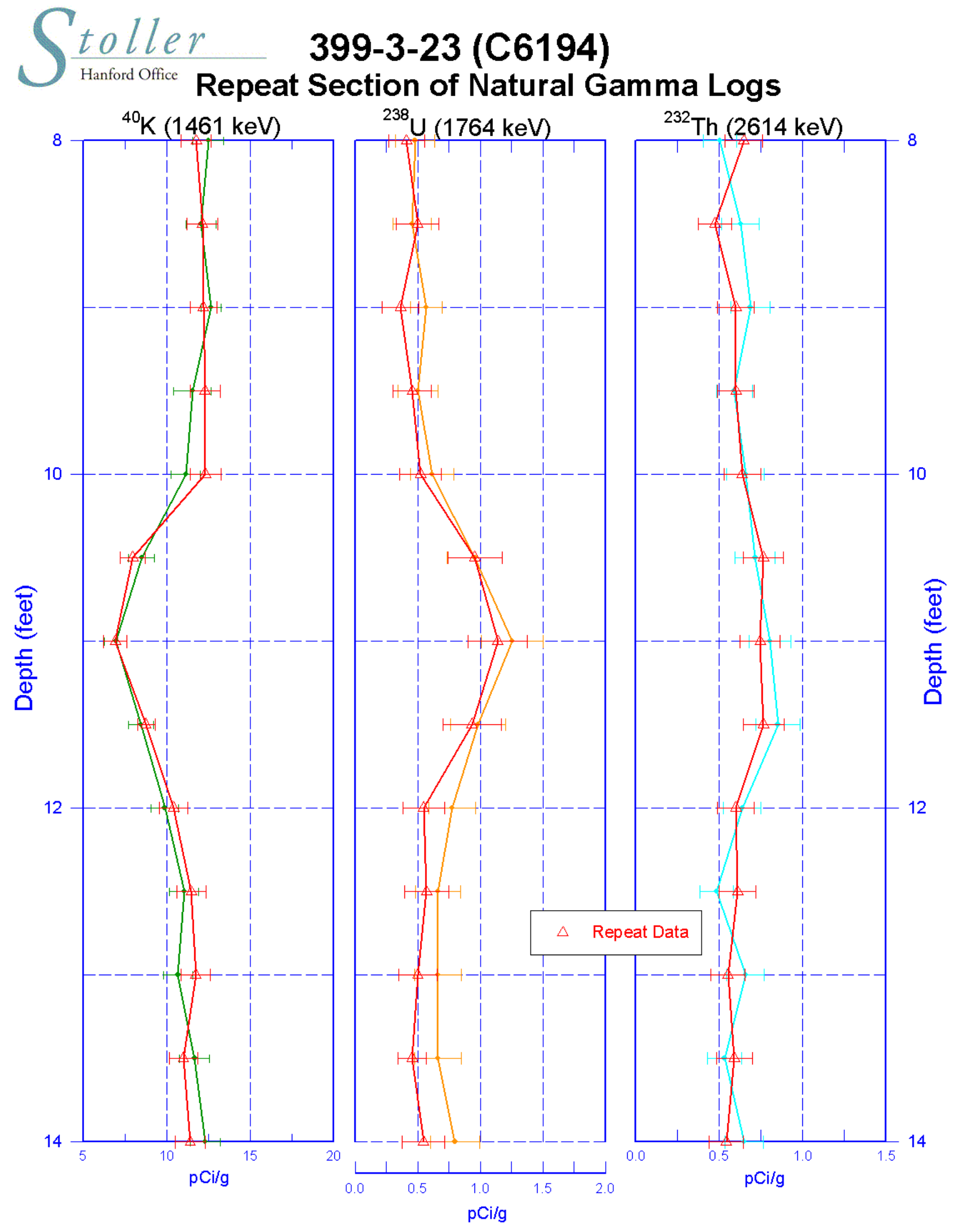




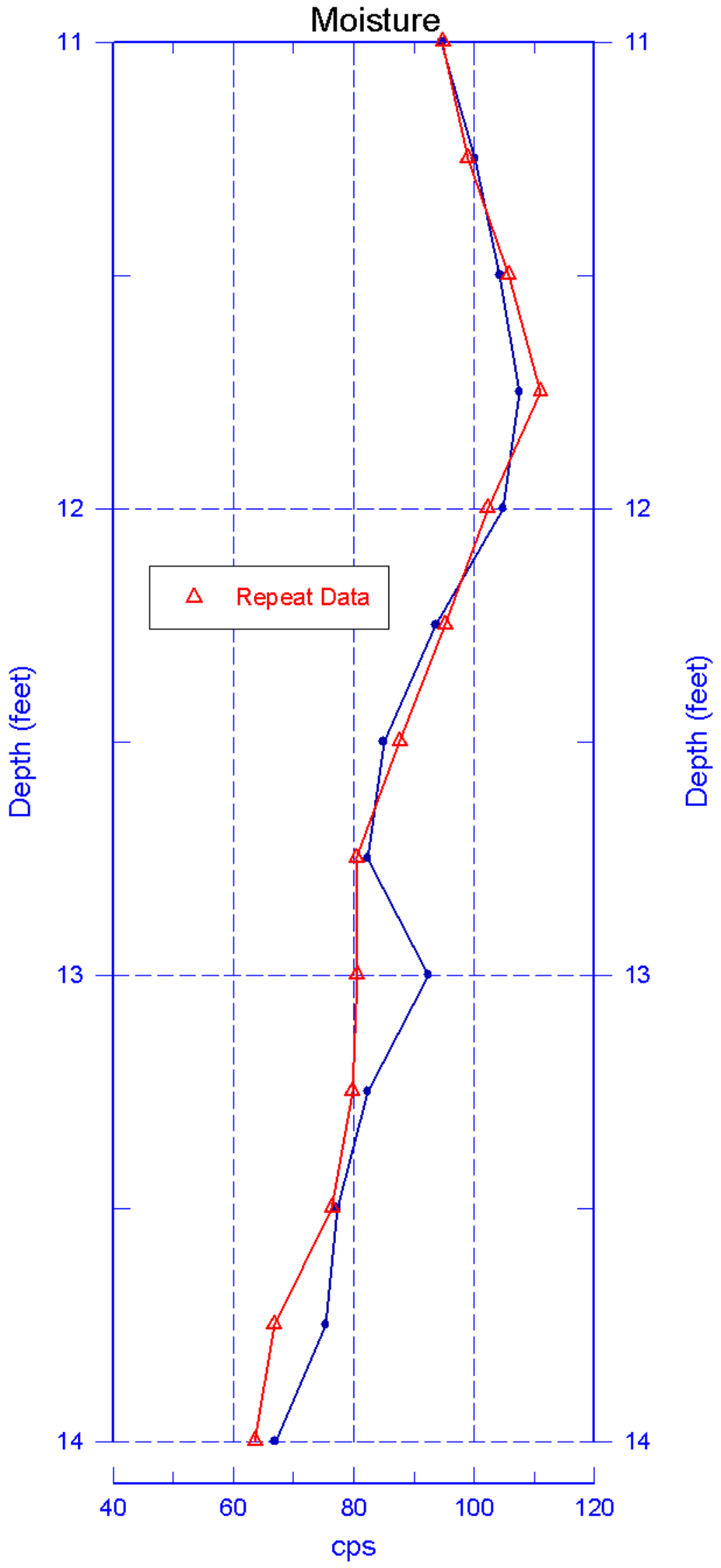




\section{9-3-24 (C6199) Log Data Report}

Borehole Information:

\begin{tabular}{|c|c|c|c|c|c|}
\hline \multicolumn{2}{|c|}{ Borehole: $\quad$ 399-3-24 (C6199) } & & \multicolumn{3}{|c|}{ 300-FF-5 } \\
\hline \multicolumn{2}{|c|}{ Coordinates (WA St Plane) } & $G_{W L}^{1}(\mathbf{f}$ & 29 & GWL Date: & $7 / 11 / 2008$ \\
\hline North (m) & East (m) & Drill Date & TOC $^{2}$ Elevation & Total Depth (ft) & Type \\
\hline 116044.6 & 594238.7 & $7 / 10 / 2008$ & Unknown & 65 & Sonic \\
\hline
\end{tabular}

\section{Casing Information:}

\begin{tabular}{|c|c|c|c|c|c|c|}
\hline Casing Type & Stickup (ft) & $\begin{array}{c}\text { Outer } \\
\text { Diameter (in.) }\end{array}$ & $\begin{array}{c}\text { Inside } \\
\text { Diameter (in.) }\end{array}$ & Thickness (in.) & Top (ft) & Bottom (ft) \\
\hline Threaded Steel & 2.6 & $77 / 8$ & $67 / 8$ & $3 / 8$ & 2.6 & 58 \\
\hline
\end{tabular}

\section{Borehole Notes:}

Casing data and total depth was reported by the site geologist. Casing diameters were measured by the logging engineer using a steel tape and rounded to the nearest 1/16 inch. The zero reference is the ground surface.

\section{Logging Equipment Information:}

\begin{tabular}{|l|l|l|l|l|}
\hline \multirow{2}{*}{ Logging System: } & \multirow{2}{*}{ Gamma 4 L } & $\begin{array}{l}\text { Type: } \\
\text { Serial No.: }\end{array}$ & $\begin{array}{l}\text { 60\% HPGE SGLS } \\
\text { 47TP32211A }\end{array}$ \\
\hline Effective Calibration Date: & $12 / 31 / 2007$ & $\begin{array}{l}\text { Calibration Reference: } \\
\text { Logging Procedure: }\end{array}$ & HGLP-CC-027 \\
\cline { 4 - 4 } & & HGLP-MAN-002; Rev. 0 \\
\hline
\end{tabular}

\begin{tabular}{|l|l|l|l|l|}
\hline \multirow{2}{*}{ Logging System: } & \multirow{2}{*}{ Gamma $4 \mathrm{H}$} & $\begin{array}{l}\text { Type: } \\
\text { Serial No.: }\end{array}$ & $\begin{array}{l}\text { NMLS } \\
\text { H310700352 }\end{array}$ \\
\hline Effective Calibration Date: & $11 / 06 / 2007$ & $\begin{array}{l}\text { Calibration Reference: } \\
\text { Logging Procedure: }\end{array}$ & HGLP-CC-021, Rev. 0 \\
\cline { 4 - 5 } & & HGLP-MAN-002; Rev. 0 \\
\hline
\end{tabular}

\section{Spectral Gamma Logging System (SGLS) Log Run Information:}

\begin{tabular}{|c|c|c|c|c|c|}
\hline Log Run & 1 & 2 Repeat & & & \\
\hline Date & $07 / 12 / 08$ & $07 / 12 / 08$ & & & \\
\hline Logging Engineer & Pearson & Pearson & & & \\
\hline Start Depth (ft) & 64.0 & 6.0 & & & \\
\hline Finish Depth (ft) & 0.0 & 12.0 & & & \\
\hline Count Time (sec) & 200 & 200 & & & \\
\hline Live/Real & $\mathrm{R}$ & $\mathrm{R}$ & & & \\
\hline Shield (Y/N) & NA & NA & & & \\
\hline MSA Interval (ft) & 0.5 & 0.5 & & & \\
\hline Log Speed (ft/min) & NA & NA & & & \\
\hline Pre-Verification & DL621CAB & DL621CAB & & & \\
\hline Start File & DL621000 & DL621129 & & & \\
\hline Finish File & DL621128 & DL621141 & & & \\
\hline Post-Verification & DL621CAA & DL621CAA & & & \\
\hline Depth Return Error (in.) & $1 / 2$ low & 0 & & & \\
\hline Comments & Repeat Section & None & & & \\
\hline
\end{tabular}


Neutron Moisture Logging System (NMLS) Log Run Information:

\begin{tabular}{|c|c|c|l|l|l|}
\hline Log Run & $\mathbf{1}$ & 2 Repeat & & & \\
\hline Date & $7 / 12 / 2008$ & $7 / 12 / 2008$ & & & \\
\hline Logging Engineer & Pearson & Pearson & & & \\
\hline Start Depth (ft) & 0.0 & 10.0 & & & \\
\hline Finish Depth (ft) & 29.0 & 13.0 & & & \\
\hline Count Time (sec) & 15 & 15 & & & \\
\hline Live/Real & R & R & & & \\
\hline Shield (Y/N) & NA & NA & & & \\
\hline MSA Interval (ft) & $0.25 \mathrm{ft}$ & $0.25 \mathrm{ft}$ & & & \\
\hline ft/min & NA & NA & & & \\
\hline Pre-Verification & DHG92CAB & DHG92CAB & & & \\
\hline Start File & DHG92000 & DHG92117 & & & \\
\hline Finish File & DHG92116 & DHG92129 & & & \\
\hline Post-Verification & DHG92CAA & DHG92CAA & & & \\
\hline Depth Return Error (in.) & NA & 1/4 high & & & \\
\hline Comments & None & Repeat section. & & & \\
\hline
\end{tabular}

\section{Logging Operation Notes:}

The SGLS data were acquired 7/12/2008 with the Gamma 4L, HO 68B-3573 detector. SGLS pre- and post-survey verification measurements were acquired in the Amersham KUTh-115 field verifier. A centralizer was installed on the sonde. Maximum logging depth was 64.2 feet before the sonde was un-weighted.

The Neutron Moisture data were acquired 7/12/2008 with Gamma 4H, HO 68B-3573 detector. NMLS pre- and post-survey verification measurements were acquired in the standard field verifier. A centralizer was installed on the sonde. Maximum logging depth was 29.0 feet before water was encountered.

\section{Analysis Notes:}

\begin{tabular}{|l|l|l|l|l|l|}
\hline Analyst: & L SPINNER & Date: & 09/10/2008 & Reference: & GJO-HGLP 1.6.3, Rev. 0 \\
\hline
\end{tabular}

The SGLS and NMLS pre- and post-survey verifications spectra met acceptance criteria.

A casing correction for a 3/8-inch casing was applied for the eight inch threaded steel casing from 0 to 58 feet, leaving seven feet of the remaining borehole uncorrected. A water correction was applied from 29 feet to end of logging depth.

SGLS spectra were processed in batch mode using APTEC SUPERVISOR to identify individual peaks and count rates. Concentrations were calculated using an EXCEL template identified as G4LDec07.xls using an efficiency function and corrections for casing, dead time and water as determined by annual calculations. NMLS spectra were processed in batch mode in APTEC SUPERVISOR to identify counts. Count rates were calculated using an EXCEL template identified as G4HNov07.xls. NMLS data are presented in counts per second (cps), because no calibration data is available for a 6 7/8 inch inner diameter borehole casing.

\section{Results and Interpretations:}

Cs -137, U-235 and U-238 (Pa-234m) were identified by the routine processing software at various depth intervals. Additional scrutiny of the individual spectra indicated no valid energy peaks and the detections are considered statistical fluctuations.

Co-60 was detected at $0.07 \mathrm{pCi} / \mathrm{g}$ at 13.5 feet. This detection is valid and the $1333 \mathrm{keV}$ energy peak is corroborated by the Co-60 energy peak at $1173 \mathrm{keV}$.

The variations in the Natural Gamma Logs may indicate a stratigraphy change at 53 feet. 
HGLP-LDR-246, Rev. 0

The Natural Gamma Logs (KUT) and the Moisture Repeat plot indicate good repeatability.

\section{List of Log Plots:}

Depth Reference is ground surface

Manmade Radionuclides

Natural Gamma Logs

Combination Plot (0-120 feet), with Moisture

Total Gamma \& Dead Time

Total Gamma \& Moisture

Repeat Section of Natural Gamma Logs (6-12 ft)

Moisture Repeat Section (10-13 ft.)

${ }^{1} \mathrm{GWL}$ - groundwater level

${ }^{2}$ TOC - top of casing 

Hanford Office Manmade Radionuclides

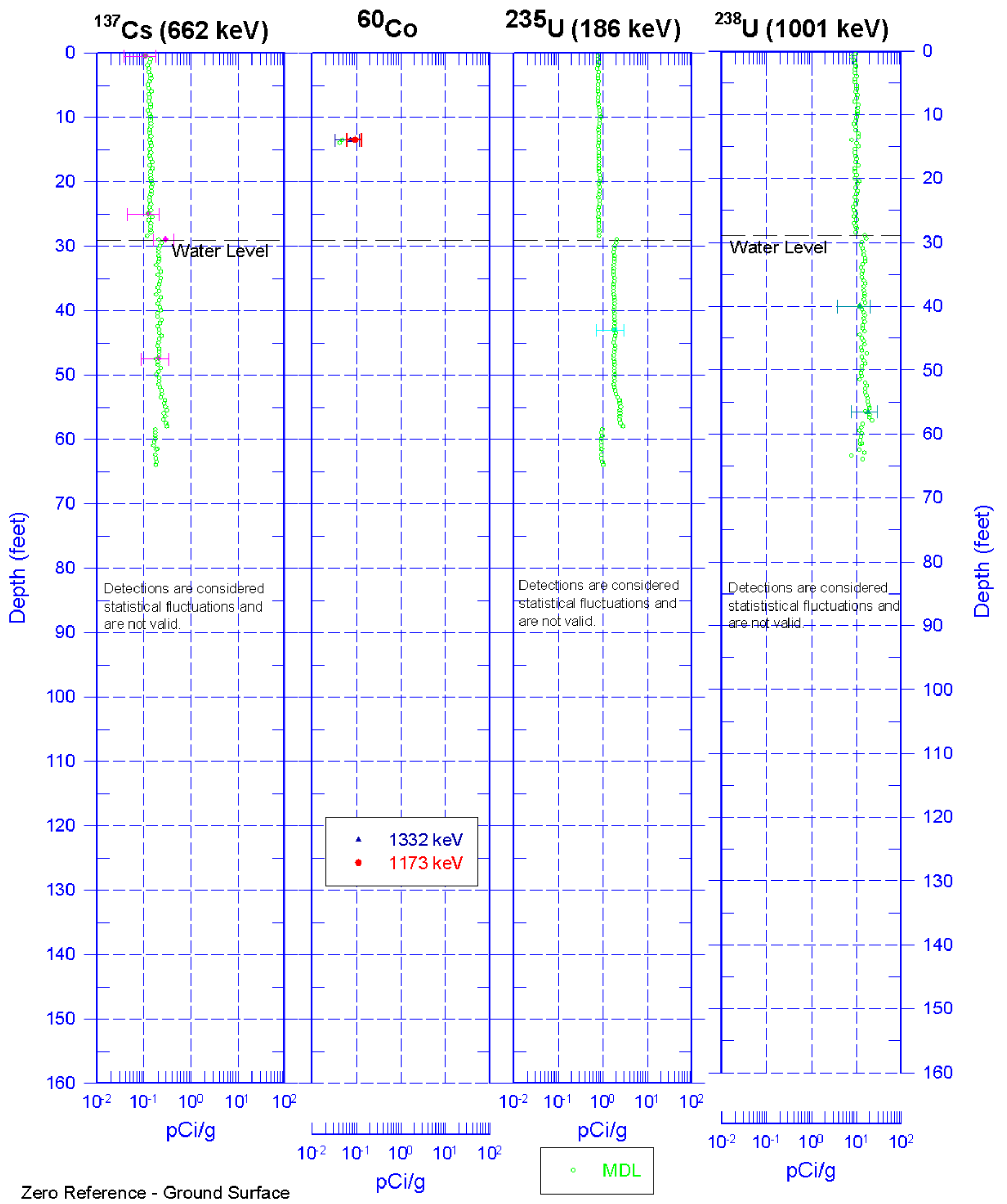




\section{$S_{\text {- }}$ toller 399-3-24 (C6199) Hanford Office Natural Gamma Logs}
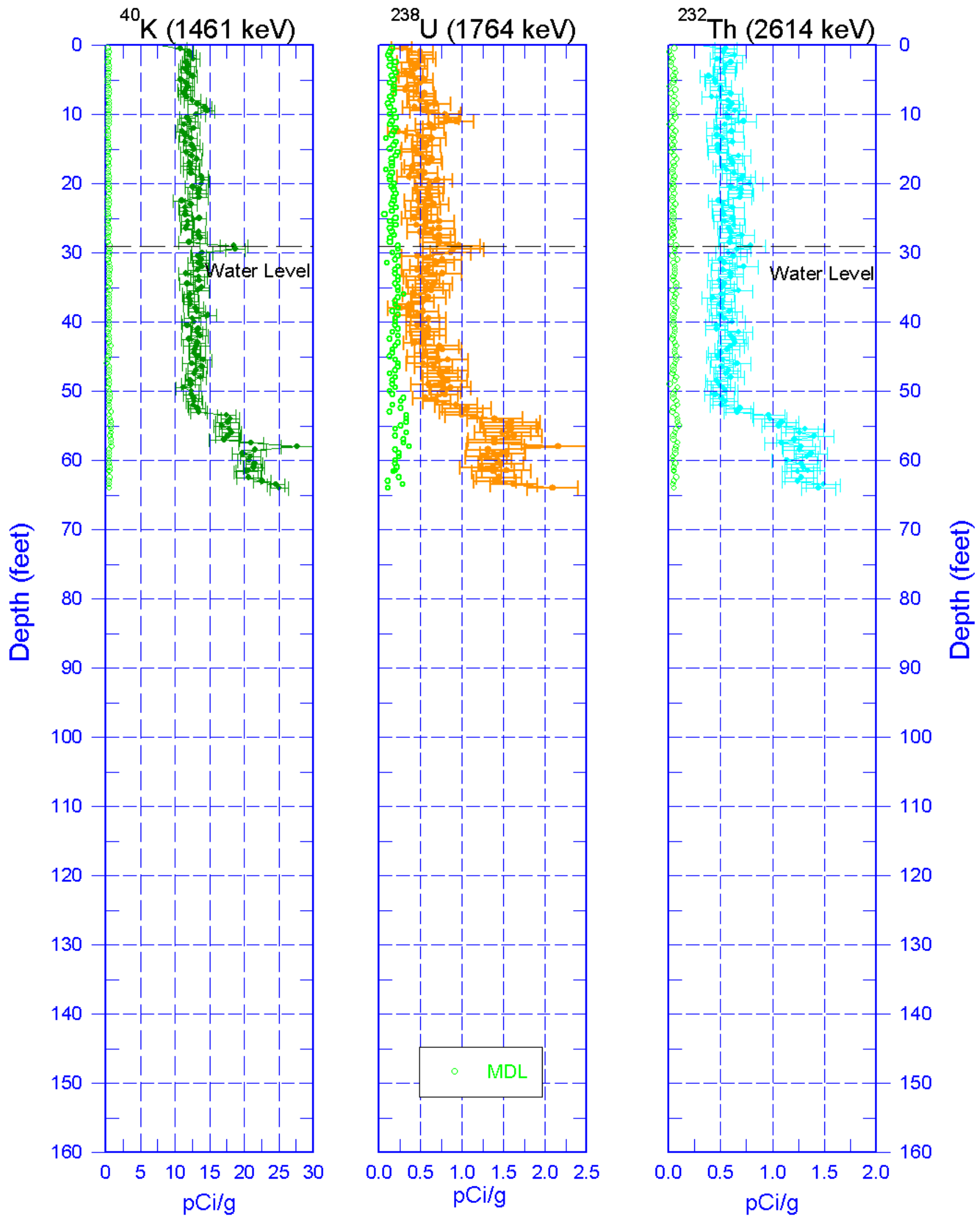
Stoller

399-3-24 (C6199) Combination Plot (0-120 ft.)

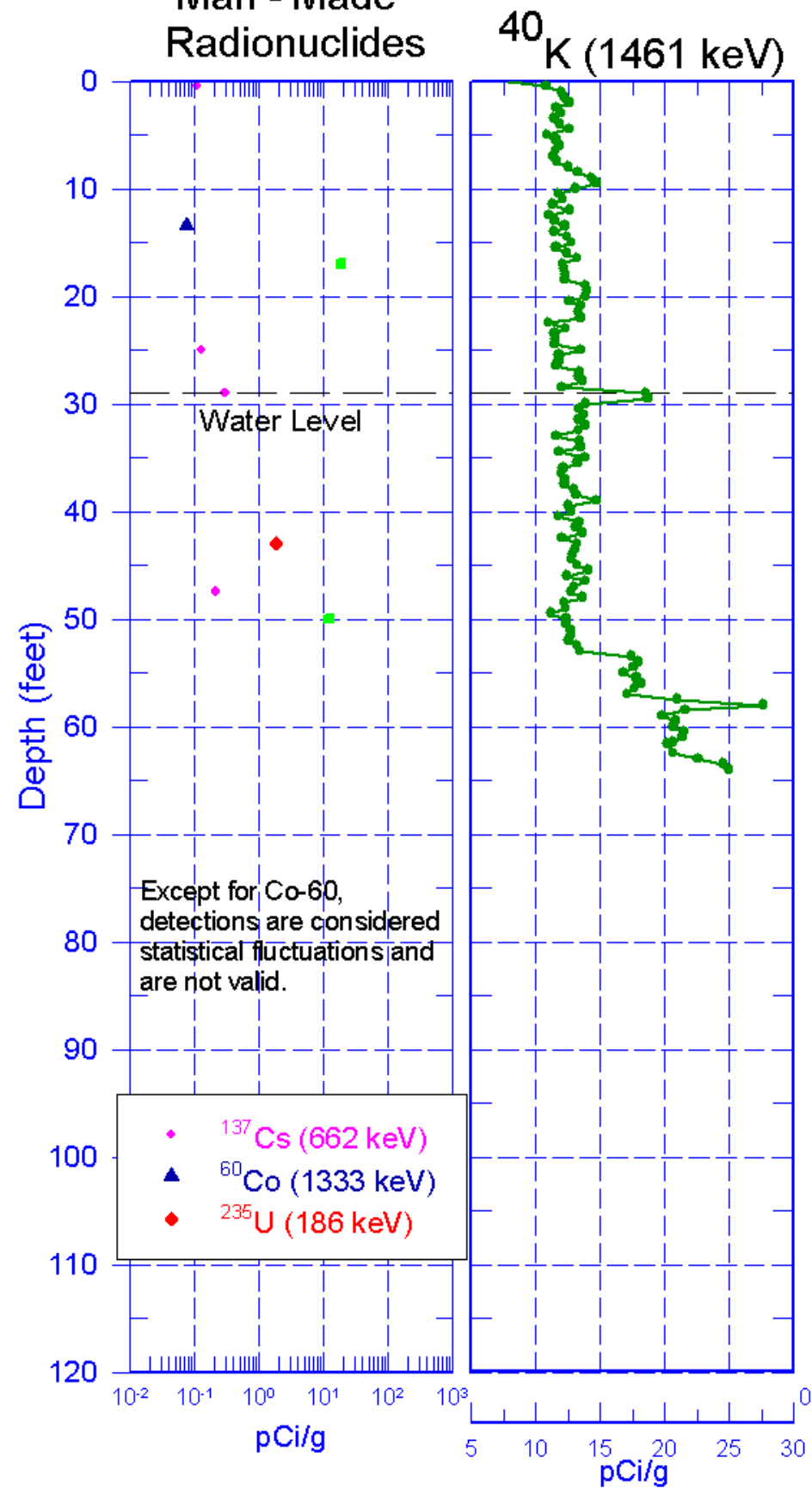

${ }^{238} \mathrm{U}(1764 \mathrm{keV}){ }^{232} \mathrm{Th}(2614 \mathrm{keV})$

Total Gamma
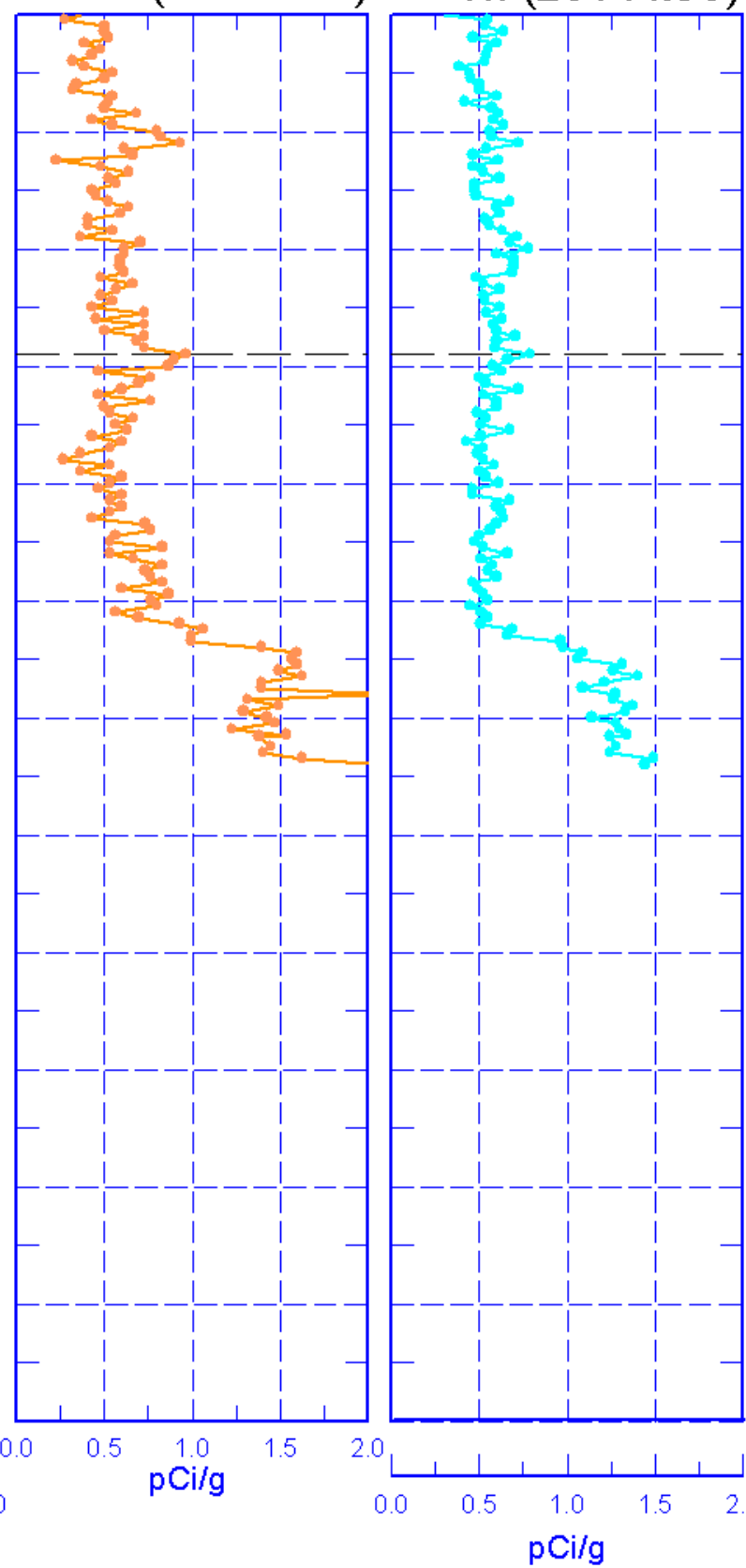

Zero Reference - Ground surface

Moisture

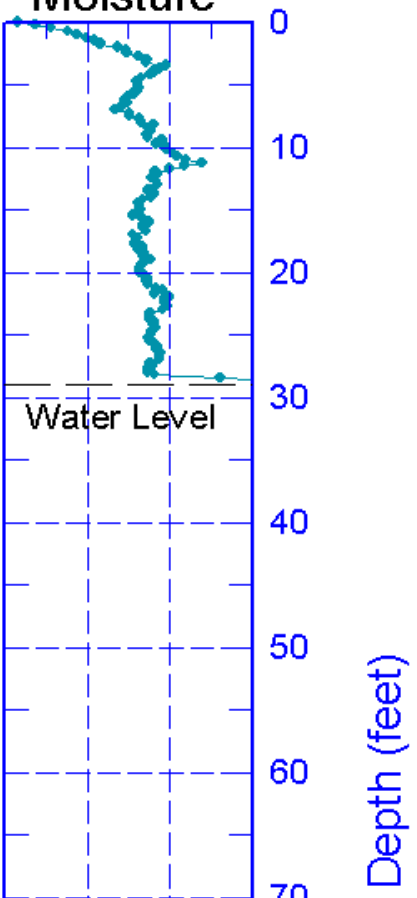




\section{Stoller}

Hanford Office

\section{Total Gamma \& Dead Time}
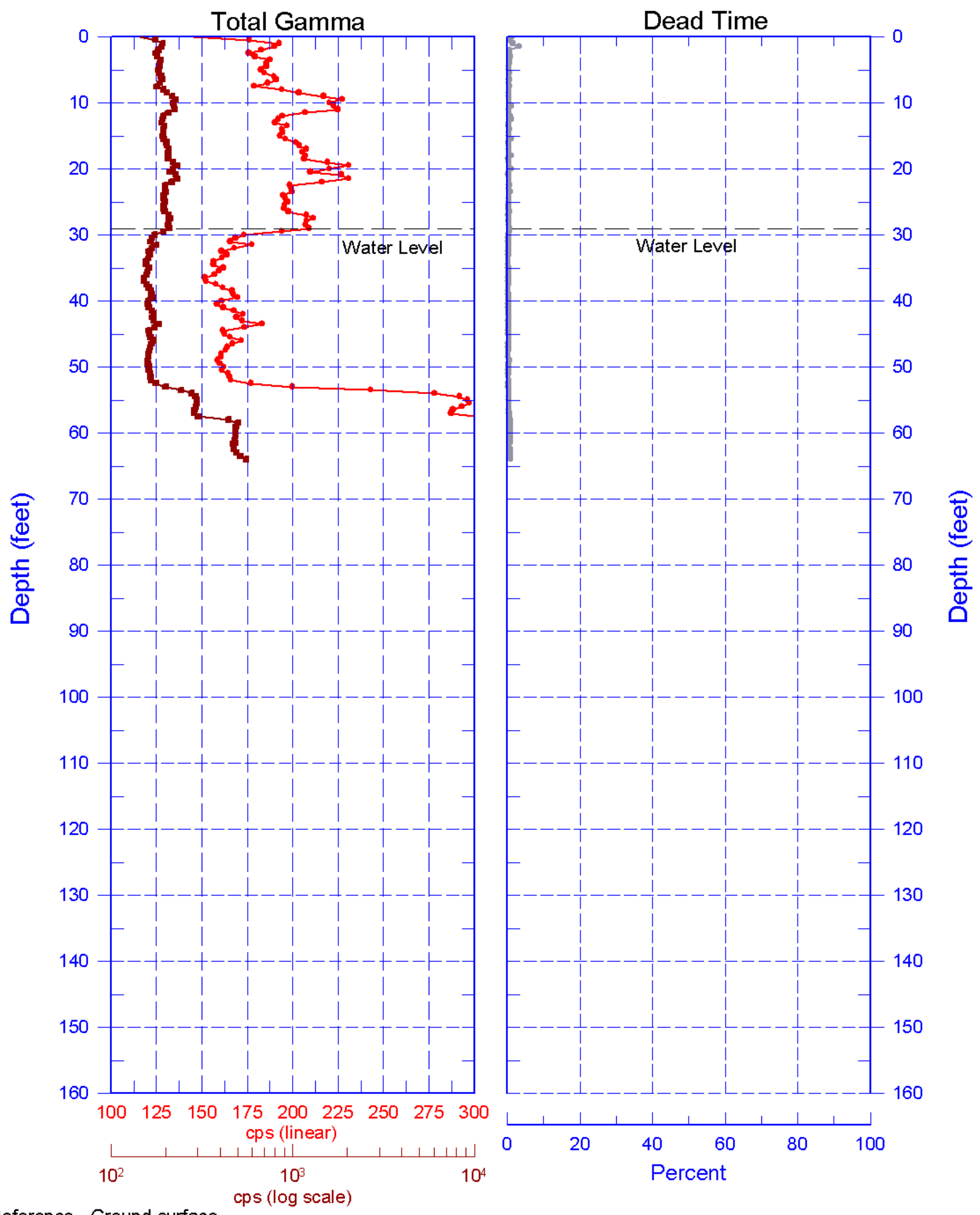

Reference - Ground surface 
toller 399-3-24 (C6199)

Hanford Office

Total Gamma \& Moisture
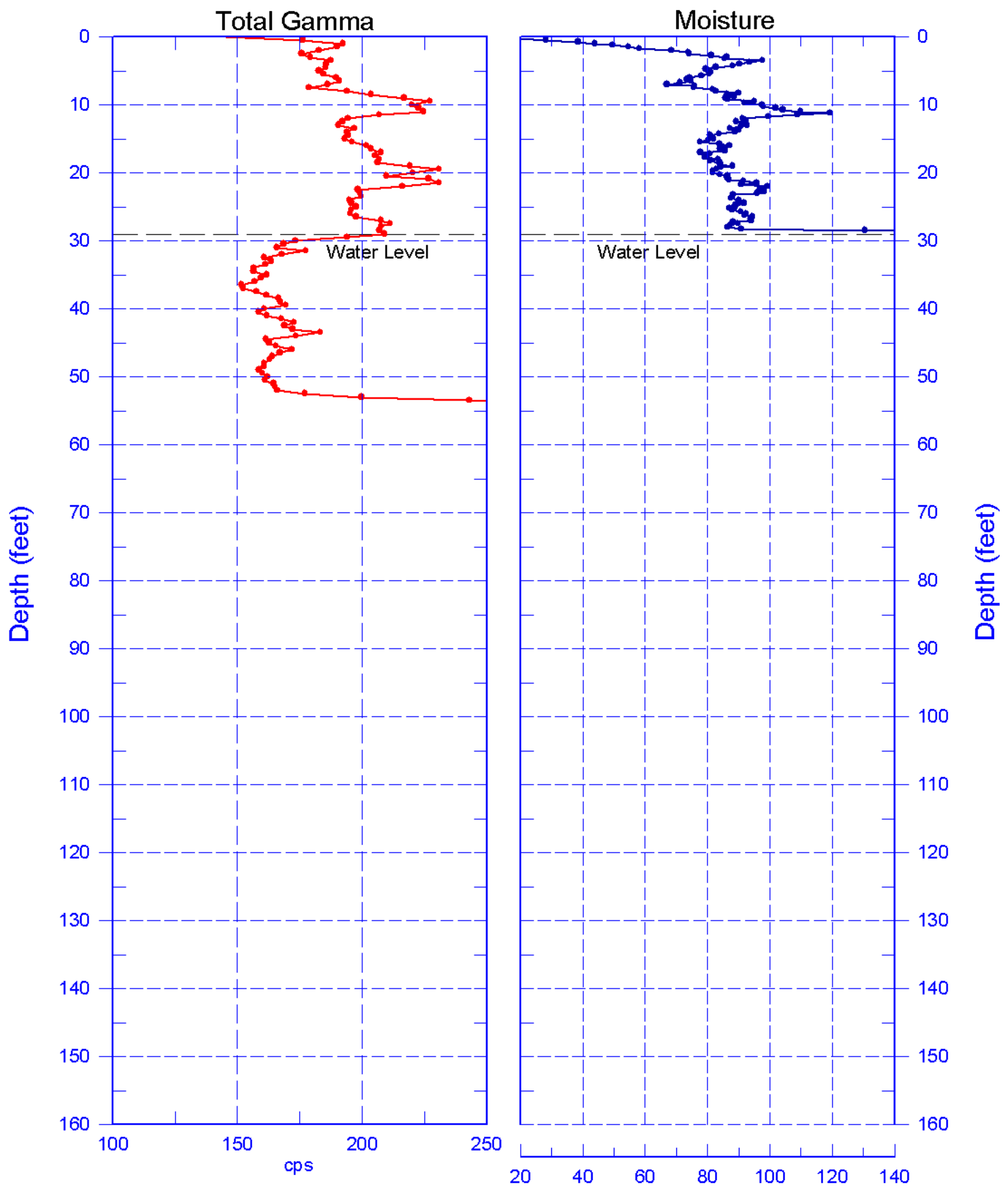

$\frac{0}{0}$
$\stackrel{0}{0}$
$\frac{5}{0}$
$\frac{0}{0}$

Reference - Ground Surface 
toller

Hanford Office

\section{9-3-24 (C6199)}

Repeat Section of Natural Gamma Logs (6-12 ft.)
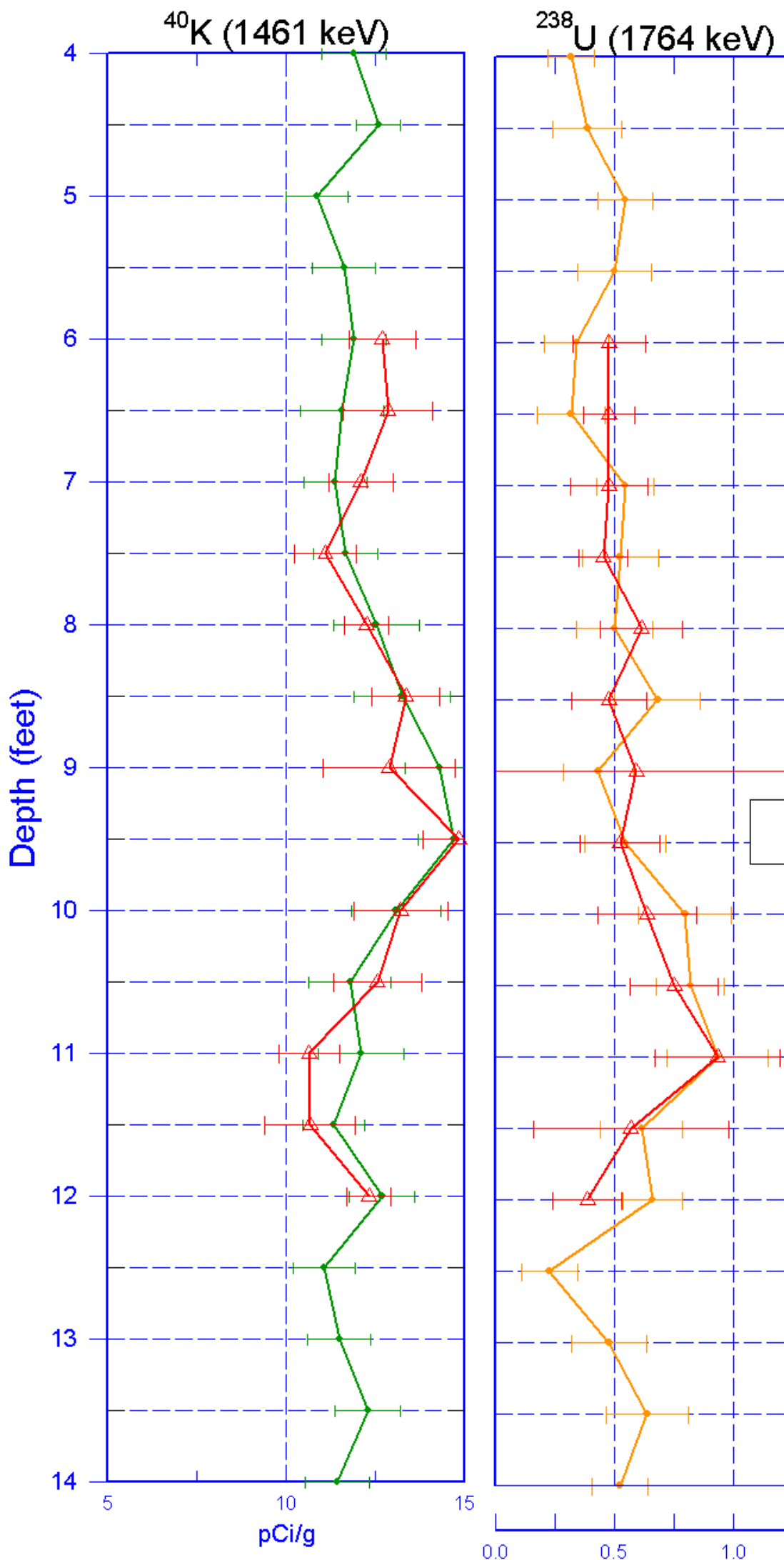

${ }^{232}$ Th (2614 keV)
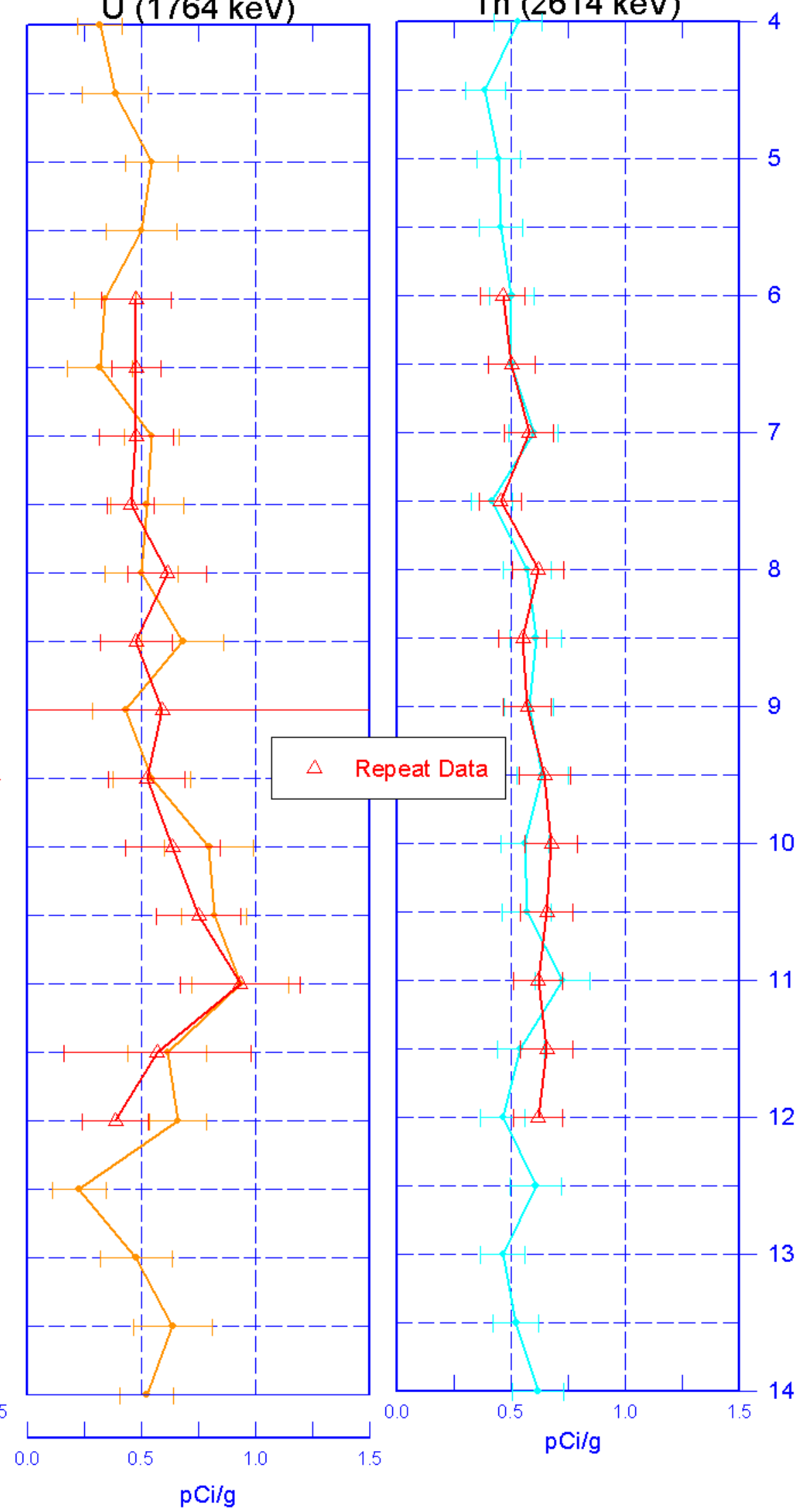
Stoller 399-3-24 (C6199)

Hanford Office

\section{Moisture Repeat Section}

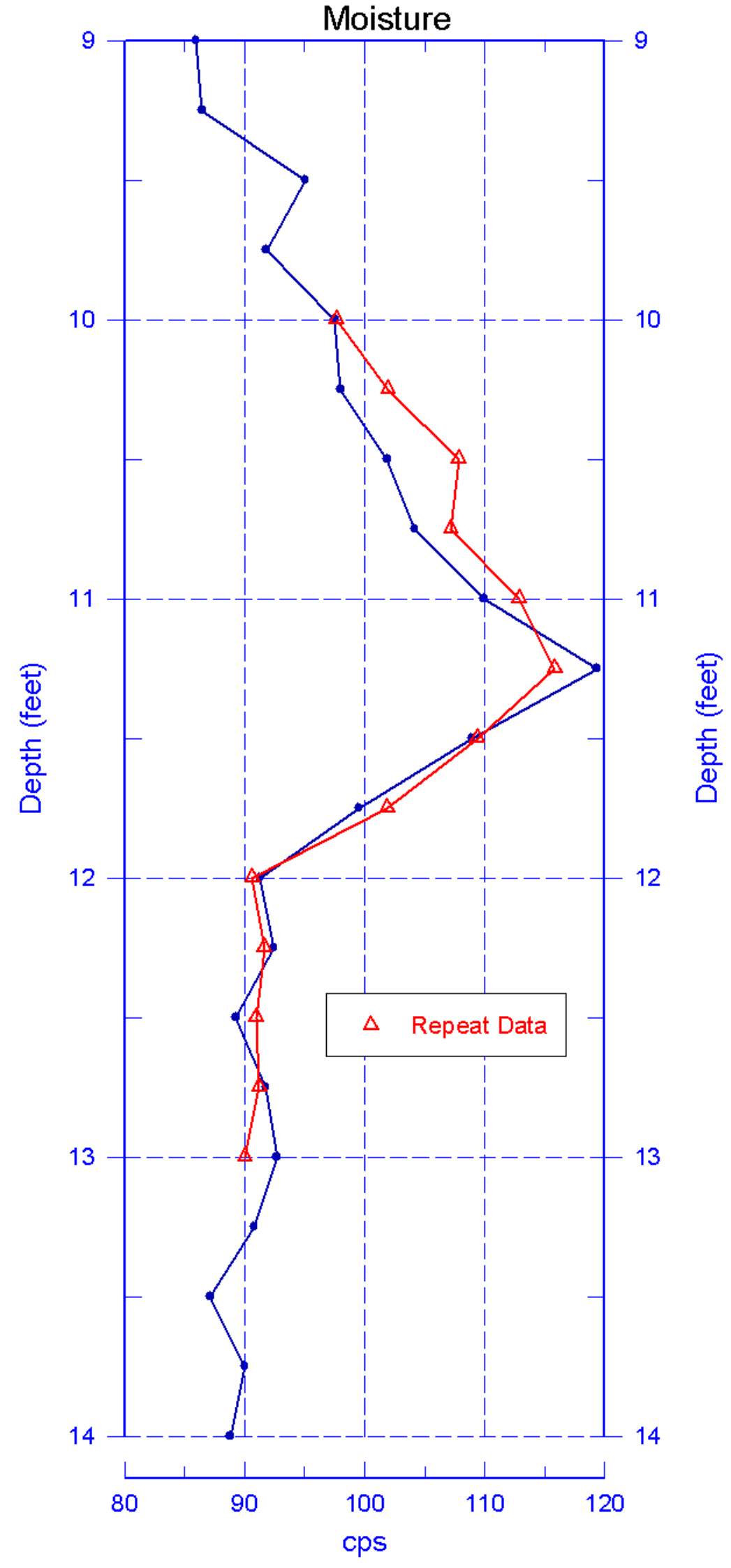

Reference - Ground Surface 


\section{9-3-25 (C6200) Log Data Report}

Borehole Information:

\begin{tabular}{|c|c|c|c|c|c|}
\hline \multicolumn{2}{|c|}{ Borehole: $\quad 399-3-25$ (C6200) } & \multicolumn{4}{|c|}{ 300-FF-5 } \\
\hline \multicolumn{2}{|c|}{ Coordinates (WA St Plane) } & GWL $^{1}$ (f & 14.5 & GWL Date: & $06 / 20 / 08$ \\
\hline North (m) & East (m) & Drill Date & TOC $^{2}$ Elevation & Total Depth (ft) & Type \\
\hline 116056.1 & 594255.1 & 06/17/08 & Unknown & 65 & Sonic \\
\hline
\end{tabular}

\section{Casing Information:}

\begin{tabular}{|c|c|c|c|c|c|c|}
\hline Casing Type & Stickup (ft) & $\begin{array}{c}\text { Outer } \\
\text { Diameter (in.) }\end{array}$ & $\begin{array}{c}\text { Inside } \\
\text { Diameter (in.) }\end{array}$ & Thickness (in.) & Top (ft) & Bottom (ft) \\
\hline Welded Steel & 3.2 & $75 / 8$ & $67 / 8$ & $3 / 8$ & -3.2 & 61 \\
\hline
\end{tabular}

\section{Borehole Notes:}

Casing data, and total depth reported by site Geologist. Logging engineer measured depth to water using an e-tape. Casing diameters were measured using a steel tape and rounded to the nearest 1/16-inch.

\section{Logging Equipment Information:}

\begin{tabular}{|c|c|c|c|c|}
\hline Logging System: & \multicolumn{2}{|c|}{ Gamma 4 L } & $\begin{array}{l}\text { Type: } \\
\text { Serial No.: }\end{array}$ & $\begin{array}{l}\text { SGLS } \\
\text { 47TP32211A }\end{array}$ \\
\hline Effective Calibration Date: & $12 / 31 / 07$ & \multirow{2}{*}{$\begin{array}{l}\text { Calibration Reference: } \\
\text { Logging Procedure: }\end{array}$} & \multicolumn{2}{|c|}{ HGLP-CC-027 } \\
\hline & & & \multicolumn{2}{|c|}{ HGLP-MAN-002, Rev. 0} \\
\hline Logging System: & \multicolumn{2}{|c|}{ Gamma $4 \mathrm{H}$} & $\begin{array}{l}\text { Type: } \\
\text { Serial No.: }\end{array}$ & $\begin{array}{l}\text { NMLS } \\
\text { H310700352 }\end{array}$ \\
\hline Effective Calibration Date: & $11 / 06 / 07$ & Calibration Reference: & \multicolumn{2}{|c|}{ HGLP-CC-021 } \\
\hline & & Logging Procedure: & \multicolumn{2}{|c|}{ HGLP-MAN-002, Rev. 0} \\
\hline
\end{tabular}

\section{Spectral Gamma Logging System (SGLS) Log Run Information:}

\begin{tabular}{|c|c|c|c|c|c|}
\hline Log Run & 1 & 2 Repeat & & & \\
\hline Date & $06 / 20 / 08$ & $06 / 20 / 08$ & & & \\
\hline Logging Engineer & Pearson & Pearson & & & \\
\hline Start Depth (ft) & 60.0 & 9.0 & & & \\
\hline Finish Depth (ft) & 0.0 & 15.0 & & & \\
\hline Count Time (sec) & 200 & 200 & & & \\
\hline Live/Real & $\mathrm{R}$ & $\mathrm{R}$ & & & \\
\hline Shield (Y/N) & $\mathrm{N}$ & $\mathrm{N}$ & & & \\
\hline MSA Interval (ft) & 0.5 & 0.5 & & & \\
\hline Log Speed (ft/min) & N/A & N/A & & & \\
\hline Pre-Verification & DL481CAB & DL481CAB & & & \\
\hline Start File & DL481000 & DL481121 & & & \\
\hline Finish File & DL481120 & DL481133 & & & \\
\hline Post-Verification & DL481CAA & DL481CAA & & & \\
\hline Depth Return Error (in.) & 0.5 high & 0 & & & \\
\hline Comments & $\begin{array}{l}\text { No fine gain } \\
\text { adjustment } \\
\text { made. }\end{array}$ & Repeat Section & & & \\
\hline
\end{tabular}


Neutron Moisture Logging System (NMLS) Log Run Information:

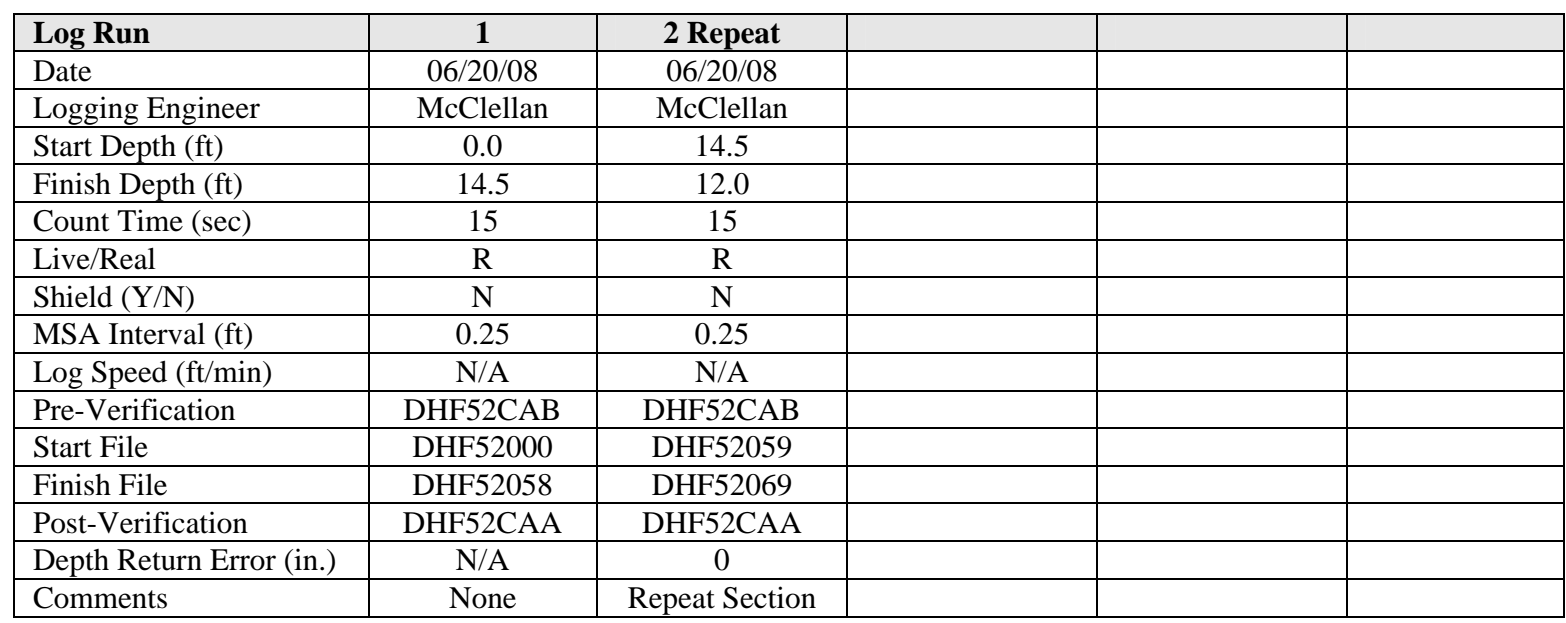

\section{Logging Operation Notes:}

Data were collected using Gamma 4, HO 68B-3573. SGLS pre - and post-survey verification measurements were acquired in the Amersham KUTh-115 field verifier. Maximum logging depth for the SGLS was $60.0 \mathrm{ft}$ before the sonde un-weighted. A centralizer was installed on the sonde. NMLS pre- and post-survey verification measurements were acquired in the standard field verifier.

\section{Analysis Notes:}

\begin{tabular}{|l|l|l|l|l|l|}
\hline Analyst: & LEGLER & Date: & 09/12/08 & Reference: & GJO-HGLP 1.6.3, Rev. 0 \\
\hline
\end{tabular}

The SGLS pre- and post-survey verification measurements met the acceptance criteria for the established system. The NMLS pre- and post-survey verification measurements met the acceptance criteria for the established system but the file, DHF52CAB, had measurements above the upper control limit for counts per second (cps). A casing correction for a 3/8-inch thick casing was applied to spectral (SGLS) log data from ground surface to total logged depth of borehole. A water correction was also applied from $14.5 \mathrm{ft}$ to total logged depth of borehole.

SGLS spectra were processed in batch mode in APTEC SUPERVISOR to identify individual energy peaks and to determine count rates. Concentrations were calculated with an EXCEL template identified as G4LDec07.xls using efficiency functions and corrections for casing, dead time and water as determined by annual calibrations.

Moisture data is presented in counts per second (cps) because no calibration data exists for a 6-7/8 inch inner diameter casing.

\section{Results and Interpretations:}

Cs-137, U-235, and U-238 (Pa-234m) were detected at several isolated depths throughout this borehole. Inspection of the individual spectra for these radionuclides at the various depths indicates these detections are statistical fluctuations associated with the processing software and are not considered valid.

The KUT plots indicate good repeatability. The moisture plot indicates good repeatability. 
HGLP-LDR-248, Rev. 0

\section{List of Log Plots:}

Depth Reference is ground surface

Manmade Radionuclides

Natural Gamma Logs

Combination Plot

Total Gamma \& Dead Time

Total Gamma \& Moisture

Repeat Section of Natural Gamma Logs

Moisture Repeat Section

${ }^{1} \mathrm{GWL}$ - groundwater level

${ }^{2}$ TOC - top of casing 

Hanford Office Manmade Radionuclides

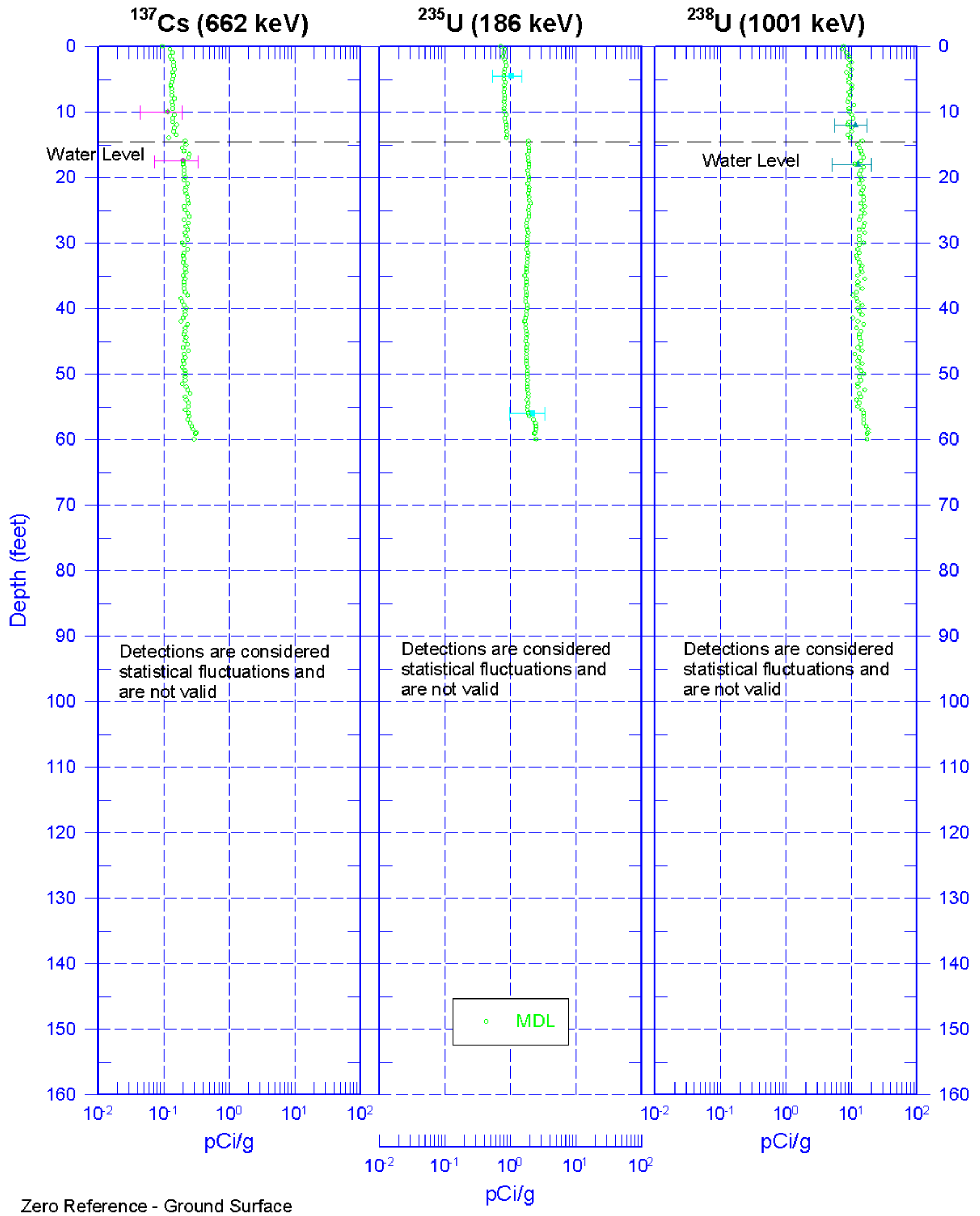


toller

399-3-25 (C6200)

Hanford Office

Natural Gamma Logs
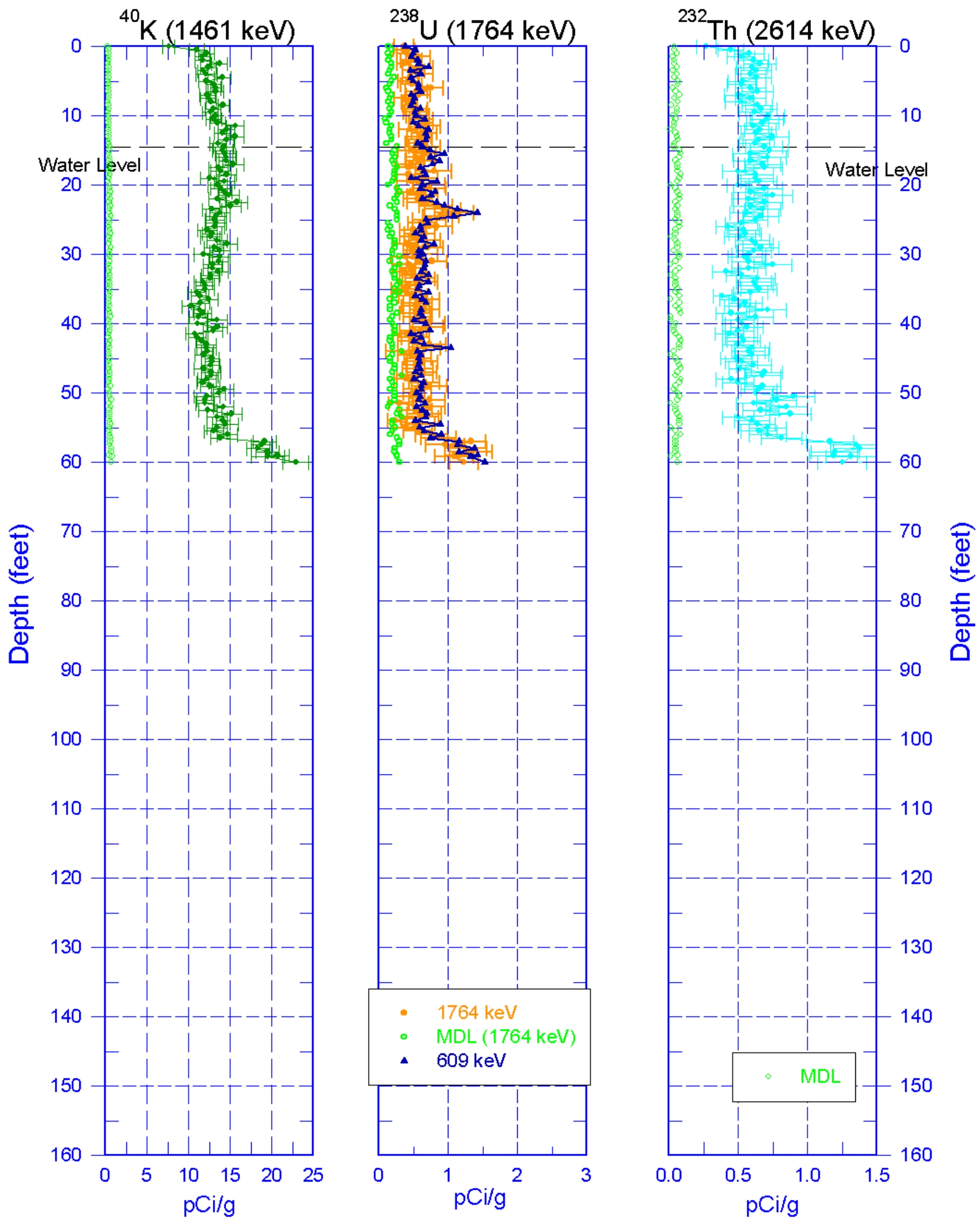

Zero Reference - Ground Surface

G.253 


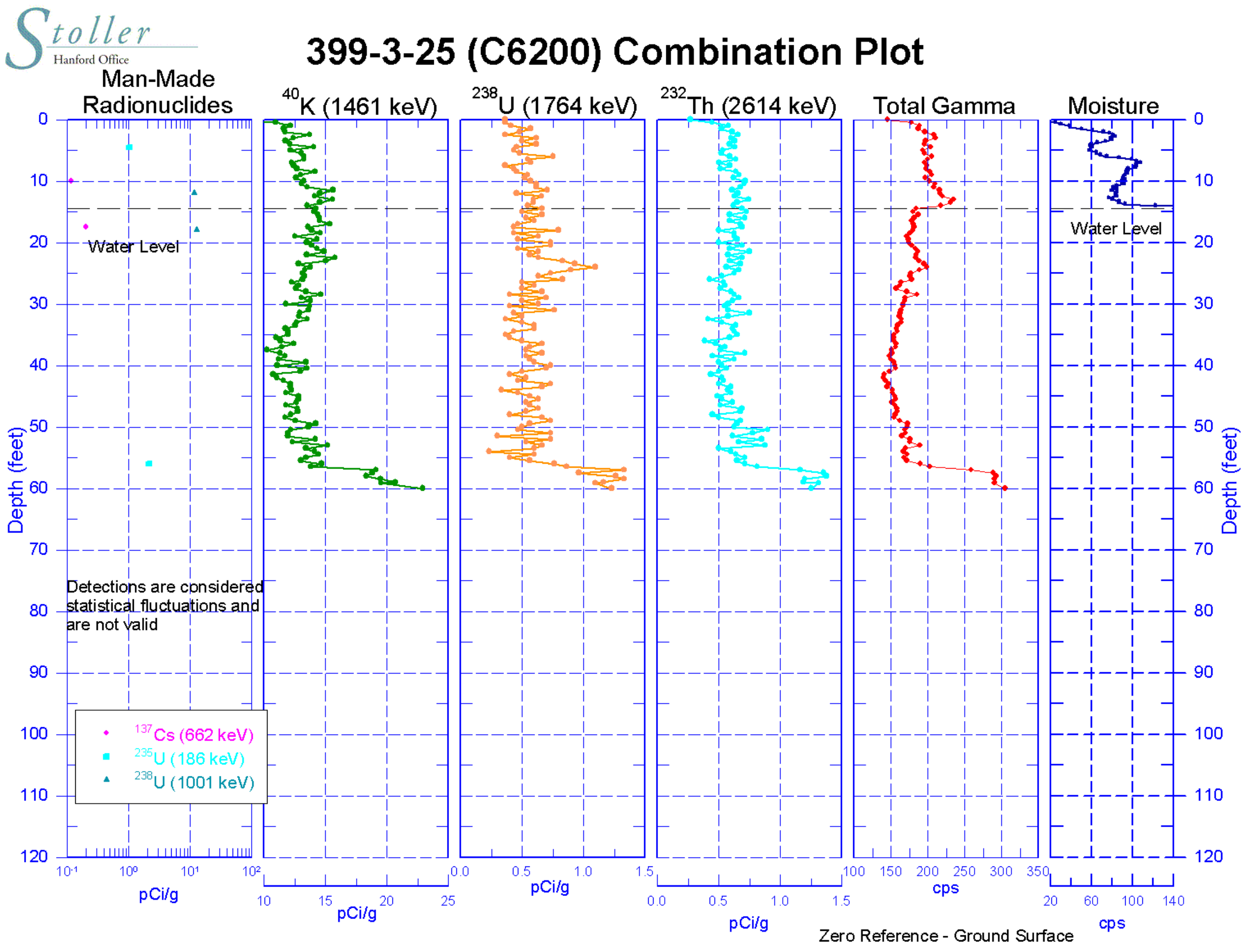


toller 399-3-25 (C6200)

Hanford Office

\section{Total Gamma \& Dead Time}
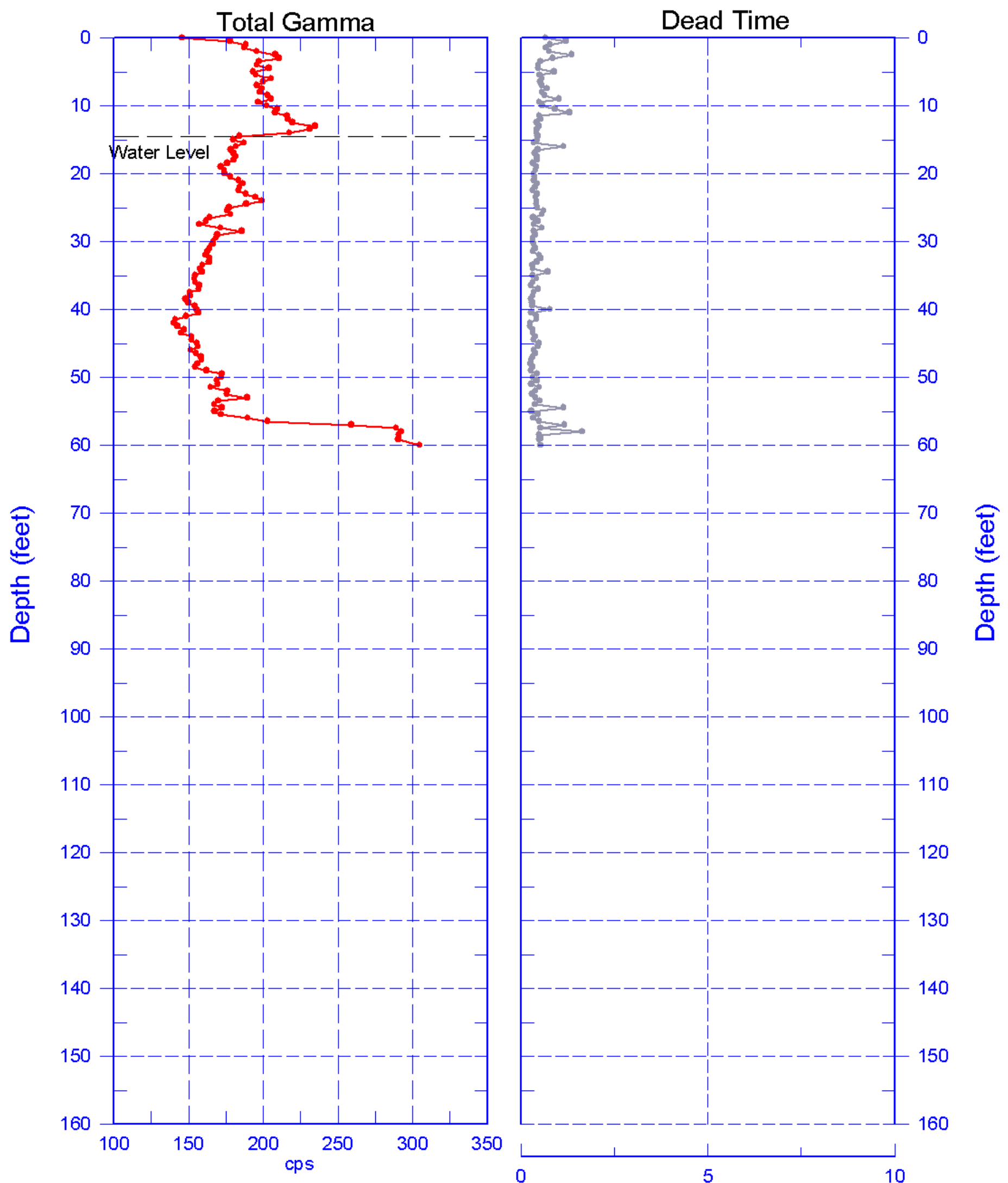

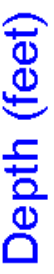

Reference - Ground Surface 
toller $\quad \mathbf{3 9 9 - 3 - 2 5}$ (C6200)

Hanford Office

\section{Total Gamma \& Moisture}
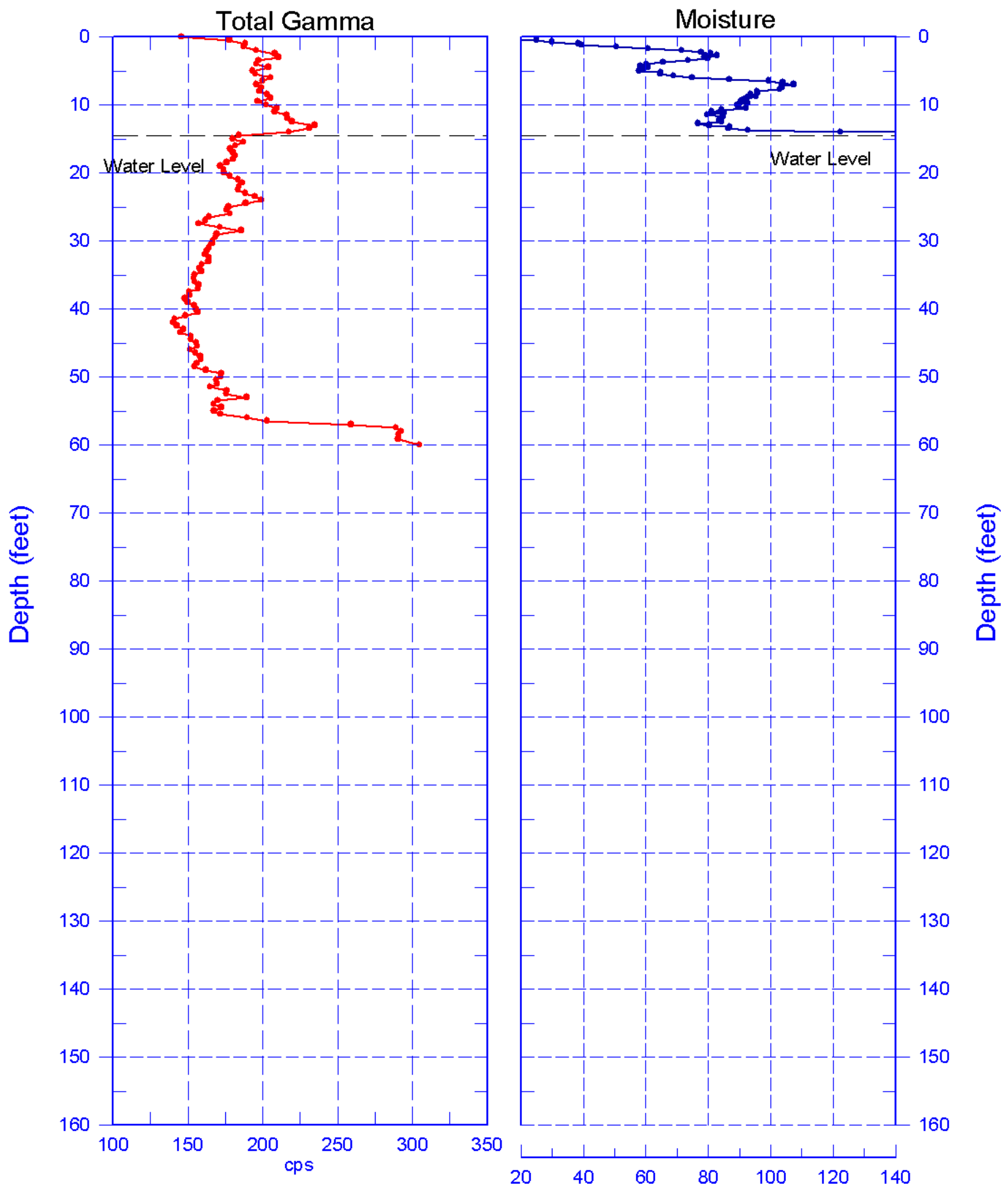

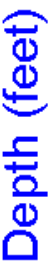

Reference - Ground Surface 


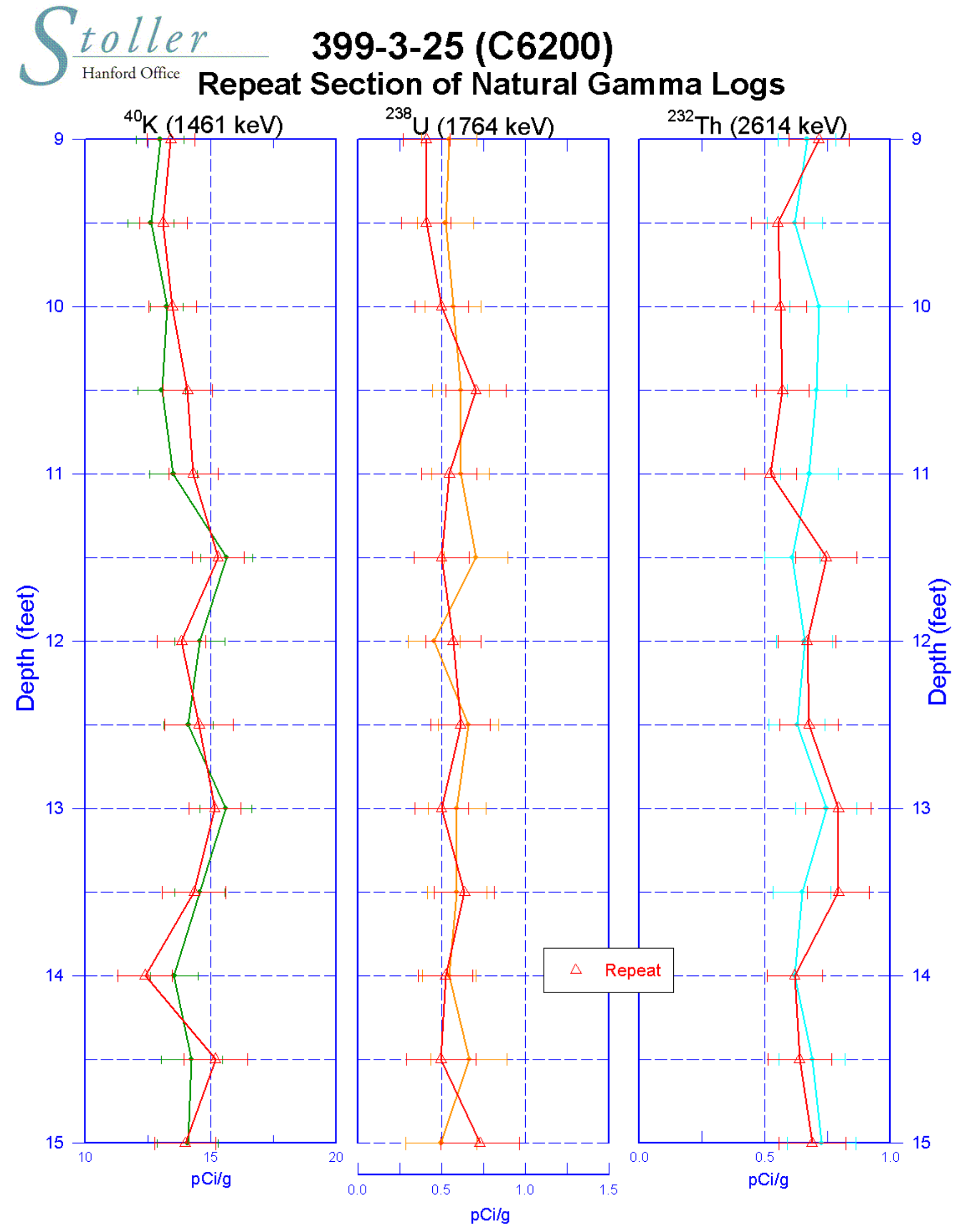

Zero Reference - Ground Surface

G.257 


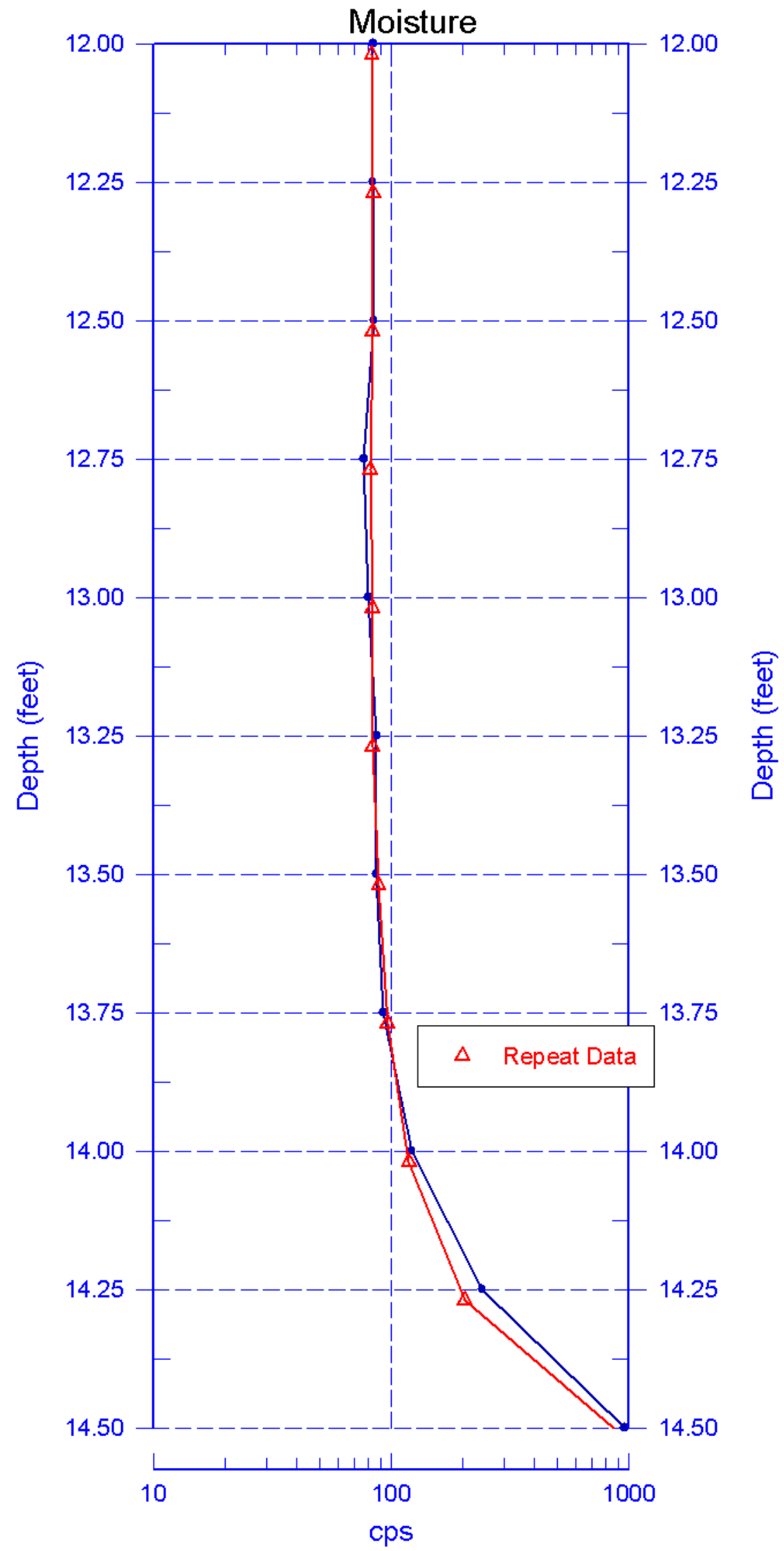




\section{9-3-26 (C6203) Log Data Report}

Borehole Information:

\begin{tabular}{|c|c|c|c|c|c|}
\hline \multirow{2}{*}{\multicolumn{2}{|c|}{ Borehole: $\quad 399-3-26$ (C6203) }} & \multicolumn{4}{|c|}{ 300-FF-5 } \\
\hline & & GWL $^{1}$ (f & 31.7 & GWL Date: & $05 / 16 / 08$ \\
\hline North (m) & East (m) & Drill Date & TOC $^{2}$ Elevation & Total Depth (ft) & Type \\
\hline 116034.6871 & 594239.606 & 05/16/08 & Unknown & 66.0 & Sonic \\
\hline
\end{tabular}

\section{Casing Information:}

\begin{tabular}{|c|c|c|c|c|c|c|}
\hline Casing Type & Stickup (ft) & $\begin{array}{c}\text { Outer } \\
\text { Diameter (in.) }\end{array}$ & $\begin{array}{c}\text { Inside } \\
\text { Diameter (in.) }\end{array}$ & Thickness (in.) & Top (ft) & Bottom (ft) \\
\hline Threaded Steel & 3.90 & $75 / 8$ & $67 / 8$ & $3 / 8$ & -3.9 & 56.8 \\
\hline
\end{tabular}

\section{Borehole Notes:}

Well site geologist reported depth to bottom, depth to water and depth of casing. Logging engineer measured casing diameter employing a steel tape and rounding to the nearest 1/16-in. The zero reference is the ground surface.

\section{Logging Equipment Information:}

\begin{tabular}{|l|l|l|l|l|}
\hline \multirow{2}{*}{ Logging System: } & \multirow{2}{*}{ Gamma $1 \mathrm{~N}$} & $\begin{array}{l}\text { Type: } \\
\text { Serial No.: }\end{array}$ & $\begin{array}{l}\text { 60\% HPGe SGLS } \\
\text { 45TP22010A }\end{array}$ \\
\hline Effective Calibration Date: & $03 / 28 / 08$ & $\begin{array}{l}\text { Calibration Reference: } \\
\text { Logging Procedure: }\end{array}$ & HGLP-CC-031 \\
\cline { 4 - 4 } & & HGLP-MAN-002, Rev. 0 \\
\hline
\end{tabular}

\begin{tabular}{|l|l|l|l|l|}
\hline Logging System: & \multirow{2}{*}{ Gamma 1 M } & $\begin{array}{l}\text { Type: } \\
\text { Serial No.: }\end{array}$ & $\begin{array}{l}\text { NMLS } \\
\text { H340207279 }\end{array}$ \\
\hline Effective Calibration Date: & $05 / 06 / 08$ & Calibration Reference: & HGLP-CC-032 \\
\hline \multicolumn{2}{|l|}{} & Logging Procedure: & HGLP-MAN-002, Rev. 0 \\
\hline
\end{tabular}

\section{Spectral Gamma Logging System (SGLS) Log Run Information:}

\begin{tabular}{|l|c|c|}
\hline Log Run & 1 & 2 Repeat \\
\hline Date & $05 / 19 / 08$ & $05 / 19 / 08$ \\
\hline Logging Engineer & McClellan & McClellan \\
\hline Start Depth (ft) & 0.0 & 60.0 \\
\hline Finish Depth (ft) & 60.0 & 54.0 \\
\hline Count Time (sec) & 200 & 200 \\
\hline Live/Real & $\mathrm{R}$ & $\mathrm{R}$ \\
\hline Shield (Y/N) & $\mathrm{N}$ & $\mathrm{N}$ \\
\hline MSA Interval (ft) & 0.5 & 0.5 \\
\hline Log Speed (ft/min) & N/A & N/A \\
\hline Pre-Verification & AN074CAB & AN074CAB \\
\hline Start File & AN074000 & AN074121 \\
\hline Finish File & AN074120 & AN074133 \\
\hline Post-Verification & AN074CAA & AN074CAA \\
\hline Depth Return Error (in.) & N/A & 0 \\
\hline Comments & $\begin{array}{c}\text { Fine gain } \\
\text { adjustment } \\
\text { made before - } \\
\text { 063 \& -103. }\end{array}$ & $\begin{array}{c}\text { No fine gain } \\
\text { adjustment } \\
\text { made. Repeat } \\
\text { Section }\end{array}$ \\
\hline
\end{tabular}




\section{Neutron Moisture Logging System (NMLS) Log Run Information:}

\begin{tabular}{|l|c|c|}
\hline Log Run & 3 & 4 Repeat \\
\hline Date & $05 / 20 / 08$ & $05 / 20 / 08$ \\
\hline Logging Engineer & McClellan & McClellan \\
\hline Start Depth (ft) & 0.0 & 29.25 \\
\hline Finish Depth (ft) & 29.25 & 26.0 \\
\hline Count Time (sec) & 15 & 15 \\
\hline Live/Real & $\mathrm{R}$ & $\mathrm{R}$ \\
\hline Shield (Y/N) & $\mathrm{N}$ & $\mathrm{N}$ \\
\hline MSA Interval (ft) & 0.25 & 0.25 \\
\hline Log Speed (ft/min) & N/A & N/A \\
\hline Pre-Verification & AM002CAB & AM002CAB \\
\hline Start File & AM002000 & AM002118 \\
\hline Finish File & AM002117 & AM002131 \\
\hline Post-Verification & AM002CAA & AM002CAA \\
\hline Depth Return Error (in.) & N/A & 0.5 high \\
\hline Comments & None & Repeat Section \\
\hline
\end{tabular}

\section{Logging Operation Notes:}

Data were collected using Gamma 1, HO 68B-3574. SGLS pre- and post-survey verification measurements were acquired in the Amersham KUTh-118 field verifier. Maximum logging depth achieved was $60.0 \mathrm{ft}$. A centralizer was installed on the sondes. NMLS pre- and post-survey verification measurements were acquired in the AmBe standard.

\section{Analysis Notes:}

\begin{tabular}{|l|l|l|l|l|l|}
\hline Analyst: & LEGLER & Date: & 6/24/08 & Reference: & GJO-HGLP 1.6.3, Rev. 0 \\
\hline
\end{tabular}

The pre- and post-survey verification met the acceptance criteria for the established systems. A casing correction for a 3/8-in. thick casing was applied to spectral log data (SGLS) from ground surface to $56.8 \mathrm{ft}$, leaving $3.2 \mathrm{ft}$ of open borehole uncorrected.

SGLS spectra were processed in batch mode using APTEC SUPERVISOR to identify individual energy peaks and determine count rates. Concentrations were calculated with an EXCEL worksheet template identified as G1NMar08.xls using efficiency functions and corrections for casing, dead time and water as determined by annual calibrations.

Moisture data are presented in counts per second (cps) because no calibration data exist for a 6 7/8-in. inner diameter casing.

\section{Results and Interpretations:}

Cs-137 and U-238 (Pa-234m) were detected at several depths in this borehole. Inspection of the individual spectra at these depths indicates that these detections are statistical fluctuations and are not valid. Plots of the MDLs for manmade radionuclides (Cs-137, U-235, and U-238) are provided.

Depth to groundwater was reported to be $31.7 \mathrm{ft}$ on 5/16/08. However, log data collected on 5/19/08 (SGLS) and 5/20/08 (NMLS) indicate a depth to ground water of approximately $29.5 \mathrm{ft}$. The depth to groundwater appears to fluctuate with the level of the river.

A fine grain sediment layer can be seen at around $15 \mathrm{ft}$, as seen by an increase in total gamma, and reflected in the K-40 and U-238 plots.

The KUT and Moisture plots indicate good repeatability. 
HGLP-LDR-231, Rev. 0

\section{List of Log Plots:}

Depth Reference is ground surface

Manmade Radionuclides

Natural Gamma Logs

Combination Plot

Total Gamma \& Dead Time

Total Gamma \& Moisture

Repeat Section of Natural Gamma Logs

Moisture Repeat Section

${ }^{1} \mathrm{GWL}$ - groundwater level

${ }^{2}$ TOC - top of casing 

Hanford Office Manmade Radionuclides

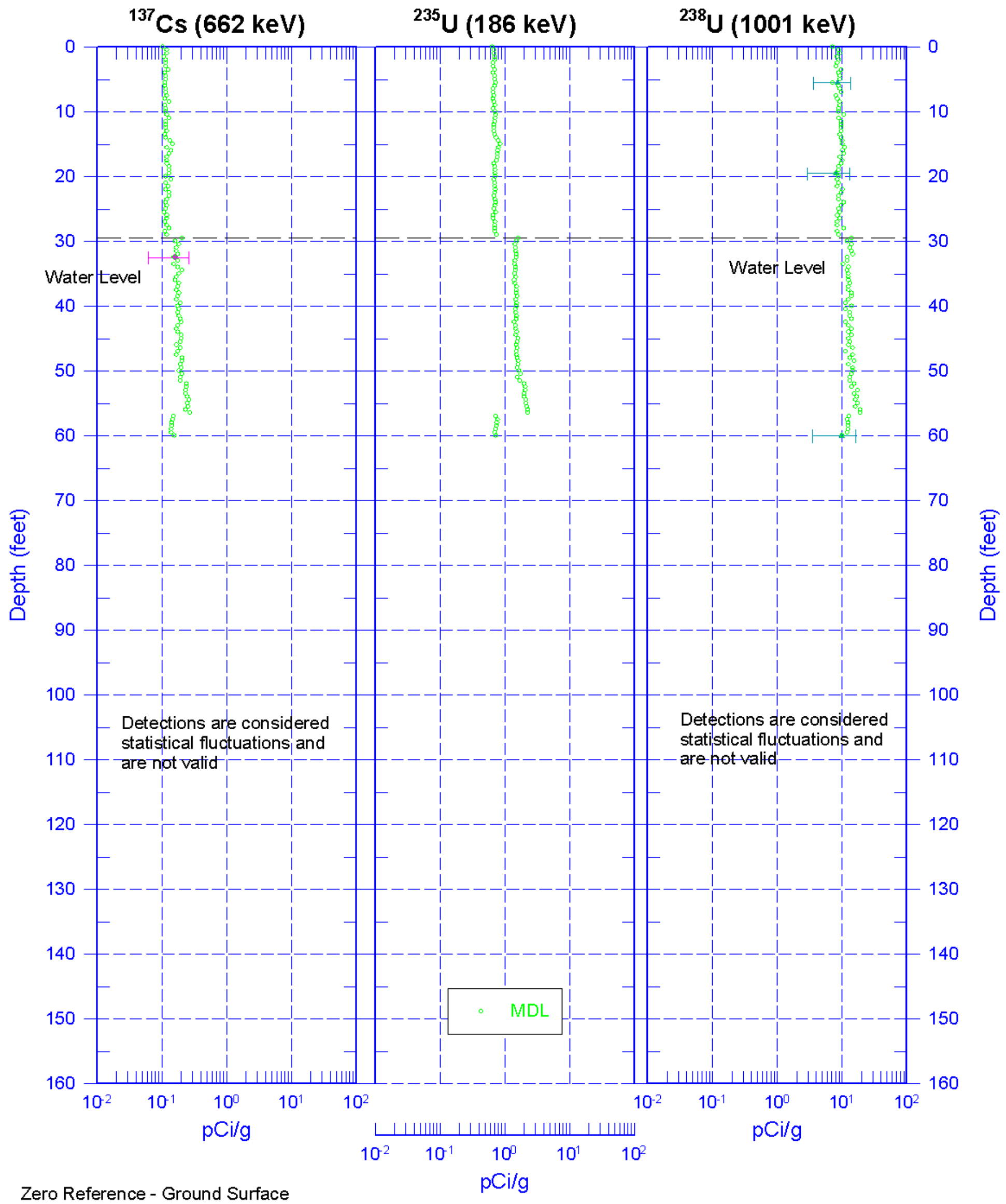



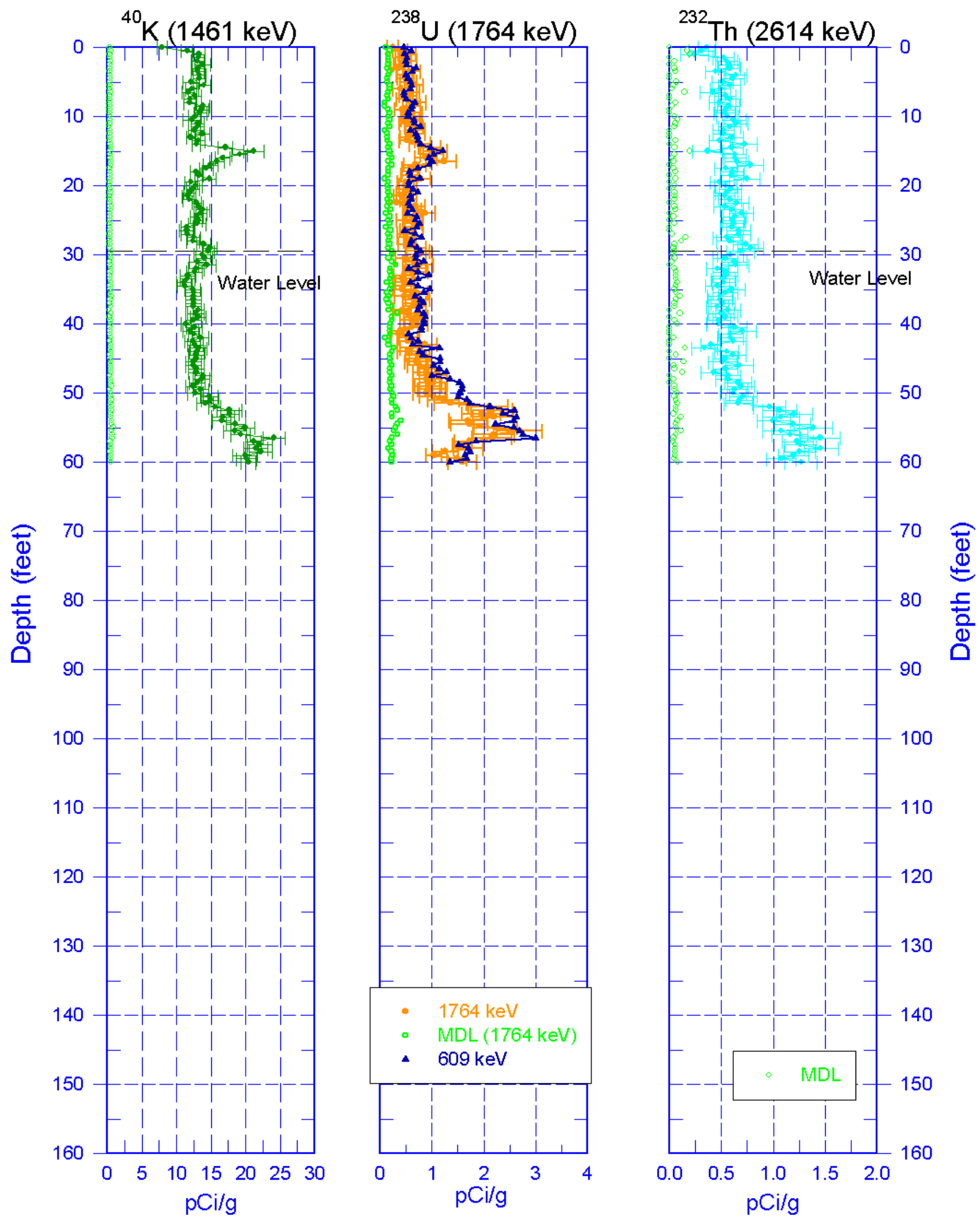


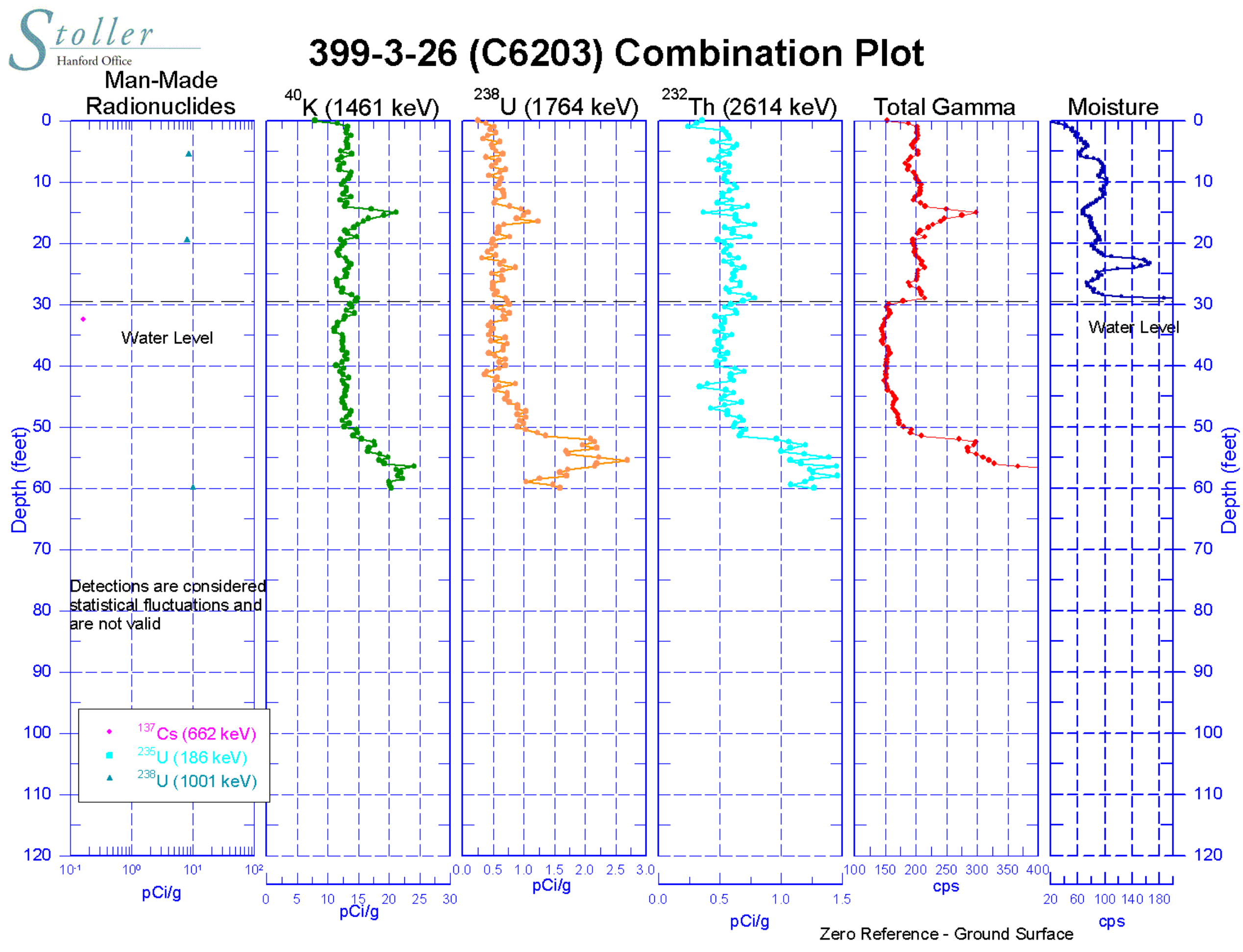


toller 399-3-26 (C6203)

Hanford Office

\section{Total Gamma \& Dead Time}
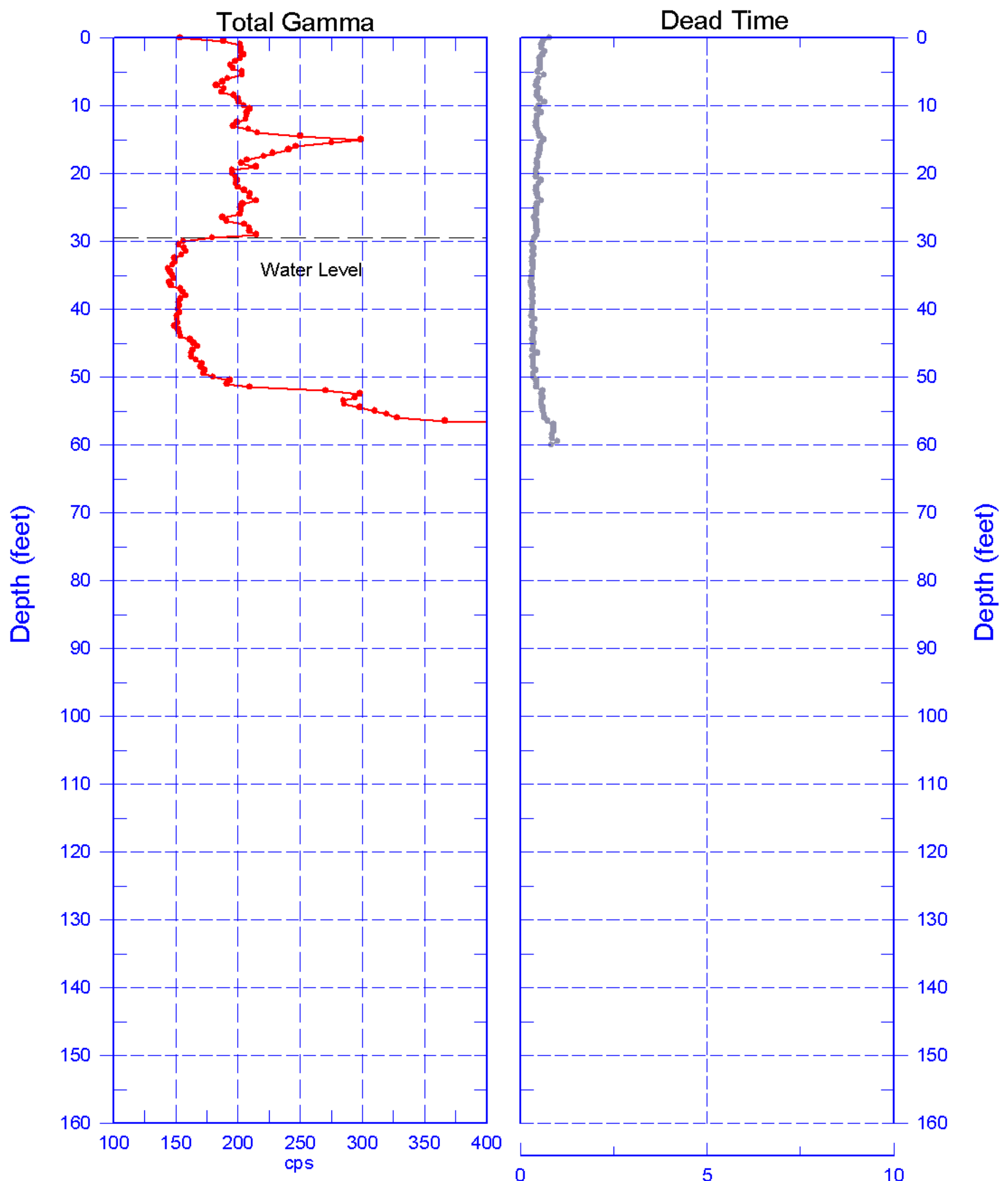

$\frac{\widehat{0}}{0}$
$\frac{0}{0}$
$\frac{0}{0}$
0

Reference - Ground Surface 


\section{S}

toller 399-3-26 (C6203)

Hanford Office

\section{Total Gamma \& Moisture}
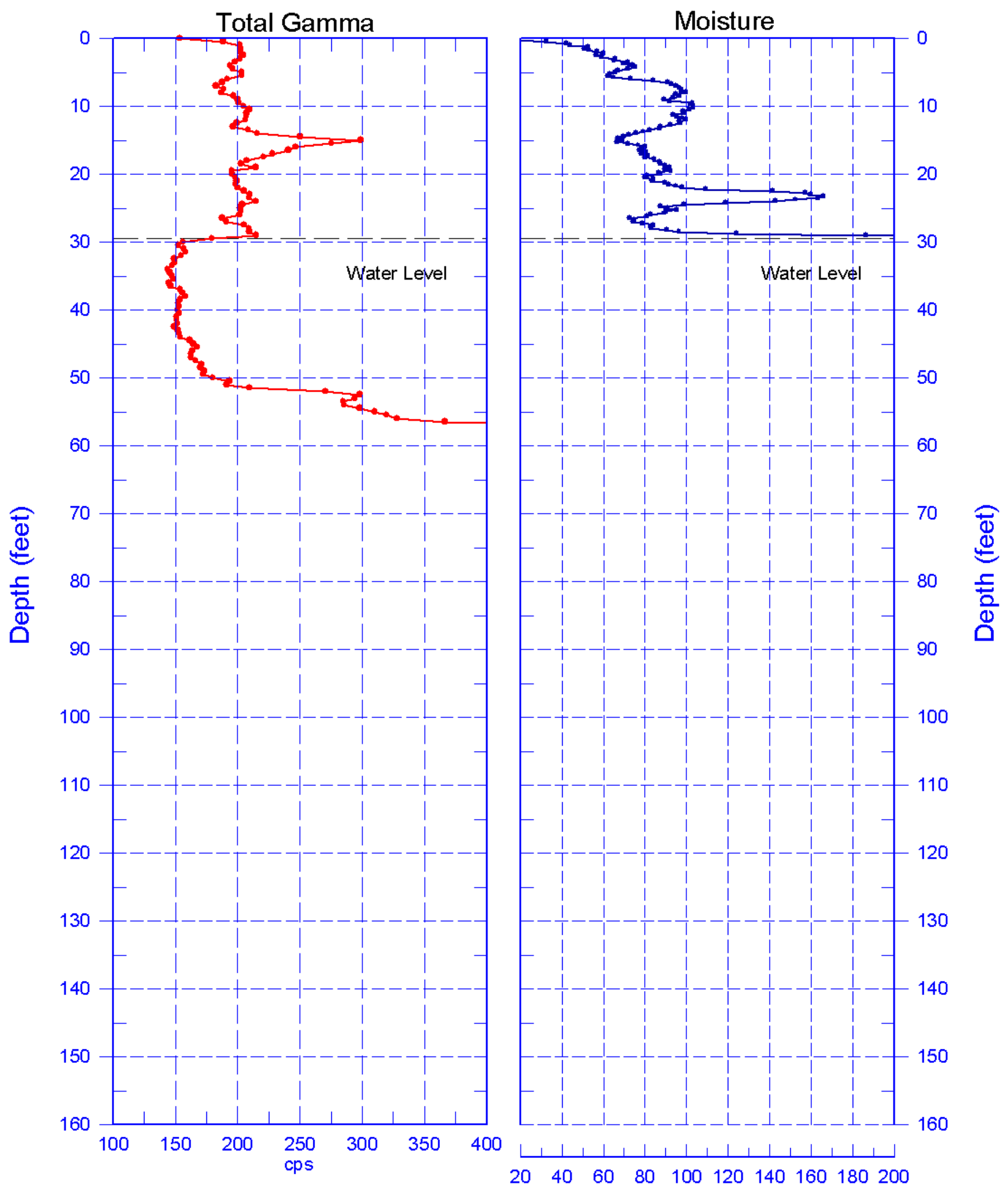


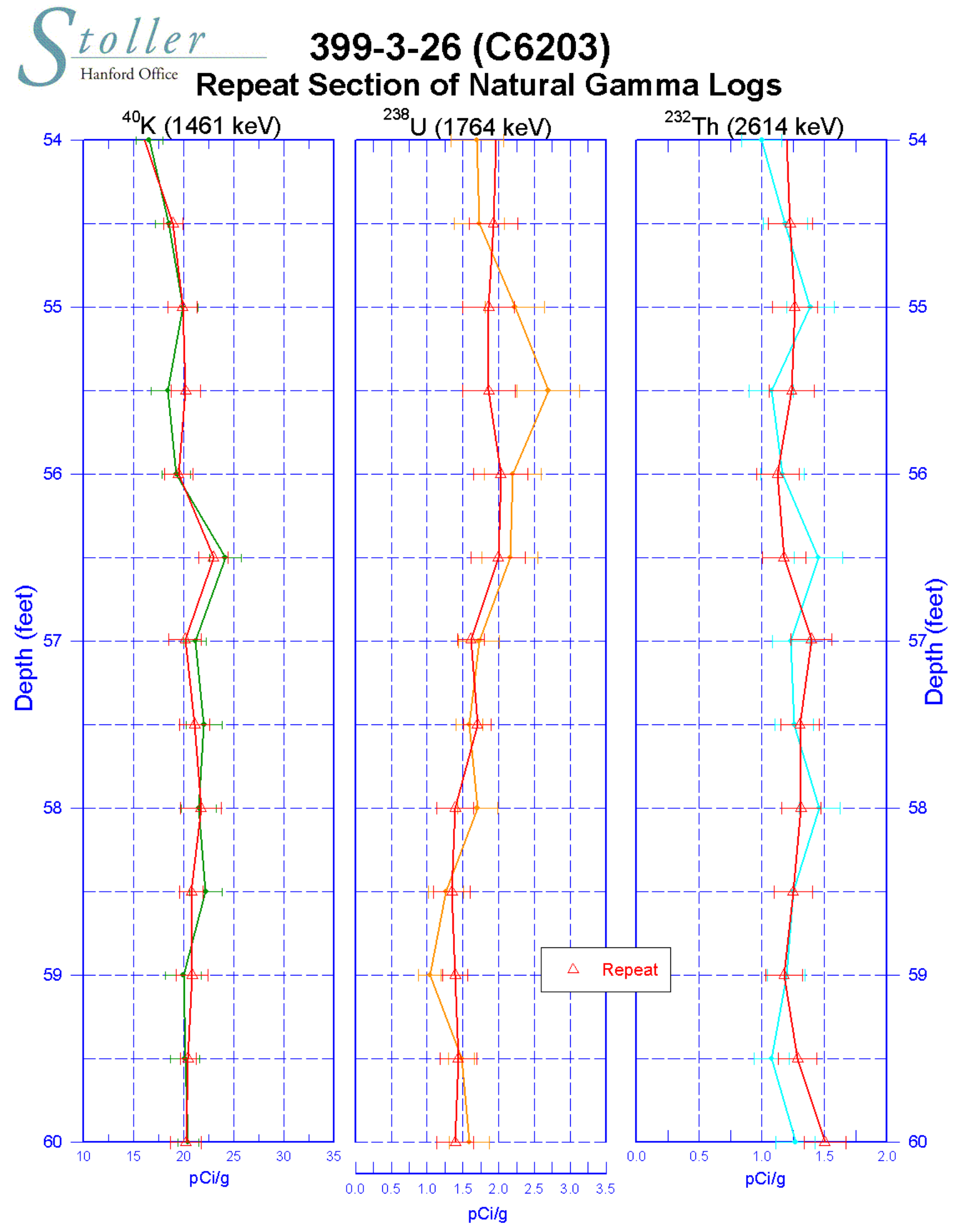




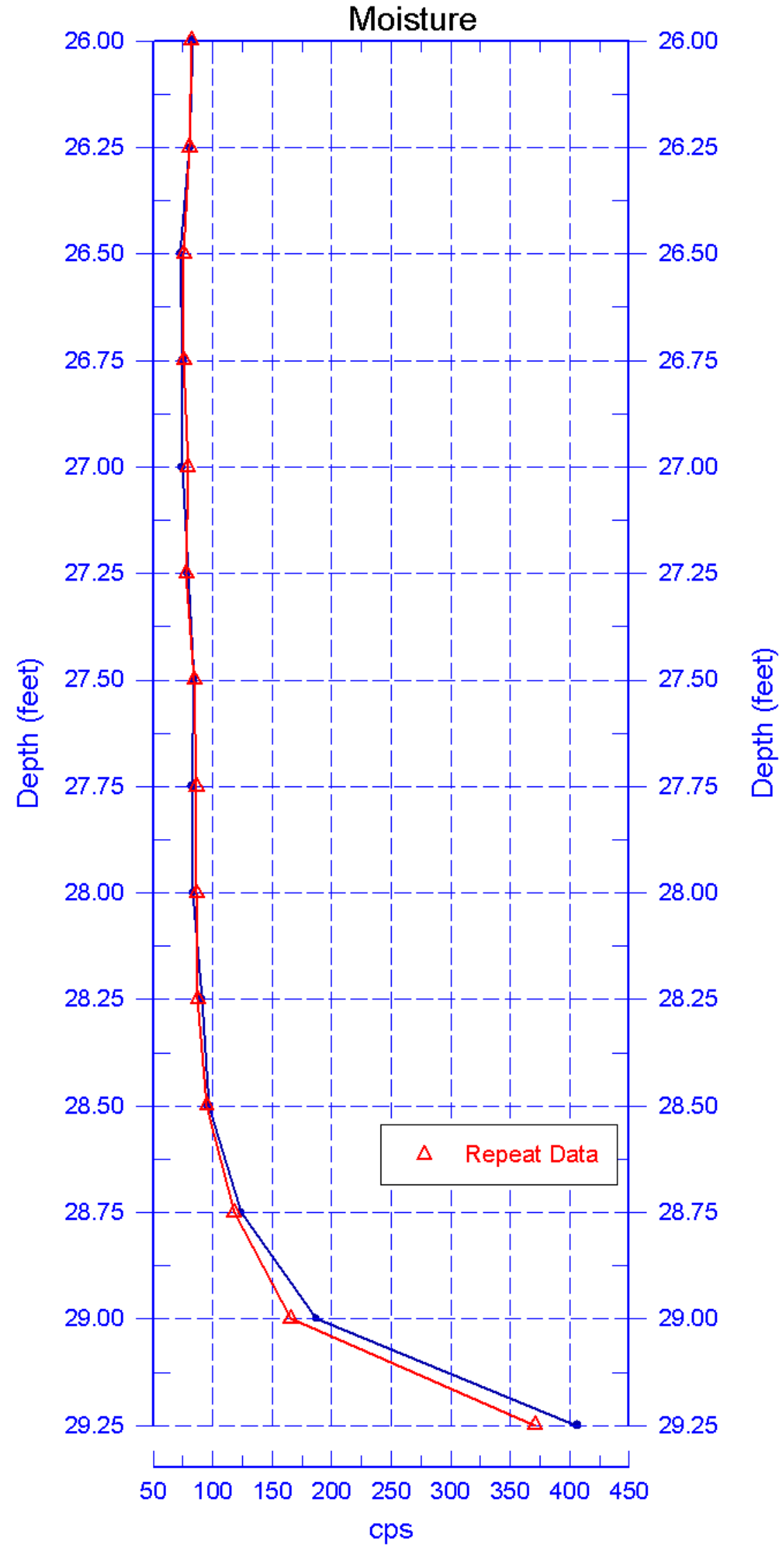




\section{9-3-27 (C6204) Log Data Report}

Borehole Information:

\begin{tabular}{|c|c|c|c|c|c|}
\hline \multicolumn{2}{|c|}{ Borehole: $\quad$ 399-3-27 (C6204) } & \multicolumn{4}{|c|}{ 300-FF-5 } \\
\hline \multicolumn{2}{|c|}{ Coordinates (WA St Plane) } & $G_{W L}^{1}(\mathbf{f}$ & 27.7 & GWL Date: & $07 / 10 / 08$ \\
\hline North (m) & East (m) & Drill Date & TOC $^{2}$ Elevation & Total Depth (ft) & Type \\
\hline 116040.4 & 594247.8 & 07/09/08 & Unknown & 62 & Sonic \\
\hline
\end{tabular}

\section{Casing Information:}

\begin{tabular}{|c|c|c|c|c|c|c|}
\hline Casing Type & Stickup (ft) & $\begin{array}{c}\text { Outer } \\
\text { Diameter (in.) }\end{array}$ & $\begin{array}{c}\text { Inside } \\
\text { Diameter (in.) }\end{array}$ & Thickness (in.) & Top (ft) & Bottom (ft) \\
\hline Threaded Steel & 3.7 & $75 / 8$ & $67 / 8$ & $3 / 8$ & -3.7 & 57.0 \\
\hline
\end{tabular}

\section{Borehole Notes:}

Casing data and total depth were reported by the site geologist. The logging engineer measured depth to water with an e-tape. Casing diameters were measured using a steel tape and rounded to the nearest 1/16 inch. The zero reference is the ground surface.

\section{Logging Equipment Information:}

\begin{tabular}{|c|c|c|c|c|}
\hline Logging System: & \multicolumn{2}{|c|}{ Gamma $4 \mathrm{~L}$} & $\begin{array}{l}\text { Type: } \\
\text { Serial No.: }\end{array}$ & $\begin{array}{l}\text { 60\% HPGe SGLS } \\
\text { 47TP32211A }\end{array}$ \\
\hline Effective Calibration Date: & $12 / 31 / 07$ & Calibration Reference: & \multicolumn{2}{|c|}{ HGLP-CC-027 } \\
\hline & & Logging Procedure: & \multicolumn{2}{|c|}{ HGLP-MAN-002, Rev. 0} \\
\hline Logging System: & \multicolumn{2}{|c|}{ Gamma $4 \mathrm{H}$} & $\begin{array}{l}\text { Type: } \\
\text { Serial No.: }\end{array}$ & $\begin{array}{l}\text { NMLS } \\
\text { H310700352 }\end{array}$ \\
\hline Effective Calibration Date: & $11 / 06 / 07$ & Calibration Reference: & \multicolumn{2}{|c|}{ HGLP-CC-021 } \\
\hline & & Logging Procedure: & \multicolumn{2}{|c|}{ HGLP-MAN-002, Rev. 0} \\
\hline
\end{tabular}

\section{Spectral Gamma Logging System (SGLS) Log Run Information:}

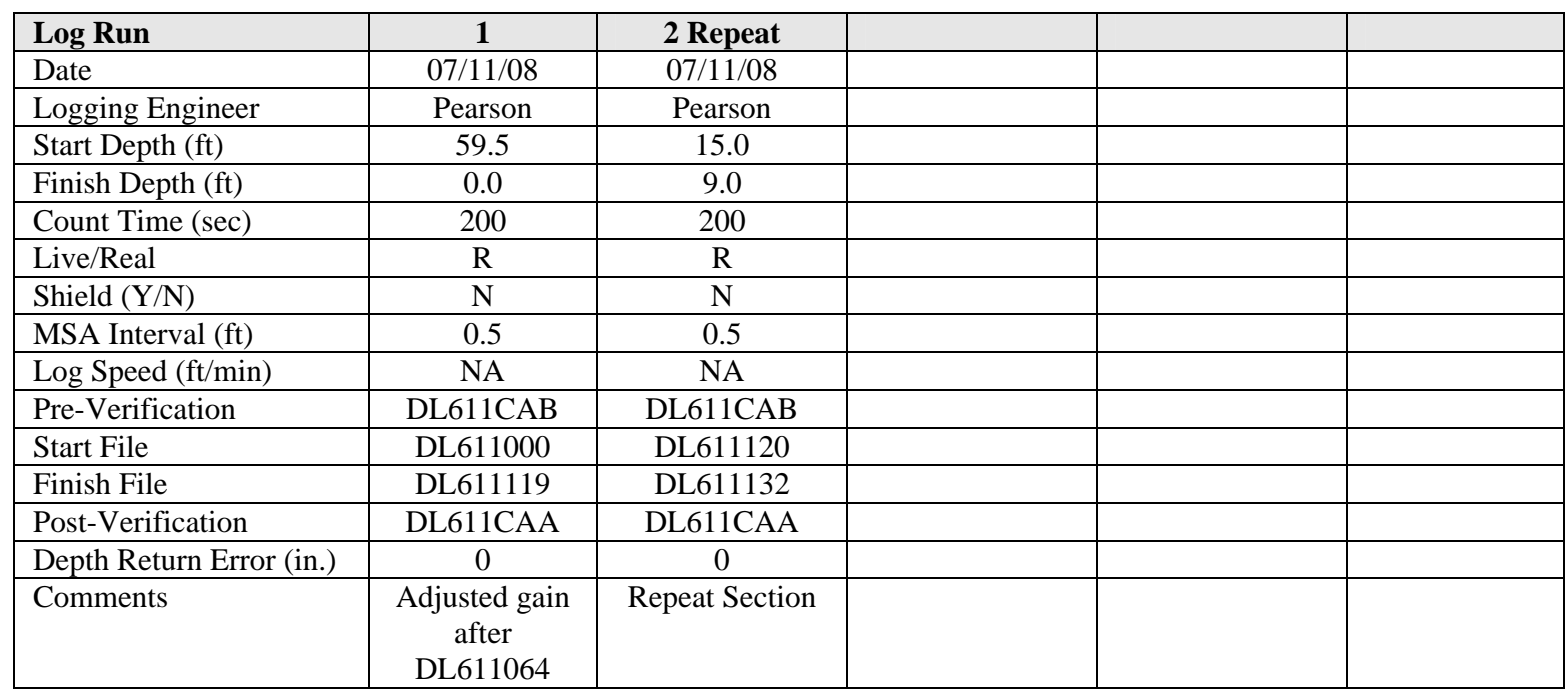


Neutron Moisture Logging System (NMLS) Log Run Information:

\begin{tabular}{|l|c|c|l|l|l|}
\hline Log Run & $\mathbf{3}$ & 4 Repeat & & & \\
\hline Date & $07 / 11 / 08$ & $07 / 11 / 08$ & & & \\
\hline Logging Engineer & Pearson & Pearson & & & \\
\hline Start Depth (ft) & 0.0 & 8.0 & & & \\
\hline Finish Depth (ft) & 28.0 & 11.0 & & & \\
\hline Count Time (sec) & 15 & 15 & & & \\
\hline Live/Real & $\mathrm{R}$ & $\mathrm{R}$ & & & \\
\hline Shield (Y/N) & $\mathrm{N}$ & $\mathrm{N}$ & & & \\
\hline MSA Interval (ft) & 0.25 & 0.25 & & & \\
\hline Log Speed (ft/min) & NA & NA & & & \\
\hline Pre-Verification & DHG82CAB & DHG82CAB & & & \\
\hline Start File & DHG82000 & DHG82113 & & & \\
\hline Finish File & DHG82112 & DHG82125 & & & \\
\hline Post-Verification & DHG82CAA & DHG82CAA & & & \\
\hline Depth Return Error (in.) & NA & 1/4 high & & & \\
\hline Comments & None & Repeat Section & & & \\
\hline
\end{tabular}

\section{Logging Operation Notes:}

Data were collected using Gamma 4, HO 68B-3573. SGLS pre- and post-survey verification measurements were acquired in the Amersham KUTh-115 field verifier. NMLS pre- and post-survey verification measurements were acquired in the standard field verifier. Maximum logging depth for the SGLS was $59.8 \mathrm{ft}$ before the sonde un-weighted. A centralizer was installed on the sonde.

Analysis Notes:

\begin{tabular}{|l|l|l|l|l|l|}
\hline Analyst: & LEGLER & Date: & 10/15/08 & Reference: & GJO-HGLP 1.6.3, Rev. 0 \\
\hline
\end{tabular}

The SGLS pre- and post-survey verification spectra met the acceptance criteria for the established system. The NMLS pre- and post-survey verification spectra met the acceptance criteria for the established system, but the verification file DHG82CAA had measurements above the upper control limit for counts per second (cps).

Analyses of log data suggest slight discrepancies with field reports of depth to water and casing depth. Corrections of log data were applied according to the analysis assumptions in the table below.

\begin{tabular}{|l|l|l|l|l|}
\hline Top (ft) & Bottom (ft) & T (in) & ID (in) & Comments \\
\hline 0 & 28.4 & $3 / 8$ & $67 / 8$ & No water correction \\
\hline 28.5 & 56.4 & $3 / 8$ & $67 / 8$ & GWL reported @ 27.7 ft; adjusted to 28.5 ft \\
\hline 56.5 & TD $^{3}$ & 0 & $67 / 8$ & Casing depth reported @ 57 ft; adjusted to 56.5ft \\
\hline
\end{tabular}

$\mathrm{T}=$ casing thickness used to calculate energy-dependent casing correction factor

ID = casing inside diameter used to calculate energy-dependent water correction factor

SGLS spectra were processed in batch mode in APTEC SUPERVISOR to identify individual peaks and count rates. Concentrations were calculated using an EXCEL template identified as G4LDec07.xls using an efficiency function and corrections for casing, dead time and water as determined by annual calibrations. NMLS spectra were processed in batch mode in APTEC SUPERVISOR to identify counts. Count rates were calculated using an EXCEL template identified as G4HNov07.xls. NMLS data are presented in counts per second (cps), because no calibration data is available for a 6 7/8-in. inner diameter borehole casing. 
HGLP-LDR-266, Rev. 0

\section{Results and Interpretations:}

Cs-137, U-235, and U-238 (Pa-234m) were detected at various isolated depths throughout this borehole. Inspection of the individual spectra indicates that these detections are statistical fluctuations associated with the processing software.

Co-60 was detected at 10.5 - $13.5 \mathrm{ft}$, and 16.0 feet. Inspection of the individual spectra indicates that the detections at $16.0 \mathrm{ft}$ are statistical fluctuations associated with the processing software. The maximum concentration was $0.61 \mathrm{pCi} / \mathrm{g}$ at $11.5 \mathrm{ft}$.

The Manmade, KUT and Moisture repeat plots indicate good repeatability.

Below the $51 \mathrm{ft}$ depth, a probable lithologic change occurs, which is indicated by the increased KUT concentrations and the Total Gamma count rates.

\section{List of Log Plots:}

Depth Reference is ground surface

Manmade Radionuclides

Natural Gamma Logs

Combination Plot

Total Gamma \& Dead Time

Total Gamma \& Moisture

Manmade Repeat Section

Repeat Section of Natural Gamma Logs

Moisture Repeat Section

${ }^{1} \mathrm{GWL}$ - groundwater level

${ }^{2}$ TOC - top of casing

${ }^{3} \mathrm{TD}$ - total depth 
S

toller 399-3-27 (C6204)

Hanford Office

Manmade Radionuclides

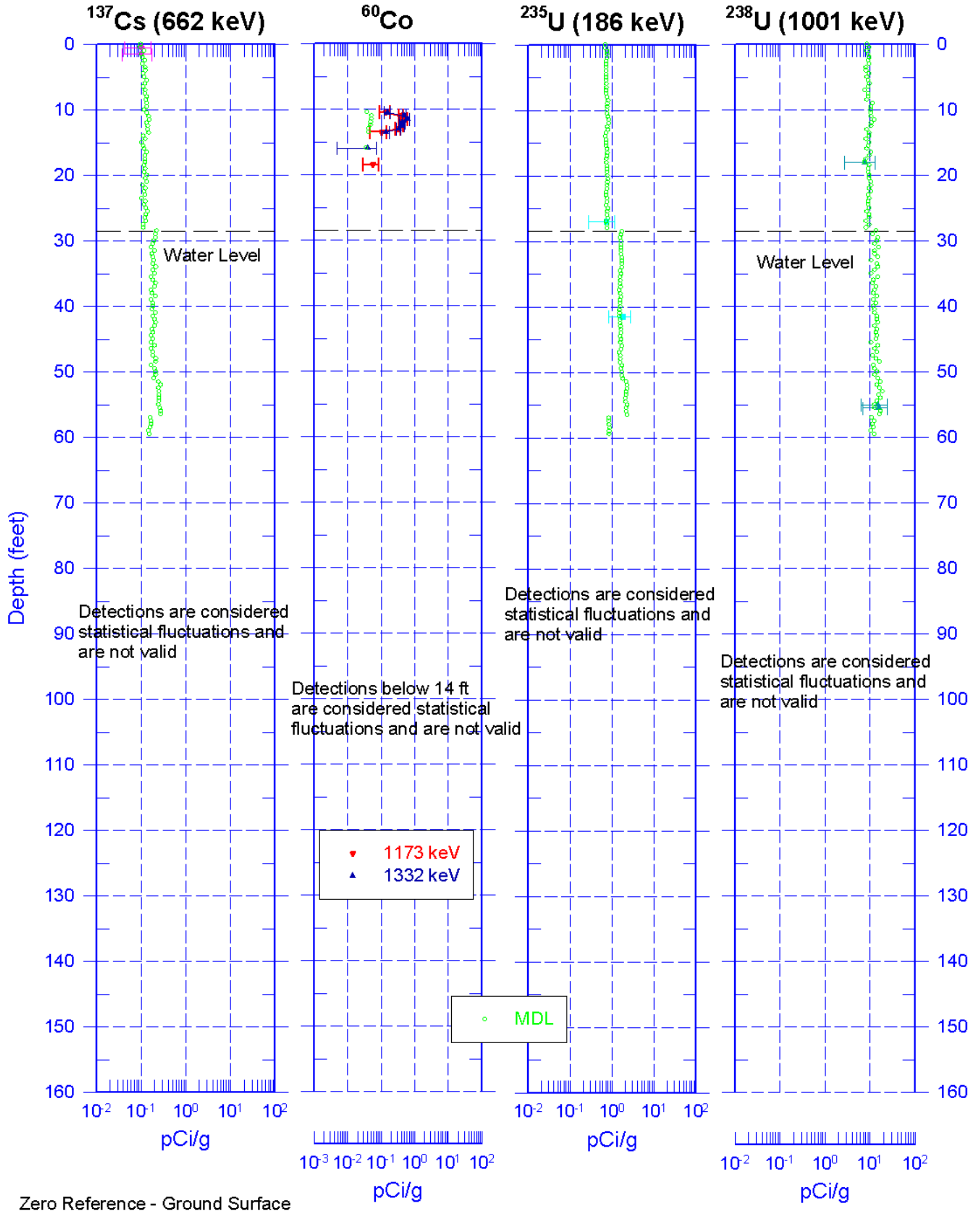

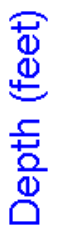

Zero Reference - Ground Surface 
$S$

toller 399-3-27 (C6204)

Hanford Office

\section{Natural Gamma Logs}
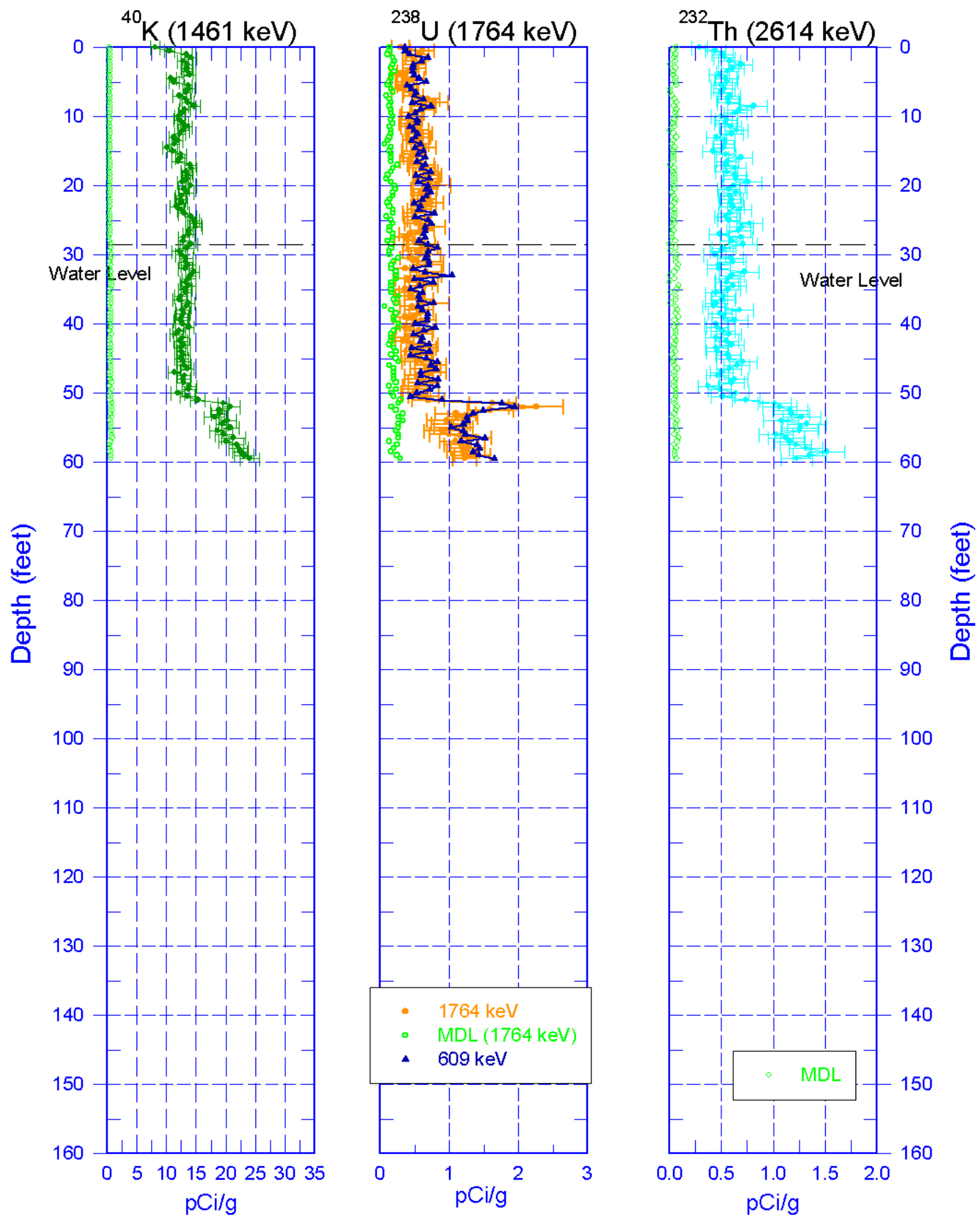

Zero Reference - Ground Surface 


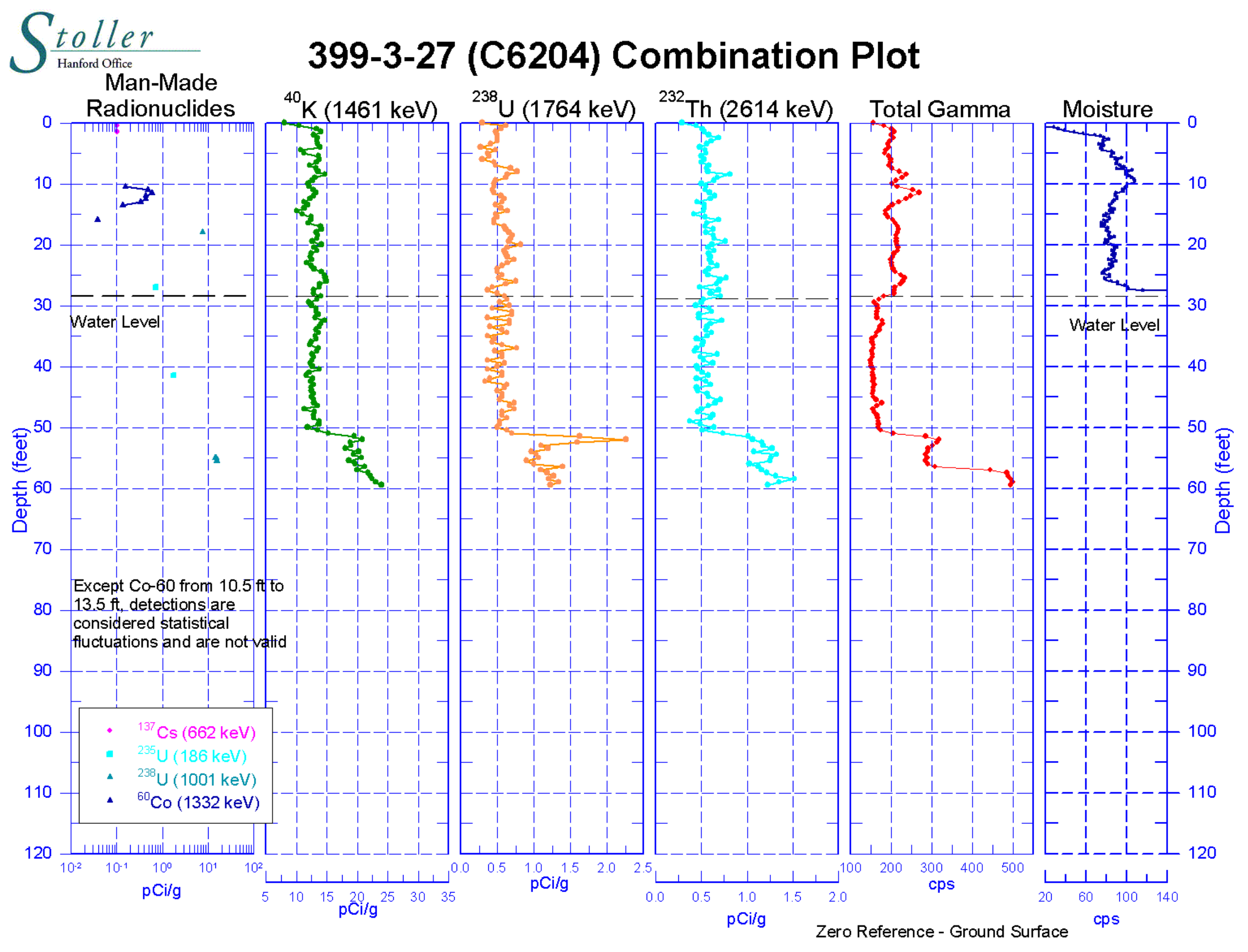


toller 399-3-27 (C6204)

Hanford Office

\section{Total Gamma \& Dead Time}
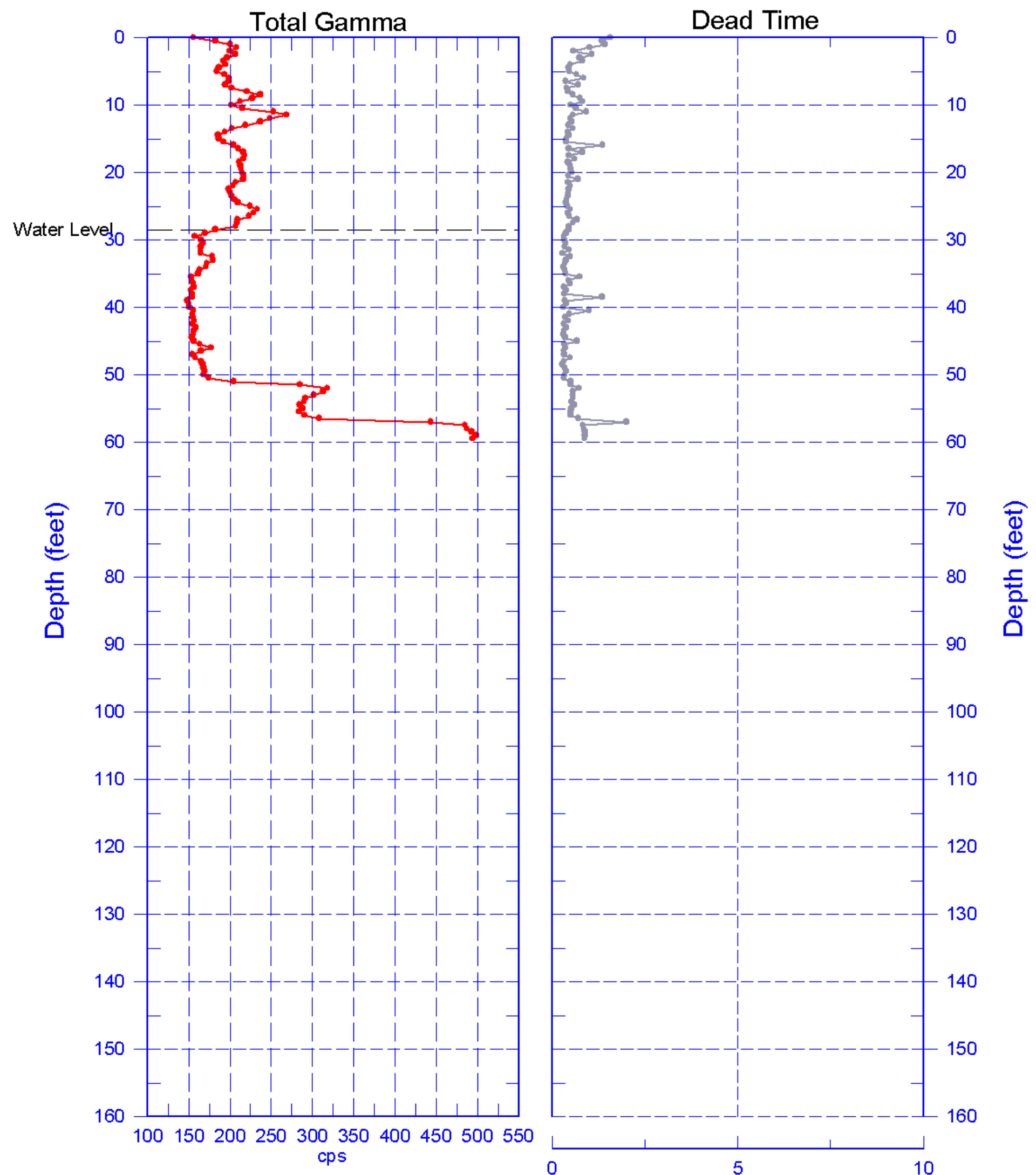

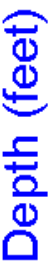

Reference - Ground Surface 


\section{S}

toller 399-3-27 (C6204)

Hanford Office

\section{Total Gamma \& Moisture}
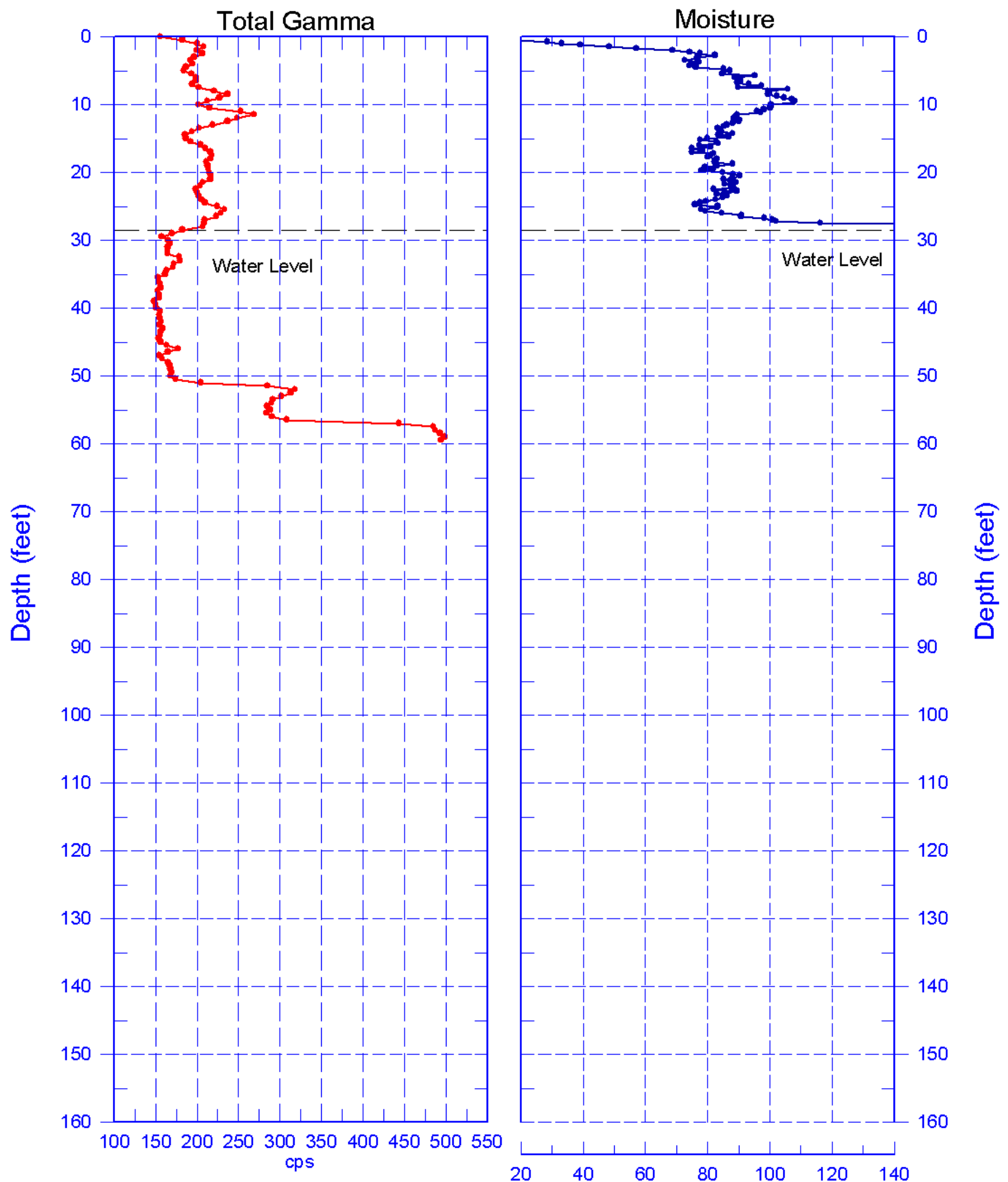

원
듬
$\frac{1}{0}$

Reference - Ground Surface 


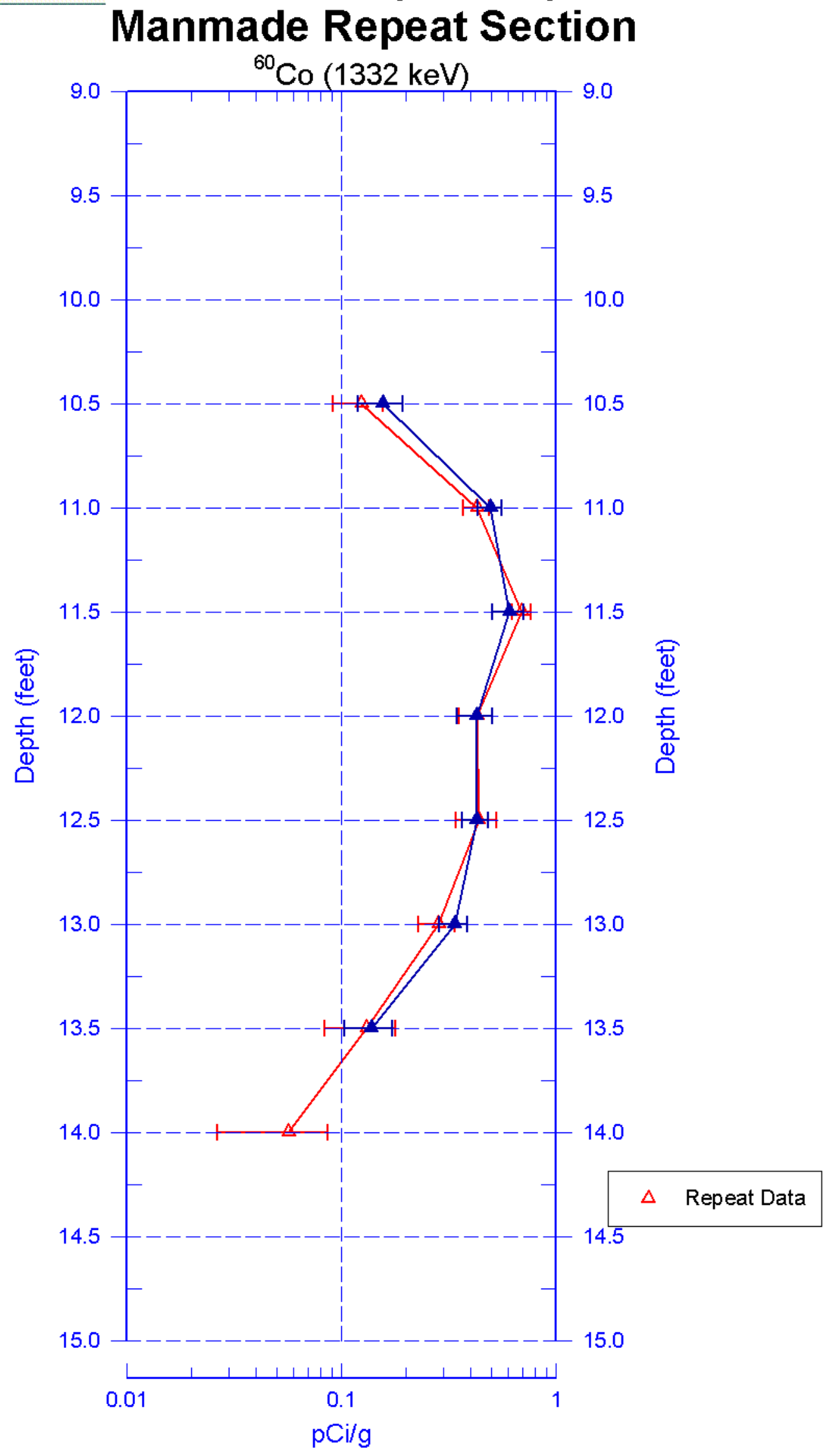




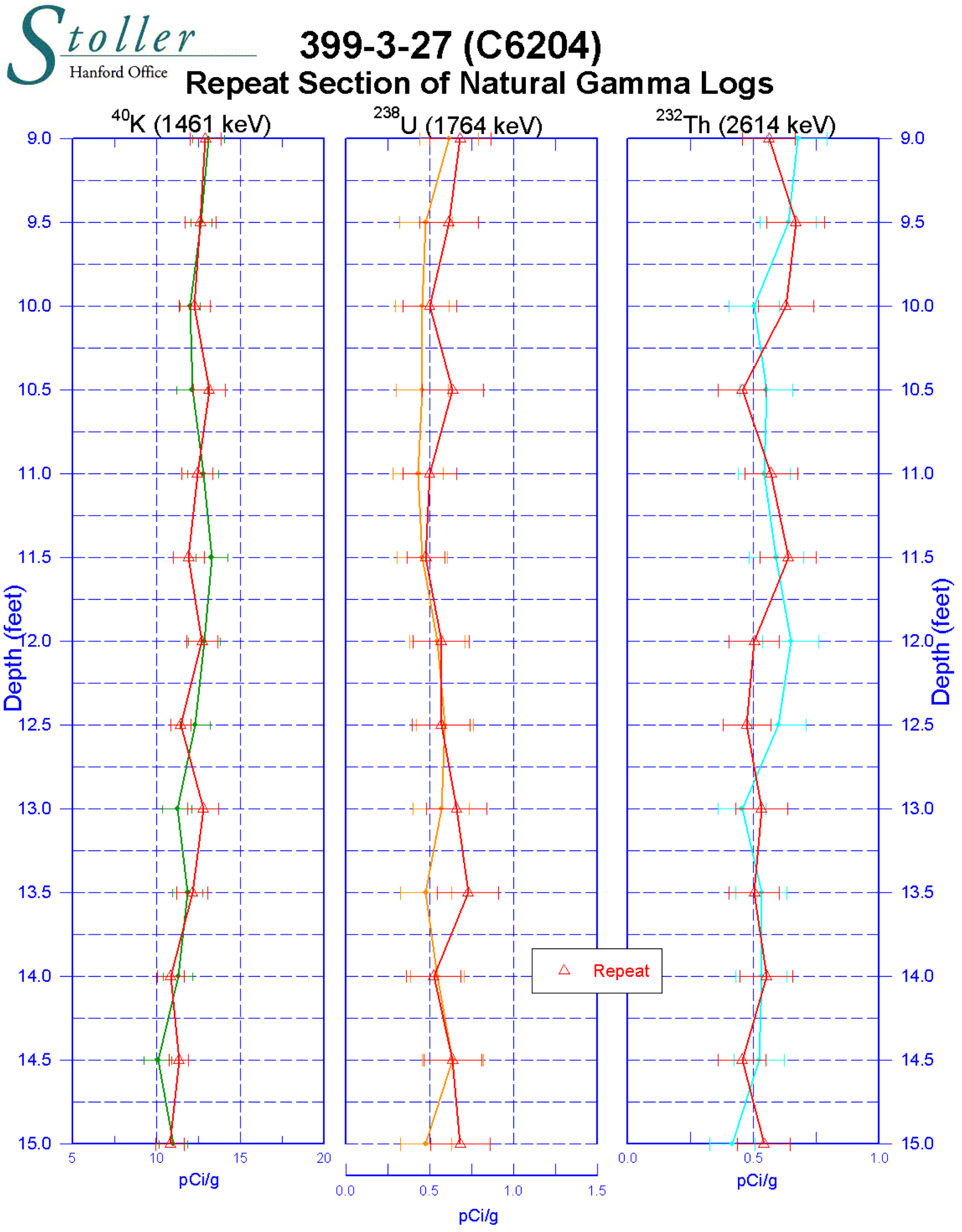

Zero Reference - Ground Surface 


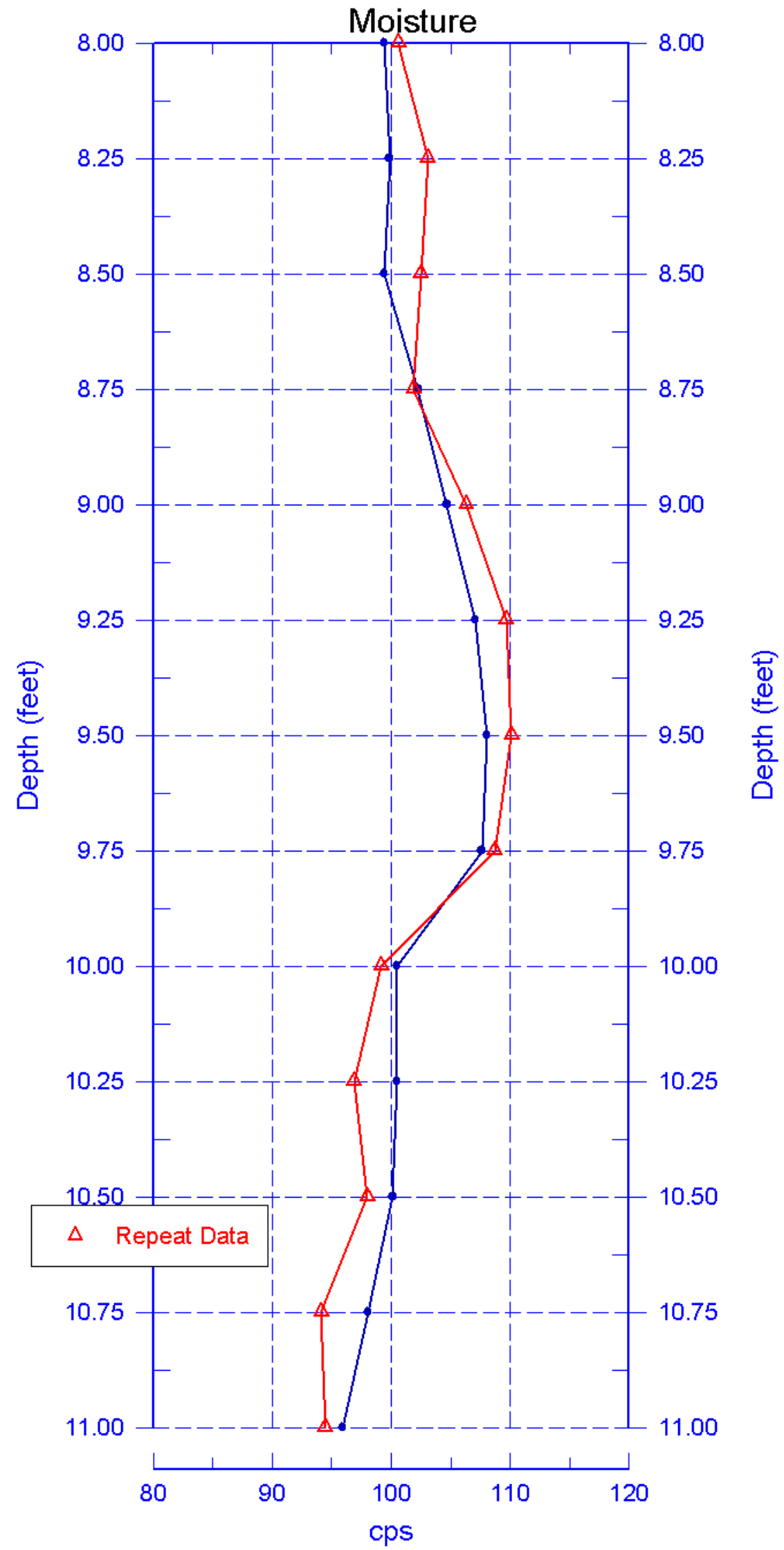




\section{9-3-28 (C6205) Log Data Report}

Borehole Information:

\begin{tabular}{|c|c|c|c|c|c|}
\hline \multirow{2}{*}{\multicolumn{2}{|c|}{ Borehole: $\quad$ 399-3-28 }} & & Site: & \multicolumn{2}{|l|}{ 300-FF-5 } \\
\hline & & $\mathrm{GWL}^{1}$ (f & 30.3 & GWL Date: & $07 / 10 / 08$ \\
\hline North (m) & East (m) & Drill Date & TOC $^{2}$ Elevation & Total Depth (ft) & Type \\
\hline 116046.2 & 594256.0 & 07/09/08 & Unknown & 64.5 & Sonic \\
\hline
\end{tabular}

\section{Casing Information:}

\begin{tabular}{|c|c|c|c|c|c|c|}
\hline Casing Type & Stickup (ft) & $\begin{array}{c}\text { Outer } \\
\text { Diameter (in.) }\end{array}$ & $\begin{array}{c}\text { Inside } \\
\text { Diameter (in.) }\end{array}$ & Thickness (in.) & Top (ft) & Bottom (ft) \\
\hline Threaded Steel & 0.8 & $75 / 8$ & $67 / 8$ & $3 / 8$ & -0.8 & 59.0 \\
\hline
\end{tabular}

\section{Borehole Notes:}

Drill site geologist reported casing information and total depth. An e-tape was employed to measure depth-to-water.

\section{Logging Equipment Information:}

\begin{tabular}{|c|c|c|c|c|}
\hline Logging System: & \multicolumn{2}{|l|}{ Gamma 4 L } & $\begin{array}{l}\text { Type: } \\
\text { Serial No.: }\end{array}$ & $\begin{array}{l}\text { 60\% HPGe SGLS } \\
\text { 47TP32211A }\end{array}$ \\
\hline Effective Calibration Date: & $12 / 31 / 2007$ & \multirow{2}{*}{$\begin{array}{l}\text { Calibration Reference: } \\
\text { Logging Procedure: }\end{array}$} & \multicolumn{2}{|c|}{ HGLP-CC-027 } \\
\hline & & & \multicolumn{2}{|c|}{ HGLP-MAN-002, Rev. 0} \\
\hline
\end{tabular}

\begin{tabular}{|l|l|l|l|l|}
\hline \multirow{2}{*}{ Logging System: } & \multirow{2}{*}{ Gamma $4 \mathrm{H}$} & $\begin{array}{l}\text { Type: } \\
\text { Serial No.: }\end{array}$ & $\begin{array}{l}\text { NMLS } \\
\text { H310700352 }\end{array}$ \\
\hline Effective Calibration Date: & $11 / 06 / 2007$ & $\begin{array}{l}\text { Calibration Reference: } \\
\text { Logging Procedure: }\end{array}$ & HGLP-CC-021 \\
\cline { 4 - 5 } & & HGLP-MAN-002, Rev. 0 \\
\hline
\end{tabular}

\section{Spectral Gamma Logging System (SGLS) Log Run Information:}

\begin{tabular}{|l|c|c|l|l|l|}
\hline Log Run & $\mathbf{1}$ & 2 Repeat & & & \\
\hline Date & $07 / 10 / 08$ & $07 / 10 / 08$ & & & \\
\hline Logging Engineer & McClellan & McClellan & & & \\
\hline Start Depth (ft) & 63.5 & 60.0 & & & \\
\hline Finish Depth (ft) & 0 & 54.0 & & & \\
\hline Count Time (sec) & 200 & 200 & & & \\
\hline Live/Real & $\mathrm{R}$ & $\mathrm{R}$ & & & \\
\hline Shield (Y/N) & $\mathrm{N}$ & $\mathrm{N}$ & & & \\
\hline MSA Interval (ft) & 0.5 & 0.5 & & & \\
\hline Log Speed (ft/min) & $\mathrm{NA}$ & $\mathrm{NA}$ & & & \\
\hline Pre-Verification & DL601CAB & DL601CAB & & & \\
\hline Start File & DL601000 & DL601128 & & & \\
\hline Finish File & DL601127 & DL601140 & & & \\
\hline Post-Verification & DL601CAA & DL601CAA & & & \\
\hline Depth Return Error (in.) & 0 & 1/4 high & & & \\
\hline Comments & None & Repeat section & & & \\
\hline
\end{tabular}


Neutron Moisture Logging System (NMLS) Log Run Information:

\begin{tabular}{|l|c|c|l|l|l|}
\hline Log Run & $\mathbf{3}$ & 4 Repeat & & & \\
\hline Date & $07 / 10 / 08$ & $07 / 10 / 08$ & & & \\
\hline Logging Engineer & Pearson & Pearson & & & \\
\hline Start Depth (ft) & 0 & 25.0 & & & \\
\hline Finish Depth (ft) & 30.25 & 28.0 & & & \\
\hline Count Time (sec) & 15 & 15 & & & \\
\hline Live/Real & $\mathrm{R}$ & $\mathrm{R}$ & & & \\
\hline Shield (Y/N) & $\mathrm{N}$ & $\mathrm{N}$ & & & \\
\hline MSA Interval (ft) & 0.25 & 0.25 & & & \\
\hline Log Speed (ft/min) & NA & NA & & & \\
\hline Pre-Verification & DHG72CAB & DHG72CAB & & & \\
\hline Start File & DHG72000 & DHG72122 & & & \\
\hline Finish File & DHG72121 & DHG72134 & & & \\
\hline Post-Verification & DHG72CAA & DHG72CAA & & & \\
\hline Depth Return Error (in.) & 0 & 1/4 high & & & \\
\hline Comments & None & Repeat section & & & \\
\hline
\end{tabular}

\section{Logging Operation Notes:}

SGLS and NMLS data were both collected using Gamma 4, HO 68B-3573.

SGLS pre- and post-survey verification measurements were acquired in the Amersham KUTh-115 field verifier. Maximum SGLS logging depth achieved was $63.7 \mathrm{ft}$ or approximately $4.7 \mathrm{ft}$ below casing before the sonde un-weighted. A centralizer was installed on the sonde.

NMLS pre- and post-survey verification measurements were acquired in the standard field verifier. Maximum logging depth achieved was $30.25 \mathrm{ft}$, at approximately groundwater level. A centralizer was installed on the sonde.

Analysis Notes:

\begin{tabular}{|l|l|l|l|l|l|}
\hline Analyst: & R. SPATZ & Date: & 10/08/08 & Reference: & GJO-HGLP 1.6.3, Rev. 0 \\
\hline
\end{tabular}

Pre- and post-survey verification spectra for both SGLS and NMLS met the acceptance criteria for the established systems.

SGLS spectra were processed in batch mode using APTEC SUPERVISOR to identify individual peaks and count rates. Concentrations were calculated using an EXCEL template identified as G4LDec07.xls using an efficiency function and corrections for casing, dead time, and water as determined by annual calibrations.

NMLS spectra were processed in batch mode using APTEC SUPERVISOR to identify counts. Count rates were calculated using an EXCEL template identified as G4HNov07.xls. NMLS data are presented in counts per second (cps), because no calibration data is available for a 6 7/8-in inside diameter borehole casing.

Analyses of log data suggest slight discrepancies with field reports of depth-to-water and casing depth. Corrections of log data were applied according to the analysis assumptions in the table below. 
HGLP-LDR-258, Rev. 0

\begin{tabular}{|c|c|c|c|l|}
\hline Top (ft) & Bottom (ft) & $\begin{array}{c}\text { T } \\
\text { (in) }\end{array}$ & $\begin{array}{l}\text { ID } \\
\text { (in) }\end{array}$ & Comments \\
\hline 0 & 29.9 & 0.375 & N/A & No water correction \\
\hline 30.0 & 59.5 & 0.375 & 6.875 & $\begin{array}{l}\text { GWL reported @ 30.3 ft; adjusted to 30.0 ft } \\
\text { Casing bottom reported @59.0 ft; adjusted to 59.5 ft }\end{array}$ \\
\hline 59.6 & TD & 0 & 0 & No adjustment made \\
\hline
\end{tabular}

$\mathrm{T}=$ casing thickness used to calculate energy-dependent casing correction factor

ID = casing inside diameter used to calculate energy-dependent water correction factor

\section{Results and Interpretations:}

Cs-137, U-235, and U-238 (Pa-234m) were detected sporadically in this borehole. Inspection of the individual spectra at these depths indicates that these detections are statistical fluctuations associated with the processing software and are not considered valid. No Co-60 was detected.

Below the $55.0 \mathrm{ft}$ depth, a probable lithologic change occurs, which is shown by the increased KUT concentrations and Total Gamma count rates. The KUT and Moisture plots both indicate good repeatability.

\section{List of Log Plots:}

Depth Reference is ground surface

Manmade Radionuclides

Natural Gamma Logs

Combination Plot

Total Gamma \& Dead Time

Total Gamma \& Moisture

Repeat Section of Natural Gamma Logs

Moisture Repeat Section

${ }^{1} \mathrm{GWL}$ - groundwater level

${ }^{2}$ TOC - top of casing 

Hanford Office Manmade Radionuclides

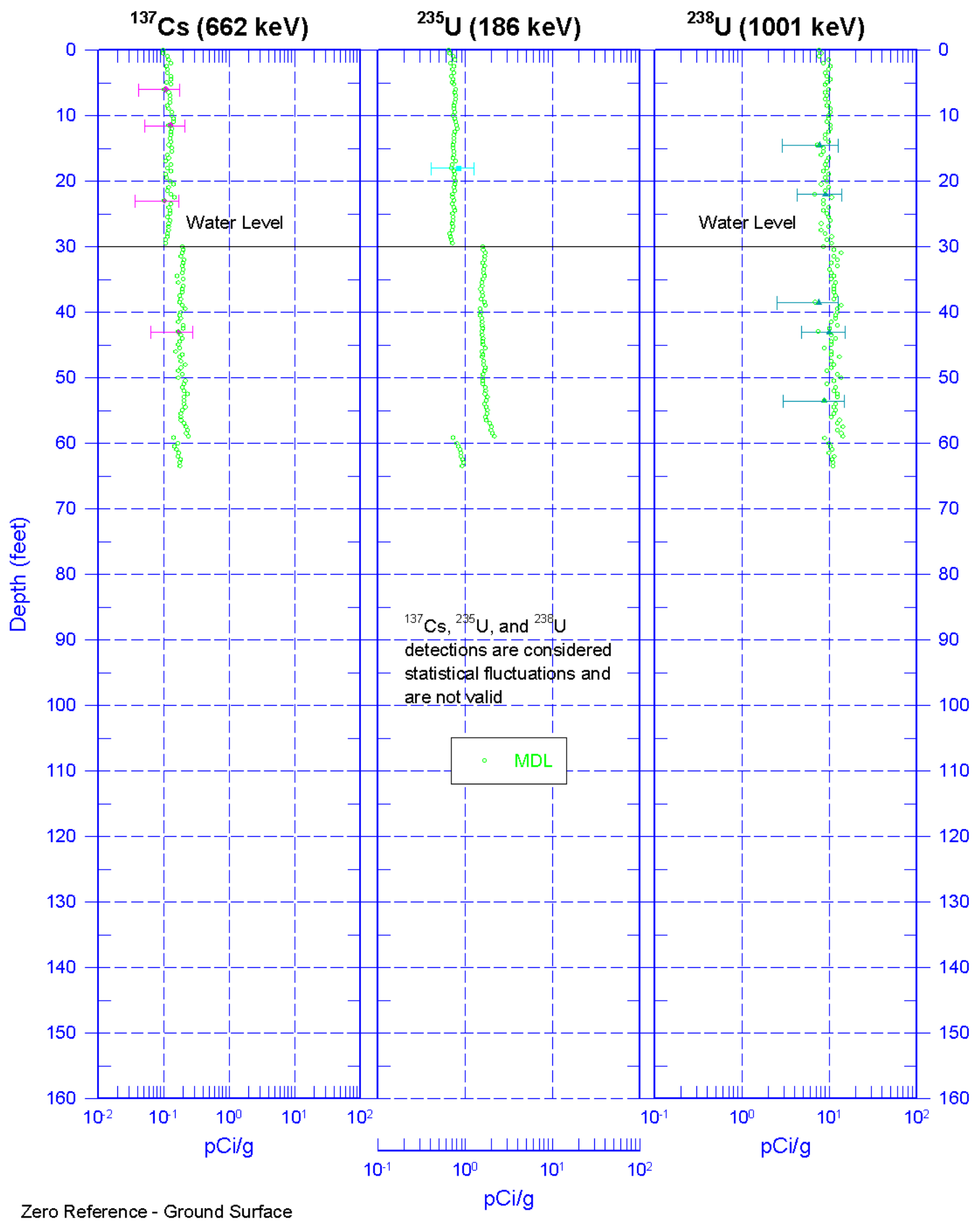


toller 399-3-28 (C6205)

Hanford Office

Natural Gamma Logs
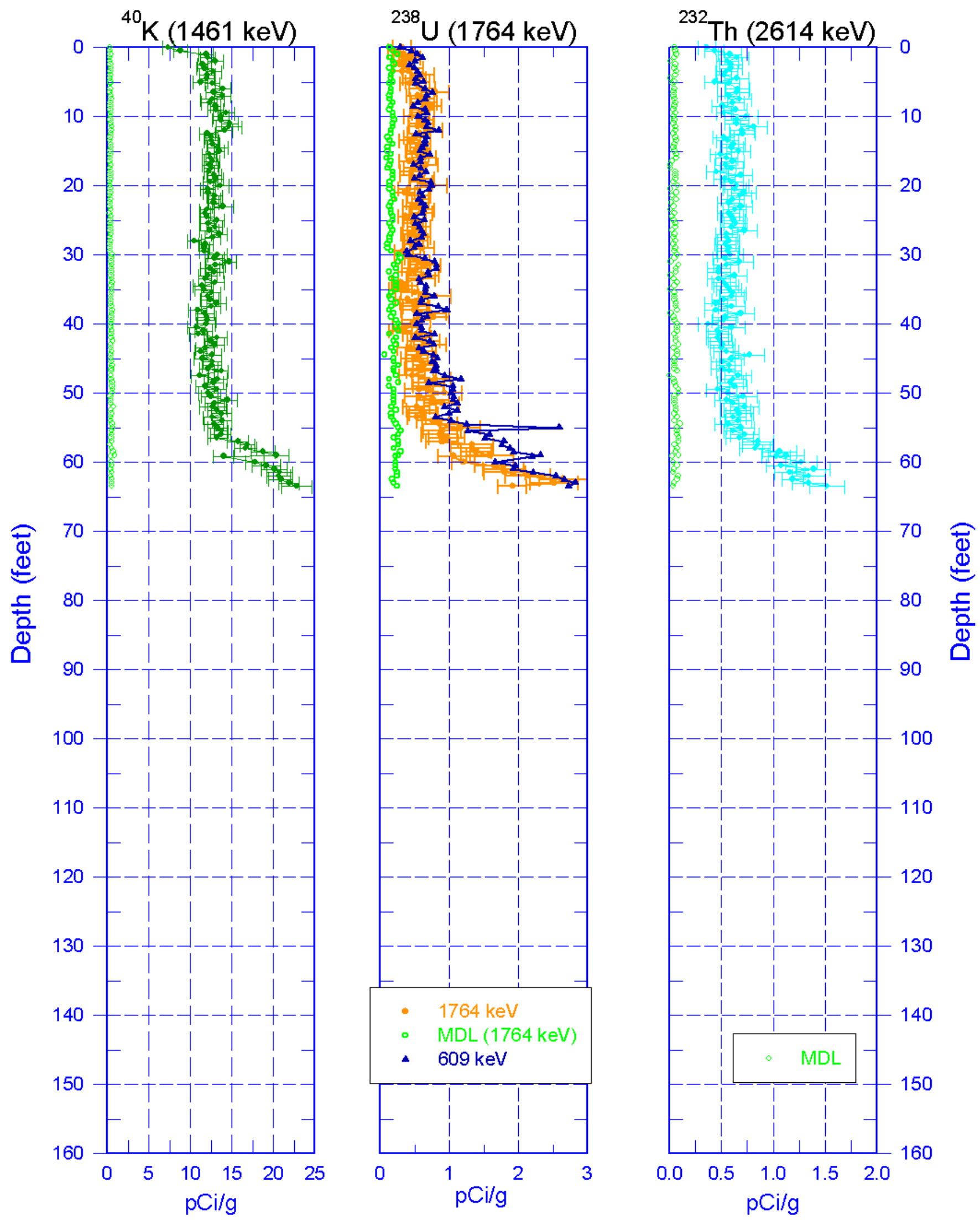

Zero Reference - Ground Surface 


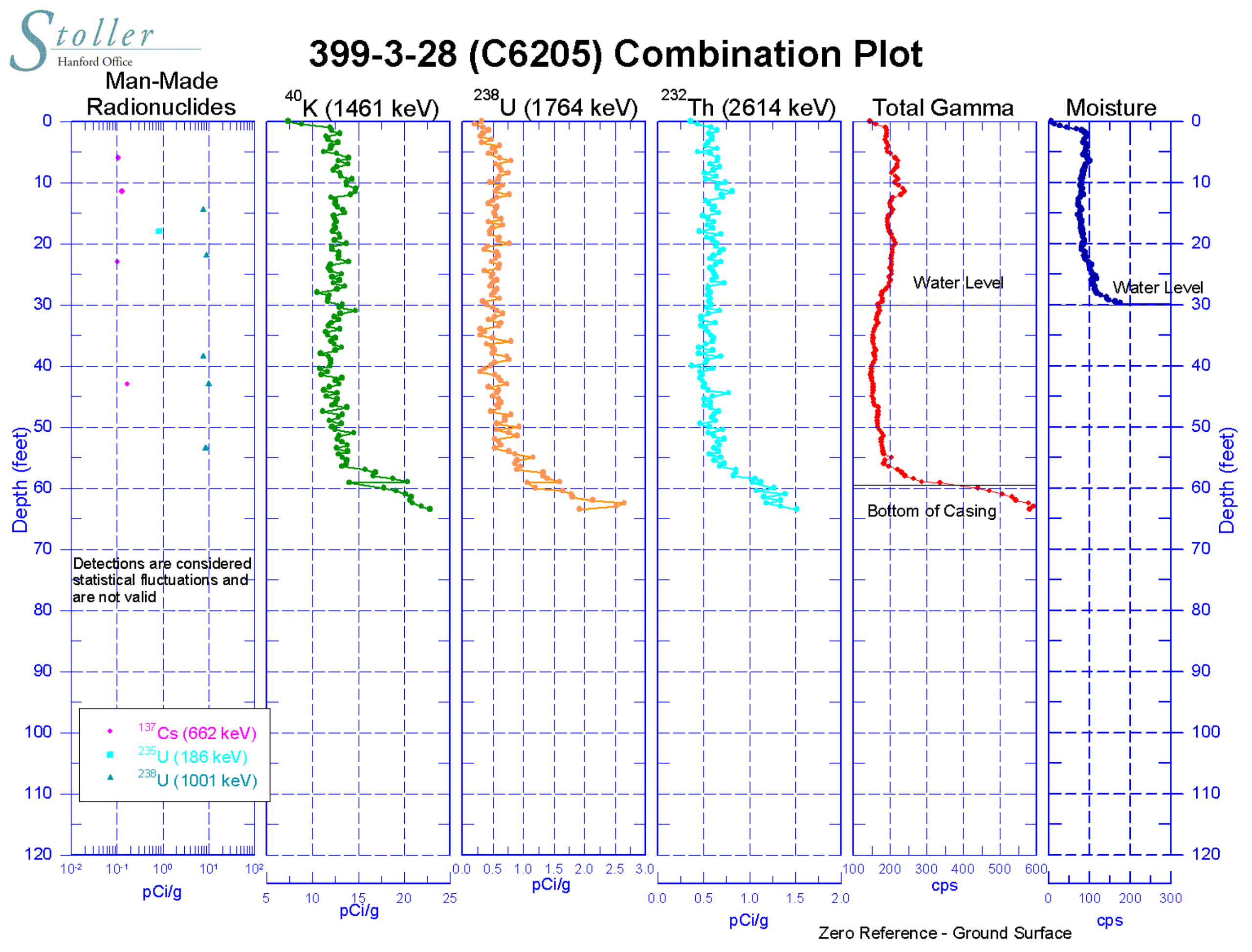


toller 399-3-28 (C6205)

Hanford Office

\section{Total Gamma \& Dead Time}
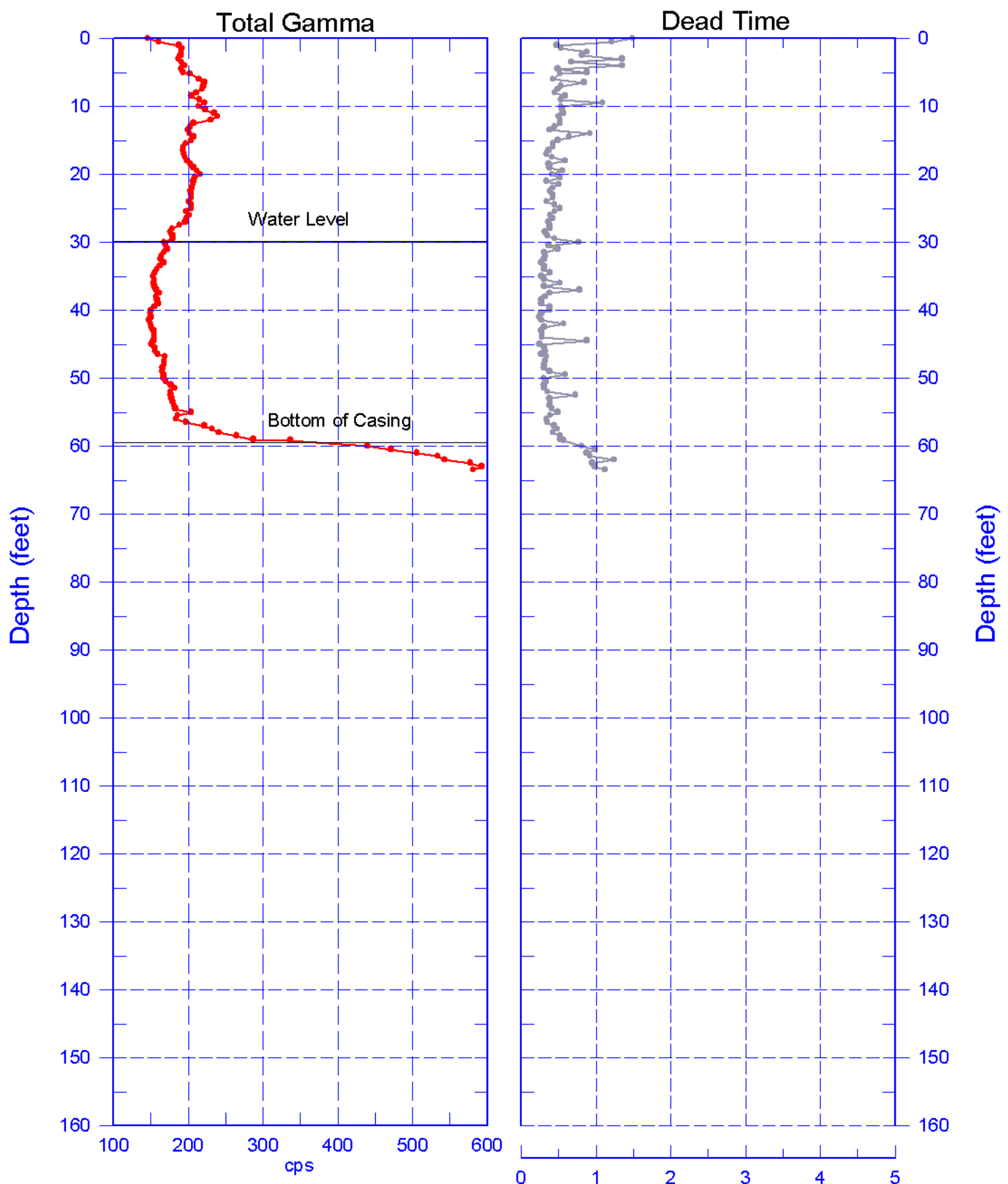

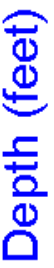

Reference - Ground Surface 
toller 399-3-28 (C6205)

Hanford Office

Total Gamma \& Moisture
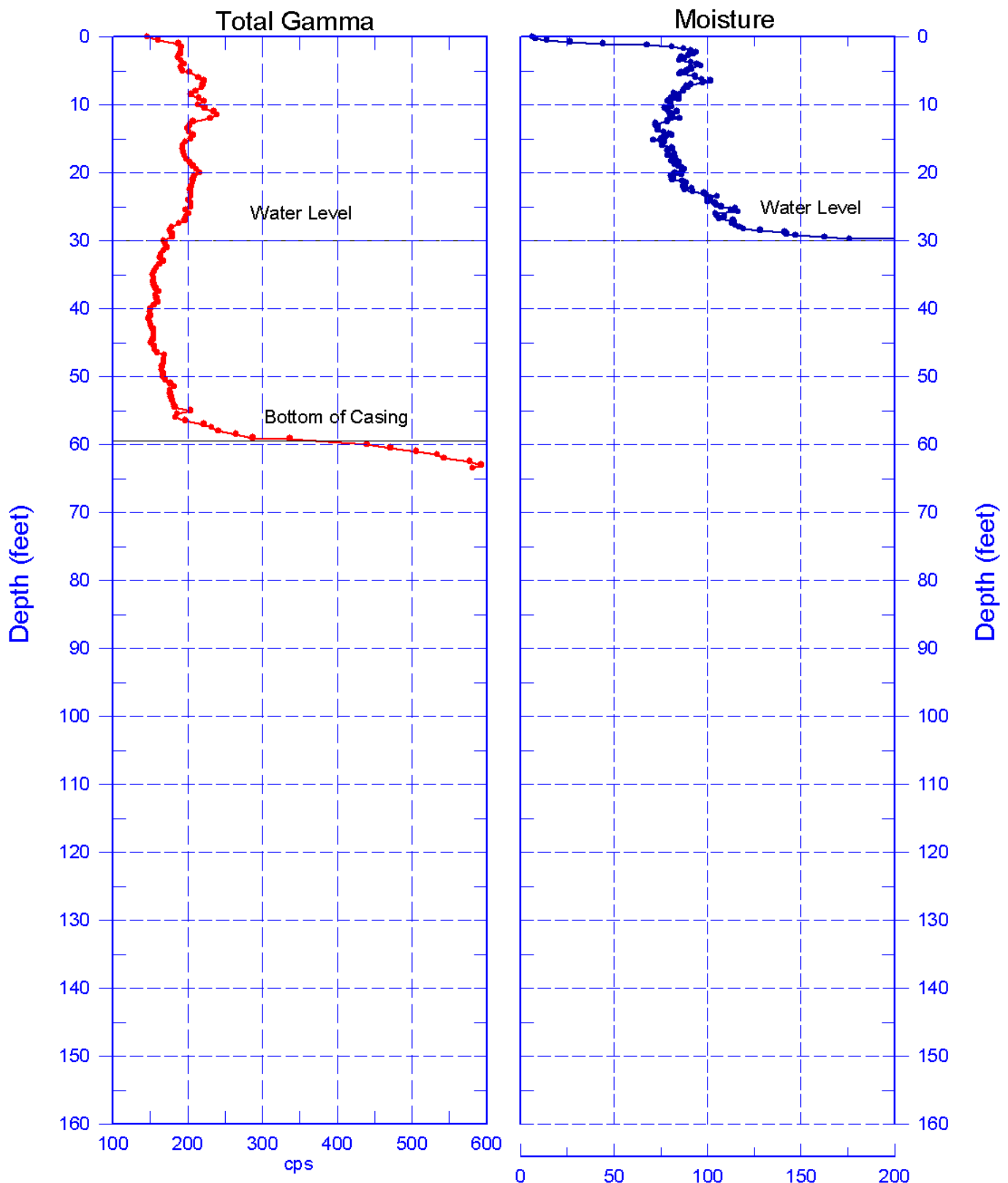

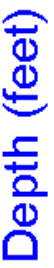

Reference - Ground Surface 


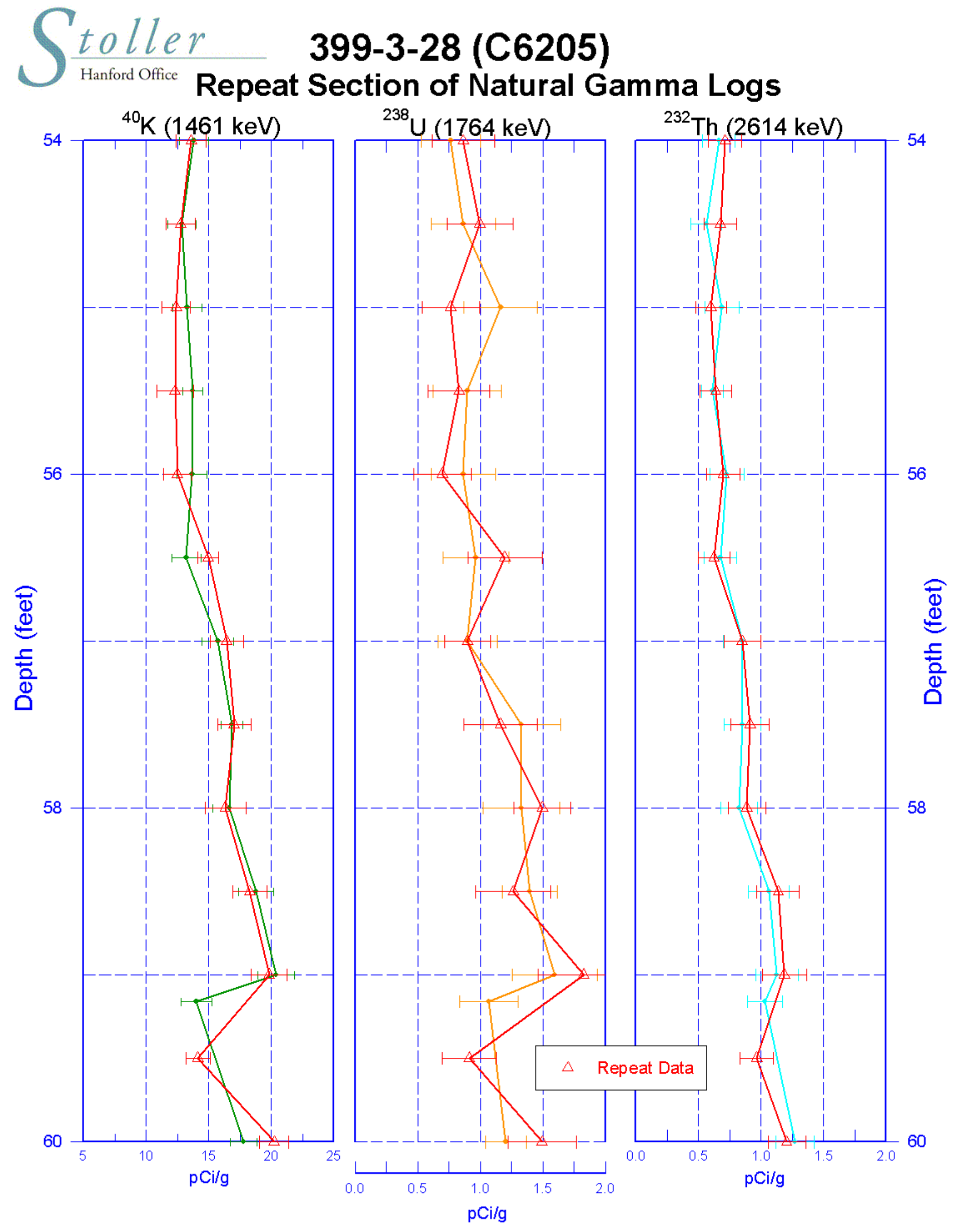




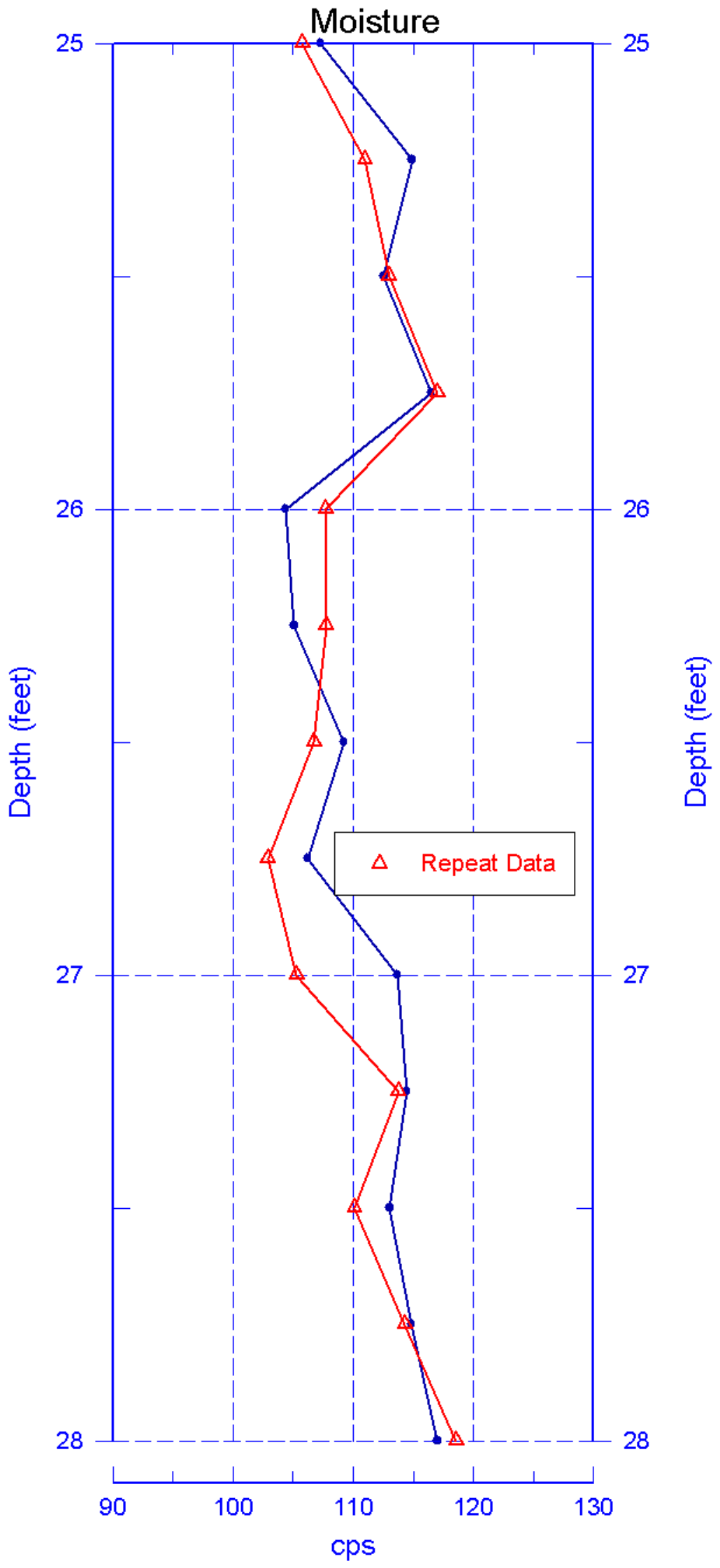




\section{9-3-29 (C6206) Log Data Report}

Borehole Information:

\begin{tabular}{|c|c|c|c|c|c|}
\hline \multirow{2}{*}{\multicolumn{2}{|c|}{ Borehole: $\quad 399-3-29$}} & & Site: & \multicolumn{2}{|l|}{ 300-FF-5 } \\
\hline & Coordinates (WA St Plane) & GWL $^{1}(\mathbf{f}$ & 26.4 & GWL Date: & 07/02/08 \\
\hline North (m) & East (m) & Drill Date & TOC $^{2}$ Elevation & Total Depth (ft) & Type \\
\hline 116051.9 & 594264.2 & 06/30/08 & Unknown & 64.0 & Sonic \\
\hline
\end{tabular}

\section{Casing Information:}

\begin{tabular}{|c|c|c|c|c|c|c|}
\hline Casing Type & Stickup (ft) & $\begin{array}{c}\text { Outer } \\
\text { Diameter (in.) }\end{array}$ & $\begin{array}{c}\text { Inside } \\
\text { Diameter (in.) }\end{array}$ & Thickness (in.) & Top (ft) & Bottom (ft) \\
\hline Threaded Steel & 2.4 & $75 / 8$ & $67 / 8$ & $3 / 8$ & -2.4 & 58.2 \\
\hline
\end{tabular}

\section{Borehole Notes:}

Drill site geologist reported casing information and total depth. An e-tape was employed to measure depth-to-water.

\section{Logging Equipment Information:}

\begin{tabular}{|c|c|c|c|c|}
\hline Logging System: & \multicolumn{2}{|l|}{ Gamma $4 \mathrm{~L}$} & $\begin{array}{l}\text { Type: } \\
\text { Serial No.: }\end{array}$ & $\begin{array}{l}\text { 60\% HPGe SGLS } \\
\text { 47TP32211A }\end{array}$ \\
\hline Effective Calibration Date: & $12 / 31 / 2007$ & \multirow{2}{*}{$\begin{array}{l}\text { Calibration Reference: } \\
\text { Logging Procedure: }\end{array}$} & \multicolumn{2}{|c|}{ HGLP-CC-027 } \\
\hline & & & \multicolumn{2}{|c|}{ HGLP-MAN-002, Rev. 0} \\
\hline Logging System: & \multicolumn{2}{|l|}{ Gamma $4 \mathrm{H}$} & $\begin{array}{l}\text { Type: } \\
\text { Serial No.: }\end{array}$ & $\begin{array}{l}\text { NMLS } \\
\text { H310700352 }\end{array}$ \\
\hline Effective Calibration Date: & $11 / 06 / 2007$ & \multirow{2}{*}{$\begin{array}{l}\text { Calibration Reference: } \\
\text { Logging Procedure: }\end{array}$} & \multicolumn{2}{|c|}{ HGLP-CC-021 } \\
\hline & & & \multicolumn{2}{|c|}{ HGLP-MAN-002, Rev. 0} \\
\hline
\end{tabular}

\section{Spectral Gamma Logging System (SGLS) Log Run Information:}

\begin{tabular}{|c|c|c|c|c|c|}
\hline Log Run & 1 & 2 Repeat & & & \\
\hline Date & $07 / 01 / 08$ & $07 / 01 / 08$ & & & \\
\hline Logging Engineer & McClellan & McClellan & & & \\
\hline Start Depth (ft) & 60.0 & 12.0 & & & \\
\hline Finish Depth (ft) & 0 & 6.0 & & & \\
\hline Count Time (sec) & 200 & 200 & & & \\
\hline Live/Real & $\mathrm{R}$ & $\mathrm{R}$ & & & \\
\hline Shield (Y/N) & $\mathrm{N}$ & $\mathrm{N}$ & & & \\
\hline MSA Interval (ft) & 0.5 & 0.5 & & & \\
\hline Log Speed (ft/min) & NA & NA & & & \\
\hline Pre-Verification & DL571CAB & DL571CAB & & & \\
\hline Start File & DL571000 & DL571121 & & & \\
\hline Finish File & DL571120 & DL571133 & & & \\
\hline Post-Verification & DL571CAA & DL571CAA & & & \\
\hline Depth Return Error (in.) & 0 & 0 & & & \\
\hline Comments & None & Repeat section & & & \\
\hline
\end{tabular}


Neutron Moisture Logging System (NMLS) Log Run Information:

\begin{tabular}{|l|c|c|l|l|l|}
\hline Log Run & $\mathbf{3}$ & 4 Repeat & & & \\
\hline Date & $07 / 02 / 08$ & $07 / 02 / 08$ & & & \\
\hline Logging Engineer & McClellan & McClellan & & & \\
\hline Start Depth (ft) & 0 & 8.0 & & & \\
\hline Finish Depth (ft) & 26.25 & 5.0 & & & \\
\hline Count Time (sec) & 15 & 15 & & & \\
\hline Live/Real & $\mathrm{R}$ & $\mathrm{R}$ & & & \\
\hline Shield (Y/N) & $\mathrm{N}$ & $\mathrm{N}$ & & & \\
\hline MSA Interval (ft) & 0.25 & 0.25 & & & \\
\hline Log Speed (ft/min) & NA & NA & & & \\
\hline Pre-Verification & DHG42CAB & DHG42CAB & & & \\
\hline Start File & DHG42000 & DHG42106 & & & \\
\hline Finish File & DHG42105 & DHG42118 & & & \\
\hline Post-Verification & DHG42CAA & DHG42CAA & & & \\
\hline Depth Return Error (in.) & 0 & NA & & & \\
\hline Comments & None & Repeat section & & & \\
\hline
\end{tabular}

\section{Logging Operation Notes:}

SGLS and NMLS data were both collected using Gamma 4, HO 68B-3573.

SGLS pre- and post-survey verification measurements were acquired in the Amersham KUTh-115 field verifier. Maximum SGLS logging depth achieved was $60.3 \mathrm{ft}$ or approximately $2 \mathrm{ft}$ below casing before the sonde un-weighted. The $5.8 \mathrm{ft}$ of reported open hole below casing was not reached. A centralizer was installed on the sonde.

NMLS pre- and post-survey verification measurements were acquired in the standard field verifier. Maximum logging depth achieved was $26.25 \mathrm{ft}$, at approximately groundwater level. A centralizer was installed on the sonde.

\section{Analysis Notes:}

\begin{tabular}{|l|l|l|l|l|l|}
\hline Analyst: & R. SPATZ & Date: & 10/08/08 & Reference: & GJO-HGLP 1.6.3, Rev. 0 \\
\hline
\end{tabular}

Pre- and post-survey verification spectra for the SGLS met the acceptance criteria for the established systems.

SGLS spectra were processed in batch mode using APTEC SUPERVISOR to identify individual peaks and count rates. Concentrations were calculated using an EXCEL template identified as G4LDec07.xls using an efficiency function and corrections for casing, dead time, and water as determined by annual calibrations.

Pre-survey verification spectra for the NMLS met the acceptance criteria. Post-survey verification was slightly above criteria but well below HASQARD limits for the established system. NMLS spectra were processed in batch mode using APTEC SUPERVISOR to identify counts. Count rates were calculated using an EXCEL template identified as G4HNov07.xls. NMLS data are presented in counts per second (cps), because no calibration data is available for a 6 7/8-in. inside diameter borehole casing.

Analyses of log data suggest slight discrepancies with field reports of depth-to-water and casing depth. Corrections of log data were applied according to the analysis assumptions in the table below. 
Hanford Office

HGLP-LDR-259, Rev. 0

\begin{tabular}{|c|c|c|c|l|}
\hline Top (ft) & Bottom (ft) & $\begin{array}{c}\text { T } \\
\text { (in) }\end{array}$ & $\begin{array}{c}\text { ID } \\
\text { (in) }\end{array}$ & Comments \\
\hline 0 & 25.9 & 0.375 & N/A & No water correction \\
\hline 26.0 & 58.2 & 0.375 & 6.875 & $\begin{array}{l}\text { GWL reported @ 26.4 ft; adjusted to 26.0 ft } \\
\text { Casing bottom reported @58.2 ft; no adjustment made }\end{array}$ \\
\hline 58.3 & TD & 0 & 0 & No adjustment made \\
\hline
\end{tabular}

$\mathrm{T}=$ casing thickness used to calculate energy-dependent casing correction factor

ID = casing inside diameter used to calculate energy-dependent water correction factor

\section{Results and Interpretations:}

Cs-137, U-235, and U-238 (Pa-234m) were detected sporadically in this borehole. Inspection of the individual spectra at these depths indicates that these detections are statistical fluctuations associated with the processing software and are not considered valid.

Co-60 was detected continuously between the depths of 9.0 and $13.5 \mathrm{ft}$. and once at $20.5 \mathrm{ft}$ just above the MDL. The maximum concentration detected was $0.18 \mathrm{pCi} / \mathrm{g}$ at the $10.5 \mathrm{ft}$ depth.

Below the $53.0 \mathrm{ft}$ depth, a probable lithologic change occurs, which is shown by the increased KUT concentrations and Total Gamma count rates. The KUT and Moisture plots both indicate good repeatability.

\section{List of Log Plots:}

Depth Reference is ground surface

Manmade Radionuclides

Natural Gamma Logs

Combination Plot

Total Gamma \& Dead Time

Total Gamma \& Moisture

Repeat Section of Natural Gamma Logs

Moisture Repeat Section

${ }^{1} \mathrm{GWL}$ - groundwater level

${ }^{2}$ TOC - top of casing 


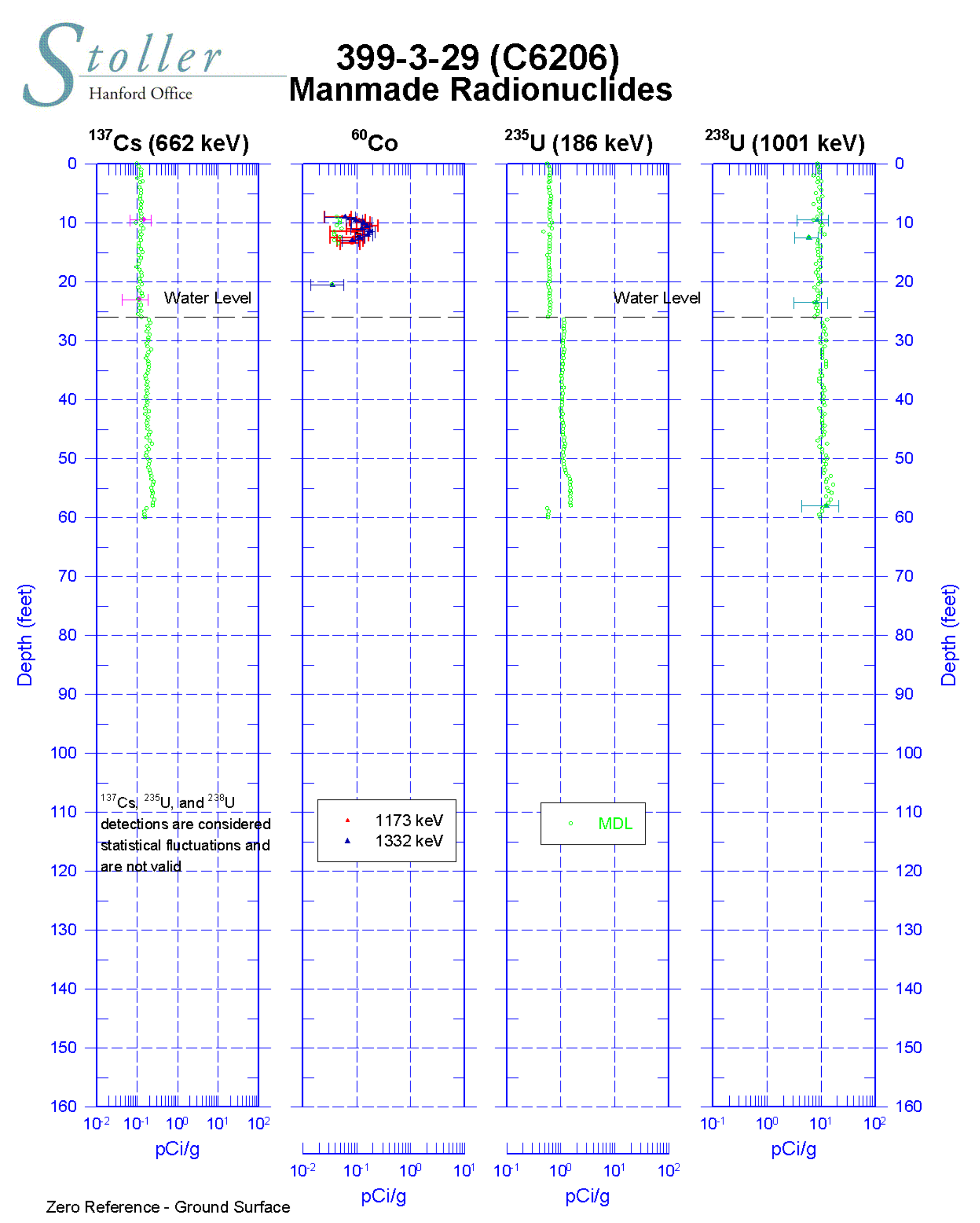


toller 399-3-29 (C6206)

Hanford Office

Natural Gamma Logs
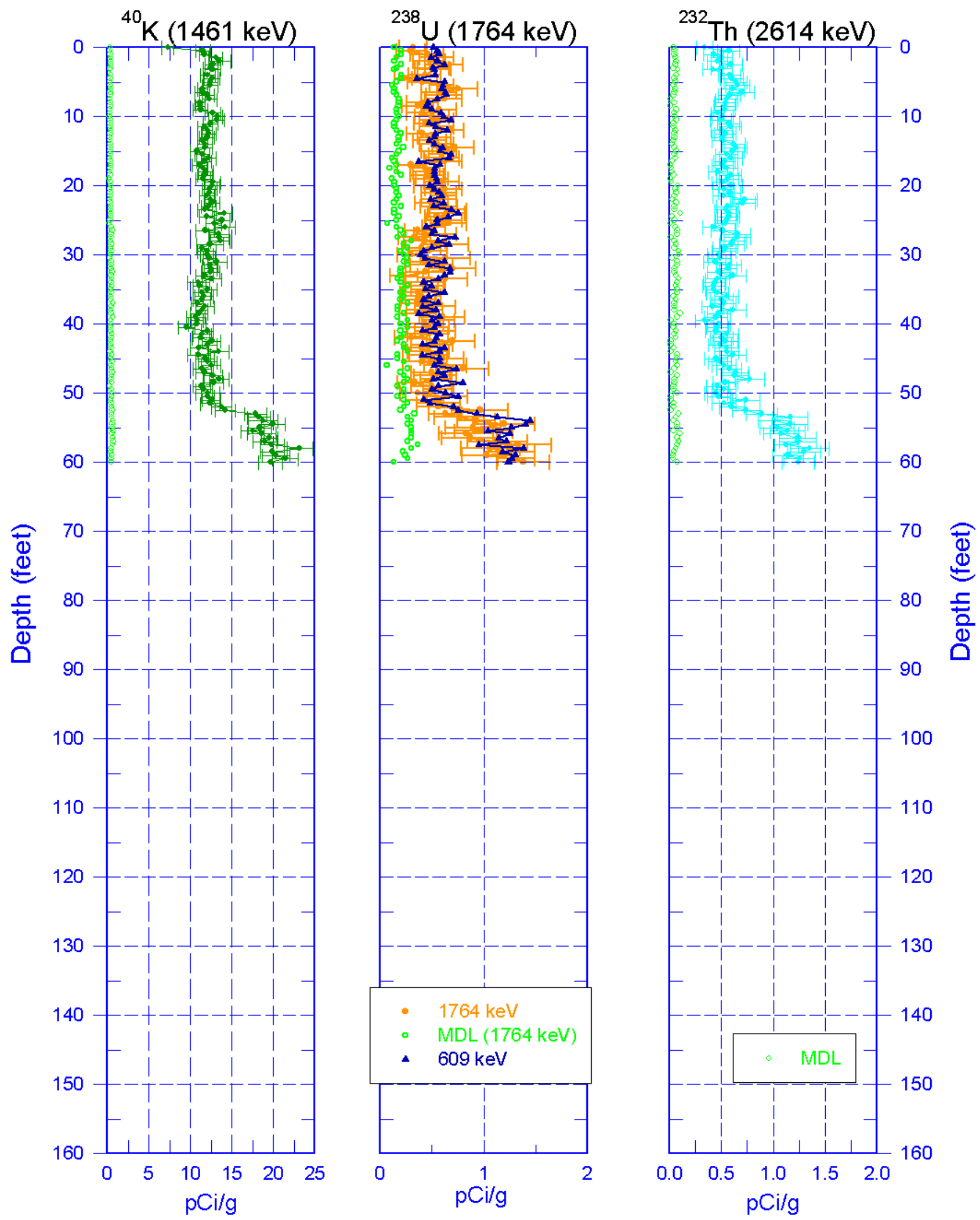

Zero Reference - Ground Surface 
Stoller

Hanford Offici

Man-Made

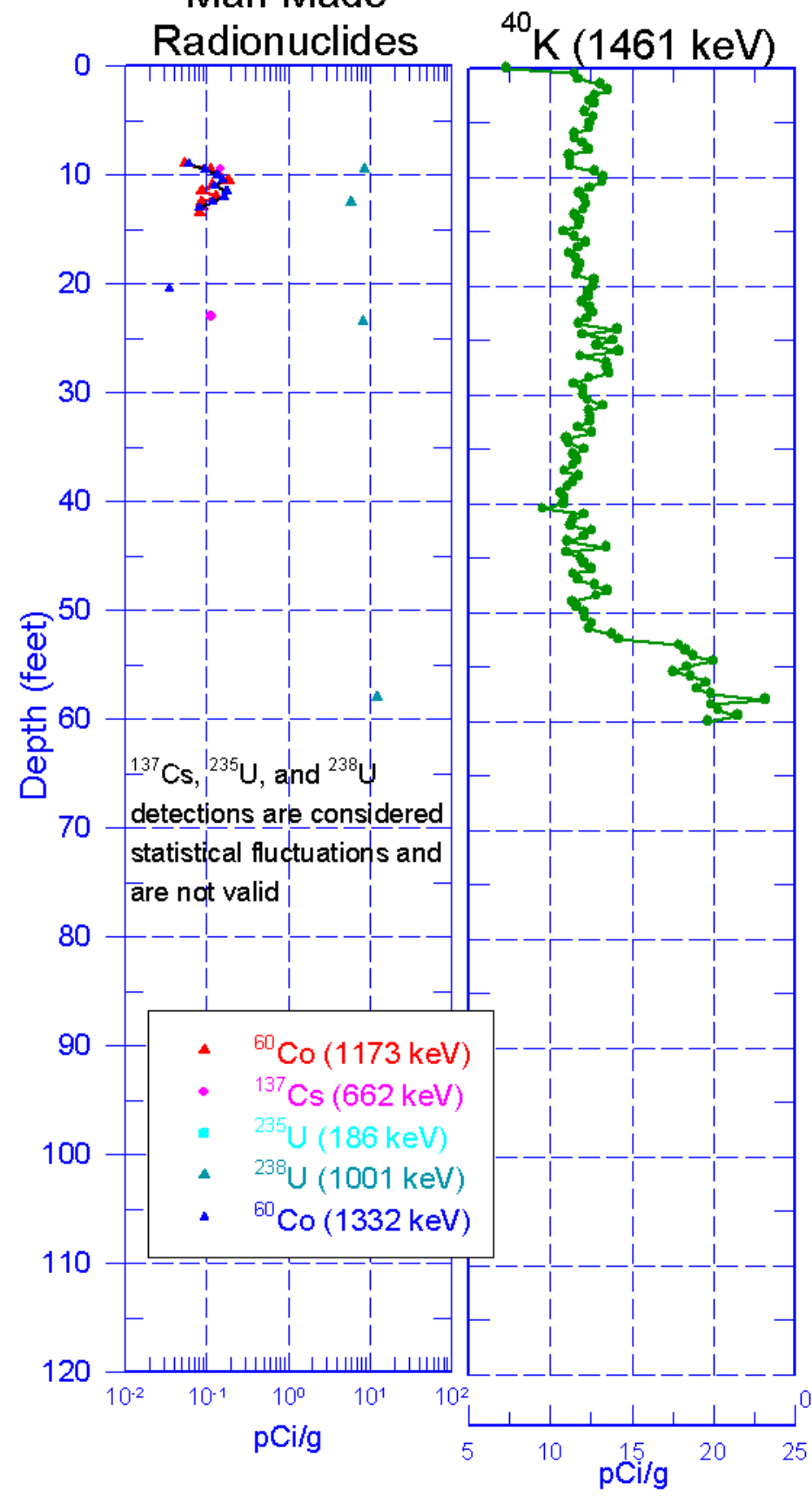

399-3-29 (C6206) Combination Plot
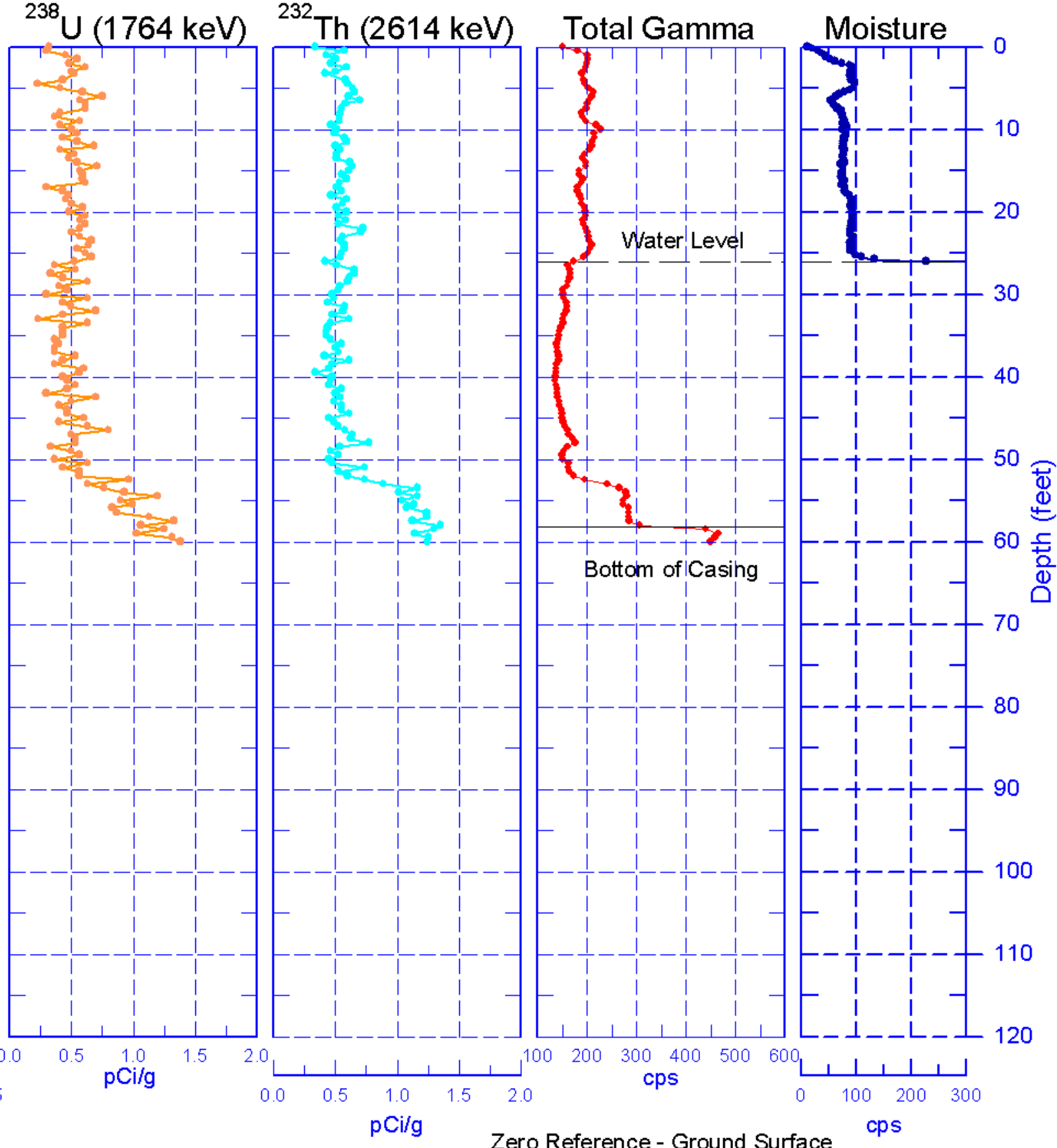


\section{toller 399-3-29 (C6206) \\ Hanford Office \\ Total Gamma \& Dead Time}
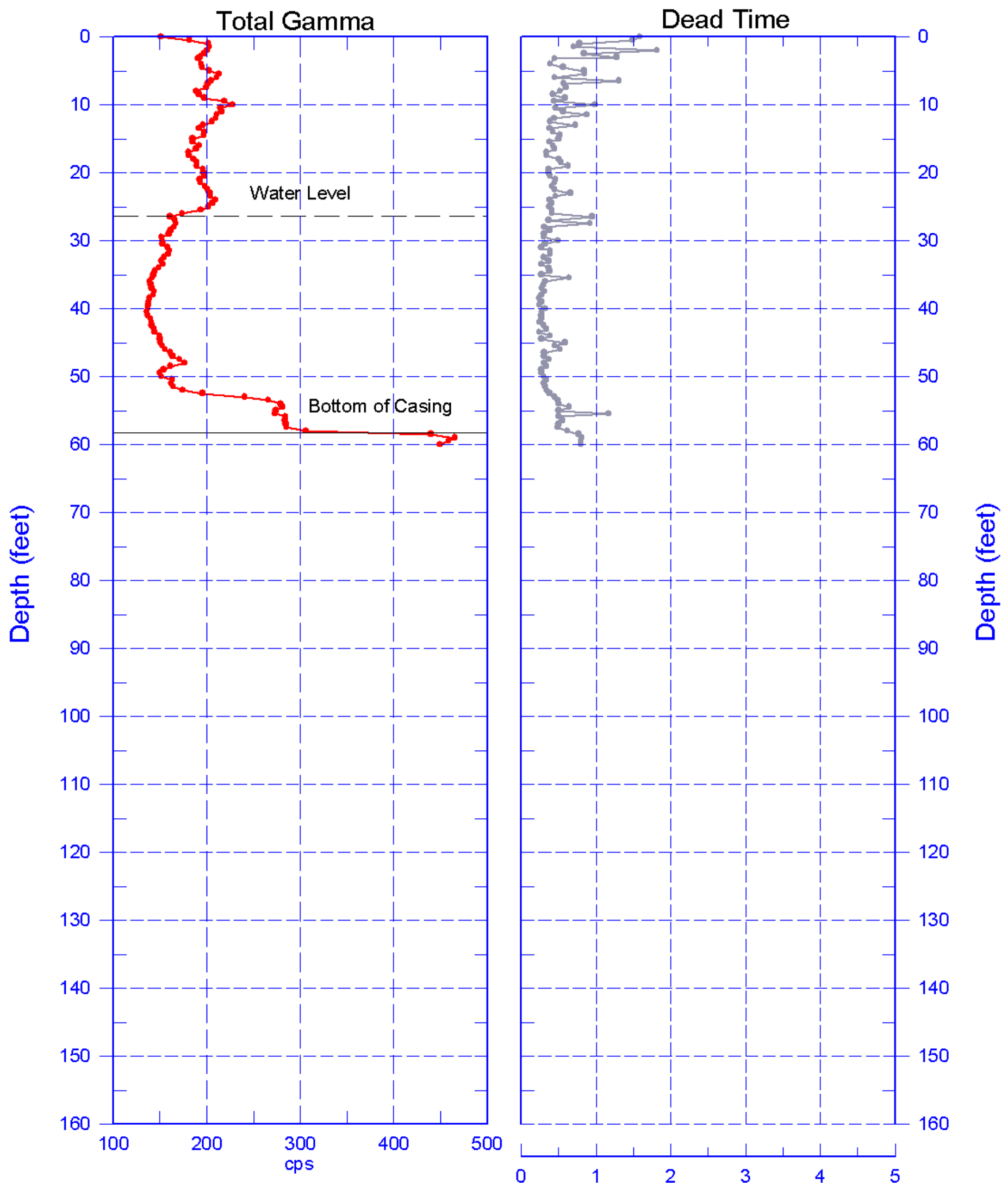
toller 399-3-29 (C6206)

Hanford Office

Total Gamma \& Moisture
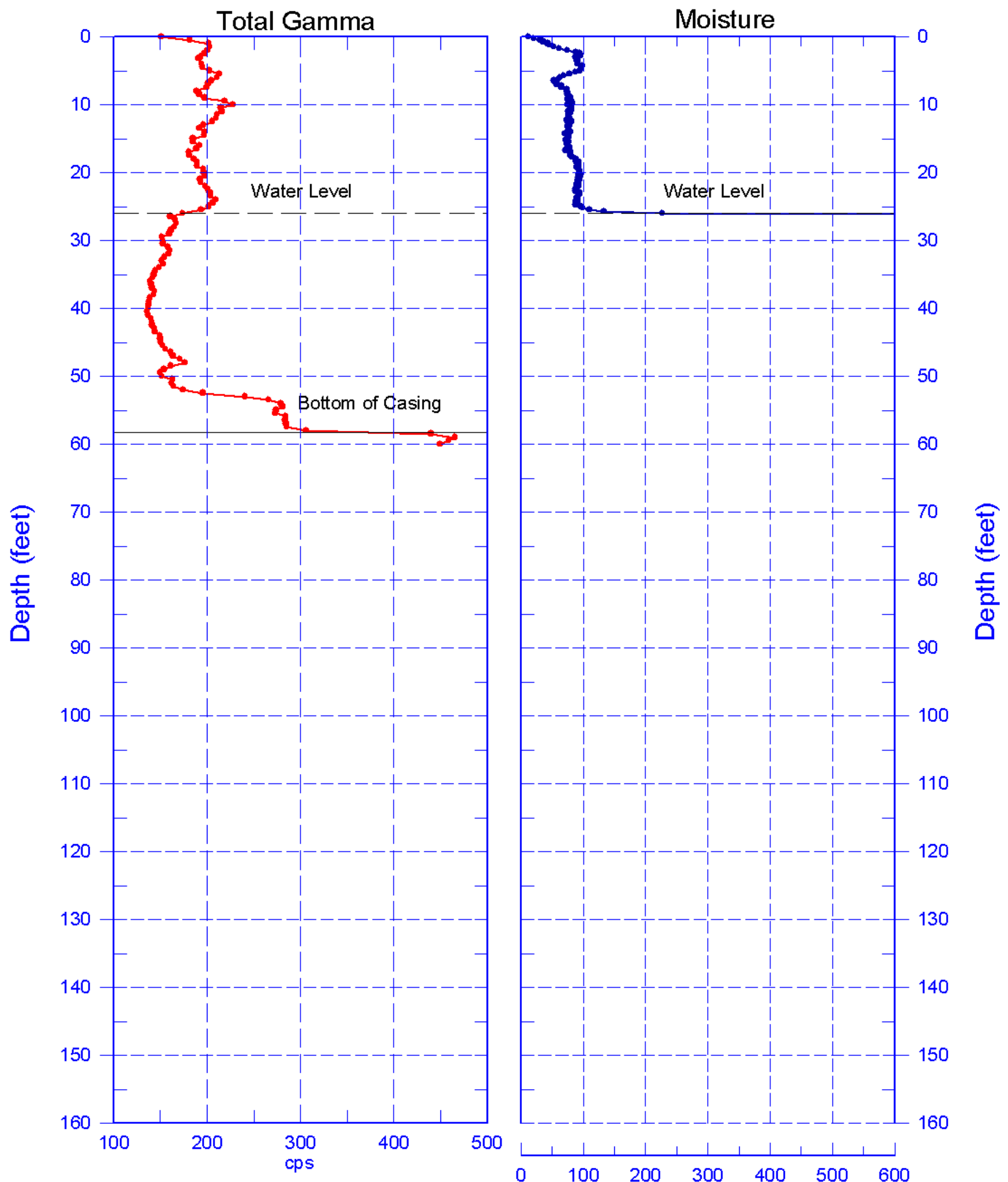

$\frac{0}{0}$
$\stackrel{0}{0}$
$\frac{5}{0}$
$\frac{0}{0}$

Reference - Ground Surface 
S

toller 399-3-29 (C6206)

Hanford Office Repeat Section of Natural Gamma Logs

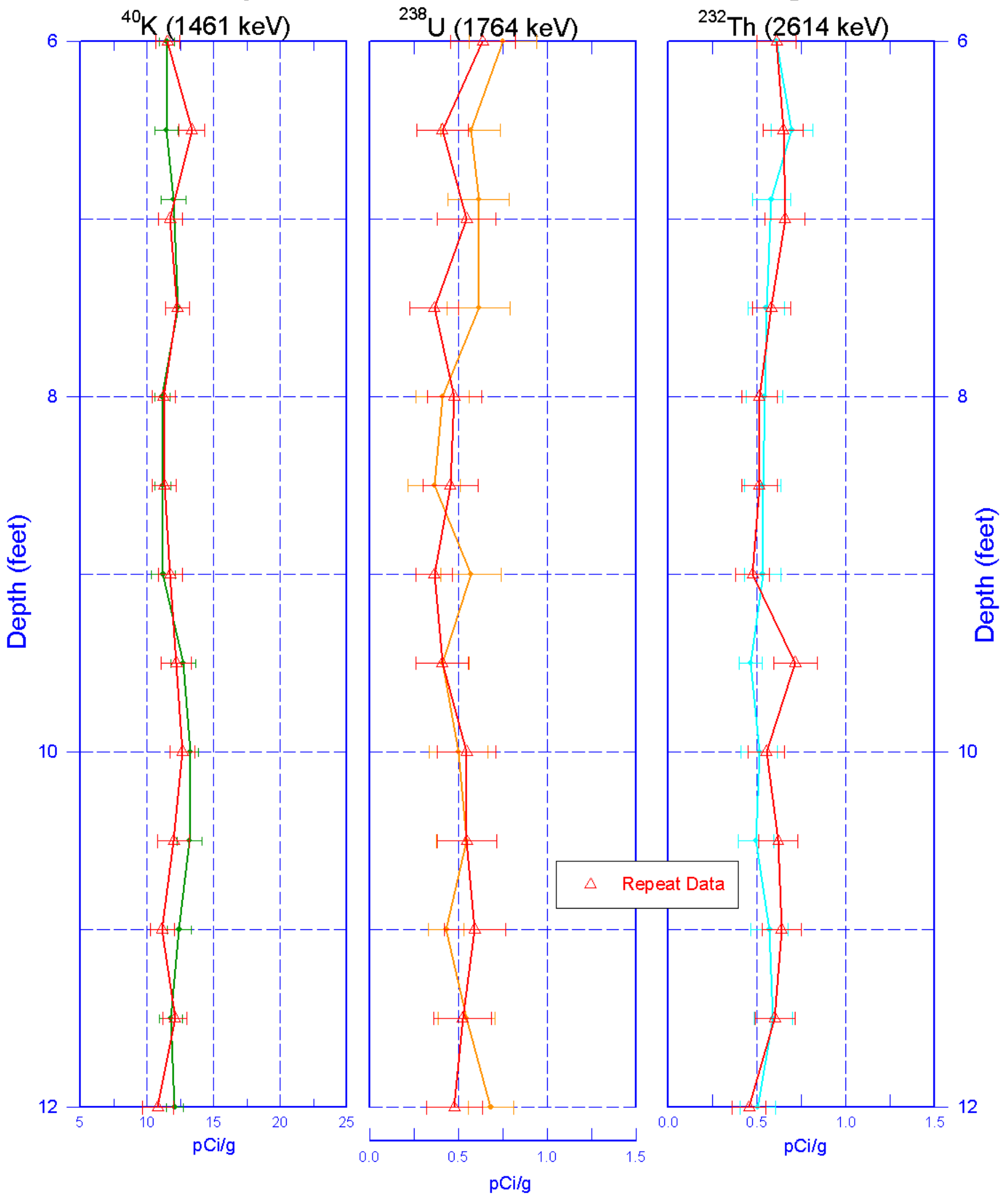


toller 399-3-29 (C6206)

Hanford Office

Moisture Repeat Section

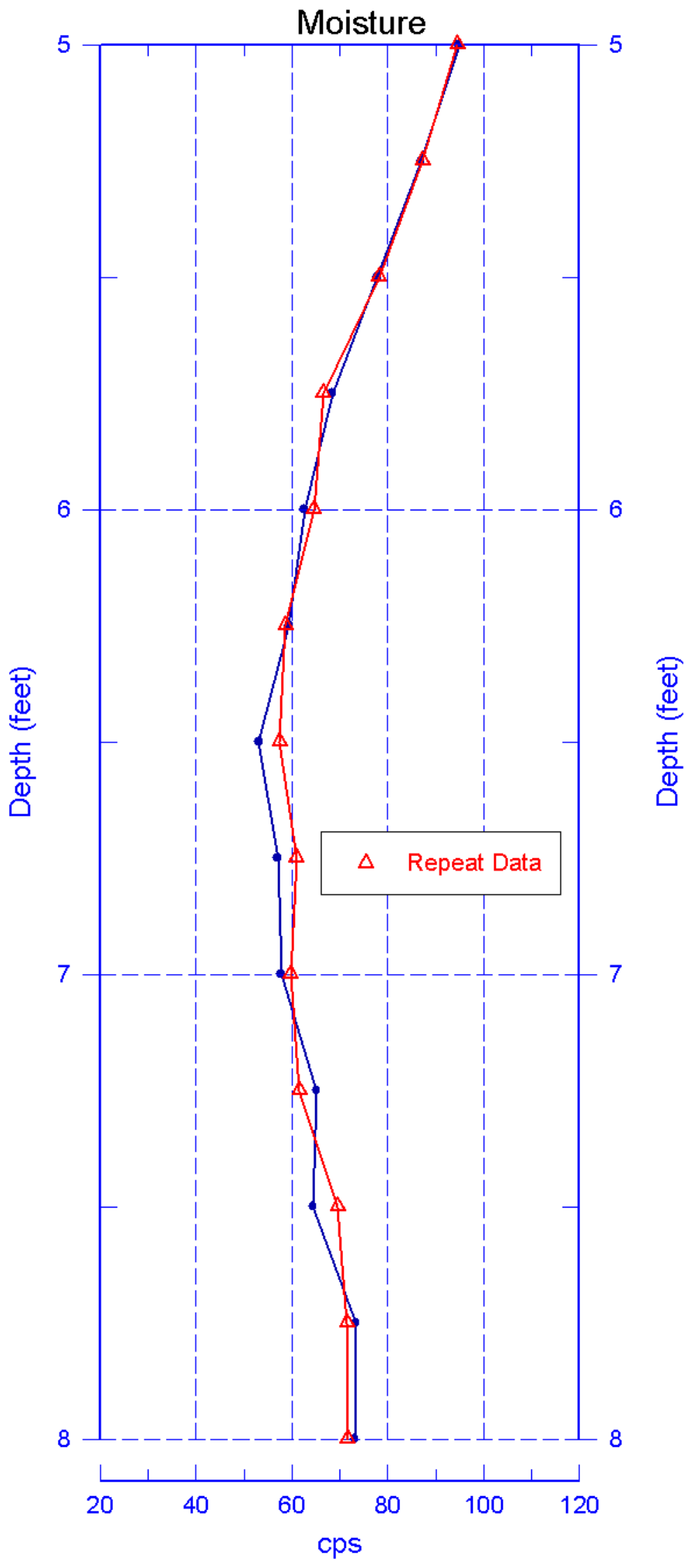

Reference - Ground Surface 


\section{9-3-31 (C6214) Log Data Report}

Borehole Information:

\begin{tabular}{|c|c|c|c|c|c|}
\hline \multirow{2}{*}{\multicolumn{2}{|c|}{$\begin{array}{c}\text { Borehole: } \\
\text { Coordinates (WA9-3-31 (C6214) }\end{array}$}} & \multicolumn{4}{|c|}{ 300-FF-5 } \\
\hline & & $\mathbf{G W L}^{1}$ (f & 28.1 & GWL Date: & $06 / 25 / 08$ \\
\hline North (m) & East (m) & Drill Date & TOC ${ }^{2}$ Elevation & Total Depth (ft) & Tyре \\
\hline 116048.3 & 594246.6 & $06 / 23 / 08$ & Unknown & 63 & Sonic \\
\hline
\end{tabular}

\section{Casing Information:}

\begin{tabular}{|c|c|c|c|c|c|c|}
\hline Casing Type & Stickup (ft) & $\begin{array}{c}\text { Outer } \\
\text { Diameter (in.) }\end{array}$ & $\begin{array}{c}\text { Inside } \\
\text { Diameter (in.) }\end{array}$ & Thickness (in.) & Top (ft) & Bottom (ft) \\
\hline Welded Steel & 0.8 & $75 / 8$ & $67 / 8$ & $3 / 8$ & -0.8 & 60.0 \\
\hline
\end{tabular}

\section{Borehole Notes:}

Casing data and total depth were reported by the site geologist. The logging engineer measured the depth to water with an e-tape. Casing diameters were measured using a steel tape and rounded to the nearest 1/16 inch. The zero reference is the ground surface

\section{Logging Equipment Information:}

\begin{tabular}{|c|c|c|c|c|}
\hline Logging System: & \multicolumn{2}{|c|}{ Gamma $4 \mathrm{~L}$} & $\begin{array}{l}\text { Type: } \\
\text { Serial No.: }\end{array}$ & $\begin{array}{l}\text { 60\% HPGe SGLS } \\
\text { 47TP32211A }\end{array}$ \\
\hline Effective Calibration Date: & $12 / 31 / 07$ & Calibration Reference: & \multicolumn{2}{|c|}{ HGLP-CC-027 } \\
\hline & & Logging Procedure: & \multicolumn{2}{|c|}{ HGLP-MAN-002, Rev. 0} \\
\hline Logging System: & \multicolumn{2}{|c|}{ Gamma $4 \mathrm{H}$} & $\begin{array}{l}\text { Type: } \\
\text { Serial No.: }\end{array}$ & $\begin{array}{l}\text { NMLS } \\
\text { H310700352 }\end{array}$ \\
\hline Effective Calibration Date: & $11 / 06 / 07$ & Calibration Reference: & \multicolumn{2}{|c|}{ HGLP-CC-021 } \\
\hline & & Logging Procedure: & \multicolumn{2}{|c|}{ HGLP-MAN-002, Rev. 0} \\
\hline
\end{tabular}

\section{Spectral Gamma Logging System (SGLS) Log Run Information:}

\begin{tabular}{|l|c|c|l|l|l|}
\hline Log Run & $\mathbf{1}$ & 2 Repeat & & & \\
\hline Date & $06 / 25 / 08$ & $06 / 25 / 08$ & & & \\
\hline Logging Engineer & McClellan & McClellan & & & \\
\hline Start Depth (ft) & 63.0 & 6.0 & & & \\
\hline Finish Depth (ft) & 0.0 & 12.0 & & & \\
\hline Count Time (sec) & 200 & 200 & & & \\
\hline Live/Real & $\mathrm{R}$ & $\mathrm{R}$ & & & \\
\hline Shield (Y/N) & $\mathrm{N}$ & $\mathrm{N}$ & & & \\
\hline MSA Interval (ft) & 0.5 & 0.5 & & & \\
\hline Log Speed (ft/min) & NA & NA & & & \\
\hline Pre-Verification & DL511CAB & DL511CAB & & & \\
\hline Start File & DL511000 & DL511127 & & & \\
\hline Finish File & DL511126 & DL511139 & & & \\
\hline Post-Verification & DL511CAA & DL511CAA & & & \\
\hline Depth Return Error (in.) & 0.5 low & NA & & & \\
\hline Comments & $\begin{array}{c}\text { No fine gain } \\
\text { adjustment } \\
\text { made. }\end{array}$ & Repeat Section. & & & \\
\hline
\end{tabular}


Neutron Moisture Logging System (NMLS) Log Run Information:

\begin{tabular}{|l|c|c|l|l|l|}
\hline Log Run & $\mathbf{3}$ & 4 Repeat & & & \\
\hline Date & $06 / 25 / 08$ & $06 / 25 / 08$ & & & \\
\hline Logging Engineer & McClellan & McClellan & & & \\
\hline Start Depth (ft) & 0.0 & 28.0 & & & \\
\hline Finish Depth (ft) & 28.0 & 25.0 & & & \\
\hline Count Time (sec) & 15 & 15 & & & \\
\hline Live/Real & $\mathrm{R}$ & $\mathrm{R}$ & & & \\
\hline Shield (Y/N) & $\mathrm{N}$ & $\mathrm{N}$ & & & \\
\hline MSA Interval (ft) & 0.25 & 0.25 & & & \\
\hline Log Speed (ft/min) & NA & NA & & & \\
\hline Pre-Verification & DHF82CAB & DHF82CAB & & & \\
\hline Start File & DHF82000 & DHF82113 & & & \\
\hline Finish File & DHF82112 & DHF82125 & & & \\
\hline Post-Verification & DHF82CAA & DHF82CAA & & & \\
\hline Depth Return Error (in.) & NA & NA & & & \\
\hline Comments & None & Repeat Section & & & \\
\hline
\end{tabular}

\section{Logging Operation Notes:}

Data were collected using Gamma 4, HO 68B-3573. SGLS pre- and post-survey verification measurements were acquired in the Amersham KUTh-115 field verifier. NMLS pre- and post-survey verification measurements were acquired in the standard field verifier. SGLS maximum logging depth was $63.14 \mathrm{ft}$ before the sonde un-weighted. A centralizer was installed on the sonde.

Analysis Notes:

\begin{tabular}{|l|l|l|l|l|l|}
\hline Analyst: & LEGLER & Date: & 09/23/08 & Reference: & GJO-HGLP 1.6.3, Rev. 0 \\
\hline
\end{tabular}

SGLS pre- and post-survey verification spectra met the acceptance criteria for the established system. NMLS preand post-survey verification spectra met the acceptance criteria for the established system, but the spectra files DHF82CAB and DHF82CAA had measurements for counts per second (cps) above the upper control limit.

A casing correction for a 3/8-in thick casing was applied to spectral data during analysis for depths up to $59.5 \mathrm{ft}$, where casing ends, leaving $3.5 \mathrm{ft}$ of open borehole uncorrected. The Borehole Data Sheet lists the casing depth as $60 \mathrm{ft}$, but data from the Total Gamma and KUT plots indicate that the casing ends at $59.5 \mathrm{ft}$.

A water correction was also applied from $28.1 \mathrm{ft}$ to total logged depth of borehole.

SGLS spectra were processed in batch mode in APTEC SUPERVISOR to identify individual peaks and count rates. Concentrations were calculated using an EXCEL template identified as G4LDec07.xls using an efficiency function and corrections for casing, dead time and water as determined by annual calibrations. NMLS spectra were processed in batch mode in APTEC SUPERVISOR to identify counts. Count rates were calculated using an EXCEL template identified as G4HNov07.xls. NMLS data are presented in counts per second (cps), because no calibration data is available for a 6 7/8-inch inner diameter borehole casing.

\section{Results and Interpretations:}

Co-60 was detected at $9-11 \mathrm{ft}$, with a maximum concentration of $0.41 \mathrm{pCi} / \mathrm{g}$ at $10 \mathrm{ft}$.

$\mathrm{U}-235$ was detected at $10.5 \mathrm{ft}$, but inspection of the spectra indicates that this detection is a statistical fluctuation associated with the processing software.

$\mathrm{U}-238$ (Pa-234m) was detected at $53.5 \mathrm{ft}$, but inspection of the spectra indicates that this detection is a statistical fluctuation associated with the processing software. 
HGLP-LDR-249, Rev. 0

The KUT, moisture and manmade plots showed good repeatability

\section{List of Log Plots:}

Depth Reference is ground surface

Manmade Radionuclides

Natural Gamma Logs

Combination Plot

Total Gamma \& Dead Time

Total Gamma \& Moisture

Manmade Repeat Section

Repeat Section of Natural Gamma Logs

Moisture Repeat Section

${ }^{1} \mathrm{GWL}$ - groundwater level

${ }^{2}$ TOC - top of casing 


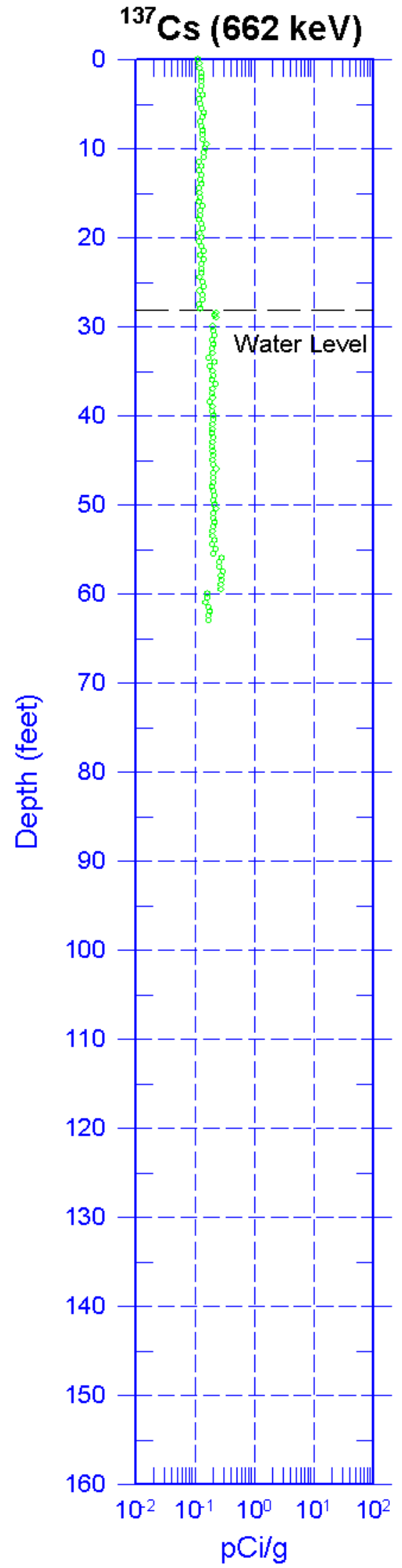

Zero Reference - Ground Surface

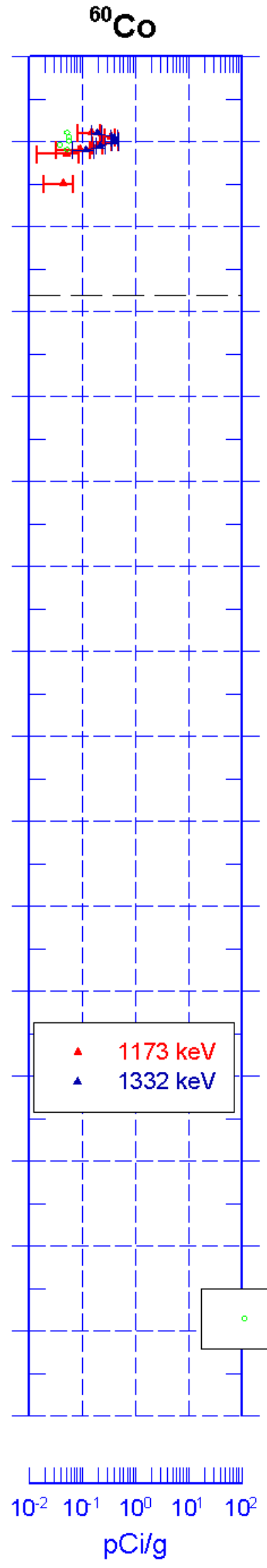

${ }^{235} \mathrm{U}(186 \mathrm{keV})$

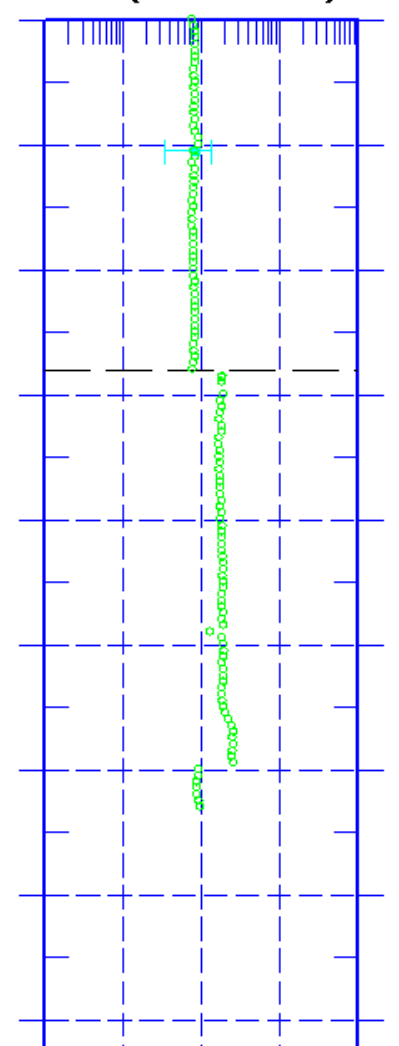

Detections are considered stat stical fluctuations and are not valid

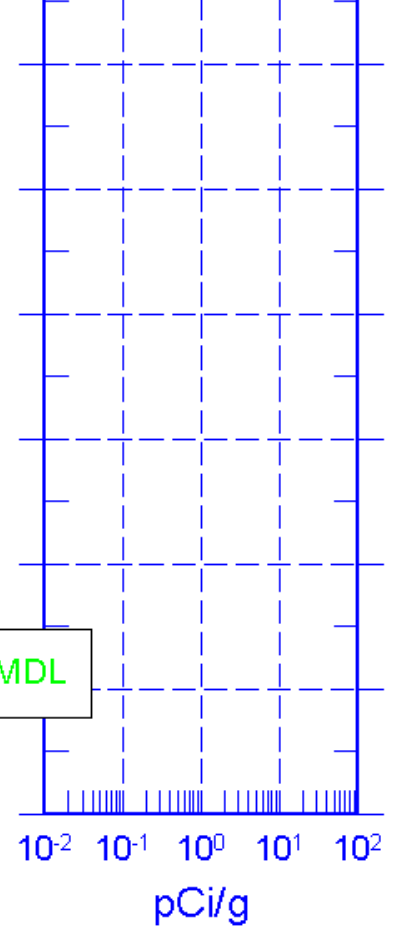

Detections are considered statistical fluctuations and are not valid
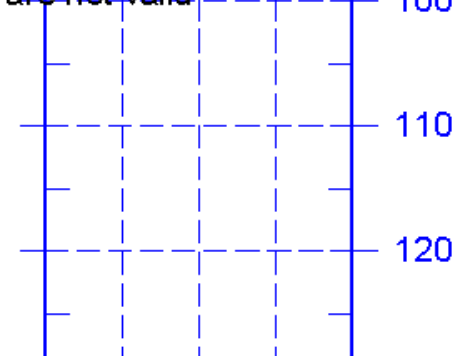

130

140 150 160

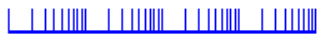
$\begin{array}{lllll}10^{-2} & 10^{-1} & 10^{0} & 10^{1} & 10^{2}\end{array}$ $\mathrm{pCi} / \mathrm{g}$ 
toller 399-3-31 (66214)

Hanford Office

\section{Natural Gamma Logs}
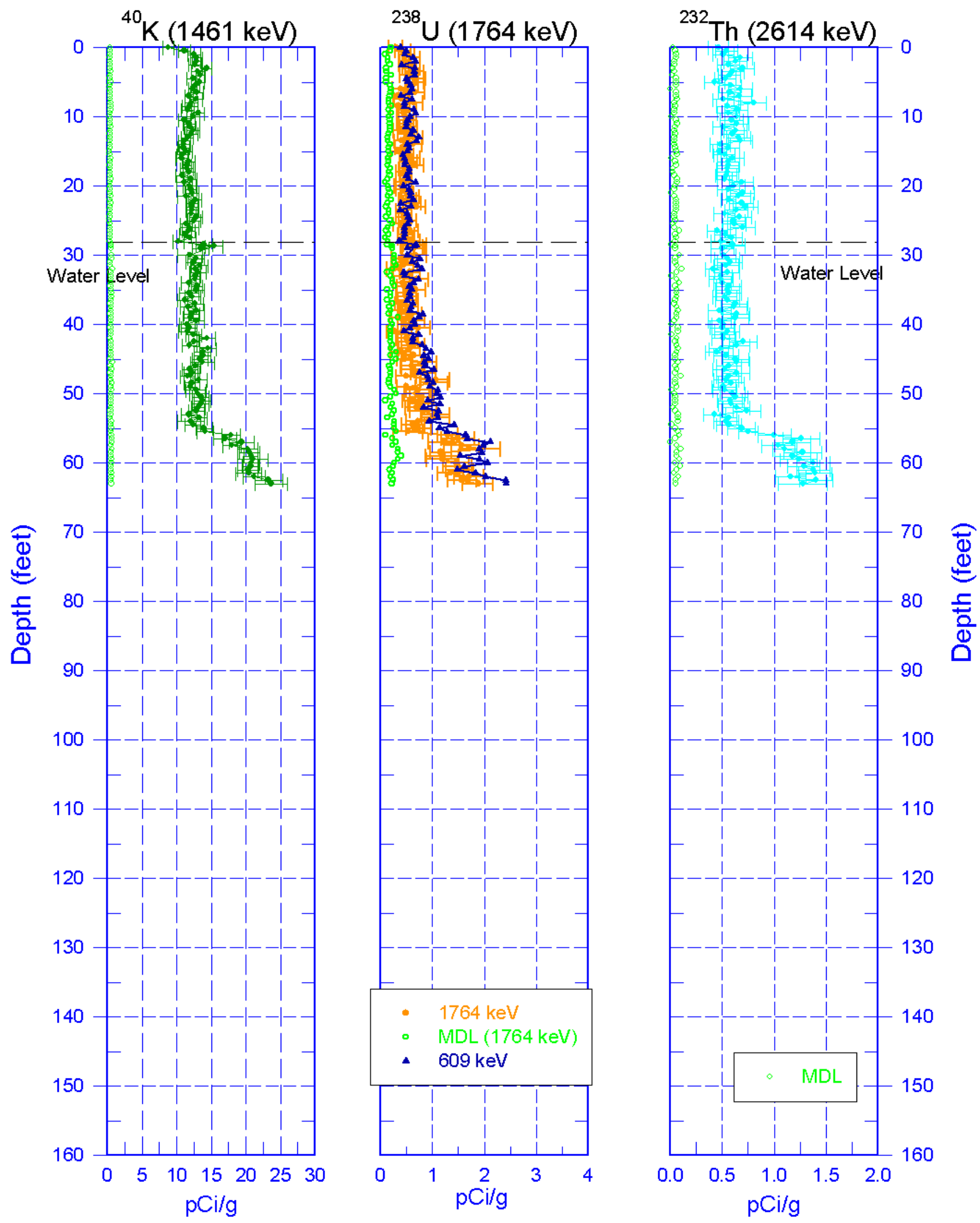

Zero Reference - Ground Surface 


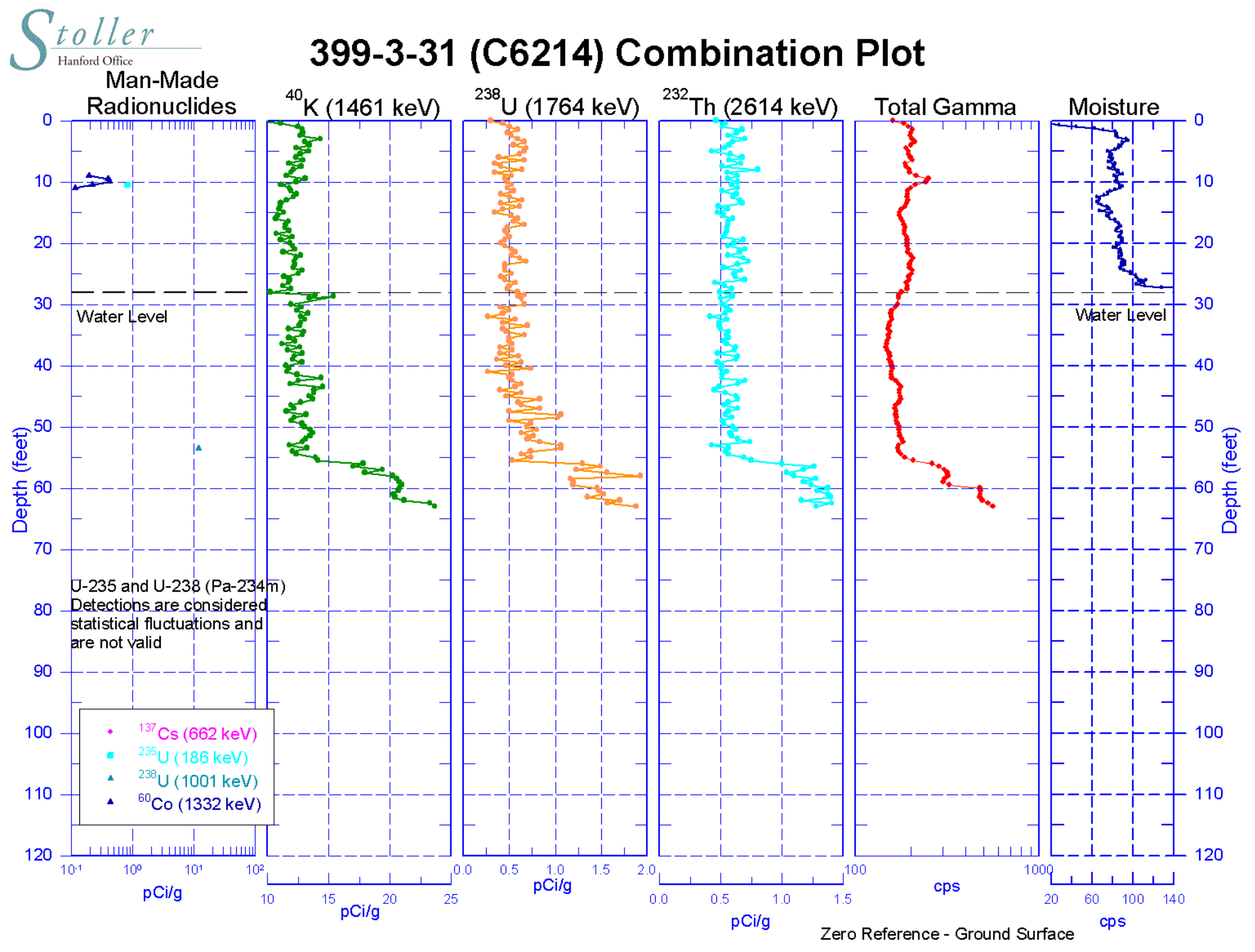


toller 399-3-31 (C6214)

Hanford Office

\section{Total Gamma \& Dead Time}
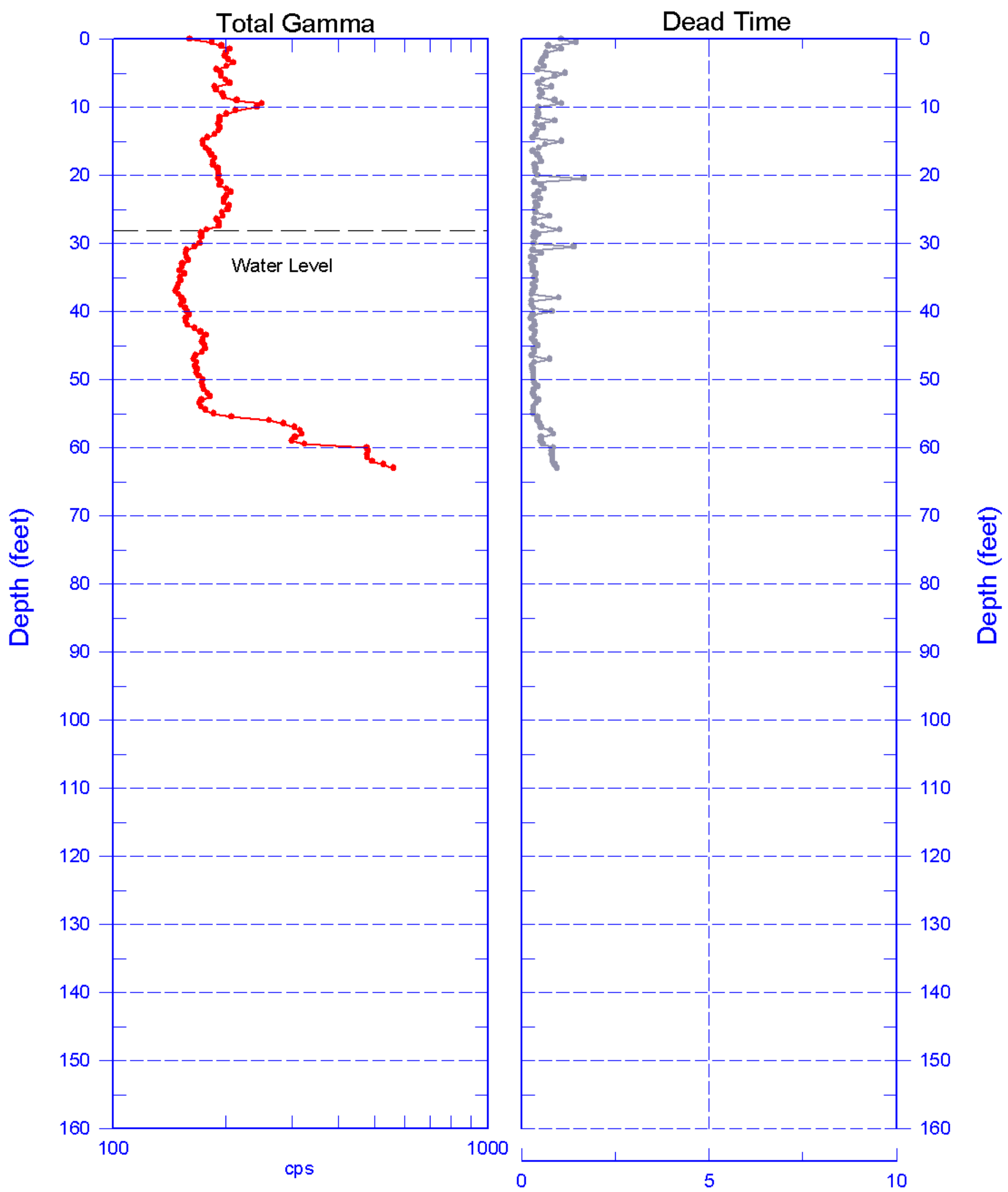

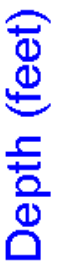

Reference - Ground Surface 
toller 399-3-31 (C6214)

Hanford Office

\section{Total Gamma \& Moisture}

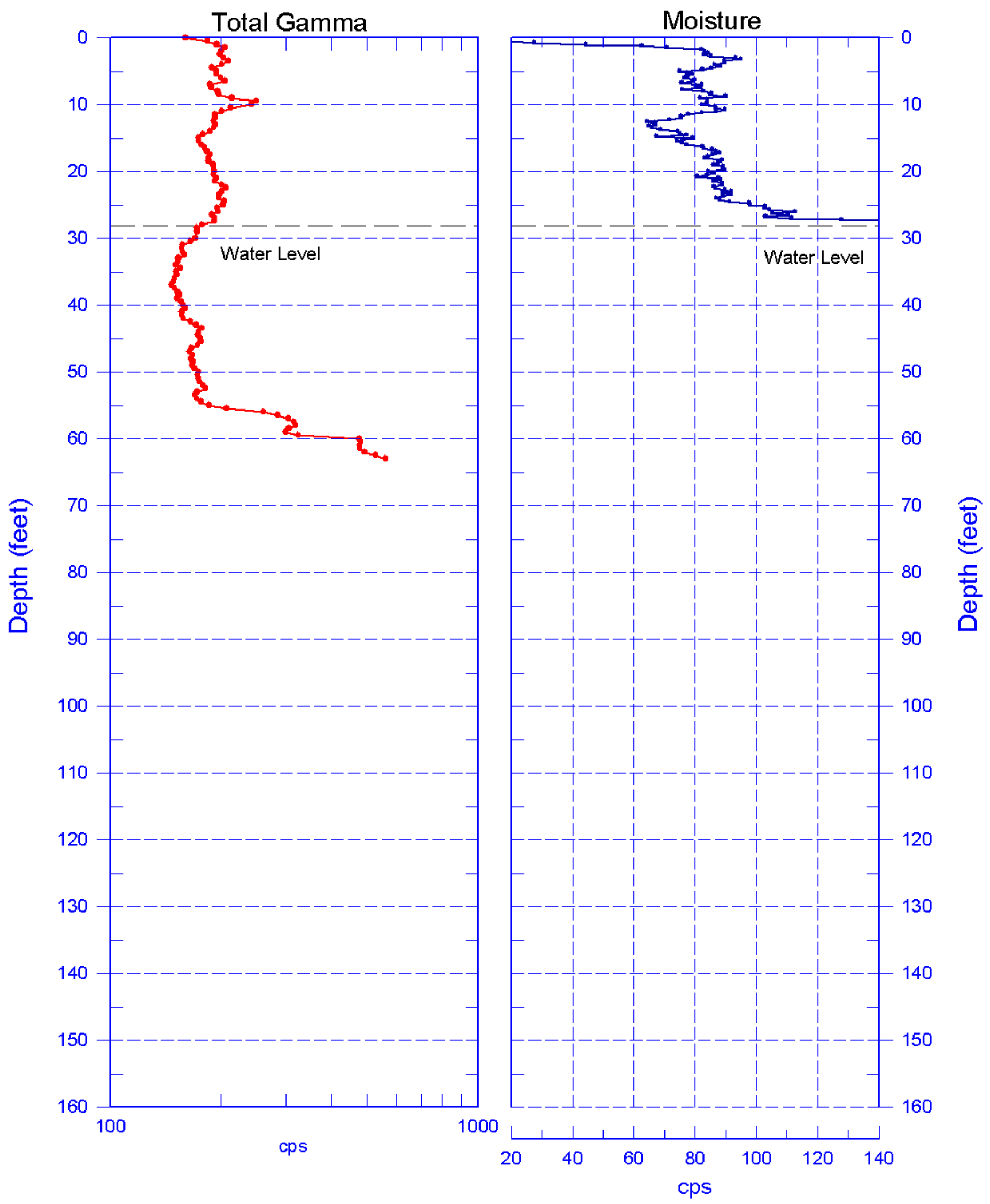

Reference - Ground Surface 
toller 399-3-31 (C6214)

Hanford Office

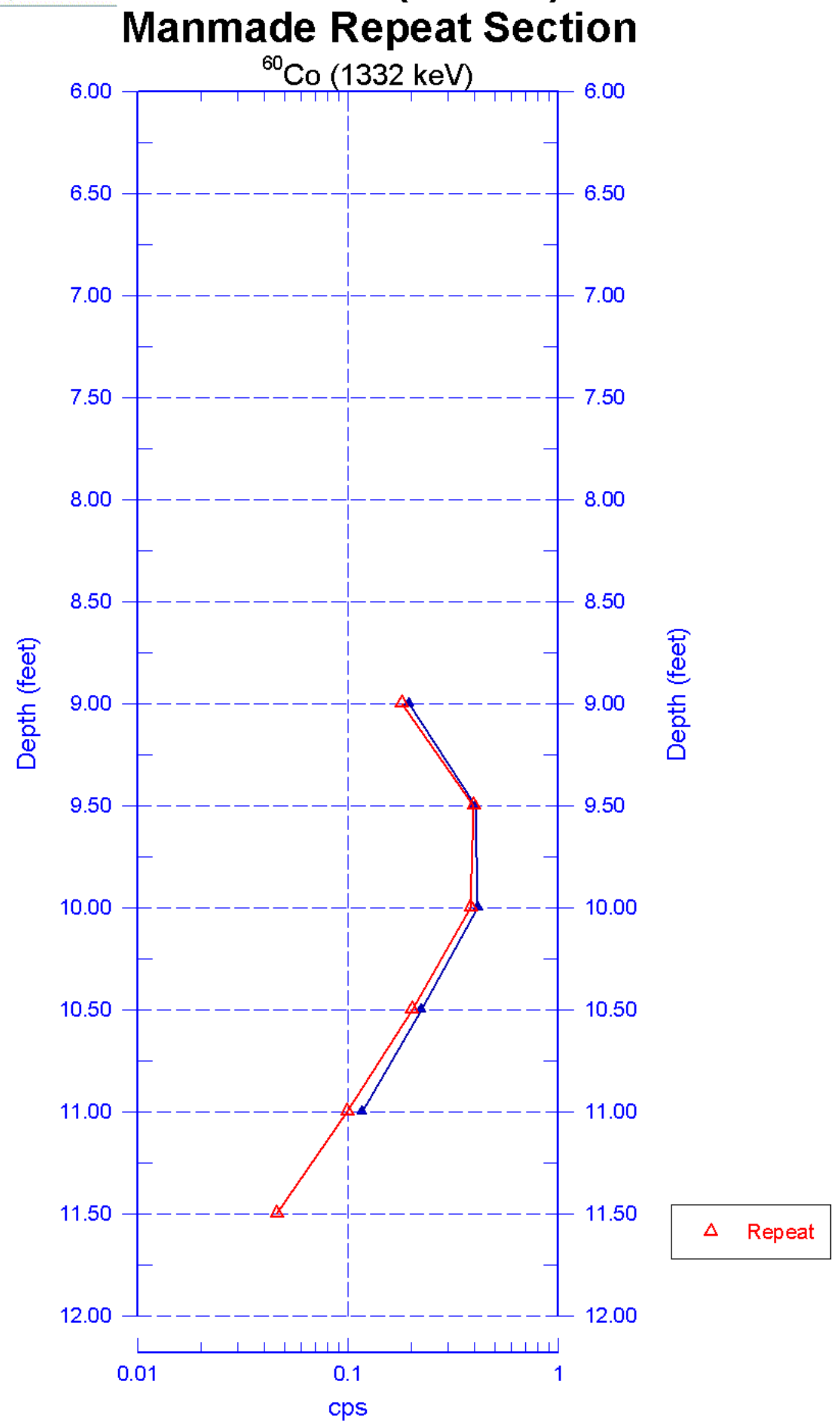

Reference - Ground Surface

G.308 


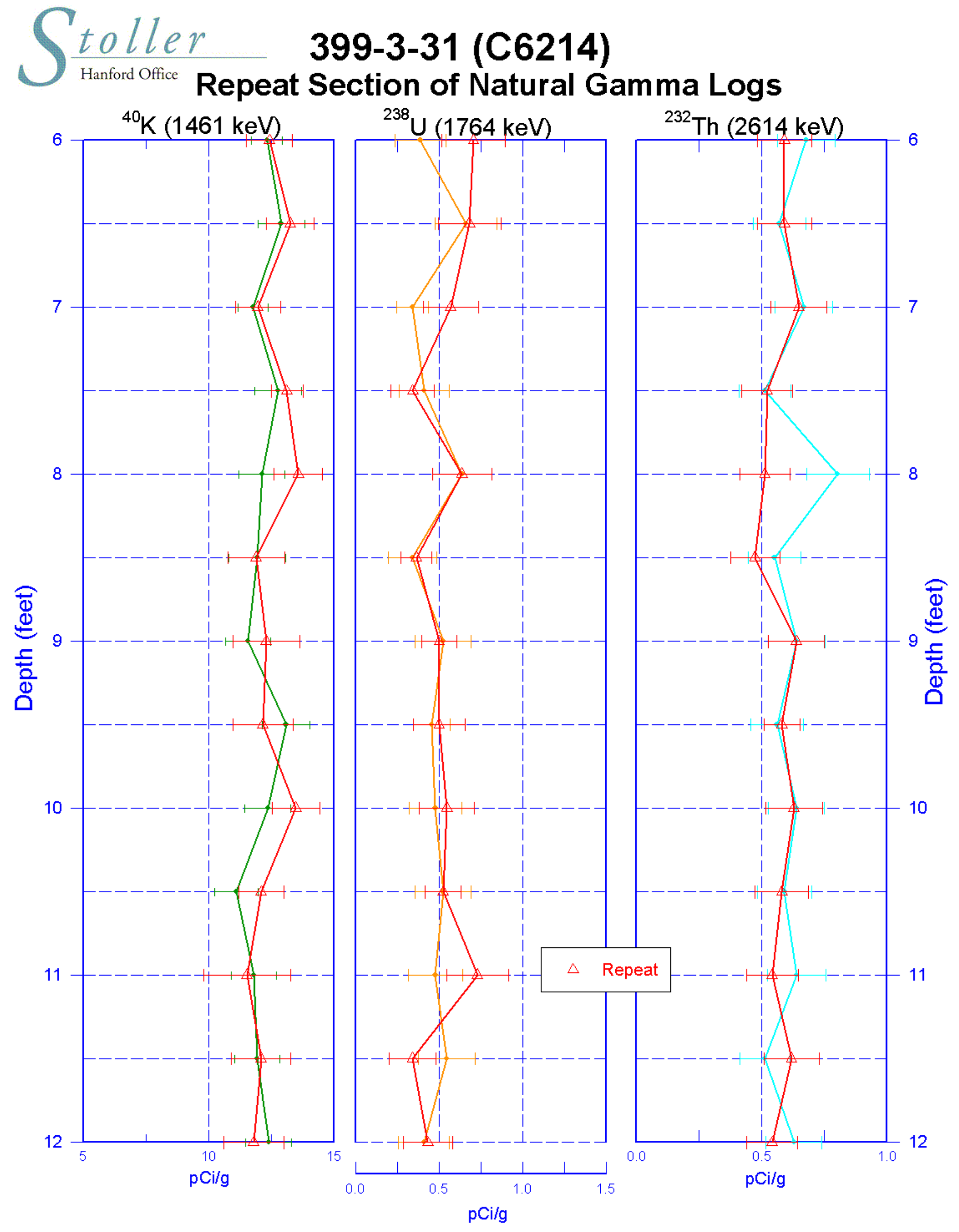

Zero Reference - Ground Surface

G.309 


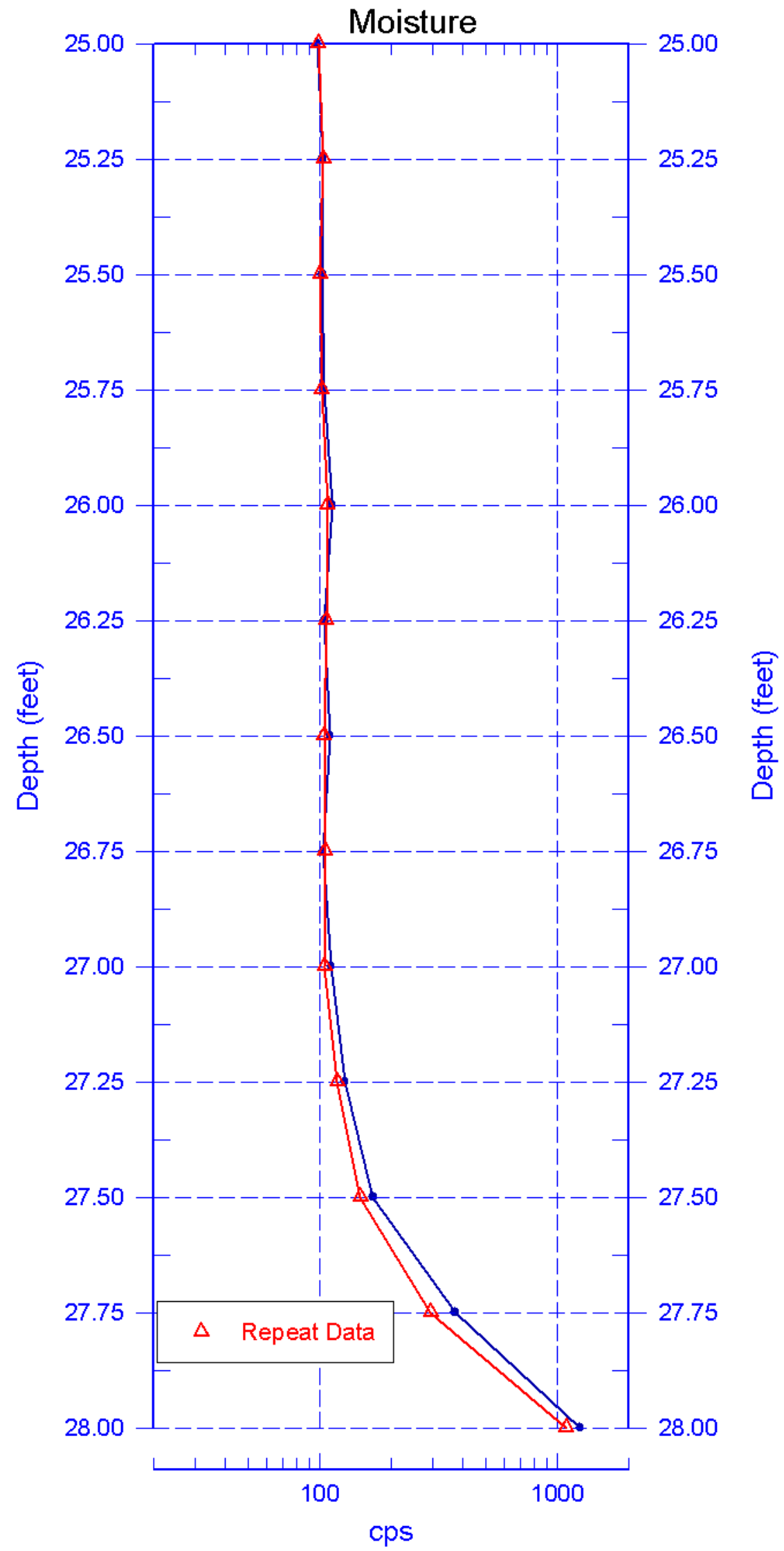

Reference - Ground Surface 
Appendix $\mathrm{H}$

Survey Reports 


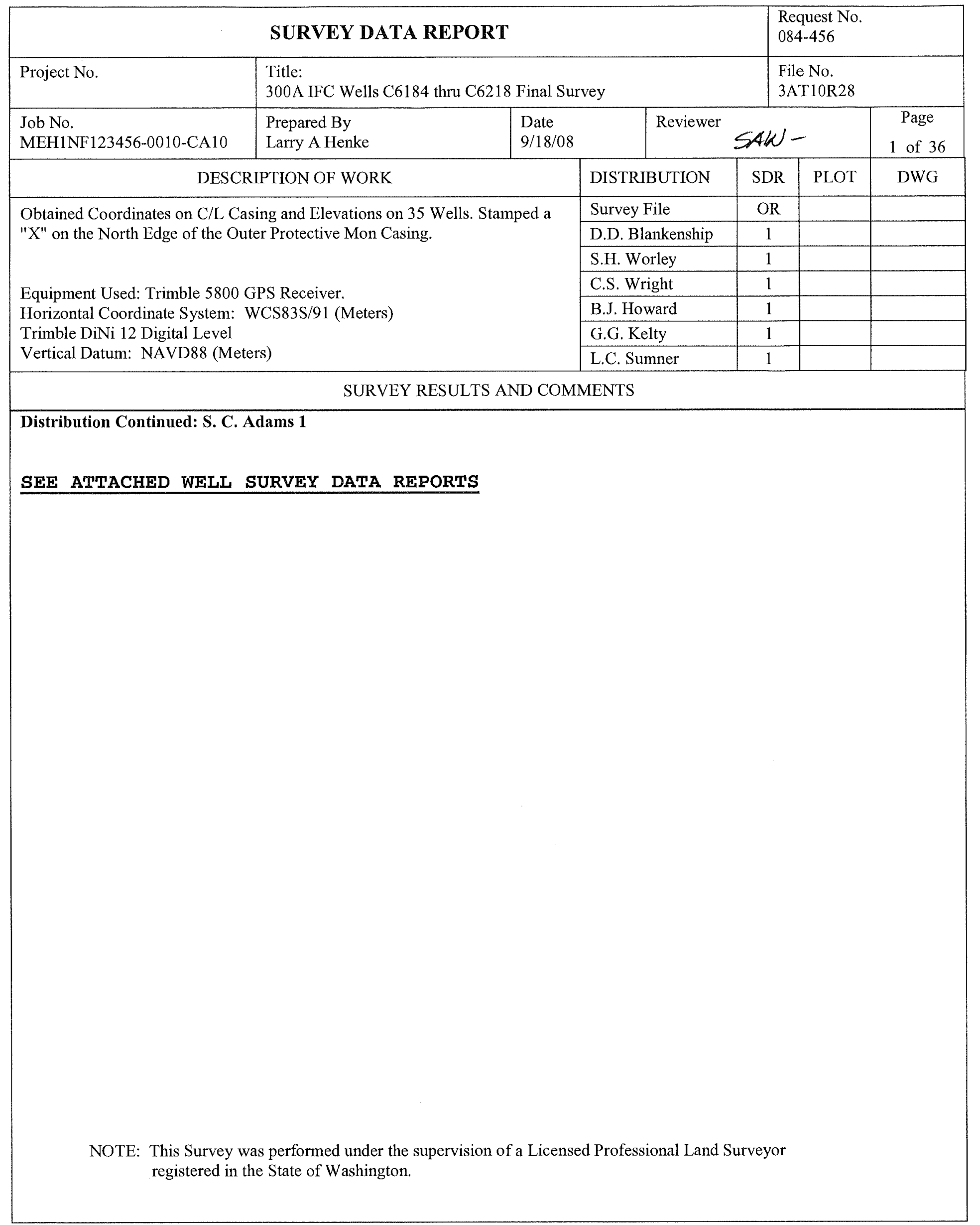


WELL SURVEY DATA REPORT

\begin{tabular}{|c|c|c|c|c|c|}
\hline \multicolumn{3}{|l|}{ Project: } & \multicolumn{3}{|c|}{$\begin{array}{ll}\text { Prepared By: } & \text { Larry A Henke } \\
\text { Company: } & \text { FGG }\end{array}$} \\
\hline \multicolumn{3}{|c|}{ Date Requested: $8 / 19 / 08$} & \multicolumn{3}{|c|}{ Requestor: Chris Wright (FH) } \\
\hline \multicolumn{3}{|c|}{ Date of Survey: $9 / 18 / 08$} & \multicolumn{3}{|c|}{ Surveyor: Larry A Henke (FGG) } \\
\hline \multicolumn{3}{|c|}{ ERC Point of Contact: } & \multicolumn{3}{|c|}{$\begin{array}{r}\text { Survey Co. Point of Contact: } \\
\text { Neil Fastabend (FGG) }\end{array}$} \\
\hline \multirow{4}{*}{\multicolumn{3}{|c|}{$\begin{array}{l}\text { Description of Work: } \\
\text { Civil Survey of Groundwater Monitoring } \\
\text { Well \#C6184 (399-2-7) }\end{array}$}} & \multicolumn{3}{|c|}{ Horizontal Datum: NAD83(91) } \\
\hline & & & \multicolumn{3}{|c|}{ Vertical Datum: NAVD88 } \\
\hline & & & \multicolumn{3}{|c|}{ Units: $\quad$ METERS } \\
\hline & & & \multicolumn{3}{|c|}{ Hanford Area Designation: $300 \mathrm{~A}$} \\
\hline \multicolumn{6}{|c|}{ Coordinate System: Washington State Plane Coordinates (South Zone) } \\
\hline \multicolumn{6}{|c|}{ Horizontal Control Monuments: $\quad$ N-323 (FGG), 300-70 (FGG) } \\
\hline \multicolumn{6}{|c|}{ Vertical Control Monuments: $300-28$ (FGG), 300-60(FGG) } \\
\hline Well ID & Well Name & Easting & Northing & Elevation & \\
\hline C6184 & $399-2-7$ & 594235.2 & 116084.49 & & Center of Casing \\
\hline & & & & 114.812 & $\begin{array}{l}\text { Top Pump Base plate. } \\
\text { N. Edge }\end{array}$ \\
\hline & & & & 114.909 & $\begin{array}{l}\text { Top Lid Frame at } \\
\text { Stamped " } X ", N \text {. Edge }\end{array}$ \\
\hline & & & & 114.910 & Brass Survey Marker \\
\hline \multicolumn{6}{|c|}{$\begin{array}{l}\text { Notes: } \\
\text { EQUIPMENT USED: TRIMBLE GPS } 5800 \text { RTK } \\
\text { TRIMBLE DiNi } 12 \text { LEVEL }\end{array}$} \\
\hline \multicolumn{6}{|c|}{$\begin{array}{l}\text { Surveyor Statement: } \\
\text { I, Larry A. Henke, a Professional Land Surveyor registered } \\
\text { in the State of Washington (Registration No. 38975), hereby } \\
\text { certify that this report is based on a field survey performed in } \\
\text { September, } 2008 \text { under my direct supervision, and that the data } \\
\text { contained here is true and correct. }\end{array}$} \\
\hline
\end{tabular}

Original to:

Distribution by DIS: 


\begin{tabular}{|c|c|c|c|c|c|c|}
\hline \multicolumn{7}{|c|}{ WELL SURVEY DATA REPORT } \\
\hline \multicolumn{3}{|l|}{ Project: } & \multicolumn{4}{|c|}{$\begin{array}{ll}\text { Prepared By: } & \text { Larry A Henke } \\
\text { Company: } & \text { FGG }\end{array}$} \\
\hline \multicolumn{3}{|c|}{ Date Requested: $8 / 19 / 08$} & \multicolumn{4}{|c|}{ Requestor: Chris Wright (FH) } \\
\hline \multicolumn{3}{|c|}{ Date of Survey: $9 / 18 / 08$} & \multicolumn{4}{|c|}{ Surveyor: Larry A Henke (FGG) } \\
\hline \multicolumn{3}{|c|}{ ERC Point of Contact: } & \multicolumn{4}{|c|}{$\begin{array}{r}\text { Survey Co. Point of Contact: } \\
\text { Neil Fastabend (FGG) }\end{array}$} \\
\hline \multirow{4}{*}{\multicolumn{3}{|c|}{$\begin{array}{l}\text { Description of Work: } \\
\text { Civil Survey of Groundwater Monitoring } \\
\text { Well \#C6185 (399-2-8) }\end{array}$}} & \multicolumn{4}{|c|}{ Horizontal Datum: NAD83(91) } \\
\hline & & & \multicolumn{4}{|c|}{ Vertical Datum: NAVD88 } \\
\hline & & & \multicolumn{4}{|c|}{ Units: $\quad$ METERS } \\
\hline & & & \multicolumn{4}{|c|}{ Hanford Area Designation: $300 \mathrm{~A}$} \\
\hline \multicolumn{7}{|c|}{ Coordinate System: Washington State Plane Coordinates (South Zone) } \\
\hline \multicolumn{7}{|c|}{ Horizontal Control Monuments: N-323(FGG), 300-70 (FGG) } \\
\hline \multicolumn{7}{|c|}{ Vertical Control Monuments: $300-28$ (FGG), 300-60(FGG) } \\
\hline Well ID & Well Name & Easting & & Northing & Elevation & \\
\hline \multirow[t]{4}{*}{ C6185 } & $399-2-8$ & 594243.4 & & 116090.31 & & Center of Casing \\
\hline & & & & & 114.812 & $\begin{array}{l}\text { Top Pump Base plate. } \\
\text { N. Edge }\end{array}$ \\
\hline & & & & & 114.923 & $\begin{array}{l}\text { Top Lid Frame at } \\
\text { Stamped "X", N. Edge }\end{array}$ \\
\hline & & & & & 114.925 & Brass Survey Marker \\
\hline \multicolumn{7}{|c|}{$\begin{array}{l}\text { Notes: } \\
\text { EQUIPMENT USED: TRIMBLE GPS } 5800 \text { RTK } \\
\text { TRIMBLE DiNi } 12 \text { LEVEL }\end{array}$} \\
\hline \multicolumn{7}{|c|}{$\begin{array}{l}\text { Surveyor Statement: } \\
\text { I, Larry A. Henke, a Professional Land Surveyor registered } \\
\text { in the State of Washington (Registration No. 38975), hereby } \\
\text { certify that this report is based on a field survey performed in } \\
\text { September, } 2008 \text { under my direct supervision, and that the data } \\
\text { contained here is true and correct. }\end{array}$} \\
\hline
\end{tabular}

Original to:

Distribution by DIS: 


\begin{tabular}{|c|c|c|c|c|c|c|}
\hline \multicolumn{7}{|c|}{ WELL SURVEY DATA REPORT } \\
\hline \multicolumn{3}{|l|}{ Project: } & \multicolumn{4}{|c|}{$\begin{array}{l}\text { Prepared By: Larry A Henke } \\
\text { Company: } \quad \text { FGG }\end{array}$} \\
\hline \multicolumn{3}{|c|}{ Date Requested: $8 / 19 / 08$} & \multicolumn{4}{|c|}{ Requestor: Chris Wright (FH) } \\
\hline \multicolumn{3}{|c|}{ Date of Survey: $9 / 18 / 08$} & \multicolumn{4}{|c|}{ Surveyor: Larry A Henke (FGG) } \\
\hline \multicolumn{3}{|c|}{ ERC Point of Contact: } & \multicolumn{4}{|c|}{$\begin{array}{l}\text { Survey Co. Point of Contact: } \\
\text { Neil Fastabend (FGG) }\end{array}$} \\
\hline \multirow{4}{*}{\multicolumn{3}{|c|}{$\begin{array}{l}\text { Description of Work: } \\
\text { Civil Survey of Groundwater Monitoring } \\
\text { Well \#C6186 (399-2-9) }\end{array}$}} & \multicolumn{4}{|c|}{ Horizontal Datum: NAD83(91) } \\
\hline & & & \multicolumn{4}{|c|}{ Vertical Datum: NAVD88 } \\
\hline & & & \multicolumn{4}{|c|}{ Units: $\quad$ METERS } \\
\hline & & & \multicolumn{4}{|c|}{ Hanford Area Designation: $300 \mathrm{~A}$} \\
\hline \multicolumn{7}{|c|}{ Coordinate System: Washington State Plane Coordinates (South Zone) } \\
\hline \multicolumn{7}{|c|}{ Horizontal Control Monuments: $\quad \mathrm{N}-323$ (FGG), $300-70$ (FGG) } \\
\hline \multicolumn{7}{|c|}{ Vertical Control Monuments: $300-28$ (FGG), 300-60(FGG) } \\
\hline Well ID & Well Name & Easting & & Northing & Elevation & \\
\hline \multirow[t]{4}{*}{ C6186 } & $399-2-9$ & 594237.7 & & 116089.72 & & Center of Casing \\
\hline & & & & & 114.772 & $\begin{array}{l}\text { Top Pump Base plate. } \\
\text { N. Edge }\end{array}$ \\
\hline & & & & & 114.907 & $\begin{array}{l}\text { Top Lid Frame at } \\
\text { Stamped "X", N. Edge }\end{array}$ \\
\hline & & & & & 114.908 & Brass Survey Marker \\
\hline \multicolumn{7}{|c|}{$\begin{array}{l}\text { Notes: } \\
\text { EQUIPMENT USED: TRIMBLE GPS } 5800 \text { RTK } \\
\text { TRIMBLE DiNi } 12 \text { LEVEL }\end{array}$} \\
\hline \multicolumn{7}{|c|}{$\begin{array}{l}\text { Surveyor Statement: } \\
\text { I, Larry A. Henke, a Professional Land Surveyor registered } \\
\text { in the State of Washington (Registration No. 38975), hereby } \\
\text { certify that this report is based on a field survey performed in } \\
\text { September, } 2008 \text { under my direct supervision, and that the data } \\
\text { contained here is true and correct. }\end{array}$} \\
\hline
\end{tabular}

Original to:

Distribution by DIS: 
WELL SURVEY DATA REPORT

\begin{tabular}{|c|c|c|c|c|c|}
\hline \multicolumn{3}{|l|}{ Project: } & \multicolumn{3}{|c|}{$\begin{array}{ll}\text { Prepared By: } & \text { Larry A Henke } \\
\text { Company: } & \text { FGG }\end{array}$} \\
\hline \multicolumn{3}{|c|}{ Date Requested: $8 / 19 / 08$} & \multicolumn{3}{|c|}{ Requestor: Chris Wright (FH) } \\
\hline \multicolumn{3}{|c|}{ Date of Survey: $9 / 18 / 08$} & \multicolumn{3}{|c|}{ Surveyor: Larry A Henke (FGG) } \\
\hline \multicolumn{3}{|c|}{ ERC Point of Contact: } & \multicolumn{3}{|c|}{$\begin{array}{r}\text { Survey Co. Point of Contact: } \\
\text { Neil Fastabend (FGG) }\end{array}$} \\
\hline \multirow{4}{*}{\multicolumn{3}{|c|}{$\begin{array}{l}\text { Description of Work: } \\
\text { Civil Survey of Groundwater Monitoring } \\
\text { Well \#C6187 (399-2-10) }\end{array}$}} & \multicolumn{3}{|c|}{ Horizontal Datum: NAD83(91) } \\
\hline & & & \multicolumn{3}{|c|}{ Vertical Datum: NAVD88 } \\
\hline & & & \multicolumn{3}{|c|}{ Units: $\quad$ METERS } \\
\hline & & & \multicolumn{3}{|c|}{ Hanford Area Designation: $300 \mathrm{~A}$} \\
\hline \multicolumn{6}{|c|}{ Coordinate System: Washington State Plane Coordinates (South Zone) } \\
\hline \multicolumn{6}{|c|}{ Horizontal Control Monuments: $\mathrm{N}-323(\mathrm{FGG}), 300-70(\mathrm{FGG})$} \\
\hline \multicolumn{6}{|c|}{ Vertical Control Monuments: $300-28$ (FGG), $300-60(\mathrm{FGG})$} \\
\hline Well ID & Well Name & Easting & Northing & Elevation & \\
\hline C6187 & $399-2-10$ & 594234.5 & 116094.49 & & Center of Casing \\
\hline & & & & 114.892 & $\begin{array}{l}\text { Top Pump Base plate. } \\
\text { N. Edge }\end{array}$ \\
\hline & & & & 114.960 & $\begin{array}{l}\text { Top Lid Frame at } \\
\text { Stamped "X", N. Edge }\end{array}$ \\
\hline & & & & 114.958 & Brass Survey Marker \\
\hline \multicolumn{6}{|c|}{$\begin{array}{l}\text { Notes: } \\
\text { EQUIPMENT USED: TRIMBLE GPS } 5800 \text { RTK } \\
\text { TRIMBLE DiNi } 12 \text { LEVEL }\end{array}$} \\
\hline \multicolumn{6}{|c|}{$\begin{array}{l}\text { Surveyor Statement: } \\
\text { I, Larry A. Henke, a Professional Land Surveyor registered } \\
\text { in the State of Washington (Registration No. 38975), hereby } \\
\text { certify that this report is based on a field survey performed in } \\
\text { September, } 2008 \text { under my direct supervision, and that the data } \\
\text { contained here is true and correct. }\end{array}$} \\
\hline
\end{tabular}

Original to:

Distribution by DIS: 


\section{WELL SURVEY DATA REPORT}

\begin{tabular}{|c|c|c|c|c|c|}
\hline \multicolumn{3}{|l|}{ Project: } & \multicolumn{3}{|c|}{$\begin{array}{l}\text { Prepared By: Larry A Henke } \\
\text { Company: } \quad \text { FGG }\end{array}$} \\
\hline \multicolumn{3}{|c|}{ Date Requested: $8 / 19 / 08$} & \multicolumn{3}{|c|}{ Requestor: Chris Wright (FH) } \\
\hline \multicolumn{3}{|c|}{ Date of Survey: $9 / 18 / 08$} & \multicolumn{3}{|c|}{ Surveyor: Larry A Henke (FGG) } \\
\hline \multicolumn{3}{|c|}{ ERC Point of Contact: } & \multicolumn{3}{|c|}{$\begin{array}{l}\text { Survey Co. Point of Contact: } \\
\text { Neil Fastabend (FGG) }\end{array}$} \\
\hline \multirow{4}{*}{\multicolumn{3}{|c|}{$\begin{array}{l}\text { Description of Work: } \\
\text { Civil Survey of Groundwater Monitoring } \\
\text { Well \#C6188 (399-2-11) }\end{array}$}} & \multicolumn{3}{|c|}{ Horizontal Datum: NAD83(91) } \\
\hline & & & \multicolumn{3}{|c|}{ Vertical Datum: NAVD88 } \\
\hline & & & \multicolumn{3}{|c|}{ Units: $\quad$ METERS } \\
\hline & & & \multicolumn{3}{|c|}{ Hanford Area Designation: $300 \mathrm{~A}$} \\
\hline \multicolumn{6}{|c|}{ Coordinate System: Washington State Plane Coordinates (South Zone) } \\
\hline \multicolumn{6}{|c|}{ Horizontal Control Monuments: $\mathrm{N}-323$ (FGG), 300-70 (FGG) } \\
\hline \multicolumn{6}{|c|}{ Vertical Control Monuments: $300-28$ (FGG), $300-60(F G G)$} \\
\hline Well ID & Well Name & Easting & Northing & Elevation & \\
\hline C6188 & $399-2-11$ & 594236.2 & 116074.49 & & Center of Casing \\
\hline & & & & 114.871 & $\begin{array}{l}\text { Top Pump Base plate. } \\
\text { N. Edge }\end{array}$ \\
\hline & & & & 114.967 & $\begin{array}{l}\text { Top Lid Frame at } \\
\text { Stamped " "X", N. Edge }\end{array}$ \\
\hline & & & & 114.963 & Brass Survey Marker \\
\hline \multirow{2}{*}{\multicolumn{6}{|c|}{$\begin{array}{l}\text { Notes: } \\
\text { EQUIPMENT USED: TRIMBLE GPS } 5800 \text { RTK } \\
\text { TRIMBLE DiNi } 12 \text { LEVEL }\end{array}$}} \\
\hline & & & & & \\
\hline \multicolumn{6}{|c|}{$\begin{array}{l}\text { Surveyor Statement: } \\
\text { I, Larry A. Henke, a Professional Land Surveyor registered } \\
\text { in the State of Washington (Registration No. 38975), hereby } \\
\text { certify that this report is based on a field survey performed in } \\
\text { September, } 2008 \text { under my direct supervision, and that the data } \\
\text { contained here is true and correct. }\end{array}$} \\
\hline
\end{tabular}

Original to:

Distribution by DIS: 


\begin{tabular}{|c|c|c|c|c|c|c|}
\hline \multicolumn{7}{|c|}{ WELL SURVEY DATA REPORT } \\
\hline \multicolumn{3}{|l|}{ Project: } & \multicolumn{4}{|c|}{$\begin{array}{l}\text { Prepared By: Larry A Henke } \\
\text { Company: } \quad \text { FGG }\end{array}$} \\
\hline \multicolumn{3}{|c|}{ Date Requested: $8 / 19 / 08$} & \multicolumn{4}{|c|}{ Requestor: Chris Wright (FH) } \\
\hline \multicolumn{3}{|c|}{ Date of Survey: $9 / 18 / 08$} & \multicolumn{4}{|c|}{ Surveyor: Larry A Henke (FGG) } \\
\hline \multicolumn{3}{|c|}{ ERC Point of Contact: } & \multicolumn{4}{|c|}{$\begin{array}{r}\text { Survey Co. Point of Contact: } \\
\text { Neil Fastabend (FGG) }\end{array}$} \\
\hline \multirow{4}{*}{\multicolumn{3}{|c|}{$\begin{array}{l}\text { Description of Work: } \\
\text { Civil Survey of Groundwater Monitoring } \\
\text { Well \#C6189 (399-2-12) }\end{array}$}} & \multicolumn{4}{|c|}{ Horizontal Datum: NAD83(91) } \\
\hline & & & \multicolumn{4}{|c|}{ Vertical Datum: NAVD88 } \\
\hline & & & \multicolumn{4}{|c|}{ Units: $\quad$ METERS } \\
\hline & & & \multicolumn{4}{|c|}{ Hanford Area Designation: $300 \mathrm{~A}$} \\
\hline \multicolumn{7}{|c|}{ Coordinate System: Washington State Plane Coordinates (South Zone) } \\
\hline \multicolumn{7}{|c|}{ Horizontal Control Monuments: $\mathrm{N}-323(\mathrm{FGG}), 300-70(\mathrm{FGG})$} \\
\hline \multicolumn{7}{|c|}{ Vertical Control Monuments: $300-28$ (FGG), $300-60(\mathrm{FGG})$} \\
\hline Well ID & Well Name & Easting & & Northing & Elevation & \\
\hline \multirow[t]{4}{*}{ C6189 } & $399-2-12$ & 594252. & & 116086.01 & & Center of Casing \\
\hline & & & & & 114.739 & $\begin{array}{l}\text { Top Pump Base plate. } \\
\text { N. Edge }\end{array}$ \\
\hline & & & & & 114.862 & $\begin{array}{l}\text { Top Lid Frame at } \\
\text { Stamped "X", N. Edge }\end{array}$ \\
\hline & & & & & 114.855 & Brass Survey Marker \\
\hline \multirow{2}{*}{\multicolumn{7}{|c|}{$\begin{array}{l}\text { Notes: } \\
\text { EQUIPMENT USED: TRIMBLE GPS } 5800 \text { RTK } \\
\text { TRIMBLE DiNi } 12 \text { LEVEL }\end{array}$}} \\
\hline & & & & & & \\
\hline \multicolumn{5}{|c|}{$\begin{array}{l}\text { Surveyor Statement: } \\
\text { I, Larry A. Henke, a Professional Land Surveyor registered } \\
\text { in the State of Washington (Registration No. 38975), hereby } \\
\text { certify that this report is based on a field survey performed in } \\
\text { September, } 2008 \text { under my direct supervision, and that the data } \\
\text { contained here is true and correct. }\end{array}$} & & 3,2009 \\
\hline
\end{tabular}

Original to:

Distribution by DIS: 


\section{WELL SURVEY DATA REPORT}

\begin{tabular}{|c|c|c|c|c|c|}
\hline \multicolumn{3}{|l|}{ Project: } & \multicolumn{3}{|c|}{$\begin{array}{l}\text { Prepared By: Larry A Henke } \\
\text { Company: } \quad \text { FGG }\end{array}$} \\
\hline \multicolumn{3}{|c|}{ Date Requested: $8 / 19 / 08$} & \multicolumn{3}{|c|}{ Requestor: Chris Wright (FH) } \\
\hline \multicolumn{3}{|c|}{ Date of Survey: $9 / 18 / 08$} & \multicolumn{3}{|c|}{ Surveyor: Larry A Henke (FGG) } \\
\hline \multicolumn{3}{|c|}{ ERC Point of Contact: } & \multicolumn{3}{|c|}{$\begin{array}{r}\text { Survey Co. Point of Contact: } \\
\text { Neil Fastabend (FGG) }\end{array}$} \\
\hline \multirow{4}{*}{\multicolumn{3}{|c|}{$\begin{array}{l}\text { Description of Work: } \\
\text { Civil Survey of Groundwater Monitoring } \\
\text { Well \#C6190 (399-2-13) }\end{array}$}} & \multicolumn{3}{|c|}{ Horizontal Datum: NAD83(91) } \\
\hline & & & \multicolumn{3}{|c|}{ Vertical Datum: NAVD88 } \\
\hline & & & \multicolumn{3}{|c|}{ Units: $\quad$ METERS } \\
\hline & & & \multicolumn{3}{|c|}{ Hanford Area Designation: $300 \mathrm{~A}$} \\
\hline \multicolumn{6}{|c|}{ Coordinate System: Washington State Plane Coordinates (South Zone) } \\
\hline \multicolumn{6}{|c|}{ Horizontal Control Monuments: $\mathrm{N}-323(\mathrm{FGG}), 300-70(\mathrm{FGG})$} \\
\hline \multicolumn{6}{|c|}{ Vertical Control Monuments: $300-28$ (FGG), $300-60(F G G)$} \\
\hline Well ID & Well Name & Easting & Northing & Elevation & \\
\hline \multirow[t]{4}{*}{ C6190 } & $399-2-13$ & 594237. & 116064.59 & & Center of Casing \\
\hline & & & & 114.813 & $\begin{array}{l}\text { Top Pump Base plate. } \\
\text { N. Edge }\end{array}$ \\
\hline & & & & 114.960 & $\begin{array}{l}\text { Top Lid Frame at } \\
\text { Stamped "X", N. Edge }\end{array}$ \\
\hline & & & & 114.958 & Brass Survey Marker \\
\hline \multicolumn{6}{|c|}{$\begin{array}{l}\text { Notes: } \\
\text { EQUIPMENT USED: TRIMBLE GPS } 5800 \text { RTK } \\
\text { TRIMBLE DiNi } 12 \text { LEVEL }\end{array}$} \\
\hline \multicolumn{6}{|c|}{$\begin{array}{l}\text { Surveyor Statement: } \\
\text { l, Larry A. Henke, a Professional Land Surveyor registered } \\
\text { in the State of Washington (Registration No. 38975), hereby } \\
\text { certify that this report is based on a field survey performed in } \\
\text { September, } 2008 \text { under my direct supervision, and that the data } \\
\text { contained here is true and correct. }\end{array}$} \\
\hline
\end{tabular}

\section{Original to:}

Distribution by DIS: 


\section{WELL SURVEY DATA REPORT}

\begin{tabular}{|c|c|c|c|c|c|}
\hline \multicolumn{3}{|l|}{ Project: } & \multicolumn{3}{|c|}{$\begin{array}{l}\text { Prepared By: Larry A Henke } \\
\text { Company: } \quad \text { FGG }\end{array}$} \\
\hline \multicolumn{3}{|c|}{ Date Requested: $8 / 19 / 08$} & \multicolumn{3}{|c|}{ Requestor: Chris Wright (FH) } \\
\hline \multicolumn{3}{|c|}{ Date of Survey: $9 / 18 / 08$} & \multicolumn{3}{|c|}{ Surveyor: Larry A Henke (FGG) } \\
\hline \multicolumn{3}{|c|}{ ERC Point of Contact: } & \multicolumn{3}{|c|}{$\begin{array}{r}\text { Survey Co. Point of Contact: } \\
\text { Neil Fastabend (FGG) }\end{array}$} \\
\hline \multirow{4}{*}{\multicolumn{3}{|c|}{$\begin{array}{l}\text { Description of Work: } \\
\text { Civil Survey of Groundwater Monitoring } \\
\text { Well \#C6191 (399-2-14) }\end{array}$}} & \multicolumn{3}{|c|}{ Horizontal Datum: NAD83(91) } \\
\hline & & & \multicolumn{3}{|c|}{ Vertical Datum: NAVD88 } \\
\hline & & & \multicolumn{3}{|c|}{ Units: $\quad$ METERS } \\
\hline & & & \multicolumn{3}{|c|}{ Hanford Area Designation: $300 \mathrm{~A}$} \\
\hline \multicolumn{6}{|c|}{ Coordinate System: Washington State Plane Coordinates (South Zone) } \\
\hline \multicolumn{6}{|c|}{ Horizontal Control Monuments: N-323 (FGG), 300-70 (FGG) } \\
\hline \multicolumn{6}{|c|}{ Vertical Control Monuments: $300-28$ (FGG), 300-60(FGG) } \\
\hline Well ID & Well Name & Easting & Northing & Elevation & \\
\hline \multirow[t]{4}{*}{ C6191 } & $399-2-14$ & 594245. & 116070.17 & & Center of Casing \\
\hline & & & & 114.741 & $\begin{array}{l}\text { Top Pump Base plate. } \\
\text { N. Edge }\end{array}$ \\
\hline & & & & 114.838 & $\begin{array}{l}\text { Top Lid Frame at } \\
\text { Stamped "X", N. Edge }\end{array}$ \\
\hline & & & & 114.834 & Brass Survey Marker \\
\hline \multicolumn{6}{|c|}{$\begin{array}{l}\text { Notes: } \\
\text { EQUIPMENT USED: TRIMBLE GPS } 5800 \text { RTK } \\
\text { TRIMBLE DiNi } 12 \text { LEVEL }\end{array}$} \\
\hline \multicolumn{6}{|c|}{$\begin{array}{l}\text { Surveyor Statement: } \\
\text { I, Larry A. Henke, a Professional Land Surveyor registered } \\
\text { in the State of Washington (Registration No. 38975), hereby } \\
\text { certify that this report is based on a field survey performed in } \\
\text { September, } 2008 \text { under my direct supervision, and that the data } \\
\text { contained here is true and correct. }\end{array}$} \\
\hline
\end{tabular}

Original to:

Distribution by DIS: 


\section{WELL SURVEY DATA REPORT}

\begin{tabular}{|c|c|c|c|c|c|c|}
\hline \multicolumn{3}{|l|}{ Project: } & \multicolumn{4}{|c|}{$\begin{array}{l}\text { Prepared By: Larry A Henke } \\
\text { Company: } \quad \text { FGG }\end{array}$} \\
\hline \multicolumn{3}{|c|}{ Date Requested: $8 / 19 / 08$} & \multicolumn{4}{|c|}{ Requestor: Chris Wright (FH) } \\
\hline \multicolumn{3}{|c|}{ Date of Survey: $9 / 18 / 08$} & \multicolumn{4}{|c|}{ Surveyor: Larry A Henke (FGG) } \\
\hline \multicolumn{3}{|c|}{ ERC Point of Contact: } & \multicolumn{4}{|c|}{$\begin{array}{r}\text { Survey Co. Point of Contact: } \\
\text { Neil Fastabend (FGG) }\end{array}$} \\
\hline \multirow{4}{*}{\multicolumn{3}{|c|}{$\begin{array}{l}\text { Description of Work: } \\
\text { Civil Survey of Groundwater Monitoring } \\
\text { Well \#C6192 (399-2-15) }\end{array}$}} & \multicolumn{4}{|c|}{ Horizontal Datum: NAD83(91) } \\
\hline & & & \multicolumn{4}{|c|}{ Vertical Datum: NAVD88 } \\
\hline & & & \multicolumn{4}{|c|}{ Units: $\quad$ METERS } \\
\hline & & & \multicolumn{4}{|c|}{ Hanford Area Designation: $300 \mathrm{~A}$} \\
\hline \multicolumn{7}{|c|}{ Coordinate System: Washington State Plane Coordinates (South Zone) } \\
\hline \multicolumn{7}{|c|}{ Horizontal Control Monuments: N-323 (FGG), 300-70 (FGG) } \\
\hline \multicolumn{7}{|c|}{ Vertical Control Monuments: $300-28$ (FGG), 300-60(FGG) } \\
\hline Well ID & Well Name & Easting & & Northing & Elevation & \\
\hline \multirow[t]{4}{*}{ C6192 } & $399-2-15$ & 594253. & & 116076.02 & & Center of Casing \\
\hline & & & & & 114.781 & $\begin{array}{l}\text { Top Pump Base plate. } \\
\text { N. Edge }\end{array}$ \\
\hline & & & & & 114.885 & $\begin{array}{l}\text { Top Lid Frame at } \\
\text { Stamped "X" N. Edqe }\end{array}$ \\
\hline & & & & & 114.880 & Brass Survey Marker \\
\hline
\end{tabular}

Notes:

EQUIPMENT USED: TRIMBLE GPS 5800 RTK TRIMBLE DiNi 12 LEVEL

\section{Surveyor Statement:}

I, Larry A. Henke, a Professional Land Surveyor registered in the State of Washington (Registration No. 38975), hereby certify that this report is based on a field survey performed in September, 2008 under my direct supervision, and that the data contained here is true and correct.

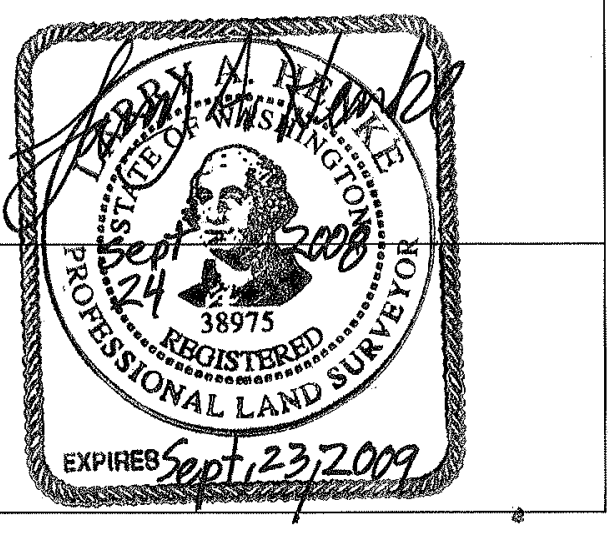

Original to:

Distribution by DIS: 


\section{WELL SURVEY DATA REPORT}

\begin{tabular}{|c|c|c|c|c|c|}
\hline \multicolumn{3}{|l|}{ Project: } & \multicolumn{3}{|c|}{$\begin{array}{l}\text { Prepared By: Larry A Henke } \\
\text { Company: }\end{array}$} \\
\hline \multicolumn{3}{|c|}{ Date Requested: $8 / 19 / 08$} & \multicolumn{3}{|c|}{ Requestor: Chris Wright (FH) } \\
\hline \multicolumn{3}{|c|}{ Date of Survey: $9 / 18 / 08$} & \multicolumn{3}{|c|}{ Surveyor: Larry A Henke (FGG) } \\
\hline \multicolumn{3}{|c|}{ ERC Point of Contact: } & \multicolumn{3}{|c|}{$\begin{array}{r}\text { Survey Co. Point of Contact: } \\
\text { Neil Fastabend (FGG) }\end{array}$} \\
\hline \multirow{4}{*}{\multicolumn{3}{|c|}{$\begin{array}{l}\text { Description of Work: } \\
\text { Civil Survey of Groundwater Monitoring } \\
\text { Well \#C6193 (399-2-16) }\end{array}$}} & \multicolumn{3}{|c|}{ Horizontal Datum: NAD83(91) } \\
\hline & & & \multicolumn{3}{|c|}{ Vertical Datum: $\quad$ NAVD88 } \\
\hline & & & \multicolumn{3}{|c|}{ Units: $\quad$ METERS } \\
\hline & & & \multicolumn{3}{|c|}{ Hanford Area Designation: $300 \mathrm{~A}$} \\
\hline \multicolumn{6}{|c|}{ Coordinate System: Washington State Plane Coordinates (South Zone) } \\
\hline \multicolumn{6}{|c|}{ Horizontal Control Monuments: N-323 (FGG), 300-70 (FGG) } \\
\hline \multicolumn{6}{|c|}{ Vertical Control Monuments: $300-28$ (FGG), 300-60(FGG) } \\
\hline Well ID & Well Name & Easting & Northing & Elevation & \\
\hline \multirow[t]{4}{*}{ C6193 } & $399-2-16$ & 594261. & 116081.92 & & Center of Casing \\
\hline & & & & 114.691 & $\begin{array}{l}\text { Top Pump Base plate. } \\
\text { N. Edge }\end{array}$ \\
\hline & & & & 114.796 & $\begin{array}{l}\text { Top Lid Frame at } \\
\text { Stamped "X", N. Edge }\end{array}$ \\
\hline & & & & 114.797 & Brass Survey Marker \\
\hline \multicolumn{6}{|c|}{$\begin{array}{l}\text { Notes: } \\
\text { EQUIPMENT USED: TRIMBLE GPS } 5800 \text { RTK } \\
\text { TRIMBLE DINi } 12 \text { LEVEL }\end{array}$} \\
\hline \multicolumn{6}{|c|}{$\begin{array}{l}\text { Surveyor Statement: } \\
\text { I, Larry A. Henke, a Professional Land Surveyor registered } \\
\text { in the State of Washington (Registration No. 38975), hereby } \\
\text { certify that this report is based on a field survey performed in } \\
\text { September, } 2008 \text { under my direct supervision, and that the data } \\
\text { contained here is true and correct. }\end{array}$} \\
\hline
\end{tabular}

Original to:

Distribution by DIS: 


\section{WELL SURVEY DATA REPORT}

\begin{tabular}{|c|c|c|c|c|c|}
\hline \multicolumn{3}{|l|}{ Project: } & \multicolumn{3}{|c|}{$\begin{array}{l}\text { Prepared By: } \\
\text { Company: }\end{array}$} \\
\hline \multicolumn{3}{|c|}{ Date Requested: $8 / 19 / 08$} & \multicolumn{3}{|c|}{ Requestor: Chris Wright (FH) } \\
\hline \multicolumn{3}{|c|}{ Date of Survey: $9 / 18 / 08$} & \multicolumn{3}{|c|}{ Surveyor: Larry A Henke (FGG) } \\
\hline \multicolumn{3}{|c|}{ ERC Point of Contact: } & \multicolumn{3}{|c|}{$\begin{array}{l}\text { Survey Co. Point of Contact: } \\
\text { Neil Fastabend (FGG) }\end{array}$} \\
\hline \multirow{4}{*}{\multicolumn{3}{|c|}{$\begin{array}{l}\text { Description of Work: } \\
\text { Civil Survey of Groundwater Monitoring } \\
\text { Well \#C6194 (399-3-23) }\end{array}$}} & \multicolumn{3}{|c|}{ Horizontal Datum: NAD83(91) } \\
\hline & & & \multicolumn{3}{|c|}{ Vertical Datum: $\quad$ NAVD88 } \\
\hline & & & \multicolumn{3}{|c|}{ Units: $\quad$ METERS } \\
\hline & & & \multicolumn{3}{|c|}{ Hanford Area Designation: $300 \mathrm{~A}$} \\
\hline \multicolumn{6}{|c|}{ Coordinate System: Washington State Plane Coordinates (South Zone) } \\
\hline \multicolumn{6}{|c|}{ Horizontal Control Monuments: N-323 (FGG), 300-70 (FGG) } \\
\hline \multicolumn{6}{|c|}{ Vertical Control Monuments: $300-28$ (FGG), $300-60(\mathrm{FGG})$} \\
\hline Well ID & Well Name & Easting & Northing & Elevation & \\
\hline \multirow[t]{4}{*}{ C6194 } & $399-3-23$ & 594237.9 & 116054.72 & & Center of Casing \\
\hline & & & & 114.940 & $\begin{array}{l}\text { Top Pump Base plate. } \\
\text { N. Edge }\end{array}$ \\
\hline & & & & 115.020 & $\begin{array}{l}\text { Top Lid Frame at } \\
\text { Stamped "X", N. Edge }\end{array}$ \\
\hline & & & & 115.014 & Brass Survey Marker \\
\hline \multicolumn{6}{|c|}{$\begin{array}{l}\text { Notes: } \\
\text { EQUIPMENT USED: TRIMBLE GPS } 5800 \text { RTK } \\
\text { TRIMBLE DiNi } 12 \text { LEVEL }\end{array}$} \\
\hline \multicolumn{6}{|c|}{$\begin{array}{l}\text { Surveyor Statement: } \\
\text { 1, Larry A. Henke, a Professional Land Surveyor registered } \\
\text { in the State of Washington (Registration No. 38975), hereby } \\
\text { certify that this report is based on a field survey performed in } \\
\text { September, } 2008 \text { under my direct supervision, and that the data } \\
\text { contained here is true and correct. }\end{array}$} \\
\hline
\end{tabular}

Original to:

Distribution by DIS: 
WELL SURVEY DATA REPORT

\begin{tabular}{|c|c|c|c|c|c|}
\hline \multicolumn{3}{|l|}{ Project: } & \multicolumn{3}{|c|}{$\begin{array}{l}\text { Prepared By: } \\
\text { Company: }\end{array}$} \\
\hline \multicolumn{3}{|c|}{ Date Requested: $8 / 19 / 08$} & \multicolumn{3}{|c|}{ Requestor: Chris Wright (FH) } \\
\hline \multicolumn{3}{|c|}{ Date of Survey: $9 / 18 / 08$} & \multicolumn{3}{|c|}{ Surveyor: Larry A Henke (FGG) } \\
\hline \multicolumn{3}{|c|}{ ERC Point of Contact: } & \multicolumn{3}{|c|}{$\begin{array}{r}\text { Survey Co. Point of Contact: } \\
\text { Neil Fastabend (FGG) }\end{array}$} \\
\hline \multirow{4}{*}{\multicolumn{3}{|c|}{$\begin{array}{l}\text { Description of Work: } \\
\text { Civil Survey of Groundwater Monitoring } \\
\text { Well \#C6195 (399-2-17) }\end{array}$}} & \multicolumn{3}{|c|}{ Horizontal Datum: NAD83(91) } \\
\hline & & & \multicolumn{3}{|c|}{ Vertical Datum: NAVD88 } \\
\hline & & & \multicolumn{3}{|c|}{ Units: $\quad$ METERS } \\
\hline & & & \multicolumn{3}{|c|}{ Hanford Area Designation: $300 \mathrm{~A}$} \\
\hline \multicolumn{6}{|c|}{ Coordinate System: Washington State Plane Coordinates (South Zone) } \\
\hline \multicolumn{6}{|c|}{ Horizontal Control Monuments: N-323 (FGG), 300-70 (FGG) } \\
\hline \multicolumn{6}{|c|}{ Vertical Control Monuments: $300-28$ (FGG), 300-60(FGG) } \\
\hline Well ID & Well Name & Easting & Northing & Elevation & \\
\hline \multirow[t]{4}{*}{ C6195 } & $399-2-17$ & 594246.1 & 116060.29 & & Center of Casing \\
\hline & & & & 114.763 & $\begin{array}{l}\text { Top Pump Base plate. } \\
\text { N. Edge }\end{array}$ \\
\hline & & & & 114.885 & $\begin{array}{l}\text { Top Lid Frame at } \\
\text { Stamped "X", N. Edge }\end{array}$ \\
\hline & & & & 114.870 & Brass Survey Marker \\
\hline \multicolumn{6}{|c|}{$\begin{array}{l}\text { Notes: } \\
\text { EQUIPMENT USED: TRIMBLE GPS } 5800 \text { RTK } \\
\text { TRIMBLE DiNi } 12 \text { LEVEL }\end{array}$} \\
\hline \multicolumn{6}{|c|}{$\begin{array}{l}\text { Surveyor Statement: } \\
\text { 1, Larry A. Henke, a Professional Land Surveyor registered } \\
\text { in the State of Washington (Registration No. 38975), hereby } \\
\text { certify that this report is based on a field survey performed in } \\
\text { September, } 2008 \text { under my direct supervision, and that the data } \\
\text { contained here is true and correct. }\end{array}$} \\
\hline
\end{tabular}

Original to:

Distribution by DIS: 


\begin{tabular}{|c|c|c|c|c|c|c|}
\hline \multicolumn{7}{|c|}{ WELL SURVEY DATA REPORT } \\
\hline \multicolumn{3}{|l|}{ Project: } & \multicolumn{4}{|c|}{$\begin{array}{l}\text { Prepared By: Larry A Henke } \\
\text { Company: } \quad \text { FGG }\end{array}$} \\
\hline \multicolumn{3}{|c|}{ Date Requested: $8 / 19 / 08$} & \multicolumn{4}{|c|}{ Requestor: Chris Wright (FH) } \\
\hline \multicolumn{3}{|c|}{ Date of Survey: $9 / 18 / 08$} & \multicolumn{4}{|c|}{ Surveyor: Larry A Henke (FGG) } \\
\hline \multicolumn{3}{|c|}{ ERC Point of Contact: } & \multicolumn{4}{|c|}{$\begin{array}{l}\text { Survey Co. Point of Contact: } \\
\text { Neil Fastabend (FGG) }\end{array}$} \\
\hline \multirow{4}{*}{\multicolumn{3}{|c|}{$\begin{array}{l}\text { Description of Work: } \\
\text { Civil Survey of Groundwater Monitoring } \\
\text { Well \#C6196 (399-2-18) }\end{array}$}} & \multicolumn{4}{|c|}{ Horizontal Datum: NAD83(91) } \\
\hline & & & \multicolumn{4}{|c|}{ Vertical Datum: NAVD88 } \\
\hline & & & \multicolumn{4}{|c|}{ Units: $\quad$ METERS } \\
\hline & & & \multicolumn{4}{|c|}{ Hanford Area Designation: $300 \mathrm{~A}$} \\
\hline \multicolumn{7}{|c|}{ Coordinate System: Washington State Plane Coordinates (South Zone) } \\
\hline \multicolumn{7}{|c|}{ Horizontal Control Monuments: N-323 (FGG), 300-70 (FGG) } \\
\hline \multicolumn{7}{|c|}{ Vertical Control Monuments: $300-28$ (FGG), 300-60(FGG) } \\
\hline Well ID & Well Name & Easting & & Northing & Elevation & \\
\hline \multirow[t]{4}{*}{ C6196 } & $399-2-18$ & 594254.1 & & 116066.13 & & Center of Casing \\
\hline & & & & & 114.733 & $\begin{array}{l}\text { Top Pump Base plate. } \\
\text { N. Edge }\end{array}$ \\
\hline & & & & & 114.861 & $\begin{array}{l}\text { Top Lid Frame at } \\
\text { Stamped " } X \text { ", N. Edge }\end{array}$ \\
\hline & & & & & 114.862 & Brass Survey Marker \\
\hline \multicolumn{7}{|c|}{$\begin{array}{l}\text { Notes: } \\
\text { EQUIPMENT USED: TRIMBLE GPS } 5800 \text { RTK } \\
\text { TRIMBLE DiNi } 12 \text { LEVEL }\end{array}$} \\
\hline \multicolumn{5}{|c|}{$\begin{array}{l}\text { Surveyor Statement: } \\
\text { I, Larry A. Henke, a Professional Land Surveyor registered } \\
\text { in the State of Washington (Registration No. 38975), hereby } \\
\text { certify that this report is based on a field survey performed in } \\
\text { September, } 2008 \text { under my direct supervision, and that the data } \\
\text { contained here is true and correct. }\end{array}$} & 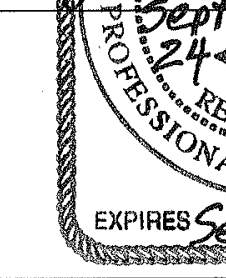 & Sars \\
\hline
\end{tabular}

Original to:

Distribution by DIS: 


\section{WELL SURVEY DATA REPORT}

\begin{tabular}{|c|c|c|c|c|c|}
\hline \multicolumn{3}{|l|}{ Project: } & \multicolumn{3}{|c|}{$\begin{array}{l}\text { Prepared By: Larry A Henke } \\
\text { Company: } \quad \text { FGG }\end{array}$} \\
\hline \multicolumn{3}{|c|}{ Date Requested: $8 / 19 / 08$} & \multicolumn{3}{|c|}{ Requestor: Chris Wright (FH) } \\
\hline \multicolumn{3}{|c|}{ Date of Survey: $9 / 18 / 08$} & \multicolumn{3}{|c|}{ Surveyor: Larry A Henke (FGG) } \\
\hline \multicolumn{3}{|c|}{ ERC Point of Contact: } & \multicolumn{3}{|c|}{$\begin{array}{l}\text { Survey Co. Point of Contact: } \\
\text { Neil Fastabend (FGG) }\end{array}$} \\
\hline \multirow{4}{*}{\multicolumn{3}{|c|}{$\begin{array}{l}\text { Description of Work: } \\
\text { Civil Survey of Groundwater Monitoring } \\
\text { Well \#C6197 (399-2-19) }\end{array}$}} & \multicolumn{3}{|c|}{ Horizontal Datum: NAD83(91) } \\
\hline & & & \multicolumn{3}{|c|}{\begin{tabular}{|l} 
Vertical Datum: NAVD88 \\
\end{tabular}} \\
\hline & & & \multicolumn{3}{|c|}{ Units: $\quad$ METERS } \\
\hline & & & \multicolumn{3}{|c|}{ Hanford Area Designation: $300 \mathrm{~A}$} \\
\hline \multicolumn{6}{|c|}{ Coordinate System: Washington State Plane Coordinates (South Zone) } \\
\hline \multicolumn{6}{|c|}{ Horizontal Control Monuments: $\mathrm{N}-323(\mathrm{FGG}), 300-70(\mathrm{FGG})$} \\
\hline \multicolumn{6}{|c|}{ Vertical Control Monuments: $300-28$ (FGG), 300-60(FGG) } \\
\hline Well ID & Well Name & Easting & Northing & Elevation & \\
\hline C6197 & $399-2-19$ & 594262.7 & 116071.76 & & Center of Casing \\
\hline & & & & 114.746 & $\begin{array}{l}\text { Top Pump Base plate. } \\
\text { N. Edge }\end{array}$ \\
\hline & & & & 114.868 & $\begin{array}{l}\text { Top Lid Frame at } \\
\text { Stamped "X", N. Edge }\end{array}$ \\
\hline & & & & 114.866 & Brass Survey Marker \\
\hline \multirow{2}{*}{\multicolumn{6}{|c|}{$\begin{array}{l}\text { Notes: } \\
\text { EQUIPMENT USED: TRIMBLE GPS } 5800 \text { RTK } \\
\text { TRIMBLE DiNi } 12 \text { LEVEL }\end{array}$}} \\
\hline & & & & & \\
\hline \multicolumn{6}{|c|}{$\begin{array}{l}\text { Surveyor Statement: } \\
\text { I, Larry A. Henke, a Professional Land Surveyor registered } \\
\text { in the State of Washington (Registration No. 38975), hereby } \\
\text { certify that this report is based on a field survey performed in } \\
\text { September, } 2008 \text { under my direct supervision, and that the data } \\
\text { contained here is true and correct. }\end{array}$} \\
\hline
\end{tabular}

Original to:

Distribution by DIS: 


\begin{tabular}{|c|c|c|c|c|c|c|}
\hline \multicolumn{7}{|c|}{ WELL SURVEY DATA REPORT } \\
\hline \multicolumn{3}{|l|}{ Project: } & \multicolumn{4}{|c|}{$\begin{array}{ll}\text { Prepared By: } & \text { Larry A Henke } \\
\text { Company: } & \text { FGG }\end{array}$} \\
\hline \multicolumn{3}{|c|}{ Date Requested: $8 / 19 / 08$} & \multicolumn{4}{|c|}{ Requestor: Chris Wright (FH) } \\
\hline \multicolumn{3}{|c|}{ Date of Survey: $9 / 18 / 08$} & \multicolumn{4}{|c|}{ Surveyor: Larry A Henke (FGG) } \\
\hline \multicolumn{3}{|c|}{ ERC Point of Contact: } & \multicolumn{4}{|c|}{$\begin{array}{r}\text { Survey Co. Point of Contact: } \\
\text { Neil Fastabend (FGG) }\end{array}$} \\
\hline \multirow{4}{*}{\multicolumn{3}{|c|}{$\begin{array}{l}\text { Description of Work: } \\
\text { Civil Survey of Groundwater Monitoring } \\
\text { Well \#C6198 (399-2-20) }\end{array}$}} & \multicolumn{4}{|c|}{ Horizontal Datum: NAD83(91) } \\
\hline & & & \multicolumn{4}{|c|}{ Vertical Datum: NAVD88 } \\
\hline & & & \multicolumn{4}{|c|}{ Units: $\quad$ METERS } \\
\hline & & & \multicolumn{4}{|c|}{ Hanford Area Designation: $300 \mathrm{~A}$} \\
\hline \multicolumn{7}{|c|}{ Coordinate System: Washington State Plane Coordinates (South Zone) } \\
\hline \multicolumn{7}{|c|}{ Horizontal Control Monuments: $\mathrm{N}-323$ (FGG), 300-70 (FGG) } \\
\hline \multicolumn{7}{|c|}{ Vertical Control Monuments: $300-28$ (FGG), 300-60(FGG) } \\
\hline Well ID & Well Name & Easting & & Northing & Elevation & \\
\hline \multirow[t]{4}{*}{ C6198 } & $399-2-20$ & 594270. & & 116077.64 & & Center of Casing \\
\hline & & & & & 114.794 & $\begin{array}{l}\text { Top Pump Base plate. } \\
\text { N. Edge }\end{array}$ \\
\hline & & & & & 114.879 & $\begin{array}{l}\text { Top Lid Frame at } \\
\text { Stamped "X" N. Edge }\end{array}$ \\
\hline & & & & & 114.886 & Brass Survey Marker \\
\hline \multicolumn{7}{|c|}{$\begin{array}{l}\text { Notes: } \\
\text { EQUIPMENT USED: TRIMBLE GPS } 5800 \text { RTK } \\
\text { TRIMBLE DiNi } 12 \text { LEVEL }\end{array}$} \\
\hline \multicolumn{7}{|c|}{$\begin{array}{l}\text { Surveyor Statement: } \\
\text { l, Larry A. Henke, a Professional Land Surveyor registered } \\
\text { in the State of Washington (Registration No. 38975), hereby } \\
\text { certify that this report is based on a field survey performed in } \\
\text { September, } 2008 \text { under my direct supervision, and that the data } \\
\text { contained here is true and correct. }\end{array}$} \\
\hline
\end{tabular}

Original to:

Distribution by DIS: 


\section{WELL SURVEY DATA REPORT}

\begin{tabular}{|c|c|c|c|c|c|}
\hline \multicolumn{3}{|l|}{ Project: } & \multicolumn{3}{|c|}{$\begin{array}{l}\text { Prepared By: Larry A Henke } \\
\text { Company: } \quad \text { FGG }\end{array}$} \\
\hline \multicolumn{3}{|c|}{ Date Requested: $8 / 19 / 08$} & \multicolumn{3}{|c|}{ Requestor: Chris Wright (FH) } \\
\hline \multicolumn{3}{|c|}{ Date of Survey: $9 / 18 / 08$} & \multicolumn{3}{|c|}{ Surveyor: Larry A Henke (FGG) } \\
\hline \multicolumn{3}{|c|}{ ERC Point of Contact: } & \multicolumn{3}{|c|}{$\begin{array}{l}\text { Survey Co. Point of Contact: } \\
\text { Neil Fastabend (FGG) }\end{array}$} \\
\hline \multirow{4}{*}{\multicolumn{3}{|c|}{$\begin{array}{l}\text { Description of Work: } \\
\text { Civil Survey of Groundwater Monitoring } \\
\text { Well \#C6199 (399-3-24) }\end{array}$}} & \multicolumn{3}{|c|}{ Horizontal Datum: NAD83(91) } \\
\hline & & & \multicolumn{3}{|c|}{ Vertical Datum: NAVD88 } \\
\hline & & & \multicolumn{3}{|c|}{ Units: $\quad$ METERS } \\
\hline & & & \multicolumn{3}{|c|}{ Hanford Area Designation: $300 \mathrm{~A}$} \\
\hline \multicolumn{6}{|c|}{ Coordinate System: Washington State Plane Coordinates (South Zone) } \\
\hline \multicolumn{6}{|c|}{ Horizontal Control Monuments: $\mathrm{N}-323$ (FGG), 300-70 (FGG) } \\
\hline \multicolumn{6}{|c|}{ Vertical Control Monuments: $300-28$ (FGG), $300-60(F G G)$} \\
\hline Well ID & Well Name & Easting & Northing & Elevation & \\
\hline \multirow[t]{4}{*}{ C6199 } & $399-3-24$ & 594238. & 116044.60 & & Center of Casing \\
\hline & & & & 114.886 & $\begin{array}{l}\text { Top Pump Base plate. } \\
\text { N. Edge }\end{array}$ \\
\hline & & & & 114.978 & $\begin{array}{l}\text { Top Lid Frame at } \\
\text { Stamped "X", N. Edge }\end{array}$ \\
\hline & & & & 114.978 & Brass Survey Marker \\
\hline \multicolumn{6}{|c|}{$\begin{array}{l}\text { Notes: } \\
\text { EQUIPMENT USED: TRIMBLE GPS } 5800 \text { RTK } \\
\text { TRIMBLE DiNi } 12 \text { LEVEL }\end{array}$} \\
\hline \multicolumn{6}{|c|}{$\begin{array}{l}\text { Surveyor Statement: } \\
\text { I, Larry A. Henke, a Professional Land Surveyor registered } \\
\text { in the State of Washington (Registration No. 38975), hereby } \\
\text { certify that this report is based on a field survey performed in } \\
\text { September, } 2008 \text { under my direct supervision, and that the data } \\
\text { contained here is true and correct. }\end{array}$} \\
\hline
\end{tabular}

Original to:

Distribution by DIS: 


\begin{tabular}{|c|c|c|c|c|c|c|}
\hline \multicolumn{7}{|c|}{ WELL SURVEY DATA REPORT } \\
\hline \multicolumn{3}{|l|}{ Project: } & \multicolumn{4}{|c|}{$\begin{array}{ll}\text { Prepared By: } & \text { Larry A Henke } \\
\text { Company: } & \text { FGG }\end{array}$} \\
\hline \multicolumn{3}{|c|}{ Date Requested: $8 / 19 / 08$} & \multicolumn{4}{|c|}{ Requestor: Chris Wright (FH) } \\
\hline \multicolumn{3}{|c|}{ Date of Survey: $9 / 18 / 08$} & \multicolumn{4}{|c|}{ Surveyor: Larry A Henke (FGG) } \\
\hline \multicolumn{3}{|c|}{ ERC Point of Contact: } & \multicolumn{4}{|c|}{$\begin{array}{r}\text { Survey Co. Point of Contact: } \\
\text { Neil Fastabend (FGG) }\end{array}$} \\
\hline \multirow{4}{*}{\multicolumn{3}{|c|}{$\begin{array}{l}\text { Description of Work: } \\
\text { Civil Survey of Groundwater Monitoring } \\
\text { Well \#C6200 (399-3-25) }\end{array}$}} & \multicolumn{4}{|c|}{ Horizontal Datum: NAD83(91) } \\
\hline & & & \multicolumn{4}{|c|}{ Vertical Datum: NAVD88 } \\
\hline & & & \multicolumn{4}{|c|}{ Units: $\quad$ METERS } \\
\hline & & & \multicolumn{4}{|c|}{ Hanford Area Designation: $300 \mathrm{~A}$} \\
\hline \multicolumn{7}{|c|}{ Coordinate System: Washington State Plane Coordinates (South Zone) } \\
\hline \multicolumn{7}{|c|}{ Horizontal Control Monuments: N-323 (FGG), 300-70 (FGG) } \\
\hline \multicolumn{7}{|c|}{ Vertical Control Monuments: $300-28(\mathrm{FGG}), \quad 300-60(\mathrm{FGG})$} \\
\hline Well ID & Well Name & Easting & & Northing & Elevation & \\
\hline \multirow[t]{4}{*}{ C6200 } & $399-3-25$ & 594255.1 & & 116056.03 & & Center of Casing \\
\hline & & & & & 114.754 & $\begin{array}{l}\text { Top Pump Base plate. } \\
\text { N. Edge }\end{array}$ \\
\hline & & & & & 114.875 & $\begin{array}{l}\text { Top Lid Frame at } \\
\text { Stamped " } \mathrm{X} \text { ", N. Edge }\end{array}$ \\
\hline & & & & & 114.875 & Brass Survey Marker \\
\hline \multicolumn{7}{|c|}{$\begin{array}{l}\text { Notes: } \\
\text { EQUIPMENT USED: TRIMBLE GPS } 5800 \text { RTK } \\
\text { TRIMBLE DiNi } 12 \text { LEVEL }\end{array}$} \\
\hline \multicolumn{7}{|c|}{$\begin{array}{l}\text { Surveyor Statement: } \\
\text { I, Larry A. Henke, a Professional Land Surveyor registered } \\
\text { in the State of Washington (Registration No. 38975), hereby } \\
\text { certify that this report is based on a field survey performed in } \\
\text { September, } 2008 \text { under my direct supervision, and that the data } \\
\text { contained here is true and correct. }\end{array}$} \\
\hline
\end{tabular}

Original to:

Distribution by DIS: 
WELL SURVEY DATA REPORT

\begin{tabular}{|c|c|}
\hline Project: & $\begin{array}{l}\text { Prepared By: Larry A Henke } \\
\text { Company: } \quad \text { FGG }\end{array}$ \\
\hline Date Requested: $8 / 19 / 08$ & Requestor: Chris Wright (FH) \\
\hline Date of Survey: $9 / 18 / 08$ & Surveyor: Larry A Henke (FGG) \\
\hline ERC Point of Contact: & $\begin{array}{r}\text { Survey Co. Point of Contact: } \\
\text { Neil Fastabend (FGG) }\end{array}$ \\
\hline Description of Work: & Horizontal Datum: NAD83(91) \\
\hline \multirow{3}{*}{$\begin{array}{l}\text { Civil Survey of Groundwater Monitoring } \\
\text { Well \#C6201 (399-2-21) }\end{array}$} & Vertical Datum: NAVD88 \\
\hline & METERS \\
\hline & Hanford Area Designation: $300 \mathrm{~A}$ \\
\hline
\end{tabular}

Coordinate System: Washington State Plane Coordinates (South Zone)

Horizontal Control Monuments: N-323 (FGG), 300-70 (FGG)

Vertical Control Monuments: $300-28$ (FGG), 300-60(FGG)

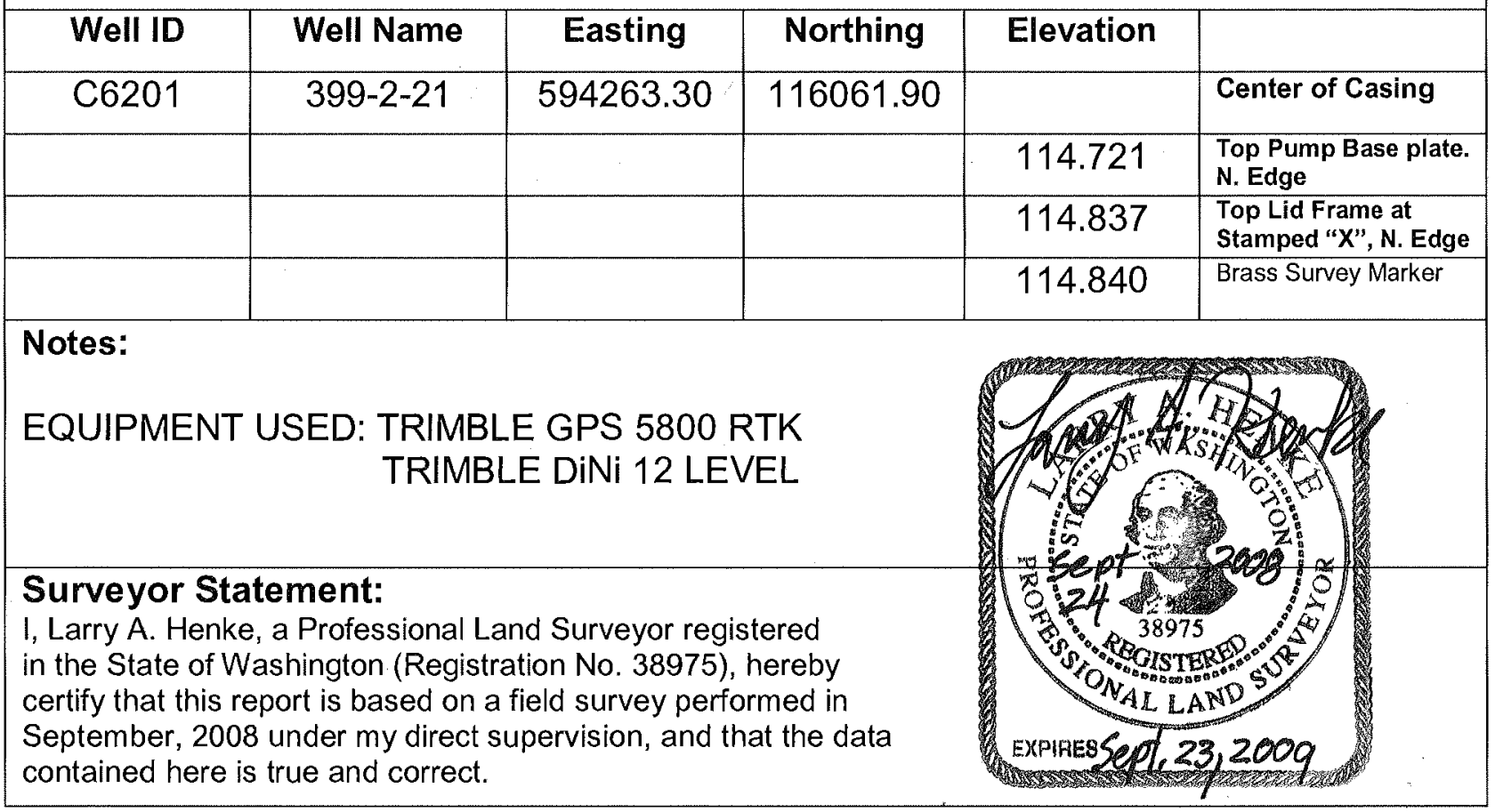

Original to:

Distribution by DIS: 


\begin{tabular}{|c|c|c|c|c|c|c|}
\hline \multicolumn{7}{|c|}{ WELL SURVEY DATA REPORT } \\
\hline \multicolumn{3}{|l|}{ Project: } & \multicolumn{4}{|c|}{$\begin{array}{l}\text { Prepared By: Larry A Henke } \\
\text { Company: } \quad \text { FGG }\end{array}$} \\
\hline \multicolumn{3}{|c|}{ Date Requested: $8 / 19 / 08$} & \multicolumn{4}{|c|}{ Requestor: Chris Wright (FH) } \\
\hline \multicolumn{3}{|c|}{ Date of Survey: $9 / 18 / 08$} & \multicolumn{4}{|c|}{ Surveyor: Larry A Henke (FGG) } \\
\hline \multicolumn{3}{|c|}{ ERC Point of Contact: } & \multicolumn{4}{|c|}{$\begin{array}{r}\text { Survey Co. Point of Contact: } \\
\text { Neil Fastabend (FGG) }\end{array}$} \\
\hline \multirow{4}{*}{\multicolumn{3}{|c|}{$\begin{array}{l}\text { Description of Work: } \\
\text { Civil Survey of Groundwater Monitoring } \\
\text { Well \#C6202 (399-2-22) }\end{array}$}} & \multicolumn{4}{|c|}{ Horizontal Datum: NAD83(91) } \\
\hline & & & \multicolumn{4}{|c|}{ Vertical Datum: NAVD88 } \\
\hline & & & \multicolumn{4}{|c|}{ Units: $\quad$ METERS } \\
\hline & & & \multicolumn{4}{|c|}{ Hanford Area Designation: $300 \mathrm{~A}$} \\
\hline \multicolumn{7}{|c|}{ Coordinate System: Washington State Plane Coordinates (South Zone) } \\
\hline \multicolumn{7}{|c|}{ Horizontal Control Monuments: $\mathrm{N}-323(\mathrm{FGG}), 300-70(\mathrm{FGG})$} \\
\hline \multicolumn{7}{|c|}{ Vertical Control Monuments: $300-28$ (FGG), 300-60(FGG) } \\
\hline Well ID & Well Name & Easting & & Northing & Elevation & \\
\hline \multirow[t]{4}{*}{ C6202 } & $399-2-22$ & 594279.6 & & 116073.37 & & Center of Casing \\
\hline & & & & & 114.806 & $\begin{array}{l}\text { Top Pump Base plate. } \\
\text { N. Edge }\end{array}$ \\
\hline & & & & & 114.871 & $\begin{array}{l}\text { Top Lid Frame at } \\
\text { Stamped "X", N. Edge }\end{array}$ \\
\hline & & & & & 114.870 & Brass Survey Marker \\
\hline \multicolumn{7}{|c|}{$\begin{array}{l}\text { Notes: } \\
\text { EQUIPMENT USED: TRIMBLE GPS } 5800 \text { RTK } \\
\text { TRIMBLE DiNi } 12 \text { LEVEL }\end{array}$} \\
\hline \multicolumn{7}{|c|}{$\begin{array}{l}\text { Surveyor Statement: } \\
\text { I, Larry A. Henke, a Professional Land Surveyor registered } \\
\text { in the State of Washington (Registration No. 38975), hereby } \\
\text { certify that this report is based on a field survey performed in } \\
\text { September, } 2008 \text { under my direct supervision, and that the data } \\
\text { contained here is true and correct. }\end{array}$} \\
\hline
\end{tabular}

Original to:

Distribution by DIS: 


\section{WELL SURVEY DATA REPORT}

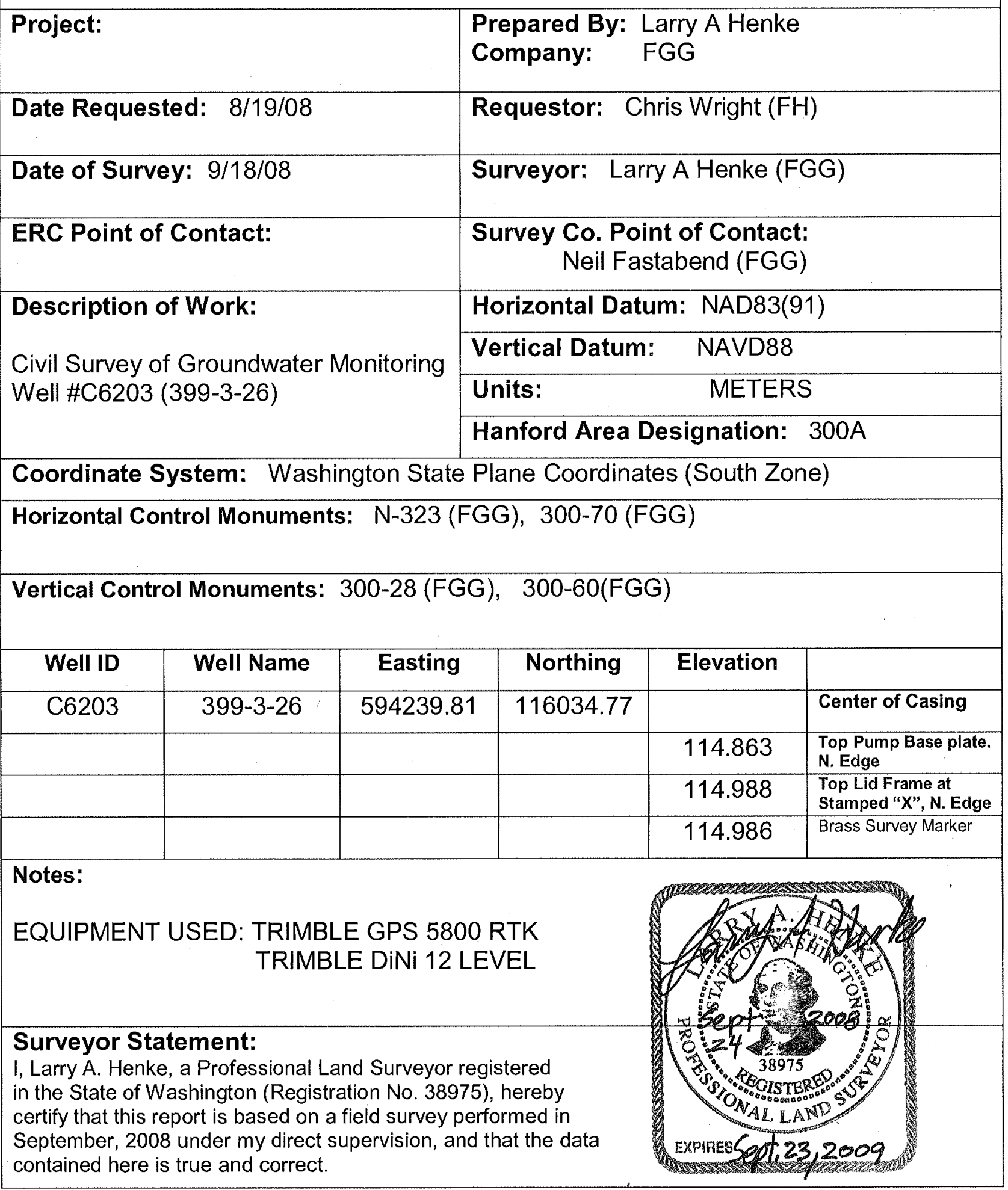

Original to:

Distribution by DIS: 


\section{WELL SURVEY DATA REPORT}

\begin{tabular}{|c|c|c|c|c|c|}
\hline \multicolumn{3}{|l|}{ Project: } & \multicolumn{3}{|c|}{$\begin{array}{l}\text { Prepared By: Larry A Henke } \\
\text { Company: } \quad \text { FGG }\end{array}$} \\
\hline \multicolumn{3}{|c|}{ Date Requested: $8 / 19 / 08$} & \multicolumn{3}{|c|}{ Requestor: Chris Wright (FH) } \\
\hline \multicolumn{3}{|c|}{ Date of Survey: $9 / 18 / 08$} & \multicolumn{3}{|c|}{ Surveyor: Larry A Henke (FGG) } \\
\hline \multicolumn{3}{|c|}{ ERC Point of Contact: } & \multicolumn{3}{|c|}{$\begin{array}{r}\text { Survey Co. Point of Contact: } \\
\text { Neil Fastabend (FGG) }\end{array}$} \\
\hline \multirow{4}{*}{\multicolumn{3}{|c|}{$\begin{array}{l}\text { Description of Work: } \\
\text { Civil Survey of Groundwater Monitoring } \\
\text { Well \#C6204 (399-3-27) }\end{array}$}} & \multicolumn{3}{|c|}{ Horizontal Datum: NAD83(91) } \\
\hline & & & \multicolumn{3}{|c|}{ Vertical Datum: NAVD88 } \\
\hline & & & \multicolumn{3}{|c|}{ Units: $\quad$ METERS } \\
\hline & & & \multicolumn{3}{|c|}{ Hanford Area Designation: $300 \mathrm{~A}$} \\
\hline \multicolumn{6}{|c|}{ Coordinate System: Washington State Plane Coordinates (South Zone) } \\
\hline \multicolumn{6}{|c|}{ Horizontal Control Monuments: $\mathrm{N}-323$ (FGG), 300-70 (FGG) } \\
\hline \multicolumn{6}{|c|}{ Vertical Control Monuments: $300-28$ (FGG), 300-60(FGG) } \\
\hline Well ID & Well Name & Easting & Northing & Elevation & \\
\hline \multirow[t]{4}{*}{ C6204 } & $399-3-27$ & 594247.9 & 116040.66 & & Center of Casing \\
\hline & & & & 114.848 & $\begin{array}{l}\text { Top Pump Base plate. } \\
\text { N. Edge }\end{array}$ \\
\hline & & & & 114.936 & $\begin{array}{l}\text { Top Lid Frame at } \\
\text { Stamped "X", N. Edge }\end{array}$ \\
\hline & & & & 114.930 & Brass Survey Marker \\
\hline \multirow{2}{*}{\multicolumn{6}{|c|}{$\begin{array}{l}\text { Notes: } \\
\text { EQUIPMENT USED: TRIMBLE GPS } 5800 \text { RTK } \\
\text { TRIMBLE DiNi } 12 \text { LEVEL }\end{array}$}} \\
\hline & & & & & \\
\hline \multicolumn{6}{|c|}{$\begin{array}{l}\text { Surveyor Statement: } \\
\text { I, Larry A. Henke, a Professional Land Surveyor registered } \\
\text { in the State of Washington (Registration No. 38975), hereby } \\
\text { certify that this report is based on a field survey performed in } \\
\text { September, } 2008 \text { under my direct supervision, and that the data } \\
\text { contained here is true and correct. }\end{array}$} \\
\hline
\end{tabular}

Original to:

Distribution by DIS: 


\begin{tabular}{|c|c|c|c|c|c|c|}
\hline \multicolumn{7}{|c|}{ WELL SURVEY DATA REPORT } \\
\hline \multicolumn{3}{|l|}{ Project: } & \multicolumn{4}{|c|}{$\begin{array}{l}\text { Prepared By: Larry A Henke } \\
\text { Company: }\end{array}$} \\
\hline \multicolumn{3}{|c|}{ Date Requested: $8 / 19 / 08$} & \multicolumn{4}{|c|}{ Requestor: Chris Wright $(\mathrm{FH})$} \\
\hline \multicolumn{3}{|c|}{ Date of Survey: $9 / 18 / 08$} & \multicolumn{4}{|c|}{ Surveyor: Larry A Henke (FGG) } \\
\hline \multicolumn{3}{|c|}{ ERC Point of Contact: } & \multicolumn{4}{|c|}{$\begin{array}{l}\text { Survey Co. Point of Contact: } \\
\text { Neil Fastabend (FGG) }\end{array}$} \\
\hline \multirow{4}{*}{\multicolumn{3}{|c|}{$\begin{array}{l}\text { Description of Work: } \\
\text { Civil Survey of Groundwater Monitoring } \\
\text { Well \#C6205 (399-3-28) }\end{array}$}} & \multicolumn{4}{|c|}{ Horizontal Datum: NAD83(91) } \\
\hline & & & \multicolumn{4}{|c|}{ Vertical Datum: NAVD88 } \\
\hline & & & \multicolumn{4}{|c|}{ Units: $\quad$ METERS } \\
\hline & & & \multicolumn{4}{|c|}{ Hanford Area Designation: $300 \mathrm{~A}$} \\
\hline \multicolumn{7}{|c|}{ Coordinate System: Washington State Plane Coordinates (South Zone) } \\
\hline \multicolumn{7}{|c|}{ Horizontal Control Monuments: $\quad \mathrm{N}-323(\mathrm{FGG}), 300-70$ (FGG) } \\
\hline \multicolumn{7}{|c|}{ Vertical Control Monuments: $300-28$ (FGG), 300-60(FGG) } \\
\hline Well ID & Well Name & Easting & & Northing & Elevation & \\
\hline \multirow[t]{4}{*}{ C6205 } & $399-3-28$ & 594256. & & 116045.98 & & Center of Casing \\
\hline & & & & & 114.803 & $\begin{array}{l}\text { Top Pump Base plate. } \\
\text { N. Edge }\end{array}$ \\
\hline & & & & & 114.909 & $\begin{array}{l}\text { Top Lid Frame at } \\
\text { Stamped "X", N. Edge }\end{array}$ \\
\hline & & & & & 114.900 & Brass Survey Marker \\
\hline \multicolumn{7}{|c|}{$\begin{array}{l}\text { Notes: } \\
\text { EQUIPMENT USED: TRIMBLE GPS } 5800 \text { RTK } \\
\text { TRIMBLE DiNi } 12 \text { LEVEL }\end{array}$} \\
\hline \multicolumn{5}{|c|}{$\begin{array}{l}\text { Surveyor Statement: } \\
\text { I, Larry A. Henke, a Professional Land Surveyor registered } \\
\text { in the State of Washington (Registration No. 38975), hereby } \\
\text { certify that this report is based on a field survey performed in } \\
\text { September, } 2008 \text { under my direct supervision, and that the data } \\
\text { contained here is true and correct. }\end{array}$} & & 2,1 \\
\hline
\end{tabular}

Original to:

Distribution by DIS: 
WELL SURVEY DATA REPORT

\begin{tabular}{|c|c|c|c|c|c|}
\hline \multicolumn{3}{|l|}{ Project: } & \multicolumn{3}{|c|}{$\begin{array}{l}\text { Prepared By: Larry A Henke } \\
\text { Company: } \quad \text { FGG }\end{array}$} \\
\hline \multicolumn{3}{|c|}{ Date Requested: $8 / 19 / 08$} & \multicolumn{3}{|c|}{ Requestor: Chris Wright (FH) } \\
\hline \multicolumn{3}{|c|}{ Date of Survey: $9 / 18 / 08$} & \multicolumn{3}{|c|}{ Surveyor: Larry A Henke (FGG) } \\
\hline \multicolumn{3}{|c|}{ ERC Point of Contact: } & \multicolumn{3}{|c|}{$\begin{array}{r}\text { Survey Co. Point of Contact: } \\
\text { Neil Fastabend (FGG) }\end{array}$} \\
\hline \multirow{4}{*}{\multicolumn{3}{|c|}{$\begin{array}{l}\text { Description of Work: } \\
\text { Civil Survey of Groundwater Monitoring } \\
\text { Well \#C6206 (399-3-29) }\end{array}$}} & \multicolumn{3}{|c|}{ Horizontal Datum: NAD83(91) } \\
\hline & & & \multicolumn{3}{|c|}{ Vertical Datum: NAVD88 } \\
\hline & & & \multicolumn{3}{|c|}{ Units: $\quad$ METERS } \\
\hline & & & \multicolumn{3}{|c|}{ Hanford Area Designation: $300 \mathrm{~A}$} \\
\hline \multicolumn{6}{|c|}{ Coordinate System: Washington State Plane Coordinates (South Zone) } \\
\hline \multicolumn{6}{|c|}{ Horizontal Control Monuments: $\mathrm{N}-323(\mathrm{FGG}), 300-70(\mathrm{FGG})$} \\
\hline \multicolumn{6}{|c|}{ Vertical Control Monuments: $300-28$ (FGG), 300-60(FGG) } \\
\hline Well ID & Well Name & Easting & Northing & Elevation & \\
\hline \multirow[t]{4}{*}{ C6206 } & $399-3-29$ & 594264. & 116051.97 & & Center of Casing \\
\hline & & & & 114.830 & $\begin{array}{l}\text { Top Pump Base plate. } \\
\text { N. Edge }\end{array}$ \\
\hline & & & & 114.903 & $\begin{array}{l}\text { Top Lid Frame at } \\
\text { Stamped "X", N. Edge }\end{array}$ \\
\hline & & & & 114.898 & Brass Survey Marker \\
\hline \multirow{2}{*}{\multicolumn{6}{|c|}{$\begin{array}{l}\text { Notes: } \\
\text { EQUIPMENT USED: TRIMBLE GPS } 5800 \text { RTK } \\
\text { TRIMBLE DiNi } 12 \text { LEVEL }\end{array}$}} \\
\hline & & & & & \\
\hline \multicolumn{6}{|c|}{$\begin{array}{l}\text { Surveyor Statement: } \\
\text { I, Larry A. Henke, a Professional Land Surveyor registered } \\
\text { in the State of Washington (Registration No. 38975), hereby } \\
\text { certify that this report is based on a field survey performed in } \\
\text { September, } 2008 \text { under my direct supervision, and that the data } \\
\text { contained here is true and correct. }\end{array}$} \\
\hline
\end{tabular}

Original to:

Distribution by DIS: 


\section{WELL SURVEY DATA REPORT}

\begin{tabular}{|c|c|c|c|c|c|}
\hline \multicolumn{3}{|l|}{ Project: } & \multicolumn{3}{|c|}{$\begin{array}{l}\text { Prepared By: Larry A Henke } \\
\text { Company: }\end{array}$} \\
\hline \multicolumn{3}{|c|}{ Date Requested: $8 / 19 / 08$} & \multicolumn{3}{|c|}{ Requestor: Chris Wright (FH) } \\
\hline \multicolumn{3}{|c|}{ Date of Survey: $9 / 18 / 08$} & \multicolumn{3}{|c|}{ Surveyor: Larry A Henke (FGG) } \\
\hline \multicolumn{3}{|c|}{ ERC Point of Contact: } & \multicolumn{3}{|c|}{$\begin{array}{l}\text { Survey Co. Point of Contact: } \\
\text { Neil Fastabend (FGG) }\end{array}$} \\
\hline \multirow{4}{*}{\multicolumn{3}{|c|}{$\begin{array}{l}\text { Description of Work: } \\
\text { Civil Survey of Groundwater Monitoring } \\
\text { Well \#C6207 (399-2-23) }\end{array}$}} & \multicolumn{3}{|c|}{ Horizontal Datum: NAD83(91) } \\
\hline & & & \multicolumn{3}{|c|}{ Vertical Datum: NAVD88 } \\
\hline & & & \multicolumn{3}{|c|}{ Units: $\quad$ METERS } \\
\hline & & & \multicolumn{3}{|c|}{ Hanford Area Designation: $300 \mathrm{~A}$} \\
\hline \multicolumn{6}{|c|}{ Coordinate System: Washington State Plane Coordinates (South Zone) } \\
\hline \multicolumn{6}{|c|}{ Horizontal Control Monuments: N-323 (FGG), 300-70 (FGG) } \\
\hline \multicolumn{6}{|c|}{ Vertical Control Monuments: $300-28$ (FGG), 300-60(FGG) } \\
\hline Well ID & Well Name & Easting & Northing & Elevation & \\
\hline C6207 & $399-2-23$ & 594272.2 & 116057.59 & & Center of Casing \\
\hline & & & & 114.805 & $\begin{array}{l}\text { Top Pump Base plate. } \\
\text { N. Edge }\end{array}$ \\
\hline & & & & 114.875 & $\begin{array}{l}\text { Top Lid Frame at } \\
\text { Stamped " } " \text { ", N. Edge }\end{array}$ \\
\hline & & & & 114.875 & Brass Survey Marker \\
\hline \multicolumn{6}{|c|}{$\begin{array}{l}\text { Notes: } \\
\text { EQUIPMENT USED: TRIMBLE GPS } 5800 \text { RTK } \\
\text { TRIMBLE DiNi } 12 \text { LEVEL }\end{array}$} \\
\hline \multicolumn{6}{|c|}{$\begin{array}{l}\text { Surveyor Statement: } \\
\text { 1, Larry A. Henke, a Professional Land Surveyor registered } \\
\text { in the State of Washington (Registration No. 38975), hereby } \\
\text { certify that this report is based on a field survey performed in } \\
\text { September, } 2008 \text { under my direct supervision, and that the data } \\
\text { contained here is true and correct. }\end{array}$} \\
\hline
\end{tabular}

Original to:

Distribution by DIS: 
WELL SURVEY DATA REPORT

\begin{tabular}{|c|c|c|c|c|c|}
\hline \multicolumn{3}{|l|}{ Project: } & \multicolumn{3}{|c|}{$\begin{array}{l}\text { Prepared By: Larry A Henke } \\
\text { Company: } \quad \text { FGG }\end{array}$} \\
\hline \multicolumn{3}{|c|}{ Date Requested: $8 / 19 / 08$} & \multicolumn{3}{|c|}{ Requestor: Chris Wright (FH) } \\
\hline \multicolumn{3}{|c|}{ Date of Survey: $9 / 18 / 08$} & \multicolumn{3}{|c|}{ Surveyor: Larry A Henke (FGG) } \\
\hline \multicolumn{3}{|c|}{ ERC Point of Contact: } & \multicolumn{3}{|c|}{$\begin{array}{r}\text { Survey Co. Point of Contact: } \\
\text { Neil Fastabend (FGG) }\end{array}$} \\
\hline \multirow{4}{*}{\multicolumn{3}{|c|}{$\begin{array}{l}\text { Description of Work: } \\
\text { Civil Survey of Groundwater Monitoring } \\
\text { Well \#C6208 (399-2-21) }\end{array}$}} & \multicolumn{3}{|c|}{ Horizontal Datum: NAD83(91) } \\
\hline & & & \multicolumn{3}{|c|}{ Vertical Datum: NAVD88 } \\
\hline & & & \multicolumn{3}{|c|}{ Units: $\quad$ METERS } \\
\hline & & & \multicolumn{3}{|c|}{ Hanford Area Designation: $300 \mathrm{~A}$} \\
\hline \multicolumn{6}{|c|}{ Coordinate System: Washington State Plane Coordinates (South Zone) } \\
\hline \multicolumn{6}{|c|}{ Horizontal Control Monuments: $\quad \mathrm{N}-323(\mathrm{FGG}), 300-70$ (FGG) } \\
\hline \multicolumn{6}{|c|}{ Vertical Control Monuments: $300-28$ (FGG), 300-60(FGG) } \\
\hline Well ID & Well Name & Easting & Northing & Elevation & \\
\hline C6208 & $399-2-21$ & 594280.6 & 116063.29 & & Center of Casing \\
\hline & & & & 114.761 & $\begin{array}{l}\text { Top Pump Base plate. } \\
\text { N. Edge }\end{array}$ \\
\hline & & & & 114.889 & $\begin{array}{l}\text { Top Lid Frame at } \\
\text { Stamped "X", N. Edge }\end{array}$ \\
\hline & & & & 114.889 & Brass Survey Marker \\
\hline \multicolumn{6}{|c|}{$\begin{array}{l}\text { Notes: } \\
\text { EQUIPMENT USED: TRIMBLE GPS } 5800 \text { RTK } \\
\text { TRIMBLE DiNi } 12 \text { LEVEL }\end{array}$} \\
\hline \multicolumn{6}{|c|}{$\begin{array}{l}\text { Surveyor Statement: } \\
\text { I, Larry A. Henke, a Professional Land Surveyor registered } \\
\text { in the State of Washington (Registration No. 38975), hereby } \\
\text { certify that this report is based on a field survey performed in } \\
\text { September, } 2008 \text { under my direct supervision, and that the data } \\
\text { contained here is true and correct. }\end{array}$} \\
\hline
\end{tabular}

Original to:

Distribution by DIS: 
WELL SURVEY DATA REPORT

\begin{tabular}{|c|c|}
\hline Project: & $\begin{array}{l}\text { Prepared By: Larry A Henke } \\
\text { Company: } \quad \text { FGG }\end{array}$ \\
\hline Date Requested: $8 / 19 / 08$ & Requestor: Chris Wright (FH) \\
\hline Date of Survey: $9 / 18 / 08$ & Surveyor: Larry A Henke (FGG) \\
\hline ERC Point of Contact: & $\begin{array}{r}\text { Survey Co. Point of Contact: } \\
\text { Neil Fastabend (FGG) }\end{array}$ \\
\hline \multirow{4}{*}{$\begin{array}{l}\text { Description of Work: } \\
\text { Civil Survey of Groundwater Monitoring } \\
\text { Well \#C6209 (399-2-25) }\end{array}$} & Horizontal Datum: NAD83(91) \\
\hline & Vertical Datum: \\
\hline & METERS \\
\hline & Hanford Area Designation: 300 \\
\hline
\end{tabular}

Coordinate System: Washington State Plane Coordinates (South Zone)

Horizontal Control Monuments: N-323 (FGG), 300-70 (FGG)

Vertical Control Monuments: $300-28$ (FGG), 300-60(FGG)

\begin{tabular}{|l|c|c|c|c|l|}
\hline Well ID & Well Name & Easting & Northing & Elevation & \\
\hline C6209 & $399-2-25$ & 594269.21 & 116088.06 & & Center of Casing \\
\hline & & & & 114.931 & $\begin{array}{l}\text { Top Pump Base plate. } \\
\text { N. Edge }\end{array}$ \\
\hline & & & & 115.534 & $\begin{array}{l}\text { Top Lid Frame at } \\
\text { Stamped "X", N. Edge }\end{array}$ \\
\hline & & & & 115.638 & Brass Survey Marker \\
\hline
\end{tabular}

Notes:

EQUIPMENT USED: TRIMBLE GPS 5800 RTK TRIMBLE DiNi 12 LEVEL

\section{Surveyor Statement:}

I, Larry A. Henke, a Professional Land Surveyor registered in the State of Washington (Registration No. 38975), hereby certify that this report is based on a field survey performed in September, 2008 under my direct supervision, and that the data contained here is true and correct.

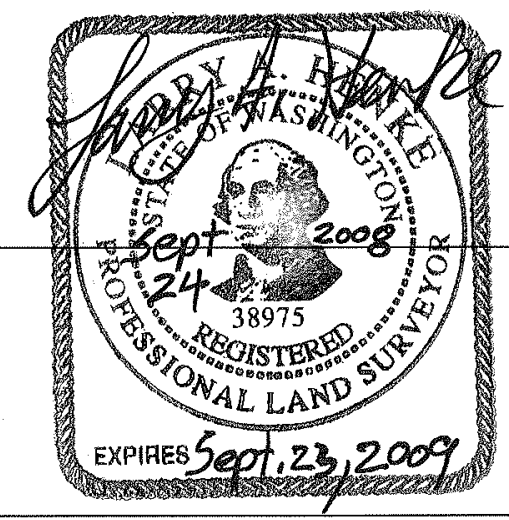

Original to:

Distribution by DIS: 
WELL SURVEY DATA REPORT

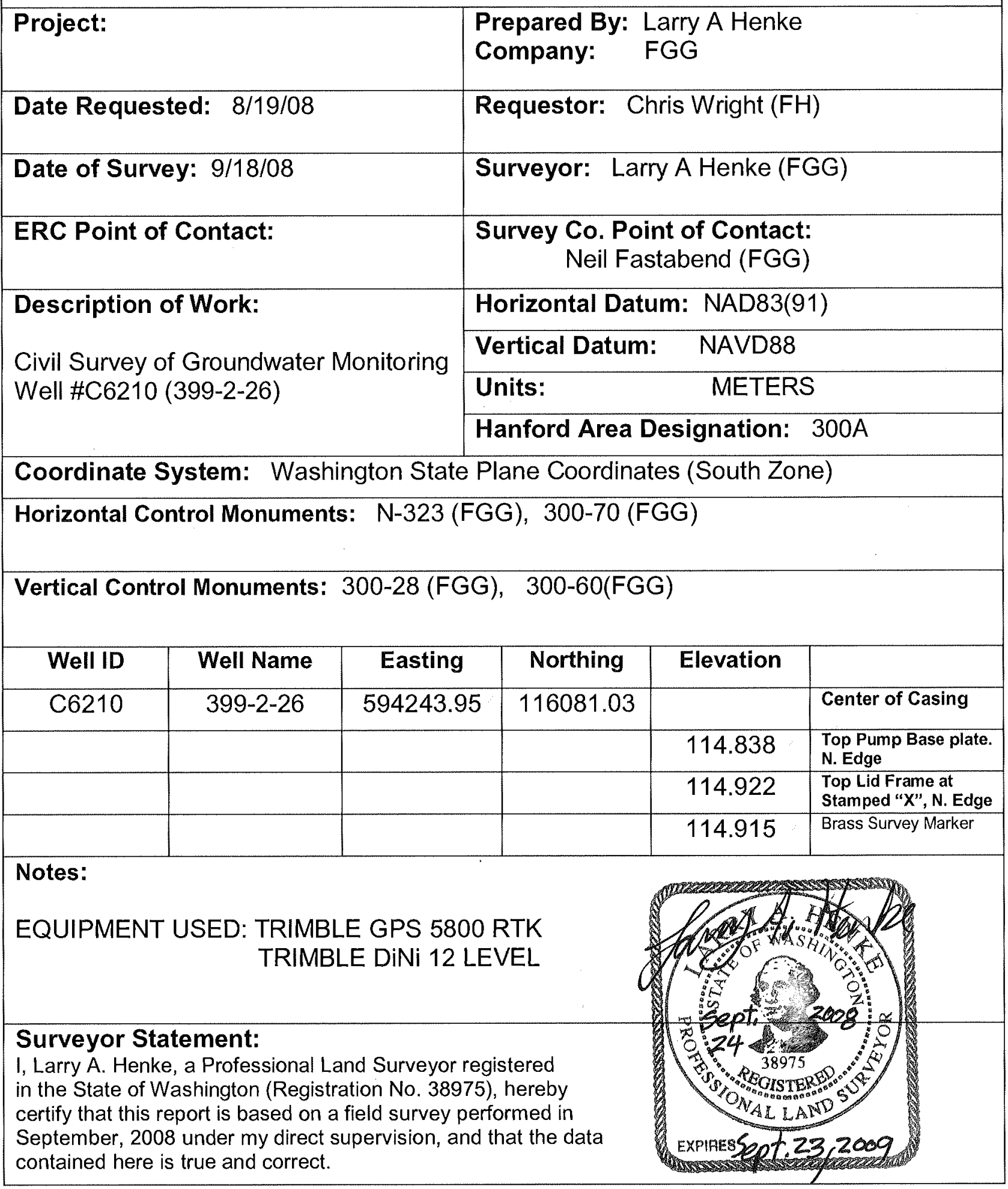

Original to:

Distribution by DIS: 


\section{WELL SURVEY DATA REPORT}

\begin{tabular}{|c|c|}
\hline Project: & $\begin{array}{l}\text { Prepared By: Larry A Henke } \\
\text { Company: } \quad \text { FGG }\end{array}$ \\
\hline Date Requested: $8 / 19 / 08$ & Requestor: Chris Wright (FH) \\
\hline Date of Survey: $9 / 18 / 08$ & Surveyor: Larry A Henke (FGG) \\
\hline ERC Point of Contact: & $\begin{array}{r}\text { Survey Co. Point of Contact: } \\
\text { Neil Fastabend (FGG) }\end{array}$ \\
\hline \multirow{4}{*}{$\begin{array}{l}\text { Description of Work: } \\
\text { Civil Survey of Groundwater Monitoring } \\
\text { Well \#C6211 (399-2-27) }\end{array}$} & Horizontal Datum: NAD83(91) \\
\hline & Vertical Datum: NAVD88 \\
\hline & METERS \\
\hline & Hanford Area Designation: $300 \mathrm{~A}$ \\
\hline \multicolumn{2}{|c|}{ Coordinate System: Washington State Plane Coordinates (South Zone) } \\
\hline
\end{tabular}

Vertical Control Monuments: $300-28$ (FGG), 300-60(FGG)

\begin{tabular}{|l|c|c|c|c|l|}
\hline Well ID & Well Name & Easting & Northing & Elevation & \\
\hline C6211 & $399-2-27$ & 594244.12 & 116078.15 & & Center of Casing \\
\hline & & & & 114.785 & $\begin{array}{l}\text { Top Pump Base plate. } \\
\text { N. Edge }\end{array}$ \\
\hline & & & & 114.899 & $\begin{array}{l}\text { Top Lid Frame at } \\
\text { Stamped " } \text { ", N. Edge }\end{array}$ \\
\hline & & & & 114.896 & Brass Survey Marker \\
\hline
\end{tabular}

Notes:

EQUIPMENT USED: TRIMBLE GPS 5800 RTK TRIMBLE DiNi 12 LEVEL

\section{Surveyor Statement:}

I, Larry A. Henke, a Professional Land Surveyor registered in the State of Washington (Registration No. 38975), hereby certify that this report is based on a field survey performed in September, 2008 under my direct supervision, and that the data contained here is true and correct.

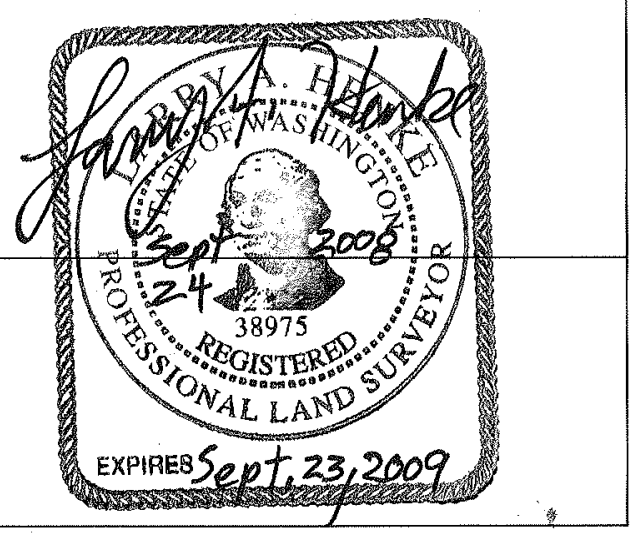

Original to:

Distribution by DIS: 


\begin{tabular}{|c|c|c|c|c|c|c|}
\hline \multicolumn{7}{|c|}{ WELL SURVEY DATA REPORT } \\
\hline \multicolumn{3}{|l|}{ Project: } & \multicolumn{4}{|c|}{$\begin{array}{l}\text { Prepared By: Larry A Henke } \\
\text { Company: } \quad \text { FGG }\end{array}$} \\
\hline \multicolumn{3}{|c|}{ Date Requested: $8 / 19 / 08$} & \multicolumn{4}{|c|}{ Requestor: Chris Wright $(\mathrm{FH})$} \\
\hline \multicolumn{3}{|c|}{ Date of Survey: $9 / 18 / 08$} & \multicolumn{4}{|c|}{ Surveyor: Larry A Henke (FGG) } \\
\hline \multicolumn{3}{|c|}{ ERC Point of Contact: } & \multicolumn{4}{|c|}{$\begin{array}{r}\text { Survey Co. Point of Contact: } \\
\text { Neil Fastabend (FGG) }\end{array}$} \\
\hline \multirow{4}{*}{\multicolumn{3}{|c|}{$\begin{array}{l}\text { Description of Work: } \\
\text { Civil Survey of Groundwater Monitoring } \\
\text { Well \#C6212 (399-2-28) }\end{array}$}} & \multicolumn{4}{|c|}{ Horizontal Datum: NAD83(91) } \\
\hline & & & \multicolumn{4}{|c|}{ Vertical Datum: NAVD88 } \\
\hline & & & \multicolumn{4}{|c|}{ Units: $\quad$ METERS } \\
\hline & & & \multicolumn{4}{|c|}{ Hanford Area Designation: $300 \mathrm{~A}$} \\
\hline \multicolumn{7}{|c|}{ Coordinate System: Washington State Plane Coordinates (South Zone) } \\
\hline \multicolumn{7}{|c|}{ Horizontal Control Monuments: $\mathrm{N}-323(\mathrm{FGG}), 300-70$ (FGG) } \\
\hline \multicolumn{7}{|c|}{ Vertical Control Monuments: $300-28$ (FGG), 300-60(FGG) } \\
\hline Well ID & Well Name & Easting & & Northing & Elevation & \\
\hline \multirow[t]{4}{*}{ C6212 } & $399-2-28$ & 594246.2 & & 116079.99 & & Center of Casing \\
\hline & & & & & 114.740 & $\begin{array}{l}\text { Top Pump Base plate. } \\
\text { N. Edge }\end{array}$ \\
\hline & & & & & 114.859 & $\begin{array}{l}\text { Top Lid Frame at } \\
\text { Stamped " } " X \text { ", N. Edge }\end{array}$ \\
\hline & & & & & 114.855 & Brass Survey Marker \\
\hline \multicolumn{7}{|c|}{$\begin{array}{l}\text { Notes: } \\
\text { EQUIPMENT USED: TRIMBLE GPS } 5800 \text { RTK } \\
\text { TRIMBLE DiNi } 12 \text { LEVEL }\end{array}$} \\
\hline \multicolumn{7}{|c|}{$\begin{array}{l}\text { Surveyor Statement: } \\
\text { I, Larry A. Henke, a Professional Land Surveyor registered } \\
\text { in the State of Washington (Registration No. 38975), hereby } \\
\text { certify that this report is based on a field survey performed in } \\
\text { September, } 2008 \text { under my direct supervision, and that the data } \\
\text { contained here is true and correct. }\end{array}$} \\
\hline
\end{tabular}

Original to:

Distribution by DIS: 


\begin{tabular}{|c|c|c|c|c|c|c|}
\hline \multicolumn{7}{|c|}{ WELL SURVEY DATA REPORT } \\
\hline \multicolumn{3}{|l|}{ Project: } & \multicolumn{4}{|c|}{$\begin{array}{ll}\text { Prepared By: } & \text { Larry A Henke } \\
\text { Company: } & \text { FGG }\end{array}$} \\
\hline \multicolumn{3}{|c|}{ Date Requested: $8 / 19 / 08$} & \multicolumn{4}{|c|}{ Requestor: Chris Wright (FH) } \\
\hline \multicolumn{3}{|c|}{ Date of Survey: $9 / 18 / 08$} & \multicolumn{4}{|c|}{ Surveyor: Larry A Henke (FGG) } \\
\hline \multicolumn{3}{|c|}{ ERC Point of Contact: } & \multicolumn{4}{|c|}{$\begin{array}{r}\text { Survey Co. Point of Contact: } \\
\text { Neil Fastabend (FGG) }\end{array}$} \\
\hline \multirow{4}{*}{\multicolumn{3}{|c|}{$\begin{array}{l}\text { Description of Work: } \\
\text { Civil Survey of Groundwater Monitoring } \\
\text { Well \#C6213 (399-3-30) }\end{array}$}} & \multicolumn{4}{|c|}{ Horizontal Datum: NAD83(91) } \\
\hline & & & \multicolumn{4}{|c|}{ Vertical Datum: NAVD88 } \\
\hline & & & \multicolumn{4}{|c|}{ Units: $\quad$ METERS } \\
\hline & & & \multicolumn{4}{|c|}{ Hanford Area Designation: $300 \mathrm{~A}$} \\
\hline \multicolumn{7}{|c|}{ Coordinate System: Washington State Plane Coordinates (South Zone) } \\
\hline \multicolumn{7}{|c|}{ Horizontal Control Monuments: $\mathrm{N}-323(\mathrm{FGG}), 300-70(\mathrm{FGG})$} \\
\hline \multicolumn{7}{|c|}{ Vertical Control Monuments: $300-28$ (FGG), 300-60(FGG) } \\
\hline Well ID & Well Name & Easting & & Northing & Elevation & \\
\hline \multirow[t]{4}{*}{ C6213 } & $399-3-30$ & 594246.3 & & 116051.35 & & Center of Casing \\
\hline & & & & & 114.791 & $\begin{array}{l}\text { Top Pump Base plate. } \\
\text { N. Edge }\end{array}$ \\
\hline & & & & & 114.879 & $\begin{array}{l}\text { Top Lid Frame at } \\
\text { Stamped "X", N. Edge }\end{array}$ \\
\hline & & & & & 114.876 & Brass Survey Marker \\
\hline \multicolumn{7}{|c|}{$\begin{array}{l}\text { Notes: } \\
\text { EQUIPMENT USED: TRIMBLE GPS } 5800 \text { RTK } \\
\text { TRIMBLE DiNi } 12 \text { LEVEL }\end{array}$} \\
\hline \multicolumn{5}{|c|}{$\begin{array}{l}\text { Surveyor Statement: } \\
\text { I, Larry A. Henke, a Professional Land Surveyor registered } \\
\text { in the State of Washington (Registration No. 38975), hereby } \\
\text { certify that this report is based on a field survey performed in } \\
\text { September, } 2008 \text { under my direct supervision, and that the data } \\
\text { contained here is true and correct. }\end{array}$} & ExpinesS & 32009 \\
\hline
\end{tabular}

Original to:

Distribution by DIS: 


\section{WELL SURVEY DATA REPORT}

\begin{tabular}{|c|c|c|c|c|c|}
\hline \multicolumn{3}{|l|}{ Project: } & \multicolumn{3}{|c|}{$\begin{array}{ll}\text { Prepared By: } & \text { Larry A Henke } \\
\text { Company: } & \text { FGG }\end{array}$} \\
\hline \multicolumn{3}{|c|}{ Date Requested: $8 / 19 / 08$} & \multicolumn{3}{|c|}{ Requestor: Chris Wright (FH) } \\
\hline \multicolumn{3}{|c|}{ Date of Survey: $9 / 18 / 08$} & \multicolumn{3}{|c|}{ Surveyor: Larry A Henke (FGG) } \\
\hline \multicolumn{3}{|c|}{ ERC Point of Contact: } & \multicolumn{3}{|c|}{$\begin{array}{r}\text { Survey Co. Point of Contact: } \\
\text { Neil Fastabend (FGG) }\end{array}$} \\
\hline \multirow{4}{*}{\multicolumn{3}{|c|}{$\begin{array}{l}\text { Description of Work: } \\
\text { Civil Survey of Groundwater Monitoring } \\
\text { Well \#C6214 (399-3-31) }\end{array}$}} & \multicolumn{3}{|c|}{ Horizontal Datum: NAD83(91) } \\
\hline & & & \multicolumn{3}{|c|}{ Vertical Datum: NAVD88 } \\
\hline & & & \multicolumn{3}{|c|}{ Units: $\quad$ METERS } \\
\hline & & & \multicolumn{3}{|c|}{ Hanford Area Designation: $300 \mathrm{~A}$} \\
\hline \multicolumn{6}{|c|}{ Coordinate System: Washington State Plane Coordinates (South Zone) } \\
\hline \multicolumn{6}{|c|}{ Horizontal Control Monuments: N-323 (FGG), 300-70 (FGG) } \\
\hline \multicolumn{6}{|c|}{ Vertical Control Monuments: $300-28$ (FGG), $300-60(F G G)$} \\
\hline \multirow{5}{*}{$\begin{array}{l}\text { Well ID } \\
\text { C6214 } \\
\end{array}$} & Well Name & Easting & Northing & Elevation & \\
\hline & $399-3-31$ & 594246.7 & 116048.27 & & Center of Casing \\
\hline & & & & 114.787 & $\begin{array}{l}\text { Top Pump Base plate. } \\
\text { N. Edge }\end{array}$ \\
\hline & & & & 114.887 & $\begin{array}{l}\text { Top Lid Frame at } \\
\text { Stamped "X", N. Edge }\end{array}$ \\
\hline & & & & 114.886 & Brass Survey Marker \\
\hline \multicolumn{6}{|c|}{$\begin{array}{l}\text { Notes: } \\
\text { EQUIPMENT USED: TRIMBLE GPS } 5800 \text { RTK } \\
\text { TRIMBLE DiNi } 12 \text { LEVEL }\end{array}$} \\
\hline \multicolumn{6}{|c|}{$\begin{array}{l}\text { Surveyor Statement: } \\
\text { 1, Larry A. Henke, a Professional Land Surveyor registered } \\
\text { in the State of Washington (Registration No. 38975), hereby } \\
\text { certify that this report is based on a field survey performed in } \\
\text { September, } 2008 \text { under my direct supervision, and that the data } \\
\text { contained here is true and correct. }\end{array}$} \\
\hline
\end{tabular}

Original to:

Distribution by DIS: 


\section{WELL SURVEY DATA REPORT}

\begin{tabular}{|c|c|c|c|c|c|}
\hline \multicolumn{3}{|l|}{ Project: } & \multicolumn{3}{|c|}{$\begin{array}{l}\text { Prepared By: } \text { Larry A Henke } \\
\text { Company: } \quad \text { FGG }\end{array}$} \\
\hline \multicolumn{3}{|c|}{ Date Requested: $8 / 19 / 08$} & \multicolumn{3}{|c|}{ Requestor: Chris Wright (FH) } \\
\hline \multicolumn{3}{|c|}{ Date of Survey: $9 / 18 / 08$} & \multicolumn{3}{|c|}{ Surveyor: Larry A Henke (FGG) } \\
\hline \multicolumn{3}{|c|}{ ERC Point of Contact: } & \multicolumn{3}{|c|}{$\begin{array}{l}\text { Survey Co. Point of Contact: } \\
\text { Neil Fastabend (FGG) }\end{array}$} \\
\hline \multirow{4}{*}{\multicolumn{3}{|c|}{$\begin{array}{l}\text { Description of Work: } \\
\text { Civil Survey of Groundwater Monitoring } \\
\text { Well \#C6215 (399-3-32) }\end{array}$}} & \multicolumn{3}{|c|}{ Horizontal Datum: NAD83(91) } \\
\hline & & & \multicolumn{3}{|c|}{ Vertical Datum: NAVD88 } \\
\hline & & & \multicolumn{3}{|c|}{ Units: $\quad$ METERS } \\
\hline & & & \multicolumn{3}{|c|}{ Hanford Area Designation: $300 \mathrm{~A}$} \\
\hline \multicolumn{6}{|c|}{ Coordinate System: Washington State Plane Coordinates (South Zone) } \\
\hline \multicolumn{6}{|c|}{ Horizontal Control Monuments: N-323 (FGG), 300-70 (FGG) } \\
\hline \multicolumn{6}{|c|}{ Vertical Control Monuments: $300-28$ (FGG), $300-60(\mathrm{FGG})$} \\
\hline Well ID & Well Name & Easting & Northing & Elevation & \\
\hline \multirow[t]{4}{*}{ C6215 } & $399-3-32$ & 594249.0 & 116050.05 & & Center of Casing \\
\hline & & & & 114.746 & $\begin{array}{l}\text { Top Pump Base plate. } \\
\text { N. Edge }\end{array}$ \\
\hline & & & & 114.884 & $\begin{array}{l}\text { Top Lid Frame at } \\
\text { Stamped "X", N. Edge }\end{array}$ \\
\hline & & & & 114.878 & Brass Survey Marker \\
\hline \multicolumn{6}{|c|}{$\begin{array}{l}\text { Notes: } \\
\text { EQUIPMENT USED: TRIMBLE GPS } 5800 \text { RTK } \\
\text { TRIMBLE DiNi } 12 \text { LEVEL }\end{array}$} \\
\hline \multicolumn{6}{|c|}{$\begin{array}{l}\text { Surveyor Statement: } \\
\text { I, Larry A. Henke, a Professional Land Surveyor registered } \\
\text { in the State of Washington (Registration No. 38975), hereby } \\
\text { certify that this report is based on a field survey performed in } \\
\text { September, } 2008 \text { under my direct supervision, and that the data } \\
\text { contained here is true and correct. }\end{array}$} \\
\hline
\end{tabular}

Original to:

Distribution by DIS: 
WELL SURVEY DATA REPORT

\begin{tabular}{|c|c|}
\hline Project: & $\begin{array}{l}\text { Prepared By: } \\
\text { Company: }\end{array}$ \\
\hline Date Requested: $8 / 19 / 08$ & Requestor: Chris Wright (FH) \\
\hline Date of Survey: $9 / 18 / 08$ & Surveyor: Larry A Henke (FGG) \\
\hline ERC Point of Contact: & $\begin{array}{r}\text { Survey Co. Point of Contact: } \\
\text { Neil Fastabend (FGG) }\end{array}$ \\
\hline \multirow{4}{*}{$\begin{array}{l}\text { Description of Work: } \\
\text { Civil Survey of Groundwater Monitoring } \\
\text { Well \#C6216 (399-2-29) }\end{array}$} & Horizontal Datum: NAD83(91) \\
\hline & Vertical Datum: NAVD88 \\
\hline & METERS \\
\hline & Hanford Area Designation: $300 \mathrm{~A}$ \\
\hline \multicolumn{2}{|c|}{ Coordinate System: Washington State Plane Coordinates (South Zone) } \\
\hline \multicolumn{2}{|c|}{ Horizontal Control Monuments: N-323 (FGG), 300-70 (FGG) } \\
\hline
\end{tabular}

\begin{tabular}{|l|c|c|c|c|l|}
\hline Well ID & Well Name & Easting & Northing & Elevation & \\
\hline C6216 & $399-2-29$ & 594270.82 & 116068.13 & & Center of Casing \\
\hline & & & & 114.792 & $\begin{array}{l}\text { Top Pump Base plate. } \\
\text { N. .dge }\end{array}$ \\
\hline & & & & 114.889 & $\begin{array}{l}\text { Top Lid Frame at } \\
\text { Stamped "X", N. Edge } \\
\text { Brass Survey Marker }\end{array}$ \\
\hline & & & & 114.891 & \\
\hline
\end{tabular}

Notes:

EQUIPMENT USED: TRIMBLE GPS 5800 RTK TRIMBLE DiNi 12 LEVEL

\section{Surveyor Statement:}

I, Larry A. Henke, a Professional Land Surveyor registered in the State of Washington (Registration No. 38975), hereby certify that this report is based on a field survey performed in September, 2008 under my direct supervision, and that the data contained here is true and correct.

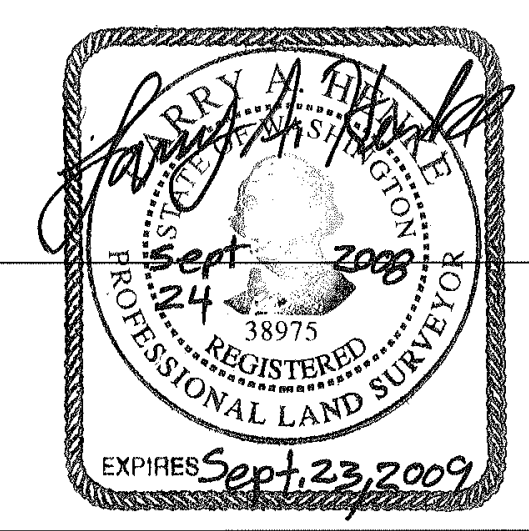

Original to:

Distribution by DIS: 
WELL SURVEY DATA REPORT

\begin{tabular}{|c|c|c|c|c|c|}
\hline \multicolumn{3}{|l|}{ Project: } & \multicolumn{3}{|c|}{$\begin{array}{l}\text { Prepared By: } \\
\text { Comparry A Henke } \\
\text { FGG }\end{array}$} \\
\hline \multicolumn{3}{|c|}{ Date Requested: $8 / 19 / 08$} & \multicolumn{3}{|c|}{ Requestor: Chris Wright (FH) } \\
\hline \multicolumn{3}{|c|}{ Date of Survey: $9 / 18 / 08$} & \multicolumn{3}{|c|}{ Surveyor: Larry A Henke (FGG) } \\
\hline \multicolumn{3}{|c|}{ ERC Point of Contact: } & \multicolumn{3}{|c|}{$\begin{array}{r}\text { Survey Co. Point of Contact: } \\
\text { Neil Fastabend (FGG) }\end{array}$} \\
\hline \multirow{4}{*}{\multicolumn{3}{|c|}{$\begin{array}{l}\text { Description of Work: } \\
\text { Civil Survey of Groundwater Monitoring } \\
\text { Well \#C6217 (399-2-30) }\end{array}$}} & \multicolumn{3}{|c|}{ Horizontal Datum: NAD83(91) } \\
\hline & & & \multicolumn{3}{|c|}{ Vertical Datum: NAVD88 } \\
\hline & & & \multicolumn{3}{|c|}{ Units: $\quad$ METERS } \\
\hline & & & \multicolumn{3}{|c|}{ Hanford Area Designation: $300 \mathrm{~A}$} \\
\hline \multicolumn{6}{|c|}{ Coordinate System: Washington State Plane Coordinates (South Zone) } \\
\hline \multicolumn{6}{|c|}{ Horizontal Control Monuments: $\mathrm{N}-323(\mathrm{FGG}), 300-70(\mathrm{FGG})$} \\
\hline \multicolumn{6}{|c|}{ Vertical Control Monuments: $300-28$ (FGG), 300-60(FGG) } \\
\hline Well ID & Well Name & Easting & Northing & Elevation & \\
\hline C6217 & $399-2-30$ & 594271.18 & 116065.52 & & Center of Casing \\
\hline & & & & 114.827 & $\begin{array}{l}\text { Top Pump Base plate. } \\
\text { N. Edge }\end{array}$ \\
\hline & & & & 114.914 & $\begin{array}{l}\text { Top Lid Frame at } \\
\text { Stamped " } \mathrm{K} \text { ", N. Edge }\end{array}$ \\
\hline & & & & 114.913 & Brass Survey Marker \\
\hline \multicolumn{6}{|c|}{$\begin{array}{l}\text { Notes: } \\
\text { EQUIPMENT USED: TRIMBLE GPS } 5800 \text { RTK } \\
\text { TRIMBLE DiNi } 12 \text { LEVEL }\end{array}$} \\
\hline \multicolumn{6}{|c|}{$\begin{array}{l}\text { Surveyor Statement: } \\
\text { 1, Larry A. Henke, a Professional Land Surveyor registered } \\
\text { in the State of Washington (Registration No. 38975), hereby } \\
\text { certify that this report is based on a field survey performed in } \\
\text { September, } 2008 \text { under my direct supervision, and that the data } \\
\text { contained here is true and correct. }\end{array}$} \\
\hline
\end{tabular}

Original to:

Distribution by DIS: 


\section{WELL SURVEY DATA REPORT}

\begin{tabular}{|c|c|c|c|c|c|}
\hline \multicolumn{3}{|l|}{ Project: } & \multicolumn{3}{|c|}{$\begin{array}{l}\text { Prepared By: Larry A Henke } \\
\text { Company: } \quad \text { FGG }\end{array}$} \\
\hline \multicolumn{3}{|c|}{ Date Requested: $8 / 19 / 08$} & \multicolumn{3}{|c|}{ Requestor: Chris Wright (FH) } \\
\hline \multicolumn{3}{|c|}{ Date of Survey: $9 / 18 / 08$} & \multicolumn{3}{|c|}{ Surveyor: Larry A Henke (FGG) } \\
\hline \multicolumn{3}{|c|}{ ERC Point of Contact: } & \multicolumn{3}{|c|}{$\begin{array}{r}\text { Survey Co. Point of Contact: } \\
\text { Neil Fastabend (FGG) }\end{array}$} \\
\hline \multirow{4}{*}{\multicolumn{3}{|c|}{$\begin{array}{l}\text { Description of Work: } \\
\text { Civil Survey of Groundwater Monitoring } \\
\text { Well \#C6218 (399-2-31) }\end{array}$}} & \multicolumn{3}{|c|}{ Horizontal Datum: NAD83(91) } \\
\hline & & & \multicolumn{3}{|c|}{ Vertical Datum: NAVD88 } \\
\hline & & & \multicolumn{3}{|c|}{ Units: $\quad$ METERS } \\
\hline & & & \multicolumn{3}{|c|}{ Hanford Area Designation: $300 \mathrm{~A}$} \\
\hline \multicolumn{6}{|c|}{ Coordinate System: Washington State Plane Coordinates (South Zone) } \\
\hline \multicolumn{6}{|c|}{ Horizontal Control Monuments: $\quad \mathrm{N}-323$ (FGG), 300-70 (FGG) } \\
\hline \multicolumn{6}{|c|}{ Vertical Control Monuments: $300-28$ (FGG), 300-60(FGG) } \\
\hline Well ID & Well Name & Easting & Northing & Elevation & \\
\hline \multirow[t]{4}{*}{ C6218 } & $399-2-31$ & 594273. & 116067.24 & & Center of Casing \\
\hline & & & & 114.788 & $\begin{array}{l}\text { Top Pump Base plate. } \\
\text { N. Edge }\end{array}$ \\
\hline & & & & 114.894 & $\begin{array}{l}\text { Top Lid Frame at } \\
\text { Stamped " } X \text { ", N. Edge }\end{array}$ \\
\hline & & & & 114.894 & Brass Survey Marker \\
\hline \multicolumn{6}{|c|}{$\begin{array}{l}\text { Notes: } \\
\text { EQUIPMENT USED: TRIMBLE GPS } 5800 \text { RTK } \\
\text { TRIMBLE DiNi } 12 \text { LEVEL }\end{array}$} \\
\hline \multicolumn{6}{|c|}{$\begin{array}{l}\text { Surveyor Statement: } \\
\text { I, Larry A. Henke, a Professional Land Surveyor registered } \\
\text { in the State of Washington (Registration No. 38975), hereby } \\
\text { certify that this report is based on a field survey performed in } \\
\text { September, } 2008 \text { under my direct supervision, and that the data } \\
\text { contained here is true and correct. }\end{array}$} \\
\hline
\end{tabular}

Original to:

Distribution by DIS: 
Appendix I

Chip Tray Photographs 


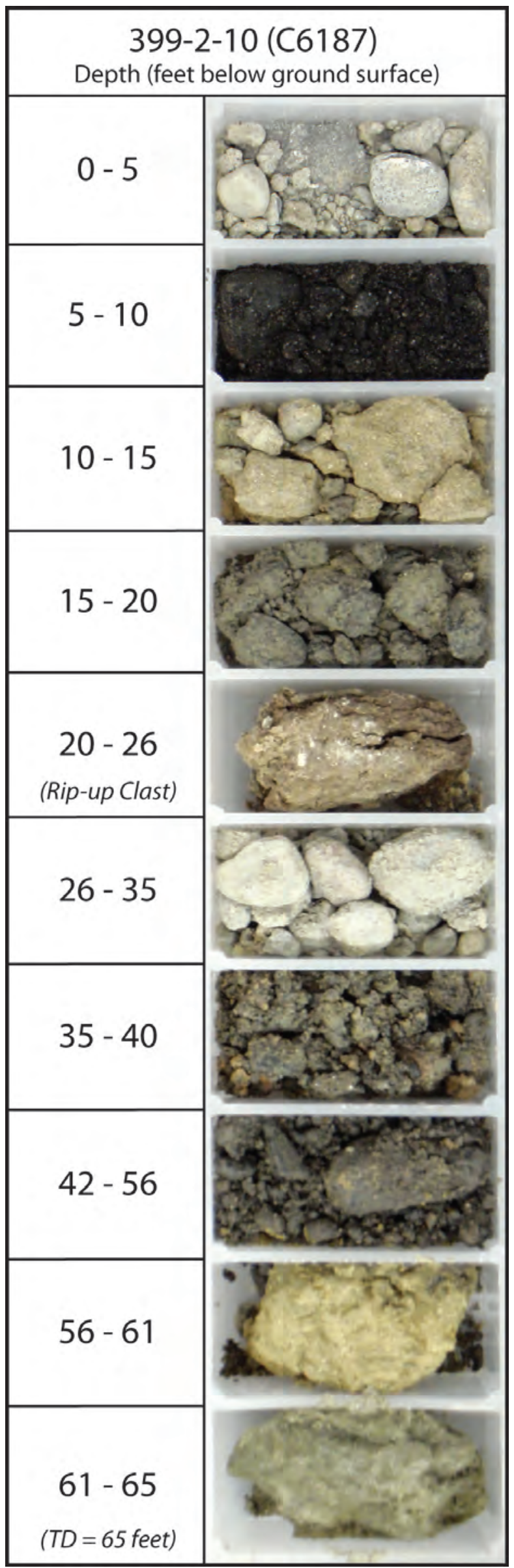




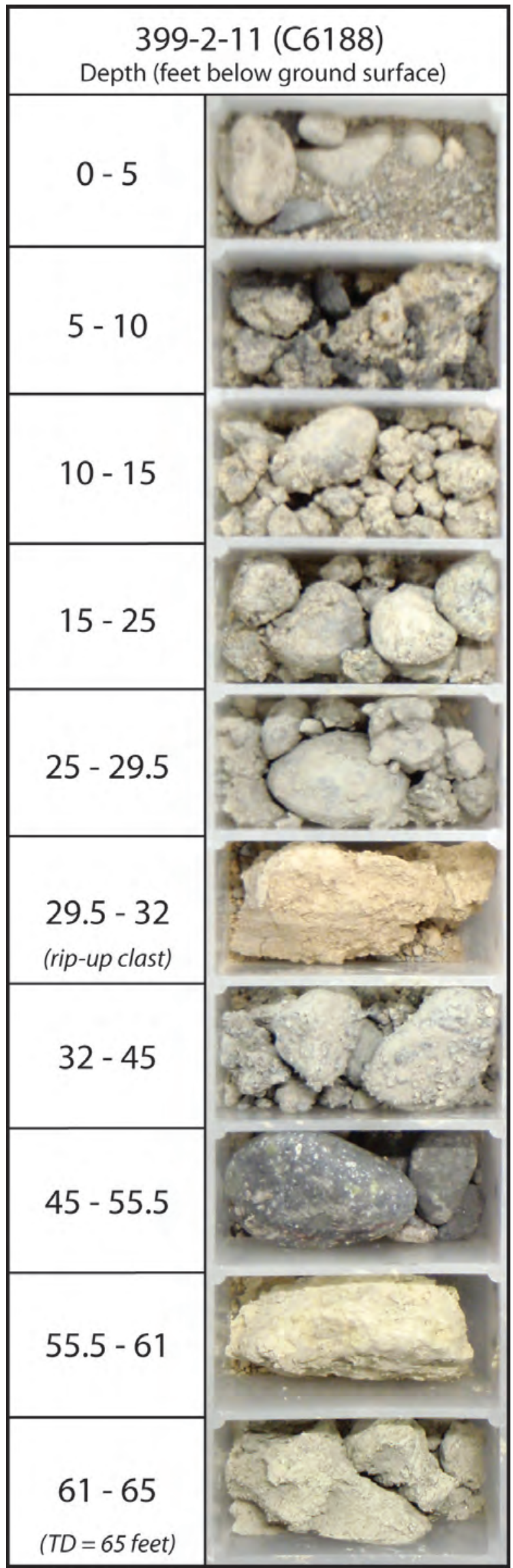




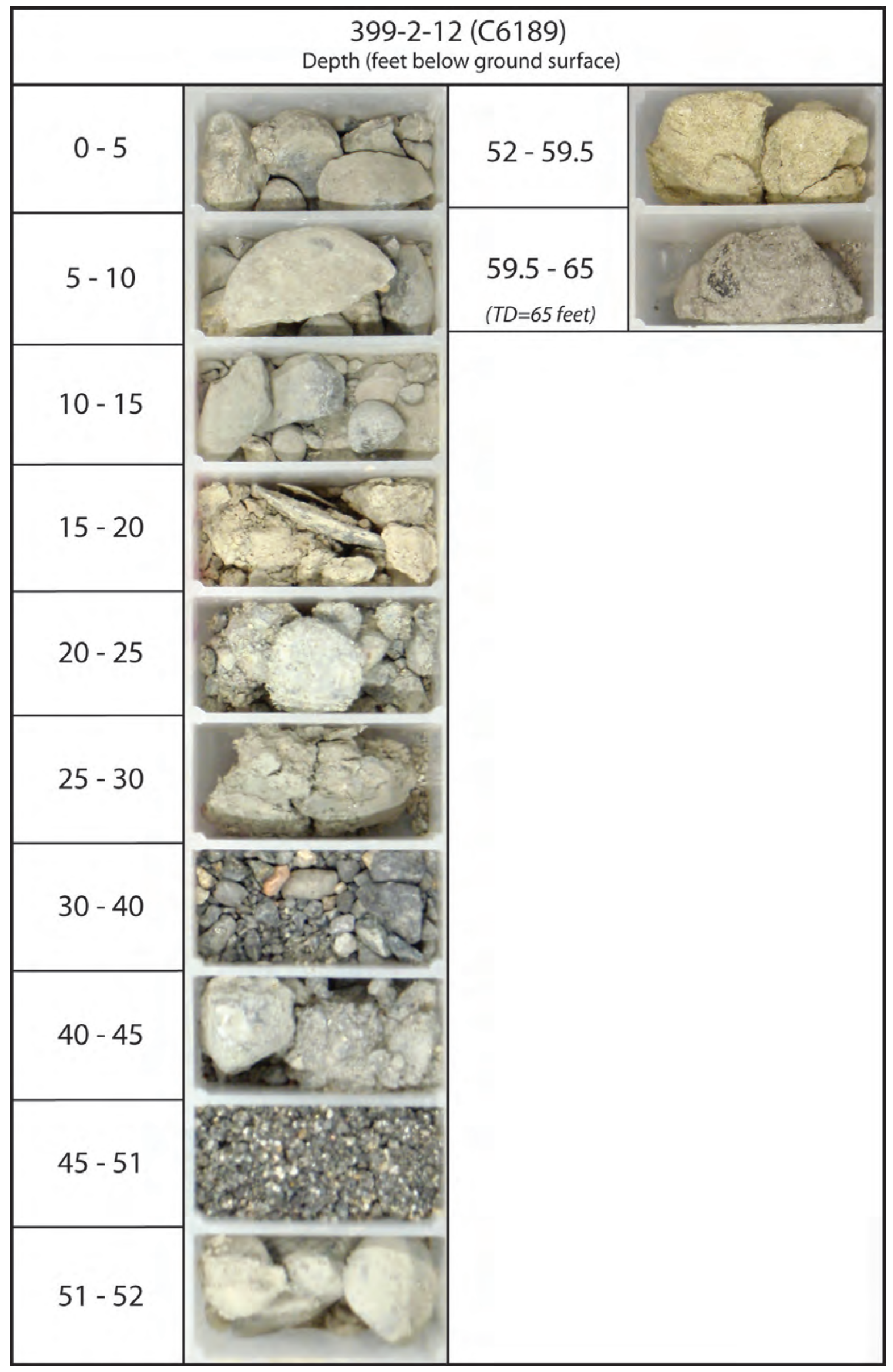




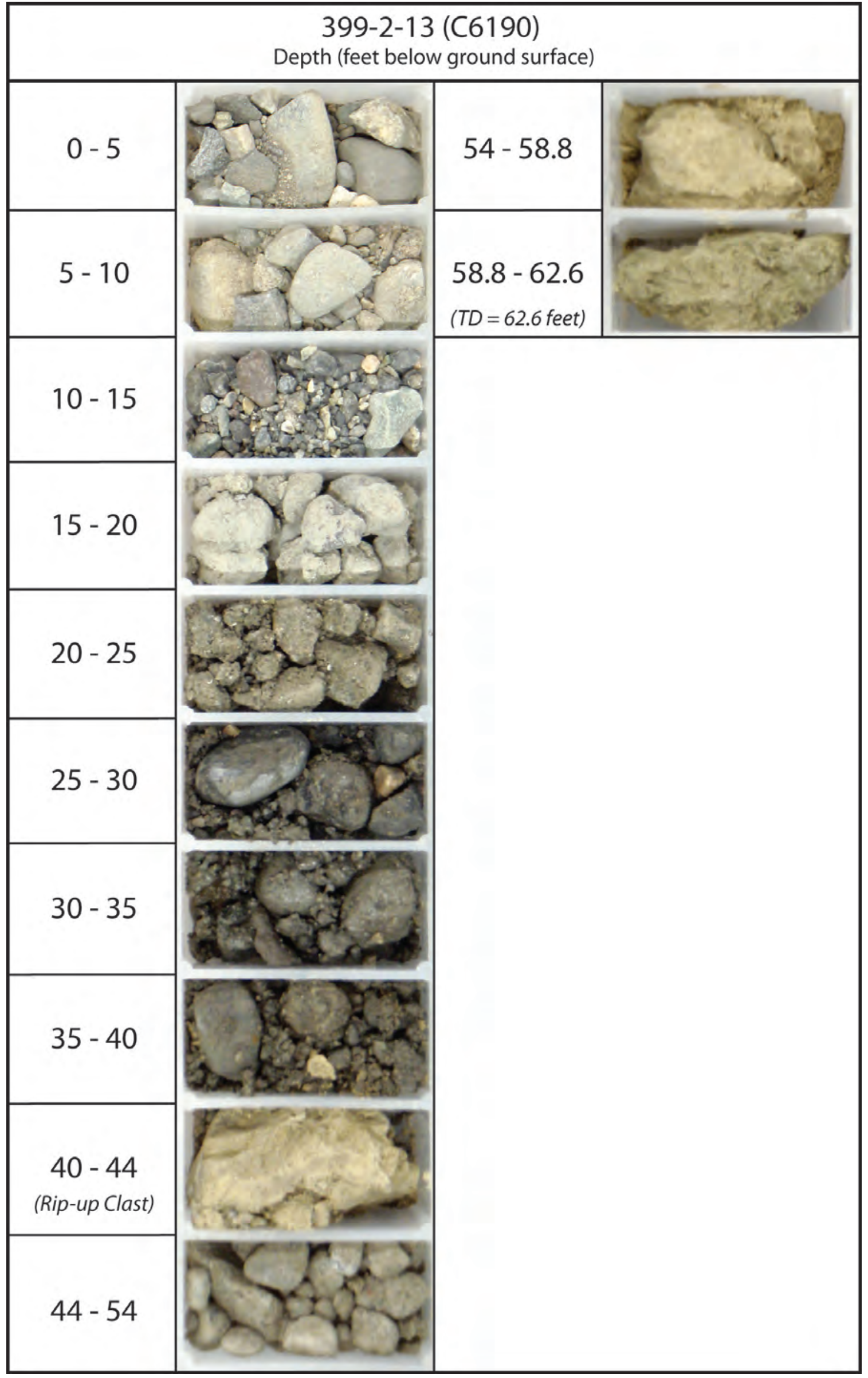




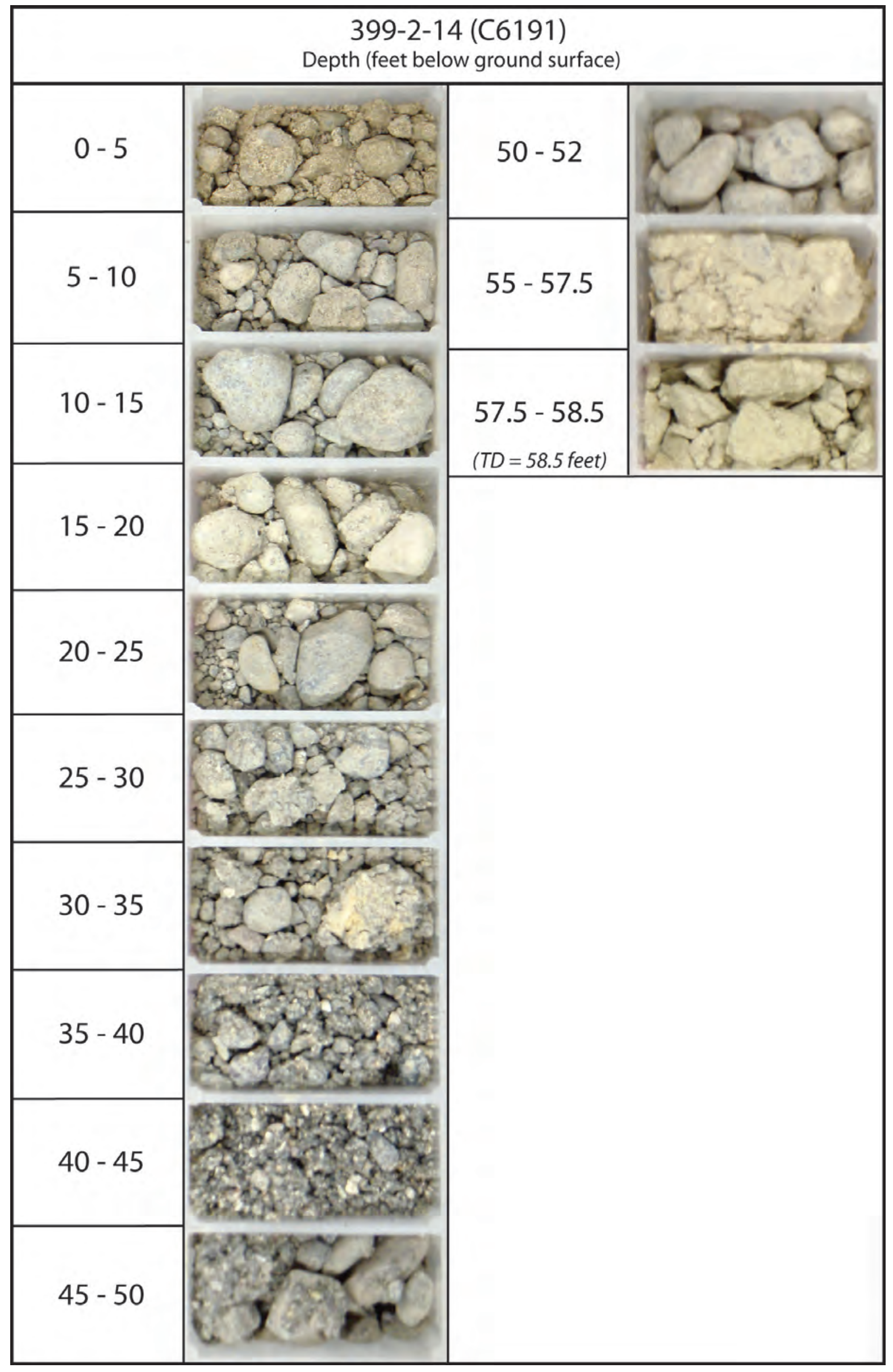




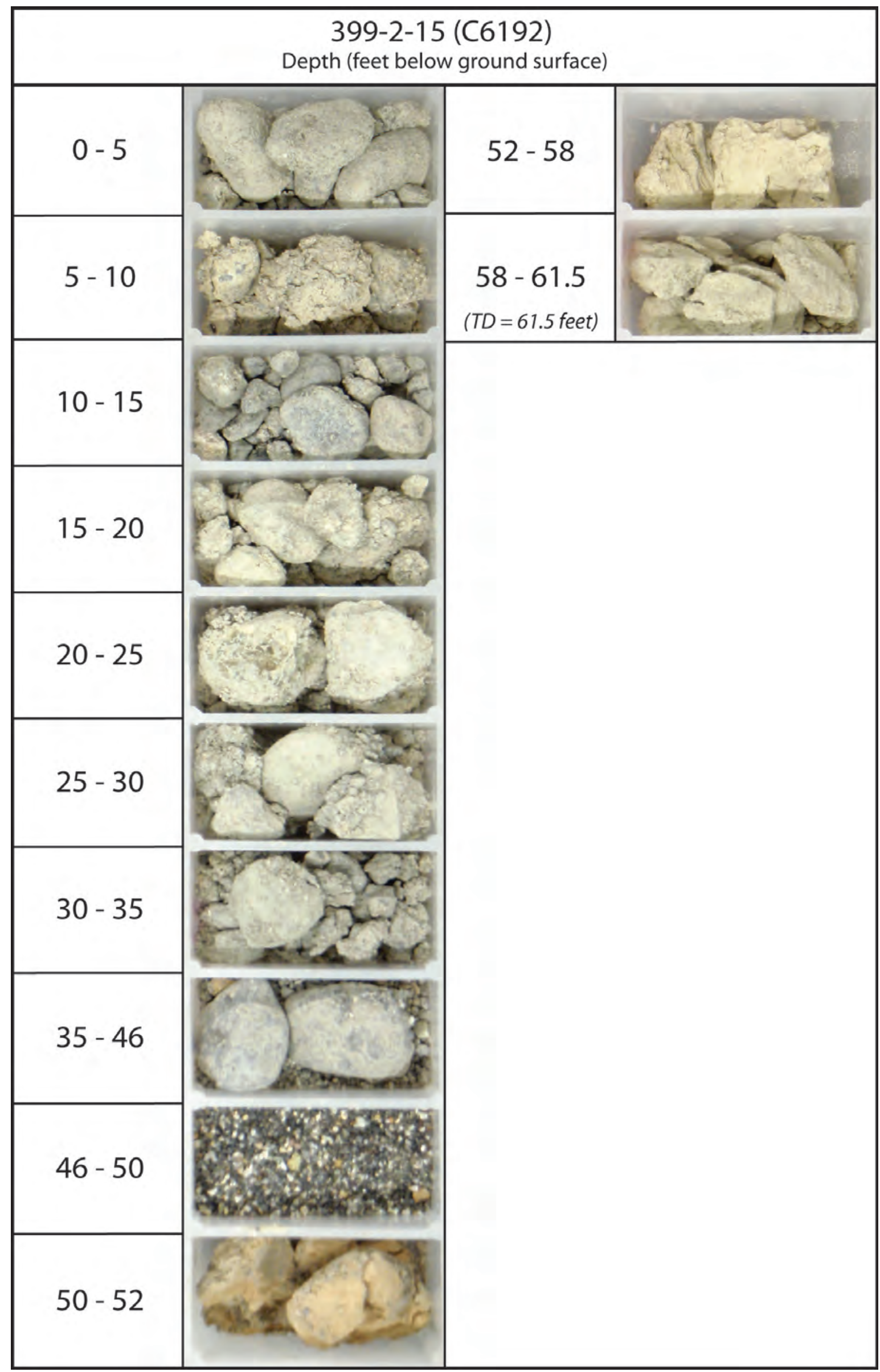




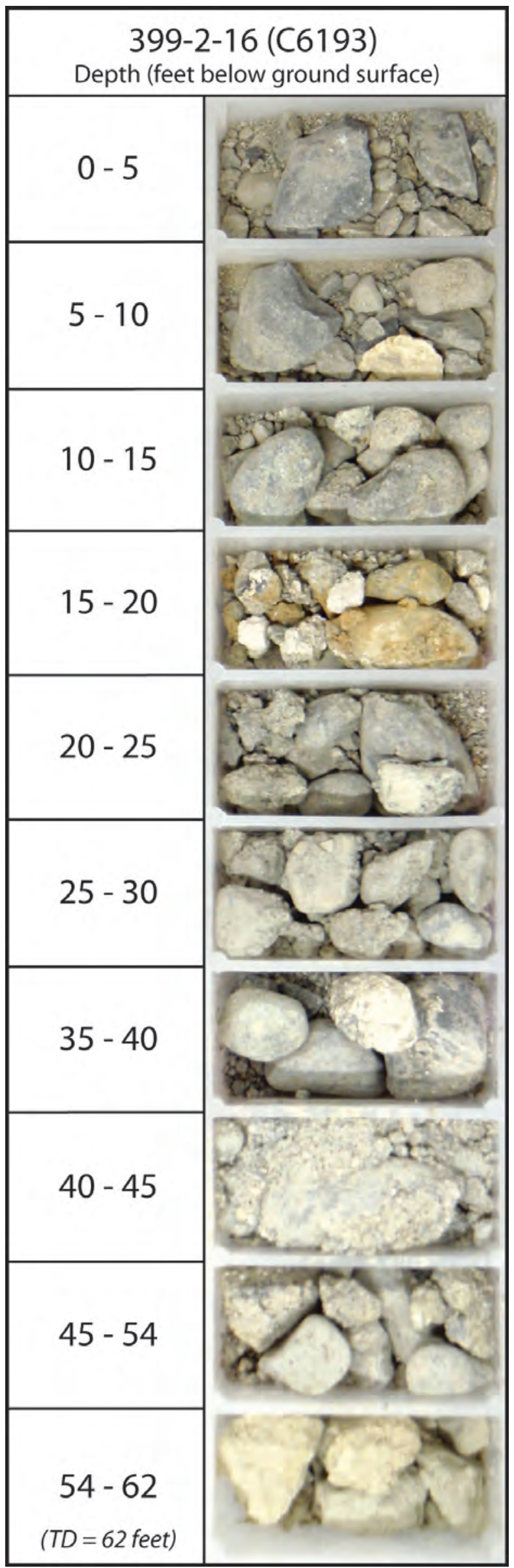




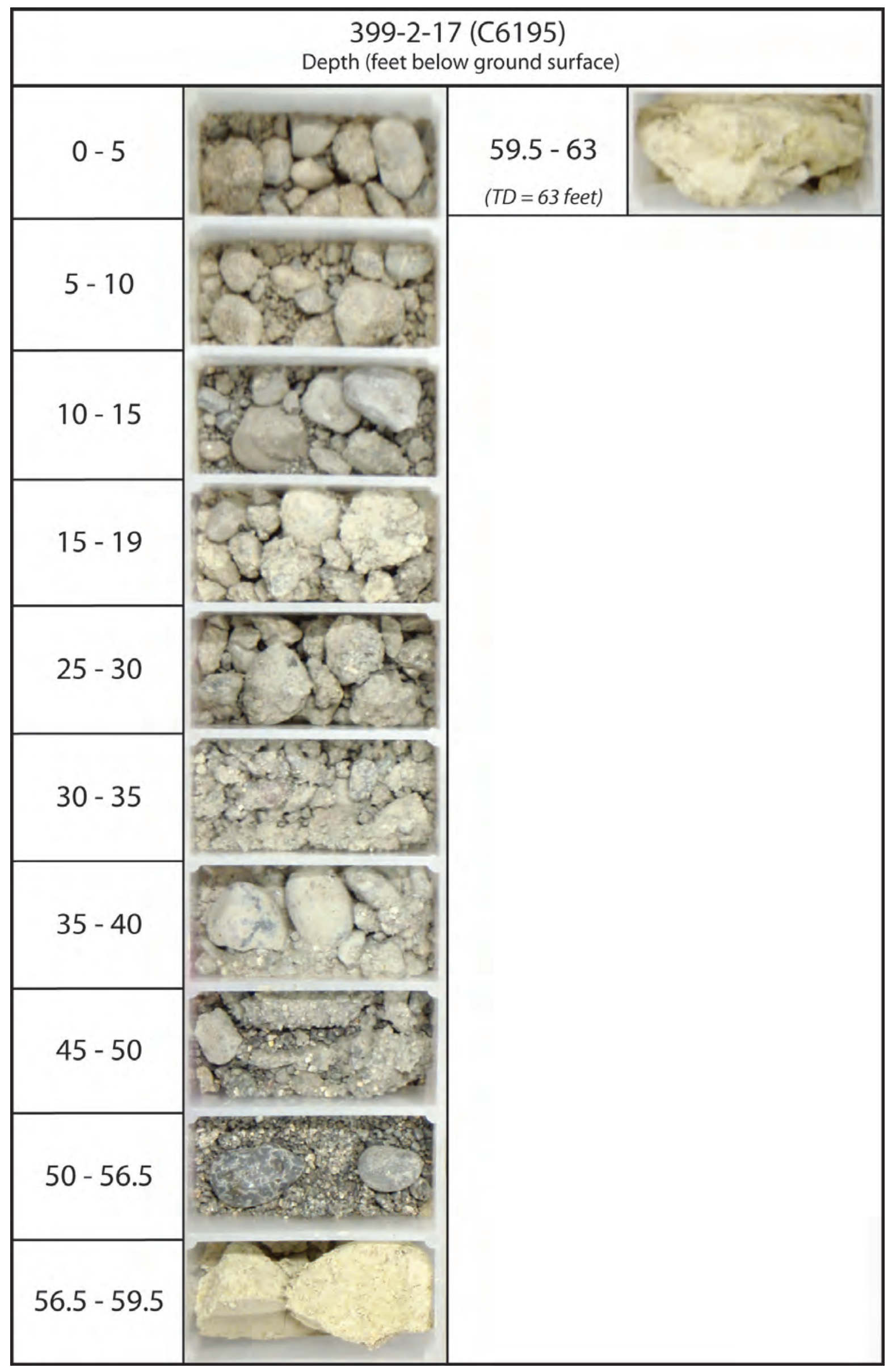




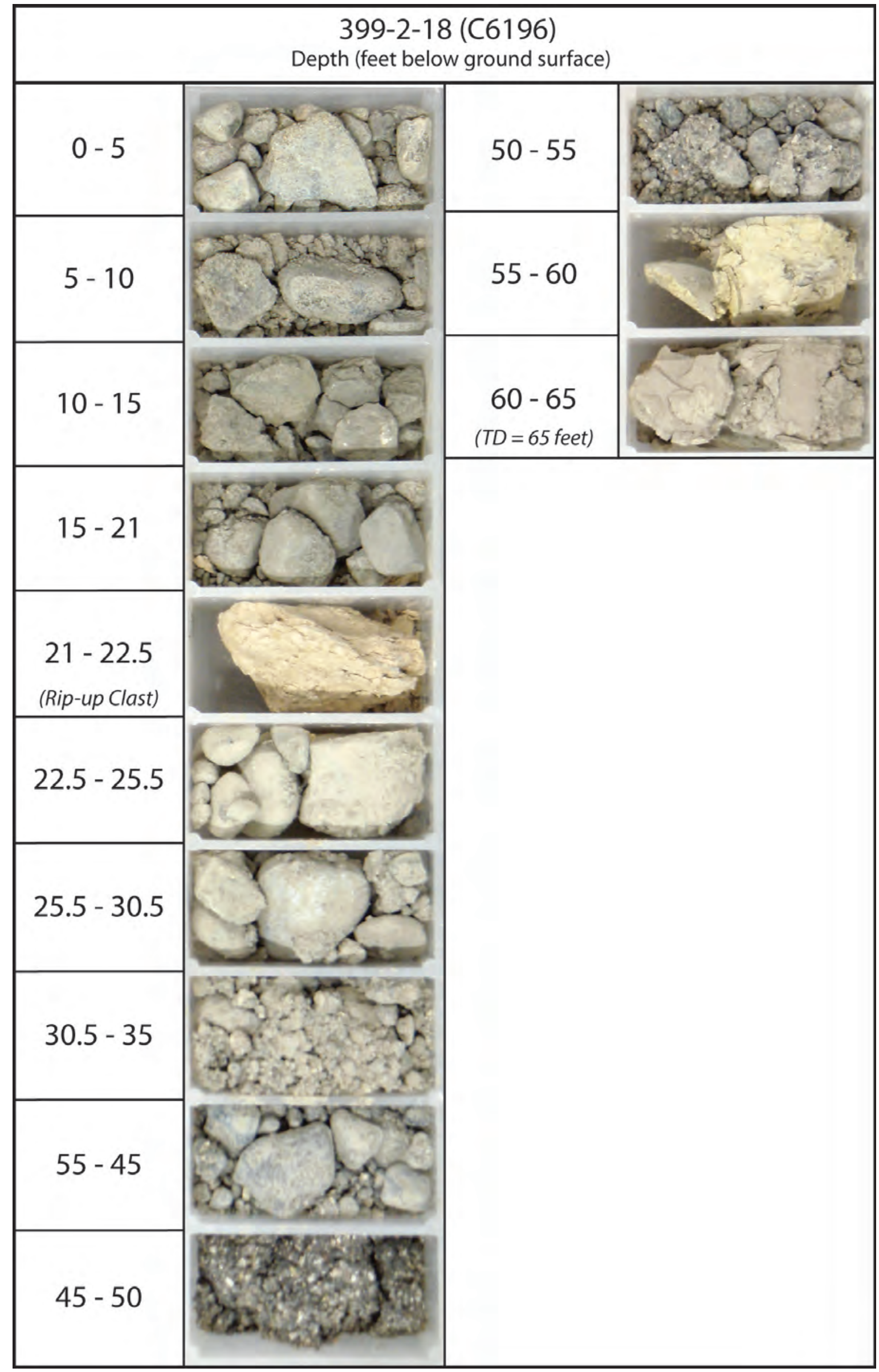




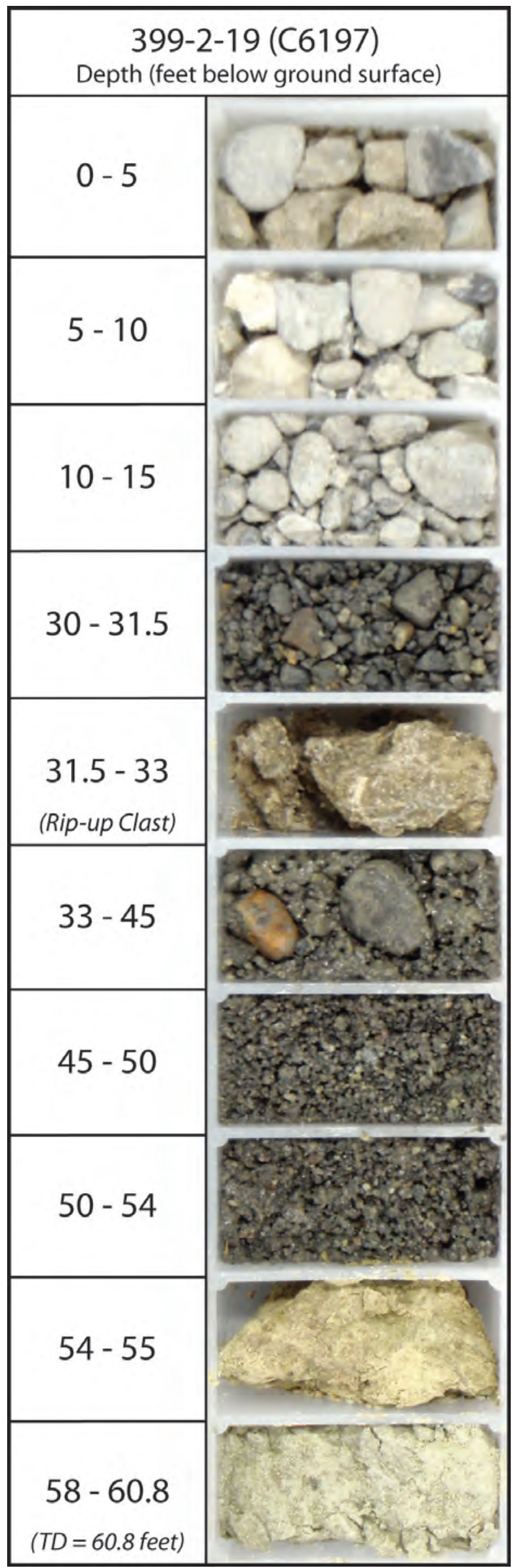

I.10 


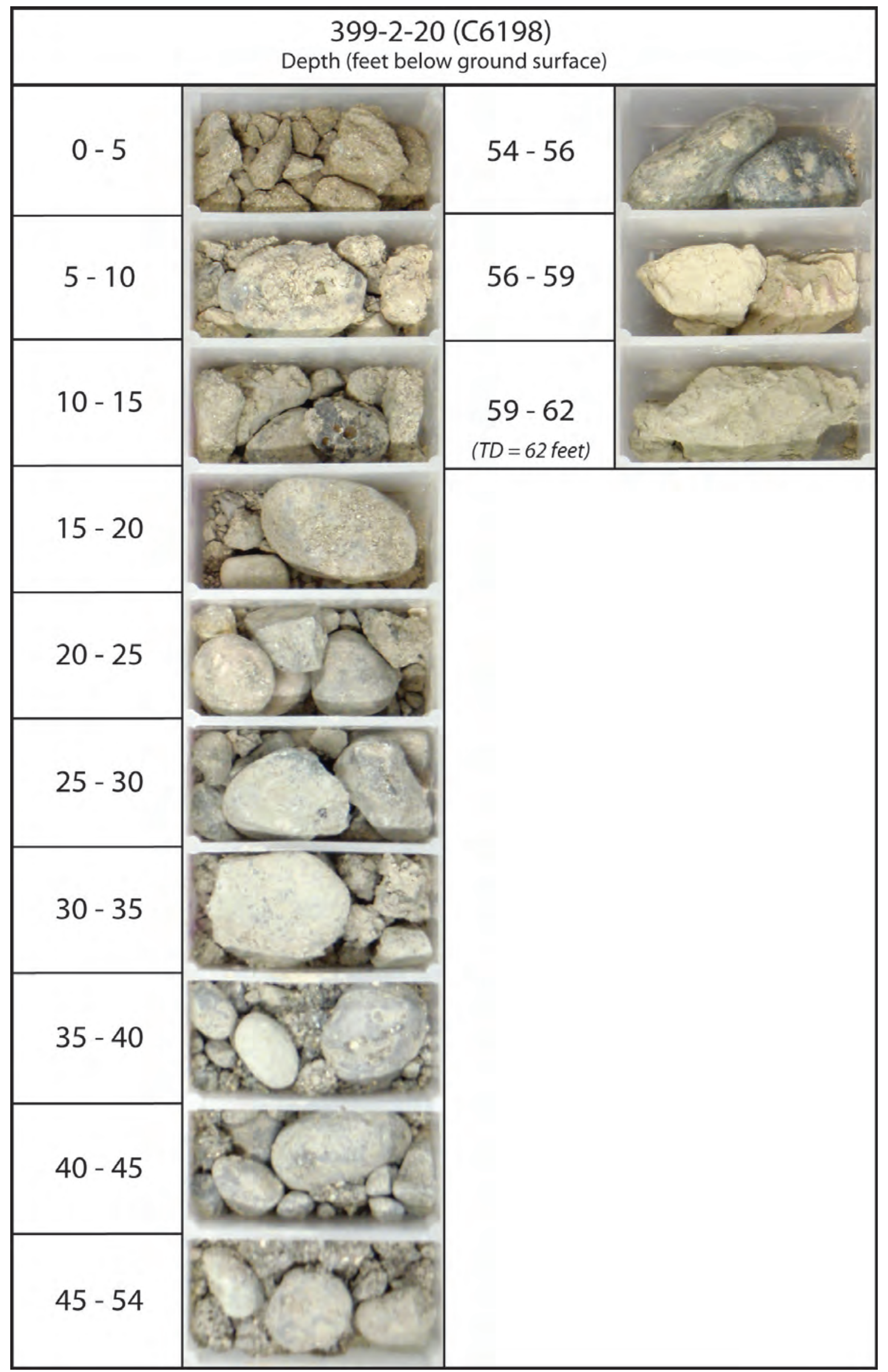




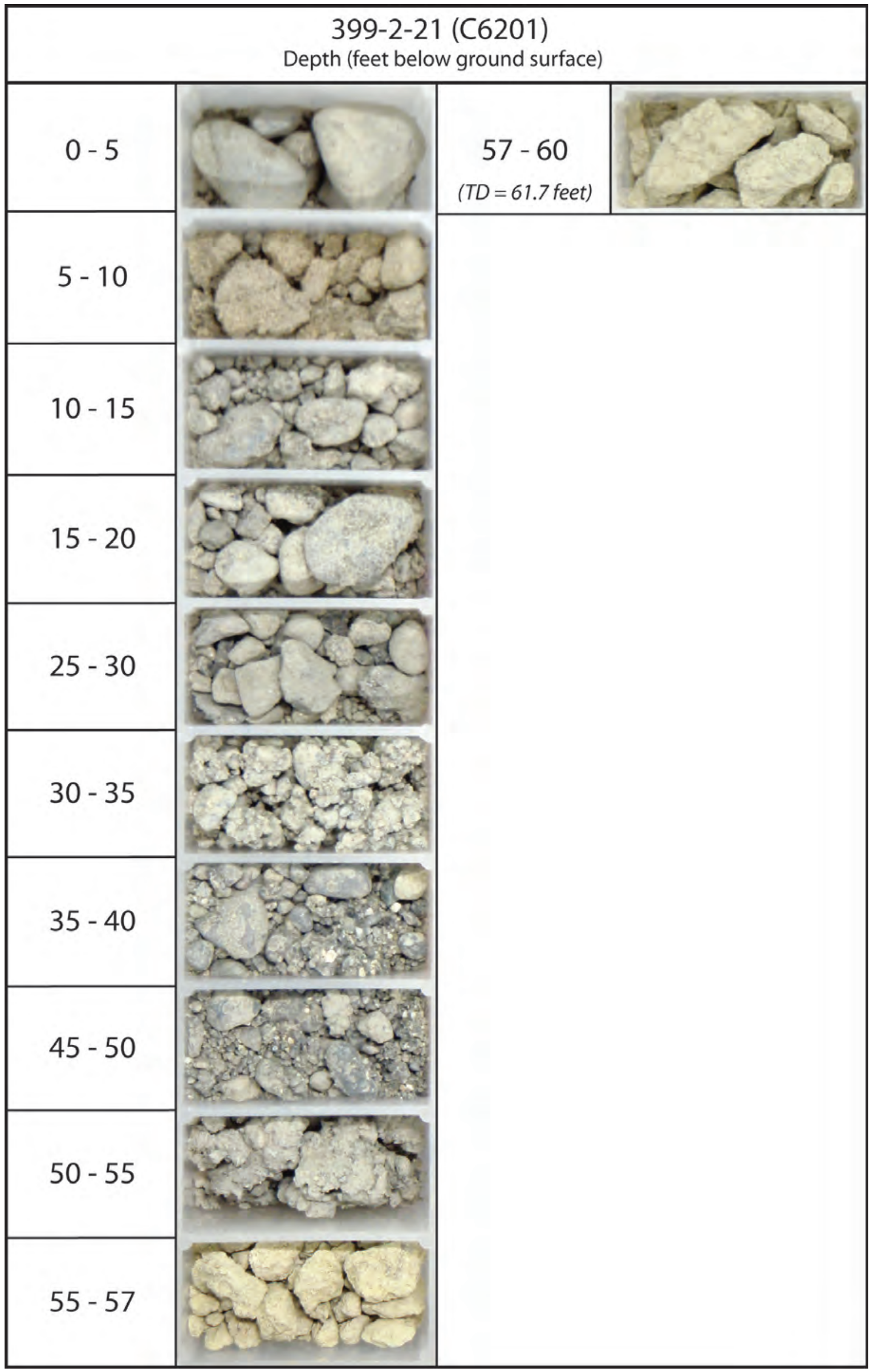




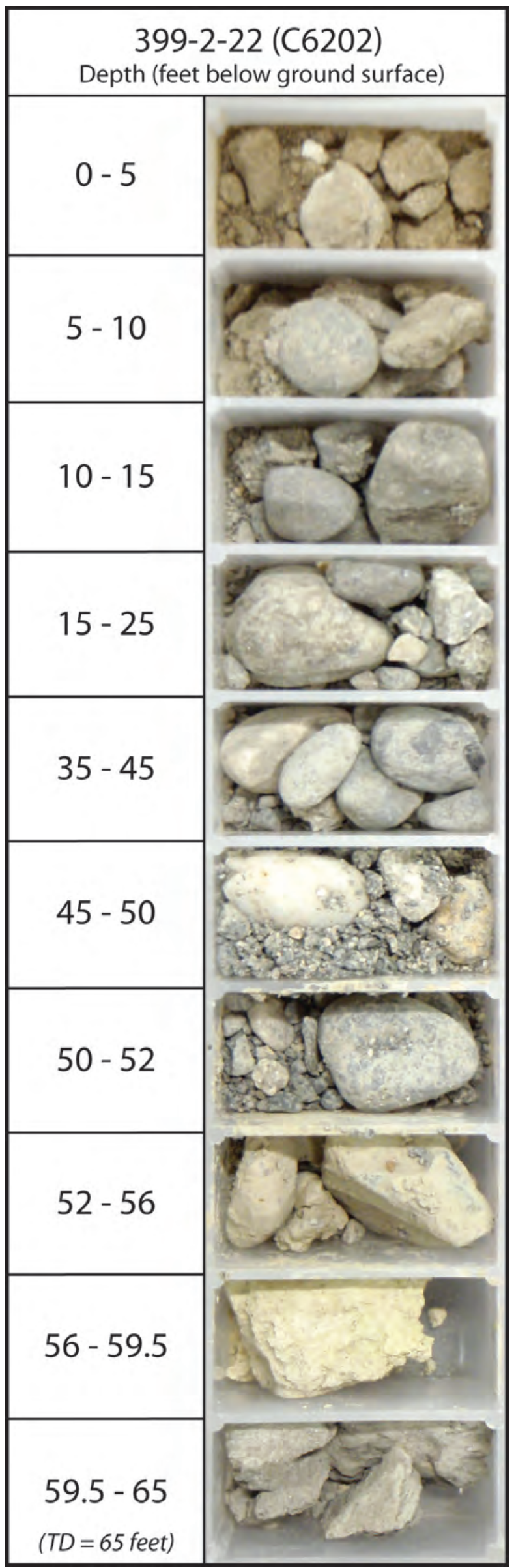




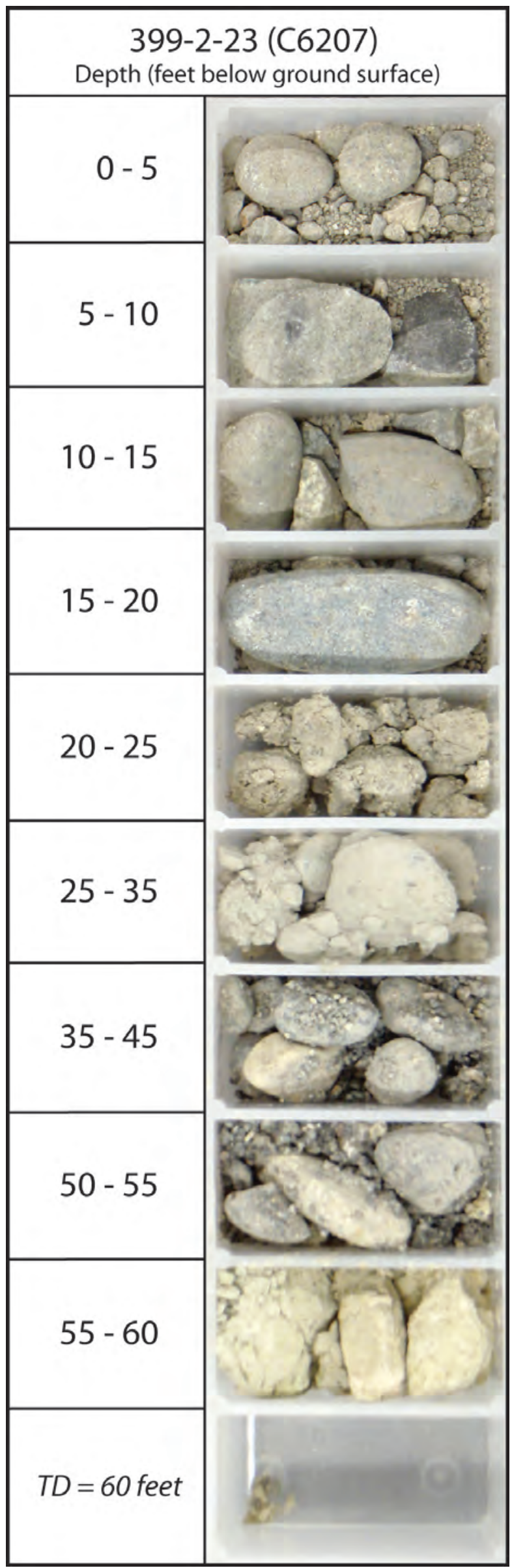




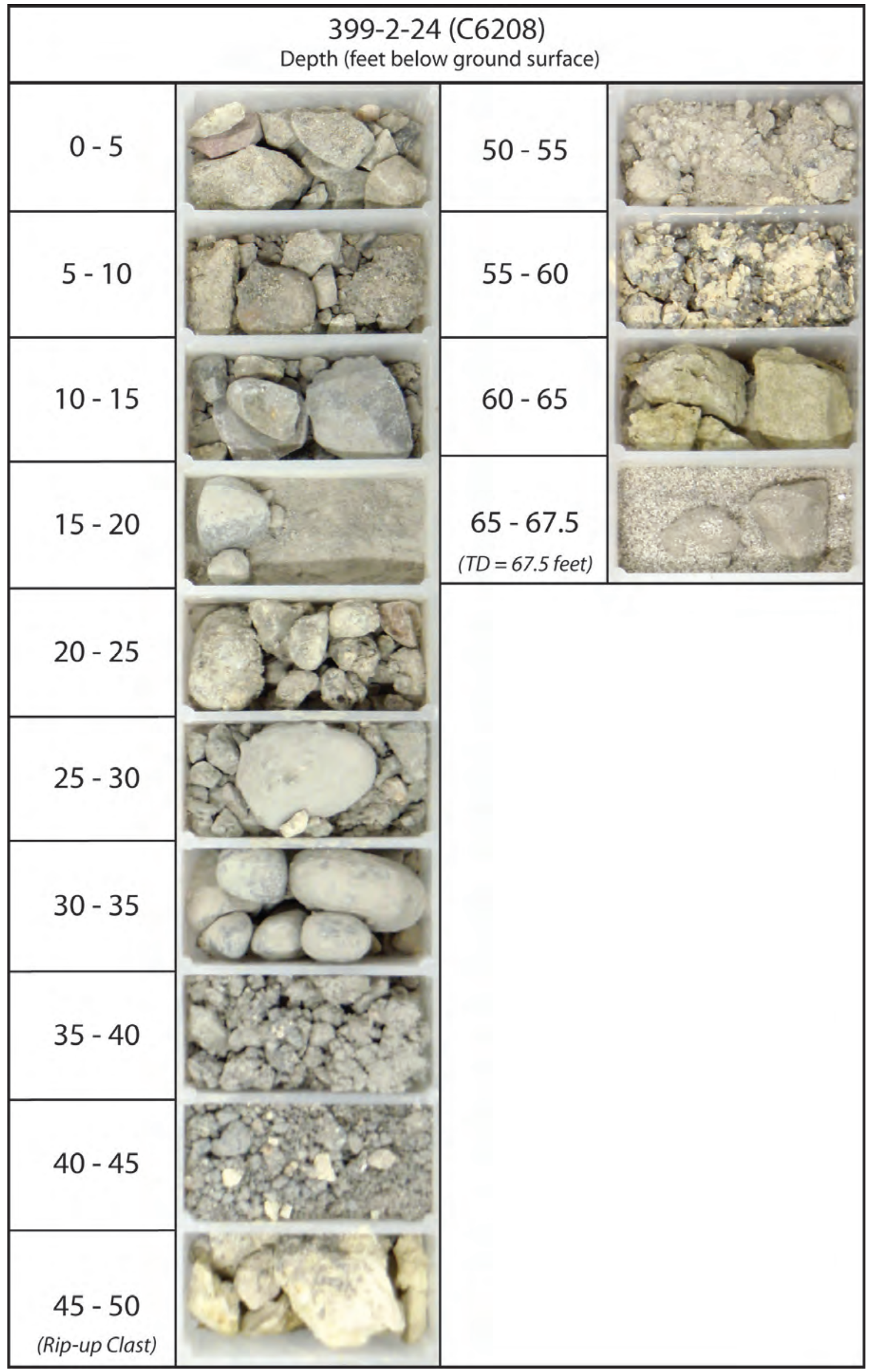




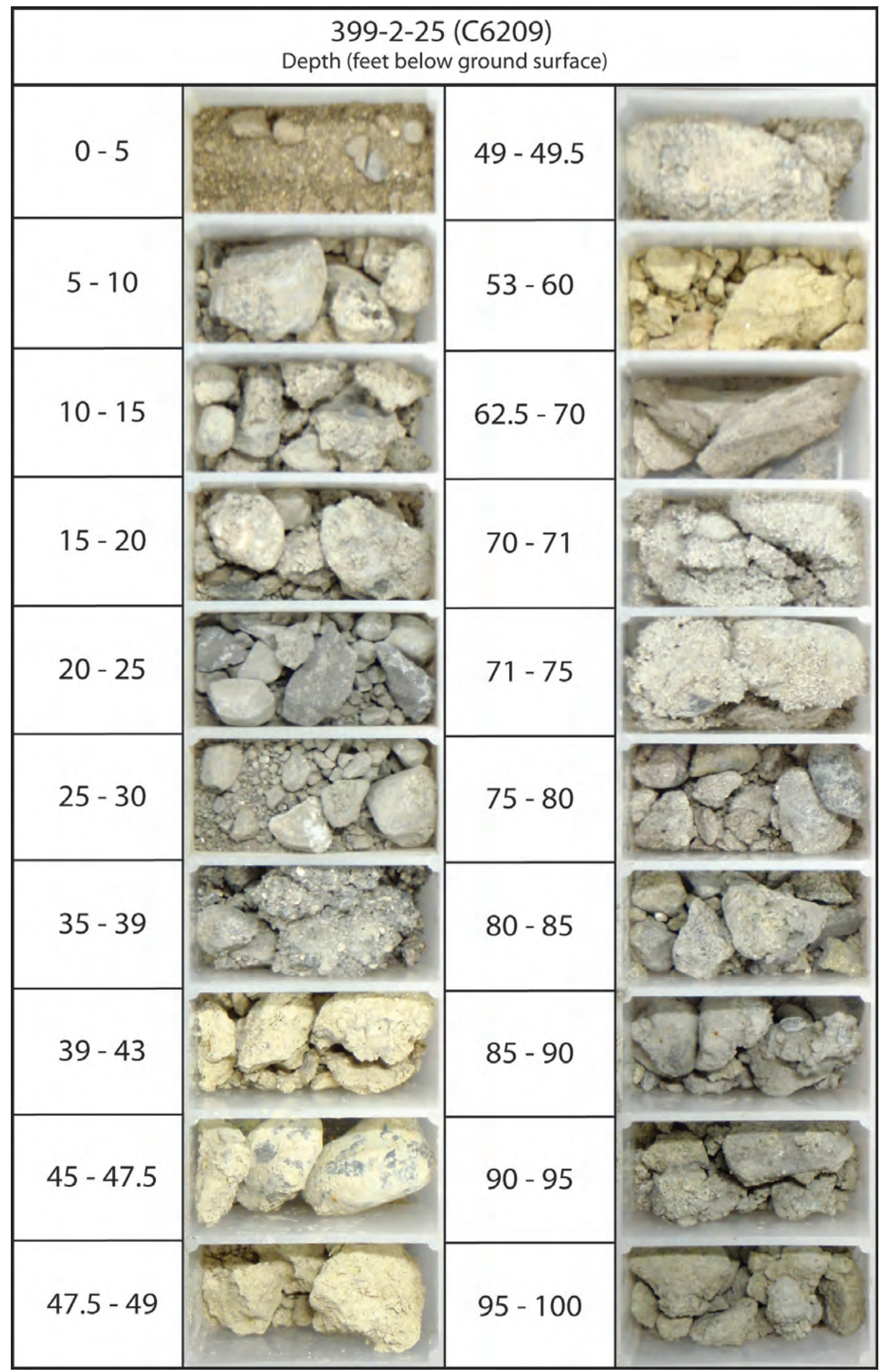




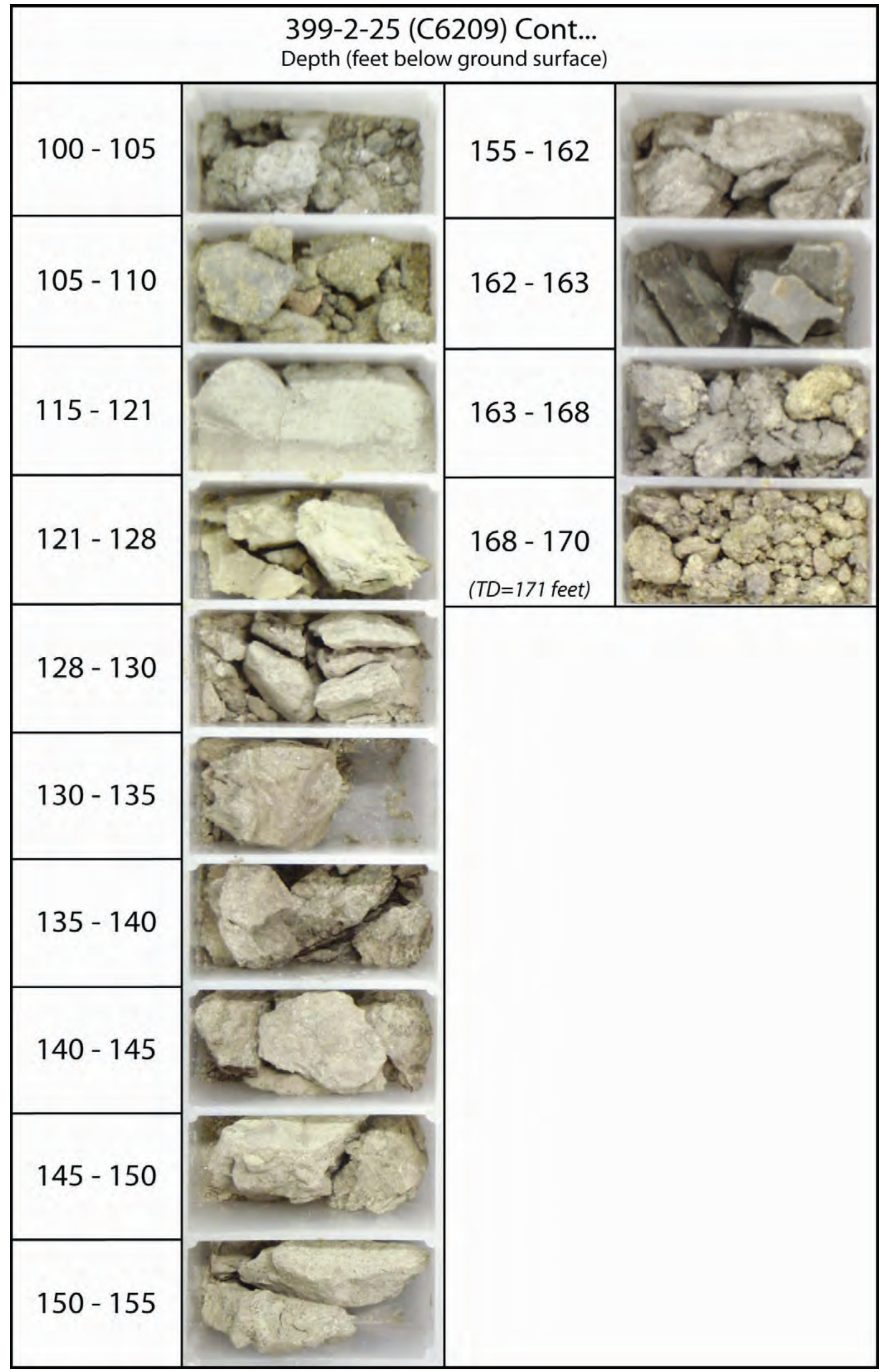




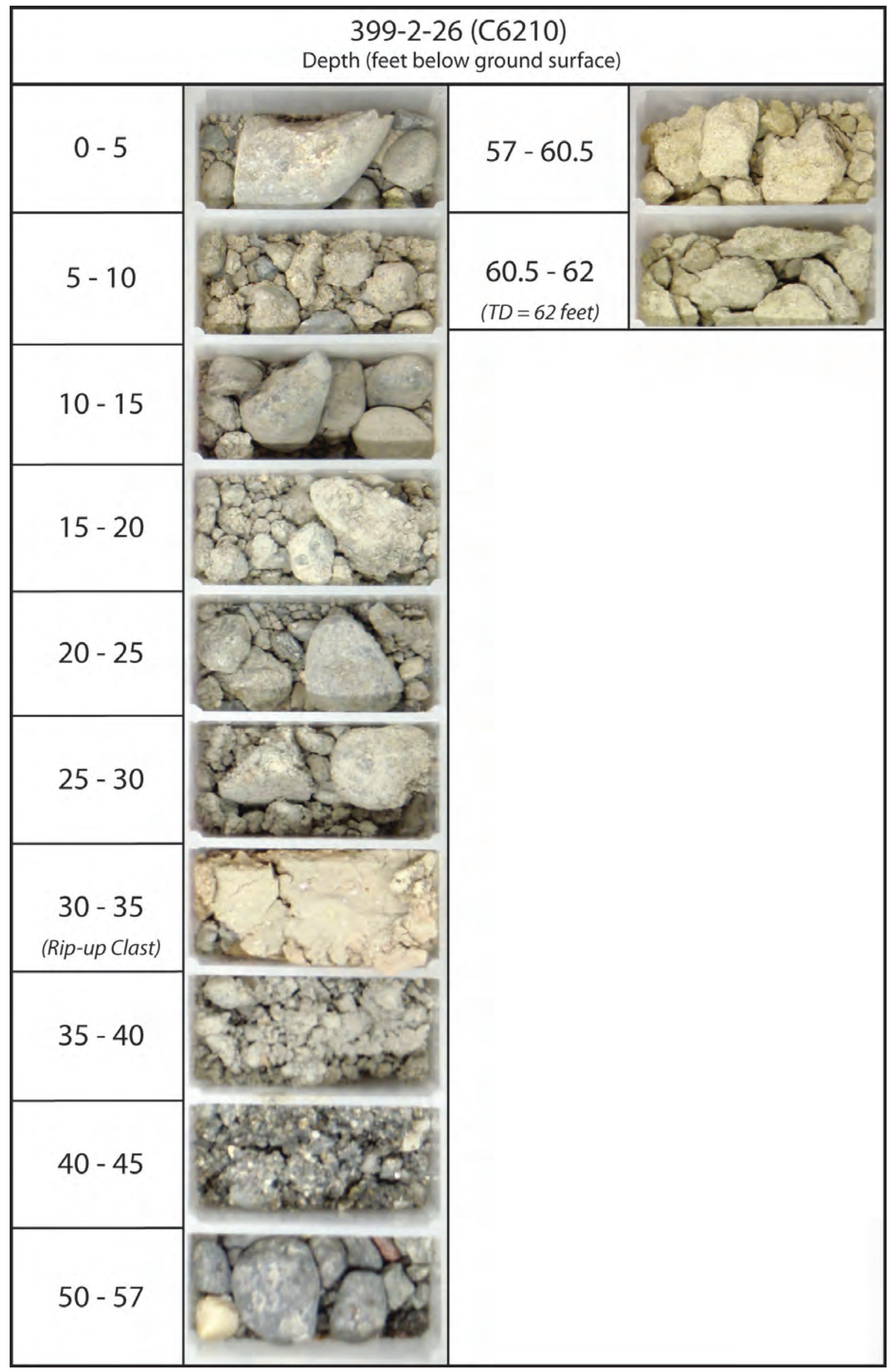




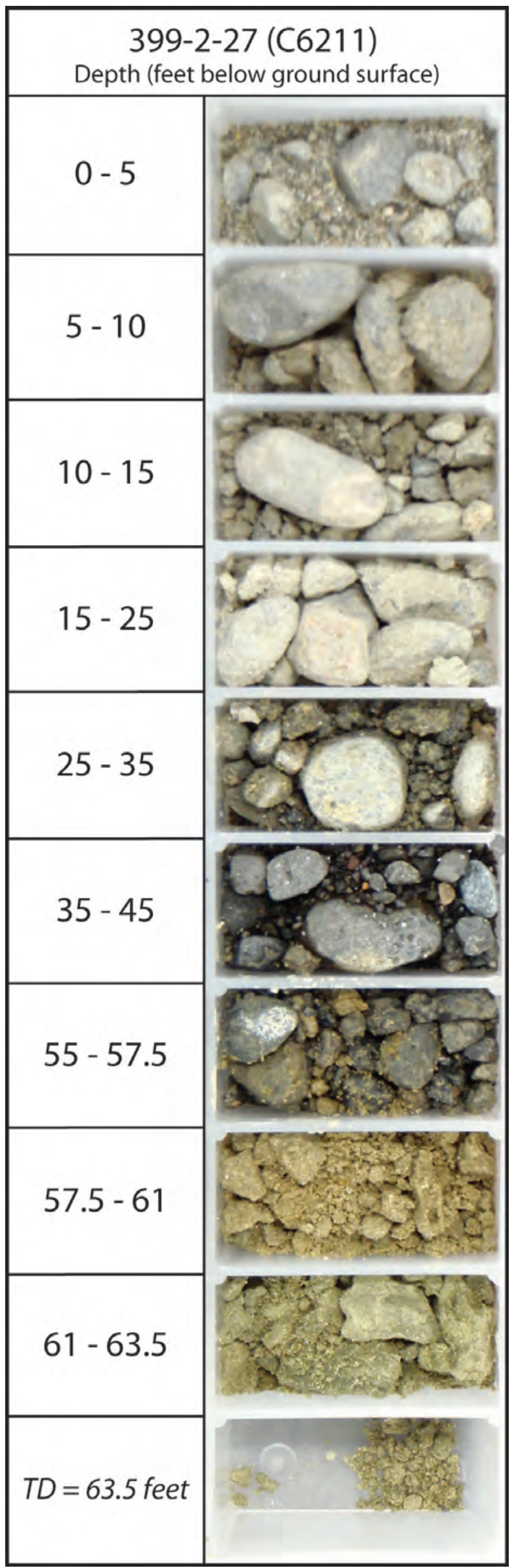

I.19 


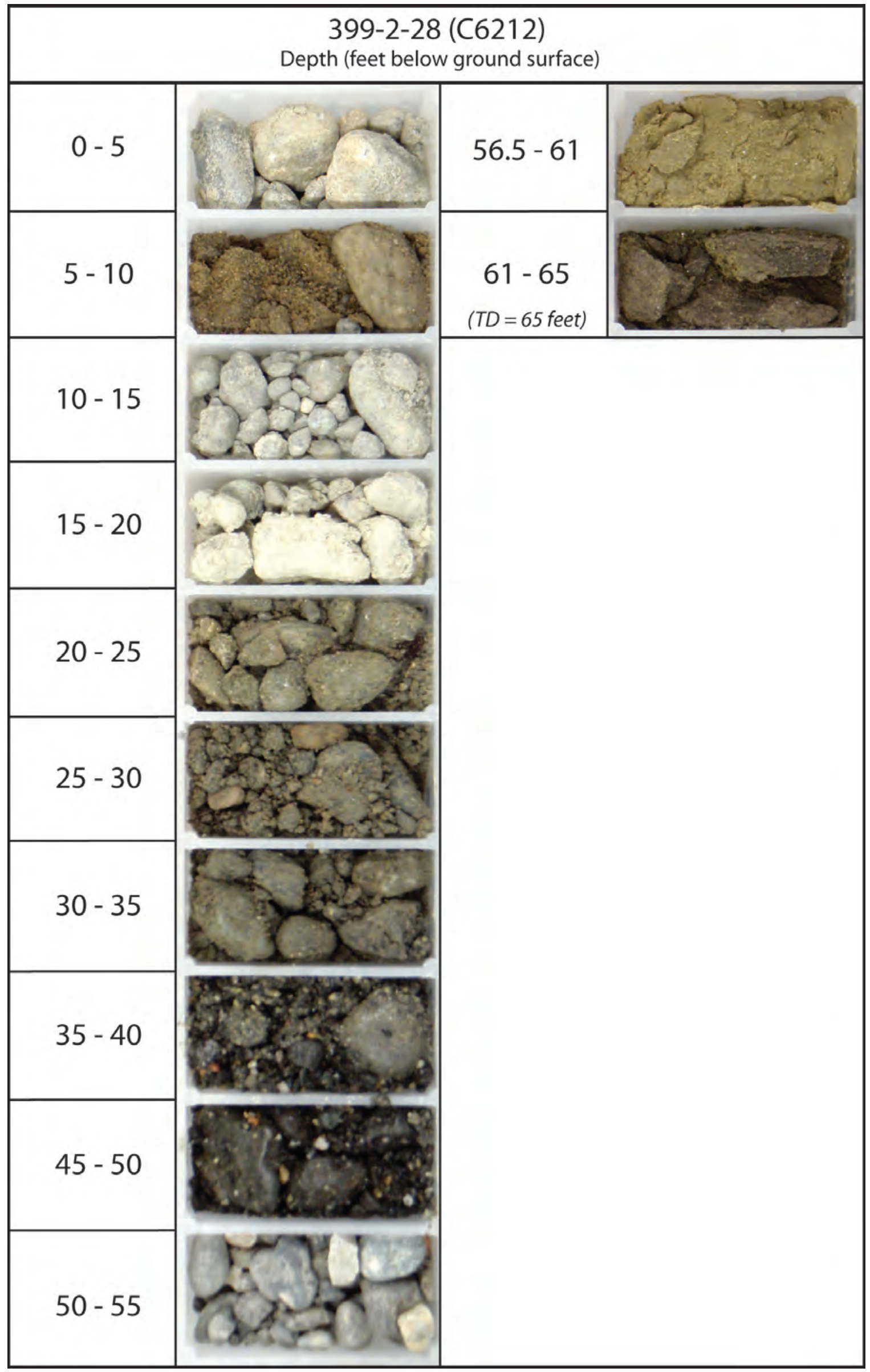




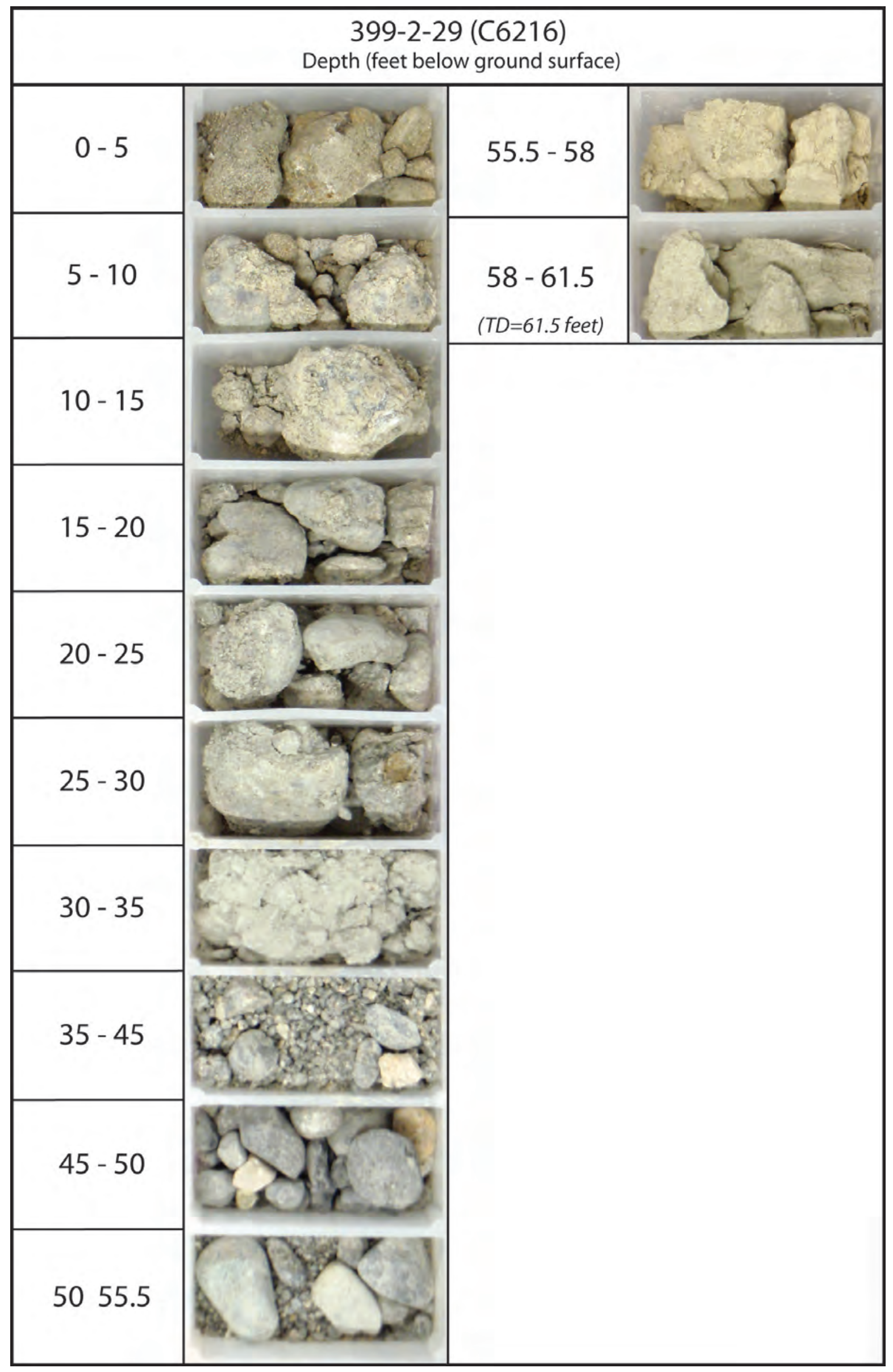




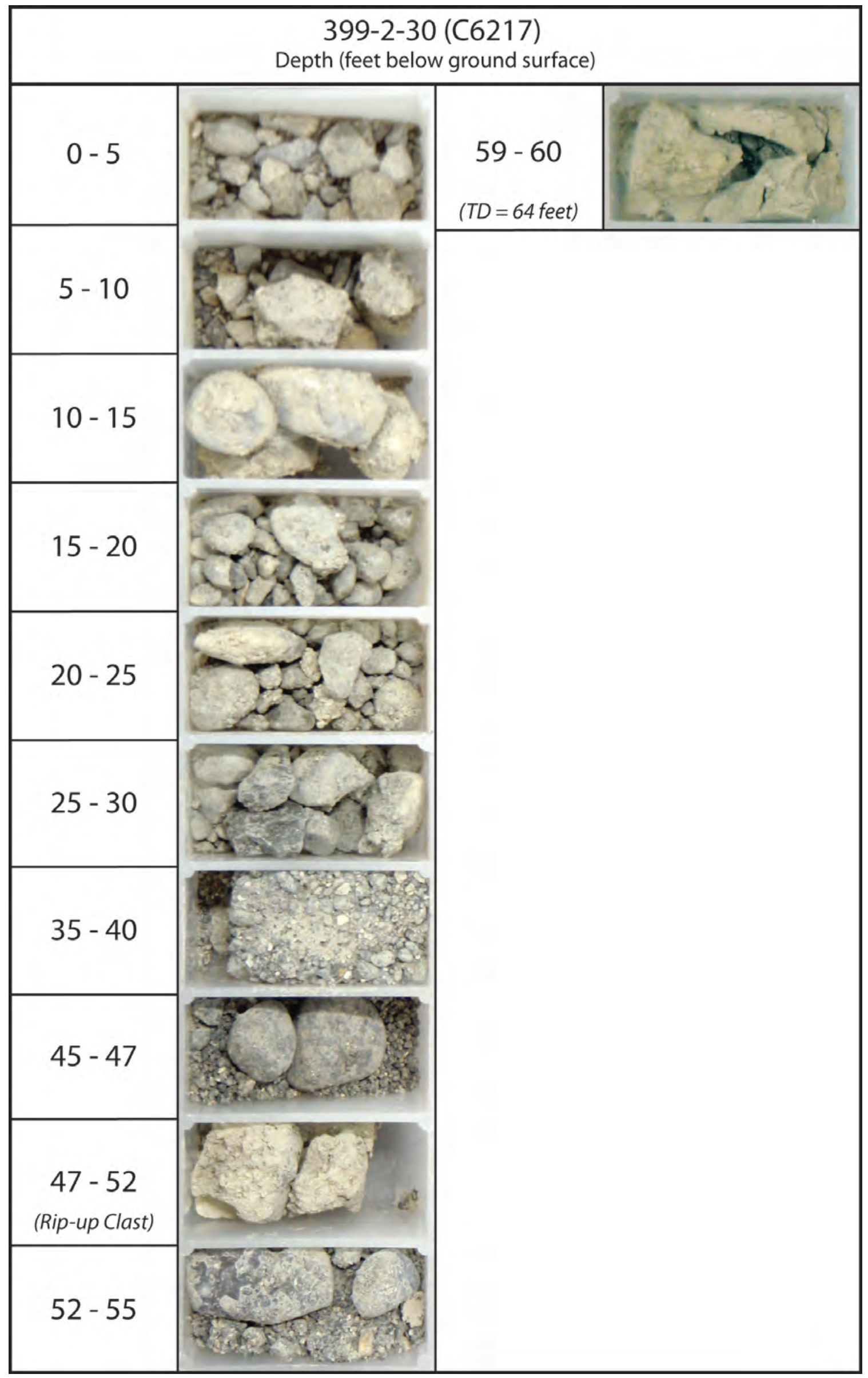




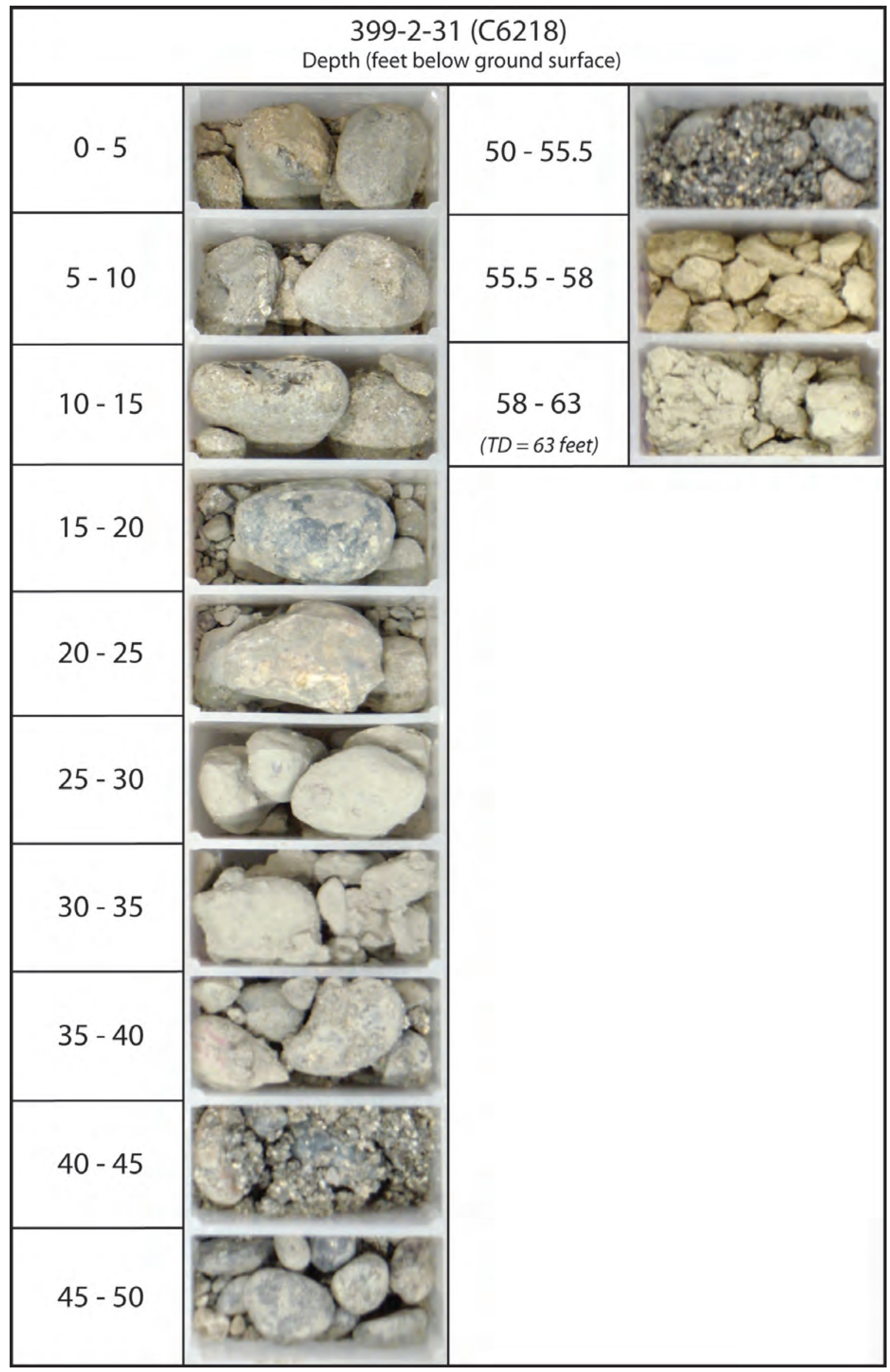




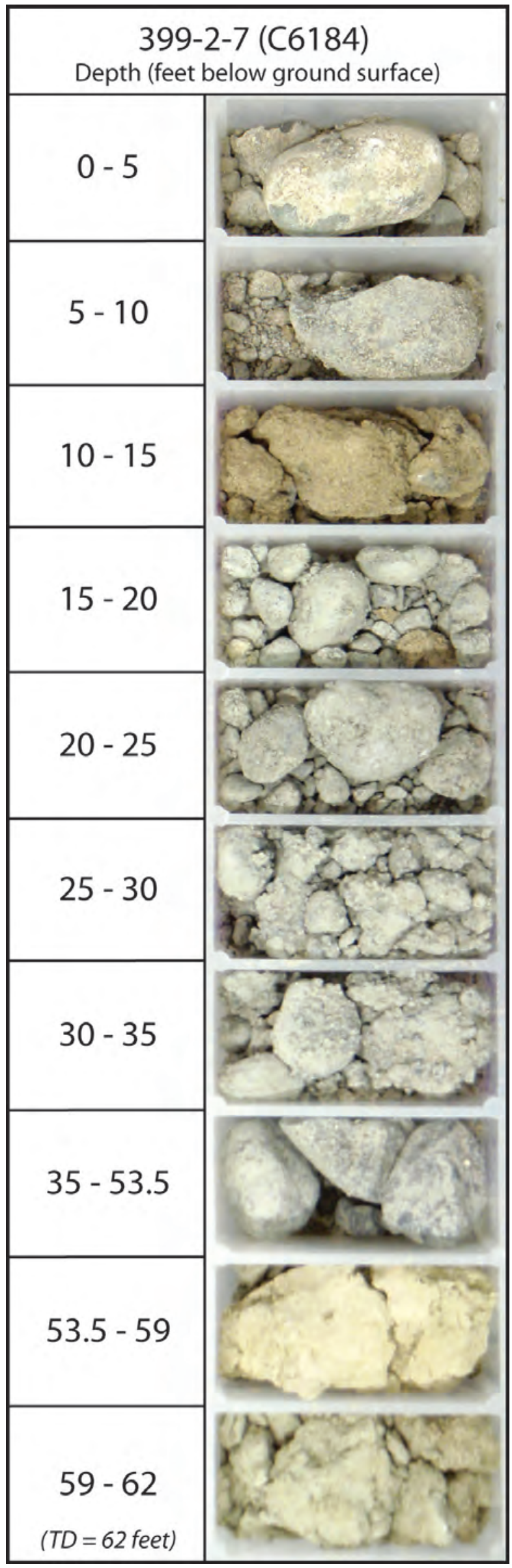




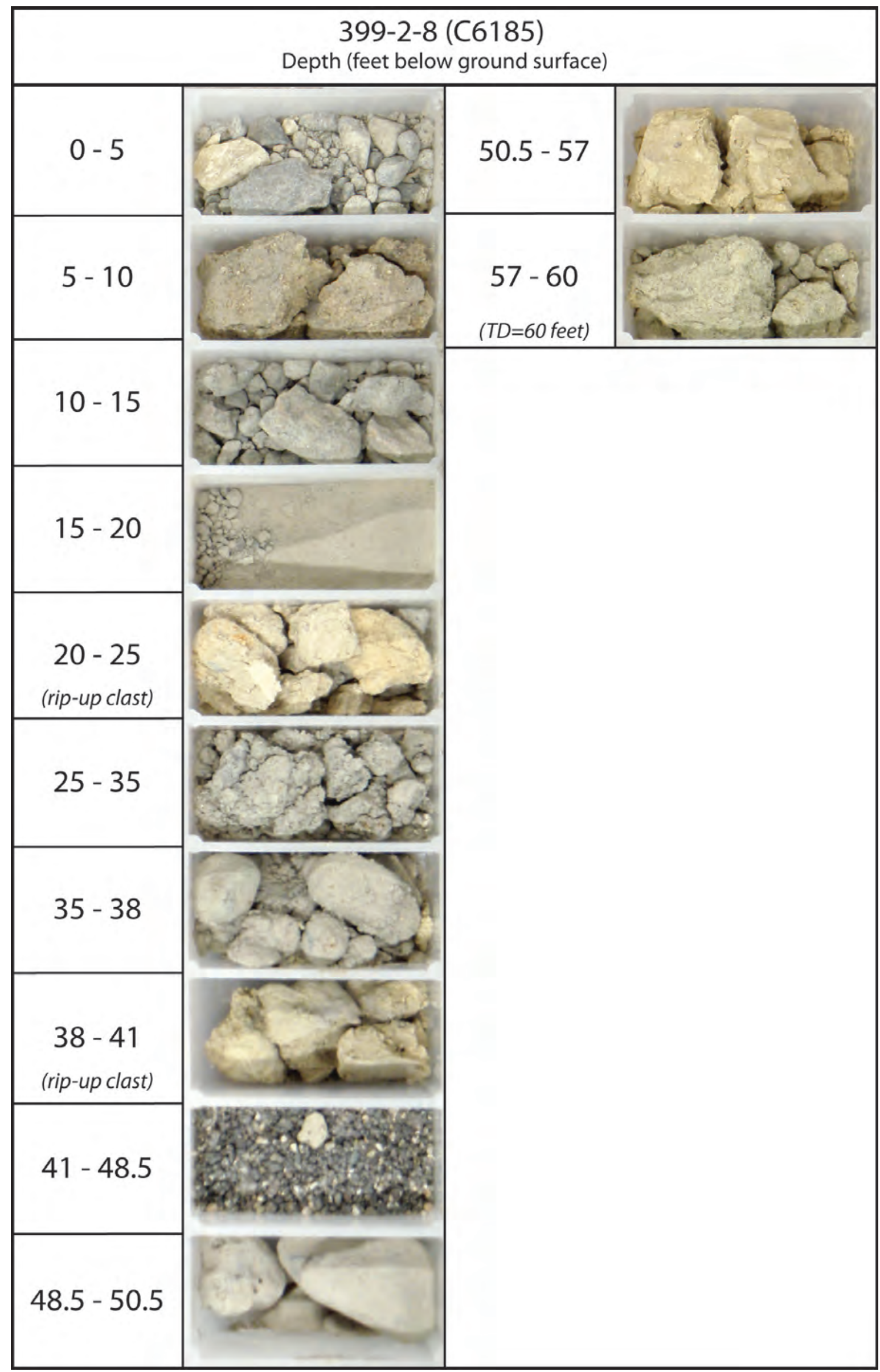




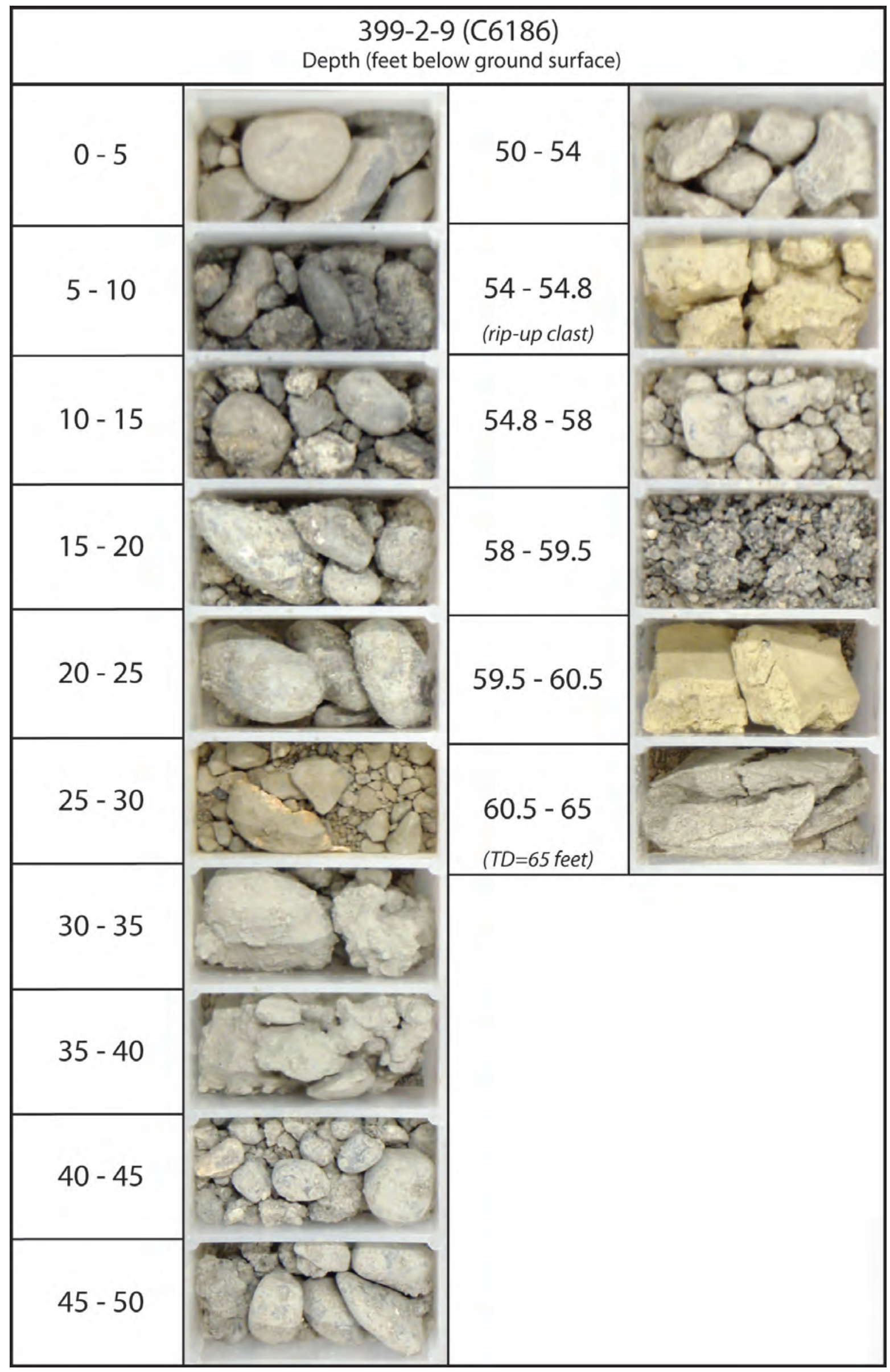




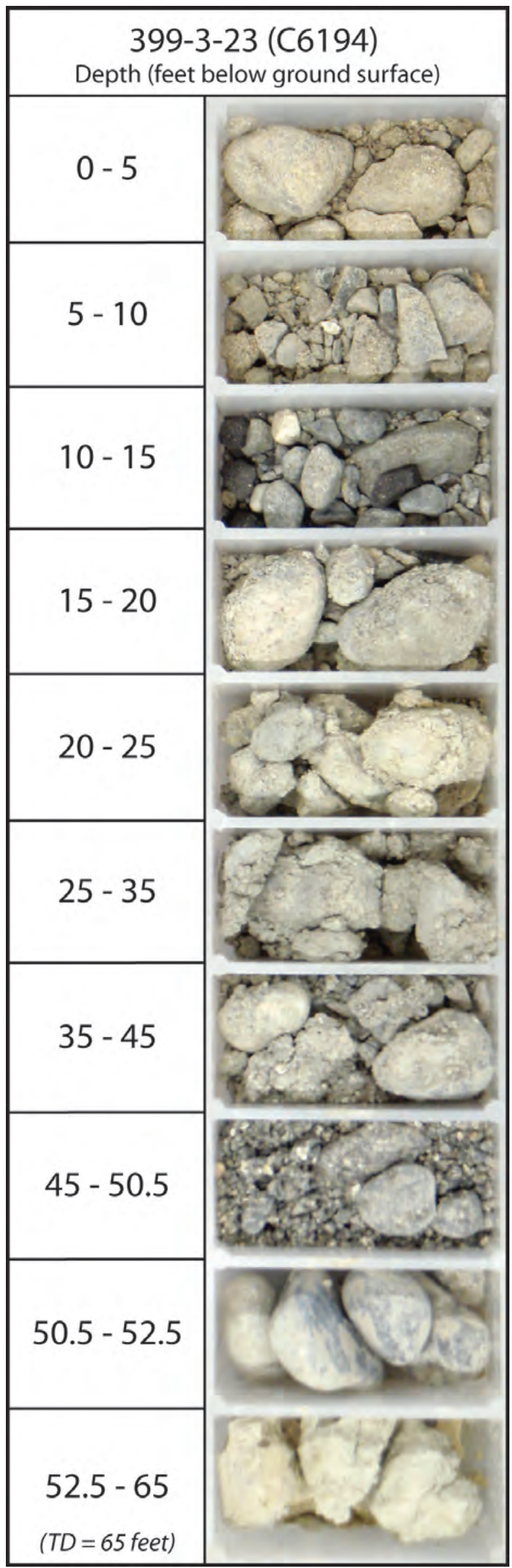




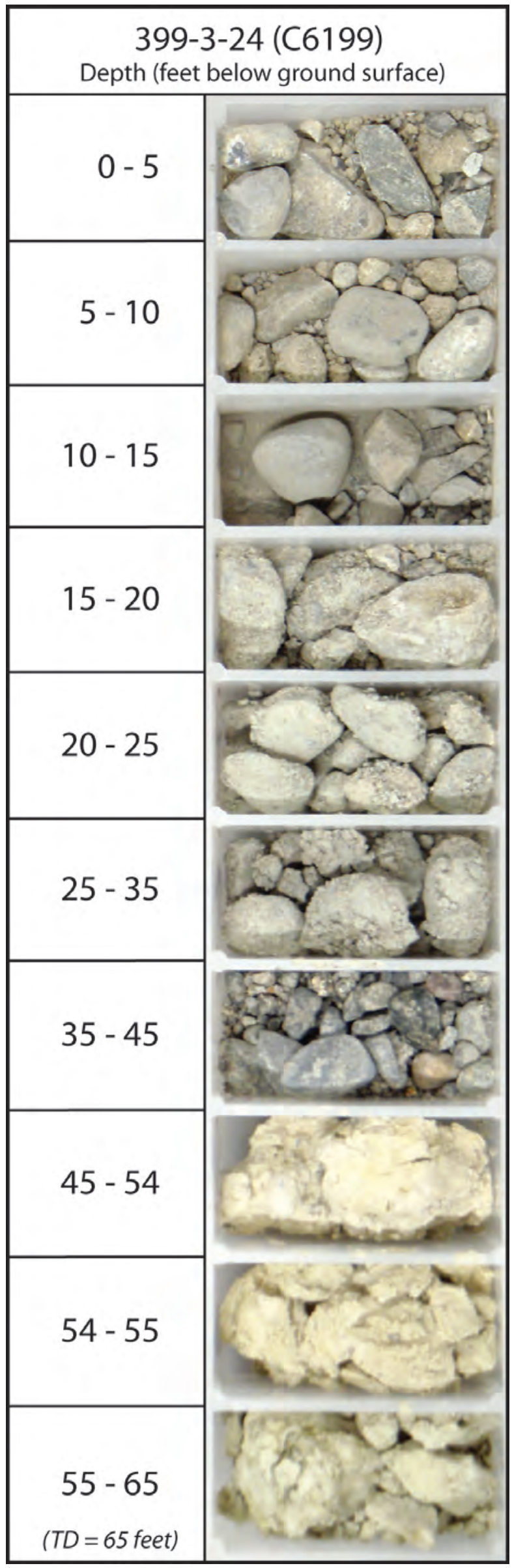




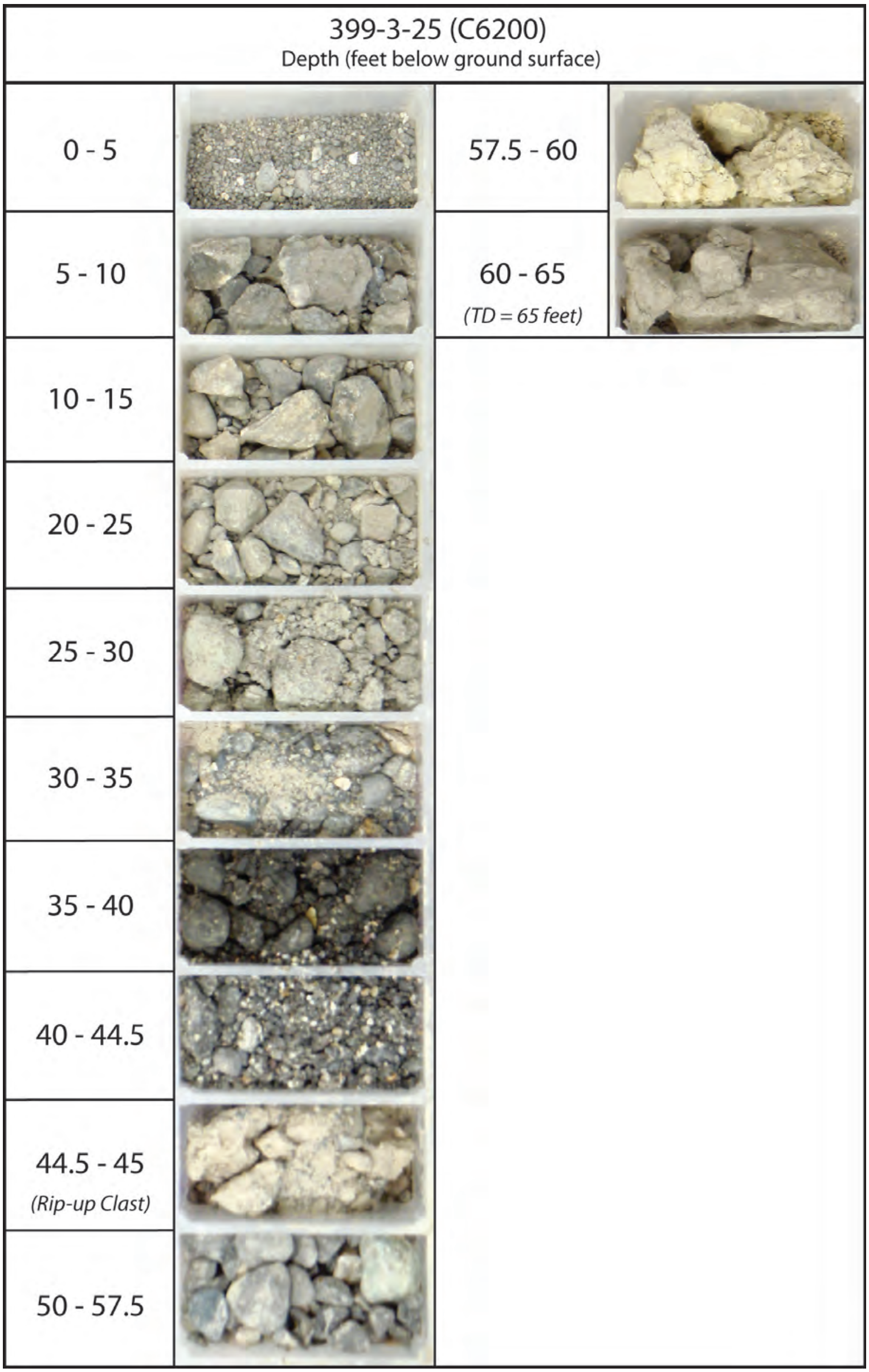




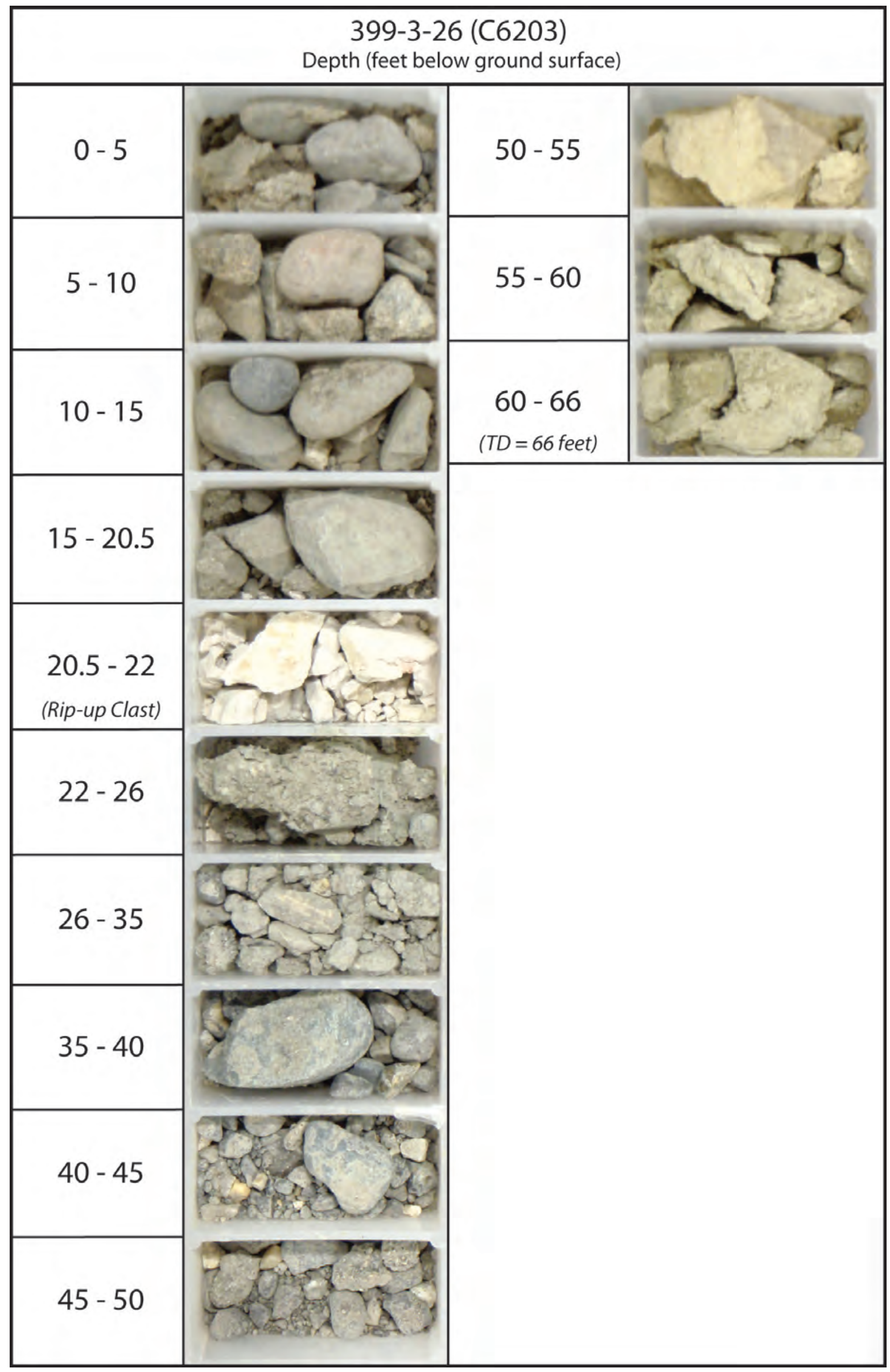




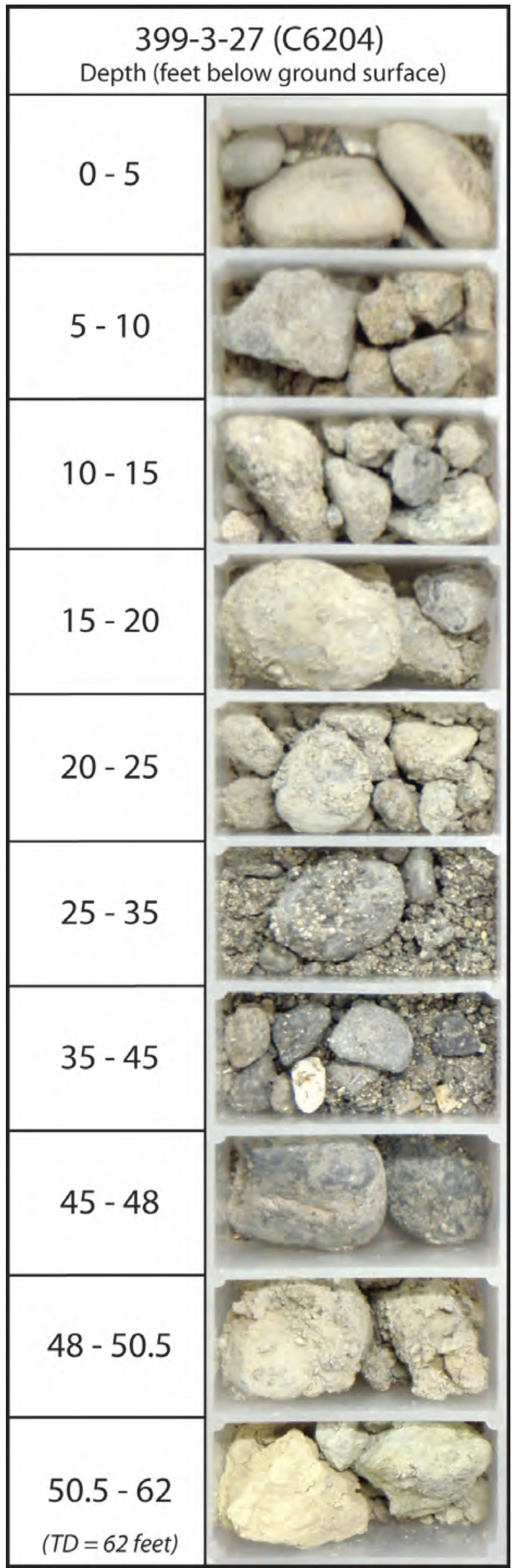




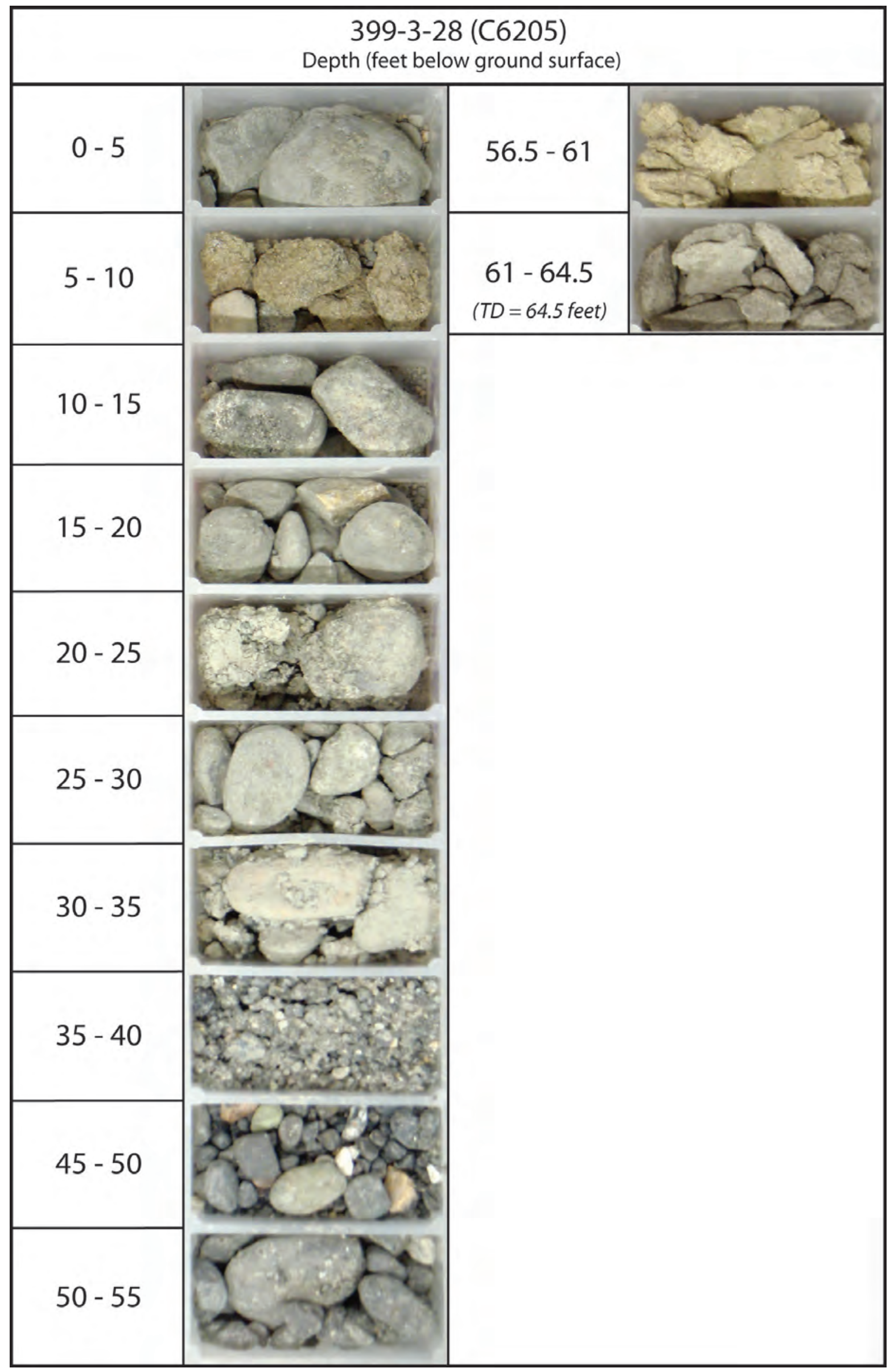




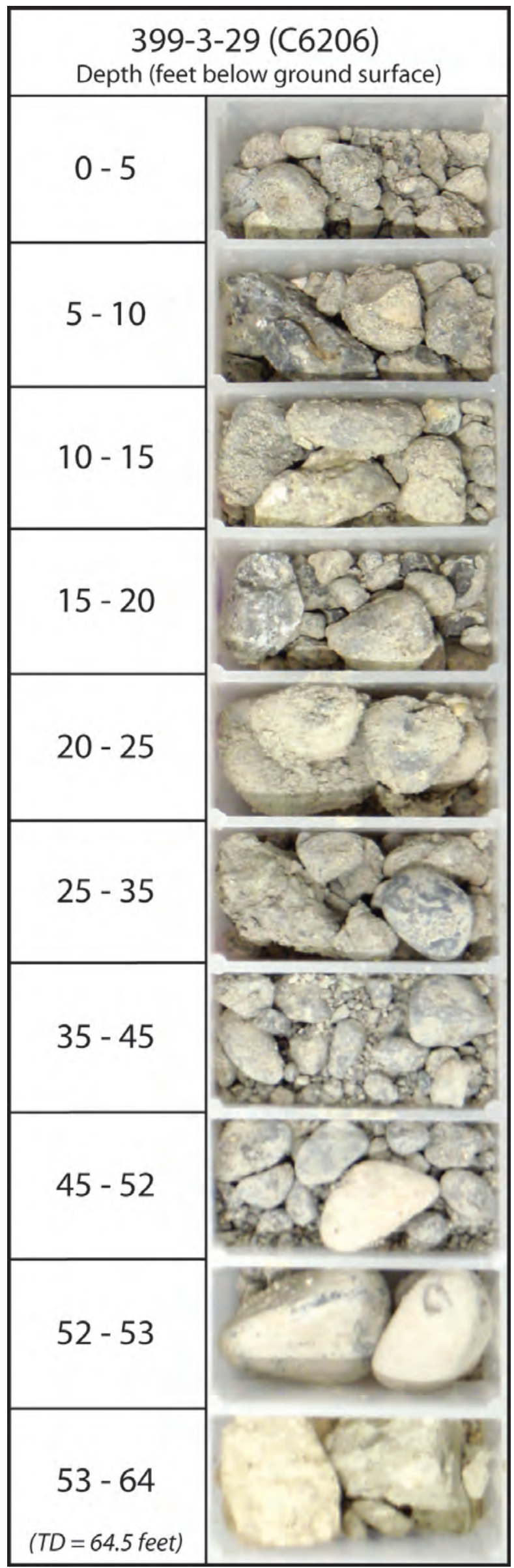




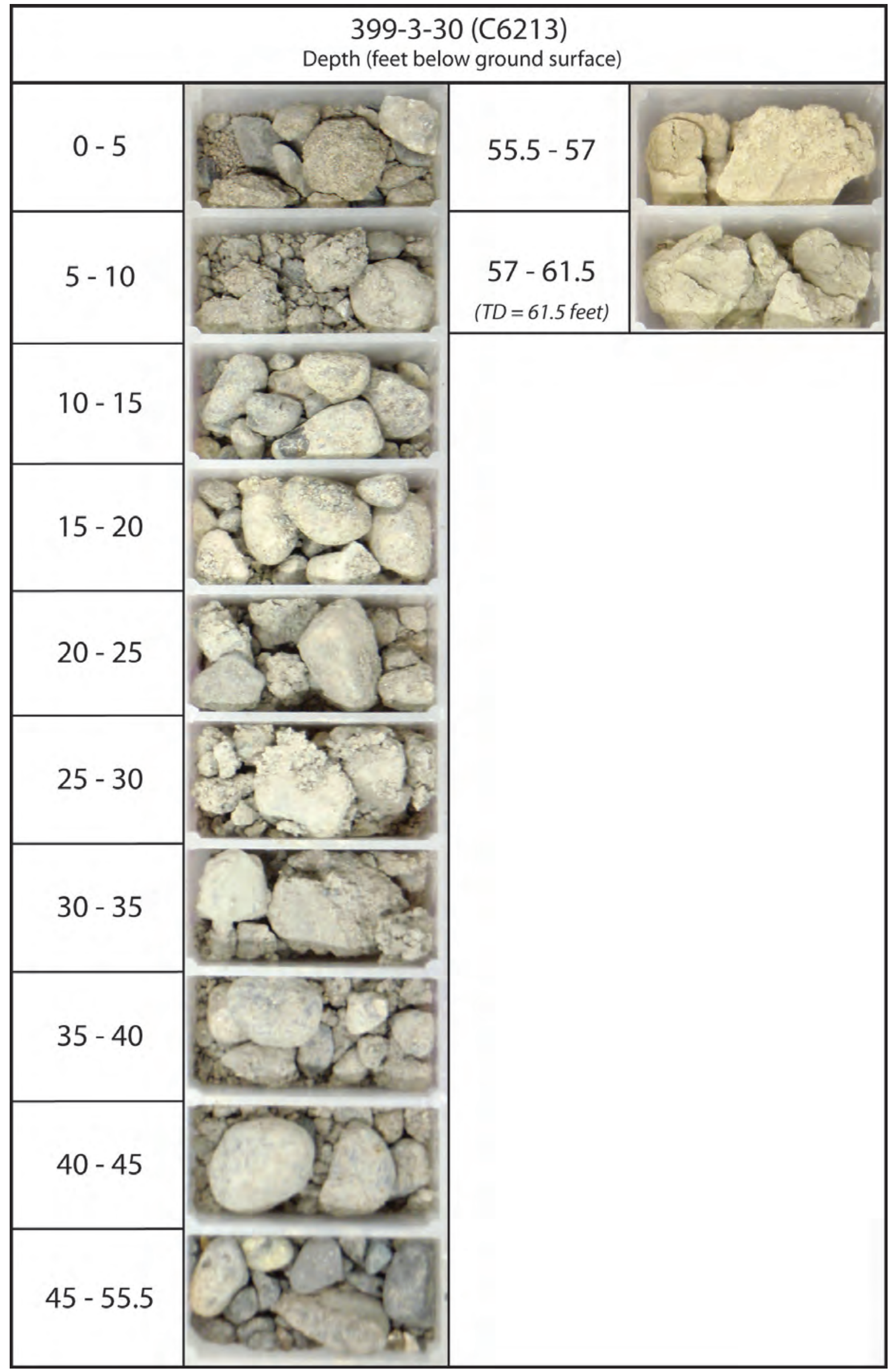




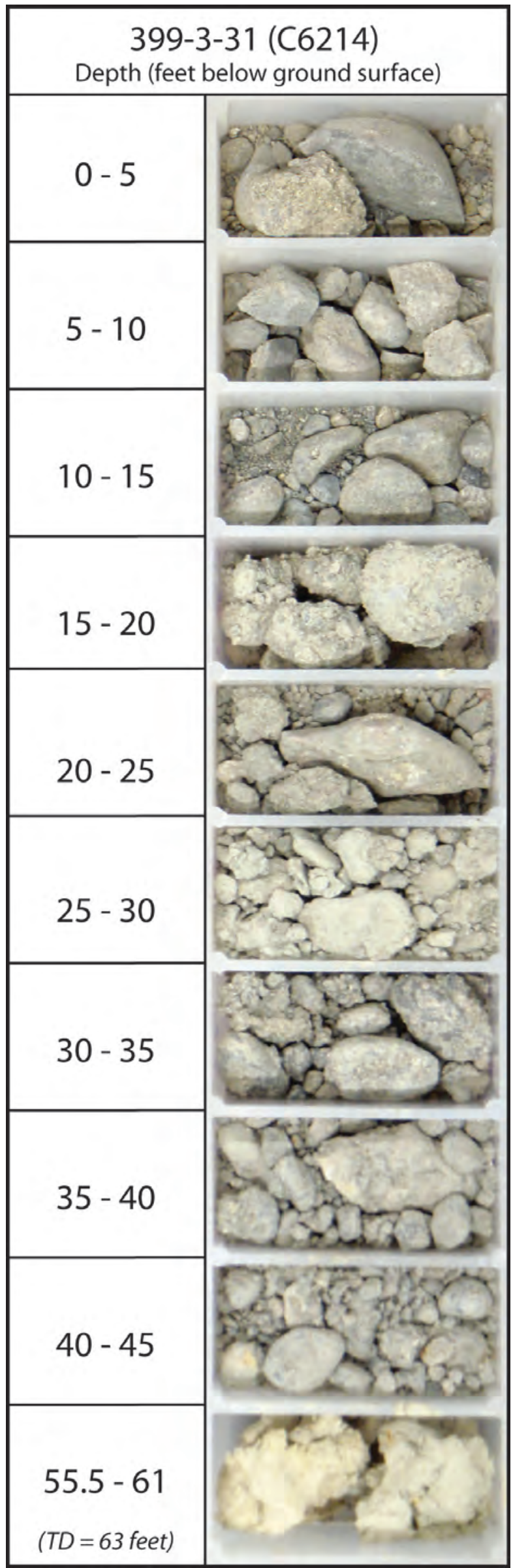




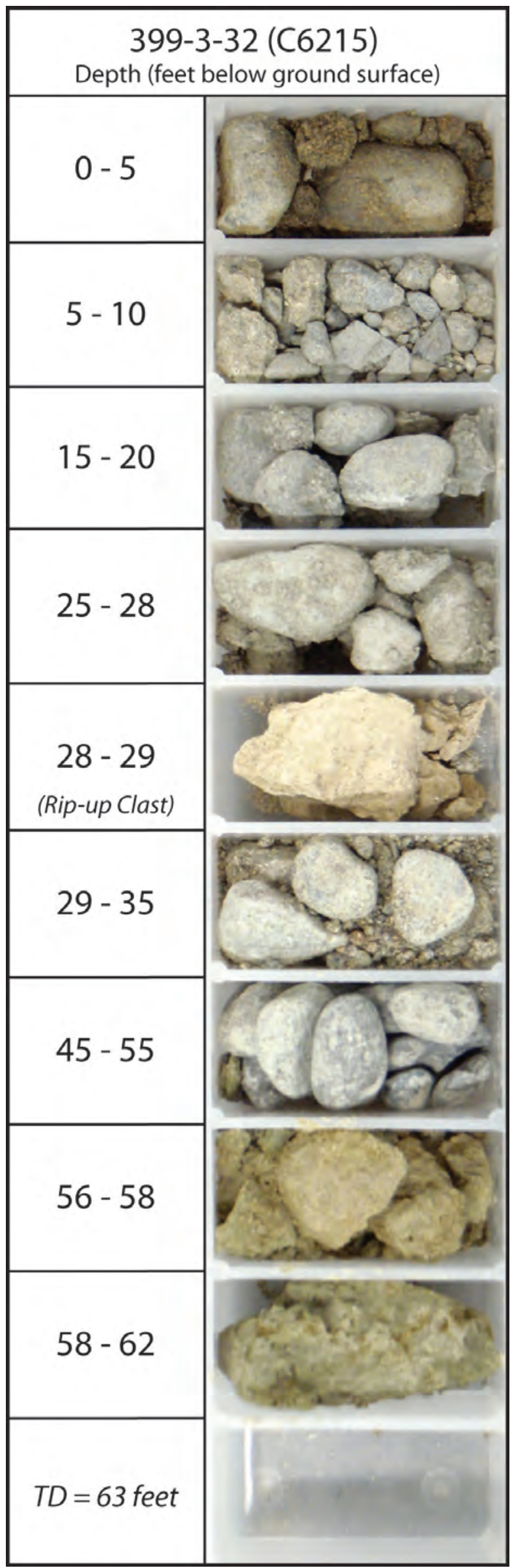

